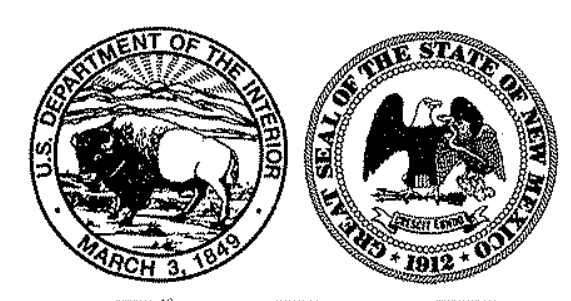

Water Resourd
New Mexico

Water Year 1996

by David Ortiz and K.M. Lange

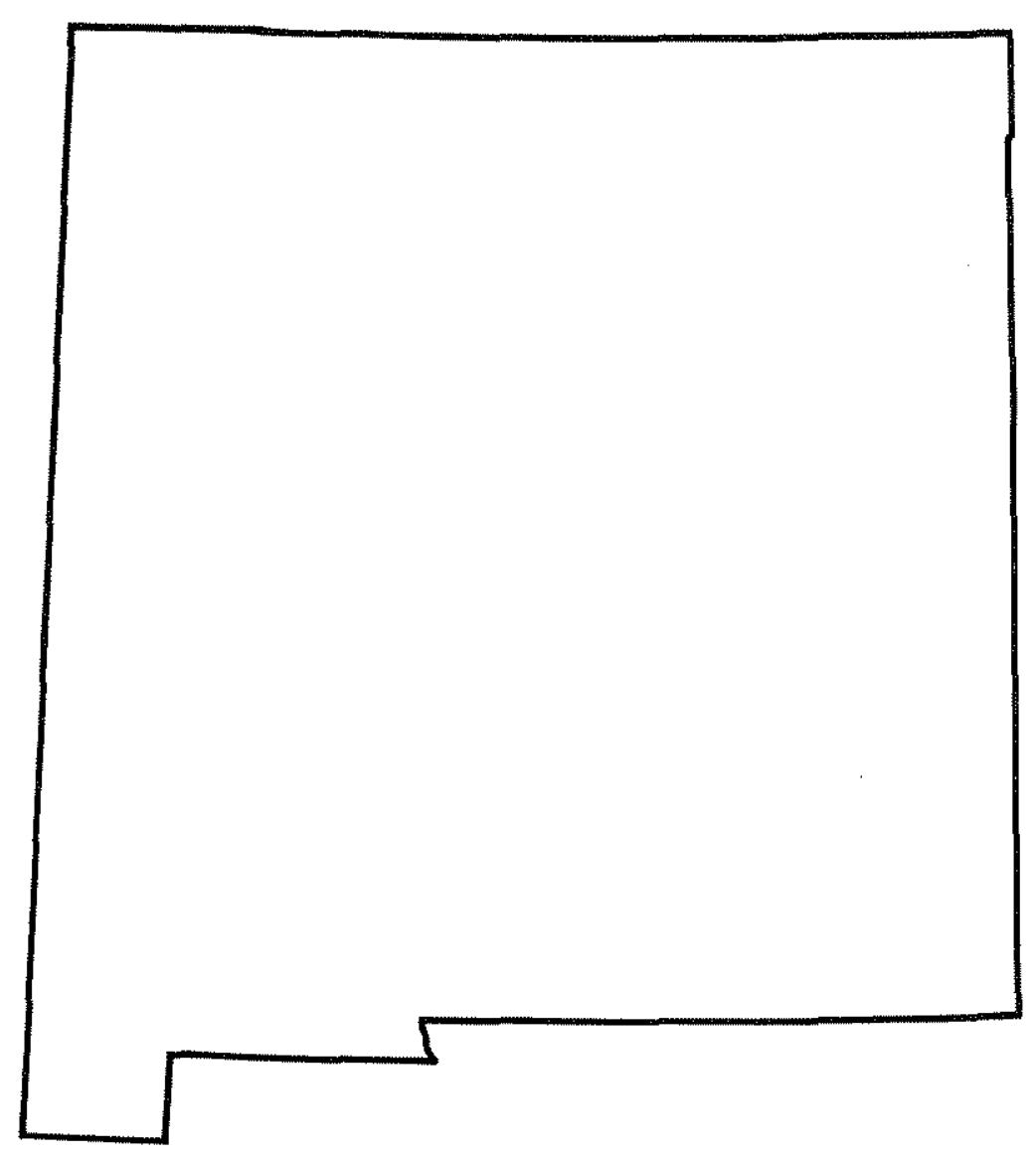

U.S. GEOLOGICAL SURVEY WATER-DATA REPORT NM-95-1

Prepared in cooperation with the State of New Mexico and with other agencies 


\section{U.S. DEPARTMENT OF THE INTERIOR}

BRUCE BABBITT, Secretary

U.S. GEOLOGICAL SURVEY

Gordon P. Eaton, Director

For additional information on the water program in New Mexico write to District Chief, Water Resources Division U.S. Geological Survey

4501 Indian School Road NE, Suite 200

Albuquerque, New Mexico 87110-3929 
This annual hydrologic data report of New Mexico is one of a sertes of annual reports that document hydrologic data gathered from the U.S. Geological Survey's surface- and ground-water data-collection networks in each State, Puerto Rico, and the Trust Territories. These records of streamflow, ground-water levels, and water quality provide the hydrologic information needed by Federal. State, and local agencies and the private sector for developing and managing our Nation's land and water resources. Bydrologic data for New Mexico are contained in this volume.

This report is the culmination of a concerted effort by dedtcated personnel of the U.S. Geological Survey who collected, complled, enalyzed, verified, and organized the data, and who typed, edited, and assembled the report. The authors had primary responsibjlity for assuring that the information contained herein is accurate, complete, and adheres to Geological Survey polfcy and established guidelines.

Personnel in charge of the Field Units:

Luis C. Madrid Las Cruces Subdistrict

David Ortiz Albuquerque Field Headquarters

Glenn A. Todd Carlsbad Field Headquarters

The following personnel collected, computed, or processed data for this report:

\begin{tabular}{|c|c|}
\hline $\begin{array}{l}\text { S.X. } \\
\text { L.V. } \\
\text { L.M. } \\
\text { R.F. } \\
\text { R.R. } \\
\text { R.K. } \\
\text { H.L. } \\
\text { D.E. } \\
\text { D.F. } \\
\text { B.A. } \\
\text { F.GE G. } \\
\text { A.C. }\end{array}$ & $\begin{array}{l}\text { Anderholm } \\
\text { Beal } \\
\text { Bexfield } \\
\text { Carter } \\
\text { Cruz } \\
\text { Dewees } \\
\text { Drekey } \\
\text { Evans } \\
\text { Funderburg } \\
\text { Healy- Menc } \\
\text { Hill } \\
\text { bhardt } 5 \text { an } \\
\text { GeIlis } \\
\text { Gold }\end{array}$ \\
\hline
\end{tabular}

S.X. Anderholm

M. Bexfield

D. E. Funderburs

A. Hill

A.C. Gellit

R.L. Gold

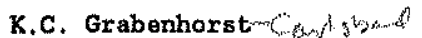 \\ T.M. Kelly \\ K.L. Hamilton - com 15 ben \\ S.A. Kimbali \\ G.W. Levings \\ S.H. Lewandowsk 1

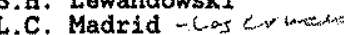 \\ R.M. McBreen \\ D.J. Milewsk \\ L.K. Milier \\ R.L. Moquino

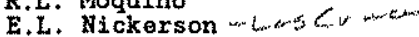 \\ T.J. Quintana \\ D.R. Rankin
}

M.D. Roark

R.J. Rogers - Nee-zonth

R.D. Ross - Lens C, romentum

Grd.-Shatrtuckw

M.L. Shewrake

G.H. Sieber

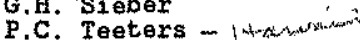

C.L. Thomes

C.R. Thorn

G.A. Todd

G.A. Todd

S.D. Waltemeyer

R.W. Wilcox

Thts report was prepared in cooperation with the state of New Mexico and other agencies under the supervision of Russell K. Livingston, District Chief, New Mexico, and D.J. Lystrom, Regtonal Hydrologist, Central. Region. 


\begin{tabular}{|c|c|c|c|c|}
\hline \multicolumn{4}{|c|}{ REPORT DOCUMENTATION PAGE } & $\begin{array}{l}\text { Form Approved } \\
\text { OMB No. } 0704-0188\end{array}$ \\
\hline \multicolumn{5}{|c|}{ 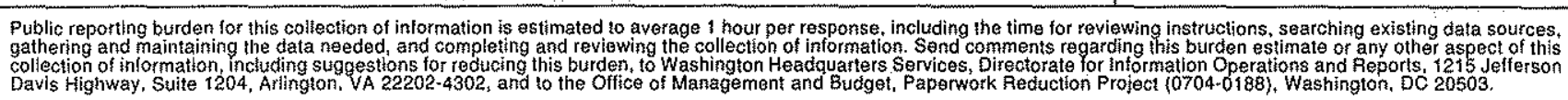 } \\
\hline 1. AGENCY USE ONLY (Leave blank) & \multicolumn{2}{|l|}{$\begin{array}{l}\text { 2. REPORT DATE } \\
\text { April } 1997\end{array}$} & \multicolumn{2}{|c|}{$\begin{array}{l}\text { 3. REPORT TYPE AND DATES COVERED } \\
\text { Annual: Oct. } 1,1995 \text {, through Sept. } 30,1996\end{array}$} \\
\hline \multicolumn{3}{|l|}{ 4. TITLE AND SUBTITLE } & \multicolumn{2}{|c|}{ 5. FUNDING NUMBERS } \\
\hline \multicolumn{3}{|c|}{ Water Resources Data, New Mexico, Water Year 1996} & \multicolumn{2}{|c|}{ 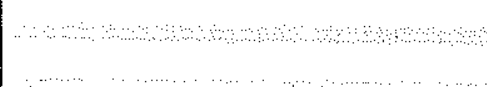 } \\
\hline \multicolumn{3}{|l|}{ 6. AUTHOR(S) } & & \\
\hline \multicolumn{3}{|c|}{ David Ortiz and K.M. Lange } & \multicolumn{2}{|c|}{ * } \\
\hline \multicolumn{3}{|c|}{ 7. PERFORMING ORGANIZATION NAME(S) AND ADDRESS(ES) } & \multicolumn{2}{|c|}{$\begin{array}{l}\text { 8. PEPFORMING ORGANIZATION } \\
\text { REPORT NUMBER }\end{array}$} \\
\hline \multicolumn{3}{|c|}{$\begin{array}{l}\text { U.S. Geological Survey, Water Resources Division } \\
4501 \text { Indian School Road NE, Suite } 200 \\
\text { Albuquerque, New Mexico } 87110-3929\end{array}$} & & GS-WDR-NM-96-1 \\
\hline \multicolumn{3}{|c|}{$\begin{array}{l}\text { U.S. Geological Survey, Water Resources Division } \\
4501 \text { Indian School Road NE, Suite } 200 \\
\text { Albuquerque, New Mexico } 87110-3929\end{array}$} & & $\begin{array}{l}\text { ONSORING /MONIORING } \\
\text { ENCY REPORT NUMBER } \\
\text { GS-WRD-NM-96-1 }\end{array}$ \\
\hline \multicolumn{5}{|l|}{ 11. SUPPLEMENTARY NOTES } \\
\hline \multicolumn{3}{|c|}{$\begin{array}{l}\text { 12a. DISTRIBUTION/AVALABBLITY STATEMENT } \\
\text { No restrictions on distributions. This report may be purchased from: } \\
\text { National Technical Information Service, Springfield, Virginia } 22161\end{array}$} & \multicolumn{2}{|c|}{ 12b. DISTRIBUTION CODE } \\
\hline \multicolumn{5}{|r|}{$\begin{array}{l}\text { is of discharge and water } \\
\text { dd water levels and water } \\
\text { ging stations; stage and } \\
\text { wells; and water levels at } \\
\text { tdditional water data were } \\
\text {, and are published as } \\
\text { ter Data System collected } \\
\text { Jew Mexico. }\end{array}$} \\
\hline \multirow{2}{*}{\multicolumn{4}{|c|}{$\begin{array}{l}\text { 14. SUBJECT TERMS } \\
\text { *New Mexico, *Hydrologic data, }{ }^{*} \text { Surface water, *Water quality, Lakes, Reservoirs, } \\
\text { Sediments, Water levels, Flow rates, Gaging stations, Chemical analyses, Water } \\
\text { analyses, Water temperature, Sampling sites }\end{array}$}} & $\begin{array}{l}\text { 15. NUMBER OF PAGES } \\
446\end{array}$ \\
\hline & & & & 16. PRICE CODE \\
\hline $\begin{array}{l}\text { 17. SECURITY CLASSIFICATION } \\
\text { OF REPORT } \\
\text { UNCLASSIFIED }\end{array}$ & $\begin{array}{l}\text { 18. SECURITY CLASSIFICATION } \\
\text { OF THIS PAGE } \\
\text { UNCLASSIFIED }\end{array}$ & $\begin{array}{l}\text { 19. SECUR } \\
\text { OFABS } \\
\text { UNCLA }\end{array}$ & & 20. LIMITATION OF ABSTRACT \\
\hline
\end{tabular}




\section{Preface.}

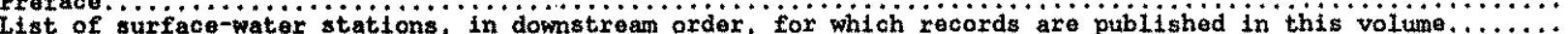

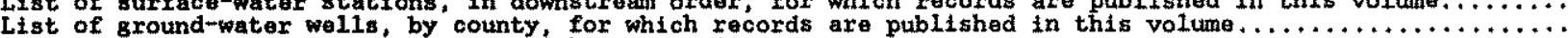

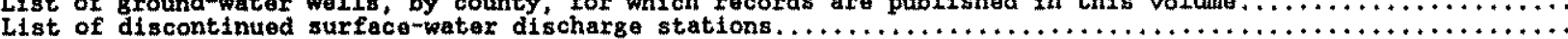

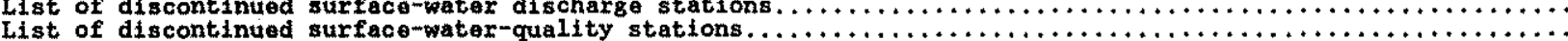
Introduction.

Cooperation.

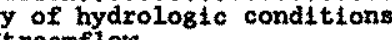

Streamflow

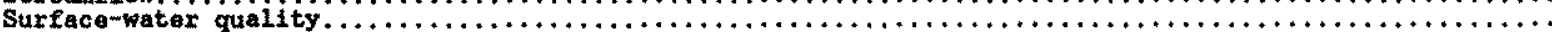

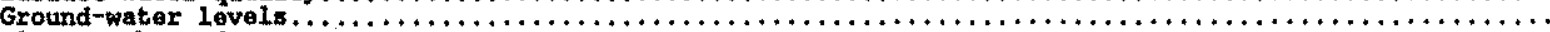

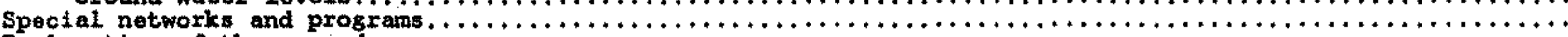
Explanation of the records.

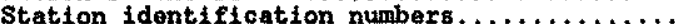

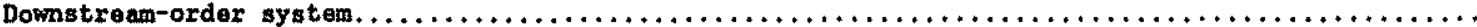

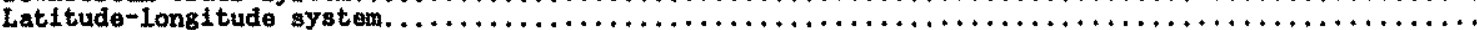

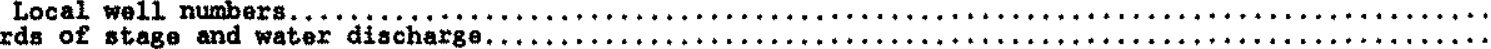
Data collection and computation.

Data presentation.

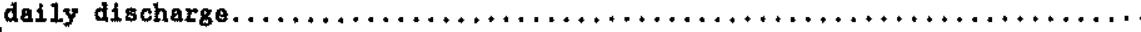

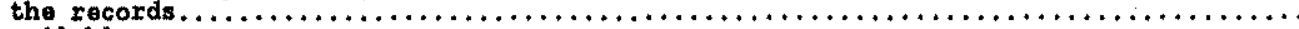

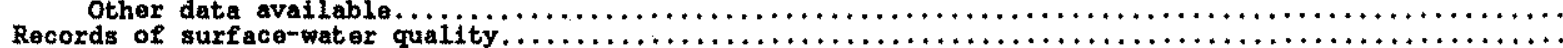

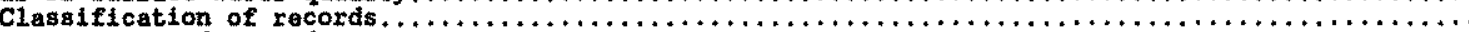

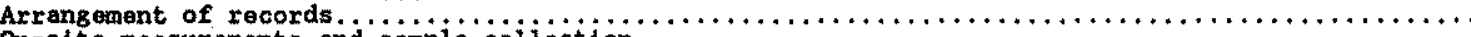

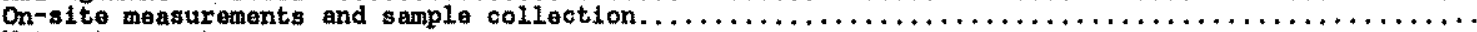
Water temperature.

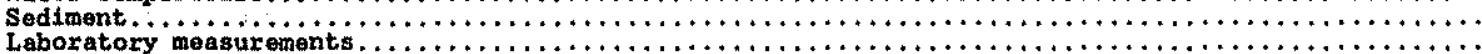

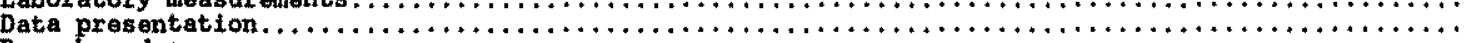
Remark codes

Records of ground watex ieveis

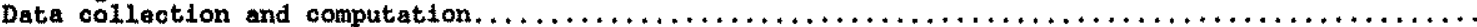

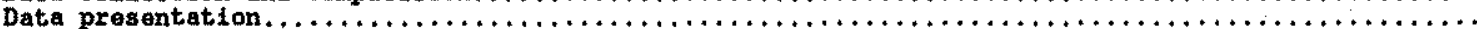

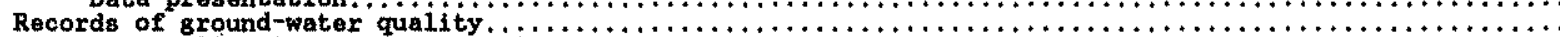

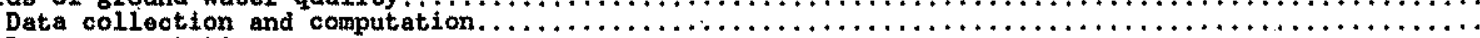

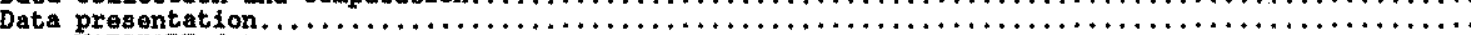

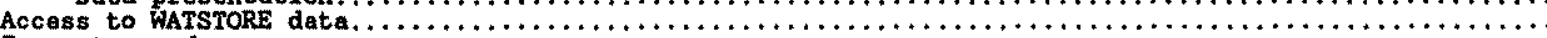

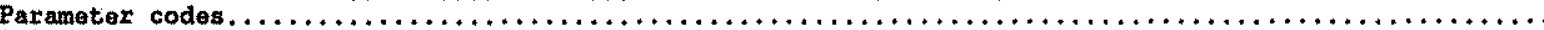

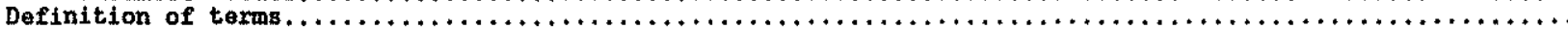

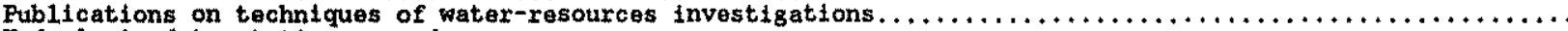

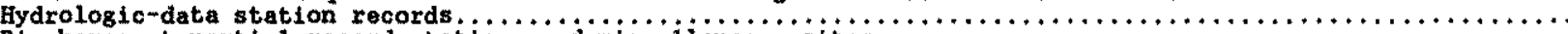
Discharge at partial-record stations and misceilianeous sites.

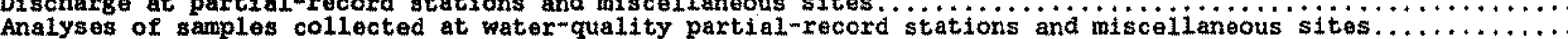

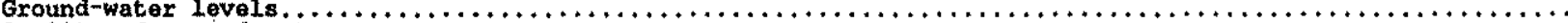

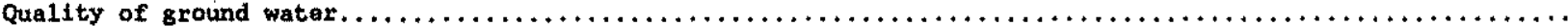

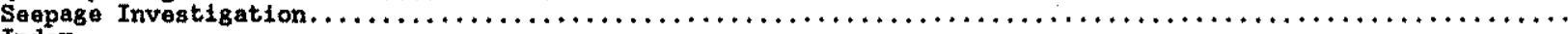

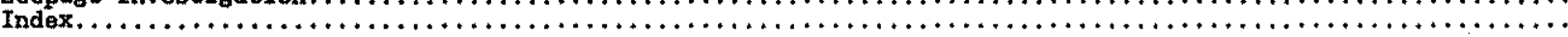

Figure 1. Areas of 5-year ground-water-level monitoring in Now Mexico showing years measured or schoduled for measuritor

2. Ground-water-level trends for last 20 years or period of record.

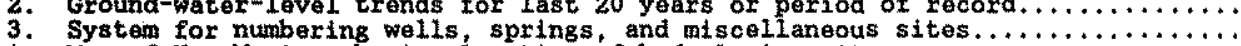

4. Map of New Mexico showing location of hydrologic units.

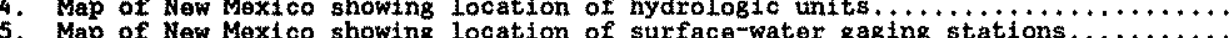

6. Map of Now Mexico showing location of water-quality gaging stations.........

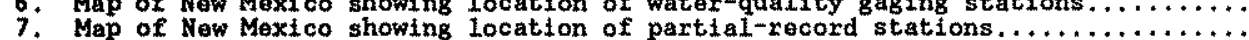

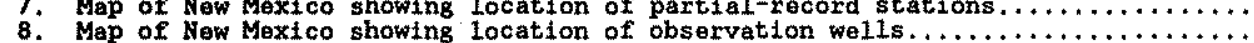

Calendar for water year $1996 \ldots \ldots \ldots \ldots \ldots \ldots \ldots \ldots \ldots \ldots \ldots \ldots \ldots \ldots \ldots \ldots \ldots$ fnside front cover Factors for converting inch-pound units to International system (si) units....... Inside back cover 
[Letters after station name designate type of data: (c) chemical; (d) discharge; (e) elevation, stage, or contents; (m) microbiological; (s) sediment; $(t$ ) water temperaturel

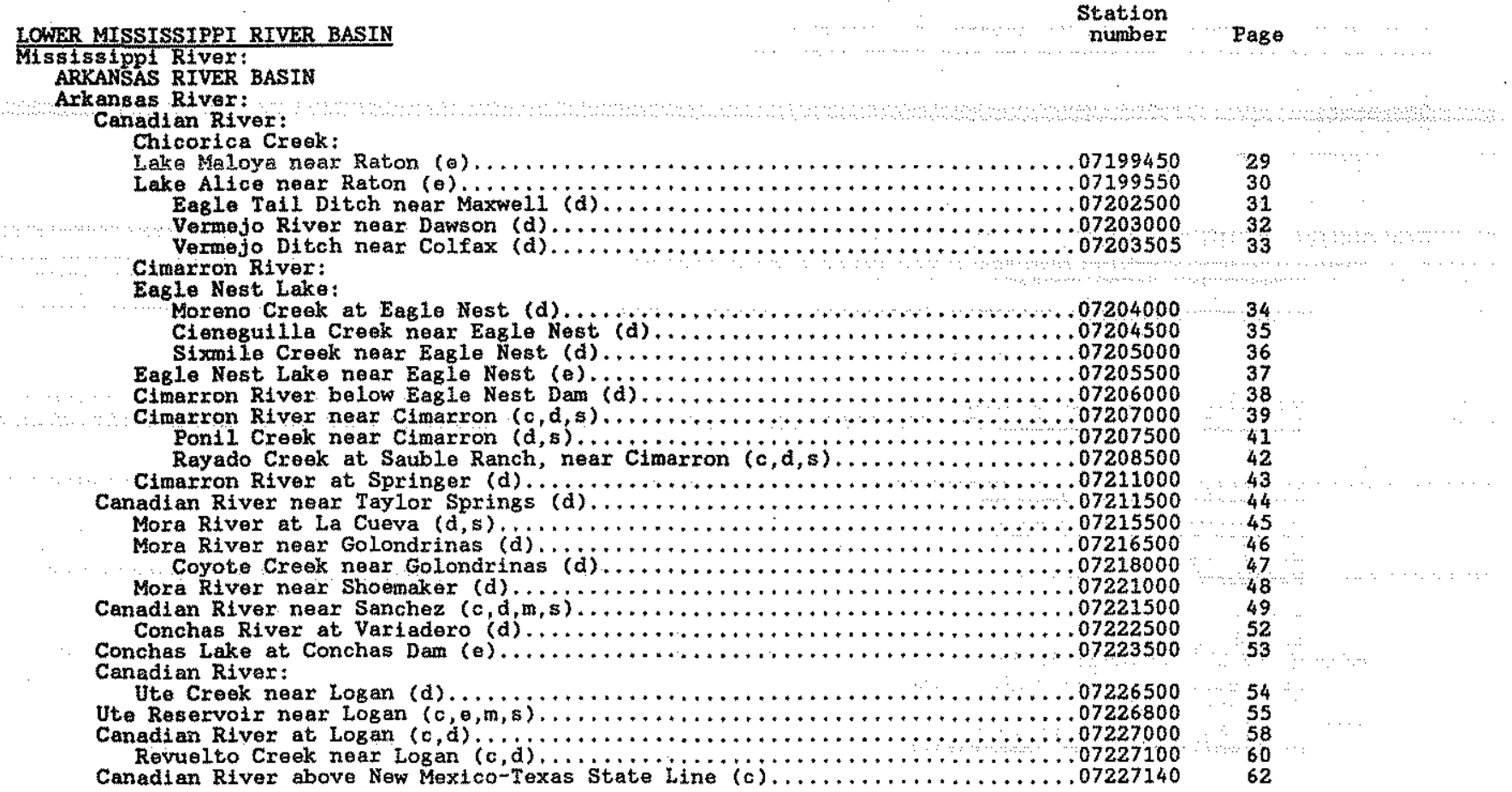

WESTERN GULF OE MEXICO BASINS

RIO GRANDE BASIN

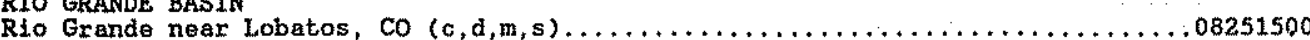

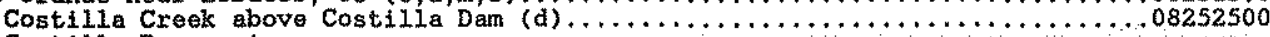
Costilla Reservoir:

Castas Croek near Costille (d) ............................... 08253000

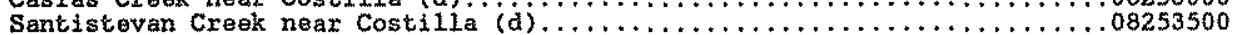

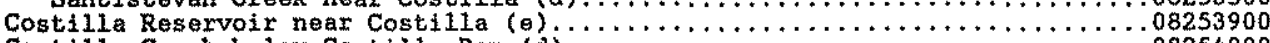

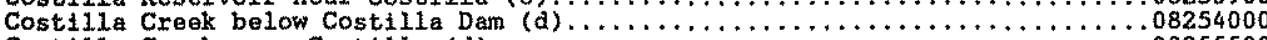

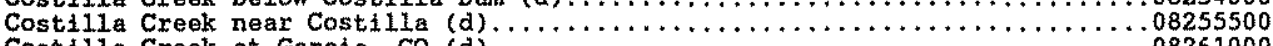

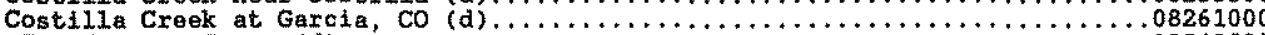

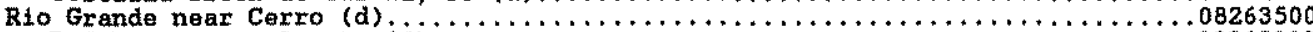

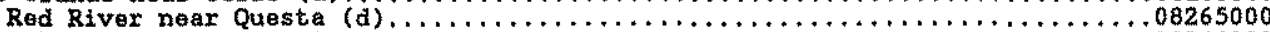

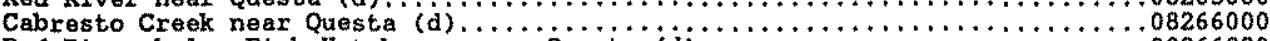

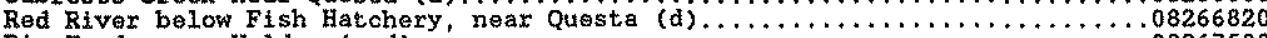

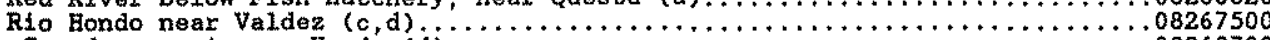

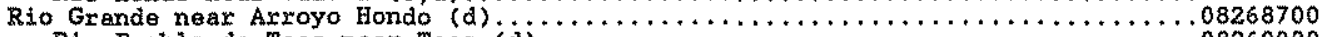

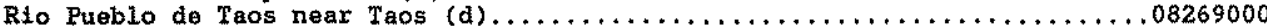

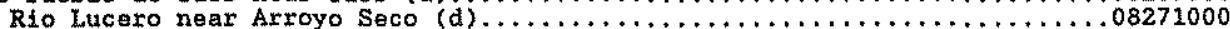

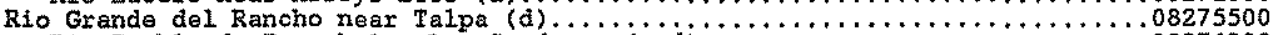

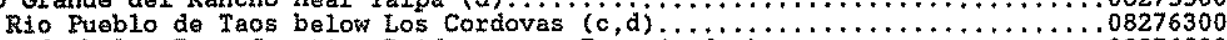

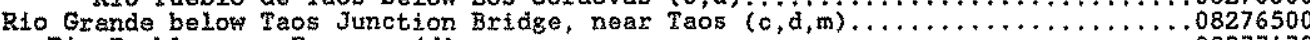

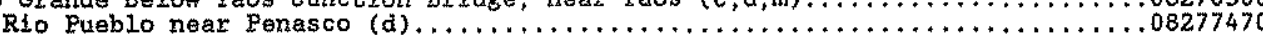

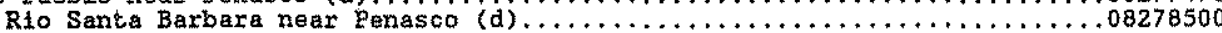

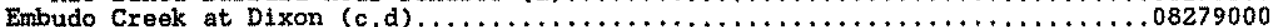

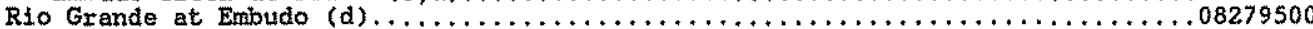

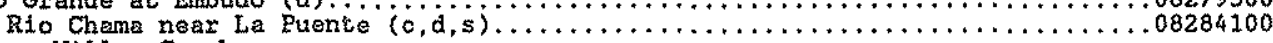
Willow Crook:

Azotea Creok:

Azotea Tunnel at Outlet, near Chana (d) .....................08284160

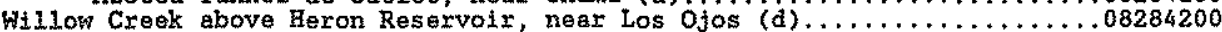
Heron Reservoir:9

Horse Lake Creek above Heron Reservoir, near los Ojos (d) . . . . . . . 08284300

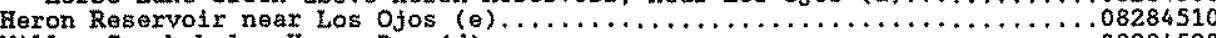

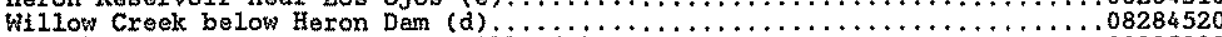

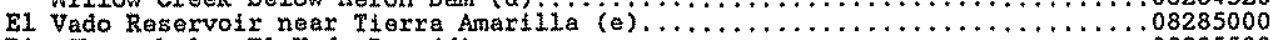

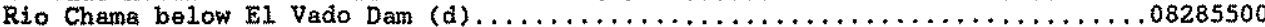

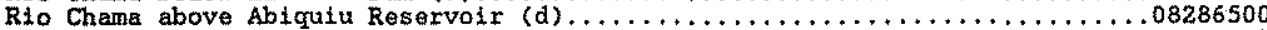

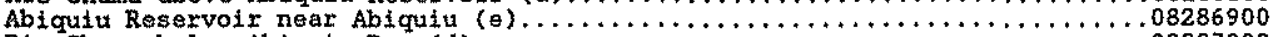

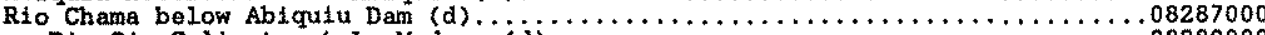

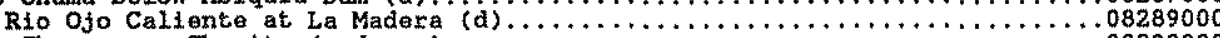

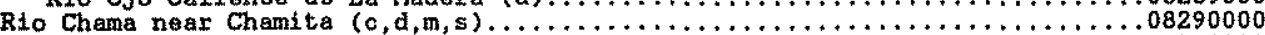

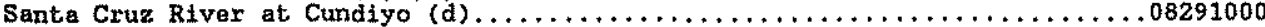

Rio Nambe (head of Pojoaque River):

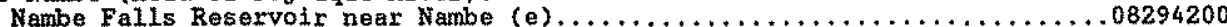

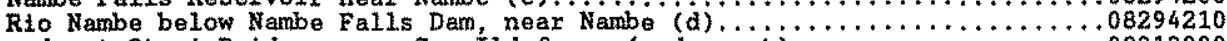

Rio Grande at otowi Bridge, near San Ildefonso ( $c, d, m, s, t) \ldots \ldots \ldots \ldots \ldots \ldots \ldots . \ldots \ldots 313000$

Rito Do Los Frijoles in Bandelier National Monument $(d) \ldots \ldots \ldots \ldots \ldots \ldots \ldots . \ldots \ldots 313350$ 
Cochiti Lake:

Santa Fe River:

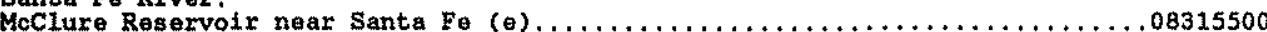

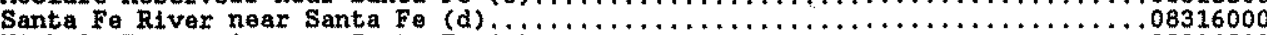

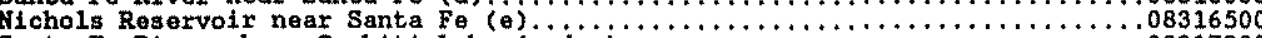

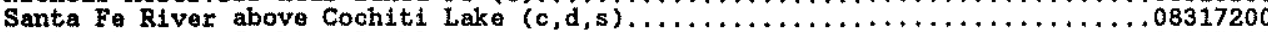

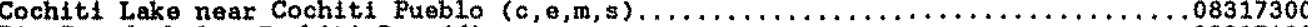

Rio Grande below Cochiti Dam (d) (a)

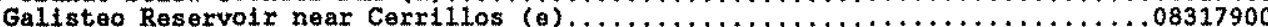

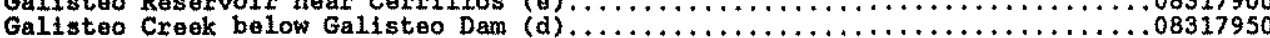

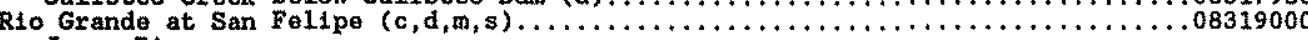
Jemez River:

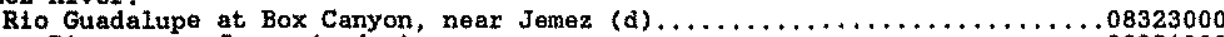

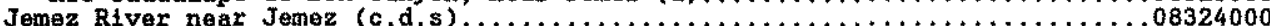

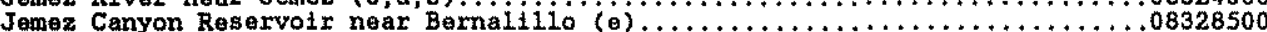

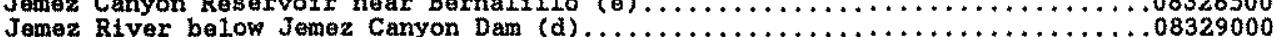
Rto Grande:

North Floodway Channel:

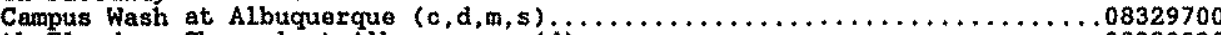

North Floodway Channel at Albuquerque (d) ............................ 08329835 Hahn Axroyo:

Hahn Arroyo;

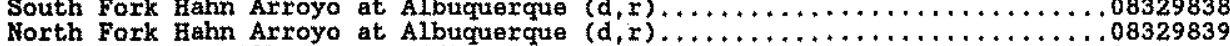

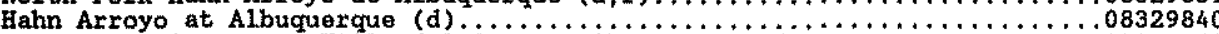

Grant Line Arroyo at Villa del Oso at Albuquerque (d).................. 08329860

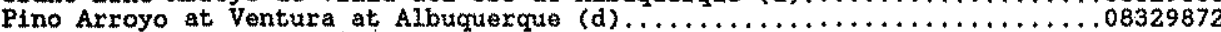

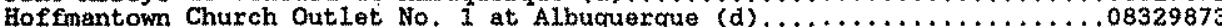

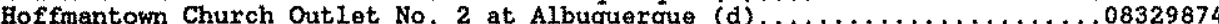

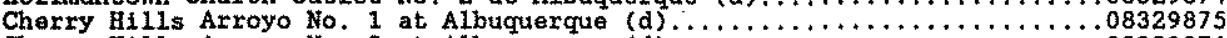

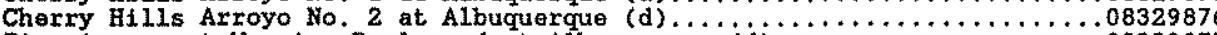

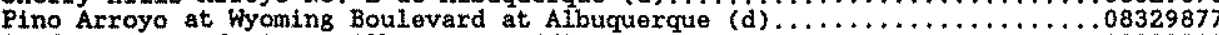

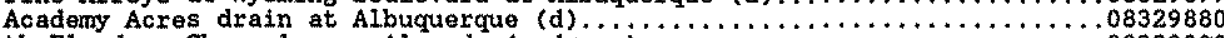

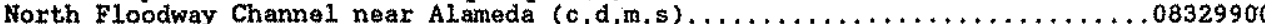

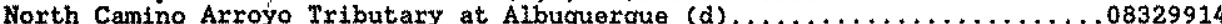

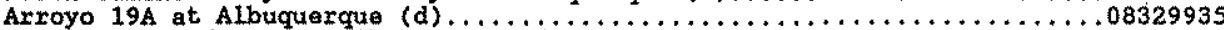

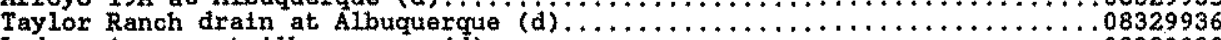

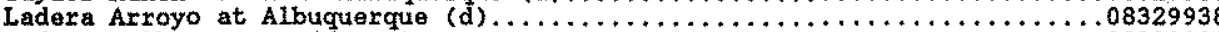

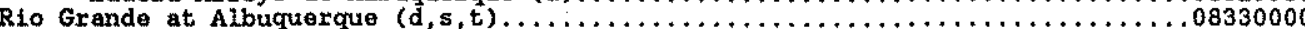
Tijeras Arroyo:

Tramway Floodway Channel at Albuquerque (d) . . . .

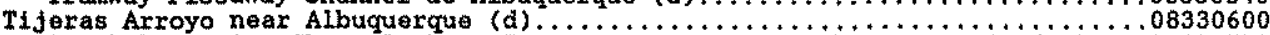
South Diverston Channel above Tijeras Arroyo near Aibuquerque $(\mathrm{c}, \mathrm{d}, \mathrm{m}) \ldots . .08330775$

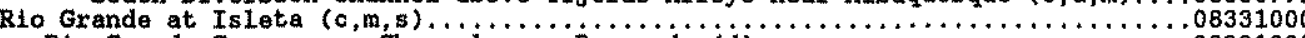

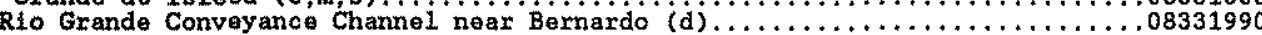

Rto Grande Floodway near Bernardo $(c, d, s, t) \ldots \ldots \ldots \ldots \ldots \ldots \ldots \ldots \ldots \ldots \ldots \ldots, \ldots \ldots \ldots 332010$

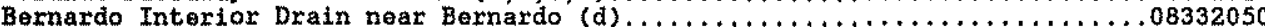

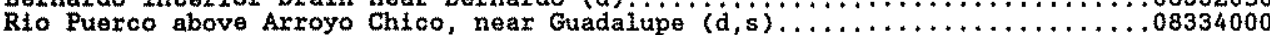
Bluewater Creek (head of Rio San Jose):

Bluewater Creek above BI uewater Dan, near Bluewater (d) . . . . . . . . 08341300

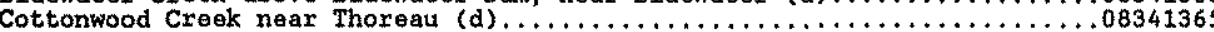

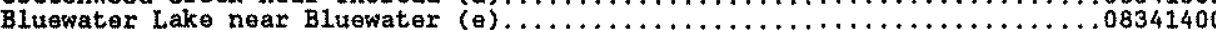
Bluewater Creek below Bluewater Dam (d) . . . . . . . .

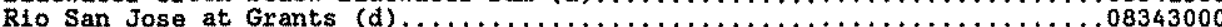

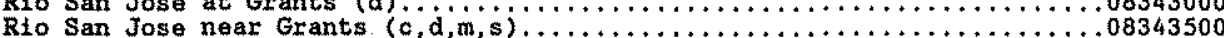

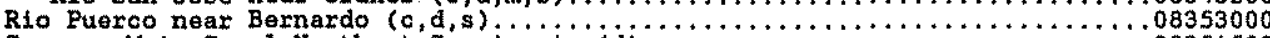
Socorro Main Canal North at San Acacta (d) ............................ 08354500

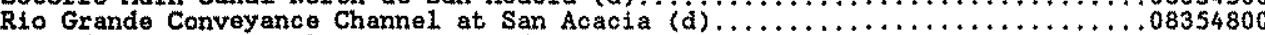

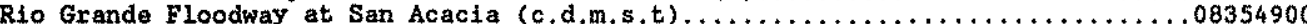

Rio Grande Conveyance Channel at San Marcial $(c, d, m, s, t) \ldots \ldots \ldots \ldots \ldots \ldots .08358300$

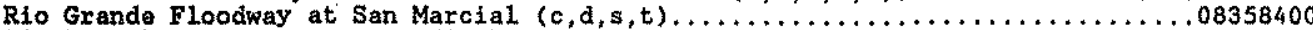

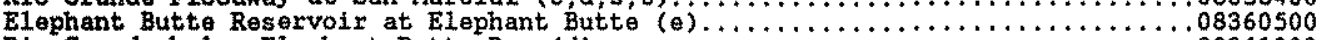

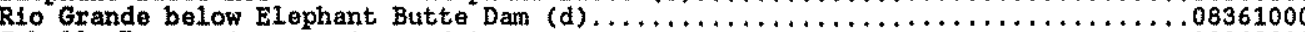

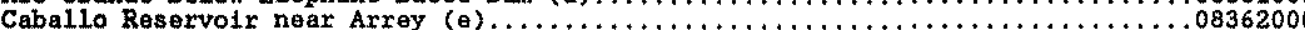

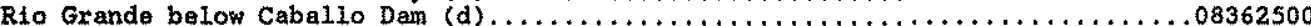

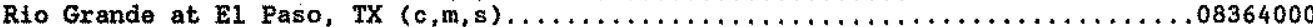
Pecos River:

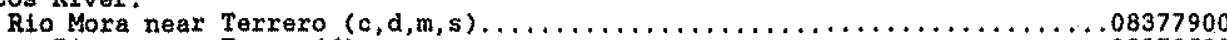

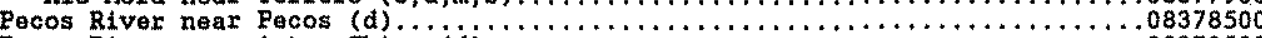

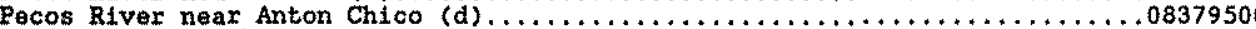

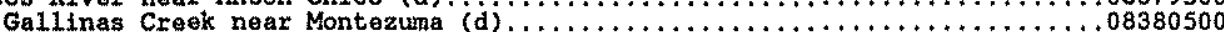




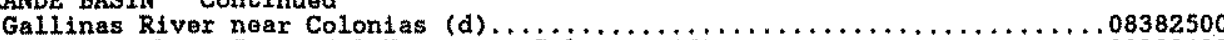

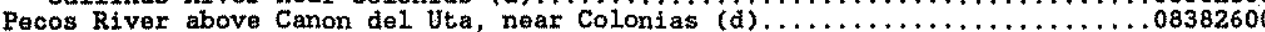

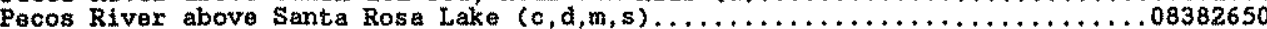

Santa Rosa lake:

Los Esteros Creek above Santa Rosa Lake (d) . . . . . . . . . . . . . . . . . 08382730

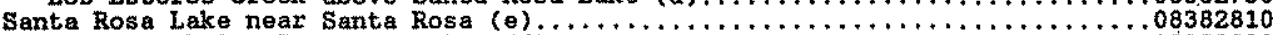

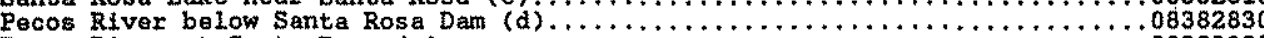

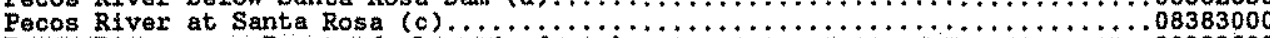

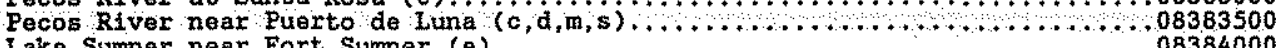

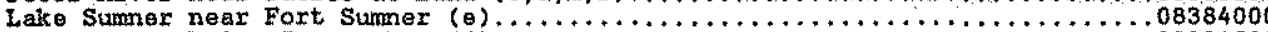

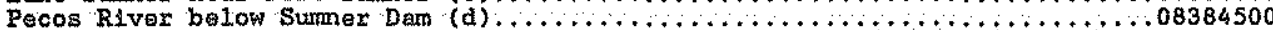

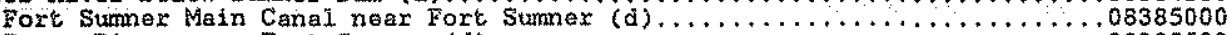

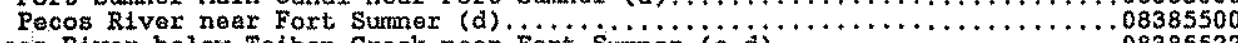

Pecos River below Taiban Creek near fort Sumer $(\mathrm{c}, \mathrm{d}) \ldots \ldots \ldots \ldots \ldots \ldots \ldots \ldots 352$

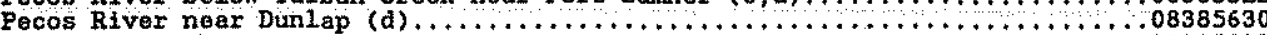

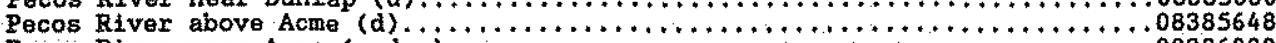

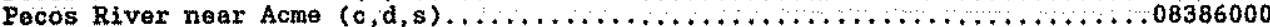

Rio Ruidoso (head of Rio Hondo):

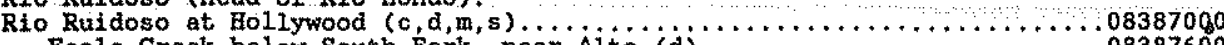

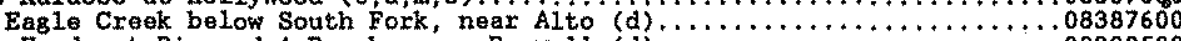

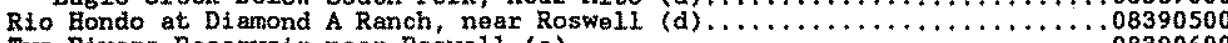

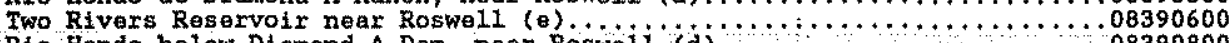

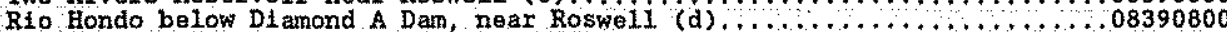

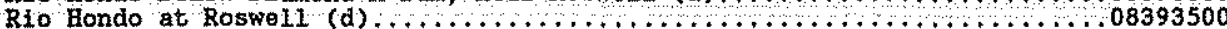

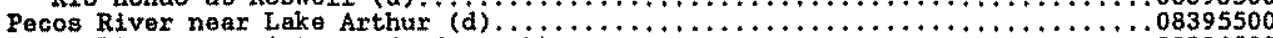

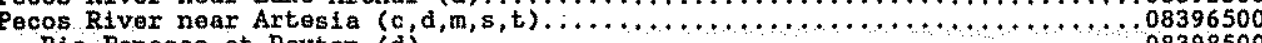

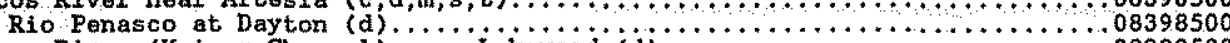

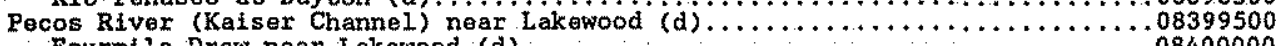

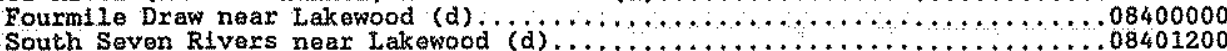

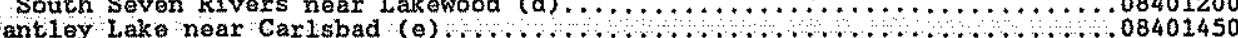

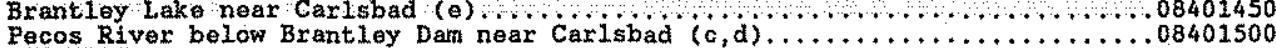

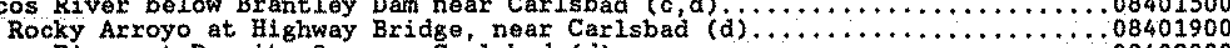

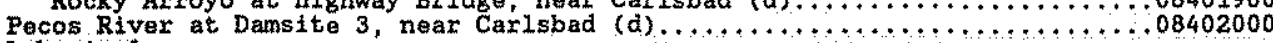

Lake Avalon:

Carlsbad Main Canal at Head, near Carlsbed (d) . . . . . . . . . . . . . . . 08403500

Lake Avalon neer Carlsbad (e)

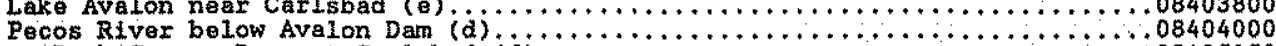

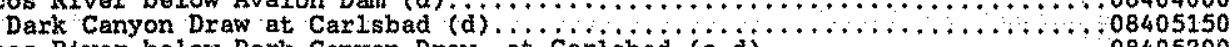

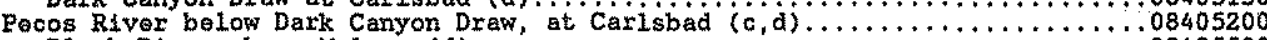

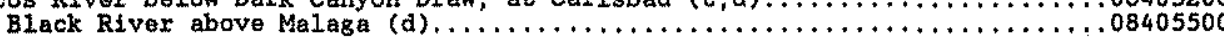

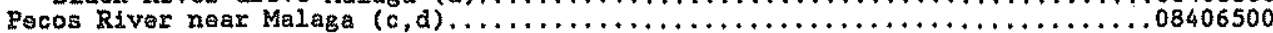

Pecos River at Pierce Canyon Crossing, near Malaga (c, d) .................08407000

Pecos River at Red Bluff ( $\mathrm{d}, \mathrm{m} s)$

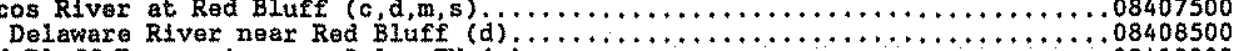

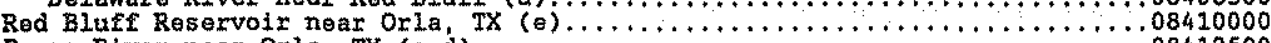

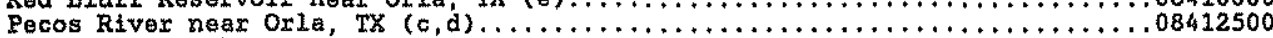
MIMBRES RIVER BASIN

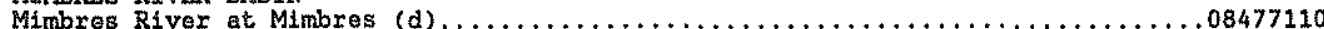

TULAROSA VALLEY BASIN

Selt Creek at Range Road 361 on White Sands Missile Range (c,d) .........08480595

Tularosa Creek near Bent $(c, d, t, s) \ldots \ldots \ldots \ldots \ldots \ldots \ldots \ldots \ldots$

\section{COLORADO RIVER BASIN}

\section{SAN JUAN RIVER BASIM}

Rio Blanco below Blanco Diverston Dam, near Pagosa Springs, Co (d)........09343300

Navajo Rivez below Oso Diverston Dam, near Chromo, Co (d) .............

Littie Navajo River below little Oso Diversion Dam, near Chromo, co (đ) . . . 09345200

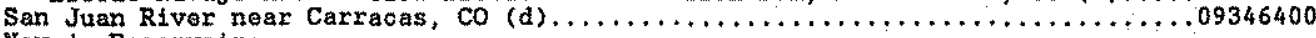

Kavajo Reservoir:

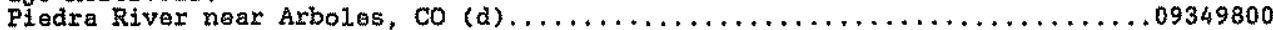

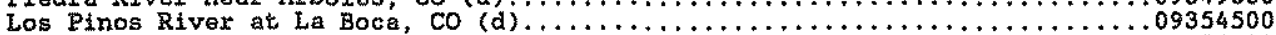

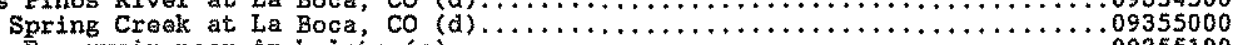

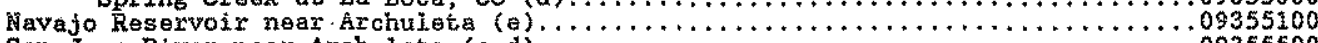

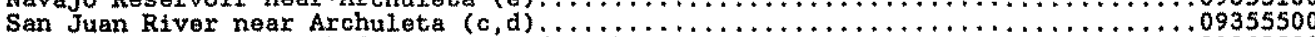

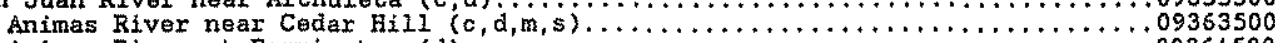

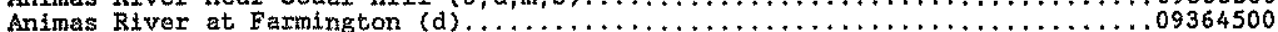

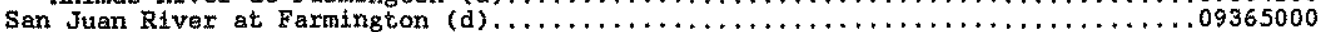

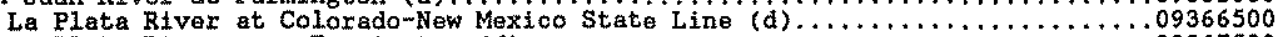

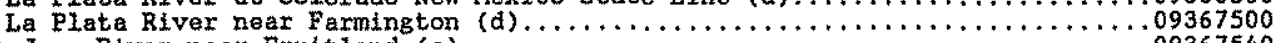

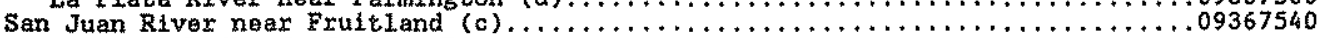
Chaco Wash (head of Chaco River):

San Juan River at Shiprock $(c, d, m, s, t) \ldots \ldots \ldots \ldots \ldots \ldots \ldots \ldots \ldots$

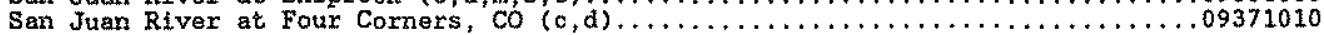
LITTLE COLORADO RIVER BASTN Zuni River:

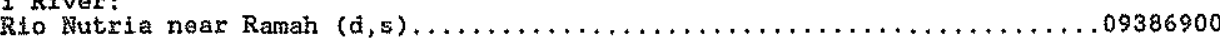

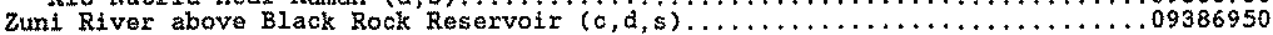
GILA RIVER BASIN

Gila Rtver near Gila (d)

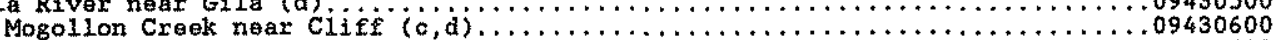

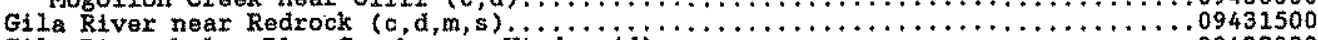

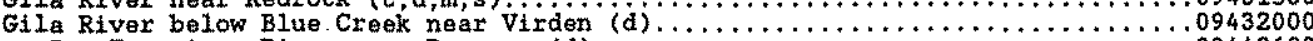

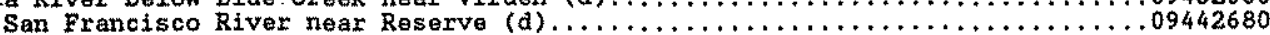

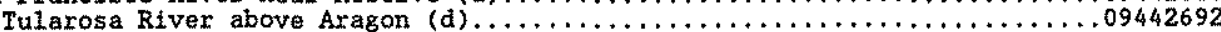

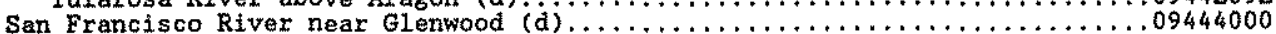


GROUND-WATER WELLS, BY COUNTY, FOR WEICH RECORDS ARE PUBLISHED

IN THIS VOLUME.

GROUND-WATER LEVELS

BERNALILLO COUNTY

WELL 350256106390801 WELL 351051106395304 CHAVES COUNTY

WELI 334138104343801 WELL 33261510430360 WELL 332255104360401 WELL 331914104253701 WELLL 331705104262801 WELL 331525104245201 WELL 331524104245101 WELL 331213104241601 WELL 331213104241601 WELL 331002104254701 WELL 330702104402401 WELL 330404104221201 CIBOLA COUNTY

WELL 350346107521201 WELL. 350923107522701 WELL 351304107543701 WELL 351630107572801 COLFAX COUNTY

WELL 364522104034501 COSTILLA COUNTY (COLORADO) WELL 370004105402201 CURRY COUNTY

WELL 341836103052001 WELL 342358103093601 WELL 342736103203701

WELL 343347103345001 WELL 343615103123801 DONA ANA COUNTY

WELL 32220310648410 WELL 321606106462901 EDDY COUNTY

WELL 325516104404601 WELL 325702104352801 WELL 325638104274801 WELL 325450104251101 WELL 324838104435301 WELI 324620104255001 WELL 324620104255101 WELL. 324325104233001 WELL 323705104225501 WELL 323542104242701 WELL 322637104142301 WELL 322636104125801 WELL 322712104074501 WELL 322120104151501 WELL 322238104101801 WELL 321741104204901 WELL 321939104113301 WELL 320604104284101 WELL 320316104294301

GRANT COUNTY

WELL 324245108175603 WELL 324600108222501 GUADALUPE COUNTY WELL 350414104485101

HARDING COUNTY

WELL 355352104054201

HIDALGO COUNTY

WELL 32405110859410 WELL 321849108392001 WELL 321248108331401 WELt 321624108504001 WELI 315630108483901 WEL 315738109004001 WELL 315048109010201 WELL, 315048109010201 WELL 312938108302301

LEA COUNTY

WELL 332115103403301 WELL 331713103283301 WELL 330458103251001 WELL 330405103194501 WELI 325730103213901 WELI 325658103200001 WELI 325132103112501 WELL 324745103082001

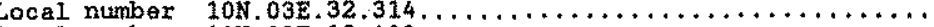

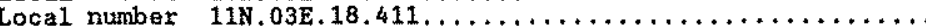

(former1y 334645104344501 ) Local number 07S.23E.23.24431...

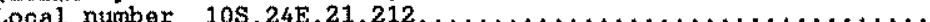

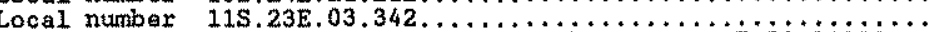
(formerty 331930104261001 ) Local number is.25E.29.34333... (formerly 332200104270001 ) Local number 12S.25E.09.422,... (formerly 331205104245101 ) Local number $12 \mathrm{~S} .25 \mathrm{E} .23 .344 \ldots$.

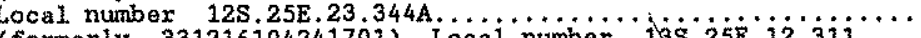
(formexly 331216104241701 ) Local number $13 \mathrm{~S} .25 \mathrm{E} .12 .311 \ldots \ldots$ (formerly 331002104272001 ) Local number 13S.25E.27.211.... (formerly 330700104402501 ) Local number $14 \mathrm{~S} .23 \mathrm{E} .08 .144 \ldots \ldots$ (formerly 330640104174501 ) Local number 14S.26E.12.43133i.

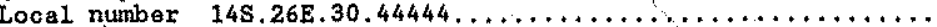

(formexly 350400107510501 ) Local number $10 \mathrm{~N} .10 \mathrm{~W} .26 .331 \ldots$... (formerly 350925107523001 ) Local number 11N.10W.27.242..... (formerly 351400107524201 ) local number $12 \mathrm{~N} .10 \mathrm{~W} .29 .434 \ldots \ldots$. (formerly 351650107535001 ) Local number $12 \mathrm{~N} .11 \mathrm{~W} .09 .424 \ldots \ldots$. (formerly 351637107584501 ) Local number $12 \mathrm{~N} .11 \mathrm{~W} .14 .213 \ldots \ldots$

(formexly 364500104031501 ) Local number 29N.27E.16.222.... (formerly 370009105410001 ) Local number $01 \mathrm{~N} .74 \mathrm{~W} .33 .322 \ldots \ldots$

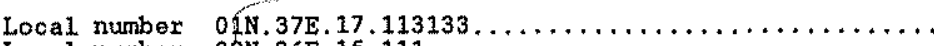

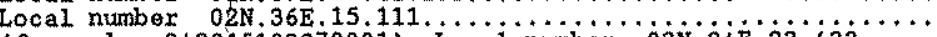
(formerly 342815103270001 ) Local number 03N.34E.23.433 ....

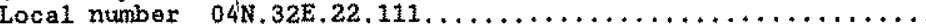

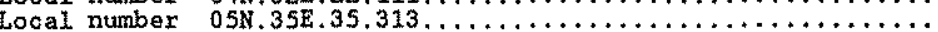

(formerly 322210106483001) Local number 22S.01E.26.411.... (formerly 321620106461501) Local number 23S.02E.31.213.....

(formerly 325510104410001 ) Local number 16S.23E.15.322333. (formerly 325735104360701 ) Local number 16S.24E.04.411341. Local number $16 \mathrm{~S} .25 \mathrm{E} .11 .11113 \mathrm{~A} . \ldots \ldots \ldots \ldots \ldots \ldots \ldots \ldots$ (formerly 325445104253501 ) Locai number ibs, $26 \mathrm{E}$.19.21i13.. (formerly 324831104435701) Local number 17S.23E.30.12344.. (formeriy 324624104244501) Local number $18 \mathrm{~S} .26 \mathrm{E} .06 .442 \mathrm{~A} \ldots$.

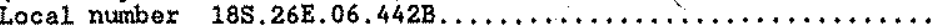

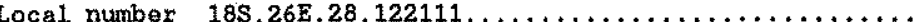

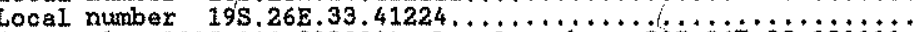
(formerly 323540104232001 ) Locai number $20 \mathrm{~s} .26 \mathrm{E} .08 .12 i i i 1 .$. (formerly 322652104141901) Local number 21S.26E.36.221... (formerly 322640104165801 ) Local number 21S.27E.32.112411. (formerly 322710104073901 ) Local number 21S.28E.30.14123.. Local number 22S.26E.25.333333 (formerly 22S 26E.36.111A) ... (formerly 322231104131001 ) Local number $22 \mathrm{~S} .27 \mathrm{E} .22 .421 \ldots \ldots$ (formeriy 321721104204801 ) Local number $23 \mathrm{~S} .25 \mathrm{E} .24 .213 \ldots \ldots$ (formerly 321930104113301 ) Local number 23S.27E.09.211124. (formerly 320602104285201 ) Local number $25 \mathrm{~S} .24 \mathrm{E} .27 .421121$. (formerly 320257104295201 ) Local number $26 \mathrm{~S} .24 \mathrm{E} .09 .443111$.

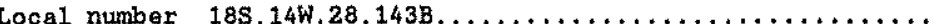

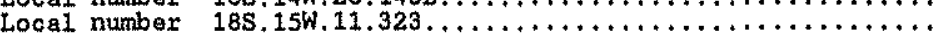

Local number

$10 \mathrm{~N}, 20 \mathrm{E} .28 .2214$

Page

400

400

400

401

401

401

402

402

402

403

403

403

404

404

404

404
405

405

405

406

406

406

407

407

408

408

408

408

409

409

410

410

410

410

411

411

412

412

433

413

413

413

414

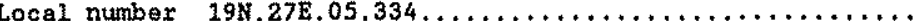

(formerly 324053108594101 ) Local number 19S.21 W.03.414.... (formerly 321848108391401) Local number 23S.18W.12.333..... (formerly 321257108331201) Local number 24S.17W.14.442,... (formerly 321540108514101) Local number 23s.20W.25.422,.... (formerly 315645108493501 ) Local number $27 \mathrm{~S} .19 \mathrm{~W} .20 .343 . \ldots$. (focal number $27 \mathrm{~S}, 21 \mathrm{~W} .17 .124 \ldots \ldots \ldots \ldots \ldots \ldots$

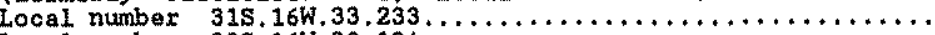

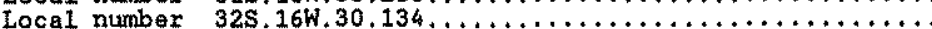

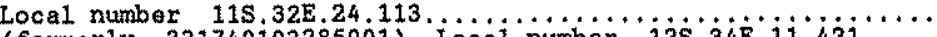
(formerly 331740103285001 ) Local number 12S.34E. 11. $421 \ldots \ldots$ (formerly 330455103251301 ) Local number $14 \mathrm{~S} .35 \mathrm{E} .28 .1111 \ldots$ (formerly 330400103193401 ) Local number 14S.36E.32.121.... (formerly 325703103213201 ) Local number $16 \mathrm{~S} .36 \mathrm{E} .04 .322 \ldots \ldots$ Local number $16 \mathrm{~S} .37 \mathrm{E} .11 .11111 \ldots \ldots \ldots \ldots \ldots \ldots \ldots \ldots \ldots \ldots$

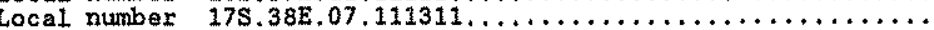

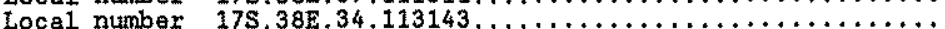

414

414

415

415

415

416

416

416 417

417

417

417

418

418

419 
GROUND-WATER WELLS, BY COUNTY, FOR WHICH RECORDS ARE PUBLISHED

IN THIS VOLUME.

GROUND-WATER LEVELS

LINCOLN COUNTY

WELL 333241105341101 WELL 332110105092501 LUNA COUNTY

WELL 322927107220101

WELL 321352107493901

WELL. 321328107565301

WELL 321010107260201

WELLL 320918107293301

WELL 320647107490701

WELL 315517107375001

WELLL 314942107361001

MCKINLEY COUNTY

WELL 352023107473201

WELL 35364510801150

WELL 353521108284901

WELL, 354235108170702

WELL 354235108170703

OTERO COUNTY

WELL 33032110601110

WELL 320657105061501

WELL 320138105063101

WELI 320008105064501

QUAY COUNTY

WELL 34384810355580

WELL 34385510348290

WELL 344406103555501

WELL 35104010343360

WELL 353239103111301 WELL 354238103

WELI 341014103264401 WELL 341037103254501 WELL 340732103145001 WELL 340732103145001 WELL. 34084410305500 WELL 334700103030601 SANDOVAL COUNTY

WELL 352121106285501

SAN JUAN COUNTY

WELL 364543108292701 WELL 36474410822500

SANTA FE COUNTY

WELL 350534106024801 WELL 350344106004601 WELL 35085910600290 WELL 35363610602100 WELL. 353516106035801 WELL 353735105581201 WELL 354013105580601

SIBRRA COUNTY

WELL 331002107150001 WELL 32592110718510 WELL 325340107183001 TAOS COUNTY

WELL 365035105360501 WELL 365644105363501 WELL 365410105345601

TORRANCE COUNTY

WELL 343443106024401 WELL 344016106070901 WELL 34423410607060 WELL 344604105574601 WELL 344842106032701 UNION COUN?Y

WELL 35514410304120 WELL 361847103064701 WELL. 362540103095001 WELL. 36341010306480 WELL 364444104000201 (formerly 333242105340701 ) Local number 09S.14E.10.13221... (formerly 332157105094101 ) Local number 11S.18E.15.33313...

(former1y 322930107221001 ) Local number 215.05W.08.444 ....

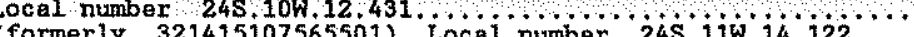
(formerly 321415107565501) Local number 24S.11W.14.122...

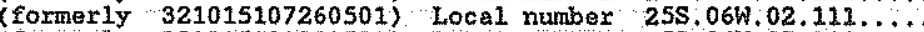
(Formerly 320915104294501 ) Local number 25S.06W.07.211....

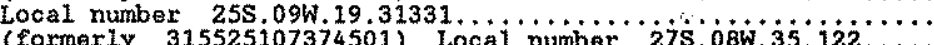
(Eormerly 315525107374501 ) Local number 275 . 09 . $01431 \ldots$ (formerly 314938107371401) Loca1 number 28S.08W:36.411......

Local number ..13N.09W.21.4123

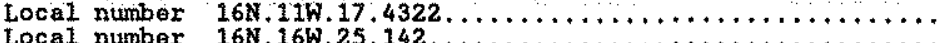

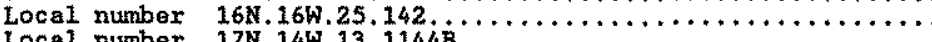

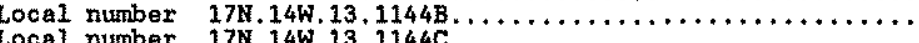

(former1y 330324106011201 ) Local number 14S, 10E.31.144.... Local number $25 \mathrm{~S} .18 \mathrm{E}, 21,233 . \ldots \ldots \ldots \ldots \ldots \ldots$

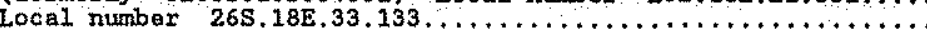

Local number $05 \mathrm{~N}, 28 \mathrm{E} .23 .222232$.

(formerly 343810103463001 ) Locai number osN 30E 18 3313ii.

Local number $06 \mathrm{~N}: 28 \mathrm{E}, 13: 33333, \ldots \ldots \ldots \ldots \ldots, \ldots, \ldots, \ldots, \ldots$

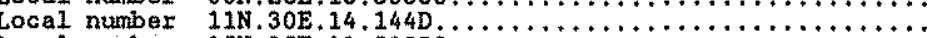

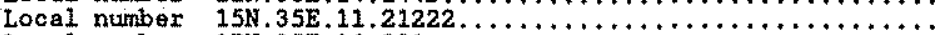

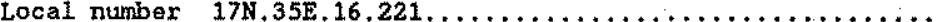

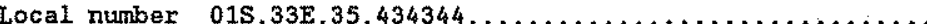

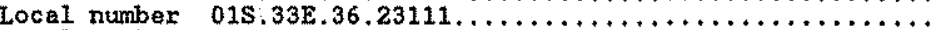

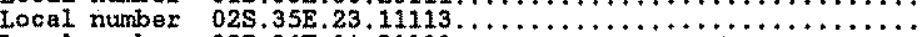

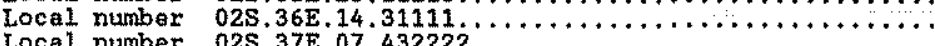

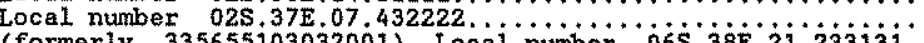

(formerly 335655103032001 ) Local number $06 \mathrm{~S} .38 \mathrm{E}, 21.233131$.

(formerly 352235106282401) Local number 13N.04E.12.112.....427

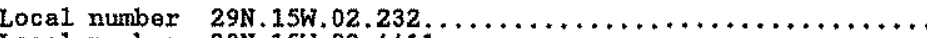

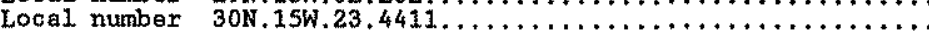

(formerly 350525106025001 ) Local number $10 \mathrm{~N} .08 \mathrm{E} .13 .1332 \ldots$ (formerly 350340106005001 ) Local number $10 \mathrm{~N} .09 \mathrm{E}, 29.1334 \ldots$.

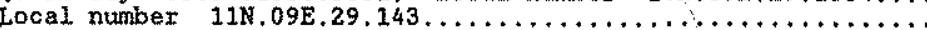

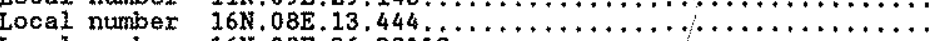

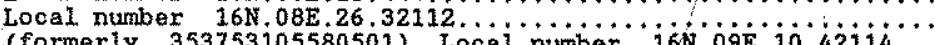
(formerly 353753105580501 ) Local number 16N.09E.10.42114.. (formerly 354005105574501 ) Local number 17N.09E.27.441...

Local number $13 \mathrm{~S} .04 \mathrm{~W} .21 .213 \ldots \ldots \ldots \ldots \ldots \ldots$ $\begin{array}{llll}\text { (formerly } & 325550107184001) \\ \text { (former1y } & 325350107175501 \text { ) } & \text { Local number } & 15 \mathrm{~S} .05 \mathrm{~W} .24 .312 \ldots \ldots \\ \text { Local number } & 16 \mathrm{~S} .05 \mathrm{~W} .25 .211 \ldots \ldots\end{array}$

(formerly 365036105355301) Local number 30N.13E.18.1121... (formerly 365650105370001 ) Local number $01 \mathrm{~S} .74 \mathrm{~W} .24 .244 \ldots$. (formerly 365410105354501 ) Local number $02 \mathrm{~S} .73 \mathrm{~W} .05 .244 \ldots$.

Local number $04 \mathrm{~N} .09 \mathrm{E} .07 .334$.

(Formerly 344016106064701 ) Local number osk.008.08.424.... (formerly 344234106074901 ) Local number $06 \mathrm{~N} .08 \mathrm{E} .32 .212 \ldots$ (formexly 344622105575501 ) Local number 06N.09E.11.211..... Loca1 number $07 \mathrm{~N} .08 \mathrm{E} .25 .121, \ldots \mathrm{Ca}$ number $06 \mathrm{~N} .09 \mathrm{E} .11 .211 \ldots \ldots$

(formerly 360940103083501 ) Local number $19 \mathrm{~N}, 36 \mathrm{E} .23 .244 \ldots \ldots$ (formerly 361910103170501 ) Local number $24 \mathrm{~N} .36 \mathrm{E} .17 .244 \ldots \ldots$

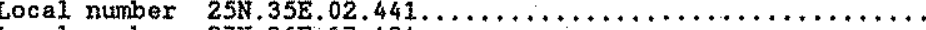

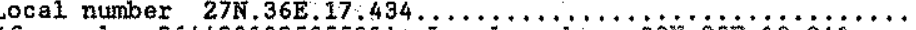

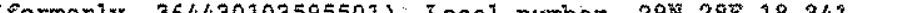

Page

419

419

420

420

420

421

421

421

422

422
422

422

423

423

423
424

424

424

424

425

425

425

426

426

426

427

427

428

428

428

429

429

429

429

430

430

430

430

431

431

431

432

432

432

433

433

433 
The following continuous-record surface-water discharge stations (gaging stations) in New Mexico have been discontinued. Daily streamflow records were collected and published for the period of record, expressed in water years, shown for each station. Those stations with an asterisk (*) after the station number are currentiy operated as crest-stage partial-record stations. Discontinued projact stations with less than 3 years of record havo not been included. Information regarding these stations may be obtained from the District office at the address given on the back side of the title page of this report.

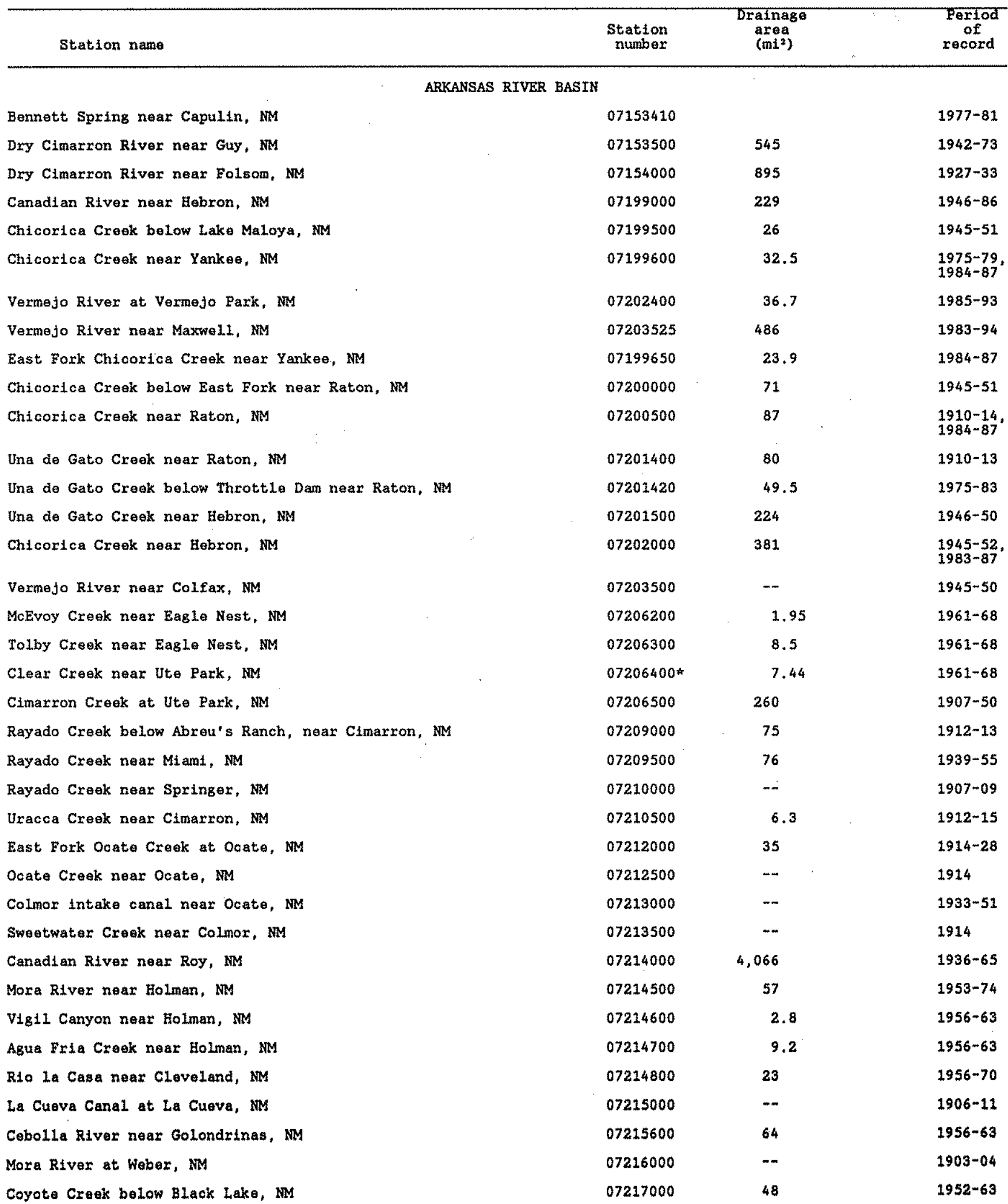


Station name

$\begin{array}{ccc}\text { Station } & \text { Drainage } & \text { Period } \\ \text { number } & \text { orea } & \text { of } \\ & \left(m 1^{2}\right) & \text { record }\end{array}$

ARKANSAS RIVER BASIN -- Continued

Coyote Creek above Guadaluptta, NM

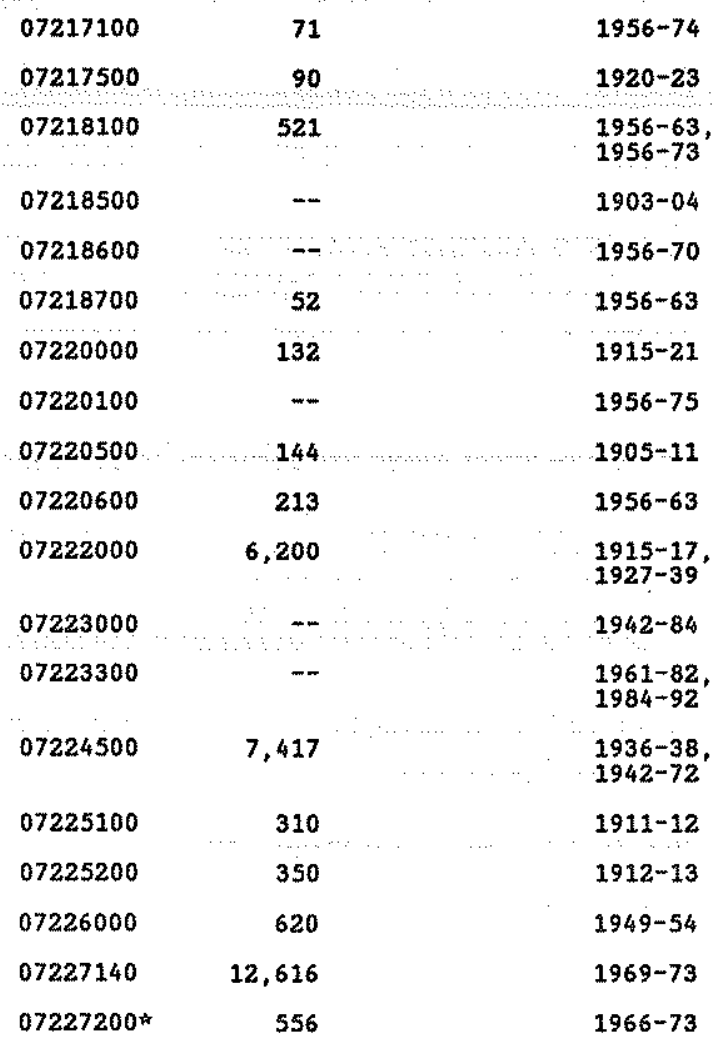

Coyota Creok at Guadalupita, NM

M

Mora River near Watrous, $M$

Sape1lo Rtver at Sape110, MM

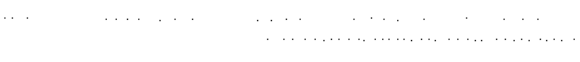

Sapello canal at Sape110, WM

Manuelitas Creok neax Rociada, MM

Sapel1o River at Sapello, MM

lake Isabel feeder canal near Sapello, MM

Sape11.o River at Los Alamos, NM

Sape110 River near Watrous, MM

Canadian River near Bell Ranch, MM

Bell Ranch Canal nar Conchas Dara, NM

Canchos Canal below Conchas Dam, NM

Canadian Rivex below Conchas Dam, RM

Pajarito Creek near Hanley, NM

Pajarito Croek near Vigil Creek, near hanley, NM

Ute Croek neax Bueyeros, NM

Canadian Rtver above New Mexico Texas State line

Tramperos Creek near Stead, $\mathrm{NM}$

BRAZOS RIVER BASTN

Running Water Draw neax Clovis, NM

RTO GRANDE BASIN

Latir Creek Outelow lake fy near Amalia, NM

08254400

$1987-88$

Latir Creek Outrlow Lake th near Amalia, NM

08254425

$1986-88$

Costilla Craek naax Arnalia, NM

08254500

152

1949-59,

Ute Creek near Amalia, NM

08255000

$1961-81$

Acequia Madre at Costilla, NM

12

1949-59

08256000

$1944-92$

Mesa ditch near Garcia, Co

08256500

1944-65.

Middle ditch at Garcla, co

08257000

$1969-83$

Cerro Canal at Costilla, m

08258000

$1944-56$

Association ditch at Costilla, NM

08258500

1944-92

Cerro Canal below Association Ditch at Costi1la, NM

08258600

$1955-71$

Corro Canal near Jaroso, co

08259000

1972-92

Cerro Canal at State Itne near Jaroso, co

08259600

$1944-72$

Penasquito ditch at Costilla, NM

08260000

1973-92

Costilla Creak below alversion dan, at Costilla, MM

08260500

1955-61

Alire ditch at Garcia, Co

08261500

08262500

$1952-86$

Costilla Creek near Jaroso, Co (near Mouth, MM)

08263000

$1944-59$

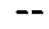




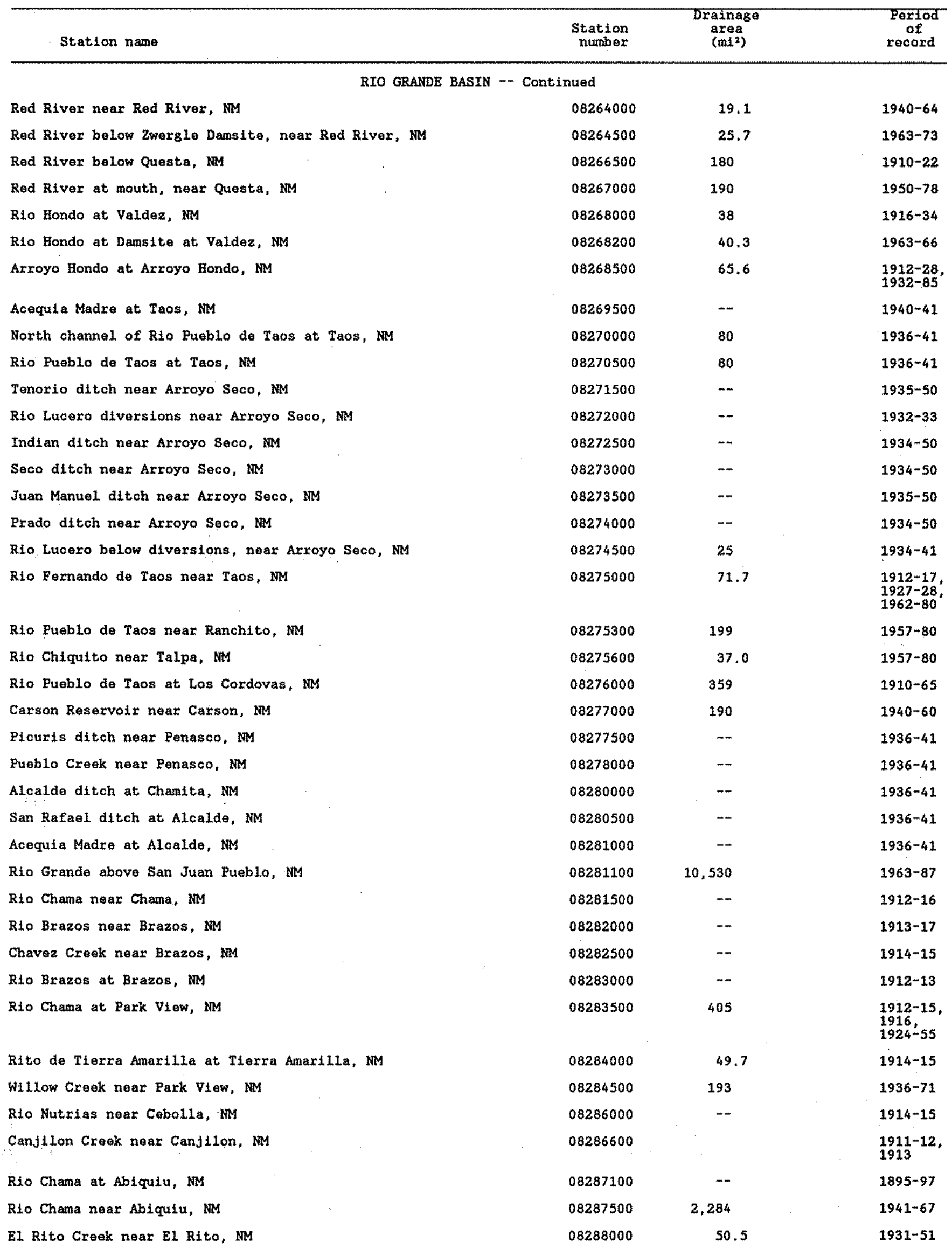




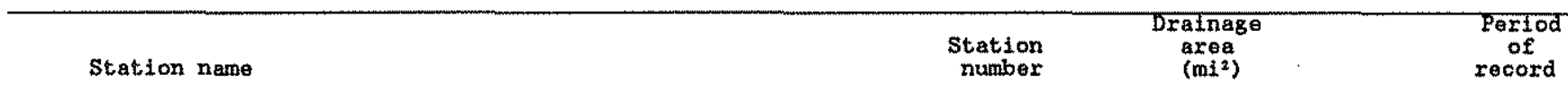

Rio Vallecitos at Vallecitos, MM Santa Clara ditch near Espanola, BM Santa Cruz River at Rlverside, NM Santa Clara Creek near Espanola, M

Hill Acequia at head, near Espanola, NM

Hill Acequia near Espanola, MM Guachupangue ditch near Espanola, NM San Ildefonso ditch near Espanola, MM Rio Nambe at Nambe Falls, IM Nambe Canal near Nambe, NM

Rio Nambe near Nambe, NM

Rto En Medio near Santa Fe, NM

Jlano Frio ditch near Nambe, NM

Llano ditch near Nambe, NM

Mioses Pene ditch near Nambe, NM

Mocha ditch at Nambe, NM

Comunidad ditch at Nambe, NM

Ortsz ditch at Nembe, NM

Canyon ditch noar Nambe, MM

Acequia Rincon near Nambe, NM

Las Joyas ditch near Nambe, MM

Truji110 ditch near Nambe, NM

Barranco Alto ditch near Nambe, NM

Pojoaque River at Pojoacue Bridge, near Nambe, MM

Jacona ditch near Nambe, NM

Jacona ditch neax San Tldefonso, RM

North Fork Tesuque Creek neax Santa Fe, NM

Middle Fork Tesuque Cxeok near Santa Fe, $M$

South Fork Tesuque Creek near Santa Fe, NM

Tesuque Creek above diversions near Sante Fe, AM

Cajon Grande ditch neax Santa Fe, MM

De La Cruz ditch near Sante Fe, NM

Acequia Madre near Sante Fo, MM

Acoquia Madre at head, near Santa Fo, MM

Littlo. Tezuque Crook noar Santa Fe, $\mathrm{FM}$

Little Tesuque Creek tributary No. 4 near Santa Fe, NA

Littio Tasuque Croek tributary No. 3 noar Sente Fo, IM

Littlo Tesuque Crook tributary No. 2 near Sante 50 , NM

Little Tosuque Croek near santa Fe, NM

Rio Tesuçue at Tesuque, near Senta $F_{0}$, NM

Acequia Modio noar Santa Fo, No

Acequia Medto at waste, near Santa Fe, NM
RIO GRANDE BASIN -- Continued

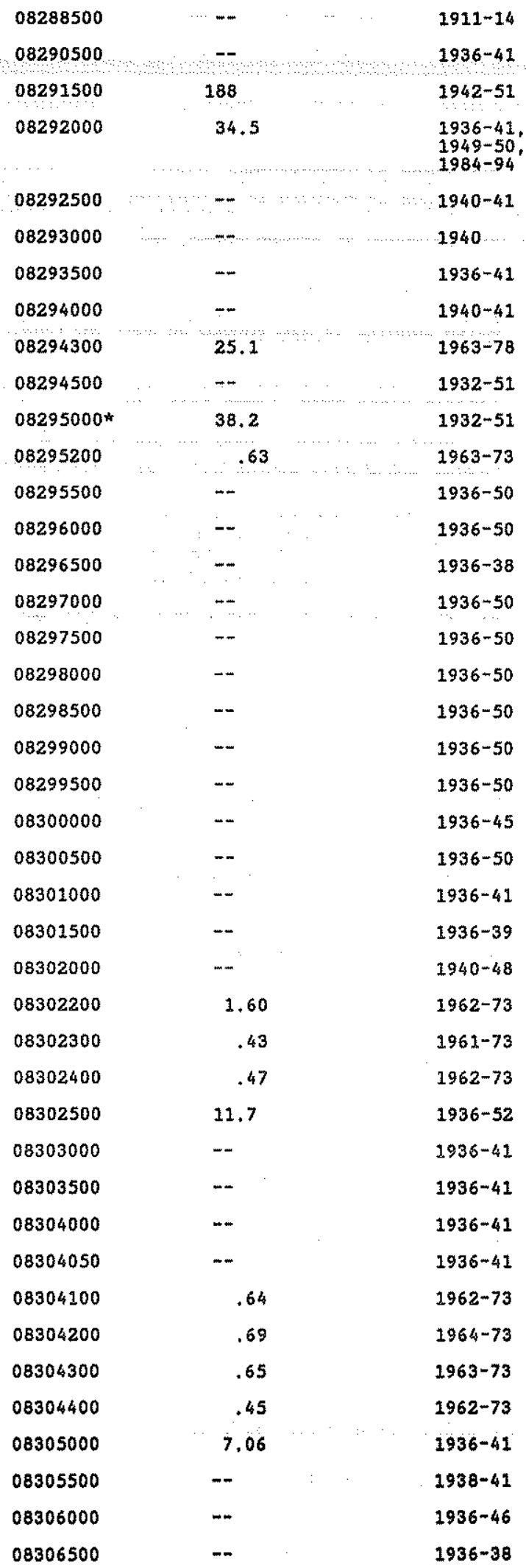




\begin{tabular}{|c|c|c|c|}
\hline Station name & $\begin{array}{l}\text { Station } \\
\text { number }\end{array}$ & $\begin{array}{c}\text { Drainage } \\
\text { area } \\
\left(m i^{2}\right)\end{array}$ & $\begin{array}{l}\text { Perfod } \\
\text { of } \\
\text { record }\end{array}$ \\
\hline Hubbard dttch near Santa Fo, NM & $\begin{array}{l}\text { - Continued } \\
08307500\end{array}$ & -- & $1938-41$ \\
\hline Mitchell ditch near Santa Ee, NM & 08308000 & -- & $1936-51$ \\
\hline Post ditch near Tesuque Pueblo, NM & 08308500 & -- & $1936-41$ \\
\hline Qwityo ditch near Tesuque Pueblo, MM & 08309000 & $-\cdots$ & $1936-41$ \\
\hline Corral ditch near Testuque Pueblo, MM & 08309500 & - & $1936-41$ \\
\hline Acequia Indtog neax San I1defonso, MM & 08310000 & - & $1936-41$ \\
\hline Acequia de la Otra Banda near San ILdefonso, NM & 08310500 & $-\infty$ & $1936-41$ \\
\hline El Rancho ditch near San Ildefonso, NM & 08311000 & - & $1936-41$ \\
\hline San Antonio ditch near San Ildefonso, MM & 08311500 & $-\infty$ & $1936-41$ \\
\hline Well ditch at San Ildefonso, $M$ & 08312000 & -- & $\begin{array}{l}1937 \\
1938-51\end{array}$ \\
\hline Ortiz ditch at San Ildefonso, NM & 08312500 & -- & $2936-41$ \\
\hline Pojoaque River near San Ildefonso Pueblo, NM & 08312600 & 184 & $1972-79$ \\
\hline Los Alamos Canyon near Los Alamos, MM & 08313042 & 9.1 & $\begin{array}{l}1970-71 \\
1991-95\end{array}$ \\
\hline Rito de los Frijoles near Los Alamos, NM & 08313300 & 8.9 & $1959-63$ \\
\hline Rio Grande at Cochit1, NM & 08314500 & 14,600 & $1924-70$ \\
\hline Santa Fe Rlver at Monument Rock, near Santa Fe, MM & 08315000 & 14 & 1910 \\
\hline Galisteo Creek above Galisteo Reservoir, NM & 08317850 & 567 & $1970-76$ \\
\hline Galisteo Creek at Domingo, MM & 08318000 & 640 & $1941-71$ \\
\hline San Felipe east stde acequia near Doningo, NM & 08318500 & - & $1936-41$ \\
\hline Rito San Antonio near Los Alamos, NM & 08319500 & - & $1949-50$ \\
\hline Redondo Creek near Jemez Springs, NM & 08319945 & 12.1 & $1982-85$ \\
\hline Sulfur Creak near Jemez Springs, NM & 08319950 & 38.0 & $1982-85$ \\
\hline Jemez River near Jamez Springs, NM & 08320000 & -- & $1949-50$ \\
\hline East Fork Jemez River near Los Alamos, NM & 08320500 & -- & $1949-50$ \\
\hline East Fork Jemez River near Jemez Springs, MM & 08321000 & $\cdots$ & $1949-50$ \\
\hline Jemez River below East Fork, near Jemez Springs, MM & 08321500 & 173 & $1951-90$ \\
\hline Rio Las Vacas near Cuba, NM & 08322000 & -- & $1939-41$ \\
\hline Rio Cebolla neax Jenez Springs, M & 08322500 & -- & 1939 \\
\hline Rio Guadalupe near Jemez Springs, NM & 08323500 & 230 & $\begin{array}{l}1938-42 \\
1949-50\end{array}$ \\
\hline Jemez east slde ditch near Jemez, MM & 08324500 & -- & $1936-41$ \\
\hline Jemez west side ditch near Jemez, NM & 08325000 & $-\cdots$ & $1936-41$ \\
\hline Antonio Pecos ditch near Jemez, MM & 08325500 & $-\cdots$ & $1936-41$ \\
\hline San Ystdro ditch near San Ystdro, NM & 08326000 & -- & $1936-41$ \\
\hline Jemez River at San Ysidro, MM & 08326500 & 854 & $1937-41$ \\
\hline Zia ditch near San Ysidro, MM & 08327000 & -+ & $1936-41$ \\
\hline Zia Reservotr near San Ysidro, MM & 08327500 & 2.4 & $1954-60$ \\
\hline Jemez River above Jemez Canyon Dam, NM & 08328000 & 961 & $1953-58$ \\
\hline Piodra Lisa Arroyo near Bernali11o, NM & 08329100 & 4.1 & $1955-74$ \\
\hline Rio Grande near Bernal11lo, NM & 08329500 & 17,300 & $1941-69$ \\
\hline Grant Line Arroyo at Albuquerque, NM & 08329865 & 0.052 & $1987-91$ \\
\hline Rio Grande near Alaneda, MM & 08329928 & 17,263 & $1989-95$ \\
\hline Rio Grande at Rio Bravo Bridge near Albuquerque, NM & 08330150 & 17,500 & $1991-95$ \\
\hline Tijeras Arroyo at Albuquerque, $\mathrm{NM}$ & $08330500^{*}$ & 75.3 & $\begin{array}{l}1921-22 \\
1943-49\end{array}$ \\
\hline
\end{tabular}




\begin{tabular}{|c|c|c|c|}
\hline Station name & $\begin{array}{c}\text { Station } \\
\text { number }\end{array}$ & $\begin{array}{c}\text { Drainage } \\
\text { area } \\
\text { (mi2) }\end{array}$ & $\begin{array}{l}\text { Pertod } \\
\text { of } \\
\text { record }\end{array}$ \\
\hline
\end{tabular}

RIO GRANDE BASIN -- Continued

Tijeras Arroyo above Four Bills Bridge at Albuquerque, MM

Tijexas Arroyo at Kirtland Air Force Base, MM

Arroyo Del Coyote near Albuquerque, NM

Arroyo De1 Coyote at Mouth near Albuquerque, NM

Tiferas Arroyo below Arroyo De1 Coyote near Albuquerque, M

Tijoras Arroyo at Montessa Park near Albuquerque, im

Tiferas Arroyo below South Diversion Channel

Inlet near Albuquerque, NM

Rio Grande near Islota, NM

North Pajarito Arroyo at Albuquerque, NM

North Pajarito Arroyo at Albuquerque, NM

Rio Grande near Belen, $\mathrm{MM}$

Rio Grande near Bernardo, NM

Lower San Juan Riverside drain near Bernardo, NM

La Jara Crook near Le Jara, NM

Rio Puerco near Cabezon, NM

Rio Puerco at Cabozon, NM

Papers Wash near Star Lake Trading Post, MM

Arroyo Chico near Guadalupe, NM

Rio Puerco near Guadalupe, MM

Bluewater Creek near Bluewater, MM

San Mateo Crook neax San Mateo, MM

Arroyo del Ruerto naer San Mateo, NM

Rio San Jose at Grants

Grants Canyon at Grants

McCartys south side ditch near San Fidel, NM

McCartys north side ditch near San Fide1, NM

Acomfta Reservoir outlet near San Fidel, NM

Rio San Jose noar San Fidel, NM

Seama-Paraje ditch near Casa Blanca, NM

Casa Blanca ditch at Casa Blanca, M

New Laguna ditch wasteway near Casa Blanca, NM

New Laguna ditch neax siow Laguna; î̀

Rio San Jose near Casa Blance, MM

Encinal Crook near Casa Blanca, MM

Laguna ditch at New Laguna, NM

Paguate Creek near Laguna, LM

Rio Paguate below Jackpile Mine near Laguna, NM

Paguate Reservoir outlet near Laguna, NM

Rio San Jose near Laguna, MM

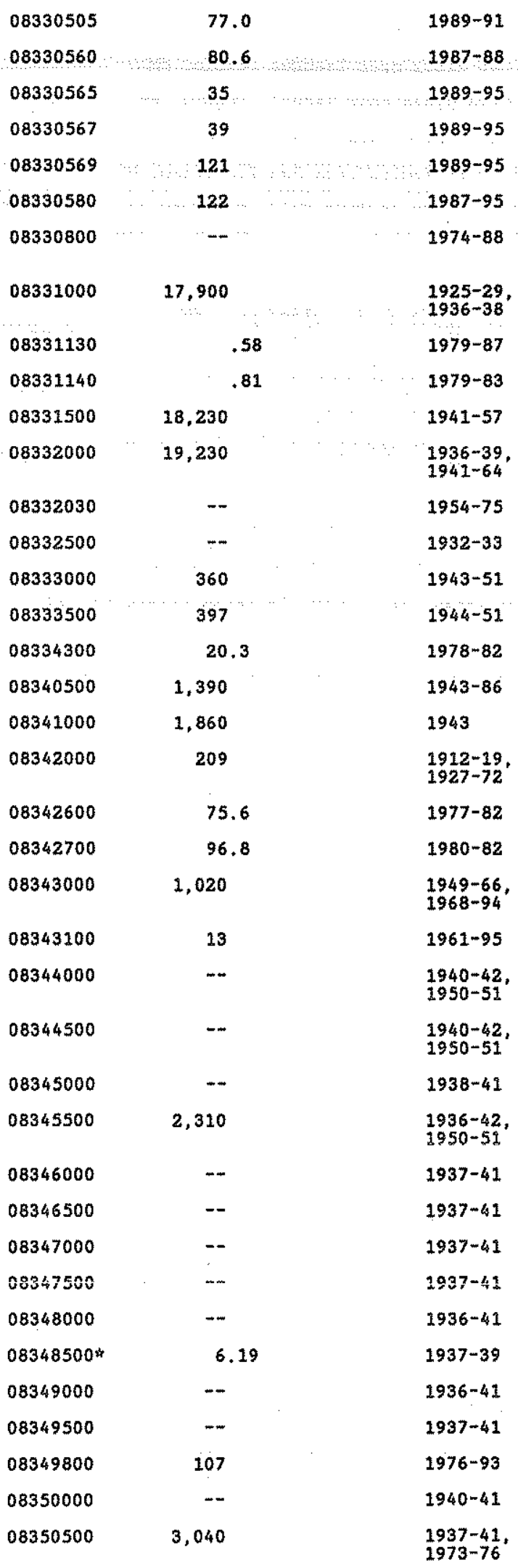




\begin{tabular}{|c|c|c|c|}
\hline Station name & $\begin{array}{l}\text { Station } \\
\text { number }\end{array}$ & $\begin{array}{l}\text { Drainage } \\
\text { area } \\
\left(\left.m\right|^{2}\right)\end{array}$ & $\begin{array}{l}\text { Period } \\
\text { of } \\
\text { record }\end{array}$ \\
\hline \multicolumn{4}{|c|}{ RIO GRANDE BASIN -- Continued } \\
\hline Mesita ditch near Laguna, NM & 08351000 & -- & $1936-41$ \\
\hline Rio San Jose at Correo, MM & 08351500 & 3,660 & $1943-94$ \\
\hline Rio Puerco at Rio Puerco, NM & 08352500 & 6,590 & $\begin{array}{l}1909-12 \\
1934-76\end{array}$ \\
\hline Alamo Creek neax Alamo, MM & 08353130 & 22.4 & $1983-85$ \\
\hline Rio Salado near Alamo, NM & 08353150 & 540 & $1983-85$ \\
\hline Rio Salado near San Acacia, NM & 08354000 & 1,380 & $1947-84$ \\
\hline Rio Grande at San Acacia, NM & 08355000 & 26,770 & $1936-64$ \\
\hline Nogal Arroyo Floodway near Socorro, NM & 08355200 & -- & $1969-77$ \\
\hline Axroyo de la Matanza near Socorro, $\mathrm{NM}$ & 08355300 & 46.0 & $1969-77$ \\
\hline Rio Grande at San Antonio, $\mathrm{NM}$ & 08355500 & 27,400 & $1951-57$ \\
\hline Socorro Main Canal South near San Antonto, NM & 08356000 & -- & $\begin{array}{l}1937-38 \\
1948-71\end{array}$ \\
\hline San Antonio Riverside Drain near San Antonio, M & 08356500 & -- & $1948-71$ \\
\hline EImendorf Interior Drain near San Antonio, NM & 08357000 & -- & $\begin{array}{l}1936-38 \\
1948-71\end{array}$ \\
\hline San Antonto Riverstde Drain near San Marcial, NM & 08357500 & -- & $1948-71$ \\
\hline $\begin{array}{l}\text { Rio Grande Conveyance Channel below heading, } \\
\text { near San Marcia1, } N M\end{array}$ & 08358000 & -- & $1953-57$ \\
\hline Rio Grande at San Marcial, NM & 08358500 & 27,700 & $1895-1964$ \\
\hline Milligan Gulch near San Marctal, NM & 08358550 & 413 & $1968-78$ \\
\hline $\begin{array}{l}\text { Rio Grande Conveyance Channel at mouth of Nogal Canyon, } \\
\text { near Truth or Consequences, WM }\end{array}$ & 08359000 & -- & $1953-57$ \\
\hline Rio Grande at the narrows, in Elephant Butte Reservoir, MM & 08359500 & 28,500 & $1951-57$ \\
\hline Alamosa Creek near Monticello, NM & $08360000 *$ & 403 & $1931-42$ \\
\hline Las Cruces Arroyo near Las Cruces, NM & 08363600 & 13.5 & $1958-66$ \\
\hline Tortugas Arroyo near Las Cruces, NM & 08363700 & 20.7 & $1962-74$ \\
\hline Rio Grande at Vinton Bridge near Anthony, TX. & 08363840 & 28,680 & $1970-74$ \\
\hline Pecos River near Cowles, NM & 08378000 & 189 & $1910-19$ \\
\hline Pecos River near San Jose, MM & 08379000 & 539 & $1939-40$ \\
\hline Tecolote Creek below Wright Canyon near E1 Porventr, MM & 08379187 & 5.42 & $1987-92$ \\
\hline Tecolote Creek near San Pablo, MM & 08379200 & 83 & $1960-65$ \\
\hline South Fork Gallinas Creek near E1. Porventr, NM & 08380000 & 25 & $1911-20$ \\
\hline Gallinas Creek at Monteztma, NM & 08381000 & 87 & $\begin{array}{l}1903, \\
1904-66\end{array}$ \\
\hline Storrie feeder canal near Las Vegas, MM & 08381.500 & -- & $1949-52$ \\
\hline Gallinas Rtver near Lourdes, MM & 08382000 & 313 & $1951-63$ \\
\hline Pecos River near Colontas, MM & 08382700 & 2,340 & $1970-74$ \\
\hline Los Esteros Creek Tributary above Santa Rosa Lake, NM & 08382760 & 13.7 & $1973-90$ \\
\hline $\begin{array}{l}\text { Pecos River above Los Esteros Dam Site, } \\
\text { near Santa Rosa, NM }\end{array}$ & 08382800 & 2,430 & $1965-77$ \\
\hline Recos Rtver at Santia Rosa, MM & 08383000 & 2,650 & $1928-92$ \\
\hline Pecos River near Fort Sumner, NM & 08385500 & 5,300 & $\begin{array}{l}1904-10, \\
1912-13 \\
1921-23\end{array}$ \\
\hline Pecos River below Fort Sumner, MM & 08385520 & 5,600 & $\begin{array}{l}1957-58 \\
1962-70\end{array}$ \\
\hline Pecos River below Yeso Arroyo, near Fort Sumer, NM & 08385620 & 7,000 & $1965-68$ \\
\hline
\end{tabular}


Station name

$\begin{array}{ccc}\text { Station } & \text { Drainage } & \text { Period } \\ \text { number } & \begin{array}{c}\text { area } \\ \left(m i^{2}\right)\end{array} & \text { of } \\ & \text { record }\end{array}$

RTO GRANDE BASIN -- Continuad

Pecos River above Buggins Creek, near Roswe11, MM

F. Herrera ditch $S$ at Hollywood, NM

Rio Ruldoso nate Glencoe, MM

Eag le Creok near Alto, NM

Rio Ruidoso at Hondo, $M$

Rio Bonito at Angus, MM

Rio Bonito at Hondo, IM

Rio Hondo at Hondo, $\mathrm{MM}$

Rio Hondo at Picacho, IM

Rio Hondo at Hondo Reservoir site, near Roswell, MM

Rio Hondo below reservoir outlet, near Roswe11, MM

Taylor-Moore ditch near Roswell, RM

Rocky Arroyo above Two Rivers Reservoir near Roswell, NM

Rocky Arroyo below Rocky Dam, near Roswell, NM

Rio Hondo at Roswell, NM

North Spring River at Roswe11, NM

Pecos River near Roswell, RM

Pecos River near Hagexman, WM

Rio Felix at old highway bridge near lagerman, NM

Rio Felix near Hagerman, MM

Cottonwood Creek near Lake Arthur, MM

Rio Penasco at Elk, NM

Rlo Penasco near Elk, MM

Rio Penasco near Dunken, NM

Pecos River below McMillan Dam, MM

Pecos River above Seven Rivers near Lakewood,

Pecos River below Avalon Dam, NM

Pecos River at Carlsbad, NOS

Rattlesnake Springs near White City, NM

Black River at Malaga, MM

8405300

08406000

MIMBRES RIVER BASTN

Mimbres River at McKnight Dam Site, near Mimbres, NM

Bear Canyon near Mimbres, NMS

Mimbres River near Mtmbres, NM

Mimbres River near Faywood, IM

Mimbres River near Spalding, NM

08477530

08477600

\begin{tabular}{|c|c|c|}
\hline 08385640 & 7,800 & $1965-68$ \\
\hline 08386900 & . & $1973-84$ \\
\hline 08387500 & -- & $1910-11$ \\
\hline 08387800 & 15.7 & $1969-80$ \\
\hline 08388000 & 290 & $1930-55$ \\
\hline 08388500 & 45.5 & $1930-31$ \\
\hline 08389500 & 295 & $1930-55$ \\
\hline 08390000 & -- & $1930-31$ \\
\hline 08390100 & 715 & $\begin{array}{l}1908-9 \\
1956-62\end{array}$ \\
\hline 08392500 & 970 & $1903-5$ \\
\hline 08393000 & - & 1908 \\
\hline 08393100 & -- & 1905 \\
\hline 08393200 & 31 & $1963-80$ \\
\hline 08393300 & 65 & $1963-80$ \\
\hline 08393500 & -- & $3903-6$ \\
\hline 08393600 & 19.5 & $1958-77$ \\
\hline 08394000 & $-m$ & $1903-6$ \\
\hline 08394100 & 13,360 & $1968-90$ \\
\hline 08394500 & 932 & $1939-87$ \\
\hline 08395000 & 934 & $1932-39$ \\
\hline 08396000 & 199 & $1932-65$ \\
\hline 08397450 & -- & $1910-11$ \\
\hline 08397500 & -- & 1911 \\
\hline $08397600^{*}$ & 583 & $1956-62$ \\
\hline 08401000 & 16,990 & $\begin{array}{l}1906-09, \\
1910-11 \\
1939-40 \\
1946-88\end{array}$ \\
\hline 08401100 & 17,000 & $1974-87$ \\
\hline 08404500 & - & 1940 \\
\hline 08405000 & 18,100 & $\begin{array}{l}1903-09, \\
1907-08, \\
1914-15, \\
1920-69\end{array}$ \\
\hline 08405300 & $-\infty$ & $1961-62$ \\
\hline 08406000 & 360 & $1939-40$ \\
\hline
\end{tabular}

08476300

08476500

97.3

$1963-72$

08477000

14.5

1937-55

152

1921-76

08477500

440

1909-11,

1912-14,

1916-17,

1920-21,

$1927-55$,

$1963-68$

San Vicente Arroyo at Silver City, MM
472

1963-68

26.5

$1953-65$ 


\begin{tabular}{|c|c|c|c|}
\hline Station name & $\begin{array}{c}\text { Station } \\
\text { number }\end{array}$ & $\begin{array}{c}\text { Drainage } \\
\text { area } \\
\left(\operatorname{mi}^{2}\right)\end{array}$ & $\begin{array}{l}\text { Fertod } \\
\text { of } \\
\text { record }\end{array}$ \\
\hline \multicolumn{4}{|c|}{ MIMBRES RIVER BASIN -- Continued } \\
\hline Rio de Arena near Hurley, NM & 08477700 & 16 & $1913-14$ \\
\hline Stevens Creek near Fort Bayard, NM & 08478004 & - & $\begin{array}{l}1907-12, \\
1912-14\end{array}$ \\
\hline Cameron Creek at Fort Bayard, NM & 08478008 & -- & $1911-13$ \\
\hline Cameron Creek near Hurley, MM & 08478012 & 46 & $1913-14$ \\
\hline Whitewater Creek at Hurley, MM & 08478016 & 35 & $1913-14$ \\
\hline Wamel Canal at head, near Deming, NM & 08478300 & -- & $1963-68$ \\
\hline Mimbres River below Warnel heading near Deming, MM & 08478400 & 1,101 & $1963-68$ \\
\hline \multicolumn{4}{|c|}{ TULAROSA VALLEY } \\
\hline Three Rivers near Three Rivers, NM & 08480600 & 6.9 & $1956-58$ \\
\hline Indian Creek neax Three Rivers, $\mathrm{NM}_{*}$ & $08480700^{*}$ & 6.8 & $1956-58$ \\
\hline Indlan Creek flume near Three Rivers, NM & 08480800 & -- & $1956-58$ \\
\hline Indian Creek at Mouth, near Three Rivers, NM & 08480900 & 10.9 & $1956-58$ \\
\hline Rto Tularosa at Mescalero, MM & 08481300 & -- & $1910-11$ \\
\hline R1o Tularosa near Tularosa, NM & 08482000 & -- & $1938-47$ \\
\hline Rio La Luz near La Luz, MM & 08483000 & 30 & $1911-12$ \\
\hline Rio Fresnal near Mountain Park, NM & 08484000 & 44 & $1911-12$ \\
\hline Rio La Luz at La Luz, NM & 08484500 & 74 & $1910-13$ \\
\hline Alamogordo-La Luz ditch at La Luz, NM & 08485000 & -- & $1934-49$ \\
\hline Alamo Creek at Woods Ranch, near Alamogordo, NM & 08485500 & - & $1931-37$ \\
\hline Alamogordo water supply near Alamogordo, NM & 08486000 & $-\cdots$ & $1932 \sim 51$ \\
\hline Tularosa Valley tributary near Whtte Sands, NM & 08486250 & 17.2 & $1965-74$ \\
\hline Tularose Valley tributary at White Sands, NM & 08486260 & 21.0 & $1965-74$ \\
\hline \multicolumn{4}{|c|}{ SAIT BASIN } \\
\hline Sacramento River near Sunspot, MM & 08492900 & 12.8 & $1984-89$ \\
\hline \multicolumn{4}{|c|}{ SAN JUAN BASIN } \\
\hline San Juan River at Rosa, NM & 09350500 & 1,990 & $\begin{array}{l}1895-99, \\
1910-65\end{array}$ \\
\hline Los Pinos River at Ignacto, CO & 09354000 & & $1910-61$ \\
\hline Martinez ditch near Archuleta, NM & 09355200 & -- & $1955-57$ \\
\hline Citizens ditch near Turley, NM & 09356000 & - & $\begin{array}{l}1938 \\
1951-58\end{array}$ \\
\hline San Juan River near Blanco, NM & 09356500 & 3,560 & $\begin{array}{l}1907-09, \\
1910, \\
1927-55\end{array}$ \\
\hline Canon Largo near Blanco, NM & 09356565 & 1,700 & $1977-81$ \\
\hline San Juan River at Bloomfield, NM & 09357000 & 5,410 & $\begin{array}{l}1909, \\
1910-11, \\
1927-31, \\
1955-63\end{array}$ \\
\hline San Juan River at Hammond Bridge neax Bloomfileld, MM & 09357100 & 5,540 & $1978-81$ \\
\hline Gallegos Canyon near Farmingtion, NM & 09357250 & 290 & $1978-81$ \\
\hline Animas Rivex at Aztec, $\mathrm{NM}$ & 09364000 & 1,270 & $\begin{array}{l}1904 \\
1907-15\end{array}$ \\
\hline Shumway Arroyo neax Fruitiand, IM & 09367555 & 62.8 & $1975-82$ \\
\hline Chaco Wash near Star Lake Trading Post, NM & 09367660 & 59.0 & $1978-82$ \\
\hline Chaco Wash at East Boundary at Chaco Canyon Natione & 09367676 & 364 & $1980-82$ \\
\hline
\end{tabular}




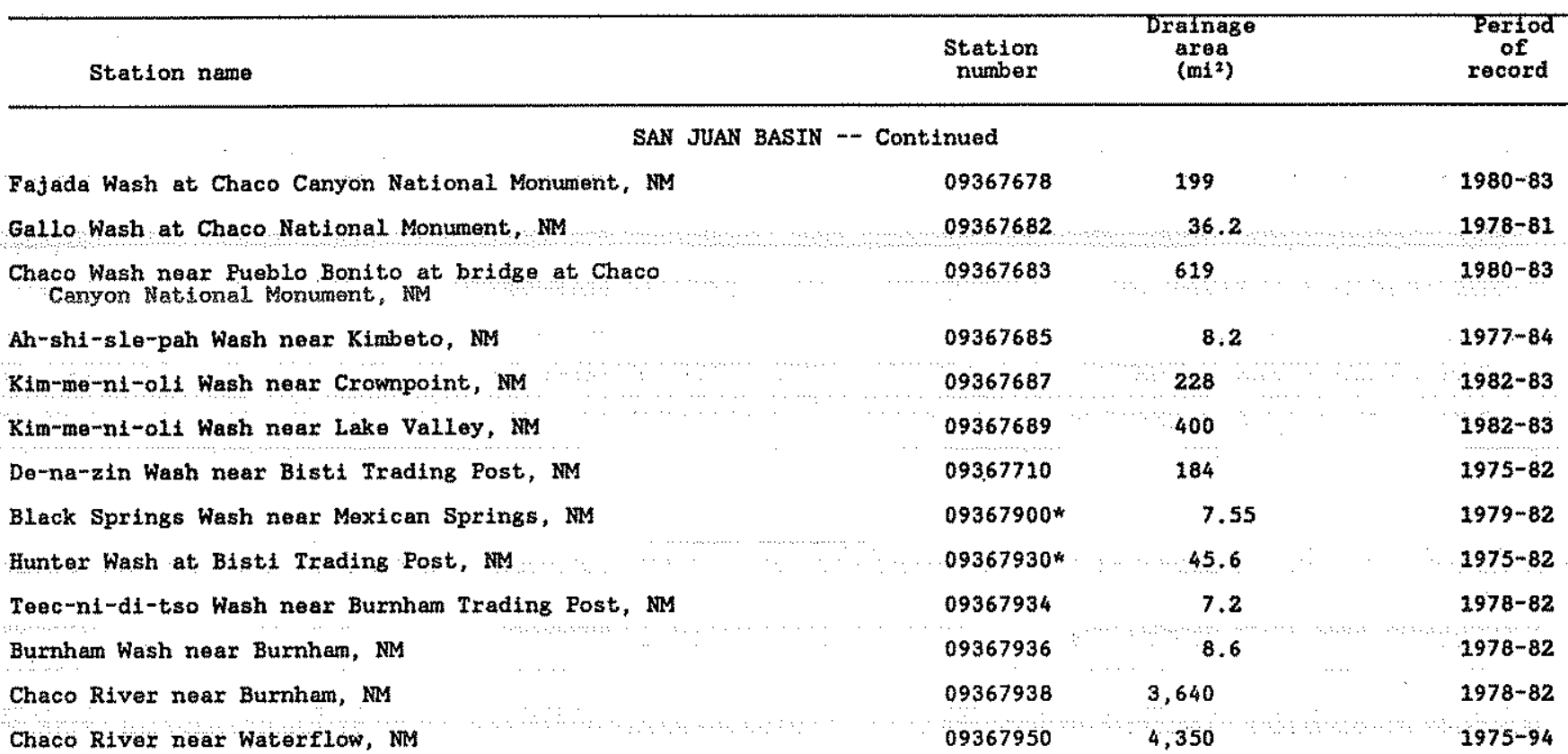

LITTLE COLORADO RIVER BASIN

Largo Creek near Mangas, MM

Zunj River at Black Rock, MM

Zunt River at New Mexico-Arizona State line

Puerco Rtver near Church Rock, NM

Puerco River at Gallup, NM

Puerco River near Manuelito, NM

Whitewater Arroyo near Cheechilgeetho, MM

Sapello Creek below Lake Roberts, near Silver City, WM

Gila River near Cliff, MM

Trout Creek near Luna, BM

San Francisco River near Alma, MM

Whitewater Creek near Mogollon, MM

$\begin{array}{lrr}09386050 & 63 & 1959-66 \\ 09387000 & 828 & 1910-30 \\ 09387300 & 1,314 & 1985-87, \\ & & 1987-89, \\ 1990-94 & 1978-82, \\ 09395350 & 193 & 1989-91 \\ 0 & & 1940-46, \\ 09395500^{*} & 558 & 1977-82 \\ & & 1989-93 \\ 09395630 & 990 & 1964-67 \\ 09395700 & 78.5 & \end{array}$

GILA RIVER BASIN

$\begin{array}{lcc}09430000 & 1,600 & 1912-19 \\ 09430150 & 78 & 1964-71 \\ 09431000 & 2,435 & 1942-51 \\ 09442653 & 27.1 & 1968-86 \\ 09443000 & 1,546 & 1904-07, \\ & & 1909-10, \\ 1912-14, & 1964-86 \\ 09443500 & 34 & 1909-23\end{array}$


The following stations were discontinued as continuous-record surface-water-quality stations prior to the 1990 water year. Records of (c) chemical, (m) microbiological, (s) sediment, or ( $t$ ) dally water temperature were collected and published for the record shown for each station.

An Inventory of chemical data analyzed prior to 1962 can be found in U.S. Geological Survey Water-Supply Paper 1786, "Inventory of Published and Unpublished Chemical Analyses of Surface Water in the Continental United States and Puerto Rico, 1961."

\begin{tabular}{|c|c|c|c|c|}
\hline Station name & $\begin{array}{r}\text { Station } \\
\text { number }\end{array}$ & $\begin{array}{c}\text { Drainage } \\
\text { area } \\
\left(\mathrm{mi}^{2}\right)\end{array}$ & $\begin{array}{l}\text { Type } \\
\text { of } \\
\text { record }\end{array}$ & $\begin{array}{l}\text { Perlod } \\
\text { of } \\
\text { record }\end{array}$ \\
\hline Dry Cimarron River near Guy, MM & 07153500 & 545 & $c, s, t$ & $1964-74$ \\
\hline Canadian River near Hebron, NM & 07199000 & 229 & c & $1966-81$ \\
\hline Chicortca Creek near Yankee, NM & 07199600 & 32.5 & & $1975-79$ \\
\hline Una de Gato Creek below Throttle Dam near Raton, NM & 07201420 & 49.5 & $\mathrm{c}, \mathbf{s}$ & $1975-84$ \\
\hline Chicorica Creok near Hebron, NM & 07202000 & 381 & $\mathrm{c}$ & $1975-81$ \\
\hline Vermejo River near Dawson, NM & 07203000 & 301 & $c, s$ & $1964-84$ \\
\hline Ctmarron River belon Eagle Nest Dam, NM & 07206000 & 167 & $\mathrm{c}, \mathrm{s}$ & $1975-84$ \\
\hline Pont1 Creok near Cimarron, NM & 07207500 & 171 & c & $1981-95$ \\
\hline Rayado Creek at Sauble Ranch, near Cimarron, NM & 07208500 & 85 & c & $1981-95$ \\
\hline Canadian River near Taylor Springs, MM & 07211500 & 2,850 & $b, c, s$ & $1966-75$ \\
\hline Mora River at La Cueva, MM & 07215500 & 173 & $\mathrm{c}$ & $1981-95$ \\
\hline Conchas Canal below Conchas Dan, NM & 07223300 & - & $\mathrm{c}$ & $1964-77$ \\
\hline Plaza Largo canal below Barranca Creek near Tucumcari, NM & 07227073 & 602 & c & $1965-66$ \\
\hline Revuelto Creek below Plaza Largo Creek near Tucumcari, NM & 07227080 & 672 & c & $1965-66$ \\
\hline Canadian River near Glenrio, NM & 07227125 & -- & $c, s, t$ & $1965-66$ \\
\hline Rto Grande above Culebra Creek near Lobatos, Co & 08249200 & & $\mathrm{~b}, \mathrm{c}, \mathrm{t}$ & $1962-69$ \\
\hline Costi1la Creok near Costilla, NM & 08255500 & 195 & $c, s$ & $1966-76$ \\
\hline Rio Grande near Cerro, NM & 08263500 & 8,440 & $c, m, s$ & $1977 ; 1979-87$ \\
\hline Rio Grande above Red Rivex near Cerro, MM & 08263510 & -- & $c, m, s$ & $1979-81$ \\
\hline Red River near Red River, NM & 08264000 & 19.1 & $\mathbf{s}$ & 1963 \\
\hline Red River below Znergle Damsite near Red River, NM & 08264500 & 28.9 & $\mathrm{c}, \mathrm{m}, \mathrm{s}$ & $1962-65 ; 1979-82$ \\
\hline Red Rtver at Molycorp Mine near Red River, NM & 08264970 & 78.3 & $c, m, s$ & $1979-82$ \\
\hline Red River near Questa, NM & 08265000 & 113 & $\mathrm{c}, \mathrm{m}, \mathrm{s}$ & $1979-87$ \\
\hline Cabresto Creek near Questa, NM & 08266000 & 36.7 & $\mathrm{c}, \mathrm{m}, \mathrm{s}$ & $1979-82$ \\
\hline Red River below Questa, NM & 08266500 & 160 & $\mathrm{c}, \mathrm{m}, \mathrm{s}$ & $1979-87$ \\
\hline Red Rtver above State Fish Hatchery near Questa, MM & 08266790 & 175 & $c, m, s$ & $1979-87,1994$ \\
\hline Red River at. Fish Hatchery near Questa, NM & 08266800 & 185 & $c, k, s, t$ & $1966-77$ \\
\hline Red River below Fish Hatchery, near Questa, NM & 08266820 & 185 & $c, m, s$ & $1978-87$ \\
\hline Red River at mouth, near Questa, NM & 08267000 & 190 & $\mathrm{c}, \mathrm{m}, \mathrm{s}$ & $1966-68 ; 1979-85$ \\
\hline R10 Grande above Rto Hondo at Dunn Bridge, MM & 08267400 & 8,690 & $c, m, s$ & $1979-87$ \\
\hline Rio Hondo at Danstte at Valdez, NM & 08268200 & 40.3 & $\mathbf{s}$ & $1962-65$ \\
\hline Arroyo Hondo at Arroyo Hondo, NM & 08268500 & 65.6 & $\mathrm{c}, \mathrm{m}, \mathrm{s}$ & $1979-82$ \\
\hline Rio Grande del Rancho near Talpa, NM & 08275500 & 83 & s & $1962-65$ \\
\hline Rio Grande above San Juan Pueblo, NM & 08281100 & 10,550 & $\mathrm{c}, \mathrm{m}, \mathrm{s}$ & $1987-88$ \\
\hline WHllow Creek above Azotea Creek near Park View, NM & 08284150 & 42 & $c, s$ & 1973 \\
\hline Azotea Tunnel at Outlet near Chama, NM & 08284160 & - & $c, s$ & $1974-75$ \\
\hline Willow Creek above Hexon Reservoir near Park View, MM & 08284200 & 112 & $\mathrm{c}, \mathrm{s}$ & $1973-74$ \\
\hline $\begin{array}{l}\text { Horse Lake Creek above Heron Reservoir near } \\
\text { Los Ojos, MM }\end{array}$ & 08284300 & 45 & $c, s$ & 1973 \\
\hline
\end{tabular}




\begin{tabular}{|c|c|c|c|c|}
\hline Station name & $\begin{array}{l}\text { Station } \\
\text { number }\end{array}$ & $\begin{array}{c}\text { Drainage } \\
\text { area } \\
\left(m i^{2}\right)\end{array}$ & $\begin{array}{l}\text { Type } \\
\text { of } \\
\text { record }\end{array}$ & $\begin{array}{l}\text { Period } \\
\text { of } \\
\text { record }\end{array}$ \\
\hline W1llow Creek near Park View, MM & 08284500 & 193 & $\mathrm{c}, \mathrm{s}$ & $1962-65$ \\
\hline Rio Chama below Heron Dam, NM & 08284540 & -- & $\mathrm{c}, \mathrm{s}$ & $1973-74$ \\
\hline E1 Vado Reservoir near Tierra Amart1la, NM & 08285000 & 873 & c & 1973 \\
\hline Rio Chama Seep below E1 Yado Dam, NM & 08285100 & 873 & c & $1973-74$ \\
\hline Rto Chama below E1 Vado Dam, NM & 08285500 & 877 & $\mathrm{c}, \mathrm{s}$ & 1974 \\
\hline Rio Chama above Abiquiu Reservotr, MM & 08286500 & 1,600 & $c, k, s, t$ & $1963-85$ \\
\hline Rio Chama below Abiquiu Dam, MM & 08287000 & 2,147 & $c, k, s, t$ & $1963-85$ \\
\hline Rio Ojo Caltente at La Madera, MM & 08289000 & 419 & $c$ & $1976-77$ \\
\hline Rio Grande at Santa Clara, NM & 08291600 & & $c, m, s$ & $1987-94$ \\
\hline Rlo Nambe at Nambe Falls, near Nambe, NM & 08294300 & 25.1 & s & $1962-65$ \\
\hline Rito de los Frijoles in Bandelier National Monument, NM & 08313350 & 18.1 & $b, c, m, s, t$ & $1977-82$ \\
\hline Rio Grande below Cochiti Dan, NM & 08317400 & 14,900 & c,s,t & $1974-84 ; 1985-88$ \\
\hline Galisteo Creek below Galisteo Dam, NM & 08317950 & 597 & $c, k, s, t$ & $1971-78$ \\
\hline Galisteo Creek at Doiningo, MM & 08318000 & 640 & $c, s, t$ & $1962-71: \cdots$ \\
\hline Jenez River below East Fork near Jemez Springs, MM & 08321500 & 173 & $\mathrm{c}, \mathrm{s}$ & $1963-67$ \\
\hline Jemez River belon Jemez Canyon Dam, NM & 08329000 & 1,038 & $\mathrm{c}, \mathrm{s}$ & $1966-88$ \\
\hline Piedra Lisa Arroyo near Bernal111o, NM & 08329100 & 4.1 & $\mathrm{c}, \mathrm{is}$ & $1962-74$ \\
\hline Rio Grande near Bernalillo, NM & 08329500 & 17,300 & $c, s, t$ & $1962-69$ \\
\hline Campus Wash at Albuquezque, NM & 08329700 & 3.80 & $c, m, s$ & $1991-94$ \\
\hline Tijeras Arroyo near Albuquerque, NM & 08330600 & 133 & c & 1979 \\
\hline Rio Grande Conveyance Channe1 near Bernardo, MM & 08331990 & - & $c, k, s, t$ & $1962-75$ \\
\hline Rio Grande near Bernardo, AM & 08332000 & 19,230 & $c, s, t$ & $1962-64$ \\
\hline Bernardo Interior Drain near Bernardo, NM & 08332050 & -- & $c, s, t$ & $1965 \sim 68$ \\
\hline San Pablo Creek near Cuba, MM & 08332700 & 12.8 & $c, s$ & 1982 \\
\hline Papers Wash near Stax Lake Trading Post, MM & 08334300 & & $\mathrm{c}, \mathrm{m}, \mathrm{s}$ & $1978-82$ \\
\hline Arroyo Chico near Guadalupe, MM & 08340500 & 1,390 & $c, s$ & $1978-86$ \\
\hline Bluewater Lake near Bluewater, NM & 08341400 & 201 & $c$ & $1966-69$ \\
\hline Rio San Jose at Grants, NM & 08343000 & 1,020 & $\mathrm{c}, \mathrm{s}$ & 1980 \\
\hline Rio Paguate below Jackpile Mine near Luguna, MM & 08349800 & 107 & c & $1977-93$ \\
\hline Rio Salado neax San Acacia, MM & 08354000 & 1,380 & $c, s$ & $1962-84$ \\
\hline Socorro Main Canal North at San Acacta, MM & 08354500 & - & $\mathbf{s}$ & 1985 \\
\hline Rio Grande Conveyance Channel at San Marciel, RM & 08358300 & - & $c, m, s, t$ & $1954-94$ \\
\hline Rio Grande below Elephant Butte Dam, NM & 08361000 & 29,450 & & $1975-82$ \\
\hline Rio Grande below Caballo Dam, NM & 08362500 & 30,700 & c & $2966-68$ \\
\hline Rto Grande at Leasburg Dam, MM & 08363500 & & $\mathrm{~b}, \mathrm{c}, \mathrm{m}$ & 1975-79 \\
\hline Tortugas Arroyo at Las Cruces, NM & 08363700 & 20.7 & $c, s$ & $1963-74$ \\
\hline Rio Grande at Vinton Bridge near Anthony, IX & 08363840 & 28,680 & $b, c, m, s$ & $1975-78$ \\
\hline Rio Grande below Old Fort Quintman, IX & 08370500 & 31,990 & $c, m, s$ & $1930-93$ \\
\hline Pecos River noar Pecos, MM & 08378500 & 189 & c & $1970-73$ \\
\hline Pecos River near Anton Chico, MM & 08379500 & 1,050 & $b, c, m, s$ & $1967-77$ \\
\hline Gallinas Creok near Montezuma, MM & 08380500 . & 84 & c & $1964-67$ \\
\hline Pecos Rtver below Sumer Dam, WM & 08384500 & 4,390 & $b, c, m, g, t$ & $1962-66 ; 1972-87$ \\
\hline Rio kondo at Diamond A Ranch noar Roswell, NM & 08390500 & 947 & 0,8 & 1962 \\
\hline
\end{tabular}




\begin{tabular}{|c|c|c|c|c|}
\hline Station name & $\begin{array}{l}\text { Station } \\
\text { number }\end{array}$ & $\begin{array}{c}\text { Dralnage } \\
\text { area } \\
\left(\mathrm{mi}^{2}\right)\end{array}$ & $\begin{array}{l}\text { Type } \\
\text { of } \\
\text { record }\end{array}$ & $\begin{array}{l}\text { Pertod } \\
\text { of } \\
\text { record }\end{array}$ \\
\hline Hagermen Canal at Dexter, NM & 08393800 & -- & $c$ & $1964-67$ \\
\hline Rio Penasco at Dayton, NM & 08398500 & 1,060 & s & $1962-72$ \\
\hline Pecos River (Kaiser Channel) near Lakewood, NM & 08399500 & & c & $1968-70 ; 1978-79$ \\
\hline Lake McMillan near Lakewood, NM & 08400500 & 16,990 & c & $1962-67 ; 1978-79$ \\
\hline Pecos River below MoMillan Dam, NM & 08401000 & 16,990 & c & $1962-66 ; 1978-79$ \\
\hline $\begin{array}{l}\text { Pecos River at Ford Crosstng above Major } \\
\text { Johnson Springs, MM }\end{array}$ & 08401300 & 16,990 & c & $1962-67$ \\
\hline Pecos River at Damsite 3 near Carlsbad, NM & 08402000 & 17,980 & $c, t$ & $1962-67$ \\
\hline Pecos River at Carlsbad, NM & 08405000 & 18,100 & $a, k, t$ & $1962-87$ \\
\hline Pecos River below Sixmile Dam near Carlsbad, NM & 08405260 & 18,650 & $b, c, m, s$ & $1975-77$ \\
\hline Black River at Harkey Crossing near Malaga, NM & 08405400 & 343 & c & $1947-66$ \\
\hline Pecos River below Red Bluff Dam, near Orla, TX & 08410100 & & $c, t$ & $1962-63$ \\
\hline Mimbres River at McKnight Dansite near Mimbres, NM & 08476300 & 97.3 & $\mathrm{c}, \mathrm{s}$ & $1967-72$ \\
\hline Mimbres River at Mimbres, NM & 08477110 & 184 & $b, c, m, s$ & $1978-86$ \\
\hline Tularosa Creek near Bent, NM & 08481500 & 120 & c & $1963-95$ \\
\hline Rio Blanco near Pagosa Springs, Co & 09343000 & 58 & $\mathbf{s}$ & $1962-65$ \\
\hline Rio Blanco at U.S. Highway 84 near Ragosa Springs, NM & 09343400 & - & $c, s$ & $1972-74$ \\
\hline Navajo River above Chromo, Co & 09344300 & 96.4 & s & $1962-65$ \\
\hline Navajo River below Oso Diverson Dam near Chromo, co & 09344450 & $\cdots$ & c.s & $1972-75$ \\
\hline Navajo River at Edith, co & 09346000 & 172 & $b, c, s$ & $1969-74$ \\
\hline San Juan River near Carracas, Co & 09346400 & 1,230 & $b, c, s$ & $1969-73$ \\
\hline Piedra River near Arboles, Co & 09349800 & 629 & $b, c, s$ & $1969-73$ \\
\hline Los Pinos River at La Boca, Co & 09354500 & 510 & $b, c, s$ & $1969-73$ \\
\hline Canon Largo near Blanco, $\mathrm{MM}$ & 09356565 & 1,700 & $c, m, s$ & $1978-81$ \\
\hline San Juan River at Bloomfleld, $M$ & 09357000 & 5,410 & $s, t$ & $1962-64$ \\
\hline San Juan River at Harmond Bridge near Bloomfield, NM & 09357100 & 5,540 & $b, c, m, s$ & $1978-81$ \\
\hline Gallegos Canyon near Farmington, NM & 09357250 & 290 & $c, m, s$ & $1978-81$ \\
\hline San Juan River above Antmas River at Farmington, NM & 09357300 & 5,800 & c & $1966-79$ \\
\hline San Juan River at Farmington, NM & 09365000 & 7,240 & $c, s, t$ & $1962-82$ \\
\hline La Plata River at Colorado-New Mexico State line & 09366500 & 331 & $\mathrm{~b}, \mathrm{c}, \mathrm{m}, \mathrm{s}$ & $1970-73$ \\
\hline La Plata River near Farmington, NM & 09367500 & 583 & c,s & $\begin{array}{l}1970-73 \\
1978-81\end{array}$ \\
\hline Shumway Arroyo near Fruitiland, NM & 09367555 & 62.8 & $b, c, m, s$ & $1976 ; 1978-82$ \\
\hline Shumway Arroyo near Waterflow, NM & 09367561 & 73.8 & $b, c, m, s$ & $1974-84 ; 1986$ \\
\hline Chaco Wash near Star Lake Trading Post, MM & 09367660 & 59 & $c, s$ & $1978-82$ \\
\hline $\begin{array}{l}\text { Chaco Wash at East Boundary at Chaco } \\
\text { Canyon National Monument, NM }\end{array}$ & 09367676 & 364 & $\mathrm{c}, \mathrm{s}$ & $1981-82$ \\
\hline Fajada Wash at Chaco Canyon National Monument, NM & 09367678 & 199 & $\mathrm{c}, \mathrm{s}$ & $1981-84$ \\
\hline Chaco Wash at Chaco Canyon National Monument, NM & 09367680 & 578 & $\mathrm{c}, \mathrm{s}$ & $1976-84$ \\
\hline Gallo Wash at Chaco Canyon National Monument, NM & 09367682 & 36.2 & $c, s$ & 1979 \\
\hline $\begin{array}{l}\text { Chaco Wash noer PB at bridge at Chaco Canyon } \\
\text { National Monument, } M M\end{array}$ & 09367683 & 619 & $c, s$ & $1981-84$ \\
\hline Ah-sh1-slo-pah Wash near Kimbeto, NM & 09367685 & 8.21 & $\mathrm{c}, \mathrm{s}$ & $1977-83$ \\
\hline Kim-me-ni-oli Wash near Crompoint, NM & 09367687 & 228 & $b, c, s$ & $1981-83$ \\
\hline Kim-me-ni-oli Wash near Lake Valley, NM & 09367689 & 400 & $b, c, s$ & $1981-83$ \\
\hline
\end{tabular}


xxiv

DISCONTINUED SURFACE-WATER-QUALITY STATIONS -- ContınUed

\begin{tabular}{|c|c|c|c|c|}
\hline Station name & $\begin{array}{c}\text { Station } \\
\text { number }\end{array}$ & $\begin{array}{c}\text { Drannage } \\
\text { area } \\
\left(m^{2}\right)\end{array}$ & $\begin{array}{c}\text { Type } \\
\text { of } \\
\text { record }\end{array}$ & $\begin{array}{l}\text { Period } \\
\text { of } \\
\text { record }\end{array}$ \\
\hline San Juan River near Fruitland, NM & 09367540 & 8,010 & c & $1978-95$ \\
\hline De-na-zin Wash near Bisti Trading Post, MM & 09367710 & 184 & $\mathrm{c}, \mathbf{s}$ & $1975-82$ \\
\hline Black Springs Wash naar Mexican Springs, NM & 09367900 & 7.05 & c, s 16 & $1981-82$ \\
\hline Hunter Wesh at Bisti Trading Fost, AM & 09367930 & 45.6 & c,s & $1975-82$ \\
\hline Teec-ni-di-tso Wash near Burnham, KM & 09367934 & 7.2 & $c, m, s, t$ & $1978-82$ \\
\hline Burnhan Wash near Burnham, NM & 09367936 & 8.6 & $c, m, s, t$ & $1978-82$ \\
\hline Chaco River near Burnham, NM & 09367938 & 3,640 & $c, m, s, t$ & $1978-82$ \\
\hline Chaco River noar Waterflow, MM & 09367950 & 4,350 & $0, \mathrm{~s}$ & $1976-89$ \\
\hline San Juan River near Bluff, UT & 09379500 & 23,000 & $c, s, t$ & $1962-68$ \\
\hline Puerco River near Church Rock, NM & 09395350 & 193. & $c, s$ & 1979 \\
\hline Foster Canyon near Continental Divide, NM & 09395381 & 16.8 & c & 1988 \\
\hline Puerco River at Gallup, MM & 09395500 & 558 & $c, k, s, t$ & $1975-77 ; 1979-84$ \\
\hline Fuerco River near Manuelito, MM & 09395630 & 990 & $c, s$ & $1989-93$ \\
\hline Gila River near Gila, NM & 09430500 & 1,864 & $c, s, t$ & $1963-67$ \\
\hline Mangas Creek below Mangas Springs, MM & 09431100 & & $c, m, s$ & $1970-86$ \\
\hline Sunset Canal above New Mextco-Artzona State line & 09433500 & -- & $b, c, s$ & $1969-72$ \\
\hline New Model Canal above New Mexico-Arizona state line & 09436500 & -- & $b, c, s$ & $1969-72$ \\
\hline Gila River at New Mexico-Arizona State line & 09438000 & 3,349 & $b, c, s$ & $1968-73$ \\
\hline San Francisco Rivex near Glenwood, NM & 09444000 & 1,653 & $b, c, s$ & $1963-85$ \\
\hline San Francisco River at Clifton, AZ & 09445000 & 2,766 & $\mathbf{s}$ & $1963-67$ \\
\hline Dry Beaver Creok neer Rimrock, $A Z$ & 09505350 & 239 & $\mathbf{s}$ & $1964-65$ \\
\hline
\end{tabular}


The Water Resources Division of the U.S. Geological Survey, in cooperation with Federal, State, and local agencies, obtains a large quantity of data pertaining to the water resources of New Mexico each water year. These data, accumulated during many water years, constitute a valuable data base for developing an improved understanding of the water resources of the State. To make these data readily avallable to interested parties outside the Geological Survey, the data are published annually in this report series entitled "Water Resources Data - New Mexico."

Water-resources data for the current year for New Mexico constst of records of discharge and water qualtty of streans; stage, contents, and water quality of lakes and reservolrs; and water levels and water quality of ground water. This report contains discharge records for 172 gaging stations and contents for 26 lakes and reservolirs; water quality for 51 gaging stations, 19 wells, and 43 partial-record stations and miscellaneous sites, and water levels at 126 observation wells. Also included are 82 crest-stage, partial-record stations. Additional water data were collected at varlous sites not involved in the systematic data-collection program and are published as miscellaneous measurements. These data represent that part of the National. Water Data System collected by the U.S. Goological Survey and cooperating Federal, State, and Local agencies in New Mexico.

Data on stream discharge and stage, and on lake or reservoir contents and stage were first published in a series of U.S. Geological Survey Water-Supply Papers entitled "Surface Water Supply of the United States." Through Septenber 30, 1960, these Water-Supply Papers were in an annual series, then in a 5-year series for 1961-65 and 1966-70. Records of chemical quality, water temperature, and suspended sediment were published from 1941 to 1970 in an annual series of Water-Suppiy Papers entitiled "Quality of Surface Waters of the United States." Records of ground-water levels were published from 1935 to 1974 in a series of Water-Supply Papers entitled "Ground-Water Levels in the United States." Water-Supply Papers generally are avallable in the libraries of the principal cities of the Untted States or may be purchased from U.S. Geological Survey, Books and Open-File Reports, Federal Center, Box 25425, Denver, Colorado 80225 .

For water years 1961 through 1974, streamflow data were released by the Geological Survey in annual reports for each State. Water-quality records for water years 1964 through 1974 were similarly released in separate reports Beginning with water year 1975, data for streamflow, water quality, and groundwater were combined in reports published annually for each State. These reports have an identification number consisting of the two-1etter State abbreviation, the last two digits of the water year, and the volume number. For example, this volume is identified as "U.S. Geologlcal Survey Water-Data Report MM-93-1.". These Water-Data Reports axe for sale by the National Technical Information Service, U.S. Department of Commerce, 5285 Port Royal Road, Springfield, Virginia 22162.

\section{COOPERATION}

The U.S. Geological Survey and State and local agencies have had joint-funding agreements for the collection of streamflow records since 1930 and for water-quality records since 1940 . Organizations that assisted in collecting the data in this report through foint-funding agreements with the survey are

New Mexico State Engineer Offlce, T,C. Turney, State Engineer,

New Mexico Interstate Stream Comission, T.C. Turney, Secretary.

Pecos River Commission, Hector Villa III, Federal representative and Chairman; Colin R. McMillan, Commissioner for New Mexico; Brad Newton, Comnissioner for Texas.

New Mexico State Bighway and Transportation Department, Pete K. Rahn, Secretary.

Canadian River Municlpal Water Authority, John C. Williams, General Manager.

Costi1la Creak Compact Commission, T.C. Turney, Commissioner for New Mexico; Hal Stmpson, Commissioner for Colorado.

Albuquerque Metropolitan Arroyo Flood Control Authority, L.A. Bla1r, Executive Engineer.

City of Albuquerque, Martin Chavez, , Mayor.

Rio San Jose Flood Control District, Alex Gonzales, Chairman.

City of Santa Rosa, Joe D. Trujt1lo Jr., Mayor.

City of Raton, Joe Apache, Mayor.

Village of Ruidoso, Gary M. Jackson, Manager.

Financial assistance for the collection of water-resources data published in this report was provided by the Corps of Engineers, U.S. Army, for 24 gaging stations; by the Bureau of Reclamation, U.S. Department of Interior, for 14 gaging stations; by the Bureau of Indian Affairs, U.S. Department of Interior, for 7 gaging stations; and by the Bureau of Land Management, U.S. Department of Interior, for 2 gaging stations.

Assistance in the form of services was provided by the Carlsbad Irrigation District.

Some data have been collected by contractors in accordance with U.S. Geological Survey specifications and under Geological Survey quality control. Organizations that provided data are recognized tn the station descrtption. 


\section{Streamflow}

Ferennial streams in New Mexico generally are in mountalnous regions in the north-central, south-central, and southwestern parts of the State. Other perennial streams include the San Juan and Animas Rivers in northwestern New Mexico, which originate in the San Juan Mountains of southwestern Colorado. When flow is not regulated by releases from dams, several reaches of the Pecos River south of Santa Rosa have perennlal flow that is maintained by relatively large spring runoff. large discharges in peremial streans normally are the result of spring snownelt in the mountasns, which way last weveral montha.

Ephemeral streams are present in the remainder of the State. Some of these streams, such as the Rio Puerco, have deeply incised channels, whereas others, especially those on the eastern plains, are swale without any welldefined channe1. Large discharges in ephemeral streams generally are caused by intense, short-duration thunderstorms (normally occurring from nid-June to mid-October); the runofe usually lasts for only a few hours.

Wacer was plentiful li most areas of New Mexico at the beginning of water year 1996 but not for the remainder of the pater year. Streamflow recorded at index gaging stations throughout the State in october generally was near or above normal, with the exception of the Pecos kiver in which flows were only 56 percent of normal. Precipitation totals indicated that the "good times" for water in New Mexico were about to end. In contrast to normal monthly precipitation, which is about 1 tnch at most recording sites, most sites recorded no precipitation in october. Precipitation total improved slightly by the end of November, but the pattern was set for an unusually small amount of precipitation. Deceptively, streanflow recorded at the various index gaging stations around the State remained near or above noxmal at the close of calendar year 1996 . The disparity between normal flows and below normel precipitation was noted in a water-supply forecast published in early January.

The forecast predicted well below normal streamflows throughout the State for March through July, based on the snowpack and precipitation conditions, and assumed that prectpitation would rebound to near-normal levels in the coming monthw. By March precipitation had not rebounded; precipitation was still 1 sss than normal for most recording sites throughout the state. For example, precipitation at Albucuerque, Cerlsbad, Gallup, and Deming was $0,27,0,35,0,16$ and 0,40 inch below normel, respectively, for the month of March. By March, reduced streamflows had proven the January streamflow forecast to be correct. 'Dry conditions reduced streamflows had proven the January streanelow forecast to be correct. Dry conditions continued through the sumer, but streanclows increased to near normal at most stations by August and precipitation amounts were back to neax and above normal at some sttes. At the ond of water year 1996 precipitation anounts varied greatly throughout the State. Frecipitation recorded at Clayton, Las Cruces, Gallup, and Albuquerque was well above normal. In contrast, precipitation recorded at Carlsbad, Tucumcari, Grants, and Deming was well below normal.

The quantity of water stored in New Mexico's reservoirs often does not represent natural hydrologic conditions because operators of those reservoirs need to meet such demands as irrigation, flood control, legal compacts and recreation. For example, during periods of heavy storm activity reservoir operators could reduco the amount of water in storage. With this in mind a review of water storage during water year 1996 indicates various trends. Brantley and Cochit. Reservoirs varied only slightly in the amount of water stored; storage at Brantley ranged from 4 to 2 percent of capacity and at Cochiti ranged from 12 to 11 percent of capacity. storage in other reservolrs, however, did reflect the reduction in water avallable during water year 1996: in El Vado Reservoir, for example the quantity of water stored was 74 percent of capacity in September and rapidiy declined to 47 percent of capacity in February as precipitation amounts continued to be below normal. Water storage in Navajo and Abiquit

Reservolrs decreased steadily during the water year. Water storage in Navafo Reservoir was 89 percent of capacity in October and ended with 71 percent of capactty in September. Similarly, water storage in Abiquitu Reservoir was 22 percent of capactty at the begining of water year 1996 but only i2 percent of capacity at the end Resarvoir was 22 percent of capactty at the begining of water year 1996 but only 12 percent of capacity at the end increased and decreased at various times during water year 1996 in response to the previously mentioned water. demands. Reservolr storage in most of the State's reservolrs at the end of water year 1996 generally was at lower levels than those recorded at the beginning of the water year. Specirically, the combined storage of 13 major reservoirs in the State decreased by 1,159,000 acre-feet during water year 1996, totaling 4,064,000 acre-feot by September 30, 1996 . The total combined capacity of these 13 reservoirs is 8,530,000 acre-feet.

Streamplow in New Maxico has been normal or greater than normal since 1979. Continuing this trend, streamflows recorded at most index gaging stations were above normal at the beginning of water year 1996 . Two exceptions were streamflow at the Gila River near Gila (station 09430500), which was 86 percent of normal, and at the Delaware River neax Red Bluff (station 08408500) which was 79 percent of normal. By the spring and sumner seasons, drought conditions had a marked exfect on streams throughout the State, and by July streamelow at most index gaging stations was well below normal. The Rto Grande below Taos Junction Bridge, near Teos (station 08276500 ) was 48 percent of normal, the Animas giver at Duxango, Co (station 09361500 ) was 52 percent of normal. and the Delaware kiver near Red Bluff (station 08408500 ) was 2 percent or normal. By the end of water year 1996 , streanflow had zebounded to nomal levels in the Pecos, Gile, and Delaware Rivere, but was still well below normal In the Rio Grande and Animas River. 
WATER RESOURCES DATA - NEW MEXICO, 1996

Discharges for water year 1996 at four index streamflow-gaging stations compared with median annual discharge for water years 1986-95 at the same stations are listed below:

\begin{tabular}{|c|c|c|c|c|}
\hline $\begin{array}{c}\text { Statton } \\
\text { number }\end{array}$ & $\begin{array}{l}\text { Station } \\
\text { name }\end{array}$ & $\begin{array}{c}\text { Medtan annual } \\
\text { Alscharge in acre-ft } \\
\begin{array}{c}\text { watter years } \\
1986-95\end{array}\end{array}$ & $\frac{\text { Annual mean }}{\text { discharge in acre-ft }}$ & $\begin{array}{l}1996 \text { discharge } \\
\text { as a percentage } \\
\text { of median }\end{array}$ \\
\hline $\begin{array}{l}08276500 \\
08378500 \\
08408500 \\
09430500\end{array}$ & $\begin{array}{l}\text { Rio Grande below Taos Junction Bridge } \\
\text { Pecos River near Pecos } \\
\text { Delaware River near Red Bluff } \\
\text { Gila River near Gila }\end{array}$ & $\begin{array}{r}541,000 \\
74,700 \\
4,220 \\
132,900\end{array}$ & $\begin{array}{r}306,100 \\
33,580 \\
5,790 \\
63,150\end{array}$ & $\begin{array}{r}57 \\
45 \\
137 \\
48\end{array}$ \\
\hline
\end{tabular}

\section{Surface-Water Quality}

Spectfic conductance in water at selected streamflow-gaging stations were near normal throughout the state during the water year. Median values of spectelc conductance for water year 1996 at selected dally stations and median values of speciflc conductance for water years 1986-95 at the same stations are listed belon:

\begin{tabular}{|c|c|c|c|c|}
\hline \multirow[b]{2}{*}{$\begin{array}{c}\text { Station } \\
\text { number }\end{array}$} & \multirow{2}{*}{$\begin{array}{c}\text { Station } \\
\text { name }\end{array}$} & \multicolumn{2}{|c|}{$\begin{array}{c}\text { Median specific conductance, in } \\
\text { microsiemens per centimeter at } \\
25 \text { Celsius }\end{array}$} & \multirow{2}{*}{$\begin{array}{l}1996 \\
\text { median as a } \\
\text { percentage } \\
\text { of } 1986-95 \\
\text { median }\end{array}$} \\
\hline & & $\begin{array}{c}\text { water years } \\
1986-95\end{array}$ & $\begin{array}{c}\text { water year } \\
1996\end{array}$ & \\
\hline $\begin{array}{l}08313000 \\
08330000 \\
08354900 \\
08358400\end{array}$ & $\begin{array}{l}\text { Rio Grande at Otowi Bridge } \\
\text { Rio Grande at Albucuerque } \\
\text { Rio Grande FW at San Acacia } \\
\text { Rio Grande FW at San Marcial }\end{array}$ & $\begin{array}{l}320 \\
384 \\
558 \\
539\end{array}$ & $\begin{array}{l}345 \\
385 \\
631 \\
626\end{array}$ & $\begin{array}{l}108 \\
100 \\
113 \\
11.6\end{array}$ \\
\hline
\end{tabular}

Suspended-sediment loads for water year 1996 at three index stations and median suspended-sediment loads for water years $1986-95$ at the same stations are listed below:

\begin{tabular}{|c|c|c|c|c|}
\hline $\begin{array}{l}\text { Station } \\
\text { number }\end{array}$ & $\begin{array}{c}\text { Station } \\
\text { name }\end{array}$ & $\begin{array}{l}\text { Median suspended- } \\
\text { sediment load } \\
\text { for } \\
\text { water years } \\
1986-95 \\
\text { in tons }\end{array}$ & $\begin{array}{l}\text { Suspended-sediment } \\
\text { Load for water year } \\
1996 \text {, in tons }\end{array}$ & $\begin{array}{l}1996 \\
\text { load } \\
\text { as a } \\
\text { percentage } \\
\text { of } 1986-95 \\
\text { median }\end{array}$ \\
\hline $\begin{array}{l}08313000 \\
08330000 \\
08358400\end{array}$ & $\begin{array}{l}\text { Rio Grande at Otowt } \\
\text { Rio Grande at Albuquerque } \\
\text { Rio Grande Floodway at San Marcial. }\end{array}$ & $\begin{array}{r}1,471,800 \\
398,100 \\
2,841,300\end{array}$ & $\begin{array}{r}399,248 \\
391,454 \\
1,488,050\end{array}$ & $\begin{array}{l}27 \\
98 \\
52\end{array}$ \\
\hline
\end{tabular}




\section{Ground-Water Levels}

Ground-water levels are measured pertodically in a network of about 6,000 observation wells in order to record changes in ground-water storage. Water levels in about 1,200 wells are measured annually and the remaining 4,800 wells are scheduled for measurement at 5-year intervals, so that wells in different areas are measured each year (fig. 1). The areas of water-level measurements are in eight of the nine major surface-water drainage basins; most are in areas where ground water is used in large quantities for irrigation, muntcipal, or industrial purposes. Twenty one selected wolls in various parts of the state are equipped with continuous water-level recorders.

Bydrographs of water levels in wells (fig. 2) In the four quadrants of the State illustrate the water-level trends for the last 20 years. A decrease in ground-water withdrawals for agriculture and mining operations may be responsible for the general rise in water levels in the well in Cibola County since ig79. The decrease in the water level in the Cibola County well since last yeer may be a result of recent withdrawals for industrial use. The wells in Luna, Union, and Chaves Counties are in areas of intensive irrigation. The water level in the Luna County well (Mimbres Valiey) decreased fron water year 1991, but continued to be higher than average for the past 20 years. The water level in the well in Union County continued to decline, which is typical of wells on the High Plains of northeastern New Mexico. The water level in the recorder well in Chaves County has yearly fluctuations. that are typtcal of water levels in welis in the Roswell artestan basin. The water levels in the vicinity of this well have also risen since the mid-1970's, probably resulting from both a decrease in withdrawals for irrigation and an increase in recharge to the aquifer.

\section{SPECIAL NETWORKS AND PROGRAMS}

Exdrologle Benchmark Network is a network of 50 sites in small dratnage basins arourd the country whose purpose is to provide consistent data on the hydxology, including water quality, and related factors in representative undeveloped watersheds nationwide, and to provide analyses on a continuing basis to compare and contrast conditions observed in basins more obviously affected by human activities.

National Strean-Quality Accounting Network (NASQAN) monitors the wator guality of large rivers within four of the Nation's largest river basins-the Mississippi, Columbia, Coloredo, and Rto Grande, The network consists of 39 stations. Samples are collected with sufficient frequency that the flux of a wide range of constituents can be estimated. The objective of NASQAN is to characterize the water quality of these large rivers by measuring concentration and mass trasport of a side range of dissolved and suspended constituents, including nutrients, major lons, dissolved and sediment-bound heavy metals, common pesticides, and inorganic and organic forms of carbon. This information will be used (1) to describe the long-term trends and changes in concentration and transport of these constituents; (2) to test findings of the National Water-Quality Assessment Program (NAWQA); (3) to characterize processes unique to large-river systems such as storage and re-mobilization of sediments and associated contaminants; and (4) to refine existing estimates of off-continent transport of water, sediment, and chemicals for assessing human effects on the world's oceans and for determining global cycles of carbon, nutrients, and other chemicals.

The National Atmosphertc Deposition Program/National Trends Network (NADP/NTN) provides continuous measurement and assessment of the chemical climate of precipitation throughout the United States. As the lead federal agency, the USGS works together with over 100 organizations to acconplish the following objectives: (1) Provide a longterm, spatial and temporal record of atmospheric deposition generated from a network of 191 prectpitation chemistry monitoring sites. (2) Provide the mechanism to evaluate the effectiveness of the significant reduction in so2 emissions that began in 1995 as implementation of the Clean Air Act Amendments (CAAA) occurred. (3) Provide the scientific basis and nationwide evaluation mechanism for implementation of the Phase II CAAA emission reductions for SO2 and NOx scheduled to begin in 2000 .

Data from the network as well as information about individual sites, are available through the world wide web at:

http://nadp.nrel.colostate,edu/NADP

The National Water-Ouality Assessment (NAWOA) Propram of the U.S. Geological Survey is a long-term program with goals to describe the status and trends of water-quality conditions for a large, representative part of the Nation's sround- and surface-weter resources; provide an improved understanding of the primary matural and human factors affecting these observed conditions and trends; and provide information that supports development and avaluation of managenent, regulatory, and monitoring decisions by other agencies.

Assessment activities are being conducted in 53 study units (major watersheds and aquifer systems) that represent a wide range of environmental settings nationwide and that account for a large percentage of the $\mathrm{Fation}$ 's water use. A wide array of chemical constituents will be measured in ground water, surface water, streambed sediments, and fish tissues. The coodinated application of comparative hydrologic studies at a wide rane of spatial and temporal scales will provide information for decision making by water-resources mangers and a foundation for aggregation and comparison of findings to address water-quality issues of regional and national interest.

Communication and coodination between USGS personnel and other local, State, and federal fnterests are critical components of the NAWQA Program. Each study unit has a local litaison committeo consisting of representatives from key federal, State, and local water resources agencies, Indian nations, and universittes tn the study unit. Liaison committees typically meet semiannually to discuss their information needs, monitoring plans and progress, dosired information products, and opportunities to collaborate efforts among tho agenctes.

Additional information about the NAWQA Program is available through the world wide web at: http://wwwrvaros , er.usgs, gov/nawqa home.htmi 


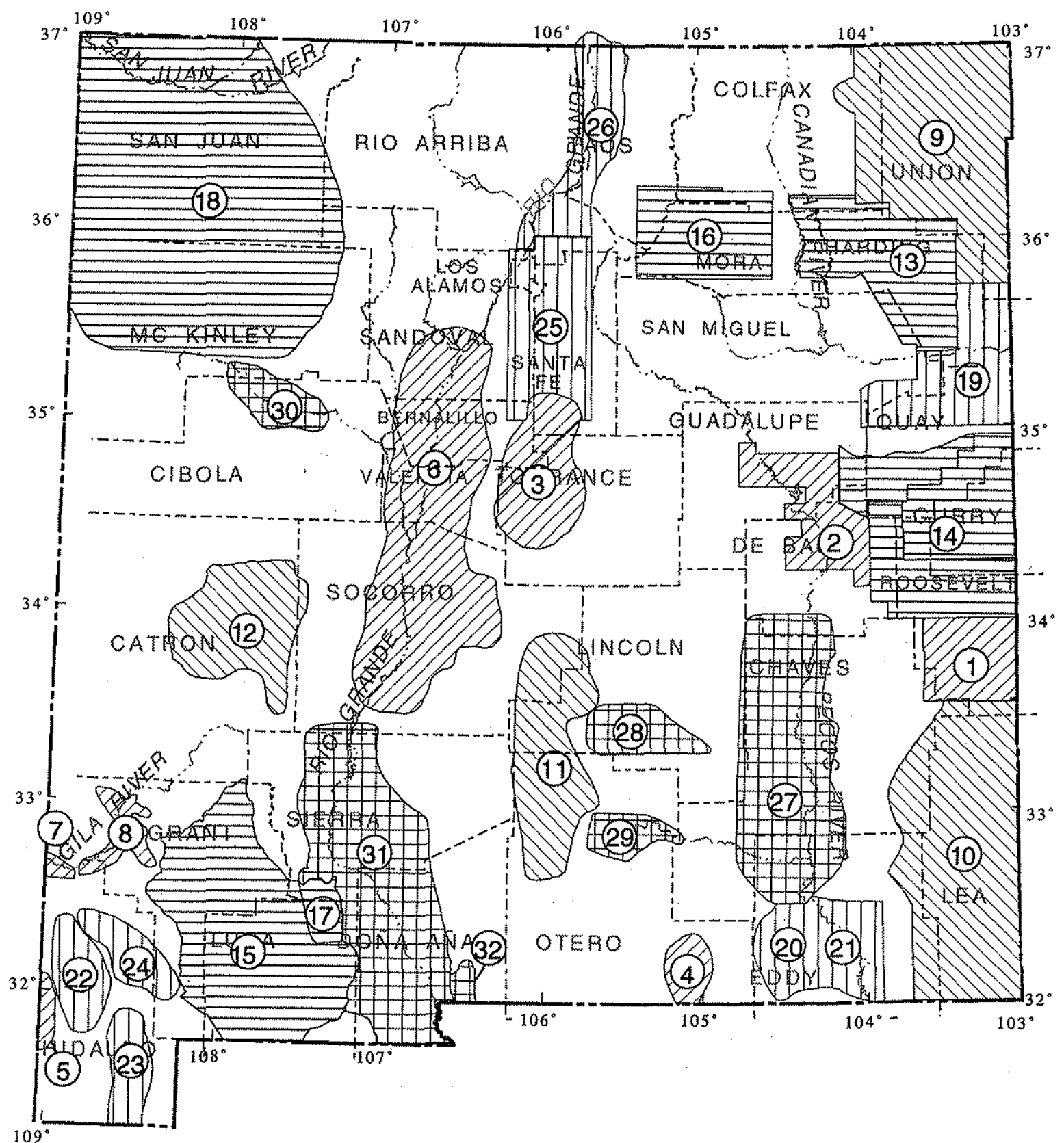
$109^{\circ}$

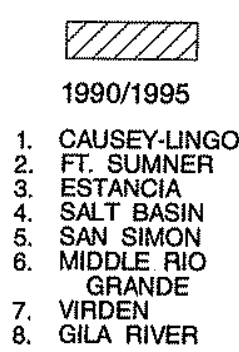

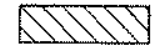

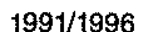

9. N. HIGH PLANS 10. LEA COUNTYHIGH PLAINS, 11. CAPITAN BASIN 12. SAN AGUSTIN PLAINS

EXPLANATION

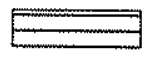

1992/1997

13. HARDING COUNTY

14. CURRY COUNTY HOUSE-PORTALES

15. MIMBRES BASIN

16. MORA AREA

17. NUTT-HOCKETT

18. SAN JUAN BASIN

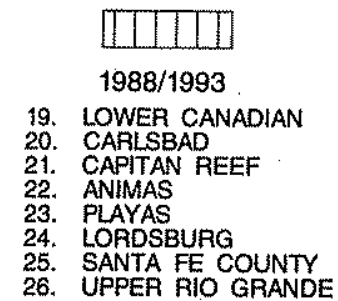

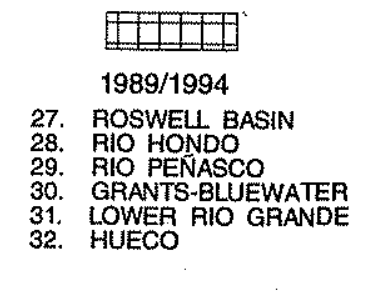

Figure 1.--Areas of 5-year ground-water-level monitoring and years measured or scheduled for measurement. 


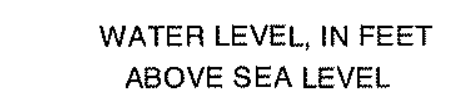

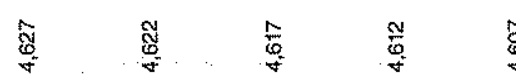

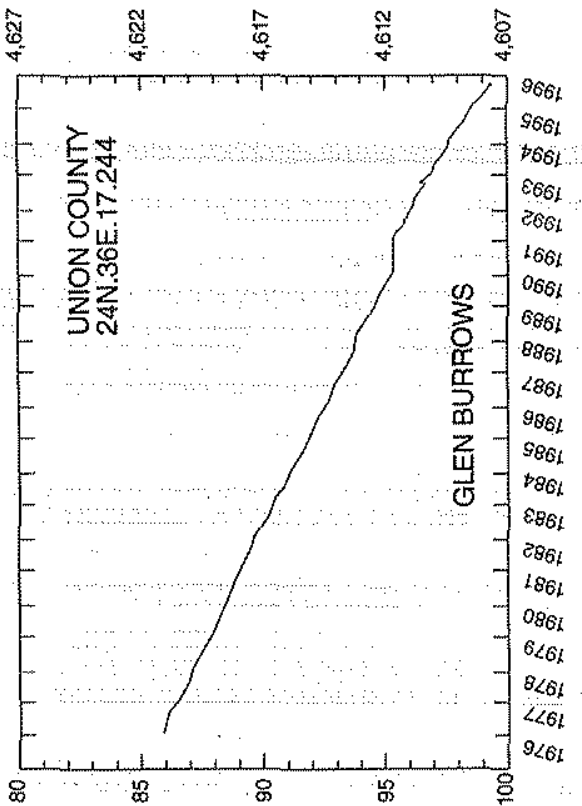

WATER LEVEL, IN FEET BELOW LAND SURFACE a........

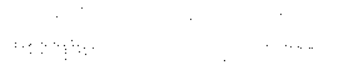

WATER LEVEL, IN FEET

ABOVE SEA LEVEL.

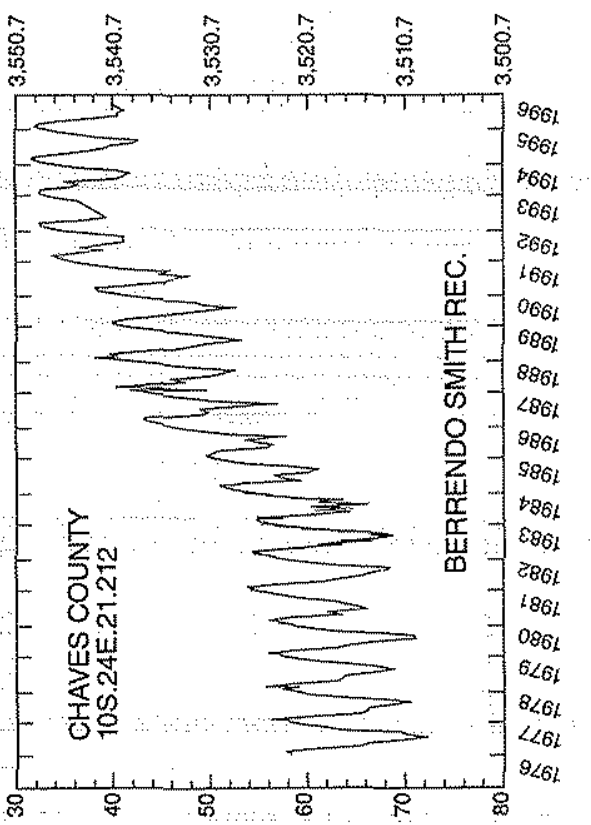

WATER LEVEL, IN FEET BELOW LAND SURFACE

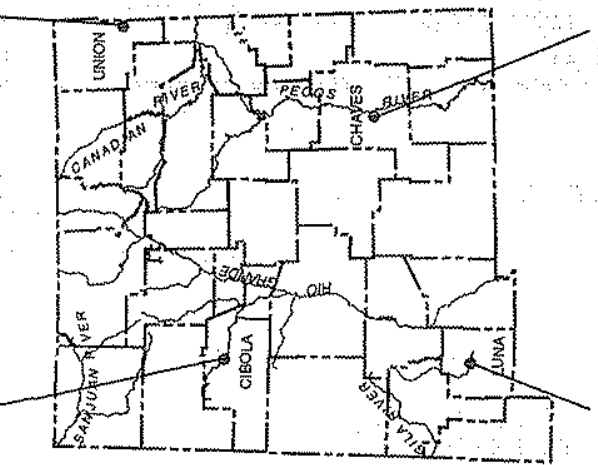

WATER LEVEL, IN FEET

ABOVE SEA LEVEL

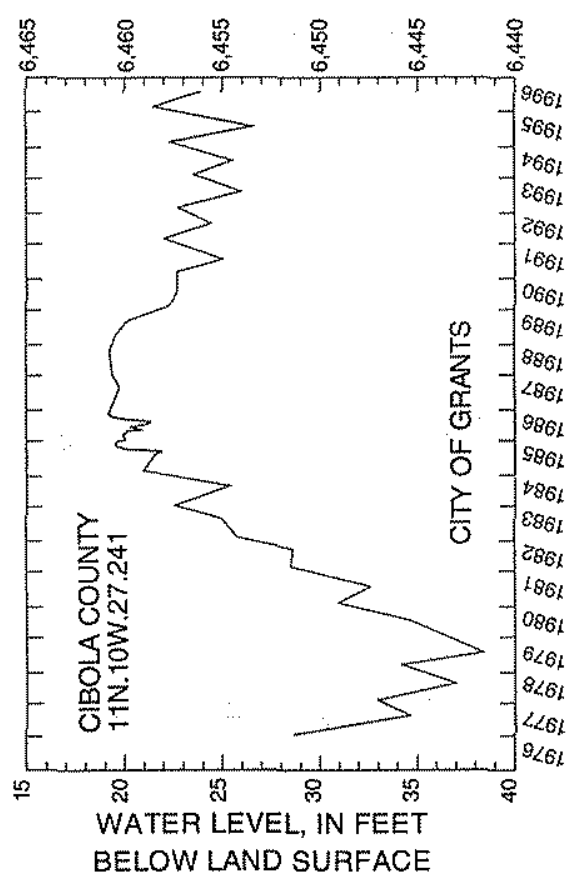

ABOVE SEA LEVEL
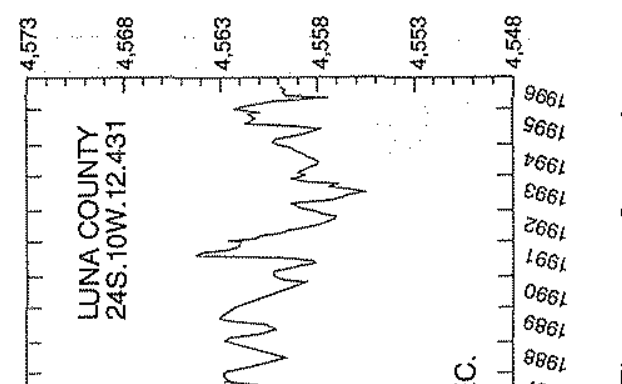

品 $\angle 806$ 
Trttiun network is a network of stations that has been established to provide baseline information on the occurrence of tritium in the Nation's surfece waters. In additton to the surface-water stations in the network, tritium data aro also obtained at a number of precipitation stations. The purpose of the precipitation stations is to provide an estimate sufficient for hydrologic studies of the tritium input to the United States. Included in the tritiun network for Now Mexico is a procipitation station that is located 1206 Field Drive NE, Albuquerque MM (Lat $35^{\circ} 05^{\circ} 35^{\prime \prime}$, long $106^{\circ} 32^{\circ} 40^{\prime}$ ).

\section{EXPLANATION OF THE RECOROS}

The surface-water and ground-water records published in this report are for the 1996 water year, which began October 1, 1995, and ended September 30, 1996. A calendar of the water year is provided on the inside of the front cover. The records contain streamflow data, stage and content data for lakes and reservoirs, water-quality data for surface and ground water, and ground-water-level data. The locations of the stations and wells where the data were collected are shown in figures 5 and 6 . The following sections of the tntroductory text are presented to provide users with a more detailed explanation of how the hydrologic data published tn this report were collected, analyzed, computed, and arranged for presentation.

\section{Station Identification Numbers}

Each data station, whether stream stte or we11, in this report is assigned a unique identiftcation number. This number is unique in that it applies spectfically to a given station and to no other. The number usually ts assigned when a station is first established and is retained for that station indefinitely. The systems used by the U.S. Geological Survey to assign identification numbers for surface water stations and for ground-water well sites differ, but both are based on geographic location. The "downstream-order" system is used for regular surface-water stations and the "latitude-longttude" system is used for welis and for surface-water stations where only miscellaneous measurements are made.

\section{Downstream-Order System}

Since October 1, 1950, the order of 1isting hydrologic-station records in Survey reports is in a downstream direction along the main stream. All stations on a tributary entering upstream from a mainstream station are listed before that station. A station on a tributary that enters between two mainstrean stations is listed between then. A simflar order is followed in listing stations on first rank, second rank, and other ranks of tributaries. The rank of any tributary on which a station is sttuated with respect to the stream to which it. 1 s immediately tributary is indicated by an indention on a list of stations in the front of this report. Each indention represents one rank. This downstream order and system of indention show which stations are on tributaries between any two stations and the rank of the tributary on which each station is situated.

The station-identification number is asstgned according to downstream order. In assigntng station numbers, no distinction is made between partial-record stations and other stations; therefore, the station number for a partial-record station indicates downstream-order position in a list made up of both types of stations. Gaps are
left in the series of numbers to allow for new stations that may be established; hence, the numbers are not consecutive. The complete oight-digit number for each station, such as 08313000 , which appears just to the left of the station name, includes the two-digit part number "oB" plus the six-digit downstream-order number "313000." The part number destgnates the major rtver basin. Records in this report are in Part 07 (Lower Mississippi River Bastn), Part 08 (Western Gulf of Mextco Bastn), and Part 09 (Colorado River Basin).

\section{Lat1 tude-Longitude Systam}

The identiftcation numbers for wells, springs, and miscellaneous sites are asstgned according to the grid system of latitude and longttude. The system provides the geographic location of the well, spring, or miscellaneous site and a untque number for each stte. The number consists of 15 digits. The first six digtts denote the degrees, minutes, and seconds of latitude, the next seven digits denote degrees, minutes, and seconds of longitude, and the last two digits (assigned secuentially) identify the wells or other sites within a 1 second grid. Seo figure 3 below.

Coordinates for wells D (334213104194801) E (334213104194802)

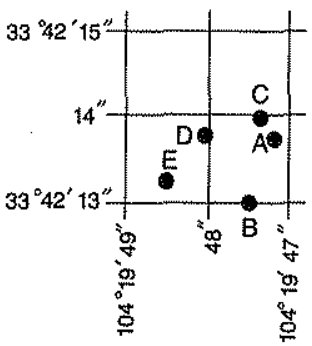

Coordinates for miscellaneous st.te C (334214104194701)

Coordinates for well A

(334213104194701) and

$(334213104194702)$

Ftgure 3.--System for numbering wells, springs, and miscellaneous sttes. 
WATER RESOURCES DATA - NEW MEXICO, 1996

Local. Well Numbers

To provide an additionel means of identification and a cross reference to records in older reports, most wells and springs have been assigned a local identifier based on the system of public land surveys. In areas covered by such surveys, the local identifier consists of a series of numbers and letters separated by periods, giving the township, range, section, and tract within section, in that order. The letters $\mathrm{N}$ or $\mathrm{S}$ locate the township north or south of the Now Mexico base line. The letters E or W locate the range east or west of the New Mexico principal meridian. A zero in a tract number indicates that the well or spring is centrally posttioned or has not been located accurately enough to be placed within a tract or quartior section. Three digits in a tract number will locate a well or spring to the nearest 10-acre tract, and six digits wili locate a site to the nearest 0.16 -acre tract. This numbering system is Illustrated in WDR kM-75-1 and WSP 1855 . On the Navajo Reservation, where public land surveys have not been made, the locel identifier is based on a different system of letters and numbers. In the examole NR032.0156x0736, the Elrst two lotters indicate that the well is on the Navajo Reservation... The thre日-digit number to the left of the decimal indicates one of a series of spocial quadrangle maps on which the well is located. The two numbers to the right of the decimal seperated by the letter $x$ are the coordinates of the well in hundredths of a mile from the northeast cornex of the area on the map. The first coordinate indicates the distance west; the second the distance south. The ebove well is located on map $032,1.56$ miles west and 7.36 miles south of the northeast comer.

\section{Records of Stage and Water Discharge}

Records of stage and water discharge may be complete or partial. Complete records of discharge are those obtained using a continuous stage-recording device through which either instantaneous or mean daily discharges may be computed for any time, or any period of time, during the period of record. Complete records of lake or reservolr content, similarly, are those for which stage or content may be computed or estimated with reasonable accuracy for any time, or period of time. They may be obtalned using a continuous stage-recording device, but need not be. Because daily mean discharges and end-of-day contents comonly are published for such stations, they are referred to as "daily stations."

By contrast, partial records are obtained through discrete measurements without using a continuous stagerecording device and pertain only to few flow characteristics, or perhaps only one. The nature of the partial record is indicated by the tablo titlo "Crest-stage pertial records. "Records of misceilaneous discharge measurements or of measurements from speclal studes, such as low-flow seepage studies, may be considerod as partial records, but they are presented separately in this report. Location of all partial-record stations for which data are given in this report is shown in figure 7.

\section{Data Collection and Computation}

The data obtained at complete-record gaging station consist of records of stage and measurements of discharge of streams or canals, and stage, surface area, and contents of lakes or reservotrs. In addition, observations of cactors effecting the stage-discharge relation or the stage-cepacity relation, weather records, and other information are used to supplement base date in determining the dally slow or volume of water in storage. Continuous records of stage are obtained from either direct readings on a nonrecording gage or from a water-stage recorder that gives alther a continuous graph of the fluctuations or a tape punched at selected time intervals. Measurements of discharge are made with a current meter, using methods adapted by the Goological Survey as a result of expertence accumulated since 1880 . These methods are described in standard textbooks, in Water-Supply Papor 2175, and in U.S. Goological Survey Techniques of Water-Resources Investigations, Book 3 , Chapter A6.

For stroam-gaging stations, rating tables giving the discharge for any stage are propared from stage-discharge relation curves. If it is nocessary to define extremes of discharge outside the range of the current meter measurements, the curves are extended using: (1) logarithmic plotting; (2) velocity-area studies; (3) results of indrect measurements of peak discharge, such as slope-area or contracted-opening measurements, and computations of flow over dams or weirs; or (4) step-beckwater techniques.

Dally mean discharges are computed by applying the dally mean stages (gage heights) to the stage-discharge curves or tables. If the stage-discharge relation is subject to change because of frequent or continual change in the physical features that form the control, the dally mean discharge is computed by the shifting-control method, in which correction factors based on individual discharge measurements and notes by the personnel making the measurements axe applied to the gage heights before discharges are determined from the curves or tables. This shifting-control method is elso used if the stage-discharge relation for a station is temporarily changed by the presence of aquatic growth or debris on the control. At some northern stream-gaging stations the stage-discharge relation is afrected by 1 ce in the winter, and it becomes impossible to compute the discharge in the usual manner. Discharge for periods of ice effect is computed on the basis of gage-height record and occasional winter discharge measurements, Consideration is given to the available information on temperature and precipitation, notes of observations, and comparable records of discharge for other stations in the same or nearby basins for comparable pertods of time.

In computing records of lake or reservoir contents, it is necessary to have curves or tables available from surveys. The application of stage to the stage-content curves or tables gives the contents from which daily, monthiy, or yearly changes in contents are dotermined.

If the stage-capacity curve changes because of deposition of sediment in a lake or reservoir, periodic resurveys of the reservoir may be necessary to define new stage-cepactty curves. During the pertod between reservolr surveys, the computed contents may be increasingly in error due to the gradual accumulation of sediment.

For some gaging stations there are periods when no gage-ineight rocord is obtalned, or the racorded gege helght is so faulty that it cannot be used to compute daily discharge or contents. This happens when the recorder stops or otherwise fails to operate properly, intakes are plugged, the float is frozen in the well, or for various other reasons. For such periods the daily discharges are estimated on the basis of recorded range in stage, prior and subsequent records, discherge measurements, weather records, and comparison with records for other stations in the same or nearby basins. Likewise, daily contents may be estimated from operator's logs, prior and subsequent records, inflow-outflow studies, and other information. Information explaining how estimated daily-discharge values are identified in station records is included in the next two sections, "Data presentation" (REMARKs paragraph) and "Identifying estimated datly discharge."

Data Presentation

Streamflow data in this report are presented in a new format that is considerably different from the format in data reports prior to the 1991 water year. The major changes are that statistical characteristics of discharge now appear in tabular summaries following the water-year data table and less information is provided in the text or station manuscript above the table. These changes represent the results of a pilot program to reformat the annual water-data report to meet curxent user needs and data preferences. 
WATER RESOURCES DATA - NEW MEXICO, 1996

The records published for each gaging station consist of two parts: the manuscript or station description and the data table for the current water year. The manuscript provides, under various headings, descriptive information, such as station location; period of record; average discharge; historical extremes; record accuracy; and other remarks pertinent to station operation and regulation. The following information, as appropriate, is provided with each continuous record of discharge or lake content. Comments to follow clarify information presented under the various headings of the station description.

LOCATION. - Information on locations is obtained from the most accurate maps avallable. The location of the gage with respect to the cultural and physical features in the vicinity and with respect to the reference place mentioned in the station name is given. River mileages, given for only a few stations, were determined by methods given in "River mileage measurement, "Bulletin 14, revision of October 1968 , prepared by the Water Resources Council or were provided by the U.S. Army Corps of Engineers.

DRAINAGE ARFA.--Drainage areas are measured using the most accurate maps available, Because the type of maps avallable varies from one drainage basin to another, the accuracy of drainage areas likewise varies.

PERIOD OF RECORD. - This indicates the period for which there are published records for the station or for an equivalent station. An equivalent station is one that was in operation at a time that the present station was not, and whose location was such that records from it can reasonably be considered equivalent with records from the present station.

REVISED RECORDS.--Fublished records, because of new information, occesionally are found to be incorrect, and revistons are printed in later reports. Listed under this heading are all the reports in whtch revisions have been published for the station and the water years to which the revisions apply. If a revision did not include datly, monthly, or annual figures of discharge, that fact is noted after the year dates as follows: "(M)" means that only the instantaneous maximum discharge was revised; "(m)" that only the instantaneous minimum was revised; and "(P)" that only peak discharges were revised. If the dratnage area has been revised, the report in which the most recently revised figure was first published is given.

GAGE. - The type of gage in current use, the datum of the current gage referred to National Geodetic Vertical Datum of 1929 (seo glossary), and a condensed history of the types, locations, and datums of previous gages are given under this heading.

REMARKS. - -All periods of estimated daily-discharge record are identified by date in this paragraph of the station description for water-discharge records. The paragraph is also used to present information relative to the accuracy of the records, to spectal methods of computation, to conditions that affect natural flow at the station, and possibly to other pertinent items. For reservoix stations, information is given on the dam forming the reservoir, the capacity, outlet works and spillway, and purpose and use of the reservoir.

COOPERATION.--Records provided by a cooperating organtzation or obtatned for the Geological Survey by a cooperating organization are identified here.

AVERAGE DISCHARGE. - The discharge value given is the axithmetic mean of the water-year mean discharges. It is computed only for stations having at least 5 water years of complete record, and only water years of complete record are included in the computetion. It is not computed for stations where diversfons, storage, or other water-use practices cause the value to be meaningless. If water developments significantly altering flow at a station are put into use after the station has been in operation for a pertod of years, a new average ts computed as soon as 5 water years of record have accumulated following the development.

EXTREMES YOR PERIOD OF RECORD. - Extremes may include maximum and mininum stages and maximum and minimum discharges or content. Uniess otherwise qualified, the maximum discharge or content is the instantaneous maximum corresponding to the highest stage that occurred. The highest stage may have been obtained from a graphic or digital recorder, a crest-stage gage, or by direct observation of a nonrecording gage. If the maximum stage did not occur on the same day as the maximum discharge or content, it is given separately. Similarly, the mintmum is the instanteneous minimum discharge, unless otherwise qualified, and was determined and is reported in the same manner as the maximum.

EXTREMES OUTSIDE PERIOD OF RECORD. --Included here is information concerning major floods or unusually low flows that occurred outside the stated period of record. The information may or may not have been obtained by the $U$. $S$. Geological Survey.

EXTREMES FOR CURRENT YEAR.--Extremes given here are similar to those for the period of record, except the peak discharge ifsting may include secondary peaks. For stations meating certain criteria, all peak discharges and stages occurring during the water year and greater than a selected base discharge are presented under this heading. The peaks greater than the base discharge, excluding the highest one, are referred to as secondary peaks. Peak discharges are not published for canals, ditches, drains, or streams for which the peaks are subject to substantial control by man. The time of occurrence for peaks is expressed in 24-hour local standard time. For example, 12:30 a.m. is 0030 , and $1: 30 \mathrm{p} . \mathrm{m}$. is 1330 . The minimum for the current water year appears below the table of peak data.

REVISIONS. - If a critical error in published records is dtscovered, a revision 1s included in the first report published following discovery of the error.

Although rare, occasionally the records of a discontinued gaging station may need revision. Because, for these stations, there would be no current or posstbly future station manuscript published to document the revision in a "Revised records" entry, users of data for these stations who obtained the record from previously published data reports may wish to contact the District office to determine if the published records were over revised after the station was discontinued. Of course, if the data were obtained by computer retrieval, the data would be current and there would be no need to check because any published reviston of data is always accompanted by revision of the corresponding data in computer storage.

Manuscript tnformation for lake or reservoix stations differs from that for stream locations in the nature of the "Renarks" and in the incluston of a skeleton stage-capacity table when daily contents are given.

\section{Data table of dally mean values}

The daily table of discharge records for streamgaging stations gives mean discharge for each day of the water year. In the monthly surmary for the table, the line headed "ToTAL" gives the sum of the daily figures for each month; the Ine headed "MEAN" gives the average flow in cubic feet per second for the month; and the lines headed "MAX" and "MIN" give the maximum and minimum daily mean discharges, respectively for each month. Discharge for the month also is usually expressed in cubic feet per second per square mile (Iine headed "CFSM"); or in lnches (1ine headed "IN"); or in acre-feet (1ine headed "AC-FT"). Figures for cubic feet pex second per square mile and runoff in inches or in acre-feet may be omitted if there is extensive regulation or diversion or if the dratnage area includes large noncontributing areas. At some stations, monthly and (or) yearly observed discharges are adjusted for reservoir storage or diversion, or diversion data or reservoir contents are given. These flgures are identified by a symbol and corresponding footnote. 
Statistics of monthly mean data

A tabulax sumnary of the mean (Ine headed "MEAN"), maximum (line headed "MAX"), ma mintmum (line headed "MIN") of monthly mean flows for each month for a destgneted period is provided below the mean values table. The water years of the first occurrence of the maximum and minimum nonthly flows are provided immediately below those figures. The designated period will be expressed as "FOR WATER YEARS - , BY WATER YEAR (WY)," and WI11 11st the first and last water years of the range of years selected from the PERTOD OF RECORD paragraph in the station manuscript. It oill consist of all of the station record within the specified water yoars, inclusive, including complete months of record for partial water years, if any, and may coincide with the period of record for the station. The water years for which the statistics are computed will be consecutive, unless a break in the station record is indicated in the manuscript.

\section{Sumnery statistics}

A table titled "SUMARY STATISTICS" follows the statisties of monthly mean data tabulation. This table consists of four colums, with the first colum containing the line headings of the statistics being reported. The table provides a statistical sumary of yearly, daily, and instantaneous flows, not only for the current water year but also for the previous calendar year and for a designated period, as appropriate. The designated period selected, "WATER YEARS - - w111 consist of all of the station record within the specified water year, inclusive, including complete months of record for partial water years, if any, and may coincide with the period of record for the station. The water years for which the statistics are computed will be consecutive, unless a break in the station record is indicated in the manuscript. All of the calculations for the statistical characteristics destgnated ANNUAL (See line headings below, ) except for the "ANNUAL 7-DAY MINIMUM" statistic, are calculated for the destgneted period using complete pater years. The other statistical characteristics may be calculated using partial water years,

The data or water year, as appropriate, of the first occurrence of each statistic reporting extreme values of discharge is provided adjacent to the statistic. Repeated occurrences may be noted in the REMARKS paragraph of the manuseript or in footmotes... Because the designated period may not be the same as the station period of record published in the manuscript, occasionally the dates of occurxence listed for the daily and instantaneous extremes in the designated-period colum may not be within the selected water years listed in the heading. When this occurs, it will bo noted in the REMARKS paragraph or in footnotes. Selected streamelow duration curve statistics and runoff data are also given. Runoff data may be omitted if there is extensive regulation or diversion of flow in the drainage basin.

The following sumary statistics data, as appropriate, are provided with each continuous record of discharge. Coments to follow clarify information presented under the various line headings of the sumary statistics table.

ANAUAL TOTAL. - The sum of the daily mean values of discharge for the year. At some stations, the annual total discharge is adjusted for reservoir storage or diversion. The adjusted pigures are identified by a symbol and corresponding footnotes.

ANMUAL MEAN. - The arithmetic mean of the individual dafly mean discharges for the year noted or for the designated period. At some stations the yearly mean discharge is adjusted for reservoir storage or diversion. The adjusted figures axe identified by a symbol and corresponding footnotes.

BIGHEST ANNUAL MEAN.--The meximum annual mean discharge occurring for the designated period.

LOWEST ANRUAL MEAN. - The minimum annual mean discharge occurring for the designated period.

EIGGEST DAILY MEAN. - - The maximum daily mean discharge for the year or for the designated period.

LOWEST DAILY MEAN. - The minimum daily mean discharge for the year or for the designated period.

ANNUAL 7-DAY MINIMUM. - - The lowest mean discharge for 7 consecutive days for a calendar year or a water year. Note that most low-flow frequency analyses of annual 7-day minimun flows use a climatic year (April 1-March 31). The date shown in the surmary statistics table is the initial date of the 7 -day period. (This value should not be confused with the 7 -day 10 -year low-flow statistic.)

INSTANTANEOUS PEAK FLOW. - The maximum instantaneous discharge occurring for the water year or for the designated period. Note that secondary instantaneous peak discharges above a selected base discharge are stored in District computer files for stations meeting cortain criteria. Those discharge values may be obtained by writing to the pistrict office. (See address on beck of title page of this report.)

INSTANTANEOUS PEAK STAGE. - - The maximum instantaneous stage occurring for the water year or for the designated period. If the dates of occurrence for the instantaneous paak flow and instentaneous peak stage difier, the REMARKS paragraph in the manuscript or a footnote may be used to provide further information.

INSTANTANEOUS LON FLOW. - The minimum instantaneous discharge occurring for the water year or for the designated period.

ANRUAL RUNOFF. - Indicates the total quantity of water in runoff for a drainage area for the year. Data reports may use any of the following untts of measurement in presenting annual runoff data:

Acre-soot (AC-FI) is the quantity of water required to cover 1 acre to a depth of 1 foot and is equal to 43,580 cubic feet or about 328,000 gallons or 1,233 cubic meters.

Cublc feet per second per square mile (CFSM) is the average number of cubic feet of water slowing per second from each square mile area drained, essuming the runoff is distributed uniformly in time and axea.

Inches (INCEES) Indicates the depth to which the drainage area would be covered if all of the runoff sor a given time perfod were uniformly distributed on ti.

10 PERCENT EXCEEDS. - The discharge that has been exceeded 10 percent of the time for the designated pertod. 50 PERCENT EXCEEDS. - The discharge that has been exceeded 50 percent of the time for the designated period. 90 PERCENT EXCEEDS.- The discharge that has been exceeded 90 percent of the time for the designated period. 
WATER RESOURCES DATA - NEW MEXICO, 1996

Footnotes to the table of daily discharge are introduced by the word "NoTe." Footnotes are used to indicate periods for which the discharge is computed or estimated by special methods because of no gage-helght record, backwater from various sources, or other unusual conditions. Feriods of no gage-helght record are indicated if the period 18 continuous for a month or more or includes the maximum discharge for the year. Pertods of backwater from an unusual source, indefinite stage relations, or any other unusual conditions at the gage site are indicated only if they are a month or more in length and the accuracy of the records is affected. Days on which the stage discharge relation is affected by ice are not indicated. The methods used in computing discharge for vartous unusual conditions have been explained in preceding paragraphs.

For most gaging stations on lakes and reservoirs, the data presented comprise a description of the station and a table showing daily contents or stage. For some reservoirs a monthly sumary table of stage and contents is given. A skeleton table of capacity at given stages is published for all reservoirs for which records are published on a daily basis, but is not published for reservoles for which only monthly data ere given, or if datly stage is published.

Data collected at partial-record stations follow the information for continuous record sites. Data for partial-record discharge stations are presented in a table of annual maximum stage and discharge at crest-stage stations. The tables of partial-record stations are followed by a listing of discharge measurements made at sites other than continuous-record or partial-record stations (miscollaneous sites). Occasionally, a series of discharge measurements axe made within a short time period to investigate the seopage gains or losses along a reach of a stream or to determine the low-flow characteristics of an area. Such measurements are also given in spectal tables following the tables of partial-record stations.

Identifying Estimated Daily Discharge

Est imated deily-discharge values published in the pater-discharge tables of annual state data reports are identified by listing the dates of the estimated record in the RMMARKS paragraph of the station description.

\section{Accuracy of the Records}

The accuracy of streamflow records depends primarily on (1) the stability of the stage-discharge relation or, if the control is unstable, the frequency of discharge measurements, and (2) the accuracy of measurements of stage, measurements of discharge, and interpretations of records.

The accuracy attributed to the records is indicated under "REMARKS." "Excellent" means that about 95 percent of the daily discharges are within 5 percent of the true value; "good" within 10 percent; and "fait" within 15 percent. Records that do not meet the criteria mentioned are rated "poor." Different accuracies may be attributed to different parts of a given record.

Daily mean discharges in this report are given to the nearest hundredth of a cubic foot per second for values of less than $1 \mathrm{ft}^{3} / \mathrm{s}$; to the nearest tenth between 1.0 and $10 \mathrm{ft} \mathrm{t}^{3 / \mathrm{s}}$; to whole numbers between 10 and $1,000 \mathrm{ft} / \mathrm{s}$. and to three significant figures above 1,000 ft $/ \mathrm{s}$. The number of significant figures used is based solely on the magnitude of the discharge value. The same rounding rules apply to discharge figures listed for partial-record stations and miscellaneous sites.

Discharge at many stations, as indicated by the monthly mean, may not reflect natural runoff due to the effects of diverston, consumption, regulation by storage, increase or decrease in evaporation due to artificial causes, or other factors. For such stations, flgures of cubic feet per second per square mile and of runoff, fn inches, are not published unless satisfactory adjustments can be made. Evaporation from a reservolr is not included tn the adjustments for changes in reservotr contents, unless it is so stated. Even at those stations where adjustments are made, large errors in computed runoff may occur if adjustments or losses are large in comparison with the observed discharge.

\section{Other Data Avallable}

Information of a more detailed nature than that published for most of the gaging stations, such as observations of water temperatures, discharge measurements, gage-helght records, and rating tables, is on file in the District office. Most gaging-station records are also available in computer-usable form and many statistical analyses have been made. office.

Information on the avallability of unpublished date or statistical analyses may be obtained from the District

\section{Records of Surface-Water Quality}

Surface-water quality samples usually are collected at or near gaging stations because interpretation of records of surface-water quality nearly always requires corresponding discharge data. Records of surface-water quality in this report may involve a variety of types of data and measurement frequencies.

\section{Classtefication of Records}

Water-quality data for surface-water sttes are grouped into one of three classifications. A continuing-record station is a site where data are collected on a regularly scheduled basts. Frequency may be once or more times daily, weekly, monthly, or quarterly. A partial-record station is a site where limtted water-quality data are collected systematicaliy over a period of years. Frequency of sampling is usualiy less than quarterly. A miscellanoous sampling stte is a location other than a continuing- or partial-record station, where random samples are collected to give better areal coverage to deftne water-qualfty conditions tn the river basin.

A careful distinction needs to be made between "continuing records" as used in this report and "continuous recordings," which refers to a continuous graph or a series of discrete values punched at short intervals on a paper tape. Some records of water quality, such as temperature and specific conductance, may be obtained through continuous recordings; however, because of costs, most data are obtained only monthly or less frequently. Locations of stations for which records of surface-water quality appear in this report are shown in figure 6 . 


\section{Arrangement of Records}

Water-quality records collected at a surface-water daily record station are published imnediately following that record, regardless of the frequency of sample collection. Station number and name are the same for both records. Where a surface-water daily record station is not avaliable or where the water quality differs significantly from that at the nearby surface-water station, the continuing water-quality record is published with its okm station number and name in the regular downstream-order sequence. Water-quality data for partial-record stations and for miscellaneous sanpling sites appear in separate tables following the table of discharge measurements at miscelianeous sites.

\section{On-Site Measurements and Sample Collection}

In obtaining water-quality data, a major conoern noods to bo assuring that the date obtained represent the in sttu quality of the water. To assure this, certain measurements, such as water temperature, pH, and dissolved oxygen, need to be made on st te when the samples are taken. To essure that measurements made in the laboratory also represent the in situ water, carefully prescribed procedures need to be followed in collecting the samples, in treating the samples to prevent chenges in quality pending analysis, and in shipping the samples to the laboratory. Procedures for on-site measurements and for collecting, treating, and shipping samples are given in publications on "Techniques of Water-Resources Investigations," Book 1, Chap. D2; Book 3, Chap. C2; Book 5, Chap. A1, A3, and A4. All of these references eppear on the list of publications following this section. Detalled information on collecting, treating, and shipping samples may also be obtained from the Geological Survey District office.

One sample can define adequately the water quality at a given time if the mixture of solutes throughout the stream cross section is homogeneous. However, the concentration of solutes at different locations in the cross section may vary widely with different rates of water discharge, depending on the source of matertal and the turbulence and mixing of the stream. Some streams must be sampled through several vertical sections to obtain a representative sample needed for an accurate mean concentration and for use in calculating load. All samples obtained for the National Stream-Quality Accounting Network (see definitions) are obtained from at least several verticals. Whether samples are obtained from the centrotd of flow or from several verticals depends on flow condttions and other factors that must be evaluated by the collector.

Chemical-quality data published in this report are constdered to be the most representative values avatiable for the stations listed. The values reported represent water-quality conditions at the time of sampling as much as possible, consistent with available sampling techniques and methods of analysis. In the rare case where an apparent inconsistency. exists between a reported pH value and the relative abundance of carbon dioxide species (carbonate and bicarbonate), the inconststency is the result of a slight uptake of carbon dioxide from the atr by the sample between measurement of $\mathrm{pH}$ in the field and determination of carbonate and bicarbonate in the laboratory.

For chemical-quality stations equipped with digital monitors, the records consist of daily maximum, minimum, and mean values for each constituent measured and are based upon hourly punches beginning at 0100 hours and ending at 2400 hours for the day of record. More detailed records (hourly values) may be obtained from the bistrict office whose address is given on the back of the title page of this report.

\section{Water Temperature}

Water temperatures are measured at most of the water-quality stations. In addition, water temperatures are taken at the time of discharge measurements for water-discharge stations. For stations where water temperatures are taken manually once or twice daily, the water temperatures are taken at about the same time each day. Large streams have small diurnal temperature changes; shallow streams may have a dally range of several degrees and may follow closely the changes in air temperature. Some streams may be affected by waste-heat discharges.

At stations where recording instruments are used, either mean temperatures or maximum and minimum temperatures for each day are published. Water temperatures measured at the time of water-discharge measurements are on file in the District office.

\section{Sediment}

Suspended-sediment concentrations are determined from samples collected by using depth-integrating samplers. Samples ustally are obtained at several verticals in the cross section, or a single sample may be obtained at a fixed point and a coefficienc applied to determine the mean concentration in the cross sections.

During periods of rapidly changing flow or rapialy changing concentration, samples may be collected more frequently (twice daily or, in some instances, hourly). The published sediment discharges for days of rapidily changing slow or concentration are computed by the subdivided-day method (time-discharge weighted average).

Therefore, for those days when the published sediment discharge value differs from the value computed as the product of discharge times mean concentration times 0.0027 , the reeder cen essume that the sediment discharge for that day has been computed by the subdivided-day method. For periods when no samples are collected, daily discharges of suspended sediment axe estimated on the basis of water discharge, sediment concentrations observed immediately before and efter the periods, and suspended-sediment loads for other periods of similar discharge.

At other stations, suspended-sediment samples axe collected periodically at many verticals tn the stream cross section. Although date collected periodicaliy may represent conditions only at the time of observations such data are useful in establishing seasonal relations between quality and streamflow in predicting long-tern sedimentdischarge characteristics of the stream.

In addition to the records of suspended-sedinent discharge, records of the periodic measurements of the particle-size distribution of the suspended sediment and bed material are included for some stations.

\section{Laboratory Measurements}

Historical and current (1992) dissolved trace-element concentrations are reported herein for water that was collected, processed, and analyzed by using either ultraclean or other than ultraclean techniques. If ultraclean techniques were used, then those concentrations are reported in nanograms per liter. If other than ultraclean techniques were used, then those concentrations are reported in micrograms per liter and could reflect contamination introduced during some phase of the procedure.

Microbiological data on coliform and streptococcal bacteria appear in this report. Methods for the collection and analysis of aquatic biologtcal and aquatic microblological samples are described by slack and others (1973). (See reference,)

Sediment samples, samples for biochemical-oxygen demand (BOD), samples for indicator bacteria, and daily samples for specific conductance are analyzed locally. All other samples are analyzed in the Geological Survey laboratory in Arvada, Colorado. Methods used in analyzing sediment samples and conputing sediment records are given in TWRI, Book 5, Chap. C1. Methods used by the Geological survey laboratories are Biven in TWRT, Book 1, Chap. D2; Book 3, Chap. C2; Book 5, Chap. A1, A3, and A4. 
For continuing-record stations, information pertinent to the history of station operation is provided in descriptive headings preceding the tabular data. These descriptive headings give details regarding location. drainago area, perlod of record, type of data available, instrumentation, general remarks, cooperation, and extremes for parameters currently measured datly. Tables of chentcal, physical, biological, radiochemical data. and so forth, obtained at a frequency less than daily, are presented first. Tables of daily values of specific conductance, pH, water temperature, dissolved oxygen, and suspended sediment then follow in sequence.

In the descriptive headings, if the location is identical to that of the discharge-gaging station, neither the LOCATION nor the DRAINAGE AREA statements are repeated. The following information, as appropriate, is provided tith each continuous-record station. Comments that follow clartfy information presented under the various headings of the station description.

LOCATION.--See "Data presentation" under "Records of stage and water discharge"; same comments apply.

DRAINAGE AREA.--See "Data presentation" under "Records of stage and water discharge"; same coments apply.

PERIOD OF RECORD. - This indicates the periods for which there are published water-quality records for the station. The pertods are shown separately for records of parameters measured daily or continuously and those measured less than dally. For those measured daliy or continuously, periods of record are given for the parameters individually.

INSTRUMENTATION.--Information on instrumentation is given only if a water-quality monitor, temperature recorder. sediment pumping sampler, or other sampling device is in operation at a station.

REMARKS, --Remarks provide added information pertinent to the collection, analysis, or computation of the records.

COOPERATION.--Records provided by a cooperating organization or obtained for the Geological Survey by a cooperating organtzation are identified here.

EXTREMES. - Maximums and minimums axe given only for parameters measured daily or more frequently. None are given for parameters measured weekly or less frequently because the true maximums or minimums may not have been sampled. Extremes, when given, are provided for both the period of record and the current year.

REVISIONS, - If errors in published water-quelity records are discovered after publication, appropriate updates are made to the Water-Quality File in the U.S. Geological Survey's computerized data system, WArSToRE, and subsequently by monthly transfer of update transactions to the U.S. Environmental Protection Agency's STORET system. Because the usual volume of updates makes it limpractical to document individual changes in the State datameport series or elsewhere, potential users of U.S. Geologieal Survey water-quality data are encouraged to obtain all required data from the appropriate computer file to ensure the most recent updates.

The surfacemater-quality records for partial-record stations and miscellaneous sampling sites are published in separate tables following the table of discharge measurements at miscalianeous sites. No descriptive statements are given for these records. Each station is published with its own station number and name in the regular downstreamorder sequence.

Remark Codes

The following remark codes may appear with the water-quality data in this report:

ERINTED OUTEUT
e
$\begin{aligned} & \text { Estimated value } \\ & \text { Actual value is known to be greater than } \\ & \text { the value shown }\end{aligned}$
Actual value 1s known to be less than
the value shown
R
Results based on colony count outside the
acceptance range (non-ideal colony count)
Biological organtsm count less than 0.5 percent
(organism may be observed rather than counted)
Biological organism count oqual to or greater
than 15 percent (dominant)
Biological organism estimated as dominant

\section{Dissolved Trace-Element Concentrations}

*NOTE. - Traditionally, dissolved trace-element concentrations have been reported at the microgram per 1iter (ug/L) leve1. Recent evidence, mostly from large rivers, indicates that actual dissolved-phase concentrations for a number of trace elements are win the range of 10 's to 100 's of nanograms per liter (ng/L). Data above the ug/L level should be viewed with caution. Such data may actually represent elevated environmental concentrations from natural or human causes; however, these data could reflect contamination introduced during sampling, processing, or

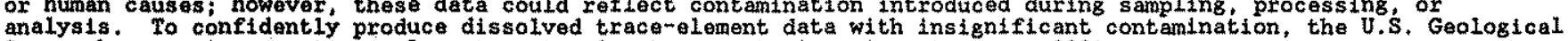
Survey began using new trace-element protocols at some stations in water year 1994.

\section{Change in National Trends Network Procedures}

*NOTE. - -Sample handling procedures at all National Trends Network stations were changed substantially on January 1 , 1994, in order to reduce contamination from the sample shipping container. The data for samples before and after that date are different and not ditectly comparable. A tabular surnary of the differences based on a spectal intercomparison study, is available from the NADP/N TN Coordination Office, Colorado State University, Fort Collins, Co 80523 (Telephone: $303-491-5643$ ). 
WATER RESOURCES DATA - NEW MEXICO, 1996

Records of Ground-Water Levels

Only water-level data from a national network of observation wells are given in this report. These data are intended to provide a sampling and historical record of water-level changes in the Nation's most important aquifers. Locations of the observation wells in this network in New Mexico are shown in figure 8.

Data Collection and Computation

Measurements of water levels are made in many types of wolls, under varying conditions of access and at different temperatures, but the methods of measurement are standardized to the extent posstble. The equipment and measuring tachniques used are those that will ensure consistent accuracy and reliability.

Tables of water-level data are presented by counties arranged in alphabecical order. The prime identification number for a given well is the 15 -digit number that appears in the upper left corner of the table. The secondary identification number is the local well number, an alphanumeric number, derived from the township-range location of the well.

Water-level records are obtatned from direct measurements with a steel tape or from the graph or punched tape of a nater-stage recorder. Water-level measurements in this report are given in feet with reference to either mean sea level (ms1) or land-surface datum (1sd). Mean sea level is the datum plane on which the national network of precise levels is based; land-surface datum is a datem plane that is approximately at land surface at each well. If known, the elevation of the land-surface datum above mean sea level is given in the well description. The height of the measuring point (MP) above or below land-surface datum is given in each well description, Water levels in wells equipped with recording gages are reported for every fifth day and the end of each month (eom),

Water levels are reported to as many significant figures as can be justified by the local conditions. For example, in measurement of a depth to water of several hundred feet, the error in determining the absolute value of the total depth to water may be a few tenths of a foot, whereas the error in determining the net change of water leve1 between successive measurements may be only a hundredth or a few hundredths of a foot. For lesser depths to water, the accuracy is greater. Accordingly, most measurements are reported to a hundredth of a foot, but some are given only to a tenth of a foot or a larger unit.

\section{Data Presentation}

Each well record consists of two parts the station description and the data table of water levels observed during the water year. The description of the well is presented first through use of descriptive headings preceding the tabular data. The coments to follow clarify information presented under the various headings.

LOCATION. - This paragraph follows the weli-identification number and reports the latitude and longitude (given in degrees, minutes, and seconds); a landiline location designation; the hydrologic unit number; the distance and direction from a geographic point of reference; and the owner's name.

AQUTFER. - This entry designates by name (if a name exists) and geologic age the aquifer(s) open to the well.

WELL CHARACTERISTICS.--This entry descrtbes the vell in terms of depth, diameter, casing depth and/or screened interval, method of construction, use, and additional information such as casing breaks, collapsed screen, and other changes since construction.

INSTRUMENTATION, - This paragraph provides information on both the frequency of measurement and the collectlon method used, allowing the user to better evaluate the reported watex-level extremes by knowing whether they are based on weekly, monthly, or some other frequency of measurement.

DATUM. - This ontry describes both the measuring potnt and the land-surface elevation at the we11. The measuring point is described physically (such as top of collar, notch in top of casing, plug in pump base, and so on), and in relation to land surface (such as $1.3 \mathrm{ft}$ above land-surface datum). The elevation of the land-surface datum is described in foet above (or below) National Geodetic Vertical Datum of 1929 (NGVD of 1929); it is reported with a precision depenatng on the method of determination.

REMARKS. - - This entry describes factors that may influence the water level in a well or the measurement of the water lovel. It should identify wells that also are water-quality observation wells, and may be used to acknowledge the assistance of local (non-Survey) observers.

PERIOD OF RECORD.--This entry indicates the period for which there are published records for the well. It reports the month and year of the start of publication of water-level records by the U.S. Geological Survey and the words "to current yeax" if the records are to be continued into the following year. Periods for which water."level. records are available, but are not published by the Geological Survey, may be noted.

EXTREMES FOR PERIOD OF RECORD.--This entry contains the highest and lowest water levels of the period of published record, with respect to iand-surface datur and the dates of their occurrence.

A table of water levels follows the station description for each well. Water levels are reported in feet below land-surface datum and al1 taped measurements of water level are listed. For wells equipped with recorders, only abbreviated tables are published; generally, only water-level lows are listed for every fifth day and at the end of the month (eom). The highest and lowest water levels of the water year and their dates of occurrence are shown on a line below the abbreviated table. Because all values are not published for wells with recorders, the extrenes way be values that are not listed in the table. Missing records are indicated by dashes in place of the water level.

\section{Records of Ground-Water Quality}

Records of ground-water quality in this report differ from other types of records in that for many sampling sites they consist of only one set of measurements for the water year. The quality of ground water ordlnarily changes only slowly; therefore, for most general purposes one annual sampling, or only a few samples taken at infrequent intervals during the year, is sufficient. Frequent measurement of the same constituents is not necessary unless one is concerned with a particular problem, such as monitoring for trends in nitrate concentration. In the spectal cases where the quality of ground water may change more rapidly, more frequent measurements are made to identify the nature of the changes. 
WATER RESOURCES DATA - NEW MEXICO, 1996

Data Collection and Computation

The records of ground-water quality in this report were obtained mostly as a part of special studies in specific areas. Consequentiy, a number of chemical analyses are presented for some counties but none are presented for others. As a result, the records for this year, by themselves, do not provide a balanced view of ground-water quality Statewide. Such a view can be attained only by considering records for this year in context with similar records obtatned for these and other counties in earlier years.

Most methods for collecting and analyzing water samples are described in the "U.S. Geologtcal Survey Techntques of Water-Resources Investigations" manuals listed on a following page. The values reported in this report represent water-quality conditions at the time of sampling as much as posstble, consistent with avallable sampling techniques and methods of analysis. All samples were obtained by trained personnel. The wells sampled were pumped long enough to assure that the water collected came directly from the aquifer and had not stood for a long time in the well casing where it would have been exposed to the atmosphere and to the material, possibly metal, comprising the casings.

\title{
Data Presentation
}

The records of ground-water quality are published in a section, "QUALITY OF GROUND WATER" Immediately following the sround-water-level records. Data for quality of ground water are listed alphabeticaliy by county. and are identified by well number. The prime identification number for wells sampled ts the 15-digit number derived from the latitude-longitude locations. No descriptive statements are given for ground-water-quality records; however, the well numbar, depth of well, date of sampling, and other pertinent data are given in the table containing the chemical analyses of the ground water. The RFMARx codes listed for surface-water-quality records are also applicable to ground-water-quelity records.

\section{ACCESS TO WATSTORE DATA}

The U.S. Geologicel Survey is the principal Federal water-data agency and, as such, collects and disseminates about 70 percent of the water data currently being used by numerous state, local, private, and other Federal agencies to develop and manage our water resources. As part of the Geological Survey's program of releasing water data to the public, a large-scale computerized system has been developed for the storage and retrieval of water data collected through its activities. The National. WATer Data STOrage and REtrieval System (WATSTORE) was established in 1972 to provide an effective and efficient means for the processing and maintenance of water data collected through the activities of the U.S. Geological Survey and to facilitate release of the data to the public. A varfety of useful products, ranging from data tables to complex statistical analyses such as Log Pearson Type III, can be produced using WATSTORE. The system resides on the central computer factilities of the U.S. Geological Survey at its National Center in Reston, Virginia, and consists of related files and data bases.

* Station Header Filo - Contains descriptive Information on more than 40,000 sites throughout the United States and its territories where the Geological Survey collects or has collected data.

- Daily Values File - Contains more than 220 million daily values of strean flow, stages, reservoir contests, water temperatures, specific conductances, sediment concentrations, sediment discharges, and ground-water levels.

* Peak Flow F1le - Contains approximately 500,000 maximum (peak) streamflow and gage-helght values at surface-watior sites.

* Water Quality File - Contains approximately 2 million analyses of water samples that describe the chemical, physical, biologicel, and radio-chemical characteristics of both surface and ground water.

* Ground-Water Site Inventory Data Base - Contains inventory data for more than 900,000 wells, springs, and other sources of ground water. The data includes stte location, geohydrologic characteristics, well-

construction history, and one-time fleld measurements such as'water temperature.

In 1976, the U.S. Geological Survey opened WATSTORE to the public for direct access. The signing of a Memorandum of Agreement ith the Survey is recuired to obtain direct access to WATSTORE. The system can be accessed edther synchronously or asynchronously. The requestor will be expected to pay all computer costs he/she incurs. Direct access may be obtained by contacting:

\author{
U.S. Geological Survey \\ Nationa1 Water Data Exchange \\ 421 USGS National Center \\ Reston, Virginia 22092
}

In addition to providing direct access to WATSTORE, data can be provided in various machine-readable formats on magnetic tape or 5-1/4 inch floppy disk; and, as noted in the introduction, on CD-ROM discs. Beginning with the 1990 water year all water-data reports will also be available on Compact Disc - Read Only Memory (CD ROM). All data reports published for the current watex year for the entire Nation, including Puerto Rico and the Trust Territortes, will be reproduced on a single CD-ROM disc. Information about the evaflability of specifle types of data or products, and user charges, can be obtained locally from each of the Water Resources Division's District office. (See address on the back of the titie page.) A linited number of CD ROM discs will be available for sale by the Books and Open-File Reports Section, U.S. Geological Survey, Federal Center, Box 25425, Denver Colorado 80225.

\section{Paraneter Codes}

The flve-digit codes shown in parentheses in the column headings of the tables in this report are parameter codes that uniquely identify a specific constituent. These are standard codes used to identify the data stored tn the files of WATSTORE. These codes are 1dentical to those used in the U.S. Environmental. Protection Agency (EPA) data system, STORET. The EPA assigns and approves all requests for new codes. 


\section{DEFINITION OF TERMS}

Terms related to streamflow, water-quality, and other hydrologic data, as used in this report, are defined below. See also the table for converting English units to International System of units (SI) on the inside of the back cover.

Acre-foot (AC-FT, acre-ft) is the quantity of water required to cover 1 acre to a depth of 1 foot and is equivalent to 43,560 cubic feet or 325,851 gallons or $1,233.49$ cubic meters.

Adenosine triphosphete (ATP) is an organic, phosphate-rloh compound laportant in the transfer of energy in organisms. Its central rolo in living celis makes tt an excellent indicator of the presence of living matertal in water. A measure of ATP therefore provides e sensitive and zapte estimate of biomass. ATP is reported in micrograms per liter of the original weter sample.

Alsae are mostly aquatic single-colled, colonial, or multicelled plants, ccintaining chlorophyll and lacking roots, stems, and leaves.

Algal growth potential (AGP) is the maximum algal dry welght biomass that can be produced in a natural water sample under standardized laboratory conditions. The growth potentiel is the algal bjomass present at stationary phase and is expressed as milligrams ary weight of algae produced per liter of sample.

Aguifer is a geologic formation, group of formations, or part of a formation that contains suffictent saturated permeable matertal to yield significant quantities of water to wells and springs.

Artesian means confined and is used to describe a well in which the water level stands above the top of the aquifer tapped by the pell. A flowing artestan well is one in which the water level is above the land surface.

Bacteria are microscopic unicellular organisms, typically spherical, rodlike, or spiral and threadlike in shape, often clumped into colonies. Some bacterla cause diseases, while others perform an essential role in nature in the recycling of materials; sor example, by decomposing organic matter into a form available for reuse by plants

Total coliform bacteria are a particular group of bacteria that are used as indicators of possible sowage pollution. They are charactexized as aerobic or facultative anaerobic, gram-negative, nonspore-forming, rodshaped bacteria which ferment lactose with ges formation within 48 hours at $35{ }^{8} \mathrm{C}$. In the laboratory these bacteria are defined as ell the organisms that produce colonies with a golden-green metallic sheen within 24 hours when incubated at $35^{\circ} \mathrm{C}+$ or $-1.0^{\circ} \mathrm{C}$ on $\mathrm{M}$-Endo medium (nutrient medium for bacterial 8 rowth). Their concentrations are expressed as number of colonies per $100 \mathrm{~mL}$ of sample.

Fecal coliform bacteria are bacteria that ere present in the intestine or feces of warmblooded animals. They are often used as indicators of the sanitary quality of the water. In the laboratory they are defined as all organisms that produce blue colonies within 24 hours when incubated at $44.5^{\circ} \mathrm{C}+$ or $-0.2{ }^{\circ} \mathrm{C}$ on $\mathrm{M}-\mathrm{FC}$ medium (nutriont medium for bacteriai growth). Their concentrations are expressed as number of colonies per $100 \mathrm{~mL}$ of sample.

Fecal streptococcal bacterla are bacteria found also in the intestine of warmblooded antmals. Their presence in pater is considered to verify fecal pollution. They are characterized as gram-positive, coccel bacterie which are capable of growth in brain-heart infusion broth. In the laboratory they are defined as all the organisms which produce red or pink colonies within 48 hours at $35^{\circ} \mathrm{C}+$ or $-1.0^{\circ} \mathrm{C}$ on $\mathrm{kF}-\mathrm{st}^{\circ}$ reptococcus medium (nutrient mediun for bacterial growth). Their concentrations are expressed as number of colonies per $100 \mathrm{~mL}$ of samplo.

Bed material is the sediment mixture of which a streambed, lake, pond, reservoir, or estuary bottom ts composed.

Biochemical oxyzen demand (BOD) is a measure of the quantity of dissolved oxygen, in milligrams per liter, necessary for the decomposition of organic matter by microorganisms, such as bacteria.

8lomess is the amount of living matter present at any given time, expressed as the mass per unit area or volume of habirat.

Ash mass is the mass or amount of residue present after the residue from the dry mass determination has been ashed in a murfle furnace at a temperature of $500^{\circ} \mathrm{C}$ for 1 hour. The ash mass values of zooplankton and phytoplankton are expressed in grams per cuble meter $\left(\mathrm{g} / \mathrm{m}^{3}\right)$, and periphyton and benthic organtsms in $\mathrm{grams}$ par squaro moter $\left(\mathrm{g} / \mathrm{m}^{2}\right)$

Dry mass refers to the mass of residue present after drying in an oven at $105^{\circ} \mathrm{C}$ for zooplankton and periphycon, until the mass remains unchanged. This mass represents the total organic matter, ash and sediment, in the sample. Dry mass values are expressed in the same units as ash mass.

Organic mass or volatile mass of the living substance is the difference between the dry mass and the ash mass and represents the actual mass of the living matter. The organic mass is expressed in the same units as for ash and ary mass.

Wet mass is the mass of living matter plus conteined water.

Bottom material: See Bed material.

Cells/volume refers to the number of cells of any organism which is counted by using a microscope and grid or counting cell. Many planktontc organisms are nulticelled and are counted according to the number of contatned cells per sample, usually milifliters (mL) or liters (L)

Cfs-day is the volume of water represented by the flow of 1 cubic foot per second for 24 hours. It 1 s equivalont to 86,400 cubic feet, 1.98347 acre-feet, 646,317 gallons, or 2,445 cubic meters.

Chemical oxygen demand (COD) is a measure of the chemically oxidizable material in the water, and furnishes an approximation of the amount of organic and reducing material present. The determined value may correlate with natural water color or with carbonaceous organic pollution from sewage or industrial wastes. 
WATER RESOURCES DATA - NEW MEXICO, 1996 in plants,

Chlorophy 11 refers to the green pigments of plants. Chlorophyll a and b are the two most common green pigments

Color untt is produced by one mllligram per liter of platinum in the form of the chloroplatinate ton. Color is expressed in untts of the platinum cobalt scale.

Contents is the volume of water in a reservotr or lake. Unless otherwise indicated, volume is computed on the basts of a level pool and does not include bank storage.

Control designates a feature downtream from the gage that determines the stage-discharge relation at the gege. This feature may be a natural constriction of the channel, an artifictal structure, or a uniform cross section over a long reach of the channel.

Control structure as used in this report is a structure on a stream or canal that is used to regulate the flow or stage of the stroam or to prevent the intrusion of salt water.

Cuble feet per second per square mile [(fts/s)/mi'] is the average number of cubtc feet of water flowing per second from each square mile of area drained, assuming that the runoff is distributed uniformly in time and area.

Cubtc foot per second (FT3/S, ft $3 / s$, cfs) is the rate of discharge xepresenting a volume of 1 cublc foot passing a given point during 1 second and is equivalent to 7,48 gallons per second or 448.8 gallons per minute or 0.02832 cubtc meters per second.

Discharge is the volume of water (or more broadly, volume of fluid plus suspended sediment), that passes a given point within a given period of time.

Mean discharge (MEAR) is the arithmettc mean of individual datly mean discharges during a specific period.

Instantaneous discharge is the discharge at a particular instant of time.

Annual 7-day mintmum is the lowest mean discharge for 7 consecutive days for a calender year or a water year. Note that most Low-Flow frequency analyses of annual 7 -day minimum flows use a c1imatic year (April 1 - March 31). The date shown in the sumary statistics table is the initial date of the $7-d a y$ period. (This value should not be confused with the 7-day 10myear low-flow statistic.)

Discharge-welghted average: See Weighted average.

Dissolved refers to that material in a representative water sample which passes through a 0.45 -un membrane filter. This is a convenient operational definition used by Federal agencies that collect water data. Determinations of "dissolved" constituents are made on subsamples of the filtrate.

D1ssolved-soltds concentration of water is determined either analyticaliy by the "residue-on-evaporation" method, or mathematicaliy by totaling the concentrations of individual constituents reported in a comprehensive chemical analysis. During the analytical determination of dissolved soltas, the bicarbonate (generaliy a majer dissolved component of water) is converted to carbonate. Therefore, in the mathematical calculation of aissolvedsolids concentration, the bicarbonate value, in milligrams per liter, is multiplied by 0.492 to reflect the change.

Diversity Index is a nunerical expresition of evenness of distribution of aquatic organisms, The formula for diverstey index is

$$
d=-\sum_{i=1}^{s} \frac{n_{1}}{n} \log _{2} \frac{n_{i}}{n}
$$

where $n_{i}$ is the number of individuals per taxon, $n$ ts the total number of individuals, and $s$ is the total. number of taxa in the sample of the community. Diversity index values range from zero, when all the organisms in the sample are the same, to some positive number, whon some or all of the organisms in the sample are different.

Drainage area of a stream at a specified location is that area, measured in a horizontal plane, enclosed by a topographic divide from which direct surface runoff from precipitation normally dratns by gravity into the stream above the specifled point. Figures of drainago area given heroin include all closed basins, or noncontributing areas, within the area unless otherwise noted.

Draingge basin is a part of the surface of the earth that is occupied by a drainage system, which consists of a surface stream or a body of impounded surface water together with all tributary surface streams and bodies of impounded sturface water.

Gape height (G.H.) is the water-surface elevation referred to some arbitrary gage datum. Gage height is often used interchangeably with the more general term "stage," although gage height is more appropriate when used with a reading on a 8 age.

Gaging station is a particular site on a stream, canal, lake, or reservoir where systematic observations of hydrologic data are obtained.

Hardness of water is a physlcal-chemical characteristic that is commonly recogntzed by the increased cuantity of soap requitred to produce lather. It is computed as the sum of equivalents of polyvalont cations and is expressed as the equivalent concentration of calcium carbonate $\left(\mathrm{CaCO}_{3}\right)$.

Bydrologic untt is a geographlc area representing part or all of a surface drainage basin or alstinct hydrologic feature as delineated by the Office of Water Data Coordination on the State Bydrologic Untt Maps; each hydrologic unit is identified by an 8-digit number.

Land-surface datum (1sd) is a datum plane thet is approximately at land surface at each ground water observation weli.

Measuring potnt (MP) is an arbitrary permanent reference point from which the distance to the water surface in a well is measured to obtain the water level. 
Matamorphic stage refers to the stage of development that an organism exhibits during fts transformation from an immature form to an adult form. This developnental process exists for most insects, and the degree of difference from the 1mmature stage to the adult forn varies from relatively slight to pronounced, with many lntermediates. Examples of metamorphic stages of insects are egg-larva-adult or egg-nymph-adult.

Methylene blue active substances (MBAS) are apparent detergents. The determination depends on the formation of a blue color when methylene blue dye reacts with synthetic anionic detergent compounds.

MBAS qualifiex MBAS deterninations made from 1970 through August 29, 1993, at the National Water Quality Laboratory in Denver (Analyzing Agency Code 80020) are biased high. These data can be corrected based on the following equation if concentrations of nitrate plus nitrite, dissolved, as $N$, and dissolved chloride, determined concurrently with the MBAS data are evallable:

MBASCOR $=M-[(0.0088) \mathrm{H}+(0.00019) \mathrm{C}] \cdots$ in which

MBASCOR = corrected MBAS concentration, in $\mathrm{mg} / \mathrm{L}$,

$M=$ reported MBAS concentration, in $\mathrm{mg} / \mathrm{L}$,

$\mathbb{N}=$ nitrate plus nitrite, dissolved, as $N$, concentration, in mg/L, and

$\mathrm{C}=$ dissolved chloride concentration, in $\mathrm{mg} / \mathrm{L}$.

The updated mothod reporting limit is $0.02 \mathrm{mg} / \mathrm{h}$. The former reporting $11 \mathrm{mit}$ was $0.01 \mathrm{mg} / \mathrm{L}$. A reporting 1 imit of $0.02 \mathrm{mg} / \mathrm{L}$ should be applied to any corrected MBAs data from 1970 Ehrough August 29, 1993 . The laboratory will automatically correct MBAS results after August 29,1993 .

Uncorrected MBAS data for New Mexico that were collected during the 1993 watex year were corrected by applying the above equation. The water-quality data files were updated with the corrected values and retrieved for publication in the 1993 edition of the annual data report. The corrected values, if greater than the updated reporting limit of $0.02 \mathrm{mg} / \mathrm{L}$ were qualified as estimated values.

Micrograms per gram (ug/g) is a unit expressing the concentration of a chenical constituent as the mass (micrograms) of the element sorbed per unit mass (gram) of material analyzed.

Micrograms per liter (UG/L, ug/L) is a unit expressing the concentration of chemical constituenes in solution as mass (micrograns) of solute per unit volume (liter) of water. One thousand micrograms per ifter is equivalent to one milligram per liter.

Milligrams per 11ter (MG/L, $\mathrm{mg} / \mathrm{L}$ ) is a unit for expressing the concentration of chemical constituents in solution. Miliigrams per liter represents the mass of solute per unit volume (1iter) of water. Concentration of suspended sediment also is expressed in $\mathrm{mg} / \mathrm{L}$, and is based on the mass of dry sediment per liter of water-sediment mixture.

National Geodetic Vertical Datum of 1929 (NGVD) is a geodetic datum derived from a general adjustment of the first order level nets of both the Unţed States and Canada. It was formerly called "Sea Leve1 Datum of 1929 " or "mean sea leval" in this serles of reports. Although the datum was derlved from the average sea level over a period of many years at 26 tide stations along the Atlantic, Gulf of Mexico, and Pacific Coasts, it does not necessarily represent local mean sea level at any particular place.

Organism is any living ontity.

Organism count/area refers to the number of organisms collected and onumerated in a sample and adjusted to the number per undt area habitat, usually square meters $\left(m^{2}\right)$, acres, or hectares. Periphyton, benthic organisms, and macrophytes are expressed in these terms.

Organtsm count/volume refers to the number of organisms collected and enumerated in a sample and adjusted to the number per sample volume, usually miliiliters (mL) or liters (L). Nurnbers of planktonic organisms can be expressed in these terms.

Total organism count is the total number of organisms collected and enumerated in any particular sample.

Partlal-record station is a partfcular site where limited streamflow and/or water-quality data aro collected systematically over a period of years for use in. hydrologic analyses.

Particle size is the dianeter, in millimeters (mm), of suspended sediment or bed material determined by either sieve ox sedimentation methods. Sedimentation methods (pipet, bottom-withdrawal tube, visual-accumulation tube) determine fall diameter of particles in either distilled water (chemdcally dispersed) or in native water (the river water at the time and point of samplings.

Farticle-size classification used in this report agrees with recoumenatatons made by the American Geophysical Union Subcommittee on Sediment Terminology. The classification is as follows:

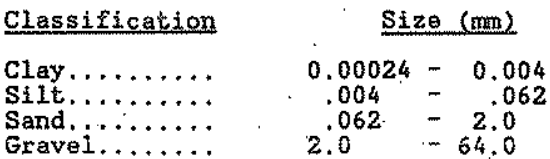

Method of analysis
Sedimentation
Sedimentation
Sedimentation or sieve
Sieve

The particle-size distributions given in this report are not necessarily representative or all particles in transport in the stream. Most of the orgenic material is removed and the sample is subjected to mechanical and chemical dispersion before analysis in distilled watex. Chenical dispersion is not used for native water analysis.

Percent composition is a unte for expressing the ratio of a particulax part of a sample or population to the total sample or population in terms of types, numbers, mass, or volume.

Periphyton are the assemblage of microorganisms attached to and growing upon solid surfaces. While primarily consisting of algae, they also, include bacteria, fungi, protozoa, rotifers, and other small organisms. 
WATER RESOURCES DATA - NEW MEXICO, 1996

Pesticides are chemical compounds used to control the growth of undestrable organtsms. Major categortes of pesticides include insecticides, miticides, fungicides, herbicides, and rodenticides. Insecticides and herbicides, which control insects and plants respectively, are the two categories reported.

Ptcocurie ( $P C, P C i)$ is one trillionth $\left(1 \times 10^{-12}\right)$ of the amount of radioactivity represented by a curite (Ci). A curie is the amount of radioactivity that yields $3.7 \times 10^{10}$ radioactive disintegrations per second. A picocurie yields 2.22 dpm (disintegrations per minute).

Plankton are the cormunity of suspended, floating, or weakly swimming organisms that live in the open water of lakes and rivers.

Phytoplankton are the plant part of the plankton. They are usually microscopic and their movement is subject to the water currents. Phytoplankton growth is dependent upon solar radiation and nutrient substances. Because they are able to incorporate as well as release materials to the surrounding water, the phytoplankton have a profound effect upon the quality of the water. They are the primary food producers in the aquatic environment, and are comonly known as algae.

Blue-green algae are a group of phytoplankton organisms having a blue ptgment, in addition to the green pigment called chlorophyl1. Blue-green algae often cause nuisance conditions in water.

Diatoms are the unicellular or colonial algae having a siliceous shell. Their concentrations axe expressed as number of cells per militiliter (cells/mL) of samplo.

Green algae have chlorophyll pigments similar in color to those of higher green plants. Some forms produce algae mats or floating "moss" in lakes. Their concentrations are expressed as number of cells per milililter (colls/mi) of sample.

Zooplankton are the antmal part of the plankton. Zooplankton are capable of extensive movements within the watex column, and are often large enough to be seen with the unaided eye. Zooplankton are secondary consumers feeding upon bacteria, phytoplanition, and detritus. Because they are the grazers in the acuatic environment, the zooplankton are a vital part of the aquatic food web. The zooplankton cosmunity is dominated by small crustaceans and rotifers.

Eolychlorinated biphenyls (PCBs) are industrial chemicals that are mlxtures of chlorinated biphenyl compounds having verious percentages of chlorine. They are similar in structure to organochlorine insecticides.

Primary productivity is a measure of the rate at which now organic matter is formed and accumulated through photosynthetic and chemosynthetic activity of producex organtsms (chiefly green plants). The rate of primary production is estimated by measuring the amount of oxygen released (oxygen method) or the amount of carbon assimilated by the plants (carbon method).

Milligrams of carbon per area or volume per untt time [mg $\mathrm{C} /\left(\mathrm{m}^{2}\right.$. time)] for pertphyton and macrophytes and [mg $\mathrm{c} /\left(\mathrm{m}^{3}\right.$.tIme) for phytoplankton are the units for expressing primary productivity. They define the amount of carbon dioxide consumed as measured by radioactive carbon (carbon 14). The carbon 14 method is of greater sensitivity than the oxygen 11 ght and dark bottle method, and is preferred for use in unenriched waters. Unit time may be either the hour or day, depending on the incubation pertod.

Milligrams of oxygen per area or volume per unit time [ms $\mathrm{O} /\left(\mathrm{m}^{2}\right.$. time)] fox periphyton and macrophytes and $[\mathrm{ms}$ $0 /\left(m^{3}\right.$. time) / fox phytoplankton are the units for expressing primary productivity. They define production and respiration rates as estimated from changes in the measured dissolved-oxygen concentration. The oxygen 11 ght and dark bottle method is preferred if the rate of primary production is sufficient for accurate measurements to be made within 24 hours. Unit time may be elther the houx or day, depending on the incubation pertod.

Recoverable from bottom material is the amount of a given constituent that is in solution after a representative sample of bottom material has been digested by a method (usually using an acid or mixture of acids) that results in dissolution of only readily soluble substances. Complete dissolution of all bottom material ts not achleved by the digestion treatment and thus the determination represents less than the total amount (that is, less than 95 percent) of the constititent in the sample. To achieve comparability of analytical data, equivalent digestion procedures would be required of all laboratories performing such analyses, because different digestion procedures are likely to produce different analytical results.

Runoff in inches (IN, in) shows the depth to which the drainage area would be covered if all the runoff for a given time period was uniformly distributed on $i t$.

Sediment is solid material that orfginates mostly from disintegrated rocks and is transported by, suspended in, or deposited from water; it includes chemical and btochemical precipitates and decomposed organic materlal, such as humus. The quantity, characteristics, and cause of the occurrence of sediment in streams are influenced by environmental factors. Some major factors are degree of slope, length of slope, soli characteristics, land usage, and quantity and intensity of precipitation.

Bed load is the sediment that is transported in a stream by rolling, sliding, or skipping along the bed and very close to $t t$. In this report, bed load is constdered to consist of particles in transit within 0.25 ft of the streambed.

Bed load discharge (tons per day) is the quantity of bed load measured by dry welght that moves past a section as bed load in a given time.

Suspended sediment is the sediment that at any given time is meintained in suspension by the upward components of turbulent currents or that exists in suspension as a colloid.

Suspended-sediment concentration is the velocity-weighted concentration of suspended sediment in the sampled zone (from the water surface to a point approximately $0.3 \mathrm{ft}$ above the bed) expressed as mililigrams of dry sediment per litter of water-sediment mixture (mg/L).

Mean concentration is the time-weighted concentration of suspended sediment passing a stream section during a 24 -hour day.

Suspended-sediment dischaxge (tons/day) is the rate at which dry wetght of sediment passes a section of a stiream or is the quantity of sediment, as measured by dry weight or volume, that passes a section in a given time. It is computed by multiplying discharge times mg/L times 0.0027 
Suspended-sediment load is a genexal term referring to material in suspension. It is not symonymous with elther discharge or concentration.

Total sediment discharge (tons/day) is the sum of the suspended-sediment discharge and the bed load discharge. It is the total quantity of sediment, as measured by dry weight or volume, that passes a section during a given time.

Total sediment load or total load is a term which refers to the total sediment (bed load plus suspendedsediment load) that is in transport. It is not synonymous with total sediment discharge.

Sodium-adsorption ratio (SAR) 1s the expression of relative activity of sodium ions in exchange reactions wthin soil and is an index of sodium or alkall hazard to the soll. Waters range, in respect to sodium hazard, from those which can be used for irrigation on almost all soils to those which are generally unsatisfactory for irrigation.

Solute is any substance that is dissolved in water.

Solution is the homogeneous mixture of solutes and water. The solutes usually comprise a very small fraction of the total peight of the mixture. For this reason, the terms "solution".. and "water" are used interchangeably.

Specific conductance is a measure of the ability of a water to conduct an electrical current, It is expressed In microsienens per centimeter at $25^{\circ} \mathrm{C}$. Specific conductance is releted to the type and concentration of ions in solution and can be used for approximating the dissolved-solids content of the water. Commonly, the concentration of dissolved solids (in milijgrams per liter) is about 65 percent of the specific conductance (in microsiemens). This relation is not constant from stream to strean, and it may vary in the same source thith changes in the composition of the water.

Stage-discharge relation is the relation between gage height (stage) and volume of water per unit of time, flowing in a channel.

Streamflow is the discharge that occurs, in a natural channel. Although the term "discharge" can be applied to the flow of a canal, the word "streamelow" uniquely describes the discharge in a surfece stream course. The term "streamflow". is more general than "runors" as streanflow may be applied to discharge whether or not it is affected by diversion or regulation.

Substrate is the physical surface upon which an organtsm lives.

Natural substrate refers to any naturally occurring emersed or submersed solid surface, such as a rock or tree, upon which an organism lives.

Artifictal substrate is a device which is purposely placed in a stream or loke for colontzatton of organisms. The artificial substrate simplifies the comonity structure by standardiaing the substrete from which each sample is taken. Examples of axtificial substrates are basket samplers (mede of wire cages filled with clean streamside rocks) and multiplate samplers (made of hardboard) for benthic organism collection, and plexiglass strips for periphyton collection.

Surface area of a lake is that area outlined on the latest U.S.G.S. topographic map as the boundery of the lake and measured by a planimeter in acres. In localities not covered by topographic maps, the areas are computed from the best maps available at the time planimetered. All areas shown are those for the stage whon the planimetered map was made.

Surficial bed material is that part ( 0.1 to 0.2 ft) of the bed material that is sampled using U.S. Serfes Bed Material Samplers.

Suspended (as used in tables of chemical analyses) refers to the amount (concentration) of undissolved material

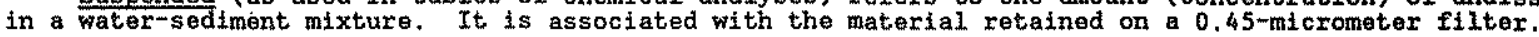

Suspended, recoverable is the amount of a given constituent that is in solution after the part of a representative wacer-suspended sediment sample that is retained on a 0.45 um membrane filter has been dibested by method (usually using a dilute acid solution) that results in dissolution of only readily soluble substances. Complete dissolution of all the paxticulate matter is not achieved by the digestion treatment and thus the determination represents something less than the "total". amount (that is, less than 95 percent) of the constituent present in the sample. To achieve comparability of analytical data, equivalent digestlon procedures pould be required of all laboratortes performing such analyses because different digestion pxocedures axe likely to produce different analytical results.

Determinations of "suspended, recoverable" constituents are made either by analyzing portions of the material collected on the filter or, more commonly, by difference, based on determinations of (1) dissolved and (2) total recoverable concentrations of the constituent.

Suspended, total is the total amount of a given constikuent in the part oz a representative watex-suspended sediment sample that is retained on a 0.45 -um menbrane filter. This term is used only when the analytical procedure assures meesurement of at least 95 percent of the constituent determined. A knowledge of the expected form of the constituent in the sample, as weli as the analytical methodology used, is required to determine when the results constituent in the sample, as well as the

Determinations of "suspended, total" constituents are made either by analyzing portions of the material collected on the filter or more comanly, by difference, based on determinations of (1) dissolved and (2) total concentrations of the constituent. 
Taxonomy is the diviston of biology concerned with the classification and naming of organisms. The classification of organisms is based upon a hierarchical scheme beginntng with Kingdom and ending with Spoctes at the base. The higher the classification level, the fewer features the organisms have in comnon. For example, the texonomy of a particular mayfly, Hexagenta limbata, is the following:

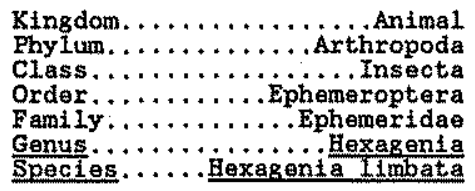

Thermograph is an instrument that continuously records vartations of temperature on a chart. The more general term "temperature recorder" is used in the table headings and refers to any instrument that records temperature, whether on a chart, tape, or any other mediun.

Time-weighted average is computed by multiplying the number of days in the sampling pertod by the concentrations of individual constituents for the corresponding period and dividing the sum of the products by the total number of days. A time-weighted average represents the composition of water that would be contained in a vessel or reservoir that had received equal quentilies of water from the stream each day for the year.

Tons per acre-foot indicates the dry mass of dissolved solids in 1 acre-foot of water. It is computed by multiplying the concentration of the constituent, in milligrams per liter, by 0.00136 .

Tons per day ( $T / D A Y$ ) is the quantity of substance in solution or suspension that passes a stream section during a 24 -hour period.

Total is the total amount of a glven constituent in a representative water-suspended sediment sample, regardiloss of the constituent's physlcal or chemical form. This term is used only when the analytical procedure assures measurement of at least 95 percent of the constituent present in both the dissolved and suspended phases of the sample. A knowledge of the expected form of the constituent in the sample, as well as the analytical methodology used, is recuired to fudge when the results should be reported as "total." (Note that the word "total" does double duty here, indicating both that the sample consists of a water-suspended sediment mixture and that the does double duty here, Indicating both that the sample consists of a water-suspended sediment mixture and that the analytical method determined all of the constituent in the sample.) Total concentrations occasionally are report sensitivity of the analytical methods used for the digested solutions. Digested solutions often contain higher concentrations of substances that interfere with analytical sensitivity through complex matrix effects.

Total discharge is the total quantity of any individual constituent, as measured by dry mass or volume, that passes through a stream cross section per unit of time. This term needs to be qualifled, such as "total sediment discharge," "total chloride discharge," and so on.

Total in bottom material is the total amount of a given constituent in a representative sample of bottom material. This term is used only when the analytical procedure assures measurement of at least 95 percent of the constituent determined. A knowledge of the expected form of the constituent in the sample, as well as the analytical methodology used, is required to judge when the results should be reported as "total in bottom material."

Total load (tons) is the total quantity of any individual constttuent, as measured by dry mass or volume, that is dissolved in a speciffc amount of water (discharge) during a given time. It is computed by multiplying the total discharge, times, the $\mathrm{mg} / \mathrm{L}$ of the constituent, times the factor 0.0027 , times the number of days.

Total recoverable is the anount of a given constituent that is in solution after a representative watersuspended sediment sample has been digested by a method (usually using a dilute acid solution) that results in dissolution of only readily soluble substances. Complete dissolution of ali particulate matter is not achieved by the digestion treatment, and thus the determination represents something less than the "total" amount (that is, less than 95 percent) of the constituent present in the dissolved and suspended phases of the sample. To achieve comparability of analytical data, equivalent digestion procedures would be required of all laboratories performing such analyses, because different digestion procedures are likely to produce different analytical results. Total recoverable concentrations occasionally are reported as less then the corresponding dissolved concentrations for a chemical constituent due to decreased analytical sensitivity of the analytical methods used for the digested solutions. Digested solutions often contain higher concentrations of substances that interfere with analytical sensitivity through complex matrix effects.

Water yeax in Geological Survey reports dealing with surface water supply ts the 12-month period, October 1 through september 30. The water year is designated by the calendar year in which it ends and which tncludes 9 of the 12 months. Thus, the year eriding September 30 , 1980 is called the "1980 water year."

Weighted average is used in this report to indicate dischargeweighted average. It is computed by multiplytng the discharge for a sampling period by the concentrations of individual constituents for the corresponding period and dividing the sum of the products by the sum of the discharges. A discharge-weighted average approxtmates the composition of water that would be found in a reservolx containing all the water passing a glven location during the water year after thorough mixing in the reservoir.

WDR is used as an abbreviation for "Water-Data Report" in the REVISED RECORDS paragraph to refer to State annual hydrologic-data reports (WRD was used as an abbreviation for "Water-Resources Data" in reports published prior to 1976).

WSP is used as an abbreviation for "Water-Supply Paper" in references to previously published reports. 


\section{PUBLICATIONS ON TECHNIQUES OF WATER-RESOURCES INVESTIGATIONS}

The U.S. Geological Survey publishes a series of manuals describing procedures for planning and conducting specialized work in water-resources investigations. The material is grouped under major subject headings called books and is further divided into sections and chapters. For example, Section A of Book 3 (Applications of Hydraulics) pertains to surface water. The chapter, the unit of publication, is limited to a narrow field of subject matter. This format permits flexibility in revision and publication as the need arises.

The reports listed below are for sale by the U.S. Geological Survey, Branch of Information Services, Box 25286, Federal Center, Denver, Colorado 80225 (authorized agent of the Superintendent of documents, Government Printing Office). Prepayment is required. Remittance should be sent by check or money order payable to the U.S. Geological Survey. Prices are not included because they are subject to change. Current prices can be obtained by writing to the above address. When ordering or inquiring about prices for any of these publications, please give the title, book number, chapter number, and "U.S. Geological Survey Techniques of WaterResources Investigations."

1-D1. Water temperature-influential factors, field measurement, and data presentation, by H.H. Stevens, Jr., J.F. Ficke and G.F. Smoot: USGS--TWRI Book 1, Chapter D1. 197565 pages.

1-D2. Guidelines for collection and field analysis of ground-water samples for selected unstable constituents, by W.W. Wood: USGS--TWRI Book 1, Chapter D2. 1976. 24 pages.

2-D1. Application of surface geophysics to ground-water investigations, by A.A.R. Zohdy, G.P. Eaton, and D.R. Mabey: USGS--TWRI Book 2, Chapter D1. 1974. 116 pages.

2-D2. Application of seismic-refraction techniques to hydrologic studies, by F.P. Haeni: USGS--TWRI Book 2, Chapter D2. 1988. 86 pages.

2-E1. Application of borehole geophysics to water-resources investigations, by W.S. Keys and L.M. MacCary: USGS--TWRI Book 2, Chapter E1. 1971. 126 pages.

2-E2. Borehole geophysics applied to ground-water investigations, by W.S. Keys: USGS--TWRI Book 2, Chapter E2. 1990. 150 pages.

2-F1. Application of drilling, coring, and sampling techniques to test holes and wells, by Eugene Shuter and W.E. Teasdale: USGS--TWRI Book 2, Chapter F1. 1989. 97 pages.

3-A1. General field and office procedures for indirect discharge measurements, by M.A. Benson and Tate Dalrymple: USGS--TWRI Book 3, Chapter A1. 1967. 30 pages.

3-A2. Measurement of peak discharge by the slope-area method, by Tate Dalrymple and M.A. Benson: USGS--TWRI Book 3, Chapter A2. 1967. 12 pages.

3-A3. Measurement of peak discharge at culverts by indirect methods, by G.L. Bodhaine: USGS-TWRI Book 3, Chapter A3. 1968. 60 pages.

3-A4. Measurement of peak discharge at width contractions by indirect methods, by H.F. Matthai: USGS-TWRI Book 3, Chapter A4. 1967. 44 pages.

3-A5. Measurement of peak discharge at dams by indirect methods, by Harry Hulsing: USGS--TWRI Book 3, Chapter A5. 1967. 29 pages.

3-A6 General procedure for gaging streams, by R.W. Carter and Jacob Davidian: USGS--TWRI Book 3, Chapter A6. 1968. 13 pages. 
PUBLICATIONS ON TECHNIQUES OF WATER-RESOURCES INVESTIGATIONS--Continued

3-A7. Stage measurement at gaging stations, by T.J. Buchanan and W.P. Somers: USGS--TWRI Book 3, Chapter A7. 1968. 28 pages.

3-A8. Discharge measurements at gaging stations, by T.J. Buchanan and W.P. Somers: USGS--TWRI Book 3, Chapter A8. 1969. 65 pages.

3-A9. Measurement of time of travel in streams by dye tracing, by F.A. Kilpatrick and J.F. Wilson, Jr.: USGS--TWRI Book 3, Chapter A9. 1989. 27 pages.

3-A10. Discharge ratings at gaging stations, by E.J. Kennedy: USGS--TWRI Book 3, Chapter A10. 1984.

59 pages.

3-A11. Measurement of discharge by the moving-boat method, by G.F. Smoot and C.E. Novak: USGS-TWRI Book 3, Chapter A11. 1969. 22 pages.

3-A12. Fluorometric procedures for dye tracing, Revised, by J.F. Wilson, Jr., E.D. Cobb, and F.A. Kilpatrick: USGS--TWRI Book 3, Chapter A12. 1986. 34 pages.

3-A13. Computation of continuous records of streamflow, by E.J. Kennedy: USGS--TWRI Book 3, Chapter A13. 1983. 53 pages.

3-A14. Use of flumes in measuring discharge, by F.A. Kilpatrick and V.R. Schneider: USGS-TWRI Book 3, Chapter A14. 1983. 46 pages.

3-A15. Computation of water-surface profles in open channels, by Jacob Davidian: USGS--TWRI Book 3, Chapter A15. 1984. 48 pages.

3-A16. Measurement of discharge using tracers, by F.A. Kilpatrick and E.D. Cobb: USGS--TWRI Book 3, Chapter A16. 1985. 52 pages.

3-A17. Acoustic velocity meter systems, by Antonius Laenen: USGS--TWRI Book 3, Chapter A17. 1985. 38 pages.

3-A18. Determination of stream reaeration coefficients by use of tracers, by F.A. Kilpatrick, R.E. Rathbun, Nobuhiro Yotsukura, G.W. Parker, and L.L. DeLong: USGS--TWRI Book 3, Chapter A18. 1989. 52 pages.

3-A19. Levels at sireamflow gaging station, by E.J. Kennedy: USGS--TWRI Book 3, Chapter A19. 1990. 31 pages

3-A20. Simulation of soluable waste transport and buildup in surface waters using tracers, by F.A. Kilpatrick: USGS--TWRI Book 3, Chapter A20. 1983. 38 pages.

3-A21. Stream-gaging cableways, by C. Russell Wagner: USGS--TWRI Book 3, Chapter A21. 1995. 56 pages.

3-B1. Aquifer-test design, observation, and data analysis, by R.W. Stallman: USGS--TWRI Book 3, Chapter B1. 1971. 26 pages.

3-B2. Introduction to ground-water hydraulics, a programed text for self-instruction, by G.D. Bennett: USGS--TWRI Book 32, Chapter B2. 1976. 172 pages. 


\section{PUBLICATIONS ON TECHNIQUES OF WATER-RESOURCES INVESTIGATIONS--Continued}

3-B3. Type curves for selected problems of flow to wells in confined aquifers, by J.E. Reed: USGS-TWRI Book 3, Chapter B3, 1980. 106 pages.

3-B4. Regression modeling of ground-water flow, by R.L. Cooley and R.L. Naff: USGS-TWRI Book 3, Chapter B4. 1990. 232 pages.

3-B4. Supplement 1. Regression modeling of ground-water-flow-Modifications to the computer code for nonlinear regression solution of stready-state ground-water-flow problems, by R.L. Cooley: USGS--TWRI Book 3, Chapter B4. 1993. 8 pages.

3-B5. Definition of boundary and initial conditions in the analysis of saturated ground-water flow systems--An introduction, by O.L. Franke, T.E. Reilly, and G.D. Bennett: USGS-TWRI Book 3, Chapter B5. 1987.

15 pages.

3-B6. The principle of superposition and its application in ground-water hydraulics, by T.E. Reilly, O.L. Franke, and G.D. Bennett: USGS--TWRI Book 3, Chapter B6. 1987. 28 pages.

3-B7. Analytical solutions for one-, two-, and three-dimensional solute transport in ground-water systems with uniform flow, by E.J. Wexler: USGS-.TWRI Book 3, Chapter B7. 1992. 190 pages.

3-C1. Fluvial sediment concepts, by H.P. Guy: USGS-TWRI Book 3, Chapter C1. 1970. 55 pages.

3-C2. Field methods for measurement of fluvial sediment, by H.P. Guy and V.W. Norman: USGS-TWRI Book 3, Chapter C2. 1970.59 pages.

3-C3. Computation of fluvial-sediment discharge, by George Porterfield: USGS--TWRI Book 3, Chapter C3. 1972.66 pages.

4-A1. Some statistical tools in hydrology, by H.C. Riggs: USGS--TWRI Book 4, Chapter A1. 1968. 39 pages.

4-A2. Frequency curves, by H.C. Riggs: USGS--TWRI Book 4, Chapter A2. 1968. 15 pages.

4-B1. Low-flow investigations, by H.C. Riggs: USGS-.TWRI Book 4, Chapter B1. 1972. 18 pages.

4-B2. Storage analyses for water-supply, by H.C. Riggs and C.H. Hardison: USGS--TWRI Book 4, Chapter B2. 1973. 20 pages.

4-B3. Regional analyses of streamflow characteristics; by H.C. Riggs: USGS--TWRI Book 4, Chapter B3. 1973. 15 pages.

4-D1. Computation of rate and volume of stream depletion by wells, by C.T. Jenkins: USGS--TWRI Book 4, Chapter D1. 1970. 17 pages.

5-A1. Methods for determination of inorganic substances in water and fluvial sediments, by M.J. Fishman and L.C. Friedman, editors: USGS--TWRI Book 5, Chapter A1. 1989. 545 pages.

5-A2. Determination of minor elements in water by emission spectroscopy, by P.R. Barnett and E.C. Mallory, Jr:: USGS--TWRI Book 5, Chapter A2. 1971. 31 pages.

5-A3. Methods for the determination of organic substances in water and fluvial sediments, edited by R.L. Wershaw, M.J. Fishman, R.R. Grabbe and L.E. Lowe: USGS--TWRI book 5, Chapter A3. 1987. 80 pages. 


\section{PUBLICATIONS ON TECHNIQUES OF WATER-RESOURCES INVESTIGATIONS--Continued}

5-A4. Methods for collection and analysis of aquatic biological and microbiological samples, by L.J. Britton and P.E. Greeson, editors: USGS-TWRI Book 5, Chapter A4. 1989. 363 pages.

5-A5. Methods for determination of radioactive substances in water and fluvial sediments, by L.L. Thatcher, V.J. Janzer, and K.W. Edwards: USGS--TWRI Book 5, Chapter A5. 1977. 95 pages.

5-A6. Quality assurance practices for the chemical and biological analyses of water and fluvial sediments, by L.C. Friedman and D.E. Erdmann: USGS--TWRI Book 5, Chapter A6. 1982. 181 pages.

5-C1. Laboratory theory and methods for sediment analysis, by H.P. Guy: USGS--TWRI Book 5, Chapter C1. 1969. 58 pages.

6-A1. A modular three-dimensional finite-difference ground-water flow model, by M.G. McDonald and A.W. Harbaugh: USGS--TWRI Book 6, Chapter A1. 1988. 586 pages.

6-A2. Documentation of a computer program to simulate aquifer-system compaction using the modular finite-difference ground-water flow model, by S.A. Leake and D.E. Prudic: USGS-TWRI Book 6, Chapter A2. 1991. 68 pages.

6-A3. A modular finite-elemental model (MODFE) for areal and axisymmetric ground-water-flow problems, Part 1: Model Description and User's Manual, by L.J. Torak: USGS--TWRI book 6, Chapter A3. 1993. 136 pages.

6-A4. A modular finite-element model (MODFE) for areal and axisymmetric ground-water-flwo problems, Part 2: Derivation of finite-element equations and comparisons with analytical solutions, by R.L. Cooley: USGS--TWRI Book 6, Chapter A4. 1992. 108 pages.

6-A5. A modular finite-element model (MODFE) for areal and axisymmetric ground-water-flow problems, Part 3: Design philosophy and programming details, by L.J. Torak: USGS--TWRI Book 6, Chapter A5. 1993. 243 pages.

7-C1. Finite difference model for aquifer simulation in two dimension with results of numerical experiments, by P.C. Trescott, G.F. Pinder, and S.P. Larson: USGS--TWRI Book 7, Chapter C1. 1976. 116 pages.

7-C2. Computer model of two-dimensional solute transport and dispersion in ground water by L.J. Konikow and J.D. Bredehoeft: USGS--TWRI Book 7, Chapter C2. 1978. 90 pages.

7-C3. A model for simulation of flow in singular and interconnected channels, by R.W. Schaffranek, R.A. Baltzer, and D.E. Goldberg: USGS--TWRI Book 7, Chapter C3. 1981. 110 pages.

8-A1. Methods of measuring water levels in deep wells, by M.S. Garber and F.C. Koopman: USGS-TWRI Book 8, Chapter A1. 1968. 23 pages.

8-A2. Installation and service manual for U.S. Geological Survey manometers, by J.D. Craig: USGS-TWRI Book 8, Chapter A2. 1983. 57 pages.

8-B2. Calibration and maintenance of veritcal-axis type current meters, by G.F. Smoot and C.E. Novak:

USGS--TWRI Book 8, Chapter B2. 1968. 15 pages. 


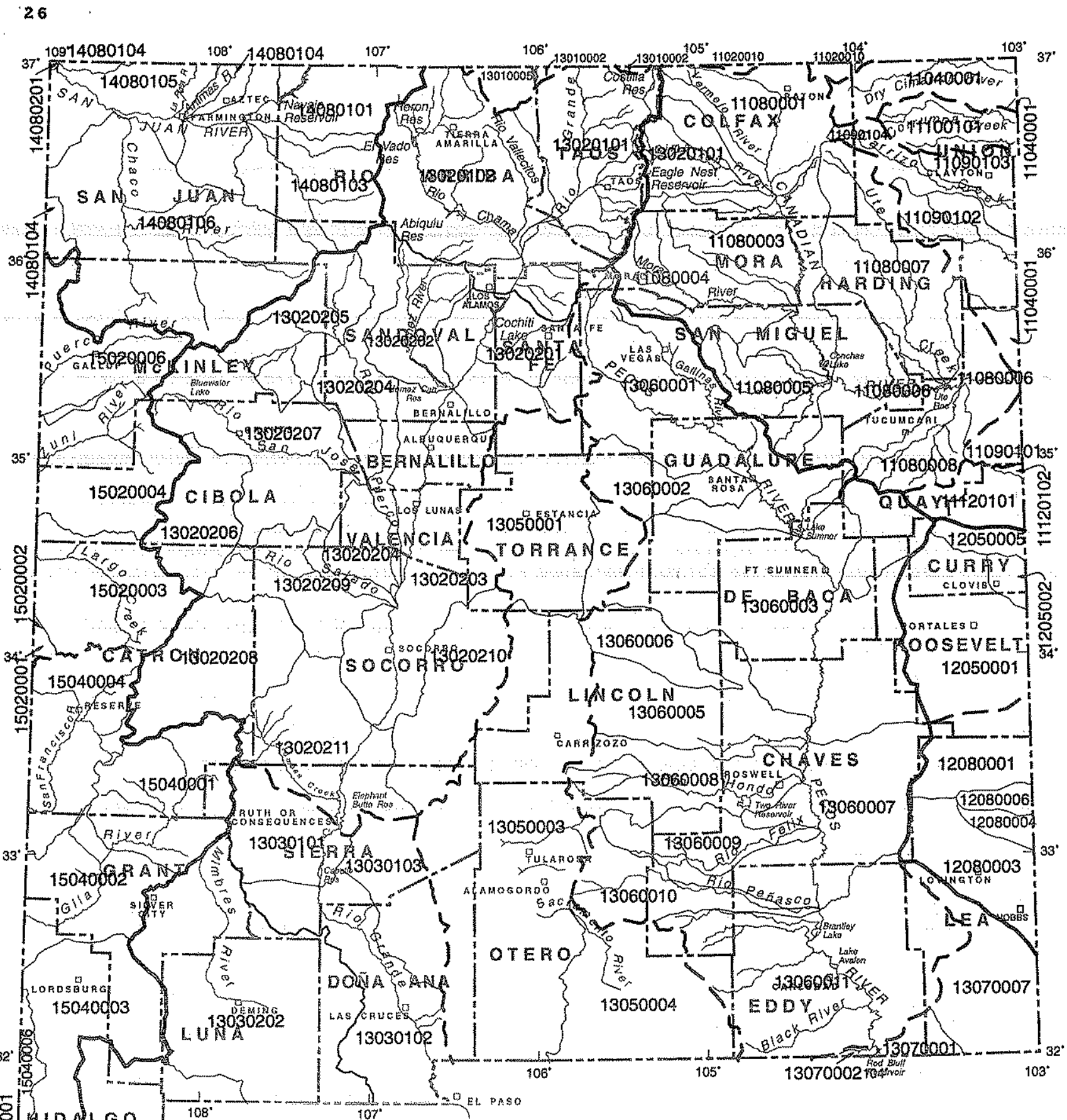

융

敬

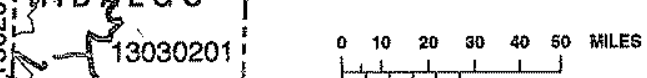

O

HYDROLOGIC UNIT CODE

$109+15080303.1 .101020304050$ KLLOMETERS

Reglon

Subreglon

13070001

Accounting Unit

U.S. Socologied Survoy Baso

\section{Regional Boundary \\ Subreglonal Boundiary}

Accounting Unî̀ Boundary

Cataloging Unit Boundary

Figure 4.--Location of hydrologic units. 


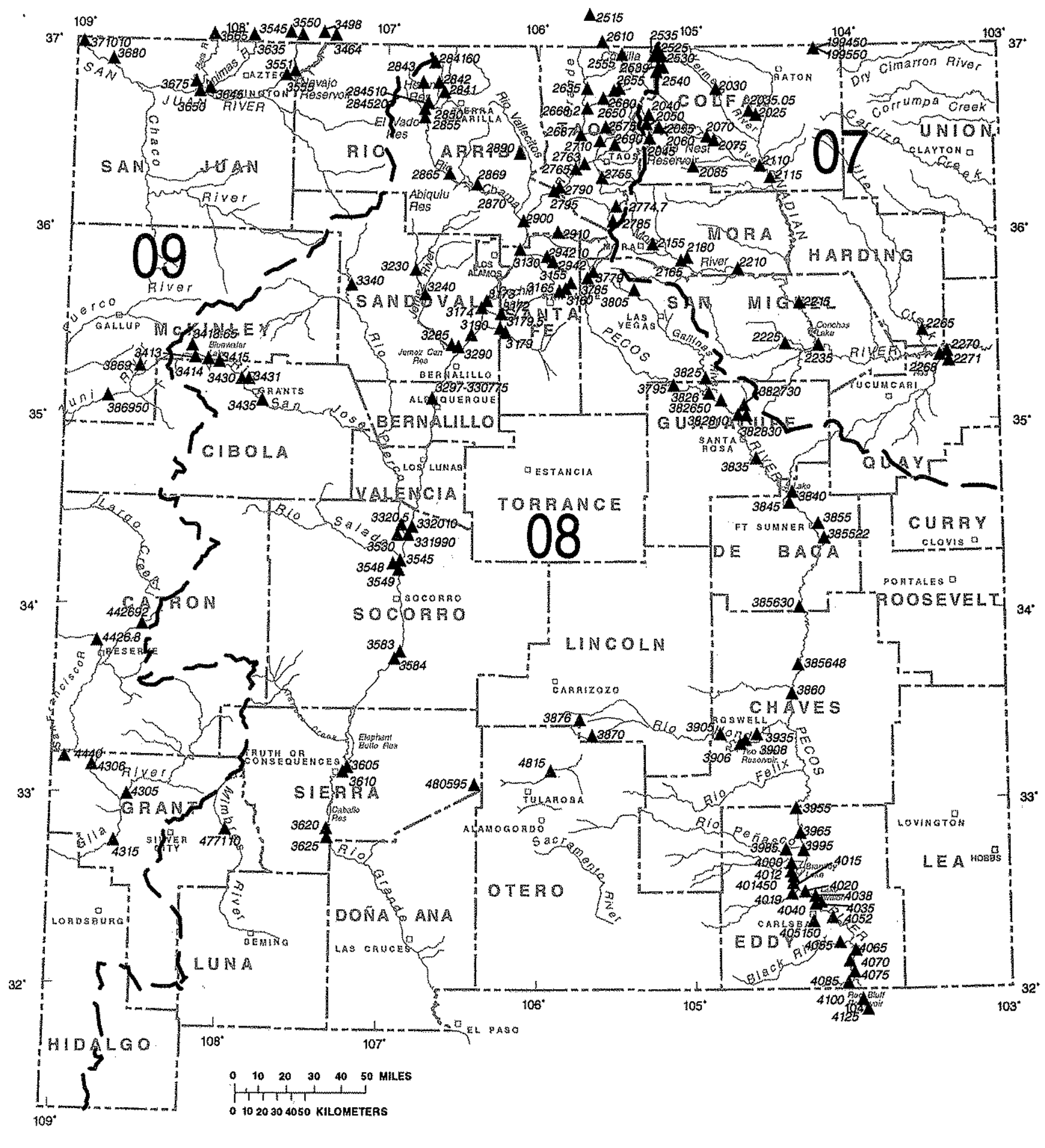

U.S. Geological Survey base

\section{EXPLANATION}

07 LOWER MISSISSIPPI RIVER BASIN NUMBER

08 WESTERN GULF OF MEXICO BASIN NUMBER

09 COLORADO RIVER BASIN NUMBER

- RIVER BASIN BOUNDARY
${ }^{401450} \triangle$ GAGING STATION AND NUMBER--

Number by symbol is abbreviated station number. Complete national station number is: 08 401450

Basin number + station number

Figure 5.--Location of surface-water gaging stations. 


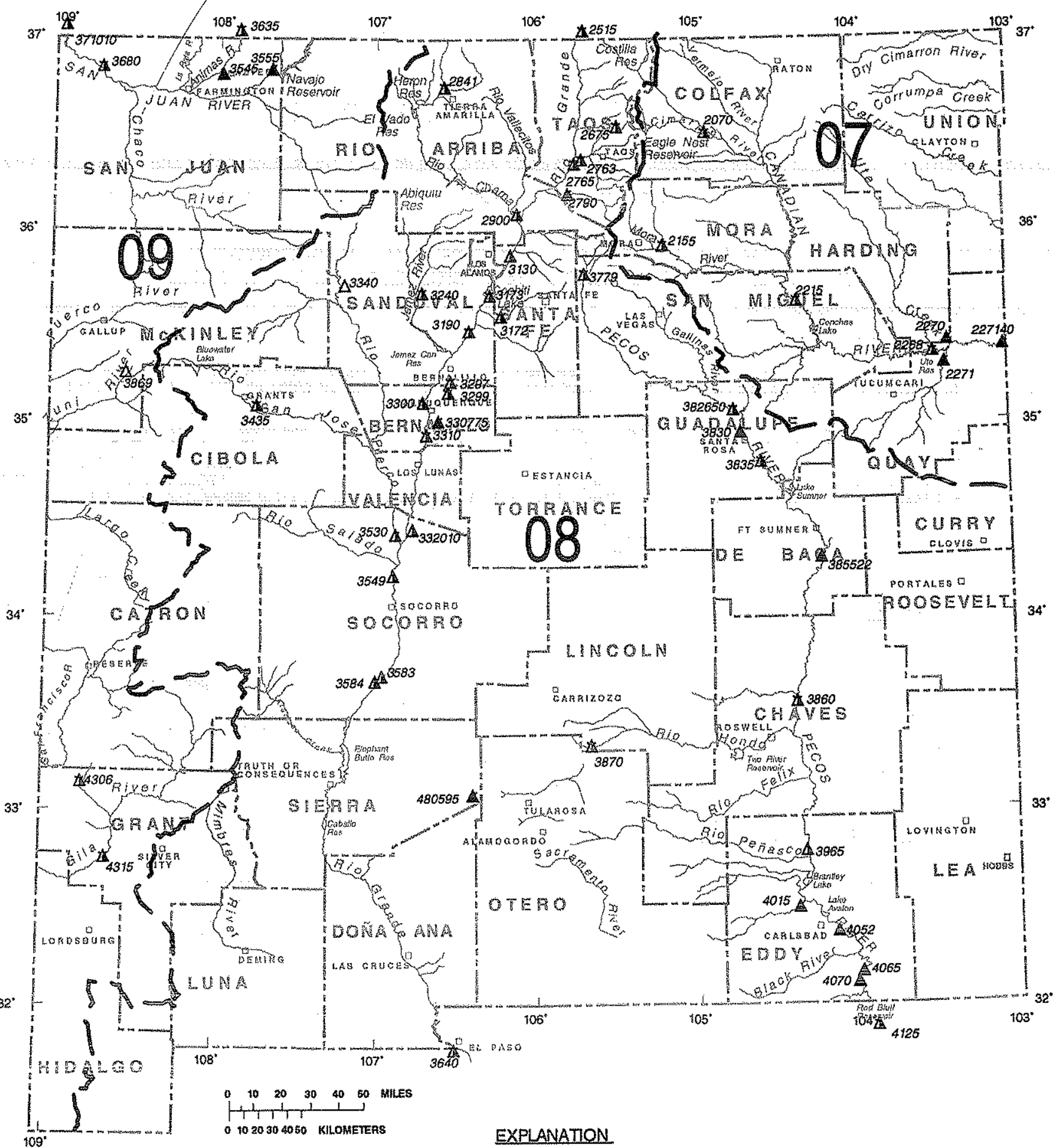

EXPLANATION

U.S. Bablogitent Sunay baw

BASIN AND STATION NLMBEER.

07 LOWER MISSISSIPPI RIVER BASIN NUMBER

08 WESTEFN QUUF OF MEXICO BASIN NUMBER

O9 COLORADO RIVER BASIN NUMBEA

330T75 BIVER BASIN BOUNDARY

330775 STATION AND NUMMBER--Number by symbol is abbrevisted station number. Complete nationa abbrowated station number. Comp
station number is: 08330775

Basin number + station number
STAMON AND SAMPIING FREOUENCY

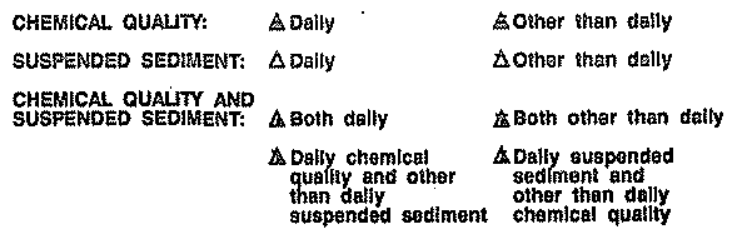

Figure 6.--Location of surface-water-quality stations. 
HYDROLOGIC-DATA STATION RECORDS

LOWER MISSISSTPPI RIVER BASIN

ARKANSAS RIVER BASIN

07199450 LAKE MALOYA NEAR RATON, NM

LOCATION, - Lat $36^{\circ} 59^{\circ} 02^{\prime \prime}$, long $104^{\circ} 22^{\prime} 24^{\prime \prime}$, Colfax County, Hydrologic Unit 11080001, in Maxwel.1 Grant, near spi11way of dam on Chicortca Creak, $6.5 \mathrm{mt}$ northeast of Raton, and at mile 21.5 .

DRAINAGE AREA. $--20.8 \mathrm{mt}^{2}$.

PERIOD OF RECORD.--May 1975 to September 1987 (monthend contents only), October 1987 to current year.

GAGE.-Water-stage recorder. Elevation of gage is National Geodetic Vertical Datum of 1929 , from topographic map.

REMARKS.--Reservoir is formed by an earthfill dam, completed in 1907; capacity, 59 acre-ft. Reservolr enlarged in 1916; capacity, 1,130 acre-ft, splilway elevation, $7,479.0 \mathrm{ft}$. Reservolr enlarged again in 1948; capacity, 3,690 acre-ft, spiliway alovation, $7,511.0$ ft. Elovation of lowest outlet, $7,439.0$ ft. No dead storage. Water is for muntcipal use of City of Raton. See table below for total monthly diversion, in acre-feet, from Lake Maloya for municipal supply for City of Raton and releases to Vermejo Conservancy District.

COORERATION, -DAversion, spillage and release data provided by City of Raton.

EXTREMES FOR PERIOD OF RECORD.-Maximum contents, 3,970 acre-ft, May 31, 1975, olevation, $7,510.79$ ft; maximum elevation observed, 7,513.01 ft, May 29, 1995; mintmum observed, 911 acre-ft, Feb. 28,1979 , elevation, $7,479.85$ ft.

EXTREMES FOR CURRENT YEAR.--Maximum contents, 3,730 acre-ft, Apr. 17-18, elevation, 7,511.34 ft; minlmum contents, 3,420 acre-ft, Aug. 10 16, elevation 7,506.43.

RESERVOIR STORAGE (ACRE-FEET), WATER YEAR OCTOBER 1995 TO SEPTEMBER 1996

\begin{tabular}{|c|c|c|c|c|c|c|c|c|c|c|c|c|}
\hline DAY & OCT & NOV & $\mathrm{DEC}$ & JAN & $\mathrm{FEB}$ & MAR & APR & MAY & JUN & TUL & AUG & $\mathrm{SEP}$ \\
\hline $\begin{array}{l}1 \\
2 \\
3 \\
4 \\
5\end{array}$ & $\begin{array}{l}3700 \\
3700 \\
3700 \\
3700 \\
3690\end{array}$ & $\begin{array}{l}3700 \\
3700 \\
3700 \\
3700 \\
3700\end{array}$ & $\begin{array}{l}3700 \\
3700 \\
3700 \\
3700 \\
3700\end{array}$ & $\begin{array}{l}3700 \\
3690 \\
3700 \\
3700 \\
3700\end{array}$ & $\begin{array}{l}3700 \\
3700 \\
3700 \\
3700 \\
3700\end{array}$ & $\begin{array}{l}3700 \\
3700 \\
3700 \\
3700 \\
3700\end{array}$ & $\begin{array}{l}3710 \\
3710 \\
3710 \\
3710 \\
3710\end{array}$ & $\begin{array}{l}3710 \\
3710 \\
3700 \\
3700 \\
3690\end{array}$ & $\begin{array}{l}3600 \\
3600 \\
3600 \\
3600 \\
3590\end{array}$ & $\begin{array}{l}3650 \\
3650 \\
3650 \\
3650 \\
3650\end{array}$ & $\begin{array}{l}3520 \\
3510 \\
3500 \\
3480 \\
3460\end{array}$ & $\begin{array}{l}3460 \\
3460 \\
3450 \\
3450 \\
3440\end{array}$ \\
\hline $\begin{array}{r}6 \\
7 \\
8 \\
9 \\
10\end{array}$ & $\begin{array}{l}3690 \\
3700 \\
3700 \\
3700 \\
3700\end{array}$ & $\begin{array}{l}3700 \\
3700 \\
3700 \\
3700 \\
3700\end{array}$ & $\begin{array}{l}3700 \\
3700 \\
3700 \\
3700 \\
3700\end{array}$ & $\begin{array}{l}3690 \\
3700 \\
3700 \\
3700 \\
3700\end{array}$ & $\begin{array}{l}e 3700 \\
-3700 \\
\oplus 3700 \\
e 3700 \\
\bullet 3700\end{array}$ & $\begin{array}{l}3690 \\
3700 \\
3700 \\
3700 \\
3700\end{array}$ & $\begin{array}{l}3710 \\
3720 \\
3710 \\
3710 \\
3720\end{array}$ & $\begin{array}{l}3690 \\
3680 \\
3680 \\
3680 \\
3670\end{array}$ & $\begin{array}{l}3580 \\
3580 \\
3570 \\
3560 \\
3560\end{array}$ & $\begin{array}{l}3650 \\
3650 \\
3650 \\
3510 \\
3510\end{array}$ & $\begin{array}{l}3450 \\
3440 \\
3430 \\
3430 \\
3420\end{array}$ & $\begin{array}{l}3440 \\
3440 \\
3440 \\
3440 \\
3440\end{array}$ \\
\hline $\begin{array}{l}11 \\
12 \\
13 \\
14 \\
15\end{array}$ & $\begin{array}{l}3700 \\
3700 \\
3700 \\
3700 \\
3700\end{array}$ & $\begin{array}{l}3700 \\
3700 \\
3700 \\
3700 \\
3700\end{array}$ & $\begin{array}{l}3700 \\
3700 \\
3700 \\
3700 \\
3700\end{array}$ & $\begin{array}{l}3700 \\
3700 \\
3700 \\
3700 \\
3700\end{array}$ & $\begin{array}{l}e 3700 \\
e 3700 \\
e 3700 \\
e 3700 \\
e 3700\end{array}$ & $\begin{array}{l}3700 \\
3700 \\
3700 \\
3700 \\
3700\end{array}$ & $\begin{array}{l}3710 \\
3710 \\
3710 \\
3700 \\
3710\end{array}$ & $\begin{array}{l}3670 \\
3660 \\
3650 \\
3650 \\
3640\end{array}$ & $\begin{array}{l}3560 \\
3550 \\
3540 \\
3560 \\
3580\end{array}$ & $\begin{array}{l}3520 \\
3510 \\
3500 \\
3500 \\
3500\end{array}$ & $\begin{array}{l}3420 \\
3420 \\
3420 \\
3420 \\
3420\end{array}$ & $\begin{array}{l}3440 \\
3440 \\
3440 \\
3450 \\
3450\end{array}$ \\
\hline $\begin{array}{l}16 \\
17 \\
18 \\
19 \\
20\end{array}$ & $\begin{array}{l}3710 \\
3710 \\
3710 \\
3700 \\
3700\end{array}$ & $\begin{array}{l}3700 \\
3690 \\
3700 \\
3700 \\
3690\end{array}$ & $\begin{array}{l}3700 \\
3700 \\
3690 \\
3690 \\
3690\end{array}$ & $\begin{array}{l}3700 \\
3700 \\
3700 \\
3700 \\
3700\end{array}$ & $\begin{array}{l}e 3700 \\
e 3700 \\
e 3700 \\
e 3700 \\
e 3700\end{array}$ & $\begin{array}{l}3700 \\
3700 \\
3700 \\
3700 \\
3700\end{array}$ & $\begin{array}{l}3720 \\
3730 \\
3730 \\
3720 \\
3710\end{array}$ & $\begin{array}{l}3630 \\
3620 \\
3610 \\
3600 \\
3600\end{array}$ & $\begin{array}{l}3650 \\
3650 \\
3650 \\
3650 \\
3650\end{array}$ & $\begin{array}{l}3490 \\
3490 \\
3480 \\
3480 \\
3490\end{array}$ & $\begin{array}{l}3420 \\
3430 \\
3430 \\
3430 \\
3430\end{array}$ & $\begin{array}{l}3460 \\
3460 \\
3460 \\
3460 \\
3460\end{array}$ \\
\hline $\begin{array}{l}21 \\
22 \\
23 \\
24 \\
25\end{array}$ & $\begin{array}{l}3700 \\
3700 \\
3690 \\
3700 \\
3700\end{array}$ & $\begin{array}{l}3700 \\
3700 \\
3700 \\
3700 \\
3700\end{array}$ & $\begin{array}{l}3700 \\
3700 \\
3690 \\
3700 \\
3700\end{array}$ & $\begin{array}{l}3700 \\
3700 \\
3700 \\
3700 \\
3700\end{array}$ & $\begin{array}{r}03710 \\
3710 \\
3700 \\
3700 \\
3700\end{array}$ & $\begin{array}{l}3700 \\
3710 \\
3710 \\
3700 \\
3710\end{array}$ & $\begin{array}{l}3710 \\
3710 \\
3720 \\
3720 \\
3720\end{array}$ & $\begin{array}{l}3580 \\
3570 \\
3560 \\
3560 \\
3580\end{array}$ & $\begin{array}{l}3650 \\
3650 \\
3650 \\
3650 \\
3650\end{array}$ & $\begin{array}{l}3500 \\
3490 \\
3500 \\
3490 \\
3490\end{array}$ & $\begin{array}{l}3430 \\
3460 \\
3470 \\
3470 \\
3470\end{array}$ & $\begin{array}{l}3460 \\
3470 \\
3470 \\
3470 \\
3460\end{array}$ \\
\hline $\begin{array}{l}26 \\
27 \\
28 \\
29 \\
30 \\
31\end{array}$ & $\begin{array}{l}3700 \\
3700 \\
3700 \\
3700 \\
3700 \\
3700\end{array}$ & $\begin{array}{l}3700 \\
3690 \\
3690 \\
3700 \\
3700 \\
-\end{array}$ & $\begin{array}{l}3700 \\
3690 \\
3700 \\
3690 \\
3690 \\
3700\end{array}$ & $\begin{array}{l}3700 \\
3700 \\
3700 \\
3700 \\
3700 \\
3700\end{array}$ & $\begin{array}{r}3700 \\
3700 \\
3700 \\
3700 \\
--- \\
---\end{array}$ & $\begin{array}{l}3700 \\
3700 \\
3710 \\
3710 \\
3710 \\
3710\end{array}$ & $\begin{array}{l}3710 \\
3720 \\
3700 \\
3700 \\
3710 \\
-\end{array}$ & $\begin{array}{l}3590 \\
3600 \\
3600 \\
3600 \\
3600 \\
3600\end{array}$ & $\begin{array}{l}3650 \\
3650 \\
3650 \\
3650 \\
3650 \\
-\end{array}$ & $\begin{array}{l}3510 \\
3520 \\
3530 \\
3520 \\
3520 \\
3520\end{array}$ & $\begin{array}{l}3470 \\
3470 \\
3480 \\
3470 \\
3470 \\
3470\end{array}$ & $\begin{array}{l}3450 \\
3450 \\
3450 \\
3450 \\
3450 \\
---\end{array}$ \\
\hline $\begin{array}{l}\text { MAX } \\
\text { MIN } \\
\text { (t) } \\
(t+) \\
\text { (t+t) } \\
\text { (ttt) }\end{array}$ & $\begin{array}{r}3710 \\
3690 \\
7511.07 \\
0 \\
0 \\
0\end{array}$ & $\begin{array}{r}3700 \\
3690 \\
7511.10 \\
0 \\
0 \\
0\end{array}$ & $\begin{array}{r}3700 \\
3690 \\
7511.09 \\
0 \\
0 \\
0\end{array}$ & $\begin{array}{r}3700 \\
3690 \\
7511.08 \\
0 \\
0 \\
0\end{array}$ & $\begin{array}{r}3710 \\
3700 \\
7511.10 \\
0 \\
0 \\
0\end{array}$ & $\begin{array}{r}3710 \\
3690 \\
7511.17 \\
+10 \\
0 \\
0\end{array}$ & $\begin{array}{r}3730 \\
3700 \\
7511.20 \\
0 \\
0 \\
0\end{array}$ & $\begin{array}{r}3710 \\
3560 \\
7510.24 \\
-110 \\
265 \\
0\end{array}$ & $\begin{array}{r}3650 \\
3540 \\
7510.65 \\
+50 \\
215 \\
0\end{array}$ & $\begin{array}{r}3650 \\
3480 \\
7509.60 \\
-130 \\
198 \\
0\end{array}$ & $\begin{array}{r}3520 \\
3420 \\
7509.17 \\
-50 \\
196 \\
0\end{array}$ & $\begin{array}{r}3470 \\
3440 \\
7509.00 \\
-20 \\
0 \\
0\end{array}$ \\
\hline
\end{tabular}

e Estimated

CAI YR 1995 MAX 3930 MIN 3210 (t†) -340 (fft) 874 (ffft) 0 WTR YR 1996 MAX 3730 MIN 3420 (tf) -250 (tff) 0 (tfft) 0

(†) ELEVATION, IN FEET, AT END OF MONTH

(t) CHANGE IN CONTENTS, IN ACRE-FEET

(tit) DIVERSION FROM LAKE MALOYA, IN ACRE-FEET

(t+t) RELEASE, IN ACRE-FEET, TO VERMEJO CONSERVANCY DISTRICT 
LOCATION.--Lat 36 $57^{\prime} 15^{\prime \prime}$, Long $104^{\circ} 23^{\prime} 06^{\prime \prime}$, Colfax County, Bydrologic unit 11080001, in Maxwell Grant, near spillway of dam on Chicorica Creek, $4.4 \mathrm{mi}$ northeast of Raton, and at mile 19.2 .

DRAINAGE AREA. $--29.4 \mathrm{mi}^{2}$.

PERIOD OF RECORD.--May 1975 to September 1996 (discontinued).

GAGE.--Nonrecording gage. Elevation of gage is Nationel Geodetio Vertical Datum of 1929, from topographic mep.

REMARKS - Reservolx is formed by an earthfili dam, completed in 1892; capacity 100 acre-ft, splilway elevation, 7,078.0 ft. Reservoir rehabilitated in 1941; capacity, 71 acre-ft, spillway elevation, 7,089.6 ft. . Elevation of

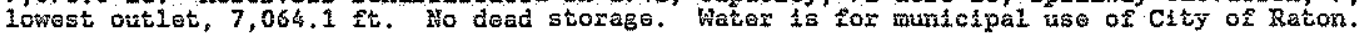

COOPERATION.--Monthend elevations and contents provided by City of Raton.

EXTREMES FOR PERIOD OF RECORD.-Maximum contents observed, 100 acre-ft, January 31, 1994, elevation, 7,090 ft; minimum observed, 0 acre-ft, Aug." . Sept. 1989, lake drained.

MONTHEND ELEVATION AND CONTENTS, WATER YEAR OCTOBER 1995 TO SEPTEMBER 1996

Sept. $30,1995 \ldots \ldots \ldots \ldots \ldots \ldots \ldots \ldots \ldots$

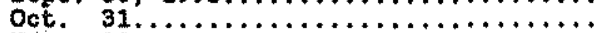

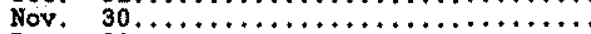

Dec, $\quad 31 \ldots \ldots \ldots \ldots \ldots \ldots \ldots \ldots \ldots \ldots$

CAL. YR 1995

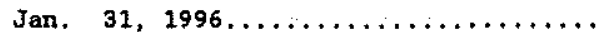

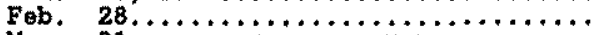

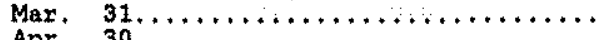

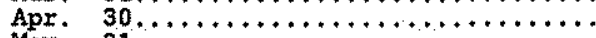

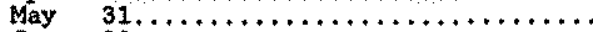

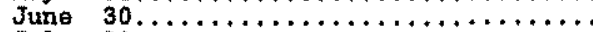

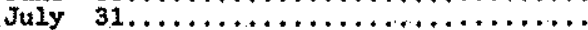

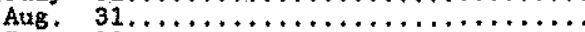

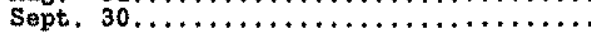

WTR YR 1996

\section{Elevation \\ (foet)}

-
$7,089.6$
$7,089.6$
$7,089.6$
$7,089.6$
$7,089.6$
$7,089.6$
$7,089.6$
$7,089.6$

$7,089.6$

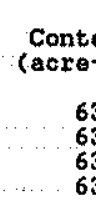

63

63
63

63

63

63

63

63
63

$-$

\section{Change in \\ (acre-feet)}

63

$\begin{array}{llll}63 & 0 \\ 63 & & 0 & 0 \\ 63 & & & 0 \\ & & & \end{array}$

$-2 \ldots+63$

0
0
0
63

63.

0

0

0

0

0

0 
ARKANSAS RIVER BASIN

07202500 EAGLE TAIL DITCH NEAR MAXWELL, NM

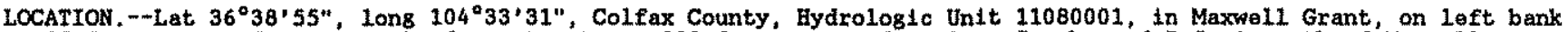
25 ft upstream from concrete drop structure, $300 \mathrm{ft}$ upstream from Crow Creek, and $7.5 \mathrm{mi}$ north of Maxwell.

PERIOD OF RECORD.--December 1944 to July 1950 (monthly discharge only October 1945 to July 1950 ), May 1975 to current year.

GAGE.--Water stage recorcier and concrete control. Elevation of gage is 6,110 ft above National Geodetic Vertical Datum of 1929. from topographic map. Prior to May 1975, at site about 200 ft upstream at different datum.

REMARKS.--Records good except for estimated dally discharges, which are poor. Eagle Tail ditch diverts water from Chicorica Creek for use near Maxwell. No diversions upstream from station. Several observations of water

temperature were made during the year. No flow at times most years, DISCHARGE, CUBIC FEET PER SECOND, MATER YEAR OCTOBER 1995 TO SEPTEMBER 1996

\begin{tabular}{|c|c|c|c|c|c|c|c|c|c|c|c|c|}
\hline DAY & $O C T$ & NOV & DEC & JAN & FEB & MAR & AER & MAY & Jun & JUL & AUG & SEP \\
\hline $\begin{array}{l}1 \\
2 \\
3 \\
4 \\
5\end{array}$ & $\begin{array}{l}2.5 \\
3.4 \\
3.4 \\
3.7 \\
3.0\end{array}$ & $\begin{array}{l}5.4 \\
5.2 \\
5.1 \\
4.9 \\
4.8\end{array}$ & $\begin{array}{l}4.6 \\
4.7 \\
4.1 \\
3.6 \\
3.6\end{array}$ & $\begin{array}{l}04.0 \\
03.7 \\
03.3 \\
03.0 \\
03.5\end{array}$ & $\begin{array}{l}04.2 \\
03.9 \\
03.7 \\
03.6 \\
03.9\end{array}$ & $\begin{array}{l}3.7 \\
3.0 \\
2.7 \\
2.2 \\
2.0\end{array}$ & $\begin{array}{l}1.1 \\
1.1 \\
.92 \\
.95 \\
1.1\end{array}$ & $\begin{array}{l}1.6 \\
1.1 \\
1.5 \\
2.4 \\
1.8\end{array}$ & $\begin{array}{l}3.1 \\
5.2 \\
3.7 \\
2.8 \\
1.4\end{array}$ & $\begin{array}{r}9.8 \\
3.9 \\
1.5 \\
.75 \\
.54\end{array}$ & $\begin{array}{l}33 \\
32 \\
4.8 \\
2.6 \\
3.2\end{array}$ & $\begin{array}{l}7.7 \\
4.2 \\
3.7 \\
3.2 \\
3.0\end{array}$ \\
\hline $\begin{array}{r}6 \\
7 \\
8 \\
9 \\
10\end{array}$ & $\begin{array}{l}4.3 \\
4.2 \\
3.7 \\
3.2 \\
2.9\end{array}$ & $\begin{array}{l}4.9 \\
4.8 \\
4.6 \\
4.6 \\
4.7\end{array}$ & $\begin{array}{l}3.4 \\
3.1 \\
2.8 \\
1.9 \\
2.6\end{array}$ & $\begin{array}{l}03.2 \\
03.2 \\
03.4 \\
03.9 \\
04.5\end{array}$ & $\begin{array}{l}03.7 \\
03.6 \\
03.6 \\
04.2 \\
04.6\end{array}$ & $\begin{array}{l}2.2 \\
1.9 \\
2.3 \\
2.2 \\
2.3\end{array}$ & $\begin{array}{l}1.3 \\
2.2 \\
1.7 \\
1.6 \\
1.2\end{array}$ & $\begin{array}{l}2.2 \\
2.1 \\
1.6 \\
1.3 \\
1.3\end{array}$ & $\begin{array}{l}.88 \\
.85 \\
.64 \\
.30 \\
.15\end{array}$ & $\begin{array}{r}.41 \\
1.27 \\
1.1 \\
12.8\end{array}$ & $\begin{array}{l}2.8 \\
1.1 \\
10^{.24} \\
40\end{array}$ & $\begin{array}{l}2.8 \\
3.8 \\
5.8 \\
3.9 \\
2.1\end{array}$ \\
\hline $\begin{array}{l}11 \\
12 \\
13 \\
14 \\
15\end{array}$ & $\begin{array}{l}2.7 \\
2.5 \\
2.3 \\
2.7 \\
3.8\end{array}$ & $\begin{array}{l}4.9 \\
5.4 \\
5.5 \\
5.3 \\
5.4\end{array}$ & $\begin{array}{l}3.3 \\
4.2 \\
3.4 \\
3.4 \\
4.1\end{array}$ & $\begin{array}{l}04.1 \\
04.8 \\
04.5 \\
e 5.4 \\
04.5\end{array}$ & $\begin{array}{l}04.3 \\
04.2 \\
04.6 \\
04.2 \\
04.0\end{array}$ & $\begin{array}{l}2.0 \\
1.8 \\
1.6 \\
1.4 \\
1.3\end{array}$ & $\begin{array}{l}1.0 \\
.63 \\
.21 \\
.19 \\
.05\end{array}$ & $\begin{array}{l}1.6 \\
1.0 \\
1.0 \\
1.2 \\
1.0\end{array}$ & $\begin{array}{l}.07 \\
.00 \\
.00 \\
.00 \\
.00\end{array}$ & $\begin{array}{l}16 \\
11 \\
3.1 \\
7.7 \\
.67\end{array}$ & $\begin{array}{r}27 \\
5.1 \\
3.2 \\
1.4 \\
.62\end{array}$ & $\begin{array}{r}3.5 \\
2.8 \\
3.4 \\
12 . \\
8.4\end{array}$ \\
\hline $\begin{array}{l}16 \\
17 \\
18 \\
19 \\
20\end{array}$ & $\begin{array}{l}5.0 \\
5.0 \\
4.7 \\
4.4 \\
3.8\end{array}$ & $\begin{array}{l}5.4 \\
5.7 \\
5.9 \\
5.7 \\
5.2\end{array}$ & $\begin{array}{l}4.6 \\
5.0 \\
7.1 \\
4.6 \\
4.0\end{array}$ & $\begin{array}{l}04.1 \\
03.8 \\
03.4 \\
03.5 \\
03.5\end{array}$ & $\begin{array}{l}03.8 \\
04.2 \\
04.8 \\
04.6 \\
04.3\end{array}$ & $\begin{array}{l}1.2 \\
1.6 \\
2.2 \\
2.3 \\
2.7\end{array}$ & $\begin{array}{l}2.2 \\
1.9 \\
1.4 \\
2.4 \\
3.0\end{array}$ & $\begin{array}{l}.63 \\
.66 \\
.40 \\
.08 \\
.00\end{array}$ & $\begin{array}{l}2.5 \\
2.6 \\
1.5 \\
1.0 \\
.44\end{array}$ & $\begin{array}{r}.27 \\
.07 \\
.03 \\
.76 \\
6.8\end{array}$ & $\begin{array}{c}.82 \\
1.0 \\
.70 \\
.92 \\
1.9\end{array}$ & $\begin{array}{r}12 \\
2.6 \\
1.4 \\
1.2 \\
2.0\end{array}$ \\
\hline $\begin{array}{l}21 \\
22 \\
23 \\
24 \\
25\end{array}$ & $\begin{array}{l}5.1 \\
4.0 \\
4.0 \\
4.0 \\
5.6\end{array}$ & $\begin{array}{l}4.9 \\
4.7 \\
4.7 \\
5.0 \\
6.0\end{array}$ & $\begin{array}{r}3.5 \\
2.9 \\
03.0 \\
02.8 \\
e 2.6\end{array}$ & $\begin{array}{l}03.5 \\
03.9 \\
03.8 \\
03.8 \\
04.1\end{array}$ & $\begin{array}{r}4.7 \\
4.8 \\
4.8 \\
4.0 \\
3.7\end{array}$ & $\begin{array}{l}2.8 \\
2.6 \\
2.1 \\
2.0 \\
1.1\end{array}$ & $\begin{array}{l}3.9 \\
3.5 \\
2.8 \\
2.4 \\
1.2\end{array}$ & $\begin{array}{l}.00 \\
.00 \\
.00 \\
.00 \\
.26\end{array}$ & $\begin{array}{l}.19 \\
.06 \\
.01 \\
.00 \\
.00\end{array}$ & $\begin{array}{l}12 \\
25 \\
8.1 \\
3.4 \\
2.4\end{array}$ & $\begin{array}{l}1.1 \\
1.2 \\
15 \\
34 \\
17\end{array}$ & $\begin{array}{r}1.1 \\
.78 \\
.64 \\
7.0 \\
13\end{array}$ \\
\hline $\begin{array}{l}26 \\
27 \\
28 \\
29 \\
30 \\
31\end{array}$ & $\begin{array}{l}4.8 \\
4.5 \\
4.5 \\
4.9 \\
5.2 \\
5.6\end{array}$ & $\begin{array}{l}4.9 \\
5.0 \\
5.0 \\
5.0 \\
4.8 \\
-.1\end{array}$ & $\begin{array}{l}e 3.0 \\
e 3.4 \\
e 3.2 \\
03.5 \\
03.8 \\
03.6\end{array}$ & $\begin{array}{l}04.5 \\
04.0 \\
03.9 \\
03.6 \\
04.0 \\
04.6\end{array}$ & $\begin{array}{l}3.5 \\
4.0 \\
4.1 \\
3.8 \\
-.-\end{array}$ & $\begin{array}{r}.78 \\
1.0 \\
.96 \\
.93 \\
.86 \\
.80\end{array}$ & $\begin{array}{r}.77 \\
.65 \\
1.2 \\
1.1 \\
.65 \\
-. .\end{array}$ & $\begin{array}{r}8.0 \\
8.2 \\
14 \\
13 \\
3.9 \\
3.2\end{array}$ & $\begin{array}{l}11 \\
17 \\
14 \\
13 \\
12 \\
\end{array}$ & $\begin{array}{l}\frac{1}{25} .5 \\
8.8 \\
2.2 \\
12 \\
41\end{array}$ & $\begin{array}{r}4.4 \\
3.1 \\
17 \\
21 \\
7.7 \\
17\end{array}$ & $\begin{array}{l}2.6 \\
4.1 \\
3.1 \\
2.5 \\
1.9 \\
-\end{array}$ \\
\hline $\begin{array}{l}\text { TOFAL } \\
\text { MEAN } \\
\text { MAX } \\
\text { MIN } \\
\text { AC-FT }\end{array}$ & $\begin{array}{r}123.4 \\
3.98 \\
5.6 \\
2.3 \\
245\end{array}$ & $\begin{array}{r}153.4 \\
5.11 \\
6.0 \\
4.6 \\
304\end{array}$ & $\begin{array}{r}113.4 \\
3.66 \\
7.1 \\
1.9 \\
22.5\end{array}$ & $\begin{array}{r}121.0 \\
3.90 \\
5.4 \\
3.0 \\
240\end{array}$ & $\begin{array}{r}119.4 \\
4.12 \\
4.8 \\
3.5 \\
237\end{array}$ & $\begin{array}{r}58.53 \\
1.89 \\
3.7 \\
.78 \\
116\end{array}$ & $\begin{array}{r}44.32 \\
1.48 \\
3.9 \\
.05 \\
88\end{array}$ & $\begin{array}{r}75.03 \\
2.42 \\
14 \\
.00 \\
149\end{array}$ & $\begin{array}{r}94.39 \\
3.15 \\
17 \\
.00 \\
187\end{array}$ & $\begin{array}{r}227.87 \\
7.35 \\
41 \\
.03 \\
452\end{array}$ & $\begin{array}{r}310.10 \\
10.0 \\
40 \\
.24 \\
615\end{array}$ & $\begin{array}{r}126.22 \\
4.21 \\
13 \\
.64 \\
250\end{array}$ \\
\hline
\end{tabular}

STATISTICS OF MONTHLY MEAN DATA FOR WATER YEARS 1945 - 1996, BY WATER YEAR (WY)

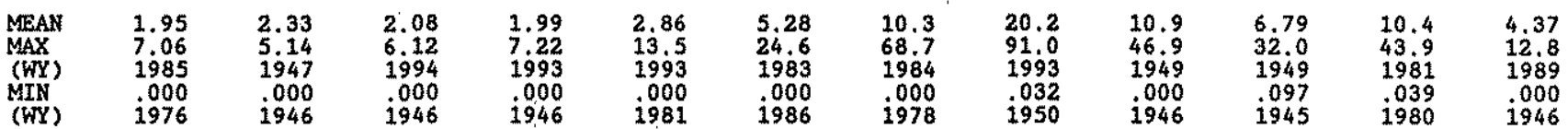

SUMARY STATISTTCS

FOR 1995 CALENDAR YEAR

FOR 1996 WATER YEAR
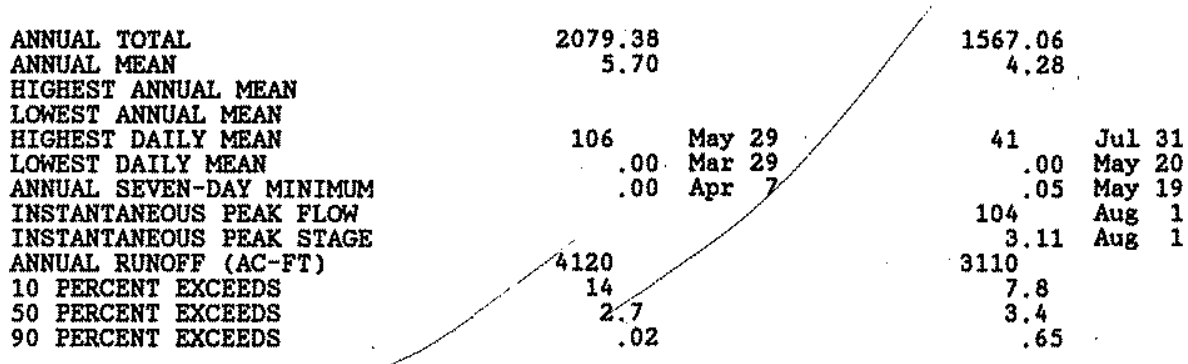

WATER YEARS 1945 - 1996

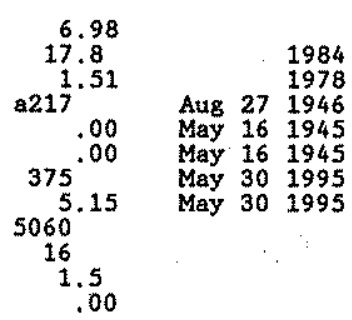

- Estimatod

a-From rating curve extended above $85 \mathrm{ft}^{3} / \mathrm{s}$. 
LOCATION,--Lat $36^{\circ} 40^{\circ} 50^{\prime \prime}$, long 104 $47^{\circ} 08^{\prime \prime}$, Colfax County, Hydrologic Unit 11080001, in Maxwell Grant, on left bank $1.3 \mathrm{mi}$ north of Dawion, $2.3 \mathrm{mi}$ upstream from Rail Canyon, and at mile 28.2 .

DRAINAGE AREA. $-301 \mathrm{mi}$.

PERIOD OF RECORD,--October 1915 to July 1918, April 1919 to May 1921, January 1927 to current year. Monthly discharge only for some periods, published in WSP 1311.

REVISED RECORDS.-WSP 11.17: 1947, dralnage area. WSP 1281: 1932(M); $1934(M), 1936-38(M), 1941-42(P), 1944-46(M)$.

GAGE.--Water-stage recorder. Elevation of gage is 6,360 pt above National Geodetic Vertical Datum of 1929 , from topographic map. See WSE 1311 or 1731 for history of changes prior to Sept. 24, 1953.

REMARKS, - Records fair except for estimated daily discharges which are poor. Diversions for trrigation of small acreage and mountain meadows upstream from station. Several observations of water temperature were made during year. U. S. Weather Service Satelitte Telemeter at gage.... Ho flow at times.

EXTREAES OUTSIDE PERIOD OF RECORD.--A majox flood occurred Aug. 2, 1921, when discherge probably exceeded 10,000 $\mathrm{ft}^{3} / \mathrm{s}$.

DISCHARGE, CUBIC FEET PER SECOND, WATER YEAR OCTOBER 1995 TO SEPTEMBER 1996

\begin{tabular}{|c|c|c|c|c|c|c|c|c|c|c|c|c|}
\hline DAY & OCT & NOV & $\mathrm{DEC}$ & JAN & FEB & MAR & APR & MAY & JUN & JUL & AUG & SEP \\
\hline $\begin{array}{l}1 \\
2 \\
3 \\
4 \\
5\end{array}$ & $\begin{array}{l}\theta 18 \\
e 17 \\
017 \\
e 16 \\
016\end{array}$ & $\begin{array}{l}12 \\
14 \\
13 \\
13 \\
14\end{array}$ & $\begin{array}{r}14 \\
14 \\
13 \\
12 \\
11\end{array}$ & $\begin{array}{l}\theta 11 \\
\text { e12 } \\
\text { e10 } \\
e 9.0 \\
\text { e11 }\end{array}$ & $\begin{array}{r}69.0 \\
e 8.0 \\
6.8 \\
7.5 \\
8.5\end{array}$ & $\begin{array}{l}12 \\
14 \\
16 \\
13 \\
12\end{array}$ & $\begin{array}{r}8.8 \\
8.8 \\
\begin{array}{l}11 \\
012 \\
16\end{array}\end{array}$ & $\begin{array}{l}17 \\
17 \\
14 \\
13 \\
14\end{array}$ & $\begin{array}{l}7.4 \\
8.3 \\
7.4 \\
4.6 \\
4.0\end{array}$ & $\begin{array}{l}27 \\
17 \\
9.5 \\
7.0 \\
8.4\end{array}$ & $\begin{array}{l}22 \\
13 \\
9.8 \\
7.7 \\
5.5\end{array}$ & $\begin{array}{l}e 55 \\
e 27 \\
e 20 \\
018 \\
e 15\end{array}$ \\
\hline $\begin{array}{r}6 \\
7 \\
8 \\
9 \\
10\end{array}$ & $\begin{array}{l}\text { e15 } \\
\text { e14 } \\
\text { e14 } \\
\text { e14 } \\
\text { B14 }\end{array}$ & $\begin{array}{l}13 \\
12 \\
11 \\
10 \\
11\end{array}$ & $\begin{array}{l}10 \\
9.5 \\
11 \\
33 \\
32\end{array}$ & $\begin{array}{l}012 \\
011 \\
010 \\
011 \\
010\end{array}$ & $\begin{array}{l}99.0 \\
810 \\
012 \\
012 \\
21\end{array}$ & $\begin{array}{l}12 \\
16 \\
13 \\
18 \\
14\end{array}$ & $\begin{array}{l}14 \\
12 \\
14 \\
13 \\
15\end{array}$ & $\begin{array}{l}15 \\
15 \\
15 \\
14 \\
13\end{array}$ & $\begin{array}{l}3.2 \\
2.8 \\
2.6 \\
4.0 \\
4.1\end{array}$ & $\begin{array}{l}10 \\
5.8 \\
40 \\
57 \\
71\end{array}$ & $\begin{array}{r}3.2 \\
7.3 \\
28 \\
6.7 \\
6.2\end{array}$ & $\begin{array}{l}\text { e15 } \\
\text { e14 } \\
\text { e14 } \\
\text { e13 } \\
\text { e13 }\end{array}$ \\
\hline $\begin{array}{l}11 \\
12 \\
13 \\
14 \\
15\end{array}$ & $\begin{array}{r}814 \\
14 \\
13 \\
13 \\
14\end{array}$ & $\begin{array}{l}14 \\
11 \\
14 \\
12 \\
13\end{array}$ & $\begin{array}{l}51 \\
22 \\
10 \\
9.9 \\
12\end{array}$ & $\begin{array}{l}e 12 \\
011 \\
09.0 \\
012 \\
\theta 15\end{array}$ & $\begin{array}{l}24 \\
18 \\
18 \\
18 \\
13\end{array}$ & $\begin{array}{l}12 \\
11 \\
11 \\
11 \\
11\end{array}$ & $\begin{array}{l}18 \\
18 \\
16 \\
19 \\
16\end{array}$ & $\begin{array}{l}15 \\
15 \\
14 \\
15 \\
13\end{array}$ & $\begin{array}{r}2.2 \\
2.3 \\
3.3 \\
6.2 \\
46\end{array}$ & $\begin{array}{l}43 \\
22 \\
16 \\
14 \\
12\end{array}$ & $\begin{array}{l}4.6 \\
2.8 \\
1.6 \\
1.2 \\
3.1\end{array}$ & $\begin{array}{l}12 \\
11 \\
27 \\
34 \\
23\end{array}$ \\
\hline $\begin{array}{l}16 \\
17 \\
18 \\
19 \\
20\end{array}$ & $\begin{array}{l}13 \\
13 \\
12 \\
12 \\
12\end{array}$ & $\begin{array}{l}12 \\
12 \\
12 \\
12 \\
12\end{array}$ & $\begin{array}{c}14 \\
9.2 \\
e 19 \\
\text { e15 } \\
\text { e14 }\end{array}$ & $\begin{array}{r}19 \\
13 \\
\mathrm{e} \\
7.9 \\
\mathrm{e} 9.0\end{array}$ & $\begin{array}{l}12 \\
12 \\
8.8 \\
9.6 \\
7.9\end{array}$ & $\begin{array}{l}12 \\
12 \\
12 \\
12 \\
10\end{array}$ & $\begin{array}{l}15 \\
14 \\
15 \\
15 \\
16\end{array}$ & $\begin{array}{l}13 \\
12 \\
12 \\
12 \\
10\end{array}$ & $\begin{array}{l}35 \\
30 \\
11 \\
6.1 \\
3.9\end{array}$ & $\begin{array}{c}15 \\
8.6 \\
7.1 \\
8.3 \\
11\end{array}$ & $\begin{array}{l}37 \\
26 \\
7.2 \\
5.0 \\
3.5\end{array}$ & $\begin{array}{l}18 \\
13 \\
13 \\
13 \\
11\end{array}$ \\
\hline $\begin{array}{l}21 \\
22 \\
23 \\
24 \\
25\end{array}$ & $\begin{array}{l}13 \\
13 \\
13 \\
12 \\
13\end{array}$ & $\begin{array}{l}11 \\
11 \\
12 \\
12 \\
12\end{array}$ & $\begin{array}{l}\text { e17 } \\
017 \\
019 \\
016 \\
014\end{array}$ & $\begin{array}{r}810 \\
011 \\
09.0 \\
09.0 \\
010\end{array}$ & $\begin{array}{r}9.3 \\
8.3 \\
8.6 \\
10 \\
9.1\end{array}$ & $\begin{array}{r}9.2 \\
8.6 \\
9.0 \\
9.9 \\
12\end{array}$ & $\begin{array}{l}17 \\
15 \\
13 \\
13 \\
15\end{array}$ & $\begin{array}{r}8.9 \\
9.6 \\
8.2 \\
7.6 \\
13\end{array}$ & $\begin{array}{l}2.8 \\
3.9 \\
6.9 \\
6.4 \\
3.0\end{array}$ & $\begin{array}{l}25 \\
15 \\
6.1 \\
4.7 \\
7.1\end{array}$ & $\begin{array}{l}2.1 \\
4.3 \\
39 \\
50 \\
28\end{array}$ & $\begin{array}{l}9.8 \\
9.1 \\
7.8 \\
7.4 \\
7.4\end{array}$ \\
\hline $\begin{array}{l}26 \\
27 \\
28 \\
29 \\
30 \\
31\end{array}$ & $\begin{array}{l}13 \\
13 \\
12 \\
13 \\
13 \\
13\end{array}$ & $\begin{array}{l}11 \\
11 \\
13 \\
11 \\
14 \\
--\end{array}$ & $\begin{array}{l}\text { e12 } \\
\text { e13 } \\
\text { e16 } \\
\text { e15 } \\
\text { e15 } \\
\text { e13 }\end{array}$ & $\begin{array}{l}011 \\
011 \\
12 \\
012 \\
13 \\
011\end{array}$ & $\begin{array}{l}10 \\
11 \\
6.0 \\
8.2 \\
-\end{array}$ & $\begin{array}{r}7.8 \\
12 \\
9.0 \\
8.4 \\
9.2 \\
8.8\end{array}$ & $\begin{array}{l}17 \\
18 \\
20 \\
22 \\
17 \\
--\end{array}$ & $\begin{array}{r}19 \\
19 \\
15 \\
12 \\
11 \\
8.6\end{array}$ & $\begin{array}{l}3.6 \\
51 \\
19 \\
21 \\
13 \\
\end{array}$ & $\begin{array}{l}30 \\
87 \\
23 \\
17 \\
53 \\
30\end{array}$ & $\begin{array}{r}95 \\
28 \\
77 \\
106 \\
0550 \\
0160\end{array}$ & $\begin{array}{r}7.1 \\
9.2 \\
12 \\
8.3 \\
7.8\end{array}$ \\
\hline $\begin{array}{l}\text { TOTAL } \\
\text { MEAN } \\
\text { MAX } \\
\text { MIR } \\
\text { AC-FT }\end{array}$ & $\begin{array}{r}426 \\
13.7 \\
18 \\
12 \\
845\end{array}$ & $\begin{array}{r}365 \\
12.2 \\
14 \\
10 \\
724\end{array}$ & $\begin{array}{r}502.6 \\
16.2 \\
51 \\
9.2 \\
997\end{array}$ & $\begin{array}{r}345.9 \\
11.2 \\
19 \\
7.9 \\
686\end{array}$ & $\begin{array}{r}325.6 \\
11.2 \\
24 \\
6.0 \\
646\end{array}$ & $\begin{array}{r}357.9 \\
11.5 \\
1.8 \\
7.8 \\
710\end{array}$ & $\begin{array}{r}453.6 \\
15.1 \\
22 \\
8.8 \\
900\end{array}$ & $\begin{array}{r}409.9 \\
13.2 \\
19 \\
7.6 \\
813\end{array}$ & $\begin{array}{r}325.0 \\
10.8 \\
51 \\
2.2 \\
645\end{array}$ & $\begin{array}{r}707.6 \\
22.8 \\
87 \\
4.7 \\
1400\end{array}$ & $\begin{array}{r}1340.8 \\
43.3 \\
550 \\
1.2 \\
2660\end{array}$ & $\begin{array}{r}464.9 \\
15.5 \\
55 \\
7.1 \\
922\end{array}$ \\
\hline
\end{tabular}

STATISTICS OF MONTHLY MEAN DATA FOR WATER YEARS 1916 - 1996, BY WATER YEAR (WY)

\begin{tabular}{|c|c|c|c|c|c|c|c|c|c|c|c|c|}
\hline $\begin{array}{l}\text { MEAN } \\
\text { MAX } \\
\text { (WY) } \\
\text { MIN } \\
\text { (WY) }\end{array}$ & $\begin{array}{l}8.84 \\
51.6 \\
1942 \\
1952 \\
1952\end{array}$ & $\begin{array}{l}6.85 \\
30.5 \\
1942 \\
1040 \\
1952\end{array}$ & $\begin{array}{r}5.65 \\
25.5 \\
1995 \\
.59 \\
1.952\end{array}$ & $\begin{array}{r}5.42 \\
15.5 \\
1921 \\
1.65 \\
1975\end{array}$ & $\begin{array}{l}6.35 \\
16.7 \\
1920 \\
1.20 \\
1952\end{array}$ & $\begin{array}{r}6.73 \\
34.8 \\
1987 \\
1951 \\
1951\end{array}$ & $\begin{array}{r}21.3 \\
370 \\
1942 \\
1.21 \\
1955\end{array}$ & $\begin{array}{r}49.5 \\
372 \\
1941 \\
196 \\
1967\end{array}$ & $\begin{array}{r}35.9 \\
179 \\
1965 \\
.65 \\
1946\end{array}$ & $\begin{array}{r}29.6 \\
138 \\
1919 \\
1.85 \\
1963\end{array}$ & $\begin{array}{r}40.5 \\
147 \\
1955 \\
4.50 \\
1951\end{array}$ & $\begin{array}{r}17.3 \\
78.4 \\
1942 \\
37 \\
1951\end{array}$ \\
\hline
\end{tabular}

SUMMARY STATISTICS

ANNUAL TOTAI

ANNUAL MEAN

HIGHEST ANNUAL MEAN

LOWEST ANNUAL MEAN

HIGHEST DAILY MEAN

LOWEST DAILY MEAN

ANNUAI SEVEN-DAY MINIMUM

INSTANTANEOUS PEAX FLOW

INSTANTANEOUS PEAK STAGE

INSTANTANEOUS LOW FLOW

ANNUAL RUNOFF (AC-FT)

10 PERCENT EXCEEDS

50 PERCENT EXCEEDS

90 PERCENT EXCEEDS

- Estimated

FOR 1995 CALENDAR YEAR

FOR 1996 WATER YEAR

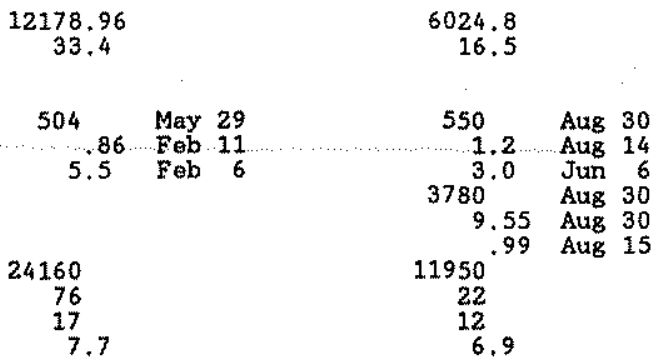

WATER YEARS 1916 - 1996

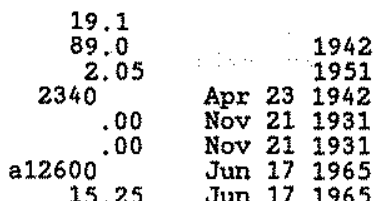

15.25 Jun 171965

13830

45.8

7.8
1.8

a-From rating curve extended above $400 \mathrm{ft} / \mathrm{s}$ on basis of slope-area measurement of peak flow. 
ARKANSAS RTVER BASIN

07203505 VERMEJO DITCH NEAR COLFAX, NM

LOCATION, - Lat $36^{\circ} 34^{\prime} 18^{\prime \prime}$, Long 104 $41^{\prime} 53^{\prime \prime}$, Colfax County, Hydrologic Untt 11080001, in Maxwell Grant, on right bank $2.0 \mathrm{mi}$ southeast of Cólfax, and 4.9 mit downstream from head.

PERIOD OF RECORD.--December 1980 to September 1996 (discontinued).

GAGE.--Water-stage recorder. Elevation of gage is $6,160 \mathrm{ft}$ above National Geodetic Vertical Datum of 1929 , from topographic map. Prior to Mar. 19, 1985 at site 0.8 mil downstream at same datum.

REMARKS.--Records falr except for estimated daily discharges which are poor. Vermejo ditch diverts water from Vermejo Rtver for use on the Vermejo Rroject. Three small diversions from Vermejo ditch upstream from gege.

Several observations of water temperature were made during the year. No flow at times most years. DISCHARGE, CUBIC FEET PER SECOND, WATER YEAR OCTOBER 1995 TO SEPTEMBER 1996

\begin{tabular}{|c|c|c|c|c|c|c|c|c|c|c|c|c|}
\hline DAY & $\infty \mathrm{CT}$ & NOV & DEC & JAN & FEB & MAR & AFR & MAY & JUN & JUL. & AUG & SEP \\
\hline $\begin{array}{l}1 \\
2 \\
3 \\
4 \\
5\end{array}$ & $\begin{array}{l}017 \\
016 \\
016 \\
015 \\
015\end{array}$ & $\begin{array}{l}12 \\
12 \\
13 \\
13 \\
12\end{array}$ & $\begin{array}{r}13 \\
12 \\
12 \\
12 \\
011\end{array}$ & $\begin{array}{r}011 \\
011 \\
09.0 \\
09.0 \\
010\end{array}$ & $\begin{array}{l}08.5 \\
07.5 \\
06.0 \\
07.0 \\
08.0\end{array}$ & $\begin{array}{l}8.3 \\
7.9 \\
8.7 \\
9.0 \\
8.5\end{array}$ & $\begin{array}{r}6.6 \\
6.5 \\
6.8 \\
9.5 \\
11\end{array}$ & $\begin{array}{l}11 \\
10 \\
9.7 \\
9.4 \\
10\end{array}$ & $\begin{array}{l}5.8 \\
5.8 \\
5.7 \\
4.9 \\
4.1\end{array}$ & $\begin{array}{l}15 \\
13 \\
8.2 \\
6.1 \\
5.4\end{array}$ & $\begin{array}{r}021 \\
11 \\
09.5 \\
7.5 \\
05.2\end{array}$ & $\begin{array}{l}051 \\
022 \\
017 \\
017 \\
014\end{array}$ \\
\hline $\begin{array}{r}6 \\
7 \\
8 \\
9 \\
10\end{array}$ & $\begin{array}{l}014 \\
013 \\
013 \\
012 \\
013\end{array}$ & $\begin{array}{l}13 \\
12 \\
12 \\
11 \\
11\end{array}$ & $\begin{array}{r}99.0 \\
08.0 \\
09.0 \\
027 \\
025\end{array}$ & $\begin{array}{l}011 \\
e 10 \\
09.0 \\
\theta 10 \\
09.0\end{array}$ & $\begin{array}{l}\theta 8.5 \\
\theta 9.5 \\
\theta 11 \\
\theta 12 \\
\theta 19\end{array}$ & $\begin{array}{l}8.1 \\
6.7 \\
8.6 \\
8.6 \\
9.4\end{array}$ & $\begin{array}{l}9.5 \\
8.6 \\
8.9 \\
8.6 \\
8.7\end{array}$ & $\begin{array}{l}10 \\
10 \\
10 \\
9.8 \\
9.6\end{array}$ & $\begin{array}{r}03.0 \\
02.5 \\
02.0 \\
3.2 \\
3.9\end{array}$ & $\begin{array}{l}6.8 \\
05.5 \\
22 \\
53 \\
66\end{array}$ & $\begin{array}{r}03.0 \\
07.0 \\
025 \\
06.5 \\
06.0\end{array}$ & $\begin{array}{l}014 \\
013 \\
013 \\
012 \\
012\end{array}$ \\
\hline $\begin{array}{l}11 \\
12 \\
13 \\
14 \\
15\end{array}$ & $\begin{array}{r}013 \\
013 \\
13 \\
12 \\
13\end{array}$ & $\begin{array}{l}13 \\
12 \\
14 \\
13 \\
13\end{array}$ & $\begin{array}{r}048 \\
019 \\
e 9.0 \\
e 8,5 \\
\text { e11. }\end{array}$ & $\begin{array}{l}011 \\
e 10 \\
e 8.5 \\
e 12 \\
014\end{array}$ & $\begin{array}{l}021 \\
016 \\
016 \\
015 \\
012\end{array}$ & $\begin{array}{l}8.9 \\
7.6 \\
7.6 \\
7.7 \\
7.9\end{array}$ & $\begin{array}{l}10 \\
11 \\
10 \\
11 \\
11\end{array}$ & $\begin{array}{r}9.7 \\
10 \\
9.8 \\
9.2 \\
8.8\end{array}$ & $\begin{array}{r}\text { ब2.0 } \\
02.0 \\
3.0 \\
3.6 \\
24\end{array}$ & $\begin{array}{r}e 40 \\
20 \\
e 15 \\
013 \\
011\end{array}$ & $\begin{array}{c}04.3 \\
02.5 \\
0.00 \\
0.00 \\
02.1\end{array}$ & $\begin{array}{l}\otimes 11 \\
\oplus 10 \\
022 \\
027 \\
019\end{array}$ \\
\hline $\begin{array}{l}16 \\
17 \\
18 \\
19 \\
20\end{array}$ & $\begin{array}{l}13 \\
12 \\
12 \\
11 \\
12\end{array}$ & $\begin{array}{l}13 \\
12 \\
12 \\
13 \\
13\end{array}$ & $\begin{array}{l}013 \\
e 8.5 \\
e 18 \\
e 14 \\
e 13\end{array}$ & $\begin{array}{l}e 18 \\
e 12 \\
e 11 \\
07.5 \\
08.0\end{array}$ & $\begin{array}{l}011 \\
011 \\
08.0 \\
09.5 \\
07.5\end{array}$ & $\begin{array}{l}8.1 \\
8.2 \\
8.0 \\
6.8 \\
6.8\end{array}$ & $\begin{array}{l}9.5 \\
9.2 \\
8.6 \\
8.6 \\
9.4\end{array}$ & $\begin{array}{l}8.2 \\
8.0 \\
7.5 \\
7.5 \\
7.3\end{array}$ & $\begin{array}{l}18 \\
24 \\
10 \\
e 6.0 \\
e 3.7\end{array}$ & $\begin{array}{r}814 \\
e 8.0 \\
07.0 \\
98.0 \\
010\end{array}$ & $\begin{array}{l}\oplus 35 \\
e 22 \\
06.8 \\
04.7 \\
03.0\end{array}$ & $\begin{array}{l}016 \\
012 \\
012 \\
012 \\
010\end{array}$ \\
\hline $\begin{array}{l}21 \\
22 \\
23 \\
24 \\
25\end{array}$ & $\begin{array}{l}12 \\
12 \\
12 \\
11 \\
11\end{array}$ & $\begin{array}{l}12 \\
12 \\
13 \\
13 \\
13\end{array}$ & $\begin{array}{l}016 \\
016 \\
017 \\
014 \\
013\end{array}$ & $\begin{array}{r}09.5 \\
011 \\
08.5 \\
08.5 \\
09.0\end{array}$ & $\begin{array}{r}99.0 \\
8.1 \\
8.0 \\
7.3 \\
7.4\end{array}$ & $\begin{array}{l}6.4 \\
6.3 \\
6.0 \\
6.5 \\
7.2\end{array}$ & $\begin{array}{r}10 \\
10 \\
8.9 \\
8.4 \\
8.8\end{array}$ & $\begin{array}{l}7.0 \\
6.6 \\
6.7 \\
6.0 \\
8.1\end{array}$ & $\begin{array}{r}62.5 \\
3.7 \\
4.2 \\
5.0 \\
02.8\end{array}$ & $\begin{array}{r}e 24 \\
e 14 \\
e 5.6 \\
04.0 \\
4.4\end{array}$ & $\begin{array}{l}01.5 \\
03.7 \\
032 \\
045 \\
025\end{array}$ & $\begin{array}{l}09.5 \\
08.2 \\
07.0 \\
06.5 \\
06.5\end{array}$ \\
\hline $\begin{array}{l}26 \\
27 \\
28 \\
29 \\
30 \\
31\end{array}$ & $\begin{array}{l}12 \\
12 \\
11 \\
12 \\
12 \\
11\end{array}$ & $\begin{array}{c}12 \\
12 \\
12 \\
9.4 \\
13 \\
-\ldots\end{array}$ & $\begin{array}{l}011 \\
e 13 \\
015 \\
e 14 \\
014 \\
012\end{array}$ & $\begin{array}{l}010 \\
010 \\
011 \\
011 \\
012 \\
010\end{array}$ & $\begin{array}{r}7.6 \\
08.0 \\
05.5 \\
08.0 \\
-\ldots \\
-.-\end{array}$ & $\begin{array}{l}6.2 \\
6.2 \\
7.0 \\
6.6 \\
6.6 \\
6.8\end{array}$ & $\begin{array}{l}9.4 \\
9.8 \\
11 \\
12 \\
11 \\
---\end{array}$ & $\begin{array}{r}10 \\
11 \\
9.3 \\
7.9 \\
7.3 \\
6.7\end{array}$ & $\begin{array}{l}3.5 \\
35 \\
17 \\
15 \\
11 \\
-\infty\end{array}$ & $\begin{array}{r}16 \\
67 \\
019 \\
14 \\
42 \\
24\end{array}$ & $\begin{array}{l}090 \\
025 \\
037 \\
046 \\
127 \\
100\end{array}$ & $\begin{array}{r}06.6 \\
08.7 \\
9.3 \\
e 5.5 \\
e 4.7 \\
\end{array}$ \\
\hline $\begin{array}{l}\text { TOTAL } \\
\text { MEAN } \\
\text { MAX } \\
\text { MIN } \\
\text { AC-FT }\end{array}$ & $\begin{array}{r}396 \\
12.8 \\
17 \\
11 \\
785\end{array}$ & $\begin{array}{r}370.4 \\
12.3 \\
14 \\
9.4 \\
735\end{array}$ & $\begin{array}{r}455.0 \\
14.7 \\
48 \\
8.0 \\
902\end{array}$ & $\begin{array}{r}321.5 \\
10.4 \\
18 \\
7.5 \\
638\end{array}$ & $\begin{array}{r}292.9 \\
10.1 \\
21 \\
5.5 \\
581\end{array}$ & $\begin{array}{r}233.2 \\
7.52 \\
9.4 \\
6.0 \\
463\end{array}$ & $\begin{array}{r}282.3 \\
9.41 \\
12 \\
6.5 \\
560\end{array}$ & $\begin{array}{r}272.1 \\
8.78 \\
11 \\
6.0 \\
540\end{array}$ & $\begin{array}{r}236.9 \\
7.90 \\
35 \\
2.0 \\
470\end{array}$ & $\begin{array}{r}581.0 \\
18.7 \\
67 \\
4.0 \\
1150\end{array}$ & $\begin{array}{r}714.30 \\
23.0 \\
127 \\
1.00 \\
1420\end{array}$ & $\begin{array}{r}408.5 \\
13.6 \\
51 \\
4.7 \\
810\end{array}$ \\
\hline
\end{tabular}

STATISTICS OF MONTHLY MEAN DATA FOR WATER YEARS 1981 - 1996, BY WATER YEAR (WY)

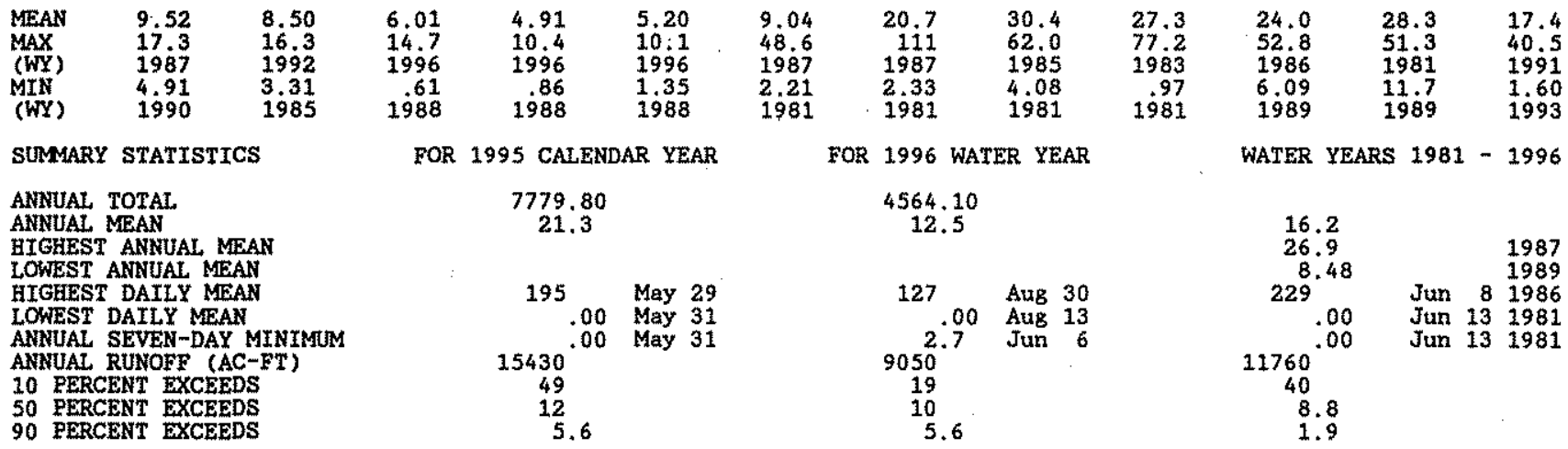

- Estimated 
ARKANSAS RIVER BASIN

07204000 MORENO CREEX AT EAGLE NEST, NM

LOCATION,--Lat $36^{\circ} 33^{\prime} 14^{\prime \prime}$, long 105"16.03", Colfax County, Hydrologic Unit 11080002, in Maxwell Grant, on right bank $175 \mathrm{ft}$ upstream from U.S. Highway 64 , 250 ft northwest of intersection of U.S. Highway 64 and State Bighway 38 , about 1,000 ft upstream from high-water line of Eagle Nest Lake at Eagle Nest.

DRAINAGE AREA. $--73.8 \mathrm{mi}^{2}$.

PERIOD OF RECORD.--April 1928 to October 1955 and June 1964 to current year (seasonal records except water year 1932). Monthiy discharge only for some perlods, published in WSP 1311. Records for December 1930 to March 1931 , published in WSP 732, are unreliable and should not bo used. Fublished as "near Therma" 1928 -34.

REVISED RECORDS.--WSP 1281: 1931(M), 1932, 1935(M), 1939-41(M), 1946-47(M). WSP 1921: Drainage area. See also PERIOD OF RECORD.

GAGE.--Water-stage recorder. Concrete control since Oct. 3, 1952. Datum of gage is 8, 197.39 ft above Nationa1 Geodetic Vertical Datum of 1929. See WSP 1921 for history of changes prior to oct, 26, 1955. 0ct. 26, 1955 to Nov. 12, 1974, water-stage recorder at site $160 \mathrm{ft}$ downstream at datum $1.41 \mathrm{ft}$ lower.

REMARKS.--Records good. Diversions for irrigation of about 1,200 acres upstream from station. Several observations of pater temperature were made during the year.

EXTREMES FOR PERTOD OF RECORD. --Maximum discharge, $240 \mathrm{ft} / \mathrm{s}$, Sept. 1, 1946, gage height, $3.10 \mathrm{ft}$, stte and datum then in use; maximum gage height, $3.55 \mathrm{ft}$, May 12,1973 ; no flow at times.

EXTREMES FOR CURRENT YEAR.--Maximum discharge during period of seasonal operation, 6.8 fts/s, at 1045 hours, Apr. 3 , gage helght, $1.78 \mathrm{ft} ;$ minimum daily discharge, $.06 \mathrm{fts} / \mathrm{s}$, Aug. 15.

DISCHARGE, CUBIC FEET PER SECOND, WATER YEAR OCTOBER 1995 TO SEFTEMBER 1996 DATIY MEAN VALUES

\begin{tabular}{|c|c|c|c|c|c|c|c|c|c|c|c|c|}
\hline DAY & OCT & nov & $\mathrm{DEC}$ & JAN & FEB & MAR & APR & MAY & JUN & JUL & AUG & SEP \\
\hline $\begin{array}{l}1 \\
2 \\
3 \\
4 \\
5\end{array}$ & $\begin{array}{l}4.5 \\
4.1 \\
3.7 \\
3.3 \\
3.2\end{array}$ & $\begin{array}{l}=- \\
-\cdots \\
-\cdots \\
--\end{array}$ & $\begin{array}{l}m- \\
\because- \\
\square-\end{array}$ & $\begin{array}{l}--- \\
\overline{--} \\
\overline{-1}\end{array}$ & $\begin{array}{l}--- \\
\overline{-} \\
\overline{--} \\
\overline{-}\end{array}$ & $\begin{array}{l}--- \\
m- \\
m- \\
--\end{array}$ & $\begin{array}{l}5.2 \\
6.0 \\
6.1 \\
5.7 \\
5.2\end{array}$ & $\begin{array}{l}3.0 \\
2.8 \\
2.7 \\
2.6 \\
2.4\end{array}$ & $\begin{array}{l}.44 \\
.43 \\
.42 \\
.37 \\
.35\end{array}$ & $\begin{array}{l}.41 \\
.29 \\
.20 \\
.17 \\
.17\end{array}$ & $\begin{array}{l}.17 \\
.22 \\
.21 \\
.23 \\
.13\end{array}$ & $\begin{array}{l}.29 \\
.37 \\
.41 \\
.33 \\
.30\end{array}$ \\
\hline $\begin{array}{r}6 \\
7 \\
8 \\
9 \\
10\end{array}$ & $\begin{array}{l}3.4 \\
-- \\
--\end{array}$ & 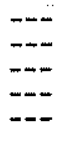 & $\begin{array}{l}--- \\
\overline{-} \\
-\overline{-} \\
-\cdots\end{array}$ & $\begin{array}{l}m- \\
-\overline{-} \\
--\end{array}$ & $\begin{array}{l}m- \\
=- \\
m- \\
---\end{array}$ & $\begin{array}{l}--1 \\
=-- \\
-- \\
--\end{array}$ & $\begin{array}{l}4.6 \\
4.5 \\
4.2 \\
3.5 \\
3.6\end{array}$ & $\begin{array}{l}2.3 \\
1.9 \\
1.8 \\
1.7 \\
1.6\end{array}$ & $\begin{array}{l}.34 \\
.34 \\
.34 \\
.33 \\
.32\end{array}$ & $\begin{array}{l}.17 \\
.28 \\
.27 \\
.41 \\
.72\end{array}$ & $\begin{array}{l}.08 \\
.09 \\
.10 \\
.11 \\
.10\end{array}$ & $\begin{array}{l}.27 \\
.29 \\
.28 \\
.26 \\
.26\end{array}$ \\
\hline $\begin{array}{l}11 \\
12 \\
13 \\
14 \\
15\end{array}$ & $=$ & 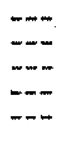 & $\begin{array}{l}--- \\
-- \\
-\cdots \\
--\end{array}$ & $\begin{array}{l}--- \\
--- \\
--- \\
--\end{array}$ & $\begin{array}{l}--- \\
-\cdots \\
-\cdots \\
-\cdots\end{array}$ & $\begin{array}{l}--- \\
--- \\
--- \\
--\end{array}$ & $\begin{array}{l}3.6 \\
3.4 \\
3.6 \\
3.3 \\
3.5\end{array}$ & $\begin{array}{l}1.5 \\
1.4 \\
1.3 \\
1.3 \\
1.1\end{array}$ & $\begin{array}{r}.32 \\
.39 \\
.40 \\
.63 \\
1.1\end{array}$ & $\begin{array}{l}.62 \\
.42 \\
.43 \\
.36 \\
.26\end{array}$ & $\begin{array}{l}.07 \\
.07 \\
.07 \\
.07 \\
.06\end{array}$ & $\begin{array}{l}.27 \\
.34 \\
.42 \\
.48 \\
.52\end{array}$ \\
\hline $\begin{array}{l}16 \\
17 \\
18 \\
19 \\
20\end{array}$ & $=-$ & $\begin{array}{l}--- \\
-\cdots \\
-- \\
--\end{array}$ & $\begin{array}{l}-- \\
-\cdots \\
-- \\
--\end{array}$ & $\begin{array}{l}m- \\
-- \\
--\end{array}$ & $\begin{array}{l}--- \\
--- \\
--- \\
--\end{array}$ & $\begin{array}{l}m-m \\
m-m \\
-m- \\
--m\end{array}$ & $\begin{array}{l}3.9 \\
3.5 \\
1.7 \\
1.6 \\
1.6\end{array}$ & $\begin{array}{l}1.1 \\
1.0 \\
1.0 \\
.91 \\
.84\end{array}$ & $\begin{array}{l}.75 \\
.60 \\
.47 \\
.38 \\
.34\end{array}$ & $\begin{array}{l}.23 \\
.23 \\
.23 \\
.20 \\
.18\end{array}$ & $\begin{array}{l}.10 \\
.10 \\
.09 \\
.10 \\
.12\end{array}$ & $\begin{array}{l}.43 \\
.41 \\
.48 \\
.47 \\
.47\end{array}$ \\
\hline $\begin{array}{l}21 \\
22 \\
23 \\
24 \\
25\end{array}$ & 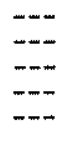 & $\begin{array}{l}--- \\
=- \\
--- \\
---\end{array}$ & 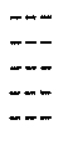 & $\begin{array}{l}-+- \\
-\cdots \\
-\cdots \\
-\infty\end{array}$ & $\begin{array}{l}m- \\
\cdots- \\
\cdots-\end{array}$ & $\overline{-}$ & $\begin{array}{l}1.6 \\
1.6 \\
1.6 \\
1.6 \\
1.6\end{array}$ & $\begin{array}{l}.86 \\
.82 \\
.76 \\
.79 \\
.83\end{array}$ & $\begin{array}{l}.34 \\
.42 \\
.36 \\
.27 \\
.27\end{array}$ & $\begin{array}{l}.15 \\
.18 \\
.17 \\
.15 \\
.15\end{array}$ & $\begin{array}{l}.13 \\
.24 \\
.31 \\
.30 \\
.31\end{array}$ & $\begin{array}{l}.43 \\
.36 \\
.36 \\
.53 \\
.64\end{array}$ \\
\hline $\begin{array}{l}26 \\
27 \\
28 \\
29 \\
30 \\
31\end{array}$ & 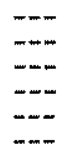 & 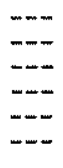 & $\begin{array}{c}\cdots-- \\
\cdots- \\
\cdots- \\
\cdots \\
\cdots-\end{array}$ & $\begin{array}{l}\cdots \\
\cdots \\
\cdots \\
\cdots\end{array}$ & $\begin{array}{c}\cdots- \\
\cdots \\
\cdots- \\
\cdots- \\
-- \\
--\end{array}$ & $\begin{array}{l}4.6 \\
4.6 \\
4.7 \\
4.6 \\
4.7\end{array}$ & $\begin{array}{l}2.5 \\
1.4 \\
1.3 \\
1.4 \\
2.0 \\
-\end{array}$ & $\begin{array}{l}.90 \\
.86 \\
.55 \\
.51 \\
.49 \\
.46\end{array}$ & $\begin{array}{r}.27 \\
.81 \\
.52 \\
.38 \\
.53 \\
\end{array}$ & $\begin{array}{r}.15 \\
.13 \\
.14 \\
.93 \\
.30 \\
.22\end{array}$ & $\begin{array}{l}.36 \\
.29 \\
.32 \\
.62 \\
.61 \\
.34\end{array}$ & $\begin{array}{r}.73 \\
.84 \\
.77 \\
1.0 \\
.95 \\
\end{array}$ \\
\hline $\begin{array}{l}\text { TOTAL } \\
\text { MIEAN } \\
\text { MAX } \\
\text { MTME } \\
\text { AC-FT }\end{array}$ & 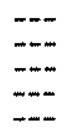 & $=-$ & $\begin{array}{l}-\cdots \\
-\cdots \\
--- \\
--\end{array}$ & $\begin{array}{l}--- \\
\cdots- \\
\cdots- \\
\cdots-\end{array}$ & $\begin{array}{l}-\cdots \\
-- \\
\cdots- \\
-\cdots\end{array}$ & $\begin{array}{l}-\overline{-} \\
-\overline{-} \\
-\overline{-} \\
-\end{array}$ & $\begin{array}{r}93.9 \\
3.13 \\
6.1 \\
1.3 \\
186\end{array}$ & $\begin{array}{r}42.08 \\
1.36 \\
3.0 \\
.46 \\
83\end{array}$ & $\begin{array}{r}13.23 \\
i .44 \\
1.27 \\
.26\end{array}$ & $\begin{array}{r}8.92 \\
.29 \\
.93 \\
.13 \\
18\end{array}$ & $\begin{array}{r}6.12 \\
.20 \\
.62 \\
.06 \\
12\end{array}$ & $\begin{array}{r}13.96 \\
4.47 \\
1.0 \\
.26 \\
28\end{array}$ \\
\hline
\end{tabular}


ARKANSAS RIVER BASIN

07204500 CIENEGUILLA CREEK NEAR EAGLE NEST, NM

OCATION, --Lat $36^{\circ} 29^{\prime} 07^{\prime \prime}$, long $105^{\circ} 15^{\prime} 54^{\prime \prime}$, Colfax County, Bydrologic Unit 11080002 , In Maxwell Grant, on right bank $0.1 \mathrm{mi}$ downstream from Schoolhouse Draw, $0.4 \mathrm{mi}$ upstream fzom high-water line of Eagle Nest Lake, $0.5 \mathrm{mi}$ east of U.S. Bighway 64 , and $4.7 \mathrm{mi}$ south of Eagle Nest.

DRAINAGE AREA. $--56 \mathrm{mi}^{2}$.

PERIOD OF RECORD.-April 1928 to September 1955 and June 1964 to current year (seasonal records except in water years 1932, 1948 and 1951). Monthly discharge only for some pertods, published in WSP 1311 and 1731 . Racords for Dacember 1930 to March 1931, published in WSP 732, are unreliable and should not be used. Published as "near Therna" 1928-34.

REVISED RECORDS. - WSP 957: 1941. WSP 1281: Drainage area. WSP 1311: 1932(M), 1935(M), 1937(M). See also PERTOD OF . RECORD.

GAGE.-Water-stage recorder. Concrete control since Sept, 25, 1947. Elovation of gage is 8,200 ft above National Goodetic Vextical Datum of 1929, from topographic map. Prior to May 8, 1928, nonrecording gage, and May 8 , 1928 to Sept. 1. 1934, water-stage recorder at stte 0.2 mil downstream at different datums.

REMARKS. --Records good except for estimated dally discharges; which are poor. Diversions for ixrigation of about 1,000 acres upstream from station. Several observations of water temperature were made during the year.

EXTREMES FOR PERIOD OF RECORD.--Maximum discharge, $505 \mathrm{ft}^{3} / \mathrm{s}$, June 16, 1965, gage height, 5.63 ft, March 19, 1994, from rating curve extended above $110 \mathrm{ft} / \mathrm{s}$, no flow at times.

EXTREMES FOR CURRENT YEAR. --Maximum discharge duxing period of seasonal operation, $21 \mathrm{ft} / \mathrm{s}$, at $0300 \mathrm{hours}$, Apr. 3 , gage height, $3: 26 \mathrm{ft}$; minimum datly discharge $0.61 \mathrm{ft} / \mathrm{s}$, Aug. 13 . DISCHARGE, CUBIC FEET PER SECOND, WATER YEAR OCTOBER 1995 TO SEPTEMBER 1996

\begin{tabular}{|c|c|c|c|c|c|c|c|c|c|c|c|c|}
\hline DAY & $\propto \mathrm{CT}$ & nov & $\mathrm{DEC}$ & JAN & FEB & MAR & AFR & MAY & JUN & JUL & AUGG & SEP \\
\hline $\begin{array}{l}1 \\
2 \\
3 \\
4 \\
5\end{array}$ & $\begin{array}{l}6.7 \\
6.2 \\
5.8 \\
5.4 \\
5.3\end{array}$ & $\begin{array}{l}m-- \\
=- \\
=- \\
m-\end{array}$ & $\begin{array}{l}-m- \\
--\overline{-} \\
-\overline{-} \\
-\cdots\end{array}$ & $\begin{array}{l}m-\cdots \\
=-\infty \\
=-- \\
-\cdots\end{array}$ & $\begin{array}{l}--- \\
--- \\
---\end{array}$ & $\begin{array}{l}--- \\
--- \\
-\cdots \\
-\cdots\end{array}$ & $\begin{array}{l}17 \\
19 \\
19 \\
14 \\
10\end{array}$ & $\begin{array}{l}7.0 \\
6.6 \\
6.4 \\
6.0 \\
5.2\end{array}$ & $\begin{array}{l}01.7 \\
e 1.6 \\
01.6 \\
01.5 \\
01.3\end{array}$ & $\begin{array}{l}3.0 \\
2.0 \\
1.5 \\
1.3 \\
1.2\end{array}$ & $\begin{array}{l}2.4 \\
1.4 \\
1.1 \\
1.1 \\
.85\end{array}$ & $\begin{array}{l}1.9 \\
2.0 \\
2.4 \\
1.8 \\
1.8\end{array}$ \\
\hline $\begin{array}{r}6 \\
7 \\
8 \\
9 \\
10\end{array}$ & $\begin{array}{c}5.4 \\
-\cdots \\
--- \\
---\end{array}$ & $\begin{array}{l}=- \\
\overline{-m} \\
\overline{-m} \\
\overline{-m}\end{array}$ & $\begin{array}{l}m- \\
m-m \\
m-m\end{array}$ & $\begin{array}{l}m- \\
m-\overline{-} \\
m-\end{array}$ & $\begin{array}{l}--\infty \\
=-- \\
-\cdots \\
-\cdots\end{array}$ & $\begin{array}{l}=-- \\
=-- \\
-\overline{-}\end{array}$ & $\begin{array}{r}8.2 \\
8.4 \\
8.5 \\
9.4 \\
10\end{array}$ & $\begin{array}{r}4.7 \\
4.7 \\
4.5 \\
04.4 \\
04.2\end{array}$ & $\begin{array}{l}\text { e1.3 } \\
\text { e1.3 } \\
\text { e1.3 } \\
\text { e1.3 } \\
\mathrm{e} 1.3\end{array}$ & $\begin{array}{l}1.3 \\
1.9 \\
1.7 \\
2.2 \\
2.7\end{array}$ & $\begin{array}{r}.72 \\
.68 \\
.88 \\
1.0 \\
1.1\end{array}$ & $\begin{array}{l}2.2 \\
1.8 \\
1.6 \\
1.4 \\
1.3\end{array}$ \\
\hline $\begin{array}{l}11 \\
12 \\
13 \\
14 \\
15\end{array}$ & $\begin{array}{l}m-- \\
-\cdots \\
-\cdots \\
-\cdots\end{array}$ & $\begin{array}{l}m-- \\
=- \\
m- \\
m-\end{array}$ & $\begin{array}{l}m- \\
m- \\
m-\end{array}$ & $\begin{array}{l}=-- \\
=-- \\
-m \\
-m\end{array}$ & $\begin{array}{l}-\cdots \\
-\overline{-} \\
-\cdots\end{array}$ & $\begin{array}{l}m- \\
--- \\
-\cdots \\
-\cdots\end{array}$ & $\begin{array}{r}9.3 \\
8.7 \\
9.2 \\
8.6 \\
10\end{array}$ & $\begin{array}{l}e 3.9 \\
93.7 \\
e 3.5 \\
e 3.2 \\
e 3.2\end{array}$ & $\begin{array}{r}01.5 \\
\mathrm{e} 1.9 \\
\mathrm{e} 1.9 \\
3.4 \\
5.6\end{array}$ & $\begin{array}{l}2.7 \\
2.2 \\
4.4 \\
6.0 \\
3.4\end{array}$ & $\begin{array}{l}.87 \\
.62 \\
.61 \\
.64 \\
.65\end{array}$ & $\begin{array}{l}1.5 \\
1.7 \\
2.3 \\
2.5 \\
4.7\end{array}$ \\
\hline $\begin{array}{l}16 \\
17 \\
18 \\
19 \\
20\end{array}$ & $\begin{array}{l}m- \\
-\cdots \\
-\cdots \\
-\infty\end{array}$ & $\begin{array}{l}-\cdots \\
-\cdots \\
-\cdots \\
-m\end{array}$ & $\begin{array}{l}m- \\
m-m \\
m-m\end{array}$ & $\begin{array}{l}m-\infty \\
-m- \\
-m \\
---\end{array}$ & $\begin{array}{l}-\sim \\
-- \\
-\cdots \\
--\end{array}$ & $\begin{array}{l}-\cdots \\
-\cdots \\
-\cdots \\
--\end{array}$ & $\begin{array}{r}11 \\
12 \\
10 \\
9.5 \\
9.0\end{array}$ & $\begin{array}{l}e 3.0 \\
e 2.8 \\
e 2.7 \\
e 2.6 \\
\theta 2.4\end{array}$ & $\begin{array}{l}2.8 \\
2.0 \\
1.6 \\
1.3 \\
.98\end{array}$ & $\begin{array}{l}3.0 \\
2.2 \\
1.8 \\
1.5 \\
1.3\end{array}$ & $\begin{array}{l}.85 \\
2.6 \\
1.5 \\
1.2 \\
2.0\end{array}$ & $\begin{array}{l}2.7 \\
2.6 \\
4.4 \\
4.2 \\
3.0\end{array}$ \\
\hline $\begin{array}{l}21 \\
22 \\
23 \\
24 \\
25\end{array}$ & $\begin{array}{l}m- \\
=- \\
-\cdots \\
-\cdots\end{array}$ & $\ddot{-\cdots}$ & 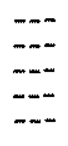 & $\begin{array}{l}m-\infty \\
-\cdots \\
-\cdots \\
-\cdots \\
m-\infty\end{array}$ & $\begin{array}{l}m-- \\
-\cdots \\
-\cdots \\
-\cdots\end{array}$ & $\begin{array}{l}-\cdots \\
--- \\
--- \\
-\cdots\end{array}$ & $\begin{array}{l}8.5 \\
8.1 \\
8.0 \\
8.5 \\
8.4\end{array}$ & $\begin{array}{l}e 2.3 \\
e 2.2 \\
e 2.2 \\
92.3 \\
02.4\end{array}$ & $\begin{array}{l}1.1 \\
1.5 \\
1.8 \\
1.2 \\
.97\end{array}$ & $\begin{array}{l}1.1 \\
1.1 \\
1.1 \\
1.0 \\
2.0\end{array}$ & $\begin{array}{l}3.3 \\
3.0 \\
4.5 \\
3.8 \\
3.3\end{array}$ & $\begin{array}{l}2.5 \\
2.4 \\
2.3 \\
2.2 \\
2.5\end{array}$ \\
\hline $\begin{array}{l}26 \\
27 \\
28 \\
29 \\
30 \\
31\end{array}$ & $\begin{array}{l}-\infty \\
-\infty \\
-\infty \\
-\infty \\
-\infty\end{array}$ & $\begin{array}{l}-\cdots \\
--- \\
-\cdots \\
-\cdots \\
-\cdots\end{array}$ & $\begin{array}{l}m- \\
m- \\
m- \\
m- \\
m\end{array}$ & 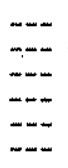 & 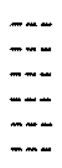 & $\begin{array}{l}-\cdots \\
99.0 \\
11 \\
12 \\
15\end{array}$ & $\begin{array}{r}8.4 \\
8.3 \\
8.2 \\
7.5 \\
7.4 \\
-7 .\end{array}$ & $\begin{array}{l}e 2.5 \\
e 2.4 \\
e 2.3 \\
e 2.1 \\
01.8 \\
01.7\end{array}$ & $\begin{array}{l}1.5 \\
7.1 \\
4.3 \\
2.4 \\
2.8\end{array}$ & $\begin{array}{l}2.0 \\
1.3 \\
2.0 \\
1.7 \\
1.9 \\
5.0\end{array}$ & $\begin{array}{l}4.0 \\
2.7 \\
2.5 \\
2.6 \\
2.5 \\
2.0\end{array}$ & $\begin{array}{l}2.7 \\
2.9 \\
2.5 \\
2.4 \\
2.3 \\
-\end{array}$ \\
\hline $\begin{array}{l}\text { TOTAL } \\
\text { MEAN } \\
\text { MAX } \\
\text { MIN } \\
\text { AC-FT }\end{array}$ & $\begin{array}{l}-- \\
-- \\
-- \\
-m\end{array}$ & $\begin{array}{l}--\infty \\
--- \\
--- \\
--\end{array}$ & $m+m$ & $\begin{array}{l}m-\infty \\
-\infty- \\
-\infty\end{array}$ & $\begin{array}{l}-- \\
-- \\
-\cdots \\
-\cdots\end{array}$ & $\begin{array}{l}--- \\
--- \\
-\cdots \\
-\cdots\end{array}$ & $\begin{array}{r}302.1 \\
10.1 \\
19 \\
7.4 \\
599\end{array}$ & $\begin{array}{r}108.9 \\
3.51 \\
7.0 \\
1.7 \\
216\end{array}$ & $\begin{array}{r}61.85 \\
2.06 \\
7.1 \\
.97 \\
i .23\end{array}$ & $\begin{array}{r}67.5 \\
2.18 \\
6.0 \\
1.0 \\
134\end{array}$ & $\begin{array}{r}56.97 \\
1.84 \\
4.5 \\
.61 \\
113\end{array}$ & $\begin{array}{r}72.5 \\
2.42 \\
4.7 \\
1.3 \\
144\end{array}$ \\
\hline
\end{tabular}

- Estimatad 
LOCATYON, --Lat $36^{\circ} 31^{\prime} 07^{\prime \prime}$, long $15^{\circ} 16^{\circ} 29^{\prime \prime}$, Colfax County, Bydrologic unit 11080002, in Maxwell Grant, on left upstream wingwall of concrete control, $250 \mathrm{ft}$ downstream from concrete box culvert on U,S. Highway 64 , and $2.6 \mathrm{mi}$ southwest of Eagle Nest.

DRAINAGE AREA. $--10.5 \mathrm{mi}^{2}$.

PERIOD OF RECORD.--April 1928 to September 1955 (seasonal records in water years 1929-31, 1933-55) July 1958 to current year (seasonal records subsequent to water year 1975). Prior to October 1930 monthly discharge only, published in WSP 1311. Records for December 1930 to March 1931, published in WSP 732 , are unreliable and should not be used. Published as "near Therma" 1928-34.

REVISED RECORDS.--WSP 1311: 1932-33(M), 1935(M), 1943(M). WSP 1681: 1937(M). WSP 1921: Drainage area. See also PERIOD OF RECORD.

GAGE.-Water-stage recorder. Concrete contro1 Sept, 11, 1931 to May 1933, and since Sept. 13, 1934. Datum of gage is $8,195.16 \mathrm{ft}$ above National Geodetic Vertical Datum of 1929. Prior to May 18, 1928, nonrecording gage at site 88 ft upstream at datum $0.98 \mathrm{ft}$ higher. May 18, 1928 to Sept. 11, 1938, water-stage recorder at site 88 ft pstream at datum $0.43 \mathrm{ft}$ hlgher.

REMARKS,--Records good. Diversions for irrigation of about 300 acres upstream from station. Several observations of water temperature were made during the year.

AVERAGE DISCEARGE -18 years (water years $1932,1959-75$ ), $2.51 \mathrm{ft} / \mathrm{s}, 1,820 \mathrm{acre}-\mathrm{ft} / \mathrm{yr}$

EXTREMES FOR PERIOD OF RECORD (1930-55 and SINCE 1957),--Maxtmum discharge, $128 \mathrm{ft} 3 / \mathrm{s}$, Aug. 5, 1969 , gage height, $2.86 \mathrm{ft}$ from rating curve extended above 32 . fts/s; maximum gage height recorded, 3.38 . ft, Apr. 2, 1937 (1ce Jam), site and datum then in use; no flow at times.

EXIREAES FOR CURRENT YEAR. --Maximum discherge during period of seasonal operation, $8.6 \mathrm{ft}^{3} / \mathrm{s}$, at $1000 \mathrm{hours,} \mathrm{Apr.}$ 29, gage height $1.68 \mathrm{ft}$; no flow many days.

DISCHARGE, CUBIC FEET PER SECOND, WATER YEAR OCTOBER 1995 TO SEPTEMBER 1996

\begin{tabular}{|c|c|c|c|c|c|c|c|c|c|c|c|c|}
\hline DAY & OCT & Nov & $\mathrm{DEC}$ & JAN & FEB & MAR & APR & MAY & JUN & JUL & AUG & $\operatorname{SEP}$ \\
\hline $\begin{array}{l}1 \\
2 \\
3 \\
4 \\
5\end{array}$ & $\begin{array}{l}3.3 \\
3.2 \\
3.1 \\
2.9 \\
2.9\end{array}$ & $\begin{array}{l}\because- \\
=- \\
-\cdots \\
-\cdots\end{array}$ & $\begin{array}{l}m- \\
m- \\
m- \\
-\end{array}$ & $\begin{array}{l}-\cdots \\
\cdots-- \\
-\cdots \\
-\cdots\end{array}$ & $\begin{array}{l}-- \\
-- \\
--\end{array}$ & $\begin{array}{l}--- \\
-\cdots \\
--- \\
---\end{array}$ & $\begin{array}{l}3.7 \\
4.0 \\
4.1 \\
3.8 \\
3.5\end{array}$ & $\begin{array}{l}5.7 \\
5.0 \\
5.0 \\
4.9 \\
4.8\end{array}$ & $\begin{array}{l}1.7 \\
1.7 \\
.96 \\
.09 \\
.06\end{array}$ & $\begin{array}{l}.80 \\
.31 \\
.14 \\
.09 \\
.09\end{array}$ & $\begin{array}{l}.07 \\
.11 \\
.06 \\
.02 \\
.02\end{array}$ & $\begin{array}{l}1.3 \\
1.2 \\
1.2 \\
1.2 \\
1.1\end{array}$ \\
\hline $\begin{array}{r}6 \\
7 \\
8 \\
9 \\
10\end{array}$ & \begin{tabular}{c}
3.0 \\
\hdashline-- \\
\hdashline- \\
--
\end{tabular} & $\begin{array}{l}--- \\
=- \\
--- \\
---\end{array}$ & $\begin{array}{l}--- \\
\overline{-m} \\
\overline{-m}\end{array}$ & $\begin{array}{l}--- \\
--- \\
--- \\
-\cdots\end{array}$ & $\begin{array}{l}m- \\
-- \\
--\end{array}$ & $\begin{array}{l}-- \\
-\cdots \\
--\end{array}$ & $\begin{array}{l}3.0 \\
2.6 \\
2.8 \\
3.0 \\
2.8\end{array}$ & $\begin{array}{l}4.6 \\
4.4 \\
4.2 \\
4.1 \\
3.9\end{array}$ & $\begin{array}{l}.02 \\
.01 \\
.03 \\
.00 \\
.00\end{array}$ & $\begin{array}{l}.09 \\
.09 \\
.14 \\
.56 \\
.94\end{array}$ & $\begin{array}{l}.00 \\
.00 \\
.00 \\
.00 \\
.00\end{array}$ & $\begin{array}{l}1.1 \\
1.1 \\
1.2 \\
1.4 \\
1.4\end{array}$ \\
\hline $\begin{array}{l}11 \\
12 \\
13 \\
14 \\
15\end{array}$ & $=$ & $\begin{array}{l}-\cdots \\
-\cdots \\
-\cdots \\
---\end{array}$ & $\begin{array}{l}m- \\
=- \\
=- \\
-\cdots\end{array}$ & $=-$ & $\begin{array}{l}z- \\
-\cdots \\
-\cdots \\
--\end{array}$ & $\begin{array}{l}--- \\
--- \\
-- \\
--\end{array}$ & $\begin{array}{l}2.9 \\
2.9 \\
3.1 \\
2.6 \\
2.5\end{array}$ & $\begin{array}{l}3.9 \\
3.8 \\
3.6 \\
3.6 \\
3.6\end{array}$ & $\begin{array}{l}.00 \\
.01 \\
.01 \\
.09 \\
.38\end{array}$ & $\begin{array}{r}.72 \\
.74 \\
1.0 \\
1.1 \\
.93\end{array}$ & $\begin{array}{l}.00 \\
.00 \\
.00 \\
.00 \\
.00\end{array}$ & $\begin{array}{l}1.4 \\
1.5 \\
1.3 \\
1.7 \\
1.9\end{array}$ \\
\hline $\begin{array}{l}16 \\
17 \\
18 \\
19 \\
20 \\
21 \\
22 \\
23 \\
24 \\
25\end{array}$ & 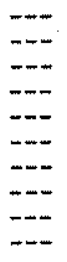 & 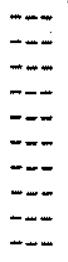 & 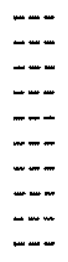 & 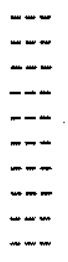 & 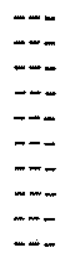 & 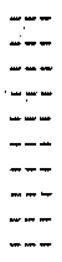 & $\begin{array}{l}2.5 \\
2.6 \\
2.9 \\
2.9 \\
2.8 \\
2.6 \\
2.4 \\
3.2 \\
5.0 \\
6.2\end{array}$ & $\begin{array}{l}3.5 \\
3.2 \\
3.1 \\
2.9 \\
2.6 \\
2.5 \\
2.4 \\
2.3 \\
2.3 \\
2.3\end{array}$ & $\begin{array}{l}.10 \\
.04 \\
.02 \\
.00 \\
.00 \\
.00 \\
.03 \\
.04 \\
.04 \\
.03\end{array}$ & $\begin{array}{r}.84 \\
1.0 \\
.93 \\
.63 \\
.27 \\
.19 \\
.13 \\
.10 \\
.08 \\
.08\end{array}$ & $\begin{array}{r}0.12 \\
.33 \\
.42 \\
.35 \\
1.2 \\
1.4 \\
2.1 \\
2.3 \\
2.0 \\
1.6\end{array}$ & $\begin{array}{l}1.6 \\
1.5 \\
1.7 \\
1.6 \\
1.5 \\
1.3 \\
1.4 \\
1.4 \\
1.4 \\
1.4\end{array}$ \\
\hline $\begin{array}{l}26 \\
27 \\
28 \\
29 \\
30 \\
31\end{array}$ & $\begin{array}{l}m-- \\
m-\infty \\
m-\infty \\
m-\infty\end{array}$ & $\begin{array}{l}=- \\
=- \\
-- \\
-\cdots \\
--\end{array}$ & 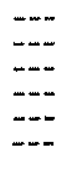 & $\begin{array}{l}-\cdots \\
-\cdots \\
-\cdots \\
-\cdots \\
\cdots\end{array}$ & $\begin{array}{l}-\cdots \\
\cdots \\
\cdots \\
\cdots- \\
\cdots-\end{array}$ & $\begin{array}{l}-.- \\
3.4 \\
3.1 \\
3.2 \\
3.3 \\
3.4\end{array}$ & $\begin{array}{l}7.5 \\
8.0 \\
8.2 \\
7.3 \\
6.4 \\
-\end{array}$ & $\begin{array}{l}1.9 \\
1.8 \\
2.0 \\
1.6 \\
1.7 \\
1.7\end{array}$ & $\begin{array}{r}.02 \\
.56 \\
.39 \\
.34 \\
.92 \\
.\end{array}$ & $\begin{array}{l}.09 \\
.07 \\
.06 \\
.08 \\
.11 \\
.10\end{array}$ & $\begin{array}{l}1.4 \\
1.3 \\
1.8 \\
1.7 \\
1.5 \\
1.4\end{array}$ & $\begin{array}{l}1.0 \\
1.4 \\
1.4 \\
1.3\end{array}$ \\
\hline $\begin{array}{l}\text { MOTAL } \\
\text { MEAN } \\
\text { MAX } \\
\text { MIN } \\
\text { AC-FT }\end{array}$ & $\begin{array}{l}--- \\
-m \\
-\cdots \\
-\infty-\end{array}$ & $\begin{array}{l}=-- \\
=-- \\
-\overline{-} \\
m-\end{array}$ & $\begin{array}{l}m- \\
-\cdots \\
-\cdots \\
--\end{array}$ & $\begin{array}{l}+-- \\
--- \\
-m- \\
---\end{array}$ & $\begin{array}{l}--- \\
--\infty \\
--- \\
---\end{array}$ & $\begin{array}{l}--- \\
-\cdots \\
-\cdots\end{array}$ & $\begin{array}{r}117.8 \\
3.93 \\
8.2 \\
2.4 \\
234\end{array}$ & $\begin{array}{r}102.9 \\
3.32 \\
5.7 \\
1.6 \\
204\end{array}$ & $\begin{array}{r}7.57 \\
1.75 \\
.00 \\
1.5\end{array}$ & $\begin{array}{r}12.50 \\
.40 \\
1.1 \\
.06 \\
25\end{array}$ & $\begin{array}{r}21.20 \\
.68 \\
2.3 \\
.00 \\
42\end{array}$ & $\begin{array}{r}42.2 \\
1.43 \\
1.9 \\
1.1 \\
84\end{array}$ \\
\hline
\end{tabular}

Estinatod 
ARKANSAS RTVER BASIN

07205500 EAGLE NEST LAKE NEAR EAGLE NEST, NM

LOCATION. -Lat $36^{\circ} 31^{\prime} 53^{\prime \prime}$, Long $105^{\circ} 13^{\prime} 44^{\prime \prime}$, Colfax County, Hydrologic Unit 11080002, in Maxweli Grant, at upstream face of Eagle Nest Dam on Cimarron River, 2.5 mi southeast of Eagle Nest, 6.7 mi west of Ute Paxk, and at mile 48.7 .

DRAINAGE AREA. $--167 \mathrm{mi}^{2}$.

PERIOD OF RECORD.--December 1927 to December 1944 (monthend contents only, publ1shed in WSP 1311 ), May 1950 to September 1965 (monthend contents only), october 1965 to June 1987 , (nonrecording gage read several times a month at randon intervals). July 1987 to current year. Frior to January 1972 published as Eagle Nest Reservolr.

REVISED RECORDS, -WSP 1281: Drainage area.

GAGE.-Water-stage recorder. Datum of gage is 8,056.8 ft above Geodetic Vertical Datum of 1929 . Prior to October 1964 gage heights were raised by addition of 8,000 ft and called elevations.

REMARKS. - Lake is formed by concrete dan with splliway cut in natural rock, completed June 30 , 1918 ; storage began In June 1917. Capacity, 79,120 acre-ft between gage heights 35.0 ft, s111 of outlet gate, and 137.0 ft, crest of ungated spłllwey. Dead storage negligible. Recordis given herein represent usable contents. Water released is used for irrigation. Lake is recreational area. Diverstons for irrigation of about 2,500 acres upstream from reservolr.

COOPERATION.--Supplemental gage readings provided by Cimarron River watermaster.

EXTREMES FOR PERIOD OF RECORD.--Maximum contents 81,360 acre-ft, May 21-29, 1994, gage height, 137.86 ft; min1mum observed, 635 acre-ft, Dec. 14, 1954, gage height, $61.33 \mathrm{ft}$.

EXTREMES FOR CURRENT YEAR,--Maximum contents, 72,280 acre-ft, Apr. 10, gage height, 134.11 ft; minimum, 55,740 acreft, Sept. 30 gage he1ght, $126,38 \mathrm{ft}$.

Capacity table (gage hejght in feet, and contents, in acre-feet)

(Based on data provided by Now Mexico State Englneer Office in 1950)

$\begin{array}{llll}125 & 53,050 & 135 & 74,350 \\ 130 & 63,170 & 140 & 86,590\end{array}$

RESERVOIR STORAGE (ACRE-FEET), WATER YEAR OCTOBER 1995 TO SEPTEMBER 1996 DAILY OBSERVATION AT 24:00 VALUES

\begin{tabular}{|c|c|c|c|c|c|c|c|c|c|c|c|c|}
\hline DAY & OCT & NOV & DEC & JAN & FEB & MAR & AFR & MAY & JUN & JUL & AUG & SEP \\
\hline $\begin{array}{l}1 \\
2 \\
3 \\
4 \\
5\end{array}$ & $\begin{array}{l}70680 \\
70710 \\
70590 \\
70460 \\
70410\end{array}$ & $\begin{array}{l}69260 \\
69310 \\
69130 \\
69310 \\
69240\end{array}$ & $\begin{array}{r}69190 \\
69190 \\
069210 \\
669200 \\
69210\end{array}$ & $\begin{array}{r}\theta 69460 \\
\theta 69450 \\
\theta 69460 \\
\theta 69470 \\
69480\end{array}$ & $\begin{array}{l}e 70260 \\
e 70280 \\
\theta 70290 \\
e 70300 \\
70300\end{array}$ & $\begin{array}{l}71160 \\
71230 \\
71440 \\
71390 \\
71370\end{array}$ & $\begin{array}{l}72050 \\
72100 \\
72050 \\
72080 \\
72050\end{array}$ & $\begin{array}{l}70820 \\
70640 \\
70590 \\
70300 \\
70160\end{array}$ & $\begin{array}{l}65830 \\
65740 \\
65630 \\
65560 \\
65520\end{array}$ & $\begin{array}{l}63190 \\
63090 \\
63030 \\
62920 \\
62880\end{array}$ & $\begin{array}{l}58790 \\
58710 \\
58650 \\
58570 \\
58370\end{array}$ & $\begin{array}{l}56600 \\
56580 \\
56580 \\
56550 \\
56540\end{array}$ \\
\hline $\begin{array}{r}6 \\
7 \\
8 \\
9 \\
10\end{array}$ & $\begin{array}{l}70640 \\
70660 \\
70520 \\
70570 \\
70500\end{array}$ & $\begin{array}{l}69190 \\
69130 \\
69280 \\
69240 \\
69150\end{array}$ & $\begin{array}{r}69210 \\
069190 \\
069190 \\
069150 \\
69130\end{array}$ & $\begin{array}{r}069560 \\
e 69580 \\
069610 \\
69600 \\
69620\end{array}$ & $\begin{array}{l}70440 \\
70440 \\
70410 \\
70480 \\
70480\end{array}$ & $\begin{array}{r}271450 \\
971450 \\
71440 \\
71570 \\
71690\end{array}$ & $\begin{array}{l}72120 \\
72170 \\
72170 \\
72190 \\
72280\end{array}$ & $\begin{array}{l}69980 \\
69730 \\
69600 \\
69460 \\
69240\end{array}$ & $\begin{array}{l}65 \\
65 \\
65 \\
65 \\
64\end{array}$ & $\begin{array}{l}62790 \\
62670 \\
62560 \\
62460 \\
62500\end{array}$ & $\begin{array}{l}58230 \\
58010 \\
57840 \\
57700 \\
57600\end{array}$ & $\begin{array}{l}56480 \\
56400 \\
56400 \\
56380 \\
56320\end{array}$ \\
\hline $\begin{array}{l}11 \\
12 \\
13 \\
14 \\
15\end{array}$ & $\begin{array}{l}70500 \\
70590 \\
70520 \\
70410 \\
70390\end{array}$ & $\begin{array}{l}68990 \\
69060 \\
69150 \\
69190 \\
69150\end{array}$ & $\begin{array}{l}69170 \\
69150 \\
69240 \\
69150 \\
69130\end{array}$ & $\begin{array}{l}69640 \\
69620 \\
69640 \\
69660 \\
69730\end{array}$ & $\begin{array}{l}70530 \\
70550 \\
70550 \\
70590 \\
70680\end{array}$ & $\begin{array}{l}71690 \\
71730 \\
71780 \\
71760 \\
71870\end{array}$ & $\begin{array}{l}72120 \\
72150 \\
72080 \\
72080 \\
72050\end{array}$ & $\begin{array}{l}69130 \\
69060 \\
68660 \\
68550 \\
68460\end{array}$ & $\begin{array}{l}64920 \\
64850 \\
64760 \\
64760 \\
64680\end{array}$ & $\begin{array}{l}62500 \\
62480 \\
62500 \\
62520 \\
62480\end{array}$ & $\begin{array}{l}57460 \\
57340 \\
57240 \\
57050 \\
56970\end{array}$ & $\begin{array}{l}56280 \\
56260 \\
56220 \\
56180 \\
56180\end{array}$ \\
\hline $\begin{array}{l}16 \\
17 \\
18 \\
19 \\
20\end{array}$ & $\begin{array}{l}70390 \\
70300 \\
70320 \\
70120 \\
70000\end{array}$ & $\begin{array}{l}69240 \\
69170 \\
69170 \\
69170 \\
69150\end{array}$ & $\begin{array}{r}69130 \\
69130 \\
669120 \\
069140 \\
669170\end{array}$ & $\begin{array}{r}69780 \\
69840 \\
069880 \\
69890 \\
069910\end{array}$ & $\begin{array}{l}70710 \\
70890 \\
70750 \\
70910 \\
71000\end{array}$ & $\begin{array}{l}71960 \\
71910 \\
71940 \\
71960 \\
72030\end{array}$ & $\begin{array}{l}72050 \\
71940 \\
71980 \\
71690 \\
71620\end{array}$ & $\begin{array}{l}68320 \\
68050 \\
68140 \\
67810 \\
67610\end{array}$ & $\begin{array}{l}64610 \\
64680 \\
64480 \\
64380 \\
64330\end{array}$ & $\begin{array}{l}62390 \\
62180 \\
61990 \\
61760 \\
61530\end{array}$ & $\begin{array}{l}56930 \\
56870 \\
56770 \\
56730 \\
56730\end{array}$ & $\begin{array}{l}56120 \\
56080 \\
56100 \\
56000 \\
55920\end{array}$ \\
\hline $\begin{array}{l}21 \\
22 \\
23 \\
24 \\
25\end{array}$ & $\begin{array}{l}70050 \\
69840 \\
69710 \\
69710 \\
69600\end{array}$ & $\begin{array}{l}69280 \\
69170 \\
69150 \\
69190 \\
69030\end{array}$ & $\begin{array}{l}069200 \\
e 69220 \\
069240 \\
069260 \\
069280\end{array}$ & $\begin{array}{r}e 69900 \\
69910 \\
069960 \\
e 69990 \\
69930\end{array}$ & $\begin{array}{l}71160 \\
71000 \\
70940 \\
70980 \\
71100\end{array}$ & $\begin{array}{l}72100 \\
72150 \\
72100 \\
71940 \\
71870\end{array}$ & $\begin{array}{l}71480 \\
71440 \\
71460 \\
71410 \\
71280\end{array}$ & $\begin{array}{l}67320 \\
67170 \\
66900 \\
66790 \\
66750\end{array}$ & $\begin{array}{l}40 \\
50 \\
90 \\
40 \\
90\end{array}$ & $\begin{array}{l}20 \\
60 \\
40 \\
50 \\
60\end{array}$ & $\begin{array}{l}56670 \\
56670 \\
56660 \\
56690 \\
56690\end{array}$ & $\begin{array}{l}55920 \\
55860 \\
55900 \\
55880 \\
55800\end{array}$ \\
\hline $\begin{array}{l}26 \\
27 \\
28 \\
29 \\
30 \\
31\end{array}$ & $\begin{array}{l}69550 \\
69550 \\
69400 \\
69420 \\
69420 \\
69420\end{array}$ & $\begin{array}{r}69150 \\
69150 \\
69120 \\
69150 \\
69190 \\
\end{array}$ & $\begin{array}{r}069310 \\
069340 \\
069360 \\
069380 \\
069410 \\
69420\end{array}$ & $\begin{array}{r}70020 \\
70080 \\
70000 \\
70120 \\
70180 \\
70210\end{array}$ & $\begin{array}{r}70980 \\
71030 \\
\mathrm{e} 71120 \\
71140 \\
-\end{array}$ & $\begin{array}{l}71940 \\
72030 \\
72030 \\
71980 \\
71960 \\
71980\end{array}$ & $\begin{array}{c}71230 \\
71230 \\
70960 \\
70980 \\
70910 \\
---\end{array}$ & $\begin{array}{l}66550 \\
66400 \\
66290 \\
66130 \\
66070 \\
65850\end{array}$ & $\begin{array}{r}63580 \\
63520 \\
63430 \\
63340 \\
63300 \\
\end{array}$ & $\begin{array}{l}60090 \\
59840 \\
59560 \\
59310 \\
59080 \\
58900\end{array}$ & $\begin{array}{l}56660 \\
56630 \\
56670 \\
56690 \\
56670 \\
56660\end{array}$ & $\begin{array}{l}55820 \\
55760 \\
55760 \\
55760 \\
55740\end{array}$ \\
\hline $\begin{array}{l}A X \\
\text { IN }\end{array}$ & $\begin{array}{r}70710 \\
69400 \\
8132.85 \\
-1420\end{array}$ & $\begin{array}{r}69310 \\
68990 \\
8132.75 \\
-230\end{array}$ & $\begin{array}{r}69420 \\
69120 \\
8132.85 \\
+230\end{array}$ & $\begin{array}{r}70210 \\
69450 \\
8133.20 \\
+790\end{array}$ & $\begin{array}{r}71160 \\
70260 \\
8133.61 \\
+930\end{array}$ & $\begin{array}{r}72150 \\
71160 \\
8133.98 \\
+840\end{array}$ & $\begin{array}{r}72280 \\
70910 \\
8133.51 \\
-1070\end{array}$ & $\begin{array}{r}70820 \\
65850 \\
8131.24 \\
-5060\end{array}$ & $\begin{array}{r}65830 \\
63300 \\
8130.06 \\
-2550\end{array}$ & $\begin{array}{r}63190 \\
58900 \\
8127.95 \\
-4400\end{array}$ & $\begin{array}{r}58790 \\
56630 \\
8126.84 \\
-2240\end{array}$ & $\begin{array}{r}56600 \\
55740 \\
8126.38 \\
-920\end{array}$ \\
\hline
\end{tabular}

CAL YR 1995 MAX 79600 MIN 68990 (†) -2170

WIR YR 1996 MAX 72280 MIN 55740 (t†) -15100

(†) GAGE HEIGHT, IN FEET, AT END OF MONTH

(ff) CHANGE IN CONTENTS, IN ACRE-FEET 
LOCATrON, - Lat $36^{\circ} 31^{\prime} 55^{\prime \prime}$, Long $105^{\circ} 13^{\prime} 43^{\prime \prime}$, Colfax County, Eydrologic Unit 11080002, in Maxwell Grant, on left bank

$300 \mathrm{ft}$ downstream Srom Eagle Nest Dam, $2.5 \mathrm{mi}$ southeast of Eagle Nest, 6.7 mi west of Ute Park, and at mile 48.6 .

DRAINAGE AREA. $-167 \mathrm{mi}^{2}$.

PERIOD OF RECORD, --May 1950 to current year. Published as Cimarron Creek below Eagle Nest Dam, October 1952 to Septanber 1965 .

REVISED RECORDS.--WSP 1281: Dreinage area.

GAGE.-Water-stage recorder. Parsha11 flume since May 15, 1951. Elevation of gage is 8,080 rí above National Godetic Vertical Datum of 1929 , from topographic map. Prior to May 15, 1951, at datum 0.81 ft higher.

REMARKS --Records good except for estimated daily discharges, which are poor. Flow regulated by Eagle Nest Lake (station 07205500) 300 ft. upstream. Diversions for irrigation of 2,500 acres upstream from station. No flow at times most years.

DISCHARGE, CUBIC FEET PER SECOND, WATER YEAR OCTOBER 1995 TO SEETEMBER 1996

\begin{tabular}{|c|c|c|c|c|c|c|c|c|c|c|c|c|}
\hline DAY & OCT & Nov & DEC & JAN & FEB & MAR & APR & MAY & JUN & JUL. & AUG & SEP \\
\hline $\begin{array}{l}1 \\
2 \\
3 \\
4 \\
5\end{array}$ & $\begin{array}{l}17 \\
17 \\
17 \\
16 \\
16\end{array}$ & $\begin{array}{l}12 \\
12 \\
12 \\
12 \\
12\end{array}$ & $\begin{array}{l}2.2 \\
2.2 \\
2.2 \\
2.0 \\
1.9\end{array}$ & $\begin{array}{l}01.2 \\
01.2 \\
01.2 \\
01.2 \\
01.2\end{array}$ & $\begin{array}{r}1.2 \\
1.2 \\
1.2 \\
\mathrm{e} 1.2 \\
01.2\end{array}$ & $\begin{array}{l}1.0 \\
1.0 \\
1.0 \\
1.0 \\
1.0\end{array}$ & $\begin{array}{r}10 \\
7.9 \\
7.5 \\
7.5 \\
7.0\end{array}$ & $\begin{array}{l}72 \\
72 \\
72 \\
77 \\
94\end{array}$ & $\begin{array}{l}43 \\
43 \\
41 \\
32 \\
32\end{array}$ & $\begin{array}{l}56 \\
56 \\
52 \\
31 \\
36\end{array}$ & $\begin{array}{l}49 \\
50 \\
50 \\
53 \\
58\end{array}$ & $\begin{array}{l}012 \\
012 \\
012 \\
012 \\
014\end{array}$ \\
\hline $\begin{array}{r}6 \\
7 \\
8 \\
9 \\
10\end{array}$ & $\begin{array}{r}16 \\
16 \\
17 \\
9.8 \\
3.2\end{array}$ & $\begin{array}{l}11 \\
11 \\
11 \\
11 \\
11\end{array}$ & $\begin{array}{l}1.9 \\
1.9 \\
1.9 \\
1.9 \\
1.9\end{array}$ & $\begin{array}{l}e 1.2 \\
01.2 \\
01.2 \\
e 1.2 \\
e 1.2\end{array}$ & $\begin{array}{l}1.2 \\
1.2 \\
1.2 \\
1.2 \\
1.2\end{array}$ & $\begin{array}{l}1.0 \\
1.0 \\
1.0 \\
1.0 \\
1.0\end{array}$ & $\begin{array}{l}8,8 \\
16 \\
16 \\
16 \\
16\end{array}$ & $\begin{array}{l}93 \\
92 \\
91 \\
91 \\
91\end{array}$ & $\begin{array}{r}36 \\
32 \\
31 \\
40 \\
44\end{array}$ & $\begin{array}{l}41 \\
46 \\
49 \\
46 \\
16\end{array}$ & $\begin{array}{l}71 \\
79 \\
79 \\
65 \\
55\end{array}$ & $\begin{array}{l}017 \\
017 \\
017 \\
017 \\
017\end{array}$ \\
\hline $\begin{array}{l}11 \\
12 \\
13 \\
14 \\
15\end{array}$ & $\begin{array}{l}3.0 \\
3.0 \\
8.4 \\
23 \\
23\end{array}$ & $\begin{array}{l}6.3 \\
3.6 \\
3.3 \\
3.3 \\
3.3\end{array}$ & $\begin{array}{l}1.9 \\
1.9 \\
1.9 \\
1.8 \\
1.7\end{array}$ & $\begin{array}{l}1.2 \\
1.2 \\
1.2 \\
1.2 \\
1.2\end{array}$ & $\begin{array}{l}1.2 \\
1.2 \\
1.2 \\
1.2 \\
1.2\end{array}$ & $\begin{array}{r}.82 \\
.80 \\
.80 \\
.81 \\
.81\end{array}$ & $\begin{array}{l}16 \\
16 \\
16 \\
34 \\
41\end{array}$ & $\begin{array}{r}88 \\
83 \\
75 \\
75 \\
75\end{array}$ & $\begin{array}{l}43 \\
44 \\
46 \\
46 \\
46\end{array}$ & $\begin{array}{r}1.5 \\
1.4 \\
3.7 \\
5.8 \\
13\end{array}$ & $\begin{array}{l}55 \\
56 \\
60 \\
53 \\
45\end{array}$ & $\begin{array}{l}17 \\
17 \\
17 \\
17 \\
17\end{array}$ \\
\hline $\begin{array}{l}16 \\
17 \\
18 \\
19 \\
20\end{array}$ & $\begin{array}{l}23 \\
31 \\
37 \\
37 \\
37\end{array}$ & $\begin{array}{l}3.3 \\
3.3 \\
3.3 \\
2.9 \\
2.7\end{array}$ & $\begin{array}{r}1.7 \\
1.7 \\
01.5 \\
01.5 \\
01.4\end{array}$ & $\begin{array}{l}1.2 \\
1.2 \\
1.2 \\
1.2 \\
1.2\end{array}$ & $\begin{array}{l}1.2 \\
1.2 \\
1.2 \\
1.2 \\
1.3\end{array}$ & $\begin{array}{l}.81 \\
.81 \\
6.4 \\
9.1 \\
8.9\end{array}$ & $\begin{array}{l}41 \\
41 \\
42 \\
42 \\
42\end{array}$ & $\begin{array}{l}75 \\
75 \\
75 \\
74 \\
80\end{array}$ & $\begin{array}{l}46 \\
40 \\
36 \\
36 \\
36\end{array}$ & $\begin{array}{r}35 \\
91 \\
101 \\
106 \\
106\end{array}$ & $\begin{array}{l}42 \\
41 \\
41 \\
39 \\
34\end{array}$ & $\begin{array}{l}017 \\
013 \\
013 \\
013 \\
013\end{array}$ \\
\hline $\begin{array}{l}21 \\
22 \\
23 \\
24 \\
25\end{array}$ & $\begin{array}{l}37 \\
37 \\
37 \\
37 \\
37\end{array}$ & $\begin{array}{l}2.7 \\
2.7 \\
2.7 \\
2.7 \\
2.7\end{array}$ & $\begin{array}{l}01.4 \\
01.4 \\
01.3 \\
01.3 \\
01.2\end{array}$ & $\begin{array}{l}1.2 \\
1.2 \\
1.2 \\
1.2 \\
1.2\end{array}$ & $\begin{array}{l}1.3 \\
1.3 \\
1.2 \\
1.2 \\
1.2\end{array}$ & $\begin{array}{l}13 \\
15 \\
15 \\
15 \\
15\end{array}$ & $\begin{array}{l}42 \\
49 \\
52 \\
43 \\
38\end{array}$ & $\begin{array}{l}83 \\
78 \\
68 \\
57 \\
39\end{array}$ & $\begin{array}{l}36 \\
36 \\
39 \\
68 \\
68\end{array}$ & $\begin{array}{l}106 \\
107 \\
109 \\
112 \\
112\end{array}$ & $\begin{array}{r}39 \\
34 \\
826 \\
e 26 \\
026\end{array}$ & $\begin{array}{r}e 13 \\
e 13 \\
013 \\
013 \\
07.4\end{array}$ \\
\hline $\begin{array}{l}26 \\
27 \\
28 \\
29 \\
30 \\
31\end{array}$ & $\begin{array}{l}37 \\
37 \\
37 \\
31 \\
19 \\
12\end{array}$ & $\begin{array}{l}2.7 \\
2.7 \\
2.7 \\
2.7 \\
2.4 \\
-\end{array}$ & $\begin{array}{l}\text { e1.2 } \\
01.2 \\
01.2 \\
\mathrm{e} 1.2 \\
\mathrm{e} 1.2 \\
01.2\end{array}$ & $\begin{array}{l}1.2 \\
1.3 \\
1.2 \\
1.2 \\
1.2 \\
1.2\end{array}$ & $\begin{array}{l}1.2 \\
1.2 \\
1.2 \\
1.1 \\
-.-\end{array}$ & $\begin{array}{l}15 \\
15 \\
15 \\
15 \\
15 \\
15\end{array}$ & $\begin{array}{l}27 \\
21 \\
33 \\
37 \\
59 \\
---\end{array}$ & $\begin{array}{l}66 \\
66 \\
66 \\
66 \\
55 \\
45\end{array}$ & $\begin{array}{r}67 \\
65 \\
46 \\
34 \\
47 \\
---\end{array}$ & $\begin{array}{r}112 \\
112 \\
123 \\
132 \\
132 \\
90\end{array}$ & $\begin{array}{r}\text { e10 } \\
\text { e8.5 } \\
08.1 \\
88.1 \\
88.5 \\
012\end{array}$ & $\begin{array}{l}07.4 \\
67.4 \\
67.4 \\
07.4 \\
07.4 \\
--\infty\end{array}$ \\
\hline $\begin{array}{l}\text { TOTAI } \\
\text { MEAN } \\
\text { MAX } \\
\text { MIN } \\
\text { AC-FT }\end{array}$ & $\begin{array}{r}728.4 \\
23.5 \\
37 \\
3.0 \\
1440\end{array}$ & $\begin{array}{r}177.0 \\
5.90 \\
12 \\
2.4 \\
351\end{array}$ & $\begin{array}{r}50.8 \\
1.64 \\
2.2 \\
1.2 \\
101\end{array}$ & $\begin{array}{r}37.3 \\
1.20 \\
1.3 \\
1.2 \\
74\end{array}$ & $\begin{array}{r}35.0 \\
1.21 \\
1.3 \\
1.1 \\
69\end{array}$ & $\begin{array}{r}203.06 \\
6.55 \\
15 \\
.80 \\
403\end{array}$ & $\begin{array}{r}844.5 \\
28.1 \\
59 \\
7.0 \\
1680\end{array}$ & $\begin{array}{r}2309 \\
74.5 \\
94 \\
39 \\
4580\end{array}$ & $\begin{array}{r}1299 \\
43.3 \\
68 \\
31 \\
2580\end{array}$ & $\begin{array}{r}2140.4 \\
69.0 \\
132 \\
1.4 \\
4250\end{array}$ & $\begin{array}{r}1281.2 \\
41.3 \\
79 \\
8.1 \\
2540\end{array}$ & $\begin{array}{r}397.4 \\
13.2 \\
17 \\
7.4 \\
788\end{array}$ \\
\hline
\end{tabular}

STATISTICS OF MONTHLY MEAN DATA FOR WATER YEARS 1950 - 1996, BY WATER YEAR (WY)

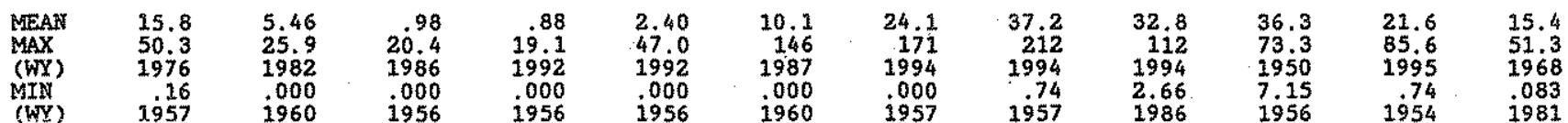

SURMARY STATISTICS

ARMUAL TOTAL

ANNUAL MEAN

HIGHEST ANNUAL MEAN

LOWEST ANNUAL MEAN

HIGHEST DAILY MEAN

LOWEST DAILY MEAN

ANWUAL SEVEN-DAY MINIMUM

ANNUAL RUNOFF (AC-ET)

10 PERCENT EXCEEDS

50 PERCENT EXCEEDS

90 PERCENT EXCEEDS

e Estimated
FOR 1995 CALENDAR YEAR

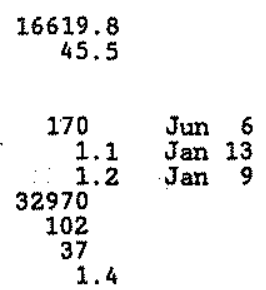

FOR 1996 WATER YEAR

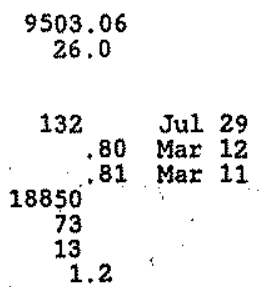

WATER YEARS 1950 - 1996

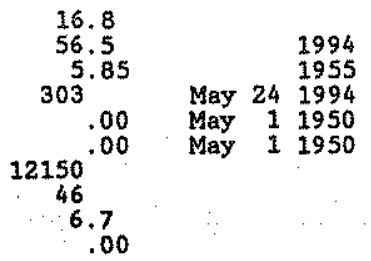


07207000 CIMARRON RIVER NEAR CIMARRON, MM

LOCATrON.--Lat $36^{\circ} 31^{\prime} 11^{\prime \prime}$, long $14^{\circ} 58^{\prime} 42^{\prime \prime}$, Colfax County, Hydrologic Unit 11080002, in Maxwell Grant, on right bank $1,200 \mathrm{ft}$ downstream from Turkey Creak Canyon, $3.6 \mathrm{mi}$ west of Cimarron, and at mile 31.6 . DRAIKAGE AREA, $--294 \mathrm{mi}^{2}$.

WATER-DISCHARGE RECORDS

PERIOD OF RECORD. --May 1950 to current year. Published as Cimarron Creek near Cimarron, October 1952 to September 1965

REVISED RECORDS. --WSP 1281: Drainage axea.

GAGE. - Water-stage recorder. Concrete control since Nov, 6, 1963. Datum of gage is 6,599.58 ft above National Geodetic Vertical Datum of 1929.

REMARKS. - Water-discharge records good except for estimeted dally discharges, which are poor. Flow regulated by Eagle Nest Lake (station 07205500). Diversions upstream from station for irrigation of about 3,500 acres, part of which is downstream from station. Philmont ditch (formerly known as Cimarroncito ditch) diverts from left bank 1.5 mi upstream from station, siphons under river 0.9 mi upstream and bypasses station for off-channel storage and irrigation downstream; Raton divarsion pipeline 300 ft upstream from station for City of Raton Water Supply started June, 1983. 'See tabulation below for monthly diversions. No flow at times.

DISCEARGE, CUBIC FEET PER SECOND, WATER YEAR OCTOBER 1995 TO SEPTEMBER 1996 DAILY MEAY VALUES

\begin{tabular}{|c|c|c|c|c|c|c|c|c|c|c|c|c|}
\hline DAY & $\infty \mathrm{Cr}$ & Nov & DEC & JAN & FEB & MAR & $A F R$ & MAY & JUN & JUL & AUUG & SEP \\
\hline $\begin{array}{l}1 \\
2 \\
3 \\
4 \\
5\end{array}$ & $\begin{array}{l}21 \\
21 \\
21 \\
20 \\
20\end{array}$ & $\begin{array}{l}11 \\
12 \\
15 \\
15 \\
15\end{array}$ & $\begin{array}{l}5.5 \\
5.2 \\
5.0 \\
4.6 \\
4.4\end{array}$ & $\begin{array}{l}03.3 \\
83.6 \\
03.1 \\
03.5 \\
03.7\end{array}$ & $\begin{array}{r}04.0 \\
04.4 \\
04.4 \\
04.5 \\
04.2\end{array}$ & $\begin{array}{l}7.4 \\
8.8 \\
4.6 \\
3.3 \\
2.8\end{array}$ & $\begin{array}{l}16 \\
12 \\
12 \\
12 \\
11\end{array}$ & $\begin{array}{l}67 \\
67 \\
66 \\
66 \\
82\end{array}$ & $\begin{array}{l}37 \\
36 \\
36 \\
29 \\
26\end{array}$ & $\begin{array}{l}47 \\
47 \\
46 \\
35 \\
31\end{array}$ & $\begin{array}{l}48 \\
47 \\
46 \\
44 \\
46\end{array}$ & $\begin{array}{l}10 \\
10 \\
10 \\
9.8 \\
9.6\end{array}$ \\
\hline $\begin{array}{r}6 \\
7 \\
8 \\
9 \\
10\end{array}$ & $\begin{array}{c}20 \\
20 \\
20 \\
16 \\
6.3\end{array}$ & $\begin{array}{l}14 \\
14 \\
13 \\
13 \\
14\end{array}$ & $\begin{array}{r}4.3 \\
4.4 \\
4.3 \\
e 3.8 \\
03.2\end{array}$ & $\begin{array}{l}03.8 \\
03.9 \\
04.2 \\
84.7 \\
04.5\end{array}$ & $\begin{array}{r}04.0 \\
04.1 \\
04.1 \\
4.2 \\
4.1\end{array}$ & $\begin{array}{l}3.0 \\
4.4 \\
6.1 \\
6.8 \\
4.3\end{array}$ & $\begin{array}{l}10 \\
14 \\
18 \\
18 \\
19\end{array}$ & $\begin{array}{l}85 \\
83 \\
81 \\
82 \\
85\end{array}$ & $\begin{array}{l}29 \\
27 \\
25 \\
28 \\
35\end{array}$ & $\begin{array}{l}38 \\
40 \\
47 \\
48 \\
39\end{array}$ & $\begin{array}{l}50 \\
64 \\
66 \\
62 \\
49\end{array}$ & $\begin{array}{l}12 \\
14 \\
14 \\
13 \\
13\end{array}$ \\
\hline $\begin{array}{l}11 \\
12 \\
13 \\
14 \\
15\end{array}$ & $\begin{array}{r}4.2 \\
3.4 \\
3.1 \\
9.3 \\
18\end{array}$ & $\begin{array}{r}13 \\
8.6 \\
7.5 \\
7.0 \\
6.7\end{array}$ & $\begin{array}{r}03.7 \\
3.9 \\
4.0 \\
3.8 \\
03.8\end{array}$ & $\begin{array}{r}04.8 \\
04.9 \\
e 4.5 \\
4.4 \\
7.1\end{array}$ & $\begin{array}{l}4.1 \\
4.1 \\
4.0 \\
4.0 \\
4.1\end{array}$ & $\begin{array}{l}4.2 \\
4.4 \\
4.5 \\
4.4 \\
5.0\end{array}$ & $\begin{array}{l}20 \\
20 \\
20 \\
25 \\
40\end{array}$ & $\begin{array}{l}84 \\
81 \\
73 \\
70 \\
68\end{array}$ & $\begin{array}{l}34 \\
36 \\
39 \\
42 \\
43\end{array}$ & $\begin{array}{r}14 \\
9.1 \\
7.4 \\
7.2 \\
7.4\end{array}$ & $\begin{array}{l}51 \\
50 \\
52 \\
52 \\
45\end{array}$ & $\begin{array}{l}13 \\
14 \\
15 \\
15 \\
16\end{array}$ \\
\hline $\begin{array}{l}16 \\
17 \\
18 \\
19 \\
20\end{array}$ & $\begin{array}{l}23 \\
25 \\
30 \\
30 \\
29\end{array}$ & $\begin{array}{l}6.8 \\
6.8 \\
6.7 \\
6.4 \\
6.2\end{array}$ & $\begin{array}{l}03.5 \\
63.9 \\
04.0 \\
63.7 \\
03.8\end{array}$ & $\begin{array}{r}4.2 \\
04.5 \\
04.7 \\
84.3 \\
04.1\end{array}$ & $\begin{array}{l}4.6 \\
4.0 \\
3.9 \\
3.7 \\
3.9\end{array}$ & $\begin{array}{l}4.8 \\
4.7 \\
5.1 \\
7.0 \\
8.4\end{array}$ & $\begin{array}{l}42 \\
42 \\
43 \\
44 \\
44\end{array}$ & $\begin{array}{l}68 \\
68 \\
68 \\
67 \\
69\end{array}$ & $\begin{array}{l}42 \\
41 \\
36 \\
34 \\
33\end{array}$ & $\begin{array}{l}14 \\
66 \\
85 \\
91 \\
93\end{array}$ & $\begin{array}{l}39 \\
39 \\
38 \\
36 \\
34\end{array}$ & $\begin{array}{l}15 \\
15 \\
17 \\
14 \\
11\end{array}$ \\
\hline $\begin{array}{l}21 \\
22 \\
23 \\
24 \\
25\end{array}$ & $\begin{array}{l}29 \\
29 \\
30 \\
30 \\
31\end{array}$ & $\begin{array}{l}6.1 \\
5.9 \\
5.8 \\
5.8 \\
5.5\end{array}$ & $\begin{array}{l}\text { e3.3 } \\
e 3.1 \\
e 3.6 \\
e 3.0 \\
e 3.2\end{array}$ & $\begin{array}{l}e 4.4 \\
04.0 \\
04.1 \\
04.3 \\
04.6\end{array}$ & $\begin{array}{l}5.2 \\
6.0 \\
5.6 \\
5.8 \\
5.9\end{array}$ & $\begin{array}{l}9.2 \\
13 \\
14 \\
15 \\
15\end{array}$ & $\begin{array}{l}44 \\
47 \\
52 \\
50 \\
41\end{array}$ & $\begin{array}{l}75 \\
70 \\
60 \\
53 \\
37\end{array}$ & $\begin{array}{l}34 \\
35 \\
36 \\
51 \\
60\end{array}$ & $\begin{array}{r}95 \\
95 \\
96 \\
101 \\
98\end{array}$ & $\begin{array}{l}32 \\
40 \\
31 \\
23 \\
23\end{array}$ & $\begin{array}{c}11 \\
7.1 \\
4.6 \\
3.8 \\
3.2\end{array}$ \\
\hline $\begin{array}{l}26 \\
27 \\
28 \\
29 \\
30 \\
31\end{array}$ & $\begin{array}{l}32 \\
32 \\
32 \\
30 \\
23 \\
12\end{array}$ & $\begin{array}{l}5.5 \\
5.6 \\
5.5 \\
5.5 \\
6.0 \\
-.-\end{array}$ & $\begin{array}{l}03.7 \\
03.7 \\
03.5 \\
02.9 \\
03.0 \\
02.8\end{array}$ & $\begin{array}{l}04.7 \\
04.9 \\
04.6 \\
04.4 \\
04.1 \\
03.8\end{array}$ & $\begin{array}{l}6.0 \\
4.7 \\
4.7 \\
5.9 \\
-.9 \\
-.-\end{array}$ & $\begin{array}{l}16 \\
17 \\
16 \\
16 \\
17 \\
17\end{array}$ & $\begin{array}{l}38 \\
27 \\
31 \\
37 \\
44 \\
-\end{array}$ & $\begin{array}{l}54 \\
58 \\
58 \\
58 \\
53 \\
42\end{array}$ & $\begin{array}{r}59 \\
68 \\
48 \\
33 \\
35 \\
-\end{array}$ & $\begin{array}{r}97 \\
98 \\
103 \\
118 \\
121 \\
107\end{array}$ & $\begin{array}{r}22 \\
20 \\
11 \\
8.8 \\
8.9 \\
9.4\end{array}$ & $\begin{array}{l}3.4 \\
4.0 \\
3.7 \\
3.6 \\
3.7\end{array}$ \\
\hline $\begin{array}{l}\text { TOTAL } \\
\text { MEAN } \\
\text { MAX } \\
\text { MIN } \\
\text { AC-FT } \\
(+) \\
(+)\end{array}$ & $\begin{array}{r}670.3 \\
21.6 \\
32 \\
3.1 \\
1330 \\
0 \\
0\end{array}$ & $\begin{array}{r}268.9 \\
8.96 \\
15 \\
5.5 \\
533 \\
0 \\
0\end{array}$ & $\begin{array}{r}118.6 \\
3.83 \\
5.5 \\
2.8 \\
235 \\
0 \\
0\end{array}$ & $\begin{array}{r}133.7 \\
4.31 \\
7.1 \\
3.1 \\
265 \\
0 \\
0\end{array}$ & $\begin{array}{r}132.2 \\
4.56 \\
6.0 \\
3.7 \\
262 \\
0 \\
0\end{array}$ & $\begin{array}{r}269.2 \\
8.68 \\
17 \\
2.8 \\
534 \\
0 \\
0\end{array}$ & $\begin{array}{r}893 \\
29.8 \\
52 \\
10 \\
1770 \\
35 \\
0\end{array}$ & $\begin{array}{r}2098 \\
67.7 \\
85 \\
37 \\
4160 \\
319 \\
0\end{array}$ & $\begin{array}{r}1147 \\
38.2 \\
68 \\
25 \\
2280 \\
97 \\
0\end{array}$ & $\begin{array}{r}1941.1 \\
62.6 \\
121 \\
7.2 \\
3850 \\
159 \\
0\end{array}$ & $\begin{array}{r}1187.1 \\
38.3 \\
66 \\
8.8 \\
2350 \\
189 \\
222\end{array}$ & $\begin{array}{r}308.5 \\
10.3 \\
17 \\
3.2 \\
612 \\
0 \\
543\end{array}$ \\
\hline
\end{tabular}

WTR YR 1995 AC-ET $(\dagger) 201 \quad$ (t⿱) 385

(†) DIVERSION, IN ACRE-FEET, BY PHILMONT DITCH, DATA EROVIDED BY CIMARRON RIVER WATERMASTER

(†) DIVERSION, IN ACRE-FEET, RATON DIVERSION, DATA PROVIDED BY CIMARRON RIVER WATERMASTER

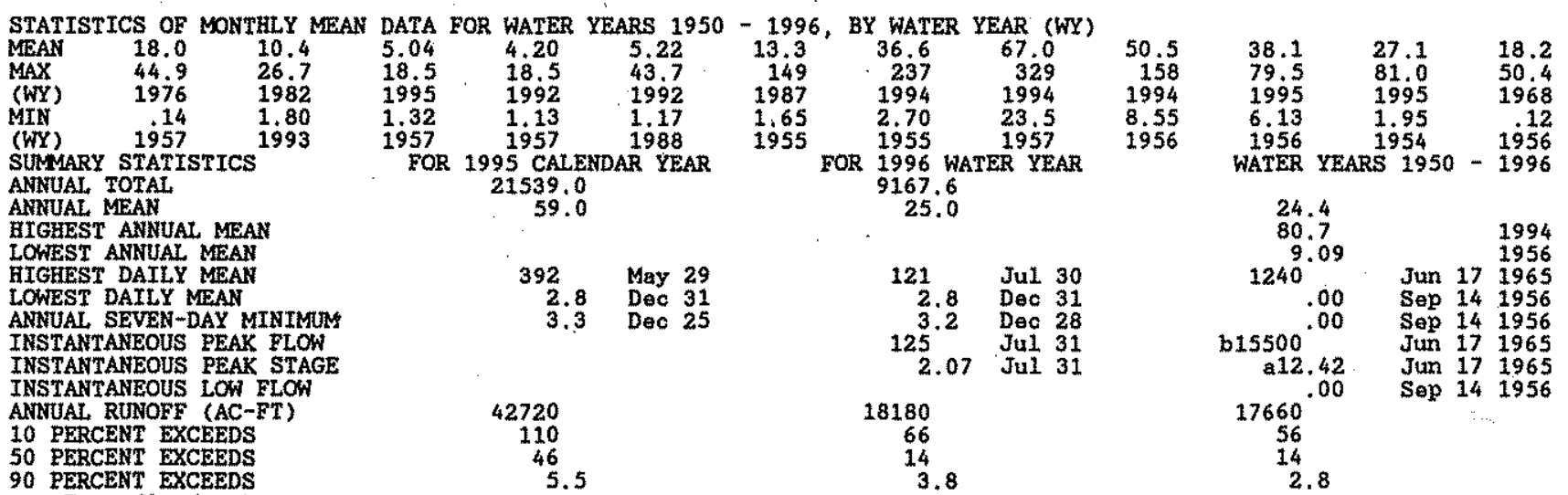

PERCENT EXCEEDS

b-From rating curve extended above $800 \mathrm{ft} / \mathrm{s}$ on basis of slope-area measurements at gage heights $4.88 \mathrm{ft}$ and 12.42 ft. 
ARKANSAS RIVER BASIN

07207000 CIMARRON RIVER NEAR CIMARRON, MM -- Continued

WATER-QUALITY RECORDS

PERIOD OF RECORD.- Water years 1979, 1981 to current year.

WATER QUALITY DATA, WATER YEAR OCTOBER 1995 TO SEPTEMBER 1996

\begin{tabular}{|c|c|c|c|c|c|c|c|c|c|c|c|c|}
\hline DATE & TIME & $\begin{array}{c}\text { DIS- } \\
\text { CAARGE, } \\
\text { INST } \\
\text { CUBIC } \\
\text { FEET } \\
\text { PER } \\
\text { SECOND } \\
(00061)\end{array}$ & $\begin{array}{l}\text { SPE- } \\
\text { CIFIC } \\
\text { CON- } \\
\text { DUCT- } \\
\text { ANCE } \\
\text { (US/CM) } \\
(00095)\end{array}$ & $\begin{array}{l}\text { PH } \\
\text { WATER } \\
\text { WHOLE } \\
\text { FIELD } \\
\text { (STAND- } \\
\text { ARD } \\
\text { WNITS) } \\
(00400)\end{array}$ & $\begin{array}{l}\text { TEMPER- } \\
\text { ATURE } \\
\text { AIR } \\
\text { (DEG C) } \\
(00020)\end{array}$ & $\begin{array}{c}\text { TEMPER- } \\
\text { ATURE } \\
\text { WATER } \\
\text { (DEG C) } \\
(00010)\end{array}$ & $\begin{array}{l}\text { BARO- } \\
\text { METRIC } \\
\text { FRES- } \\
\text { SURE } \\
\text { (MM } \\
\text { OF } \\
\text { HG) } \\
(00025)\end{array}$ & $\begin{array}{c}\text { OXYGEN, } \\
\text { DIS- } \\
\text { SOLVED } \\
(\text { HG/L) } \\
(00300)\end{array}$ & $\begin{array}{l}\text { OXYGEN, } \\
\text { DIS- } \\
\text { SOLVED } \\
\text { (PER- } \\
\text { CENT } \\
\text { SATUR- } \\
\text { ATION) } \\
\text { (OO301) }\end{array}$ & $\begin{array}{l}\text { HARD- } \\
\text { NESS } \\
\text { TOTAL } \\
\text { (MG/I } \\
\text { AS } \\
\text { CACO3) } \\
(00900)\end{array}$ & $\begin{array}{l}\text { FARD- } \\
\text { NESS } \\
\text { NONCARB } \\
\text { DISSOLV } \\
\text { FLD. AS } \\
\text { CACOB } \\
(\text { MG/L) } \\
(00904)\end{array}$ & $\begin{array}{l}\text { CALCIUM } \\
\text { DIS- } \\
\text { SOLVED } \\
\text { (AGG/L } \\
\text { AS CA) } \\
\text { (00915) }\end{array}$ \\
\hline $\begin{array}{l}\text { DEC } 1995 \\
141996 \\
\text { FEB }\end{array}$ & 0930 & 4.0 & 454 & 8.3 & 2.0 & 2.0 & 598 & 10.4 & 96 & 180 & 28 & 54 \\
\hline $15 \ldots$ & 0915 & 4.0 & 396 & 8.5 & 1.0 & 1.5 & 600 & 11.1 & 101 & - & - & -- \\
\hline $10 \ldots$ & 0945 & 84 & 307 & 8.2 & 18.0 & 10.5 & 605 & 9.0 & 102 & 130 & -- & 40 \\
\hline
\end{tabular}

\begin{tabular}{|c|c|c|c|c|c|c|c|c|c|c|c|c|}
\hline DATE & $\begin{array}{c}\text { MAGNE- } \\
\text { SIUM, } \\
\text { DIS- } \\
\text { SOLVED } \\
\text { (MG/L } \\
\text { AS MG) } \\
(00925)\end{array}$ & $\begin{array}{c}\text { SODIUM, } \\
\text { DIS- } \\
\text { SOLVED } \\
\text { (NGSLL) } \\
\text { AS NA ) } \\
(00930)\end{array}$ & $\begin{array}{c}\text { SODIUM } \\
\text { AD- } \\
\text { SORP- } \\
\text { TION } \\
\text { RATIO } \\
(00931)\end{array}$ & $\begin{array}{c}\text { POTAS- } \\
\text { SIUM, } \\
\text { DIS- } \\
\text { SOLVED } \\
\text { (NSG/L } \\
\text { AS K) } \\
(00935)\end{array}$ & $\begin{array}{c}\text { BICAR- } \\
\text { BONATE } \\
\text { WATER } \\
\text { DIS IT } \\
\text { FIELD } \\
\text { NG/L AS } \\
\text { ECOO3 } \\
(00453)\end{array}$ & $\begin{array}{c}\text { CAR - } \\
\text { BONATE } \\
\text { WATER } \\
\text { DIS IT } \\
\text { FIELD } \\
\text { MG/L AS } \\
\text { CO3 } \\
(00452)\end{array}$ & $\begin{array}{l}\text { ALKA- } \\
\text { LINITY } \\
\text { WAT DIS } \\
\text { TOT IT } \\
\text { FIELD } \\
\text { MG/L AS } \\
\text { CACO3 } \\
(39086)\end{array}$ & $\begin{array}{c}\text { ALKA- } \\
\text { LINITY } \\
\text { IAAB } \\
\text { (MG/L } \\
\text { AS } \\
\text { CACO3) } \\
(90410)\end{array}$ & $\begin{array}{c}\text { SULFATE } \\
\text { DIS- } \\
\text { SOLVED } \\
(M G / L \\
\text { AS SO4) } \\
(00945)\end{array}$ & $\begin{array}{l}\text { CHLO- } \\
\text { RIDE, } \\
\text { DIS- } \\
\text { SOLVED } \\
\text { (NG/L } \\
\text { AS CL) } \\
(00940)\end{array}$ & $\begin{array}{c}\text { FLUO- } \\
\text { RIDE, } \\
\text { DIS- } \\
\text { SOLVED } \\
\text { (MG/L } \\
\text { AS F) } \\
(00950)\end{array}$ & $\begin{array}{c}\text { SILICA, } \\
\text { DIS- } \\
\text { SOLVED } \\
\text { (MG/L } \\
\text { AS } \\
\text { SIO2) } \\
(00955)\end{array}$ \\
\hline $\begin{array}{l}\mathrm{DEC} 1995 \\
14 \mathrm{FEB} \\
\mathrm{FE}\end{array}$ & 10 & 18 & 0.6 & 1.4 & 178 & 1 & 147 & 153 & 54 & 7.0 & 0.40 & 10 \\
\hline MAY & - & -- & -- & -- & - & - & -- & -- & -- & $-\rightarrow$ & - & - \\
\hline $10 \ldots$ & 8.1 & 12 & 0.5 & 1.7 & $\cdots$ & - & - & 139 & 15 & 5.9 & 0.40 & 2.3 \\
\hline
\end{tabular}

DEC 1995

FEB 1996

$15 \ldots$

MAY ...

DATE

DEC 1995

$\operatorname{EEB}^{14} 1996$

$15 .$.

$10 \ldots$
SOLIDS, NITRO-

NTRO- NTTRO-

SuM of

CONSTI- HITRTTE

SOIS- SOLVED

(70301) AS

(00613)

$243<0.010$

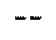

169

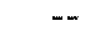

$<0.050$

GEN GEN,AM-

AMONA MON,AN- GEN,AM-

DIS- DIS- ORGANIC MONIA

DOLVED SOLVED TOTAL ORGANIC

MOLVED TOTAL DIS.

(MG/L)

AS N)

$(00623)$

$<0.20$

$<0.20$

-.

$-\cdots$

$-$
TTTRITE NO2+HOS

SOLVED (MG/L

(00608) AS N)

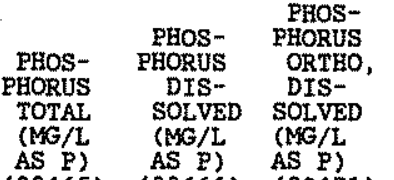

ALUM- ANTI-

INUM, MONY,

SOLVED SOLVED

(UG/L (UG/L

AS AL) AS SB)

(00665) (00666) (00671)

$<0.010<0.010$

$<0.010$

10

(01095)

$-\cdots$

$-\cdots$

$-\infty \quad-$

$<1.0$

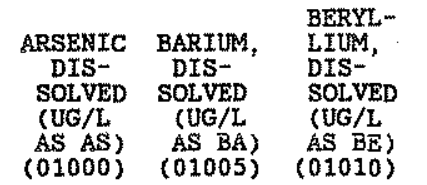

BORON, CADMIUM

CHRO- COBALT, COPPER, DIS- DIS-' DIS-

SOLVED SOLVED SOLVED

(UG/L

SOLVED SOLVED

(UG/L (UG/L

$A S$ B)
$(01020)$ (AS CD)

AS CR)

SOLVED

AS CO)

(UG/L

(US CU)

AS CU)

IRON,
DTS-
SOLVED
(UG/L
AS FE)
$(01046)$

$\cdot$

$<1.0<1.0<1.0$

$2.0 \quad 12$

12

36

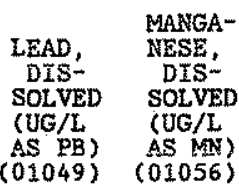

$<1.0 \quad 7.0$

$\begin{array}{cccc}1 & 74 & <1.0 & 30 \\ -- & -- & -- & -- \\ - & -- & -- & 20\end{array}$

$\begin{array}{cccc}\text { MERCURY } & \text { NOLYB- } & & \\ \text { TOTAL } & \text { DENUM, } & \text { NICKEL, } & \text { SELE- } \\ \text { RECOV- } & \text { DIS- } & \text { DIS- } & \text { NIUM, } \\ \text { ERABLE } & \text { SOLVED } & \text { SOLVED } & \text { TOTAL } \\ \text { (UG/L } & \text { (UG/L } & \text { (UG/L } & \text { (UG/L } \\ \text { AS HG) } & \text { AS MO) } & \text { AS NI) } & \text { AS SE) } \\ (71900) & (01060) & (01065) & (01147)\end{array}$

$\begin{array}{lc}\text { SELE- } & \\ \text { NIUM, } & \text { SILVER, } \\ \text { DIS- } & \text { DIS- } \\ \text { SOLVED } & \text { SOLVED } \\ \text { (UG/L } & \text { (UG/L } \\ \text { AS SE) } & \text { AS AG) }\end{array}$ (01145)
$A S$ AG)
$(01075)$

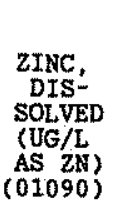

URANIUM NATURAL
DISDOIS(UG/L AS U)
$(22703)$

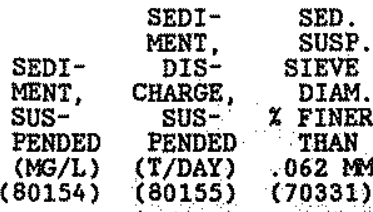

DEC 1995

2.0

2.0

1

$<1<1.0 \quad 15$

2.0

0.09

$\therefore 94$

$15 .$.

$--$ 
ARKAKSAS RIVHR BASIN

07207500 PONIL CREEK NEAR CIMARRON, NM

LOCATION. - Lat $36^{\circ} 34^{\circ} 25^{\prime \prime}$, long $104^{\circ} 56^{\circ} 46^{\prime \prime}$, Colfax County, Hydrologic Unit 11080002, in Maxwe11 Grant, on left bank $1.6 \mathrm{mi}$ domatream from confluence of North and South Ponil Creeks, and $4.7 \mathrm{mi}$ northwest of Cimarron.

DRAINAGE AREA. --171 mín. $^{2}$.

PERIOD OF RECORD.--November 1915 to June 1919, August 1919 to July 1925, September 1925, September 1927 to July 1929, May 1950 to current year. Prior to May 1950 monthly discharge only, published in WSP 1311.

REVISED RECORDS.--WSP 1281: Drainage area. WSP 1731: 1920.

GAGE.--Water-stage recorder. Elevation of gage is 6,630 ft above National Geodetic Vertical Datum of 1929 , from topographic map. Prior to May 8, 1922, at site $0.1 \mathrm{mi}$ downstream at different datum. May 8, 1922 to Aug. 8, 1929, at site 0.4 mi upstream at different datum.

REMARKS. --Records good except for estimated daily discharges, which are poor. Diversions for trrigation of about 250 acres upstream from station. Diversion 1,000 ft domstrean from station for irrigation of about 300 acres. No flow at times most years.

EXTREMES OUTSIDE PERTOD OF RECORD.--Discharge for flood of Aug. 8, 1929, which destroyed gage, was estimated as 5, $200 \mathrm{ft} / \mathrm{s}$ by New Mexico State Engineer.

DISCHARGE, CUBIC FEET PER SECOND, WATER YEAR OCTOBER 1995 TO SEPTEMBER 1996

\begin{tabular}{|c|c|c|c|c|c|c|c|c|c|c|c|c|}
\hline DAY & $\infty C T$ & NOV & DEC & JAN & FEB & MAR & APR & MAY & JUN & JUL & AUG & SEP \\
\hline $\begin{array}{l}1 \\
2 \\
3 \\
4 \\
5\end{array}$ & $\begin{array}{c}10 \\
9.4 \\
8.8 \\
7.8 \\
7.3\end{array}$ & $\begin{array}{l}4.6 \\
4.7 \\
4.7 \\
4.7 \\
4.7\end{array}$ & $\begin{array}{l}4.8 \\
4.6 \\
4.2 \\
4.2 \\
4.1\end{array}$ & $\begin{array}{l}e 3.2 \\
e 3.1 \\
02.9 \\
03.4 \\
03.7\end{array}$ & $\begin{array}{l}\mathrm{e} .8 \\
\mathrm{e} .9 \\
\mathrm{e} .9 \\
\mathrm{e} .1 \\
\mathrm{e} .2 .9\end{array}$ & $\begin{array}{l}3.4 \\
3.3 \\
2.9 \\
2.8 \\
2.8\end{array}$ & $\begin{array}{l}3.6 \\
3.9 \\
4.4 \\
5.0 \\
4.9\end{array}$ & $\begin{array}{l}5.3 \\
4.9 \\
4.5 \\
4.3 \\
3.9\end{array}$ & $\begin{array}{l}.72 \\
.67 \\
.63 \\
.60 \\
.52\end{array}$ & $\begin{array}{l}.65 \\
.53 \\
.25 \\
.19 \\
.17\end{array}$ & $\begin{array}{l}1.5 \\
1.3 \\
1.3 \\
2.0 \\
1.3\end{array}$ & $\begin{array}{l}5.5 \\
5.5 \\
5.1 \\
4.5 \\
4.1\end{array}$ \\
\hline $\begin{array}{r}6 \\
7 \\
8 \\
9 \\
10\end{array}$ & $\begin{array}{l}7.2 \\
7.1 \\
6.7 \\
6.2 \\
5.9\end{array}$ & $\begin{array}{l}4.7 \\
4.7 \\
4.6 \\
4.4 \\
4.8\end{array}$ & $\begin{array}{r}4.1 \\
3.9 \\
3.8 \\
03.3 \\
02.6\end{array}$ & $\begin{array}{l}\text { e3.1 } \\
\text { e2.8 } \\
\text { e3.0 } \\
\text { e3.2 } \\
\text { e3.4 }\end{array}$ & $\begin{array}{l}\mathrm{e} .1 \\
\mathrm{e} 2.8 \\
\mathrm{e} 2.7 \\
\mathrm{e} 2.9 \\
\mathrm{e} 2.7\end{array}$ & $\begin{array}{l}2.8 \\
2.6 \\
2.9 \\
2.8 \\
2.9\end{array}$ & $\begin{array}{l}4.1 \\
4.3 \\
4.4 \\
4.9 \\
6.0\end{array}$ & $\begin{array}{l}3.8 \\
3.6 \\
3.5 \\
3.3 \\
3.1\end{array}$ & $\begin{array}{l}.46 \\
.39 \\
.34 \\
.32 \\
.34\end{array}$ & $\begin{array}{r}.15 \\
.27 \\
.39 \\
.95 \\
4.5\end{array}$ & $\begin{array}{l}.93 \\
2.0 \\
2.1 \\
1.4 \\
1.2\end{array}$ & $\begin{array}{l}4.0 \\
4.8 \\
4.0 \\
3.5 \\
3.2\end{array}$ \\
\hline $\begin{array}{l}11 \\
12 \\
13 \\
14 \\
15\end{array}$ & $\begin{array}{l}5.7 \\
5.5 \\
5.3 \\
5.2 \\
5.2\end{array}$ & $\begin{array}{l}4.7 \\
4.9 \\
5.0 \\
4.9 \\
4.8\end{array}$ & $\begin{array}{r}03.7 \\
03.4 \\
3.2 \\
3.3 \\
\mathrm{e} .1\end{array}$ & $\begin{array}{l}03.3 \\
03.7 \\
03.8 \\
04.0 \\
03.9\end{array}$ & $\begin{array}{l}e 2.6 \\
e 2.6 \\
e 2.7 \\
e 2.7 \\
e 2.6\end{array}$ & $\begin{array}{l}3.0 \\
3.0 \\
3.0 \\
3.1 \\
3.5\end{array}$ & $\begin{array}{l}6.5 \\
6.4 \\
6.4 \\
6.5 \\
6.1\end{array}$ & $\begin{array}{l}3.0 \\
2.8 \\
2.6 \\
2.5 \\
2.2\end{array}$ & $\begin{array}{l}.32 \\
.34 \\
.51 \\
.58 \\
.65\end{array}$ & $\begin{array}{l}4.3 \\
2.3 \\
2.2 \\
2.2 \\
1.5\end{array}$ & $\begin{array}{c}1.2 \\
1.0 \\
.79 \\
.66 \\
.60\end{array}$ & $\begin{array}{l}3.5 \\
3.5 \\
5.1 \\
4.5 \\
5.0\end{array}$ \\
\hline $\begin{array}{l}16 \\
17 \\
18 \\
19 \\
20\end{array}$ & $\begin{array}{l}5.2 \\
5.1 \\
5.0 \\
5.1 \\
5.1\end{array}$ & $\begin{array}{l}4.8 \\
4.7 \\
4.5 \\
4.5 \\
4.3\end{array}$ & $\begin{array}{l}e 3.0 \\
e 3.1 \\
e 2.7 \\
e 3.0 \\
02.8\end{array}$ & $\begin{array}{r}03.7 \\
3.8 \\
2.9 \\
02.6 \\
02.6\end{array}$ & $\begin{array}{r}\mathrm{e} 2.6 \\
2.9 \\
2.8 \\
2.8 \\
2.9\end{array}$ & $\begin{array}{l}3.3 \\
3.2 \\
3.0 \\
3.0 \\
2.9\end{array}$ & $\begin{array}{l}6.3 \\
6.0 \\
5.8 \\
5.6 \\
5.8\end{array}$ & $\begin{array}{l}2.0 \\
1.8 \\
1.6 \\
1.4 \\
1.3\end{array}$ & $\begin{array}{l}.56 \\
.66 \\
.57 \\
.32 \\
.22\end{array}$ & $\begin{array}{l}1.2 \\
1.1 \\
1.2 \\
2.2 \\
1.1\end{array}$ & $\begin{array}{r}.72 \\
1.0 \\
.89 \\
.86 \\
.82\end{array}$ & $\begin{array}{l}4.1 \\
3.5 \\
3.6 \\
3.5 \\
3.1\end{array}$ \\
\hline $\begin{array}{l}21 \\
22 \\
23 \\
24 \\
25\end{array}$ & $\begin{array}{l}5.2 \\
4.9 \\
4.9 \\
4.9 \\
5.1\end{array}$ & $\begin{array}{l}4.2 \\
4.4 \\
4.3 \\
4.1 \\
4.0\end{array}$ & $\begin{array}{l}02.9 \\
03.1 \\
83.0 \\
03.2 \\
03.4\end{array}$ & $\begin{array}{l}02.9 \\
02.9 \\
02.8 \\
02.9 \\
\mathrm{e} 3.0\end{array}$ & $\begin{array}{l}3.0 \\
3.3 \\
3.2 \\
2.8 \\
3.0\end{array}$ & $\begin{array}{l}3.1 \\
3.2 \\
3.4 \\
3.4 \\
3.1\end{array}$ & $\begin{array}{l}5.7 \\
5.3 \\
4.9 \\
4.8 \\
4.9\end{array}$ & $\begin{array}{l}1.2 \\
1.1 \\
1.1 \\
1.0 \\
1.1\end{array}$ & $\begin{array}{l}.20 \\
.23 \\
.24 \\
.17 \\
.14\end{array}$ & $\begin{array}{c}1.2 \\
2.2 \\
.96 \\
.88 \\
1.8\end{array}$ & $\begin{array}{l}.74 \\
1.5 \\
4.2 \\
6.8 \\
7.6\end{array}$ & $\begin{array}{l}3.6 \\
3.4 \\
2.8 \\
2.7 \\
2.4\end{array}$ \\
\hline $\begin{array}{l}26 \\
27 \\
28 \\
29 \\
30 \\
31\end{array}$ & $\begin{array}{l}5.1 \\
4.9 \\
4.8 \\
4.8 \\
4.8 \\
4.7\end{array}$ & $\begin{array}{l}4.1 \\
4.1 \\
3.7 \\
3.7 \\
4.8 \\
-\cdots\end{array}$ & $\begin{array}{l}03.4 \\
03.5 \\
03.1 \\
03.3 \\
03.6 \\
03.7\end{array}$ & $\begin{array}{l}02.8 \\
02.9 \\
02.7 \\
02.9 \\
02.7 \\
02.6\end{array}$ & $\begin{array}{l}2.9 \\
2.8 \\
2.7 \\
2.9 \\
-.-\end{array}$ & $\begin{array}{l}2.8 \\
3.1 \\
3.4 \\
3.4 \\
3.4 \\
3.4\end{array}$ & $\begin{array}{l}5.5 \\
5.5 \\
5.5 \\
6.0 \\
5.6 \\
-\end{array}$ & $\begin{array}{r}1.2 \\
1.2 \\
1.1 \\
.94 \\
.88 \\
.83\end{array}$ & $\begin{array}{l}.16 \\
.78 \\
.39 \\
.27 \\
.44 \\
-.-\end{array}$ & $\begin{array}{l}1.6 \\
2.0 \\
1.1 \\
1.4 \\
1.7 \\
1.9\end{array}$ & $\begin{array}{l}8.2 \\
7.1 \\
7.6 \\
8.1 \\
7.1 \\
6.5\end{array}$ & $\begin{array}{l}2.3 \\
2.6 \\
2.5 \\
2.3 \\
2.0\end{array}$ \\
\hline $\begin{array}{l}\text { TOTAL } \\
\text { MEAN } \\
\text { MAX } \\
\text { MIN } \\
\text { AC-FT }\end{array}$ & $\begin{array}{r}182.9 \\
5.90 \\
10 \\
4.7 \\
363\end{array}$ & $\begin{array}{r}135.1 \\
4.50 \\
5.0 \\
3.7 \\
268\end{array}$ & $\begin{array}{r}107.1 \\
3.45 \\
4.8 \\
2.6 \\
212\end{array}$ & $\begin{array}{r}97.2 \\
3.14 \\
4.0 \\
2.6 \\
1.93\end{array}$ & $\begin{array}{r}82.9 \\
2.86 \\
3.3 \\
2.6 \\
164\end{array}$ & $\begin{array}{r}95.9 \\
3.09 \\
3.5 \\
2.6 \\
190\end{array}$ & $\begin{array}{r}160.6 \\
5.35 \\
6.5 \\
3.6 \\
319\end{array}$ & $\begin{array}{r}73.05 \\
2.36 \\
5.3 \\
.83 \\
145\end{array}$ & $\begin{array}{r}12.74 \\
.42 \\
.78 \\
.14 \\
25\end{array}$ & $\begin{array}{r}44.09 \\
1.42 \\
4.5 \\
.15 \\
87\end{array}$ & $\begin{array}{r}89.01 \\
2.87 \\
8.2 \\
.60 \\
177\end{array}$ & $\begin{array}{r}110.2 \\
3.67 \\
5.5 \\
2.0 \\
219\end{array}$ \\
\hline $\begin{array}{l}\text { STATIS } \\
\text { MEAN } \\
\text { MAX } \\
(\text { WY }) \\
\text { MIN } \\
\text { (WY })\end{array}$ & $\begin{array}{l}\text { CS OF } \\
3.76 \\
23.2 \\
1961 \\
1000 \\
1952\end{array}$ & $\begin{array}{l}\text { NTHLY MAAN } \\
3.35 \\
12.3 \\
1920 \\
000 \\
1952\end{array}$ & $\begin{array}{l}\text { DATA FOR } \\
2.42 \\
8.80 \\
1920 \\
1.13 \\
1957\end{array}$ & $\begin{array}{l}\text { WATER } \\
2.15 \\
8.04 \\
1920 \\
1029 \\
1957\end{array}$ & $\begin{array}{c}\text { YEARS } 1916 \\
2.31 \\
7.35 \\
1987 \\
1.14 \\
1957\end{array}$ & $\begin{array}{c}-1996 \\
5.10 \\
25.5 \\
1987 \\
.33 \\
1955\end{array}$ & $\begin{array}{c}\text { BY WATER } \\
25.4 \\
126 \\
1924 \\
1.94 \\
1925\end{array}$ & $\begin{array}{c}\text { YEAR (WY) } \\
46.7 \\
196 \\
1924 \\
97 \\
1963\end{array}$ & $\begin{array}{r}18.9 \\
122 \\
1979 \\
198 \\
1963\end{array}$ & $\begin{array}{l}7.31 \\
31.9 \\
1921 \\
003 \\
1964\end{array}$ & $\begin{array}{r}14.9 \\
159 \\
1991 \\
1971 \\
1974\end{array}$ & $\begin{array}{l}5.61 \\
51.7 \\
1991 \\
000 \\
1951\end{array}$ \\
\hline
\end{tabular}

SUMAARY STATTSTICS ANNUAL TOTAL

ANNUAL. MEAN

HIGEEST ANNUAL MEAN

LOWEST ANNUAL MEAN

GIGEEST DAILY MEAN

LOWEST DAILY MEAN

ANRUAL SEVEN-DAY MINIMUM

INSTANTANEOUS PEAX FLOW

INSTANTANEOUS PEAK STAGE

INSTANTANEOUS LOW FLOW

ANNUAL RUNOFF (AC-FT)

10 PERCENT EXCEEDS

50 PERCENT EXCEEDS

90 PERCENT EXCEEDS

- Estimated

3.5

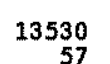
DAIUY MEAN VALUES

Q2.

3.0

3.0

3.1

3.3

3.2
3.0

3.0

2.9

3.1

3.4

3.1

1.1

.14

.39
.27

2.74

.42

.14

1963

FOR 1995 CALENDAR YEAR 6821.8
18.7

FOR 1996 WATER YEAR 1190.79

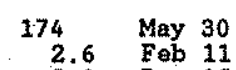

$\begin{array}{ll}2.6 & \text { Fab } 11 \\ 2.9 & \text { Dec } 15\end{array}$

57.2

3.5

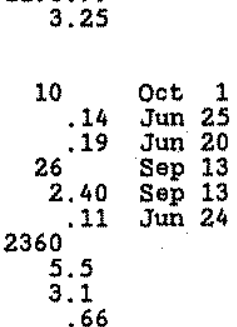

WATER YEARS 1916 - 1996

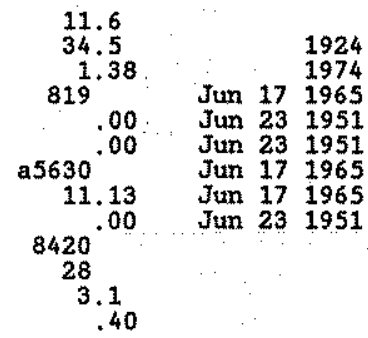

a-From rating curve extended above $230 \mathrm{ft} / \mathrm{s}$ on basis of slope-area measurements at gage heights $3.56 \mathrm{ft}, 5.80$ $\mathrm{ft}, 7.15 \mathrm{ft}$ and $11.13 \mathrm{ft}$. 
07208500 RAYADO CREEK AT SAUBLE RANCB, NEAR CIMARRON, NM

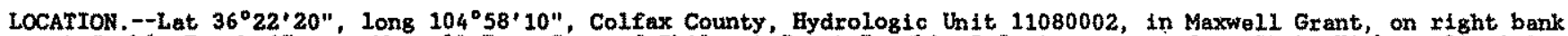
at Sauble Ranch (Carson-Maxwoll Base Camp of Philmont Scout Ranch), 2.5 mi upstream from State Highway 21, 4.0 mi downstream from Bonito Creek, and $9.8 \mathrm{mi}$ southwest of Cimarron.

DRAINAGE AREA. $--65 \mathrm{mi}^{2}$.

PERIOD OF RECORD.--January 1909 to February 1910, June to August 1910, May 1911 to May 1913 , July 1913 to February 1915, October 1915 to September 1918, March 1919 to September 1920, June 1923 to Septenber 1924, March to May 1927, August 1927 to current yeax. Monthly discharge only for some pertods, published in wSP 1311. Records for April and May 1910, published in WSP 267, are unreliablo and should not be used. "Published as Rayado River "at," "near," or "above" Abreu's Ranch near Cimerron prior to October 1925 and as Rayado River at Sauble Ranch, near Cimarron, October 1925 to September 1952

REVISED RECORDS. - WSP 1281: 1914, 1934-35(M), 1937 (M), 1941(P), 1942(M), 1944(M), drainage area.

GAGE.--Water-stage recorder. Concrete control since Oct. 13, 1976 . Elevation of gage is 6, 720 ft above National Geodetic Vertical Datum or 1929, from topographic map. See WSP 1921 for history of changes prior to Oct. 1, 1954. Oct... 1, 1954 to Jume 16, 1965, at site $270 \mathrm{ft}$ downstream at datum 2.79 ft lower.

REMARKS,--Records good excopt for estimated dally discharges, which are poor. No diverston upstream from station. EXTREMES OUTSIDE EERIOD OF RECORD. - The major flood of June 10, 1913 destroyed the gage (stage and discharge not determined). Another major flood probably occurred Sept. 29 or $30,1904$.

DISCHARGE, CUBIC FEET PER SECOMD, WATER YEAR OCTOBER 1995 TO SEPTEMBER 1996

\begin{tabular}{|c|c|c|c|c|c|c|c|c|c|c|c|c|}
\hline DAY & OCT & Nov & $\mathrm{DEC}$ & JAN & EEB & MAR & AFR & MAY & JUN & JUL & AUG & SEP \\
\hline $\begin{array}{l}1 \\
2 \\
3 \\
4 \\
5\end{array}$ & $\begin{array}{l}10 \\
9.6 \\
9.4 \\
8.3 \\
8.0\end{array}$ & $\begin{array}{l}6.2 \\
6.8 \\
6.2 \\
6.0 \\
6.4\end{array}$ & $\begin{array}{l}6.5 \\
6.1 \\
5.7 \\
5.5 \\
5.7\end{array}$ & $\begin{array}{l}04.5 \\
24.7 \\
05.1 \\
05.0 \\
05.0\end{array}$ & $\begin{array}{r}5.6 \\
e 5.8 \\
e 5.7 \\
e 6.1 \\
66.0\end{array}$ & $\begin{array}{l}8.8 \\
8.6 \\
7.3 \\
5.9 \\
5.6\end{array}$ & $\begin{array}{l}11 \\
12 \\
13 \\
12 \\
11\end{array}$ & $\begin{array}{l}12 \\
12 \\
12 \\
11 \\
11\end{array}$ & $\begin{array}{l}3.7 \\
3.6 \\
3.5 \\
3.4 \\
3.1\end{array}$ & $\begin{array}{l}7.5 \\
5.0 \\
4.2 \\
3.8 \\
4.5\end{array}$ & $\begin{array}{l}5.1 \\
5.1 \\
4.6 \\
3.3 \\
2.8\end{array}$ & $\begin{array}{l}5.4 \\
4.5 \\
5.7 \\
4.0 \\
3.7\end{array}$ \\
\hline $\begin{array}{r}6 \\
7 \\
8 \\
9 \\
10\end{array}$ & $\begin{array}{l}8.0 \\
7.9 \\
7.7 \\
7.2 \\
7.0\end{array}$ & $\begin{array}{l}6.2 \\
6.2 \\
6.2 \\
6.2 \\
7.2\end{array}$ & $\begin{array}{r}5.6 \\
5.6 \\
5.7 \\
e 5.6 \\
5.5\end{array}$ & $\begin{array}{l}e 5.3 \\
05.5 \\
e 5.6 \\
05.7 \\
05.4\end{array}$ & $\begin{array}{l}6.3 \\
5.9 \\
5.7 \\
6.0 \\
6.3\end{array}$ & $\begin{array}{l}5.2 \\
9.7 \\
6.4 \\
8.3 \\
6.8\end{array}$ & $\begin{array}{l}9.8 \\
11 \\
10 \\
11 \\
12\end{array}$ & $\begin{array}{l}11 \\
10 \\
9.8 \\
8.9 \\
8.9\end{array}$ & $\begin{array}{l}2.9 \\
3.0 \\
2.9 \\
2.8 \\
3.0\end{array}$ & $\begin{array}{l}4.0 \\
5.2 \\
5.2 \\
6.1 \\
7.5\end{array}$ & $\begin{array}{l}2.5 \\
2.5 \\
3.3 \\
3.7 \\
3.5\end{array}$ & $\begin{array}{l}3.8 \\
3.9 \\
3.6 \\
3.4 \\
3.3\end{array}$ \\
\hline $\begin{array}{l}11 \\
12 \\
13 \\
14 \\
15\end{array}$ & $\begin{array}{l}7.0 \\
6.7 \\
6.7 \\
6.7 \\
6.4\end{array}$ & $\begin{array}{l}5.6 \\
6.8 \\
6.7 \\
6.5 \\
6.2\end{array}$ & $\begin{array}{l}5.3 \\
4.9 \\
4.9 \\
4.8 \\
4.2\end{array}$ & $\begin{array}{r}95.5 \\
05.3 \\
5.6 \\
5.6 \\
6.4\end{array}$ & $\begin{array}{l}6.5 \\
6.0 \\
5.7 \\
6.2 \\
6.7\end{array}$ & $\begin{array}{l}7.2 \\
7.5 \\
8.3 \\
8.9 \\
8.7\end{array}$ & $\begin{array}{l}12 \\
12 \\
12 \\
9.8 \\
12\end{array}$ & $\begin{array}{l}8.8 \\
8.3 \\
8.0 \\
7.6 \\
7.0\end{array}$ & $\begin{array}{l}2.9 \\
2.8 \\
3.1 \\
4.5 \\
5.3\end{array}$ & $\begin{array}{r}9.0 \\
7.4 \\
11 \\
8.3 \\
6.8\end{array}$ & $\begin{array}{l}3.1 \\
2.5 \\
2.4 \\
2.6 \\
3.6\end{array}$ & $\begin{array}{l}3.9 \\
3.8 \\
6.4 \\
4.6 \\
7.4\end{array}$ \\
\hline $\begin{array}{l}16 \\
17 \\
18 \\
19 \\
20\end{array}$ & $\begin{array}{l}6.4 \\
6.4 \\
6.3 \\
6.1 \\
6.1\end{array}$ & $\begin{array}{l}6.2 \\
5.9 \\
5.7 \\
5.5 \\
5.5\end{array}$ & $\begin{array}{r}4.2 \\
04.2 \\
04.0 \\
03.8 \\
03.5\end{array}$ & $\begin{array}{r}5.5 \\
05.3 \\
7.0 \\
7.0 \\
7.7\end{array}$ & $\begin{array}{l}7.5 \\
7.2 \\
7.4 \\
6.9 \\
7.6\end{array}$ & $\begin{array}{l}8.0 \\
7.8 \\
7.4 \\
7.2 \\
7.6\end{array}$ & $\begin{array}{l}14 \\
13 \\
12 \\
12 \\
12\end{array}$ & $\begin{array}{l}6.6 \\
6.3 \\
6.0 \\
5.6 \\
5.4\end{array}$ & $\begin{array}{l}3.9 \\
3.7 \\
2.9 \\
2.4 \\
2.2\end{array}$ & $\begin{array}{l}5.7 \\
4.8 \\
4.4 \\
4.0 \\
3.8\end{array}$ & $\begin{array}{l}3.6 \\
3.9 \\
3.0 \\
2.5 \\
3.0\end{array}$ & $\begin{array}{l}5.0 \\
4.4 \\
7.8 \\
7.2 \\
5.2\end{array}$ \\
\hline $\begin{array}{l}21 \\
22 \\
23 \\
24 \\
25\end{array}$ & $\begin{array}{l}6.1 \\
6.4 \\
6.1 \\
6.5 \\
6.5\end{array}$ & $\begin{array}{l}5.6 \\
5.6 \\
5.3 \\
5.0 \\
5.1\end{array}$ & $\begin{array}{l}03.7 \\
84.1 \\
04.0 \\
04.1 \\
03.7\end{array}$ & $\begin{array}{l}9.1 \\
6.5 \\
8.4 \\
8.7 \\
9.6\end{array}$ & $\begin{array}{l}9.1 \\
9.2 \\
7.6 \\
6.8 \\
7.2\end{array}$ & $\begin{array}{l}7.8 \\
8.8 \\
9.4 \\
9.3 \\
8.7\end{array}$ & $\begin{array}{l}12 \\
12 \\
11 \\
12 \\
12\end{array}$ & $\begin{array}{l}5.3 \\
5.2 \\
4.8 \\
4.7 \\
5.2\end{array}$ & $\begin{array}{l}2.1 \\
2.9 \\
3.7 \\
2.7 \\
2.6\end{array}$ & $\begin{array}{l}4.0 \\
5.9 \\
4.8 \\
4.0 \\
4.0\end{array}$ & $\begin{array}{l}4.4 \\
4.4 \\
15 \\
11 \\
8.5\end{array}$ & $\begin{array}{l}4.5 \\
4.1 \\
3.8 \\
3.7\end{array}$ \\
\hline $\begin{array}{l}26 \\
27 \\
28 \\
29 \\
30 \\
31\end{array}$ & $\begin{array}{l}6.2 \\
6.1 \\
6.1 \\
6.4 \\
6.2 \\
6.1\end{array}$ & $\begin{array}{l}5.5 \\
4.2 \\
3.4 \\
6.5 \\
6.9 \\
-\cdots\end{array}$ & $\begin{array}{l}e 3.9 \\
e 3.8 \\
04.1 \\
04.2 \\
04.6 \\
04.9\end{array}$ & $\begin{array}{r}e 7.6 \\
66.8 \\
e 6.5 \\
e 5.7 \\
5.1 \\
6.2\end{array}$ & $\begin{array}{l}6.0 \\
5.2 \\
6.0 \\
9.4 \\
-.-\end{array}$ & $\begin{array}{r}8.8 \\
9.0 \\
8.9 \\
9.4 \\
9.5 \\
10\end{array}$ & $\begin{array}{l}13 \\
13 \\
14 \\
13 \\
13 \\
-\end{array}$ & $\begin{array}{l}5.0 \\
4.5 \\
4.1 \\
3.9 \\
4.6 \\
4.2\end{array}$ & $\begin{array}{c}4.0 \\
13 \\
8.1 \\
5.6 \\
6.8\end{array}$ & $\begin{array}{l}4.2 \\
4.0 \\
4.7 \\
4.1 \\
4.7 \\
7.9\end{array}$ & $\begin{array}{l}7.5 \\
6.0 \\
7.5 \\
5.3 \\
4.8 \\
4.9\end{array}$ & $\begin{array}{l}4.1 \\
3.8\end{array}$ \\
\hline $\begin{array}{l}\text { TOTAR } \\
\text { MEAN } \\
\text { MAX } \\
\text { MIN } \\
\text { AC-FI }\end{array}$ & $\begin{array}{r}216.6 \\
6.99 \\
10 \\
6+\frac{1}{430}\end{array}$ & $\begin{array}{r}177.3 \\
5.91 \\
7.2 \\
3.4 \\
352\end{array}$ & $\begin{array}{r}145.44 \\
4.72 \\
6.5 \\
3.5 \\
290\end{array}$ & $\begin{array}{r}192.9 \\
6.22 \\
9.6 \\
4.5 \\
383\end{array}$ & $\begin{array}{r}193.5 \\
6.68 \\
9.4 \\
5.2 \\
384\end{array}$ & $\begin{array}{r}250.8 \\
8.09 \\
10 \\
5.2 \\
497\end{array}$ & $\begin{array}{r}358.6 \\
12.0 \\
14 \\
9.8 \\
711\end{array}$ & $\begin{array}{r}227.7 \\
7.35 \\
12 \\
3.9 \\
452\end{array}$ & $\begin{array}{r}11.7 .1 \\
3.90 \\
13 \\
2.1 \\
232\end{array}$ & $\begin{array}{r}170.5 \\
5.50 \\
11 \\
3.8 \\
330\end{array}$ & $\begin{array}{r}145.9 \\
4.71 \\
15 \\
2.4 \\
289\end{array}$ & $\begin{array}{r}136.7 \\
4.56 \\
7.8 \\
3.3 \\
271\end{array}$ \\
\hline $\begin{array}{l}\text { STATIS' } \\
\text { MEAN } \\
\text { MAX } \\
\text { (WIY }) \\
\text { MIN } \\
(W Y)\end{array}$ & $\begin{array}{l}\text { CS OF } \\
5.85 \\
30.4 \\
1942 \\
1.23 \\
1957\end{array}$ & $\begin{array}{r}\text { ONTEL.Y M } \\
4.98 \\
20.0 \\
1.42 \\
1.40 \\
1.957\end{array}$ & $\begin{array}{l}\text { DATA } \\
3.94 \\
12.4 \\
1987 \\
1.27 \\
1957\end{array}$ & $\begin{array}{c}\text { FOR WATGR } \\
3.61 \\
8.01 \\
1.942 \\
1.58 \\
1957\end{array}$ & $\begin{array}{c}\text { YEARS } 1912 \\
4.04 \\
8.68 \\
1987 \\
1.95 \\
1951 .\end{array}$ & $\begin{array}{c}-1996 \\
7.67 \\
23.7 \\
1939 \\
2.98 \\
1951\end{array}$ & $\begin{array}{c}\text { BY WATER } \\
31.1 \\
144 \\
1987 \\
5.20 \\
1956\end{array}$ & $\begin{array}{c}\text { YEAR (WY) } \\
52.1 \\
287 \\
1941 \\
3.65 \\
1967\end{array}$ & $\begin{array}{r}23.0 \\
231 \\
1965 \\
1.79 \\
1956\end{array}$ & $\begin{array}{l}10.3 \\
54.7 \\
1959 \\
1.42 \\
1956\end{array}$ & $\begin{array}{l}11.5 \\
71.5 \\
1965 \\
2.10 \\
1956\end{array}$ & $\begin{array}{r}7.28 \\
33.0 \\
1991 \\
.88 \\
1956\end{array}$ \\
\hline
\end{tabular}

SURATARY STATISTICS ANWUAL TOTAI

ANNUAL MEAN

BIGHEST ANNUAL MEAN

LOWEST ANNUAL MEAN

GIGHEST DAILY MEAN

LOWEST DAILY MEAN

ANNUAL SEVEN-DAY MINTMUM

INSTANTANEOUS PEAK FLON

INSTANTANEOUS PEAK STAGE

INSTANTANEOUS LOW FLON

ANNUAL RUNOFF (AC-FT)

10 PERCENT EXCEEDS

50 PERCENT EXCEEDS

90 PERCENT EXCEEDS

a Estimated

a-From floodmarks

b-From rating curve extended above $70 \mathrm{ft}^{\mathrm{s}} / \mathrm{s}$ on basis of field estimate of peak flow.

c-Also may have been less during periods of tice effect.

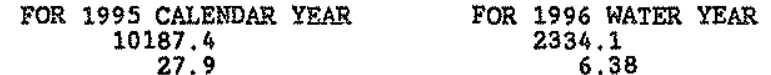

2334.13

$$
\begin{aligned}
& \begin{array}{r}
10187.4 \\
27.9
\end{array} \\
& 357 \text { Jun } 18 \\
& \begin{array}{lll}
2.5 & \text { Feb } & 11 \\
3.8 & \text { Dec } & 19
\end{array}
\end{aligned}
$$

20210

12

5.0

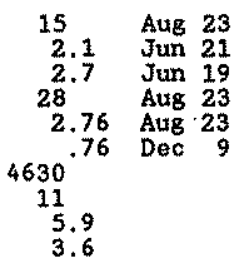

WATER YEARS 1912 - 1996

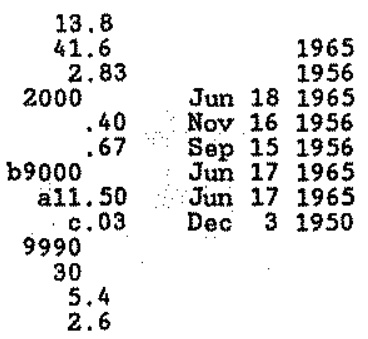


07211000 CTMARRON RIVER AT SPRINGER, MM

LOCATION. -lat $36^{\circ} 21^{\prime} 37^{\prime \prime}$, long $104^{\circ} 35^{\prime} 53^{*}$, Colfax County, Eydrologle Untt 11080002, in Maxwell Grant, on left bank in Springer, $400 \mathrm{ft}$ downstrean from bridge on State Highway $21,0.3$ mi upstrean fron Salado Creek, and at mile

DRAINAGE AREA. $--1,032 \mathrm{mi}$.

PERIOD OF RECORD. --August 1907 to December 1909, January 1921 to February 1922 , October 1924 to January 1926, September 1926 to current year. Monthly discharge only for some periods, published in WsP 1311 . Published as Cimarron Creek at Springer, October 1952 to September 1965.

REVISED RECORDS. - WSP 827: 1934-36(M). WSP 1281: 1942, 1945-46(M).

GAGE.-Water-stage recorder. Concrate control since Nov. 5, 1954. Elevation of 8 age 15 ; 770 ft above National Geodetic Vertical Datum of 1929, from topographic map. See WSp 1311 or 1731 for history of changes prior to July 17,1942 .

REMARKS. - -Records good except for estimated dally discharges, which are poor. Flow partly regulated by Eagle Nest Lake (atation 07205500). Diversions for 1rrigation of about 23,000 acres upstream from station and a few hundred acres between station and mouth. Several observations of water temperature were made during the year. No flow at times.

EXTREMES OUTSIDE PERIOD OF RECORD.-Maximum stage, about $22 \mathrm{ft}$, Sept, 29, 1904 (backwater from debris on railroad bridge). Another major flood occurred June 11, 1913. Maximum discharge of these floods probably extended 10,000 $\mathrm{ft}^{3} / \mathrm{s}$, but probably were less than the 1965 flood. DISCHARGE, CIBIC FEET PER SECOND, MATER YEAR OCTOBER 1995 TO SEPTEMBER 1996

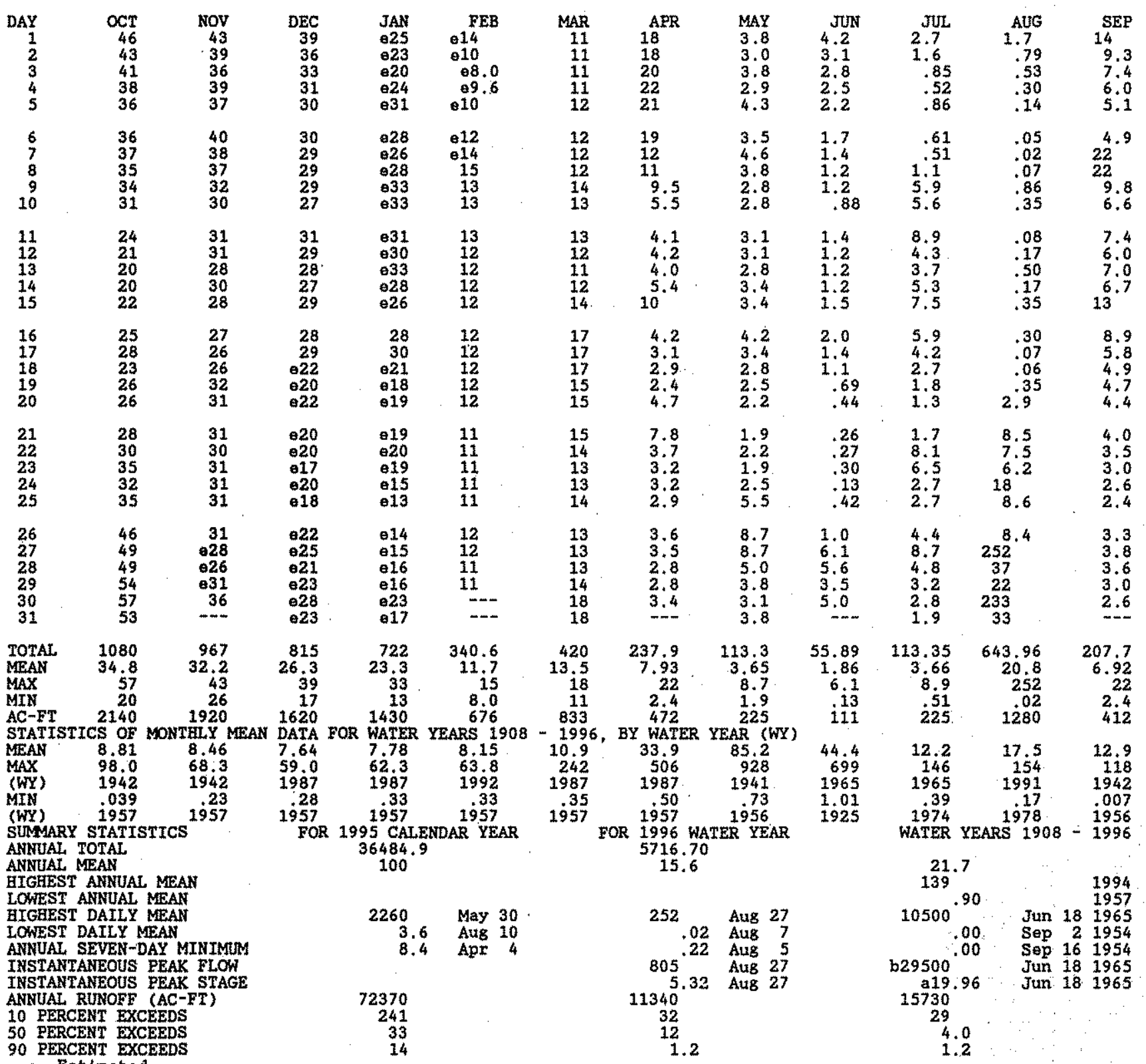




\section{ARKANSAS RIVER BASIN}

07211500 CANADIAN RIVER NEAR TAYLOR SPRINGS, MM

LOCATION, - Lat $36^{\circ} 17^{\prime} 49^{\prime \prime}$, long $14^{\circ} 29^{\prime} 36^{\prime \prime}$, in NWKSE sec.21, T.24 N., R.23 E., Colfax County, Hydrologic Unit

11080003 , on left bank at head of gorge, $2.0 \mathrm{mi}$ south of Taylor Springs, $2.3 \mathrm{mi}$ downstream from Cimerron River, $2.4 \mathrm{mi}$ upstream from Chico Crook, $7.1 \mathrm{mi}$ southeast of Springer, and at mile 847.9 .

DRAINAGE AREA. $--2,850 \mathrm{mt}^{2}$.

PERIOD OF RECORD. - January 1940 to September 1958, and annua1 maximum, water years 1959-63, June 1964 to current yoar. Water yoar estimato for 1940, published in WSP 1311.

REVISED RECORDS.-WSP 1177: Drainage area. WSP 1281: $1941-42(P), 1945-47(M), 1948-50(P)$,

GAGE. - Water-stage xecorder. Elevation of gage is 5,640 ft above National Geodetic Vertical Datum of 1929 , from topographic map. Frior to June 10, 1964, water-8tage recorder at site $1.7 \mathrm{mi}$ downstream at different datun; operated as crest-stage gage at that site and datum during water years 1959-64.

RERARKS --Rocords good except for estimated daily discharges which are poor. Diversions for frrigation of about 30,000 acres upstream from station. Several observations of water temperatore were made during the year. U.S. Army Corps of Engineers satellite telemeter at station. No flow at times some years.

EXTRERES OUTSIDE PERIOD OF RECORD. --Maximum flood prior to 1965 occurred Sept, 29, 1904, discharge published as $91,100 \mathrm{ft} 3 / \mathrm{s}$ in WSP 842,847 .

DISCHARGE, CUBIC FEET PER SECOND, WATER YEAR OCTOBER 1995 TO SEPTEMBER 1996

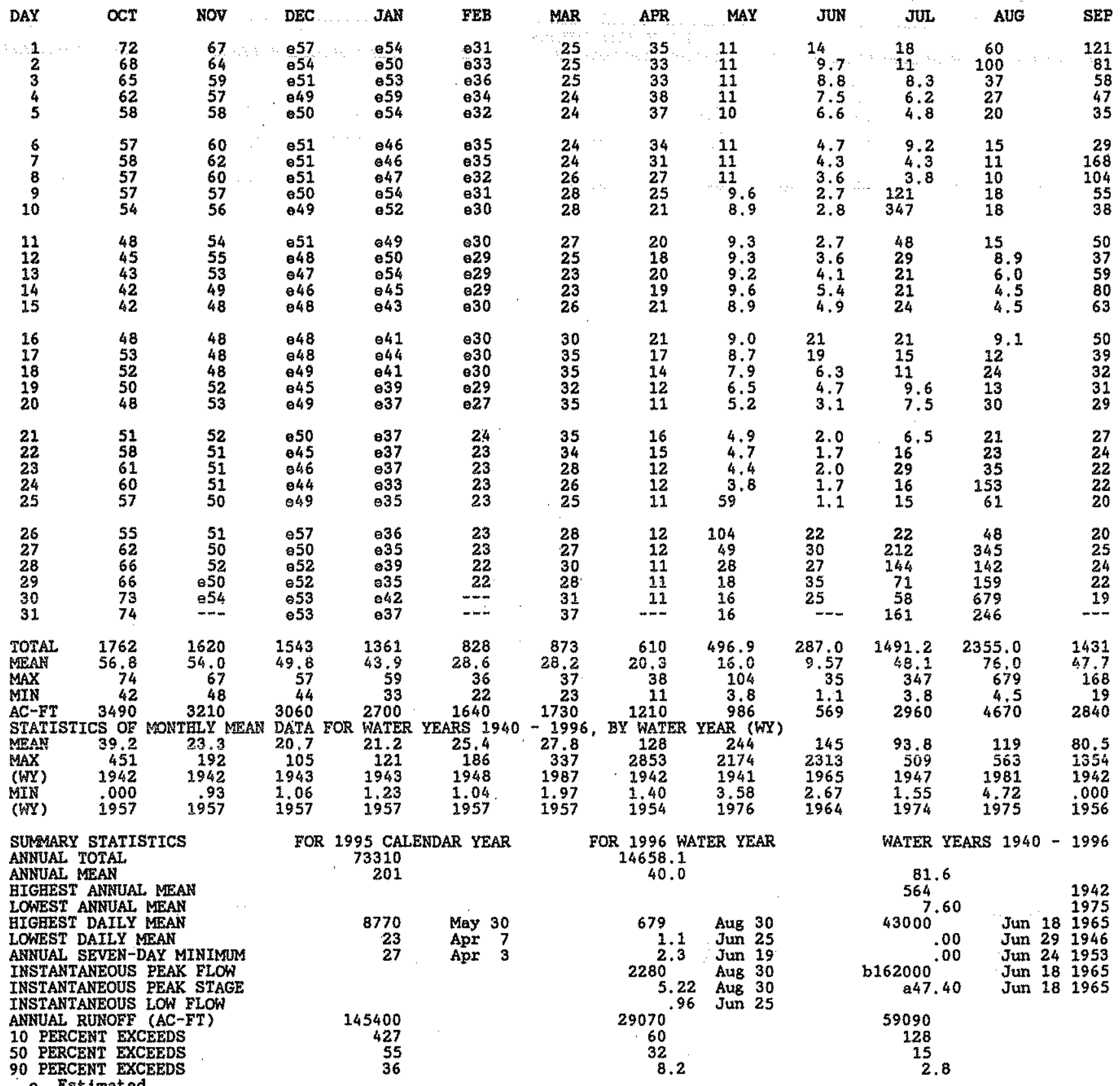

e Estimated

a-From floodmarks.

b-From rating curve extended above $7,000 \mathrm{fts} / \mathrm{s}$ on basis of slope-area measurement of peak flow. 
LOCATION.--Lat $35^{\circ} 56^{\circ} 27^{\prime \prime}$, Long $105^{\circ} 14^{\prime} 59^{\prime \prime}$, Mora County, Hydrologic Unit 11080004, in Mora Grant, on left bank 45 ft upstream from bridge on State Bighway 518 at La Cueva, $0.3 \mathrm{mi}$ downstream from La Cueva damsite, and at mile 86.8 . DRAINAGE AREA $-173 \mathrm{mi}^{2}$

PERIOD OF RECORD.--August 1903 to Apri1 1905 (gage helghts and discharge measurements only), May to December 1905, May 1906 to July 1911, April 1931 to current year. Monthly discharge only for some pertods, published in WSP 1311. Records for February to April 1905, published in WSP 173 are unreliable and should not be used.

REVISED RECORDS. - WSP 857: 1937. WSP 1281: 1931(M) 1932. WSP 1511: Drainage area, See also RERIOD OF RECORD

GAGE.-Water-stage recorder. Elevation of gage is 7,000 ft above National Geodetic Vertical Datum of 1929 , from topographic map. Mar. 10,1915 to June 4,1921 pater-stage recorder at site 2.8 mi upstream at different datum. July 6,1921 to Jan. 5, 1929 , nonrecording gage or water-stage recorder at site 0.7 mi downstream at datum about $14 \mathrm{ft}$ lower and Jan. 6, 1929 to Apr. 1, 1972, water"stage recorder at site $0.7 \mathrm{mi}$ downstream at datum about $15 \mathrm{ft}$ lower.

REMARKS.--Records good except for estimated daily discharges, which are poor. Diversions upstream from station for irrigation of about 7,000 acres, part of which are downstream from station. See tabulation below for monthly and yearly diversion of La Cueva Canal, which bypasses gage on left bank. Several observations of water temperature were made during the year. No flow at times.

EXTREMES OUTSIDE PERIOD OF RECORD.--FLood of Sept, 29, 1904, may have exceeded 20,000 fts/s; another major flood occurred June 11, 1913, but is believed less than that of 1904.

DISCHARGE, CUBIC FEET PER SECOND, WATER YEAR OCTOBER 1995 TO SEPTEMBER 1996

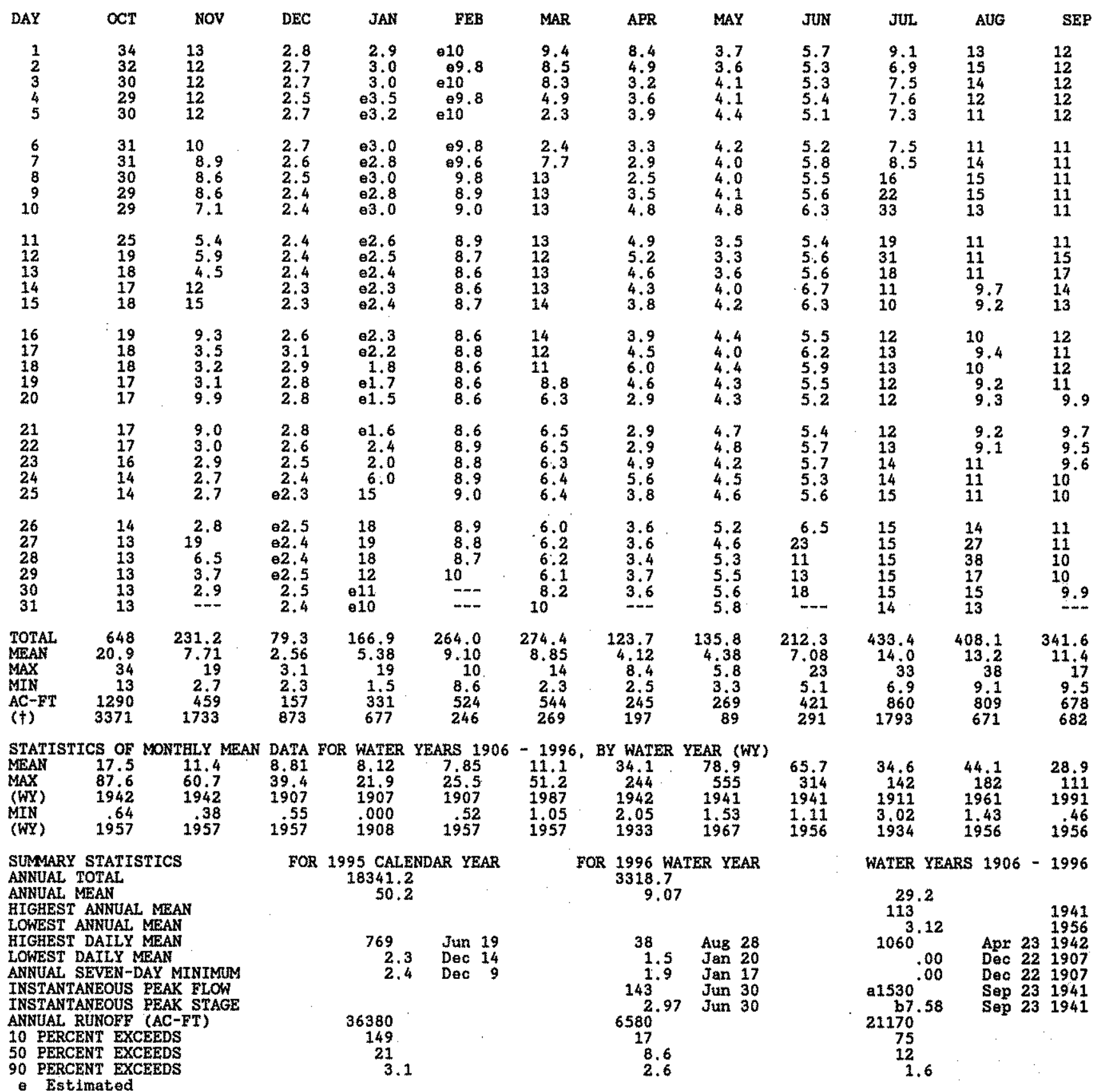

Estimated

a-From rating curve extended above $400 \mathrm{ft}^{3} / \mathrm{s}$.

b-Site and datum then in use.

CAl YR 1995 ( $\dagger$ ) 9820 WRT YR 1996 ( $\dagger$ ) 10590

(†) DIVERSION, IN ACRE-FEET, BY LA CUEVA CANAL 


\section{ARKANSAS RIVER BASIN}

07216500 MORA RIVER MEAR GOLONDRINAS, NM

LOCATION. -Lat $35^{\circ} 53^{\prime 2} 27^{\prime \prime}$, Long $105^{\circ} 09^{\prime} 47^{\prime \prime}$, Mora County, Hydrologic Unit 11080004, in Mora Grant, on right bank 0.7 mi upstream from bridge on State Highway 161, 1.2 mi east of Golondrinas, 1.9 mi upstream from Coyote Creek, 4.7 mi downstream from Rito Cebol1a, and at mile 75.8 .

DRATNAGE AREA. -267 mi. 2 .

PERIOD OF RECORD.-March 1915 to May 1921, October 1921 to March 1922, May, August, September 1922 , July 1923 to July 1924, December 1924 to September 1986, March 1988 to current year. Monthly discharge only $1915-30$, published in WSP 1311.

REVISED RECORDS,-WSP 1281, 1951(M).WSP 1311: 1935(M), 1937-38(M), 1940-42(M), 1949(M), WSP 1511: Drainage area. WSP 1731: $1958(\mathrm{M})$.

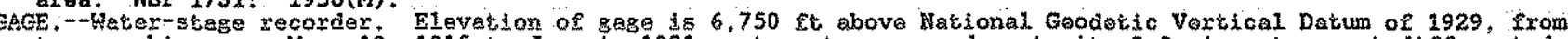
topographic map. Mar. 10, 1915 to June 4, 1921, water-stage recorder at site 2.8 mi upstream at different datum. July 6, 1921 to Jan. 5, 1929 , nonrecording 8 age or water-stage recorder at site 0.7 mi downstream at datum about 14 ft lower and Jan. 6, 1929 to Apx 1, 1972, watex-stage recorder at sito 0.7 mi domstream datum about 15 ft lower

REMARKS --Records good except for estimated datly discharges, which are poor. Diverstons for irrigation of about 12,000 acres upstream from station. Off-channel lakes meke it possible to divert and store water during nonirrigation sesson. Several observations of water temperature were made during the year... National Weather Service satellite telemeter at station. No flow at times some years.

EXTREMES OUTSIDE PERIOD OF RECORD.--Floods of Sept. 29, 1904, and June 11, 1913, probably exceeded 25,000 ft3/8. DISCHARGE, CUBIC FEET PER SECOND, WATER YEAR OCTOBER 1995 TO SEPTEMBER 1996

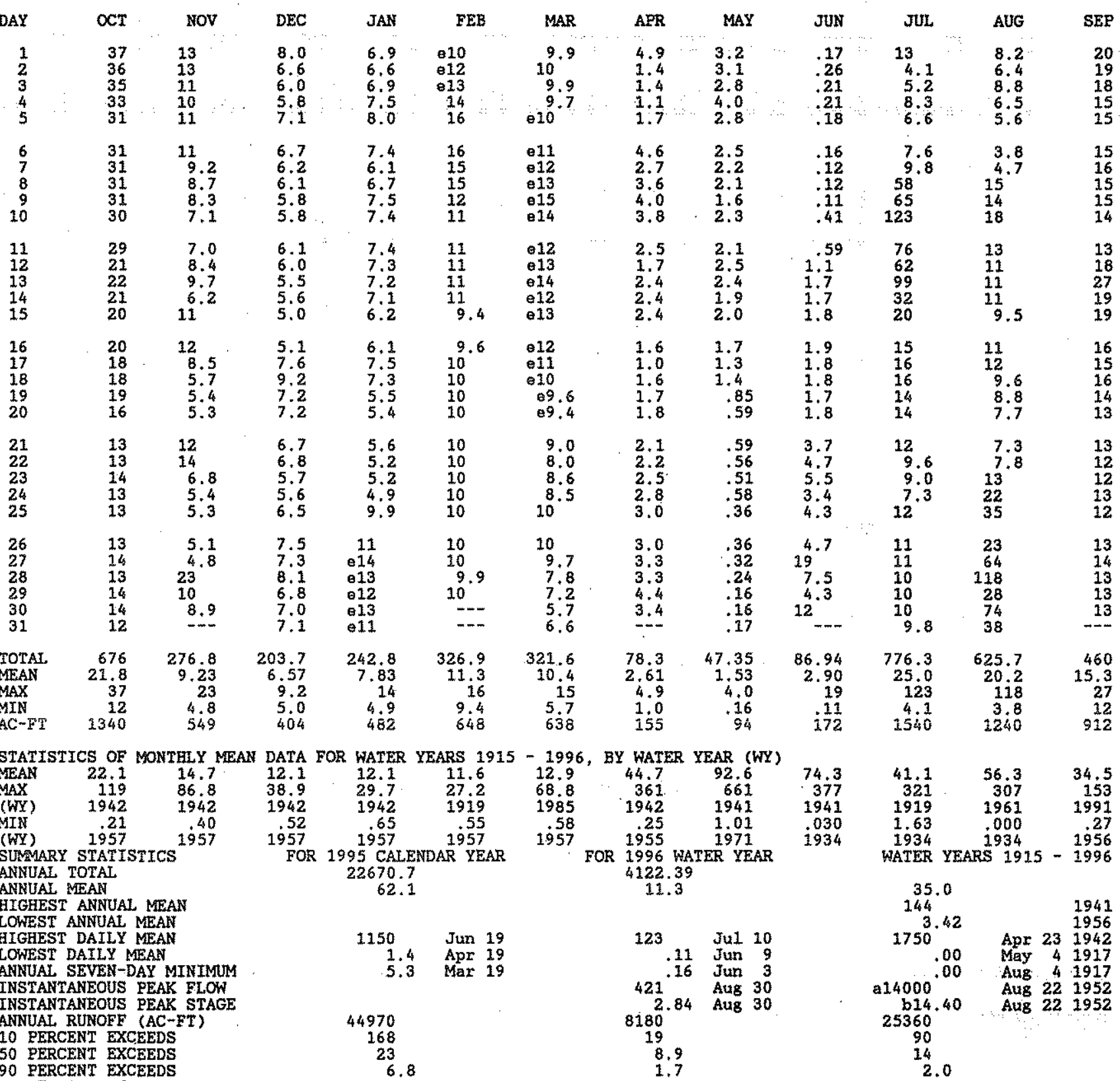

90 PERCENT EXCEEDS

a-From rating curve extended above $660 \mathrm{ft}^{3} / \mathrm{s}$ on basis of slope-area measurements of peak flow.

$b$-Site and datum then in use. 
ARKANSAS RIVER BASIN

07218000 COYOTE CREEK NEAR GOLONDRINAS, NM

LOCATION, -Lat $35^{\circ} 55^{\circ} 00^{\prime \prime}$, Long $105^{\circ} 09^{\prime} 49^{\prime \prime}$, Mora County, Hydrologic Unit 11080004, in Mora Grant, on left bank 0.5 mi downstream from Coyoté Creek damsite, 2.3 mi northeast of Golondrinas, and at mile 2.7 .

DRATNAGE AREA. $--215 \mathrm{mi}^{2}$.

PERIOD OF RECORD.--Apri1 1928 to September 1930 (monthly discharge only, published in WSP 1311 ), October 1930 to current year.

REVISED RECORDS.-WSP 1281: 1939-40(M), 1941-42, 1945-47. WSP 1511: Drainage area.

GAGE.-Water-stage recorder. Elevation of gage is 6,780 ft above National. Geodetic Vertical Datum of 1929 , from topographle map. Prior to Apr. 26, 1938 , at site 0.4 mi domstream at different datum (nonrecording gage prior to Apr. 20, 1929). Apr. 26, 1938 to Sept. 25, 1946, at site 139 ft downstream at same datum.

REMARKS.--Records sood except for estimated daily discharges, which are poor. Diversions (1ncluding off-channel storage) for irrigation of about 4,000 acres upstream from station. Several observations of water temperature were made during the year. No flow at times.

DISCAARGE, CUBIC FEET PER SECOND, WATER YEAR OCTOBER 1995 TO SEPTEMBKR 1996

\begin{tabular}{|c|c|c|c|c|c|c|c|c|c|c|c|c|}
\hline DAY & OCT & NOV & DEC & JAN & FEB & MAR & APR & MAY & JUN & JUL & AUG & SEP \\
\hline $\begin{array}{l}1 \\
2 \\
3 \\
4 \\
5\end{array}$ & $\begin{array}{l}13 \\
15 \\
15 \\
13 \\
15\end{array}$ & $\begin{array}{r}10 \\
12 \\
9.8 \\
9.4 \\
9.8\end{array}$ & $\begin{array}{l}16 \\
15 \\
14 \\
14 \\
13\end{array}$ & $\begin{array}{l}015 \\
014 \\
012 \\
011 \\
012\end{array}$ & $\begin{array}{l}7.9 \\
8.8 \\
12 \\
16 \\
10\end{array}$ & $\begin{array}{l}9.0 \\
8.8 \\
8.6 \\
8.1 \\
8.0\end{array}$ & $\begin{array}{l}8.8 \\
7.5 \\
7.3 \\
7.0 \\
7.0\end{array}$ & $\begin{array}{l}2.8 \\
2.8 \\
2.8 \\
2.6 \\
2.6\end{array}$ & $\begin{array}{l}3.2 \\
3.2 \\
2.9 \\
2.8 \\
2.8\end{array}$ & $\begin{array}{l}2.9 \\
2.5 \\
2.3 \\
2.2 \\
2.1\end{array}$ & $\begin{array}{l}3.9 \\
3.4 \\
2.8 \\
2.6 \\
2.3\end{array}$ & $\begin{array}{l}9.9 \\
8.5 \\
8.0 \\
7.4 \\
6.9\end{array}$ \\
\hline $\begin{array}{r}6 \\
7 \\
8 \\
9 \\
10\end{array}$ & $\begin{array}{l}15 \\
16 \\
16 \\
15 \\
16\end{array}$ & $\begin{array}{l}8.9 \\
8.9 \\
9.1 \\
9.0 \\
9.6\end{array}$ & $\begin{array}{l}13 \\
13 \\
14 \\
14 \\
14\end{array}$ & $\begin{array}{l}e 14 \\
e 12 \\
e 15 \\
e 13 \\
e 14\end{array}$ & $\begin{array}{r}10 \\
9.0 \\
9.1 \\
9.3 \\
8.9\end{array}$ & $\begin{array}{l}7.0 \\
6.4 \\
6.1 \\
6.5 \\
7.0\end{array}$ & $\begin{array}{l}8.4 \\
7.6 \\
7.2 \\
6.9 \\
6.2\end{array}$ & $\begin{array}{l}2.5 \\
2.3 \\
2.3 \\
2.3 \\
2.4\end{array}$ & $\begin{array}{r}2.9 \\
2.9 \\
\mathrm{e} 2.3 \\
\mathrm{e} 2.7 \\
\mathrm{e} 1.9\end{array}$ & $\begin{array}{r}2.0 \\
1.8 \\
1.9 \\
6.0 \\
12\end{array}$ & $\begin{array}{l}2.0 \\
2.6 \\
2.4 \\
2.6 \\
3.5\end{array}$ & $\begin{array}{r}16 \\
9.6 \\
7.4 \\
7.2 \\
6.8\end{array}$ \\
\hline $\begin{array}{l}11 \\
12 \\
13 \\
14 \\
15\end{array}$ & $\begin{array}{l}18 \\
16 \\
15 \\
14 \\
13\end{array}$ & $\begin{array}{l}9.7 \\
9.8 \\
8.9 \\
8.8 \\
9.2\end{array}$ & $\begin{array}{l}14 \\
14 \\
13 \\
13 \\
13\end{array}$ & $\begin{array}{l}16 \\
17 \\
17 \\
15 \\
16\end{array}$ & $\begin{array}{l}9.1 \\
10 \\
12 \\
13 \\
12\end{array}$ & $\begin{array}{l}7.1 \\
6.5 \\
6.1 \\
6.0 \\
7.1\end{array}$ & $\begin{array}{l}5.8 \\
5.7 \\
6.2 \\
6.3 \\
6.3\end{array}$ & $\begin{array}{l}2.4 \\
2.4 \\
2.5 \\
2.5 \\
2.6\end{array}$ & $\begin{array}{l}01.7 \\
01.8 \\
01.7 \\
02.1 \\
01.6\end{array}$ & $\begin{array}{r}10 \\
9.9 \\
9.6 \\
8.4 \\
7.6\end{array}$ & $\begin{array}{l}2.4 \\
2.0 \\
2.0 \\
1.9 \\
6.7\end{array}$ & $\begin{array}{l}6.8 \\
7.6 \\
8.5 \\
8.5 \\
8.7\end{array}$ \\
\hline $\begin{array}{l}16 \\
17 \\
18 \\
19 \\
20\end{array}$ & $\begin{array}{l}14 \\
14 \\
15 \\
14 \\
14\end{array}$ & $\begin{array}{l}9.2 \\
9.9 \\
9.9 \\
9.5 \\
9.5\end{array}$ & $\begin{array}{l}14 \\
15 \\
16 \\
14 \\
13\end{array}$ & $\begin{array}{l}14 \\
15 \\
14 \\
13 \\
11\end{array}$ & $\begin{array}{l}13 \\
13 \\
13 \\
16 \\
15\end{array}$ & $\begin{array}{l}7.9 \\
8.6 \\
8.6 \\
7.5 \\
7.2\end{array}$ & $\begin{array}{l}5.9 \\
5.6 \\
5.5 \\
5.2 \\
4.8\end{array}$ & $\begin{array}{l}2.8 \\
2.8 \\
2.8 \\
2.8 \\
2.8\end{array}$ & $\begin{array}{r}01.8 \\
e 1.6 \\
e 2.2 \\
01.8 \\
1.9\end{array}$ & $\begin{array}{l}7.3 \\
7.0 \\
7.4 \\
6.4 \\
6.0\end{array}$ & $\begin{array}{l}29 \\
13 \\
8.1 \\
6.3 \\
5.6\end{array}$ & $\begin{array}{r}8.7 \\
8.7 \\
9.7 \\
10 \\
10\end{array}$ \\
\hline $\begin{array}{l}21 \\
22 \\
23 \\
24 \\
25\end{array}$ & $\begin{array}{c}11 \\
10 \\
9.4 \\
11.4 \\
9.2\end{array}$ & $\begin{array}{l}10 \\
10 \\
10 \\
9.0 \\
6.1\end{array}$ & $\begin{array}{r}13 \\
15 \\
14 \\
16 \\
017\end{array}$ & $\begin{array}{l}13 \\
12 \\
10 \\
11 \\
15\end{array}$ & $\begin{array}{c}15 \\
15 \\
14 \\
11 \\
9.3\end{array}$ & $\begin{array}{l}6.0 \\
5.6 \\
5.5 \\
5.3 \\
5.0\end{array}$ & $\begin{array}{l}4.7 \\
4.6 \\
4.4 \\
4.0 \\
3.9\end{array}$ & $\begin{array}{l}2.9 \\
2.8 \\
2.7 \\
2.6 \\
2.7\end{array}$ & $\begin{array}{l}1.9 \\
1.9 \\
2.0 \\
2.0 \\
1.5\end{array}$ & $\begin{array}{l}4.4 \\
3.7 \\
3.5 \\
3.3 \\
3.6\end{array}$ & $\begin{array}{l}5.2 \\
5.1 \\
7.6 \\
8.4 \\
6.3\end{array}$ & $\begin{array}{l}8.8 \\
7.6 \\
6.9 \\
6.4 \\
6.3\end{array}$ \\
\hline $\begin{array}{l}26 \\
27 \\
28 \\
29 \\
30 \\
31\end{array}$ & $\begin{array}{r}9.2 \\
9.6 \\
9.4 \\
11 \\
12 \\
11\end{array}$ & $\begin{array}{r}5.4 \\
5.0 \\
9.7 \\
8.5 \\
15 \\
-.--\end{array}$ & $\begin{array}{l}e 14 \\
015 \\
017 \\
e 13 \\
e 12 \\
e 14\end{array}$ & $\begin{array}{l}11 \\
14 \\
14 \\
12 \\
9.9 \\
9.3\end{array}$ & $\begin{array}{l}9.1 \\
9.8 \\
10 \\
11 \\
-\ldots\end{array}$ & $\begin{array}{l}7.1 \\
8.0 \\
8.9 \\
9.4 \\
9.9 \\
9.9\end{array}$ & $\begin{array}{l}3.6 \\
3.5 \\
3.5 \\
3.1 \\
3.0 \\
--\end{array}$ & $\begin{array}{l}2.8 \\
3.0 \\
3.1 \\
3.0 \\
3.0 \\
3.2\end{array}$ & $\begin{array}{l}1.3 \\
4.0 \\
2.6 \\
2.9 \\
2.8 \\
\end{array}$ & $\begin{array}{l}8.0 \\
7.6 \\
5.9 \\
4.4 \\
3.3 \\
3.6\end{array}$ & $\begin{array}{l}6.1 \\
19 \\
9.8 \\
8.3 \\
20 \\
21\end{array}$ & $\begin{array}{l}6.9 \\
6.4 \\
6.2 \\
6.4 \\
6.3 \\
-\end{array}$ \\
\hline $\begin{array}{l}\text { TOTAL } \\
\text { MEAN } \\
\text { MAX } \\
\text { MIN } \\
\text { AC-WI }\end{array}$ & $\begin{array}{r}408.8 \\
13.2 \\
18 \\
9.2 \\
811\end{array}$ & $\begin{array}{r}279.6 \\
9.32 \\
15 \\
5.0 \\
555\end{array}$ & $\begin{array}{r}439 \\
14.2 \\
17 \\
12 \\
871\end{array}$ & $\begin{array}{r}411.2 \\
13.3 \\
17 \\
9.3 \\
816\end{array}$ & $\begin{array}{r}331.3 \\
11.4 \\
16 \\
7.9 \\
657\end{array}$ & $\begin{array}{r}228.7 \\
7.38 \\
9.9 \\
5.0 \\
454\end{array}$ & $\begin{array}{r}169.5 \\
5.65 \\
8.8 \\
3.0 \\
336\end{array}$ & $\begin{array}{r}83.6 \\
2.70 \\
3.2 \\
2.3 \\
1.66\end{array}$ & $\begin{array}{r}68.7 \\
2.29 \\
4.0 \\
1.3 \\
136\end{array}$ & $\begin{array}{r}166.6 \\
5.37 \\
12 \\
1.8 \\
330\end{array}$ & $\begin{array}{r}221.9 \\
7.16 \\
29 \\
1.9 \\
440\end{array}$ & $\begin{array}{r}243.1 \\
8.10 \\
16 \\
6.2 \\
482\end{array}$ \\
\hline
\end{tabular}

STATISTICS OF MONTHLY MEAN DATA FOR WATER YEARS 1930 - 1996, BY WATER YEAR (WY)

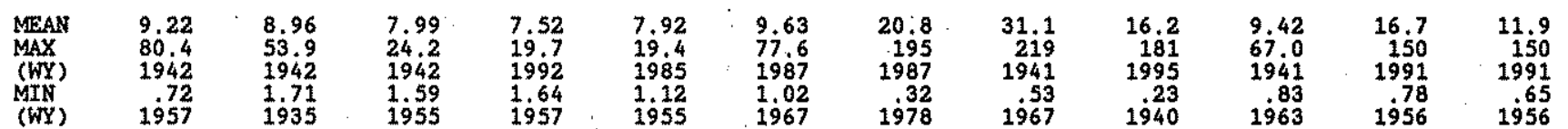

SUMMARY STATISTICS

ANNUAL TOTAL

ANNUAL MEAN

HIGHEST ANNUAL MEAN

LOWEST ANNUAL MEAN

HIGHEST DAILY MEAN

LOWEST DAILY MEAN

ANNUAL SEVEN-DAY MINIMIM

INSTANTANEOUS PEAK FLOW

INSTANTANEOUS PEAK STAGE

ANNUAL RUNOFF (AC-FT)

10 EERCENT EXCEEDS

50 PERCENT EXCEEDS

90 PERCENT EXCEEDS

- Estimated

a-Fron rating curve extended above $250 \mathrm{ft} / 3 / \mathrm{s}$ on basis of slopemarea measurements at gage heights $5.54 \mathrm{ft}, 7.74$ ft and $9.60 \mathrm{ft}$.

b-site and datum then in use.
FOR 1995 CALENDAR YEAR

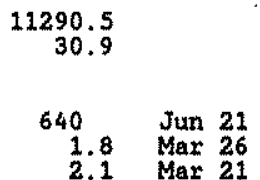

22390

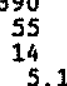

FOR 1996 WATER YEAR

$$
3052.0
$$

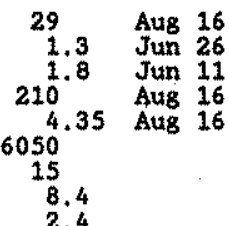

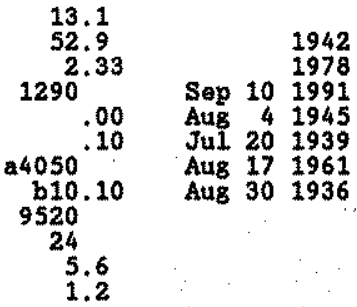




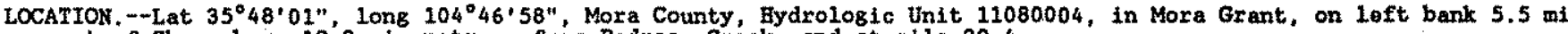
east of Shoenaker, 12.3 mi upstream from Pedroso Creek, and at mile 39.4 .

DRATNAGE AREA. $-1,104 \mathrm{mit}^{2}$, of which $71 \mathrm{mi}^{2}$ is probably noncontributing.

PERIOD OF RECORD.--October 1914 to July 1915 , October 1915 to August 1918 , May 1919 to July 1924 , September to November 1924, March to July 1925, June 1927 to September 1996 (discontinued). Prior to October 1930 monthly discharge only, published in WSP 1311.

REVISED RECORDS,-WSP 1117: Drainage area, WSP 1281:, 1931(M), 1933-1934(M), 1937(M), 1938(P), 1939-40(M), 194142(P). WSR 1731: 1921, 1928, 1951(M). WRD NM-75-1: 1974. WRD NM-78-1: 1977.

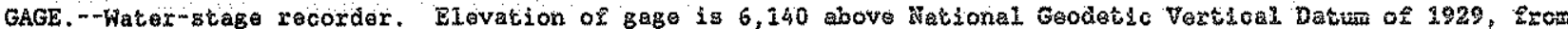
topographic map. Prior to Oct. 10, 1934, at site 2,000 ft upstream at different datum.

REMARKS,-Records good except for estimated dally discharges which are poor Diversions for irrigation of about 26,000 acres upstream from station. Off-channel lakes make it possible to divert and stoxe water during nonirrigation season. Several observations of water temperature were made during the year. No flow at times some years.

EXTREMES OUTSIDE FERIOD OF RECORD.--Floods of Sept, 29, 1904, and June 11, 1913, probably exceeded 30,000 ft3/s. DISCHARGE, CUBIC FEET PER SECOND, WATER YEAR OCTOBER 1995 TO SEPTIMMER 1996

\begin{tabular}{|c|c|c|c|c|c|c|c|c|c|c|c|c|c|c|}
\hline DAY & $\propto C T$ & NOV & & DEC & & JAN & FEB & MAR & AFR & MAY & JUN & JuL & AUG & SEP \\
\hline $\begin{array}{l}1 \\
2 \\
3 \\
4 \\
5\end{array}$ & $\begin{array}{l}55 \\
54 \\
55 \\
54 \\
49\end{array}$ & $\begin{array}{l}28 \\
26 \\
30 \\
30 \\
31\end{array}$ & & $\begin{array}{l}36 \\
40 \\
39 \\
34 \\
29\end{array}$ & & $\begin{array}{l}e 39 \\
027 \\
025 \\
e 27 \\
e 29\end{array}$ & $\begin{array}{r}035 \\
\text { e32 } \\
\text { e34 } \\
\quad 35 \\
\quad 040\end{array}$ & $\begin{array}{c}14 \\
11 . \\
7.9 \\
12 \\
9.4\end{array}$ & $\begin{array}{r}e 3.0 \\
03.1 \\
03.2 \\
03.3 \\
03.4\end{array}$ & $\begin{array}{l}02.9 \\
02.8 \\
02.6 \\
02.7 \\
02.5\end{array}$ & $\begin{array}{r}1.7 \\
2.1 \\
2.6 \\
3.0 \\
2.0\end{array}$ & $\begin{array}{l}5.7 \\
4.5 \\
3.7 \\
3.6 \\
3.2\end{array}$ & $\begin{array}{r}45 \\
14 \\
8.0 \\
\\
5.7 \\
4.8\end{array}$ & $\begin{array}{l}93 \\
61 \\
53 \\
47 \\
38\end{array}$ \\
\hline $\begin{array}{r}6 \\
7 \\
8 \\
9 \\
10\end{array}$ & $\begin{array}{l}53 \\
62 \\
61 \\
60 \\
61\end{array}$ & $\begin{array}{l}32 \\
32 \\
33 \\
32 \\
34\end{array}$ & & $\begin{array}{l}18 \\
16 \\
16 \\
25 \\
26\end{array}$ & & $\begin{array}{l}031 \\
e 34 \\
037 \\
041 \\
37\end{array}$ & $\begin{array}{r}039 \\
44 \\
47 \\
45 \\
45\end{array}$ & $\begin{array}{l}7.8 \\
13 \\
9.5 \\
1.3 \\
12\end{array}$ & $\begin{array}{l}e 3.0 \\
e 3.2 \\
\text { e3.1 } \\
\text { e3.2 } \\
\text { e3.0 }\end{array}$ & $\begin{array}{r}2.4 \\
3.1 \\
2.9 \\
3.1 \\
3.0\end{array}$ & $\begin{array}{l}01.9 \\
01.8 \\
01.8 \\
01.9 \\
02.1\end{array}$ & $\begin{array}{r}3.4 \\
3.1 \\
20 \\
404 \\
861\end{array}$ & $\begin{array}{l}4.7 \\
4.4 \\
4.6 \\
4.8 \\
5.1\end{array}$ & 35 \\
\hline $\begin{array}{l}11 \\
12 \\
13 \\
14 \\
15\end{array}$ & $\begin{array}{l}56 \\
55 \\
47 \\
45 \\
45\end{array}$ & $\begin{array}{l}36 \\
37 \\
37 \\
40 \\
38\end{array}$ & & $\begin{array}{l}25 \\
27 \\
26 \\
25 \\
25\end{array}$ & & $\begin{array}{l}40 \\
38 \\
38 \\
31 \\
29\end{array}$ & $\begin{array}{l}41 \\
40 \\
40 \\
41 \\
40\end{array}$ & $\begin{array}{l}9.8 \\
8.5 \\
8.2 \\
7.0 \\
8.7\end{array}$ & $\begin{array}{l}\text { e3.1 } \\
\text { e3.2 } \\
\text { e3.3 } \\
\text { e3.0 } \\
\text { e3.1 }\end{array}$ & $\begin{array}{l}2.9 \\
2.9 \\
2.9 \\
3.1 \\
2.8\end{array}$ & $\begin{array}{l}01.9 \\
e 1.6 \\
e 1.7 \\
01.8 \\
01.9\end{array}$ & $\begin{array}{r}880 \\
166 \\
155 \\
91 \\
50\end{array}$ & $\begin{array}{l}4.6 \\
4.1 \\
4.1 \\
4.0 \\
4.2\end{array}$ & $\begin{array}{l}35 \\
34 \\
49 \\
53\end{array}$ \\
\hline $\begin{array}{l}16 \\
17 \\
18 \\
19 \\
20\end{array}$ & $\begin{array}{l}42 \\
42 \\
29 \\
24 \\
25\end{array}$ & $\begin{array}{l}37 \\
38 \\
38 \\
36 \\
35\end{array}$ & & $\begin{array}{l}26 \\
26 \\
32 \\
35 \\
29\end{array}$ & & $\begin{array}{l}29 \\
29 \\
34 \\
39 \\
33\end{array}$ & $\begin{array}{l}37 \\
34 \\
30 \\
20 \\
19\end{array}$ & $\begin{array}{l}11 \\
14 \\
16 \\
14 \\
9.5 .\end{array}$ & $\begin{array}{l}e 2.6 \\
02.1 \\
02.8 \\
02.3 \\
02.9\end{array}$ & $\begin{array}{l}2.6 \\
2.5 \\
2.3 \\
2.2 \\
2.1\end{array}$ & $\begin{array}{r}02.0 \\
01.9 \\
01.9 \\
01.9 \\
1.7\end{array}$ & $\begin{array}{r}35 \\
012 \\
10 \\
69.0 \\
68.1\end{array}$ & $\begin{array}{l}5.0 \\
6.3 \\
6.3 \\
4.4 \\
4.4\end{array}$ & 43 \\
\hline $\begin{array}{l}21 \\
22 \\
23 \\
24 \\
25\end{array}$ & $\begin{array}{l}26 \\
22 \\
25 \\
30 \\
30\end{array}$ & $\begin{array}{l}35 \\
36 \\
38 \\
36 \\
34\end{array}$ & & $\begin{array}{r}31 \\
30 \\
35 \\
e 33 \\
e 32\end{array}$ & & $\begin{array}{r}e 34 \\
31 \\
e 33 \\
029 \\
027\end{array}$ & $\begin{array}{l}25 \\
21 \\
25 \\
24 \\
19\end{array}$ & $\begin{array}{l}6.9 \\
6.3 \\
4.9 \\
4.3 \\
5.8 .\end{array}$ & $\begin{array}{l}e 3.1 \\
e 2.5 \\
e 2.1 \\
e 1.9 \\
e 2.3\end{array}$ & $\begin{array}{l}2.3 \\
2.2 \\
1.7 \\
1.5 \\
3.5\end{array}$ & $\begin{array}{l}1.7 \\
1.8 \\
2.5 \\
2.0 \\
2.2\end{array}$ & $\begin{array}{l}99.8 \\
99.1 \\
09.7 \\
08.2 \\
09.6\end{array}$ & $\begin{array}{r}4.3 \\
4.2 \\
5.5 \\
11 \\
38\end{array}$ & $\begin{array}{l}6 \\
2 \\
3 \\
1\end{array}$ \\
\hline $\begin{array}{l}26 \\
27 \\
28 \\
29 \\
30 \\
31\end{array}$ & $\begin{array}{l}28 \\
29 \\
36 \\
35 \\
33 \\
35\end{array}$ & $\begin{array}{r}30 \\
29 \\
29 \\
31 \\
36 \\
-\end{array}$ & & $\begin{array}{l}\text { e30 } \\
e 31 \\
e 32 \\
e 30 \\
e 40 \\
e 38\end{array}$ & & $\begin{array}{l}e 24 \\
e 29 \\
e 41 \\
e 38 \\
e 35 \\
e 37\end{array}$ & $\begin{array}{r}16 \\
14 \\
14 \\
19 \\
-m-\end{array}$ & $\begin{array}{r}7.7 \\
5.5 \\
4.5 \\
5.3 \\
e 3.1 \\
e 3.2\end{array}$ & $\begin{array}{l}83.1 \\
\mathrm{e} 2.8 \\
\mathrm{e} .9 \\
\mathrm{e} .2 .2 \\
\mathrm{e} .0 \\
-\end{array}$ & $\begin{array}{r}3.0 \\
2.5 \\
2.0 \\
2.1 \\
2.2 \\
2.0\end{array}$ & $\begin{array}{l}2.7 \\
4.9 \\
5.2 \\
6.2 \\
6.8 \\
\end{array}$ & $\begin{array}{r}09.1 \\
011 \\
09.3 \\
010 \\
09.5 \\
09.2\end{array}$ & $\begin{array}{r}37 \\
114 \\
293 \\
126 \\
93 \\
746\end{array}$ & 20 \\
\hline $\begin{array}{l}\text { TOTAL } \\
\text { MEAK } \\
\text { MAX } \\
\text { MIN } \\
\text { AC- }\end{array}$ & $\begin{array}{r}1303 \\
42.0 \\
62 \\
22 \\
2580\end{array}$ & $\begin{array}{r}1014 \\
33.8 \\
40 \\
26 \\
2010\end{array}$ & & $\begin{array}{r}917 \\
29.6 \\
40 \\
16 \\
1820\end{array}$ & & $\begin{array}{r}1025 \\
33.1 \\
41 \\
24 \\
2030\end{array}$ & $\begin{array}{r}915 \\
31.6 \\
47 \\
14 \\
1810\end{array}$ & $\begin{array}{r}273.8 \\
3.83 \\
16 \\
3.1 \\
543\end{array}$ & $\begin{array}{r}86.8 \\
2.89 \\
3.4 \\
1.9 \\
172\end{array}$ & $\begin{array}{l}79.3 \\
2.56 \\
.3 .5 \\
1.5 \\
157\end{array}$ & $\begin{array}{r}75.2 \\
2.51 \\
6.8 \\
1.6 \\
149\end{array}$ & $\begin{array}{r}2832.9 \\
92.4 \\
880 \\
3.1 \\
5620\end{array}$ & $\begin{array}{r}1620.5 \\
52.3 \\
746 \\
4.0 \\
3210\end{array}$ & $\begin{array}{r}1071 \\
35.7 \\
93 \\
12 \\
2120\end{array}$ \\
\hline $\begin{array}{l}\text { STATI } \\
\text { MEAN } \\
\text { MAX } \\
\text { (WY) } \\
\text { MiLX } \\
\text { (WX) }\end{array}$ & $\begin{array}{r}\text { ICS OF } \\
38.4 \\
326 \\
1942 \\
.000 \\
1957\end{array}$ & $\begin{array}{r}\text { MONTELY } \\
30.3 \\
212 \\
1942 \\
.35 \\
1957\end{array}$ & MEAN & $\begin{array}{r}\text { DATA } \\
28.6 \\
134 \\
1924 \\
164 \\
1957\end{array}$ & FOR & $\begin{array}{l}\text { WATER } \\
28.6 \\
94.3 \\
1924 \\
.98 \\
1957\end{array}$ & $\begin{array}{c}\text { YEARS } 1920 \\
24.4 \\
126 \\
1987 \\
175 \\
1957\end{array}$ & $\begin{array}{c}1996 \\
25.4 \\
.318 \\
1987 \\
.58 \\
1955\end{array}$ & $\begin{array}{c}\text { BY WATER } \\
72.9 \\
842 \\
1942 \\
.34 \\
1955\end{array}$ & $\begin{array}{c}\text { YEAR (WY) } \\
131 \\
1437 \\
1941 \\
1.63 \\
1954\end{array}$ & $\begin{array}{r}108 \\
937 \\
1921 \\
440 \\
1954\end{array}$ & $\begin{array}{r}60.7 \\
700 \\
1921 \\
1920\end{array}$ & $\begin{array}{r}93.2 \\
587 \\
1961 \\
094 \\
1964\end{array}$ & $\begin{array}{r}62.0 \\
404 \\
1991 \\
1920 \\
1954\end{array}$ \\
\hline
\end{tabular}

SUMARY STATISTICS

ANNUAL, TOTAL

ANNUAL MEAN

BIGHEST ARNUAL MEAN

LOWEST ANNUAL MRAN

HTGEEST DAILY MEAN

LOWEST DAILY MEAN

ANNUAL SEVEN-DAY MINIMUM

IXSTANTANEOUS PEAK FLOW

IRSTANTANEOUS PEAK STAGE

ANNUAL RUNOFF (AC-FT)

10 PERCENT EXCEEDS

50 PERCENT EXCEEDS

90 PERCENT EXCEEDS

- Estimated

a-Fon rating cur and 12,79 ft.
FOR 1995 CALENDAR YEAR

$$
381.45 .1
$$

105

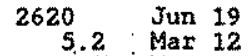

5.2
7.9

75660

204
43

15
FOR 1996. WATER YEAR

$$
\begin{array}{r}
11213.5 \\
30.6
\end{array}
$$

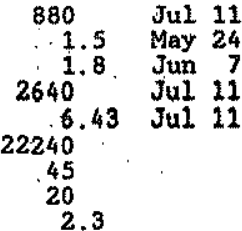

WATER YEARS $1920-1996$

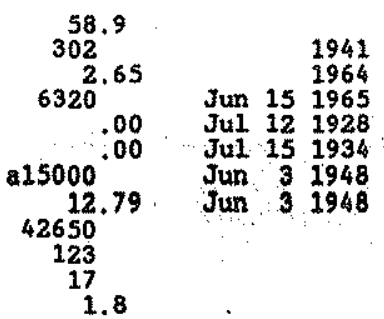


ARKANSAS RIVER BASIN

07221500 CANADIAN RIVER NEAR SANCHEZ, NM

LOCATION,--Lat $35^{\circ} 39^{\circ} 08^{\prime \prime}$, Long $104^{\circ} 22^{\prime} 39^{\prime \prime}$, In SWK sec.34, T.17 N., R.24 E., San Miguel County, Hydrologic Unit 11080003 , on rtght bank 1,000 ft downstream from bridge on State Highway $419,0.9$ mi upstream from Lagartifa Creak, 3.2 mi northeast of Sanchez, $10 \mathrm{mi}$ downstream frcm Mora River, $25 \mathrm{mi}$ southwest of Mosquero, and at mile 777.0 .

DRAINAGE AREA. $--6,015 \mathrm{mi}^{2}$, of whtch $303 \mathrm{mi}^{2}$ is probably noncontributing.

WATER-DISCHARGE RECORDS

PERIOD OF RECORD.--May 1912 to Decembar 1914, October 1935 to current year. Monthiy discharge only for some

periods, published tn WSP 1311. 82-1: 1965(M), 1979(M). The revised figures of discharge for September 1942, as pubilished in wSP 1281 supersede those published in WSP 1311.

GAGE. - Water-stage recorder. Elevation of gage is 4,500 ft above National Geodetic Vertical Datum of 1929 , from topographic map. See WSP 2121 for history of changes orior to November 1966.

RFMARKS.-Water-discharge records good. Diversions for irrigation of about 56,000 acres upstream from station. U.S. Aruy Corps of Englneers satellite telemeter at station. No flow at times some years.

EXTREMES OUTSIDE PERIOD OF RECORD.--The flood of Sept. 29 , or 30,1904 , probably exceeded 100,000 fts/s, but 1s belleved to have been less than the peak of June $18,1965$.

DISCEARGE, CUBIC FEET PER SECOND, WATER YEAR OCTOBER 1995 TO SEPTEMBER 1996

\begin{tabular}{|c|c|c|c|c|c|c|c|c|c|c|c|c|}
\hline DAY & OCT & Nov & DEC & JAN & FEB & MAR & APR & MAY & JUN & JUL & AUG & SEP \\
\hline $\begin{array}{l}1 \\
2 \\
3 \\
4 \\
5\end{array}$ & $\begin{array}{l}127 \\
122 \\
118 \\
112 \\
107\end{array}$ & $\begin{array}{l}98 \\
99 \\
94 \\
84 \\
83\end{array}$ & $\begin{array}{l}82 \\
90 \\
91 \\
99 \\
95\end{array}$ & $\begin{array}{l}78 \\
77 \\
72 \\
79 \\
84\end{array}$ & $\begin{array}{l}72 \\
77 \\
88 \\
83 \\
68\end{array}$ & $\begin{array}{l}38 \\
37 \\
36 \\
37 \\
38\end{array}$ & $\begin{array}{l}35 \\
33 \\
35 \\
37 \\
37\end{array}$ & $\begin{array}{l}11 \\
11 \\
10 \\
9.9 \\
9.2\end{array}$ & $\begin{array}{l}36 \\
29 \\
26 \\
24 \\
21\end{array}$ & $\begin{array}{r}150 \\
100 \\
52 \\
38 \\
28\end{array}$ & $\begin{array}{r}71 \\
107 \\
104 \\
92 \\
72\end{array}$ & $\begin{array}{l}755 \\
352 \\
302 \\
187 \\
135\end{array}$ \\
\hline $\begin{array}{r}6 \\
7 \\
8 \\
9 \\
10\end{array}$ & $\begin{array}{r}104 \\
99 \\
101 \\
107 \\
108\end{array}$ & $\begin{array}{l}83 \\
83 \\
85 \\
87 \\
86\end{array}$ & $\begin{array}{l}88 \\
80 \\
77 \\
68 \\
65\end{array}$ & $\begin{array}{l}82 \\
86 \\
83 \\
78 \\
78\end{array}$ & $\begin{array}{l}70 \\
83 \\
89 \\
86 \\
89\end{array}$ & $\begin{array}{l}36 \\
35 \\
36 \\
37 \\
35\end{array}$ & $\begin{array}{l}36 \\
38 \\
42 \\
40 \\
37\end{array}$ & $\begin{array}{l}8.9 \\
8.8 \\
9.3 \\
9.3 \\
8.5\end{array}$ & $\begin{array}{l}18 \\
16 \\
13 \\
11 \\
11\end{array}$ & $\begin{array}{r}25 \\
63 \\
23 \\
123 \\
1820\end{array}$ & $\begin{array}{l}49 \\
37 \\
31 \\
37 \\
51\end{array}$ & $\begin{array}{l}328 \\
248 \\
207 \\
212 \\
159\end{array}$ \\
\hline $\begin{array}{l}11 \\
12 \\
13 \\
14 \\
15\end{array}$ & $\begin{array}{r}106 \\
105 \\
98 \\
92 \\
85\end{array}$ & $\begin{array}{l}84 \\
83 \\
83 \\
84 \\
85\end{array}$ & $\begin{array}{l}64 \\
57 \\
70 \\
79 \\
80\end{array}$ & $\begin{array}{l}79 \\
86 \\
91 \\
91 \\
93\end{array}$ & $\begin{array}{l}85 \\
79 \\
74 \\
71 \\
70\end{array}$ & $\begin{array}{l}33 \\
36 \\
38 \\
39 \\
37\end{array}$ & $\begin{array}{l}35 \\
31 \\
28 \\
26 \\
24\end{array}$ & $\begin{array}{l}8.0 \\
7.0 \\
6.9 \\
6.5 \\
6.1\end{array}$ & $\begin{array}{l}9.0 \\
8.0 \\
7.7 \\
7.1 \\
6.5\end{array}$ & $\begin{array}{r}1450 \\
603 \\
483 \\
367 \\
204\end{array}$ & $\begin{array}{l}38 \\
38 \\
27 \\
20 \\
17\end{array}$ & $\begin{array}{l}147 \\
110 \\
106 \\
150 \\
198\end{array}$ \\
\hline $\begin{array}{l}16 \\
17 \\
18 \\
19 \\
20\end{array}$ & $\begin{array}{l}81 \\
80 \\
77 \\
81 \\
74\end{array}$ & $\begin{array}{l}84 \\
82 \\
81 \\
81 \\
81\end{array}$ & $\begin{array}{l}74 \\
73 \\
83 \\
84 \\
77\end{array}$ & $\begin{array}{l}90 \\
93 \\
89 \\
89 \\
68\end{array}$ & $\begin{array}{l}68 \\
68 \\
65 \\
62 \\
61\end{array}$ & $\begin{array}{l}37 \\
36 \\
36 \\
37 \\
42\end{array}$ & $\begin{array}{l}22 \\
20 \\
20 \\
20 \\
20\end{array}$ & $\begin{array}{l}5.3 \\
5.4 \\
4.9 \\
3.8 \\
3.0\end{array}$ & $\begin{array}{r}5.9 \\
6.3 \\
5.4 \\
6.2 \\
15\end{array}$ & $\begin{array}{r}119 \\
83 \\
70 \\
80 \\
65\end{array}$ & $\begin{array}{l}18 \\
16 \\
96 \\
89 \\
78\end{array}$ & $\begin{array}{r}170 \\
132 \\
111 \\
94 \\
83\end{array}$ \\
\hline $\begin{array}{l}21 \\
22 \\
23 \\
24 \\
25\end{array}$ & $\begin{array}{l}69 \\
64 \\
63 \\
69 \\
73\end{array}$ & $\begin{array}{l}80 \\
81 \\
82 \\
82 \\
84\end{array}$ & $\begin{array}{l}74 \\
73 \\
78 \\
72 \\
65\end{array}$ & $\begin{array}{l}67 \\
66 \\
66 \\
70 \\
55\end{array}$ & $\begin{array}{l}56 \\
51 \\
48 \\
49 \\
46\end{array}$ & $\begin{array}{l}46 \\
46 \\
44 \\
44 \\
42\end{array}$ & $\begin{array}{l}20 \\
19 \\
18 \\
17 \\
15\end{array}$ & $\begin{array}{r}2.8 \\
2.7 \\
2.2 \\
1.7 \\
163\end{array}$ & $\begin{array}{r}11 \\
8.5 \\
6.8 \\
5.0 \\
3.4\end{array}$ & $\begin{array}{r}56 \\
38 \\
42 \\
155 \\
74\end{array}$ & $\begin{array}{r}45 \\
40 \\
166 \\
89 \\
191\end{array}$ & $\begin{array}{l}75 \\
70 \\
62 \\
55 \\
51\end{array}$ \\
\hline $\begin{array}{l}26 \\
27 \\
28 \\
29 \\
30 \\
31\end{array}$ & $\begin{array}{l}79 \\
81 \\
78 \\
83 \\
91 \\
94\end{array}$ & $\begin{array}{r}85 \\
80 \\
82 \\
80 \\
80 \\
-\end{array}$ & $\begin{array}{l}61 \\
58 \\
58 \\
68 \\
70 \\
80\end{array}$ & $\begin{array}{l}56 \\
48 \\
62 \\
62 \\
64 \\
68\end{array}$ & $\begin{array}{r}46 \\
44 \\
42 \\
39 \\
-2 \\
\hdashline-\end{array}$ & $\begin{array}{l}40 \\
37 \\
35 \\
33 \\
34 \\
35\end{array}$ & $\begin{array}{r}14 \\
13 \\
14 \\
13 \\
12 \\
---\end{array}$ & $\begin{array}{l}755 \\
280 \\
123 \\
77 \\
52 \\
45\end{array}$ & $\begin{array}{r}2.8 \\
3.8 \\
7.5 \\
136 \\
244 \\
\end{array}$ & $\begin{array}{r}54 \\
43 \\
36 \\
118 \\
168 \\
99\end{array}$ & $\begin{array}{r}851 \\
316 \\
2020 \\
787 \\
424 \\
937\end{array}$ & $\begin{array}{r}47 \\
45 \\
45 \\
43 \\
39 \\
-\end{array}$ \\
\hline $\begin{array}{l}\text { TOTAL } \\
\text { MEAN } \\
\text { MAX } \\
\text { MIN } \\
\text { AC-FT }\end{array}$ & $\begin{array}{r}2828 \\
91.2 \\
127 \\
63 \\
5610\end{array}$ & $\begin{array}{r}2526 \\
84.2 \\
99 \\
80 \\
5010\end{array}$ & $\begin{array}{r}2333 \\
75.3 \\
99 \\
57 \\
4630\end{array}$ & $\begin{array}{r}2350 \\
75.8 \\
93 \\
48 \\
4660\end{array}$ & $\begin{array}{r}1929 \\
66.5 \\
89 \\
39 \\
3830\end{array}$ & $\begin{array}{r}1172 \\
37.8 \\
46 \\
33 \\
2320\end{array}$ & $\begin{array}{r}771 \\
25.7 \\
42 \\
12 \\
1.530\end{array}$ & $\begin{array}{r}1657.2 \\
53.5 \\
755 \\
1.7 \\
3290\end{array}$ & $\begin{array}{r}710.9 \\
23.7 \\
244 \\
2.8 \\
1410\end{array}$ & $\begin{array}{r}6829 \\
220 \\
1820 \\
23 \\
13550\end{array}$ & $\begin{array}{r}6954 \\
224 \\
2020 \\
16 \\
13790\end{array}$ & $\begin{array}{r}4718 \\
157 \\
755 \\
39 \\
9360\end{array}$ \\
\hline $\begin{array}{l}\text { STATIS } \\
\text { MEAN } \\
\text { MAX } \\
\text { (WY) } \\
\text { MIN } \\
\text { (WY) }\end{array}$ & $\begin{array}{r}\text { CS OF } \\
110 \\
870 \\
1942 \\
000 \\
1957\end{array}$ & $\begin{array}{c}\text { MONTHLY MEAN } \\
58.6 \\
506 \\
1942 \\
1.43 \\
1957\end{array}$ & $\begin{array}{l}\text { DATA FOR } \\
50.7 \\
252 \\
1942 \\
1.97 \\
1957\end{array}$ & $\begin{array}{l}\text { WATER } \\
52.9 \\
183 \\
1943 \\
1.42 \\
1957\end{array}$ & $\begin{array}{c}\text { YEARS } 1913 \\
63.7 \\
363 \\
1961 \\
1.46 \\
1957\end{array}$ & $\begin{array}{c}1996 \\
60.0 \\
737 \\
1987 \\
.74 \\
1957\end{array}$ & $\begin{array}{c}\text { BY WATER } \\
215 \\
5573 \\
1942 \\
000 \\
1936\end{array}$ & $\begin{array}{c}\text { YEAR (WY) } \\
400 \\
4721 \\
1941 \\
000 \\
1967\end{array}$ & $\begin{array}{r}397 \\
4260 \\
1965 \\
000 \\
1974\end{array}$ & $\begin{array}{r}239 \\
1129 \\
1914 \\
000 \\
1964\end{array}$ & $\begin{array}{r}320 \\
1173 \\
1946 \\
8.39 \\
1980\end{array}$ & $\begin{array}{r}249 \\
4079 \\
1942 \\
197 \\
1956\end{array}$ \\
\hline
\end{tabular}

SUMMARY STATISTICS

ANNUAL TOTAL

ANNIUAT MEA

HIGHEST ANNUAL, MEAN

LOWEST ANNUAL MEAN

HIGHEST DAILY MEAN

LOWEST DAILY MEAN

ANNUAL SEVEN-DAY MINIMUM

INSTANTANEOUS REAK FLOW

INSTANTANEOUS PEAK STAGE

INSTANTANEOUS LOW FLOW

ANNUAL RUNOFE (AC-FT)

10 PERCENT EXCEEDS

50 PERCENT EXCEEDS

90 EBRCENT

a-From floodmarks, present site and datum.

b-From rating curve extended above $91,000 \mathrm{ft} / \mathrm{s}$ on basts of slope-area measurement of peak flow.

\section{DAILY MEAN VALUES}


PERIOD OF RECORD,--Water years 1975 to current year.

WATER QUALITY DATA, WATER YEAR OCTOBER 1995 TO SEPTEMBER 1996

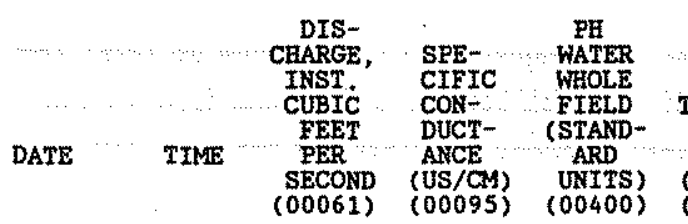

0095)
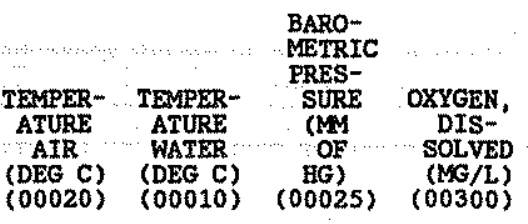

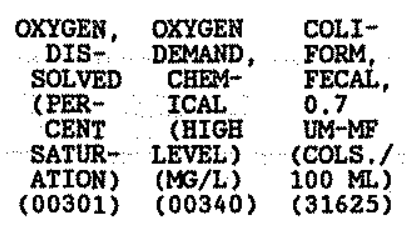

$(00301)$

\begin{tabular}{|c|c|c|c|c|c|c|c|c|c|c|c|}
\hline $\begin{array}{l}\text { DEC } 1995 \\
1330 \\
\text { FEB } 1996\end{array}$ & 0930 & 69 & 1450 & 8.3 & 17.0 & 3.0 & 644 & 11.1 & 98 & $<10$ & $\mathrm{~K} 1$ \\
\hline MAY $14 . \cdots$ & 1015 & 73 & 1240 & 8.4 & 15.0 & 6.0 & 647 & 10.4 & 99 & $<10$ & $<1$ \\
\hline AUG 09 & 0915 & 4.0 & 1720 & 8.1 & 22.5 & 18.5 & 648 & 6.8 & 86 & 17 & 55 \\
\hline $21 \ldots$ & 0845 & 35 & 702 & 8.2 & 21.5 & 23.0 & 653 & 6.5 & 89 & 19 & - \\
\hline
\end{tabular}

\begin{tabular}{|c|c|c|c|c|c|c|c|c|c|c|c|}
\hline DATE & $\begin{array}{c}\text { STREP- } \\
\text { TOCOCCI } \\
\text { FECAL, } \\
\text { KF AGAR } \\
\text { (COLS } \\
\text { PER } \\
100 \mathrm{ML}) \\
(31673)\end{array}$ & $\begin{array}{l}\text { HARD- } \\
\text { NESS } \\
\text { TOTAL } \\
\text { (NG/L } \\
\text { AS } \\
\text { CACO3) } \\
(00900)\end{array}$ & $\begin{array}{l}\text { HARD- } \\
\text { NESS } \\
\text { NONCARB } \\
\text { DISSOLV } \\
\text { FLD. AS } \\
\text { CACO3 } \\
(\text { MG } / \mathrm{L}) \\
(00904)\end{array}$ & $\begin{array}{l}\text { CALCIUM } \\
\text { DIS- } \\
\text { SOLVED } \\
\text { (MG } \\
\text { AS CA) } \\
(00915)\end{array}$ & $\begin{array}{c}\text { MAGNE- } \\
\text { SIUM, } \\
\text { DIS- } \\
\text { SOLVED } \\
\text { (MG/L } \\
\text { AS MG) } \\
(00925) .\end{array}$ & $\begin{array}{l}\text { SODIUM, } \\
\text { DIS- } \\
\text { SOLVED } \\
\text { (MG/L, } \\
\text { AS NA }) \\
(00930)\end{array}$ & $\begin{array}{c}\text { SODIUM } \\
\text { AD- } \\
\text { SORP- } \\
\text { TION } \\
\text { RATIO } \\
(00931)\end{array}$ & $\begin{array}{c}\text { POTAS- } \\
\text { SIUM, } \\
\text { DIS- } \\
\text { SOLVED } \\
\text { (NG/L } \\
\text { AS K) } \\
(00935)\end{array}$ & $\begin{array}{l}\text { BICAR- } \\
\text { BONATE } \\
\text { WATER } \\
\text { DIS IT } \\
\text { FIELD } \\
\text { MG/L AS } \\
\text { HCO3 } \\
(00453)\end{array}$ & $\begin{array}{c}\text { CAR- } \\
\text { BONATE } \\
\text { WATER } \\
\text { DIS IT } \\
\text { FIELD } \\
\text { MG/L AS } \\
\text { CO3 } \\
(00452)\end{array}$ & $\begin{array}{l}\text { ALKA- } \\
\text { LINITY } \\
\text { WAT DIS } \\
\text { TOT IT } \\
\text { FIELD } \\
\text { NG/L AS } \\
\text { CACOB } \\
(39086)\end{array}$ \\
\hline $\begin{array}{l}\text { DEC } 1995 \\
333906\end{array}$ & K7 & 590 & 390 & 120 & 70 & 110 & 2 & 2.6 & 236 & 0 & 193 \\
\hline $\operatorname{MAY}^{14} \because$ & $<1$ & 490 & 310 & 100 & 59 & 87 & 2 & 2.3 & 211 & 8 & 186 \\
\hline $\operatorname{AUG}^{09} \ldots$ & 680 & 650 & 470 & 120 & 86 & 150 & 3 & 4.2 & 219 & 0 & 180 \\
\hline 21. & -- & 220 & 120 & 52 & 23 & 42 & 1 & 3.5 & 123 & 3 & 106 \\
\hline DATE & $\begin{array}{c}\text { ALKA- } \\
\text { LINITYY } \\
\text { LAB } \\
\text { (NGG/L } \\
\text { AS } \\
\text { CACO3) } \\
(90410)\end{array}$ & $\begin{array}{l}\text { SULFATE } \\
\text { DIS- } \\
\text { SOLVED } \\
(\text { (MG } / L \\
\text { AS SO4) } \\
(00945)\end{array}$ & $\begin{array}{l}\text { CHLO- } \\
\text { RIDE, } \\
\text { DIS-- } \\
\text { SOLVED } \\
\text { (MSG/L } \\
\text { AS CL) } \\
(00940)\end{array}$ & $\begin{array}{l}\text { FLUO- } \\
\text { RIDE, } \\
\text { DIS- } \\
\text { SOLVED } \\
\text { (MG/L } \\
\text { AS F) } \\
(00950)\end{array}$ & $\begin{array}{l}\text { SILICA, } \\
\text { DIS- } \\
\text { SOLVED } \\
\text { (ME/L } \\
\text { AS } \\
\text { SIO2) } \\
(00955)\end{array}$ & $\begin{array}{c}\text { SOLIDS, } \\
\text { SUM OF } \\
\text { CONSTI- } \\
\text { TUENTS, } \\
\text { DIS- } \\
\text { SOLVED } \\
\text { (MG/L) } \\
(70301)\end{array}$ & $\begin{array}{c}\text { NITRO- } \\
\text { GEN, } \\
\text { NITRITE } \\
\text { DIS- } \\
\text { SOLVED } \\
\text { (ME/L } \\
\text { AS N) } \\
(00613)\end{array}$ & $\begin{array}{c}\text { NITRO- } \\
\text { GEN, } \\
\text { NO2+NÓ3 } \\
\text { DIS- } \\
\text { SOLVED } \\
\text { (MG/L } \\
\text { AS N) } \\
(00631)\end{array}$ & $\begin{array}{c}\text { NITRO- } \\
\text { GEN, } \\
\text { AMONIA } \\
\text { DIS- } \\
\text { SOLVED } \\
\text { (MG/L } \\
\text { AS N) } \\
(00608)\end{array}$ & $\begin{array}{c}\text { NITRO- } \\
\text { GEN, } \\
\text { ORGANIC } \\
\text { DIS- } \\
\text { SOLVED } \\
\text { (WG/L } \\
\text { AS H) } \\
(00607)\end{array}$ & $\begin{array}{l}\text { NITRO- } \\
\text { GEN, AM- } \\
\text { MONIA + } \\
\text { ORGANIC } \\
\text { TOTAL } \\
\text { (MG/L } \\
\text { AS H) } \\
(00625)\end{array}$ \\
\hline $\begin{array}{l}13 \\
\mathrm{FEC} \\
\mathrm{FEB}\end{array}$ & 395 & 570 & 22 & 0.50 & 8.9 & 1020 & $<0.010$ & $<0.050$ & $<0.015$ & -- & $<0,20$ \\
\hline${ }_{\mathrm{MAY}}^{14} \cdots$ & 185 & 460 & 18 & 0 & 8.7 & 847 & -- & -- & -- & - & -- \\
\hline $\begin{array}{c}09 \\
\text { AUG }\end{array}$ & 180 & 720 & 31 & 0.60 & 7.2 & 1230 & $<0.010$ & $<0.050$ & 0.020 & 0.28 & 0.40 \\
\hline $21 \ldots$ & 108 & 200 & 7.8 & 0.30 & 6.9 & 400 & $<0.010$ & 0.100 & $<0.015$ & - & $<0.20$ \\
\hline
\end{tabular}

\begin{tabular}{|c|c|c|c|c|c|c|c|c|c|c|c|}
\hline DATE & $\begin{array}{l}\text { NITRO- } \\
\text { GEN,AM- } \\
\text { MONIA + } \\
\text { ORGANIC } \\
\text { DIS. } \\
\text { (WSG/L } \\
\text { AS H) } \\
(00623)\end{array}$ & $\begin{array}{l}\text { PHOS- } \\
\text { PHORUS } \\
\text { TOTAL } \\
\text { (MG/L } \\
\text { AS P) } \\
(00665)\end{array}$ & $\begin{array}{c}\text { PHOS- } \\
\text { PHORUS } \\
\text { DIS- } \\
\text { SOLVED } \\
\text { (MGLL } \\
\text { AS P) } \\
(00666)\end{array}$ & $\begin{array}{l}\text { PHOS- } \\
\text { PHORUS } \\
\text { ORTHO, } \\
\text { DIS- } \\
\text { SOLVED } \\
\text { (NSG/L } \\
\text { AS P) } \\
(00671)\end{array}$ & $\begin{array}{c}\text { CARBON, } \\
\text { ORGANIC } \\
\text { TOTAL } \\
\text { CME/L } \\
\text { AS C) } \\
(00680)\end{array}$ & $\begin{array}{l}\text { ALUM- } \\
\text { INUM, } \\
\text { DIS- } \\
\text { SOLVED } \\
\text { (UL/L } \\
\text { AS AL) } \\
\text { (01106) }\end{array}$ & $\begin{array}{l}\text { ANTI- } \\
\text { MONY, } \\
\text { DIS- } \\
\text { SOLVED } \\
\text { (UG/L } \\
\text { AS SB) } \\
\text { (01095) }\end{array}$ & $\begin{array}{c}\text { ARSENIC } \\
\text { DIS- } \\
\text { SOLVED } \\
\text { (USL } \\
\text { AS AS }) \\
(01000)\end{array}$ & $\begin{array}{l}\text { BARIUM, } \\
\text { DIS- } \\
\text { SOLVED } \\
\text { (US /L } \\
\text { AS BA }) \\
(01005)\end{array}$ & $\begin{array}{l}\text { BERYL- } \\
\text { LIUM, } \\
\text { DIS- } \\
\text { SOLVED } \\
\text { (UG/L } \\
\text { AS BE) } \\
(01010)\end{array}$ & $\begin{array}{l}\text { BORON, } \\
\text { DIS- } \\
\text { SOLVED } \\
\text { (UG/L } \\
\text { AS B) } \\
(01020)\end{array}$ \\
\hline & $<0.20$ & $<0.010$ & $<0.010$ & $<0.010$ & 4.2 & 5.0 & $<1.0$ & $<1$ & 62 & $<1.0$ & 90 \\
\hline & -- & -- & -- & - & 2.1 & -- & -- & -- & -- & -- & 70 \\
\hline & 0.30 & 0.020 & $<0.010$ & $<0.010$ & 4.7 & 5.0 & $<1.0$ & $<1$ & 74 & $<1.0$ & 130 \\
\hline & 0.20 & $<0.010$ & $<0.010$ & .010 & 7.7 & -- & - & -- & -- & - & 58 \\
\hline
\end{tabular}


ARKANSAS RIVER BASIN

07221500 CANADIAN RIVER NEAR SANCBEZ, MM -- Continued

WATER-QUALITY RECORDS

WATER QUALITY DATA, WATER YEAR OCTOBER 1995 TO SEFTEMBER 1996

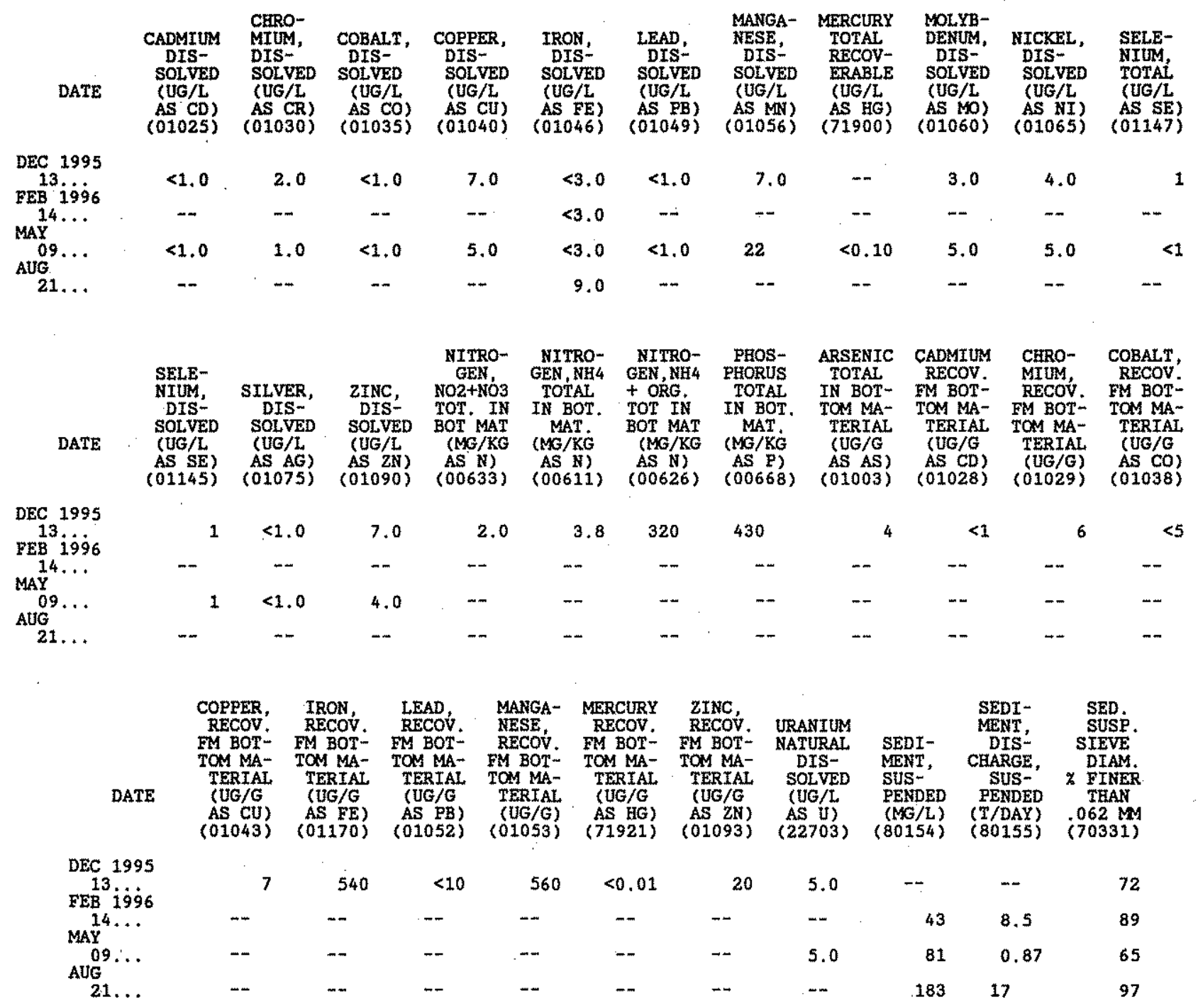


ARKANSAS RIVER BASIN

07222500 CONCHAS RIVER AT VARIADERO, NM

LOCATION, - - Let $35^{\circ} 24^{\prime} 10^{\prime \prime}$ long $104^{\circ} 26^{\prime} 35^{\prime \prime}$, in NEKNE sec.36, T.14 N., R.23 E., San Miguel County Hydrologic Unit 11080005 , on left bank $1.5 \mathrm{mi}$ northeast of Variadero, $14 \mathrm{mi}$ west of Conchas Dam, and at mile 15.0 .

DRAINAGE AREA, $--523 \mathrm{mi}^{2}$, of which $130 \mathrm{mi}^{2}$ is probably noncontributing.

PERIOD OF RECORD.--October 1936 to September 1996 (discontinued).

REVISED RECORDS.--WSP 1281: 1937-39, 1941-47.

GAGE.-Water-stage recorder. Elevation of gage 18 4,390 ft above National Geodetic vertical Datum of 1929 , from topographic map. PrLor to Mar. 30, 1942, at site 1.5 mi upstream at different datum. Mar. 30, 1942 to May 18, 1950, at prosent aite at datur 0.5 et higher.

REMARKS --Records fair except for estimated daily discharge which are poox. Diversions for trrigation of about 300 acres upstream from station. ... Several observations of water temperature were made during the year... No flow many ... days each year.

DISCHARGE, CUBIC FEET PER SECOND, WATER YEAR OCTOBER 1995 TO SEPTEMBER 1996

\begin{tabular}{|c|c|c|c|c|c|c|c|c|c|c|c|c|}
\hline DAY & OCT & now. & $\mathrm{DEC}$ & JAN & FEB & MAR & AER & MAY & Jun & JUL & AUG & SEP \\
\hline $\begin{array}{l}1 \\
2 \\
3 \\
4 \\
5\end{array}$ & $\begin{array}{l}.04 \\
.03 \\
.03 \\
.03 \\
.03\end{array}$ & $\begin{array}{l}.00 \\
.00 \\
.00 \\
.02 \\
.02\end{array}$ & $\begin{array}{l}.04 \\
.04 \\
.04 \\
.04 \\
.03\end{array}$ & $\begin{array}{l}.03 \\
.03 \\
.03 \\
.03 \\
.03\end{array}$ & $\begin{array}{l}.03 \\
.03 \\
.03 \\
.03 \\
.03\end{array}$ & $\begin{array}{l}.03 \\
.03 \\
.03 \\
.03 \\
.03\end{array}$ & $\begin{array}{l}.03 \\
.03 \\
.03 \\
.03 \\
.03\end{array}$ & $\begin{array}{l}.00 \\
.00 \\
.00 \\
.00 \\
.00\end{array}$ & $\begin{array}{l}0.00 \\
0.00 \\
0.00 \\
0.00 \\
0.00\end{array}$ & $\begin{array}{l}88 \\
8.3 \\
2.8 \\
1.7 \\
1.5\end{array}$ & $\begin{array}{l}77 \\
27 \\
9.2 \\
4.0 \\
2.1\end{array}$ & $\begin{array}{l}68 \\
28 \\
14 \\
9 \cdot 3 \\
16\end{array}$ \\
\hline $\begin{array}{r}6 \\
7 \\
8 \\
9 \\
10\end{array}$ & $\begin{array}{l}.03 \\
.03 \\
.03 \\
.03 \\
.02\end{array}$ & $\begin{array}{l}.02 \\
.01 \\
.01 \\
.01 \\
.01\end{array}$ & $\begin{array}{l}.02 \\
.02 \\
.02 \\
.02 \\
.02\end{array}$ & $\begin{array}{r}.03 \\
.03 \\
.03 \\
.03 \\
.03\end{array}$ & $\begin{array}{r}.03 \\
.03 \\
.03 \\
.03 \\
.03\end{array}$ & $\begin{array}{l}.03 \\
.03 \\
.04 \\
.04 \\
.04\end{array}$ & $\begin{array}{r}.03 \\
.03 \\
.03 \\
.03 \\
.03\end{array}$ & $\begin{array}{r}.00 \\
00 \\
000 \\
0.00 \\
0.00\end{array}$ & $\begin{array}{r}.00 \\
00 \\
.00 \\
.00 \\
.00\end{array}$ & $\begin{array}{r}1.4 \\
1.4 \\
1.4 \\
231 \\
1820\end{array}$ & $\begin{array}{r}1.3 \\
199^{\circ} \\
42\end{array}$ & $\begin{array}{c}10 \\
13 \\
35 \\
17 \\
7.5\end{array}$ \\
\hline $\begin{array}{l}11 \\
12 \\
13 \\
14 \\
15\end{array}$ & $\begin{array}{l}.01 \\
.01 \\
.00 \\
.00 \\
.00\end{array}$ & $\begin{array}{l}.00 \\
.00 \\
.00 \\
.00 \\
.01\end{array}$ & $\begin{array}{l}.02 \\
.03 \\
.02 \\
.02 \\
.02\end{array}$ & $\begin{array}{l}.03 \\
.03 \\
.03 \\
.03 \\
.03\end{array}$ & $\begin{array}{l}.03 \\
.03 \\
.03 \\
.03 \\
.03\end{array}$ & $\begin{array}{l}.04 \\
.04 \\
.03 \\
.04 \\
.04\end{array}$ & $\begin{array}{l}.02 \\
.00 \\
.00 \\
.00 \\
.00\end{array}$ & $\begin{array}{l}0.00 \\
0.00 \\
0.00 \\
0.00 \\
0.00\end{array}$ & $\begin{array}{l}.00 \\
.00 \\
.00 \\
.00 \\
.00\end{array}$ & $\begin{array}{r}69 \\
30 \\
12 \\
261 \\
47\end{array}$ & $\begin{array}{c}12 \\
4.5 \\
2.4 \\
1.5 \\
.93\end{array}$ & $\begin{array}{r}18 \\
330 \\
89 \\
60 \\
86\end{array}$ \\
\hline $\begin{array}{l}16 \\
17 \\
18 \\
19 \\
20\end{array}$ & $\begin{array}{l}.00 \\
.00 \\
.00 \\
.00 \\
.00\end{array}$ & $\begin{array}{l}.02 \\
.02 \\
.02 \\
.03 \\
.04\end{array}$ & $\begin{array}{l}.03 \\
.04 \\
.05 \\
.03 \\
.03\end{array}$ & $\begin{array}{l}.02 \\
.03 \\
.02 \\
.03 \\
.03\end{array}$ & $\begin{array}{l}.03 \\
.03 \\
.03 \\
.03 \\
.03\end{array}$ & $\begin{array}{l}.04 \\
.03 \\
.03 \\
.04 \\
.04\end{array}$ & $\begin{array}{l}.00 \\
.00 \\
.00 \\
.00 \\
.00\end{array}$ & $\begin{array}{l}0.00 \\
e .00 \\
0.00 \\
0.00 \\
0.00\end{array}$ & $\begin{array}{l}.00 \\
.00 \\
.00 \\
.00 \\
.00\end{array}$ & $\begin{array}{c}18 \\
7.6 \\
5.5 \\
7.9 \\
19\end{array}$ & $\begin{array}{l}.61 \\
.46 \\
.35 \\
.54 \\
.39\end{array}$ & $\begin{array}{l}26 \\
10 \\
6.0 \\
4.4 \\
3.4\end{array}$ \\
\hline $\begin{array}{l}21 \\
22 \\
23 \\
24 \\
25\end{array}$ & $\begin{array}{l}.00 \\
.00 \\
.00 \\
.00 \\
.00\end{array}$ & $\begin{array}{l}.04 \\
.04 \\
.03 \\
.03 \\
.03\end{array}$ & $\begin{array}{l}.03 \\
.03 \\
.03 \\
.03 \\
.03\end{array}$ & $\begin{array}{l}.03 \\
.03 \\
.03 \\
.03 \\
.03\end{array}$ & $\begin{array}{l}.03 \\
.03 \\
.03 \\
.03 \\
.03\end{array}$ & $\begin{array}{l}.04 \\
.04 \\
.04 \\
.03 \\
.03\end{array}$ & $\begin{array}{l}.00 \\
.00 \\
.00 \\
.00 \\
.00\end{array}$ & $\begin{array}{l}0.00 \\
0.00 \\
0.00 \\
0.00 \\
0.00\end{array}$ & $\begin{array}{l}.00 \\
.00 \\
.00 \\
.00 \\
.00\end{array}$ & $\begin{array}{c}8.8 \\
4.1 \\
8.3 \\
25 \\
5.8\end{array}$ & $\begin{array}{l}5.23 \\
5.88 \\
88^{.18} \\
20\end{array}$ & $\begin{array}{l}2.7 \\
2.3 \\
1.9 \\
1.6 \\
1.5\end{array}$ \\
\hline $\begin{array}{l}26 \\
27 \\
28 \\
29 \\
30 \\
31\end{array}$ & $\begin{array}{l}.00 \\
.00 \\
.00 \\
.00 \\
.00 \\
.00\end{array}$ & $\begin{array}{l}.04 \\
.04 \\
.04 \\
.04 \\
.04 \\
-\end{array}$ & $\begin{array}{l}.03 \\
.03 \\
.03 \\
.03 \\
.03 \\
.03\end{array}$ & $\begin{array}{l}.02 \\
.02 \\
.02 \\
.02 \\
.02 \\
.02\end{array}$ & $\begin{array}{l}.03 \\
.03 \\
.03 \\
.03 \\
-.03 \\
-. .7\end{array}$ & $\begin{array}{l}.03 \\
.03 \\
.03 \\
.03 \\
.03 \\
.04\end{array}$ & $\begin{array}{l}.00 \\
.00 \\
.00 \\
.00 \\
.00 \\
.0\end{array}$ & $\begin{array}{l}e .00 \\
\theta .00 \\
e .00 \\
e .00 \\
0.00 \\
0.00\end{array}$ & 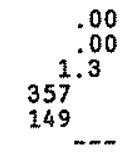 & $\begin{array}{r}2.0 \\
145 \\
35 \\
10 \\
189 \\
21\end{array}$ & $\begin{array}{r}77 \\
31 \\
1340 \\
97 \\
37 \\
53\end{array}$ & $\begin{array}{l}1.3 \\
1.2 \\
1.1 \\
1.1 \\
1.0\end{array}$ \\
\hline $\begin{array}{l}\text { TOTAL } \\
\text { MEAN } \\
\text { MAX } \\
\text { MIN } \\
\text { AC-FE }\end{array}$ & $\begin{array}{l}0.32 \\
.010 \\
.04 \\
.00 \\
.6\end{array}$ & $\begin{array}{r}0.61 \\
.020 \\
.04 \\
.00 \\
i .2\end{array}$ & $\begin{array}{r}0.91 \\
.029 \\
.05 \\
.02 \\
1.8\end{array}$ & $\begin{array}{r}0.85 \\
.027 \\
.03 \\
.02 \\
1.7\end{array}$ & $\begin{array}{l}0.87 \\
.030 \\
.03 \\
.03 \\
1.7\end{array}$ & $\begin{array}{l}1.07 \\
.035 \\
.04 \\
.03 \\
2.1\end{array}$ & $\begin{array}{r}0.32 \\
.011 \\
.03 \\
.00 \\
.6\end{array}$ & $\begin{array}{l}0.00 \\
.000 \\
.00 \\
.00 \\
.00\end{array}$ & $\begin{array}{r}507.30 \\
16.9 \\
357 \\
.00 \\
1010\end{array}$ & $\begin{array}{r}3088.5 \\
99.6 \\
1820 \\
1.4 \\
6130\end{array}$ & $\begin{array}{r}2136.11 \\
68.9 \\
1340 \\
4.18 \\
4240\end{array}$ & $\begin{array}{r}866.3 \\
28.9 \\
330 \\
1.0 \\
1720\end{array}$ \\
\hline
\end{tabular}

STATISTICS OF MONTHLY MEAN DATA FOR WATER YEARS 1937 - 1996, BY WATER YEAR (WY)

\begin{tabular}{|c|c|c|c|c|c|c|c|c|c|c|c|c|}
\hline $\begin{array}{l}\text { MEAN } \\
\text { MAX } \\
\text { (WY } \\
\text { MIN } \\
\text { (WY) }\end{array}$ & $\begin{array}{l}8.27 \\
90.5 \\
1942 \\
1000 \\
1937\end{array}$ & $\begin{array}{l}1.61 \\
31.6 \\
1987 \\
1000 \\
1937\end{array}$ & $\begin{array}{r}85 \\
1 i^{8.0} \\
1943 \\
000 \\
1937\end{array}$ & $\begin{array}{l}.70 \\
8.65 \\
1943 \\
1000 \\
1937\end{array}$ & $\begin{array}{r}.74 \\
8.26 \\
1987 \\
1000 \\
1953\end{array}$ & $\begin{array}{l}1.23 \\
22.0 \\
1987 \\
1900 \\
1949\end{array}$ & $\begin{array}{l}2.88 \\
63.8 \\
1942 \\
1900 \\
1938\end{array}$ & $\begin{array}{r}11.7 \\
302 \\
1941 \\
1000 \\
1938\end{array}$ & $\begin{array}{r}25.2 \\
503 \\
1937 \\
1000 \\
1945\end{array}$ & $\begin{array}{r}30.9 \\
144 \\
1972 \\
1900 \\
1980\end{array}$ & $\begin{array}{r}33.5 \\
154 \\
1977 \\
1980\end{array}$ & $\begin{array}{r}37.9 \\
549 \\
1941 \\
1000 \\
1948\end{array}$ \\
\hline
\end{tabular}

\section{SUMAAPY STATISTICS}

ANNUAL TOTAL

HIGHEST ANNUAL MEAN

LOWEST ANNUAL MEAN

BIGHEST DAILY MEAN

LOWEST DAILY MEAN

ANNUAL SEVEN-DAY MINIMUM

INSTANTANEOUS PEAK FLON

INSTANTANEOUS PEAK STAGE

ANNUAL RUNOFF (AC-FT)

10 PERCENT EXCEEDS

50 PERCENT EXCEEDS

90 PERCENT EXCEEDS

- Estimated
FOR 1995 CALENDAR YEAR

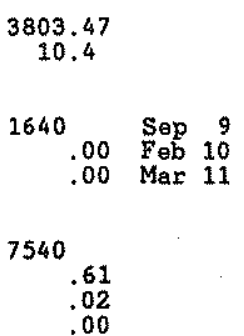

FOR 1996 WATER YEAR

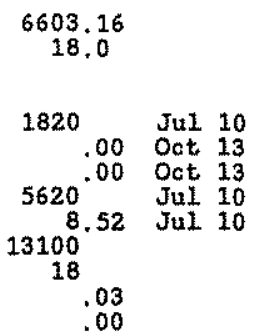

WATER YEARS 1937 - 1996

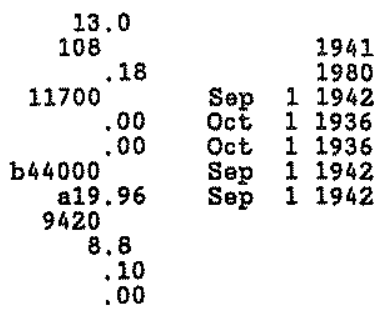

a-Present detum.

b-From rating curve extended above $760 \mathrm{ft} / \mathrm{s}$ on basis of slope-area measurements at gage helghts 12.5 ft and 19.96, present datum. 
ARKANSAS RIVER BASIN

07223500 CONCBAS LAKE AT CONCHAS DAM, NM

LOCATION.--Lat $35^{\circ} 24^{\prime} 10^{\prime \prime}$, Long $104^{\circ} 11^{\prime} 25^{\prime \prime}$, San Miguel County, Eydrologic Unit 11080003, in Pablo Montoya Grant, stiling well within concrete portion of Conchas Dam on Canadian River, $24 \mathrm{mi}$ north of Newkirk, and at mile 746.0 .

DRAINAGE AREA. $-7,409 \mathrm{mt}^{2}$, of which $433 \mathrm{mt}^{2}$, is probably noncontributing.

PERIOD OF RECORD. --December 1938 to September 1965 (monthend contents only), October 1965 to current year. Brior to October 1965, published as Conchas Reservoir near Conchas Dam.

GAGE.-Water-stage recorder. Datum of gage is National Goodetic Vertical Datum of 1929.

REMARKS. - - Lake is formed by dam consisting of concrete main section and earthftil wings, completed Sept. 15, 1939; storage began Dec. 29, 1938 Capacity, 330,100 acre-ft between elevations $4,060,0$ ft and 4,201.0 ft, crest of $300 \mathrm{ft}$ ungated service spiliway. Inactive storage, 70,490 acre-ft, at elevation $4,155.0$ ft. Lake usually not drawn below elevation, 4,157.35 ft, sill of irrigation outlet, capacity, 77,790 acre-ft, except for minor slutcing; at times irrigation water is pumped into Conchas Canal. Capacity of 198,800 acre-ft between elevations $4,201.0 \mathrm{ft}$ crest of $300 \mathrm{ft}$ ungated service spillway, and $4,218.0 \mathrm{ft}$ crest of $3,000 \mathrm{ft}$ ungated emergency spillway, acts as detention storage in the control of floods. Figures given herein represent total contents. Lake is used for irrigation, flood control, and recreation. Diversions upstream from station for irrigation of about 57,000 acres. Direct diverstons through Conchas Dam to Beli Ranch Canal and Conchas Canal (stations 07223000 , 07223300; irrigate about 36,000 acres near Tucumcari, and on Bell Ranch. U.S. Army Corps of Engineers satellite telemeter at station.

COOPERATION.--Records provided by U.S. Arny Corps of Engineers.

EXTREMES FOR PERIOD OF RECORD,--Maximum contents, 479,600 acre-ft, Apr. 24, 1942, elevation, 4, 208.41 ft; minimum after inftial filling, 78,080 acre-ft, Sept. 18, 1976, elevation, 4,157.44 ft; mintrum elevation, 4,155.80 ft, Sept. 24,1954 .

EXTREMES FOR CURRENT YEAR.--Maximum contents, 312,290 acre-ft, Oct. 1, elevation, 4,200.64 ft; minimum, 244,630 acre-ft, June 26,28 elevation, $4,192.83 \mathrm{ft}$.

Capactiy table (elovation, in feet, and contents, in acre-feet)

(Based on Survey by U.S. Army Corps of Engtneers in 1970)

$\begin{array}{ll}4,180 & 160,600 \\ 4,190 & 223,400 \\ 4,200 & 306,200 \\ 4,210 & 412,100\end{array}$

RESERVOIR STORAGE (ACRE-FEET), WATER YEAR OCTOBER 1995 TO SEPTEMBER 1996

\begin{tabular}{|c|c|c|c|c|c|c|c|c|c|c|c|c|}
\hline DAY & $\infty \mathrm{CT}$ & Nov & $D E C$ & JAN & FEB & MAR & AFR & MAY & JỤN & JUL & AuG & SEP \\
\hline $\begin{array}{l}1 \\
2 \\
3 \\
4 \\
5\end{array}$ & $\begin{array}{l}312290 \\
312100 \\
311910 \\
311150 \\
310770\end{array}$ & $\begin{array}{l}299180 \\
298810 \\
298620 \\
298620 \\
298620\end{array}$ & $\begin{array}{l}299540 \\
299730 \\
299730 \\
299730 \\
299730\end{array}$ & $\begin{array}{l}302040 \\
302130 \\
302220 \\
302320 \\
302320\end{array}$ & $\begin{array}{l}304090 \\
304090 \\
304090 \\
304180 \\
304550\end{array}$ & $\begin{array}{l}305680 \\
305580 \\
305580 \\
305490 \\
305400\end{array}$ & $\begin{array}{l}298160 \\
297700 \\
297240 \\
296790 \\
296420\end{array}$ & $\begin{array}{l}140 \\
260 \\
480 \\
700 \\
920\end{array}$ & $\begin{array}{l}258830 \\
258420 \\
258010 \\
257520 \\
256860\end{array}$ & $\begin{array}{l}248890 \\
249050 \\
249050 \\
248730 \\
247940\end{array}$ & $\begin{array}{l}281270 \\
281190 \\
280920 \\
280840 \\
280400\end{array}$ & $\begin{array}{l}289890 \\
290700 \\
291150 \\
291330 \\
291150\end{array}$ \\
\hline $\begin{array}{r}6 \\
7 \\
8 \\
9 \\
10\end{array}$ & $\begin{array}{l}310390 \\
310010 \\
309630 \\
309440 \\
309060\end{array}$ & $\begin{array}{l}298620 \\
298620 \\
298810 \\
298710 \\
298530\end{array}$ & $\begin{array}{l}299820 \\
299820 \\
299820 \\
299910 \\
299910\end{array}$ & $\begin{array}{l}302510 \\
302600 \\
302690 \\
302780 \\
302880\end{array}$ & $\begin{array}{l}304650 \\
304930 \\
304840 \\
304930 \\
305110\end{array}$ & $\begin{array}{l}305400 \\
305300 \\
305300 \\
305210 \\
305210\end{array}$ & $\begin{array}{l}295960 \\
295600 \\
295140 \\
294770 \\
294680\end{array}$ & $\begin{array}{l}276060 \\
275450 \\
274590 \\
273480 \\
272630\end{array}$ & $\begin{array}{l}256130 \\
255400 \\
254670 \\
253940 \\
253210\end{array}$ & $\begin{array}{l}247540 \\
247460 \\
247140 \\
248410 \\
268220\end{array}$ & & $\begin{array}{l}290970 \\
292050 \\
292140 \\
292230 \\
292050\end{array}$ \\
\hline $\begin{array}{l}14 \\
15\end{array}$ & $\begin{array}{l}308680 \\
308120 \\
307740 \\
307550 \\
307180\end{array}$ & $\begin{array}{l}298530 \\
298530 \\
298620 \\
298810 \\
298810\end{array}$ & $\begin{array}{l}300100 \\
300100 \\
300100 \\
300100 \\
300280\end{array}$ & $\begin{array}{l}303060 \\
303150 \\
303250 \\
303440 \\
303530\end{array}$ & $\begin{array}{l}305210 \\
305300 \\
305490 \\
305580 \\
305580\end{array}$ & $\begin{array}{l}305210 \\
305110 \\
305020 \\
305110 \\
305300\end{array}$ & $\begin{array}{l}294230 \\
293770 \\
293140 \\
292600 \\
291960\end{array}$ & $\begin{array}{l}272200 \\
271350 \\
270670 \\
269820 \\
268980\end{array}$ & $\begin{array}{l}252330 \\
251530 \\
250960 \\
250560 \\
250000\end{array}$ & & & $\begin{array}{l}291870 \\
293500 \\
293690 \\
294050 \\
294230\end{array}$ \\
\hline 16 & $\begin{array}{l}306800 \\
306610 \\
305860 \\
305300 \\
304740\end{array}$ & $\begin{array}{l}298810 \\
298810 \\
298990 \\
298990 \\
298990\end{array}$ & $\begin{array}{l}300470 \\
301020 \\
301020 \\
301020 \\
301210\end{array}$ & $\begin{array}{l}303620 \\
303710 \\
303900 \\
304090 \\
304090\end{array}$ & $\begin{array}{l}305680 \\
305770 \\
305770 \\
305860 \\
305960\end{array}$ & $\begin{array}{l}305110 \\
305110 \\
304650 \\
303990 \\
303530\end{array}$ & $\begin{array}{l}291240 \\
290520 \\
289800 \\
289080 \\
288360\end{array}$ & $\begin{array}{l}268050 \\
267210 \\
266370 \\
265450 \\
264620\end{array}$ & $\begin{array}{l}249690 \\
249290 \\
248730 \\
248330 \\
247940\end{array}$ & $\begin{array}{l}277610 \\
277610 \\
277700 \\
277610 \\
277610\end{array}$ & & $\begin{array}{l}294320 \\
293960 \\
294140 \\
293690 \\
293320\end{array}$ \\
\hline $\begin{array}{l}21 \\
22 \\
23 \\
24 \\
25\end{array}$ & $\begin{array}{l}304370 \\
303810 \\
303250 \\
303060 \\
302220\end{array}$ & $\begin{array}{l}299180 \\
299180 \\
299180 \\
299180\end{array}$ & $\begin{array}{l}301210 \\
301300 \\
301490 \\
301490\end{array}$ & $\begin{array}{l}304090 \\
304090 \\
304180 \\
304180 \\
304270\end{array}$ & $\begin{array}{l}305960 \\
305960 \\
306050 \\
306050 \\
306050\end{array}$ & $\begin{array}{l}302780 \\
302410 \\
301490 \\
301020\end{array}$ & $\begin{array}{l}28 \\
28 \\
28 \\
28\end{array}$ & $\begin{array}{l}26 \\
26 \\
26 \\
26 \\
26\end{array}$ & & & & $\begin{array}{l}870 \\
680 \\
050 \\
690 \\
150\end{array}$ \\
\hline 26 & $\begin{array}{l}301490 \\
301210 \\
300650 \\
300470 \\
299910 \\
299540\end{array}$ & $\begin{array}{l}299180 \\
299180 \\
299180 \\
299270 \\
299360\end{array}$ & $\begin{array}{l}301580 \\
301670 \\
301670 \\
301670 \\
301760 \\
301850\end{array}$ & $\begin{array}{l}304180 \\
304180 \\
304090 \\
303990 \\
304090 \\
304090\end{array}$ & $\begin{array}{r}305770 \\
305680 \\
305680 \\
305680 \\
\ldots \\
\ldots-\end{array}$ & $\begin{array}{l}300740 \\
300280 \\
299910 \\
299540 \\
298990 \\
298620\end{array}$ & $\begin{array}{l}284090 \\
283380 \\
282590 \\
281710 \\
280840\end{array}$ & $\begin{array}{l}260800 \\
260720 \\
260470 \\
259980 \\
259820 \\
259400\end{array}$ & $\begin{array}{l}244 \\
244 \\
244 \\
246 \\
248\end{array}$ & $\begin{array}{l}276490 \\
280400 \\
280570 \\
280050 \\
280840 \\
281190\end{array}$ & $\begin{array}{l}271940 \\
272710 \\
279260 \\
281190 \\
285950 \\
288000\end{array}$ & $\begin{array}{l}610 \\
070 \\
710 \\
260 \\
630\end{array}$ \\
\hline t) & $\begin{array}{r}312290 \\
299540 \\
4199.28 \\
-13220 \\
\text { R. } 1995\end{array}$ & $\begin{array}{c}299360 \\
298530 \\
4199.26 \\
-180 \\
\operatorname{MAX} 318150\end{array}$ & $\begin{array}{r}301850 \\
299540 \\
4199.53 \\
+2490 \\
0 \quad \text { MIN 27 }\end{array}$ & $\begin{array}{r}304270 \\
302040 \\
4199.77 \\
+2240 \\
2970\end{array}$ & $\begin{array}{r}306050 \\
304090 \\
4199.94 \\
+1590 \\
+26310\end{array}$ & $\begin{array}{r}305680 \\
298620 \\
4199.18 \\
-7060\end{array}$ & $\begin{array}{r}298160 \\
280840 \\
4197.20 \\
-17780\end{array}$ & $\begin{array}{r}280140 \\
259400 \\
4194.67 \\
-21440\end{array}$ & $\begin{array}{r}258830 \\
244630 \\
4193.30 \\
-11070\end{array}$ & $\begin{array}{r}281190 \\
247140 \\
4197.24 \\
+32860\end{array}$ & $\begin{array}{r}288000 \\
271010 \\
4198.01 \\
+6810\end{array}$ & $\begin{array}{r}294320 \\
288630 \\
4198.08 \\
+630\end{array}$ \\
\hline
\end{tabular}

WTR YR 1996 MAX 312290 MIN 244630 (t†) -24130

(t) ELEVATION, IN FEET, AT END OF MONTH

(t) CEANGE IN CONTENTS, IN ACRE-FEET 
LOCATION - Lat $35^{\circ} 26^{\prime} 18^{\prime \prime}$, long $103^{\circ} 31^{\prime} 31^{\prime \prime}$, in NWHSE\% sec.15, T.14 N, R.32 E. Barding County, Bydrologic Untt 11090007 , on right bank $19 \mathrm{mi}$ downstream from Alamosa Creak, 4.5 mi upstream from $5 t a t \theta$ Road 155 , 4.7 mi upstrean from high-water itne of vte Reservolx, 8.2 mi northwest of Logan, and at mile 10.0 .

DRAINAGE AREA, - $-2,060 \mathrm{mi}^{2}$, of which $617 \mathrm{mi}^{2}$ is probably noncontributing.

PERIOD OF RECORD,--January 1912 to May 1914 (gage heights and discharge measurements only), January 1942 to current year Records of discharge for August 1904 to June 1906, April 1909 to December 1911 , published in WSP 307 , are unreitable and should not be used.

REVISED RECORDS, -WSP 1281: 1942-48, 1950, 1951(P), WDR MM-81-1: 1965(P), 1967-68(M), 1969(P), 1971(M), 1972, $1975(\mathrm{M}), 1977,1979$, Se) aIso PERIOD OF RECORD.

GAGE.- Water-stage recorder. Elevation of gage is 3,820 It above National Geodetic Vertical Datum of 1929, from topographic map. See WSP 2121 for history of changes prior to Oct., 1, 1964....

REMARKS, - Records felr except for estimeted daily discharges which are poor. piversions for irrigation of a fen hundred acres upstream from station. No flow most of time.

EXIREMES OUTSIDE PERIOD OF RECORD. Flood of May 1, 1914, reached a stage of 22.95 ft $s i t e$ and datum then in use. Another mafor flood reached a stage of 16.0 ft. 1942 datum, sometime in 1941, from information furntshed by Bureau of Reclamation; dischargo, about $70,000^{\circ} \mathrm{ft} / \mathrm{s}$.

DISCHARGE, CUBIC FEET PER SECOND, WATER YEAR OCTOBER 1995 TO SEPTEMBER 1996

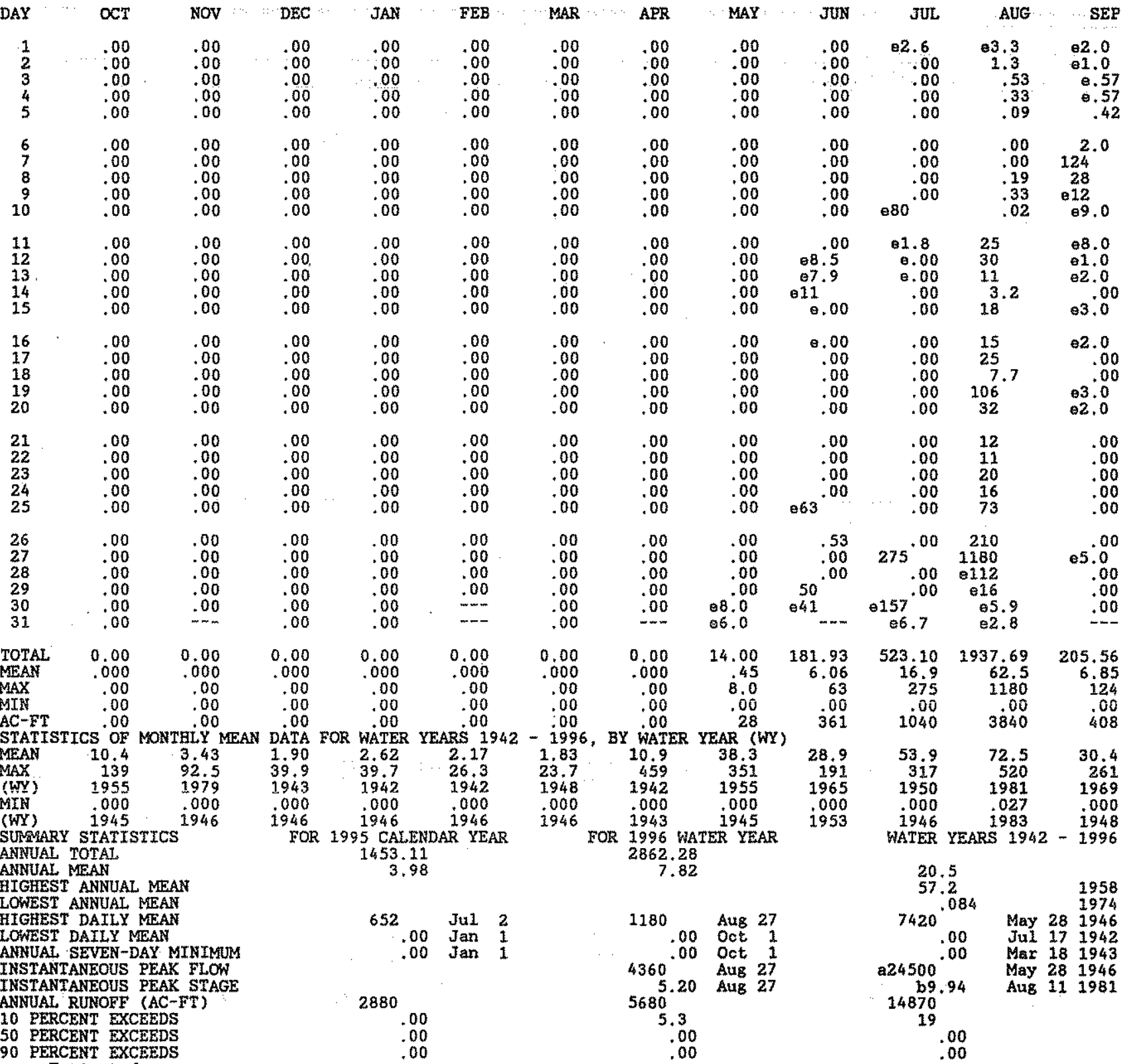

a-Fron rating curve extended above $7,700 \mathrm{ft}^{3} / \mathrm{s}$ on basts of slope-area measurements at 8 age heights 5.2 ft and $7.2 \mathrm{ft}$.

b-Site and datum then in use. 
ARKANSAS RIVER BASIN

07226800 UTE RESERVOIR NEAR LOGAN, NM

LOCATrON. --Lat $35^{\circ} 20^{\circ} 35^{\prime \prime}$, Long $103^{\circ} 26^{\circ} 37 "$, in NWK sec.21, T.13 N., R.33 E., Quay County, Bydrologic Unit 11080006, on face of Ute Dam on Canadian Rtver, 2.5 mi southwest of Logan, $3.5 \mathrm{mi}$ downstream from Ute Creek, and at mile 673.1 .

DRAINAGE AREA. - - $11,110 \mathrm{mi}^{2}$, of which $1,110 \mathrm{mi}^{2}$ is probably noncontributing.

WATER-DISCEARGE RECORDS

PERIOD OF RECORD.--May 1963 to September 1965 (monthend contents only), October 1965 to current year.

REVISED RECORDS. - WDR NM-78-1: 1977.

GAGE.- Water-stage recorder. Datum of gage 18 National Geodetic Vertical Datum of 1929 (levels by Interstate Stream Comission). Prior to Feb. 25, 1974, nonrecording gage at same site and datum.

REMARKS. --Reservolx is formed by an earthfill dam $132 \mathrm{ft}$ high above streambed, 2,050 ft 1ong; an earthen dike section on north bank of Canadian River $3,640 \mathrm{ft}$ long with a maximum height of $38 \mathrm{ft}$; a concrete 1 abyrinth spillway section with an equivalent weir length of $3,360 \mathrm{ft}$ is located upstream of an $840 \mathrm{ft}$ long ogee section between the main embankment and dike. Original construction completed in May 1963, storage began Dec. 13, 1962; modification project to construct labyrinth spillway and increase height of dam and dike completed April i984. Capactty, 244,960 acre-ft at elovation 3,787.0 ft, crest of labyrinth spillway from capacity table dated November 1992. Original capacity at elevation $3,787.0 \mathrm{ft}$ was 272,770 acre-ft, Top of dam 1 s at elevation $3,812.0 \mathrm{ft}$. Dead storage, 10,780 acre-ft at elevation 3,725.0 ft, sili of outlet intake tower; inactive pool of 25,070 acreft between elevations $3,725.0$ and $3,741.6 \mathrm{ft}$, maintained for sediment control and fish and wildilfe. Figures given herein represent total contents. Reservoir storage is for municipal and industrial uses, recreational purposes, sediment control and some incidental flood control. Diversions upstream from station for trrigation about 90,000 acres.

EXTREMES FOR PERIOD OF RECORD. - Maximum contents observed, 250,000 acre-ft, May 20, 21, 1987, elevation, 3,787.40 $\mathrm{ft}$; minimum since reservoir first filled in September 1965, 31,320 acre-ft, June 6, 1984 , elevation, $3,739.10 \mathrm{ft}$.

EXTREMES FOR CURRENT YEAR.--Maximum contents, 194,230 acre-ft, July 31, elevation, 3,780.16 ft; minimum, 170,440 acre-ft, June 11, elevation, $3,776.50 \mathrm{ft}$.

Capacity table (elevation, in feet, and contents, in acre-feet)

(Based on survey by U. S. Bureau of Reclamation and New Mexico Interstate Stroam Commlssion 1992)

$$
\begin{array}{llll}
3,744 & 41,030 & 3,780 & 193,100 \\
3,760 & 88,760 & 3,788 & 253,100
\end{array}
$$

RESERVOIR STORAGE (ACRE-FEET), WATER YEAR OCTOBER 1995 TO SEPTEMBER 1996 DAILY OBSERVATION AT 24:00 VALUES

\begin{tabular}{|c|c|c|c|c|c|c|c|c|c|c|c|c|}
\hline & OCT & Nov & DEC & JAN & FEB & MAR & APR & MAY & JUN & JUL & AUG & EP \\
\hline $\begin{array}{l}1 \\
2 \\
3 \\
4 \\
5\end{array}$ & $\begin{array}{l}185280 \\
184110 \\
183910\end{array}$ & $\begin{array}{l}178850 \\
178590 \\
178460 \\
178590 \\
178910\end{array}$ & $\begin{array}{l}178 \\
17 \\
178 \\
17 \\
17\end{array}$ & $\begin{array}{l}1 \\
1 \\
1 \\
1 \\
1\end{array}$ & & & & & & & & $\begin{array}{l}140 \\
760 \\
340 \\
770 \\
470\end{array}$ \\
\hline $\begin{array}{r}6 \\
7 \\
8 \\
9 \\
10\end{array}$ & $\begin{array}{l}180650 \\
180060 \\
179740 \\
179740\end{array}$ & $\begin{array}{l}178460 \\
178780 \\
178910\end{array}$ & $\begin{array}{l}178 \\
178 \\
177 \\
177 \\
178\end{array}$ & $\begin{array}{l}17 \\
17 \\
17\end{array}$ & & 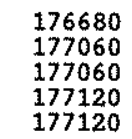 & & & & & & \\
\hline & $\begin{array}{l}179740 \\
179610 \\
179480 \\
179680 \\
179610\end{array}$ & $\begin{array}{l}178340 \\
178590 \\
178460 \\
178590 \\
178460\end{array}$ & $\begin{array}{l}177 \\
178 \\
178 \\
177 \\
177\end{array}$ & $\begin{array}{l}1782 \\
1783 \\
1783 \\
1783 \\
1784\end{array}$ & 178 & $\begin{array}{l}177 \\
177 \\
177 \\
177\end{array}$ & & & & & & \\
\hline 16 & $\begin{array}{l}179480 \\
179480 \\
180580 \\
181300\end{array}$ & $\begin{array}{l}1 \\
1 \\
1 \\
1\end{array}$ & $\begin{array}{l}17 \\
17 \\
17 \\
17\end{array}$ & $\begin{array}{l}178 \\
178 \\
178 \\
178\end{array}$ & 17 & & & & & & & \\
\hline $\begin{array}{l}21 \\
22 \\
23\end{array}$ & $\begin{array}{l}185550 \\
185550 \\
179290 \\
178970 \\
178970\end{array}$ & $\begin{array}{l}17 \\
17 \\
17 \\
17 \\
17\end{array}$ & $\begin{array}{l}17 \\
17 \\
17 \\
17 \\
17\end{array}$ & $\begin{array}{l}17 \\
17 \\
17 \\
17 \\
17\end{array}$ & $\begin{array}{l}17 \\
17 \\
17\end{array}$ & $\begin{array}{l}1 \\
1 \\
1\end{array}$ & & & & & & \\
\hline 26 & $\begin{array}{l}180650 \\
180650 \\
178850 \\
178910 \\
178720 \\
178850\end{array}$ & $\begin{array}{l}1 \\
1 \\
1 \\
1\end{array}$ & $\begin{array}{l}178 \\
178 \\
178 \\
178 \\
178 \\
178\end{array}$ & $\begin{array}{l}17 \\
17 \\
17 \\
17 \\
17 \\
17\end{array}$ & $\begin{array}{r}177250 \\
177120 \\
176810 \\
176810 \\
=-\end{array}$ & $\begin{array}{l}176 \\
176 \\
176 \\
176 \\
176\end{array}$ & & & & & & \\
\hline$A X$ & $\begin{array}{r}18555 \\
17872 \\
3777.8 \\
-630\end{array}$ & $\begin{array}{r}18013 \\
17821 \\
3777.7 \\
-64\end{array}$ & $\begin{array}{r}17859 \\
17789 \\
3777.7 \\
+250\end{array}$ & $\begin{array}{r}1791 \\
1779 \\
3777 \\
+66\end{array}$ & $\begin{array}{r}179050 \\
176810 \\
3777.51 \\
-2310\end{array}$ & $\begin{array}{r}177630 \\
176420 \\
3777.47 \\
-260\end{array}$ & $\begin{array}{r}176930 \\
174190 \\
3777.10 \\
-236\end{array}$ & $\begin{array}{r}171190 \\
3776.69 \\
-2560\end{array}$ & $\begin{array}{r}173680 \\
170440 \\
3777.02 \\
+2050\end{array}$ & $\begin{array}{r}194230 \\
173750 \\
3780.16 \\
+20550\end{array}$ & $\begin{array}{r}194100 \\
186720 \\
3779.98 \\
-1220\end{array}$ & $\begin{array}{r}193760 \\
180650 \\
3778,12 \\
-12300\end{array}$ \\
\hline
\end{tabular}

CAL YR 1995 MAX 215060 MIN 172120 (††) +2230

WTR YR 1996 MAX 194230 MIN 170440 (†) -4440

(†) ELEVATTON, IN FEET, AT END OF MONTH

$(\dagger \dagger)$ CHANGE IN CONTENTS, IN ACRE-FEET 
ARKANSAS RIVER BASIN

07226800 UTE RESERVOIR NEAR LOGAN, MM -- Continued

WATER-QUALITY RECORDS

LOCATION. --Samples collected in Ute Reservoir impounded by Ute Dam on the Canadian River.

PERIOD OF RECORD. --Water years 1963 to current year.

REMARKS. --Samples for chemical analyses are collected annually at Site B which is located 0.6 mi upstream from Ute Dam. Samples are collected 5 feet above the bottom of the reservoix.

07226560 - UTE RE AT SITE B, 0.6 MILES AB UTE DAM, MM (LAT $35^{\circ} 20^{\prime} 32^{\prime \prime}$ LONG $103^{\circ} 27^{\prime} 16^{\prime \prime}$ )

WATER QUALITY DATA, WATER YRAR OCTOBER 1995 TO SEFTEMBER 1996

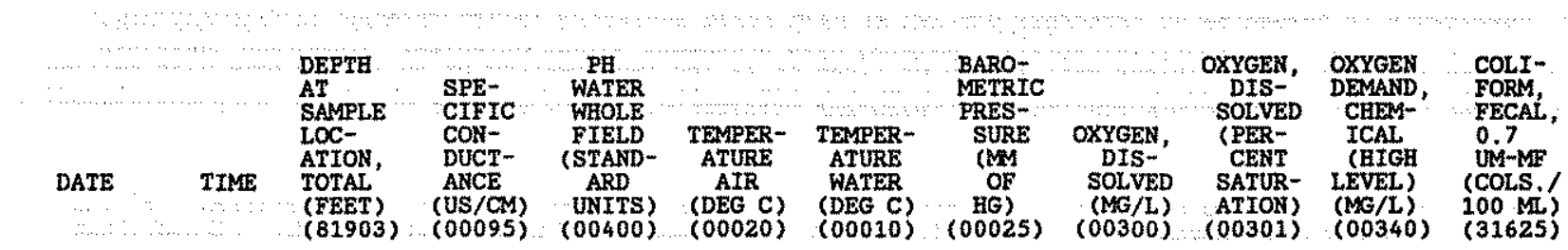

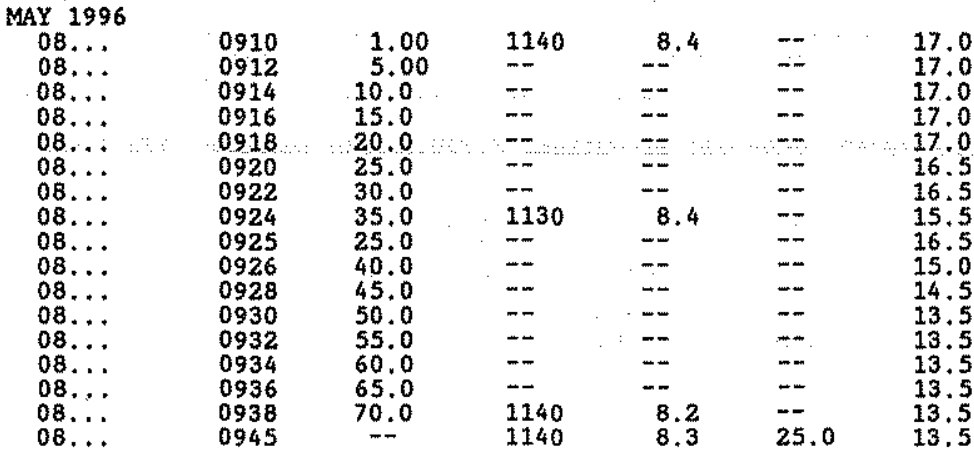

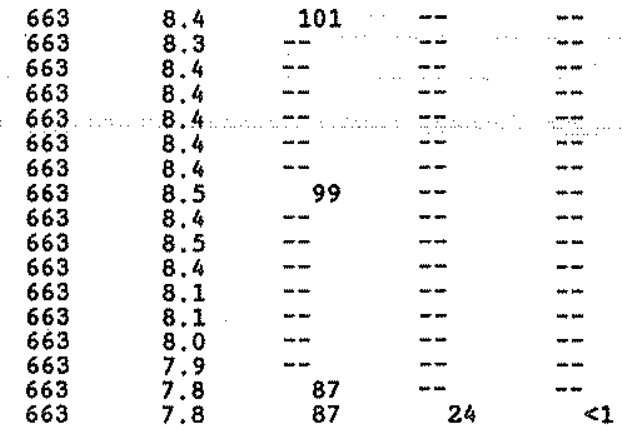

\begin{tabular}{|c|c|c|c|c|c|c|c|c|c|c|}
\hline $\begin{array}{l}\text { STREP- } \\
\text { TOCOCCI } \\
\text { FECAL, } \\
\text { XF AGAR } \\
\text { (COLS. } \\
\text { PER }\end{array}$ & $\begin{array}{l}\text { HAR } \\
\text { NES } \\
\text { TOT } \\
\text { CMG } \\
\text { AS } \\
\text { CAC } \\
\text { COO9 }\end{array}$ & $\begin{array}{l}\text { HARD- } \\
\text { NESS } \\
\text { NONCARB } \\
\text { DISSOLV } \\
\text { FLD.AS } \\
\text { CACO3 } \\
\text { (MG/L) } \\
(00904)\end{array}$ & $\begin{array}{l}\text { CAL } \\
\text { DI } \\
\text { SOI } \\
\text { MM } \\
\text { AS } \\
\text { COO }\end{array}$ & $\begin{array}{c}\text { MAG } \\
\text { SI } \\
D I \\
S O L \\
\text { SMG }\end{array}$ & s, & $\begin{array}{r}\text { SODI } \\
\text { AD } \\
\text { SORP } \\
\text { TIOI } \\
\text { RATIC } \\
(0093\end{array}$ & $\begin{array}{c}\mathrm{POI} \\
\mathrm{S} \\
\mathrm{DI} \\
\mathrm{SOI} \\
\text { (MS } \\
\mathrm{AS}\end{array}$ & $\begin{array}{c}\text { BON } \\
\text { WA } \\
\text { DIS } \\
\text { FI } \\
\text { MG/ } \\
\text { HC } \\
600\end{array}$ & $\begin{array}{c}\text { CAR- } \\
\text { BONATE } \\
\text { WATER } \\
\text { DIS IT } \\
\text { FIELD } \\
\text { MG/L AS } \\
\text { CO3 } \\
(00452)\end{array}$ & $\begin{array}{l}\text { IS } \\
\text { IS } \\
\text { D } \\
\text { AS } \\
3 \\
\text { 3) }\end{array}$ \\
\hline
\end{tabular}

MAY 1996

\begin{tabular}{|c|c|c|c|c|c|c|c|c|c|c|c|}
\hline $\begin{array}{c}a x+\ldots 90 \\
08 . .\end{array}$ & -- & -- & $\cdots$ & -- & -- & -- & -- & - & -- & -- & -- \\
\hline & -- & -- & -- & + & -- & $-\cdots$ & -- & -- & $\rightarrow$ & -- & -- \\
\hline $08 \ldots$ & -- & -- & -- & - & $\rightarrow$ & -- & -- & $\therefore$ & -- & -- & - \\
\hline $08 \ldots$ & -- & -- & -- & -- & -- & - & -- & -- & -- & -- & -- \\
\hline $08 \ldots$ & $\cdots$ & - & $\mathrm{mm}$ & -- & -- & -- & - & -- & -- & $-\cdots$ & - \\
\hline $08 \ldots$ & -- & -- & - & -- & -- & - & $\cdots$ & -- & -- & -- & $\cdots$ \\
\hline $08 \ldots$ & -- & -- & -- & -- & -- & -- & -- & - & -- & -- & - \\
\hline $08 \ldots$ & -- & -- & -- & -- & - & -- & $\cdots$ & $-\cdots$ & $\cdots$ & -- & $m$ \\
\hline $08 \ldots$ & -- & -- & -- & -- & - & -- & -- & -- & -- & -- & $\cdots$ \\
\hline$\ldots$ & $\cdots$ & -- & -- & $\cdots$ & -- & $-\infty$ & -- & -- & - & -- & - \\
\hline 08 & $\cdots$ & -- & -- & -- & -- & -- & $\rightarrow$ & -- & - & - & -- \\
\hline $3 .$. & $\cdots$ & $-\infty$ & -- & -- & -- & -- & - & -- & - & -- & $m$ \\
\hline $08 \ldots$ & $=-$ & $=$ & $\overline{-}$ & $=$ & $=$ & $\overline{-}$ & $=$ & $=-$ & $\overline{-}$ & $=$ & $=$ \\
\hline & $=$ & $=$ & $=$ & - & 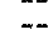 & $=$ & $=$ & $=$ & $=$ & - & $=$ \\
\hline & -- & -- & -- & $m$ & - & - & $\rightarrow$ & -- & -- & -- & - - \\
\hline & & 31 & 12 & & & & 3 & 6.4 & 228 & & 18 \\
\hline
\end{tabular}


ARKANSAS RIVER BASIN

07226800 UTE RESERVOIR NEAR LOGAN, NM -- Continued

WATER-QUALITY RECORDS

07226560 - UTE RE AT STTE B, 0.6 MILES AB UTE DAM, NM (LAT $35^{\circ} 20^{\circ} 32^{\prime \prime}$ LONG $103^{\circ} 27^{\prime} 16^{\prime \prime}$ )

WATER QUALITY DATA, WATER YEAR OCTOBER 1995 TO SEPTEMBER 1996

\begin{tabular}{|c|c|c|c|c|c|c|c|c|c|c|c|}
\hline DATE & $\begin{array}{c}\text { ALKA- } \\
\text { LINITY } \\
\text { LAB } \\
\text { (MG/L } \\
\text { AS } \\
\text { CACO3) } \\
(90410)\end{array}$ & $\begin{array}{c}\text { SULFATE } \\
\text { DIS- } \\
\text { SOLVED } \\
\text { (MG/L } \\
\text { AS SO4) } \\
(00945)\end{array}$ & $\begin{array}{l}\text { CHLO- } \\
\text { RIDE, } \\
\text { DIS- } \\
\text { SOLVED } \\
\text { (MG/L } \\
\text { AS CL) } \\
(00940)\end{array}$ & $\begin{array}{l}\text { FLUO- } \\
\text { RIDE, } \\
\text { DIS- } \\
\text { SOLVED } \\
\text { (MG/L } \\
\text { AS F) } \\
(00950)\end{array}$ & $\begin{array}{l}\text { SILICA, } \\
\text { DIS- } \\
\text { SOLVED } \\
\text { (MG/L } \\
\text { AS } \\
\text { SIO2) } \\
(00955)\end{array}$ & $\begin{array}{c}\text { SOLIDS, } \\
\text { SUM OF } \\
\text { CONSTI- } \\
\text { TUENTS, } \\
\text { DIS- } \\
\text { SOLVED } \\
\text { (MG/L) } \\
\text { (70301) }\end{array}$ & $\begin{array}{c}\text { NITRO- } \\
\text { GEN, } \\
\text { NITRIYE } \\
\text { DIS- } \\
\text { SOLVED } \\
\text { (MG/L } \\
\text { AS N) } \\
(00613)\end{array}$ & $\begin{array}{c}\text { NITRO- } \\
\text { GEN } \\
\text { NO2+NO3 } \\
\text { DIS- } \\
\text { SOLVED } \\
\text { (MG/L } \\
\text { AS N) } \\
(00631)\end{array}$ & $\begin{array}{c}\text { NITRO- } \\
\text { GEN, } \\
\text { AMMONIA } \\
\text { DIS- } \\
\text { SOLVED } \\
\text { (MG } / \mathrm{L} \\
\text { AS N) } \\
\text { (00608) }\end{array}$ & $\begin{array}{c}\text { NITRO- } \\
\text { GEN, } \\
\text { ORGANIC } \\
\text { DIS- } \\
\text { SOLVED } \\
\text { (MG/L } \\
\text { AS N } \\
(00607)\end{array}$ & $\begin{array}{l}\text { NITRO- } \\
\text { GEN, AM- } \\
\text { MONIA + } \\
\text { ORGANIC } \\
\text { TOTAL } \\
\text { (MG/L } \\
\text { AS N } \\
\text { (00625) }\end{array}$ \\
\hline 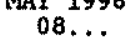 & 196 & 330 & 47 & 0.70 & 4.9 & 730 & $<0.010$ & $<0.050$ & 0.030 & 0.27 & 0.40 \\
\hline DATE & $\begin{array}{l}\text { NITRO- } \\
\text { GEN,AM- } \\
\text { MONIA + } \\
\text { ORGANIC } \\
\text { DIS. } \\
\text { (MG/L } \\
\text { AS N) } \\
\text { (00623) }\end{array}$ & $\begin{array}{c}\text { PROS- } \\
\text { PHORUS } \\
\text { TOTAL } \\
\text { (MG/L } \\
\text { AS P) } \\
(00665)\end{array}$ & $\begin{array}{c}\text { PHOS- } \\
\text { PHORUS } \\
\text { DIS- } \\
\text { SOLVED } \\
\text { (MG/L } \\
\text { AS P) } \\
(00666)\end{array}$ & $\begin{array}{c}\text { PHOS- } \\
\text { PHORUS } \\
\text { ORTHO, } \\
\text { DIS- } \\
\text { SOLVED } \\
\text { (MG/L } \\
\text { AS P) } \\
(00671)\end{array}$ & $\begin{array}{l}\text { CARBON, } \\
\text { ORGANIC } \\
\text { TOTAL } \\
\text { (MG/L } \\
\text { AS C) } \\
(00680)\end{array}$ & $\begin{array}{l}\text { ALUM- } \\
\text { INUM, } \\
\text { DIS- } \\
\text { SOLVED } \\
\text { (UG/L. } \\
\text { AS AL) } \\
(01106)\end{array}$ & $\begin{array}{l}\text { ANTI- } \\
\text { MONY, } \\
\text { DIS- } \\
\text { SOLVED } \\
\text { (UG/L } \\
\text { AS SB) } \\
(01095)\end{array}$ & $\begin{array}{c}\text { ARSENIC } \\
\text { DIS- } \\
\text { SOLVED } \\
\text { (UG/L } \\
\text { AS AS) } \\
(01000)\end{array}$ & $\begin{array}{l}\text { BARIUM, } \\
\text { DIS- } \\
\text { SOLVED } \\
\text { (UG/L } \\
\text { AS BA) } \\
\text { (01005) }\end{array}$ & $\begin{array}{l}\text { BERYL- } \\
\text { LIUM, } \\
\text { DIS- } \\
\text { SOLVED } \\
\text { (UG/L } \\
\text { AS BE) } \\
(01010)\end{array}$ & $\begin{array}{l}\text { BORON, } \\
\text { DIS- } \\
\text { SOLVED } \\
\text { (UG/L } \\
\text { AS B) } \\
(01020)\end{array}$ \\
\hline $08 \ldots$ & 30 & $<0,010$ & $<0.010$ & $<0.010$ & 4.8 & 5.0 & $<1,0$ & 1 & 238 & 0 & 170 \\
\hline
\end{tabular}

\begin{tabular}{|c|c|c|c|c|c|c|c|c|c|c|c|}
\hline ATE & $\begin{array}{c}\text { CADMIUM } \\
\text { DIS- } \\
\text { SOLVED } \\
\text { (UG/L } \\
\text { AS CD) } \\
(01025)\end{array}$ & $\begin{array}{l}\text { CHRO- } \\
\text { MIUM, } \\
\text { DIS- } \\
\text { SOLVED } \\
\text { (UG/L } \\
\text { AS CR) } \\
(01030)\end{array}$ & $\begin{array}{c}\text { COBALT, } \\
\text { DIS- } \\
\text { SOLVED } \\
\text { (UG/L } \\
\text { AS CO) } \\
(01035)\end{array}$ & $\begin{array}{l}\text { COPPER, } \\
\text { DIS- } \\
\text { SOLVED } \\
\text { (UG/L } \\
\text { AS CU) } \\
(01040)\end{array}$ & $\begin{array}{l}\text { IRON, } \\
\text { DIS- } \\
\text { SOLVED } \\
\text { (UG/L } \\
\text { AS FE) } \\
\text { (01046) }\end{array}$ & $\begin{array}{l}\text { LEAD, } \\
\text { DIS- } \\
\text { SOLVED } \\
\text { (UG/L } \\
\text { AS PB) } \\
(01049)\end{array}$ & $\begin{array}{l}\text { MANGA- } \\
\text { NESE, } \\
\text { DIS- } \\
\text { SOLVED } \\
\text { (UG/L } \\
\text { AS MN) } \\
\text { (01056) }\end{array}$ & $\begin{array}{c}\text { MERCURY } \\
\text { TOTAL } \\
\text { RECOV- } \\
\text { ERABLE } \\
\text { (UG/L } \\
\text { AS HG) } \\
\text { (71900) }\end{array}$ & $\begin{array}{c}\text { MOLYB- } \\
\text { DENUM, } \\
\text { DIS- } \\
\text { SOLVED } \\
\text { (UG /L } \\
\text { AS MO) } \\
(01060)\end{array}$ & $\begin{array}{c}\text { NICKEL, } \\
\text { DIS- } \\
\text { SOLVED } \\
\text { (IUG/L } \\
\text { AS NI) } \\
(01065)\end{array}$ & $\begin{array}{c}\text { SELE- } \\
\text { NIUM, } \\
\text { TOTAL } \\
\text { (UG/L } \\
\text { AS SE }) \\
(01147)\end{array}$ \\
\hline $\begin{array}{c}191996 \\
08 . \ldots\end{array}$ & $<1,0$ & $<1.0$ & $<1.0$ & 4.0 & $<3.0$ & $<1.0$ & $<1.0$ & $<0.10$ & 5.0 & 2.0 & $<1$ \\
\hline
\end{tabular}

\begin{tabular}{|c|c|c|c|c|c|c|c|c|c|c|}
\hline DATE & $\begin{array}{c}\text { SELE- } \\
\text { NIUM, } \\
\text { DIS- } \\
\text { SOLVED } \\
\text { (UG/L } \\
\text { AS SE) } \\
(01145)\end{array}$ & $\begin{array}{c}\text { SILVER, } \\
\text { DIS- } \\
\text { SOLVED } \\
\text { (UG/L } \\
\text { AS AG) } \\
(01075)\end{array}$ & $\begin{array}{l}\text { ZINC, } \\
\text { DIS- } \\
\text { SOLVED } \\
\text { (UG/L } \\
\text { AS ZN) } \\
\text { (01090) }\end{array}$ & $\begin{array}{c}\text { NITRO- } \\
\text { GEN } \\
\text { NO2+NO3 } \\
\text { TOT. IN } \\
\text { BOT MAT } \\
\text { (MG/KG } \\
\text { AS N } \\
(00633)\end{array}$ & 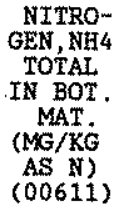 & $\begin{array}{l}\text { NITRO- } \\
\text { GEN, NH4 } \\
+ \text { ORG. } \\
\text { TOT IN } \\
\text { BOT MAT } \\
\text { (MG /KG } \\
\text { AS N) } \\
\text { (O0626) }\end{array}$ & $\begin{array}{c}\text { PHOS- } \\
\text { PHORUS } \\
\text { TOTAL } \\
\text { IN BOT. } \\
\text { MAT. } \\
\text { (MG /KG } \\
\text { AS P) } \\
(00668)\end{array}$ & $\begin{array}{c}\text { ARSENIC } \\
\text { TOTAL } \\
\text { IN BOT- } \\
\text { TOM MA- } \\
\text { TERIAL } \\
\text { (UG/G } \\
\text { AS AS) } \\
\text { (01003) }\end{array}$ & $\begin{array}{c}\text { CADMIUM } \\
\text { RECOV. } \\
\text { FM BOT- } \\
\text { TOM MAm } \\
\text { TERIAL. } \\
\text { (UG/G } \\
\text { AS CD) } \\
(01028)\end{array}$ & $\begin{array}{c}\text { CERO- } \\
\text { MIJM, } \\
\text { RECOV. } \\
\text { FM BOT- } \\
\text { TOM MA- } \\
\text { TERIAI } \\
\text { (UG/G) } \\
(01029)\end{array}$ \\
\hline $08 \ldots$ & $<1$ & $<1.0$ & 3.0 & 2.0 & 32 & 1200 & 600 & to the & $<10$ & $10+0,8$ \\
\hline
\end{tabular}

\begin{tabular}{|c|c|c|c|c|c|c|c|c|c|c|}
\hline DATE & $\begin{array}{c}\text { COBALT, } \\
\text { RECOV. } \\
\text { FM BOT- } \\
\text { TOM MA- } \\
\text { TERIAL } \\
\text { (UG/G } \\
\text { AS CO) } \\
(01038)\end{array}$ & $\begin{array}{c}\text { COPPER, } \\
\text { RECOV. } \\
\text { FM BOT- } \\
\text { TOM MA- } \\
\text { TERIAL } \\
\text { (UG /G } \\
\text { AS CU) } \\
\text { (01043) }\end{array}$ & $\begin{array}{l}\text { IRON, } \\
\text { RECOV. } \\
\text { FM BOT- } \\
\text { TOM MA- } \\
\text { TERIAL } \\
\text { (UG/G } \\
\text { AS FE) } \\
(01170)\end{array}$ & $\begin{array}{c}\text { LEAD, } \\
\text { RECOV. } \\
\text { FM BOT- } \\
\text { TOM MA- } \\
\text { TERIAL } \\
\text { (UG/G } \\
\text { AS PB) } \\
(01052)\end{array}$ & $\begin{array}{c}\text { MANGA- } \\
\text { NESE, } \\
\text { RECOV. } \\
\text { FM BOT- } \\
\text { TOM MA- } \\
\text { TERIALL } \\
\text { (UG/G) } \\
\text { (01053) }\end{array}$ & $\begin{array}{c}\text { MERCURY } \\
\text { RECOV. } \\
\text { FM BOT- } \\
\text { TOM MA- } \\
\text { TERIAL } \\
\text { (UG/G } \\
\text { AS HG) } \\
\text { (71921) }\end{array}$ & $\begin{array}{c}\text { ZINC, } \\
\text { RECOV. } \\
\text { FM BOT- } \\
\text { TOM MA } \\
\text { TERIAL } \\
\text { (UGG/G } \\
\text { AS ZN) } \\
(01093)\end{array}$ & $\begin{array}{l}\text { URANIUM } \\
\text { NATURAL } \\
\text { DIS- } \\
\text { SOLVED } \\
\text { (UG/L } \\
\text { AS U) } \\
\text { (22703) }\end{array}$ & $\begin{array}{l}\text { SEDI- } \\
\text { MENT, } \\
\text { SUS- } \\
\text { PENDED } \\
\text { (MG/L) } \\
(80154)\end{array}$ & $\begin{array}{l}\text { SED. } \\
\text { SUSP. } \\
\text { SIEVE } \\
\text { DIAM. } \\
\% \text { FINER } \\
\text { THAN } \\
\text { THE2 MM } \\
\text { (70331) }\end{array}$ \\
\hline $08 \ldots$ & 10 & 20 & 9300 & $<100$ & 400 & $<0.10$ & 40 & 6.0 & 27 & 78 \\
\hline
\end{tabular}


LOCATION, --Lat $35^{\circ} 21^{\prime} 25^{\prime \prime}$, long $103^{\circ} 25^{\prime} 03^{\prime \prime}$, in NE\%NE sec.15, T.13 N., R.33 E., Quay County, Eydrologic Unit 11080006 , on left bank 1,110 ft upstream from bridge on U.S. H1ghway 54, 0.7 mi south of Logan, 1.4 mi upstream from Chicago, Rock Island \& Eacific Railroad Co. bridge, $2.0 \mathrm{mi}$ downstream from Ute Dam, $4.3 \mathrm{mi}$ upstream from Revuel to Creak, and at mile 672.0

DRAINAGE AREA, $-11,141 \mathrm{mi}^{3}$, of which $1,110 \mathrm{mi}^{3}$ is probably noncontributing. September 1909. February 1910, April to July 1910, August 1910 to September 1911 (gage heights and discharge measurements only), October 1911 to May 1914, January to May 1924, September 1924 to July 1925 , January 1927 to Apri1 1934. August 1934 to current year. Monthly discharge only for some periods published in WSP 1311. Records for December 1909, Januery 1910, and May to July 1934, published in WSP 267, 287, and 762 are unrel1able and should not be used. Published as "South Canads an Rver" June to September 1904

REVISED RECORDS,--WSP 1087: 1935-36, WSP 1117 : Drainage area, WSP 1281: 1912, 1932(N), 1934, $1945-47,1949-50$. WSP 1311: 1931(M). See also PERIOD OF RECORD.

GAGE, - Water-stage recorder. Datum of gage is 3,667.1 ft above National Geodetic Vertical Datum of 1929 . Prior to Jan. 1, 1987 same site at datum 1.0 ft higher. Soe WSP 1311 or 1731 for history of changes prior to Oct. 1 , 1934.

REMARKS - Water-discharge records good except for estimated discharges which are poor. Flow regulated by Conchas Lake, 45 mi upstream (station 07223500 ) and Ute Reservoir, 2 mi upsiream (station 07226800 ). Diversions for irrigation of about 90,000 acres upstream from station. Several observations of water temperature were made during the year. No flow at times prior to completion of Ute Dam.

AVERAGE DISCBARGE, - -15 years (water years $1909,1912-13,1927-38$ ), $392 \mathrm{ft} / \mathrm{s}, 284,000$ acre-ft/yr, prior to completion of Conchas dam. 24 years (water years 1939-62), 257 ft3/s, 186, 200 acre-ft/yr, prior to completion of Ute Dam.

EXTREMES OUTSIDE PERIOD OF RECORD. - Maximum discharge, 278,000 $\mathrm{st} / \mathrm{s}$, Sopt, 30, 1904, gage hotght, about $36.5 \mathrm{ft}$, site and datum used in 1909 , from rating curve extended above $14,000 \mathrm{ft}^{3} / \mathrm{s}$, from finth Biennial Report of New Mexico State Engineer.

DISCHARGE, CUBIC FEET PER SECOND, WATER YEAR OCTOBER 1995 TO SEPTEMBER 1996

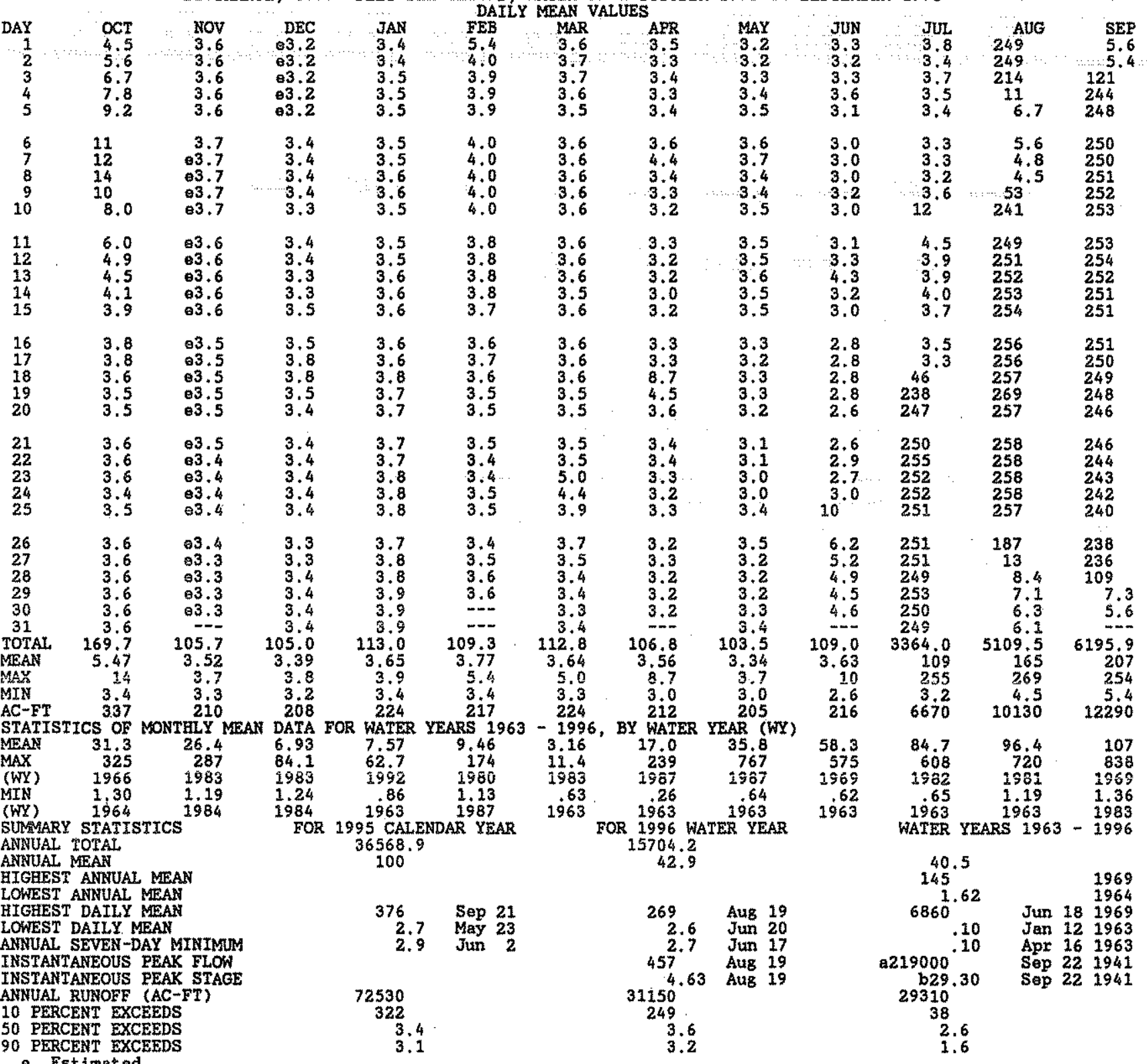

a-From rating curve extended above 75,000

b-From floodmarks. 
ARKANSAS RIVER BASIN

07227000 CANADTAN RIVER AT LOGAN, NM -- Continued

WATER-QUALITY RECORDS

PERIOD OF RECORD.--Water years 1957-62, 1992 to current year.

WATER QUALITY DATA, WATER YEAR OCTOBER 1995 TO SEPTEMBER 1996

\begin{tabular}{|c|c|c|c|c|c|c|c|c|c|c|c|c|}
\hline ATE & TIME & $\begin{array}{c}\text { DIS- } \\
\text { CHARGE, } \\
\text { INST. } \\
\text { CUBIC } \\
\text { FEET } \\
\text { PER } \\
\text { SECOND } \\
(00061)\end{array}$ & $\begin{array}{l}\text { SPE- } \\
\text { CIFIC } \\
\text { CON- } \\
\text { DUCT- } \\
\text { ANCE } \\
\text { (US/CM) } \\
\text { (00095) }\end{array}$ & $\begin{array}{c}\text { PH } \\
\text { WATER } \\
\text { WHOLE } \\
\text { FIELD } \\
\text { (STAND- } \\
\text { ARD } \\
\text { UNITS) } \\
(00400)\end{array}$ & $\begin{array}{c}\text { TEMPER- } \\
\text { ATURE } \\
\text { AIR } \\
(\text { DEG C) } \\
(00020)\end{array}$ & $\begin{array}{c}\text { TEMRER- } \\
\text { ATURE } \\
\text { WATER } \\
(\text { DEG C) } \\
(00010)\end{array}$ & $\begin{array}{c}\text { BARO- } \\
\text { METRIC } \\
\text { FRES- } \\
\text { SURE } \\
\text { (MY } \\
\text { OF } \\
\text { EGG) } \\
(00025)\end{array}$ & $\begin{array}{c}\text { OXYGEN, } \\
\text { DIS } \\
\text { SOLVED } \\
\text { (MG/L) } \\
(00300)\end{array}$ & $\begin{array}{c}\text { OXYGEN, } \\
\text { DIS- } \\
\text { SOLVED } \\
\text { (PER- } \\
\text { CENT } \\
\text { SATUR- } \\
\text { ATION) } \\
\text { (O0301) }\end{array}$ & $\begin{array}{l}\text { HARD- } \\
\text { NESS } \\
\text { TOTAL } \\
(M G / L \\
\text { AS } \\
\text { CACO3) } \\
(00900)\end{array}$ & $\begin{array}{l}\text { CALCIUM } \\
\text { DIS- } \\
\text { SOLVED } \\
\text { (MG/L } \\
\text { AS CA) } \\
\text { (00915) }\end{array}$ & $\begin{array}{c}\text { MAGNE- } \\
\text { SIUM, } \\
\text { DIS- } \\
\text { SOLVED } \\
\text { (MG/L. } \\
\text { AS MG) } \\
\text { (00925) }\end{array}$ \\
\hline
\end{tabular}

\begin{tabular}{|c|c|c|c|c|c|c|c|c|c|c|c|c|}
\hline $\begin{array}{c}\text { NoV } 1995 \\
201996 \\
\text { FEB } 1996\end{array}$ & 1545 & 3.1 & 9360 & 7.9 & 15.0 & 11.5 & 675 & 10.5 & 113 & 630 & 130 & 74 \\
\hline$\underset{M A Y}{20} \cdots$ & 1430 & 3.5 & 8510 & 8.0 & 25.5 & 14.5 & 662 & 10.7 & 125 & -- & -- & -- \\
\hline $\begin{array}{l}07 . . \\
\text { AUSG }\end{array}$ & 1430 & 3.7 & 8900 & 7.9 & 25.5 & 30.5 & 665 & 8.2 & 130 & 560 & 120 & 63 \\
\hline $22 \ldots$ & 1000 & 260 & 1230 & 8.0 & 23.0 & 21.5 & 673 & 8.3 & 107 & 300 & 54 & 41 \\
\hline
\end{tabular}

$\begin{array}{ccccc}\text { SODILM, } & \text { AD- } & \text { SIUM, } & \text { LINITY } & \text { SULFATE } \\ \text { DIS- } & \text { SORP- } & \text { DIS- } & \text { LAB } & \text { DIS } \\ \text { SOLVED } & \text { TION } & \text { SOLVED } & \text { (MG/L } & \text { SOLVED } \\ \text { (MG/L } & \text { RATIO } & \text { (MG/L } & \text { AS } & \text { (MG/L } \\ \text { AS NA) } & & \text { AS K) } & \text { CACO3) } & \text { AS SO4) } \\ (00930) & (00931) & (00935) & (90410) & (00945)\end{array}$

\begin{tabular}{|c|c|c|c|}
\hline $\begin{array}{l}\text { SODILM, } \\
\text { DIS- } \\
\text { SOLVED } \\
\text { (WG/L } \\
\text { AS NA) } \\
(00930)\end{array}$ & $\begin{array}{c}\text { SODIUM } \\
\text { AD- } \\
\text { SORP- } \\
\text { TION } \\
\text { RATIO } \\
(00931)\end{array}$ & $\begin{array}{c}\text { POTAS- } \\
\text { SIUM, } \\
\text { DIS- } \\
\text { SOLVED } \\
\text { (MG/L } \\
\text { AS K) } \\
\text { (00935) }\end{array}$ & $\begin{array}{c}\text { ALKA- } \\
\text { LINITY } \\
\text { LAB } \\
\text { (MG/L } \\
\text { AS } \\
\text { CACO3) } \\
(90410)\end{array}$ \\
\hline
\end{tabular}

31

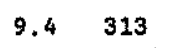

$--$

$8.9 \quad 322$

$6.7 \quad 197$
3
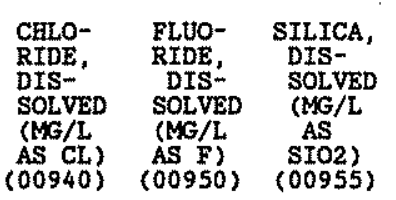

SOLIDS,

SUM OF

CONSTI-

TUENTS,

DIS-

SOLVED

(MG/L)

(70301)

$\begin{array}{lr}20 \\ 20 \\ 20 \\ M A 96 & \\ 07 \ldots & 1700 \\ \text { AUG } \ldots & \\ 22 & 140\end{array}$

$\begin{array}{cc}490 & 2500 \\ 510 & 2600 \\ 330 & 62 .\end{array}$

$0.90 \quad 11$

$5200 \quad 360<10$

$m-$

$\begin{array}{lll}5210 & 380 & 29\end{array}$

$\begin{array}{lll}759 & 185 & <3.0\end{array}$ 


\section{ARKANSAS RIVER BASIN}

07227100 REVUELTO CREEK NEAR LOGAN, RM

LOCATION,--Lat $35^{\circ} 20^{\prime} 29^{\prime \prime}$, long $103^{\circ} 23^{\prime} 37^{\prime \prime}$, in SW/4NW/ sec.24, T.13 N., R.33 E., Quay County, Eydrologic Unit

11080008 , on right bank $0.3 \mathrm{mi}$ upstream from bridge on State Bighway $469,1.9$ mi southeast of Logan, and at mile 2.3.

DRAINAGE AREA. $-786 \mathrm{mi}^{2}$.

\section{WATER-DISCHARGE RECORDS}

PERTOD OF RECORD. --August 1959 to current year.

GAGE.--Water-8tage recorder. Elovation of gage is 3,660 ft above National. Geodetic Vertical Datum of 1929 , from topographic map. Prior to Jan. 16, 1981, at site 320 ft upstrean at datum 0.56 ft higher.

REMARKS.--Water-discharge records good except for estimated discharges, which are poor... Low flows supplomented by surface and ground-water return from irrigation in vicinity of Tucumcart. Several observations of water temperature pere made during the year.

EXTREXES OUTSIDE PERIOD OF RECORD (1941-47).--Maximum discharge determined, about 13,400 fts/s, Sept. 18, 1946, gage height, $9.04 \mathrm{ft}$, at site $180 \mathrm{ft}$ downstream at different datum, from unpublished records collected by Bureau of Reclamation. A peak of $26,100 \mathrm{fts} / \mathrm{s}$, date unknown, gage height, 12.9 ft at former stte and datum, was measured by slope-area mothod in May 1957. DISCHARGE, CUBIC FEET PER SECOND, WATER YEAR OCTOBER 1995 TO SEFTEMBER 1996

\begin{tabular}{|c|c|c|c|c|c|c|c|c|c|c|c|c|}
\hline$A Y$ & $\alpha C$ & NOV & DEC & JAN & FEB & MAR & AFR & MAY & JUN & JUL & AUG & SEP \\
\hline $\begin{array}{l}1 \\
2 \\
3 \\
4 \\
5\end{array}$ & $\begin{array}{l}7.7 \\
65 \\
40 \\
11 \\
22\end{array}$ & $\begin{array}{l}36 \\
33 \\
37 \\
45 \\
34\end{array}$ & $\begin{array}{l}2.6 \\
2.4 \\
1.8 \\
1.8 \\
1.6\end{array}$ & $\begin{array}{l}2.7 \\
1.9 \\
1.6 \\
2.8 \\
2.1\end{array}$ & $\begin{array}{l}04.2 \\
04.2 \\
04.4 \\
04.3 \\
04.0\end{array}$ & $\begin{array}{r}.36 \\
.99 \\
2.6 \\
.99 \\
.53\end{array}$ & $\begin{array}{l}11 \\
18 \\
19 \\
16 \\
17\end{array}$ & $\begin{array}{l}14 \\
12 \\
9.9 \\
7.6 \\
6.4\end{array}$ & $\begin{array}{l}11 \\
8.1 \\
9.8 \\
25 \\
23\end{array}$ & $\begin{array}{r}126 \\
89 \\
178 \\
70 \\
2.5\end{array}$ & $\begin{array}{r}4.2 \\
.55 \\
.39 \\
.10 \\
.06\end{array}$ & $\begin{array}{r}68 \\
47 \\
227 \\
66 \\
27\end{array}$ \\
\hline $\begin{array}{r}6 \\
7 \\
8 \\
9 \\
10\end{array}$ & $\begin{array}{r}8.5 \\
6.9 \\
7.2 \\
9.4 \\
11\end{array}$ & $\begin{array}{l}17 \\
14 \\
30 \\
11 \\
7.2\end{array}$ & $\begin{array}{l}1.7 \\
1.8 \\
1.8 \\
1.4 \\
1.7\end{array}$ & $\begin{array}{r}1.4 \\
2.0 \\
1.9 \\
+5.2 \\
\times 5.0\end{array}$ & $\begin{array}{r}4.1 \\
4.0 \\
3.5 \\
3.0 \\
2.8\end{array}$ & $\begin{array}{c}.70 \\
1.0 \\
.66 \\
1.65 \\
1.0\end{array}$ & $\begin{array}{l}28 \\
19 \\
16 \\
14 \\
14\end{array}$ & $\begin{array}{l}18 \\
17 \\
14 \\
13 \\
11\end{array}$ & $\begin{array}{r}8.2 \\
5.5 \\
6.5 \\
5.5 \\
27\end{array}$ & $\begin{array}{r}7.4 \\
5.1 \\
5.1 \\
8.3 \\
3750\end{array}$ & $\begin{array}{l}.06 \\
.04 \\
.04 \\
.01 \\
3.1\end{array}$ & $\begin{array}{r}17 \\
188 \\
98 \\
115 \\
70\end{array}$ \\
\hline $\begin{array}{l}11 \\
12 \\
13 \\
14 \\
15\end{array}$ & $\begin{array}{r}10 \\
8.4 \\
4.6 \\
8.8 \\
13\end{array}$ & $\begin{array}{l}5.1 \\
30 \\
9.0 \\
20 \\
13\end{array}$ & $\begin{array}{l}2.8 \\
2.5 \\
1.5 \\
.90 \\
1.3\end{array}$ & $\begin{array}{l}e 5.1 \\
05.2 \\
05.3 \\
04.9 \\
04.4\end{array}$ & $\begin{array}{l}1.7 \\
2.0 \\
2.1 \\
1.9 \\
1.6\end{array}$ & $\begin{array}{l}.98 \\
.44 \\
.23 \\
.19 \\
.19\end{array}$ & $\begin{array}{l}6.6 \\
4.7 \\
5.2 \\
4.5 \\
8.0\end{array}$ & $\begin{array}{l}17 \\
36 \\
30 \\
46 \\
21\end{array}$ & $\begin{array}{r}17 \\
89 \\
758 \\
469 \\
446\end{array}$ & $\begin{array}{r}1200 \\
575 \\
821 \\
278 \\
112\end{array}$ & $\begin{array}{l}.02 \\
.01 \\
.03 \\
.05 \\
.04\end{array}$ & $\begin{array}{l}78 \\
27 \\
50 \\
30 \\
53\end{array}$ \\
\hline $\begin{array}{l}36 \\
17 \\
18 \\
19 \\
20\end{array}$ & $\begin{array}{l}12 \\
8.9 \\
9.6 \\
16 \\
41\end{array}$ & $\begin{array}{l}4.8 \\
3.3 \\
2.5 \\
2.5 \\
2.5\end{array}$ & $\begin{array}{l}1.7 \\
4.7 \\
21 \\
92 \\
36\end{array}$ & $\begin{array}{l}04.8 \\
84.5 \\
04.3 \\
04.6 \\
04.9\end{array}$ & $\begin{array}{l}1.4 \\
1.4 \\
1.5 \\
1.3 \\
1.0\end{array}$ & $\begin{array}{l}.18 \\
.22 \\
.21 \\
.18 \\
.18\end{array}$ & $\begin{array}{l}20 \\
15 \\
9.4 \\
7.1 \\
7.5\end{array}$ & $\begin{array}{l}15 \\
11 \\
18 \\
9.8 \\
3.1\end{array}$ & $\begin{array}{r}285 \\
103 \\
50 \\
23 \\
23\end{array}$ & $\begin{array}{r}36 \\
5.5 \\
.94 \\
.16 \\
.07\end{array}$ & $\begin{array}{c}77 \\
59 \\
9.3 \\
89 \\
286\end{array}$ & $\begin{array}{l}46 \\
42 \\
36 \\
12 \\
16\end{array}$ \\
\hline $\begin{array}{l}21 \\
22 \\
23 \\
24 \\
25\end{array}$ & $\begin{array}{l}21 \\
14 \\
13 \\
16 \\
18\end{array}$ & $\begin{array}{l}2.7 \\
2.7 \\
2.6 \\
2.4 \\
2.0\end{array}$ & $\begin{array}{r}14 \\
8.0 \\
5.6 \\
4.7 \\
5.0\end{array}$ & $\begin{array}{l}04.7 \\
04.6 \\
e 4.4 \\
04.5 \\
04.3\end{array}$ & $\begin{array}{r}.77 \\
.44 \\
.33 \\
.33 \\
.33\end{array}$ & $\begin{array}{r}.19 \\
.18 \\
42.81 \\
24\end{array}$ & $\begin{array}{l}6.8 \\
6.6 \\
9.2 \\
6.8 \\
8.7\end{array}$ & $\begin{array}{l}3.7 \\
4.6 \\
2.4 \\
1.5 \\
3.6\end{array}$ & $\begin{array}{r}7.4 \\
1.4 \\
57 \\
61 \\
377\end{array}$ & $\begin{array}{l}.02 \\
2.2 \\
2.0 \\
.28 \\
.08\end{array}$ & $\begin{array}{r}695 \\
106 \\
5.5 \\
60 \\
436\end{array}$ & $\begin{array}{l}15 \\
5.6 \\
23 \\
13 \\
43\end{array}$ \\
\hline $\begin{array}{l}26 \\
27 \\
28 \\
29 \\
30 \\
31\end{array}$ & $\begin{array}{l}25 \\
23 \\
27 \\
41 \\
43 \\
39\end{array}$ & $\begin{array}{l}1.8 \\
1.4 \\
1.3 \\
1.9 \\
2.4 \\
-.-\end{array}$ & $\begin{array}{l}5.9 \\
5.5 \\
4.7 \\
4.2 \\
2.6 \\
3.0\end{array}$ & $\begin{array}{l}e 4.3 \\
04.3 \\
e 4.4 \\
04.4 \\
04.3 \\
04.3\end{array}$ & $\begin{array}{r}.37 \\
.29 \\
.29 \\
.32 \\
-.- \\
--\infty\end{array}$ & $\begin{array}{l}42 \\
38 \\
34 \\
10 \\
15 \\
10\end{array}$ & $\begin{array}{l}22 \\
17 \\
12 \\
16 \\
19 \\
--\end{array}$ & $\begin{array}{l}53 \\
18 \\
10 \\
7.3 \\
9.3 \\
12.3\end{array}$ & $\begin{array}{r}3170 \\
1630 \\
629 \\
623 \\
249 \\
---\end{array}$ & $\begin{array}{l}224^{.21} \\
358 \\
130 \\
488 \\
121\end{array}$ & $\begin{array}{c}567 \\
696 \\
379 \\
74 \\
4.5 \\
30\end{array}$ & $\begin{array}{l}4.3 \\
1.2 \\
3.4 \\
9.0 \\
3.2 \\
-\end{array}$ \\
\hline $\begin{array}{l}\text { TALI } \\
\text { ANA } \\
\text { X } \\
N \\
N-E T\end{array}$ & $\begin{array}{r}507.0 \\
19.4 \\
65 \\
4.6 \\
11.90\end{array}$ & $\begin{array}{r}378.1 \\
12.6 \\
45 \\
1.3 \\
750\end{array}$ & $\begin{array}{r}246.20 \\
7.94 \\
92 \\
.90 \\
488\end{array}$ & $\begin{array}{r}123.1 \\
3.97 \\
5.3 \\
1.4 \\
244\end{array}$ & $\begin{array}{r}57.87 \\
2.00 \\
4.4 \\
.29 \\
115\end{array}$ & $\begin{array}{r}228.66 \\
7.38 \\
42 \\
.18 \\
454\end{array}$ & $\begin{array}{r}384.1 \\
12.8 \\
28 \\
4.5 \\
762\end{array}$ & $\begin{array}{r}455.2 \\
14.7 \\
53 \\
1.5 \\
903\end{array}$ & $\begin{array}{r}9197.4 \\
307 \\
3170 \\
1.4 \\
18240\end{array}$ & $\begin{array}{r}8618.36 \\
278 \\
3750 \\
1702 \\
17090\end{array}$ & $\begin{array}{r}3582.00 \\
116 \\
696 \\
.01 \\
7100\end{array}$ & $\begin{array}{r}1433.7 \\
47.8 \\
227 \\
1.2 \\
2840\end{array}$ \\
\hline 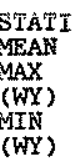 & $\begin{array}{r}\text { CS OF } \\
34.7 \\
320 \\
1961 \\
.000 \\
1965\end{array}$ & $\begin{array}{r}\text { MONTII } \\
9.18 \\
34.1 \\
1.962 \\
i 056 \\
1978\end{array}$ & $\begin{array}{r}\text { DATA } \\
9.86 \\
129 \\
1960 \\
.001 \\
1976\end{array}$ & $\begin{array}{c}\text { FOR WAYRR } \\
5.58 \\
27.9 \\
1990 \\
.000 \\
1965\end{array}$ & $\begin{array}{c}\text { WARS } 1959 \\
7.39 \\
42.5 \\
1983 \\
.000 \\
1965\end{array}$ & $\begin{array}{c}9-19.95 \\
6.44 \\
52.1 \\
1985 \\
.003 \\
1980\end{array}$ & $\begin{array}{c}\text { DY WATEP } \\
23.9 \\
346 \\
1970 \\
32 \\
1981\end{array}$ & $\begin{array}{c}\text { YEAP (WY } \\
46.0 \\
203 \\
1991 \\
.085 \\
1976\end{array}$ & $\begin{array}{r}75.9 \\
492 \\
1960 \\
889 \\
1990\end{array}$ & $\begin{array}{r}122 \\
1203 \\
1960 \\
.42 \\
1983\end{array}$ & $\begin{array}{r}123 \\
575 \\
1981 \\
.93 \\
1978\end{array}$ & $\begin{array}{r}73.7 \\
515 \\
1969 \\
1.72 \\
1978\end{array}$ \\
\hline
\end{tabular}

SUMMARY STATISTICS ANNUAL, TOTAL

ANNUAL MEAB

EIGHEST ANNUAL MEAN

LOWEST ANNUAL MEAN

HIGEEST DAILY MEAN

LOWEST DATLY MEAN

ANMUAL SEVEN-DAY MINIMLM

INSTANTANEOUS PEAK FLOW

INSTANTANEOUS PEAK STAGE

ANNUAL RUNOFE (AC-FT)

10 PERCENT EXCEEDS

50 PERCENT EXCEEDS

90 PERCENT EXCEEDS

Estimated

a-From slope-erea measurement of peak Elow.
FOR 1995 CALENDAR YEAR FOR 1996 WATER YEAR

$$
\begin{array}{cc}
14529.80 & 25305.69 \\
39.8 & 69.1
\end{array}
$$

$1010 \quad$ Sep 10 .02 Jun 16
.08 Mar 21

28820

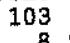

8.5

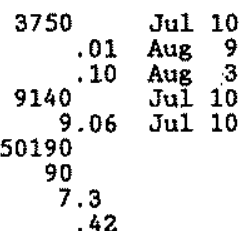

WATER YEARS $1959-1996$

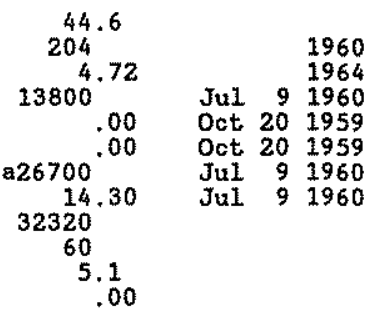


ARKANSAS RIVER BASIN

07227100 REVUELTO CREEK NEAR LOGAN, NM -- ContInued

WATER-QUALITY RECORDS

PERIOD OF RECORD.--Water years 1959 to current year.

WATER QUALITY DATA, WATER YEAR OCTOBER 1995 TO SEFTEMBER 1996

\begin{tabular}{|c|c|c|c|c|c|c|c|c|c|c|c|c|}
\hline DATE & TIME & $\begin{array}{c}\text { DIS- } \\
\text { CHARGE, } \\
\text { INST, } \\
\text { CUBIC } \\
\text { FEET } \\
\text { PER } \\
\text { SECOND } \\
(00061)\end{array}$ & $\begin{array}{l}\text { SFE- } \\
\text { CIFIC } \\
\text { CON- } \\
\text { DUCT- } \\
\text { ANCE } \\
(\text { US/CM) } \\
(00095)\end{array}$ & $\begin{array}{c}\text { PE } \\
\text { WATER } \\
\text { WAOLE } \\
\text { FIELD } \\
\text { (STAND- } \\
\text { ARD } \\
\text { UNITS) } \\
(00400)\end{array}$ & $\begin{array}{c}\text { TEMPER- } \\
\text { ATURE } \\
\text { AIR } \\
\text { (DEG C) } \\
(00020)\end{array}$ & $\begin{array}{l}\text { TEMPER- } \\
\text { ATURE } \\
\text { WATER } \\
\text { (DEG C) } \\
(00010)\end{array}$ & $\begin{array}{c}\text { BARO- } \\
\text { METRIC } \\
\text { FRES- } \\
\text { SURE } \\
\text { (MM } \\
\text { OF } \\
\text { BG) } \\
(00025)\end{array}$ & $\begin{array}{r}\text { OXYGEN, } \\
\text { DIS- } \\
\text { SOLVED } \\
(M G / L) \\
(00300)\end{array}$ & $\begin{array}{c}\text { OXYGEN, } \\
\text { DIS- } \\
\text { SOLVED } \\
\text { (PER- } \\
\text { CENT } \\
\text { SATUR- } \\
\text { ATION) } \\
\text { (00301) }\end{array}$ & $\begin{array}{l}\text { HARD- } \\
\text { NESS } \\
\text { TOTAL } \\
\text { (MG/L } \\
\text { AS } \\
\text { CACO3) } \\
(00900)\end{array}$ & $\begin{array}{l}\text { CALCIUM } \\
\text { DIS- } \\
\text { SOLVED } \\
\text { (MG/L } \\
\text { AS CA) } \\
\text { (00915) }\end{array}$ & $\begin{array}{c}\text { MAGNE- } \\
\text { SIUM, } \\
\text { DIS- } \\
\text { SOLVED } \\
\text { (NG/L } \\
\text { AS MG) } \\
(00925)\end{array}$ \\
\hline $\begin{array}{l}V_{1995} \\
20\end{array}$ & 141.5 & 2.5 & 1870 & 8,3 & 15.0 & 15.0 & 675 & 8.7 & 98 & 360 & 71 & 44 \\
\hline $20 \ldots$ & 1245 & 1.0 & 3060 & 8.3 & 17.5 & 23.0 & 661 & 8.5 & 116 & -- & -- & -- \\
\hline $07 \ldots$ & 1300 & 17 & 1620 & 8.2 & 31.0 & 30.0 & 666 & 6.8 & 104 & 400 & 73 & 54 \\
\hline & 0845 & 120 & 546 & 8.4 & 30.0 & 20.5 & 673 & 7.8 & 99 & 66 & 17 & 5.6 \\
\hline
\end{tabular}

\begin{tabular}{|c|c|c|c|c|c|c|c|c|c|c|c|}
\hline DATE & $\begin{array}{c}\text { SODIUM, } \\
\text { DIS- } \\
\text { SOLVED } \\
\text { (MG/L } \\
\text { AS NA }) \\
(00930)\end{array}$ & $\begin{array}{c}\text { SODIUM } \\
\text { AD- } \\
\text { SORP- } \\
\text { TION } \\
\text { RATIO } \\
(00931)\end{array}$ & $\begin{array}{c}\text { POTAS- } \\
\text { SIUM, } \\
\text { DIS- } \\
\text { SOLVED } \\
\text { (MG/L } \\
\text { AS K) } \\
(00935)\end{array}$ & $\begin{array}{c}\text { ALKA- } \\
\text { LINITY } \\
\text { LAB } \\
\text { (MG/L } \\
\text { AS } \\
\text { CACO3) } \\
(90410)\end{array}$ & $\begin{array}{c}\text { SULFATE } \\
\text { DIS- } \\
\text { SOLVED } \\
\text { (MG/L } \\
\text { AS SO4) } \\
(00945)\end{array}$ & $\begin{array}{l}\text { CELO- } \\
\text { RIDE, } \\
\text { DIS- } \\
\text { SOLVED } \\
\text { (MG/L } \\
\text { AS CL) } \\
(00940)\end{array}$ & $\begin{array}{l}\text { FLUO- } \\
\text { RIDE, } \\
\text { DIS- } \\
\text { SOLVED } \\
(M G / L \\
\text { AS F) } \\
(00950)\end{array}$ & $\begin{array}{c}\text { SILICA, } \\
\text { DIS- } \\
\text { SOLVED } \\
\text { (MG/L } \\
\text { AS } \\
\text { SIO2) } \\
(00955)\end{array}$ & $\begin{array}{l}\text { SOLIDS, } \\
\text { SUM OF } \\
\text { CONSTI- } \\
\text { TUENTS, } \\
\text { DIS- } \\
\text { SOLVED } \\
\text { (MG/L) } \\
(70301)\end{array}$ & $\begin{array}{c}\text { BORON, } \\
\text { DIS- } \\
\text { SOLVED } \\
\text { (UG/L } \\
\text { AS B) } \\
(01020)\end{array}$ & $\begin{array}{l}\text { IRON, } \\
\text { DIS - } \\
\text { SOL VED } \\
\text { (UG/L } \\
\text { AS FE) } \\
(01046)\end{array}$ \\
\hline & 270 & 6 & 4.7 & 1.1 & 450 & 180 & 0.50 & 9.0 & 1030 & 280 & $<3.0$ \\
\hline$\cdots$ & -- & - & $m$ & - & $\rightarrow$ & $\omega$ & $-\infty$ & $\cdots$ & $-m$ & -- & -- \\
\hline & 200 & 4 & 7.4 & 221 & 520 & 74 & 0.80 & 7.2 & 1070 & 250 & $<3.0$ \\
\hline & 65 & 3 & 2.4 & 133 & 79 & 9.3 & 0.40 & 8.3 & 267 & 139 & $<3.0$ \\
\hline
\end{tabular}




\section{ARKANSAS RIVER BASIN}

07227140 CANADIAN RIVER ABOVE NEW MEXICO-TEXAS STATE LINE, MM WATER-QUALITY RECORDS

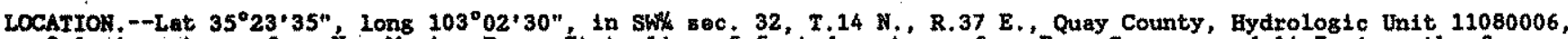

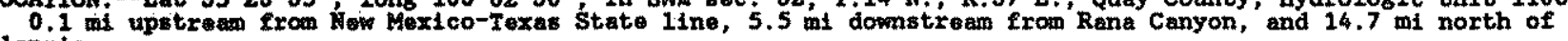
Glonrio.

PERIOD OF RXCORD,-Water years 1969-73,1975-86, 1992 to current year.

WATER QUALTTY DATA, WATER YEAR OCYOBAR 1995 TO SEPTEMBER 1996

\begin{tabular}{|c|c|c|c|c|c|c|c|c|c|c|c|c|}
\hline DATE & TIME & $\begin{array}{c}\text { DIS- } \\
\text { CAARGE, } \\
\text { INST } \\
\text { CUBIC } \\
\text { FEET } \\
\text { PER } \\
\text { SECOND } \\
(00061)\end{array}$ & $\begin{array}{l}\text { SPE- } \\
\text { CIFIC } \\
\text { CON- } \\
\text { DUCE- } \\
\text { AKCE } \\
\text { (US/CH) } \\
\text { (OOO95) }\end{array}$ & $\begin{array}{c}\text { PR } \\
\text { WATER } \\
\text { WEOLE } \\
\text { FIELD } \\
\text { (STAND- } \\
\text { ARD } \\
\text { UNITS) } \\
\text { (00400) }\end{array}$ & $\begin{array}{c}\text { TEUPER- } \\
\text { ATURE } \\
\text { AIR } \\
\text { (DEG C) } \\
\text { (00020) }\end{array}$ & $\begin{array}{l}\text { TEMPER- } \\
\text { ATURE } \\
\text { WATER } \\
\text { (DEG C) } \\
\text { (00010) }\end{array}$ & $\begin{array}{c}\text { BARO- } \\
\text { METRIC } \\
\text { FRES- } \\
\text { SURE } \\
\text { (MII } \\
\text { OF } \\
\text { HG) } \\
(00025)\end{array}$ & $\begin{array}{c}\text { OXYGEN, } \\
\text { DIS- } \\
\text { SOLVED } \\
(\mathrm{MG} / \mathrm{L}) \\
(00300)\end{array}$ & $\begin{array}{c}\text { OXYGEN, } \\
\text { DIS- } \\
\text { SOLVED } \\
\text { (PER- } \\
\text { CENT } \\
\text { SATUR- } \\
\text { ATION) } \\
\text { (00301) }\end{array}$ & $\begin{array}{l}\text { HARD- } \\
\text { NESS } \\
\text { TOTAL } \\
\text { (MS/L } \\
\text { AS } \\
\text { CACO3) } \\
(00900)\end{array}$ & $\begin{array}{l}\text { CALCIUM } \\
\text { DIS- } \\
\text { SOLVED } \\
\text { (ME /L } \\
\text { AS CA) } \\
\text { (00915) }\end{array}$ & $\begin{array}{c}\text { MAGNE- } \\
\text { SIUM, } \\
\text { DIS- } \\
\text { SOLVED } \\
\text { (MG /L } \\
\text { AS NG) } \\
\text { (00925) }\end{array}$ \\
\hline V 1995 & 0945 & 13 & 12500 & 8.2 & 10.5 & 7.0 & 678 & 10.2 & 99 & 690 & 140 & 83 \\
\hline 21950 & 1130 & 9.2 & 10300 & 8.3 & 21.0 & 10.0 & 667 & 9.9 & 104 & -- & -- & - \\
\hline $07 \ldots$ & 0945 & 11 & 8150 & 8.2 & 20.0 & 17.5 & 670 & 8.5 & 104 & 580 & 110 & 73 \\
\hline & 1445 & 1020 & 866 & 8.2 & 33.5 & 25.5 & 674 & 6.1 & 85 & 110 & 24 & 11 \\
\hline
\end{tabular}

$21 . .1445 \quad 1020$

$\begin{array}{llll}8.2 & 33.5 & 25.5 & 674\end{array}$

6.1

85

SOLIDS,

\begin{tabular}{|c|c|c|c|c|c|c|c|c|c|}
\hline $\begin{array}{l}\text { SODIUM } \\
\text { AD- } \\
\text { SORP- } \\
\text { TION } \\
\text { RATIO }\end{array}$ & $\begin{array}{c}\text { POTAS- } \\
\text { SIUM, } \\
\text { DIS- } \\
\text { SOLVED } \\
\text { (MG/L } \\
\text { AS K) } \\
(00935)\end{array}$ & $\begin{array}{c}\text { ALKA- } \\
\text { LINITY } \\
\text { IAB } \\
\text { (NG/L } \\
\text { AS } \\
\text { CACO3) } \\
(90410)\end{array}$ & $\begin{array}{r}\text { SULE } \\
\text { DIS } \\
\text { SOL } \\
\text { (ME } \\
\text { AS S } \\
1009\end{array}$ & $\begin{array}{l}\text { CHLO- } \\
\text { RIDE, } \\
\text { DIS- } \\
\text { SOLVED } \\
\text { (ME/L } \\
\text { AS CL) } \\
(00940)\end{array}$ & $\begin{array}{c}\text { FLUO- } \\
\text { RIDE, } \\
\text { DIS- } \\
\text { SOLVED } \\
\text { (MG/L } \\
\text { AS F) } \\
(00950)\end{array}$ & $\begin{array}{c}\text { SILICA, } \\
\text { DIS- } \\
\text { SOLVED } \\
\text { (NG/L } \\
\text { AS } \\
\text { SIO2) } \\
(00955)\end{array}$ & $\begin{array}{r}\text { TUE } \\
\text { D } \\
\text { SO } \\
(70 \\
6\end{array}$ & $\begin{array}{r}\mathrm{BO} \\
\mathrm{D} \\
\mathrm{SO} \\
(\mathrm{U} \\
\mathrm{AS} \\
\mathrm{O}\end{array}$ & $\begin{array}{l}\text { If } \\
\text { D } \\
\text { (1) } \\
\text { AS }\end{array}$ \\
\hline
\end{tabular}

ROV 1995

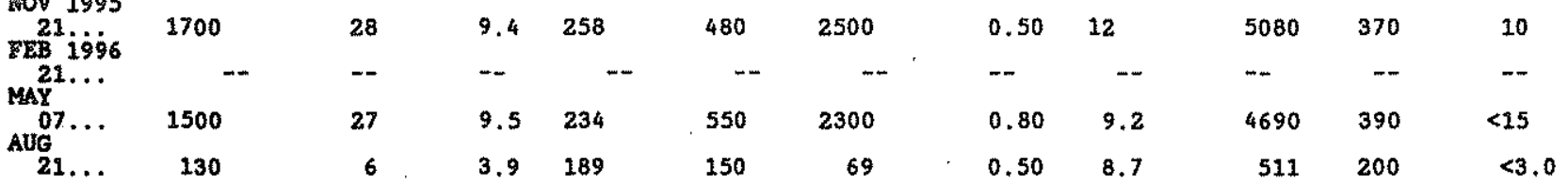


WESTERN GULF OF MEXICO BASINS

RIO GRANDE BASIN

08251500 RIO GRANDE NEAR LOBATOS, CO

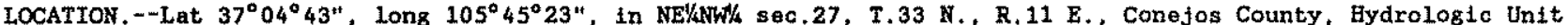
13010002 , on right benk at highway bridge, $5.7 \mathrm{mi}$ north of Colorado-New Mexico State line, $8 \mathrm{mi}$ downstream from Culebra Creek, $11 \mathrm{mi}$ east of Lobatos, and $14 \mathrm{mi}$ east of Antonito.

DRAINAGE AREA. $-7,700 \mathrm{mi}^{2}$, approximately, includes $2,940 \mathrm{mt}^{2}$ in closed basin in northerm part of San Luis Valley, CO.

WATER-DISCHARGE RECORDS

PERIOD OF RECORD --June 1899 to current year. Monthly discharge only for some periods, published in WSP 1312. Fublished as "at Cenicero" 1899-1901, and as "near Cenicero" 1902-4. Statistical sumary computed for 1931 to current year.

REVISED RECORDS, - -WSP 1312: 1919 (monthly runoff) WSP 210: Dratnage area WDR CO-78-1: 1976.

GAGE, - Water-stage recorder with satellite telemetry. Datum of gage is $7,427.63$ ft above sea level. Prior to 1910 , nonrecording gages at same site and datum.

REMARKS.--Estimated daily discharges: Oct. 30-31, Nov, 16-17, and Nov. 25 to Mar. 2, Records good except for estimated datiy discharges, which are fair. Natural flow of stream affected by transmountain diversions, storage reservoirs, groundwater of thdraw.

COOPERATION.--Records collected and computed by Colorado Division of Water Resources and reviewed by Geological

EXTREMES OUTSIDE PERIOD OF RECORD.--Maximum stage since at least 1828, that of June 8, 1905.

DISCHARGE, CUIBIC FEET PER SECOND, WATER YEAR OCTOBER 1995 TO SEPTEMBER 1996

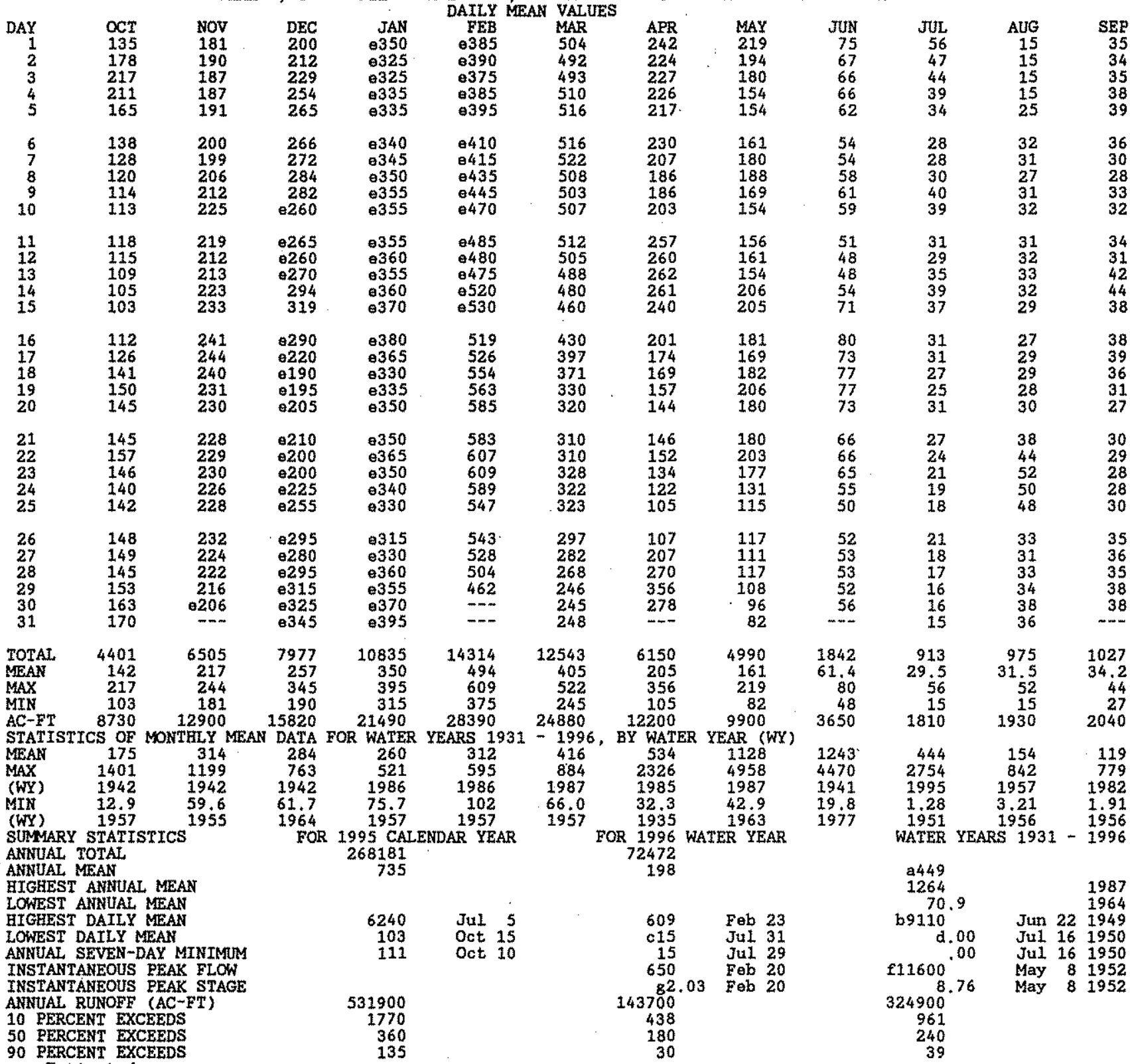

90 PERCENT EXCEEDS

a-Average discharge for 31 years (water years $1900-30$ ), $846 \mathrm{ft} 3 / \mathrm{s} ; 612900 \mathrm{acr} \theta-\mathrm{ft} / \mathrm{yr}$, includes period of extensive development for Irrigation.

b-Maximum daily discharge for pertod of record, $13100 \mathrm{ft}^{3} / \mathrm{s}$, Jun 8, 1905.

c:-Also occurred Aug 1-4.

d-No flow at times in 1950-51, 1956.

f-Maximum discharge and stage for period of record, $13200 \mathrm{ft} / \mathrm{s}$, Jun 8,1905 , gage height, 9.1 ft, from rating curve extended above $8000 \mathrm{ft}^{3} / \mathrm{s}$.

8-Maxtmun gage height, $3.63 \mathrm{ft}$. Feb 14, backwater from ice. 
64

RIO GRANDE BASIN

08251500 RTO GRANDE NEAR LOBATOS, CO - - Continued

WATER-QUALITY RECORDS

PERIOD OF RECORD. --Water years 1969 to current year.

REMARKS. --Replaces station 08249200 Rio Grande above Culebra Creek, near Lobatos, Colo., which was discontinued Juiy 1969. This station operated by the Colorado District.

WATER-QUALITY DATA, WATER YEAR OCTOBER 1995 TO SEPTEMABER 1996

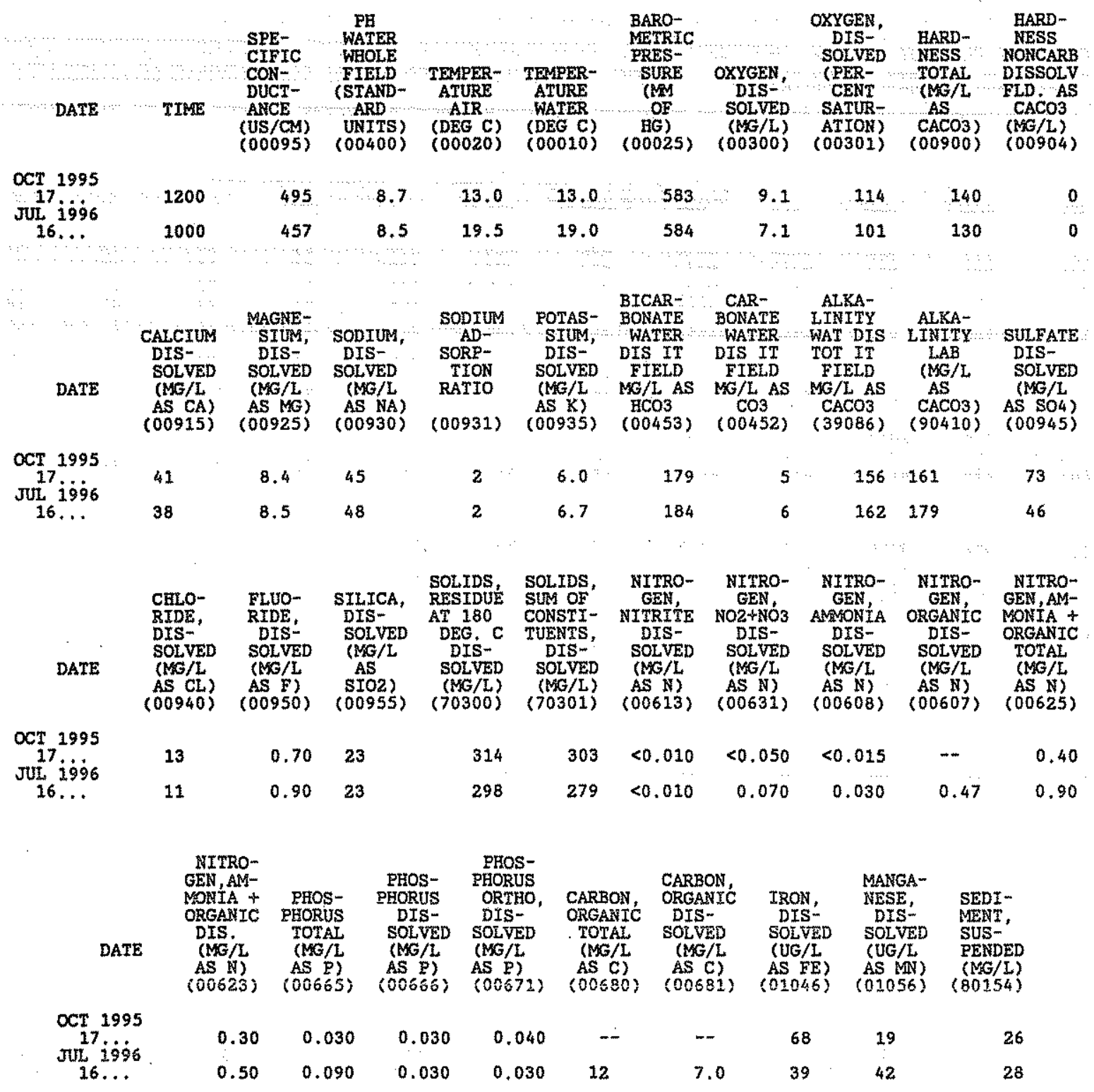


RIO GRANDE BASTN

08251500 RIO GRANDE NEAR LOBATOS, CO--Continued

WATER-QUALITY RECORDS

WATER-QUALITY DATA, WATER YEAR OCTOBER 1995 TO SEPTEMBER 1996

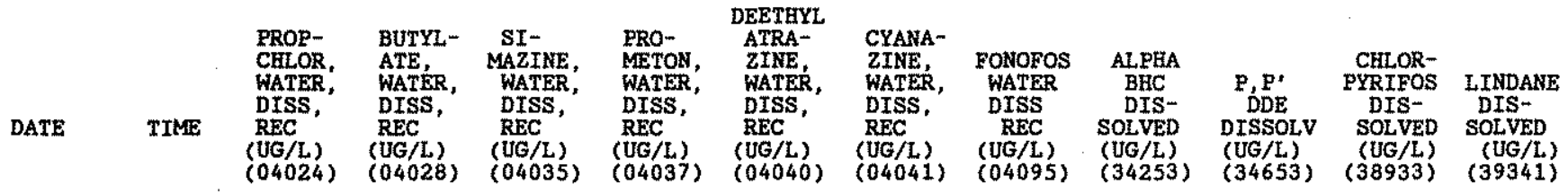

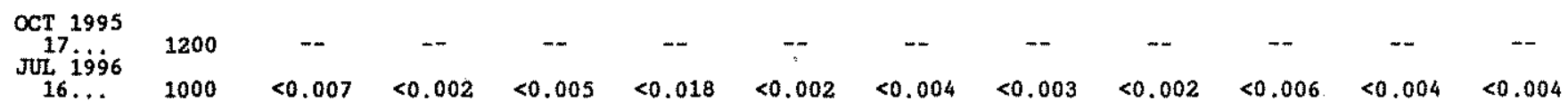

ELDRTN LACHLOR

\begin{tabular}{|c|c|c|c|}
\hline $\begin{array}{l}\text { MALA- } \\
\text { TRION, } \\
\text { DIS- } \\
\text { SOLVED } \\
\text { (UG/L) } \\
39532 \text { ). }\end{array}$ & $\begin{array}{l}\text { PARA- } \\
\text { THION, } \\
\text { DIS- } \\
\text { SOLVED } \\
\text { (UG/L) } \\
(39542)\end{array}$ & $\begin{array}{c}\text { DI- } \\
\text { AZINON, } \\
\text { DIS- } \\
\text { SOLVED } \\
\text { (UG/L) } \\
(39572)\end{array}$ & $\begin{array}{l}\text { ATRA- } \\
\text { ZINE, } \\
\text { WATER, } \\
\text { DISS, } \\
\text { REC } \\
\text { (UG/L) } \\
(39632)\end{array}$ \\
\hline
\end{tabular}

$\begin{array}{lll} & \\ \text { ALA- } & \text { ACETO- } & \text { METRI- } \\ \text { CHLOR, } & \text { CHLOR, } & \text { BUZIN } \\ \text { WATER, } & \text { WATER } & \text { SENCOR } \\ \text { DISS, } & \text { FLTRD } & \text { WATER } \\ \text { REC } & \text { REC } & \text { DISSOLV } \\ \text { (UG/L) } & \text { (UG/L) } & \text { (UG/L) } \\ (46342) & (49260) & (82630)\end{array}$

$2,6-\mathrm{DI}-$

TRI- ETHAL-

ETHYL FLUR- FLUR-

ANILINE ALIN ALIN

WAT FLT WAT FLT WAT FLT

$\begin{array}{llll}0.7 \mathrm{U} & 0.7 \mathrm{U} & 0.7 \mathrm{U}\end{array}$

SOLVED DISSOLV

$\begin{array}{ll}(\mathrm{UG} / \mathrm{L}) & (\mathrm{UG} / \mathrm{L}) \\ (39381) & (39415)\end{array}$

(39532)

(UG/L) (UG/L)
$(39542)(39572)$

$(39632)$

GF, REC

GF, REC

(UG/L) (UG/L)

OCT 1995

JUL 179

16 ...

$<0,001$

$-$
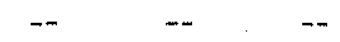

0.005

$+-$

$-$

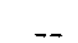

2660

82661) (82663)

\begin{tabular}{|c|c|c|c|c|c|c|c|c|c|c|c|c|}
\hline $\mathbf{E}$ & $\begin{array}{c}\text { PHORATE } \\
\text { WATER } \\
\text { FLTRD } \\
0.7 \text { U } \\
\text { GF, REC } \\
\text { (UG/L) } \\
\text { (82664) }\end{array}$ & $\begin{array}{c}\text { TER- } \\
\text { BACIL } \\
\text { WATER } \\
\text { FLTRD } \\
0.7 U \\
\text { GF REC } \\
\text { (UG/L) } \\
(82665)\end{array}$ & $\begin{array}{l}\text { LIN- } \\
\text { URON } \\
\text { WATER } \\
\text { FLTRD } \\
0.7 \text { U } \\
\text { GF. REC } \\
\text { (UG/L) } \\
(82666)\end{array}$ & $\begin{array}{c}\text { METHYL } \\
\text { PARA- } \\
\text { THION } \\
\text { WAT FLT } \\
0.7 \text { U } \\
\text { GF, REC } \\
\text { (UG/L) } \\
\text { ( } 82667)\end{array}$ & $\begin{array}{l}\text { EPTC } \\
\text { WATER } \\
\text { FLTRD } \\
0.7 \text { U } \\
\text { GF, REC } \\
\text { (UG/L) } \\
(82668)\end{array}$ & $\begin{array}{c}\text { BEB- } \\
\text { ULATE } \\
\text { WATER } \\
\text { FILTRD } \\
0.7 U \\
\text { GF, REC } \\
\text { (UG/L) } \\
(82669)\end{array}$ & $\begin{array}{l}\text { TEBU- } \\
\text { THIIURON } \\
\text { WATER } \\
\text { ELTRD } \\
0.7 \mathrm{U} \\
\text { GE, REC } \\
\text { (UG/L) } \\
(82670)\end{array}$ & $\begin{array}{c}\text { MOL-- } \\
\text { INATE } \\
\text { WATER } \\
\text { FLTRD } \\
0.7 \mathrm{U} \\
\text { GF, REC } \\
(\mathrm{UG} / L) \\
(82671)\end{array}$ & $\begin{array}{c}\text { ETHO- } \\
\text { DROP } \\
\text { WATER } \\
\text { FLTRD } \\
0.7 \mathrm{U} \\
\text { GF, REC } \\
\text { (UG/L) } \\
(82672)\end{array}$ & $\begin{array}{c}\text { BEN- } \\
\text { FLUR- } \\
\text { ALIN } \\
\text { WAT FLD } \\
0.7 \mathrm{UJ} \\
\text { GF, REC } \\
(\mathrm{UG} / \mathrm{L}) \\
(82673)\end{array}$ & $\begin{array}{c}\text { CARBO- } \\
\text { FURAN } \\
\text { WATER } \\
\text { FLTRD } \\
0.7 \text { U } \\
\text { GF, REC } \\
\text { (UG/L) } \\
(82674)\end{array}$ & $\begin{array}{c}\text { TER- } \\
\text { BUFOS } \\
\text { WATER } \\
\text { FLTRD } \\
0.7 \text { U } \\
\text { GF, REC } \\
\text { (UG/L) } \\
\text { (82675) }\end{array}$ \\
\hline
\end{tabular}

OCT 1995

JUL 1996

16 ...
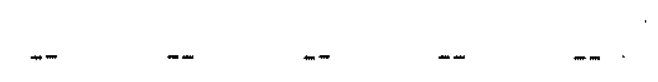

$<0.002$

$<0.007<0.002<0.006$

$<0.002<0.004$
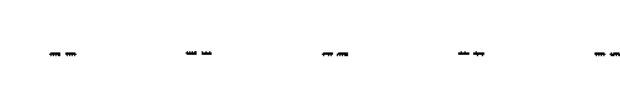

-

\begin{tabular}{|c|c|c|c|c|c|c|c|c|c|c|c|}
\hline $\begin{array}{c}\text { FRON- } \\
\text { AMIDE } \\
\text { WATER } \\
\text { FLTRD } \\
0.7 \text { U } \\
\text { GE, REC } \\
\text { (UG/L) } \\
(82676)\end{array}$ & $\begin{array}{l}\text { DISUL- } \\
\text { FOTON } \\
\text { WATER } \\
\text { FLTRD } \\
0.7 \text { U } \\
\text { GF, REC } \\
\text { (UG/L) } \\
\text { (82677) }\end{array}$ & $\begin{array}{l}\text { TRTAL- } \\
\text { LATE } \\
\text { WATER } \\
\text { FLTRD } \\
0.7 \mathrm{U} \\
\text { GF, REC } \\
\text { (UG/L) } \\
(82678)\end{array}$ & $\begin{array}{c}\text { ERO- } \\
\text { PANIL } \\
\text { WATER } \\
\text { FLTRD } \\
0.7 \text { U } \\
\text { GF, REC } \\
\text { (UG/L) } \\
\text { (82679) }\end{array}$ & $\begin{array}{c}\text { CAR- } \\
\text { BARYL } \\
\text { WATER } \\
\text { FLTRD } \\
0.7 \mathrm{U} \\
\text { GE, REC } \\
(\mathrm{UG} / \mathrm{L}) \\
(82680)\end{array}$ & $\begin{array}{c}\text { THIO- } \\
\text { BENCARB } \\
\text { WATER } \\
\text { FLTRD } \\
0.7 \text { U } \\
\text { GF, REC } \\
\text { (UG/L) } \\
\text { ( } 82681)\end{array}$ & $\begin{array}{c}\text { DCPA } \\
\text { WATER } \\
\text { FLTRD } \\
0.7 \mathrm{U} \\
\text { GF, REC } \\
\text { (UG/L) } \\
(82682)\end{array}$ & $\begin{array}{c}\text { PENDI- } \\
\text { METHE- } \\
\text { ALIN } \\
\text { WAT FLT } \\
0.7 \text { U } \\
\text { GE, REC } \\
\text { (UG/L) } \\
\text { (82683) }\end{array}$ & $\begin{array}{c}\text { NAPROP- } \\
\text { AMIDE } \\
\text { WATER } \\
\text { FLTRD } \\
0.7 \text { U } \\
\text { GF, REC } \\
\text { (UG/L) } \\
(82684)\end{array}$ & $\begin{array}{c}\text { PRO- } \\
\text { PARGITE } \\
\text { WATER } \\
\text { FLTRD } \\
0.7 \mathrm{U} \\
\text { GE. REC } \\
\text { (UG/L) } \\
\text { (82685) }\end{array}$ & $\begin{array}{c}\text { METHYL } \\
\text { AZIN- } \\
\text { PHOS } \\
\text { WAT FLT } \\
0.7 \mathrm{U} \\
\text { GF, REC } \\
\text { (UG/L) } \\
(82686)\end{array}$ & $\begin{array}{c}\text { PER- } \\
\text { METHRIN } \\
\text { CIS } \\
\text { WAT FLT } \\
0.7 \text { U } \\
\text { GF, REC } \\
\text { (UG/L) } \\
\text { (82687) }\end{array}$ \\
\hline
\end{tabular}

DATE

GF, REC

$(\mathrm{UG} / \mathrm{L})$
$(82676)$

(82677)

(82678) (82679)

82680 )

OCT 1995

गये 1796

16 ...

$<0.003$

$+$

$-$

$--$

$<0.003 \quad<0.002$

E0.002

$<0.004$

$<0,003$

$<0.013$

$<0.001$

$<0.005$ 
RIO GRANDE BASIN

08252500 COSTILLA CREEK ABOVE COSTILLA DAM, NM

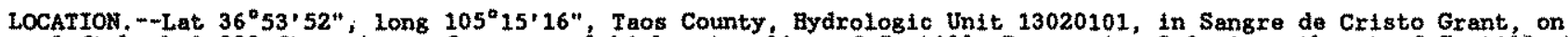
left bank 1,900 ft upstream from normal high-water line of Costilla Reservoir, 2.1 mi northeast of Costilia Dam, 16 mi southeast of Costilla, and at mile 36.9 .

DRAINAGE AREA. $--25.1 \mathrm{mi}^{2}$.

PERIOD OF RECORD -April 1937 to current year (seasonal records). Monthly discharge only for some periods, published in WSP 1312 and 1732. Prior to October 1951, published as "above reservoir, near Costilla,"

REVISED RECORDS, -WSE 878: 1937. WSF 1923: 1937-50, drainage area.

GAGE. - -Water-stage recorder. Concrete control since Sept. 17, 1965. Elevation of gage is 9,430 ft above National Geodetic Vertical Datum of 1929, from topographic map. See WSP 1923 for history of changes prior to Sept. 17 , 1965.

REMARKS.--Records good except for estimated daily discharges, which are poor. Natural flow may be augmented by transbasin diversions or irrigation returns from about 1,300 acres irrigated from Casias Creok (station $08253000)$. Several observations of water temperature were made during the year.

EXTREMES FOR PERIOD OF RECORD.--Maximum discharge 3,870 fts/s, July 22, 1954, gage height, about 4.8 ft, from Iloodmarks, site and datum then in use, on basis of slope-area measurement of peak flow; minimum not detexmined. The flood in 1954 destroyed the gaging station and is highest since about 1909 from information by local range rider. A portion of this flow may have originated in Castas Creek basin (see REMARKS).

EXTREMES FOR CURRENT YEAR.--Maximum discharge during period of seasonal operation, 25 its/s, at 1345 hours Apr. 29, gage height $3.40 \mathrm{ft} ;$ minimum daily discharge $1.5 \mathrm{ft} / \mathrm{s}$, Aug. 12,13 .

DISCHARGE, CUBIC FEET PER SECOND, WATER YEAR OCTOBER 1995 TO SEFTEMBER 1996

\begin{tabular}{|c|c|c|c|c|c|c|c|c|c|c|c|c|}
\hline DAY & $\propto \mathrm{CT}$ & NOV & DEC & JAN & FEB & MAR & APR & MAY & JUN & JUL & AUG & SEF \\
\hline $\begin{array}{l}1 \\
2 \\
3 \\
4 \\
5\end{array}$ & $\begin{array}{l}7.6 \\
7.0 \\
6.8 \\
-.-\end{array}$ & $\bar{m}$ & $\begin{array}{c}--- \\
-\cdots \\
-- \\
-\cdots\end{array}$ & $=$ & $\overline{-}$ & $\begin{array}{l}--- \\
-\cdots \\
-\cdots \\
---\end{array}$ & $\begin{array}{l}011 \\
011 \\
010 \\
010 \\
010\end{array}$ & $\begin{array}{l}13 \\
13 \\
14 \\
15 \\
16\end{array}$ & $\begin{array}{l}5.0 \\
4.8 \\
4.5 \\
4.2 \\
4.0\end{array}$ & $\begin{array}{l}4.4 \\
3.4 \\
3.0 \\
2.8 \\
2.8\end{array}$ & $\begin{array}{r}1.9 \\
2.7 \\
3.1 \\
2.4 \\
1.8\end{array}$ & $\begin{array}{l}2.3 \\
2.4 \\
2.5 \\
2.1 \\
2.0\end{array}$ \\
\hline $\begin{array}{r}6 \\
7 \\
8 \\
9 \\
10\end{array}$ & 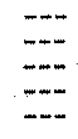 & $=$ & $=$ & 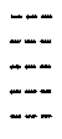 & 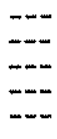 & $=$ & $\begin{array}{l}012 \\
010 \\
11 \\
12 \\
10\end{array}$ & $\begin{array}{l}17 \\
17 \\
16 \\
16 \\
15\end{array}$ & $\begin{array}{l}3.8 \\
3.7 \\
3.4 \\
3.8 \\
3.9\end{array}$ & $\begin{array}{l}2.4 \\
2.5 \\
3.5 \\
6.0 \\
3.9\end{array}$ & $\begin{array}{l}1.6 \\
1.7 \\
2.3 \\
2.3 \\
2.0\end{array}$ & $\begin{array}{l}2.5 \\
2.7 \\
2.3 \\
2.0 \\
2.2\end{array}$ \\
\hline $\begin{array}{l}11 \\
12 \\
13 \\
14 \\
15\end{array}$ & $\begin{array}{l}-\cdots \\
\overline{-} \\
\cdots- \\
-\cdots\end{array}$ & $m$ & 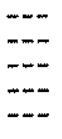 & $\begin{array}{l}m-- \\
\overline{--} \\
--- \\
---\end{array}$ & $\begin{array}{l}-m \\
m-\infty \\
-\cdots\end{array}$ & $\begin{array}{l}-\cdots \\
-\cdots \\
-\cdots \\
-\cdots\end{array}$ & $\begin{array}{r}8.9 \\
9.0 \\
7.5 \\
6.3 \\
13\end{array}$ & $\begin{array}{l}14 \\
14 \\
14 \\
14 \\
13\end{array}$ & $\begin{array}{l}3.6 \\
3.4 \\
4.7 \\
5.9 \\
8.0\end{array}$ & $\begin{array}{l}3.1 \\
3.6 \\
3.6 \\
3.3 \\
3.6\end{array}$ & $\begin{array}{l}1.7 \\
1.5 \\
1.5 \\
1.6 \\
1.7\end{array}$ & $\begin{array}{l}2.1 \\
2.3 \\
3.3 \\
3.0 \\
3.4\end{array}$ \\
\hline $\begin{array}{l}16 \\
17 \\
18 \\
19 \\
20\end{array}$ & $\begin{array}{l}--- \\
-\square \\
-\cdots \\
-\cdots\end{array}$ & 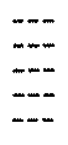 & $\begin{array}{l}m- \\
-- \\
-- \\
--\end{array}$ & $\begin{array}{l}m-- \\
m- \\
m-\end{array}$ & $\begin{array}{l}\cdots-- \\
-\cdots \\
-\cdots\end{array}$ & $=$ & $\begin{array}{c}12 \\
11 \\
10 \\
9.8 \\
9.2\end{array}$ & $\begin{array}{c}12 \\
12 \\
11 \\
10 \\
9.7\end{array}$ & $\begin{array}{l}4.6 \\
3.7 \\
3.3 \\
2.9 \\
2.7\end{array}$ & $\begin{array}{l}2.6 \\
2.6 \\
2.5 \\
2.2 \\
2.0\end{array}$ & $\begin{array}{l}1.9 \\
2.1 \\
2.3 \\
1.9 \\
2.1\end{array}$ & $\begin{array}{l}3.0 \\
2.4 \\
3.3 \\
3.0 \\
2.6\end{array}$ \\
\hline $\begin{array}{l}21 \\
22 \\
23 \\
24 \\
25\end{array}$ & $\begin{array}{l}-- \\
-\cdots \\
-\cdots \\
--\end{array}$ & $\begin{array}{l}-\cdots \\
\cdots \\
\cdots-\end{array}$ & $\begin{array}{l}--- \\
-\overline{-} \\
-\overline{-} \\
--\end{array}$ & $\begin{array}{l}-- \\
-- \\
-- \\
--\end{array}$ & $\begin{array}{l}-- \\
-\cdots \\
--\end{array}$ & $\begin{array}{l}--- \\
- \\
--\end{array}$ & $\begin{array}{l}9.5 \\
9.2 \\
10^{\circ} \\
13 \\
14\end{array}$ & $\begin{array}{l}9.2 \\
8.5 \\
8.0 \\
7.7 \\
7.7\end{array}$ & $\begin{array}{l}2.9 \\
4.7 \\
4.0 \\
2.7 \\
2.9\end{array}$ & $\begin{array}{l}2.0 \\
2.1 \\
1.9 \\
1.8 \\
2.0\end{array}$ & $\begin{array}{l}2.1 \\
3.5 \\
4.1 \\
3.8 \\
4.8\end{array}$ & $\begin{array}{l}2.3 \\
2.2 \\
2.1 \\
2.2 \\
2.1\end{array}$ \\
\hline $\begin{array}{l}26 \\
27 \\
28 \\
29 \\
30 \\
31\end{array}$ & $\begin{array}{l}-m \\
-\infty \\
-- \\
-m \\
-m\end{array}$ & $\begin{array}{l}=- \\
-\cdots \\
=-\infty \\
=-\infty \\
-\cdots\end{array}$ & 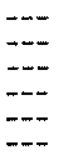 & $\begin{array}{l}--- \\
--- \\
-- \\
-- \\
-- \\
-\end{array}$ & $\begin{array}{l}--- \\
-\cdots \\
-- \\
-- \\
--\end{array}$ & $\begin{array}{r}e 9.6 \\
e 9.8 \\
e 9.9 \\
e 10 \\
010\end{array}$ & $\begin{array}{l}15 \\
16 \\
15 \\
14 \\
12 \\
-\end{array}$ & $\begin{array}{l}7.8 \\
7.6 \\
6.7 \\
6.0 \\
5.6 \\
5.3\end{array}$ & $\begin{array}{l}3.1 \\
5.8 \\
3.5 \\
3.0 \\
4.6 \\
\end{array}$ & $\begin{array}{l}2.0 \\
2.0 \\
2.9 \\
3.5 \\
2.8 \\
2.2\end{array}$ & $\begin{array}{l}3.9 \\
3.4 \\
4.7 \\
3.1 \\
2.5 \\
2.2\end{array}$ & $\begin{array}{l}2 \\
2 \\
2 \\
2 \\
2\end{array}$ \\
\hline $\begin{array}{l}\text { TOTAL } \\
\text { MEAN } \\
\text { MAX } \\
\text { MIN } \\
\text { AC-FT }\end{array}$ & $\begin{array}{r}21.4 \\
7.13 \\
7.6 \\
6.8 \\
42\end{array}$ & $\begin{array}{l}=- \\
=- \\
-m \\
--\end{array}$ & $\begin{array}{l}--- \\
-- \\
-\cdots \\
-\cdots\end{array}$ & $\begin{array}{l}m- \\
m- \\
-- \\
m-\end{array}$ & $\begin{array}{c}m-- \\
m- \\
m- \\
-\cdots \\
m-\end{array}$ & $\begin{array}{r}49.3 \\
9.86 \\
10 \\
9.6 \\
98\end{array}$ & $\begin{array}{r}331.4 \\
11.0 \\
16 \\
6.3 \\
657\end{array}$ & $\begin{array}{r}355.8 \\
11.5 \\
17 \\
5.3 \\
706\end{array}$ & $\begin{array}{r}121.1 \\
4.04 \\
8.0 \\
2.7 \\
240\end{array}$ & $\begin{array}{r}89.0 \\
2.87 \\
6.0 \\
1.8 \\
177\end{array}$ & $\begin{array}{r}78.2 \\
2.52 \\
4.8 \\
1.5 \\
155\end{array}$ & $\begin{array}{l}73 \\
2 . \\
3\end{array}$ \\
\hline
\end{tabular}

- Estimatod 
RIO GRANDE BASIN

08253000. CASIAS CREEK NEAR COSTILLA, NM

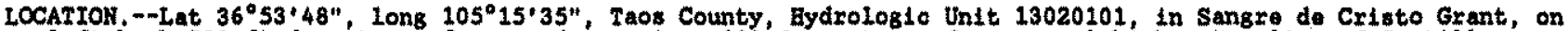
Ieft bank 200 ft downstream from road crossing, 900 ft upstroam from nomal high-water line of costilis Reservolx, $1.8 \mathrm{mi}$ northeast of Costilia Dam, and 16 mi southeast of Costilla.

DRAINAGE AREA. $--16.6 \mathrm{mi}^{2}$.

DERTOD OF RECORD,--April 1937 to current year (aessonal records). Monthly discherge only for some periods, published in WSP 1312 and 1732. Records for Nov, 1-7, 1947 and Nov, 1-16, 1948, published in WSE 1118 and 1148 , are unreliable and should not be used.

REVISED RECORDS, -WSP 1282: 1948-51, WSP 1923; Drainage area,

GAGE.--Water-stage recorder and conerete control. Elevation of gage is 9,400 ft above National Geodetio Vertical Datum of 1929, erom topographic map. Frlor to July 18, 1940, water-stage recorder and wooden control 100 ft downstream at datum 1.56 ft lower.

REMARKS.--Records fair. Diversion 3.5 mi upstream for irrigation of about 1,300 acres, part of which is th Costilla Creek basin. Several observations of water teaperature were made during the year.

EXTREMES FOR RERIOD OF RECORD.--Maximum discharge, $181 \mathrm{ft} / \mathrm{s}$, July 20, 1971, gage helght, 2.07 ft, from rating curve extended above $85 \mathrm{ft}^{3} / \mathrm{s}$; minimum not determined.

EXTREYES FOR CURRENT YLAR. --Maximum discharge during pertod of seasonal operation, 27 fts/8, at 2300 hours July 8 , gage haight $0.90 \mathrm{ft}$; minimum daily discharge $4.0 \mathrm{ft} / \mathrm{s}$, Sept. 11 .

DISCHARGE, CUBIC FEET PER SECOND, WATER YEAR OCTOBER 1995 TO SEPTERBER 1996 DAILY MEAN VALUES

\begin{tabular}{|c|c|c|c|c|c|c|c|c|c|c|c|c|}
\hline DAY & $\infty \mathrm{CT}$ & Nov & DEC & JAN & FEB & MAR & APR & MAY & JUN & JUL & AUG & SEP \\
\hline $\begin{array}{l}1 \\
2 \\
3 \\
4 \\
5\end{array}$ & $\begin{array}{r}11 \\
10 \\
9.8 \\
-\end{array}$ & $\begin{array}{l}=- \\
=-- \\
=-- \\
---\end{array}$ & $=-$ & $\begin{array}{l}=- \\
=- \\
-\square\end{array}$ & 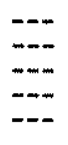 & $\begin{array}{l}m-\overline{-} \\
\overline{-}-\overline{-} \\
\overline{--}\end{array}$ & $\begin{array}{l}9.5 \\
7.8 \\
6.6 \\
5.6 \\
5.7\end{array}$ & $\begin{array}{l}11 \\
11 \\
13 \\
14 \\
15\end{array}$ & $\begin{array}{l}9.0 \\
8.9 \\
9.0 \\
8.9 \\
8.8\end{array}$ & $\begin{array}{l}9.1 \\
8.2 \\
8.0 \\
8.3 \\
7.8\end{array}$ & $\begin{array}{l}5.0 \\
6.1 \\
6.3 \\
5.4 \\
4.8\end{array}$ & $\begin{array}{l}4.6 \\
5.0 \\
5.0 \\
4.4 \\
4.3\end{array}$ \\
\hline $\begin{array}{r}6 \\
7 \\
8 \\
9 \\
10\end{array}$ & $=-$ & 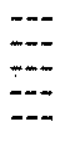 & $\begin{array}{l}m- \\
\cdots \\
\cdots \\
\cdots\end{array}$ & 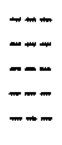 & $=-$ & $\begin{array}{l}m-\overline{-} \\
\overline{-} \\
\overline{-}\end{array}$ & $\begin{array}{l}6.0 \\
7.7 \\
7.8 \\
9.3 \\
8.2\end{array}$ & $\begin{array}{l}15 \\
16 \\
17 \\
16 \\
16\end{array}$ & $\begin{array}{l}8.3 \\
8.5 \\
8.5 \\
8.7 \\
8.6\end{array}$ & $\begin{array}{c}7.3 \\
7.3 \\
10 \\
13 \\
9.4\end{array}$ & $\begin{array}{l}4.5 \\
4.6 \\
5.8 \\
5.4 \\
4.9\end{array}$ & $\begin{array}{l}4.6 \\
4.7 \\
4.4 \\
4.1 \\
4.2\end{array}$ \\
\hline $\begin{array}{l}11 \\
12 \\
13 \\
14 \\
15\end{array}$ & $=-\cdots$ & $=-\cdots$ & $\begin{array}{l}\cdots-- \\
-\cdots \\
-\cdots\end{array}$ & 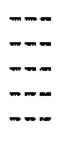 & $=$ & $=$ & $\begin{array}{l}8.0 \\
8.0 \\
6.5 \\
7.6 \\
7.8\end{array}$ & $\begin{array}{l}15 \\
17 \\
17 \\
17 \\
17\end{array}$ & $\begin{array}{l}8.9 \\
8.3 \\
10 \\
11 \\
14\end{array}$ & $\begin{array}{l}9.0 \\
9.8 \\
9.3 \\
8.5 \\
9.7\end{array}$ & $\begin{array}{l}4.6 \\
4.3 \\
4.2 \\
4.1 \\
4.2\end{array}$ & $\begin{array}{l}4.0 \\
4.4 \\
5.3 \\
5.0 \\
5.2\end{array}$ \\
\hline $\begin{array}{l}16 \\
17 \\
18 \\
19 \\
20\end{array}$ & 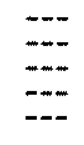 & $=-$ & $=$ & 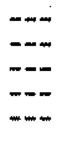 & $\begin{array}{l}m-- \\
=- \\
=- \\
m-\end{array}$ & $=$ & $\begin{array}{l}8.0 \\
8.0 \\
7.0 \\
6.1 \\
5.9\end{array}$ & $\begin{array}{l}17 \\
17 \\
16 \\
16 \\
16\end{array}$ & $\begin{array}{r}10 \\
9.6 \\
8.9 \\
8.7 \\
8.3\end{array}$ & $\begin{array}{l}7.6 \\
7.6 \\
7.2 \\
6.6 \\
6.2\end{array}$ & $\begin{array}{l}4.3 \\
4.2 \\
4.5 \\
4.2 \\
4.2\end{array}$ & $\begin{array}{l}4.6 \\
4.5 \\
5.5 \\
4.9 \\
4.5\end{array}$ \\
\hline $\begin{array}{l}21 \\
22 \\
23 \\
24 \\
25\end{array}$ & $\begin{array}{l}--\infty \\
--- \\
m-\infty \\
m-\infty\end{array}$ & $=$ & $=-$ & 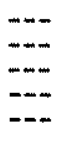 & $=$ & 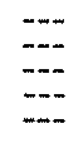 & $\begin{array}{l}5.7 \\
5.7 \\
6.4 \\
8.0 \\
9.4\end{array}$ & $\begin{array}{l}16 \\
16 \\
15 \\
14 \\
13\end{array}$ & $\begin{array}{r}9.3 \\
12 \\
9.7 \\
8.7 \\
9.1\end{array}$ & $\begin{array}{l}6.1 \\
6.0 \\
5.8 \\
5.6 \\
5.7\end{array}$ & $\begin{array}{l}4.3 \\
5.8 \\
6.0 \\
7.4 \\
7.2\end{array}$ & $\begin{array}{l}4.3 \\
4.3 \\
4.1 \\
4.1 \\
4.1\end{array}$ \\
\hline $\begin{array}{l}26 \\
27 \\
28 \\
29 \\
30 \\
31\end{array}$ & $\begin{array}{l}m \\
=-\infty \\
=-\infty \\
=-\infty \\
m-\infty\end{array}$ & 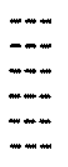 & $\begin{array}{l}\cdots \\
\cdots \\
\cdots \\
\cdots\end{array}$ & \begin{tabular}{l}
--- \\
\hdashline- \\
$-\square$ \\
$-\square$ \\
$-\square$
\end{tabular} & $\begin{array}{l}--- \\
=- \\
-\square \\
--- \\
--\end{array}$ & $\begin{array}{l}-.6 \\
9.6 \\
9.1 \\
9.0 \\
6.5 \\
8.0\end{array}$ & $\begin{array}{c}10 \\
11 \\
9.7 \\
10 \\
9.0 \\
-\end{array}$ & $\begin{array}{l}13 \\
12 \\
11 \\
10 \\
10 \\
9.3\end{array}$ & $\begin{array}{r}8.7 \\
14 \\
9.5 \\
8.5 \\
10 \\
---\end{array}$ & $\begin{array}{l}5.4 \\
5.6 \\
6.4 \\
6.4 \\
5.8 \\
5.6\end{array}$ & $\begin{array}{l}5.8 \\
6.2 \\
6.7 \\
5.7 \\
5.0 \\
4.7\end{array}$ & $\begin{array}{l}5.0 \\
4.6 \\
4.6 \\
4.6 \\
4.4\end{array}$ \\
\hline $\begin{array}{l}\text { TOTAL } \\
\text { MEAN } \\
\text { MAX } \\
\text { MIN } \\
\text { AC-FT }\end{array}$ & $\begin{array}{r}30.8 \\
10.3 \\
11 \\
9.8 \\
61\end{array}$ & $\begin{array}{l}--- \\
--\cdots \\
-\cdots+ \\
-\cdots\end{array}$ & $\begin{array}{l}--- \\
-- \\
--- \\
---\end{array}$ & $\begin{array}{l}-- \\
-- \\
--- \\
--\end{array}$ & 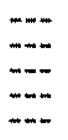 & $\begin{array}{r}42.2 \\
8.44 \\
9.6 \\
6.5 \\
84\end{array}$ & $\begin{array}{r}232.0 \\
7.73 \\
11 \\
5.6 \\
460\end{array}$ & $\begin{array}{r}448.3 \\
14.5 \\
17 \\
9.3 \\
889\end{array}$ & $\begin{array}{r}284.4 \\
9.48 \\
14 \\
8.3 \\
564\end{array}$ & $\begin{array}{r}234.3 \\
7.56 \\
13 \\
5.4 \\
465\end{array}$ & $\begin{array}{r}160.4 \\
5.17 \\
7.4 \\
4.1 \\
318\end{array}$ & $\begin{array}{r}137.3 \\
4.58 \\
5.5 \\
4.0 \\
272\end{array}$ \\
\hline
\end{tabular}


LOCArron, - Lat $36^{\circ} 53^{\prime} 03^{\prime \prime}$, long $105^{\circ} 16^{\circ} 50^{\prime \prime}$, Taos Coumty, Hydrologic Unit 13020101, in Sangre de Cristo Grant, on left bank 200 ft upstreem from road crossing, 1,300 ft upstream from normal high-water line of Costilla Rmervoir, 0.6 mi north of Costille Dem, and 16 mi southest of Costille.

DRAIHAGE AREA. $--2.15 \mathrm{mi}^{2}$.

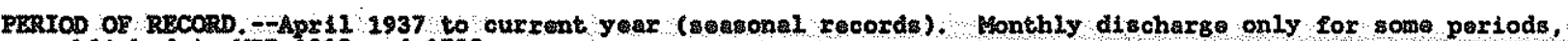
publimhod in WSP 1312 and 1732 .

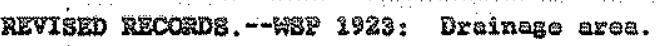

GAFE,-Water-stage recorder and Parmhe11 Elume. Elevetion of gage is 9,490 2t sove National Geodetic Vertical. Datum of 1929, Exc topographic map. Prior to Jun 27, 1940, pator-8tage recordar and wooden control at datum 0.99 2t lower.

REAARKS, --Records good except for ostimeted dasly discharges, which are falr. Several observations of water temperature were made during the year.

EXTREAES FOR PERIOD OR RECORD.--Maximun discharge, $20 \mathrm{ft} / 8$, Jume 29, 1995; maximum gage height, 1.73 ft, Aug. 11, 1941; mintmen not determined.

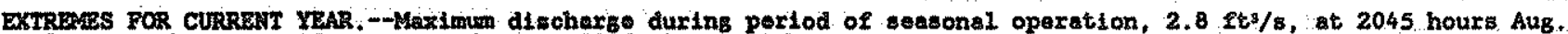
27, gage height 0.55 ; minimus daily $0.58 \mathrm{fts} / \mathrm{s}$, Mar. 29 .

DISCEARGE, CUBIC FEET PER SECORD, WATER YEAR CCTOBER 1995 TO SEPTEABER 1996

\begin{tabular}{|c|c|c|c|c|c|c|c|c|c|c|c|c|}
\hline DAZ & $\operatorname{coc} t$ & ROV & DEC & JAYH & $5 \mathrm{~s}$ & MAR & API & MAY & SUP & JUL & AUte & SEP \\
\hline $\begin{array}{l}1 \\
2 \\
3 \\
4 \\
5\end{array}$ & $\begin{array}{l}1.9 \\
1.8 \\
1.6 \\
\end{array}$ & $=$ & $\overline{-}=\overline{-}$ & $=-$ & $=$ & $\begin{array}{l}=- \\
=- \\
=- \\
=-\end{array}$ & $\begin{array}{r}0.73 \\
.73 \\
.68 \\
0.64 \\
0.64\end{array}$ & $\begin{array}{l}.90 \\
.95 \\
.99 \\
1.0 \\
1.1\end{array}$ & $\begin{array}{r}1.7 \\
01.8 \\
1.7 \\
1.6 \\
1.5\end{array}$ & $\begin{array}{l}93 \\
.91 \\
.90 \\
.95 \\
.88\end{array}$ & $\begin{array}{l}.62 \\
0.70 \\
0.70 \\
0.66 \\
0.63\end{array}$ & $\begin{array}{l}.73 \\
.85 \\
.77 \\
.69 \\
.70\end{array}$ \\
\hline $\begin{array}{r}6 \\
7 \\
8 \\
9 \\
10\end{array}$ & 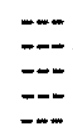 & $=$ & $\begin{array}{l}=- \\
=- \\
=-\end{array}$ & 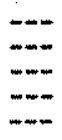 & $=-$ & $\begin{array}{l}=- \\
\square \\
\square- \\
-\end{array}$ & $\begin{array}{l}0.64 \\
.70 \\
0.76 \\
.97 \\
.90\end{array}$ & $\begin{array}{l}1.1 \\
1.1 \\
1.1 \\
1.1 \\
1.1\end{array}$ & $\begin{array}{l}1.5 \\
1.5 \\
1.4 \\
1.4 \\
1.4\end{array}$ & $\begin{array}{r}.88 \\
.91 \\
1.1 \\
1.1 \\
.94\end{array}$ & $\begin{array}{l}.63 \\
.65 \\
.71 \\
.69 \\
.66\end{array}$ & $\begin{array}{l}.72 \\
.71 \\
.69 \\
.69 \\
.68\end{array}$ \\
\hline $\begin{array}{l}11 \\
12 \\
13 \\
14 \\
15\end{array}$ & $=$ & $\bar{m}$ & 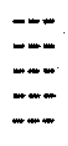 & $\begin{array}{l}--- \\
-- \\
-\cdots \\
-\cdots\end{array}$ & $\begin{array}{l}m-m \\
m-- \\
---\end{array}$ & $=$ & $\begin{array}{r}.88 \\
.92 \\
.80 \\
.80 \\
.90\end{array}$ & $\begin{array}{l}1.1 \\
1.2 \\
1.4 \\
1.4 \\
1.5\end{array}$ & $\begin{array}{l}1.3 \\
1.3 \\
1.4 \\
1.4 \\
1.5\end{array}$ & $\begin{array}{c}.93 \\
1.1 \\
1.0 \\
.94 \\
.93\end{array}$ & $\begin{array}{l}.62 \\
.62 \\
.62 \\
.61 \\
.66\end{array}$ & $\begin{array}{l}.70 \\
.78 \\
.87 \\
.82 \\
.83\end{array}$ \\
\hline $\begin{array}{l}16 \\
17 \\
18 \\
19 \\
20\end{array}$ & $=$ & $=$ & $\begin{array}{l}-- \\
-\cdots \\
-\cdots \\
m-\end{array}$ & $\begin{array}{l}=-- \\
--- \\
---\end{array}$ & $=$ & $=$ & $\begin{array}{r}0.90 \\
.96 \\
.90 \\
.79 \\
.85\end{array}$ & $\begin{array}{l}1.5 \\
1.5 \\
1.5 \\
1.5 \\
1.5\end{array}$ & $\begin{array}{l}1.2 \\
1.1 \\
1.1 \\
1.1 \\
1.0\end{array}$ & $\begin{array}{l}.90 \\
.89 \\
.86 \\
.82 \\
.81\end{array}$ & $\begin{array}{l}.63 \\
.68 \\
.66 \\
.63 \\
.62\end{array}$ & $\begin{array}{l}.73 \\
.75 \\
.82 \\
.75 \\
.72\end{array}$ \\
\hline $\begin{array}{l}21 \\
22 \\
23 \\
24 \\
25\end{array}$ & \begin{tabular}{l}
$m-$ \\
$\square--$ \\
\hdashline- \\
--- \\
\end{tabular} & 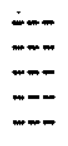 & $=$ & $=-$ & $\begin{array}{l}--- \\
--- \\
-- \\
--\end{array}$ & 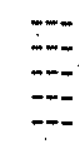 & $\begin{array}{l}.79 \\
.79 \\
.82 \\
.89 \\
.99\end{array}$ & $\begin{array}{l}1.5 \\
1.4 \\
1.6 \\
1.7 \\
1.8\end{array}$ & $\begin{array}{l}01.0 \\
01.3 \\
81.1 \\
01.0 \\
01.0\end{array}$ & $\begin{array}{l}.81 \\
.80 \\
.77 \\
.74 \\
.72\end{array}$ & $\begin{array}{l}.71 \\
.81 \\
.89 \\
.96 \\
.77\end{array}$ & $\begin{array}{r}.70 \\
.70 \\
0.70 \\
0.71 \\
0.72\end{array}$ \\
\hline $\begin{array}{l}26 \\
27 \\
28 \\
29 \\
30 \\
31\end{array}$ & $\begin{array}{l}--- \\
-- \\
-\cdots \\
- \\
-\end{array}$ & $\begin{array}{l}--- \\
-\cdots \\
-- \\
-\square \\
-\end{array}$ & $\begin{array}{l}m-\cdots \\
m-\infty \\
-\infty \\
-\infty\end{array}$ & 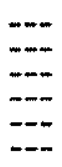 & $=$ & $\begin{array}{l}--- \\
0.61 \\
0.61 \\
0.58 \\
0.60 \\
0.65\end{array}$ & $\begin{array}{r}1.0 \\
1.0 \\
.85 \\
.80 \\
.91 \\
-90\end{array}$ & $\begin{array}{r}1.8 \\
1.8 \\
1.7 \\
1.7 \\
1.7 \\
1.7\end{array}$ & $\begin{array}{r}.96 \\
1.3 \\
.92 \\
\mathrm{e.92} \\
01.0 \\
\end{array}$ & $\begin{array}{l}.68 \\
.69 \\
.77 \\
.73 \\
.67 \\
.65\end{array}$ & $\begin{array}{l}.71 \\
1.1 \\
.93 \\
1.0 \\
.84 \\
.76\end{array}$ & $\begin{array}{r}0.73 \\
.73 \\
.72 \\
.70 \\
.70 \\
-.-\end{array}$ \\
\hline $\begin{array}{l}\text { TOLAL } \\
\text { MiBAN } \\
\text { MAX } \\
\text { MIN } \\
\text { AC-FY }\end{array}$ & $\begin{array}{r}5.3 \\
1.77 \\
1.9 \\
1.6 \\
11\end{array}$ & ב- & $=$ & $\begin{array}{l}-- \\
-- \\
-- \\
--\end{array}$ & $m$ & $\begin{array}{r}3.05 \\
.61 \\
.65 \\
58 \\
6.0\end{array}$ & $\begin{array}{r}24.93 \\
.83 \\
1.0 \\
.64 \\
49\end{array}$ & $\begin{array}{r}42.94 \\
1.39 \\
1.8 \\
.90 \\
85\end{array}$ & $\begin{array}{r}38.40 \\
1.28 \\
1.8 \\
.92 \\
76\end{array}$ & $\begin{array}{r}26.71 \\
i .86 \\
1.1 \\
.65 \\
53\end{array}$ & $\begin{array}{r}22.48 \\
.73 \\
i 1 \\
.61 \\
45\end{array}$ & $\begin{array}{r}22.11 \\
.74 \\
.87 \\
.68 \\
44\end{array}$ \\
\hline
\end{tabular}

- Estimatad 
RIO GRANDE BASTN

08253900 COSTILLA RESERVOIR MEAR COSTILLA, MM

LOCATION, --'hat $36^{\circ} 52^{\prime} 36^{\prime \prime}$, Long $105^{\circ} 16^{\prime} 45^{\prime \prime}$, Taos County, Hydrologic Unit 13020101, In Sangre de Cristo Grant, on face of Costilia Dan on Cositilla Crook, $16 \mathrm{mi}$ southoast of Costilla, and at mile 34.8 .

DRAINAGE AREA. $--54.6 \mathrm{mi}^{2}$.

PERIOD OF RECORD.--May 1922 to Septamber 1965 (monthend contents only), October 1965 to September 1983 , April 1990 to ourrent yoax. Records prior to October 1960 published in WSP 1732. Prior to October 1966, published as Costilla Lake near Costilia.

REVISED RECORDS.--WSE 1923: Dratnage area.

GAGE.-Watar-stage recorder with satellite telemeter. Elovation of gage is 9,300 above National Geodetic Vertical datum of 1929, from topographic map.

REMpKS.--Records good except for estimated periods which are poor. Reservoir is formed by eaxthfill dam faced with rock. Storage began in 1920. Diversions for irr $\$ g a t i o n$ of about 1,300 acres above Reservoir. Reservolr is used for 1rrigation. Satelifte telemeter at otation.

EXTREXES FOR EERIOD OF RECORD, - Maximum contents, 16, 500 acre-ft June 1-4, 1994, June 19-22, 1995, gage helght, 107.61 ft; no stoxage October 1925 to February 1926, September 1956, Aug. 22' to Sept. 24, 1972 , July 29 to Sept. 7. 1977 .

EXTREMES FOR CURRENT YEAR. -Maximum contents, 13,300 acre-ft, Apr, 27-30, gage-he 1 ght; 99,89 ft, Apr. 29; minimum contents, 1,020 acre-ft, Sept. 23-25, gage height, 44.10 ft.

RSSERVOIR STORAGE (ACRE FEET), WATER YEAR OCTOBER 1995 TO SEPTEMBER 1996 DAILY OBSERVATION AT 24:00 VALUES

\begin{tabular}{|c|c|c|c|c|c|c|c|c|c|c|c|c|}
\hline DAY & $\infty \mathrm{T}$ & NOV & DEC & JAN & FEB & MAR & AFR & MAY & JUN & JUL & AUG & SER \\
\hline $\begin{array}{l}1 \\
2 \\
3 \\
4 \\
5\end{array}$ & $\begin{array}{l}8790 \\
8760 \\
8520 \\
8420 \\
8460\end{array}$ & $\begin{array}{r}9200 \\
e 9230 \\
99260 \\
\theta 9290 \\
99310\end{array}$ & $\begin{array}{l}9910 \\
9940 \\
9950 \\
9970 \\
9990\end{array}$ & $\begin{array}{l}10500 \\
10500 \\
10500 \\
10500 \\
10600\end{array}$ & $\begin{array}{l}11000 \\
11000 \\
11100 \\
11100 \\
11100\end{array}$ & 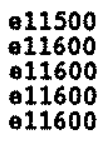 & $\begin{array}{l}\theta 12300 \\
\theta 12300 \\
012400 \\
012400 \\
-12500\end{array}$ & $\begin{array}{l}13200 \\
13200 \\
13200 \\
13200 \\
13200\end{array}$ & $\begin{array}{r}10200 \\
10200 \\
9950 \\
9720 \\
9500\end{array}$ & $\begin{array}{l}6800 \\
6690 \\
6620 \\
6540 \\
6510\end{array}$ & $\begin{array}{l}4440 \\
4360 \\
4350 \\
4270 \\
4050\end{array}$ & $\begin{array}{l}1530 \\
1450 \\
1390 \\
1350 \\
1310\end{array}$ \\
\hline $\begin{array}{r}6 \\
7 \\
8 \\
9 \\
10\end{array}$ & $\begin{array}{l}8490 \\
8520 \\
8560 \\
8590 \\
8630\end{array}$ & $\begin{array}{l}99340 \\
99370 \\
99400 \\
99420 \\
+9440\end{array}$ & $\begin{array}{l}10000 \\
10000 \\
10100 \\
10100 \\
10100\end{array}$ & $\begin{array}{l}10600 \\
10600 \\
10600 \\
10600 \\
10600\end{array}$ & $\begin{array}{l}11100 \\
11100 \\
11100 \\
11200 \\
11200\end{array}$ & $\begin{array}{l}011600 \\
111700 \\
011700 \\
611700 \\
011700\end{array}$ & $\begin{array}{l}12500 \\
12500 \\
12600 \\
12600 \\
12700\end{array}$ & $\begin{array}{l}13100 \\
13000 \\
12900 \\
12700 \\
12700\end{array}$ & $\begin{array}{l}9290 \\
9210 \\
9220 \\
9170 \\
8950\end{array}$ & $\begin{array}{l}6520 \\
6490 \\
6430 \\
6380 \\
6320\end{array}$ & $\begin{array}{l}3830 \\
3640 \\
3460 \\
3390 \\
3380\end{array}$ & $\begin{array}{l}1310 \\
1310 \\
1280 \\
1230 \\
1180\end{array}$ \\
\hline $\begin{array}{l}11 \\
12 \\
13 \\
14 \\
15\end{array}$ & $\begin{array}{l}8660 \\
8690 \\
8720 \\
8750 \\
8780\end{array}$ & $\begin{array}{l}99460 \\
99480 \\
99500 \\
69530 \\
69550\end{array}$ & $\begin{array}{l}10100 \\
10100 \\
10100 \\
10200 \\
10200\end{array}$ & $\begin{array}{l}10700 \\
10700 \\
10700 \\
10700 \\
10700\end{array}$ & $\begin{array}{r}11200 \\
11200 \\
11200 \\
+11200 \\
011200\end{array}$ & $\begin{array}{l}011800 \\
-11800 \\
-11800 \\
\cdot 11800 \\
-11800\end{array}$ & $\begin{array}{l}12700 \\
12700 \\
12800 \\
12800 \\
12800\end{array}$ & $\begin{array}{l}12800 \\
12800 \\
12600 \\
12400 \\
12200\end{array}$ & $\begin{array}{l}8750 \\
8570 \\
8410 \\
8360 \\
8390\end{array}$ & $\begin{array}{l}6270 \\
6250 \\
6260 \\
6250 \\
6180\end{array}$ & $\begin{array}{l}3340 \\
3170 \\
3020 \\
2860 \\
2710\end{array}$ & $\begin{array}{l}01140 \\
01110 \\
-1110 \\
\mathrm{e} 1110 \\
\mathrm{e} 1080\end{array}$ \\
\hline $\begin{array}{l}16 \\
17 \\
18 \\
19 \\
20\end{array}$ & $\begin{array}{l}8800 \\
8840 \\
8860 \\
8880 \\
8910\end{array}$ & $\begin{array}{l}9580 \\
9600 \\
9630 \\
9650 \\
9680\end{array}$ & $\begin{array}{l}10200 \\
10200 \\
10200 \\
10300 \\
10300\end{array}$ & $\begin{array}{l}10700 \\
10800 \\
10800 \\
10800 \\
10800\end{array}$ & $\begin{array}{l}11300 \\
611300 \\
+11300 \\
-11300 \\
-11300\end{array}$ & $\begin{array}{l}011900 \\
011900 \\
011900 \\
011900 \\
011900\end{array}$ & $\begin{array}{l}012800 \\
612900 \\
012900 \\
13000 \\
13000\end{array}$ & $\begin{array}{l}12000 \\
12000 \\
12000 \\
11900 \\
11800\end{array}$ & $\begin{array}{l}8340 \\
8140 \\
7960 \\
7780 \\
7620\end{array}$ & $\begin{array}{l}6100 \\
6030 \\
5950 \\
5920 \\
5920\end{array}$ & $\begin{array}{l}2650 \\
2660 \\
2620 \\
2460 \\
2300\end{array}$ & $\begin{array}{l}+1060 \\
\theta 1050 \\
\theta 1040 \\
-1030 \\
e 1030\end{array}$ \\
\hline $\begin{array}{l}21 \\
22 \\
23 \\
24 \\
25\end{array}$ & $\begin{array}{l}8930 \\
8960 \\
8980 \\
9010 \\
9030\end{array}$ & $\begin{array}{l}9700 \\
9730 \\
9750 \\
9770 \\
9790\end{array}$ & $\begin{array}{l}10300 \\
10300 \\
10300 \\
10300 \\
10400\end{array}$ & $\begin{array}{l}10800 \\
10900 \\
10900 \\
10900 \\
10900\end{array}$ & $\begin{array}{l}\mathrm{Q} 11300 \\
\mathrm{e} 11400 \\
\mathrm{\theta} 11400 \\
\mathrm{\theta} 11400 \\
\mathrm{\theta} 11400\end{array}$ & $\begin{array}{l}\theta 12000 \\
\theta 12000 \\
-12000 \\
e 12000 \\
\theta 12100\end{array}$ & $\begin{array}{l}13000 \\
13100 \\
13100 \\
13200 \\
13200\end{array}$ & $\begin{array}{l}11600 \\
11500 \\
11300 \\
11200 \\
11200\end{array}$ & $\begin{array}{l}7580 \\
7600 \\
7550 \\
7370 \\
7210\end{array}$ & $\begin{array}{l}5880 \\
5760 \\
5630 \\
5500 \\
5370\end{array}$ & $\begin{array}{l}2130 \\
2000 \\
1960 \\
1970 \\
1950\end{array}$ & $\begin{array}{l}01030 \\
e 1030 \\
01020 \\
01020 \\
-1020\end{array}$ \\
\hline $\begin{array}{l}26 \\
27 \\
28 \\
29 \\
30 \\
31\end{array}$ & $\begin{array}{l}9060 \\
9080 \\
9110 \\
9140 \\
9170 \\
9190\end{array}$ & $\begin{array}{l}9810 \\
9830 \\
9840 \\
9860 \\
9890 \\
--\cdots\end{array}$ & $\begin{array}{l}10400 \\
10400 \\
10400 \\
10400 \\
10400 \\
10500\end{array}$ & $\begin{array}{l}10900 \\
10900 \\
11000 \\
11000 \\
11000 \\
11000\end{array}$ & $\begin{array}{r}611400 \\
611500 \\
011500 \\
611500 \\
\end{array}$ & $\begin{array}{l}012100 \\
012100 \\
012100 \\
012200 \\
012200 \\
012200\end{array}$ & $\begin{array}{l}13200 \\
13300 \\
13300 \\
13300 \\
13300\end{array}$ & $\begin{array}{l}11200 \\
11000 \\
10800 \\
10500 \\
10300 \\
10200\end{array}$ & $\begin{array}{l}7060 \\
6950 \\
6900 \\
6910 \\
6900 \\
---\end{array}$ & $\begin{array}{l}5300 \\
5300 \\
5250 \\
5040 \\
4840 \\
4640\end{array}$ & $\begin{array}{l}1840 \\
1750 \\
1660 \\
1590 \\
1550 \\
1550\end{array}$ & $\begin{array}{l}1030 \\
1030 \\
1030 \\
1040 \\
1050 \\
-\end{array}$ \\
\hline $\begin{array}{l}\operatorname{MAX} \\
\operatorname{MIN} \\
(t) \\
(t+)\end{array}$ & $\begin{array}{r}9190 \\
8420 \\
87.71 \\
+440\end{array}$ & $\begin{array}{r}9890 \\
9200 \\
89.97 \\
+700\end{array}$ & $\begin{array}{r}10500 \\
9910 \\
91.79 \\
+610\end{array}$ & $\begin{array}{r}11000 \\
10500 \\
93.37 \\
+500\end{array}$ & $\begin{array}{r}11500 \\
11000 \\
+500\end{array}$ & $\begin{array}{r}12200 \\
11500 \\
+700\end{array}$ & $\begin{array}{r}13300 \\
12300 \\
99.68 \\
+1100\end{array}$ & $\begin{array}{r}13200 \\
10200 \\
91.03 \\
-3100\end{array}$ & $\begin{array}{r}10200 \\
6900 \\
79.70 \\
-3300\end{array}$ & $\begin{array}{r}6800 \\
4640 \\
70.09 \\
-2260\end{array}$ & $\begin{array}{r}4440 \\
1550 \\
51.05 \\
-3090\end{array}$ & $\begin{array}{r}1530 \\
1020 \\
44.74 \\
-500\end{array}$ \\
\hline
\end{tabular}

CAL YR 1995 MAX 16500 MIN 6360 AC-FT (tt) +4140 WTR YR 1996 MAX 13300 MIN 1020 AC-FT (t+) -7700

- Estimated

(†) GAGE BEIGHT, IN FEET, AT END OF MONTH

(t $t)$ CHANGE IN CONTENTS, IN ACRE-FEET 


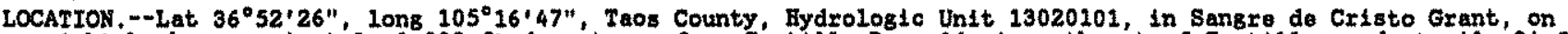
right bank approximately 1,000 ft downstream from Costilila Dam, 16 mil southeast of Costilila, and at mile 34,5 . DRAINAGE AREA. $--54.6 \mathrm{mi}^{3}$,

PERIOD OF RECORD.--April 1937 to current year (sossonal records 1937-44, 1947-49, 1988-95). Monthly discharge only for Bome periods, publishod In WSP 1312. Prior to Ootober 1951, pubilshed as "below reservoir near Costilia, "s REVISED RECORDS. - WSE 1923: DrELnege area.

GAGE.--Water-stage recorder and concrete control. Elovation of gage is 9,290 ft above National Goodetio Vertioal Datum of 1929 , from topogro ft upstream at different datum.

REMARKS:--Records good. Flow regulated by Costilie Reservoir (station 08253900). Diversions for irrigation of about. 1, 300 acres upstrean from reservoir. Severel observations of weter temperature were made during the year. AVRRAGE DISCEARGE. -41 years (water years $1945-47,1950-87$ ), 18,6 fts/s, 13,480 acre-rt/yr.

EXTREMS FOR PERIOD OF RECORD, --Maximum discharge, $366 \mathrm{fts} / \mathrm{s}$, July 29, 1994, gage height, $3.57 \mathrm{ft}$; no flow at times.

EXTREMES FOR CURRENT YEAR,--Maximum daily discharge during period of seasonel operation, 14S fts/8, Oct. 3; minimum daily, $0.12 \mathrm{ft} / \mathrm{s}$, April 18-21. DISCEARGE, CUBIC FEET PER SECOND, WATER YEAR OCTOBER 1995 TO SEPTEMBER 1996

\begin{tabular}{|c|c|c|c|c|c|c|c|c|c|c|c|c|}
\hline DAY & OCT & NOV & DEC & JAN & FEB & MAR & APR & MAY & JUN & JUL & AUUC & SEP \\
\hline $\begin{array}{l}1 \\
2 \\
3 \\
4 \\
5\end{array}$ & $\begin{array}{r}33^{1.7} \\
145 \\
65 \\
\end{array}$ & $\begin{array}{l}m-- \\
\overline{--} \\
\overline{--} \\
-\overline{-}\end{array}$ & $\begin{array}{l}-- \\
-\overline{-} \\
--\end{array}$ & $\begin{array}{l}-- \\
-- \\
-- \\
--\end{array}$ & 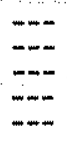 & $=-$ & $\begin{array}{l}.17 \\
.18 \\
.15 \\
.13 \\
.13\end{array}$ & $\begin{array}{c}46 \\
46 \\
20 \\
2.0 \\
28\end{array}$ & $\begin{array}{r}15 \\
47 \\
139 \\
138 \\
134\end{array}$ & $\begin{array}{l}64 \\
58 \\
54 \\
52 \\
25\end{array}$ & $\begin{array}{r}110 \\
57 \\
14 \\
47 \\
124\end{array}$ & $\begin{array}{l}20 \\
43 \\
34 \\
29 \\
29\end{array}$ \\
\hline $\begin{array}{r}6 \\
7 \\
8 \\
9 \\
10\end{array}$ & $\begin{array}{l}--- \\
-- \\
-- \\
--\end{array}$ & 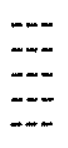 & $\begin{array}{l}--- \\
-- \\
-- \\
-m\end{array}$ & $\begin{array}{l}=- \\
=- \\
--\end{array}$ & $=$ & $\begin{array}{l}=- \\
=- \\
-\cdots\end{array}$ & $\begin{array}{l}.16 \\
.17 \\
.18 \\
.17 \\
.17\end{array}$ & $\begin{array}{l}93 \\
90 \\
88 \\
87 \\
29\end{array}$ & $\begin{array}{c}130 \\
51 \\
8.8 \\
41 \\
129\end{array}$ & $\begin{array}{l}10 \\
23 \\
50 \\
43 \\
40\end{array}$ & $\begin{array}{c}117 \\
110 \\
105 \\
46 \\
7.8\end{array}$ & $\begin{array}{c}16 \\
6 \\
19 \\
33 \\
30\end{array}$ \\
\hline $\begin{array}{l}11 \\
12 \\
13 \\
14 \\
15\end{array}$ & 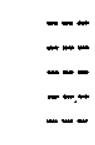 & $\bar{m}$ & $\begin{array}{l}--- \\
-\overline{-} \\
-\overline{-} \\
-\cdots\end{array}$ & $\begin{array}{l}--- \\
-- \\
--- \\
---\end{array}$ & 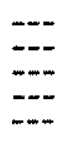 & 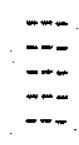 & $\begin{array}{l}.17 \\
.17 \\
.28 \\
.16 \\
.16\end{array}$ & $\begin{array}{l}2.0 \\
44 \\
133 \\
130 \\
126\end{array}$ & $\begin{array}{r}119 \\
111 \\
105 \\
49 \\
12\end{array}$ & $\begin{array}{c}40 \\
20 \\
8.5 \\
19 \\
48\end{array}$ & $\begin{array}{l}30 \\
87 \\
85 \\
84 \\
84\end{array}$ & $\begin{array}{l}28 \\
24 \\
15 \\
7.5 \\
13\end{array}$ \\
\hline $\begin{array}{l}16 \\
17 \\
18 \\
19 \\
20\end{array}$ & 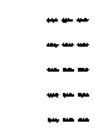 & $=$ & $m+m$ & $=$ & $\begin{array}{l}=- \\
=- \\
m- \\
m-\end{array}$ & $\begin{array}{l}-\cdots \\
\cdots- \\
\cdots-\end{array}$ & $\begin{array}{l}.14 \\
.13 \\
.12 \\
.12 \\
.12\end{array}$ & $\begin{array}{r}122 \\
66 \\
21 \\
40 \\
109\end{array}$ & $\begin{array}{r}41 \\
124 \\
113 \\
104 \\
93\end{array}$ & $\begin{array}{c}47 \\
45 \\
45 \\
24 \\
9.2\end{array}$ & $\begin{array}{l}36 \\
5.1 \\
28 \\
86 \\
90\end{array}$ & $\begin{array}{l}21 \\
18 \\
18 \\
17 \\
12\end{array}$ \\
\hline $\begin{array}{l}21 \\
22 \\
23 \\
24 \\
25\end{array}$ & - & $=$ & $\begin{array}{l}m- \\
\ddot{-}-\overline{-} \\
-\square \\
--\end{array}$ & 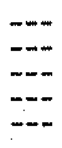 & $\begin{array}{l}-\cdots \\
--- \\
-- \\
-m\end{array}$ & $\begin{array}{l}--- \\
-\cdots \\
-\cdots \\
-\cdots\end{array}$ & $\begin{array}{r}.12 \\
.79 \\
1.8 \\
1.9 \\
1.9\end{array}$ & $\begin{array}{r}114 \\
113 \\
113 \\
57 \\
16\end{array}$ & $\begin{array}{c}36 \\
9.6 \\
36 \\
108 \\
96\end{array}$ & $\begin{array}{l}28 \\
70 \\
73 \\
72 \\
71\end{array}$ & $\begin{array}{l}88 \\
82 \\
38 \\
7.3 \\
26\end{array}$ & $\begin{array}{l}7.4 \\
7.4 \\
7.4 \\
6.9 \\
6.4\end{array}$ \\
\hline $\begin{array}{l}26 \\
27 \\
28 \\
29 \\
30 \\
31\end{array}$ & $\begin{array}{l}-\cdots \\
-\cdots \\
-\cdots \\
-\cdots \\
-\cdots\end{array}$ & $\begin{array}{l}--- \\
-\cdots \\
-\cdots \\
-\cdots \\
--\end{array}$ & $\begin{array}{l}-- \\
-- \\
-- \\
-- \\
--\end{array}$ & $\begin{array}{l}--- \\
--- \\
--- \\
-\cdots \\
--\end{array}$ & $\begin{array}{l}m-- \\
m-- \\
m- \\
-- \\
--\end{array}$ & $\begin{array}{l}.20 \\
.18 \\
.15 \\
.13 \\
.14\end{array}$ & $\begin{array}{r}1.9 \\
1.9 \\
\frac{3}{17} .9 \\
46\end{array}$ & $\begin{array}{r}48 \\
132 \\
135 \\
135 \\
132 \\
62\end{array}$ & $\begin{array}{l}93 \\
82 \\
34 \\
9.5 \\
25 \\
-. .-\end{array}$ & $\begin{array}{l}43 \\
7.2 \\
39 \\
125 \\
116 \\
111\end{array}$ & $\begin{array}{l}66 \\
62 \\
58 \\
56 \\
27 \\
8.5\end{array}$ & $\begin{array}{l}6.3 \\
6.2 \\
6.2 \\
6.1 \\
3.6\end{array}$ \\
\hline $\begin{array}{l}\text { TOTAL } \\
\text { MEAN } \\
\text { MAX } \\
\text { MIN } \\
\text { AC-FT }\end{array}$ & $\begin{array}{r}244.7 \\
61.2 \\
145 \\
1.7 \\
485\end{array}$ & $=$ & $=$ & 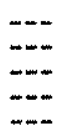 & $=-$ & $\begin{array}{r}0.80 \\
.16 \\
.20 \\
.13 \\
1.6\end{array}$ & $\begin{array}{r}78.39 \\
2.61 \\
46 \\
.12 \\
155\end{array}$ & $\begin{array}{r}2381.0 \\
76.8 \\
135 \\
2.0 \\
4720\end{array}$ & $\begin{array}{r}2233.0 \\
74.4 \\
139 \\
8.8 \\
4430\end{array}$ & $\begin{array}{r}1479.9 \\
47.7 \\
125 \\
7.2 \\
2940\end{array}$ & $\begin{array}{r}1871.7 \\
60.4 \\
124 \\
5.1 \\
3710\end{array}$ & $\begin{array}{r}496.8 \\
16.6 \\
43 \\
3.6 \\
985\end{array}$ \\
\hline
\end{tabular}


RIO GRANDE BASIN

08255500 COSTILLA CREEK NEAR COSTILLA, NM

LOCATION.-Lat $36^{\circ} 58^{\prime} 01^{\prime \prime}$, long $105^{\circ} 30^{\prime} 23^{\prime \prime}$. Taos County, Hydrologio Unit 13020101, In Sangre de Cristo Grant, on right bank $70 \mathrm{ft}$ downstream from bridge on State Bighway 196, 0.5 mi upstream from diversion dam, 1.6 mi southeast of Costilla, and at mile 15.9 .

DRATNAGE AREA. $--195 \mathrm{~m}^{2}$.

PERIOD OF RECORD.--March 1936 to current year (no winter records 1936-41, 1943), Monthly discharge for March 1943 and water-year estimate for 1943, published in WSP 1312.

REVISED RECORDS, - -WSP 1312: $1937-39(\mathrm{M})$.

GAGE.--Water-stage recordex. Concrete control since Oct. 13, 1952. Elevation of 8age 1s 7,900 ft above National. Geodetic Vertical Datum of 1929, from topographic map. Prior to June 18, 1944 , at site 200 ft downstream at different datum. June 18, $1944^{\prime}$ to Sept. 30, 1964 , at site $0.4 \mathrm{mt}$ upstream at different datum.

REMARKS, --Records good except for estimated daily discharges, which are poor. Flow regulated by Costilla Reservoir (station 08253900) $19 \mathrm{mi}$ upstream. Diversions for irrigation of about 2,000 acres upstream from station. Several observations of water temperature were made during the year. Satellite telemeter at station.

EXTREMES OUTSIDE FERIOD OF RECORD.--A major flood occurred in 1886, from information by local restdents. DISCHARGE, CUBIC FEET PER SECOND, WATER YEAR OCTOBER 1995 TO SEPTEMBER 1996

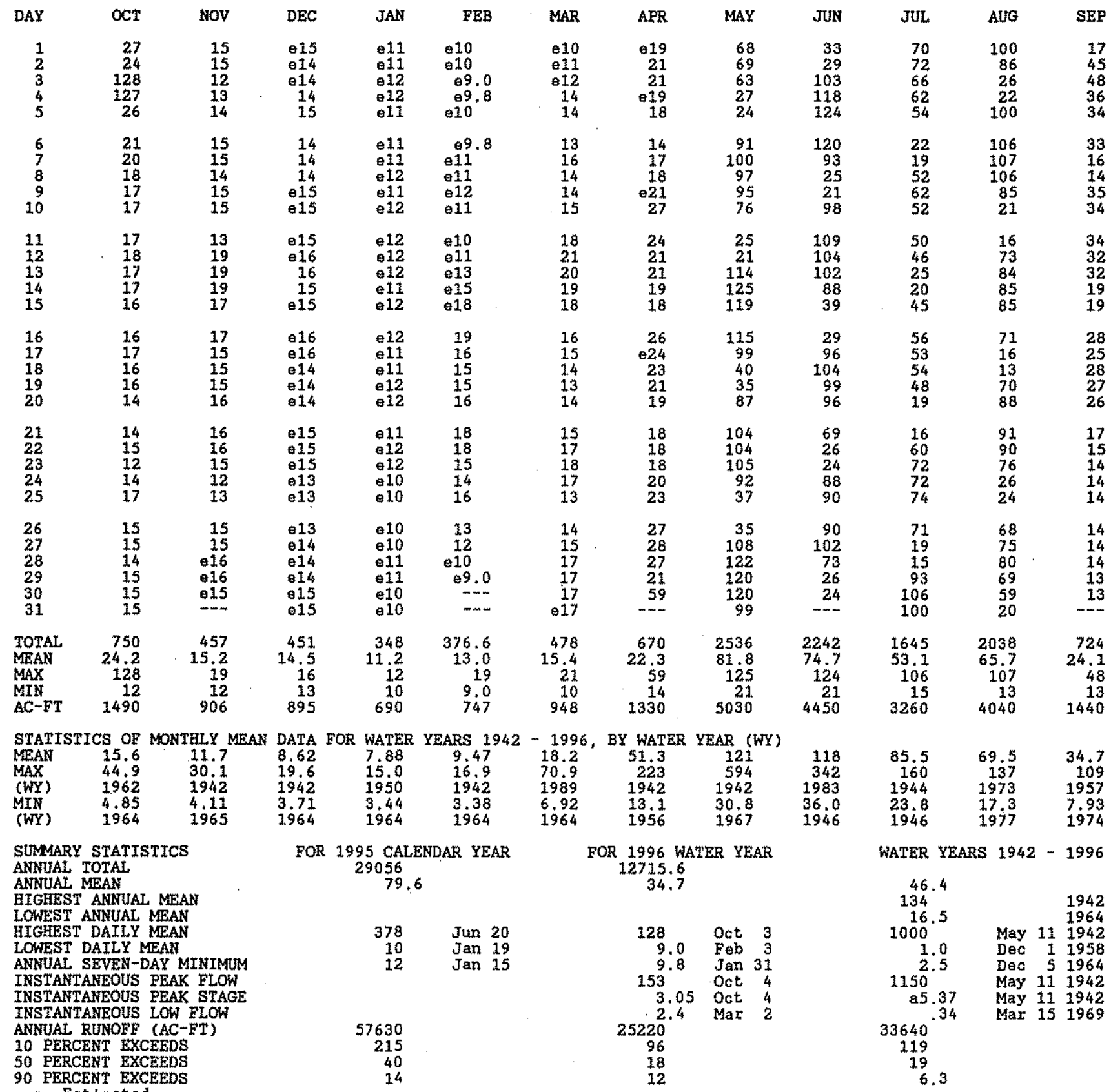

\footnotetext{
Estimated
} 
LOCATION,--Lat $36^{\circ} 59^{\prime} 21^{\prime \prime}$, long 105\%31'54". Taos County, Hydrologic Unit 13020101, in Sangre de Cristo Grant, on left bank $0.4 \mathrm{mi}$ downstream from old State Highway $3,0.5 \mathrm{mi}$ upstream from New Mexico-Colorado State itne, 0.9 mi south of Garcia, and at mile 13.3 .

DRAINAGE AREA. - $-200 \mathrm{mi}^{2}$, approximately.

PERTOD OF RECORD --June 1944 to current year (seasonal records).

GAGE. - Water-stage recorder. Concrete control stnce Oct. 9, 1956. Elevation of gage is 7,760 ft above National

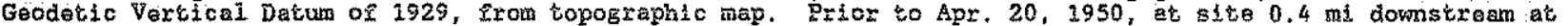
different datum.

REMARS.-Records good. Flow partly regulated by Costilla Reservoir (station 08253900) 22 mi upstrean. Several observations of water temperature were made during the year.

EXIREMES FOR PERTOD OF RFCORD.-Maximum discharge, 444 Its/s, June 1, 1983, gage helght, 4.91 ft; no flow for many days most years.

EXTREMES OUTSIDE PERIOD OF RECORD.--A major flood occurred in 1886, from information by local residents. Flood of May 11, 1942, probably reached a discharge of $1,000 \mathrm{ft}^{3} / \mathrm{s}$.

EXTREMES FOR CURRENT YEAR.-Maximum discharge during period of seasonal operation, 127 fts/s, at 1600 hours Oct. 3 , gage height, $3.69 \mathrm{ft}$; no flow at times.

DISCEARGE, CUBIC FEET PER SECOND, WATER YEAR OCTOBER 1995 TO SEPTEMBER 1996

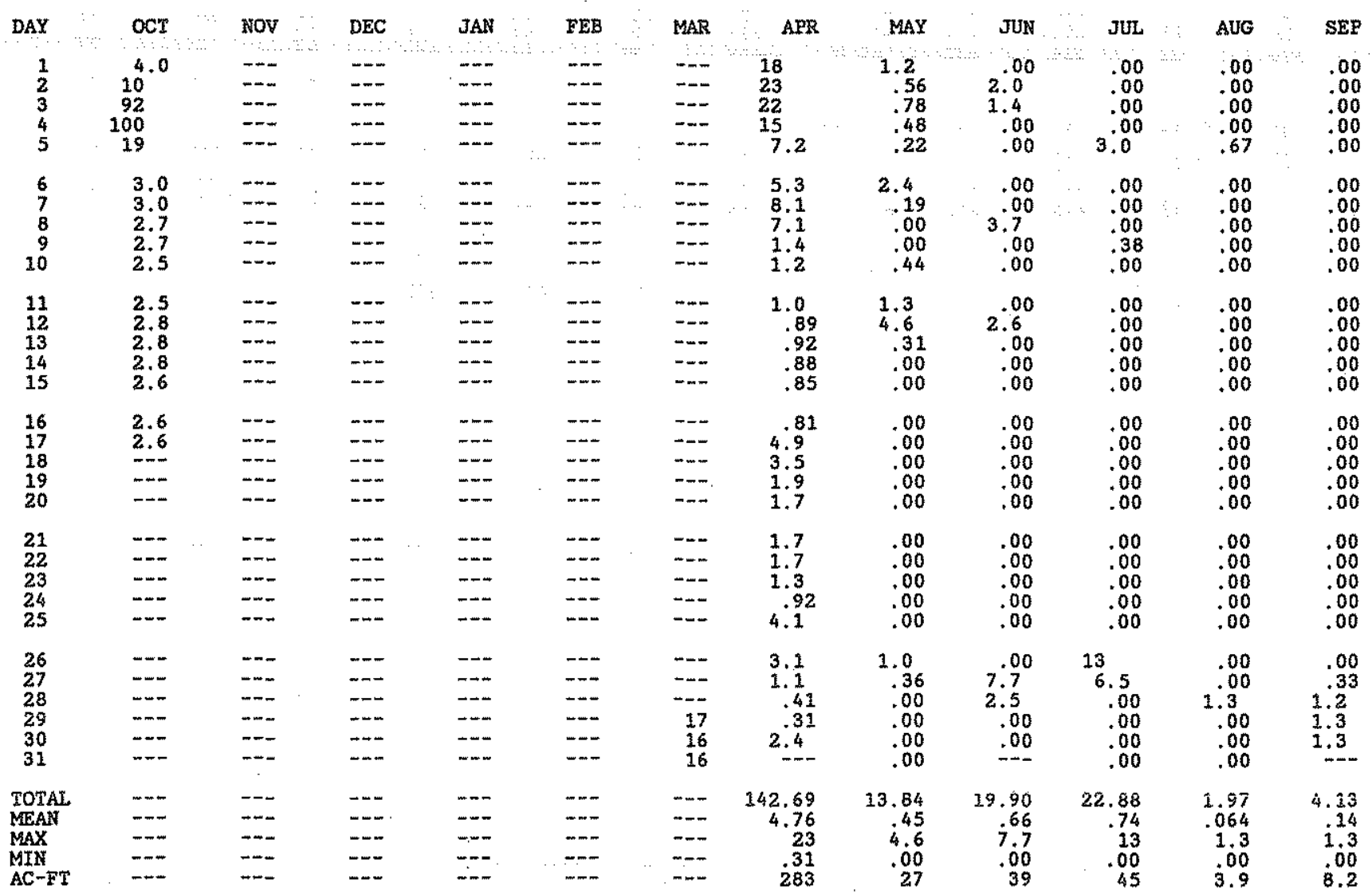


RIO GRANDE BASIN

08263500 RIO GRANDE NEAR CERRO, NM

LOCATION --Lat $36^{\circ} 44^{\prime} 24^{\prime \prime}$, long $105^{\circ} 40^{\circ} 59^{\prime \prime}$, In NW/NEX sec.20, T.29 N., R.12 E., Taos County, Hydrologic Unit 13020101 , on left bank $4 \mathrm{mi}$ southwest of Cerro, $5.5 \mathrm{mi}$ northwest of Questa, $7.4 \mathrm{mi}$ upstream from Red Rtver, and at $\mathrm{mile} 1,693,1$.

DRATNAGE AREA. $--8,440 \mathrm{mi}^{2}$, approximately, tncluding $2,940 \mathrm{mi}^{2}$ in closed basin in San Luis valley, co.

PERIOD OF RECORD. - May 1948 to September 1994, October 1995 to current year.

REVISED RECORDS. - WDR NM-80-1: $1978(M)$.

GAGE.--Water-stage recorder. Elevation of gage is 7,110 ft above National Geodetic Vertical Datum of 1929 , from topographic map.

REMARKS.--Recordi fair except for estimated daily discharges, which are poor. Diversions upstream from station for irrigation of about 620,000 acres in Colorado and 7,000 acres in New Mexico. Several observations of water temperature pere made during year.

DISCEARGE, CUBIC FEET PER SECOND, WATER YEAR OCTOBER 1995 TO SEPTEMBER 1996 DATLY MEAN VALUES

\begin{tabular}{|c|c|c|c|c|c|c|c|c|c|c|c|c|}
\hline DAY & $\alpha \mathrm{TT}$ & NOV & DEC & JAN & FEB & MAR & $A E R$ & MAY & JuN & JuL & AUG & SEP \\
\hline $\begin{array}{l}1 \\
2 \\
3 \\
4 \\
5\end{array}$ & $\begin{array}{l}0178 \\
0181 \\
0201 \\
e 237 \\
e 242\end{array}$ & $\begin{array}{l}231 \\
239 \\
250 \\
250 \\
247\end{array}$ & $\begin{array}{l}267 \\
259 \\
266 \\
284 \\
304\end{array}$ & $\begin{array}{l}381 \\
395 \\
372 \\
373 \\
391\end{array}$ & $\begin{array}{l}463 \\
453 \\
455 \\
443 \\
449\end{array}$ & $\begin{array}{l}527 \\
576 \\
580 \\
590 \\
596\end{array}$ & $\begin{array}{l}300 \\
283 \\
267 \\
274 \\
272\end{array}$ & $\begin{array}{l}322 \\
258 \\
231 \\
216 \\
196\end{array}$ & $\begin{array}{l}0146 \\
0137 \\
0133 \\
e 130 \\
0125\end{array}$ & $\begin{array}{r}0101 \\
0101 \\
098 \\
096 \\
094\end{array}$ & $\begin{array}{l}064 \\
063 \\
058 \\
066 \\
060\end{array}$ & $\begin{array}{r}e 76 \\
e 76 \\
77 \\
76 \\
78\end{array}$ \\
\hline $\begin{array}{r}6 \\
7 \\
8 \\
9 \\
10\end{array}$ & $\begin{array}{l}e 217 \\
0187 \\
e 174 \\
0170 \\
e 164\end{array}$ & $\begin{array}{l}251 \\
257 \\
257 \\
262 \\
265\end{array}$ & $\begin{array}{l}313 \\
314 \\
318 \\
332 \\
323\end{array}$ & $\begin{array}{l}391 \\
402 \\
406 \\
411 \\
425\end{array}$ & $\begin{array}{l}469 \\
483 \\
488 \\
514 \\
524\end{array}$ & $\begin{array}{l}608 \\
604 \\
609 \\
598 \\
601\end{array}$ & $\begin{array}{l}265 \\
280 \\
250 \\
227 \\
227\end{array}$ & $\begin{array}{l}195 \\
199 \\
21.6 \\
227 \\
218\end{array}$ & $\begin{array}{l}\text { e127 } \\
\text { e } 121 \\
\text { e } 116 \\
\text { e119 } \\
\text { e121 }\end{array}$ & $\begin{array}{r}096 \\
e 86 \\
086 \\
91 \\
92\end{array}$ & $\begin{array}{l}60 \\
67 \\
73 \\
73 \\
69\end{array}$ & $\begin{array}{l}81 \\
81 \\
78 \\
74 \\
73\end{array}$ \\
\hline $\begin{array}{l}11 \\
12 \\
13 \\
14 \\
15\end{array}$ & $\begin{array}{l}0161 \\
0163 \\
0164 \\
0160 \\
0156\end{array}$ & $\begin{array}{l}283 \\
269 \\
265 \\
270 \\
279\end{array}$ & $\begin{array}{l}301 \\
306 \\
302 \\
311 \\
337\end{array}$ & $\begin{array}{l}425 \\
425 \\
435 \\
433 \\
440\end{array}$ & $\begin{array}{l}554 \\
575 \\
571 \\
565 \\
620\end{array}$ & $\begin{array}{l}603 \\
607 \\
589 \\
574 \\
566\end{array}$ & $\begin{array}{l}242 \\
315 \\
312 \\
316 \\
317\end{array}$ & $\begin{array}{l}200 \\
208 \\
206 \\
197 \\
261\end{array}$ & $\begin{array}{l}0121 \\
0121 \\
0116 \\
e 114 \\
0118\end{array}$ & $\begin{array}{l}e 94 \\
094 \\
\text { e93 } \\
\text { e88 } \\
\text { e86 }\end{array}$ & $\begin{array}{l}73 \\
73 \\
73 \\
74 \\
74\end{array}$ & $\begin{array}{l}76 \\
76 \\
79 \\
77 \\
91\end{array}$ \\
\hline $\begin{array}{l}16 \\
17 \\
18 \\
19 \\
20\end{array}$ & $\begin{array}{r}0155 \\
0157 \\
168 \\
183 \\
195\end{array}$ & $\begin{array}{l}288 \\
297 \\
299 \\
296 \\
287\end{array}$ & $\begin{array}{l}354 \\
325 \\
265 \\
233 \\
242\end{array}$ & $\begin{array}{l}451 \\
472 \\
448 \\
407 \\
410\end{array}$ & $\begin{array}{l}635 \\
624 \\
645 \\
681 \\
655\end{array}$ & $\begin{array}{l}539 \\
501 \\
465 \\
431 \\
394\end{array}$ & $\begin{array}{l}287 \\
238 \\
204 \\
198 \\
196\end{array}$ & $\begin{array}{l}258 \\
229 \\
214 \\
221 \\
248\end{array}$ & $\begin{array}{r}0122 \\
0124 \\
0123 \\
0117 \\
120\end{array}$ & $\begin{array}{l}e 89 \\
\oplus 88 \\
084 \\
083 \\
078\end{array}$ & $\begin{array}{l}74 \\
71 \\
70 \\
71 \\
72\end{array}$ & $\begin{array}{l}85 \\
83 \\
80 \\
81 \\
76\end{array}$ \\
\hline $\begin{array}{l}21 \\
22 \\
23 \\
24 \\
25\end{array}$ & $\begin{array}{r}190 \\
189 \\
0194 \\
0192 \\
0186\end{array}$ & $\begin{array}{l}284 \\
283 \\
286 \\
285 \\
281\end{array}$ & $\begin{array}{l}251 \\
253 \\
242 \\
244 \\
266\end{array}$ & $\begin{array}{l}427 \\
427 \\
444 \\
428 \\
414\end{array}$ & $\begin{array}{l}681 \\
675 \\
710 \\
701 \\
667\end{array}$ & $\begin{array}{l}383 \\
373 \\
364 \\
372 \\
379\end{array}$ & $\begin{array}{l}191 \\
191 \\
191 \\
191 \\
188\end{array}$ & $\begin{array}{r}221 \\
221 \\
238 \\
220 \\
0200\end{array}$ & $\begin{array}{l}115 \\
113 \\
109 \\
108 \\
104\end{array}$ & $\begin{array}{l}e 74 \\
e 74 \\
e 72 \\
e 70 \\
e 76\end{array}$ & $\begin{array}{r}73 \\
77 \\
879 \\
082 \\
080\end{array}$ & $\begin{array}{l}72 \\
69 \\
69 \\
68 \\
68\end{array}$ \\
\hline $\begin{array}{l}26 \\
27 \\
28 \\
29 \\
30 \\
31\end{array}$ & $\begin{array}{r}0194 \\
e 209 \\
207 \\
202 \\
213 \\
223\end{array}$ & $\begin{array}{l}282 \\
281 \\
278 \\
264 \\
268 \\
---\end{array}$ & $\begin{array}{l}294 \\
330 \\
317 \\
335 \\
352 \\
361\end{array}$ & $\begin{array}{l}394 \\
458 \\
394 \\
427 \\
421 \\
437\end{array}$ & $\begin{array}{l}622 \\
605 \\
577 \\
559 \\
---\end{array}$ & $\begin{array}{l}376 \\
351 \\
334 \\
313 \\
297 \\
300\end{array}$ & $\begin{array}{l}184 \\
183 \\
248 \\
335 \\
431 \\
---\end{array}$ & $\begin{array}{l}0178 \\
0173 \\
0170 \\
e 166 \\
0162 \\
0155\end{array}$ & $\begin{array}{r}97 \\
0102 \\
0102 \\
096 \\
098 \\
--\end{array}$ & $\begin{array}{l}071 \\
070 \\
e 70 \\
069 \\
069 \\
067\end{array}$ & $\begin{array}{l}e 81 \\
077 \\
074 \\
076 \\
076 \\
076\end{array}$ & $\begin{array}{l}66 \\
67 \\
69 \\
72 \\
72 \\
---\end{array}$ \\
\hline $\begin{array}{l}\text { TOPAL } \\
\text { MEAN } \\
\text { MAX } \\
\text { MIN } \\
\text { AC-ET }\end{array}$ & $\begin{array}{r}5812 \\
187 \\
242 \\
155 \\
11530\end{array}$ & $\begin{array}{r}8134 \\
271 \\
299 \\
231 \\
16130\end{array}$ & $\begin{array}{r}9201 \\
297 \\
361 \\
233 \\
18250\end{array}$ & $\begin{array}{r}12964 \\
418 \\
472 \\
372 \\
25710\end{array}$ & $\begin{array}{r}16663 \\
575 \\
710 \\
443 \\
33050\end{array}$ & $\begin{array}{r}15000 \\
484 \\
609 \\
297 \\
29750\end{array}$ & $\begin{array}{r}7603 \\
253 \\
431 \\
183 \\
15080\end{array}$ & $\begin{array}{r}6624 \\
214 \\
322 \\
155 \\
13140\end{array}$ & $\begin{array}{r}3515 \\
117 \\
146 \\
96 \\
6970\end{array}$ & $\begin{array}{r}2600 \\
83.9 \\
101 \\
67 \\
5160\end{array}$ & $\begin{array}{r}2229 \\
71.9 \\
82 \\
58 \\
4420\end{array}$ & $\begin{array}{r}2266 \\
75.5 \\
91 \\
66 \\
4490\end{array}$ \\
\hline
\end{tabular}

STATISTICS OF MONTHLY MEAN DATA FOR WATER YEARS 1949 - 1996, BY WATER YEAR (WY)

\begin{tabular}{|c|c|c|c|c|c|c|c|c|c|c|c|c|}
\hline $\begin{array}{l}\text { MEAN } \\
\text { MAX } \\
\text { (WY) } \\
\text { MIN } \\
\text { (WY) }\end{array}$ & $\begin{array}{r}199 \\
720 \\
1970 \\
52.7 \\
1957\end{array}$ & $\begin{array}{r}355 \\
1073 \\
1987 \\
88.1 \\
1957\end{array}$ & $\begin{array}{r}301 \\
774 \\
1987 \\
100 \\
1964\end{array}$ & $\begin{array}{r}292 \\
566 \\
1987 \\
116 \\
1957\end{array}$ & $\begin{array}{r}353 \\
657 \\
1987 \\
140 \\
1957\end{array}$ & $\begin{array}{r}471 \\
1010 \\
1987 \\
110 \\
1957\end{array}$ & $\begin{array}{r}555 \\
2335 \\
1987 \\
107 \\
1955\end{array}$ & $\begin{array}{r}956 \\
4577 \\
1987 \\
84.1 \\
1963\end{array}$ & $\begin{array}{l}1130 \\
4400 \\
1949 \\
58.1 \\
1977\end{array}$ & $\begin{array}{r}463 \\
2181 \\
1986 \\
51.5 \\
1951\end{array}$ & $\begin{array}{r}230 \\
957 \\
1957 \\
48.1 \\
1956\end{array}$ & $\begin{array}{r}173 \\
804 \\
1982 \\
44.8 \\
1956\end{array}$ \\
\hline
\end{tabular}

SUMMARY STATISTICS

FOR 1996 WATER YEAR

WATER YEARS 1949 - 1996

ANNUAL TOTAL

ANNUAL MEAN

HIGHEST ANNUAL MEAN

LOWEST ANNUAL MEAN

HIGHEST DAILY MEAN

LOWEST DAILY MEAN

ANNUAL SEVEN-DAY MINTMUM

INSTANTANEOUS PEAK FLOW

INSTANTANEOUS REAK STAGE

INSTANTANEOUS LOW FLOW

ANNUAL RUNOFF (AC-FT)

10 PERCENT EXCEEDS

50 PERCENT EXCEEDS

90 PERCENT EXCEEDS

- Estinated

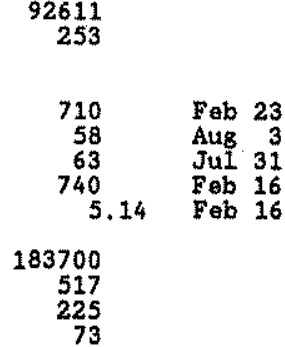

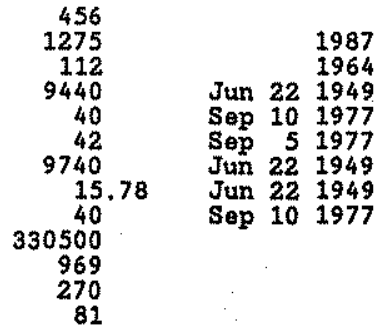


LOCATION, -Lat $36^{\circ} 42^{\prime} 12^{\prime \prime}$, long $105^{\circ} 34^{\prime} 04^{\prime \prime}$, in NE $/ 4$ SE $/ 4$ sec.32, T.29 N., R. 13 E. (projected), Taos County, Hydrologic Unit 13020101, in Carson National Forest, on left bank 1.3 mi upstream from Cabresto Creok, 1.5 mi east of Questa, and at milis 9,0

DRATXAGE AREA $-113 \mathrm{mi}^{2}$

PERIOD OF RECORD.-April to October 1910 and January to September 1911 (gage heights end discharge measurements only), October 1912 to March 1924, May 1924 to September 1925, January to March 1926 , September 1926 to current year. Monthly discharge only for some periods, published in WSP 1312. Published as "Rlo Colorado above Questa" 1910-11, 1926-30, and as "Rto Colorado near Questa" 1912-25, 1930-48.

REVISED RECORDS. -WSP BOB: 1935. WSP 1392: 1913, 1932, 1941, 1947-48, WSP 1712: Dratnage area,

GAGE. -Water-stage recorder. Wood or concrete control since Mar 20, 1936 . Datun of gage is $7,451.92$ ft above National Geodatic Vertical Datim of 1929 . See WSP 1923 for history of changes prior to oct. 4 , 1938 .

REMARKS.--Records good except for estimated dally discharges, which are fair. Dtversions for irrigation of a few hundred acres upstrem from station. Egures of discharge do not tnclude flow in South ditch which divarts from left bank $1,500 \mathrm{ft}$ upstream and bypasses gage for trrigation and stock water downstream. January 1966 to December 1991 surface and ground-water diversions by Molybdenum Corp. of Amertca (Molycorp) refinery 5.5 mi upstream bypass 8 age in tallings pipelines on left bank and discharge into settilng pond 3 mi downstream. Effluent from this pond enters Red River as surface water and is included in discharge at Red River below Ftsh Hatchery, near Questa (station 08266820 ). Several observations of water temperature were made during year. AVERAGE DISCHARGE. - -52 years (water years 1913-25, 1927-65), 55.9 fts/s, 40,500 acre-ft/yr, prior to extensive upstroan divorsions by Molycorp.

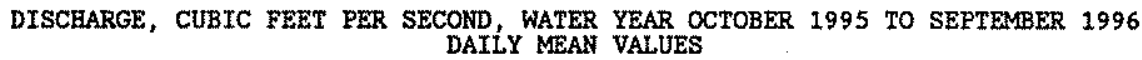

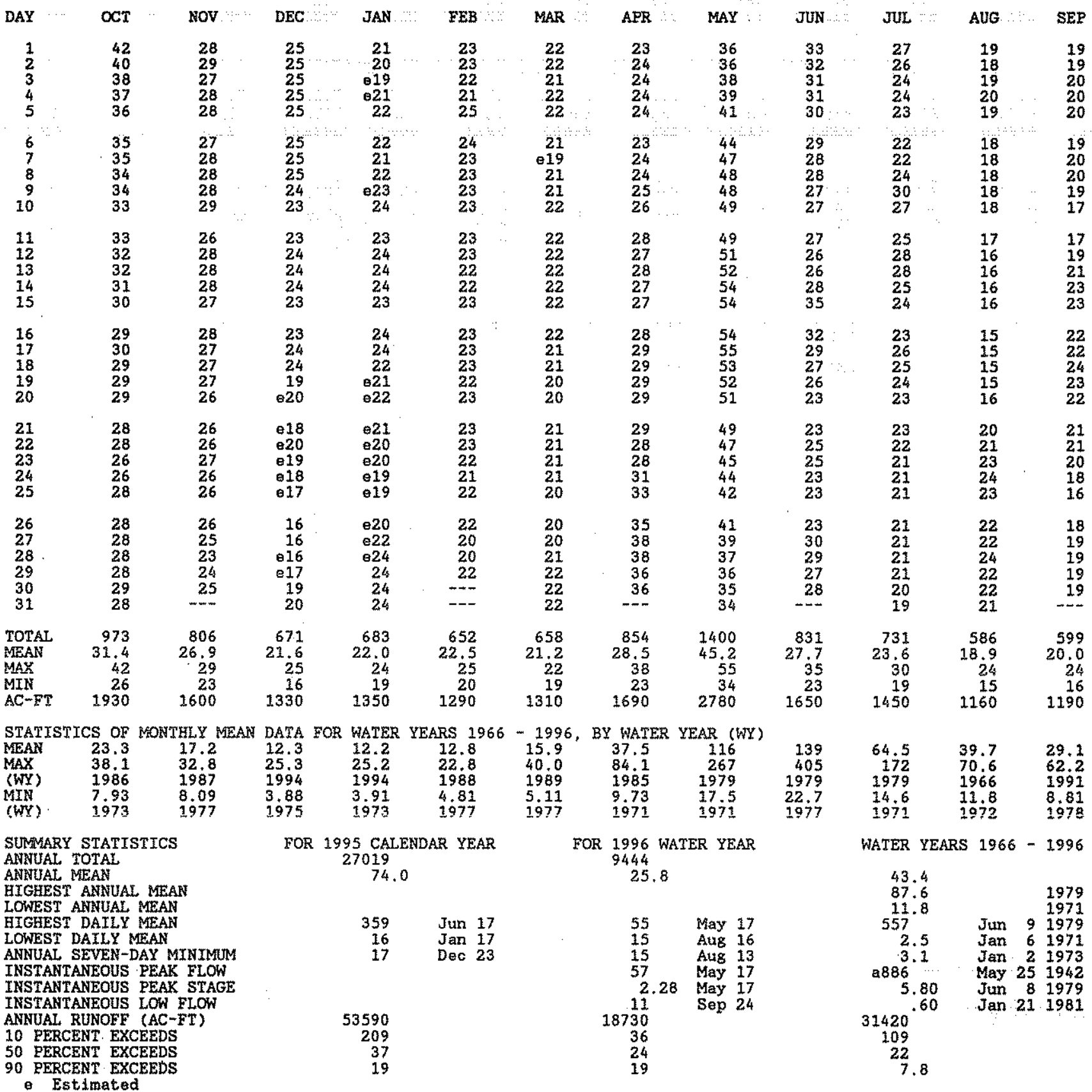


LOCATION.--Lat $36^{\circ} 43^{\circ} 50^{\prime \prime}$, long $105^{\circ} 33^{\prime} 12^{\prime \prime}$, in SEkSEK sec.21, T.29 N., R.13 E., Taos County, Hydrologic Unit 13020101 , In Carson National Forest, on right bank 900 ft downstream from Llano ditch heading, $2.6 \mathrm{mi}$ downstream from Lake Fork, $3 \mathrm{mi}$ northeast of Questa, and at mile 3.5 .

DRAINAGE AREA. $--36.7 \mathrm{mi}^{2}$.

PERTOD OF RECORD.--September 1943 to September 1996 (discontinued).

REVISED RECORDS.--WSP 1712: Drainage area.

GAGE.- Water-stage recorder and concrete control. Elevation of gage is 7,845 ft above National Geodetic Vertical Datum of 1929 , from river-profile map.

REMARKS.--Records fair excopt for estimated dally discharges, which are poor. Llano ditch (station 08265500), the only diversion upstream from station, diverts from right bank $900 \mathrm{ft}$ upstream from gage for irrigation of about 800 acres downstream. See tabulation below for monthly diversion of Llano ditch (records of daily discharge available in District files). Flow regulated by Cabresto Reservotr (capacity, 732 acre-feet, after

reconstruction in 1928) on Lake Fork $1 \mathrm{mi}$ upstream from mouth. Present capacity of Cabresto Reservoir is 1,100 acre-feet after further rehabilitation between 1959 and 1961 . Several observations of water temperature were made durtng year.

EXTREMES OUTSIDE PERIOD OF RECORD. --The flood of May 25, 1942, may have exceeded the maximum of record. DISCEARGE, CUBIC FEET PER SECOND, WATER YEAR OCTOBER 1995 TO SEPTEMBER 1996

\begin{tabular}{|c|c|c|c|c|c|c|c|c|c|c|c|c|}
\hline DAY & $\infty \mathrm{CT}$ & Nov & DEC & JAN & FEB & MAR & AER & MAY & $J W N$ & JUL & AUG & SEP \\
\hline $\begin{array}{l}1 \\
2 \\
3 \\
4 \\
5\end{array}$ & $\begin{array}{l}9.3 \\
9.2 \\
9.1 \\
8.9 \\
8.6\end{array}$ & $\begin{array}{l}8.3 \\
8.3 \\
8.1 \\
8.2 \\
8.2\end{array}$ & $\begin{array}{l}6.1 \\
6.1 \\
6.1 \\
6.1 \\
6.0\end{array}$ & $\begin{array}{l}06.7 \\
06.6 \\
06.6 \\
06.7 \\
06.7\end{array}$ & $\begin{array}{l}5.8 \\
5.6 \\
4.7 \\
5.3 \\
5.9\end{array}$ & $\begin{array}{l}6.2 \\
6.2 \\
6.1 \\
6.3 \\
6.1\end{array}$ & $\begin{array}{l}5.6 \\
7.7 \\
8.2 \\
8.4 \\
8.0\end{array}$ & $\begin{array}{l}12 \\
11 \\
11 \\
11 \\
11\end{array}$ & $\begin{array}{l}7.6 \\
7.4 \\
7.2 \\
7.1 \\
7.1\end{array}$ & $\begin{array}{l}7.8 \\
7.5 \\
7.0 \\
6.6 \\
6.4\end{array}$ & $\begin{array}{l}4.5 \\
4.3 \\
4.4 \\
4.7 \\
4.4\end{array}$ & $\begin{array}{l}1.7 \\
1.6 \\
1.8 \\
2.0 \\
2.0\end{array}$ \\
\hline $\begin{array}{r}6 \\
7 \\
8 \\
9 \\
10\end{array}$ & $\begin{array}{l}8.4 \\
8.9 \\
9.1 \\
8.9 \\
8.9\end{array}$ & $\begin{array}{l}8.3 \\
8.1 \\
7.9 \\
8.0 \\
8.0\end{array}$ & $\begin{array}{l}6.0 \\
6.0 \\
6.1 \\
6.0 \\
5.8\end{array}$ & $\begin{array}{r}e 6.8 \\
\mathrm{e} .8 .8 \\
\mathrm{e} 6.8 \\
6.8 \\
6.8\end{array}$ & $\begin{array}{l}5.8 \\
5.6 \\
5.6 \\
5.7 \\
5.8\end{array}$ & $\begin{array}{l}6.2 \\
5.6 \\
6.4 \\
6.4 \\
6.5\end{array}$ & $\begin{array}{l}7.5 \\
7.8 \\
7.7 \\
8.6 \\
9.9\end{array}$ & $\begin{array}{l}11 \\
12 \\
11 \\
11 \\
11\end{array}$ & $\begin{array}{l}7.0 \\
7.0 \\
7.5 \\
8.5 \\
8.5\end{array}$ & $\begin{array}{l}6.2 \\
6.1 \\
6.4 \\
7.2 \\
6.7\end{array}$ & $\begin{array}{l}4.2 \\
4.1 \\
4.3 \\
4.4 \\
4.2\end{array}$ & $\begin{array}{l}1.9 \\
1.8 \\
1.9 \\
1.8 \\
2.2\end{array}$ \\
\hline $\begin{array}{l}11 \\
12 \\
13 \\
14 \\
15\end{array}$ & $\begin{array}{l}8.8 \\
8.7 \\
8.7 \\
8.8 \\
8.6\end{array}$ & $\begin{array}{l}7.5 \\
7.6 \\
7.6 \\
7.5 \\
7.5\end{array}$ & $\begin{array}{l}5.8 \\
6.4 \\
6.6 \\
6.6 \\
6.6\end{array}$ & $\begin{array}{l}6.8 \\
6.8 \\
6.8 \\
6.8 \\
6.7\end{array}$ & $\begin{array}{l}5.8 \\
5.8 \\
5.8 \\
5.8 \\
6.0\end{array}$ & $\begin{array}{l}6.6 \\
6.9 \\
6.7 \\
6.7 \\
6.6\end{array}$ & $\begin{array}{l}11 \\
11 \\
11 \\
8.8 \\
9.2\end{array}$ & $\begin{array}{l}11 \\
11 \\
11 \\
11 \\
10\end{array}$ & $\begin{array}{l}8.3 \\
8.2 \\
8.0 \\
8.6 \\
9.6\end{array}$ & $\begin{array}{l}6.4 \\
7.0 \\
7.0 \\
6.3 \\
5.9\end{array}$ & $\begin{array}{l}3.9 \\
3.8 \\
3.6 \\
3.5 \\
3.5\end{array}$ & $\begin{array}{l}3.0 \\
3.2 \\
4.5 \\
5.3 \\
5.6\end{array}$ \\
\hline $\begin{array}{l}16 \\
17 \\
18 \\
19 \\
20\end{array}$ & $\begin{array}{l}8.6 \\
8.8 \\
8.5 \\
8.5 \\
8.5\end{array}$ & $\begin{array}{l}7.3 \\
7.3 \\
7.3 \\
7.2 \\
7.1\end{array}$ & $\begin{array}{l}6.6 \\
6.8 \\
6.8 \\
6.3 \\
6.4\end{array}$ & $\begin{array}{l}6.6 \\
6.9 \\
6.4 \\
6.5 \\
7.1\end{array}$ & $\begin{array}{l}6.1 \\
6.2 \\
6.2 \\
6.3 \\
6.4\end{array}$ & $\begin{array}{l}6.5 \\
6.5 \\
6.3 \\
5.9 \\
6.1\end{array}$ & $\begin{array}{l}9.6 \\
9.8 \\
9.5 \\
9.5 \\
9.6\end{array}$ & $\begin{array}{c}10 \\
9.9 \\
9.8 \\
9.6 \\
9.6\end{array}$ & $\begin{array}{l}8.6 \\
8.2 \\
7.8 \\
7.4 \\
7.1\end{array}$ & $\begin{array}{l}5.7 \\
5.7 \\
5.7 \\
5.5 \\
5.3\end{array}$ & $\begin{array}{l}3.4 \\
3.3 \\
3.2 \\
3.2 \\
3.2\end{array}$ & $\begin{array}{l}4.9 \\
4.7 \\
5.3 \\
5.3 \\
5.1\end{array}$ \\
\hline $\begin{array}{l}21 \\
22 \\
23 \\
24 \\
25\end{array}$ & $\begin{array}{l}8.4 \\
8.5 \\
8.3 \\
8.3 \\
8.5\end{array}$ & $\begin{array}{l}7.1 \\
7.1 \\
7.1 \\
7.0 \\
6.9\end{array}$ & $\begin{array}{l}6.5 \\
6.8 \\
6.8 \\
5.7 \\
5.3\end{array}$ & $\begin{array}{l}6.8 \\
7.2 \\
7.1 \\
7.0 \\
7.1\end{array}$ & $\begin{array}{l}6.5 \\
6.5 \\
6.4 \\
6.1 \\
6.6\end{array}$ & $\begin{array}{l}6.6 \\
6.9 \\
7.0 \\
7.1 \\
6.6\end{array}$ & $\begin{array}{r}9.2 \\
9.0 \\
9.8 \\
11 \\
2.6\end{array}$ & $\begin{array}{l}9.5 \\
9.3 \\
9.1 \\
9.1 \\
9.0\end{array}$ & $\begin{array}{l}7.4 \\
8.7 \\
8.5 \\
7.8 \\
7.5\end{array}$ & $\begin{array}{l}5.1 \\
5.1 \\
5.1 \\
5.0 \\
4.9\end{array}$ & $\begin{array}{l}3.7 \\
4.1 \\
4.1 \\
4.1 \\
4.1\end{array}$ & $\begin{array}{l}5.0 \\
4.9 \\
4.7 \\
4.6 \\
4.7\end{array}$ \\
\hline $\begin{array}{l}26 \\
27 \\
28 \\
29 \\
30 \\
31\end{array}$ & $\begin{array}{l}8.5 \\
8.5 \\
8.4 \\
8.4 \\
8.3 \\
8.3\end{array}$ & $\begin{array}{l}7.0 \\
6.9 \\
6.8 \\
6.2 \\
6.1 \\
-. .\end{array}$ & $\begin{array}{l}5.8 \\
6.7 \\
6.9 \\
6.9 \\
6.6 \\
6.6\end{array}$ & $\begin{array}{l}7.0 \\
7.0 \\
7.1 \\
7.1 \\
7.0 \\
6.1\end{array}$ & $\begin{array}{l}6.3 \\
5.7 \\
6.2 \\
6.5 \\
-.- \\
---\end{array}$ & $\begin{array}{l}6.6 \\
6.6 \\
6.8 \\
7.0 \\
5.5 \\
3.8\end{array}$ & $\begin{array}{c}3.6 \\
9.3 \\
13 \\
11 \\
11 \\
\end{array}$ & $\begin{array}{l}9.1 \\
8.9 \\
8.5 \\
8.4 \\
8.0 \\
7.8\end{array}$ & $\begin{array}{l}7.6 \\
9.1 \\
8.4 \\
7.9 \\
7.9 \\
-9\end{array}$ & $\begin{array}{l}4.9 \\
4.9 \\
4.9 \\
5.0 \\
4.9 \\
4.7\end{array}$ & $\begin{array}{l}3.9 \\
3.8 \\
4.0 \\
2.4 \\
2.0 \\
1.8\end{array}$ & $\begin{array}{l}4.8 \\
5.1 \\
5.0 \\
4.9 \\
4.8\end{array}$ \\
\hline $\begin{array}{l}\text { TOTAL } \\
\text { MEAN } \\
\text { MAX } \\
\text { MIN } \\
\text { AC-FT } \\
\dagger)\end{array}$ & $\begin{array}{r}268.2 \\
8.65 \\
9.3 \\
8.3 \\
532 \\
0\end{array}$ & $\begin{array}{r}223.5 \\
7.45 \\
8.3 \\
6.1 \\
443 \\
0\end{array}$ & $\begin{array}{r}195.8 \\
6.32 \\
6.9 \\
5.3 \\
388 \\
0\end{array}$ & $\begin{array}{r}211.2 \\
6.81 \\
7.2 \\
6.1 \\
419 \\
0\end{array}$ & $\begin{array}{r}173.0 \\
5.97 \\
6.6 \\
4.7 \\
343 \\
5.4\end{array}$ & $\begin{array}{r}197.3 \\
6.36 \\
7.1 \\
3.8 \\
391 \\
70\end{array}$ & $\begin{array}{r}267.9 \\
8.93 \\
13 \\
2.6 \\
531 \\
0\end{array}$ & $\begin{array}{r}311.6 \\
10.1 \\
12 \\
7.8 \\
618 \\
0\end{array}$ & $\begin{array}{r}237.5 \\
7.92 \\
9.6 \\
7.0 \\
471 \\
0\end{array}$ & $\begin{array}{r}182.9 \\
5.90 \\
7.8 \\
4.7 \\
363 \\
0\end{array}$ & $\begin{array}{r}116.1 \\
3.75 \\
4.7 \\
1.8 \\
230 \\
0\end{array}$ & $\begin{array}{r}114.1 \\
3.80 \\
5.6 \\
1.6 \\
226 \\
0\end{array}$ \\
\hline
\end{tabular}

STATISTICS OF MONTHLY MEAN DATA FOR WATER YEARS 1944 - 1996, BY WATER YEAR (WY)

\begin{tabular}{|c|c|c|c|c|c|c|c|c|c|c|c|c|}
\hline $\begin{array}{l}\text { MEAN } \\
\text { MAX } \\
(W Y) \\
M I N \\
\text { (WY) }\end{array}$ & $\begin{array}{l}6.64 \\
13.1 \\
1986 \\
2.68 \\
1957\end{array}$ & $\begin{array}{l}5.44 \\
10.1 \\
1994 \\
2.73 \\
1978\end{array}$ & $\begin{array}{l}4.67 \\
8.14 \\
1958 \\
2.43 \\
1964\end{array}$ & $\begin{array}{l}4.56 \\
8.11 \\
1991 \\
2.30 \\
1964\end{array}$ & $\begin{array}{l}4.64 \\
7.19 \\
1991 \\
2.32 \\
1964\end{array}$ & $\begin{array}{l}5.56 \\
12.8 \\
1989 \\
2.96 \\
1964\end{array}$ & $\begin{array}{l}13.4 \\
29.5 \\
1985 \\
6.70 \\
1973\end{array}$ & $\begin{array}{l}33.8 \\
99.5 \\
1994 \\
7.88 \\
1963\end{array}$ & $\begin{array}{l}23.6 \\
99.4 \\
1995 \\
5.77 \\
1946\end{array}$ & $\begin{array}{l}11.5 \\
27.4 \\
1979 \\
4.55 \\
1946\end{array}$ & $\begin{array}{l}9.67 \\
20.9 \\
1957 \\
3.75 \\
1996\end{array}$ & $\begin{array}{l}7.68 \\
13.8 \\
1957 \\
2.94 \\
1972\end{array}$ \\
\hline
\end{tabular}

SUMMARY STATISTICS

FOR 1995 CALENDAR YEAR

FOR 1996 WATER YEAR

$\begin{array}{rr}7707.1 & \\ 21.1 & \\ 126 & \text { Jun } 7 \\ 4.5 & \text { Jan } 18 \\ 5.7 & \text { Feb } 14 \\ & \\ & \\ 15290 & \\ 65 & \\ 9.3 & \\ 6.1 & \end{array}$

HIGHEST DAILY MEAN

$$
\begin{array}{r}
2499.1 \\
6.83
\end{array}
$$

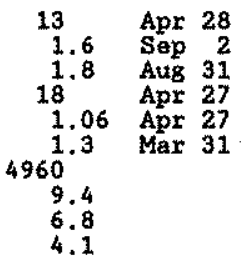

ANNUAL. TOTAL.

AMTUAEST MEANUAL, MEAN

LOWEST ANNUAL MEAN

LOWEST DAILY MEAN

ANNUAL SEVEN-DAY MINIMUM

INSTANTANEOUS PEAK FLOW

INSTANTANEOUS PEAK STAGE

INSTANTANEOUS LOW FLOW

ANNUAL RUNOFF (AC-FT)

10 PERCENT EXCEEDS

50 PERCENT EXCEEDS

90 PERCENT EXCE

CAL YR 1995 AC-FT (t) 3420

$(\uparrow)$ DIVERSIONS, IN ACRE-FEET, BY LIANO DITCH
WATER YEARS 1944 - 1996

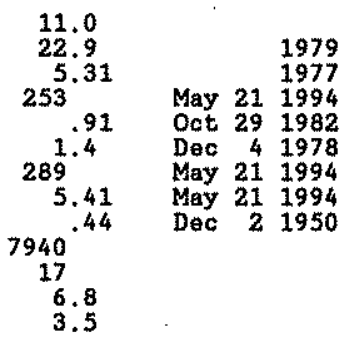


RIO GRANDE BASIN

08266820 RED RIVER BELOW FISH HATCHERY, NEAR QUESTA, NM

LOCATrON --Lat $36^{\circ} 40^{\prime} 54^{\prime \prime}$, long $105^{\circ} 39^{\prime} 21^{\prime \prime}$, in NWhNW sec.10, T.28 N, R.12 E., Taos County, Bydrologic Unit 13020101 , on right bank $0.3 \mathrm{mi}$ downstrean from State Fish Hatchery, 3.5 mi upstream from mouth, and 3.7 mi southwest of Questa.

DRAINAGE AREA, $-185 \mathrm{mi}^{2}$.

PERIOD OF RECORD.--August 1969 to July 1978 (discharge measurements only), August 1978 to current year.

GAGE, - Water-8tage recorder. Elevation of gage is 7,070 ft above National Geodetic Vertical. Datum of 1929, Irom topographic map. Prior to Aug 16,1979 , ot dto about 250 ft upstram at datum 5.55 ft higher.

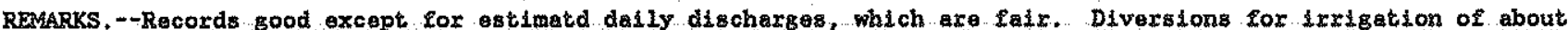

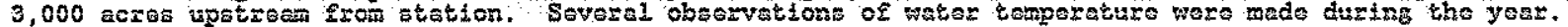

DISCEARGE, CUBIC FEET PER SECOND, WATER YEAR OCTOBER 1995 TO SEPTEMBER 1996

DAILY MEAN VALUES

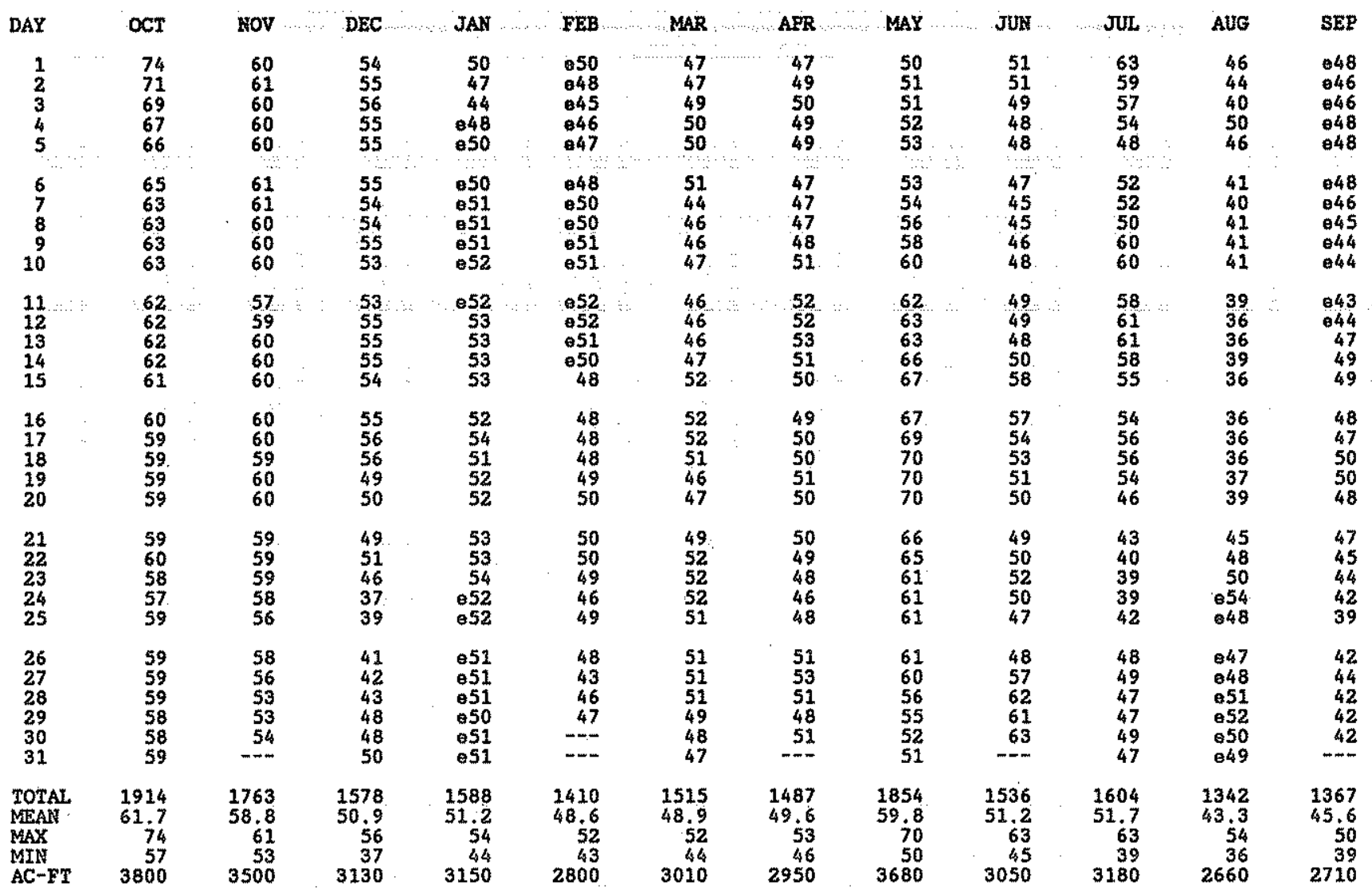

STATISTICS OF MONTHLY MEAN DATA FOR WATER YEARS 1978 - 1996, BY WATER YEAR (WY)

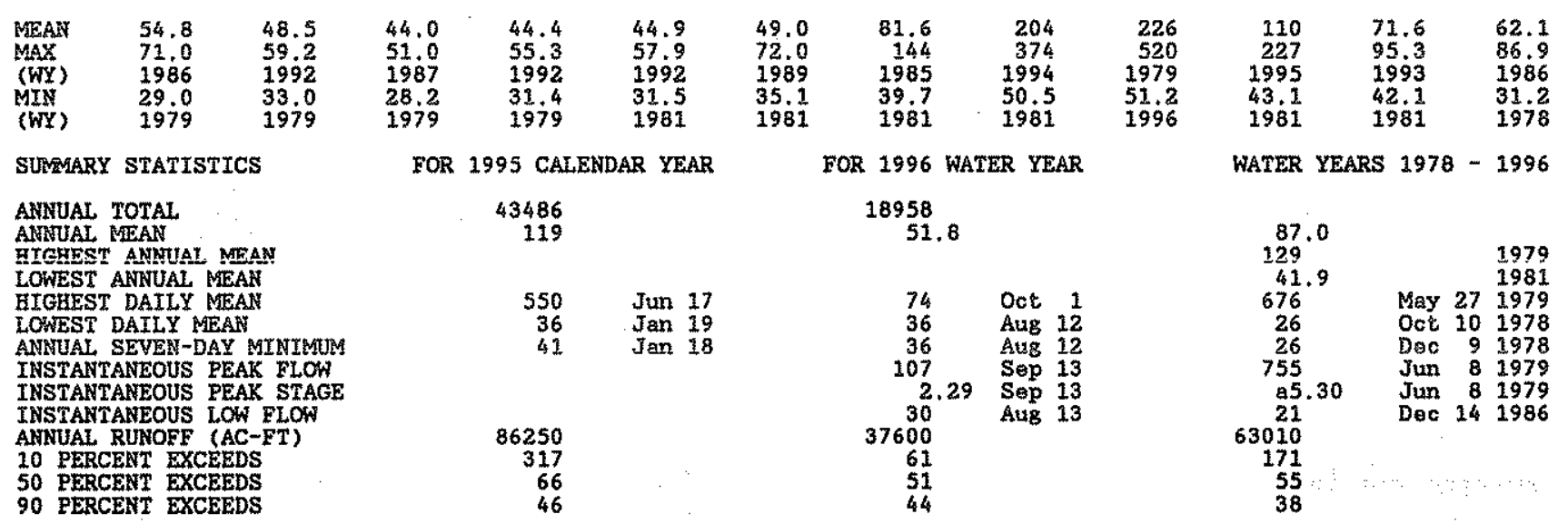

- Estimated

a-Site and datum then in use. 


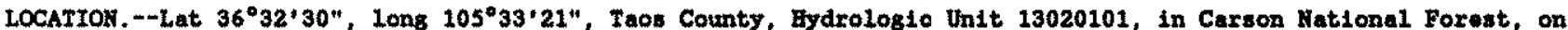
right bank 500 ft upstroam from firwt diversion, 1.6 mi enst of Valder, 3.8 mi downtrean from South Fork, and at $m i 109.2$.

DRAINAGE AREA. $--36.2 \mathrm{~mL}^{2}$.

\section{WATER-DISCHARGE RECORDS}

PERIOD OR RECORD.--August 1934 to current year.

REVISED RECORDS.-WSP 1342: 1935. WSP 1712: Dratnage area. WSP 1732: 1942(M).

GAGE.--Water-stage recordex. Concrete control stnce Oct. 28, 1938. Elevation of gage is 7,650 ft above National Geodetic Vertical Datum of 1929, from topographic map. Frfor to Oct. 28, 1938, at datum $1.92 \mathrm{ft} 10 \mathrm{wer}$.

REMARKS. -Water-discharge records good except for estimated dafly discharges, which are poor. DISCEARGE, CUBIC FEET PER SECOND, WATER YEAR OCTOBER 1995 TO SEPTEMEKR 1996 DAILY EIRMI VALUES

\begin{tabular}{|c|c|c|c|c|c|c|c|c|c|c|c|c|}
\hline DAY & $O C T$ & Nov & DEC & JAN & FEB & $M A R$ & $\mathbf{A R R}$ & MAX & JUN & Jut. & AUSG & SEP \\
\hline $\begin{array}{l}1 \\
2 \\
3 \\
4 \\
5\end{array}$ & $\begin{array}{r}32 \\
31 \\
30 \\
024 \\
025\end{array}$ & $\begin{array}{l}20 \\
20 \\
18 \\
18 \\
18\end{array}$ & $\begin{array}{l}14 \\
14 \\
14 \\
14 \\
14\end{array}$ & $\begin{array}{l}12 \\
012 \\
013 \\
012 \\
112 \\
012\end{array}$ & $\begin{array}{l}.11 \\
.12 \\
.12 \\
012 \\
015\end{array}$ & $\begin{array}{l}13 \\
13 \\
13 \\
11 \\
11\end{array}$ & $\begin{array}{l}14 \\
15 \\
15 \\
14 \\
14\end{array}$ & $\begin{array}{l}27 \\
28 \\
31 \\
34 \\
36\end{array}$ & $\begin{array}{l}28 \\
26 \\
25 \\
24 \\
24\end{array}$ & $\begin{array}{l}19 \\
18 \\
18 \\
18 \\
18\end{array}$ & $\begin{array}{l}14 \\
14 \\
14 \\
14 \\
13\end{array}$ & $\begin{array}{l}15 \\
15 \\
15 \\
15 \\
15\end{array}$ \\
\hline $\begin{array}{r}6 \\
7 \\
8 \\
9 \\
10\end{array}$ & $\begin{array}{l}\text { e24 } \\
\text { e23 } \\
\text { e25 } \\
\text { e23 } \\
\text { e24 }\end{array}$ & $\begin{array}{r}18 \\
19 \\
18 \\
.18 \\
18\end{array}$ & $\begin{array}{l}14 \\
14 \\
13 \\
12 \\
12\end{array}$ & $\begin{array}{r}12 \\
012 \\
12 \\
12 \\
011\end{array}$ & $\begin{array}{l}013 \\
012 \\
011 \\
013 \\
012\end{array}$ & $\begin{array}{l}11 \\
15 \\
12 \\
11 \\
12\end{array}$ & $\begin{array}{l}13 \\
14 \\
15 \\
17 \\
18\end{array}$ & $\begin{array}{l}39 \\
42 \\
43 \\
44 \\
44\end{array}$ & $\begin{array}{l}23 \\
22 \\
21 \\
21 \\
20\end{array}$ & $\begin{array}{l}17 \\
18 \\
18 \\
19 \\
18\end{array}$ & $\begin{array}{l}13 \\
13 \\
14 \\
13 \\
13\end{array}$ & $\begin{array}{l}15 \\
15 \\
15 \\
15 \\
15\end{array}$ \\
\hline $\begin{array}{l}11 \\
12 \\
13 \\
14 \\
15\end{array}$ & $\begin{array}{l}\text { e25 } \\
\text { e24 } \\
\text { e23 } \\
\text { e24 } \\
\text { e25 }\end{array}$ & $\begin{array}{l}16 \\
17 \\
17 \\
17 \\
17\end{array}$ & $\begin{array}{l}13 \\
13 \\
14 \\
14 \\
13\end{array}$ & $\begin{array}{l}11 \\
11 \\
11 \\
11 . \\
11\end{array}$ & $\begin{array}{r}011 \\
12 \\
12 \\
12 \\
12\end{array}$ & $\begin{array}{l}13 \\
13 \\
12 \\
12 \\
12\end{array}$ & $\begin{array}{l}19 \\
18 \\
18 \\
17 \\
16\end{array}$ & $\begin{array}{l}44 \\
45 \\
47 \\
49 \\
49\end{array}$ & $\begin{array}{l}20 \\
20 \\
19 \\
20 \\
25\end{array}$ & $\begin{array}{l}17 \\
18 \\
18 \\
17 \\
16\end{array}$ & $\begin{array}{l}13 \\
13 \\
13 \\
13 \\
12\end{array}$ & $\begin{array}{l}15 \\
16 \\
18 \\
20 \\
22\end{array}$ \\
\hline $\begin{array}{l}16 \\
17 \\
18 \\
19 \\
20\end{array}$ & $\begin{array}{r}024 \\
025 \\
25 \\
24 \\
24\end{array}$ & $\begin{array}{l}17 \\
17 \\
16 \\
16 \\
16\end{array}$ & $\begin{array}{l}13 \\
13 \\
13 \\
11 \\
12\end{array}$ & $\begin{array}{r}11 \\
11 \\
012 \\
012 \\
13\end{array}$ & $\begin{array}{l}12 \\
12 \\
12 \\
12 \\
12\end{array}$ & $\begin{array}{l}12 \\
12 \\
11 \\
12 \\
11\end{array}$ & $\begin{array}{l}17 \\
17 \\
18 \\
17 \\
17\end{array}$ & $\begin{array}{l}49 \\
50 \\
49 \\
49 \\
48\end{array}$ & $\begin{array}{l}21 \\
20 \\
19 \\
18 \\
17\end{array}$ & $\begin{array}{l}17 \\
23 \\
18 \\
17 \\
15\end{array}$ & $\begin{array}{l}12 \\
12 \\
12 \\
12 \\
12\end{array}$ & $\begin{array}{l}19 \\
18 \\
19 \\
19 \\
18\end{array}$ \\
\hline $\begin{array}{l}21 \\
22 \\
23 \\
24 \\
25\end{array}$ & $\begin{array}{l}23 \\
23 \\
20 \\
21 \\
22\end{array}$ & $\begin{array}{l}16 \\
16 \\
16 \\
16 \\
15\end{array}$ & $\begin{array}{r}14 \\
12 \\
14 \\
014 \\
015\end{array}$ & $\begin{array}{r}013 \\
12 \\
12 \\
012 \\
013\end{array}$ & $\begin{array}{l}12 \\
12 \\
12 \\
11 \\
11\end{array}$ & $\begin{array}{l}12 \\
13 \\
13 \\
12 \\
12\end{array}$ & $\begin{array}{l}17 \\
17 \\
17 \\
19 \\
21\end{array}$ & $\begin{array}{l}48 \\
45 \\
43 \\
41 \\
40\end{array}$ & $\begin{array}{l}17 \\
19 \\
18 \\
17 \\
17\end{array}$ & $\begin{array}{l}15 \\
15 \\
14 \\
15 \\
15\end{array}$ & $\begin{array}{l}16 \\
19 \\
18 \\
17 \\
16\end{array}$ & $\begin{array}{l}17 \\
16 \\
16 \\
16 \\
16\end{array}$ \\
\hline $\begin{array}{l}26 \\
27 \\
28 \\
29 \\
30 \\
31\end{array}$ & $\begin{array}{l}21 \\
21 \\
21 \\
21 \\
20 \\
20\end{array}$ & $\begin{array}{r}15 \\
14 \\
13 \\
13 \\
14 \\
-\end{array}$ & $\begin{array}{r}014 \\
013 \\
013 \\
12 \\
12 \\
12\end{array}$ & $\begin{array}{l}012 \\
011 \\
013 \\
e 12 \\
e 11 \\
e 13\end{array}$ & $\begin{array}{r}11 \\
16 \\
14 \\
12 \\
-- \\
-\end{array}$ & $\begin{array}{l}12 \\
12 \\
12 \\
12 \\
12 \\
13\end{array}$ & $\begin{array}{r}24 \\
27 \\
29 \\
27 \\
27 \\
-\end{array}$ & $\begin{array}{l}39 \\
37 \\
35 \\
32 \\
31 \\
29\end{array}$ & $\begin{array}{r}18 \\
28 \\
22 \\
20 \\
20 \\
--\end{array}$ & $\begin{array}{l}15 \\
15 \\
15 \\
15 \\
15 \\
14\end{array}$ & $\begin{array}{l}15 \\
16 \\
17 \\
15 \\
16 \\
15\end{array}$ & $\begin{array}{r}19 \\
18 \\
17 \\
16 \\
16 \\
\end{array}$ \\
\hline $\begin{array}{l}\text { TOTAL } \\
\text { MEAN } \\
\text { MAX } \\
\text { MIN } \\
\text { AC-FT }\end{array}$ & $\begin{array}{r}737 \\
23.8 \\
32 \\
20 \\
1460\end{array}$ & $\begin{array}{r}499 \\
16.6 \\
20 \\
13 \\
990\end{array}$ & $\begin{array}{r}409 \\
13.2 \\
15 \\
11 \\
811\end{array}$ & $\begin{array}{r}368 \\
11.9 \\
13 \\
11 \\
730\end{array}$ & $\begin{array}{r}353 \\
12,2 \\
16 \\
11 \\
700\end{array}$ & $\begin{array}{r}377 \\
12.2 \\
15 \\
11 \\
748\end{array}$ & $\begin{array}{r}548 \\
18.3 \\
29 \\
13 \\
1090\end{array}$ & $\begin{array}{r}1267 \\
40.9 \\
50 \\
27 \\
2510\end{array}$ & $\begin{array}{r}629 \\
21.0 \\
28 \\
17 \\
1250\end{array}$ & $\begin{array}{r}520 \\
16.8 \\
23 \\
14 \\
1030\end{array}$ & $\begin{array}{r}439 \\
14.2 \\
19 \\
12 \\
871\end{array}$ & $\begin{array}{r}501 \\
16.7 \\
22 \\
15 \\
994\end{array}$ \\
\hline
\end{tabular}

STATISTICS OF MONTHLY MEAN DATA FOR WATER YEARS 1935 - 1996, BY WATER YEAR (WY)

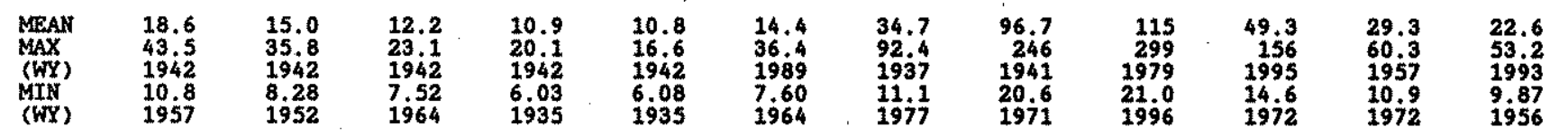

SUMMARY STATISTICS

ANNUAL TOTAL

ANNUAL MEAN

BIGAEST ANNUAL MEAN

LOWEST ANNUAL MEAN

BIGHEST DAILY MAAN

LOWEST DAILY MEAN

ANNUAL SEVEN-DAY MINIMTM

INSTANTANEOUS PEAK FLON

INSTANTANEOUS PEAK STAGE

INSTANTANEOUS LOW FLOW

ANNUAL RUNOFF (AC-FT)

10 PERCENT EXCEEDS

50 PERCENT EXCEEDS

90 PERCENT EXCEEDS

FOR 1995 CALENDAR YEAR

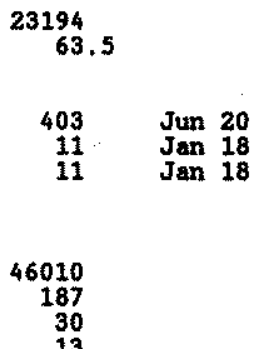

FOR 1996 WATER YEAR

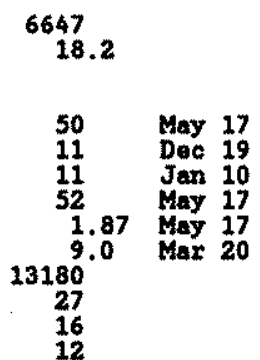

MATER YEARS 1935 - 1996

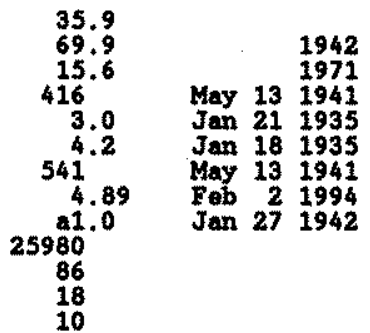

- Estimated

a-Result of freeze-up. 
RIO GRANDE BASIN

08267500 RIO HONDO NEAR VALDEZ, NM -- Continued

WATER-QUALITY RECORDS

PERIOD OF RECORD to current year.--Water years 1963, 1986 to current year.

WATER QUALITY DATA, WATER YEAR OCTOBER 1995 TO SEPTEMBER 1996

\begin{tabular}{|c|c|c|c|c|c|c|c|c|c|}
\hline DATE & TIME & $\begin{array}{c}\text { DIS- } \\
\text { CHARGE, } \\
\text { INST } \\
\text { CUBIC } \\
\text { FEET } \\
\text { PER } \\
\text { SECOND } \\
(00061)\end{array}$ & $\begin{array}{l}\text { SPE- } \\
\text { CIFIC } \\
\text { CON- } \\
\text { DUCT- } \\
\text { ANCE } \\
\text { (US/CM) } \\
(00095)\end{array}$ & $\begin{array}{l}\text { FYG } \\
\text { WATER } \\
\text { WHOLE } \\
\text { FIELD } \\
\text { (STAND- } \\
\text { ARD } \\
\text { UNITS) } \\
(00400)\end{array}$ & $\begin{array}{l}\text { TEMPER - } \\
\text { ATURE } \\
\text { AIR } \\
\text { (DEG C) } \\
(00020)\end{array}$ & $\begin{array}{l}\text { TEMPER- } \\
\text { ATURE } \\
\text { WATER } \\
\text { (DEG C) } \\
(00010)\end{array}$ & $\begin{array}{c}\text { BARO- } \\
\text { METRIC } \\
\text { PRES - } \\
\text { SURE } \\
\text { (MM } \\
\text { OF } \\
\text { HG) } \\
(00025)\end{array}$ & $\begin{array}{c}\text { OXYGEN, } \\
\text { DIS- } \\
\text { SOLVED } \\
\text { (MG/L) } \\
(00300)\end{array}$ & $\begin{array}{c}\text { OXYGER, } \\
\text { DIS- } \\
\text { SOLVED } \\
\text { (PER- } \\
\text { CENT } \\
\text { SATUR- } \\
\text { ATION) } \\
\text { (O0301) }\end{array}$ \\
\hline $\begin{array}{l}1995 \\
j 996\end{array}$ & 1520 & 14 & 150 & 7.7 & 8.0 & 2.5 & 576 & 9.3 & 90 \\
\hline $\begin{array}{l}1996 \\
\ldots\end{array}$ & 1210 & 12 & 183 & 8,6 & 6.0 & 3.0 & 567 & $\quad 10.8$ & $\ldots$ \\
\hline$\cdots$ & 1625 & 19 & 140 & 8.5 & 19.5 & 11.5 & 580 & 8.5 & 103 \\
\hline$\ldots$ & 0630 & 12 & 198 & 8.4 & 11.0 & 9.5 & 583 & 8.4 & 96 \\
\hline & & & & & & $\begin{array}{l}\cdots \\
\therefore \therefore\end{array}$ & & & \\
\hline DATE & $\begin{array}{c}\text { NITRO- } \\
\text { GEN } \\
\text { NITRATE } \\
\text { DIS- } \\
\text { SOLVED } \\
\text { (RG/L } \\
\text { AS N) } \\
\text { (00618) }\end{array}$ & $\begin{array}{c}\text { NITRO- } \\
\text { GEN } \\
\text { NITRITE } \\
\text { DIS- } \\
\text { SOLVED } \\
\text { (MGG/L } \\
\text { AS N) } \\
\text { (00613) }\end{array}$ & $\begin{array}{c}\text { NIMRO- } \\
\text { GEN } \\
\text { NO2+NO3 } \\
\text { DIS- } \\
\text { SOLVED } \\
\text { (MG/L } \\
\text { AS N) } \\
(00631)\end{array}$ & $\begin{array}{c}\text { NITRO- } \\
\text { GEN, } \\
\text { ARMONIA } \\
\text { DIS- } \\
\text { SOLVED } \\
\text { CNG/L } \\
\text { AS N } \\
(00608)\end{array}$ & $\begin{array}{l}\text { NYTRO- } \\
\text { GEN,AM- } \\
\text { MONIA + } \\
\text { ORGANIC } \\
\text { TOTAL } \\
\text { (MG L } \\
\text { AS N) } \\
(00625)\end{array}$ & $\begin{array}{l}\text { NITRO- } \\
\text { GEN,AM- } \\
\text { MONIA + } \\
\text { ORGANIC } \\
\text { DIS } \\
\text { (MG/L } \\
\text { AS N) } \\
(00623)\end{array}$ & $\begin{array}{l}\text { PHOS-- } \\
\text { PHORUS } \\
\text { TOTAL } \\
\text { (MG/L } \\
\text { AS P) } \\
(00665)\end{array}$ & $\begin{array}{c}\text { FHOS- } \\
\text { PHORUS } \\
\text { DIS- } \\
\text { SOLVED } \\
\text { (MG/L } \\
\text { AS P) } \\
(00666)\end{array}$ & $\begin{array}{l}\text { PHOS- } \\
\text { PHORUS } \\
\text { ORTHO, } \\
\text { DIS- } \\
\text { SOLVED } \\
\text { SOMG/L } \\
\text { AS P) } \\
(00671)\end{array}$ \\
\hline $\begin{array}{l}1995 \\
1996\end{array}$ & - & $-\cdots$ & - & -- & - & $-\cdots$ & - & -- & -- \\
\hline . & - & $\cdots$ & -- & -- & -- & $-\infty$ & - & "- & $\cdots$ \\
\hline & -- & -- & - & -- & -- & -- & -- & -- & -- \\
\hline & 0.210 & 0.010 & 0.220 & 0.030 & $<0.20$ & $<0.20$ & $<0.010$ & $<0.010$ & 0.010 \\
\hline
\end{tabular}


RTO GRANDE BASIN

08268700 RIO GRANDE NEAR ARROYO HONDO, NM

LOCATION. --Lat $36^{\circ} 32^{\prime} 04^{\prime \prime}$, long $105^{\circ} 42^{\prime} 34^{\prime \prime}$, in NWW sec.31, T.27 N., R. 12 E., Taos County, Bydrologic Unit, 13020101, on right bank $350 \mathrm{ft}$ downstream from Arroyo Hondo, $400 \mathrm{ft}$ downstream from bridge on county road, $2.2 \mathrm{mi}$ west of Arroyo Hondo, $11.6 \mathrm{mi}$ northwest of $\mathrm{Taos}$, and at mile $1,677.4$.

DRAINAGE AREA. $--8,760 \mathrm{mi}^{2}$, approximately, tncluding $2,940 \mathrm{mi}^{2}$ in closed basin in San Luis Va11ey, Co.

PERTOD OF RECORD. --February 1963 to September 1996 (discontinued).

GAGE.--Water-stage recorder. Elevation of gage is 6,470 ft above National Geodetic Vertical Datum of 1929 , from topographic map.

REMARKS --Records good. Diverstons upstream from station for irrigation of about 620,000 acres in Colorado and 15,000 acres in New Mexico. Several observations of water temperature were made during the year.

DISCHARGE, CUBIC FEET PER SECOND, WATER YEAR OCTOBER 1995 TO SEPTEMBER 1996

\begin{tabular}{|c|c|c|c|c|c|c|c|c|c|c|c|c|}
\hline DAY & OCT & nov & DEC & JAN & FEB & MAR & $A P R$ & MAY & JUN & JUL & AUG & SEE \\
\hline $\begin{array}{l}1 \\
2 \\
3 \\
4 \\
5\end{array}$ & $\begin{array}{l}343 \\
348 \\
386 \\
455 \\
465\end{array}$ & $\begin{array}{l}381 \\
396 \\
402 \\
407 \\
399\end{array}$ & $\begin{array}{l}402 \\
394 \\
398 \\
419 \\
440\end{array}$ & $\begin{array}{l}516 \\
533 \\
504 \\
531 \\
530\end{array}$ & $\begin{array}{l}607 \\
599 \\
576 \\
547 \\
581\end{array}$ & $\begin{array}{l}613 \\
700 \\
727 \\
722 \\
733\end{array}$ & $\begin{array}{l}434 \\
415 \\
389 \\
399 \\
398\end{array}$ & $\begin{array}{l}493 \\
410 \\
367 \\
348 \\
326\end{array}$ & $\begin{array}{l}265 \\
253 \\
246 \\
240 \\
236\end{array}$ & $\begin{array}{l}240 \\
237 \\
233 \\
224 \\
213\end{array}$ & $\begin{array}{l}182 \\
179 \\
172 \\
194 \\
180\end{array}$ & $\begin{array}{l}200 \\
199 \\
198 \\
197 \\
197\end{array}$ \\
\hline $\begin{array}{r}6 \\
7 \\
8 \\
9 \\
10\end{array}$ & $\begin{array}{l}417 \\
360 \\
335 \\
327 \\
316\end{array}$ & $\begin{array}{l}403 \\
410 \\
409 \\
417 \\
418\end{array}$ & $\begin{array}{l}464 \\
463 \\
463 \\
479 \\
478\end{array}$ & $\begin{array}{l}536 \\
538 \\
542 \\
555 \\
565\end{array}$ & $\begin{array}{l}606 \\
624 \\
628 \\
643 \\
664\end{array}$ & $\begin{array}{l}744 \\
734 \\
740 \\
737 \\
733\end{array}$ & $\begin{array}{l}386 \\
401 \\
380 \\
350 \\
350\end{array}$ & $\begin{array}{l}310 \\
318 \\
340 \\
356 \\
354\end{array}$ & $\begin{array}{l}239 \\
229 \\
223 \\
225 \\
233\end{array}$ & $\begin{array}{l}213 \\
209 \\
215 \\
216 \\
226\end{array}$ & $\begin{array}{l}169 \\
169 \\
192 \\
191 \\
187\end{array}$ & $\begin{array}{l}199 \\
202 \\
200 \\
197 \\
191\end{array}$ \\
\hline $\begin{array}{l}11 \\
12 \\
13 \\
14 \\
15\end{array}$ & $\begin{array}{l}309 \\
313 \\
315 \\
308 \\
300\end{array}$ & $\begin{array}{l}435 \\
424 \\
419 \\
414 \\
428\end{array}$ & $\begin{array}{l}451 \\
453 \\
457 \\
457 \\
489\end{array}$ & $\begin{array}{l}567 \\
569 \\
577 \\
581 \\
575\end{array}$ & $\begin{array}{l}687 \\
712 \\
709 \\
707 \\
752\end{array}$ & $\begin{array}{l}736 \\
743 \\
727 \\
711 \\
711\end{array}$ & $\begin{array}{l}364 \\
431 \\
454 \\
448 \\
453\end{array}$ & $\begin{array}{l}337 \\
330 \\
361 \\
337 \\
400\end{array}$ & $\begin{array}{l}238 \\
237 \\
231 \\
227 \\
235\end{array}$ & $\begin{array}{l}221 \\
225 \\
221 \\
210 \\
210\end{array}$ & $\begin{array}{l}182 \\
183 \\
179 \\
184 \\
181\end{array}$ & $\begin{array}{l}196 \\
197 \\
241 \\
215 \\
219\end{array}$ \\
\hline $\begin{array}{l}16 \\
17 \\
18 \\
19 \\
20\end{array}$ & $\begin{array}{l}298 \\
301 \\
320 \\
340 \\
357\end{array}$ & $\begin{array}{l}437 \\
449 \\
455 \\
452 \\
442\end{array}$ & $\begin{array}{l}506 \\
494 \\
444 \\
344 \\
372\end{array}$ & $\begin{array}{l}591 \\
624 \\
593 \\
556 \\
539\end{array}$ & $\begin{array}{l}760 \\
766 \\
789 \\
811 \\
800\end{array}$ & $\begin{array}{l}683 \\
642 \\
604 \\
567 \\
529\end{array}$ & $\begin{array}{l}427 \\
372 \\
333 \\
316 \\
314\end{array}$ & $\begin{array}{l}427 \\
400 \\
381 \\
376 \\
413\end{array}$ & $\begin{array}{l}244 \\
254 \\
252 \\
239 \\
245\end{array}$ & $\begin{array}{l}217 \\
220 \\
211 \\
208 \\
200\end{array}$ & $\begin{array}{l}181 \\
180 \\
175 \\
181 \\
182\end{array}$ & $\begin{array}{l}220 \\
212 \\
217 \\
214 \\
212\end{array}$ \\
\hline $\begin{array}{l}21 \\
22 \\
23 \\
24 \\
25\end{array}$ & $\begin{array}{l}358 \\
353 \\
350 \\
355 \\
351\end{array}$ & $\begin{array}{l}436 \\
433 \\
435 \\
433 \\
429\end{array}$ & $\begin{array}{l}387 \\
365 \\
386 \\
329 \\
382\end{array}$ & $\begin{array}{l}550 \\
583 \\
569 \\
523 \\
557\end{array}$ & $\begin{array}{l}826 \\
812 \\
838 \\
841 \\
815\end{array}$ & $\begin{array}{l}519 \\
514 \\
503 \\
508 \\
516\end{array}$ & $\begin{array}{l}297 \\
296 \\
299 \\
291 \\
268\end{array}$ & $\begin{array}{l}402 \\
378 \\
404 \\
386 \\
351\end{array}$ & $\begin{array}{l}240 \\
241 \\
236 \\
233 \\
229\end{array}$ & $\begin{array}{l}191 \\
191 \\
189 \\
183 \\
201\end{array}$ & $\begin{array}{l}197 \\
223 \\
221 \\
220 \\
228\end{array}$ & $\begin{array}{l}206 \\
201 \\
201 \\
200 \\
198\end{array}$ \\
\hline $\begin{array}{l}26 \\
27 \\
28 \\
29 \\
30 \\
31\end{array}$ & $\begin{array}{l}351 \\
357 \\
360 \\
353 \\
355 \\
370\end{array}$ & $\begin{array}{l}429 \\
426 \\
417 \\
397 \\
405 \\
\end{array}$ & $\begin{array}{l}407 \\
461 \\
464 \\
478 \\
491 \\
519\end{array}$ & $\begin{array}{l}528 \\
511 \\
533 \\
561 \\
570 \\
570\end{array}$ & $\begin{array}{l}769 \\
744 \\
726 \\
669 \\
--.\end{array}$ & $\begin{array}{l}513 \\
493 \\
477 \\
460 \\
439 \\
429\end{array}$ & $\begin{array}{l}249 \\
246 \\
313 \\
456 \\
558 \\
\end{array}$ & $\begin{array}{l}313 \\
309 \\
303 \\
297 \\
294 \\
281\end{array}$ & $\begin{array}{l}228 \\
238 \\
242 \\
235 \\
238\end{array}$ & $\begin{array}{l}193 \\
188 \\
188 \\
191 \\
192 \\
186\end{array}$ & $\begin{array}{l}216 \\
218 \\
208 \\
200 \\
201 \\
200\end{array}$ & $\begin{array}{l}200 \\
201 \\
203 \\
204 \\
205 \\
-\cdots\end{array}$ \\
\hline $\begin{array}{l}\text { TOTAL } \\
\text { MEAN } \\
\text { MAX } \\
\text { MIN } \\
\text { AC-FT }\end{array}$ & $\begin{array}{r}10826 \\
349 \\
465 \\
298 \\
21470\end{array}$ & $\begin{array}{r}12637 \\
421 \\
455 \\
381 \\
25070\end{array}$ & $\begin{array}{r}13536 \\
437 \\
519 \\
329 \\
26850\end{array}$ & $\begin{array}{r}17177 \\
554 \\
624 \\
504 \\
34070\end{array}$ & $\begin{array}{r}20608 \\
711 \\
841 \\
547 \\
40880\end{array}$ & $\begin{array}{r}19207 \\
620 \\
744 \\
429 \\
38100\end{array}$ & $\begin{array}{r}11087 \\
370 \\
558 \\
246 \\
21990\end{array}$ & $\begin{array}{r}11102 \\
358 \\
493 \\
281 \\
22020\end{array}$ & $\begin{array}{r}7151 \\
238 \\
265 \\
223 \\
14180\end{array}$ & $\begin{array}{r}6462 \\
208 \\
240 \\
183 \\
12820\end{array}$ & $\begin{array}{r}5955 \\
192 \\
228 \\
169 \\
11810\end{array}$ & $\begin{array}{r}6142 \\
205 \\
241 \\
191 \\
12180\end{array}$ \\
\hline
\end{tabular}

STATISTICS OF MONTHLY MEAN DATA FOR WATER YEARS 1963 - 1996, BY WATER YEAR (WY)

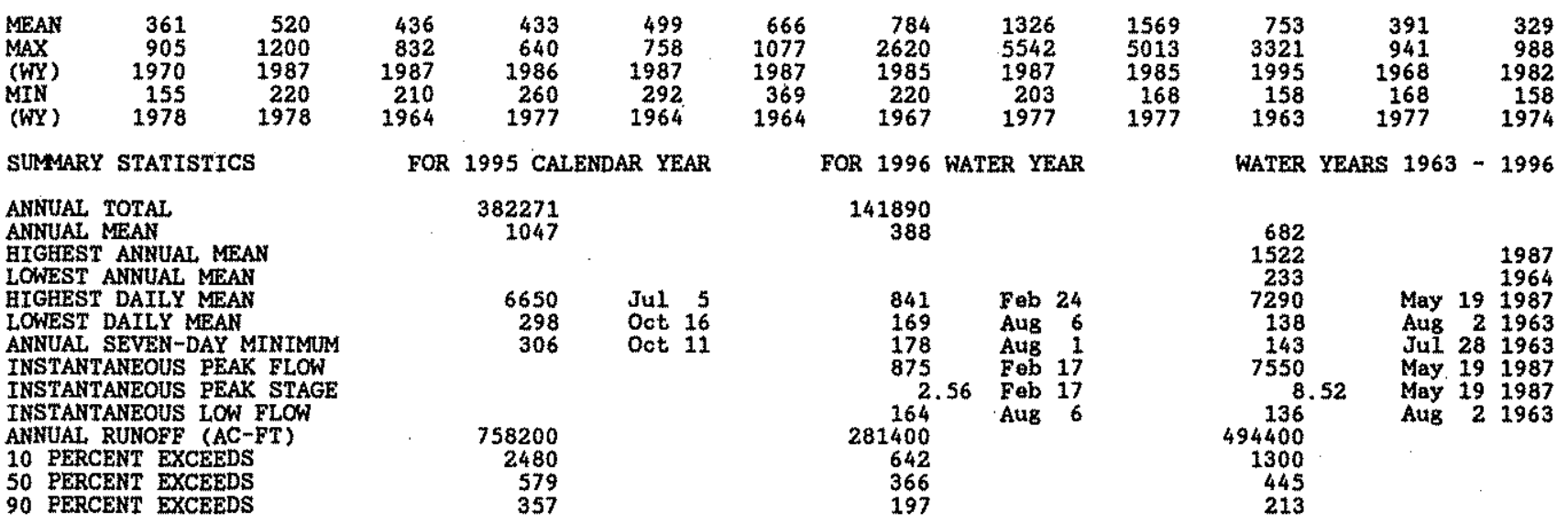


RIO GRANDE BASIN

08269000 RIO PUEBLO DE TAOS NEAR TAOS, NM

LOCATION, -lat $36^{\circ} 26^{\circ} 22^{\prime \prime}$, long $105^{\circ} 30^{\prime} 11^{\prime \prime}$, in SW/6Ek sec.36, T.26 N, R.13 E., Teos County, Hydrologic Unit 13020101, in Taos Pueblo Grant, on right bank $2.3 \mathrm{mi}$ east of Tros Pueblo, $4.5 \mathrm{mi}$ northeast of Taos, 5.8 mi upstream from Rio Lucero, and at mile 15.1 .

DRAINAGE AREA. $--66,6 \mathrm{mi}^{2}$.

PERTOD OF RECORD. -January 1911 to December 1916, January 1940 to December 1951, (annual maximum), water years 1952-62, October 1962 (monthly discharge only), November 1962 to current year. Monthly discharge only for some pertods, published in WSP 1312.

REVISED RECORDS.-WSP 1312: 1911-12, 1914. WSP 1732: Drainage area.

GAGE, -Water-stage recorder Concrete control since Wov. 20, 1962 . Elovation of gage is 7,380 ft above kational Geodettc Vertical Datum of 1929, from topographic map. See. WSP 1923 for history of changes prior to Nov. 20, 1962 .

REMARKS, --Records good except for estimated daily discharges; which are poor. No diversions upstrean from station. Several observetions of water temperature were made during the year. Satellite teleneter at station DISCHARGE, CUBIC FEET PER SECOND, WATER YEAR OCTOBER 1995 TO SEFTEMBER 1996

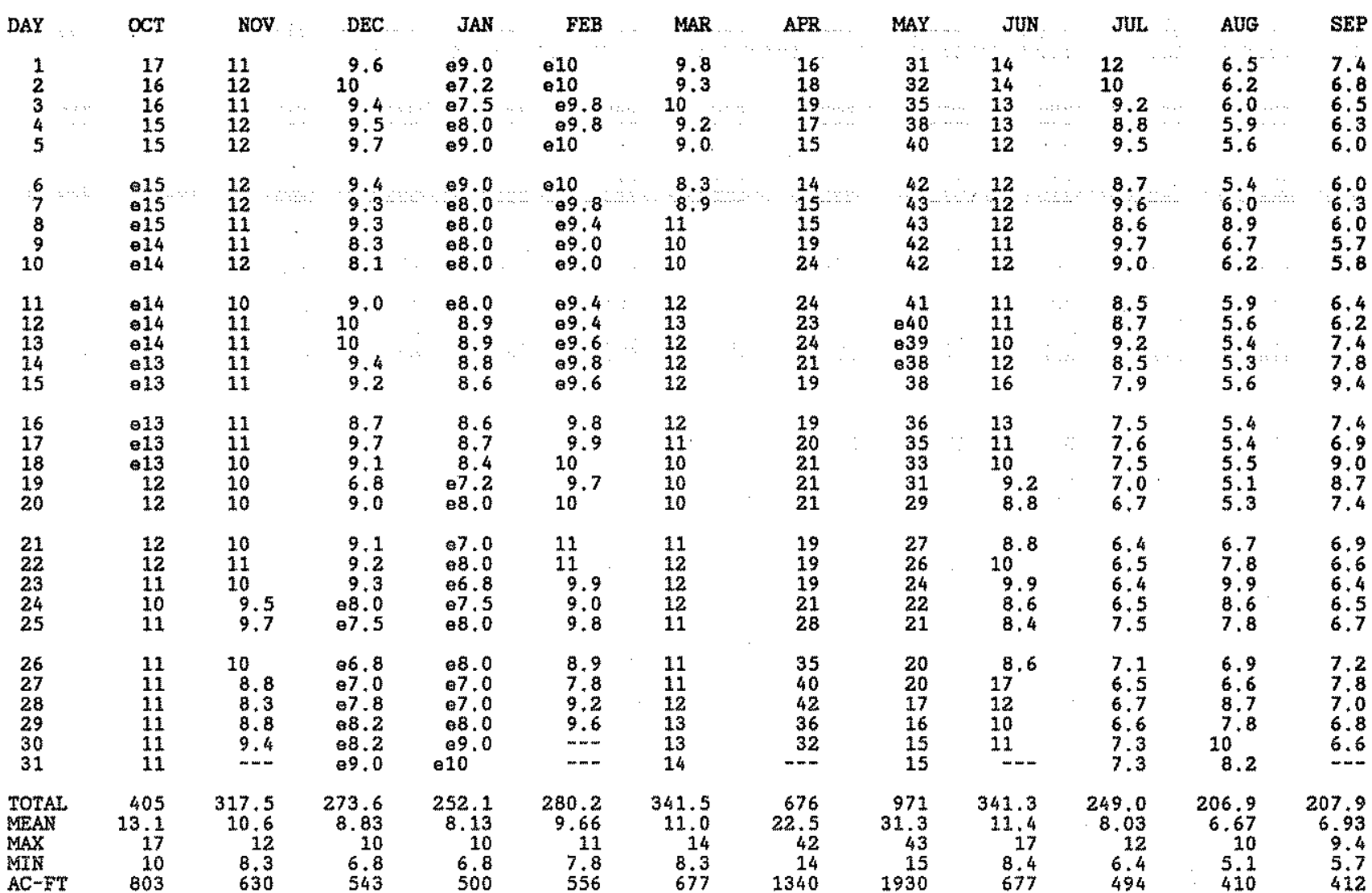

STATISTICS OF NONTHLY MEAN DATA FOR WATER YEARS 1913 - 1996, BY WATER YEAR (WY)

\begin{tabular}{|c|c|c|c|c|c|c|c|c|c|c|c|c|c|c|c|c|c|c|}
\hline $\begin{array}{l}\text { MEAN } \\
\text { MAX } \\
\text { (WY) } \\
\text { MIN } \\
\text { (WY) }\end{array}$ & $\begin{array}{l}9.94 \\
19.1 \\
1942 \\
4.84 \\
1965\end{array}$ & $\begin{array}{l}9.13 \\
17.5 \\
1942 \\
4.80 \\
1982\end{array}$ & $\begin{array}{l}7.98 \\
12.5 \\
1992 \\
4.05 \\
1964\end{array}$ & & $\begin{array}{r}99 \\
1.1 \\
984 \\
39 \\
964\end{array}$ & $\begin{array}{l}7.59 \\
13.3 \\
1995 \\
3.64 \\
1964\end{array}$ & $\begin{array}{l}13.6 \\
39.7 \\
1989 \\
5.58 \\
1964\end{array}$ & & $\begin{array}{r}51.3 \\
155 \\
1942 \\
13.1 \\
1971\end{array}$ & & $\begin{array}{r}121 \\
356 \\
1941 \\
11.3 \\
1972\end{array}$ & & $\begin{array}{r}76.1 \\
268 \\
1979 \\
8.64 \\
1972\end{array}$ & $\begin{array}{l}23.7 \\
75.4 \\
1995 \\
4.60 \\
1972\end{array}$ & & $\begin{array}{l}15.6 \\
32.2 \\
1991 \\
4.45 \\
1972\end{array}$ & & $\begin{array}{l}11.7 \\
32.4 \\
1982 \\
4.17 \\
1972\end{array}$ \\
\hline SUMAARY & STATIS & & FOR & 1995 & CALEND & DAR YEAR & & $\mathrm{FOR}$ & 1996 & WAT & $E R$ YE & EAR & & WATER & YEARS & 1913 & $3-$ & 1996 \\
\hline $\begin{array}{l}\text { ANNUAL } \\
\text { ANNUAL } \\
\text { HIGHEST } \\
\text { LOWEST } \\
\text { HIGHEST } \\
\text { LOWEST } \\
\text { ANNUAL } \\
\text { INSTANT } \\
\text { INSTANT } \\
\text { INSTANT } \\
\text { ANNUAL } \\
10 \text { PERC } \\
50 \text { PERC } \\
90 \text { EERC }\end{array}$ & $\begin{array}{l}\text { TOTAL } \\
\text { MEAN } \\
\text { ANNUAL } \\
\text { ANNUAL } \\
\text { DAILY } \\
\text { DAILY } \\
\text { SEVEN-D } \\
\text { ANEOUS } \\
\text { ANEOUS } \\
\text { ANEOUS } \\
\text { RUNOFF } \\
\text { ENT EXC } \\
\text { ENT EXC } \\
\text { ENT EXC }\end{array}$ & $\begin{array}{l}\text { AN } \\
\text { N } \\
\text { N } \\
\text { MINIMEM } \\
\text { K FLOW } \\
\text { K STAGE } \\
\text { ELOW } \\
\text {-ET) } \\
\text { S } \\
\text { S } \\
\text { S }\end{array}$ & & 20 & $\begin{array}{r}120 \\
201 \\
20 \\
8.2\end{array}$ & $\begin{array}{ll}\text { May } & 22 \\
\text { Dec } & 19 \\
\text { Jen } & 26\end{array}$ & & & $\begin{array}{r}43 \\
5 \\
5 \\
46 \\
1 \\
5 \\
8970 \\
21 \\
9 \\
6\end{array}$ & $\begin{array}{l}1 \\
.4 \\
.21 \\
.0 \\
.8 \\
.6\end{array}$ & $\begin{array}{l}\text { May } \\
\text { Aug } \\
\text { Aug } \\
\text { May } \\
\text { May } \\
\text { Dec }\end{array}$ & $\begin{array}{r}7 \\
19 \\
14 \\
6 \\
6 \\
19\end{array}$ & & $\begin{array}{r}30 \\
72 \\
7 \\
926 \\
2 \\
2 \\
21050 \\
\text { b3 } \\
22110 \\
72 \\
11 \\
5\end{array}$ & $\begin{array}{l}.5 \\
.3 \\
.74 \\
.0 \\
.8 \\
.90 \\
.69 \\
.8\end{array}$ & $\begin{array}{l}\text { May } \\
\text { Dec } \\
\text { Jan } \\
\text { May } \\
\text { May } \\
\text { Deb }\end{array}$ & $\begin{array}{r}26 \\
3 \\
29 \\
26 \\
14 \\
27\end{array}$ & $\begin{array}{l}1979 \\
1972 \\
1979 \\
1950 \\
1990 \\
1979 \\
1941 \\
1991\end{array}$ \\
\hline
\end{tabular}

- Estimated

a-From rating curve axtended above $370 \mathrm{ft} / \mathrm{s}$.

b-From floodmark, site and datum then in use. 
RTO GRANDE BASTN

08271000 RIO LUCERO NEAR ARROYO SECO, NM

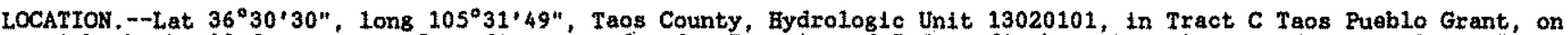
right bank $200 \mathrm{ft}$ upstream from diversion dem for Tenoxio and Indian ditches, $2.2 \mathrm{mi}$ east of Arroyo Seco, $7.4 \mathrm{mt}$ northeast of Taos, and at mile 8.1 .

DRATNAGE AREA. $-16.6 \mathrm{mi}^{2}$.

PERIOD OF RDCORD,--Aprt1 to December 1910 (discharge measurements and occasional gage hetghts), January 1911 to September 1915, March to December 1916 (fragmentary), October 1933 to December 1951, (annual maximum), water years 1952-62, October 1962 (monthly discharge only), November 1962 to current year. Monthly discharge only for some periods, published in WSP 1312. Fragmentary records for October 1915 to February 1916, published in WSP 438, are unrelifable and should not be used. Published as "near Taos," 1910-16.

REVISED RECORDS. -WSP 1512: 1912, 1916, 1949. WSP 1732: Dratnage area. WDR NM-75-1: 1973. See also PERIOD OF RECORD.

GAGE. - Water-stage recorder. Concrete control since Nov, 21, 1962. Datum of gage 1s $8,051.44$ ft above National Geodetic Vertical Datum of 1929. See WSP 1923 for history of changes prior to Nov. 21 , 1962.

REMARKS.--Records good except for estimated daily discharges, which are poor. No diversion upstream from station. DISCEARGE, CUBIC FEET FER SECOND, WATER YEAR OCTOBER 1995 TO SEPTEMBER 1996

\begin{tabular}{|c|c|c|c|c|c|c|c|c|c|c|c|c|}
\hline DAY & $\alpha \mathrm{cr}$ & NOV & DEC & JAN & FEB & MAR & APR & MAY & JUN & JUL & AUG & SEP \\
\hline $\begin{array}{l}1 \\
2 \\
3 \\
4 \\
5\end{array}$ & $\begin{array}{l}16 \\
15 \\
15 \\
14 \\
14\end{array}$ & $\begin{array}{l}9.5 \\
9.6 \\
8.8 \\
8.9 \\
8.8\end{array}$ & $\begin{array}{l}7.8 \\
7.7 \\
7.4 \\
7.5 \\
7.4\end{array}$ & $\begin{array}{r}5.9 \\
5.8 \\
e 5.8 \\
05.8 \\
06.0\end{array}$ & $\begin{array}{r}6.2 \\
6.1 \\
06.0 \\
06.0 \\
06.1\end{array}$ & $\begin{array}{r}e 6.1 \\
e 6.1 \\
06.2 \\
6.3 \\
5.9\end{array}$ & $\begin{array}{r}8.8 \\
9.6 \\
9.4 \\
8.8 \\
8.1\end{array}$ & $\begin{array}{l}14 \\
15 \\
17 \\
20 \\
22\end{array}$ & $\begin{array}{l}15 \\
14 \\
14 \\
14 \\
13\end{array}$ & $\begin{array}{l}12 \\
12 \\
11 \\
11 \\
11\end{array}$ & $\begin{array}{l}8.2 \\
8.0 \\
9.0 \\
8.4 \\
7.8\end{array}$ & $\begin{array}{l}8.4 \\
8.3 \\
8.4 \\
7.7 \\
7.4\end{array}$ \\
\hline $\begin{array}{r}6 \\
7 \\
8 \\
9 \\
10\end{array}$ & $\begin{array}{l}14 \\
13 \\
13 \\
13 \\
13\end{array}$ & $\begin{array}{l}9.2 \\
9.0 \\
8.8 \\
8.7 \\
8.4\end{array}$ & $\begin{array}{l}7.1 \\
6.9 \\
6.8 \\
7.5 \\
6.3\end{array}$ & $\begin{array}{l}e 6.0 \\
e 6.2 \\
e 6.0 \\
06.4 \\
06.4\end{array}$ & $\begin{array}{l}6.3 \\
6.1 \\
6.1 \\
6.2 \\
6.3\end{array}$ & $\begin{array}{l}5.9 \\
6.2 \\
6.2 \\
6.1 \\
6.4\end{array}$ & $\begin{array}{r}8.0 \\
8.3 \\
9.1 \\
11.1 \\
13\end{array}$ & $\begin{array}{l}25 \\
28 \\
29 \\
29 \\
30\end{array}$ & $\begin{array}{l}13 \\
13 \\
12 \\
12 \\
12\end{array}$ & $\begin{array}{l}11 \\
11 \\
12 \\
12 \\
12\end{array}$ & $\begin{array}{l}7.6 \\
8.3 \\
8.3 \\
7.9 \\
7.5\end{array}$ & $\begin{array}{l}7.8 \\
7.4 \\
7.2 \\
7.0 \\
7.3\end{array}$ \\
\hline $\begin{array}{l}11 \\
12 \\
13 \\
14 \\
15\end{array}$ & $\begin{array}{l}12 \\
12 \\
12 \\
12 \\
11\end{array}$ & $\begin{array}{l}8.3 \\
9.0 \\
8.4 \\
8.4 \\
8.2\end{array}$ & $\begin{array}{l}6.8 \\
7.5 \\
6.8 \\
6.7 \\
6.5\end{array}$ & $\begin{array}{r}06.4 \\
6.4 \\
6.4 \\
6.3 \\
6.3\end{array}$ & $\begin{array}{l}6.3 \\
6.4 \\
6.4 \\
6.6 \\
6.8\end{array}$ & $\begin{array}{l}6.9 \\
7.2 \\
7.1 \\
7.2 \\
7.2\end{array}$ & $\begin{array}{l}12 \\
11 \\
11 \\
10 \\
9.8\end{array}$ & $\begin{array}{l}31 \\
34 \\
37 \\
38 \\
37\end{array}$ & $\begin{array}{l}12 \\
12 \\
12 \\
12 \\
14\end{array}$ & $\begin{array}{l}12 \\
1.2 \\
1.2 \\
1.1 \\
9.8\end{array}$ & $\begin{array}{l}7.3 \\
7.1 \\
7.1 \\
7.0 \\
6.8\end{array}$ & $\begin{array}{r}7.3 \\
7.3 \\
8.3 \\
9.6 \\
11\end{array}$ \\
\hline $\begin{array}{l}16 \\
17 \\
18 \\
19 \\
20\end{array}$ & $\begin{array}{l}11 \\
11 \\
11 \\
11 \\
11\end{array}$ & $\begin{array}{l}8.1 \\
8.0 \\
7.9 \\
7.9 \\
7.8\end{array}$ & $\begin{array}{l}6.7 \\
6.4 \\
6.3 \\
6.5 \\
6.6\end{array}$ & $\begin{array}{l}6.3 \\
6.4 \\
6.4 \\
6.5 \\
6.3\end{array}$ & $\begin{array}{l}6.9 \\
7.0 \\
7.0 \\
6.9 \\
6.9\end{array}$ & $\begin{array}{l}7.1 \\
6.9 \\
6.8 \\
6.9 \\
7.0\end{array}$ & $\begin{array}{r}9.6 \\
10 \\
9.8 \\
9.5 \\
9.2\end{array}$ & $\begin{array}{l}37 \\
38 \\
37 \\
35 \\
34\end{array}$ & $\begin{array}{l}12 \\
12 \\
11 \\
11 \\
11\end{array}$ & $\begin{array}{l}9.8 \\
10 \\
10 \\
9.5 \\
9.1\end{array}$ & $\begin{array}{l}6.6 \\
6.6 \\
6.5 \\
6.5 \\
6.7\end{array}$ & $\begin{array}{l}9.8 \\
9.6 \\
11 \\
11 \\
10\end{array}$ \\
\hline $\begin{array}{l}21 \\
22 \\
23 \\
24 \\
25\end{array}$ & $\begin{array}{l}11 \\
10 \\
9.5 \\
10 \\
10\end{array}$ & $\begin{array}{l}7.8 \\
7.7 \\
7.6 \\
7.3 \\
7.8\end{array}$ & $\begin{array}{r}6.7 \\
7.1 \\
6.9 \\
e 6.0 \\
05.4\end{array}$ & $\begin{array}{l}6.3 \\
6.3 \\
6.3 \\
6.2 \\
6.2\end{array}$ & $\begin{array}{l}6.9 \\
6.6 \\
6.4 \\
6.7 \\
6.3\end{array}$ & $\begin{array}{l}7.1 \\
7.7 \\
7.9 \\
7.7 \\
7.3\end{array}$ & $\begin{array}{c}8.7 \\
8.4 \\
8.8 \\
10 \\
12\end{array}$ & $\begin{array}{l}31 \\
29 \\
27 \\
24 \\
23\end{array}$ & $\begin{array}{l}11 \\
12 \\
11 \\
10 \\
10\end{array}$ & $\begin{array}{l}9.0 \\
8.8 \\
8.7 \\
9.1 \\
9.5\end{array}$ & $\begin{array}{r}7.4 \\
8.8 \\
9.8 \\
10 \\
9.0\end{array}$ & $\begin{array}{l}10 \\
9.7 \\
9.4 \\
9.3 \\
9.2\end{array}$ \\
\hline $\begin{array}{l}26 \\
27 \\
28 \\
29 \\
30 \\
31\end{array}$ & $\begin{array}{l}9.9 \\
9.8 \\
9.7 \\
9.6 \\
9.4 \\
9.4\end{array}$ & $\begin{array}{l}7.6 \\
6.8 \\
6.5 \\
7.2 \\
7.9 \\
-.9\end{array}$ & $\begin{array}{r}e 5.0 \\
e 5.2 \\
e 5.2 \\
05.2 \\
e 5.4 \\
5.9\end{array}$ & $\begin{array}{r}6.2 \\
06.0 \\
06.0 \\
6.2 \\
6.1 \\
6.1\end{array}$ & $\begin{array}{r}5.7 \\
05.5 \\
06.0 \\
06.0 \\
-.-. \\
-.-\end{array}$ & $\begin{array}{l}7.0 \\
6.9 \\
7.1 \\
7.3 \\
7.6 \\
8.0\end{array}$ & $\begin{array}{l}14 \\
16 \\
16 \\
14 \\
14 \\
-m-m\end{array}$ & $\begin{array}{l}22 \\
20 \\
18 \\
17 \\
16 \\
16\end{array}$ & $\begin{array}{r}11 \\
17 \\
14 \\
12 \\
12 \\
---\end{array}$ & $\begin{array}{l}8.9 \\
8.7 \\
8.7 \\
8.7 \\
8.6 \\
8.5\end{array}$ & $\begin{array}{l}8.3 \\
8.7 \\
9.5 \\
8.7 \\
9.4 \\
8.5\end{array}$ & $\begin{array}{l}9.8 \\
9.3 \\
9.2 \\
9.4 \\
9.2 \\
---\end{array}$ \\
\hline $\begin{array}{l}\text { TOTAL } \\
\text { MEAN } \\
\text { MAX } \\
\text { MIN } \\
\text { AC-FT }\end{array}$ & $\begin{array}{r}362.3 \\
11.7 \\
16 \\
9.4 \\
719\end{array}$ & $\begin{array}{r}245.9 \\
8.20 \\
9.6 \\
6.5 \\
488\end{array}$ & $\begin{array}{r}203.2 \\
6.55 \\
7.8 \\
5.0 \\
403\end{array}$ & $\begin{array}{r}191.9 \\
6.19 \\
6.5 \\
5.8 \\
381\end{array}$ & $\begin{array}{r}184.7 \\
6.37 \\
7.0 \\
5.5 \\
366\end{array}$ & $\begin{array}{r}213.3 \\
6.88 \\
8.0 \\
5.9 \\
423\end{array}$ & $\begin{array}{r}317.9 \\
10.6 \\
16 \\
8.0 \\
631\end{array}$ & $\begin{array}{r}830 \\
26.8 \\
38 \\
14 \\
1650\end{array}$ & $\begin{array}{r}371 \\
12.4 \\
17 \\
10 \\
736\end{array}$ & $\begin{array}{r}317.4 \\
10.2 \\
12 \\
8.5 \\
630\end{array}$ & $\begin{array}{r}247.3 \\
7.98 \\
10 \\
6.5 \\
491\end{array}$ & $\begin{array}{r}266.3 \\
8.88 \\
11 \\
7.0 \\
528\end{array}$ \\
\hline
\end{tabular}

STATISTICS OF MONTHLY MEAN DATA FOR WATER YEARS 1913 - 1996, BY WATER YEAR (WY)

\begin{tabular}{|c|c|c|c|c|c|c|c|c|c|c|c|c|}
\hline $\begin{array}{l}\text { MEAN } \\
\text { MAX } \\
(W Y) \\
\text { MIN } \\
(W X)\end{array}$ & $\begin{array}{l}11.7 \\
27.8 \\
1942 \\
6.29 \\
1979\end{array}$ & $\begin{array}{l}9.12 \\
22.0 \\
1942 \\
5.37 \\
1977\end{array}$ & $\begin{array}{l}7.28 \\
14.8 \\
1991 \\
4.26 \\
1951\end{array}$ & $\begin{array}{l}6.08 \\
10.0 \\
1942 \\
3.51 \\
1951\end{array}$ & $\begin{array}{l}6.08 \\
9.92 \\
1991 \\
3.47 \\
1964\end{array}$ & $\begin{array}{l}9.20 \\
21.2 \\
1989 \\
4.11 \\
1977\end{array}$ & $\begin{array}{l}22.6 \\
47.5 \\
1937 \\
8.77 \\
1977\end{array}$ & $\begin{array}{r}59.4 \\
1.56 \\
1941 \\
14.5 \\
1972\end{array}$ & $\begin{array}{r}72.0 \\
178 \\
1941 \\
12.4 \\
1996\end{array}$ & $\begin{array}{r}30.8 \\
101 \\
1995 \\
7.86 \\
1972\end{array}$ & $\begin{array}{l}18.5 \\
37.5 \\
1967 \\
6.55 \\
1972\end{array}$ & $\begin{array}{l}13.9 \\
34.5 \\
1982 \\
6.74 \\
1972\end{array}$ \\
\hline
\end{tabular}

SUMMARY STATISTICS

ANNUAL TOTAL

ANNUAL MEAN

HIGHEST ANNUAL MEAN

LOWEST ANNUAL MEAN

HIGHEST DAILY MEAN

LOWEST DAILY MEAN

ANNUAL SEVEN-DAY MINIMUM

INSTANTANEOUS PEAK FLOW

INSTANTANEOUS PEAK STAGE

INSTANTANEOUS LOW FLOW

ANNUAL RUNOFF (AC-FT)

10 EERCENT EXCEEDS

50 PERCENT EXCEEDS

90 PERCENT EXCEEDS

- Estimatod
FOR 1995 CALENDAR YEAR

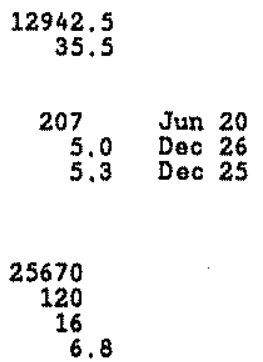

FOR 1996 WATER YEAR

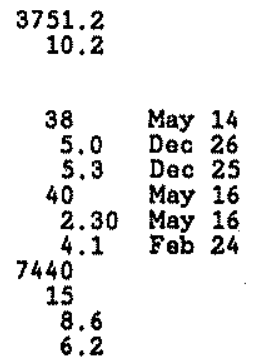

WATER YEARS $1913-1996$

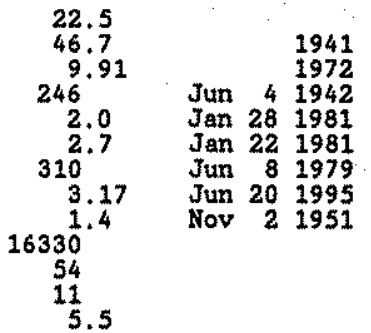




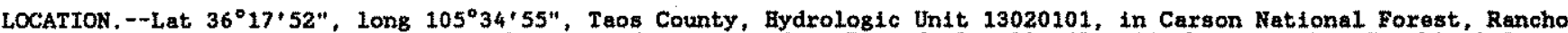
del Rio Grande Grant, on right bank $1.4 \mathrm{mi}$ downstream from Rito de la olla (locally known at Pot Creek), $3.2 \mathrm{mi}$ south of Talpa, 4.3 mi upstream from Rio Chiquito, and at mile 6.9 .

DRAINAGE AREA. $\rightarrow-83 \mathrm{mi}^{2}$, approximately.

PERIOD OF RECORD, --October 1952 to September 1982, October 1983 to September 1985 (annual maximum only), October 1985 to current year. Prior to October 1955, published as "Rio Grande del Rancho nr Taos" and October 1955 to September 1960 as Rio Grande de Ranchos nr Talpa."

GAGE.-Water-stage recorder. Elevation of gage $187,240 \mathrm{ft}$ above National Goodetic Vertical Datum of 1929 , from topographic map. Frior to Nov. 11, 1952, nonrocording gage at sitta 1,035 ft downstream at lower datum. Hov. 11. 1952 to Nov. 5, 1968 , water-stage recorder at stte 1,000 ft downsteam at lower datum. Hov. 6 . 1968 to Aus. 28, 1980 , water-stage recorder at present site on left bank at same datum.

REMARKS.---Records good except for estimated daily discharges, which are poor. Minor diversions for irrigation above station. Several observations of water temperature were made during the year.

DISCHARGE, CUBIC FEET PER SECOND, WATER YEAR OCTOBER 1995 TO SEPTEMBER 1996

\begin{tabular}{|c|c|c|c|c|c|c|c|c|c|c|c|c|}
\hline DAY & OCT & Nov & DEC & JAN & FEB & MAR & APR & MAY & JUN & JUL. & AUG & SEF \\
\hline $\begin{array}{l}1 \\
2 \\
3 \\
4 \\
5\end{array}$ & $\begin{array}{l}9.8 \\
9.2 \\
8.9 \\
8.6 \\
8.5\end{array}$ & $\begin{array}{l}6.8 \\
7.0 \\
7.0 \\
7.0 \\
7.1\end{array}$ & $\begin{array}{l}6.8 \\
6.7 \\
6.7 \\
6.9 \\
7.0\end{array}$ & $\begin{array}{l}6.9 \\
7.2 \\
7.4 \\
7.8 \\
7.2\end{array}$ & $\begin{array}{l}6.0 \\
6.1 \\
5.6 \\
5.7 \\
6.6\end{array}$ & $\begin{array}{l}6.0 \\
6.0 \\
6.3 \\
7.0 \\
7.0\end{array}$ & $\begin{array}{l}9.7 \\
11 \\
12 \\
12 \\
11\end{array}$ & $\begin{array}{l}20 \\
20 \\
21 \\
23 \\
25\end{array}$ & $\begin{array}{l}8.7 \\
8.1 \\
7.6 \\
7.2 \\
6.8\end{array}$ & $\begin{array}{l}6.7 \\
5.9 \\
5.2 \\
5.3 \\
5.9\end{array}$ & $\begin{array}{l}4.0 \\
3.5 \\
4.0 \\
3.8 \\
3.4\end{array}$ & $\begin{array}{l}5.0 \\
4.6 \\
4.7 \\
4.2 \\
3.7\end{array}$ \\
\hline $\begin{array}{r}6 \\
7 \\
8 \\
9 \\
10\end{array}$ & $\begin{array}{l}8.4 \\
8.4 \\
8.2 \\
8.1 \\
8.0\end{array}$ & $\begin{array}{l}7.0 \\
7.0 \\
7.2 \\
7.2 \\
7.6\end{array}$ & $\begin{array}{r}7.0 \\
7.0 \\
7.0 \\
6.7 \\
6.7\end{array}$ & $\begin{array}{l}7.0 \\
7.2 \\
7.6 \\
7.5 \\
7.3\end{array}$ & $\begin{array}{l}6.4 \\
6.2 \\
6.1 \\
6.0 \\
5.9\end{array}$ & $\begin{array}{l}6.8 \\
6.1 \\
6.8 \\
7.0 \\
7.3\end{array}$ & $\begin{array}{l}10 \\
11 \\
11 \\
12 \\
14\end{array}$ & $\begin{array}{l}26 \\
26 \\
27 \\
28 \\
28\end{array}$ & $\begin{array}{r}6.4 \\
6.3 \\
5.9 \\
5.6 \\
5.5\end{array}$ & $\begin{array}{l}4.6 \\
4.4 \\
4.6 \\
6.4 \\
6.4\end{array}$ & $\begin{array}{l}3.0 \\
2.9 \\
3.2 \\
3.1 \\
3.3\end{array}$ & $\begin{array}{r}4.1 \\
4.0 \\
3.6 \\
3.4 \\
3.2\end{array}$ \\
\hline $\begin{array}{l}11 \\
12 \\
13 \\
14 \\
15\end{array}$ & $\begin{array}{l}7.7 \\
7.5 \\
7.5 \\
7.5 \\
7.4\end{array}$ & $\begin{array}{l}6.8 \\
6.9 \\
7.0 \\
7.0 \\
7.0\end{array}$ & $\begin{array}{l}6.6 \\
6.9 \\
6.8 \\
6.8 \\
7.0\end{array}$ & $\begin{array}{l}7.1 \\
6.9 \\
6.9 \\
6.8 \\
6.8\end{array}$ & $\begin{array}{l}5.9 \\
5.8 \\
5.8 \\
6.0 \\
5.9\end{array}$ & $\begin{array}{l}7.3 \\
7.3 \\
7.3 \\
7.5 \\
7.8\end{array}$ & $\begin{array}{l}15 \\
14 \\
15 \\
13 \\
12\end{array}$ & $\begin{array}{l}27 \\
27 \\
27 \\
26 \\
26\end{array}$ & $\begin{array}{l}5.3 \\
5.2 \\
5.2 \\
6.2 \\
6.5\end{array}$ & $\begin{array}{l}5.4 \\
4.9 \\
5.7 \\
6.4 \\
5.3\end{array}$ & $\begin{array}{l}2.9 \\
2.6 \\
2.3 \\
2.2 \\
2.3\end{array}$ & $\begin{array}{l}3.2 \\
3.3 \\
4.2 \\
4.7 \\
6.9\end{array}$ \\
\hline $\begin{array}{l}16 \\
17 \\
18 \\
19 \\
20\end{array}$ & $\begin{array}{l}7.3 \\
7.0 \\
7.0 \\
6.8 \\
6.7\end{array}$ & $\begin{array}{l}7.0 \\
7.0 \\
7.0 \\
6.9 \\
6.9\end{array}$ & $\begin{array}{l}6.6 \\
6.9 \\
7.1 \\
6.5 \\
7.0\end{array}$ & $\begin{array}{l}6.8 \\
7.2 \\
6.7 \\
6.3 \\
6.8\end{array}$ & $\begin{array}{l}5.9 \\
6.1 \\
6.3 \\
6.2 \\
6.3\end{array}$ & $\begin{array}{l}7.8 \\
7.7 \\
7.0 \\
6.7 \\
7.0\end{array}$ & $\begin{array}{l}12 \\
13 \\
14 \\
15 \\
15\end{array}$ & $\begin{array}{l}25 \\
23 \\
22 \\
21 \\
19\end{array}$ & $\begin{array}{l}5.7 \\
5.1 \\
4.7 \\
4.4 \\
4.1\end{array}$ & $\begin{array}{l}4.8 \\
4.5 \\
4.7 \\
4.2 \\
3.8\end{array}$ & $\begin{array}{l}2.2 \\
2.9 \\
3.4 \\
2.7 \\
2.5\end{array}$ & $\begin{array}{l}6.6 \\
6.1 \\
7.8 \\
9.3\end{array}$ \\
\hline $\begin{array}{l}21 \\
22 \\
23 \\
24 \\
25\end{array}$ & $\begin{array}{l}6.7 \\
6.7 \\
6.6 \\
6.5 \\
6.7\end{array}$ & $\begin{array}{l}7.2 \\
7.2 \\
7.0 \\
6.9 \\
6.8\end{array}$ & $\begin{array}{l}6.5 \\
7.4 \\
7.1 \\
6.3 \\
6.3\end{array}$ & $\begin{array}{l}6.1 \\
7.0 \\
6.7 \\
6.5 \\
7.1\end{array}$ & $\begin{array}{l}6.5 \\
6.5 \\
6.5 \\
6.2 \\
6.4\end{array}$ & $\begin{array}{l}7.5 \\
7.7 \\
8.0 \\
8.0 \\
8.0\end{array}$ & $\begin{array}{l}14 \\
13 \\
13 \\
14 \\
16\end{array}$ & $\begin{array}{l}18 \\
17 \\
15 \\
14 \\
13\end{array}$ & $\begin{array}{l}4.0 \\
4.3 \\
4.8 \\
4.2 \\
3.9\end{array}$ & $\begin{array}{l}3.5 \\
3.2 \\
3.1 \\
3.1 \\
3.2\end{array}$ & $\begin{array}{l}3.4 \\
3.9 \\
5.7 \\
7.2 \\
7.1\end{array}$ & $\begin{array}{l}7.6 \\
6.8 \\
5.7 \\
5.1 \\
4.9\end{array}$ \\
\hline $\begin{array}{l}26 \\
27 \\
28 \\
29 \\
30 \\
31\end{array}$ & $\begin{array}{l}7.0 \\
7.0 \\
7.0 \\
6.9 \\
6.8 \\
6.8\end{array}$ & $\begin{array}{l}7.0 \\
6.9 \\
6.4 \\
6.4 \\
6.7 \\
.+.\end{array}$ & $\begin{array}{l}7.1 \\
7.6 \\
7.6 \\
7.6 \\
7.2 \\
6.9\end{array}$ & $\begin{array}{l}6.9 \\
7.0 \\
6.9 \\
6.6 \\
6.3 \\
6.0\end{array}$ & $\begin{array}{l}6.8 \\
6.2 \\
5.7 \\
6.4 \\
-.-\end{array}$ & $\begin{array}{l}8.2 \\
8.1 \\
8.5 \\
8.9 \\
9.0 \\
9.1\end{array}$ & $\begin{array}{l}20 \\
22 \\
24 \\
22 \\
21 \\
-\end{array}$ & $\begin{array}{l}13 \\
12 \\
11 \\
11 \\
9.6 \\
9.2\end{array}$ & $\begin{array}{r}4.2 \\
10 \\
8.7 \\
6.8 \\
6.7 \\
\end{array}$ & $\begin{array}{l}3.1 \\
3.1 \\
3.2 \\
3.6 \\
4.5 \\
4.4\end{array}$ & $\begin{array}{l}6.3 \\
5.7 \\
5.9 \\
6.6 \\
7.3 \\
6.1\end{array}$ & $\begin{array}{l}5.6 \\
6.0 \\
5.7 \\
5.1 \\
4.6\end{array}$ \\
\hline $\begin{array}{l}\text { TOTAL } \\
\text { MEAN } \\
\text { MAX } \\
\text { MIN } \\
A C-F T\end{array}$ & $\begin{array}{r}233.2 \\
7.52 \\
9.8 \\
6.5 \\
463\end{array}$ & $\begin{array}{r}208.9 \\
6.96 \\
7.6 \\
6.4 \\
41.4\end{array}$ & $\begin{array}{r}214.3 \\
6.91 \\
7.6 \\
6.3 \\
425\end{array}$ & $\begin{array}{r}214.5 \\
6.92 \\
7.8 \\
6.0 \\
425\end{array}$ & $\begin{array}{r}178.0 \\
6.14 \\
6.8 \\
5.6 \\
353\end{array}$ & $\begin{array}{r}230.7 \\
7.44 \\
9.1 \\
6.0 \\
458\end{array}$ & $\begin{array}{r}430.7 \\
14.4 \\
24 \\
9.7 \\
854\end{array}$ & $\begin{array}{r}629.8 \\
20.3 \\
28 \\
9.2 \\
1250\end{array}$ & $\begin{array}{r}178.1 \\
5.94 \\
10 \\
3.9 \\
353\end{array}$ & $\begin{array}{r}143.1 \\
4.62 \\
6.7 \\
3.1 \\
284\end{array}$ & $\begin{array}{r}125.4 \\
4.05 \\
7.3 \\
2.2 \\
24.9\end{array}$ & $\begin{array}{r}158.0 \\
5.27 \\
9.3 \\
3.2 \\
313\end{array}$ \\
\hline
\end{tabular}

STATISTICS OF MONTHLY MEAN DATA FOR WATER YEARS 1953 - 1996, BY WATER YEAR (WY)

\begin{tabular}{|c|c|c|c|c|c|c|c|c|c|c|c|}
\hline $\begin{array}{l}\text { MEAN } \\
\text { MAN } \\
\text { (WY }) \\
\text { MIN } \\
(W Y)\end{array}$ & $\begin{array}{l}7.38 \\
1.42 \\
1958 \\
2.12 \\
1957\end{array}$ & $\begin{array}{l}6.70 \\
13.9 \\
1995 \\
2.95 \\
1957\end{array}$ & $\begin{array}{l}5.82 \\
10.4 \\
1958 \\
2.97 \\
1957\end{array}$ & $\begin{array}{l}5.31 \\
9.19 \\
1958 \\
2.06 \\
1955\end{array}$ & $\begin{array}{l}5.62 \\
9.31 \\
1989 \\
2.65 \\
1955\end{array}$ & $\begin{array}{l}9.41 \\
22.9 \\
1994 \\
4.65 \\
1955\end{array}$ & $\begin{array}{l}32.1 \\
91.9 \\
1962 \\
9.61 \\
1981\end{array}$ & $\begin{array}{r}92.6 \\
264 \\
1994 \\
12.9 \\
1981\end{array}$ & $\begin{array}{r}52.9 \\
174 \\
1995 \\
5.94 \\
1996\end{array}$ & $\begin{array}{l}14.6 \\
42.9 \\
1986 \\
3.14 \\
1956\end{array}$ & $\begin{array}{l}12.4 \\
35.7 \\
1957 \\
2.33 \\
1972\end{array}$ \\
\hline
\end{tabular}

SUMMARY STATISTICS

ANNUAL TOTAL

ANNUAL MEAN

HIGHEST ANNUAL MEAN

LOWEST ANNUAL MEAN

HIGHEST DAILY MEAN

LOWEST DAILY MEAN

ANNUAL SEVEN-DAY MINIMUM

INSTANTANEOUS PEAK FLOW

INSTANTANEOUS PEAK STAGE

INSTANTANEOUS LOW FLOW

ANNUAL RUNOFF (AC-FT)

10 PERCENT EXCEEDS

50 PERCENT EXCEEDS

90 PERCENT EXCEEDS
FOR 1995 CALENDAR YEAR

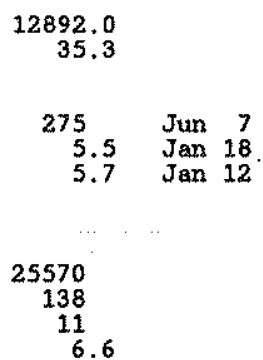

FOR 1996 WATER YEAR

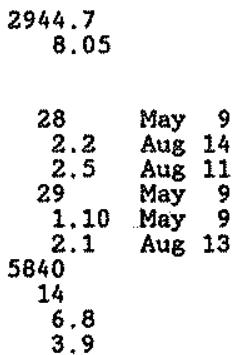

WATER YEARS 1953 - 1996

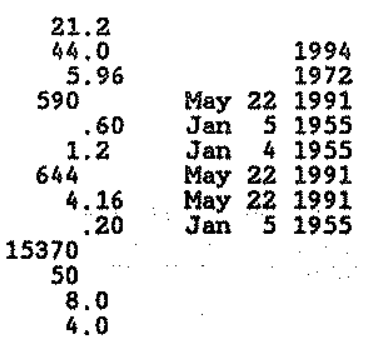


RIO GRANDE BASIN

08276300 RIO PUEBLO DE TAOS BELOW LOS CORDOVAS, NM

LOCATION.--Lat $36^{\circ} 22^{\prime} 39^{\prime \prime}$, Long $105^{\circ} 40^{\prime} 05^{\prime \prime}$. Taos County, Bydrologic Unit 13020101, In Gijosa Grant, on loft bank 1.9 mi southwest of Los Cordovas, $2.5 \mathrm{mi}$ downstream from Rio Grande del Rancho, and at mile 5.1 .

DRAINAGE AREA. $--380 \mathrm{mi}^{2}$.

WATER-DISCEARGE RECORDS

RERIOD OF RECORD. - March 1957 to current yeax.

REVISED RECORDS, -WSP 1732: 1957(M), WSP 1923: 1957(P), 1958, WDR MM-81-1: 1979(P).

GAGE. - Water-stage recorder. Elevation of gage is $6,650 \mathrm{ft}$ above National Geodetic Vertical Datum of 1929 , from topographic map. Prior to Sept. 4, 1984 at site 700 ft downstream at same datum.

REMARKS. - Water-discharge records good except for estimated daily discharges, which are fair. Diverstons for irrigation of about 12,000 acres upstream from station, of which about 1,700 acres are irrigated by water from Rto tiondo. Several observations of water temperature were made during the year. Satelifte telemeter at station.

DISCBARGE, CUBIC FEET PER SECOND, WATER YEAR OCTOBER 1995 TO SEPTEMBER 1996

\begin{tabular}{|c|c|c|c|c|c|c|c|c|c|c|c|c|}
\hline DAY & OCT & Nov & DEC & JAN & FEB & MAR & APR & MAY & JUN & Jun. & AUG & SEP \\
\hline $\begin{array}{l}1 \\
2 \\
3 \\
4 \\
5\end{array}$ & $\begin{array}{l}54 \\
50 \\
48 \\
45 \\
45\end{array}$ & $\begin{array}{l}42 \\
43 \\
41 \\
43 \\
45\end{array}$ & $\begin{array}{l}40 \\
40 \\
40 \\
39 \\
39\end{array}$ & $\begin{array}{l}43 \\
41 \\
46 \\
45 \\
46\end{array}$ & $\begin{array}{l}53 \\
49 \\
43 \\
44 \\
55\end{array}$ & $\begin{array}{l}48 \\
46 \\
44 \\
47 \\
45\end{array}$ & $\begin{array}{l}42 \\
42 \\
46 \\
49 \\
47\end{array}$ & $\begin{array}{l}24 \\
24 \\
25 \\
23 \\
23\end{array}$ & $\begin{array}{l}7.3 \\
7.5 \\
7.5 \\
7.2 \\
7.3\end{array}$ & $\begin{array}{l}9.0 \\
8.7 \\
7.7 \\
7.2 \\
6.8\end{array}$ & $\begin{array}{l}7.2 \\
6.8 \\
6.7 \\
6.3 \\
6.1\end{array}$ & $\begin{array}{l}8.1 \\
7.9 \\
7.6 \\
7.0 \\
6.4\end{array}$ \\
\hline $\begin{array}{r}6 \\
7 \\
8 \\
9 \\
10\end{array}$ & $\begin{array}{l}46 \\
46 \\
46 \\
40 \\
42\end{array}$ & $\begin{array}{l}42 \\
43 \\
43 \\
44 \\
45\end{array}$ & $\begin{array}{l}39 \\
37 \\
37 \\
35 \\
34\end{array}$ & $\begin{array}{l}45 \\
43 \\
45 \\
45 \\
44\end{array}$ & $\begin{array}{l}64 \\
55 \\
54 \\
52 \\
52\end{array}$ & $\begin{array}{l}46 \\
45 \\
55 \\
68 \\
62\end{array}$ & $\begin{array}{l}41 \\
39 \\
39 \\
33 \\
34\end{array}$ & $\begin{array}{l}19 \\
21 \\
21 \\
20 \\
19\end{array}$ & $\begin{array}{l}6.9 \\
6.9 \\
6.1 \\
6.1 \\
6.4\end{array}$ & $\begin{array}{l}7.0 \\
6.6 \\
7.1 \\
8.7 \\
8.8\end{array}$ & $\begin{array}{r}6.0 \\
24 \\
6.5 \\
6.7 \\
6.1\end{array}$ & $\begin{array}{l}6.4 \\
6.5 \\
7.2 \\
7.3 \\
6.8\end{array}$ \\
\hline $\begin{array}{l}11 \\
12 \\
13 \\
14 \\
15\end{array}$ & $\begin{array}{l}41 \\
44 \\
40 \\
38 \\
40\end{array}$ & $\begin{array}{l}47 \\
45 \\
45 \\
44 \\
43\end{array}$ & $\begin{array}{l}33 \\
36 \\
40 \\
39 \\
42\end{array}$ & $\begin{array}{l}44 \\
46 \\
46 \\
48 \\
46\end{array}$ & $\begin{array}{l}50 \\
50 \\
51 \\
50 \\
50\end{array}$ & $\begin{array}{l}58 \\
55 \\
51 \\
50 \\
59\end{array}$ & $\begin{array}{l}40 \\
39 \\
41 \\
41 \\
36\end{array}$ & $\begin{array}{l}17 \\
17 \\
17 \\
18 \\
19\end{array}$ & $\begin{array}{l}6.3 \\
6.1 \\
6.1 \\
6.6 \\
7.0\end{array}$ & $\begin{array}{l}7.7 \\
7.7 \\
9.3 \\
8.2 \\
7.5\end{array}$ & $\begin{array}{l}6.0 \\
5.2 \\
5.2 \\
5.4 \\
5.2\end{array}$ & $\begin{array}{l}7.1 \\
7.9 \\
8.5 \\
8.9 \\
9.8\end{array}$ \\
\hline $\begin{array}{l}16 \\
17 \\
18 \\
19 \\
20\end{array}$ & $\begin{array}{l}39 \\
37 \\
35 \\
36 \\
36\end{array}$ & $\begin{array}{l}40 \\
41 \\
41 \\
39 \\
37\end{array}$ & $\begin{array}{l}40 \\
41 \\
44 \\
39 \\
40\end{array}$ & $\begin{array}{l}46 \\
56 \\
47 \\
41 \\
49\end{array}$ & $\begin{array}{l}51 \\
50 \\
50 \\
49 \\
49\end{array}$ & $\begin{array}{l}62 \\
53 \\
49 \\
45 \\
45\end{array}$ & $\begin{array}{l}32 \\
29 \\
30 \\
31 \\
32\end{array}$ & $\begin{array}{l}17 \\
15 \\
14 \\
12 \\
11\end{array}$ & $\begin{array}{l}6.8 \\
6.5 \\
5.8 \\
5.6 \\
5.1\end{array}$ & $\begin{array}{l}6.8 \\
9.2 \\
9.6 \\
8.0 \\
7.6\end{array}$ & $\begin{array}{r}5.4 \\
32.5 \\
7.5 \\
5.2 \\
5.1\end{array}$ & $\begin{array}{c}9.6 \\
9.7 \\
11 \\
11 \\
9.8\end{array}$ \\
\hline $\begin{array}{l}21 \\
22 \\
23 \\
24 \\
25\end{array}$ & $\begin{array}{l}36 \\
36 \\
35 \\
36 \\
40\end{array}$ & $\begin{array}{l}37 \\
37 \\
35 \\
37 \\
38\end{array}$ & $\begin{array}{l}40 \\
41 \\
42 \\
35 \\
45\end{array}$ & $\begin{array}{l}40 \\
48 \\
43 \\
41 \\
44\end{array}$ & $\begin{array}{l}51 \\
52 \\
45 \\
39 \\
48\end{array}$ & $\begin{array}{l}48 \\
49 \\
47 \\
47 \\
45\end{array}$ & $\begin{array}{l}32 \\
28 \\
23 \\
21 \\
22\end{array}$ & $\begin{array}{l}10 \\
10 \\
9.2 \\
9.0 \\
8.7\end{array}$ & $\begin{array}{l}5.1 \\
5.5 \\
5.7 \\
5.1 \\
5.3\end{array}$ & $\begin{array}{l}7.4 \\
7.3 \\
6.8 \\
6.7 \\
6.6\end{array}$ & $\begin{array}{r}6.6 \\
06.8 \\
96.9 \\
97.0 \\
07.2\end{array}$ & $\begin{array}{l}9.4 \\
9.6 \\
9.1 \\
9.7 \\
9.7\end{array}$ \\
\hline $\begin{array}{l}26 \\
27 \\
28 \\
29 \\
30 \\
31\end{array}$ & $\begin{array}{l}43 \\
43 \\
43 \\
43 \\
41 \\
41\end{array}$ & $\begin{array}{r}37 \\
38 \\
37 \\
34 \\
37 \\
-\end{array}$ & $\begin{array}{l}40 \\
39 \\
40 \\
43 \\
41 \\
44\end{array}$ & $\begin{array}{l}45 \\
61 \\
45 \\
46 \\
49 \\
53\end{array}$ & $\begin{array}{r}46 \\
40 \\
43 \\
48 \\
--. \\
-\end{array}$ & $\begin{array}{l}45 \\
44 \\
43 \\
43 \\
43 \\
40\end{array}$ & $\begin{array}{r}26 \\
30 \\
30 \\
27 \\
25 \\
-\end{array}$ & $\begin{array}{l}8.7 \\
9.7 \\
9.1 \\
8.3 \\
7.7 \\
7.6\end{array}$ & $\begin{array}{r}12 \\
16 \\
11 \\
9.1 \\
9.1\end{array}$ & $\begin{array}{l}6.4 \\
6.2 \\
6.9 \\
7.4 \\
8.3 \\
7.8\end{array}$ & $\begin{array}{r}97.5 \\
97.7 \\
7.9 \\
9.4 \\
9.5 \\
8.2\end{array}$ & $\begin{array}{r}10 \\
10 \\
10 \\
9.9 \\
9.0\end{array}$ \\
\hline $\begin{array}{l}\text { TOTAL. } \\
\text { MEAN } \\
\text { MAX } \\
\text { MIN } \\
\text { AC-FT }\end{array}$ & $\begin{array}{r}1285 \\
41.5 \\
54 \\
35 \\
2550\end{array}$ & $\begin{array}{r}1220 \\
40.7 \\
47 \\
34 \\
2420\end{array}$ & $\begin{array}{r}1224 \\
39.5 \\
45 \\
33 \\
2430\end{array}$ & $\begin{array}{r}1427 \\
46.0 \\
61 \\
40 \\
2830\end{array}$ & $\begin{array}{r}1433 \\
49.4 \\
64 \\
39 \\
2840\end{array}$ & $\begin{array}{r}1527 \\
49.3 \\
68 \\
40 \\
3030\end{array}$ & $\begin{array}{r}1027 \\
34.2 \\
49 \\
21 \\
2040\end{array}$ & $\begin{array}{r}474.0 \\
15.3 \\
25 \\
7.6 \\
940\end{array}$ & $\begin{array}{r}215.2 \\
7.17 \\
16 \\
5.1 \\
427\end{array}$ & $\begin{array}{r}237.0 \\
7.65 \\
9.6 \\
6.2 \\
470\end{array}$ & $\begin{array}{r}249.3 \\
8.04 \\
32 \\
5.1 \\
494\end{array}$ & $\begin{array}{r}260.9 \\
8.70 \\
11 \\
6.4 \\
517\end{array}$ \\
\hline
\end{tabular}

STATISTICS OF MONTHLY MEAN DATA FOR WATER YEARS 1957 - 1996, BY WATER YEAR (WY)

\begin{tabular}{|c|c|c|c|c|c|c|c|c|c|c|c|c|}
\hline $\begin{array}{l}\text { MEAN } \\
\text { MAX } \\
(W Y) \\
M I N \\
(W Y)\end{array}$ & $\begin{array}{l}27.1 \\
74.9 \\
1958 \\
7.88 \\
1964\end{array}$ & $\begin{array}{l}33.3 \\
71.9 \\
1958 \\
14.3 \\
1973\end{array}$ & $\begin{array}{l}34.2 \\
56.8 \\
1987 \\
13.5 \\
1973\end{array}$ & $\begin{array}{l}32.9 \\
48.4 \\
1995 \\
14.0 \\
1973\end{array}$ & $\begin{array}{l}38.4 \\
60.3 \\
1987 \\
21.5 \\
1973\end{array}$ & $\begin{array}{r}49.8 \\
113 \\
1995 \\
23.9 \\
1971\end{array}$ & $\begin{array}{r}117 \\
440 \\
1994 \\
8.32 \\
1972\end{array}$ & $\begin{array}{r}257 \\
1063 \\
1994 \\
5.71 \\
1972\end{array}$ & $\begin{array}{r}145 \\
708 \\
1979 \\
4.69 \\
1971\end{array}$ & $\begin{array}{r}31.2 \\
169 \\
1995 \\
3.89 \\
1972\end{array}$ & $\begin{array}{l}25.0 \\
97.9 \\
1957 \\
4.28 \\
1972\end{array}$ & $\begin{array}{l}23.8 \\
67.5 \\
1993 \\
4.26 \\
1972\end{array}$ \\
\hline
\end{tabular}

SUMMARY STATISTICS

ANNUAL TOTAL

ANNUAL MEAN

HIGHEST ANNUAL MEAN

LOWEST ANNUAL MEAN

BIGHEST DAILY MEAN

LOWEST DATLY MEAN

ANNUAL SEVEN-DAY MINIMUM

INSTANTANEOUS PEAK FLOW

INSTANTANEOUS PEAK STAGE

INSTANTANEOUS LOW FLOW

ANNUAL RUNOFF (AC-FT)

10 PERCENT EXCEEDS

50 PERCENT EXCEEDS

90 PERCENT EXCEEDS
FOR 1995 CALENDAR YEAR

$\begin{aligned} 50589 & \\ 139 & \\ & \\ 690 & \text { May } 31 \\ 18 & \text { Aug } 7 \\ 22 & \text { Aug } 5 \\ & \\ & \\ 100300 & \\ 496 & \\ 51 & \\ 33 & \end{aligned}$

FOR 1996 WATER YEAR

$$
\begin{array}{r}
10579.4 \\
28.9
\end{array}
$$

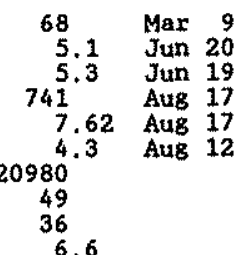

WATER YEARS 1957 - 1996

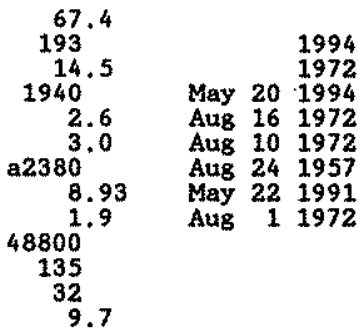

- Estimated

a-From rating curve extended above $900 \mathrm{fts} / \mathrm{s}$. 
PERTOD OF RECORD 1986 to current year.-Water years 1981, 1986 to current year.

\section{WATER QUALITY DATA, WATER YEAR OCTOBER 1995 TO SEPTEMBER 1996}
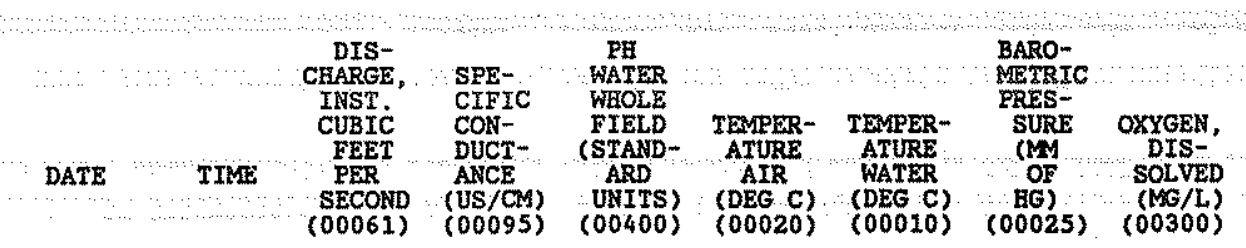

OXYGEN,
DIS-
SOLVED
(PER-
CENT
SATUR-
ATION)
(OOBO1)

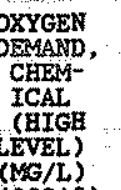

COLI-

FERA,

0.7

(COLS.,

$100 \mathrm{ML})$

\begin{tabular}{|c|c|c|c|c|c|c|c|c|c|c|c|}
\hline Nov 1995 & 0900 & 36 & 488 & 8.0 & -3.0 & 0.5 & 601 & 12.1 & 107 & $<10$ & $-\cdots$ \\
\hline $01 \ldots$ & 0900 & 45 & 454 & 8.6 & 2.5 & 2.5 & 588 & 10.4 & 99 & $<10$ & - \\
\hline $12 \ldots$ & 0845 & 45 & 388 & 8.4 & 4.0 & 5.5 & 597 & 10.8 & 110 & 12 & \\
\hline & 0815 & 5.0 & 647 & 8.3 & 15.5 & 15.5 & 606 & 8.3 & 105 & 22 & 87 \\
\hline
\end{tabular}

\begin{tabular}{|c|c|c|c|c|c|}
\hline $\begin{array}{c}\text { STREP- } \\
\text { TOCOCCI } \\
\text { FECAL, } \\
\text { KF AGAR } \\
\text { (COLS, } \\
\text { PER } \\
100 \text { ML) } \\
(31673)\end{array}$ & $\begin{array}{l}\text { HARD- } \\
\text { NESS } \\
\text { TOTAL } \\
\text { (NG/L } \\
\text { AS } \\
\text { CACO3) }\end{array}$ & $\begin{array}{l}\text { HARD- } \\
\text { NESS } \\
\text { NONCARB } \\
\text { DISSOLV } \\
\text { FLD. AS } \\
\text { CACO3 } \\
\text { (MG/L) }\end{array}$ & $\begin{array}{l}\text { CALCIUM } \\
\text { DIS- } \\
\text { SOLVED } \\
\text { (MG/L } \\
\text { AS CA) }\end{array}$ & $\begin{array}{l}\text { MAGNE- } \\
\text { SIUM, } \\
\text { DIS- } \\
\text { SOLVED } \\
\text { (MG/L } \\
\text { AS MG) }\end{array}$ & $\begin{array}{l}\text { SODIUM, } \\
\text { DIS- } \\
\text { SOLVED } \\
\text { (WG } \\
\text { AS NAA) } \\
\text { (00930) }\end{array}$ \\
\hline
\end{tabular}

$\begin{array}{ccc} & & \text { BICAR- } \\ \text { SODIUM } & \text { POTAS- } & \text { BONATE } \\ \text { AD- } & \text { SIUM, WATER } \\ \text { SORP- } & \text { DIS- } & \text { DIS IT } \\ \text { TION } & \text { SOLVED } & \text { FIELD } \\ \text { RATIO } & \text { (MG/L } & \text { MG/L AS } \\ & \text { AS K } & \text { HCOS }\end{array}$

CAR-

CONATE ALKA-

WATER WAT DIS

DIS IT TOT IT

FIELD FIELD

$M G / L$ AS $M G / L$ AS

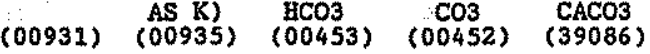

\begin{tabular}{|c|c|c|c|c|c|c|}
\hline $\begin{array}{c}\text { Nov } 1995 \\
30 \ldots\end{array}$ & -- & 210 & 21 & 64 & 13 & 17 \\
\hline EB 1996 & -- & 200 & 24 & 61 & 12 & 16 \\
\hline $12 \ldots$ & $-\rightarrow$ & 180 & 23 & S5 & 11 & 12 \\
\hline & 4700 & 250 & 26 & 73 & 17 & 27 \\
\hline
\end{tabular}

$\begin{array}{lll}0.5 & 1.2 & 235 \\ 0.5 & 1.5 & 198 \\ 0.4 & 1.1 & 186 \\ 0.7 & 1.7 & 276\end{array}$

SOLIDS, NITRO- NITRO- NITRO- NITRO- NITROSUM OF GEN, GEN, GEN, GEN, GEN,

AINITY- SULFATE

$\begin{array}{lll}\text { CHLO } & \text { FLUO- SILICA, SUM OF } \\ \text { RIDE, } & \text { RIDE, } & \text { DIS- } \\ \text { DIS- } & \text { DISTI- }\end{array}$

TUERTS,

(MAB/L SOLVED

SOLVED

(MG/L

AS (MG/L

$\begin{array}{ll}(90410) & (00945) \\ (00940)\end{array}$

SOLVED $\quad(M G / L$

(MG/L

AS F) SIO2)

SOLVED

$(\mathrm{MG} / \mathrm{L})$

DIS-
SOLVED

(MG/L

AS N)

DIS- DrS-

SOLVED SOLVED DIS- DIS-

MG/L MG/L SOE SOLED

AS N AS N AS N) ASN)

NOV 1995

301996

$\begin{array}{lllll}168 & 49 & 7.7 & 0.40 & 14 \\ 188 & 47 & 7.5 & 0.50 & 13 \\ 162 & 42 & 5.3 & 0.30 & 11 \\ 211 & 76 & 10 & 0.70 & 19\end{array}$

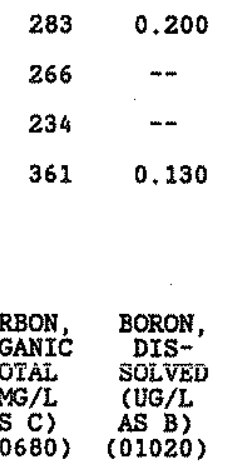

0.020

0.220

0.080

$01 . .$.

APR ... 162

$20, \ldots \quad 211$

76

10

$0.70 \quad 19$

$<0.010$

0.210

0.180

0.12

NITRO- NITRO-

GEN,AM- GEN,AM-

MONIA + MONIA +

OROAA + PHOS-

PHOS- EHORUS

DATE

$\begin{array}{lll}\text { TOTAL } & \text { DIS. } & \text { TOTAL } \\ \text { (MG/L } & \text { (MG/L } & \text { (MG/L } \\ \text { ASN } & \text { AS N) } & \text { AS P }\end{array}$

$\begin{array}{ccc}\text { (00625) } & \text { (00623) } & \text { (AS P) } \\ (00665)\end{array}$

DIS- ORTHO,

SOL VED

AS P) AS P)

$\begin{array}{lll}(00666) & \text { AS P) } & \text { AS C) } \\ (00671) & (00680)\end{array}$

$<0.010$

0.100

$<0.015$

--

NOV 1995

FEB 1996

APR 1 .
$0.20<0.20$
0.050
0.030
0.030
0.40
0.30
0.030
0.020
0.020
$0.20<0.20$
0.020
0.010
$<0.010$

1.740

3.010

0.010

0.140

0.040

$12,$.

3.320

$0.040<0.010$

0.020

$7.0 \quad 83$

13
57
17
10

21
14
20

2.0

66

$1.7 \quad 75$

264

2.4

75

84 
RIO GRANDE BASIN

08276500 RIO GRANDE BELOW TAOS JUNCTION BRIDGE, NEAR TAOS, NM

LOCATION. --Lat $36^{\circ} 19 \cdot 12^{\prime \prime}$, long $105^{\circ} 45^{\prime} 14^{\prime \prime}$, in NWKNEK sec.15, T.24 N., R.11 E. Taos County, Hydrologic Untt 13020101 , on left bank $1.7 \mathrm{mi}$ downstream from bridge on State Highway $567,2.0 \mathrm{mi}$ downstream from Rio Rueblo de Taos, $11.8 \mathrm{mt}$ southwest of Taos, and at mile 1,657.7.

DRATNAGE AREA. $-9,730 \mathrm{mt}^{2}$, approximately, Including 2,940 $\mathrm{mi}^{2}$ in closed basin in San Luis Valley, Co.

WATER-DISCHARGE RECORDS

PERIOD OF RECORD --July 1925 to current year. Prior to October 1930 monthly discharge only, published in WSP 1312 . Published as "at Taos Junction Bridge, noar Taos" prior to 1934.

REVISED RECORDS.--WSP 788: 1934(M). WSP 828: Drainage area, WSP 1392: 1931-1932, 1935, $1937,1945,1950$.

GAGE. - Water-stage recorder. Datum of gage $186,050.3 \mathrm{ft}$ above National Geodettc Vertical Datum of 1929 . Prior to Apr. 14, 1934, at bridge $1.7 \mathrm{mi}$ upstream at different datum.

REMARKS. - Water-discharge records good except for estimated daily discharges which axe fair. Diversions upstream from station for irrigation of about 620,000 acres in Colorado and 30,000 acres in New Mexico. Satellite telemeter at station.

EXTREMES OUTSTDE PERIOD OF RECORD.--Maximum flood since at least 1888 , about $14,000 \mathrm{ft}^{3} / \mathrm{s}$ June 19 , 1903 , from records for Rio Grande at Embudo and estimated inflow. Other floods exceeding $10,000 \mathrm{ft}^{3} / \mathrm{s}$ occurred June 9 1905, May 28, 1920, and June 16, 1921, from comparison of records for stations near Lobatos and at Embudo. DISCHARGE, CUBIC FEET PER SECOND, WATER YEAR OCTOBER 1995 TO SEPTEMBER 1996

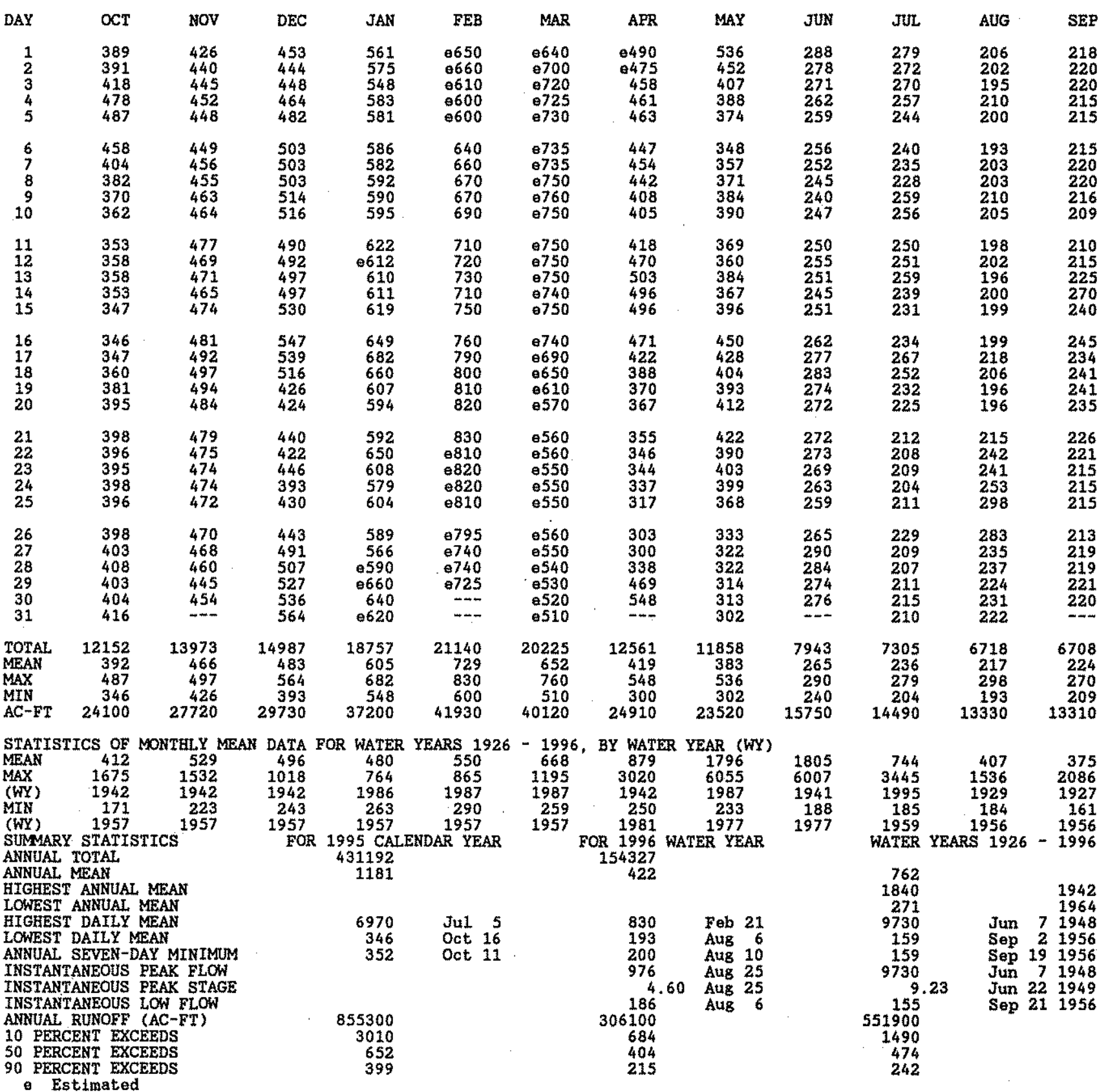


PERIOD OF RECORD. - Water years 1975 to current year.

WATER QUALITY DATA, WATER YEAR OCTOBER 1995 TO SEPTEMBER 1996

\begin{tabular}{|c|c|c|c|c|c|c|c|c|c|c|c|c|}
\hline DATE & TIME & $\begin{array}{c}\text { DIS- } \\
\text { CEARGE, } \\
\text { INSST } \\
\text { CUBIC } \\
\text { FEET } \\
\text { PER } \\
\text { SECOND } \\
(00061)\end{array}$ & $\begin{array}{l}\text { SPE- } \\
\text { CIFIC } \\
\text { CON- } \\
\text { DUCT- } \\
\text { ANCE } \\
\text { (US/CM) } \\
\text { (00095) }\end{array}$ & $\begin{array}{l}\text { PA } \\
\text { WATER } \\
\text { WHOLE } \\
\text { FIELD } \\
\text { (STAND- } \\
\text { ARD } \\
\text { UNITS) } \\
\text { (O0400) }\end{array}$ & $\begin{array}{c}\text { TEPIPER- } \\
\text { ATURE } \\
\text { AIR } \\
\text { (DEG C) } \\
(00020)\end{array}$ & $\begin{array}{l}\text { TEMIPER- } \\
\text { ATURE } \\
\text { WATER } \\
\text { (DEG C) } \\
(00010)\end{array}$ & $\begin{array}{c}\text { TUR- } \\
\text { BID- } \\
\text { ITY } \\
(\text { NTU) } \\
(00076)\end{array}$ & $\begin{array}{c}\text { BARO- } \\
\text { METRIC } \\
\text { PRES - } \\
\text { SURE } \\
\text { (MA } \\
\text { OE } \\
\text { GG) } \\
(00025)\end{array}$ & $\begin{array}{c}\text { OXYGEN, } \\
\text { DIS- } \\
\text { SOLVED } \\
(\text { MG/L }) \\
(00300)\end{array}$ & $\begin{array}{c}\text { OXYGEN- } \\
\text { DIS- } \\
\text { SOLVED } \\
\text { (PER- } \\
\text { CENT } \\
\text { SATUR- } \\
\text { ATION) } \\
\text { (00301) }\end{array}$ & $\begin{array}{c}\text { OXYGEN } \\
\text { DEMAND, } \\
\text { CHESH- } \\
\text { ICAL } \\
\text { (EIGH } \\
\text { LEVEL) } \\
(M E L / L) \\
(00340)\end{array}$ & $\begin{array}{l}\text { COLI- } \\
\text { FORM, } \\
\text { FECAL, } \\
0.7 \\
\text { UM-MF } \\
\text { (COLS. } \\
100 \mathrm{ML}) \\
(31625)\end{array}$ \\
\hline $\begin{array}{l}\text { Nov } 1995 \\
29 \\
\text { JAN } 1996\end{array}$ & 1030 & 445 & 37.4 & 8.1 & -5.0 & 3.5 & $-\cdots$ & 616 & 11.1 & 104 & 12 & . . . . \\
\hline $\mathrm{APR}^{31} \cdots$ & 0945 & 640 & 306 & 8.4 & 3.0 & 4.0 & -- & 606 & 10.7 & 103 & $<10$ & $<4$ \\
\hline $11 \ldots$ & 101 & 418 & 382 & 8.6 & 1 & 12.0 & 2. & 60 & 9.4 & 110 & 14 & $K_{4}$ \\
\hline $16 \ldots$ & 1415 & 234 & 3 & 8 & 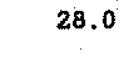 & 23.0 & - & 612 & 7. & 104 & - & - \\
\hline $20 \ldots$ & 1145 & 196 & 327 & 8.5 & 25.5 & 20.5 & 9.7 & 619 & 8.1 & 112 & 17 & -- \\
\hline
\end{tabular}

\begin{tabular}{|c|c|c|c|c|c|c|c|c|c|c|c|c|}
\hline DATE & $\begin{array}{c}\text { STREP- } \\
\text { TOCOCCI } \\
\text { FECAL, } \\
\text { KF AGAR } \\
\text { (COLS. } \\
\text { PER } \\
100 \mathrm{ML}) \\
(31673)\end{array}$ & $\begin{array}{l}\text { HARD } \\
\text { NESS } \\
\text { TOTAL } \\
\text { (MG/L } \\
\text { AS } \\
\text { CACO3) } \\
(00900)\end{array}$ & $\begin{array}{l}\text { HARD- } \\
\text { NESS } \\
\text { NONCARB } \\
\text { DISSOLV } \\
\text { FLD. AS } \\
\text { CACOO } \\
(M G / L) \\
(00904)\end{array}$ & $\begin{array}{l}\text { CALCIUM } \\
\text { DIS- } \\
\text { SOLVED } \\
\text { (MG/L } \\
\text { AS CA) } \\
(00915)\end{array}$ & $\begin{array}{c}\text { MAGNE- } \\
\text { SIUM, } \\
\text { DIS- } \\
\text { SOLVED } \\
\text { (MG/L } \\
\text { AS MG) } \\
(00925)\end{array}$ & $\begin{array}{c}\text { SODIUM, } \\
\text { DIS- } \\
\text { SOLVED } \\
\text { (MG/L } \\
\text { AS NA) } \\
(00930)\end{array}$ & $\begin{array}{c}\text { SODIUM } \\
\text { AD- } \\
\text { SORP- } \\
\text { TION } \\
\text { RATIO } \\
(00931)\end{array}$ & $\begin{array}{c}\text { POTAS- } \\
\text { SIUM, } \\
\text { DIS- } \\
\text { SOLVED } \\
\text { (MG/L } \\
\text { AS K) } \\
(00935)\end{array}$ & $\begin{array}{l}\text { BICAR- } \\
\text { BONATE } \\
\text { WATER } \\
\text { DIS IT } \\
\text { FIELD } \\
\text { MG/L AS } \\
\text { ECO3 } \\
(00453)\end{array}$ & $\begin{array}{c}\text { CAR- } \\
\text { BONATE } \\
\text { WATER } \\
\text { DIS IT } \\
\text { FIELD } \\
\text { MG/L AS } \\
\text { CO3 } \\
(00452)\end{array}$ & $\begin{array}{c}\text { ALKA- } \\
\text { LINITY } \\
\text { WAT DIS } \\
\text { TOT IT } \\
\text { FIELD } \\
\text { MG/L AS } \\
\text { CACO3 } \\
(39086)\end{array}$ & $\begin{array}{c}\text { ALKA- } \\
\text { LINITY } \\
\text { LAB } \\
\text { (MG } \\
\text { AS } \\
\text { CACO3) } \\
(90410)\end{array}$ \\
\hline $\begin{array}{l}\text { NOV } 1995 \\
29 \\
\text { JAN } 19996\end{array}$ & 24 & 130 & 0 & 37 & 8.3 & 31 & 1 & 3.7 & 147 & 4 & 126 & 132 \\
\hline $\begin{array}{l}31 \\
\mathrm{APR}\end{array}$ & 41 & 98 & 0 & 29 & 6.3 & 22 & 1 & 3.9 & 117 & 3 & 101 & 108 \\
\hline JUL $11 \ldots$ & 50 & 130 & 7 & 38 & 7.9 & 28 & 1 & 4.1 & 135 & 6 & 120 & 124 \\
\hline AUG $16 \ldots$ & $-\cdots$ & 110 & 9 & 30 & 7.4 & 22 & 0.9 & 3.3 & 118 & 0 & 96 & 106 \\
\hline $20 \ldots$ & -- & 100 & 0 & 29 & 7.2 & 28 & 1 & 3.5 & 121 & 3 & 104 & 109 \\
\hline DATE & $\begin{array}{c}\text { SULFATE } \\
\text { DIS - } \\
\text { SOLVED } \\
\text { (NG/1 } \\
\text { AS SO4) } \\
(00945)\end{array}$ & $\begin{array}{l}\text { CHLO- } \\
\text { RIDE, } \\
\text { DIS- } \\
\text { SOLVED } \\
\text { (MG/L } \\
\text { AS CL) } \\
(00940)\end{array}$ & $\begin{array}{l}\text { FLUO- } \\
\text { RIDE. } \\
\text { DIS- } \\
\text { SOLVED } \\
\text { (NG/L } \\
\text { AS F) } \\
(00950)\end{array}$ & $\begin{array}{l}\text { SILICA, } \\
\text { DIS- } \\
\text { SOLVED } \\
\text { (MG/L } \\
\text { AS } \\
\text { SIO2) } \\
(00955)\end{array}$ & $\begin{array}{l}\text { SOLIDS, } \\
\text { RESIDUE } \\
\text { AT } 180 \\
\text { DEG.C } \\
\text { DIS- } \\
\text { SOLVED } \\
(M G / L) \\
(70300)\end{array}$ & $\begin{array}{c}\text { SOLIDS, } \\
\text { SUM OF } \\
\text { CONSTI- } \\
\text { TUENTS, } \\
\text { DIS- } \\
\text { SOLVED } \\
\text { (MG/L) } \\
\text { (70301) }\end{array}$ & $\begin{array}{c}\text { NITRO- } \\
\text { GEN, } \\
\text { NITRATE } \\
\text { DIS- } \\
\text { SOLVED } \\
\text { (MG/L } \\
\text { AS N) } \\
(00618)\end{array}$ & $\begin{array}{c}\text { NITRO- } \\
\text { GEN, } \\
\text { NITRITE } \\
\text { DIS- } \\
\text { SOLVED } \\
\text { (NG/L } \\
\text { AS N) } \\
(00513)\end{array}$ & $\begin{array}{c}\text { NITRO- } \\
\text { GEN } \\
\text { NO2+NÓ3 } \\
\text { DIS- } \\
\text { SOLVED } \\
\text { (NG/L } \\
\text { AS N) } \\
(00631)\end{array}$ & $\begin{array}{c}\text { NITRO- } \\
\text { GEN, } \\
\text { AMNONIA } \\
\text { DIS- } \\
\text { SOLVED } \\
\text { (MG/L } \\
\text { AS N) } \\
(00608)\end{array}$ & $\begin{array}{l}\text { NITRO- } \\
\text { GEA,AM- } \\
\text { MONIA + } \\
\text { ORGANIC } \\
\text { TOTAL } \\
\text { (MG/L } \\
\text { AS N) } \\
(00625)\end{array}$ & $\begin{array}{l}\text { NITRO- } \\
\text { GEN,AM- } \\
\text { MONIA + } \\
\text { ORGANIC } \\
\text { DIS. } \\
\text { (MGG/L } \\
\text { AS N) } \\
(00623)\end{array}$ \\
\hline $\begin{array}{l}\text { NOV } 1995 \\
29 \\
\text { JAN } 19966\end{array}$ & 54 & 5 & .60 & 25 & $m$ & 246 & - & $<0.010$ & 0.170 & $<0.015$ & 0.30 & $<0.20$ \\
\hline APR & 34 & 7.4 & 0.50 & 29 & -- & 195 & $\cdots$ & $<0.010$ & 0.490 & 0.020 & $<0.20$ & $<0.20$ \\
\hline JuL & 59 & 8.4 & 0.80 & 22 & -- & 241 & - & $<0.010$ & $<0.050$ & $<0.015$ & 0.40 & $<0.20$ \\
\hline${ }_{\text {AUG }}^{16} \ldots$ & 48 & 7.4 & 0.80 & 28 & 224 & 206 & 0.290 & 0.010 & 0.300 & 0.030 & 0.60 & $<0.20$ \\
\hline $20 \ldots$ & 43 & 8.8 & 0.80 & $3 \hat{i}$ & - & 214 & 0.050 & 0.010 & 0.070 & 0.020 & $<0.20$ & $<0.20$ \\
\hline
\end{tabular}

\begin{tabular}{|c|c|c|c|c|c|c|c|c|c|c|}
\hline $\begin{array}{c}\text { PHOS- } \\
\text { PHORUS } \\
\text { TOTAL } \\
\text { (MG/L } \\
\text { AS E) } \\
(00665)\end{array}$ & $\begin{array}{c}\text { PHOS- } \\
\text { PHORUS } \\
\text { DIS- } \\
\text { SOLVED } \\
\text { (ME/L } \\
\text { AS P) } \\
(00666)\end{array}$ & $\begin{array}{l}\text { PHOS- } \\
\text { PHORUS } \\
\text { ORTHO, } \\
\text { DIS } \\
\text { SOLVED } \\
(\text { MG/L } \\
\text { AS P) } \\
(00671)\end{array}$ & $\begin{array}{l}\text { CARBON, } \\
\text { ORGANIC } \\
\text { TOTAL } \\
\text { (MG/L } \\
\text { AS C) } \\
(00680)\end{array}$ & $\begin{array}{l}\text { CARBON } \\
\text { ORGANIC } \\
\text { DIS- } \\
\text { SOLVED } \\
\text { (MG/L } \\
\text { AS C) } \\
(00681)\end{array}$ & $\begin{array}{c}\text { CYANIDE } \\
\text { TOTAL } \\
\text { (MG/L } \\
\text { AS CN }) \\
(00720)\end{array}$ & $\begin{array}{l}\text { ALUM- } \\
\text { INUM, } \\
\text { DIS- } \\
\text { SOLVED } \\
\text { (UG/L } \\
\text { AS AL) } \\
\text { (01106) }\end{array}$ & $\begin{array}{l}\text { ANTI- } \\
\text { MONY, } \\
\text { DIS- } \\
\text { SOLVEDD } \\
\text { (UG/L } \\
\text { AS SB) } \\
(01095)\end{array}$ & $\begin{array}{c}\text { ARSENIC } \\
\text { DIS- } \\
\text { SOLVED } \\
\text { (UG/L. } \\
\text { AS AS) } \\
(01000)\end{array}$ & $\begin{array}{l}\text { BARIUM, } \\
\text { DIS- } \\
\text { SOLVED } \\
\text { (UG/L } \\
\text { AS BA) } \\
(01005)\end{array}$ & $\begin{array}{l}\text { BERYL- } \\
\text { LIUM, } \\
\text { DIS- } \\
\text { SOLVED } \\
\text { (UG } \\
\text { AS BE) } \\
(01010)\end{array}$ \\
\hline$<0.010$ & $<0.010$ & $<0.010$ & 2.3 & -- & $<0.010$ & 27 & $<1.0$ & 2 & 32 & $<1.0$ \\
\hline 0.040 & 0.030 & 0.030 & 2.7 & $\ldots$ & -- & -- & $\cdots$ & -- & $-\cdots$ & -- \\
\hline 0.040 & $<0.010$ & $<0.010$ & 4.1 & -- & $<0.010$ & 27 & $<1.0$ & 2 & 33 & $<1.0$ \\
\hline 0.110 & 0.050 & 0.050 & 5.7 & 1.9 & -- & -- & $m$ & -- & -- & -- \\
\hline 0.010 & $<0.010$ & 0.020 & 3.6 & -- & $<0.010$ & -- & -- & $\cdots$ & - & -- \\
\hline
\end{tabular}


RIO GRANDE BASIN

08276500 RIO GRANDE BELOW TAOS JUNCTION BRIDGE, NEAR TAOS, NM -- Continued WATER-QUALITY RECORDS

WATER QUALITY DATA, WATER YEAR OCTOBER 1995 TO SEPTEMBER 1996

ATE

\begin{tabular}{|c|c|}
\hline $\begin{array}{c}\text { BORON, } \\
\text { DIS- } \\
\text { SOLVED } \\
\text { (UG/4. } \\
\text { AS B) } \\
(01020)\end{array}$ & $\begin{array}{c}\text { CADMIUM } \\
\text { DIS- } \\
\text { SOLVED } \\
\text { (UG/L } \\
\text { AS CD) } \\
\text { (01025) }\end{array}$ \\
\hline
\end{tabular}

$\begin{array}{lc}\text { CHRO- } & \\ \text { MIUM, } & \text { COBALT, } \\ \text { DIS- } & \text { DIS- } \\ \text { SOLVED } & \text { SOLVED } \\ \text { (UG/L } & (U G / L \\ \text { AS CR) } & \text { AS CO) } \\ (01030) & (01035)\end{array}$

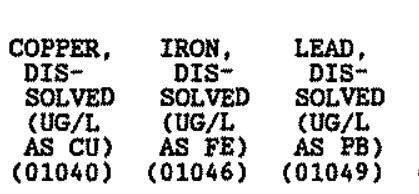

MANGA- MARCURY MOLYB-

NESE, TOTAL DENUM, NICKSL,

DIS-

SOLVED

(UG/L

AS MN)

RECOV-

ERABLE

(UG/L

DIS- DIS-

SOLVED SOLVED

(UG/L

AS $\mathrm{NO)}$ AS NI)

$(01020)(01025)(01030)(01035)$

(01046) (01049)

(71900) (01060) (01065)

NOV 1995
29.996
JAN 1996
APR $\ldots$
$11 \ldots$
JUL
$16 \ldots$
AUG
$20 \ldots$

\begin{tabular}{|c|c|c|c|}
\hline 60 & $<1.0$ & $<1.0$ & $<1.0$ \\
\hline 20 & - & - & - \\
\hline 70 & $<1.0$ & 1.0 & $<1.0$ \\
\hline$-\cdots$ & - & -- & -- \\
\hline 58 & - & - & -- \\
\hline $\begin{array}{l}\text { SELE- } \\
\text { WIUM, } \\
\text { TOTAL } \\
(U G / L \\
\text { US SE) } \\
01147)\end{array}$ & $\begin{array}{c}\text { SELE- } \\
\text { NIUM, } \\
\text { DIS- } \\
\text { SOLVED } \\
\text { (UG/L } \\
\text { AS SE) } \\
\text { (01145) }\end{array}$ & $\begin{array}{c}\text { SILVSR, } \\
\text { DIS- } \\
\text { SOLVED } \\
\text { (UG/L. } \\
\text { AS AG) } \\
(01075)\end{array}$ & $\begin{array}{l}\text { ZINC, } \\
\text { DIS- } \\
\text { SOLVED } \\
\text { (UG/L } \\
\text { AS ZN) } \\
(01090)\end{array}$ \\
\hline
\end{tabular}

\begin{abstract}
$2.0 \quad 21$
\end{abstract}
$<1.0$

8.0

$<0.10$

8.0

2.0

- 17

2.

8.0

$<1.0$

6.0

$<0.10$

$-$

-- 13

$--$

10

$<0.10$

$8.0 \quad 2.0$

DATE

(01147)

10

10

$\begin{array}{cc}\text { NITRO- } & \text { NITRO- } \\ \text { GEN, } & \text { GEN,NH4 } \\ \text { NO2+NO3 } & \text { TOTAL } \\ \text { TOT. IN } & + \\ \text { IN BOT. } & \text { TOT.MAT } \\ \text { MAT. } & \text { MAT. } \\ \text { (MG/KG } & \text { (MG/KG } \\ \text { AS N) } & \text { AS N) } \\ (00633) & (00611)\end{array}$

NITRO- PHOSGEN,NH4 PHORUS + ORG. TOTAL TOT IN BOT MAT MG/KG MAT. (MG/KG (MG/KG

AS N) AS P)

(00668)

NOV 1995
29.1996
JAN 1996
$31 \ldots$
APR
$11 \ldots$
JUL $\ldots \ldots$
$16 \ldots$
AUG $\ldots \ldots$
$20 \ldots$
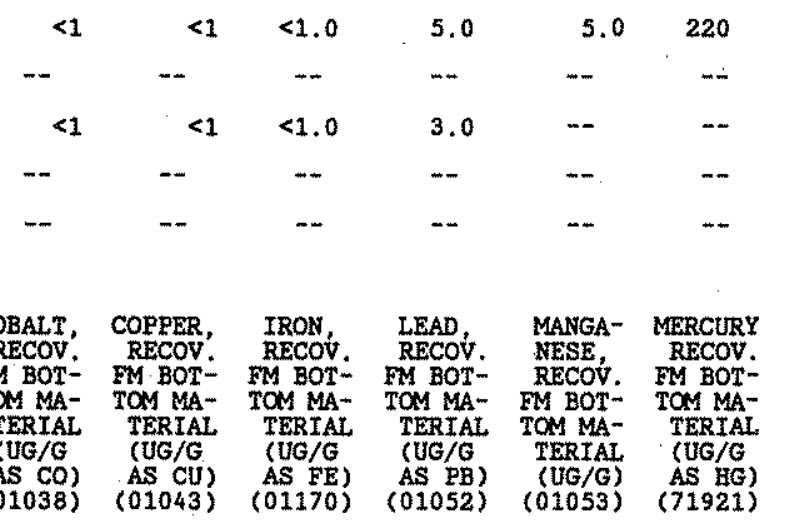

$3200 \quad 1300$

ARSENIC CADMIUM CHROTOTAL RECOV. MIUM, IN BOT- FM BOT- RECOV. TOM MA- TOM MA- FM BOT(UG/G (UG/G TERIAL AS AS) AS CD) (UG/G)$$
\sqrt{2}+x^{2}
$$

ATE

$\begin{array}{cccccc}\text { COBALT, } & \text { COPPER, } & \text { IRON, } & \text { LEAD, } & \text { MANGA- } & \text { MERCURY } \\ \text { RECOV, } & \text { RECOV: } & \text { RECOV. } & \text { RECOV. } & \text { NESE, } & \text { RECOV. } \\ \text { FM BOT- } & \text { FMBOT- } & \text { FM BOT- } & \text { FM BOT- } & \text { RECOV. } & \text { FM BOT- } \\ \text { TOM MA- } & \text { TOM MA- } & \text { TOM MA- } & \text { TOM MA- } & \text { FM BOT- } & \text { TOM MA- } \\ \text { TERIAL } & \text { TERIAL } & \text { TERTAL } & \text { TERIAL } & \text { TOM MA- } & \text { TERTAL. } \\ \text { (UG/G } & \text { (UG/G } & \text { (UG/G } & (U G / G & \text { TERIAL. } & (U G / G \\ \text { AS CO) } & \text { AS CU) } & \text { AS FE) } & \text { AS PB) } & (U G / G) & \text { AS HG) } \\ (01038) & (01043) & (01170) & (01052) & (01053) & (71921)\end{array}$
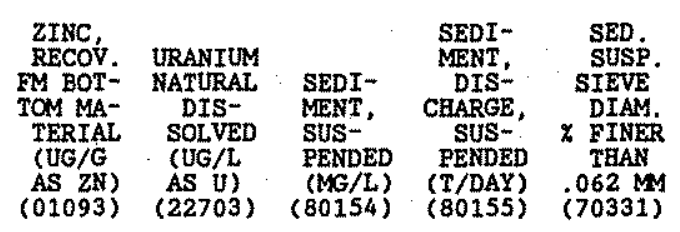

\footnotetext{
NOV 1995

JAN 29996

$31 \ldots$.

APR.

$11 \ldots$

AUG $20 .$.
}

$\begin{array}{cccccccc}<5 & 19 & 2500 & 10 & 2300 & 0.02 & 220 & 3.0 \\ -- & -- & -- & -- & -- & -- & -- & -- \\ -- & -- & -- & -- & -- & -- & -- & 3.0 \\ -- & -- & -- & -- & -- & -- & -- & -- \\ -- & -- & -- & - & -- & -- & -- & --\end{array}$

$\begin{array}{ll}4 & 4.8 \\ 10 & 17 \\ 15 & 17 \\ 86 & 54 \\ 67 & 35\end{array}$

87

66

60. 
RIO GRANDE BASIN

08276500 RIO GRANDE BELOW TAOS JUNCTION BRIDGE, NEAR TAOS, NM - - Continued

WATER-QUALITY RECORDS

CHEMICAL ANALYSES OF ORGANIC COMPOUNDS, WATER YEAR OCTOBER 1995 TO SEPTEMBER 1996

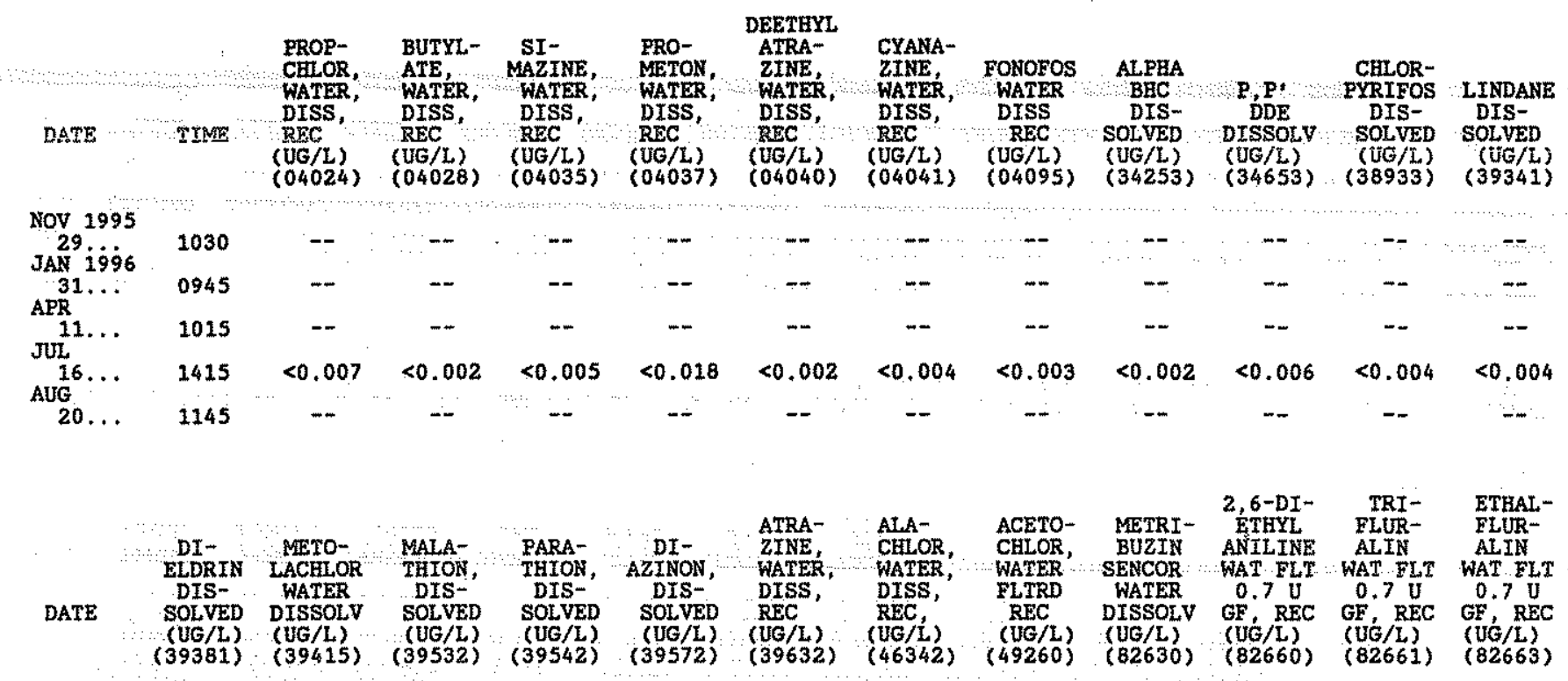

NoV 1995

JAN 1996

$31 \ldots$

11 .. $\begin{array}{cccccc}11 \ldots & \ldots & \ldots & \ldots & \ldots \\ \text { JUL } & & & & & \\ 16 \ldots & <0.001 & <0.002 & <0.005 & <0.004 & <0.002 \\ \text { AUG } & \ldots & \ldots & \ldots & \ldots & \ldots\end{array}$

$<0$

$-$

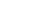

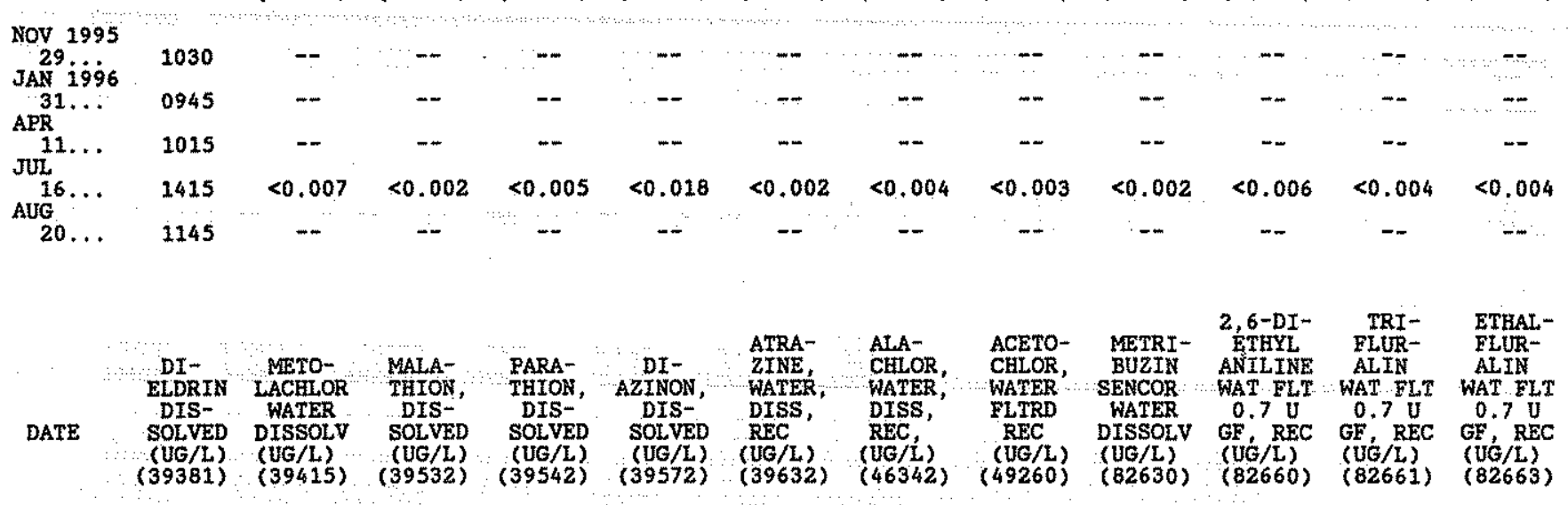

.

$-$

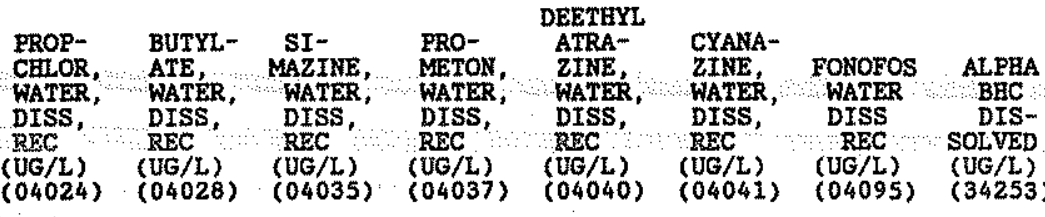

$\begin{array}{ccccc} & \text { TER- } & \text { LIN- } & \text { METHYL } & \\ \text { PHORATE } & \text { BACIL } & \text { URON } & \text { PARA- } & \text { EPTC } \\ \text { WATER } & \text { WATER } & \text { WATER } & \text { THION } & \text { WATER } \\ \text { FLTRD } & \text { FLTRD } & \text { FLTRD } & \text { WAT FLT } & \text { FLTRD } \\ 0.7 \text { U } & 0.7 \text { U } & 0.7 \text { U } & 0.7 \mathrm{U} & 0.7 \mathrm{U} \\ \text { GF REC } & \text { GF, REC } & \text { GF, REC } & \text { GF, REC } & \text { GF, REC } \\ (U G / L) & (U G / L) & (U G / L) & (U G / L) & (U G / L) \\ (82664) & (82665) & (82666) & (82667) & (82668)\end{array}$

$\begin{array}{ccc}\text { PEB- } & \text { TEBU- } & \text { MOL- } \\ \text { ULATE } & \text { THIURON } & \text { INATE } \\ \text { WATER } & \text { WATER } & \text { WATER } \\ \text { FILTRD } & \text { FLTRD } & \text { FLTRD } \\ 0.7 \text { UL } & 0.7 \text { U } & 0.7 \text { U } \\ \text { GF, REL } & \text { GE, REC } & \text { GF, REC } \\ (U G / L) & (\text { UG/L) } & (\text { UG } / L) \\ (82669) & (82670) & (82671)\end{array}$
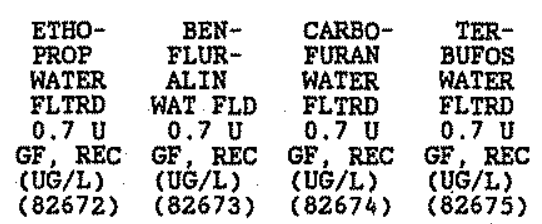

Nov 1995

JAN 1996

APR

11.

AUG

$<0.002<0.007<0.002<0.006<0.002$

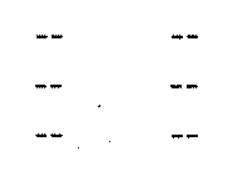

$-$

20...

PRON
AMIDE
WATER
FLTRD
0.7 U
GF REC
$(U G / L)$
$(82676)$

DISUL-
FOTON
WATER
FLTRD
0.7 U
GF, REC
$(U G / L)$
$(82677)$
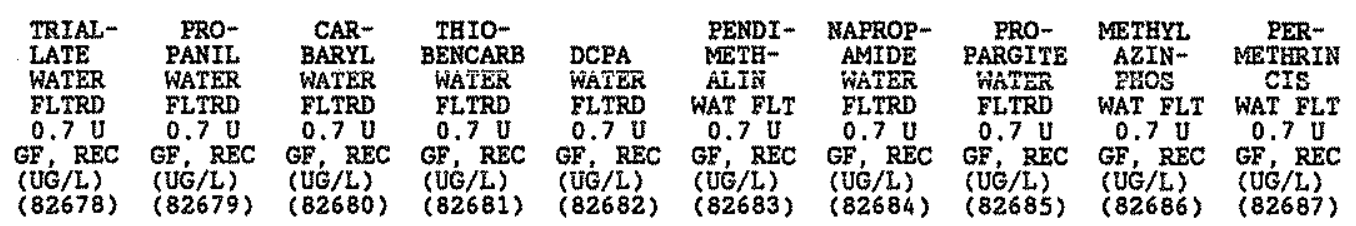

NoV 1995

JAN $29 \dot{9} 6$

$31 \ldots$

$11 .$.

JUL

AUG
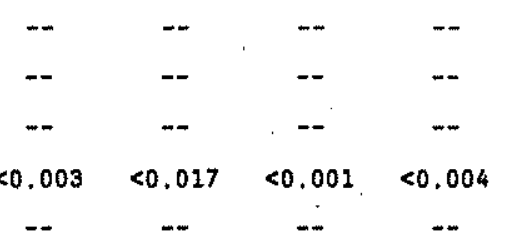$$
--
$$

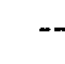

$20 .$. 
RIO GRANDE BASIN

08277470 RIO PUEBLO NEAR PENASCO, MM

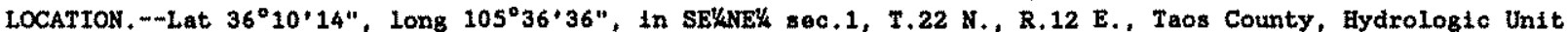
13020101 , on left bank $10 \mathrm{ft}$ downstream from bridge on private road, 0.5 mi upstream from junction of State Highways 518 and $75,1.0 \mathrm{mi}$ domstream from Osha Canyon and $6.0 \mathrm{mi}$ east of Penasco.

DRAINAGE AREA. $--101 \mathrm{mi}^{2}$.

PERIOD OF RECORD. --December 1991 to current year.

GAGE.--Water-stage recorder. Datum of gage is 7,760 ft above National Geodetic Vertical Datum of 1929 from, topographic map.

REMARKS.--Records fair except for estimated daily discharges, which are poor. Several observations of water temperature where made during year.

DISCEARGE, CUBIC FEET PER SECOND, WATER YEAR OCTOBER 199.5 TO SEPTEMBER 1996

\begin{tabular}{|c|c|c|c|c|c|c|c|c|c|c|c|c|}
\hline DAY & $\infty \mathrm{CT}$ & NOV & $\mathrm{DEC}$ & JAN & FEB & MAR & APR & MAY & JUN & JUL & AUG & SEP \\
\hline $\begin{array}{l}1 \\
2 \\
3 \\
4 \\
5\end{array}$ & $\begin{array}{l}20 \\
18 \\
16 \\
16 \\
15\end{array}$ & $\begin{array}{l}14 \\
15 \\
1.3 \\
14 \\
14\end{array}$ & $\begin{array}{l}08.8 \\
09.6 \\
010 \\
011 \\
011\end{array}$ & $\begin{array}{l}08.0 \\
68.0 \\
\mathbf{8 8 . 0} \\
69.0 \\
09.0\end{array}$ & $\begin{array}{l}012 \\
011 \\
610 \\
09.0 \\
010\end{array}$ & $\begin{array}{l}11 \\
010 \\
99.9 \\
010 \\
010\end{array}$ & $\begin{array}{l}21 \\
26 \\
28 \\
26 \\
23\end{array}$ & $\begin{array}{l}57 \\
55 \\
57 \\
59 \\
55\end{array}$ & $\begin{array}{l}6.7 \\
5.8 \\
5.4 \\
5.2 \\
4.8\end{array}$ & $\begin{array}{l}8.7 \\
6.4 \\
5.2 \\
4.6 \\
4.6\end{array}$ & $\begin{array}{r}5.3 \\
8.6 \\
13 \\
6.5 \\
5.1\end{array}$ & $\begin{array}{l}8.6 \\
7.7 \\
7.4 \\
7.4 \\
7.4\end{array}$ \\
\hline $\begin{array}{r}6 \\
7 \\
8 \\
9 \\
10\end{array}$ & $\begin{array}{l}15 \\
15 \\
15 \\
14 \\
14\end{array}$ & $\begin{array}{l}14 \\
14 \\
15 \\
15 \\
15\end{array}$ & $\begin{array}{l}012 \\
e 12 \\
011 \\
012 \\
e 12\end{array}$ & $\begin{array}{l}99.0 \\
910 \\
010 \\
910 \\
910\end{array}$ & $\begin{array}{r}\text { e11 } \\
\text { e12 } \\
013 \\
14 \\
14\end{array}$ & $\begin{array}{r}013 \\
14 \\
17 \\
16 \\
16\end{array}$ & $\begin{array}{l}20 \\
22 \\
24 \\
31 \\
38\end{array}$ & $\begin{array}{l}52 \\
52 \\
51 \\
49 \\
47\end{array}$ & $\begin{array}{l}4.4 \\
4.4 \\
4.4 \\
4.3 \\
3.9\end{array}$ & $\begin{array}{r}4.8 \\
4.8 \\
6.2 \\
8.5 \\
18\end{array}$ & $\begin{array}{l}4.7 \\
4.4 \\
4.8 \\
4.5 \\
5.2\end{array}$ & $\begin{array}{l}7.7 \\
6.9 \\
5.8 \\
5.3 \\
5.4\end{array}$ \\
\hline $\begin{array}{l}11 \\
12 \\
13 \\
14 \\
15\end{array}$ & $\begin{array}{l}14 \\
14 \\
14 \\
13 \\
13\end{array}$ & $\begin{array}{l}12 \\
15 \\
15 \\
15 \\
14\end{array}$ & $\begin{array}{r}012 \\
011 \\
012 \\
13 \\
12\end{array}$ & $\begin{array}{l}99.8 \\
99.7 \\
99.6 \\
99.5 \\
99.4\end{array}$ & $\begin{array}{l}14 \\
14 \\
13 \\
14 \\
15\end{array}$ & $\begin{array}{l}16 \\
16 \\
16 \\
16 \\
16\end{array}$ & $\begin{array}{l}36 \\
34 \\
37 \\
30 \\
31\end{array}$ & $\begin{array}{l}44 \\
42 \\
40 \\
38 \\
36\end{array}$ & $\begin{array}{l}4.0 \\
4.0 \\
4.0 \\
6.1 \\
5.2\end{array}$ & $\begin{array}{l}11 \\
11 \\
16 \\
14 \\
11\end{array}$ & $\begin{array}{l}4.8 \\
4.5 \\
4.5 \\
4.6 \\
5.0\end{array}$ & $\begin{array}{r}6.0 \\
6.0 \\
9.3 \\
8.9 \\
11\end{array}$ \\
\hline $\begin{array}{l}16 \\
17 \\
18 \\
19 \\
20\end{array}$ & $\begin{array}{l}14 \\
14 \\
14 \\
13 \\
13\end{array}$ & $\begin{array}{l}14 \\
14 \\
13 \\
13 \\
13\end{array}$ & $\begin{array}{r}12 \\
13 \\
12 \\
12 \\
011\end{array}$ & $\begin{array}{r}99.4 \\
99.3 \\
99.4 \\
99.6 \\
010\end{array}$ & $\begin{array}{l}15 \\
15 \\
15 \\
15 \\
16\end{array}$ & $\begin{array}{l}16 \\
15 \\
14 \\
14 \\
15\end{array}$ & $\begin{array}{l}31 \\
34 \\
37 \\
40 \\
38\end{array}$ & $\begin{array}{l}33 \\
31 \\
25 \\
20 \\
18\end{array}$ & $\begin{array}{l}4.3 \\
4.1 \\
3.8 \\
3.7 \\
3.4\end{array}$ & $\begin{array}{r}10 \\
9.1 \\
8.4 \\
8.5 \\
7.3\end{array}$ & $\begin{array}{l}4.8 \\
4.9 \\
5.6 \\
5.6 \\
5.0\end{array}$ & $\begin{array}{c}8.7 \\
7.4 \\
13 \\
14 \\
9.8\end{array}$ \\
\hline $\begin{array}{l}21 \\
22 \\
23 \\
24 \\
25\end{array}$ & $\begin{array}{l}13 \\
14 \\
12 \\
13 \\
13\end{array}$ & $\begin{array}{r}13 \\
14 \\
14 \\
12 \\
011\end{array}$ & $\begin{array}{r}010 \\
09.6 \\
08.8 \\
08.0 \\
08.0\end{array}$ & $\begin{array}{l}e 9.0 \\
010 \\
\text { e10 } \\
99.0 \\
99.0\end{array}$ & $\begin{array}{l}18 \\
17 \\
15 \\
17 \\
15\end{array}$ & $\begin{array}{l}16 \\
16 \\
16 \\
15 \\
16\end{array}$ & $\begin{array}{l}35 \\
34 \\
35 \\
42 \\
52\end{array}$ & $\begin{array}{l}17 \\
15 \\
13 \\
13 \\
12\end{array}$ & $\begin{array}{l}3.3 \\
3.7 \\
4.1 \\
3.8 \\
3.9\end{array}$ & $\begin{array}{l}6.8 \\
6.6 \\
5.8 \\
5.4 \\
5.7\end{array}$ & $\begin{array}{l}5.4 \\
5.5 \\
5.7 \\
6.1 \\
7.2\end{array}$ & $\begin{array}{l}8.7 \\
8.0 \\
7.6 \\
7.8 \\
7.6\end{array}$ \\
\hline $\begin{array}{l}26 \\
27 \\
28 \\
29 \\
30 \\
31\end{array}$ & $\begin{array}{l}13 \\
14 \\
13 \\
14 \\
13 \\
13\end{array}$ & $\begin{array}{l}09.6 \\
98.4 \\
06.7 \\
97.5 \\
08.0 \\
-.-\end{array}$ & $\begin{array}{l}e 7.8 \\
e 7.7 \\
e 7.6 \\
e 7.7 \\
08.2 \\
08.8\end{array}$ & $\begin{array}{l}\theta 9.0 \\
010 \\
\theta 10 \\
\theta 11 \\
\theta 12 \\
\theta 12\end{array}$ & $\begin{array}{r}14 \\
13 \\
013 \\
011 \\
\ldots \\
-\ldots\end{array}$ & $\begin{array}{l}15 \\
16 \\
16 . \\
18 \\
18 \\
19\end{array}$ & $\begin{array}{r}65 \\
75 \\
79 \\
68 \\
60 \\
-\end{array}$ & $\begin{array}{r}12 \\
12 \\
9.3 \\
8.1 \\
7.8 \\
7.5\end{array}$ & $\begin{array}{l}4.4 \\
23 \\
11 \\
8.5 \\
8.2\end{array}$ & $\begin{array}{l}5.3 \\
5.7 \\
6.1 \\
8.3 \\
6.4 \\
5.2\end{array}$ & $\begin{array}{l}8.5 \\
8.8 \\
8.8 \\
8.8 \\
8.8 \\
9.0\end{array}$ & $\begin{array}{l}9.0 \\
9.7 \\
8.4 \\
8.2 \\
7.9\end{array}$ \\
\hline $\begin{array}{l}\text { TOTAL } \\
\text { MRAN } \\
\text { MAX } \\
\text { MIN } \\
\text { AC-ET }\end{array}$ & $\begin{array}{r}439 \\
14.2 \\
20 \\
12 \\
871\end{array}$ & $\begin{array}{r}385.2 \\
12.8 \\
15 \\
6.7 \\
764\end{array}$ & $\begin{array}{r}321.6 \\
10.4 \\
13 \\
7.6 \\
638\end{array}$ & $\begin{array}{r}297.7 \\
9.60 \\
12 \\
8.0 \\
590\end{array}$ & $\begin{array}{r}395.0 \\
13.6 \\
18 \\
9.0 \\
783\end{array}$ & $\begin{array}{r}461.9 \\
14.9 \\
19 \\
9.9 \\
916\end{array}$ & $\begin{array}{r}1152 \\
38,4 \\
79 \\
20 \\
2280\end{array}$ & $\begin{array}{r}987.7 \\
31.9 \\
59 \\
7.5 \\
1960\end{array}$ & $\begin{array}{r}165.8 \\
5.53 \\
23 \\
3.3 \\
329\end{array}$ & $\begin{array}{r}245.4 \\
7.92 \\
18 \\
4.6 \\
487\end{array}$ & $\begin{array}{r}194.0 \\
6.26 \\
13 \\
4.4 \\
385\end{array}$ & $\begin{array}{r}246.6 \\
8.22 \\
14 \\
5.3 \\
489\end{array}$ \\
\hline
\end{tabular}

STATISTICS OF MONTHLY MEAN DATA FOR WATER YEARS 1992 - 1996, BY WATER YEAR (WY)

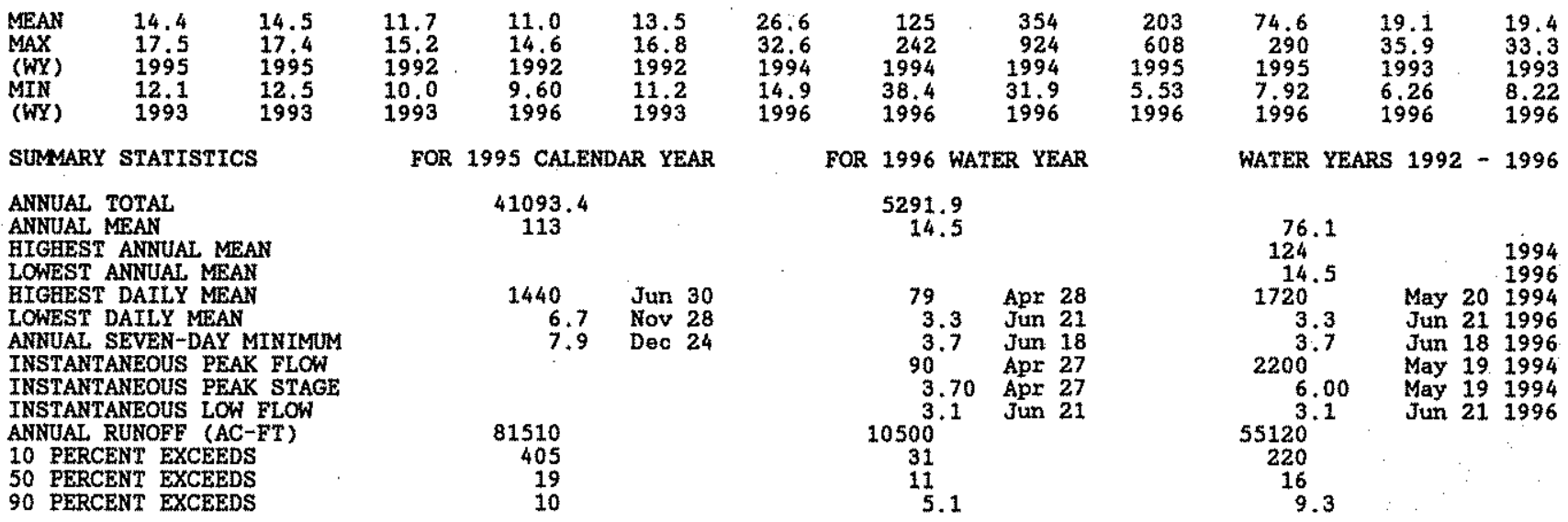

e Estimated 
08278500 RIO SANTA BARBARA NR PENASCO, NM

LOCATrON, --Lat $36^{\circ} 06^{\prime} 13^{\prime \prime}$, long $105^{\circ} 37^{\prime} 14^{\prime \prime}$, Taos County, Hydrologic Unit 13020101, in Santa Barbara Grant, on right bank at bridge on U.S. Forest Service Road $116,1,4 \mathrm{mi}$ below Santa Barbara Campground and 6.5 mi southeast of

Penasco

DRAINAGE AREA. $--38 \mathrm{mi}^{2}$ (approximately).

PERIOD OF RECORD.--November 1991 to current year. October 1952 to December 1957 published as Rio Santa Barbara nr Llano, NMA (08278500).

GAGE.--Water-stage recorder, Elevation of gege 158,640 It above National Geodetic Vertical Datum, from topographic map.

REMARKS.--Records fair except for estimated dally discharges, which are poor. Several observations of water temperature woxe made during year.

DISCHARGE, CUBIC FEET PER SECOND, WATER YEAR OCTOBER 1995 TO SEPTEMBER 1996

\begin{tabular}{|c|c|c|c|c|c|c|c|c|c|c|c|c|}
\hline DAY & $\propto C T$ & nov & DEC & JAN & FEB & MAR & APR & MAY & JUN & JUL & AUIG & SEP \\
\hline $\begin{array}{l}1 \\
2 \\
3 \\
4 \\
5\end{array}$ & $\begin{array}{l}26 \\
24 \\
22 \\
21 \\
21\end{array}$ & $\begin{array}{l}15 \\
15 \\
14 \\
15 \\
14\end{array}$ & $\therefore \begin{array}{c}09.0 \\
12 \\
11 \\
11 \\
10\end{array}$ & $\begin{array}{r}e 6.0 \\
06.0 \\
06.0 \\
06.0 \\
96.0\end{array}$ & $\begin{array}{l}09.0 \\
08.0 \\
88.0 \\
68.0 \\
08.0\end{array}$ & $\begin{array}{r}e 7.0 \\
07.0 \\
e 7.0 \\
6.7 \\
6.1\end{array}$ & $\begin{array}{l}14 \\
17 \\
18 \\
15 \\
14\end{array}$ & $\begin{array}{r}69 \\
072 \\
074 \\
e 75 \\
e 78\end{array}$ & $\begin{array}{l}34 \\
33 \\
31 \\
30 \\
28\end{array}$ & $\begin{array}{r}28 \\
\ldots . .25 \\
23 \\
22 \\
21\end{array}$ & $\begin{array}{l}19 \\
19 \\
25 \\
19 \\
18\end{array}$ & $\begin{array}{l}19 \\
19 \\
19 \\
18 \\
18\end{array}$ \\
\hline $\begin{array}{r}6 \\
7 \\
8 \\
9 \\
10\end{array}$ & $\begin{array}{l}20 \\
20 \\
20 \\
19 \\
19\end{array}$ & $\begin{array}{l}14 \\
14 \\
12 \\
13 \\
15\end{array}$ & $\begin{array}{r}10 \\
9.9 \\
9.3 \\
96.8 \\
66.8\end{array}$ & $\begin{array}{l}e 6.0 \\
e 7.0 \\
08.0 \\
e 8.0 \\
e 8.0\end{array}$ & $\begin{array}{r}88.0 \\
08.0 \\
08.0 \\
08.0 \\
7.1\end{array}$ & $\begin{array}{r}6.4 \\
07.0 \\
7.1 \\
8.3 \\
8.5\end{array}$ & $\begin{array}{l}17 \\
17 \\
22 \\
30 \\
28\end{array}$ & $\begin{array}{r}978 \\
976 \\
075 \\
\quad 78 \\
78\end{array}$ & $\begin{array}{r}27 \\
\therefore \quad 26 \\
25 \\
23 \\
22\end{array}$ & $\begin{array}{l}20 \\
22 \\
24 \\
30 \\
46\end{array}$ & $\begin{array}{l}17 \\
17 \\
17 \\
18 \\
18\end{array}$ & $\begin{array}{l}18 \\
17 \\
17 \\
16 \\
16\end{array}$ \\
\hline $\begin{array}{l}11 \\
12 \\
13 \\
14 \\
15\end{array}$ & $\begin{array}{l}19 \\
19 \\
18 \\
18 \\
18\end{array}$ & $\begin{array}{l}14 \\
13 \\
13 \\
13 \\
12\end{array}$ & $\begin{array}{r}66.8 \\
66.8 \\
8.8 \\
8.7 \\
8.8\end{array}$ & $\begin{array}{l}e 7.0 \\
e 7.0 \\
e 7.0 \\
e 8.0 \\
88.0\end{array}$ & $\begin{array}{l}7.1 \\
7.0 \\
7.1 \\
7.3 \\
7.5\end{array}$ & $\begin{array}{l}8.3 \\
8.1 \\
8.4 \\
8.4 \\
8.1\end{array}$ & $\begin{array}{l}27 \\
30 \\
24 \\
24 \\
26\end{array}$ & $\begin{array}{l}77 \\
78 \\
79 \\
78 \\
77\end{array}$ & $\begin{array}{l}21 \\
21 \\
23 \\
37 \\
30\end{array}$ & $\begin{array}{l}33 \\
32 \\
35 \\
33 \\
32\end{array}$ & $\begin{array}{l}16 \\
15 \\
15 \\
15 \\
15\end{array}$ & $\begin{array}{l}16 \\
16 \\
16 \\
18 \\
21\end{array}$ \\
\hline $\begin{array}{l}16 \\
17 \\
18 \\
19 \\
20\end{array}$ & $\begin{array}{l}18 \\
17 \\
16 \\
16 \\
16\end{array}$ & $\begin{array}{l}12 \\
12 \\
12 \\
12 \\
12\end{array}$ & $\begin{array}{r}e 6.5 \\
06.5 \\
8.7 \\
06.0 \\
66.0\end{array}$ & $\begin{array}{l}e 8.0 \\
88.0 \\
88.0 \\
06.0 \\
98.0\end{array}$ & $\begin{array}{l}7.7 \\
7.5 \\
7.7 \\
7.3 \\
7.7\end{array}$ & $\begin{array}{l}7.9 \\
08.0 \\
08.0 \\
08.0 \\
08.0\end{array}$ & $\begin{array}{l}28 \\
29 \\
30 \\
27 \\
27\end{array}$ & $\begin{array}{l}76 \\
76 \\
73 \\
69 \\
66\end{array}$ & $\begin{array}{l}29 \\
28 \\
27 \\
20 \\
15\end{array}$ & $\begin{array}{l}31 \\
31 \\
30 \\
29 \\
27\end{array}$ & $\begin{array}{l}14 \\
14 \\
16 \\
13 \\
14\end{array}$ & $\begin{array}{l}17 \\
17 \\
22 \\
21 \\
18\end{array}$ \\
\hline $\begin{array}{l}21 \\
22 \\
23 \\
24 \\
25\end{array}$ & $\begin{array}{l}16 \\
16 \\
14 \\
16 \\
16\end{array}$ & $\begin{array}{l}12 \\
10 \\
67.9 \\
87.9 \\
07.9\end{array}$ & $\begin{array}{l}66.0 \\
66.0 \\
65.0 \\
65.0 \\
05.0\end{array}$ & $\begin{array}{l}86.0 \\
87.0 \\
07.0 \\
67.0 \\
07.0\end{array}$ & $\begin{array}{l}8.2 \\
7.6 \\
6.7 \\
8.6 \\
6.9\end{array}$ & $\begin{array}{r}88.0 \\
9.0 \\
9.0 \\
8.6 \\
8.5\end{array}$ & $\begin{array}{l}29 \\
39 \\
50 \\
56 \\
52\end{array}$ & $\begin{array}{l}63 \\
60 \\
56 \\
53 \\
51\end{array}$ & $\begin{array}{l}14 \\
16 \\
15 \\
13 \\
13\end{array}$ & $\begin{array}{l}26 \\
25 \\
24 \\
23 \\
23\end{array}$ & $\begin{array}{l}16 \\
21 \\
26 \\
23 \\
20\end{array}$ & $\begin{array}{l}17 \\
17\end{array}$ \\
\hline $\begin{array}{l}26 \\
27 \\
28 \\
29 \\
30 \\
31\end{array}$ & $\begin{array}{l}15 \\
15 \\
15 \\
15 \\
15 \\
15\end{array}$ & $\begin{array}{l}67.2 \\
67.2 \\
97.2 \\
07.4 \\
88.0 \\
-\end{array}$ & $\begin{array}{l}05.0 \\
05.0 \\
e 6.0 \\
07.0 \\
07.0 \\
07.0\end{array}$ & $\begin{array}{l}87.0 \\
88.0 \\
e 8.0 \\
88.0 \\
99.0 \\
89.0\end{array}$ & $\begin{array}{r}6.3 \\
07.0 \\
07.0 \\
07.0 \\
-\ldots\end{array}$ & $\begin{array}{c}9.1 \\
11 \\
9.3 \\
9.9 \\
10 \\
12\end{array}$ & $\begin{array}{r}48 \\
51 \\
56 \\
61 \\
65 \\
---\end{array}$ & $\begin{array}{l}49 \\
46 \\
43 \\
40 \\
39 \\
36\end{array}$ & $\begin{array}{r}16 \\
44 \\
27 \\
29 \\
29 \\
-\end{array}$ & $\begin{array}{l}22 \\
25 \\
26 \\
27 \\
22 \\
21\end{array}$ & $\begin{array}{l}20 \\
23 \\
21 \\
20 \\
21 \\
20\end{array}$ & 38 \\
\hline $\begin{array}{l}\text { TOTAL } \\
\text { MEAN } \\
\text { MAX } \\
\text { MIN } \\
\text { AC-FT }\end{array}$ & $\begin{array}{r}555 \\
17.9 \\
26 \\
14 \\
1100\end{array}$ & $\begin{array}{r}348.7 \\
11.6 \\
15 \\
7.2 \\
692\end{array}$ & $\begin{array}{r}233.4 \\
7.53 \\
12 \\
5.0 \\
463\end{array}$ & $\begin{array}{r}225.0 \\
7.26 \\
9.0 \\
6.0 \\
446\end{array}$ & $\begin{array}{r}219.3 \\
7.56 \\
9.0 \\
6.3 \\
435\end{array}$ & $\begin{array}{r}256.7 \\
8.28 \\
12 \\
6.1 \\
509\end{array}$ & $\begin{array}{r}971 \\
32.4 \\
65 \\
34 \\
1930\end{array}$ & $\begin{array}{r}2038 \\
65.7 \\
79 \\
36 \\
4040\end{array}$ & $\begin{array}{r}746 \\
24.9 \\
44 \\
13 \\
1480\end{array}$ & $\begin{array}{r}838 \\
27.0 \\
46 \\
20 \\
1660\end{array}$ & $\begin{array}{r}565 \\
18.2 \\
26 \\
13 \\
1120\end{array}$ & $\begin{array}{r}524 \\
17.5 \\
22 \\
16 \\
1040\end{array}$ \\
\hline
\end{tabular}

STATISTICS OF MONTHLY MEAH DATA FOR WATER YEARS 1953 - 1996, BY WATER TEAR (WY)

\begin{tabular}{|c|c|c|c|c|c|c|c|c|c|c|c|c|}
\hline $\begin{array}{l}\text { MEAN } \\
\text { MAX } \\
\text { (WY }) \\
\text { MIN } \\
(W Y)\end{array}$ & $\begin{array}{l}13.5 \\
17.9 \\
1996 \\
4.95 \\
1957\end{array}$ & $\begin{array}{l}11.1 \\
17.3 \\
1992 \\
5.13 \\
1957\end{array}$ & $\begin{array}{l}8.15 \\
13.6 \\
1992 \\
4.18 \\
1957\end{array}$ & $\begin{array}{l}6.86 \\
9.24 \\
1953 \\
4.10 \\
1954\end{array}$ & $\begin{array}{l}6.89 \\
9.13 \\
1992 \\
3.93 \\
1957\end{array}$ & $\begin{array}{l}10.9 \\
17.5 \\
1992 \\
6.46 \\
1957\end{array}$ & $\begin{array}{l}38.3 \\
75.3 \\
1992 \\
18.6 \\
1956\end{array}$ & $\begin{array}{r}104 \\
199 \\
1994 \\
35.6 \\
1956\end{array}$ & $\begin{array}{r}116 \\
211 \\
1995 \\
17.0 \\
1956\end{array}$ & $\begin{array}{l}36.7 \\
62.1 \\
1957 \\
8.13 \\
1956\end{array}$ & $\begin{array}{r}38.6 \\
129 \\
1957 \\
8.11 \\
1956\end{array}$ & $\begin{array}{l}25.3 \\
66.5 \\
1957 \\
4.50 \\
1956\end{array}$ \\
\hline
\end{tabular}

SUMARY STATISTICS

ANNUAL. TOTAL

ANMUAY MEAN

HIGLEST ANNUAL MEAN

LOWEST ANHUAL NEAH

BIGESEST DAILY MEAN

LOWEST DAILY MEAN

ANNUAL SEVEN-DAY MINIMUM

INSTANTANEOUS PEAK FLOW

INSTANTANEOUS PEAK STAGE

INSTANTANEOUS LOW FLOW

ANNUAL RUNOEE (AC-FT)

10 PERCENT EXCEEDS

50 PERCENT EXCEEDS

90 PERCENT EXCEEDS

- Estimated
FOR 1995 CALENDAR YEAR

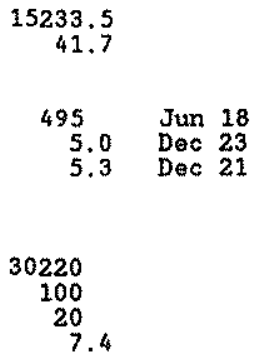

FOR 1996 WATER YEAR

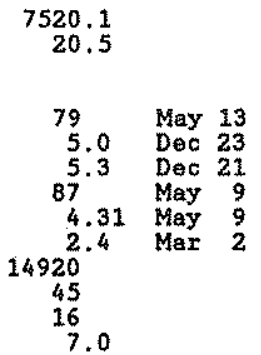

WATER YEARS 1953 - 1996

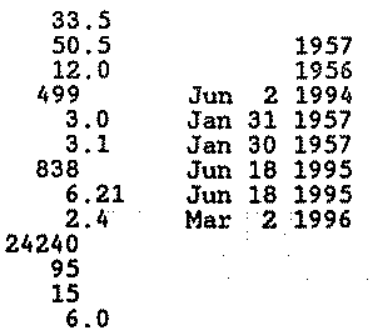


RIO GRANDE BASIN

08279000. EMBUDO CREEK AT DIXON, NM

LOCATION.--Lat $36^{\circ} 12^{\prime} 39^{\prime \prime}$, Long $105^{\circ} 54^{\prime} 47^{\prime \prime}$, in NE\%/SE\% soc.19, T.23 N., R. 10 E., Rlo Arriba County, Hydrologic Unit 13020101 on right bank 750 ft upstream from State Highway $68,0.5$ mi upstroam from mouth, 0.5 mi east of Embudo Post Office, and $1.7 \mathrm{mi}$ northwest of Dixon.

DRATNAGE AREA, $-m 305 \mathrm{~ms}^{2}$.

WATER-DISCEARGE RECORD

PERIOD OF RECORD.--October 1923 to February 1926 October 1926 to September 1955 (annual maximum), water years 1956-62, September 1962 to current year. Monthly discharge only for some perlods, published in WSP 1312.

F1gures of dally discharge for July 6-25, 1932, published in WSP 733, and maximum discharges for water years $1931-33,1935,1937-38,1941$, are unreliable and should not be used.

REVISED RECORDS.--WSP 1512; 1931-32, 1941, 1947 (M). Also see PERIOD OF RECORD.

GAGE, - Water-stage recorder. Datum of gage is $5,858.60 \mathrm{ft}$ above National Geodetic Vertical Datum of 1929 . Prior to Nov. 30,1938 at site about 1 mi upstream at different datum. Nov. 30,1938 to Aug, 1 , 1941, at sito about 0.9 in1 upstream at datum about 59.9 ft higher. Aug. 2, 1941 to Sept. 1, 1971, at site 750 ft downstream at datum 9.10 ft lower. Apri1 1956 to Sept. 21, 1962 , crest-stage gage.

REMARKS. --Water-discharge records good. Diversions upstream from station for 1rrigation of about 6,600 acres, a small part of which are downstream from gage.

DISCHARGE, CUBIC FEET PER SECOND, WATER YEAR OCTOBER 1995 TO SEPTEMBER 1996

\begin{tabular}{|c|c|c|c|c|c|c|c|c|c|c|c|c|}
\hline DAY & $\infty T$ & NOV & DEC & JAN & FEB & MAR & APR & MAY & JUN & JUL & AUS & SEP \\
\hline $\begin{array}{l}1 \\
2 \\
3 \\
4 \\
5\end{array}$ & $\begin{array}{l}58 \\
51 \\
47 \\
45 \\
44\end{array}$ & $\begin{array}{l}34 \\
37 \\
39 \\
40 \\
43\end{array}$ & $\begin{array}{l}39 \\
37 \\
37 \\
39 \\
39\end{array}$ & $\begin{array}{l}39 \\
36 \\
30 \\
38 \\
40\end{array}$ & $\begin{array}{l}38 \\
36 \\
27 \\
30 \\
38\end{array}$ & $\begin{array}{l}32 \\
32 \\
34 \\
37 \\
36\end{array}$ & $\begin{array}{l}34 \\
37 \\
43 \\
45 \\
37\end{array}$ & $\begin{array}{l}45 \\
45 \\
42 \\
45 \\
41\end{array}$ & $\begin{array}{l}8.7 \\
8.4 \\
8.4 \\
7.8 \\
8.0\end{array}$ & $\begin{array}{l}9.0 \\
9.9 \\
8.9 \\
7.3 \\
7.2\end{array}$ & $\begin{array}{r}5.5 \\
9.2 \\
15 \\
12 \\
7.2\end{array}$ & $\begin{array}{l}15 \\
12 \\
11 \\
12 \\
12\end{array}$ \\
\hline $\begin{array}{r}6 \\
7 \\
8 \\
9 \\
10\end{array}$ & $\begin{array}{l}43 \\
44 \\
45 \\
43 \\
43\end{array}$ & $\begin{array}{l}45 \\
43 \\
42 \\
43 \\
43\end{array}$ & $\begin{array}{l}38 \\
38 \\
39 \\
33 \\
31\end{array}$ & $\begin{array}{l}37 \\
33 \\
35 \\
36 \\
37\end{array}$ & $\begin{array}{l}40 \\
39 \\
38 \\
38 \\
40\end{array}$ & $\begin{array}{l}35 \\
26 \\
36 \\
38 \\
39\end{array}$ & $\begin{array}{l}32 \\
34 \\
34 \\
34 \\
45\end{array}$ & $\begin{array}{l}36 \\
36 \\
33 \\
30 \\
29\end{array}$ & $\begin{array}{l}7.8 \\
7.7 \\
7.2 \\
6.5 \\
6.2\end{array}$ & $\begin{array}{l}7.1 \\
21 \\
18 \\
21 \\
33\end{array}$ & $\begin{array}{l}5.3 \\
5.1 \\
5.2 \\
5.7 \\
5.8\end{array}$ & $\begin{array}{c}12 \\
12 \\
10 \\
10 \\
9.2\end{array}$ \\
\hline $\begin{array}{l}11 \\
12 \\
13 \\
14 \\
15\end{array}$ & $\begin{array}{l}41 \\
40 \\
35 \\
33 \\
32\end{array}$ & $\begin{array}{l}38 \\
43 \\
42 \\
40 \\
41\end{array}$ & $\begin{array}{l}33 \\
35 \\
40 \\
40 \\
39\end{array}$ & $\begin{array}{l}36 \\
36 \\
37 \\
37 \\
36\end{array}$ & $\begin{array}{l}40 \\
38 \\
36 \\
36 \\
38\end{array}$ & $\begin{array}{l}40 \\
39 \\
38 \\
38 \\
41\end{array}$ & $\begin{array}{l}49 \\
48 \\
55 \\
46 \\
40\end{array}$ & $\begin{array}{l}33 \\
34 \\
32 \\
30 \\
33\end{array}$ & $\begin{array}{l}6.5 \\
6.6 \\
6.8 \\
6.8 \\
6.8\end{array}$ & $\begin{array}{l}33 \\
23 \\
28 \\
23 \\
17\end{array}$ & $\begin{array}{l}5.1 \\
4.9 \\
4.8 \\
4.4 \\
4.6\end{array}$ & $\begin{array}{l}8.1 \\
9.0 \\
11 \\
15 \\
21\end{array}$ \\
\hline $\begin{array}{l}16 \\
17 \\
18 \\
19 \\
20\end{array}$ & $\begin{array}{l}34 \\
36 \\
38 \\
38 \\
36\end{array}$ & $\begin{array}{l}41 \\
42 \\
40 \\
38 \\
39\end{array}$ & $\begin{array}{l}34 \\
36 \\
36 \\
34 \\
32\end{array}$ & $\begin{array}{l}35 \\
44 \\
36 \\
26 \\
38\end{array}$ & $\begin{array}{l}38 \\
38 \\
39 \\
37 \\
39\end{array}$ & $\begin{array}{l}40 \\
39 \\
34 \\
33 \\
33\end{array}$ & $\begin{array}{l}36 \\
35 \\
35 \\
41 \\
40\end{array}$ & $\begin{array}{l}35 \\
35 \\
42 \\
30 \\
27\end{array}$ & $\begin{array}{l}6.3 \\
6.2 \\
5.8 \\
5.9 \\
5.7\end{array}$ & $\begin{array}{c}13 \\
13 \\
11 \\
10 \\
8.7\end{array}$ & $\begin{array}{l}4.8 \\
4.7 \\
4.7 \\
6.9 \\
6.0\end{array}$ & $\begin{array}{l}18 \\
15 \\
23 \\
38 \\
31\end{array}$ \\
\hline $\begin{array}{l}21 \\
22 \\
23 \\
24 \\
25\end{array}$ & $\begin{array}{l}36 \\
35 \\
36 \\
34 \\
34\end{array}$ & $\begin{array}{l}38 \\
37 \\
36 \\
35 \\
34\end{array}$ & $\begin{array}{l}29 \\
35 \\
30 \\
26 \\
27\end{array}$ & $\begin{array}{l}29 \\
42 \\
36 \\
28 \\
35\end{array}$ & $\begin{array}{l}42 \\
42 \\
38 \\
33 \\
39\end{array}$ & $\begin{array}{l}36 \\
37 \\
35 \\
35 \\
33\end{array}$ & $\begin{array}{l}36 \\
33 \\
28 \\
31 \\
41\end{array}$ & $\begin{array}{l}25 \\
22 \\
19 \\
18 \\
17\end{array}$ & $\begin{array}{l}5.3 \\
5.3 \\
4.8 \\
4.7 \\
4.7\end{array}$ & $\begin{array}{r}8.2 \\
6.9 \\
6.1 \\
4.6 \\
4.0\end{array}$ & $\begin{array}{l}5.4 \\
15 \\
18 \\
20 \\
19\end{array}$ & $\begin{array}{l}30 \\
29 \\
28 \\
26 \\
25\end{array}$ \\
\hline $\begin{array}{l}26 \\
27 \\
28 \\
29 \\
30 \\
31\end{array}$ & $\begin{array}{l}33 \\
33 \\
33 \\
33 \\
33 \\
34\end{array}$ & $\begin{array}{r}36 \\
34 \\
31 \\
30 \\
37 \\
-\end{array}$ & $\begin{array}{l}27 \\
29 \\
30 \\
39 \\
36 \\
42\end{array}$ & $\begin{array}{l}34 \\
29 \\
41 \\
36 \\
37 \\
40\end{array}$ & $\begin{array}{r}36 \\
29 \\
31 \\
35 \\
-9 \\
--9\end{array}$ & $\begin{array}{l}34 \\
34 \\
35 \\
34 \\
36 \\
34\end{array}$ & $\begin{array}{r}48 \\
54 \\
66 \\
59 \\
51 \\
-\end{array}$ & $\begin{array}{c}16 \\
15 \\
14 \\
13 \\
10 \\
9.6\end{array}$ & $\begin{array}{r}4.9 \\
5.8 \\
10 \\
7.5 \\
7.0 \\
\end{array}$ & $\begin{array}{l}4.4 \\
5.5 \\
7.9 \\
7.0 \\
7.1 \\
6.2\end{array}$ & $\begin{array}{l}16 \\
24 \\
21 \\
16 \\
14 \\
16\end{array}$ & $\begin{array}{l}23 \\
27 \\
29 \\
27 \\
24 \\
-\end{array}$ \\
\hline $\begin{array}{l}\text { TOTAL } \\
\text { MEAN } \\
\text { MAX } \\
\text { MIN } \\
\text { AC-ET }\end{array}$ & $\begin{array}{r}1200 \\
38.7 \\
58 \\
32 \\
2380\end{array}$ & $\begin{array}{r}1161 \\
38.7 \\
45 \\
30 \\
2300\end{array}$ & $\begin{array}{r}1079 \\
34.8 \\
42 \\
26 \\
2140\end{array}$ & $\begin{array}{r}1109 \\
35.8 \\
44 \\
26 \\
2200\end{array}$ & $\begin{array}{r}1068 \\
36.8 \\
42 \\
27 \\
2120\end{array}$ & $\begin{array}{r}1103 \\
35.6 \\
41 \\
26 \\
2190\end{array}$ & $\begin{array}{r}1247 \\
41.6 \\
66 \\
28 \\
2470\end{array}$ & $\begin{array}{r}891.6 \\
28.8 \\
45 \\
9.6 \\
1770\end{array}$ & $\begin{array}{r}200.1 \\
6.67 \\
10 \\
4.7 \\
397\end{array}$ & $\begin{array}{r}390.0 \\
12.6 \\
33 \\
4.0 \\
774\end{array}$ & $\begin{array}{r}311.3 \\
10.0 \\
24 \\
4.4 \\
617\end{array}$ & $\begin{array}{r}572.3 \\
19.1 \\
38 \\
8.1 \\
1140\end{array}$ \\
\hline
\end{tabular}

STATISTICS OF MONTHLY MEAN DATA FOR WATER YEARS 1924 - 1996, BY WATER YEAR (WY)

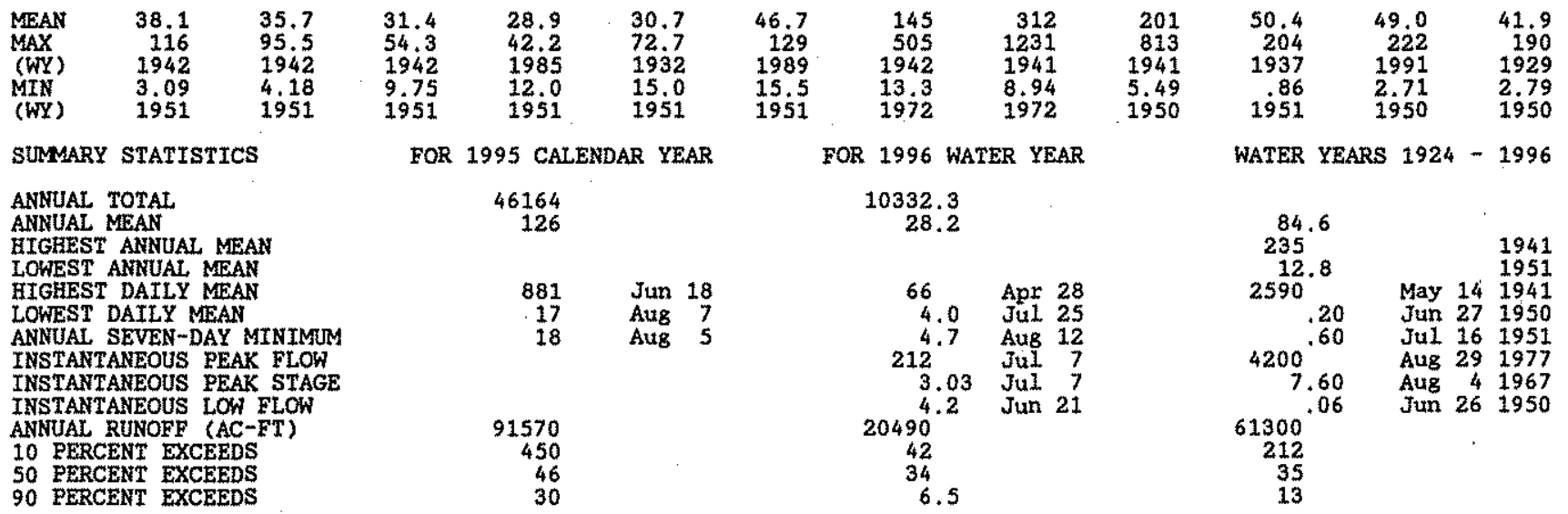


RIO GRANDE BASIN

08279000 EMBUDO CREEK AT DIXON, MM --Continued

WATER-QULAITY RECORDS

FERIOD OF RECORD.-Water years 1970 to current year.

WATER QUALITY DATA, WATER YEAR OCTOBER 1995 TO SEPTEMBER 1996

\begin{tabular}{|c|c|c|c|c|c|c|c|c|c|}
\hline DATE & TMME & $\begin{array}{c}\text { DHS } \\
\text { CEARGE, } \\
\text { INST } \\
\text { CUBIC } \\
\text { EEET } \\
\text { WER } \\
\text { SECOND } \\
(00061)\end{array}$ & $\begin{array}{l}\text { SPE- } \\
\text { CIFIC } \\
\text { CON- } \\
\text { DUCT- } \\
\text { ARCE } \\
\text { (US/CR } \\
\text { (DOO95) }\end{array}$ & $\begin{array}{l}\text { FH } \\
\text { WATER } \\
\text { WHOLE } \\
\text { FIELD } \\
\text { (STAND- } \\
\text { ARD } \\
\text { UNITS) } \\
(00400)\end{array}$ & $\begin{array}{c}\text { THMPER- } \\
\text { ATURE } \\
\text { AIR } \\
(D E G C) \\
(00020)\end{array}$ & $\begin{array}{l}\text { TEMPER- } \\
\text { ATURE } \\
\text { HATER } \\
\text { (DEG C) } \\
\text { (00010) }\end{array}$ & $\begin{array}{c}\text { BARO- } \\
\text { NEIRIC } \\
\text { FRES- } \\
\text { SURE } \\
\text { (MY } \\
\text { OF } \\
\text { HG) } \\
(00025)\end{array}$ & $\begin{array}{c}\text { OXYGEN; } \\
\text { DIS- } \\
\text { SOLVED } \\
\text { (NG/L) } \\
(00300)\end{array}$ & $\begin{array}{c}\text { OKYGEN, } \\
\text { DIS- } \\
\text { SOLVED } \\
\text { (PER- } \\
\text { CENT } \\
\text { SATUR- } \\
\text { ATION) } \\
\text { (00301) }\end{array}$ \\
\hline
\end{tabular}

\begin{tabular}{|c|c|c|c|c|c|c|c|c|c|c|}
\hline $\begin{array}{l}\text { DEC } 1995 \\
01.906\end{array}$ & 1106 & 37 & . & 420 & 8.0 & 10.5 & 4.0 & 617 & 10.1 & 95 \\
\hline $\begin{array}{l}\text { MAR } 1996 \\
29 \ldots \\
\text { JUH }\end{array}$ & 1445 & 34 & & 332 & 8.5 & 10.5 & 11.5 & 607 & 9.0 & 104 \\
\hline $\operatorname{sep}^{17} \cdots$ & 1100 & 6.2 & & 44.4 & 8.4 & 23.5 & 17.5 & 618 & 8.9 & 115 \\
\hline $09 \ldots$ & 1045 & 10 & & 458 & 8.4 & 22.5 & 16.0 & 620 & 9.5 & 119 \\
\hline
\end{tabular}


RIO GRANDE BASIN

08279500 RTO GRANDE AT EMBUDO, MM

LOCATION, --Lat $36^{\circ} 12^{\prime} 20^{\prime \prime}$, long $105^{\circ} 57^{\prime} 49^{\prime \prime}$, In SWKSW s.oc.23, T.23 N. R.9 E. Rio Arriba County, Bydrologic Untt 13020101 , on r1ght bank 0.2 mi downstream from bridge at Embudo, $2.8 \mathrm{mi}$ downstream from Embudo Creak, and at mile $1,643.1$.

DRAINAGE AREA. - -10,400 $\mathrm{mt}^{2}$, approximately, Including 2,940 $\mathrm{mt}^{2}$ in closed basin in San Luts Valley, co.

PERTOD OF RECORD - - January 1889 to current year. Nonthly discharge only for some periods, published in wSP 1312. Figures of daily discharge for Oct. 4 to Nov. 30, 1896, published in WSP 358 , are unreliable and should not be used.

REVISED RECORDS.--WSP 358: 1900-1902. WSP 828: Drainage area. WSP 878: 1915-16. WSP 1512: 1892-99, 1904, 1916, 1931-32, 1939, 1944 45, 1950. WSP 1712: 1903(M). See also PERIOD OF RECORD.

GAGE. -Water-stage recorder. Datum of gage is 5,789.14 $\mathrm{ft}$ above National Geodetic Vertical Datum of 1929. Jan. 1 to $\mathrm{Feb} .28$, 1889 , nonrecording gage 1.2 mi upstream at different datum. March 1889 to Decomber 1903 nonrecording gage $1,300 \mathrm{ft}$ upstream at different datum. September 1912 to June 1914 , water-stage recorder on downstream ond of bridge plex at sito 200 ft upstream at present datum.

REMARKS. --Records good. Dlversions upstream from station for irrigation of about 620,000 acres in Colorado and 40,000 acres in New Mexico. Several observattons of pater temperature pere made during the year. Nationel Weather Service gage-height telemeter and U.S. Army Corps of Engineers satelilte telemeter at station.

AVERAGE DISCHARGE. - -41 years (water years 1890-1930), 1,238 fts/s, 896, 900 acra-ft/yr.

DISCEARGE, CUBIC FEET PER SECOND, WATER YEAR OCTOBER 1995 TO SEPTEMBER 1996

\begin{tabular}{|c|c|c|c|c|c|c|c|c|c|c|c|c|}
\hline DAY & $\propto C r$ & NON & DEC & JAN & FEB & MAR & APR & MAY & JUN & JUL & AUG & SEP \\
\hline $\begin{array}{l}1 \\
2 \\
3 \\
4 \\
5\end{array}$ & $\begin{array}{r}464 \\
470 \\
524 \\
538 \\
545\end{array}$ & $\begin{array}{l}469 \\
484 \\
491 \\
502 \\
508\end{array}$ & $\begin{array}{l}507 \\
498 \\
494 \\
506 \\
526\end{array}$ & $\begin{array}{l}605 \\
627 \\
593 \\
620 \\
629\end{array}$ & $\begin{array}{l}701 \\
708 \\
663 \\
656 \\
664\end{array}$ & $\begin{array}{l}682 \\
742 \\
767 \\
769 \\
777\end{array}$ & $\begin{array}{l}510 \\
506 \\
495 \\
488 \\
495\end{array}$ & $\begin{array}{l}608 \\
513 \\
460 \\
442 \\
420\end{array}$ & $\begin{array}{l}284 \\
274 \\
266 \\
258 \\
253\end{array}$ & $\begin{array}{l}274 \\
264 \\
260 \\
246 \\
230\end{array}$ & $\begin{array}{l}226 \\
224 \\
228 \\
232 \\
221\end{array}$ & $\begin{array}{l}254 \\
254 \\
248 \\
243 \\
241\end{array}$ \\
\hline $\begin{array}{r}6 \\
7 \\
8 \\
9 \\
10\end{array}$ & $\begin{array}{l}486 \\
454 \\
436 \\
426 \\
410\end{array}$ & $\begin{array}{l}505 \\
506 \\
508 \\
512 \\
516\end{array}$ & $\begin{array}{l}549 \\
552 \\
554 \\
554 \\
563\end{array}$ & $\begin{array}{l}635 \\
619 \\
634 \\
648 \\
658\end{array}$ & $\begin{array}{l}715 \\
731 \\
726 \\
735 \\
760\end{array}$ & $\begin{array}{l}788 \\
773 \\
783 \\
798 \\
791\end{array}$ & $\begin{array}{l}482 \\
467 \\
483 \\
453 \\
440\end{array}$ & $\begin{array}{l}394 \\
393 \\
397 \\
403 \\
412\end{array}$ & $\begin{array}{l}250 \\
250 \\
245 \\
242 \\
245\end{array}$ & $\begin{array}{l}218 \\
231 \\
252 \\
267 \\
269\end{array}$ & $\begin{array}{l}211 \\
208 \\
228 \\
232 \\
226\end{array}$ & $\begin{array}{l}242 \\
246 \\
247 \\
245 \\
240\end{array}$ \\
\hline $\begin{array}{l}11 \\
12 \\
13 \\
14 \\
15\end{array}$ & $\begin{array}{l}412 \\
413 \\
396 \\
395 \\
392\end{array}$ & $\begin{array}{l}517 \\
529 \\
521 \\
516 \\
521\end{array}$ & $\begin{array}{l}544 \\
535 \\
548 \\
546 \\
574\end{array}$ & $\begin{array}{l}666 \\
666 \\
670 \\
680 \\
671\end{array}$ & $\begin{array}{l}772 \\
792 \\
799 \\
790 \\
824\end{array}$ & $\begin{array}{l}783 \\
784 \\
779 \\
762 \\
765\end{array}$ & $\begin{array}{l}454 \\
476 \\
547 \\
539 \\
529\end{array}$ & $\begin{array}{l}402 \\
393 \\
403 \\
397 \\
404\end{array}$ & $\begin{array}{l}253 \\
257 \\
260 \\
258 \\
259\end{array}$ & $\begin{array}{l}269 \\
257 \\
274 \\
255 \\
234\end{array}$ & $\begin{array}{l}217 \\
215 \\
213 \\
212 \\
217\end{array}$ & $\begin{array}{l}237 \\
250 \\
261 \\
309 \\
281\end{array}$ \\
\hline $\begin{array}{l}16 \\
17 \\
18 \\
19 \\
20\end{array}$ & $\begin{array}{l}393 \\
400 \\
423 \\
437 \\
438\end{array}$ & $\begin{array}{l}529 \\
540 \\
546 \\
546 \\
540\end{array}$ & $\begin{array}{l}590 \\
600 \\
567 \\
484 \\
470\end{array}$ & $\begin{array}{l}684 \\
726 \\
712 \\
651 \\
654\end{array}$ & $\begin{array}{l}839 \\
853 \\
854 \\
867 \\
891\end{array}$ & $\begin{array}{l}754 \\
716 \\
673 \\
630 \\
596\end{array}$ & $\begin{array}{l}518 \\
476 \\
441 \\
415 \\
408\end{array}$ & $\begin{array}{l}472 \\
456 \\
438 \\
410 \\
417\end{array}$ & $\begin{array}{l}269 \\
283 \\
290 \\
286 \\
276\end{array}$ & $\begin{array}{l}228 \\
239 \\
293 \\
251 \\
240\end{array}$ & $\begin{array}{l}217 \\
216 \\
251 \\
214 \\
216\end{array}$ & $\begin{array}{l}283 \\
272 \\
283 \\
296 \\
281\end{array}$ \\
\hline $\begin{array}{l}21 \\
22 \\
23 \\
24 \\
25\end{array}$ & $\begin{array}{l}450 \\
446 \\
446 \\
443 \\
447\end{array}$ & $\begin{array}{l}530 \\
522 \\
519 \\
519 \\
516\end{array}$ & $\begin{array}{l}469 \\
482 \\
492 \\
442 \\
452\end{array}$ & $\begin{array}{l}631 \\
687 \\
660 \\
633 \\
636\end{array}$ & $\begin{array}{l}883 \\
884 \\
881 \\
892 \\
879\end{array}$ & $\begin{array}{l}580 \\
573 \\
558 \\
554 \\
559\end{array}$ & $\begin{array}{l}400 \\
380 \\
370 \\
372 \\
364\end{array}$ & $\begin{array}{l}438 \\
403 \\
404 \\
411 \\
381\end{array}$ & $\begin{array}{l}283 \\
279 \\
278 \\
266 \\
260\end{array}$ & $\begin{array}{l}225 \\
212 \\
214 \\
214 \\
223\end{array}$ & $\begin{array}{l}233 \\
260 \\
302 \\
307 \\
295\end{array}$ & $\begin{array}{l}269 \\
257 \\
247 \\
245 \\
242\end{array}$ \\
\hline $\begin{array}{l}26 \\
27 \\
28 \\
29 \\
30 \\
31\end{array}$ & $\begin{array}{l}445 \\
449 \\
457 \\
456 \\
449 \\
458\end{array}$ & $\begin{array}{l}515 \\
517 \\
508 \\
496 \\
495 \\
-9-\end{array}$ & $\begin{array}{l}474 \\
516 \\
546 \\
568 \\
581 \\
616\end{array}$ & $\begin{array}{l}644 \\
591 \\
635 \\
635 \\
670 \\
674\end{array}$ & $\begin{array}{l}843 \\
794 \\
788 \\
768 \\
-0 .\end{array}$ & $\begin{array}{l}559 \\
547 \\
558 \\
546 \\
534 \\
509\end{array}$ & $\begin{array}{l}360 \\
355 \\
369 \\
494 \\
576\end{array}$ & $\begin{array}{l}349 \\
327 \\
330 \\
314 \\
309 \\
300\end{array}$ & $\begin{array}{l}262 \\
302 \\
295 \\
277 \\
273 \\
-\end{array}$ & $\begin{array}{l}252 \\
230 \\
243 \\
233 \\
241 \\
230\end{array}$ & $\begin{array}{l}392 \\
289 \\
292 \\
269 \\
271 \\
263\end{array}$ & $\begin{array}{l}235 \\
247 \\
248 \\
250 \\
245\end{array}$ \\
\hline $\begin{array}{l}\text { TOTAL } \\
\text { MEAN } \\
\text { MAX } \\
\text { MIN } \\
\text { AC-FT }\end{array}$ & $\begin{array}{r}13800 \\
445 \\
545 \\
392 \\
27370\end{array}$ & $\begin{array}{r}15443 \\
515 \\
546 \\
469 \\
30630\end{array}$ & $\begin{array}{r}16399 \\
529 \\
616 \\
442 \\
32530\end{array}$ & $\begin{array}{r}20144 \\
650 \\
726 \\
591 \\
39960\end{array}$ & $\begin{array}{r}22952 \\
791 \\
892 \\
656 \\
45530\end{array}$ & $\begin{array}{r}20989 \\
677 \\
798 \\
509 \\
41630\end{array}$ & $\begin{array}{r}13662 \\
455 \\
576 \\
355 \\
27100\end{array}$ & $\begin{array}{r}12600 \\
406 \\
608 \\
300 \\
24990\end{array}$ & $\begin{array}{r}8033 \\
268 \\
302 \\
242 \\
15930\end{array}$ & $\begin{array}{r}7568 \\
244 \\
293 \\
212 \\
15010\end{array}$ & $\begin{array}{r}7597 \\
245 \\
392 \\
208 \\
15070\end{array}$ & $\begin{array}{r}7698 \\
257 \\
309 \\
235 \\
15270\end{array}$ \\
\hline $\begin{array}{l}\text { STATIS } \\
\text { MEAK } \\
\text { MAX } \\
\text { (WY) } \\
\text { MIN } \\
\text { (WY) }\end{array}$ & $\begin{array}{r}\text { ICS of } \\
419 \\
1795 \\
1942 \\
182 \\
1957\end{array}$ & $\begin{array}{c}\text { MONTHLY MEAN } \\
554 \\
1611 \\
1942 \\
243 \\
1957\end{array}$ & $\begin{array}{r}\text { DATA } \\
523 \\
1052 \\
1942 \\
269 \\
1957\end{array}$ & $\begin{array}{c}\text { FOR WATER } \\
508 \\
799 \\
1942 \\
300 \\
1957\end{array}$ & $\begin{array}{c}\text { YEARS } 1931 \\
579 \\
888 \\
1987 \\
323 \\
1957\end{array}$ & $\begin{array}{c}-1996, \\
712 \\
1290 \\
1989 \\
286 \\
1957\end{array}$ & $\begin{array}{c}\text { BY WATER } \\
1023 \\
3544 \\
1942 \\
274 \\
1981\end{array}$ & $\begin{array}{c}\text { YEAR (WY) } \\
2071 \\
7228 \\
1941 \\
249 \\
1972\end{array}$ & $\begin{array}{l}1989 \\
6837 \\
1941 \\
199 \\
1977\end{array}$ & $\begin{array}{r}786 \\
3540 \\
1995 \\
188 \\
1963\end{array}$ & $\begin{array}{r}442 \\
1699 \\
1957 \\
186 \\
1956\end{array}$ & $\begin{array}{r}377 \\
1132 \\
1982 \\
171 \\
1956\end{array}$ \\
\hline
\end{tabular}

SUMMARY STATISTICS ANRUAL, TOTAL

ANNUAL, MEAN

HIGHEST ANNUAL MEAN

LOWEST AMNUAL MEAN

BIGHEST DAILY MEAN

LOWEST DAILY MEAN

ANNUAL SEVEN-DAY MINIMUM

INSTANTANEOUS PEAK FLON

IMSTANTANEOUS PEAK STAGE

INSTANTANEOUS LOW FLOW

ANNUAL RUNOFE (AC-FT)

10 PERCENT EXCEEDS

50 PERCENT EXCEEDS

90 PERCENT EXCEEDS
FOR 1995 CALENDAR YEAR 465889

1276

FOR 1996 WATER YEAR 166885

$\begin{array}{rrrrr}7240 & \text { Ju1 } 6 & 892 & \text { Fob } 24 \\ 392 & \text { Oct } 15 & 208 & \text { Aug } & 7 \\ 400 & \text { Oct } 11 & 215 & \text { Aug } 11 \\ & & 936 & \text { Fob } 17 \\ & & 4.06 & \text { Fob } 17 \\ 924100 & & 198 & \text { Aug } & 6 \\ 3400 & & 331000 & & \\ 674 & & 732 & \\ 452 & & 452 & \end{array}$

WATER YEARS 1931 - 1996

\begin{tabular}{|c|c|c|}
\hline $\begin{array}{r}832 \\
2077 \\
308 \\
11700 \\
165 \\
166 \\
16200 \\
15.90 \\
198 \\
602900 \\
1620 \\
510 \\
261\end{array}$ & $\begin{array}{l}\text { May } \\
\text { Sep } \\
\text { Sep } \\
\text { Jun } \\
\text { Jun } \\
\text { Aug }\end{array}$ & $\begin{array}{r}16 \\
2 \\
1 \\
19 \\
19 \\
6\end{array}$ \\
\hline
\end{tabular}


RIO GRANDE BASIN

08284100 RIO CHAMA NEAR LA PUENTE, MM

LOCATION,--Lat 36 $39.45^{\prime \prime}$, long $106^{\circ} 37 \cdot 57^{\prime \prime}$, Rio Arriba County, Eydrolog lo Unit 13020102, in Tlerra Amarilla Grant on right bank $0.7 \mathrm{mi}$ downstream from Rito de Tierrs Amartils, 3.1 southwest of La puente, $6.7 \mathrm{mi}$ upstream from flow line of EI Vedo Reservoir, and at mile 91.4 .

DRAINAGE AREA. $-480 \mathrm{mi}^{2}$, approximately.

WATER-DISCHAREE RECORDS

PERTOD OF RECORD. - - October 1955 to current year.

GAGE.-Water-gtage recorder, Concrete control since Nov, 9, 1965. Elovation of gago 1s 7,083 ft above Natdonal Geodetic Vertical Datu of 1929, frow river prokilo map.

REMARKS. - Water-discharge records good except for estimated daily discharges, which aro poor. Diverstons Lor irrigation of about 10,300 acres upstream from station (1962 determination). Several observations of water temperature pere mede during the year. Bureau of Reclamation satellite telemetex in gage.

EXTREMES OUTSIDE PERIOD OF RECORDS. - A discharge of about 9,000 fts/s occurred Apr. 16, 1937, based on flow of Rlo Chams at Los OJos (Park View) with allowance for tributary inflow, A poak on May 21 or 22, 1926, may have exceeded 10,000 f.ts $/ \mathrm{s}$.

DISCEARGE, CUBIC FEET PER SECOND, WATER YEAR OCTOBER 1995 TO SEPTEMBER 1996

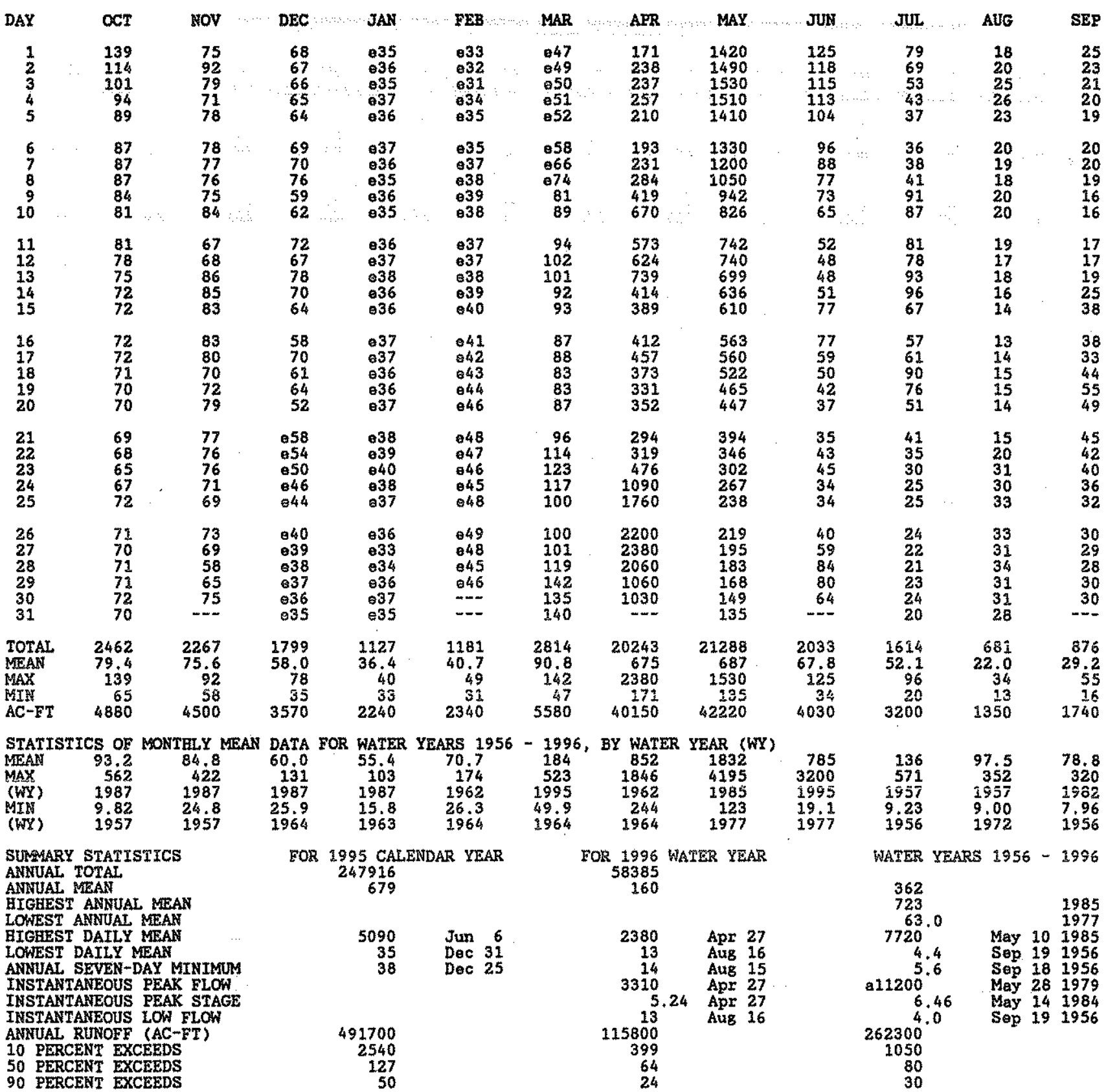

PERCENT EXCEBDS

Estimated

a-From rating curve extended above $5,400 \mathrm{fts} / \mathrm{s}$, 
RIO GRANDE BASIN

08284100 RIO CHAMA NEAR LA PUENTE, MM -- Continued

WATER-QUALITY RECORDS

PERIOD OF RECORD.--Water years 1974, 1986 to current year,

WATER QUALITY DATA, WATER YEAR OCTOBER 1995 TO SEPTEMBER 1996

\begin{tabular}{|c|c|c|c|c|c|c|c|c|c|c|}
\hline DATE & TIME & $\begin{array}{c}\text { DIS- } \\
\text { CEARGE, } \\
\text { INSST. } \\
\text { CUBIC } \\
\text { FEET } \\
\text { PER } \\
\text { SECOND } \\
(00061)\end{array}$ & $\begin{array}{l}\text { SPE- } \\
\text { CIFIC } \\
\text { CON- } \\
\text { DUCT- } \\
\text { ANCE } \\
\text { (US/CM) } \\
(00095)\end{array}$ & $\begin{array}{c}\text { PH } \\
\text { WATER } \\
\text { WHOLE } \\
\text { FIELD } \\
\text { (STAND- } \\
\text { ARD } \\
\text { URITS) } \\
\text { (00400) }\end{array}$ & $\begin{array}{l}\text { TEMPER- } \\
\text { ATURE } \\
\text { AIR } \\
\text { (DEG C) } \\
(00020)\end{array}$ & $\begin{array}{l}\text { TEMPER- } \\
\text { ATURE } \\
\text { WATER } \\
\text { (DEG C) } \\
(00010)\end{array}$ & $\begin{array}{c}\text { BARO- } \\
\text { METRIC } \\
\text { PRES } \\
\text { SURE } \\
\text { (MM } \\
\text { OF } \\
\text { HG) } \\
(00025)\end{array}$ & $\begin{array}{c}\text { OXYGEN, } \\
\text { DIS- } \\
\text { SOLVED } \\
\text { (MG/L) } \\
(00300)\end{array}$ & $\begin{array}{c}\text { OXYGEN, } \\
\text { DIS- } \\
\text { SOLVED } \\
\text { (PER- } \\
\text { CENT } \\
\text { SATUR- } \\
\text { ATTON) } \\
\text { (00301) }\end{array}$ & $\begin{array}{l}\text { HARD- } \\
\text { NESS } \\
\text { TOTAL } \\
\text { (MG/L } \\
\text { AS } \\
\text { CACO3) } \\
(00900)\end{array}$ \\
\hline $\begin{array}{l}\text { ov } 1995 \\
30 \text { B } 1996\end{array}$ & 1330 & E73 & 191 & 7.3 & 9.5 & 1.5 & 585 & 11.3 & 105 & 75 \\
\hline $01 \ldots$ & 1345 & E47 & 158 & 7.8 & 1.5 & 0.0 & 573 & 11.1 & 101 & 65 \\
\hline 09. & 1030 & 419 & 117 & 8.2 & 12.0 & 5.0 & 588 & 10.5 & 107 & 49 \\
\hline & 1030 & 19 & 262 & 8.0 & 19.5 & 16.0 & 586 & 7.6 & 101 & 110 \\
\hline
\end{tabular}

\begin{tabular}{|c|c|c|c|c|c|c|c|c|c|c|}
\hline & $\begin{array}{l}\text { KARD- } \\
\text { NESS } \\
\text { NONCARB } \\
\text { DISSOLV } \\
\text { FLD. AS } \\
\text { CACO3 } \\
\text { (MG/L) } \\
(00904)\end{array}$ & $\begin{array}{c}\text { CALCIUM } \\
\text { DIS- } \\
\text { SOLVED } \\
\text { (MG/L } \\
\text { AS CA }) \\
(00915)\end{array}$ & $\begin{array}{c}\text { MAGNE- } \\
\text { SHUM, } \\
\text { DIS- } \\
\text { SOLVED } \\
\text { (MG/L } \\
\text { AS MG) } \\
(00925)\end{array}$ & $\begin{array}{c}\text { SODIUM, } \\
\text { DIS- } \\
\text { SOLVED } \\
\text { (MG/L } \\
\text { AS NA) } \\
(00930)\end{array}$ & $\begin{array}{c}\text { SODILM } \\
\text { AD- } \\
\text { SORP- } \\
\text { TION } \\
\text { RATIO } \\
(00931)\end{array}$ & $\begin{array}{c}\text { POTAS- } \\
\text { SIUM, } \\
\text { DIS- } \\
\text { SOLVED } \\
\text { (MG/L } \\
\text { AS K }) \\
(00935)\end{array}$ & $\begin{array}{c}\text { BICAR- } \\
\text { BONATE } \\
\text { WATER } \\
\text { DIS IT } \\
\text { FIELD } \\
\text { MG/L AS } \\
\text { HCO3 } \\
(00453)\end{array}$ & $\begin{array}{c}\text { CAR- } \\
\text { BONATE } \\
\text { WATER } \\
\text { DIS IT } \\
\text { FIELD } \\
\text { MG/L AS } \\
\text { CO3 } \\
(00452)\end{array}$ & $\begin{array}{c}\text { ALKA- } \\
\text { LINITY } \\
\text { WAT DIS } \\
\text { TOT IT } \\
\text { FIELD } \\
\text { MG AL AS } \\
\text { CACO3 } \\
(39086)\end{array}$ & $\bar{y}$ \\
\hline
\end{tabular}

\begin{tabular}{|c|c|c|c|c|c|c|c|c|c|c|}
\hline $\begin{array}{l}\text { Nov } 1995 \\
3001996\end{array}$ & -- & 23 & 4.3 & 6.4 & 0.3 & 1.6 & $-\infty$ & - & -- & 75 \\
\hline O1... & - & 20 & 3.6 & 5.6 & 0.3 & 1.5 & -- & - & $\rightarrow$ & 67 \\
\hline $\begin{array}{l}09 . . \\
\text { SEP }\end{array}$ & 2 & 15 & 2.7 & 3.6 & 0.2 & 1.5 & 57 & 0 & 46 & 51 \\
\hline $05 \ldots$ & $\because$ & 34 & 6.1 & 8.8 & 0.4 & 2.1 & -- & -- & - & 110 \\
\hline DATE & $\begin{array}{l}\text { SULFATE } \\
\text { DIS- } \\
\text { SOLVED } \\
\text { (MG/L } \\
\text { AS SO4) } \\
(00945)\end{array}$ & $\begin{array}{l}\text { CHLO- } \\
\text { RIDE, } \\
\text { DIS - } \\
\text { SOLVED } \\
\text { (MG/L } \\
\text { AS CL) } \\
(00940)\end{array}$ & $\begin{array}{l}\text { FLUO- } \\
\text { RIDE, } \\
\text { DIS- } \\
\text { SOLVED } \\
\text { (MG/L } \\
\text { AS F) } \\
(00950)\end{array}$ & $\begin{array}{c}\text { SILICA, } \\
\text { DIS- } \\
\text { SOLVED } \\
\text { (MG/L } \\
\text { AS } \\
\text { SIO2) } \\
(00955)\end{array}$ & $\begin{array}{c}\text { SOLIDS, } \\
\text { SUM OF' } \\
\text { CONSTI- } \\
\text { TUENTS, } \\
\text { DIS- } \\
\text { SOLVED } \\
(M G / L) \\
(70301)\end{array}$ & $\begin{array}{l}\text { BORON, } \\
\text { DIS- } \\
\text { SOLVED } \\
\text { (UG/L } \\
\text { AS B) } \\
(01020)\end{array}$ & $\begin{array}{l}\text { IRON, } \\
\text { DIS- } \\
\text { SOLVED } \\
\text { (UG/L } \\
\text { AS FE) } \\
(01046)\end{array}$ & $\begin{array}{l}\text { SEDI- } \\
\text { MENT, } \\
\text { SUS- } \\
\text { PENDED } \\
(M G / L) \\
(80154)\end{array}$ & $\begin{array}{c}\text { SEDI- } \\
\text { MENT, } \\
\text { DIS- } \\
\text { CHARGE, } \\
\text { SUS- } \\
\text { PENDED } \\
\text { (T/DAY } \\
(80155)\end{array}$ & $\begin{array}{c}\text { SED. } \\
\text { SUSP. } \\
\text { SIEVE } \\
\text { DIAM. } \\
\text { \% FINER } \\
\text { THAAN } \\
\text { T062 MM } \\
(70331)\end{array}$ \\
\hline
\end{tabular}

\begin{tabular}{|c|c|c|c|c|c|c|c|c|c|}
\hline $\begin{array}{l}\text { Nov } 1995 \\
30 \\
\text { 30 }\end{array}$ & 14 & 1.7 & 0.10 & 22 & 118 & 20 & 23 & 11 & - \\
\hline APR & 12 & 1.5 & 0.10 & 20 & 104 & $<10$ & 14 & 2 & - \\
\hline$\underset{\text { SEP }}{09} .$. & 8.2 & 1.1 & $<0.10$ & 14 & 74 & $<10$ & 94 & 59 & 67 \\
\hline 05. & 23 & 1.8 & 0.10 & 20 & 162 & 26 & 51 & 12 & 0.62 \\
\hline
\end{tabular}


RIO GRANDE BASIN

08284160 AZOTEA TUNNEL AT OUTLET, NEAR CRAMA, MM

LOCATION, --Lat 36 $51^{\prime} 12^{\prime \prime}$, long 106 40 18", Rio Arriba County, Hydrologic Unit 13020102, in Tierra Amarilla Grant, on left bank at south portel, $0.2 \mathrm{mf}$ upstream from Azotea Creek, and $6.2 \mathrm{mi}$ southwest of Chama.

PERIOD OF RECORD, --October 1970 to current year.

GAGE. - Water-stage recorder and Parshall flume. Datum of gage is 7,519.87 ft above National Geodetic Vertical Datum of 1929 (levels by Bureau of Reclamation).

REMARKS. -Records represent regulated diversions from Rlo Blanco, Little Navajo River, and Navajo River in San Juan River Basin.

COOPERATION, --Records provided by Bureau of Reclemation.

AVERAGE DISCHARGE. - 26 years, $128 \mathrm{ft} / \mathrm{s}, 92,740 \mathrm{acre-st} / \mathrm{yr}$.

EXTREMES FOR PERIOD OF RECORD,-Maximun discharge, 1,170 fts/s, May 17; 1978 ; 8 age helght, 7.85 ft; no flow many days most years.

EXTREMES FOR CURRENT YEAR.--Maximum daily dischargo, $831 \mathrm{ft} / \mathrm{s}$, May 17; no flow many days.

DISCEARGE, CUBIC FEET PER SECOND, WATER YEAR OCTOBER 1995 TO SEPTEMBER 1996 DAIIY MEAN VALUES

\begin{tabular}{|c|c|c|c|c|c|c|c|c|c|c|c|c|}
\hline DAY & $\alpha C T$ & NOV & $\mathrm{DEC}$ & JAN & FEB & MAR & APR & MAY & JUN & JUL & AUG & SEP \\
\hline $\begin{array}{l}1 \\
2 \\
3 \\
4 \\
5\end{array}$ & $\begin{array}{l}.00 \\
.00 \\
.00 \\
.00 \\
.00\end{array}$ & $\begin{array}{l}.00 \\
.00 \\
.00 \\
.00 \\
.00\end{array}$ & $\begin{array}{l}.00 \\
.00 \\
.00 \\
.00 \\
.00\end{array}$ & $\begin{array}{l}.00 \\
.00 \\
.00 \\
.00 \\
.00\end{array}$ & $\begin{array}{l}.00 \\
.00 \\
.00 \\
00 \\
00\end{array}$ & $\begin{array}{l}.00 \\
.00 \\
.00 \\
.00 \\
.00\end{array}$ & $\begin{array}{l}174 \\
214 \\
169 \\
148 \\
128\end{array}$ & $\begin{array}{l}364 \\
379 \\
475 \\
525 \\
571\end{array}$ & $\begin{array}{l}180 \\
167 \\
167 \\
166 \\
171\end{array}$ & $\begin{array}{l}84 \\
56 \\
42 \\
29 \\
24\end{array}$ & $\begin{array}{r}3.0 \\
3.0 \\
5.0 \\
3.5 \\
3.0\end{array}$ & $\begin{array}{l}.00 \\
.00 \\
.00 \\
.00 \\
.00\end{array}$ \\
\hline $\begin{array}{r}6 \\
7 \\
8 \\
9 \\
10\end{array}$ & $\begin{array}{l}.00 \\
.00 \\
.00 \\
.00 \\
.00\end{array}$ & $\begin{array}{l}.00 \\
.00 \\
.00 \\
.00 \\
.00\end{array}$ & $\begin{array}{r}.00 \\
.00 \\
.00 \\
.00 \\
.00\end{array}$ & $\begin{array}{l}.00 \\
.00 \\
.00 \\
.00 \\
.00\end{array}$ & $\begin{array}{r}.00 \\
.00 \\
.00 \\
.00 \\
.00\end{array}$ & $\begin{array}{l}.00 \\
.00 \\
.00 \\
.00 \\
.00\end{array}$ & $\begin{array}{l}133 \\
160 \\
210 \\
350 \\
374\end{array}$ & $\begin{array}{r}611 \\
614 \\
537 \\
540 \\
617\end{array}$ & $\begin{array}{l}170 \\
167 \\
151 \\
123 \\
102\end{array}$ & $\begin{array}{c}19 \\
28 \\
44 \\
188 \\
109\end{array}$ & $\begin{array}{r}2.5 \\
1.5 \\
1.0 \\
1.0 \\
1.0\end{array}$ & $\begin{array}{l}.00 \\
.00 \\
.00 \\
.00 \\
.00\end{array}$ \\
\hline $\begin{array}{l}11 \\
12 \\
13 \\
14 \\
15\end{array}$ & $\begin{array}{l}.00 \\
.00 \\
.00 \\
.00 \\
.00\end{array}$ & $\begin{array}{l}.00 \\
.00 \\
.00 \\
.00 \\
.00\end{array}$ & $\begin{array}{l}.00 \\
.00 \\
.00 \\
.00 \\
.00\end{array}$ & $\begin{array}{l}.00 \\
.00 \\
.00 \\
.00 \\
.00\end{array}$ & $\begin{array}{l}.00 \\
.00 \\
.00 \\
.00 \\
.00\end{array}$ & $\begin{array}{l}.00 \\
.00 \\
.00 \\
.00 \\
.00\end{array}$ & $\begin{array}{l}385 \\
291 \\
299 \\
207 \\
175\end{array}$ & $\begin{array}{l}559 \\
599 \\
654 \\
663 \\
761\end{array}$ & $\begin{array}{r}91 \\
86 \\
95 \\
95 \\
124\end{array}$ & $\begin{array}{r}113 \\
72 \\
71 \\
49 \\
76\end{array}$ & $\begin{array}{l}1.0 \\
.00 \\
.00 \\
.00 \\
.50\end{array}$ & $\begin{array}{l}.00 \\
.00 \\
.00 \\
.00 \\
.00\end{array}$ \\
\hline $\begin{array}{l}16 \\
17 \\
18 \\
39 \\
20\end{array}$ & $\begin{array}{l}.00 \\
.00 \\
.00 \\
.00 \\
.00\end{array}$ & $\begin{array}{l}.00 \\
.00 \\
.00 \\
.00 \\
.00\end{array}$ & $\begin{array}{l}.00 \\
.00 \\
.00 \\
.00 \\
.00\end{array}$ & $\begin{array}{l}.00 \\
.00 \\
.00 \\
.00 \\
.00\end{array}$ & $\begin{array}{l}.00 \\
.00 \\
.00 \\
.00 \\
.00\end{array}$ & $\begin{array}{l}.00 \\
.00 \\
.00 \\
.00 \\
.00\end{array}$ & $\begin{array}{l}188 \\
199 \\
160 \\
158 \\
161\end{array}$ & $\begin{array}{l}769 \\
831 \\
780 \\
782 \\
730\end{array}$ & $\begin{array}{l}93 \\
76 \\
66 \\
58 \\
44\end{array}$ & $\begin{array}{l}46 \\
59 \\
61 \\
46 \\
25\end{array}$ & $\begin{array}{r}.50 \\
.50 \\
1.5 \\
2.5 \\
1.5\end{array}$ & $\begin{array}{r}.00 \\
.00 \\
34.00 \\
31\end{array}$ \\
\hline $\begin{array}{l}21 \\
22 \\
23 \\
24 \\
25\end{array}$ & $\begin{array}{l}.00 \\
.00 \\
.00 \\
.00 \\
.00\end{array}$ & $\begin{array}{l}.00 \\
.00 \\
.00 \\
.00 \\
.00\end{array}$ & $\begin{array}{l}.00 \\
.00 \\
.00 \\
.00 \\
.00\end{array}$ & $\begin{array}{l}.00 \\
.00 \\
.00 \\
.00 \\
.00\end{array}$ & $\begin{array}{l}.00 \\
.00 \\
.00 \\
.00 \\
.00\end{array}$ & $\begin{array}{l}22 \\
82 \\
91 \\
65 \\
41\end{array}$ & $\begin{array}{r}142 \\
128 \\
203 \\
348 \\
491\end{array}$ & $\begin{array}{r}563 \\
\quad 544 \\
475 \\
383 \\
266\end{array}$ & $\begin{array}{l}37 \\
48 \\
42 \\
26 \\
25\end{array}$ & $\begin{array}{r}16 \\
15 \\
7.1 \\
4.0 \\
3.0\end{array}$ & $\begin{array}{r}1.0 \\
.00 \\
.00 \\
.00 \\
.00\end{array}$ & $\begin{array}{r}21 \\
9.6 \\
4.0 \\
2.0 \\
1.0\end{array}$ \\
\hline $\begin{array}{l}26 \\
27 \\
28 \\
29 \\
30 \\
31\end{array}$ & $\begin{array}{l}.00 \\
.00 \\
.00 \\
.00 \\
.00 \\
.00\end{array}$ & $\begin{array}{l}.00 \\
.00 \\
.00 \\
.00 \\
.00 \\
.--\end{array}$ & $\begin{array}{l}.00 \\
.00 \\
.00 \\
.00 \\
.00 \\
.00\end{array}$ & $\begin{array}{l}.00 \\
.00 \\
.00 \\
.00 \\
.00 \\
.00\end{array}$ & $\begin{array}{l}.00 \\
.00 \\
.00 \\
.00 \\
-.-\end{array}$ & $\begin{array}{l}36 \\
54 \\
73 \\
84 \\
62 \\
94\end{array}$ & $\begin{array}{l}624 \\
670 \\
620 \\
404 \\
341 \\
--\end{array}$ & $\begin{array}{l}222 \\
167 \\
128 \\
101 \\
141 \\
159\end{array}$ & $\begin{array}{r}18 \\
88 \\
124 \\
84 \\
49 \\
--\end{array}$ & $\begin{array}{r}4.0 \\
3.0 \\
4.0 \\
5.5 \\
11.5\end{array}$ & $\begin{array}{l}.00 \\
.00 \\
.00 \\
.00 \\
.00 \\
.00\end{array}$ & $\begin{array}{r}1.0 \\
.50 \\
.00 \\
.00 \\
.00 \\
.\end{array}$ \\
\hline $\begin{array}{l}\text { TOTAI } \\
\text { MEAN } \\
\text { MAX } \\
\text { MIN } \\
\text { AC- FE }\end{array}$ & $\begin{array}{r}0.00 \\
.000 \\
.00 \\
.00 \\
.00\end{array}$ & $\begin{array}{l}0.00 \\
.000 \\
.00 \\
.00 \\
.00\end{array}$ & $\begin{array}{r}0.00 \\
.000 \\
.00 \\
.00 \\
.00\end{array}$ & $\begin{array}{l}0.00 \\
.000 \\
.00 \\
.00 \\
.00\end{array}$ & $\begin{array}{r}0.00 \\
.000 \\
.00 \\
.00 \\
.00\end{array}$ & $\begin{array}{r}704.00 \\
22.7 \\
94 \\
.00 \\
1400\end{array}$ & $\begin{array}{r}8254 \\
275 \\
670 \\
128 \\
16370\end{array}$ & $\begin{array}{r}15510 \\
500 \\
831 \\
101 \\
30760\end{array}$ & $\begin{array}{r}2933 \\
97.8 \\
180 \\
18 \\
5820\end{array}$ & $\begin{array}{r}1318.1 \\
42.5 \\
188 \\
3.0 \\
2610\end{array}$ & $\begin{array}{r}33.50 \\
1.08 \\
5.0 \\
.00 \\
66\end{array}$ & $\begin{array}{r}104.10 \\
3.47 \\
34 \\
.00 \\
206\end{array}$ \\
\hline
\end{tabular}

CAL YR 1995 TOTAL 43493.00 MEAN 119 MAX 981 MIN .00 AC-FT 86270 WTR YR 1996 TOTAL 28856.70 MEAN 78.8 MAX 831 MIN .00 AC-FT 57240 
RIO GRANDE BASIN

08284200 WILLOW CREEK ABOVE HERON RESERVOIR, NEAR LOS OJOS, NM

LOCATION.--Lat $36^{\circ} 44^{\prime} 33^{\prime \prime}$, long $106^{\circ} 37^{\prime} 34^{\prime \prime}$, Rio Arriba County, Hydrologio Unit 13020102, in Tierra Amarilla Grant, on right bank 200 ft downstream from bridge, 0.2 mi downstream from Iron Spring Creek, 3.3 mi west of Los Ojos, and at mile 9.7 .

DRAINAGE AREA. --112 mi2.

PERIOD OF RECORD.--October and November 1962 (monthly discharge only), December 1962 to current year. Published as "near Park View" prior to 1976.

GAGE.--Water-stage recorder. Concrete control since June 6, 1963. Datum of gage is 7,196.29 ft above National. Geodetic Vertical Datum of 1929 (levels by Bureau of Reciamation). Prior to Apr. 1, 1971, at site 900 ft downstream at lower datum.

REMARKS.--Records represent inflow to Heron Reservoir and since Nov. 17, 1970, include San Juan Rtver water imported through Azotea tunnel (station 08284160).

COOPERATION. --Recorda provided by Bureau of Reclamation.

AVERAGE DISCRARGE. - - 8 years (water years 1963-70), $10.5 \mathrm{ft} / \mathrm{s}, 7,610$ acre-ft/yr, prior to completion of Azotea tunnel. 26 years (pater years 1971-96), $141 \mathrm{ft} / \mathrm{s}, 102,200$ acre-ft/yr.

EXTREMES FOR PERIOD OF RECORD.--Maximum discharge, 1,610 fts/s, Mar. 12, 1985, gage height, $6.65 \mathrm{ft}$; no flow at times most years.

EXTREMES FOR CURRENT YEAR.--Maximum daily discharge, $814 \mathrm{ft} 3 / \mathrm{s}$, May 17-19; no flow Mar. 1-20.

DISCHARGE, CUBIC FEET PER SECOND, WATER YEAR OCTOBER 1995 TO SEPTEMBER 1996

\begin{tabular}{|c|c|c|c|c|c|c|c|c|c|c|c|c|}
\hline DAY & $\propto C T$ & Nov & DEC & JAN & FEB & MAR & APR & MAY & JUN & JUL & AUG & SEP \\
\hline $\begin{array}{l}1 \\
2 \\
3 \\
4 \\
5\end{array}$ & $\begin{array}{l}2.0 \\
1.9 \\
1.8 \\
.96 \\
.76\end{array}$ & $\begin{array}{l}.50 \\
.50 \\
.50 \\
.50 \\
.50\end{array}$ & $\begin{array}{l}.20 \\
.20 \\
.20 \\
.20 \\
.20\end{array}$ & $\begin{array}{l}.11 \\
.11 \\
.11 \\
.11 \\
.11\end{array}$ & $\begin{array}{l}.12 \\
.12 \\
.12 \\
.15 \\
.12\end{array}$ & $\begin{array}{l}.00 \\
.00 \\
.00 \\
.00 \\
.00\end{array}$ & $\begin{array}{l}150 \\
261 \\
201 \\
168 \\
141\end{array}$ & $\begin{array}{l}363 \\
373 \\
475 \\
532 \\
572\end{array}$ & $\begin{array}{l}191 \\
176 \\
162 \\
176 \\
176\end{array}$ & $\begin{array}{l}90 \\
60 \\
49 \\
38 \\
30\end{array}$ & $\begin{array}{l}4.0 \\
3.0 \\
3.0 \\
3.0 \\
3.5\end{array}$ & $\begin{array}{l}2.0 \\
2.0 \\
2.0 \\
2.0 \\
1.5\end{array}$ \\
\hline $\begin{array}{r}6 \\
7 \\
8 \\
9 \\
10\end{array}$ & $\begin{array}{l}.40 \\
.34 \\
.30 \\
.27 \\
.24\end{array}$ & $\begin{array}{l}.50 \\
.50 \\
.50 \\
.50 \\
.50\end{array}$ & $\begin{array}{l}.20 \\
.20 \\
.20 \\
.20 \\
.20\end{array}$ & $\begin{array}{l}.11 \\
.11 \\
.11 \\
.11 \\
.11\end{array}$ & $\begin{array}{l}.11 \\
.12 \\
.11 \\
.12 \\
.12\end{array}$ & $\begin{array}{l}.00 \\
.00 \\
.00 \\
.00 \\
.00\end{array}$ & $\begin{array}{l}148 \\
173 \\
217 \\
349 \\
378\end{array}$ & $\begin{array}{l}582 \\
532 \\
570 \\
599 \\
572\end{array}$ & $\begin{array}{l}180 \\
176 \\
127 \\
113 \\
109\end{array}$ & $\begin{array}{r}25 \\
24 \\
55 \\
246 \\
120\end{array}$ & $\begin{array}{l}3.0 \\
2.0 \\
1.0 \\
1.5 \\
.50\end{array}$ & $\begin{array}{l}1.5 \\
1.5 \\
1.0 \\
1.0 \\
.50\end{array}$ \\
\hline $\begin{array}{l}11 \\
12 \\
13 \\
14 \\
15\end{array}$ & $\begin{array}{r}.24 \\
.24 \\
.23 \\
.21 \\
1.1\end{array}$ & $\begin{array}{l}.50 \\
.50 \\
.50 \\
.50 \\
.50\end{array}$ & $\begin{array}{l}.20 \\
.20 \\
.20 \\
.20 \\
.20\end{array}$ & $\begin{array}{l}.11 \\
.11 \\
.11 \\
.11 \\
.11\end{array}$ & $\begin{array}{l}.12 \\
.19 \\
.29 \\
.80 \\
1.4\end{array}$ & $\begin{array}{l}.00 \\
.00 \\
.00 \\
.00 \\
.00\end{array}$ & $\begin{array}{l}378 \\
288 \\
300 \\
220 \\
189\end{array}$ & $\begin{array}{l}615 \\
654 \\
711 \\
696 \\
751\end{array}$ & $\begin{array}{r}110 \\
92 \\
105 \\
94 \\
128\end{array}$ & $\begin{array}{r}137 \\
87 \\
89 \\
71 \\
76\end{array}$ & $\begin{array}{l}4.5 \\
2.5 \\
1.5 \\
1.0 \\
1.0\end{array}$ & $\begin{array}{r}.50 \\
.50 \\
.50 \\
1.5 \\
3.0\end{array}$ \\
\hline $\begin{array}{l}16 \\
17 \\
18 \\
19 \\
20\end{array}$ & $\begin{array}{l}1.5 \\
1.3 \\
1.2 \\
.80 \\
.56\end{array}$ & $\begin{array}{r}.50 \\
.50 \\
.50 \\
.50 \\
.50\end{array}$ & $\begin{array}{l}.20 \\
.20 \\
.20 \\
.20 \\
.20\end{array}$ & $\begin{array}{l}.11 \\
.11 \\
.11 \\
.11 \\
.11\end{array}$ & $\begin{array}{l}1.2 \\
1.6 \\
2.3 \\
2.0 \\
3.2\end{array}$ & $\begin{array}{l}.00 \\
.00 \\
.00 \\
.00 \\
.00\end{array}$ & $\begin{array}{l}189 \\
221 \\
176 \\
159 \\
160\end{array}$ & $\begin{array}{l}736 \\
814 \\
814 \\
814 \\
734\end{array}$ & $\begin{array}{r}101 \\
82 \\
73 \\
65 \\
50\end{array}$ & $\begin{array}{l}57 \\
68 \\
73 \\
57 \\
36\end{array}$ & $\begin{array}{l}1.0 \\
1.0 \\
2.0 \\
2.5 \\
2.0\end{array}$ & $\begin{array}{l}3.0 \\
3.0 \\
4.5 \\
48 \\
36\end{array}$ \\
\hline $\begin{array}{l}21 \\
22 \\
23 \\
24 \\
25\end{array}$ & $\begin{array}{l}.38 \\
.36 \\
.34 \\
.36 \\
.59\end{array}$ & $\begin{array}{l}.50 \\
.50 \\
.50 \\
.50 \\
.50\end{array}$ & $\begin{array}{l}.20 \\
.20 \\
.20 \\
.20 \\
.20\end{array}$ & $\begin{array}{l}.11 \\
.11 \\
\vdots 11 \\
.11 \\
.11\end{array}$ & $\begin{array}{l}5.4 \\
7.0 \\
3.7 \\
3.7 \\
4.1\end{array}$ & $\begin{array}{c}5.6 \\
95 \\
117 \\
89 \\
59\end{array}$ & $\begin{array}{l}135 \\
135 \\
239 \\
338 \\
469\end{array}$ & $\begin{array}{l}476 \\
504 \\
464 \\
375 \\
277\end{array}$ & $\begin{array}{l}40 \\
43 \\
51 \\
29 \\
24\end{array}$ & $\begin{array}{r}23 \\
22 \\
9.6 \\
5.6 \\
4.0\end{array}$ & $\begin{array}{r}2.0 \\
5.6 \\
13 \\
4.0 \\
6.6\end{array}$ & $\begin{array}{r}25 \\
5.0 \\
8.6 \\
5.0 \\
3.5\end{array}$ \\
\hline $\begin{array}{l}26 \\
27 \\
28 \\
29 \\
30 \\
31\end{array}$ & $\begin{array}{l}.59 \\
.59 \\
.56 \\
.52 \\
.72 \\
.59\end{array}$ & $\begin{array}{r}.50 \\
.50 \\
.50 \\
.50 \\
.50 \\
\end{array}$ & $\begin{array}{l}.20 \\
.20 \\
.20 \\
.20 \\
.20 \\
.20\end{array}$ & $\begin{array}{l}.11 \\
.11 \\
.11 \\
.11 \\
.11 \\
.11\end{array}$ & $\begin{array}{l}2.8 \\
1.8 \\
1.5 \\
1.4 \\
.--\end{array}$ & $\begin{array}{l}46 \\
48 \\
86 \\
75 \\
76 \\
95\end{array}$ & $\begin{array}{l}538 \\
655 \\
635 \\
416 \\
362 \\
-2\end{array}$ & $\begin{array}{l}233 \\
188 \\
140 \\
106 \\
137 \\
162\end{array}$ & $\begin{array}{r}21 \\
91 \\
131 \\
100 \\
60 \\
-\end{array}$ & $\begin{array}{l}3.5 \\
3.5 \\
4.5 \\
5.0 \\
7.6 \\
5.6\end{array}$ & $\begin{array}{r}4.0 \\
4.5 \\
10 \\
4.5 \\
3.5 \\
2.5\end{array}$ & $\begin{array}{l}2.0 \\
2.0 \\
1.0 \\
1.0 \\
1.0\end{array}$ \\
\hline $\begin{array}{l}\text { TOTAL } \\
\text { MEAN } \\
\text { MAX } \\
\text { MIN } \\
\text { AC }-F T\end{array}$ & $\begin{array}{r}21.95 \\
.71 \\
2.0 \\
.21 \\
44\end{array}$ & $\begin{array}{r}15.00 \\
.50 \\
.50 \\
.50 \\
30\end{array}$ & $\begin{array}{r}6.20 \\
.20 \\
.20 \\
.20 \\
12\end{array}$ & $\begin{array}{r}3.41 \\
.11 \\
.11 \\
.11 \\
6.8\end{array}$ & $\begin{array}{r}45.71 \\
1.58 \\
7.0 \\
.11 \\
91\end{array}$ & $\begin{array}{r}791.60 \\
25.5 \\
117 \\
.00 \\
1570\end{array}$ & $\begin{array}{r}8388 \\
280 \\
655 \\
135 \\
16640\end{array}$ & $\begin{array}{r}15571 \\
502 \\
814 \\
106 \\
30890\end{array}$ & $\begin{array}{r}3076 \\
103 \\
191 \\
21 \\
6100\end{array}$ & $\begin{array}{r}1581.9 \\
51.0 \\
246 \\
3.5 \\
3140\end{array}$ & $\begin{array}{r}103.70 \\
3.35 \\
13 \\
.50 \\
206\end{array}$ & $\begin{array}{r}169.60 \\
5.65 \\
48 \\
.50 \\
336\end{array}$ \\
\hline
\end{tabular}

CAL. YR 1995 TOTAL 53653.24 MEAN 147 MAX 1060 MIN .00 AC-FT 106400 WTR YR 1996 TOTAL 29774.07 MEAN 81.3 MAX 814 MIN .00 AC-FT 59060 
RIO GRANDE BASIN

08284300 HORSE LAKE CREEK ABOVE HERON RESERVOTR, NEAR LOS OJOS, MM

LOCATION, --Lat $36^{\circ} 42^{\prime} 24^{\prime \prime}$, long $106^{\circ} 44^{\circ} 42^{\prime \prime}$, Rio Arriba County, Eydrologic Unit 13020102, In Tierra Amarilla Grant, on right bank $3.7 \mathrm{mi}$ northwest of Beron Dam, $7.8 \mathrm{mi}$ downstream from Horse Lake, and $9.9 \mathrm{mi}$ west of Los OJos.

DRATNAGE AREA. $--45 \mathrm{mi}^{2}$, approximately.

PERIOD OF RECORD.--October and November 1962 (monthly discharge only), December 1962 to current year. No winter records subsequent to 1973. Published as "near Park View" prior to 1976.

GAGE. - Water-stage recorder, Concrete control since June 10, 1963. Datum of gage is 7, 188, 85 ft above National. Geodetic Vertical Datum of 1929 (levals by Bureau of Reclamation). Frior to July 1, 1971, at site 1,100 ft upstream at higher datims.

REMARKS.--Diversions upstrean from station for irrigation of meadows and for off-channel stock tanks.

COOPERATION. --Records provided by Bureau of Reclamation.

AVERAGE DISCAARGE--11 years (water years $1963-73$ ), $1.10 \mathrm{ft} / \mathrm{s}, 797$ acre- $\mathrm{ft} / \mathrm{yr}$.

EXTREMES FOR PERTOD OF RECORD.--Maximum discharge, 3,960 fts/s, July 30, 1968, gage height, 4.9 ft, site and datum then in use, from rating curve extended above $37 \mathrm{ft}^{3 / \mathrm{s}}$ on bests of slope-area measurements at gage helghts 3.20 ft and 4.9 ft; no flow most of time.

EXTREMES FOR CURRENT YEAR.--Maximum daily dischargo, $3.2 \mathrm{ft} / \mathrm{s}$, Apr, 19, no flow most of time.

DISCBARGE, CUBIC FEET PER SECOND, WATER YEAR OCTOBER 1995 TO SEPTEMBER 1996

\begin{tabular}{|c|c|c|c|c|c|c|c|c|c|c|c|c|}
\hline DAY & OCT & NOV & DEC & JAN & FEB & MAR & APR & MAY & JUN & JUL & AUGG & SEP \\
\hline $\begin{array}{l}1 \\
2 \\
3 \\
4 \\
5\end{array}$ & $\begin{array}{l}-- \\
=- \\
-\cdots \\
-\cdots\end{array}$ & $\begin{array}{l}=-1 \\
m-\infty \\
m-\end{array}$ & $\begin{array}{l}-m \\
-- \\
- \\
--\end{array}$ & $\begin{array}{l}-\cdots \\
\cdots- \\
\cdots- \\
\cdots-\end{array}$ & 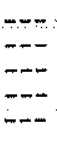 & $\begin{array}{l}-- \\
-- \\
\cdots \\
\cdots\end{array}$ & $\begin{array}{l}2.5 \\
2.5 \\
2.4 \\
2.3 \\
2.2\end{array}$ & $\begin{array}{l}.00 \\
.00 \\
.00 \\
.00 \\
.00\end{array}$ & $\begin{array}{l}.00 \\
.00 \\
.00 \\
.00 \\
.00\end{array}$ & $\begin{array}{l}.00 \\
.00 \\
.00 \\
.00 \\
.00\end{array}$ & $\begin{array}{l}.00 \\
.00 \\
.00 \\
.00 \\
.00\end{array}$ & $\begin{array}{l}.00 \\
.00 \\
.00 \\
.00 \\
.00\end{array}$ \\
\hline $\begin{array}{r}6 \\
7 \\
8 \\
9 \\
10\end{array}$ & $=-$ & $\begin{array}{l}--- \\
-\infty \\
-\infty\end{array}$ & $=-$ & $\begin{array}{l}m-- \\
=- \\
-\cdots \\
-\cdots\end{array}$ & $-\infty$ & $\begin{array}{l}m- \\
m- \\
m-\end{array}$ & $\begin{array}{l}2.3 \\
2.5 \\
2.5 \\
2.6 \\
2.7\end{array}$ & $\begin{array}{l}.00 \\
.00 \\
.00 \\
.00 \\
.00\end{array}$ & $\begin{array}{l}.00 \\
.00 \\
.00 \\
.00 \\
.00\end{array}$ & $\begin{array}{l}.00 \\
.00 \\
.00 \\
.00 \\
.00\end{array}$ & $\begin{array}{l}.00 \\
.00 \\
.00 \\
.00 \\
.00\end{array}$ & $\begin{array}{l}.00 \\
.00 \\
.00 \\
.00 \\
.00\end{array}$ \\
\hline $\begin{array}{l}11 \\
12 \\
13 \\
14 \\
15\end{array}$ & $\begin{array}{l}-- \\
\cdots- \\
\cdots\end{array}$ & $\begin{array}{l}m-\infty \\
=-- \\
---\end{array}$ & 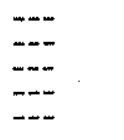 & $=$ & 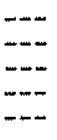 & $\begin{array}{l}-. . \\
1.3 \\
1.5 \\
1.7 \\
2.0\end{array}$ & $\begin{array}{l}2.7 \\
2.9 \\
2.6 \\
.50 \\
2.8\end{array}$ & $\begin{array}{l}.00 \\
.00 \\
.00 \\
.00 \\
.00\end{array}$ & $\begin{array}{l}.00 \\
.00 \\
.00 \\
.00 \\
.00\end{array}$ & $\begin{array}{l}.00 \\
.00 \\
.00 \\
.00 \\
.00\end{array}$ & $\begin{array}{l}.00 \\
.00 \\
.00 \\
.00 \\
.00\end{array}$ & $\begin{array}{l}.00 \\
.00 \\
.00 \\
.00 \\
.00\end{array}$ \\
\hline $\begin{array}{l}16 \\
17 \\
18 \\
19 \\
20\end{array}$ & $\begin{array}{l}-\cdots \\
\cdots- \\
\cdots- \\
-\cdots\end{array}$ & $\begin{array}{l}m \\
--- \\
--\end{array}$ & $\begin{array}{l}-\cdots \\
=-- \\
=--\end{array}$ & $\begin{array}{l}m- \\
-\cdots \\
-\cdots \\
---\end{array}$ & 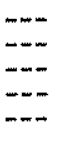 & $\begin{array}{l}2.1 \\
2.3 \\
2.5 \\
2.6 \\
2.7\end{array}$ & $\begin{array}{l}2.9 \\
3.0 \\
3.3 \\
3.2 \\
2.5\end{array}$ & $\begin{array}{l}.00 \\
.00 \\
.00 \\
.00 \\
.00\end{array}$ & $\begin{array}{l}.00 \\
.00 \\
.00 \\
.00 \\
.00\end{array}$ & $\begin{array}{l}.00 \\
.00 \\
.00 \\
.00 \\
.00\end{array}$ & $\begin{array}{l}.00 \\
.00 \\
.00 \\
.00 \\
.00\end{array}$ & $\begin{array}{l}.00 \\
.00 \\
.00 \\
.00 \\
.00\end{array}$ \\
\hline $\begin{array}{l}21 \\
22 \\
23 \\
24 \\
25\end{array}$ & $\begin{array}{l}m- \\
m- \\
m-\end{array}$ & $=-$ & $m$ & $\begin{array}{l}--- \\
\cdots- \\
\cdots-\end{array}$ & $-\cdots$ & $\begin{array}{l}2.8 \\
2.9 \\
2.9 \\
2.9 \\
2.9\end{array}$ & $\begin{array}{l}2.0 \\
1.0 \\
1.0 \\
.50 \\
.50\end{array}$ & $\begin{array}{l}.00 \\
.00 \\
.00 \\
.00 \\
.00\end{array}$ & $\begin{array}{l}.00 \\
.00 \\
.00 \\
.00 \\
.00\end{array}$ & $\begin{array}{l}.00 \\
.00 \\
.00 \\
.00 \\
.00\end{array}$ & $\begin{array}{l}.00 \\
.00 \\
.00 \\
.00 \\
.00\end{array}$ & $\begin{array}{l}.00 \\
.00 \\
.00 \\
.00 \\
.00\end{array}$ \\
\hline $\begin{array}{l}26 \\
27 \\
28 \\
29 \\
30 \\
31\end{array}$ & $\begin{array}{l}\cdots \\
\cdots \\
\cdots \\
\cdots\end{array}$ & $\begin{array}{l}m- \\
m- \\
m- \\
m- \\
m-\end{array}$ & $\begin{array}{l}--- \\
--- \\
--- \\
--- \\
-\cdots\end{array}$ & $\begin{array}{c}\cdots \\
\cdots \\
\cdots \\
\cdots \\
\cdots-\end{array}$ & \begin{tabular}{c}
$\overline{--}$ \\
$\overline{-1}$ \\
\hdashline$-\overline{-}$ \\
\hdashline-- \\
\hdashline-
\end{tabular} & $\begin{array}{l}3.0 \\
3.0 \\
2.8 \\
2.0 \\
1.9 \\
2.4\end{array}$ & $\begin{array}{l}.50 \\
.00 \\
.00 \\
.00 \\
.00 \\
.-\end{array}$ & $\begin{array}{l}.00 \\
.00 \\
.00 \\
.00 \\
.00 \\
.00\end{array}$ & $\begin{array}{l}.00 \\
.00 \\
.00 \\
.00 \\
.00 \\
-\end{array}$ & $\begin{array}{l}.00 \\
.00 \\
.00 \\
.00 \\
.00 \\
.00\end{array}$ & $\begin{array}{l}.00 \\
.00 \\
.00 \\
.00 \\
.00 \\
.00\end{array}$ & $\begin{array}{l}.00 \\
.00 \\
.00 \\
.00 \\
.00\end{array}$ \\
\hline $\begin{array}{l}\text { TOTAL } \\
\text { MEAN } \\
\text { MAX } \\
\text { MIN } \\
\text { AC-FT }\end{array}$ & $\begin{array}{l}--- \\
\cdots- \\
-- \\
--\end{array}$ & $\begin{array}{l}--\infty \\
-- \\
-\cdots \\
-\infty\end{array}$ & $\begin{array}{l}-\cdots \\
-\cdots \\
m- \\
m- \\
---\end{array}$ & $\begin{array}{l}z-- \\
-- \\
-- \\
-\cdots\end{array}$ & $-\cdots$ & 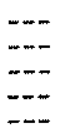 & $\begin{array}{r}56.20 \\
1.87 \\
3.2 \\
.00 \\
111\end{array}$ & $\begin{array}{l}0.00 \\
.000 \\
.00 \\
.00 \\
.00\end{array}$ & $\begin{array}{r}0.00 \\
.000 \\
.00 \\
.00 \\
.00\end{array}$ & $\begin{array}{l}0.00 \\
.000 \\
.00 \\
.00 \\
.00\end{array}$ & $\begin{array}{l}0.00 \\
.000 \\
.00 \\
.00 \\
.00\end{array}$ & $\begin{array}{l}0.00 \\
.000 \\
.00 \\
.00 \\
.00\end{array}$ \\
\hline
\end{tabular}


RIO GRANDE BASIN

08284510 HERON RESERVOIR NEAR LOS OJOS, NM

LOCATION. -Lat $36^{\circ} 39.56^{\prime \prime}$, Long $106^{\circ} 42 \cdot 13^{\prime \prime}$, R10 Arriba County, Hydrologic Unit 13020102, in Tierra Amarilla Grant, at Heron Dam on Willow Creek, $0.2 \mathrm{mi}$ upstream from Rio Chama, $5.1 \mathrm{mi}$ northeast of Ei Vado Dam, and 8.7 mi southwest of Los OJos.

DRAINAGE AREA. $--193 \mathrm{mi}^{2}$.

PERIOD OF RECORD. - October 1970 to current year. Published as "near Park Vtew" prior to 1976.

GAGE.--Water-stage recorder. Datum of gage is National Geodetic Vertical Datum of 1929 (levels by Bureau of Reclamation). Prior to Mar. 24, 1971, nonrecording gage.

REMARKS. --Reservoir is formed by earthfill dam; storage bagan Oct. 21, 1970. Total capacity 401,300 acre-ft at elevation 7,186.1 ft, low point on crest of uncontrolled splliway, including 1,340 acre-ft of dead storage at elevation 7,003.0 ft invert of gate sill of outlet tunnel. Reservolr is used for storage of transmountain water from. San Juan Rtver basin and for recreation. Figures given herein represent total storage.

COOPERATION.--Records provided by Bureau of Reclamation.

EXTRDMES FOR PERIOD OF RECORD,--Maximum contents, 401,800 acre-ft, July 28, 1982, elevation, 7, 186.19 ft; no storage prior to Oct. 21, 1970 .

EXTREMES FOR CURRENT YEAR.--Maximum contents, 388,690 acremf, Oct, 1, elevation, 7,183.94 ft; minimum, 335,150 acre-ft, Sept, 30 , elevation, 7,174.32 ft.

Capacity table (elevation, in feet, and contents, in acre-feet)

(Based on survey by Bureau of Reclamation in 1986)

$$
\begin{array}{ll}
7,170 & 312,600 \\
7,180 & 366,200 \\
7,190 & 424,700
\end{array}
$$

RESERVOIR STORAGE (ACRE-FEET), WATER YEAR OCTOBER 1995 TO SEPTEMBER 1996

\begin{tabular}{|c|c|c|c|c|c|c|c|c|c|c|c|c|}
\hline DAY & OCT & NOV & $D E C$ & JAN & FEB & MAR & AFR & MAY & JUN & JUL & ALG & SEP \\
\hline $\begin{array}{l}1 \\
2 \\
3 \\
4 \\
5\end{array}$ & $\begin{array}{l}388690 \\
388580 \\
388460 \\
388230 \\
388110\end{array}$ & $\begin{array}{l}386 \\
386 \\
386 \\
386 \\
386\end{array}$ & $\begin{array}{l}377590 \\
377590 \\
377540 \\
377480 \\
377420\end{array}$ & $\begin{array}{l}37 \\
37 \\
37 \\
37 \\
37\end{array}$ & $\begin{array}{l}37 \\
37 \\
37 \\
37\end{array}$ & $\begin{array}{l}37 \\
37 \\
37 \\
37 \\
37\end{array}$ & $\begin{array}{l}36 \\
36 \\
36 \\
35 \\
35\end{array}$ & $\begin{array}{l}3 \\
3 \\
35 \\
3 \\
3\end{array}$ & & & & $\begin{array}{l}342590 \\
342540 \\
342430 \\
342320 \\
342270\end{array}$ \\
\hline $\begin{array}{r}6 \\
7 \\
8 \\
9 \\
10\end{array}$ & $\begin{array}{l}388170 \\
388050 \\
387940 \\
387820 \\
387710\end{array}$ & $\begin{array}{l}385970 \\
385910 \\
385910 \\
385740 \\
385680\end{array}$ & $\begin{array}{l}377420 \\
377420 \\
377310 \\
377250 \\
377190\end{array}$ & $\begin{array}{l}37640 \\
37640 \\
37640 \\
37634 \\
37634\end{array}$ & $\begin{array}{l}373840 \\
373840 \\
373840 \\
373840 \\
373840\end{array}$ & $\begin{array}{l}37 \\
37 \\
37 \\
37 \\
37\end{array}$ & $\begin{array}{l}35843 \\
35782 \\
35727 \\
35694 \\
35666\end{array}$ & $\begin{array}{l}3424 \\
3436 \\
3447 \\
3459 \\
3468\end{array}$ & $\begin{array}{l}36 \\
36 \\
36 \\
36 \\
36\end{array}$ & & $\begin{array}{l}3 \\
3 \\
3 \\
3 \\
3\end{array}$ & $\begin{array}{l}160 \\
050 \\
000 \\
890\end{array}$ \\
\hline $\begin{array}{l}11 \\
12 \\
13 \\
14 \\
15\end{array}$ & $\begin{array}{l}387710 \\
387710 \\
387590 \\
387530 \\
387480\end{array}$ & $\begin{array}{l}385630 \\
385570 \\
385390 \\
385050 \\
384650\end{array}$ & $\begin{array}{l}377250 \\
377190 \\
377190 \\
377140 \\
377080\end{array}$ & $\begin{array}{l}376 \\
376 \\
376 \\
37 \\
37\end{array}$ & $\begin{array}{l}37 \\
37 \\
37 \\
37 \\
37\end{array}$ & $\begin{array}{l}37 \\
37 \\
37 \\
37 \\
37\end{array}$ & $\begin{array}{l}35 \\
35 \\
35 \\
35 \\
35\end{array}$ & & $\begin{array}{l}36 \\
36 \\
36 \\
36 \\
36\end{array}$ & $\begin{array}{l}3 \\
3 \\
3 \\
3 \\
3\end{array}$ & $\begin{array}{l}3 \\
3 \\
3 \\
3 \\
3\end{array}$ & $\begin{array}{l}3 \\
3 \\
3 \\
3 \\
3\end{array}$ \\
\hline $\begin{array}{l}16 \\
17 \\
18 \\
19 \\
20\end{array}$ & $\begin{array}{l}387420 \\
387300 \\
387240 \\
387190 \\
387010\end{array}$ & $\begin{array}{l}384130 \\
383670 \\
383210 \\
382750 \\
382290\end{array}$ & $\begin{array}{l}377080 \\
377020 \\
377020 \\
377020 \\
377020\end{array}$ & $\begin{array}{l}375 \\
37 \\
37 \\
375 \\
37\end{array}$ & $\begin{array}{l}37378 \\
37378 \\
37378 \\
37373 \\
37378\end{array}$ & $\begin{array}{l}37 \\
37 \\
37 \\
37 \\
37\end{array}$ & $\begin{array}{l}35 \\
35 \\
35 \\
35 \\
35\end{array}$ & & & & & $\begin{array}{l}510 \\
080 \\
650 \\
280 \\
900\end{array}$ \\
\hline $\begin{array}{l}21 \\
22 \\
23 \\
24 \\
25\end{array}$ & $\begin{array}{l}386950 \\
386780 \\
386670 \\
386610 \\
386550\end{array}$ & $\begin{array}{l}381770 \\
381310 \\
380850 \\
380340 \\
379820\end{array}$ & $\begin{array}{l}377020 \\
376910 \\
376850 \\
376790 \\
376790\end{array}$ & $\begin{array}{l}375260 \\
375090 \\
374920 \\
374750 \\
374630\end{array}$ & $\begin{array}{l}373 \\
373 \\
373 \\
373\end{array}$ & $\begin{array}{l}371240 \\
370390 \\
369430 \\
368590 \\
367690\end{array}$ & $\begin{array}{l}349 \\
348 \\
346 \\
345 \\
344\end{array}$ & $\begin{array}{l}3 \\
36 \\
36 \\
36 \\
36\end{array}$ & & $\begin{array}{l}3 \\
3 \\
3 \\
3 \\
3\end{array}$ & $\begin{array}{l}3 \\
3 \\
3 \\
3 \\
3\end{array}$ & $\begin{array}{l}3394 \\
3389 \\
3386 \\
3381 \\
3376\end{array}$ \\
\hline $\begin{array}{l}26 \\
27 \\
28 \\
29 \\
30 \\
31\end{array}$ & $\begin{array}{l}386610 \\
386430 \\
386320 \\
386260 \\
386260 \\
386200\end{array}$ & $\begin{array}{r}379250 \\
378850 \\
378390 \\
377820 \\
377540 \\
\end{array}$ & $\begin{array}{l}376740 \\
376740 \\
376740 \\
376680 \\
376680 \\
376680\end{array}$ & $\begin{array}{l}374410 \\
374180 \\
374070 \\
374010 \\
373840 \\
373840\end{array}$ & $\begin{array}{r}373610 \\
373610 \\
373730 \\
373780 \\
- \\
--\infty\end{array}$ & $\begin{array}{l}366790 \\
366170 \\
365050 \\
364380 \\
363550 \\
362760\end{array}$ & $\begin{array}{l}342650 \\
341300 \\
339690 \\
337600 \\
337120\end{array}$ & $\begin{array}{l}365000 \\
365280 \\
365450 \\
365610 \\
365840 \\
366060\end{array}$ & $\begin{array}{l}365280 \\
364890 \\
364660 \\
364380 \\
363880\end{array}$ & $\begin{array}{l}35 \\
34 \\
34 \\
34 \\
34 \\
34\end{array}$ & $\begin{array}{l}3432 \\
3432 \\
3432 \\
3429 \\
3428 \\
3427\end{array}$ & $\begin{array}{l}337010 \\
336420 \\
336050 \\
335630 \\
335150\end{array}$ \\
\hline $\begin{array}{l}11) \\
(\neq \dagger\end{array}$ & $\begin{array}{r}388690 \\
386200 \\
7183.51 \\
-2550\end{array}$ & $\begin{array}{r}386200 \\
377540 \\
7182.01 \\
-8660\end{array}$ & $\begin{array}{r}377590 \\
376680 \\
7181.84 \\
-860\end{array}$ & $\begin{array}{r}376680 \\
373840 \\
7181.35 \\
-2840\end{array}$ & $\begin{array}{r}373900 \\
373610 \\
7181.34 \\
-60\end{array}$ & $\begin{array}{r}373900 \\
362760 \\
7179.38 \\
-11020\end{array}$ & $\begin{array}{r}361760 \\
337120 \\
7174.68 \\
-25640\end{array}$ & $\begin{array}{r}366060 \\
337710 \\
7179.87 \\
+28940\end{array}$ & $\begin{array}{r}369880 \\
363880 \\
7179.57 \\
-2180\end{array}$ & $\begin{array}{r}363320 \\
347030 \\
7176.52 \\
-16850\end{array}$ & $\begin{array}{r}346540 \\
342700 \\
7175.72 \\
-4330\end{array}$ & $\begin{array}{r}342590 \\
335150 \\
7174.31 \\
-7550\end{array}$ \\
\hline
\end{tabular}

DATLY OBSERVATION AT 24:00 VALUES

CAL YR 1995 MAX 400330 MIN 325330 (f

WTR YR 1996 MAX 388690 MIN 335150 (tt) -53600

(†) ELEVATION, IN FEET, AT END OF MONTE

(it) CHANGE IN CONTENTS, IN ACRE-EEET 
08284520 WILLOW CREEK BELOW GERON DAM, NM

LOCATYON, --Lat $36^{\circ} 39^{\circ} 56^{\prime \prime}$, Long $106^{\circ} 42^{\prime} 13^{\prime \prime}$, Rio Arriba County, Bydrologic Unit 13020102, in Tierra Amarilla Grant, in outlet conduits of Heron Dam, $0.2 \mathrm{mi}$ upstream from Rto Chama, 5.1 mi northeast of El Vado Dam, and 8.7 mi southwest of Los Ofos.

DRAINAGE AREA. $--193 \mathrm{mi}^{2}$.

PERIOD OF RECORD.---January 1971 to current year.

GAGE.--Totalizing flowneters in each of two outlet conduits in Heron Dam.

REAARKS, - Flow ragulated by Heron Reservoix (atation 06264510), Outlot condutts are 14-in. and 120-in. in diameter.

COORERATION. - -Records provided by Bureau of Reclamation,

AVERAGE DISCHARGE, -26 years, $125 \mathrm{ft} / \mathrm{s}, 90,560 \mathrm{acre}-\mathrm{ft} / \mathrm{yr}$,

EXTREMES FOR PERIOD OF RECORD,--Maximum daily discharge, 2,780 fts/s, Dec, 18, 19, 1982 ; no flow many days each year.

EXTREMES FOR CURRENT YEAR,--Maximum daily dischargo, 1,400 ft3/s, Apr, 26-29; no flow many days.

DISCEARGE, CUBIC FEET PER SECOND, WATER YEAR OCTOBER 19.95 TO SEPTEMBER 1996

\begin{tabular}{|c|c|c|c|c|c|c|c|c|c|c|c|c|}
\hline DAY & $\infty \mathrm{Cr}$ & Nov & DEC & JAN & FEB & MAR & APR & MAY & JuN & JUL & AUG & SEP \\
\hline $\begin{array}{l}1 \\
2 \\
3 \\
4 \\
5\end{array}$ & $\begin{array}{l}.00 \\
.00 \\
.00 \\
.00 \\
.00\end{array}$ & $\begin{array}{l}.00 \\
.00 \\
.00 \\
.00 \\
.00\end{array}$ & $\begin{array}{l}.00 \\
.00 \\
.00 \\
.00 \\
.00\end{array}$ & $\begin{array}{l}.00 \\
.00 \\
00 \\
.00 \\
.00\end{array}$ & $\begin{array}{l}41 \\
18 \\
.00 \\
.00 \\
.00\end{array}$ & $\begin{array}{l}.00 \\
.00 \\
.00 \\
.00 \\
.00\end{array}$ & $\begin{array}{l}500 \\
500 \\
500 \\
500 \\
500\end{array}$ & $\begin{array}{l}.00 \\
.00 \\
.00 \\
.00 \\
.00\end{array}$ & $\begin{array}{l}.00 \\
.00 \\
.00 \\
.00 \\
.00\end{array}$ & $\begin{array}{l}346 \\
346 \\
346 \\
346 \\
346\end{array}$ & $\begin{array}{l}266 \\
198 \\
198 \\
198 \\
198\end{array}$ & $\begin{array}{l}.00 \\
.00 \\
.00 \\
.00 \\
.00\end{array}$ \\
\hline $\begin{array}{r}6 \\
7 \\
8 \\
9 \\
10\end{array}$ & $\begin{array}{l}.00 \\
.00 \\
.00 \\
.00 \\
.00\end{array}$ & $\begin{array}{l}.00 \\
.00 \\
.00 \\
.00 \\
.00\end{array}$ & $\begin{array}{l}.00 \\
.00 \\
.00 \\
.00 \\
.00\end{array}$ & $\begin{array}{l}.00 \\
.00 \\
.00 \\
.00 \\
.00\end{array}$ & $\begin{array}{l}.00 \\
.00 \\
.00 \\
.00 \\
.00\end{array}$ & $\begin{array}{l}.00 \\
.00 \\
.00 \\
.00 \\
.00\end{array}$ & $\begin{array}{l}500 \\
500 \\
500 \\
500 \\
500\end{array}$ & $\begin{array}{l}.00 \\
.00 \\
.00 \\
.00 \\
.00\end{array}$ & $\begin{array}{l}.00 \\
.00 \\
.00 \\
.00 \\
.00\end{array}$ & $\begin{array}{l}346 \\
346 \\
346 \\
344 \\
344\end{array}$ & $\begin{array}{r}198 \\
80 \\
.00 \\
.00 \\
.00 \\
.00\end{array}$ & $\begin{array}{l}.00 \\
.00 \\
.00 \\
.00 \\
.00\end{array}$ \\
\hline $\begin{array}{l}11 \\
12 \\
13 \\
14 \\
15\end{array}$ & $\begin{array}{l}.00 \\
.00 \\
.00 \\
.00 \\
.00\end{array}$ & ${ }_{131^{.00}}^{.00}$ & $\begin{array}{l}.00 \\
.00 \\
.00 \\
.00 \\
.00\end{array}$ & $\begin{array}{l}25^{.00} \\
41 \\
41 \\
41\end{array}$ & $\begin{array}{l}.00 \\
.00 \\
.00 \\
.00 \\
.00\end{array}$ & $\begin{array}{l}.00 \\
.00 \\
.00 \\
.00 \\
.00\end{array}$ & $\begin{array}{l}500 \\
500 \\
500 \\
500 \\
500\end{array}$ & $\begin{array}{l}.00 \\
.00 \\
.00 \\
.00 \\
.00\end{array}$ & $\begin{array}{l}.00 \\
.00 \\
.00 \\
.00 \\
.00\end{array}$ & $\begin{array}{l}344 \\
344 \\
344 \\
344 \\
343\end{array}$ & $\begin{array}{l}.00 \\
.00 \\
.00 \\
.00 \\
.00\end{array}$ & $\begin{array}{l}.00 \\
.00 \\
.00 \\
.00 \\
.00\end{array}$ \\
\hline $\begin{array}{l}16 \\
17 \\
18 \\
19 \\
20\end{array}$ & $\begin{array}{l}.00 \\
.00 \\
.00 \\
.00 \\
.00\end{array}$ & $\begin{array}{l}243 \\
243 \\
243 \\
243 \\
243\end{array}$ & $\begin{array}{l}.00 \\
.00 \\
.00 \\
.00 \\
.00\end{array}$ & $\begin{array}{l}41 \\
41 \\
41 \\
41 \\
41\end{array}$ & $\begin{array}{l}.00 \\
.00 \\
.00 \\
.00 \\
.00\end{array}$ & $\begin{array}{r}.00 \\
.00 \\
309^{.00} \\
500\end{array}$ & $\begin{array}{l}500 \\
500 \\
500 \\
500 \\
500\end{array}$ & $\begin{array}{l}.00 \\
.00 \\
.00 \\
.00 \\
.00\end{array}$ & $\begin{array}{l}.00 \\
172.00 \\
344 \\
346\end{array}$ & $\begin{array}{l}343 \\
343 \\
343 \\
343 \\
343\end{array}$ & $\begin{array}{l}.00 \\
.00 \\
.00 \\
.00 \\
.00\end{array}$ & $\begin{array}{l}132 \\
237 \\
237 \\
237 \\
237\end{array}$ \\
\hline $\begin{array}{l}21 \\
22 \\
23 \\
24 \\
25\end{array}$ & $\begin{array}{l}.00 \\
.00 \\
.00 \\
.00 \\
.00\end{array}$ & $\begin{array}{l}242 \\
242 \\
243 \\
242 \\
242\end{array}$ & $\begin{array}{l}.00 \\
.00 \\
.00 \\
.00 \\
.00\end{array}$ & $\begin{array}{l}41 \\
41 \\
41 \\
41 \\
41\end{array}$ & $\begin{array}{l}.00 \\
.00 \\
.00 \\
.00 \\
.00\end{array}$ & $\begin{array}{l}500 \\
500 \\
500 \\
500 \\
500\end{array}$ & $\begin{array}{r}500 \\
728 \\
900 \\
900 \\
1190\end{array}$ & $\begin{array}{l}.00 \\
.00 \\
.00 \\
.00 \\
.00\end{array}$ & $\begin{array}{l}346 \\
346 \\
346 \\
346 \\
346\end{array}$ & $\begin{array}{l}343 \\
343 \\
343 \\
343 \\
343\end{array}$ & $\begin{array}{l}.00 \\
.00 \\
.00 \\
.00 \\
.00\end{array}$ & $\begin{array}{l}237 \\
237 \\
237 \\
237 \\
237\end{array}$ \\
\hline $\begin{array}{l}26 \\
27 \\
28 \\
29 \\
30 \\
31\end{array}$ & $\begin{array}{l}.00 \\
.00 \\
.00 \\
.00 \\
.00 \\
.00\end{array}$ & $\begin{array}{r}242 \\
241 \\
263 \\
233 \\
78\end{array}$ & $\begin{array}{l}.00 \\
.00 \\
.00 \\
.00 \\
.00 \\
.00\end{array}$ & $\begin{array}{l}41 \\
41 \\
41 \\
41 \\
41 \\
41\end{array}$ & \begin{tabular}{l}
.00 \\
.00 \\
.00 \\
.00 \\
\hdashline. \\
.
\end{tabular} & $\begin{array}{l}500 \\
500 \\
500 \\
500 \\
500 \\
500\end{array}$ & $\begin{array}{r}1400 \\
1400 \\
1400 \\
1400 \\
554 \\
-\end{array}$ & $\begin{array}{l}.00 \\
.00 \\
.00 \\
.00 \\
.00 \\
.00\end{array}$ & $\begin{array}{l}346 \\
346 \\
346 \\
346 \\
346\end{array}$ & $\begin{array}{l}343 \\
343 \\
343 \\
343 \\
343 \\
343\end{array}$ & $\begin{array}{l}46^{.00} \\
79 \\
79 \\
33 \\
.00\end{array}$ & $\begin{array}{r}237 \\
237 \\
237 \\
237 \\
237 \\
\end{array}$ \\
\hline $\begin{array}{l}\text { TOTAL } \\
\text { MEAN } \\
\text { MAAK } \\
\text { MIN } \\
\text { AC-FT }\end{array}$ & $\begin{array}{l}0.00 \\
.000 \\
.00 \\
.00 \\
.00\end{array}$ & $\begin{array}{r}4069.00 \\
136 \\
263 \\
.00 \\
8070\end{array}$ & $\begin{array}{l}0.00 \\
.000 \\
.00 \\
.00 \\
.00\end{array}$ & $\begin{array}{r}804,00 \\
25.9 \\
41 \\
150 \\
1590\end{array}$ & $\begin{array}{r}59.00 \\
2.03 \\
41 \\
.00 \\
117\end{array}$ & $\begin{array}{r}6309.00 \\
204 \\
500 \\
.00 \\
12.510\end{array}$ & $\begin{array}{r}20372 \\
679 \\
1400 \\
500 \\
40410\end{array}$ & $\begin{array}{l}0.00 \\
.000 \\
.00 \\
.00 \\
.00\end{array}$ & $\begin{array}{r}4322.00 \\
144 \\
346 \\
850 \\
8570\end{array}$ & $\begin{array}{r}10663 \\
344 \\
346 \\
343 \\
21150\end{array}$ & $\begin{array}{r}1573.00 \\
50.7 \\
266 \\
3120\end{array}$ & $\begin{array}{r}3450.00 \\
115 \\
237 \\
600 \\
6840\end{array}$ \\
\hline
\end{tabular}

CAL YR 1995 TOTAL 62629.00 MEAN 172 MAX 1530 MIN .00 AC-FT 124200 WTR YR 1996 TOTAL 51621.00 MEAN 141 MAX 1400 MIN .00 AC-FT 102400 
RIO GRANDE BASIN

08285000 EL VADO RESERVOIR NEAR TIERRA AMARILLA, NM

LOCATION, -Lat 36 $35^{\prime} 39^{\prime \prime}$, long 106 $44^{\circ} 00^{\prime \prime}$, Rio Arriba County, Hydrologic Unit 13020102, Tierra Amar $\$ 11$ a Grant, at outlet tower of dam on Rio Chama, at village of E1 Vado, $12.4 \mathrm{mi}$ southwest of Tierra Amarilla, and at mile 77.7 .

DRAINAGE AREA. --873 $\mathrm{mi}^{2}$, of which about $100 \mathrm{mi}^{2}$ probably is noncontributing.

PERIOD OF RECORD.--January 1935 to September 1965 (monthend contents only), October 1965 to current year. Prior to October 1967, contents at about 0730 hours.

GAGE.--Water-stage recorder. Prior to October 1967, nonrecording gage only below gage height $6,879.3$ ft. Datum of gage 18 8.21 ft above National Geodetic Vertical Datum of 1929.

REMARKS, - -Reservoir is formed by rockfill dam, steel faced.' Storage began in January 1935. Capacity 186, 250 acreft between 8 age heights $6,759.0 \mathrm{ft}$ and $6,902.0 \mathrm{ft}$, top of spiliway gate. Dead storage, 1,480 acre-ft below $6,775.0 \mathrm{ft}$, sill of outlet works. Figures given herein represent total contents. Reservoif is used to impound water for irrigation by Middle Rio Grande Conservancy District and, since December 1972, for storage of contract water from San Juan-Chama Project. Rehabilitation of outlet works, completed in December 1966, increased valvecontrolled release from about $1,750 \mathrm{ft} / \mathrm{s}$ to about $6,000 \mathrm{ft} / \mathrm{s}$.

COOPERATION. --Records provided by Bureau of Reclamation.

EXTREMES FOR PERIOD OF RECORD.--Maximum contents, 204,900 acre-ft, of whtch 7,400 acre-ft was uncontrolled storage, June 4, 5, 1948, gage height, 6,904.2 ft; no storage at times prior to December 1966.

EXTREMES FOR CURRENT YEAR.--Maximum contents, 159,920 acre-ft, May 7, elevation, 6,893.27 ft; minimum, 45,160 acreft, Sept. 30, elevation $6,837.15$.

Capacity table (gage height, in feet, and contents, in acre-feet)

(Based on survey by Bureau of Reclamation in 1987)

$\begin{array}{llll}6,845 & 56,100 & 6,875 & 111,000 \\ 6,850 & 63,730 & 6,885 & 135,900 \\ 6,860 & 80,510 & 6,895 & 164,400 \\ 6,865 & 89,870 & 6,900 & 179,800\end{array}$

RESERVOIR STORAGE (ACRE-FEET), WATER YEAR OCTOBER 1995 TO SEETEMBER 1996 DAILY OBSERVATION AT 24:00 VALUES

\begin{tabular}{|c|c|c|c|c|c|c|c|c|c|c|c|c|}
\hline DAY & $\mathrm{OCT}$ & Nov & DEC & JAN & $\mathrm{FEB}$ & MAR & APR & MAY & JUN & JUL & ALG & SEP \\
\hline $\begin{array}{l}1 \\
2 \\
3 \\
4 \\
5\end{array}$ & $\begin{array}{l}138130 \\
138020 \\
137810 \\
137620 \\
137350\end{array}$ & $\begin{array}{l}125680 \\
125150 \\
124590 \\
124090 \\
123520\end{array}$ & $\begin{array}{l}117430 \\
117100 \\
116740 \\
116360 \\
116050\end{array}$ & $\begin{array}{l}106260 \\
105860 \\
105420 \\
105070 \\
104650\end{array}$ & $\begin{array}{l}96500 \\
96200 \\
95790 \\
95400 \\
95070\end{array}$ & $\begin{array}{l}87900 \\
87590 \\
87270 \\
86940 \\
86660\end{array}$ & $\begin{array}{l}94600 \\
95660 \\
96730 \\
97740 \\
98580\end{array}$ & $\begin{array}{l}150400 \\
152410 \\
154380 \\
156430 \\
158140\end{array}$ & $\begin{array}{l}138210 \\
136790 \\
135410 \\
133960 \\
132620\end{array}$ & $\begin{array}{l}107600 \\
107980 \\
108110 \\
108070 \\
107930\end{array}$ & $\begin{array}{l}97290 \\
96130 \\
94650 \\
93510 \\
92810\end{array}$ & $\begin{array}{l}60010 \\
59100 \\
58030 \\
57060 \\
56020\end{array}$ \\
\hline $\begin{array}{r}6 \\
7 \\
8 \\
9 \\
10\end{array}$ & $\begin{array}{l}137090 \\
136870 \\
136660 \\
136440 \\
136230\end{array}$ & $\begin{array}{l}122990 \\
122420 \\
121900 \\
121310 \\
120810\end{array}$ & $\begin{array}{l}115690 \\
115360 \\
115000 \\
114650 \\
114230\end{array}$ & $\begin{array}{l}104260 \\
103890 \\
103470 \\
103100 \\
102740\end{array}$ & $\begin{array}{l}94710 \\
94340 \\
94020 \\
93720 \\
93450\end{array}$ & $\begin{array}{l}86370 \\
86050 \\
85750 \\
85450 \\
85190\end{array}$ & $\begin{array}{r}99410 \\
100320 \\
101230 \\
102460 \\
104280\end{array}$ & $\begin{array}{l}159440 \\
159920 \\
159770 \\
159230 \\
158430\end{array}$ & $\begin{array}{l}131000 \\
129300 \\
127680 \\
125980 \\
124240\end{array}$ & $\begin{array}{l}107800 \\
107660 \\
107530 \\
107750 \\
108160\end{array}$ & $\begin{array}{l}92160 \\
91230 \\
90180 \\
88800 \\
86910\end{array}$ & $\begin{array}{l}55160 \\
53580 \\
52050 \\
51250 \\
50600\end{array}$ \\
\hline $\begin{array}{l}11 \\
12 \\
13 \\
14 \\
15\end{array}$ & $\begin{array}{l}135990 \\
135810 \\
135570 \\
135330 \\
135090\end{array}$ & $\begin{array}{l}120270 \\
119690 \\
119390 \\
119270 \\
119200\end{array}$ & $\begin{array}{l}113870 \\
113520 \\
113200 \\
112890 \\
112520\end{array}$ & $\begin{array}{l}102350 \\
102000 \\
101750 \\
101450 \\
101190\end{array}$ & $\begin{array}{l}93230 \\
92990 \\
92710 \\
92450 \\
92200\end{array}$ & $\begin{array}{l}84870 \\
84630 \\
84420 \\
84230 \\
84050\end{array}$ & $\begin{array}{l}105970 \\
107750 \\
109760 \\
111180 \\
112520\end{array}$ & $\begin{array}{l}157460 \\
156550 \\
155610 \\
155290 \\
155610\end{array}$ & $\begin{array}{l}122540 \\
120840 \\
119150 \\
117720 \\
116260\end{array}$ & $\begin{array}{l}108540 \\
108880 \\
109310 \\
109700 \\
110010\end{array}$ & $\begin{array}{l}85430 \\
84360 \\
83290 \\
82240 \\
81190\end{array}$ & $\begin{array}{l}49980 \\
49350 \\
48690 \\
48110 \\
47500\end{array}$ \\
\hline $\begin{array}{l}16 \\
17 \\
18 \\
19 \\
20\end{array}$ & $\begin{array}{l}134880 \\
134670 \\
134400 \\
134140 \\
133380\end{array}$ & $\begin{array}{l}119100 \\
118960 \\
118860 \\
118740 \\
118620\end{array}$ & $\begin{array}{l}112170 \\
111850 \\
111550 \\
111180 \\
110950\end{array}$ & $\begin{array}{r}100890 \\
100790 \\
100490 \\
100190 \\
99940\end{array}$ & $\begin{array}{l}91860 \\
91620 \\
91380 \\
91130 \\
90790\end{array}$ & $\begin{array}{l}83860 \\
83640 \\
83440 \\
83730 \\
84510\end{array}$ & $\begin{array}{l}113690 \\
114960 \\
116100 \\
116860 \\
117680\end{array}$ & $\begin{array}{l}155840 \\
155400 \\
154560 \\
153600 \\
153050\end{array}$ & $\begin{array}{l}114840 \\
113380 \\
112150 \\
111300 \\
110330\end{array}$ & $\begin{array}{l}110520 \\
110720 \\
111250 \\
111000 \\
109900\end{array}$ & $\begin{array}{l}79790 \\
77960 \\
76410 \\
75570 \\
74390\end{array}$ & $\begin{array}{l}47150 \\
47010 \\
46890 \\
46770 \\
46610\end{array}$ \\
\hline $\begin{array}{l}21 \\
22 \\
23 \\
24 \\
25\end{array}$ & $\begin{array}{l}132330 \\
131320 \\
130200 \\
129270 \\
128990\end{array}$ & $\begin{array}{l}118500 \\
118420 \\
118420 \\
118350 \\
118330\end{array}$ & $\begin{array}{l}110450 \\
110080 \\
109700 \\
109260 \\
108900\end{array}$ & $\begin{array}{l}99650 \\
99350 \\
99060 \\
98740 \\
98450\end{array}$ & $\begin{array}{l}90580 \\
90400 \\
90150 \\
89780 \\
89480\end{array}$ & $\begin{array}{l}85300 \\
86150 \\
86980 \\
87820 \\
88610\end{array}$ & $\begin{array}{l}118370 \\
119150 \\
120490 \\
123020 \\
127120\end{array}$ & $\begin{array}{l}153220 \\
153140 \\
152210 \\
151000 \\
149400\end{array}$ & $\begin{array}{l}109560 \\
108970 \\
108450 \\
107570 \\
106730\end{array}$ & $\begin{array}{l}109150 \\
108250 \\
106860 \\
105490 \\
104100\end{array}$ & $\begin{array}{l}73320 \\
72340 \\
71110 \\
69330 \\
68000\end{array}$ & $\begin{array}{l}46470 \\
46290 \\
46150 \\
46000 \\
45830\end{array}$ \\
\hline $\begin{array}{l}26 \\
27 \\
28 \\
29 \\
30 \\
31\end{array}$ & $\begin{array}{l}128660 \\
128350 \\
127860 \\
127230 \\
126670 \\
126160\end{array}$ & $\begin{array}{l}118250 \\
118280 \\
118230 \\
118110 \\
117840\end{array}$ & $\begin{array}{l}108470 \\
108090 \\
107690 \\
107330 \\
106950 \\
106610\end{array}$ & $\begin{array}{l}98140 \\
97830 \\
97560 \\
97250 \\
96980 \\
96730\end{array}$ & $\begin{array}{r}89210 \\
88840 \\
88530 \\
88230 \\
-\end{array}$ & $\begin{array}{l}89390 \\
90180 \\
90990 \\
91940 \\
92810 \\
93680\end{array}$ & $\begin{array}{r}132020 \\
137410 \\
142350 \\
146120 \\
148460 \\
\end{array}$ & $\begin{array}{l}147750 \\
145870 \\
144340 \\
142840 \\
141330 \\
139720\end{array}$ & $\begin{array}{c}105990 \\
105660 \\
106390 \\
106770 \\
107170\end{array}$ & $\begin{array}{r}102740 \\
101360 \\
100030 \\
99140 \\
98600 \\
98030\end{array}$ & $\begin{array}{l}67050 \\
66070 \\
65230 \\
64440 \\
63180 \\
61400\end{array}$ & $\begin{array}{r}45660 \\
45490 \\
45400 \\
45280 \\
45160 \\
-\end{array}$ \\
\hline $\begin{array}{l}\text { MAX } \\
\text { MIN } \\
(t) \\
(t+)\end{array}$ & $\begin{array}{r}138130 \\
126160 \\
6881.25 \\
-12020\end{array}$ & $\begin{array}{r}125680 \\
117840 \\
6877.89 \\
-8320\end{array}$ & $\begin{array}{r}117430 \\
106610 \\
6873.04 \\
-11230\end{array}$ & $\begin{array}{r}106260 \\
96730 \\
6868.42 \\
-9880\end{array}$ & $\begin{array}{r}96500 \\
88230 \\
6864.15 \\
-8500\end{array}$ & $\begin{array}{r}93680 \\
83440 \\
6866.92 \\
-5450\end{array}$ & $\begin{array}{r}148460 \\
94600 \\
6889.58 \\
+54780\end{array}$ & $\begin{array}{r}159920 \\
139720 \\
6886.43 \\
-8740\end{array}$ & $\begin{array}{r}138210 \\
105660 \\
6873.29 \\
-32550\end{array}$ & $\begin{array}{r}111250 \\
98030 \\
6869.05 \\
-9140\end{array}$ & $\begin{array}{r}97290 \\
61400 \\
6848.51 \\
-36630\end{array}$ & $\begin{array}{r}60010 \\
45160 \\
6837.15 \\
-16240\end{array}$ \\
\hline
\end{tabular}

CAL YR 1995 MAX 171490 MIN 69950 (fj) +27180

WTR YR 1996 MAX 159920 MIN 45160 (†) -93020

( $\dagger$ ) ELEVATION, IN FEET, AT END OF MONTH

(tt) CHANGE IN CONTENTS, IN ACRE-FEET 
08285500 RIO CHAMA BELOW EL VADO DAM, NM

LOCATION, --Lat $36^{\circ} 34^{\prime} 48^{\prime \prime}$, Long $106^{\circ} 43^{\prime 2} 4^{\prime \prime}$, Rio Arriba County, Bydrologic Untt 13020102, in Tierra Amarilla Grant, on left bank $1.5 \mathrm{mi}$ downstream from El Vado Dam, $2.8 \mathrm{mi}$ upstream from Rio Nutrias, 13 mi southwest of Tierra Amarilla, and at mile 76.2 .

DRAINAGE AREA. $-877 \mathrm{mi}^{3}$ of which about $100 \mathrm{mi}^{2}$ is probably noncontributing.

FERIOD OF RECORD.--October 1913 to November 1915, Apri1 to November 1916, March, Apri1 1920, September 1920 to August 1924, October 1935 to current year. Monthly discharge only for some periods, published in WSP 1312. Published as "Chama River" prior to 1935, as "near Tierra Amarilla" 1913-14, 1935-47, as "near E1 Vado" 1915-16, and "as "at E1 Vado" 1920-24

REVISED RECORDS, - WSP 1312: 1914, 1949. WSP 1392: 1949. WDR-NM-90: 1989

GAGE.-Watox-stage recorder. "Datum of gage is $6,696.12$ et above National Geodetic Vortical Datum of 1929 . Prior to October 1935, at site $1.5 \mathrm{mi}$ upstream at different datum. Detober 1935 to September 1938 at sto $1.2 \mathrm{mi}$ upstream at datum 30.34 rt higher.

REMARKS.-Records good. Flow regulated by El Vado Reservolr (station 08285000) since 1935. Flow affected by release of transmountain water from Beron Reservolr (station 08284510) since May 1971. Diversions for irrigation of about 10,600 acres upstream from station, Several observations of water temperature were made during the year. Satellite telemeter at station.

AVERAGE DISCHARGE, - -5 years (water yoars 1914-15, 1921-23), $448 \mathrm{ft} / \mathrm{s}, 324,600$ acre-ft/yr, prior to completion of E1 Vado Dam. 35 years (water years $1936-70$ ), $373 \mathrm{ft} / \mathrm{s}, 270,200$ acre-et/yx, prior to release of transmountain water.

EXTREMES FOR FERIOD OF RECORD.--Meximum discharge, 9,000 fts/s, May 22, 1920, gage height, $12 \mathrm{ft}$, site and datum then in use, from rating curve extended above $3,500 \mathrm{ft} 3 / 8$; no flow Mar., 25, 26, 31, 1955 . Naximum discharge since construction of E1 Vado Dam in $1935,6,610 \mathrm{ft} / \mathrm{s}$, May 7,1985 , gage helght, $7.08 \mathrm{ft}$

EXTREMES OUTSIDE PERIOD OF RECORD.--Flood of Oct. 4 or 5,1911 , was greater than floods in September 3904 and May 1920, from informetion by local residents.

DISCHARGE, CUBIC FEET PER SECOND, WATER YEAR OCTOBER 1995 TO SEPTEMBER 1996

\begin{tabular}{|c|c|c|c|c|c|c|c|c|c|c|c|c|}
\hline DAY & $\operatorname{Crt}$ & NOV & $\mathrm{DEC}$ & JAN & $F E B$ & MAR & $\mathrm{APR}$ & MAY & JUN & JUL & AUG & SEP \\
\hline $\begin{array}{l}1 \\
2 \\
3 \\
4 \\
5\end{array}$ & $\begin{array}{l}187 \\
190 \\
190 \\
190 \\
1.90\end{array}$ & $\begin{array}{l}349 \\
348 \\
349 \\
348 \\
348\end{array}$ & $\begin{array}{l}253 \\
253 \\
253 \\
253 \\
253\end{array}$ & $\begin{array}{l}245 \\
257 \\
258 \\
256 \\
256\end{array}$ & $\begin{array}{l}260 \\
258 \\
256 \\
255 \\
255\end{array}$ & $\begin{array}{l}229 \\
229 \\
229 \\
229 \\
228\end{array}$ & $\begin{array}{l}186 \\
188 \\
190 \\
232 \\
257\end{array}$ & $\begin{array}{l}200 \\
200 \\
200 \\
200 \\
200\end{array}$ & $\begin{array}{l}877 \\
856 \\
854 \\
854 \\
852\end{array}$ & $\begin{array}{l}218 \\
218 \\
349 \\
431 \\
432\end{array}$ & $\begin{array}{r}656 \\
885 \\
1030 \\
790 \\
594\end{array}$ & $\begin{array}{l}743 \\
557 \\
557 \\
561 \\
563\end{array}$ \\
\hline $\begin{array}{r}6 \\
7 \\
8 \\
9 \\
10\end{array}$ & $\begin{array}{l}190 \\
190 \\
192 \\
193 \\
194\end{array}$ & $\begin{array}{l}347 \\
346 \\
343 \\
343 \\
343\end{array}$ & $\begin{array}{l}252 \\
252 \\
252 \\
252 \\
252\end{array}$ & $\begin{array}{l}256 \\
255 \\
253 \\
253 \\
252\end{array}$ & $\begin{array}{l}255 \\
254 \\
254 \\
231 \\
210\end{array}$ & $\begin{array}{l}228 \\
228 \\
228 \\
227 \\
228\end{array}$ & $\begin{array}{l}260 \\
264 \\
264 \\
264 \\
215\end{array}$ & $\begin{array}{r}463 \\
815 \\
1010 \\
1120 \\
1150\end{array}$ & $\begin{array}{l}895 \\
952 \\
950 \\
949 \\
946\end{array}$ & $\begin{array}{l}433 \\
434 \\
435 \\
302 \\
229\end{array}$ & $\begin{array}{r}595 \\
597 \\
595 \\
820 \\
1030\end{array}$ & $\begin{array}{l}561 \\
839 \\
820 \\
427 \\
342\end{array}$ \\
\hline $\begin{array}{l}11 \\
12 \\
13 \\
14 \\
15\end{array}$ & $\begin{array}{l}189 \\
290 \\
190 \\
190 \\
191\end{array}$ & $\begin{array}{l}343 \\
343 \\
343 \\
343 \\
343\end{array}$ & $\begin{array}{l}252 \\
252 \\
252 \\
252 \\
252\end{array}$ & $\begin{array}{l}252 \\
252 \\
252 \\
252 \\
252\end{array}$ & $\begin{array}{l}210 \\
222 \\
229 \\
228 \\
228\end{array}$ & $\begin{array}{l}228 \\
225 \\
199 \\
186 \\
186\end{array}$ & $\begin{array}{l}194 \\
191 \\
194 \\
195 \\
199\end{array}$ & $\begin{array}{r}1150 \\
1150 \\
1110 \\
681 \\
448\end{array}$ & $\begin{array}{l}945 \\
943 \\
943 \\
884 \\
830\end{array}$ & $\begin{array}{l}229 \\
230 \\
233 \\
235 \\
236\end{array}$ & $\begin{array}{l}751 \\
579 \\
578 \\
577 \\
576\end{array}$ & $\begin{array}{l}337 \\
333 \\
344 \\
343 \\
342\end{array}$ \\
\hline $\begin{array}{l}16 \\
17 \\
18 \\
19 \\
20\end{array}$ & $\begin{array}{l}189 \\
188 \\
195 \\
197 \\
491\end{array}$ & $\begin{array}{l}343 \\
343 \\
343 \\
343 \\
346\end{array}$ & $\begin{array}{l}252 \\
252 \\
252 \\
252 \\
252\end{array}$ & $\begin{array}{l}252 \\
253 \\
259 \\
260 \\
260\end{array}$ & $\begin{array}{l}228 \\
229 \\
228 \\
228 \\
298\end{array}$ & $\begin{array}{l}186 \\
186 \\
184 \\
179 \\
179\end{array}$ & $\begin{array}{l}329 \\
278 \\
311 \\
402 \\
428\end{array}$ & $\begin{array}{l}448 \\
847 \\
953 \\
959 \\
673\end{array}$ & $\begin{array}{l}830 \\
829 \\
827 \\
827 \\
828\end{array}$ & $\begin{array}{l}237 \\
236 \\
232 \\
607 \\
928\end{array}$ & $\begin{array}{l}799 \\
995 \\
800 \\
563 \\
563\end{array}$ & $\begin{array}{l}341 \\
343 \\
343 \\
342 \\
343\end{array}$ \\
\hline $\begin{array}{l}21 \\
22 \\
23 \\
24 \\
25\end{array}$ & $\begin{array}{l}572 \\
570 \\
566 \\
470 \\
229\end{array}$ & $\begin{array}{l}347 \\
315 \\
298 \\
298 \\
298\end{array}$ & $\begin{array}{l}250 \\
250 \\
249 \\
249 \\
249\end{array}$ & $\begin{array}{l}260 \\
260 \\
260 \\
260 \\
260\end{array}$ & $\begin{array}{l}218 \\
214 \\
226 \\
233 \\
233\end{array}$ & $\begin{array}{l}179 \\
179 \\
179 \\
179 \\
179\end{array}$ & $\begin{array}{l}428 \\
586 \\
639 \\
545 \\
422\end{array}$ & $\begin{array}{r}320 \\
396 \\
759 \\
963 \\
1010\end{array}$ & $\begin{array}{l}744 \\
698 \\
696 \\
740 \\
822\end{array}$ & $\begin{array}{r}718 \\
864 \\
1050 \\
1050 \\
1040\end{array}$ & $\begin{array}{l}561 \\
562 \\
786 \\
983 \\
751\end{array}$ & $\begin{array}{l}343 \\
344 \\
345 \\
344 \\
337\end{array}$ \\
\hline $\begin{array}{l}26 \\
27 \\
28 \\
29 \\
30 \\
31\end{array}$ & $\begin{array}{l}229 \\
229 \\
323 \\
349 \\
349 \\
349\end{array}$ & $\begin{array}{l}298 \\
298 \\
298 \\
305 \\
276 \\
---\end{array}$ & $\begin{array}{l}248 \\
248 \\
248 \\
248 \\
248 \\
248\end{array}$ & $\begin{array}{l}259 \\
260 \\
259 \\
258 \\
258 \\
260\end{array}$ & $\begin{array}{l}232 \\
233 \\
233 \\
230 \\
-\cdots \\
-\end{array}$ & $\begin{array}{l}179 \\
182 \\
183 \\
185 \\
186 \\
186\end{array}$ & $\begin{array}{l}422 \\
428 \\
390 \\
274 \\
200 \\
-\end{array}$ & $\begin{array}{r}1140 \\
1130 \\
1000 \\
956 \\
956 \\
952\end{array}$ & $\begin{array}{l}770 \\
417 \\
218 \\
218 \\
218 \\
---\end{array}$ & $\begin{array}{r}1040 \\
1040 \\
1040 \\
794 \\
642 \\
653\end{array}$ & $\begin{array}{l}560 \\
559 \\
558 \\
559 \\
751 \\
972\end{array}$ & $\begin{array}{l}331 \\
319 \\
307 \\
306 \\
296\end{array}$ \\
\hline $\begin{array}{l}\text { TOTAL } \\
\text { MEAN } \\
\text { MAX } \\
\text { MIN } \\
\text { AC-FT }\end{array}$ & $\begin{array}{r}8351 \\
269 \\
572 \\
187 \\
16560\end{array}$ & $\begin{array}{r}9928 \\
331 \\
349 \\
276 \\
19690\end{array}$ & $\begin{array}{r}7780 \\
251 \\
253 \\
248 \\
15430\end{array}$ & $\begin{array}{r}7939 \\
256 \\
260 \\
245 \\
15750\end{array}$ & $\begin{array}{r}6868 \\
237 \\
298 \\
210 \\
13620\end{array}$ & $\begin{array}{r}6217 \\
201 \\
229 \\
179 \\
12330\end{array}$ & $\begin{array}{r}9375 \\
312 \\
639 \\
186 \\
18600\end{array}$ & $\begin{array}{r}23559 \\
760 \\
1150 \\
200 \\
46730\end{array}$ & $\begin{array}{r}23212 \\
774 \\
952 \\
218 \\
46040\end{array}$ & $\begin{array}{r}16815 \\
542 \\
1050 \\
218 \\
33350\end{array}$ & $\begin{array}{r}21975 \\
709 \\
1030 \\
558 \\
43590\end{array}$ & $\begin{array}{r}12653 \\
422 \\
839 \\
296 \\
25100\end{array}$ \\
\hline
\end{tabular}

STATISTICS OF MONTHLY MEAN DATA FOR WATER YEARS 1971 - 1996, BY WATER YEAR (WY)

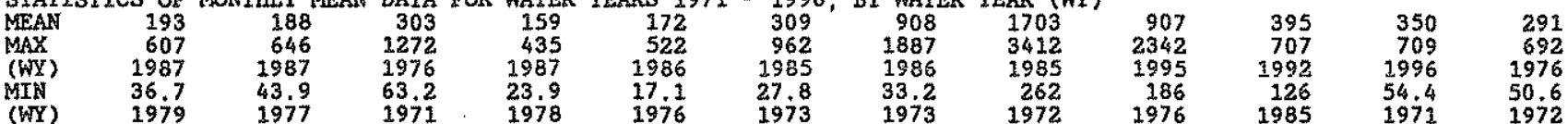

SURMARY STATISTICS ANNUAL TOTAL

ANNUAL MEAN

BIGAEST ANNUAL MEAN

LOWEST ANINUAL MEAN

HIGHEST DAILY MEAN

LOWEST DAILY MEAN

ANNUAL SEVEN-DAY MINIMUM

ANNUAL RUNOFE (AC-FT)

10 PERCENT EXCEEDS

50 PERCENT EXCEEDS

90 PERCENT EXCEEDS
FOR 1995 CALENDAR YEAR

$$
\begin{array}{r}
271869 \\
745
\end{array}
$$

FOR 1996 WATER YEAR 154672
423
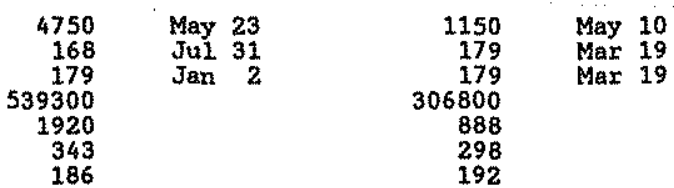

May 10

Mar 19
1976

WATER YEARS 1971 - 1996

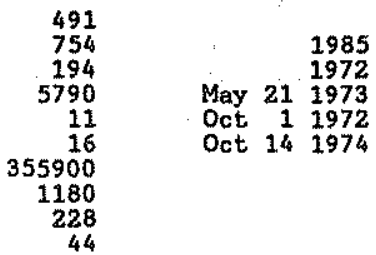


RTO GRANDE BASIN

08286500 RIO CHAMA ABOVE ABIQUTU RESERVOTR, NM

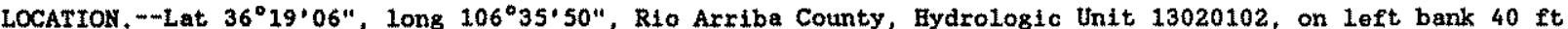
downstream from site of former bridge, 7.7 mi downstrean from Rio Gallina, 9 mi northwest of Youngsville, 15.6 mi. upstream from Abiquiu Dam, 30.3 mi downstream from E1 Vado Dam, and at mile 47.4

DRAINAGE AREA. $-1,600 \mathrm{mi}^{2}$, of which about $100 \mathrm{mi}^{2}$ is probably noncontributing.

PERIOD OF RECORD. --August 1961 to current year.

GAGE.-Water-stage recorder. Elevation of gage is 6,280 ft above National Geodetic Vertical Datum of 1929 , from topographic map.

REMARKS. --Records good except for daily discharges, which are fair. Flow regulated by E1 Vado Reservotr $(08285000)$. Since May 1971 flow affected by release of transmountain water from Heron Reservolr (station 08284510). Diversions for irrigation of about 15,000 acres upstream from station. Several observations of water temperature were made during the year. U.s. Army Corps of Engineers satellite telemeter at station.

AVERAGE DISCAARGE. --9 years (water years 1962-70), 358 fts/s, 259,400 acre-ft/yr, prior to release of transmountain watex.

EXTREMES OUTSIDE PERIOD OF RECORD.-Major floods probably occurred on Sept, 29, 1904, Oct. 4 or 5, 1911, and May 22. 1920 .

DISCHARGE, CUBIC FEET PER SECOND, WATER YEAR OCTOBER 1995 TO SEPTEMBER 1996

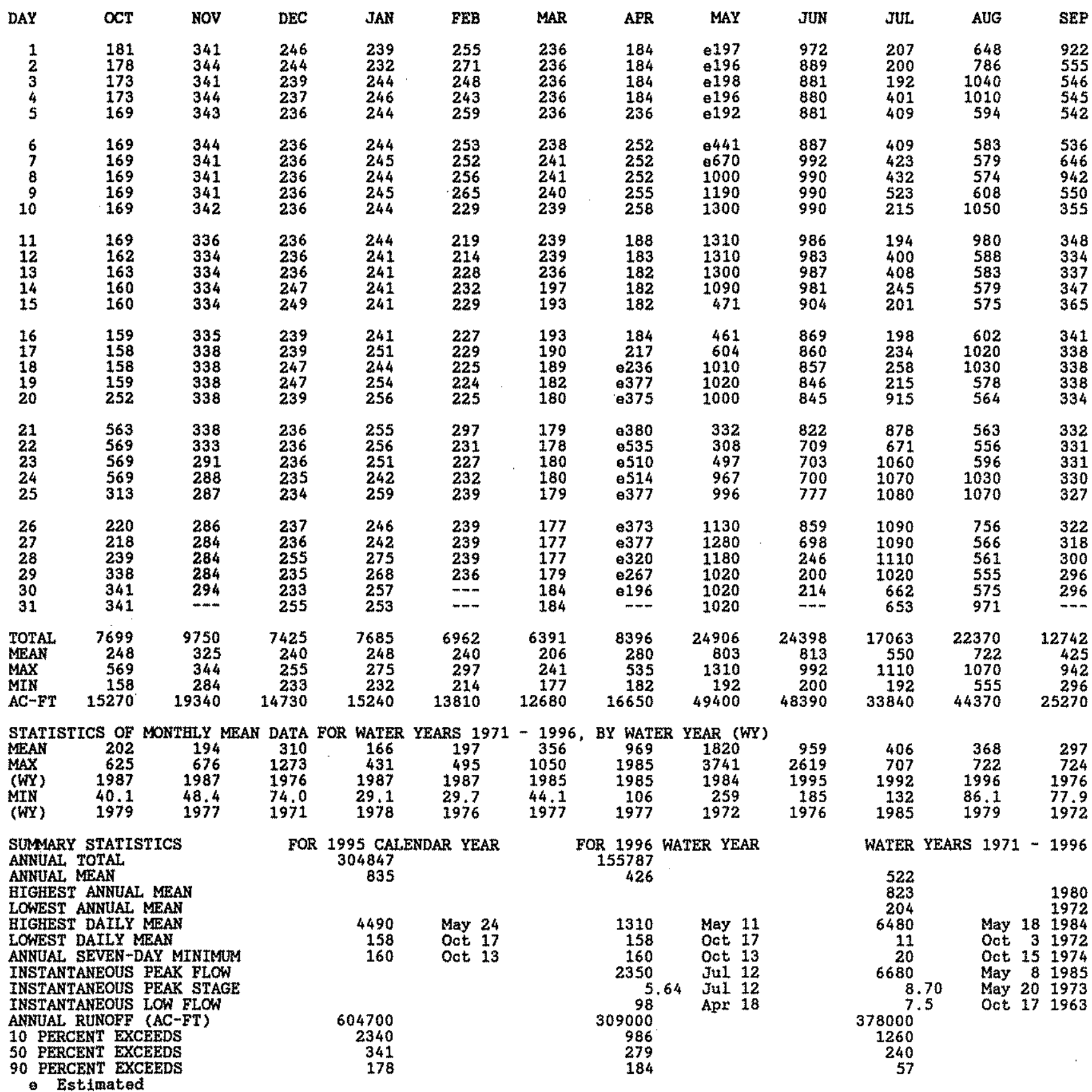




\section{RIO GRANDE BASIN}

08286900 ABIQUTU RESERVOIR MEAR ABIQUIU, NM

LOCATION, - Lat $36^{\circ} 14^{\circ} 24^{\prime \prime}$, long $106^{\circ} 25^{\prime} 4^{\prime \prime}$, Rio Arriba County, Hydrologic Unit 13020102, in Piedra Lumbre Grant, in operations building at Abiquiu Dem on Rio Chama, 6.6 mi northwest of Abiquiu, and at mile 32.1.

DRAINAGE AREA, $--2,146 \mathrm{mi}^{2}$, of which about $100 \mathrm{mi}$ is probably noncontributing.

PERTOD OF RECORD. - February 1963 to September 1965 (monthend contents only), October 1965 to current year. October 1969 to Docember 1975 , contents at 0800 hours.

GAGE. - Water-stage recorder. Datum of gage is Nationel Geodetic Vertical Datum of 1929 (1evels by U.S. Corps of Engineers).

REMARKS.--Regervoir is Formed by earthfill dam, completed Fob. 5, 1963. Capacity, 1,198,500 acre-ft between levations 6,060 ft, invert of outlet tunnel, and $6,350 \mathrm{ft}$, crest of spiliway, based on capacity table from survey 1990, No dead storage. Reservoir 18 used for elood control and, since March 1976, for recreation. A desilting pool of about 2,000 acre-ft mas maintained from May 1968 to 1974, when it was increased to 4,000 acreft and continued until. December 1975. U.S. Army Corps of Engineors satellite telemeter at station.

COOPERATION, -Records provided by U.S. Army Corps of Engineers.

EXTREATS FOR PERIOD OF RECORD,--Maximum contents, 382,720 acre-ft, June 11, 1985, elevation, 6,256.22 ft; no storage at times prior to May 1968 and Jan. 11 to Mar. 25, 1976.

EXTREMES FOR CURRENT YEAR.-Maximum contents, 267,150 acre-It, oct, 1, elevation, 6,237.35 ft; minimum, 145,510 acre-ft, Sopt. 30, elevation, 6,208.69 ft.

Capacity table (elevation, in feet, and contents, in acre-feet)

(Based on survey by U.S. Army Corps of Engineers in 1990)

$\begin{array}{llll}6,200 & 115,360 & 6,240 & 280,470 \\ 6,220 & 189,310 & 6,250 & 333,840 \\ 6,230 & 232,160 & 6,260 & 392,280\end{array}$

RESERVOIR STORAGE (ACRE-FEET), WATER YEAR OCTOBER 1995 TO SEPTEMBER 1996

DAILY OBSERVATION AT 24:00 VALUES

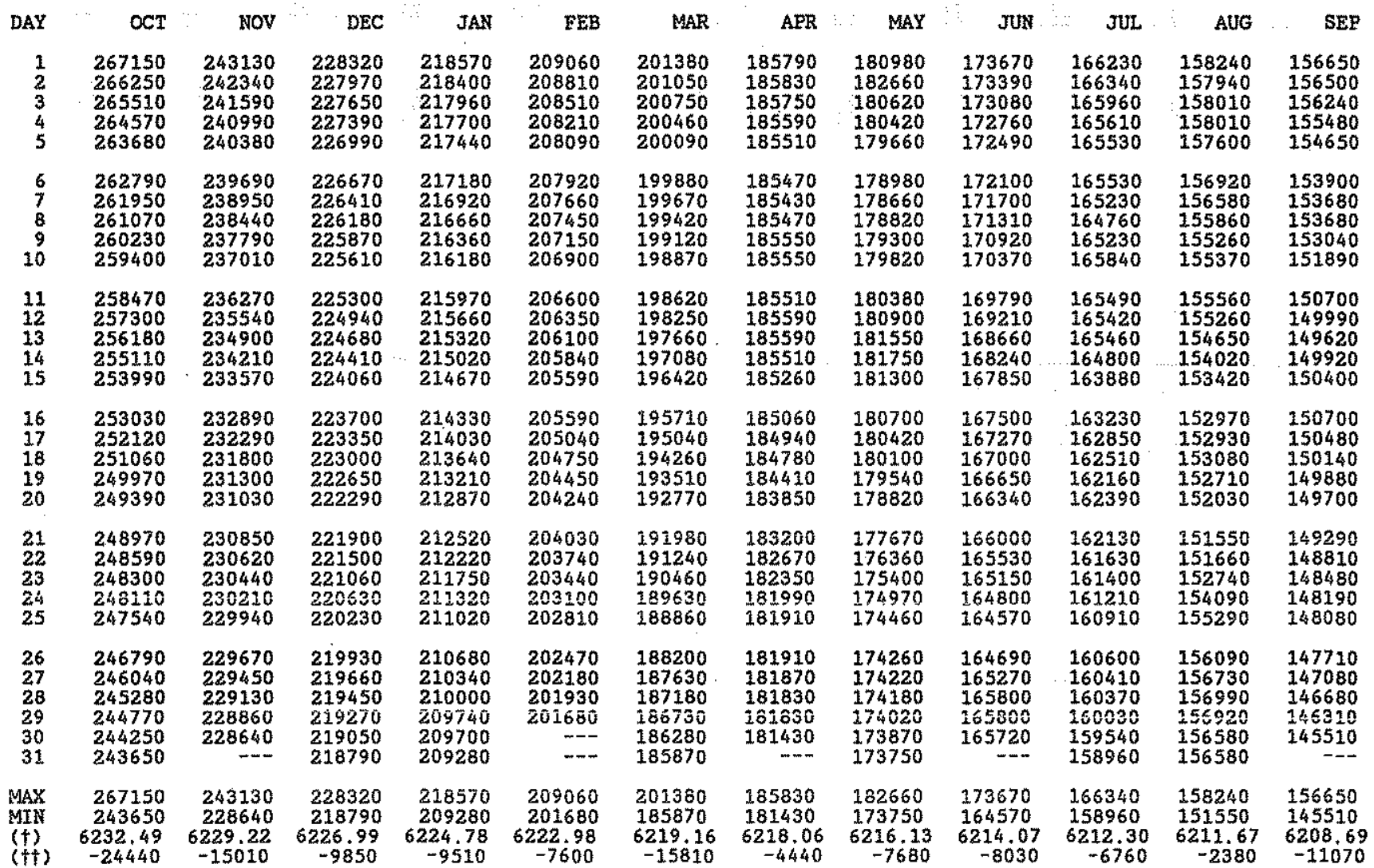

CAL YR 1995 MAX 305940 MIN 156950 (††) +61570

WTR YR 1996 MAX 267150 MIN 145510 (†) -122580 
RIO GRANDE BASIN

08287000 RIO CHAMA BELOW ABIQUTU DAM, MM

LOCATrON,--Lat $36^{\circ} 14^{\circ} 12^{\prime \prime}$, Long $106^{\circ} 24^{\circ} 59^{\prime \prime}$, in SE\%SE\% sec.8, T.23 N., R.5 E., Rto Arriba County, Hydrologic Unit

13020102, on right bark $0.8 \mathrm{mt}$ downstream from Abiquiu Dam, $5.9 \mathrm{mi}$ northwest of Abiquiu, and at mile 31.3 .

DRAINAGE AREA, - 2,147 $\mathrm{mi}^{2}$, of which about $100 \mathrm{mi}^{2}$ is probably noncontributing.

PERTOD OF RECORD.--October 1961 to current year (monthly discharge only, October 1961).

REVISED RECORDS. - -WDR-NM-90: 1989.

GAGE.--Water-stage recorder. Concrete control since Jan, 25, 1966. Elevation of gage is 6,040 ft above National Geodetic Vertical Datum of 1929, from topographic map. Prior to Jan. 25, 1966, at datum 1.60 ft lowar.

REMARKS, --Records good. FIow controlled by E1 Vado Reservoir (station 08285000) 46.4 mi upstream and Abiquitu Reservoir (station 08286900) $0.8 \mathrm{mi}$ upstream since February 1963. Since May 1971 flow affected by release of transmountain water from Heron Reservoix (station 08284510) 54.5 mi upstream. Diversions for irrigation of about 17,600 acres upstream from station. U.S. Army Corps of Engineers sateilite telemetex at station. Several observations of pater temperature talien during year.

AVERAGE DISCHARGE. --9 years (water years 1962-70), $384 \mathrm{ft} / \mathrm{s}, 278,200$ acre-ft/yr, prior to release of transmountain water.

DISCHARGE, CUBIC FEET PER SECOND, WATER YEAR OCTOBER 1995 TO SEPTEMBER 1996

\begin{tabular}{|c|c|c|c|c|c|c|c|c|c|c|c|c|}
\hline DAY & OCT & Nov & DEC & JAN & FEB & MAR & $\mathrm{ARR}$ & MAY & JUN & JUL & AUG & SEP \\
\hline $\begin{array}{l}1 \\
2 \\
3 \\
4 \\
5\end{array}$ & $\begin{array}{l}625 \\
607 \\
552 \\
559 \\
603\end{array}$ & $\begin{array}{l}672 \\
713 \\
712 \\
706 \\
706\end{array}$ & $\begin{array}{l}450 \\
450 \\
450 \\
450 \\
450\end{array}$ & $\begin{array}{l}417 \\
408 \\
409 \\
424 \\
412\end{array}$ & $\begin{array}{l}408 \\
408 \\
407 \\
403 \\
409\end{array}$ & $\begin{array}{l}407 \\
408 \\
408 \\
408 \\
399\end{array}$ & $\begin{array}{l}263 \\
177 \\
262 \\
282 \\
309\end{array}$ & $\begin{array}{l}441 \\
357 \\
321 \\
401 \\
531\end{array}$ & $\begin{array}{r}989 \\
993 \\
992 \\
992 \\
1010\end{array}$ & $\begin{array}{l}281 \\
314 \\
420 \\
570 \\
427\end{array}$ & $\begin{array}{l}952 \\
946 \\
946 \\
886 \\
842\end{array}$ & $\begin{array}{l}740 \\
687 \\
776 \\
884 \\
906\end{array}$ \\
\hline $\begin{array}{r}6 \\
7 \\
8 \\
9 \\
10\end{array}$ & $\begin{array}{l}613 \\
584 \\
584 \\
584 \\
601\end{array}$ & $\begin{array}{l}706 \\
706 \\
718 \\
718 \\
714\end{array}$ & $\begin{array}{l}448 \\
415 \\
426 \\
429 \\
429\end{array}$ & $\begin{array}{l}412 \\
412 \\
416 \\
411 \\
403\end{array}$ & $\begin{array}{l}408 \\
408 \\
408 \\
408 \\
408\end{array}$ & $\begin{array}{l}396 \\
398 \\
398 \\
398 \\
398\end{array}$ & $\begin{array}{l}295 \\
297 \\
297 \\
297 \\
292\end{array}$ & $\begin{array}{l}653 \\
792 \\
863 \\
884 \\
946\end{array}$ & $\begin{array}{l}1050 \\
1120 \\
1120 \\
1160 \\
1240\end{array}$ & $\begin{array}{l}394 \\
612 \\
697 \\
419 \\
270\end{array}$ & $\begin{array}{l}847 \\
924 \\
921 \\
859 \\
825\end{array}$ & $\begin{array}{l}893 \\
749 \\
825 \\
902 \\
947\end{array}$ \\
\hline $\begin{array}{l}11 \\
12 \\
13 \\
14 \\
15\end{array}$ & $\begin{array}{l}672 \\
716 \\
735 \\
746 \\
745\end{array}$ & $\begin{array}{l}710 \\
706 \\
707 \\
708 \\
706\end{array}$ & $\begin{array}{l}457 \\
466 \\
432 \\
429 \\
473\end{array}$ & $\begin{array}{l}419 \\
423 \\
443 \\
466 \\
469\end{array}$ & $\begin{array}{l}403 \\
403 \\
403 \\
403 \\
403\end{array}$ & $\begin{array}{l}411 \\
455 \\
508 \\
499 \\
563\end{array}$ & $\begin{array}{l}267 \\
245 \\
244 \\
226 \\
248\end{array}$ & $\begin{array}{r}995 \\
1070 \\
922 \\
819 \\
838\end{array}$ & $\begin{array}{l}1260 \\
1210 \\
1200 \\
1110 \\
1030\end{array}$ & $\begin{array}{l}401 \\
518 \\
443 \\
629 \\
715\end{array}$ & $\begin{array}{l}761 \\
804 \\
885 \\
909 \\
880\end{array}$ & $\begin{array}{l}895 \\
746 \\
544 \\
310 \\
208\end{array}$ \\
\hline $\begin{array}{l}16 \\
17 \\
18 \\
19 \\
20\end{array}$ & $\begin{array}{l}649 \\
625 \\
689 \\
710 \\
690\end{array}$ & $\begin{array}{l}706 \\
717 \\
716 \\
598 \\
463\end{array}$ & $\begin{array}{l}482 \\
482 \\
482 \\
482 \\
482\end{array}$ & $\begin{array}{l}469 \\
469 \\
469 \\
468 \\
464\end{array}$ & $\begin{array}{l}403 \\
403 \\
400 \\
400 \\
403\end{array}$ & $\begin{array}{l}568 \\
570 \\
570 \\
570 \\
570\end{array}$ & $\begin{array}{l}350 \\
344 \\
410 \\
513 \\
700\end{array}$ & $\begin{array}{r}765 \\
703 \\
998 \\
1480 \\
1240\end{array}$ & $\begin{array}{l}998 \\
948 \\
928 \\
925 \\
923\end{array}$ & $\begin{array}{l}608 \\
531 \\
532 \\
548 \\
695\end{array}$ & $\begin{array}{l}866 \\
856 \\
788 \\
839 \\
929\end{array}$ & $\begin{array}{l}304 \\
456 \\
513 \\
485 \\
447\end{array}$ \\
\hline $\begin{array}{l}21 \\
22 \\
23 \\
24 \\
25\end{array}$ & $\begin{array}{l}679 \\
679 \\
675 \\
676 \\
648\end{array}$ & $\begin{array}{l}449 \\
450 \\
450 \\
450 \\
450\end{array}$ & $\begin{array}{l}482 \\
482 \\
482 \\
482 \\
482\end{array}$ & $\begin{array}{l}464 \\
462 \\
461 \\
464 \\
464\end{array}$ & $\begin{array}{l}403 \\
404 \\
398 \\
398 \\
400\end{array}$ & $\begin{array}{l}579 \\
592 \\
582 \\
579 \\
579\end{array}$ & $\begin{array}{l}740 \\
757 \\
775 \\
729 \\
493\end{array}$ & $\begin{array}{r}990 \\
998 \\
1010 \\
1050 \\
1090\end{array}$ & $\begin{array}{l}921 \\
920 \\
914 \\
821 \\
901\end{array}$ & $\begin{array}{r}958 \\
1080 \\
1090 \\
1110 \\
1160\end{array}$ & $\begin{array}{l}858 \\
680 \\
266 \\
234 \\
523\end{array}$ & $\begin{array}{l}567 \\
593 \\
495 \\
429 \\
441\end{array}$ \\
\hline $\begin{array}{l}26 \\
27 \\
28 \\
29 \\
30 \\
31\end{array}$ & $\begin{array}{l}625 \\
631 \\
619 \\
619 \\
625 \\
636\end{array}$ & $\begin{array}{l}449 \\
445 \\
445 \\
455 \\
455\end{array}$ & $\begin{array}{l}455 \\
424 \\
424 \\
428 \\
429 \\
429\end{array}$ & $\begin{array}{l}459 \\
459 \\
459 \\
443 \\
408 \\
408\end{array}$ & $\begin{array}{l}400 \\
400 \\
403 \\
403 \\
--- \\
--\end{array}$ & $\begin{array}{l}560 \\
454 \\
413 \\
418 \\
418 \\
418\end{array}$ & $\begin{array}{l}419 \\
456 \\
454 \\
413 \\
438 \\
-\end{array}$ & $\begin{array}{r}1150 \\
1190 \\
1070 \\
1010 \\
1000 \\
989\end{array}$ & $\begin{array}{l}806 \\
397 \\
245 \\
245 \\
243\end{array}$ & $\begin{array}{r}1200 \\
1150 \\
1090 \\
1070 \\
966 \\
909\end{array}$ & $\begin{array}{l}406 \\
231 \\
440 \\
655 \\
774 \\
822\end{array}$ & $\begin{array}{l}484 \\
513 \\
548 \\
570 \\
608\end{array}$ \\
\hline $\begin{array}{l}\text { TOTAL } \\
\text { MEAN } \\
\text { MAX } \\
\text { MIN } \\
\text { AC-FT }\end{array}$ & $\begin{array}{r}20001 \\
645 \\
746 \\
552 \\
39670\end{array}$ & $\begin{array}{r}18306 \\
610 \\
718 \\
445 \\
36310\end{array}$ & $\begin{array}{r}14063 \\
454 \\
482 \\
415 \\
27890\end{array}$ & $\begin{array}{r}13634 \\
440 \\
469 \\
403 \\
27040\end{array}$ & $\begin{array}{r}11708 \\
404 \\
409 \\
398 \\
23220\end{array}$ & $\begin{array}{r}14894 \\
480 \\
592 \\
396 \\
29540\end{array}$ & $\begin{array}{r}11992 \\
400 \\
775 \\
177 \\
23790\end{array}$ & $\begin{array}{r}27566 \\
889 \\
1480 \\
321 \\
54680\end{array}$ & $\begin{array}{r}27611 \\
920 \\
1260 \\
243 \\
54770\end{array}$ & $\begin{array}{r}21807 \\
703 \\
1200 \\
270 \\
43250\end{array}$ & $\begin{array}{r}23354 \\
753 \\
952 \\
231 \\
46320\end{array}$ & $\begin{array}{r}18465 \\
615 \\
947 \\
208 \\
36630\end{array}$ \\
\hline
\end{tabular}

STATISTICS OF MONTHLY MEAN DATA FOR WATER YEARS 1971 - 1996, BY WATER YEAR (WY)

\begin{tabular}{|c|c|c|c|c|c|c|c|c|c|c|c|c|c|}
\hline $\begin{array}{l}\text { MEAN } \\
\text { MAX } \\
\text { (WY) } \\
\text { MIN } \\
\text { (WY) }\end{array}$ & $\begin{array}{r}287 \\
1261 \\
1988 \\
44.9 \\
1979\end{array}$ & $\begin{array}{r}319 \\
1181 \\
1980 \\
45.8 \\
1990\end{array}$ & $\begin{array}{r}327 \\
1308 \\
1976 \\
43.9 \\
1975\end{array}$ & $\begin{array}{r}195 \\
860 \\
1986 \\
35.7 \\
1978\end{array}$ & $\begin{array}{r}256 \\
1708 \\
1987 \\
38.0 \\
1978\end{array}$ & $\begin{array}{r}438 \\
1668 \\
1987 \\
52.4 \\
1977\end{array}$ & $\begin{array}{r}920 \\
1894 \\
1985 \\
111 \\
1977\end{array}$ & $\begin{array}{r}1206 \\
2055 \\
1983 \\
242 \\
1972\end{array}$ & \multirow[t]{2}{*}{$\begin{array}{r}1100 \\
2418 \\
1984 \\
184 \\
1976\end{array}$} & $\begin{array}{r}657 \\
1488 \\
1973 \\
201 \\
1972\end{array}$ & & $\begin{array}{r}469 \\
1084 \\
1973 \\
98.4 \\
1979\end{array}$ & $\begin{array}{r}406 \\
1199 \\
1987 \\
64.4 \\
1972\end{array}$ \\
\hline \multicolumn{3}{|c|}{ SUMMARY STATISTICS } & FOR & $1995 \mathrm{CAL}$ & AR YEAR & \multicolumn{3}{|c|}{ FOR 1996 WATER YEAR } & & WATER & YEARS & S 1971 & -1996 \\
\hline $\begin{array}{l}\text { ANNUAL } \\
\text { ANNUAL } \\
\text { HIGEES } \\
\text { LOWEST } \\
\text { HIGHEST } \\
\text { LOWEST } \\
\text { ANNUAL } \\
\text { ANNUAL } \\
10 \text { PERC } \\
50 \text { PERO } \\
90 \text { PERC }\end{array}$ & $\begin{array}{l}\text { TOTAL } \\
\text { MEAN } \\
\text { ANNUAL } \\
\text { ANNUAL } \\
\text { DAILY } \\
\text { DAILY } \\
\text { SEVEN- } \\
\text { RUNOFF } \\
\text { ENT EXO } \\
\text { ENT EX } \\
\text { ENT EXC EO }\end{array}$ & $\begin{array}{l}\text { AN } \\
\text { MINIMUM } \\
- \text { ET) } \\
\text { NTI }\end{array}$ & & $\begin{array}{r}1810 \\
95 \\
147 \\
566100 \\
1770 \\
611 \\
181\end{array}$ & $\begin{array}{ll}\text { Jun } & 24 \\
\text { Feb } & 24 \\
\text { Feb } & 20\end{array}$ & & $\begin{array}{r}1480 \\
177 \\
260 \\
443100 \\
992 \\
531 \\
398\end{array}$ & $\begin{array}{rr}\text { May } & 19 \\
\text { Apr } & 2 \\
\text { Apr } & 9\end{array}$ & & $\begin{array}{r}549 \\
872 \\
213 \\
2660 \\
10 \\
21 \\
397800 \\
1620 \\
318 \\
52\end{array}$ & & $\begin{array}{ll}\text { May } & 1 \\
\text { Sep } & 1 \\
\text { Sep } & 3\end{array}$ & 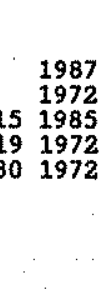 \\
\hline
\end{tabular}


LOCATION,--Lat $36^{\circ} 20^{\circ} 59^{\prime \prime}$, long $106^{\circ} 02^{\prime} 37^{\prime \prime}$, in NWHEEk sec.1, T.24 N., R. 8 E., Rio Arriba County, Hydrologic Unit

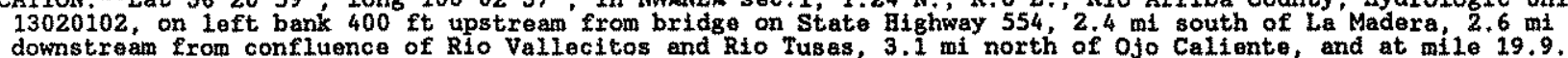

DRAINAGE AREA. $--419 \mathrm{mi}^{2}$.

PERIOD OF RECORD.--April 1932 to current year.

REVISED RECORDS. --WSP 1712: 1959.

GAGE.--Water-stage recorder.... Datum of gage is 6,358.84 ft above National Geodetic Vertical Datum or 1929. Frior

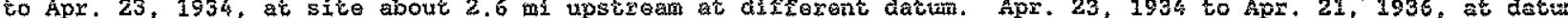

12.58 ft lower and Apr. 22, 1936 to Oct, 26, 1956, at datum 13.84 ft lower, both at site 1,400 ft downstream.

REMARKS, - Records good except for estimated daily discharges, which are fair.. Diversions upstream from station for Irrigation of about 3,500 acres (1962 determination). Several observations of water temperature were made during the year.

EXTREMES OUTSIDE PERTOD OF RECORD.-The flood of Apr, 21, 1958, may have been oxceeded by a flood in May 1920, Irom information by local resident.

DISCHARGE, CUBIC FEET PER SECOND, WATER YEAR OCTOBER 1995 TO SEPTEMBER 1996

\begin{tabular}{|c|c|c|c|c|c|c|c|c|c|c|c|c|}
\hline DAY & OCT & NOV & DEC & JAN & FEB & MAR & APR & MAY & JUN & JUL & AUG & SEP \\
\hline $\begin{array}{l}1 \\
2 \\
3 \\
4 \\
5\end{array}$ & $\begin{array}{l}17 \\
15 \\
14 \\
13 \\
13\end{array}$ & $\begin{array}{l}14 \\
14 \\
14 \\
15 \\
15\end{array}$ & $\begin{array}{l}16 \\
16 \\
16 \\
16 \\
17\end{array}$ & $\begin{array}{l}16 \\
15 \\
15 \\
15 \\
15\end{array}$ & $\begin{array}{l}25 \\
24 \\
22 \\
22 \\
23\end{array}$ & $\begin{array}{l}23 \\
23 \\
24 \\
27 \\
25\end{array}$ & $\begin{array}{l}38 \\
48 \\
55 \\
60 \\
53\end{array}$ & $\begin{array}{l}84 \\
87 \\
82 \\
76 \\
70\end{array}$ & $\begin{array}{l}7.3 \\
7.1 \\
6.6 \\
6.5 \\
6.1\end{array}$ & $\begin{array}{l}8.4 \\
6.1 \\
5.8 \\
6.0 \\
5.7\end{array}$ & $\begin{array}{l}6.6 \\
6.4 \\
6.1 \\
6.2 \\
5.7\end{array}$ & $\begin{array}{l}6.2 \\
6.5 \\
5.8 \\
5.6 \\
5.1\end{array}$ \\
\hline $\begin{array}{r}6 \\
7 \\
8 \\
9 \\
10\end{array}$ & $\begin{array}{l}13 \\
13 \\
13 \\
13 \\
13\end{array}$ & $\begin{array}{l}15 \\
15 \\
15 \\
15 \\
16\end{array}$ & $\begin{array}{l}18 \\
18 \\
17 \\
16 \\
14\end{array}$ & $\begin{array}{l}15 \\
15 \\
14 \\
14 \\
15\end{array}$ & $\begin{array}{l}25 \\
26 \\
26 \\
25 \\
25\end{array}$ & $\begin{array}{l}26 \\
21 \\
25 \\
26 \\
25\end{array}$ & $\begin{array}{r}47 \\
63 \\
83 \\
122 \\
156\end{array}$ & $\begin{array}{l}63 \\
58 \\
52 \\
48 \\
44\end{array}$ & $\begin{array}{l}5.7 \\
5.6 \\
5.6 \\
5.4 \\
5.1\end{array}$ & $\begin{array}{l}5.2 \\
5.3 \\
10 \\
11 \\
9.1\end{array}$ & $\begin{array}{l}5.5 \\
5.7 \\
5.9 \\
6.0 \\
5.7\end{array}$ & $\begin{array}{l}5.3 \\
5.7 \\
5.4 \\
5.0 \\
5.3\end{array}$ \\
\hline $\begin{array}{l}11 \\
12 \\
13 \\
14 \\
15\end{array}$ & $\begin{array}{l}13 \\
13 \\
12 \\
12 \\
12\end{array}$ & $\begin{array}{l}16 \\
15 \\
16 \\
17 \\
17\end{array}$ & $\begin{array}{l}15 \\
16 \\
19 \\
18 \\
18\end{array}$ & $\begin{array}{l}15 \\
16 \\
17 \\
18 \\
19\end{array}$ & $\begin{array}{l}25 \\
25 \\
24 \\
25 \\
26\end{array}$ & $\begin{array}{l}26 \\
28 \\
29 \\
29 \\
29\end{array}$ & $\begin{array}{r}136 \\
149 \\
165 \\
85 \\
86\end{array}$ & $\begin{array}{l}41 \\
37 \\
35 \\
30 \\
28\end{array}$ & $\begin{array}{l}4.9 \\
4.7 \\
4.7 \\
4.8 \\
4.7\end{array}$ & $\begin{array}{l}7.1 \\
7.1 \\
9.7 \\
7.6 \\
6.9\end{array}$ & $\begin{array}{l}5.4 \\
5.3 \\
5.2 \\
5.1 \\
5.2\end{array}$ & $\begin{array}{l}5.4 \\
5.1 \\
5.3 \\
6.0 \\
6.6\end{array}$ \\
\hline $\begin{array}{l}16 \\
17 \\
18 \\
19 \\
20\end{array}$ & $\begin{array}{l}13 \\
12 \\
12 \\
12 \\
1.2\end{array}$ & $\begin{array}{l}16 \\
16 \\
17 \\
16 \\
16\end{array}$ & $\begin{array}{l}16 \\
18 \\
18 \\
15 \\
15\end{array}$ & $\begin{array}{l}19 \\
23 \\
21 \\
20 \\
22\end{array}$ & $\begin{array}{l}25 \\
26 \\
27 \\
26 \\
28\end{array}$ & $\begin{array}{l}28 \\
27 \\
26 \\
26 \\
26\end{array}$ & $\begin{array}{r}89 \\
108 \\
97 \\
94 \\
87\end{array}$ & $\begin{array}{l}26 \\
23 \\
23 \\
20 \\
18\end{array}$ & $\begin{array}{l}4.6 \\
4.3 \\
4.1 \\
4.1 \\
4.2\end{array}$ & $\begin{array}{l}6.4 \\
6.4 \\
6.2 \\
6.4 \\
6.1\end{array}$ & $\begin{array}{l}5.1 \\
5.0 \\
5.1 \\
4.7 \\
4.9\end{array}$ & $\begin{array}{l}6.6 \\
6.7 \\
7.3 \\
7.4 \\
7.8\end{array}$ \\
\hline $\begin{array}{l}21 \\
22 \\
23 \\
24 \\
25\end{array}$ & $\begin{array}{l}12 \\
12 \\
12 \\
13 \\
13\end{array}$ & $\begin{array}{l}17 \\
17 \\
17 \\
16 \\
16\end{array}$ & $\begin{array}{l}14 \\
15 \\
13 \\
13 \\
13\end{array}$ & $\begin{array}{r}21 \\
23 \\
22 \\
e 23 \\
e 22\end{array}$ & $\begin{array}{l}30 \\
34 \\
32 \\
27 \\
31\end{array}$ & $\begin{array}{l}26 \\
29 \\
32 \\
33 \\
30\end{array}$ & $\begin{array}{r}67 \\
70 \\
90 \\
177 \\
209\end{array}$ & $\begin{array}{l}17 \\
13 \\
12 \\
13 \\
11\end{array}$ & $\begin{array}{l}4.1 \\
4.7 \\
4.7 \\
4.5 \\
4.8\end{array}$ & $\begin{array}{l}5.8 \\
5.8 \\
5.9 \\
5.9 \\
5.9\end{array}$ & $\begin{array}{l}5.8 \\
7.4 \\
8.6 \\
8.3 \\
7.6\end{array}$ & $\begin{array}{l}5.7 \\
6.0 \\
5.5 \\
5.5 \\
5.9\end{array}$ \\
\hline $\begin{array}{l}26 \\
27 \\
28 \\
29 \\
30 \\
31\end{array}$ & $\begin{array}{l}13 \\
13 \\
13 \\
13 \\
13 \\
14\end{array}$ & $\begin{array}{r}16 \\
16 \\
14 \\
13 \\
14 \\
-\end{array}$ & $\begin{array}{l}14 \\
14 \\
14 \\
15 \\
15 \\
15\end{array}$ & $\begin{array}{l}21 \\
22 \\
24 \\
22 \\
23 \\
25\end{array}$ & $\begin{array}{r}27 \\
23 \\
22 \\
24 \\
-\ldots \\
-\end{array}$ & $\begin{array}{l}29 \\
27 \\
29 \\
34 \\
37 \\
35\end{array}$ & $\begin{array}{r}218 \\
203 \\
174 \\
93 \\
81 \\
-\end{array}$ & $\begin{array}{r}10 \\
9.0 \\
9.0 \\
8.8 \\
8.2 \\
7.8\end{array}$ & $\begin{array}{l}5.0 \\
5.9 \\
5.7 \\
5.8 \\
7.2 \\
-.\end{array}$ & $\begin{array}{l}6.9 \\
6.0 \\
6.7 \\
6.6 \\
6.4 \\
6.5\end{array}$ & $\begin{array}{l}8.3 \\
7.7 \\
6.8 \\
7.3 \\
6.4 \\
6.1\end{array}$ & $\begin{array}{l}5.8 \\
5.8 \\
6.0 \\
6.0 \\
6.0\end{array}$ \\
\hline $\begin{array}{l}\text { TOTAL } \\
\text { MEAHT } \\
\text { MAX } \\
\text { MIN } \\
\text { AC-FT }\end{array}$ & $\begin{array}{r}401 \\
12.9 \\
17 \\
12 \\
795\end{array}$ & $\begin{array}{r}466 \\
15.5 \\
17 \\
13 \\
924\end{array}$ & $\begin{array}{r}487 \\
15.7 \\
19 \\
13 \\
966\end{array}$ & $\begin{array}{r}587 \\
18.9 \\
25 \\
14 \\
1160\end{array}$ & $\begin{array}{r}750 \\
25.9 \\
34 \\
22 \\
1490\end{array}$ & $\begin{array}{r}860 \\
27.7 \\
37 \\
21 \\
1710\end{array}$ & $\begin{array}{r}3203 \\
107 \\
218 \\
38 \\
6350\end{array}$ & $\begin{array}{r}1063.8 \\
34.3 \\
87 \\
7.8 \\
2110\end{array}$ & $\begin{array}{r}158.5 \\
5.28 \\
7.3 \\
4.1 \\
314\end{array}$ & $\begin{array}{r}210.9 \\
6.80 \\
11 \\
5.2 \\
418\end{array}$ & $\begin{array}{r}191.1 \\
6.16 \\
8.6 \\
4.7 \\
379\end{array}$ & $\begin{array}{r}178.3 \\
5.94 \\
7.8 \\
5.0 \\
354\end{array}$ \\
\hline
\end{tabular}

STATISTICS OF MONTHLY MEAN DATA FOR WATER YEARS 1933 - 1996, BY WATER YEAR (WY)

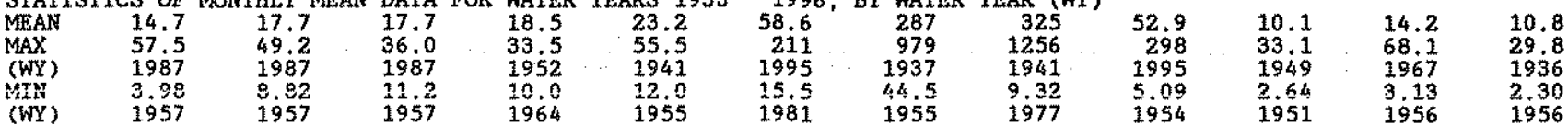

SUMMARY STATISTYCS

ANNUAL TOTAL

ANNUAL MEAN

HIGHEST ANNUAL, MEAN

LOWEST ANNUAL MEAN

HIGHEST DAILY MEAN

LOWEST DAILY MEAN

ANNUAL SEVEN-DAY MINIMUM

INSTANTANEOUS PEAK FLOW

INSTANTANEOUS PEAK STAGE

INSTANTANEOUS LOW FLOW

ANNUAL RUNOFF (AC-FT)

10 PERCENT 50 PERCENT EXCEEDS

50 PERCENT EXCEEDS

99210

FOR 1995 CALENDAR YEAR

50019.9

$\begin{array}{cc}1100 & \text { May } 16 \\ 7.1 & \text { Aug } 10\end{array}$

7.1
8.1 Aug 10

172

24
12

- Estimated

a-From slope-area measurement of peak flow.
FOR 1996 WATER YEAR 8556.6
23.4

218 Apr 26

$\begin{array}{ll}4.1 & \text { Jun } 18 \\ 4.3 & \text { Jun } 15\end{array}$

$\begin{array}{ll}372 & \text { Apr } 26 \\ 4.82 & \text { Apr } 26\end{array}$

16970

15
5.4
.2

5.6

5.3

5.4

5.3

5.4

5.3

6.6

6.6

7.7

7.4

5.7

6.0

5.5

5.8

5.8

6.0

6.0

8.3

7.8

354

WATER YEARS 1933 - 1996

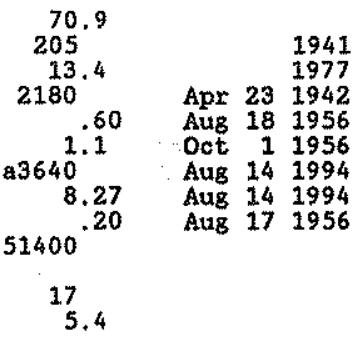


RIO GRANDE BASIN

08290000 RIO CBAMA REAR CBAMTTA, RM

LOCATION.--Lat $36^{\circ} 04^{\circ} 26^{\prime \prime}$, long $106^{\circ} 06^{\circ} 40^{\circ}$, in NEXKEX sec.8, T.21 N., R.8 E., Rio Arriba County, Bydrologic Unit 13020102 , in San Juan pueblo Grant, near left downstream corner of bridge on U.S. Highway 285, $0.5 \mathrm{mi}$ west of Chamita, $2.5 \mathrm{mi}$ northwest of San Juan Pueblo, and at mile 2.8

DRAINAGE AREA. $--3,144 \mathrm{mi}^{2}$, of which about $100 \mathrm{mi}^{2}$ is probably noncontributing. WATKR-DISCHARGE RECORDS

RERIOD OF RECORD. - October 1912 to current year. Monthly discharge only for some periods, published in WSP 1312. Published as "Chama River near Chamita" prior to 1928, and "Chama River at Chamita" 1929-30.

REVISED RECORDS. --WSP 1512: 1913-15, 1934, 1936, WSP 1632: 1929(M). WSP 1732: 1931(M). WSP 1923: Dratnage area.

GAGE.--Water-gtage recorder. Concrete contro1 since Jan. 1, 1964 . Datum of gage is 5,653.61 ft above National Geodetic Vertical Datum of 1929. Prior to oct. 4, 1933, at railroad bridge 2.3 mi domstream at different datums. Oct. 4, 1933 to Mar. 1, 1942, at site 50 ft downstream at datum 0.22 ft higher. Mar. 2, 1942 to Dec. 31,1963 , at site 200 ft downstream, present datum.

RaMARKS --Records good. Diversions upstream from station for irrigation of about 27,600 acres. Chantta ditch (station 08289500), on left bank, and Eernandez ditch (station 08289800), on right bank, bypass gage fox irrigation of several hundred acres downstream from station. Flow regulated by El Vado Reservoir (station $08285000) 74.9 \mathrm{mi}$ upstream since January 1935 and Abiquilu Reservolx (station 08286900), $29.3 \mathrm{mi}$ upstream since February 1963. Since May 1971 flow affected by rolease of trsnsmountain water from Heron Reservoir (station 08284510) 83.0 mi upstream. U.S. Army Corps of Engineers Satellite telemeter at station. No flow at times some years.

AVERAGE DISCHARGE, - -58 years (fater years 1913-70), $541 \mathrm{ft} / \mathrm{s}, 392,000$ acre-ft/yr, prior to release of transmountain patar

EXTREMES OUTSIDE PERIOD OF RECORD.--The floods of Sept, 29, 1904, and Oct. 4 or 5, 1911, probably exceeded 15,000 fts/s. Another major flood occurred in 1884, from newspaper accounts.

DISCHARGE, CUBIC FEET PER SECOND, WATER YEAR OCTOBER 1995 TO SEPTEMBER 1996

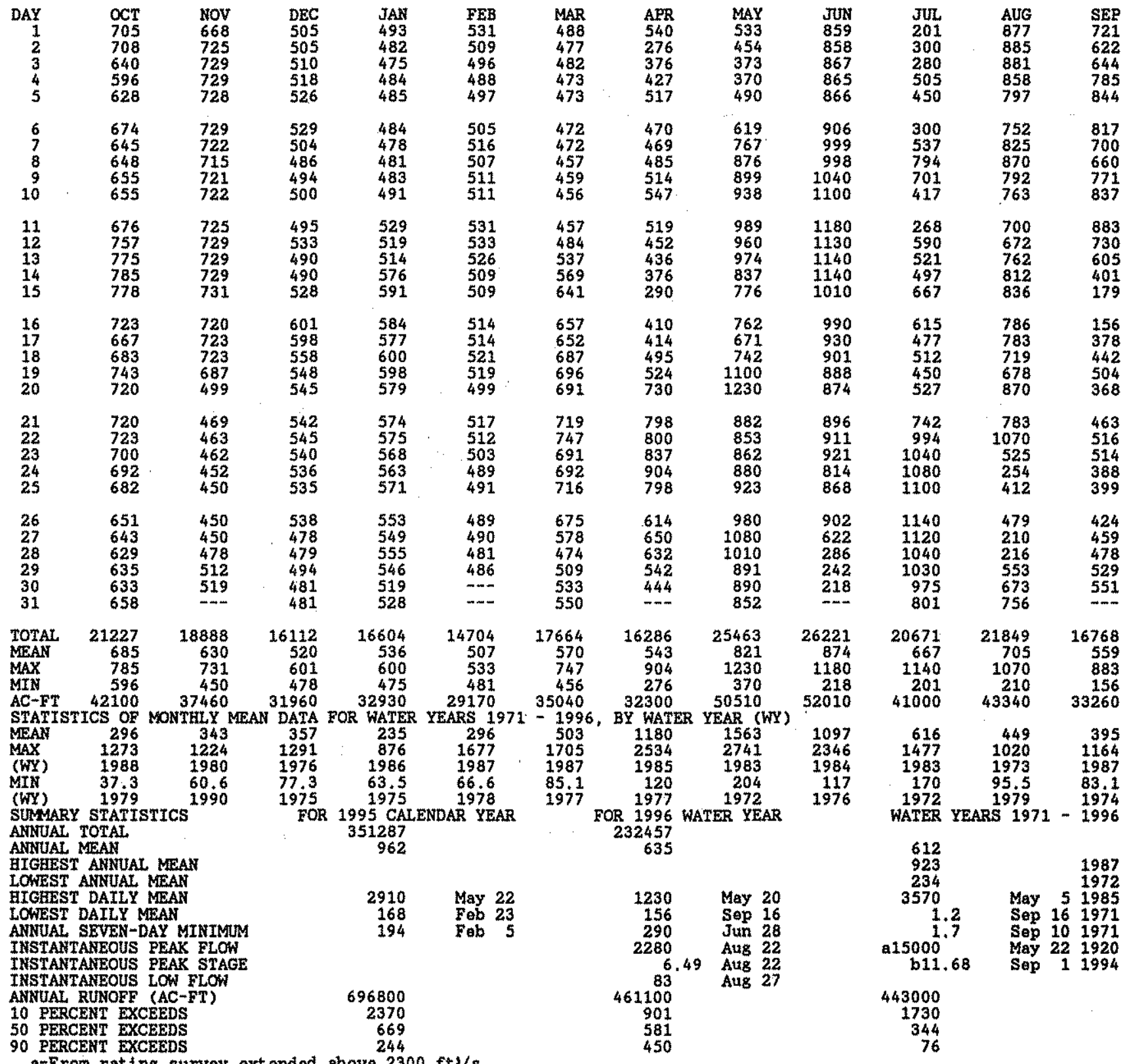

a-From rating survey extended above $2300 \mathrm{ft}^{3} / \mathrm{s}$.

b-From floodmarks of slope-area measurement of peak flow. 
PERIOD OF RECORD.--Watex years 1948 to current year.

WATER QUALITY DATA, WATER YEAR OCTOBER 1995 TO SEPTEMBER 1996

\begin{tabular}{|c|c|c|c|c|c|c|c|c|c|c|c|}
\hline DATE & TIME & $\begin{array}{c}\text { DIS- } \\
\text { CHARGE } \\
\text { INST, } \\
\text { CUPIC } \\
\text { FEET } \\
\text { PER } \\
\text { SECOND } \\
(00061)\end{array}$ & $\begin{array}{l}\text { SPE- } \\
\text { CIFIC } \\
\text { CON- } \\
\text { DUCT- } \\
\text { ANCE } \\
(\text { US /CM }) \\
(00095)\end{array}$ & $\begin{array}{c}\text { PB } \\
\text { WATER } \\
\text { WHOLEE } \\
\text { FIELD } \\
\text { (STARD- } \\
\text { ARD } \\
\text { UNITS) } \\
(00400)\end{array}$ & $\begin{array}{l}\text { TERPPER- } \\
\text { ATURE } \\
\text { AIR } \\
(D E G \text { C) } \\
(00020)\end{array}$ & $\begin{array}{l}\text { TERPSR- } \\
\text { ATURE } \\
\text { WATER } \\
(\text { DEG C) } \\
(00010)\end{array}$ & $\begin{array}{c}\text { BARO- } \\
\text { METRIC } \\
\text { PRES } \\
\text { SURE } \\
\text { (MA } \\
\text { OF } \\
\text { HG) } \\
(00025)\end{array}$ & $\begin{array}{c}\text { OXYGES, } \\
\text { DIS- } \\
\text { SOLVD } \\
\text { (NGG/L) } \\
(00300)\end{array}$ & $\begin{array}{c}\text { OXYGEN } \\
\text { DISS- } \\
\text { SOLVED } \\
\text { (PER - } \\
\text { CERT } \\
\text { SATUR- } \\
\text { ATION) } \\
\text { (00301) }\end{array}$ & 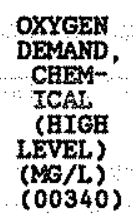 & $\begin{array}{c}\text { COLI- } \\
\text { FORM } \\
\text { FECAL, } \\
0.7 \\
\text { UM-MF } \\
(\mathrm{COLS}, \\
100 \mathrm{ML}) \\
(31625)\end{array}$ \\
\hline 995 & 1000 & 462 & 306 & 7.8 & -2.5 & 4.0 & 623 & 10.7 & 100 & 12 & $<1$ \\
\hline .. & 0945 & 490 & 297 & 8.3 & -3.0 & 1.5 & 620 & 11.3 & 99 & $<10$ & $<7$ \\
\hline$\cdots$ & 1300 & 554 & 297 & 8.1 & 18.0 & 13.5 & 623 & 8.5 & 100 & 63 & 730 \\
\hline & 0815 & 477 & 318 & 8.0 & 16.5 & 16.5 & 621 & 7.9 & 100 & - & - \\
\hline$\cdots$ & 1145 & 674 & 363 & 8.3 & 24.0 & 18.0 & 625 & 8.2 & 106 & 21 & 70 \\
\hline DATE & $\begin{array}{c}\text { STREP- } \\
\text { TOCOCCI } \\
\text { FECAL, } \\
\text { KF AGAR } \\
\text { (COLS. } \\
\text { PER } \\
100 \mathrm{ML}) \\
(31673)\end{array}$ & $\begin{array}{c}\text { HARD- } \\
\text { NESS } \\
\text { TOTAL } \\
\text { (MG/L } \\
\text { AS } \\
\text { CACO3) } \\
(00900)\end{array}$ & $\begin{array}{l}\text { RARD- } \\
\text { NESS } \\
\text { NONCARB } \\
\text { DISSOLV } \\
\text { FLD. AS } \\
\text { CACOS } \\
\text { (MG/L) } \\
(00904)\end{array}$ & $\begin{array}{c}\text { CALCIUM } \\
\text { DIS- } \\
\text { SOLVED } \\
\text { (NSEL/ } \\
\text { AS CA }) \\
(00915)\end{array}$ & $\begin{array}{c}\text { MAGE- } \\
\text { SIUM, } \\
\text { DIS- } \\
\text { SOLVED } \\
\text { (MG } \\
\text { AS MGG } \\
(00925)\end{array}$ & $\begin{array}{l}\text { SODIUM, } \\
\text { DIS" } \\
\text { SOLVED } \\
\text { (MG/L } \\
\text { AS NA) } \\
(00930)\end{array}$ & $\begin{array}{c}\text { SODIUM } \\
\text { ADD- } \\
\text { SORP- } \\
\text { TON } \\
\text { RATIO } \\
(00931)\end{array}$ & $\begin{array}{l}\text { POTAS- } \\
\text { SIIN, } \\
\text { DIS- } \\
\text { SOLVD } \\
\text { (MG/L. } \\
\text { AS K) } \\
(00935)\end{array}$ & $\begin{array}{c}\text { BICAR- } \\
\text { BONATE } \\
\text { WATER } \\
\text { DIS IT } \\
\text { FIELD } \\
\text { MG/L AS } \\
\text { HCOO3 } \\
(00453)\end{array}$ & $\begin{array}{c}\text { CAR- } \\
\text { BONAP } \\
\text { WATER } \\
\text { DIS IT } \\
\text { FIILD } \\
\text { MG/L AS } \\
\text { COS } \\
(00452)\end{array}$ & $\begin{array}{l}\text { ALKA- } \\
\text { LINITY } \\
\text { WAT DIS } \\
\text { TOT IT } \\
\text { FIELD } \\
\text { MG/L AS } \\
\text { CACO3 } \\
(39086)\end{array}$ \\
\hline 395 & 22 & 120 & 28 & 35 & 7.4 & 16 & 0.6 & 1.8 & 110 & 0 & 90 \\
\hline $30 \ldots$ & $<14$ & 120 & 33 & 35 & 6.9 & 14 & 0.6 & 2.0 & 101 & 0 & 82 \\
\hline $09 .$. & 1000 & 110 & 21 & 32 & 6.8 & 17 & 0.7 & 2.4 & 106 & 0 & 87 \\
\hline $17 \ldots$ & -- & 110 & 23 & 34 & 6.6 & 15 & 0.6 & 1.8 & 108 & 0 & 88 \\
\hline$\ldots$ & 880 & -- & -- & -- & -. & -- & -- & -- & 104 & 3 & 91 \\
\hline DATE & $\begin{array}{c}\text { ALKA- } \\
\text { LINITY } \\
\text { LAB } \\
\text { (MAS } \\
\text { AS } \\
\text { CACO3) } \\
(90410)\end{array}$ & $\begin{array}{c}\text { SULFATE } \\
\text { DIS- } \\
\text { SOLVED } \\
\text { (MG/L) } \\
\text { AS SO4) } \\
(00945)\end{array}$ & $\begin{array}{l}\text { CHLO- } \\
\text { RIDE, } \\
\text { DIS- } \\
\text { SOLVDD } \\
\text { (MG/L } \\
\text { AS CL ) } \\
(00940)\end{array}$ & $\begin{array}{l}\text { FLUO- } \\
\text { RIDEE, } \\
\text { DIS. } \\
\text { SOLED } \\
\text { (MG/L) } \\
\text { AS F }) \\
(00950)\end{array}$ & $\begin{array}{l}\text { SILICA, } \\
\text { DIS- } \\
\text { SOLVED } \\
\text { (MGGL } \\
\text { AS } \\
\text { SIO2) } \\
(00955)\end{array}$ & $\begin{array}{l}\text { SOLIDS, } \\
\text { RESIDUE } \\
\text { AT } 180 \\
\text { DEG C } \\
\text { DIS- } \\
\text { SOLVED } \\
(\text { MGG/L) } \\
(70300)\end{array}$ & $\begin{array}{c}\text { SOLIDS, } \\
\text { SUM OFF } \\
\text { CONSTI- } \\
\text { TUENTS- } \\
\text { DIS- } \\
\text { SOLVED } \\
\text { (MGGL) } \\
(70301)\end{array}$ & $\begin{array}{c}\text { NITRO- } \\
\text { GEN } \\
\text { NTRATE } \\
\text { DIS } \\
\text { SOLVED } \\
\text { (MG/L) } \\
\text { AS N } \\
(00618)\end{array}$ & $\begin{array}{c}\text { NITRO- } \\
\text { GENI } \\
\text { NITRIE } \\
\text { DIS- } \\
\text { SOVVD } \\
\text { (MG/L } \\
\text { AS N) } \\
(00613)\end{array}$ & $\begin{array}{c}\text { NITRO- } \\
\text { GENA } \\
\text { NO2+NÓ3 } \\
\text { DIS- } \\
\text { SOVED } \\
\text { (MG/L) } \\
\text { AS NI) } \\
(00631)\end{array}$ & $\begin{array}{c}\text { NITRO- } \\
\text { GEN } \\
\text { ARYONIA } \\
\text { DIS- } \\
\text { SOLVED } \\
\text { (MG / } \\
\text { AS H) } \\
(00608)\end{array}$ \\
\hline 6 & 97 & 53 & 3.7 & 0.20 & 15 & -- & 186 & -- & $<0.010$ & $<0.050$ & $<0.015$ \\
\hline $30 \ldots$ & 90 & 54 & 3.4 & 0.20 & 14 & - & 179 & -- & $<0.010$ & $<0.050$ & $<0.015$ \\
\hline $09 .$. & 109 & 49 & 5.9 & 0.20 & 14 & -- & 180 & -- & $<0.010$ & $<0.050$ & 0.020 \\
\hline UG & 105 & 60 & 3.3 & 0.20 & 13 & 203 & 187 & -- & $<0.010$ & 0.070 & 0.030 \\
\hline & - & $\tilde{n}$ & -- & -- & -- & -- & -- & 0.310 & 0.020 & 0.330 & 0.040 \\
\hline
\end{tabular}

NITRO- NITRO- NITROGEN GEN,AM- GEH,AMOREANIC MOAIA + MONTA + DIS- ORGANIC ORGANIC PHORUS

DATE

Nov 1995 JAN 1996

$30 \ldots$

APR 09

JuL $17 \ldots$

AUG

$19 \ldots$

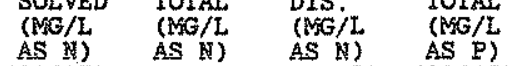
(00607) (00625) $(00623)$

$$
\ldots<0.20
$$$$
<0.20
$$$$
--<0.20
$$$$
<0.20
$$$$
<0.20
$$$$
1.0
$$$$
<0.20
$$$$
\text { - } \quad 0.40
$$$$
<0.20
$$$$
0.16<0.20
$$$$
0.20
$$

$$
\begin{gathered}
\text { PHOS- } \\
\text { PHOS- } \\
\text { PHODUSU } \\
\text { DIS- } \\
\text { DITSO } \\
\text { DIS- }
\end{gathered}
$$$$
\text { DIS- }
$$$$
\text { SOLVE }
$$$$
\text { (MS P) }
$$

(MG /L
AS P) AS P)

(00666)

(0. P)

$$
0.010
$$

$<0.010$

$<0.010$

0.020

$<0.010$

0.010

0.290

0.010

$<0.010$

0.200

$<0.010$

0.060

0.040

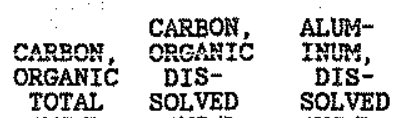

(MG/L) (NG/L

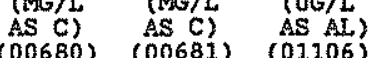

(011.06)$$
4.4 \quad-
$$$$
4.0
$$$$
5.0
$$$$
6.0
$$

ANTI-

ANAY, ARSEYYC

DIS- DIS-

SOLVED SOLVED

(US/L, (UG/L

AS SB) $(A S$ AS $)$
$(01095)(01000)$

$<1.0 \quad 2$

3.2

$<1.0$

$<1.0$

2

2 
RIO GRANDE BASIN

08290000 RIO CHAMA NEAR CHAMITA, NM -- Continued

WATER-QUALITY RECORDS

WATER QUALITY DATA, WATER YEAR OCTOBER 1995 TO SEPTEMBER 1996

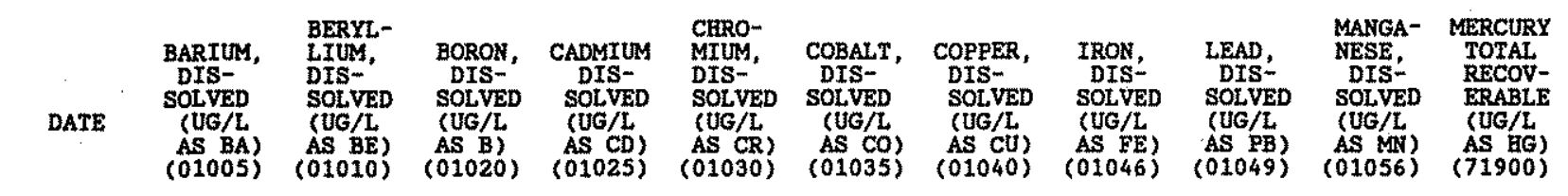

\begin{tabular}{|c|c|c|c|c|c|c|c|c|c|c|c|}
\hline Nov 1995 & 65 & $<1.0$ & 20 & $<1.0$ & $<1.0$ & $<1.0$ & 2.0 & 7.0 & $<1.0$ & 5.0 & -- \\
\hline $\begin{array}{l}\text { JAN } 1996 \\
30 \ldots \\
\text { APR }\end{array}$ & 63 & $<1.0$ & $<10$ & $<1.0$ & $<1.0$ & $<1.0$ & 2.0 & 4.0 & $<1.0$ & 4.0 & $<0.10$ \\
\hline $\begin{array}{l}09 . . . \\
\text { JUL }\end{array}$ & 62 & $<1.0$ & 40 & $<1.0$ & $<1.0$ & $<1.0$ & 1.0 & 12 & $<1.0$ & 8.0 & $<0.10$ \\
\hline $\begin{array}{l}17 \\
\text { AUG }\end{array}$ & - & -- & - & -- & - & -- & -- & 6.0 & -- & 12 & -- \\
\hline $19 \ldots$ & 86 & $<1,0$ & -- & $<1.0$ & $<1.0$ & $<1.0$ & 1.0 & -- & $<1.0$ & 3.0 & $<0.10$ \\
\hline
\end{tabular}

\begin{tabular}{|c|c|c|c|c|c|c|c|c|c|c|}
\hline DATE & $\begin{array}{c}\text { MOLYB- } \\
\text { DENUM, } \\
\text { DIS - } \\
\text { SOLVED } \\
\text { (UG/L } \\
\text { AS MO) } \\
\text { (01060) }\end{array}$ & $\begin{array}{c}\text { NICKEL, } \\
\text { DIS- } \\
\text { SOLVED } \\
\text { (UG/L } \\
\text { AS NI) } \\
\text { (01065) }\end{array}$ & $\begin{array}{c}\text { SELE- } \\
\text { NIUM, } \\
\text { TOTAi } \\
\text { (UG/L } \\
\text { AS SE) } \\
(01147 \text { ) }\end{array}$ & $\begin{array}{c}\text { SELE- } \\
\text { NIUM, } \\
\text { DIS- } \\
\text { SOLVED } \\
\text { (UG/L } \\
\text { AS SE) } \\
\text { (OI145) }\end{array}$ & $\begin{array}{c}\text { SILVER, } \\
\text { DIS- } \\
\text { SOLVED } \\
\text { (UG/L } \\
\text { AS AG) } \\
\text { (01075) }\end{array}$ & $\begin{array}{l}\text { ZINC, } \\
\text { DIS } \\
\text { SOLVED } \\
\text { (UG/L } \\
\text { AS ZN) } \\
(01090)\end{array}$ & $\begin{array}{c}\text { URANIUM } \\
\text { NATURAL } \\
\text { DIS- } \\
\text { SOLVED } \\
\text { (UG/L } \\
\text { AS U) } \\
(22703)\end{array}$ & $\begin{array}{l}\text { SEDI- } \\
\text { MENT, } \\
\text { SUS- } \\
\text { PENDED } \\
\text { (MG/L) } \\
(80154)\end{array}$ & $\begin{array}{c}\text { SEDI- } \\
\text { MENT, } \\
\text { DIS- } \\
\text { CHARGE, } \\
\text { SUS- } \\
\text { PENDED } \\
\text { (T/DAY) } \\
\text { (80155) }\end{array}$ & $\begin{array}{r}\text { SED. } \\
\text { SUSP. } \\
\text { SIEVE } \\
\text { DIAM. } \\
\times \text { FINER } \\
\text { THAA } \\
062 \text { MM } \\
\text { (70331) }\end{array}$ \\
\hline
\end{tabular}

\begin{tabular}{|c|c|c|c|c|c|c|c|c|c|c|}
\hline Nov 1995 & $<1.0$ & 2.0 & $<1$ & $<1$ & $<1.0$ & 5.0 & 1.0 & 35 & 44 & 38 \\
\hline $30 \ldots$ & $<1.0$ & 1.0 & $<1$ & $<1$ & $<1.0$ & 12 & 1.0 & 37 & 49 & 23 \\
\hline APR $09 .$. & 1.0 & 1.0 & $<1$ & $<1$ & $<1.0$ & 2.0 & 2.0 & 875 & 1310 & 87 \\
\hline AUG $17 \ldots$ & - & -- & -- & $-m$ & -- & - & -- & 536 & 690 & - \\
\hline $19 \ldots$ & 1.0 & 2.0 & $<1$ & $<1$ & $<1.0$ & $<1.0$ & $<1.0$ & 113 & 206 & 55 \\
\hline
\end{tabular}

CHEMICAL ANALYSES OF ORGANIC COMPOUNDS, WATER YEAR OCTOBER 1995 TO SEPTEMBER 1996

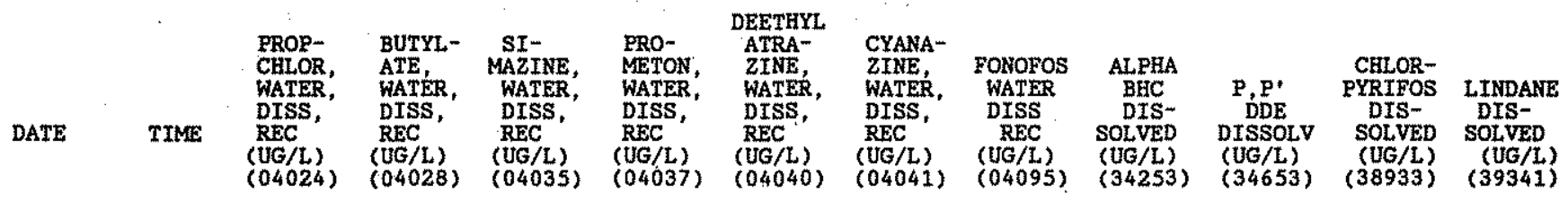

\begin{tabular}{|c|c|c|c|c|c|c|c|c|c|c|c|c|}
\hline 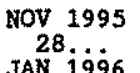 & 1000 & -- & + & $m$ & -- & - & -- & - & $\cdots$ & -- & $\cdots$ & -- \\
\hline APR & 0945 & - & - & -- & -- & -- & - & $\cdots$ & -- & $\cdots$ & - & -- \\
\hline suL & 1300 & -- & - & - & -- & -- & -- & - & -- & -- & - & - \\
\hline AUG 17 & 0815 & $<0.007$ & $<0.002$ & $<0.005$ & $<0.018$ & $<0.002$ & $<0.004$ & $<0.003$ & $<0.002$ & $<0.006$ & $<0.004$ & $<0.004$ \\
\hline 19. & 1145 & -- & -- & $-m$ & -- & -- & -- & -- & -+ & -- & -- & $-\infty$ \\
\hline
\end{tabular}




\section{RIO GRANDE BASIN}

08290000 RIO CHAMA NEAR CHAMITA, NM -- Continued

WATER-QUALITY RECORDS

WATER QUALITY DATA, WATER YEAR OCTOBER 1995 TO SEPTEMBER 1996

\begin{tabular}{|c|c|c|c|c|c|c|c|c|c|c|c|c|}
\hline DATE & $\begin{array}{c}\text { DI- } \\
\text { ELDRIN } \\
\text { DIS- } \\
\text { SOLVED } \\
\text { (UG/L) } \\
\text { (39381) }\end{array}$ & $\begin{array}{l}\text { METO- } \\
\text { LACBILOR } \\
\text { WATER } \\
\text { DISSOLV } \\
\text { (UG/L) } \\
\text { (39415) }\end{array}$ & $\begin{array}{c}\text { MALA- } \\
\text { THION, } \\
\text { DIS- } \\
\text { SOLVED } \\
\text { (UG/L) } \\
(39532)\end{array}$ & $\begin{array}{c}\text { PARA- } \\
\text { THYON, } \\
\text { DIS- } \\
\text { SOLVED } \\
\text { (UG/Li }) \\
(39542)\end{array}$ & $\begin{array}{c}\text { DI- } \\
\text { AZINON, } \\
\text { DIS- } \\
\text { SOLVED } \\
\text { (UG/L) } \\
(39572)\end{array}$ & $\begin{array}{l}\text { ATRA- } \\
\text { ZIAE, } \\
\text { WATER, } \\
\text { DISS, } \\
\text { REC } \\
(\text { UG/L) } \\
(39632)\end{array}$ & $\begin{array}{l}\text { ALA- } \\
\text { CHLOR, } \\
\text { HATER, } \\
\text { DISS, } \\
\text { REC, } \\
\text { (UG/L) } \\
\text { (46342) }\end{array}$ & $\begin{array}{c}\text { ACETO- } \\
\text { CHLOR, } \\
\text { WATER } \\
\text { FLTRD } \\
\text { REC } \\
\text { (UG/L) } \\
(49260)\end{array}$ & $\begin{array}{l}\text { METRI- } \\
\text { BUZIN } \\
\text { SENCOR } \\
\text { WATER } \\
\text { DISSOLV } \\
(\mathrm{UE} / \mathrm{L}) \\
(82630)\end{array}$ & $\begin{array}{c}\text { 2,6-DT- } \\
\text { ETEY } \\
\text { ANILINE } \\
\text { WAT FLT } \\
0,7 \mathrm{U} \\
\text { GE, REC } \\
\text { (UG/L) } \\
(82660)\end{array}$ & $\begin{array}{l}\text { TRI- } \\
\text { FLUR- } \\
\text { ALIN } \\
\text { WAT FLT } \\
0.7 \mathrm{U} \\
\text { GE REC } \\
\text { (UG/L) } \\
(82661)\end{array}$ & $\begin{array}{c}\text { ETHAL- } \\
\text { FLUR- } \\
\text { ALII } \\
\text { WAT FLT } \\
0.7 \mathrm{U} \\
\text { GF, REC } \\
\text { (UÉ } 1 \mathrm{~L}) \\
(82663)\end{array}$ \\
\hline
\end{tabular}

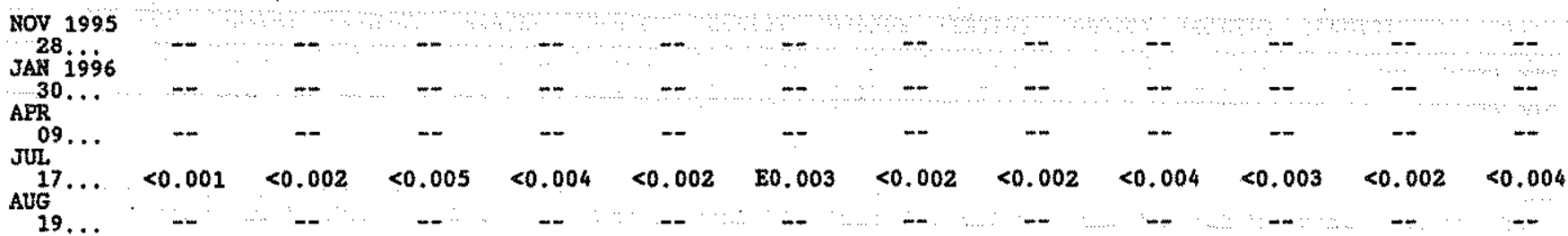

\begin{tabular}{|c|c|c|c|c|c|c|c|c|c|c|c|}
\hline $\begin{array}{l}\text { TER } \\
\text { TRD } \\
7 \mathrm{U} \\
\text { REC } \\
\text { (1L) } \\
6664)\end{array}$ & $\begin{array}{l}\text { TER- } \\
\text { BACIL } \\
\text { HATER } \\
\text { FLTRD } \\
0.7 \mathrm{U} \\
\text { GF, REC } \\
\text { (UG/L) } \\
\text { (82665) }\end{array}$ & $\begin{array}{c}\text { LIN- } \\
\text { URON } \\
\text { WATER } \\
\text { FLTRD } \\
0.7 \text { U } \\
\text { GF, REC } \\
\text { (UG/L) } \\
\text { (U2666) }\end{array}$ & $\begin{array}{c}\text { METHYL } \\
\text { PARA- } \\
\text { THION } \\
\text { WAT FLT } \\
0.7 \mathrm{U} \\
\text { GE. REC } \\
\text { (UGG/L) } \\
(82667)\end{array}$ & $\begin{array}{l}\text { EPTC } \\
\text { WATER } \\
\text { ELTRD } \\
0.7 \mathrm{U} \\
\text { GF, REC } \\
\text { (UG/L) } \\
(82668)\end{array}$ & $\begin{array}{c}\text { PEB- } \\
\text { VLATE } \\
\text { WATER } \\
\text { FILTRD } \\
0.7 \mathrm{U} \\
\text { GF, REC } \\
(\mathrm{UG} / \mathrm{L}) \\
(82669)\end{array}$ & $\begin{array}{c}\text { TEBU- } \\
\text { THIURON } \\
\text { WATER } \\
\text { FLTRD } \\
0.7 \mathrm{U} \\
\text { GF, REC } \\
\text { (UG/L) } \\
(82670)\end{array}$ & $\begin{array}{c}\text { MOL- } \\
\text { INATE } \\
\text { WATERR } \\
\text { FLTKD } \\
0.7 \mathrm{U} \\
\text { GF, REC } \\
\text { (UGG/L) } \\
\text { (B2671) }\end{array}$ & $\begin{array}{c}\text { ETHO- } \\
\text { PROP } \\
\text { WATER } \\
\text { FLTRD } \\
0.7 \text { U } \\
\text { GF, REC } \\
\text { (UG/L) } \\
\text { ( } 82672)\end{array}$ & $\begin{array}{c}\text { BEN- } \\
\text { FLUR- } \\
\text { ALIN } \\
\text { WAT FLD } \\
0.7 \mathrm{U} \\
\text { GE, REC } \\
\text { (UG/L) } \\
(82673)\end{array}$ & $\begin{array}{c}\text { CARBO- } \\
\text { FURAN } \\
\text { WATER } \\
\text { FLTRD } \\
0.7 \mathrm{~V} \\
\text { GE, REC } \\
\text { (UG/L) } \\
(82674)\end{array}$ & $\begin{array}{l}\text { TER- } \\
\text { BUFOS } \\
\text { WATER } \\
\text { FETRD } \\
0.7 \mathrm{U}\end{array}$ \\
\hline
\end{tabular}

NoV 1995

JAN 1986

$30 .$.

APR.

09.

$17, \ldots$

$+\cdots$

$19 .$.

$<0.002<0.007$

$<0.002<0.006$

$<0.002<0.004$

$80.005<0.004$

$<0,003$

$<0.002<0.003<0.013$

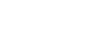

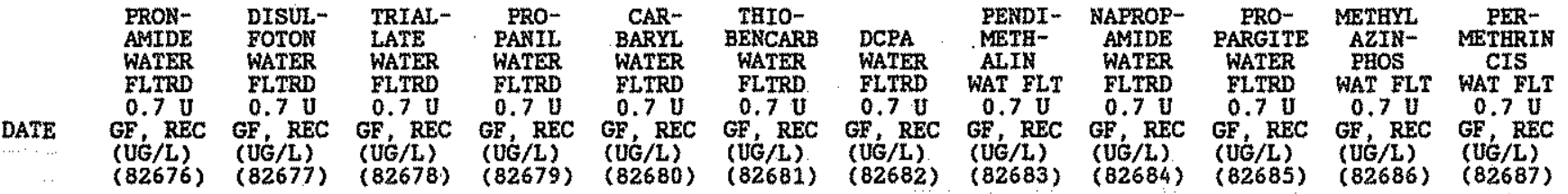

NoV 1995

\begin{tabular}{|c|c|c|c|c|c|c|c|c|c|c|c|c|}
\hline 28 & -- & - & - & -- & $-\cdots$ & -- & $-\infty$ & -- & -- & - & -- & -- \\
\hline $30 \ldots$ & -- & $=-$ & -- & -- & $\cdots$ & - & - & - & -- & $\cdots$ & -- & -- \\
\hline $\begin{array}{l}\text { APR } \\
09 . . .\end{array}$ & - & -- & - & - & - & -- & -- & - & -- & - & - & - \\
\hline $17 \ldots$ & $<0.003$ & $<0.017$ & $<0.001$ & $<0.004$ & $<0.003$ & $<0.002$ & E0.003 & $<0.004$ & $<0.003$ & $<0.013$ & $<0.001$ & $<0.005$ \\
\hline & -- & $-\infty$ & - & -- & -- & $m$ & -- & - & -- & - & - & - \\
\hline
\end{tabular}


RIO GRANDE BASIN

08291000 SANTA CRUZ RIVER AT CUNDIYO, NM

LOCATTON --Lat $35^{\circ} 57^{\prime} 53^{\prime \prime}$, long $105^{\circ} 54^{\circ} 14^{\prime \prime}$, in SEMWW sec.17, T.20 N., R.10 E., Santa Fe County, Hydrologic Unit 13020101 , on left bank $135 \mathrm{ft}$ downstream from bridge on State Highway 503,200 ft downstream from confluence of Rto Medio and Rio Frijoles, $0.6 \mathrm{mi}$ northwest of Cundiyo, $1.8 \mathrm{mi}$ upstream from Santa Cruz Dam, and at mile 11.9 .

DRAINAGE AREA. $-86 \mathrm{mi}^{2}$, approximately.

PERIOD OF RECORD.--October 1930 to current year. Monthly discharge only from some perlods, published in wSP 1312. Prior to October 1953, published as Rio Santa Cruz at Cundiyo.

REVISED RECORDS.-WSP 1392: 1931(M), 1932-33, 1934-39(M), 1942, $1943(\mathrm{M})$.

GAGE.--Water-stage recorder. Concrete control since Jan. 3, 1954 . Elevation of gage is 6,460 ft above National Geodetic Vertical Datum of 1929, from topographic map. Sept. 1, 1930 to Aug. 12, 1932, water-stage recorder at site about $1 \mathrm{mi}$ downstream at different datum. Aug. 13, 1932 to Oct. 29, 1934, water-stage recorder at site 35 ft upstream at datum $0.42 \mathrm{ft}$ higher. Oct. 30, 1934 to Jan. 2, 1954, water-stage recorder at present site at datum $0.64 \mathrm{ft}$ lower.

REMARKS. --Records good except for estimated dally discharges, which are poor. Diversions for trrigation of about 1,000 ecres upstream from station. Several observations of water temperature were made during the year.

DISCHARGE, CUBIC FEET PER SECOND, WATER YEAR OCTOBER 1995 TO SEPTEMBER 1996

\begin{tabular}{|c|c|c|c|c|c|c|c|c|c|c|c|c|}
\hline DAY & OCT & Nov & DEC & JAN & FEB & MAR & AER & MAY & JUN & JUL & AUG & SEP \\
\hline $\begin{array}{l}1 \\
2 \\
3 \\
4 \\
5\end{array}$ & $\begin{array}{l}18 \\
16 \\
15 \\
14 \\
13\end{array}$ & $\begin{array}{c}11 \\
12 \\
9,9 \\
11 \\
11\end{array}$ & $\begin{array}{l}12 \\
11 \\
11 \\
9.8 \\
9.6\end{array}$ & $\begin{array}{l}07.0 \\
67.0 \\
06.0 \\
67.0 \\
07.0\end{array}$ & $\begin{array}{r}8.5 \\
8.3 \\
e 7.0 \\
07.0 \\
88.0\end{array}$ & $\begin{array}{l}9.2 \\
9.9 \\
9.3 \\
8.7 \\
8.7\end{array}$ & $\begin{array}{l}13 \\
15 \\
17 \\
16 \\
14\end{array}$ & $\begin{array}{l}20 \\
22 \\
22 \\
23 \\
23\end{array}$ & $\begin{array}{l}11 \\
10 \\
10 \\
10 \\
10\end{array}$ & $\begin{array}{l}27 \\
20 \\
16 \\
14 \\
13\end{array}$ & $\begin{array}{l}7.7 \\
7.7 \\
7.6 \\
7.3 \\
7.2\end{array}$ & $\begin{array}{l}6.9 \\
6.7 \\
7.0 \\
7.7 \\
7.0\end{array}$ \\
\hline $\begin{array}{r}6 \\
7 \\
8 \\
9 \\
10\end{array}$ & $\begin{array}{l}13 \\
13 \\
13 \\
13 \\
12\end{array}$ & $\begin{array}{l}11 \\
11 \\
10 \\
11 \\
11\end{array}$ & $\begin{array}{r}9.6 \\
9.2 \\
9.1 \\
8.0 \\
07.0\end{array}$ & $\begin{array}{l}\text { e7.0 } \\
e 7.0 \\
e 7.0 \\
e 7.0 \\
e 7.0\end{array}$ & $\begin{array}{l}8.5 \\
8.7 \\
8.5 \\
8.7 \\
9.2\end{array}$ & $\begin{array}{l}8.2 \\
8.5 \\
9.3 \\
9.0 \\
9.1\end{array}$ & $\begin{array}{l}12 \\
12 \\
12 \\
17 \\
17\end{array}$ & $\begin{array}{l}25 \\
26 \\
27 \\
26 \\
26\end{array}$ & $\begin{array}{l}8.0 \\
8.6 \\
8.8 \\
9.4 \\
8.3\end{array}$ & $\begin{array}{l}12 \\
12 \\
12 \\
20 \\
20\end{array}$ & $\begin{array}{l}5.4 \\
4.5 \\
5.8 \\
6.7 \\
7.3\end{array}$ & $\begin{array}{l}8.2 \\
8.3 \\
8.0 \\
6.5 \\
5.5\end{array}$ \\
\hline $\begin{array}{l}11 \\
12 \\
13 \\
14 \\
15\end{array}$ & $\begin{array}{l}12 \\
12 \\
12 \\
12 \\
12\end{array}$ & $\begin{array}{l}{ }_{12}^{6.5} \\
11 \\
11 \\
10\end{array}$ & $\begin{array}{r}97.0 \\
07.0 \\
87.0 \\
08.0 \\
8.5\end{array}$ & $\begin{array}{r}\text { e7.0 } \\
88.0 \\
8.9 \\
8.6 \\
9.9\end{array}$ & $\begin{array}{l}9.1 \\
8.9 \\
9.1 \\
9.5 \\
9.6\end{array}$ & $\begin{array}{l}9.9 \\
10 \\
10 \\
10 \\
10\end{array}$ & $\begin{array}{l}14 \\
14 \\
15 \\
12 \\
11\end{array}$ & $\begin{array}{l}26 \\
26 \\
25 \\
25 \\
25\end{array}$ & $\begin{array}{r}8.6 \\
9.2 \\
9.1 \\
22 \\
14\end{array}$ & $\begin{array}{l}17 \\
14 \\
16 \\
14 \\
12\end{array}$ & $\begin{array}{l}6.3 \\
5.4 \\
4.9 \\
5.3 \\
6.1\end{array}$ & $\begin{array}{c}7.1 \\
7.8 \\
9.3 \\
10 \\
16\end{array}$ \\
\hline $\begin{array}{l}16 \\
17 \\
18 \\
19 \\
20\end{array}$ & $\begin{array}{l}11 \\
10 \\
11 \\
11 \\
11\end{array}$ & $\begin{array}{l}10 \\
10 \\
9.4 \\
8.7 \\
8.9\end{array}$ & $\begin{array}{r}8.7 \\
8.4 \\
8.5 \\
7.1 \\
87.0\end{array}$ & $\begin{array}{r}9.4 \\
8.7 \\
7.9 \\
08.0 \\
8.9\end{array}$ & $\begin{array}{l}9.6 \\
9.5 \\
9.8 \\
9.4 \\
9.9\end{array}$ & $\begin{array}{l}9.9 \\
5.6 \\
8.8 \\
8.8 \\
9.8\end{array}$ & $\begin{array}{r}12 \\
12 \\
14 \\
15 \\
16\end{array}$ & $\begin{array}{l}24 \\
22 \\
20 \\
18\end{array}$ & $\begin{array}{l}11 \\
8.7 \\
8.5 \\
8.6\end{array}$ & $\begin{array}{l}14 \\
12 \\
12 \\
9.6\end{array}$ & $\begin{array}{l}5.9 \\
5.8 \\
5.3 \\
3.2\end{array}$ & $\begin{array}{l}13 \\
15 \\
18 \\
13\end{array}$ \\
\hline $\begin{array}{l}21 \\
22 \\
23 \\
24 \\
25\end{array}$ & $\begin{array}{l}11 \\
11 \\
8.8 \\
11 \\
12\end{array}$ & $\begin{array}{l}8.9 \\
9.0 \\
9.5 \\
7.4 \\
8.7\end{array}$ & $\begin{array}{l}67.0 \\
87.0 \\
67.0 \\
96.0 \\
66.0\end{array}$ & $\begin{array}{r}e 8.0 \\
8.9 \\
e 9.0 \\
e 8.0 \\
e 8.0\end{array}$ & $\begin{array}{c}10 \\
10 \\
8.7 \\
8.3 \\
9.4\end{array}$ & $\begin{array}{l}10 \\
11 \\
12 \\
11 \\
10\end{array}$ & $\begin{array}{l}14 \\
13 \\
15 \\
18 \\
20\end{array}$ & $\begin{array}{l}18 \\
17 \\
15 \\
15 \\
14\end{array}$ & $\begin{array}{l}8.1 \\
8.0 \\
8.7 \\
7.1 \\
6.9\end{array}$ & $\begin{array}{l}8.1 \\
8.8 \\
7.4 \\
6.7 \\
6.6\end{array}$ & $\begin{array}{l}6.1 \\
8.0 \\
11 \\
10 \\
11\end{array}$ & $\begin{array}{l}11 \\
10 \\
9.4 \\
8.8 \\
8.8\end{array}$ \\
\hline $\begin{array}{l}26 \\
27 \\
28 \\
29 \\
30 \\
31\end{array}$ & $\begin{array}{l}12 \\
11 \\
11 \\
11 \\
11 \\
11\end{array}$ & $\begin{array}{r}9.8 \\
7.0 \\
4.4 \\
9.3 \\
12 \\
\end{array}$ & $\begin{array}{l}07.0 \\
08.0 \\
08.0 \\
08.0 \\
08.0 \\
08.0\end{array}$ & $\begin{array}{r}08.0 \\
08.0 \\
08.0 \\
08.0 \\
08.0 \\
8.5\end{array}$ & $\begin{array}{l}8.2 \\
5.9 \\
8.3 \\
8.8 \\
-.- \\
---\end{array}$ & $\begin{array}{l}10 \\
10 \\
11 \\
12 \\
11 \\
12\end{array}$ & $\begin{array}{r}24 \\
28 \\
27 \\
22 \\
20 \\
--\end{array}$ & $\begin{array}{l}14 \\
14 \\
13 \\
13 \\
12 \\
12\end{array}$ & $\begin{array}{l}7.1 \\
35 \\
20 \\
23 \\
25 \\
-\end{array}$ & $\begin{array}{l}7.5 \\
8.1 \\
8.3 \\
7.7 \\
8.3 \\
8.4\end{array}$ & $\begin{array}{l}9.8 \\
12 \\
19 \\
13 \\
10 \\
8.7\end{array}$ & $\begin{array}{c}10 \\
10 \\
8.9 \\
8.8 \\
8.7\end{array}$ \\
\hline $\begin{array}{l}\text { TOTAL } \\
\text { MEAN } \\
\text { MAX } \\
\text { MIN } \\
\text { AC }-F T\end{array}$ & $\begin{array}{r}374.8 \\
12.1 \\
18 \\
8.8 \\
743\end{array}$ & $\begin{array}{r}292.4 \\
9.75 \\
12 \\
4.4 \\
580\end{array}$ & $\begin{array}{r}253.5 \\
8.18 \\
12 \\
6.0 \\
503\end{array}$ & $\begin{array}{r}244.7 \\
7.89 \\
9.9 \\
6.0 \\
485\end{array}$ & $\begin{array}{r}254.4 \\
8.77 \\
10 \\
5.9 \\
505\end{array}$ & $\begin{array}{r}306.7 \\
9.89 \\
12 \\
8.2 \\
608\end{array}$ & $\begin{array}{r}482 \\
16.1 \\
28 \\
11 \\
956\end{array}$ & $\begin{array}{r}632 \\
20.4 \\
27 \\
12 \\
1250\end{array}$ & $\begin{array}{r}351.4 \\
11.7 \\
35 \\
6.9 \\
697\end{array}$ & $\begin{array}{r}386.5 \\
12.5 \\
27 \\
6.6 \\
767\end{array}$ & $\begin{array}{r}239.6 \\
7.73 \\
19 \\
3.2 \\
475\end{array}$ & $\begin{array}{r}286.4 \\
9.55 \\
18 \\
5.5 \\
568\end{array}$ \\
\hline
\end{tabular}

STATISTICS OF MONTHLY MEAN DATA FOR WATER YEARS 1933 - 1996, BY WATER YEAR (WY)

\begin{tabular}{|c|c|c|c|c|c|c|c|c|c|c|c|c|}
\hline $\begin{array}{l}\text { MEAN } \\
\text { MAX } \\
\text { (WY) } \\
\text { MIN } \\
\text { (WY) }\end{array}$ & $\begin{array}{l}15.5 \\
61.3 \\
1942 \\
3.88 \\
1957\end{array}$ & $\begin{array}{l}12.2 \\
43.4 \\
1942 \\
4.69 \\
1957\end{array}$ & $\begin{array}{l}10.4 \\
25.2 \\
1987 \\
3.82 \\
1951\end{array}$ & $\begin{array}{l}9.44 \\
19.5 \\
1987 \\
4.75 \\
1951\end{array}$ & $\begin{array}{l}10.2 \\
23.9 \\
1995 \\
5.44 \\
1981\end{array}$ & $\begin{array}{l}20.0 \\
51.1 \\
1985 \\
6.97 \\
1981\end{array}$ & $\begin{array}{r}51.5 \\
205 \\
1942 \\
13.2 \\
1951\end{array}$ & $\begin{array}{r}98.4 \\
329 \\
1941 \\
15.9 \\
1950\end{array}$ & $\begin{array}{r}76.6 \\
293 \\
1979 \\
7.05 \\
1956\end{array}$ & $\begin{array}{r}28.4 \\
115 \\
1986 \\
5.64 \\
1956\end{array}$ & $\begin{array}{r}25.4 \\
109 \\
1991 \\
4.57 \\
1956\end{array}$ & $\begin{array}{l}19.4 \\
78.6 \\
1988 \\
2.47 \\
1956\end{array}$ \\
\hline
\end{tabular}

\section{SUMMARY STATISTICS}

ANNUAL TOTAL.

AIGHEST ANNUAL MEAN

LOWEST ANNUAL MEAN

HIGHEST DAILY MEAN

LOWEST DAILY MEAN

ANNUAL, SEVEN-DAY MTNIMUM

INSTANTANEOUS PEAK FLON

INSTANTANEOUS PEAR STAGE

INSTANTANEOUS LOW FLOW

ANNUAL RUNOFF (AC-FT)

10 PERCENT EXCEEDS

50 PERCENT EXCEEDS

90 PERCENT EXCEEDS

e Estimated
FOR 1995 CALENDAR YEAR

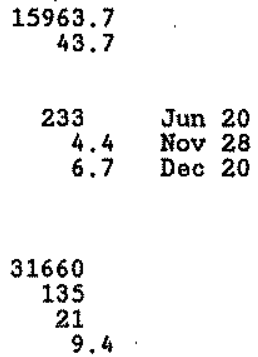

FOR 1996 WATER YEAR

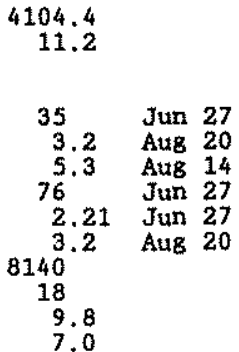

WATER YEARS 1933 - 1996

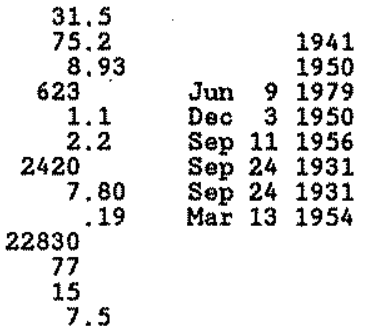


LOCATION, - Lat $35^{\circ} 50^{\prime} 46^{\prime \prime}$, Long $105^{\circ} 54^{\prime} 17^{\prime \prime}$, in NEthsW, sec.29, T.19 N., R.10 E., Santa Fe County, Hydrologic Unit 13020101 , on Nambe Indian Reservation, 300 ft upstream from Nanbe Falis, 2.6 mi upstream from Rio En Medio, 4.4 mi southeast of Nambe Pueblo, and $5.4 \mathrm{mi}$ southeast of Nambe.

DRAIHAGE AREA, $--34.1 \mathrm{mi}^{2}$.

PERIOD OF RECORD. - February 1976 to current year.

REVISED RECORDS, - WDR KMY-77-1: Drainage area.

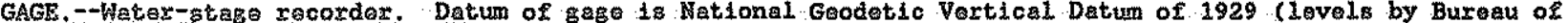
Reclamation), Prior to July 22, 1976 , nonrocorcing gage at seme sito dind datum.

REMARKS,--Reservoir is formed by a concrete arch and eartheill dam, storage began Feb. 23, 1976. Total capacity, 2,020 acre-ft at ilevetion $6,826,6 \mathrm{ft}$, crest of ogee weir spilipay, including 237 ecre-ft of storage in a permanent pool betwen elevation $6,760.9$ ft, invert of outlet condutts, and $6,780,0$ rt. Dead 8 torage 121 acreft below elevation $6,760.9$ ft. Outlet conduits aro one 6-in and two 12-in diameter pipes. Reservoir is used for storage of irrigetion pator and for recreation. Figures given herein represent total storage.

EXTRBAES FOR PERIOD OF RECORD.--Maximum contents, 2,060 acre-ft June 9, 1979, elevation, 6,827.24 ft; no storage prior to $\mathrm{Feb}, 23,1976$.

EXTREMES FOR CURRENT YEAR.-Maximum contents, 1,940 acre-ft, Mar. 25, elovation 6,825.18 ft; minimum, 429 acre-It, Sept. 12, elevets on $6,178.75 \mathrm{ft}$.

Capacity table (elovation, in feet, and contents, in acre-feet)

(Based on survey by Bureau of Reclamation in 1976)

$\begin{array}{rrrrr}6,801 & 870 & & 6,820 & 1,660 \\ 6,810 & 1,201 & & 6,825 & 1,930 \\ 6,815 & 1,420 & & 6,830 & 2,230\end{array}$

RESHRVOIR STORAGE (ACRE-FEET), WATER YEAR OCTOBER 1995 TO SEPTEMBER 1996

\begin{tabular}{|c|c|c|c|c|c|c|c|c|c|c|c|c|}
\hline DAY & $\mathrm{OCT}$ & HOV & DEC & JAN & FEB & MAR & APR & MAY & JUN & JU & AUG & SEP \\
\hline $\begin{array}{l}1 \\
2 \\
3 \\
4 \\
5\end{array}$ & $\begin{array}{l}1240 \\
1250 \\
1260 \\
1270 \\
1280\end{array}$ & $\begin{array}{l}1230 \\
1220 \\
1220 \\
1220 \\
1220\end{array}$ & $\begin{array}{l}1300 \\
1300 \\
1310 \\
1310 \\
1320\end{array}$ & $\begin{array}{l}1480 \\
1490 \\
1490 \\
1500 \\
1500\end{array}$ & $\begin{array}{l}1650 \\
1660 \\
1660 \\
1670 \\
1670\end{array}$ & $\begin{array}{l}1810 \\
1810 \\
1820 \\
1820 \\
1830\end{array}$ & $\begin{array}{r}1880 \\
1870 \\
1870 \\
\cdot 1860 \\
11840\end{array}$ & $\begin{array}{l}1470 \\
1480 \\
1490 \\
1500 \\
1500\end{array}$ & $\begin{array}{l}940 \\
940 \\
937 \\
936 \\
928\end{array}$ & $\begin{array}{l}782 \\
796 \\
806 \\
817 \\
830\end{array}$ & $\begin{array}{l}1050 \\
1050 \\
1050 \\
1040 \\
1040\end{array}$ & $\begin{array}{l}456 \\
450 \\
445 \\
441 \\
440\end{array}$ \\
\hline $\begin{array}{r}6 \\
7 \\
8 \\
9 \\
10\end{array}$ & $\begin{array}{l}1280 \\
1290 \\
1290 \\
1310 \\
1320\end{array}$ & $\begin{array}{l}1210 \\
1210 \\
1210 \\
1200 \\
1190\end{array}$ & $\begin{array}{l}1330 \\
1340 \\
1340 \\
1350 \\
1350\end{array}$ & $\begin{array}{l}1510 \\
1510 \\
1520 \\
1520 \\
1530\end{array}$ & $\begin{array}{l}1680 \\
1680 \\
1690 \\
1690 \\
1700\end{array}$ & $\begin{array}{l}1840 \\
1840 \\
1840 \\
1850 \\
1860\end{array}$ & $\begin{array}{l}1820 \\
1800 \\
1780 \\
1760 \\
1740\end{array}$ & $\begin{array}{l}1520 \\
1520 \\
1530 \\
1500 \\
1480\end{array}$ & $\begin{array}{r}898 \\
866 \\
834 \\
802 \\
0788\end{array}$ & $\begin{array}{r}837 \\
845 \\
853 \\
881 \\
9905\end{array}$ & $\begin{array}{r}1030 \\
1020 \\
1010 \\
973 \\
906\end{array}$ & $\begin{array}{l}442 \\
441 \\
439 \\
436 \\
433\end{array}$ \\
\hline $\begin{array}{l}11 \\
12 \\
13 \\
14 \\
15\end{array}$ & $\begin{array}{l}1330 \\
1340 \\
1340 \\
1340 \\
1340\end{array}$ & $\begin{array}{l}1190 \\
1180 \\
1180 \\
1170 \\
1180\end{array}$ & $\begin{array}{l}1360 \\
1370 \\
1370 \\
1380 \\
1380\end{array}$ & $\begin{array}{l}1530 \\
1540 \\
1550 \\
1550 \\
1560\end{array}$ & $\begin{array}{l}1700 \\
1710 \\
1720 \\
1720 \\
1730\end{array}$ & $\begin{array}{r}1860 \\
1870 \\
1870 \\
-1870 \\
e 1880\end{array}$ & $\begin{array}{l}1720 \\
1710 \\
1690 \\
1670 \\
1650\end{array}$ & $\begin{array}{l}1450 \\
1410 \\
1360 \\
1320 \\
1280\end{array}$ & $\begin{array}{r}0758 \\
707 \\
698 \\
706 \\
704\end{array}$ & $\begin{array}{l}931 \\
948 \\
963 \\
981 \\
985\end{array}$ & $\begin{array}{l}837 \\
769 \\
701 \\
633 \\
565\end{array}$ & $\begin{array}{l}430 \\
429 \\
430 \\
434 \\
445\end{array}$ \\
\hline $\begin{array}{l}16 \\
17 \\
18 \\
19 \\
20\end{array}$ & $\begin{array}{l}1330 \\
1320 \\
1310 \\
1300 \\
1300\end{array}$ & $\begin{array}{l}1190 \\
1200 \\
1210 \\
1210 \\
1220\end{array}$ & $\begin{array}{l}1390 \\
1400 \\
1400 \\
1410 \\
1410\end{array}$ & $\begin{array}{l}1560 \\
1570 \\
1580 \\
1580 \\
1590\end{array}$ & $\begin{array}{l}1730 \\
1740 \\
1740 \\
1750 \\
1760\end{array}$ & $\begin{array}{l}1890 \\
1900 \\
1900 \\
1900 \\
1910\end{array}$ & $\begin{array}{l}1630 \\
1610 \\
1590 \\
1570 \\
1540\end{array}$ & $\begin{array}{l}1270 \\
1270 \\
1270 \\
1260 \\
1260\end{array}$ & $\begin{array}{l}698 \\
695 \\
696 \\
697 \\
695\end{array}$ & $\begin{array}{r}01000 \\
1020 \\
1040 \\
1050 \\
1050\end{array}$ & $\begin{array}{r}0550 \\
0535 \\
0520 \\
505 \\
496\end{array}$ & $\begin{array}{r}-450 \\
456 \\
470 \\
481 \\
489\end{array}$ \\
\hline $\begin{array}{l}21 \\
22 \\
23 \\
24 \\
25\end{array}$ & $\begin{array}{l}1290 \\
1280 \\
1270 \\
1260 \\
1250\end{array}$ & $\begin{array}{l}1230 \\
1240 \\
1240 \\
1250 \\
1260\end{array}$ & $\begin{array}{l}1420 \\
1420 \\
1430 \\
1430 \\
1430\end{array}$ & $\begin{array}{l}1590 \\
1600 \\
1600 \\
1610 \\
1610\end{array}$ & $\begin{array}{l}1760 \\
1770 \\
1770 \\
1780 \\
1790\end{array}$ & $\begin{array}{r}1910 \\
\times 1920 \\
\times 1930 \\
1930 \\
1940\end{array}$ & $\begin{array}{l}1500 \\
1410 \\
1410 \\
1410 \\
1430\end{array}$ & $\begin{array}{l}1250 \\
2230 \\
1230 \\
1130 \\
1100\end{array}$ & $\begin{array}{l}695 \\
695 \\
696 \\
695 \\
694\end{array}$ & $\begin{array}{l}1060 \\
1070 \\
1070 \\
1070 \\
1080\end{array}$ & $\begin{array}{l}489 \\
481 \\
478 \\
474 \\
470\end{array}$ & $\begin{array}{l}495 \\
500 \\
504 \\
511 \\
523\end{array}$ \\
\hline $\begin{array}{l}26 \\
27 \\
28 \\
29 \\
30 \\
31\end{array}$ & $\begin{array}{l}1250 \\
1240 \\
1230 \\
1230 \\
1240 \\
1230\end{array}$ & $\begin{array}{l}1270 \\
1270 \\
1270 \\
1280 \\
1250 \\
---\end{array}$ & $\begin{array}{l}1440 \\
1450 \\
1450 \\
1460 \\
1470 \\
1470\end{array}$ & $\begin{array}{l}1620 \\
1620 \\
1630 \\
1630 \\
1640 \\
1640\end{array}$ & $\begin{array}{r}1790 \\
1790 \\
1800 \\
1800 \\
\ldots \ldots\end{array}$ & $\begin{array}{l}1930 \\
1920 \\
1920 \\
1910 \\
1900 \\
1890\end{array}$ & $\begin{array}{l}1440 \\
1450 \\
1460 \\
1460 \\
1470 \\
=0\end{array}$ & $\begin{array}{r}1070 \\
1030 \\
993 \\
956 \\
938 \\
940\end{array}$ & $\begin{array}{l}693 \\
716 \\
729 \\
748 \\
765 \\
---\end{array}$ & $\begin{array}{l}1080 \\
1070 \\
1060 \\
1050 \\
1050 \\
1050\end{array}$ & $\begin{array}{l}464 \\
465 \\
468 \\
467 \\
465 \\
461\end{array}$ & $\begin{array}{l}532 \\
534 \\
536 \\
538 \\
539\end{array}$ \\
\hline $\begin{array}{l}\text { MAX } \\
\text { MIN } \\
(\dagger)\end{array}$ & $\begin{array}{r}1340 \\
1230 \\
6810.76 \\
0\end{array}$ & $\begin{array}{r}1290 \\
1370 \\
6812.05 \\
+60\end{array}$ & $\begin{array}{r}1470 \\
1300 \\
6816.13 \\
+180\end{array}$ & $\begin{array}{r}1640 \\
1480 \\
6819.63 \\
+170\end{array}$ & $\begin{array}{r}1800 \\
1650 \\
6822.71 \\
+160\end{array}$ & $\begin{array}{r}1940 \\
1810 \\
6824.26 \\
+90\end{array}$ & $\begin{array}{r}1880 \\
1410 \\
6815.99 \\
-420\end{array}$ & $\begin{array}{r}1530 \\
938 \\
6803.12 \\
-530\end{array}$ & $\begin{array}{r}940 \\
693 \\
6797.57 \\
-175\end{array}$ & $\begin{array}{r}1080 \\
782 \\
6806.28 \\
+285\end{array}$ & $\begin{array}{r}1050 \\
461 \\
6785.32 \\
-589\end{array}$ & $\begin{array}{r}53 \\
42 \\
6788.9 \\
+7\end{array}$ \\
\hline
\end{tabular}

CAL IR 1995 MAX 2040 MIN 1120 (tt) -300

WTR YR 1996 MAX 1940. MIN 429 (th) -691

- Estimated

(†) ELEVATION, IN FEET, AT END OF MONTE

(t) CHANGE IN CONTENTS, IN ACRE-FEET 
RIO GTRANDE BASIN

08294210 RIO NAMBE BELOW NAMBE FALLS DAM, NEAR NAMBE, MM

LOCATTON.--Lat $35^{\circ} 50^{\prime} 46^{\prime \prime}$, long $105^{\circ} 54^{\prime} 17^{\prime \prime}$, In NEKSWK sec.29, T. 19 N., R.10 E., Santa Fe County, Hydrologic Unit

13020101, on Nambe Indian Reservation, in outlet conduits of Nambi Falls Dam, 300 ft upstream from Nambe Palls, 2.6 mi upstream from Rto En Medio, 4.4 mi southeast of Nambe Pueblo and 5.4 mi southeast of Nambe.

DRAINAGE AREA. $--34.1 \mathrm{mi}^{2}$.

PERIOD OF RECORD. --January 1979 to current year.

GAGE.--Totalizing flowmeters in each of three outlet conduits in Nambe Falls Dam.

REMARKS. - Records good except for estimated dally dishcarges, which are fair. Flow regulated by Nambe Falls

Reservoir (station 08294200): Outlet conduts are one 6-in. and two 12-in. diameter plpes. During periods of

spi11 at Nambe Falls Dam, record computed at site 1,100 ft downstream, site of discontinued station 08294300 ,

Rio Nambe at Nambe Falls.

DISCHARGE, CUBIC FEET PER SECOND, WATER YEAR OCTOBER 1995 TO SEPTEMBER 1996

\begin{tabular}{|c|c|c|c|c|c|c|c|c|c|c|c|c|}
\hline DAY & $\propto C T$ & Nov & $D E C$ & JAN & EEB & MAR & APR & MAY & JUN & JuL & AUUG & SEP \\
\hline $\begin{array}{l}1 \\
2 \\
3 \\
4 \\
5\end{array}$ & $\begin{array}{l}5.3 \\
2.5 \\
2.5 \\
2.5 \\
2.5\end{array}$ & $\begin{array}{l}8.4 \\
8.4 \\
7.4 \\
6.7 \\
6.8\end{array}$ & $\begin{array}{l}1.1 \\
1.1 \\
1.1 \\
1.1 \\
1.1\end{array}$ & $\begin{array}{l}1.1 \\
1.0 \\
1.1 \\
1.1 \\
1.1\end{array}$ & $\begin{array}{l}1.0 \\
1.0 \\
1.0 \\
1.99 \\
1.0\end{array}$ & $\begin{array}{l}.98 \\
1.0 \\
1.0 \\
1.0 \\
1.0\end{array}$ & $\begin{array}{r}8.7 \\
8.6 \\
8.7 \\
08.3 \\
14\end{array}$ & $\begin{array}{l}4.5 \\
4.4 \\
4.4 \\
4.3 \\
4.2\end{array}$ & $\begin{array}{l}4.5 \\
4.4 \\
4.5 \\
4.5 \\
7.6\end{array}$ & $\begin{array}{l}4.1 \\
4.1 \\
4.1 \\
4.0 \\
4.0\end{array}$ & $\begin{array}{r}5.9 \\
5.9 \\
5.9 \\
5.9 \\
5.8\end{array}$ & $\begin{array}{l}11 \\
11 \\
11 \\
9.1 \\
7.4\end{array}$ \\
\hline $\begin{array}{r}6 \\
7 \\
8 \\
9 \\
10\end{array}$ & $\begin{array}{l}2.6 \\
2.6 \\
2.6 \\
1.4 \\
.62\end{array}$ & $\begin{array}{l}6.8 \\
7.3 \\
7.9 \\
7.9 \\
7.8\end{array}$ & $\begin{array}{l}1.0 \\
1.0 \\
1.0 \\
1.99 \\
1.0\end{array}$ & $\begin{array}{l}1.1 \\
1.1 \\
1.1 \\
1.1 \\
1.0\end{array}$ & $\begin{array}{l}1.0 \\
1.1 \\
1.1 \\
1.1 \\
1.1\end{array}$ & $\begin{array}{l}1.0 \\
1.0 \\
1.0 \\
1.1 \\
1.0\end{array}$ & $\begin{array}{l}16 \\
16 \\
16 \\
16 \\
16\end{array}$ & $\begin{array}{l}4.3 \\
4.3 \\
7.6 \\
21 \\
21\end{array}$ & $\begin{array}{l}20 \\
20 \\
20 \\
20 \\
20\end{array}$ & $\begin{array}{l}4.0 \\
4.1 \\
4.1 \\
4.3 \\
4.9\end{array}$ & $\begin{array}{l}7.3 \\
10 \\
10 \\
21 \\
32\end{array}$ & $\begin{array}{l}7.4 \\
7.4 \\
7.5 \\
7.4 \\
7.4\end{array}$ \\
\hline $\begin{array}{l}11 \\
12 \\
13 \\
14 \\
15\end{array}$ & $\begin{array}{r}.68 \\
.70 \\
2.3 \\
6.2 \\
9.9\end{array}$ & $\begin{array}{l}7.8 \\
7.8 \\
7.9 \\
7.8 \\
4.2\end{array}$ & $\begin{array}{l}1.0 \\
1.0 \\
1.0 \\
1.0 \\
1.0\end{array}$ & $\begin{array}{l}.98 \\
.97 \\
.98 \\
.98 \\
.99\end{array}$ & $\begin{array}{l}1.1 \\
1.1 \\
1.1 \\
1.1 \\
1.1\end{array}$ & $\begin{array}{l}1.0 \\
1.1 \\
1.0 \\
1.1 \\
1.1\end{array}$ & $\begin{array}{l}16 \\
16 \\
16 \\
16 \\
16\end{array}$ & $\begin{array}{l}21 \\
21 \\
21 \\
21 \\
21\end{array}$ & $\begin{array}{l}20 \\
20 \\
10 \\
9.1 \\
9.1\end{array}$ & $\begin{array}{l}5.7 \\
5.7 \\
5.6 \\
5.6 \\
5.4\end{array}$ & $\begin{array}{l}35 \\
36 \\
37 \\
36 \\
37\end{array}$ & $\begin{array}{l}7.5 \\
7.5 \\
7.7 \\
7.7 \\
7.7\end{array}$ \\
\hline $\begin{array}{l}16 \\
17 \\
18 \\
19 \\
20\end{array}$ & $\begin{array}{r}9.8 \\
9.8 \\
9.9 \\
9.9 \\
10\end{array}$ & $\begin{array}{l}1.1 \\
1.1 \\
1.1 \\
1.1 \\
1.1\end{array}$ & $\begin{array}{l}1.0 \\
1.0 \\
1.0 \\
1.0 \\
1.0\end{array}$ & $\begin{array}{r}.98 \\
1.0 \\
-1.0 \\
.96 \\
.97\end{array}$ & $\begin{array}{l}1.1 \\
1.1 \\
1.1 \\
1.1 \\
1.1\end{array}$ & $\begin{array}{l}1.1 \\
1.1 \\
1.0 \\
1.0 \\
1.0\end{array}$ & $\begin{array}{l}16 \\
16 \\
16 \\
16 \\
20\end{array}$ & $\begin{array}{l}21 \\
21 \\
21 \\
21 \\
21\end{array}$ & $\begin{array}{l}9.0 \\
6.2 \\
4.4 \\
4.3 \\
4.2\end{array}$ & $\begin{array}{l}5.3 \\
5.3 \\
5.3 \\
5.3 \\
5.2\end{array}$ & $\begin{array}{l}18 \\
10 \\
10 \\
10 \\
10\end{array}$ & $\begin{array}{l}7.6 \\
7.6 \\
7.7 \\
7.6 \\
7.6\end{array}$ \\
\hline $\begin{array}{l}21 \\
22 \\
23 \\
24 \\
25\end{array}$ & $\begin{array}{r}9.9 \\
10 \\
9.9 \\
9.9 \\
10\end{array}$ & $\begin{array}{l}1.1 \\
1.1 \\
1.1 \\
1.1 \\
1.1\end{array}$ & $\begin{array}{l}1.0 \\
1.0 \\
1.0 \\
1.0 \\
1.0\end{array}$ & $\begin{array}{l}.97 \\
.98 \\
.97 \\
.95 \\
.97\end{array}$ & $\begin{array}{l}1.1 \\
1.1 \\
1.0 \\
1.99 \\
1.0\end{array}$ & $\begin{array}{l}1.1 \\
1.1 \\
1.1 \\
1.1 \\
1.1\end{array}$ & $\begin{array}{c}28 \\
29 \\
29 \\
14 \\
3.2\end{array}$ & $\begin{array}{l}21 \\
24 \\
32 \\
31 \\
24\end{array}$ & $\begin{array}{l}4.1 \\
4.1 \\
4.1 \\
4.1 \\
4.1\end{array}$ & $\begin{array}{l}5.1 \\
5.1 \\
5.1 \\
5.1 \\
5.1\end{array}$ & $\begin{array}{l}10 \\
10 \\
10 \\
11 \\
11\end{array}$ & $\begin{array}{l}7.7 \\
7.7 \\
7.7 \\
5.3 \\
3.7\end{array}$ \\
\hline $\begin{array}{l}26 \\
27 \\
28 \\
29 \\
30 \\
31\end{array}$ & $\begin{array}{r}9.9 \\
10 \\
8.6 \\
4.5 \\
4.5 \\
6.5\end{array}$ & $\begin{array}{l}1.1 \\
1.1 \\
1.1 \\
1.1 \\
1.1\end{array}$ & $\begin{array}{l}1.0 \\
1.0 \\
1.0 \\
1.1 \\
1.1 \\
1.1\end{array}$ & $\begin{array}{r}.96 \\
.95 \\
.97 \\
.98 \\
.99 \\
1.0\end{array}$ & $\begin{array}{r}1.0 \\
.98 \\
.97 \\
.99 \\
-0 . \\
-.-\end{array}$ & $\begin{array}{l}4.2 \\
9.1 \\
9.2 \\
9.0 \\
8.7 \\
8.7\end{array}$ & $\begin{array}{l}3.2 \\
3.7 \\
4.3 \\
4.4 \\
4.4 \\
-\end{array}$ & $\begin{array}{c}24 \\
24 \\
24 \\
24 \\
14 \\
4.5\end{array}$ & $\begin{array}{l}4.1 \\
4.1 \\
4.1 \\
4.2 \\
4.0 \\
\end{array}$ & $\begin{array}{l}7.3 \\
9.8 \\
9.9 \\
7.7 \\
5.9 \\
5.9\end{array}$ & $\begin{array}{l}11 \\
11 \\
11 \\
11 \\
11 \\
11\end{array}$ & $\begin{array}{l}5.5 \\
7.5 \\
7.4 \\
7.4 \\
7.4 \\
-\cdots\end{array}$ \\
\hline $\begin{array}{l}\text { TOTAL } \\
\text { MEAN } \\
\text { MAX } \\
\text { MIN } \\
\text { AC-FT }\end{array}$ & $\begin{array}{r}188.00 \\
6.06 \\
10 \\
.62 \\
373\end{array}$ & $\begin{array}{r}127.4 \\
4.25 \\
8.4 \\
1.1 \\
253\end{array}$ & $\begin{array}{r}31.79 \\
1.03 \\
1.1 \\
.99 \\
63\end{array}$ & $\begin{array}{r}31.30 \\
1.01 \\
1.1 \\
.95 \\
62\end{array}$ & $\begin{array}{r}30.52 \\
1.05 \\
1.1 \\
.97 \\
61\end{array}$ & $\begin{array}{r}74.98 \\
2.42 \\
9.2 \\
i 98 \\
149\end{array}$ & $\begin{array}{r}415.5 \\
13.8 \\
29 \\
3.2 \\
82.4\end{array}$ & $\begin{array}{r}536.5 \\
17.3 \\
32 \\
4.2 \\
1060\end{array}$ & $\begin{array}{r}262.8 \\
8.76 \\
20 \\
4.0 \\
521\end{array}$ & $\begin{array}{r}168.1 \\
5.42 \\
9.9 \\
4.0 \\
333\end{array}$ & $\begin{array}{r}466.7 \\
15.1 \\
37 \\
5.8 \\
926\end{array}$ & $\begin{array}{r}230.1 \\
7.67 \\
11 \\
3.7 \\
456\end{array}$ \\
\hline
\end{tabular}

STATISTICS OF MONTHLY MEAN DATA FOR WATER YEARS 1979 - 1996, BY WATER YEAR (WY)

\begin{tabular}{|c|c|c|c|c|c|c|c|c|c|c|c|c|}
\hline $\begin{array}{l}\text { MEAN } \\
\text { MAX } \\
\text { (WY) } \\
\text { MIN } \\
\text { (WY) }\end{array}$ & $\begin{array}{l}7.49 \\
19.5 \\
1989 \\
2.83 \\
1991\end{array}$ & $\begin{array}{l}4.79 \\
11.9 \\
1987 \\
1.30 \\
1980\end{array}$ & $\begin{array}{r}2.65 \\
8.70 \\
1987 \\
1.45 \\
1980\end{array}$ & $\begin{array}{r}2.32 \\
5.29 \\
1992 \\
195 \\
1980\end{array}$ & $\begin{array}{r}3.08 \\
7.68 \\
1995 \\
.45 \\
1980\end{array}$ & $\begin{array}{l}6.04 \\
17.4 \\
1985 \\
1979\end{array}$ & $\begin{array}{l}17.2 \\
42.3 \\
1985 \\
1.60 \\
1.981\end{array}$ & $\begin{array}{l}41.1 \\
85.4 \\
1985 \\
9.89 \\
1981\end{array}$ & $\begin{array}{r}50.6 \\
125 \\
1979 \\
8.76 \\
1996\end{array}$ & $\begin{array}{l}23.4 \\
48.4 \\
1983 \\
5.42 \\
1996\end{array}$ & $\begin{array}{l}17.0 \\
51.9 \\
1983 \\
2.86 \\
1989\end{array}$ & $\begin{array}{l}11.6 \\
45.4 \\
1988 \\
1.47 \\
1994\end{array}$ \\
\hline
\end{tabular}

SUMMARY STATISTICS

ANNUAL TOTAL

ANNUAL MEAN

HIGHEST ANNUAL MEAN

LOWEST ANNUAL MEAN

BIGEIEST DAILY MEAN

LOWEST DAILY MEAN

ANNUAL SEVEN-DAY MINIMUM

INSTANTANEOUS PEAK FLOW

INSTANTANEOUS PEAK STAGE

INSTANTANEOUS LOW FLOW

ANNUAL, RUNOFF (AC-FT)

10. PERCENT EXCEEDS

50 PERCENT EXCEEDS

90 PERCENT EXCEEDS
FOR 1995 CALENDAR YEAR

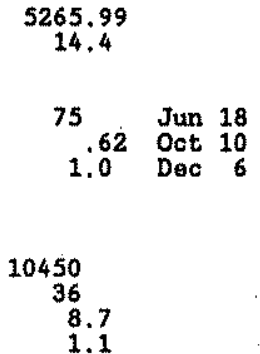

FOR 1996 WATER YEAR

$$
2563.69
$$

$$
7.00
$$

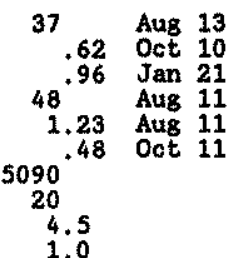

WATER YEARS 1979 - 1996

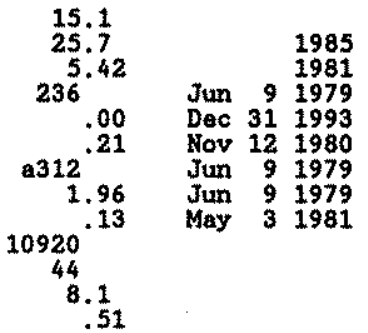

- Estimatoc

a-At site 1,100 ft downstream (maximum release and spi11 computed at Nambe Fa1ls Dam, 250 fts/s, June 9, 1979). 
LOCATION.--Lat $35^{\circ} 52^{\prime 2} 29^{\prime \prime}$, long 106 $06^{\circ} 30^{\prime \prime}$, in SW/SW/ sec.18, T.19 N., R.8 E., Santa Fe County, Eydrologic Unit 13020101 , on San Ildefonso Pueblo Grant, near right bank on downstream end of pier of former railway bridge, 400 ft downstream from bridge on State Highway $502,1.8 \mathrm{mi}$ southwest of San Ildefonso Pueblo, $2.5 \mathrm{mi}$ downstream from Pojoaque River, $6.8 \mathrm{mi}$ west of Pojoaque, and at mile $1,614.2$.

DRAINAGE AREA. - - 14,300 $\mathrm{mi}^{2}$, approxtmately, including $2,940^{\circ 1^{2}}$ in closed basin in San Luis Valley, Co.

WATER-DISCHARGE RECORDS

EERIOD OF RECORD. --February 1895 to December 1905, June 1909 to current year. Monthly discharge only for sone periods, published in WSP 1312. In early reports this record was published as "at Water Tank," as "at Rio

REVISED RECORDS. -WSP 828: Dratnage area. WSP 1512: 1895-99, 1904-6, 1911-12, 1914, 1931(M), 1935, WSP 1712: $1904(M)$. WDR-MM-90: 1989

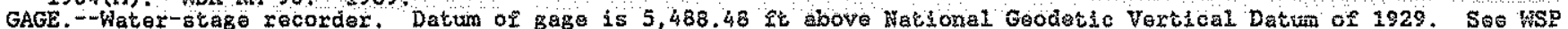
1312,1732 , or 1923 for history of changes prior to June 1,1910

REMARKS. - Water-discharge records good. Considerable regulation by Heron Reservoir (station 08284510 ), El Vado Reservoir (station 08285000) and Abiquiu Reservotr (station 08286900) on Rio Chame, which can contribute a major portion of the total flow. Flow affected by release of transmountain water from Heron Reservoir since May 1971. Diversions upstream from station for irrigation of about 620,000 acres in Colorado and 75,000 acres in New Mexico Gage-height telemeter and U.S. Army Corps of Engineers satellite telemeter at station.

AVERAGE DISCEARGE. - -71 years (water years $1895-1914,1916,1920-70$ ), $1,530 \mathrm{ft} / \mathrm{s}, 1,108,000$ acre-ft/yr. Prior to release of transmountain water.

EXTREMES OUTSIDE PERIOD OF RECORD. - - The 1920 flood is greatest since at least 1884 and probably since 1741 ; information from $W$. B. Yeo's file on floods.

DISCHARGE, CUBIC FEET PER SECOND, WATER YEAR OCTOBER 1995 TO SEPTEMBER 1996

\begin{tabular}{|c|c|c|c|c|c|c|c|c|c|c|c|c|}
\hline DAY & $\propto C_{T}^{T}$ & Nov & DEC & JAN & FEB & MAR & APR & MAY & JUN & JUL. & AUG & SEP \\
\hline $\begin{array}{l}1 \\
2 \\
3 \\
4 \\
5\end{array}$ & $\begin{array}{l}1160 \\
1130 \\
1050 \\
1030 \\
1140\end{array}$ & $\begin{array}{l}1240 \\
1340 \\
1360 \\
1370 \\
1380\end{array}$ & $\begin{array}{r}1010 \\
1000 \\
991 \\
998 \\
1030\end{array}$ & $\begin{array}{l}1110 \\
1130 \\
1080 \\
1080 \\
1140\end{array}$ & $\begin{array}{l}1190 \\
1230 \\
1170 \\
1130 \\
1130\end{array}$ & $\begin{array}{l}1240 \\
1210 \\
1290 \\
1300 \\
1310\end{array}$ & $\begin{array}{l}949 \\
697 \\
699 \\
748 \\
852\end{array}$ & $\begin{array}{r}1050 \\
887 \\
738 \\
670 \\
746\end{array}$ & $\begin{array}{l}1130 \\
1130 \\
1110 \\
1120 \\
1100\end{array}$ & $\begin{array}{l}394 \\
423 \\
393 \\
577 \\
628\end{array}$ & $\begin{array}{r}979 \\
1020 \\
1100 \\
1060 \\
946\end{array}$ & $\begin{array}{r}955 \\
799 \\
796 \\
981 \\
1050\end{array}$ \\
\hline $\begin{array}{r}6 \\
7 \\
8 \\
9 \\
10\end{array}$ & $\begin{array}{l}1220 \\
1140 \\
1090 \\
1060 \\
1030\end{array}$ & $\begin{array}{l}1380 \\
1390 \\
1380 \\
1380 \\
1400\end{array}$ & $\begin{array}{l}1050 \\
1060 \\
1020 \\
1030 \\
1030\end{array}$ & $\begin{array}{l}1130 \\
1110 \\
1120 \\
1130 \\
1130\end{array}$ & $\begin{array}{l}1200 \\
1210 \\
1230 \\
1230 \\
1250\end{array}$ & $\begin{array}{l}1310 \\
1330 \\
1310 \\
1330 \\
1340\end{array}$ & $\begin{array}{l}822 \\
769 \\
766 \\
782 \\
797\end{array}$ & $\begin{array}{r}873 \\
959 \\
1140 \\
1160 \\
1170\end{array}$ & $\begin{array}{l}1130 \\
1210 \\
1230 \\
1220 \\
1280\end{array}$ & $\begin{array}{r}358 \\
420 \\
1010 \\
1100 \\
736\end{array}$ & $\begin{array}{l}871 \\
907 \\
993 \\
940 \\
890\end{array}$ & $\begin{array}{r}1080 \\
962 \\
796 \\
940 \\
. \quad 1010\end{array}$ \\
\hline $\begin{array}{l}11 \\
12 \\
13 \\
14 \\
15\end{array}$ & $\begin{array}{l}1010 \\
1140 \\
1170 \\
1180 \\
1160\end{array}$ & $\begin{array}{l}1400 \\
1400 \\
1400 \\
1400 \\
1400\end{array}$ & $\begin{array}{l}1030 \\
1060 \\
1060 \\
1030 \\
1070\end{array}$ & $\begin{array}{l}1160 \\
1160 \\
1160 \\
1220 \\
1240\end{array}$ & $\begin{array}{l}1270 \\
1280 \\
1310 \\
1300 \\
1300\end{array}$ & $\begin{array}{l}1330 \\
1360 \\
1430 \\
1450 \\
1470\end{array}$ & $\begin{array}{l}806 \\
774 \\
839 \\
825 \\
717\end{array}$ & $\begin{array}{l}1220 \\
1230 \\
1270 \\
1090 \\
1060\end{array}$ & $\begin{array}{l}1400 \\
1360 \\
1300 \\
1360 \\
1290\end{array}$ & $\begin{array}{l}448 \\
666 \\
836 \\
635 \\
870\end{array}$ & $\begin{array}{l}769 \\
690 \\
792 \\
686 \\
935\end{array}$ & $\begin{array}{r}1080 \\
938 \\
830 \\
665 \\
420\end{array}$ \\
\hline $\begin{array}{l}16 \\
17 \\
18 \\
19 \\
20\end{array}$ & $\begin{array}{r}1120 \\
1020 \\
986 \\
1140 \\
1150\end{array}$ & $\begin{array}{l}1410 \\
1410 \\
1430 \\
1430 \\
1150\end{array}$ & $\begin{array}{r}1130 \\
1150 \\
1150 \\
1090 \\
991\end{array}$ & $\begin{array}{l}1240 \\
1300 \\
1310 \\
1250 \\
1220\end{array}$ & $\begin{array}{l}1360 \\
1370 \\
1360 \\
1370 \\
1410\end{array}$ & $\begin{array}{l}1540 \\
1500 \\
1450 \\
1390 \\
1350\end{array}$ & $\begin{array}{r}757 \\
783 \\
813 \\
825 \\
1030\end{array}$ & $\begin{array}{l}1110 \\
1050 \\
1030 \\
1450 \\
1750\end{array}$ & $\begin{array}{l}1270 \\
1190 \\
1140 \\
1100 \\
1060\end{array}$ & $\begin{array}{l}896 \\
661 \\
687 \\
554 \\
561\end{array}$ & $\begin{array}{l}892 \\
897 \\
855 \\
750 \\
946\end{array}$ & $\begin{array}{l}330 \\
496 \\
631 \\
832 \\
619\end{array}$ \\
\hline $\begin{array}{l}21 \\
22 \\
23 \\
24 \\
25\end{array}$ & $\begin{array}{l}1170 \\
1180 \\
1180 \\
1160 \\
1180\end{array}$ & $\begin{array}{l}1070 \\
1060 \\
1040 \\
1030 \\
1030\end{array}$ & $\begin{array}{r}1000 \\
1010 \\
1010 \\
988 \\
923\end{array}$ & $\begin{array}{l}1190 \\
1210 \\
1240 \\
1200 \\
1170\end{array}$ & $\begin{array}{l}1390 \\
1420 \\
1420 \\
1420 \\
1420\end{array}$ & $\begin{array}{l}1310 \\
1310 \\
1310 \\
1310 \\
1310\end{array}$ & $\begin{array}{l}1150 \\
1100 \\
1130 \\
1220 \\
1160\end{array}$ & $\begin{array}{l}1360 \\
1330 \\
1300 \\
1310 \\
1400\end{array}$ & $\begin{array}{r}1030 \\
1070 \\
1070 \\
969 \\
929\end{array}$ & $\begin{array}{r}773 \\
1090 \\
1080 \\
1110 \\
1150\end{array}$ & $\begin{array}{r}926 \\
1110 \\
991 \\
529 \\
519\end{array}$ & $\begin{array}{l}655 \\
777 \\
753 \\
557 \\
530\end{array}$ \\
\hline $\begin{array}{l}26 \\
27 \\
28 \\
29 \\
30 \\
31\end{array}$ & $\begin{array}{l}1140 \\
1140 \\
1160 \\
1140 \\
1160 \\
1190\end{array}$ & $\begin{array}{l}1030 \\
1030 \\
1010 \\
1000 \\
1000\end{array}$ & $\begin{array}{r}984 \\
972 \\
1010 \\
1050 \\
1060 \\
1070\end{array}$ & $\begin{array}{l}1210 \\
1130 \\
1160 \\
1190 \\
1170 \\
1170\end{array}$ & $\begin{array}{r}1400 \\
1340 \\
1310 \\
1290 \\
- \\
-\end{array}$ & $\begin{array}{r}1300 \\
1230 \\
1080 \\
1030 \\
1020 \\
991\end{array}$ & $\begin{array}{l}854 \\
870 \\
877 \\
864 \\
887 \\
-\end{array}$ & $\begin{array}{l}1390 \\
1450 \\
1420 \\
1250 \\
1220 \\
1160\end{array}$ & $\begin{array}{r}1340 \\
983 \\
1430 \\
400 \\
503\end{array}$ & $\begin{array}{r}1250 \\
1260 \\
1170 \\
1170 \\
1150 \\
927\end{array}$ & $\begin{array}{l}785 \\
442 \\
330 \\
700 \\
841 \\
978\end{array}$ & $\begin{array}{l}552 \\
615 \\
632 \\
716 \\
736\end{array}$ \\
\hline $\begin{array}{l}\text { TOTAL } \\
\text { MEAN } \\
\text { MAX } \\
\text { MIN } \\
\text { AC-FT }\end{array}$ & $\begin{array}{r}34926 \\
1127 \\
1220 \\
986 \\
69280\end{array}$ & $\begin{array}{r}37750 \\
1258 \\
1430 \\
1000 \\
74880\end{array}$ & $\begin{array}{r}32057 \\
1034 \\
1150 \\
923 \\
63590\end{array}$ & $\begin{array}{r}36460 \\
1176 \\
1310 \\
1080 \\
72320\end{array}$ & $\begin{array}{r}37710 \\
1300 \\
1420 \\
1130 \\
74800\end{array}$ & $\begin{array}{r}40441 \\
1305 \\
1540 \\
991 \\
80210\end{array}$ & $\begin{array}{r}26162 \\
872 \\
1220 \\
697 \\
51890\end{array}$ & $\begin{array}{r}36243 \\
1169 \\
1750 \\
670 \\
71890\end{array}$ & $\begin{array}{r}33654 \\
1122 \\
1430 \\
400 \\
66750\end{array}$ & $\begin{array}{r}24983 \\
806 \\
2260 \\
358 \\
49550\end{array}$ & $\begin{array}{r}26269 \\
847 \\
1110 \\
330 \\
52100\end{array}$ & $\begin{array}{r}22733 \\
758 \\
1080 \\
330 \\
45090\end{array}$ \\
\hline $\begin{array}{l}\text { STATIS } \\
\text { MEAN } \\
\text { MAAX } \\
\text { (WY }) \\
\text { MIN } \\
\text { (WY) }\end{array}$ & $\begin{array}{r}\text { Cs OF } \\
782 \\
1554 \\
1988 \\
361 \\
1975\end{array}$ & $\begin{array}{c}\text { MONTHLY M } \\
1016 \\
2034 \\
1987 \\
401 \\
1978\end{array}$ & $\begin{array}{r}\text { DATA } \\
971 \\
1959 \\
1976 \\
450 \\
1975\end{array}$ & $\begin{array}{c}\text { FOR WATER } \\
825 \\
1757 \\
1986 \\
436 \\
1977\end{array}$ & $\begin{array}{c}\text { EARS } 1971 \\
957 \\
2641 \\
1987 \\
499 \\
1978\end{array}$ & $\begin{array}{c}-1996, \\
1427 \\
3127 \\
1987 \\
612 \\
1977\end{array}$ & $\begin{array}{c}\text { BY WATER } \\
2400 \\
6412 \\
1985 \\
489 \\
1977\end{array}$ & $\begin{array}{c}\text { YEAR (WY) } \\
3774 \\
8390 \\
1985 \\
433 \\
1972\end{array}$ & $\begin{array}{r}3382 \\
7014 \\
1979 \\
470 \\
1972\end{array}$ & $\begin{array}{r}1620 \\
4548 \\
1995 \\
394 \\
1972\end{array}$ & $\begin{array}{r}943 \\
1612 \\
1973 \\
391 \\
1972\end{array}$ & $\begin{array}{r}845 \\
1547 \\
1982 \\
263 \\
1974\end{array}$ \\
\hline
\end{tabular}

SURMARY STATISTICS ANNUAL TOTAL

ANNUAL, MEAN

BIGHEST ANNUAL MEAN

LOWEST ANNUAL MEAN

HIGHEST DAILY MEAI

LOWEST DAILY MEAN

ANNUAL SEVEN-DAY MINIMUM

INSTANTANEOUS PEAK FLOW

INSTANTANEOUS PEAK STAGE

INSTANTANEOUS LOW FLOW

ANNUAL RUNOFF (AC-FT)

10 PERCENT EXCEEDS

50 PERCENT EXCEEDS

90 PERCENT EXCEEDS

a-Present site and datum.
FOR 1995 CALENDAR YEAR 842369

2308

FOR 1996 WATER YEAR 389388

1064

$\begin{array}{rrr}8650 & \text { Jul } & 6 \\ 702 & \text { Aug } & 3 \\ 816 & \text { Aug } & 2\end{array}$

1671000

5950

973

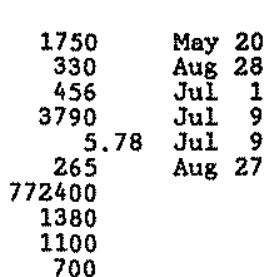

WATER YEARS 1971 - 1996

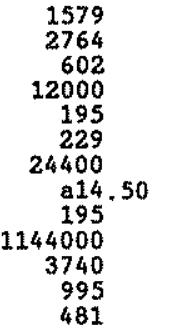

1987

May 111985

Aus 41977

Sep 111971

May 231920

$\begin{array}{lrr}\text { Sep } & 29 & 1904 \\ \text { Aug } & 4 & 1977\end{array}$ 
RIO GRANDE BASIN

08313000 RTO GRANDE AT OTOWI BRIDGE, NEAR SAN ILDEFONSO, NM -- Continued

WATER-QUALITY RECORDS

PERTOD OF RECORD.--Water years 1947 to current year.

PERIOD OF DAILY RECORD.--

SPECIFIC CONDUCTANCE: October 1946 to current year.

SUSPENDED-SEDIMENT DISCEARGE: October 1947 to current year.

EXTREMES FOR PERIOD OF DAILY RECORD.--

SPECIFIC CONDUCTANCE: Maximum daily, 1,310 microsiemens, Aug. 5, 1963; minimum dally, 88 microsiemens, May 12, 1984 .

SEDIMENT CONCENTRATION: Maximum dally mean, 43,500 mg/L, Aug. 21, 1955; mintmum dally mean, $11 \mathrm{mg} / \mathrm{L}$, July 27, 1963 and Fob. 7, 1974.

SEDIMENT LOAD: Maximum daily, 386,000 tons, July 6, 1995; minimum dally, 3 tons, July 27, 1963.

EXTREMES FOR CURRENT YEAR. --

SPECIFIC CONDUCTAKCE: Maximum dally, 450 microsiemens, Aug. 23; minimum datly, 297 microsiemens, Fob. 28. SEDIMENT CONCENTRATION: Maximum daily moan, 22,200 mg/L, Aug. 23; minimum datiy mean, $41 \mathrm{mg} / \mathrm{L}$, Nov. 29 . SEDIMENT LOAD: Maximum dally, 54,400 tons, Aug. 23; minimum daily, 110 tons, Nov. 29.

REMARKS. - Once dally specific conductance values pere determined in the laboratory from datly suspended sediment samples collected by the field observer.

WATER QUALITY DATA, WATER YEAR OCTOBER 1995 TO SEPTEMBER 1996

\begin{tabular}{|c|c|c|c|c|c|c|c|c|c|c|c|c|}
\hline DATE & TIME & $\begin{array}{c}\text { DIS- } \\
\text { CHARGE, } \\
\text { INST. } \\
\text { CUBIC } \\
\text { FEET } \\
\text { PER } \\
\text { SECOND } \\
(00061)\end{array}$ & $\begin{array}{l}\text { SPE- } \\
\text { CIFIC } \\
\text { CON- } \\
\text { DUCT- } \\
\text { ANCE } \\
\text { (US/CM) } \\
(00095)\end{array}$ & $\begin{array}{c}\text { PH } \\
\text { WATER } \\
\text { WHOLE } \\
\text { FIELD } \\
\text { (STAND- } \\
\text { ARD } \\
\text { UNITS) } \\
\text { (00400) }\end{array}$ & $\begin{array}{c}\text { TEMPER- } \\
\text { ATURE } \\
\text { AIR } \\
\text { (DEG C) } \\
(00020)\end{array}$ & $\begin{array}{c}\text { TEMPER- } \\
\text { ATURE } \\
\text { WATER } \\
\text { (DEG C) } \\
(00010)\end{array}$ & $\begin{array}{l}\text { TUR- } \\
\text { BID- } \\
\text { ITY } \\
\text { (NTU) } \\
(00076)\end{array}$ & $\begin{array}{l}\text { BARO- } \\
\text { METRIC } \\
\text { PRES- } \\
\text { SURE } \\
\text { (MM } \\
\text { OF } \\
\text { BG) } \\
(00025)\end{array}$ & $\begin{array}{c}\text { OXYGEN, } \\
\text { DIS- } \\
\text { SOLVED } \\
\text { (ME/L) } \\
(00300)\end{array}$ & $\begin{array}{c}\text { OXYGEN, } \\
\text { DIS- } \\
\text { SOLVED } \\
\text { (PER- } \\
\text { CENT } \\
\text { SATUR- } \\
\text { ATION) } \\
\text { (00301) }\end{array}$ & $\begin{array}{l}\text { OXYGEN } \\
\text { DEMAND, } \\
\text { CHEM- } \\
\text { ICAI } \\
\text { (HIGH } \\
\text { LEVEL) } \\
\text { (MG/L) } \\
(00340)\end{array}$ & $\begin{array}{l}\text { COLI- } \\
\text { FORM } \\
\text { FECAL, } \\
0.7 \\
\text { UM-MF } \\
\text { (COLS.' } \\
\text { 100 ML) } \\
\text { (31625) }\end{array}$ \\
\hline $\begin{array}{l}\text { OV } 1995 \\
27\end{array}$ & 1315 & 1040 & 362 & 8.2 & 9.0 & 7.0 & 7.0 & 621 & 10.9 & 111 & $<10$ & $<11$ \\
\hline $29 . \ldots$ & 1200 & 1180 & 319 & 8.3 & 4.5 & 1.5 & 3.8 & 625 & 11.7 & 102 & 10 & $<2$ \\
\hline $10 \ldots$ & 0845 & 750 & 347 & 8.2 & 6.5 & 12.0 & 24 & 625 & 8.2 & 93 & 15 & 73 \\
\hline $17 \ldots$ & 1030 & 688 & 338 & 7.9 & 24.0 & 21.0 & $\therefore$ & 625 & 7.5 & 103 & -- & -- \\
\hline 28 & 0845 & 359 & 384 & 8.2 & 16.5 & 19.0 & 180 & 630 & 7.4 & 97 & 33 & 2100 \\
\hline TIE & $\begin{array}{c}\text { STREP- } \\
\text { TOCOCCI } \\
\text { FECAL, } \\
\text { KF AGAR } \\
\text { (COLS, } \\
\text { PER } \\
100 \text { ML) } \\
(31673)\end{array}$ & $\begin{array}{l}\text { HARD- } \\
\text { NESS } \\
\text { TOTAL } \\
\text { (MG/L } \\
\text { AS } \\
\text { CACO3) } \\
(00900)\end{array}$ & $\begin{array}{l}\text { HARD- } \\
\text { NESS } \\
\text { NONCARB } \\
\text { DISSOLV } \\
\text { FLD. AS } \\
\text { CACO3 } \\
\text { (MG/L) } \\
(00904)\end{array}$ & $\begin{array}{c}\text { CALCIUM } \\
\text { DIS- } \\
\text { SOLVED } \\
\text { (MG/L } \\
\text { AS CA) } \\
(00915)\end{array}$ & $\begin{array}{c}\text { MAGNE- } \\
\text { SIUM, } \\
\text { DIS- } \\
\text { SOLVED } \\
\text { (MG/L } \\
\text { AS MG) } \\
(00925)\end{array}$ & $\begin{array}{c}\text { SODTUM, } \\
\text { DIS- } \\
\text { SOLVED } \\
\text { (MG/L } \\
\text { AS NA) } \\
(00930)\end{array}$ & $\begin{array}{c}\text { SODIUM } \\
\text { AD- } \\
\text { SORP- } \\
\text { TION } \\
\text { RATIO } \\
(00931)\end{array}$ & $\begin{array}{c}\text { POTAS- } \\
\text { SIUM, } \\
\text { DIS- } \\
\text { SOLVED } \\
(M G / L \\
\text { AS K) } \\
(00935)\end{array}$ & $\begin{array}{l}\text { BICAR- } \\
\text { BONATE } \\
\text { WATER } \\
\text { DIS IT } \\
\text { FIELD } \\
\text { MG/L AS } \\
\text { HCOS } \\
(00453)\end{array}$ & $\begin{array}{c}\text { CAR- } \\
\text { BONATE } \\
\text { WATER } \\
\text { DIS IT } \\
\text { FIELD } \\
\text { MG/L AS } \\
\text { CO3 } \\
(00452)\end{array}$ & $\begin{array}{c}\text { ALKA- } \\
\text { LINITY } \\
\text { WAT DIS } \\
\text { TOT IT } \\
\text { FIELD } \\
\text { MG/L AS } \\
\text { CACOB } \\
(39086)\end{array}$ & $\begin{array}{c}\text { ALKA- } \\
\text { LINITY } \\
\text { LAB } \\
\text { (MG/L } \\
\text { AS } \\
\text { CACO3) } \\
(90410)\end{array}$ \\
\hline
\end{tabular}

Nov 1995

\begin{tabular}{|c|c|c|c|c|c|}
\hline 27 & 54 & 130 & 17 & 38 & 7.7 \\
\hline $\begin{array}{c}29 \\
\mathrm{APR}\end{array}$ & $<16$ & 110 & 12 & 34 & 6.6 \\
\hline & 170 & 120 & 15 & 38 & 7.2 \\
\hline AUG 17. & & 120 & 22 & 37 & 7.1 \\
\hline 28 & 2600 & 140 & 12 & 43 & 71 \\
\hline
\end{tabular}

$\begin{array}{llllll}0.9 & 2.7 & 134 & 0 & 110 & 122 \\ 0.8 & 2.9 & 122 & 0 & 100 & 110 \\ 0.9 & 2.8 & 133 & 0 & 109 & 115 \\ 0.7 & 2.3 & 122 & 0 & 100 & 127 \\ 0.9 & 3.3 & 136 & 8 & 123 & 126\end{array}$




\section{RIO GRANDE BASIN}

08313000 RIO GRANDE AT OTOWI BRIDGE, NEAR SAN ILDEFONSO, NM -- Continued

WATER-QUALITY RECORDS

WATER QUALITY DATA, WATER YEAR OCTOBER 1995 TO SEPTEMBER 1996

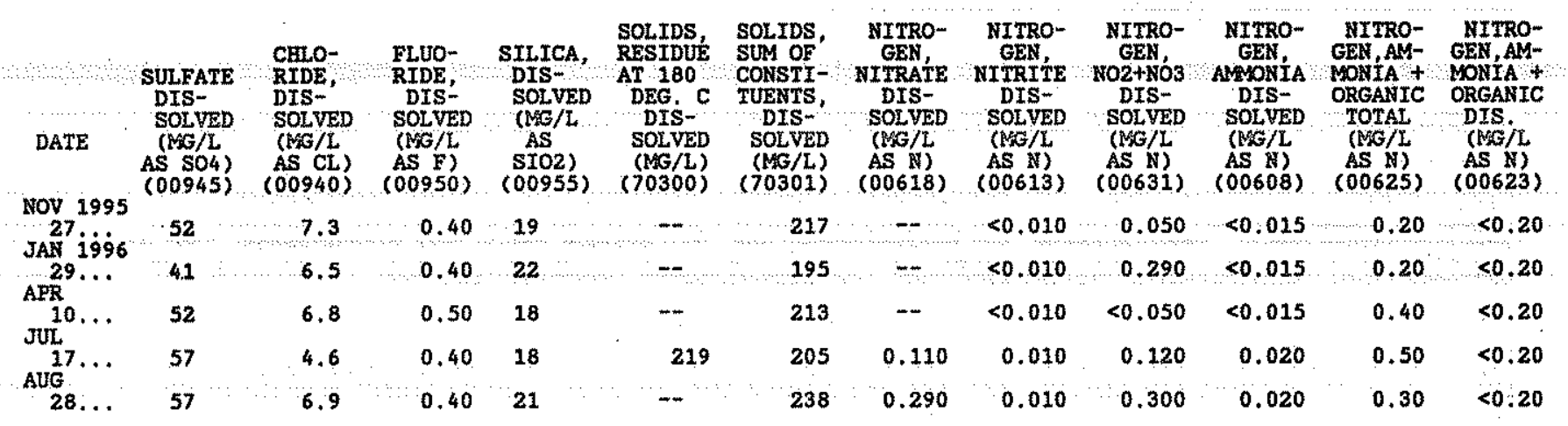

\begin{tabular}{|c|c|c|c|c|c|c|c|c|c|c|c|}
\hline DATE & $\begin{array}{c}\text { PHOS- } \\
\text { PHORUS } \\
\text { TOTAL } \\
\text { (MG/L } \\
\text { AS P) } \\
(00665)\end{array}$ & $\begin{array}{c}\text { FHOS- } \\
\text { PHORUS } \\
\text { DTS- } \\
\text { SOLVED } \\
\text { (NG/I } \\
\text { AS P) } \\
(00666)\end{array}$ & $\begin{array}{l}\text { PHOS- } \\
\text { PHORUS } \\
\text { ORTHO, } \\
\text { DIS- } \\
\text { SOLVED } \\
\text { (MELL } \\
\text { AS P) } \\
(00671)\end{array}$ & $\begin{array}{l}\text { CARBON, } \\
\text { ORGANIC } \\
\text { TOTAL } \\
\text { (MG/L } \\
\text { AS C) } \\
(00680)\end{array}$ & $\begin{array}{l}\text { CARBON, } \\
\text { ORGANIC } \\
\text { DIS- } \\
\text { SOLVED } \\
\text { (MG/L } \\
\text { AS C) } \\
(00681)\end{array}$ & $\begin{array}{l}\text { CYANTDE } \\
\text { TOTAL, } \\
\text { (ME/L } \\
\text { AS CN) } \\
(00720)\end{array}$ & $\begin{array}{l}\text { ALUM- } \\
\text { INUM, } \\
\text { DIS- } \\
\text { SOLVED } \\
\text { (UG/L } \\
\text { AS AL) } \\
(01106)\end{array}$ & $\begin{array}{l}\text { ANTI- } \\
\text { MONY, } \\
\text { DIS- } \\
\text { SOLVED } \\
\text { (UG/L } \\
\text { AS SB) } \\
\text { (01095) }\end{array}$ & $\begin{array}{l}\text { ARSENIC } \\
\text { DIS- } \\
\text { SOLVED } \\
\text { (UG/L } \\
\text { AS AS) } \\
(01000)\end{array}$ & $\begin{array}{l}\text { BARIUM, } \\
\text { DIS- } \\
\text { SOLVED } \\
\text { (UG/L } \\
\text { AS BA) } \\
(01005)\end{array}$ & $\begin{array}{l}\text { BERYL- } \\
\text { LIUM, } \\
\text { DIS- } \\
\text { SOLVED } \\
\text { (UG/L } \\
\text { AS BE) } \\
(01010)\end{array}$ \\
\hline V 1995 & 0.020 & $<0.010$ & $<0.010$ & 3.3 & -- & $<0.010$ & 11 & $<1.0$ & 2 & 54 & $<1.0$ \\
\hline $29 \ldots$ & 0.030 & $<0.010$ & 0.020 & 2.7 & $-\cdots$ & $<0.010$ & -- & $\rightarrow$ & $-m$ & - & -- \\
\hline $10 \ldots$ & 0.080 & 0.020 & $<0.010$ & 5.4 & - & $<0.010$ & -- & -- & -- & -- & -- \\
\hline $17 \ldots$ & 0.110 & 0.020 & 0.030 & 7.1 & 3.2 & - & -- & - & $-\rightarrow$ & -- & -- \\
\hline $20 . \cdots$ & 0.060 & 0.030 & 0.050 & 9.6 & - & $<0.010$ & 6.0 & $<1.0$ & 2 & 106 & $<1.0$ \\
\hline
\end{tabular}

DATE

NoV 1995

JAN 1996

$29 .$.

AER 10.

JUL $10 .$.

AUG 17.

$28 .$.

$\begin{array}{cc}\text { BORON, } & \text { CADMIUM } \\ \text { DIS- } & \text { DIS- } \\ \text { SOLVED } & \text { SOLVED } \\ \text { (UG/L } & \text { (UG/L } \\ \text { AS B) } & \text { AS CD) }\end{array}$

$A S B)$
$(01020) \quad\left(\begin{array}{c}A S \\ 0\end{array}\right)$

50

$<1.0$

30

50

$-$

51

--

$-$

$--$

$<1.0$

CERO- COBALT, COPPER,

MIUM, COBALT, COPPER

SOLVED SOLVED

AS CR) AS CO)

$(01030)(01035)$

SOLVED

(UG/L

AS CU)

$<1.0<1.0$

2.0

$-$

--

$--$$$
--
$$$$
--
$$$$
--
$$

$<1.0$

$<1.0$

2.0

NITRO-
GEN,
SILVER,
DIS-

SELE-

NITRO

4 PHOS-

TOTAL + ORG.

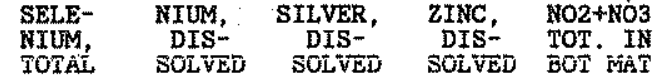

TUGA

DATE

AS SE)

SOLVED

AS SE)

SOL VED

(UG/L (UG/L

(UG $\mathrm{ZW}$

(MG/KG

AS N)
$(00633)$

IN BOT, TOT IN

BOT

(MG/KG $\quad(M G / K G$

(MS

AS N)
$(00611) \quad(00626)$

8.0

\begin{abstract}
$4.0 \quad 32$
\end{abstract}
460

JAN $279 \dot{9} 6$

JAN 29.

APR 10.

JuL 10.

AUG

28 ... 
RIO GRANDE BASTN

08313000 RIO GRANDE AT OTOWI BRTDGE, NEAR SAN ILDEFONSO, NM -- Continued

WATER-QUALITY RECORDS

WATER QUALITY DATA, WATER YEAR OCTOBER 1995 TO SEPTEMBER 1996

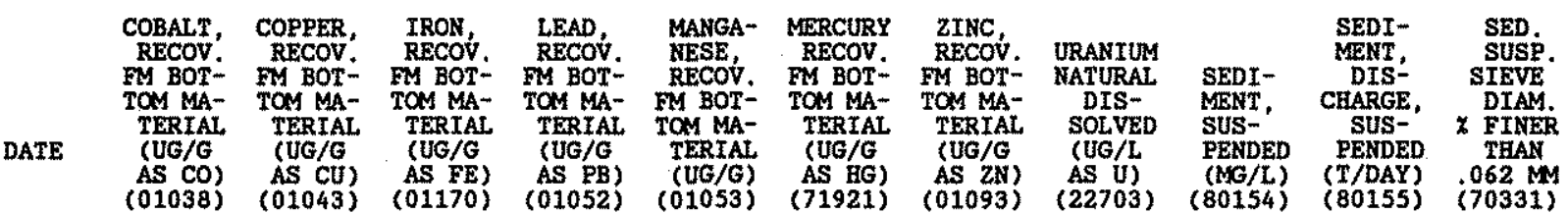

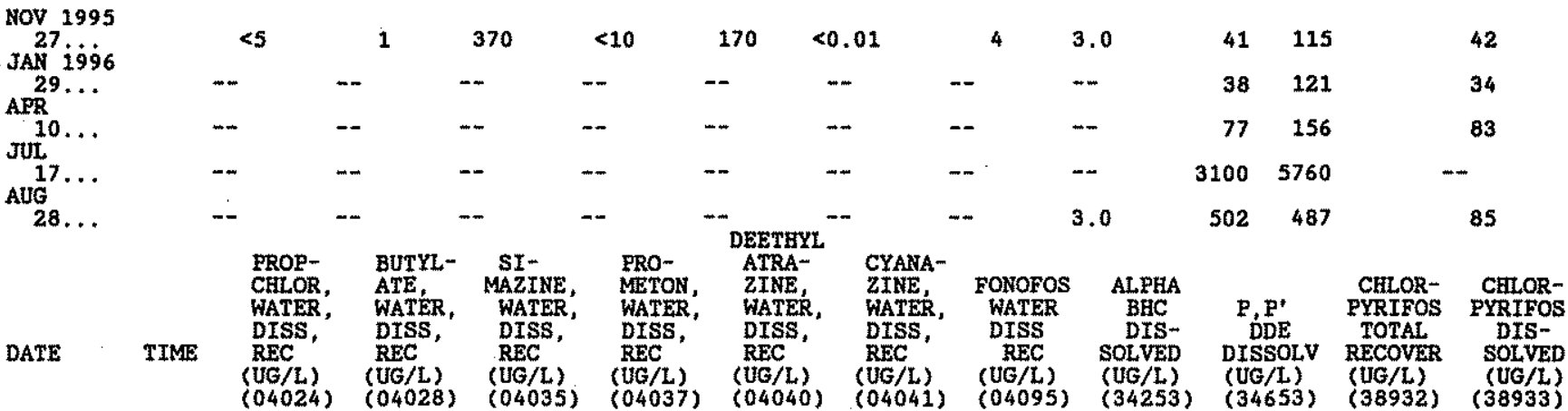

\begin{tabular}{|c|c|c|c|c|c|c|c|c|c|c|c|c|}
\hline ov 1995 & 1315 & - & $-\cdots$ & -- & -- & -- & -- & -- & - & - & -- & -- \\
\hline $29 \ldots$ & 1200 & -- & - & -- & -- & -- & -- & -- & - & -- & - & -- \\
\hline . & 0845 & -- & $-\cdots$ & -- & - & -- & - & -- & - & -- & $-\sim$ & - \\
\hline $17 \ldots$ & 1030 & $<0.007$ & $<0.002$ & $<0.005$ & $<0.018$ & $<0.002$ & $<0.004$ & $<0.003$ & $<0.002$ & $<0.006$ & -- & $<0.004$ \\
\hline & 0845 & - & - & - & $-\infty$ & -- & -- & -- & $-\cdots$ & -- & $<0.010$ & $-\cdots$ \\
\hline
\end{tabular}

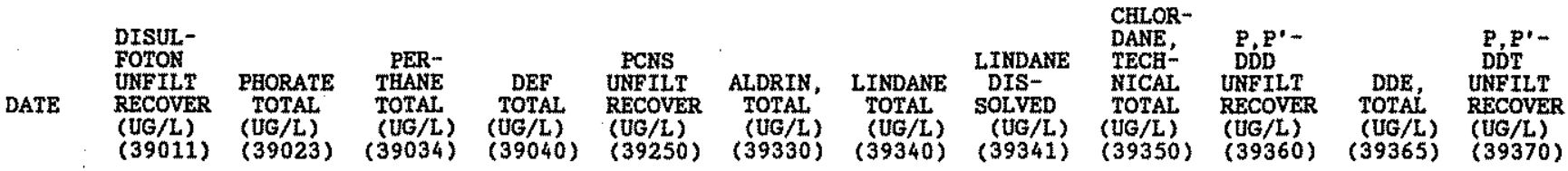

Nov 1995

JAN $27 \dot{9} \dot{9}$

$29 .$.

APR
$10 .$.
JUL .
$17 .$.

$28 \ldots<0.010<0.010<0.100<0.010<0.100<0.010<0.010$

$<0.010$

$<0.010$

$<0.010$

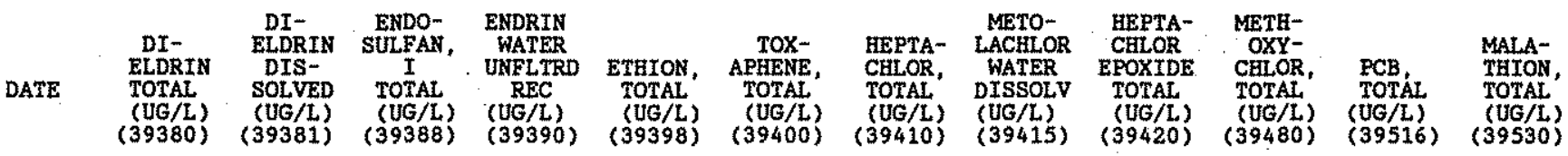

Nov 1995

$\begin{aligned} & \text { NOV } 1995 \\ & 27 \\ & \text { JAN } 1996\end{aligned}$
$\quad-$


RTO GRANDE BASIN

08313000 RIO GRANDE AT OTOWI BRIDGE, NEAR SAN ILDEFONSO, MM - Cont Inued

WATER-QUALITY RECORDS

WATER QUALITY DATA, WATER YEAR OCTOBER 1995 TO SEPTEABBER 1996

\begin{tabular}{|c|c|c|c|c|c|c|c|c|c|c|c|}
\hline DATE & $\begin{array}{l}\text { MALA- } \\
\text { THIOA, } \\
\text { DIS- } \\
\text { SOLVED } \\
\text { (UG/L) } \\
\text { (39532) }\end{array}$ & $\begin{array}{l}\text { PARA- } \\
\text { TEION, } \\
\text { TOTAL } \\
\text { (UG/L) } \\
\text { (39540) }\end{array}$ & $\begin{array}{c}\text { PARA- } \\
\text { TEION, } \\
\text { DIS- } \\
\text { SOLVED } \\
\text { (UG/L) } \\
(39542)\end{array}$ & $\begin{array}{c}\text { DI- } \\
\text { AZINOR, } \\
\text { TOTAL } \\
\text { (UG/L) } \\
(39570)\end{array}$ & $\begin{array}{c}\text { DI- } \\
\text { AZINON, } \\
\text { DIS } \\
\text { SOLVED } \\
\text { (UG/L) } \\
(39572)\end{array}$ & $\begin{array}{l}\text { METHYL } \\
\text { PARA- } \\
\text { TEIOD, } \\
\text { TOTAL, } \\
\text { (UG/L) } \\
(39600)\end{array}$ & $\begin{array}{l}\text { ATRA- } \\
\text { ZTNE, } \\
\text { WATER, } \\
\text { DISS, } \\
\text { REC } \\
\text { (UG/L) } \\
(39632)\end{array}$ & $\begin{array}{c}2,4-D, \\
\text { TOTAL, } \\
\text { (UG/L) } \\
(39730)\end{array}$ & $\begin{array}{c}2,4,5-T \\
\text { TOTAL } \\
(U G / L) \\
(39740)\end{array}$ & $\begin{array}{c}\text { NTREA, } \\
\text { TOTAL } \\
\text { (UG/L) } \\
(39755)\end{array}$ & $\begin{array}{c}\text { SILVEX, } \\
\text { TOTAL } \\
\text { (UG/L) } \\
(39760)\end{array}$ \\
\hline $\begin{array}{c}\text { Nov } 1995 \\
27 \ldots\end{array}$ & $\cdots$ & - & -- & - & $-m$ & - & $-\infty$ & -- & -- & -- & - \\
\hline JAN 1996 & & & & & & & & & & & \\
\hline${ }_{\mathrm{APR}}^{29} \cdot \cdots$ & - & -- & -- & -- & -- & $-\infty$ & -- & -- & -- & -- & -- \\
\hline ЛL. & -- & - & -- & - & - & $-m$ & - & $<0.010$ & $<0.010$ & -- & $<0.010$ \\
\hline AUG 17 & $<0.005$ & - & $<0.004$ & $-\cdots$ & $<0.002$ & $-\cdots$ & $<0.001$ & -- & - & $\cdots-m$ & $\because \quad=-$ \\
\hline $28 \ldots$ & - & $<0.010$ & -- & $<0.010$ & -- & $<0.010$ & -- & -- & -- & $<0.010$ & -- \\
\hline DATE & $\begin{array}{c}\text { TOTAL } \\
\text { TRI- } \\
\text { THION } \\
\text { (UG/L) } \\
(39786)\end{array}$ & $\begin{array}{l}\text { ALA } \\
\text { CHLOR, } \\
\text { WATER, } \\
\text { DISS, } \\
\text { REC , } \\
\text { (UG/L) } \\
\text { (46342) }\end{array}$ & $\begin{array}{l}\text { ACETO } \\
\text { CHLOR, } \\
\text { WATER } \\
\text { FLTRD } \\
\text { REC } \\
(U G / L) \\
(49260)\end{array}$ & $\begin{array}{l}2, \quad 4-\mathrm{DP} \\
\text { TOTAL } \\
(\mathrm{UG} / \mathrm{L}) \\
(82183)\end{array}$ & $\begin{array}{l}\text { FONOFOS } \\
\text { (DY- } \\
\text { FONATE) } \\
\text { WATER } \\
\text { WHOLE } \\
\text { TOT.REC } \\
\text { (UG/L) } \\
\text { (B2614) }\end{array}$ & $\begin{array}{l}\text { METRI- } \\
\text { BUZIN } \\
\text { SENCOR } \\
\text { WATER } \\
\text { DISSOLV } \\
(U G / L) \\
(82630)\end{array}$ & $\begin{array}{c}2,6-\mathrm{DI}- \\
\text { ETHYL } \\
\text { ANILINE } \\
\text { WAT FLT } \\
0.7 \mathrm{U} \\
\text { GF, REC } \\
(\mathrm{UG} / \mathrm{L}) \\
(82660)\end{array}$ & $\begin{array}{c}\text { TRI- } \\
\text { FLUR- } \\
\text { ALIN } \\
\text { WAT FLT } \\
0.7 \text { U } \\
\text { GF, REC } \\
\text { (UG /L) } \\
(82661)\end{array}$ & $\begin{array}{c}\text { ETHAL- } \\
\text { FLUR- } \\
\text { ALTN } \\
\text { WAT FLT } \\
0.7 \mathrm{U} \\
\text { GF, REC } \\
(\mathrm{UG} / \mathrm{L}) \\
(82663)\end{array}$ & $\begin{array}{c}\text { PHORATE } \\
\text { WATER } \\
\text { FLTRD } \\
0.7 \mathrm{U} \\
\text { GF, REC } \\
(\mathrm{UG} / \mathrm{L}) \\
(82664)\end{array}$ & $\begin{array}{c}\text { TER- } \\
\text { BACTL } \\
\text { WATER } \\
\text { FLTRD } \\
0.7 \text { U } \\
\text { GF, REC } \\
\text { (UG/L) } \\
\text { (82665) }\end{array}$ \\
\hline $\begin{array}{l}\text { NOV } 1995 \\
27 \\
\text { JAN } 1996\end{array}$ & -- & $-m$ & -- & -- & $-m$ & -- & $-m$ & $\rightarrow$ & -- & -- & -- \\
\hline $\begin{array}{l}29 \\
\text { APR }\end{array}$ & - & -- & -- & -- & -- & -- & -- & -- & 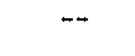 & - & $m$ \\
\hline $\begin{array}{l}10 \ldots \\
\text { JuL }\end{array}$ & -- & - & $\cdots$ & $<0.010$ & - & $-\infty$ & -- & -- & -- & $-m$ & - \\
\hline AUG $17 \ldots$ & - & $<0.002$ & $<0.002$ & - & -- & $<0.004$ & $<0.003$ & $<0.002$ & $<0.004$ & $<0.002$ & $<0.007$ \\
\hline $28 \ldots$ & $<0,010$ & - & -- & - & $<0.010$ & -- & -- & -- & -- & $m$ & $\sim$ \\
\hline DATE & $\begin{array}{l}\text { LIN- } \\
\text { URON } \\
\text { WATER } \\
\text { FLTRD } \\
0.7 \mathrm{U} \\
\text { GF, REC } \\
\text { (UG/L) } \\
\text { (82666) }\end{array}$ & $\begin{array}{c}\text { METEYL } \\
\text { PARA- } \\
\text { THION } \\
\text { WAT FLT } \\
0.7 \text { U } \\
\text { GF, REC } \\
\text { (UG'/L) } \\
\text { (82667) }\end{array}$ & $\begin{array}{l}\text { EPTC } \\
\text { WATER } \\
\text { FLTRD } \\
0.7 \text { U } \\
\text { GE, REC } \\
(\mathrm{UG} / \mathrm{L}) \\
(82668)\end{array}$ & $\begin{array}{l}\text { PEB- } \\
\text { ULATE } \\
\text { WATER } \\
\text { FILTRD } \\
0.7 \text { U } \\
\text { GF, REC } \\
\text { (UG/L) } \\
(82669)\end{array}$ & $\begin{array}{l}\text { TEBU- } \\
\text { THIURON } \\
\text { WATER } \\
\text { FLTRD } \\
0.7 \mathrm{U} \\
\text { GF, REC } \\
\text { (UG/L) } \\
(82670)\end{array}$ & $\begin{array}{l}\text { MOL- } \\
\text { INATE } \\
\text { WATER } \\
\text { FLTRD } \\
0.7 \mathrm{U} \\
\text { GF, REC } \\
\text { (UG/L) } \\
(82671)\end{array}$ & $\begin{array}{l}\text { ETHO- } \\
\text { PROP } \\
\text { WATER } \\
\text { FLTRD } \\
0.7 \mathrm{U} \\
\text { GF, REC } \\
\text { (UG/L) } \\
(82672)\end{array}$ & $\begin{array}{c}\text { BEN- } \\
\text { FLUR- } \\
\text { ALIN } \\
\text { WAT FLD } \\
0.7 \text { U } \\
\text { GF, REC } \\
\text { (UG/L) } \\
(82673)\end{array}$ & $\begin{array}{l}\text { CARBO- } \\
\text { FURAN } \\
\text { WATER } \\
\text { FLTRD } \\
0.7 \mathrm{U} \\
\text { GF, REC } \\
(\mathrm{UG} / \mathrm{L}) \\
(82674)\end{array}$ & $\begin{array}{l}\text { TER- } \\
\text { BUFOS } \\
\text { WATER } \\
\text { FLTRD } \\
0.7 \mathrm{U} \\
\text { GF, REC } \\
(\mathrm{UG} / \mathrm{L}) \\
(\mathrm{B2} 675)\end{array}$ & $\begin{array}{c}\text { FRON- } \\
\text { AMIDE } \\
\text { WATER } \\
\text { BLTRD } \\
0.7 \mathrm{U} \\
\text { GF, REC } \\
(\mathrm{UG} / \mathrm{L}) \\
(82676)\end{array}$ \\
\hline $\begin{array}{l}\text { NOV } 1995 \\
27 \\
\text { JAN } i 996\end{array}$ & -- & -- & -- & $\cdots$ & -- & -- & -- & -- & -- & -- & -- \\
\hline $29 .$. & - & - & -- & -- & $-m$ & $m-$ & $-\infty$ & $-\infty$ & -- & -- & -- \\
\hline $\operatorname{JUL}^{10 \ldots}$ & $\cdots$ & -- & -- & -- & -- & -- & -- & - & $-\infty$ & $-\rightarrow$ & - \\
\hline${ }_{A U G}^{17} \ldots$ & $<0.002$ & $<0.006$ & $<0.002$ & $<0.004$ & $<0.010$ & $<0,004$ & $<0.003$ & $<0.002$ & $<0.003$ & $<0.013$ & $<0.003$ \\
\hline $28 \ldots$ & -- & - & -- & -- & -- & $-\cdots$ & $-m$ & $m$ & -- & -- & -- \\
\hline DATE & $\begin{array}{l}\text { DISUL- } \\
\text { FOTOH } \\
\text { WATER } \\
\text { FLTRD } \\
0.7 \text { U } \\
\text { GE, REC } \\
(U G / L) \\
(82677)\end{array}$ & $\begin{array}{l}\text { TRIAL- } \\
\text { LATE } \\
\text { WATER } \\
\text { FLTRD } \\
0.7 \mathrm{U} \\
\text { GE, REC } \\
\text { (UG/L) } \\
(82678)\end{array}$ & $\begin{array}{c}\text { PRO- } \\
\text { PANIL } \\
\text { WATER } \\
\text { FLTRD } \\
0.7 \text { U } \\
\text { GF, REC } \\
(U G / L) \\
(82679)\end{array}$ & $\begin{array}{c}\text { CAR- } \\
\text { BARYL } \\
\text { WATER } \\
\text { FLWRD } \\
0.7 \mathrm{U} \\
\text { GE; REC } \\
(\mathrm{UG} / \mathrm{L}) \\
(82680)\end{array}$ & $\begin{array}{c}\text { THIO- } \\
\text { BENCARB } \\
\text { WATER } \\
\text { FLTRD } \\
0.7 \text { U } \\
\text { GF, REC } \\
\text { (UG/L) } \\
\text { (82681) }\end{array}$ & $\begin{array}{l}\text { DCPA } \\
\text { WATER } \\
\text { FLTRD } \\
0.7 \mathrm{U} \\
\text { GE, REC } \\
(\mathrm{UG} / \mathrm{L}) \\
(82682)\end{array}$ & $\begin{array}{c}\text { PENDI- } \\
\text { METH- } \\
\text { ALIN } \\
\text { WAT FLT } \\
0.7 \mathrm{U} \\
\text { GF, REC } \\
\text { (UG/L) } \\
(82683)\end{array}$ & $\begin{array}{l}\text { NAPROP- } \\
\text { AMIDE } \\
\text { WATER } \\
\text { FLTRD } \\
0.7 \text { U } \\
\text { GF, REC } \\
(\mathrm{UG} / \mathrm{L}) \\
(82684)\end{array}$ & $\begin{array}{c}\text { PRO- } \\
\text { PARGITE } \\
\text { WATER } \\
\text { FLTRD } \\
0.7 \mathrm{U} \\
\text { GE, REC } \\
(\mathrm{UG} / \mathrm{L}) \\
(82685)\end{array}$ & $\begin{array}{c}\text { METHYL } \\
\text { AZIN- } \\
\text { RHOS } \\
\text { WAT FLT } \\
0.7 \mathrm{U} \\
\text { GE, REC } \\
(\mathrm{UE} / \mathrm{L}) \\
(82686)\end{array}$ & $\begin{array}{c}\text { PER- } \\
\text { METRRIN } \\
\text { CIS } \\
\text { WAT ELT } \\
0.7 \mathrm{U} \\
\text { GE, REC } \\
\text { (UE/L) } \\
(82687)\end{array}$ \\
\hline $\begin{array}{l}\text { NOV } 1995 \\
270996 \\
\text { JAN } 1996\end{array}$ & - & - & -- & -- & $\ldots$ & $-\infty$ & $-\infty$ & -- & -- & -- & -- \\
\hline $29 \cdots$ & - & - & -- & $-\cdots$ & - & $\cdots$ & - & - & -- & -- & -- \\
\hline JuL $10 \ldots$ & $m$ & $m$ & -- & $-m$ & $-\cdots$ & $\cdots$ & -- & -- & -- & -- & -- \\
\hline AUG $^{17 \ldots}$ & $<0.017$ & $<0.001$ & $<0,004$ & $<0.003$ & $<0.002$ & $<0.002$ & $<0.004$ & $<0.003$ & $<0.013$ & $<0.001$ & $<0.005$ \\
\hline $28 \ldots$ & -- & -- & -- & -- & -- & - & - & -- & $-m$ & $-\cdots$ & $\cdots$ \\
\hline
\end{tabular}


RTO GRANDE BASIN

08313000 RIO GRANDE AT OTOWI BRIDGE, NM -- Continued

WATER-QUAL.ITY RECORDS

SPECTFIC CONDUCTANCE, US/CM 25 DEGREES CENTIGRADE, WATER YEAR OCTOBER 1995 TO SEPTEMBER 1996 DAILY INSTANTANEOUS VALUES

\begin{tabular}{|c|c|c|c|c|c|c|c|c|c|c|c|c|}
\hline DAY & $\infty \mathrm{CT}$ & nov & DEC & JAN & FEB & MAR & APR & MAY & JUN & JuL & AUG & SEP \\
\hline $\begin{array}{l}1 \\
2 \\
3 \\
4 \\
5\end{array}$ & $\begin{array}{l}m \\
m-m \\
-m \\
-m\end{array}$ & $\begin{array}{l}339 \\
330 \\
337 \\
340 \\
338\end{array}$ & $\begin{array}{l}362 \\
365 \\
353 \\
350 \\
352\end{array}$ & $\begin{array}{l}342 \\
331 \\
326 \\
\end{array}$ & $\begin{array}{l}324 \\
317 \\
314 \\
309 \\
312\end{array}$ & $\begin{array}{l}312 \\
313 \\
314 \\
316 \\
324\end{array}$ & $\begin{array}{l}361 \\
390 \\
404 \\
380 \\
365\end{array}$ & $\begin{array}{l}420 \\
358 \\
340 \\
336 \\
338\end{array}$ & $\begin{array}{l}349 \\
342 \\
340 \\
346 \\
343\end{array}$ & $\begin{array}{l}\cdots \\
=- \\
-\cdots\end{array}$ & 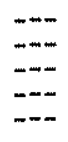 & $\begin{array}{l}359 \\
364 \\
364 \\
360 \\
359\end{array}$ \\
\hline $\begin{array}{r}6 \\
7 \\
8 \\
9 \\
10\end{array}$ & $\begin{array}{l}m \\
-\cdots \\
-\cdots \\
-\cdots\end{array}$ & $\begin{array}{l}340 \\
336 \\
336 \\
339 \\
341\end{array}$ & $\begin{array}{l}356 \\
358 \\
357 \\
349 \\
345\end{array}$ & $\begin{array}{l}\cdots \\
328 \\
324 \\
327\end{array}$ & $\begin{array}{l}313 \\
310 \\
320 \\
314 \\
309\end{array}$ & $\begin{array}{l}322 \\
319 \\
316 \\
318 \\
320\end{array}$ & $\begin{array}{l}366 \\
378 \\
372 \\
362 \\
358\end{array}$ & $\begin{array}{l}341 \\
347 \\
343 \\
346 \\
345\end{array}$ & $\begin{array}{l}336 \\
333 \\
334 \\
329 \\
328\end{array}$ & $\begin{array}{l}-m \\
-m \\
-m\end{array}$ & $\begin{array}{l}355 \\
348 \\
351 \\
353 \\
348\end{array}$ & $\begin{array}{l}359 \\
362 \\
366 \\
361 \\
360\end{array}$ \\
\hline $\begin{array}{l}11 \\
12 \\
13 \\
14 \\
15\end{array}$ & $\begin{array}{l}325 \\
320 \\
322 \\
322 \\
324\end{array}$ & $\begin{array}{l}341 \\
343 \\
340 \\
336 \\
330\end{array}$ & $\begin{array}{l}347 \\
345 \\
344 \\
345 \\
343\end{array}$ & $\begin{array}{l}327 \\
321 \\
322 \\
320 \\
317\end{array}$ & $\begin{array}{l}309 \\
306 \\
303 \\
306 \\
307\end{array}$ & $\begin{array}{l}323 \\
327 \\
330 \\
327 \\
324\end{array}$ & $\begin{array}{l}349 \\
354 \\
367 \\
383 \\
396\end{array}$ & $\begin{array}{l}344 \\
346 \\
344 \\
350 \\
363\end{array}$ & $\begin{array}{l}323 \\
325 \\
324 \\
323 \\
326\end{array}$ & $\begin{array}{l}=- \\
=- \\
=-- \\
=--\end{array}$ & $\begin{array}{l}351 \\
355 \\
355 \\
360 \\
357\end{array}$ & $\begin{array}{l}357 \\
363 \\
365 \\
364 \\
376\end{array}$ \\
\hline $\begin{array}{l}16 \\
17 \\
18 \\
19 \\
20\end{array}$ & $\begin{array}{l}332 \\
341 \\
342 \\
336 \\
338\end{array}$ & $\begin{array}{l}335 \\
335 \\
337 \\
341 \\
344\end{array}$ & $\begin{array}{l}344 \\
346 \\
350 \\
346 \\
337\end{array}$ & $\begin{array}{l}318 \\
316 \\
318 \\
314 \\
313\end{array}$ & $\begin{array}{l}308 \\
311 \\
306 \\
310 \\
309\end{array}$ & $\begin{array}{l}326 \\
326 \\
325 \\
329 \\
342\end{array}$ & $\begin{array}{l}367 \\
355 \\
342 \\
338 \\
333\end{array}$ & $\begin{array}{l}362 \\
371 \\
374 \\
354 \\
342\end{array}$ & $\begin{array}{l}328 \\
326 \\
-126 \\
326\end{array}$ & $\begin{array}{l}343 \\
351 \\
352 \\
359 \\
353\end{array}$ & $\begin{array}{l}359 \\
360 \\
363 \\
364 \\
360\end{array}$ & $\begin{array}{l}414 \\
398 \\
372 \\
365 \\
370\end{array}$ \\
\hline $\begin{array}{l}21 \\
22 \\
23 \\
24 \\
25\end{array}$ & $\begin{array}{l}345 \\
347 \\
346 \\
342 \\
338\end{array}$ & $\begin{array}{l}360 \\
337 \\
357 \\
355 \\
354\end{array}$ & $\begin{array}{l}335 \\
337 \\
342 \\
339 \\
344\end{array}$ & $\begin{array}{l}314 \\
309 \\
319 \\
314 \\
316\end{array}$ & $\begin{array}{l}308 \\
306 \\
311 \\
301 \\
298\end{array}$ & $\begin{array}{l}349 \\
351 \\
356 \\
357 \\
356\end{array}$ & $\begin{array}{l}335 \\
342 \\
351 \\
351 \\
345\end{array}$ & $\begin{array}{l}345 \\
350 \\
358 \\
354 \\
351\end{array}$ & $\begin{array}{l}328 \\
330 \\
328 \\
331 \\
330\end{array}$ & \begin{tabular}{l}
340 \\
331 \\
\hdashline- \\
$\cdots$
\end{tabular} & $\begin{array}{l}359 \\
357 \\
450 \\
378 \\
393\end{array}$ & $\begin{array}{l}372 \\
363 \\
364 \\
372 \\
374\end{array}$ \\
\hline $\begin{array}{l}26 \\
27 \\
28 \\
29 \\
30 \\
31\end{array}$ & $\begin{array}{l}346 \\
341 \\
340 \\
339 \\
339 \\
340\end{array}$ & $\begin{array}{l}355 \\
356 \\
360 \\
358 \\
358 \\
-\end{array}$ & $\begin{array}{l}346 \\
354 \\
354 \\
359 \\
350 \\
345\end{array}$ & $\begin{array}{l}319 \\
314 \\
320 \\
321 \\
324 \\
326\end{array}$ & $\begin{array}{l}301 \\
300 \\
297 \\
305 \\
-\end{array}$ & $\begin{array}{l}359 \\
366 \\
367 \\
363 \\
360 \\
362\end{array}$ & $\begin{array}{l}356 \\
351 \\
344 \\
343 \\
365 \\
--\end{array}$ & $\begin{array}{l}360 \\
353 \\
347 \\
347 \\
348 \\
352\end{array}$ & $\begin{array}{l}=- \\
=- \\
=- \\
=- \\
=-\end{array}$ & $\begin{array}{l}\cdots \\
\cdots \\
\cdots \\
\cdots\end{array}$ & $\begin{array}{l}377 \\
380 \\
395 \\
377 \\
368 \\
366\end{array}$ & $\begin{array}{l}374 \\
373 \\
370 \\
368 \\
367 \\
\end{array}$ \\
\hline $\begin{array}{l}\text { MEAN } \\
\text { MAX } \\
\text { MIN }\end{array}$ & $=$ & $\begin{array}{l}344 \\
360 \\
330\end{array}$ & $\begin{array}{l}348 \\
365 \\
335\end{array}$ & $m$ & $\begin{array}{l}308 \\
324 \\
297\end{array}$ & $\begin{array}{l}335 \\
367 \\
312\end{array}$ & $\begin{array}{l}360 \\
404 \\
333\end{array}$ & $\begin{array}{l}353 \\
420 \\
336\end{array}$ & $=-$ & $\begin{array}{l}-\cdots \\
--- \\
---\end{array}$ & $\begin{array}{l}m-\infty \\
-m\end{array}$ & $\begin{array}{l}368 \\
414 \\
357\end{array}$ \\
\hline
\end{tabular}


RIO GRANDE BASTN

08313000 RIO GRANDE AT OTOWI BRIDGE, MM - Continued

WATER-QUALITY RECORDS

SUSPENDED-SEDTMENT, WATER YEAR OCYOBER 1995 TO SEPTEMBER 1996

\begin{tabular}{|c|c|c|c|c|c|c|c|c|c|c|c|c|}
\hline DAY & $\begin{array}{l}\text { MIRAN } \\
\text { CONCEN- } \\
\text { IRATION } \\
\text { (MG/L) }\end{array}$ & $\begin{array}{l}\text { LOADS } \\
(\mathrm{J} / \mathrm{DAY})\end{array}$ & $\begin{array}{l}\text { MEAN } \\
\text { CONCEN- } \\
\text { TRATYON } \\
\text { (MG/L) }\end{array}$ & $\begin{array}{l}\text { LOADS } \\
(T / D A Y)\end{array}$ & $\begin{array}{l}\text { MEAN } \\
\text { CONCEN- } \\
\text { TRATION } \\
\text { (MG/L) }\end{array}$ & $\begin{array}{l}\text { LOADS } \\
(T / D A X)\end{array}$ & $\begin{array}{l}\text { MEAN } \\
\text { CONCEN- } \\
\text { MRATION } \\
\text { (KO/L) }\end{array}$ & $\begin{array}{l}\text { LOADS } \\
(\mathrm{T} / \mathrm{DAY})\end{array}$ & $\begin{array}{l}\text { MFAN } \\
\text { CONCEN- } \\
\text { TRATION } \\
(M G / L)\end{array}$ & $\begin{array}{l}\text { LOADS } \\
\text { (T/DAY) }\end{array}$ & $\begin{array}{c}\text { MEAN } \\
\text { CONCEN- } \\
\text { MRATION } \\
\text { (LE/L) }\end{array}$ & $\begin{array}{l}\text { LOADS } \\
(\mathrm{T} / \mathrm{DAY})\end{array}$ \\
\hline $\begin{array}{l}1 \\
2 \\
3 \\
4 \\
5\end{array}$ & $\begin{array}{l}139 \\
155 \\
102 \\
91 \\
93\end{array}$ & $\begin{array}{r}\text { TOBER } \\
438 \\
473 \\
292 \\
254 \\
286\end{array}$ & $\begin{array}{l}\text { Nov } \\
140 \\
183 \\
292 \\
194 \\
142\end{array}$ & $\begin{array}{r}\text { EMBEA } \\
469 \\
663 \\
1070 \\
716 \\
527\end{array}$ & $\begin{array}{l}\text { DEC } \\
99 \\
185 \\
114 \\
146 \\
120\end{array}$ & $\begin{array}{r}\text { OMBER } \\
272 \\
500 \\
305 \\
395 \\
333\end{array}$ & $\begin{array}{c}104^{J} \\
94 \\
120 \\
203 \\
111\end{array}$ & $\begin{array}{r}\text { NUARY } \\
313 \\
286 \\
349 \\
607 \\
343\end{array}$ & $\begin{array}{l}\text { FEE } \\
112 \\
225 \\
115 \\
79 \\
153\end{array}$ & $\begin{array}{l}\text { RUARY } \\
360 \\
744 \\
365 \\
243 \\
465\end{array}$ & $\begin{array}{l}179 \\
178 \\
282 \\
197 \\
245\end{array}$ & $\begin{array}{r}\text { MARCE } \\
594 \\
581 \\
983 \\
692 \\
869\end{array}$ \\
\hline $\begin{array}{r}6 \\
7 \\
8 \\
9 \\
10\end{array}$ & $\begin{array}{r}179 \\
125 \\
114 \\
122 \\
96\end{array}$ & $\begin{array}{l}594 \\
389 \\
336 \\
348 \\
266\end{array}$ & $\begin{array}{l}174 \\
297 \\
193 \\
124 \\
121\end{array}$ & $\begin{array}{r}647 \\
1110 \\
722 \\
462 \\
458\end{array}$ & $\begin{array}{r}113 \\
113 \\
89 \\
85 \\
92\end{array}$ & $\begin{array}{l}328 \\
322 \\
244 \\
237 \\
257\end{array}$ & $\begin{array}{l}106 \\
124 \\
113 \\
101 \\
103\end{array}$ & $\begin{array}{l}322 \\
372 \\
340 \\
309 \\
314\end{array}$ & $\begin{array}{l}144 \\
161 \\
164 \\
153\end{array}$ & $\begin{array}{l}468 \\
528 \\
544 \\
508 \\
590\end{array}$ & $\begin{array}{l}214 \\
164 \\
192 \\
277 \\
306\end{array}$ & $\begin{array}{r}758 \\
590 \\
676 \\
996 \\
1110\end{array}$ \\
\hline $\begin{array}{l}11 \\
12 \\
13 \\
14 \\
15\end{array}$ & $\begin{array}{l}155 \\
301 \\
427 \\
380 \\
324\end{array}$ & $\begin{array}{r}424 \\
929 \\
1340 \\
1210 \\
1010\end{array}$ & $\begin{array}{l}102 \\
140 \\
208 \\
211 \\
162\end{array}$ & $\begin{array}{l}384 \\
530 \\
785 \\
795 \\
612\end{array}$ & $\begin{array}{r}89 \\
122 \\
194 \\
110 \\
92\end{array}$ & $\begin{array}{l}247 \\
351 \\
555 \\
307 \\
266\end{array}$ & $\begin{array}{l}110 \\
112 \\
110 \\
120 \\
131\end{array}$ & $\begin{array}{l}343 \\
350 \\
345 \\
396 \\
439\end{array}$ & $\begin{array}{l}157 \\
145 \\
181 \\
203 \\
176\end{array}$ & $\begin{array}{l}537 \\
502 \\
636 \\
712 \\
618\end{array}$ & $\begin{array}{l}352 \\
264 \\
288 \\
349 \\
386\end{array}$ & $\begin{array}{r}1270 \\
965 \\
1110 \\
1370 \\
1540\end{array}$ \\
\hline $\begin{array}{l}16 \\
17 \\
18 \\
19 \\
20\end{array}$ & $\begin{array}{l}262 \\
216 \\
170 \\
188 \\
183\end{array}$ & $\begin{array}{l}794 \\
593 \\
453 \\
579 \\
570\end{array}$ & $\begin{array}{l}131 \\
133 \\
132 \\
206 \\
108\end{array}$ & $\begin{array}{l}500 \\
507 \\
511 \\
794 \\
342\end{array}$ & $\begin{array}{l}118 \\
105 \\
88 \\
97 \\
73\end{array}$ & $\begin{array}{l}362 \\
326 \\
274 \\
284 \\
197\end{array}$ & $\begin{array}{r}153 \\
261 \\
148 \\
120 \\
96\end{array}$ & $\begin{array}{l}510 \\
563 \\
524 \\
405 \\
315\end{array}$ & $\begin{array}{l}176 \\
306 \\
263 \\
216\end{array}$ & $\begin{array}{r}646 \\
1130 \\
969 \\
799 \\
707\end{array}$ & $\begin{array}{l}437 \\
313 \\
256 \\
217 \\
197\end{array}$ & $\begin{array}{r}1810 \\
1260 \\
1000 \\
817 \\
718\end{array}$ \\
\hline $\begin{array}{l}21 \\
22 \\
23 \\
24 \\
25\end{array}$ & $\begin{array}{l}204 \\
182 \\
143 \\
130 \\
183\end{array}$ & $\begin{array}{l}643 \\
579 \\
458 \\
409 \\
584\end{array}$ & $\begin{array}{l}78 \\
81 \\
77 \\
87 \\
66\end{array}$ & $\begin{array}{l}231 \\
214 \\
242 \\
182\end{array}$ & $\begin{array}{l}70 \\
73 \\
82 \\
81 \\
92\end{array}$ & $\begin{array}{l}189 \\
199 \\
224 \\
216 \\
230\end{array}$ & $\begin{array}{r}97 \\
103 \\
93 \\
88 \\
110\end{array}$ & $\begin{array}{l}312 \\
338 \\
311 \\
285 \\
348\end{array}$ & $\begin{array}{l}253 \\
270 \\
226 \\
224 \\
225\end{array}$ & $\begin{array}{r}948 \\
1040 \\
867 \\
859 \\
864\end{array}$ & $\begin{array}{l}243 \\
265 \\
230 \\
183 \\
150\end{array}$ & $\begin{array}{l}858 \\
940 \\
813 \\
643 \\
534\end{array}$ \\
\hline $\begin{array}{l}26 \\
27 \\
28 \\
29 \\
30 \\
31\end{array}$ & $\begin{array}{l}362 \\
183 \\
176 \\
254 \\
214 \\
96\end{array}$ & $\begin{array}{r}1110 \\
562 \\
552 \\
785 \\
671 \\
307\end{array}$ & $\begin{array}{r}71 \\
63 \\
42 \\
41 \\
59 \\
-4\end{array}$ & $\begin{array}{l}196 \\
176 \\
114 \\
110 \\
161 \\
-\infty\end{array}$ & $\begin{array}{r}107 \\
114 \\
125 \\
123 \\
99 \\
101\end{array}$ & $\begin{array}{l}286 \\
300 \\
340 \\
348 \\
283 \\
293\end{array}$ & $\begin{array}{l}117 \\
169 \\
133 \\
139 \\
157 \\
107\end{array}$ & $\begin{array}{l}385 \\
514 \\
417 \\
447 \\
496 \\
339\end{array}$ & $\begin{array}{l}210 \\
171 \\
168 \\
164 \\
-\end{array}$ & $\begin{array}{l}797 \\
620 \\
592 \\
570 \\
\ldots \ldots \\
\ldots \ldots\end{array}$ & $\begin{array}{l}144 \\
197 \\
183 \\
145 \\
130 \\
150\end{array}$ & $\begin{array}{l}505 \\
650 \\
535 \\
404 \\
357 \\
402\end{array}$ \\
\hline TOTAL & $\cdots$ & 18238 & $\cdots$ & 14454 & $-\infty$ & 9260 & $-\infty$ & 11937 & $-m$ & 19228 & $m$ & 26350 \\
\hline & $\begin{array}{l}\text { MEAI } \\
\text { CONCEN- } \\
\text { TRATION }\end{array}$ & IDAY & $\begin{array}{l}\text { MEAN } \\
\text { CONCEN- } \\
\text { TRATION }\end{array}$ & Tin & $\begin{array}{l}\text { MEAN } \\
\text { CONCEN- } \\
\text { TRATION }\end{array}$ & TTUAY & $\begin{array}{l}\text { MEAN } \\
\text { CONCEN- } \\
\text { TRATION }\end{array}$ & OADS & $\begin{array}{l}\text { MEAN } \\
\text { CONCEN- } \\
\text { TRATION }\end{array}$ & CT TDAY & $\begin{array}{c}\text { MFAN } \\
\text { CONCEN- } \\
\text { TRATION }\end{array}$ & LOADS \\
\hline
\end{tabular}

DA

(MG/L) (T/DAY)

(MG/L) (T/DAY)

(NG/L) (T/DAY)

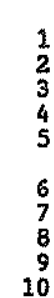

74
61
70
76
85

190
116
135
154
196

$\begin{array}{rr} & \text { MAY } \\ 216 & 60 \\ 286 & 68 \\ 174 & 35 \\ 95 & 17 \\ 150 & 305\end{array}$

$\begin{array}{rr}6 & 71 \\ 7 & 66 \\ 9 & 76 \\ 9 & 128\end{array}$

$\begin{array}{lll}159 & 142 & 335 \\ 136 & 177\end{array}$

11
12
13
14
15

191

157
275

413

188
203
175

463
577
640

552

$\begin{array}{ll} & \text { JUNE } \\ 160 & 487 \\ 289 & 882 \\ 237 & 710 \\ 128 & 387 \\ 216 & 642\end{array}$

$\begin{array}{ll}128 & \text { JULY } \\ 143 & \\ 153 & \\ 232 & 3 \\ 223 & 37\end{array}$

169
178
162
376
379

$\begin{array}{ll}231 & \text { AUGUST } \\ 230 & 61 \\ 260 & 63 \\ 238 & 67 \\ 215 & 58\end{array}$

$241 \quad 736 \quad 131$

$\begin{array}{lll}203 & 665 & 227 \\ 242 & 803 & 343 \\ 219 & 722 & 298\end{array}$

219

219

914

298

$$
\begin{aligned}
& 132 \\
& 263
\end{aligned}
$$

263

981

$\begin{array}{rrr}372 & 1410 & 221 \\ 271 & 999 & 230\end{array}$

$\begin{array}{ll}190 & 415 \\ 124 & 256 \\ 132 & 303\end{array}$

$\begin{array}{lll}415 & 195 & 640 \\ 256 & 266 & 882\end{array}$

$303 \quad 361 \quad 1240$

143

139
134

410

271
167

167
149
285

999
621
570

570

221
230
283
187
218

274

16

17

$\begin{array}{rr}84 & 178 \\ 111 & 234 \\ 103 & 2 \\ 78 & 173\end{array}$

$178 \quad 197 \quad 597$

234
226

411

211
183
427

601
509
1710

1710

212

146
170

166

728
470
52
490

1110

5310

$\begin{array}{rr}1450 & 5310 \\ 776 & 2810 \\ 185 & 645\end{array}$

$\begin{array}{ll}185 & 645 \\ 178 & 630\end{array}$

$\begin{array}{ll}121 & 335 \\ 105 & 303\end{array}$

100

276
262

$\begin{array}{rr}761 & 187 \\ 1150 & 205 \\ 1660 & 308 \\ 602 & 89 \\ 392 & 595\end{array}$

433
664

328

510

260
471
1050
652
669

612
1170
2820
1670
1600

675

563
436

410
477

1400

1050
937

1220

341

341
245
313
311

82

82
59
73
63
91

$1680 \quad 3510$

$\begin{array}{ll}6860 & 20200 \\ 4500 & 13100\end{array}$

332

5990

477
579
22200
17500
8150

$\begin{array}{lll}147 & 451 & 266 \\ 154 & 409 & 257\end{array}$

146

$123 \quad 163$

1512200

257
221
223

223

901
876

876
700
707

707
693

3850

3850
2000

1250

1600

1690

1180
1740
54400
25200
10500

7970
2480

1110
3070

4190

4470

-- 63426

$-\cdots$

136928

17021

TOTAL LOTAL LOAD FOR YEAR:
TOT9 399248 TONS. 
RIO GRANDE BASIN

08313350 RITO DE LOS FRIJOLES IN BANDELIER NATIONAL MONUMENT, NM

LOCATION, --Lat $35^{\circ} 46^{\circ} 35^{\prime \prime}$, long $106^{\circ} 16^{\circ} 06^{\prime \prime}$, Sandoval County, Eydrologic Unit 13020201, in Bandelier National Monument, on right bank $800 \mathrm{ft}$ downstream from Monument headquarters, $6.5 \mathrm{mi}$ south of Los Alamos, $18.5 \mathrm{mi}$ northwest of Santa $\mathrm{Fe}$, and at mile 2.0 .

DRAINAGE AREA. $-18.1 \mathrm{mi}^{2}$.

PERIOD OF RECORD.--July 1963 to September 1969, July 1977 to September 1982, May 1993 to September 1996 (discontinued).

GAGE.--Water-stage recorder and concrete control. Altitude of gage is 6,035 ft, from topographic map. Prior to Oct. 3,1979 , at site upstream at different datum.

REMARKS.--Records good except those for winter period, which are poor. One small diversion from left bank about 1.0 mi upstream for irrigation of small orchard. The La Mesa forest fire which occurred during mid-June 1977 burned about $40 \%$ of the forest cover of thts watershed and evidently changed the flow characteristics.

DISCHARGE, CUBIC FEET PER SECOND, WATER YEAR OCTOBER 1995 TO SEPTEMBER 1996

\begin{tabular}{|c|c|c|c|c|c|c|c|c|c|c|c|c|}
\hline DAY & OCT & NOV & DEC & JAN & FEB & MAR & APR & MAY & JUN & JUL, & AUG & SEP \\
\hline $\begin{array}{l}1 \\
2 \\
3 \\
4 \\
5\end{array}$ & $\begin{array}{l}.82 \\
.77 \\
.76 \\
.74 \\
.73\end{array}$ & $\begin{array}{l}.76 \\
.76 \\
.76 \\
.81 \\
.87\end{array}$ & $\begin{array}{l}.86 \\
.86 \\
.86 \\
.86 \\
.86\end{array}$ & $\begin{array}{r}0.90 \\
0.90 \\
.86 \\
0.80 \\
.98\end{array}$ & $\begin{array}{l}0.80 \\
6.70 \\
6.70 \\
6.80 \\
\theta .80\end{array}$ & $\begin{array}{l}1.3 \\
1.2 \\
1.1 \\
1.0 \\
1.0\end{array}$ & $\begin{array}{r}.98 \\
.98 \\
.96 \\
.97 \\
1.0\end{array}$ & $\begin{array}{l}.70 \\
.66 \\
.65 \\
.61 \\
.58\end{array}$ & $\begin{array}{l}.28 \\
.27 \\
.29 \\
.33 \\
.27\end{array}$ & $\begin{array}{l}1.0 \\
1.0 \\
.81 \\
.62 \\
.65\end{array}$ & $\begin{array}{l}.97 \\
.76 \\
.78 \\
.82 \\
.61\end{array}$ & $\begin{array}{r}.72 \\
.98 \\
1.5 \\
1.0 \\
.85\end{array}$ \\
\hline $\begin{array}{r}6 \\
7 \\
8 \\
9 \\
10\end{array}$ & $\begin{array}{l}.77 \\
.78 \\
.76 \\
.76 \\
.76\end{array}$ & $\begin{array}{l}.86 \\
.82 \\
.81 \\
.81 \\
.81\end{array}$ & $\begin{array}{l}.86 \\
.86 \\
.87 \\
.95 \\
.94\end{array}$ & $\begin{array}{r}.97 \\
.95 \\
0.99 \\
.97 \\
.96\end{array}$ & $\begin{array}{l}0.90 \\
1.0 \\
1.0 \\
1.0 \\
1.0\end{array}$ & $\begin{array}{l}1.0 \\
1.0 \\
1.0 \\
1.0 \\
1.0\end{array}$ & $\begin{array}{l}1.0 \\
1.0 \\
.98 \\
.96 \\
.94\end{array}$ & $\begin{array}{l}.58 \\
.57 \\
.58 \\
.58 \\
.56\end{array}$ & $\begin{array}{l}.23 \\
.23 \\
.23 \\
.25 \\
.27\end{array}$ & $\begin{array}{r}.79 \\
.51 \\
.60 \\
1.0 \\
.93\end{array}$ & $\begin{array}{l}.53 \\
.53 \\
.59 \\
.64 \\
.63\end{array}$ & .8 \\
\hline $\begin{array}{l}11 \\
12 \\
13 \\
14 \\
15\end{array}$ & $\begin{array}{l}.75 \\
.73 \\
.71 \\
.75 \\
.76\end{array}$ & $\begin{array}{l}.81 \\
.83 \\
.81 \\
.81 \\
.81\end{array}$ & $\begin{array}{l}.90 \\
.91 \\
.89 \\
.87 \\
.87\end{array}$ & $\begin{array}{l}.94 \\
.94 \\
.93 \\
.94 \\
.92\end{array}$ & $\begin{array}{l}.98 \\
.98 \\
.98 \\
.99 \\
.99\end{array}$ & $\begin{array}{r}.99 \\
.98 \\
.97 \\
.98 \\
1.0\end{array}$ & $\begin{array}{l}.94 \\
.94 \\
.96 \\
.99 \\
.98\end{array}$ & $\begin{array}{r}.54 \\
.52 \\
.50 \\
.49 \\
.45\end{array}$ & $\begin{array}{l}.24 \\
.25 \\
.31 \\
.56 \\
.54\end{array}$ & $\begin{array}{l}.97 \\
.85 \\
.93 \\
.84 \\
.73\end{array}$ & $\begin{array}{l}.50 \\
.45 \\
.44 \\
.41 \\
.42\end{array}$ & $\begin{array}{r}.70 \\
.88 \\
1.0 \\
1.2 \\
1.4\end{array}$ \\
\hline $\begin{array}{l}16 \\
17 \\
18 \\
19 \\
20\end{array}$ & $\begin{array}{l}.76 \\
.76 \\
.76 \\
.76 \\
.76\end{array}$ & $\begin{array}{l}.81 \\
.81 \\
.81 \\
.81 \\
.81\end{array}$ & $\begin{array}{l}.92 \\
.96 \\
.98 \\
.90 \\
.95\end{array}$ & $\begin{array}{r}.93 \\
0.80 \\
0.90 \\
0.80 \\
0.80\end{array}$ & $\begin{array}{r}.98 \\
.99 \\
1.0 \\
1.0 \\
1.1\end{array}$ & $\begin{array}{c}1.0 \\
1.0 \\
.98 \\
.98 \\
1.0\end{array}$ & $\begin{array}{l}.97 \\
.92 \\
.84 \\
.83 \\
.83\end{array}$ & $\begin{array}{l}.45 \\
.42 \\
.41 \\
.38 \\
.36\end{array}$ & $\begin{array}{r}.40 \\
.35 \\
.33 \\
.26 \\
.22\end{array}$ & $\begin{array}{l}.69 \\
.76 \\
.81 \\
.70 \\
.62\end{array}$ & $\begin{array}{r}.50 \\
.49 \\
.52 \\
.45 \\
.48\end{array}$ & $\begin{array}{c}1.1 \\
1.95 \\
1.0 \\
1.0 \\
.88\end{array}$ \\
\hline $\begin{array}{l}21 \\
22 \\
23 \\
24 \\
25\end{array}$ & $\begin{array}{r}.80 \\
.87 \\
1.1 \\
.94 \\
.76\end{array}$ & $\begin{array}{l}.81 \\
.81 \\
.81 \\
.81 \\
.81\end{array}$ & $\begin{array}{l}1.0 \\
.85 \\
0.80 \\
9.80 \\
0.80\end{array}$ & $\begin{array}{l}0.80 \\
0.80 \\
0.80 \\
0.70 \\
0.70\end{array}$ & $\begin{array}{l}1.1 \\
1.0 \\
1.0 \\
.98 \\
.98\end{array}$ & $\begin{array}{r}1.0 \\
1.0 \\
.99 \\
.99 \\
1.0\end{array}$ & $\begin{array}{r}.83 \\
.81 \\
.79 \\
.78 \\
.74\end{array}$ & $\begin{array}{l}.36 \\
.36 \\
.35 \\
.36 \\
.37\end{array}$ & $\begin{array}{l}.21 \\
.29 \\
.36 \\
.25 \\
.24\end{array}$ & $\begin{array}{l}.56 \\
.52 \\
.49 \\
.46 \\
.51\end{array}$ & $\begin{array}{l}.53 \\
1.1 \\
1.6 \\
1.4 \\
1.1\end{array}$ & $\begin{array}{r}.72 \\
.74\end{array}$ \\
\hline $\begin{array}{l}26 \\
27 \\
28 \\
29 \\
30 \\
31\end{array}$ & $\begin{array}{l}.76 \\
.76 \\
.76 \\
.74 \\
.72 \\
.75\end{array}$ & $\begin{array}{l}.82 \\
.81 \\
.84 \\
.89 \\
.91 \\
\end{array}$ & $\begin{array}{r}.82 \\
0.80 \\
0.80 \\
0.80 \\
0.80 \\
0.90\end{array}$ & $\begin{array}{l}0.80 \\
0.80 \\
0.80 \\
0.70 \\
0.70 \\
0.80\end{array}$ & $\begin{array}{r}.98 \\
.98 \\
.92 \\
1.1 \\
-0\end{array}$ & $\begin{array}{c}1.0 \\
1.0 \\
1.0 \\
.99 \\
1.0 \\
.98\end{array}$ & $\begin{array}{l}.71 \\
.70 \\
.69 \\
.70 \\
.73 \\
.-\end{array}$ & $\begin{array}{l}.42 \\
.45 \\
.38 \\
.33 \\
.32 \\
.30\end{array}$ & $\begin{array}{r}.32 \\
1.2 \\
.64 \\
1.0 \\
.70 \\
-0\end{array}$ & $\begin{array}{r}.55 \\
.58 \\
.71 \\
.84 \\
.74 \\
1.1\end{array}$ & $\begin{array}{r}1.1 \\
1.0 \\
.89 \\
.80 \\
.82 \\
.74\end{array}$ & .69 \\
\hline $\begin{array}{l}\text { TOTAL } \\
\text { MEAN } \\
\text { MAX } \\
\text { MIN } \\
\text { AC }-F T\end{array}$ & $\begin{array}{r}24.11 \\
.78 \\
i .1 \\
.71 \\
48\end{array}$ & $\begin{array}{r}24.51 \\
.82 \\
.91 \\
.76 \\
49\end{array}$ & $\begin{array}{r}27.10 \\
.87 \\
1.0 \\
.80 \\
54\end{array}$ & $\begin{array}{r}26.58 \\
.86 \\
.99 \\
.70 \\
53\end{array}$ & $\begin{array}{r}27.73 \\
.96 \\
i .1 \\
.70 \\
55\end{array}$ & $\begin{array}{r}31.43 \\
1.01 \\
1.3 \\
.97 \\
62\end{array}$ & $\begin{array}{r}26.45 \\
.88 \\
1.0 \\
.69 \\
52\end{array}$ & $\begin{array}{r}14.59 \\
.47 \\
.70 \\
.30 \\
29\end{array}$ & $\begin{array}{r}11.32 \\
.38 \\
1.2 \\
.21 \\
22\end{array}$ & $\begin{array}{r}22.87 \\
.74 \\
1.1 \\
.46 \\
45\end{array}$ & $\begin{array}{r}22.60 \\
.73 \\
1.6 \\
.41 \\
45\end{array}$ & $\begin{array}{r}26.27 \\
.88 \\
1.5 \\
.63 \\
52\end{array}$ \\
\hline
\end{tabular}

STATISTICS OF MONTHLY MEAN DATA FOR WATER YEARS 1983 - 1996, BY WATER YEAR (WY)

\begin{tabular}{|c|c|c|c|c|c|c|c|c|c|c|c|}
\hline $\begin{array}{l}\text { MEAN } \\
\text { MAX } \\
\text { (WY) } \\
\text { MIN } \\
\text { (WY) }\end{array}$ & $\begin{array}{r}1.32 \\
2.53 \\
1986 \\
.74 \\
1993\end{array}$ & $\begin{array}{l}1.71 \\
5.04 \\
1987 \\
1982 \\
1996\end{array}$ & $\begin{array}{r}1.43 \\
3.10 \\
1987 \\
198 \\
1990\end{array}$ & $\begin{array}{l}1.27 \\
2.35 \\
1995 \\
.75 \\
1990\end{array}$ & $\begin{array}{r}1.49 \\
2.53 \\
1995 \\
.60 \\
1990\end{array}$ & $\begin{array}{l}3.19 \\
7.29 \\
1987 \\
1.01 \\
1996\end{array}$ & $\begin{array}{r}5.80 \\
16.6 \\
1985 \\
198 \\
1996\end{array}$ & $\begin{array}{r}3.82 \\
11.0 \\
1985 \\
4.47 \\
1996\end{array}$ & $\begin{array}{l}1.56 \\
3.98 \\
1986 \\
1998 \\
1996\end{array}$ & $\begin{array}{r}1.22 \\
4.40 \\
1986 \\
198 \\
1990\end{array}$ & $\begin{array}{r}1.40 \\
3.97 \\
1991 \\
1963\end{array}$ \\
\hline
\end{tabular}

SUMMARY STATISTICS

ANNUAL. TOTAL.

ANRUAL MEAN

BIGHEST ANNUAL MEAN

LOWEST ANNUAL MEAN

HIGHEST DAILY MEAN

LOWEST DAILY MEAN

ANNUAL SEVEN-DAY MINIMUM

INSTANTANEOUS PEAK FLOW

INSTANTANEOUS PEAK STAGE

INSTANTANEOUS LOW FLOW

ANRUAL RUNOFF (AC-FT)

10 PERCENT EXCEEDS

50 PERCENT EXCEEDS

90 PERCENT EXCEEDS

- Estimated

a-Site and datum then in use

FOR 1995 CALENDAR YEAR

FOR 1996 WATER YEAR

WATER YEARS 1983 - 1996

$\begin{array}{rrrr}672.17 & & 285.56 & \\ 1.84 & & .78 & \\ & & & \\ 5.1 & \text { May } 1 & 1.6 & \text { Aug 23 } \\ .67 & .21 & \text { Jun 21 } \\ .71 & \text { JuI 25 } & .24 & \text { Jun } 6 \\ & & 9.4 & \text { Jun } 29 \\ & 2.19 & \text { Jun 29 } \\ 1330 & 566 & \text { Jun 20 } \\ 3.5 & 1.0 & \\ 1.4 & .81 & \\ .76 & .41 & \end{array}$

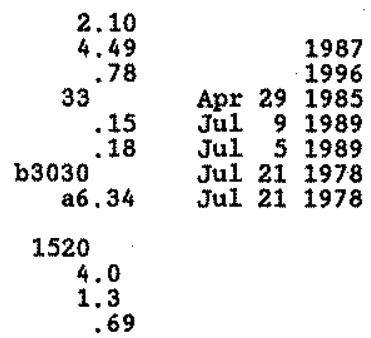

b-From rating curve extended above $20 \mathrm{ft} / \mathrm{s}$, on basis of slope-area measurements at gage height 3.88 , $5.02 \mathrm{ft}$ and 6.34. Maximum discharge prior to the forest fire of June 1977 was $19 \mathrm{ft}^{3} / \mathrm{s}$, June 18,1965 , gage height 1.49 ft; site and datum then in use, from rating curve extended above $7.6 \mathrm{ft} / \mathrm{s}$, on basts of theoretical rating. 
LOCATION,--Lat $35^{\circ} 41^{\prime} 18^{\prime \prime}$, Long $105^{\circ} 50^{\prime} 06^{\prime \prime}$, in NEKSWK, sec.24, T.17 N., R.10 E., Santa Fe County, Bydrolog Ic Unit 13020201 , in Santa Fe'National Forest, at McClure Dam on Santa Fe River, 2.1 mi upstream from Nichols Reservotr, 5.8 mi east of Santa $F \theta$, and at mile 37.1 .

DRATNAGE AREA, $--17.4 \mathrm{mi}^{2}$.

PERIOD OF RECORD, --September 1929, July to October 1930, April 1931 to June 2946 , September 1947 to current year Prior to October 1947, published in WSP 1312. Prior to October 1965, monthend contents only. Prior to January 1980 at site on outlet tower.

GAGE. - Water-stage recordex. Elevation of gage is 7,790 ft above National Geodetic Vertical Datum of 1929 , from topographic mep. Prior to Oct. 1, 1947, nonrecording gages at same sito and various datums all referred to the Public Service Co. of New Mexico assumed datum, 165.9 ft lower.

REMARKS.--Reservoir is formed by earthfill dam, completed in 1926, capacity, 561 acre-1t, raised 3 ft in 1935, capacity, 650 acre-ft, and raised $36.5 \mathrm{ft}$ more in 1947, capacity, 2,615 acre-ft at gage height 96.6 ft, crest of concrete spillway. Between October 1947 and May 1953 varying emounts of sandbag bulkheads were placed on crest of spillway to increase capacity. Batween May 1953 and Decenber 1971 spillway was equipped with radial gates that opened automatically thereby increasing capacity to over 3,000 acre-ft. Rediel gates were removed during 1972 , capacity, 2,615 acre-ft. In 1995 , modifications to the dam and spiliway increased capacity to 3,257 acreft. Only the storage of Rio Grande water in excess of 1,061 acre-ft is subject to texns of the Rio Grande Compact. No dead storage. Water is for municipal use of City of Santa Fe.

COOPERATION,--Capacity table and supplementary gage readings, provided by Public Service Co. of New Mexico.

EXTREMES FOR PERIOD OF RECORD.--Maximum contents, 3,140 acre-ft, June 25, 1960, gage height, 103.7 ft; no contents Jan. 25 to May $8,1951$.

EXIREMES FOR CURRENT YEAR.-Maximum contents observed, 2,250 acre-ft; Oct. 1, elevation, 7,872.00 ft; minimum, 485 acre-ft, July 6 , gage height, 7834.34

Capacity table (gage height, in feet, and contents, in acre-feet)

(Based on survey by Public Service Co. of New Mexico in 1995)

$\begin{array}{rrrr}7,834 & 476 & 7,864 & 1740 \\ 7,844 & 780 & 7,874 & 2380 \\ 7,854 & 1200 & 7,884 & 3120\end{array}$

RESERVOTR STORAGE (ACRE-FEET), WATER YEAR OCTOBER 1995 TO SEPTEMBER 1996 DAILY OBSERVATION AT 24:00 VALUES

\begin{tabular}{|c|c|c|c|c|c|c|c|c|c|c|c|c|}
\hline$A Y$ & OCT & Nov & DEC & JAN & FEB & MAR & APR & MAY & JUN & JUL & AUG & SEP \\
\hline $\begin{array}{l}1 \\
2 \\
3 \\
4 \\
5\end{array}$ & $\begin{array}{l}2250 \\
2230 \\
2210 \\
2190 \\
2170\end{array}$ & $\begin{array}{l}1970 \\
1970 \\
1960 \\
1960 \\
1950\end{array}$ & $\begin{array}{l}1900 \\
1900 \\
1900 \\
1900 \\
1900\end{array}$ & $\begin{array}{l}1910 \\
1900 \\
1900 \\
1900 \\
1900\end{array}$ & $\begin{array}{r}1910 \\
\text { e1910 } \\
\mathrm{e} 1910 \\
\mathrm{e} 1910 \\
\mathrm{e} 1910\end{array}$ & $\begin{array}{l}01920 \\
\theta 1920 \\
\theta 1920 \\
01920 \\
1920\end{array}$ & $\begin{array}{l}1920 \\
1930 \\
1930 \\
1920 \\
1920\end{array}$ & $\begin{array}{l}1650 \\
1640 \\
1610 \\
1570 \\
1530\end{array}$ & $\begin{array}{r}0700 \\
0696 \\
687 \\
0680 \\
6660\end{array}$ & $\begin{array}{l}494 \\
492 \\
489 \\
487 \\
487\end{array}$ & $\begin{array}{l}699 \\
693 \\
687 \\
680 \\
674\end{array}$ & $\begin{array}{l}542 \\
539 \\
537 \\
533 \\
530\end{array}$ \\
\hline $\begin{array}{r}6 \\
7 \\
8 \\
9 \\
10\end{array}$ & $\begin{array}{l}2150 \\
2130 \\
2110 \\
2090 \\
2080\end{array}$ & $\begin{array}{l}1940 \\
1940 \\
1930 \\
1930 \\
1930\end{array}$ & $\begin{array}{l}1900 \\
1900 \\
1900 \\
1900 \\
1900\end{array}$ & $\begin{array}{r}1900 \\
1900 \\
1900 \\
81910 \\
1910\end{array}$ & $\begin{array}{l}\text { e1910 } \\
\text { O1910 } \\
\text { e1910 } \\
\text { e1910 } \\
\text { e1920 }\end{array}$ & $\begin{array}{l}\text { e1920 } \\
01920 \\
01920 \\
01920 \\
01920\end{array}$ & $\begin{array}{l}1920 \\
1920 \\
1920 \\
1920 \\
1910\end{array}$ & $\begin{array}{l}1490 \\
1450 \\
1410 \\
1380 \\
1340\end{array}$ & $\begin{array}{r}0640 \\
0620 \\
8605 \\
0595 \\
587\end{array}$ & $\begin{array}{l}485 \\
488 \\
496 \\
539 \\
577\end{array}$ & $\begin{array}{l}667 \\
661 \\
656 \\
650 \\
645\end{array}$ & $\begin{array}{l}531 \\
530 \\
506 \\
523 \\
520\end{array}$ \\
\hline $\begin{array}{l}11 \\
12 \\
13 \\
14 \\
15\end{array}$ & $\begin{array}{l}2080 \\
2070 \\
2070 \\
2060 \\
2050\end{array}$ & $\begin{array}{l}1920 \\
1910 \\
1910 \\
1900 \\
1900\end{array}$ & $\begin{array}{l}1900 \\
1900 \\
1900 \\
1900 \\
1900\end{array}$ & $\begin{array}{l}1910 \\
1910 \\
1900 \\
1900 \\
1910\end{array}$ & $\begin{array}{l}01920 \\
\theta 1920 \\
01920 \\
01920 \\
01920\end{array}$ & $\begin{array}{l}\text { e1920 } \\
\text { e1920 } \\
01920 \\
01920 \\
\mathrm{e} 1920\end{array}$ & $\begin{array}{l}1910 \\
1910 \\
1910 \\
1910 \\
1900\end{array}$ & $\begin{array}{l}1310 \\
1270 \\
1230 \\
1200 \\
1160\end{array}$ & $\begin{array}{l}0583 \\
0578 \\
0575 \\
0572 \\
0568\end{array}$ & $\begin{array}{l}605 \\
627 \\
644 \\
657 \\
670\end{array}$ & $\begin{array}{l}638 \\
630 \\
623 \\
616 \\
611\end{array}$ & $\begin{array}{l}516 \\
513 \\
511 \\
514 \\
518\end{array}$ \\
\hline $\begin{array}{l}16 \\
17 \\
18 \\
19 \\
20\end{array}$ & $\begin{array}{l}2050 \\
2050 \\
2040 \\
2040 \\
2030\end{array}$ & $\begin{array}{l}1900 \\
1900 \\
1900 \\
1900 \\
1900\end{array}$ & $\begin{array}{l}1900 \\
1900 \\
1900 \\
1900 \\
1900\end{array}$ & $\begin{array}{l}1910 \\
1910 \\
1910 \\
1910 \\
1910\end{array}$ & $\begin{array}{l}01920 \\
01920 \\
01920 \\
\theta 1920 \\
01920\end{array}$ & $\begin{array}{l}\text { e1920 } \\
01920 \\
\text { e1920 } \\
\text { e1920 } \\
01920\end{array}$ & $\begin{array}{l}1890 \\
1870 \\
1860 \\
1840 \\
1820\end{array}$ & $\begin{array}{r}1120 \\
1090 \\
1050 \\
1020 \\
979\end{array}$ & $\begin{array}{r}e 564 \\
e 560 \\
556 \\
0546 \\
e 536\end{array}$ & $\begin{array}{l}683 \\
697 \\
704 \\
712 \\
715\end{array}$ & $\begin{array}{l}602 \\
593 \\
507 \\
581 \\
573\end{array}$ & $\begin{array}{l}523 \\
527 \\
536 \\
544 \\
551\end{array}$ \\
\hline $\begin{array}{l}21 \\
22 \\
23 \\
24 \\
25\end{array}$ & $\begin{array}{l}2030 \\
2020 \\
2020 \\
2010 \\
2000\end{array}$ & $\begin{array}{l}1900 \\
1900 \\
1900 \\
1900 \\
1900\end{array}$ & $\begin{array}{l}1900 \\
1900 \\
1900 \\
1900 \\
1900\end{array}$ & $\begin{array}{r}1910 \\
1910 \\
1910 \\
01910 \\
01910\end{array}$ & $\begin{array}{r}01920 \\
01920 \\
01920 \\
1920 \\
1920\end{array}$ & $\begin{array}{l}01920 \\
\text { e1920 } \\
\text { e1920 } \\
\text { e1920 } \\
01920\end{array}$ & $\begin{array}{l}1810 \\
1790 \\
1770 \\
1760 \\
1740\end{array}$ & $\begin{array}{r}944 \\
910 \\
890 \\
8860 \\
8810\end{array}$ & $\begin{array}{l}524 \\
517 \\
509 \\
502 \\
496\end{array}$ & $\begin{array}{l}718 \\
720 \\
722 \\
722 \\
722\end{array}$ & $\begin{array}{l}566 \\
560 \\
558 \\
556 \\
555\end{array}$ & $\begin{array}{l}556 \\
560 \\
563 \\
565 \\
566\end{array}$ \\
\hline $\begin{array}{l}26 \\
27 \\
28 \\
29 \\
30 \\
31\end{array}$ & $\begin{array}{l}2000 \\
1990 \\
1990 \\
1980 \\
1980 \\
1970\end{array}$ & $\begin{array}{l}1900 \\
1900 \\
1900 \\
1900 \\
1900\end{array}$ & $\begin{array}{l}1900 \\
1900 \\
1900 \\
1900 \\
1900 \\
1900\end{array}$ & $\begin{array}{r}\text { e1910 } \\
\text { e1910 } \\
1910 \\
1910 \\
1910 \\
1910\end{array}$ & $\begin{array}{r}1920 \\
1920 \\
1920 \\
1920 \\
- \\
-\end{array}$ & $\begin{array}{r}1920 \\
1920 \\
1920 \\
1920 \\
1920 \\
1930\end{array}$ & $\begin{array}{l}1730 \\
1710 \\
1700 \\
1680 \\
1670\end{array}$ & $\begin{array}{r}\theta 780 \\
748 \\
0740 \\
e 730 \\
\theta 720 \\
e 710\end{array}$ & $\begin{array}{l}491 \\
492 \\
490 \\
494 \\
496\end{array}$ & $\begin{array}{l}719 \\
716 \\
713 \\
710 \\
708 \\
704\end{array}$ & $\begin{array}{l}551 \\
547 \\
545 \\
546 \\
546 \\
544\end{array}$ & $\begin{array}{l}569 \\
569 \\
570 \\
569 \\
569\end{array}$ \\
\hline $\begin{array}{l}\operatorname{MAX} \\
\operatorname{MIN} \\
(\dagger) \\
(t+)\end{array}$ & $\begin{array}{r}2250 \\
1970 \\
867.80 \\
-300\end{array}$ & $\begin{array}{r}1970 \\
1900 \\
7866.61 \\
-70\end{array}$ & $\begin{array}{r}1900 \\
1900 \\
7866.69 \\
0\end{array}$ & $\begin{array}{r}1910 \\
1900 \\
7866.78 \\
+10\end{array}$ & $\begin{array}{r}1920 \\
1910 \\
7866.97 \\
+10\end{array}$ & $\begin{array}{r}1930 \\
1920 \\
7867.04 \\
+10\end{array}$ & $\begin{array}{r}1930 \\
1670 \\
7862.85 \\
-260\end{array}$ & $\begin{array}{r}1650 \\
710 \\
7841.97 \\
-960\end{array}$ & $\begin{array}{r}700 \\
490 \\
7834.74 \\
-214\end{array}$ & $\begin{array}{r}722 \\
485 \\
7841.79 \\
+208\end{array}$ & $\begin{array}{r}699 \\
544 \\
7836.54 \\
-160\end{array}$ & $\begin{array}{r}570 \\
506 \\
7837.43 \\
+25\end{array}$ \\
\hline
\end{tabular}

CAL YR 1995 MAX $3280^{\circ}$ MIN 1720 (t†) +20 WTR YR 1996 MAX 2250 MIN 485 (t

e Estimated

( $\dagger$ GAGE BEIGHT, IN FEET, AT END OF MONTH (OCT TO DEC), ELEVATION IN FEET, AT END OF MONTH (JAN-SEPT).

(ti) CHANGE IN CONTENTS, IN ACRE-FEET 
RIO GRANDE BASIN

08316000 SARTA FE RIVER NEAR SANTA FE, NM

LOCATYON.--Lat $35^{\circ} 41^{\prime} 12^{\prime \prime}$, Long $105^{\circ} 50^{\prime} 35^{\prime \prime}$, in NEXsEK sec.23, T.17 N., R.10 E., Santa Fe County, Bydrologic Unit 13020201 , in Santa Fe National Forest, on left bank 0.4 mi domstrean from McClure Dam, 5.3 mi east of Santa Fe, and at mile 36.6 .

DRAINAGE AREA, $-18,2 \mathrm{mi}^{2}$.

PERIOD of RECORD,--June 1910, January 1913 to current year. Monthly discharge only for some pertods, published in WSP 1312. Prior to October 1953, publishod as. "Santa Fe Creek near Santa" Fe."

REVISED RECORDS.--WSP 1512: 1933, 1936-37(M), 1942, drainage area. WSP 1732: 1923, 1925. WDR NM-75-1: 1927.

GAGE. -Water-stage recorder and concrete control. Elevation of gage is 7,720 ft above National Geodetic Vertical Datum of 1929, from topographic map. See WSP 1312 for history of changes prior to Oct. 1 , 1947.

REMARKS,--Records sood. Flow regulated by McClure Reservoir (station 08315500), completed in 1926, raised in 1935 1947, and again tn 1989. Several observations of water tempexature pere made during year.

EXTREMES OUTSIDE PERIOD OF RECORD, -mPeaks which probably exceeded 1,000 ft3/s occurred Aug. 19, 1872, and Sept, 29 or 30, 1904. Without regulation the flood of Sept. 23, 1929, might have exceeded 1,500 ft's/s. DTSCBARGE, CUBIC FEET PER SECOND, WATER YIAAR OCTOBER 1995 TO SEFTEMBER 1996

\begin{tabular}{|c|c|c|c|c|c|c|c|c|c|c|c|c|}
\hline DAY & $\operatorname{OcT}$ & Hov & DEC & JAN & FEB & MAR & A $\mathbf{A F R}$ & MAY & $\pi N$ & JUL & AUG & SEP \\
\hline $\begin{array}{l}1 \\
2 \\
3 \\
4 \\
5\end{array}$ & $\begin{array}{l}11 \\
11 \\
11 \\
11 \\
11\end{array}$ & $\begin{array}{l}3.8 \\
3.8 \\
3.8 \\
3.8 \\
3.8\end{array}$ & $\begin{array}{l}1.1 \\
1.1 \\
1.0 \\
1.1 \\
1.1\end{array}$ & $\begin{array}{l}1.0 \\
1.0 \\
1.0 \\
1.0 \\
1.0\end{array}$ & $\begin{array}{l}1.1 \\
1.1 \\
1.1 \\
1.1 \\
1.1\end{array}$ & $\begin{array}{l}1.0 \\
1.1 \\
1.1 \\
1.1 \\
1.1\end{array}$ & $\begin{array}{l}1.2 \\
1.2 \\
1.2 \\
1.7 \\
2.5\end{array}$ & $\begin{array}{l}11 \\
11 \\
18 \\
24 \\
23\end{array}$ & $\begin{array}{l}6.2 \\
6.1 \\
6.1 \\
5.9 \\
5.9\end{array}$ & $\begin{array}{l}5.2 \\
5.2 \\
5.2 \\
5.2 \\
5.2\end{array}$ & $\begin{array}{l}5.9 \\
5.9 \\
5.9 \\
5.7 \\
5.7\end{array}$ & $\begin{array}{l}4.9 \\
4.9 \\
4.9 \\
4.9 \\
4.9\end{array}$ \\
\hline $\begin{array}{r}6 \\
7 \\
8 \\
9 \\
10\end{array}$ & $\begin{array}{l}11 \\
11 \\
11 \\
11 \\
8.4\end{array}$ & $\begin{array}{l}3.8 \\
3.8 \\
3.8 \\
3.8 \\
3.8\end{array}$ & $\begin{array}{l}1.1 \\
1.0 \\
1.0 \\
1.0 \\
1.0\end{array}$ & $\begin{array}{l}1.0 \\
1.0 \\
1.0 \\
1.0 \\
1.0\end{array}$ & $\begin{array}{l}1.1 \\
1.1 \\
1.1 \\
1.1 \\
1.1\end{array}$ & $\begin{array}{l}1.1 \\
1.1 \\
1.1 \\
1.1 \\
1.1\end{array}$ & $\begin{array}{l}2.6 \\
2.6 \\
2.6 \\
2.6 \\
2.6\end{array}$ & $\begin{array}{l}23 \\
23 \\
22 \\
22 \\
22\end{array}$ & $\begin{array}{l}5.9 \\
5.9 \\
5.9 \\
5.9 \\
5.9\end{array}$ & $\begin{array}{l}5.2 \\
5.2 \\
5.2 \\
6.2 \\
6.2\end{array}$ & $\begin{array}{l}5.4 \\
5.4 \\
5.4 \\
5.4 \\
5.4\end{array}$ & $\begin{array}{l}4.9 \\
4.8 \\
4.7 \\
4.7 \\
4.7\end{array}$ \\
\hline $\begin{array}{l}11 \\
12 \\
13 \\
14 \\
15\end{array}$ & $\begin{array}{l}4.2 \\
4.2 \\
4.1 \\
4.0 \\
4.0\end{array}$ & $\begin{array}{l}3.8 \\
3.8 \\
3.8 \\
3.8 \\
3.8\end{array}$ & $\begin{array}{l}1.0 \\
1.0 \\
1.0 \\
1.0 \\
1.0\end{array}$ & $\begin{array}{l}1.0 \\
1.0 \\
1.0 \\
1.0 \\
1.0\end{array}$ & $\begin{array}{l}1.1 \\
1.1 \\
1.1 \\
1.1 \\
1.1\end{array}$ & $\begin{array}{l}1.1 \\
1.1 \\
1.1 \\
1.1 \\
1.1\end{array}$ & $\begin{array}{l}2.6 \\
2.6 \\
2.6 \\
2.6 \\
4.3\end{array}$ & $\begin{array}{l}22 \\
22 \\
22 \\
21 \\
21\end{array}$ & $\begin{array}{l}5.9 \\
5.8 \\
5.7 \\
5.7 \\
5.7\end{array}$ & $\begin{array}{l}6.0 \\
5.9 \\
5.9 \\
5.9 \\
5.9\end{array}$ & $\begin{array}{l}5.4 \\
5.4 \\
5.4 \\
5.4 \\
5.4\end{array}$ & $\begin{array}{l}4.7 \\
4.8 \\
4.9 \\
4.9 \\
4.9\end{array}$ \\
\hline $\begin{array}{l}16 \\
17 \\
18 \\
19 \\
20\end{array}$ & $\begin{array}{l}4.0 \\
4.0 \\
4.0 \\
4.0 \\
4.0\end{array}$ & $\begin{array}{l}2.5 \\
1.1 \\
1.1 \\
\frac{1}{1} \cdot 1 \\
1.1\end{array}$ & $\begin{array}{l}1.0 \\
1.0 \\
1.0 \\
1.0 \\
1.0\end{array}$ & $\begin{array}{l}1.0 \\
1.1 \\
1.1 \\
1.1 \\
1.0\end{array}$ & $\begin{array}{l}1.1 \\
1.1 \\
1.1 \\
1.1 \\
1.1\end{array}$ & $\begin{array}{l}1.1 \\
1.1 \\
1.2 \\
1.2 \\
1.2\end{array}$ & $\begin{array}{l}8.0 \\
8.8 \\
10 \\
10 \\
10\end{array}$ & $\begin{array}{l}21 \\
21 \\
21 \\
20 \\
20\end{array}$ & $\begin{array}{l}5.5 \\
5.4 \\
5.3 \\
5.2 \\
5.2\end{array}$ & $\begin{array}{l}5.9 \\
5.9 \\
5.9 \\
5.9 \\
5.9\end{array}$ & $\begin{array}{l}5.2 \\
5.2 \\
5.2 \\
5.2 \\
5.2\end{array}$ & $\begin{array}{l}4.9 \\
4.9 \\
5.0 \\
4.9 \\
4.9\end{array}$ \\
\hline $\begin{array}{l}21 \\
22 \\
23 \\
24 \\
25\end{array}$ & $\begin{array}{l}4.0 \\
4.0 \\
3.9 \\
3.8 \\
3.8\end{array}$ & $\begin{array}{l}1.1 \\
1.1 \\
1.1 \\
1.1 \\
1.1\end{array}$ & $\begin{array}{l}1.0 \\
1.0 \\
1.0 \\
1.1 \\
1.1\end{array}$ & $\begin{array}{l}1.0 \\
1.0 \\
1.0 \\
1.0 \\
1.0\end{array}$ & $\begin{array}{l}1.1 \\
1.1 \\
1.1 \\
1.0 \\
1.0\end{array}$ & $\begin{array}{l}1.2 \\
1.2 \\
1.2 \\
1.2 \\
1.2\end{array}$ & $\begin{array}{l}10 \\
11 \\
11 \\
11 \\
11\end{array}$ & $\begin{array}{l}20 \\
19 \\
19 \\
19 \\
19\end{array}$ & $\begin{array}{l}5.2 \\
5.2 \\
5.2 \\
5.1 \\
5.0\end{array}$ & $\begin{array}{l}5.9 \\
5.9 \\
5.9 \\
5.9 \\
5.9\end{array}$ & $\begin{array}{l}5.2 \\
5.2 \\
5.2 \\
5.2 \\
5.2\end{array}$ & $\begin{array}{l}5.0 \\
5.0 \\
5.1 \\
5.2 \\
5.2\end{array}$ \\
\hline $\begin{array}{l}26 \\
27 \\
28 \\
29 \\
30 \\
31\end{array}$ & $\begin{array}{l}3.8 \\
3.8 \\
3.8 \\
3.8 \\
3.8 \\
3.8\end{array}$ & $\begin{array}{l}1.1 \\
1.1 \\
1.1 \\
1.1 \\
1.1 \\
=-\infty\end{array}$ & $\begin{array}{l}1.1 \\
1.0 \\
1.0 \\
1.0 \\
1.0 \\
1.0\end{array}$ & $\begin{array}{l}1.0 \\
1.1 \\
1.1 \\
1.1 \\
1.1 \\
1.1\end{array}$ & $\begin{array}{l}1.0 \\
1.0 \\
1.0 \\
1.0 \\
\ldots \\
\ldots\end{array}$ & $\begin{array}{l}1.2 \\
1.2 \\
1.2 \\
1.2 \\
1.2 \\
1.2\end{array}$ & $\begin{array}{l}11 \\
11 \\
11 \\
11 \\
11 \\
-\infty\end{array}$ & $\begin{array}{l}18 \\
13 \\
6.2 \\
6.2 \\
6.2 \\
6.2\end{array}$ & $\begin{array}{l}4.9 \\
5.2 \\
5.2 \\
5.2 \\
5.2 \\
-.-\end{array}$ & $\begin{array}{l}5.9 \\
5.9 \\
5.9 \\
5.9 \\
5.9 \\
5.9\end{array}$ & $\begin{array}{l}5.2 \\
5.2 \\
5.2 \\
5.2 \\
5.2 \\
4.9\end{array}$ & $\begin{array}{l}5.2 \\
5.2 \\
5.2 \\
5.2 \\
5.2 \\
-.-1\end{array}$ \\
\hline $\begin{array}{l}\text { TOFAL } \\
\text { MEAN } \\
\text { MAX } \\
\text { MIN } \\
\text { AC-FT }\end{array}$ & $\begin{array}{r}190.2 \\
6.14 \\
11 \\
3.8 \\
377\end{array}$ & $\begin{array}{r}74.9 \\
2.50 \\
3.8 \\
1.1 \\
149\end{array}$ & $\begin{array}{r}31.8 \\
1.03 \\
1.1 \\
1.0 \\
63\end{array}$ & $\begin{array}{r}31.8 \\
1.03 \\
1.1 \\
1.0 \\
63\end{array}$ & $\begin{array}{r}31.3 \\
1.08 \\
1.1 \\
1.0 \\
62\end{array}$ & $\begin{array}{r}35.4 \\
1.14 \\
1.2 \\
1.0 \\
70\end{array}$ & $\begin{array}{r}191.3 \\
6.38 \\
11 \\
1.2 \\
379\end{array}$ & $\begin{array}{r}561.8 \\
18.1 \\
24 \\
6.2 \\
1110\end{array}$ & $\begin{array}{r}166.5 \\
5.55 \\
6.2 \\
4.9 \\
330\end{array}$ & $\begin{array}{r}178.0 \\
5.74 \\
6.2 \\
5.2 \\
353\end{array}$ & $\begin{array}{r}166.0 \\
5.35 \\
5.9 \\
4.9 \\
329\end{array}$ & $\begin{array}{r}148.6 \\
4.95 \\
5.2 \\
4.7 \\
295\end{array}$ \\
\hline
\end{tabular}

STATISTICS OF MONTHLY MEAN DATA FOR WATER YEARS 1913 - 1996, BY WATER YEAR (WY)

\begin{tabular}{|c|c|c|c|c|c|c|c|c|}
\hline $\begin{array}{l}\text { MFAN } \\
\text { MAX } \\
(W Y) \\
\text { MIN } \\
(W Y)\end{array}$ & $\begin{array}{r}4.68 \\
22.6 \\
1942 \\
\end{array}$ & $\begin{array}{r}3.02 \\
13.5 \\
1942 \\
.26\end{array}$ & $\begin{array}{r}2.58 \\
7.19 \\
1959 \\
.28\end{array}$ & $\begin{array}{r}2.40 \\
6.87 \\
1970 \\
.50\end{array}$ & $\begin{array}{r}2.71 \\
14.2 \\
1916 \\
.37\end{array}$ & $\begin{array}{r}4.91 \\
30.0 \\
1916 \\
.34\end{array}$ & $\begin{array}{r}12.6 \\
68.5 \\
1915 \\
.23\end{array}$ & $\begin{array}{r}23.4 \\
92.9 \\
1941 \\
1053\end{array}$ \\
\hline
\end{tabular}

\section{SURMARY STATISTICS}

ARNUAL TOTAY

ANNUAL MEAN

GIGHEST ANNUAL MEAN

LOWEST ANNUIAL MEAN

HIGHEST DAILY MEAN

LOWEST DAILY MEAT

ANRUAL SEVEN-DAY MINIMIM

INSTANTANEOUS PEAK FLON

INSTANTANEOUS PEAK STAGE

IHSTANTANEOUS LOW FLOW

ANNUAL RUNOFF (AC-FT)

10 PERCENT EXCEEDS

50 PERCENT EXCEEDS

90 PARCENT EXCEEDS

a-Site and datum then in use.
FOR 1995 CALENDAR YEAR

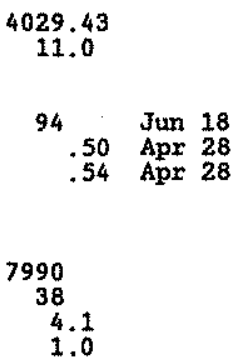

FOR 1996 WATER YEAR

WATER YEARS 1913 - 1996

1807.6

b-From rating curve extended above $150 \mathrm{ft}^{\mathrm{s}} / \mathrm{s}$. 


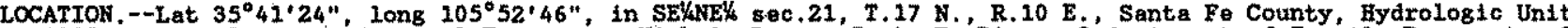
13020201 , in Santa Fe' National Forest, at Nichols Dam on Santa Fe Rtver, 0.6 mi east of Twomile Reservoir, 3,3 mi east of Santa Fe, and at mile 34.4 .

DRAIHAGE AREA. $--22.8 \mathrm{mi}^{2}$.

PERIOD OF RECORD. --March 1943 to September 1965 (monthend contents only), October 1965 to current year. Prior to January 1980 at site on outlet towex.

GACE.-Watar-gesge recorder. Detum of 8 age is 7.313.2 zt above National Goodetic Vertical Datum of 1929.

REMARXS,--Reservoir is formed by earthfill deri. No contents prior to Mar. 16, 1943 , Capacity, 685 acre-ft between gage heights 121,2 ft, bottom of lower operational gate and 167.0 . ft, crest of spillway. Dead storage, 14 acreft. Water is for municipal use of City of Santa Fe.

COOPERATION.--Survey to compute capacity table and supplementary gage readings, provided by Public Service Co. of New Merico.

EXTREMES FOR PERIOD OF RECORD, - Raximum contents, 836 acre-ft, June 8, 1952, gage height, 171,8 ft; minimum, 16 acre-ft, Feb. 11 to Mar. 10, 1944, Feb, 1-19, 1948.

EXTREMES FOR CURRENT YEAR,-Maximum contents, 591 acre-ft, Nov. 17; maximum gage height, 163.72 ft; winimum, 233 acre-ft, Apr, 16, gage height, 147.02

Capacity table (gage height, in leet, and contents, in acre-feet)

(Based on survey by Public Service Co, of New Mexico in 1943)

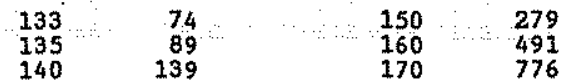

RESERVOIR STORAGE (ACRE-FEET), WATER YEAR OCTOBER 1995 TO SEPTEMBER 1996

\begin{tabular}{|c|c|c|c|c|c|c|c|c|c|c|c|c|}
\hline DAY & OCT & HOV & DEC & JAN & $\therefore$ FEB & MAR & AFR & MAY & JUW & JUL & AUG & SEF \\
\hline $\begin{array}{l}1 \\
2 \\
3 \\
4 \\
5\end{array}$ & $\begin{array}{l}514 \\
526 \\
535 \\
545 \\
555\end{array}$ & $\begin{array}{l}524 \\
528 \\
533 \\
538 \\
543\end{array}$ & $\begin{array}{l}569 \\
570 \\
571 \\
573 \\
574\end{array}$ & $\begin{array}{l}549 \\
548 \\
548 \\
550 \\
551\end{array}$ & $\begin{array}{l}553 \\
554 \\
556 \\
554 \\
549\end{array}$ & $\begin{array}{l}519 \\
520 \\
522 \\
523 \\
525\end{array}$ & $\begin{array}{l}343 \\
336 \\
327 \\
331 \\
329\end{array}$ & $\begin{array}{l}248 \\
254 \\
272 \\
284 \\
305\end{array}$ & $\begin{array}{r}462 \\
454 \\
454 \\
0448 \\
0442\end{array}$ & $\begin{array}{l}368 \\
376 \\
383 \\
380 \\
376\end{array}$ & $\begin{array}{l}501 \\
501 \\
501 \\
498 \\
493\end{array}$ & $\begin{array}{l}474 \\
472 \\
469 \\
467 \\
465\end{array}$ \\
\hline $\begin{array}{r}6 \\
7 \\
8 \\
9 \\
10\end{array}$ & $\begin{array}{l}563 \\
573 \\
583 \\
589 \\
585\end{array}$ & $\begin{array}{l}547 \\
552 \\
557 \\
562 \\
568\end{array}$ & $\begin{array}{l}574 \\
573 \\
574 \\
575 \\
574\end{array}$ & $\begin{array}{l}548 \\
545 \\
546 \\
548 \\
548\end{array}$ & $\begin{array}{l}543 \\
538 \\
533 \\
535 \\
535\end{array}$ & $\begin{array}{l}522 \\
514 \\
508 \\
509 \\
507\end{array}$ & $\begin{array}{l}319 \\
313 \\
299 \\
284 \\
273\end{array}$ & $\begin{array}{r}322 \\
340 \\
355 \\
0367 \\
0380\end{array}$ & $\begin{array}{r}436 \\
8428 \\
0420 \\
8414 \\
410\end{array}$ & $\begin{array}{l}372 \\
369 \\
378 \\
399 \\
418\end{array}$ & $\begin{array}{l}480 \\
481 \\
484 \\
481 \\
478\end{array}$ & $\begin{array}{l}463 \\
468 \\
476 \\
483 \\
479\end{array}$ \\
\hline $\begin{array}{l}11 \\
12 \\
13 \\
14 \\
15\end{array}$ & $\begin{array}{l}578 \\
574 \\
567 \\
561 \\
558\end{array}$ & $\begin{array}{l}574 \\
581 \\
587 \\
589 \\
588\end{array}$ & $\begin{array}{l}573 \\
570 \\
568 \\
565 \\
566\end{array}$ & $\begin{array}{l}543 \\
542 \\
543 \\
543 \\
544\end{array}$ & $\begin{array}{l}529 \\
524 \\
522 \\
516 \\
510\end{array}$ & $\begin{array}{l}498 \\
489 \\
482 \\
476 \\
477\end{array}$ & $\begin{array}{l}265 \\
254 \\
250 \\
242 \\
238\end{array}$ & $\begin{array}{r}0392 \\
8404 \\
8416 \\
426 \\
436\end{array}$ & $\begin{array}{l}0406 \\
0400 \\
0397 \\
0393 \\
0390\end{array}$ & $\begin{array}{l}427 \\
440 \\
452 \\
463 \\
474\end{array}$ & $\begin{array}{l}482 \\
482 \\
474 \\
466 \\
458\end{array}$ & $\begin{array}{l}476 \\
479 \\
489 \\
499 \\
508\end{array}$ \\
\hline $\begin{array}{l}16 \\
17 \\
18 \\
19 \\
20\end{array}$ & $\begin{array}{l}553 \\
549 \\
544 \\
540 \\
533\end{array}$ & $\begin{array}{l}589 \\
591 \\
588 \\
582 \\
576\end{array}$ & $\begin{array}{l}567 \\
567 \\
564 \\
561 \\
561\end{array}$ & $\begin{array}{r}542 \\
e 543 \\
535 \\
536 \\
537\end{array}$ & $\begin{array}{l}508 \\
510 \\
511 \\
513 \\
515\end{array}$ & $\begin{array}{l}478 \\
477 \\
469 \\
459 \\
450\end{array}$ & $\begin{array}{l}233 \\
237 \\
240 \\
243 \\
249\end{array}$ & $\begin{array}{l}446 \\
454 \\
457 \\
465 \\
472\end{array}$ & $\begin{array}{r}8385 \\
382 \\
375 \\
0371 \\
367\end{array}$ & $\begin{array}{l}479 \\
488 \\
499 \\
501 \\
498\end{array}$ & $\begin{array}{l}445 \\
433 \\
418 \\
416 \\
413\end{array}$ & $\begin{array}{l}509 \\
509 \\
519\end{array}$ \\
\hline $\begin{array}{l}21 \\
22 \\
23 \\
24 \\
25\end{array}$ & $\begin{array}{l}524 \\
519 \\
516 \\
512 \\
514\end{array}$ & $\begin{array}{l}573 \\
570 \\
570 \\
571 \\
572\end{array}$ & $\begin{array}{l}562 \\
561 \\
560 \\
559 \\
557\end{array}$ & $\begin{array}{r}539 \\
0540 \\
0541 \\
542 \\
0542\end{array}$ & $\begin{array}{l}517 \\
518 \\
520 \\
521 \\
519\end{array}$ & $\begin{array}{l}441 \\
430 \\
418 \\
405 \\
393\end{array}$ & $\begin{array}{l}254 \\
256 \\
255 \\
253 \\
250\end{array}$ & $\begin{array}{l}478 \\
484 \\
490 \\
501 \\
521\end{array}$ & $\begin{array}{r}8359 \\
350 \\
343 \\
333 \\
330\end{array}$ & $\begin{array}{l}495 \\
496 \\
497 \\
493 \\
491\end{array}$ & $\begin{array}{l}413 \\
424 \\
434 \\
440 \\
445\end{array}$ & 5 \\
\hline $\begin{array}{l}26 \\
27 \\
28 \\
29 \\
30 \\
31\end{array}$ & $\begin{array}{r}516 \\
514 \\
513 \\
5 \quad 515 \\
517 \\
519\end{array}$ & $\begin{array}{l}573 \\
573 \\
570 \\
570 \\
569 \\
-\end{array}$ & $\begin{array}{l}557 \\
556 \\
552 \\
548 \\
550 \\
550\end{array}$ & $\begin{array}{l}544 \\
546 \\
547 \\
549 \\
550 \\
552\end{array}$ & $\begin{array}{r}515 \\
516 \\
\therefore \quad 517 \\
518 \\
--- \\
-\cdots\end{array}$ & $\begin{array}{l}381 \\
370 \\
363 \\
364 \\
363 \\
353\end{array}$ & $\begin{array}{l}248 \\
241 \\
243 \\
253 \\
254 \\
--4\end{array}$ & $\begin{array}{l}534 \\
528 \\
511 \\
493 \\
478 \\
468\end{array}$ & $\begin{array}{l}327 \\
336 \\
346 \\
356 \\
360 \\
-\end{array}$ & $\begin{array}{l}501 \\
506 \\
510 \\
507 \\
505 \\
503\end{array}$ & $\begin{array}{l}452 \\
461 \\
470 \\
474 \\
480 \\
478\end{array}$ & 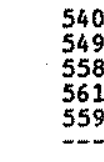 \\
\hline $\begin{array}{l}\text { MAX } \\
\text { MIN } \\
(Y) \\
(Y Y)\end{array}$ & $\begin{array}{r}589 \\
512 \\
161.06 \\
+19\end{array}$ & $\begin{array}{r}591 \\
524 \\
162.88 \\
+50\end{array}$ & $\begin{array}{r}575 \\
548 \\
162.19 \\
-19\end{array}$ & $\begin{array}{r}552 \\
535 \\
162.28 \\
+2\end{array}$ & $\begin{array}{r}556 \\
508 \\
161.00 \\
-34\end{array}$ & $\begin{array}{r}525 \\
353 \\
153.86 \\
-165\end{array}$ & $\begin{array}{r}343 \\
233 \\
148.35 \\
-99\end{array}$ & $\begin{array}{r}534 \\
248 \\
159.03 \\
+214\end{array}$ & $\begin{array}{r}462 \\
327 \\
154.22 \\
-108\end{array}$ & $\begin{array}{r}510 \\
368 \\
160.45 \\
+143\end{array}$ & $\begin{array}{r}501 \\
413 \\
159.45 \\
-25\end{array}$ & $\begin{array}{r}56 \\
46 \\
162.5 \\
+8\end{array}$ \\
\hline
\end{tabular}

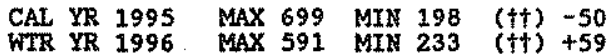

- Estimated

(†) GAGE HEIGAT, IN FEET, AT END OF MONTH

(††) CHANGE IN CONTENTS, IN ACRE-FEET 
RTO GRANDE BASIN

08317200 SANTA FE RIVER ABOVE COCHITI LAKE, NM

LOCATION.--Lat $35^{\circ} 32 \times 49^{\prime \prime}$, long $106^{\circ} 13^{\prime} 41^{\prime \prime}$, in NWK sec.8, T.15 N., R.7 E. Santa Fe County, Bydrologic Unit 1302.0201 In Mesita de Juana Lopez Grant, on right bank at foot of La Bajada Hili, 5.0 mi upstream from Cochiti Dam, 6.3 mi east of Pena Blanca, and at mile 7.9 .

DRAINAGE AREA. $--232 \mathrm{mi}^{3}$.

PERIOD OF RECORD, - March 1970 to current year.

WATER-DISCEARGE RECORDS

GAGE.--Water-stage recorder and concrete control. Elevation of gage is 5,505 ft above National Geodetic Vertical Datum of 1929 from topographic map.

RDMARKS. --Water-discharge records good except for estimated daily discharges, which are poor. Surface-and groundwater diversions and returns for municipal supply of City of Santa Fe in upper part of basin. Diverstons for irrigation of about 400 acres upstream from station. See tabulation below for the results of discharge

measurements made during year at point adjacent to gage of an unnamed ditch on right bank which diverts water 0.4 mi upstream and bypasses gage; ditch flow not included in record. Lowest flow for period of record, no flow July $16-18,1971$.

DISCHARGE MEASUREMENTS, IN CUBIC FEET PER SECOND, OF DITCA, WATER YEAR OCTOBER 1995 TO SEPTEMBER 1996

$\begin{array}{cccc}\text { Date } & \text { Discharge } & \text { Date } & \text { Discharge } \\ 10 / 12 / 95 & 0.20 & 03 / 12 / 96 & 0 \\ 12 / 07 / 95 & 0 & 07 / 16 / 96 & 0 \\ 02 / 06 / 96 & 0 & 08 / 06 / 96 & .38\end{array}$

DISCEARGE, CUBIC FEET PER SECOND, WATER YEAR OCTOBER 1995 TO SEPTEMBER 1996

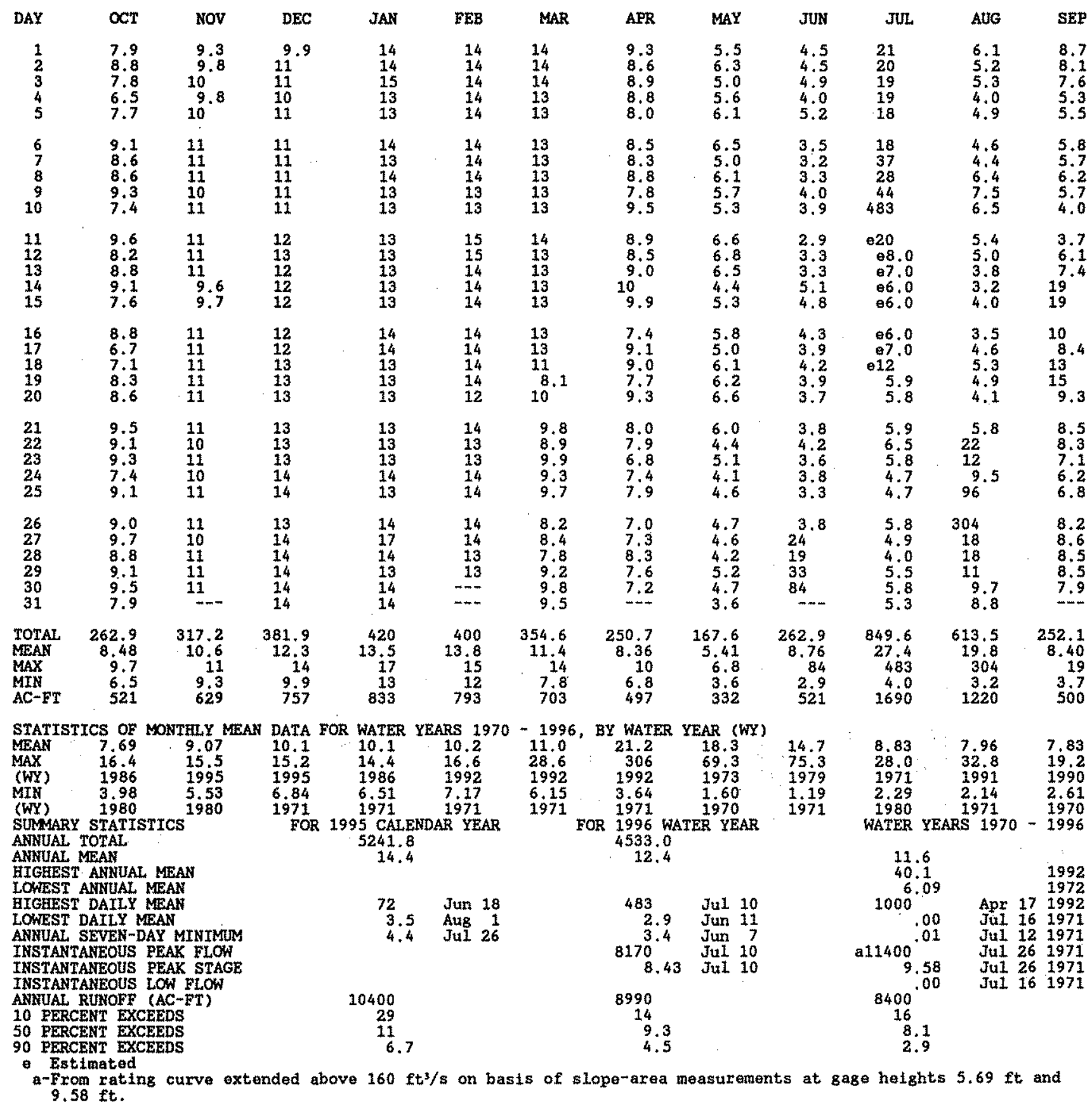


RIO GRANDE BASIN

08317200 SANTA FE RIVER ABOVE COCAITI LAKE, NM -- Continued

WATER-QUALITY RECORDS

PERIOD OF RECORD.--Water years 1974-75, 1979, 1981 to current year.

WATHR QUALITY DATA, WATER YEAR OCTOBER 1995 TO SEPTEMBER 1996

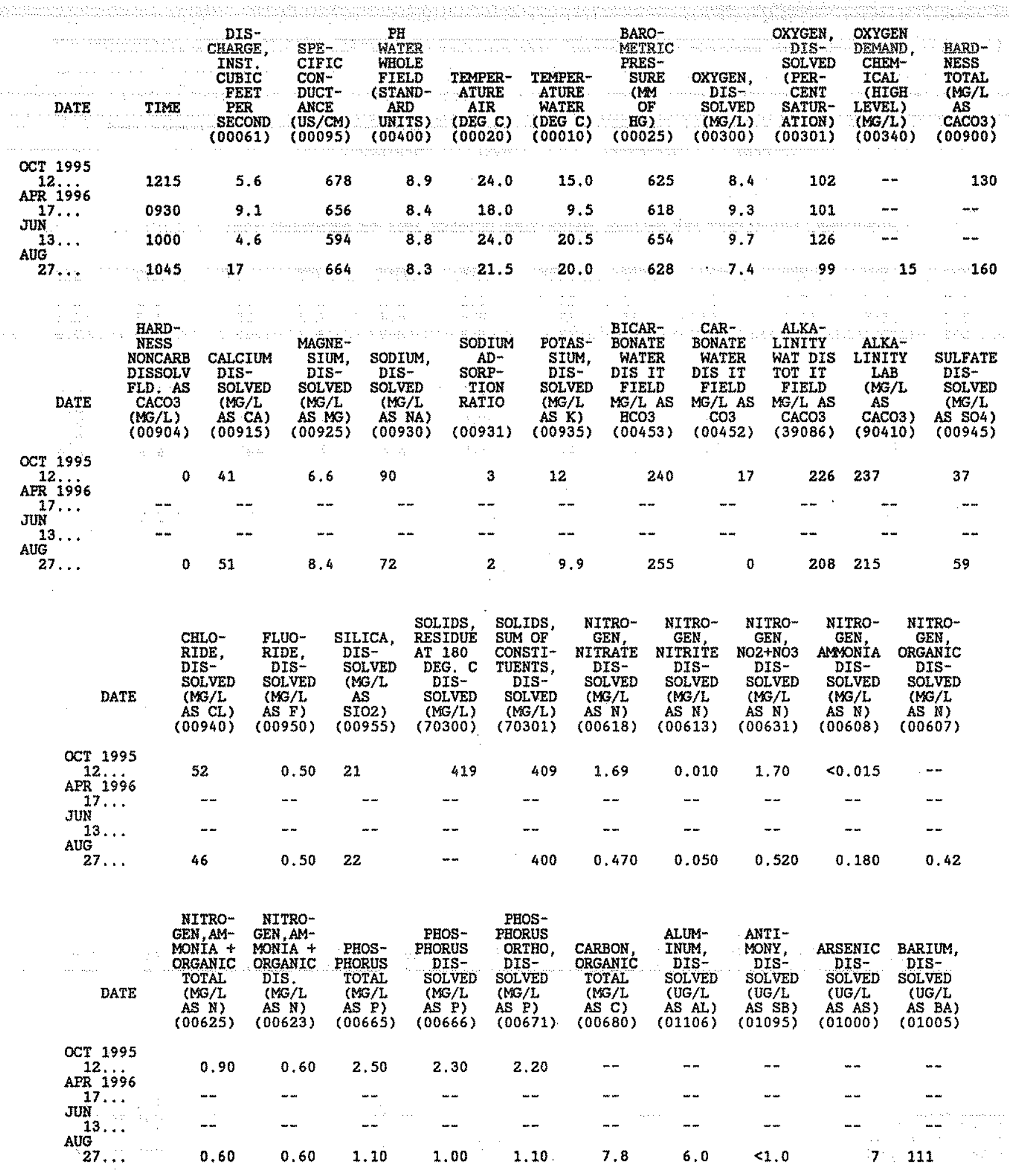


RIO GRANDE BASIN

08317200 SANTA FE RIVER ABOVE COCHITI LAKE, NM -- Continued

WATER-QUALITY RECORDS

WATER QUALITY DATA, WATER YEAR OCTOBER 1995 TO SEETEMBER 1996

\begin{tabular}{|c|c|c|c|c|c|c|c|c|c|c|}
\hline ATE & $\begin{array}{l}\text { BERYL- } \\
\text { LIUM, } \\
\text { DIS- } \\
\text { SOLVED } \\
\text { (UG/L } \\
\text { AS BE) } \\
\text { (01010) }\end{array}$ & $\begin{array}{c}\text { BORON, } \\
\text { DIS- } \\
\text { SOLVED } \\
\text { (UG/L } \\
\text { AS B }) \\
(01020)\end{array}$ & $\begin{array}{c}\text { CADMIUM } \\
\text { DIS- } \\
\text { SOLVED } \\
\text { (UG/L } \\
\text { AS CD) } \\
(01025)\end{array}$ & $\begin{array}{l}\text { CERO- } \\
\text { MIUM, } \\
\text { DIS- } \\
\text { SOLVED } \\
\text { (UG/L } \\
\text { AS CR) } \\
\text { (01030) }\end{array}$ & $\begin{array}{l}\text { COBALT, } \\
\text { DIS- } \\
\text { SOLVED } \\
\text { (UG/L } \\
\text { AS CO) } \\
(01035)\end{array}$ & $\begin{array}{c}\text { COPPER, } \\
\text { DIS- } \\
\text { SOLVED } \\
\text { (UG/L } \\
\text { AS CU) } \\
(01040)\end{array}$ & $\begin{array}{c}\text { IRON, } \\
\text { DIS - } \\
\text { SOLVED } \\
\text { (UG/L } \\
\text { AS FE) } \\
(01046)\end{array}$ & $\begin{array}{c}\text { LEAD, } \\
\text { DIS: } \\
\text { SOLVED } \\
\text { (UG/L } \\
\text { AS PB) } \\
(01049)\end{array}$ & $\begin{array}{l}\text { MANGA- } \\
\text { NESE, } \\
\text { DIS- } \\
\text { SOLVED } \\
\text { (UG/L } \\
\text { AS MN) } \\
\text { (01056) }\end{array}$ & $\begin{array}{c}\text { MERCURY } \\
\text { TOTAL } \\
\text { RECOV- } \\
\text { ERABLE } \\
\text { (UG/L } \\
\text { AS HG) } \\
\text { (71900) }\end{array}$ \\
\hline
\end{tabular}

\begin{tabular}{|c|c|c|c|c|c|c|c|c|c|c|}
\hline OCT 1995 & $-\cdots$ & -- & -- & $\cdots$ & $\cdots$ & -- & 8,0 & $\cdots$ & 11 & - \\
\hline $\begin{array}{c}A P R \\
17 \ldots 96\end{array}$ & $-\cdots$ & -- & -- & -- & $-\cdots$ & - & -- & -- & - & -- \\
\hline $13 \ldots$ & -- & -- & -- & $-m$ & -- & -- & $\rightarrow$ & - & - & -- \\
\hline 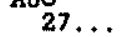 & $<1.0$ & 189 & $<1.0$ & 2.0 & 2.0 & 3.0 & 3.0 & $<1.0$ & 19 & $<0.10$ \\
\hline
\end{tabular}

$\begin{array}{lcc} & \\ \text { MOLYB- } & & \\ \text { DENUM, NICKEL, } & \text { SELE- } \\ \text { DIS- } & \text { DIS- } & \text { NIUM, } \\ \text { SOLVED } & \text { SOLVED } & \text { TOTAL } \\ \text { (UG/L } & \text { (UG/L } & \text { (UG/L } \\ \text { AS MO) } & \text { AS NI) } & \text { AS SE }) \\ (01060) & (01065) & (01147)\end{array}$

\begin{tabular}{|c|c|c|c|c|c|c|}
\hline $\begin{array}{l}\text { SELEE- } \\
\text { NIUM, } \\
\text { DIS- } \\
\text { SOLVED } \\
\text { (UG/L } \\
\text { AS SE) } \\
(01145)\end{array}$ & $\begin{array}{c}\text { SILVER, } \\
\text { DIS- } \\
\text { SOLVED } \\
\text { (UG/L } \\
\text { AS AG) } \\
(01075)\end{array}$ & $\begin{array}{c}\text { ZINC, } \\
\text { DIS } \\
\text { SOLVED } \\
\text { (UG/L } \\
\text { AS ZN) } \\
(01090)\end{array}$ & $\begin{array}{c}\text { URANIUM } \\
\text { NATURAL } \\
\text { DIS- } \\
\text { SOLVED } \\
\text { (UG/L } \\
\text { AS U) } \\
(22703)\end{array}$ & $\begin{array}{l}\text { SEDI- } \\
\text { MENT, } \\
\text { SUS- } \\
\text { PENDED } \\
\text { (MG/L) } \\
(80154)\end{array}$ & $\begin{array}{c}\text { SEDI- } \\
\text { MENT, } \\
\text { DIS- } \\
\text { CHARGE, } \\
\text { SUS- } \\
\text { PENDED } \\
\text { (T/DAY) } \\
\text { (80155) }\end{array}$ & $\begin{array}{c}\text { SED } \\
\text { SUSE. } \\
\text { SIEVE } \\
\text { DIAM. } \\
\% \text { FINER } \\
\text { THAN } \\
.062 \mathrm{MM} \\
(70331)\end{array}$ \\
\hline
\end{tabular}

\begin{tabular}{|c|c|c|c|c|}
\hline \multicolumn{4}{|l|}{$\begin{array}{l}\text { OCT } 1995 \\
12 \\
\text { AFR } 1996 \\
17 \ldots \\
\text { JUN } \\
13 \ldots \\
\text { AUG }\end{array}$} & $\begin{array}{l}\text { OCT } 1995 \\
12 \\
\text { AFR } 1996 \\
17 \ldots \\
\text { JUN } \\
13 \ldots \\
\text { AUG }\end{array}$ \\
\hline
\end{tabular}

$\begin{array}{cccccc}-- & -- & - & -- & -- & -- \\ -- & -- & -- & -- & -- & -- \\ -- & -- & -- & -- & -- & -- \\ 10 & 6.0 & <1 & <1 & <1,0 & 19\end{array}$

$+-$

172
-
-
-
1390

2.6

$--$

$--$

$27 \ldots$

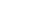


RIO GRANDE BASIN

08317300 COCHITI LAKE NEAR COCHITI PUEBLO, NM

LOCATION,--Lat $35^{\circ} 37^{\prime} 01^{\prime \prime}$, long $106^{\circ} 18^{\circ} 58^{\prime \prime}$, In NWWSWk sec.16, T.16 N., R.6 E. Sandoval County, Bydrologic Unit 13020201 , in Pueblo de Cochiti Grant, in control tower at Cochiti Dam, 1.7 mi northeast of Cochiti Pueblo, and at mile $1,588,1$.

DRAINAGE AREA. - - 14,900 $\mathrm{mi}^{2}$ approximately, including $2,940 \mathrm{mi}^{2}$, in closed basin in $5 a n$ Luis Velley, Co.

WATER-DISCHARGE RECORDS

PERIOD OF RECORD, - November 1973 to current year.

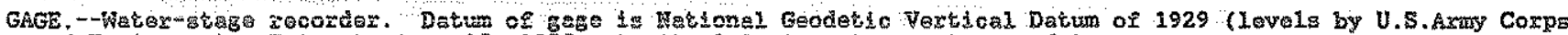
of Engineers). Prior to Apr. 15, 1975, at site 1.3 mi upstream at same datum.

REMARKS.--Lake is formed by an earthfill dam on Rio Grande and Santa Fe River. Storage began on Nov, 12 , 1973. Capacity, based on capacity tablo effective Jan, 1, 1992, 502, 330 acre-ft between elevations $5,247.0$ fit and $5,450.0 \mathrm{ft}$, crest of service spillmay. Dad storage 560 acre-ft below elevation $5,255.0 \mathrm{ft}$, invert of outlot structure. Lake created primarily for flood and gediment control. A 50,000-acre-ft permanent pool is authorized for recreationel purposes. U.S. Army Corps of Engineers satellite telemeter at station.

COOPERATION.--Records provided by U.S. Army Corps of Engineers.

EXTREMES FOR PERIOD OF RECORD,--Maximum contents, 301,000 acre-ft, July 3, 1986, elovation, 5,417.32 ft; no storage prior to Nov. $12,1973$.

EXTREAES FOR CURRENT YEAR. --Maximum contents, 59,570 acre-ft, July 10, elevation, 5,342.36 ft; minimum, 54,670 acre-ft, Oct. 12, Olovgtion, 5,338.99 ft.

Capacity table (elevation, in feet, and contents, in acre-feet)

(Based on survey by Corps of Engineers in 1992)

\begin{tabular}{|c|c|c|}
\hline $\begin{array}{l}5,325 \\
5,335 \\
5,345 \\
5,355\end{array}$ & $\begin{array}{r}39,108 \\
49,770 \\
63,520 \\
81,310\end{array}$ & $\begin{array}{l}5,365 \\
5,375 \\
5,385 \\
5,395\end{array}$ \\
\hline
\end{tabular}

RESERVOIR STORAGE (ACRE-FEET), WATER YEAR OCTOBER 1995 TO SEPTEMBER 1996 DAILY OBSERVATION AT 24:00 VALUES

\begin{tabular}{|c|c|c|c|c|c|c|c|c|c|c|c|c|}
\hline DAY & OCT & Nov & $\mathrm{DEC}$ & JAN & FEB & MAR & APR & MAY & JUN & JUL & AUG & SEP \\
\hline $\begin{array}{l}1 \\
2 \\
3 \\
4 \\
5\end{array}$ & $\begin{array}{l}55190 \\
55030 \\
54970 \\
54830 \\
54850\end{array}$ & $\begin{array}{l}55310 \\
55560 \\
55660 \\
55470 \\
55510\end{array}$ & $\begin{array}{l}55450 \\
55400 \\
55310 \\
55230 \\
55310\end{array}$ & $\begin{array}{l}56960 \\
56960 \\
56770 \\
56630 \\
56850\end{array}$ & $\begin{array}{l}57940 \\
57900 \\
57860 \\
57690 \\
57730\end{array}$ & $\begin{array}{l}58280 \\
57900 \\
57610 \\
57620 \\
57800\end{array}$ & $\begin{array}{l}57640 \\
57550 \\
57410 \\
57340 \\
57410\end{array}$ & $\begin{array}{l}57480 \\
57550 \\
57310 \\
56890 \\
56500\end{array}$ & $\begin{array}{l}57520 \\
57450 \\
57370 \\
57280 \\
57170\end{array}$ & $\begin{array}{l}57860 \\
57750 \\
57900 \\
58250 \\
58520\end{array}$ & $\begin{array}{l}57310 \\
57310 \\
57420 \\
57480 \\
57420\end{array}$ & $\begin{array}{l}56840 \\
56950 \\
56840 \\
56770 \\
56860\end{array}$ \\
\hline $\begin{array}{r}6 \\
7 \\
8 \\
9 \\
10\end{array}$ & $\begin{array}{l}55010 \\
55110 \\
55050 \\
54970 \\
54860\end{array}$ & $\begin{array}{l}55530 \\
55420 \\
55410 \\
55480 \\
55550\end{array}$ & $\begin{array}{l}55530 \\
55620 \\
55620 \\
55510 \\
55420\end{array}$ & $\begin{array}{l}57060 \\
57200 \\
57190 \\
56940 \\
56710\end{array}$ & $\begin{array}{l}57970 \\
58130 \\
58140 \\
58130\end{array}$ & $\begin{array}{l}57820 \\
57850 \\
57820 \\
57820 \\
57790\end{array}$ & $\begin{array}{l}57590 \\
57650 \\
57710 \\
57730 \\
57730\end{array}$ & $\begin{array}{l}56350 \\
56280 \\
56450 \\
56700 \\
56910\end{array}$ & $\begin{array}{l}57060 \\
56920 \\
56840 \\
56740 \\
56700\end{array}$ & $\begin{array}{l}58380 \\
57870 \\
58130 \\
58520 \\
59570\end{array}$ & $\begin{array}{l}57200 \\
56920 \\
56950 \\
57230 \\
57370\end{array}$ & $\begin{array}{l}57000 \\
57000 \\
56570 \\
56390 \\
56490\end{array}$ \\
\hline $\begin{array}{l}11 \\
12 \\
13 \\
14 \\
15\end{array}$ & $\begin{array}{l}54710 \\
54670 \\
54780 \\
54890 \\
55010\end{array}$ & $\begin{array}{l}55590 \\
55620 \\
55530 \\
55560 \\
55560\end{array}$ & $\begin{array}{l}55370 \\
55380 \\
55450 \\
55420 \\
55400\end{array}$ & $\begin{array}{l}56530 \\
56640 \\
56680 \\
56770 \\
56940\end{array}$ & $\begin{array}{l}58130 \\
58150 \\
58130 \\
57940 \\
57940\end{array}$ & $\begin{array}{l}57790 \\
57760 \\
57820 \\
57930 \\
57900\end{array}$ & $\begin{array}{l}57710 \\
57680 \\
57710 \\
57830 \\
57800\end{array}$ & $\begin{array}{l}57 \\
57 \\
57 \\
58 \\
58\end{array}$ & $\begin{array}{l}56 \\
56 \\
57 \\
57 \\
57\end{array}$ & $\begin{array}{l}58 \\
57 \\
58 \\
57 \\
57\end{array}$ & & $\begin{array}{l}70 \\
30 \\
60 \\
30 \\
30\end{array}$ \\
\hline $\begin{array}{l}16 \\
17 \\
18 \\
19 \\
20\end{array}$ & $\begin{array}{l}55110 \\
55000 \\
54820 \\
54780 \\
54920\end{array}$ & $\begin{array}{l}55590 \\
55590 \\
55510 \\
55480 \\
55510\end{array}$ & $\begin{array}{l}55560 \\
55670 \\
55730 \\
55730 \\
55810\end{array}$ & $\begin{array}{l}57100 \\
57240 \\
57270 \\
57240 \\
57160\end{array}$ & $\begin{array}{l}58140 \\
58300 \\
58510 \\
58720 \\
58780\end{array}$ & $\begin{array}{l}57990 \\
57870 \\
57680 \\
57510 \\
57540\end{array}$ & $\begin{array}{l}573 \\
570 \\
568 \\
565 \\
564\end{array}$ & $\begin{array}{l}58 \\
57 \\
57 \\
57 \\
57\end{array}$ & $\begin{array}{l}57 \\
57 \\
57 \\
57 \\
57\end{array}$ & $\begin{array}{l}57 \\
57 \\
57 \\
57 \\
57\end{array}$ & & $\begin{array}{l}90 \\
60 \\
90 \\
80 \\
60\end{array}$ \\
\hline $\begin{array}{l}21 \\
22 \\
23 \\
24 \\
25\end{array}$ & $\begin{array}{l}55080 \\
54940 \\
54870 \\
54970 \\
55040\end{array}$ & $\begin{array}{l}55370 \\
55350 \\
55490 \\
55450 \\
55490\end{array}$ & $\begin{array}{l}56000 \\
56220 \\
56360 \\
56500 \\
56500\end{array}$ & $\begin{array}{l}57100 \\
57060 \\
57240 \\
57480 \\
57680\end{array}$ & $\begin{array}{l}58510 \\
58170 \\
57990 \\
57970 \\
57960\end{array}$ & $\begin{array}{l}57 \\
57 \\
57 \\
57 \\
57\end{array}$ & $\begin{array}{l}56 \\
56 \\
56 \\
57 \\
57\end{array}$ & $\begin{array}{l}57 \\
57 \\
57 \\
57 \\
57\end{array}$ & $\begin{array}{l}56 \\
56 \\
57 \\
56 \\
56\end{array}$ & & & $\begin{array}{l}60 \\
40 \\
50 \\
50 \\
70\end{array}$ \\
\hline $\begin{array}{l}26 \\
27 \\
28 \\
29 \\
30 \\
31\end{array}$ & $\begin{array}{l}54940 \\
54890 \\
54970 \\
54960 \\
54850 \\
54830\end{array}$ & $\begin{array}{r}55410 \\
55300 \\
55310 \\
55340 \\
55420\end{array}$ & $\begin{array}{l}56590 \\
56710 \\
56750 \\
56880 \\
56910 \\
56910\end{array}$ & $\begin{array}{l}57860 \\
57940 \\
58030 \\
58110 \\
58140 \\
58110\end{array}$ & $\begin{array}{r}57860 \\
57790 \\
57870 \\
58390 \\
- \\
\cdots\end{array}$ & $\begin{array}{l}57590 \\
57510 \\
57450 \\
57340 \\
57450 \\
57590\end{array}$ & $\begin{array}{l}57620 \\
57510 \\
57420 \\
57370 \\
57410\end{array}$ & $\begin{array}{l}57310 \\
57590 \\
57790 \\
57680 \\
57560 \\
57590\end{array}$ & $\begin{array}{l}57090 \\
57710 \\
58180 \\
58140 \\
57900\end{array}$ & $\begin{array}{l}57300 \\
57480 \\
57490 \\
57480 \\
57450 \\
57420\end{array}$ & $\begin{array}{l}57130 \\
57610 \\
57090 \\
56600 \\
56480 \\
56640\end{array}$ & $\begin{array}{l}56630 \\
56560 \\
56530 \\
56500 \\
56560\end{array}$ \\
\hline 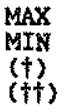 & $\begin{array}{r}55190 \\
54670 \\
338.97 \\
-500\end{array}$ & $\begin{array}{r}55660 \\
55300 \\
5339.40 \\
+590\end{array}$ & $\begin{array}{r}56910 \\
55230 \\
5340.47 \\
+1490\end{array}$ & $\begin{array}{r}58140 \\
56530 \\
5341.33 \\
+1200\end{array}$ & $\begin{array}{r}58780 \\
57690 \\
5341.53 \\
+280\end{array}$ & $\begin{array}{r}58280 \\
57340 \\
5340.96 \\
-800\end{array}$ & $\begin{array}{r}57830 \\
56410 \\
5340.83 \\
-180\end{array}$ & $\begin{array}{r}58070 \\
56280 \\
5340.96 \\
+180\end{array}$ & $\begin{array}{r}58180 \\
56700 \\
5341.18 \\
+310\end{array}$ & $\begin{array}{r}59570 \\
57170 \\
5340.84 \\
-480\end{array}$ & $\begin{array}{r}57610 \\
56480 \\
5340.28 \\
-780\end{array}$ & $\begin{array}{r}57330 \\
56390 \\
5340.22 \\
-80\end{array}$ \\
\hline
\end{tabular}

CAL YR 1995 MAX 131810 MIN 53970 (t) -2300

WTR YR 2996 MAX 59570 MIX 54670 ( $+\uparrow)+1230$

(†) ELEVATION, IN FEET, AT END OF MONTH.

(†) CHANGE IN CONTENTS, IN ACRE-FEET. 
RIO GRANDE BASIN

08317300 COCHITI LAKE NEAR COCHITI PUEBLO, NM -- Continued

WATER-QUALITX RECORDS

LOCATION. - Samples collected in Cochit1 Lake Impounded by Cochit1 Dam on the Rio Grande. PERIOD OF RECORD.-WAter years 1981 to current year.

REMARKS. - Samples for chemical analyses are collected annually at Site A which is located 500 ft upstrean from the Outlet Tower (Riser). Samples are collected 5 feet above the bottom of the lake.

WATER QUALTTY DATA, WATER YEAR OCTOBER 1995 TO SEPTEMBER 1996

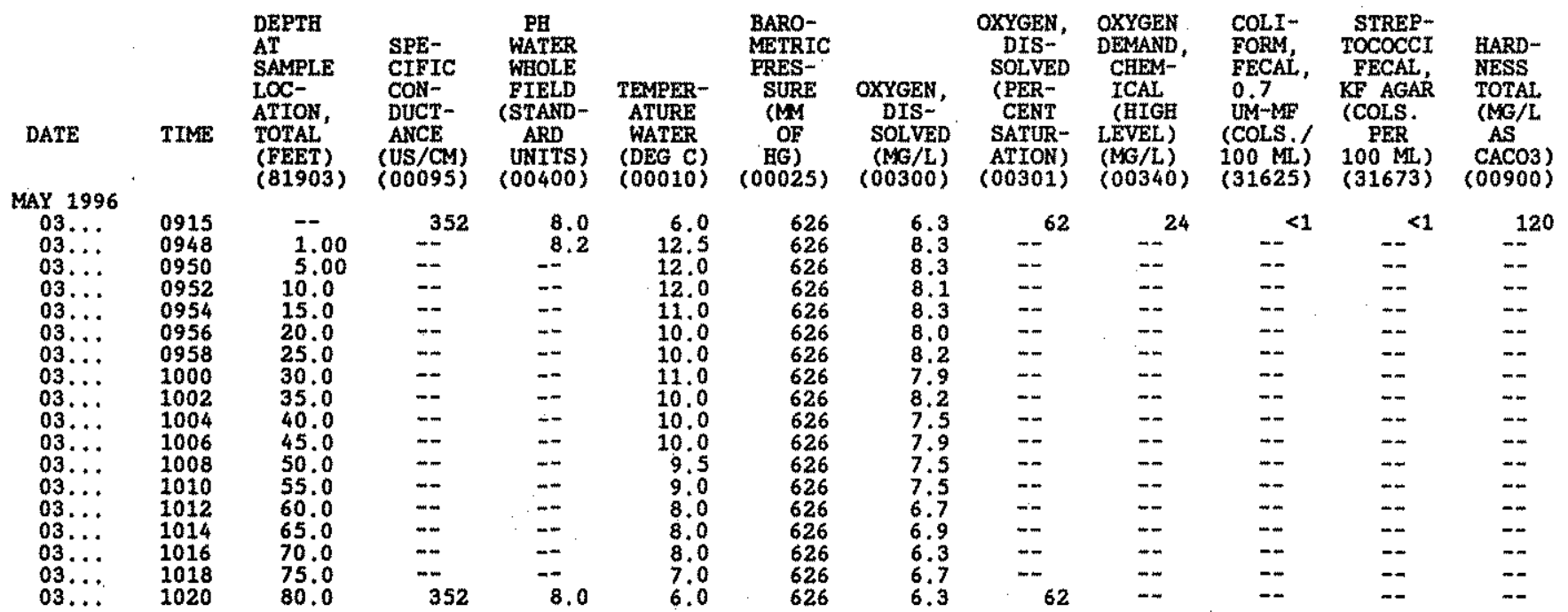

$\begin{array}{cccccccc} & & \text { MAGNE- } & & \text { SODIUM } & \text { POTAS- } & \text { ALKA- } & \\ & \text { CALCIUM } & \text { SIUM, } & \text { SODIUM, } & \text { AD- } & \text { SIUM, LINITY } & \text { SULFATE } \\ \text { DIS- } & \text { DIS- } & \text { DIS- } & \text { SORP- } & \text { DIS- } & \text { LAB } & \text { DIS- } \\ \text { SOLVED } & \text { SOLVED } & \text { SOLVED } & \text { TION } & \text { SOLVED } & (M G / L & \text { SOLVED } \\ \text { DATE } & (M G / L & (M G / L & (M G / L & \text { RATIO } & (M G / L & \text { AS } & (M G / L \\ & \text { AS CA }) & \text { AS MK) } & \text { AS NA) } & & \text { AS K) } & \text { CACO3) AS SO4) } \\ & (00915) & (00925) & (00930) & (00931) & (00935) & (90410) & (00945)\end{array}$

$\begin{array}{lcccc}\text { CHLO- } & \text { FLUO- } & \text { SILICA, } & \text { SUM OF } & \text { GEN, } \\ \text { RIDE, } & \text { RIDE, } & \text { DIS- } & \text { CONSTI- } & \text { NITRITE } \\ \text { DIS- } & \text { DIS } & \text { SOLVED } & \text { TUENTS, } & \text { DIS- } \\ \text { SOLVED } & \text { SOLVED } & \text { (MG/L } & \text { DIS - } & \text { SOLVDD } \\ (M G / L & (M G / L & \text { AS } & \text { SOLVED } & (\text { MG } / L \\ \text { AS CL) } & \text { AS F) } & \text { SIO2) } & (\text { MG/L) } & \text { AS N) } \\ (00940) & (00950) & (00955) & (70301) & (00613)\end{array}$

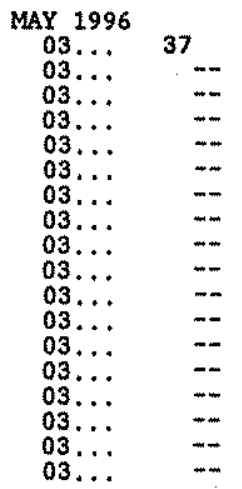

\begin{tabular}{|c|c|}
\hline 7.0 & 21 \\
\hline$m$ & - \\
\hline$-m$ & $-\cdots$ \\
\hline$m-$ & $-\cdots$ \\
\hline-- & - \\
\hline$-m$ & - \\
\hline$-m$ & - \\
\hline$m$ & - \\
\hline$=-$ & - \\
\hline-- & 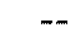 \\
\hline-- & $-m$ \\
\hline-- & $m$ \\
\hline-- & - \\
\hline-- & 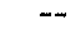 \\
\hline$m$ & 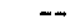 \\
\hline-- & \\
\hline-- & \\
\hline
\end{tabular}

\begin{tabular}{|c|c|c|}
\hline 0.8 & 2.9 & 116. \\
\hline$-\infty$ & -- & \\
\hline- & -- & - \\
\hline-- & $=-$ & - \\
\hline-- & -- & - \\
\hline- & $-m$ & - \\
\hline 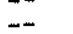 & $\ldots$ & \\
\hline- & $m$ & \\
\hline-- & -- & \\
\hline$-m$ & سب & \\
\hline$-m$ & $m$ & \\
\hline-- & $-m$ & \\
\hline$-\pi$ & -- & \\
\hline- & -- & \\
\hline$-\infty$ & $=$ & \\
\hline- & - & \\
\hline
\end{tabular}

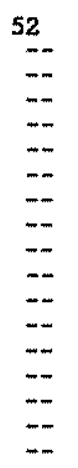

\begin{tabular}{|c|c|c|}
\hline 7.0 & 0.40 & 19 \\
\hline-- & -- & $\omega$ \\
\hline$-m$ & $\rightarrow$ & -+ \\
\hline$-m$ & - & -- \\
\hline$\rightarrow$ & -- & $-\infty$ \\
\hline- & -- & $m$ \\
\hline$-m$ & -- & -- \\
\hline-- & -- & - \\
\hline$-\rightarrow$ & -- & $m-$ \\
\hline-- & -- & -- \\
\hline$-m$ & $-m$ & $-m$ \\
\hline- & - & -- \\
\hline سـ- & -. & $-m$ \\
\hline-- & - & - س \\
\hline-- & - & $m$ \\
\hline- & - & $-\infty$ \\
\hline$-\infty$ & - & $\cdots$ \\
\hline
\end{tabular}

\begin{tabular}{|c|c|}
\hline 216 & $<0,010$ \\
\hline$m$ & -- \\
\hline- & - \\
\hline$-\infty$ & $m$ \\
\hline-- & -- \\
\hline$m-$ & $-m$ \\
\hline- & - \\
\hline- & -- \\
\hline- & -- \\
\hline 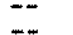 & $\cdots$ \\
\hline- & $=$ \\
\hline$-m$ & - \\
\hline-- & $-m$ \\
\hline-- & -- \\
\hline-- & -- \\
\hline 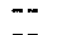 & $-m$ \\
\hline & -- \\
\hline
\end{tabular}


RIO GRANDE BASIN

08317300 COCHITI LAKE NEAR COCHITI PUEBLO, NM -- Continued

WATER-QUALITY RECORDS

COCHITI LAKE AT SITE A (Lat $35^{\circ} 38^{\prime} 11^{\prime \prime}$, Long $106^{\circ} 19^{\prime} 05^{\prime \prime}$ )

WATER QUALITY DATA, WATER YEAR OCTOBER 1995 TO SEPTERBER 1996

\begin{tabular}{|c|c|c|c|c|c|c|c|c|c|c|c|c|}
\hline DATE & $\begin{array}{c}\text { MITRO- } \\
\text { GEN } \\
\text { MO2+NO3 } \\
\text { DIS- } \\
\text { SOLVED } \\
\text { (MSG/L } \\
\text { AS N) } \\
\text { (00631) }\end{array}$ & $\begin{array}{c}\text { MITRO- } \\
\text { GEN } \\
\text { ARNONIA } \\
\text { DIS- } \\
\text { SOLVED } \\
\text { (MEG/L } \\
\text { AS N) } \\
(00608)\end{array}$ & $\begin{array}{c}\text { NITRO- } \\
\text { GEN, ANS- } \\
\text { MONIA + } \\
\text { ORGANIC } \\
\text { TOTAI } \\
\text { (MG/L } \\
\text { AS N) } \\
(00625)\end{array}$ & $\begin{array}{l}\text { MITRO- } \\
\text { GEN,AHI- } \\
\text { MONIA + } \\
\text { ORGANIC } \\
\text { DIS. } \\
\text { (MG/L } \\
\text { AS N) } \\
(00623)\end{array}$ & $\begin{array}{c}\text { FHOS- } \\
\text { PEORUS } \\
\text { TOTAL } \\
\text { (WS/L } \\
\text { AS P) } \\
(00665)\end{array}$ & $\begin{array}{c}\text { FHOS- } \\
\text { PHORUS } \\
\text { DIS- } \\
\text { SOLVE } \\
\text { (MG/L } \\
\text { AS P) } \\
(00666)\end{array}$ & $\begin{array}{l}\text { EBOS- } \\
\text { ERORUS } \\
\text { ORTHO, } \\
\text { DIS- } \\
\text { SOLVED } \\
\text { (MG/L } \\
\text { AS P) } \\
(00671)\end{array}$ & $\begin{array}{c}\text { CARBON, } \\
\text { ORGANIC } \\
\text { TOTAL } \\
\text { (MG/L } \\
\text { AS C) } \\
(00680)\end{array}$ & $\begin{array}{l}\text { ALUM- } \\
\text { INUM, } \\
\text { DIS- } \\
\text { SOLVED } \\
\text { (UG/L } \\
\text { AS AL) } \\
\text { (01106) }\end{array}$ & $\begin{array}{l}\text { ANET- } \\
\text { MONY, } \\
\text { DIS- } \\
\text { SOLVED } \\
\text { (UG /L } \\
\text { AS SB) } \\
\text { (01095) }\end{array}$ & $\begin{array}{c}\text { ARSENIC } \\
\text { DIS- } \\
\text { SOLVED } \\
\text { (UG/L } \\
\text { AS AS) } \\
(01000)\end{array}$ & $\begin{array}{l}\text { BARIUM, } \\
\text { DIS- } \\
\text { SOLVED } \\
\text { (UG/L } \\
\text { AS BA) } \\
(01005)\end{array}$ \\
\hline $\begin{array}{l}y 1996 \\
03 \ldots\end{array}$ & 0.050 & 0.110 & 0.60 & $<0.20$ & 0.180 & 0.030 & 0.020 & 3.6 & 12 & $<1.0$ & 2 & 59 \\
\hline & $\cdots$ & an & $\cdots \cdot$ & $\cdots$ & $\cdots$ & $\because$ & a & a & & $\begin{array}{l}\cdots \\
\cdots \\
\cdots\end{array}$ & & \\
\hline DATE & $\begin{array}{l}\text { BERYL- } \\
\text { LIUM, } \\
\text { DIS- } \\
\text { SOLVED } \\
\text { (UG/L } \\
\text { AS BE) } \\
(01010)\end{array}$ & $\begin{array}{l}\text { BORON, } \\
\text { DIS- } \\
\text { SOLVED } \\
\text { (UG/L } \\
\text { AS B) } \\
(01020)\end{array}$ & $\begin{array}{c}\text { CADMIUM } \\
\text { DIS- } \\
\text { SOLVED } \\
\text { (UG/L } \\
\text { AS CD) } \\
(01025)\end{array}$ & $\begin{array}{l}\text { CHRO- } \\
\text { MIUM, } \\
\text { DIS- } \\
\text { SOLVED } \\
\text { (US/L } \\
\text { AS CR }) \\
(01030)\end{array}$ & $\begin{array}{c}\text { COBALT, } \\
\text { DIS - } \\
\text { SOLVED } \\
(U G / 1 \\
\text { AS CO) } \\
(01035)\end{array}$ & $\begin{array}{l}\text { COPPER, } \\
\text { DIS- } \\
\text { SOLVED } \\
\text { (UG/L } \\
\text { AS CU) } \\
(01040)\end{array}$ & $\begin{array}{c}\text { IRON, } \\
\text { DIS- } \\
\text { SOLVED } \\
\text { (UG/L } \\
\text { AS FE) } \\
(01046)\end{array}$ & $\begin{array}{c}\text { LEAD, } \\
\text { DIS } \\
\text { SOLVED } \\
\text { (UG/L } \\
\text { AS PB) } \\
(01049)\end{array}$ & $\begin{array}{c}\text { MANGA- } \\
\text { NESE, } \\
\text { DIS- } \\
\text { SOLVED } \\
\text { (UG/L } \\
\text { AS MH) } \\
(01056)\end{array}$ & $\begin{array}{l}\text { MERCURY } \\
\text { TOTAL } \\
\text { RECOV- } \\
\text { ERABLE } \\
\text { (UG/L } \\
\text { AS BG) } \\
(71900)\end{array}$ & $\begin{array}{l}\text { MOLYB- } \\
\text { DENUM, } \\
\text { DIS- } \\
\text { SOLVED } \\
\text { (UG/L } \\
\text { AS MO) } \\
\text { (01060) }\end{array}$ & $\begin{array}{l}\text { NICKEL, } \\
\text { DIS- } \\
\text { SOLVED } \\
\text { (UC/1. } \\
\text { AS NI) } \\
(01065 \text { ) }\end{array}$ \\
\hline Y 1996 & $<$ & 50 & $<1.0$ & $<1.0$ & $<1$. & .0 & 5.0 & 1.0 & 49 & 0.10 & 0 & 2.0 \\
\hline DATE & $\begin{array}{l}\text { SELE- } \\
\text { NIUM, } \\
\text { TOTAL } \\
\text { (UG/L } \\
\text { AS SE) } \\
\text { (01147) }\end{array}$ & $\begin{array}{c}\text { SELE- } \\
\text { NIUM, } \\
\text { DIS- } \\
\text { SOLVED } \\
\text { (UG/L } \\
\text { AS SE) } \\
(01145)\end{array}$ & $\begin{array}{c}\text { SILVER, } \\
\text { DIS- } \\
\text { SOLVED } \\
\text { (UG/L } \\
\text { AS AG) } \\
(01075)\end{array}$ & $\begin{array}{l}\text { ZINC, } \\
\text { DIS- } \\
\text { SOLVED } \\
\text { (UG/L } \\
\text { AS ZN) } \\
(01090)\end{array}$ & $\begin{array}{c}\text { NITRO- } \\
\text { GEN, } \\
\text { NO2+NÓ3 } \\
\text { TOT. IN } \\
\text { BOT MAT } \\
\text { (MG/KG } \\
\text { AS N) } \\
(00633)\end{array}$ & $\begin{array}{l}\text { MITRO- } \\
\text { GEN,NH4 } \\
\text { TOTAI } \\
\text { IN BOT. } \\
\text { MAT. } \\
\text { (MG/KG } \\
\text { AS NI) } \\
\text { (00611) }\end{array}$ & $\begin{array}{l}\text { NITRO- } \\
\text { GEN, NHA } \\
\text { +ORG, } \\
\text { TOT IN } \\
\text { BOT MAT } \\
\text { (MGG/KG } \\
\text { AS N) } \\
(00626)\end{array}$ & $\begin{array}{c}\text { PHOS- } \\
\text { PHORUS } \\
\text { TOTALL } \\
\text { IN BOT, } \\
\text { MAT. } \\
\text { (MG/KG } \\
\text { AS P) } \\
(00668)\end{array}$ & $\begin{array}{l}\text { ARSENIC } \\
\text { TOTAL } \\
\text { IN BOT- } \\
\text { TOM MA- } \\
\text { TERIAL } \\
\text { (UG/G } \\
\text { AS AS) } \\
\text { (01003) }\end{array}$ & $\begin{array}{l}\text { CADMIUM } \\
\text { RECOV. } \\
\text { FM BOT- } \\
\text { TOM MA- } \\
\text { TERIAL } \\
\text { (UAG/G } \\
\text { AS CD) } \\
\text { (01028) }\end{array}$ & $\begin{array}{c}\text { CERO- } \\
\text { MIUM, } \\
\text { RECOV. } \\
\text { FM BOT- } \\
\text { TOQI MA- } \\
\text { TERIAL } \\
\text { (UG/G) } \\
(01029)\end{array}$ & $\begin{array}{c}\text { COBALT, } \\
\text { RECOV'. } \\
\text { FM BOT- } \\
\text { TOA MA- } \\
\text { TERIAL } \\
\text { (UG/G } \\
\text { AS CO) } \\
(01038)\end{array}$ \\
\hline $\begin{array}{l}\text { AY } 199 \\
03 \ldots\end{array}$ & $<1$ & $<1$ & $<1,0$ & $<1.0$ & $<2.0$ & 48 & 640 & 87 & 8 & $<10$ & 30 & $<5$ \\
\hline DA? & $\begin{array}{c}\text { COPPER, } \\
\text { RECOV'. } \\
\text { FM BOT- } \\
\text { TOM MA- } \\
\text { TERIAL } \\
\text { (UG/G } \\
\text { AS CVI) } \\
(01043)\end{array}$ & $\begin{array}{c}\text { IRON } \\
\text { RECOV. } \\
\text { FM BOT- } \\
\text { TOM MA- } \\
\text { TERIAL } \\
\text { (UG/G } \\
\text { AS FE) } \\
(01170)\end{array}$ & $\begin{array}{c}\text { LEAD, } \\
\text { RECOV. } \\
\text { FM BOT- } \\
\text { TOM MA- } \\
\text { TERIAL } \\
\text { (UG/E } \\
\text { AS PB) } \\
\text { (01052) }\end{array}$ & $\begin{array}{c}\text { MANGA- } \\
\text { NESE, } \\
\text { RECOV. } \\
\text { FM BOT- } \\
\text { TOM MA- } \\
\text { TERIAL } \\
(U G / G) \\
(01053)\end{array}$ & $\begin{array}{c}\text { MERCURY } \\
\text { RECOV } \\
\text { FM BOT- } \\
\text { TOM MA- } \\
\text { TERIAL } \\
\text { (UG /G } \\
\text { AS HG) } \\
\text { (71921) }\end{array}$ & $\begin{array}{c}\text { ZINC, } \\
\text { RECOV. } \\
\text { FM BOT- } \\
\text { TOM MA- } \\
\text { TERIAL } \\
\text { (UG/G } \\
\text { AS } Z \mathrm{ZN}) \\
(01093)\end{array}$ & $\begin{array}{c}\text { RADIUM } \\
\text { 226, } \\
\text { DIS- } \\
\text { SOLVED, } \\
\text { RADON } \\
\text { METHOD } \\
(\text { PCI /L) } \\
(09511)\end{array}$ & $\begin{array}{l}\text { RA-226 } \\
2 \text { SIGMA } \\
\text { WATER, } \\
\text { DISS, } \\
(\text { PCI/L) } \\
(76001)\end{array}$ & $\begin{array}{l}\text { URANIUM } \\
\text { NATURAL } \\
\text { DIS- } \\
\text { SOLVED } \\
\text { (UG/L } \\
\text { AS U) } \\
(22703)\end{array}$ & $\begin{array}{l}\text { URANIUM } \\
\text { NATURAL } \\
2 \text { SIGMA } \\
\text { WATER, } \\
\text { DISS, } \\
\text { (UG/L) } \\
(75990)\end{array}$ & $\begin{array}{l}\text { SEDI- } \\
\text { MENT. } \\
\text { SUS-- } \\
\text { PENDED } \\
(\text { MG/L) } \\
(80154)\end{array}$ & $\begin{array}{c}\text { SED, } \\
\text { SUSP. } \\
\text { SIEVE } \\
\text { DIAM. } \\
\text { D FIRER } \\
\text { FHAN } \\
\text { THE2 } \\
(70331)\end{array}$ \\
\hline
\end{tabular}

MAY 1996

$03 .$.

110

$0.07 \quad 0.020$

1.8

0.0

41

60 
RIO GRANDE BASIN

08317300 COCHITI LAKE NEAR COCHITI PUEBLO, NM -- Continued

WATER-QUALITY RECORDS

COCHITI LAKE AT SITE A (Lat $35^{\circ} 38^{\prime} 11^{\prime \prime}$, Long $106^{\circ} 19^{\circ} 05^{\prime \prime}$ )

CHEMICAL ANALYSES OF ORGANIC COMPOUNDS, WATER YEAR OCTOBER 1995 TO SEPTEMBER 1996

\begin{tabular}{|c|c|c|c|c|c|c|c|c|c|c|c|c|}
\hline DATE & TIME & $\begin{array}{l}\text { PROP- } \\
\text { CHLOR, } \\
\text { WATER, } \\
\text { DISS, } \\
\text { REC } \\
\text { (UG/L) } \\
(04024)\end{array}$ & $\begin{array}{l}\text { BUTYL- } \\
\text { ATE } \\
\text { WATER, } \\
\text { DISS, } \\
\text { REC } \\
(\text { UG/L) } \\
(04028)\end{array}$ & $\begin{array}{l}\text { SI-' } \\
\text { MAZINE, } \\
\text { WATER, } \\
\text { DISS, } \\
\text { REC } \\
\text { (UG/L) } \\
(04035)\end{array}$ & $\begin{array}{l}\text { PRO- } \\
\text { METON, } \\
\text { WATER, } \\
\text { DISS, } \\
\text { REC } \\
\text { (UG/L) } \\
(04037)\end{array}$ & $\begin{array}{l}\text { DEETHYL } \\
\text { ATRA- } \\
\text { ZINE, } \\
\text { WATER, } \\
\text { DISS, } \\
\text { REC } \\
\text { (UG/L) } \\
(04040)\end{array}$ & $\begin{array}{l}\text { CYANA- } \\
\text { ZINE, } \\
\text { WATER, } \\
\text { DISS, } \\
\text { REC } \\
\text { (UG/L) } \\
(04041)\end{array}$ & $\begin{array}{l}\text { FONOFOS } \\
\text { WATER } \\
\text { DISS } \\
\text { REC } \\
(\text { UG/L) } \\
(04095)\end{array}$ & $\begin{array}{c}\text { ALPHA } \\
\text { BHC } \\
\text { DIS- } \\
\text { SOLVED } \\
\text { (UG/L) } \\
(34253)\end{array}$ & $\begin{array}{c}\text { P, } P^{\prime} \\
\text { DDE } \\
\text { DISSOLV } \\
\text { (UE/L) } \\
(34653)\end{array}$ & $\begin{array}{c}\text { CHLOR- } \\
\text { PYRIFOS } \\
\text { DIS- } \\
\text { SOLVED } \\
\text { (UG/L) } \\
(38933)\end{array}$ & $\begin{array}{l}\text { LINDANE } \\
\text { DIS- } \\
\text { SOLVED } \\
(\text { UG/L) } \\
(39341)\end{array}$ \\
\hline $\begin{array}{l}\pi .1996 \\
18 \ldots\end{array}$ & 0930 & $<0.007$ & $<0.002$ & $<0.005$ & E0.012 & $<0.002$ & $<0.004$ & $<0.003$ & $<0.002$ & $<0.006$ & $<0.004$ & $<0.004$ \\
\hline DATE & $\begin{array}{l}\text { DI- } \\
\text { ELDRIN } \\
\text { DIS- } \\
\text { SOLVED } \\
\text { (UG/L) } \\
(39381)\end{array}$ & $\begin{array}{l}\text { METO- } \\
\text { LACKLOR } \\
\text { HATERR } \\
\text { DISSOLV } \\
\text { (UG/L) } \\
(39415)\end{array}$ & $\begin{array}{l}\text { MALA- } \\
\text { THION, } \\
\text { DIS-- } \\
\text { SOLVED } \\
\text { (UG/L) } \\
(39532)\end{array}$ & $\begin{array}{l}\text { PARA- } \\
\text { THION, } \\
\text { DIS- } \\
\text { SOLVFED } \\
\text { (UG/L) } \\
\text { (39542) }\end{array}$ & $\begin{array}{c}\text { DI- } \\
\text { AZINON, } \\
\text { DIS- } \\
\text { SOLVED } \\
\text { (UG/L) } \\
(39572)\end{array}$ & $\begin{array}{l}\text { ATRA- } \\
\text { ZINE, } \\
\text { WATER, } \\
\text { DISS, } \\
\text { REC } \\
(\text { UG/L) } \\
(39632) .\end{array}$ & $\begin{array}{l}\text { ALA- } \\
\text { CHLOR, } \\
\text { WATER, } \\
\text { DISS, } \\
\text { REC, } \\
\text { (UG/L) } \\
(46342)\end{array}$ & $\begin{array}{c}\text { ACETO- } \\
\text { CHLOR, } \\
\text { WATER } \\
\text { FLTRD } \\
\text { REC } \\
\text { (UG/L) } \\
(49260)\end{array}$ & $\begin{array}{l}\text { METRI- } \\
\text { BUZIN } \\
\text { SENCOR } \\
\text { WATER } \\
\text { DISSOLV } \\
\text { (UG/L) } \\
(82630)\end{array}$ & $\begin{array}{c}2,6 \text {-DI- } \\
\text { ETHYL } \\
\text { ANILINE } \\
\text { WAT FLT } \\
0.7 \text { U } \\
\text { GF, REC } \\
(U G / L) \\
(82660)\end{array}$ & $\begin{array}{l}\text { TRI- } \\
\text { FLUR- } \\
\text { ALIN } \\
\text { WAT FL.T } \\
0.7 \text { U } \\
\text { GF, REC } \\
(U G / L) \\
(82661)\end{array}$ & $\begin{array}{l}\text { ETRAL- } \\
\text { FLUR- } \\
\text { ALIN } \\
\text { WAT ELT } \\
0.7 \text { U } \\
\text { GF, REC } \\
\text { (UG/L) } \\
(82663)\end{array}$ \\
\hline $\begin{array}{c}\text { TuL } 1996 \\
18 \ldots\end{array}$ & $<0.001$ & $<0.002$ & $<0.005$ & $<0.004$ & $<0.002$ & E0.003 & $<0.002$ & $<0.002$ & $<0.004$ & $<0.003$ & $<0.002$ & $<0.004$ \\
\hline DATE & $\begin{array}{l}\text { PHORATE } \\
\text { WATER } \\
\text { FLTRD } \\
0.7 \text { U } \\
\text { GF, REC } \\
\text { (UG/L) } \\
(82664)\end{array}$ & $\begin{array}{c}\text { TER- } \\
\text { BACIL } \\
\text { WATER } \\
\text { FLTRD } \\
0.7 \mathrm{U} \\
\text { GF, REC } \\
(\mathrm{UG} / \mathrm{L}) \\
(82665)\end{array}$ & $\begin{array}{c}\text { LIN- } \\
\text { URON } \\
\text { WATER } \\
\text { FLTRD } \\
0.7 \mathrm{U} \\
\text { GF, REC } \\
(\mathrm{UG} / \mathrm{L}) \\
(82666)\end{array}$ & $\begin{array}{c}\text { METHYL } \\
\text { PARA- } \\
\text { THYON } \\
\text { WAT FLT } \\
0.7 \mathrm{U} \\
\text { GF, REC } \\
\text { (UG/L) } \\
(82667)\end{array}$ & $\begin{array}{l}\text { EPTC } \\
\text { WATER } \\
\text { FLTRD } \\
0.7 \mathrm{U} \\
\text { GF, REC } \\
\text { (UG/L) } \\
(82668)\end{array}$ & $\begin{array}{l}\text { FEB- } \\
\text { ULATE } \\
\text { WATER } \\
\text { FILTRD } \\
0.7 \text { IJ } \\
\text { GF, REC } \\
\text { (UG/L) } \\
(82669)\end{array}$ & $\begin{array}{l}\text { TEBU- } \\
\text { THIURON } \\
\text { WATER } \\
\text { FLTRD } \\
0.7 \mathrm{U} \\
\text { GF, REC } \\
(\mathrm{UG} / \mathrm{L}) \\
(82670)\end{array}$ & $\begin{array}{c}\text { MOL- } \\
\text { INATE } \\
\text { WATER } \\
\text { FLTRD } \\
0.7 \mathrm{U} \\
\text { GF, REC } \\
\text { (UG/L) } \\
(82671)\end{array}$ & $\begin{array}{l}\text { ETHO- } \\
\text { PROP } \\
\text { WATER } \\
\text { FLTRD } \\
0.7 \mathrm{U} \\
\text { GF, REC } \\
\text { (UG/L) } \\
(82672)\end{array}$ & $\begin{array}{c}\text { BEN- } \\
\text { FLUR- } \\
\text { ALIN } \\
\text { WAT FLD } \\
0.7 \text { U } \\
\text { GF, REC } \\
(\mathrm{UG} / \mathrm{L}) \\
(82673)\end{array}$ & $\begin{array}{l}\text { CARBO- } \\
\text { FURAN } \\
\text { WATER } \\
\text { FLTRD } \\
0.7 \mathrm{U} \\
\text { GE, REC } \\
\text { (UG/L) } \\
(82674)\end{array}$ & $\begin{array}{c}\text { TER- } \\
\text { BUFOS } \\
\text { WATER } \\
\text { FLTRD } \\
0.7 \mathrm{U} \\
\text { GF, REC } \\
(U G / L) \\
(82675)\end{array}$ \\
\hline $\begin{array}{c}\text { JUL } 1996 \\
18 \ldots\end{array}$ & $<0.002$ & $<0.007$ & $<0.002$ & $<0.006$ & $<0.002$ & $<0.004$ & $<0.010$ & $<0.004$ & $<0.003$ & $<0.002$ & $<0.003$ & $<0.013$ \\
\hline DATE & $\begin{array}{l}\text { PRON- } \\
\text { AMIDE } \\
\text { WATER } \\
\text { FLTRD } \\
0.7 \mathrm{U} \\
\text { GF, REC } \\
(\mathrm{UG} / \mathrm{L}) \\
(82676)\end{array}$ & $\begin{array}{l}\text { DISUL- } \\
\text { FOTON } \\
\text { WATER } \\
\text { FLTRD } \\
0.7 \text { U } \\
\text { GF, REC } \\
\text { (UG/L) } \\
(82677)\end{array}$ & $\begin{array}{c}\text { TRIAL- } \\
\text { IATE } \\
\text { WATER } \\
\text { FLTRD } \\
0.7 \mathrm{U} \\
\text { GF, REC } \\
(\mathrm{UG} / \mathrm{L}) \\
(82678)\end{array}$ & $\begin{array}{c}\text { FRO- } \\
\text { PANIL } \\
\text { WATER } \\
\text { FLTRD } \\
0.7 \mathrm{U} \\
\text { GF, REC } \\
\text { (UE } / \mathrm{L}) \\
(82679)\end{array}$ & $\begin{array}{c}\text { CAR- } \\
\text { BARYL } \\
\text { WATER } \\
\text { FLTRD } \\
0.7 \text { U } \\
\text { GF, REC } \\
(\mathrm{UG} / \mathrm{L}) \\
(82680)\end{array}$ & $\begin{array}{c}\text { THIO- } \\
\text { BENCARB } \\
\text { WATER } \\
\text { FLTRD } \\
0.7 \mathrm{U} \\
\text { GF, REC } \\
\text { (UG/L) } \\
\text { (82681) }\end{array}$ & $\begin{array}{l}\text { DCEA } \\
\text { WATER } \\
\text { FLTRD } \\
0.7 \mathrm{U} \\
\text { GF, REC } \\
\text { (UG/L.) } \\
(82682)\end{array}$ & $\begin{array}{l}\text { PENDI- } \\
\text { METH- } \\
\text { ALIN } \\
\text { WAT FLT } \\
0.7 \mathrm{U} \\
\text { GF, REC } \\
\text { (UG/L) } \\
(82683)\end{array}$ & $\begin{array}{c}\text { NAPROP- } \\
\text { AMIDE } \\
\text { WATER } \\
\text { FLTRD } \\
0.7 \mathrm{U} \\
\text { GE, REC } \\
(\mathrm{UG} / \mathrm{L}) \\
(82684)\end{array}$ & $\begin{array}{c}\text { FRO- } \\
\text { PARGITE } \\
\text { WATER } \\
\text { FLTRD } \\
0.7 \mathrm{U} \\
\text { GF, REC } \\
\text { (UG/L) } \\
(82685)\end{array}$ & $\begin{array}{c}\text { METHYL } \\
\text { AZIN- } \\
\text { PHOS } \\
\text { WAT FLT } \\
0.7 \text { U } \\
\text { GE, REC } \\
\text { (UG/L) } \\
\text { (82686) }\end{array}$ & $\begin{array}{c}\text { PER- } \\
\text { METHRIN } \\
\text { CIS } \\
\text { WAT FLT } \\
0.7 \mathrm{U} \\
\text { GE, REC } \\
(\mathrm{UG} / \mathrm{L}) \\
(82687)\end{array}$ \\
\hline $\begin{array}{c}\text { UL } 1996 \\
18 \ldots\end{array}$ & $<0.003$ & $<0.017$ & $<0.001$ & $<0.004$ & $<0.003$ & $<0.002$ & $<0.0 .02$ & $<0.004$ & $<0.003$ & $<0.013$ & $<0.001$ & $<0.005$ \\
\hline
\end{tabular}




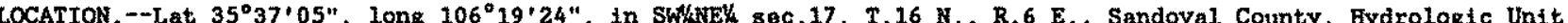
13020201 , in Pueblo de Cochiti Grant, on right bank 320 it upstream from bridge on State 1 ighway 22 , 700 ft downstream from Cochiti Dam, 1.4 mi northeast of Cochiti Pueblo, and at mile 1.587 .6$.

DRAINAGE AREA. -14,900 $\mathrm{mi}^{2}$, approximately, including $2,940 \mathrm{~m}^{2}$ in closed basin in San Luis Valley, Co.

PERIOD OF RECORD. - October 1970 to current year.

GAGE.--Water-stage recorder, Daturn of gage is $5,226.08$ ft above National Geodetic Vertical Datum of 1929 (U.S. Army Corps of Engineers bench mark). Prior to Nov. 14, 1973, at site 2.4 mi downstream at elovation 5,210 ft, Irom topographic map. Nov, 14, 1973, to Jan, 8,1976 , at site 320 ft downstream at datun 1.79 ft 10 wer

RDMARKS. --Records good except for estimated daliy discharges, which are rair. Discharges include flow of Santa Fe Wiver, which is intercepted by Cochtt. Dan and released through the combined outlet works Flow regulated by

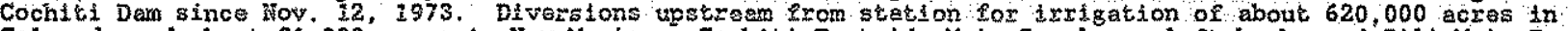

Colorado and about 81,000 acres in New Mexico. Cochitt Eastside Main Canal, on left bank, and Sili Main Canal, on right bank, head at Cochiti Dam and bypass gage for irrigation of about 6,000 acres downstream from station see tabulation below for monthly and yearly diversion. U.S. Army Corps of Engineers satellite telemeter at stiotion.

EXTREMES OUTSIDE PERIOD OF RECORD.--The flood of Nay 15, 1941 , reached a discharge of 23,400 ft3/s at a nearby site upstream from mouth of Santa Fe River. The flood of May 23, 1920, probably exceeded 23,400 ft $3 / 8$, and is Itkely the highest since 1905 .

DISCEARGE, CUBIC FEET PER SECOND, WATER YEAR OCTOBER 1995 TO SEPTEMBER 1996 DAILY MEAN VALUES

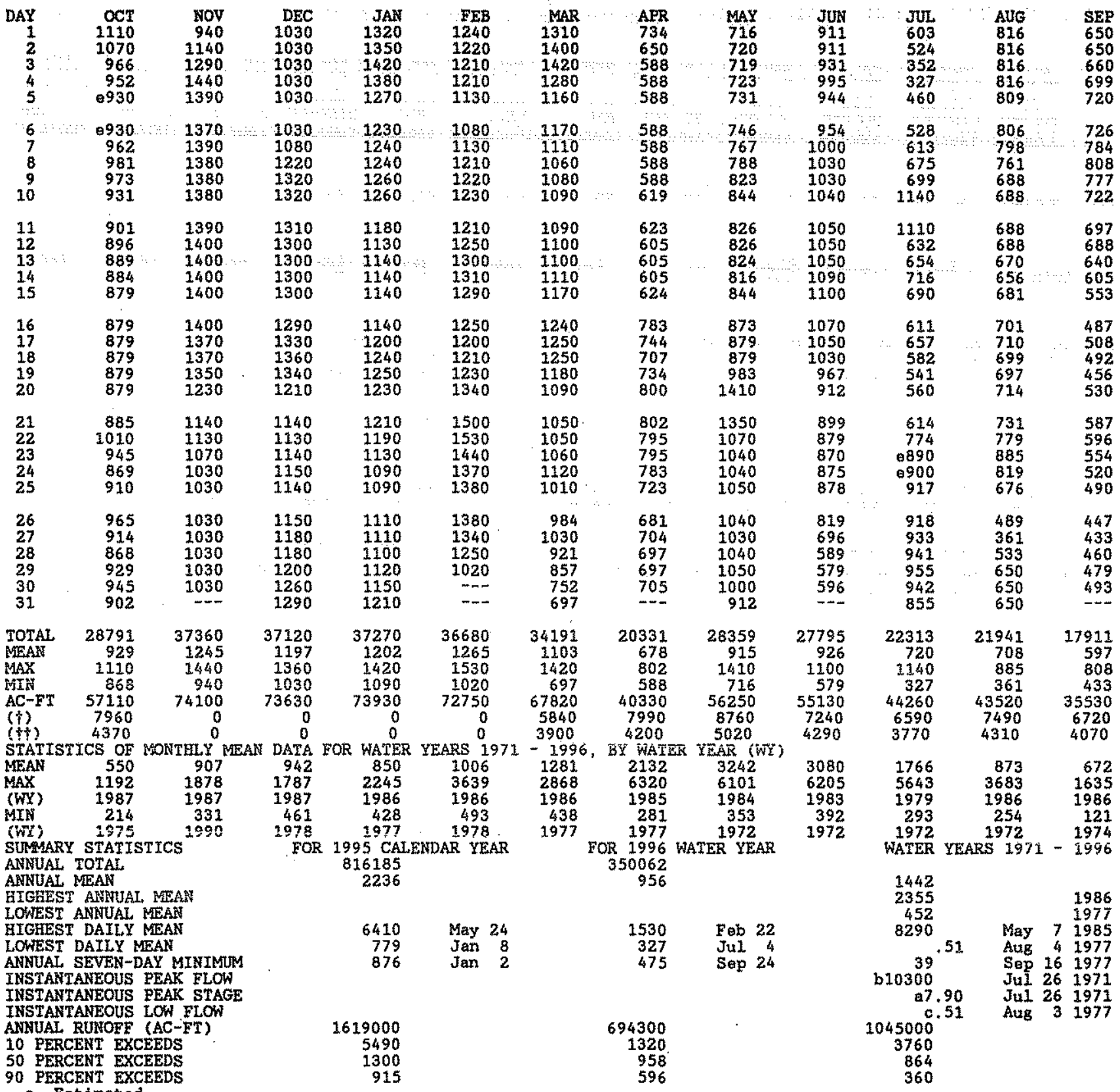

Estimated

a-Site and datum then in use.

b-From rating curve extended above $2,600 \mathrm{ft} 3 / \mathrm{s}$.

c-Aug, 3-5, 1977 , Aug. 27, 28, 1978 , result of regulation.

(†) DIVERSION, IN ACRE-FEET, BY COCHITI EASTSIDE MAIN CANAL AT HEAD

(††) DIVERSION, IN ACRE-FEET, BY SILI MAIN CANAL AT HEAD 
RIO GRANDE BASIN

08317900 GALISTEO RESERVOIR MEAR CERRILLOS, NM

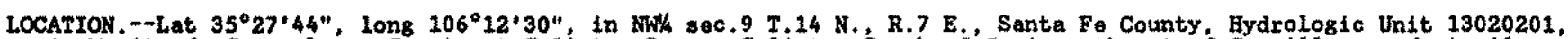
in Mesita de Juana Lopez Grant, at Galisteo Dam on Galisteo Creek, 5.0 mi northwest of Cerrillos, and at mile 11.8 .

DRAINAGE AREA, $-596 \mathrm{mi}^{2}$.

PERIOD OF RECORDS.--October 1970 to current year.

GAGE. - Water-stage recorder above elevation 5,500.3 ft, nonrecording below. Datun of gage is National Geodetic Vertical Datim of 1929 (Levels by U.S. Army Corps of Engineers).

REMARKS, - Reservolr is formed by an earthftli. dam, completed oct. 11, 1970. Capactty, based on capacity table effective January $1972,88,990$ acre-ft between elevations $5,496.0$ ft, sill of ungated outlet conduit, and $5,608.0 \mathrm{ft}$, crest of uncontrolled splllway. No dead storage. Reservolr is used for flood control. U.S. Aray Corps of Hingtnears satellite telemeter at station.

COOPERATION,--Records provided by U.8. Axmy Corps of Engineers.

EXTREAES FOR PERIOD OF RECORD.--Maximum contents, 2,510 acre-ft, July 26, 1971, elevation, 5,517.00; no storage most of time.

EXIREMES FOR CURRENT YEAR. - No storage all year.

Capacity table (elovation, in feet, and contents, in acre-feet)

(Based on survey by U.S. Army Corps of Englneers in 1972)

$\begin{array}{rrrr}5,500 & 0 & 5,504 & 41 \\ 5,501 & 2 & 5,505 & 69 \\ 5,502 & 9 & 5,506 & 109 \\ 5,503 & 21 & 5,508 & 244\end{array}$


LOCATION.--Lat $35^{\circ} 27^{\prime} 53^{\prime \prime}$, long $106^{\circ} 12^{\prime} 49^{\prime \prime}$, in NEWNEK sec.8, T.14 N., R.7 E., Santa Fe County, Bydrologic Unit 13020201 , in Mesite de Juana Lopez Grant, on right bank $0.4 \mathrm{mi}$ downstream from Galisteo Dam, $5.3 \mathrm{mi}$ northwest of Cerrillos, and at mile 11.4 .

DRAINAGE AREA. $--597 \mathrm{~m}^{2}$.

PERIOD OF RECORD. - March 1970 to current year.

GAGE. - Water-stage recorder. Elevation of gage is 5,450 ft above National Geodetic Vertical Datum of 1929 , from toposraphic map. Prior to Dec. 21, 1981, at site 1,200 st domotream at different datum.

REMARKS.--Records fair except for estimated dally discherges, which are poor. Flow regulated by Gallsteo Reservois 0.4 mi upstream. Diversions for irrigation of about 50 acres upstream from station. Several observations of pater temperature were made during the year. U.S. Army Corps of Englneers satellite telemeter at station. No Slow for many days.

DISCHARGE, CUBIC FEET PER SECOND, WATER YEAR OCTOBER 1995 TO SEPTEMBER 1996 DAILY MEAN VALUES

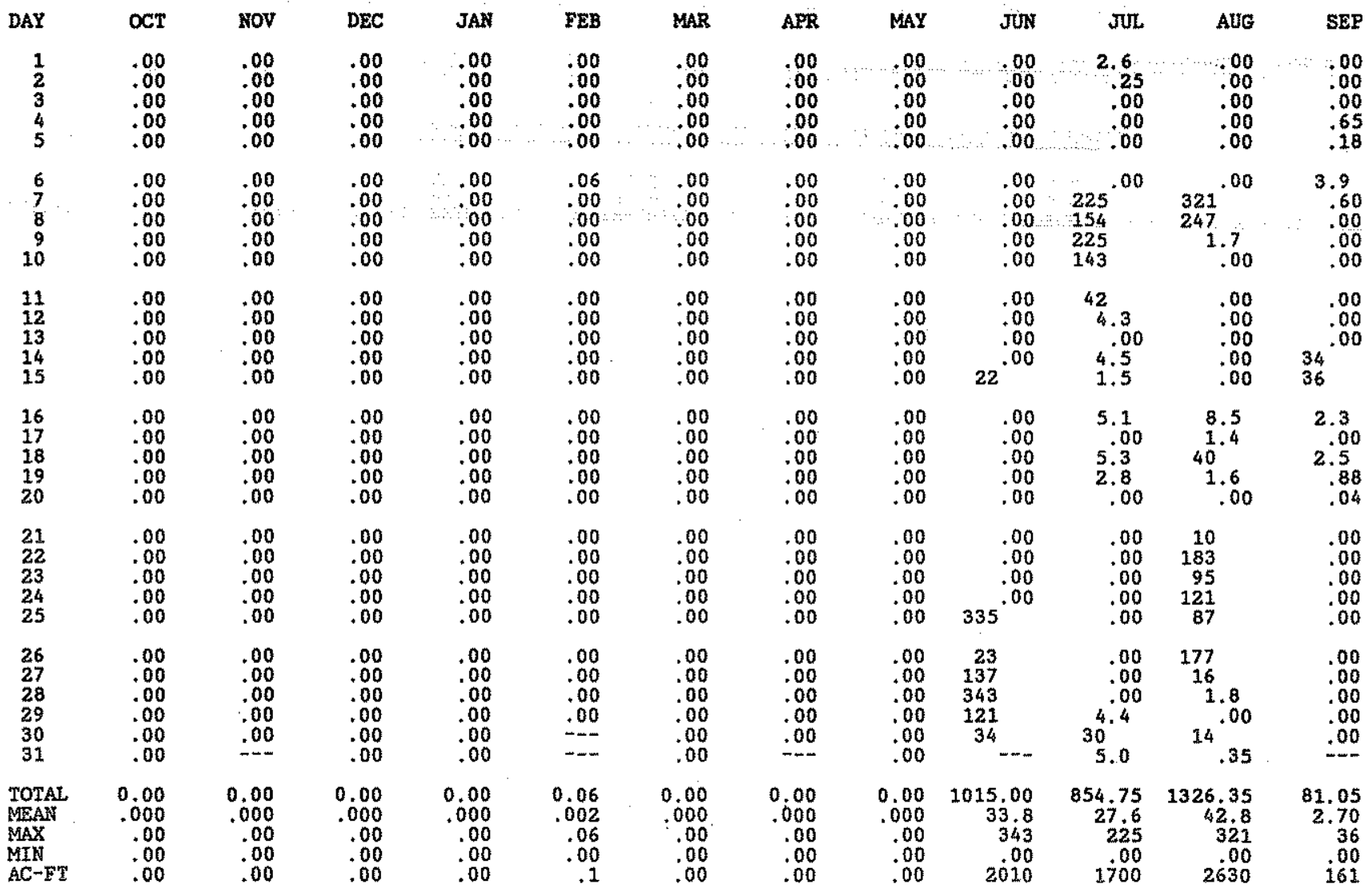

STATISTICS OF MONTHLY MEAN DATA FOR WATER YEARS 1970 - 1996, BY WATER YEAR (WY)

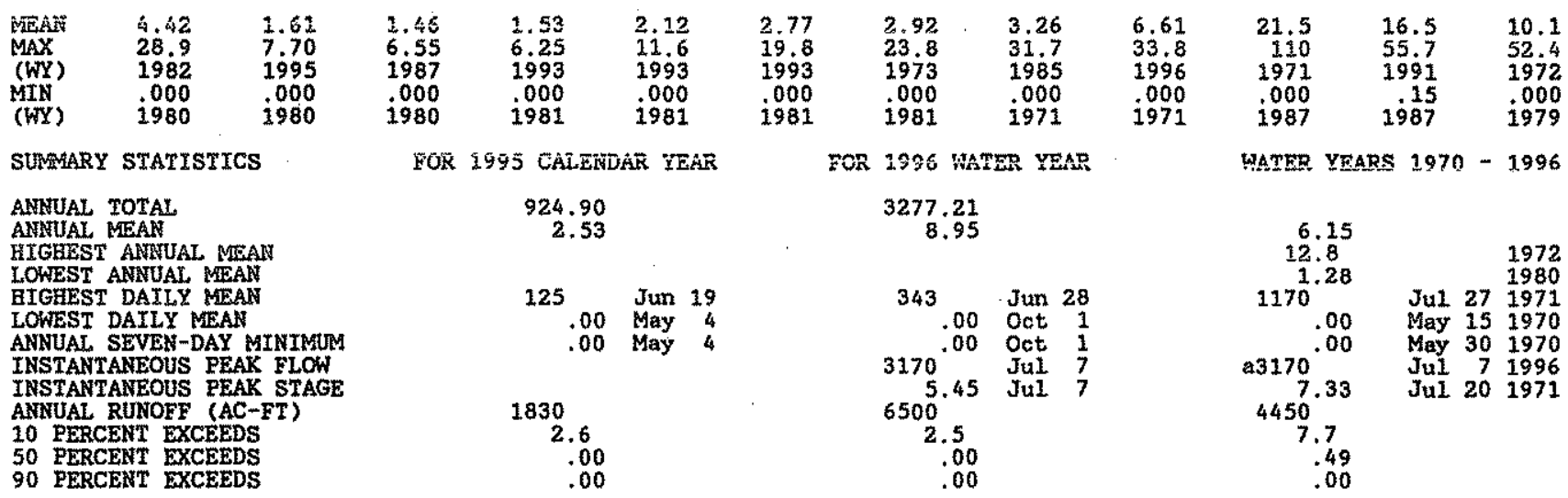

a-From rating curve axtended above $1,400 \mathrm{ft} / \mathrm{s}$. 
RIO GRANDE BASIN

08319000 RIO GRANDE AT SAN FELIPE, MM

LOCATION, - - Lat $35^{\circ} 26^{\circ} 39^{\prime \prime}$, Long $106^{\circ} 26^{\prime} 23^{\prime \prime}$, in SW'MWW sec.17, T.14 N., R. 5 E., Sandoval County, Bydrologic Unit

13020201 , in San Follpe Grant, on right bank $200 \mathrm{ft}$ downstroam from Tonque Arroyo, 1,700 ft upstream from steel

highway bridge, $0.8 \mathrm{mi}$ upstream from San Felipe Pueblo, $11 \mathrm{mi}$ northeast of Bernali1lo, and at mile $1,572.7$.

DRAINAGE AREA. - -16,100 $\mathrm{mi}^{2}$, approximately, including 2,940 $\mathrm{mi}^{2}$ in closed basin in San Luis Valley, Co.

WATER-DISCEARGE RECORDS

PERIOD OF RECORD. - - October 1925 to current year. Monthly discharge only for some perlods, published in WSP 1312. REVISED RECORDS. --WSP 1312: 1926-30, WSP 1392: 1937(M), WSP 1512: 1931-32, 1933(M), 1934-36, 1938(M).

GAGE. - Water-stage recorder. Datum of gage is $5,115.73$ ft above National Geodetic Vertical Datum of 1929 Prior to Sapt. 27 . 1957, at 8 tite 1,800 ft downstream at dat

REMARKS, - Water-discharge records good except for estimated dally discharges, ohich are poor. Flow completely

reguiated since Novernber 1973 by Cochiti Dam (station 08317300) 17 mi upstream. Prtor to Noveraber 1973 some

regulation of flow by El Vado Reservoir (station 08285000) and Abiquiu Reservoir (station 08286900). Since May

1971 flow affected by release of transmountain pater from Heron Reservoir (station 08284510), Diverstons for

irrigation of about 705,000 acres upstream from station, some of which is irrigated downstream by Cochiti.

Eastgide Main Canal and San Felipe eastitie acequia, which bypass station.

EXTRFPMES OUTSIDE PERIOD OF RECORD.--Other major floods occurred in 1874, 1884, and 1904. DISCAARGE, CUBIC FEET PER SECOND, WATER YEAR OCTOBER 1995 TO SEPTEMBER 1996

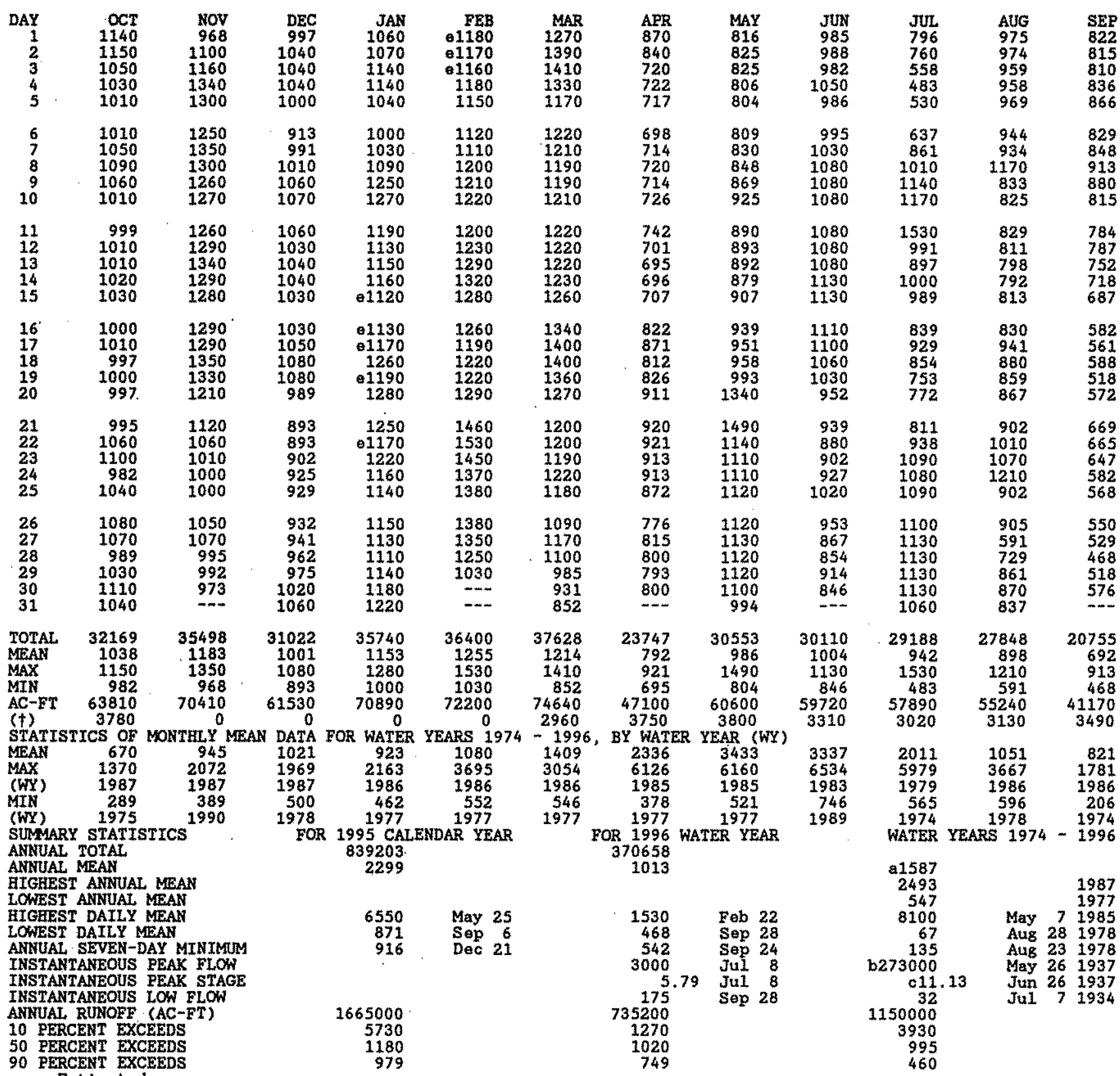

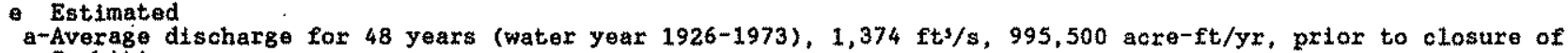
Cochiti.

b-From rating curve extended above $15,000 \mathrm{ft}^{3} / \mathrm{s}$.

c-Site and datum then in use.

( $\uparrow$ ) MONTHLY DIVERSIONS, IN ACRE-FEET, OF COCHITY EASTSIDE CANAL, RECORDS OF THE FLOW FURNISHED BY MIDDLE RIO GRANDE CONSERVANCY DISTRICT. 
136

RIO GRANDE BASIN

08319000 RIO GRANDE AT SAN FELIPE, NM -- Continued

WATER-QUALITY RECORDS

PERIOD OF RECORD. - -Water years 1975 to current year.

WATER QUALITY DATA, WATER YEAR OCTOBER 1995 TO SEPTEMBER 1996

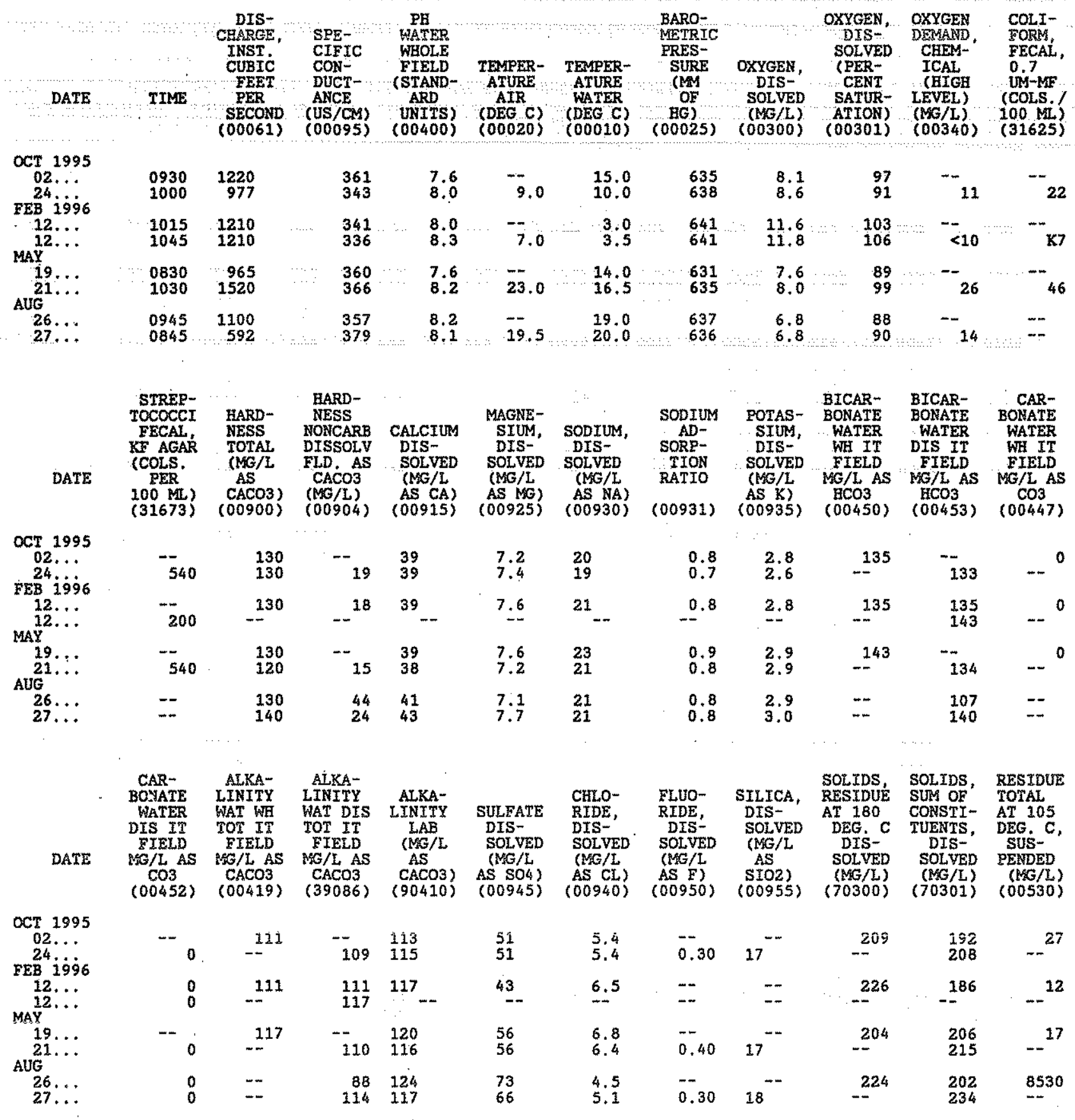


RIO GRANDE BASIN

08319000 RIO GRANDE AT SAN FELTPE, NM -- Continuad

WATER-QUALITY RECORDS

WATER QUALITY DATA, WATER YEAR OCTOBER 1995 TO SEPTEMBER 1996

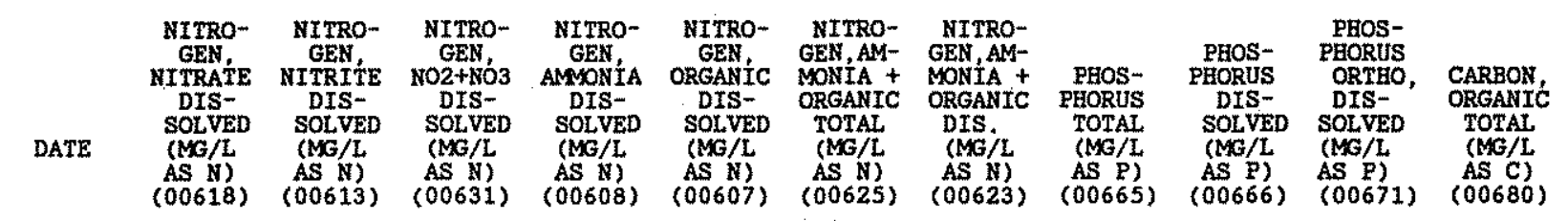

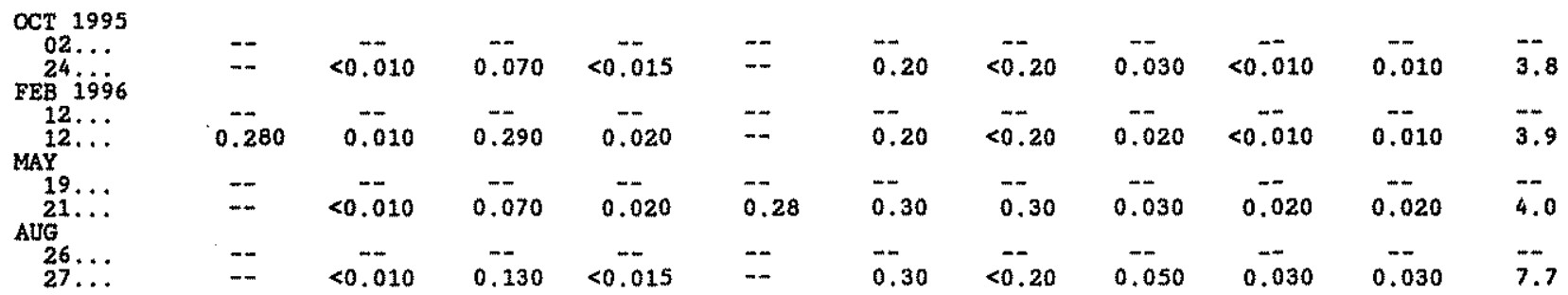

\begin{tabular}{|c|c|c|c|c|c|c|c|c|c|c|}
\hline $\begin{array}{c}\text { CYANIDE } \\
\text { TOTAL } \\
\text { (MG/L } \\
\text { AS CN }) \\
(00720)\end{array}$ & $\begin{array}{l}\text { ALUM- } \\
\text { INUM, } \\
\text { TOTAL } \\
\text { RECOV- } \\
\text { ERABLE } \\
\text { (UG/L } \\
\text { AS AL) } \\
\text { (01105) }\end{array}$ & $\begin{array}{l}\text { Al.tM- } \\
\text { INIM, } \\
\text { DIS- } \\
\text { SOLVED } \\
\text { (UG/L } \\
\text { AS AL) } \\
\text { (01106) }\end{array}$ & $\begin{array}{l}\text { ANTI- } \\
\text { MONY, } \\
\text { DIS- } \\
\text { SOLVED } \\
\text { (UG/L } \\
\text { AS SB) } \\
(01095)\end{array}$ & $\begin{array}{c}\text { ARSENIC } \\
\text { TOTAL } \\
\text { (UG/L } \\
\text { AS ASS) } \\
(01002)\end{array}$ & $\begin{array}{c}\text { ARSENIC } \\
\text { DIS- } \\
\text { SOLVED } \\
\text { (UG/L } \\
\text { AS AS }) \\
(01000)\end{array}$ & $\begin{array}{c}\text { BARTUM, } \\
\text { DIS- } \\
\text { SOLVED } \\
\text { (UG/L } \\
\text { AS BA) } \\
(01005)\end{array}$ & $\begin{array}{l}\text { BERYL- } \\
\text { LIUM, } \\
\text { DIS- } \\
\text { SOLVED } \\
\text { (UG/L } \\
\text { AS BE) } \\
(01010)\end{array}$ & $\begin{array}{l}\text { BORON, } \\
\text { DIS- } \\
\text { SOLVED } \\
\text { (UG/L } \\
\text { AS B) } \\
(01020)\end{array}$ & $\begin{array}{c}\text { CADMIUM } \\
\text { DIS- } \\
\text { SOLVED } \\
\text { (UG/L } \\
\text { AS CD }) \\
\text { (01025) }\end{array}$ & $\begin{array}{l}\text { CERO- } \\
\text { MIUM, } \\
\text { DIS- } \\
\text { SOLVED } \\
\text { (UG/L } \\
\text { AS CR) } \\
(01030)\end{array}$ \\
\hline
\end{tabular}

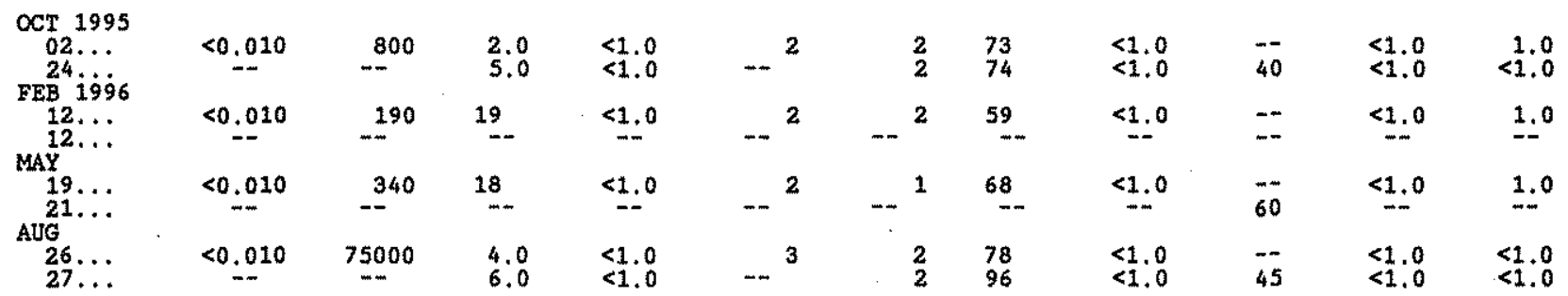

$\begin{array}{cccccc} & & & \text { MANGA- } & \text { MERCURY } \\ \text { COBALT, } & \text { COPPER, } & \text { IRON, } & \text { LEAD, } & \text { NESE, } & \text { TOTAL } \\ \text { DIS- } & \text { DIS- } & \text { DIS- } & \text { DIS- } & \text { DIS- } & \text { RECOV- } \\ \text { SOLVED } & \text { SOLVED } & \text { SOLVED } & \text { SOLVED } & \text { SOLVED } & \text { ERABLE } \\ (U G / L & \text { (UG } / L & (U G / L & (U G / L & (U G / L & \text { (UGG/L } \\ \text { AS CO) } & \text { AS CU) } & \text { AS FE) } & \text { AS PB) } & \text { AS MN) } & \text { AS HG) } \\ (01035) & (01040) & (01046) & (01049) & (01056) & (71900)\end{array}$

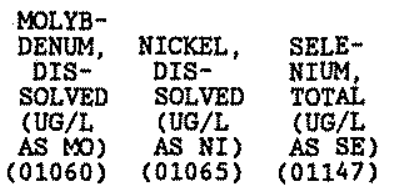

$\begin{array}{cc}\text { SELE- } & \text { SILVER, } \\ \text { NIUM, } & \text { TOTAL } \\ \text { DIS- } & \text { RECOV- } \\ \text { SOLVED } & \text { ERABLE } \\ \text { (UG/L, } & \text { (UG/L } \\ \text { AS SE) } & \text { AS AG) } \\ (01145) & (01077)\end{array}$

\begin{tabular}{|c|c|c|c|c|c|c|c|c|c|c|c|}
\hline $\begin{array}{c}\text { OCT } 1995 \\
02 \ldots \\
24 \ldots \\
241996\end{array}$ & $\begin{array}{l}<1.0 \\
<1.0\end{array}$ & $\begin{array}{l}1.0 \\
2.0\end{array}$ & $\ddot{<3.0}$ & $\begin{array}{l}<1.0 \\
<1.0\end{array}$ & $\begin{array}{l}7.0 \\
7.0\end{array}$ & $<0.10$ & $\begin{array}{l}4.0 \\
4.0\end{array}$ & $\begin{array}{r}<1.0 \\
1.0\end{array}$ & $--<1$ & $--<$ & $-\infty$ \\
\hline $12 \ldots$ & $<1.0$ & 1.0 & $=$ & $<1.0$ & 14 & $=$ & 4.0 & 2.0 & -- & $\because-$ & \\
\hline $\begin{array}{r}\text { MAY } \\
19 \ldots \\
21 \ldots \\
\text { AUG }\end{array}$ & $<1.0$ & 1.0 & $-\overline{4.0}$ & $<1.0$ & 12 & $=$ & 4.0 & 2.0 & $=-$ & $\because$ & $--^{<1}$ \\
\hline $\begin{array}{l}26 \ldots \\
27 \ldots\end{array}$ & $\begin{array}{l}<1.0 \\
<1.0\end{array}$ & $\begin{array}{l}1.0 \\
1.0\end{array}$ & $<-\frac{1}{<3.0}$ & $\begin{array}{l}<1.0 \\
<1.0\end{array}$ & $<1.0$ & $<0.10$ & $\begin{array}{l}3.0 \\
4.0\end{array}$ & $\begin{array}{l}2.0 \\
2.0\end{array}$ & $--<1$ & $-<1$ & $<1$ \\
\hline
\end{tabular}


RIO GRANDE BASTN

08319000 RIO GRANDE AT SAN FELIPE, MM -- Continued

WATER-QUALITY RECORDS

WATER QUALITY DATA, WATER YEAR OCTOBER 1995 TO SEPTEMBER 1996

\begin{tabular}{|c|c|c|c|c|c|c|c|c|c|c|}
\hline ATE & $\begin{array}{c}\text { SILVER, } \\
\text { DDS- } \\
\text { SOLVED } \\
\text { (GG/ } \\
\text { AS AG) } \\
(01075)\end{array}$ & $\begin{array}{l}\text { ZINC, } \\
\text { DIS- } \\
\text { SOLVED } \\
\text { (UEL } \\
\text { AS ZN) } \\
(01090)\end{array}$ & $\begin{array}{l}\text { MITRO- } \\
\text { GEN, } \\
\text { NO2+NOS } \\
\text { TOT. IN } \\
\text { BOT ROAT } \\
\text { (MG/KG } \\
\text { AS N } \\
(00633)\end{array}$ & 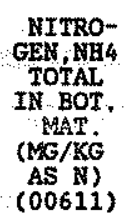 & 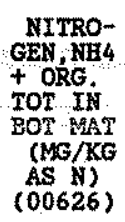 & 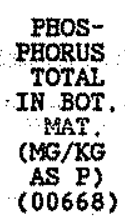 & $\begin{array}{l}\text { ARSENIC } \\
\text { TOPALL } \\
\text { IN BOT- } \\
\text { TOM MAA- } \\
\text { TERIAL } \\
\text { USG } \\
\text { AS AS) } \\
\text { (01003) }\end{array}$ & $\begin{array}{c}\text { CADMIUM } \\
\text { RECOV. } \\
\text { FM BOT- } \\
\text { TOM MA- } \\
\text { TERAL } \\
\text { (UG/G } \\
\text { AS CD) } \\
\text { (01028) }\end{array}$ & 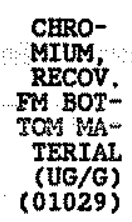 & $\begin{array}{l}\text { COBALT, } \\
\text { RECOV: } \\
\text { FM BOT- } \\
\text { TOM MA- } \\
\text { TERIAL } \\
\text { (UG/G } \\
\text { AS CO) } \\
\text { (01038) }\end{array}$ \\
\hline & $<0$ & $<1: 0$ & $<2,0$ & 21 & 380 & & $\because$ & $-<<1$ & - & \\
\hline$y$ & $<0.20$ & 2.0 & $=$ & 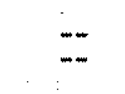 & $=$ & -- & $=$ & -- & $z$ & $=$ \\
\hline 19. & $<0.20$ & 3.0 & -- & - & $\cdots$ & - & -- & - & - & ... \\
\hline & $<0.20$ & $<\begin{array}{r}6.0 \\
<1.0\end{array}$ & $\because$ & $=$ & $\because$ & $\because$ & $\because-$ & $\because$ & $\because$ & \\
\hline
\end{tabular}

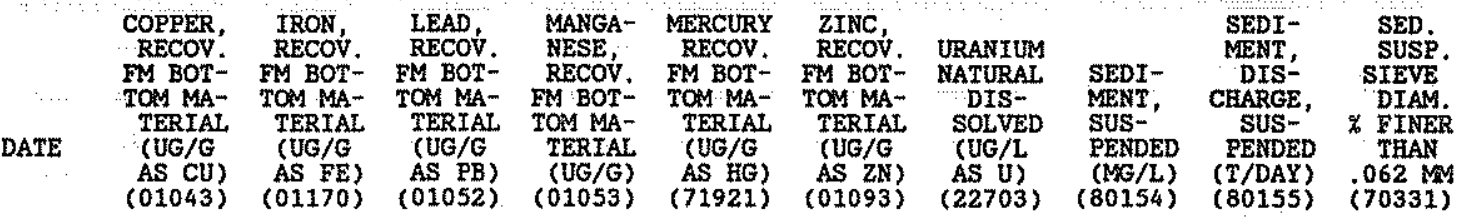

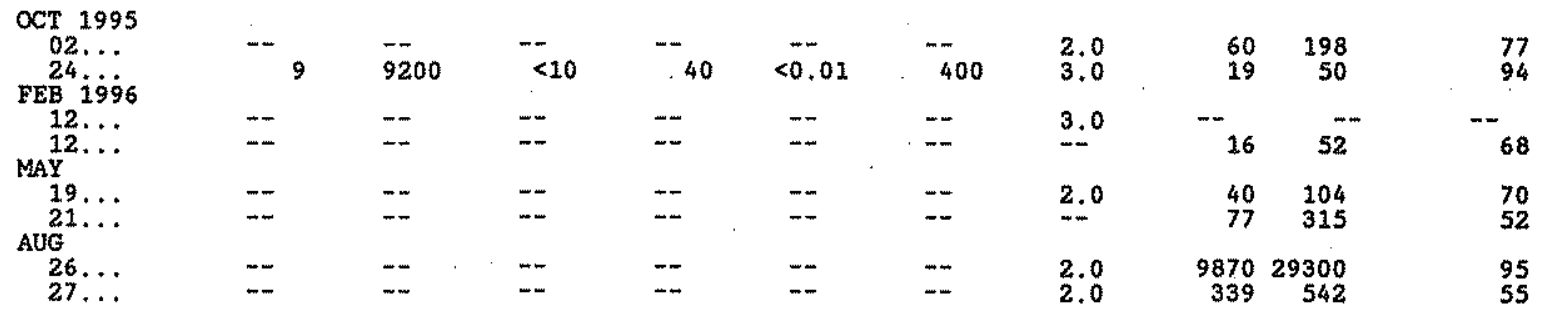

CHEMICAL ANALYSES OF ORGANIC COMPOUNDS, WATER YEAR OCTOBER 1995 TO SEPTEMBER 1996

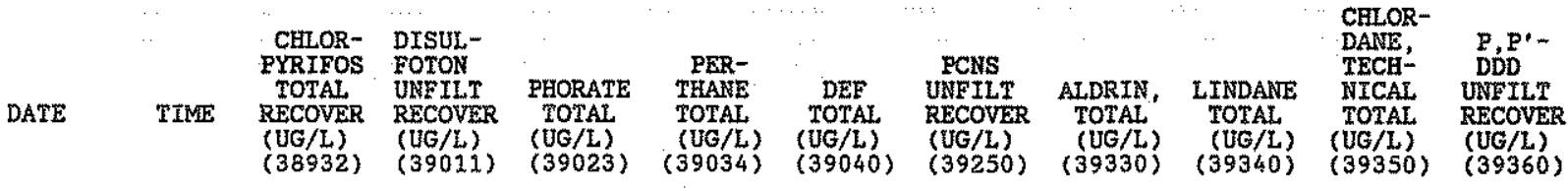

\begin{tabular}{|c|c|c|c|c|c|c|c|c|c|c|c|}
\hline $\begin{array}{c}\text { OCT } 199.5 \\
02 . .\end{array}$ & & -- & -- & - & $-m$ & -- & $\rightarrow$ & -- & $-m$ & -- & $m$ \\
\hline${ }_{\mathrm{FEB}}^{24} 1996$ & 1000 & - & - & -- & $m$ & - & -- & -- & -- & -- & -- \\
\hline $12 \ldots$ & 1015 & -- & $m$ & - & $\rightarrow$ & $\cdots$ & -- & -- & -- & - & - - \\
\hline $\operatorname{MAX}^{12} \cdots$ & 1045 & - & $\rightarrow$ & $m$ & -- & + & $m$ & $-r$ & $m$ & - & -- \\
\hline $19 \cdots$ & 0830 & - & -- & -- & - & $-m$ & $m$ & $-m$ & $m$ & - & -- \\
\hline AUG $21 \ldots$ & 1030 & $\cdots$ & -- & $-m$ & -- & -- & $-m$ & -- & -- & -- & 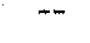 \\
\hline $\begin{array}{l}26 \ldots \\
27 \ldots\end{array}$ & $\begin{array}{l}0945 \\
0845\end{array}$ & $<0 . \overline{0} 0$ & $<0.010$ & $<0.010$ & $<0.100$ & $<0.010$ & $<0 . \overline{100}$ & $<0 . \overline{0} 10$ & $<0 . \overline{0} 10$ & $<0.100$ & $<0.010$ \\
\hline
\end{tabular}


RIO GRANDE BASIN

08319000 RIO GRANDE AT SAN FELIPE, NM -- Continued

WATER-QUALITY RECORDS

WATER QUALITY DATA, WATER YEAR COTOBER 1995 TO SEPTEMBER 1996

\begin{tabular}{|c|c|c|c|c|c|c|c|c|c|c|c|}
\hline DATE & $\begin{array}{c}\text { DDE, } \\
\text { TOTAL } \\
\text { (UG/L) } \\
(39365)\end{array}$ & $\begin{array}{l}\text { P,P' - } \\
\text { DDT } \\
\text { UNFILT } \\
\text { RECOVR } \\
\text { REG/L) } \\
\text { (UG9370) } \\
\text { (3937) }\end{array}$ & $\begin{array}{c}\text { DI- } \\
\text { ELDRIN } \\
\text { TOTAL } \\
\text { (UG/L) } \\
(39380)\end{array}$ & $\begin{array}{c}\text { ENDO- } \\
\text { SULFAN, } \\
\text { I } \\
\text { TUTAI } \\
\text { (UG/L) } \\
(39388)\end{array}$ & $\begin{array}{l}\text { ENDRIN } \\
\text { WATER } \\
\text { UNFLTRD } \\
\text { REC } \\
\text { (UEGL) } \\
(39390)\end{array}$ & $\begin{array}{c}\text { ETHION, } \\
\text { TORAL } \\
\text { (UGGL) } \\
(39398)\end{array}$ & $\begin{array}{c}\text { TOX- } \\
\text { APHENE, } \\
\text { TOTALI } \\
\text { (UG/L) } \\
(39400)\end{array}$ & $\begin{array}{c}\text { HEPTA- } \\
\text { CHLOR, } \\
\text { TOTAL } \\
\text { (UG/L) } \\
(39410)\end{array}$ & $\begin{array}{c}\text { HEPTA- } \\
\text { CHLOR } \\
\text { EPOXIDE } \\
\text { TOTAL } \\
\text { (UG/L) } \\
(39420)\end{array}$ & $\begin{array}{c}\text { METH- } \\
\text { OXY- } \\
\text { CHLOR, } \\
\text { TOTAL, } \\
\text { (UG/L) } \\
(39480)\end{array}$ & $\begin{array}{c}\text { PCB } \\
\text { TOTAL } \\
\text { (UG/L) } \\
(39516)\end{array}$ \\
\hline 1995 & -- & -- & -- & -- & -- & -- & -- & -- & -- & $\ldots$ & - \\
\hline $24:$ & - & - & -. & -- & -- & - & -- & -- & - & -- & -- \\
\hline 12. & $-\cdots$ & - & - & -- & -- & - & - & - & - & $\cdots$ & - \\
\hline${ }_{A Y}^{12} \cdots$ & $\cdots$ & - & -- & - & - & -- & -- & $m$ & - & 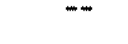 & -- \\
\hline $19 \ldots$ & -- & -- & -- & -- & -- & $=$ & $-\cdots$ & - & - & -- & $=$ \\
\hline AUG & - & - & - & - & $\ldots$ & - & 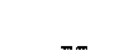 & 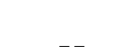 & 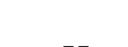 & 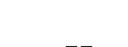 & $\ldots$ \\
\hline $27 \ldots$ & $<0.010$ & $<0.010$ & $<0.010$ & $<0.010$ & $<0.010$ & $<0.010$ & $<1.00$ & $<0.010$ & $<0.010$ & $<0.010$ & $<0.100$ \\
\hline DATE & $\begin{array}{l}\text { MALA- } \\
\text { THION, } \\
\text { TOTAL, } \\
\text { (UG/L/L) } \\
(39530)\end{array}$ & $\begin{array}{c}\text { PARA- } \\
\text { THION, } \\
\text { TOTALA } \\
\text { (UG/L) } \\
(39540)\end{array}$ & $\begin{array}{c}\text { DI- } \\
\text { AZINON, } \\
\text { TOTAL, } \\
\text { (UG/L }) \\
(39570)\end{array}$ & $\begin{array}{l}\text { METHYL } \\
\text { PARA- } \\
\text { THION, } \\
\text { TOTAL, } \\
\text { (UG/L) } \\
(39600)\end{array}$ & $\begin{array}{c}2,4-D, \\
\text { TOTAL } \\
\text { (UG/L) } \\
(39730)\end{array}$ & $\begin{array}{c}2,4,5-T \\
\text { TOOALL } \\
\text { (UGGL) } \\
(39740)\end{array}$ & $\begin{array}{c}\text { MIREX, } \\
\text { TOTAL } \\
\text { (UGGL) } \\
(39755)\end{array}$ & $\begin{array}{c}\text { SILVEX, } \\
\text { TOTALL } \\
\text { (UG/L) } \\
(39760)\end{array}$ & $\begin{array}{c}\text { TOTAL } \\
\text { TRI - } \\
\text { THITN } \\
\text { (UG/L) } \\
\text { (39786) }\end{array}$ & $\begin{array}{l}2,4-D P \\
\text { ToTAL } \\
\text { (UG/L) } \\
(82183)\end{array}$ & $\begin{array}{l}\text { FONOFOS } \\
\text { (DY- } \\
\text { FONATE) } \\
\text { WATER } \\
\text { WHOLE } \\
\text { TOT.REC } \\
\text { (UG/L) } \\
(82614)\end{array}$ \\
\hline OCT 1995 & & & & & & & & & & & \\
\hline & $=$ & $=$ & $=$ & $\because$ & $=$ & $\because$ & $=$ & $=$ & $=$ & $z$ & $=$ \\
\hline $\begin{array}{c}\text { FEB } 1996 \\
12 \ldots\end{array}$ & & $-\infty$ & -- & $-\cdots$ & -- & -- & -- & - & -- & -- & 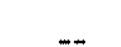 \\
\hline & -- & -- & -- & -- & -- & -- & -- & -- & -- & -- & $=$ \\
\hline & - & - & - & -- & -- & -- & - & -- & - & -- & -- \\
\hline & $\ldots$ & - & -- & 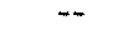 & $<0.010$ & $<0.010$ & - & $<0.010$ & - & $<0.010$ & -- \\
\hline $\begin{array}{r}26 \\
27 \ldots\end{array}$ & $\overline{0}$ & 010 & -010 & $-\overline{01}$ & 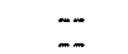 & $\because$ & $-\overline{0} 0$ & $=-$ & $-\overline{0} 0$ & $\overline{--}$ & - \\
\hline & & & & & & & & & & & .010 \\
\hline
\end{tabular}


RIO GRANDE BASIN

08323000 RIO GUADALUPE AT BOX CANYON, NEAR JEMEZ, NM

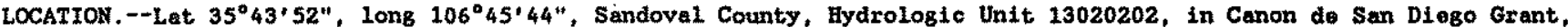
on left bank at downstream ond of Guadalupe Box Canyon, 4.8 mi upstream from mouth, 5 m1 southwest of Jemez Springs, and $7 \mathrm{mi}$ north of Jemez.

DRAINAGE AREA. $--235 \mathrm{mi}^{2}$.

PERIOD OF RECORD.--November 1938 to September 1942, August 1949 to September 1950 (monthly discharge only for November. December 1938 and August 1949 published in WSP 1312), May 1951 to September 1957 (ixrigation seasons only), May 1958 to September 1976, July 1981 to September 1996 (discontinued). Prior to 1951 published as "08323500 Rio Guadalupe near Jemez Springs."

REVISED RECORDS, - WWS 1712: Drainage area.

GAGE, - -Watex-gtage recorder. Datum of gage is 6,015 ft above National Geodetic Vertical Datum of 1929 (planetable survey by Topograph1c Division, U.S. Geological Survey, 1952), Prtor to 1951 , at site 2.4 mi downtream at lower datums.

REMARKS.--Records good except for estimated datly discharges, which are poor. Flow reguleted to some extent since October 1958 by San Gregorto Reservoir on Clear Creek, $24 \mathrm{mi}$ upatream (capacity, 345 ecremet, and by transmountain diversion into Rio Puerco basin for irrigation of about 300 acres in vicinity of Cuba. Several observations of water temperature were made during the year.

DISCHARGE, CUBTC FEET PER SECOND, WATER YEAR OCTOBER 1995 TO SEPTERBER 1996

\begin{tabular}{|c|c|c|c|c|c|c|c|c|c|c|c|}
\hline OCT & NOV & DEC & JAN & FEB. & MAR & APR & MAY & JUN & JUL & AUG & SEE \\
\hline $\begin{array}{r}26 \\
22 \\
20 \\
19 \\
19\end{array}$ & $\begin{array}{l}14 \\
16 \\
17 \\
18 \\
19\end{array}$ & $\begin{array}{l}11 \\
11 \\
11 \\
11 \\
12\end{array}$ & $\begin{array}{l}11 \\
9.6 \\
e 10 \\
11 \\
e 11\end{array}$ & $\begin{array}{r}11 \\
11 \\
12 \\
013 \\
013\end{array}$ & $\begin{array}{l}11 \\
12 \\
12 \\
14 \\
13\end{array}$ & $\begin{array}{l}17 \\
18 \\
19 \\
19 \\
19\end{array}$ & $\begin{array}{l}016 \\
016 \\
016 \\
016 \\
016\end{array}$ & $\begin{array}{l}7.5 \\
7.2 \\
7.1 \\
7.3 \\
7.1\end{array}$ & $\begin{array}{l}13 \\
13 \\
12 \\
11 \\
10\end{array}$ & $\begin{array}{r}8.8 \\
8.8 \\
8.7 \\
10 \\
9.8\end{array}$ & $\begin{array}{l}13 \\
12 \\
11 \\
9.9 \\
9.3\end{array}$ \\
\hline $\begin{array}{l}19 \\
19 \\
19 \\
19 \\
19\end{array}$ & $\begin{array}{r}18 \\
18 \\
\therefore \quad 17 \\
16 \\
16\end{array}$ & $\begin{array}{r}11 \\
11 \\
12 \\
10 \\
11\end{array}$ & $\begin{array}{l}011 \\
010 \\
010 \\
\theta 10 \\
\theta 10\end{array}$ & $\begin{array}{r}12 \\
13 \\
13 \\
13 \\
14\end{array}$ & $\begin{array}{l}13 \\
11 \\
12 \\
14 \\
14\end{array}$ & $\begin{array}{r}18 \\
18 \\
20 \\
024 \\
027\end{array}$ & $\begin{array}{l}015 \\
015 \\
014 \\
014 \\
015\end{array}$ & $\begin{array}{r}6.9 \\
6.8 \\
7.3 \\
7.7 \\
7.2\end{array}$ & $\begin{array}{c}9.2 \\
9.8 \\
11 \\
17 \\
19\end{array}$ & $\begin{array}{r}8.8 \\
8.0 \\
8.4 \\
8.8 \\
8.3\end{array}$ & $\begin{array}{l}9.1 \\
8.8 \\
6.2 \\
7.8 \\
8.1\end{array}$ \\
\hline $\begin{array}{l}19 \\
19 \\
18 \\
18 \\
18\end{array}$ & $\begin{array}{l}15 \\
15 \\
15 \\
14 \\
14\end{array}$ & $\begin{array}{l}10 \\
11 \\
12 \\
12 \\
11\end{array}$ & $\begin{array}{r}10 \\
10 \\
10 \\
=10 \\
e 10\end{array}$ & $\begin{array}{l}15 \\
17 \\
16 \\
17 \\
17\end{array}$ & $\begin{array}{l}15 \\
18 \\
12 \\
11 \\
12\end{array}$ & $\begin{array}{l}028 \\
029 \\
e 26 \\
e 23 \\
023\end{array}$ & $\begin{array}{r}015 \\
614 \\
13 \\
12 \\
12\end{array}$ & $\begin{array}{l}7.1 \\
7.0 \\
7.1 \\
8.5 \\
9.1\end{array}$ & $\begin{array}{l}18 \\
14 \\
13 \\
13 \\
13\end{array}$ & $\begin{array}{l}8.0 \\
7.7 \\
7.3 \\
7.2 \\
7.1\end{array}$ & $\begin{array}{r}7.8 \\
8.2 \\
8.8 \\
13 \\
14\end{array}$ \\
\hline $\begin{array}{l}18 \\
18 \\
18 \\
18 \\
18\end{array}$ & $\begin{array}{l}22 \\
17 \\
16 \\
15 \\
14\end{array}$ & $\begin{array}{l}11 \\
13 \\
12 \\
11 \\
11\end{array}$ & $\begin{array}{l}e 10 \\
010 \\
e 11 \\
010 \\
09.0\end{array}$ & $\begin{array}{l}17 \\
16 \\
16 \\
16 \\
17\end{array}$ & $\begin{array}{l}11 \\
10 \\
9.7 \\
9.6 \\
9.7\end{array}$ & $\begin{array}{l}e 23 \\
e 22 \\
e 22 \\
e 21 \\
e 20\end{array}$ & $\begin{array}{l}11 \\
11 \\
10 \\
9.4 \\
8.7\end{array}$ & $\begin{array}{l}9.8 \\
8.9 \\
8.1 \\
7.3 \\
6.6\end{array}$ & $\begin{array}{l}12 \\
12 \\
11 \\
10 \\
10\end{array}$ & $\begin{array}{r}7.2 \\
11.5 \\
9.5 \\
8.8 \\
8.9\end{array}$ & $\begin{array}{l}16 \\
14 \\
15 \\
15 \\
16\end{array}$ \\
\hline $\begin{array}{l}18 \\
18 \\
17 \\
23 \\
27\end{array}$ & $\begin{array}{l}14 \\
14 \\
14 \\
13 \\
13\end{array}$ & $\begin{array}{l}\text { e11 } \\
012 \\
012 \\
013 \\
014\end{array}$ & $\begin{array}{l}09.0 \\
011 \\
010 \\
09.0 \\
010\end{array}$ & $\begin{array}{l}18 \\
17 \\
15 \\
13 \\
14\end{array}$ & $\begin{array}{l}11 \\
33 \\
16 \\
16 \\
15\end{array}$ & $\begin{array}{l}820 \\
020 \\
020 \\
023 \\
028\end{array}$ & $\begin{array}{l}8.7 \\
8.4 \\
8.1 \\
7.9 \\
7.9\end{array}$ & $\begin{array}{l}6.4 \\
6.6 \\
6.8 \\
6.6 \\
6.5\end{array}$ & $\begin{array}{l}9.2 \\
8.5 \\
8.1 \\
7.8 \\
7.7\end{array}$ & $\begin{array}{l}13 \\
13 \\
14 \\
21 \\
26\end{array}$ & $\begin{array}{l}14 \\
12 \\
10 \\
9.8 \\
9.7\end{array}$ \\
\hline $\begin{array}{l}19 \\
16 \\
15 \\
15 \\
15 \\
14\end{array}$ & $\begin{array}{l}13 \\
12 \\
11 \\
11 \\
11 \\
-\end{array}$ & $\begin{array}{l}015 \\
014 \\
013 \\
012 \\
013 \\
11\end{array}$ & $\begin{array}{l}011 \\
012 \\
010 \\
e 11 \\
012 \\
012\end{array}$ & $\begin{array}{l}13 \\
11 \\
12 \\
13 \\
- \\
--\end{array}$ & $\begin{array}{l}15 \\
14 \\
14 \\
16 \\
17 \\
18\end{array}$ & $\begin{array}{l}028 \\
025 \\
020 \\
017 \\
017 \\
--\end{array}$ & $\begin{array}{l}7.9 \\
8.2 \\
8.5 \\
8.2 \\
7.8 \\
7.7\end{array}$ & $\begin{array}{l}9.1 \\
10 \\
13 \\
12 \\
13 \\
-\cdots\end{array}$ & $\begin{array}{l}7.6 \\
7.7 \\
8.2 \\
9.4 \\
9.0 \\
8.8\end{array}$ & $\begin{array}{l}20 \\
18 \\
22 \\
24 \\
17 \\
14\end{array}$ & $\begin{array}{l}7.1 \\
6.3 \\
6.1 \\
6.0 \\
5.6\end{array}$ \\
\hline $\begin{array}{r}580 \\
18.7 \\
27 \\
14 \\
1150\end{array}$ & $\begin{array}{r}452 \\
15,1 \\
22 \\
11 \\
897\end{array}$ & $\begin{array}{r}365 \\
11.8 \\
15 \\
10 \\
724\end{array}$ & $\begin{array}{r}320.6 \\
10.3 \\
12 \\
9.0 \\
636\end{array}$ & $\begin{array}{r}415 \\
14.3 \\
18 \\
11 \\
823\end{array}$ & $\begin{array}{r}409.0 \\
13.2 \\
18 \\
9.6 \\
811\end{array}$ & $\begin{array}{r}654 \\
21.8 \\
29 \\
17 \\
1300\end{array}$ & $\begin{array}{r}356.4 \\
11.6 \\
16 \\
7.7 \\
711\end{array}$ & $\begin{array}{r}241.6 \\
8.05 \\
13 \\
6.4 \\
479\end{array}$ & $\begin{array}{r}343.0 \\
11.1 \\
79 \\
680\end{array}$ & $\begin{array}{r}373.1 \\
12.0 \\
266 \\
7.1 \\
740\end{array}$ & $\begin{array}{r}310.6 \\
10.4 \\
16 \\
5.6 \\
616\end{array}$ \\
\hline
\end{tabular}

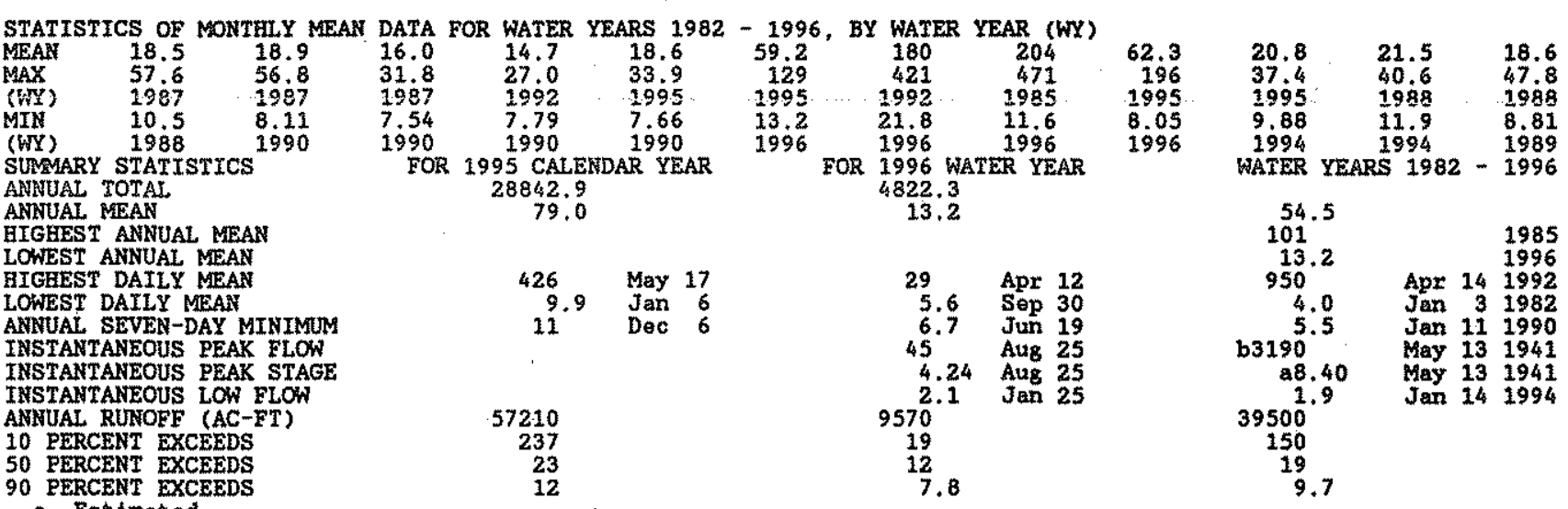

Estimated

a-From floodmarks, site and datum in use June 1941 to September 1942.

b-Fron rating curve extended above $1,000 \mathrm{ft}^{\mathrm{s}} / \mathrm{s}$. 
RIO GRANDE BASIN

08324000 JEMEZ RIVER NEAR JEMEZ, NM

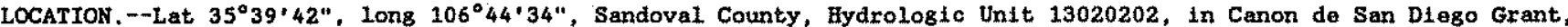
on left bank $0.7 \mathrm{mt}$ downstream from Rio Guadalupe, $3.5 \mathrm{mi}$ north of Jemez, and at mile 29.5 .

DRATNAGE AREA. $--470 \mathrm{mi}^{2}$.

WATER-DISCEARGE RECORDS

PGRIOD OF RECORD.--June 1936 to May 1941, August 1949 to October 1950, May 1951 to September 1952 (1rrigation seasons only), March 1953 to current year. Monthly discharge only for some periods, published in wsp 1732 . Published as Jemez Creok near Jemez, 1936-41.

REVTSED RFCORDS, - WSP 1712: Drainage area WSP $1923,1957-58$

GAGE.-Water-stage recorder. Concrete control since bec. 6, 1965. Datum of 8980 is 5,622 ft above National Geodetic Vertical Datum of 1929 (plane-table survey by Topographic Diviston, U.S. Geological Survey, 1952 ). June 22, 1936, to Mar. 11, 1937, at site 60 ft upstrean at datum 0.50 ft higher. Mar. 12, 1937 , to July 8 , 1938, at present site at datum 0.7 ft higher. July 9,1938 , to May 6,1941 , at site 60 ft upstream at datum $0.70 \mathrm{ft}$ higher.

REMARKS, -Water-discharge records good except for estimated daily discharges which are fair. Diverston for irrigation of about 300 acres upstxeam from station. Several observations of water temperature were made durting the year. U.S. Army Corps of Engineers satellite telometer at station.

EXTREMES OUTSIDE PERIOD OF RECORD,-Maximum flood since at least 1890 occurred between May 6 and 15 , 1941 , after gage was destroyed (discharge probably exceeded $6,000 \mathrm{ft} / \mathrm{s}$ ), from information by local residents.

DISCEARGE, CUBIC FEET PER SECOND, WATER YEAR OCTOBER 1995 TO SEPTEMBER 1996 DAIIY MEAN VALUES

\begin{tabular}{|c|c|c|c|c|c|c|c|c|c|c|c|c|}
\hline DAY & $\infty \mathrm{CT}$ & NOV & DEC & JAN & FEB & MAR & APR & MAY & JUN & JUL & AUG & SEP \\
\hline $\begin{array}{l}1 \\
2 \\
3 \\
4 \\
5\end{array}$ & $\begin{array}{l}37 \\
32 \\
31 \\
30 \\
29\end{array}$ & $\begin{array}{l}34 \\
34 \\
34 \\
35 \\
35\end{array}$ & $\begin{array}{l}25 \\
26 \\
26 \\
27 \\
28\end{array}$ & $\begin{array}{l}27 \\
24 \\
23 \\
25 \\
25\end{array}$ & $\begin{array}{l}24 \\
24 \\
22 \\
20 \\
25\end{array}$ & $\begin{array}{l}24 \\
25 \\
26 \\
27 \\
24\end{array}$ & $\begin{array}{l}27 \\
27 \\
29 \\
29 \\
28\end{array}$ & $\begin{array}{l}20 \\
18 \\
18 \\
18 \\
18\end{array}$ & $\begin{array}{l}11 \\
9.9 \\
10 \\
10 \\
9.3\end{array}$ & $\begin{array}{l}15 \\
19 \\
18 \\
18 \\
16\end{array}$ & $\begin{array}{l}12 \\
13 \\
12 \\
11 \\
7.8\end{array}$ & $\begin{array}{l}18 \\
16 \\
16 \\
14 \\
14\end{array}$ \\
\hline $\begin{array}{r}6 \\
7 \\
8 \\
9 \\
10\end{array}$ & $\begin{array}{l}28 \\
32 \\
31 \\
31 \\
29\end{array}$ & $\begin{array}{l}35 \\
35 \\
34 \\
33 \\
32\end{array}$ & $\begin{array}{l}28 \\
28 \\
29 \\
26 \\
29\end{array}$ & $\begin{array}{l}24 \\
23 \\
23 \\
23 \\
23\end{array}$ & $\begin{array}{l}23 \\
24 \\
25 \\
26 \\
26\end{array}$ & $\begin{array}{l}24 \\
20 \\
24 \\
28 \\
30\end{array}$ & $\begin{array}{l}27 \\
27 \\
29 \\
28 \\
35\end{array}$ & $\begin{array}{l}17 \\
16 \\
16 \\
15 \\
16\end{array}$ & $\begin{array}{l}9.3 \\
9.2 \\
7.9 \\
7.4 \\
8.5\end{array}$ & $\begin{array}{l}17 \\
17 \\
14 \\
37 \\
37\end{array}$ & $\begin{array}{l}7.4 \\
19 \\
15 \\
15 \\
14\end{array}$ & $\begin{array}{l}14 \\
17 \\
16 \\
14 \\
14\end{array}$ \\
\hline $\begin{array}{l}11 \\
12 \\
13 \\
14 \\
15\end{array}$ & $\begin{array}{l}28 \\
29 \\
30 \\
30 \\
31\end{array}$ & $\begin{array}{l}31 \\
30 \\
30 \\
29 \\
28\end{array}$ & $\begin{array}{l}28 \\
28 \\
31 \\
31 \\
30\end{array}$ & $\begin{array}{l}22 \\
22 \\
23 \\
23 \\
23\end{array}$ & $\begin{array}{l}28 \\
29 \\
29 \\
30 \\
31\end{array}$ & $\begin{array}{l}31 \\
34 \\
31 \\
28 \\
27\end{array}$ & $\begin{array}{l}37 \\
37 \\
40 \\
34 \\
33\end{array}$ & $\begin{array}{l}16 \\
15 \\
15 \\
14 \\
12\end{array}$ & $\begin{array}{l}8.0 \\
9.8 \\
10 \\
11 \\
12\end{array}$ & $\begin{array}{l}43 \\
29 \\
29 \\
28 \\
22\end{array}$ & $\begin{array}{l}12 \\
10 \\
10 \\
10 \\
12\end{array}$ & $\begin{array}{l}15 \\
17 \\
19 \\
24 \\
27\end{array}$ \\
\hline $\begin{array}{l}16 \\
17 \\
18 \\
19 \\
20\end{array}$ & $\begin{array}{l}30 \\
29 \\
29 \\
29 \\
28\end{array}$ & $\begin{array}{l}31 \\
29 \\
28 \\
26 \\
25\end{array}$ & $\begin{array}{l}29 \\
33 \\
31 \\
27 \\
29\end{array}$ & $\begin{array}{l}24 \\
25 \\
22 \\
19 \\
22\end{array}$ & $\begin{array}{l}32 \\
32 \\
35 \\
35 \\
37\end{array}$ & $\begin{array}{l}25 \\
25 \\
24 \\
23 \\
23\end{array}$ & $\begin{array}{l}33 \\
32 \\
31 \\
28 \\
28\end{array}$ & $\begin{array}{l}11 \\
11 \\
10 \\
11 \\
10\end{array}$ & $\begin{array}{r}12 \\
11 \\
9.3 \\
8.9 \\
8.0\end{array}$ & $\begin{array}{l}19 \\
19 \\
17 \\
16 \\
16\end{array}$ & $\begin{array}{l}11 \\
19 \\
13 \\
10 \\
10\end{array}$ & $\begin{array}{l}26 \\
23 \\
24 \\
26 \\
27\end{array}$ \\
\hline $\begin{array}{l}21 \\
22 \\
23 \\
24 \\
25\end{array}$ & $\begin{array}{l}29 \\
31 \\
33 \\
35 \\
40\end{array}$ & $\begin{array}{l}25 \\
24 \\
23 \\
22 \\
21\end{array}$ & $\begin{array}{l}23 \\
24 \\
23 \\
26 \\
27\end{array}$ & $\begin{array}{l}18 \\
24 \\
22 \\
20 \\
21\end{array}$ & $\begin{array}{l}38 \\
42 \\
35 \\
31 \\
32\end{array}$ & $\begin{array}{l}23 \\
25 \\
27 \\
27 \\
26\end{array}$ & $\begin{array}{l}28 \\
28 \\
31 \\
39 \\
39\end{array}$ & $\begin{array}{l}11 \\
10 \\
11 \\
11 \\
12\end{array}$ & $\begin{array}{r}08.1 \\
7.6 \\
7.2 \\
7.6 \\
8.2\end{array}$ & $\begin{array}{r}12 \\
9.6 \\
8.7 \\
9.4 \\
11\end{array}$ & $\begin{array}{l}22 \\
25 \\
70 \\
35 \\
35\end{array}$ & $\begin{array}{l}24 \\
20 \\
19 \\
19 \\
19\end{array}$ \\
\hline $\begin{array}{l}26 \\
27 \\
28 \\
29 \\
30 \\
31\end{array}$ & $\begin{array}{l}35 \\
34 \\
33 \\
34 \\
34 \\
34\end{array}$ & $\begin{array}{l}21 \\
20 \\
16 \\
19 \\
23 \\
---\end{array}$ & $\begin{array}{l}11 \\
24 \\
23 \\
27 \\
26 \\
26\end{array}$ & $\begin{array}{r}12 \\
23 \\
24 \\
21 \\
22 \\
23\end{array}$ & $\begin{array}{r}11 \\
25 \\
26 \\
26 \\
- \\
-\infty\end{array}$ & $\begin{array}{l}30 \\
24 \\
25 \\
26 \\
27 \\
27\end{array}$ & $\begin{array}{r}18 \\
32 \\
30 \\
26 \\
21 \\
-\end{array}$ & $\begin{array}{l}11 \\
11 \\
11 \\
11 \\
11\end{array}$ & $\begin{array}{r}16 \\
020 \\
20 \\
15 \\
\end{array}$ & $\begin{array}{l}12 \\
13 \\
13 \\
13 \\
12\end{array}$ & $\begin{array}{l}24 \\
28 \\
29 \\
23 \\
20\end{array}$ & $\begin{array}{l}17 \\
16 \\
15 \\
15 \\
-1-m\end{array}$ \\
\hline $\begin{array}{l}\text { TOTAL } \\
\text { MEAN } \\
\text { MAX } \\
\text { MIN } \\
\text { AC-FT }\end{array}$ & $\begin{array}{r}975 \\
31.5 \\
40 \\
28 \\
1930\end{array}$ & $\begin{array}{r}842 \\
28,1 \\
35 \\
16 \\
1670\end{array}$ & $\begin{array}{r}847 \\
27.3 \\
33 \\
23 \\
1680\end{array}$ & $\begin{array}{r}704 \\
22.7 \\
27 \\
18 \\
1400\end{array}$ & $\begin{array}{r}842 \\
29.0 \\
42 \\
20 \\
1670\end{array}$ & $\begin{array}{r}805 \\
26.0 \\
34 \\
20 \\
1600\end{array}$ & $\begin{array}{r}928 \\
30.9 \\
40 \\
21 \\
1840\end{array}$ & $\begin{array}{r}418 \\
13.5 \\
20 \\
10 \\
829\end{array}$ & $\begin{array}{r}314.2 \\
10.5 \\
20 \\
7.2 \\
623\end{array}$ & $\begin{array}{r}570.7 \\
18.4 \\
43 \\
8.7 \\
1130\end{array}$ & $\begin{array}{r}584.2 \\
18.8 \\
70 \\
7.4 \\
1160\end{array}$ & $\begin{array}{r}563 \\
18.8 \\
27 \\
14 \\
1120\end{array}$ \\
\hline
\end{tabular}

STATISTICS OF MONTELY MEAN DATA FOR WATER YEARS 1954 - 1996, BY WATER YEAR (WY)

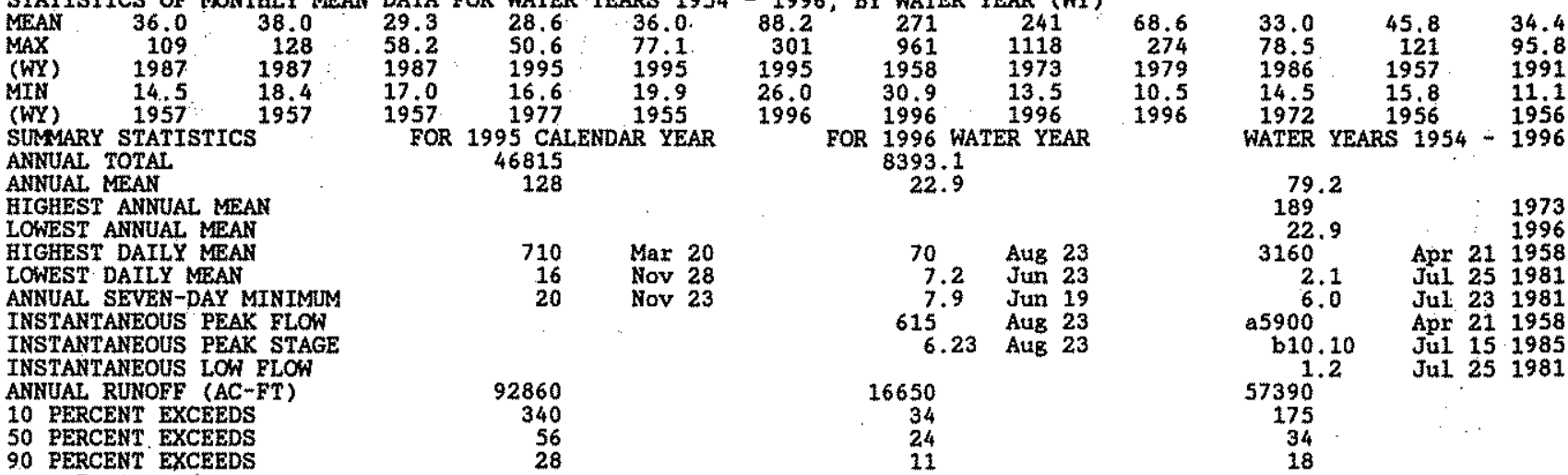

\section{EXCEEDS}

Estimated

bresent datum. 
PERIOD OF RECORD. - -Water years 1981 to current year.

WATER QUALITY DATA, WATER YEAR OCTOBER 1995 TO SEPTEMBER 1996

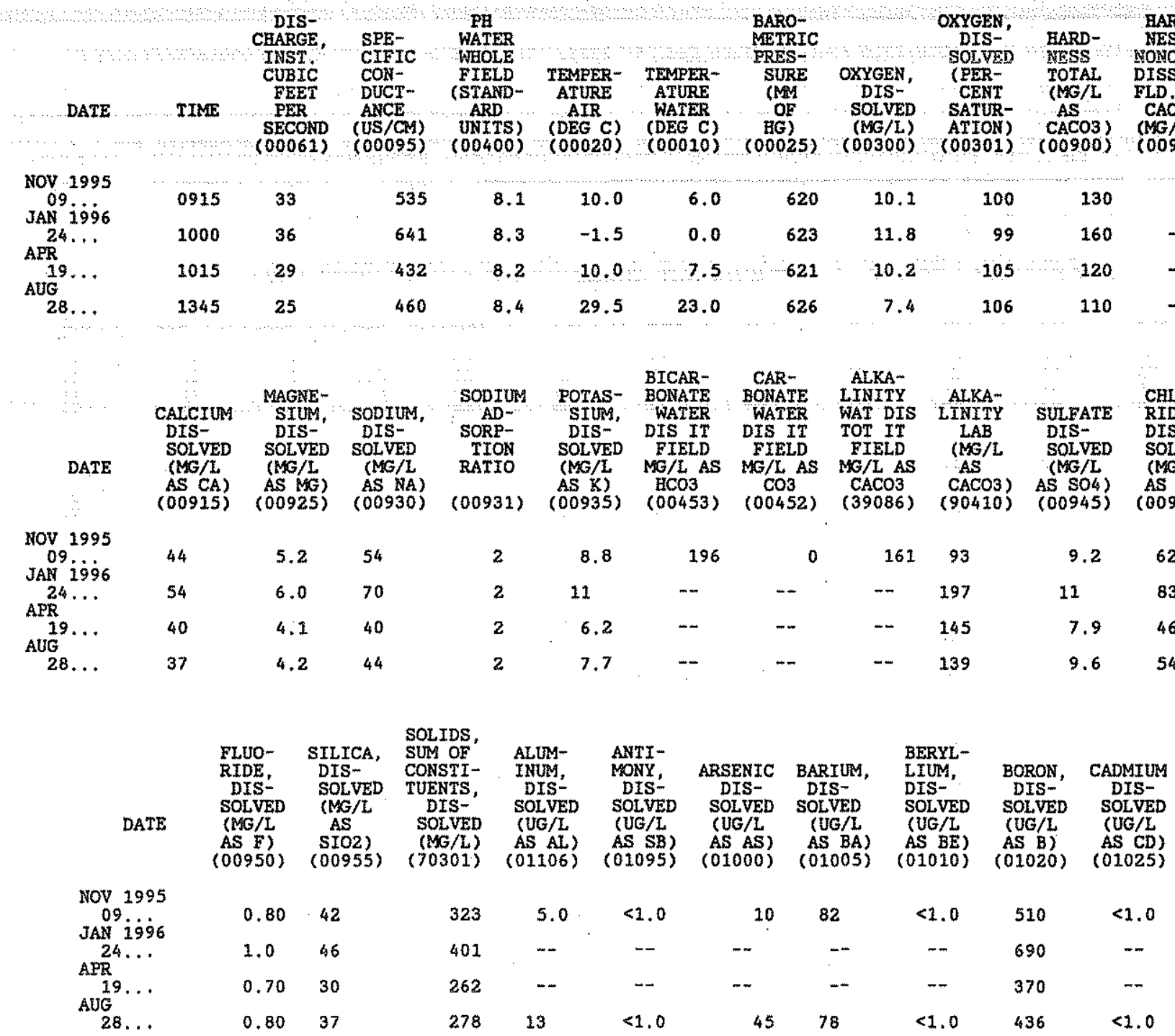

DATE

\begin{tabular}{|c|c|c|c|c|c|c|c|c|c|}
\hline $\begin{array}{l}\text { CHRO- } \\
\text { MILM, } \\
\text { DIS-- } \\
\text { SOLVED } \\
\text { (UE/L } \\
\text { AS CR) } \\
\text { (01030) }\end{array}$ & $\begin{array}{l}\text { COBALT, } \\
\text { DIS } \\
\text { SOLVED } \\
\text { (UG/L } \\
\text { AS CO) } \\
(01095)\end{array}$ & $\begin{array}{l}\text { COPPER, } \\
\text { DIS- } \\
\text { SOLVED } \\
\text { (UC/L } \\
\text { AS CUI) } \\
\text { (01040) }\end{array}$ & $\begin{array}{c}\text { IRON, } \\
\text { DIS } \\
\text { SOLVED } \\
\text { (UG/L. } \\
\text { AS FE) } \\
\text { (01046) }\end{array}$ & $\begin{array}{l}\text { LEAD, } \\
\text { DIS- } \\
\text { SOLVED } \\
\text { (UG/L } \\
\text { AS PB) } \\
\text { (01049) }\end{array}$ & $\begin{array}{l}\text { MANGA- } \\
\text { WESE, } \\
\text { DIS- } \\
\text { SOLVDD } \\
\text { (UC/L } \\
\text { AS MN) } \\
\text { (01056) }\end{array}$ & $\begin{array}{l}\text { MERCURY } \\
\text { TOTAL } \\
\text { RECOV- } \\
\text { ERABLE } \\
\text { (UG/L } \\
\text { AS BG) } \\
(71900)\end{array}$ & $\begin{array}{l}\text { MOLYB- } \\
\text { DENUM, } \\
\text { DIS- } \\
\text { SOLVED } \\
\text { (UG/L } \\
\text { AS MO) } \\
\text { (O1060) }\end{array}$ & $\begin{array}{l}\text { NICKEL, } \\
\text { DIS } \\
\text { SOLVED } \\
\text { (UG/L } \\
\text { AS NI) } \\
\text { (01065) }\end{array}$ & $\begin{array}{l}\text { SEILE- } \\
\text { NLUM, } \\
\text { TOMAL } \\
\text { (UG/L } \\
\text { AS SE) } \\
\text { (01147) }\end{array}$ \\
\hline
\end{tabular}

\begin{tabular}{|c|c|c|c|c|c|c|c|c|c|c|}
\hline $\begin{array}{l}\text { NOV } 1995 \\
09 \\
\text { JAN } 1996\end{array}$ & $<1.0$ & $<2,0$ & 1.0 & 29 & $<1,0$ & 14 & $<0.10$ & 4.0 & 1.0 & $<1$ \\
\hline $\operatorname{AER} 24 \ldots$ & -- & - & $m$ & 50 & $-m$ & +- & $m$ & $m$ & $-\cdots$ & -- \\
\hline AUG $19 .$. & $m$ & $\omega$ & - & 36 & - & $-m$ & $m$ & -- & $m$ & \\
\hline $28 \ldots$ & 1.0 & $<1,0$ & $<1.0$ & 39 & $<1.0$ & 16 & $<0.10$ & 4.0 & 2.0 & $\therefore<1$ \\
\hline
\end{tabular}


RIO GRANDE BASTN

08324000 JEMEZ RIVER NEAR JEMEZ, NM -- Continued

WATER-QUALTTY RECORDS

WATER QUALITY DATA, WATER YEAR OCTOBER 1995 TO SEPTEMBER 1996

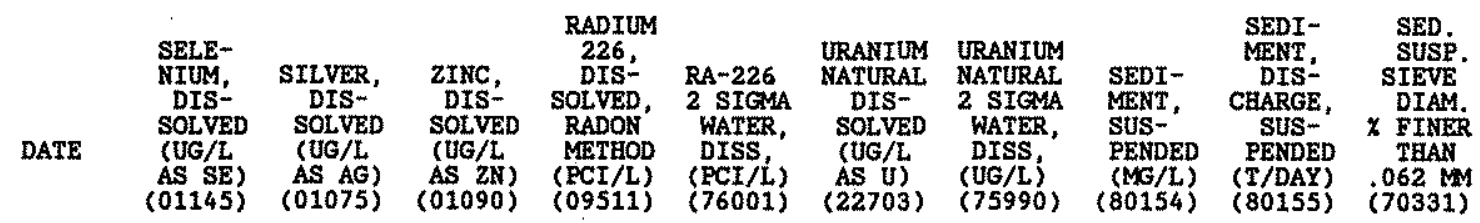

Nov 1995

JAN 09

$\begin{array}{ccccc}<1 & <1.0 & 3.0 & 2.2 & 0.370 \\ -- & -- & -- & -- & -- \\ -\cdots & -- & -- & -- & -- \\ <1 & <1.0 & 2.0 & -- & --\end{array}$

1.1

0.0

11

0.98

67

$24 \ldots$

$19 .$.

AUG
28 ...

$<1<1.0$

2.0

$--$

$-$

-

1.0 
08328500 JEMEZ CANYON RESERVOIR NEAR BERNALILLO, NM

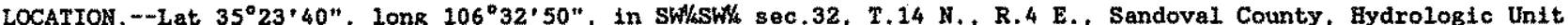
13020202 , at comer of outlet porks control tower of Jemez Canyon Dam on Jemez River, 2.8 ini upstream rrom mouth, and 6 mi north of Bernalilio.

DRAINAGE AREA. $--1,034 \mathrm{mt}^{2}$.

PERIOD OF RECORD.--October 1953 to September 1965 (monthend contents only), October 1965 to current year.

GAGE, - Water-stage recordex Datum of gage is National Geodetic Vertical Datum of 1929 (10vels by U.S. Army Corps of Engineers).

REMARKS, -Reservoir is formed by earchrill dam, completed October 19, 1953. Capacity, 172, 800 acre-ri, from capacity table adapted January 1, 1992, between elevations $5,125,0$ ft, sill of outlet gates, and $5,252.3$ ft,

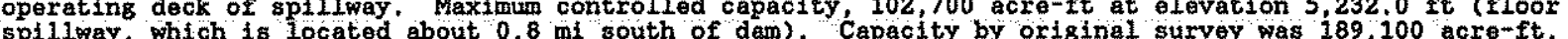
original plan for reservoir operation was to desilt all flow above 30 fts/s by storage for one day before releasing to Rio Grande, and for possible detention during flood stage on Rio Grande. U.S. Antay Corps of Engineers satelit te telemeter at station.

COOPERATION.--Records provided by U.S. Army Corps of Engineers.

EXTREMES FOR PERIOD OF RECORD.-Maximum contents, 72,110 acre-ft, June 1, 1987, elevation, 5,220.24 ft; no storage most of time priox to March 1979.

EXTREAES FOR CURRENT YEAR,-Maximum contents, 23,700 acre-ft, Oct. 2, 3, elevation, 5,193.90 ft; minimum contents, 18,110 acre $-\mathrm{ft}$, Sept. 30, elevetion, $5,189.34 \mathrm{ft}$.

Capacity tablo, (elevation, in feet, and contents, in acre-feet)

(Based on survey by U.S. Amm Corps of Engineers in 1992)

$\begin{array}{llll}5,193.0 & 22,540 & 5,208.0 & 44,810 \\ 5,198.0 & 29,260 & 5,213.0 & 54,080 \\ 5,203.0 & 36,560 & 5,218.0 & 64,720\end{array}$

RESERVOIR STORAGE (ACRE-FEET), WATER YEAR OCTOBER 1995 TO SEPTEMBER 1996

\begin{tabular}{|c|c|c|c|c|c|c|c|c|c|c|c|c|}
\hline $\mathrm{DAY}$ & $\cos$ & NOV & DEC & JAN & $\mathrm{FEB}$ & MAR & AFR & $M A Y$ & JUN & JUL & AUE & SEP \\
\hline $\begin{array}{l}1 \\
2 \\
3 \\
4 \\
5\end{array}$ & $\begin{array}{l}23690 \\
23700 \\
23700 \\
23660 \\
23580\end{array}$ & $\begin{array}{l}22800 \\
22830 \\
22810 \\
22840 \\
22860\end{array}$ & $\begin{array}{l}22470 \\
22470 \\
22470 \\
22470 \\
22470\end{array}$ & $\begin{array}{l}22560 \\
22620 \\
22610 \\
22570 \\
22560\end{array}$ & $\begin{array}{l}22330 \\
22380 \\
22400 \\
22370 \\
22380\end{array}$ & $\begin{array}{l}22090 \\
22120 \\
22170 \\
22170 \\
22060\end{array}$ & $\begin{array}{l}21810 \\
21810 \\
21800 \\
21770 \\
21800\end{array}$ & $\begin{array}{l}22180 \\
22170 \\
22170 \\
22120 \\
22090\end{array}$ & $\begin{array}{l}20980 \\
20960 \\
20930 \\
20880 \\
20850\end{array}$ & $\begin{array}{l}20120 \\
20120 \\
20100 \\
20070 \\
20020\end{array}$ & $\begin{array}{l}19620 \\
19540 \\
19510 \\
19460 \\
19400\end{array}$ & $\begin{array}{l}18880 \\
18810 \\
18760 \\
18720 \\
18640\end{array}$ \\
\hline $\begin{array}{r}6 \\
7 \\
8 \\
9 \\
10\end{array}$ & $\begin{array}{l}23580 \\
23570 \\
23560 \\
23550 \\
23530\end{array}$ & $\begin{array}{l}22890 \\
22780 \\
22670 \\
22670 \\
22710\end{array}$ & $\begin{array}{l}22470 \\
22470 \\
22460 \\
22460 \\
22460\end{array}$ & $\begin{array}{l}22500 \\
22470 \\
22410 \\
22350 \\
22310\end{array}$ & $\begin{array}{l}22380 \\
22350 \\
22330 \\
22310 \\
22300\end{array}$ & $\begin{array}{l}21940 \\
21870 \\
21860 \\
21910 \\
21990\end{array}$ & $\begin{array}{l}21830 \\
21860 \\
21880 \\
21890 \\
21910\end{array}$ & $\begin{array}{l}22070 \\
22020 \\
21990 \\
21960 \\
21920\end{array}$ & $\begin{array}{l}20790 \\
20710 \\
20700 \\
20650 \\
20600\end{array}$ & $\begin{array}{l}19970 \\
19990 \\
20160 \\
20830 \\
20770\end{array}$ & $\begin{array}{l}19370 \\
19500 \\
19640 \\
19600 \\
19560\end{array}$ & $\begin{array}{l}18580 \\
18510 \\
18450 \\
18370 \\
18320\end{array}$ \\
\hline $\begin{array}{l}11 \\
12 \\
13 \\
14 \\
15\end{array}$ & $\begin{array}{l}23520 \\
23510 \\
23480 \\
23470 \\
23460\end{array}$ & $\begin{array}{l}22710 \\
22740 \\
22720 \\
22690 \\
22660\end{array}$ & $\begin{array}{l}22460 \\
22460 \\
22450 \\
22460 \\
22460\end{array}$ & $\begin{array}{l}22230 \\
22180 \\
22180 \\
22180 \\
22180\end{array}$ & $\begin{array}{l}22250 \\
22230 \\
22220 \\
22220 \\
22180\end{array}$ & $\begin{array}{l}22010 \\
21990 \\
21920 \\
21840 \\
21820\end{array}$ & $\begin{array}{l}21920 \\
21960 \\
22020 \\
22030 \\
22080\end{array}$ & $\begin{array}{l}21890 \\
21870 \\
21830 \\
21800 \\
21760\end{array}$ & $\begin{array}{l}20550 \\
20530 \\
20480 \\
20490 \\
20470\end{array}$ & $\begin{array}{l}20370 \\
20230 \\
20240 \\
20240 \\
20260\end{array}$ & $\begin{array}{l}19510 \\
19470 \\
19420 \\
19370 \\
19320\end{array}$ & $\begin{array}{l}18230 \\
18150 \\
18110 \\
18300 \\
18390\end{array}$ \\
\hline $\begin{array}{l}16 \\
17 \\
18 \\
19 \\
20\end{array}$ & $\begin{array}{l}23430 \\
23440 \\
23430 \\
23380 \\
23350\end{array}$ & $\begin{array}{l}22650 \\
22650 \\
22640 \\
22620 \\
22570\end{array}$ & $\begin{array}{l}22480 \\
22470 \\
22500 \\
22510 \\
22510\end{array}$ & $\begin{array}{l}22140 \\
22140 \\
22110 \\
22120 \\
22170\end{array}$ & $\begin{array}{l}22190 \\
22190 \\
22180 \\
22190 \\
22230\end{array}$ & $\begin{array}{l}21830 \\
21800 \\
21800 \\
21780 \\
21720\end{array}$ & $\begin{array}{l}22090 \\
22130 \\
22120 \\
22120 \\
22130\end{array}$ & $\begin{array}{l}21720 \\
21670 \\
21650 \\
21570 \\
21510\end{array}$ & $\begin{array}{l}20430 \\
20410 \\
20370 \\
20350 \\
20320\end{array}$ & $\begin{array}{l}20340 \\
20350 \\
20540 \\
20530 \\
20480\end{array}$ & $\begin{array}{l}19260 \\
19210 \\
19190 \\
19130 \\
19090\end{array}$ & $\begin{array}{l}18340 \\
18340 \\
18390 \\
18370 \\
18340\end{array}$ \\
\hline $\begin{array}{l}21 \\
22 \\
23 \\
24 \\
25\end{array}$ & $\begin{array}{l}23330 \\
23310 \\
23250 \\
23250 \\
23240\end{array}$ & $\begin{array}{l}22560 \\
22560 \\
22590 \\
2.590 \\
22590\end{array}$ & $\begin{array}{l}22510 \\
22470 \\
22450 \\
22400 \\
22360\end{array}$ & $\begin{array}{l}22190 \\
22230 \\
22270 \\
22320 \\
22300\end{array}$ & $\begin{array}{l}22220 \\
22190 \\
22160 \\
22110 \\
22070\end{array}$ & $\begin{array}{l}21710 \\
21730 \\
21770 \\
21760 \\
21750\end{array}$ & $\begin{array}{l}22140 \\
22120 \\
22170 \\
22180 \\
22190\end{array}$ & $\begin{array}{l}21470 \\
21450 \\
21390 \\
21320 \\
21280\end{array}$ & $\begin{array}{l}20250 \\
20230 \\
20200 \\
20160 \\
20100\end{array}$ & $\begin{array}{l}20420 \\
20370 \\
20310 \\
20220 \\
20170\end{array}$ & $\begin{array}{l}19050 \\
19150 \\
19420 \\
19620 \\
19600\end{array}$ & $\begin{array}{l}18340 \\
18330 \\
18310 \\
18270 \\
18230\end{array}$ \\
\hline $\begin{array}{l}26 \\
27 \\
28 \\
29 \\
30\end{array}$ & $\begin{array}{l}23120 \\
23010 \\
23020 \\
23020 \\
23020 \\
22890\end{array}$ & $\begin{array}{c}22550 \\
22510 \\
22480 \\
22470 \\
22470 \\
-\cdots\end{array}$ & $\begin{array}{l}22280 \\
22260 \\
22300 \\
22360 \\
22380 \\
22470\end{array}$ & $\begin{array}{l}22260 \\
22220 \\
22230 \\
22250 \\
22250 \\
22280\end{array}$ & $\begin{array}{r}22020 \\
22010 \\
22010 \\
22030 \\
\ldots \ldots \\
\ldots\end{array}$ & $\begin{array}{l}21770 \\
21800 \\
21800 \\
21800 \\
21820 \\
21810\end{array}$ & $\begin{array}{c}22210 \\
22210 \\
22190 \\
22210 \\
22190 \\
=\end{array}$ & $\begin{array}{l}21230 \\
21190 \\
21140 \\
21100 \\
21080 \\
21040\end{array}$ & $\begin{array}{c}20100 \\
20140 \\
20120 \\
20120 \\
20100 \\
\ldots-\end{array}$ & $\begin{array}{l}20110 \\
20050 \\
19990 \\
19340 \\
19890 \\
19830\end{array}$ & $\begin{array}{l}19680 \\
19270 \\
19020 \\
19020 \\
19010 \\
18940\end{array}$ & $\begin{array}{l}18220 \\
18180 \\
18160 \\
18120 \\
18110 \\
-\end{array}$ \\
\hline$y$ & $\begin{array}{r}23700 \\
22890 \\
5193.28 \\
-800\end{array}$ & $\begin{array}{r}22890 \\
22470 \\
5192.95 \\
-420\end{array}$ & $\begin{array}{r}22510 \\
22260 \\
5192.95 \\
0\end{array}$ & $\begin{array}{r}22620 \\
22110 \\
5192,80 \\
-190\end{array}$ & $\begin{array}{r}22400 \\
22010 \\
5192.60 \\
-250\end{array}$ & $\begin{array}{r}22170 \\
21710 \\
5192.42 \\
-220\end{array}$ & $\begin{array}{r}22210 \\
21770 \\
5192.73 \\
+380\end{array}$ & $\begin{array}{r}22180 \\
21040 \\
5191.80 \\
-1150\end{array}$ & $\begin{array}{r}20980 \\
20100 \\
5191.02 \\
-940\end{array}$ & $\begin{array}{r}20830 \\
19830 \\
5190,80 \\
-270\end{array}$ & $\begin{array}{r}19680 \\
18940 \\
5190.05 \\
-890\end{array}$ & $\begin{array}{r}18880 \\
18110 \\
5189.34 \\
-830\end{array}$ \\
\hline
\end{tabular}

CAL YR 1995 MAX 30700 MIN 21530 (t) +860

WTR YR 1996 MAX 23700 MIN 18110 (tt) -5580

(†) ELEVATION, IN FEET, AT END OF MONZH.

(†) CHANGE IN CONTENTS, IN ACRE-FEET. 
RIO GRANDE BASIN

08329000 JEMEZ RIVER BELOW JEMEZ CANYON DAM, NM

LOCATION, - Lat $35^{\circ} 23^{\prime} 24^{\prime \prime}$, long $106^{\circ} 32^{\prime} 03^{\prime \prime}$, in NEK sec.5, T.13 \%, R.4 E., Sandoval County, Hydrologic Unit 13020202 , on right bank $0.8 \mathrm{mi}$ downstream from Jemez Canyon Dam, 2.0 mi upstream from mouth, and 6 mi north of Bernalilio. DRAINAGE AREA $--1,038 \mathrm{mi}^{3}$

PERIOD OF RECORD.-March 1936 to January 1938, March 1943 to current year. Published as "Jemez Creak" prior to 1948, and as "near Bernalil1o" prior to 1954

REVISED'RECORDS.--WSP 1178: 1949."WSP 1212: 1950. WSP 1512: 1936, 1943, 1945, 1947-48, 1949(M), 1950. WSP 1732: Drainage araa.

GAGE. - Water-stage recorder. Datum of gage is $5,095.60 \mathrm{ft}$ above National Geodettc Vertical Datum of 1929 (U. $\mathrm{s}$ Army Corps of Engineers bench mark). Prior to Apr. 24, 1951 , at site 0.8 mi upstream at datum 24.51 ft higher Apr. 24, 1951, to June 25, 1958 , at site 37 ft upstream at datum 4.40 ft above present datum. Supplementary water-stage recorder at gages on Jemez Canyon Dam at datum $5,125.00$ ft above National Geodetic Vertical Datum of 1929 (U.S. Army Corps of Engineers bench mark) used at times since January 1953 .

RDMARKS - Records good except for estimated daily discharges, which are poor. Subsequent to October 1953, flow at this station can be completely regulated by Jemez Canyon Reservolx (station 08328500). However, reservoir is designed essentially for desilting and flood control rather than storage. Diversions for irrigation of about 3,000 acres upstream from station. U.S. Army Corps of Enginears satelitte telemeter at station. No flow for many days

EXTREMES OUTSIDE PERIOD OF RECORD, - A flood in 1900 was probably less than 16,000 fts/s, but highest observed outside period of record.

DISCEARGE, CUBIC FEET PER SECOND, WATER YEAR OCTOBER 1995 TO SEPTEMBER 1996

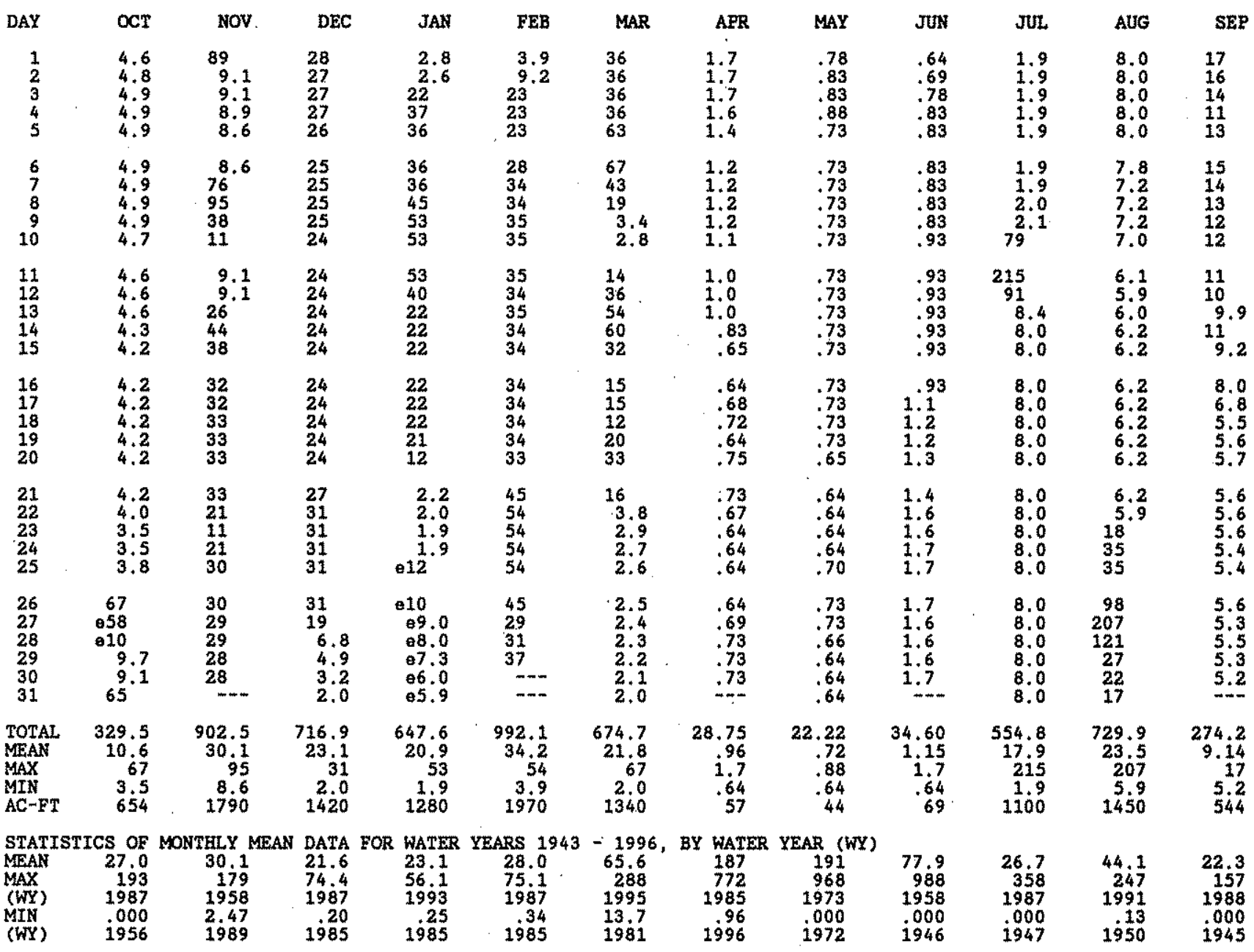

SUMMARY STATISTICS ANNUAL TOTAL. ANNUAL MEAN

BIGHEST ANNUAL MEAN

LOWEST ANNUAL MEAN

LOWEST ANI

FIGHEST DAILY MEAN

LOWEST DAILY MEAN

INSTANTANEOUS PEAK FLON

INSTANTANEOUS REAK STAGE

ANNUAL RUNOFF (AC-ET)

10 PERCENT EXCEEDS

50 PERCENT EXCEEDS

90 PERCEHT EXCEEDS

- Estimated

a-site and datum then in use.

FOR 1995 CALENDAR YEAR 40977.84

FOR 1996 WATER YEAR 5907.77

815 Max 20 .00
.00 Fob 1

81280

340

.63
16.1

215 Jul 11 .64 Apr 16
.66 May 19

11720

8.0
WATER YEARS 1943 - 1996

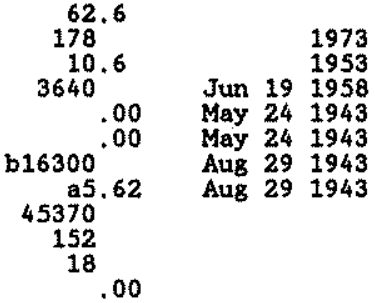

b-From rating curve extended above 3,000 f.t $3 / \mathrm{s}$. 
RTO GRANDE BASTN

08329700 CAMPUS WASH AT ALBUQUERQUE, MM

LOCATION,--Lat $35^{\circ} 05^{\prime} 40^{\prime \prime}$, long $106^{\circ} 37^{\prime} 22^{\prime \prime}$, in SEK sec.16, T.10 N., R.3 E., Bernali1lo County, Bydrologic Unit 13020203 , on right bank $100 \mathrm{ft}$ west of southwest comer of university of New Mexico North Golf Course, 200 ft downstream from Barelas Stormater Pumping Station outfall, 600 ft downstream from Tucker Rosd bridge, and 1, 500 ft northeast of intersection of Lomas and biviversity Boulevards " in Albuquerque.

DRAINAGE AREA. $--3.80 \mathrm{mi}^{2}$.

EERIOD OF RECORD,-Apri 1982 to current year (seasonal records).

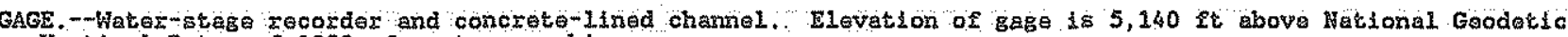
Vertical Datu of 1929 , Irom topographic map.

REMARKS.--Water-discharge records poor. Recording rain gage at station. Some minor streamflow may exist on days where daily mean discherges have been recorded as zero due to the sensitivity limits of the streamflow monttoring oquipment.

EXTREMS FOR PERIOD OF RECORD.--Maximum discharge, 1,230 fts/s, July 14, 1990, gage helght, 4.50 ft, from rating curve developed by step-backwater analysis of channel; no flow most of time.

EXTREMES FOR CURRENT YEAR,--Maximum discharge during period of seasonal operation, 593 ft"s, at 2335 hours Aug. 25, gage hefght, $2.95 \mathrm{ft}$; no flow most of time. DISCAARGE, CUBIC FEET PER SECOND, WATER YEAR OCTOBER 1995 TO SEPTEMBER 1996

\begin{tabular}{|c|c|c|c|c|c|c|c|c|c|c|c|c|}
\hline DAY & OCF & HOV & DEC & $\therefore$ JAN & $\therefore$ FEB & $\cdots M A R$ & APR & MAY & $\therefore$ JUN & JUL & AUG & SEP \\
\hline $\begin{array}{l}1 \\
2 \\
3 \\
4 \\
5\end{array}$ & $\begin{array}{l}.00 \\
.03 \\
.00 \\
.00 \\
.00\end{array}$ & $\begin{array}{l}.00 \\
.01 \\
.00 \\
.00 \\
.00\end{array}$ & $\begin{array}{l}m- \\
m- \\
m- \\
m- \\
---\end{array}$ & 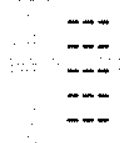 & 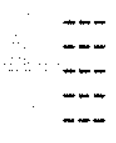 & $\begin{array}{l}-m \\
-m \\
m-m \\
-m\end{array}$ & $\begin{array}{l}.00 \\
.00 \\
.00 \\
.00 \\
.00\end{array}$ & $\begin{array}{l}.00 \\
.39 \\
.00 \\
.00 \\
.00\end{array}$ & $\begin{array}{l}.00 \\
.00 \\
.00 \\
.00 \\
.00\end{array}$ & $\begin{array}{l}.00 \\
.00 \\
00 \\
00 \\
.00\end{array}$ & $\begin{array}{r}.00 \\
.00 \\
.00 \\
.02\end{array}$ & $\begin{array}{l}.00 \\
.00 \\
.00 \\
.00 \\
.00\end{array}$ \\
\hline $\begin{array}{r}6 \\
7 \\
8 \\
9 \\
10\end{array}$ & $\begin{array}{l}.00 \\
.00 \\
.00 \\
.00 \\
.00\end{array}$ & $\begin{array}{l}.01 \\
.00 \\
.00 \\
.00 \\
.00\end{array}$ & $\begin{array}{c}-\infty \\
-\infty \\
-m \\
-\infty \\
-\infty\end{array}$ & 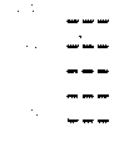 & ט- & $\begin{array}{c}m-\infty \\
-\infty \\
-\infty \\
-\infty\end{array}$ & $\begin{array}{l}.00 \\
.00 \\
.00 \\
.00 \\
.00\end{array}$ & $\begin{array}{l}.00 \\
.00 \\
.00 \\
.00 \\
.00\end{array}$ & $\begin{array}{l}.00 \\
.00 \\
.00 \\
.00 \\
.00\end{array}$ & $\begin{array}{c}.00 \\
3.6 \\
.02 \\
.81 \\
9.1\end{array}$ & $\begin{array}{l}.00 \\
1.2 \\
.86 \\
.00 \\
.00\end{array}$ & $\begin{array}{l}.45 \\
.00 \\
.00 \\
.00 \\
.09\end{array}$ \\
\hline $\begin{array}{l}11 \\
12 \\
13 \\
14 \\
15\end{array}$ & $\begin{array}{l}.00 \\
.00 \\
.00 \\
.00 \\
.69\end{array}$ & $\begin{array}{l}.00 \\
.00 \\
.00 \\
.00 \\
.00\end{array}$ & $\begin{array}{l}-w \\
-w \\
-w \\
--\infty \\
--m\end{array}$ & $\begin{array}{l}--\infty \\
-\cdots \\
-\cdots \\
-\infty\end{array}$ & $\begin{array}{l}m-- \\
=-- \\
-m-\end{array}$ & $\begin{array}{l}.00 \\
.00 \\
.00 \\
.00\end{array}$ & $\begin{array}{l}.00 \\
.00 \\
.00 \\
.00 \\
.00\end{array}$ & $\begin{array}{l}.00 \\
.00 \\
.00 \\
.00 \\
.00\end{array}$ & $\begin{array}{l}.00 \\
.00 \\
.00 \\
.71 \\
.00\end{array}$ & $\begin{array}{l}.00 \\
.00 \\
.00 \\
.00 \\
.00\end{array}$ & $\begin{array}{l}.00 \\
.00 \\
.01 \\
.00 \\
.08\end{array}$ & $\begin{array}{l}.00 \\
.00 \\
.00 \\
.-\end{array}$ \\
\hline $\begin{array}{l}16 \\
17 \\
18 \\
19 \\
20\end{array}$ & $\begin{array}{l}.00 \\
.00 \\
.00 \\
.00 \\
.00\end{array}$ & $\begin{array}{l}.01 \\
.00 \\
.00 \\
.00 \\
.0\end{array}$ & $\begin{array}{l}m- \\
-m- \\
m- \\
m-m \\
m-\infty\end{array}$ & $\begin{array}{l}m- \\
m- \\
m- \\
m-\end{array}$ & $=$ & $\begin{array}{l}.00 \\
.00 \\
.00 \\
.00 \\
.00\end{array}$ & $\begin{array}{l}.00 \\
.00 \\
.00 \\
.00 \\
.00\end{array}$ & $\begin{array}{l}.00 \\
.00 \\
.00 \\
.00 \\
.00\end{array}$ & $\begin{array}{l}.00 \\
.00 \\
.00 \\
.00 \\
.00\end{array}$ & $\begin{array}{l}4.5 \\
3.5 \\
5.2 \\
.00 \\
.00\end{array}$ & $\begin{array}{r}.00 \\
.00 \\
.00 \\
.00 \\
1.7\end{array}$ & $\begin{array}{l}--- \\
-0 \\
-0 \\
-0\end{array}$ \\
\hline $\begin{array}{l}21 \\
22 \\
23 \\
24 \\
25\end{array}$ & $\begin{array}{l}.00 \\
.00 \\
.00 \\
.00 \\
.00\end{array}$ & $=-$ & $\begin{array}{l}--m \\
--\infty \\
--- \\
-m \\
--\end{array}$ & $\begin{array}{l}m-\infty \\
-\infty \\
-\infty\end{array}$ & $=-$ & $\begin{array}{l}.00 \\
.00 \\
.00 \\
.00 \\
.00\end{array}$ & $\begin{array}{l}.00 \\
.00 \\
.00 \\
.00 \\
.00\end{array}$ & $\begin{array}{l}.00 \\
.00 \\
.00 \\
.01 \\
.05\end{array}$ & $\begin{array}{l}.00 \\
.00 \\
.00 \\
.00 \\
.00\end{array}$ & $\begin{array}{l}.00 \\
.00 \\
-00 \\
.00\end{array}$ & $\begin{array}{c}4.00 \\
7.7 \\
11^{.04}\end{array}$ & $\begin{array}{l}.00 \\
.00 \\
.00 \\
.00 \\
.00\end{array}$ \\
\hline $\begin{array}{l}26 \\
27 \\
28 \\
29 \\
30 \\
31\end{array}$ & $\begin{array}{l}.00 \\
.00 \\
.00 \\
.00 \\
.00 \\
.00\end{array}$ & $\begin{array}{l}m-m \\
m-m \\
m-m \\
-m\end{array}$ & $\begin{array}{l}-\cdots \\
-\infty \\
-\infty \\
-\infty \\
-\infty\end{array}$ & $\begin{array}{l}--- \\
--- \\
-- \\
-- \\
-- \\
---\end{array}$ & $\begin{array}{l}m+m \\
m-\infty \\
--\infty\end{array}$ & $\begin{array}{l}.00 \\
.00 \\
.00 \\
.00 \\
.00 \\
.00\end{array}$ & $\begin{array}{l}.00 \\
.00 \\
.00 \\
.00 \\
.00 \\
.\end{array}$ & $\begin{array}{l}.14 \\
.00 \\
.00 \\
.00 \\
.00 \\
.00\end{array}$ & $\begin{array}{l}32 \\
11 \\
13 \\
2.00 \\
2.8\end{array}$ & $\begin{array}{l}.00 \\
.00 \\
.00 \\
.00 \\
.00 \\
.00\end{array}$ & $\begin{array}{r}18 \\
.00 \\
.00 \\
9.8 \\
.00 \\
.00\end{array}$ & $\begin{array}{r}.36 \\
.00 \\
1.0 \\
.00 \\
.00 \\
.\end{array}$ \\
\hline $\begin{array}{l}\text { TOTAL } \\
\text { MEAN } \\
\text { MAX } \\
\text { MEN } \\
\text { AC-FT } \\
(t)\end{array}$ & $\begin{array}{r}0.72 \\
.023 \\
.69 \\
.00 \\
1.4 \\
0.01\end{array}$ & $\begin{array}{c}-w \\
- \\
--- \\
- \\
-\overline{-} \\
0.05\end{array}$ & $\begin{array}{l}-\cdots \\
-\cdots \\
-\cdots \\
-\cdots \\
0.11\end{array}$ & $\begin{array}{l}m- \\
--m \\
m- \\
--m \\
0.25\end{array}$ & $\begin{array}{c}\cdots \\
-\infty \\
--- \\
-\cdots \\
0.03\end{array}$ & $\begin{array}{l}-\cdots \\
\cdots \\
\cdots \\
-\cdots \\
0.00\end{array}$ & $\begin{array}{l}0.00 \\
.000 \\
.00 \\
.00 \\
.00 \\
0.01\end{array}$ & $\begin{array}{r}0.59 \\
.019 \\
.39 \\
.00 \\
1.2 \\
0.09\end{array}$ & $\begin{array}{r}59.51 \\
1.98 \\
32 \\
.00 \\
118 \\
1.68\end{array}$ & $\begin{array}{l}-- \\
-- \\
-1 . \\
-19\end{array}$ & 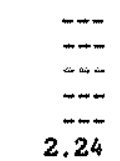 & $\begin{array}{l}\cdots \\
\cdots \\
0.11 \\
0 .\end{array}$ \\
\hline
\end{tabular}

(†) Total rainfall accumulation in inches. 
RIO GRANDE BASIN

08329835 NORTH FLOODWAY CHANNEL AT ALBUQUERQUE', NM

LOCATrON, -Iat $35^{\circ} 07^{\circ} 03^{\prime \prime}$, Long $106^{\circ} 36^{\circ} 42^{* \prime}$, in SEK sec.3, T.10 N., R. 3 E., Bernalillo County, Bydrologic Unit 13020203 , on right bank of concrete-1ined drainage channel, 300 ft downstream (north) of bridge on Candelarta Boulevard. NE, and 3,000 ft downstream from confluence of Campus Wash and Embudo Arroyo in Albuquerque.

DRAINAGE AREA. $-40.0 \mathrm{~mL}^{2}$.

PERTOD OF RECORD, --May 1982 to current year (seasonal records).

GAGE.--Water-stage recorder and concrete-lined channe1. Elevation of gage is 5,110 ft above National Geodetic Vertical: Datum of 1929 , from topographic map.

REMARKS.--Records fair except for estimated daily discharges, which are poor. Some minor streamflow may exist on days where daily meen discharges have been recorded as zero due to the sensitivity limits of the streamflow monitoring equipment.

EXTREMES FOR PERIOD OF RECORD.--Maximum discharge, 7,250 fts/s, July 9, 1988, gage height, $12.10 \mathrm{ft}$, from floodmarks from step- backwater analysis of channel; no flow most of time.

EXTREMES FOR CURRENT YEAR.-Maximum discharge during period of seasonal operation, $4,200 \mathrm{ft} / \mathrm{s}$, at $2400 \mathrm{hours}$ Aug. 25, gage height, $9.13 \mathrm{ft}$; no flow most of time.

DISCAARGE, CUBIC FEET PER SECOND, WATER YEAR OCTOBER 1995 TO SEPTEMBER 1996

\begin{tabular}{|c|c|c|c|c|c|c|c|c|c|c|c|c|}
\hline DAY & OCT & Nov & DEC & JAN & FEB & MAR & APR & MAY & JUN & JULL & AUG & SEP \\
\hline $\begin{array}{l}1 \\
2 \\
3 \\
4 \\
5\end{array}$ & $\begin{array}{l}m-- \\
--- \\
m-- \\
---\end{array}$ & $\begin{array}{l}-+ \\
- \\
-\infty \\
-\infty\end{array}$ & $m-m$ & $\begin{array}{l}m-m \\
-m-m \\
-m-m \\
-m\end{array}$ & $\begin{array}{l}m-m \\
-m-m \\
-m-\infty\end{array}$ & $\begin{array}{l}-m \\
--- \\
m-m \\
m- \\
--\end{array}$ & $\begin{array}{l}.00 \\
.00 \\
.00 \\
.00 \\
.00\end{array}$ & $\begin{array}{l}.00 \\
.00 \\
.00 \\
.00 \\
.00\end{array}$ & $\begin{array}{l}.00 \\
.00 \\
.00 \\
.00 \\
.00\end{array}$ & $\begin{array}{l}.00 \\
.00 \\
.00 \\
.00 \\
.00\end{array}$ & ${ }_{\mathrm{e}}^{\mathrm{e}} \mathrm{3}^{.00} .00$ & $\begin{array}{l}.00 \\
.00 \\
.00 \\
.00 \\
.00\end{array}$ \\
\hline $\begin{array}{r}6 \\
7 \\
8 \\
9 \\
10\end{array}$ & $\begin{array}{l}--- \\
-- \\
--- \\
-m-\end{array}$ & $\begin{array}{l}-m \\
-\infty \\
-\infty \\
-\infty\end{array}$ & $\begin{array}{l}m- \\
m-- \\
-- \\
-\infty\end{array}$ & $\begin{array}{l}m-m \\
m-\infty \\
-m- \\
-m-m\end{array}$ & $\begin{array}{l}-m \\
--\infty \\
m-m \\
m-m\end{array}$ & $\begin{array}{l}-m \\
-m- \\
m-m \\
-m-m \\
--m\end{array}$ & $\begin{array}{l}.00 \\
.00 \\
.00 \\
.00 \\
.00\end{array}$ & $\begin{array}{l}.00 \\
.00 \\
.00 \\
.00 \\
.00\end{array}$ & $\begin{array}{l}.00 \\
.00 \\
.00 \\
.00 \\
.00\end{array}$ & $\begin{array}{l}13 \\
4.00 \\
33^{4} \\
89\end{array}$ & $\begin{aligned} & e .00 \\
& 050^{-5} \\
& .00 \\
& .00\end{aligned}$ & $\begin{array}{r}05.0 \\
0.00 \\
.00 \\
.00 \\
.00\end{array}$ \\
\hline $\begin{array}{l}11 \\
12 \\
13 \\
14 \\
15\end{array}$ & $\begin{array}{l}--- \\
--- \\
--- \\
--- \\
--\infty\end{array}$ & $\begin{array}{l}m-- \\
--- \\
--\infty\end{array}$ & $\begin{array}{l}m-\infty \\
-m- \\
-m\end{array}$ & $\begin{array}{l}-m \\
--m \\
-m-m \\
-m-\infty\end{array}$ & $\begin{array}{l}m-\infty \\
-\infty \\
-\infty \\
-\infty\end{array}$ & $\begin{array}{l}.00 \\
.00 \\
.00 \\
.00\end{array}$ & $\begin{array}{l}.00 \\
.00 \\
.00 \\
.00 \\
.00\end{array}$ & $\begin{array}{l}.00 \\
.00 \\
.00 \\
.00 \\
.00\end{array}$ & $\begin{array}{r}.00 \\
.40 \\
1.1 \\
.94\end{array}$ & $\begin{array}{r}19 \\
.44 \\
.00 \\
.00 \\
8.9\end{array}$ & $\begin{array}{l}.00 \\
.00 \\
.00 \\
.78 \\
.00\end{array}$ & $\begin{array}{r}05.0 \\
228 \\
15\end{array}$ \\
\hline $\begin{array}{l}16 \\
17 \\
18 \\
19 \\
20\end{array}$ & $\begin{array}{l}m-- \\
m-- \\
--- \\
-- \\
--\end{array}$ & $\begin{array}{l}m-- \\
-\cdots \\
-\cdots \\
-\infty\end{array}$ & $\begin{array}{l}m-\infty \\
m-m \\
m-m\end{array}$ & $\begin{array}{l}-m \\
-m-\infty \\
-m-m\end{array}$ & $\begin{array}{l}m-\infty \\
m-m \\
m-\infty\end{array}$ & $\begin{array}{l}.00 \\
.00 \\
.00 \\
.00 \\
.00\end{array}$ & $\begin{array}{l}.00 \\
.00 \\
.00 \\
.00 \\
.00\end{array}$ & $\begin{array}{l}.00 \\
.00 \\
.00 \\
.00 \\
.00\end{array}$ & $\begin{array}{l}.00 \\
.00 \\
.00 \\
.00 \\
.00\end{array}$ & $\begin{array}{l}17 \\
34 \\
28 \\
4.1 \\
4.0\end{array}$ & $\begin{array}{r}.00 \\
.00 \\
.00 \\
21.00\end{array}$ & $\begin{array}{l}6.8 \\
81 \\
79 \\
2.4 \\
.00\end{array}$ \\
\hline $\begin{array}{l}21 \\
22 \\
23 \\
24 \\
25\end{array}$ & $\begin{array}{l}m-m \\
-m-m \\
--m \\
m-m \\
-m-\end{array}$ & $\begin{array}{l}m-- \\
-m- \\
-m- \\
--\end{array}$ & $\begin{array}{l}m-\infty \\
=-\infty \\
-\infty-\end{array}$ & - & $\begin{array}{l}-m \\
-m \\
-\infty \\
-\infty\end{array}$ & $\begin{array}{l}.00 \\
.00 \\
.00 \\
.00 \\
.00\end{array}$ & $\begin{array}{l}.00 \\
.00 \\
.00 \\
.00 \\
.00\end{array}$ & $\begin{array}{l}.00 \\
.00 \\
.00 \\
.00 \\
.00\end{array}$ & $\begin{array}{l}.00 \\
.00 \\
.00 \\
.00 \\
.00\end{array}$ & $\begin{array}{l}3.1 \\
2.3 \\
2.0 \\
.28 \\
.04\end{array}$ & $\begin{array}{r}2.4 \\
70 \\
0100 \\
05.0 \\
010\end{array}$ & $\begin{array}{l}.00 \\
.00 \\
.00 \\
.00 \\
.28\end{array}$ \\
\hline $\begin{array}{l}26 \\
27 \\
28 \\
29 \\
30 \\
31\end{array}$ & $\begin{array}{l}+- \\
-\infty \\
--\infty \\
--\infty\end{array}$ & - & $\begin{array}{l}-\infty \\
-\infty \\
-\infty \\
-\infty\end{array}$ & $\begin{array}{l}m-m \\
-m-m \\
-m-m \\
-m\end{array}$ & $\begin{array}{l}m-m \\
m-m \\
m-m \\
m-m\end{array}$ & $\begin{array}{l}.00 \\
.00 \\
.00 \\
.00 \\
.00 \\
.00\end{array}$ & $\begin{array}{l}.00 \\
.00 \\
.00 \\
.00 \\
.00 \\
.0\end{array}$ & $\begin{array}{r}16.00 \\
.00 \\
.00 \\
.00 \\
.00\end{array}$ & $\begin{array}{r}268 \\
83 \\
113 \\
.27 \\
8.6\end{array}$ & $\begin{array}{l}.00 \\
.00 \\
.00 \\
.00 \\
.00 \\
.00\end{array}$ & $\begin{array}{r}0400 \\
020 \\
0.00 \\
0150 \\
.00 \\
.00\end{array}$ & $\begin{array}{r}16 \\
.00 \\
2.0 \\
.00 \\
.00 \\
=-\end{array}$ \\
\hline $\begin{array}{l}\text { TOTAL } \\
\text { MEAN } \\
\text { MAX } \\
\text { MIN } \\
\text { AC-FT }\end{array}$ & $=$ & $=$ & $=$ & 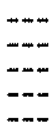 & $=-$ & $=$ & $\begin{array}{l}0.00 \\
.000 \\
.00 \\
.00 \\
.00\end{array}$ & $\begin{array}{r}16.00 \\
.52 \\
16 \\
.00 \\
32\end{array}$ & $\begin{array}{r}505.31 \\
16.8 \\
268 \\
.00 \\
1000\end{array}$ & $\begin{array}{r}262.36 \\
8.46 \\
89 \\
.00 \\
520\end{array}$ & $\begin{array}{r}847.18 \\
27.3 \\
400 \\
100 \\
1680\end{array}$ & $\begin{array}{r}440.48 \\
14.7 \\
228 \\
.00 \\
874\end{array}$ \\
\hline
\end{tabular}

- Estimated 
RIO GRANDE BASIN

08329838 SOUTH FORK HAHN ARROYO AT ALBUQUERQUE, NM

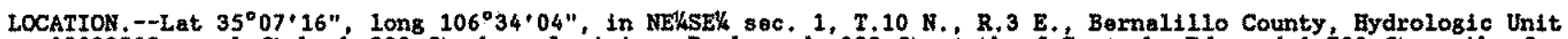
13020203 , on left bank $300 \mathrm{ft}$ above Louisiana Boulevard, 900 ft south of Comanche Rd, and 1,700 ft north of Candelaria Rd, in Albuquerque.

DRATNAGE AREA. $--2,03 \mathrm{mi}^{2}$.

PERIOD OF RECORD.--June 1978 to December 1983, June 1992 to current year (seasonal record).

GAGE--Water-stage rocorder and concrete lined channel. Elevation of gage is 5,300 lt above Netional Geodetic Vertical Datum of 1929, from topographic map. Prior to 1983 at sito 300 ft downstream on louisiana Boulevard bxidge, at distexane datum.

REMARKS, --Records good. Some minor streamflow may exist on days where dally mean discharges have been recorded as zero due to the sensitivity linits of the streamflow monitoring equipment. Recording rain gege at station.

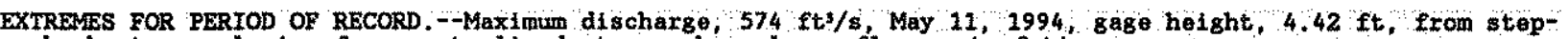
backwater analysis of concrete lined stream channel; no flow most of time.

EXTREMES FOR CURRENT YEAR,--Maximum discharge during period of seasonal operation, 526 fts/s, at 1705 hours, June 28 , gage height, $4.21 \mathrm{st}$; no flow most of time.

DISCHARGE, CUBIC FEET PER SECOND, WATER YEAR OCTOBER 1995 TO SEPTEMBER 1996

\begin{tabular}{|c|c|c|c|c|c|c|c|c|c|c|c|c|}
\hline DAY & oCT & Nov & DEC & JAN & FEB & MAR & APR & MAY & JUN & JUL & AUG & SEP \\
\hline $\begin{array}{l}1 \\
2 \\
3 \\
4 \\
5\end{array}$ & $\begin{array}{l}.06 \\
.25 \\
.27 \\
.26 \\
.37\end{array}$ & $\begin{array}{r}.41 \\
.81 \\
.15 \\
.05 \\
.04\end{array}$ & $\cdots$ & $\underset{-\infty}{-\infty}$ & 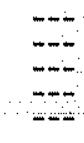 & $\overline{--}$ & $\begin{array}{l}.00 \\
.00 \\
.00 \\
.00 \\
.00\end{array}$ & $\begin{array}{l}.00 \\
.00 \\
.00 \\
.04 \\
.00\end{array}$ & $\begin{array}{l}.00 \\
.04 \\
.05 \\
08 \\
.11\end{array}$ & $\begin{array}{r}.16 \\
.27 \\
.15 \\
.15 \\
.10\end{array}$ & $\begin{array}{l}-- \\
--- \\
--- \\
-\square \\
-\overline{-}\end{array}$ & $\begin{array}{l}--- \\
--- \\
--- \\
.11 \\
15\end{array}$ \\
\hline $\begin{array}{r}6 \\
7 \\
8 \\
9 \\
10\end{array}$ & $\begin{array}{l}.29 \\
.01 \\
.06 \\
.34 \\
.21\end{array}$ & $\begin{array}{r}.00 \\
.00 \\
.00 \\
.00 \\
.15\end{array}$ & $\therefore$ & $\begin{array}{l}--- \\
-m \\
-m \\
-m \\
-\infty\end{array}$ & $=$ & $\begin{array}{l}m-- \\
-\cdots \\
-\cdots\end{array}$ & $\begin{array}{l}.00 \\
.00 \\
.00 \\
.00 \\
.00\end{array}$ & $\begin{array}{l}.04 \\
.03 \\
.03 \\
.24 \\
.04\end{array}$ & $\begin{array}{l}.07 \\
.08 \\
.04 \\
.05 \\
.06\end{array}$ & $\begin{array}{l}.06 \\
.67 \\
.93 \\
3.1 \\
1.0\end{array}$ & $\begin{array}{l}.02 \\
.01 \\
.33 \\
.10 \\
.11\end{array}$ & $\begin{array}{l}1.1 \\
.07 \\
.05 \\
.12 \\
.10\end{array}$ \\
\hline $\begin{array}{l}11 \\
12 \\
13 \\
14 \\
15\end{array}$ & $\begin{array}{l}.22 \\
.30 \\
.19 \\
.04 \\
.07\end{array}$ & $\begin{array}{l}.05 \\
.05 \\
.00 \\
.00 \\
.00\end{array}$ & $\begin{array}{l}--- \\
=- \\
--- \\
--\end{array}$ & $\begin{array}{l}m-\infty \\
-m- \\
-m- \\
-m-\infty \\
-m-\end{array}$ & 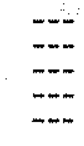 & $\begin{array}{l}.00 \\
.00 \\
.00 \\
.00\end{array}$ & $\begin{array}{l}.04 \\
.03 \\
.03 \\
.06 \\
.00\end{array}$ & $\begin{array}{l}.00 \\
.01 \\
.03 \\
.04 \\
.48\end{array}$ & $\begin{array}{r}.08 \\
.10 \\
.06 \\
3.2 \\
.04\end{array}$ & $\begin{array}{r}.25 \\
.00 \\
-\ldots \\
-\ldots\end{array}$ & $\begin{array}{l}.02 \\
.03 \\
.00 \\
.03 \\
.04\end{array}$ & 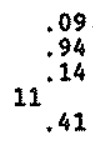 \\
\hline $\begin{array}{l}16 \\
17 \\
18 \\
19 \\
20\end{array}$ & $\begin{array}{l}.07 \\
.11 \\
.00 \\
.00 \\
.13\end{array}$ & $\begin{array}{l}.00 \\
.13 \\
.07 \\
.03 \\
.00\end{array}$ & $\begin{array}{l}m-m \\
m-m \\
m-\infty \\
m-m\end{array}$ & $\begin{array}{l}-m- \\
-m- \\
-m \\
m-m \\
m-m\end{array}$ & $\begin{array}{l}--- \\
-m \\
-m \\
-\infty\end{array}$ & $\begin{array}{l}.00 \\
.00 \\
.04 \\
.04 \\
.06\end{array}$ & $\begin{array}{l}.00 \\
.00 \\
.01 \\
.00 \\
.00\end{array}$ & $\begin{array}{l}.05 \\
.13 \\
.02 \\
.01 \\
.05\end{array}$ & $\begin{array}{l}.06 \\
.08 \\
.06 \\
.06 \\
.05\end{array}$ & $\begin{array}{l}- \\
--m \\
-\infty \\
--\infty \\
--\infty\end{array}$ & $\begin{array}{r}.05 \\
.00 \\
.00 \\
.05 \\
2.7\end{array}$ & $\begin{array}{l}.18 \\
4.1 \\
3.4 \\
.32 \\
.12\end{array}$ \\
\hline $\begin{array}{l}21 \\
22 \\
23 \\
24 \\
25\end{array}$ & $\begin{array}{l}.01 \\
.29 \\
.00 \\
.00 \\
.00\end{array}$ & $\begin{array}{l}.00 \\
.00 \\
.00 \\
.15 \\
.06\end{array}$ & $m-m$ & $\begin{array}{l}--m \\
--- \\
-m- \\
--m \\
--\infty\end{array}$ & $\begin{array}{l}m-- \\
-- \\
-- \\
--\end{array}$ & $\begin{array}{l}.06 \\
.10 \\
.00 \\
.07 \\
.08\end{array}$ & $\begin{array}{l}.00 \\
.02 \\
.04 \\
.03 \\
.01\end{array}$ & $\begin{array}{l}.18 \\
.09 \\
.03 \\
.04 \\
.00\end{array}$ & $\begin{array}{l}.06 \\
.00 \\
.11 \\
.09 \\
.12\end{array}$ & $\begin{array}{l}m-m \\
--- \\
--- \\
-m \\
-m\end{array}$ & $\begin{array}{l}6.44 \\
5.9 \\
1.8 \\
11\end{array}$ & $\begin{array}{l}.04 \\
.07 \\
.16 \\
.16 \\
.08\end{array}$ \\
\hline $\begin{array}{l}26 \\
27 \\
28 \\
29 \\
30 \\
31\end{array}$ & $\begin{array}{l}.03 \\
.16 \\
.04 \\
.13 \\
.00 \\
.00\end{array}$ & $\begin{array}{l}.11 \\
.00 \\
.00 \\
-0- \\
--7\end{array}$ & $\begin{array}{l}m-m \\
-m-m \\
-m-m \\
-m-m \\
-m-m\end{array}$ & $\begin{array}{c}w- \\
m-\infty \\
m-\infty \\
m-\infty \\
m-\infty\end{array}$ & $\begin{array}{l}-- \\
-- \\
-- \\
-- \\
--\end{array}$ & $\begin{array}{l}.09 \\
.09 \\
.07 \\
.00 \\
.14 \\
.00\end{array}$ & $\begin{array}{l}.02 \\
.00 \\
.00 \\
.00 \\
.00 \\
.\end{array}$ & $\begin{array}{l}1.1 \\
.04 \\
.14 \\
.06 \\
.04 \\
.05\end{array}$ & $\begin{array}{r}22 \\
3.5 \\
12.90 \\
.61 \\
-=-\end{array}$ & $\begin{array}{l}-\cdots \\
-\infty \\
-\infty \\
-\infty \\
-\infty \\
-\infty\end{array}$ & $\begin{array}{r}7.2 \\
.18 \\
-18 \\
- \\
-\cdots \\
-\end{array}$ & $\begin{array}{l}.98 \\
.08 \\
.01 \\
.00 \\
.15 \\
.\end{array}$ \\
\hline $\begin{array}{l}\text { TOTAL } \\
\text { MEAN } \\
\text { MAX } \\
\text { MIN } \\
\text { AC-FI } \\
(\dagger)\end{array}$ & $\begin{array}{r}3.91 \\
.13 \\
.37 \\
.00 \\
7.6 \\
0.07\end{array}$ & $\begin{array}{c}m-m \\
-m \\
-m \\
0.31\end{array}$ & $\frac{1}{m-05}$ & $\begin{array}{c}m-\cdots \\
\cdots \\
\cdots \\
\cdots\end{array}$ & $\begin{array}{l}-- \\
-- \\
-\cdots \\
-\infty \\
0.11\end{array}$ & $\begin{array}{l}--- \\
--- \\
--- \\
0.21\end{array}$ & $\begin{array}{r}0.29 \\
.010 \\
.06 \\
.00 \\
0.15\end{array}$ & $\begin{array}{l}3.01 \\
.097 \\
1.1 \\
.00 \\
6.0 \\
0.00\end{array}$ & $\begin{array}{r}43.76 \\
1.46 \\
22 \\
.00 \\
87 \\
2.12\end{array}$ & $\begin{array}{c}\cdots \\
\cdots \\
0.04 \\
-\cdots\end{array}$ & $\begin{array}{c}-- \\
-- \\
-\cdots \\
-\overline{-} \\
2.50\end{array}$ & $\begin{array}{c}-m \\
-m \\
-m \\
1.55\end{array}$ \\
\hline
\end{tabular}

(f) Total rainfall accumulation in inches. 
RIO GRANDE BASIN

08329839 NORTK FORK EAFN ARROYO AT ALBUQUERQUE, NM

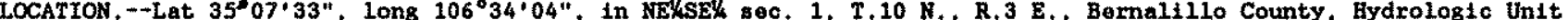
13020203 , on right bank $200 \mathrm{ft}$ above Louisiana Boulevard, $1,150 \mathrm{ft}$ north of Comanche $\mathrm{Rd}$, and 1 , $450 \mathrm{ft}$ south of Montgomery Boulevard, in Albuquerque.

DRAINAGE AREA. $--1.51 \mathrm{mi}^{3}$.

PERIOD OF RECORD."-May 1979 to December 1983, June 1992 to current year (soasonal records).

GAGE. - Water-stage recorder and concrete lined channel. Elevation of gage is 5,290 ft above National Geodetio Vertical Datum of 1929 , from topographic map, Prior to 1983 at site 200 ft downstream on Louisiana Boulevard bridge, at different datum.

REMARKS.--Records falx. Some minor streamflow may exist on days where dally mean discharges have been recorded as zero due to the sensitivity limits of the streamflow monitoring equiponent. Recording rain gage at station.

EXIREMES FOR PERIOD OF RECORD.--Maximum discharge, $439 \mathrm{ft}^{\mathrm{s}} / \mathrm{s}$; Aug. 14, 1980, gage height, 1.94 ft, from stepbackwater analysts of concreto lined stream channel; no flom most of time.

EXTRIMES FOR CURRENT YEAR.-Maximum discharge during period of seasonal operation, .30 fts/s, at $1700 \mathrm{hours,}$ June 28 , gage height, $1.68 \mathrm{ft}$; no flow most of time.

DISCHARGE, CUBIC FEET PER SECOND, WATER YEAR OCTOBER 1995 TO SEPTEMBER 1996

\begin{tabular}{|c|c|c|c|c|c|c|c|c|c|c|c|c|}
\hline DAY & OCT & NOV & DEC & JAN & FEB & MAR & APR & MAY & JuN & JUL & AUG & SEP \\
\hline $\begin{array}{l}1 \\
2 \\
3 \\
4 \\
5\end{array}$ & $\begin{array}{l}.00 \\
.00 \\
.00 \\
.00 \\
.00\end{array}$ & $\begin{array}{r}.00 \\
.00 \\
.00 \\
---\end{array}$ & $\begin{array}{l}m-m \\
-m \\
-m- \\
-m- \\
-m-\end{array}$ & $\begin{array}{l}m-m \\
m-\infty \\
-m \\
-m \\
-m-\end{array}$ & $\begin{array}{l}m-m \\
m-m \\
m-m \\
m-m\end{array}$ & $\begin{array}{l}m-m \\
m-m \\
m-m \\
m-m\end{array}$ & $\begin{array}{l}.00 \\
.00 \\
.00 \\
.00 \\
.00\end{array}$ & $\begin{array}{l}.00 \\
.00 \\
.00 \\
.00 \\
.00\end{array}$ & $\begin{array}{l}.00 \\
.00 \\
.00 \\
.00 \\
.00\end{array}$ & $\begin{array}{l}.00 \\
.00 \\
.00 \\
.00 \\
.00\end{array}$ & $\begin{array}{l}-m \\
-\cdots \\
--\infty \\
--\infty\end{array}$ & $\begin{array}{l}.00 \\
.00\end{array}$ \\
\hline $\begin{array}{r}6 \\
7 \\
8 \\
9 \\
10\end{array}$ & $\begin{array}{l}.00 \\
.00 \\
.00 \\
.00 \\
.00\end{array}$ & $\begin{array}{l}-m \\
m-m \\
m-m \\
-m \\
-m\end{array}$ & $\begin{array}{l}-m \\
-m- \\
m-m \\
m-m \\
m-m\end{array}$ & $\begin{array}{l}m- \\
m- \\
m-m \\
m-m \\
m-m\end{array}$ & $\begin{array}{l}m-m \\
-m- \\
-m \\
-m-m\end{array}$ & $\begin{array}{l}m-m \\
m-m \\
m-m \\
m-m\end{array}$ & $\begin{array}{l}.00 \\
.00 \\
.00 \\
.00 \\
.00\end{array}$ & $\begin{array}{l}.00 \\
.00 \\
.00 \\
.00 \\
.00\end{array}$ & $\begin{array}{l}.00 \\
.00 \\
.00 \\
.00 \\
.00\end{array}$ & $\begin{array}{l}.00 \\
.01 \\
.00 \\
.00 \\
.00\end{array}$ & $\begin{array}{l}\cdots \\
m- \\
.00 \\
.00\end{array}$ & $\begin{array}{l}.01 \\
.04 \\
.00 \\
.00 \\
.00\end{array}$ \\
\hline $\begin{array}{l}11 \\
12 \\
13 \\
14 \\
15\end{array}$ & $\begin{array}{l}.00 \\
.00 \\
.00 \\
.00 \\
.00\end{array}$ & $\begin{array}{l}--- \\
-m- \\
--\infty \\
-m- \\
-m-\end{array}$ & $\begin{array}{l}-m \\
--- \\
-m \\
-m- \\
-m-\end{array}$ & $\begin{array}{l}-\infty \\
-- \\
--- \\
--\infty \\
---\end{array}$ & 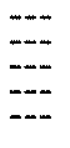 & $\begin{array}{l}.00 \\
.00 \\
.00 \\
.00\end{array}$ & $\begin{array}{l}.00 \\
.00 \\
.00 \\
.00 \\
.00\end{array}$ & $\begin{array}{l}.00 \\
.00 \\
.00 \\
.00 \\
.00\end{array}$ & $\begin{array}{l}.00 \\
.00 \\
.00 \\
.00 \\
.00\end{array}$ & $\begin{array}{l}.00 \\
.00 \\
.00 \\
.00 \\
.00\end{array}$ & $\begin{array}{l}.00 \\
.00 \\
.00 \\
.00 \\
.00\end{array}$ & $\begin{array}{l}.00 \\
.00 \\
.00 \\
.11 \\
.06\end{array}$ \\
\hline $\begin{array}{l}16 \\
17 \\
18 \\
19 . \\
20\end{array}$ & $\begin{array}{l}.00 \\
.00 \\
.00 \\
.00 \\
.00\end{array}$ & 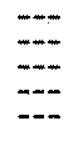 & $\begin{array}{l}-m \\
m-m \\
-m \\
-m \\
-m\end{array}$ & $\begin{array}{l}m-m \\
m-m \\
m-m \\
m-m \\
m-m\end{array}$ & $\begin{array}{l}m \\
m-m \\
m-m\end{array}$ & $\begin{array}{l}.00 \\
.00 \\
.00 \\
.00 \\
.00\end{array}$ & $\begin{array}{l}.00 \\
.00 \\
.00 \\
.00 \\
.00\end{array}$ & $\begin{array}{l}.00 \\
.00 \\
.00 \\
.00 \\
.00\end{array}$ & $\begin{array}{l}.00 \\
.00 \\
.00 \\
.00 \\
.00\end{array}$ & $\begin{array}{r}.01 \\
.00 \\
+\ldots \\
-\ldots\end{array}$ & $\begin{array}{l}.00 \\
.00 \\
.00 \\
.00 \\
.02\end{array}$ & $\begin{array}{l}.01 \\
.10 \\
.05 \\
.00 \\
.00\end{array}$ \\
\hline $\begin{array}{l}21 \\
22 \\
23 \\
24 \\
25\end{array}$ & $\begin{array}{l}.00 \\
.00 \\
.00 \\
.00 \\
.00\end{array}$ & 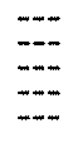 & $\begin{array}{l}-m \\
--- \\
m-m \\
-m-\end{array}$ & $\begin{array}{l}m-m \\
-m \\
-m \\
m-m \\
-m-m\end{array}$ & $\begin{array}{l}m-- \\
m-m \\
m-- \\
m-m\end{array}$ & $\begin{array}{l}.00 \\
.00 \\
.00 \\
.00 \\
.00\end{array}$ & $\begin{array}{l}.00 \\
.00 \\
.00 \\
.00 \\
.00\end{array}$ & $\begin{array}{l}.00 \\
.00 \\
.00 \\
.00 \\
.00\end{array}$ & $\begin{array}{l}.00 \\
.00 \\
.00 \\
.00 \\
.00\end{array}$ & $\begin{array}{l}m-m \\
-m- \\
m-m \\
m-m\end{array}$ & $\begin{array}{l}.05 \\
.13 \\
.10 \\
.07 \\
.11\end{array}$ & $\begin{array}{l}.00 \\
.00 \\
.00 \\
.00 \\
.00\end{array}$ \\
\hline $\begin{array}{l}26 \\
27 \\
28 \\
29 \\
30 \\
31\end{array}$ & $\begin{array}{l}.00 \\
.00 \\
.00 \\
.00 \\
.00 \\
.00\end{array}$ & $m-m$ & $\begin{array}{l}m-m \\
m-m \\
m-m \\
m-m\end{array}$ & $\begin{array}{l}m- \\
m- \\
m- \\
m- \\
m-\end{array}$ & $\begin{array}{l}m-- \\
-\overline{-} \\
-\cdots \\
-\cdots \\
-\cdots \\
--\end{array}$ & $\begin{array}{l}.00 \\
.00 \\
.00 \\
.00 \\
.00 \\
.00\end{array}$ & $\begin{array}{l}.00 \\
.00 \\
.00 \\
.00 \\
.00 \\
.0\end{array}$ & $\begin{array}{l}.00 \\
.00 \\
.00 \\
.00 \\
.00 \\
.00\end{array}$ & $\begin{array}{l}.29 \\
.01 \\
.20 \\
.00 \\
.00 \\
.\end{array}$ & $\begin{array}{l}m-m \\
m-m \\
m-m \\
m-m\end{array}$ & $\begin{array}{l}.12 \\
.04 \\
-\cdots \\
--- \\
---\end{array}$ & $\begin{array}{l}.00 \\
.00 \\
.00 \\
.00 \\
.00 \\
0\end{array}$ \\
\hline $\begin{array}{l}\text { TOTAL } \\
\text { MEAN } \\
\text { MAX } \\
\text { MIN } \\
\text { AC-FT } \\
\text { (t) }\end{array}$ & $\begin{array}{l}0.00 \\
.000 \\
.00 \\
.00 \\
.00 \\
0.02\end{array}$ & $\begin{array}{l}m-m \\
m-m \\
m-m \\
m-m \\
0.18\end{array}$ & $\begin{array}{l}m-m \\
m-m \\
m-2 \\
m .16\end{array}$ & $\begin{array}{l}m \\
--. \\
-\ldots \\
-- \\
0.24\end{array}$ & $\begin{array}{l}-\cdots \\
\cdots \\
\cdots- \\
-\infty \\
-\infty \\
-11\end{array}$ & $\frac{-m}{-11}$ & $\begin{array}{l}0.00 \\
.000 \\
.00 \\
.00 \\
.00 \\
0.19\end{array}$ & $\begin{array}{r}0.00 \\
.000 \\
.00 \\
.00 \\
.00 \\
0.27\end{array}$ & $\begin{array}{r}0.50 \\
.017 \\
.29 \\
.00 \\
1.0 \\
2.39\end{array}$ & $\begin{array}{l}m-- \\
--- \\
-m \\
-m \\
79\end{array}$ & $\begin{array}{l}m- \\
--- \\
-\cdots- \\
-\cdots \\
2.12\end{array}$ & 1.88 \\
\hline
\end{tabular}

(t) Total rainfall accumulation in inches. 
LOCATTON, --Lat $35^{\circ} 07^{\prime} 33^{\prime \prime}$, long $106^{\circ} 35^{\prime} 23^{\prime \prime}$, in SEKNEK sec.2, T.10 N., R.3 E., Bernalillo County, Bydrologic Unit $13020203,860 \mathrm{ft}$ below San Mateo Boulevard Bridge on right bank, $750 \mathrm{ft}$ north of Comanche Road, and $2,050 \mathrm{ft}$ south of Montgomery Boulevard in Albuquerque.

DRAINAGE AREA. $--4.23 \mathrm{~m}^{2}$.

PERIOD OF RECORD. --June 1978 to current year (seasonal records).

GAGE - Water-stage recorder and concrete-11ned channel, Elevation of gago 1 s 5,400 ft above National Geodetic Vertical Datum of 1929 , from topographic map. Frior to 1992 at site on downstram side of San Mateo Boulevard Bridge, at different detum.

RERARKS. --Records faix. Some minor streanclow may exist on days where dally mean discharges have been recorded as zero due to the sensitivity limits of the streanflow monitoring equipment, Recording rain gage at station. Development within basin is predominantly residential, but there are some commercial areas. See tabulation below for monthly precipitation in inches.

EXTREMES FOR PERIOD OF RECORD,--Maximum discharge, 1,080 fts/s, Aug. 14, 1980, gage height, $2.54 \mathrm{ft}$, from rating curve extended above $10 \mathrm{ft} / \mathrm{s}$ on basis of step" forward analysis of channel; no flow most of time.

EXTREMES FOR CURREN YEAR. --Maximum discharge during period of seasonal operation, 420 fts/s, at 1700 hours June 28 , gage height, $1.74 \mathrm{ft}$, from rating curve extended above $10 \mathrm{ft} / \mathrm{s}$ on bas 13 of step-forward analysis of channel; no flow most of time. DISCBARGE, CUBTC FEET PER SECOND, WATER YEAR OCTOBER 1995 TO SEPTEMBER 1996

\begin{tabular}{|c|c|c|c|c|c|c|c|c|c|c|c|c|}
\hline DAY & OCT & Nov & $\mathrm{DEC}$ & JAN & FEB & MAR & APR & MAY & Jux & JUL & AUG & SEP \\
\hline $\begin{array}{l}1 \\
2 \\
3 \\
4 \\
5\end{array}$ & $\begin{array}{l}.00 \\
.00 \\
.00 \\
.00 \\
.00\end{array}$ & $\begin{array}{l}.79 \\
.97 \\
.13 \\
.03 \\
.00\end{array}$ & $\begin{array}{l}-\cdots \\
-- \\
--- \\
-\cdots\end{array}$ & $\begin{array}{l}-\cdots \\
-\cdots \\
--- \\
--\end{array}$ & $\begin{array}{c}-- \\
-- \\
-- \\
--\end{array}$ & $\begin{array}{l}\cdots- \\
\cdots \\
\cdots\end{array}$ & $\begin{array}{l}.00 \\
.00 \\
.00 \\
.00 \\
.00\end{array}$ & $\begin{array}{l}.29 \\
.26 \\
.00 \\
.00 \\
.00\end{array}$ & $\begin{array}{l}.77 \\
.00 \\
.00 \\
.00 \\
.00\end{array}$ & $\begin{array}{r}2.9 \\
3.9 \\
.87 \\
.41 \\
.37\end{array}$ & $\begin{array}{c}- \\
- \\
- \\
1.3 \\
.85\end{array}$ & $\begin{array}{r}.42 \\
.37 \\
.52 \\
1.5 \\
1.1\end{array}$ \\
\hline $\begin{array}{r}6 \\
7 \\
8 \\
9 \\
10\end{array}$ & $\begin{array}{l}.00 \\
.00 \\
.00 \\
.08 \\
.00\end{array}$ & $\begin{array}{l}.00 \\
.00 \\
.00 \\
.00 \\
.88\end{array}$ & 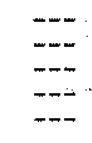 & $\begin{array}{l}\cdots \\
\cdots \\
\cdots \\
\cdots-\end{array}$ & $\begin{array}{l}m-m \\
-\cdots \\
-\cdots \\
-\cdots\end{array}$ & $\begin{array}{l}m- \\
\cdots- \\
m- \\
--\end{array}$ & $\begin{array}{l}.57 \\
.05 \\
.00 \\
.00 \\
.00\end{array}$ & $\begin{array}{l}.00 \\
.00 \\
.00 \\
.00 \\
.00\end{array}$ & $\begin{array}{l}.00 \\
.00 \\
.00 \\
.00 \\
.00\end{array}$ & $\begin{array}{l}.24 \\
2.4 \\
2.4 \\
8.2 \\
4.4\end{array}$ & $\begin{array}{r}.36 \\
.88 \\
3.1 \\
1.7 \\
.99\end{array}$ & $\begin{array}{l}4.2 \\
2.9 \\
1.1 \\
1.0 \\
.51\end{array}$ \\
\hline $\begin{array}{l}11 \\
12 \\
13 \\
14 \\
15\end{array}$ & $\begin{array}{l}.00 \\
.00 \\
.00 \\
.00 \\
.00\end{array}$ & $\begin{array}{l}.56 \\
.00 \\
.00 \\
.00 \\
.00\end{array}$ & $m$ & $\begin{array}{l}--- \\
-- \\
-- \\
--\end{array}$ & $=$ & $\begin{array}{l}.00 \\
.00 \\
.00\end{array}$ & $\begin{array}{l}.65 \\
.56 \\
.53 \\
.78 \\
.00\end{array}$ & $\begin{array}{l}.00 \\
.00 \\
.00 \\
.00 \\
.00\end{array}$ & $\begin{array}{l}2.9 \\
3.1 \\
4.1 \\
8.2 \\
.27\end{array}$ & $\begin{array}{c}1.5 \\
.64 \\
.02 \\
.15 \\
.90\end{array}$ & $\begin{array}{c}1.4 \\
1.0 \\
.50 \\
.40 \\
1.0\end{array}$ & $\frac{.00}{18^{.82}}$ \\
\hline $\begin{array}{l}16 \\
17 \\
18 \\
19 \\
20\end{array}$ & $\begin{array}{l}.00 \\
.00 \\
.00 \\
.00 \\
.09\end{array}$ & $\begin{array}{l}.00 \\
.00 \\
.00 \\
.00 \\
.00\end{array}$ & $\overline{--}$ & $\begin{array}{l}--- \\
-- \\
--- \\
---\end{array}$ & $\begin{array}{l}\cdots- \\
\cdots \\
\cdots- \\
\cdots-\end{array}$ & $\begin{array}{l}.01 \\
.01 \\
.04 \\
.06 \\
.26\end{array}$ & $\begin{array}{l}.60 \\
.00 \\
.00 \\
.00 \\
.54\end{array}$ & $\begin{array}{l}.00 \\
.00 \\
.00 \\
.00 \\
.00\end{array}$ & $\begin{array}{l}.55 \\
.40 \\
.23 \\
.35 \\
.42\end{array}$ & $\begin{array}{r}3.9 \\
.62 \\
2.7 \\
.72 \\
.16\end{array}$ & $\begin{array}{r}.65 \\
.39 \\
.23 \\
.81 \\
4.3\end{array}$ & $\begin{array}{c}7.71 \\
10.9 \\
1.4 \\
.41\end{array}$ \\
\hline $\begin{array}{l}21 \\
22 \\
23 \\
24 \\
25\end{array}$ & $\begin{array}{l}.00 \\
.00 \\
.13 \\
.00 \\
.00\end{array}$ & $\begin{array}{l}.00 \\
.00 \\
.00 \\
.00 \\
.00\end{array}$ & $\begin{array}{l}-\cdots \\
--- \\
-\cdots \\
m-\infty\end{array}$ & $\begin{array}{l}m-- \\
=- \\
--- \\
---\end{array}$ & $\begin{array}{l}-\cdots \\
-\cdots \\
-\cdots \\
-- \\
--\end{array}$ & $\begin{array}{l}.06 \\
.32 \\
.14 \\
.42 \\
.76\end{array}$ & $\begin{array}{l}.49 \\
.87 \\
.81 \\
.00 \\
.00\end{array}$ & $\begin{array}{l}.00 \\
.00 \\
.00 \\
.00 \\
.00\end{array}$ & $\begin{array}{r}.82 \\
.32 \\
1.0 \\
1.2 \\
.47\end{array}$ & $\begin{array}{l}.15 \\
.45 \\
.00 \\
-.- \\
.--\end{array}$ & $\begin{array}{l}2.3 \\
14 \\
13 \\
5.5 \\
11\end{array}$ & $\begin{array}{l}.27 \\
.27 \\
.61 \\
.52 \\
.59\end{array}$ \\
\hline $\begin{array}{l}26 \\
27 \\
28 \\
29 \\
30 \\
31\end{array}$ & $\begin{array}{l}.00 \\
.00 \\
.00 \\
.00 \\
.00 \\
.01\end{array}$ & \begin{tabular}{l}
.03 \\
.00 \\
.00 \\
0. \\
\hdashline- \\
\hdashline-
\end{tabular} & $\begin{array}{l}m-\infty \\
-\cdots \\
--- \\
--- \\
--\infty\end{array}$ & $\begin{array}{l}-\cdots \\
-- \\
-\cdots \\
-\cdots \\
-\cdots\end{array}$ & 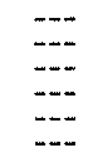 & $\begin{array}{l}.00 \\
.00 \\
.00 \\
.00 \\
.00 \\
.00\end{array}$ & $\begin{array}{l}.00 \\
.39 \\
.74 \\
.00 \\
.54 \\
.\end{array}$ & $\begin{array}{l}4.1 \\
.87 \\
1.3 \\
1.6 \\
1.86 \\
1.5\end{array}$ & $\begin{array}{r}30 \\
9.2 \\
35 \\
3.8 \\
2.8\end{array}$ & 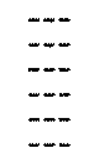 & $\begin{array}{r}15 \\
.00 \\
.58 \\
8.3 \\
1.1 \\
.47\end{array}$ & $\begin{array}{r}3.1 \\
.29 \\
.05 \\
.06 \\
.40 \\
-. .\end{array}$ \\
\hline $\begin{array}{l}\text { TOTAL } \\
\text { MEAAH } \\
\text { MAX } \\
\text { MIN } \\
\text { AC-FT } \\
(t)\end{array}$ & $\begin{array}{r}0.31 \\
.010 \\
.13 \\
.00 \\
0.60\end{array}$ & 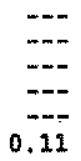 & $\begin{array}{c}\cdots \\
\cdots \\
\cdots \\
\cdots \\
0.10\end{array}$ & $\begin{array}{c}=- \\
\overline{-} \\
\overline{-}-- \\
0.28\end{array}$ & $\begin{array}{c}--- \\
\cdots \\
\cdots \\
0.19\end{array}$ & 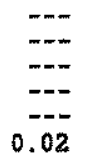 & $\begin{array}{r}8.12 \\
.27 \\
.87 \\
.00 \\
16 \\
0.00\end{array}$ & $\begin{array}{r}10.78 \\
.35 \\
4.1 \\
.00 \\
21 \\
0.04\end{array}$ & $\begin{array}{r}85.90 \\
2.86 \\
30 \\
100 \\
170 \\
1.94\end{array}$ & $\begin{array}{c}--- \\
\cdots-- \\
\cdots-- \\
0.80\end{array}$ & 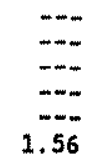 & \begin{tabular}{r}
--- \\
--- \\
\hdashline-- \\
$-\square$ \\
2.12
\end{tabular} \\
\hline
\end{tabular}

(†) Total rainfall accunulation in inches. 
RIO GRANDE BASIN

08329860 GRANT LINE ARROYO AT VILLA DEL OSO AT ALBUQUERQUE, NM

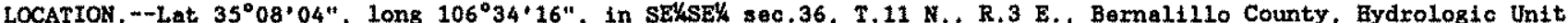
13020203, on left bank of rock-11ned channel, and 60 ft west of northwest comer of apartment parking lot at 4215 Louts iana Boulevard NE in Albuquerque.

DRATNAGE AREA. $--0.052 \mathrm{mi} \mathbf{1}^{2}$.

PERIOD OF RECORD. - June 1976 to current year (seasonal records).

GAGE.-Water-stage recorder and concrete control. Elevation of gage is 5,300 ft above National Geodetic Vertical Datum of 1929, from topographic map.

REMARKS.--Records good. Recording rain gage at station. Development within bastn is predominantiy residenttal. Soe tabulation below for monthly precipitation in tnches. No flow most of time.

EXTREMES FOR PERIOD OF RECORD.--Maximum discharge, $13 \mathrm{ft} / \mathrm{s}$, Aug, 13,1993, gage helght, 1.76 ft, from rating curve extended above $5.0 \mathrm{fts} / \mathrm{s}$ on basis of slope-grea measurements at gage hoight 2.08 ; no flow most of time,

EXTREMES FOR CURRENT YEAR.--Maximum discharge during period of seasonal operation, 14 ft3/s, at 1935 hours Sept. 17 , gage height, $1.78 \mathrm{ft}$. No flow most of time.

DISCHARGE, CUBTC FEET PER SECOND, WATER YEAR OCTOBER 1995 TO SEPTEMBER 1996

\begin{tabular}{|c|c|c|c|c|c|c|c|c|c|c|c|c|}
\hline DAX & $\alpha \mathrm{T}$ & NOV & DEC & JAN & FEB & MAR & AFR & MAY & JUN & JUL & AUG & SEP \\
\hline $\begin{array}{l}1 \\
2 \\
3 \\
4 \\
5\end{array}$ & $\begin{array}{l}.00 \\
.00 \\
.00 \\
.00 \\
.00\end{array}$ & $\begin{array}{l}.00 \\
.01 \\
.00 \\
.00 \\
.00\end{array}$ & $m$ & $m$ & $\begin{array}{l}m-m \\
-m- \\
-m-m\end{array}$ & $\begin{array}{l}m-m \\
-\infty \\
-m \\
--\end{array}$ & $\begin{array}{l}.00 \\
.00 \\
.00 \\
.00 \\
.00\end{array}$ & $\begin{array}{l}.00 \\
.00 \\
.00 \\
.00 \\
.00\end{array}$ & $\begin{array}{l}.00 \\
.00 \\
.00 \\
.00 \\
.00\end{array}$ & $\begin{array}{l}.00 \\
.01 \\
.00 \\
.00 \\
.00\end{array}$ & $\begin{array}{r}.00 \\
.00 \\
-00 \\
.00 \\
.00\end{array}$ & $\begin{array}{l}.00 \\
.00 \\
.00 \\
.00 \\
.00\end{array}$ \\
\hline $\begin{array}{r}6 \\
7 \\
8 \\
9 \\
10\end{array}$ & $\begin{array}{l}.00 \\
.00 \\
.00 \\
.00 \\
.00\end{array}$ & $\begin{array}{l}.00 \\
.00 \\
.00 \\
.00 \\
.00\end{array}$ & $\begin{array}{l}-m-m \\
-m-m \\
-m- \\
-m-m \\
-m-m\end{array}$ & $\begin{array}{l}m \\
m- \\
m- \\
m-\end{array}$ & 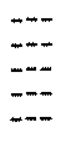 & $\begin{array}{l}m \\
m-m \\
m-m \\
m-m \\
m-m\end{array}$ & $\begin{array}{l}.00 \\
.00 \\
.00 \\
.00 \\
.00\end{array}$ & $\begin{array}{l}.00 \\
.00 \\
.00 \\
.00 \\
.00\end{array}$ & $\begin{array}{l}.00 \\
.00 \\
.00 \\
.00 \\
.00\end{array}$ & $\begin{array}{l}.00 \\
.01 \\
.01 \\
.01 \\
.02\end{array}$ & $\begin{array}{r}.00 \\
.00 \\
.01 \\
.00 \\
.00\end{array}$ & $\begin{array}{l}.01 \\
.00 \\
.00 \\
.00 \\
.00\end{array}$ \\
\hline $\begin{array}{l}11 \\
12 \\
13 \\
14 \\
15\end{array}$ & $\begin{array}{l}.00 \\
.00 \\
.00 \\
.00 \\
.00\end{array}$ & $\begin{array}{l}.00 \\
.00 \\
.00 \\
.00 \\
.00\end{array}$ & $=-$ & $\begin{array}{l}--- \\
=-\infty \\
=- \\
--\end{array}$ & 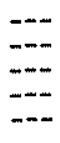 & $\begin{array}{l}.-- \\
.00 \\
.00 \\
.00\end{array}$ & $\begin{array}{l}.00 \\
.00 \\
.00 \\
.00 \\
.00\end{array}$ & $\begin{array}{l}.00 \\
.00 \\
.00 \\
.00 \\
.00\end{array}$ & $\begin{array}{l}.00 \\
.00 \\
.00 \\
.00 \\
.00\end{array}$ & $\begin{array}{l}.00 \\
.00 \\
.00 \\
.00 \\
.00\end{array}$ & $\begin{array}{l}.00 \\
.00 \\
.00 \\
.00 \\
.00\end{array}$ & $\begin{array}{l}.00 \\
.01 \\
.00 \\
.31 \\
.00\end{array}$ \\
\hline $\begin{array}{l}16 \\
17 \\
18 \\
19 \\
20\end{array}$ & $\begin{array}{l}.00 \\
.00 \\
.00 \\
.00 \\
.00\end{array}$ & $\begin{array}{l}.00 \\
.00 \\
.00 \\
.00 \\
.00\end{array}$ & $\begin{array}{l}m-m \\
m-m \\
-m \\
-m\end{array}$ & $\begin{array}{l}m- \\
=-- \\
m-m\end{array}$ & $\begin{array}{l}-m \\
-\cdots \\
-\cdots \\
-\cdots \\
-\cdots\end{array}$ & $\begin{array}{l}.00 \\
.00 \\
.00 \\
.00 \\
.00\end{array}$ & $\begin{array}{l}.00 \\
.00 \\
.00 \\
.00 \\
.00\end{array}$ & $\begin{array}{l}.00 \\
.00 \\
.00 \\
.00 \\
.00\end{array}$ & $\begin{array}{l}.00 \\
.00 \\
.00 \\
.00 \\
.00\end{array}$ & $\begin{array}{l}.00 \\
.00 \\
.00 \\
.00 \\
.00\end{array}$ & $\begin{array}{r}.00 \\
.00 \\
.00 \\
.00 \\
.01\end{array}$ & $\begin{array}{l}.00 \\
.20 \\
.11 \\
.00 \\
.00\end{array}$ \\
\hline $\begin{array}{l}21 \\
22 \\
23 \\
24 \\
25\end{array}$ & $\begin{array}{l}.00 \\
.00 \\
.00 \\
.00 \\
.00\end{array}$ & $\begin{array}{l}.00 \\
.00 \\
.00 \\
.00 \\
.00\end{array}$ & $\begin{array}{l}m- \\
m- \\
m- \\
m-\end{array}$ & $m$ & 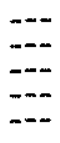 & $\begin{array}{l}.00 \\
.00 \\
.00 \\
.00 \\
.00\end{array}$ & $\begin{array}{l}.00 \\
.00 \\
.00 \\
.00 \\
.00\end{array}$ & $\begin{array}{l}.00 \\
.00 \\
.00 \\
.00 \\
.00\end{array}$ & $\begin{array}{l}.00 \\
.00 \\
.00 \\
.00 \\
.00\end{array}$ & $\begin{array}{l}.00 \\
.00 \\
.00 \\
.00 \\
.00\end{array}$ & $\begin{array}{r}.00 \\
.16 \\
.10 \\
.13 \\
.15\end{array}$ & $\begin{array}{l}.00 \\
.00 \\
.00 \\
.00 \\
.00\end{array}$ \\
\hline $\begin{array}{l}26 \\
27 \\
28 \\
29 \\
30 \\
31\end{array}$ & $\begin{array}{l}.00 \\
.00 \\
.00 \\
.00 \\
.00 \\
.00\end{array}$ & $\begin{array}{l}.00 \\
.00 \\
.00 \\
.00 \\
.0 \\
.-\end{array}$ & 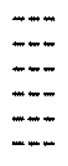 & $\begin{array}{l}m-- \\
-\infty \\
-m \\
-m \\
-\infty\end{array}$ & $\begin{array}{l}m-m \\
-m-m \\
-m-m \\
-m-m\end{array}$ & $\begin{array}{l}.00 \\
.00 \\
.00 \\
.00 \\
.00 \\
.00\end{array}$ & $\begin{array}{l}.00 \\
.00 \\
.00 \\
.00 \\
.00 \\
-0-\end{array}$ & $\begin{array}{l}.00 \\
.00 \\
.00 \\
.00 \\
.00 \\
.00\end{array}$ & $\begin{array}{l}.23 \\
.04 \\
.02 \\
.00 \\
.00 \\
---\end{array}$ & $\begin{array}{l}.00 \\
.00 \\
.00 \\
.00 \\
.00 \\
.00\end{array}$ & $\begin{array}{l}.18 \\
.00 \\
-0.00 \\
.00 \\
.00\end{array}$ & $\begin{array}{l}.01 \\
.00 \\
.00 \\
.00 \\
.00 \\
.0\end{array}$ \\
\hline $\begin{array}{l}\text { TOTAL } \\
\text { MEAN } \\
\text { MAX } \\
\text { MIN } \\
\text { AC-FT } \\
(\dagger)\end{array}$ & $\begin{array}{l}0.00 \\
.000 \\
.00 \\
.00 \\
.00 \\
-\ldots\end{array}$ & $\begin{array}{l}m-m \\
m-m \\
m-m \\
m-m\end{array}$ & $\begin{array}{l}m- \\
m-m \\
m-m \\
m-m \\
m-m\end{array}$ & $=$ & $\begin{array}{l}m-m \\
m-m \\
m-m \\
m-m\end{array}$ & $\begin{array}{l}-m \\
m-\infty \\
-m \\
-\infty \\
0.00\end{array}$ & $\begin{array}{l}0.00 \\
.000 \\
.00 \\
.00 \\
.00 \\
0.00\end{array}$ & $\begin{array}{l}0.00 \\
.000 \\
.00 \\
.00 \\
.00 \\
0.00\end{array}$ & $\begin{array}{r}0.29 \\
.010 \\
.23 \\
.00 \\
.6 \\
0.14\end{array}$ & $\begin{array}{r}0.06 \\
.002 \\
.02 \\
.00 \\
.1 \\
0.47\end{array}$ & 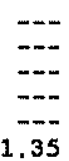 & $\begin{array}{r}0.65 \\
.022 \\
.31 \\
.00 \\
1.3 \\
1.23\end{array}$ \\
\hline
\end{tabular}

(t) Total rainfall accumulation in inches. 
RIO GRANDE BASIN

08329872 PINO ARROYO AT VENTURA BOULEVARD AT ALBUQUERQUE, NM.

LOCATrox,--Lat $35^{\circ} 08^{\circ} 40^{\prime \prime}$, long $106^{\circ} 32^{\prime} 50^{\prime \prime}$, Bernal1110 County, Bydrologic Unit 132020203, in Elena Gallegos Grant, on left bank in Tanoan Country Club, and $30 \mathrm{ft}$ upstream from Ventura Boulevard in Albuquerque.

DRAINAGE AREA. $--5.40 \mathrm{mi}^{2}$.

PERIOD OF RECORD,--August 1990 to current year (geasonal records).

GAGE. - Water-stage recorder. Elovation of gage 18 5,490 ft above National Geodetic Vertical Datum of 1929 , from topographic map.

REMARKS, - -Records good.

EXTREMES FOR PERIOD OF RECORD, - Maximum discharge, $126 \mathrm{ft}$ /s, July 23, 1992, gage height, 1.98 ft, from rating curve extended above 12 ft/s on basis of slope-area measurement of peak flow; no flow part of many days.

EXTREMES FOR CURRENY YEAR.--Maximum discharge during period of seasonal operation, 53 Its/s, at 1645 hours Sept, 14 , gage height, $1.50 \mathrm{ft}$, from rating curve extended above $12 \mathrm{ft} / \mathrm{s}$ on basis of slope-area measurement of peak flow; no flow part of many days.

DISCEARGE, CUBIC FEET PER SECOND, WATER YEAR OCTOBER 1995 TO SEPTEMBER 1996 DAILY MEAN VALUES

\begin{tabular}{|c|c|c|c|c|c|c|c|c|c|c|c|c|c|c|c|}
\hline DAY & $\therefore \therefore$ OCT & NOY & $\therefore$ DEC & & JAN & & FEB & & MAR & APR & MAY & JUN & 지느. & AUG & SEP \\
\hline $\begin{array}{l}1 \\
2 \\
3 \\
4 \\
5\end{array}$ & $\begin{array}{r}.02 \\
.02 \\
.02 \\
.01 \\
.01\end{array}$ & $\begin{array}{l}.00 \\
.01 \\
.00 \\
.00 \\
.00\end{array}$ & 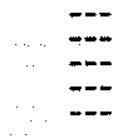 & & 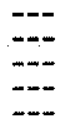 & & $\begin{array}{l}m-\infty \\
-m \\
-m-\infty \\
m-\infty\end{array}$ & $\begin{array}{l} \\
\because \\
\cdots \\
\because \\
\cdots\end{array}$ & $m$ & $\begin{array}{l}.44 \\
.41 \\
.42 \\
.40 \\
.68\end{array}$ & $\begin{array}{l}.40 \\
.30 \\
.20 \\
.17 \\
.21\end{array}$ & $\begin{array}{l}.73 \\
.62 \\
.71 \\
.75 \\
.95\end{array}$ & $\begin{array}{l}m-\infty \\
--- \\
-m \\
m-m \\
m-\infty\end{array}$ & $\begin{array}{l}.44 \\
.33 \\
.76 \\
.30 \\
.34\end{array}$ & $\begin{array}{l}.20 \\
.26 \\
.27 \\
.34 \\
.27\end{array}$ \\
\hline $\begin{array}{r}6 \\
7 \\
8 \\
9 \\
10\end{array}$ & $\begin{array}{l}.03 \\
.02 \\
.00 \\
.03 \\
.01\end{array}$ & $\begin{array}{l}.00 \\
.00 \\
.04 \\
.02 \\
.01\end{array}$ & $\begin{array}{l}m \\
=- \\
-- \\
-m\end{array}$ & & $\begin{array}{c}-- \\
-- \\
--\end{array}$ & & $\begin{array}{l}-\infty \\
-\infty \\
-\infty \\
-\infty \\
--\infty\end{array}$ & & $\begin{array}{l}--- \\
-m \\
-\cdots \\
-\cdots\end{array}$ & $\begin{array}{l}52 \\
.58 \\
.46 \\
.17 \\
.16\end{array}$ & $\begin{array}{l}.23 \\
.24 \\
.28 \\
.45 \\
.35\end{array}$ & $\begin{array}{l}.40 \\
.40 \\
.39 \\
.41 \\
.49\end{array}$ & 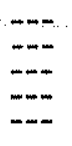 & $\begin{array}{r}.27 \\
.32 \\
.61 \\
.39 \\
.36\end{array}$ & $\begin{array}{r}.23 \\
.23 \\
.22 \\
.24 \\
.37\end{array}$ \\
\hline $\begin{array}{l}11 \\
12 \\
13 \\
14 \\
15\end{array}$ & $\begin{array}{l}.01 \\
.01 \\
.01 \\
.01 \\
.01\end{array}$ & $\begin{array}{l}.00 \\
.01 \\
.02 \\
.02 \\
.01\end{array}$ & $\therefore \quad m$ & & $=$ & $\therefore$ & $\begin{array}{l}--- \\
--- \\
m-- \\
-\infty \\
--\infty\end{array}$ & & $\begin{array}{l}. .- \\
.13 \\
.60 \\
.64\end{array}$ & $\begin{array}{l}.19 \\
.16 \\
.27 \\
.21 \\
.20\end{array}$ & $\begin{array}{l}.39 \\
.34 \\
.28 \\
.23 \\
.26\end{array}$ & $\begin{array}{r}.67 \\
.56 \\
.53 \\
1.3 \\
.64\end{array}$ & $\begin{array}{l}--- \\
--- \\
-\infty- \\
-m \\
-\infty\end{array}$ & $\begin{array}{r}.21 \\
.24 \\
.25 \\
.22 \\
.22\end{array}$ & $\begin{array}{r}.27 \\
.45 \\
.17 \\
4.7 \\
6.1\end{array}$ \\
\hline $\begin{array}{l}16 \\
17 \\
18 \\
19 \\
20\end{array}$ & $\begin{array}{l}.03 \\
.04 \\
.05 \\
.03 \\
.01\end{array}$ & $\begin{array}{r}1.6 \\
.18 \\
.01 \\
.00 \\
.02\end{array}$ & $m$ & & $m$ & & $\begin{array}{l}-+- \\
-m \\
-\infty \\
-\infty \\
-\infty\end{array}$ & & $\begin{array}{l}.23 \\
.21 \\
.23 \\
.19 \\
.34\end{array}$ & $\begin{array}{l}.31 \\
.44 \\
.81 \\
.84 \\
.73\end{array}$ & $\begin{array}{l}.33 \\
.32 \\
.42 \\
.49 \\
.43\end{array}$ & $\begin{array}{l}.53 \\
.43 \\
.52 \\
.50 \\
.40\end{array}$ & $\begin{array}{l}m-m \\
--- \\
m-m \\
m-\infty \\
m-\infty\end{array}$ & $\begin{array}{l}.33 \\
.25 \\
.20 \\
.15 \\
.15\end{array}$ & $\begin{array}{r}1.5 \\
1.6 \\
2.1 \\
.26 \\
--\end{array}$ \\
\hline $\begin{array}{l}21 \\
22 \\
23 \\
24 \\
25\end{array}$ & $\begin{array}{l}.00 \\
.00 \\
.03 \\
.03 \\
.04\end{array}$ & $\begin{array}{l}.02 \\
.07 \\
.00 \\
.01 \\
.01\end{array}$ & $=$ & & $\begin{array}{l}--- \\
--- \\
--- \\
m-\end{array}$ & & $\begin{array}{l}-\infty \\
--- \\
--- \\
---\end{array}$ & & $\begin{array}{l}.34 \\
.24 \\
.26 \\
.27 \\
.25\end{array}$ & $\begin{array}{l}.40 \\
.40 \\
.40 \\
.40 \\
.40\end{array}$ & $\begin{array}{l}.36 \\
.52 \\
.43 \\
.33 \\
.40\end{array}$ & $\overline{--}$ & - & $\begin{array}{l}.36 \\
3.0 \\
2.8 \\
.39 \\
.17\end{array}$ & .07 \\
\hline $\begin{array}{l}26 \\
27 \\
28 \\
29 \\
30 \\
31\end{array}$ & $\begin{array}{l}.02 \\
.02 \\
.00 \\
.01 \\
.01 \\
.01\end{array}$ & $\begin{array}{l}.01 \\
.01 \\
.03 \\
-.- \\
.-\end{array}$ & $\begin{array}{l}m- \\
--- \\
-- \\
--\end{array}$ & & $\begin{array}{l}m-\infty \\
=-\infty \\
=-\infty \\
=-\infty\end{array}$ & & $\begin{array}{l}--- \\
m-\infty \\
-\cdots \\
-- \\
--\end{array}$ & & $\begin{array}{l}.23 \\
.17 \\
.21 \\
.33 \\
.33 \\
.35\end{array}$ & $\begin{array}{l}.40 \\
.40 \\
.40 \\
.40 \\
.40 \\
-.-\end{array}$ & $\begin{array}{l}.35 \\
.32 \\
.40 \\
.38 \\
.55 \\
.63\end{array}$ & $\begin{array}{l}m \\
m-- \\
m-m \\
m-\end{array}$ & $\begin{array}{l}m-\infty \\
-\cdots \\
--- \\
-\infty \\
-\infty\end{array}$ & $\begin{array}{c}.20 \\
.16 \\
.35 \\
1.2 \\
.22 \\
.30\end{array}$ & .3 \\
\hline $\begin{array}{l}\text { TOTAL } \\
\text { MEARY } \\
\text { MAX } \\
\text { MIN } \\
\text { AC-FT }\end{array}$ & $\begin{array}{r}0.55 \\
.018 \\
.05 \\
.00 \\
1.1\end{array}$ & $=$ & 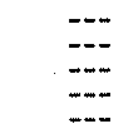 & & $m$ & & $\begin{array}{l}-w \\
-m \\
-m \\
-- \\
--\end{array}$ & & 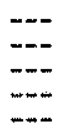 & $\begin{array}{r}12.40 \\
.41 \\
.84 \\
.16 \\
25\end{array}$ & $\begin{array}{r}10.99 \\
.35 \\
.63 \\
.17 \\
22\end{array}$ & $\begin{array}{l}-m \\
-m- \\
-m \\
--m \\
--\infty\end{array}$ & $\begin{array}{l}--- \\
--- \\
-m \\
-m \\
-m-\end{array}$ & $\begin{array}{r}15.39 \\
.50 \\
3.0 \\
.15 \\
31\end{array}$ & 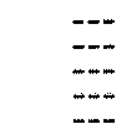 \\
\hline
\end{tabular}


RTO GRANDE BASIN

08329873 HOFFMANTOWN CHURCH OUTLET NO, 1 AT ALBUQUERQUE, MM.

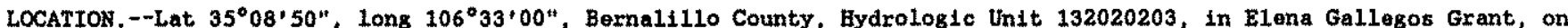
right bank at dratnage outlot of east parking lot of Hofinantown Baptist Church, at northarn boundary of Albuquerque Acadeny and $0.1 \mathrm{mi}$ downstream from Ventura Boulevard. in Albuquerque.

DRAINAGE AREA. $--.00859 \mathrm{mt}^{2}$

PERIOD OF RECORD. --August 1990 to current year (seasonal records).

GAGE.-Water-gtage recorder and Palmer-Bowlus flume. Elevation of gage is 5,490 ft above National Geodetic Vertical Datum of 1929 , from topographte map.

REMARKS. - -Records good.

EXTREMES FOR PERIOD OF RECORD.-Maximum discharge, $18 \mathrm{ft} / \mathrm{s}$, Aug. 1, 1993, gage height, 1.86 ft; no flow most of time.

EXTREMES FOR CURRENT YRAR.--Maximum discharge during period of seasonal operation, $0.13 \mathrm{ft} / \mathrm{s}$, at $2400 \mathrm{hours}$ June 26, gage height, $1.42 \mathrm{ft}$; no flow most of time.

DISCEARGE, CUBIC FEET PER SECOND, WATER YEAR OCTOBER 1995 TO SEPTEMBER 1996

\begin{tabular}{|c|c|c|c|c|c|c|c|c|c|c|c|c|}
\hline DAY & $\infty C T$ & NOV & DEC & JAN & FEB & MAR & APR & MAY & JUN & JUL. & AUG & SEP \\
\hline $\begin{array}{l}1 \\
2 \\
3 \\
4 \\
5\end{array}$ & $\begin{array}{l}.00 \\
.00 \\
.00 \\
.00 \\
.00\end{array}$ & $\begin{array}{l}.00 \\
.00 \\
.00 \\
.00 \\
.00\end{array}$ & 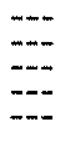 & $\begin{array}{l}--- \\
--- \\
m-\infty \\
m-\infty\end{array}$ & $m-m$ & $\begin{array}{l}m-m \\
m-m \\
-m- \\
m-- \\
m-m\end{array}$ & $\begin{array}{l}.00 \\
.00 \\
.00 \\
.00 \\
.00\end{array}$ & $\begin{array}{l}.00 \\
.00 \\
.00 \\
.00 \\
.00\end{array}$ & $\begin{array}{l}.00 \\
.00 \\
.00 \\
.00 \\
.00\end{array}$ & $\begin{array}{l}.03 \\
.02 \\
.01 \\
.00 \\
.00\end{array}$ & $\begin{array}{l}.00 \\
.00 \\
.00 \\
.00 \\
.00\end{array}$ & $\begin{array}{l}.04 \\
.03 \\
.01 \\
.01 \\
.01\end{array}$ \\
\hline $\begin{array}{r}6 \\
7 \\
8 \\
9 \\
10\end{array}$ & $\begin{array}{l}.00 \\
.00 \\
.00 \\
.00 \\
.00\end{array}$ & $\begin{array}{l}.00 \\
.00 \\
.00 \\
.00 \\
.00\end{array}$ & $m=$ & 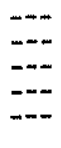 & $m=$ & $\begin{array}{l}m-- \\
--- \\
-m- \\
---\end{array}$ & $\begin{array}{l}.00 \\
.00 \\
.00 \\
.00 \\
.00\end{array}$ & $\begin{array}{l}.00 \\
.00 \\
.00 \\
.00 \\
.00\end{array}$ & $\begin{array}{l}.00 \\
.00 \\
.00 \\
.00 \\
.00\end{array}$ & $\begin{array}{l}.00 \\
.00 \\
.00 \\
.02 \\
.02\end{array}$ & $\begin{array}{l}.00 \\
.00 \\
.00 \\
.00 \\
.00\end{array}$ & $\begin{array}{l}.01 \\
.00 \\
.00 \\
.00 \\
.00\end{array}$ \\
\hline $\begin{array}{l}11 \\
12 \\
13 \\
14 \\
15\end{array}$ & $\begin{array}{l}.00 \\
.00 \\
.00 \\
.00 \\
.00\end{array}$ & $\begin{array}{l}.00 \\
.00 \\
.00 \\
.00 \\
.00\end{array}$ & $=-$ & $=$ & $\begin{array}{l}-= \\
-- \\
--- \\
--\end{array}$ & $\begin{array}{l}.-- \\
.00 \\
.00\end{array}$ & $\begin{array}{l}.00 \\
.00 \\
.00 \\
.00 \\
.00\end{array}$ & $\begin{array}{l}.00 \\
.00 \\
.00 \\
.00 \\
.00\end{array}$ & $\begin{array}{l}.00 \\
.00 \\
.00 \\
.00 \\
.00\end{array}$ & $\begin{array}{l}.03 \\
.01 \\
.00 \\
.00 \\
.00\end{array}$ & $\begin{array}{l}.00 \\
.00 \\
.00 \\
.00 \\
.00\end{array}$ & $\begin{array}{l}.00 \\
.02 \\
.01 \\
.04 \\
.07\end{array}$ \\
\hline $\begin{array}{l}16 \\
17 \\
18 \\
19 \\
20\end{array}$ & $\begin{array}{l}.00 \\
.00 \\
.00 \\
.00 \\
.00\end{array}$ & $\begin{array}{l}.00 \\
.00 \\
.00 \\
.00 \\
.00\end{array}$ & $\begin{array}{l}m \\
-\cdots \\
m- \\
m-\end{array}$ & $\begin{array}{l}--m \\
--\overline{-} \\
m-m\end{array}$ & $\begin{array}{l}--- \\
m-m \\
m-m \\
m-m \\
m-\infty\end{array}$ & $\begin{array}{l}.00 \\
.00 \\
.00 \\
.00 \\
.00\end{array}$ & $\begin{array}{l}.00 \\
.00 \\
.00 \\
.00 \\
.00\end{array}$ & $\begin{array}{l}.00 \\
.00 \\
.00 \\
.00 \\
.00\end{array}$ & $\begin{array}{l}.00 \\
.00 \\
.00 \\
.00 \\
.00\end{array}$ & $\begin{array}{l}.00 \\
.00 \\
.00 \\
.00 \\
.00\end{array}$ & $\begin{array}{l}.00 \\
.00 \\
.00 \\
.00 \\
.00\end{array}$ & $\begin{array}{l}.06 \\
.05 \\
.08 \\
.09 \\
.08\end{array}$ \\
\hline $\begin{array}{l}21 \\
22 \\
23 \\
24 \\
25\end{array}$ & $\begin{array}{l}.00 \\
.00 \\
.00 \\
.00 \\
.00\end{array}$ & $\begin{array}{l}.00 \\
.00 \\
.00 \\
.00 \\
.00\end{array}$ & $m$ & $\begin{array}{l}--m \\
--m \\
m-m \\
-m\end{array}$ & $m$ & $\begin{array}{l}.00 \\
.00 \\
.00 \\
.00 \\
.00\end{array}$ & $\begin{array}{l}.00 \\
.00 \\
.00 \\
.00 \\
.00\end{array}$ & $\begin{array}{l}.00 \\
.00 \\
.00 \\
.00 \\
.00\end{array}$ & $\begin{array}{l}.00 \\
.00 \\
.00 \\
.00 \\
.00\end{array}$ & $\begin{array}{l}.00 \\
.00 \\
.00 \\
.00 \\
.00\end{array}$ & $\begin{array}{l}.01 \\
.05 \\
.08 \\
.08 \\
.07\end{array}$ & $\begin{array}{l}.06 \\
.05 \\
.04 \\
.03 \\
.03\end{array}$ \\
\hline $\begin{array}{l}26 \\
27 \\
28 \\
29 \\
30 \\
31\end{array}$ & $\begin{array}{l}.00 \\
.00 \\
.00 \\
.00 \\
.00 \\
.00\end{array}$ & $\begin{array}{l}.00 \\
.00 \\
.00 \\
-. \\
-\infty\end{array}$ & $=-$ & $\begin{array}{l}m- \\
--\overline{-} \\
--- \\
--\overline{-}\end{array}$ & $m=$ & $\begin{array}{l}.00 \\
.00 \\
.00 \\
.00 \\
.00 \\
.00\end{array}$ & $\begin{array}{l}.00 \\
.00 \\
.00 \\
.00 \\
.00 \\
.-\end{array}$ & $\begin{array}{l}.00 \\
.00 \\
.00 \\
.00 \\
.00 \\
.00\end{array}$ & $\begin{array}{l}.03 \\
.09 \\
.08 \\
.06 \\
.04 \\
.\end{array}$ & $\begin{array}{l}.00 \\
.00 \\
.00 \\
.00 \\
.00 \\
.00\end{array}$ & $\begin{array}{l}.09 \\
.08 \\
.07 \\
.07 \\
.06 \\
.05\end{array}$ & $\begin{array}{l}.03 \\
.03 \\
.01 \\
.01 \\
.00 \\
\end{array}$ \\
\hline $\begin{array}{l}\text { TOTAZ } \\
\text { MEAN } \\
\text { MAX } \\
\text { MIN } \\
\text { AC-ET }\end{array}$ & $\begin{array}{l}0.00 \\
.000 \\
.00 \\
.00 \\
.00\end{array}$ & $=$ & 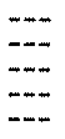 & $\begin{array}{l}m- \\
m- \\
--- \\
---\end{array}$ & $m$ & $\begin{array}{l}m-m \\
-\cdots \\
-\cdots \\
-\cdots\end{array}$ & $\begin{array}{r}0.00 \\
.000 \\
.00 \\
.00 \\
.00\end{array}$ & $\begin{array}{r}0.00 \\
.000 \\
.00 \\
.00 \\
.00\end{array}$ & $\begin{array}{r}0.30 \\
.010 \\
.09 \\
.00 \\
.6\end{array}$ & $\begin{array}{r}0.14 \\
.005 \\
.03 \\
.00 \\
.3\end{array}$ & $\begin{array}{r}0.71 \\
.023 \\
.09 \\
.00 \\
1.4\end{array}$ & $\begin{array}{r}0.90 \\
.030 \\
.09 \\
.00 \\
1.8\end{array}$ \\
\hline
\end{tabular}


08329874 HOFFMANTOWN CHURCH OUTLET NO. 2 AT ALBUQUERQUE, NM.

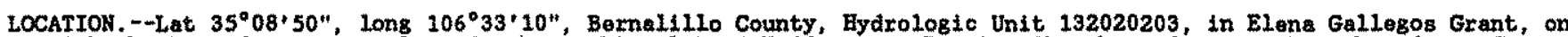
right bank at drainage outlet of wost parking lot of Hoffmantown Baptist Church, and at northern boundary of Albuquerque Academy and $0.3 \mathrm{mi}$ south of Harper Boulevard. in Albuquerque.

DRAINAGE AREA. - - .0413 $\mathrm{mi}^{2}$

PERTOD OF RECORD. - August 1990 to current year (seasonal records).

GAGE.--Water-stage recorder and concrete-lined channel. Elevation of gage is 5,480 ft above National Geodetic Verticel Detum of 1929, Srom topographic mep.

REMARKS, --Records fair.

EXTREMES FOR PERIOD OF RECORD.--Maximum dischargo, 46 fts/s, Aug, 1, 1993, gage height, 3.18 ft, from rating curve extended above $7.0 \mathrm{ft}^{3 / \mathrm{s}}$ on basts of theoreteral rating for open box culvert; no flow most of time.

EXTREMES FOR CURRENT YEAR.--Maximum discharge during period of seasonal operation, 38 fts/s, at 1135 hours June 26 , gage height, $3.02 \mathrm{ft}$, from rating curve extended above $7.0 \mathrm{ft} / \mathrm{s}$ on basis of theoreteral rating for open box

culvert; no slow most of time.

DISCHARGE, CUBIC FEET PER SECOND, WATER YEAR OCTOBER 1995 TO SEPTEMBER 1996 DAIIY MEAH VALUES

\begin{tabular}{|c|c|c|c|c|c|c|c|c|c|c|c|c|}
\hline DAY & OCT & Nov & $\mathrm{DEC}$ & JAN & FEB & MAR & APR & MAY & JUN & JUL & AUE & SEP \\
\hline $\begin{array}{l}1 \\
2 \\
3 \\
4 \\
5\end{array}$ & $\begin{array}{l}.00 \\
.00 \\
.00 \\
.00 \\
.00\end{array}$ & $\begin{array}{l}.00 \\
.00 \\
.00 \\
.00 \\
.00\end{array}$ & $\cdots$ & $\cdots$ & $\begin{array}{l}=- \\
-\square \\
-\square\end{array}$ & $\begin{array}{l}m- \\
--- \\
-- \\
--\end{array}$ & $\begin{array}{l}.00 \\
.00 \\
.00 \\
.00 \\
.00\end{array}$ & $\begin{array}{l}.00 \\
.00 \\
.00 \\
.00 \\
.00\end{array}$ & $\begin{array}{l}.00 \\
.00 \\
.00 \\
.00 \\
.00\end{array}$ & $\begin{array}{l}.00 \\
.00 \\
.00 \\
.00 \\
.00\end{array}$ & $\begin{array}{l}.00 \\
.00 \\
.10 \\
.00 \\
.00\end{array}$ & $\begin{array}{l}.00 \\
.00 \\
.00 \\
.00 \\
.00\end{array}$ \\
\hline $\begin{array}{r}6 \\
7 \\
8 \\
9 \\
10\end{array}$ & $\begin{array}{l}.00 \\
.00 \\
.00 \\
.00 \\
.00\end{array}$ & $\begin{array}{l}.00 \\
.00 \\
.00 \\
.00 \\
.00\end{array}$ & $\begin{array}{l}\cdots \\
=- \\
-\cdots\end{array}$ & $\begin{array}{c}\cdots \\
\cdots \\
\cdots\end{array}$ & $\begin{array}{l}-\cdots- \\
=-- \\
-\because- \\
-\square\end{array}$ & 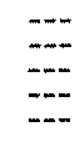 & $\begin{array}{l}.00 \\
.00 \\
.00 \\
.00 \\
.00\end{array}$ & $\begin{array}{l}.00 \\
.00 \\
.00 \\
.00 \\
.00\end{array}$ & $\begin{array}{l}.00 \\
.00 \\
.00 \\
.00 \\
.00\end{array}$ & $\begin{array}{l}.00 \\
.10 \\
.23 \\
.35 \\
.34\end{array}$ & $\begin{array}{l}.00 \\
.02 \\
.00 \\
.00 \\
.00\end{array}$ & $\begin{array}{l}.00 \\
.00 \\
.00 \\
.00 \\
.00\end{array}$ \\
\hline $\begin{array}{l}11 \\
12 \\
13 \\
14 \\
15\end{array}$ & $\begin{array}{l}.00 \\
.00 \\
.00 \\
.00 \\
.00\end{array}$ & $\begin{array}{l}.00 \\
.00 \\
.00 \\
.00 \\
.00\end{array}$ & $\begin{array}{l}-\overline{-} \\
-\overline{-} \\
-\square \\
--\end{array}$ & $\begin{array}{l}--- \\
-\cdots \\
-\cdots \\
-\cdots\end{array}$ & $\begin{array}{l}--- \\
=- \\
-\square \\
--\end{array}$ & $\begin{array}{l}.00 \\
.00 \\
.00\end{array}$ & $\begin{array}{l}.00 \\
.00 \\
.00 \\
.00 \\
.00\end{array}$ & $\begin{array}{l}.00 \\
.00 \\
.00 \\
.00 \\
.00\end{array}$ & $\begin{array}{l}.00 \\
.00 \\
.00 \\
.13 \\
.00\end{array}$ & $\begin{array}{l}.19 \\
.10 \\
.00 \\
.00 \\
.00\end{array}$ & $\begin{array}{l}.00 \\
.00 \\
.00 \\
.00 \\
.00\end{array}$ & $\begin{array}{l}.00 \\
.23 \\
.12 \\
.80 \\
.12\end{array}$ \\
\hline $\begin{array}{l}16 \\
17 \\
18 \\
19 \\
20\end{array}$ & $\begin{array}{l}.00 \\
.00 \\
.00 \\
.00 \\
.00\end{array}$ & $\begin{array}{l}.00 \\
.00 \\
.00 \\
.00 \\
.00\end{array}$ & $\begin{array}{l}-- \\
=- \\
=-\end{array}$ & 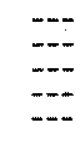 & $\begin{array}{l}=- \\
-\cdots \\
-\cdots \\
---\end{array}$ & $\begin{array}{l}.00 \\
.00 \\
.00 \\
.00 \\
.00\end{array}$ & $\begin{array}{l}.00 \\
.00 \\
.00 \\
.00 \\
.00\end{array}$ & $\begin{array}{l}.00 \\
.00 \\
.00 \\
.00 \\
.00\end{array}$ & $\begin{array}{l}.00 \\
.00 \\
.00 \\
.00 \\
.00\end{array}$ & $\begin{array}{l}.09 \\
.19 \\
.27 \\
.19 \\
.03\end{array}$ & $\begin{array}{l}.00 \\
.00 \\
.00 \\
.00 \\
.26\end{array}$ & $\begin{array}{l}.00 \\
.30 \\
.45 \\
.13 \\
.00\end{array}$ \\
\hline $\begin{array}{l}21 \\
22 \\
23 \\
24 \\
25\end{array}$ & $\begin{array}{l}.00 \\
.00 \\
.00 \\
.00 \\
.00\end{array}$ & $\begin{array}{l}.00 \\
.00 \\
.00 \\
.00 \\
.00\end{array}$ & 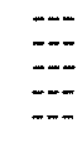 & $\begin{array}{l}=- \\
=- \\
-\cdots \\
---\end{array}$ & $\begin{array}{l}=-: \\
=- \\
=- \\
=-\end{array}$ & $\begin{array}{l}.00 \\
.00 \\
.00 \\
.00 \\
.00\end{array}$ & $\begin{array}{l}.00 \\
.00 \\
.00 \\
.00 \\
.00\end{array}$ & $\begin{array}{l}.00 \\
.00 \\
.00 \\
.00 \\
.00\end{array}$ & $\begin{array}{l}.00 \\
.00 \\
.00 \\
.00 \\
.00\end{array}$ & $\begin{array}{l}.00 \\
.00 \\
.00 \\
.00 \\
.00\end{array}$ & $\begin{array}{l}.07 \\
.40 \\
.33 \\
.20 \\
.15\end{array}$ & $\begin{array}{l}.00 \\
.00 \\
.00 \\
.00 \\
.00\end{array}$ \\
\hline $\begin{array}{l}26 \\
27 \\
28 \\
29 \\
30 \\
31\end{array}$ & $\begin{array}{l}.00 \\
.00 \\
.00 \\
.00 \\
.00 \\
.00\end{array}$ & $\begin{array}{l}.00 \\
.00 \\
.00 \\
-.- \\
-.-\end{array}$ & $\begin{array}{l}=- \\
=- \\
=- \\
-\square- \\
-\square \\
---\end{array}$ & 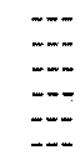 & $\begin{array}{l}--- \\
--- \\
--- \\
--- \\
---\end{array}$ & $\begin{array}{l}.00 \\
.00 \\
.00 \\
.00 \\
.00 \\
.00\end{array}$ & $\begin{array}{l}.00 \\
.00 \\
.00 \\
.00 \\
.00 \\
.\end{array}$ & $\begin{array}{l}.00 \\
.00 \\
.00 \\
.00 \\
.00 \\
.00\end{array}$ & $\begin{array}{r}1.1 \\
.35 \\
.45 \\
.21 \\
.12 \\
-\cdots\end{array}$ & $\begin{array}{l}.00 \\
.00 \\
.00 \\
.00 \\
.00 \\
.00\end{array}$ & $\begin{array}{l}.34 \\
-\cdots \\
-\cdots \\
-\cdots \\
-\cdots\end{array}$ & $\begin{array}{l}.21 \\
.00 \\
.00 \\
.00 \\
.00 \\
-\end{array}$ \\
\hline $\begin{array}{l}\text { TOTAL } \\
\text { MEAN } \\
\text { MAX } \\
\text { MIN } \\
\text { AC-FI } \\
(\dagger)\end{array}$ & $\begin{array}{r}0.00 \\
.000 \\
.00 \\
.00 \\
.00 \\
0.00\end{array}$ & $\begin{array}{c}-\cdots \\
-- \\
-- \\
-\cdots \\
0.12\end{array}$ & $\begin{array}{c}--- \\
--- \\
\overline{--} \\
0.13\end{array}$ & 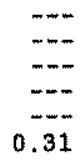 & 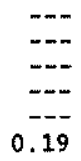 & $\begin{array}{l}-- \\
-\overline{-} \\
\overline{-0} \\
0.06\end{array}$ & $\begin{array}{r}0.00 \\
.000 \\
.00 \\
.00 \\
.00 \\
0.00\end{array}$ & $\begin{array}{r}0.00 \\
.000 \\
.00 \\
.00 \\
.00 \\
0.01\end{array}$ & $\begin{array}{r}2.36 \\
.079 \\
1.1 \\
.00 \\
4.7 \\
2.49\end{array}$ & $\begin{array}{r}2.08 \\
.067 \\
.35 \\
.00 \\
4.2 \\
0.86\end{array}$ & 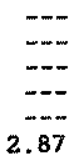 & $\begin{array}{r}2.36 \\
.079 \\
.80 \\
.00 \\
6.7 \\
2.01\end{array}$ \\
\hline
\end{tabular}

(†) Total rainfall accumulation in inches. 
RIO GRANDE BASIN

08329875 CHERRY HILLS ARROYO NO. 1 AT ALBUQUERQUE, MM.

LOCATION. - Lat $35^{\circ} 08^{\prime} 50^{\prime \prime}$, long $106^{\circ} 33^{\prime} 10^{\prime \prime}$, Bernalillo County, Hydrologic Unit 132020203, in Elena Gallegos Grant, on left bank, on grounds of the Albuquerque Academy, and $300^{\prime}$ ft downstream from Harper Road in Albuquercue.

DRAIKAGE AREA. $-.0147 \mathrm{~mL}^{2}$

PERIOD OF RECORD.--August 1990 to current year (seasonal records).

GAGE.-Water-gtage recorder and Parshall flume. Elevation of gage is 5,470 ft above National Geodetic Vertical Datiun of 1929, from topographio map.

REMARKS. - -Records fair.

EXTREMES FOR PERIOD OF RECORD.--Maximun dlscharge, $17 \mathrm{fts} / \mathrm{s}$, Aug. 2, 1994, gage helght, 3.99 ft; no flow most of timo.

EXTREMES FOR CURRENT YEAR. - Maximum discharge during period of seasonal operation, 9.5 fts/s, at 1255 hours June 26 , gage height, $3.35 \mathrm{ft}$; no flow most of the time.

DISCEARGE, CUBIC FEET FER SECOND, WATER YEAR OCTOBER 1995 TO SEPTEMBER 1996 DAILY MEAN VALUES

\begin{tabular}{|c|c|c|c|c|c|c|c|c|c|c|c|c|}
\hline DAY & $\alpha C$ & NOV & DEC & JAN & FEB & MAR & AFR & MAY & JUN & JUL & AUG & SEP \\
\hline $\begin{array}{l}1 \\
2 \\
3 \\
4 \\
5\end{array}$ & $\begin{array}{l}.00 \\
.00 \\
.00 \\
.00 \\
.00\end{array}$ & $\begin{array}{l}.00 \\
.00 \\
.00 \\
.00 \\
.00\end{array}$ & $\begin{array}{l}m-m \\
-m \\
-m \\
-m-m\end{array}$ & 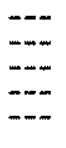 & ב- & $\begin{array}{l}-\infty \\
-m-\infty \\
-\infty \\
-\infty\end{array}$ & $\begin{array}{l}.00 \\
.00 \\
.00 \\
.00 \\
.00\end{array}$ & $\begin{array}{l}.00 \\
.00 \\
.00 \\
.00 \\
.00\end{array}$ & $\begin{array}{l}.00 \\
.00 \\
.00 \\
.00 \\
.00\end{array}$ & $\begin{array}{l}.00 \\
.00 \\
.00 \\
.00 \\
.00\end{array}$ & $\begin{array}{l}.00 \\
.00 \\
.01 \\
.00 \\
.00\end{array}$ & $\begin{array}{l}.00 \\
.00 \\
.00 \\
.00 \\
.00\end{array}$ \\
\hline $\begin{array}{r}6 \\
7 \\
8 \\
9 \\
10\end{array}$ & $\begin{array}{l}.00 \\
.00 \\
.00 \\
.00 \\
.00\end{array}$ & $\begin{array}{l}.00 \\
.00 \\
.00 \\
.00 \\
.00\end{array}$ & $\begin{array}{l}-m \\
-- \\
m- \\
--m \\
--\end{array}$ & $\begin{array}{l}m- \\
-\rightarrow- \\
-m \\
-m- \\
-m\end{array}$ & $\begin{array}{l}m- \\
m- \\
m-\end{array}$ & $\begin{array}{l}-m \\
-m- \\
-m- \\
--- \\
-m-m\end{array}$ & $\begin{array}{l}.00 \\
.00 \\
.00 \\
.00 \\
.00\end{array}$ & $\begin{array}{l}.00 \\
.00 \\
.00 \\
.00 \\
.00\end{array}$ & $\begin{array}{l}.00 \\
.00 \\
.00 \\
.00 \\
.00\end{array}$ & $\begin{array}{l}.00 \\
.01 \\
.00 \\
.02 \\
.04\end{array}$ & $\begin{array}{l}.00 \\
.00 \\
.00 \\
.00 \\
.00\end{array}$ & $\begin{array}{l}.00 \\
.00 \\
.00 \\
.00 \\
.00\end{array}$ \\
\hline $\begin{array}{l}11 \\
12 \\
13 \\
14 \\
15\end{array}$ & $\begin{array}{l}.00 \\
.00 \\
.00 \\
.00 \\
.00\end{array}$ & $\begin{array}{r}.00 \\
.00 \\
.00 \\
.00 \\
.00\end{array}$ & 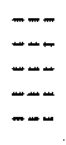 & $\begin{array}{l}m-\infty \\
-m \\
-m \\
-m\end{array}$ & $\begin{array}{l}m- \\
-m \\
-m \\
--m\end{array}$ & $\begin{array}{l}. .- \\
.00 \\
.00 \\
.00\end{array}$ & $\begin{array}{l}.00 \\
.00 \\
.00 \\
.00 \\
.00\end{array}$ & $\begin{array}{l}.00 \\
.00 \\
.00 \\
.00 \\
.00\end{array}$ & $\begin{array}{l}.00 \\
.00 \\
.00 \\
.00 \\
.00\end{array}$ & $\begin{array}{l}.00 \\
.00 \\
.00 \\
.00 \\
.00\end{array}$ & $\begin{array}{l}.00 \\
.00 \\
.00 \\
.00 \\
.00\end{array}$ & $\begin{array}{l}.00 \\
.00 \\
.00 \\
.18 \\
.00\end{array}$ \\
\hline $\begin{array}{l}16 \\
17 \\
18 \\
19 \\
20\end{array}$ & $\begin{array}{l}.00 \\
.00 \\
.00 \\
.00 \\
.00\end{array}$ & $\begin{array}{l}.00 \\
.00 \\
.00 \\
.00 \\
.00\end{array}$ & $\begin{array}{l}m-m \\
m-m \\
m-m \\
-m-m\end{array}$ & $\begin{array}{l}-\cdots \\
-\cdots \\
-- \\
-- \\
--\end{array}$ & $\begin{array}{l}-- \\
-- \\
-- \\
-m \\
-\infty\end{array}$ & $\begin{array}{l}.00 \\
.00 \\
.00 \\
.00 \\
.00\end{array}$ & $\begin{array}{l}.00 \\
.00 \\
.00 \\
.00 \\
.00\end{array}$ & $\begin{array}{l}.00 \\
.00 \\
.00 \\
.00 \\
.00\end{array}$ & $\begin{array}{l}.00 \\
.00 \\
.00 \\
.00 \\
.00\end{array}$ & $\begin{array}{l}.00 \\
.00 \\
.01 \\
.00 \\
.00\end{array}$ & $\begin{array}{l}.00 \\
.00 \\
.00 \\
.00 \\
.05\end{array}$ & $\begin{array}{l}.00 \\
.06 \\
.03 \\
.00 \\
.00\end{array}$ \\
\hline $\begin{array}{l}21 \\
22 \\
23 \\
24 \\
25\end{array}$ & $\begin{array}{l}.00 \\
.00 \\
.00 \\
.00 \\
.00\end{array}$ & $\begin{array}{l}.00 \\
.00 \\
.00 \\
.00 \\
.00\end{array}$ & $\begin{array}{l}m \\
m-m \\
m-m \\
-m- \\
m-m\end{array}$ & 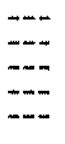 & $\begin{array}{l}-m \\
-\cdots \\
-\cdots \\
-\cdots\end{array}$ & $\begin{array}{l}.00 \\
.00 \\
.00 \\
.00 \\
.00\end{array}$ & $\begin{array}{l}.00 \\
.00 \\
.00 \\
.00 \\
.00\end{array}$ & $\begin{array}{l}.00 \\
.00 \\
.00 \\
.00 \\
.00\end{array}$ & $\begin{array}{l}.00 \\
.00 \\
.00 \\
.00 \\
.00\end{array}$ & $\begin{array}{l}.00 \\
.00 \\
.00 \\
.00 \\
.00\end{array}$ & $\begin{array}{l}.00 \\
.06 \\
.06 \\
.01 \\
.03\end{array}$ & $\begin{array}{l}.00 \\
.00 \\
.00 \\
.00 \\
.00\end{array}$ \\
\hline $\begin{array}{l}26 \\
27 \\
28 \\
29 \\
30 \\
31 .\end{array}$ & $\begin{array}{l}.00 \\
.00 \\
.00 \\
.00 \\
.00 \\
.00\end{array}$ & $\begin{array}{l}.00 \\
.00 \\
.00 \\
--- \\
--\infty\end{array}$ & 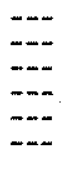 & 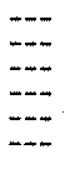 & $\begin{array}{l}m-- \\
=-\infty \\
=-\infty \\
=-\infty\end{array}$ & $\begin{array}{l}.00 \\
.00 \\
.00 \\
.00 \\
.00 \\
.00\end{array}$ & $\begin{array}{l}.00 \\
.00 \\
.00 \\
.00 \\
.00 \\
.\end{array}$ & $\begin{array}{l}.00 \\
.00 \\
.00 \\
.00 \\
.00 \\
.00\end{array}$ & $\begin{array}{r}.27 \\
-2-0 \\
-00 \\
.00 \\
-0-\end{array}$ & $\begin{array}{l}.00 \\
.00 \\
.00 \\
.00 \\
.00 \\
.00\end{array}$ & $\begin{array}{l}\cdots \\
.00 \\
.00 \\
0\end{array}$ & $\begin{array}{l}.00 \\
.00 \\
.00 \\
.00 \\
.00 \\
.\end{array}$ \\
\hline $\begin{array}{l}\text { TOTAL } \\
\text { MEAN } \\
\text { MAX } \\
\text { MIN } \\
\text { AC-FT }\end{array}$ & $\begin{array}{l}0.00 \\
.000 \\
.00 \\
.00 \\
.00\end{array}$ & $\frac{--m}{m-m}+m$ & $\begin{array}{c}--- \\
-\infty \\
-\infty \\
-\infty \\
-\infty\end{array}$ & $\begin{array}{l}m-\infty \\
m-\infty \\
m-\infty\end{array}$ & $\begin{array}{l}m-m \\
-m- \\
-m- \\
-m-\end{array}$ & $\begin{array}{l}-m \\
--m \\
-m- \\
-m-m\end{array}$ & $\begin{array}{r}0.00 \\
.000 \\
.00 \\
.00 \\
.00\end{array}$ & $\begin{array}{l}0.00 \\
.000 \\
.00 \\
.00 \\
.00\end{array}$ & $\begin{array}{l}m-- \\
=-- \\
=-- \\
-m\end{array}$ & $\begin{array}{r}0.08 \\
.003 \\
.04 \\
.00 \\
.2\end{array}$ & 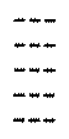 & $\begin{array}{r}0.27 \\
.009 \\
.18 \\
.00 \\
.5\end{array}$ \\
\hline
\end{tabular}


08329876 CHERRY BILLS ARROYO NO. 2 AT ALBUQUERQUE, NM.

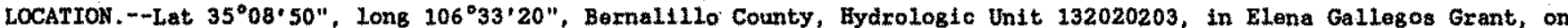
right bank, on grounds of the Albuquerque Academy, and 390 ft dowstream from Harper Road in Albuquerque.

DRAINAGE AREA. $--.0796 \mathrm{mi}^{2}$

PERIOD OF RECORD. --August 1990 to current year (seasonal records).

GAGE. - Water-stage recorder and Parshall flume. Elovation of gage is 5,440 ft above National Geodetic Vert loal Datua of 1929 , from topographte map.

RARARKS. --Records good.

EXTREAES FOR PERIOD OF RECORD, - Maximum discharge, 21 fts/s; Sept. 22, 1990, gage hejght, 1.85 ft; no flow most of time.

EXTREAES FOR CURREXT YFAR,--Maxtmum discharge during period of seasonal operation, 8.9 It $3 / 8$, at 1130 hours June 26, gage height, 1.07 ft; no flow most of time.

DISCHARGE, CUBIC FEET PER SECOND, WATER YEAR OCTOBER 1995 TO SEPTEMBER 1996 DAILY MEAN VALUES

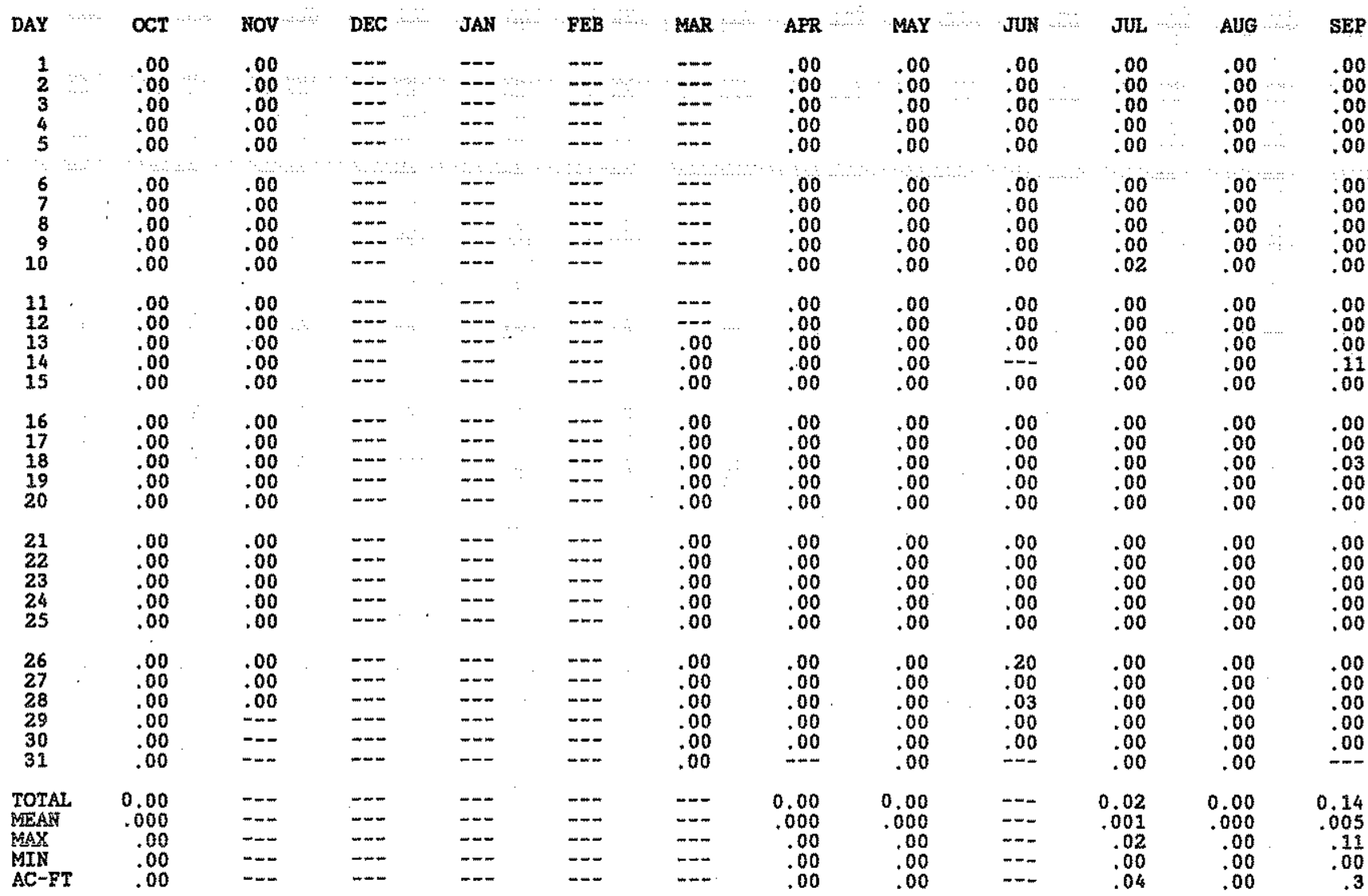


RIO GRANDE BASIN

08329877 PINO ARROYO AT WYOMING BOULEVARD AT ALBUQUERQUE, MM.

LOCATION.--Lat $35^{\circ} 09^{\circ} 25^{\prime \prime}$, long $106^{\circ} 33^{\prime} 29^{\prime \prime}$, Bernalillo County, Hydrologic Unit 132020203, in Elena Gallegos Grant, on the grounds of the Albuquerque Acadeny, on left bank, and 560 ft upstream from Wyoming Boulevard in Albuquerque.

DRAIKAGE AREA, $--5,80 \mathrm{mi}$.

PERIOD OF RECORD. - August 1990 to current year (seasonal records).

GAGE.- Water-stage recordex and concrete control. Elevation of gage is 5,540 ft above National Geodetic Vertical Datum of 1929, from topographic map.

REMARKS, --Records poox.

EXTREMS FOR PERIOD OF RBCORD.--Maximum discharge, $120 \mathrm{ft} / \mathrm{s}$, Aug. 28, 1994, gage helght, 2.14 ft, from floodmarks; no flow part of many days.

EXIREASS FOR CURRENT YEAR.--Maximum d1scharge during perlod of seasonal operation, 104 ft'/s, at 1735 hours June 28 , gage height, $1.78 \mathrm{ft}$; no flow most of time.

DISCEARGE, CUBIC FEET PER SECOND, WATER YEAR OCTOBER 1995 TO SEPTEMBER 1996

DAILY MEAN VALUES

\begin{tabular}{|c|c|c|c|c|c|c|c|c|c|c|c|c|}
\hline DAY & $\infty \mathrm{CT}$ & Nov & DEC & JAN & FEB & MAR & APR & MAX & JuN & JUL & AUG & SER \\
\hline $\begin{array}{l}1 \\
2 \\
3 \\
4 \\
5\end{array}$ & $\begin{array}{l}.00 \\
.00 \\
.00 \\
.00 \\
.00\end{array}$ & $\begin{array}{l}.00 \\
.00 \\
.00 \\
.00 \\
.00\end{array}$ & $m-m$ & $\begin{array}{c}m-m \\
m-m \\
m-m \\
-m-\end{array}$ & 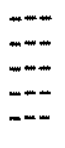 & $\begin{array}{l}-m \\
-m \\
--m \\
---\end{array}$ & $\begin{array}{l}.00 \\
.00 \\
.00 \\
.00 \\
.00\end{array}$ & $\begin{array}{l}.00 \\
.00 \\
.00 \\
.00 \\
.00\end{array}$ & $\begin{array}{l}.00 \\
.00 \\
.00 \\
.00 \\
.00\end{array}$ & $\begin{array}{l}.00 \\
.00 \\
.00 \\
.00 \\
.00\end{array}$ & $\begin{array}{l}.00 \\
.00 \\
.00 \\
.00 \\
.00\end{array}$ & $\begin{array}{l}.00 \\
.00 \\
.00 \\
.00 \\
.00\end{array}$ \\
\hline $\begin{array}{r}6 \\
7 \\
8 \\
9 \\
10\end{array}$ & $\begin{array}{l}.00 \\
.00 \\
.00 \\
.00 \\
.00\end{array}$ & $\begin{array}{l}.00 \\
.00 \\
.00 \\
.00 \\
.00\end{array}$ & $\begin{array}{l}m-m \\
m-m \\
m-m \\
m-m\end{array}$ & $m$ & $\begin{array}{l}m-\infty \\
-\cdots \\
-\cdots \\
-\cdots\end{array}$ & $\begin{array}{l}=- \\
-\cdots \\
-\cdots \\
--\infty\end{array}$ & $\begin{array}{l}.00 \\
.00 \\
.00 \\
.00 \\
.00\end{array}$ & $\begin{array}{l}.00 \\
.00 \\
.00 \\
.00 \\
.00\end{array}$ & $\begin{array}{l}.00 \\
.00 \\
.00 \\
.00 \\
.00\end{array}$ & $\begin{array}{l}.00 \\
.00 \\
.00 \\
.00 \\
.00\end{array}$ & $\begin{array}{l}.00 \\
.00 \\
.00 \\
.00 \\
.00\end{array}$ & $\begin{array}{l}.00 \\
.00 \\
.00 \\
.00 \\
.00\end{array}$ \\
\hline $\begin{array}{l}11 \\
12 \\
13 \\
14 \\
15\end{array}$ & $\begin{array}{l}.00 \\
.00 \\
.00 \\
.00 \\
.00\end{array}$ & $\begin{array}{l}.00 \\
.00 \\
.00 \\
.00 \\
.00\end{array}$ & $\begin{array}{l}m-- \\
-m \\
-m \\
-m\end{array}$ & $\begin{array}{l}m- \\
-m \\
-m \\
-m-\end{array}$ & $\begin{array}{l}m-m \\
-m \\
-m \\
--m\end{array}$ & $\begin{array}{l}.00 \\
.00 \\
.00\end{array}$ & $\begin{array}{l}.00 \\
.00 \\
.00 \\
.00 \\
.00\end{array}$ & $\begin{array}{l}.00 \\
.00 \\
.00 \\
.00 \\
.00\end{array}$ & $\begin{array}{l}.00 \\
.00 \\
.00 \\
.00 \\
.00\end{array}$ & $\begin{array}{l}.00 \\
.00 \\
.00 \\
.00 \\
.00\end{array}$ & $\begin{array}{l}.00 \\
.00 \\
.00 \\
.00 \\
.00\end{array}$ & $\begin{array}{r}.00 \\
.00 \\
.00 \\
2.0 \\
.00\end{array}$ \\
\hline $\begin{array}{l}16 \\
17 \\
18 \\
19 \\
20\end{array}$ & $\begin{array}{l}.00 \\
.00 \\
.00 \\
.00 \\
.00\end{array}$ & $\begin{array}{l}.00 \\
.00 \\
.00 \\
.00 \\
.00\end{array}$ & $\begin{array}{l}--- \\
--- \\
-\infty- \\
m-\end{array}$ & $\begin{array}{l}m \\
-m \\
-m \\
-\infty\end{array}$ & $\begin{array}{l}m-m \\
m-m \\
-\cdots\end{array}$ & $\begin{array}{l}.00 \\
.00 \\
.00 \\
.00 \\
.00\end{array}$ & $\begin{array}{l}.00 \\
.00 \\
.00 \\
.00 \\
.00\end{array}$ & $\begin{array}{l}.00 \\
.00 \\
.00 \\
.00 \\
.00\end{array}$ & $\begin{array}{l}.00 \\
.00 \\
.00 \\
.00 \\
.00\end{array}$ & $\begin{array}{l}.00 \\
.00 \\
.00 \\
.00 \\
.00\end{array}$ & $\begin{array}{l}.00 \\
.00 \\
.00 \\
.00 \\
.00\end{array}$ & $\begin{array}{l}.00 \\
.00 \\
.00 \\
.00 \\
.00\end{array}$ \\
\hline $\begin{array}{l}21 \\
22 \\
23 \\
24 \\
25\end{array}$ & $\begin{array}{l}.00 \\
.00 \\
.00 \\
.00 \\
.00\end{array}$ & $\begin{array}{l}.00 \\
.00 \\
.00 \\
.00 \\
.00\end{array}$ & $m$ & 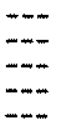 & $\begin{array}{l}-\cdots \\
-\cdots \\
-\cdots \\
-\cdots\end{array}$ & $\begin{array}{l}.00 \\
.00 \\
.00 \\
.00 \\
.00\end{array}$ & $\begin{array}{l}.00 \\
.00 \\
.00 \\
.00 \\
.00\end{array}$ & $\begin{array}{l}.00 \\
.00 \\
.00 \\
.00 \\
.00\end{array}$ & $\begin{array}{l}.00 \\
.00 \\
.00 \\
.00 \\
.00\end{array}$ & $\begin{array}{l}.00 \\
.00 \\
.00 \\
.00 \\
.00\end{array}$ & $\begin{array}{l}.00 \\
.00 \\
.00 \\
.00 \\
.00\end{array}$ & $\begin{array}{l}.00 \\
.00 \\
.00 \\
.00 \\
.00\end{array}$ \\
\hline $\begin{array}{l}26 \\
27 \\
28 \\
29 \\
30 \\
31\end{array}$ & $\begin{array}{l}.00 \\
.00 \\
.00 \\
.00 \\
.00 \\
.00\end{array}$ & $\begin{array}{l}.00 \\
.00 \\
.00 \\
.00 \\
.-\end{array}$ & $\begin{array}{c}-m \\
m-m \\
m-m \\
m-m\end{array}$ & 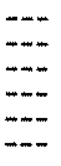 & $\begin{array}{l}-\cdots \\
-\cdots \\
-\cdots \\
-\cdots \\
-\cdots\end{array}$ & $\begin{array}{l}.00 \\
.00 \\
.00 \\
.00 \\
.00 \\
.00\end{array}$ & $\begin{array}{l}.00 \\
.00 \\
.00 \\
.00 \\
.00 \\
\end{array}$ & $\begin{array}{l}.00 \\
.00 \\
.00 \\
.00 \\
.00 \\
.00\end{array}$ & $\begin{array}{r}.84 \\
.00 \\
1.0 \\
.00 \\
.00 \\
-.-7\end{array}$ & $\begin{array}{l}.00 \\
.00 \\
.00 \\
.00 \\
.00 \\
.00\end{array}$ & $\begin{array}{l}.00 \\
.00 \\
.00 \\
.00 \\
.00 \\
.00\end{array}$ & $\begin{array}{l}.00 \\
.00 \\
.00 \\
.00 \\
.00 \\
.\end{array}$ \\
\hline $\begin{array}{l}\text { TOTAL } \\
\text { MEAN } \\
\text { MAX } \\
\text { MIN } \\
\text { AC-FT }\end{array}$ & $\begin{array}{r}0.00 \\
.000 \\
.00 \\
.00 \\
.00\end{array}$ & 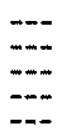 & $\begin{array}{l}m-m \\
m-m \\
m-m \\
m-m\end{array}$ & $\begin{array}{l}-\infty \\
-\infty \\
-\infty \\
--\infty\end{array}$ & 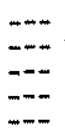 & $m$ & $\begin{array}{r}0.00 \\
.000 \\
.00 \\
.00 \\
.00\end{array}$ & $\begin{array}{r}0.00 \\
.000 \\
.00 \\
.00 \\
.00\end{array}$ & $\begin{array}{r}1.84 \\
.061 \\
1.0 \\
.00 \\
3.6\end{array}$ & $\begin{array}{r}0.00 \\
.000 \\
.00 \\
.00 \\
.00\end{array}$ & $\begin{array}{r}0.00 \\
.000 \\
.00 \\
.00 \\
.00\end{array}$ & $\begin{array}{r}2.00 \\
.067 \\
2.0 \\
.00 \\
4.0\end{array}$ \\
\hline
\end{tabular}


RIO GRANDE BASIN

08329880 ACADEMY ACRES DRAIN AT ALBUQUERQUE, MM

LOCATION. - Lat $35^{\circ} 09^{\circ} 02^{\prime \prime}$, long $106^{\circ} 34^{\circ} 18^{*}$, in NEHSEX sec.25, T.12 N., R.3 E., Bernalillo County, Hydrologic Unit 13020203 , on left bank of concrete-1ined channe1, 250 ft north of intersection of Esther Avenue and Burlison Drive, and $0.4 \mathrm{mi}$ north of Academy Road in Albuquerqua.

DRAINAGE AREA. $--0.124 \mathrm{mi}^{2}$.

PERTOD OF RECORD. --June 1976 to current yeax (seasonal records)

GAGE. - Water-stage recorder and V-notch weir. Elevation of gage is 5,310 rt above National Geodetic vertical Datum of 1929, Exom topographic map.

REMARKS.--Records feir. Recording rain gage at station. The basin is primarily urban residential. Some minor streanflow may exist on days where dally mean discharges have been recorded as zero due to the sensitivity limits of the streamflow monftoring equipment. See tabulation below for monthly prectpitation in inches. No flow most. of time.

EXIREMES FOR PERIOD OF RECORD,-Maximum discharge, 103 fts/s, Aug. 3, 1978, gage he1ght, 4.09 ft, from rating curve extended above $10 \mathrm{ft} / \mathrm{s}$ on basis of slope-area measurement of peak flow; no flow most time.

EXTRDAES FOR CURRENT YEAR,-Maximum discharge during period of seasonal operation, 22 fts/s, at 1145 hours June 26 , gage helght, $2.92 \mathrm{ft}$; no flow most of time,

DISCHARGE, CUBIC FEET PER SECOND, WATER YEAR OCTOBER 1995 TO SEPTEMBER 1996

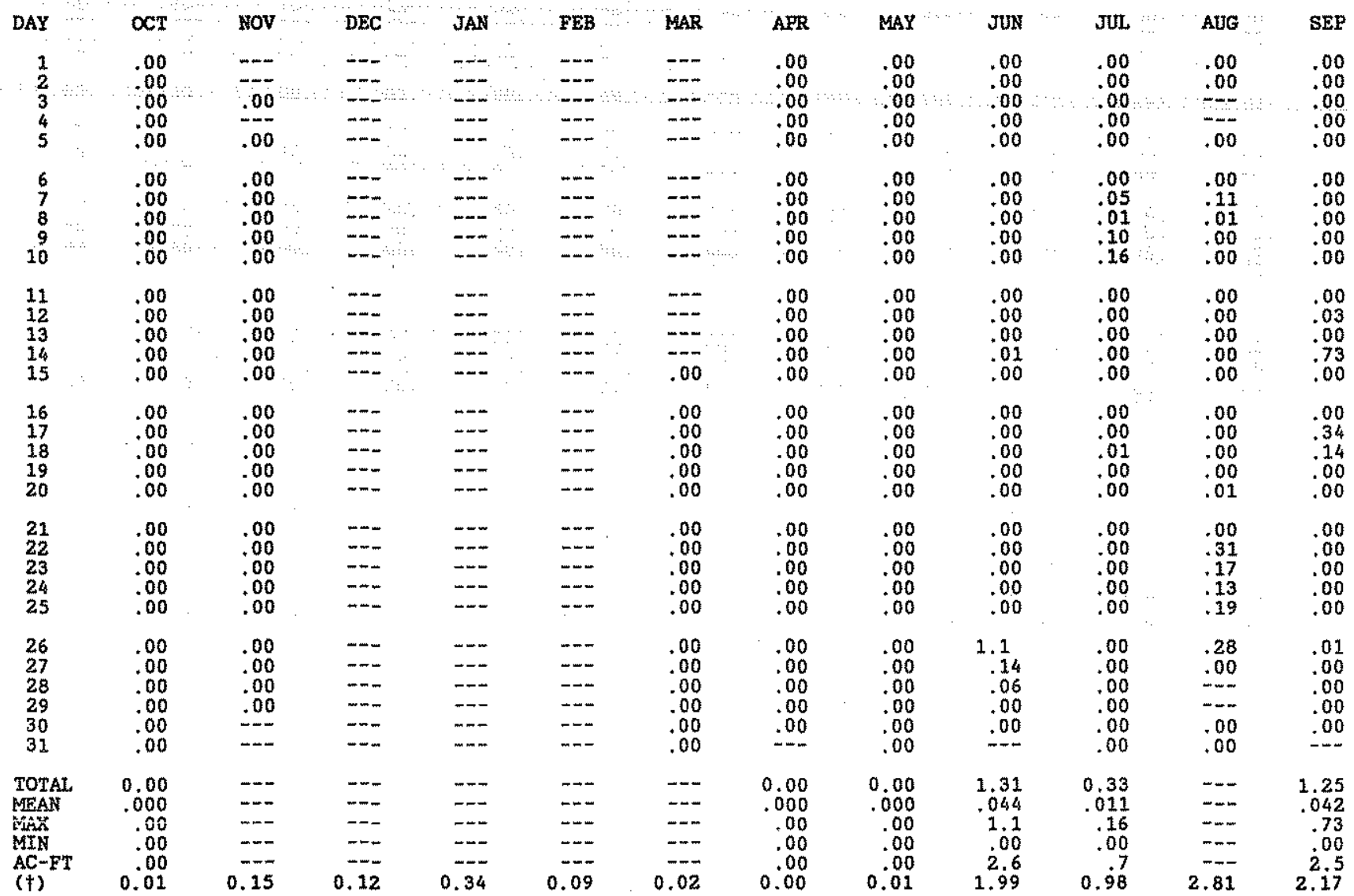

(†) Total rainfall accumulation in inches. 
RIO GRANDE BASIN

08329900 NORTE FLOODWAY CEANAEL NEAR ALAMEDA, NM

LOCATION.--Lat $35^{\circ} 11^{\prime} 58^{\prime \prime}$, Long $106^{\circ} 35^{\circ} 53^{\prime \prime}$, Bernalillo County, Hydrologic Untt 13020203, in Elena Gallegos Grant, on left bank $0.5 \mathrm{mi}$ upstream from Edith Boulevard, 1.1 mi upstream from mouth, and $1.2 \mathrm{mi}$ northeast of Alameda.

WATER-DISCHARGE RECORDS

PERIOD OF RECORD.--July 1968 to current year (no winter records in water years 1969-89).

GAGE - Water-stage recorder and concrete-1ined channel. Elevation of gage is 5,015 ft above National Geodetic Vertical Datum of 1929, from U.S. Army Corps of Engineers plan and proftle map.

REMARKS.--Records poor except for estimated daily discharges, which are poor. Some minor streamflow may exist on days where daily mean discharges have been recorded as zero due to the sensttivity limits of the streamflow monitoring equipment. Floodway channel intercepts flow of numerous arroyos in northeast Albuquerque and discharges into the R10 Grande at a point 1.6 wi north of Alaneda. No flow most of time.

DISCHARGE, CUBIC FEET PER SECOND, WATER YEAR OCTOBER 1995 TO SEPTEMBER 1996

\begin{tabular}{|c|c|c|c|c|c|c|c|c|c|c|c|c|}
\hline DAY & OCT & Nov & DEC & JAN & FEB & MAR & APR & MAY & JUN & JUL. & AUJG & SEP \\
\hline $\begin{array}{l}1 \\
2 \\
3 \\
4 \\
5\end{array}$ & $\begin{array}{l}.00 \\
.00 \\
0.00 \\
0.00 \\
0.00\end{array}$ & $17^{.00}$ & $\begin{array}{l}.00 \\
.00 \\
.00 \\
.00 \\
.00\end{array}$ & $\begin{array}{r}58 \\
.00 \\
.00 \\
.00 \\
.00\end{array}$ & $\begin{array}{l}0.00 \\
0.00 \\
0.00 \\
0.00 \\
0.00\end{array}$ & $\begin{array}{l}0.00 \\
0.00 \\
0.00 \\
0.00 \\
0.00\end{array}$ & $\begin{array}{r}.00 \\
.00 \\
.00 \\
0.00 \\
.00\end{array}$ & $\begin{array}{l}.00 \\
0.00 \\
0.00 \\
0.00 \\
0.00\end{array}$ & $\begin{array}{l}.00 \\
.00 \\
.00 \\
.00 \\
.00\end{array}$ & $\begin{array}{l}0.00 \\
0.00 \\
0.00 \\
0.00 \\
0.00\end{array}$ & $\begin{array}{r}0.00 \\
0.00 \\
015 \\
0.00 \\
0.00\end{array}$ & $\begin{array}{r}.00 \\
.00 \\
.00 \\
.00 \\
.00\end{array}$ \\
\hline $\begin{array}{r}6 \\
7 \\
8 \\
9 \\
10\end{array}$ & $\begin{array}{l}0.00 \\
0.00 \\
0.00 \\
0.00 \\
0.00\end{array}$ & $\begin{array}{l}.00 \\
.00 \\
.00 \\
.00 \\
.00\end{array}$ & $\begin{array}{l}11^{.00} \\
.00 \\
.00\end{array}$ & $\begin{array}{r}0.00 \\
.00 \\
0.00 \\
0.00 \\
0.00\end{array}$ & $\begin{array}{l}0.00 \\
0.00 \\
0.00 \\
0.00 \\
0.00\end{array}$ & $\begin{array}{l}0.00 \\
0.00 \\
0.00 \\
0.00 \\
0.00\end{array}$ & $\begin{array}{r}.00 \\
.00 \\
.00 \\
.00 \\
0.00\end{array}$ & $\begin{array}{l}0.00 \\
0.00 \\
0.00 \\
0.00 \\
0.00\end{array}$ & $\begin{array}{r}0.00 \\
.00 \\
0.00 \\
0.00 \\
0.00\end{array}$ & $\begin{array}{c}0.00 \\
018 \\
0.00 \\
040^{\circ} \\
0166\end{array}$ & $\begin{array}{r}0.00 \\
0.00 \\
0.00 \\
020^{0.00}\end{array}$ & $\begin{array}{r}e .00 \\
0.00 \\
.00 \\
.00 \\
.00\end{array}$ \\
\hline $\begin{array}{l}11 \\
12 \\
13 \\
14 \\
15\end{array}$ & $\begin{array}{l}0.00 \\
0.00 \\
0.00 \\
0.00 \\
0.00\end{array}$ & $\begin{array}{l}.00 \\
.00 \\
.00 \\
.00 \\
.00\end{array}$ & $\begin{array}{l}.00 \\
.00 \\
.00 \\
.00 \\
.00\end{array}$ & $\begin{array}{l}0.00 \\
0.00 \\
0.00 \\
0.00 \\
0.00\end{array}$ & $\begin{array}{r}.00 \\
.00 \\
.00 \\
.00 \\
.00\end{array}$ & $\begin{array}{l}0.00 \\
0.00 \\
0.00 \\
0.00 \\
0.00\end{array}$ & $\begin{array}{r}0.00 \\
.00 \\
.00 \\
.00 \\
.00\end{array}$ & $\begin{array}{l}0.00 \\
e .00 \\
0.00 \\
0.00 \\
0.00\end{array}$ & $\begin{array}{r}0.00 \\
0.00 \\
0.00 \\
040 \\
0.00\end{array}$ & $\begin{array}{r}e 30 \\
\theta .00 \\
0.00 \\
\theta .00 \\
010\end{array}$ & $\begin{array}{r}46 \\
.00 \\
.00 \\
.00 \\
.00\end{array}$ & $\begin{array}{r}0.00 \\
0.00 \\
\theta .00 \\
0300 \\
020\end{array}$ \\
\hline $\begin{array}{l}16 \\
17 \\
18 \\
19 \\
20\end{array}$ & $\begin{array}{l}0.00 \\
0.00 \\
0.00 \\
0.00 \\
0.00\end{array}$ & $\begin{array}{l}.00 \\
.00 \\
.00 \\
.00 \\
.00\end{array}$ & $\begin{array}{l}.00 \\
.00 \\
.00 \\
.00 \\
.00\end{array}$ & $\begin{array}{l}0.00 \\
0.00 \\
0.00 \\
0.00 \\
0.00\end{array}$ & $\begin{array}{l}.00 \\
.00 \\
.00 \\
0.00 \\
0.00\end{array}$ & $\begin{array}{l}0.00 \\
0.00 \\
0.00 \\
0.00 \\
0.00\end{array}$ & $\begin{array}{r}.00 \\
.00 \\
.00 \\
0.00 \\
.00\end{array}$ & $\begin{array}{l}0.00 \\
0.00 \\
0.00 \\
0.00 \\
0.00\end{array}$ & $\begin{array}{l}e .00 \\
0.00 \\
e .00 \\
e .00 \\
0.00\end{array}$ & $\begin{array}{l}020 \\
040 \\
\ominus 30 \\
\odot .00 \\
\theta .00\end{array}$ & $\begin{array}{r}0.00 \\
0.00 \\
1.5 \\
0.00 \\
050\end{array}$ & $\begin{array}{r}0.00 \\
0213 \\
0.00 \\
0.00 \\
0.00\end{array}$ \\
\hline $\begin{array}{l}21 \\
22 \\
23 \\
24 \\
25\end{array}$ & $\begin{array}{l}0.00 \\
0.00 \\
0.00 \\
0.00 \\
0.00\end{array}$ & $\begin{array}{l}0.00 \\
0.00 \\
0.00 \\
0.00 \\
0.00\end{array}$ & $\begin{array}{l}.00 \\
0.00 \\
0.00 \\
0.00 \\
0.00\end{array}$ & $\begin{array}{l}0.00 \\
0.00 \\
0.00 \\
0.00 \\
0.00\end{array}$ & $\begin{array}{r}82 \\
.00 \\
.00 \\
.00 \\
.00\end{array}$ & $\begin{array}{l}0.00 \\
0.00 \\
0.00 \\
0.00 \\
0.00\end{array}$ & $\begin{array}{l}.00 \\
.00 \\
.00 \\
.00 \\
.00\end{array}$ & $\begin{array}{l}0.00 \\
0.00 \\
0.00 \\
0.00 \\
0.00\end{array}$ & $\begin{array}{l}0.00 \\
0.00 \\
0.00 \\
0.00 \\
0.00\end{array}$ & $\begin{array}{l}0.00 \\
0.00 \\
0.00 \\
0.00 \\
0.00\end{array}$ & $\begin{array}{r}25 \\
91 \\
\text { e120 } \\
6.5 \\
.00\end{array}$ & $\begin{array}{l}0.00 \\
0.00 \\
0.00 \\
0.00 \\
0.00\end{array}$ \\
\hline $\begin{array}{l}26 \\
27 \\
28 \\
29 \\
30 \\
31\end{array}$ & $\begin{array}{l}0.00 \\
0.00 \\
0.00 \\
0.00 \\
0.00 \\
.00\end{array}$ & $\begin{array}{l}0.00 \\
0.00 \\
0.00 \\
0.00 \\
0.00 \\
-0\end{array}$ & $\begin{array}{l}0.00 \\
0.00 \\
0.00 \\
0.00 \\
.00 \\
.00\end{array}$ & $\begin{array}{l}0.00 \\
0.00 \\
0.00 \\
0.00 \\
0.00 \\
0.00\end{array}$ & $\begin{array}{r}.00 \\
0.00 \\
0.00 \\
0.00 \\
-\ldots \\
-.+\end{array}$ & $\begin{array}{r}0.00 \\
.00 \\
.00 \\
0.00 \\
e .00 \\
.00\end{array}$ & $\begin{array}{l}.00 \\
.00 \\
.00 \\
0.00 \\
0.00 \\
.\end{array}$ & $\begin{array}{l}0.00 \\
0.00 \\
0.00 \\
0.00 \\
.00 \\
.00\end{array}$ & $\begin{array}{r}e 350 \\
186 \\
222 \\
920 \\
910\end{array}$ & $\begin{array}{l}0.00 \\
e .00 \\
\theta .00 \\
\theta .00 \\
\theta .00 \\
0.00\end{array}$ & $\begin{array}{r}0700 \\
105 \\
0220.00 \\
6.00 \\
.00\end{array}$ & $\begin{array}{r}020 \\
0.00 \\
0.00 \\
0.00 \\
0.00 \\
-\end{array}$ \\
\hline $\begin{array}{l}\text { TOTAL } \\
\text { MEAN } \\
\text { MAX } \\
\text { MIN } \\
\text { AC-FT }\end{array}$ & $\begin{array}{l}0.00 \\
.000 \\
.00 \\
.00 \\
.00\end{array}$ & $\begin{array}{r}17.00 \\
.57 \\
17 \\
.00 \\
34\end{array}$ & $\begin{array}{r}22.00 \\
.71 \\
11 \\
.00 \\
44\end{array}$ & $\begin{array}{r}58.00 \\
1.87 \\
58 \\
.00 \\
115\end{array}$ & $\begin{array}{r}82.00 \\
2.83 \\
82 \\
.00 \\
163\end{array}$ & $\begin{array}{l}0.00 \\
.000 \\
.00 \\
.00 \\
.00\end{array}$ & $\begin{array}{r}0.00 \\
.000 \\
.00 \\
.00 \\
.00\end{array}$ & $\begin{array}{r}0.00 \\
.000 \\
.00 \\
.00 \\
.00\end{array}$ & $\begin{array}{r}828.00 \\
27.6 \\
350 \\
.00 \\
1640\end{array}$ & $\begin{array}{r}354.00 \\
11.4 \\
166 \\
.00 \\
702\end{array}$ & $\begin{array}{r}1513.50 \\
48.8 \\
700 \\
.00 \\
3000\end{array}$ & $\begin{array}{r}553.00 \\
18.4 \\
300 \\
100 \\
1100\end{array}$ \\
\hline
\end{tabular}

STATISTICS OF MONTHLY MEAN DATA FOR WATER YEARS 1968 - 1996, BY WATER YEAR (WY)

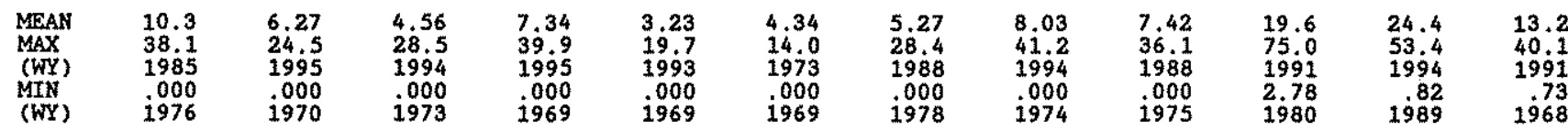

SUMARY STATISTICS

ANNUAL TOTAI

ANNUAL MEAN

EIGHEST ANNUAL MEAN

LOWEST ANNUAL MEAN

HIGHEST DAILY MEA

LOWEST DATLY MFAN

ANNUJAL SEVEN-DAY MINIMUM

TNSTANTANEOUS PEAK FLOH

INSTANTANEOUS PEAK STAGE

ANNUAL RUNOFF (AC-FT)

10 PERCENT EXCEEDS

50 PBRCENT EXCEEDS

FOR 1995 CALENDAR YEAR

3380.04
9.26

FOR 1996 WATER YEAR

3427.50
9.36

800 Jan 6

6700

12.00

.00
$700 \quad$ Aug 26

.00 Oct 1

$7700^{\circ}$ Aug 26

6800

1.9

.00
HATER YEARS $1968-1996$

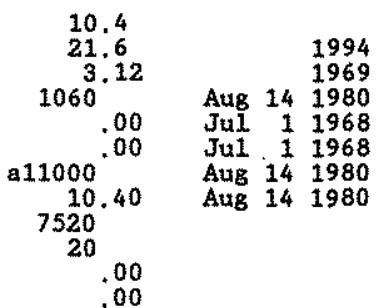

Estimated

a-From rating curve extended above $2,900 \mathrm{ft}^{3} / \mathrm{s}$. 
PERIOD OF RECORD. - Water year8 1982-83, 1991 to current year.

RDMARXS --Selected compogite gamples were collected with an automatic parastaltic pump sampler that was activated whenever the Ilow stage exceeded 1.5 foet. Samples were punped into a refrigerated chamber manualiy retrieved within 12 hours, and expaditiously processed for delivery to the analytical laboratories. An automatic waterquality minimonitor recorder was usod to obtain maximum, minimum, and mean dally values of water temperature and specific conductance of flow in the channel

WATER QUALITY DATA; WATER YEAR OCTOBER 1995 TO SEPTEMBER 1996

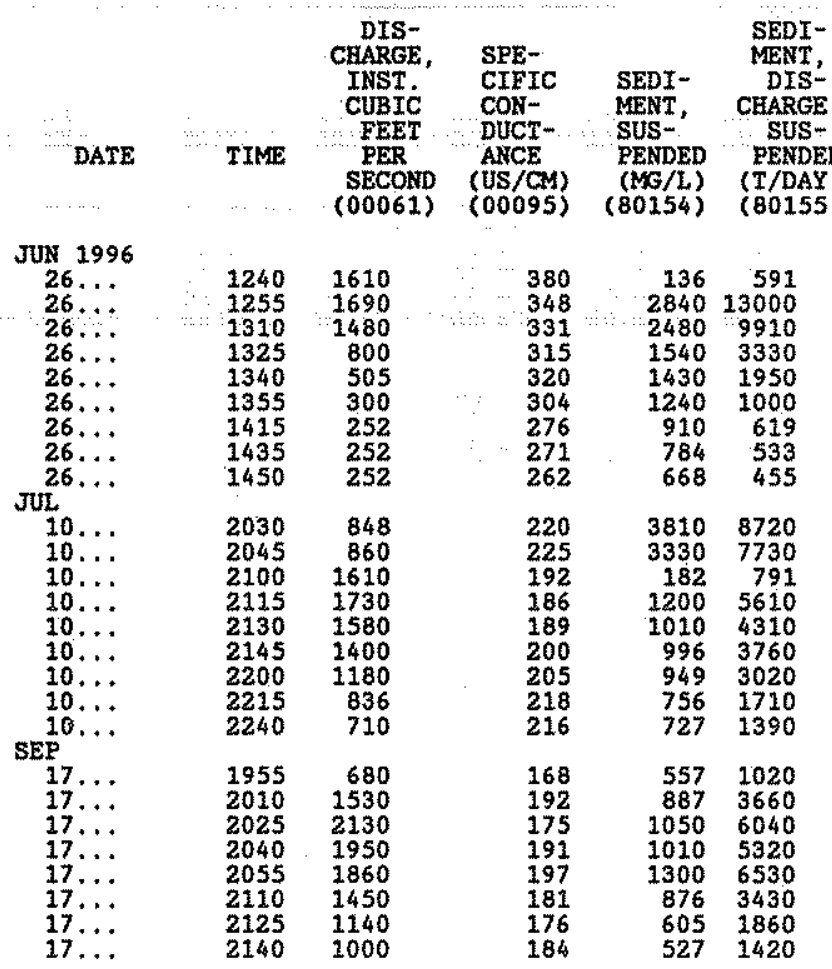


RIO GRANDE BASIN

08329900 NORTH FLOODWAY CHANNEL MEAR ALAMEDA, NM -- ContInUed

WATER-QUALITY RECORDS

SPECIFIC CONDUCTANCE (MICROSIDAENS/CM AT 25 DEG. C), WATER YEAR OCTOBER 1993 TO SEPTEMBER 1994 FROM AUTOMATYC WATER-QUALITY MINIMOHITOR RECORDER

\begin{tabular}{|c|c|c|c|c|c|c|c|c|c|c|c|c|}
\hline DAY & \multicolumn{3}{|c|}{ OCTOBER } & \multicolumn{3}{|c|}{ MARCH } & \multicolumn{3}{|c|}{ APRIL } & \multicolumn{2}{|r|}{ MAY } & MEAN \\
\hline $\begin{array}{l}2 \\
2 \\
3 \\
4 \\
5\end{array}$ & $\begin{array}{l}202 \\
221 \\
228 \\
551 \\
554\end{array}$ & $\begin{array}{l}183 \\
201 \\
213 \\
218 \\
454\end{array}$ & $\begin{array}{l}195 \\
211 \\
220 \\
318 \\
483\end{array}$ & $\begin{array}{l}=- \\
=- \\
-- \\
--\end{array}$ & $\begin{array}{l}m-\cdots \\
-\cdots \\
-\cdots\end{array}$ & 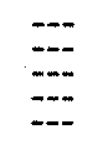 & $\begin{array}{l}--- \\
=-- \\
=-- \\
---\end{array}$ & 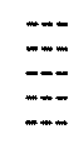 & 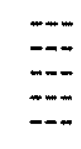 & \begin{tabular}{c}
$-\cdots$ \\
\hdashline-- \\
897 \\
$>999$
\end{tabular} & \begin{tabular}{l}
--- \\
\hdashline-- \\
572 \\
769
\end{tabular} & $\begin{array}{l}--- \\
-0 \\
699 \\
887\end{array}$ \\
\hline $\begin{array}{r}6 \\
7 \\
8 \\
9 \\
10\end{array}$ & 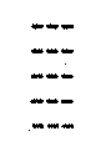 & $=$ & $\begin{array}{l}--- \\
--- \\
--\end{array}$ & $\begin{array}{l}=- \\
=- \\
=- \\
--\end{array}$ & 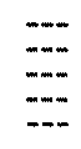 & $\begin{array}{l}-m \\
-\cdots \\
---\end{array}$ & $\begin{array}{l}--- \\
--- \\
--- \\
---\end{array}$ & 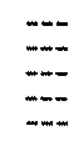 & $=$ & $\begin{array}{l}870 \\
894 \\
929 \\
958 \\
976\end{array}$ & $\begin{array}{l}702 \\
670 \\
766 \\
766 \\
610\end{array}$ & $\begin{array}{l}781 \\
776 \\
851 \\
835 \\
726\end{array}$ \\
\hline $\begin{array}{l}11 \\
12 \\
13 \\
14 \\
15\end{array}$ & $\begin{array}{l}=- \\
=-- \\
=-\end{array}$ & 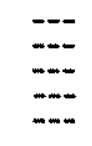 & 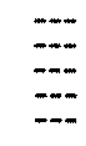 & $\begin{array}{l}=-\infty \\
=-- \\
=-\end{array}$ & $\begin{array}{l}=- \\
=- \\
--- \\
-\cdots\end{array}$ & 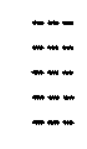 & $=$ & $\begin{array}{l}-\cdots \\
-\cdots \\
-\cdots \\
--- \\
--\end{array}$ & 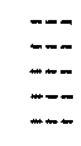 & $\begin{array}{r}759 \\
753 \\
922 \\
>999 \\
>999\end{array}$ & $\begin{array}{l}608 \\
641 \\
738 \\
858 \\
790\end{array}$ & $\begin{array}{l}690 \\
706 \\
815 \\
945 \\
928\end{array}$ \\
\hline $\begin{array}{l}16 \\
17 \\
18 \\
19 \\
20\end{array}$ & $\begin{array}{l}--- \\
--- \\
-\cdots \\
m-\infty\end{array}$ & $=$ & $\begin{array}{l}--- \\
-- \\
--- \\
---\end{array}$ & $\begin{array}{l}=- \\
=- \\
=- \\
---\end{array}$ & $=$ & $\begin{array}{l}-\cdots \\
-\cdots \\
-\cdots \\
---\end{array}$ & $\begin{array}{l}--- \\
--- \\
---\end{array}$ & $\begin{array}{l}m \\
m-\overline{-} \\
m-\end{array}$ & $\begin{array}{l}m- \\
\square- \\
-\cdots\end{array}$ & $\begin{array}{l}>999 \\
>999 \\
>999 \\
>999 \\
>999\end{array}$ & $\begin{array}{r}730 \\
882 \\
887 \\
>999 \\
>999\end{array}$ & $\begin{array}{r}854 \\
975 \\
977 \\
>999 \\
>999\end{array}$ \\
\hline $\begin{array}{l}21 \\
22 \\
23 \\
24 \\
25\end{array}$ & $\begin{array}{l}--- \\
m-\infty \\
m-\infty \\
---\end{array}$ & 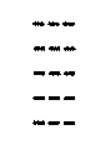 & 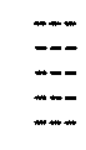 & $\begin{array}{l}=- \\
=-- \\
=-\end{array}$ & $\begin{array}{l}\cdots-- \\
=- \\
-\cdots \\
-\cdots\end{array}$ & $\begin{array}{l}m- \\
-\cdots \\
-\cdots \\
--\end{array}$ & $\begin{array}{l}=-\infty \\
=-- \\
-\square\end{array}$ & 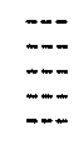 & $\begin{array}{l}--- \\
-\cdots \\
-\cdots \\
---\end{array}$ & $\begin{array}{l}>999 \\
>999 \\
>999 \\
>999 \\
>999\end{array}$ & $\begin{array}{r}>999 . \\
>999 \\
>999 \\
965 \\
858\end{array}$ & $\begin{array}{r}>999 \\
>999 \\
>999 \\
>999 \\
999\end{array}$ \\
\hline $\begin{array}{l}26 \\
27 \\
28 \\
29 \\
30 \\
31\end{array}$ & 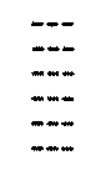 & 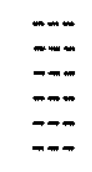 & $\begin{array}{l}=-- \\
=- \\
=- \\
--\end{array}$ & $\begin{array}{l}=- \\
=- \\
=- \\
=- \\
=-\end{array}$ & 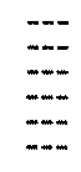 & 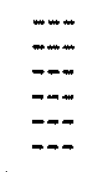 & $\begin{array}{l}=- \\
=- \\
=- \\
--\end{array}$ & $\begin{array}{l}=- \\
=- \\
=- \\
m- \\
m-\end{array}$ & $\begin{array}{c}=- \\
-\cdots \\
-\cdots \\
-\cdots\end{array}$ & $\begin{array}{r}>999 \\
726 \\
>999 \\
>999 \\
>999 \\
928\end{array}$ & $\begin{array}{l}607 \\
638 \\
719 \\
881 \\
754 \\
646\end{array}$ & $\begin{array}{l}826 \\
687 \\
798 \\
960 \\
907 \\
806\end{array}$ \\
\hline \multirow[t]{2}{*}{$\begin{array}{l}\text { NONTH } \\
\text { DAY }\end{array}$} & $\begin{array}{l}554 \\
\text { MAX }\end{array}$ & $\begin{array}{l}183 \\
\mathrm{MIN}\end{array}$ & $\begin{array}{r}285 \\
\text { MEAN }\end{array}$ & $\overline{\operatorname{MAX}}$ & $\overrightarrow{\text { MIN }}$ & MEAN & MAX & $\overrightarrow{M I N}$ & MEAN & $\begin{array}{r}>999 \\
\operatorname{MAX}\end{array}$ & $\begin{array}{l}572 \\
\text { MIN }\end{array}$ & $\begin{array}{r}872 \\
\text { MEAN }\end{array}$ \\
\hline & \multicolumn{3}{|c|}{ JUNE } & \multicolumn{3}{|c|}{ JULY } & \multicolumn{3}{|c|}{ AUGUST } & \multicolumn{3}{|c|}{ SEPTEMBER } \\
\hline $\begin{array}{l}1 \\
2 \\
3 \\
4 \\
5\end{array}$ & $\begin{array}{r}783 \\
>999 \\
903 \\
840 \\
830\end{array}$ & $\begin{array}{l}661 \\
764 \\
692 \\
644 \\
588\end{array}$ & $\begin{array}{l}720 \\
953 \\
823 \\
745 \\
725\end{array}$ & $\begin{array}{l}266 \\
368 \\
367 \\
510 \\
831\end{array}$ & $\begin{array}{l}131 \\
129 \\
129 \\
367 \\
477\end{array}$ & $\begin{array}{l}194 \\
31.2 \\
239 \\
461 \\
625\end{array}$ & $\begin{array}{r}>999 \\
>999 \\
>999 \\
531 \\
550\end{array}$ & $\begin{array}{r}895 \\
>999 \\
531 \\
449 \\
449\end{array}$ & $\begin{array}{r}962 \\
>999 \\
955 \\
475 \\
499\end{array}$ & $\begin{array}{l}234 \\
262 \\
303 \\
335 \\
361\end{array}$ & $\begin{array}{l}201 \\
231 \\
260 \\
302 \\
333\end{array}$ & $\begin{array}{l}218 \\
248 \\
285 \\
321 \\
347\end{array}$ \\
\hline $\begin{array}{r}6 \\
7 \\
8 \\
9 \\
10\end{array}$ & $\begin{array}{r}772 \\
>999 \\
>999 \\
>999 \\
>999\end{array}$ & $\begin{array}{r}636 \\
735 \\
>999 \\
>999 \\
>999\end{array}$ & $\begin{array}{r}702 \\
947 \\
>999 \\
>999 \\
>999\end{array}$ & $\begin{array}{r}>999 \\
>999 \\
246 \\
313 \\
278\end{array}$ & $\begin{array}{r}778 \\
132 \\
119 \\
88 \\
72\end{array}$ & $\begin{array}{l}875 \\
890 \\
207 \\
168 \\
171\end{array}$ & $\begin{array}{l}607 \\
628 \\
314 \\
313 \\
424\end{array}$ & $\begin{array}{l}526 \\
314 \\
282 \\
291 \\
303\end{array}$ & $\begin{array}{l}565 \\
553 \\
292 \\
304 \\
363\end{array}$ & $\begin{array}{l}391 \\
212 \\
230 \\
245 \\
268\end{array}$ & $\begin{array}{l}188 \\
187 \\
210 \\
226 \\
242\end{array}$ & $\begin{array}{l}373 \\
203 \\
220 \\
235 \\
255\end{array}$ \\
\hline $\begin{array}{l}11 \\
12 \\
13 \\
14 \\
15\end{array}$ & $\begin{array}{r}>999 \\
>999 \\
>999 \\
>999 \\
518\end{array}$ & $\begin{array}{r}>999 \\
>999 \\
>999 \\
325 \\
335\end{array}$ & $\begin{array}{r}>999 \\
>999 \\
>999 \\
627 \\
408\end{array}$ & $\begin{array}{l}172 \\
271 \\
371 \\
423 \\
455\end{array}$ & $\begin{array}{r}80 \\
172 \\
271 \\
325 \\
158\end{array}$ & $\begin{array}{l}134 \\
220 \\
332 \\
387 \\
370\end{array}$ & $\begin{array}{r}495 \\
647 \\
626 \\
981 \\
>999\end{array}$ & $\begin{array}{l}419 \\
442 \\
555 \\
535 \\
915\end{array}$ & $\begin{array}{l}460 \\
542 \\
584 \\
704 \\
983\end{array}$ & $\begin{array}{l}301 \\
330 \\
329 \\
272 \\
107\end{array}$ & $\begin{array}{r}264 \\
273 \\
271 \\
78 \\
70\end{array}$ & $\begin{array}{r}285 \\
299 \\
290 \\
140 \\
90\end{array}$ \\
\hline $\begin{array}{l}16 \\
17 \\
18 \\
19 \\
20\end{array}$ & $\begin{array}{l}665 \\
614 \\
688 \\
851 \\
938\end{array}$ & $\begin{array}{l}456 \\
559 \\
608 \\
688 \\
763\end{array}$ & $\begin{array}{l}543 \\
590 \\
651 \\
738 \\
809\end{array}$ & $\begin{array}{l}274 \\
389 \\
331 \\
610 \\
701\end{array}$ & $\begin{array}{l}142 \\
102 \\
113 \\
237 \\
481\end{array}$ & $\begin{array}{l}218 \\
201 \\
202 \\
422 \\
629\end{array}$ & $\begin{array}{l}>999 \\
>999 \\
>999 \\
>999 \\
>999\end{array}$ & $\begin{array}{r}485 \\
610 \\
>999 \\
>999 \\
>999\end{array}$ & $\begin{array}{r}933 \\
867 \\
>999 \\
>999 \\
>999\end{array}$ & $\begin{array}{r}131 \\
173 \\
97 \\
130 \\
178\end{array}$ & $\begin{array}{r}103 \\
52 \\
56 \\
62 \\
126\end{array}$ & $\begin{array}{r}115 \\
134 \\
74 \\
103 \\
151\end{array}$ \\
\hline $\begin{array}{l}21 \\
22 \\
23 \\
24 \\
25\end{array}$ & $\begin{array}{l}>999 \\
>999 \\
>999 \\
>999 \\
>999\end{array}$ & $\begin{array}{r}811 \\
>999 \\
872 \\
848 \\
999\end{array}$ & $\begin{array}{r}952 \\
>999 \\
954 \\
951 \\
>999\end{array}$ & $\begin{array}{l}>999 \\
>999 \\
>933 \\
>999 \\
>999\end{array}$ & $\begin{array}{l}681 \\
833 \\
720 \\
761 \\
906\end{array}$ & $\begin{array}{l}895 \\
948 \\
865 \\
938 \\
982\end{array}$ & $\begin{array}{r}>999 \\
894 \\
174 \\
172 \\
211\end{array}$ & $\begin{array}{r}845 \\
57 \\
67 \\
88 \\
73\end{array}$ & $\begin{array}{l}912 \\
256 \\
101 \\
131 \\
159\end{array}$ & $\begin{array}{l}195 \\
229 \\
255 \\
273 \\
287\end{array}$ & $\begin{array}{l}175 \\
189 \\
226 \\
254 \\
263\end{array}$ & $\begin{array}{l}184 \\
212 \\
241 \\
264 \\
277\end{array}$ \\
\hline $\begin{array}{l}26 \\
27 \\
28 \\
29 \\
30 \\
31\end{array}$ & $\begin{array}{r}>999 \\
152 \\
149 \\
202 \\
226 \\
---\end{array}$ & $\begin{array}{r}68 \\
72 \\
64 \\
106 \\
85 \\
--\end{array}$ & $\begin{array}{l}570 \\
109 \\
111 \\
155 \\
169 \\
-\end{array}$ & $\begin{array}{r}>999 \\
>999 \\
>999 \\
981 \\
746 \\
964\end{array}$ & $\begin{array}{l}971 \\
970 \\
883 \\
252 \\
433 \\
739\end{array}$ & $\begin{array}{l}996 \\
991 \\
978 \\
719 \\
659 \\
860\end{array}$ & $\begin{array}{l}150 \\
338 \\
664 \\
657 \\
156 \\
201\end{array}$ & $\begin{array}{r}53 \\
141 \\
315 \\
59 \\
89 \\
156\end{array}$ & $\begin{array}{r}76 \\
228 \\
486 \\
128 \\
133 \\
179\end{array}$ & $\begin{array}{l}277 \\
235 \\
203 \\
202 \\
732 \\
-\end{array}$ & $\begin{array}{l}166 \\
171 \\
179 \\
191 \\
194 \\
-\cdots\end{array}$ & $\begin{array}{l}193 \\
190 \\
187 \\
195 \\
339\end{array}$ \\
\hline $\begin{array}{l}\text { MONTH } \\
\text { YEAR }\end{array}$ & $\begin{array}{l}>999 \\
<999\end{array}$ & $\begin{array}{l}64 \\
52\end{array}$ & $\begin{array}{l}731 \\
570\end{array}$ & $>999$ & 72 & 551 & $>999$ & 53 & 543 & 732 & 52 & 222 \\
\hline
\end{tabular}


RIO GRANDE BASIN

08329900 NORTE FLOODWAY CHANLEL NEAR ALAMEDA, MM - - Continued

WATER-QUALITY RECORDS

TEMPERATURE, WATER (DEG. C) WATER YEAR OCTOBER 1993 TO SEPTEMBER 1994 EROA AUTONATIC WATER-QUALITY MINIMONITOR RECORDER

\begin{tabular}{|c|c|c|c|c|c|c|c|c|c|c|c|c|}
\hline DAY & $\mathrm{MAX}$ & MIN & MAAN & MAX & MIN & MEAN & $\operatorname{Max}$ & MIN & MEAN & MAX & MIN & MEAN \\
\hline & & OCTOBER & & & MARCH & & \multicolumn{3}{|c|}{ APRIL } & \multicolumn{3}{|c|}{ MAY } \\
\hline $\begin{array}{l}1 \\
2 \\
3 \\
4 \\
5\end{array}$ & $\begin{array}{l}23.8 \\
25.0 \\
23.1 \\
19.9 \\
16.5\end{array}$ & $\begin{array}{r}11.2 \\
11.3 \\
10.2 \\
6.5 \\
4.4\end{array}$ & $\begin{array}{l}16.8 \\
16.9 \\
15.9 \\
13.0 \\
12.1\end{array}$ & - & $\begin{array}{l}m \\
m-\infty \\
m-\infty \\
m- \\
m-\infty\end{array}$ & $\begin{array}{l}-- \\
--\infty \\
--\end{array}$ & $\underset{-\cdots}{-\cdots}$ & 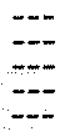 & $=-$ & 35.0 & 8.4 & 17.4 \\
\hline $\begin{array}{r}6 \\
7 \\
8 \\
9 \\
10\end{array}$ & - & $\begin{array}{l}--- \\
=-\infty \\
-m \\
-m-\infty \\
-\infty-\infty\end{array}$ & $\begin{array}{l}-+\cdots \\
-\infty \\
--- \\
--\infty\end{array}$ & 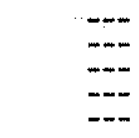 & $\begin{array}{l}--- \\
--\infty \\
--\infty \\
-m\end{array}$ & $m$ & 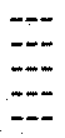 & 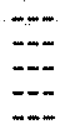 & $\begin{array}{l}-- \\
--- \\
-\infty\end{array}$ & $\begin{array}{l}30.8 \\
27.8 \\
28.1 \\
29.2 \\
35.0\end{array}$ & $\begin{array}{l}7.0 \\
8.2 \\
6.0 \\
9.8 \\
6.8\end{array}$ & $\begin{array}{l}16.9 \\
15.9 \\
16.7 \\
18.0 \\
18.5\end{array}$ \\
\hline $\begin{array}{l}11 \\
12 \\
13 \\
14 \\
15\end{array}$ & $\begin{array}{l}-\infty \\
-\infty \\
-\infty \\
-\infty\end{array}$ & $\begin{array}{l}-\infty \\
m-\infty \\
m-\infty \\
-\infty-\infty\end{array}$ & - & 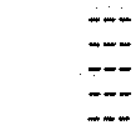 & $\begin{array}{l}-- \\
-m \\
m- \\
--m \\
--\end{array}$ & $\begin{array}{l}-\cdots \\
-\cdots \\
--\infty \\
-\infty\end{array}$ & $\begin{array}{l}-m \\
=- \\
--\end{array}$ & $=$ & $=$ & $\begin{array}{l}27.5 \\
32.5 \\
26.1 \\
27.5 \\
29.4\end{array}$ & $\begin{array}{l}6.8 \\
5.3 \\
7.2 \\
7.5 \\
6.8\end{array}$ & $\begin{array}{l}16.2 \\
17.8 \\
16.1 \\
17.1 \\
18.9\end{array}$ \\
\hline $\begin{array}{l}16 \\
17 \\
18 \\
19 \\
20\end{array}$ & $-\cdots$ & $=$ & $\begin{array}{c}- \\
- \\
- \\
- \\
- \\
-\end{array}$ & $\begin{array}{l}- \\
-- \\
-- \\
-- \\
--\end{array}$ & $\begin{array}{l}-- \\
--m \\
--m \\
-m-\end{array}$ & $\begin{array}{c}- \\
- \\
- \\
-\end{array}$ & $\underset{-m}{-m}$ & $\begin{array}{c}-\cdots \\
-\cdots \\
-\cdots \\
--\end{array}$ & - & $\begin{array}{r}34.9 \\
29.4 \\
-28.8 \\
29.7\end{array}$ & $\begin{array}{r}13.0 \\
7.9 \\
13.3 \\
8.6\end{array}$ & $\begin{array}{r}22.1 \\
18.7 \\
-22.2 \\
17.7\end{array}$ \\
\hline $\begin{array}{l}21 \\
22 \\
23 \\
24 \\
25\end{array}$ & $\overline{-}$ & $\begin{array}{l}-m \\
--- \\
--- \\
--\infty \\
--\end{array}$ & $-\overline{-}$ & 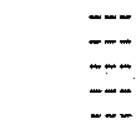 & $m$ & $\begin{array}{c}--- \\
-- \\
-- \\
--\end{array}$ & $=$ & 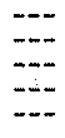 & $=-$ & $\begin{array}{r}29.0 \\
31.5 \\
30.0 \\
- \\
\end{array}$ & $\begin{array}{r}10.0 \\
12.5 \\
6.3 \\
\ldots . .-\end{array}$ & $\begin{array}{r}17.4 \\
19.7 \\
19.2 \\
- \\
-\end{array}$ \\
\hline $\begin{array}{l}26 \\
27 \\
28 \\
29 \\
30 \\
31\end{array}$ & 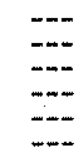 & $m$ & 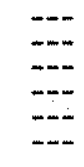 & $=-$ & $=-$ & 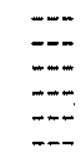 & $\begin{array}{l}m-m \\
m- \\
-m \\
-m \\
-\cdots\end{array}$ & 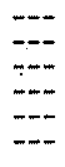 & $\begin{array}{l}m- \\
m- \\
-\cdots \\
- \\
-\end{array}$ & $\begin{array}{r}29.6 \\
29.0 \\
30.0 \\
27.0\end{array}$ & $\begin{array}{r}--- \\
13.7 \\
9.8 \\
11.2 \\
10.3\end{array}$ & $\begin{array}{r}19.1 \\
18.6 \\
18.8 \\
18.5\end{array}$ \\
\hline \multirow[t]{2}{*}{$\begin{array}{l}\text { MONTH } \\
\text { DAY }\end{array}$} & $\begin{array}{r}25.0 \\
\text { MAX }\end{array}$ & $\begin{array}{l}4.4 \\
\text { MIN }\end{array}$ & MEA 9 & $\overrightarrow{M A X}$ & MIN & MEAN & MAX & $\overrightarrow{\text { MIN }}$ & MEAN & $\begin{array}{r}35.0 \\
\text { MAX }\end{array}$ & $\begin{array}{r}5.3 \\
\text { MIN }\end{array}$ & $\begin{array}{l}18.2 \\
\text { MEAN }\end{array}$ \\
\hline & \multicolumn{3}{|c|}{ JUNE } & \multicolumn{3}{|c|}{ JULY } & \multicolumn{3}{|c|}{ AUGUST } & \multicolumn{3}{|c|}{ SEPTEMBER } \\
\hline $\begin{array}{l}1 \\
2 \\
3 \\
4 \\
5\end{array}$ & $\begin{array}{l}30.0 \\
29.1 \\
33.6 \\
34.0 \\
33.3\end{array}$ & $\begin{array}{l}11.8 \\
11.4 \\
10.4 \\
10.4 \\
15.7\end{array}$ & $\begin{array}{l}19.7 \\
20.1 \\
20.7 \\
23.0 \\
23.2\end{array}$ & $\begin{array}{r}31.7 \\
29.3 \\
--- \\
-30.1\end{array}$ & $\begin{array}{r}23.1 \\
22.1 \\
20.4\end{array}$ & $\begin{array}{r}29.6 \\
27.8 \\
--- \\
28.5\end{array}$ & 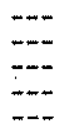 & \begin{tabular}{l}
$\cdots$ \\
$\cdots$ \\
\hdashline- \\
$-\cdots$
\end{tabular} & $\begin{array}{l}m-m \\
m-m \\
m-m \\
-m\end{array}$ & $\overline{-}$ & $\begin{array}{l}=- \\
m-m \\
m-\infty \\
-m\end{array}$ & 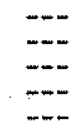 \\
\hline $\begin{array}{r}6 \\
7 \\
8 \\
9 \\
10\end{array}$ & $\begin{array}{l}30.0 \\
28.7 \\
30.0 \\
28.1 \\
28.1\end{array}$ & $\begin{array}{l}12.1 \\
11.0 \\
13.7 \\
13.0 \\
11.4\end{array}$ & $\begin{array}{l}21.1 \\
21.9 \\
21.2 \\
22.4 \\
21.5\end{array}$ & $\begin{array}{r}38.4 \\
36.9 \\
30.4 \\
32.2 \\
\ldots .\end{array}$ & $\begin{array}{r}17.8 \\
18.7 \\
14.1 \\
20.2 \\
-\end{array}$ & $\begin{array}{r}24.4 \\
26.8 \\
25.3 \\
25.4 \\
-. .\end{array}$ & 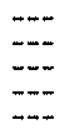 & $=$ & $\begin{array}{l}m-m \\
-m \\
-m \\
-m\end{array}$ & $\begin{array}{l}- \\
- \\
-- \\
- \\
-\end{array}$ & $\begin{array}{l}m-m \\
=- \\
-m \\
m-\end{array}$ & 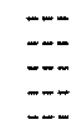 \\
\hline $\begin{array}{l}11 \\
12 \\
13 \\
14 \\
15\end{array}$ & $\begin{array}{r}27.8 \\
-2 \\
26.8 \\
32.5\end{array}$ & $\begin{array}{r}14.1 \\
-15.0 \\
12.5\end{array}$ & $\begin{array}{r}23.2 \\
-2 \\
20.9 \\
22.2\end{array}$ & 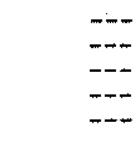 & 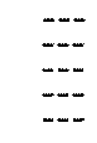 & 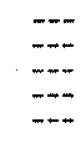 & $\begin{array}{l}-- \\
-- \\
--- \\
--\end{array}$ & $\begin{array}{l}m-m \\
m-m \\
m- \\
m-\end{array}$ & $\begin{array}{l}-- \\
- \\
- \\
- \\
- \\
-\end{array}$ & $\begin{array}{r}-5 \\
20.9 \\
19.9 \\
19.9 \\
24.0\end{array}$ & $\begin{array}{r}14.8 \\
12.0 \\
12.0 \\
14.2\end{array}$ & $\begin{array}{r}--- \\
16.2 \\
17.0 \\
16.9 \\
17.5\end{array}$ \\
\hline $\begin{array}{l}16 \\
17 \\
18 \\
19 \\
20\end{array}$ & $\begin{array}{r}35.0 \\
- \\
- \\
-\end{array}$ & $\begin{array}{c}10.8 \\
-. \\
-- \\
--\end{array}$ & $\begin{array}{r}21.0 \\
- \\
- \\
-\end{array}$ & 38.5 & $\begin{array}{c}- \\
-\infty \\
-\infty \\
-\infty\end{array}$ & $\begin{array}{r}-0- \\
-0 \\
25.1\end{array}$ & $=$ & $\begin{array}{l}-m \\
-m \\
-- \\
--\end{array}$ & - & $\begin{array}{l}24.4 \\
25.0 \\
16.4 \\
22.4 \\
22.4\end{array}$ & $\begin{array}{r}10.9 \\
10.7 \\
14.0 \\
9.3 \\
10.9\end{array}$ & $\begin{array}{l}17.4 \\
18.3 \\
15.5 \\
15.5 \\
15.7\end{array}$ \\
\hline $\begin{array}{l}21 \\
22 \\
23 \\
24 \\
25\end{array}$ & 27.8 & $\begin{array}{c}-\cdots \\
18.9 \\
-\end{array}$ & 23.9 & $\begin{array}{l}32.6 \\
27.5 \\
34.7 \\
34.6 \\
32.8\end{array}$ & $\begin{array}{l}15.0 \\
20.7 \\
17.6 \\
17.0 \\
18.7\end{array}$ & $\begin{array}{l}24.9 \\
26.4 \\
26.3 \\
22.2 \\
23.8\end{array}$ & $\begin{array}{l}m- \\
m- \\
m- \\
--\end{array}$ & $\begin{array}{l}-- \\
-- \\
- \\
-\end{array}$ & $\begin{array}{l}m-m \\
m-m \\
m--\end{array}$ & $\begin{array}{l}24.3 \\
20.6 \\
23.1 \\
20.9 \\
21.3\end{array}$ & $\begin{array}{l}10.6 \\
12.0 \\
10.7 \\
14.7 \\
12.7\end{array}$ & $\begin{array}{l}16.5 \\
16.5 \\
18.4 \\
18.0 \\
16.7\end{array}$ \\
\hline $\begin{array}{l}26 \\
27 \\
28 \\
29 \\
30 \\
31\end{array}$ & $\begin{array}{r}24.4 \\
- \\
-\end{array}$ & $\begin{array}{r}19.6 \\
- \\
-\end{array}$ & $\begin{array}{r}22.7 \\
- \\
- \\
-\end{array}$ & $\begin{array}{r}32.5 \\
32.0 \\
29.7 \\
32.5 \\
- \\
-\end{array}$ & $\begin{array}{r}13.5 \\
17.7 \\
13.7 \\
15.1 \\
-\end{array}$ & $\begin{array}{r}22.4 \\
22.7 \\
23.8 \\
26.3 \\
-. . \\
-.-\end{array}$ & 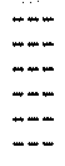 & $\begin{array}{l}=- \\
--- \\
--- \\
--m \\
--\end{array}$ & - & $\begin{array}{r}20.0 \\
18.2 \\
18.6 \\
19.8 \\
18.9 \\
-\end{array}$ & $\begin{array}{r}14.0 \\
11.6 \\
7.0 \\
7.6 \\
12.2 \\
\end{array}$ & $\begin{array}{r}16.6 \\
14.2 \\
14.3 \\
13.7 \\
16.6 \\
\end{array}$ \\
\hline $\begin{array}{l}\text { MONTE } \\
\text { YEAR }\end{array}$ & $\begin{array}{l}35.0 \\
38.5\end{array}$ & $\begin{array}{r}10.4 \\
4.4\end{array}$ & $\begin{array}{l}21.8 \\
19.9\end{array}$ & 38.5 & 13.5 & 25.4 & $m$ & $-\cdots$ & -+- & 25.0 & 7.0 & 16.4 \\
\hline
\end{tabular}


RIO GRANDE BASIN

08329914 NORTH CAMINO ARROYO TRIBUTARY AT ALBUQUERQUE, NM

LOCATION, - Lat $35^{\circ} 11^{\prime} 47^{\prime \prime}$, long $106^{\circ} 33^{\prime} 57^{\prime \prime}$, Bernalillo County, Eydrologtc Untt 13020203, in Elena Gallegos Grant, on left bank in right-of-way for extenston of Wyoming Boulevard, 150 ft south of Ventce Avenue, 15 ft north of Beverly Bills Avenue, and $1.5 \mathrm{mi}$ north of intersection of Paseo del Norte and Wyoming Boulevard in Albuquerque.

DRAINAGE AREA. $-0.06 \mathrm{mi}^{2}$.

PERIOD OF RECORD.--June 1979 to current year (seasonal records).

GAGE.-Water-stage recorder. Elevation of gage is $5,360 \mathrm{ft}$ above National Geodetic Vertical Datum of 1929 , from topographic map.

REMARKS.--Records good. Recording rain gage at station. The basin is totally undeveloped. See tabulation below for monthly precipitation in inches.

EXTREMES FOR PERIOD OF RECORD.-Maximum discharge, $134 \mathrm{ft}^{3 / s,}$ July 7,1981 , gage height, 2.10 ft, from slopemares measurement of peak flow; no flow most of time.

EXTREMES FOR CURRENI YEAR. - Maximum discharge during period of seasonal operation, 80 ft $3 / \mathrm{s}$, at 0005 hours July 18 , gage height, $1.72 \mathrm{ft}$; no flow most of time.

DISCEARGE, CUBIC FEET PER SECOND, WATER YEAR OCTOBER 1995 TO SEPTEMBER 1996

\begin{tabular}{|c|c|c|c|c|c|c|c|c|c|c|c|c|}
\hline DAY & OCT & Nov & $\mathrm{DEC}$ & JAN & FEB & MAR & APR & MAY & JUN & JUL & AUG & SEP \\
\hline $\begin{array}{l}1 \\
2 \\
3 \\
4 \\
5\end{array}$ & $\begin{array}{l}.00 \\
.00 \\
.00 \\
.00 \\
.00\end{array}$ & $\begin{array}{c}--- \\
.00 \\
.00\end{array}$ & $\begin{array}{l}-m \\
--m \\
-m- \\
-m-\end{array}$ & $\begin{array}{l}m \\
-\infty \\
-\infty \\
-\infty\end{array}$ & $\begin{array}{l}m \\
m-m \\
m-m \\
-m\end{array}$ & $\begin{array}{l}m-m \\
-m-m \\
-m-\end{array}$ & $\begin{array}{l}.00 \\
.00 \\
.00 \\
.00 \\
.00\end{array}$ & $\begin{array}{l}.00 \\
.00 \\
.00 \\
.00 \\
.00\end{array}$ & $\begin{array}{l}.00 \\
.00 \\
.00 \\
.00 \\
.00\end{array}$ & $\begin{array}{l}.00 \\
.00 \\
.00 \\
.00 \\
.00\end{array}$ & $\begin{array}{r}.00 \\
.00 \\
-00 \\
.00 \\
.00\end{array}$ & $\begin{array}{l}.00 \\
.00 \\
.00 \\
.00 \\
.00\end{array}$ \\
\hline $\begin{array}{r}6 \\
7 \\
8 \\
9 \\
10\end{array}$ & $\begin{array}{l}.00 \\
.00 \\
.00 \\
.00 \\
.00\end{array}$ & $\begin{array}{l}.00 \\
.00 \\
.00 \\
.00 \\
.00\end{array}$ & 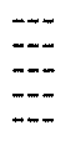 & $\begin{array}{l}m-m \\
=-m \\
-m \\
---\end{array}$ & $\begin{array}{l}m-\infty \\
=-\infty \\
-m\end{array}$ & 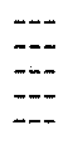 & $\begin{array}{l}.00 \\
.00 \\
.00 \\
.00 \\
.00\end{array}$ & $\begin{array}{l}.00 \\
.00 \\
.00 \\
.00 \\
.00\end{array}$ & $\begin{array}{l}.00 \\
.00 \\
.00 \\
.00 \\
.00\end{array}$ & $\begin{array}{l}.00 \\
6.2 \\
1.7 \\
6.6 \\
9.8\end{array}$ & $\begin{array}{r}.00 \\
.20 \\
2.2 \\
.00 \\
.00\end{array}$ & $\begin{array}{l}.00 \\
.00 \\
.00 \\
.00 \\
.00\end{array}$ \\
\hline $\begin{array}{l}11 \\
12 \\
13 \\
14 \\
15\end{array}$ & $\begin{array}{l}.00 \\
.00 \\
.00 \\
.00 \\
.00\end{array}$ & $\begin{array}{l}.00 \\
.00 \\
.00 \\
.00 \\
.00\end{array}$ & $\begin{array}{l}+-\infty \\
-\infty- \\
-\infty \\
-\infty\end{array}$ & $\begin{array}{l}-m \\
-m \\
-m \\
-m \\
-m\end{array}$ & $\begin{array}{l}=- \\
m- \\
m- \\
-m \\
-\end{array}$ & $\begin{array}{l}. \\
.00 \\
.00 \\
.00 \\
.00\end{array}$ & $\begin{array}{l}.00 \\
.00 \\
.00 \\
.00 \\
.00\end{array}$ & $\begin{array}{l}.00 \\
.00 \\
.00 \\
.00 \\
.00\end{array}$ & $\begin{array}{r}.00 \\
.00 \\
.00 \\
1.7 \\
.00\end{array}$ & $\begin{array}{l}.55 \\
.00 \\
.00 \\
.00 \\
.00\end{array}$ & $\begin{array}{l}.00 \\
.00 \\
.00 \\
.00 \\
.00\end{array}$ & $\begin{array}{r}.00 \\
4.30 \\
.00 \\
.00\end{array}$ \\
\hline $\begin{array}{l}16 \\
17 \\
18 \\
19 \\
20\end{array}$ & $\begin{array}{l}.00 \\
.00 \\
.00 \\
.00 \\
.00\end{array}$ & $\begin{array}{l}.00 \\
.00 \\
.00 \\
.00 \\
.00\end{array}$ & 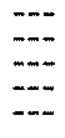 & $\begin{array}{l}-- \\
-- \\
m-m \\
-m \\
--\end{array}$ & 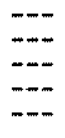 & $\begin{array}{l}.00 \\
.00 \\
.00 \\
.00 \\
.00\end{array}$ & $\begin{array}{l}.00 \\
.00 \\
.00 \\
.00 \\
.00\end{array}$ & $\begin{array}{l}.00 \\
.00 \\
.00 \\
.00 \\
.00\end{array}$ & $\begin{array}{l}.00 \\
.00 \\
.00 \\
.00 \\
.00\end{array}$ & $\begin{array}{r}.00 \\
1.9 \\
9.5 \\
.00 \\
.00\end{array}$ & $\begin{array}{r}.00 \\
.00 \\
.00 \\
.00 \\
3.8\end{array}$ & $\begin{array}{l}12^{.00} \\
28 \\
11.00 \\
.00\end{array}$ \\
\hline $\begin{array}{l}21 \\
22 \\
23 \\
24 \\
25\end{array}$ & $\begin{array}{l}.00 \\
.00 \\
.00 \\
.00 \\
.00\end{array}$ & $\begin{array}{l}.00 \\
.00 \\
.00 \\
.00 \\
.00\end{array}$ & $\begin{array}{l}=-\infty \\
=-\infty \\
=-\infty\end{array}$ & $\begin{array}{l}m-m \\
m-m \\
m-m \\
m-m \\
m-m\end{array}$ & $\begin{array}{l}m-m \\
-\cdots \\
-\cdots \\
-\cdots\end{array}$ & $\begin{array}{l}.00 \\
.00 \\
.00 \\
.00 \\
.00\end{array}$ & $\begin{array}{l}.00 \\
.00 \\
.00 \\
.00 \\
.00\end{array}$ & $\begin{array}{l}.00 \\
.00 \\
.00 \\
.00 \\
.00\end{array}$ & $\begin{array}{l}.00 \\
.00 \\
.00 \\
.00 \\
.00\end{array}$ & $\begin{array}{l}.00 \\
.00 \\
.00 \\
.00 \\
.00\end{array}$ & $\begin{array}{l}11^{.00} \\
18 \\
4.8 \\
1.8\end{array}$ & $\begin{array}{l}.00 \\
.00 \\
.00 \\
.00 \\
.00\end{array}$ \\
\hline $\begin{array}{l}26 \\
27 \\
28 \\
29 \\
30 \\
31\end{array}$ & $\begin{array}{l}.00 \\
.00 \\
.00 \\
.00 \\
.00 \\
.00\end{array}$ & $\begin{array}{l}.00 \\
.00 \\
.00 \\
.00 \\
. .\end{array}$ & $\begin{array}{l}m-\infty \\
-\cdots \\
-m \\
m- \\
m-\end{array}$ & 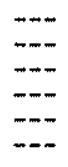 & $\begin{array}{l}m-m \\
m-m \\
m-\infty \\
m-\infty \\
m-\end{array}$ & $\begin{array}{l}.00 \\
.00 \\
.00 \\
.00 \\
.00 \\
.00\end{array}$ & $\begin{array}{l}.00 \\
.00 \\
.00 \\
.00 \\
.00 \\
-.-1\end{array}$ & $\begin{array}{l}.00 \\
.00 \\
.00 \\
.00 \\
.00 \\
.00\end{array}$ & $\begin{array}{l}16 \\
11 \\
9.7 \\
.00 \\
.00 \\
-=-\end{array}$ & $\begin{array}{r}.00 \\
.00 \\
.00 \\
1.9 \\
.00 \\
.00\end{array}$ & $\begin{array}{r}10 \\
.00 \\
-0.00 \\
.00 \\
.00\end{array}$ & $\begin{array}{l}3.7 \\
.00 \\
.00 \\
.00 \\
.00 \\
-\end{array}$ \\
\hline $\begin{array}{l}\text { TOTAL } \\
\text { MEAN } \\
\text { MAX } \\
\text { MIN } \\
\text { AC-FT } \\
(\dagger)\end{array}$ & $\begin{array}{r}0.00 \\
.000 \\
.00 \\
.00 \\
.00 \\
0.01\end{array}$ & 0 & $\begin{array}{l}=-- \\
=-- \\
--- \\
-23\end{array}$ & 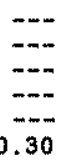 & 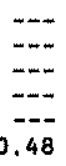 & 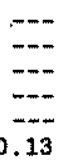 & $\begin{array}{l}0.00 \\
.000 \\
.00 \\
.00 \\
.00 \\
0.01\end{array}$ & $\begin{array}{l}0.00 \\
.000 \\
.00 \\
.00 \\
.00 \\
0.00\end{array}$ & $\begin{array}{r}38.40 \\
1.28 \\
16 \\
.00 \\
76 \\
3.31\end{array}$ & $\begin{array}{r}38.15 \\
1.23 \\
9.8 \\
.00 \\
76 \\
2.85\end{array}$ & $\begin{array}{c}m-\infty \\
m-3 \\
m-3\end{array}$ & $\begin{array}{r}89.00 \\
2.97 \\
30 \\
.00 \\
177 \\
3.77\end{array}$ \\
\hline
\end{tabular}

(†) Total rainfall accumulation in inches. 
RIO GRANDE BASIN

08329935 ARROYO 19A AT ALBUQUERQUE, NM

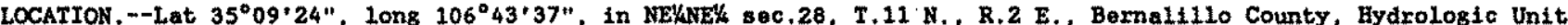
13020203 , on right bank 900 ft upstream from culvert under 81 st Street, 1,200 ft south of city water tank, and 13020203 , on right bank 900 ft upstream from culvert under 81 st Street, 1,200
0.6 mi south of intersection of 81 st Street and Atrisco Drive at Albuquerque.

DRAIKAGE AREA, $-1.50 \mathrm{mi*}$.

PERIOD OF RECORD, --June 1977 to current year (seasonal records).

GAGE.--Water-stage xecorder and Parshall flume, Elevation of gage is 5 , 330 ft ebove National Geodetic Vertical Datur of 1929, exom topographic map. Prior to June 19, 1986 at site 450 ft downstrean at different datun,

REMARKS,--Records good. Recording rain gage at station. The basin drains undeveloped semidesert terrain above the ascarpment west of Albuquerque. See tabulation below for monthly precipitation in inches. No flow nost of time.

EXIREMES FOR PERIOD OF RECORD.-MaXimum discharge, 100 fts/s, Oct. 2, 1981, gage ho1ght, 4.03 ft, 8 ite and datum then in use, Ixom slope-area measurement of peak flow; no flow most time.

EXTREMES FOR CURRENT YEAR, - No flow during wetor year.

DISCHARGE, CUBIC FEET PER SECOND, WATER YEAR OCTOBER 1995 TO SEETEMBER 1996

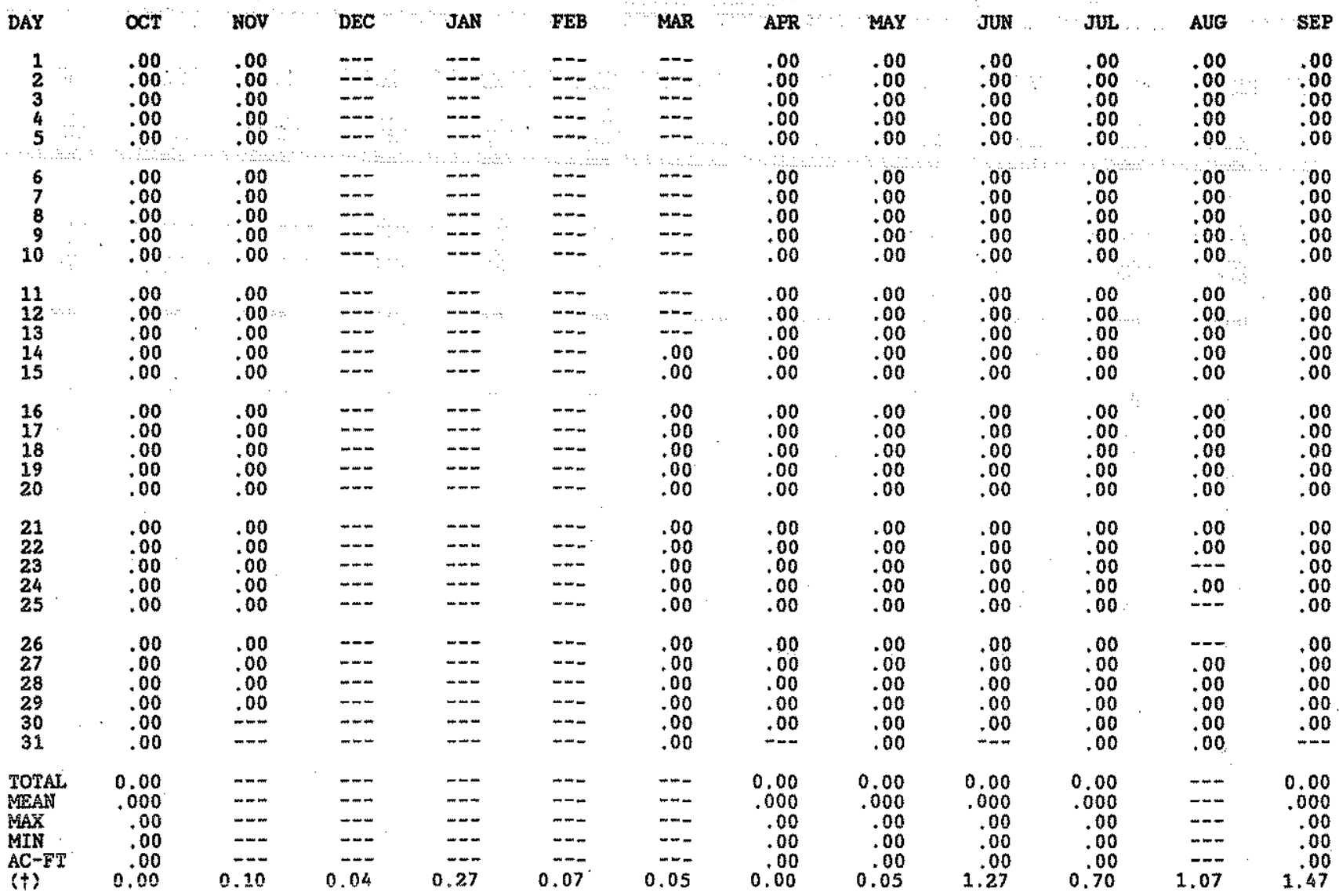

(†) Total rainfall accumulation in inches. 
RIO GRANDE BASIN

08329936 TAYLOR RANCH DRAIN AT ALBUQUERQUE, NM

LOCATION --Lat $35^{\circ} 08^{\prime} 56^{\prime \prime}$, Long $106^{\circ} 42^{\prime} 03^{\prime \prime}$, in SEKSWK sec.26, T.11 N., R.2 E., Bernalillo County, Hydrologic Unit 13020203 on loft bank of drainage outlot for Taylor Ranch subdivision, 120 ft west of intersection of Calle Nuestra and Cabri110 Circle, and $1,850 \mathrm{ft}$ southwest of intersection of Montano Road and Valle V1sta Drive in Albuquerque.

DRAINAGE AREA. $--0.132 \mathrm{mt}^{2}$.

FERIOD OF RECORD.--August 1978 to current year (seasonal records).

GAGE. --Water-stage recorder and Parshall flume. Elevation of gage is 5,120 ft above Nattonal Geodetic Vertical Datum of 1929, from topographtc map.

REMARKS. - -Records good. Recording rain gage at station. The basin is primarily urban residential. See tabulation below for monthily precipitation in inches.

EXTREMES FOR PERIOD OR RECORD.--Maximum discharge, $43 \mathrm{ft} / \mathrm{s}$, Sept, 8, 1980, gage height, 3.26 ft; no flow most most of time.

EXTREAES FOR CURRENT YEAR.--Maximum discharge during period of seasonal operation, 14 fts/s, sept. 14 , at 1555 hours gage height, $2.09 \mathrm{ft}$; no flow most of time.

DISCEARGE, CUBIC FEET PER SECOND, WATER YEAR OCTOBER 1995 TO SEPTEMBER 1996

\begin{tabular}{|c|c|c|c|c|c|c|c|c|c|c|c|c|}
\hline DAY & $\alpha C T$ & Hov & $\mathrm{DEC}$ & JAN & FEB & MAR & AFR & MAY & JUN & JUL & AUG & $\mathrm{SEP}$ \\
\hline $\begin{array}{l}1 \\
2 \\
3 \\
4 \\
5\end{array}$ & $\begin{array}{l}.00 \\
.00 \\
.00 \\
.00 \\
.00\end{array}$ & $\begin{array}{l}.00 \\
.00 \\
.00 \\
.00 \\
.00\end{array}$ & $\begin{array}{l}--- \\
--- \\
--- \\
---\end{array}$ & $\begin{array}{l}--- \\
-\cdots \\
-\cdots \\
-\cdots\end{array}$ & $=$ & $\begin{array}{l}m-- \\
-\cdots \\
-\cdots\end{array}$ & $\begin{array}{l}.00 \\
.00 \\
.00 \\
.00 \\
.00\end{array}$ & $\begin{array}{l}.00 \\
.00 \\
.00 \\
.00 \\
.00\end{array}$ & $\begin{array}{l}.00 \\
.00 \\
.00 \\
.00 \\
.00\end{array}$ & $\begin{array}{l}.00 \\
.00 \\
.00 \\
.00 \\
.00\end{array}$ & $\begin{array}{l}.00 \\
.00 \\
.01 \\
.01 \\
.00\end{array}$ & $\begin{array}{l}.00 \\
.00 \\
.00 \\
.00 \\
.00\end{array}$ \\
\hline $\begin{array}{r}6 \\
7 \\
8 \\
9 \\
10\end{array}$ & $\begin{array}{l}.00 \\
.00 \\
.00 \\
.00 \\
.00\end{array}$ & $\begin{array}{l}.00 \\
.00 \\
.00 \\
.00 \\
.00\end{array}$ & $\begin{array}{l}m-\square \\
=-- \\
=-- \\
=--\end{array}$ & $\begin{array}{l}--- \\
--- \\
--- \\
--\end{array}$ & $\begin{array}{l}--- \\
--- \\
--- \\
---\end{array}$ & $\begin{array}{l}--- \\
--- \\
-\square \\
--\end{array}$ & $\begin{array}{l}.00 \\
.00 \\
.00 \\
.00 \\
.00\end{array}$ & $\begin{array}{l}.00 \\
.00 \\
.00 \\
.00 \\
.00\end{array}$ & $\begin{array}{l}.00 \\
.00 \\
.00 \\
.00 \\
.00\end{array}$ & $\begin{array}{l}.00 \\
.06 \\
.00 \\
.09 \\
.00\end{array}$ & $\begin{array}{l}.00 \\
.29 \\
.02 \\
.00 \\
.00\end{array}$ & $\begin{array}{l}.00 \\
.00 \\
.00 \\
.00 \\
.00\end{array}$ \\
\hline $\begin{array}{l}11 \\
12 \\
13 \\
14 \\
15\end{array}$ & $\begin{array}{l}.00 \\
.00 \\
.00 \\
.00 \\
.00\end{array}$ & $\begin{array}{l}.00 \\
.00 \\
.00 \\
.00 \\
.00\end{array}$ & 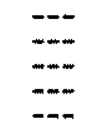 & $\begin{array}{l}=-0 \\
=-- \\
=--\end{array}$ & $\begin{array}{l}m-- \\
--- \\
---\end{array}$ & $\begin{array}{l}-\cdots \\
-\cdots \\
.00 \\
.00\end{array}$ & $\begin{array}{l}.00 \\
.00 \\
.00 \\
.00 \\
.00\end{array}$ & $\begin{array}{l}.00 \\
.00 \\
.00 \\
.00 \\
.00\end{array}$ & $\begin{array}{l}.00 \\
.00 \\
.00 \\
.00 \\
.00\end{array}$ & $\begin{array}{l}.00 \\
.00 \\
.00 \\
.00 \\
.00\end{array}$ & $\begin{array}{l}.00 \\
.00 \\
.00 \\
.00 \\
.00\end{array}$ & $\begin{array}{l}.00 \\
.00 \\
.09 \\
.45 \\
.00\end{array}$ \\
\hline $\begin{array}{l}16 \\
17 \\
18 \\
19 \\
20\end{array}$ & $\begin{array}{l}.00 \\
.00 \\
.00 \\
.00 \\
.00\end{array}$ & $\begin{array}{l}.00 \\
.00 \\
.00 \\
.01 \\
.00\end{array}$ & $\begin{array}{l}--- \\
=-- \\
--- \\
-\cdots\end{array}$ & $\begin{array}{l}=- \\
=- \\
-\cdots\end{array}$ & $\begin{array}{l}m-\cdots \\
-\cdots \\
-\cdots\end{array}$ & $\begin{array}{l}.00 \\
.00 \\
.00 \\
.00 \\
.00\end{array}$ & $\begin{array}{l}.00 \\
.00 \\
.00 \\
.00 \\
.00\end{array}$ & $\begin{array}{l}.00 \\
.00 \\
.00 \\
.00 \\
.00\end{array}$ & $\begin{array}{l}.00 \\
.00 \\
.00 \\
.00 \\
.00\end{array}$ & $\begin{array}{l}.00 \\
.16 \\
.11 \\
.00 \\
.00\end{array}$ & $\begin{array}{l}.00 \\
.00 \\
.00 \\
.00 \\
.00\end{array}$ & $\begin{array}{l}.00 \\
.17 \\
.09 \\
.00 \\
.00\end{array}$ \\
\hline $\begin{array}{l}21 \\
22 \\
23 \\
24 \\
25\end{array}$ & $\begin{array}{l}.00 \\
.00 \\
.00 \\
.00 \\
.00\end{array}$ & $\begin{array}{l}.00 \\
.00 \\
.00 \\
.00 \\
.00\end{array}$ & $\begin{array}{l}m-- \\
=-- \\
-\because- \\
---\end{array}$ & $\begin{array}{l}=-- \\
--- \\
--- \\
--\end{array}$ & $\begin{array}{l}=- \\
=- \\
-\square \\
---\end{array}$ & $\begin{array}{l}.00 \\
.00 \\
.00 \\
.00 \\
.00\end{array}$ & $\begin{array}{l}.00 \\
.00 \\
.00 \\
.00 \\
.00\end{array}$ & $\begin{array}{l}.00 \\
.00 \\
.00 \\
.00 \\
.00\end{array}$ & $\begin{array}{l}.00 \\
.00 \\
.00 \\
.00 \\
.00\end{array}$ & $\begin{array}{l}.00 \\
.00 \\
.00 \\
.00 \\
.00\end{array}$ & $\begin{array}{l}.00 \\
.00 \\
.01 \\
.00 \\
-\end{array}$ & $\begin{array}{l}.00 \\
.00 \\
.00 \\
.00 \\
.00\end{array}$ \\
\hline $\begin{array}{l}26 \\
27 \\
28 \\
29 \\
30 \\
31\end{array}$ & $\begin{array}{l}.00 \\
.00 \\
.00 \\
.00 \\
.00 \\
.00\end{array}$ & \begin{tabular}{l}
.00 \\
.00 \\
.00 \\
.01 \\
\hdashline$-\cdots$
\end{tabular} & 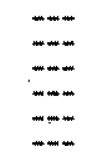 & $\begin{array}{l}=- \\
=- \\
=- \\
=-\end{array}$ & 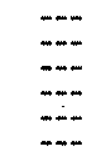 & $\begin{array}{l}.00 \\
.00 \\
.00 \\
.00 \\
.00 \\
.00\end{array}$ & $\begin{array}{l}.00 \\
.00 \\
.00 \\
.00 \\
.00 \\
.\end{array}$ & $\begin{array}{l}.00 \\
.00 \\
.00 \\
.00 \\
.00 \\
.00\end{array}$ & $\begin{array}{l}.36 \\
.15 \\
.04 \\
.00 \\
.00 \\
-.-\end{array}$ & $\begin{array}{l}.00 \\
.00 \\
.00 \\
.00 \\
.00 \\
.00\end{array}$ & $\begin{array}{l}.00 \\
.00 \\
.00 \\
.00 \\
.00\end{array}$ & $\begin{array}{l}.00 \\
.00 \\
.00 \\
.00 \\
.00 \\
-.\end{array}$ \\
\hline $\begin{array}{l}\text { TOTAL } \\
\text { MEAN } \\
\text { MAX } \\
\text { MIN } \\
\text { AC-FT } \\
(\dagger)\end{array}$ & $\begin{array}{r}0.00 \\
.000 \\
.00 \\
.00 \\
.00 \\
0.02\end{array}$ & $\begin{array}{l}0-- \\
\overline{-11} \\
\overline{0.11}\end{array}$ & 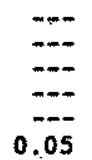 & 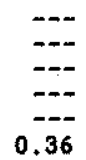 & $\begin{array}{c}--- \\
--- \\
--- \\
0.16\end{array}$ & $\begin{array}{c}-- \\
-- \\
-- \\
0.05\end{array}$ & $\begin{array}{r}0.00 \\
.000 \\
.00 \\
.00 \\
.00 \\
0.01\end{array}$ & $\begin{array}{r}0.00 \\
.000 \\
.00 \\
.00 \\
.00 \\
0.07\end{array}$ & $\begin{array}{r}0.55 \\
.018 \\
.36 \\
.00 \\
1.1 \\
1.97\end{array}$ & $\begin{array}{r}0.42 \\
.014 \\
.16 \\
.00 \\
1.81\end{array}$ & 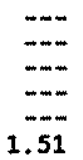 & $\begin{array}{r}0.80 \\
.027 \\
.45 \\
.00 \\
i .6 \\
2.35\end{array}$ \\
\hline
\end{tabular}

(†) Total rainfall accunulation in inches. 
RTO GRANDE BASIN

08329938 LADERA ARROYO AT ALBUQUERQUE, NM

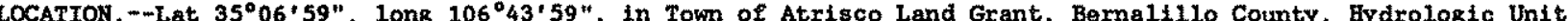
13020203 on left bank, $0.25 \mathrm{mi}$ northwest of City of Albuquerque water storage tank, on dirt road extension of Ouray Road, and $2.3 \mathrm{mi}$ west of North Coors Road in Albuquerque.

DRAIRAGE AREA. $--0.34 \mathrm{mi}^{2}$.

PERIOD OF RECORD.-May 1981 to current year (soasonal records).

GAGE. -Watex-stage recorder. Elevation of gage is 5,220 ft above National Geodetic Vertical Datum of 1929, from

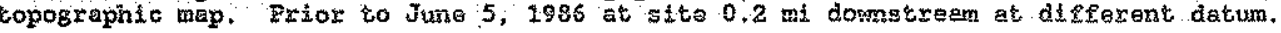

REMARKS.--Records good. Recording rain gage at station. The basin ts undeveloped semidesert terrain, part of which, is wove the escarpment west of Albuquerque see tabulation below for monthly precipitation in inches.

EXTREMES FOR PBRIOD OF RECORD.-Maximum discharge, 182 fts/s, Aug. 27, 1993, gage hejght, 4, 11 ft, from stepbackpater analysis of channel; no flow most of time.

EXIREMES FOR CURRENT YEAR.--Maximum discharge during period of soasonal operation, $1.3 \mathrm{ft} / \mathrm{s}$, Sept. 14, at 1655 hours, gage height, $2.14 \mathrm{ft}$; no flow most of time.

DISCHARGE, CUBIC FEET PER SECOND, WATER YEAR OCTOBER 1995 TO SEPTEMBER 1996

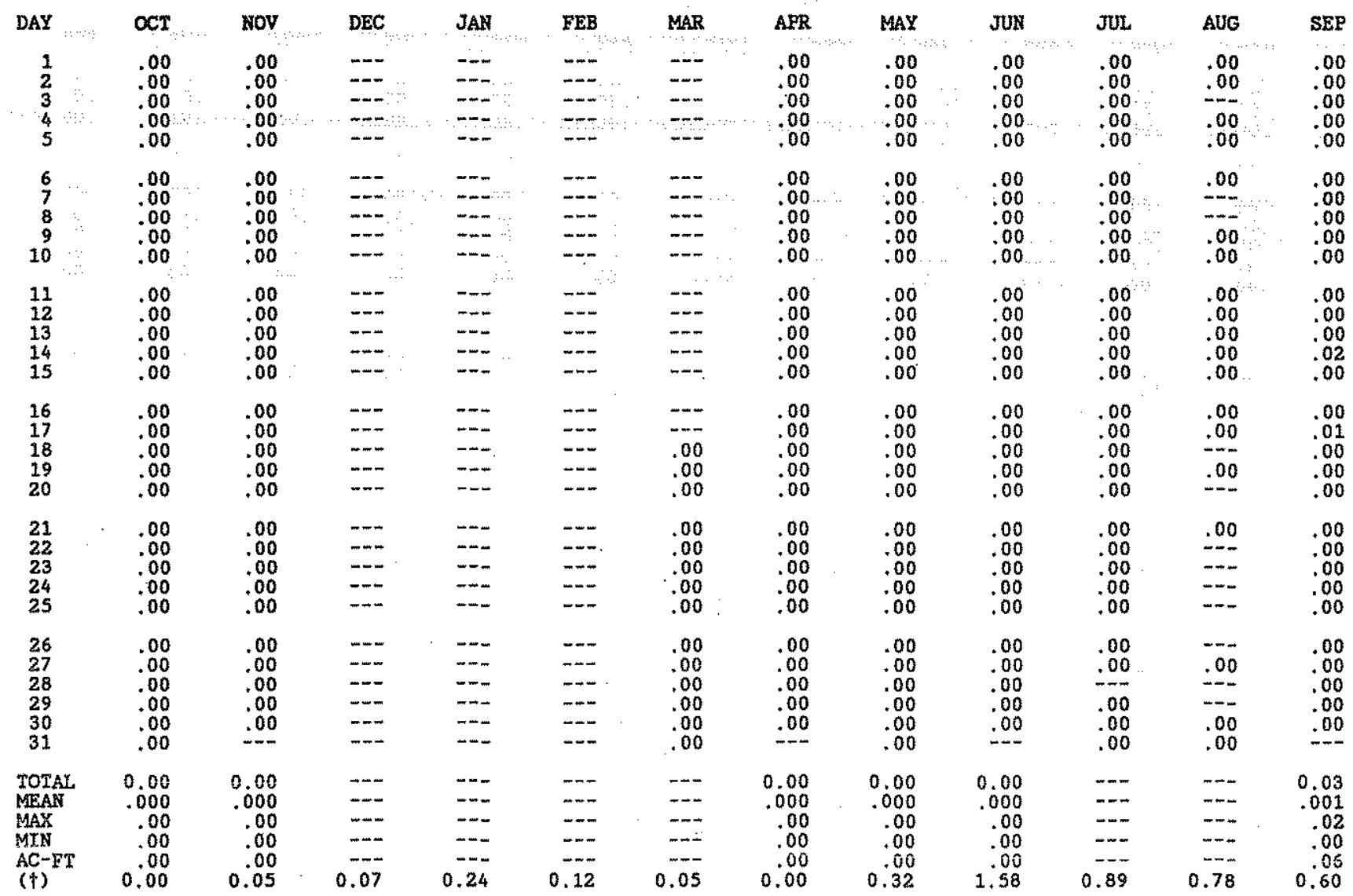

(†) Total rainfall accunulation in inches. 
RIO GRANDE BASIN

08330000 RIO GRANDE AT ALBUQUERQUE, NM

LOCATION.-Lat $35^{\circ} 05^{\prime} 21^{\prime \prime}$, Iong $106^{\circ} 40^{\prime 4} 48^{\prime \prime}$, Bernalillo County, Eydrologic Unit 13020203, in Atrisco Grant, on downstream side of Central Ave, Bridge in Albuquerque, and at mile $1,540.0$.

DRAINAGE AREA. $-17,440 \mathrm{mi}^{2}$, approximately, including $2,940 \mathrm{mi}^{2}$ in closed basin in San Luis Valley, Co.

WATER-DISCHARGE RECORDS

PERIOD OF RECORD, - October 1941 to current year. Monthly discharge only for sone periods, published in WSP 1312.

REVISED RECORDS - - HSP 1312. $1946(\mathrm{M})$

GAGE. -Water-gtege recorder. Datum of gage is 4,946.16 ft above National Geodetic Vertical Datum of 1929 . Prior to Sept. 18, 1947, at varfous sites at datum about 2.00 ft higher; Sept. 15, 1982, to Sept. 20, 1983 , at site $1.0 \mathrm{mi}$ upstream at different datum.

REMARKS.-Water-discharge records good except for estimated daily discharges, which are poor, Flon completely regulated since November 1973 by Cochiti Dam (station 08317300) 50 mi upstream. Possible regulation by operation of reservotrs on R1o Chama and by flood and silt-detention reservoirs on Galisteo Creek and Jemez River (stations $08285000,08286900,08317900,08328500)$. Since May 1971 flow affected by release of transmountain water from

Heron Reservoir (station 08284510). Diverstons upstrean from station for irrigation of about 718,000 acres several hundred of which are downstxeam from station. National Weather Service gage-hetght telemeter, and is.s. Army Corps of Engineers satelilte telemeter at station.

COOPERATION.--Records for Albuquerque Riverside drain and Arenal, Armijo, and Atrisco canals provided by Middle Rio Grande Coneservancy District.

DISCHARGE, CUBIC FEET PER SECOND, WATER YRAR OCTOBER 1995 TO SEPTEMBER 1996

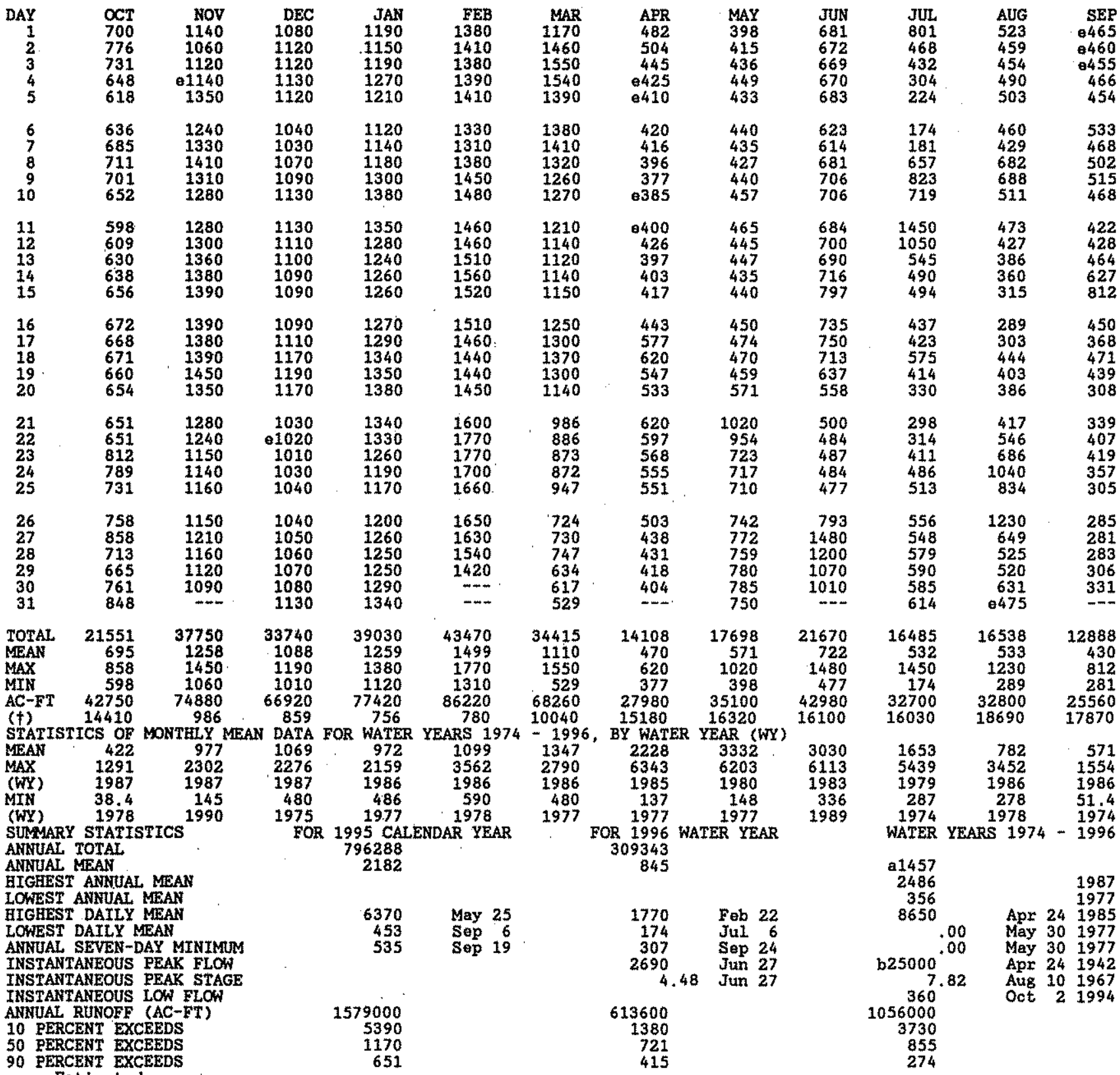

e Estimated

a-Average discharge for 33 years (water year 1942-74), 1,440 fts/s, 1,043,000 acre-ft, prtor to clostre of Cochiti Dam.

b-From rating curve extended above $13,900 \mathrm{ft} 3 / \mathrm{s}$

( $\dagger$ ) COMBINED FLOW, IN ACRE-FEET, OF ALBUQUERQUE RIVERSIDE DRATN, AND ARENAL, ARMIJO AND ATRISCO CANALS THIS FLOW, WHICE BYPASSES RIVER GAGE, CAN BE ADDED TO RIVER RECORDS TO GET THE ENTIRE FLOW IN VALLEY CROSS SECTION 
PERIOD OF RECORD.--Watex years 1969 to current year.

PERIOD OF DAILY RECORD. --

SPECIFIC CONDUCTANCE: October 1969 to current year.

HATER TEMPERATURE: October 1969 to current year.

SUSPEADED-SEDTMERT DISCHARGE: May 1969 to September 1969 (partial-record station), Octobar 1969 to cuxxent, youx.

REAARS.--Daily sediment total-1oads were calculated for one day of nearly every month. Daily total-1oad values were determined using equation from double-mass relationship plot for period of record. Once-daily temperature readings were made by U.S. Geological Survey, and once-daily specifie conductance values were determined in the laboratory from daily suspended sediment samples.

EXTREATES FOR PERTOD OF DAILY RECORD.--

SPECIFIC CONDUCTARCE: Maximum daily, 1,840 microstemens, Oct. 12, 1974; minimum daily, 115 microsiemens, Aug. 14, 1980 .

WATER TEMPERATURE: Maximum daily, $34.0^{\circ} \mathrm{C}$, July 12, 1970; minimum dally, $0.0^{\circ} \mathrm{C}$ on meny days during winter periods.

SEDIATATT CONCERTRATION: Maximum dally mean, $45,500 \mathrm{mg} / \mathrm{L}$, July 21, 1971; minimum daily mean, no flow on many days in 1971, 1972, and 1977 .

SEDINENT LOAD: Maximu daily, 275,000 tons, July 27, 1971; minimum dally, 0 ton on many days in 1971 , 1972 , and 1977 .

EXTREAES FOR CURREAT YEAR.--

SPECIFIC CONDUCTARCE: Maximum dally, undetermined; minimum daily, undetermined.

WATER TEMPERATURE: Maximun daily, undetermined; minimum daliy, undetertained.

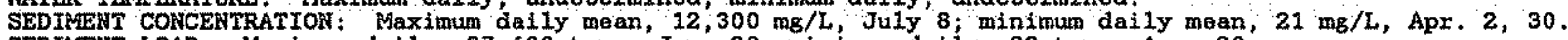
SEDIRENT LOAD: Maximum dafly, 27,600 tons, June 30; minimun daily, 22 tons, Apr. 30 .'

WATER QUALITY DATA, WATER YEAR OCTOBER 1995 TO SEPTEMBER 1996

\begin{tabular}{|c|c|c|c|c|c|c|c|c|c|c|}
\hline DATE & IIME & $\begin{array}{c}\text { DIS- } \\
\text { CHARGE, } \\
\text { INSTT. } \\
\text { CUBIC } \\
\text { EEET } \\
\text { PER } \\
\text { SECOND } \\
(00061)\end{array}$ & $\begin{array}{c}\text { STREAM } \\
\text { WIDTH } \\
\text { (FT) } \\
(00004)\end{array}$ & $\begin{array}{c}\text { STREAM } \\
\text { DEPTE, } \\
\text { MEAN } \\
\text { (FT) } \\
(00064)\end{array}$ & $\begin{array}{c}\text { STREAPA } \\
\text { VELOC- } \\
\text { ITY } \\
\text { MEAN } \\
(\text { F/S }) \\
(00055)\end{array}$ & $\begin{array}{c}\text { TEMPER- } \\
\text { ATURE } \\
\text { WATER } \\
\text { (DEG C) } \\
(00010)\end{array}$ & $\begin{array}{l}\text { SEDI- } \\
\text { MENT, } \\
\text { SUS- } \\
\text { PENDED } \\
\text { (MG/L) } \\
\text { (BO154) }\end{array}$ & $\begin{array}{c}\text { SEDI- } \\
\text { MENT, } \\
\text { DIS- } \\
\text { CHARGE, } \\
\text { SUS- } \\
\text { PENDED } \\
(\text { T/DAY }) \\
(80155)\end{array}$ & $\begin{array}{c}\text { SEDI- } \\
\text { MENT, } \\
\text { DISCE, } \\
\text { SUSP. } \\
\text { BED MA- } \\
\text { TERIAL } \\
(\text { TIDAY }) \\
(80156)\end{array}$ & $\begin{array}{r}\text { SED. } \\
\text { SUSP. } \\
\text { SIEVE } \\
\text { DIAN. } \\
\% \text { FINER } \\
\text { THAN } \\
2.00 \mathrm{M} \\
(70336)\end{array}$ \\
\hline
\end{tabular}

\begin{tabular}{|c|c|c|c|c|c|c|c|c|c|c|}
\hline $\begin{array}{l}\text { OCT } 1995 \\
06, \ldots\end{array}$ & 1215 & 660 & 254 & 1.2 & 2.10 & 14.5 & 60 & 107 & 175 & -- \\
\hline $03 \ldots$ & 0840 & 11.20 & 297 & 1.6 & 2.34 & 11.0 & 153 & 463 & 721 & -- \\
\hline 05 & 1115 & 1130 & 206 & 2.2 & 2.49 & -- & 102 & 311 & 491 & - \\
\hline $19 . \cdots$ & 1000 & 1310 & $\because$ & -- & -- & 2.0 & 179 & 633 & 975 & - \\
\hline MAR & 0815 & 1520 & 322 & 1.9 & 2.53 & 5.0 & 247 & 603 & 930 & -- \\
\hline AER & 1115 & 1290 & 318 & 1.7 & 2.46 & 7.0 & 158 & 550 & 851 & -- \\
\hline MAY & 0920 & 437 & 129 & 1.7 & 1.95 & 8.5 & 920 & 1090 & 1650 & 100 \\
\hline JU木 $03 .$. & 1027 & 471 & 119 & 2.0 & 1.99 & 15.5 & 367 & 467 & 727 & -- \\
\hline${ }_{\text {JUL }}^{20} .$. & 0927 & 572 & 266 & 1.2 & 1.78 & 19.0 & 86 & 133 & 216 & - \\
\hline AUG & 2000 & 267 & 183 & 0.93 & 1.55 & 22.0 & 786 & 567 & 677 & $m$ \\
\hline $\operatorname{seP} \cdots$ & 0820 & 528 & 260 & 1.2 & 1.76 & 21.0 & 901 & 1280 & 1920 & -- \\
\hline $06 \ldots$ & 0930 & 593 & 258 & 1.3 & 1.81 & 20.0 & 382 & 612 & 944 & - \\
\hline
\end{tabular}


RIO GRANDE BASIN

08330000 RIO GRANDE AT ALBUQUERQUE, MM - Continued

WATER-QUALITY RECORDS

WATER QUALITY DATA, WATER YEAR OCTOBER 1995 TO SEFTEMBER 1996

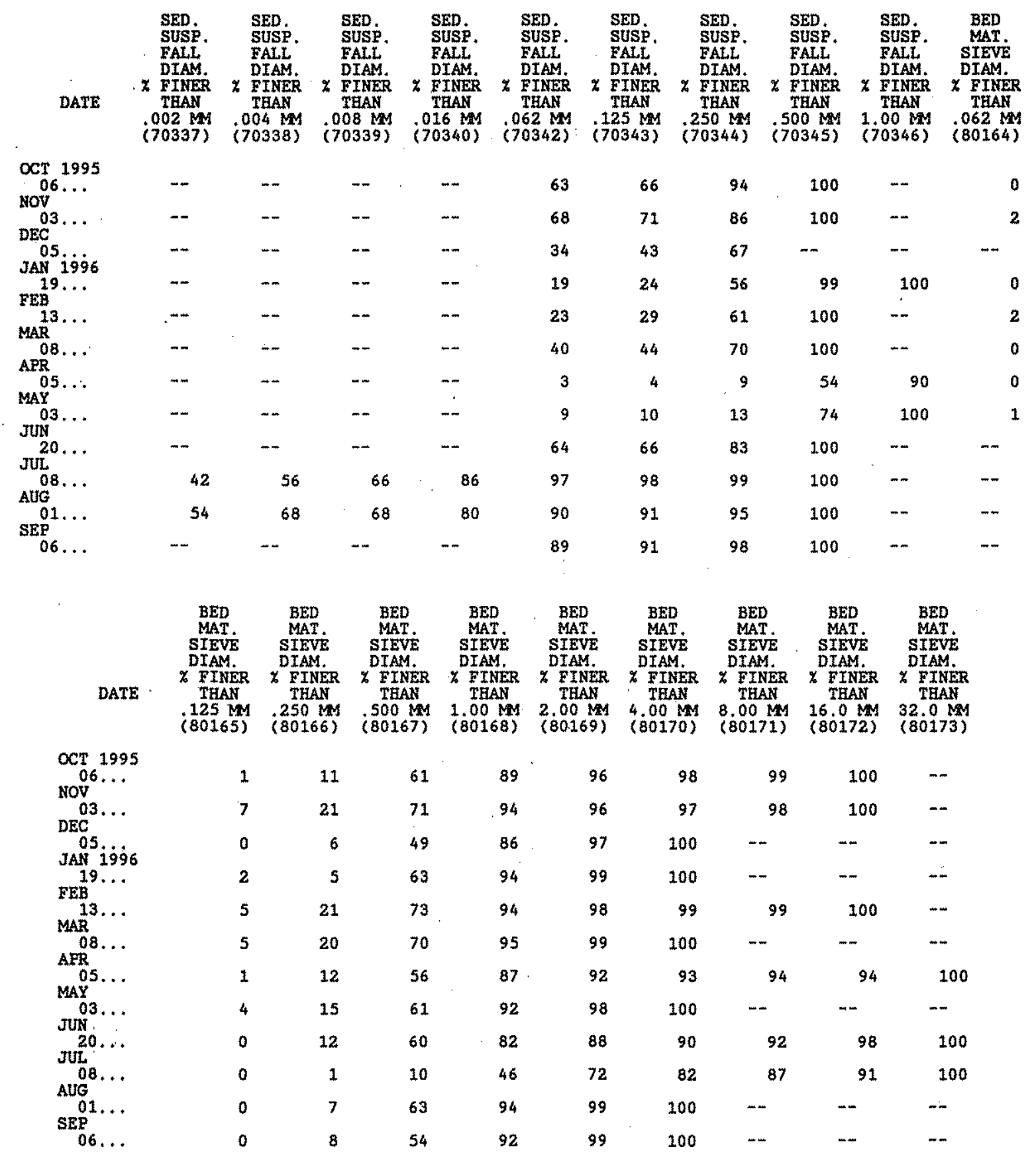


RIO GRANDE BÁSIN

08330000 RIO GRANDE AT ALBUQUERQUE, NM -- Continued

WATER-QUALITY RECORDS

SPECIFIC CONDUCTANCE, US/CA 225 DEGREES CENTIGRADE, WATER YEAR OCTOBER 1995 TO SEETEMBER 1996 DAILY INSTANTANEOUS VALUES

\begin{tabular}{|c|c|c|c|c|c|c|c|c|c|c|c|c|}
\hline DAY & OCT & NOV & DEC & JAN & FEB & MAR & APR & MAY & JUN & JUL & AUG & SER \\
\hline $\begin{array}{l}1 \\
2 \\
3 \\
4 \\
5\end{array}$ & $\begin{array}{l}160 \\
360 \\
374\end{array}$ & $\frac{401}{369}$ & \begin{tabular}{l}
383 \\
\hdashline 381 \\
-
\end{tabular} & $=$ & $\frac{358}{365}$ & 352 & $\frac{\frac{361}{361}}{370}$ & $\frac{384}{385}$ & $\begin{array}{l}376 \\
233 \\
-\end{array}$ & $\frac{498}{382}$ & $\frac{---}{375}$ & $\begin{array}{r}-- \\
413 \\
413\end{array}$ \\
\hline $\begin{array}{r}6 \\
7 \\
8 \\
9 \\
10\end{array}$ & $\underset{365}{\cdots}$ & $\begin{array}{l}366 \\
384 \\
379 \\
-\end{array}$ & $\frac{384}{385}$ & $\frac{388}{389}$ & 371 & $\begin{array}{l}366 \\
363 \\
-\end{array}$ & $\frac{m-}{375}$ & $\begin{array}{l}389 \\
-383 \\
383 \\
-\end{array}$ & ב... & $\frac{480}{454}$ & 386 & $\frac{440}{402}$ \\
\hline $\begin{array}{l}13 \\
15 \\
15\end{array}$ & $\begin{array}{c}362 \\
362 \\
--- \\
-\end{array}$ & $\overline{365}$ & $\frac{387}{389}$ & \begin{tabular}{l}
$--m$ \\
388 \\
\hdashline- \\
$-\square$
\end{tabular} & $\frac{-367}{366}$ & $\begin{array}{l}351 \\
360 \\
-\infty 60\end{array}$ & \begin{tabular}{l}
374 \\
\hdashline 383 \\
-
\end{tabular} & $\begin{array}{l}-\cdots \\
380 \\
382\end{array}$ & $\begin{array}{l}373 \\
-367 \\
-\end{array}$ & \begin{tabular}{l}
- \\
388 \\
\hdashline
\end{tabular} & $\begin{array}{l}391 \\
397 \\
-\end{array}$ & $=$ \\
\hline $\begin{array}{l}19 \\
20\end{array}$ & $\frac{360}{356}$ & $\begin{array}{l}\overline{-} \\
\overline{-} \\
\overline{-}\end{array}$ & $\begin{array}{l}-\cdots \\
-\cdots \\
-\cdots \\
-\cdots\end{array}$ & $\frac{374}{375}$ & \begin{tabular}{l}
367 \\
\hdashline- \\
$\cdots$
\end{tabular} & $\begin{array}{l}--- \\
-- \\
350 \\
355\end{array}$ & $\begin{array}{l}--- \\
385 \\
387 \\
-\cdots\end{array}$ & $\begin{array}{c}--- \\
804 \\
-\cdots \\
383\end{array}$ & $\begin{array}{l}\overline{953} \\
-- \\
363\end{array}$ & $\frac{392}{385}$ & $\begin{array}{l}393 \\
390 \\
\end{array}$ & $\begin{array}{l}426 \\
356 \\
-\end{array}$ \\
\hline $\begin{array}{l}21 \\
22 \\
23 \\
24 \\
25\end{array}$ & $\frac{-\cdots}{362}$ & \begin{tabular}{l}
379 \\
378 \\
\hdashline 376 \\
-
\end{tabular} & $\begin{array}{l}- \\
- \\
- \\
=-\end{array}$ & $\begin{array}{l}-5 \\
376 \\
370\end{array}$ & $\begin{array}{l}369 \\
361 \\
-\end{array}$ & $\begin{array}{l}-75 \\
345 \\
348\end{array}$ & $\begin{array}{l}-34 \\
384 \\
382\end{array}$ & $\begin{array}{l}370 \\
380 \\
--\end{array}$ & \begin{tabular}{l}
368 \\
\hdashline 365 \\
-2 \\
-
\end{tabular} & $\begin{array}{l}395 \\
230 \\
\end{array}$ & \begin{tabular}{l}
374 \\
362 \\
\hdashline-
\end{tabular} & $\frac{--.}{417}$ \\
\hline $\begin{array}{l}26 \\
27 \\
28 \\
29 \\
30 \\
31\end{array}$ & $\frac{388}{375}$ & $\begin{array}{l}386 \\
-382 \\
-- \\
--\end{array}$ & $\begin{array}{l}=- \\
=- \\
=- \\
-\cdots \\
--\end{array}$ & $\begin{array}{l}380 \\
-- \\
-365 \\
366\end{array}$ & $\begin{array}{l}363 \\
352 \\
355 \\
- \\
-\end{array}$ & $\begin{array}{l}349 \\
-- \\
350 \\
--\end{array}$ & \begin{tabular}{c}
389 \\
\hdashline 389 \\
\hdashline-9 \\
-9
\end{tabular} & \begin{tabular}{l}
$-\cdots$ \\
$-\cdots$ \\
$-\cdots$ \\
375 \\
\hdashline 376
\end{tabular} & \begin{tabular}{l}
364 \\
449 \\
\hdashline- \\
\hdashline-
\end{tabular} & $\frac{374}{370}$ & $\frac{--}{648}$ & $\begin{array}{c}\cdots-\cdots \\
\cdots \\
\cdots \\
\cdots-\end{array}$ \\
\hline $\begin{array}{l}\text { MEAN } \\
\text { MAX } \\
\text { MIN }\end{array}$ & $\begin{array}{l}--- \\
-\cdots\end{array}$ & $=$ & $=$ & $=$ & $=$ & $=$ & $=$ & $=$ & $=$ & - & $=$ & \\
\hline
\end{tabular}

WATER TEMPERATURE, DEGREES CELSIUS, WATER YEAR OCTOBER 1995 TO SEFTEMBER 1996 DAILY INSTANTANEOUS VALUES

\begin{tabular}{|c|c|c|c|c|c|c|c|c|c|c|c|c|}
\hline DAY & $\propto T$ & Nov & DEC & JAN & FEB & MAR & APR & MAY & JUN & JUL. & AUG & SEP \\
\hline $\begin{array}{l}1 \\
2 \\
3 \\
4 \\
5\end{array}$ & $\begin{array}{r}16.0 \\
15.0 \\
-\end{array}$ & \begin{tabular}{r}
12.5 \\
11.0 \\
\hdashline$\ldots$ \\
$\cdots$
\end{tabular} & \begin{tabular}{r}
10.5 \\
\hdashline 0.0 \\
\end{tabular} & $\overline{--}$ & $\frac{3.0}{6.8}$ & \begin{tabular}{l}
-- \\
7.4 \\
\hdashline-4
\end{tabular} & $\frac{10.7}{12.0} \frac{7}{8.3}$ & $\begin{array}{r}12.2 \\
15.4 \\
\hdashline\end{array}$ & $\begin{array}{r}20.2 \\
23.3 \\
-\end{array}$ & $\begin{array}{r}27.0 \\
23.0 \\
\cdots\end{array}$ & $\frac{20.5}{20.0}$ & $\begin{array}{r}22.0 \\
22.0\end{array}$ \\
\hline $\begin{array}{r}6 \\
7 \\
8 \\
9 \\
10\end{array}$ & $\begin{array}{c}33.5 \\
\cdots\end{array}$ & $\begin{array}{r}12.5 \\
10.5 \\
10.5 \\
\end{array}$ & $\frac{7.5}{7.0}$ & $\frac{6.0}{5.5}$ & $\begin{array}{l}5.7 \\
5.7 \\
5.5\end{array}$ & $\begin{array}{l}--.5 \\
5.5 \\
6.9 \\
-.-\end{array}$ & 11.4 & $\begin{array}{r}15.2 \\
20.5 \\
15.9\end{array}$ & $\frac{}{m-}$ & 23.0 & 20.5 & $\frac{20.0}{23.0}$ \\
\hline $\begin{array}{l}11 \\
12 \\
13 \\
14 \\
15\end{array}$ & $\begin{array}{r}15.0 \\
15.0 \\
-\end{array}$ & 10.0 & $\begin{array}{l}\frac{6.5}{0.5} \\
7.0 \\
6.5\end{array}$ & 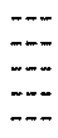 & $\begin{array}{l}5.4 \\
5.4 \\
-5 \\
-\infty\end{array}$ & $\begin{array}{l}7.6 \\
7.8 \\
6.7\end{array}$ & \begin{tabular}{r}
10.3 \\
\hdashline 9.2 \\
9.2
\end{tabular} & $\frac{18.1}{18.4}$ & $\begin{array}{r}24.6 \\
19.0\end{array}$ & \begin{tabular}{c}
$\cdots$ \\
26.0 \\
\hdashline-
\end{tabular} & $\begin{array}{r}25.0 \\
22.0 \\
\end{array}$ & \begin{tabular}{r}
15.0 \\
\hdashline-1 \\
$\cdots$
\end{tabular} \\
\hline $\begin{array}{l}16 \\
17 \\
18 \\
19 \\
20\end{array}$ & $\begin{array}{r}16.0 \\
13.0 \\
12.0\end{array}$ & $\begin{array}{r}=- \\
= \\
= \\
12.0\end{array}$ & $\begin{array}{l}-\cdots \\
5.5 \\
\cdots\end{array}$ & $\begin{array}{l}--5 \\
5.5 \\
2.0 \\
-.\end{array}$ & $\begin{array}{l}5.8 \\
-\infty \\
-\infty\end{array}$ & $\begin{array}{r}7.3 \\
70.0 \\
10.0\end{array}$ & $\begin{array}{r}11.8 \\
15.6 \\
\cdots\end{array}$ & $\begin{array}{r}24.9 \\
\hdashline 16.8\end{array}$ & $\frac{20.0}{19.2}$ & $\frac{21.0}{22.0}$ & $\begin{array}{r}19.5 \\
20.0 \\
-.0\end{array}$ & $\frac{16.0}{25.0}$ \\
\hline $\begin{array}{l}21 \\
22 \\
23 \\
24 \\
25\end{array}$ & $\begin{array}{r}-- \\
9.5 \\
11.5\end{array}$ & $\begin{array}{r}12.0 \\
9.5 \\
-. . \\
\hdashline-.\end{array}$ & $m$ & $\begin{array}{l}4.0 \\
1.0 \\
1.0\end{array}$ & $\begin{array}{l}--9 \\
8.9 \\
7.4 \\
---\end{array}$ & \begin{tabular}{r}
20.7 \\
9.0 \\
\hdashline 7.1 \\
7.1
\end{tabular} & $\frac{13.0}{17.8}$ & $\frac{21.5}{16.7}$ & $\begin{array}{r}20.7 \\
24.7 \\
-.7\end{array}$ & $\frac{26.0}{23.0}$ & $\begin{array}{r}26.0 \\
22.0 \\
\hdashline\end{array}$ & 19.0 \\
\hline $\begin{array}{l}26 \\
27 \\
28 \\
29 \\
30 \\
31\end{array}$ & $\frac{11.0}{11.5}$ & $\begin{array}{l}7.5 \\
7.5 \\
6.5 \\
\cdots \\
-\cdots\end{array}$ & $\begin{array}{l}\cdots- \\
\cdots \\
\cdots \\
\cdots\end{array}$ & \begin{tabular}{l}
1.5 \\
\hdashline. .5 \\
2.5 \\
5.0
\end{tabular} & $\begin{array}{l}6.6 \\
5.8 \\
4.5 \\
-\ldots-\end{array}$ & $\begin{array}{r}6.3 \\
9.5 \\
9.5\end{array}$ & \begin{tabular}{r}
12.3 \\
\hdashline 8.8 \\
8.8 \\
-
\end{tabular} & $\begin{array}{r}16.7 \\
17.7\end{array}$ & $\begin{array}{r}23.0 \\
23 \\
\cdots\end{array}$ & $\begin{array}{r}-- \\
\overline{z-} \\
27.0 \\
26.0\end{array}$ & $\begin{array}{r}- \\
26.0 \\
23.0\end{array}$ & $=$ \\
\hline $\begin{array}{l}\text { MEAN } \\
\text { MAX }\end{array}$ & $\cdots$ & $=-$ & $\overline{--}$ & $\overline{-}$ & $=$ & \begin{tabular}{l}
--- \\
\hdashline-- \\
--
\end{tabular} & $=$ & $\ddot{--}$ & $=$ & $=-$ & $\overline{--}$ & $=$ \\
\hline
\end{tabular}


RIO GRANDE BASIN

08330000 RIO GRANDE AT ALBUQUERQUE, MM -- Continued

WATER-OUALITY RECORDS

SUSPENDED-SEDIMENT, WATER YEAR OCTOBER 1995 TO SEPTEMRER 1996

\begin{tabular}{|c|c|c|c|c|c|c|c|c|c|c|c|c|}
\hline DAY & $\begin{array}{c}\text { MEAN } \\
\text { CONCEN- } \\
\text { TRATION } \\
\text { (MG/L) }\end{array}$ & $\begin{array}{l}\text { LOADS } \\
\text { (T/DAY) }\end{array}$ & $\begin{array}{c}\text { MEAN } \\
\text { CONCEN- } \\
\text { TRATION } \\
\text { (MG/L) }\end{array}$ & $\begin{array}{l}\text { LOADS } \\
\text { (T/DAY) }\end{array}$ & $\begin{array}{c}\text { MEAN } \\
\text { CONCEN- } \\
\text { TRATTOA } \\
(M G / L)\end{array}$ & $\begin{array}{l}\text { LOADS } \\
\text { (T/DAY) }\end{array}$ & $\begin{array}{l}\text { MEAN } \\
\text { CONCEN- } \\
\text { TRATION } \\
\text { (MG/L) }\end{array}$ & $\begin{array}{l}\text { LOADS } \\
\text { (T/DAY })\end{array}$ & $\begin{array}{c}\text { MEAN } \\
\text { CONCEN- } \\
\text { TRATION } \\
\text { (MG/L) }\end{array}$ & $\begin{array}{l}\text { LOADS } \\
\text { (T/DAY) }\end{array}$ & $\begin{array}{c}\text { MEAN } \\
\text { CONCEN- } \\
\text { TRATYON } \\
\text { (MG/L) }\end{array}$ & $\begin{array}{l}\text { LOADS } \\
\text { (T/DAY }\end{array}$ \\
\hline $\begin{array}{l}1 \\
2 \\
3 \\
4 \\
5\end{array}$ & $\begin{array}{l}145^{\alpha} \\
149 \\
131 \\
114 \\
112\end{array}$ & $\begin{array}{r}\text { TOBER } \\
274 \\
319 \\
259 \\
199 \\
187\end{array}$ & $\begin{array}{l}\text { NOV } \\
279 \\
202 \\
151 \\
161 \\
177\end{array}$ & $\begin{array}{c}\text { EMBER } \\
855 \\
573 \\
454 \\
496 \\
646\end{array}$ & $\begin{array}{c}\text { DE } \\
126 \\
114 \\
99 \\
86 \\
73\end{array}$ & $\begin{array}{r}\text { FMBER } \\
366 \\
344 \\
301 \\
263 \\
221\end{array}$ & $\begin{array}{l}140^{\mathrm{J}} \\
141 \\
142 \\
142 \\
143\end{array}$ & $\begin{array}{r}\text { NUARY } \\
450 \\
439 \\
456 \\
488 \\
468\end{array}$ & $\begin{array}{l}\text { FE } \\
146 \\
157 \\
149 \\
138 \\
130\end{array}$ & $\begin{array}{r}\text { RUARY } \\
544 \\
601 \\
554 \\
519 \\
495\end{array}$ & $\begin{array}{l}171 \\
169 \\
168 \\
166 \\
165\end{array}$ & $\begin{array}{r}\text { MARCH } \\
542 \\
666 \\
701 \\
692 \\
619\end{array}$ \\
\hline $\begin{array}{r}6 \\
7 \\
8 \\
9 \\
10\end{array}$ & $\begin{array}{r}108 \\
104 \\
100 \\
96 \\
89\end{array}$ & $\begin{array}{l}186 \\
193 \\
192 \\
183 \\
157\end{array}$ & $\begin{array}{l}192 \\
188 \\
246 \\
675 \\
613\end{array}$ & $\begin{array}{r}641 \\
677 \\
933 \\
2380 \\
2120\end{array}$ & $\begin{array}{r}65 \\
74 \\
86 \\
93 \\
100\end{array}$ & $\begin{array}{l}182 \\
207 \\
248 \\
273 \\
304\end{array}$ & $\begin{array}{l}144 \\
144 \\
145 \\
148 \\
153\end{array}$ & $\begin{array}{l}436 \\
445 \\
462 \\
519 \\
569\end{array}$ & $\begin{array}{l}142 \\
156 \\
153 \\
148 \\
140\end{array}$ & $\begin{array}{l}508 \\
551 \\
572 \\
579 \\
559\end{array}$ & $\begin{array}{l}163 \\
164 \\
176 \\
200 \\
228\end{array}$ & $\begin{array}{l}625 \\
626 \\
678 \\
782\end{array}$ \\
\hline $\begin{array}{l}11 \\
12 \\
13 \\
14 \\
15\end{array}$ & $\begin{array}{r}77 \\
138 \\
236 \\
202 \\
159\end{array}$ & $\begin{array}{l}124 \\
229 \\
401 \\
349 \\
282\end{array}$ & $\begin{array}{l}483 \\
381 \\
306 \\
267 \\
236\end{array}$ & $\begin{array}{r}1680 \\
1330 \\
1120 \\
993 \\
886\end{array}$ & $\begin{array}{r}105 \\
97 \\
88 \\
86 \\
87\end{array}$ & $\begin{array}{l}320 \\
290 \\
262 \\
253 \\
255\end{array}$ & $\begin{array}{l}175 \\
199 \\
176 \\
147 \\
123\end{array}$ & $\begin{array}{l}639 \\
685 \\
586 \\
497 \\
418\end{array}$ & $\begin{array}{l}133 \\
132 \\
163 \\
197 \\
192\end{array}$ & $\begin{array}{l}524 \\
518 \\
666 \\
833 \\
791\end{array}$ & $\begin{array}{l}285 \\
591 \\
935 \\
379 \\
147\end{array}$ & $\begin{array}{r}922 \\
1810 \\
2830 \\
1160 \\
455\end{array}$ \\
\hline $\begin{array}{l}16 \\
17 \\
18 \\
19 \\
20\end{array}$ & $\begin{array}{l}127 \\
112 \\
107 \\
123 \\
146\end{array}$ & $\begin{array}{l}230 \\
202 \\
194 \\
220 \\
258\end{array}$ & $\begin{array}{l}208 \\
184 \\
162 \\
143 \\
130\end{array}$ & $\begin{array}{l}783 \\
685 \\
609 \\
558 \\
474\end{array}$ & $\begin{array}{r}99 \\
116 \\
130 \\
132 \\
132\end{array}$ & $\begin{array}{l}294 \\
347 \\
411 \\
423 \\
417\end{array}$ & $\begin{array}{r}102 \\
94 \\
129 \\
166 \\
136\end{array}$ & $\begin{array}{l}352 \\
329 \\
466 \\
608 \\
508\end{array}$ & $\begin{array}{l}181 \\
161 \\
143 \\
126 \\
117\end{array}$ & $\begin{array}{l}737 \\
637 \\
557 \\
492 \\
458\end{array}$ & $\begin{array}{l}133 \\
135 \\
137 \\
146 \\
176\end{array}$ & $\begin{array}{l}450 \\
475 \\
508 \\
509 \\
538\end{array}$ \\
\hline $\begin{array}{l}21 \\
22 \\
23 \\
24 \\
25\end{array}$ & $\begin{array}{l}178 \\
218 \\
243 \\
181 \\
142\end{array}$ & $\begin{array}{l}313 \\
383 \\
532 \\
389 \\
281\end{array}$ & $\begin{array}{l}146 \\
130 \\
136 \\
147 \\
158\end{array}$ & $\begin{array}{l}504 \\
434 \\
424 \\
451 \\
495\end{array}$ & $\begin{array}{l}133 \\
134 \\
134 \\
135 \\
136\end{array}$ & $\begin{array}{l}371 \\
369 \\
367 \\
376 \\
382\end{array}$ & $\begin{array}{r}104 \\
81 \\
68 \\
59 \\
61\end{array}$ & $\begin{array}{l}379 \\
291 \\
230 \\
189 \\
191\end{array}$ & $\begin{array}{l}157 \\
256 \\
618 \\
420 \\
220\end{array}$ & $\begin{array}{r}682 \\
1230 \\
2940 \\
1940 \\
991\end{array}$ & $\begin{array}{l}447 \\
889 \\
532 \\
274 \\
150\end{array}$ & $\begin{array}{r}1170 \\
2130 \\
1250 \\
647 \\
384\end{array}$ \\
\hline $\begin{array}{l}26 \\
27 \\
28 \\
29 \\
30 \\
31\end{array}$ & $\begin{array}{l}176 \\
214 \\
204 \\
189 \\
186 \\
238\end{array}$ & $\begin{array}{l}361 \\
498 \\
393 \\
339 \\
385 \\
544\end{array}$ & $\begin{array}{l}170 \\
172 \\
130 \\
103 \\
113\end{array}$ & $\begin{array}{l}528 \\
559 \\
408 \\
310 \\
332\end{array}$ & $\begin{array}{l}136 \\
137 \\
138 \\
138 \\
139 \\
139\end{array}$ & $\begin{array}{l}382 \\
387 \\
394 \\
399 \\
403 \\
426\end{array}$ & $\begin{array}{r}65 \\
75 \\
87 \\
101 \\
116 \\
132\end{array}$ & $\begin{array}{l}211 \\
256 \\
295 \\
341 \\
404 \\
474\end{array}$ & $\begin{array}{l}127 \\
113 \\
116 \\
166 \\
---\end{array}$ & $\begin{array}{l}565 \\
497 \\
483 \\
637 \\
-0 \\
-\end{array}$ & $\begin{array}{r}114 \\
96 \\
105 \\
112 \\
97 \\
82\end{array}$ & $\begin{array}{l}223 \\
189 \\
212 \\
193 \\
162 \\
118\end{array}$ \\
\hline $2 \pi$ & --- & 9056 & $-\infty$ & 23338 & $m$ & 10237 & -- & 13081 & $-m$ & 21660 & $m$ & 228 \\
\hline
\end{tabular}

SUSPENDED-SEDIMENT, WATER YEAR OCTOBER 1995 TO SEPTEMBER 1996

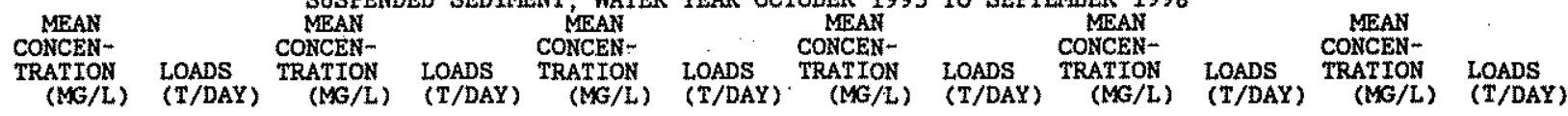

DAY

$$
\text { (Wo/L) (ITDA }
$$
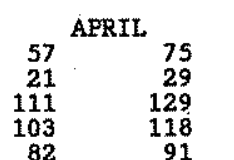

$\begin{array}{rrr} & \text { MAY } & \\ 79 & 86 & 140 \\ 104 & 116 & 126 \\ 113 & 133 & 116 \\ 99 & 119 & 117 \\ 83 & 97 & 115\end{array}$

JINE

$\begin{array}{rr}89 & 101 \\ 100 & 112 \\ 106 & 113 \\ 94 & 95 \\ 84 & 87\end{array}$

$\begin{array}{rr}72 & 8 \\ 73 & 8 \\ 77 & 8 \\ 90 & 10 \\ 90 & 11\end{array}$

$\begin{array}{rr}86 & 112 \\ 86 & 110 \\ 88 & 107 \\ 107 & 105 \\ 112 & 102\end{array}$

258
229
209
212

\begin{tabular}{rr} 
& \multicolumn{2}{c}{ JULY } \\
7540 & 16500 \\
2460 & 3100 \\
987 & 1150 \\
1460 & 1180 \\
2540 & 1500
\end{tabular}

AUGUST

$\begin{array}{ll}292 & 41 \\ 262 & 32 \\ 241 & 29 \\ 221 & 29 \\ 201 & 27\end{array}$

413
324
295
293
272

$400 \quad 502$

212

2540

2080

189
182

7650
12300
11800

3700
23000
25800

172

153
164
178

214

178
305
328

$\begin{array}{ll}400 & 502 \\ 310 & 385 \\ 250 & 307 \\ 234 & 287\end{array}$

$\begin{array}{ll}234 & 287 \\ 667 & 828\end{array}$

$\begin{array}{lr}89 & 96 \\ 93 & 108\end{array}$

$\begin{array}{ll}84 & 91 \\ 75 & 82 \\ 74 & 83\end{array}$

$\begin{array}{lll}89 & 112 & 102\end{array}$

$\begin{array}{rrr}87 & 105 & 105 \\ 88 & 107 & 142\end{array}$

$\begin{array}{lll}101 & 119 & 193 \\ 115 & 136 & 220\end{array}$

$5450 \quad 21000$

194

328
267

1530

$1180 \quad 2220$

195

$\begin{array}{rr}114 & 137 \\ 151 & 234 \\ 76 & 129 \\ 75 & 110 \\ 89 & 129\end{array}$

$\begin{array}{ll}115 & 139 \\ 124 & 158 \\ 133 & 169 \\ 123 & 152\end{array}$

$\begin{array}{ll}139 & 245 \\ 158 & 297\end{array}$

169
152

297
501
873
909

187
198
264
375

$\begin{array}{rr}2960 & 8720 \\ 1610 & 2410\end{array}$

211
226
213

269

$\begin{array}{rr}872 & 1140 \\ 487 & 652\end{array}$

184

269
260
222
190

$814 \quad 1100$

47

$\begin{array}{ll}347 & 412 \\ 274 & 313\end{array}$

$\begin{array}{ll}274 & 313 \\ 255 & 397 \\ 245 & 273\end{array}$

224
598
1670
3220

156

328

772
416

$107 \quad 179$

118

21
22
23
24
25

$\begin{array}{rl}107 & 179 \\ 121 & 194 \\ 100 & 154 \\ 83 & 124 \\ 87 & 130\end{array}$

$\begin{array}{ll}134 & 372 \\ 152 & 390 \\ 151 & 294 \\ 147 & 284 \\ 149 & 285\end{array}$

372
390
294
284
285

$\begin{array}{rr}108 & 147 \\ 83 & 109 \\ 83 & 109 \\ 93 & 120 \\ 216 & 279\end{array}$

245

1480

175
495
2070
3520
1550

223
329

$329 \quad 383$

$834 \quad 650$

834
$1330 \quad 2770$

\section{$95 \quad 128 \quad 151 \quad 303$}

26
27
28
29
30
31

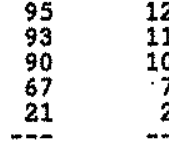

$\begin{array}{ll}151 & 303 \\ 154 & 32 \\ 157 & 32 \\ 159 & 334 \\ 157 & 33 \\ 154 & 31\end{array}$

$303 \quad 576$

$\begin{array}{ll}1970 & 1410 \\ 20 & 7110\end{array}$

$\begin{array}{lll}334 & 6260 & 20800 \\ & 8770 & 24900\end{array}$

$10300-24900$

27600

$248 \quad 200$

251

261

271

213

291
356
379

631

1030
1890

1890
1730
1430

706

1540
3540

4850

$1740 \quad 2120$

$275 \quad 413 \quad 1180$

275
277
278

278
282

282
301
318

410

435

449
476
528

1180
975
805
663
54
473

3960

1720

Tomat

3370

$-5959$

91104

-... 136496

$-\cdots \quad 34954$

951
459

$204 \quad 169$

TOTAL LOAD FOR YEAR: 391454 TONS. 
LOCATION.--Lat $35^{\circ} 04^{\prime} 43^{\prime \prime}$, Long $106^{\circ} 29^{\prime} 51^{\prime \prime}$, Bernalillo County, Bydrologic Unit 13020203, on right bank 300 ft

downstream from Copper Boulevard Bridge, near corner of Trameay and Copper Boulevards $\mathrm{kE}$ in Albuquerque.

DRAIMAGE AREA. $-1.60 \mathrm{~mL}^{2}$.

PERIOD OF RECORD,--July 1987 to current year (seasonal record).

GAGE.--Water-stage recorder and concrete-lined channel. Elovation of gage is 5,760 ft above fational Geodetic Vertical Datum of 1929, from topographic map.

RAMARES, - Recordz poor. Some minor streamflow may exist on days where datly mean discharges have been recorded as

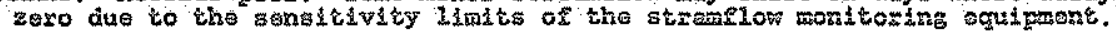

EXIREMES FOR FERIOD OF RECORD.--Maximum discharge, 3,250 ft\$/s, July 9, 1988, gage height, 7,62 ft, Erom 2100dmarks, from stop backpater analysis of channel; no flow most of time.

EXTREIES FOR CURREET YEAR.-Maximum discharge during period of seasonal operation, 164 fts/s, at 1750 hours Aug. 23, gage haight $1.28 \mathrm{et}$; no Llow most of time.

DISCHARGE, CUBIC FEET PER SECOND, WATER YEAR OCTOBER 1995 TO SEPTEMBER 1996

\begin{tabular}{|c|c|c|c|c|c|c|c|c|c|c|c|c|}
\hline DAY & OCI & HOV & DEC & JAN & FEB & $M A R$ & APR & MAY & Jun & JUL & AUE & SEP \\
\hline $\begin{array}{l}1 \\
2 \\
3 \\
4 \\
5\end{array}$ & $\begin{array}{l}-- \\
-- \\
-- \\
-- \\
--\end{array}$ & $\begin{array}{l}m- \\
--- \\
--- \\
---\end{array}$ & $\begin{array}{c}-\cdots \\
-\infty \\
-\infty \\
-\infty \\
-\infty\end{array}$ & $=$ & $\begin{array}{l}--- \\
-- \\
--- \\
-\square \\
-\square\end{array}$ & 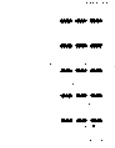 & $\begin{array}{l}.00 \\
.00 \\
.00 \\
.00 \\
.00\end{array}$ & $\begin{array}{l}.00 \\
.00 \\
.00 \\
.00 \\
.00\end{array}$ & $\begin{array}{l}.00 \\
.00 \\
.00 \\
.00 \\
.00\end{array}$ & $\begin{array}{l}.00 \\
.00 \\
.00 \\
.00 \\
.00\end{array}$ & $\begin{array}{l}.00 \\
.00 \\
.00 \\
.00 \\
.00\end{array}$ & $\begin{array}{l}.00 \\
.00 \\
.00 \\
.00 \\
.00\end{array}$ \\
\hline $\begin{array}{r}6 \\
7 \\
8 \\
9 \\
10\end{array}$ & $\begin{array}{l}--m \\
-- \\
-m \\
--\infty \\
-m\end{array}$ & 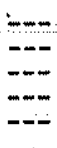 & 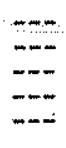 & $\begin{array}{l}-- \\
-\infty \\
-- \\
--\end{array}$ & $\begin{array}{l}--- \\
--\infty \\
-\infty \\
-- \\
-\end{array}$ & $\begin{array}{l}-m \\
-m \\
--- \\
-m \\
-m\end{array}$ & $\begin{array}{l}.00 \\
.00 \\
.00 \\
.00 \\
.00\end{array}$ & $\begin{array}{l}.00 \\
.00 \\
.00 \\
.00 \\
.00\end{array}$ & $\begin{array}{l}.00 \\
.00 \\
.00 \\
.00 \\
.00\end{array}$ & $\begin{array}{r}.00 \\
6.30 \\
0.00 \\
61.0 \\
02.0\end{array}$ & $\begin{array}{l}.00 \\
.00 \\
.00 \\
.00 \\
.00\end{array}$ & $\begin{array}{l}.00 \\
.00 \\
.00 \\
.00 \\
.00\end{array}$ \\
\hline $\begin{array}{l}11 \\
12 \\
13 \\
14 \\
15\end{array}$ & $\begin{array}{l}--\infty \\
-\infty+\cdots \\
-\infty \\
-\infty\end{array}$ & $\begin{array}{l}--- \\
--\overline{-} \\
--\overline{-} \\
-\cdots\end{array}$ & $\begin{array}{l}-\cdots \\
-\infty \\
m- \\
m-\infty \\
m-\infty\end{array}$ & $\begin{array}{l}m-\infty \\
-\infty \\
-\infty \\
-\infty\end{array}$ & 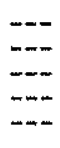 & $\begin{array}{l}-.- \\
.00 \\
.00 \\
.00\end{array}$ & $\begin{array}{l}.00 \\
.00 \\
.00 \\
.00 \\
.00\end{array}$ & $\begin{array}{l}.00 \\
.00 \\
.00 \\
.00 \\
.00\end{array}$ & $\begin{array}{l}.00 \\
.00 \\
.00 \\
.02 \\
.00\end{array}$ & $\begin{array}{r}.00 \\
.00 \\
.00 \\
.00 \\
.10\end{array}$ & $\begin{array}{r}.00 \\
.00 \\
.00 \\
1.0 \\
.35\end{array}$ & $\begin{array}{r}.00 \\
.00 \\
.00 \\
4.6 \\
.02\end{array}$ \\
\hline $\begin{array}{l}16 \\
17 \\
18 \\
19 \\
20\end{array}$ & $\begin{array}{l}m-m \\
m-\infty \\
-\cdots \\
-\infty\end{array}$ & $\begin{array}{l}--- \\
m-\infty \\
-\infty \\
-\infty \\
-\infty\end{array}$ & $\begin{array}{l}--- \\
--\infty \\
-\infty-\infty \\
-\infty\end{array}$ & $\begin{array}{l}-\infty \\
-\infty \\
-\infty \\
-\infty\end{array}$ & - & $\begin{array}{l}.00 \\
.00 \\
.00 \\
.00 \\
.00\end{array}$ & $\begin{array}{l}.00 \\
.00 \\
.00 \\
.00 \\
.00\end{array}$ & $\begin{array}{l}.00 \\
.00 \\
.00 \\
.00 \\
.00\end{array}$ & $\begin{array}{l}.00 \\
.00 \\
.00 \\
.00 \\
.00\end{array}$ & $\begin{array}{l}.00 \\
.00 \\
.00 \\
.00 \\
.00\end{array}$ & $\begin{array}{l}.00 \\
.00 \\
.00 \\
.00 \\
.00\end{array}$ & $\begin{array}{r}.00 \\
02.0 \\
02.0 \\
.00 \\
.00\end{array}$ \\
\hline $\begin{array}{l}21 \\
22 \\
23 \\
24 \\
25\end{array}$ & $\begin{array}{l}m-m \\
m-m \\
-\infty \\
-m\end{array}$ & $\begin{array}{l}--- \\
--\infty \\
-m- \\
---\end{array}$ & $\begin{array}{l}-- \\
-\infty \\
-\infty \\
-\infty \\
-\infty\end{array}$ & $=$ & $\begin{array}{l}--- \\
-m \\
-m \\
--\end{array}$ & $\begin{array}{r}.00 \\
.00 \\
.00 \\
.00 \\
.00\end{array}$ & $\begin{array}{l}.00 \\
.00 \\
.00 \\
.00 \\
.00\end{array}$ & $\begin{array}{l}.00 \\
.00 \\
.00 \\
.00 \\
.00\end{array}$ & $\begin{array}{l}.00 \\
.00 \\
.00 \\
.00 \\
.00\end{array}$ & $\begin{array}{l}.00 \\
.00 \\
.00 \\
.00 \\
.00\end{array}$ & $\begin{array}{c}.40 \\
.15 \\
9.2 \\
0.10 \\
01.0\end{array}$ & $\begin{array}{l}.00 \\
.00 \\
.00 \\
.00 \\
.00\end{array}$ \\
\hline $\begin{array}{l}26 \\
27 \\
28 \\
29 \\
30 \\
31\end{array}$ & $=$ & $\begin{array}{l}-- \\
-- \\
-- \\
-\infty \\
-\infty\end{array}$ & $\begin{array}{l}\cdots \\
\cdots- \\
\cdots- \\
\cdots- \\
-\cdots\end{array}$ & 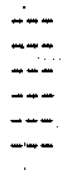 & $\begin{array}{l}-m \\
-m- \\
-m- \\
m-m \\
m-m\end{array}$ & $\begin{array}{l}.00 \\
.00 \\
.00 \\
.00 \\
.00 \\
.00\end{array}$ & $\begin{array}{l}.00 \\
.00 \\
.00 \\
.00 \\
.00 \\
.-\end{array}$ & $\begin{array}{l}.50 \\
.00 \\
.00 \\
.00 \\
.00 \\
.00\end{array}$ & $\begin{array}{l}.00 \\
01.0 \\
02.0 \\
.00 \\
.00 \\
-1 .\end{array}$ & $\begin{array}{l}.00 \\
.00 \\
.00 \\
.00 \\
.00 \\
.00\end{array}$ & $\begin{array}{r}02.0 \\
.00 \\
8.50 \\
.10 \\
.00 \\
.00\end{array}$ & $\begin{array}{l}.06 \\
.00 \\
.00 \\
.00 \\
.00 \\
.--\end{array}$ \\
\hline $\begin{array}{l}\text { TOTAL } \\
\text { MEAN } \\
\text { MAX } \\
\text { MIIE } \\
\text { AC-FT }\end{array}$ & 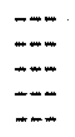 & $\begin{array}{l}--- \\
--- \\
--\infty \\
---\end{array}$ & 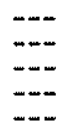 & $\begin{array}{l}m-\infty \\
-\rightarrow \\
-\rightarrow\end{array}$ & $\begin{array}{l}-\cdots \\
-- \\
-\infty \\
-\infty\end{array}$ & $\begin{array}{l}-m \\
-m \\
-\infty \\
-\infty \\
-\infty\end{array}$ & $\begin{array}{l}0.00 \\
.000 \\
.00 \\
.00 \\
.00\end{array}$ & $\begin{array}{r}0.50 \\
.016 \\
.50 \\
.00 \\
1.0\end{array}$ & $\begin{array}{r}3.02 \\
10 \\
2.0 \\
60 \\
6.0\end{array}$ & $\begin{array}{r}3.40 \\
.11 \\
2.0 \\
.00 \\
6.7\end{array}$ & $\begin{array}{r}14.80 \\
.48 \\
9.2 \\
.00 \\
29\end{array}$ & $\begin{array}{r}8.68 \\
.29 \\
4.6 \\
.00 \\
17\end{array}$ \\
\hline
\end{tabular}

e Estimated 
RIO GRANDE BASIN

08330600 TIJERAS ARROYO NEAR ALBUQUERQUE, NM

LOCATION, - Lat $35^{\circ} 00^{\circ} 09^{\prime \prime}$, long $106^{\circ} 38^{\circ} 57^{\prime \prime}$, in SW/SWk sec.17, T.9 N., R.3 E., Bornali110 County, Eydrologic Unit 13020203 , on left bank $800 \mathrm{ft}$ upstream from bridge on Broadway Boulevard SE, $0.2 \mathrm{mi}$ downstream from bridge on Interstate Highway 25 , and $3.0 \mathrm{mi}$ south of Albuquerque:

DRATNAGE AREA, $--128 \mathrm{mi}^{2}$.

EERIOD OF RECORD.--October 1951 to September 1968 (annual maximum only), August 1974 to current year (seasonal records).

GAGE. -Water-stage recorder. Elevation of gage is 5,000 ft above National Geodetic Vertical Datum of 1929, from topographic map. Prior to Mar. 10, 1988, at site 1,700 ft downstream at different datum.

REMARKS. - -Records fair.

EXTREMES FOR PERIOD OF RECORD,-Maximun discharge, 2,930 fts/s, July 9, 1988, gage height, 9.6 ft, from floodmarks, from slope-area measurement of peak flow; no fiow most of time.

EXTREAES FOR CURRENT YEAR.--Maxtmum discharge during period of seasonal operation, 985 fts/s, at 0638 hours July 9. gage height, $6.60 \mathrm{ft}$ from rating curve extended above $10 \mathrm{cfs}$ on basis of step-backwater analysis of channel; no flow most of time.

DISCEARGE, CUBIC FEET PER SECOND, WATER YEAR OCTOBER 1995 TO SEPTEMBER 1996

\begin{tabular}{|c|c|c|c|c|c|c|c|c|c|c|c|c|}
\hline DAY & $\alpha r$ & Nov & DEC & JAN & FEB & MAR & APR & MAY & JUN & JUL & AUG & SEP \\
\hline $\begin{array}{l}1 \\
2 \\
3 \\
4 \\
5\end{array}$ & $m$ & 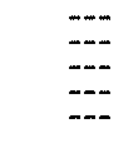 & $\begin{array}{l}-m-m \\
-m \\
\rightarrow-m \\
-m- \\
m-\end{array}$ & 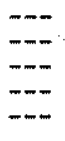 & $\begin{array}{l}m- \\
m-\infty \\
m-m \\
m-m\end{array}$ & $\begin{array}{l}-+- \\
- \\
- \\
- \\
-\infty \\
-\infty\end{array}$ & $\begin{array}{l}.00 \\
.00 \\
.00 \\
.00 \\
.00\end{array}$ & $\begin{array}{l}.00 \\
.00 \\
.00 \\
.00 \\
.00\end{array}$ & $\begin{array}{l}.00 \\
.00 \\
.00 \\
.00 \\
.00\end{array}$ & $\begin{array}{l}.00 \\
.00 \\
.00 \\
.00 \\
.00\end{array}$ & $\begin{array}{l}.00 \\
.00 \\
.00 \\
.00 \\
.00\end{array}$ & $\begin{array}{l}.00 \\
.00 \\
.00 \\
.00 \\
.00\end{array}$ \\
\hline $\begin{array}{r}6 \\
7 \\
8 \\
9 \\
10\end{array}$ & $\begin{array}{l}m-m \\
m-m \\
-m\end{array}$ & $m-m$ & $\begin{array}{l}-- \\
- \\
-- \\
-- \\
--\end{array}$ & $\begin{array}{l}-\cdots \\
-\cdots \\
-\cdots \\
-\cdots\end{array}$ & $\begin{array}{l}m-m \\
m-m \\
m-m \\
m-m \\
m-m\end{array}$ & $\begin{array}{l}-\infty \\
-m \\
m- \\
m-m \\
m-m\end{array}$ & $\begin{array}{l}.00 \\
.00 \\
.00 \\
.00 \\
.00\end{array}$ & $\begin{array}{l}.00 \\
.00 \\
.00 \\
.00 \\
.00\end{array}$ & $\begin{array}{l}.00 \\
.00 \\
.00 \\
.00 \\
.00\end{array}$ & $\begin{array}{r}.00 \\
e .00 \\
01.5 \\
e 40 \\
e 15\end{array}$ & $\begin{array}{l}.00 \\
.00 \\
.00 \\
.00 \\
.00\end{array}$ & $\begin{array}{l}.00 \\
.00 \\
.00 \\
.00 \\
.00\end{array}$ \\
\hline $\begin{array}{l}11 \\
12 \\
13 \\
14 \\
15\end{array}$ & $\begin{array}{l}--\infty \\
--\infty \\
-\infty\end{array}$ & $=$ & $\begin{array}{l}m-m \\
m-m \\
m-m \\
m-m\end{array}$ & $m$ & $\begin{array}{l}m- \\
m-\infty \\
m-m \\
m-\infty \\
m-\infty\end{array}$ & $\begin{array}{l}-m-m \\
-m- \\
.00 \\
.00\end{array}$ & $\begin{array}{l}.00 \\
.00 \\
.00 \\
.00 \\
.00\end{array}$ & $\begin{array}{l}.00 \\
.00 \\
.00 \\
.00 \\
.00\end{array}$ & $\begin{array}{l}.00 \\
.00 \\
.00 \\
.00 \\
.00\end{array}$ & $\begin{array}{r}035 \\
.00 \\
.00 \\
.00 \\
.05\end{array}$ & $\begin{array}{l}.00 \\
.00 \\
.00 \\
.00 \\
.00\end{array}$ & $1 . ._{.00}^{.00}$ \\
\hline $\begin{array}{l}16 \\
17 \\
18 \\
19 \\
20\end{array}$ & $=$ & $\begin{array}{l}m-\infty \\
m-\infty \\
m-\infty\end{array}$ & $\begin{array}{l}--- \\
-m \\
-- \\
-- \\
--\end{array}$ & $m$ & $\begin{array}{l}m-m \\
m-m \\
-m- \\
-m- \\
-m-m\end{array}$ & $\begin{array}{l}.00 \\
.00 \\
.00 \\
.00 \\
.00\end{array}$ & $\begin{array}{l}.00 \\
.00 \\
.00 \\
.00 \\
.00\end{array}$ & $\begin{array}{l}.00 \\
.00 \\
.00 \\
.00 \\
.00\end{array}$ & $\begin{array}{l}.00 \\
.00 \\
.00 \\
.00 \\
.00\end{array}$ & $\begin{array}{l}.00 \\
.00 \\
.00 \\
.00 \\
.00\end{array}$ & $\begin{array}{l}.00 \\
.00 \\
.00 \\
.00 \\
.00\end{array}$ & $\begin{array}{r}.00 \\
6.5 \\
03.5 \\
.00\end{array}$ \\
\hline $\begin{array}{l}21 \\
22 \\
23 \\
24 \\
25\end{array}$ & 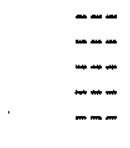 & $m$ & $\begin{array}{l}m-m \\
m-m \\
m-m \\
m-\end{array}$ & $m$ & $\begin{array}{l}-m \\
-+- \\
m- \\
-m \\
-\infty\end{array}$ & $\begin{array}{l}.00 \\
.00 \\
.00 \\
.00 \\
.00\end{array}$ & $\begin{array}{l}.00 \\
.00 \\
.00 \\
.00 \\
.00\end{array}$ & $\begin{array}{l}.00 \\
.00 \\
.00 \\
.00 \\
.00\end{array}$ & $\begin{array}{l}.00 \\
.00 \\
.00 \\
.00 \\
.00\end{array}$ & $\begin{array}{l}.00 \\
.00 \\
.00 \\
.00 \\
.00\end{array}$ & $\begin{array}{r}.00 \\
.29 \\
03.0 \\
02.2 \\
0.00\end{array}$ & $\begin{array}{l}.00 \\
.00 \\
.00 \\
.00 \\
.00\end{array}$ \\
\hline $\begin{array}{l}26 \\
27 \\
28 \\
29 \\
30 \\
31\end{array}$ & - & 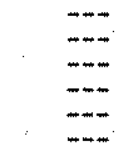 & $\begin{array}{l}m-m \\
m-m \\
m-m \\
m-m \\
m-m\end{array}$ & $\begin{array}{l}m-- \\
m-\infty \\
-\infty \\
-\infty\end{array}$ & $\begin{array}{l}m \\
m-\infty \\
m-\infty \\
m-\infty\end{array}$ & $\begin{array}{l}.00 \\
.00 \\
.00 \\
.00 \\
.00 \\
.00\end{array}$ & $\begin{array}{l}.00 \\
.00 \\
.00 \\
.00 \\
.00 \\
.\end{array}$ & $\begin{array}{l}.00 \\
.00 \\
.00 \\
.00 \\
.00 \\
.00\end{array}$ & $\begin{array}{l}1.3 \\
1.2 \\
40 \\
85.0 \\
19 \\
\end{array}$ & $\begin{array}{l}.00 \\
.00 \\
.00 \\
.00 \\
.00 \\
.00\end{array}$ & $\begin{array}{r}014.06 \\
.00 \\
7.7 \\
.00 \\
.00\end{array}$ & $\begin{array}{l}.00 \\
.00 \\
.00 \\
.00 \\
.00 \\
\end{array}$ \\
\hline $\begin{array}{l}\text { TOTAL } \\
\text { MEAN } \\
\text { MAX } \\
\text { MIN } \\
\text { AC-FT }\end{array}$ & $\begin{array}{l}m- \\
--\infty \\
m-\infty \\
-\infty\end{array}$ & $=$ & $\begin{array}{l}m-- \\
--5 \\
-m- \\
m-m\end{array}$ & $\begin{array}{l}m-\infty \\
m-\infty \\
-\infty \\
m-\infty\end{array}$ & $\begin{array}{l}m \\
m-\infty \\
m-m \\
m-\end{array}$ & $\begin{array}{l}m-m \\
m-\infty \\
m-\infty \\
m-\infty\end{array}$ & $\begin{array}{l}0.00 \\
.000 \\
.00 \\
.00 \\
.00\end{array}$ & $\begin{array}{r}0.00 \\
.000 \\
.00 \\
.00 \\
.00\end{array}$ & $\begin{array}{r}66.50 \\
2.22 \\
40 \\
.00 \\
132\end{array}$ & $\begin{array}{r}91.55 \\
2.95 \\
40 \\
.00 \\
182\end{array}$ & $\begin{array}{r}27.25 \\
.88 \\
14 \\
.00 \\
54\end{array}$ & $\begin{array}{r}29.00 \\
.97 \\
16 \\
.00 \\
58\end{array}$ \\
\hline
\end{tabular}


08330775 SOUTH DIVERSION CHANNEL ABOVE TIJERAS ARROYO NEAR ALBUQUERQUE, NM

LOCATION, -Lat 3500'09", Iong $106^{\circ} 39^{\prime} 02^{\prime \prime}$, Bernalillo County, Hydrologic Unit 13020203, on right bank 600 ft upstream from confluence with Tijeras Arroyo, and $2.5 \mathrm{mi}$ south of Albuquerque.

DRAINAGE AREA, $--11.0 \mathrm{~mL}^{2}$.

PERIOD OF RECORD. - June 1988 to current year.

GAGE. - Water stage recorder and concrete control. Elevation of gage is 4,930 ft above National Geodetic Vertical Datum of 1929 , from topographic map.

REMARKs.--Records Salr except For estimated daily discharges, which are poor.

EXTREMES FOR FERIOD OF RECORD. -Maximum discharge, 1,960 ft\$s, July 14, 1990, gage height, 6.30 Pt Erom Iloodmarks, from rating curve extended above 30 cfs on basis of step-backwater analys of channel; no flow most of time.

EXIREMES FOR CURRENT YTAR, -Maximum discharge, 589 fts/s, at 2055 hours June 26, gage helght, 3.13 ft; no flow most of time.

DISCHARGE, CUBIC FEET PER SECOND, WATER YEAR OCTOBER 1995 TO SEPTEMBER 1996

\begin{tabular}{|c|c|c|c|c|c|c|c|c|c|c|c|c|}
\hline DAY & OCT & NOV & DEC & JAN & FEB & MAR & $\mathbf{A F R}$ & MAY & JUN & JUL & AUG & SEP \\
\hline $\begin{array}{l}1 \\
2 \\
3 \\
4 \\
5\end{array}$ & $\begin{array}{l}.00 \\
.00 \\
.00 \\
.00 \\
.00\end{array}$ & $\begin{array}{l}.00 \\
.00 \\
.00 \\
.00 \\
.00\end{array}$ & $\begin{array}{l}.00 \\
.00 \\
.00 \\
.00 \\
.00\end{array}$ & $\begin{array}{l}.28 \\
.00 \\
.00 \\
.00 \\
.00\end{array}$ & $\begin{array}{r}.00 \\
.00 \\
.00 \\
.00 \\
.00\end{array}$ & $\begin{array}{l}.00 \\
.00 \\
.00 \\
.00 \\
.00\end{array}$ & $\begin{array}{l}.00 \\
.00 \\
.00 \\
.00 \\
.00\end{array}$ & $\begin{array}{l}0.00 \\
0.00 \\
0.00 \\
0.00 \\
0.00\end{array}$ & $\begin{array}{l}.00 \\
.00 \\
.00 \\
.00 \\
.00\end{array}$ & $\begin{array}{l}.00 \\
.00 \\
.00 \\
.00 \\
.00\end{array}$ & $\begin{array}{r}.00 \\
.00 \\
.00 \\
\times 1.7 \\
02.2\end{array}$ & $\begin{array}{l}.00 \\
.00 \\
.00 \\
.00 \\
.00\end{array}$ \\
\hline $\begin{array}{r}6 \\
7 \\
8 \\
9 \\
10\end{array}$ & $\begin{array}{l}.00 \\
.00 \\
.00 \\
.00 \\
.00\end{array}$ & $\begin{array}{l}.00 \\
.00 \\
.00 \\
.00 \\
.00\end{array}$ & $\begin{array}{l}.00 \\
.00 \\
.00 \\
.00 \\
.00\end{array}$ & $\begin{array}{l}.00 \\
.00 \\
.00 \\
.00 \\
.00\end{array}$ & $\begin{array}{l}.00 \\
.00 \\
.00 \\
.00 \\
.00\end{array}$ & $\begin{array}{l}.00 \\
.00 \\
.00 \\
.00 \\
.00\end{array}$ & $\begin{array}{l}.00 \\
.00 \\
.00 \\
.00 \\
.00\end{array}$ & $\begin{array}{r}0.00 \\
.00 \\
.00 \\
.00 \\
.00\end{array}$ & $\begin{array}{l}.00 \\
.00 \\
.00 \\
.00 \\
.00\end{array}$ & $\begin{array}{r}.00 \\
.00 \\
.00 \\
.00 \\
.00\end{array}$ & $\begin{array}{l}.00 \\
.00 \\
.00 \\
.00 \\
.00\end{array}$ & $\begin{array}{l}.00 \\
.00 \\
.00 \\
.00 \\
.00\end{array}$ \\
\hline $\begin{array}{l}11 \\
12 \\
13 \\
14 \\
15\end{array}$ & $\begin{array}{l}.00 \\
.00 \\
.01 \\
.00 \\
.00\end{array}$ & $\begin{array}{l}.00 \\
.00 \\
.00 \\
.00 \\
.00\end{array}$ & $\begin{array}{r}.00 \\
.00 \\
.00 \\
.00 \\
.00\end{array}$ & $\begin{array}{l}.00 \\
.00 \\
.00 \\
.00 \\
.00\end{array}$ & $\begin{array}{l}.00 \\
.00 \\
.00 \\
.00 \\
.00\end{array}$ & $\begin{array}{l}.00 \\
.00 \\
.00 \\
.00 \\
.00\end{array}$ & $\begin{array}{l}.00 \\
.00 \\
.00 \\
.00 \\
.00\end{array}$ & $\begin{array}{l}.00 \\
.00 \\
.00 \\
.00 \\
.00\end{array}$ & $\begin{array}{l}.00 \\
.00 \\
.00 \\
.00 \\
.00\end{array}$ & $\begin{array}{l}.00 \\
.00 \\
.00 \\
.00 \\
.00\end{array}$ & $\begin{array}{l}.00 \\
.00 \\
.00 \\
.00 \\
.00\end{array}$ & $\begin{array}{r}.00 \\
.00 \\
.00 \\
07.3\end{array}$ \\
\hline $\begin{array}{l}16 \\
17 \\
18 \\
19 \\
20\end{array}$ & $\begin{array}{r}.00 \\
.00 \\
.00 \\
e .00 \\
.00\end{array}$ & $\begin{array}{r}.00 \\
.00 \\
.00 \\
.00 \\
.00\end{array}$ & $\begin{array}{l}.50 \\
.00 \\
.00 \\
.00 \\
.00\end{array}$ & $\begin{array}{l}.00 \\
.00 \\
.00 \\
.00 \\
.00\end{array}$ & $\begin{array}{l}.00 \\
.00 \\
.00 \\
.00 \\
.00\end{array}$ & $\begin{array}{l}.00 \\
.00 \\
.00 \\
.00 \\
.00\end{array}$ & $\begin{array}{l}.00 \\
.00 \\
.00 \\
.00 \\
.00\end{array}$ & $\begin{array}{l}.00 \\
.00 \\
.00 \\
.00 \\
.00\end{array}$ & $\begin{array}{l}.00 \\
.00 \\
.00 \\
.00 \\
0.00\end{array}$ & $\begin{array}{l}.00 \\
0.00 \\
4.6 \\
.00 \\
.00\end{array}$ & $\begin{array}{l}.00 \\
.00 \\
.00 \\
.00 \\
.00\end{array}$ & $\begin{array}{r}0.00 \\
1.6 \\
3.5 \\
01.1 \\
.00\end{array}$ \\
\hline $\begin{array}{l}21 \\
22 \\
23 \\
24 \\
25\end{array}$ & $\begin{array}{l}.00 \\
.00 \\
.00 \\
.00 \\
.00\end{array}$ & $\begin{array}{l}.00 \\
.00 \\
.00 \\
.00 \\
.00\end{array}$ & $\begin{array}{l}.50 \\
.00 \\
.00 \\
.00 \\
.00\end{array}$ & $\begin{array}{l}.00 \\
.00 \\
.00 \\
.00 \\
.00\end{array}$ & $\begin{array}{l}.00 \\
.00 \\
.00 \\
.00 \\
.00\end{array}$ & $\begin{array}{l}.00 \\
.00 \\
.00 \\
.00 \\
.00\end{array}$ & $\begin{array}{r}.00 \\
.00 \\
.00 \\
.00 \\
0.00\end{array}$ & $\begin{array}{l}.00 \\
.00 \\
.00 \\
.00 \\
.00\end{array}$ & $\begin{array}{l}.00 \\
0.00 \\
.00 \\
.00 \\
.00\end{array}$ & $\begin{array}{l}.00 \\
.00 \\
.00 \\
.00 \\
.00\end{array}$ & $\begin{array}{r}.00 \\
.00 \\
.4 \\
.00 \\
.00\end{array}$ & $\begin{array}{l}.00 \\
.00 \\
.00 \\
.00 \\
.00\end{array}$ \\
\hline $\begin{array}{l}26 \\
27 \\
28 \\
29 \\
30 \\
31\end{array}$ & $\begin{array}{l}.00 \\
.00 \\
.00 \\
.00 \\
.00 \\
.00\end{array}$ & $\begin{array}{l}.00 \\
.00 \\
.00 \\
.00 \\
.00 \\
.0\end{array}$ & $\begin{array}{l}.00 \\
.00 \\
.00 \\
.00 \\
.00 \\
.00\end{array}$ & $\begin{array}{l}.00 \\
.00 \\
.00 \\
.00 \\
.00 \\
.00\end{array}$ & $\begin{array}{l}.00 \\
.00 \\
.00 \\
.00 \\
--- \\
---\end{array}$ & $\begin{array}{l}.00 \\
.00 \\
.00 \\
.00 \\
.00 \\
.00\end{array}$ & $\begin{array}{l}0.00 \\
0.00 \\
0.00 \\
0.00 \\
0.00 \\
-.-\end{array}$ & $\begin{array}{l}.00 \\
.00 \\
.00 \\
.00 \\
.00 \\
.00\end{array}$ & $\begin{array}{r}37 \\
35 \\
\text { e15 } \\
e 5.5 \\
\text { e1.7 }\end{array}$ & $\begin{array}{l}.00 \\
.00 \\
.00 \\
.00 \\
.00 \\
.00\end{array}$ & $\begin{array}{r}041 \\
.00 \\
.00 \\
11 . \\
06.9 \\
.00\end{array}$ & $\begin{array}{l}.00 \\
.00 \\
.00 \\
.00 \\
.00 \\
.\end{array}$ \\
\hline $\begin{array}{l}\text { MEAN } \\
\text { MAX } \\
\text { MIN } \\
\text { AC-ET }\end{array}$ & $\begin{array}{l}.000 \\
.01 \\
.00 \\
.02\end{array}$ & $\begin{array}{l}.000 \\
.00 \\
.00 \\
.00\end{array}$ & $\begin{array}{r}.032 \\
.50 \\
.00 \\
2.0\end{array}$ & $\begin{array}{r}.009 \\
.28 \\
.00 \\
.6\end{array}$ & $\begin{array}{r}.000 \\
.00 \\
.00 \\
.00\end{array}$ & $\begin{array}{r}.000 \\
.00 \\
.00 \\
.00\end{array}$ & $\begin{array}{l}.000 \\
.00 \\
.00 \\
.00\end{array}$ & $\begin{array}{r}.000 \\
.00 \\
.00 \\
.00\end{array}$ & $\begin{array}{r}3.14 \\
37 \\
.00 \\
187\end{array}$ & $\begin{array}{l}23 \\
4.6 \\
.00 \\
14\end{array}$ & $\begin{array}{r}2.26 \\
41 \\
.00 \\
139\end{array}$ & $\begin{array}{r}1.45 \\
30 \\
.00 \\
86\end{array}$ \\
\hline
\end{tabular}

CAL YR 1995 MEAN .30 MAX 25 MIN .00 AC $-F T 216$

WTR YR 1996 MEAN .59 MAX 41 MIN .00 AC-FT 429

- Estimated 
RIO GRANDE BASIN

08331000 RIO GRANDE AT ISLETA, NM

LOCATION,--Lat $34^{\circ} 55^{\prime} 4^{\prime \prime}$, long $106^{\circ} 40^{\circ} 44^{\prime \prime}$, in NE/KSE $/ 4$ SWK sec, 13, T.8 N., R.2 E., Valencia County, Hydrologic Unit 13020203 , in Isleta Pueblo Grant, on right bank $0.5 \mathrm{mi}$ upstream from Isleta Diversion Dam, 1.0 mi west from State Highway $47,1.2 \mathrm{mi}$ from Isleta Pueblo, and at mile 1527.7 .

DRATNAGE AREA. - -18,100 $\mathrm{mi}^{2}$ (estimated), including 2,940 $\mathrm{mi}^{2}$ in closed basin in San Luis valley, Co.

WATER-DISCHARGE RECORDS

PERIOD OF RECORD. --October 1995 to current year.

GAGE. - Water-stage recorder. Elevation of gage is 4,890 ft above National Vertical Datum of 1929, from topographic map.

REMARKS. -Water-discharge recorder good except for estimated datly discharges, which are poor. Flow completely

regulated since November 1993 by (Cochitt Dam station 08317300) 11 mi upstream. Posstble regulation by operation of regulated on Rio Chama and by flood and silt-detention reservotrs on Galisteo Creek and Jemez River (stations
$08285000,08286900,083179000,08328500)$. Diversion upstream from station for irrigation of about 752,000 acres.

DISCEARGE, CUBIC FEET PER SECOND, WATER YEAR OCTOBER 1995 TO SEPTEMBER 1996

\begin{tabular}{|c|c|c|c|c|c|c|c|c|c|c|c|c|}
\hline DAY & $\operatorname{oct}$ & Hov & $\mathrm{DEC}$ & JAN & FEB & MAR & $A P R$ & MAY & JUN & JUL & AUG & SEP \\
\hline $\begin{array}{l}1 \\
2 \\
3 \\
4 \\
5\end{array}$ & $\begin{array}{l}915 \\
976 \\
974 \\
935 \\
922\end{array}$ & $\begin{array}{l}1110 \\
1070 \\
1060 \\
1070 \\
1060\end{array}$ & $\begin{array}{l}1090 \\
1100 \\
1130 \\
1140 \\
1130\end{array}$ & $\begin{array}{l}1190 \\
1190 \\
1200 \\
1270 \\
1270\end{array}$ & $\begin{array}{l}1290 \\
1320 \\
1300 \\
1290 \\
1280\end{array}$ & $\begin{array}{l}1240 \\
1320 \\
1390 \\
1390 \\
1350\end{array}$ & $\begin{array}{l}728 \\
708 \\
703 \\
698 \\
655\end{array}$ & $\begin{array}{l}546 \\
556 \\
556 \\
553 \\
541\end{array}$ & $\begin{array}{l}1680 \\
1630 \\
1630 \\
1630 \\
1700\end{array}$ & $\begin{array}{l}1940 \\
1650 \\
1550 \\
1500 \\
1400\end{array}$ & $\begin{array}{l}1920 \\
1810 \\
1800 \\
1880 \\
1930\end{array}$ & $\begin{array}{l}1940 \\
1900 \\
1880 \\
1890 \\
1920\end{array}$ \\
\hline $\begin{array}{r}6 \\
7 \\
8 \\
9 \\
10\end{array}$ & $\begin{array}{l}922 \\
947 \\
963 \\
968 \\
963\end{array}$ & $\begin{array}{l}1040 \\
1080 \\
1110 \\
1110 \\
1100\end{array}$ & $\begin{array}{l}1110 \\
1070 \\
1110 \\
1120 \\
1150\end{array}$ & $\begin{array}{l}1240 \\
1220 \\
1250 \\
1290 \\
1360\end{array}$ & $\begin{array}{l}1240 \\
1180 \\
1180 \\
1230 \\
1220\end{array}$ & $\begin{array}{l}1300 \\
1340 \\
1330 \\
1310 \\
1320\end{array}$ & $\begin{array}{l}615 \\
590 \\
564 \\
550 \\
543\end{array}$ & $\begin{array}{l}533 \\
538 \\
533 \\
533 \\
553\end{array}$ & $\begin{array}{l}1620 \\
1610 \\
1660 \\
1720 \\
1750\end{array}$ & $\begin{array}{l}1350 \\
1350 \\
1580 \\
1840 \\
2230\end{array}$ & $\begin{array}{l}1920 \\
1860 \\
2000 \\
2240 \\
1910\end{array}$ & $\begin{array}{l}2030 \\
2040 \\
1990 \\
2080 \\
2010\end{array}$ \\
\hline $\begin{array}{l}11 \\
12 \\
13 \\
14 \\
15\end{array}$ & $\begin{array}{l}934 \\
928 \\
938 \\
926 \\
930\end{array}$ & $\begin{array}{l}1080 \\
1060 \\
1050 \\
1000 \\
1060\end{array}$ & $\begin{array}{l}1150 \\
1140 \\
1130 \\
1140 \\
1170\end{array}$ & $\begin{array}{l}1370 \\
1320 \\
1260 \\
1260 \\
1260\end{array}$ & $\begin{array}{l}1230 \\
1210 \\
1250 \\
1330 \\
1400\end{array}$ & $\begin{array}{l}1340 \\
1330 \\
1330 \\
1340 \\
1350\end{array}$ & $\begin{array}{l}536 \\
576 \\
541 \\
532 \\
540\end{array}$ & $\begin{array}{l}556 \\
555 \\
559 \\
555 \\
556\end{array}$ & $\begin{array}{l}1760 \\
1800 \\
1770 \\
1820 \\
1970\end{array}$ & $\begin{array}{l}2580 \\
2580 \\
1930 \\
1740 \\
1810\end{array}$ & $\begin{array}{l}1830 \\
1770 \\
1690 \\
1640 \\
1590\end{array}$ & $\begin{array}{l}1910 \\
1840 \\
1940 \\
1980 \\
2520\end{array}$ \\
\hline $\begin{array}{l}16 \\
17 \\
18 \\
19 \\
20\end{array}$ & $\begin{array}{l}930 \\
940 \\
949 \\
947 \\
939\end{array}$ & $\begin{array}{r}1020 \\
1020 \\
1010 \\
1010 \\
995\end{array}$ & $\begin{array}{l}1180 \\
1190 \\
1210 \\
1230 \\
1220\end{array}$ & $\begin{array}{l}1260 \\
1270 \\
1290 \\
1310 \\
1290\end{array}$ & $\begin{array}{l}1360 \\
1320 \\
1270 \\
1280 \\
1290\end{array}$ & $\begin{array}{l}1370 \\
1400 \\
1430 \\
1410 \\
1390\end{array}$ & $\begin{array}{l}545 \\
574 \\
643 \\
590 \\
574\end{array}$ & $\begin{array}{l}561 \\
590 \\
580 \\
564 \\
596\end{array}$ & $\begin{array}{l}1930 \\
1860 \\
1880 \\
1870 \\
1880\end{array}$ & $\begin{array}{l}1810 \\
1750 \\
1870 \\
1740 \\
1580\end{array}$ & $\begin{array}{l}1550 \\
1540 \\
1630 \\
1720 \\
1690\end{array}$ & $\begin{array}{l}2090 \\
1930 \\
2070 \\
2120 \\
1920\end{array}$ \\
\hline $\begin{array}{l}21 \\
22 \\
23 \\
24 \\
25\end{array}$ & $\begin{array}{r}943 \\
946 \\
978 \\
1020 \\
968\end{array}$ & $\begin{array}{l}1060 \\
1170 \\
1120 \\
1080 \\
1080\end{array}$ & $\begin{array}{l}1160 \\
1100 \\
1090 \\
1100 \\
1110\end{array}$ & $\begin{array}{l}1280 \\
1280 \\
1280 \\
1260 \\
1240\end{array}$ & $\begin{array}{l}1340 \\
1450 \\
1460 \\
1420 \\
1390\end{array}$ & $\begin{array}{l}1340 \\
1300 \\
1280 \\
1280 \\
1310\end{array}$ & $\begin{array}{l}641 \\
647 \\
642 \\
641 \\
623\end{array}$ & $\begin{array}{l}1910 \\
2090 \\
1780 \\
1750 \\
1750\end{array}$ & $\begin{array}{l}1720 \\
1670 \\
1680 \\
1680 \\
1630\end{array}$ & $\begin{array}{l}1540 \\
1560 \\
1600 \\
1730 \\
1730\end{array}$ & $\begin{array}{l}1710 \\
1860 \\
2190 \\
2450 \\
2430\end{array}$ & $\begin{array}{l}1910 \\
2090 \\
2090 \\
2050 \\
1840\end{array}$ \\
\hline $\begin{array}{l}26 \\
27 \\
28 \\
29 \\
30 \\
31\end{array}$ & $\begin{array}{r}1010 \\
1050 \\
1030 \\
972 \\
993 \\
1050\end{array}$ & $\begin{array}{l}1080 \\
1120 \\
1150 \\
1110 \\
1100\end{array}$ & $\begin{array}{l}1110 \\
1120 \\
1110 \\
1110 \\
1120 \\
1140\end{array}$ & $\begin{array}{l}1240 \\
1240 \\
1260 \\
1250 \\
1250 \\
1270\end{array}$ & $\begin{array}{r}1390 \\
1400 \\
1400 \\
1360 \\
--0\end{array}$ & $\begin{array}{r}1260 \\
1160 \\
1010 \\
946 \\
858 \\
810\end{array}$ & $\begin{array}{l}616 \\
551 \\
546 \\
544 \\
548\end{array}$ & $\begin{array}{l}1800 \\
1870 \\
1870 \\
1850 \\
1820 \\
1780\end{array}$ & $\begin{array}{l}1750 \\
2980 \\
2500 \\
2420 \\
2190\end{array}$ & $\begin{array}{l}1780 \\
1840 \\
1910 \\
1960 \\
1960 \\
1960\end{array}$ & $\begin{array}{l}2980 \\
2300 \\
2080 \\
2160 \\
2330 \\
2050\end{array}$ & $\begin{array}{l}1730 \\
1700 \\
1710 \\
1690 \\
1710\end{array}$ \\
\hline $\begin{array}{l}\text { TOTAL } \\
\text { MEAN } \\
\text { MAX } \\
\text { MIN } \\
\text { AC-FT }\end{array}$ & $\begin{array}{r}29806 \\
961 \\
1050 \\
915 \\
59120\end{array}$ & $\begin{array}{r}32185 \\
1073 \\
1170 \\
995 \\
63840\end{array}$ & $\begin{array}{r}35180 \\
1135 \\
1230 \\
1070 \\
69780\end{array}$ & $\begin{array}{r}39220 \\
1265 \\
1370 \\
1190 \\
77790\end{array}$ & $\begin{array}{r}38080 \\
1313 \\
1460 \\
1180 \\
75530\end{array}$ & $\begin{array}{r}39534 \\
1275 \\
1430 \\
810 \\
78420\end{array}$ & $\begin{array}{r}18004 \\
600 \\
728 \\
532 \\
35710\end{array}$ & $\begin{array}{r}31384 \\
1012 \\
2090 \\
533 \\
62250\end{array}$ & $\begin{array}{r}55390 \\
1846 \\
2980 \\
1610 \\
109900\end{array}$ & $\begin{array}{r}55350 \\
1785 \\
2580 \\
1350 \\
109800\end{array}$ & $\begin{array}{r}60460 \\
1950 \\
2980 \\
1540 \\
119900\end{array}$ & $\begin{array}{r}58520 \\
1951 \\
2520 \\
1690 \\
116100\end{array}$ \\
\hline
\end{tabular}

STATISTICS OF MONTHLY MEAN DATA FOR WATER YEARS 1995 - 1996, BX WATER YEAR (WY)

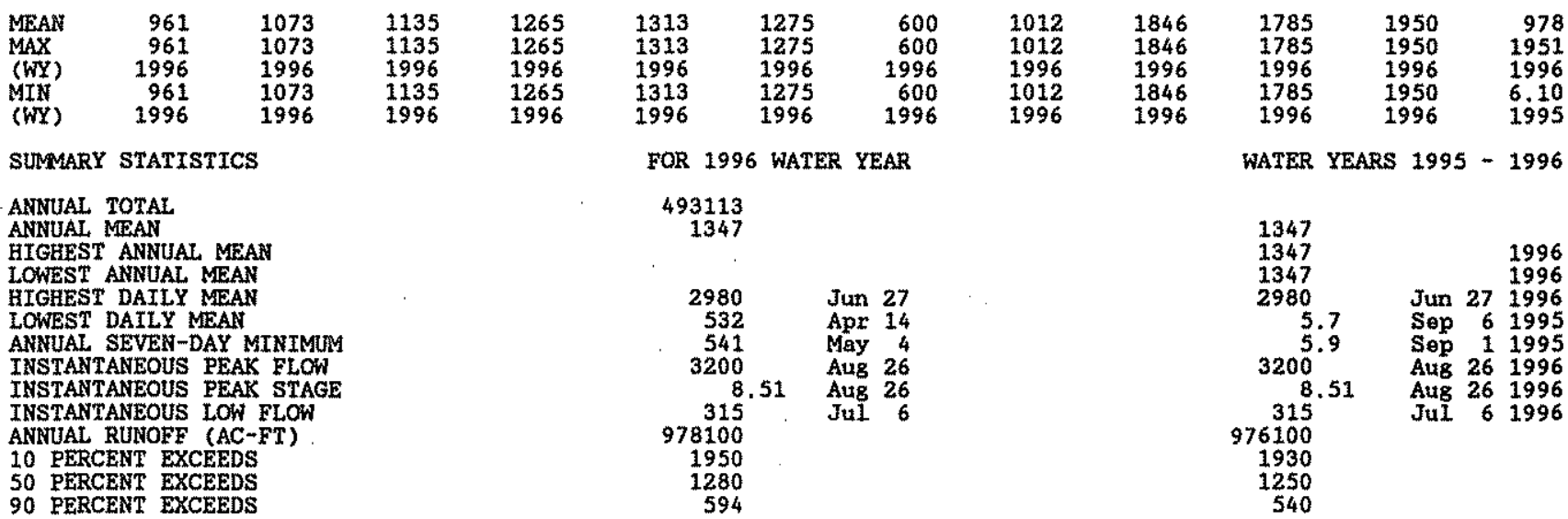


LOCATION.--Lat $34^{\circ} 55^{\prime} 14^{\prime \prime}$, Long $106^{\circ} 40^{\prime} 44^{\prime \prime}$, in NEUSELSWh sec. 13, T.8 N, R. 2 E., Valoncta County, Hydrologic Unit 13020203 , in Isleta Públo Grant, on right bank 0.5 mi upstream Erom Isieta Diversion Dam, 1.0 mi west from State Highway $47,1.2 \mathrm{mi}$ from Isleta Pueblo, and at mile 1527.7 .

DRAINAGE AREA. $--18,100 \mathrm{ml}^{2}$ (estimated).

PERIOD OF RECORD. --Water years 1972 to current year,

REMARKS.-Water-discharge measurements were made at the time water-quality samples were collected. Samples collectod upstream of bridge during periods of low flow.

WATER QUALITY DATA, WATER YEAR OCTOBER 1995 TO SEPTEMBER 1996

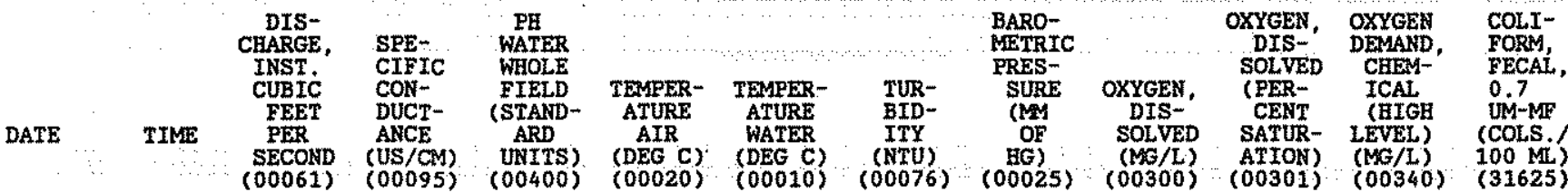

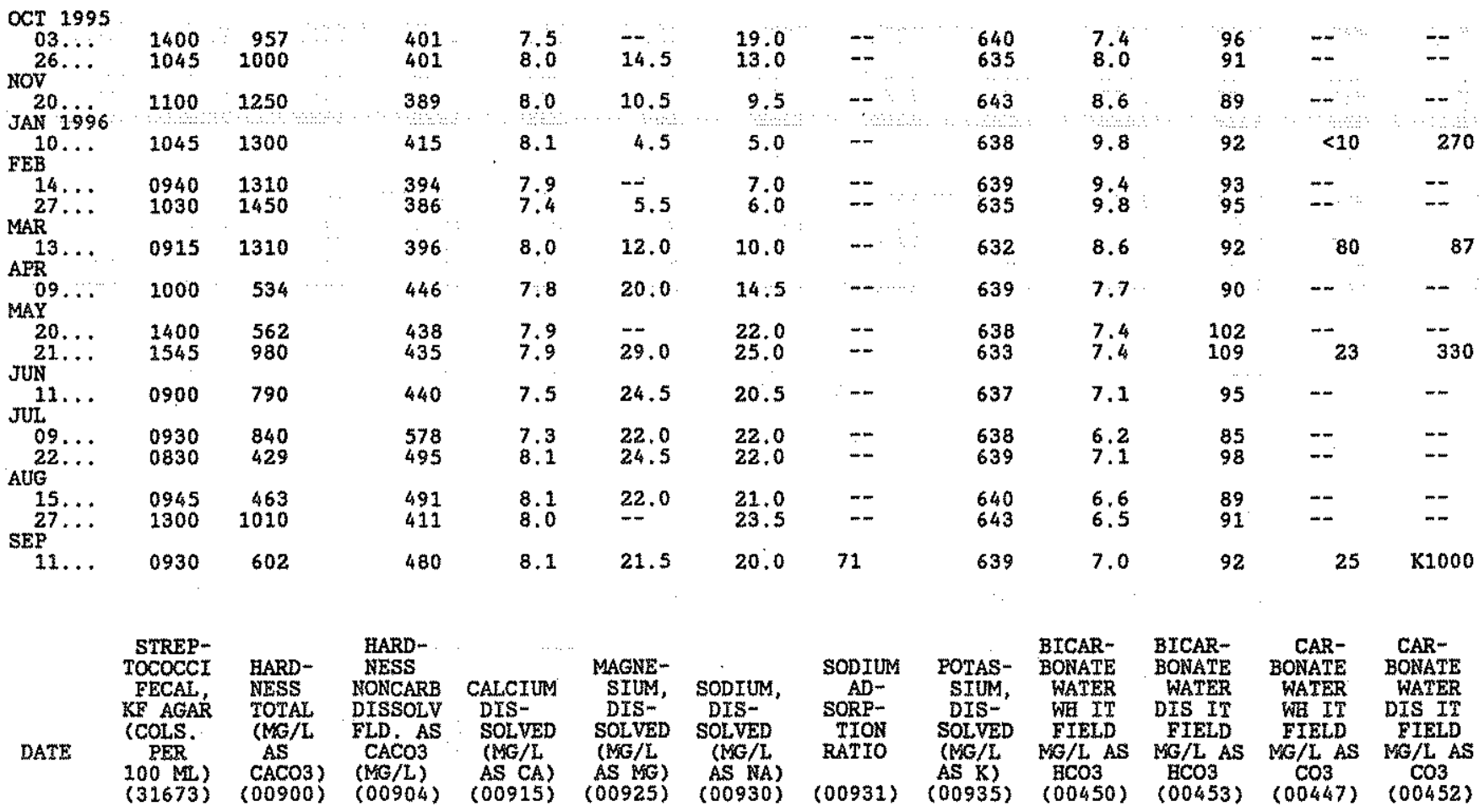

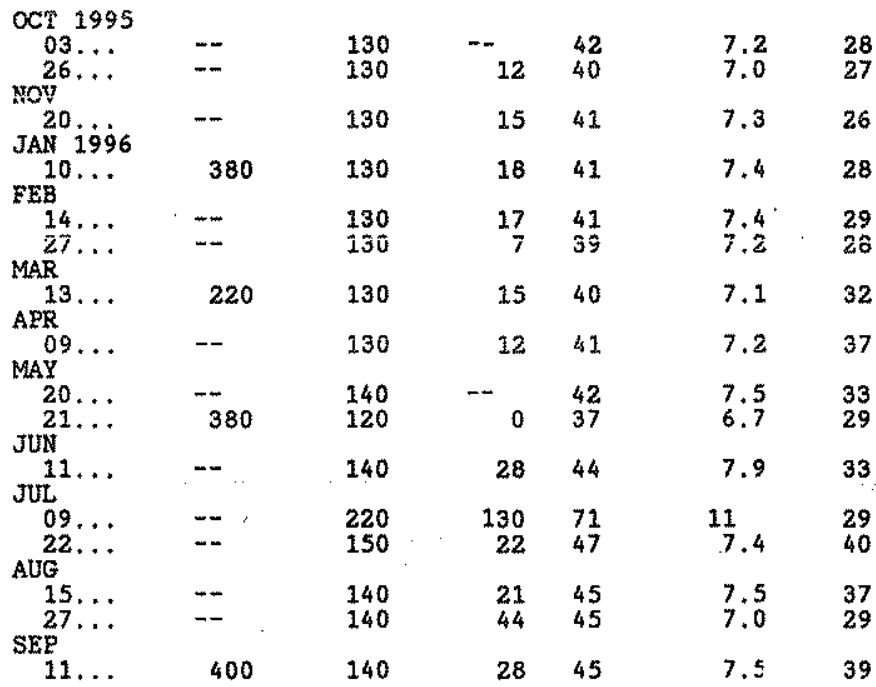

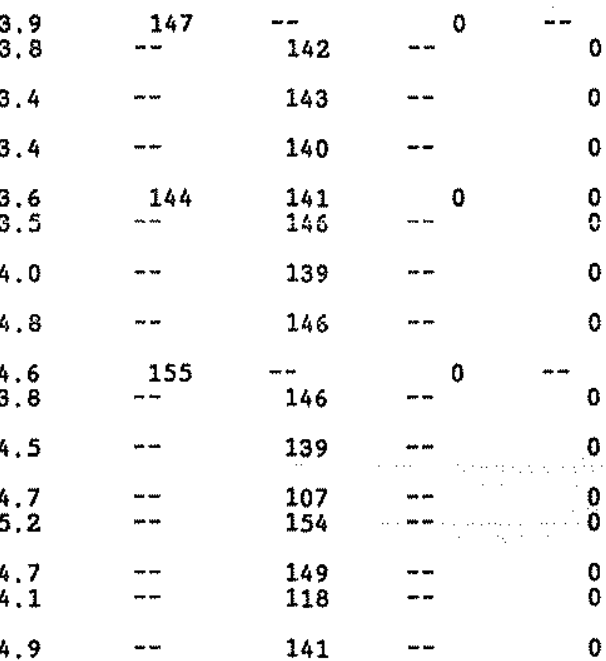


RIO GRANDE BASIN

08331000 RIO GRANDE AT ISLETA, NM -- Continued

WATER-QUAL,ITY RECORDS

WATER QUALITY DATA, WATER YEAR OCTOBER 1995 TO SEPTEMBER 1996

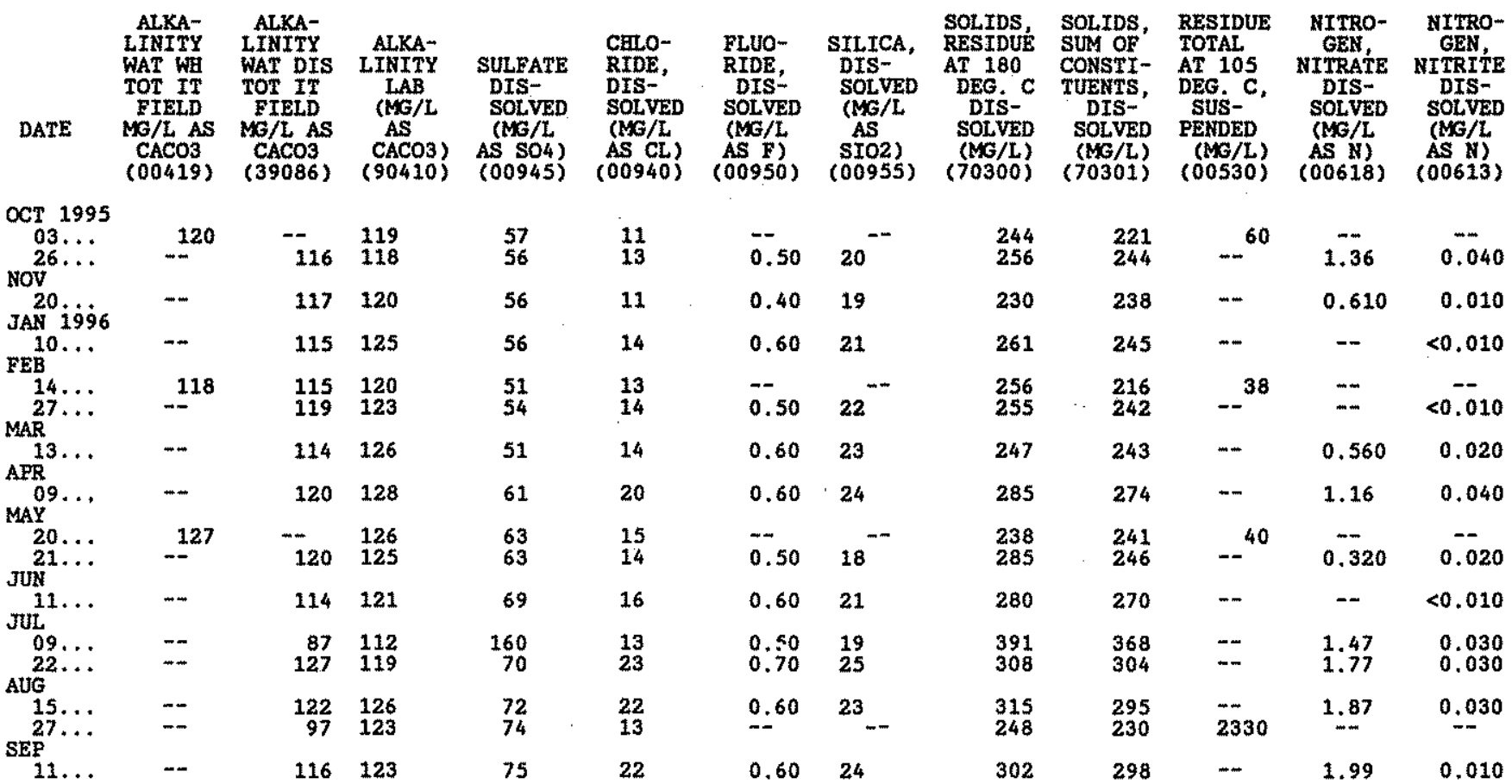

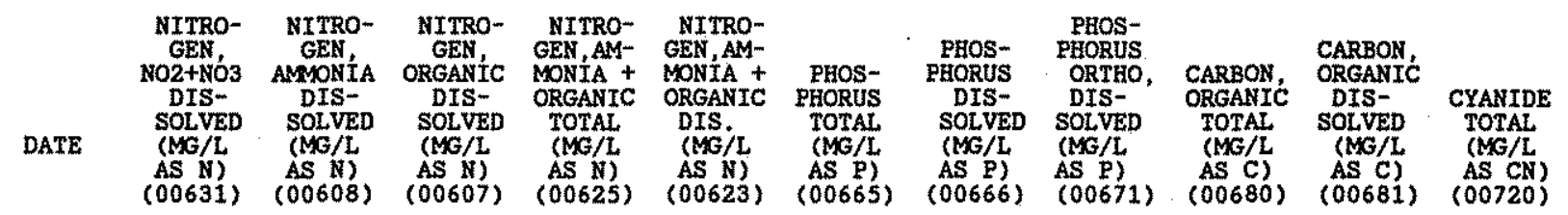

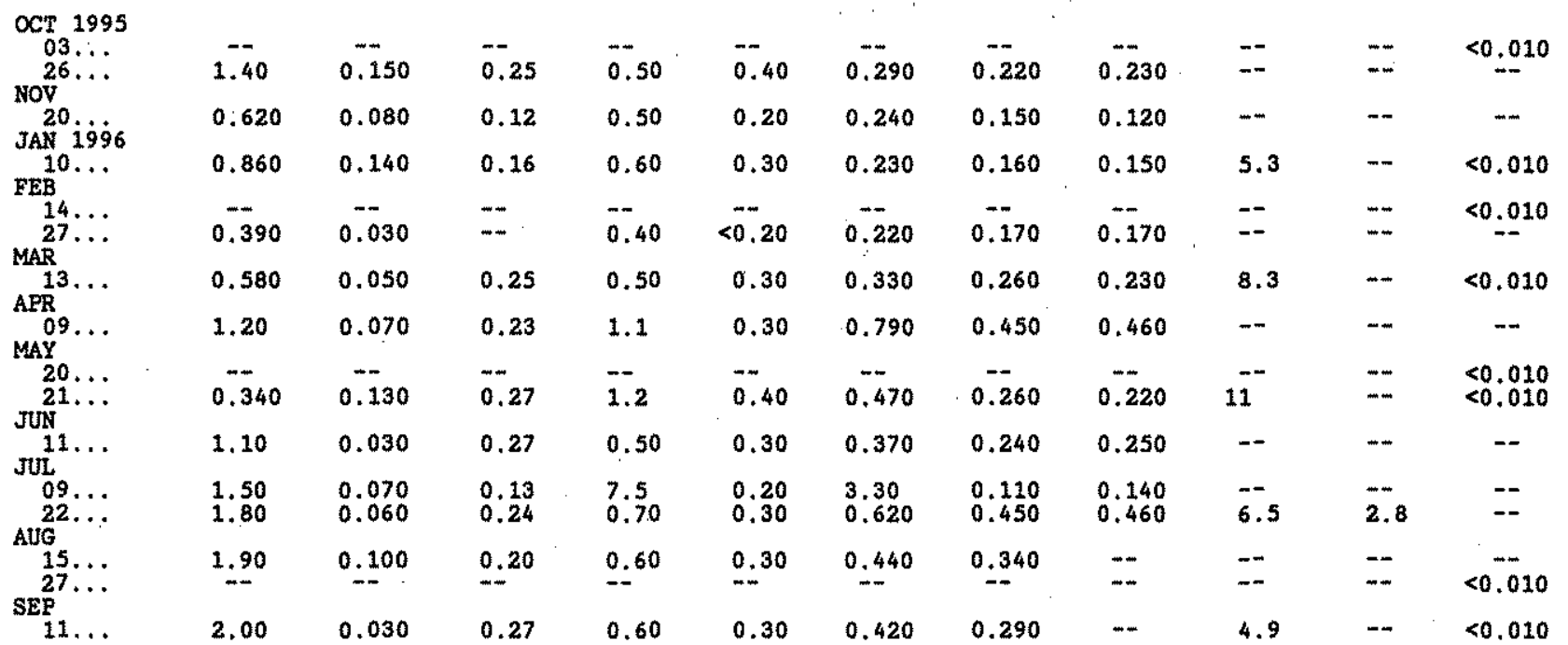


RIO GRANDE BASIN

08331000 RIO GRANDE AT ISLETA, NM -- Continued

WATER-QUALITY RECORDS

WATER QUALITY DATA, WATER YEAR OCTOBER 1995 TO SEPTEMBER 1996

\begin{tabular}{|c|c|c|c|c|c|c|c|c|c|c|c|}
\hline DATE & $\begin{array}{c}\text { ALUM- } \\
\text { INUMA } \\
\text { TOOAL } \\
\text { RECOV- } \\
\text { ERALE } \\
\text { (USL/L } \\
\text { AS AL) } \\
(01105)\end{array}$ & $\begin{array}{l}\text { ALUM- } \\
\text { INUM, } \\
\text { DIS- } \\
\text { SoOVED } \\
\text { (US/L } \\
\text { AS AL) } \\
(01106)\end{array}$ & $\begin{array}{l}\text { ANTI- } \\
\text { MONY } \\
\text { DIS- } \\
\text { SOLED } \\
\text { (UG/L } \\
\text { AS SB }) \\
(01095)\end{array}$ & $\begin{array}{c}\text { ARSENIC } \\
\text { TOTAL } \\
\text { (US/L } \\
\text { AS AS }) \\
(01002)\end{array}$ & $\begin{array}{c}\text { ARSENIC } \\
\text { DIS- } \\
\text { SOLVED } \\
\text { CUG/L } \\
\text { AS AS } \\
(01000)\end{array}$ & $\begin{array}{c}\text { BARIUM, } \\
\text { DIS- } \\
\text { SOLVED } \\
\text { (UELL } \\
\text { AS BA }) \\
(01005)\end{array}$ & $\begin{array}{l}\text { BERYL- } \\
\text { IIIM- } \\
\text { DIS- } \\
\text { SOLVED } \\
\text { (UG/L } \\
\text { AS BE) } \\
(01010)\end{array}$ & $\begin{array}{c}\text { BORON, } \\
\text { DIS- } \\
\text { SOLVED } \\
\text { (UG } \\
\text { AS B } \\
(01020)\end{array}$ & $\begin{array}{c}\text { CADMIUM } \\
\text { DIS- } \\
\text { SOLVED } \\
\text { (USGL } \\
\text { AS CD) } \\
(01025)\end{array}$ & $\begin{array}{c}\text { CARO- } \\
\text { MIUM- } \\
\text { DIS- } \\
\text { SOLVED } \\
\text { (UG/L } \\
\text { AS CR) } \\
\text { (01030) }\end{array}$ & $\begin{array}{c}\text { COBALT, } \\
\text { DIS- } \\
\text { SOLVED } \\
\text { (UG/L } \\
\text { AS CO) } \\
(01035)\end{array}$ \\
\hline $\begin{array}{c}\mathrm{OCT}_{0} 1995 \\
03 \ldots \\
26 \ldots\end{array}$ & 1600 & 4.0 & $<1.0$ & $--^{3}$ & $\ldots^{3}$ & 68 & $<1,0$ & $\because$ & $<1.0$ & 2.0 & $<1.0$ \\
\hline & $\cdots$ & 28 & $<1.0$ & $\cdots$ & 3 & 69 & $<1.0$ & -- & $<1.0$ & 2.0 & $<1.0$ \\
\hline $10 \ldots$ & -- & -- & -- & $\cdots$ & - & -- & -- & 70 & - & - & -- \\
\hline $\begin{array}{r}14 \ldots \\
27 \ldots \\
\operatorname{MAR}\end{array}$ & 360 & 20 & $<1.0$ & $--^{3}$ & $n^{3}$ & 67 & $<1.0$ & $=-$ & $<1.0$ & 1.0 & $<1.0$ \\
\hline $13 \ldots$ & - & - & -- & -- & -- & -- & -- & 60 & $\because$ & - & -- \\
\hline${ }_{\mathrm{MAY}}{ }^{\prime} \ldots$ & $\cdots$ & -- & $\because$ & -- & -- & $\cdots$ & -- & -- & - & -- & -- \\
\hline & -730 & 3.0 & $<1.0$ & $--^{3}$ & $--^{3}$ & 64 & $<1.0$ & $\ddot{80}$ & $<1.0$ & $<1.0$ & $<1.0$ \\
\hline $11 \ldots$ & -- & -- & - & -- & -- & -- & -- & $\because$ & $-\cdots$ & $-m$ & -- \\
\hline $09 \ldots$ & $=-$ & $=$ & $=$ & $=$ & - & $=$ & $=$ & $=$ & $\therefore$ & $=$ & $=$ \\
\hline $\begin{array}{l}\text { AUG } \\
15 \% \ldots\end{array}$ & 31000 & 3.0 & $<\overline{1.0}$ & ${ }_{3}$ & $-{ }_{3}$ & 88 & $<\overline{1.0}$ & $=$ & $<-i .0$ & $<1.0$ & $<\ddot{1.0} 0$ \\
\hline $11 \ldots$ & -- & 18 & $<1.0$ & -- & 4 & 89 & $<1.0$ & 102 & $<1.0$ & $<1.0$ & $<1.0$ \\
\hline DATE & $\begin{array}{c}\text { COPPER, } \\
\text { DIS- } \\
\text { SOLVED } \\
\text { (USG/L } \\
\text { AS CU) } \\
(01040)\end{array}$ & $\begin{array}{l}\text { IRON, } \\
\text { DIS- } \\
\text { SOLVED } \\
\text { (UG/L } \\
\text { AS EE) } \\
(01046)\end{array}$ & $\begin{array}{l}\text { LEAD, } \\
\text { DII- } \\
\text { SOLVED } \\
\text { (US/L } \\
\text { AS PB }) \\
(01049)\end{array}$ & $\begin{array}{l}\text { MANGA- } \\
\text { NESEA, } \\
\text { DIS- } \\
\text { SOLVED } \\
\text { (US MLL } \\
\text { AS MN) } \\
(01056)\end{array}$ & $\begin{array}{l}\text { MERCURY } \\
\text { TOTAL } \\
\text { RECOV- } \\
\text { ERABLE } \\
\text { GUG/L } \\
\text { AS HG) } \\
(71900)\end{array}$ & $\begin{array}{c}\text { MOLYB- } \\
\text { DENUM- } \\
\text { DLS- } \\
\text { SOLED } \\
\text { (UG } / 1 \\
\text { AS WO } \\
(01060)\end{array}$ & $\begin{array}{l}\text { NICKEL, } \\
\text { DIS- } \\
\text { SOLVED } \\
\text { (UG/L } \\
\text { AS NI }) \\
(01065)\end{array}$ & $\begin{array}{l}\text { SELE- } \\
\text { NUMA } \\
\text { TOTAL } \\
\text { UG /L } \\
\text { AS SE) } \\
(01147)\end{array}$ & $\begin{array}{l}\text { SELE- } \\
\text { NIUM, } \\
\text { DIS } \\
\text { SoLVD } \\
\text { (UUG/L } \\
\text { AS SE) } \\
(01145)\end{array}$ & $\begin{array}{c}\text { SILVER, } \\
\text { TOTALI } \\
\text { RECOV- } \\
\text { ERABLE } \\
\text { (UG/L } \\
\text { AS AG) } \\
\text { (01077) }\end{array}$ & $\begin{array}{c}\text { SILVER, } \\
\text { DIS- } \\
\text { SOLVDD } \\
\text { (UG/L } \\
\text { AS AG) } \\
(01075)\end{array}$ \\
\hline $\begin{array}{c}\text { OCT } 1995 \\
03 \ldots \\
26 \ldots\end{array}$ & 1.0 & 18 & $<1.0$ & 18.0 & $=$ & 6.0 & 1.0 & $=$ & $\because$ & $\ldots 1$ & $<0.20$ \\
\hline 20 & 2.0 & 16 & $<1,0$ & 16 & $<0.10$ & 5.0 & $<1.0$ & $<1$ & $<1$ & - & $<1.0$ \\
\hline $10 \ldots$ & -- & 7.0 & -- & 18 & - & -- & -- & -- & - & $\cdots$ & -- \\
\hline $\begin{array}{l}14 \ldots \\
27 \ldots\end{array}$ & 2.0 & 8.0 & $<1,0$ & $\begin{array}{l}18 \\
16\end{array}$ & $=$ & 5.0 & 1.0 & $=$ & $=$ & $--^{<1}$ & $<0.20$ \\
\hline $13 \ldots$ & - & 16 & -- & 12 & -- & -- & - & $\cdots$ & -- & -- & - \\
\hline $09 \ldots$ & -- & 31 & -- & 21 & -- & -- & -- & -- & -- & -- & - \\
\hline $\begin{array}{l}20 \ldots \\
21 \ldots\end{array}$ & 2.0 & $\overline{4.0}$ & $<1.0$ & $\begin{array}{l}6.0 \\
4.0\end{array}$ & $=$ & 9.0 & 2.0 & -- & $=$ & $-{ }_{-1}$ & $<0.20$ \\
\hline is... & -- & 8.0 & - & 7.0 & $\cdots$ & - & -- & -- & -- & -- & - \\
\hline $22 \ldots$ & -- & $\begin{array}{l}16 \\
12\end{array}$ & $=$ & $\begin{array}{l}<1.0 \\
12\end{array}$ & $=$ & $\tilde{m}$ & $=$ & $=$ & $=$ & $=$ & $=$ \\
\hline${ }_{\mathrm{SEP}}^{27}$ & $\overline{1.0}$ & 15 & 3.0 & $\begin{array}{l}12 \\
<1.0\end{array}$ & -- & 5.0 & $\overline{2.0}$ & $=$ & $=-$ & $-<1$ & $<0.20$ \\
\hline 11. & 1.0 & 13 & $<1.0$ & 5.0 & $<0.10$ & 9.0 & 1.0 & $<1$ & $<1$ & -- & $<1.0$ \\
\hline
\end{tabular}


RIO GRANDE BASIN

08331000 RTO GRANDE AT ISLETA, MM -- Continued

WATER-QUALITY RECORDS

WATER QUALITY DATA, WATER YEAR OCTOBER 1995 TO SEPTEMBER 1996

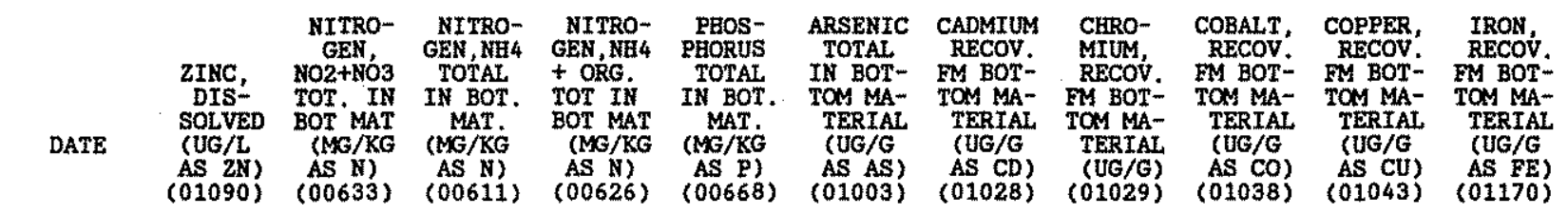

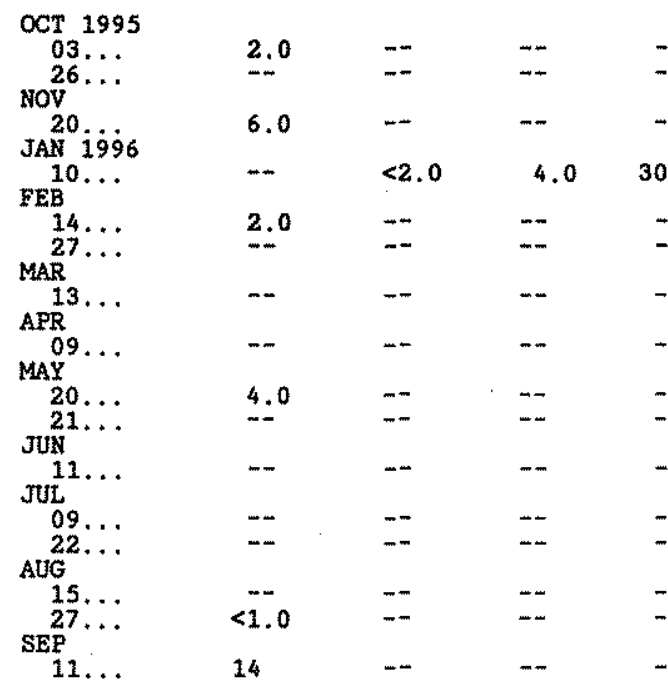

$\begin{array}{ll}-- & \\ -- & \\ - & \\ 00 & 480 \\ -- & \\ -- & \\ -- & \\ -- & \\ -- & \\ -- & - \\ -- & - \\ -- & - \\ -- & -\end{array}$

$$
\begin{array}{llllll}
-- & -- & - & -- & = & = \\
-- & -- & -- & -- & -- & -
\end{array}
$$


08331000 RIO GRANDE AT ISLETA, IM -- Continued

WATER-QUALITY RECORDS

CHEMICAL ANALYSES OF ORGANIC COMFOUNDS, WATER YEAR OCTOBER 1995 TO SEPTEMBER 1996

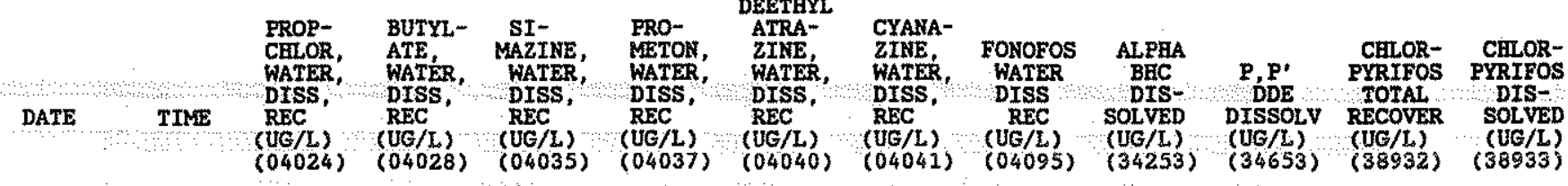

\begin{tabular}{|c|c|c|c|c|c|c|c|c|c|c|c|c|}
\hline $\begin{array}{l}C \mathrm{CT} 1995 \\
03 \ldots \ldots \\
26 \ldots\end{array}$ & $\begin{array}{l}1400 \\
1045\end{array}$ & - & - & $=$ & - & $-\cdots$ & $=$ & $=$ & $-\infty$ & $=$ & $m$ & - \\
\hline 201996 & 1100 & $<0.007$ & $<0.002$ & $<0.005$ & $<0.02$ & $<0.002$ & $<0.004$ & $<0.003$ & $<0.002$ & $<0.006$ & $\div$ & 0.006 \\
\hline $10 \ldots$ & 1045 & -- & -- & -- & -- & -- & -- & -- & $-\cdots$ & -- & -- & -- \\
\hline $14 \ldots$ & $\begin{array}{l}0940 \\
1030\end{array}$ & $=$ & $\because$ & $=$ & $\overline{-}$ & $=$ & -- & -- & $m$ & -- & $=$ & -- \\
\hline $13 \ldots$ & 0915 & $<0.007$ & $<0.002$ & $<0.005$ & E0.007 & $<0.002$ & $<0.004$ & $<0.003$ & $<0.002$ & $<0.006$ & -- & $<0.004$ \\
\hline${ }_{\mathrm{MAY}}^{09} \cdot$. & 1000 & $<0.007$ & $<0.002$ & 0.014 & $<0.018$ & $<0.002$ & $<0.004$ & $<0.003$ & $<0.002$ & $<0.006$ & -- & 0.008 \\
\hline $\begin{array}{r}20 \\
21 \\
J^{2}\end{array}$ & $\begin{array}{l}1400 \\
1545\end{array}$ & $<0.007$ & $<0.002$ & 0.005 & 50.007 & $<0.002$ & $<0.004$ & $<0 . \overline{0}$ & $<0.002$ & $<0 . \overline{006}$ & $=$ & $<0.004$ \\
\hline${ }_{\mathrm{JUL}}^{11} \ldots$ & 0900 & $<0.007$ & $<0.002$ & 0.019 & E0.006 & $<0.002$ & $<0.004$ & $<0,003$ & $<0.002$ & $<0.006$ & -- & $<0.004$ \\
\hline $\begin{array}{r}09 . . \\
22 . \cdots \\
\text { AUG }\end{array}$ & $\begin{array}{l}0930 \\
0830\end{array}$ & $\begin{array}{l}<0.007 \\
<0.007\end{array}$ & $\begin{array}{l}<0.002 \\
<0.002\end{array}$ & $\begin{array}{l}0.009 \\
0.008\end{array}$ & $\begin{array}{r}0.038 \\
E 0.010\end{array}$ & $\begin{array}{l}<0.002 \\
<0.002\end{array}$ & $\begin{array}{l}<0.004 \\
<0.004\end{array}$ & $\begin{array}{l}<0.003 \\
<0.003\end{array}$ & $\begin{array}{l}<0.002 \\
<0.002\end{array}$ & $\begin{array}{l}<0.006 \\
<0.006\end{array}$ & $=$ & $\begin{array}{l}<0.004 \\
<0.004\end{array}$ \\
\hline $\begin{array}{r}15 \ldots \\
27 \ldots \\
\operatorname{SEP}\end{array}$ & $\begin{array}{l}0945 \\
1300\end{array}$ & $<0.007$ & $<0.002$ & 0.026 & 0.097 & $<0.002$ & $<0.004$ & $<0.003$ & $<0.002$ & $<0.006$ & $\because \cdots$ & $<0.004$ \\
\hline 11 & 0930 & $<0.007$ & $<0.002$ & 0.016 & $E 0.016$ & $<0.002$ & $<0.004$ & $<0.003$ & $<0.002$ & $<0.006$ & $<0.010$ & 0.007 \\
\hline
\end{tabular}

\begin{tabular}{|c|c|c|c|c|c|c|c|c|c|c|c|c|}
\hline DATE & $\begin{array}{l}\text { PHORATE } \\
\text { TOTAL } \\
\text { (UG/L) } \\
\text { (39023) }\end{array}$ & $\begin{array}{c}\text { PER- } \\
\text { THANE } \\
\text { TOTAL } \\
(\text { UG/L) } \\
(39034)\end{array}$ & $\begin{array}{c}\text { DEF } \\
\text { TOTAL } \\
\text { (UG/L) } \\
\text { (39040) }\end{array}$ & $\begin{array}{l}\text { PCNS } \\
\text { UNFILT } \\
\text { RECOVER } \\
\text { (UG/L) } \\
(39250)\end{array}$ & $\begin{array}{c}\text { ALDRIN, } \\
\text { TOTAL } \\
\text { (UG/L) } \\
(39330)\end{array}$ & $\begin{array}{c}\text { LINDANE } \\
\text { TOTAL } \\
\text { (UG/L) } \\
(39340)\end{array}$ & $\begin{array}{l}\text { LINDANE } \\
\text { DIS- } \\
\text { SOLVED } \\
\text { (UG/L) } \\
(39341)\end{array}$ & $\begin{array}{l}\text { CHLOR- } \\
\text { DANE, } \\
\text { TECH- } \\
\text { NICAL } \\
\text { TOTAL } \\
\text { (UG/L) } \\
(39350)\end{array}$ & $\begin{array}{l}\text { P,P'- } \\
\text { DDD } \\
\text { UNEILT } \\
\text { RECOVER } \\
(U G / L) \\
(39360)\end{array}$ & $\begin{array}{c}\text { DDE, } \\
\text { TOTAL } \\
\text { (UG/L) } \\
(39365)\end{array}$ & $\begin{array}{l}\text { P,P'- } \\
\text { DDT } \\
\text { UNFILF } \\
\text { RECOVER } \\
\text { (UG/L) } \\
(39370)\end{array}$ & $\begin{array}{l}\text { DI- } \\
\text { ELDRIN } \\
\text { TOTAL, } \\
\text { (UG/L) } \\
\text { (39380) }\end{array}$ \\
\hline
\end{tabular}

\begin{tabular}{|c|c|c|c|c|c|c|c|c|c|c|c|c|}
\hline OCT 1995 & '. & & & & & & & & & & & \\
\hline $\begin{array}{l}03 \ldots \\
26 \ldots\end{array}$ & $=$ & $m$ & - & $\overline{-}$ & $=$ & $=$ & $\cdot=$ & $=$ & $=$ & $=$ & $\therefore$ & $=$ \\
\hline Nov & -- & -- & -- & & $-e_{0}$ & & & & & & & \\
\hline JAN ${ }^{20} \ddot{9} 96$ & $-{ }^{-}$ & - & $-\pi$ & - & - & -- & $<0.004$ & -- & - & - & - & - \\
\hline $10 \ldots$ & - & -- & $-m$ & -- & $-\infty$ & - & $-\cdots$ & -- & - & - & -- & -- \\
\hline $14 \ldots$ & -- & -- & -- & -- & -- & -7 & $m$ & -- & -- & -- & -- & $=-$ \\
\hline $27 \ldots$ & -- & -- & $-m$ & -- & -- & -- & -- & -- & - & -- & - & -- \\
\hline $\mathrm{MAR}_{13}$ & $-\pi$ & -- & $m$ & -7 & -- & & Ca & & & & & \\
\hline APR & $m$ & -- & $m-\infty$ & -- & - & - & $<0.004$ & -- & -- & $\cdots$ & -- & -- \\
\hline$\stackrel{09}{M A Y}$ & -- & -- & -- & - & -- & -- & $<0.004$ & - & -- & -- & $-m$ & -- \\
\hline $20 \ldots$ & -- & $-\infty$ & -- & -- & - & -- & -- & -- & -- & -- & -- & -- \\
\hline$\underset{\text { JuN }}{21} \cdots$ & -- & -- & - & -- & -- & -- & $<0.004$ & $-m$ & - & -- & $-\cdots$ & -- \\
\hline $11 .$. & $-\infty$ & -- & -- & $m$ & -- & - & 0.004 & - & -- & -- & $-\infty$ & - \\
\hline JUL & -- & $-m$ & -- & -- & -- & $-m$ & $<0.004$ & $\ldots$ & - & - & - & \\
\hline $2 \bar{Z}, \ldots$ & -- & -- & -- & $m m$ & -- & -- & $<0,0004$ & -- & -- & $=$ & - & - \\
\hline AUG & & & & & & & & & & & & \\
\hline $\begin{array}{l}15 \ldots \\
27 \ldots\end{array}$ & - & - & $=$ & - & $=$ & $=$ & $<0.004$ & $=$ & $=$ & $=$ & $=$ & $=$ \\
\hline & & & & & & & & & & & & \\
\hline $11 \ldots$ & $<0.010$ & $<0.100$ & $<0.010$ & $<0.100$ & $<0.010$ & $<0.010$ & $<0.004$ & $<0.100$ & $<0.010$ & $<0.010$ & $<0.010$ & 0.010 \\
\hline
\end{tabular}


RIO GRANDE BASIN

08331000 RIO GRANDE AT ISLETA, MM -- Continued

WATER-QUALITY RECORDS

CHEMICAL ANALYSES OF ORGANIC COMPOUNDS, WATER YEAR OCTOBER 1995 TO SEPTEMBER 1996

DATE

$\begin{array}{ccc}\text { DI- } & \text { ENDO- } & \text { ENDRIN } \\ \text { ELDRIN } & \text { SULFAN, } & \text { WATER } \\ \text { DIS- } & \text { I } & \text { UNFLTRD } \\ \text { SOLVED } & \text { TOTAL } & \text { REC } \\ \text { (UG/L) } & \text { (UG/L) } & \text { (UG/L) } \\ (39381) & \text { (USG } & \end{array}$
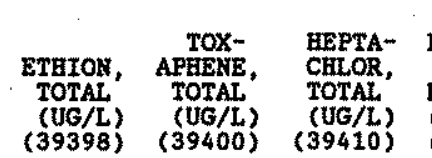

METO-

LACELOR HEPTA-

HEPTA- METH-

WATER EPOXIDE CHLOR,

DISSOLV TOTAL

(UG/L) (UG/L) (UG/L)

(UG/L) (UG/L) (UG/L)

PCB, MALA-

$\begin{array}{lllllllllll}(39381) & (39388) & (39390) & (39398) & (39400) & (39410) & (39415) & (39420) & (39480) & (39516) & (39530)\end{array}$

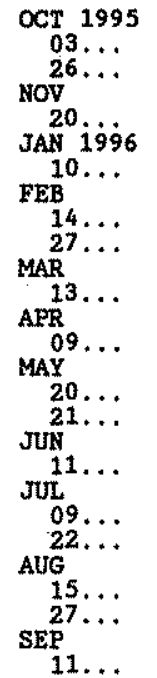

$\begin{array}{ccc}- & - & - \\ -- & - & - \\ <0.001 & -- & - \\ -- & -- & - \\ -- & - & - \\ -- & -- & - \\ <0.001 & -- & -- \\ <0.001 & -- & -- \\ <0.001 & -- & -- \\ <0.001 & -- & - \\ <0.001 & -- & -- \\ <0.001 & -- & - \\ <0.001 & -- & -- \\ -- & - & - \\ <0.001 & <0.010 & <0.010 \quad<0.01\end{array}$

$\because-$
$\therefore-$
--
--
--
--
--
--
--
--
--

$=-$

$=\quad-\because \quad-\cdots \quad-$

$\begin{array}{lll}- & - & -\end{array}$

--

$<0.002$

$-$$$
-
$$$$
--
$$$$
-
$$$$
\text { -. }-
$$$$
\div \quad-\cdots
$$$$
-\cdots
$$

$<0.002$

$<0.002$

$-m, \quad-m$

$+0.000$

--

$<0.002$

$--$

$+\infty$

$<0.002$

$+-$

$<0.002$

0.010

$<0.002<0.010$

$<0.010$

DATE

MALA-

PARA- PARA-

SOLVED

(UG/L)

THION, THION,

(39532)

(39540)

THION,

(UG/L)

(39542) (UG/L)

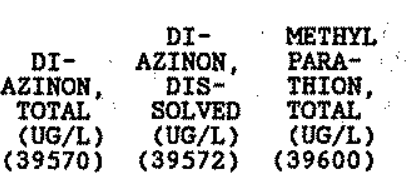

ATRA -

ZINE,

WATER,

REC

(UG/L)

2,4-D, 2,4,5-T MIREX, SILVEX, TOTAL TOTAL TOTAL.

$\begin{array}{llllll}(39632) & (39730) & (39740) & (39755) & (39760)\end{array}$

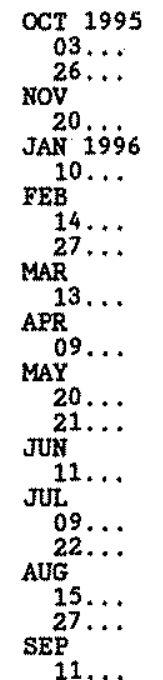

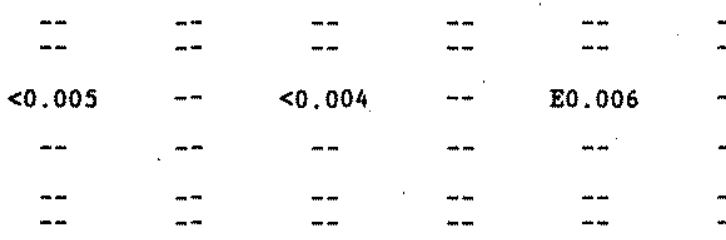

$<0.005 \quad--$

$<0.004$

0.009

$-$

$\begin{array}{ll}m & -m \\ -\infty & -- \\ -\infty & -m \\ -\infty & -m\end{array}$

$$
\begin{array}{lll}
- & - & -- \\
- & - & - \\
- & - & -- \\
- & -- & -- \\
-- & -- & --
\end{array}
$$

$<0.005$

$<0.004$

$<0.001$

$<0.010$

$<0.010$

$-\infty$

$<0.010$

$<0.005 \quad--$

$<0.005$

$<0.004$

0.026

$<0.001$

$0.019 \quad=-$

$<0 .-1001$

$-$

$\begin{array}{lll}<0.005 & -\infty & <0.004\end{array} \quad<0.005 \quad \ldots$

$<0.002 \quad--$

$<0.001$

$-$

$<0.005$

0.014

$<0.005$

$<0.004 \quad--$

0.036

$<0.001$

$<0.001$

$<0.005<0.010$

$<0.004$

0.070

$0.071<0.010$

$<0.001$

$<0.001$

$-$

$--$

$=$ 


\section{RIO GRANDE BASIN}

08331000 RIO GRANDE AT ISLETA, NM -- Continued

WATER-QUALITY RECORDS

CHEMICAL ANALYSES OF ORGANIC COMPOUNDS, WATER YEAR OCTOBER 1995 TO SEPTEMBER 1996

DATE $\begin{gathered}\text { TOTAL } \\ \text { TRI- } \\ \text { THION } \\ \text { (UG/L) } \\ (39786)\end{gathered}$
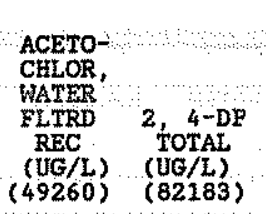

FONOFOS (DYFONATE)

CEIOR

WATER

DISS,

(UGG/L)

(46342)

$\operatorname{OCr} 1995$

$03 . .$.

NoV

20

JAN $19 \dot{9} \dot{6}$

$10 \ldots$

FEB $14 \ldots$

$27 \ldots$

$13 .$.

APR.

MAY 99

20 ...

$21 .$.

JUN

JuL 11

$09,$.

UuG

$15 \ldots$

$27 \ldots$

$11 .$.

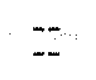

$--$

$--$

$--$

TOT.REC

(UG/L)

(82614)
$<0.002$

$--\quad<0.002$

$-$

$-$

$--$

$--\quad<0.002$

$\ldots \quad<0.002$

$=\quad-0.002$

$<0.002$

$<0.002$

$=\quad<0.002$

$--\quad<0.002$

$<0.010$

$<0,002$

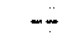

$\div$

$<0.002$

$<0.002$

$--$

$<0.002$

$<0.002$

$<0.002$

$<0.002$

$<0.002$

$<0.002$

LIN- METHYL
URON

URO

WATER

FLTRD

DATE

$$
\text { GF, REC }
$$

(UG/L)

$(82666)$ WAT FLT $0.7 \mathrm{U}$ GF $0.7 \mathrm{U}$ UG/L) GF, REC

$\begin{array}{lcc} & \text { PEB- } & \text { TEBU- } \\ \text { EFTC } & \text { ULATE } & \text { THIURON } \\ \text { WATER } & \text { WATER } & \text { WATER } \\ \text { FLTRD } & \text { FILTRD } & \text { FLTRD } \\ 0.7 \mathrm{U} & 0.7 \mathrm{U} & 0.7 \mathrm{U} \\ \text { GF, REC } & \text { GF REC } & \text { GE. REC } \\ (\mathrm{UG} / \mathrm{L}) & (\mathrm{UG} / \mathrm{L}) & (\mathrm{UG} / \mathrm{L}) \\ (82668) & (82669) & (82670)\end{array}$

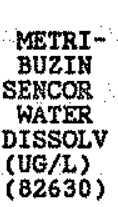
$\begin{array}{cc}\text { 2,6-DI- } & \text { TRI- } \\ \text { ETHYL } & \text { FLUR } \\ \text { ANILINE ALIN } \\ \text { WAT FLT WAT FLI } \\ 0.7 \text { U } 0.7 \mathrm{U} \\ \text { GF, REC GF, REC } \\ \text { (UG/L) } & \text { (UG/L) } \\ (82660) & (82661)\end{array}$
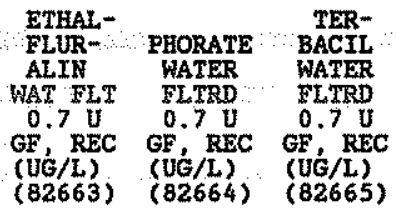<smiles>C#C[As](C)(=O)O</smiles>

$26 \ldots$

20

JAN 1996

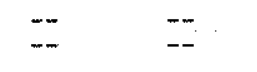

$<0.002$

$-$

$-$

$--$

$\ldots$

-

$\rightarrow$

$\ldots$

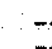

$<0.004$

$<0.003$

$<0.002$

$<0.004$

$<0.002<0.007$

$10 \ldots$

FEB

$14 \ldots$

MAR

$13 \ldots$

$\triangle P P$
09.

MAY

$20 .$.

$21 .$.

II...

$09 .$.

$22 .$.

AUG

$15 .$.

27.

$11 .$.
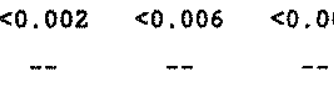

$<0.004$

$<0.01$

--

MOL-

INATE

WATER

FLTRD

GF, REC

(UG/L) (UG/L)

(UG/L)

ETHO- BEN- CARBO-

FLUR-

TER ALIN

WAT FLD

$0.7 \mathrm{U}$

0.7 v

(UG/L)

(82674)

CARAO

WATER

$0.7 \mathrm{U}$

(82671)

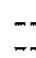

$--$

$-$

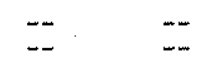

$<0.002$

$<0.006$

$+$

$<0.002$

$<0.006$

$<0.002$

-

$-$

0.

$<0.006$

$<0.00$

$<0.004$

$<0.010$

$<0.002$

$<0.004$

$<0.010$

$<0.004$

$<0.004$

$<0.004$

$<0.010$

$<0.004$

$<0.00 \hat{z}$

$<0.006$

$<0.002$

$<0.00 \mathrm{~A}$

$<0.0100$

$<0.0004$

$<0.002$

$<0.006$

$<0.002<0.004<0.010$

$<0.004$

$<0.002$

$<0.006$

$<0.002<0.004$

$<0.010$

$<0.004$

$<0.004$

$<0.002$

$<0.004$

$<0.010$

$+$

$<0.006$

$<0.002<0.004<0.010$

$<0.010<0.004$

$<0.003$

$<0.002$

$-$

$<0.003$

$<0.003$

$<0.002$

$<0.002$

$<0.003$

$<0.00 \mathrm{~s}$

$<0.002$

$<0.002$

$<0.003$

$<0.003$

$<0.002$

$<0.002$

$<0.003$

$<0.003$

$<0.002$

$<0.003$

TER-

BUFO

FATER

$0.7 \mathrm{U}$

GF REC $0.7 \mathrm{U}$

(UG/L) GE, REC

(82675) (82676)

$$
<0.002
$$


RIO GRANDE BASIN

08331000 RIO GRANDE AT ISLETA, NM -- Continued

MATER-QUALITY RECORDS

CEFMICAL ANALYSES OF ORGANIC COMPOUNDS, WATER YEAR OCTOBER 1995 TO SEPTEMBER 1996

$\begin{array}{llcccc}\text { DISUL- } & \text { TRIAL- } & \text { PRO- } & \text { CAR- } & \text { THYO- } & \\ \text { FOTON } & \text { LATE } & \text { PANTL } & \text { BARYL } & \text { BENCARB } & \text { DCPA } \\ \text { WATER } & \text { WATER } & \text { WATHR } & \text { WATER } & \text { WATER } & \text { WATER } \\ \text { FLTRD } & \text { FLTRD } & \text { FLTRD } & \text { FLTRD } & \text { FLTRD } & \text { FLTRD } \\ 0.7 \text { U } & 0.7 \text { U } & 0.7 U & 0.7 U & 0.7 \text { U } & 0.7 U \\ \text { GF, REC } & \text { GF, REC } & \text { GF, REC } & \text { GF, REC } & \text { GF, REC } & \text { GF, REC } \\ \text { (UG/L) } & (U G / L) & (U G / L) & (U G / L) & (U G / L) & (U G / L) \\ (82677) & (82678) & (82679) & (82680) & (82681) & (82682)\end{array}$

$\begin{array}{ll}\text { PENDI- } & \text { WAPROP- } \\ \text { METH- } & \text { AMIDE } \\ \text { ALIN } & \text { WATER } \\ \text { WAT FLT } & \text { FLTRD } \\ 0.7 \mathrm{U} & 0.7 \mathrm{U} \\ \text { GF, REC } & \text { GF, REC } \\ \text { (UG/L) } & (\mathrm{UG} / \mathrm{L}) \\ (82683) & (82684)\end{array}$

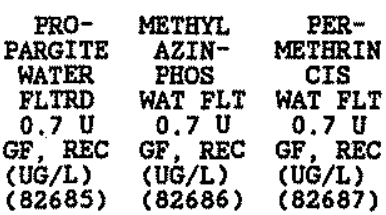

OCT 1995

$26 \ldots$

$10 \mathrm{~V}$

20.

JAN 1996

10 .

$27 \ldots$

MAR

13...
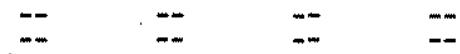

$\because$

$\because$

$\because$

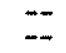

$=$

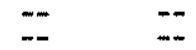

$<0.02<0.001<0.004$

E0.01.

$<0.002$

50.001

$<0.004$

$<0.003$

$<0.01$

$<0.001<0.005$

-

$-\infty$

$--$

$-$

$--$

$--$

$-\infty$

$-$

$\cdots$

$<0.017$

$<0.001<0.004$

$-$

$-\overline{-}$

$-$

$-m$

$-$

$-\infty$

$--$

$09 . .$.

$<0.017$

$<0.001$

$<0.003$

$<0.002$

$<0.002$

$<0.004$

$<0.003$

$<0.013$

$<0.001$

$<0.005$

$20 .$.

$<0.017$

$<0.001<0.004$

0.004

$<0.003$

$<0.002$

$<0.002$

$<0.004$

$<0.003$

$<0.013$

$<0.001$

$<0.005$

JuN

$11 .$.

$<0.017$

$<0.003$

$<0.002$

$<0.002$

$<0,004$

$<0.003$

$<0.013$

$<0,001$

$<0.005$

$\begin{array}{lll}<0.017 & <0.001 & <0.004 \\ <0.017 & <0.001 & <0.004\end{array}$

$<0.001<0.004$

$<0.003$

$<0.002$

$<0.002$

$<0.004$

$<0.003$

$<0.013$

$<0.001$

$<0.005$

$22 \ldots$

AUG

$15 . .$.

SEP

$<0.060$
$\mathrm{E0} 0.028$

$<0,002$

E0.003

$<0.004$

$<0.003$

$<0.013$

$<0.001$

$<0.005$

$<0.017<0.001<0.004$

$<0.003$

$<0,002$

$<0.003$

$<0.013$

$<0.001$

$<0,005$

$<0.017<0.001<0.004$

$<0.003$

$<0.002<0.002$

$<0.004$

$<0.003$

$<0.013$

$<0.001$

$<0.005$

CROSS SECTION ANALYSES

\begin{tabular}{|c|c|c|c|c|c|c|c|}
\hline$A ?$ & TIME & $\begin{array}{l}\text { SAMPLE } \\
\text { LOC- } \\
\text { ATION, } \\
\text { CROSS } \\
\text { SECTION } \\
\text { (FT FM } \\
\text { L BANK) } \\
\text { (00009) }\end{array}$ & $\begin{array}{l}\text { DEPTH } \\
\text { AT } \\
\text { SAMPLE } \\
\text { LOC- } \\
\text { ATION, } \\
\text { TOTAL } \\
\text { (FEET) } \\
\text { (81903) }\end{array}$ & $\begin{array}{l}\text { SPE- } \\
\text { CIFIC } \\
\text { CON- } \\
\text { DUCT- } \\
\text { ANCE } \\
\text { (US/CM) } \\
(00095)\end{array}$ & $\begin{array}{c}\text { PH } \\
\text { WATER } \\
\text { WHOLE } \\
\text { FIELD } \\
\text { (STAND- } \\
\text { ARD } \\
\text { UNITS) } \\
(00400)\end{array}$ & $\begin{array}{c}\text { TEMPER- } \\
\text { ATURE } \\
\text { WATER } \\
\text { (DEG C) } \\
(00010)\end{array}$ & $\begin{array}{c}\text { OXYGEN, } \\
\text { DIS- } \\
\text { SOLVED } \\
(M G / L) \\
(00300)\end{array}$ \\
\hline
\end{tabular}

SEP 1996

.

$11 \ldots$

$11 \ldots$

$11 .$.

$11 \ldots$

$11 \ldots$

$11 \ldots$

$11 .$.

$11 .$.

$11 \ldots$

0901
0902
0903
0904
0905
0906
0907
0908
0909
0910
0911
0912

10.0
30.0
50.0
70.0
90.0
110
130
150
170
190
230
270

3.50
3.40
2.90
3.10
1.30
2.40
2.50
2.60
2.80
3.20
1.00
1.20

458
463
481
490
492
500
493
487
479
477
474
471

$\begin{array}{ll}8.0 & 19.5 \\ 8.1 & 19.5 \\ 8.1 & 20.0 \\ 8.1 & 20.0 \\ 8.1 & 20.0 \\ 8.1 & 20.0 \\ 8.2 & 20.0 \\ 8.2 & 20.0 \\ 8.2 & 20.0 \\ 8.2 & 20.0 \\ 8.1 & 19.5 \\ 8.1 & 19.5\end{array}$

6.8
6.8
6.9
7.0
7.0
7.1
7.1
7.1
7.1
7.0
7.0
6.9 
RIO GRANDE BASIN

08331990 RIO GRANDE CONVEYANCE CRANNEL NEAR BERNARDO, MM

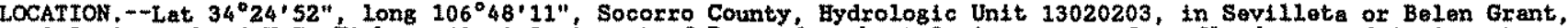
$0.2 \mathrm{mi}$ south of U.S. Highway $60,1.8 \mathrm{mi}$ east of Bernardo, about 3 mi upstream from floodway, and $4 \mathrm{mi}$ upstream from Rio Puerco.

PERIOD OF RECORD.--June 1936 to September 1937, October 1964 to current year. July 1943 to September 1964 , included in composite flow of "Rio Grande neax Bernardo." Octobex 1960 to September 1964, monthly acre-feet pubifished in WSP 1923 (daily records available in district iles). Beginning October igsa, fíow in conveyance channel represents controlled diversion from Rio Grande. Prior to October 1952, records called "San Francisco Riverstde drain near Bernardo" are not equivalent.

GAGE. - Water-stage recorder and concrete control, Datum of gage is 4, 720.00 ft above National Geodetic Vertical Datum of 1929. Friox to October 1964, 0.2 ins upstrean ad various datums.

REMARKS. -Records good. Conveyance channel is 1 of 4 channels (stations 08332010, 08332030, and 08332050) carrying flow in valley cross section. Original degign and plan were for conveyance channel to carry flows up to about $2,000 \mathrm{ft} / \mathrm{s}$. For combined monthly flow in acre-ft of this channel, floodway, Bernardo interior drain, and Lower San Juan Riverside drain, see tabulation below dally table for station 08332010. Several observations of water temperature were made during the year. No flow many days most years.

DISCEARGE, CUBIC FEET PIR SECOND, WATER YEAR OCTOBER 1995 TO SEPTEMBER 1996

\begin{tabular}{|c|c|c|c|c|c|c|c|c|c|c|c|c|}
\hline $\mathrm{DAX}$ & $O C T$ & NOY & DEC & JAN & FEB & MAR & APR & MAY & JUN & JUL & AUG & SEP \\
\hline $\begin{array}{l}1 \\
2 \\
3 \\
4 \\
5\end{array}$ & $\begin{array}{l}4.4 \\
3.5 \\
3.1 \\
2.9 \\
2.8\end{array}$ & $\begin{array}{l}5.3 \\
4.4 \\
4.2 \\
4.2 \\
4.1\end{array}$ & $\begin{array}{l}4.4 \\
4.4 \\
4.4 \\
4.4 \\
4.4\end{array}$ & $\begin{array}{r}6.2 \\
6.3 \\
6.2 \\
6.2 \\
6.2\end{array}$ & $\begin{array}{r}5.0 \\
5.1 \\
5.1 \\
5.2 \\
5.3\end{array}$ & $\begin{array}{r}5.4 \\
5.1 \\
4.8 \\
5.0 \\
20\end{array}$ & $\begin{array}{r}3.4 \\
3.4 \\
3.4 \\
4.9 \\
5.5\end{array}$ & $\begin{array}{r}6.0 \\
6.1 \\
11 \\
6.1 \\
4.6\end{array}$ & $\begin{array}{l}20 \\
18 \\
14 \\
15 \\
16\end{array}$ & $\begin{array}{r}42 \\
44 \\
43 \\
40 \\
35\end{array}$ & $\begin{array}{r}24 \\
25 \\
27 \\
35 \\
36\end{array}$ & $\begin{array}{l}51 \\
55 \\
52 \\
45 \\
35\end{array}$ \\
\hline $\begin{array}{r}6 \\
7 \\
8 \\
9 \\
10\end{array}$ & $\begin{array}{l}2.9 \\
3.0 \\
3.0 \\
3.3 \\
3.9\end{array}$ & $\begin{array}{l}4.3 \\
4.3 \\
4.2 \\
4.3 \\
4.3\end{array}$ & $\begin{array}{l}4.4 \\
4.4 \\
4.4 \\
4.4 \\
4.4\end{array}$ & $\begin{array}{r}6.3 \\
6.3 \\
6.2 \\
6.0 \\
6.1\end{array}$ & $\begin{array}{l}5.3 \\
5.3 \\
5.5 \\
5.4 \\
5.5\end{array}$ & $\begin{array}{l}56 \\
52 \\
57 \\
44 \\
57\end{array}$ & $\begin{array}{r}4.1 \\
8.2 \\
7.9 \\
7.1 \\
7.8\end{array}$ & $\begin{array}{l}5.1 \\
4.2 \\
7.0 \\
7.6 \\
5.1\end{array}$ & $\begin{array}{l}12 \\
12 \\
13 \\
13 \\
11\end{array}$ & $\begin{array}{l}25 \\
25 \\
23 \\
26 \\
29\end{array}$ & $\begin{array}{l}35 \\
40 \\
36 \\
46 \\
47\end{array}$ & $\begin{array}{l}35 \\
35 \\
38 \\
31 \\
28\end{array}$ \\
\hline $\begin{array}{l}11 \\
12 \\
13 \\
14 \\
15\end{array}$ & $\begin{array}{l}3.3 \\
3.0 \\
4.4 \\
3.4 \\
3.2\end{array}$ & $\begin{array}{l}4.4 \\
4.4 \\
4.4 \\
4.4 \\
4.4\end{array}$ & $\begin{array}{r}18 \\
9.8 \\
7.6 \\
6.8 \\
6.4\end{array}$ & $\begin{array}{r}5.4 \\
5.1 \\
5.1 \\
5.0 \\
5.0\end{array}$ & $\begin{array}{l}5.5 \\
5.5 \\
5.7 \\
8.7 \\
5.3\end{array}$ & $\begin{array}{c}54 \\
53 \\
14 \\
35 \\
7.3\end{array}$ & $\begin{array}{r}7.6 \\
6.3 \\
5.8 \\
5.1 \\
7.2\end{array}$ & $\begin{array}{r}5.1 \\
5.5 \\
6.8 \\
7.3 \\
7.0\end{array}$ & $\begin{array}{l}11 \\
15 \\
16 \\
17 \\
16\end{array}$ & $\begin{array}{l}44 \\
45 \\
47 \\
47 \\
42\end{array}$ & $\because \begin{array}{r}46 \\
51 \\
51 \\
47 \\
42\end{array}$ & $\begin{array}{l}26 \\
39 \\
47 \\
57 \\
60\end{array}$ \\
\hline $\begin{array}{l}16 \\
17 \\
18 \\
19 \\
20\end{array}$ & $\begin{array}{l}7.4 \\
5.7 \\
6.7 \\
7.0 \\
9.8\end{array}$ & $\begin{array}{l}4.4 \\
4.4 \\
4.4 \\
4.5 \\
4.5\end{array}$ & $\begin{array}{l}6.4 \\
6.1 \\
5.8 \\
5.8 \\
9.2\end{array}$ & $\begin{array}{l}5.1 \\
5.2 \\
5.1 \\
5.2 \\
5.1\end{array}$ & $\begin{array}{l}6.5 \\
5.8 \\
5.6 \\
5.5 \\
5.4\end{array}$ & $\begin{array}{l}5.4 \\
5.0 \\
4.3 \\
4.4 \\
4.6\end{array}$ & $\begin{array}{l}7.0 \\
6.8 \\
6.7 \\
7.8 \\
8.0\end{array}$ & $\begin{array}{l}5.6 \\
4.1 \\
4.2 \\
3.8 \\
3.6\end{array}$ & $\begin{array}{l}10 \\
5.5 \\
16 \\
28 \\
26\end{array}$ & $\begin{array}{l}50 \\
49 \\
45 \\
44 \\
37\end{array}$ & $\begin{array}{l}38 \\
33 \\
37 \\
36 \\
40\end{array}$ & $\begin{array}{l}62 \\
59 \\
62 \\
61 \\
57\end{array}$ \\
\hline $\begin{array}{l}23 \\
22 \\
23 \\
24 \\
25\end{array}$ & $\begin{array}{l}9.8 \\
5.5 \\
4.7 \\
9.5 \\
5.4\end{array}$ & $\begin{array}{l}4.5 \\
4.2 \\
4.3 \\
4.3 \\
4.1\end{array}$ & $\begin{array}{l}8.3 \\
7.8 \\
7.2 \\
6.3 \\
7.7\end{array}$ & $\begin{array}{l}5.3 \\
5.4 \\
5.1 \\
4.7 \\
4.8\end{array}$ & $\begin{array}{l}5.6 \\
5.7 \\
5.9 \\
6.0 \\
6.1\end{array}$ & $\begin{array}{l}4.5 \\
7.6 \\
6.0 \\
6.5 \\
5.9\end{array}$ & $\begin{array}{l}6.3 \\
6.4 \\
6.1 \\
7.1 \\
6.4\end{array}$ & $\begin{array}{l}4.2 \\
5.7 \\
7.3 \\
6.7 \\
7.4\end{array}$ & $\begin{array}{l}31 \\
35 \\
38 \\
41 \\
40\end{array}$ & $\begin{array}{l}40 \\
34 \\
25 \\
23 \\
23\end{array}$ & $\begin{array}{l}40 \\
48 \\
47 \\
52 \\
54\end{array}$ & $\begin{array}{l}62 \\
54 \\
43 \\
54 \\
67\end{array}$ \\
\hline $\begin{array}{l}26 \\
27 \\
28 \\
29 \\
30 \\
31\end{array}$ & $\begin{array}{l}9.5 \\
4.9 \\
4.7 \\
5.9 \\
5.6 \\
6.1\end{array}$ & $\begin{array}{l}4.1 \\
4.1 \\
4.2 \\
4.4 \\
4.4 \\
-.-\end{array}$ & $\begin{array}{l}7.2 \\
5.8 \\
5.9 \\
6.1 \\
6.2 \\
6.1\end{array}$ & $\begin{array}{l}4.8 \\
4.7 \\
4.8 \\
4.8 \\
4.9 \\
5.1\end{array}$ & $\begin{array}{l}6.3 \\
6.0 \\
8.6 \\
5.5 \\
-. .2 \\
-.-\end{array}$ & $\begin{array}{l}5.5 \\
5.1 \\
4.7 \\
4.4 \\
4.0 \\
4.0\end{array}$ & $\begin{array}{c}8.5 \\
10 \\
5.8 \\
6.1 \\
7.5 \\
-. .\end{array}$ & $\begin{array}{l}9.5 \\
9.8 \\
8.6 \\
12 \\
10 \\
12\end{array}$ & $\begin{array}{l}41 \\
44 \\
45 \\
43 \\
45 \\
---\end{array}$ & $\begin{array}{l}29 \\
28 \\
28 \\
28 \\
26 \\
28\end{array}$ & $\begin{array}{l}57 \\
61 \\
52 \\
40 \\
39 \\
43\end{array}$ & $\begin{array}{r}58 \\
53 \\
52 \\
50 \\
40 \\
---\end{array}$ \\
\hline $\begin{array}{l}\text { TOTAL } \\
\text { MEAN } \\
\text { MAX } \\
\text { MEN } \\
\text { AC-ET }\end{array}$ & $\begin{array}{r}158.3 \\
5.11 \\
9.8 \\
2.8 \\
314\end{array}$ & $\begin{array}{r}130.4 \\
4.35 \\
5.3 \\
4.1 \\
259\end{array}$ & $\begin{array}{r}200.5 \\
6.47 \\
18 \\
4.4 \\
398\end{array}$ & $\begin{array}{r}167.7 \\
5.41 \\
6.3 \\
4.7 \\
333\end{array}$ & $\begin{array}{r}167.9 \\
5.79 \\
8.7 \\
5.0 \\
333\end{array}$ & $\begin{array}{r}551.5 \\
17.8 \\
57 \\
4.0 \\
1090\end{array}$ & $\begin{array}{r}194.2 \\
6: 47 \\
10 \\
3.4 \\
385\end{array}$ & $\begin{array}{r}209.0 \\
6.74 \\
12 \\
3.6 \\
415\end{array}$ & $\begin{array}{r}707.5 \\
23.6 \\
45 \\
5.5 \\
1400\end{array}$ & $\begin{array}{r}1094 \\
35.3 \\
50 \\
23 \\
2170\end{array}$ & $\begin{array}{r}1305 \\
42.1 \\
61 \\
24 \\
2590\end{array}$ & $\begin{array}{r}1468 \\
48.9 \\
67 \\
26 \\
2910\end{array}$ \\
\hline
\end{tabular}

STATISTICS OF MONTHLY MEAN DATA FOR WATER YEARS 1964 - 1996, BY WATER YEAR (WY)

\begin{tabular}{|c|c|c|c|c|c|c|c|c|c|}
\hline $\begin{array}{l}\text { MEAN } \\
\text { MAX } \\
\text { (WY) } \\
\text { MIN } \\
\text { (WWY })\end{array}$ & $\begin{array}{r}87.0 \\
682 \\
1970 \\
000\end{array}$ & $\begin{array}{r}316 \\
1395 \\
1971 \\
1.54\end{array}$ & $\begin{array}{r}284 \\
1579 \\
1974 \\
2.62\end{array}$ & $\begin{array}{r}260 \\
1417 \\
1974 \\
2.42\end{array}$ & $\begin{array}{r}259 \\
1006 \\
1970 \\
2.55\end{array}$ & $\begin{array}{r}215 \\
1028 \\
1966 \\
3.93\end{array}$ & $\begin{array}{r}221 \\
1354 \\
1966 \\
2.92\end{array}$ & $\begin{array}{r}222 \\
1259 \\
1973 \\
164 \\
1977\end{array}$ & \\
\hline
\end{tabular}

SURMARY STATISTICS

ANNUAL TOTAL

ANNUAL MEAE

HIGHEST ANNUAL MEAN

LOWEST ANNUAE REAN

HIGHEST DAILY MEAN

LOWEST DAILY MEAN

ANNUAL SEVEN-DAY MINTMUM

INSTANTANEOUS PEAK FLOW

ANNUAL RUNOFF (AC-FT)

10 PERCENT EXCEEDS

50 PERCENT EXCEEDS

90 PERCENT EXCEEDS
FOR 1995 CALENDAR YEAR

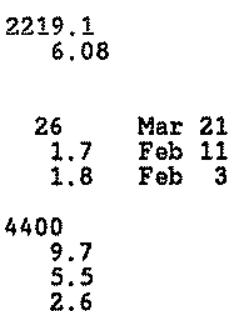

FOR 1996 WATER YEAR

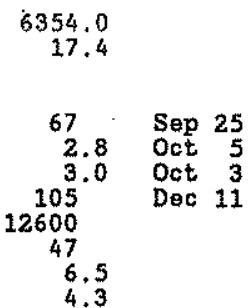

WATER YEARS 1964 - 1996

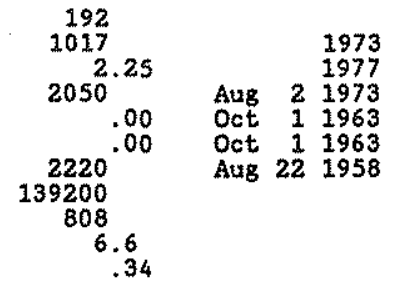


RIO GRANDE BASIN

08332010 RIO GRANDE FLOODWAY NEAR BERNARDO, NM

LOCATION,--Lat $34^{\circ} 25^{\circ} 01^{\prime \prime}$, Long $106^{\circ} 48^{\circ} 00^{\prime \prime}$. Socorro County, Bydrologic Unit 13020203, in Belen or Sevilleta Grant, on downstream side of bridge on U.S. Elghway $60,2.0$ mi east of Bernardo, and at mile $1,487.2$. and $5.0 \mathrm{mi}$ dowmstream from heading of conveyance channel.

DRAINAGE AREA.--19,230 $\mathrm{mi}^{2}$, approximately, Including 2,940 $\mathrm{mi}^{2}$ in closed basin in San Luis Valley, Co.

\section{WATER-DISCEARGE RECORDS}

FERIOD OF RECORD --June 1936 to January 1939, October 1941 to current year. Monthly discharge only October 1942 to June 1943, published in WSP 1312 , and October 1960 to September 1964 , published In WSP 1923 (daily records availabla in district files). Published as "Rio Grande near Bernardo" prior to October 1964. Prior to October included from October 1952 to September 1964. Flow in the conveyance channel, formerly "San Francisco Riverside drain," has been included In records prior to October 1964.

GAGE.--Water-stage recorder. Datum of gage is $4,722.55$ ft above National Geodetic Vertical Datum of 1929.

REMARKS. -Water-discharge records good except for estimated daily discharges, which are fair. Since November 1973 flow completely regulated by Cochiti Dam (station 08317300) $100 \mathrm{mi}$ upstream. Floodway is 1 of 4 channels (stations 08331990,08332030 , and 08332050 ) carrying flow in valley cross section. For combined monthly flow in acre-ft of floodway, conveyance channel, Bernardo intertor drain and Lower San Juan Riverside drain see tabulation below. Diversions for Irrigation of about 740,000 acres upstream from station. No flow for many days most years.

AVERAGE DISCHARGE. - -19 years (water years 1937-38, 1942-58), 1,125 fts/s, 815, 100 acre-ft/yr. Includes flow of floodway, conveyance channel, and Bernardo interior drain. 15 years (water years 1959-73), 898 ft's/s, Riverside floodway, conveyance channe 1 , and Bernardo interior drain. 15 years (water years 1959-73), 898 fts/s, Riverst includes flow of floodway, conveyance channel, Bernardo interior drain, and lowex San Juan Riverstde drain, since closure of Cochiti Dam.

EXTREMES FOR PERIOD OF RECORD (1936-39 AND SINCE 1941).--Maximum dtscharge, 21,000 ft3/s, Apr. 25, 1942, gage height, $6.90 \mathrm{ft}$; no flow most years.

EXTREMES FOR CURRENT YEAR.--Maximum daily discharge, 1,720 fts/s, Nov, 2; minimum daily, no flow May 14-16. DISCHARGE, CUBIC FEET RER SECOND, WATER YEAR OCTOBER 1995 TO SEPTEMBER 1996

\begin{tabular}{|c|c|c|c|c|c|c|c|c|c|c|c|c|}
\hline DAY & $\alpha C T$ & NOV & DEC & JAN & FEB & MAR & APR & MAY & JuN & JUL & AUG & SEP \\
\hline $\begin{array}{l}1 \\
2 \\
3 \\
4 \\
5\end{array}$ & $\begin{array}{l}e 857 \\
0847 \\
e 819 \\
e 857 \\
e 765\end{array}$ & $\begin{array}{r}1340 \\
1720 \\
01670 \\
-1700 \\
e 1690\end{array}$ & $\begin{array}{r}01300 \\
+1220 \\
-1210 \\
1270 \\
1290\end{array}$ & $\begin{array}{l}01280 \\
\theta 1300 \\
e 1210 \\
\theta 1160 \\
\theta 1350\end{array}$ & $\begin{array}{l}01290 \\
01320 \\
01340 \\
\theta 1360 \\
e 1370\end{array}$ & $\begin{array}{l}01180 \\
+1140 \\
\theta 1230 \\
01260 \\
01090\end{array}$ & $\begin{array}{l}0646 \\
0500 \\
0388 \\
0300 \\
0266\end{array}$ & $\begin{array}{r}0.00 \\
0.00 \\
0.38 \\
52 . \\
.54\end{array}$ & $\begin{array}{r}180 \\
144 \\
94 \\
52 \\
24\end{array}$ & $\begin{array}{r}1150 \\
896 \\
686 \\
539 \\
338\end{array}$ & $\begin{array}{l}33 \\
28 \\
34 \\
32 \\
38\end{array}$ & $\begin{array}{r}151 \\
127 \\
88 \\
80 \\
67\end{array}$ \\
\hline $\begin{array}{r}6 \\
7 \\
8 \\
9 \\
10\end{array}$ & $\begin{array}{l}e 774 \\
0646 \\
0599 \\
0518 \\
0584\end{array}$ & $\begin{array}{l}01680 \\
01660 \\
01640 \\
01620 \\
01600\end{array}$ & $\begin{array}{l}1290 \\
1260 \\
1180 \\
1210 \\
1180\end{array}$ & $\begin{array}{l}01290 \\
01260 \\
\theta 1230 \\
01190 \\
+1230\end{array}$ & $\begin{array}{r}01390 \\
1320 \\
1290 \\
\theta 1260 \\
-1210\end{array}$ & $\begin{array}{r}1030 \\
975 \\
886 \\
756 \\
838\end{array}$ & $\begin{array}{l}01.96 \\
0184 \\
0135 \\
0124 \\
0112\end{array}$ & $\begin{array}{l}0.71 \\
0.88 \\
01.1 \\
01.2 \\
01.4\end{array}$ & $\begin{array}{l}24 \\
42 \\
37 \\
12 \\
17\end{array}$ & $\begin{array}{l}216 \\
171 \\
132 \\
133 \\
400\end{array}$ & $\begin{array}{l}28 \\
20 \\
39 \\
66 \\
64\end{array}$ & $\begin{array}{l}83 \\
76 \\
90 \\
76 \\
74\end{array}$ \\
\hline $\begin{array}{l}11 \\
12 \\
13 \\
14 \\
15\end{array}$ & $\begin{array}{l}e 577 \\
e 554 \\
e 615 \\
0623 \\
0562\end{array}$ & $\begin{array}{l}\text { e1580 } \\
\text { e1560 } \\
\text { e1540 } \\
e 1520 \\
\text { e } 1500\end{array}$ & $\begin{array}{l}1190 \\
1170 \\
1150 \\
1110 \\
1120\end{array}$ & $\begin{array}{r}01290 \\
1180 \\
1220 \\
\theta 1130 \\
\theta 1020\end{array}$ & $\begin{array}{r}e 1150 \\
01100 \\
935 \\
971 \\
994\end{array}$ & $\begin{array}{l}0730 \\
0696 \\
0655 \\
0638 \\
0696\end{array}$ & $\begin{array}{r}0100 \\
090 \\
086 \\
074 \\
070\end{array}$ & $\begin{array}{l}1.4 \\
1.6 \\
.74 \\
.00 \\
.00\end{array}$ & $\begin{array}{r}16 \\
40 \\
28 \\
57 \\
117\end{array}$ & $\begin{array}{l}605 \\
534 \\
672 \\
316 \\
184\end{array}$ & $\begin{array}{l}62 \\
48 \\
44 \\
37 \\
32\end{array}$ & $\begin{array}{r}98 \\
64 \\
129 \\
275 \\
289\end{array}$ \\
\hline $\begin{array}{l}16 \\
17 \\
18 \\
19 \\
20\end{array}$ & $\begin{array}{l}0451 \\
0480 \\
0486 \\
0491 \\
0496\end{array}$ & $\begin{array}{l}01480 \\
01460 \\
01440 \\
01420 \\
01400\end{array}$ & $\begin{array}{l}1120 \\
1120 \\
1080 \\
1170 \\
1480\end{array}$ & $\begin{array}{r}\theta 1000 \\
8975 \\
e 996 \\
e 1020 \\
e 1050\end{array}$ & $\begin{array}{r}01010 \\
0996 \\
1010 \\
982 \\
995\end{array}$ & $\begin{array}{l}0730 \\
0765 \\
0810 \\
8829 \\
0857\end{array}$ & $\begin{array}{r}060 \\
28 \\
38 \\
75 \\
70\end{array}$ & $\begin{array}{l}.00 \\
.53 \\
1.4 \\
10 \\
27\end{array}$ & $\begin{array}{l}190 \\
311 \\
257 \\
212 \\
153\end{array}$ & $\begin{array}{l}200 \\
205 \\
186 \\
174 \\
216\end{array}$ & $\begin{array}{l}20 \\
22 \\
17 \\
17 \\
22\end{array}$ & $\begin{array}{l}552 \\
369 \\
301 \\
310 \\
335\end{array}$ \\
\hline $\begin{array}{l}21 \\
22 \\
23 \\
24 \\
25\end{array}$ & $\begin{array}{l}e 500 \\
e 505 \\
e 509 \\
e 332 \\
0326\end{array}$ & $\begin{array}{l}01390 \\
01380 \\
\theta 1370 \\
\theta 1360 \\
\theta 1350\end{array}$ & $\begin{array}{l}\oplus 1450 \\
\theta 1400 \\
\otimes 1350 \\
\bullet 1300 \\
\theta 1260\end{array}$ & $\begin{array}{r}01070 \\
01100 \\
01120 \\
1130 \\
01170\end{array}$ & $\begin{array}{r}1230 \\
\theta 1240 \\
\theta 1550 \\
01410 \\
\theta 1350\end{array}$ & $\begin{array}{l}0810 \\
0783 \\
0792 \\
0783 \\
0747\end{array}$ & $\begin{array}{l}e 67 \\
064 \\
e 60 \\
e 57 \\
e 55\end{array}$ & $\begin{array}{c}11 \\
8.5 \\
91 \\
177 \\
132\end{array}$ & $\begin{array}{r}122 \\
136 \\
121 \\
113 \\
82\end{array}$ & $\begin{array}{r}147 \\
102 \\
79 \\
0120 \\
0202\end{array}$ & $\begin{array}{r}49 \\
63 \\
109 \\
145 \\
220\end{array}$ & $\begin{array}{l}205 \\
117 \\
116 \\
111 \\
145\end{array}$ \\
\hline $\begin{array}{l}26 \\
27 \\
28 \\
29 \\
30 \\
31\end{array}$ & $\begin{array}{l}0270 \\
0359 \\
0365 \\
0305 \\
0388 \\
0575\end{array}$ & $\begin{array}{r}1340 \\
01320 \\
01310 \\
01300 \\
01290 \\
-\end{array}$ & $\begin{array}{l}\theta 1220 \\
01200 \\
01210 \\
e 1170 \\
\theta 1160 \\
-1150\end{array}$ & $\begin{array}{r}1250 \\
+1290 \\
01240 \\
1200 \\
+1250 \\
1270\end{array}$ & $\begin{array}{r}01340 \\
01290 \\
01240 \\
01200 \\
-\ldots\end{array}$ & $\begin{array}{l}0739 \\
0747 \\
0704 \\
0747 \\
0713 \\
0655\end{array}$ & $\begin{array}{l}052 \\
050 \\
048 \\
045 \\
046 \\
-\end{array}$ & $\begin{array}{l}161 \\
201 \\
216 \\
199 \\
186 \\
190\end{array}$ & $\begin{array}{r}123 \\
174 \\
1400 \\
1270 \\
1410 \\
-0\end{array}$ & $\begin{array}{l}94 \\
91 \\
72 \\
63 \\
47 \\
36\end{array}$ & $\begin{array}{l}335 \\
485 \\
438 \\
251 \\
230 \\
275\end{array}$ & $\begin{array}{r}117 \\
91 \\
69 \\
37 \\
55 \\
\end{array}$ \\
\hline $\begin{array}{l}\text { TOTAL } \\
\text { MEAN } \\
\text { MAX } \\
\text { MIN } \\
\text { AC-FT } \\
(t)\end{array}$ & $\begin{array}{r}17035 \\
550 \\
857 \\
270 \\
33790 \\
52800\end{array}$ & $\begin{array}{r}44630 \\
1488 \\
1720 \\
1290 \\
88520 \\
94950\end{array}$ & $\begin{array}{r}37990 \\
1225 \\
1480 \\
1080 \\
75350 \\
80600\end{array}$ & $\begin{array}{r}36451 \\
1176 \\
1350 \\
975 \\
72300 \\
77460\end{array}$ & $\begin{array}{r}35143 \\
1212 \\
1550 \\
935 \\
69710 \\
74450\end{array}$ & $\begin{array}{r}26011 \\
839 \\
1260 \\
638 \\
51590 \\
62820\end{array}$ & $\begin{array}{r}4086 \\
136 \\
646 \\
28 \\
8100 \\
20330\end{array}$ & $\begin{array}{r}1673.38 \\
54.0 \\
216 \\
3.00 \\
3320 \\
17600\end{array}$ & $\begin{array}{r}6958 \\
232 \\
1410 \\
12 \\
13800 \\
28840\end{array}$ & $\begin{array}{r}9006 \\
291 \\
1150 \\
36 \\
17860 \\
31710\end{array}$ & $\begin{array}{r}3303 \\
107 \\
485 \\
17 \\
6550 \\
22820\end{array}$ & $\begin{array}{r}4697 \\
157 \\
552 \\
37 \\
9320 \\
27130\end{array}$ \\
\hline
\end{tabular}

CAL YR 1995 TOTAL 723867 MEAN 1983 MAX 5500 MIN $202 \quad$ AC-FT 1436000 ( $\dagger$ ) MEAN 2170 AC-FT 1572000 WTR YR 1996 TOTAL 226983.38 MEAN 620 MAX 1720 MIN .0. AC-FT 450200 ( $\dagger$ ) MEAN 815 AC-FT 591500

( $f$ COStinated COMBINED FLOW, IN ACRE-FT, AND MEAN, IN CUBIC FEET PER SECOND, OF FLOODWAY, CONVEYANCE CHANNEL, BERNARDO INTERIOR DRAIN AND LOWER SAN JUAN RIVERSIDE DRAIN, 
PERIOD OF RECORD. --Water years 1957 to current year.

PERIOD OF DAILY RECORD.--

SPECIFIC CONDUCTANCE; Octobar 1956 to current year.

WATER TEMPERATURE: Octobex 1964 to current year.

SUSPESDED-SEDIMENT DISCEARGE: October 1964 to current year.

REMARKS. --Sediment total-loads (suspended sediment plus bed material discharge), in tons per day, pere determined from the regression equation developed for the period of record. Once-dally water tempereture readings were made by the field observer, and once-daily specific conductance values were determined in the laboratory from daily suspended sediment samples collected by the field observer.

EXTREMIIS FOR PERIOD OF DAILY RECORD.--

SPECIFIC CONDUCTANCE (pater years 1964-96): Maximum daily, 1,410 microsiemens, July 23, 1976; minimum dally, 224 microsiemens, June 5, 1980.

WATER TEMPERATURE: Maximum daily, 35.0 ${ }^{\circ} \mathrm{C}$, June 5, 6, July 5, 23, 1996; minimum daily, $0.0{ }^{\circ} \mathrm{C}$ on several days during 1971-72, 1976-77, 1979, and 1983-87.

SEDIMENT CONCENTRATIÓN (watex years 1975-96): Maximum daily mean, 21, $400 \mathrm{mg} / \mathrm{L}$, Aug. 11, 1979; minimum

daily mean, no flow on many days of most yoars.

SEDIMENT LOAD: Maximum datly, 356,000 tons, Aug. 11, 1967; minimum daily, 0 ton on many days each year.

EXTREMUES FOR CURRENT YEAR.--

SPECIFIC CONDUCTARCE: Maximum daily, 2,440 microstemens, July 25; minimum dally, 341 microsiemens, Nov. 21. WATER TEMPERATURE: Maximum dally, $35.0^{\circ} \mathrm{C}$, June 5,6 , July 5,23 , minimum daily, $6.0^{\circ} \mathrm{C}, \mathrm{Jan} .2$.

SEDIMENT CONCENTRATION: Maximum daily mean, 18,700 mg/L, June 28; minimum daily mean, no flow, May $14-16$. SEDIMENT LOAD: Maximum daily, 70,800 tons, June 28; minimun daily, 0 ton, May 14-16.

WATER QUALITY DATA, WATER YEAR OCTOBER 1995 TO SEPTEMBER 1996

\begin{tabular}{|c|c|c|c|c|c|c|c|c|c|c|c|}
\hline & & $\begin{array}{c}\text { DIS- } \\
\text { CBARGE, }\end{array}$ & +4 & $\begin{array}{c}\text { FH } \\
\text { WATER }\end{array}$ & & & $\begin{array}{l}\text { BARO- } \\
\text { METRIC }\end{array}$ & & $\begin{array}{c}\text { OXYGEN, } \\
\text { DIS- }\end{array}$ & & \\
\hline $\mathbf{T E}$ & IRIE & $\begin{array}{c}\text { INST } \\
\text { CUBIC } \\
\text { FEET } \\
\text { PER } \\
\text { SECOND } \\
(00061)\end{array}$ & $\begin{array}{l}\text { CIFIC } \\
\text { CON- } \\
\text { DUCT- } \\
\text { ANCE } \\
\text { (US/CM) } \\
(00095)\end{array}$ & $\begin{array}{c}\text { WHOLE } \\
\text { FIELD } \\
\text { (STAND- } \\
\text { ARD } \\
\text { UNITS) } \\
(00400 \text { ) }\end{array}$ & $\begin{array}{c}\text { TEMPER- } \\
\text { ATURE } \\
\text { AIR } \\
\text { (DEG C) } \\
(00020)\end{array}$ & $\begin{array}{c}\text { TEMPER- } \\
\text { ATURE } \\
\text { WATER } \\
\text { (DEG C) } \\
(00010)\end{array}$ & $\begin{array}{l}\text { PRES- } \\
\text { SURE } \\
\text { (MM } \\
\text { OF } \\
\text { HG) } \\
(00025)\end{array}$ & $\begin{array}{c}\text { OXYGEN, } \\
\text { DIS- } \\
\text { SOLVED } \\
(M G / L) \\
(00300)\end{array}$ & $\begin{array}{l}\text { SOLVED } \\
\text { (PER- } \\
\text { CENT } \\
\text { SATUR- } \\
\text { ATION) } \\
\text { (00301) }\end{array}$ & $\begin{array}{l}\text { ICAL } \\
\text { (BIGH } \\
\text { LEVEL) } \\
(\mathrm{HG} / \mathrm{L}) \\
(00340)\end{array}$ & $\begin{array}{c}\text { TORAL } \\
\text { (MG/L } \\
\text { AS } \\
\text { CACO3) } \\
(00900)\end{array}$ \\
\hline
\end{tabular}

\begin{tabular}{|c|c|c|c|c|c|c|c|c|c|c|c|}
\hline $\begin{array}{l}\text { OCT } 1995 \\
17\end{array}$ & 1330 & 471 & 462 & 8.4 & 17.0 & 16.0 & 645 & 8.8 & 106 & 22 & 150 \\
\hline $13 \ldots$ & 0900 & 550 & 421 & 8.3 & 12.0 & 10.0 & 638 & 9.1 & 97 & 18 & 130 \\
\hline $30 \ldots$ & 0945 & 205 & 488 & 8.8 & -- & 18.5 & 642 & 9.8 & 125 & 14 & \\
\hline & 0930 & 52 & 566 & 8.3 & 27.5 & 21.0 & 649 & 7.4 & 98 & 44 & \\
\hline
\end{tabular}

DATE

$\begin{array}{lc}\text { HARD- } & \\ \text { NESS } & \\ \text { NONCARB } & \text { CALCTUM } \\ \text { DISSOLV } & \text { DIS- } \\ \text { FLD AS } & \text { SOLVED } \\ \text { CACO3 } & \text { (MSG/L } \\ (\text { MGG } / L) & \text { AS CA) } \\ (00904) & (00915)\end{array}$

$\begin{array}{cc}\text { MAGNE- } & \\ \text { SIUM, } & \text { SODIUM, } \\ \text { DIS- } & \text { DIS- } \\ \text { SOLVED } & \text { SOLVED } \\ \text { (NGG/L } & \text { (MG/L } \\ \text { AS MGG) } & \text { AS NAA) } \\ (00925) & (00930)\end{array}$

$\begin{array}{cc}\text { SODIUM } & \text { POTAS- } \\ \text { AD- } & \text { SIUM, } \\ \text { SORP- } & \text { DIS- } \\ \text { TION } & \text { SOLVED } \\ \text { RATIO } & \text { (ME/L } \\ (00932) & (00935)\end{array}$

BICAR-
BONATE
WATER
DIS IT
FIELD
MG $/ \mathrm{L}$ AS
HCO3
$(00453)$

CAR-
BONATE
WATER
DIS IT
FIELD
WE/L AS
CO3
$(00452)$

ALKA-
LINITY
WAT DIS
TOT IT
FIELD
MG/L AS
CACO3
(39086)

ALKALINITY $\underset{I A B}{I A G / L}$ (MG/L AS (ACO3)

\begin{tabular}{|c|c|c|c|}
\hline OCT 1995 & 16 & 47 & 8.0 \\
\hline $13 \ldots$ & 10 & 41 & 7.2 \\
\hline $\begin{array}{l}30 . \\
\text { AUG }\end{array}$ & 19 & 48 & 8.6 \\
\hline & 26 & SE & 9.0 \\
\hline
\end{tabular}

$\begin{array}{rrrrrr}1 & 4.1 & 154 & 5 & 133 & 135 \\ 1 & 4.0 & 143 & 3 & 122 & 130 \\ 1 & 4.7 & 140 & 13 & 136 & 145 \\ 3 & 4.9 & 174 & 5 & 150 & 159\end{array}$


RIO GRANDE BASIN

08332010 RIO GRANDE FLOODWAY NEAR BERNARDO, NM -- Continued

WATER-QUALITY RECORDS

WATER QUALITY DATA, WATER YEAR OCTOBER 1995 TO SEPTEMBER 1996

DATE

SULFATE

RIS-

FLUO- SILICA,

DIS-

RIDE,

DIS-

NITRO- NITRO

GEN,

(MG/L (MG/L

AS $\mathrm{SO}_{4}$ )

SOLVED TUENTS

DISGEN,

$\begin{array}{lllllllll}\text { SOLVED } & \text { SOLVED } & \text { (MG/L } & \text { DIS- } & \text { SOLVED } & \text { SOLVED } & \text { SOLVED } & \text { SOLVED } & \text { SOLVED } \\ (M G / L & (M G / L & \text { AS } & \text { SOLVED } & \text { (MG/L } & \text { (MG/L } & \text { (MG/L } & \text { (MG/L } & \text { (MG/L } \\ \text { AS CL) } & \text { AS F) } & \text { SIO2) } & (M G / L) & \text { AS N) } & \text { AS N) } & \text { AS N) } & \text { AS N }) & \text { AS N) }\end{array}$

$\begin{array}{lllllllll}\text { SOLVED } & \text { SOLVED } & \text { (MG/L } & \text { DIS- } & \text { SOLVED } & \text { SOLVED } & \text { SOLVED } & \text { SOLVED } & \text { SOLVED } \\ (M G / L & (M G / L & \text { AS } & \text { SOLVED } & \text { (MG/L } & \text { (MG/L } & \text { (MG/L } & \text { (MG/L } & \text { (MG/L } \\ \text { AS CL) } & \text { AS F) } & \text { SIO2) } & (M G / L) & \text { AS N) } & \text { AS N) } & \text { AS N) } & \text { AS N }) & \text { AS N) }\end{array}$

DIS- DIS-

GEN NTTRO-

AMMONIA ORGANIC

DIS- DIS-

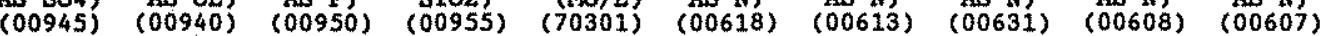

OCT 1995

MAR $19 \dot{9} \dot{6}$

13 ...

MAY

AUG

$\begin{array}{ll}66 & 17 \\ 61 & 14 \\ 78 & 17 \\ 95 & 19\end{array}$

0.5021

286

1.39

0.010

1.40

$<0.015$

$0.50 \quad 24$

$261 \quad<0.010$

0.740

$<0.015 \quad \ldots$

$12 \ldots$

0.6019

297

$\ldots \quad<0.010$

0.270

0.020

0.38

$0.60 \quad 24$

$349 \quad 0.830$

0.020

0.850

0.030

NITRO- NTTRO-

MONIA + MONIA + ORGANIC ORGANIC

DATE TOTAL DIS (MG/L $\quad$ (MG/L

AS N)
$(00625)$

AS N)
$(00623)$

PHOS-

PHOS- PHOS-

PHOS- PRORUS ALIM- ANTI-

ORTHO, CARBON, INUM, MONY, ARSENIC BARILM,

DIS- DIS-

SOIVID

DIS-

TORAL

TOTAL

(MG/L $\quad(M G / L$

(MG/L (MG/L

(MG/L

ORGANIC TIS,

IS- DIS-

TOTAL SOLVED

(MG/L

(UG/L

$\left(\begin{array}{c}\text { AS P) } \\ (00665)\end{array}\right.$

$(00666)$

$(00680) \quad(01106)$

SOLVED DIS- DIS-

(UG/L ULGD SOLVED

AS SB) AS AS) AS BA)

OCT 1995

MAR 1796

$13 . .$.

MAY

AUG

$12,$.

$\begin{array}{rrrrr}0.40 & 0.30 & 0.280 & 0.230 & 0.210 \\ 0.40 & 0.20 & 0.300 & 0.240 & 0.230 \\ 0.80 & 0.40 & 0.240 & 0.150 & 0.140 \\ 0.30 & <0.20 & 0.220 & 0.130 & 0.180\end{array}$

6.5

5.

$<1$

(01005)

\begin{tabular}{|c|c|c|}
\hline $\begin{array}{l}\text { BERYL- } \\
\text { LIUM, } \\
\text { DIS- } \\
\text { SOLVED } \\
\text { (UG/L } \\
\text { AS BE) }\end{array}$ & $\begin{array}{l}\text { BORON, } \\
\text { DIS- } \\
\text { SOLVED } \\
\text { (UG/L } \\
\text { AS B) }\end{array}$ & $\begin{array}{c}\text { CADMIUM } \\
\text { DIS- } \\
\text { SOLVED } \\
\text { (UGG/L } \\
\text { AS CD) }\end{array}$ \\
\hline
\end{tabular}

MIUM, COBALT, COPPER, IRON, LEAD, MANGA- MERCURY

DIS- DIS- DIS- $^{-}$DIS- LIS- DIS- RECAL-

SOLVED SOLVED SOLVED SOLVED SOLVED SOLVED ERABLE

(UG/L (UG/L (UG/L (UG/L (UG/L (UG/L

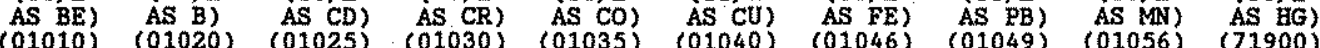

OCT 1995

MAR 1996

13
MAY....
30

AUG

$12 .$.

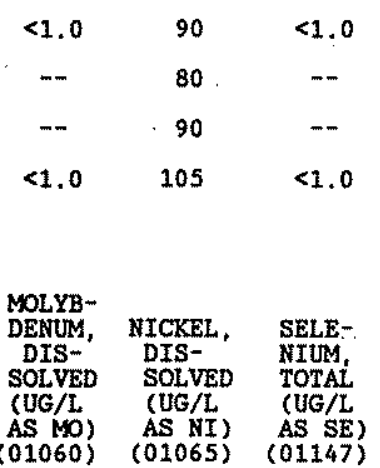

$1.0<1.0$

2. 0

$<3.0<1.0$

$3.0<0.10$

$-\infty$

.--

$-$

$<3.0$

-.

--

$--$

$-$

$+$

2.0

$<3.0$

$\rightarrow-$

$2.0<1.0$

$<3.0$

$<1.0$

$<1.0<0.10$

OCT 1995

$\operatorname{MAR}_{13}^{17} 1996$
MAY
AUG

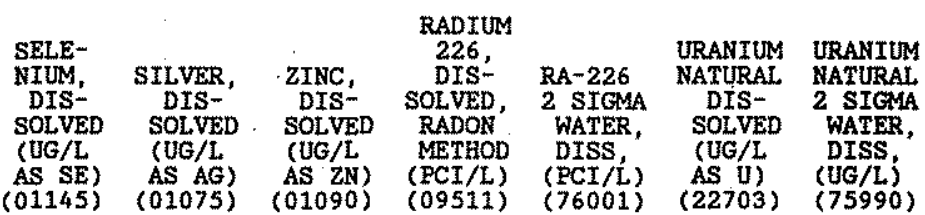

$\begin{array}{llllcccccc}7.0 & 2.0 & <1 & <1 & <1.0 & 2.0 & 0.08 & 0.020 & 1.9 & 0.1 \\ -- & -- & -- & -- & -- & -- & -- & -- & -- & - \\ -- & -- & -- & -- & -- & -- & -- & -- & -- & -- \\ 8.0 & 3.0 & <1 & <1 & <1.0 & <1.0 & -- & -- & 3.0 & --\end{array}$


RIO GRANDE BASIN

08332010 RIO GRANDE FLOODWAY NEAR BERNARDO, NM -- Continued

WATER-QUALITY RECORDS

WATER QUALITY DATA, WATER YEAR OCTOBER 1995 TO SEPTEMBER 1996

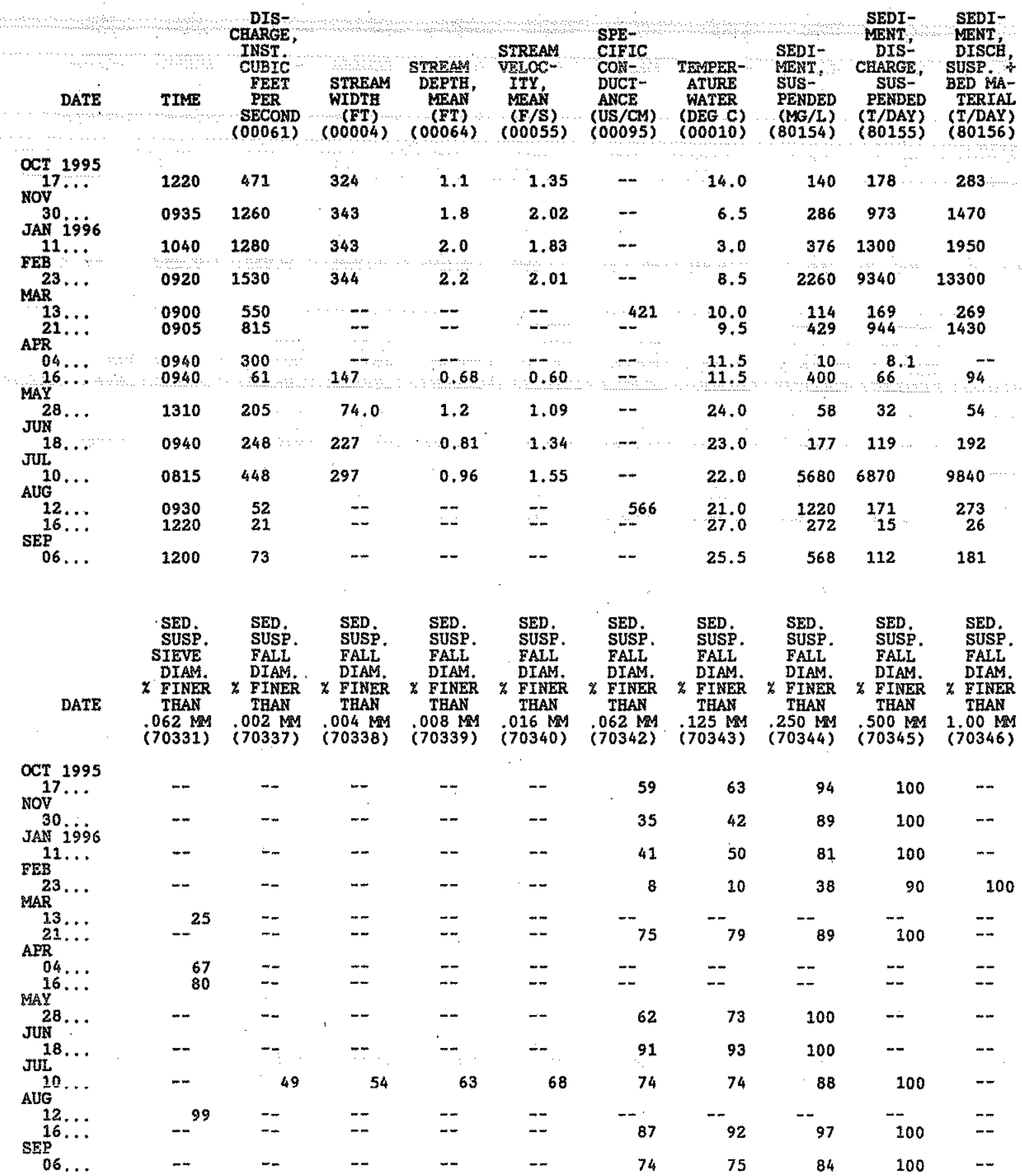


RIO GRANDE BASIN

08332010 RIO GRANDE FLOODWAY NEAR BERNARDO, NM -- Continued

WATER-QUALITY RECORDS

WATER QUALITY DATA, WATER YEAR OCTOBER 1995 TO SEPTEMBER 1996

$\begin{array}{lllllllll}\text { BED } & \text { BED } & \text { BED } & \text { BED } & \text { BED } & \text { BED } & \text { BED } & \text { BED } & \text { BED }\end{array}$

MAT. MAT. MAT. MAT. MAT. MAT. MAT. MAT. MAT. MAT. SIEVE SIEVE SIEVE SIEVE SIEVE SIEVE SIEVE SIEVE SIEVE SIEVE

DIA. DIAM. DIAM. DIAM. DIAM. DIAM. DIAM. DIAM. DIAM. DIAM.

T FTER

$x$ FINIXR $x$ FINER $X$ EINER

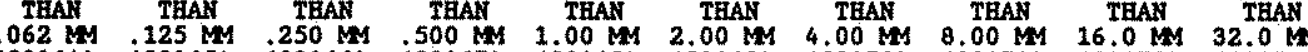

DATE

$(80164)$

$125 \mathrm{MM}, 250 \mathrm{M}$

(80170) (80171)

$\begin{array}{ll}16.0 \mathrm{M} & 32.0 \mathrm{MM} \\ (80172) & (80173)\end{array}$

\begin{tabular}{|c|c|c|c|c|c|c|c|c|c|c|}
\hline $\begin{array}{l}\text { OCT } 1995 \\
17 \ldots\end{array}$ & $m$ & $-\cdots$ & -- & -- & -- & - & -- & -- & -- & $m$ \\
\hline${ }_{\text {JAN }}^{30} 1996$ & 0 & 1 & 18 & 75 & 95 & 97 & 97 & 98 & 100 & -- \\
\hline $\operatorname{san}^{12} \ldots$ & $\ldots$ & 0 & 1 & 20 & 74 & 96 & 100 & -- & -- & $m$ \\
\hline${ }_{\text {MAR }}^{23} \ldots$ & 0 & 1 & 18 & 75 & 90 & 92 & 94 & 94 & 98 & 100 \\
\hline $\begin{array}{r}13 \ldots \\
21 \ldots \\
A P R\end{array}$ & -- & --5 & $-\frac{}{32}$ & 88 & .99 & $-\overline{100}$ & $\because-$ & $m$ & $m$ & $m$ \\
\hline $\begin{array}{r}04 \ldots \\
16 \ldots \\
M A Y\end{array}$ & $m 3$ & --12 & 37 & --80 & 96 & 98 & -79 & 99 & -- & $=$ \\
\hline${ }_{\text {JUN }}^{28} \ldots$ & 2 & 8 & 48 & 89 & 99 & 100 & -- & -- & $m$ & -- \\
\hline $\mathrm{JUL}^{18 \ldots}$ & 0 & 2 & 37 & 91. & 99 & 100 & -+ & $-\cdots$ & -- & -- \\
\hline AUG $10 \ldots$ & 1 & 4 & 33 & 85 & 97 & 99 & 99 & 100 & $-m$ & $-m$ \\
\hline $\begin{array}{r}12 \ldots \\
16 \ldots\end{array}$ & $\because 2$ & -5 & 23 & 81 & 97 & 100 & $m$ & $m$ & -- & $m$ \\
\hline $06 \ldots$ & 5 & 8 & 25 & 82 & 98 & 100 & -- & $-m$ & -- & -- \\
\hline
\end{tabular}


RIO GRANDE BASIN

08332010 RIO GRANDE FLOODWAY NEAR BERNARDO, NM -- Continued

WATER-QUALITY RECORDS

SPECIFIC CONDUCTANCE, US/CA $\$ 25$ DEGREES CENTIGRADE, WATER YEAR OCTOBER 1995 TO SEPTEMBER 1996

DAILY INSTANTAREOUS VALUES

\begin{tabular}{|c|c|c|c|c|c|c|c|c|c|c|c|c|}
\hline DAY & OCT & Nov & $\mathrm{DEC}$ & JAN & FEB & MAR & APR & MAY & JUN & JUL & AUG & SEP \\
\hline $\begin{array}{l}1 \\
2 \\
3 \\
4 \\
5\end{array}$ & $\begin{array}{l}466 \\
474 \\
465 \\
461 \\
458\end{array}$ & $\begin{array}{l}465 \\
473 \\
487 \\
488 \\
477\end{array}$ & $\begin{array}{l}-- \\
-- \\
-\end{array}$ & $\begin{array}{l}457 \\
456 \\
543 \\
465 \\
452\end{array}$ & $\begin{array}{l}438 \\
440 \\
433 \\
433 \\
428\end{array}$ & $\begin{array}{l}426 \\
438 \\
428 \\
420 \\
412\end{array}$ & $\begin{array}{l}509 \\
543 \\
569 \\
586 \\
584\end{array}$ & $\begin{array}{l}605 \\
677 \\
624 \\
636 \\
615\end{array}$ & $\begin{array}{l}530 \\
530 \\
529 \\
533 \\
559\end{array}$ & $\begin{array}{l}494 \\
505 \\
522 \\
526 \\
519\end{array}$ & $\begin{array}{l}574 \\
594 \\
564 \\
557 \\
531\end{array}$ & $\begin{array}{l}526 \\
556 \\
566 \\
567 \\
583\end{array}$ \\
\hline $\begin{array}{r}6 \\
7 \\
8 \\
9 \\
10\end{array}$ & $\begin{array}{l}466 \\
474 \\
483 \\
473 \\
478\end{array}$ & $\begin{array}{l}454 \\
456 \\
460 \\
470 \\
169\end{array}$ & $\begin{array}{l}-520 \\
477 \\
473 \\
472\end{array}$ & $\begin{array}{l}462 \\
465 \\
471 \\
462 \\
457\end{array}$ & $\begin{array}{l}427 \\
427 \\
426 \\
427 \\
-\end{array}$ & $\begin{array}{l}434 \\
433 \\
440 \\
435 \\
436\end{array}$ & $\begin{array}{l}584 \\
601 \\
598 \\
607 \\
629\end{array}$ & $\begin{array}{l}638 \\
619 \\
633 \\
631 \\
649\end{array}$ & $\begin{array}{l}575 \\
550 \\
578 \\
595 \\
-\end{array}$ & $\begin{array}{l}544 \\
542 \\
535 \\
478 \\
525\end{array}$ & $\begin{array}{l}507 \\
574 \\
613 \\
572 \\
541\end{array}$ & $\begin{array}{l}547 \\
541 \\
538 \\
575 \\
551\end{array}$ \\
\hline $\begin{array}{l}11 \\
12 \\
13 \\
14 \\
15\end{array}$ & $\begin{array}{l}469 \\
490 \\
493 \\
481 \\
470\end{array}$ & $\begin{array}{l}471 \\
461 \\
464 \\
454 \\
462\end{array}$ & $\begin{array}{l}469 \\
464 \\
466 \\
469 \\
473\end{array}$ & $\begin{array}{l}452 \\
449 \\
455 \\
456 \\
452\end{array}$ & $\begin{array}{l}-\cdots \\
--- \\
436 \\
436 \\
436\end{array}$ & $\begin{array}{l}435 \\
428 \\
442 \\
440 \\
446\end{array}$ & $\begin{array}{l}633 \\
659 \\
731 \\
699 \\
690\end{array}$ & $\begin{array}{l}622 \\
595 \\
594 \\
--\end{array}$ & $\begin{array}{l}617 \\
551 \\
613 \\
609 \\
536\end{array}$ & $\begin{array}{r}1060 \\
483 \\
524 \\
569 \\
561\end{array}$ & $\begin{array}{l}543 \\
564 \\
589 \\
635 \\
621\end{array}$ & $\begin{array}{l}526 \\
559 \\
505 \\
581 \\
525\end{array}$ \\
\hline $\begin{array}{l}16 \\
17 \\
18 \\
19 \\
20\end{array}$ & $\begin{array}{l}488 \\
471 \\
461 \\
480 \\
474\end{array}$ & $\begin{array}{l}457 \\
460 \\
459 \\
458 \\
457\end{array}$ & $\begin{array}{l}470 \\
468 \\
464 \\
464 \\
467\end{array}$ & $\begin{array}{l}447 \\
444 \\
442 \\
448 \\
444\end{array}$ & $\begin{array}{r}435 \\
435 \\
-\cdots \\
--\end{array}$ & $\begin{array}{l}445 \\
442 \\
444 \\
432 \\
431\end{array}$ & $\begin{array}{l}605 \\
617 \\
592 \\
616 \\
585\end{array}$ & $\begin{array}{l}-- \\
602 \\
612 \\
720 \\
644\end{array}$ & $\begin{array}{l}544 \\
553 \\
509 \\
521 \\
519\end{array}$ & $\begin{array}{l}586 \\
595 \\
615 \\
601 \\
592\end{array}$ & $\begin{array}{l}646 \\
605 \\
665 \\
666 \\
673\end{array}$ & $\begin{array}{l}409 \\
523 \\
549 \\
516 \\
498\end{array}$ \\
\hline $\begin{array}{l}21 \\
22 \\
23 \\
24 \\
25\end{array}$ & $\begin{array}{l}478 \\
482 \\
490 \\
461 \\
400\end{array}$ & $\begin{array}{l}341 \\
463 \\
462 \\
471 \\
471\end{array}$ & $\begin{array}{l}465 \\
465 \\
475 \\
476 \\
477\end{array}$ & $\begin{array}{l}446 \\
441 \\
438 \\
440 \\
454\end{array}$ & $\begin{array}{l}419 \\
450 \\
419 \\
421 \\
428\end{array}$ & $\begin{array}{l}439 \\
-467 \\
480 \\
472\end{array}$ & $\begin{array}{l}572 \\
558 \\
553 \\
601 \\
589\end{array}$ & $\begin{array}{l}568 \\
577 \\
-194 \\
560\end{array}$ & $\begin{array}{l}530 \\
556 \\
549 \\
573 \\
576\end{array}$ & $\begin{array}{r}559 \\
560 \\
567 \\
2440\end{array}$ & $\begin{array}{l}589 \\
571 \\
546 \\
533 \\
528\end{array}$ & $\begin{array}{l}529 \\
553 \\
546 \\
537 \\
570\end{array}$ \\
\hline $\begin{array}{l}26 \\
27 \\
28 \\
29 \\
30 \\
31\end{array}$ & $\begin{array}{l}481 \\
488 \\
471 \\
472 \\
502 \\
527\end{array}$ & \begin{tabular}{l}
467 \\
468 \\
\hdashline- \\
\hdashline- \\
\hdashline
\end{tabular} & $\begin{array}{l}470 \\
468 \\
473 \\
476 \\
470 \\
469\end{array}$ & $\begin{array}{l}452 \\
465 \\
456 \\
450 \\
452\end{array}$ & $\begin{array}{l}420 \\
424 \\
426 \\
425 \\
- \\
-\end{array}$ & $\begin{array}{l}459 \\
462 \\
497 \\
476 \\
487 \\
487\end{array}$ & $\begin{array}{l}579 \\
559 \\
630 \\
604 \\
566 \\
-\end{array}$ & $\begin{array}{l}541 \\
528 \\
503 \\
507 \\
495 \\
524\end{array}$ & $\begin{array}{l}565 \\
533 \\
583 \\
452 \\
515 \\
\end{array}$ & $\begin{array}{r}871 \\
1370 \\
571 \\
566 \\
562 \\
587\end{array}$ & $\begin{array}{l}478 \\
528 \\
463 \\
516 \\
607 \\
584\end{array}$ & $\begin{array}{l}582 \\
603 \\
601 \\
596 \\
568\end{array}$ \\
\hline $\begin{array}{l}\text { MEAN } \\
\text { MAX } \\
\text { MIN }\end{array}$ & $\begin{array}{l}475 \\
527 \\
400\end{array}$ & $\overline{--}$ & $=$ & $\overline{--}$ & $\overline{-\cdots}$ & $\overline{-}$ & $\begin{array}{l}602 \\
731 \\
509\end{array}$ & $=$ & $\begin{array}{l}\overline{-} \\
-\cdots-\end{array}$ & $=$ & $\begin{array}{l}573 \\
673 \\
463\end{array}$ & $\begin{array}{l}548 \\
603 \\
409\end{array}$ \\
\hline
\end{tabular}

WATER TEMPERATURE, DEGREES CELSIUS, WATER YEAR OCTOBER 1995 TO SEPTEMBER 1996

\begin{tabular}{|c|c|c|c|c|c|c|c|c|c|c|c|c|}
\hline DAY & OCT & nov & DEC & JAN & FEB & MAR & AFR & MAY & JUN & JUL & AUG & SEP \\
\hline $\begin{array}{l}1 \\
2 \\
3 \\
4 \\
5\end{array}$ & $\begin{array}{l}25.0 \\
23.0 \\
23.0 \\
20.0 \\
20.0\end{array}$ & $\begin{array}{l}16.0 \\
15.0 \\
17.0 \\
15.0 \\
14.0\end{array}$ & $\begin{array}{l}--- \\
--- \\
--- \\
---\end{array}$ & $\begin{array}{r}7.0 \\
6.0 \\
7.0 \\
9.0 \\
12.0\end{array}$ & $\begin{array}{r}10.0 \\
9.0 \\
\hdashline 10.0\end{array}$ & $\begin{array}{r}12.0 \\
13.0 \\
13.0 \\
11.0 \\
14.0\end{array}$ & $\begin{array}{l}21.0 \\
22.0 \\
23.0 \\
18.0 \\
14.0\end{array}$ & $\begin{array}{l}27.0 \\
28.0 \\
28.0 \\
27.0 \\
30.0\end{array}$ & $\begin{array}{l}29.0 \\
30.0 \\
32.0 \\
33.0 \\
35.0\end{array}$ & $\begin{array}{l}31.0 \\
32.0 \\
30.0 \\
30.0 \\
35.0\end{array}$ & $\begin{array}{l}30.0 \\
30.0 \\
31.0 \\
32.0 \\
31.0\end{array}$ & $\begin{array}{l}26.0 \\
29.0 \\
28.0 \\
26.0\end{array}$ \\
\hline $\begin{array}{r}6 \\
7 \\
8 \\
9 \\
10\end{array}$ & $\begin{array}{l}20.0 \\
14.0 \\
20.0 \\
22.0 \\
22.0\end{array}$ & $\begin{array}{l}15.0 \\
15.0 \\
17.0 \\
19.0 \\
14.0\end{array}$ & $\begin{array}{l}13.0 \\
13.0 \\
13.0 \\
14.0\end{array}$ & $\begin{array}{l}10.0 \\
10.0 \\
13.0 \\
11.0 \\
12.0\end{array}$ & $\begin{array}{r}11.0 \\
11.0 \\
12.0 \\
-\end{array}$ & $\begin{array}{l}10.0 \\
10.0 \\
13.0 \\
15.0 \\
16.0\end{array}$ & $\begin{array}{l}21.0 \\
21.0 \\
25.0 \\
26.0 \\
18.0\end{array}$ & $\begin{array}{l}30.0 \\
30.0 \\
31.0 \\
30.0 \\
30.0\end{array}$ & $\begin{array}{l}35.0 \\
34.0 \\
30.0 \\
32.0 \\
34.0\end{array}$ & $\begin{array}{l}30.0 \\
30.0 \\
31.0 \\
29.0 \\
30.0\end{array}$ & $\begin{array}{l}33.0 \\
23.0 \\
22.0 \\
25.0 \\
26.0\end{array}$ & $\begin{array}{l}29.0 \\
29.0 \\
30.0 \\
28.0 \\
27.0\end{array}$ \\
\hline $\begin{array}{l}11 \\
12 \\
13 \\
14 \\
15\end{array}$ & $\begin{array}{l}25.0 \\
23.0 \\
23.0 \\
20.0 \\
15.0\end{array}$ & $\begin{array}{l}10.0 \\
14.0 \\
14.0 \\
15.0 \\
16.0\end{array}$ & $\begin{array}{r}10.0 \\
12.0 \\
14.0 \\
12.0 \\
9.0\end{array}$ & $\begin{array}{r}12.0 \\
13.0 \\
8.0 \\
9.0 \\
9.0\end{array}$ & $\begin{array}{r}--- \\
18.0 \\
18.0 \\
19.0\end{array}$ & $\begin{array}{l}17.0 \\
17.0 \\
12.0 \\
13.0 \\
15.0\end{array}$ & $\begin{array}{l}18.0 \\
23.0 \\
18.0 \\
23.0 \\
22.0\end{array}$ & $\begin{array}{r}30.0 \\
33.0 \\
30.0 \\
- \\
-\end{array}$ & $\begin{array}{l}30.0 \\
27.0 \\
28.0 \\
26.0 \\
30.0\end{array}$ & $\begin{array}{r}33.0 \\
31.0 \\
23.0\end{array}$ & $\begin{array}{l}31.0 \\
23.0 \\
30.0 \\
30.0 \\
28.0\end{array}$ & $\begin{array}{l}24.0 \\
22.0 \\
20.0 \\
18.0 \\
22.0\end{array}$ \\
\hline $\begin{array}{l}16 \\
17 \\
18 \\
19 \\
20\end{array}$ & $\begin{array}{l}20.0 \\
20.0 \\
22.0 \\
20.0 \\
21.0\end{array}$ & $\begin{array}{l}17.0 \\
17.0 \\
13.0 \\
17.0 \\
17: 0\end{array}$ & $\begin{array}{l}10.0 \\
13.0 \\
12.0 \\
10.0 \\
10.0\end{array}$ & $\begin{array}{l}13.0 \\
13.0 \\
12.0 \\
12.0 \\
12.0\end{array}$ & $\begin{array}{r}19.0 \\
20.0 \\
-2\end{array}$ & $\begin{array}{l}16.0 \\
15.0 \\
15.0 \\
16.0 \\
17.0\end{array}$ & $\begin{array}{l}22.0 \\
23.0 \\
23.0 \\
22.0 \\
22.0\end{array}$ & \begin{tabular}{c}
$2 \overline{8} .5$ \\
\hdashline- \\
\hdashline-
\end{tabular} & $\begin{array}{l}30.0 \\
30.0 \\
30.0 \\
33.0 \\
34.0\end{array}$ & $\begin{array}{l}\cdots \\
\cdots \\
\cdots \\
\cdots-\end{array}$ & $\begin{array}{r}28.0 \\
29.0 \\
28.0 \\
-\end{array}$ & $\begin{array}{l}23.0 \\
24.0 \\
21.0 \\
22.0 \\
26.0\end{array}$ \\
\hline $\begin{array}{l}21 \\
22 \\
23 \\
24 \\
25\end{array}$ & $\begin{array}{l}20.0 \\
22.0 \\
15.0 \\
17.0 \\
18.0\end{array}$ & $\begin{array}{l}16.0 \\
16.0 \\
15.0 \\
16.0 \\
16.0\end{array}$ & $\begin{array}{r}9.0 \\
12.0 \\
11.0 \\
17.0 \\
18.0\end{array}$ & $\begin{array}{r}8.0 \\
12.0 \\
10.0 \\
12.0 \\
11.0\end{array}$ & $\begin{array}{l}15.0 \\
18.0 \\
13.0 \\
11.0 \\
10.0\end{array}$ & $\begin{array}{r}17.0 \\
19.0 \\
16.0 \\
15.0\end{array}$ & $\begin{array}{l}22.0 \\
23.0 \\
25.0 \\
27.0 \\
27.0\end{array}$ & $\begin{array}{r}31.0 \\
30.0 \\
26.0 \\
23.0\end{array}$ & $\begin{array}{l}32.0 \\
28.0 \\
33.0 \\
31.0 \\
31.0\end{array}$ & $\begin{array}{r}33.0 \\
-35.0 \\
34.0 \\
27.0\end{array}$ & $\begin{array}{r}--- \\
-\cdots \\
29.0\end{array}$ & $\begin{array}{l}24.0 \\
26.0 \\
25.0 \\
21.0 \\
25.0\end{array}$ \\
\hline $\begin{array}{l}26 \\
27 \\
28 \\
29 \\
30 \\
31\end{array}$ & $\begin{array}{l}18.0 \\
20.0 \\
21.0 \\
15.0 \\
20.0 \\
17.0\end{array}$ & $\begin{array}{r}15.0 \\
15.0 \\
0 \\
\end{array}$ & $\begin{array}{r}13.0 \\
11.0 \\
9.0 \\
8.0 \\
9.0 \\
10.0\end{array}$ & $\begin{array}{r}8.0 \\
10.0 \\
10.0 \\
11.0 \\
12.0\end{array}$ & $\begin{array}{r}10.0 \\
11.0 \\
9.0 \\
9.0 \\
\end{array}$ & $\begin{array}{l}16.0 \\
17.0 \\
19.0 \\
15.0 \\
18.0\end{array}$ & $\begin{array}{l}28.0 \\
28.0 \\
19.0 \\
22.0 \\
25.0\end{array}$ & $\begin{array}{l}21.0 \\
29.0 \\
30.0 \\
28.0 \\
25.0\end{array}$ & $\begin{array}{l}29.0 \\
28.0 \\
28.0 \\
26.0 \\
29.0\end{array}$ & $\begin{array}{l}34.0 \\
34.0 \\
31.0 \\
25.0 \\
28.0 \\
30.0\end{array}$ & $\begin{array}{r}26.0 \\
28.0 \\
29.0 \\
. . \\
\end{array}$ & $\begin{array}{l}15.0 \\
20.0 \\
23.0 \\
25.0 \\
25.0\end{array}$ \\
\hline $\begin{array}{l}\text { MEAN } \\
\text { MAX } \\
\text { MIN }\end{array}$ & $\begin{array}{l}20.0 \\
25.0 \\
14.0\end{array}$ & $=-$ & $=-$ & 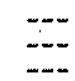 & $\because \cdots$ & 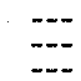 & $\begin{array}{l}22.4 \\
28.0 \\
14.0\end{array}$ & $\begin{array}{l}--- \\
-\cdots \\
-\cdots\end{array}$ & $\begin{array}{l}30.6 \\
35.0 \\
26.0\end{array}$ & 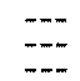 & $=$ & $=$ \\
\hline
\end{tabular}


RIO GRANDE BASIN

08332010 RIO GRANDE FLOODWAY NEAR BERNARDO, NM -- Continued

WATER-QUALITY RECORDS

SUSPENDED-SEDIMENT, WATER YEAR OCTOBER 1995 TO SEPTEMBER 1996

\begin{tabular}{|c|c|c|c|c|c|c|c|c|c|c|c|c|}
\hline DAY & $\begin{array}{c}\text { MEAN } \\
\text { CONCEN- } \\
\text { TRATION } \\
\text { (MG/L) }\end{array}$ & $\begin{array}{l}\text { LOADS } \\
\text { (T/DAY) }\end{array}$ & $\begin{array}{l}\text { MEAN } \\
\text { CONCEN- } \\
\text { TRATION } \\
(M G / L)\end{array}$ & $\begin{array}{l}\text { LOADS } \\
\text { (T/DAY })\end{array}$ & $\begin{array}{c}\text { MEAN } \\
\text { CONCEN- } \\
\text { TRATION } \\
\text { (MG/L) }\end{array}$ & $\begin{array}{l}\text { LOADS } \\
(\mathrm{T} / \mathrm{DAY})\end{array}$ & $\begin{array}{c}\text { MEAN } \\
\text { CONCEN- } \\
\text { TRATION } \\
\text { (MG } / L)\end{array}$ & $\begin{array}{l}\text { LOADS } \\
\text { (T/DAY) }\end{array}$ & $\begin{array}{c}\text { MEAN } \\
\text { CONCEN- } \\
\text { TRATION } \\
\text { (MG/L) }\end{array}$ & $\begin{array}{l}\text { LOADS } \\
\text { (T/DAY) }\end{array}$ & $\begin{array}{c}\text { MEAN } \\
\text { CONCEN- } \\
\text { TRATION } \\
(M G / L)\end{array}$ & $\begin{array}{l}\text { LOADS } \\
\text { (T/DAY) }\end{array}$ \\
\hline $\begin{array}{l}1 \\
2 \\
3 \\
4 \\
5\end{array}$ & $\begin{array}{c}1270 \\
667 \\
538 \\
395 \\
324\end{array}$ & $\begin{array}{r}\text { TOBER } \\
2930 \\
1470 \\
1230 \\
914 \\
669\end{array}$ & $\begin{array}{c}\text { Nov } \\
2520 \\
1110 \\
466 \\
433 \\
430\end{array}$ & $\begin{array}{r}\text { BMER } \\
9100 \\
5150 \\
2100 \\
1990 \\
1960\end{array}$ & $\begin{array}{l}D E \\
871 \\
746 \\
639 \\
752 \\
806\end{array}$ & $\begin{array}{l}\text { DMER } \\
3060 \\
2460 \\
2090 \\
2580 \\
2810\end{array}$ & $\begin{array}{l}485^{J /} \\
1920 \\
868 \\
900 \\
1100\end{array}$ & $\begin{array}{l}\text { NUARY } \\
1680 \\
6740 \\
2840 \\
2820 \\
4010\end{array}$ & $\begin{array}{c}\text { FE } \\
702 \\
1110 \\
900 \\
950 \\
1500\end{array}$ & $\begin{array}{r}\text { RUARY } \\
2450 \\
3960 \\
3260 \\
3490 \\
5550\end{array}$ & $\begin{array}{r}1030 \\
700 \\
1010 \\
900 \\
616\end{array}$ & $\begin{array}{r}\text { MARCB } \\
3280 \\
2150 \\
3350 \\
3060 \\
1810\end{array}$ \\
\hline $\begin{array}{r}6 \\
7 \\
8 \\
9 \\
10\end{array}$ & $\begin{array}{l}341 \\
362 \\
259 \\
200 \\
543\end{array}$ & $\begin{array}{l}713 \\
631 \\
419 \\
280 \\
856\end{array}$ & $\begin{array}{r}420 \\
556 \\
750 \\
1250 \\
1300\end{array}$ & $\begin{array}{l}1910 \\
2490 \\
3320 \\
5470 \\
5620\end{array}$ & $\begin{array}{r}782 \\
879 \\
1710 \\
1250 \\
812\end{array}$ & $\begin{array}{l}2720 \\
2990 \\
5440 \\
4090 \\
2590\end{array}$ & $\begin{array}{l}1300 \\
1200 \\
1200 \\
1400 \\
1040\end{array}$ & $\begin{array}{l}4530 \\
4080 \\
3990 \\
4500 \\
3400\end{array}$ & $\begin{array}{r}1210 \\
919 \\
708 \\
650 \\
900\end{array}$ & $\begin{array}{l}4520 \\
3280 \\
2470 \\
2210 \\
2940\end{array}$ & $\begin{array}{l}602 \\
734 \\
518 \\
495 \\
501\end{array}$ & $\begin{array}{l}1670 \\
1930 \\
1240 \\
1010 \\
1130\end{array}$ \\
\hline $\begin{array}{l}11 \\
12 \\
13 \\
14 \\
15\end{array}$ & $\begin{array}{l}363 \\
184 \\
186 \\
621 \\
916\end{array}$ & $\begin{array}{r}566 \\
275 \\
309 \\
1040 \\
1390\end{array}$ & $\begin{array}{r}1470 \\
391 \\
252 \\
251 \\
700\end{array}$ & $\begin{array}{l}6270 \\
1650 \\
1050 \\
1030 \\
2840\end{array}$ & $\begin{array}{l}998 \\
534 \\
797 \\
806 \\
794\end{array}$ & $\begin{array}{l}3210 \\
1690 \\
2470 \\
2420 \\
2400\end{array}$ & $\begin{array}{l}1300 \\
1890 \\
1900 \\
1670 \\
1640\end{array}$ & $\begin{array}{l}4530 \\
6020 \\
6270 \\
5100 \\
4510\end{array}$ & $\begin{array}{r}1100 \\
1200 \\
1100 \\
900 \\
1000\end{array}$ & $\begin{array}{l}3420 \\
3560 \\
2780 \\
2360 \\
2680\end{array}$ & $\begin{array}{l}450 \\
475 \\
506 \\
127 \\
374\end{array}$ & $\begin{array}{l}887 \\
893 \\
895 \\
219 \\
703\end{array}$ \\
\hline $\begin{array}{l}16 \\
17 \\
18 \\
19 \\
20\end{array}$ & $\begin{array}{l}422 \\
147 \\
317 \\
333 \\
300\end{array}$ & $\begin{array}{l}514 \\
191 \\
416 \\
441 \\
402\end{array}$ & $\begin{array}{r}1370 \\
214 \\
416 \\
974 \\
1430\end{array}$ & $\begin{array}{r}5470 \\
844 \\
1620 \\
3730 \\
5410\end{array}$ & $\begin{array}{r}1140 \\
868 \\
941 \\
706 \\
632\end{array}$ & $\begin{array}{l}3440 \\
2620 \\
2740 \\
2230 \\
2530\end{array}$ & $\begin{array}{l}1760 \\
2280 \\
1820 \\
2500 \\
2000\end{array}$ & $\begin{array}{l}4740 \\
5990 \\
4890 \\
6880 \\
5670\end{array}$ & $\begin{array}{r}800 \\
1000 \\
700 \\
900 \\
1100\end{array}$ & $\begin{array}{l}2180 \\
2690 \\
1910 \\
2390 \\
2960\end{array}$ & $\begin{array}{r}700 \\
1100 \\
1090 \\
1500 \\
1600\end{array}$ & $\begin{array}{l}1380 \\
2270 \\
2380 \\
3360 \\
3700\end{array}$ \\
\hline $\begin{array}{l}21 \\
22 \\
23 \\
24 \\
25\end{array}$ & $\begin{array}{l}318 \\
401 \\
194 \\
380 \\
340\end{array}$ & $\begin{array}{l}429 \\
547 \\
267 \\
341 \\
299\end{array}$ & $\begin{array}{l}790 \\
543 \\
309 \\
333 \\
394\end{array}$ & $\begin{array}{l}2960 \\
2020 \\
1140 \\
1220 \\
1440\end{array}$ & $\begin{array}{r}863 \\
1000 \\
900 \\
1110 \\
1200\end{array}$ & $\begin{array}{l}3380 \\
3780 \\
3280 \\
3900 \\
4080\end{array}$ & $\begin{array}{r}1600 \\
1220 \\
1030 \\
949 \\
1330\end{array}$ & $\begin{array}{l}4620 \\
3620 \\
3110 \\
2900 \\
4200\end{array}$ & $\begin{array}{r}505 \\
1200 \\
1130 \\
1170 \\
1130\end{array}$ & $\begin{array}{l}1680 \\
4020 \\
4710 \\
4440 \\
4120\end{array}$ & $\begin{array}{r}1340 \\
1300 \\
1240 \\
281 \\
400\end{array}$ & $\begin{array}{r}2930 \\
2750 \\
2650 \\
594 \\
807\end{array}$ \\
\hline $\begin{array}{l}26 \\
27 \\
28 \\
29 \\
30 \\
31\end{array}$ & $\begin{array}{r}384 \\
1210 \\
980 \\
357 \\
160 \\
289\end{array}$ & $\begin{array}{r}280 \\
1180 \\
966 \\
294 \\
168 \\
449\end{array}$ & $\begin{array}{l}656 \\
845 \\
781 \\
713 \\
785 \\
-\end{array}$ & $\begin{array}{l}2370 \\
3010 \\
2760 \\
2500 \\
2730\end{array}$ & $\begin{array}{r}1340 \\
616 \\
1350 \\
826 \\
2760 \\
3730\end{array}$ & $\begin{array}{r}4410 \\
2000 \\
4410 \\
2610 \\
8640 \\
11600\end{array}$ & $\begin{array}{r}1510 \\
2010 \\
1290 \\
1260 \\
822 \\
600\end{array}$ & $\begin{array}{l}5090 \\
6990 \\
4310 \\
4090 \\
2770 \\
2060\end{array}$ & $\begin{array}{r}1110 \\
917 \\
1500 \\
1240 \\
-\cdots\end{array}$ & $\begin{array}{r}4020 \\
3190 \\
5020 \\
4020 \\
- \\
--\end{array}$ & $\begin{array}{l}651 \\
500 \\
484 \\
500 \\
786 \\
400\end{array}$ & $\begin{array}{r}1300 \\
1010 \\
920 \\
1010 \\
1510 \\
707\end{array}$ \\
\hline OTAL & --- & 20876 & $-m$ & 91174 & $-\cdots$ & 108670 & -- & 136950 & -- & 96280 & $-\cdots$ & 53505 \\
\hline & $\begin{array}{l}\text { MEAN } \\
\text { CONCEN- } \\
\text { TRATION } \\
\text { (MG/L) }\end{array}$ & $\begin{array}{l}\text { LOADS } \\
\text { (T/DAY) }\end{array}$ & $\begin{array}{l}\text { MEAN } \\
\text { CONCEN- } \\
\text { TRATION } \\
\text { (MG } / L \text { ) }\end{array}$ & $\begin{array}{l}\text { LOADS } \\
\text { (T/DAY }\end{array}$ & $\begin{array}{l}\text { MEAN } \\
\text { CONCEN- } \\
\text { TRATION } \\
\text { (MG/L) }\end{array}$ & $\begin{array}{l}\text { LOADS } \\
\text { (T/DAY }\end{array}$ & $\begin{array}{l}\text { MEAN } \\
\text { CONCEN- } \\
\text { TRATION } \\
\text { (MG/L) }\end{array}$ & $\begin{array}{l}\text { LOADS } \\
\text { CT/DAY }\end{array}$ & $\begin{array}{l}\text { MEAN } \\
\text { CONCEN- } \\
\text { TRATION } \\
\text { (MG } / \mathrm{L} \text { ) }\end{array}$ & $\begin{array}{l}\text { LOADS } \\
\text { (T/DAY }\end{array}$ & $\begin{array}{l}\text { MEAN } \\
\text { CONCEN- } \\
\text { TRATION } \\
\text { (MG/L) }\end{array}$ & $\begin{array}{l}\text { LOADS } \\
\text { (T/DAY) }\end{array}$ \\
\hline
\end{tabular}

$\begin{array}{lr} & \text { APRIL } \\ 364 & 63 \\ 252 & 34 \\ 190 & 199 \\ 218 & 17 \\ 220 & 15 \\ & \\ 189 & 100 \\ 208 & 10 \\ 347 & 12 \\ 242 & 8 \\ 200 & 6\end{array}$

(I/Dy)

$\begin{array}{rr}68 & \text { MAY } \\ 100 & .00 \\ 71 & .01 \\ 70 & .07 \\ 75 & .8 \\ & .11 \\ 64 & .12 \\ 46 & .11 \\ 53 & .16 \\ 116 & .38 \\ 91 & .34\end{array}$

$\begin{array}{ll}155 & 42 \\ 239 & 58 \\ 322 & 75 \\ 179 & 36 \\ 519 & 98\end{array}$

407

439

110

141

263

103
259
83

66
33
21
22
27
48
18
42
13
11
12
9.5
6.9
6.4
8.1
.--

$83 \quad 12$

70
53
53
65
-

$47 \quad .18$

$\begin{array}{rr}47 & .18 \\ 43 & .19 \\ 64 & .13 \\ 0 & .00 \\ 0 & .00\end{array}$

$\begin{array}{rr}0 & .00 \\ 1030 & 1.5 \\ 483 & 1.8 \\ 165 & 4.5 \\ 97 & 7.1\end{array}$

7.1

$\begin{array}{rr}82 & 2.4 \\ 188 & 4.3 \\ 287 & 71 \\ 355 & 170 \\ 214 & 76\end{array}$

$402 \quad 175$

$\begin{array}{rr}254 & 138 \\ 161 & 94 \\ 166 & 89\end{array}$

$639 \quad 321$

70 JUNE

34 .00
.01
.07
.8

$\begin{array}{rr}70 & 34 \\ 69 & 27 \\ 55 & 14 \\ 48 & 6 .\end{array}$

4
6.7
3.0

8290 JULY

25700

$\begin{array}{rr}9910 & 24000 \\ 6430 & 11900 \\ 3910 & 5700\end{array}$

\begin{tabular}{l}
3910 \\
2100 \\
\hline
\end{tabular}

$\begin{array}{lll}59 & 3.8 & 1380\end{array}$

12

59
62

50
49
56

3.8
7.0
5.0
1.6
2.6

1380
1030

402
604
3620

804
474
143
217

$49 \quad 2.1 \quad 17700$

$\begin{array}{rrr}49 & 2.1 & 17700 \\ 59 & 6.4 & 12600 \\ 39 & 2.9 & 8000 \\ 50 & 7.7 & 3780 \\ 3790 & 1200 & 2660\end{array}$

28900

28900
18200
14500

$\begin{array}{lll}.00 & 940 & 482 \\ 1.5 & 564 & 474\end{array}$

$\begin{array}{lll}1.5 & 564 & 474 \\ 1.8 & 219 & 152\end{array}$

179
130

102
54

$\begin{array}{rr}116 & 38 \\ 105 & 39 \\ 85 & 28\end{array}$

$\begin{array}{ll}85 & 28 \\ 78 & 24 \\ 79 & 17\end{array}$

2660

3230
1320

6530

6990
4510

6900

2390

3530

3870
2260

3240

$\begin{array}{ll}1130 & 448 \\ 1080 & 298\end{array}$

371

162
938

298
76
35

35
261

26
27
28
29
30

31

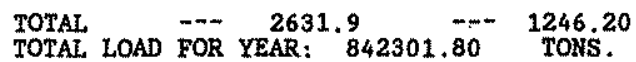

\begin{tabular}{rr}
68 & 23 \\
194 & 91 \\
18700 & 70800 \\
7140 & 24500 \\
10700 & 40700 \\
\hline
\end{tabular}

1270
1700
1890

1890
917

917
1320
2170

323
417
367

367
156

167

1830 AUGUST

$\begin{array}{rr}1830 & 168 \\ 1130 & 89 \\ 1210 & 114 \\ 516 & 47 \\ 2490 & 269\end{array}$

88
89
47
69

$\begin{array}{rr}1480 & 120 \\ 1450 & 86\end{array}$

$\begin{array}{rr}1450 & 86 \\ 1350 & 157\end{array}$

$\begin{array}{ll}2200 & 421 \\ 2460 & 465\end{array}$

SEPTEMBER

-.- 157972

\section{$2810 \quad 523$}

1540229

$\begin{array}{rr}929 & 125 \\ 868 & 98\end{array}$

868
914

346
260

230

168

360

242

189

478
1170

$4280 \quad 1390$

$6690 \quad 3310$

$5180 \quad 2740$

262021290

$2840 \quad 1660$ $\begin{array}{ll}1450 & 431 \\ 719 & 171\end{array}$

$\begin{array}{rr}719 & 171 \\ 569 & 86 \\ 464 & 61 \\ 353 & 36\end{array}$

$954 \quad 126$

$1030 \quad 128$

$\begin{array}{ll}268 & 48 \\ 380 & 52\end{array}$

$300 \quad 59$

212
2280

$\begin{array}{ll}5120 & 3350 \\ 3720 & 2620\end{array}$

$2870 \quad 4030$

$2000 \quad 1900$

$1010 \quad 843$

$\begin{array}{rr}1010 & 843 \\ 1270 & 1150\end{array}$

$636 \quad 354$

$\begin{array}{ll}349 & 113 \\ 736 & 234\end{array}$

$\begin{array}{ll}331 & 102 \\ 294 & 117\end{array}$

$309 \quad 102$

$\begin{array}{rr}234 & 61 \\ 186 & 37\end{array}$

$150 . \quad 16$

TOTAL LOAD FOR YEAR: 842301.80 TONS.

-.. 15869.9

-- 18279 
08332050 BERNARDO INTERIOR DRAIN MEAR BERNARDO, NM

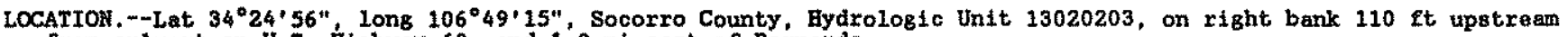
from culvert on U.S. Highway 60 , and $1.0 \mathrm{mi}$ east of Bernardo.

PERIOD OF RECORD.--June 1936 to May 1937, Octobex 1943 to current year. Monthly discharge only June 1936 to May 1937, publ1shed in WSP 828. October 1943 to September 1960 included in composite records for station 08332000 "Rio Grande near Bernardo." October 1960 to September 1964, monthly acre-ft published in WSP 1923. Daily records available in district files beginning October 1943.

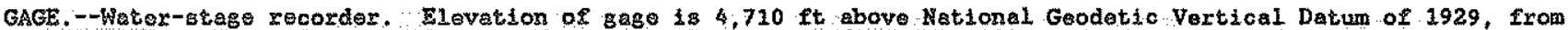
topographic map. Juna 4, 1936 , to Way 17,1937 , nonrecording gago 300 st downstrean, and Oct. 1 , 2943 to Jan. 12, 1978, water-atago recorder at site 150 ft downstream at different datum.

REAARK, --Records good except for estimated daily discharges, which are poor. This drain is 1 of 4 channels (8tations 08331990,08332010 , and 08332030 ) carrying flow in valley cross section... For combined monthly flow in ecre-ft of this drain, conveyance channel loodvgy and Lover San Juen Riverside drain seo tabulation below daily table for station 08332010 . Several observations of water temperature were made during the year. Prior to 1952, drain was subject to overfiow from floodway.

DISCAARGE, CUBIC FEET PER SECOND, WATER YEAR OCTOBER 1995 TO SEPTEMBER 1996

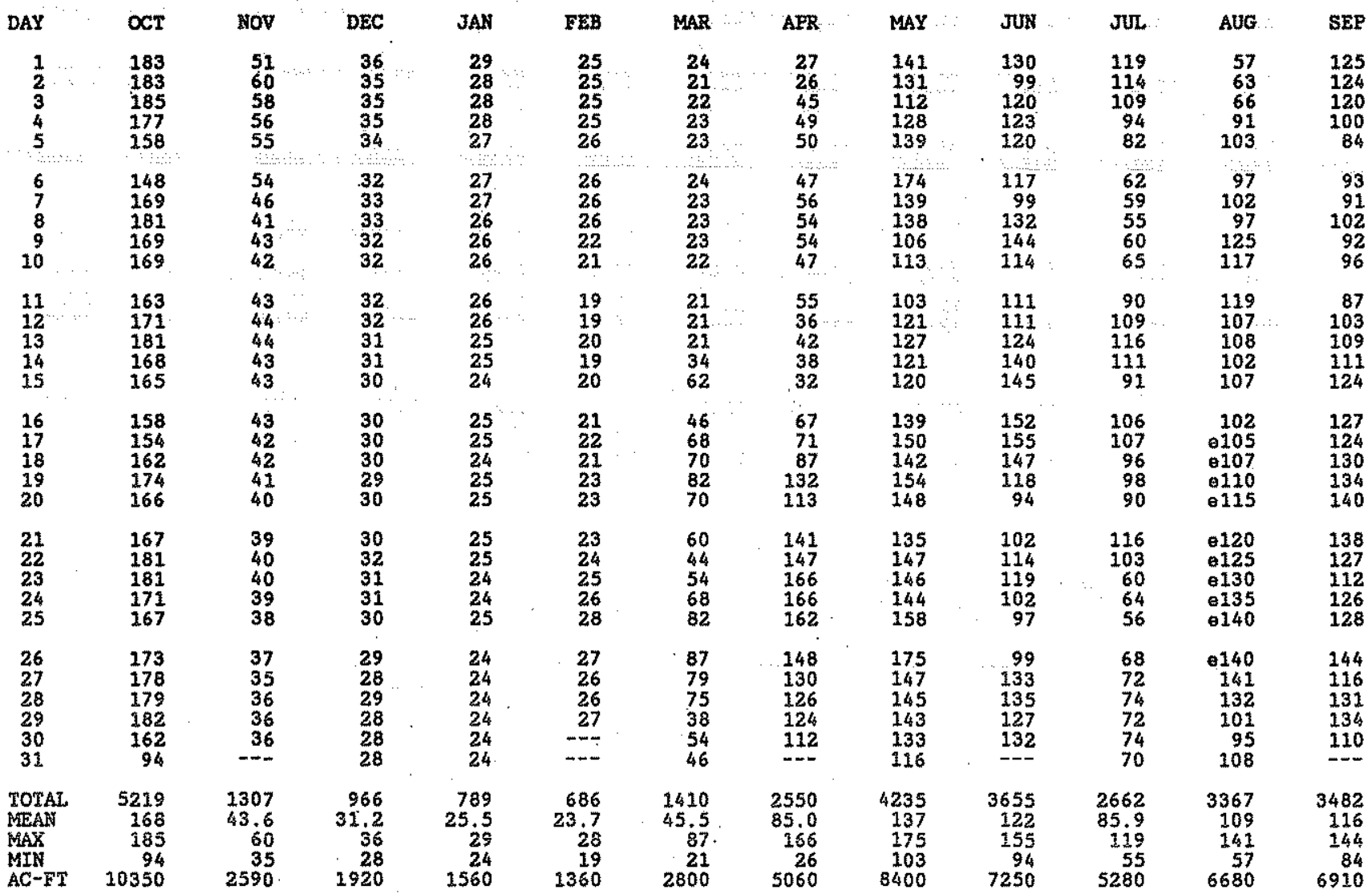

STATISTICS OF WONTEIY MEAN DATA FOR WATER YEARS 1954 - 1996, BY WATER YEAR (WY)

\begin{tabular}{|c|c|c|c|c|c|c|c|c|c|c|c|c|}
\hline $\begin{array}{l}\text { MEAN } \\
\text { MAX } \\
(W Y) \\
\text { MiIX } \\
(W Y)\end{array}$ & $\begin{array}{r}74.7 \\
168 \\
1996 \\
1957\end{array}$ & $\begin{array}{l}31.2 \\
87.9 \\
1987 \\
1.57 \\
1957\end{array}$ & $\begin{array}{l}27.5 \\
74.2 \\
1987 \\
3.50 \\
1955\end{array}$ & $\begin{array}{l}26.1 \\
87.7 \\
1990 \\
3.30 \\
1957\end{array}$ & $\begin{array}{l}26.3 \\
74.5 \\
1990 \\
3.90 \\
1957\end{array}$ & $\begin{array}{l}48.5 \\
96.9 \\
1985 \\
5.61 \\
1954\end{array}$ & $\begin{array}{r}59.0 \\
118 \\
1969 \\
4.82 \\
1955\end{array}$ & $\begin{array}{r}62.8 \\
137 \\
1996 \\
4.84 \\
1954\end{array}$ & $\begin{array}{r}57.3 \\
134 \\
1992 \\
1.64 \\
1954\end{array}$ & $\begin{array}{r}58.2 \\
146 \\
1992 \\
1956\end{array}$ & $\begin{array}{r}68.9 \\
146 \\
1992 \\
1906 \\
1954\end{array}$ & $\begin{array}{r}72.1 \\
164 \\
1995 \\
010 \\
1956\end{array}$ \\
\hline
\end{tabular}

SUMRARY STATISTICS

ANNUAL TOTAL

ANINUAL MEAN

HIGHEST ANNUAL MEAN

LOWEST ANNUAL MEAN

HIGHEST DAILY MEAN

LOWEST DAILY MEAP

ANNUAL SEVEN-DAY MINIMUM

INSTANTANEOUS PEAK FLON

INSTANTANEOUS PEAK STAGE

INSTANTANEOUS LOW FLOW

ANNUAL RUNOFF (AC-FT)

10 PERCENT EXCEEDS

SO PERCENT EXCEEDS

90 PERCENT EXCEEDS

- Estimated
FOR 1995 CALENDAR YEAR

$\begin{aligned} 25939 & \\ 71.1 & \\ & \\ 189 & \text { Sep } 1 \\ 22 & \operatorname{Jan} 10 \\ 22 & \text { Jen } 10 \\ & \\ & \\ 51450 & \\ 168 & \\ 57 & \\ 25 & \end{aligned}$

FOR 1996 WATER YEAR

30328

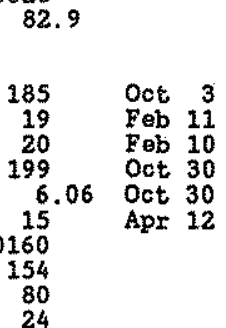

WATER YEARS 1954 - 1996

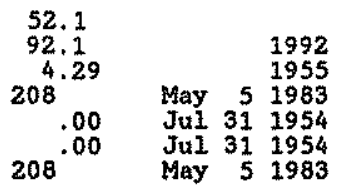

37730

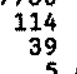


RIO GRANDE BASIN

08334000 RIO PUERCO ABOVE ARROYO CHICO, NEAR GUADALUPE, MM

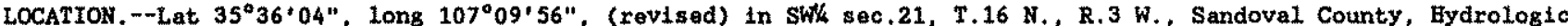
Unit 13020204 , on right bank 1.6 mi upstream from Arroyo Chico, 5.5 mi northeast of village of Guadalupe, and at mile 106.8 .

DRAINAGE AREA. $--420 \mathrm{mi}^{2}$, approximately.

WATER-DISCEARGE RECORDS

PERTOD OF RECORD. --July 1951 to current year.

GAGE. - Water-gtage recorder. Datum of gage is 5,950 ft above National Geodetic Vertical Datum of 1929 . Prior to July 14,1966 , at datum $1.01 \mathrm{ft}$ higher.

REMARKS, - Water-discharge records good except for estimated dally discharges, which are poor. Diverstons for irrigation of about 3,700 acres upstream from station in past years, but present diversion negligible. Several. observations of pater temperature were made during the year. Satelitte telemeter at station. No flop for many days most yoars.

EXTREMES OUTSIDE PERIOD OF RECORD.--Flood of June 29, 1943, probably exceeded 5,000 ft3/s based on records for stations upstream and domstream.

DISCAARGE, CUBTC FEET PER SECOND, WATER YEAR OCTOBER 1995 TO SEPTEMBER 1996

\begin{tabular}{|c|c|c|c|c|c|c|c|c|c|c|c|c|}
\hline DAY & $\infty r$ & Hov & DEC & JAN & FEB & MAR & APR & MAY & JUN & JUL & AUG & SEP \\
\hline $\begin{array}{l}1 \\
2 \\
3 \\
4 \\
5\end{array}$ & $\begin{array}{l}7.0 \\
1.8 \\
.43 \\
.14 \\
.07\end{array}$ & $\begin{array}{l}.03 \\
.03 \\
.02 \\
.05 \\
.06\end{array}$ & $\begin{array}{l}.03 \\
.03 \\
.04 \\
.03 \\
.04\end{array}$ & $\begin{array}{l}.01 \\
.01 \\
.01 \\
.01 \\
.01\end{array}$ & $\begin{array}{l}0.00 \\
0.00 \\
0.00 \\
0.00 \\
0.00\end{array}$ & $\begin{array}{l}.13 \\
.13 \\
.14 \\
.14 \\
.13\end{array}$ & $\begin{array}{l}.00 \\
.00 \\
.00 \\
.00 \\
.00\end{array}$ & $\begin{array}{l}.00 \\
.00 \\
.00 \\
.00 \\
.00\end{array}$ & $\begin{array}{l}.00 \\
.00 \\
.00 \\
.00 \\
.00\end{array}$ & $\begin{array}{l}51 \\
47 \\
47 \\
47 \\
45\end{array}$ & $\begin{array}{l}.00 \\
.00 \\
.00 \\
.21 \\
.00\end{array}$ & $\begin{array}{l}810 \\
83.0 \\
e 50 \\
48 \\
34\end{array}$ \\
\hline $\begin{array}{r}6 \\
7 \\
8 \\
9 \\
10\end{array}$ & $\begin{array}{l}.06 \\
.06 \\
.05 \\
.04 \\
.03\end{array}$ & $\begin{array}{l}.04 \\
.03 \\
.02 \\
.03 \\
.05\end{array}$ & $\begin{array}{l}.04 \\
.04 \\
.04 \\
.03 \\
.03\end{array}$ & $\begin{array}{l}.00 \\
.01 \\
.00 \\
.00 \\
.00\end{array}$ & $\begin{array}{l}0.00 \\
0.00 \\
0.00 \\
0.00 \\
0.00\end{array}$ & $\begin{array}{r}.19 \\
.19 \\
.17 \\
.19 \\
.23\end{array}$ & $\begin{array}{l}.00 \\
.00 \\
.00 \\
.00 \\
.00\end{array}$ & $\begin{array}{l}.00 \\
.00 \\
.00 \\
.00 \\
.00\end{array}$ & $\begin{array}{l}.00 \\
.00 \\
.00 \\
.00 \\
.00\end{array}$ & $\begin{array}{r}35 \\
45 \\
177 \\
1170 \\
865\end{array}$ & $\begin{array}{l}.00 \\
2.5 \\
.75 \\
1.9 \\
.53\end{array}$ & $\begin{array}{r}e 5.0 \\
e .50 \\
e .00 \\
0.00 \\
e 5.0\end{array}$ \\
\hline $\begin{array}{l}11 \\
12 \\
13 \\
14 \\
15\end{array}$ & $\begin{array}{l}.03 \\
.03 \\
.02 \\
.01 \\
.01\end{array}$ & $\begin{array}{l}.03 \\
.03 \\
.02 \\
.03 \\
.03\end{array}$ & $\begin{array}{l}.01 \\
.01 \\
.01 \\
.01 \\
.01\end{array}$ & $\begin{array}{l}.00 \\
.00 \\
.00 \\
.00 \\
.00\end{array}$ & $\begin{array}{l}0.00 \\
0.00 \\
0.00 \\
0.00 \\
0.00\end{array}$ & $\begin{array}{l}.20 \\
0.10 \\
0.05 \\
0.05 \\
0.20\end{array}$ & $\begin{array}{l}.00 \\
.00 \\
.00 \\
.00 \\
.00\end{array}$ & $\begin{array}{l}.00 \\
.00 \\
.00 \\
.00 \\
.00\end{array}$ & $\begin{array}{l}.00 \\
.00 \\
.00 \\
.00 \\
.00\end{array}$ & $\begin{array}{l}.00 \\
9.2 \\
.00 \\
.00 \\
.00\end{array}$ & $\begin{array}{l}.03 \\
.00 \\
.00 \\
.00 \\
.00\end{array}$ & $\begin{array}{r}\text { e.50 } \\
\text { e5.0 } \\
\text { e10 } \\
254 \\
236\end{array}$ \\
\hline $\begin{array}{l}16 \\
17 \\
18 \\
19 \\
20\end{array}$ & $\begin{array}{l}.02 \\
.02 \\
.01 \\
.00 \\
.00\end{array}$ & $\begin{array}{l}.03 \\
.03 \\
.03 \\
.03 \\
.04\end{array}$ & $\begin{array}{r}.01 \\
.03 \\
0.01 \\
0.00 \\
0.00\end{array}$ & $\begin{array}{l}0.00 \\
0.00 \\
0.00 \\
0.00 \\
0.00\end{array}$ & $\begin{array}{l}0.00 \\
0.00 \\
0.00 \\
0.00 \\
0.01\end{array}$ & $\begin{array}{l}0.10 \\
.10 \\
.05 \\
.05 \\
.20\end{array}$ & $\begin{array}{l}.00 \\
.00 \\
.02 \\
.00 \\
.01\end{array}$ & $\begin{array}{l}.00 \\
.00 \\
.00 \\
.00 \\
.00\end{array}$ & $\begin{array}{l}.00 \\
.00 \\
.00 \\
.00 \\
.00\end{array}$ & $\begin{array}{l}.00 \\
85.0 \\
16 \\
8.1 \\
3.5\end{array}$ & $\begin{array}{l}.00 \\
.00 \\
.00 \\
.00 \\
.00\end{array}$ & $\begin{array}{l}e 10 \\
e 60 \\
304 \\
261 \\
e 25\end{array}$ \\
\hline $\begin{array}{l}21 \\
22 \\
23 \\
24 \\
25\end{array}$ & $\begin{array}{l}.01 \\
.01 \\
.00 \\
.01 \\
.02\end{array}$ & $\begin{array}{l}.04 \\
.03 \\
.03 \\
.03 \\
.03\end{array}$ & $\begin{array}{l}\theta .00 \\
\theta .00 \\
0.00 \\
0.00 \\
e .00\end{array}$ & $\begin{array}{l}0.00 \\
0.00 \\
0.00 \\
0.00 \\
0.00\end{array}$ & $\begin{array}{r}0.02 \\
0.05 \\
0.10 \\
.18 \\
.16\end{array}$ & $\begin{array}{l}.10 \\
.10 \\
.05 \\
.05 \\
.03\end{array}$ & $\begin{array}{l}.01 \\
.01 \\
.01 \\
.02 \\
.00\end{array}$ & $\begin{array}{l}.00 \\
.00 \\
.00 \\
.00 \\
.00\end{array}$ & $\begin{array}{l}.00 \\
.00 \\
.00 \\
.00 \\
.00\end{array}$ & $\begin{array}{l}1.6 \\
.96 \\
.27 \\
.05 \\
.00\end{array}$ & $\begin{array}{r}4.6 \\
13 \\
4.8 \\
4.5 \\
64\end{array}$ & $\begin{array}{l}2.0 \\
0.50 \\
0.00 \\
0.00 \\
.00\end{array}$ \\
\hline $\begin{array}{l}26 \\
27 \\
28 \\
29 \\
30 \\
31\end{array}$ & $\begin{array}{l}.01 \\
.00 \\
.01 \\
.02 \\
.02 \\
.02\end{array}$ & $\begin{array}{r}.03 \\
.02 \\
0.01 \\
0.01 \\
.03 \\
.\end{array}$ & $\begin{array}{l}0.00 \\
0.00 \\
0.00 \\
0.00 \\
0.00 \\
0.00\end{array}$ & $\begin{array}{l}.00 \\
.00 \\
.00 \\
.00 \\
.00 \\
.00\end{array}$ & $\begin{array}{l}.12 \\
.15 \\
.21 \\
.17 \\
. .- \\
. .-\end{array}$ & $\begin{array}{l}.02 \\
.01 \\
.00 \\
.00 \\
.00 \\
.00\end{array}$ & $\begin{array}{l}.00 \\
.00 \\
.00 \\
.00 \\
.00 \\
.\end{array}$ & $\begin{array}{l}.00 \\
.00 \\
.00 \\
.00 \\
.00 \\
.00\end{array}$ & $\begin{array}{l}14 \\
63 \\
66 \\
17 \\
73 \\
\end{array}$ & $\begin{array}{l}.00 \\
.00 \\
.00 \\
.00 \\
.00 \\
.00\end{array}$ & $\begin{array}{r}23.74 \\
\quad .74 \\
225 \\
191 \\
43\end{array}$ & $\begin{array}{l}.00 \\
.00 \\
.00 \\
.00 \\
.00 \\
.\end{array}$ \\
\hline $\begin{array}{l}\text { TOTAZ } \\
\text { MEAN } \\
\text { MAX } \\
\text { MIN } \\
\text { AC-FT }\end{array}$ & $\begin{array}{r}9.96 \\
.32 \\
7.0 \\
.00 \\
20\end{array}$ & $\begin{array}{r}0.92 \\
.031 \\
.06 \\
.01 \\
1.8\end{array}$ & $\begin{array}{r}0.45 \\
.015 \\
.04 \\
.00 \\
.9\end{array}$ & $\begin{array}{r}0.06 \\
.002 \\
.01 \\
.00 \\
.1\end{array}$ & $\begin{array}{r}1.17 \\
.040 \\
.21 \\
.00 \\
2.3\end{array}$ & $\begin{array}{r}3.10 \\
.10 \\
.23 \\
.00 \\
6.1\end{array}$ & $\begin{array}{r}0.08 \\
.003 \\
.02 \\
.00 \\
.2\end{array}$ & $\begin{array}{l}0.00 \\
.000 \\
.00 \\
.00 \\
.00\end{array}$ & $\begin{array}{r}233.00 \\
7.77 \\
73 \\
.00 \\
462\end{array}$ & $\begin{array}{r}2573.68 \\
83.0 \\
1170 \\
5100\end{array}$ & $\begin{array}{r}579.67 \\
18.7 \\
225 \\
.00 \\
1150\end{array}$ & $\begin{array}{r}1323.50 \\
44.1 \\
304 \\
.00 \\
2630\end{array}$ \\
\hline
\end{tabular}

STATISTICS OF MONTHLY MEAN DATA FOR WATER YEARS 1952 - 1996, BY WATER YEAR (WY)

\begin{tabular}{|c|c|c|c|c|c|c|c|c|c|c|}
\hline $\begin{array}{l}\text { MEAN } \\
\text { MAX } \\
\text { (WY) } \\
\text { MIN } \\
\text { (WY) }\end{array}$ & $\begin{array}{r}7.60 \\
129 \\
1958 \\
000 \\
1953\end{array}$ & $\begin{array}{r}3.09 \\
28.2 \\
1987 \\
1000 \\
1953\end{array}$ & $\begin{array}{l}1.46 \\
15.9 \\
1987 \\
1000 \\
1953\end{array}$ & $\begin{array}{l}3.02 \\
48.2 \\
1993 \\
1000 \\
1953\end{array}$ & $\begin{array}{l}12.6 \\
79.2 \\
1979 \\
1000 \\
1953\end{array}$ & $\begin{array}{r}17.5 \\
161 \\
1960 \\
1000 \\
1953\end{array}$ & $\begin{array}{r}19.7 \\
99.3 \\
1958 \\
000 \\
1964\end{array}$ & $\begin{array}{r}40.2 \\
236 \\
1973 \\
000 \\
1964\end{array}$ & $\begin{array}{r}16.6 \\
113 \\
1995 \\
000 \\
1953\end{array}$ & \\
\hline
\end{tabular}

SUMARY STATISTICS

ANNUAL TOTAL.

ANNUAL MEAN
HIGHEST ANNUAL MEAN

HIGHEST ANNUAL MEAN

LOWEST ANNUAL MEAN

LOWEST DAILY MEAN

ANWUAL SEVEN-DAY MINIMIM

INSTANTANEOUS PEAK FLOW

INSTANTANEOUS PEAK STAGE

ANWUAL RINOFE (AC-FT)

10 PERCENT EXCEEDS

50 PERCENT EXCEEDS

90 PERCENT EXCEEDS

a-From rating curve extended above $1,300 \mathrm{ft} / \mathrm{s}$ on basis of slope-axea measurements at gage hetghts 7.75 ft and $10.60 \mathrm{ft}$.
FOR 1995 CALENDAR YEAR FOR 1996 WATER YEAR

$$
4725.59
$$

$\begin{array}{cc}9739.62 & 4725.59 \\ 26.7 & 12.9\end{array}$

316 Aug $27 \quad 1170 \quad$ Ju1 9

$\begin{array}{rrr}.00 & \text { Oct } 19 \\ .00 & \text { Dec } 19 \\ 2520^{\circ} & \text { Jul } & 8\end{array}$

19320

98.4

$\gamma .3$

.01 $\begin{array}{rrr}8.10 & \text { Jul } & 8\end{array}$
WATER YEARS 1952 - 1996

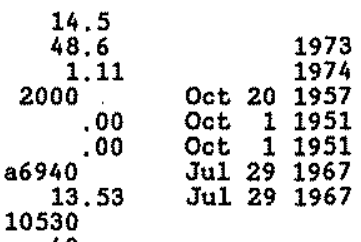

.12 
RIO GRANDE BASIN

08334000 RIO PUERCO ABOVE ARROYO CAICO, NEAR GUADALUPE, MM - Continued

WATER-QUALITY RECORDS

PERIOD OF RECORD.--Water years 1948-56 (published as "below Cabezon"), 1981 to current year.

PERIOD OE DAILY RECORD.--

SUSPENDED-SEDIMENT DISCHARGES: July 1948 to June 1956., October 1981 to current year.

INSTRUMERTATION, --Automatic pumping sediment sampler since August 1981.

EXTRENES FOR PERIOD OF DAILY RECORD.--

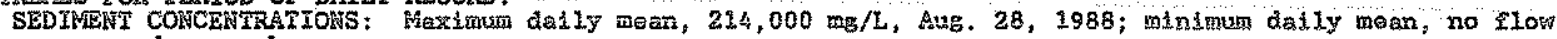

SEDIMFENT LOADS: Maximum daily, 730,000 tons, July 27, 1955; mintmum daily, 0 con on many days each yeax.

EXTREMES FOR CURRENT YEAR.--

SEDIMENT CONCEHTRATIONS: Maximum daily mean, 110,000 mg/L, Aug. 7; minimum daily mean, no flow on many days. SEDIMENT LOADS: Maximum daily, 232,000 tons, July 9; minimum dally, 0 ton on many days.

SUSPENDED-SEDIMENT, WATER YEAR OCTOBER 1995 TO SEPTEMBER 1996

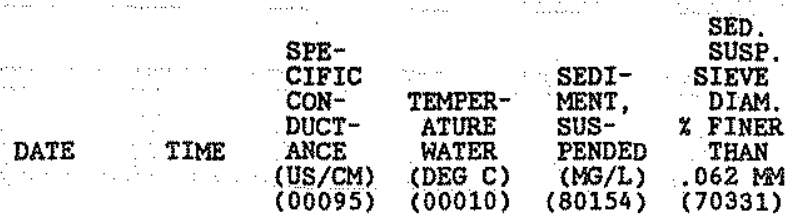

OCT 1995

$03 . .$.

$05 .$.

MAR 1996

$0950 \quad 1810$

$05 . .$.

1143

1810
2290

5.0
11.5

50700

70331)

1000

4440

7.0

173

100

100

83 
RIO GRANDE BASIN

08334000 RIO PUERCO ABOVE ARROYO CHICO, NEAR GUADALUPE, NM -- Cont InUed

WATER QUALITY RECORDS

SUSPENDED-SEDIMENT, WATER YEAR OCTOBER 1995 TO SEFTEMBER 1996

\begin{tabular}{|c|c|c|c|c|c|c|c|c|c|c|c|c|}
\hline DAY & $\begin{array}{l}\text { MEAN } \\
\text { CONCEN- } \\
\text { TRATION } \\
\text { (ME/L) }\end{array}$ & $\begin{array}{l}\text { LOADS } \\
\text { (T/DAY) }\end{array}$ & $\begin{array}{c}\text { MEAN } \\
\text { CONCEN- } \\
\text { TRATION } \\
(M G / L)\end{array}$ & $\begin{array}{l}\text { LOADS } \\
\text { (T/DAY) }\end{array}$ & $\begin{array}{c}\text { MEAN } \\
\text { CONCEN- } \\
\text { TRATION } \\
\text { (MG/L) }\end{array}$ & $\begin{array}{l}\text { LOADS } \\
(\mathrm{T} / \mathrm{DAY})\end{array}$ & $\begin{array}{c}\text { MEAN } \\
\text { CONCEN- } \\
\text { TRATION } \\
\text { (MG } / L)\end{array}$ & $\begin{array}{l}\text { LOADS } \\
\text { (T/DAY) }\end{array}$ & $\begin{array}{l}\text { MEAN } \\
\text { CONCEN- } \\
\text { TRATION } \\
\text { (MG/L) }\end{array}$ & $\begin{array}{l}\text { LOADS } \\
(T / D A Y)\end{array}$ & $\begin{array}{l}\text { MEAN } \\
\text { CONCEN- } \\
\text { TRATION } \\
(\mathrm{ME} / \mathrm{L})\end{array}$ & $\begin{array}{l}\text { LOADS } \\
\text { (T/DAY) }\end{array}$ \\
\hline $\begin{array}{l}1 \\
2 \\
3 \\
4 \\
5\end{array}$ & $\begin{array}{l}{ }^{\alpha} \alpha \\
34100 \\
38600 \\
48100 \\
49600 \\
55200\end{array}$ & $\begin{array}{c}\text { TOBER } \\
644 \\
188 \\
56 \\
19 \\
10\end{array}$ & $\begin{array}{c}\text { NOV } \\
45 \\
60 \\
60 \\
550 \\
1500\end{array}$ & $\begin{array}{r}\text { GMBER } \\
.00 \\
.00 \\
.00 \\
.07 \\
.24\end{array}$ & $\begin{array}{l}\text { DEC } \\
150 \\
100 \\
300 \\
200 \\
350\end{array}$ & $\begin{array}{r}\text { EMBER } \\
.01 \\
.01 \\
.03 \\
.02 \\
.04\end{array}$ & $\begin{array}{l}70 \\
70 \\
70 \\
50 \\
50 \\
30\end{array}$ & $\begin{array}{r}.00 \\
.00 \\
.00 \\
.00 \\
.00\end{array}$ & $\begin{array}{l}F E \\
0 \\
0 \\
0 \\
0 \\
0\end{array}$ & $\begin{array}{r}\text { RUARY } \\
.00 \\
.00 \\
.00 \\
.00 \\
.00\end{array}$ & $\begin{array}{l}133 \\
129 \\
135 \\
139 \\
160\end{array}$ & $\begin{array}{r}\text { ARCH } \\
.05 \\
.05 \\
.05 \\
.05 \\
.06\end{array}$ \\
\hline $\begin{array}{r}6 \\
7 \\
8 \\
9 \\
10\end{array}$ & $\begin{array}{r}1090 \\
155 \\
37 \\
25 \\
20\end{array}$ & $\begin{array}{l}.18 \\
.03 \\
.00 \\
.00 \\
.00\end{array}$ & $\begin{array}{r}800 \\
100 \\
80 \\
150 \\
500\end{array}$ & $\begin{array}{l}.09 \\
.01 \\
.00 \\
.01 \\
.07\end{array}$ & $\begin{array}{l}200 \\
175 \\
175 \\
125 \\
100\end{array}$ & $\begin{array}{l}.02 \\
.02 \\
.02 \\
.01 \\
.01\end{array}$ & $\begin{array}{r}0 \\
30 \\
0 \\
0 \\
0\end{array}$ & $\begin{array}{l}.00 \\
.00 \\
.00 \\
.00 \\
.00\end{array}$ & $\begin{array}{l}0 \\
0 \\
0 \\
0 \\
0\end{array}$ & $\begin{array}{l}.00 \\
.00 \\
.00 \\
.00 \\
.00\end{array}$ & $\begin{array}{r}132 \\
119 \\
124 \\
118 \\
97\end{array}$ & $\begin{array}{r}.07 \\
.06 \\
.06 \\
.06 \\
.06\end{array}$ \\
\hline $\begin{array}{l}11 \\
12 \\
13 \\
14 \\
15\end{array}$ & $\begin{array}{l}20 \\
20 \\
25 \\
20 \\
20\end{array}$ & $\begin{array}{l}.00 \\
.00 \\
.00 \\
.00 \\
.00\end{array}$ & $\begin{array}{r}100 \\
80 \\
100 \\
80 \\
80\end{array}$ & $\begin{array}{l}.01 \\
.01 \\
.01 \\
.01 \\
.01\end{array}$ & $\begin{array}{l}80 \\
50 \\
50 \\
50 \\
40\end{array}$ & $\begin{array}{l}.00 \\
.00 \\
.00 \\
.00 \\
.00\end{array}$ & $\begin{array}{l}0 \\
0 \\
0 \\
0 \\
0\end{array}$ & $\begin{array}{l}.00 \\
.00 \\
.00 \\
.00 \\
.00\end{array}$ & $\begin{array}{l}0 \\
0 \\
0 \\
0 \\
0\end{array}$ & $\begin{array}{l}.00 \\
.00 \\
.00 \\
.00 \\
.00\end{array}$ & $\begin{array}{r}34 \\
28 \\
27 \\
-66\end{array}$ & $\begin{array}{r}.02 \\
.01 \\
.00 \\
.04\end{array}$ \\
\hline $\begin{array}{l}16 \\
17 \\
18 \\
19 \\
20\end{array}$ & $\begin{array}{r}37 \\
37 \\
15 \\
0 \\
0\end{array}$ & $\begin{array}{l}.00 \\
.00 \\
.00 \\
.00 \\
.00\end{array}$ & $\begin{array}{r}70 \\
70 \\
70 \\
60 \\
250\end{array}$ & $\begin{array}{l}.01 \\
.01 \\
.01 \\
.00 \\
.03\end{array}$ & $\begin{array}{r}40 \\
40 \\
200 \\
100 \\
0\end{array}$ & $\begin{array}{l}.00 \\
.00 \\
.01 \\
.00 \\
.00\end{array}$ & $\begin{array}{l}0 \\
0 \\
0 \\
0 \\
0\end{array}$ & $\begin{array}{l}.00 \\
.00 \\
.00 \\
.00 \\
.00\end{array}$ & $\begin{array}{r}0 \\
0 \\
0 \\
0 \\
53\end{array}$ & $\begin{array}{l}.00 \\
.00 \\
.00 \\
.00 \\
.00\end{array}$ & $\begin{array}{l}41 \\
38 \\
34 \\
35 \\
35\end{array}$ & $\begin{array}{l}.01 \\
.01 \\
.00 \\
.00 \\
.02\end{array}$ \\
\hline $\begin{array}{l}21 \\
22 \\
23 \\
24 \\
25\end{array}$ & $\begin{array}{r}72 \\
58 \\
0 \\
30 \\
50\end{array}$ & $\begin{array}{l}.00 \\
.00 \\
.00 \\
.00 \\
.00\end{array}$ & $\begin{array}{r}200 \\
150 \\
100 \\
80 \\
80\end{array}$ & $\begin{array}{l}.02 \\
.01 \\
.01 \\
.01 \\
.01\end{array}$ & $\begin{array}{l}0 \\
0 \\
0 \\
0 \\
0\end{array}$ & $\begin{array}{l}.00 \\
.00 \\
.00 \\
.00 \\
.00\end{array}$ & $\begin{array}{l}0 \\
0 \\
0 \\
0 \\
0\end{array}$ & $\begin{array}{l}.00 \\
.00 \\
.00 \\
.00 \\
.00\end{array}$ & $\begin{array}{l}123 \\
200 \\
159 \\
145 \\
139\end{array}$ & $\begin{array}{l}.01 \\
.03 \\
.04 \\
.07 \\
.06\end{array}$ & $\begin{array}{l}26 \\
45 \\
55 \\
25 \\
25\end{array}$ & $\begin{array}{l}.01 \\
.01 \\
.01 \\
.00 \\
.00\end{array}$ \\
\hline $\begin{array}{l}26 \\
27 \\
28 \\
29 \\
30 \\
31\end{array}$ & $\begin{array}{r}25 \\
0 \\
25 \\
35 \\
35 \\
35\end{array}$ & $\begin{array}{l}.00 \\
.00 \\
.00 \\
.00 \\
.00 \\
.00\end{array}$ & $\begin{array}{r}80 \\
60 \\
40 \\
40 \\
175 \\
\end{array}$ & $\begin{array}{l}.01 \\
.00 \\
.00 \\
.00 \\
.01 \\
.01\end{array}$ & $\begin{array}{l}0 \\
0 \\
0 \\
0 \\
0 \\
0\end{array}$ & $\begin{array}{l}.00 \\
.00 \\
.00 \\
.00 \\
.00 \\
.00\end{array}$ & $\begin{array}{l}0 \\
0 \\
0 \\
0 \\
0 \\
0\end{array}$ & $\begin{array}{l}.00 \\
.00 \\
.00 \\
.00 \\
.00 \\
.00\end{array}$ & $\begin{array}{l}116 \\
131 \\
173 \\
146 \\
-\end{array}$ & $\begin{array}{r}.04 \\
.05 \\
.10 \\
.07 \\
.0 \\
.\end{array}$ & $\begin{array}{r}22 \\
22 \\
0 \\
0 \\
0 \\
0\end{array}$ & $\begin{array}{l}.00 \\
.00 \\
.00 \\
.00 \\
.00 \\
.00\end{array}$ \\
\hline TOTAL & $\cdots$ & 917.21 & $-\infty$ & 0.68 & $-m$ & 0.20 & $-\infty$ & 0.00 & $m-m$ & 0.47 & $-m$ & 0.71 \\
\hline & $\begin{array}{c}\text { MEAN } \\
\text { CONCEN- } \\
\text { TRATION } \\
(M G / L)\end{array}$ & $\begin{array}{l}\text { LOADS } \\
\text { (T/DAY) }\end{array}$ & $\begin{array}{c}\text { MEAN } \\
\text { CONCEN- } \\
\text { TRATION } \\
\text { (MG/L) }\end{array}$ & $\begin{array}{l}\text { LOADS } \\
(\mathrm{T} / \mathrm{DAY})\end{array}$ & $\begin{array}{c}\text { MEAN } \\
\text { CONCEN- } \\
\text { TRATION } \\
\text { (MG/L) }\end{array}$ & $\begin{array}{l}\text { LOADS } \\
(\mathrm{T} / \mathrm{DAY})\end{array}$ & $\begin{array}{l}\text { MEAN } \\
\text { CONCEN- } \\
\text { TRATION } \\
\text { (MG/L) }\end{array}$ & $\begin{array}{l}\text { OADS } \\
\text { T/DAY) }\end{array}$ & $\begin{array}{l}\text { MEAN } \\
\text { CONCEN- } \\
\text { TRATION } \\
(M G / L)\end{array}$ & $\begin{array}{l}\text { OADS } \\
\text { T/DAY) }\end{array}$ & $\begin{array}{l}\text { MEAN } \\
\text { CONCEN- } \\
\text { TRATION } \\
\text { (ME/L) }\end{array}$ & $\begin{array}{l}\text { LOADS } \\
\text { (T/DAY) }\end{array}$ \\
\hline
\end{tabular}

$\begin{array}{llllll} & & & & & \text { MAY } \\ 1 & 0 & .00 & 0 & & .00 \\ 2 & 0 & .00 & 0 & .00 \\ 3 & 0 & .00 & 0 & .00 \\ 4 & 0 & .00 & 0 & .00 \\ 5 & 0 & .00 & 0 & .00\end{array}$

$\begin{array}{ll}.00 & 0 \\ .00 & 0 \\ .00 & 0 \\ .00 & 0 \\ .00 & 0\end{array}$

$\begin{array}{llr}.00 & 72000 & 9910 \\ .00 & 33100 & 4200 \\ .00 & 22800 & 2900 \\ .00 & 17900 & 2270 \\ .00 & 11800 & 1440\end{array}$

\begin{tabular}{rrrr} 
& \multicolumn{2}{c}{ AUGUST } & \multicolumn{2}{c}{ SEPTEMBER } \\
0 & .0 & 25400 & 686 \\
0 & .0 & 13300 & 108 \\
0 & .0 & 13300 & 1800 \\
9420 & 5.3 & 11200 & 1450 \\
0 & .0 & 5940 & 545
\end{tabular}

$\begin{array}{rll}6 & 0 & .00 \\ 7 & 0 & .00 \\ 8 & 0 & .00 \\ 9 & 0 & .00 \\ 10 & 0 & .00\end{array}$

.00
.00
.00
.00
.00

$\begin{array}{rrr}.00 & 3380 & 320 \\ .00 & 28000 & 3400 \\ 00 & 33900 & 16200\end{array}$

$\begin{array}{llr}.00 & 33900 & 16200 \\ .00 & 70600 & 223000\end{array}$

$\begin{array}{rrr}.00 & 70600 & 223000 \\ .00 & 10700 & 24900\end{array}$

$\begin{array}{rr}0 & \\ 111000 & 748^{\circ} \\ 10100 & 20 \\ 28900 & 148 \\ 22700 & 32\end{array}$

$2610 \quad 35$

$\begin{array}{lll}11 & 0 & .00\end{array}$

11
12
13

14
15

$\begin{array}{ll}0 & .00 \\ 0 & .00 \\ 0 & .00 \\ 0 & .00 \\ 0 & .00\end{array}$

$\begin{array}{rrr}.00 & 0 & .0 \\ .00 & 30800 & 766{ }^{\circ} \\ .00 & 0 & .0 \\ .00 & 0 & .0 \\ .00 & 0 & .0\end{array}$

4020

32

$\begin{array}{rr}578 & .78 \\ 0 & .00 \\ 0 & .00 \\ 2930 & 40\end{array}$

.00
.00
.00
.00
.00

16
17
19
19

$\begin{array}{rr}0 & .00 \\ 0 & : 00 \\ 175 & .00 \\ 0 & : 00 \\ 70 & : 00\end{array}$

$\begin{array}{rrr}.00 & 0 & \\ .00 & 19300 & 260^{\circ} \\ .00 & 80300 & 3470 \\ .00 & 8320 & 182 \\ .00 & 2650 & 25\end{array}$

4020
0
0
0
0

$\begin{array}{rrr}.3 & 930 & 1.3 \\ .0 & 1930 & 26 \\ .0 & 823 & 22 \\ .0 & 21600 & 14800 \\ .0 & 26000 & 16600\end{array}$

.00

$\begin{array}{ll}0 & .00 \\ 0 & .00 \\ 0 & .00 \\ 0 & .00\end{array}$

$\begin{array}{ll}0 & .00 \\ 0 & .00 \\ 0 & .00 \\ 0 & .00 \\ 0 & .00\end{array}$

$\begin{array}{rrr}.00 & 1370 & 5.9 \\ .00 & 509 & 1.3 \\ .00 & 79 & .1 \\ .00 & 60 & .0 \\ .00 & 0 & .0\end{array}$

$\begin{array}{rrr}5.9 & 38100 & 473 \\ 1.3 & 52800 & 1850 \\ .1 & 36400 & 471 \\ .0 & 30100 & 366 \\ .0 & 53100 & 9170\end{array}$

$\begin{array}{rrrr}0 & .0 & 9400 & 254 \\ 0 & .0 & 12500 & 2030 \\ 0 & .0 & 9130 & 7490\end{array}$

$\begin{array}{rrr}.0 & 7250 & 5110 \\ .0 & 7790 & 525\end{array}$

$\begin{array}{lrl}21 & 70 & .00 \\ 22 & 70 & .00 \\ 23 & 60 & .00 \\ 24 & 150 & .01 \\ 25 & 0 & .00\end{array}$

.00
.00
.00
.00
00

$\begin{array}{ll}0 & .00 \\ 0 & .00 \\ 0 & .00 \\ 0 & .00 \\ 0 & .00\end{array}$

$26100 \quad 988$

8670014800

6320011300

$49000 \quad 2250$

5370010600

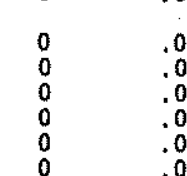

$\begin{array}{cc}74100 & 4600 \\ 38100 & 76 \\ 19000 & 5.6 \\ 63900 & 38800 \\ 74200 & 38300 \\ 56400 & 6550\end{array}$

$\begin{array}{cc}5130 & 28 \\ 3160 & 4.3\end{array}$

$\begin{array}{rr}3160 & 4.3 \\ 0 & .00 \\ 0 & .00 \\ 0 & .00\end{array}$

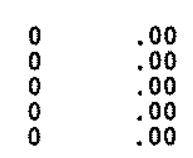

TOTAL LOAD FOR YEAR: 487278.17 TONS. 
RIO GRANDE BASIN

08341300 BLUEWATER CREEK ABOVE BLUEWATER DAM, NEAR BLUEWATER, MM

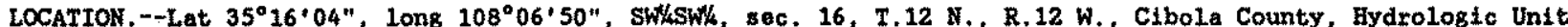

13020207, on left bank $2.0 \mathrm{ml}$, south of Bluewater Dam, $7.0 \mathrm{mi}$ west of Bluewater, and 11 mi east of Thoreau.

DRAIHAGE AREA, $--75.0 \mathrm{mi}^{2}$.

PERIOD OF RECORD,--October 1953 to September 1978 (annual maximum only), July 1989 to current year.

GAGE,-Water-stage recorder, Elevation of gage is 7,410 ft above National Geodetic Vertical Datum of 1929, from topographic map.

REMARKS, --Records good except for estimered dally discherges, whoh are poor.

DISCRARGE, CUBIC FEET PER SECOND, WATER YEAR OCTOBER 1995 TO SEFTEMBER 1996

\begin{tabular}{|c|c|c|c|c|c|c|c|c|c|c|c|c|}
\hline DAY & $\propto C T$ & HOV & DEC & JAN & FEB & MAR & APR & MAY & JUN & JUL. & AUG & SEP \\
\hline $\begin{array}{l}1 \\
2 \\
3 \\
4 \\
5\end{array}$ & $\begin{array}{l}.22 \\
.22 \\
.22 \\
.20 \\
.20\end{array}$ & $\begin{array}{l}.88 \\
1.1 \\
1.0 \\
1.2 \\
1.3\end{array}$ & $\begin{array}{l}.88 \\
.89 \\
.90 \\
.92 \\
.94\end{array}$ & $\begin{array}{l}0.90 \\
e .92 \\
0.90 \\
e .92 \\
0.91\end{array}$ & $\begin{array}{l}0.94 \\
0.92 \\
0.91 \\
e .90 \\
0.89\end{array}$ & $\begin{array}{l}.92 \\
.92 \\
.92 \\
.93 \\
.94\end{array}$ & $\begin{array}{l}.77 \\
.77 \\
.77 \\
.73 \\
.72\end{array}$ & $\begin{array}{l}.41 \\
.42 \\
.44 \\
.46 \\
.44\end{array}$ & $\begin{array}{l}.20 \\
.19 \\
.18 \\
.16 \\
.16\end{array}$ & $\begin{array}{l}.11 \\
.06 \\
.09 \\
.05 \\
.04\end{array}$ & $\begin{array}{l}.02 \\
.02 \\
.07 \\
.06 \\
.01\end{array}$ & $\begin{array}{l}.06 \\
.06 \\
.09 \\
.04 \\
.01\end{array}$ \\
\hline $\begin{array}{r}6 \\
7 \\
8 \\
9 \\
10\end{array}$ & $\begin{array}{l}.20 \\
.20 \\
.18 \\
.18 \\
.18\end{array}$ & $\begin{array}{l}1.3 \\
1.4 \\
1.3 \\
1.3 \\
1.2\end{array}$ & $\begin{array}{l}.96 \\
.94 \\
.95 \\
.97 \\
.94\end{array}$ & $\begin{array}{l}0.89 \\
0.90 \\
0.91 \\
0.90 \\
0.88\end{array}$ & $\begin{array}{l}.88 \\
.90 \\
.88 \\
.86 \\
.84\end{array}$ & $\begin{array}{r}.92 \\
.90 \\
.95 \\
1.0 \\
.98\end{array}$ & $\begin{array}{l}.72 \\
.67 \\
.68 \\
.62 \\
.57\end{array}$ & $\begin{array}{l}.42 \\
.43 \\
.44 \\
.44 \\
.46\end{array}$ & $\begin{array}{l}.13 \\
.13 \\
.14 \\
.14 \\
.16\end{array}$ & $\begin{array}{l}.03 \\
.05 \\
.07 \\
.09 \\
.07\end{array}$ & $\begin{array}{r}.00 \\
.00 \\
.00 \\
1.2 \\
.38\end{array}$ & $\begin{array}{l}.03 \\
.02 \\
.01 \\
.00 \\
.00\end{array}$ \\
\hline $\begin{array}{l}11 \\
12 \\
13 \\
14 \\
15\end{array}$ & $\begin{array}{l}.18 \\
.19 \\
.21 \\
.20 \\
.20\end{array}$ & $\begin{array}{l}1.1 \\
1.2 \\
1.1 \\
.96 \\
0.89\end{array}$ & $\begin{array}{l}.95 \\
.95 \\
.97 \\
.98 \\
.99\end{array}$ & $\begin{array}{l}0.89 \\
e .86 \\
e .88 \\
e .94 \\
0.92\end{array}$ & $\begin{array}{l}.83 \\
.82 \\
.88 \\
.87 \\
.86\end{array}$ & $\begin{array}{l}.96 \\
.97 \\
.98 \\
.90 \\
.92\end{array}$ & $\begin{array}{l}.55 \\
.50 \\
.52 \\
.53 \\
.52\end{array}$ & $\begin{array}{l}.45 \\
.42 \\
.43 \\
.44 \\
.46\end{array}$ & $\begin{array}{l}.12 \\
.12 \\
.16 \\
.23 \\
.27\end{array}$ & $\begin{array}{l}.04 \\
.04 \\
.25 \\
.07 \\
.05\end{array}$ & $\begin{array}{l}.15 \\
.06 \\
.01 \\
.00 \\
.00\end{array}$ & $\begin{array}{l}.00 \\
.02 \\
.16 \\
.34 \\
.35\end{array}$ \\
\hline $\begin{array}{l}16 \\
17 \\
18 \\
19 \\
20\end{array}$ & $\begin{array}{l}.22 \\
.22 \\
.22 \\
.22 \\
.21\end{array}$ & $\begin{array}{l}0.92 \\
0.91 \\
0.95 \\
0.92 \\
.95\end{array}$ & $\begin{array}{l}.98 \\
.99 \\
.98 \\
.96 \\
.97\end{array}$ & $\begin{array}{l}0.90 \\
0.91 \\
0.92 \\
0.91 \\
0.94\end{array}$ & $\begin{array}{r}.85 \\
.87 \\
.86 \\
.85 \\
e .84\end{array}$ & $\begin{array}{l}.88 \\
.86 \\
.84 \\
.82 \\
.64\end{array}$ & $\begin{array}{l}.46 \\
.44 \\
.43 \\
.42 \\
.43\end{array}$ & $\begin{array}{l}.40 \\
.42 \\
.43 \\
.42 \\
.40\end{array}$ & $\begin{array}{l}.22 \\
.17 \\
.11 \\
.06 \\
.03\end{array}$ & $\begin{array}{l}.07 \\
.06 \\
.04 \\
.03 \\
.03\end{array}$ & $\begin{array}{l}.00 \\
.00 \\
.01 \\
.01 \\
.01\end{array}$ & $\begin{array}{l}.22 \\
.18 \\
.50 \\
.45 \\
.32\end{array}$ \\
\hline $\begin{array}{l}21 \\
22 \\
23 \\
24 \\
25\end{array}$ & $\begin{array}{l}.22 \\
.27 \\
.32 \\
.36 \\
.38\end{array}$ & $\begin{array}{l}.91 \\
.89 \\
.89 \\
.90 \\
.94\end{array}$ & $\begin{array}{l}0.98 \\
0.99 \\
0.98 \\
0.96 \\
0.94\end{array}$ & $\begin{array}{l}0.92 \\
0.90 \\
0.91 \\
0.92 \\
0.91\end{array}$ & $\begin{array}{l}0.92 \\
0.93 \\
0.92 \\
0.94 \\
0.92\end{array}$ & $\begin{array}{l}.66 \\
.66 \\
.66 \\
.73 \\
.76\end{array}$ & $\begin{array}{l}.44 \\
.43 \\
.41 \\
.41 \\
.42\end{array}$ & $\begin{array}{l}.41 \\
.30 \\
.28 \\
.26 \\
.26\end{array}$ & $\begin{array}{l}.04 \\
.09 \\
.02 \\
.01 \\
.00\end{array}$ & $\begin{array}{l}.02 \\
.01 \\
.01 \\
.01 \\
.01\end{array}$ & $\begin{array}{l}.13 \\
.36 \\
.44 \\
.40 \\
.81\end{array}$ & $\begin{array}{l}.25 \\
.18 \\
.14 \\
.09 \\
.21\end{array}$ \\
\hline $\begin{array}{l}26 \\
27 \\
28 \\
29 \\
30 \\
31\end{array}$ & $\begin{array}{l}.59 \\
.87 \\
.83 \\
.86 \\
.84 \\
.83\end{array}$ & $\begin{array}{l}0.98 \\
0.95 \\
0.91 \\
0.90 \\
0.95\end{array}$ & $\begin{array}{l}0.96 \\
8.98 \\
0.97 \\
8.98 \\
0.92 \\
0.91\end{array}$ & $\begin{array}{l}0.90 \\
0.91 \\
0.92 \\
0.91 \\
0.96 \\
0.98\end{array}$ & $\begin{array}{r}0.90 \\
e .92 \\
0.92 \\
0.90 \\
\hdashline-\end{array}$ & $\begin{array}{l}.72 \\
.62 \\
.66 \\
.69 \\
.77 \\
.81\end{array}$ & $\begin{array}{r}.40 \\
.41 \\
.38 \\
.40 \\
.42 \\
. .\end{array}$ & $\begin{array}{l}.27 \\
.27 \\
.26 \\
.22 \\
.21 \\
.21\end{array}$ & $\begin{array}{l}.01 \\
.46 \\
.19 \\
.07 \\
.11\end{array}$ & $\begin{array}{l}.01 \\
.00 \\
.03 \\
.06 \\
.06 \\
.06\end{array}$ & $\begin{array}{l}.56 \\
.35 \\
.35 \\
.27 \\
.20 \\
.14\end{array}$ & $\begin{array}{r}.40 \\
.30 \\
.23 \\
.21 \\
.15\end{array}$ \\
\hline $\begin{array}{l}\text { TOTAL } \\
\text { MEAN } \\
\text { MAX } \\
\text { MIN } \\
\text { AC-FT }\end{array}$ & $\begin{array}{r}10.44 \\
.34 \\
.87 \\
.18 \\
.21\end{array}$ & $\begin{array}{r}31.10 \\
1.04 \\
1.4 \\
.88 \\
62\end{array}$ & $\begin{array}{r}29.58 \\
.95 \\
.99 \\
.88 \\
59\end{array}$ & $\begin{array}{r}28.24 \\
.91 \\
.98 \\
.86 \\
56\end{array}$ & $\begin{array}{r}25.72 \\
.89 \\
.94 \\
.82 \\
51\end{array}$ & $\begin{array}{r}25.89 \\
84 \\
1.0 \\
.62 \\
51\end{array}$ & $\begin{array}{r}15.94 \\
.53 \\
.77 \\
.38 \\
32\end{array}$ & $\begin{array}{r}11.58 \\
.37 \\
.46 \\
.21 \\
23\end{array}$ & $\begin{array}{r}4.08 \\
.14 \\
.46 \\
.00 \\
8.1\end{array}$ & $\begin{array}{r}1.62 \\
.052 \\
.25 \\
.00 \\
3.2\end{array}$ & $\begin{array}{r}6.02 \\
i 19 \\
i .2 \\
.00 \\
12\end{array}$ & $\begin{array}{r}5.02 \\
.17 \\
.50 \\
.00 \\
10\end{array}$ \\
\hline
\end{tabular}

STATISTICS OF MONTHLY MEAN DATA FOR WATER YEARS 1989 - 1996, BY WATER YEAR (WY)

\begin{tabular}{|c|c|c|c|c|c|c|c|c|c|c|c|c|}
\hline $\begin{array}{l}\text { MEAN } \\
\text { MAX } \\
\text { (WY) } \\
\text { MIN } \\
\text { (WY) }\end{array}$ & $\begin{array}{r}.48 \\
1.90 \\
1994 \\
.093 \\
1991\end{array}$ & $\begin{array}{l}1.11 \\
3.47 \\
1994 \\
i 055 \\
1991\end{array}$ & $\begin{array}{r}.97 \\
2.54 \\
1995 \\
.050 \\
1991\end{array}$ & $\begin{array}{l}3.17 \\
19.9 \\
1993 \\
1091 \\
1991\end{array}$ & $\begin{array}{r}12.0 \\
42.1 \\
1995 \\
.48 \\
1990\end{array}$ & $\begin{array}{r}62.5 \\
227 \\
1993 \\
195 \\
1990\end{array}$ & $\begin{array}{r}46.3 \\
225 \\
1993 \\
.43 \\
1990\end{array}$ & $\begin{array}{r}4.50 \\
34.6 \\
1993 \\
1996\end{array}$ & $\begin{array}{l}1.03 \\
1.78 \\
1993 \\
1077 \\
1990\end{array}$ & $\begin{array}{r}.48 \\
.88 \\
1993 \\
1052 \\
1996\end{array}$ & $\begin{array}{l}2.08 \\
19.7 \\
1993 \\
1023 \\
1990\end{array}$ & $\begin{array}{l}.83 \\
3.48 \\
1993 \\
1059 \\
1990\end{array}$ \\
\hline
\end{tabular}

\section{SURMARY STATISTICS}

ANEUAL TOTAL

ANAUAL REAN

LOWEST ANNUAL MEAN

LOWEST ANNUAL MEAN

BIGEEST DATLY MEAN

LONEST DAILY MEAN

IASTANTANEOUS PEAK FLOW

IRSTANTANEOUS PEAK STAGE

ANNUAL RUNOFF (AC-FT)

10 PERCENT EXCEEDS

50 PERCENT EXCEEDS

90 PERCENT EXCEEDS

- Estimated
FOR 1995 CALENDAR YEAR

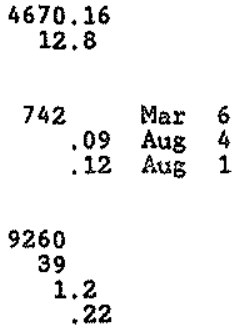

FOR 1996 WATER YEAR

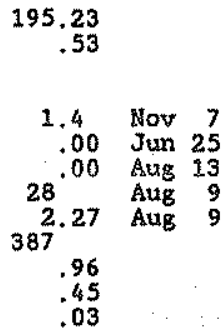

WATER YEARS 1989 - 1996

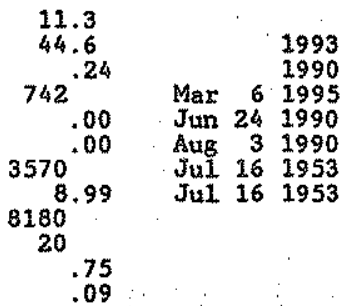


RIO GRANDE BASTK

08341365 COTTONHOOD CREEK NEAR THOREAU, MM

LOCATION. - lat $35^{\circ} 20^{\prime} 32^{\prime \prime}$, Long $106^{\circ} 12^{\prime} 42^{\prime \prime}$, In NEKSE sec.21, T.13 N., R. 13, McKinley County, Hydrologic Unit 13020207 , on left bank 4.0 mi southeast of Thoreau, and $4.0 \mathrm{mi}$ southwest of north ond of Biuewater Lake.

DRAINAGE AREA. $--77.0 \mathrm{ml}$.

RERTOD OF RECORD.- - July 1989 to current year.

GAGE.--Water-stage recoxder. Elevation of gage is 7,420 ft above National Geodetic Vertical Datum of 1929 , 2rom topographic map.

REMARKS. --Records good except for astimated dally discharges, which are poor. No flow most of time. DISCEARGE, CUBIC FEET PER SECOND, WATER YEAR OCTOBER 1995 TO SEPTEMBER 1996

\begin{tabular}{|c|c|c|c|c|c|c|c|c|c|c|c|c|}
\hline DAY & $\infty \mathbf{C T}$ & NOV & DEC & JAN & EED & MAR. & APR & MAY & JUN & JUL & AUG & SER \\
\hline $\begin{array}{l}1 \\
2 \\
3 \\
4 \\
5\end{array}$ & $\begin{array}{l}.00 \\
.00 \\
.00 \\
.00 \\
.00\end{array}$ & $\begin{array}{l}.00 \\
.00 \\
.00 \\
.11 \\
.04\end{array}$ & $\begin{array}{l}.17 \\
.20 \\
.22 \\
.21 \\
.25\end{array}$ & $\begin{array}{l}0.10 \\
0.10 \\
0.00 \\
0.00 \\
0.00\end{array}$ & $\begin{array}{l}0.20 \\
0.40 \\
0.30 \\
0.20 \\
0.20\end{array}$ & $\begin{array}{l}.09 \\
.09 \\
.08 \\
.07 \\
.03\end{array}$ & $\begin{array}{l}.11 \\
.07 \\
.06 \\
.06 \\
.11\end{array}$ & $\begin{array}{l}.00 \\
.00 \\
.00 \\
.00 \\
.00\end{array}$ & $\begin{array}{l}.00 \\
.00 \\
.00 \\
.00 \\
.00\end{array}$ & $\begin{array}{l}.00 \\
.00 \\
.00 \\
.00 \\
.00\end{array}$ & $\begin{array}{l}.00 \\
.00 \\
.00 \\
.00 \\
.00\end{array}$ & $\begin{array}{l}.00 \\
.00 \\
.00 \\
.00 \\
.00\end{array}$ \\
\hline $\begin{array}{r}6 \\
7 \\
8 \\
9 \\
10\end{array}$ & $\begin{array}{l}.00 \\
.00 \\
.00 \\
.00 \\
.00\end{array}$ & $\begin{array}{l}.02 \\
.01 \\
.00 \\
.00 \\
.00\end{array}$ & $\begin{array}{l}.25 \\
.15 \\
.16 \\
.12 \\
.12\end{array}$ & $\begin{array}{l}0.00 \\
0.00 \\
0.00 \\
0.00 \\
0.00\end{array}$ & $\begin{array}{l}.17 \\
.12 \\
.11 \\
.08 \\
.06\end{array}$ & $\begin{array}{l}.03 \\
.03 \\
.03 \\
.02 \\
.02\end{array}$ & $\begin{array}{l}.12 \\
.09 \\
.08 \\
.09 \\
.10\end{array}$ & $\begin{array}{l}.00 \\
.00 \\
.00 \\
.00 \\
.00\end{array}$ & $\begin{array}{l}.00 \\
.00 \\
.00 \\
.00 \\
.00\end{array}$ & $\begin{array}{l}.00 \\
.00 \\
.00 \\
.00 \\
4.3\end{array}$ & $\begin{array}{r}.00 \\
.00 \\
15.00 \\
1.7\end{array}$ & $\begin{array}{l}.00 \\
.00 \\
.00 \\
.00 \\
.00\end{array}$ \\
\hline $\begin{array}{l}11 \\
12 \\
13 \\
14 \\
15\end{array}$ & $\begin{array}{l}.00 \\
.00 \\
.00 \\
.00 \\
.00\end{array}$ & $\begin{array}{l}.00 \\
.01 \\
.02 \\
.03 \\
.04\end{array}$ & $\begin{array}{l}.12 \\
.12 \\
.12 \\
.18 \\
.22\end{array}$ & $\begin{array}{l}0.00 \\
0.00 \\
0.00 \\
0.00 \\
0.00\end{array}$ & $\begin{array}{l}.05 \\
.03 \\
.03 \\
.03 \\
.02\end{array}$ & $\begin{array}{l}.02 \\
.01 \\
.01 \\
.02 \\
.02\end{array}$ & $\begin{array}{l}.08 \\
.08 \\
15 \\
.20 \\
.16\end{array}$ & $\begin{array}{l}.00 \\
.00 \\
.00 \\
.00 \\
.00\end{array}$ & $\begin{array}{l}.00 \\
.00 \\
.00 \\
.00 \\
.00\end{array}$ & $\begin{array}{l}1.4 \\
.03 \\
3.0 \\
1.0 \\
.03\end{array}$ & $\begin{array}{l}.04 \\
.00 \\
.00 \\
.00 \\
.00\end{array}$ & $\begin{array}{l}.00 \\
.00 \\
.00 \\
.00 \\
.00\end{array}$ \\
\hline $\begin{array}{l}16 \\
17 \\
18 \\
19 \\
20\end{array}$ & $\begin{array}{l}.00 \\
.00 \\
.00 \\
.00 \\
.00\end{array}$ & $\begin{array}{l}.04 \\
.04 \\
.04 \\
.04 \\
.07\end{array}$ & $\begin{array}{r}.20 \\
.23 \\
-.20 \\
0.20 \\
-.10\end{array}$ & $\begin{array}{l}0.00 \\
0.20 \\
0.10 \\
0.10 \\
0.10\end{array}$ & $\begin{array}{l}.01 \\
.01 \\
.01 \\
.01 \\
.01\end{array}$ & $\begin{array}{l}.05 \\
.03 \\
.01 \\
.01 \\
.01\end{array}$ & $\begin{array}{l}.12 \\
.03 \\
.00 \\
.01 \\
.02\end{array}$ & $\begin{array}{l}.00 \\
.00 \\
.00 \\
.00 \\
.00\end{array}$ & $\begin{array}{l}.00 \\
.00 \\
.00 \\
.00 \\
.00\end{array}$ & $\begin{array}{l}.00 \\
.00 \\
.00 \\
.00 \\
.00\end{array}$ & $\begin{array}{l}.00 \\
.00 \\
.00 \\
.00 \\
.92\end{array}$ & $\begin{array}{l}.00 \\
.00 \\
.00 \\
.00 \\
.00\end{array}$ \\
\hline $\begin{array}{l}21 \\
22 \\
23 \\
24 \\
25\end{array}$ & $\begin{array}{l}.00 \\
.00 \\
.00 \\
.00 \\
.00\end{array}$ & $\begin{array}{r}.10 \\
.09 \\
.09 \\
.10 \\
.13\end{array}$ & $\begin{array}{l}0.10 \\
0.10 \\
0.10 \\
0.00 \\
0.00\end{array}$ & $\begin{array}{l}0.00 \\
e .00 \\
0.00 \\
0.00 \\
0.20\end{array}$ & $\begin{array}{l}.01 \\
.02 \\
.02 \\
.01 \\
.02\end{array}$ & $\begin{array}{l}.01 \\
.03 \\
.02 \\
.01 \\
.01\end{array}$ & $\begin{array}{l}.00 \\
.02 \\
.02 \\
.02 \\
.00\end{array}$ & $\begin{array}{l}.00 \\
.00 \\
.00 \\
.00 \\
.00\end{array}$ & $\begin{array}{l}.00 \\
.00 \\
.00 \\
.00 \\
.00\end{array}$ & $\begin{array}{l}.00 \\
.00 \\
.00 \\
.00 \\
.00\end{array}$ & $\begin{array}{r}.30 \\
.10 \\
.00 \\
.00 \\
9.7\end{array}$ & $\begin{array}{l}.00 \\
.00 \\
.00 \\
.00 \\
.00\end{array}$ \\
\hline $\begin{array}{l}26 \\
27 \\
28 \\
29 \\
30 \\
31\end{array}$ & $\begin{array}{l}.00 \\
.00 \\
.00 \\
.00 \\
.00 \\
.00\end{array}$ & $\begin{array}{l}.09 \\
.09 \\
.09 \\
.12 \\
.16 \\
-1 .\end{array}$ & $\begin{array}{l}0.00 \\
0.00 \\
0.20 \\
0.10 \\
0.10 \\
0.10\end{array}$ & $\begin{array}{l}0.10 \\
0.10 \\
0.10 \\
0.00 \\
0.00 \\
0.00\end{array}$ & $\begin{array}{l}.02 \\
.04 \\
.06 \\
.08 \\
.-.\end{array}$ & $\begin{array}{r}.03 \\
.05 \\
.06 \\
.10 \\
.17 \\
.18\end{array}$ & $\begin{array}{l}.00 \\
.00 \\
.00 \\
.00 \\
.00\end{array}$ & $\begin{array}{l}.00 \\
.00 \\
.00 \\
.00 \\
.00 \\
.00\end{array}$ & $\begin{array}{l}.00 \\
.00 \\
.00 \\
.00 \\
.00 \\
.\end{array}$ & $\begin{array}{l}.00 \\
.00 \\
.00 \\
.00 \\
.00 \\
.00\end{array}$ & $\begin{array}{r}3.6 \\
4.4 \\
.44 \\
.00 \\
.00 \\
.00\end{array}$ & $\begin{array}{l}.00 \\
.00 \\
.00 \\
.00 \\
.00 \\
.\end{array}$ \\
\hline $\begin{array}{l}\text { TOTAL } \\
\text { MEAN } \\
\text { MAX } \\
\text { MIN } \\
\text { AC-FT }\end{array}$ & $\begin{array}{r}0.00 \\
.000 \\
.00 \\
.00 \\
.00\end{array}$ & $\begin{array}{r}1.57 \\
.052 \\
.16 \\
.00 \\
3.1\end{array}$ & $\begin{array}{r}4.34 \\
.14 \\
.25 \\
.00 \\
8.6\end{array}$ & $\begin{array}{r}1.20 \\
.039 \\
.20 \\
.00 \\
2.4\end{array}$ & $\begin{array}{r}2.33 \\
.080 \\
.40 \\
.01 \\
4.6\end{array}$ & $\begin{array}{r}1.35 \\
.044 \\
.18 \\
.01 \\
2.7\end{array}$ & $\begin{array}{r}1.80 \\
.060 \\
.20 \\
.00 \\
3.6\end{array}$ & $\begin{array}{r}0.00 \\
.000 \\
.00 \\
.00 \\
.00\end{array}$ & $\begin{array}{l}0.00 \\
.000 \\
.00 \\
.00 \\
.00\end{array}$ & $\begin{array}{r}9.76 \\
.31 \\
4.3 \\
.00 \\
19\end{array}$ & $\begin{array}{r}36.20 \\
1.17 \\
15 \\
.00 \\
72\end{array}$ & $\begin{array}{r}0.00 \\
.000 \\
.00 \\
.00 \\
.00\end{array}$ \\
\hline
\end{tabular}

STATISTICS OF MONTHLY MEAN DATA FOR WATER YEARS 1989 - 1996, BY WATER YEAR (WY)

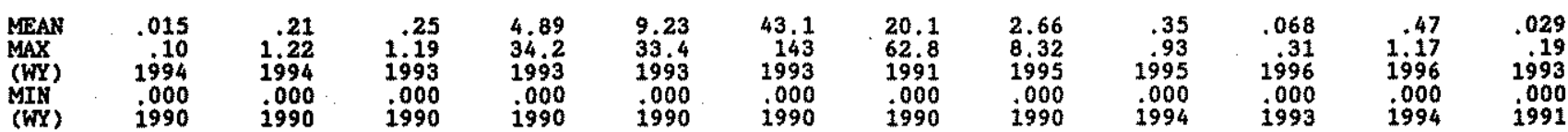

SUMAARY STATISTICS

AMNUAL. TOTAL

HIGHEST ANNUAL MEAN

LOWEST ANIULAT MTAX

HIGHEST DAILY MEAN

LOWEST DAILY MEAN

ANNUAL SEVEN-DAY MTNIMUM

INSTANTANEOUS PEAK FLOW

INSTANTANEOUS PBAK STAGE

ANNUAL RUNOFF (AC-FT)

10 PARCEHT EXCEEDS

50 PARCENT EXCEEDS

90 PERCENT EXCEEDS
FOR 1995 CALENDAR YEAR

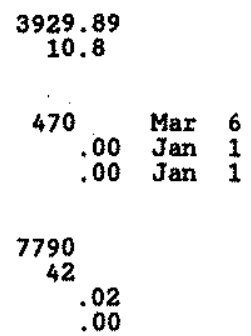

FOR 1996 WATER YEAR

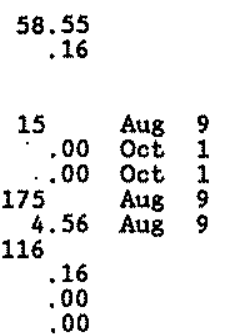

WATER YEARS 1989 - 1996

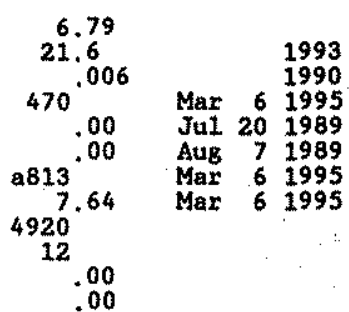

- Estimated

a-From rating curve extended above $2000 \mathrm{ft}^{3} / \mathrm{s}$. 
LOCATION.--Lat $35^{\circ} 17^{\prime} 31^{\prime \prime}$, Long $108^{\circ} 06^{\circ} 40^{*}$, in SE\% sec.9, T.12 N., R.12 W., Cibola County, Bydrologic Unit 13020207, at left end of Bluewater Dam on Bluewater Creek, and' $9.5 \mathrm{mi}$ west of Bliewater.

DRAINAGE AREA. --201 mi².

PERIOD OF RECORD.--June 1927 to December 1950 (monthend contents only, published tn wSP 1732), April 1958 to current year (monthend contents only).

GAGE. - Water-stag o recorder. Datum of gege is 7,345.57 ft above National Geodetic vertical Datum of 1929 . July 1958 to Jenuery. 1961, nonxecording gage et neaxby sita, same datum. Gage hoights have boen converted to sealeve1 olevations.

REMARXS.--Lake is formed by concrete aroh dam. Storage began in 1927. Capacity, 38,500 acre-ft, survey of 1945 at elevation 7,402.6 ft, crest of uncontrolled siphon spillway, which is vented to avold drawdown below crest, and 44,200 acre-ft at levation 7,405.6 ft, crest of ungated spillway over dam. Capacity table used through ig44 showed a capacity of 50,300 acre-et at crest of unsated spilifway over dam, and that used erom 1945-50, 43,500 acre-ft. Tables used prior to 1958 are not avaliablo and no adjustments are made for changes in tables. Dead storage, 3.4 acre-ft at elevation $7,345.4 \mathrm{ft}$, sill of lower out let tube. Lake not usualiy drawn below conservation-pool level elevation, $7,365.36 \mathrm{ft}$, below which ownership is by State Game and Fish Department. Above this level, pater is owned and used by Bluewater-Toltec Irrigation Co. Figures given herein represent total contents at 2400 hours.

EXIREMES FOR PERIOD OF RECORD.--Meximum contents determined, 47,100 acre-ft, Apr, 30, 1941. Contents may have been greater on Apr. 28, 1941, when peak discharge of $800 \mathrm{ft} / \mathrm{s}$ occurred at station $8 \mathrm{mi}$ downstream; no storage at times prior to 1947 .

EXTREMES FOR CURRENT YZAR,--Maximum contents, 11,500 acre-ft, March 28, elevation, 7,380.75 ft; minimum, 3,380 acreft, Sspt. 30, elevation, $7,365,45 \mathrm{ft}$.

NONTHEND ELEVATION AND CONTENTS, AT 2400, WATER YEAR OCTOBER 1995 TO SEPTEMBER 1996

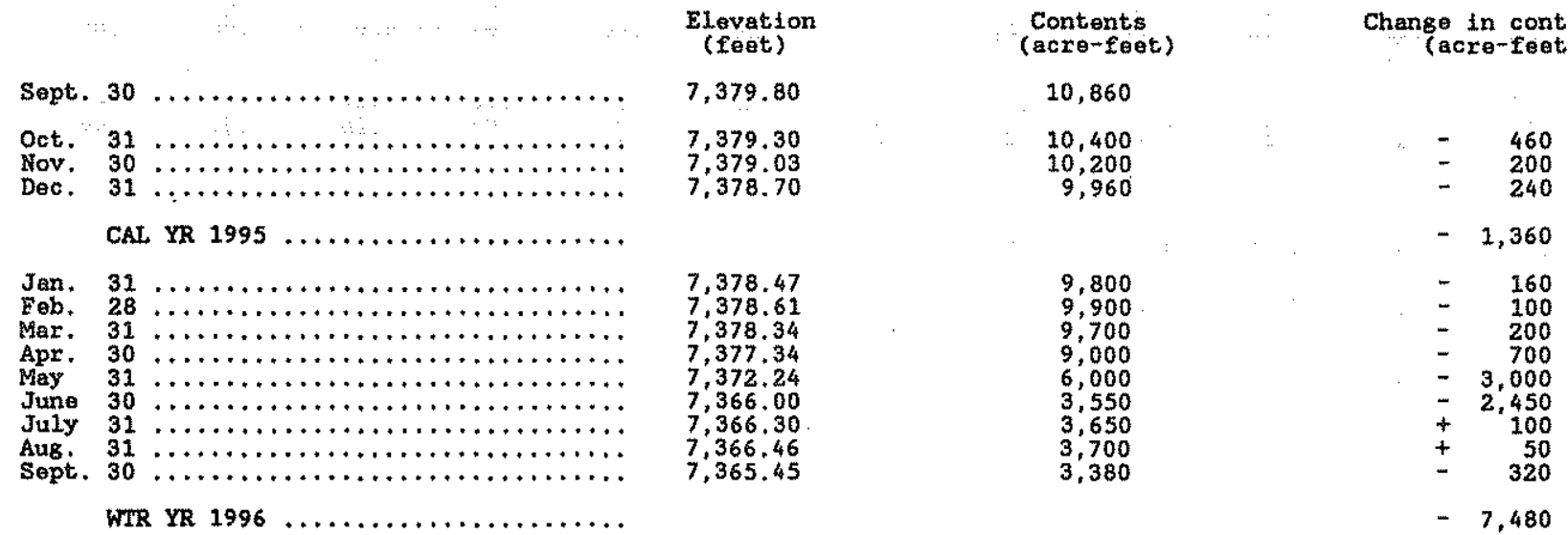


RIO GRANDE BASIN

08341500 BLUEWATER CREEX BELON BLUEWATER DAM, NM

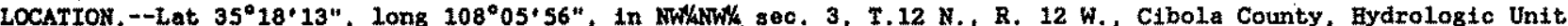
13020207, on left bank $0.5 \mathrm{mi}$ downstream from Bluevater Dam and il mi west of Bluewater.

DRAINAGE AREA. $--201 \mathrm{mi}^{2}$.

PERIOD OF RECORD. -March 1951 to September 1960, July 1989 to current year.

GAGE. - Water-stage recorder. Elevation of gage is 7,290 ft above National Geodetic Vertical Datum of 1929, from topographic map. March 14, 1951 to September 30, 1960 at site 0.5 mi upstream at different datum.

REMARKS.--Records good except for estimated daily discharges, which are poor. Flow regulated by Bluewater Lake (station 08341400 ) $0.5 \mathrm{mi}$ upstream, since 1927. No flow at times in $1955,1957$.

EXIREMES OUTSIDE PERTOD OF RECORD.-Maximum flood known occurxed Sept. 6, 1909, where Bluewater Dam washed out stage and discharge not determined. Another major flood probably occurred July 12-19, 1919 when a stage of 13.5 was reached at station (08342000) $8.0 \mathrm{ml}$ downstream.

DISCHARGE, CUBIC FEET PER SECOND, WATER YFAR OCTOBER 1995 TO SEPTEMBER 1996

\begin{tabular}{|c|c|c|c|c|c|c|c|c|c|c|c|c|}
\hline DAY & oct & Nov & DEC & JAN & FEB & MAR & APR & MAY & JUN & JUL & AUG & SEP \\
\hline $\begin{array}{l}1 \\
2 \\
3 \\
4 \\
5\end{array}$. & $\begin{array}{l}.85 \\
.84 \\
.84 \\
.81 \\
.83\end{array}$ & $\begin{array}{l}1.1 \\
1.2 \\
1.1 \\
1.2 \\
1.2\end{array}$ & $\begin{array}{l}1.1 \\
1.1 \\
1.1 \\
1.1 \\
1.1\end{array}$ & $\begin{array}{l}0.98 \\
0.96 \\
0.97 \\
0.98 \\
0.97\end{array}$ & $\begin{array}{r}.94 \\
.93 \\
.93 \\
.93 \\
.93\end{array}$ & $\begin{array}{l}.87 \\
.88 \\
.85 \\
.84 \\
.84\end{array}$ & $\begin{array}{r}1.0 \\
.99 \\
.99 \\
.98 \\
1.0\end{array}$ & $\begin{array}{l}16 \\
19 \\
43 \\
43 \\
54\end{array}$ & $\begin{array}{l}79 \\
76 \\
75 \\
74 \\
73\end{array}$ & $\begin{array}{r}.63 \\
.57 \\
.57 \\
.61 \\
.72\end{array}$ & $\begin{array}{r}.47 \\
.48 \\
.50 \\
.51 \\
.46\end{array}$ & $\begin{array}{l}.41 \\
.42 \\
.44 \\
.43 \\
.43\end{array}$ \\
\hline $\begin{array}{r}6 \\
7 \\
8 \\
9 \\
10\end{array}$ & $\begin{array}{l}.84 \\
.84 \\
.83 \\
.82 \\
.83\end{array}$ & $\begin{array}{l}1.2 \\
1.1 \\
1.1 \\
1.2 \\
1.2\end{array}$ & $\begin{array}{r}1.1 \\
1.0 \\
.98 \\
.98 \\
.98\end{array}$ & $\begin{array}{l}0.96 \\
0.97 \\
0.98 \\
0.98 \\
0.97\end{array}$ & $\begin{array}{r}.93 \\
.93 \\
.93 \\
.93 \\
.93\end{array}$ & $\begin{array}{l}.87 \\
.88 \\
.89 \\
.89 \\
.89\end{array}$ & $\begin{array}{l}1.0 \\
1.0 \\
1.0 \\
1.0 \\
1.0\end{array}$ & $\begin{array}{l}59 \\
59 \\
61 \\
61 \\
61\end{array}$ & $\begin{array}{l}72 \\
69 \\
67 \\
65 \\
62\end{array}$ & $\begin{array}{r}.75 \\
.80 \\
.81 \\
.93 \\
.89\end{array}$ & $\begin{array}{l}.41 \\
.42 \\
.42 \\
.58 \\
.48\end{array}$ & $\begin{array}{r}.46 \\
.47 \\
.46 \\
.47 \\
.49\end{array}$ \\
\hline $\begin{array}{l}11 \\
12 \\
13 \\
14 \\
15\end{array}$ & $\begin{array}{r}.84 \\
.84 \\
.84 \\
.84 \\
.84\end{array}$ & $\begin{array}{l}1.2 \\
1.2 \\
1.2 \\
1.2 \\
1.2\end{array}$ & $\begin{array}{r}.98 \\
.98 \\
.98 \\
1.0 \\
.98\end{array}$ & $\begin{array}{l}0.96 \\
0.97 \\
0.98 \\
0.99 \\
0.98\end{array}$ & $\begin{array}{r}.91 \\
.89 \\
.89 \\
.89 \\
.89\end{array}$ & $\begin{array}{l}.89 \\
.87 \\
.88 \\
.90 \\
.90\end{array}$ & $\begin{array}{l}1.0 \\
1.0 \\
1.1 \\
1.0 \\
1.0\end{array}$ & $\begin{array}{l}62 \\
62 \\
61 \\
62 \\
62\end{array}$ & $\begin{array}{l}61 \\
52 \\
47 \\
36 \\
18\end{array}$ & $\begin{array}{l}.79 \\
.78 \\
.88 \\
.86 \\
.74\end{array}$ & $\begin{array}{r}.41 \\
.41 \\
.41 \\
.41 \\
.42\end{array}$ & $\begin{array}{l}.48 \\
.52 \\
.53 \\
.59 \\
.52\end{array}$ \\
\hline $\begin{array}{l}16 \\
17 \\
18 \\
19 \\
20\end{array}$ & $\begin{array}{r}.86 \\
.84 \\
.84 \\
.86 \\
.89\end{array}$ & $\begin{array}{l}1.2 \\
1.2 \\
1.2 \\
1.2 \\
1.2\end{array}$ & $\begin{array}{r}.98 \\
.99 \\
.98 \\
.96 \\
.95\end{array}$ & $\begin{array}{r}0.99 \\
01.0 \\
.97 \\
.98 \\
.98\end{array}$ & $\begin{array}{l}.89 \\
.89 \\
.89 \\
.87 \\
.88\end{array}$ & $\begin{array}{l}.90 \\
.87 \\
.87 \\
.88 \\
.89\end{array}$ & $\begin{array}{l}1.0 \\
1.0 \\
1.1 \\
\frac{1}{1.1} \\
1.1\end{array}$ & $\begin{array}{l}62 \\
64 \\
64 \\
59 \\
55\end{array}$ & $\begin{array}{r}12 \\
02.8 \\
0.62 \\
.61 \\
.60\end{array}$ & $\begin{array}{l}.78 \\
.84 \\
.78 \\
.71 \\
.59\end{array}$ & $\begin{array}{r}.42 \\
.43 \\
.43 \\
.44 \\
.47\end{array}$ & $\begin{array}{r}.48 \\
.48 \\
0.50 \\
0.48 \\
0.48\end{array}$ \\
\hline $\begin{array}{l}21 \\
22 \\
23 \\
24 \\
25\end{array}$ & $\begin{array}{l}.89 \\
.89 \\
.91 \\
.93 \\
.93\end{array}$ & $\begin{array}{l}1.2 \\
1.2 \\
1.1 \\
1.1 \\
1.1\end{array}$ & $\begin{array}{r}.95 \\
.95 \\
.94 \\
.93 \\
.95\end{array}$ & $\begin{array}{l}.96 \\
.97 \\
.97 \\
.95 \\
.94\end{array}$ & $\begin{array}{l}.89 \\
.87 \\
.88 \\
.87 \\
.85\end{array}$ & $\begin{array}{r}.87 \\
.88 \\
.87 \\
.92 \\
.93\end{array}$ & $\begin{array}{l}1.2 \\
1.2 \\
1.2 \\
1.3 \\
1.3\end{array}$ & $\begin{array}{l}57 \\
59 \\
56 \\
57 \\
56\end{array}$ & $\begin{array}{l}.62 \\
.64 \\
.61 \\
.61 \\
.61\end{array}$ & $\begin{array}{l}.42 \\
.44 \\
.43 \\
.42 \\
.44\end{array}$ & $\begin{array}{r}.53 \\
.48 \\
.44 \\
.60 \\
1.8\end{array}$ & $\begin{array}{r}e .47 \\
e .47 \\
0.48 \\
0.48 \\
.49\end{array}$ \\
\hline $\begin{array}{l}26 \\
27 \\
28 \\
29 \\
30 \\
31\end{array}$ & $\begin{array}{l}.93 \\
1.0 \\
1.1 \\
1.1 \\
1.1 \\
1.1\end{array}$ & $\begin{array}{l}1.1 \\
1.1 \\
1.1 \\
1.1 \\
1.1 \\
-.=\end{array}$ & $\begin{array}{r}.94 \\
.94 \\
.95 \\
.96 \\
0.98 \\
0.98\end{array}$ & $\begin{array}{l}.93 \\
.96 \\
.97 \\
.96 \\
.95 \\
.95\end{array}$ & $\begin{array}{r}.90 \\
.93 \\
.88 \\
.89 \\
-.0 \\
. .-\end{array}$ & $\begin{array}{r}.94 \\
.96 \\
.97 \\
1.0 \\
1.0 \\
1.0\end{array}$ & $\begin{array}{l}1.3 \\
1.2 \\
.99 \\
1.0 \\
3.6 \\
-.-\end{array}$ & $\begin{array}{l}54 \\
62 \\
76 \\
84 \\
82 \\
80\end{array}$ & $\begin{array}{l}.66 \\
1.0 \\
.65 \\
.57 \\
.66 \\
.\end{array}$ & $\begin{array}{l}.45 \\
.45 \\
.49 \\
.47 \\
.47 \\
.47\end{array}$ & $\begin{array}{l}.77 \\
.47 \\
.45 \\
.42 \\
.41 \\
.41\end{array}$ & $\begin{array}{r}.51 \\
.49 \\
.49 \\
.47 \\
.47 \\
.-1\end{array}$ \\
\hline $\begin{array}{l}\text { TOTAL } \\
\text { MAAN } \\
\text { MAX } \\
\text { MIN } \\
\text { AC-FT }\end{array}$ & $\begin{array}{r}27.70 \\
.89 \\
1.1 \\
.81 \\
55\end{array}$ & $\begin{array}{r}34.8 \\
1.16 \\
1.2 \\
1.1 \\
69\end{array}$ & $\begin{array}{r}30.79 \\
.99 \\
1.1 \\
.93 \\
61\end{array}$ & $\begin{array}{r}30.03 \\
.97 \\
1.0 \\
.93 \\
60\end{array}$ & $\begin{array}{r}26.16 \\
.90 \\
.94 \\
.85 \\
52\end{array}$ & $\begin{array}{r}27.89 \\
.90 \\
1.0 \\
.84 \\
55\end{array}$ & $\begin{array}{r}34.65 \\
1.15 \\
3.6 \\
.98 \\
69\end{array}$ & $\begin{array}{r}1812 \\
58.5 \\
84 \\
16 \\
3590\end{array}$ & $\begin{array}{r}949.26 \\
31.6 \\
79 \\
.57 \\
1880\end{array}$ & $\begin{array}{r}19.98 \\
.64 \\
.93 \\
.42 \\
40\end{array}$ & $\begin{array}{r}15.76 \\
.51 \\
1.8 \\
.41 \\
31\end{array}$ & $\begin{array}{r}14.36 \\
.48 \\
.59 \\
.41 \\
28\end{array}$ \\
\hline
\end{tabular}

STATISTICS OF MONTHLY MEAN DATA FOR WATER YEARS 1989 - 1996, BY WATER YEAR (WY)

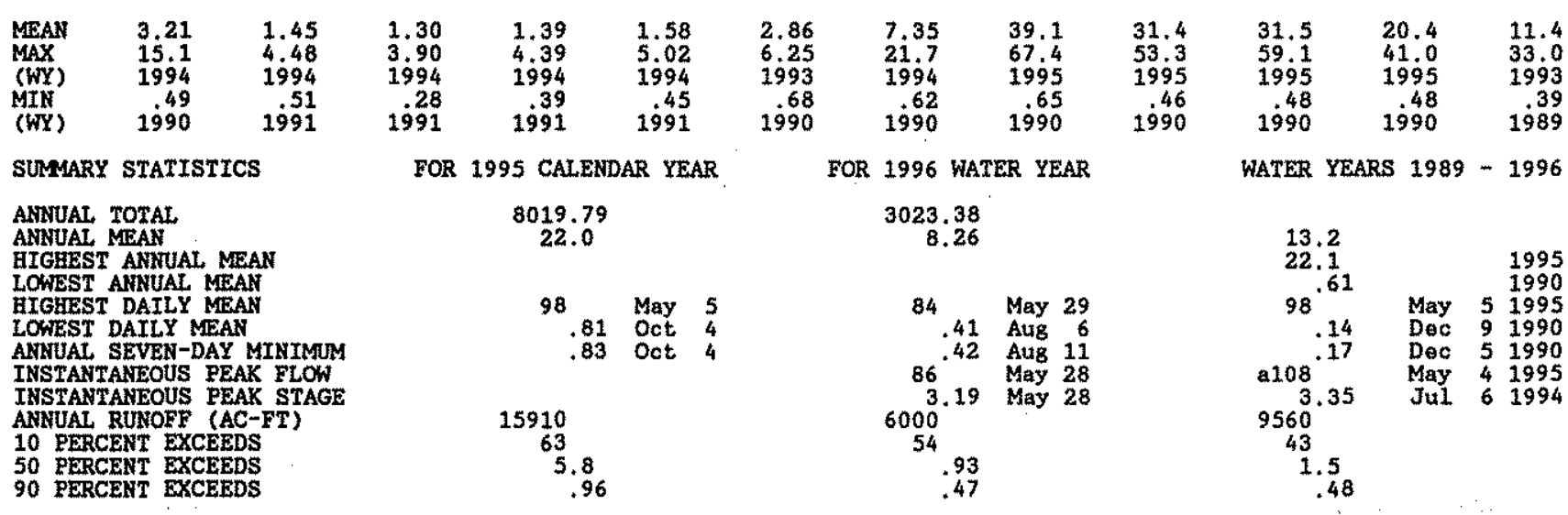

- Estimated

a-From rating curve extended above $50 \mathrm{ft}^{\mathrm{s}} / \mathrm{s}$. 


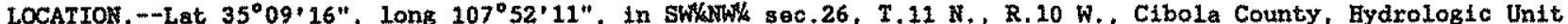
13020207 , on right bank upstream 1,500 ft from El korro St., 0.2 mi south of Santa Fe Ave. in Grants, and at mile 67.8 .

DRAINAGE AREA, $-1,020 \mathrm{mt}^{2}$, approximately.

PERIOD OF RECORD,-Octobar 1912 to February 1914, June 1914, October 1914 to February 1915 , May 1915 to June 1921, September 1921 to June 1923, October 1923 to May 1926, September to December 1926, May 1949 to September 1966 , June 1968 to current year. Monthly discharge only for some periods published in WSP 1312. Prior to October 1967. publtshed "Bluewater Creak at Grants."

REVISED RECORDS, - -WSP 1512: 1913-14. WSP 1712: Drainage area.

GAGE, -Water-stage recorder. Datum of gage is $6,468.34$ ft above Hational Geodetic Vertical Datum of 1929 (1evels by U.S. Army Corps of Engineers). See Wsp 1732 or 1923 for history of changes prtor to Jan. 1 , 1926 . Prior to 1992 at site on right bank at bridge at El Morro $S t$, at same datum.

REMARKS.--Records poor. Flow s1ightly regulatad by Bluewater Lalce (station 08341400) 24 mi upstream. Diversions and ground-water withdrawals for irrigation of about 4,500 acres upstream from station. Several observations of water temperature were made during the year. No flow most of time.

EXIREAES OUTSIDE PERIOD OF RECORD.-Heximum flood observed occurred Sept. 6 or 7 , 1909, when Bluewater Dam washed out. A flood in July 1919 probably exceeded the one in 1952.

DISCHARGE, CUBIC FEET PER SECOND, WATER YEAR OCTOBER 1995 TO SEPTEMBER 1996

\begin{tabular}{|c|c|c|c|c|c|c|c|c|c|c|c|c|c|}
\hline $\begin{array}{r}\text { DAX } \\
1 \\
2 \\
3 \\
4 \\
5\end{array}$ & $\begin{array}{l}O C T \\
.00 \\
.00 \\
.00 \\
.00 \\
.00\end{array}$ & $\begin{array}{l}\text { NoV } \\
.00 \\
.00 \\
.00 \\
.00 \\
.00\end{array}$ & $\begin{array}{l}\text { DEC } \\
.00 \\
.00 \\
.00 \\
.00 \\
.00\end{array}$ & 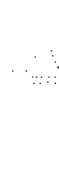 & $\begin{array}{l}\text { JAN } \\
.00 \\
.00 \\
.00 \\
.00 \\
.00\end{array}$ & $\begin{array}{l}\text { FEB } \\
.00 \\
.00 \\
.00 \\
.00 \\
.00\end{array}$ & $\begin{array}{l}\text { MAR } \\
.00 \\
.00 \\
.00 \\
.00 \\
.00\end{array}$ & $\begin{array}{l}\text { AFR } \\
.00 \\
.00 \\
.00 \\
.00 \\
.00\end{array}$ & $\begin{array}{l}\text { MAY } \\
.00 \\
.00 \\
.00 \\
.00 \\
.00\end{array}$ & $\begin{array}{l}\text { JUN } \\
.00 \\
.00 \\
.00 \\
.00 \\
.00\end{array}$ & $\begin{array}{l}\text { JUL } \\
.00 \\
.00 \\
.00 \\
.00 \\
.00\end{array}$ & $\begin{array}{l}\text { AUG } \\
0.00 \\
0.00 \\
e .00 \\
0.00 \\
0.00\end{array}$ & $\begin{array}{l}\text { SEP } \\
.00 \\
.00 \\
.00 \\
.00 \\
.00\end{array}$ \\
\hline $\begin{array}{r}6 \\
7 \\
8 \\
9 \\
10\end{array}$ & $\begin{array}{l}.00 \\
.00 \\
.00 \\
.00 \\
.00\end{array}$ & $\begin{array}{l}.00 \\
.00 \\
.00 \\
.00 \\
.00\end{array}$ & $\begin{array}{l}.00 \\
.00 \\
.00 \\
.00 \\
.00\end{array}$ & & $\begin{array}{l}.00 \\
.00 \\
.00 \\
.00 \\
.00\end{array}$ & $\begin{array}{l}.00 \\
.00 \\
.00 \\
.00 \\
.00\end{array}$ & $\begin{array}{l}.00 \\
.00 \\
.00 \\
.00 \\
.00\end{array}$ & $\begin{array}{l}.00 \\
.00 \\
.00 \\
.00 \\
.00\end{array}$ & $\begin{array}{l}.00 \\
.00 \\
.00 \\
.00 \\
.00\end{array}$ & $\begin{array}{l}.00 \\
.00 \\
.00 \\
.00 \\
.00\end{array}$ & $\begin{array}{l}.00 \\
.00 \\
.00 \\
.00 \\
.00\end{array}$ & $\begin{array}{l}0.00 \\
e .00 \\
0.00 \\
0.00 \\
0.00\end{array}$ & $\begin{array}{l}.00 \\
.00 \\
.00 \\
.00 \\
.00\end{array}$ \\
\hline $\begin{array}{l}11 \\
12 \\
13 \\
14 \\
15\end{array}$ & $\begin{array}{l}.00 \\
.00 \\
.00 \\
.00 \\
.00\end{array}$ & $\begin{array}{l}.00 \\
.00 \\
.00 \\
.00 \\
.00\end{array}$ & $\begin{array}{l}.00 \\
.00 \\
.00 \\
.00 \\
.00\end{array}$ & & $\begin{array}{l}.00 \\
.00 \\
.00 \\
.00 \\
.00\end{array}$ & $\begin{array}{l}.00 \\
.00 \\
.00 \\
.00 \\
.00\end{array}$ & $\begin{array}{l}.00 \\
.00 \\
.00 \\
.00 \\
.00\end{array}$ & $\begin{array}{l}.00 \\
.00 \\
.00 \\
.00 \\
.00\end{array}$ & $\begin{array}{l}.00 \\
.00 \\
.00 \\
.00 \\
.00\end{array}$ & $\begin{array}{l}.00 \\
.00 \\
.00 \\
.00 \\
.00\end{array}$ & $\begin{array}{l}.00 \\
.00 \\
.00 \\
.00 \\
.00\end{array}$ & $\begin{array}{l}e .00 \\
e .00 \\
.00 \\
.00 \\
.00\end{array}$ & $\begin{array}{l}.00 \\
.84 \\
1.2 \\
2.6 \\
1.1\end{array}$ \\
\hline $\begin{array}{l}16 \\
17 \\
18 \\
19 \\
20\end{array}$ & $\begin{array}{l}.00 \\
.00 \\
.00 \\
.00 \\
.00\end{array}$ & $\begin{array}{l}.00 \\
.00 \\
.00 \\
.00 \\
.00\end{array}$ & $\begin{array}{l}.00 \\
.00 \\
.00 \\
.00 \\
.00\end{array}$ & & $\begin{array}{l}.00 \\
.00 \\
.00 \\
.00 \\
.00\end{array}$ & $\begin{array}{l}.00 \\
.00 \\
.00 \\
.00 \\
.00\end{array}$ & $\begin{array}{l}.00 \\
.00 \\
.00 \\
.00 \\
.00\end{array}$ & $\begin{array}{l}.00 \\
.00 \\
.00 \\
.00 \\
.00\end{array}$ & $\begin{array}{l}.00 \\
.00 \\
.00 \\
.00 \\
.00\end{array}$ & $\begin{array}{l}.00 \\
.00 \\
.00 \\
.00 \\
.00\end{array}$ & $\begin{array}{l}.00 \\
.00 \\
.00 \\
.00 \\
.00\end{array}$ & $\begin{array}{l}.00 \\
.00 \\
.00 \\
.00 \\
.00\end{array}$ & $\begin{array}{r}.43 \\
.12 \\
.90 \\
1.2 \\
.53\end{array}$ \\
\hline $\begin{array}{l}21 \\
22 \\
23 \\
24 \\
25\end{array}$ & $\begin{array}{l}.00 \\
.00 \\
.00 \\
.00 \\
.00\end{array}$ & $\begin{array}{l}.00 \\
.00 \\
.00 \\
.00 \\
.00\end{array}$ & $\begin{array}{l}.00 \\
.00 \\
.00 \\
.00 \\
.00\end{array}$ & & $\begin{array}{l}.00 \\
.00 \\
.00 \\
.00 \\
.00\end{array}$ & $\begin{array}{l}.00 \\
.00 \\
.00 \\
.00 \\
.00\end{array}$ & $\begin{array}{l}.00 \\
.00 \\
.00 \\
.00 \\
.00\end{array}$ & $\begin{array}{l}.00 \\
.00 \\
.00 \\
.00 \\
.00\end{array}$ & $\begin{array}{l}.00 \\
.00 \\
.00 \\
.00 \\
.00\end{array}$ & $\begin{array}{l}.00 \\
.00 \\
.00 \\
.00 \\
.00\end{array}$ & $\begin{array}{l}.00 \\
.00 \\
.00 \\
.00 \\
.00\end{array}$ & $\begin{array}{l}3.00 \\
30 \\
11 \\
4.6\end{array}$ & $\begin{array}{r}.18 \\
.02 \\
0.00 \\
0.00 \\
0.00\end{array}$ \\
\hline $\begin{array}{l}26 \\
27 \\
28 \\
29 \\
30 \\
31\end{array}$ & $\begin{array}{l}.00 \\
.00 \\
.00 \\
.00 \\
.00 \\
.00\end{array}$ & $\begin{array}{l}.00 \\
.00 \\
.00 \\
.00 \\
.00 \\
\end{array}$ & $\begin{array}{l}.00 \\
.00 \\
.00 \\
.00 \\
.00 \\
.00\end{array}$ & & $\begin{array}{l}.00 \\
.00 \\
.00 \\
.00 \\
.00 \\
.00\end{array}$ & $\begin{array}{l}.00 \\
.00 \\
.00 \\
.00 \\
.0\end{array}$ & $\begin{array}{l}.00 \\
.00 \\
.00 \\
.00 \\
.00 \\
.00\end{array}$ & $\begin{array}{l}.00 \\
.00 \\
.00 \\
.00 \\
.00 \\
.\end{array}$ & $\begin{array}{l}.00 \\
.00 \\
.00 \\
.00 \\
.00 \\
.00\end{array}$ & $\begin{array}{l}.00 \\
.00 \\
.00 \\
.00 \\
.00 \\
.-1\end{array}$ & $\begin{array}{l}.00 \\
.00 \\
.00 \\
.00 \\
.00 \\
.00\end{array}$ & $\begin{array}{l}1.8 \\
.43 \\
.07 \\
.00 \\
.00 \\
.00\end{array}$ & $\begin{array}{l}0.00 \\
0.00 \\
0.00 \\
0.00 \\
0.00 \\
-\end{array}$ \\
\hline $\begin{array}{l}\text { TOTAL } \\
\text { MEAN } \\
\text { MAX } \\
\text { MIN } \\
\text { AC-ET }\end{array}$ & $\begin{array}{r}0.00 \\
.000 \\
.00 \\
.00 \\
.00\end{array}$ & $\begin{array}{l}0.00 \\
.000 \\
.00 \\
.00 \\
.00\end{array}$ & $\begin{array}{l}0.00 \\
.000 \\
.00 \\
.00 \\
.00\end{array}$ & & $\begin{array}{l}0.00 \\
.000 \\
.00 \\
.00 \\
.00\end{array}$ & $\begin{array}{l}0.00 \\
.000 \\
.00 \\
.00 \\
.00\end{array}$ & $\begin{array}{l}0.00 \\
.000 \\
.00 \\
.00 \\
.00\end{array}$ & $\begin{array}{l}0.00 \\
.000 \\
.00 \\
.00 \\
.00\end{array}$ & $\begin{array}{l}0.00 \\
.000 \\
.00 \\
.00 \\
.00\end{array}$ & $\begin{array}{l}0.00 \\
.000 \\
.00 \\
.00 \\
.00\end{array}$ & $\begin{array}{l}0.00 \\
.000 \\
.00 \\
.00 \\
.00\end{array}$ & $\begin{array}{r}51.00 \\
1.65 \\
30 \\
.00 \\
101\end{array}$ & $\begin{array}{r}9.12 \\
.30 \\
2.6 \\
.00 \\
18\end{array}$ \\
\hline $\begin{array}{l}\text { STATIS } \\
\text { MANAN } \\
\text { MAX } \\
\text { (WY) } \\
\text { MYN } \\
\text { (WY) }\end{array}$ & $\begin{array}{l}\text { CS OF } \\
.19 \\
2.51 \\
1970 \\
.000 \\
1969\end{array}$ & $\begin{array}{c}\text { MONIFY AEAN } \\
.002 \\
.061 \\
1980 \\
.000 \\
1969\end{array}$ & $\begin{array}{l}\text { DATA } \\
.000 \\
.000 \\
1969 \\
.000 \\
1969\end{array}$ & FOR & $\begin{array}{l}\text { WATER } \\
.000 \\
.000 \\
1969 \\
.000 \\
1969\end{array}$ & $\begin{array}{c}\text { YEARS } 1968 \\
.000 \\
.000 \\
1969 \\
.000 \\
3969\end{array}$ & $\begin{array}{l}-1996 \\
.23 \\
6.30 \\
1985 \\
.000 \\
1969\end{array}$ & $\begin{array}{c}\text { BY WATER } \\
6: 02 \\
87.0 \\
1980 \\
.000 \\
1969\end{array}$ & $\begin{array}{l}\text { YEAR (WY) } \\
1.92 \\
22.5 \\
1983 \\
.000 \\
1969\end{array}$ & $\begin{array}{l}.001 \\
.017 \\
1981 \\
.000 \\
1968\end{array}$ & $\begin{array}{l}.099 \\
1.20 \\
1981 \\
000 \\
1968\end{array}$ & $\begin{array}{r}.49 \\
7.79 \\
1993 \\
000 \\
1969\end{array}$ & $\begin{array}{r}.29 \\
5.49 \\
1972 \\
.000 \\
1968\end{array}$ \\
\hline
\end{tabular}

SURMARY STATISTICS

ANNUAL, TOTAL

ANIUAL MEAN

HTGEEST ANWUAL MAET

LOWEST ANNUAL MAN

HIGHEST DAILY MEAK

LOWEST DAILY MEAN

ANNUAL SEVEN-DAY MINIMUM

INSTAWTANEOUS PEAK FLOW

INSTANTANEOUS PEAK STAGE

ANNUAL RUNOFF (AC-FT)

10 PERCENT EXCEEDS

50 PERCENT EXCEEDS

90 PERCENT EXCEEDS

$\theta$ Estimated

a-From rating curve extended above $300 \mathrm{ft}^{3} / \mathrm{s}$, on basis of velocity-area studies.
FOR 1995 CALENDAR YEAR FOR 1996 WATER YEAR

$$
60.12
$$

$30 \quad$ Aug 23

$\begin{array}{rll}.00 & \text { Oct } 1 \\ .00 & \text { Oct } 1 \\ 73 & \end{array}$

73
2.59 Aug 22

119

.00

.00
WATER YEARS $1968-1996$

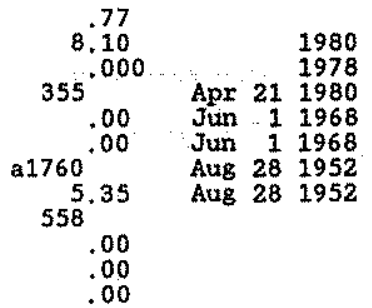


RIO GRANDE BASTN

08343500 RIO SAN JOSE NEAR GRANTS, NM

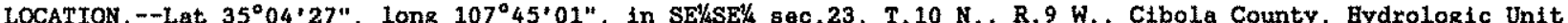

13020207 , on right bank at west boundary of Acoma Pubblo Grant, 8.5 mil southeast of Grants, and at mile 57.4 . DRAINAGE AREA. - $-2,300 \mathrm{~mL}^{2}$, approximately, of which $1,130 \mathrm{mi}^{2}$ does not contribute directly to surface runoff.

WATER-DISCBARGE RECORDS

PERIOD OF RECORD.--June 1936 to current year, Prior to October 1955, published as "San Jose River near Grants." REVISED RECORDS.--WSP 898: 1936-39(M). WSP 1512: 1943. WSP 1712: Drainage area.

GAGE.-Watex-stage recorder and concrete control. Datum of gage is 6,269,47 ft above National Geodetic Vertical Datum of 1929 .

REMARKS.--Water-discharge records good. Flow slightly regulated by Bluewater Lake (atatton 08341400), 34 mi upstream. Diversions and ground-water withdrawal for irrigation of about 5,100 acres upstream from station.

EXTREMES OUTSIDE PERIOD OF RECORD, --Maximum flood probably occurred Sept. 6 or 7 , 1909, following destruction of Bluevater Dam. The peak of Sept. 20, 1963, may have been exceeded by those of July 1919, August and September 1929, and August 1935. DISCEARGE, CUBIC FEET PER SECOND, WATER YEAR OCTOBER 1995 TO SEPTEMBER 1996

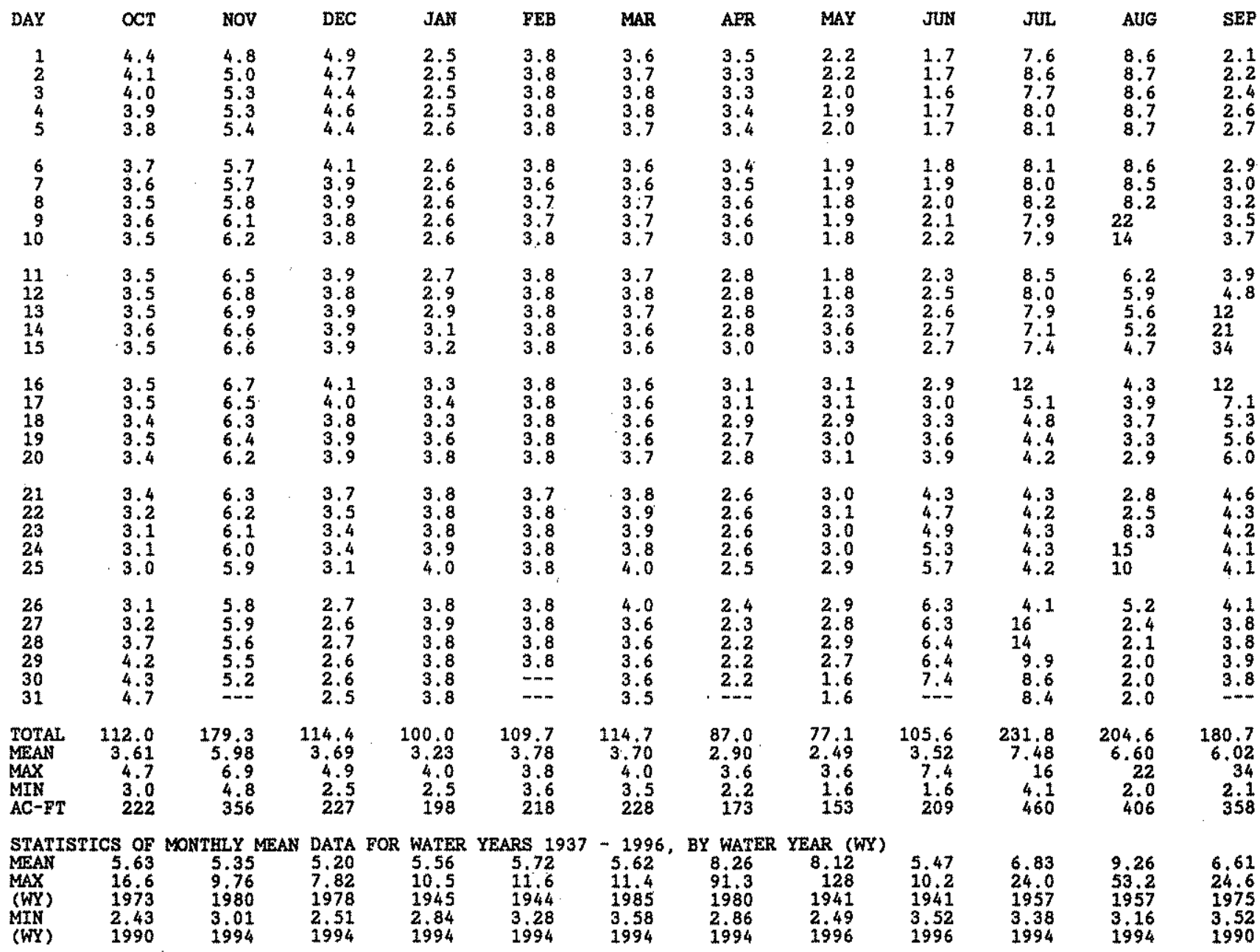

SUMMARY STATISTICS ANNUAL TOTAL

ANMUAL MEAN

HIGEEST ANNUAL MEAN

LOWEST ANNUAL MEAN

HIGAEST DAILY MEAN

LOWEST DATLY MEAN

ANNUAL SEVEN-DAY MINIMLN

INSTANTANEOUS PEAK FLOW

INSTANTANEOUS PEAK STAGE

INSTANTANEOUS LOW FLOW

ANNUAL RUNOFF (AC-FT)
10

50 PERCENT EXCEEDS

90 PERCENT EXCEEDS
FOR 1995 CALENDAR YEAR 1680.7

FOR 1996 WATER YEAR 1616.9

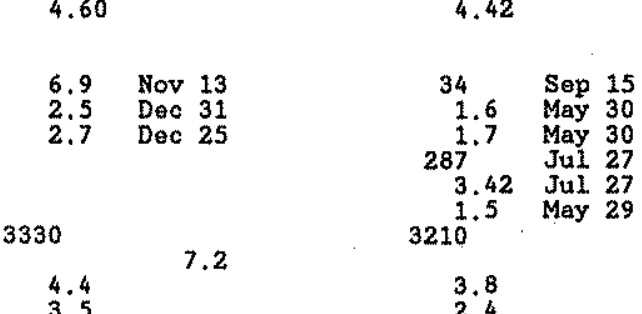

WATER YEARS $1937-1996$

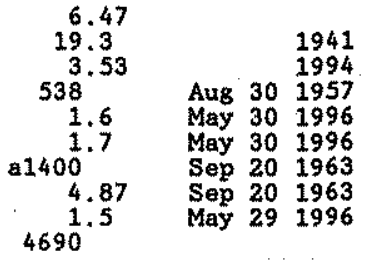

5.1

- rom ratir 
RIO GRANDE BASIN

08343500 RIO SAN JOSE MEAR GRANTS, NM - - Continued

WATER-QUALITY RECORDS

PERIOD OF RECORD.--Water years 1980-82, 1986 to current year,

WATER QUALITY DATA, WATER YEAR OCTOBER 1995 TO SEFTEMBER 1996

\begin{tabular}{|c|c|c|c|c|c|c|c|c|c|c|c|}
\hline DATE & TIME & $\begin{array}{c}\text { DIS- } \\
\text { CEARGS, } \\
\text { INST } \\
\text { CUBTC } \\
\text { FEET } \\
\text { PER } \\
\text { SECOND } \\
(00061)\end{array}$ & $\begin{array}{l}\text { SPE- } \\
\text { CIFIC } \\
\text { CON- } \\
\text { DUCT- } \\
\text { ANCE } \\
(\text { US/CM) } \\
(00095)\end{array}$ & $\begin{array}{c}\text { PE } \\
\text { WATER } \\
\text { WHOLE } \\
\text { FIELD } \\
\text { (STAND- } \\
\text { ARD } \\
\text { UNITS) } \\
(00400)\end{array}$ & $\begin{array}{l}\text { TEMPER- } \\
\text { ATURE } \\
\text { AIR } \\
(D E G C) \\
(00020)\end{array}$ & $\begin{array}{l}\text { TEMPER- } \\
\text { ATURE } \\
\text { WATER } \\
\text { (DEG C ) } \\
(00010)\end{array}$ & $\begin{array}{c}\text { BARO- } \\
\text { MERRIC } \\
\text { PRES- } \\
\text { SURE } \\
\text { (RM } \\
\text { OF } \\
\text { HG) } \\
(00025)\end{array}$ & $\begin{array}{c}\text { OXYGEN, } \\
\text { DIS } \\
\text { SOLVED } \\
(\mathrm{NE} / \mathrm{I}) \\
(00300)\end{array}$ & $\begin{array}{c}\text { OXYGEN } \\
\text { DIS- } \\
\text { SOLVED } \\
\text { (PER- } \\
\text { CENT } \\
\text { SATUR- } \\
\text { AIION) } \\
\text { (OOB01) }\end{array}$ & $\begin{array}{l}\text { EARD- } \\
\text { NESS } \\
\text { TOTAL } \\
\text { (MG/L } \\
\text { AS } \\
\text { CACO3) } \\
(00900)\end{array}$ & $\begin{array}{c}\text { HARD- } \\
\text { AESS } \\
\text { NONCARB } \\
\text { DISSOLV } \\
\text { FLD AS } \\
\text { CACO3 } \\
\text { (MG/L) } \\
(00904)\end{array}$ \\
\hline $\begin{array}{c}\text { DCT } 1995 \\
25.9 \\
\text { JAN } 1996\end{array}$ & 1100 & 3.0 & 1.050 & 7.9 & 15.0 & 13.0 & 611 & 8.9 & 106 & 320 & 130 \\
\hline $25 \cdots$ & 1045 & 4.5 & 986 & 8.2 & 5.5 & 11.0 & 602 & 10.4 & 120 & 310 & $-m$ \\
\hline $18 \ldots$ & 1000 & 3.0 & 1090 & 8.3 & 12.5 & 14.0 & 608 & 10.2 & 125 & 320 & - \\
\hline & & & & & & & & & & & \\
\hline DATE & $\begin{array}{l}\text { CALCIUM } \\
\text { DIS- } \\
\text { SOLVED } \\
\text { (MG/L } \\
\text { AS CA) } \\
(00915)\end{array}$ & $\begin{array}{c}\text { MAGNE- } \\
\text { SIUM, } \\
\text { DIS- } \\
\text { SOLVED } \\
\text { (MG/L } \\
\text { AS MG) } \\
\text { (00925) }\end{array}$ & $\begin{array}{c}\text { SODTUM, } \\
\text { DIS-- } \\
\text { SOLVED } \\
\text { (MG/L } \\
\text { AS NA) } \\
(00930)\end{array}$ & $\begin{array}{l}\text { SODIUM } \\
\text { AD- } \\
\text { SORP- } \\
\text { TION } \\
\text { RATIO } \\
(00931)\end{array}$ & $\begin{array}{l}\text { POTAS- } \\
\text { SIUM, } \\
\text { DIS- } \\
\text { SOLVED } \\
\text { (ME/L } \\
\text { AS K) } \\
(00935)\end{array}$ & $\begin{array}{c}\text { BICAR- } \\
\text { BONATE } \\
\text { WATER } \\
\text { DIS IT } \\
\text { EIELD } \\
\text { MG/L AS } \\
\text { BCO3 } \\
(00453)\end{array}$ & $\begin{array}{c}\text { CAR- } \\
\text { BONATE } \\
\text { WATER } \\
\text { DIS IT } \\
\text { FIELD } \\
\text { WG/L AS } \\
\text { CO3 } \\
(00452)\end{array}$ & $\begin{array}{c}\text { ALKA- } \\
\text { LINITY } \\
\text { WAT DIS } \\
\text { TOT IT } \\
\text { FIELD } \\
\text { MG/L AS } \\
\text { CACO3 } \\
(39086)\end{array}$ & $\begin{array}{c}\text { ALKA } \\
\text { LINITY } \\
\text { LAB } \\
(\mathrm{MG} / \mathrm{L} \\
\text { AS } \\
\text { CACO3) } \\
(90410)\end{array}$ & $\begin{array}{l}\text { SULFATE } \\
\text { DIS- } \\
\text { SOLVED } \\
\text { (1EG/L } \\
\text { AS SO4) } \\
(00945)\end{array}$ & $\begin{array}{l}\text { CHLO- } \\
\text { RIDE, } \\
\text { DIS- } \\
\text { SOLVED } \\
\text { (ME/L } \\
\text { AS CL) } \\
(00940)\end{array}$ \\
\hline $\begin{array}{l}\text { OCT } 1995 \\
25 \\
\text { JAN } 19966\end{array}$ & 74 & 34 & 99 & 2 & 5.0 & 234 & 0 & 192 & 203 & 240 & 69 \\
\hline${ }_{A F R}^{25} \cdots$ & 72 & 32 & 92 & 2 & 4.7 & -- & -- & $-m$ & 201 & 230 & 63 \\
\hline 18 & 74 & 34 & 100 & 2 & 4.7 & $-\infty$ & -- & -- & 201 & 250 & 73 \\
\hline
\end{tabular}

DATE

OCT 1995

JAN 1996

$25 \ldots$

AFR.

$18 \ldots$

OCI 1995

JAN 1996

$25 .$.

$18 \ldots$

\section{FLUO- SILICA, SOLIDS, \\ RIDE, DIS- CONSTI- \\ DIS: SOLVED TUENTS, \\ $\begin{array}{lll}\text { SOLVED } & \text { (MG/L } & \text { DIS- } \\ \text { (HG/L } & \text { AS } & \text { SOLVED }\end{array}$ \\ AS F) SIO2) ( 1 SG/L)}

$\begin{array}{ccc}\text { As } & \text { (00950) } & (00955) \quad(70301)\end{array}$

$\begin{array}{ll}0.70 & 32 \\ 0.80 & 31 \\ 0.90 & 30\end{array}$

669
646
687

ALUM- ANTI-

INUM, MONY, ARSENIC BARIUM, BERYL-

DIS- DIS- DIS- DIS-

SOLVED SOLVED SOLVED SOLVED SOLVED

(UG/L (UG/I. (UG/L (UG/L (UG/L

AS AL) AS SB) AS AS) AS BA) AS BE

$(01106) \quad(01095)$

$3.0<1.0$

$5 \quad 43$

$(01010)$
BORON, CADMIUM

DIS- DIS-

SOLVED SOLVED

(UG/L (UG/L

AS B)
$(01020) \quad(01025)$

\begin{tabular}{|c|c|c|c|c|c|c|c|c|c|c|}
\hline DATE & $\begin{array}{l}\text { CFRO- } \\
\text { MIUM, } \\
\text { DIS- } \\
\text { SOLVED } \\
\text { (UG/L } \\
\text { AS CR) } \\
\text { (O1030) }\end{array}$ & $\begin{array}{c}\text { COBAL:T, } \\
\text { DIS- } \\
\text { SOLVED } \\
\text { (UG/L } \\
\text { AS CO) } \\
\text { (01035) }\end{array}$ & $\begin{array}{c}\text { COPPER, } \\
\text { DIS- } \\
\text { SOLVED } \\
\text { (UYG/L } \\
\text { AS CU) } \\
\text { (OIU40) }\end{array}$ & $\begin{array}{l}\text { IROA , } \\
\text { DIS - } \\
\text { SOLVED } \\
\text { (UG/L } \\
\text { AS FE) } \\
(01046)\end{array}$ & $\begin{array}{l}\text { LEAD, } \\
\text { DIS - } \\
\text { SOLVED } \\
\text { (UG/L } \\
\text { AS RB) } \\
(01049)\end{array}$ & $\begin{array}{l}\text { MANGA- } \\
\text { NESE, } \\
\text { DIS- } \\
\text { SOLVED } \\
\text { (UG/L } \\
\text { AS MN) } \\
(01056)\end{array}$ & $\begin{array}{l}\text { MIRCURY } \\
\text { TOTAL } \\
\text { RECOV- } \\
\text { ERABLE } \\
\text { (UG/L } \\
\text { AS IHG) } \\
(71900)\end{array}$ & $\begin{array}{l}\text { MOLYB- } \\
\text { DENUM, } \\
\text { DIS } \\
\text { SOLVED } \\
\text { (UG/L } \\
\text { AS MO) } \\
(01060)\end{array}$ & $\begin{array}{c}\text { PYCKEL, } \\
\text { DIS- } \\
\text { SOLVED } \\
\text { (UE/L } \\
\text { AS NI ) } \\
(01065)\end{array}$ & $\begin{array}{c}\text { SELEO- } \\
\text { NIINS, } \\
\text { TOTAL } \\
\text { (UG/L } \\
\text { AS SE) } \\
(02147)\end{array}$ \\
\hline $\begin{array}{c}\text { OCI } 1995 \\
25 \\
\text { JAN } 1996\end{array}$ & 2.0 & $<1.0$ & 1.0 & $<3.0$ & $<1,0$ & 4.0 & $<0.10$ & 3.0 & $<1,0$ & 4 \\
\hline${ }_{A R R}^{25} \cdots$ & -- & - & -- & 3.0 & $\sim$ & -- & $m$ & -- & -- & $m$ \\
\hline $18 \ldots$ & -- & -- & $m-$ & 5.0 & - & -- & -- & $\sim$ & $-\sim$ & -- \\
\hline
\end{tabular}

OCT 1995 250.96 APR 25

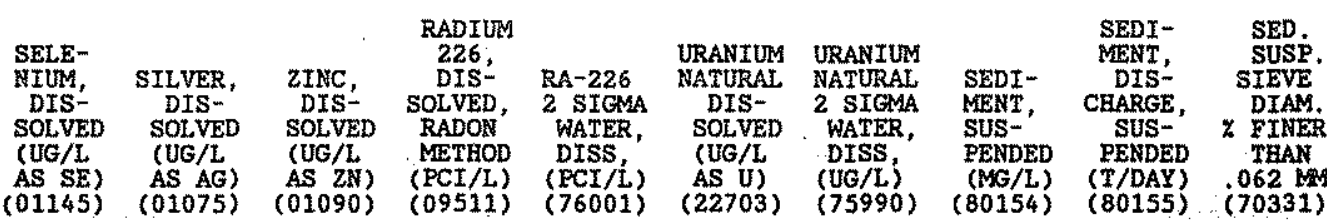
18 ...

$\begin{array}{rrr}3 & <1.0 & 2.0 \\ - & -- & -\end{array}$

\begin{abstract}
$0.07 \quad 0.020$
\end{abstract}
2.3

0.0 -.
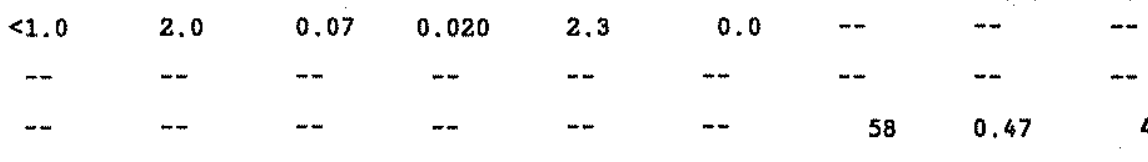
RIO GRANDE BASIN

08353000 RIO PUERCO NEAR BERNARDO, NM

LOCATION, - - Lat $34^{\circ} 24^{\prime} 33^{\prime \prime}$, long $106^{\circ} 51^{\prime} 09^{\prime \prime}$, in SEM sec.8, T.2 N., R. 1 E., Socorro County, Eydrologic Unit 13020204, on bridge on former U'.s. Highway 85, 0.2 mi upstream from Interstate Bighway $25,1.2$ mi southwest of Bernardo, $3.0 \mathrm{mi}$ upstream from mouth, and $18 \mathrm{mi}$ south of Belen.

DRAINAGE AREA. $-7,350 \mathrm{mi}^{2}$, approximately, of which at least $1,130 \mathrm{mt}^{2}$ does not contribute directly to surface runoff.

WATER-DISCEARGE RECORDS

PERIOD OF RECORD.-November 1939 to current year. Fragmentary gage-hetght record and footnotes concerning no flow for the pertod September 1910 to August 1914 published in WSP 358 and 388 are in error and should not be used. REVISED RECORDS,--WSP 1512: 1941-42, 1944-45, 1946(P), 1947-49. WSP 1632: 1957. WSP 1732: Dratnage area. See also PERIOD OF RECORD.

GAGE. - Water-stage recorder. Datum of gage is 4,722.34 ft above National Geodetic Verttcal Datum of 1929 . Prior to Jan. 24,1969 , at datum 3.10 ft highor.

REMARKS. -Water-discharge records poor except for estimated datly discharges, which are poor. Divergions for irrigation of about 11,500 acres upstrean from station (includes 3,700 acres 1 rrigated wholly or partiy from wel1s).

EXTREXES OUTSIDE PERIOD OF RECORD.--The greatest flood since about 1880 occurred Sept. 23, 1929, from information by local residents (discharge, about $35,000 \mathrm{ft} / \mathrm{s}$, estimated on basis of peak at Rio Puerco). Another flood occurred Aug. 12, 1929 (discharge, 30,600 fts/s, by slope-area measurement, from reports of Ner Mexico State Engtinear).

DISCEARGE, CUBIC FEET PER SECOND, WATER YEAR OCTOBER 1995 TO SEPTEMBER 1996

\begin{tabular}{|c|c|c|c|c|c|c|c|c|c|c|c|c|c|}
\hline DAY & $\propto C T$ & nov & DEC & JAN & FEB & MAR & APR & MAX & JUN & JUL & \multicolumn{2}{|r|}{ AUTG } & SEP \\
\hline $\begin{array}{l}1 \\
2 \\
3 \\
4 \\
5\end{array}$ & $\begin{array}{r}14 \\
16 \\
7.5 \\
5.9 \\
2.1\end{array}$ & $\begin{array}{l}.00 \\
.00 \\
.00 \\
.00 \\
.00\end{array}$ & $\begin{array}{l}.00 \\
.00 \\
.00 \\
.00 \\
.00\end{array}$ & $\begin{array}{l}.00 \\
.00 \\
.00 \\
.00 \\
.00\end{array}$ & $\begin{array}{l}.00 \\
.00 \\
.00 \\
.00 \\
.00\end{array}$ & $\begin{array}{l}.00 \\
.00 \\
.00 \\
.00 \\
.00\end{array}$ & $\begin{array}{l}.00 \\
.00 \\
.00 \\
.00 \\
.00\end{array}$ & $\begin{array}{l}.00 \\
.00 \\
.00 \\
.00 \\
.00\end{array}$ & $\begin{array}{l}.00 \\
.00 \\
.00 \\
.00 \\
.00\end{array}$ & $\begin{array}{r}e 80 \\
e 20 \\
e 200 \\
e 300 \\
e 200\end{array}$ & & $\begin{array}{l}.00 \\
.00 \\
.00 \\
.00 \\
.00\end{array}$ & $\begin{array}{r}399 \\
59 \\
18 \\
8.9 \\
3.6\end{array}$ \\
\hline $\begin{array}{r}6 \\
7 \\
8 \\
9 \\
10\end{array}$ & $\begin{array}{l}.61 \\
.03 \\
.00 \\
.00 \\
.00\end{array}$ & $\begin{array}{l}.00 \\
.00 \\
.00 \\
.00 \\
.00\end{array}$ & $\begin{array}{l}.00 \\
.00 \\
.00 \\
.00 \\
.00\end{array}$ & $\begin{array}{l}.00 \\
.00 \\
.00 \\
.00 \\
.10\end{array}$ & $\begin{array}{l}.00 \\
.00 \\
.00 \\
.00 \\
.00\end{array}$ & $\begin{array}{l}.00 \\
.00 \\
.00 \\
.00 \\
.00\end{array}$ & $\begin{array}{l}.00 \\
.00 \\
.00 \\
.00 \\
.00\end{array}$ & $\begin{array}{l}.00 \\
.00 \\
.00 \\
.00 \\
.00\end{array}$ & $\begin{array}{l}.00 \\
.00 \\
.00 \\
.00 \\
.00\end{array}$ & $\begin{array}{c}0100 \\
040 \\
01.6 \\
e 50 \\
0160\end{array}$ & $\begin{array}{l} \\
0800 \\
0360\end{array}$ & $\begin{array}{l}.00 \\
.00 \\
.00 \\
0\end{array}$ & $26^{.00} .57$ \\
\hline $\begin{array}{l}11 \\
12 \\
13 \\
14 \\
15\end{array}$ & $\begin{array}{l}.00 \\
.00 \\
.00 \\
.00 \\
.00\end{array}$ & $\begin{array}{l}.00 \\
.00 \\
.00 \\
.00 \\
.00\end{array}$ & $\begin{array}{l}.00 \\
.00 \\
.00 \\
.00 \\
.00\end{array}$ & $\begin{array}{l}.20 \\
.20 \\
.10 \\
.00 \\
.00\end{array}$ & $\begin{array}{l}.00 \\
.00 \\
.00 \\
.00 \\
.00\end{array}$ & $\begin{array}{l}.00 \\
.00 \\
.00 \\
.00 \\
.00\end{array}$ & $\begin{array}{l}.00 \\
.00 \\
.00 \\
.00 \\
.00\end{array}$ & $\begin{array}{l}.00 \\
.00 \\
.00 \\
.00 \\
.00\end{array}$ & $\begin{array}{l}.00 \\
.00 \\
.00 \\
.00 \\
.00\end{array}$ & $\begin{array}{r}\theta 100 \\
070 \\
0120 \\
0160 \\
0190\end{array}$ & $\begin{array}{r}e 130 \\
060 \\
018 \\
01 \\
\theta\end{array}$ & $\begin{array}{l}0 \\
0 \\
8 \\
1.1 \\
0.20\end{array}$ & $\begin{array}{c}4.8 \\
13^{.90} \\
68 \\
330\end{array}$ \\
\hline $\begin{array}{l}16 \\
17 \\
18 \\
19 \\
20\end{array}$ & $\begin{array}{l}.00 \\
.00 \\
.00 \\
.00 \\
.00\end{array}$ & $\begin{array}{l}.00 \\
.00 \\
.00 \\
.00 \\
.00\end{array}$ & $\begin{array}{l}.00 \\
.00 \\
.00 \\
.00 \\
.00\end{array}$ & $\begin{array}{l}.00 \\
.00 \\
.00 \\
.00 \\
.00\end{array}$ & $\begin{array}{l}.00 \\
.00 \\
.00 \\
.00 \\
.00\end{array}$ & $\begin{array}{l}.00 \\
.00 \\
.00 \\
.00 \\
.00\end{array}$ & $\begin{array}{l}.00 \\
.00 \\
.00 \\
.00 \\
.00\end{array}$ & $\begin{array}{l}.00 \\
.00 \\
.00 \\
.00 \\
.00\end{array}$ & $\begin{array}{l}.00 \\
.00 \\
.00 \\
.00 \\
.00\end{array}$ & $\begin{array}{r}8110 \\
040 \\
050 \\
070 \\
e 50\end{array}$ & & $\begin{array}{l}.00 \\
.00 \\
.00 \\
.00 \\
.00\end{array}$ & $\begin{array}{r}664 \\
400 \\
101 \\
58 \\
39\end{array}$ \\
\hline $\begin{array}{l}21 \\
22 \\
23 \\
24 \\
25\end{array}$ & $\begin{array}{l}.00 \\
.00 \\
.00 \\
.00 \\
.00\end{array}$ & $\begin{array}{l}.00 \\
.00 \\
.00 \\
.00 \\
.00\end{array}$ & $\begin{array}{l}.00 \\
.00 \\
.00 \\
.00 \\
.00\end{array}$ & $\begin{array}{l}.00 \\
.00 \\
.00 \\
.00 \\
.00\end{array}$ & $\begin{array}{l}.00 \\
.00 \\
.00 \\
.00 \\
.00\end{array}$ & $\begin{array}{l}.00 \\
.00 \\
.00 \\
.00 \\
.00\end{array}$ & $\begin{array}{l}.00 \\
.00 \\
.00 \\
.00 \\
.00\end{array}$ & $\begin{array}{l}.00 \\
.00 \\
.00 \\
.00 \\
.00\end{array}$ & $\begin{array}{l}.00 \\
.00 \\
.00 \\
.00 \\
.00\end{array}$ & $\begin{array}{l}e 20 \\
03.0 \\
01.0 \\
0.50 \\
0.00\end{array}$ & $\begin{array}{r}01250 \\
01100\end{array}$ & $\begin{array}{r}.00 \\
.00 \\
.00 \\
0\end{array}$ & $\begin{array}{r}22 \\
13 \\
4.5 \\
1.1 \\
.07\end{array}$ \\
\hline $\begin{array}{l}26 \\
27 \\
28 \\
29 \\
30 \\
31\end{array}$ & $\begin{array}{l}.00 \\
.00 \\
.00 \\
.00 \\
.00 \\
.00\end{array}$ & $\begin{array}{l}.00 \\
.00 \\
.00 \\
.00 \\
.00 \\
.\end{array}$ & $\begin{array}{l}.00 \\
.00 \\
.00 \\
.00 \\
.00 \\
.00\end{array}$ & $\begin{array}{l}.00 \\
.00 \\
.00 \\
.00 \\
.00 \\
.00\end{array}$ & $\begin{array}{l}.00 \\
.00 \\
.00 \\
.00 \\
.--\end{array}$ & $\begin{array}{l}.00 \\
.00 \\
.00 \\
.00 \\
.00 \\
.00\end{array}$ & $\begin{array}{l}.00 \\
.00 \\
.00 \\
.00 \\
.00 \\
.0\end{array}$ & $\begin{array}{l}.00 \\
.00 \\
.00 \\
.00 \\
.00 \\
.00 \\
.00\end{array}$ & $\begin{array}{r}.00 \\
01150 \\
01300 \\
0200\end{array}$ & $\begin{array}{l}060 \\
051 \\
010 \\
03.0 \\
0.50 \\
0.00\end{array}$ & $\begin{array}{r}0850 \\
0690 \\
0290 \\
040 \\
69 \\
634\end{array}$ & & $\begin{array}{l}.00 \\
.00 \\
.00 \\
.00 \\
.00 \\
.\end{array}$ \\
\hline $\begin{array}{l}\text { TOTAL } \\
\text { MEAN } \\
\text { MAX } \\
\text { MIN } \\
\text { AC-ET }\end{array}$ & $\begin{array}{r}46.14 \\
1.49 \\
16 \\
.00 \\
92\end{array}$ & $\begin{array}{l}0.00 \\
.000 \\
.00 \\
.00 \\
.00\end{array}$ & $\begin{array}{l}0.00 \\
.000 \\
.00 \\
.00 \\
.00\end{array}$ & $\begin{array}{r}0.60 \\
.019 \\
.20 \\
.00 \\
1.2\end{array}$ & $\begin{array}{l}0.00 \\
.000 \\
.00 \\
.00 \\
.00\end{array}$ & $\begin{array}{l}0.00 \\
.000 \\
.00 \\
.00 \\
.00\end{array}$ & $\begin{array}{l}0.00 \\
.000 \\
.00 \\
.00 \\
.00\end{array}$ & $\begin{array}{l}0.00 \\
.000 \\
.00 \\
.00 \\
.00\end{array}$ & $\begin{array}{r}2650.00 \\
88.3 \\
1300 \\
.00 \\
5260\end{array}$ & $\begin{array}{r}2260.60 \\
72.9 \\
300 \\
.00 \\
4480\end{array}$ & $\begin{array}{r}6292 \\
1 \\
12\end{array}$ & $\begin{array}{r}2.30 \\
203 \\
1250 \\
.00 \\
2480\end{array}$ & $\begin{array}{r}2249.44 \\
75.0 \\
664 \\
.00 \\
4460\end{array}$ \\
\hline $\begin{array}{l}\text { SUMMAR } \\
\text { ANNUAL } \\
\text { ANNUAL } \\
\text { HIGHES } \\
\text { LOWEST } \\
\text { HIGHES } \\
\text { LOWEST } \\
\text { ANNUAL } \\
\text { INSTAN } \\
\text { INSTAN } \\
\text { ANNUAL } \\
10 \text { PER } \\
50 \text { PERC } \\
90 \text { PERO }\end{array}$ & $\begin{array}{l}\text { STATIS } \\
\text { TOTAL } \\
\text { MEAN } \\
\text { ANNUAL } \\
\text { ANNUAL } \\
\text { DAILY } \\
\text { DAILY MI } \\
\text { SEVEN-D } \\
\text { ANEOUS } \\
\text { ANEOUS } \\
\text { RUNOEF } \\
\text { ENT EXC } \\
\text { ENT EXC } \\
\text { ENT EXCI }\end{array}$ & $\begin{array}{l}\text { TICS } \\
\text { MEAN } \\
\text { MEAN } \\
\text { MEAN } \\
\text { IEAN } \\
\text { OAY MINIMIM } \\
\text { PEAK FLOW } \\
\text { PEAK STAGE } \\
\text { (AC FT) } \\
\text { DEEDS } \\
\text { BEEDS } \\
\text { EEEDS }\end{array}$ & & $\begin{array}{r}1995 \mathrm{CAt} \\
6697 \\
18 \\
325 \\
\end{array}$ & $\begin{array}{lr} & \\
\text { Aug } & 29 \\
\text { Jul } & 8 \\
\text { Jul } & 8\end{array}$ & & $\begin{array}{r}1996 \text { WA } \\
13499.08 \\
36.9 \\
1300 \\
.00 \\
.00 \\
1330 \\
10.25 \\
26780 \\
53 \\
.00 \\
.00\end{array}$ & $\begin{array}{rrr} & \text { Jun } 29 \\
0 & \text { Oct } 8 \\
0 & \text { Oct } 8 \\
& \text { Jun } 28 \\
5 & \text { Jun } 28\end{array}$ & $\begin{array}{l}9 \\
8 \\
8 \\
8 \\
8\end{array}$ & $\begin{array}{r}\text { WATER } \\
43 . \\
171^{\circ} \\
5980^{\circ} \\
. \\
\text { a } 10800^{\circ} \\
\text { b16. } \\
31310^{\circ} \\
68\end{array}$ & $\begin{array}{l}\text { YEARS } \\
.2 \\
.47 \\
.00 \\
.00 \\
.90 \\
.00 \\
.00\end{array}$ & $\begin{array}{l}\text { May } \\
\text { Nov } \\
\text { Nov } \\
\text { Sep } \\
\text { Aug }\end{array}$ & 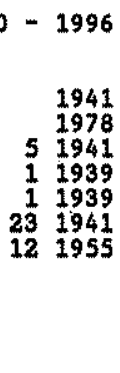 \\
\hline
\end{tabular}

a-From rating ourve extionded above $7,800 \mathrm{ft} / \mathrm{s}$,

b-Maximum gage hejght, 16.9 ft, present datum, Aug. 12, 1955. 


\section{RIO GRANDE BASIN}

08353000 RTO PUERCO NEAR BERNARDO, MM - - Continued

WATER-QUALITY RECORDS

PERIOD OF RECORD. --Water years 1947 to current year.

SUSPENDED-SEDIMENT DISCAARGE: October 1947 to current yeax.

PERIOD OF DAILY RECORD --

SUSPENDED-SEDIMENT DISCRARGE: October 1947 to current year.

INSTRUMETTATION.-Automatic pumping sampler June 1995.

REMARKS.--Deily suspended-sediment samples are collectod when flow is observad on thts opheperal atream.

EXTRERES FOR PERTOD OF DAILY RECORD.--

SEDIMENI CONCENTRATION: Maximum daily mean, 267,000 mg/L, July 26, 1957; minimum daily mean, no flow on many

ays of each yoar.

SEDIMENT LOAD: Maximum daliy, 2,240,000 tons, Aug. 7, 1957; minimum daily, 0 ton on many days of oach year.

EXTREMES FOR CURRENT YBAR -

SED TMENT CONCENTRATION: Maximum daily mean, 125,000 mg/L, July 4 ; minimum daily mean, no $\mathrm{clow}$ on many days. SEDIMEN LOAD: Maximum dafly, 251,000 tons, Aus. 25; minimum daily, 0 ton on many days.

WATER QUALITY DATA, WATER YEAR OCTOBER 1995 TO SEPTEMBER 1996

\begin{tabular}{|c|c|c|c|c|c|c|c|c|c|c|c|}
\hline DAIE & TIME & $\begin{array}{c}\text { DIS- } \\
\text { CRARGE, } \\
\text { INST, } \\
\text { CUBIC } \\
\text { FEET } \\
\text { PER } \\
\text { SECOND } \\
\text { (00061) }\end{array}$ & $\begin{array}{l}\text { SPE- } \\
\text { CIFIC } \\
\text { CON- } \\
\text { DUCT- } \\
\text { ANCE } \\
\text { (US/CM) } \\
\text { (00095) }\end{array}$ & $\begin{array}{c}\text { PH } \\
\text { WATER } \\
\text { WROLE } \\
\text { FIELD } \\
\text { (STAND- } \\
\text { ARD } \\
\text { UNITS) } \\
(00400)\end{array}$ & $\begin{array}{l}\text { TEMPER- } \\
\text { ATURE } \\
\text { AIR } \\
\text { (DEG C) } \\
(00020)\end{array}$ & $\begin{array}{l}\text { TEMPER- } \\
\text { ATURE } \\
\text { WATER } \\
\text { (DEG C) } \\
(00010)\end{array}$ & $\begin{array}{c}\text { BARO- } \\
\text { RETRIC } \\
\text { PRES- } \\
\text { SURE } \\
\text { (RNA } \\
\text { OF } \\
\text { HG) } \\
(00025)\end{array}$ & $\begin{array}{c} \\
\text { OXYGEX, } \\
\text { DIS- } \\
\text { SOLYED } \\
(\text { MO } / L) \\
(00300)\end{array}$ & $\begin{array}{l}\text { OXYGEN, } \\
\text { DIS- } \\
\text { SOLVED } \\
\text { (PER- } \\
\text { CENT } \\
\text { SATUR- } \\
\text { ATION) } \\
\text { (00301) }\end{array}$ & $\begin{array}{c}\text { EARD- } \\
\text { NESS } \\
\text { TOTAL } \\
\text { (MGG/L } \\
\text { AS } \\
\text { CACO3) } \\
(00900)\end{array}$ & $\begin{array}{l}\text { CALCIUM } \\
\text { DIS- } \\
\text { SOLVED } \\
\text { (MESLL } \\
\text { AS CA) } \\
\text { (00915) }\end{array}$ \\
\hline
\end{tabular}

\begin{tabular}{|c|c|c|c|c|c|c|c|c|c|c|c|}
\hline $\begin{array}{l}19.15 \\
15 .\end{array}$ & 1015 & -- & $-\cdots$ & -- & -- & $-\infty$ & - & -- & -- & -- & -- \\
\hline תर् $i 996$ & 0830 & - & - & -- & -- & 4.0 & $-\cdots$ & $m$ & -- & - & -- \\
\hline AUG & 0930 & 180 & 2480 & 7.6 & 24.0 & 21.5 & 645 & 4.7 & 64 & - & -- \\
\hline $12 \ldots$ & 1230 & 66 & 1060 & 7.7 & 36.5 & 23.0 & 648 & 7.0 & 97 & 290 & 88 \\
\hline
\end{tabular}

\begin{tabular}{|c|c|c|c|c|c|}
\hline $\begin{array}{c}\text { MAGNE- } \\
\text { SIUM, } \\
\text { DIS-- } \\
\text { SOLVED } \\
\text { (ME /L } \\
\text { AS MS) } \\
\text { (00925) }\end{array}$ & $\begin{array}{c}\text { SODIUM, } \\
\text { DIS- } \\
\text { SOLVED } \\
\text { (MG/L } \\
\text { AS NA) } \\
(00930)\end{array}$ & $\begin{array}{c}\text { SODIUM } \\
\text { AD- } \\
\text { SORP } \\
\text { TION } \\
\text { RATIO } \\
(00931)\end{array}$ & $\begin{array}{c}\text { POTAS- } \\
\text { SIUM, } \\
\text { DIS- } \\
\text { SOLVED } \\
\text { (MG/L } \\
\text { AS K) } \\
(00935)\end{array}$ & $\begin{array}{c}\text { ALKA- } \\
\text { LINITY } \\
\text { LAB } \\
\text { (MG/L } \\
\text { AS } \\
\text { CACO3) } \\
(90410)\end{array}$ & $\begin{array}{c}\text { SULFATE } \\
\text { DIS- } \\
\text { SOLVED } \\
\text { (MG/L } \\
\text { AS SO4) } \\
(00945)\end{array}$ \\
\hline
\end{tabular}

\begin{tabular}{|c|c|c|c|c|}
\hline $\begin{array}{l}\text { CHLO- } \\
\text { RIDE, } \\
\text { DIS- } \\
\text { SOLVED } \\
\text { (ME/L }\end{array}$ & $\begin{array}{l}\text { FLUO- } \\
\text { RIDE, } \\
\text { DIS- } \\
\text { SOLVED } \\
\text { (MG/L } \\
\text { AS F ) }\end{array}$ & $\begin{array}{c}\text { SILICA, } \\
\text { DIS- } \\
\text { SOL.VED } \\
\text { (MEC/L } \\
\text { AS }\end{array}$ & $\begin{array}{c}\text { SOLIDS, } \\
\text { SUM OF } \\
\text { CONSTI- } \\
\text { TUENTS, } \\
\text { DIS- } \\
\text { SOLVED } \\
\text { (Net/L) }\end{array}$ & $\begin{array}{l}\text { ALUM- } \\
\text { INUM, } \\
\text { DIS- } \\
\text { SOLVED } \\
\text { (UG/L }\end{array}$ \\
\hline
\end{tabular}

\section{OCT 1995 \\ $15 \ldots$ \\ DEC \\ JuL 1996}

AUG

$12 .$.

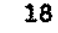

98
$+$

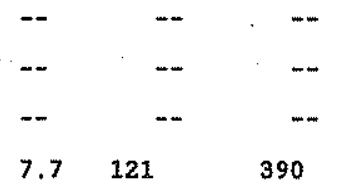

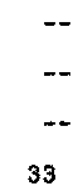

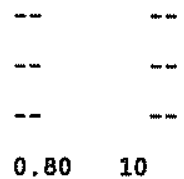

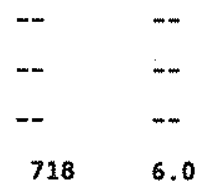

DATE

$\begin{array}{lcc}\text { AATI- } & & \\ \text { NONY, } & \text { ARSENTC } & \text { BARIUM, } \\ \text { DIS- } & \text { DIS- } & \text { DIS- } \\ \text { SOLVED } & \text { SOLVED } & \text { SOLVED } \\ \text { (UG/L } & \text { (UG/L } & \text { (UG/1 } \\ \text { AS SB) } & \text { AS AS) } & \text { AS BA }) \\ (01095) & (01000) & (01005)\end{array}$

BERYL-

CERO-

LITM; BORON: CADMTUM MXUM

$\begin{array}{lll}\text { DIS- } & \text { DIS- } & \text { DIS- } \\ \text { SOLVED } & \text { SOLVED } & \text { SOLVED }\end{array}$

(UG/L

$\mathrm{AS} \mathrm{BE}$ )

(01010)
(UG/L

AS B) AS CD

(01020) (01025)
MXUM,
DIS-
SOLVED
(UG/L
AS CR)

(AS CR)
COBAXT, COPPER, IRON,

SOLVED SOLVED SOL

(UG/L (UG/L U $/ \mathrm{L} / \mathrm{L}$

(US CO)

AS CO) AS CU)
$(01035)(01040)(01046)$

OCT 1995
$15 \ldots$
DEC
TUL 1996
$03 \ldots$

AUG 12 ...

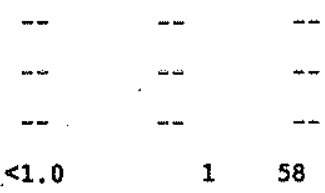

--
--
-
$<1.0$

$--$
--
--
--
$<1.0$

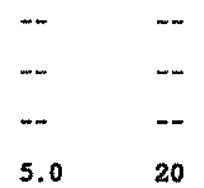


RTO GRANDE BASIN

08353000 RIO PUERCO NEAR BERNARDO, NM - Cont Inued

WATER-QUALITY RECORDS

WATER QUALITY DATA, WATER YEAR OCTCBER 1995 TO SEPTEMBER 1996

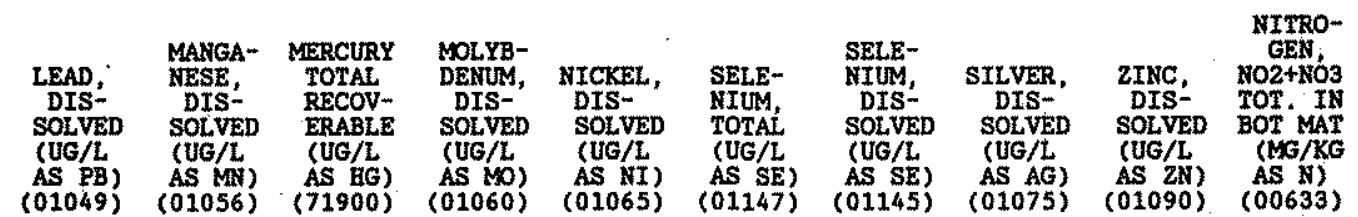

oct 1995

DEC

Jut 0796

Aug 03

$$
\begin{array}{cc}
-- & -- \\
-- & <1,0
\end{array}
$$$$
--
$$

$-$

$-$

\begin{tabular}{|c|c|c|c|c|c|}
\hline $\begin{array}{c}\text { MITRO- } \\
\text { GEN, NH4 } \\
\text { TOTAL } \\
\text { IN BOT. } \\
\text { MAT. } \\
(M G / K G \\
\text { AS N } \\
(00611)\end{array}$ & $\begin{array}{l}\text { NITRO- } \\
\text { GEN, NH4 } \\
+ \text { ORG } \\
\text { TOT IN } \\
\text { BOT MAT } \\
\text { (MG/KG } \\
\text { AS N) } \\
(00626)\end{array}$ & $\begin{array}{c}\text { PHOS- } \\
\text { PHORUS } \\
\text { TOTAL } \\
\text { IN BOT. } \\
\text { MAT. } \\
\text { (MG/KG } \\
\text { AS P) } \\
(00668)\end{array}$ & $\begin{array}{c}\text { ARSENIC } \\
\text { TOTAL } \\
\text { IN BOT- } \\
\text { TOM MA- } \\
\text { TERIAL } \\
\text { (UG/G } \\
\text { AS AS) } \\
\text { (01003) }\end{array}$ & $\begin{array}{c}\text { CADMIUM } \\
\text { RECOV } \\
\text { FM BOT- } \\
\text { TOM MA- } \\
\text { TERIAL } \\
\text { (UG/G } \\
\text { AS CD) } \\
(01028)\end{array}$ & $\begin{array}{l}\text { CEI } \\
\text { RI } \\
\text { RM } \\
\text { TOM } \\
\text { TE } \\
(\mathrm{U} \\
\text { CO1 }\end{array}$ \\
\hline
\end{tabular}
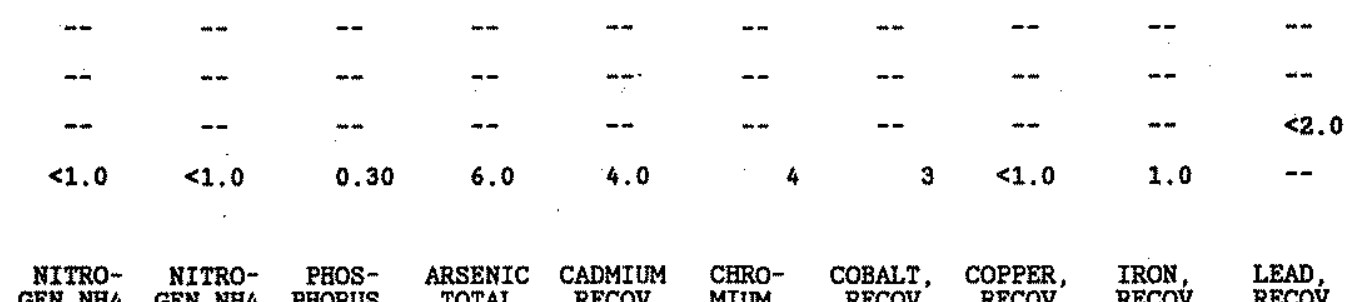

COBALT, COPPER, IRON, LEAD,

RECOV. RECOV. RECOV. RECOV.

TOM MA- TOM MA- TOM MA- TOM MA-

TERTAL TERIAL TERIAL TERTAI

(UG/G (UG/G (UG/G (UG/G

DATE

(00611) $\quad(00626)$

AS P)

(01003)

AS CO)

AS CU) AS FE)

AS EE) AS PB)

OCT 1995

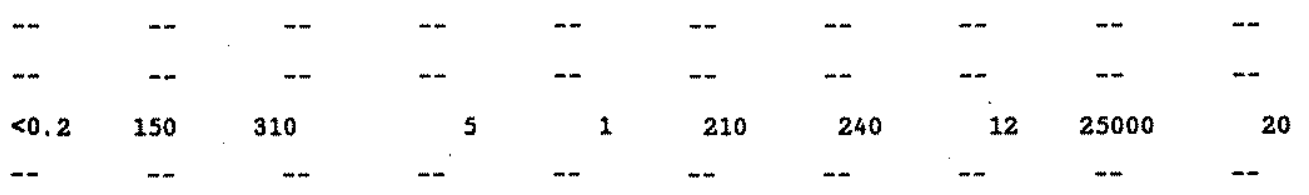

AUG 12.

DEC

JuL 19796

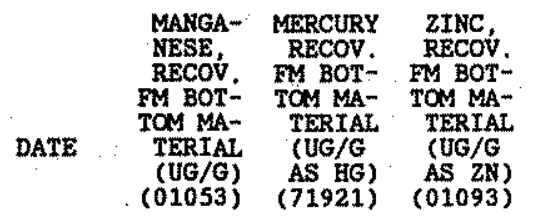

RADTUM

$\begin{array}{ccc}226, & & \text { URAWIUM URANIUM } \\ \text { DIS'- } & \text { RA-226 } & \text { NATURAL NATURAL, } \\ \text { SOLVED } & 2 \text { SIGMA } & \text { DIS- }\end{array}$

RADON

2 SIGMA

WATER

DIS- 2 STGMA

DISS, (UG/L DISS,

(PCI/L) AS U) (UG/L)

(75990) ( $(80154)$

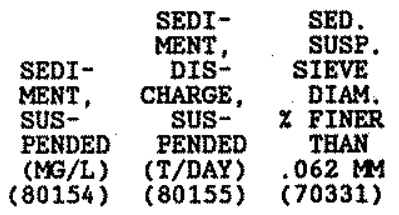

OCT 1995

$15 \ldots$
DEC
JuL
03

$$
\begin{array}{lll}
- & \cdots & \cdots \\
240 & 0.01 & 30
\end{array}
$$

$$
\begin{array}{ll}
- \\
-- \\
0.10
\end{array}
$$$$
+\cdots
$$

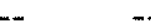

$\begin{array}{lrr}-- & 325 & - \\ -- & 659 & - \\ 0.3 & 122000 & 59300 \\ 0.0 & 27700 & 4940\end{array}$

100

0.020

3.9

$0.0 \quad 27700 \quad 4940$

99

12 ...

$\rightarrow$

$-\infty$

0.12

0.030

1.3

(1) 
RIO GRANDE BASIN

08353000 RIO PUERCO NEAR BERNARDO, MM -- Continued

WATER-QUALITY RECORDS

SUSPENDED-SEDIMENT, WATER YEAR OCTOBER 1995 TO SEPTEMBER 1996

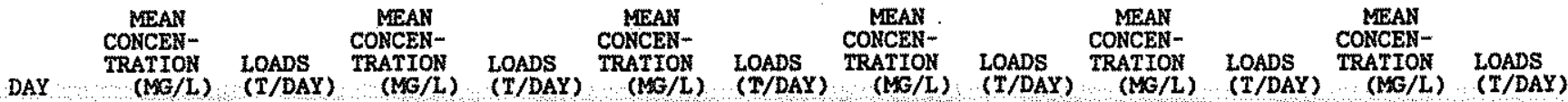

$\begin{array}{cccc} & \text { OCTOBER } & \text { NOVEMBER } \\ 74200 & 2810 & 0 & 0 \\ 49100 & 2120 & 0 & .00 \\ 22300 & 452 & 0 & 0 \\ 20700 & 329 & 0 & 0 \\ 8420 & 48 & 0 & 0 \\ & & 0 & .00\end{array}$

.00
.00
.00
.00
.00
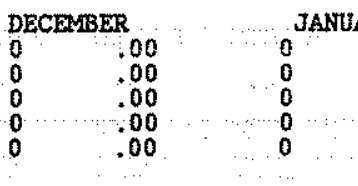

.00
.00
00
.00
.00

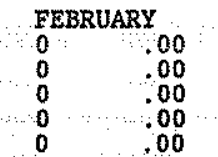

$\begin{array}{ll} & \text { MARCH } \\ 0 & .00 \\ 0 & .00 \\ 0 & .00 \\ 0 & .00 \\ 0 & .00\end{array}$

6
7
8
9
10

$\begin{array}{rc}1970 & 3.3 \\ 630 & .05 \\ 0 & .00 \\ 0 & .00 \\ 0 & .00\end{array}$

$\begin{array}{ll}0 & .00 \\ 0 & .00 \\ 0 & .00 \\ 0 & .00 \\ 0 & .00\end{array}$

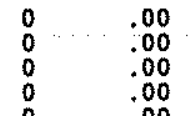

$\begin{array}{rl}0 & .00 \\ 0 & .00 \\ 0 & .00 \\ 20 & .01 \\ 53 & .01\end{array}$

11
12
13
14
15
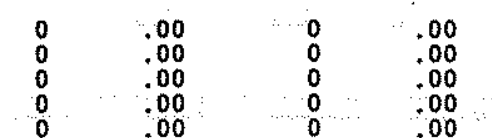

$$
\begin{aligned}
& 0 \\
& 0 \\
& 0 \\
& 0 \\
& 0
\end{aligned}
$$

.00

15

TOTAL.

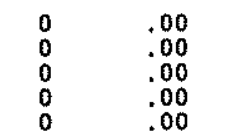

$\begin{array}{ll}0 & .00 \\ 0 & .00 \\ 0 & .00 \\ 0 & .00 \\ 0 & .00\end{array}$

$\begin{array}{ll}0 & .00 \\ 0 & .00 \\ 0 & .00 \\ 0 & .00 \\ 0 & .00 \\ 0 & .00\end{array}$

$\begin{array}{rr}0 & .00 \\ 0 & .00 \\ 0 & .00 \\ 0 & .00 \\ 0 & .00 \\ - & -\end{array}$

$$
\begin{array}{ll}
0 & 00 \\
0 & 00
\end{array}
$$

53

$\begin{array}{ll}0 & .00 \\ 0 & .00 \\ 0 & .00 \\ 0 & .00 \\ 0 & .00\end{array}$

$\begin{array}{rr}75 & .04 \\ 70 & .04 \\ 45 & .01 \\ 10 & .00 \\ 0 & .00\end{array}$

$\begin{array}{cc}0 & .00 \\ 0 & .00 \\ 0 & .00 \\ 0 & .00 \\ 0 & .00\end{array}$

$$
\begin{aligned}
& \begin{array}{ll}
0 & .00 \\
0 & 00
\end{array} \\
& \begin{array}{ll}
0 & .00 \\
0 & .00 \\
0 & .00 \\
0 & .00
\end{array} \\
& \begin{array}{ll}
0.00 \\
0
\end{array}
\end{aligned}
$$$$
\begin{array}{lllllll}
.00 & 0 & .00 & 0 & .00 & 0 & .00 \\
.00 & 0 & .00 & 0 & .00 & 0 & .00 \\
.00 & 0 & .00 & 0 & .00 & 0 & .00 \\
.00 & 0 & .00 & 0 & .00 & 0 & .00 \\
.00 & 0 & .00 & 0 & .00 & 0 & .00 \\
.00 & 0 & .00 & 0 & .00 & 0 & .00
\end{array}
$$

.00
.00
.00
.00
.00
.00

$\begin{array}{ll}0 & .00 \\ 0 & .00 \\ 0 & .00 \\ 0 & .00 \\ 0 & .00 \\ 0 & .00\end{array}$

$$
\begin{array}{ll}
0 & .00 \\
0 & .00 \\
0 & .00 \\
0 & .00 \\
0 & .00
\end{array}
$$

$\begin{array}{ll}0 & .00 \\ 0 & .00 \\ 0 & .00 \\ 0 & .00 \\ 0 & .00\end{array}$

$\begin{array}{ll}0 & .00 \\ 0 & .00 \\ 0 & .00 \\ 0 & .00 \\ 0 & .00\end{array}$

$\begin{array}{ll}0 & .00 \\ 0 & .00 \\ 0 & .00 \\ 0 & .00 \\ 0 & .00\end{array}$

$$
\begin{array}{ll}
0 & .00 \\
0 & .00 \\
0 & .00 \\
0 & .00 \\
0 & .00
\end{array}
$$

$\begin{array}{ll}0 & .00 \\ 0 & .00 \\ 0 & .00 \\ 0 & .00 \\ 0 & .00 \\ 0 & .00\end{array}$

0
0
0
0
0
0

$\begin{array}{lllll}.00 & 0 & .00 & 0 & .00 \\ .00 & 0 & .00 & 0 & .00 \\ .00 & 0 & .00 & 0 & .00 \\ .00 & 0 & .00 & 0 & .00 \\ .00 & -00 & -.0 & 0 & .00 \\ .00 & -0 & -.00 & 0 & .00\end{array}$
0.00

0.00

-...

0.11

0.00

$--$

0.00

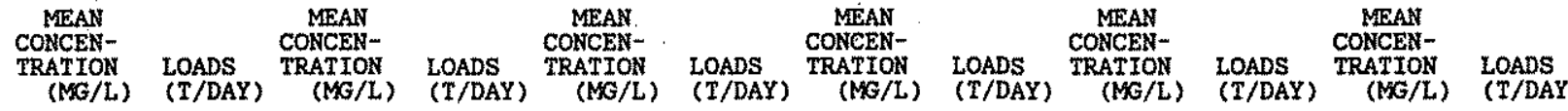

DAY

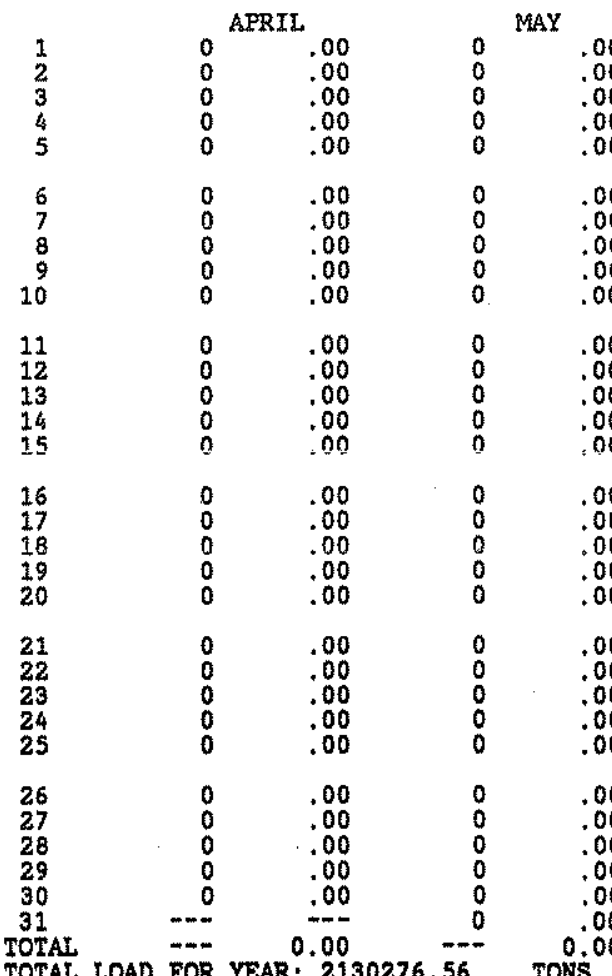

$\begin{array}{lll} & \text { JUNE } & \\ 0 & .0 & \\ 0 & .0 & 1 \\ 0 & .0 & 1 \\ 0 & .0 & 1 \\ 0 & .0 & \\ 0 & .0 \\ 0 & .0 \\ 0 & .0 \\ 0 & .0 \\ 0 & .0 & 1 \\ 0 & .0 \\ 0 & .0 \\ 0 & .0 \\ 0 & .0 \\ 0 & .0 \\ 0 & .0 \\ 0 & .0 \\ 0 & .0 \\ 0 & .0 \\ 0 & .0 \\ 0 & .0 \\ 0 & .0 \\ 0 & .0 \\ 0 & .0 \\ 0 & .0 \\ 0 & .0\end{array}$
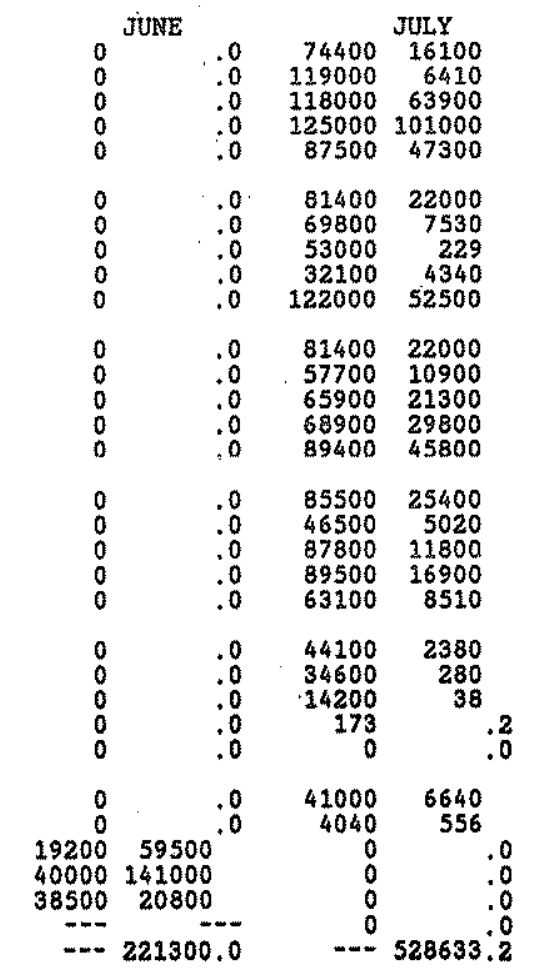
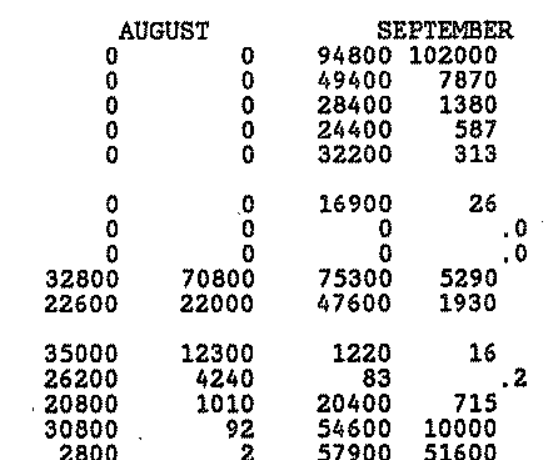

$2800 \quad 22 \quad 54600 \quad 10000$

$0-0 \quad 31200 \quad 55900$

$$
\begin{array}{llll}
0 & 0 & 31200 & 55900 \\
0 & 0 & 67500 & 72800 \\
0 & 0 & 42200 & 11500 \\
0 & 0 & 27500 & 4310 \\
0 & 0 & 32500 & 3420
\end{array}
$$

TOTAL LOAD FOR YEAR: 2130276,56 TONS. 
RIO GRANDE BASIN

08354500 SOCORRO MAIN CANAL NORTH AT SAN ACACIA, NM

LOCATION,-Lat $34^{\circ} 15^{\prime} 17^{\prime \prime}$, Long $106^{\circ} 53^{\prime} 43^{\prime \prime}$, in SEKAWt/ sec.1, T.1 S., R.1 W, Socorro County, Bydrologic Unit 13020203 , on right bank at San Acacia, 'and 0.5 mil downstream frón point' of diversion.

PERIOD OF RECORD. - April 1936 to September 1964 (anonthly discharge only), October 1964 to current year.

REVISED RECORDS, - WSR 1242: 1951.

GAGE - Water-gtage recorder. Datum of gage is 4.660.16 ft above National Geodetic Vertical Datum of 1929 (U.S. Bureau of Reclamation bench mark). Prior to Mar. 8,1958 , at site 300 ft upstream (in old channe1) at datum 0.42 ft lower.

REMARKS.-Records good except for estimatod datly discharges, which are fatr. This canal is 1 of 3 channels (stations 08354800,08354900 ) carrying flow in valley cross section. For combined monthly flow in acre-ft of this canal, conveyance channel, and floodway, see tabulation below daily table for 08354900. Canal diverts water from right bank of Rio Grande for irrigation of about 8,000 acres. Alamillo acequia and 3 other smaller ditches divert water from canal upstream from station for irrigation of about 400 acres. Discharge records collected at the canal heading from October 1964 to September 1965 indicate that 7,770 acre- ft or $9 \times$ reaching the regular gaging station, Several observations of water temperature were made during the year. No flow at times. DISCHARGE, CUBIC FEET PER SECOND, WATER YEAR OCTOBER 1995 TO SEPTEMBER 1996

\begin{tabular}{|c|c|c|c|c|c|c|c|c|c|c|c|c|}
\hline DAY & oct & NOV & DEC & JAN & FEB & MAR & APR & MAY & JUN & JUL & AUG & SEP \\
\hline $\begin{array}{l}1 \\
2 \\
3 \\
4 \\
5\end{array}$ & $\begin{array}{l}175 \\
216 \\
223 \\
225 \\
221\end{array}$ & $\begin{array}{r}27 \\
.00 \\
.00 \\
.00 \\
.00\end{array}$ & $\begin{array}{l}.00 \\
.00 \\
.00 \\
.00 \\
.00\end{array}$ & $\begin{array}{l}.00 \\
.00 \\
.00 \\
.00 \\
.00\end{array}$ & $\begin{array}{l}.00 \\
.00 \\
.00 \\
.00 \\
.00\end{array}$ & $\begin{array}{r}37 \\
86 \\
91 \\
128 \\
153\end{array}$ & $\begin{array}{l}283 \\
280 \\
284 \\
288 \\
280\end{array}$ & $\begin{array}{l}192 \\
209 \\
166 \\
157 \\
182\end{array}$ & $\begin{array}{l}302 \\
283 \\
219 \\
196 \\
160\end{array}$ & $\begin{array}{r}161 \\
185 \\
0135 \\
0120 \\
0115\end{array}$ & $\begin{array}{l}114 \\
148 \\
173 \\
243 \\
244\end{array}$ & $\begin{array}{l}171 \\
173 \\
177 \\
172 \\
165\end{array}$ \\
\hline $\begin{array}{r}6 \\
7 \\
8 \\
9 \\
10\end{array}$ & $\begin{array}{l}217 \\
217 \\
217 \\
208 \\
206\end{array}$ & $\begin{array}{l}.00 \\
.00 \\
.00 \\
.00 \\
.00\end{array}$ & $\begin{array}{l}.00 \\
.00 \\
.00 \\
.00 \\
.00\end{array}$ & $\begin{array}{l}.00 \\
.00 \\
.00 \\
.00 \\
.00\end{array}$ & $\begin{array}{l}.00 \\
.00 \\
.00 \\
.00 \\
.00\end{array}$ & $\begin{array}{l}178 \\
202 \\
251 \\
261 \\
263\end{array}$ & $\begin{array}{l}276 \\
274 \\
271 \\
258 \\
246\end{array}$ & $\begin{array}{l}198 \\
243 \\
238 \\
179 \\
185\end{array}$ & $\begin{array}{l}124 \\
122 \\
150 \\
182 \\
140\end{array}$ & $\begin{array}{l}169 \\
192 \\
241 \\
252 \\
243\end{array}$ & $\begin{array}{l}236 \\
221 \\
209 \\
182 \\
163\end{array}$ & $\begin{array}{l}206 \\
223 \\
224 \\
220 \\
226\end{array}$ \\
\hline $\begin{array}{l}11 \\
12 \\
13 \\
14 \\
15\end{array}$ & $\begin{array}{l}202 \\
194 \\
199 \\
197 \\
203\end{array}$ & $\begin{array}{l}.00 \\
.00 \\
.00 \\
.00 \\
.00\end{array}$ & $\begin{array}{l}.00 \\
.00 \\
.00 \\
.00 \\
.00\end{array}$ & $\begin{array}{l}.00 \\
.00 \\
.00 \\
.00 \\
.00\end{array}$ & $\begin{array}{l}.00 \\
.00 \\
.00 \\
.00 \\
.00\end{array}$ & $\begin{array}{l}266 \\
266 \\
256 \\
255 \\
257\end{array}$ & $\begin{array}{l}243 \\
221 \\
167 \\
205 \\
239\end{array}$ & $\begin{array}{l}181 \\
166 \\
107 \\
111 \\
124\end{array}$ & $\begin{array}{l}117 \\
119 \\
135 \\
155 \\
255\end{array}$ & $\begin{array}{l}206 \\
242 \\
213 \\
179 \\
158\end{array}$ & $\begin{array}{l}178 \\
178 \\
196 \\
210 \\
212\end{array}$ & $\begin{array}{l}237 \\
186 \\
141 \\
116 \\
111\end{array}$ \\
\hline $\begin{array}{l}16 \\
17 \\
18 \\
19 \\
20\end{array}$ & $\begin{array}{l}239 \\
245 \\
245 \\
238 \\
230\end{array}$ & $\begin{array}{l}.00 \\
.00 \\
.00 \\
.00 \\
.00\end{array}$ & $\begin{array}{l}.00 \\
.00 \\
.00 \\
.00 \\
.00\end{array}$ & $\begin{array}{l}.00 \\
.00 \\
.00 \\
.00 \\
.00\end{array}$ & $\begin{array}{l}.00 \\
.00 \\
.00 \\
.00 \\
.00\end{array}$ & $\begin{array}{l}253 \\
254 \\
254 \\
253 \\
248\end{array}$ & $\begin{array}{l}226 \\
205 \\
218 \\
232 \\
242\end{array}$ & $\begin{array}{l}181 \\
194 \\
188 \\
170 \\
182\end{array}$ & $\begin{array}{l}268 \\
253 \\
271 \\
276 \\
276\end{array}$ & $\begin{array}{r}0137 \\
0116 \\
108 \\
105 \\
133\end{array}$ & $\begin{array}{l}195 \\
161 \\
148 \\
153 \\
142\end{array}$ & $\begin{array}{l}141 \\
184 \\
169 \\
188 \\
194\end{array}$ \\
\hline $\begin{array}{l}21 \\
22 \\
23 \\
24 \\
25\end{array}$ & $\begin{array}{l}231 \\
219 \\
214 \\
220 \\
222\end{array}$ & $\begin{array}{l}.00 \\
.00 \\
.00 \\
.00 \\
.00\end{array}$ & $\begin{array}{l}.00 \\
.00 \\
.00 \\
.00 \\
.00\end{array}$ & $\begin{array}{l}.00 \\
.00 \\
.00 \\
.00 \\
.00\end{array}$ & $\begin{array}{l}.00 \\
.00 \\
.00 \\
.00 \\
.00\end{array}$ & $\begin{array}{r}248 \\
253 \\
259 \\
266 \\
268\end{array}$ & $\begin{array}{l}269 \\
275 \\
269 \\
276 \\
264\end{array}$ & $\begin{array}{l}179 \\
152 \\
177 \\
234 \\
259\end{array}$ & $\begin{array}{l}267 \\
257 \\
264 \\
258 \\
232\end{array}$ & $\begin{array}{r}194 \\
198 \\
147 \\
114 \\
95\end{array}$ & $\begin{array}{l}149 \\
190 \\
204 \\
205 \\
166\end{array}$ & $\begin{array}{l}175 \\
164 \\
202 \\
222 \\
218\end{array}$ \\
\hline $\begin{array}{l}26 \\
27 \\
28 \\
29 \\
30 \\
31\end{array}$ & $\begin{array}{l}220 \\
220 \\
220 \\
222 \\
219 \\
212\end{array}$ & $\begin{array}{l}.00 \\
.00 \\
.00 \\
.00 \\
.00 \\
.0\end{array}$ & $\begin{array}{l}.00 \\
.00 \\
.00 \\
.00 \\
.00 \\
.00\end{array}$ & $\begin{array}{l}.00 \\
.00 \\
.00 \\
.00 \\
.00 \\
.00\end{array}$ & $\begin{array}{r}.00 \\
.00 \\
.00 \\
.00 \\
. .- \\
. .\end{array}$ & $\begin{array}{l}275 \\
294 \\
290 \\
299 \\
283 \\
275\end{array}$ & $\begin{array}{l}279 \\
270 \\
232 \\
197 \\
206 \\
-\end{array}$ & $\begin{array}{l}284 \\
280 \\
274 \\
269 \\
287 \\
299\end{array}$ & $\begin{array}{l}200 \\
269 \\
163 \\
165 \\
163 \\
-\infty\end{array}$ & $\begin{array}{l}190 \\
167 \\
132 \\
180 \\
199 \\
119\end{array}$ & $\begin{array}{l}153 \\
158 \\
164 \\
141 \\
146 \\
164\end{array}$ & $\begin{array}{l}217 \\
216 \\
226 \\
224 \\
225 \\
---\end{array}$ \\
\hline $\begin{array}{l}\text { TOTAL } \\
\text { MEAN } \\
\text { MAX } \\
\text { MIN } \\
\text { AC-FT }\end{array}$ & $\begin{array}{r}6736 \\
217 \\
245 \\
175 \\
13360\end{array}$ & $\begin{array}{r}27.00 \\
.90 \\
27 \\
.00 \\
54\end{array}$ & $\begin{array}{l}0.00 \\
.000 \\
.00 \\
.00 \\
.00\end{array}$ & $\begin{array}{l}0.00 \\
.000 \\
.00 \\
.00 \\
.00\end{array}$ & $\begin{array}{l}0.00 \\
.000 \\
.00 \\
.00 \\
.00\end{array}$ & $\begin{array}{r}7222 \\
233 \\
299 \\
. \quad 37 \\
14320\end{array}$ & $\begin{array}{r}7475 \\
249 \\
288 \\
167 \\
14830\end{array}$ & $\begin{array}{r}6247 \\
202 \\
299 \\
107 \\
12390\end{array}$ & $\begin{array}{r}6241 \\
208 \\
302 \\
117 \\
12380\end{array}$ & $\begin{array}{r}5145 \\
166 \\
252 \\
95 \\
10210\end{array}$ & $\begin{array}{r}5546 \\
179 \\
244 \\
114 \\
11000\end{array}$ & $\begin{array}{r}5713 \\
190 \\
237 \\
111 \\
11330\end{array}$ \\
\hline
\end{tabular}

STATISTICS OF MONTHLY MEAN DATA FOR WATER YEARS 1964 - 1996, BY WATER YEAR (WY)

\begin{tabular}{|c|c|c|c|c|c|c|c|c|c|c|c|c|}
\hline $\begin{array}{l}\text { MEAN } \\
\text { MAX } \\
\text { (WY) } \\
\text { MIN } \\
\text { (WY) }\end{array}$ & $\begin{array}{r}126 \\
257 \\
1994 \\
17.1 \\
1964\end{array}$ & $\begin{array}{l}9.35 \\
86.0 \\
1989 \\
1000 \\
1967\end{array}$ & $\begin{array}{l}7.79 \\
79.0 \\
1976 \\
1900 \\
1964\end{array}$ & $\begin{array}{l}7.13 \\
56.7 \\
1976 \\
1000 \\
1964\end{array}$ & $\begin{array}{l}5.25 \\
52.4 \\
1979 \\
.000 \\
1964\end{array}$ & $\begin{array}{r}148 \\
234 \\
1995 \\
39.4 \\
1983\end{array}$ & $\begin{array}{r}198 \\
249 \\
1996 \\
121 \\
1967\end{array}$ & $\begin{array}{r}196 \\
269 \\
1993 \\
81.0 \\
1977\end{array}$ & $\begin{array}{r}191 \\
298 \\
1994 \\
49.9 \\
1977\end{array}$ & $\begin{array}{r}172 \\
291 \\
1995 \\
43.8 \\
1964\end{array}$ & $\begin{array}{r}148 \\
277 \\
1995 \\
56.2 \\
1964\end{array}$ & $\begin{array}{r}128 \\
223 \\
1992 \\
12.6\end{array}$ \\
\hline
\end{tabular}

SUMMARY STATISTICS

ANNUAL TOTAL

ANNUAL MEAN

HIGHEST ANNUAL MEABT

LOWEST ANNUAL MEAN

HIGEEST DAILY MEAN

LOWEST DAILY MEAN

ANNUAL SEVEN-DAY MINIMUM

ANNUAL RUNOFF (AC-FT)

10 PERCENT EXCEEDS

50 PERCENT EXCEEDS

90 PERCENT EXCEEDS

- Estimated
FOR 1995 CALENDAR YEAR

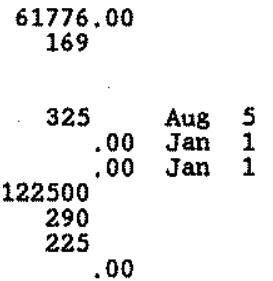

FOR 1996 WATER YEAR

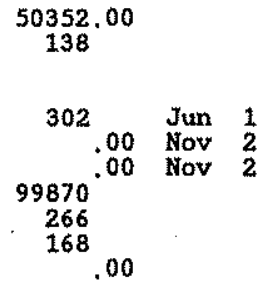

WATER YEARS 1964 - 1996

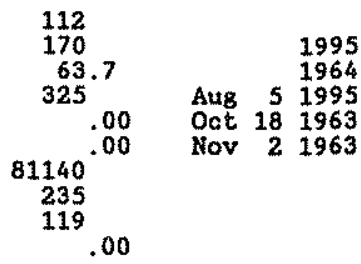


RIO GRANDE BASIN

08354800 RIO GRANDE CONVEYANCE CHANNEL AT SAN ACACIA, NM

LOCATION, -Lat $34^{\circ} 14^{\prime} 54^{\prime \prime}$, Long $106^{\circ} 54^{\circ} 04^{\prime \prime}$, in SW' sec.1, T.1 S., R. 1 W. Socorro County, Bydrologic Unit 13020203 , on right bank $75 \mathrm{ft}$ upstream from railway crossing, 0.5 mi south of San Acacia, and $1.2 \mathrm{mi}$ downstream from San Acacia diversion dam.

PERIOD OF RECORD,--October 1958 to September 1964 included in composite flow of station 08355000 , "Rio Grande at San Acacia," October 1960 to September 1964 (monthly discharge published in WSP 1923 with records for station 08355000 ), October 1964 to January 1994, October 1994 to current year. Dally records 1958-64 are available in flles at district office.

GAGE.-Water-stage recorder. Datum of gago is 4,652,50 ft above National Geodetic Vertical Datun of 1929 (1ovels by U.S. Burawis of Reclamation).

REMARKS. --Records good except estimated dally discharges, which are poor. Conveyance channel, constructed in 1958, is 1 of 3 channels (stations 08354500,08354900 ) carrying flow in valiey cross section. Original design and plan were for conveyance channel to carry all flows up to about 2,000 ft $3 / \mathrm{s}$. For combined monthly flow in acre-ft of this channel, floodway, and Socorro main canal north, seo tabuletion below dally table for station 0835490 . No flow at times.

DISCHARGE, CUBIC FEET PER SECOND, WATER YEAR OCTOBER 1994 TO SEPTEMBER 1995

\begin{tabular}{|c|c|c|c|c|c|c|c|c|c|c|c|c|c|}
\hline DAY & OCT & ROV & DEC & JAN & FEB & MAR & AFR & MAY & Jun & Ли & AvG & & SEP \\
\hline $\begin{array}{l}1 \\
2 \\
3 \\
4 \\
5\end{array}$ & $\begin{array}{r}6.4 \\
6.4 \\
6.4 \\
5.9 \\
68.4\end{array}$ & $\begin{array}{l}011 \\
012 \\
010 \\
08.2 \\
07.6\end{array}$ & $\begin{array}{l}99.3 \\
98.2 \\
98.0 \\
97.1 \\
87.6\end{array}$ & $\begin{array}{l}011 \\
012 \\
011 \\
011 \\
09.8\end{array}$ & $\begin{array}{r}010 \\
011 \\
010 \\
011 \\
012\end{array}$ & $\begin{array}{c}010 \\
09.6 \\
010 \\
010 \\
011\end{array}$ & $\begin{array}{l}14 \\
13 \\
14 \\
18 \\
18\end{array}$ & $\begin{array}{l}23 \\
14 \\
10 \\
9.1 \\
8.9\end{array}$ & $\begin{array}{l}25 \\
21 \\
20 \\
17 \\
14\end{array}$ & $\begin{array}{r}07.1 \\
06.7 \\
07.0 \\
09.9 \\
013\end{array}$ & $\begin{array}{l}07.2 \\
08.1 \\
012 \\
08.9 \\
06.9\end{array}$ & & $\begin{array}{l}24 \\
21 \\
16 \\
13 \\
11\end{array}$ \\
\hline $\begin{array}{r}6 \\
7 \\
8 \\
9 \\
10\end{array}$ & $\begin{array}{r}08.6 \\
09.6 \\
99.0 \\
99.8 \\
08.8\end{array}$ & $\begin{array}{l}08.0 \\
07.6 \\
08.2 \\
08.0 \\
98.7\end{array}$ & $\begin{array}{l}e 8.2 \\
e 8.4 \\
e 9.0 \\
e 10 \\
\theta 9.6\end{array}$ & $\begin{array}{l}011 \\
012 \\
013 \\
011 \\
010\end{array}$ & $\begin{array}{l}e 11 \\
e 10 \\
e 9.7 \\
e 11 \\
\text { e12 }\end{array}$ & $\begin{array}{c}010 \\
09.7 \\
010 \\
\theta 10 \\
11\end{array}$ & $\begin{array}{c}15 \\
12 \\
9.1 \\
6.1 \\
7.0\end{array}$ & $\begin{array}{l}16 \\
16 \\
12 \\
11 \\
9.5\end{array}$ & $\begin{array}{l}13 \\
12 \\
10 \\
9.8 \\
11\end{array}$ & $\begin{array}{l}016 \\
021 \\
020 \\
019 \\
027\end{array}$ & $\begin{array}{r}05.9 \\
07.4 \\
08.1 \\
06.3 \\
05.7\end{array}$ & & $\begin{array}{r}10 \\
9.5 \\
019 \\
017 \\
014\end{array}$ \\
\hline $\begin{array}{l}11 \\
12 \\
13 \\
14 \\
15\end{array}$ & $\begin{array}{l}09.0 \\
99.6 \\
99.0 \\
98.8 \\
99.7\end{array}$ & $\begin{array}{l}e 9.4 \\
e 12 \\
e 14 \\
015 \\
e 14\end{array}$ & $\begin{array}{l}\text { e10 } \\
011 \\
013 \\
\text { e11 } \\
011\end{array}$ & $\begin{array}{l}e 9.4 \\
e 10 \\
=11 \\
e 12 \\
e 11\end{array}$ & $\begin{array}{l}11 \\
011 \\
010 \\
e 11 \\
\text { e13 }\end{array}$ & $\begin{array}{r}9.9 \\
10 \\
9.7 \\
8.2 \\
8.1\end{array}$ & $\begin{array}{l}7.1 \\
7.4 \\
9.4 \\
7.6 \\
7.8\end{array}$ & $\begin{array}{l}8.3 \\
8.4 \\
8.9 \\
7.8 \\
5.5\end{array}$ & $\begin{array}{r}12 \\
8.6 \\
8.5 \\
6.9 \\
010\end{array}$ & $\begin{array}{l}018 \\
014 \\
012 \\
013 \\
011\end{array}$ & $\begin{array}{l}06.8 \\
011 \\
018 \\
022 \\
12\end{array}$ & & $\begin{array}{l}013 \\
014 \\
034 \\
024 \\
019\end{array}$ \\
\hline $\begin{array}{l}16 \\
17 \\
18 \\
19 \\
20\end{array}$ & $\begin{array}{r}09.0 \\
08.4 \\
09.0 \\
011 \\
010\end{array}$ & $\begin{array}{l}\text { e12 } \\
\text { e12 } \\
\text { e11 } \\
\text { e11 } \\
\text { e10 }\end{array}$ & $\begin{array}{l}011 \\
012 \\
014 \\
013 \\
014\end{array}$ & $\begin{array}{l}e 10 \\
e 9.4 \\
e 11 \\
e 8.9 \\
e 10\end{array}$ & $\begin{array}{l}\text { e14 } \\
\text { e13 } \\
e 12 \\
\text { e12 } \\
\text { e11 }\end{array}$ & $\begin{array}{l}8.4 \\
8.5 \\
8.0 \\
7.9 \\
7.7\end{array}$ & $\begin{array}{l}8.9 \\
11 \\
12 \\
11 \\
14\end{array}$ & $\begin{array}{l}8.6 \\
9.4 \\
12 \\
15 \\
15\end{array}$ & $\begin{array}{r}9.3 \\
14 \\
187 \\
067 \\
036\end{array}$ & $\begin{array}{l}010 \\
012 \\
010 \\
015 \\
027\end{array}$ & $\begin{array}{c}18 \\
12 \\
9.9 \\
108 \\
18\end{array}$ & & $\begin{array}{l}012 \\
015 \\
012 \\
\otimes 8.9 \\
08.1\end{array}$ \\
\hline $\begin{array}{l}21 \\
22 \\
23 \\
24 \\
25\end{array}$ & $\begin{array}{l}\otimes 10 \\
\oplus 11 \\
012 \\
813 \\
012\end{array}$ & $\begin{array}{l}09.6 \\
09.3 \\
09.0 \\
08.1 \\
07.3\end{array}$ & $\begin{array}{l}013 \\
011 \\
010 \\
09.3 \\
010\end{array}$ & $\begin{array}{l}07.1 \\
08.0 \\
09.1 \\
08.3 \\
09.0\end{array}$ & $\begin{array}{r}e 10 \\
09.8 \\
09.7 \\
09.3 \\
09.0\end{array}$ & $\begin{array}{l}7.1 \\
6.3 \\
7.0 \\
8.4 \\
9.0\end{array}$ & $\begin{array}{l}16 \\
19 \\
20 \\
28 \\
27\end{array}$ & $\begin{array}{r}9.5 \\
8.3 \\
8.9 \\
11 \\
13\end{array}$ & $\begin{array}{l}021 \\
019 \\
015 \\
012 \\
011\end{array}$ & $\begin{array}{r}059 \\
222 \\
353 \\
0150 \\
094\end{array}$ & $\begin{array}{c}11 \\
9.7 \\
143 \\
30 \\
11\end{array}$ & & $\begin{array}{l}07.3 \\
07.0 \\
06.3 \\
06.2 \\
05.9\end{array}$ \\
\hline $\begin{array}{l}26 \\
27 \\
28 \\
29 \\
30 \\
31\end{array}$ & $\begin{array}{l}09.2 \\
88.3 \\
08.1 \\
09.4 \\
011 \\
011\end{array}$ & $\begin{array}{r}07.0 \\
97.4 \\
09.0 \\
010 \\
99.6 \\
\end{array}$ & $\begin{array}{r}012 \\
011 \\
08.6 \\
07.1 \\
07.6 \\
09.0\end{array}$ & $\begin{array}{l}\text { e10 } \\
012 \\
011 \\
010 \\
012 \\
011\end{array}$ & $\begin{array}{r}e 9.7 \\
010 \\
09.4 \\
--.- \\
---\end{array}$ & $\begin{array}{l}8.5 \\
8.5 \\
8.8 \\
10 \\
13 \\
16\end{array}$ & $\begin{array}{l}28 \\
24 \\
23 \\
25 \\
24 \\
\end{array}$ & $\begin{array}{l}18 \\
18 \\
17 \\
20 \\
22 \\
39\end{array}$ & $\begin{array}{r}09.2 \\
08.5 \\
010 \\
012 \\
08.4 \\
-\end{array}$ & $\begin{array}{l}060 \\
044 \\
018 \\
014 \\
011 \\
010\end{array}$ & $\begin{array}{l}9.6 \\
63 \\
38 \\
29 \\
23 \\
75\end{array}$ & & $\begin{array}{l}05.8 \\
06.1 \\
06.9 \\
06.3 \\
06.1 \\
\ldots\end{array}$ \\
\hline $\begin{array}{l}\text { TOTAL } \\
\text { MEAN } \\
\text { MAK } \\
\text { MIN } \\
\text { AC-FT }\end{array}$ & $\begin{array}{r}287.8 \\
9.28 \\
13 \\
5.9 \\
571\end{array}$ & $\begin{array}{r}296.0 \\
9.87 \\
15 \\
7.0 \\
587\end{array}$ & $\begin{array}{r}314.0 \\
10.1 \\
14 \\
7.1 \\
623\end{array}$ & $\begin{array}{r}322.0 \\
10.4 \\
13 \\
7.1 \\
639\end{array}$ & $\begin{array}{r}302.6 \\
10.8 \\
14 \\
9.0 \\
600\end{array}$ & $\begin{array}{r}290.3 \\
9.36 \\
16 \\
6.3 \\
576\end{array}$ & $\begin{array}{r}456.4 \\
15.2 \\
28 \\
6.1 \\
905\end{array}$ & $\begin{array}{r}413.1 \\
13.3 \\
39 \\
5.5 \\
819\end{array}$ & $\begin{array}{r}638.2 \\
21.3 \\
187 \\
6.9 \\
1270\end{array}$ & $\begin{array}{r}2323.7 \\
42.7 \\
353 \\
6.7 \\
2630\end{array}$ & $\begin{array}{r}754.5 \\
24.3 \\
143 \\
5.7 \\
1500\end{array}$ & & $\begin{array}{r}378.4 \\
12.6 \\
34 \\
5.8 \\
751\end{array}$ \\
\hline
\end{tabular}

CAL YR 1994 TOTAL 6754.50 MEAN 18.5 MAX 729 MIN .01 AC-FT 13400 WTR YR 1995 TOTAL 5777.0 MEAN 15.8 MAX 353 MIA 5.5 AC-FY 12460 
RIO GRANDE BASIN

08354800 RIO GRANDE CONVEYANCE CHANNEL AT SAN ACACIA, NM - - Continued DISCKARGE, CUBIC FEET PER SECOND, WATER YEAR OCTOBER 1995 TO SEPTEMBER 1996

\begin{tabular}{|c|c|c|c|c|c|c|c|c|c|c|c|c|}
\hline DAY & oct & NOV & DEC & JAN & FEB & MAR & APR & MAY & JUN & JUL & AUG & SEP \\
\hline $\begin{array}{l}1 \\
2 \\
3 \\
4 \\
5\end{array}$ & $\begin{array}{l}16 \\
30 \\
31 \\
31 \\
31\end{array}$ & $\begin{array}{l}.00 \\
.00 \\
.00 \\
.00 \\
.00\end{array}$ & $\begin{array}{l}.00 \\
.00 \\
.00 \\
.00 \\
.00\end{array}$ & $\begin{array}{l}.00 \\
.00 \\
.00 \\
.00 \\
.00\end{array}$ & $\begin{array}{l}.00 \\
.00 \\
.00 \\
.00 \\
.00\end{array}$ & $\begin{array}{l}.00 \\
.00 \\
.00 \\
.00 \\
.00\end{array}$ & $\begin{array}{l}.00 \\
.00 \\
.00 \\
.00 \\
.00\end{array}$ & $\begin{array}{l}34 \\
35 \\
34 \\
34 \\
34\end{array}$ & $\begin{array}{ll}27 & \\
27 & \\
25 & \\
11 & \\
& .00\end{array}$ & $\begin{array}{l}.00 \\
.00 \\
.00 \\
.00 \\
.00\end{array}$ & $\begin{array}{l}.00 \\
.00 \\
.00 \\
.00 \\
.00\end{array}$ & $\begin{array}{l}.00 \\
.00 \\
.00 \\
.00 \\
.00\end{array}$ \\
\hline $\begin{array}{r}6 \\
7 \\
8 \\
9 \\
10\end{array}$ & $\begin{array}{l}31 \\
31 \\
31 \\
31 \\
31\end{array}$ & $\begin{array}{l}.00 \\
.00 \\
.00 \\
.00 \\
.00\end{array}$ & $\begin{array}{l}.00 \\
.00 \\
.00 \\
.00 \\
.00\end{array}$ & $\begin{array}{l}.00 \\
.00 \\
.00 \\
.00 \\
.00\end{array}$ & $\begin{array}{l}.00 \\
.00 \\
.00 \\
.00 \\
.00\end{array}$ & $\begin{array}{l}.00 \\
.00 \\
.00 \\
.00 \\
.00\end{array}$ & $\begin{array}{l}.00 \\
.00 \\
.00 \\
.00 \\
.00\end{array}$ & $\begin{array}{l}34 \\
35 \\
35 \\
34 \\
35\end{array}$ & $\begin{array}{l}.00 \\
.00 \\
.00 \\
.00 \\
.00\end{array}$ & $\begin{array}{l}.00 \\
.00 \\
.00 \\
.00 \\
.00\end{array}$ & $\begin{array}{l}.00 \\
.00 \\
.00 \\
.00 \\
.00\end{array}$ & $\begin{array}{l}.00 \\
.00 \\
.00 \\
.00 \\
.00\end{array}$ \\
\hline $\begin{array}{l}11 \\
12 \\
13 \\
14 \\
15\end{array}$ & $\begin{array}{l}31 \\
31 \\
23 \\
13 \\
\end{array}$ & $\begin{array}{l}.00 \\
.00 \\
.00 \\
.00 \\
.00\end{array}$ & $\begin{array}{l}.00 \\
.00 \\
.00 \\
.00 \\
.00\end{array}$ & $\begin{array}{l}.00 \\
.00 \\
.00 \\
.00 \\
.00\end{array}$ & $\begin{array}{l}.00 \\
.00 \\
.00 \\
.00 \\
.00\end{array}$ & $\begin{array}{l}.00 \\
.00 \\
.00 \\
.00 \\
.00\end{array}$ & $\begin{array}{l}.00 \\
.00 \\
.00 \\
.00 \\
.00\end{array}$ & $\begin{array}{l}34 \\
34 \\
31 \\
23 \\
23\end{array}$ & $\begin{array}{l}.00 \\
.00 \\
.00 \\
.00 \\
.00\end{array}$ & $\begin{array}{l}.00 \\
.00 \\
.00 \\
.00 \\
.00\end{array}$ & $\begin{array}{l}.00 \\
.00 \\
.00 \\
.00 \\
.00\end{array}$ & $\begin{array}{l}.00 \\
.00 \\
.00 \\
.00 \\
.00\end{array}$ \\
\hline $\begin{array}{l}16 \\
17 \\
18 \\
19 \\
20\end{array}$ & $\begin{array}{l}.00 \\
.00 \\
.00 \\
.00 \\
.00\end{array}$ & $\begin{array}{l}.00 \\
.00 \\
.00 \\
.00 \\
.00\end{array}$ & $\begin{array}{l}.00 \\
.00 \\
.00 \\
.00 \\
.00\end{array}$ & $\begin{array}{l}.00 \\
.00 \\
.00 \\
.00 \\
.00\end{array}$ & $\begin{array}{l}.00 \\
.00 \\
.00 \\
.00 \\
.00\end{array}$ & $\begin{array}{l}.00 \\
.00 \\
.00 \\
.00 \\
.00\end{array}$ & $\begin{array}{r}.00 \\
.00 \\
.00 \\
5.7 \\
18\end{array}$ & $\begin{array}{l}23 \\
23 \\
23 \\
23 \\
24\end{array}$ & $\begin{array}{l}.00 \\
.00 \\
.00 \\
.00 \\
.00\end{array}$ & $\begin{array}{l}.00 \\
.00 \\
.00 \\
.00 \\
.00\end{array}$ & $\begin{array}{l}.00 \\
.00 \\
.00 \\
.00 \\
.00\end{array}$ & $\begin{array}{l}.00 \\
.00 \\
.00 \\
.00 \\
.00\end{array}$ \\
\hline $\begin{array}{l}21 \\
22 \\
23 \\
24 \\
25\end{array}$ & $\begin{array}{l}.00 \\
.00 \\
.00 \\
.00 \\
.00\end{array}$ & $\begin{array}{l}.00 \\
.00 \\
.00 \\
.00 \\
.00\end{array}$ & $\begin{array}{l}.00 \\
.00 \\
.00 \\
.00 \\
.00\end{array}$ & $\begin{array}{l}.00 \\
.00 \\
.00 \\
.00 \\
.00\end{array}$ & $\begin{array}{l}.00 \\
.00 \\
.00 \\
.00 \\
.00\end{array}$ & $\begin{array}{l}.00 \\
.00 \\
.00 \\
.00 \\
.00\end{array}$ & $\begin{array}{l}19 \\
23 \\
32 \\
35 \\
35\end{array}$ & $\begin{array}{l}24 \\
21 \\
21 \\
15 \\
30\end{array}$ & $\begin{array}{l}.00 \\
.00 \\
.00 \\
.00 \\
.00\end{array}$ & $\begin{array}{l}.00 \\
.00 \\
.00 \\
.00 \\
.00\end{array}$ & $\begin{array}{l}.00 \\
.00 \\
.00 \\
.00 \\
.00\end{array}$ & $\begin{array}{l}.00 \\
.00 \\
.00 \\
.00 \\
.00\end{array}$ \\
\hline $\begin{array}{l}26 \\
27 \\
28 \\
29 \\
30 \\
31\end{array}$ & $\begin{array}{l}.00 \\
.00 \\
.00 \\
.00 \\
.00 \\
.00\end{array}$ & $\begin{array}{l}.00 \\
.00 \\
.00 \\
.00 \\
.00 \\
.-\end{array}$ & $\begin{array}{l}.00 \\
.00 \\
.00 \\
.00 \\
.00 \\
.00\end{array}$ & $\begin{array}{l}.00 \\
.00 \\
.00 \\
.00 \\
.00 \\
.00\end{array}$ & $\begin{array}{l}.00 \\
.00 \\
.00 \\
.00 \\
-. .-\end{array}$ & $\begin{array}{l}.00 \\
.00 \\
.00 \\
.00 \\
.00 \\
.00\end{array}$ & $\begin{array}{l}35 \\
35 \\
34 \\
33 \\
35\end{array}$ & $\begin{array}{l}34 \\
49 \\
75 \\
96 \\
65 \\
27\end{array}$ & $\begin{array}{l}.00 \\
.00 \\
.00 \\
.00 \\
.00 \\
\end{array}$ & $\begin{array}{l}.00 \\
.00 \\
.00 \\
.00 \\
.00 \\
.00\end{array}$ & $\begin{array}{l}.00 \\
.00 \\
.00 \\
.00 \\
.00 \\
.00\end{array}$ & $\begin{array}{l}.00 \\
.00 \\
.00 \\
.00 \\
.00 \\
.\end{array}$ \\
\hline $\begin{array}{l}\text { TOTAL } \\
\text { MEAN } \\
\text { MAX } \\
\text { MIN } \\
\text { AC-FT }\end{array}$ & $\begin{array}{r}392.00 \\
12.6 \\
31 \\
.00 \\
778\end{array}$ & $\begin{array}{l}0.00 \\
.000 \\
.00 \\
.00 \\
.00\end{array}$ & $\begin{array}{l}0.00 \\
.000 \\
.00 \\
.00 \\
.00\end{array}$ & $\begin{array}{l}0.00 \\
.000 \\
.00 \\
.00 \\
.00\end{array}$ & $\begin{array}{l}0.00 \\
.000 \\
.00 \\
.00 \\
.00\end{array}$ & $\begin{array}{r}0.00 \\
.000 \\
.00 \\
.00 \\
.00\end{array}$ & $\begin{array}{r}339.70 \\
11.3 \\
35 \\
.00 \\
674\end{array}$ & $\begin{array}{r}1062 \\
34,3 \\
96 \\
15 \\
2110\end{array}$ & $\begin{array}{r}90.00 \\
3.00 \\
27 \\
.00 \\
179\end{array}$ & $\begin{array}{r}0.00 \\
.000 \\
.00 \\
.00 \\
.00\end{array}$ & $\begin{array}{l}0.00 \\
.000 \\
.00 \\
.00 \\
.00\end{array}$ & $\begin{array}{r}0.00 \\
.000 \\
.00 \\
.00 \\
.00\end{array}$ \\
\hline
\end{tabular}

STATISTICS OF MONTHLY MEAN DATA FOR HATER YEARS 1959 - 1996, BY WATER YEAR (WY)

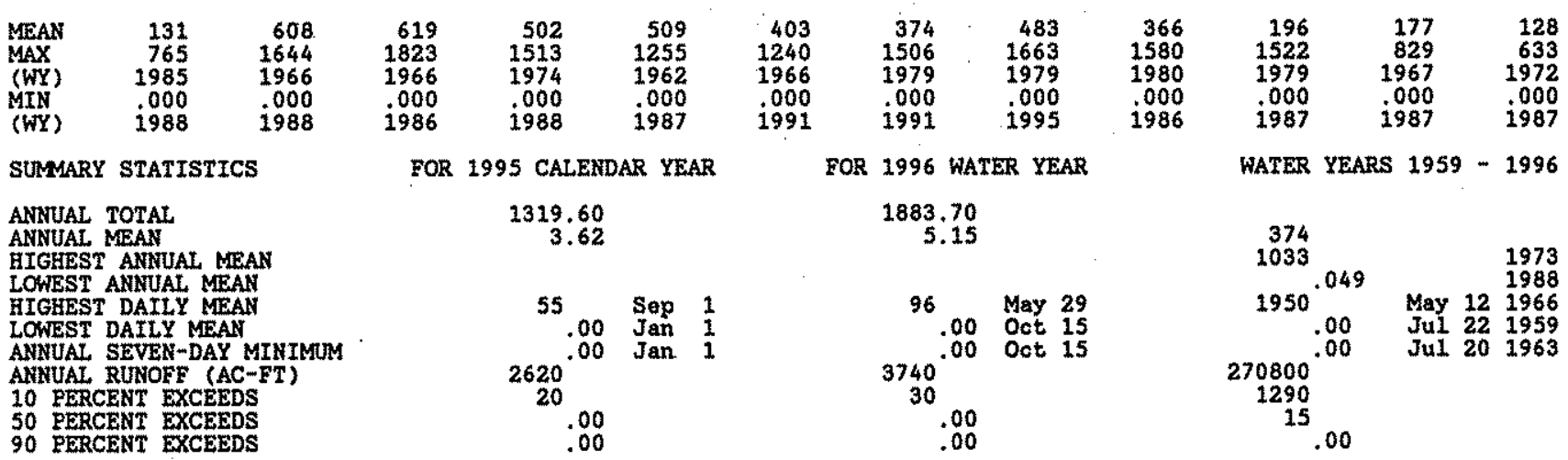




\section{RIO GRANDE BASIN}

08354900 RIO GRANDE FLOODWAY AT SAN ACACIA, MM

LOCATION, -lat $34^{\circ} 15^{\prime} 23^{\prime \prime}$, Long $106^{\circ} 53^{\prime} 18^{\prime \prime}$, Socorro County, Bydrologic Unit 13020203, in Sevilleta Grant, on right bank $0.2 \mathrm{mi}$ downstream from San Acacia diversion dam, $0.3 \mathrm{mi}$ east of San Acacia, 2 mi downstream from Rio Salado, and at mile 1,472.6.

DRAINAGE AREA, --26,770 mi², approximately, including 2,940 mi² in closed basin in San Luis Valley, Co.

WATER-DISCEARGE RECORDS

PERIOD OF RECORD,-Apri1 1936 to September 1958 (prior to construction of conveyance channel), october 1958 to September 1964 (flow in conveyanco channel included), October 1964 to current year. Prior to October 1964 . published as 08355000 "Rlo Grande st Sar Acacta" and rocosda axe not oguivalont.

REVISED RECORDS:-WSP 1242: 1951. WSP 1732: 1958(M). WRD 1969: 1967.

GAGE. - Water-stage recorder. Datum of gage is 4,654,50 ft above National Geodetic Vertical Datum of 1929 . Prior to Mar. 19, 19.53, at several sites 0.1 mi upstrean at different datums Mar, 19, 1953, to Aug. 19, 1965, at sito $0.4 \mathrm{mi}$ downstream at datum $3.60 \mathrm{ft}$ higher. Aug. 19, 1965 , to Aug. is, 1967 , at sane site at datum 1.89 ft higher. Detum on Aug, 21, 1987 , was lowered $2.00 \mathrm{ft}$. Floodway is bypassed by Socorro main canal north and since Oct. 1958 by conveyance channel.

REMARKS. - Water-discharge records fair except for estimated daily discharges, which are poor. Floodway is 1 of 3 channels (stations 08354500 , 08354800) carrying flow in valley cross section. For combtned monthly flow in acreft of floodway, conveyance channel, and Socorro main canal north, soo tabulation bolow. Normal plan is for floodway to carry flow when combined capacities of conveyance channel (about 2,000 fts/s) and Socorro main canal north (about $200 \mathrm{ft} / \mathrm{s}$ ) is exceeded, during periods of silt sluicing, and when river silt load is excessive. Diversions upstream from station for irrigation of about 760,000 acres; this includes Socorro main canal north, which bypasses station and irrigates about 8,000 acres. U.S. Bureau or Reclamation satellite telemeter at station. No flow at times.

AVERAGE DISCHARGE.--22 years (water years 1937-58), 1,192 1ts/s, 863,000 acre-1t/yr, prior to construction of conveyance channel; does not include Socorro main canal north. is years (water years 1959-73), 911 fts/s, 660,000 acre-1t/yr, combined flow of floodway, conveyance channel and Socorro main canal north, prior to closure of Cochiti Dan. 23 years (watex years 1974-96). 1,482 fts/s, 1,074,000 acre-ft/yr, combined flow of floodway, conveyance channel, and Socorro Main Canel North, since closure of Cochiti: Dan.

EXTREMES FOR FERIOD OF RECORD,--Maximun discharge, 27,400 fts/s, Aug. 5, 1936, gage height, 10.75 ft, site and datum then in use; no flow at times

EXTREMES FOR CURRENT YEAR.-Maximun dafly discharge, 5, 500 2t3/s, Juns 28; minimum daily, 17 Its/s, Apr. 22. DISCHARGE, CUBTC FEET PER SECOND, WATER YEAR OCTOBER 1995 TO SEPTEMBER 1996

\begin{tabular}{|c|c|c|c|c|c|c|c|c|c|c|c|c|}
\hline DAY & $\mathrm{OCT}$ & NOV & DEC & JAN & FEB & MAR & AER & MAY & JUN & JuL. & AUUG & SEP \\
\hline $\begin{array}{l}1 \\
2 \\
3 \\
4 \\
5\end{array}$ & $\begin{array}{l}\theta 520 \\
\theta 610 \\
\theta 920 \\
\theta 810 \\
\theta 570\end{array}$ & $\begin{array}{r}699 \\
1270 \\
1300 \\
01100 \\
01050\end{array}$ & $\begin{array}{r}8940 \\
8920 \\
e 938 \\
\mathrm{e} 1200 \\
1010\end{array}$ & $\begin{array}{l}876 \\
746 \\
736 \\
725 \\
715\end{array}$ & $\begin{array}{l}11430 \\
-1440 \\
01500 \\
01490 \\
-1480\end{array}$ & $\begin{array}{r}1020 \\
989 \\
1130 \\
1240 \\
1110\end{array}$ & $\begin{array}{l}0320 \\
e 280 \\
0250 \\
0210 \\
0165\end{array}$ & $\begin{array}{l}57 \\
63 \\
60 \\
60 \\
61\end{array}$ & $\begin{array}{l}81 \\
77 \\
57 \\
70 \\
79\end{array}$ & $\begin{array}{r}028 \\
034 \\
0135 \\
0180 \\
0172\end{array}$ & $\begin{array}{r}82 \\
96 \\
120 \\
138 \\
130\end{array}$ & $\begin{array}{l}784 \\
440 \\
272 \\
174 \\
134\end{array}$ \\
\hline $\begin{array}{r}6 \\
7 \\
8 \\
9 \\
10\end{array}$ & $\begin{array}{l}490 \\
548 \\
527 \\
532 \\
544\end{array}$ & $\begin{array}{l}01060 \\
01070 \\
01080 \\
01070 \\
01060\end{array}$ & $\begin{array}{r}965 \\
1020 \\
997 \\
1030 \\
959\end{array}$ & $\begin{array}{r}705 \\
695 \\
685 \\
745 \\
01220\end{array}$ & $\begin{array}{l}01410 \\
01410 \\
01420 \\
01440 \\
01480\end{array}$ & $\begin{array}{r}1110 \\
01180 \\
01150 \\
885 \\
787\end{array}$ & $\begin{array}{l}e 144 \\
0130 \\
0105 \\
094 \\
.086\end{array}$ & $\begin{array}{l}61 \\
61 \\
60 \\
51 \\
41\end{array}$ & $\begin{array}{l}75 \\
83 \\
87 \\
87 \\
85\end{array}$ & $\begin{array}{l}6140 \\
0122 \\
0100 \\
070 \\
074\end{array}$ & $\begin{array}{l}143 \\
152 \\
174 \\
526 \\
476\end{array}$ & $\begin{array}{r}134 \\
93 \\
78 \\
73 \\
48\end{array}$ \\
\hline $\begin{array}{l}11 \\
12 \\
13 \\
14 \\
15\end{array}$ & $\begin{array}{l}561 \\
507 \\
532 \\
511 \\
531\end{array}$ & $\begin{array}{l}1060 \\
01050 \\
1010 \\
01000 \\
01080\end{array}$ & $\begin{array}{l}955 \\
942 \\
924 \\
864 \\
809\end{array}$ & $\begin{array}{l}01400 \\
01500 \\
01500 \\
01500 \\
\theta 1480\end{array}$ & $\begin{array}{l}01490 \\
\theta 1500 \\
e 1510 \\
\theta 1490 \\
\theta 1500\end{array}$ & $\begin{array}{l}786 \\
820 \\
788 \\
797 \\
806\end{array}$ & $\begin{array}{l}\theta 80 \\
\theta 66 \\
\theta 60 \\
e 48 \\
040\end{array}$ & $\begin{array}{l}41 \\
42 \\
42 \\
42 \\
42\end{array}$ & $\begin{array}{r}85 \\
86 \\
90 \\
91 \\
725\end{array}$ & $\begin{array}{r}0170 \\
0196 \\
0170 \\
\theta 150 \\
070\end{array}$ & $\begin{array}{l}336 \\
278 \\
159 \\
132 \\
111\end{array}$ & $\begin{array}{r}29 \\
384 \\
143 \\
191 \\
918\end{array}$ \\
\hline $\begin{array}{l}16 \\
17 \\
18 \\
19 \\
20\end{array}$ & $\begin{array}{r}501 \\
0395 \\
0415 \\
0440 \\
0450\end{array}$ & $\begin{array}{l}e 1100 \\
\theta 1150 \\
\theta 1110 \\
e 1100 \\
e 1090\end{array}$ & $\begin{array}{l}786 \\
757 \\
719 \\
646 \\
666\end{array}$ & $\begin{array}{l}01480 \\
01450 \\
01430 \\
01480 \\
-1500\end{array}$ & $\begin{array}{l}11550 \\
01540 \\
01530 \\
01500 \\
-1490\end{array}$ & $\begin{array}{l}816 \\
838 \\
839 \\
878 \\
910\end{array}$ & $\begin{array}{l}035 \\
024 \\
022 \\
020 \\
018\end{array}$ & $\begin{array}{l}45 \\
54 \\
67 \\
79 \\
96\end{array}$ & $\begin{array}{l}137 \\
194 \\
246 \\
214 \\
121\end{array}$ & $\begin{array}{l}\text { e190 } \\
e 250 \\
0340 \\
0430 \\
0500\end{array}$ & $\begin{array}{l}86 \\
77 \\
70 \\
64 \\
58\end{array}$ & $\begin{array}{l}894 \\
909 \\
720 \\
818 \\
815\end{array}$ \\
\hline $\begin{array}{l}21 \\
22 \\
23 \\
24 \\
25\end{array}$ & $\begin{array}{r}0470 \\
452 \\
460 \\
468 \\
475\end{array}$ & $\begin{array}{l}01090 \\
01100 \\
61080 \\
01060 \\
-1040\end{array}$ & $\begin{array}{r}681 \\
657 \\
01000 \\
0996 \\
0970\end{array}$ & $\begin{array}{l}01510 \\
-1560 \\
01380 \\
01300 \\
\theta 1300\end{array}$ & $\begin{array}{r}1480 \\
1380 \\
1520 \\
1550 \\
1430\end{array}$ & $\begin{array}{l}6940 \\
0910 \\
0860 \\
e 800 \\
8790\end{array}$ & $\begin{array}{l}18 \\
17 \\
50 \\
29 \\
30\end{array}$ & $\begin{array}{r}115 \\
132 \\
151 \\
6170 \\
52\end{array}$ & $\begin{array}{l}84 \\
83 \\
89 \\
99 \\
73\end{array}$ & $\begin{array}{r}6400 \\
6270 \\
67 \\
75 \\
74\end{array}$ & $\begin{array}{r}53 \\
54 \\
117 \\
138 \\
428\end{array}$ & $\begin{array}{r}712 \\
0464 \\
0414 \\
e 374 \\
e 363\end{array}$ \\
\hline $\begin{array}{l}26 \\
27 \\
28 \\
29 \\
30 \\
31\end{array}$ & $\begin{array}{l}483 \\
491 \\
499 \\
507 \\
515 \\
524\end{array}$ & $\begin{array}{r}01020 \\
01000 \\
0990 \\
9980 \\
0965 \\
-\end{array}$ & $\begin{array}{r}8960 \\
8980 \\
e 1010 \\
01040 \\
81060 \\
1030\end{array}$ & $\begin{array}{l}01320 \\
01340 \\
01300 \\
01330 \\
01380 \\
01420\end{array}$ & $\begin{array}{r}1340 \\
1310 \\
1270 \\
1160 \\
-\ldots \\
\ldots-m\end{array}$ & $\begin{array}{l}e 780 \\
0760 \\
e 700 \\
e 580 \\
0460 \\
e 390\end{array}$ & $\begin{array}{r}33 \\
48 \\
51 \\
50 \\
51 \\
---\end{array}$ & $\begin{array}{r}75 \\
108 \\
113 \\
79 \\
87 \\
84\end{array}$ & $\begin{array}{r}47 \\
136 \\
e 5500 \\
0350 \\
0200 \\
---\end{array}$ & $\begin{array}{r}93 \\
80 \\
76 \\
85 \\
103 \\
79\end{array}$ & $\begin{array}{r}752 \\
920 \\
922 \\
724 \\
637 \\
1040\end{array}$ & $\begin{array}{l}0325 \\
0203 \\
0190 \\
0168 \\
-142\end{array}$ \\
\hline $\begin{array}{l}\text { TOTAL } \\
\text { MEAN } \\
\text { MAX } \\
\text { MIN } \\
\text { AC-FT } \\
\text { (†) }\end{array}$ & $\begin{array}{r}16258 \\
524 \\
920 \\
395 \\
32250 \\
46390\end{array}$ & $\begin{array}{r}31834 \\
1061 \\
1300 \\
699 \\
63140 \\
63190\end{array}$ & $\begin{array}{r}28431 \\
917 \\
1200 \\
646 \\
56390 \\
56390\end{array}$ & $\begin{array}{r}37708 \\
1216 \\
1560 \\
685 \\
74790 \\
74790\end{array}$ & $\begin{array}{r}42040 \\
1450 \\
1550 \\
1160 \\
83390 \\
83390\end{array}$ & $\begin{array}{r}26849 \\
866 \\
1240 \\
390 \\
53250 \\
67570\end{array}$ & $\begin{array}{r}2574 \\
85.8 \\
320 \\
17 \\
5110 \\
20610\end{array}$ & $\begin{array}{r}2291 \\
73.9 \\
170 \\
41 \\
4540 \\
19040\end{array}$ & $\begin{array}{r}9431 \\
314 \\
5500 \\
47 \\
18710 \\
31270\end{array}$ & $\begin{array}{r}4923 \\
159 \\
500 \\
28 \\
9760 \\
19970\end{array}$ & $\begin{array}{r}9193 \\
297 \\
1040 \\
53 \\
18230 \\
29230\end{array}$ & $\begin{array}{r}11406 \\
380 \\
918 \\
29 \\
22620 \\
33950\end{array}$ \\
\hline
\end{tabular}

CAL YR 1995 TOTAL 695050 MEAN 1904 MAX 6000 MIN 80 AC-FT 1379000 ( WTR YR 1996 TOTAL 222938 MEAN 609 MAX 5500 MIN 17 AC-FT 442200 (t) MEAN 752 AC-FT 545800

(†) COMBINED FLOW, IN ACRE-FEET, AND MEAN, IN CUBTC FEET PER SECOND, OF FLOODWAY, CONVEYANCE CHANWEL, AND SOCORRO MAIN CANAL NORTH. 
RIO GRANDE BASIN

08354900 RIO GRANDE FLOODWAY AT SAN ACACIA, MM -- Continued

WATER-QUALITY RECORDS

PERIOD OF RECORD. -Water years 1937-56, 1959 to current year.

PERTOD OF DAILY RECORD --

SPECIFIC CONDUCTANCE: July to December 1937, March 1939 to September 1956 , October 1964 to current year. WATER TEMPERATURE: October 1947 to August 1956, January 1959 to current year.

SUSPENDED-SEDIMENT DISCHARGE: July 1946 to June 1956, January 1959 to current year.

REMARKS.--Sediment total-loads (suspended sediment plus bed material discharge), in tons per day, were determined from the regression equation for the period of record.

EXTREMGS FOR PERIOD OF DAILY RECORD .--

SFECIFIC CONDUCTAKCE: Maxtmum daily, 3,700 microsiemens, July 14, 1940; minimum daliy, 236 microsiemens, June 1,

WATER TEMPERATURE: (1947-56, 1959-62, 1964-96): Maximum daily, $34.5^{\circ} \mathrm{C}$, July 13, 1971; mintmum datly,

$0.0^{\circ} \mathrm{C}$ on many days during winter months of most yaars.

SEDIMENT CONCENTRATION: Maxtmum dally mean, 223,000 ms/L, Aug. 11, 1946; minimum daily mean, no flow on many days of most years.

SEDIMENT LOAD: Maximum dafly, 1,760,000 tons, Aug. 12, 1955; minimum dafly, 0 ton on many days of most years.

EXTREMES FOR CURRENT YEAR. -

SPECIFIC CONDUCTANCE: Maxtmun daily, 1,170 microstemens, July 3; mintmum dally, 360 microstenens, Oct. 23. WATER TEMPERATURE: Maximum daily, $28.0^{\circ} \mathrm{C}$, on July 18 ; minimum dally, $2.00^{\circ} \mathrm{C}, \mathrm{Dec}, 28$.

SEDIMENT CONCENTRATION: Maximum dally mean, 31,800 mg/L, June 28; minimum daily mean, $33 \mathrm{mg} / \mathrm{L}$, May 4 .

SEDIMENT LOAD: Maximum daily, 472,000 tons, June 28; minimum daily, 2.1 tons Apr. 21 .

WATER QUALITY DATA, WATER YEAR OCTOBER 1995 TO SEPTEMBER 1996

\begin{tabular}{|c|c|c|c|c|c|c|c|c|c|c|c|c|}
\hline DATE & TIME & $\begin{array}{c}\text { DIS- } \\
\text { CHARGE, } \\
\text { INST. } \\
\text { CUBIC } \\
\text { FEE } \\
\text { PER } \\
\text { SECOND } \\
(00061)\end{array}$ & $\begin{array}{l}\text { SPE- } \\
\text { CIFIC } \\
\text { CON- } \\
\text { DUCT- } \\
\text { ANCE } \\
\text { (US/CM) } \\
(00095)\end{array}$ & $\begin{array}{c}\text { PH } \\
\text { WATER } \\
\text { WHOLE } \\
\text { FIELD } \\
\text { (STAND- } \\
\text { ARD } \\
\text { UNTTS) } \\
(00400)\end{array}$ & $\begin{array}{c}\text { TEMPER- } \\
\text { ATURE } \\
\text { AIR } \\
\text { (DEG C) } \\
(00020)\end{array}$ & $\begin{array}{l}\text { TEMPER - } \\
\text { ATURE } \\
\text { WATER } \\
\text { (DEG C) } \\
(00010)\end{array}$ & $\begin{array}{l}\text { BARO- } \\
\text { METRIC } \\
\text { BRES- } \\
\text { SURE } \\
\text { (MI } \\
\text { OF } \\
\text { HG) } \\
(00025)\end{array}$ & $\begin{array}{c}\text { OXYGEN, } \\
\text { DIS- } \\
\text { SOLVED } \\
(M \times / L) \\
(00300)\end{array}$ & $\begin{array}{c}\text { OXYGEN, } \\
\text { DIS- } \\
\text { SOLVED } \\
\text { (PER- } \\
\text { CENT } \\
\text { SATUR- } \\
\text { ATION) } \\
\text { (00301) }\end{array}$ & $\begin{array}{l}\text { OXYGEN } \\
\text { DEMAND, } \\
\text { CHEM- } \\
\text { ICAL } \\
\text { (HIGH } \\
\text { LEVEL) } \\
\text { (MG/L) } \\
(00340)\end{array}$ & $\begin{array}{l}\text { COLI- } \\
\text { FORM, } \\
\text { FECAL, } \\
0.7 \\
\text { UM-MF } \\
\text { (COLS. } \\
100 \mathrm{ML}) \\
\text { (31625) }\end{array}$ & $\begin{array}{c}\text { STREP- } \\
\text { TOCOCCI } \\
\text { FECAL, } \\
\text { KF AGAR } \\
\text { (COLS. } \\
\text { PER } \\
100 \mathrm{ML} \text { ) } \\
(31673)\end{array}$ \\
\hline $\begin{array}{l}1995 \\
171996\end{array}$ & 0945 & 391 & 508 & 8.2 & 14.5 & 12.5 & 646 & 8.9 & 99 & 17 & -- & - \\
\hline $11 \ldots$ & 1200 & 790 & 479 & 8.3 & 20.0 & 10.5 & 646 & 9.8 & 104 & 20 & 73 & K350 \\
\hline $04 \ldots$ & 0945 & 75 & 620 & 8.7 & 24.0 & 22.0 & 648 & 7.3 & 99 & 36 & 87 & 640 \\
\hline $23 \ldots$ & .0845 & 67 & 730 & 8.4 & 28.0 & 22.0 & 646 & 7.4 & 101 & -- & -- & -- \\
\hline $13 \ldots$ & 1200 & 155 & 749 & 8.2 & 30.0 & 22.5 & 648 & 7.1 & 97 & 99 & $m$ & -- \\
\hline
\end{tabular}

$\begin{array}{cccccc} & \text { HARD- } & & & \\ & \text { HARD- } & \text { NESS } & & \text { MAGNE- } & \\ & \text { NESS } & \text { NONCARB } & \text { CALCIUM } & \text { SIIIM, } & \text { SODIUM, } \\ & \text { TOTAL } & \text { DISSOLV } & \text { DIS- } & \text { DIS- } & \text { DIS- } \\ \text { DATE } & (M G / L & \text { FLD.AS } & \text { SOLVED } & \text { SOLVED } & \text { SOLVED } \\ & \text { AS } & \text { CACO3 } & (M G / L & (M G / L & (M G / L \\ & \text { CACO3) } & (M G / L) & \text { AS CA) } & \text { AS MG) } & \text { AS NA) } \\ & (00900) & (00904) & (00915) & (00925) & (00930)\end{array}$

$\begin{array}{ccc} & & \text { BICAR- } \\ \text { SODIUM } & \text { POTAS- } & \text { BONATE } \\ \text { AD- } & \text { SIUM, } & \text { WATER } \\ \text { SORP- } & \text { DIS- } & \text { DIS IT } \\ \text { TION } & \text { SOLVED } & \text { FIELD } \\ \text { RATIO } & (\text { MG/L } & \text { MG/L. AS } \\ & \text { AS K K) } & \text { HCO3 } \\ \text { (00931) } & (00935) & (00453)\end{array}$
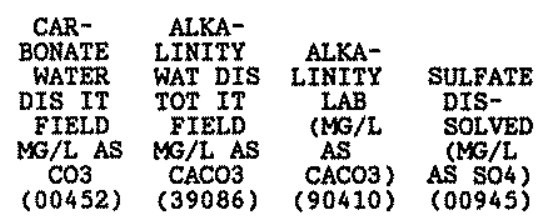

$\begin{array}{cc}\text { OCT } 1995 \\ 17 \ldots 996 \\ \begin{array}{c}\text { MAR } 1996 \\ 11 \ldots\end{array} \\ \begin{array}{c}\text { JUN } \\ 04 \ldots\end{array} \\ \text { JUL } \ldots & 140 \\ 23 \ldots & 200 \\ \text { AUG } \ldots & 210 \\ 13 \ldots . & 220\end{array}$

$\begin{array}{ll}20 & 50 \\ 13 & 44 \\ 36 & 60 \\ 46 & 66 \\ 66 & 67\end{array}$

$\begin{array}{rr}8.7 & 41 \\ 7.7 & 39 \\ 11 & 51 \\ 12 & 63 \\ 12 & 62\end{array}$

$\begin{array}{lll}1 & 4.3 & 172 \\ 1 & 4.2 & 157 \\ 2 & 5.1 & 162 \\ 2 & 5.5 & 195 \\ 2 & 5.8 & 184\end{array}$

$\begin{array}{rrr}0 & 141 & 143 \\ 0 & 129 & 135 \\ 16 & 159 & 166 \\ 5 & 168 & 171 \\ 0 & 150 & 159\end{array}$


RIO GRANDE BASIN

08354900 RIO GRANDE FLOODWAY AT SAN ACACIA, MM -- Continued

WATER-QUALITY RECORDS

WATER QUALITY DATA, WATER YEAR OCTOBER 1995 TO SEPTEMBER 1996

\begin{tabular}{|c|c|c|c|c|c|c|c|c|c|c|c|}
\hline ATE & $\begin{array}{c}\text { CHLO- } \\
\text { RIDE, } \\
\text { DIS- } \\
\text { SOLVED } \\
\text { (BSE/L } \\
\text { AS CL) } \\
(00940)\end{array}$ & $\begin{array}{c}\text { FLUO- } \\
\text { RIDE, } \\
\text { DIS- } \\
\text { SOLVED } \\
(\text { NG/L } \\
\text { AS F) } \\
(00950)\end{array}$ & $\begin{array}{l}\text { SILICA, } \\
\text { DIS- } \\
\text { SOLVED } \\
\text { (MG/L } \\
\text { AS } \\
\text { SIO2) } \\
(00955)\end{array}$ & $\begin{array}{c}\text { SOLIDS, } \\
\text { RESIDUE } \\
\text { AT } 180 \\
\text { DEG.C } \\
\text { DIS- } \\
\text { SOLYED } \\
(M G / L) \\
(70300)\end{array}$ & $\begin{array}{c}\text { SOLIDS, } \\
\text { SUM OF } \\
\text { CONSTI- } \\
\text { TUENTS, } \\
\text { DIS- } \\
\text { SOLVED } \\
\text { (MG/L) } \\
(70301)\end{array}$ & $\begin{array}{c}\text { NITRO- } \\
\text { GEN, } \\
\text { NITRATE } \\
\text { DIS- } \\
\text { SOLVED } \\
\text { (REG/ } \\
\text { AS N) } \\
\text { (00618) }\end{array}$ & $\begin{array}{c}\text { NITRO- } \\
\text { GEN, } \\
\text { NITRITE } \\
\text { DIS- } \\
\text { SOLVED } \\
\text { (ESG/I } \\
\text { AS N) } \\
(00613)\end{array}$ & $\begin{array}{c}\text { NITRO- } \\
\text { GEN, } \\
\text { NO2+NÓ3 } \\
\text { DIS- } \\
\text { SOLVED } \\
\text { (RG/L } \\
\text { AS N) } \\
(00631)\end{array}$ & $\begin{array}{c}\text { NITRO- } \\
\text { GEN, } \\
\text { AMNONIA } \\
\text { DIS- } \\
\text { SOLVED } \\
\text { (RGG/I. } \\
\text { AS N } \\
\text { (00608) }\end{array}$ & $\begin{array}{c}\text { NITRO- } \\
\text { GEN, } \\
\text { ORGANIC } \\
\text { DIS- } \\
\text { SOLVED } \\
\text { (BEG/L } \\
\text { AS \&4) } \\
(00607)\end{array}$ & $\begin{array}{l}\text { NITRO- } \\
\text { GEN,AM- } \\
\text { MONIA + } \\
\text { ORGANIC } \\
\text { TOTAL } \\
\text { (BES/L } \\
\text { AS N) } \\
\text { (00625) }\end{array}$ \\
\hline & & & 22 & -1 & & $-\infty$ & & 12 & & - & .40 \\
\hline & & 0.6 & 24 & -- & 2 & - & $<0.010$ & 0.590 & $<0.015$ & -- & $<0.20$ \\
\hline & 32 & .6 & 23 & $-m$ & 37 & - & $<0.010$ & $<0.0$ & 020 & 0.28 & 1.0 \\
\hline & 3 & 0.70 & 24 & 480 & 454 & 0.180 & 0.020 & 0.200 & 0.050 & 0.15 & 0.90 \\
\hline & 27 & 0.70 & 22 & 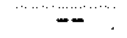 & 451 & 0.770 & 0.010 & 0.780 & 0.030 & 0.17 & 0.40 \\
\hline
\end{tabular}

NITRO-

GEN, AM-

MOAIA + PHOS- PHORUS ORTHO

DATE

ORGANIC

(MG/L (MG/L

(00623)

AS P) AS P) (MG/L

AS P) ASC) (MS/L

(00671) $(00680)$

(00681)

CARBON, ALUM- ANTI-

ALUM- ANTI- INUM, MONY, ARSENIC BARIUM, BERYL-

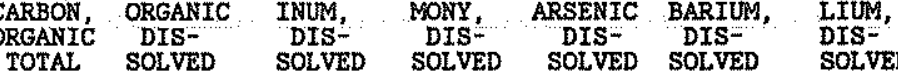

(UIG/L

(UG/L

(UG/L (UG/L (UG/L

(00623) (00665)

OCT 1995

MAR 17996

$11 \ldots$

JUN

$04 \ldots$

$0.20 \therefore$

0.260

0.180

0.180

$4.4 \ldots=$

AS AS) AS BA

AS BE)

JUL. $23 .$.

AUG 23.

$<0.20$

0.260

0.250

0.180

4.6 --

$--$

564

0.30

0.270

0.100

0.090

0.20

0.380

0.060

0.090

0.20

0.190

0.050

0.080

8.

--

$9.0<1.0$

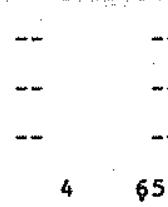

--

CHRO-

BORON, CADMIUM MIUM, COBALT, COPPER,

$\begin{array}{ccccc}\text { DIS- } & \text { DIS- } & \text { DIS- } & \text { DIS- } & \text { DIS- } \\ \text { SOLVED } & \text { SOLVED } & \text { SOLVED } & \text { SOLVED } & \text { SOLVED }\end{array}$

(UG/L (UG/L

$\begin{array}{lllll}(\mathrm{UG} / \mathrm{L} & \text { (UG/L } & \text { (UG/L } & \text { (UG/L } & \text { (UG/L } \\ \mathrm{AS} B) & \mathrm{AS} C D & \mathrm{AS} \mathrm{CR}) & \mathrm{AS} \mathrm{CO}) & \mathrm{AS} \mathrm{CU})\end{array}$

(01020) (01025)

As CO) AS CU)

(01030)

(01035)

2.0

$(01046)$

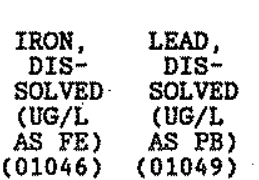

MANGA- MERCURY

DESE, TOTAL DENUM, NICKEL,

DIS RECOV-

SOEVD ERABLE

AS $M$ (UN) AS $/ L$

SOLVED SOLVED

(UG/L (UG/L

OCT 1995 100

$<1.0<1$

$<1.0$

2.0

$4.0<1.0$

5.0

$<0.10$

01060

(01065)

MAR 199

JUN $11 \ldots$

$04 .$.

JUL

$23, \ldots$

$13 .$.

90

116

$--$

$-$

--

4.0 --

$-$

$-\infty$

7.0

2.0

130

$<1.0$

$-$

--

$-$

5.0

-

$<1.0 \quad 4.0$

$7.0<1.0<1.0$

NITRO- NITRO- NITRO- PHOS- ARSENIC CADMIUM CHRO-

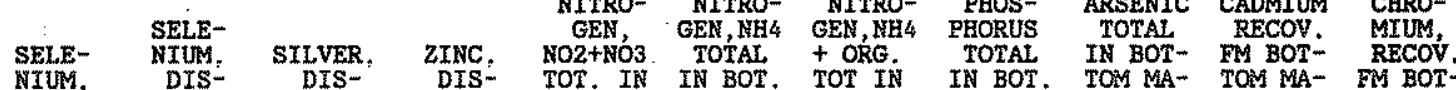
$\begin{array}{llllllll}\text { NIUM, } & \text { DIS- } & \text { DIS- } & \text { DIS- } & \text { TOT. IN IN BOT. TOT IN } & \text { IN BOT. TOM MA- TOA MA- FM BOT- } \\ \text { TOTAL } & \text { SOLVED } & \text { SOLVED } & \text { SOLVED } & \text { BOT MAT } & \text { MAT. BOT MAT } & \text { MAT. } & \text { TERIAL TERIAL TOM MA- }\end{array}$

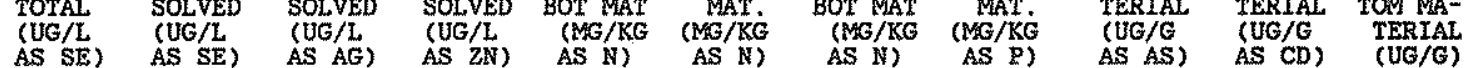

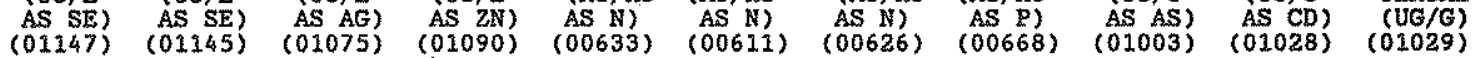

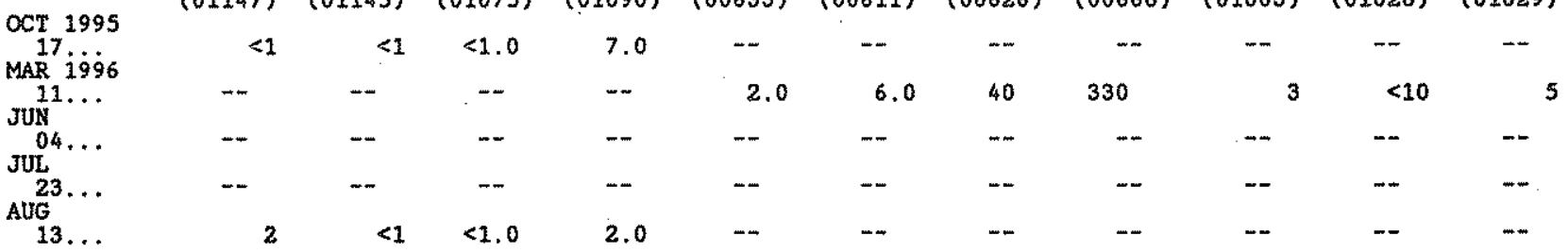


RIO GRANDE BASIN

08354900 RTO GRANDE FLOODWAY AT SAN ACACIA, NM -- Continued

WATER-QUALITY RECORDS

WATER QUALITY DATA, WATER YEAR OCTOBER 1995 TO SEPTEMBER 1996

\begin{tabular}{|c|c|c|c|c|c|c|c|c|c|c|c|}
\hline TE & $\begin{array}{c}\text { COBALT, } \\
\text { RECOV: } \\
\text { FM BOT- } \\
\text { TOM MA- } \\
\text { TERTAL } \\
\text { (UG/G } \\
\text { AS CO) } \\
\text { (01038) }\end{array}$ & $\begin{array}{c}\text { COPPER, } \\
\text { RECOV. } \\
\text { FM BOT- } \\
\text { TQM MA- } \\
\text { TERTAL } \\
\text { (UG/G } \\
\text { AS CUS) } \\
\text { (01043) }\end{array}$ & $\begin{array}{c}\text { IRON, } \\
\text { RECOV. } \\
\text { FM BOT- } \\
\text { TOM MA- } \\
\text { TERIAL } \\
\text { (UG/G } \\
\text { AS FE) } \\
\text { (01170) }\end{array}$ & $\begin{array}{c}\text { LEAD } \\
\text { RECOV. } \\
\text { FM BOT- } \\
\text { TOM MA- } \\
\text { TERIAL } \\
\text { (UG/G } \\
\text { AS PB) } \\
(01052)\end{array}$ & $\begin{array}{c}\text { MANGA- } \\
\text { NESE, } \\
\text { RECOV. } \\
\text { FM BOT- } \\
\text { TOM MA- } \\
\text { TERIAL } \\
(\text { UG/G) } \\
(01053)\end{array}$ & $\begin{array}{c}\text { MERCURY } \\
\text { RECOV. } \\
\text { EM BOT- } \\
\text { TOM MA- } \\
\text { TERIAL } \\
\text { (UG/G } \\
\text { AS BG) } \\
\text { (71921) }\end{array}$ & $\begin{array}{c}\text { ZINC, } \\
\text { RECOV. } \\
\text { FM BOT- } \\
\text { TOM MA- } \\
\text { TERIAL } \\
\text { (UG/G } \\
\text { AS ZN } \\
\text { (01093) }\end{array}$ & $\begin{array}{c}\text { RADIUM } \\
\text { 226, } \\
\text { DIS- } \\
\text { SOLVED, } \\
\text { RADON } \\
\text { METHOD } \\
\text { (FCI/L) } \\
(09511)\end{array}$ & $\begin{array}{c}\text { RA-226 } \\
2 \text { SIGMA } \\
\text { WATER, } \\
\text { DISS, } \\
(\mathrm{BCI} / \mathrm{L}) \\
(76001)\end{array}$ & $\begin{array}{c}\text { URANTUM } \\
\text { NATURAL } \\
\text { DIS- } \\
\text { SOLVED } \\
\text { (UG/L } \\
\text { AS U) } \\
(22703)\end{array}$ & $\begin{array}{c}\text { URANIUM } \\
\text { NATURAL } \\
2 \text { STGMA } \\
\text { WATER, } \\
\text { DISS, } \\
\text { (UG/L) } \\
(75990)\end{array}$ \\
\hline
\end{tabular}

\begin{tabular}{|c|c|c|c|c|c|c|c|c|c|c|c|}
\hline $\begin{array}{l}\text { OCT } 1995 \\
177 \text { g }\end{array}$ & $=$ & -- & -- & -- & -- & $\cdots$ & $-\cdots$ & 0.08 & 0.020 & 2.1 & 0.1 \\
\hline $11 \ldots$ & $<50$ & $<10$ & 5700 & $<10$ & 160 & $<0.10$ & 20 & - & -- & -- & -- \\
\hline $04 \ldots$ & -- & -- & -- & -- & -- & $m$ & -- & -- & -- & $\cdots$ & -- \\
\hline $23 \ldots$ & - & - & - & -- & - & -- & -- & -- & $-m$ & $\cdots$ & -- \\
\hline . & - & -- & -- & -- & - & -- & -- & -- & $-\cdots$ & 4.0 & -- \\
\hline
\end{tabular}

\begin{tabular}{|c|c|c|c|c|c|c|c|c|c|c|c|}
\hline REC & REC & $\begin{array}{l}\text { PROP- } \\
\text { CHLOR, } \\
\text { WATER, } \\
\text { DISS, } \\
\text { REC } \\
\text { (UG/L) } \\
(04024)\end{array}$ & $\begin{array}{c}\text { BUTYL- } \\
\text { ATE, } \\
\text { WATER, } \\
\text { DISS, } \\
\text { REC } \\
\text { (UG/L) } \\
(04028)\end{array}$ & $\begin{array}{l}\text { SI- } \\
\text { MAZINE, } \\
\text { WATER, } \\
\text { DISS, } \\
\text { SOLVED } \\
\text { (UG/L) } \\
\text { (0403S) }\end{array}$ & $\begin{array}{l}\text { PRO- } \\
\text { METON, } \\
\text { WATER, } \\
\text { DISS, } \\
\text { DISSOLV } \\
\text { (UG/L) } \\
(04037)\end{array}$ & $\begin{array}{l}\text { DEETHYL } \\
\text { ATRA- } \\
\text { ZINE, } \\
\text { WATER, } \\
\text { DISS, } \\
\text { SOLVED } \\
\text { (UG/L) } \\
(04040)\end{array}$ & $\begin{array}{l}\text { CYANA- } \\
\text { ZINE, } \\
\text { WATER, } \\
\text { DISS, } \\
\text { (UG/L) } \\
(04041)\end{array}$ & $\begin{array}{l}\text { FONOFOS } \\
\text { WATER } \\
\text { DISS } \\
\text { (UG/L) } \\
(04095)\end{array}$ & $\begin{array}{c}\text { ALPHA } \\
\text { BHC } \\
\text { DIS- } \\
\text { (UG/L) } \\
\text { (34253) }\end{array}$ & $\begin{array}{c}P_{{ }^{\prime} P^{\prime}} \\
(\mathrm{DEE} / \mathrm{L}) \\
(34653)\end{array}$ & $\begin{array}{c}\text { CHLOR- } \\
\text { PYRIFOS } \\
\text { DIS- } \\
(U G / L) \\
(38933)\end{array}$ \\
\hline $\begin{array}{l}\text { OCT } 1995 \\
17 \\
M A R\end{array}$ & 0945 & $m$ & -- & - & -- & $-m$ & - & -- & -- & -- & - \\
\hline Jun $11 \cdots$ & 1200 & -- & -- & -- & $-\infty$ & -- & -- & -- & - & $-\cdots$ & -- \\
\hline JuL $04 \ldots$ & 0945 & -- & -- & - & - & -- & - & -- & - & -- & -- \\
\hline$\underset{A U G}{23} \ldots$ & 0845 & $<0.007$ & $<0.002$ & 0.007 & E0.008 & $<0.002$ & $<0.004$ & $<0.003$ & $<0.002$ & $<0.006$ & $<0.004$ \\
\hline $13 .$. & 1200 & $-\cdots$ & - & -- & -- & - & - & -- & -- & -- & $-\cdots$ \\
\hline
\end{tabular}

\begin{tabular}{|c|c|c|c|c|c|c|c|c|c|c|}
\hline $\begin{array}{l}\text { LINDANE } \\
\text { DIS- } \\
\text { SOLVED } \\
\text { (UG/L) } \\
\text { (39341) }\end{array}$ & $\begin{array}{c}\text { DI- } \\
\text { ELDRIN } \\
\text { DIS- } \\
\text { SOLVED } \\
\text { (UG/L) } \\
\text { (39381) }\end{array}$ & $\begin{array}{l}\text { METO- } \\
\text { LACHLOR } \\
\text { WATER } \\
\text { DISSOLV } \\
\text { (UG/L) } \\
\text { (39415) }\end{array}$ & $\begin{array}{l}\text { MALA- } \\
\text { THION, } \\
\text { DIS- } \\
\text { SOLVED } \\
\text { (UG/L) } \\
\text { (39532) }\end{array}$ & $\begin{array}{l}\text { PARA- } \\
\text { THION, } \\
\text { DIS- } \\
\text { SOLVED } \\
\text { (UG/L) } \\
(39542)\end{array}$ & $\begin{array}{c}\text { DI- } \\
\text { AZINON, } \\
\text { DIS- } \\
\text { SOLVED } \\
\text { (UG/L) } \\
(39572)\end{array}$ & $\begin{array}{l}\text { ATRA- } \\
\text { ZINE, } \\
\text { WATER, } \\
\text { DISS, } \\
\text { REC } \\
\text { (UG/L) } \\
(39632)\end{array}$ & $\begin{array}{l}2,4-D \\
\text { TOTAL } \\
(U G / L) \\
(39730)\end{array}$ & $\begin{array}{c}2,4,5-T \\
\text { TOTAL. } \\
\text { (UG/L) } \\
(39740)\end{array}$ & $\begin{array}{c}\text { SILVEX, } \\
\text { TOTAL } \\
\text { (UG/L) } \\
(39760)\end{array}$ & $\begin{array}{l}\text { ALA- } \\
\text { CHLOR, } \\
\text { WATER, } \\
\text { DISS, } \\
\text { REC, } \\
\text { (UG/L) } \\
\text { (46342) }\end{array}$ \\
\hline
\end{tabular}

\begin{tabular}{|c|c|c|c|c|c|c|c|c|c|c|c|}
\hline $\begin{array}{l}\text { OCT } 1995 \\
1779 \\
\text { MAR } 1996\end{array}$ & -- & -- & $\rightarrow$ & - & $-m$ & -- & -- & $\rightarrow$ & -- & $-\infty$ & -- \\
\hline${ }_{\mathrm{JUN}}^{11} \cdots$ & -- & -+ & - & -- & $-m$ & -+ & -- & -- & $-m$ & -- & $-m$ \\
\hline $\begin{array}{c}04 \ldots \\
\text { JuL }\end{array}$ & -- & -- & -- & $-\infty$ & -- & -- & $\cdots$ & 0.060 & $<0.010$ & $<0.010$ & -- \\
\hline$\underset{A U G}{23} \ldots$ & $<0.004$ & $<0.001$ & $<0.002$ & $<0.005$ & $<0.004$ & $\mathrm{E} 0.004$ & 0.004 & $-m$ & $-\infty$ & $-\rightarrow$ & $<0.002$ \\
\hline $13 \ldots$ & $m$ & -- & $-m$ & $-m$ & $m$ & -- & $-\infty$ & $-m$ & -- & $m$ & $-\infty$ \\
\hline
\end{tabular}

\begin{tabular}{|c|c|c|c|c|c|c|c|c|c|c|}
\hline & ACETO- & & METRI- & 2, 6-DI- & $\begin{array}{l}\text { TRI- } \\
\text { FLUR- }\end{array}$ & ETHAL- & PHORATE & $\begin{array}{l}\text { TER- } \\
\text { BACIL }\end{array}$ & $\begin{array}{l}\text { LIN- } \\
\text { URON }\end{array}$ & $\begin{array}{c}\text { METHYL } \\
\text { PARA- }\end{array}$ \\
\hline DATE & $\begin{array}{l}\text { CHLOR, } \\
\text { WATER } \\
\text { FLTRD } \\
\text { REC } \\
\text { (UG/L) } \\
(49260)\end{array}$ & $\begin{array}{l}2, \quad 4-D P \\
\text { TOTAL } \\
\text { (UG/L) } \\
\text { (82183) }\end{array}$ & $\begin{array}{l}\text { BUZIN } \\
\text { SENCOR } \\
\text { WATER } \\
\text { DISSOLV } \\
(U G / L) \\
(82630)\end{array}$ & $\begin{array}{c}\text { ANTLINE } \\
\text { WAT FLT } \\
0.7 \text { U } \\
\text { GF, REC } \\
(U G / L) \\
(82660)\end{array}$ & $\begin{array}{c}\text { ALIN } \\
\text { WAT ELT } \\
0.7 \text { U } \\
\text { GF. REC } \\
\text { (UG/L) } \\
\text { (82661). }\end{array}$ & $\begin{array}{c}\text { ALIN } \\
\text { WAT FLT } \\
0.7 \text { U } \\
\text { GF, REC } \\
(U G / L) \\
(82663)\end{array}$ & $\begin{array}{c}\text { WATER } \\
\text { FLTRD } \\
0.7 \mathrm{U} \\
\text { GF, REC } \\
(\mathrm{UG} / \mathrm{L}) \\
(82664)\end{array}$ & $\begin{array}{c}\text { WAFER } \\
\text { FLTRD } \\
0.7 \mathrm{U} \\
\text { GF, REC } \\
(\mathrm{UG} / \mathrm{L}) \\
(82665)\end{array}$ & $\begin{array}{c}\text { WATER } \\
\text { FLTRD } \\
0.7 \mathrm{U} \\
\text { GF; REC } \\
(\mathrm{UG} / \mathrm{L}) \\
(\mathrm{B2} 666)\end{array}$ & $\begin{array}{c}\text { THION } \\
\text { WAT FLT } \\
0.7 \mathrm{U} \\
\text { GE, REC } \\
(\mathrm{UG} / \mathrm{L}) \\
(82667)\end{array}$ \\
\hline
\end{tabular}

\begin{tabular}{|c|c|c|c|c|c|c|c|c|c|c|}
\hline $\begin{array}{l}\text { OCT } 1995 \\
177 \\
\operatorname{MAR}_{1996}\end{array}$ & -- & -- & $m$. & -- & $-\infty$ & - & -- & $m$ & $-m$ & $\cdots$ \\
\hline$\frac{11}{\text { JuN }}$ & -- & -- & $-m$ & -- & - & -- & $-m$ & -- & - & -- \\
\hline $04 \ldots$ & - & $<0.010$ & $m$ & $-m$ & - & - & $m$ & -- & $-\cdots$ & -- \\
\hline $\begin{array}{c}23 \\
\text { AvG }\end{array}$ & $<0.002$ & -- & $<0.004$ & $<0.003$ & $<0.002$ & $<0.004$ & $<0.002$ & $<0.007$ & $<0.002$ & $<0.006$ \\
\hline $13 \ldots$ & $\cdots$ & - w & -- & $\rightarrow$ & - & -- & $-m$ & $\rightarrow$ & - - & -- \\
\hline
\end{tabular}


RIO GRANDE BASIN

08354900 RIO GRANDE FLOODWAY AT SAN ACACIA, MM -- Continued

WATER-QUALITY RECORDS

WATER QUALITY DATA, WATER YEAR OCTOBER 1995 TO SEPTEMBER 1996

\begin{tabular}{|c|c|c|c|c|c|c|c|c|c|c|}
\hline DATE & $\begin{array}{c}\text { EPTC } \\
\text { WATER } \\
\text { FLTRD } \\
0.7 \mathrm{U} \\
\text { GE, REC } \\
\text { (UG/L) } \\
(82668)\end{array}$ & $\begin{array}{c}\text { PEB- } \\
\text { ULATE } \\
\text { WATER } \\
\text { FILTRD } \\
0.7 \mathrm{U} \\
\text { GF, REC } \\
\text { (UGGL) } \\
(82669)\end{array}$ & $\begin{array}{c}\text { TEBU- } \\
\text { THIURON } \\
\text { WATER } \\
\text { RLTRD } \\
0.70 \\
\text { GE; REC } \\
\text { (UG/L) } \\
(82670)\end{array}$ & $\begin{array}{l}\text { MOL- } \\
\text { INATE } \\
\text { WATER } \\
\text { GLTRD } \\
0.7 \text { V } \\
\text { GF REC } \\
\text { (UGG/) } \\
(82671)\end{array}$ & $\begin{array}{l}\text { ETHO- } \\
\text { PROP } \\
\text { WATER } \\
\text { FLTRD } \\
0.7 \text { U } \\
\text { GF, REC } \\
\text { (UG/L) } \\
(82672)\end{array}$ & $\begin{array}{c}\text { BEN- } \\
\text { FLUR } \\
\text { ALIN } \\
\text { WAT FLD } \\
0.7 \mathrm{Y} \\
\mathrm{GE}, \mathrm{REC} \\
(\mathrm{UG} / \mathrm{L}) \\
(82673)\end{array}$ & $\begin{array}{l}\text { CARBO- } \\
\text { FURAN } \\
\text { WATER } \\
\text { FLTRD } \\
0.7 \text { V } \\
\text { GE REC } \\
\text { (UGG/L) } \\
(82674)\end{array}$ & $\begin{array}{c}\text { TER- } \\
\text { BUFOS } \\
\text { WUAER } \\
\text { FLTRD } \\
0.7 \mathrm{U} \\
\text { GF REC } \\
\text { (U⿺𠃊) RE) } \\
(82675)\end{array}$ & $\begin{array}{c}\text { PRON- } \\
\text { PRIDE } \\
\text { AMATER } \\
\text { FLTRD } \\
0.7 \text { V } \\
\text { GE REC } \\
\text { GEG/L) } \\
(82676)\end{array}$ & $\begin{array}{l}\text { DISUL- } \\
\text { FOTON } \\
\text { WATER } \\
\text { FLTRD } \\
0.7 \text { U } \\
\text { GF, REC } \\
\text { (UG LL) } \\
\text { (82677) }\end{array}$ \\
\hline $\begin{array}{l}1995 \\
\ldots .\end{array}$ & -- & -- & - & - & -- & $-m$ & - & - & 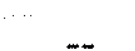 & -- \\
\hline 8996 & -. & -. & - & -. & -. & -. & -. & .. & -- & - \\
\hline$\cdots$ & - & $=-$ & -- & -- & -- & -- & -- & -- & - & - \\
\hline$\cdots$ & E0.003 & $<0,004$ & $E 0.004$ & $<0.004$ & $<0.003$ & $<0.002$ & $<0.003$ & $<0.013$ & $<0.003$ & $<0.017$ \\
\hline . & -- & -- & - & - & -- & $\cdots$ & -- & - & -- & -- \\
\hline DATE & $\begin{array}{c}\text { TRIAL- } \\
\text { LATE } \\
\text { WATER } \\
\text { FLTRD } \\
0.7 \mathrm{y} \\
\text { GE, REC } \\
\text { (UG/L) } \\
(82678)\end{array}$ & $\begin{array}{l}\text { PRO- } \\
\text { PANIL } \\
\text { WATER } \\
\text { FLTRD } \\
0.7 \mathrm{U} \\
\text { GE, REC } \\
\text { (UGGL) } \\
(82679)\end{array}$ & $\begin{array}{c}\text { CAR- } \\
\text { BARYL } \\
\text { WAER } \\
\text { FLTRD } \\
0.7 \mathrm{U} \\
\text { GF REC } \\
\text { (UG,L) } \\
(82680)\end{array}$ & $\begin{array}{c}\text { THIO- } \\
\text { BENCARB } \\
\text { WATR } \\
\text { FLTRD } \\
0.7 \mathrm{U} \\
\text { GF REC } \\
\text { (UGG/L) } \\
(82681)\end{array}$ & $\begin{array}{l}\text { DCPA } \\
\text { WATER } \\
\text { FLTRD } \\
0,7 \mathrm{U} \\
\text { GF, REC } \\
\text { (UG/L) } \\
(82682)\end{array}$ & $\begin{array}{l}\text { PENDI- } \\
\text { METH- } \\
\text { ALIN } \\
\text { WAT FLT } \\
0.7 \mathrm{U} \\
\text { GE. REC } \\
\text { (UG/L) } \\
(82683)\end{array}$ & $\begin{array}{l}\text { NAPROP- } \\
\text { AMIDE } \\
\text { WATER } \\
\text { FLTRD } \\
0.7 \text { U } \\
\text { GF, REC } \\
\text { (UG } / L) \\
(82684)\end{array}$ & $\begin{array}{c}\text { PRO- } \\
\text { PARGITE } \\
\text { WATER } \\
\text { FLTRD } \\
0.7 \text { U } \\
\text { GE. REC } \\
\text { (U⿺尢) } \\
(82685)\end{array}$ & $\begin{array}{c}\text { METHYL } \\
\text { AZTNN- } \\
\text { PHOS } \\
\text { WAT FLT } \\
0.7 \mathrm{U} \\
\text { GE REC } \\
(\mathrm{UGGL}) \\
(82686)\end{array}$ & $\begin{array}{l}\text { PER- } \\
\text { METHRIN } \\
\text { CIS } \\
\text { WAT FLT } \\
0.7 \mathrm{U} \\
\text { GF REC } \\
\text { (UG/L) } \\
(82687)\end{array}$ \\
\hline $\begin{array}{l}1995 \\
17\end{array}$ & - & -- & $\cdots$ & $\cdots$ & -- & -- & -- & -- & -- & - \\
\hline $\begin{array}{l}11996 \\
11 \ldots\end{array}$ & -- & -- & -- & $\begin{array}{ll}-- & -1\end{array}$ & -- & -- & -- & -- & -- & -- \\
\hline $04 \ldots$ & -- & -- & -- & -- & -- & -- & -- & -- & -- & -- \\
\hline & $<0.001$ & $<0.004$ & $<0.003$ & $<0.002$ & $<0.002$ & $<0.004$ & $<0.003$ & $<0.013$ & $<0.002$ & $<0.005$ \\
\hline $13 \ldots$ & -- & -- & -- & $\therefore$ & -- & -- & -- & -- & -- & $\cdots$ \\
\hline DATE & TIME & $\begin{array}{c}\text { DIS- } \\
\text { CHARGE, } \\
\text { INST. } \\
\text { CUBIC } \\
\text { FEET } \\
\text { PER } \\
\text { SECOND } \\
\text { SO0061) }\end{array}$ & $\begin{array}{c}\text { STREAM } \\
\text { WIDTH } \\
\text { (FT) } \\
(00004)\end{array}$ & $\begin{array}{c}\text { STREAM } \\
\text { DEFTH, } \\
\text { NEAN } \\
\text { (FT) } \\
(00064)\end{array}$ & $\begin{array}{l}\text { STREAM } \\
\text { VELOC- } \\
\text { ITY } \\
\text { MEAN } \\
(E / S) \\
(00055)\end{array}$ & $\begin{array}{l}\text { SPE- } \\
\text { CIFIC } \\
\text { CON- } \\
\text { DUCT- } \\
\text { ANCE } \\
\text { (US/CM) } \\
\text { (O0095) }\end{array}$ & $\begin{array}{l}\text { TEMPER- } \\
\text { ATURE } \\
\text { WATER } \\
\text { (DEG C) } \\
(00010)\end{array}$ & $\begin{array}{l}\text { SEDI- } \\
\text { MENT, } \\
\text { SUS- } \\
\text { PENDED } \\
\text { (MG/L) } \\
(80154)\end{array}$ & $\begin{array}{c}\text { SEDI- } \\
\text { MEENT, } \\
\text { DAIS- } \\
\text { CHAREE, } \\
\text { SUS- } \\
\text { PENDED } \\
(T / D A Y) \\
(80155) \\
(8015)\end{array}$ & $\begin{array}{l}\text { SEDI- } \\
\text { MENT: } \\
\text { DISCE, } \\
\text { SUSP . } \\
\text { BED MA- } \\
\text { TERTAL } \\
\text { (T/DAY) } \\
(80156)\end{array}$ \\
\hline$\frac{1}{19} 1995$ & 0945 & 391 & 82.0 & 1.5 & 3.16 & 508 & 12.5 & 121 & 128 & 206 \\
\hline & 1130 & 1220 & 155 & 2.1 & 3.81 & -- & 7.5 & 1810 & 5960 & 20900 \\
\hline 促 & 0950 & 1200 & 141 & 2.6 & $3.25^{\circ}$ & -- & 3.0 & 506 & 1640 & 2370 \\
\hline${ }_{\mathbb{R}}^{22} \ldots$ & 1030 & 1380 & 153 & 2.3 & 3.97 & -- & 10.0 & 836 & 3110 & 4370 \\
\hline$\frac{11}{20} \ldots$ & $\begin{array}{l}1200 \\
0920\end{array}$ & $\begin{array}{l}790 \\
918\end{array}$ & 147 & 2.8 & $\begin{array}{l}-2.24 \\
2.4\end{array}$ & $\begin{array}{l}479 \\
\cdots\end{array}$ & $\begin{array}{r}10.5 \\
9.5\end{array}$ & $\begin{array}{r}159 \\
75\end{array}$ & $\begin{array}{l}339 \\
186\end{array}$ & $\begin{array}{l}523 \\
294\end{array}$ \\
\hline $17 \ldots$ & 0930 & 27 & 46.0 & 1.2 & 0.46 & $\cdots$ & 13.0 & 86 & 6.3 & 10 \\
\hline $04 \ldots$ & $\begin{array}{l}0945 \\
1310\end{array}$ & $\begin{array}{r}75 \\
224\end{array}$ & 74.0 & $\overline{1.3}$ & 2.23 & 620 & $\begin{array}{l}22.0 \\
23.5\end{array}$ & $\begin{array}{r}45 \\
213\end{array}$ & $129^{9.1}$ & $\begin{array}{r}16 \\
207\end{array}$ \\
\hline $\begin{array}{l}18 \\
23\end{array}$ & $\begin{array}{l}0930 \\
0845\end{array}$ & $\begin{array}{r}336 \\
67\end{array}$ & 95.0 & 0.89 & 3.90 & 730 & $\begin{array}{l}22.0 \\
22.0\end{array}$ & $\begin{array}{r}6960 \\
707\end{array}$ & $\begin{array}{r}6310 \\
128\end{array}$ & $\begin{array}{r}8620 \\
206\end{array}$ \\
\hline $\begin{array}{l}13 \\
15\end{array}$ & $\begin{array}{l}1200 \\
0950\end{array}$ & $\begin{array}{l}155 \\
119\end{array}$ & 66.0 & $\overline{1.2}$ & $\ddot{2.45}$ & 749 & 22.5 & $\begin{array}{r}4550 \\
830\end{array}$ & $\begin{array}{r}1900 \\
267\end{array}$ & $\begin{array}{r}2730 \\
416\end{array}$ \\
\hline & 1005 & 192 & 76.0 & 1.2 & 2.13 & -- & 20.5 & 2550 & 1320 & 1920 \\
\hline
\end{tabular}


RIO GRANDE BASIN

08354900 RIO GRANDE FLOODWAX AT SAN ACACIA, NM - - Cont Inued

WATER-QUALITY RECORDS

WATER QUALTTY DATA, WATER YEAR OCTOBER 1995 TO SEPTEMBER 1996

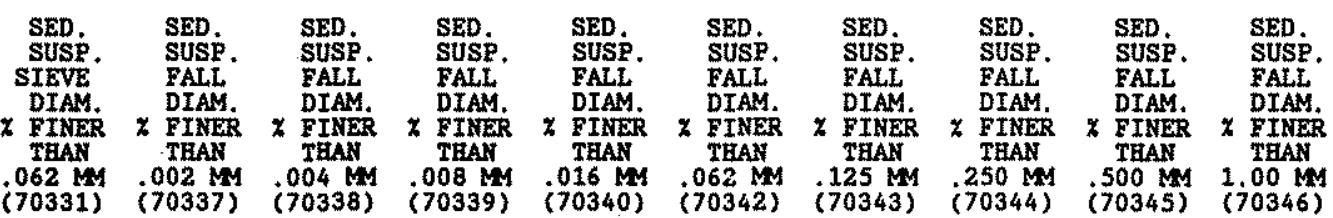

OCT 1995
$17 \ldots$
DEC
$04 \ldots$
JAN 1996
$10 \ldots$
FEB
$22 \ldots$
MAR $\ldots$
$11 \ldots$
$20 \ldots$
AER $\ldots$
$17 \ldots$
JUI
$04 \ldots$
$19 \ldots$
JUL $\ldots$
$18 \ldots$
$23 \ldots$
AUG $\ldots$
$13 \ldots$
$15 \ldots$
SEP
$04 \ldots$

$$
78
$$$$
87
$$$$
90 \quad 92
$$

\begin{tabular}{|c|c|c|c|c|}
\hline 84 & 85 & 96 & 100 & $\cdots$ \\
\hline 14 & 20 & 51 & 88 & 100 \\
\hline 39 & 51 & 93 & 100 & -- \\
\hline 23 & 34 & 84 & 100 & -- \\
\hline-81 & $-{ }^{-} 82$ & 98 & $-\overline{100}$ & $=$ \\
\hline 55 & 58 & 84 & 100 & -- \\
\hline-86 & $--_{87}$ & ${ }_{97}$ & 100 & $=$ \\
\hline$--^{86}$ & 95 & 99 & 100 & $=$ \\
\hline$-{ }_{88}$ & ${ }_{91}^{-}$ & -700 & $=$ & $=$ \\
\hline 96 & 97 & 99 & 100 & -- \\
\hline
\end{tabular}

\section{DATE}

\begin{tabular}{|c|c|c|c|c|c|c|c|c|c|}
\hline $\begin{array}{l}\text { SI } \\
\text { DI }\end{array}$ & $\begin{array}{l}\text { SIEVE } \\
\text { DIAM. } \\
\text { T FINER } \\
\text { THAN }\end{array}$ & $\begin{array}{c}\text { SIEVE } \\
\text { DIAM, } \\
\times \text { FINGR } \\
\text { THAN } \\
\text { TSO MM }\end{array}$ & $\begin{array}{l}\text { SIEVE } \\
\text { DIAM. } \\
\times \text { FINER } \\
\text { THAN } \\
500\end{array}$ & $\begin{array}{l}\text { STEVE } \\
\text { DIAM. } \\
\text { X FINER }\end{array}$ & $\begin{array}{l}\text { SIEVE } \\
\text { DIAM. } \\
\text { \% FINER }\end{array}$ & $\begin{array}{r}\text { SIE } \\
\text { DIA } \\
\times \quad \mathrm{FI} \\
\mathrm{TH}\end{array}$ & $\begin{array}{r}\text { SI } \\
\text { DI } \\
\times \quad F \\
T\end{array}$ & $\mathbf{R}$ & \\
\hline
\end{tabular}

\begin{tabular}{|c|c|c|c|c|c|c|c|c|c|c|}
\hline $\begin{array}{c}\text { OCT } 1995 \\
17 \ldots \\
\operatorname{Drc}^{2}\end{array}$ & 51 & 70 & 84 & 88 & 88 & 89 & 90 & 93 & 98 & 100 \\
\hline${ }^{04} \mathfrak{4} 996$ & 0 & 1 & 35 & 96 & 100 & -- & -- & -- & - & -- \\
\hline${ }_{\mathrm{FEB}}^{10} \ldots$ & 3 & 11 & 53 & 90 & 92 & 92 & 92 & 93 & 93 & 100 \\
\hline${ }_{\operatorname{MAR}}^{22} \ldots$ & 1 & 8 & 39 & 90 & 99 & 99 & 100 & -- & - & -- \\
\hline $\begin{array}{r}\frac{11}{20} \ldots \\
A P R\end{array}$ & $-{ }_{1}$ & $-{ }_{3}$ & -- & 60 & 67 & --68 & 70 & ${ }^{--} 74$ & 82 & 100 \\
\hline${ }_{\text {JuN }} 17 \ldots$ & 0 & 1 & 13 & 48 & 57 & 59 & 61 & 65 & 76 & 86 \\
\hline$\stackrel{04 \ldots}{19 . \ldots}$ & $\cdots 1$ & -- & 27 & 76 & .81 & 82 & -83 & -86 & $-{ }_{95}$ & 100 \\
\hline $\begin{array}{r}18 . . \\
23 \\
\text { AUG }\end{array}$ & & $--^{13}$ & $-m^{65}$ & 98 & 100 & $=$ & $=-$ & $=$ & $=$ & $=$ \\
\hline $\begin{array}{r}13 \ldots \\
15 \ldots \\
\operatorname{SEP}\end{array}$ & $-{ }_{3}$ & $\cdots 9$ & $-{ }^{--} 22$ & -69 & $-{ }_{92}$ & --96 & 97 & -98 & $\overline{100}$ & $=$ \\
\hline $04 \ldots$ & 2 & 5 & 20 & 89 & 97 & 99 & 100 & -- & $-m$ & - \\
\hline
\end{tabular}


RIO GRANDE BASIN

08354900 RIO GRANDE FLOODWAY AT SAN ACACTA, MM - Continued

WATER-QUALITY RECORDS SPECIFIC CONDUCTANCE, US/CM 25 DEGREES CENTIGRADE, WATER YEAR OCTOBER 1995 TO SEPTEMBER 1996

\begin{tabular}{|c|c|c|c|c|c|c|c|c|c|c|c|c|}
\hline DAY & OCT & HOV & DEC & JAN & FEB & MAR & APR & MAY & JUN & JUL & AUG & SEP \\
\hline $\begin{array}{l}1 \\
2 \\
3 \\
4 \\
5\end{array}$ & $\begin{array}{l}481 \\
525 \\
516 \\
510\end{array}$ & $\begin{array}{l}568 \\
519 \\
497 \\
503 \\
519\end{array}$ & $\begin{array}{l}546 \\
500 \\
552 \\
446 \\
553\end{array}$ & $\begin{array}{l}536 \\
563 \\
563 \\
538 \\
576\end{array}$ & $\begin{array}{l}540 \\
565 \\
528 \\
537 \\
525\end{array}$ & $\begin{array}{l}--1 \\
527 \\
494 \\
516 \\
495\end{array}$ & $\begin{array}{l}585 \\
586 \\
556 \\
763 \\
657\end{array}$ & $\begin{array}{l}667 \\
685 \\
753 \\
650\end{array}$ & $\begin{array}{l}591 \\
706 \\
705 \\
644 \\
679\end{array}$ & $\begin{array}{r}715 \\
1060 \\
1170 \\
-953\end{array}$ & $\begin{array}{l}687 \\
796 \\
730 \\
794 \\
-\end{array}$ & $\begin{array}{l}775 \\
716 \\
690 \\
772 \\
811\end{array}$ \\
\hline $\begin{array}{r}6 \\
7 \\
8 \\
9 \\
10\end{array}$ & $\begin{array}{l}505 \\
508 \\
515 \\
503 \\
523\end{array}$ & $\begin{array}{l}505 \\
511 \\
510 \\
501 \\
493\end{array}$ & $\begin{array}{l}547 \\
546 \\
556 \\
547 \\
556\end{array}$ & $\begin{array}{l}559 \\
536 \\
524 \\
536 \\
534\end{array}$ & $\begin{array}{l}550 \\
497 \\
528 \\
526 \\
542\end{array}$ & $\begin{array}{l}521 \\
515 \\
523 \\
523 \\
496\end{array}$ & $\begin{array}{l}780 \\
783 \\
638 \\
641 \\
783\end{array}$ & $\begin{array}{r}686 \\
681 \\
722 \\
722 \\
676\end{array}$ & $\begin{array}{l}699 \\
680 \\
724 \\
582 \\
678\end{array}$ & $\begin{array}{l}735 \\
678 \\
689 \\
724\end{array}$ & $\begin{array}{l}-m \\
-\cdots- \\
--- \\
729\end{array}$ & $\frac{735}{--\infty}$ \\
\hline $\begin{array}{l}11 \\
12 \\
13 \\
14 \\
15\end{array}$ & $\begin{array}{l}515 \\
513 \\
497 \\
518 \\
510\end{array}$ & $\begin{array}{l}494 \\
504 \\
501 \\
494 \\
540\end{array}$ & $\begin{array}{l}556 \\
545 \\
546 \\
551 \\
521\end{array}$ & $\begin{array}{l}525 \\
517 \\
534 \\
541 \\
544\end{array}$ & $\begin{array}{l}530 \\
544 \\
543 \\
495 \\
548\end{array}$ & $\begin{array}{l}538 \\
516 \\
529 \\
517 \\
515\end{array}$ & $\begin{array}{l}784 \\
700 \\
769 \\
793 \\
785\end{array}$ & $\begin{array}{l}753 \\
718 \\
740 \\
702 \\
734\end{array}$ & $\begin{array}{l}662 \\
634 \\
829 \\
890 \\
794\end{array}$ & $\begin{array}{r}728 \\
964 \\
815 \\
1010 \\
969\end{array}$ & $\begin{array}{l}710 \\
688 \\
707 \\
697 \\
710\end{array}$ & $\begin{array}{l}--- \\
--- \\
708 \\
685\end{array}$ \\
\hline $\begin{array}{l}16 \\
17 \\
18 \\
19 \\
20\end{array}$ & $\begin{array}{l}498 \\
526 \\
517 \\
536 \\
520\end{array}$ & $\begin{array}{l}500 \\
498 \\
506 \\
501 \\
506\end{array}$ & $\begin{array}{l}522 \\
551 \\
536 \\
524 \\
530\end{array}$ & $\begin{array}{l}535 \\
538 \\
520 \\
543 \\
543\end{array}$ & $\begin{array}{l}541 \\
536 \\
540 \\
496 \\
450\end{array}$ & $\begin{array}{l}567 \\
518 \\
531 \\
531 \\
549\end{array}$ & $\begin{array}{l}790 \\
787 \\
701 \\
792 \\
769\end{array}$ & $\begin{array}{l}605 \\
650 \\
715 \\
718 \\
620\end{array}$ & $\begin{array}{l}731 \\
641 \\
641 \\
733 \\
728\end{array}$ & $\begin{array}{r}1060 \\
1100 \\
1160 \\
722 \\
715\end{array}$ & $\begin{array}{l}724 \\
707 \\
957 \\
904 \\
797\end{array}$ & $\begin{array}{l}722 \\
683 \\
608 \\
684 \\
746\end{array}$ \\
\hline $\begin{array}{l}21 \\
22 \\
23 \\
24 \\
25\end{array}$ & $\begin{array}{l}521 \\
519 \\
360 \\
521 \\
525\end{array}$ & $\begin{array}{l}501 \\
546 \\
503 \\
493 \\
538\end{array}$ & $\begin{array}{l}576 \\
552 \\
546 \\
529 \\
\end{array}$ & $\begin{array}{l}521 \\
544 \\
537 \\
545 \\
541\end{array}$ & $\begin{array}{l}496 \\
541 \\
501 \\
539 \\
544\end{array}$ & $\begin{array}{l}566 \\
550 \\
520 \\
567 \\
520\end{array}$ & $\begin{array}{l}-1- \\
663 \\
663 \\
711 \\
728\end{array}$ & $\frac{--}{584}$ & $\begin{array}{l}787 \\
629 \\
632 \\
742 \\
599\end{array}$ & $\begin{array}{r}713 \\
958 \\
1100 \\
1110 \\
932\end{array}$ & $\begin{array}{l}821 \\
787 \\
825 \\
878 \\
940\end{array}$ & $\begin{array}{l}627 \\
607 \\
683 \\
630 \\
---\end{array}$ \\
\hline $\begin{array}{l}26 \\
27 \\
28 \\
29 \\
30 \\
31\end{array}$ & $\begin{array}{l}508 \\
514 \\
499 \\
536 \\
513 \\
528\end{array}$ & $\begin{array}{l}541 \\
532 \\
533 \\
538 \\
543 \\
-\end{array}$ & $\begin{array}{l}-1- \\
564 \\
545 \\
562 \\
538 \\
563\end{array}$ & $\begin{array}{l}541 \\
-545 \\
541 \\
546 \\
547\end{array}$ & $\begin{array}{l}502 \\
494 \\
501 \\
521 \\
-\end{array}$ & $\begin{array}{l}560 \\
522 \\
549 \\
557 \\
517 \\
585\end{array}$ & $\begin{array}{l}-7 \\
668 \\
688 \\
688 \\
-- \\
--\end{array}$ & $\begin{array}{l}591 \\
591 \\
624 \\
605 \\
746 \\
710\end{array}$ & $\begin{array}{l}725 \\
888 \\
910 \\
840 \\
534 \\
-\end{array}$ & $\begin{array}{l}974 \\
988 \\
746 \\
742 \\
754 \\
755\end{array}$ & $\begin{array}{l}731 \\
734 \\
737 \\
720 \\
818 \\
748\end{array}$ & $\begin{array}{l}757 \\
695 \\
711 \\
764 \\
683 \\
-\end{array}$ \\
\hline $\begin{array}{l}\text { MEAN } \\
\text { MAX } \\
\text { MIN }\end{array}$ & $m$ & $\begin{array}{l}515 \\
568 \\
493\end{array}$ & $=$ & $\begin{array}{l}--- \\
-w- \\
---\end{array}$ & $\begin{array}{l}524 \\
565 \\
450\end{array}$ & $\begin{array}{l}--- \\
-m \\
---\end{array}$ & $=-$ & - & $\begin{array}{l}709 \\
910 \\
534\end{array}$ & 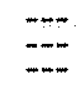 & $\overline{--}$ & $m+\cdots$ \\
\hline
\end{tabular}

WATER TEMPERATURE, DEGREES CELSIUS, WATER YEAR OCTOBER 1995 TO SEPTEMBER 1996 DAILY INSTANTANEOUS VALUES

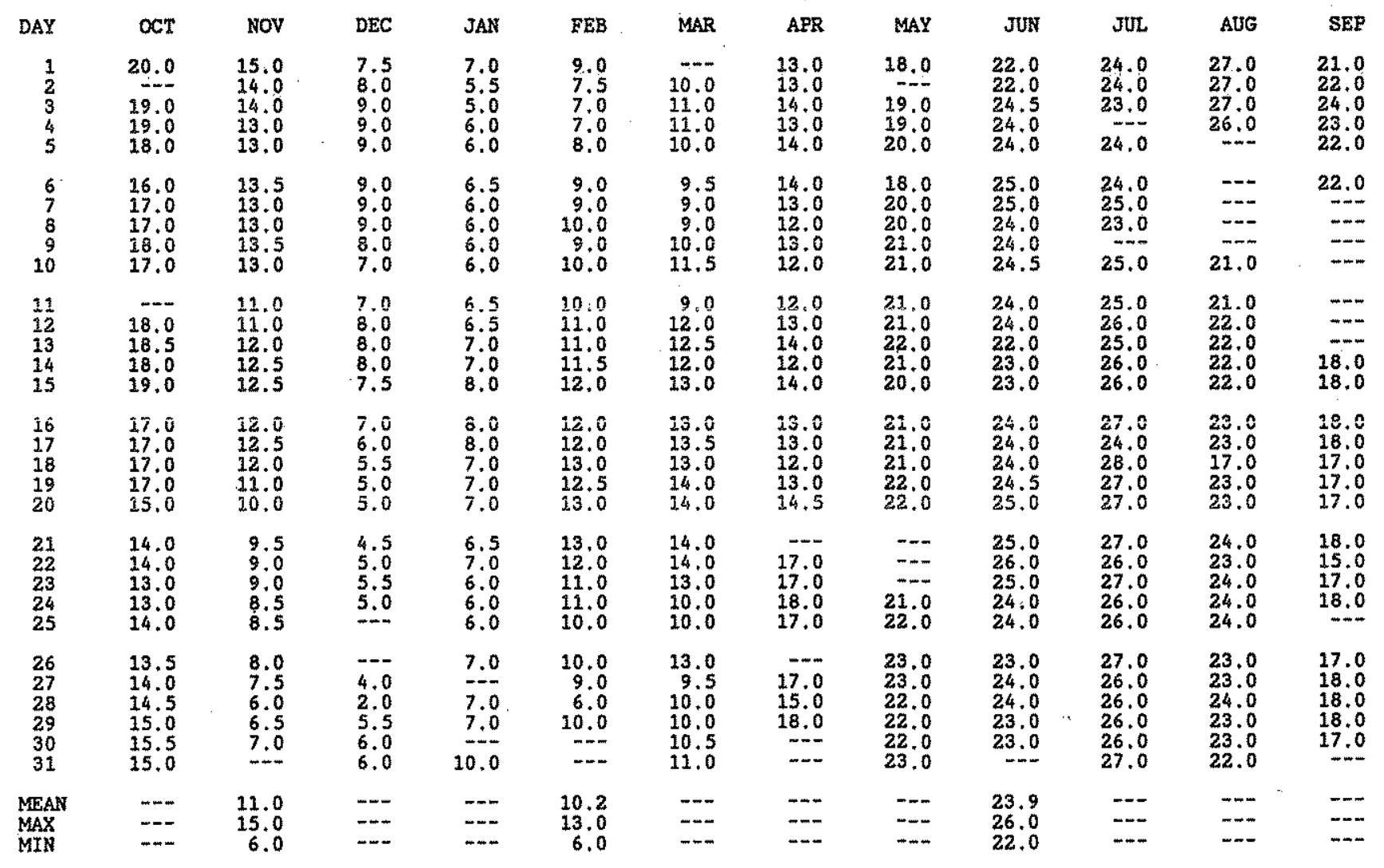


RIO GRANDE BASIN

08354900 RIO GRANDE FLOODWAY AT SAN ACACIA, NM -- Continued

WATER-QUALITY RECORDS

SUSPENDED-SEDIMENT, WATER YEAR OCTOBER 1995 TO SEPTEMBER 1996

\begin{tabular}{|c|c|c|c|c|c|c|c|c|c|c|c|c|}
\hline DAY & $\begin{array}{l}\text { MEAN } \\
\text { CONCEN- } \\
\text { TRATION } \\
\text { (MG/L) }\end{array}$ & $\begin{array}{l}\text { LOADS } \\
(\mathrm{T} / \mathrm{DAY})\end{array}$ & $\begin{array}{l}\text { MEAN } \\
\text { CONCEN- } \\
\text { TRATIOA } \\
\text { (MG/L) }\end{array}$ & $\begin{array}{l}\text { LOADS } \\
(T / D A Y)\end{array}$ & $\begin{array}{l}\text { MEAN } \\
\text { CONCEN- } \\
\text { IRATIOH } \\
\text { (MG/L) }\end{array}$ & $\begin{array}{l}\text { LOADS } \\
\text { (T/DAY) }\end{array}$ & $\begin{array}{l}\text { MEAN } \\
\text { CONCEN- } \\
\text { TRATION } \\
\text { (MG/L) }\end{array}$ & $\begin{array}{l}\text { LOADS } \\
(T / D A Y)\end{array}$ & $\begin{array}{c}\text { MEAN } \\
\text { CONCEN- } \\
\text { TRATION } \\
\text { (MG/L) }\end{array}$ & $\begin{array}{l}\text { LOADS } \\
\text { (T/DAY) }\end{array}$ & $\begin{array}{l}\text { MEAN } \\
\text { CONCEN- } \\
\text { MRATION } \\
\text { (MG/L) }\end{array}$ & $\begin{array}{l}\text { LOADS } \\
\text { (T/DAY) }\end{array}$ \\
\hline $\begin{array}{l}1 \\
2 \\
3 \\
4 \\
5\end{array}$ & $\begin{array}{l}3900 \\
2760 \\
1710 \\
2550 \\
1820\end{array}$ & $\begin{array}{r}\text { TOBER } \\
5470 \\
4540 \\
4240 \\
5580 \\
2810\end{array}$ & $\begin{array}{l}\text { NO } \\
900 \\
1860 \\
1000 \\
1070 \\
1420\end{array}$ & $\begin{array}{l}\text { EXBER } \\
1700 \\
6370 \\
3520 \\
3170 \\
4040\end{array}$ & $\begin{array}{l}\text { DEC } \\
103 \\
307 \\
197 \\
154 \\
66\end{array}$ & $\begin{array}{c}\text { EMBER } \\
261 \\
763 \\
499 \\
499 \\
180\end{array}$ & $\begin{array}{l}210^{3 A} \\
195 \\
111 \\
107 \\
120\end{array}$ & $\begin{array}{r}\text { NUARY } \\
497 \\
393 \\
221 \\
209 \\
232\end{array}$ & $\begin{array}{l}\text { FEE } \\
126 \\
127 \\
136 \\
140 \\
150\end{array}$ & $\begin{array}{r}\text { RUARY } \\
486 \\
494 \\
551 \\
563 \\
599\end{array}$ & $\begin{array}{r}156 \\
144 \\
119 \\
127 \\
92\end{array}$ & $\begin{array}{r}\text { ARCE } \\
430 \\
385 \\
363 \\
425 \\
276\end{array}$ \\
\hline $\begin{array}{r}6 \\
7 \\
8 \\
9 \\
10\end{array}$ & $\begin{array}{r}323 \\
602 \\
1260 \\
909 \\
132\end{array}$ & $\begin{array}{r}427 \\
891 \\
1790 \\
1310 \\
194\end{array}$ & $\begin{array}{l}488 \\
576 \\
682 \\
566 \\
610\end{array}$ & $\begin{array}{l}1400 \\
1660 \\
1990 \\
1640 \\
1750\end{array}$ & $\begin{array}{l}162 \\
198 \\
212 \\
240 \\
149\end{array}$ & $\begin{array}{l}422 \\
545 \\
571 \\
667 \\
386\end{array}$ & $\begin{array}{l}176 \\
162 \\
148 \\
178 \\
168\end{array}$ & $\begin{array}{l}335 \\
304 \\
274 \\
358 \\
553\end{array}$ & $\begin{array}{l}170 \\
221 \\
165 \\
170 \\
289\end{array}$ & $\begin{array}{r}647 \\
841 \\
633 \\
661 \\
1150\end{array}$ & $\begin{array}{r}105 \\
76 \\
113 \\
112 \\
79\end{array}$ & $\begin{array}{l}315 \\
242 \\
351 \\
268 \\
168\end{array}$ \\
\hline $\begin{array}{l}11 \\
12 \\
13 \\
14 \\
15\end{array}$ & $\begin{array}{l}147 \\
146 \\
127 \\
135 \\
144\end{array}$ & $\begin{array}{l}223 \\
200 \\
182 \\
186 \\
206\end{array}$ & $\begin{array}{l}371 \\
309 \\
423 \\
366 \\
153\end{array}$ & $\begin{array}{r}1060 \\
876 \\
1150 \\
988 \\
446\end{array}$ & $\begin{array}{l}165 \\
181 \\
167 \\
156 \\
107\end{array}$ & $\begin{array}{l}425 \\
460 \\
417 \\
364 \\
234\end{array}$ & $\begin{array}{l}162 \\
139 \\
143 \\
134 \\
166\end{array}$ & $\begin{array}{l}612 \\
563 \\
579 \\
543 \\
663\end{array}$ & $\begin{array}{r}306 \\
288 \\
185 \\
97 \\
118\end{array}$ & $\begin{array}{r}1230 \\
1170 \\
754 \\
390 \\
478\end{array}$ & $\begin{array}{r}108 \\
79 \\
68 \\
70 \\
73\end{array}$ & $\begin{array}{l}229 \\
175 \\
145 \\
151 \\
159\end{array}$ \\
\hline $\begin{array}{l}16 \\
17 \\
18 \\
19 \\
20\end{array}$ & $\begin{array}{r}132 \\
127 \\
112 \\
98 \\
100\end{array}$ & $\begin{array}{l}179 \\
154 \\
132 \\
116 \\
121\end{array}$ & $\begin{array}{l}236 \\
475 \\
837 \\
398 \\
227\end{array}$ & $\begin{array}{r}701 \\
1470 \\
2510 \\
1180 \\
668\end{array}$ & $\begin{array}{l}120 \\
148 \\
223 \\
192 \\
129\end{array}$ & $\begin{array}{l}255 \\
302 \\
433 \\
335 \\
232\end{array}$ & $\begin{array}{l}194 \\
208 \\
172 \\
145 \\
168\end{array}$ & $\begin{array}{l}775 \\
814 \\
664 \\
579 \\
680\end{array}$ & $\begin{array}{r}113 \\
98 \\
224 \\
244 \\
166\end{array}$ & $\begin{array}{l}473 \\
407 \\
925 \\
988 \\
668\end{array}$ & $\begin{array}{l}73 \\
69 \\
70 \\
72 \\
72\end{array}$ & $\begin{array}{l}161 \\
156 \\
159 \\
171 \\
177\end{array}$ \\
\hline $\begin{array}{l}21 \\
22 \\
23 \\
24 \\
25\end{array}$ & $\begin{array}{l}143 \\
128 \\
103 \\
151 \\
152\end{array}$ & $\begin{array}{l}181 \\
156 \\
128 \\
191 \\
195\end{array}$ & $\begin{array}{l}255 \\
152 \\
293 \\
519 \\
229\end{array}$ & $\begin{array}{r}750 \\
451 \\
854 \\
1490 \\
643\end{array}$ & $\begin{array}{l}111 \\
135 \\
156 \\
134 \\
138\end{array}$ & $\begin{array}{l}204 \\
239 \\
421 \\
360 \\
361\end{array}$ & $\begin{array}{r}197 \\
185 \\
210 \\
160 \\
85\end{array}$ & $\begin{array}{l}803 \\
779 \\
782 \\
562 \\
298\end{array}$ & $\begin{array}{l}108 \\
109 \\
191 \\
164 \\
131\end{array}$ & $\begin{array}{l}432 \\
406 \\
784 \\
686 \\
506\end{array}$ & $\begin{array}{l}63 \\
57 \\
74 \\
69 \\
74\end{array}$ & $\begin{array}{l}160 \\
140 \\
172 \\
149 \\
158\end{array}$ \\
\hline $\begin{array}{l}26 \\
27 \\
28 \\
29 \\
30 \\
31\end{array}$ & $\begin{array}{l}141 \\
157 \\
154 \\
129 \\
126 \\
150\end{array}$ & $\begin{array}{l}184 \\
208 \\
207 \\
177 \\
175 \\
212\end{array}$ & $\begin{array}{l}134 \\
131 \\
140 \\
195 \\
190 \\
-\end{array}$ & $\begin{array}{l}369 \\
354 \\
374 \\
516 \\
495 \\
-\end{array}$ & $\begin{array}{l}155 \\
123 \\
176 \\
197 \\
127 \\
149\end{array}$ & $\begin{array}{l}402 \\
325 \\
480 \\
553 \\
363 \\
414\end{array}$ & $\begin{array}{r}90 \\
115 \\
156 \\
156 \\
190 \\
105\end{array}$ & $\begin{array}{l}321 \\
416 \\
548 \\
560 \\
708 \\
403\end{array}$ & $\begin{array}{l}195 \\
124 \\
154 \\
151 \\
-\cdots \\
-\end{array}$ & $\begin{array}{l}706 \\
439 \\
528 \\
473 \\
--- \\
---\end{array}$ & $\begin{array}{l}62 \\
65 \\
54 \\
56 \\
66 \\
51\end{array}$ & $\begin{array}{r}131 \\
133 \\
102 \\
88 \\
82 \\
54\end{array}$ \\
\hline TOTAL & $m=-$ & 30965 & $m$ & 44585 & --- & 12372 & $m-m$ & 16028 & $m$ & 19068 & $-\cdots$ & 6275 \\
\hline & $\begin{array}{c}\text { MEAN } \\
\text { CONCEN- } \\
\text { TRATION }\end{array}$ & LOADS & $\begin{array}{l}\text { MEAN } \\
\text { CONCEN- } \\
\text { TRATION }\end{array}$ & LOADS & $\begin{array}{l}\text { MEAN } \\
\text { CONCEN- } \\
\text { TRATION }\end{array}$ & LOADS & $\begin{array}{l}\text { MEAN } \\
\text { CONCEN- } \\
\text { TRATION }\end{array}$ & LOADS & $\begin{array}{l}\text { MEAN } \\
\text { CONCEN- } \\
\text { TRATION }\end{array}$ & LOADS & $\begin{array}{l}\text { MEAN } \\
\text { CONCEN- } \\
\text { TRATION }\end{array}$ & LOADS \\
\hline
\end{tabular}

DAY

\begin{tabular}{lrrr} 
& \multicolumn{2}{c}{ AFRIL } & \multicolumn{2}{c}{ MAY } \\
49 & 42 & 86 & 13 \\
48 & 36 & 61 & 10 \\
52 & 35 & 50 & 8 \\
52 & 29 & 33 & 5 \\
52 & 23 & 49 & 8
\end{tabular}

13
10
8.1
8.3
5.3
8.1

$\begin{array}{lr}87 & 14 \\ 93 & 15 \\ 57 & 9\end{array}$

11

13

15

16

18

19
20

21

24

26
27

28
29

30 $\begin{array}{ll}58 & 23 \\ 40 & 14 \\ 52 & 15 \\ 55 & 14 \\ 52 & 12\end{array}$

$\begin{array}{ll}38 & 8.2 \\ 52 & 9.3 \\ 43 & 7.0 \\ 38 & 4.9 \\ 47 & 5.1\end{array}$

$\begin{array}{ll}48 & 4.5 \\ 60 & 2.8 \\ 51 & 3.3 \\ 38 & 2.3 \\ 41 & 2.2\end{array}$

$\begin{array}{ll}44 & 2.1 \\ 58 & 2.7 \\ 64 & 9.0 \\ 49 & 3.8\end{array}$

$\begin{array}{ll}49 & 3.8 \\ 45 & 3.6\end{array}$

$\begin{array}{rr}47 & 4 \\ 79 & 10 \\ 80 & 11 \\ 52 & 7 \\ 52 & 7 \\ - & -\end{array}$
14
15
9.2
6.3
7.2

\section{2}

$$
\begin{aligned}
& 4.9 \\
& 5.2
\end{aligned}
$$

5.2
5.6

$$
\begin{aligned}
& 5.2 \\
& 4.6 \\
& 5.2
\end{aligned}
$$

5.6
5.2

7.7
4.7

\section{3.:4}

3.7

19.2
7.2
6.8

51
46
51
49
47
47
47
48
49
54 11
9.6
7.8
9.3

11.
9.6
7.8
9.3

8440
17100
28400
30700
22700 JULY

638
1570
10400
14900
10500

9.5
11
11
12
12

9570

9570
349
225
171

171
228

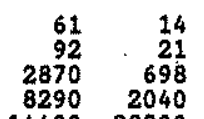

$\begin{array}{rr}8290 & 2040 \\ 14400 & 28200\end{array}$

$10900 \quad 4030$

$\begin{array}{ll}252 & 132 \\ 169 & 112\end{array}$

$193 \quad 112$

112
106

311

311
139
107
174

71
31
26
47
95

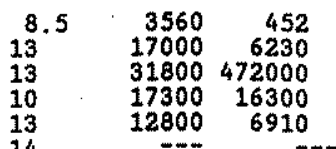

452
6230
2000
6300
6910

291
16400
10800
16400

16400
18700

25100

27200

5020

2810

1250
766
1220
1050
1820

1820

5370

9890
5640

2470

4920
2750
3620

3620
115
61
32
46

134
8650
4960
6660

3540

12900
15400
25000
5830

3790

1350
558
85
21

212

1350

2140

1160
566

1370
587
1340
4620
7690
10800
3540

UST

298
1200
2490
4020

1240

2340

870
826
12800
10300

905
357
388
18200
13200

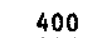

400

481
369

363
335

335
206

206
132

132
197

895

895
477
21900

213
102

102
4320

4800

4800
2870

16900

15700

19900
18800

17600
27100

2450
328
6450
7180

26900

21300

20100

16800

16200

-n

387096 \begin{tabular}{rr}
\multicolumn{2}{c}{ SEFTEMBER } \\
7920 & 18000 \\
6780 & 8920 \\
6000 & 5040 \\
7600 & 3860 \\
7700 & 2790 \\
& \\
11000 & 3970 \\
1700 & 428 \\
1270 & 267 \\
1930 & 947 \\
2410 & 312
\end{tabular}

$3210 \quad 251$

$\begin{array}{rr}9570 & 9920 \\ 9640 & 3720\end{array}$

$18300 \quad 27500$

$\begin{array}{ll}18300 & 27500 \\ 17600 & 38200\end{array}$

$14500 \quad 28600$ $9880 \quad 20400$

$4160 \quad 6820$

$\begin{array}{rr}2790 & 5340 \\ 5570 & 10700\end{array}$

$4010 \quad 6760$

$\begin{array}{ll}3310 & 4440 \\ 3620 & 4570\end{array}$

$1850 \quad 2040$

$\begin{array}{lll}33200 & 2720 & 3250\end{array}$

$57300 \quad 4490 \quad 4760$

$\begin{array}{lll}55500 & 3490 & 4760 \\ 5000 & 2590\end{array}$

$52700 \quad 10100 \quad 6520$

$\begin{array}{rrr}35000 & 9950 \quad 7410\end{array}$

$\begin{array}{lll}30000 & 3210 & 2050 \\ 48200 & \end{array}$

TOTAL
TOTAL LOAD FOR YEAR: 1434362.6

-.- $\quad 139256$

240375 
08358300 RIO GRANDE CONVEYANCE CHANNEL AT SAN MARCTAL, NM

LOCATION. --Lat $33^{\circ} 41^{\prime} 15^{\prime \prime}$, Long $106^{\circ} 59^{\circ} 40^{\prime \prime}$, Socorro County, Eydrologle Unit 13020203, in Pedro Armendaris Grant No. 34 , on right bank $0.4 \mathrm{mi}$ northwest of Atchtson, Topeks and Santa Fe Railway Co. bridge over floodwey channel, i. mi southwest of former site of San Marcial, $3.5 \mathrm{mi}$ downstream from rallroad bridge near Tiffany siding, and 51 mi downstream from hoading at San Acacia.

WATER-DISCHARGE RECORDS

PERIOD of RECORD - October 1958 to September 1959 , October 1964 to current year Prior to October 1964 monthly dischaxge only published with record for Rio Grande at San Marcial (station 08358500 ).

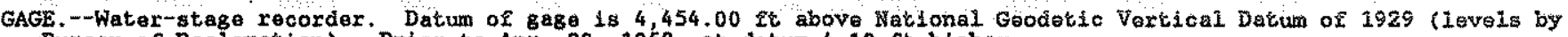
Bureau of Reclamation). Prior to Apr. 29, 1958, at datum 4.19 ft higher.

REAARKS. -Water-discharge records good except for estimated daily discharges, which are fair, orisinat design and plan were for conveyence channel to carry all flows up to about 2,000 ft $/ 8$. Conveyance channel is 1 of 2 channels (station 08358400 ) carrying flow in valley cross section. For combined monthly flow in acre-ft of this channel and floodway, see tabulation below daliy table for station 08358400. Bureau of Raclamation satellite telemeter at station. No flow from River since 1965.

DISCHARGE, CUBIC FEET PER SECORD, WATER YEAR OCTOBER 1995 TO SEPTEMER 1996

\begin{tabular}{|c|c|c|c|c|c|c|c|c|c|c|c|c|}
\hline DAY & $o c r$ & nov & DEC & JAN & $\mathrm{FEB}$ & MAR & $A P R$ & MAY & JUN & JuL & AUG & SEP \\
\hline $\begin{array}{l}1 \\
2 \\
3 \\
4 \\
5\end{array}$ & $\begin{array}{l}288 \\
298 \\
335 \\
338 \\
342\end{array}$ & $\begin{array}{l}377 \\
319 \\
272 \\
271 \\
270\end{array}$ & $\begin{array}{l}257 \\
256 \\
256 \\
255 \\
255\end{array}$ & $\begin{array}{l}248 \\
248 \\
246 \\
246 \\
246\end{array}$ & $\begin{array}{l}269 \\
269 \\
270 \\
270 \\
271\end{array}$ & $\begin{array}{l}255 \\
277 \\
295 \\
293 \\
300\end{array}$ & $\begin{array}{l}382 \\
363 \\
351 \\
354 \\
354\end{array}$ & $\begin{array}{l}242 \\
240 \\
220 \\
206 \\
178\end{array}$ & $\begin{array}{r}138 \\
182 \\
199 \\
177 \\
97\end{array}$ & $\begin{array}{l}326 \\
326 \\
319 \\
330 \\
307\end{array}$ & $\begin{array}{l}153 \\
147 \\
144 \\
146 \\
160\end{array}$ & $\begin{array}{l}258 \\
273 \\
255 \\
241 \\
236\end{array}$ \\
\hline $\begin{array}{r}6 \\
7 \\
8 \\
9 \\
10\end{array}$ & $\begin{array}{l}343 \\
337 \\
330 \\
353 \\
323\end{array}$ & $\begin{array}{l}269 \\
269 \\
269 \\
268 \\
268\end{array}$ & $\begin{array}{l}255 \\
255 \\
255 \\
255 \\
255\end{array}$ & $\begin{array}{l}246 \\
245 \\
245 \\
245 \\
245\end{array}$ & $\begin{array}{l}271 \\
273 \\
275 \\
272 \\
273\end{array}$ & $\begin{array}{l}312 \\
327 \\
341 \\
332 \\
329\end{array}$ & $\begin{array}{l}356 \\
368 \\
375 \\
385 \\
342\end{array}$ & $\begin{array}{l}182 \\
149 \\
092 \\
114 \\
135\end{array}$ & $\begin{array}{r}91 \\
93 \\
91 \\
91 \\
109\end{array}$ & $\begin{array}{l}275 \\
293 \\
287 \\
224 \\
259\end{array}$ & $\begin{array}{l}157 \\
143 \\
139 \\
144 \\
191\end{array}$ & $\begin{array}{l}229 \\
218 \\
201 \\
211 \\
228\end{array}$ \\
\hline $\begin{array}{l}11 \\
12 \\
13 \\
14 \\
15\end{array}$ & $\begin{array}{l}317 \\
334 \\
328 \\
328 \\
322\end{array}$ & $\begin{array}{l}268 \\
267 \\
265 \\
266 \\
265\end{array}$ & $\begin{array}{l}254 \\
253 \\
253 \\
253 \\
252\end{array}$ & $\begin{array}{l}244 \\
244 \\
246 \\
247 \\
248\end{array}$ & $\begin{array}{l}273 \\
274 \\
275 \\
275 \\
275\end{array}$ & $\begin{array}{l}345 \\
351 \\
314 \\
344 \\
352\end{array}$ & $\begin{array}{l}298 \\
288 \\
270 \\
267 \\
272\end{array}$ & $\begin{array}{l}162 \\
156 \\
153 \\
150 \\
102\end{array}$ & $\begin{array}{r}122 \\
89 \\
78 \\
82 \\
147\end{array}$ & $\begin{array}{l}242 \\
247 \\
225 \\
266 \\
280\end{array}$ & $\begin{array}{l}202 \\
200 \\
183 \\
159 \\
176\end{array}$ & $\begin{array}{l}206 \\
246 \\
315 \\
323 \\
275\end{array}$ \\
\hline $\begin{array}{l}16 \\
17 \\
18 \\
19 \\
20\end{array}$ & $\begin{array}{l}336 \\
320 \\
336 \\
339 \\
341\end{array}$ & $\begin{array}{l}264 \\
263 \\
263 \\
263 \\
262\end{array}$ & $\begin{array}{l}252 \\
253 \\
253 \\
252 \\
251\end{array}$ & $\begin{array}{l}250 \\
251 \\
252 \\
253 \\
255\end{array}$ & $\begin{array}{l}275 \\
277 \\
285 \\
278 \\
279\end{array}$ & $\begin{array}{l}356 \\
346 \\
379 \\
370 \\
357\end{array}$ & $\begin{array}{l}249 \\
178 \\
191 \\
163 \\
146\end{array}$ & $\begin{array}{r}91 \\
89 \\
129 \\
135 \\
135\end{array}$ & $\begin{array}{l}195 \\
222 \\
205 \\
177 \\
159\end{array}$ & $\begin{array}{l}293 \\
240 \\
232 \\
238 \\
225\end{array}$ & $\begin{array}{l}202 \\
183 \\
167 \\
161 \\
143\end{array}$ & $\begin{array}{l}214 \\
215 \\
270 \\
263 \\
250\end{array}$ \\
\hline $\begin{array}{l}21 \\
22 \\
23 \\
24 \\
25\end{array}$ & $\begin{array}{l}330 \\
335 \\
344 \\
369 \\
349\end{array}$ & $\begin{array}{l}262 \\
262 \\
261 \\
259 \\
259\end{array}$ & $\begin{array}{l}251 \\
250 \\
249 \\
249 \\
249\end{array}$ & $\begin{array}{l}256 \\
257 \\
258 \\
259 \\
261\end{array}$ & $\begin{array}{l}279 \\
278 \\
274 \\
271 \\
268\end{array}$ & $\begin{array}{l}348 \\
362 \\
358 \\
345 \\
385\end{array}$ & $\begin{array}{l}145 \\
174 \\
174 \\
193 \\
207\end{array}$ & $\begin{array}{r}105 \\
101 \\
126 \\
85 \\
81\end{array}$ & $\begin{array}{l}239 \\
231 \\
227 \\
266 \\
227\end{array}$ & $\begin{array}{l}221 \\
216 \\
193 \\
191 \\
173\end{array}$ & $\begin{array}{l}149 \\
165 \\
199 \\
237 \\
203\end{array}$ & $\begin{array}{l}242 \\
250 \\
252 \\
234 \\
239\end{array}$ \\
\hline $\begin{array}{l}26 \\
27 \\
28 \\
29 \\
30 \\
31\end{array}$ & $\begin{array}{l}336 \\
339 \\
335 \\
347 \\
363 \\
352\end{array}$ & $\begin{array}{l}259 \\
259 \\
258 \\
258 \\
258 \\
--\infty\end{array}$ & $\begin{array}{l}248 \\
248 \\
249 \\
249 \\
248 \\
248\end{array}$ & $\begin{array}{l}262 \\
262 \\
263 \\
264 \\
265 \\
266\end{array}$ & $\begin{array}{l}265 \\
263 \\
259 \\
261 \\
-m- \\
m-m\end{array}$ & $\begin{array}{l}366 \\
333 \\
333 \\
341 \\
363 \\
392\end{array}$ & $\begin{array}{l}227 \\
230 \\
263 \\
288 \\
248 \\
-\end{array}$ & $\begin{array}{l}100 \\
142 \\
133 \\
158 \\
171 \\
109\end{array}$ & $\begin{array}{l}213 \\
250 \\
330 \\
362 \\
332 \\
---\end{array}$ & $\begin{array}{l}183 \\
169 \\
192 \\
191 \\
174 \\
184\end{array}$ & $\begin{array}{l}244 \\
265 \\
271 \\
276 \\
266 \\
245\end{array}$ & $\begin{array}{l}253 \\
277 \\
277 \\
273 \\
291 \\
-\end{array}$ \\
\hline $\begin{array}{l}\text { TOTAI } \\
\text { MEAN } \\
\text { MAX } \\
\text { MIN } \\
\text { AC-FT }\end{array}$ & $\begin{array}{r}10387 \\
335 \\
369 \\
288 \\
20600\end{array}$ & $\begin{array}{r}8093 \\
270 \\
377 \\
258 \\
16050\end{array}$ & $\begin{array}{r}7818 \\
252 \\
257 \\
248 \\
15510\end{array}$ & $\begin{array}{r}7823 \\
252 \\
266 \\
244 \\
15520\end{array}$ & $\begin{array}{r}7897 \\
272 \\
285 \\
259 \\
15660\end{array}$ & $\begin{array}{r}10501 \\
339 \\
392 \\
255 \\
20830\end{array}$ & $\begin{array}{r}8101 \\
270 \\
385 \\
145 \\
16070\end{array}$ & $\begin{array}{r}4371 \\
141 \\
242 \\
81 \\
8670\end{array}$ & $\begin{array}{r}5421 \\
181 \\
362 \\
78 \\
10750\end{array}$ & $\begin{array}{r}7521 \\
243 \\
330 \\
169 \\
14920\end{array}$ & $\begin{array}{r}5820 \\
188 \\
276 \\
139 \\
11540\end{array}$ & $\begin{array}{r}7515 \\
250 \\
323 \\
201 \\
14910\end{array}$ \\
\hline
\end{tabular}

STATISTICS OF MONTEIY MEAN DATA FOR WATER YEARS 1965 - 1996, BY WATER YEAR (WY)

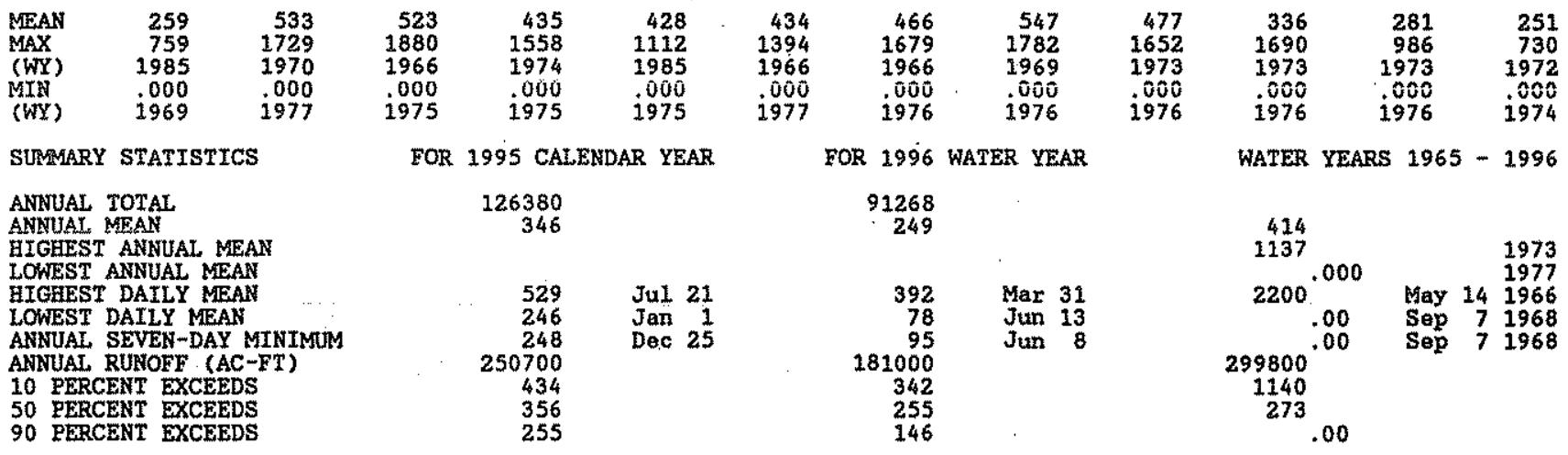

- Estimated 
RTO GRANDE BASIN

08358300 RIO GRANDE CONVEYANCE CHANNEL AT SAN MARCTAL, MM -- Continued

WATER-QUALTTY RECORDS

PERIOD OF RECORD. - -Water years 1954 to current year.

WATER QUALITY DATA, WATER YEAR OCTOBER 1995 TO SEPTEMBER 1996

\begin{tabular}{|c|c|c|c|c|c|c|c|c|c|c|c|c|}
\hline DATE & TIME & $\begin{array}{c}\text { DIS- } \\
\text { CEARGE, } \\
\text { INST } \\
\text { CUBIC } \\
\text { FEET } \\
\text { PER } \\
\text { SECOND } \\
(00061)\end{array}$ & $\begin{array}{l}\text { SPE- } \\
\text { CIFIC } \\
\text { CON- } \\
\text { DUCT- } \\
\text { ANCE } \\
\text { (US/CM) } \\
(00095)\end{array}$ & $\begin{array}{c}\text { PH } \\
\text { WATER } \\
\text { WHOLE } \\
\text { FIELD } \\
\text { (STAND- } \\
\text { ARD } \\
\text { UNITS) } \\
(00400)\end{array}$ & $\begin{array}{c}\text { TEMPER- } \\
\text { ATURE } \\
\text { AIR } \\
(D E G C) \\
(00020)\end{array}$ & $\begin{array}{c}\text { TEMPER- } \\
\text { ATURE } \\
\text { WATER } \\
\text { (DEG C) } \\
(00010)\end{array}$ & $\begin{array}{c}\text { BARO- } \\
\text { METRIC } \\
\text { PRES- } \\
\text { SURE } \\
\text { (MM } \\
\text { OF } \\
\text { HG) } \\
(00025)\end{array}$ & $\begin{array}{c}\text { OXYGEN, } \\
\text { DIS- } \\
\text { SOLVED } \\
(M G / L) \\
(00300)\end{array}$ & $\begin{array}{c}\text { OXYGEN, } \\
\text { DIS- } \\
\text { SOLVED } \\
\text { (PER- } \\
\text { CENT } \\
\text { SATUR- } \\
\text { ATION) } \\
\text { (00301) }\end{array}$ & $\begin{array}{l}\text { OXYGEN } \\
\text { DEMAND, } \\
\text { CHEM- } \\
\text { ICAL } \\
\text { (HIGH } \\
\text { LEVEL) } \\
\text { (NG/L) } \\
(00340)\end{array}$ & $\begin{array}{l}\text { COLI- } \\
\text { FORM, } \\
\text { FECAL, } \\
0.7 \\
\text { UM-MF } \\
\text { (COLS.' } \\
\text { 100 ML) } \\
(31625)\end{array}$ & $\begin{array}{c}\text { STREP- } \\
\text { TOCOCCI } \\
\text { FECAL, } \\
\text { KF.AGAR } \\
\text { (COLS. } \\
\text { PER } \\
100 \mathrm{ML}) \\
(31673)\end{array}$ \\
\hline
\end{tabular}

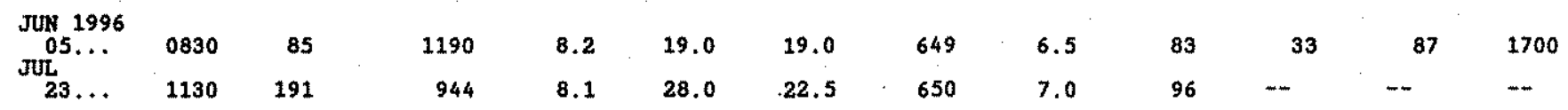

\begin{tabular}{|c|c|c|c|c|c|c|c|c|c|c|c|c|}
\hline TE & $\begin{array}{l}\text { HARD- } \\
\text { NESS } \\
\text { TOTAL } \\
\text { (NG/L } \\
\text { AS } \\
\text { CACO3) } \\
(00900)\end{array}$ & $\begin{array}{l}\text { HARD- } \\
\text { NESS } \\
\text { NONCARB } \\
\text { DISSOLV } \\
\text { FLD. AS } \\
\text { CACOB } \\
\text { (MG/L) } \\
(00904)\end{array}$ & $\begin{array}{c}\text { CALCIUM } \\
\text { DIS- } \\
\text { SOLVED } \\
\text { (MG/L } \\
\text { AS CA }) \\
\text { (00915) }\end{array}$ & $\begin{array}{c}\text { MAGNE- } \\
\text { SIUM, } \\
\text { DIS- } \\
\text { SOLVED } \\
\text { (MG/L } \\
\text { AS MG) } \\
(00925)\end{array}$ & $\begin{array}{l}\text { SODIUM, } \\
\text { DIS- } \\
\text { SOLVED } \\
\text { (NG/L } \\
\text { AS NA) } \\
(00930)\end{array}$ & $\begin{array}{c}\text { SODIUM } \\
\text { AD- } \\
\text { SORP- } \\
\text { TION } \\
\text { RATIO } \\
(00931)\end{array}$ & $\begin{array}{c}\text { POTAS- } \\
\text { SIUM, } \\
\text { DIS- } \\
\text { SOLVED } \\
\text { (MG/L } \\
\text { AS K) } \\
(00935)\end{array}$ & $\begin{array}{c}\text { BICAR- } \\
\text { BONATE } \\
\text { WATER } \\
\text { DIS IT } \\
\text { FIELD } \\
\text { MG/L AS } \\
\text { HCO3 } \\
(00453)\end{array}$ & $\begin{array}{c}\text { CAR- } \\
\text { BONATE } \\
\text { WATER } \\
\text { DIS IT } \\
\text { FIELD } \\
\text { MG/L AS } \\
\text { COS } \\
(00452)\end{array}$ & $\begin{array}{l}\text { ALKA- } \\
\text { LINITY } \\
\text { WAT DIS } \\
\text { TOT IT } \\
\text { FIELD } \\
\text { MG/L AS } \\
\text { CACO3 } \\
(39086)\end{array}$ & $\begin{array}{c}\text { ALKA- } \\
\text { LINITY } \\
\text { IAAB } \\
\text { (MG/L } \\
\text { AS } \\
\text { CACO3) } \\
(90410)\end{array}$ & $\begin{array}{c}\text { SULFATE } \\
\text { DIS- } \\
\text { SOLVED } \\
\text { (MG/L } \\
\text { AS SO4) } \\
\text { (00945) }\end{array}$ \\
\hline
\end{tabular}

\begin{tabular}{|c|c|c|c|c|c|c|c|c|c|c|c|c|}
\hline \multirow{2}{*}{$\begin{array}{c}\text { JUN } 1996 \\
05, \ldots \\
\operatorname{JuL}_{23} \ldots\end{array}$} & 250 & 53 & 77 & 15 & 140 & 4 & 6.9 & 245 & 0 & 200 & 189 & 200 \\
\hline & 220 & 59 & 66 & 13 & 100 & 3 & 5.7 & 194 & 0 & 159 & 206 & 170 \\
\hline
\end{tabular}

\begin{tabular}{|c|c|c|c|c|c|c|c|c|c|c|c|c|}
\hline ATE & $\begin{array}{l}\text { CHLO- } \\
\text { RIDE, } \\
\text { DIS- } \\
\text { SOLVED } \\
\text { (MG/L } \\
\text { AS CL) } \\
(00940)\end{array}$ & $\begin{array}{l}\text { FLUO- } \\
\text { RIDE: } \\
\text { DIS- } \\
\text { SOLVED } \\
\text { (MG/L } \\
\text { AS F) } \\
(00950)\end{array}$ & $\begin{array}{c}\text { SILICA, } \\
\text { DIS- } \\
\text { SOLVED } \\
\text { (NG/L } \\
\text { AS } \\
\text { SIO2) } \\
\text { (00955) }\end{array}$ & $\begin{array}{c}\text { SOLIDS, } \\
\text { RESIDUUE } \\
\text { AT } 180 \\
\text { DEG. C } \\
\text { DIS- } \\
\text { SOLVED } \\
(M G / L) \\
(70300)\end{array}$ & $\begin{array}{c}\text { SOLIDS, } \\
\text { SUM OF } \\
\text { CONSTI- } \\
\text { TUENTS, } \\
\text { DIS- } \\
\text { SOLVED } \\
\text { (MG/L) } \\
(70301)\end{array}$ & $\begin{array}{c}\text { NITRO- } \\
\text { GEN, } \\
\text { NITRATE } \\
\text { DIS- } \\
\text { SOLVED } \\
\text { (MG/L } \\
\text { AS N) } \\
\text { (00618) }\end{array}$ & $\begin{array}{c}\text { MITRO- } \\
\text { GEN, } \\
\text { NITRITE } \\
\text { DIS- } \\
\text { SOLVED } \\
\text { (MG/L } \\
\text { AS N) } \\
\text { (00613) }\end{array}$ & $\begin{array}{c}\text { NITRO- } \\
\text { GEN, } \\
\text { NO2+NO3 } \\
\text { DIS- } \\
\text { SOLVED } \\
\text { (MG/L } \\
\text { AS N) } \\
(00631)\end{array}$ & $\begin{array}{c}\text { NITRO- } \\
\text { GEN, } \\
\text { AMNONIA } \\
\text { DIS- } \\
\text { SOLVED } \\
\text { (MG/L } \\
\text { AS N) } \\
(00608)\end{array}$ & $\begin{array}{c}\text { NITRO- } \\
\text { GEN, } \\
\text { ORGANIC } \\
\text { DIS - } \\
\text { SOLVED } \\
\text { (MG/L } \\
\text { AS N) } \\
(00607)\end{array}$ & $\begin{array}{c}\text { NITRO- } \\
\text { GEN,AM- } \\
\text { MONIA + } \\
\text { ORGANIC } \\
\text { TOTAL } \\
\text { (MG/L } \\
\text { AS N) } \\
(00625)\end{array}$ & $\begin{array}{l}\text { NITRO- } \\
\text { GEN,AM- } \\
\text { MONIA + } \\
\text { ORGANIC } \\
\text { DIS. } \\
\text { (MG/L } \\
\text { AS N) } \\
(00623)\end{array}$ \\
\hline $05 .$. & 120 & 60 & 25 & $\cdots$ & 705 & -- & $<0.010$ & $<0.050$ & 0.030 & 0.27 & 0.60 & 0.30 \\
\hline & 74 & 0.60 & 24 & 594 & 550 & 0.220 & 0.010 & 0.230 & 0.040 & - & 0.50 & $<0.20$ \\
\hline
\end{tabular}

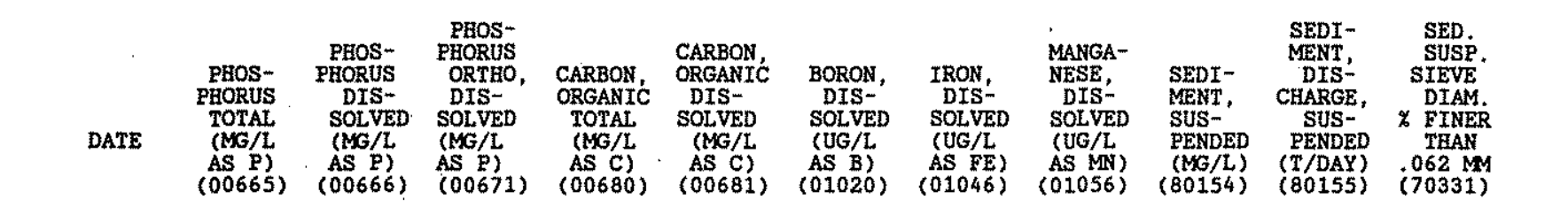

\begin{tabular}{|c|c|c|c|c|c|c|c|c|c|c|c|}
\hline $\begin{array}{c}\text { JUN } 1996 \\
05 . \cdots\end{array}$ & 0.150 & 0.050 & 0.050 & -- & -- & 212 & 3.0 & -- & 98 & 22 & 77 \\
\hline $23 \ldots$ & 0.280 & 0.060 & 0.080 & 6.1 & 3.2 & - & $<3.0$ & 2.0 & 386 & 199 & - \\
\hline
\end{tabular}


RIO GRANDE BASIN

08358400 RIO GRANDE FLOODWAY AT SAN MARCIAL, NM

LOCATION.--"Lat $33^{\circ} 40^{\circ} 50^{\prime \prime}$, long $106^{\circ} 59^{\prime} 30^{\prime \prime}$. Socorro County, Hydrologic Unit 13020203, in Pedro Armendaris Grant No. 33 , on pier of the Atchison, Topeka, and Santa Ee Railway Co. bridge, 1.1 mi downstream from formex site of San Marcial, $18.5 \mathrm{mi}$ southwest of $\operatorname{San}$ Antonio, and at mile 1,425.2.

DRAINAGE AREA. --27,700 $\mathrm{mi}^{2}$, approximately, including 2,940 $\mathrm{mi}^{2}$ in closed basin in San Luis Valley, Co.

WATER-DISCHARGE RECORDS

PERTOD OF RECORD. - October 1964 to current year. Records collected at this site January 1895 to September 1964 represented total flow of the river med were published es Rio Grande at San Marclal (station o8358500). Rocords of dally discharge for floodway only, Apr11 1950, to September 1964, are avallable in files of district office.

GAGE.-Water-stage recorder. Datum of gage is 4,455.19 ft above National Geodetic Vertical Datum of 1929.

REMARKS.--Water-discharge records fair except for estimated dally dischargez, which are poor, Floodway 18 of 2 channels (station 08358300) carrying flow in valley cross section. Prior to 1950 all flow was in floodway channel. Normal plan is for floodway to carry slow when capacity of conveyance channel (about 2,000 ft $3 / \mathrm{s}$ ) is . exceeded. Combined monthly discharge in acre-ft is given at ond of each yoar table. Diversion for irrigation of about 775,000 acres upstream from station (includes about 13,800 acre-ft diverted from conveyance channel, as based on peekly measurements, data provided by Bureau of Reciamation). Bureau of Reclamation satelifte telemeter at station.

AVRRAGE DISCHARGE, - 32 years (water years 1965-96), $807 \mathrm{ft}$ s/s, 584,700 acre-ft/yr. Total flow of river, 101 years (water years 1895-1996), 1,273 fts/s, 922,300 acre-1t/yr.

EXTREXIXS FOR PERIOD OF RECORD,-Maximum discharge, since January 1895, about 50,000 fts/s, 0ct. 11, 1904; no Ilow at times.

EXTREES FOR CURREAT YEAR.-Maximum daily discharge, $1,690 \mathrm{ft} 3 / \mathrm{s}$, June 30, no flow at rimes, DISCHARGE, CUBIC FEET PER SECOND, WATER YEAR OCTOBER 1995 TO SEPTEMBER 1996

\begin{tabular}{|c|c|c|c|c|c|c|c|c|c|c|c|c|}
\hline DAY & $\operatorname{CCT}$ & Nov & $\mathrm{DEC}$ & JAN & FEB & MAR & ARR & MAY & JUN & JUL & AUG & SEP \\
\hline $\begin{array}{l}1 \\
2 \\
3 \\
4 \\
5\end{array}$ & $\begin{array}{l}416 \\
537 \\
399 \\
427 \\
445\end{array}$ & $\begin{array}{r}286 \\
472 \\
851 \\
1010 \\
971\end{array}$ & $\begin{array}{l}928 \\
897 \\
870 \\
856 \\
872\end{array}$ & $\begin{array}{r}899 \\
894 \\
955 \\
1010 \\
985\end{array}$ & $\begin{array}{r}912 \\
932 \\
991 \\
1070 \\
1070\end{array}$ & $\begin{array}{r}1120 \\
985 \\
893 \\
779 \\
898\end{array}$ & $\begin{array}{r}213 \\
118 \\
96 \\
82 \\
e 13\end{array}$ & $\begin{array}{l}.00 \\
.00 \\
.00 \\
.00 \\
.00\end{array}$ & $\begin{array}{l}.00 \\
.00 \\
.00 \\
.00 \\
.00\end{array}$ & $\begin{array}{l}951 \\
916 \\
780 \\
950 \\
796\end{array}$ & $\begin{array}{l}.00 \\
.00 \\
.00 \\
.00 \\
.00\end{array}$ & $\begin{array}{r}585 \\
816 \\
422 \\
209 \\
55\end{array}$ \\
\hline $\begin{array}{r}6 \\
7 \\
8 \\
9 \\
10\end{array}$ & $\begin{array}{l}425 \\
315 \\
268 \\
261 \\
246\end{array}$ & $\begin{array}{l}1020 \\
1130 \\
1180 \\
1090 \\
1140\end{array}$ & $\begin{array}{l}885 \\
887 \\
898 \\
876 \\
849\end{array}$ & $\begin{array}{r}982 \\
1050 \\
1020 \\
968 \\
969\end{array}$ & $\begin{array}{r}1020 \\
997 \\
993 \\
931 \\
892\end{array}$ & $\begin{array}{r}859 \\
826 \\
765 \\
681 \\
684\end{array}$ & $\begin{array}{l}.00 \\
.00 \\
.00 \\
.00 \\
.00\end{array}$ & $\begin{array}{l}.00 \\
.00 \\
.00 \\
.00 \\
.00\end{array}$ & $\begin{array}{l}.00 \\
.00 \\
.00 \\
.00 \\
.00\end{array}$ & $\begin{array}{r}565 \\
230 \\
211 \\
19 \\
\quad .00\end{array}$ & $\begin{array}{r}.00 \\
.00 \\
.00 \\
0210^{.00}\end{array}$ & $\begin{array}{r}8.8 \\
.00 \\
.00 \\
.00 \\
.00\end{array}$ \\
\hline $\begin{array}{l}11 \\
12 \\
13 \\
14 \\
15\end{array}$ & $\begin{array}{l}238 \\
247 \\
231 \\
203 \\
193\end{array}$ & $\begin{array}{l}1170 \\
1130 \\
1110 \\
1100 \\
1080\end{array}$ & $\begin{array}{l}886 \\
905 \\
933 \\
929 \\
928\end{array}$ & $\begin{array}{r}975 \\
1050 \\
1120 \\
1100 \\
1040\end{array}$ & $\begin{array}{r}931 \\
983 \\
1000 \\
997 \\
996\end{array}$ & $\begin{array}{l}604 \\
494 \\
485 \\
490 \\
394\end{array}$ & $\begin{array}{l}.00 \\
.00 \\
.00 \\
.00 \\
.00\end{array}$ & $\begin{array}{l}.00 \\
.00 \\
.00 \\
.00 \\
.00\end{array}$ & $\begin{array}{l}.00 \\
.00 \\
.00 \\
.00 \\
.00\end{array}$ & $\begin{array}{r}384 \\
1160 \\
924 \\
837 \\
487\end{array}$ & $\begin{array}{r}552 \\
312 \\
266 \\
135 \\
39\end{array}$ & $\begin{array}{l}389^{.00} \\
791 \\
823\end{array}$ \\
\hline $\begin{array}{l}16 \\
17 \\
18 \\
19 \\
20\end{array}$ & $\begin{array}{l}202 \\
191 \\
164 \\
181 \\
179\end{array}$ & $\begin{array}{l}1130 \\
1120 \\
1120 \\
1120 \\
1120\end{array}$ & $\begin{array}{l}920 \\
926 \\
934 \\
942 \\
941\end{array}$ & $\begin{array}{r}1020 \\
999 \\
1020 \\
1050 \\
1030\end{array}$ & $\begin{array}{l}1020 \\
1060 \\
1050 \\
1030 \\
1000\end{array}$ & $\begin{array}{l}367 \\
406 \\
425 \\
451 \\
507\end{array}$ & $\begin{array}{l}.00 \\
.00 \\
.00 \\
.00 \\
.00\end{array}$ & $\begin{array}{l}.00 \\
.00 \\
.00 \\
.00 \\
.00\end{array}$ & $\begin{array}{l}.00 \\
.00 \\
.00 \\
.00 \\
.00\end{array}$ & $\begin{array}{r}191 \\
453 \\
194 \\
96 \\
126\end{array}$ & $\begin{array}{r}022 \\
.00 \\
.00 \\
.00 \\
.00\end{array}$ & $\begin{array}{r}1220 \\
1160 \\
1050 \\
622 \\
456\end{array}$ \\
\hline $\begin{array}{l}21 \\
22 \\
23 \\
24 \\
25\end{array}$ & $\begin{array}{l}185 \\
160 \\
131 \\
126 \\
153\end{array}$ & $\begin{array}{r}1130 \\
1160 \\
1100 \\
1040 \\
994\end{array}$ & $\begin{array}{r}957 \\
1010 \\
982 \\
922 \\
896\end{array}$ & $\begin{array}{l}1050 \\
1070 \\
1070 \\
1040 \\
1030\end{array}$ & $\begin{array}{r}965 \\
963 \\
970 \\
1090 \\
1310\end{array}$ & $\begin{array}{l}573 \\
588 \\
537 \\
429 \\
359\end{array}$ & $\begin{array}{l}.00 \\
.00 \\
.00 \\
.00 \\
.00\end{array}$ & $\begin{array}{l}.00 \\
.00 \\
.00 \\
.00 \\
.00\end{array}$ & $\begin{array}{l}.00 \\
.00 \\
.00 \\
.00 \\
.00\end{array}$ & $\begin{aligned} & 213 \\
& 38 \\
& .00 \\
& .00 \\
& .00\end{aligned}$ & $\begin{array}{r}.00 \\
.00 \\
.00 \\
118\end{array}$ & $\begin{array}{r}413 \\
372 \\
169 \\
12 \\
.00\end{array}$ \\
\hline $\begin{array}{l}26 \\
27 \\
28 \\
29 \\
30 \\
31\end{array}$ & $\begin{array}{l}218 \\
317 \\
270 \\
279 \\
309 \\
298\end{array}$ & $\begin{array}{l}933 \\
888 \\
885 \\
892 \\
941 \\
---\end{array}$ & $\begin{array}{l}881 \\
885 \\
891 \\
890 \\
883 \\
890\end{array}$ & $\begin{array}{r}1010 \\
953 \\
933 \\
915 \\
935 \\
919\end{array}$ & $\begin{array}{l}1200 \\
1160 \\
1150 \\
1140 \\
--\end{array}$ & $\begin{array}{l}316 \\
325 \\
331 \\
289 \\
137 \\
241\end{array}$ & $\begin{array}{l}.00 \\
.00 \\
.00 \\
.00 \\
.00 \\
.\end{array}$ & $\begin{array}{l}.00 \\
.00 \\
.00 \\
.00 \\
.00 \\
.00\end{array}$ & $\begin{array}{r}.00 \\
654^{.00} \\
1690\end{array}$ & $\begin{array}{l}.00 \\
.00 \\
.00 \\
.00 \\
.00 \\
.00\end{array}$ & $\begin{array}{r}361 \\
881 \\
1070 \\
1020 \\
628 \\
381\end{array}$ & $\begin{array}{l}.00 \\
.00 \\
.00 \\
.00 \\
.00 \\
.\end{array}$ \\
\hline $\begin{array}{l}\text { TOTAL } \\
\text { MEAN } \\
\text { MAX } \\
\text { MIN } \\
\text { AC-FT } \\
(\dagger)\end{array}$ & $\begin{array}{r}8214 \\
265 \\
537 \\
126 \\
16290 \\
36890\end{array}$ & $\begin{array}{r}30313 \\
1010 \\
1180 \\
286 \\
60130 \\
76180\end{array}$ & $\begin{array}{r}28149 \\
908 \\
1010 \\
849 \\
55830 \\
71340\end{array}$ & $\begin{array}{r}31061 \\
1002 \\
1120 \\
894 \\
61610 \\
77130\end{array}$ & $\begin{array}{r}29823 \\
1028 \\
1310 \\
892 \\
59150 \\
74810\end{array}$ & $\begin{array}{r}17238 \\
556 \\
1120 \\
137 \\
34190 \\
55020\end{array}$ & $\begin{array}{r}522.00 \\
17.4 \\
213 \\
.00 \\
1040 \\
17110\end{array}$ & $\begin{array}{r}0.00 \\
.000 \\
.00 \\
.00 \\
.00 \\
8670\end{array}$ & $\begin{array}{r}2344.00 \\
78.1 \\
1690 \\
.00 \\
4650 \\
15400\end{array}$ & $\begin{array}{r}10521.00 \\
339 \\
1160 \\
.00 \\
20870 \\
35790\end{array}$ & $\begin{array}{r}5995.00 \\
193 \\
1070 \\
100 \\
11890 \\
23430\end{array}$ & $\begin{array}{r}9572.80 \\
319 \\
1220 \\
.00 \\
18990 \\
33900\end{array}$ \\
\hline
\end{tabular}

CAL YR 1995. TOTAL 579540.00 MEAN 1588 MAX 4880 MrN .00 AC-FT 1150000 (†) MEAN 1934 AC-FT 1,401,00 WTR YR 1996 TOTAL 173752.80 MEAN 475 MAX 1690 MIN .00 AC-FT 344600 (t) MEAN 724 AC-FT 525,700

Estimated

( $\uparrow$ COABINED FLOW, IN ACRE-FEET, AND MEAN, IN CUBIC FEET PER SECOND, OF FLOODWAY AND CONVEYANCE CHANNEL. 
RIO GRANDE BASIN

08358400 RIO GRANDE FLOODWAY AT SAN MARCIAL, NM -- Continued

WATER-QUALITY RECORDS

PERIOD OF RECORD. -Water years 1905-07, 1946 to current year.

PERIOD OF DAILY RECORD ..-

SPECIFIC CONDUCTANCE: May 1905 to Apri1 1907, July 1946 to current year.

WATER TEMPERATURE: January 1949 to September 1994 (discontinued)

SUSPENDED-SEDIMENT DISCEARGE: JuIy 1946 to current year.

IHSTRUMENTATION. --Automatic pump sampler since September 1994.

REMARKS.--Records of chemical analyses and sediment discharge for years prior to 1946 have been published in Water Bulletins of International Boundary and Water Comission. Sediment total-1oads (suspended plus bed material discharge), In tons per day, were determined from the regression equation developed for the period of record. Once-daily specific conductance values were determined in the laboratory from daily suspended sediment samples collected by Isco sampler.

EXTREMES FOR PERTOD OF DAILY RECORD -

SPECIFIC CONDUCTANCE: Maximum daily, 2,730 microsiemens, Apr. 8, 1953; minimum dally, 276 microsiemens, May 11, 1994.

SEDIMENT CONCENTRATION: Maximum daily mean, 135,000 ms/L, July 23, 1977; minimum daily mean, no flow on many days each year.

SEDIMENT LOAD: Maximum dally, 1,200,000 tons, Sept. 21, 1982; mintmum dally, 0 ton on many days each year.

EXTREMES FOR CURRENT YEAR.--

SPECIFIC CONDUCTANCE: Maximum daily, 1,620 microsiemens, July 13; minimum daily, 410 microstemens, Fob. 6. SEDIMENT CONCENTRATION: Maximum daily mean, $38,100 \mathrm{mg} / \mathrm{L}$, Sept. $14 ;$ minimum daily mean, no flow on many days . SEDIMENT LOAD: Maximum daily, 114,000 tons, Sept. 16; minimum daliy, 0 ton on many days.

WATER QUALITY DATA, WATER YEAR OCTOBER 1995 TO SEPTEMBER 1996

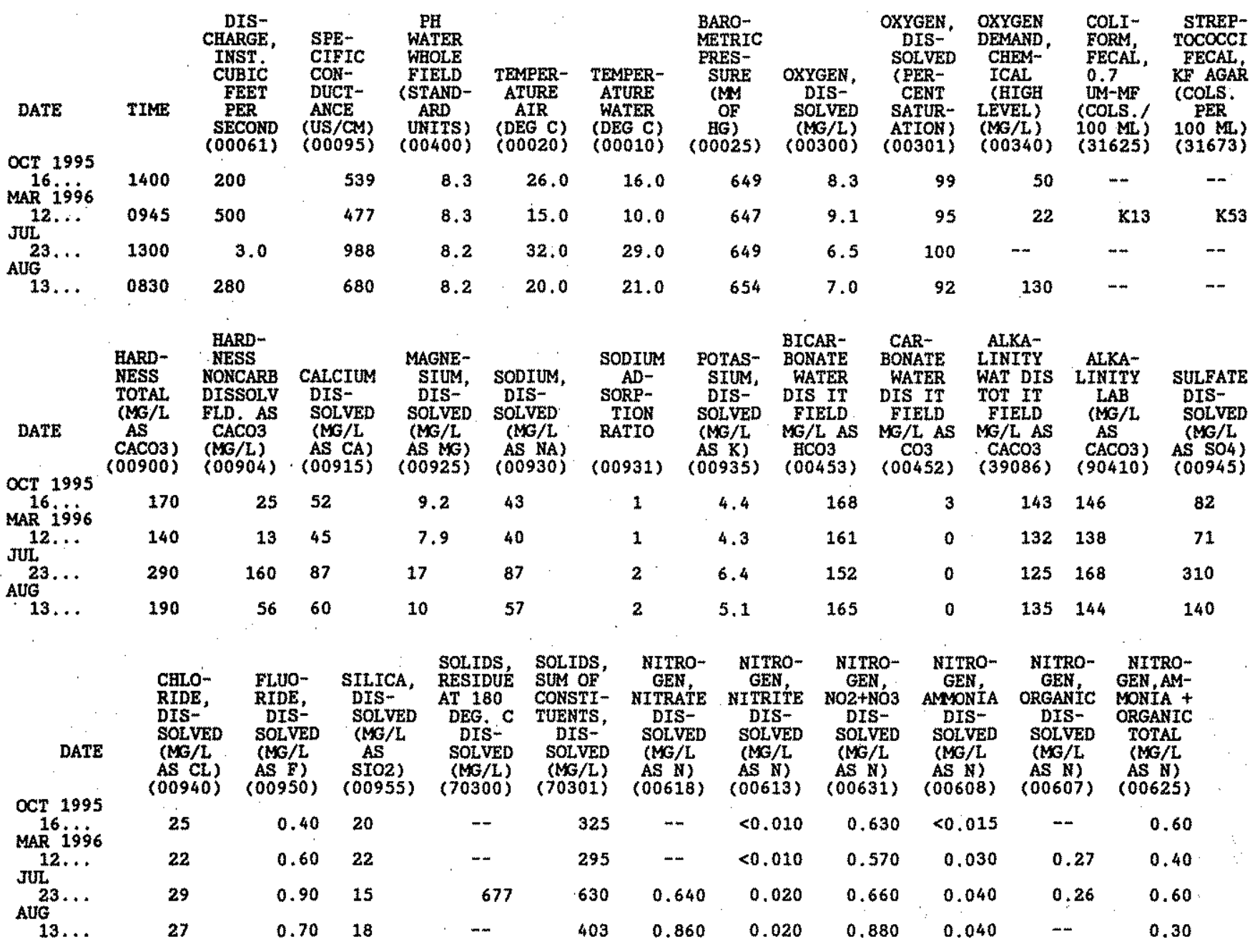


RIO GRANDE BASIN

08358400 RIO GRANDE FLOODWAY AT SAN MARCIAI, MM - Continued

WATER-QUALITY RECORDS

WATER QUALITY DATA; WATER YEAR OCTOBER 1995 TO SEPTERBER 1996

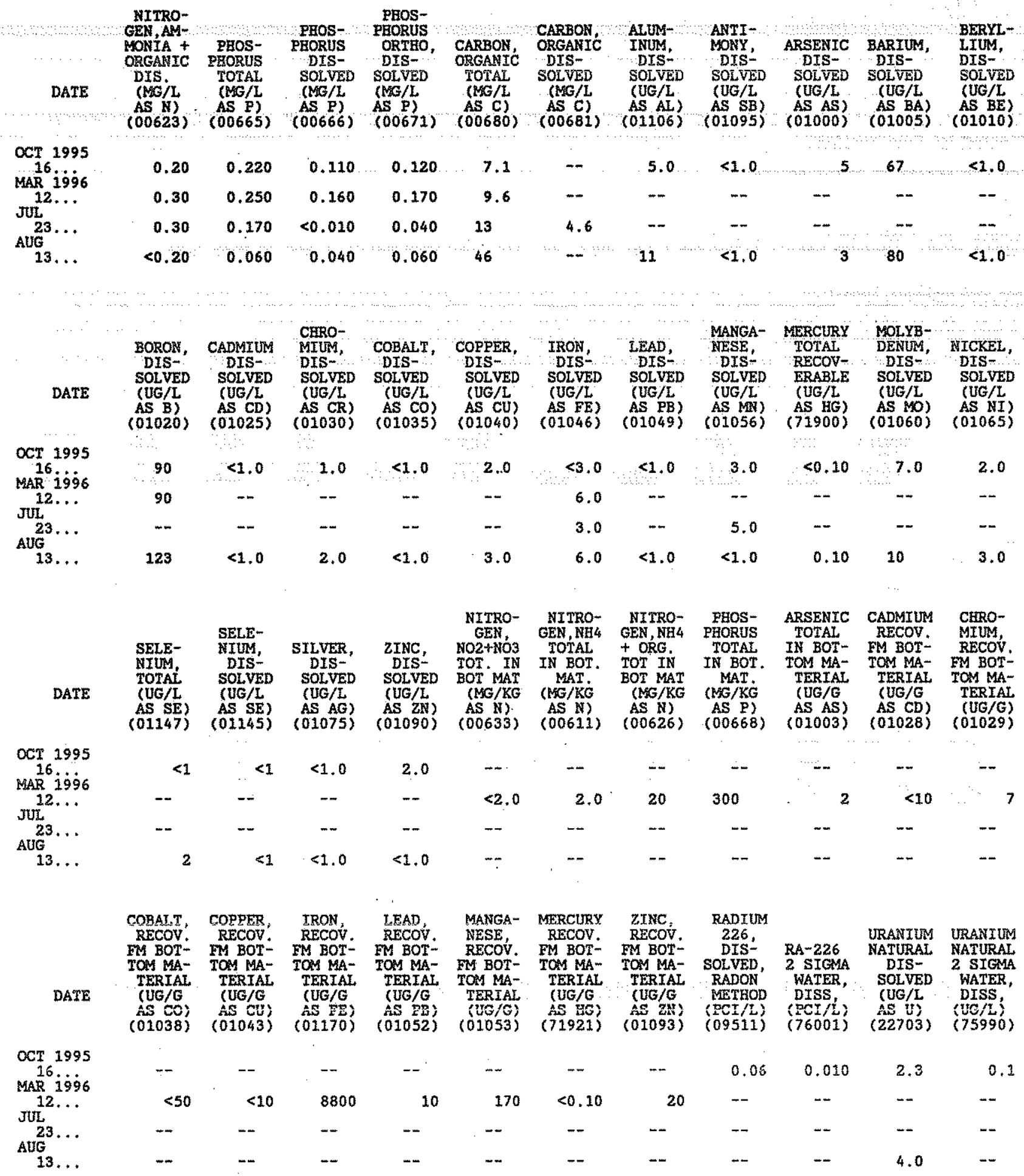


RIO GRANDE BASIN

08358400 RIO GRANDE FLOODWAY AT SAY MARCIAL, NM -- Continued

WATER-QUALITY RECORDS

CHEMICAL ANALYSES OF ORGANIC COMPOUNDS, WATER YEAR OCTOBER 1995. TO SEPTEMBER 1996

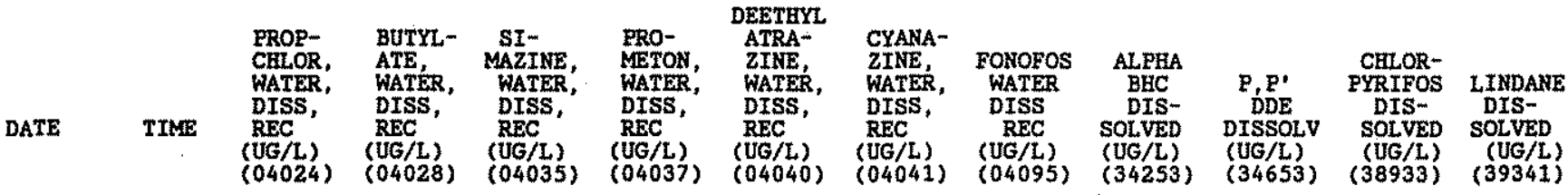

\begin{tabular}{|c|c|c|c|c|c|c|c|c|c|c|c|c|}
\hline $\begin{array}{l}\text { OCT } 1995 \\
16 \\
\text { MAR } 1996\end{array}$ & 1400 & $m$ & -- & $m$ & -- & $m$ & -- & -- & $-m$ & $-m$ & -- & $m$ \\
\hline JuL & 0945 & - & $m-$ & - & $-m$ & -- & -- & -- & . & -- & -- & -- \\
\hline UG $^{23} \ldots$ & 1300 & $<0.007$ & $<0,002$ & 0.008 & E0.014 & $\mathrm{E} 0.003$ & $<0,004$ & $<0.003$ & $<0.002$ & $<0.006$ & $<0.004$ & $<0.004$ \\
\hline 13 & 0830 & -- & -- & $-m$ & -- & $m$ & -- & -- & -- & $m$ & -- & $-m$ \\
\hline
\end{tabular}

\begin{tabular}{|c|c|c|c|c|}
\hline $\begin{array}{l}\text { DI- } \\
\text { ELDRIN } \\
\text { DIS- } \\
\text { SOLVED } \\
\text { (UG/L) } \\
39381 \text { ) }\end{array}$ & $\begin{array}{l}\text { METO- } \\
\text { LACHLOR } \\
\text { WATER } \\
\text { DISSOLV } \\
\text { (UG/L) } \\
\text { (39415) }\end{array}$ & $\begin{array}{l}\text { MALA- } \\
\text { THION, } \\
\text { DIS- } \\
\text { SOLVED } \\
\text { (UG/L) } \\
\text { (39532) }\end{array}$ & $\begin{array}{c}\text { PARA- } \\
\text { THION, } \\
\text { DIS - } \\
\text { SOLVED } \\
\text { (UG/L) } \\
(39542)\end{array}$ & $\begin{array}{c}\text { DI- } \\
\text { AZINON, } \\
\text { DIS- } \\
\text { SOLVDD } \\
\text { (UG/L) } \\
\text { (39572) }\end{array}$ \\
\hline
\end{tabular}

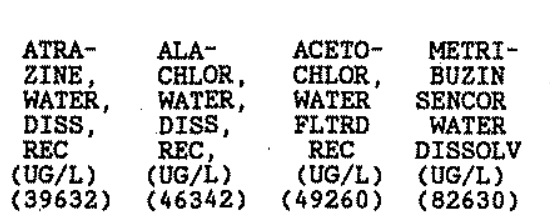

2, 6-DIETHYL ANILINE FLURWAT FLT WAT FLT $0.7 \mathrm{U}$ GF REC (UF, REC ( $(\mathrm{U} G / \mathrm{L})$

$\begin{array}{cc}\text { TRI- } & \text { ETHAL- } \\ \text { FLUR- } & \text { FLUR- } \\ \text { ALIN } & \text { ALIN } \\ \text { HAT FLT } & \text { WAT FLT } \\ 0.7 \text { U } & 0.7 \text { U } \\ \text { GE, REC } & \text { GF, REC } \\ (U G / L) & (U G / L) \\ (82661) & (82663)\end{array}$

\section{OCT 1995}

MAR 1696

$12 \ldots$

$\begin{array}{cc}\underset{12}{\mathrm{MAR}} 19 \dot{9} 6 & \\ 12 \ldots & -- \\ \text { JUL } & \\ 23 \ldots & <0.001 \\ \text { AUUG } & \\ 13 . . & --\end{array}$

PHORATE
WATER
FLTRD
0.7 U
GF, REC GPA
(UG/L) (

TER-

$\begin{array}{ccc}\text { TER- } & \text { LIN- } & \text { METHYL } \\ \text { BACIL } & \text { URON } & \text { PARA- } \\ \text { WATER } & \text { WATER } & \text { THION } \\ \text { FLTRD } & \text { FLTRD } & \text { WAT FLT } \\ 0.7 \mathrm{U} & 0.7 \mathrm{U} & 0.7 \mathrm{U} \\ \text { GF, REC } & \text { GF, REC } & \text { GF, REC } \\ (\mathrm{UG} / \mathrm{L}) & (\mathrm{UG} / \mathrm{L}) & (\mathrm{UG} / L) \\ (82665) & (82666) & (82667)\end{array}$

$\ldots$
0.006
$\cdots$


EPTC
WATER
FLTRD
$0.7 \mathrm{U}$
F, REC
UG/L)
(2668)

$\begin{array}{cc}\cdots & - \\ -- & -- \\ 0.005 & <0.002 \\ -- & - \\ & \\ \text { PEB- } & \text { TEBU- } \\ \text { ULATEE } & \text { THIURON } \\ \text { WATER } & \text { WATER } \\ \text { FILTRD } & \text { FLTRD } \\ 0.7 \mathrm{U} & 0.7 \mathrm{U} \\ \text { GF, REC } & \text { GF, REC } \\ (\mathrm{UGG} / \mathrm{L}) & (\mathrm{UG} / \mathrm{L}) \\ (82669) & (82670) \\ & \end{array}$

--
$<0.002$
--

MOL-
INATE
WATER
FLTRD
0.7 U
GF REC
(UG/L)
$(82671)$

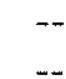

OCT 1995

MAR 1696

JUL $12 \ldots$

AJG $^{23}$

$<0.002$

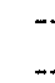

$-$

13.
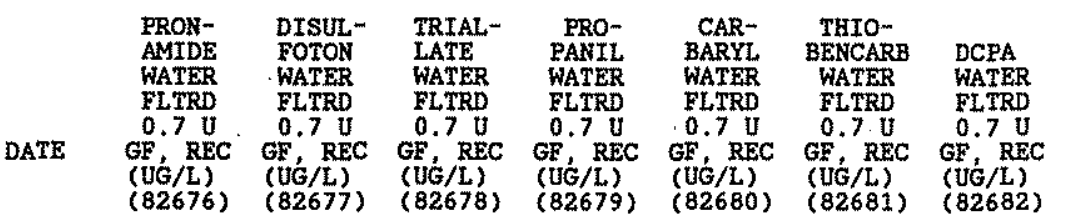

PENDI - NAPROP-

PRO- METHYL PER-

ALTN WATER

ALIN WATER

WAT FLT FLTRD

$0.7 \mathrm{U}$

$0.7 \mathrm{U}$
$\mathrm{GF}, \mathrm{REC}$
$(\mathrm{UG} / \mathrm{L})$

(IGG/L)
$(82683)$

(82684)

FLTRD WAT ELT WAT FLT
$0.7 \mathrm{U}$
$0.7 \mathrm{U}$
$0.7 \mathrm{U}$

GF, REC GF, REC

$\begin{array}{lll}(82685) & (\text { UG } / L) & (\text { UG } / L) \\ & (82686) & (82687)\end{array}$

OCT 1995

$16+996$

JuL $12 \ldots$

$23 \ldots<0.003$

AUG

13.

$<0.003$

$<0.017$

$<0.001$

$--$

00.003

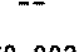

$-$

$--$

$-$

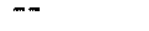

-. 
RIO GRANDE BASIN

08358400 RIO GRANDE FLOODWAY AT SAN MARCIAL, MM - Continued

WATER-QUALITY RECORDS

WATER QUALITY DATA, WATER YEAR OCTOBER 1995 TO SEPTEMBER 1996

\begin{tabular}{|c|c|c|c|c|c|c|c|c|c|c|c|c|}
\hline DATE & TIAE & $\begin{array}{c}\text { DIS- } \\
\text { CHARGE, } \\
\text { INST } \\
\text { CUBYC } \\
\text { PEEI } \\
\text { ZEE } \\
\text { SECOHD } \\
(00061)\end{array}$ & $\begin{array}{c}\text { STREAM } \\
\text { WIDTE } \\
\text { (FT) } \\
(00004)\end{array}$ & $\begin{array}{c}\text { STREAM } \\
\text { DEPTH, } \\
\text { HEATH } \\
\text { (FT) } \\
(00064)\end{array}$ & $\begin{array}{l}\text { STREAM } \\
\text { VELOC } \\
\text { ITY } \\
\text { STAE } \\
(F / S) \\
(00055)\end{array}$ & $\begin{array}{l}\text { SPE- } \\
\text { CIFIC } \\
\text { CON- } \\
\text { DUCT- } \\
\text { ARCE } \\
\text { (US/CM) } \\
(00095)\end{array}$ & $\begin{array}{c}\text { TEMPER- } \\
\text { ATURE } \\
\text { NATER } \\
\text { (DEG C) } \\
(00010)\end{array}$ & $\begin{array}{l}\text { SEDT- } \\
\text { MENT, } \\
\text { SUS- } \\
\text { EENDED } \\
(M G / L) \\
(80154)\end{array}$ & $\begin{array}{c}\text { SEDI- } \\
\text { MENT, } \\
\text { DIS- } \\
\text { CHARGE, } \\
\text { SUS- } \\
\text { PEIWDED } \\
\text { (T/DAY) } \\
(80155)\end{array}$ & $\begin{array}{l}\text { SEDI- } \\
\text { MENT, } \\
\text { DISCH, } \\
\text { SUSP, } \\
\text { BED MA- } \\
\text { TERIAL } \\
(\text { T/DAY) } \\
(80156)\end{array}$ & $\begin{array}{c}\text { SED } \\
\text { SUSP. } \\
\text { SIEVE } \\
\text { DTAM. } \\
\text { X FINER } \\
\text { TEAS } \\
\text { O62 MM } \\
(70331)\end{array}$ & $\begin{array}{l}\text { SED. } \\
\text { SUSP. } \\
\text { FALL } \\
\text { DIAM. } \\
\% \text { FINER } \\
\text { FruAN } \\
\text { (062 MM } \\
(70342)\end{array}$ \\
\hline $\begin{array}{l}1995 \\
16 \ldots\end{array}$ & 1400 & 200 & 180 & 0.86 & 1.29 & 539 & 16.0 & 340 & 184 & 316 & 90 & $\cdots$ \\
\hline EC & 1030 & 1200 & -- & - & -- & 577 & 19.5 & 2300 & 7450 & 9420 & 91 & $-\cdots$ \\
\hline $14 \ldots$ & $\begin{array}{l}1045 \\
1030\end{array}$ & $\begin{array}{l}910 \\
929\end{array}$ & 176 & 2.7 & 1.92 & $-\overline{551}$ & $\begin{array}{l}5.5 \\
4.0\end{array}$ & $\begin{array}{r}159 \\
1270\end{array}$ & $\begin{array}{r}391 \\
3190\end{array}$ & $\begin{array}{r}631 \\
4330\end{array}$ & 58 & \\
\hline $12 \ldots$ & 1025 & 1020 & 183 & 2.7 & 2.09 & -- & -- & 127 & 350 & 570 & $\cdots$ & 79 \\
\hline $21 \ldots$ & 1244 & 967 & 175 & 2. & 1.93 & - & 12.0 & 134 & 350 & 570 & - & 83 \\
\hline $\begin{array}{l}12 \ldots \\
19\end{array}$ & $\begin{array}{l}0945 \\
1105\end{array}$ & $\begin{array}{l}500 \\
444\end{array}$ & $180^{--}$ & -- & 2.52 & 477 & 10. & $\begin{array}{r}226 \\
80\end{array}$ & $\begin{array}{r}305 \\
96\end{array}$ & $\begin{array}{l}503 \\
174\end{array}$ & $\begin{array}{l}93 \\
95\end{array}$ & $=$ \\
\hline $19 \ldots$ & $\begin{array}{l}1319 \\
1300\end{array}$ & 3.0 & 56.0 & 0.32 & 1.10 & 988 & $\begin{array}{l}25.0 \\
29.0\end{array}$ & $\begin{array}{r}664 \\
1020\end{array}$ & $\begin{array}{l}36 \\
8.3\end{array}$ & 71 & 99 & -- \\
\hline & 0830 & 280 & -- & $\cdots$ & -- & 680 & 21.0 & 5140 & 3890 & 5190 & 100 & $m$ \\
\hline
\end{tabular}

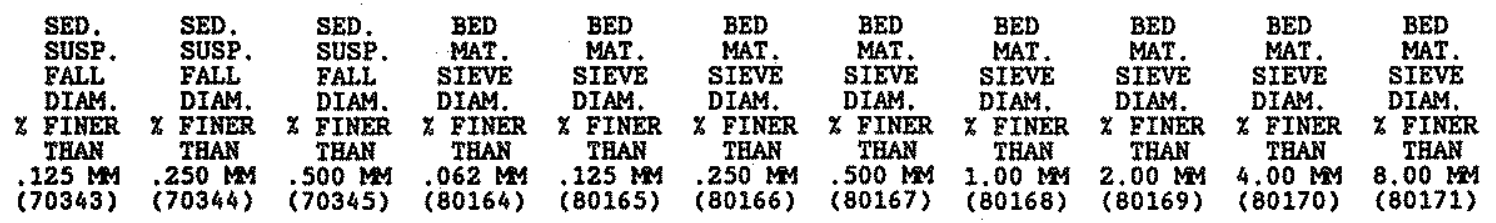

$$
\begin{gathered}
\text { OCT } 1995 \\
16 \ldots \\
\text { NOV } \ldots \\
08 \ldots \\
\text { DEC } \ldots \\
01 \ldots \\
14 \ldots \\
\text { JAN } 1996 \\
12 \ldots \\
\text { FEB } \\
21 \ldots \\
\text { MAR } \ldots \\
12 \ldots \\
19 \ldots \\
\text { JUL } \ldots \\
09 \ldots \\
23 \ldots \\
\text { AvE } \ldots \\
13 \ldots
\end{gathered}
$$

$$
\begin{aligned}
& \begin{array}{cccccccccccc}
-- & - & - & & 1 & 2 & 30 & 96 & 100 & \ldots & -.
\end{array} \\
& \text {-.. } \\
& \begin{array}{lll}
90 \quad 100 \quad \ldots- & -
\end{array} \\
& 8198 \quad 100 \\
& 8599 \quad 100 \\
& =\quad-\cdots \quad- \\
& --
\end{aligned}
$$


RIO GRANDE BASIN

08358400 RIO GRANDE FLOODWAY AT SAN MARCTAL, NM -- Continued

WATER-QUALITY RECORDS

SPECIFIC CONDUCTANCE, US/CM 25 DEGREES CENTIGRADE, WATER YEAR OCTOBER 1995 TO SEPTEMAER 1996 DAILY INSTANTANEOUS VALUES

\begin{tabular}{|c|c|c|c|c|c|c|c|c|c|c|c|c|}
\hline DAY & $\propto C T$ & nov & DEC & JAN & FEB & MAR & AFR & MAY & JUN & JUL & AUG & SEP \\
\hline $\begin{array}{l}1 \\
2 \\
3 \\
4 \\
5\end{array}$ & $\begin{array}{l}531 \\
490 \\
491 \\
601 \\
539\end{array}$ & $\begin{array}{l}524 \\
562 \\
533 \\
533 \\
520\end{array}$ & $\begin{array}{l}499 \\
501 \\
504 \\
504 \\
505\end{array}$ & $\begin{array}{l}505 \\
502 \\
498 \\
490 \\
486\end{array}$ & $\begin{array}{l}487 \\
491 \\
493 \\
485 \\
475\end{array}$ & $\begin{array}{l}474 \\
474 \\
474 \\
490 \\
480\end{array}$ & $\begin{array}{l}534 \\
535 \\
552 \\
590 \\
605\end{array}$ & $\begin{array}{l}- \\
-- \\
-- \\
-- \\
--m\end{array}$ & $\begin{array}{l}m-- \\
-\cdots \\
-\cdots\end{array}$ & $\begin{array}{r}617 \\
899 \\
1470 \\
1230\end{array}$ & $\begin{array}{l}1050 \\
1060 \\
1060 \\
1070 \\
\end{array}$ & $\begin{array}{r}1090 \\
1250 \\
1090 \\
855 \\
764\end{array}$ \\
\hline $\begin{array}{r}6 \\
7 \\
8 \\
9 \\
10\end{array}$ & $\begin{array}{l}--\cdots \\
--15 \\
515 \\
510 \\
513\end{array}$ & $\begin{array}{l}509 \\
498 \\
486 \\
488 \\
489\end{array}$ & $\begin{array}{l}502 \\
498 \\
500 \\
502 \\
505\end{array}$ & $\begin{array}{l}491 \\
-1 \\
511 \\
515\end{array}$ & $\begin{array}{l}410 \\
479 \\
479 \\
486 \\
491\end{array}$ & $\begin{array}{l}472 \\
459 \\
464 \\
474 \\
485\end{array}$ & $\begin{array}{l}507 \\
607 \\
497 \\
596 \\
595\end{array}$ & $\begin{array}{l}m-m \\
m-m \\
m-m\end{array}$ & $\begin{array}{l}-\infty \\
=- \\
-\infty \\
-\infty\end{array}$ & $\begin{array}{r}1260 \\
1050 \\
755 \\
761 \\
791\end{array}$ & $\begin{array}{r}870 \\
690 \\
1150\end{array}$ & $\begin{array}{l}734 \\
769 \\
797 \\
800 \\
740\end{array}$ \\
\hline $\begin{array}{l}11 \\
12 \\
13 \\
14 \\
15\end{array}$ & $\begin{array}{l}509 \\
519 \\
519 \\
524 \\
527\end{array}$ & $\begin{array}{l}492 \\
491 \\
490 \\
486 \\
482\end{array}$ & $\begin{array}{l}500 \\
498 \\
499 \\
499\end{array}$ & $\begin{array}{l}510 \\
502 \\
498 \\
496 \\
497\end{array}$ & $\begin{array}{l}492 \\
483 \\
481 \\
477 \\
479\end{array}$ & $\begin{array}{l}485 \\
484 \\
489 \\
486 \\
495\end{array}$ & $\begin{array}{l}601 \\
603 \\
507 \\
611 \\
612\end{array}$ & $\begin{array}{l}m-m \\
m-m \\
m-m \\
m-m\end{array}$ & $\begin{array}{l}m-m \\
m-m \\
m-m \\
m-m\end{array}$ & $\begin{array}{r}1340 \\
1380 \\
1620 \\
875 \\
680\end{array}$ & $\begin{array}{l}963 \\
419 \\
668 \\
862 \\
779\end{array}$ & $\begin{array}{l}629 \\
664 \\
901 \\
--7 \\
--\end{array}$ \\
\hline $\begin{array}{l}16 \\
17 \\
18 \\
19 \\
20\end{array}$ & $\begin{array}{l}532 \\
528 \\
524 \\
526 \\
521\end{array}$ & $\begin{array}{l}478 \\
479 \\
480 \\
480 \\
482\end{array}$ & $\begin{array}{l}502 \\
505 \\
500 \\
502 \\
496\end{array}$ & $\begin{array}{l}501 \\
497 \\
499 \\
500 \\
498\end{array}$ & $\begin{array}{l}480 \\
473 \\
472 \\
476 \\
477\end{array}$ & $\begin{array}{l}501 \\
499 \\
495 \\
501 \\
491\end{array}$ & $\begin{array}{l}614 \\
618 \\
622 \\
624 \\
527\end{array}$ & $\begin{array}{l}m-m \\
m-m \\
m-m \\
m-m \\
m-m\end{array}$ & $\begin{array}{l}--- \\
1200 \\
-\end{array}$ & $\begin{array}{l}754 \\
885 \\
860 \\
720 \\
927\end{array}$ & $\begin{array}{l}791 \\
807 \\
807 \\
812 \\
814\end{array}$ & $\begin{array}{l}m-1 \\
--7 \\
807 \\
404 \\
678\end{array}$ \\
\hline $\begin{array}{l}21 \\
22 \\
23 \\
24 \\
25\end{array}$ & $\begin{array}{l}-7- \\
530 \\
544 \\
547 \\
539\end{array}$ & $\begin{array}{l}484 \\
477 \\
481 \\
488 \\
493\end{array}$ & $\begin{array}{l}497 \\
498 \\
495 \\
506 \\
506\end{array}$ & $\begin{array}{l}494 \\
493 \\
490 \\
491 \\
488\end{array}$ & $\begin{array}{l}488 \\
487 \\
486 \\
484 \\
469\end{array}$ & $\begin{array}{l}482 \\
477 \\
486 \\
500 \\
520\end{array}$ & $\begin{array}{l}630 \\
631 \\
632 \\
636 \\
634\end{array}$ & $\begin{array}{l}-m \\
--- \\
--\infty \\
-m \\
-m-\end{array}$ & $\begin{array}{r}978 \\
1030 \\
1070 \\
1080 \\
1090\end{array}$ & $\begin{array}{r}1440 \\
1110 \\
966 \\
951 \\
953\end{array}$ & $\begin{array}{l}831 \\
813 \\
-m \\
-m- \\
732\end{array}$ & $\begin{array}{l}703 \\
635 \\
640 \\
655 \\
674\end{array}$ \\
\hline $\begin{array}{l}26 \\
27 \\
28 \\
29 \\
30 \\
31\end{array}$ & $\begin{array}{l}537 \\
512 \\
508 \\
523 \\
508 \\
506\end{array}$ & $\begin{array}{l}496 \\
500 \\
503 \\
504 \\
500\end{array}$ & $\begin{array}{l}512 \\
513 \\
509 \\
505 \\
509 \\
508\end{array}$ & $\begin{array}{l}490 \\
494 \\
495 \\
496 \\
496 \\
496\end{array}$ & $\begin{array}{l}469 \\
472 \\
473 \\
471 \\
--- \\
---\end{array}$ & $\begin{array}{l}531 \\
527 \\
522 \\
516 \\
520 \\
541\end{array}$ & $\begin{array}{l}m-- \\
m-m \\
m-m \\
m-\end{array}$ & $\begin{array}{l}-\infty \\
m- \\
m- \\
-- \\
- \\
-\end{array}$ & $\begin{array}{r}1070 \\
1070 \\
1070 \\
822 \\
415 \\
---\end{array}$ & \begin{tabular}{l}
956 \\
960 \\
959 \\
\hdashline-2 \\
$-\ldots$
\end{tabular} & $\begin{array}{r}1170 \\
1070 \\
850 \\
723 \\
708 \\
758\end{array}$ & $\begin{array}{l}696 \\
660 \\
627 \\
603 \\
627 \\
-\end{array}$ \\
\hline $\begin{array}{l}\text { MEAN } \\
\text { MAX } \\
\text { MIN }\end{array}$ & $=$ & $\begin{array}{l}497 \\
562 \\
477\end{array}$ & 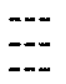 & $m$ & $\begin{array}{l}478 \\
493 \\
410\end{array}$ & $\begin{array}{l}493 \\
541 \\
459\end{array}$ & $m-m$ & $\begin{array}{l}-- \\
-- \\
-\end{array}$ & $m-m$ & $=$ & 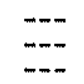 & 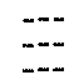 \\
\hline
\end{tabular}


RIO GRANDE BASIN

08358400 RIO GRANDE FLOODWAY AT SAN MARCIAL, MM -- Continued

WATER-QUALITY RECORDS

SUSPENDED-SEDIMENT, WATER YEAR OCTOBER 1995 TO SEPTEMBER 1996

\begin{tabular}{|c|c|c|c|c|c|c|c|c|c|c|c|c|}
\hline$A Y$ & $\begin{array}{l}\text { MEAN } \\
\text { CONCEN- } \\
\text { TRATION } \\
\text { (MG/L) }\end{array}$ & $\begin{array}{l}\text { LOADS } \\
(T / D A Y)\end{array}$ & $\begin{array}{l}\text { MEAN } \\
\text { CONCEN- } \\
\text { TRATION } \\
\text { (NE/L) }\end{array}$ & $\begin{array}{l}\text { LOADS } \\
(\mathrm{T} / \mathrm{DAY})\end{array}$ & $\begin{array}{l}\text { MEAN } \\
\text { CONCEN- } \\
\text { IRATION } \\
\text { (NG/L) }\end{array}$ & $\begin{array}{l}\text { LOADS } \\
\text { (T/DAY) }\end{array}$ & $\begin{array}{l}\text { MEAN } \\
\text { CONCEN- } \\
\text { TRATION } \\
\text { (AS/L) }\end{array}$ & $\begin{array}{l}\text { LOADS } \\
\text { (T/DAY) }\end{array}$ & $\begin{array}{l}\text { MEAN } \\
\text { CORCEN- } \\
\text { TRATION } \\
\text { (MG/L) }\end{array}$ & $\begin{array}{l}\text { LOADS } \\
\text { (T/DAY) }\end{array}$ & $\begin{array}{l}\text { MEAN } \\
\text { CONCEN- } \\
\text { TRATION } \\
\text { (NG/L) }\end{array}$ & $\begin{array}{l}\text { LOADS } \\
(\mathrm{I} / \mathrm{DAY})\end{array}$ \\
\hline $\begin{array}{l}1 \\
2 \\
3 \\
4 \\
5\end{array}$ & $\begin{array}{l}1290 \\
1010 \\
708 \\
2180 \\
2140\end{array}$ & $\begin{array}{r}\text { Togng } \\
1450 \\
1460 \\
763 \\
2520 \\
2580\end{array}$ & $\begin{array}{l}\text { pyox } \\
238 \\
374 \\
881 \\
883 \\
681\end{array}$ & $\begin{array}{r}\text { EAPER } \\
184 \\
477 \\
2020 \\
2410 \\
1790\end{array}$ & $\begin{array}{l}\text { DEC } \\
193 \\
193 \\
209 \\
235 \\
226\end{array}$ & $\begin{array}{r}484 \\
467 \\
491 \\
491 \\
543 \\
532\end{array}$ & $\begin{array}{l}\text { JA } \\
273 \\
277 \\
271 \\
320 \\
326\end{array}$ & $\begin{array}{r}\text { MUAPY } \\
663 \\
669 \\
699 \\
873 \\
867\end{array}$ & $\begin{array}{l}769 \\
168 \\
192 \\
370 \\
477 \\
536\end{array}$ & $\begin{array}{r}414 \\
483 \\
990 \\
1380 \\
1550\end{array}$ & $\begin{array}{l}627 \\
486 \\
162 \\
380 \\
517\end{array}$ & $\begin{array}{r}\text { MARCE } \\
1900 \\
1290 \\
391 \\
799 \\
1250\end{array}$ \\
\hline $\begin{array}{r}6 \\
7 \\
8 \\
9 \\
10\end{array}$ & $\begin{array}{l}992 \\
792 \\
793 \\
601 \\
462\end{array}$ & $\begin{array}{r}1140 \\
674 \\
574 \\
424 \\
307\end{array}$ & $\begin{array}{l}661 \\
666 \\
640 \\
542 \\
561\end{array}$ & $\begin{array}{l}1820 \\
2030 \\
2040 \\
1600 \\
1730\end{array}$ & $\begin{array}{l}236 \\
247 \\
218 \\
185 \\
183\end{array}$ & $\begin{array}{l}564 \\
592 \\
529 \\
438 \\
419\end{array}$ & $\begin{array}{l}250 \\
284 \\
243 \\
133 \\
191\end{array}$ & $\begin{array}{l}663 \\
805 \\
669 \\
348 \\
500\end{array}$ & $\begin{array}{l}510 \\
383 \\
332 \\
277 \\
236\end{array}$ & $\begin{array}{r}1400 \\
1030 \\
890 \\
696 \\
568\end{array}$ & $\begin{array}{l}406 \\
345 \\
383 \\
287 \\
266\end{array}$ & $\begin{array}{l}942 \\
769 \\
791 \\
528 \\
491\end{array}$ \\
\hline $\begin{array}{l}11 \\
12 \\
13 \\
14 \\
15\end{array}$ & $\begin{array}{l}404 \\
368 \\
407 \\
386 \\
347\end{array}$ & $\begin{array}{l}260 \\
245 \\
254 \\
212 \\
181\end{array}$ & $\begin{array}{l}534 \\
442 \\
413 \\
421 \\
438\end{array}$ & $\begin{array}{l}1690 \\
1350 \\
1240 \\
1250 \\
1280\end{array}$ & $\begin{array}{l}203 \\
198 \\
175 \\
170 \\
163\end{array}$ & $\begin{array}{l}486 \\
484 \\
441 \\
426 \\
408\end{array}$ & $\begin{array}{l}224 \\
229 \\
302 \\
442 \\
379\end{array}$ & $\begin{array}{r}590 \\
649 \\
913 \\
1310 \\
1060\end{array}$ & $\begin{array}{l}273 \\
293 \\
262 \\
281 \\
348\end{array}$ & $\begin{array}{l}686 \\
778 \\
707 \\
756 \\
936\end{array}$ & $\begin{array}{l}366 \\
393 \\
485 \\
295 \\
256\end{array}$ & $\begin{array}{l}597 \\
524 \\
635 \\
390 \\
272\end{array}$ \\
\hline $\begin{array}{l}16 \\
17 \\
18 \\
19 \\
20\end{array}$ & $\begin{array}{l}345 \\
309 \\
301 \\
285 \\
293\end{array}$ & $\begin{array}{l}188 \\
159 \\
133 \\
139 \\
142\end{array}$ & $\begin{array}{l}443 \\
402 \\
392 \\
392 \\
390\end{array}$ & $\begin{array}{l}1350 \\
1220 \\
1190 \\
1190 \\
1180\end{array}$ & $\begin{array}{l}241 \\
263 \\
261 \\
299 \\
332\end{array}$ & $\begin{array}{l}599 \\
658 \\
658 \\
760 \\
844\end{array}$ & $\begin{array}{l}293 \\
302 \\
322 \\
245 \\
258\end{array}$ & $\begin{array}{l}807 \\
815 \\
887 \\
695 \\
717\end{array}$ & $\begin{array}{l}321 \\
341 \\
375 \\
340 \\
368\end{array}$ & $\begin{array}{r}884 \\
976 \\
1060 \\
946 \\
994\end{array}$ & $\begin{array}{l}408 \\
509 \\
545 \\
438 \\
416\end{array}$ & $\begin{array}{l}404 \\
558 \\
625 \\
533 \\
569\end{array}$ \\
\hline $\begin{array}{l}21 \\
22 \\
23 \\
24 \\
25\end{array}$ & $\begin{array}{l}347 \\
361 \\
269 \\
165 \\
182\end{array}$ & $\begin{array}{r}173 \\
156 \\
95 \\
56 \\
75\end{array}$ & $\begin{array}{l}305 \\
241 \\
251 \\
257 \\
235\end{array}$ & $\begin{array}{l}931 \\
755 \\
745 \\
722 \\
631\end{array}$ & $\begin{array}{l}353 \\
343 \\
363 \\
380 \\
394\end{array}$ & $\begin{array}{l}912 \\
935 \\
962 \\
946 \\
953\end{array}$ & $\begin{array}{l}261 \\
265 \\
303 \\
300 \\
343\end{array}$ & $\begin{array}{l}740 \\
766 \\
875 \\
842 \\
954\end{array}$ & $\begin{array}{l}283 \\
165 \\
394 \\
416 \\
672\end{array}$ & $\begin{array}{r}737 \\
429 \\
1030 \\
1220 \\
2380\end{array}$ & $\begin{array}{l}512 \\
634 \\
662 \\
639 \\
571\end{array}$ & $\begin{array}{r}792 \\
1010 \\
960 \\
740 \\
553\end{array}$ \\
\hline $\begin{array}{l}26 \\
27 \\
28 \\
29 \\
30 \\
31\end{array}$ & $\begin{array}{l}244 \\
330 \\
396 \\
299 \\
280 \\
302\end{array}$ & $\begin{array}{l}144 \\
282 \\
289 \\
225 \\
234 \\
243\end{array}$ & $\begin{array}{l}261 \\
255 \\
242 \\
226 \\
192 \\
-\end{array}$ & $\begin{array}{l}657 \\
611 \\
578 \\
544 \\
488 \\
---\end{array}$ & $\begin{array}{l}396 \\
408 \\
442 \\
411 \\
324 \\
261\end{array}$ & $\begin{array}{r}942 \\
975 \\
1060 \\
988 \\
772 \\
627\end{array}$ & $\begin{array}{l}314 \\
281 \\
295 \\
216 \\
213 \\
230\end{array}$ & $\begin{array}{l}856 \\
723 \\
743 \\
534 \\
538 \\
571\end{array}$ & $\begin{array}{l}904 \\
631 \\
613 \\
578 \\
\ldots \ldots \\
-\ldots\end{array}$ & $\begin{array}{r}2930 \\
1980 \\
1900 \\
1780 \\
- \\
-\end{array}$ & $\begin{array}{l}465 \\
343 \\
321 \\
382 \\
295 \\
245\end{array}$ & $\begin{array}{l}397 \\
301 \\
287 \\
298 \\
109 \\
159\end{array}$ \\
\hline TOTAL. & -- & 15777 & $m$ & 35703 & --- & 20895 & -- & 23341 & --- & 32510 & --- & 19864 \\
\hline & $\begin{array}{l}\text { MEAN } \\
\text { CONCEN- } \\
\text { TRATION } \\
\text { (ME/L) }\end{array}$ & $\begin{array}{l}\text { LOADS } \\
\text { (T/DAY) }\end{array}$ & $\begin{array}{l}\text { MEAN } \\
\text { CONCEN- } \\
\text { TRATION } \\
\text { (MG/L) }\end{array}$ & $\begin{array}{l}\text { LOADS } \\
\text { (T/DA) }\end{array}$ & $\begin{array}{l}\text { MEAN } \\
\text { CONCEN- } \\
\text { TRATION } \\
\text { (MG/L) }\end{array}$ & $\begin{array}{l}\text { LOADS } \\
\text { (T/DAY) }\end{array}$ & $\begin{array}{l}\text { MEAN } \\
\text { CONCEN- } \\
\text { TRATION } \\
\text { (MG/L) }\end{array}$ & $\begin{array}{l}\text { LOADS } \\
\text { (T/DAY }\end{array}$ & $\begin{array}{l}\text { MEAN } \\
\text { CONCEN- } \\
\text { TRATION } \\
\text { (MG/L) }\end{array}$ & $\begin{array}{l}\text { OADS } \\
\text { T/DAY) }\end{array}$ & $\begin{array}{l}\text { MEAN } \\
\text { CONCEN- } \\
\text { TRATION } \\
\text { (MG/L) }\end{array}$ & $\begin{array}{l}\text { LOADS } \\
\text { (T/DAY) }\end{array}$ \\
\hline
\end{tabular}

DAY $(\mathrm{MG} / \mathrm{L}) \quad$ (T/DAY) (MG/L) (T/

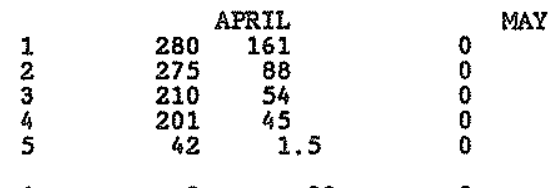

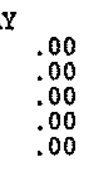

.00
.00
.00
.00
.00

10

11

12
13
14

15

16
17
18
19
20

21

22
23

24
25

26

27
28
29

30
31

TOTAL.
.00
.00
.00

.00
.00

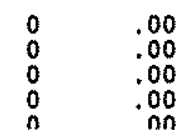

.00
.00
.00
.00

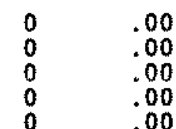

.00
.00

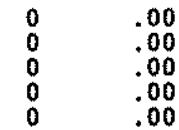

.00
.00
.00
.00
.00

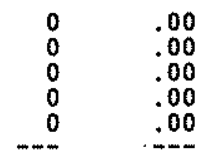

.00
.00

.00

.00
.00
.00
.00
.00
.00

.00

.00
.00

.00
.00

.00
.00

.00

.00

.00
.00
.00

.00

.00
.00

.00

.00

0.00

TONS.

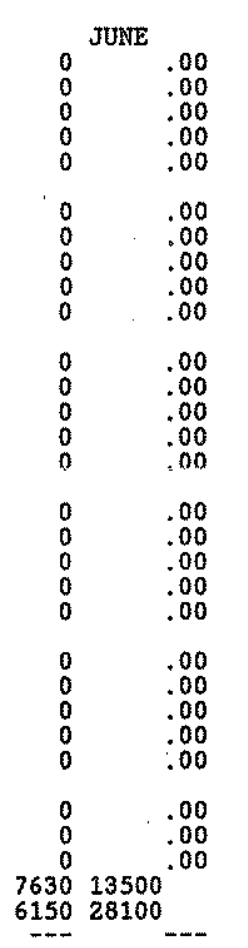

$\begin{array}{rc} & \text { JULX } \\ 4220 & 10800 \\ 4110 & 10200 \\ 5870 & 12400 \\ 11800 & 30300 \\ 19500 & 41900\end{array}$

(MG/L)

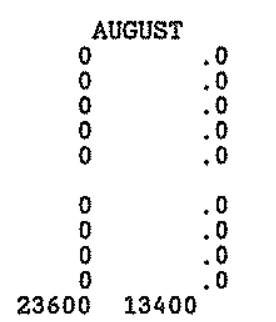

(MG/L) (T/DAY)

$19100 \quad 29100$

$\begin{array}{rr}13600 & 8420 \\ 4260 & 2430\end{array}$

$\begin{array}{rr}355 & 18 \\ 0 & .0\end{array}$

$\begin{array}{rr}408 & 423 \\ 23800 & 74700 \\ 24300 & 60700 \\ 20300 & 45900 \\ 9580 & 12600\end{array}$

35000
7920
3740
2200

52200
6670
2680

800

$\begin{array}{rr}7110 & 3670 \\ 16400 & 20000 \\ 18500 & 9710 \\ 11600 & 3020 \\ 11200 & 3820\end{array}$

6

65
0
0
0
0

$30600 \quad 17600$

$17300 \quad 1770$

2330
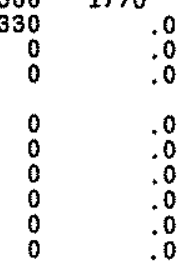

$\begin{array}{rr}0 & \\ 18100 & 5780 \\ 23200 & 22600 \\ 33400 & 79500 \\ 23800 & 68700 \\ 7790 & 21500 \\ 9520 & 16100 \\ 9870 & 10100\end{array}$

- 399481.0

$--300257.9$
SEPTEMBER

$24000 \quad 52800$

$16700 \quad 19000$

$10900 \quad 6170$

$651 \quad 15$

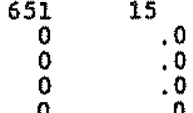

$2550026800^{\circ}$

$88100 \quad 81400$

$35900 \quad 79700$

34500114000

32800103000

$17300 \quad 49100$

$\begin{array}{rr}11600 & 19400 \\ 7970 & 9810\end{array}$

$\begin{array}{rr}6080 & 6780 \\ 3920 & 3940 \\ 1930 & 879 \\ 1530 & 49 \\ 0 & .0\end{array}$

$--41600.00$ 
RIO GRANDE BASIN

08360500 ELEPHANT BUTTE RESERVOIR AT ELEPHANT BUTTE, NM

LOCATION --Lat $33^{\circ} 09^{\prime} 15^{\prime \prime}$, Long $107^{\circ} 11^{\prime} 28^{*}$, in NWK sec.30, T.13 S, R.3 W., Sierra County, Hydrologic Unit 13020211 , at dam on Rio Grande, 1 mi west of Elephant Butte, 4 mi northeast of Truth or Consecuences (Hot Springs), and at mile $1,383.2$

DRAINAGE AREA, - $-29,445 \mathrm{mt}^{2}$, approximately, including 2,940 mi2 in closed basin in San Luis Valley, Co.

PERIOD OF RECORD,-March 1915 to December 1939 (monthend contents only published in WSP 1312), January 1940 to September 1965 (monthend contents only), October 1965 to current year.

REVISED RECORDS.--WSP 1442: 1954(m). WSP 1632: Drainage area.

GAGE.--Water-stage recorder. Datum of gage is $43,3 \mathrm{ft}$ above National Geodetic Vertical Datum of 1929 . Oct. 16, 1939, to May 2, 1940, and prior to September 1930, nonrecording gages.

REMARLS - Reservolr is formed by concrete dam. Storage began Jan. 6, 1915. Dam completed May 13 , 1916. Capacity, $2,065,000$ acre- ft, gurvey of 1988 at 8 age height $4,407.0$ ft crest of spillway. Capacity by orisinal survey was $2,638,900$ acre-ft. No adjustment made for decrease in capacity due to sedimentation between effective dates of capacity tables. No dead storage. No storage allocated to flood control. Water is used for power development and irrigation on Rio Grande Project of Bureau of Reclamation. A 50,000-acre-ft permanent pool is authorized for recreational purposes.

COOPERATION.---Records provided by Bureau of Reclamation.

EXTREMIS FOR PERIOD OF RECORD.--Maximum daily contents, 2,303,000 acre-ft, June 16-18, 1942, gage height, 4, 409.19 ft; mintmum daily contents after inttial filing, 9,900 acre-ft, Aug, 6, 1954, gage hetght, 4,258,03 ft.

EXTREMES FOR CURRENT YEAR.-Maximum contents, 2,045,300 acre-ft, Jan. 2, gage height, 4,406.46 ft; minimum contents, $1,634,100$ acre-ft, Sept. 11, gage height, 4,394.21 ft.

Capactty table (gage height, in feet, and contents, in thousands of acre-feet)

$\begin{array}{llll}4,380 & 1,241.2 & 4,400 & 1,819.7 \\ 4,390 & 1,509.1 & 4,410 & 2,177.0\end{array}$

RESERVOIR STORAGE (ACRE-FEET), WATER YEAR OCTOBER 1995 TO SEPTEMBER 1996 DAILY OBSERVATION AT 24:00 VALUES

\begin{tabular}{|c|c|c|c|c|c|c|c|c|c|c|c|c|}
\hline$A \mathbf{Y}$ & OCT & nov & DEC & JAN & FEB & MAR & APR & MAY & JUN & JUL & AUG & SEP \\
\hline $\begin{array}{l}1 \\
2 \\
3 \\
4 \\
5\end{array}$ & $\begin{array}{l}1956400 \\
1955700 \\
1954300 \\
1953300\end{array}$ & $\begin{array}{l}1932200 \\
1932900 \\
1934300 \\
1935700\end{array}$ & $\begin{array}{l}1990 \\
1991 \\
1993 \\
1994\end{array}$ & $\begin{array}{l}2043 \\
2045 \\
20402 \\
2041 \\
2041\end{array}$ & $\begin{array}{l}2036600 \\
2037300 \\
2036600 \\
2035800 \\
2035100\end{array}$ & $\begin{array}{l}2040 \\
2040 \\
2040 \\
2040\end{array}$ & $\begin{array}{l}200 \\
200 \\
200 \\
200\end{array}$ & $\begin{array}{l}1946 \\
1944 \\
1942 \\
1941\end{array}$ & & & & $\begin{array}{l}1642700 \\
1642400 \\
1642400 \\
1642400 \\
1641200\end{array}$ \\
\hline $\begin{array}{r}6 \\
7 \\
8 \\
9 \\
10\end{array}$ & $\begin{array}{l}195 \\
195 \\
194 \\
194\end{array}$ & $\begin{array}{l}1940 \\
1943 \\
1944 \\
1947\end{array}$ & $\begin{array}{l}0 \\
0 \\
0 \\
0 \\
0\end{array}$ & $\begin{array}{l}20 \\
20 \\
20 \\
20\end{array}$ & $\begin{array}{l}20 \\
20 \\
20 \\
20\end{array}$ & & & & & & & $\begin{array}{l}600 \\
000 \\
400 \\
500 \\
000\end{array}$ \\
\hline $\begin{array}{l}11 \\
12 \\
15 \\
14\end{array}$ & $\begin{array}{l}194 \\
194 \\
194\end{array}$ & $\begin{array}{l}195 \\
195 \\
195\end{array}$ & $\begin{array}{l}20 \\
20 \\
20\end{array}$ & $\begin{array}{l}2042700 \\
2042400 \\
2042400 \\
2042000\end{array}$ & & & & & & & & $\begin{array}{l}300 \\
300 \\
900 \\
600\end{array}$ \\
\hline $\begin{array}{l}16 \\
17 \\
18 \\
19\end{array}$ & $\begin{array}{l}1943800 \\
1943100 \\
1942400 \\
1941300\end{array}$ & $\begin{array}{l}196 \\
196 \\
196 \\
196\end{array}$ & $\begin{array}{l}20 \\
20 \\
20\end{array}$ & & & & & & & & & \\
\hline $\begin{array}{l}21 \\
22 \\
23 \\
24 \\
25\end{array}$ & $\begin{array}{l}1938500 \\
1937500 \\
1935700\end{array}$ & $\begin{array}{l}1974 \\
1976 \\
1978\end{array}$ & $\begin{array}{l}202 \\
202 \\
202\end{array}$ & $\begin{array}{l}20 \\
20 \\
20 \\
20\end{array}$ & & & & & & & & \\
\hline $\begin{array}{l}26 \\
27 \\
28 \\
29 \\
30\end{array}$ & $\begin{array}{l}1934100 \\
1933300 \\
1932900 \\
1932900 \\
1932600 \\
1931900\end{array}$ & $\begin{array}{r}1904000 \\
1984800 \\
1985100 \\
1986600 \\
1987600 \\
\end{array}$ & $\begin{array}{l}2032200 \\
2033300 \\
2034400 \\
2037300 \\
2040200\end{array}$ & $\begin{array}{l}203 \\
203 \\
203 \\
203 \\
203\end{array}$ & $\begin{array}{r}2039100 \\
2041300 \\
2042000 \\
=- \\
=-\end{array}$ & $\begin{array}{l}2008 \\
2008 \\
2006 \\
2005 \\
2004\end{array}$ & $\begin{array}{l}1955 \\
1952 \\
1950 \\
1946\end{array}$ & $\begin{array}{l}18 \\
18 \\
18 \\
18 \\
18 \\
18\end{array}$ & $\begin{array}{l}17616 \\
17603 \\
1756 \\
17649 \\
17635\end{array}$ & $\begin{array}{l}0 \\
0 \\
0 \\
0 \\
0 \\
0\end{array}$ & $\begin{array}{l}10 \\
0 \\
0 \\
10 \\
0 \\
0\end{array}$ & $\begin{array}{l}00 \\
00 \\
00 \\
00\end{array}$ \\
\hline 4) & $\begin{array}{r}1956400 \\
1931900 \\
4403.28 \\
-25200\end{array}$ & $\begin{array}{r}1987600 \\
1932200 \\
4404.86 \\
+55700\end{array}$ & $\begin{array}{r}1990100 \\
4406.32 \\
+52600\end{array}$ & $\begin{array}{r}2045300 \\
2033300 \\
4406.13 \\
-6900\end{array}$ & $\begin{array}{r}2043800 \\
2034800 \\
4406.37 \\
+8700\end{array}$ & $\begin{array}{r}2004500 \\
4405.33 \\
-37500\end{array}$ & $\begin{array}{r}2003700 \\
1946200 \\
4403.69 \\
-58300\end{array}$ & $\begin{array}{r}1946200 \\
1858200 \\
4401.14 \\
-88000\end{array}$ & $\begin{array}{r}1855100 \\
1756400 \\
4398.31 \\
-94300\end{array}$ & $\begin{array}{l}3900 \\
6600 \\
5.90 \\
7300\end{array}$ & $\begin{array}{r}1682600 \\
1639300 \\
4394.49 \\
-43900\end{array}$ & $\begin{array}{r}1648300 \\
1634100 \\
4394.60 \\
+3400\end{array}$ \\
\hline
\end{tabular}

CAL YR $1995 \mathrm{MAX} 2041300$ MIN 1931900 (tt) +1500

WIR YR 1996 MAX 2045300 MIN 1634100 (ff) -311000

(†) ELEVATION, IN FEET, AT END OF MONTH.

$(t+)$ CHANGE IN CONTENTS, IN ACRE-FEET 
08361000 RIO GRANDE BELOW ELEPBANT BUTTE DAM, NM

LoCATroN,-Lat $33^{\circ} 08^{\prime} 54^{\prime \prime}$, Long $107^{\circ} 12^{\prime} 22^{\prime \prime}$, Sierra County, Bydrologic Unit 13030101, in Pedro Armendaris Grant, on

left bank $1.0 \mathrm{mi}$ domstream from dam, $1.5 \mathrm{mi}$ upstream from Cuchillo Negro River, and at mile $1,382.2$.

DRAINAGE AREA. $-29,450 \mathrm{mi}^{2}$, approximately, tncluding 2,940 $\mathrm{mi}^{2}$ in closed basin in San Luis Valley, Co.

PERIOD OF RECORD.--January 1915 to current year. Monthly or annual discharge only for some periods, published in

WSP 1732. Figures of daily discharge, published in WSP 458 for october to December 1916 , are unreliable.

REVISED RECORDS.-WSP 1562: 1920. WSP 1632: Drainage area. WSP 1732: 1917, 1920. Sea also FaRIOD OE RECORD:

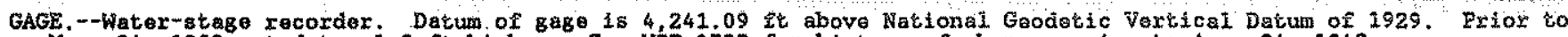

Pfar. 24, 1980, te datum 1.0 ft highar. Seo WSp 1732 for history of changes prior to Apr. 24, 1942.

REAARKS, -Recordis good except for estimated daily discharges, which are fair. Flow regulated by Elephant Butte

Rasorvoir (station 08360500) D. Diversion for irrigation of about 800,000 acres upstream from station. U.S. U.

Góplogtcal Survey satellite telemeter et station. No elow at times prior to 1929, Mar. 2-4, 1979.

DISCEARGE, CUBTC FEET EER SECOND, WATER YEAR OCTOBER 1995 TO SEPTEMAER 1996

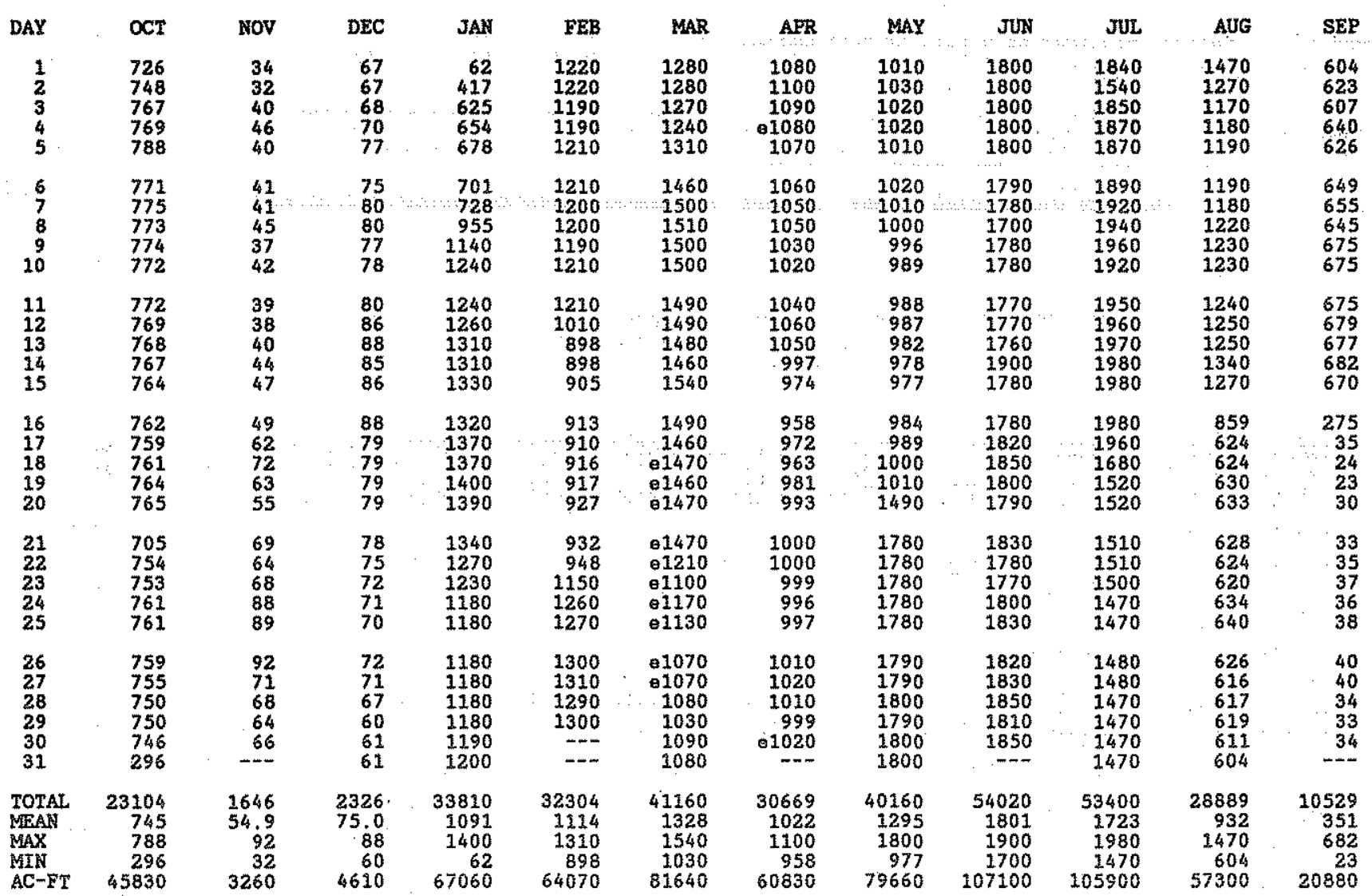

STATISTICS OF MONTHLY MEAN DATA FOR WATER YEARS 1917 - 1996, BY WATER YEAR (WY)

$\begin{array}{lrrrrrrrrrrrr}\text { MSAN } & 336 & 270 & 320 & 340 & 718 & 1168 & 1527 & 1607 & 1822 & 1724 & 1400 & 791 \\ \text { MAX } & 2040 & 2662 & 2110 & 1944 & 3026 & 2297 & 2717 & 7601 & 6098 & 4032 & 2623 & 2169 \\ \text { (WY) } & 1987 & 1942 & 1987 & 1987 & 1986 & 1989 & 1942 & 1942 & 1942 & 1995 & 1924 & 1939 \\ \text { MIN } & 2.41 & 1.25 & 1.38 & 000 & 3.38 & 16.6 & 188 & 8.32 & 284 & 673 & 155 & 2.73 \\ \text { (WY) } & 1986 & 1972 & 1994 & 1918 & 1955 & 1983 & 1977 & 1957 & 1964 & 1964 & 1954 & 1954\end{array}$

SURAARY STATISTICS

ANNUAL TOTAL

ANWUAL RIEAN

HIGHEST ANNUAL MEAN

LOWEST ANNUAL BEAN

HYGBEST DAILY MEAN

LONEST DAILY MEAN

INSTANTANEOUS PEAK FLOW

INSTANTANEOUS PEAK STAGE

IRSTANTANEOUS LOW FLOW

ANSUAL RUNOFF (AC-FT)

10 PERCENT EXCEEDS

50 PERCENT EXCEEDS

90 RERCENT EXCEEDS

FOR 1995 CALENDAR YEAR

$\begin{aligned} 590274 & \\ 1617 & \\ 4600 & \text { Ju1 } 14 \\ 32 & \text { Nov } 2 \\ 39 & \text { Nov } 1\end{aligned}$

1171000

4030
966

966
71
FOR 1996 WATER YEAR<smiles>[193Pb]</smiles>

962

1980
23

.31 Sep 17

698200

1790
1010

63
WATER YEARS 1917 - 1996

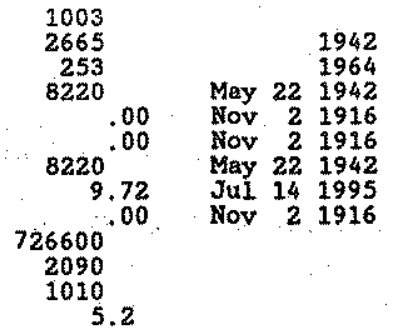

e Estimatod 
RIO GRANDE BASIN

08362000 CABALLO RESERVOIR NEAR ARREY, NM

LOCATION.--Lat $32^{\circ} 53^{\circ} 47^{\prime \prime}$, Long $107^{\circ} 17^{\prime} 30^{\prime \prime}$, In SEMSW sec.19, T.16 S., R.4 W. , Sterra County, Hydrologic Unit

13030101, In control tower of Caballo Dam on Rto Grande, $0.5 \mathrm{mi}$ downstream from mouth of' Apache Canyon, 0.9 mi upstream from Bofarquez Bridge, 2 mi. upstream from Rercha diversion dam, 3.5 mi northeast of Arrey, 5.2 mi south of Caballo, and at mile $1,356.6$.

DRAIKAGE AREA. $--30,700 \mathrm{mt}^{2}$, approximately, tncluding $2,940 \mathrm{mi}^{*}$ in closed basin in San Luis Valley, Co.

PERIOD OF RECORD.-mebruaxy 1938 to September 1965 (monthend contents only), October 1965 to current yeax.

REVISED RECORDS.--WSP 978: 1942. WSP 1632: Dratnage area.

GAGE. - Water-stage recorder. Datum of gage is $43.3 \mathrm{ft}$ above National Geodetic Vertical Datum of 1929.

REMARKS. --Reservoir 1s foxmed by earthfil1 dam, completed Sept, 19, 1938. Storage began Feb. 8, 1938. Capac1ty by 1983 survey. 331,500 acremt between gage heights $4,104 \mathrm{ft}$, bottom of tunnel entrance of gates and 4,182 ft, gage height above which spillway gates operate automatically. Capacity by ortginal survey pas 345,900 acre-ft. No dead storage. Storage held for flood control, 100,000 acre-ft. Water released from Elephant Butce Reservoir fox power developenent is stored in Caballo Reservotr and reloased for irrigation on Rio Grande Project of Bureau of Reclamation.

COOPERATION.--Records provided by Bureau of Reclamation.

EXTREMES FOR PERIOD OF RECORD.--Maximum contents, 347,000 acre-ft, Mar. 4, 1942, gage height, 4,182.06 ft; minimum contents, 118 acre-ft, Oct. 14, 1938, gage height, $4,108.1 \mathrm{ft}$.

EXTREMES FOR CURRENT YEAR.-Maximum contents, 223,790 acre-ft, Fob. 20, 23, 27 gage height, 4,171.61 ft; mintmum contents, 28,240 acre-ft, Sept. 30 , gage height, $4,137.94$ ft.

Capacity table (gage height, in feot, and contents, in acre-feet)

$\begin{array}{rrrr}4,125 & 4,810 & 4,160 & 131,200 \\ 4,130 & 11,680 & 4,170 & 209,400 \\ 4,140 & 33,770 & 4,180 & 308,900 \\ 4,150 & 71,800 & & \end{array}$

RESERVOIR STORAGE (ACRE-FEET), WATER YEAR OCTOBER 1995 TO SEPTEMBER 1996

DAILY OBSERVATION AT 24:00 VALUES

\begin{tabular}{|c|c|c|c|c|c|c|c|c|c|c|c|c|}
\hline AY & $\alpha T$ & NOV & DEC & JAN & FEB & MAR & APR & MAY & JUN & JUL & AUG & SEP \\
\hline $\begin{array}{l}1 \\
2 \\
3 \\
4 \\
5\end{array}$ & $\begin{array}{l}158110 \\
157740 \\
156980 \\
156380 \\
156080\end{array}$ & $\begin{array}{l}151820 \\
152040 \\
152340 \\
152340 \\
152340\end{array}$ & $\begin{array}{l}155330 \\
155480 \\
155630 \\
155850 \\
156000\end{array}$ & $\begin{array}{l}161600 \\
161980 \\
162210 \\
163660 \\
164880\end{array}$ & $\begin{array}{l}207870 \\
208140 \\
209190 \\
210240 \\
211300\end{array}$ & $\begin{array}{l}221 \\
220 \\
220 \\
220 \\
219\end{array}$ & $\begin{array}{l}180910 \\
179140 \\
177700 \\
176670\end{array}$ & $\begin{array}{l}15 \\
15 \\
15 \\
15 \\
15\end{array}$ & $\begin{array}{l}1289 \\
1287 \\
1286 \\
1286 \\
1284\end{array}$ & $\begin{array}{l}250 \\
970 \\
310 \\
970\end{array}$ & $\begin{array}{l}118780 \\
116800 \\
115230 \\
113680 \\
112070\end{array}$ & $\begin{array}{l}69680 \\
68540 \\
68020 \\
66430 \\
65060\end{array}$ \\
\hline $\begin{array}{r}6 \\
7 \\
8 \\
9 \\
10\end{array}$ & $\begin{array}{l}155 \\
154 \\
153 \\
153 \\
152\end{array}$ & $\begin{array}{l}1.52270 \\
152570 \\
152710 \\
152860 \\
152860\end{array}$ & $\begin{array}{l}15 \\
15 \\
15 \\
15 \\
15\end{array}$ & $\begin{array}{l}16 \\
16 \\
17 \\
17\end{array}$ & $\begin{array}{l}21 \\
21 \\
21 \\
21 \\
21\end{array}$ & $\begin{array}{l}21 \\
21 \\
21 \\
21 \\
21\end{array}$ & $\begin{array}{l}17 \\
17 \\
17 \\
17 \\
17\end{array}$ & & $\begin{array}{l}12 \\
12 \\
12 \\
12 \\
12\end{array}$ & $\begin{array}{l}1 \\
1 \\
1 \\
1 \\
1\end{array}$ & $\begin{array}{l}560 \\
590 \\
520 \\
510 \\
70\end{array}$ & $\begin{array}{l}63740 \\
62580 \\
61660 \\
60700 \\
59790\end{array}$ \\
\hline $\begin{array}{l}11 \\
12 \\
13 \\
14 \\
15\end{array}$ & $\begin{array}{l}152040 \\
151080 \\
149890 \\
148710 \\
147540\end{array}$ & $\begin{array}{l}152940 \\
153010 \\
153090 \\
153460 \\
153610\end{array}$ & $\begin{array}{l}157210 \\
157510 \\
157660 \\
157810 \\
158260\end{array}$ & $\begin{array}{l}175320 \\
177540 \\
179300 \\
180990 \\
182770\end{array}$ & $\begin{array}{l}21 \\
22 \\
22 \\
22 \\
22\end{array}$ & $\begin{array}{l}21 \\
21 \\
21 \\
21 \\
20\end{array}$ & $\begin{array}{l}17 \\
17 \\
17 \\
17 \\
17\end{array}$ & $\begin{array}{l}1 \\
1 \\
1 \\
1 \\
13\end{array}$ & $\begin{array}{l}12 \\
12 \\
12 \\
12 \\
12\end{array}$ & $\begin{array}{l}12 \\
12 \\
12 \\
12 \\
12\end{array}$ & $\begin{array}{l}0 \\
10 \\
0 \\
0 \\
0\end{array}$ & $\begin{array}{l}80 \\
70 \\
70 \\
20\end{array}$ \\
\hline $\begin{array}{l}16 \\
17 \\
18 \\
19 \\
20\end{array}$ & $\begin{array}{l}146360 \\
145120 \\
143660 \\
142210\end{array}$ & $\begin{array}{l}153390 \\
152860 \\
153760 \\
153830\end{array}$ & $\begin{array}{l}15 \\
15 \\
15 \\
15 \\
15\end{array}$ & $\begin{array}{l}184 \\
186 \\
188 \\
189 \\
191\end{array}$ & $\begin{array}{l}22 \\
22 \\
22 \\
22 \\
22\end{array}$ & $\begin{array}{l}20 \\
20 \\
20 \\
20\end{array}$ & $\begin{array}{l}17 \\
16 \\
16 \\
16\end{array}$ & & $\begin{array}{l}12 \\
12 \\
12 \\
12\end{array}$ & $\begin{array}{l}12 \\
12 \\
13 \\
12 \\
12\end{array}$ & $\begin{array}{r}1009 \\
984 \\
960 \\
937 \\
911\end{array}$ & $\begin{array}{l}460 \\
840 \\
850 \\
920\end{array}$ \\
\hline $\begin{array}{l}21 \\
22 \\
23 \\
24 \\
25\end{array}$ & $\begin{array}{l}142860 \\
143590 \\
144390 \\
144170 \\
144970\end{array}$ & $\begin{array}{l}153910 \\
154060 \\
154210 \\
154430 \\
154660\end{array}$ & $\begin{array}{l}159170 \\
159320 \\
159470 \\
159700 \\
159850\end{array}$ & $\begin{array}{l}193 \\
194 \\
194 \\
198 \\
199\end{array}$ & $\begin{array}{l}22370 \\
22333 \\
22379 \\
22361 \\
22333\end{array}$ & $\begin{array}{l}200 \\
198 \\
196 \\
194 \\
193\end{array}$ & $\begin{array}{l}164040 \\
162820 \\
161600 \\
160690 \\
160000\end{array}$ & & & $\begin{array}{l}12 \\
12 \\
12 \\
12 \\
12\end{array}$ & & $\begin{array}{l}52030 \\
50240 \\
48520 \\
46530 \\
43430\end{array}$ \\
\hline $\begin{array}{l}26 \\
27 \\
28 \\
29 \\
30 \\
31\end{array}$ & $\begin{array}{l}145630 \\
146440 \\
147610 \\
148790 \\
149890 \\
151150\end{array}$ & $\begin{array}{l}154880 \\
155030 \\
155100 \\
154950 \\
155100\end{array}$ & $\begin{array}{l}160080 \\
160380 \\
160460 \\
160690 \\
160990 \\
161290\end{array}$ & $\begin{array}{l}201300 \\
202330 \\
203360 \\
204570 \\
205700 \\
206310\end{array}$ & $\begin{array}{r}223150 \\
223790 \\
221510 \\
221420 \\
\\
-\end{array}$ & $\begin{array}{l}191910 \\
189580 \\
187760 \\
185870 \\
184240 \\
182610\end{array}$ & $\begin{array}{l}159170 \\
158410 \\
157660 \\
156830 \\
155700\end{array}$ & $\begin{array}{l}12 \\
12 \\
12 \\
12 \\
12\end{array}$ & $\begin{array}{l}1183 \\
1178 \\
1193 \\
1206 \\
1241\end{array}$ & $\begin{array}{l}12 \\
12 \\
12 \\
12 \\
12 \\
12\end{array}$ & $\begin{array}{l}350 \\
110 \\
220 \\
410 \\
730 \\
390\end{array}$ & $\begin{array}{l}39960 \\
36140 \\
33380 \\
30760 \\
28240 \\
\end{array}$ \\
\hline $\begin{array}{l}\text { MAX } \\
\text { MIX } \\
(t)\end{array}$ & $\begin{array}{r}158110 \\
142140 \\
4162.76 \\
-7420\end{array}$ & $\begin{array}{r}155100 \\
151820 \\
4163.29 \\
+3950\end{array}$ & $\begin{array}{r}161290 \\
155330 \\
4164.11 \\
+6190\end{array}$ & $\begin{array}{r}206310 \\
161600 \\
4169.65 \\
+45020\end{array}$ & $\begin{array}{r}223790 \\
207870 \\
4171.35 \\
+15110\end{array}$ & $\begin{array}{r}221420 \\
182610 \\
4166.83 \\
-38810\end{array}$ & $\begin{array}{r}180910 \\
155700 \\
4163.37 \\
-26910\end{array}$ & $\begin{array}{r}155180 \\
128140 \\
415969 \\
-26660\end{array}$ & $\begin{array}{r}128910 \\
117850 \\
4158.98 \\
-4880\end{array}$ & $\begin{array}{r}130230 \\
120380 \\
4158.42 \\
-3780\end{array}$ & $\begin{array}{r}118780 \\
70390 \\
4149.71 \\
-49990\end{array}$ & $\begin{array}{r}69680 \\
28240 \\
4137.94 \\
-42150\end{array}$ \\
\hline
\end{tabular}

CAL YR 1995 MAX 279720 MIN 86990 (†t) +76920

WTR YR 1996 MAX 223790 MIN 28240 (tt) -130330

(†) ELEVATION, IN FEET, AT END OF MONTH.

(†) CHANGE IN CONTENTS, IN ACRE-FEET. 


\section{RIO GRANDE BASIN}

08362500 RIO GRANDE BELON CABALLO DAM, NM

LOCATION, --Lat $32^{\circ} 53^{\prime} 05^{\prime \prime}$, Long $107^{\circ} 17^{\prime} 31^{\prime \prime}$, in NEKSW sec.30, T. 16 S., R. 4 W. Sierra County, Hydrologic Unit

13030102 , on loft bank 2,000 ft upstream from Interstate Highway $25,4,200$ ft downstream from Caballo Dam, $1.2 \mathrm{mi}$ downstream from Apache Canyon, $1.3 \mathrm{mi}$ upstream Erom. Perche diversion dam, $3 \mathrm{mi}$ northeast of Arrey, 5 mi south of Caballo, and at mile $1,355.6$.

DRAINAGE AREA. $--30,700 \mathrm{mi}^{2}$, approximately, including 2,940 $\mathrm{mi}^{2}$ in closed basin in San Luis Valley, Co.

PERTOD OF RECORD.-January 1938 to current year.

GACE.-Wgter-gtage recordex. Datum or gage is 4,140.9 ft above National Geodortc Vortical Detur of 1929. Prior to Dct. 7, 1938, at datum 7,0 ft higher, Oct. 7-12, 1938, at datum 6.0 ft higher, and Oct. 13, 1938, to Dac. 31, 1945 , at datum 5.0 Et higher then present datum.

REMARKS, -Flow regulated by Caballo Reservolx (station 08362000), capacity, 331,500 acre-ft, 1981 survey and Elephant Butte Reservoir (station 08360500 ), capacity, 2,065,000 acre-ft, 1988 survey. Diversions for irrigetion of about 800,000 acres upstream erom station. Figures of daily discharge do not include Bonita ditch, which diverts from Cabalio Dam and bypasses station for irrigation downstrean. See monthly table below for rocord of ditch. Bureau of Reclamation satelilite telemeter at station.

COOPERATYON. --Records provided by Bureau of Reclamation.

AVERAGE DISCHARGE. -58 years, $928 \mathrm{ft}^{3} / \mathrm{s}, 672,300$ acre-ft/yx.

EXTREMIS FOR PERTOD OF RECORD,--Maximum daily discharge, 7,650 ft/3/s, May 20, 1942; minimum daily, $0.1 \mathrm{ft} / \mathrm{s}, 0 \mathrm{ct}$. 31 to Nov, 14,1954 , Nov. 7 to Dec. 31,1955, Fob. 15-29, 1972 .

EXTREMES FOR CURRENT YEAR.--Maximum daily aischarge, 2,380 fts/s, July 31 ; minimum daily $1.0 \mathrm{ft} / \mathrm{s}, \mathrm{Jan}$. 5-11. DISCHARGE, CUBIC FEET PER SECOND, WATER YEAR OCTOBER 1995 TO SEPTEMBER 1996

\begin{tabular}{|c|c|c|c|c|c|c|c|c|c|c|c|c|}
\hline DAY & OCT & NOV & DEC & JAN & FEB & MAR & AFR & MAY & JUN & JUL & AUG & SEP \\
\hline $\begin{array}{l}1 \\
2 \\
3 \\
4 \\
5\end{array}$ & $\begin{array}{l}1030 \\
1020 \\
1010 \\
1000 \\
1000\end{array}$ & $\begin{array}{l}8.0 \\
7.0 \\
7.0 \\
6.0 \\
6.0\end{array}$ & $\begin{array}{l}7.0 \\
6.0 \\
6.0 \\
6.0 \\
6.0\end{array}$ & $\begin{array}{l}2.0 \\
2.0 \\
2.0 \\
2.0 \\
1.0\end{array}$ & $\begin{array}{l}659 \\
607 \\
557 \\
558 \\
559\end{array}$ & $\begin{array}{l}1200 \\
1280 \\
1290 \\
1280 \\
1510\end{array}$ & $\begin{array}{l}1820 \\
1770 \\
1720 \\
1720 \\
1250\end{array}$ & $\begin{array}{l}1550 \\
1540 \\
1590 \\
1660 \\
1660\end{array}$ & $\begin{array}{l}1930 \\
1930 \\
1930 \\
1970 \\
2110\end{array}$ & $\begin{array}{l}1180 \\
1310 \\
1480 \\
1480 \\
1580\end{array}$ & $\begin{array}{l}2370 \\
2260 \\
2140 \\
2000 \\
1830\end{array}$ & $\begin{array}{l}1270 \\
1260 \\
1400 \\
1500 \\
1410\end{array}$ \\
\hline $\begin{array}{r}6 \\
7 \\
8 \\
9 \\
10\end{array}$ & $\begin{array}{r}1000 \\
1000 \\
998 \\
997 \\
1080\end{array}$ & $\begin{array}{r}6.0 \\
5.0 \\
5.0 \\
5.0 \\
5.0\end{array}$ & $\begin{array}{r}5.0 \\
5.0 \\
5.0 \\
5.0 \\
5.0\end{array}$ & $\begin{array}{l}1.0 \\
1.0 \\
1.0 \\
1.0 \\
1.0\end{array}$ & $\begin{array}{l}559 \\
558 \\
542 \\
307 \\
133\end{array}$ & $\begin{array}{l}1720 \\
1720 \\
1770 \\
1820 \\
1830\end{array}$ & $\begin{array}{r}760 \\
774 \\
772 \\
1080 \\
1370\end{array}$ & $\begin{array}{l}1650 \\
1700 \\
1730 \\
1640 \\
1530\end{array}$ & $\begin{array}{l}2100 \\
2050 \\
2000 \\
2000 \\
1990\end{array}$ & $\begin{array}{l}1660 \\
1660 \\
1650 \\
1910 \\
2230\end{array}$ & $\begin{array}{l}1780 \\
1730 \\
1720 \\
1660 \\
1590\end{array}$ & $\begin{array}{l}1300 \\
1180 \\
1170 \\
1160 \\
1290\end{array}$ \\
\hline $\begin{array}{l}11 \\
12 \\
13 \\
14 \\
15\end{array}$ & $\begin{array}{l}1240 \\
1210 \\
1230 \\
1340 \\
1330\end{array}$ & $\begin{array}{l}5.0 \\
5.0 \\
5.0 \\
5.0 \\
5.0\end{array}$ & $\begin{array}{l}5.0 \\
5.0 \\
4.0 \\
4.0 \\
4.0\end{array}$ & $\begin{array}{l}169 \\
169 \\
323 \\
323 \\
323\end{array}$ & $\begin{array}{l}110 \\
114 \\
409 \\
642 \\
637\end{array}$ & $\begin{array}{l}1860 \\
2070 \\
2260 \\
2240 \\
2170\end{array}$ & $\begin{array}{l}1370 \\
1370 \\
1380 \\
1360 \\
1380\end{array}$ & $\begin{array}{l}1380 \\
1380 \\
1380 \\
1630 \\
1900\end{array}$ & $\begin{array}{l}1990 \\
1990 \\
2000 \\
1980 \\
1960\end{array}$ & $\begin{array}{l}2150 \\
2100 \\
2230 \\
2150 \\
1650\end{array}$ & $\begin{array}{l}1590 \\
1580 \\
1740 \\
1890 \\
1870\end{array}$ & $\begin{array}{r}1440 \\
1260 \\
957 \\
849 \\
847\end{array}$ \\
\hline $\begin{array}{l}16 \\
17 \\
18 \\
19 \\
20\end{array}$ & $\begin{array}{r}1330 \\
1390 \\
1500 \\
980 \\
612\end{array}$ & $\begin{array}{r}6.0 \\
6.0 \\
6.0 \\
6.0 \\
6.0\end{array}$ & $\begin{array}{l}4.0 \\
4.0 \\
4.0 \\
3.0 \\
3.0\end{array}$ & $\begin{array}{l}324 \\
32.5 \\
322 \\
324 \\
323\end{array}$ & $\begin{array}{l}684 \\
752 \\
755 \\
761 \\
826\end{array}$ & $\begin{array}{l}2110 \\
2110 \\
2160 \\
2220 \\
2270\end{array}$ & $\begin{array}{l}1740 \\
2020 \\
1820 \\
1700 \\
1590\end{array}$ & $\begin{array}{l}1890 \\
1930 \\
1980 \\
1970 \\
1970\end{array}$ & $\begin{array}{l}1960 \\
1960 \\
2080 \\
2190 \\
2200\end{array}$ & $\begin{array}{l}1380 \\
1500 \\
1670 \\
1620 \\
1570\end{array}$ & $\begin{array}{l}1930 \\
1990 \\
1980 \\
1970 \\
1980\end{array}$ & $\begin{array}{l}850 \\
725 \\
624 \\
620 \\
845\end{array}$ \\
\hline $\begin{array}{l}21 \\
22 \\
23 \\
24 \\
25\end{array}$ & $\begin{array}{l}302 \\
283 \\
280 \\
282 \\
281\end{array}$ & $\begin{array}{l}6.0 \\
6.0 \\
6.0 \\
6.0 \\
6.0\end{array}$ & $\begin{array}{l}3.0 \\
3.0 \\
3.0 \\
3.0 \\
3.0\end{array}$ & $\begin{array}{l}324 \\
326 \\
325 \\
325 \\
326\end{array}$ & $\begin{array}{r}890 \\
1070 \\
1170 \\
1370 \\
1180\end{array}$ & $\begin{array}{l}2270 \\
2110 \\
1980 \\
1960 \\
1970\end{array}$ & $\begin{array}{l}1580 \\
1580 \\
1520 \\
1470 \\
1460\end{array}$ & $\begin{array}{l}1920 \\
1840 \\
1740 \\
1700 \\
1680\end{array}$ & $\begin{array}{l}2220 \\
2250 \\
2250 \\
2240 \\
2210\end{array}$ & $\begin{array}{l}1570 \\
1570 \\
1730 \\
1860 \\
1840\end{array}$ & $\begin{array}{l}1990 \\
1980 \\
1750 \\
1530 \\
1530\end{array}$ & $\begin{array}{l}1070 \\
1060 \\
1120 \\
1440 \\
2850\end{array}$ \\
\hline $\begin{array}{l}26 \\
27 \\
28 \\
29 \\
30 \\
31\end{array}$ & $\begin{array}{r}281 \\
219 \\
13 \\
12 \\
11 \\
10\end{array}$ & $\begin{array}{l}6.0 \\
6.0 \\
7.0 \\
7.0 \\
7.0 \\
\end{array}$ & $\begin{array}{l}3.0 \\
3.0 \\
3.0 \\
3.0 \\
3.0 \\
3.0\end{array}$ & $\begin{array}{l}409 \\
502 \\
503 \\
505 \\
614 \\
705\end{array}$ & $\begin{array}{l}1180 \\
1160 \\
1150 \\
1140 \\
-\cdots\end{array}$ & $\begin{array}{l}1950 \\
1960 \\
1970 \\
1970 \\
1880 \\
1820\end{array}$ & $\begin{array}{l}1410 \\
1370 \\
1360 \\
1370 \\
1460 \\
-\end{array}$ & $\begin{array}{l}1660 \\
1660 \\
1730 \\
1830 \\
1830 \\
1880\end{array}$ & $\begin{array}{l}2180 \\
1720 \\
1430 \\
1360 \\
1250 \\
-\end{array}$ & $\begin{array}{l}1870 \\
1900 \\
1890 \\
1890 \\
2110 \\
2380\end{array}$ & $\begin{array}{l}1520 \\
1590 \\
1670 \\
1600 \\
1400 \\
1280\end{array}$ & $\begin{array}{r}2030 \\
1980 \\
1670 \\
1880 \\
697 \\
\end{array}$ \\
\hline $\begin{array}{l}\text { TOTAL } \\
\text { MEAN } \\
\text { MAX } \\
\text { MIN } \\
\text { AC-FT } \\
(\dagger)\end{array}$ & $\begin{array}{r}24271 \\
783 \\
1500 \\
10 \\
48140 \\
0\end{array}$ & $\begin{array}{r}178.0 \\
5.93 \\
8.0 \\
5.0 \\
353 \\
0\end{array}$ & $\begin{array}{r}129.0 \\
4.16 \\
7.0 \\
3.0 \\
256 \\
11\end{array}$ & $\begin{array}{r}7635.0 \\
246 \\
705 \\
1.0 \\
15140 \\
0\end{array}$ & $\begin{array}{r}20839 \\
719 \\
1180 \\
110 \\
41330 \\
0\end{array}$ & $\begin{array}{r}58730 \\
1895 \\
2270 \\
1200 \\
116500 \\
178\end{array}$ & $\begin{array}{r}43346 \\
1445 \\
2020 \\
760 \\
85980 \\
85\end{array}$ & $\begin{array}{r}53130 \\
1714 \\
1980 \\
1380 \\
105400 \\
202\end{array}$ & $\begin{array}{r}59430 \\
1981 \\
2250 \\
1250 \\
117900 \\
199\end{array}$ & $\begin{array}{r}54770 \\
1767 \\
2300 \\
1180 \\
108600 \\
146\end{array}$ & $\begin{array}{r}55440 \\
1788 \\
2370 \\
1280 \\
110000 \\
111\end{array}$ & $\begin{array}{r}36754 \\
1225 \\
2030 \\
620 \\
72900 \\
163\end{array}$ \\
\hline
\end{tabular}

CAL YR 1995 TOTAL 552534.0 MEAN 1514 MAX 4540 MIN 2.0 AC-FT 1096000 WTR YR 1996 TOTAI 414652.0 MEAN 1133 MAX 2380 MIN 1.0 AC-FT 822500

(†) DIVERSION, IN ACRE-FEET, BY BONITA DITCH; DIVERTS DIRECTLY FROM CABALLO DAM AND THIS DIVERSION IS NOT INCLUDED IN THE RIVER RECORDS. 
RIO GRANDE BASIN

08364000 RTO GRANDE AT EL PASO, TX

National stroam quality accounting network

and Rational Water-Quality Assessment Program Station)

WATER-QUALITY RECORDS

LOCATrow,--Lat $31^{\circ} 48^{\prime} 10^{\prime \prime}$, Long $106^{\circ} 32^{\prime} 2^{\prime \prime}$, El Paso County, Bydrologic Untt 13030102, on downstream side of first pter from left abutment of Courchesne Bridge at El Paso, 1.7 mi upstream from American Dam, 5.6 mi upstrean from Santa Fe Street-Juarez Avenue Bridge between El Paso and Cd. Juarez, Chihuahua, and at mile $1,249$.

DRAINAGE AREA.-32,207 $\mathrm{mL}^{2}$, approximately, including $2,940 \mathrm{mi}^{2}$ in closed basin in San Luis Valley, Co.

PERIOD OR RBCORD.--Water years 1930 to current year.

REMARKS.---Records of discharge are given in International Boundary and Water Commission Water BuLletins.

WATER QUALITY DATA, WATER YEAR OCTOBER 1995 TO SEPTEMBER 1996

\begin{tabular}{|c|c|c|c|c|c|c|c|c|c|c|c|}
\hline DATE & TIME & 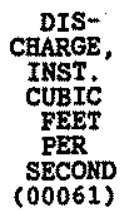 & $\begin{array}{l}\text { SPE- } \\
\text { CIFIC } \\
\text { CON- } \\
\text { DUCT- } \\
\text { ANCE } \\
\text { (US /CM }) \\
(00095)\end{array}$ & 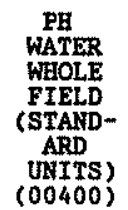 & $\begin{array}{l}\text { TEMPER- } \\
\text { ATREE } \\
\text { AIR } \\
\text { (DEG C) } \\
(00020)\end{array}$ & $\begin{array}{l}\text { TEMPER- } \\
\text { ATURE } \\
\text { WATER } \\
\text { (DEG C) } \\
(00010)\end{array}$ & $\begin{array}{c}\text { TUR- } \\
\text { 琞D- } \\
\text { ITY } \\
\text { (NTU) } \\
(00076)\end{array}$ & $\begin{array}{c}\text { BARO- } \\
\text { METRIC } \\
\text { PRES - } \\
\text { SURE } \\
\text { SUM } \\
\text { OF } \\
\text { OG) } \\
(00025)\end{array}$ & $\begin{array}{c}\text { OXYGEN, } \\
\text { DIS- } \\
\text { SOLVED } \\
\text { (NG/L) } \\
(00300)\end{array}$ & $\begin{array}{c}\text { OXYGEN, } \\
\text { DIS- } \\
\text { SOLED } \\
\text { (ERR- } \\
\text { CERT } \\
\text { SATRR- } \\
\text { ATION) } \\
(00301)\end{array}$ & $\begin{array}{l}\text { HARD- } \\
\text { NESS } \\
\text { TOTALL } \\
\text { (MGS/L } \\
\text { AS } \\
\text { CACO3) } \\
(00900)\end{array}$ \\
\hline
\end{tabular}

\begin{tabular}{|c|c|c|c|c|c|c|c|c|c|c|c|}
\hline $\begin{array}{l}\text { Nov } 1995 \\
02 \ldots \\
D E C\end{array}$ & 1400 & 268 & 1830 & 8.5 & 20.0 & 17.5 & 18 & 664 & 8.5 & 103 & 380 \\
\hline JAN $079 \dot{9} 6$ & 1245 & 174 & 2300 & 8.4 & 20.5 & 14.5 & -- & 661 & 9.4 & 107 & 430 \\
\hline $\begin{array}{c}17 \ldots \\
24 \ldots\end{array}$ & $\begin{array}{l}0815 \\
1530\end{array}$ & $\begin{array}{l}338 \\
354\end{array}$ & $\begin{array}{l}1310 \\
1270\end{array}$ & $\begin{array}{l}8.3 \\
8.2\end{array}$ & $\begin{array}{l}15.0 \\
12.5\end{array}$ & 9.5 & $16^{--}$ & $\begin{array}{l}652 \\
668\end{array}$ & $\begin{array}{r}9.2 \\
11.9\end{array}$ & $\begin{array}{r}95 \\
118\end{array}$ & $\begin{array}{l}260 \\
260\end{array}$ \\
\hline$\underset{\text { MAR }}{29}$... & 0830 & E406 & 1110 & 8.2 & 7.5 & 6.5 & -- & 670 & 9.5 & 88. & 230 \\
\hline$\underset{A P R}{26} \cdot \cdots$ & 0900 & 999 & 878 & 8.3 & 4.0 & 10.0 & 24 & 665 & 9.4 & 96 & 190 \\
\hline${ }_{M A Y}^{23} \ldots$ & 0750 & 738 & 1020 & 8.2 & 10.5 & 16.5 & -- & 670 & 8.0 & 94 & 220 \\
\hline${ }_{J U N}^{29} \ldots$ & 0845 & 731 & 1060 & 8.4 & 24.0 & 20.5 & 30 & 663 & 7.6 & 98 & 210 \\
\hline${ }_{\text {JUL. }}^{27 . .}$ & 0815 & 1280 & 801 & 7.9 & 22.5 & 23.5 & -- & 664 & 5.3 & 72 & 170 \\
\hline AUG $25 \ldots$ & 0800 & 858 & 1000 & 8.3 & 24.5 & 24.0 & -- & 668 & 7.0 & 96 & 210 \\
\hline${ }_{\mathrm{SEP}}^{13}+\ldots$ & 0830 & 858 & 963 & 8.0 & 26.5 & 24.0 & 170 & 668 & 6.4 & 87 & 200 \\
\hline $10 \ldots$ & 0900 & 597 & 1220 & 8.4 & 25.5 & 22.5 & - & 666 & 7.2 & 96 & 250 \\
\hline DATE & $\begin{array}{l}\text { HARD- } \\
\text { NESS } \\
\text { NONCARB } \\
\text { DISSOLV } \\
\text { FLD AS } \\
\text { CACOS } \\
\text { (ME/L) } \\
(00904)\end{array}$ & $\begin{array}{c}\text { CALCIUM } \\
\text { DIS- } \\
\text { SOLVED } \\
\text { (ME/L } \\
\text { AS CA) } \\
(00915)\end{array}$ & $\begin{array}{c}\text { MAGNE- } \\
\text { SIUM, } \\
\text { DIS- } \\
\text { SOLVED } \\
(M G / L \\
\text { AS MG) } \\
(00925)\end{array}$ & $\begin{array}{c}\text { SODIUM, } \\
\text { DIS- } \\
\text { SOLVED } \\
\text { (MG/L } \\
\text { AS NA) } \\
(00930)\end{array}$ & $\begin{array}{c}\text { SODIUM } \\
\text { AD- } \\
\text { SORP- } \\
\text { TION } \\
\text { RATIO } \\
(00931)\end{array}$ & $\begin{array}{c}\text { POTAS- } \\
\text { SIUM, } \\
\text { DIS- } \\
\text { SOLVED } \\
\text { (MG/L } \\
\text { AS K) } \\
(00935)\end{array}$ & $\begin{array}{c}\text { BICAR- } \\
\text { BONATE } \\
\text { WATER } \\
\text { DIS IT } \\
\text { FIELD } \\
\text { MG/L AS } \\
\text { BCO3 } \\
(00453)\end{array}$ & $\begin{array}{c}\text { CAR- } \\
\text { BONATE } \\
\text { WATER } \\
\text { DIS IT } \\
\text { FIELD } \\
\text { NG/L AS } \\
\text { CO3 } \\
(00452)\end{array}$ & $\begin{array}{l}\text { ALKA- } \\
\text { LINITY } \\
\text { WAT DIS } \\
\text { TOT IT } \\
\text { FIELD } \\
\text { MG/L AS } \\
\text { CACOB } \\
(39086)\end{array}$ & $\begin{array}{c}\text { ALKA- } \\
\text { LINYTYY } \\
\text { LAB } \\
\text { (MG/L } \\
\text { AS } \\
\text { CACO3) } \\
(90410)\end{array}$ & $\begin{array}{c}\text { SULFATE } \\
\text { DIS- } \\
\text { SOLVED } \\
\text { (MG/L } \\
\text { AS SO4) } \\
(00945)\end{array}$ \\
\hline
\end{tabular}

\begin{tabular}{|c|c|c|c|c|}
\hline $\begin{array}{l}\text { Nov }_{02} 1995 \\
02 .\end{array}$ & 130 & 110 & 24 & 230 \\
\hline JAN 1976 & 160 & 120 & 31 & 330 \\
\hline $\begin{array}{c}17 \cdots \\
24 \cdots \\
F E B\end{array}$ & $\begin{array}{l}69 \\
77\end{array}$ & $\begin{array}{l}73 \\
74\end{array}$ & $\begin{array}{l}18 \\
19\end{array}$ & $\begin{array}{l}160 \\
160\end{array}$ \\
\hline$\underset{M A R}{29} \ldots$ & 58 & 66 & 17 & 130 \\
\hline$\underset{\mathrm{APR}}{26} \ldots$ & 33 & 56 & 1.3 & 95 \\
\hline$\underset{\text { MAY }}{23} \cdots$ & - & 65 & 15 & 110 \\
\hline Jun $29 .$. & 39 & 62 & 14 & 120 \\
\hline $27 \ldots$ & 41 & 49 & 11 & 86 \\
\hline$\underset{A U G}{25}$. & 41 & 60 & 14 & 110 \\
\hline SEP 13 & 48 & 57 & 13 & 100 \\
\hline & 65 & 72 & 17 & 150 \\
\hline
\end{tabular}

$\begin{array}{rlrrrrr}5 & 11 & 281 & 8 & 243 & 252 & 370 \\ 7 & 11 & 281 & 25 & 272 & 260 & 480 \\ 4 & 8.3 & 228 & 0 & 187 & 194 & 260 \\ 4 & 7.4 & 228 & 0 & 187 & 189 & 240 \\ 4 & 7.1 & 197 & 9 & 177 & 178 & 200 \\ 3 & 5.8 & 196 & 0 & 161 & -- & 160 \\ 3 & 5.2 & - & -- & -- & 180 & 190 \\ 4 & 6.8 & 198 & .7 & 174 & -- & 200 \\ 3 & 6.3 & 155 & 0 & 127 & 148 & 150 \\ 3 & 7.1 & 195 & 4 & 167 & 191 & 190 \\ 3 & 7.4 & 181 & 0 & 148 & -- & 180 \\ 4 & 7.5 & 217 & 4 & 185 & 193 & 240\end{array}$


RIO GRANDE BASIN

08364000 RIO GRANDE AT EL PASO, TX -- Continued

WATER-QUALITY RECORDS

WATER QUALITY DATA, WATER YEAR OCTOBER 1995 TO SEPTEMBER 1996

\begin{tabular}{|c|c|c|c|c|c|c|c|c|c|c|c|}
\hline DATE & $\begin{array}{c}\text { CELO- } \\
\text { RYDE, } \\
\text { DIS- } \\
\text { SOLVED } \\
\text { (MG/L } \\
\text { AS CL) } \\
(00940)\end{array}$ & $\begin{array}{l}\text { FLUO- } \\
\text { QIDE, } \\
\text { DIS- } \\
\text { SOLVED } \\
\text { (MG/L } \\
\text { AS F) } \\
(00950)\end{array}$ & $\begin{array}{c}\text { SILICA, } \\
\text { DIS- } \\
\text { SOLVED } \\
\text { (MG/L } \\
\text { AS } \\
\text { SIO2) } \\
(00955)\end{array}$ & $\begin{array}{c}\text { SOLIDS, } \\
\text { RESIDUE } \\
\text { AT } 180 \\
\text { DEG. C } \\
\text { DIS- } \\
\text { SOLVED } \\
(M G / L) \\
(70300)\end{array}$ & $\begin{array}{c}\text { SOLIDS, } \\
\text { SUMA OF } \\
\text { COASSTI- } \\
\text { TUENTS, } \\
\text { DIS- } \\
\text { SOLVED } \\
\text { ((MG/L) } \\
\text { (70301) }\end{array}$ & $\begin{array}{c}\text { NITRO- } \\
\text { GEN, } \\
\text { IITRATS } \\
\text { DIS- } \\
\text { SOLVED } \\
\text { (MG/L } \\
\text { AS N) } \\
(00618)\end{array}$ & $\begin{array}{l}\text { NITRO- } \\
\text { GEN, } \\
\text { NITRITE } \\
\text { DIS- } \\
\text { SOLVED } \\
\text { (MG/L } \\
\text { AS N) } \\
(00613)\end{array}$ & $\begin{array}{c}\text { NITRO- } \\
\text { GEN, } \\
\text { BO2HNO3 } \\
\text { DIS- } \\
\text { SOLVED } \\
\text { (MS/L } \\
\text { AS N) } \\
\text { (00631) }\end{array}$ & $\begin{array}{l}\text { NITRO- } \\
\text { GEN, } \\
\text { AREON } \\
\text { DIS- } \\
\text { SOLVED } \\
\text { (MGG/L } \\
\text { AS N) } \\
(00608)\end{array}$ & $\begin{array}{c}\text { NITRO- } \\
\text { GEN } \\
\text { ORGANIC } \\
\text { DIS- } \\
\text { SOLVED } \\
\text { (MG/L } \\
\text { AS N } \\
(00607)\end{array}$ & $\begin{array}{l}\text { NITRO- } \\
\text { GEN, AM- } \\
\text { RONISA - } \\
\text { ORGANIC } \\
\text { TOTAL } \\
\text { (MG/L } \\
\text { AS N) } \\
(00625)\end{array}$ \\
\hline $\begin{array}{l}\text { Nov } 1995 \\
\text { o2 } \ldots\end{array}$ & 190 & 0.60 & 20 & 1190 & 1110 & 0.860 & 0.050 & 0.910 & 0.020 & 0.18 & 0.40 \\
\hline${ }_{\mathrm{JAN}}^{07} \mathrm{i} 9{ }^{2}$ & 310 & 0.70 & 25 & 1510 & 1480 & 1.07 & 0.030 & 1.10 & 0,070 & 0.23 & 0.50 \\
\hline $\begin{array}{r}17 \ldots \ldots \\
24 \ldots\end{array}$ & $\begin{array}{l}150 \\
140\end{array}$ & $\begin{array}{l}0.60 \\
0.70\end{array}$ & $\begin{array}{l}15 \\
14\end{array}$ & $\begin{array}{l}828 \\
780\end{array}$ & $\begin{array}{l}801 \\
772\end{array}$ & $\begin{array}{l}0.890 \\
0.650\end{array}$ & $\begin{array}{l}0.020 \\
0.020\end{array}$ & $\begin{array}{l}0.910 \\
0.670\end{array}$ & $\begin{array}{l}0.020 \\
0.060\end{array}$ & 0.28 & $\begin{array}{l}0.80 \\
0.40\end{array}$ \\
\hline $\operatorname{MAR}_{29}^{29}$ & 120 & 0.70 & 12 & 698 & 661 & 0.470 & 0.010 & 0.480 & $<0.015$ & $\because$ & 0.60 \\
\hline${ }_{A \mathrm{AR}}^{26} \ldots$ & 79 & 0.70 & 11 & 557 & 519 & 0.310 & 0.010 & 0.320 & $<0.015$ & - & 0.70 \\
\hline${ }_{\mathrm{MAY}}^{23} \cdots$ & 95 & 0.70 & 11 & 624 & 601 & 0.220 & 0.020 & 0.240 & 0.020 & 0.28 & 0.50 \\
\hline JUN & 95 & 0.60 & 12 & $\cdots$ & -- & -- & & & & & \\
\hline${ }_{\text {JUL }}^{27} \cdots$ & 66 & 0.60 & 12 & 498 & 459 & 0.360 & 0.020 & 0.380 & $<0.015$ & - & 2.4 \\
\hline AUG $25 \ldots$ & 89 & 0.70 & 17 & 627 & 589 & 0.180 & 0.020 & 0.200 & 0.040 & -5 & 1.0 \\
\hline $\operatorname{sep}^{13} \ldots$ & 85 & 0.60 & 16 & 588 & 551 & 0.460 & 0.020 & 0.480 & 0.030 & -- & 1.3 \\
\hline $10 \ldots$ & 120 & 0.70 & 18 & 766 & 737 & 0.320 & 0.010 & 0.330 & $<0.015$ & -- & 0.90 \\
\hline DATE & $\begin{array}{l}\text { YITRO- } \\
\text { GEN,ABY- } \\
\text { MONIA + } \\
\text { ORGANIC } \\
\text { DIS. } \\
\text { (MAS NL } \\
\text { AS N) } \\
\text { (00623) }\end{array}$ & $\begin{array}{c}\text { PEOS- } \\
\text { PEORUS } \\
\text { TOTAI } \\
\text { (NG /L } \\
\text { AS P) } \\
(00665)\end{array}$ & $\begin{array}{c}\text { PHOS- } \\
\text { PHORUS } \\
\text { DIS- } \\
\text { SOLVED } \\
\text { (MG/L } \\
\text { AS P) } \\
(00666)\end{array}$ & $\begin{array}{l}\text { PEOS- } \\
\text { PHORUS } \\
\text { ORTHO, } \\
\text { DIS- } \\
\text { SOLVED } \\
\text { (NGLL } \\
\text { AS P) } \\
(00671)\end{array}$ & $\begin{array}{c}\text { CARBON, } \\
\text { ORGANIC } \\
\text { TOTAI } \\
\text { (WSG/L } \\
\text { AS C) } \\
(00680)\end{array}$ & $\begin{array}{c}\text { CARBON, } \\
\text { ORGANIC } \\
\text { DIS- } \\
\text { SOLVED } \\
\text { (MG/L } \\
\text { AS C) } \\
(00681)\end{array}$ & $\begin{array}{l}\text { CARBON, } \\
\text { ORGANIC } \\
\text { SUS- } \\
\text { PENDED } \\
\text { TOTAL } \\
\text { (MGG/L } \\
\text { AS C) } \\
(00689)\end{array}$ & $\begin{array}{l}\text { ALUM- } \\
\text { INUM, } \\
\text { DIS- } \\
\text { SOLVED } \\
\text { (UG/L } \\
\text { AS AL) } \\
\text { (01106) }\end{array}$ & $\begin{array}{c}\text { ANTI- } \\
\text { MONY, } \\
\text { DIS- } \\
\text { SOLVED } \\
\text { (UG/L } \\
\text { AS SB) } \\
(01095)\end{array}$ & $\begin{array}{c}\text { ARSENIC } \\
\text { DIS- } \\
\text { SOLVED } \\
\text { (UG/L } \\
\text { AS AS) } \\
(01000)\end{array}$ & $\begin{array}{c}\text { BARIUM, } \\
\text { DIS- } \\
\text { SOLVED } \\
\text { (UG/L } \\
\text { AS BA) } \\
(01005)\end{array}$ \\
\hline $\begin{array}{l}\text { Nov } 1995 \\
02 \ldots\end{array}$ & 0.20 & 0.110 & 0.100 & 0.050 & - & 13 & 0.70 & $<10$ & -- & 3 & 100 \\
\hline JAN 1996 & 0.30 & 0.100 & 0.060 & 0.050 & -- & - & - & -- & -- & -- & -- \\
\hline $\begin{array}{c}17 \ldots \\
24 \ldots\end{array}$ & 0.30 & $\begin{array}{l}0.170 \\
0.090\end{array}$ & & $\begin{array}{l}0.050 \\
0.050\end{array}$ & 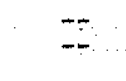 & $\cdots$ & - & $10^{--}$ & -- & -2 & $60^{--}$ \\
\hline $29 \ldots$ & $<0.20$ & 0.150 & 0.030 & 0.040 & -- & - & $\rightarrow$ & 4.0 & $<1.0$ & 2 & 63 \\
\hline$\underset{A P R}{26} \cdot \cdots$ & 0.20 & 0.200 & 0.030 & 0.030 & $-\cdots$ & 6.1 & 2.5 & 3.0 & $<1.0$ & 2 & 57 \\
\hline$\underset{\operatorname{MAY}}{23} \cdots$ & 0.30 & 0.110 & 0.020 & 0.020 & - & - & -- & - & -- & $\cdots$ & -- \\
\hline$\underset{\mathrm{JUN}}{29} \cdot \cdots$ & - & - & -- & - & -- & -- & $-\infty$ & 3.0 & $<1.0$ & 2 & 69 \\
\hline JUL $27 .$. & 0.40 & 0.890 & 0.030 & 0.040 & -- & -- & $\because-$ & -- & -- & -- & -- \\
\hline${ }_{\text {AUE }}^{25} \ldots$ & $<0.20$ & 0.210 & $<0.010$ & 0.030 & 8.6 & 3.4 & -- & -- & - & - & - \\
\hline $\operatorname{SEP}^{13} \ldots$ & $<0.20$ & 0.500 & 0.050 & 0.040 & - & 3 & 3.2 & 13 & $<1.0$ & 4 & 4 \\
\hline $10 \ldots$ & $<0.20$ & 0.190 & 0.020 & 0.020 & - & -- & -- & - & - & $\cdots$ & - \\
\hline
\end{tabular}


RIO GRANDE BASIN

$08364000^{\circ}$ RIO GRANDE AT EL PASO, TX -- Continued

WATER-QUALITY RECORDS

WATER QUALITY DATA, WATER YEAR OCTOBER 1995 TO SEPTEMBER 1996

\begin{tabular}{|c|c|c|c|c|c|c|c|c|c|c|c|}
\hline DATE & $\begin{array}{l}\text { BERYL- } \\
\text { LIUM, } \\
\text { DIS- } \\
\text { SOLVED } \\
\text { (UG/L } \\
\text { AS BE) } \\
(01010)\end{array}$ & $\begin{array}{l}\text { BORON, } \\
\text { DIS- } \\
\text { SOLVED } \\
\text { (UG/L } \\
\text { AS B) } \\
(01020)\end{array}$ & $\begin{array}{c}\text { CADMIUM } \\
\text { DIS- } \\
\text { SOLVED } \\
\text { (UG/L } \\
\text { AS CD }) \\
(01025)\end{array}$ & $\begin{array}{l}\text { CERO- } \\
\text { MIUM, } \\
\text { DIS- } \\
\text { SOLVED } \\
\text { (UG/L } \\
\text { AS CR) } \\
(01030)\end{array}$ & $\begin{array}{c}\text { COBALT, } \\
\text { DIS- } \\
\text { SOLVED } \\
\text { (UG/L } \\
\text { AS CO) } \\
(01035)\end{array}$ & $\begin{array}{c}\text { COPPER, } \\
\text { DIS- } \\
\text { SOLVED } \\
\text { (UG/L } \\
\text { AS CU) } \\
\text { (01040) }\end{array}$ & $\begin{array}{c}\text { IRON, } \\
\text { DIS } \\
\text { SOLVED } \\
\text { (UG/L } \\
\text { AS FE) } \\
(01046)\end{array}$ & $\begin{array}{l}\text { LEAD, } \\
\text { DIS- } \\
\text { SOLVED } \\
\text { (UG/L } \\
\text { AS PB) } \\
(01049)\end{array}$ & $\begin{array}{c}\text { LITHIUM } \\
\text { DIS- } \\
\text { SOLVED } \\
\text { (UG/L } \\
\text { AS LI) } \\
\text { (01130) }\end{array}$ & $\begin{array}{l}\text { MANGA- } \\
\text { NESE, } \\
\text { DIS- } \\
\text { SOLVED } \\
\text { (UG/L } \\
\text { AS MN) } \\
(01056)\end{array}$ & $\begin{array}{c}\text { MERCURY } \\
\text { DIS- } \\
\text { SOLVED } \\
\text { (UG/L } \\
\text { AS HG) } \\
\text { (71890) }\end{array}$ \\
\hline $\begin{array}{l}\text { Nov } 1995 \\
02 \ldots \\
\text { pre }\end{array}$ & $<0.50$ & -- & $<1.0$ & $<5.0$ & $<3.0$ & 1.0 & 4.0 & $<1.0$ & 160 & 15 & -- \\
\hline $\mathrm{JAN}^{07} \mathrm{i} \ddot{9}_{96}$ & -- & $-m$ & -- & -- & -- & $\cdots$ & $<9.0$ & -- & - & 37 & -- \\
\hline $\begin{array}{r}17 \ldots \\
24 \ldots \\
\operatorname{FEB}\end{array}$ & $=-$ & $=$ & $<\overline{1.0}$ & $<1.0$ & $<3.0$ & 1.0 & $\begin{array}{r}<3.0 \\
3.0\end{array}$ & $<1.0$ & 120 & $\begin{array}{l}16 \\
29\end{array}$ & $<0.1$ \\
\hline${ }_{\operatorname{MAR}}^{29} \cdot \cdots$ & $<1.0$ & -- & $<1.0$ & 2.0 & $<1.0$ & 2.0 & $<3.0$ & $<1.0$ & -- & 9.0 & $<0.1$ \\
\hline$\underset{\mathrm{APR}}{26 \ldots}$ & $<1,0$ & 160 & $<1.0$ & 1.0 & $<1.0$ & 2.0 & $<3.0$ & $<1.0$ & 82 & 2.0 & - \\
\hline$\underset{\mathrm{MAY}}{23} \ldots$ & - & -- & -- & -- & - & -- & 31 & - & -- & 5.0 & - \\
\hline$\underset{\text { JuN }}{29} \ldots$ & $<1.0$ & 180 & $<1.0$ & $<1.0$ & $<1.0$ & 3.0 & $<3.0$ & $<1.0$ & 93 & 4.0 & - \\
\hline${ }_{\text {JuL }}^{27 . .}$ & - & -- & -- & -- & - & -- & 9.0 & -- & -- & 3.0 & - \\
\hline${ }_{A U G}^{25} \ldots$ & -- & -- & -- & -- & - & -- & $<3.0$ & -- & -- & $<1.0$ & $-\infty$ \\
\hline $\operatorname{sep} \ldots$ & $<1.0$ & $175^{\circ}$ & $<1.0$ & 2.0 & $<1.0$ & 2.0 & 4.0 & $<1.0$ & 81 & $<1.0$ & -- \\
\hline $10 \ldots$ & -- & -- & - & $-m$. & -- & $m$ & $<3.0$ & -- & -- & 1.0 & -- \\
\hline DATE & $\begin{array}{l}\text { MOLYB- } \\
\text { DENUM, } \\
\text { DIS- } \\
\text { SOLVED } \\
\text { (UG/L } \\
\text { AS MO) } \\
(01060)\end{array}$ & $\begin{array}{l}\text { NICKEL, } \\
\text { DIS- } \\
\text { SOLVED } \\
\text { (UG/L } \\
\text { AS NI) } \\
(01065)\end{array}$ & $\begin{array}{l}\text { SELE- } \\
\text { NIUM, } \\
\text { DIS- } \\
\text { SOLVED } \\
\text { (UG/L } \\
\text { AS -SE) } \\
\text { (01145) }\end{array}$ & $\begin{array}{c}\text { SILVER, } \\
\text { DIS-' } \\
\text { SOLVED } \\
\text { (UG/L } \\
\text { AS AG) } \\
(01075)\end{array}$ & $\begin{array}{c}\text { SFRON- } \\
\text { TIUM, } \\
\text { DIS- } \\
\text { SOLVED } \\
\text { (UG/L } \\
\text { AS SR) } \\
(01080)\end{array}$ & $\begin{array}{l}\text { VANA- } \\
\text { DIUM, } \\
\text { DIS- } \\
\text { SOLVED } \\
\text { (UG/L } \\
\text { AS V) } \\
(01085)\end{array}$ & $\begin{array}{c}\text { ZINC, } \\
\text { DIS } \\
\text { SOLVED } \\
\text { (UG/L } \\
\text { AS ZN) } \\
(01090)\end{array}$ & $\begin{array}{c}\text { URANIUM } \\
\text { NATURAL } \\
\text { DIS- } \\
\text { SOLVED } \\
\text { (UG/L } \\
\text { AS U) } \\
(22703)\end{array}$ & $\begin{array}{l}\text { SEDI- } \\
\text { MENT, } \\
\text { SUS- } \\
\text { PENDED } \\
\text { (MG/L) } \\
(80154)\end{array}$ & $\begin{array}{c}\text { SEDI- } \\
\text { MENT, } \\
\text { DIS- } \\
\text { CHARGE, } \\
\text { SUS- } \\
\text { PENDED } \\
\text { (T/DAY) } \\
(80155)\end{array}$ & $\begin{array}{r}\text { SED. } \\
\text { SUSP. } \\
\text { SIEVE } \\
\text { DIAM. } \\
\text { \% FINER } \\
\text { THAAN } \\
\text { T062 MM } \\
(70331)\end{array}$ \\
\hline $\begin{array}{l}\text { Nov } 1995 \\
022 \ldots \\
D E C\end{array}$ & $<10$ & $<1.0$ & $<1$ & $<1.0$ & 1500 & $<6$ & 12 & -- & 250 & 181 & 26 \\
\hline JAN 079 & - & -- & -- & - & -- & -- & - & -- & 109 & 51 & -- \\
\hline $\begin{array}{r}17 \\
24\end{array}$ & $20^{--}$ & $<1.0$ & $<1$ & $<\overline{1.0}$ & $1000^{--}$ & $-<6$ & $<10^{--}$ & $\because-$ & 127 & 116 & $--^{64}$ \\
\hline$\stackrel{29 \ldots}{\operatorname{MAR}}$ & 8.0 & 3.0 & $<1$ & $<1.0$ & -- & -- & 6.0 & 3.0 & 92 & $\cdots$ & 68 \\
\hline$\underset{A P R}{26 \ldots}$ & 7.0 & 1.0 & $<1$ & $<1.0$ & 730 & $<6$ & 2.0 & 3.0 & 252 & 680 & 49 \\
\hline${ }_{\mathrm{MAY}}^{23} \ldots$ & - & - & -- & -- & -- & -- & -- & - & 1360 & 2710 & 5 \\
\hline $\operatorname{sun}^{29} \cdot$ & 8.0 & 3.0 & $<1$ & $<1.0^{\circ}$ & 810 & $<6$ & 3.0 & 3.0 & 189 & 373 & 48 \\
\hline${ }_{\text {JuL }}^{27 . .}$ & -- & - & - & -- & -- & -- & - & -- & 2070 & 7160 & 36 \\
\hline${ }_{A \cup G}^{25} \ldots$ & -- & -- & - & -- & -- & $\cdots$ & -- & -- & 292 & 676 & -- \\
\hline $\operatorname{SEg}_{10}^{13} \cdots$ & 8.0 & 3.0 & $<1$ & $<1.0$ & 750. & $<6$ & 9.0 & 3.0 & -- & -- & -- \\
\hline $10, \ldots$ & - & -- & -- & $-\cdots$ & -- & -- & - & $\rightarrow$ & 322 & 519 & 81 \\
\hline
\end{tabular}


RIO GRANDE BASIN

08364000 RIO GRANDE AT EL PASO, TX -- Continued

WATER-QUALITY RECORDS

CHEMICAL ANALYSES OF ORGANIC COMPOUNDS, WATER YEAR OCTOBER 1995 TO SEPTEMBER 1996

\begin{tabular}{|c|c|c|c|c|c|c|c|c|c|c|c|c|}
\hline DATE & TIME & $\begin{array}{l}\text { PROP- } \\
\text { CHLOR, } \\
\text { WATER, } \\
\text { DISS, } \\
\text { REC } \\
\text { (UG/L) } \\
(04024)\end{array}$ & $\begin{array}{l}\text { BUTYL- } \\
\text { ATE, } \\
\text { WATER, } \\
\text { DISS, } \\
\text { REC } \\
\text { (UG/L) } \\
(04028)\end{array}$ & $\begin{array}{l}\text { SI- } \\
\text { MAZINE, } \\
\text { WATER, } \\
\text { DISS, } \\
\text { REC } \\
\text { (1⿴G/L) } \\
(04035)\end{array}$ & $\begin{array}{l}\text { PRO- } \\
\text { METON, } \\
\text { WATER, } \\
\text { DISS, } \\
\text { REC } \\
\text { (UG/L) } \\
\text { (04037) }\end{array}$ & $\begin{array}{c}\text { DEETHYL } \\
\text { ATRA- } \\
\text { ZINE, } \\
\text { WATER, } \\
\text { DISS, } \\
\text { REC } \\
\text { (UEG/L) } \\
(04040)\end{array}$ & $\begin{array}{l}\text { CYANA- } \\
\text { ZINE, } \\
\text { HATER, } \\
\text { DISS, } \\
\text { REC } \\
\text { (UEG/L) } \\
(04041)\end{array}$ & $\begin{array}{l}\text { FONOFOS } \\
\text { WATER } \\
\text { DISS } \\
\text { REC } \\
\text { (UEG/L) } \\
\text { (04095) }\end{array}$ & $\begin{array}{l}\text { ALPHA } \\
\text { BHC } \\
\text { DIS- } \\
\text { SOLVED } \\
\text { (UG/L) } \\
(34253)\end{array}$ & $\begin{array}{l}\mathbf{P}_{\text {, PDE }}^{\prime} \\
\text { DDE } \\
\text { DISSOLV } \\
(U \in / L) \\
(34653)\end{array}$ & $\begin{array}{c}\text { CAROR- } \\
\text { PYRIFOS } \\
\text { DIS- } \\
\text { SOLVED } \\
\text { rUG/L } \\
\text { (38933) }\end{array}$ & $\begin{array}{l}\text { LINDANE } \\
\text { DIS- } \\
\text { SOLVED } \\
\text { (UE /L }) \\
\text { (39341) }\end{array}$ \\
\hline $02 \ldots$ & 1400 & $<0.007$ & $<0.002$ & E0.004 & E0.005 & $<0.002$ & $<0.004$ & $<0.003$ & $<0.002$ & $<0.006$ & $E 0.003$ & $<0.004$ \\
\hline${ }^{07} j i 9$ & 1245 & -- & - & $-m$ & $m-$ & - & $\cdots$ & - & $-\cdots$ & - & $\cdots$ & -- \\
\hline $\begin{array}{l}17 \\
24\end{array}$ & $\begin{array}{l}0815 \\
1530\end{array}$ & $=$ & $=$ & $=$ & $=$ & $=$ & $\overline{--}$ & $=$ & $=-$ & $=$ & $=-$ & $=$ \\
\hline 29. & 0830 & $<0.007$ & $<0,002$ & 0.007 & 50.006 & $<0.002$ & $<0.004$ & $<0.003$ & $<0.002$ & $<0.006$ & $<0.004$ & $<0.004$ \\
\hline 26. & 0900 & $<0.007$ & $<0.002$ & 0.010 & 20.008 & $<0.002$ & $<0.004$ & $<0.003$ & $<0.002$ & $<0.006$ & $<0.004$ & $<0.004$ \\
\hline$\underset{A Y}{23} \cdots$ & 0750 & -- & -- & -- & -- & - & $\cdots \cdot-$ & -- & $\cdots$ & - & $\because m$ & - \\
\hline $29 \cdots$ & 0845 & $<0.007$ & $<0.002$ & 0.006 & $\mathrm{E} 0.007$ & $<0.002$ & $<0.004$ & $<0.003$ & $<0.002$ & $<0.006$ & 0,029 & $<0.004$ \\
\hline $27 \ldots$ & .0815 & $<0.007$ & $<0.002$ & 0.011 & 0.330 & $<0.002$ & $<0,004$ & $<0,003$ & $<0.002$ & $<0,006$ & 0.006 & $<0.004$ \\
\hline $25 \ldots$ & 0800 & $<0.007$ & $<0.002$ & 0.010 & E0.009 & $E 0.003$ & 0.009 & $<0.003$ & $<0.002$ & $<0.006$ & $<0.004$ & $<0.004$ \\
\hline$\frac{13}{\mathrm{EP}}$ & 0830 & -- & - & $-m$ & -- & $m-$ & $-\cdots$ & -- & $-m$ & -- & - & -- \\
\hline $10 \ldots$ & 0900 & $<0.007$ & $<0.002$ & $E 0.004$ & 0.018 & $<0.002$ & $<0.004$ & $<0.003$ & $<0.002$ & $<0.006$ & $\mathrm{EO}, 0.04$ & $<0.004$ \\
\hline DATE & $\begin{array}{l}\text { DI- } \\
\text { ELDRIN } \\
\text { DIS- } \\
\text { SOLVED } \\
\text { (UG/L) } \\
\text { (39381) }\end{array}$ & $\begin{array}{l}\text { METO- } \\
\text { LACHLOR } \\
\text { WATER } \\
\text { DISSOLV } \\
\text { (UG/L) } \\
(39415)\end{array}$ & $\begin{array}{l}\text { MALA- } \\
\text { THION, } \\
\text { DIS- } \\
\text { SOLVED } \\
\text { (UG/L) } \\
(39532)\end{array}$ & $\begin{array}{c}\text { PARA- } \\
\text { THION, } \\
\text { DIS- } \\
\text { SOLVED } \\
\text { (UG/L) } \\
(39542)\end{array}$ & $\begin{array}{c}\text { DI- } \\
\text { AZINON, } \\
\text { DIS-- } \\
\text { SOLVED } \\
\text { (UG/L) } \\
(39572)\end{array}$ & $\begin{array}{l}\text { ATRA- } \\
\text { ZINE, } \\
\text { WATER, } \\
\text { DISS, } \\
\text { REC } \\
\text { (UG/L) } \\
\text { (39632) }\end{array}$ & $\begin{array}{l}\text { ALA- } \\
\text { CHLOR, } \\
\text { WATER, } \\
\text { DISS, } \\
\text { REC, } \\
\text { (UG/L) } \\
(46342)\end{array}$ & $\begin{array}{c}\text { ACETO- } \\
\text { CHLOR, } \\
\text { WATER } \\
\text { FLTRD } \\
\text { REC } \\
(U G / L) \\
(49260)\end{array}$ & $\begin{array}{l}\text { METRI- } \\
\text { BUZIIN } \\
\text { SENCOR } \\
\text { WATHR } \\
\text { DISSOLV } \\
(U G / L) \\
(82630)\end{array}$ & $\begin{array}{c}2,6-\mathrm{DI}- \\
\text { ETgYL } \\
\text { ANILINE } \\
\text { WAT FLT } \\
0.7 \mathrm{U} \\
\text { GE, REC } \\
\text { (UG /L) } \\
(B 2660)\end{array}$ & $\begin{array}{c}\text { TRI- } \\
\text { FLUR- } \\
\text { ALIN } \\
\text { WAT FLT } \\
0.7 \mathrm{~V} \\
\text { GF, REC } \\
\text { (UG/L) } \\
(82661)\end{array}$ & $\begin{array}{l}\text { ETHAL- } \\
\text { FLUR- } \\
\text { ALIN } \\
\text { WAT FLT } \\
0.7 \text { U } \\
\text { GF REC } \\
\text { (UG/L) } \\
(82663)\end{array}$ \\
\hline 02. & 01 & 0.006 & $<0.005$ & $<0.004$ & 0.004 & $<0.001$ & $<0.002$ & $<0.002$ & $<0.004$ & $<0.003$ & $<0.002$ & $<0.004$ \\
\hline 07 & - & - & -- & -- & $=$ & -- & - & - & -- & $-\infty$ & $-\cdots$ & - \\
\hline 17 & $=$ & $=$ & $\because$ & $=$ & $=$ & $=-$ & $\because-$ & $=-$ & $=$ & - & - & -- \\
\hline $29 \ldots$ & $<0.001$ & 0.004 & $<0.005$ & $<0.004$ & $<0.002$ & $<0.001$ & $<0.002$ & $<0.002$ & $<0.004$ & $<0.003$ & $<0.002$ & $<0.004$ \\
\hline $26 \ldots$ & $<0.001$ & 0.011 & $<0.005$ & $<0.004$ & $<0.002$ & $<0.001$ & $<0.002$ & $<0.002$ & $<0.004$ & $<0.003$ & $<0.002$ & $<0.004$ \\
\hline $23 \ldots$ & -- & -- & -- & - & -- & -- & - & $-\infty$ & - & - & - & -- \\
\hline $29 \ldots$ & $<$ & 0.008 & $<0.005$ & $<0.004$ & $<0.002$ & E0.004 & $<0.002$ & $<0.002$ & $<0.004$ & $<0.003$ & $<0.002$ & $<0.004$ \\
\hline $27 \ldots$ & $<0.001$ & 0.014 & 0.013 & $<0.004$ & 0.052 & EC & $<0.002$ & $<0.002$ & 004 & $<0.003$ & $<0.002$ & $<0.004$ \\
\hline $25 \ldots$ & $<0.0$ & 0.009 & $<0.005$ & $<0.004$ & 50.003 & 0.005 & $<0.002$ & $<0.002$ & $<0.004$ & $<0,003$ & 0.005 & $<0.004$ \\
\hline $\begin{array}{l}13 \ldots \\
10 . .\end{array}$ & $<0.001$ & 0.005 & $<0.005$ & $\begin{array}{c}-- \\
<0.004\end{array}$ & $<0.002$ & $<0.001$ & $<0.002$ & $<0.002$ & $<0.004$ & $<0.003$ & $\begin{array}{c}-\cdots \\
<0.002\end{array}$ & $<0.004$ \\
\hline
\end{tabular}


RIO GRANDE BASIN

08364000 RIO GRANDE AT EL PASO, TX -- Continued

WATER-QUALITY RECORDS

CHEMICAL ANALYSES OF ORGANIC COMPOUNDS, WATER YEAR OCTOBER 1995 TO SEPTEMBER 1996

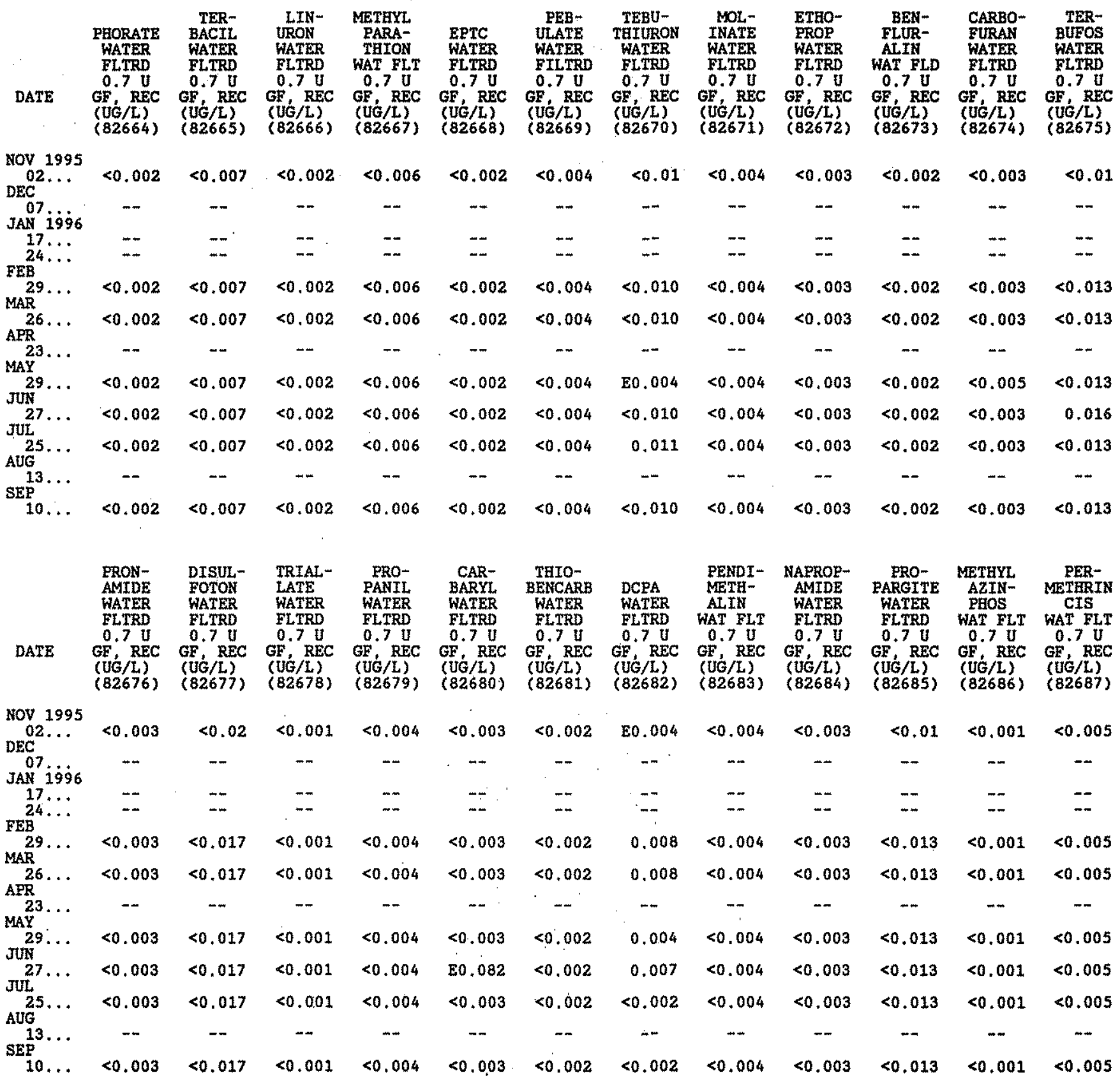


RTO GRANDE BASTN

08364000 RIO GRANDE AT EL RASO, TX - - Continued

WATER-QUALITY RECORDS

WATER QUALITY DATA, WATER YEAR OCTOBER 1995 TO SEPTEMBER 1996

CROSS SECTION ANALYSES

\begin{tabular}{|c|c|c|c|c|c|}
\hline $\begin{array}{l}\text { SAMPLE } \\
\text { LOC- } \\
\text { ATION, } \\
\text { CROSS } \\
\text { SECTION } \\
\text { (FT FM } \\
\text { L BARK) }\end{array}$ & $\begin{array}{l}\text { DEPTH } \\
\text { AT } \\
\text { SALYLE } \\
\text { JOC- } \\
\text { ATION, } \\
\text { TOTAL, } \\
\text { (FET) }\end{array}$ & $\begin{array}{l}\mathrm{SI} \\
\mathrm{CO} \\
\mathrm{DI} \\
\text { AN } \\
\text { CUS } \\
\mathrm{CO}\end{array}$ & $\begin{array}{l}\text { FH } \\
\text { WATER } \\
\text { WROLD } \\
\text { FIELD } \\
\text { SSTAND } \\
\text { ARD } \\
\text { UNITS } \\
\text { COLODO }\end{array}$ & $\begin{array}{l}\mathbb{R}- \\
\mathbf{R} \\
\mathbf{R} \\
\mathbf{R} \\
\text { C) } \\
(0)\end{array}$ & 5 \\
\hline
\end{tabular}

$\begin{array}{ccccccccc}\text { SEP } 1996 & & 0822 & 155 & 3.80 & 1070 & 8.3 & 22.5 & 7.4 \\ 10 \ldots & 0826 & 145 & 4.10 & 1140 & 8.4 & 22.5 & 7.4 \\ 10 \ldots & 0828 & 135 & 2.90 & 1230 & 8.4 & 22.5 & 7.2 \\ 10 \ldots & 0830 & 125 & 0.60 & 1220 & 8.4 & 22.5 & 7.1 \\ 10 \ldots & 0832 & 115 & 1.30 & 1240 & 8.4 & 22.5 & 7.2 \\ 10 \ldots & 0833 & 105 & 1.40 & 1260 & 8.4 & 22.5 & 7.2 \\ 10 \ldots & 083.5 & 12.5 & 7.2 \\ 10 \ldots & 0835 & 95.0 & 1.50 & 1250 & 8.4 & 22.5 & 7.2 \\ 10 \ldots & 0837 & 85.0 & 1.60 & 1260 & 8.4 & 22.5 & 7.2 \\ 10 \ldots & 0838 & 75.0 & 1.90 & 1260 & 8.4 & 22.5 & 7.5 \\ 10 \ldots & 0839 & 65.0 & 1.60 & 1270 & 8.4 & 22.5 & 7.2 \\ 10 \ldots & 0840 & 55.0 & 1.70 & 1270 & 8.4 & 22.5 & 7.2 \\ 10 \ldots & 0841 & 45.0 & 1.50 & 1270 & 8.4 & 22.5 & 7.2 \\ 10 \ldots & 0842 & 35.0 & 1.60 & 1270 & 8.4 & 22.5 & 7.2 \\ 10 \ldots & 0843 & 25.0 & 1.60 & 1280 & 8.4 & 22.5 & 7.2 \\ 10 \ldots & 0844 & 15.0 & 1.60 & 1280 & 8.4 & 22.5 & 7.2 \\ 10 \ldots & 0845 & 5.00 & 1.60 & 1280 & 8.4 & 22.5 & 7.2\end{array}$


RIO GRANDE BASIN

08377900 RIO MORA NEAR TERRERO, NM

(Aydrologic bench-mark station)

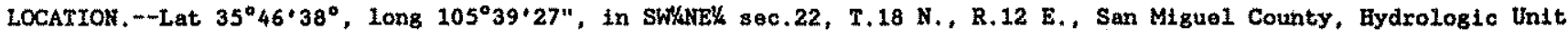
13060001 , in Santa Fe'National Forest, on left bank 450 ft upstream from bridge on State Highway 63 , 600 ft upstream fxom mouth, and $2.6 \mathrm{ml}$ north of Terrero.

DRATNAGE AREA, $--53,2$ mi2.

WATER-DISCEARGE RECORDS

PERIOD OF RECORD.--October 1963 to current year.

GAGE. -Water-stage recorder and concrete control. Elevation of gage is 7,890 ft above National Geodetic Vertical Datum of 1929 , from topographic map.

REMARKS.-Water-discharge records good except for estimated daily discharges, which are poor, About 90 percent of the drainage $t \mathrm{~s}$ in the Pecos Wilderness Area and not subject to development, watershed managenent, ox the building of highways; there is limited cattle grazing by pertith.

EXTREMES OUTSIDE FERIOD OF RECORD.--Greatest flood since 1886 probably occurred Sept. 29, 1904 (based on statement for Pecos River near Pocos and history of that flood period).

DISCHARGE, CUBIC FEET PER SECOND, WATER YEAR OCTOBER 1994 TO SEPTEMBER 1995

\begin{tabular}{|c|c|c|c|c|c|c|c|c|c|c|c|c|}
\hline DAY & OCT & Nov & DEC & JAN & FEB & MAR & $\mathrm{APR}$ & MAY & JUN & JUL & AUG & SEP \\
\hline $\begin{array}{l}1 \\
2 \\
3 \\
4 \\
5\end{array}$ & $\begin{array}{l}16 \\
16 \\
15 \\
15 \\
15\end{array}$ & $\begin{array}{l}23 \\
22 \\
26 \\
24 \\
21\end{array}$ & $\begin{array}{l}012 \\
013 \\
014 \\
e 13 \\
012\end{array}$ & $\begin{array}{l}7.2 \\
7.2 \\
7.3 \\
7.0 \\
7.5\end{array}$ & $\begin{array}{l}6.6 \\
7.7 \\
10 \\
11 \\
11\end{array}$ & $\begin{array}{l}20 \\
19 \\
17 \\
16 \\
16\end{array}$ & $\begin{array}{l}27 \\
22 \\
21 \\
20 \\
20\end{array}$ & $\begin{array}{r}74 \\
84 \\
96 \\
95 \\
109\end{array}$ & $\begin{array}{l}278 \\
277 \\
284 \\
286 \\
296\end{array}$ & $\begin{array}{l}98 \\
89 \\
82 \\
81 \\
71\end{array}$ & $\begin{array}{l}24 \\
21 \\
20 \\
18 \\
17\end{array}$ & $\begin{array}{l}49 \\
43 \\
39 \\
35 \\
32\end{array}$ \\
\hline $\begin{array}{r}6 \\
7 \\
8 \\
9 \\
10\end{array}$ & $\begin{array}{l}14 \\
15 \\
16 \\
15 \\
16\end{array}$ & $\begin{array}{l}22 \\
21 \\
21 \\
20 \\
19\end{array}$ & $\begin{array}{l}011 \\
011 \\
011 \\
9.4 \\
09.2\end{array}$ & $\begin{array}{l}7.9 \\
7.6 \\
7.3 \\
7.1 \\
7.3\end{array}$ & $\begin{array}{l}11 \\
12 \\
12 \\
12 \\
12\end{array}$ & $\begin{array}{l}19 \\
22 \\
28 \\
26 \\
19\end{array}$ & $\begin{array}{l}20 \\
23 \\
30 \\
41 \\
40\end{array}$ & $\begin{array}{r}114 \\
109 \\
104 \\
92 \\
94\end{array}$ & $\begin{array}{l}328 \\
342 \\
323 \\
307 \\
288\end{array}$ & $\begin{array}{l}64 \\
59 \\
54 \\
48 \\
45\end{array}$ & $\begin{array}{l}16 \\
16 \\
15 \\
16 \\
18\end{array}$ & $\begin{array}{l}30 \\
32 \\
42 \\
32 \\
31\end{array}$ \\
\hline $\begin{array}{l}11 \\
12 \\
13 \\
14 \\
15\end{array}$ & $\begin{array}{l}15 \\
14 \\
13 \\
14 \\
14\end{array}$ & $\begin{array}{r}21 \\
99 \\
108 \\
60 \\
48\end{array}$ & $\begin{array}{l}99.6 \\
99.6 \\
99.8 \\
010 \\
010\end{array}$ & $\begin{array}{r}7.2 \\
7.0 \\
6.6 \\
06.5 \\
06.2\end{array}$ & $\begin{array}{l}11 \\
11 \\
11 \\
10 \\
11\end{array}$ & $\begin{array}{l}21 \\
25 \\
23 \\
23 \\
25\end{array}$ & $\begin{array}{l}35 \\
31 \\
33 \\
40 \\
41\end{array}$ & $\begin{array}{l}110 \\
128 \\
138 \\
156 \\
222\end{array}$ & $\begin{array}{l}273 \\
265 \\
261 \\
260 \\
245\end{array}$ & $\begin{array}{l}41 \\
39 \\
37 \\
40 \\
41\end{array}$ & $\begin{array}{l}16 \\
21 \\
20 \\
17 \\
18\end{array}$ & $\begin{array}{l}32 \\
28 \\
27 \\
26 \\
28\end{array}$ \\
\hline $\begin{array}{l}16 \\
17 \\
18 \\
19 \\
20\end{array}$ & $\begin{array}{l}19 \\
34 \\
24 \\
24 \\
25\end{array}$ & $\begin{array}{r}39 \\
32 \\
e 26 \\
e 21 \\
-20\end{array}$ & $\begin{array}{c}e 11 \\
11 \\
10 \\
10 \\
9.7\end{array}$ & $\begin{array}{r}6.5 \\
6.7 \\
06.3 \\
6.8 \\
6.3\end{array}$ & $\begin{array}{l}11 \\
09.8 \\
09.6 \\
010 \\
14\end{array}$ & $\begin{array}{l}26 \\
26 \\
28 \\
38 \\
49\end{array}$ & $\begin{array}{l}39 \\
37 \\
34 \\
31 \\
28\end{array}$ & $\begin{array}{l}300 \\
327 \\
260 \\
222 \\
236\end{array}$ & $\begin{array}{l}234 \\
248 \\
366 \\
482 \\
416\end{array}$ & $\begin{array}{l}40 \\
39 \\
38 \\
40 \\
34\end{array}$ & $\begin{array}{l}18 \\
25 \\
18 \\
18 \\
16\end{array}$ & $\begin{array}{l}25 \\
24 \\
23 \\
22 \\
21\end{array}$ \\
\hline $\begin{array}{l}21 \\
22 \\
23 \\
24 \\
25\end{array}$ & $\begin{array}{l}26 \\
26 \\
26 \\
27 \\
26\end{array}$ & $\begin{array}{l}e 18 \\
e 18 \\
e 15 \\
e 14 \\
e 13\end{array}$ & $\begin{array}{l}9.4 \\
9.1 \\
9.3 \\
8.8 \\
8.2\end{array}$ & $\begin{array}{r}6.2 \\
6.3 \\
6.0 \\
5.7 \\
5.7\end{array}$ & $\begin{array}{l}18 \\
18 \\
18 \\
19 \\
19\end{array}$ & $\begin{array}{l}54 \\
68 \\
61 \\
54 \\
50\end{array}$ & $\begin{array}{l}26 \\
23 \\
24 \\
22 \\
23\end{array}$ & $\begin{array}{l}287 \\
349 \\
371 \\
335 \\
293\end{array}$ & $\begin{array}{l}333 \\
292 \\
245 \\
199 \\
163\end{array}$ & $\begin{array}{l}31 \\
30 \\
28 \\
26 \\
25\end{array}$ & $\begin{array}{l}16 \\
18 \\
22 \\
28 \\
43\end{array}$ & $\begin{array}{l}21 \\
20 \\
19 \\
20 \\
23\end{array}$ \\
\hline $\begin{array}{l}26 \\
27 \\
28 \\
29 \\
30 \\
31\end{array}$ & $\begin{array}{r}25 \\
025 \\
25 \\
26 \\
26 \\
25\end{array}$ & $\begin{array}{l}010 \\
011 \\
011 \\
010 \\
011 \\
-\cdots-\end{array}$ & $\begin{array}{l}8.1 \\
7.8 \\
7.7 \\
7.4 \\
7.7 \\
7.6\end{array}$ & $\begin{array}{r}5.7 \\
5.7 \\
5.5 \\
5.8 \\
05.8 \\
6.0\end{array}$ & $\begin{array}{l}21 \\
22 \\
21 \\
-\cdots \\
-\cdots\end{array}$ & $\begin{array}{l}44 \\
39 \\
31 \\
27 \\
26 \\
34\end{array}$ & $\begin{array}{r}32 \\
39 \\
44 \\
52 \\
67 \\
--\infty\end{array}$ & $\begin{array}{l}280 \\
235 \\
222 \\
249 \\
277 \\
296\end{array}$ & $\begin{array}{l}142 \\
126 \\
112 \\
111 \\
109 \\
-\end{array}$ & $\begin{array}{l}25 \\
23 \\
22 \\
21 \\
21 \\
22\end{array}$ & $\begin{array}{l}76 \\
73 \\
64 \\
58 \\
60 \\
55\end{array}$ & $\begin{array}{r}19 \\
18 \\
19 \\
21 \\
20 \\
-\end{array}$ \\
\hline $\begin{array}{l}\text { TOTAL } \\
\text { MEAN } \\
\text { MAX } \\
\text { MIN } \\
\text { AC-ET }\end{array}$ & $\begin{array}{r}632 \\
20.4 \\
34 \\
13 \\
1250\end{array}$ & $\begin{array}{r}824 \\
27.5 \\
108 \\
10 \\
1630\end{array}$ & $\begin{array}{r}307.4 \\
9.92 \\
14 \\
7.4 \\
610\end{array}$ & $\begin{array}{r}203.9 \\
6.58 \\
7.9 \\
5.5 \\
404\end{array}$ & $\begin{array}{r}369.7 \\
13.2 \\
22 \\
6.6 \\
733\end{array}$ & $\begin{array}{r}974 \\
31.4 \\
68 \\
1.6 \\
1930\end{array}$ & $\begin{array}{r}965 \\
32.2 \\
67 \\
20 \\
1910\end{array}$ & $\begin{array}{r}6264 \\
202 \\
371 \\
74 \\
12420\end{array}$ & $\begin{array}{r}7891 \\
263 \\
482 \\
109 \\
15650\end{array}$ & $\begin{array}{r}1354 \\
43.7 \\
98 \\
21 \\
2690\end{array}$ & $\begin{array}{r}881 \\
28.4 \\
76 \\
15 \\
1750\end{array}$ & $\begin{array}{r}821 \\
27.4 \\
49 \\
18 \\
1630\end{array}$ \\
\hline
\end{tabular}

STATISTICS OF MONTHLY MEAN DATA FOR WATER YEARS 1964 - 1995, BY WATER YEAR (WY)

\begin{tabular}{|c|c|c|c|c|c|c|c|c|c|c|}
\hline $\begin{array}{l}\text { MEAN } \\
\text { MAX } \\
\text { (WY) } \\
\text { MIN } \\
\text { (WY) }\end{array}$ & $\begin{array}{l}14.4 \\
25.2 \\
1986 \\
5.73 \\
1965\end{array}$ & $\begin{array}{l}10.3 \\
27.5 \\
1995 \\
3.72 \\
1990\end{array}$ & $\begin{array}{l}7.08 \\
13.3 \\
1985 \\
2.90 \\
1990\end{array}$ & $\begin{array}{l}5.95 \\
9.82 \\
1986 \\
1.72 \\
1964\end{array}$ & $\begin{array}{l}6.28 \\
13.2 \\
1995 \\
2.43 \\
1964\end{array}$ & $\begin{array}{l}12.2 \\
41.3 \\
1989 \\
3.40 \\
1964\end{array}$ & $\begin{array}{l}36.4 \\
88.4 \\
1985 \\
11.2\end{array}$ & $\begin{array}{r}126 \\
319 \\
1973 \\
14.2 \\
1967\end{array}$ & $\begin{array}{r}87.9 \\
263 \\
1995 \\
8.25 \\
1967\end{array}$ & \\
\hline
\end{tabular}

SUMARY STATISTICS

ANNUAL TOTAL

ANNUAL MEAN

HIGHEST ANNUAL MEAN

LOWEST ANNUAL MEAN

HIGHEST DAILY MEAN

LOWEST DAILY MEAN

ANNUAL SEVEN-DAY MINIMUM

INSTANTANEOUS PEAX FLOW

INSTANTANEOUS PEAK STAGE

INSTANTANEOUS LOW FLOW

ANNUAL RUNOFF (AC-FT)

10 PERCENT EXCEEDS

50 PERCENT EXCEEDS

90 PERCENT EXCEEDS

- Estimated
FOR 1994 CALENDAR YEAR

$\begin{aligned} & 16871.9 \\ & 46.2 \vdots \\ & 391 \text { May } 21 \\ & 4.0 \text { Fob } \frac{1}{4.7} \\ & \text { Jan } 28\end{aligned}$

33470

107
20

6.0
FOR 1995 WATER YEAR

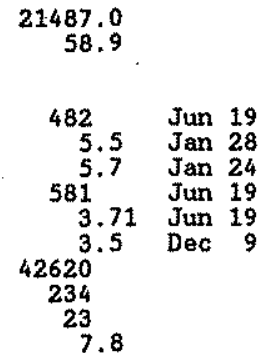

WATER YEARS 1964 - 1995

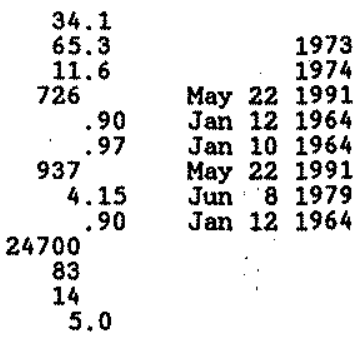




\section{RIO GRANDE BASIN}

08377900 RIO MORA NEAR TERRERO, MM -- Continued

WATER-QUALITY RECORDS

PERIOD OF RECORD. - Water years 1963 to current year.

WATER QUALITY DATA, WATER YEAR OCTOBER 1995 TO SEPTEMBER 1996

\begin{tabular}{|c|c|c|c|c|c|c|c|c|c|c|c|}
\hline DATE & TIME & $\begin{array}{c}\text { DIS- } \\
\text { CEARGE, } \\
\text { INST: } \\
\text { CUBIC } \\
\text { FEET } \\
\text { PER } \\
\text { SECOND } \\
(00061)\end{array}$ & $\begin{array}{l}\text { SPE- } \\
\text { CIFIC } \\
\text { CON- } \\
\text { DUCT- } \\
\text { ANCE } \\
\text { (US/CM) } \\
(00095)\end{array}$ & $\begin{array}{c}\text { PH } \\
\text { WATER } \\
\text { WHOLE } \\
\text { FIELD } \\
\text { (STAND- } \\
\text { ARD } \\
\text { UNITS) } \\
\text { (00400) }\end{array}$ & $\begin{array}{c}\text { TERPFER- } \\
\text { ATURE } \\
\text { AIR } \\
\text { (DEG C) } \\
(00020)\end{array}$ & $\begin{array}{l}\text { TEMPER- } \\
\text { ATURE } \\
\text { WATER } \\
\text { (DEG C) } \\
\text { (00010) }\end{array}$ & $\begin{array}{c}\text { TUR- } \\
\text { BID } \\
\text { ITY } \\
\text { (ATU) } \\
(00076)\end{array}$ & $\begin{array}{c}\text { BARO- } \\
\text { MAETRIC } \\
\text { PRES- } \\
\text { SURE } \\
\text { (MM } \\
\text { OF } \\
\text { HG) } \\
(00025)\end{array}$ & $\begin{array}{c}\text { OXYGEN, } \\
\text { DIS } \\
\text { SOLVED } \\
(M G / L) \\
(00300)\end{array}$ & $\begin{array}{c}\text { OXYGER, } \\
\text { DIS- } \\
\text { SOLVED } \\
\text { (PER- } \\
\text { CENT } \\
\text { SATUR- } \\
\text { ATION) } \\
\text { (00301) }\end{array}$ & $\begin{array}{l}\text { COLI- } \\
\text { FORM, } \\
\text { FECAL, } \\
0,7 \\
\text { WM-MF } \\
\text { (COLS, } \\
100 \mathrm{ML}) \\
\text { (31625) }\end{array}$ \\
\hline $\begin{array}{l}\text { DEC } 1995 \\
12 \\
\text { FEB } 1996\end{array}$ & 1130 & 11 & 143 & 7.9 & 9.0 & 0.0 & 0.20 & 570 & 10.9 & 100 & $<1$ \\
\hline $13 \ldots$ & 0945 & 6.0 & 126 & 8.1 & -3.5 & 0.0 & -- & 574 & 10.9 & 99 & $<1$ \\
\hline DATE & $\begin{array}{c}\text { STREP- } \\
\text { TOCOCCI } \\
\text { FECAL, } \\
\text { KF AGAR } \\
\text { (COLS. } \\
\text { PER } \\
100 \mathrm{ML}) \\
(31673)\end{array}$ & $\begin{array}{c}\text { HARD- } \\
\text { NESS } \\
\text { TOTAL } \\
\text { (HG/L } \\
\text { AS } \\
\text { CACO3) } \\
(00900)\end{array}$ & $\begin{array}{l}\text { HARD- } \\
\text { NESS } \\
\text { NONCARB } \\
\text { DISSOLV } \\
\text { FLD. AS } \\
\text { CACO3 } \\
(\text { MGG/L) } \\
(00904)\end{array}$ & $\begin{array}{c}\text { CALCIUM } \\
\text { DIS- } \\
\text { SOLVED } \\
\text { (NGG/L } \\
\text { AS CA) } \\
\text { (00915) }\end{array}$ & $\begin{array}{c}\text { MAGNE- } \\
\text { SIUM, } \\
\text { DIS-- } \\
\text { SOLVED } \\
\text { (MG M } \\
\text { AS MG) } \\
(00925)\end{array}$ & $\begin{array}{c}\text { SODIUM, } \\
\text { DIS- } \\
\text { SOLVED } \\
\text { (MGG/L } \\
\text { AS AA) } \\
(00930)\end{array}$ & $\begin{array}{c}\text { SODIUM } \\
\text { AD- } \\
\text { SORP- } \\
\text { TION } \\
\text { RATTO } \\
(00931)\end{array}$ & $\begin{array}{l}\text { POTAS- } \\
\text { SIUM, } \\
\text { DIS- } \\
\text { SOLVED } \\
\text { (MG/L } \\
\text { AS K) } \\
(00935)\end{array}$ & $\begin{array}{c}\text { BICAR- } \\
\text { BONATE } \\
\text { WATER } \\
\text { DIS IT } \\
\text { FIELD } \\
\text { MG/L AS } \\
\text { HCO3 } \\
(00453)\end{array}$ & $\begin{array}{c}\text { CAR- } \\
\text { BONATE } \\
\text { WATER } \\
\text { DIS IT } \\
\text { FIELD } \\
\text { MG/L AS } \\
\text { COS } \\
(00452)\end{array}$ & $\begin{array}{l}\text { ALKA- } \\
\text { LINITY } \\
\text { WAT DIS } \\
\text { TOT IT } \\
\text { FIELD } \\
\text { MG IL AS } \\
\text { CACO3 } \\
\text { (39086) }\end{array}$ \\
\hline $\begin{array}{l}\text { DEC } 1995 \\
12 \text { FEB } 1996\end{array}$ & 18 & 64 & 6 & 22 & 2.3 & 1.9 & 0.1 & 0.50 & 71 & 0 & 58 \\
\hline $13 \ldots$ & 400 & - & $\cdots$ & -- & -- & -- & -- & - & 69 & 0 & 56 \\
\hline DATE & $\begin{array}{c}\text { ALKA- } \\
\text { LINITY } \\
\text { IAAB } \\
\text { (NG/L } \\
\text { AS } \\
\text { CACO3) } \\
(90410)\end{array}$ & $\begin{array}{c}\text { SULFATE } \\
\text { DIS- } \\
\text { SOLVED } \\
\text { (NGG/L } \\
\text { AS SO4) } \\
(00945)\end{array}$ & $\begin{array}{l}\text { CHLO- } \\
\text { RIDE, } \\
\text { DIS- } \\
\text { SOLVED } \\
\text { (MSG/L } \\
\text { AS CL) } \\
(00940)\end{array}$ & $\begin{array}{l}\text { FLUO- } \\
\text { RIDE, } \\
\text { DIS- } \\
\text { SOLVED } \\
\text { (MG/L } \\
\text { AS F) } \\
(00950)\end{array}$ & $\begin{array}{c}\text { SILICA, } \\
\text { DIS- } \\
\text { SOLVED } \\
\text { (NSG/L } \\
\text { AS } \\
\text { SIO2) } \\
(00955)\end{array}$ & $\begin{array}{l}\text { SOLIDS, } \\
\text { RESIDUE } \\
\text { AT } 180 \\
\text { DEG. C } \\
\text { DIS- } \\
\text { SOLVED } \\
\text { (MG/L) } \\
(70300)\end{array}$ & $\begin{array}{c}\text { SOLIDS, } \\
\text { SUM OF } \\
\text { CONSTI- } \\
\text { TUENTS, } \\
\text { DIS- } \\
\text { SOLVED } \\
\text { (NG/L) } \\
(70301)\end{array}$ & $\begin{array}{c}\text { NITRO- } \\
\text { GEN, } \\
\text { NITRITE } \\
\text { DIS- } \\
\text { SOLVED } \\
\text { (MG/L } \\
\text { AS N) } \\
(00613)\end{array}$ & $\begin{array}{c}\text { NITRO- } \\
\text { GEN, } \\
\text { NO2+NO3 } \\
\text { DIS- } \\
\text { SOLVED } \\
\text { (MG/L } \\
\text { AS N) } \\
(00631)\end{array}$ & $\begin{array}{c}\text { NITRO- } \\
\text { GEN } \\
\text { AMONIA } \\
\text { DIS- } \\
\text { SOLVED } \\
\text { (MGLL } \\
\text { AS N) } \\
(00608)\end{array}$ & $\begin{array}{l}\text { NITRO- } \\
\text { GEN, ARY- } \\
\text { MONIA + } \\
\text { ORGAMIC } \\
\text { TOTAL } \\
\text { (NG /L } \\
\text { AS N) } \\
\text { (00625) }\end{array}$ \\
\hline $\begin{array}{l}\text { DEC } 1995 \\
121996 \\
\text { FEB } 1996\end{array}$ & 57 & 7.9 & 0.60 & 0.30 & 7.0 & 81 & 78 & $<0.010$ & 0.050 & $<0.015$ & $<0.20$ \\
\hline & - & -- & $m$ & -- & -- & -- & -- & $<0.010$ & 0.110 & $<0.015$ & $<0.20$ \\
\hline DATE & $\begin{array}{c}\text { PHOS- } \\
\text { PHORUS } \\
\text { TOTAL } \\
\text { (NG/L } \\
\text { AS P) } \\
(00665)\end{array}$ & $\begin{array}{c}\text { PHOS- } \\
\text { PHORUS } \\
\text { DIS- } \\
\text { SOLVED } \\
\text { (MG/L } \\
\text { AS P) } \\
(00666)\end{array}$ & $\begin{array}{l}\text { PHOS- } \\
\text { PHORUS } \\
\text { ORTHO, } \\
\text { DIS- } \\
\text { SOLVED } \\
\text { (HSG/L } \\
\text { AS P) } \\
(00671)\end{array}$ & $\begin{array}{l}\text { ALUM- } \\
\text { INUM, } \\
\text { DIS - } \\
\text { SOLVED } \\
\text { (UG/I, } \\
\text { AS AI) } \\
\text { (01106) }\end{array}$ & $\begin{array}{l}\text { BARIUM, } \\
\text { DIS- } \\
\text { SOLVED } \\
\text { (UG/L } \\
\text { AS BA) } \\
(01005)\end{array}$ & $\begin{array}{c}\text { COBALT, } \\
\text { DIS- } \\
\text { SOLVED } \\
\text { (UG/L } \\
\text { AS CO) } \\
\text { (01035) }\end{array}$ & $\begin{array}{l}\text { IRON, } \\
\text { DIS- } \\
\text { SOLVED } \\
\text { (UGLL } \\
\text { AS FE) } \\
(01046)\end{array}$ & $\begin{array}{c}\text { LITHIUM } \\
\text { DIS- } \\
\text { SOLVED } \\
\text { (UG/L } \\
\text { AS LI) } \\
\text { (01130) }\end{array}$ & $\begin{array}{l}\text { MANGA- } \\
\text { NESE, } \\
\text { DIS } \\
\text { SOLVED } \\
\text { (UG/L } \\
\text { AS NAN) } \\
\text { (01056) }\end{array}$ & $\begin{array}{l}\text { MOLYB- } \\
\text { DENUM, } \\
\text { DIS- } \\
\text { SOLVED } \\
\text { (UG/L } \\
\text { AS MO) } \\
(01060)\end{array}$ & $\begin{array}{c}\text { NICKEL, } \\
\text { DIS- } \\
\text { SOLVED } \\
\text { (UE/L } \\
\text { AS HI) } \\
\text { (01065) }\end{array}$ \\
\hline $\begin{array}{l}\mathrm{DEC} 1995 \\
12 \\
\mathrm{FEB} \\
1996\end{array}$ & $<0.010$ & $<0.010$ & $<0.010$ & $<10$ & 29 & $<3.0$ & $<3.0$ & $<\dot{4}$ & $<1.00$ & 10 & $<1.0$ \\
\hline 13. & $<0.010$ & $<0.010$ & $<0.010$ & $\rightarrow$ & -- & $-\infty$ & -- & $-m$ & -- & -- & $-\cdots$ \\
\hline DATE & $\begin{array}{l}\text { SELE- } \\
\text { NIUM, } \\
\text { DIS- } \\
\text { SOLVED } \\
\text { (UG/L } \\
\text { AS SE) } \\
\text { (OI145) }\end{array}$ & $\begin{array}{c}\text { SILVER, } \\
\text { DIS- } \\
\text { SOLVED } \\
\text { (UG/L } \\
\text { AS AG) } \\
\text { (01075) }\end{array}$ & $\begin{array}{c}\text { STRON- } \\
\text { TIUM, } \\
\text { DIS- } \\
\text { SOLVED } \\
\text { (UG/L } \\
\text { AS SR) } \\
(01080)\end{array}$ & $\begin{array}{l}\text { VANA- } \\
\text { DIUM, } \\
\text { DIS- } \\
\text { SOLVED } \\
\text { (UEG/L } \\
\text { AS V) } \\
(01085)\end{array}$ & $\begin{array}{c}\text { RADIUM } \\
\text { 226, } \\
\text { DIS- } \\
\text { SOLVED, } \\
\text { RADON } \\
\text { METHOD } \\
\text { (RCI/L) } \\
(09511)\end{array}$ & $\begin{array}{l}\text { RA-226 } \\
2 \text { SIGMA } \\
\text { WATER, } \\
\text { DISS } \\
\text { (PCI/L) } \\
(76001)\end{array}$ & $\begin{array}{l}\text { URANIUM } \\
\text { NATURAL } \\
\text { DIS- } \\
\text { SOLVED } \\
\text { (UG/L } \\
\text { AS U) } \\
(22703)\end{array}$ & $\begin{array}{l}\text { URANIUM } \\
\text { NATURAL } \\
2 \text { SIGMA } \\
\text { WATER, } \\
\text { DISS, } \\
\text { (UC/L) } \\
(75990)\end{array}$ & $\begin{array}{l}\text { SEDI- } \\
\text { MENT, } \\
\text { SUS- } \\
\text { PENDED } \\
\text { (ME/L) } \\
(80154)\end{array}$ & $\begin{array}{c}\text { SEDI- } \\
\text { MENY, } \\
\text { DIS- } \\
\text { CEARGE, } \\
\text { SUS-- } \\
\text { PENDED } \\
(\mathrm{T} / \mathrm{DAY}) \\
(\mathrm{BO} 155)\end{array}$ & $\begin{array}{l}\text { SED. } \\
\text { SUSE. } \\
\text { SIEVE } \\
\text { DIAM. } \\
\% \text { FINEK } \\
\text { THAAN } \\
\text { OST RE } \\
(70331)\end{array}$ \\
\hline $\begin{array}{l}\text { DEC } 1995 \\
12 \\
\text { FEB } 1996 \\
13 . .\end{array}$ & $-<$ & $<1.0$ & 51 & $\begin{array}{l}<6 \\
--\end{array}$ & 0.03 & $\begin{array}{l}0.010 \\
--\end{array}$ & $\begin{array}{l}0.47 \\
-\infty\end{array}$ & $\begin{array}{l}0.0 \\
--\end{array}$ & 56 & $\begin{array}{l}1.7 \\
0.10\end{array}$ & 24 \\
\hline
\end{tabular}


RIO GRANDE BASIN

08378500 PECOS RIVER NEAR PECOS, NM

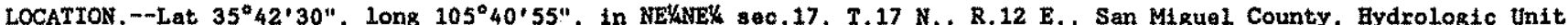
13060001 , In Santa Fe'National Forest, on left bank 30 ft downstrean from bridge on private road, 270 ft upstream from Indían Creak, $2.4 \mathrm{mi}$ downstroam from Boly Ghost Creok, $9.0 \mathrm{mi}$ north of Pecos, and at mile 896.6 .

DRAINAGE AREA. $-189 \mathrm{~mL}^{2}$.

PERIOD OF RECORD.--August 1919 to current year. Monthly discharge only for some periods, publ1shed tn WSP 1312.

Published as "near Comles" 1919-25, "at Irvins Ranch" 1926-29, and as "at Irvins Ranch near Pecos" 1930-39.

REVISED RECORDS, - WSP 898: Dratnage area, WSP 1312: $1932(\mathrm{M})$.

GAGE.-Watex-stage recorder. Datum of gage is 7,502.94 ft above National Geodetic Vertical Datum of 1929 . Prior to Oct. 27,1977 , at site 30 ft upstrean at same datum.

REMARKS. --Records good except for estimated daily discharges, which are poor. Diversions for ixrlgation of about 75 acres, 1959 determinations, upstream from station. Several observations of pater temporature wore made during the year. National Weather Service satelifte telemater at atation.

EXTREMES OUTSIDE RERIOD OF RECORD.--Flood of Sept. 29, 1904, was greatest since 1886, from infoxmation by local ress dents.

DISCEARGE, CUBIC FEET PER SECOND, WATER YEAR OCTOBER 1995 TO SEPTEMBER 1996

\begin{tabular}{|c|c|c|c|c|c|c|c|c|c|c|c|c|}
\hline DAY & OCI & MOV & DEC & JAI & FEB & MAR & $\mathrm{APR}$ & MAY & JUN & JUL & AUG & SEP \\
\hline $\begin{array}{l}1 \\
2 \\
3 \\
4 \\
5\end{array}$ & $\begin{array}{l}64 \\
60 \\
57 \\
54 \\
51\end{array}$ & $\begin{array}{l}37 \\
41 \\
36 \\
38 \\
38\end{array}$ & $\begin{array}{l}34 \\
35 \\
34 \\
34 \\
33\end{array}$ & $\begin{array}{l}\Theta 21 \\
\theta 22 \\
022 \\
e 22 \\
\theta 22\end{array}$ & $\begin{array}{l}e 20 \\
e 20 \\
e 22 \\
e 22 \\
e 22\end{array}$ & $\begin{array}{r}\theta 30 \\
031 \\
\theta 30 \\
29 \\
29\end{array}$ & $\begin{array}{l}37 \\
43 \\
48 \\
48 \\
42\end{array}$ & $\begin{array}{r}99 \\
108 \\
116 \\
125 \\
130\end{array}$ & $\begin{array}{l}44 \\
43 \\
41 \\
40 \\
38\end{array}$ & $\begin{array}{l}70 \\
55 \\
49 \\
46 \\
48\end{array}$ & $\begin{array}{l}42 \\
42 \\
39 \\
36 \\
34\end{array}$ & $\begin{array}{l}82 \\
78 \\
75 \\
70 \\
63\end{array}$ \\
\hline $\begin{array}{r}6 \\
7 \\
8 \\
9 \\
10\end{array}$ & $\begin{array}{l}51 \\
52 \\
50 \\
48 \\
47\end{array}$ & $\begin{array}{l}40 \\
38 \\
37 \\
38 \\
41\end{array}$ & $\begin{array}{r}32 \\
31 \\
32 \\
30 \\
e 32\end{array}$ & $\begin{array}{l}\theta 20 \\
\theta 20 \\
\theta 20 \\
\theta 19 \\
\theta 19\end{array}$ & $\begin{array}{r}\text { e25 } \\
029 \\
32 \\
33 \\
33\end{array}$ & $\begin{array}{r}28 \\
031 \\
-33 \\
34 \\
31\end{array}$ & $\begin{array}{l}36 \\
38 \\
39 \\
50 \\
62\end{array}$ & $\begin{array}{l}132 \\
131 \\
129 \\
127 \\
126\end{array}$ & $\begin{array}{l}37 \\
36 \\
35 \\
34 \\
33\end{array}$ & $\begin{array}{r}47 \\
51 \\
70 \\
136 \\
116\end{array}$ & $\begin{array}{l}31 \\
31 \\
39 \\
37 \\
36\end{array}$ & $\begin{array}{l}64 \\
59 \\
55 \\
52 \\
51\end{array}$ \\
\hline $\begin{array}{l}11 \\
12 \\
13 \\
14 \\
15\end{array}$ & $\begin{array}{l}46 \\
45 \\
44 \\
43 \\
43\end{array}$ & $\begin{array}{l}33 \\
43 \\
41 \\
39 \\
38\end{array}$ & $\begin{array}{l}34 \\
35 \\
32 \\
31 \\
28\end{array}$ & $\begin{array}{l}019 \\
019 \\
019 \\
022 \\
025\end{array}$ & $\begin{array}{l}32 \\
30 \\
32 \\
33 \\
34\end{array}$ & $\begin{array}{l}32 \\
33 \\
33 \\
33 \\
31\end{array}$ & $\begin{array}{l}62 \\
57 \\
64 \\
52 \\
48\end{array}$ & $\begin{array}{l}121 \\
119 \\
116 \\
113 \\
108\end{array}$ & $\begin{array}{l}32 \\
32 \\
33 \\
55 \\
45\end{array}$ & $\begin{array}{l}80 \\
77 \\
75 \\
66 \\
62\end{array}$ & $\begin{array}{l}31 \\
29 \\
28 \\
31 \\
35\end{array}$ & $\begin{array}{l}52 \\
52 \\
63 \\
58 \\
70\end{array}$ \\
\hline $\begin{array}{l}16 \\
17 \\
18 \\
19 \\
20\end{array}$ & $\begin{array}{l}41 \\
41 \\
40 \\
39 \\
38\end{array}$ & $\begin{array}{l}37 \\
37 \\
35 \\
35 \\
35\end{array}$ & $\begin{array}{r}28 \\
\oplus 28 \\
e 28 \\
028 \\
028\end{array}$ & $\begin{array}{l}e 25 \\
e 25 \\
e 25 \\
e 17 \\
e 17\end{array}$ & $\begin{array}{l}35 \\
33 \\
33 \\
33 \\
34\end{array}$ & $\begin{array}{r}31 \\
30 \\
29 \\
28 \\
e 18\end{array}$ & $\begin{array}{l}53 \\
59 \\
63 \\
65 \\
61\end{array}$ & $\begin{array}{r}103 \\
99 \\
93 \\
87 \\
83\end{array}$ & $\begin{array}{l}37 \\
33 \\
31 \\
29 \\
28\end{array}$ & $\begin{array}{l}55 \\
54 \\
46 \\
43 \\
45\end{array}$ & $\begin{array}{l}24 \\
24 \\
29 \\
24 \\
24\end{array}$ & $\begin{array}{l}61 \\
55 \\
69 \\
77 \\
65\end{array}$ \\
\hline $\begin{array}{l}21 \\
22 \\
23 \\
24 \\
25\end{array}$ & $\begin{array}{l}37 \\
37 \\
34 \\
37 \\
40\end{array}$ & $\begin{array}{l}35 \\
36 \\
35 \\
30 \\
34\end{array}$ & $\begin{array}{l}e 28 \\
e 25 \\
e 25 \\
e 25 \\
e 25\end{array}$ & $\begin{array}{l}019 \\
020 \\
020 \\
019 \\
019\end{array}$ & $\begin{array}{l}38 \\
38 \\
33 \\
32 \\
35\end{array}$ & $\begin{array}{l}016 \\
017 \\
016 \\
016 \\
015\end{array}$ & $\begin{array}{l}55 \\
57 \\
61 \\
78 \\
91\end{array}$ & $\begin{array}{l}77 \\
73 \\
69 \\
65 \\
63\end{array}$ & $\begin{array}{l}27 \\
28 \\
31 \\
27 \\
26\end{array}$ & $\begin{array}{l}56 \\
50 \\
43 \\
44 \\
48\end{array}$ & $\begin{array}{r}37 \\
58 \\
104 \\
82 \\
79\end{array}$ & $\begin{array}{l}59 \\
56 \\
53 \\
51 \\
50\end{array}$ \\
\hline $\begin{array}{l}26 \\
27 \\
28 \\
29 \\
30 \\
31\end{array}$ & $\begin{array}{l}38 \\
37 \\
37 \\
38 \\
37 \\
37\end{array}$ & $\begin{array}{r}36 \\
28 \\
25 \\
32 \\
36 \\
\end{array}$ & $\begin{array}{l}e 20 \\
e 20 \\
020 \\
e 20 \\
\theta 20 \\
\theta 20\end{array}$ & $\begin{array}{l}019 \\
019 \\
019 \\
019 \\
020 \\
020\end{array}$ & $\begin{array}{r}31 \\
27 \\
029 \\
030 \\
--- \\
---\end{array}$ & $\begin{array}{r}017 \\
016 \\
018 \\
030 \\
32 \\
34\end{array}$ & $\begin{array}{r}108 \\
123 \\
127 \\
108 \\
98 \\
-9\end{array}$ & $\begin{array}{l}61 \\
59 \\
55 \\
52 \\
49 \\
47\end{array}$ & $\begin{array}{r}32 \\
98 \\
64 \\
80 \\
66 \\
-\end{array}$ & $\begin{array}{l}47 \\
48 \\
52 \\
56 \\
48 \\
44\end{array}$ & $\begin{array}{r}79 \\
87 \\
93 \\
99 \\
109 \\
91\end{array}$ & $\begin{array}{r}52 \\
53 \\
49 \\
48 \\
46 \\
-\end{array}$ \\
\hline $\begin{array}{l}\text { TOTAL } \\
\text { MEAN } \\
\text { MAX } \\
\text { MIN } \\
\text { AC-FT }\end{array}$ & $\begin{array}{r}1363 \\
44.0 \\
64 \\
34 \\
2700\end{array}$ & $\begin{array}{r}1084 \\
36.1 \\
43 \\
25 \\
2150\end{array}$ & $\begin{array}{r}875 \\
28.2 \\
35 \\
20 \\
1740\end{array}$ & $\begin{array}{r}633 \\
20.4 \\
25 \\
17 \\
1260\end{array}$ & $\begin{array}{r}880 \\
30.3 \\
38 \\
20 \\
1750\end{array}$ & $\begin{array}{r}831 \\
26.8 \\
34 \\
15 \\
1650\end{array}$ & $\begin{array}{r}1933 \\
64.4 \\
127 \\
36 \\
3830\end{array}$ & $\begin{array}{r}2935 \\
94.7 \\
132 \\
47 \\
5820\end{array}$ & $\begin{array}{r}1215 \\
40.5 \\
98 \\
26 \\
2410\end{array}$ & $\begin{array}{r}1827 \\
58.9 \\
136 \\
43 \\
3620\end{array}$ & $\begin{array}{r}1564 \\
50.5 \\
109 \\
24 \\
3100\end{array}$ & $\begin{array}{r}1788 \\
59.6 \\
82 \\
46 \\
3550\end{array}$ \\
\hline
\end{tabular}

STATISTICS OF MONTHLY MEAN DATA FOR WATER YEARS 1920 - 1996, BY WATER YEAR (WY)

\begin{tabular}{|c|c|c|c|c|c|c|c|c|c|}
\hline $\begin{array}{l}\text { MEAN } \\
\text { MAX } \\
\text { (WY) } \\
\text { MIN } \\
(W Y)\end{array}$ & $\begin{array}{r}51.9 \\
21.7 \\
1942 \\
11.9 \\
1957\end{array}$ & $\begin{array}{r}38.0 \\
138 \\
1942 \\
11.6 \\
1957\end{array}$ & $\begin{array}{l}29.5 \\
61.9 \\
1942 \\
9.52 \\
1957\end{array}$ & $\begin{array}{l}26.3 \\
49.7 \\
1942 \\
11.2 \\
1957\end{array}$ & $\begin{array}{l}26.7 \\
45.6 \\
1995 \\
14.8 \\
1951\end{array}$ & $\begin{array}{l}40.7 \\
97.3 \\
1995 \\
18.1 \\
1951\end{array}$ & $\begin{array}{r}133 \\
366 \\
1942 \\
40.1 \\
1951\end{array}$ & $\begin{array}{r}336 \\
1158 \\
1941 \\
43.7 \\
1950\end{array}$ & $\begin{array}{r}252 \\
950 \\
1979 \\
28.6 \\
1956\end{array}$ \\
\hline
\end{tabular}

SUMAARY STATISTICS

ANNUAL TOTAL

ANNUALE MEAN

HIGHEST ANNUAL MEAN

LOWEST ANNUAL MEAN

HIGHEST DAILY MEAN

LOWEST DAILY MEAN

ANNUAL SEVEN-DAY MINIMEM

INSTANTANEOUS PEAK FLOW

INSTANTANEOUS PEAK STAGE

INSTANTANEOUS LOW FLOW

ANNUAL RUNOFF (AC-FT)

10 PERCENT EXCEEDS

50 PERCENT EXCEEDS

90 PERCENT EXCEEDS

- Estimated
FOR 1995 CALENDAR YEAR

61607
169

$\begin{array}{rr}1370 & \text { Jun } 18 \\ 20 & \text { Dec } 26\end{array}$

122200

576
77
FOR 1996 WATER YEAR

$$
\begin{array}{r}
16928 \\
46.3
\end{array}
$$

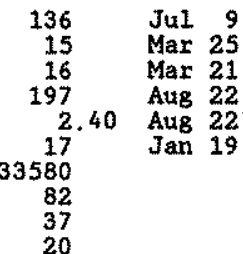

WATER YEARS $1920-1996$

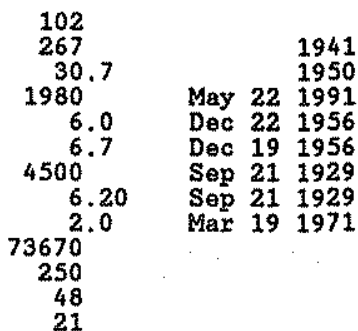


RIO GRANDE BASIN

08379500 PECOS RIVER NEAR ANTON CHICO, MM

LOCATION.--Lat $35^{\circ} 10^{\prime 4} 4^{\prime \prime}$, Long $105^{\circ} 06^{\prime} 30^{\prime \prime}$, Guadalupe County, Bydrologic Undt 13060001, in Anton Chico Grant, on right bank $2.1 \mathrm{mi}$ upstream frow Canon Blanco, 2.3 mi southeast of Anton Chico, 9.7 mi domstream from Tecolote Creek, and at mile 808.0

DRAINAGE AREA - - 1,050 mi2, approximately (contributing ares).

PERIOD OF RECORD,--April 1910 to May 1916, October 1916 to September 1924, August to December 1925 , January 1927 to current year. Monthly discharge only for some periods, published in WSP 1312

REVISED RECORDS:-WSP 1342: 1951(M), 1952-53. WSP 1512: 1912-14, 1931, 1933(M), 1935-36(M), 1938(P), 1939-40, 41$42(\mathrm{P}), 1945(\mathrm{M}), 1946(\mathrm{P})$. WSP 1712: 1942(P).

GAGE. - Water-stage recorder Elevation of 8 age is 5,130 ft above National Geodetic Vertical Datum of 1929 , from river-profile map. See WSP 1732 for history of changes prior to June 21, 1951.

RERARKS, - Records good except for estimated dally discharges, which are poor. Diversions upstream from station for

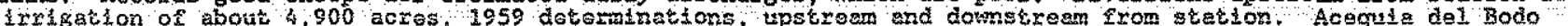
Juan Paiz (see table below) diverts water 8 mi upstream from gage and bypasses this station on left bank; ditch flow not included in record measurements made at point opposite regular gage. A portion of this flow may be returned to the river about $5.0 \mathrm{mi}$ dowstream. Several observations of pater temperature pere mede durtng the year. No flow at times some years.

EXIREMES OUTSIDE PERIOD OF RECORD, - The greatest flood since 1879 occurred Sept, 29, 1904 , discharge about 73,000 $\mathrm{ft}^{3} / \mathrm{s}$, from information by a local resident.

DISCBARGE, CUBIC FEET PER SECOND, WATER YEAR OCTOBER 1995 TO SEPTEMBER 1996

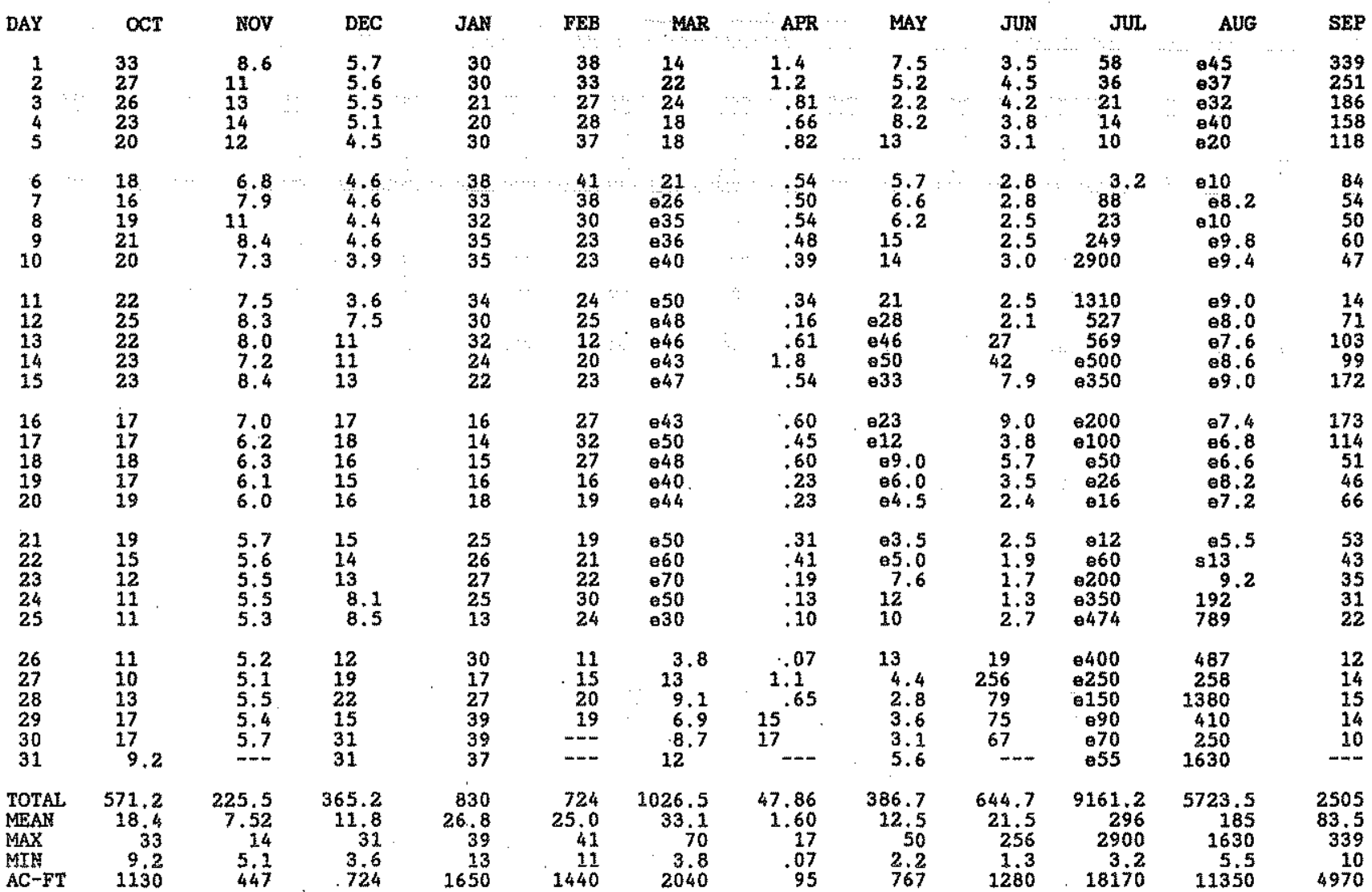

STATISTICS OF MOATHLY MEAN DATA FOR WATER YEARS 1929 - 1996, BY WATER YEAR (WY)

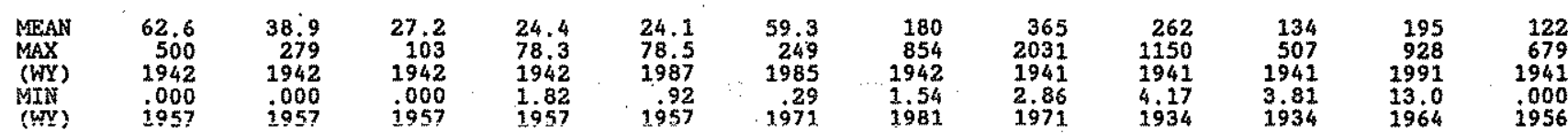

SUMARY STATISTICS

ARHUAE TOTAZ

ANNUAL MEAN

HIGHEST ANRUAZ, MEAN

LOWEST ANEUAL REAN

HIGHEST DAILY MEAN

LOWEST DAILY MEAN

ANNUAL SEVEN-DAY MINTMUM

INSTANTAREOUS PEAK FLOW

INSTANTANEOUS PEAK STAGE

INSTANTANEOUS LOW FLOW

ANNUAI RUNOFE (AC-FT)

10 PERCENT EXCEEDS

50 PERCENT EXCEEDS

90 PERCENT EXCEEDS

e Estimated
FOR 1995 CALENDAR YEAR

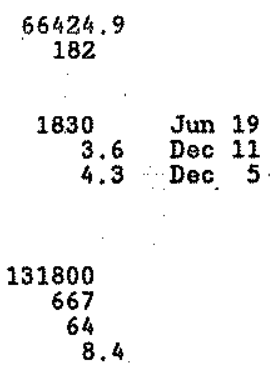

FOR 1996 WATER YEAR

22211.36
60.7

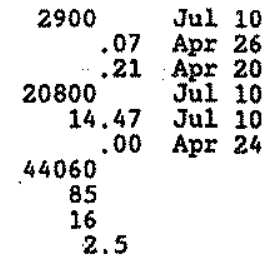

WATIR YEARS 1929 - 1996

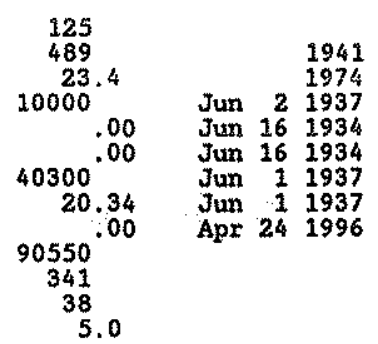


RIO GRANDE BASTN

08380500 GALLINAS CREEK NEAR MONTEZUMA, NM

LOCATION.-Lat $35^{\circ} 39^{\circ} 07^{\prime \prime}$, long $105^{\circ} 19^{\prime} 06^{*}$, San Miguel County, Eydrologie Untt 13060001, in Las Vegas Grant, on Left bank $2.4 \mathrm{mi}$ west of Montezuma, $6.9 \mathrm{mi}$ northwest of Las Vegas, and at mile 74.4.

DRAINAGE AREA. - $-84 \mathrm{mt}^{2}$, approximately.

PERIOD OF RECORD. - March to September 1915, June 1916 to current year. Monthly discharge only for some perlods. publ1shed in WSP 1312. Prior to October 1964, published as Gallinas River near Hontezuma.

REVISED RECORDS, --WSP 898: Dratnage area. WSP 1562: 1951(P), 1952(M), 1955(P), 1957. WSP 1632: 1931-32, $1933(\mathrm{M}), 1934,1935(\mathrm{M}), 1938,1939-40(\mathrm{M}), 1941-42,1945,1949-50(\mathrm{M})$.

GAGE.--Water-gtage recorder. Elevation of gage is 6,880 ft above National Geodetic Vertical Datum of 1929, from topographic map. Prior to Sept. 21 , 1934, at different datum.

REMARKS, - Records good except for estimated daily discharges, which are poor Diversions for irrigation of about 80 acres, 1959 determination, upstream from station. Several observations of water temperature were made during the year. 1916-1925 not included in statistics.

EXTREMES OUTSIDE PERIOD OF RECORD.- The greatest flood since about 1900 occurred the night of Sept. 29 , 1904 (discharge not determined), from information by local residents and $G$. B. Monk's report on floods,

DISCEARGE, CUBIC FEET PER SECOND, WATER YEAR OCTOBER 1995 TO SEPTEMBER 1996

\begin{tabular}{|c|c|c|c|c|c|c|c|c|c|c|c|c|}
\hline DAY & $\infty \mathrm{Cr}$ & Nov & DEC & JAN & FEB & MAR & AFR & MAY & JuN & JUL. & AUG & SEP \\
\hline $\begin{array}{l}1 \\
2 \\
3 \\
4 \\
5\end{array}$ & $\begin{array}{l}7.2 \\
7.2 \\
6.9 \\
6.6 \\
6.4\end{array}$ & $\begin{array}{l}4.3 \\
4.6 \\
4.8 \\
5.0 \\
5.4\end{array}$ & $\begin{array}{l}5.3 \\
5.2 \\
4.9 \\
5.6 \\
5.0\end{array}$ & $\begin{array}{l}04.3 \\
04.0 \\
04.5 \\
04.5 \\
04.8\end{array}$ & $\begin{array}{l}02.6 \\
02.5 \\
02.4 \\
02.5 \\
02.2\end{array}$ & $\begin{array}{l}4.6 \\
4.6 \\
4.4 \\
4.8 \\
4.5\end{array}$ & $\begin{array}{l}4.9 \\
5.0 \\
5.2 \\
5.3 \\
5.1\end{array}$ & $\begin{array}{l}5.5 \\
5.2 \\
4.4 \\
4.0 \\
4.9\end{array}$ & $\begin{array}{l}1.9 \\
1.9 \\
1.8 \\
1.8 \\
1.7\end{array}$ & $\begin{array}{r}17 \\
8.3 \\
5.2 \\
4.0 \\
3.4\end{array}$ & $\begin{array}{c}12 \\
10 \\
9.3 \\
11 \\
8.4\end{array}$ & $\begin{array}{l}44 \\
41 \\
42 \\
32 \\
27\end{array}$ \\
\hline $\begin{array}{r}6 \\
7 \\
8 \\
9 \\
10\end{array}$ & $\begin{array}{l}6.4 \\
6.4 \\
6.2 \\
5.9 \\
5.7\end{array}$ & $\begin{array}{l}5.5 \\
5.2 \\
5.2 \\
5.2 \\
5.1\end{array}$ & $\begin{array}{l}4.7 \\
4.6 \\
4.7 \\
4.2 \\
4.2\end{array}$ & $\begin{array}{r}e 5.2 \\
e 5.6 \\
06.0 \\
06.4 \\
5.1\end{array}$ & $\begin{array}{l}e 2.0 \\
e 3.5 \\
06.2 \\
e 7.4 \\
07.2\end{array}$ & $\begin{array}{l}4.6 \\
4.4 \\
4.5 \\
4.4 \\
5.0\end{array}$ & $\begin{array}{l}5.1 \\
5.1 \\
5.0 \\
5.2 \\
5.4\end{array}$ & $\begin{array}{l}4.9 \\
4.8 \\
4.4 \\
3.4 \\
3.1\end{array}$ & $\begin{array}{l}1.7 \\
1.7 \\
1.7 \\
1.4 \\
1.5\end{array}$ & $\begin{array}{r}3.6 \\
5.0 \\
56 \\
319 \\
282\end{array}$ & $\begin{array}{r}7.2 \\
6.6 \\
8.0 \\
9.0 \\
16\end{array}$ & $\begin{array}{l}26 \\
23 \\
20 \\
18 \\
17\end{array}$ \\
\hline $\begin{array}{l}11 \\
12 \\
13 \\
14 \\
15\end{array}$ & $\begin{array}{l}5.6 \\
5.5 \\
5.4 \\
5.5 \\
5.4\end{array}$ & $\begin{array}{l}5.4 \\
5.2 \\
5.4 \\
5.3 \\
5.3\end{array}$ & $\begin{array}{l}4.6 \\
4.7 \\
5.2 \\
4.8 \\
4.6\end{array}$ & $\begin{array}{l}5.3 \\
5.0 \\
4.9 \\
5.0 \\
4.7\end{array}$ & $\begin{array}{l}06.8 \\
06.6 \\
06.4 \\
06.0 \\
06.0\end{array}$ & $\begin{array}{l}5.2 \\
5.2 \\
5.3 \\
4.9 \\
5.0\end{array}$ & $\begin{array}{l}5.7 \\
6.2 \\
6.0 \\
6.1 \\
5.7\end{array}$ & $\begin{array}{l}3.5 \\
3.4 \\
3.3 \\
3.2 \\
3.0\end{array}$ & $\begin{array}{l}1.4 \\
1.8 \\
1.7 \\
2.2 \\
2.3\end{array}$ & $\begin{array}{r}133 \\
110 \\
151 \\
74 \\
48\end{array}$ & $\begin{array}{r}12 \\
9.3 \\
8.0 \\
7.1 \\
9.8\end{array}$ & $\begin{array}{l}17 \\
22 \\
37 \\
28 \\
27\end{array}$ \\
\hline $\begin{array}{l}16 \\
17 \\
18 \\
19 \\
20\end{array}$ & $\begin{array}{l}5.2 \\
5.2 \\
5.1 \\
5.2 \\
5.0\end{array}$ & $\begin{array}{l}5.1 \\
5.0 \\
5.1 \\
4.9 \\
4.9\end{array}$ & $\begin{array}{r}4.3 \\
4.7 \\
4.2 \\
4.0 \\
04.3\end{array}$ & $\begin{array}{r}4.9 \\
5.5 \\
4.5 \\
03.0 \\
02.9\end{array}$ & $\begin{array}{l}06.2 \\
06.0 \\
05.8 \\
05.8 \\
06.0\end{array}$ & $\begin{array}{l}4.9 \\
4.9 \\
4.7 \\
5.0 \\
4.7\end{array}$ & $\begin{array}{l}5.5 \\
5.3 \\
5.3 \\
5.5 \\
5.2\end{array}$ & $\begin{array}{l}3.0 \\
2.8 \\
2.6 \\
2.4 \\
2.2\end{array}$ & $\begin{array}{l}2.4 \\
2.1 \\
1.9 \\
1.8 \\
1.6\end{array}$ & $\begin{array}{l}32 \\
26 \\
21 \\
18 \\
15\end{array}$ & $\begin{array}{r}138 \\
207 \\
65 \\
43 \\
44\end{array}$ & $\begin{array}{l}22 \\
20 \\
19 \\
22 \\
19\end{array}$ \\
\hline $\begin{array}{l}21 \\
22 \\
23 \\
24 \\
25\end{array}$ & $\begin{array}{l}4.9 \\
4.8 \\
4.8 \\
4.8 \\
5.0\end{array}$ & $\begin{array}{l}5.0 \\
4.9 \\
4.7 \\
4.9 \\
4.8\end{array}$ & $\begin{array}{l}04.5 \\
04.6 \\
04.8 \\
05.0 \\
04.8\end{array}$ & $\begin{array}{l}e 2.7 \\
03.0 \\
63.0 \\
e 2.5 \\
e 2.5\end{array}$ & $\begin{array}{r}05.6 \\
05.4 \\
4.9 \\
4.8 \\
4.9\end{array}$ & $\begin{array}{l}5.0 \\
5.2 \\
5.1 \\
4.8 \\
4.7\end{array}$ & $\begin{array}{l}5.3 \\
5.1 \\
5.0 \\
5.0 \\
5.2\end{array}$ & $\begin{array}{l}2.2 \\
2.1 \\
2.0 \\
1.9 \\
1.8\end{array}$ & $\begin{array}{l}1.5 \\
1.5 \\
1.5 \\
1.5 \\
1.7\end{array}$ & $\begin{array}{l}13 \\
11 \\
10 \\
12 \\
10\end{array}$ & $\begin{array}{r}45 \\
31 \\
77 \\
170 \\
103\end{array}$ & $\begin{array}{l}17 \\
16 \\
14 \\
13 \\
13\end{array}$ \\
\hline $\begin{array}{l}26 \\
27 \\
28 \\
29 \\
30 \\
31\end{array}$ & $\begin{array}{l}5.0 \\
4.9 \\
4.7 \\
4.8 \\
4.6 \\
4.6\end{array}$ & $\begin{array}{l}4.9 \\
4.8 \\
4.5 \\
4.5 \\
5.2 \\
-\end{array}$ & $\begin{array}{l}04.0 \\
04.0 \\
04.2 \\
04.5 \\
04.4 \\
04.5\end{array}$ & $\begin{array}{l}e 2.4 \\
01.6 \\
01.5 \\
e 2.1 \\
01.8 \\
e 2.0\end{array}$ & $\begin{array}{l}4.9 \\
5.0 \\
3.8 \\
4.6 \\
-.-- \\
-.-\end{array}$ & $\begin{array}{l}4.3 \\
4.5 \\
4.8 \\
4.8 \\
4.9 \\
4.9\end{array}$ & $\begin{array}{l}5.2 \\
5.5 \\
5.7 \\
6.0 \\
5.9 \\
-\end{array}$ & $\begin{array}{l}1.5 \\
1.7 \\
1.8 \\
1.8 \\
1.9 \\
1.9\end{array}$ & $\begin{array}{l}1.6 \\
5.4 \\
5.9 \\
3.9 \\
7.5 \\
\end{array}$ & $\begin{array}{l}8.9 \\
8.7 \\
8.1 \\
8.5 \\
50 \\
28\end{array}$ & $\begin{array}{r}76 \\
351 \\
191 \\
99 \\
71 \\
57\end{array}$ & $\begin{array}{l}12 \\
12 \\
12 \\
11 \\
11 \\
- \\
\end{array}$ \\
\hline $\begin{array}{l}\text { TOTAL } \\
\text { MEAN } \\
\text { MAX } \\
\text { MIN } \\
\text { AC-FT }\end{array}$ & $\begin{array}{r}170.9 \\
5.51 \\
7.2 \\
4.6 \\
339\end{array}$ & $\begin{array}{r}150.1 \\
5.00 \\
5.5 \\
4.3 \\
298\end{array}$ & $\begin{array}{r}143.1 \\
4.62 \\
5.6 \\
4.0 \\
284\end{array}$ & $\begin{array}{r}121.2 \\
3.91 \\
6.4 \\
1.5 \\
240\end{array}$ & $\begin{array}{r}144.0 \\
4.97 \\
7.4 \\
2.0 \\
286\end{array}$ & $\begin{array}{r}148.6 \\
4.79 \\
5.3 \\
4.3 \\
295\end{array}$ & $\begin{array}{r}161.7 \\
5.39 \\
6.2 \\
4.9 \\
321\end{array}$ & $\begin{array}{r}94.6 \\
3.05 \\
5.5 \\
1.5 \\
1.88\end{array}$ & $\begin{array}{r}68.3 \\
2.28 \\
7.5 \\
1.4 \\
135\end{array}$ & $\begin{array}{r}1499.7 \\
48.4 \\
319 \\
3.4 \\
2970\end{array}$ & $\begin{array}{r}1911.7 \\
61.7 \\
351 \\
6.6 \\
3790\end{array}$ & $\begin{array}{r}654 \\
21.8 \\
44 \\
11 \\
1300\end{array}$ \\
\hline
\end{tabular}

STATISTICS OF MONTHLY MEAN DATA FOR WATER YEARS 1926 - 1996, BY WATER YEAR (WY)

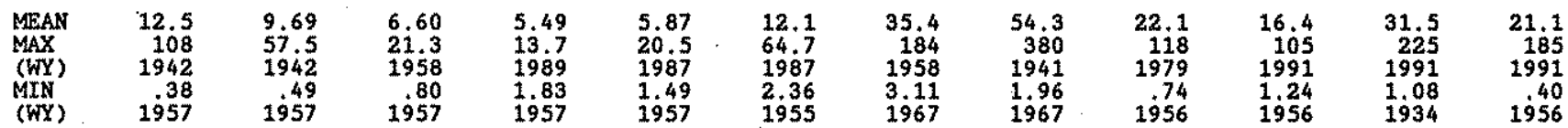

SUMMARY STATISTICS

ANNUAL TOTAL

ANNUAL MEAN

HTGHEST ANNUAL. MEAN

LONEST ANNUAL MEAN

HTGHEST DATLY MEAI

LOWEST DATIY MEAN

ANNUAL SEVEN-DAY MINIMUM

INSTANTANEOUS PEAX FLOW

INSTANTANEOUS PEAK STAGE

INSTANTANEOUS LOW FLON

ANNUAL RUNOFF (AC-FT)

10 PERCENT EXCEEDS

50 PERCENT EXCEEDS

90 PKRCENT EXCEEDS

- Estimated

FOR 1995 CALENDAR YEAR

$$
\begin{array}{r}
7869.1 \\
21.6
\end{array}
$$

127 May 30

$\begin{array}{lll}4.0 & \text { Dec } 19 \\ 4.3 & \text { Dec } 25\end{array}$

15610

70
11
4.
FOR 1996 WATER YEAR

5267.9

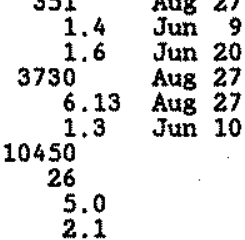

WATER YEARS $1926-1996$

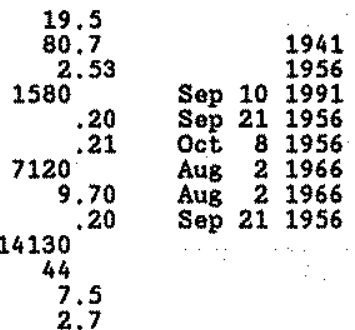


LOCATION.--Lat $35^{\circ} 10^{\prime} 55^{\prime \prime}$, Long 104 53'59", Guadalupe County, Hydrologic Unit 13060001, in Anton Chico Grant, and Preston Beck Grants, on right bank $2.3 \mathrm{mi}$ south of San Miguel-Guadalupe County 11ne, $2.4 \mathrm{mi}$ upstream from mouth, $5.8 \mathrm{mi}$ northwest of Colonias, and $9.0 \mathrm{mi}$ east of Dilia. Houth at Pecos River milo 789.2 .

DRATKAGE AREA. $--610 \mathrm{mit}^{2}$, approximately.

PERTOD OF RECORD.--January 1951 to current year.

GAGE.-Water-stage recorder Elevation of gago is 4,940 ft above National Geodetic Vartical Datum of 1929 , from topographic map.

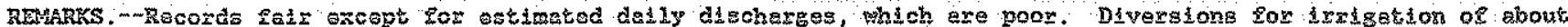
7,000 acres, 1959 determination, upstream from station. Soveral observations of watex temperature were anade 7,000 acres, 1959 determination, upstream exom

EXTREMES OUTSIDE PERIOD OF RECORD.-Flood of about June 1, 1937, reached a stage of about 27.2 ft; discharge determined as $26,700 \mathrm{ft}^{3 / \mathrm{s}}$ by slope-area measurement mede in 1951 . A flood of about the same magnitude occurred Sept. 29-30, 1904 .

DISCHARGE, CUBIC FEET PER SECOND, WATER YEAR OCTOBER 1995 TO SEPTEMBER 1996

\begin{tabular}{|c|c|c|c|c|c|c|c|c|c|c|c|c|}
\hline DAY & OCT & NOV & DEC & JAN & FEB & MAR & APR & MAY & JUN & JUL & AUUG & SEP \\
\hline $\begin{array}{l}1 \\
2 \\
3 \\
4 \\
5\end{array}$ & $\begin{array}{r}06.5 \\
06.2 \\
05.7 \\
4.6 \\
3.5\end{array}$ & $\begin{array}{r}2.1 \\
1.9 \\
1.7 \\
2.0 \\
2.8\end{array}$ & $\begin{array}{r}2.6 \\
2.5 \\
2.4 \\
2.3 \\
1.8\end{array}$ & $\begin{array}{r}3.8 \\
4.4 \\
5.2 \\
4.0 \\
5.2\end{array}$ & $\begin{array}{l}3.6 \\
3.8 \\
3.1 \\
2.4 \\
3.3\end{array}$ & $\begin{array}{r}2.1 \\
2.3 \\
2.3 \\
2.3 \\
2.0\end{array}$ & $\begin{array}{c}1.3 \\
1.2 \\
1.3 \\
.82 \\
.56\end{array}$ & $\begin{array}{l}.00 \\
.00 \\
.00 \\
.00 \\
.00\end{array}$ & $\begin{array}{l}.00 \\
.00 \\
.00 \\
.00 \\
.00\end{array}$ & $\begin{array}{r}49 \\
44 \\
16 \\
8.8 \\
6.0\end{array}$ & $\begin{array}{r}273 \\
20 \\
10 \\
17 \\
8.3\end{array}$ & $\begin{array}{r}115 \\
52 \\
35 \\
26 \\
21\end{array}$ \\
\hline $\begin{array}{r}6 \\
7 \\
8 \\
9 \\
10\end{array}$ & $\begin{array}{l}2.8 \\
2.3 \\
2.5 \\
3.3 \\
2.2\end{array}$ & $\begin{array}{l}3.4 \\
3.3 \\
3.1 \\
3.1 \\
2.8\end{array}$ & $\begin{array}{r}1.4 \\
1.4 \\
1.3 \\
1.3 \\
1.4\end{array}$ & $\begin{array}{l}5.9 \\
5.6 \\
5.4 \\
4.9 \\
4.8\end{array}$ & $\begin{array}{l}4.5 \\
5.5 \\
4.9 \\
4.2 \\
3.7\end{array}$ & $\begin{array}{l}1.5 \\
1.5 \\
1.5 \\
1.6 \\
2.0\end{array}$ & $\begin{array}{r}.44 \\
.00 \\
.00 \\
.00 \\
.00\end{array}$ & $\begin{array}{r}.00 \\
.00 \\
.00 \\
.00 \\
.00\end{array}$ & $\begin{array}{l}.00 \\
.00 \\
.00 \\
.00 \\
.00\end{array}$ & $\begin{array}{c}4.6 \\
8.9 \\
101 \\
83 \\
1080\end{array}$ & $\begin{array}{r}5.7 \\
4.4 \\
4.3 \\
246 \\
13\end{array}$ & $\begin{array}{l}17 \\
14 \\
17 \\
16 \\
25\end{array}$ \\
\hline $\begin{array}{l}11 \\
12 \\
13 \\
14 \\
15\end{array}$ & $\begin{array}{l}2.9 \\
2.9 \\
1.6 \\
1.3 \\
1.3\end{array}$ & $\begin{array}{l}2.6 \\
2.6 \\
2.7 \\
3.1 \\
3.0\end{array}$ & $\begin{array}{l}2.5 \\
2.0 \\
1.6 \\
1.4 \\
1.7\end{array}$ & $\begin{array}{l}4.8 \\
4.7 \\
4.7 \\
4.5 \\
4.4\end{array}$ & $\begin{array}{r}3.5 \\
3.3 \\
3.1 \\
3.1 \\
3.0\end{array}$ & $\begin{array}{l}2.0 \\
1.8 \\
1.5 \\
1.1 \\
1.0\end{array}$ & $\begin{array}{l}.00 \\
.00 \\
.00 \\
.00 \\
.00\end{array}$ & $\begin{array}{l}.00 \\
.00 \\
.00 \\
.00 \\
.00\end{array}$ & $\begin{array}{r}.00 \\
.00 \\
.00 \\
15^{.00}\end{array}$ & $\begin{array}{r}572 \\
338 \\
339 \\
84 \\
67\end{array}$ & $\begin{array}{l}6.9 \\
6.0 \\
5.4 \\
3.8 \\
3.2\end{array}$ & $\begin{array}{l}17 \\
53 \\
28 \\
46 \\
43\end{array}$ \\
\hline $\begin{array}{l}16 \\
17 \\
18 \\
19 \\
20\end{array}$ & $\begin{array}{l}1.2 \\
1.1 \\
.87 \\
.41 \\
.47\end{array}$ & $\begin{array}{l}2.6 \\
2.8 \\
2.8 \\
2.8 \\
2.8\end{array}$ & $\begin{array}{l}2.3 \\
3.2 \\
4.3 \\
4.3 \\
4.3\end{array}$ & $\begin{array}{l}4.1 \\
3.9 \\
3.9 \\
3.9 \\
3.9\end{array}$ & $\begin{array}{l}2.9 \\
2.9 \\
2.9 \\
2.6 \\
2.5\end{array}$ & $\begin{array}{l}1.1 \\
1.4 \\
1.7 \\
1.7 \\
1.7\end{array}$ & $\begin{array}{l}.00 \\
.00 \\
.00 \\
.00 \\
.00\end{array}$ & $\begin{array}{l}.00 \\
.00 \\
.00 \\
.00 \\
.00\end{array}$ & $\begin{array}{r}4.8 \\
1.6 \\
.43 \\
.00 \\
.00\end{array}$ & $\begin{array}{l}32 \\
20 \\
14 \\
10 \\
11\end{array}$ & $\begin{array}{l}2.3 \\
1.4 \\
1.97 \\
14\end{array}$ & $\begin{array}{l}25 \\
18 \\
14 \\
12 \\
11\end{array}$ \\
\hline $\begin{array}{l}21 \\
22 \\
23 \\
24 \\
25\end{array}$ & $\begin{array}{l}.25 \\
.00 \\
.00 \\
.00 \\
.00\end{array}$ & $\begin{array}{l}2.8 \\
2.8 \\
2.8 \\
2.6 \\
2.6\end{array}$ & $\begin{array}{l}4.3 \\
4.5 \\
4.5 \\
4.5 \\
4.3\end{array}$ & $\begin{array}{l}4.3 \\
4.3 \\
3.8 \\
3.9 \\
3.2\end{array}$ & $\begin{array}{l}2.2 \\
2.1 \\
1.8 \\
1.6 \\
1.8\end{array}$ & $\begin{array}{l}1.7 \\
1.3 \\
1.2 \\
1.3 \\
1.4\end{array}$ & $\begin{array}{l}.00 \\
.00 \\
.00 \\
.00 \\
.00\end{array}$ & $\begin{array}{l}.00 \\
.00 \\
.00 \\
.00 \\
.00\end{array}$ & $\begin{array}{l}.00 \\
.00 \\
.00 \\
.00 \\
.00\end{array}$ & $\begin{array}{r}22 \\
7.4 \\
5.5 \\
4.6 \\
4.2\end{array}$ & $\begin{array}{c}27 \\
6.9 \\
18 \\
102 \\
72\end{array}$ & $\begin{array}{r}10 \\
9.6 \\
8.7 \\
7.9 \\
7.3\end{array}$ \\
\hline $\begin{array}{l}26 \\
27 \\
28 \\
29 \\
30 \\
31\end{array}$ & $\begin{array}{l}1.2 \\
1.2 \\
1.4 \\
1.8 \\
2.0 \\
2.1\end{array}$ & $\begin{array}{l}2.6 \\
2.3 \\
2.1 \\
2.3 \\
2.6 \\
.--\end{array}$ & $\begin{array}{l}3.1 \\
2.6 \\
2.9 \\
3.1 \\
3.9 \\
4.5\end{array}$ & $\begin{array}{l}1.6 \\
3.2 \\
2.5 \\
2.9 \\
4.1 \\
4.0\end{array}$ & $\begin{array}{l}1.9 \\
1.9 \\
2.0 \\
2.0 \\
-. . \\
-.\end{array}$ & $\begin{array}{l}1.4 \\
1.5 \\
1.7 \\
1.6 \\
1.5 \\
1.4\end{array}$ & $\begin{array}{l}.00 \\
.00 \\
.00 \\
.00 \\
.00 \\
.0\end{array}$ & $\begin{array}{l}.00 \\
.00 \\
.00 \\
.00 \\
.00 \\
.00\end{array}$ & $\begin{array}{r}.00 \\
42^{.00} \\
362 \\
39 \\
.-.\end{array}$ & $\begin{array}{c}57 \\
22 \\
9.1 \\
179 \\
166 \\
85\end{array}$ & $\begin{array}{r}161 \\
222 \\
1350 \\
114 \\
70 \\
475\end{array}$ & $\begin{array}{l}6.0 \\
5.6 \\
5.5 \\
5.0 \\
4.3 \\
--\end{array}$ \\
\hline $\begin{array}{l}\text { TOTAL } \\
\text { MEAN } \\
\text { MAX } \\
\text { MIN } \\
\text { AC-FT }\end{array}$ & $\begin{array}{r}63.60 \\
2.05 \\
6.5 \\
.00 \\
126\end{array}$ & $\begin{array}{r}79.5 \\
2.65 \\
3.4 \\
1.7 \\
158\end{array}$ & $\begin{array}{r}88.2 \\
2.85 \\
4.5 \\
1.3 \\
175\end{array}$ & $\begin{array}{r}129.8 \\
4.19 \\
5.9 \\
1.6 \\
257\end{array}$ & $\begin{array}{r}86.1 \\
2.97 \\
5.5 \\
1.6 \\
171\end{array}$ & $\begin{array}{r}50.1 \\
1.62 \\
2.3 \\
1.0 \\
99\end{array}$ & $\begin{array}{r}5.62 \\
.19 \\
1.3 \\
.00 \\
11\end{array}$ & $\begin{array}{l}0.00 \\
.000 \\
.00 \\
.00 \\
.00\end{array}$ & $\begin{array}{r}464.83 \\
15.5 \\
362 \\
.00 \\
922\end{array}$ & $\begin{array}{r}3450.1 \\
111 \\
1080 \\
4.2 \\
6840\end{array}$ & $\begin{array}{r}3278.57 \\
106 \\
1350 \\
6.97 \\
6500\end{array}$ & $\begin{array}{r}674.9 \\
22.5 \\
115 \\
4.3 \\
1340\end{array}$ \\
\hline
\end{tabular}

STATISTICS OF MONTHLY MEAN DATA FOR WATER YEARS 1951 - 1996, BY WATER YEAR (WY)

\begin{tabular}{|c|c|c|c|c|c|c|c|c|c|}
\hline $\begin{array}{l}\text { MEAN } \\
\text { MAX } \\
(W Y) \\
\text { MIN } \\
\text { (WY })\end{array}$ & $\begin{array}{r}12.2 \\
166 \\
1958 \\
i 000 \\
1953\end{array}$ & $\begin{array}{l}4.99 \\
50.0 \\
1987 \\
.000\end{array}$ & $\begin{array}{l}3.23 \\
18.3 \\
1987 \\
.000\end{array}$ & $\begin{array}{l}2.97 \\
18.9 \\
1992 \\
000\end{array}$ & $\begin{array}{l}3.77 \\
58.9 \\
1987 \\
000\end{array}$ & $\begin{array}{l}4.55 \\
48.2 \\
1958 \\
.000\end{array}$ & $\begin{array}{r}16.5 \\
269 \\
1958 \\
000\end{array}$ & $\begin{array}{r}17.8 \\
261 \\
1973 \\
000\end{array}$ & $\begin{array}{l}18.2 \\
91.4 \\
1986 \\
000 \\
1951\end{array}$ \\
\hline
\end{tabular}

SUMMARY STATISTICS

ANRUA TOTAL

ANNUAL MEAN

HIGHEST ANNUAL. MEAN

LOWEST ANWUAL MEAN

HIGHEST DAILY MEAN

LOWEST DAILY MFAN

ANNUAZ SEVEN-DAY MINTMUM

INSTANTANEOUS PEAK FLOW

INSTANTANEOUS PEAK STAGE

INSTANTANEOUS LOW FLOW

ANNUAL RUNOFF (AC-FT)

10 PERCENT EXCEEDS

50 PERCENT EXCEEDS

90 PERCENT EXCEEDS

FOR 1995 CALENDAR YEAR

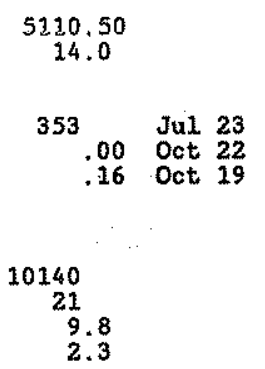

FOR 1996 WATER YEAR

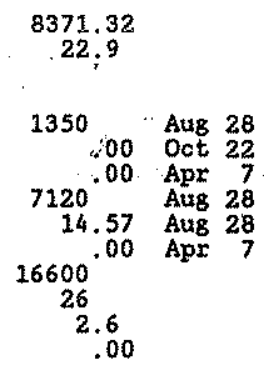

WATER YEARS 1951 - 1996

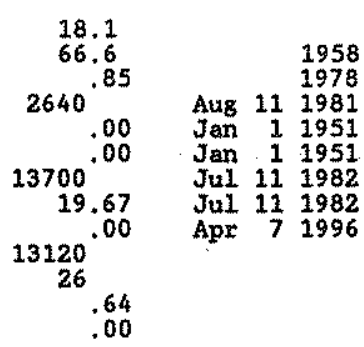


RIO GRANDE BASIN

08382600 PECOS RIVER ABOVE CANON DEL UTA NEAR COLONIAS, NM

LOCATION. - Lat $35^{\circ} 05^{\circ} 29^{\prime \prime}$, long $104^{\circ} 48^{\prime} 00^{\prime \prime}$, in T.10 N., R.20 E., Guadalupe County, Hydrologic Unit 13060001, in Anton

Chico Grant, on right bank $0.4 \mathrm{mi}$ upstrean from Canon del Uta, 2,9 mi southeast of Colonias, and at mile 775.8 .

DRAINAGE AREA. $--2,330 \mathrm{mi}$, approximately.

PERIOD OF RECORD.--January 1976 to current year.

GAGE.--Water-stage recorder. Elevation of gage is 4,800 ft above National Geodetic Vertical Datum of 1929 , from U.S. Army Corps of Engineers plan and profile map.

REMARKS.--Records fair. Diversions and ground-water pithdrawals for irrigation for about 11,800 acres, 1959

determination, upstream from station. Several observations of water temperature were made during the year. U.S. Army Corps of Engineers satellite telemeter at station. No flow many days most years.

DISCEARGE, CUBIC FEET PER SECOND, WATER YEAR OCTOBER 1995 TO SEPTEMBER 1996

\begin{tabular}{|c|c|c|c|c|c|c|c|c|c|c|c|c|}
\hline DAY & $\alpha x$ & Nov & DEC & JAN & FEB & MAR & APR & MAY & JUN & JUL & AUGG & SEP \\
\hline $\begin{array}{l}1 \\
2 \\
3 \\
4 \\
5\end{array}$ & $\begin{array}{l}18 \\
17 \\
16 \\
15 \\
15\end{array}$ & $\begin{array}{l}8.8 \\
8.8 \\
8.8 \\
9.3 \\
8.9\end{array}$ & $\begin{array}{l}7.9 \\
8.0 \\
7.9 \\
7.9 \\
7.6\end{array}$ & $\begin{array}{l}6.0 \\
6.1 \\
5.8 \\
5.6 \\
5.6\end{array}$ & $\begin{array}{l}4.8 \\
4.8 \\
4.8 \\
4.7 \\
4.7\end{array}$ & $\begin{array}{l}4.0 \\
4.0 \\
4.0 \\
3.7 \\
3.4\end{array}$ & $\begin{array}{l}1.9 \\
1.9 \\
1.8 \\
1.9 \\
1.9\end{array}$ & $\begin{array}{l}1.8 \\
1.6 \\
1.6 \\
1.7 \\
1.6\end{array}$ & $\begin{array}{l}.73 \\
.73 \\
.73 \\
.73 \\
.73\end{array}$ & $\begin{array}{l}85 \\
98 \\
41 \\
26 \\
27\end{array}$ & $\begin{array}{r}185 \\
24 \\
4.6 \\
4.1 \\
3.5\end{array}$ & $\begin{array}{r}379 \\
167 \\
106 \\
68 \\
46\end{array}$ \\
\hline $\begin{array}{r}6 \\
7 \\
8 \\
9 \\
10\end{array}$ & $\begin{array}{l}15 \\
15 \\
14 \\
13 \\
13\end{array}$ & $\begin{array}{l}8.6 \\
8.3 \\
8.3 \\
8.1 \\
7.3\end{array}$ & $\begin{array}{l}7.4 \\
7.4 \\
7.4 \\
7.0 \\
6.9\end{array}$ & $\begin{array}{l}5.6 \\
5.5 \\
5.0 \\
4.8 \\
4.8\end{array}$ & $\begin{array}{l}4.8 \\
5.0 \\
5.2 \\
5.2 \\
5.2\end{array}$ & $\begin{array}{l}3.9 \\
3.6 \\
3.4 \\
3.2 \\
3.2\end{array}$ & $\begin{array}{l}1.8 \\
1.8 \\
1.6 \\
1.6 \\
1.7\end{array}$ & $\begin{array}{l}1.6 \\
1.6 \\
1.6 \\
1.6 \\
1.8\end{array}$ & $\begin{array}{r}.73 \\
.73 \\
.73 \\
.85 \\
1.4\end{array}$ & $\begin{array}{r}23 \\
28 \\
30 \\
185 \\
2300\end{array}$ & $\begin{array}{r}2.8 \\
2.8 \\
2.8 \\
141 \\
18\end{array}$ & $\begin{array}{r}21 \\
7.7 \\
2.6 \\
2.5 \\
2.6\end{array}$ \\
\hline $\begin{array}{l}11 \\
12 \\
13 \\
14 \\
15\end{array}$ & $\begin{array}{l}13 \\
12 \\
12 \\
12 \\
11\end{array}$ & $\begin{array}{l}7.9 \\
7.8 \\
7.9 \\
7.9 \\
7.9\end{array}$ & $\begin{array}{l}6.7 \\
6.5 \\
7.0 \\
7.4 \\
7.9\end{array}$ & $\begin{array}{l}4.6 \\
4.3 \\
4.3 \\
4.3 \\
4.3\end{array}$ & $\begin{array}{l}5.2 \\
4.8 \\
4.8 \\
4.8 \\
4.8\end{array}$ & $\begin{array}{l}3.2 \\
2.9 \\
2.9 \\
2.9 \\
3.0\end{array}$ & $\begin{array}{l}1.5 \\
1.7 \\
1.0 \\
1.91 \\
1.5\end{array}$ & $\begin{array}{l}1.9 \\
1.9 \\
1.9 \\
1.9 \\
1.7\end{array}$ & $\begin{array}{l}1.1 \\
1.1 \\
\frac{1}{1} \cdot 2 \\
25 \\
17\end{array}$ & $\begin{array}{r}2490 \\
362 \\
532 \\
322 \\
145\end{array}$ & $\begin{array}{l}6.0 \\
5.6 \\
5.6 \\
5.4 \\
5.0\end{array}$ & $\begin{array}{c}2.3 \\
82 \\
56 \\
53 \\
108\end{array}$ \\
\hline $\begin{array}{l}16 \\
17 \\
18 \\
19 \\
20\end{array}$ & $\begin{array}{l}11 \\
11 \\
11 \\
10 \\
10\end{array}$ & $\begin{array}{l}7.9 \\
7.9 \\
8.2 \\
7.9 \\
7.9\end{array}$ & $\begin{array}{l}7.9 \\
8.3 \\
8.3 \\
7.9 \\
7.9\end{array}$ & $\begin{array}{l}4.2 \\
3.9 \\
4.0 \\
4.0 \\
4.2\end{array}$ & $\begin{array}{l}4.8 \\
4.8 \\
4.2 \\
3.9 \\
3.7\end{array}$ & $\begin{array}{l}3.1 \\
3.0 \\
2.9 \\
2.7 \\
2.5\end{array}$ & $\begin{array}{l}1.6 \\
.96 \\
.73 \\
.50 \\
.89\end{array}$ & $\begin{array}{l}1.6 \\
3.3 \\
1.1 \\
1.1 \\
1.1\end{array}$ & $\begin{array}{l}.49 \\
.29 \\
.00 \\
.00 \\
.04\end{array}$ & $\begin{array}{l}80 \\
46 \\
25 \\
12 \\
\\
.63\end{array}$ & $\begin{array}{l}4.3 \\
4.3 \\
5.3 \\
4.9 \\
4.3\end{array}$ & $\begin{array}{r}65 \\
54 \\
17 \\
4.4 \\
2.1\end{array}$ \\
\hline $\begin{array}{l}21 \\
22 \\
23 \\
24 \\
25\end{array}$ & $\begin{array}{l}9.6 \\
9.5 \\
9.3 \\
9.3 \\
9.3\end{array}$ & $\begin{array}{l}8.3 \\
8.0 \\
7.9 \\
7.6 \\
7.9\end{array}$ & $\begin{array}{l}7.6 \\
7.4 \\
7.4 \\
7.4 \\
7.0\end{array}$ & $\begin{array}{l}4.3 \\
4.3 \\
4.1 \\
4.1 \\
4.3\end{array}$ & $\begin{array}{l}3.6 \\
3.6 \\
3.7 \\
4.0 \\
4.2\end{array}$ & $\begin{array}{l}2.3 \\
2.7 \\
2.4 \\
2.0 \\
2.3\end{array}$ & $\begin{array}{l}.98 \\
1.1 \\
1.1 \\
1.1 \\
1.5\end{array}$ & $\begin{array}{l}1.1 \\
1.6 \\
1.6 \\
1.6 \\
1.6\end{array}$ & $\begin{array}{r}.35 \\
.75 \\
.94 \\
.73 \\
.60\end{array}$ & $\begin{array}{l}.00 \\
.00 \\
.00 \\
.00 \\
.00\end{array}$ & $\begin{array}{r}4.3 \\
5.4 \\
4.3 \\
94 \\
322\end{array}$ & $\begin{array}{l}8.6 \\
7.2 \\
4.7 \\
4.0 \\
4.3\end{array}$ \\
\hline $\begin{array}{l}26 \\
27 \\
28 \\
29 \\
30 \\
31\end{array}$ & $\begin{array}{l}8.9 \\
9.3 \\
9.3 \\
9.3 \\
9.3 \\
9.1\end{array}$ & $\begin{array}{l}7.5 \\
7.8 \\
7.8 \\
7.6 \\
7.9 \\
-\end{array}$ & $\begin{array}{l}6.9 \\
6.9 \\
6.9 \\
6.6 \\
6.1 \\
5.9\end{array}$ & $\begin{array}{l}4.3 \\
4.3 \\
4.3 \\
4.7 \\
4.7 \\
4.8\end{array}$ & $\begin{array}{l}3.8 \\
3.6 \\
3.8 \\
4.0 \\
-.- \\
--.\end{array}$ & $\begin{array}{l}1.8 \\
1.7 \\
1.9 \\
1.6 \\
1.5 \\
1.9\end{array}$ & $\begin{array}{l}1.8 \\
1.5 \\
1.7 \\
1.6 \\
1.3 \\
--m\end{array}$ & $\begin{array}{r}1.3 \\
1.1 \\
.82 \\
.35 \\
.48 \\
.73\end{array}$ & $\begin{array}{l}136^{.45} \\
323 \\
269\end{array}$ & $\begin{array}{c}220 \\
41 \\
1.4 \\
185^{.34} \\
26\end{array}$ & $\begin{array}{r}341 \\
485 \\
1370 \\
450 \\
178 \\
911\end{array}$ & $\begin{array}{l}4.5 \\
6.3 \\
2.8 \\
3.5 \\
3.6 \\
-.--\end{array}$ \\
\hline $\begin{array}{l}\text { TOTAL } \\
\text { MEAN } \\
\text { MAX } \\
\text { MIN } \\
\text { AC-ET }\end{array}$ & $\begin{array}{r}366.2 \\
11.8 \\
18 \\
8.9 \\
726\end{array}$ & $\begin{array}{r}242.7 \\
8.09 \\
9.3 \\
7.3 \\
481\end{array}$ & $\begin{array}{r}227.3 \\
7.33 \\
8.3 \\
5.9 \\
451\end{array}$ & $\begin{array}{r}145.1 \\
4.68 \\
6.1 \\
3.9 \\
288\end{array}$ & $\begin{array}{r}129.3 \\
4.46 \\
5.2 \\
3.6 \\
256\end{array}$ & $\begin{array}{r}87: 6 \\
2.83 \\
4.0 \\
1.5 \\
174\end{array}$ & $\begin{array}{r}42.87 \\
1.43 \\
1.9 \\
.50 \\
85\end{array}$ & $\begin{array}{r}44.28 \\
1.43 \\
1.9 \\
.35 \\
88\end{array}$ & $\begin{array}{r}786.34 \\
26.2 \\
323 \\
.00 \\
1560\end{array}$ & $\begin{array}{r}7331.37 \\
236 \\
2490 \\
1450 \\
14540\end{array}$ & $\begin{array}{r}4604.3 \\
149 \\
1370 \\
2.8 \\
9130\end{array}$ & $\begin{array}{r}1295.7 \\
43.2 \\
379 \\
2.1 \\
2570\end{array}$ \\
\hline
\end{tabular}

STATISTICS OF MONTHLY MEAN DATA FOR WATER YEARS 1976 - 1996, BY WATER XEAR (WY)

\begin{tabular}{|c|c|c|c|c|c|c|c|c|}
\hline $\begin{array}{l}\text { MEAN } \\
\text { MAX } \\
\text { (WY) } \\
\text { MIN } \\
\text { (WX) }\end{array}$ & $\begin{array}{r}22.9 \\
139 \\
1986 \\
1000 \\
1978\end{array}$ & $\begin{array}{r}22.8 \\
148 \\
1995 \\
000 \\
1977\end{array}$ & $\begin{array}{l}7.63 \\
42.0 \\
1987 \\
1000 \\
1977\end{array}$ & $\begin{array}{l}4.10 \\
19.0 \\
1987 \\
1000 \\
1976\end{array}$ & $\begin{array}{r}6.19 \\
73.4 \\
1987 \\
1900 \\
1976\end{array}$ & $\begin{array}{r}33.4 \\
192 \\
1985 \\
000 \\
1976\end{array}$ & $\begin{array}{r}107 \\
382 \\
1987 \\
000 \\
1976\end{array}$ & $\begin{array}{r}314 \\
736 \\
1979 \\
126 \\
1981\end{array}$ \\
\hline
\end{tabular}

SUMMARY STATISTICS

ANNUAL TOTAL

ANNUAL MEAN

HIGHEST ANNUAL MEAN

LOWEST ANNUAL MEAN

HIGHEST DAILY MEAN

LOWEST DAILY MEAN

ANNUAL SEVEN-DAY MINIMEM

INSTANTANEOUS PEAK FLOW

INSTANTANEOUS PEAK STAGE

INSTANTANEOUS LOW FLOW

ANNUAL RUNOFF (AC-FT)

10 PERCENT EXCEEDS

50 PERCENT EXCEEDS

90 PERCENT EXCEEDS
FOR 1995 CALENDAR YEAR

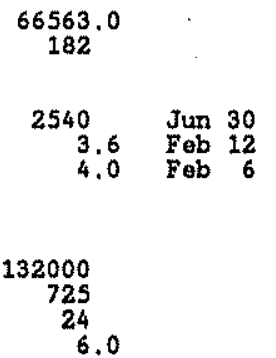

FOR 1996 WATER YEAR

$$
\begin{gathered}
15303.06 \\
41.8
\end{gathered}
$$

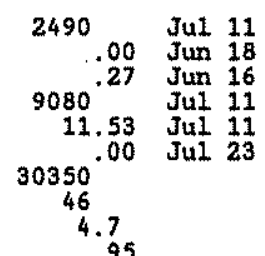

WATER YEARS 1976 - 1996

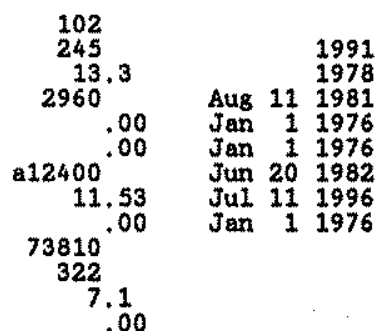

a-From rating curve extended above $1,200 \mathrm{ft} / \mathrm{s}$, on basis of step-backwater analysis of channel. 


\section{RIO GRANDE BASIN}

08382650 PECOS RIVER ABOVE SANTA ROSA LAKE, MM

LOCATION,-Lat $35^{\circ} 03^{\prime} 35^{\prime \prime}$, Iong $104^{\circ} 45^{\prime} 41^{\prime \prime}$, in KEKSEKSEH sec.25, T.10 N., R.20 E., Guadalupe County, Bydrologic Unit 13060001 , at south boundary Preston Beck Grant, on left bank $1.6 \mathrm{mi}$ upstream from River Ranch, 5.8 mi southeast of Colonias, 9.1 mi northwest of Santa Rosa, and at mile 770.8 .

DRAINAGE AREA. $-2,340 \mathrm{mi}^{2}$, approximately.

WATER-DISCAARGE RECORDS

PERIOD OF RECORD.-February 1976 to current year. Prior to October 1979, published as above Los Esteros Roservoix,"

GAGE.-Water-gtage recorder. Elevation of gage is 4,760 ft above National Geodetic Verticai Datum of 1929 (lovels by U.S. Army Coxps of Engineers).

RDMARKS. - Water-discharge records fair except for estimated daily discharges, which are poor, Diversions and groundwater althdrawals for irrigation of about 11,800 acres, 1959 determination, upstroam from station. Several observations of water temperature were made during the year.

DISCHARGE, CUBTC FEET FER SECOND, WATER YEAR OCTOBER 1995 TO SEPTEYBER 1996

\begin{tabular}{|c|c|c|c|c|c|c|c|c|c|c|c|c|}
\hline DAY & OCT & NOV & DEC & JAN & FEB & MAR & ARR & MAY & JUR & JUL & AUG & SEP \\
\hline $\begin{array}{l}1 \\
2 \\
3 \\
4 \\
5\end{array}$ & $\begin{array}{l}37 \\
35 \\
33 \\
33 \\
34\end{array}$ & $\begin{array}{l}32 \\
32 \\
31 \\
30 \\
29\end{array}$ & $\begin{array}{r}35 \\
35 \\
34 \\
33 \\
33\end{array}$ & $\begin{array}{l}30 \\
31 \\
32 \\
32 \\
32\end{array}$ & $\begin{array}{l}36 \\
35 \\
34 \\
32 \\
30\end{array}$ & $\begin{array}{r}29 \\
29 \\
28 \\
27 \\
27\end{array}$ & $\begin{array}{l}24 \\
25 \\
24 \\
24 \\
23\end{array}$ & $\begin{array}{l}23 \\
23 \\
23 \\
24 \\
24\end{array}$ & $\begin{array}{l}24 \\
24 \\
24 \\
23 \\
23\end{array}$ & $\begin{array}{l}84 \\
73 \\
42 \\
29 \\
28\end{array}$ & $\begin{array}{r}0205 \\
070 \\
022 \\
021 \\
020\end{array}$ & $\begin{array}{r}0450 \\
0240 \\
0128 \\
\times 82 \\
056\end{array}$ \\
\hline $\begin{array}{r}6 \\
7 \\
8 \\
9 \\
10\end{array}$ & $\begin{array}{l}34 \\
33 \\
35 \\
35 \\
34\end{array}$ & $\begin{array}{l}30 \\
30 \\
30 \\
30 \\
30\end{array}$ & $\begin{array}{r}32 \\
32 \\
33 \\
33 \\
33\end{array}$ & $\begin{array}{l}31 \\
32 \\
32 \\
32 \\
32\end{array}$ & $\begin{array}{l}29 \\
28 \\
28 \\
27 \\
27\end{array}$ & $\begin{array}{l}28 \\
28 \\
28 \\
28 \\
26\end{array}$ & $\begin{array}{l}22 \\
22 \\
22 \\
24 \\
24\end{array}$ & $\begin{array}{l}24 \\
23 \\
22 \\
22 \\
22\end{array}$ & $\begin{array}{l}21 \\
21 \\
21 \\
21 \\
20\end{array}$ & $\begin{array}{r}27 \\
26 \\
25 \\
148 \\
4310\end{array}$ & $\begin{array}{r}020 \\
020 \\
020 \\
0172 \\
e 59\end{array}$ & $\begin{array}{l}045 \\
034 \\
030 \\
027 \\
031\end{array}$ \\
\hline $\begin{array}{l}11 \\
12 \\
13 \\
14 \\
15\end{array}$ & $\begin{array}{l}35 \\
36 \\
37 \\
37 \\
36\end{array}$ & $\begin{array}{l}30 \\
30 \\
30 \\
30 \\
30\end{array}$ & $\begin{array}{l}32 \\
32 \\
32 \\
33 \\
33\end{array}$ & $\begin{array}{l}32 \\
33 \\
35 \\
34 \\
34\end{array}$ & $\begin{array}{l}26 \\
26 \\
26 \\
26 \\
28\end{array}$ & $\begin{array}{l}27 \\
28 \\
28 \\
28 \\
27\end{array}$ & $\begin{array}{r}23 \\
23 \\
22 \\
23 \\
22\end{array}$ & $\begin{array}{l}21 \\
21 \\
21 \\
21 \\
22\end{array}$ & $\begin{array}{l}21 \\
21 \\
21 \\
31 \\
38\end{array}$ & $\begin{array}{r}3930 \\
461 \\
\quad \quad 723 \\
542 \\
273\end{array}$ & $\begin{array}{l}023 \\
022 \\
020 \\
019 \\
019\end{array}$ & $\begin{array}{r}025 \\
8103 \\
078 \\
85 \\
142\end{array}$ \\
\hline $\begin{array}{l}16 \\
17 \\
18 \\
19 \\
20\end{array}$ & $\begin{array}{l}35 \\
35 \\
42 \\
40 \\
37\end{array}$ & $\begin{array}{l}30 \\
30 \\
30 \\
30 \\
30\end{array}$ & $\begin{array}{l}33 \\
34 \\
33 \\
32 \\
32\end{array}$ & $\begin{array}{l}34 \\
33 \\
33 \\
34 \\
33\end{array}$ & $\begin{array}{l}28 \\
29 \\
29 \\
28 \\
28\end{array}$ & $\begin{array}{l}28 \\
30 \\
31 \\
30 \\
29\end{array}$ & $\begin{array}{l}22 \\
22 \\
22 \\
23 \\
23\end{array}$ & $\begin{array}{l}21 \\
21 \\
21 \\
21 \\
21\end{array}$ & $\begin{array}{l}19 \\
17 \\
16 \\
16 \\
17\end{array}$ & $\begin{array}{l}0112 \\
048 \\
029 \\
019 \\
017\end{array}$ & $\begin{array}{l}018 \\
018 \\
023 \\
019 \\
018\end{array}$ & $\begin{array}{l}88 \\
77 \\
46 \\
28 \\
24\end{array}$ \\
\hline $\begin{array}{l}21 \\
22 \\
23 \\
24 \\
25\end{array}$ & $\begin{array}{l}36 \\
34 \\
33 \\
34 \\
34\end{array}$ & $\begin{array}{l}31 \\
30 \\
29 \\
30 \\
31\end{array}$ & $\begin{array}{l}32 \\
32 \\
33 \\
33 \\
34\end{array}$ & $\begin{array}{l}33 \\
32 \\
33 \\
34 \\
34\end{array}$ & $\begin{array}{l}27 \\
27 \\
29 \\
29 \\
27\end{array}$ & $\begin{array}{l}29 \\
29 \\
28 \\
28 \\
28\end{array}$ & $\begin{array}{l}23 \\
24 \\
23 \\
23 \\
24\end{array}$ & $\begin{array}{l}21 \\
22 \\
21 \\
21 \\
22\end{array}$ & $\begin{array}{l}17 \\
17 \\
17 \\
17 \\
17\end{array}$ & $\begin{array}{l}016 \\
016 \\
015 \\
15 \\
16\end{array}$ & $\begin{array}{r}018 \\
024 \\
019 \\
0200 \\
0530\end{array}$ & $\begin{array}{l}29 \\
25 \\
19 \\
18 \\
18\end{array}$ \\
\hline $\begin{array}{l}26 \\
27 \\
28 \\
29 \\
30 \\
31\end{array}$ & $\begin{array}{l}33 \\
33 \\
33 \\
34 \\
34 \\
33\end{array}$ & $\begin{array}{r}32 \\
35 \\
40 \\
39 \\
35 \\
-\end{array}$ & $\begin{array}{l}33 \\
33 \\
33 \\
32 \\
31 \\
30\end{array}$ & $\begin{array}{l}34 \\
34 \\
34 \\
35 \\
34 \\
36\end{array}$ & $\begin{array}{r}27 \\
27 \\
28 \\
28 \\
-- \\
---\end{array}$ & $\begin{array}{l}28 \\
26 \\
24 \\
25 \\
24 \\
23\end{array}$ & $\begin{array}{r}24 \\
23 \\
23 \\
23 \\
23 \\
-\end{array}$ & $\begin{array}{l}22 \\
22 \\
22 \\
22 \\
22 \\
22\end{array}$ & $\begin{array}{r}16 \\
18 \\
146 \\
330 \\
309 \\
--\end{array}$ & $\begin{array}{r}226 \\
77 \\
31 \\
23 \\
0215 \\
092\end{array}$ & $\begin{array}{r}0640 \\
0540 \\
03210 \\
8560 \\
8340 \\
01140\end{array}$ & $\begin{array}{r}18 \\
19 \\
22 \\
21 \\
21 \\
--\end{array}$ \\
\hline $\begin{array}{l}\text { TOTAL } \\
\text { MEAN } \\
\text { MAX } \\
\text { MIN } \\
\text { AC-FT }\end{array}$ & $\begin{array}{r}1084 \\
35.0 \\
42 \\
33 \\
2150\end{array}$ & $\begin{array}{r}936 \\
31.2 \\
40 \\
29 \\
1860\end{array}$ & $\begin{array}{r}1015 \\
32.7 \\
35 \\
30 \\
2010\end{array}$ & $\begin{array}{r}1024 \\
33.0 \\
36 \\
30 \\
2030\end{array}$ & $\begin{array}{r}829 \\
28.6 \\
36 \\
26 \\
1640\end{array}$ & $\begin{array}{r}856 \\
27.6 \\
31 \\
23 \\
1700\end{array}$ & $\begin{array}{r}692 \\
23.1 \\
25 \\
22 \\
1370\end{array}$ & $\begin{array}{r}680 \\
21.9 \\
24 \\
21 \\
1350\end{array}$ & $\begin{array}{r}1343 \\
44.8 \\
330 \\
16 \\
2660\end{array}$ & $\begin{array}{r}11688 \\
377 \\
4310 \\
15 \\
23180\end{array}$ & $\begin{array}{r}8049 \\
260 \\
3210 \\
18 \\
15970\end{array}$ & $\begin{array}{r}2029 \\
67.6 \\
450 \\
18 \\
4020\end{array}$ \\
\hline
\end{tabular}

STATISTICS OE MONTHLY MEAN DATA FOR WATER YEARS 1976 - 1996, BY WATER YEAR (WY)

\begin{tabular}{|c|c|c|c|c|c|c|c|c|c|c|c|c|}
\hline $\begin{array}{l}\text { MEAN } \\
\text { MAX } \\
\text { (WY) } \\
\text { MIN } \\
\text { (WY) }\end{array}$ & $\begin{array}{r}42.8 \\
147 \\
1986 \\
6.50 \\
1979\end{array}$ & $\begin{array}{r}40.9 \\
176 \\
1995 \\
9.53 \\
1982\end{array}$ & $\begin{array}{l}24.8 \\
68.7 \\
1987 \\
7.77 \\
1978\end{array}$ & $\begin{array}{l}22.1 \\
46.1 \\
1987 \\
7.74 \\
1978\end{array}$ & $\begin{array}{r}23.6 \\
106 \\
1987 \\
6.40 \\
1978\end{array}$ & $\begin{array}{r}48.9 \\
207 \\
1985 \\
5: 69 \\
1978\end{array}$ & $\begin{array}{r}120 \\
415 \\
1987 \\
4.99 \\
1978\end{array}$ & $\begin{array}{r}328 \\
768 \\
1985 \\
7.93 \\
1981\end{array}$ & $\begin{array}{r}299 \\
945 \\
1979 \\
8.87 \\
1977\end{array}$ & $\begin{array}{r}151 \\
440 \\
1991 \\
18.6 \\
1980\end{array}$ & $\begin{array}{r}221 \\
1077 \\
1991 \\
16,1 \\
1978\end{array}$ & $\begin{array}{r}127 \\
683 \\
1991 \\
6.12 \\
1978\end{array}$ \\
\hline
\end{tabular}

SUMARY STATISTICS

ANHUAL. TOTAL.

ANNUAL MEAN

HIGHEST ANAUAL MEAN

LONEST ANHUAL MEAN

HIGHEST DAILY MEAN

LOWEST DAILY MEAN

ANNUAL, SEVEN-DAY MINIMIM

INSTANTANEOUS PEAK FLON

INSTANTANEOUS PEAK STAGE

INSTANTANEOUS LOW FLOW

ANWUAL RUNOFF (AC-FT)

10 PERCENT EXCEEDS

50 PERCENT EXCEEDS

90 PERCENT EXCEEDS

Estimated
FOR 1995 CALENDAR YEAR

66789
183

3120
24
27

132500

595
54
30
FOR 1996 WATER YEAR
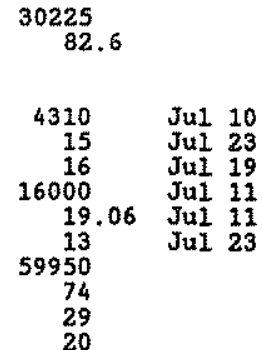

WATER YEARS 1976 - 1996

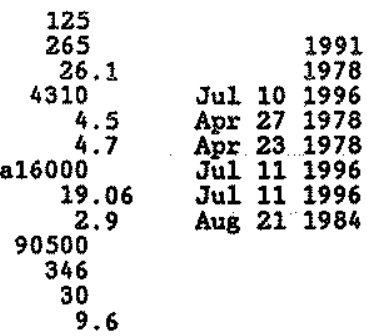


RIO GRANDE BASIN

08382650 PECOS RIVER ABOVE SANTA ROSA LAKE, MM - Continued

WATER-QUALITY RECORDS

PERIOD OF RECORD. -Water years 1976, 1981 to current year.

WATER QUALITY DATA, WATER YEAR OCTOBER 1995 TO SEPTEMBER 1996

\begin{tabular}{|c|c|c|c|c|c|c|c|c|c|c|c|}
\hline DATE & TIME & $\begin{array}{c}\text { DIS- } \\
\text { CHARGE, } \\
\text { INST, } \\
\text { CUBIC } \\
\text { FEET } \\
\text { PER } \\
\text { SECOND } \\
(00061)\end{array}$ & $\begin{array}{l}\text { SPE- } \\
\text { CIFIC } \\
\text { CON- } \\
\text { DUCT- } \\
\text { ANCE } \\
\text { (US/CM) } \\
(00095)\end{array}$ & $\begin{array}{c}\text { PH } \\
\text { WATER } \\
\text { WHOLE } \\
\text { FIELD } \\
\text { (STAND- } \\
\text { ARD } \\
\text { UNITS) } \\
\text { (00400) }\end{array}$ & $\begin{array}{c}\text { TEMPER- } \\
\text { ATURE } \\
\text { AIR } \\
\text { (DEG C) } \\
(00020)\end{array}$ & $\begin{array}{c}\text { TEMPER- } \\
\text { ATURE } \\
\text { WATER } \\
\text { (DEG C) } \\
(00010)\end{array}$ & $\begin{array}{l}\text { BARO- } \\
\text { METRIC } \\
\text { ERES- } \\
\text { SURE } \\
\text { (MM } \\
\text { OF } \\
\text { HG) } \\
(00025)\end{array}$ & $\begin{array}{c}\text { OXYGEN, } \\
\text { DIS - } \\
\text { SOLVED } \\
(\mathrm{MG/L}) \\
(00300)\end{array}$ & $\begin{array}{c}\text { OXYGEN, } \\
\text { DIS- } \\
\text { SOLVED } \\
\text { (PER- } \\
\text { CENT } \\
\text { SATUR- } \\
\text { ATION) } \\
\text { (OO301) }\end{array}$ & $\begin{array}{l}\text { HARD- } \\
\text { NESS } \\
\text { TOTAL } \\
\text { (MG/L } \\
\text { AS } \\
\text { CACO3) } \\
(00900)\end{array}$ & $\begin{array}{c}\text { CALC } \\
\text { DIS } \\
\text { SOL } \\
\text { (NG } \\
\text { AS } \\
C 009\end{array}$ \\
\hline $\begin{array}{l}\mathrm{BC} 1995 \\
\text { OS } \\
\text { IAR } 1996\end{array}$ & 1415 & 33 & 890 & 8.1 & 15.0 & 15.0 & 644 & 9.8 & 116 & 450 & 150 \\
\hline $06 \ldots$ & 0900 & 28 & 930 & 7.9 & 1.5 & 8.0 & 640 & 9.8 & 99 & 490 & 160 \\
\hline $\mathrm{EP}^{12} \ldots$ & 0930 & 21 & 970 & 8.1 & 23.0 & 19.0 & 645 & 12.8 & 164 & 570 & 190 \\
\hline $05 . .$. & 0930 & 56. & 4350 & 8.1 & 24.5 & 20.0 & 643 & 9.2 & 122 & 220 & 73 \\
\hline
\end{tabular}

MAGNE-

STUM, SODIUM,

DIS-- DIS-

SOLVED SOLVED

(MG/L (MG/L

AS $M G)$ AS NA)

(00925)

$(00930)$

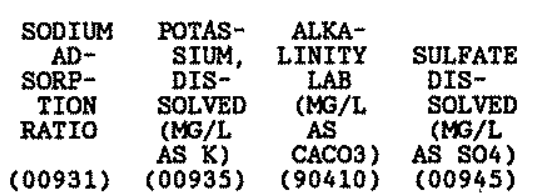

CHLO-
RIDE,
DIS-
SOLVED
(ME
AS CL
$(00940)$

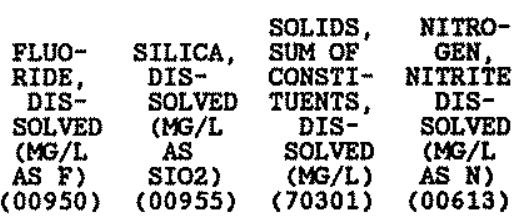

DEC 1995

\begin{tabular}{|c|c|}
\hline${ }_{\text {MAR }}^{05} 1996$ & 19 \\
\hline JUN $06 \cdots$ & 21 \\
\hline${ }_{\operatorname{SEP}}^{12} \ldots$ & 23 \\
\hline 05. & 9.7 \\
\hline
\end{tabular}

$\begin{array}{llll}0.2 & 1.3 & 128 & 310 \\ 0.2 & 1.2 & 144 & 340 \\ 0.2 & 1.5 & 123 & 400 \\ 0.3 & 2.1 & 128 & 130\end{array}$

$\begin{array}{lll}5.8 & 0.30 & 11 \\ 6.7 & 0.30 & 11 \\ 6.9 & 0.30 & 12 \\ 5.4 & 0.20 & 10\end{array}$

585

$<0.010$

11.

130

5.4

$0.20 \quad 10$

638

719

$319<0.010$

NITRO- NITRO- NITRO- NITRO-

NITRO- NITRO- NITRO- NITRO- RHOS-

GEN, GEN, GEN,AM- GEN,AM-

GEN,
GEN,
MO2+NO3 AMN, AM- GEN,AM-
AMONA MONIA +

DIS- DIS- ORGANIC ORGANIC

SOLVED SOLVED TOTAL DIS (MG/L $\quad(M G / L$

AS

(00631)

(MG/L (MG/L

DIS-

DIS-

SOLVED SOLVED

(MG/L $\quad(M G / L$

(00666) $(00671)$ AS AL)

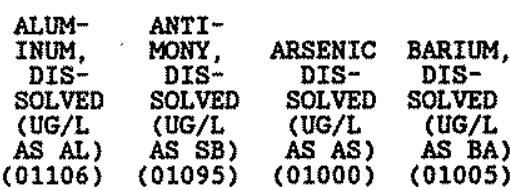

DEC 199

05

$$
0.110<0.015<0.20<0.20<0.010
$$

$<0.010$

$<0.010$

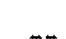

(01095) (01005)

$$
\begin{array}{cc}
-- & -m \\
-\infty & -- \\
0.090 & <0.015
\end{array}
$$$$
-
$$$$
--
$$$$
-
$$$$
\rightarrow
$$$$
-
$$

05...

0.50

$<0.20$

0.070

\begin{tabular}{|c|c|c|c|c|c|c|c|c|c|}
\hline $\begin{array}{l}\text { BERYL- } \\
\text { LIUM, } \\
\text { DIS- } \\
\text { SOLVED } \\
\text { (UG/L } \\
\text { AS BE) } \\
\text { (01010) }\end{array}$ & $\begin{array}{l}\text { BORON, } \\
\text { DIS- } \\
\text { SOLVED } \\
\text { (UG/L } \\
\text { AS B) } \\
(01020)\end{array}$ & $\begin{array}{c}\text { CADMIUM } \\
\text { DIS- } \\
\text { SOLVED } \\
\text { (UG/L } \\
\text { AS CD) } \\
(01025)\end{array}$ & $\begin{array}{l}\text { CHRO- } \\
\text { MIUM, } \\
\text { DIS- } \\
\text { SOLVED } \\
\text { (UG/L } \\
\text { AS CR) } \\
(01030)\end{array}$ & $\begin{array}{l}\text { COBALT, } \\
\text { DIS- } \\
\text { SOLVED } \\
\text { (UG/L. } \\
\text { AS CO) } \\
\text { (01035) }\end{array}$ & $\begin{array}{c}\text { CORPER, } \\
\text { DIS- } \\
\text { SOLVED } \\
\text { (UG/L } \\
\text { AS CU) } \\
(01040 \text { ) }\end{array}$ & $\begin{array}{l}\text { IRON, } \\
\text { DIS- } \\
\text { SOLVED } \\
\text { (UG/L } \\
\text { AS FE) } \\
(01046)\end{array}$ & $\begin{array}{l}\text { LEAD, } \\
\text { DIS - } \\
\text { SOLVED } \\
\text { (UG/L } \\
\text { AS PB) } \\
\text { (01049) }\end{array}$ & $\begin{array}{l}\text { MANGA- } \\
\text { NESE, } \\
\text { DIS- } \\
\text { SOLVED } \\
\text { (UG/L } \\
\text { AS MN) } \\
\text { (01056) }\end{array}$ & $\begin{array}{c}\text { MGRCURY } \\
\text { TOTAL } \\
\text { RECOV- } \\
\text { ERABLE } \\
\text { (UG/L } \\
\text { AS HG) } \\
\text { (71900) }\end{array}$ \\
\hline
\end{tabular}

$<0.010$

$<0.010$

$<1.0$

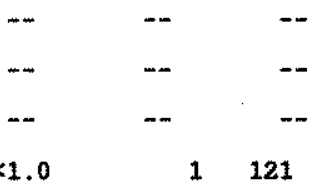

DATE

DEC 1995

MAR
MOS
JUN
12
SEP

$\begin{array}{ll}-- & 60 \\ -- & 40 \\ -- & 47 \\ <1.0 & 32\end{array}$

60
40
47
32

$\begin{array}{ll}-- & -- \\ -- & -- \\ -- & -- \\ <1.0 & <1.0\end{array}$

$$
\begin{array}{cc}
-- & -- \\
-- & -- \\
-- & -- \\
<1.0 & 2.0
\end{array}
$$$$
\begin{array}{lll}
16 & -- & - \\
<3.0 & -- & - \\
<3.0 & -- & - \\
6.0 & <1.0 & 10
\end{array}
$$

$<0.10$

$-$

$-$

$05 .$.

$<1.0 \quad 32$

$<1.0<1.0<1.0$

$6.0<1.0 \quad 10$

$<0.10$ 
RIO GRANDE BASIN

08382650 PECOS RIVER ABOVE SANTA ROSA LAKE, NM -- Continued

WATER-QUALITY RECORDS

WATER QUALITY DATA, WATER YEAR OCTOBER 1995 TO SEPTEMBER 1996

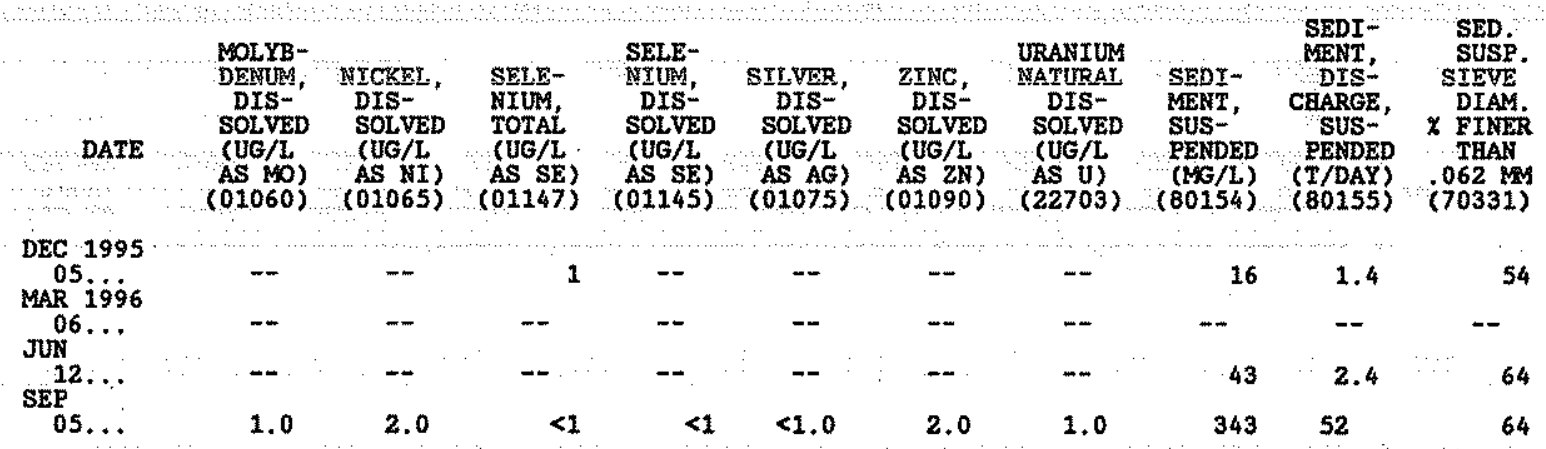


RIO GRANDE BASTN

08382730 LOS ESTEROS CREEK ABOVE SANTA ROSA LAKE, NM

LOCATION. - Lat 35 05'42", Long 104'39'49", Guadalupe County, Hydrologic Unit 13060001, in Preston Beck Grant, on left bank $3.7 \mathrm{ml}$ upstream from mouth, 4.9 mi northeast of Santa Rosa Dam, and $10.4 \mathrm{mi}$ northeast of Santa Rosa. Mouth at Pecos River mile 763,0 .

DRAINAGE AREA. $--65.6 \mathrm{mt}^{2}$.

PERIOD OF RECORD.--July 1973 to current year. Prtor to October 1979, published as "above Los Esteros Reservoli." GAGE.-Water-stage recorder and concrete control. Elevation of gage is 4,770 ft above National Geodetic Vertical. Datum of 1929, from topographto map.

REMARKS, - Records fatr except for estimated discharges, which are poor. No known diversions or ground-water withdrawals for irrigation upstream from station. Soveral observations of water temperature were made during the year. No flow most of time.

DISCHARGE, CUETC FEET PER SECOND, WATER YEAR OCTOBER 1995 TO SEPTEMBER 1996

\begin{tabular}{|c|c|c|c|c|c|c|c|c|c|c|c|c|}
\hline DAY & $\operatorname{OCT}$ & Nov & DEC & JAN & FEB & MAR & APR & MAY & JUN & JUL & AUG & SEP \\
\hline $\begin{array}{l}1 \\
2 \\
3 \\
4 \\
5\end{array}$ & $\begin{array}{l}.01 \\
.14 \\
.04 \\
.01 \\
.00\end{array}$ & $\begin{array}{l}.00 \\
.00 \\
.00 \\
.00 \\
.00\end{array}$ & $\begin{array}{l}.00 \\
.00 \\
.00 \\
.00 \\
.00\end{array}$ & $\begin{array}{l}.00 \\
.00 \\
.00 \\
.00 \\
.00\end{array}$ & $\begin{array}{l}.00 \\
.00 \\
.00 \\
.00 \\
.00\end{array}$ & $\begin{array}{l}.00 \\
.00 \\
.00 \\
.00 \\
.00\end{array}$ & $\begin{array}{r}.00 \\
.00 \\
.00 \\
.00 \\
.00\end{array}$ & $\begin{array}{l}.00 \\
.00 \\
.00 \\
.00 \\
.00\end{array}$ & $\begin{array}{l}.00 \\
.00 \\
.00 \\
.00 \\
.00\end{array}$ & $\begin{array}{r}15 \\
.69 \\
.37 \\
.17 \\
.00\end{array}$ & $\begin{array}{l}0.00 \\
0.00 \\
0.00 \\
0.00 \\
0.00\end{array}$ & $\begin{array}{l}.08 \\
.00 \\
.00 \\
.00 \\
.00\end{array}$ \\
\hline $\begin{array}{r}6 \\
7 \\
8 \\
9 \\
10\end{array}$ & $\begin{array}{l}.00 \\
.00 \\
.00 \\
.00 \\
.00\end{array}$ & $\begin{array}{l}.00 \\
.00 \\
.00 \\
.00 \\
.00\end{array}$ & $\begin{array}{l}.00 \\
.00 \\
.00 \\
.00 \\
.00\end{array}$ & $\begin{array}{l}.00 \\
.00 \\
.00 \\
.00 \\
.00\end{array}$ & $\begin{array}{l}.00 \\
.00 \\
.00 \\
.00 \\
.00\end{array}$ & $\begin{array}{l}.00 \\
.00 \\
.00 \\
.00 \\
.00\end{array}$ & $\begin{array}{l}.00 \\
.00 \\
.00 \\
.00 \\
.00\end{array}$ & $\begin{array}{l}.00 \\
.00 \\
.00 \\
.00 \\
.00\end{array}$ & $\begin{array}{l}.00 \\
.00 \\
.00 \\
.00 \\
.00\end{array}$ & $\begin{array}{r}.00 \\
.00 \\
.00 \\
770^{.00}\end{array}$ & $\begin{array}{l}0.00 \\
0.00 \\
0.00 \\
0.00 \\
0.00\end{array}$ & $\begin{array}{l}.00 \\
.00 \\
.00 \\
.00 \\
.00\end{array}$ \\
\hline $\begin{array}{l}11 \\
12 \\
13 \\
14 \\
15\end{array}$ & $\begin{array}{l}.00 \\
.00 \\
.00 \\
.00 \\
.00\end{array}$ & $\begin{array}{l}.00 \\
.00 \\
.00 \\
.00 \\
.00\end{array}$ & $\begin{array}{l}.00 \\
.00 \\
.00 \\
.00 \\
.00\end{array}$ & $\begin{array}{l}.00 \\
.00 \\
.00 \\
.00 \\
.00\end{array}$ & $\begin{array}{l}.00 \\
.00 \\
.00 \\
.00 \\
.00\end{array}$ & $\begin{array}{l}.00 \\
.00 \\
.00 \\
.00 \\
.00\end{array}$ & $\begin{array}{l}.00 \\
.00 \\
.00 \\
.00 \\
.00\end{array}$ & $\begin{array}{l}.00 \\
.00 \\
.00 \\
.00 \\
.00\end{array}$ & $\begin{array}{l}.00 \\
.00 \\
.00 \\
.00 \\
.00\end{array}$ & $\begin{array}{r}3.2 \\
341^{.71} \\
e 22 \\
\quad 0.00\end{array}$ & $\begin{array}{l}0.00 \\
0.00 \\
0.00 \\
0.00 \\
0.00\end{array}$ & $\begin{array}{l}.00 \\
.00 \\
.00 \\
.00 \\
.94\end{array}$ \\
\hline $\begin{array}{l}16 \\
17 \\
18 \\
19 \\
20\end{array}$ & $\begin{array}{l}.00 \\
.00 \\
.00 \\
.00 \\
.00\end{array}$ & $\begin{array}{l}.00 \\
.00 \\
.00 \\
.00 \\
.00\end{array}$ & $\begin{array}{l}.00 \\
.00 \\
.00 \\
.00 \\
.00\end{array}$ & $\begin{array}{l}.00 \\
.00 \\
.00 \\
.00 \\
.00\end{array}$ & $\begin{array}{l}.00 \\
.00 \\
.00 \\
.00 \\
.00\end{array}$ & $\begin{array}{l}.00 \\
.00 \\
.00 \\
.00 \\
.00\end{array}$ & $\begin{array}{l}.00 \\
.00 \\
.00 \\
.00 \\
.00\end{array}$ & $\begin{array}{l}.00 \\
.00 \\
.00 \\
.00 \\
.00\end{array}$ & $\begin{array}{l}.00 \\
.00 \\
.00 \\
.00 \\
.00\end{array}$ & $\begin{array}{l}0.00 \\
0.00 \\
0.00 \\
0.00 \\
0.00\end{array}$ & $\begin{array}{l}.00 \\
.00 \\
.00 \\
.00 \\
.00\end{array}$ & $\begin{array}{l}.58 \\
.03 \\
.00 \\
.00 \\
.00\end{array}$ \\
\hline $\begin{array}{l}21 \\
22 \\
23 \\
24 \\
25\end{array}$ & $\begin{array}{l}.00 \\
.00 \\
.00 \\
.00 \\
.00\end{array}$ & $\begin{array}{l}.00 \\
.00 \\
.00 \\
.00 \\
.00\end{array}$ & $\begin{array}{l}.00 \\
.00 \\
.00 \\
.00 \\
.00\end{array}$ & $\begin{array}{l}.00 \\
.00 \\
.00 \\
.00 \\
.00\end{array}$ & $\begin{array}{l}.00 \\
.00 \\
.00 \\
.00 \\
.00\end{array}$ & $\begin{array}{l}.00 \\
.00 \\
.00 \\
.00 \\
.00\end{array}$ & $\begin{array}{l}.00 \\
.00 \\
.00 \\
.00 \\
.00\end{array}$ & $\begin{array}{l}.00 \\
.00 \\
.00 \\
.00 \\
.00\end{array}$ & $\begin{array}{l}.00 \\
.00 \\
.00 \\
.00 \\
.00\end{array}$ & $\begin{array}{l}0.00 \\
0.00 \\
0.00 \\
0.00 \\
0.00\end{array}$ & $\begin{array}{l}.00 \\
.00 \\
.00 \\
.00 \\
.00\end{array}$ & $\begin{array}{l}.00 \\
.00 \\
.00 \\
.00 \\
.00\end{array}$ \\
\hline $\begin{array}{l}26 \\
27 \\
28 \\
29 \\
30 \\
31\end{array}$ & $\begin{array}{l}.00 \\
.00 \\
.00 \\
.00 \\
.00 \\
.00\end{array}$ & $\begin{array}{l}.00 \\
.00 \\
.00 \\
.00 \\
.00 \\
.0\end{array}$ & $\begin{array}{l}.00 \\
.00 \\
.00 \\
.00 \\
.00 \\
.00\end{array}$ & $\begin{array}{l}.00 \\
.00 \\
.00 \\
.00 \\
.00 \\
.00\end{array}$ & $\begin{array}{l}.00 \\
.00 \\
.00 \\
.00 \\
- \\
-\end{array}$ & $\begin{array}{l}.00 \\
.00 \\
.00 \\
.00 \\
.00 \\
.00\end{array}$ & $\begin{array}{l}.00 \\
.00 \\
.00 \\
.00 \\
.00 \\
.+-\end{array}$ & $\begin{array}{l}.00 \\
.00 \\
.00 \\
.00 \\
.00 \\
.00\end{array}$ & $\begin{array}{l}.00 \\
.00 \\
34.00 \\
33 \\
.---\end{array}$ & $\begin{array}{l}0.00 \\
0.00 \\
0.00 \\
0.00 \\
0.00 \\
0.00\end{array}$ & $\begin{array}{l}.00 \\
.12 \\
5.9 \\
3.5 \\
.20 \\
.11\end{array}$ & $\begin{array}{l}.00 \\
.00 \\
.00 \\
.00 \\
.00 \\
-0\end{array}$ \\
\hline $\begin{array}{l}\text { TOTAL } \\
\text { MFAN } \\
\text { MAX } \\
\text { MIN } \\
\text { AC-FT }\end{array}$ & $\begin{array}{r}0.20 \\
.006 \\
.14 \\
.00 \\
.4\end{array}$ & $\begin{array}{l}0.00 \\
.000 \\
.00 \\
.00 \\
.00\end{array}$ & $\begin{array}{l}0.00 \\
.000 \\
.00 \\
.00 \\
.00\end{array}$ & $\begin{array}{l}0.00 \\
.000 \\
.00 \\
.00 \\
.00\end{array}$ & $\begin{array}{r}0.00 \\
.000 \\
.00 \\
.00 \\
.00\end{array}$ & $\begin{array}{l}0.00 \\
.000 \\
.00 \\
.00 \\
.00\end{array}$ & $\begin{array}{l}0.00 \\
.000 \\
.00 \\
.00 \\
.00\end{array}$ & $\begin{array}{l}0.00 \\
.000 \\
.00 \\
.00 \\
.00\end{array}$ & $\begin{array}{r}67.00 \\
2.23 \\
34 \\
.00 \\
133\end{array}$ & $\begin{array}{r}1153.14 \\
37.2 \\
770 \\
.00 \\
2290\end{array}$ & $\begin{array}{r}9.83 \\
.32 \\
5.9 \\
.00 \\
19\end{array}$ & $\begin{array}{r}1.63 \\
.054 \\
.94 \\
.00 \\
3.2\end{array}$ \\
\hline
\end{tabular}

STATISTICS OF MONTELY MEAN DATA FOR WATER YEARS 1973 - 1996, BY WATER YEAR (WY)

\begin{tabular}{|c|c|c|c|c|c|c|c|c|c|c|c|c|}
\hline $\begin{array}{l}\text { MEAN } \\
\text { MAX } \\
\text { (WY) } \\
\text { MIN } \\
\text { (WY) }\end{array}$ & $\begin{array}{r}.33 \\
5.20 \\
1986 \\
.000 \\
1974\end{array}$ & $\begin{array}{l}.19 \\
2.26 \\
1979 \\
1000 \\
1974\end{array}$ & $\begin{array}{r}.000 \\
0007 \\
1979 \\
000 \\
1974\end{array}$ & $\begin{array}{l}.003 \\
1028 \\
1987 \\
1000 \\
1974\end{array}$ & $\begin{array}{r}.18 \\
3.74 \\
1987 \\
1000 \\
1974\end{array}$ & $\begin{array}{r}.003 \\
.045 \\
1987 \\
1000 \\
1974\end{array}$ & $\begin{array}{l}.070 \\
1.57 \\
1985 \\
1000 \\
1974\end{array}$ & $\begin{array}{r}.85 \\
10.9 \\
1994 \\
000 \\
1974\end{array}$ & $\begin{array}{l}4.30 \\
37.9 \\
1995 \\
1000 \\
1974\end{array}$ & $\begin{array}{l}4.70 \\
37.2 \\
1996 \\
1000 \\
1980\end{array}$ & $\begin{array}{r}6.48 \\
48.1 \\
1977 \\
1000 \\
1979\end{array}$ & $\begin{array}{l}2.77 \\
18.1 \\
1988 \\
1000 \\
1973\end{array}$ \\
\hline
\end{tabular}

SUMMARY STATISTICS

AMUAL TOTAL

ANNUAL MBAN

HIGHEST ANNUAL MEAN

LOWEST ANNUAL MEAN

LOWEST ANNUAL MEAN

EIGHEST DAILY MEAN
LOWEST DAILY MEAN

ANNUAL SEVEN-DAY MINIMIM

INSTANTANEOUS PEAK FLOW

INSTANTANEOUS PEAK STAGE

INSTANTANEOUS LOW FLOW

ANNUAL RUNOFE (AC-FT)

10 PERCENT EXCEEDS

50. PERCENT EXCEEDS

90 PERCENT EXCEEDS
FOR 1995 CALENDAR YEAR

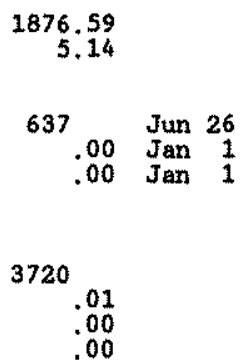

FOR 1996 WATER YEAR

$$
\begin{array}{r}
1231.80 \\
3.37
\end{array}
$$

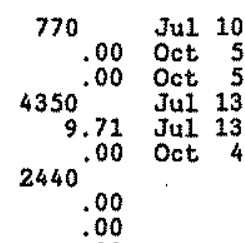

WATER YEARS 1973 - 1996

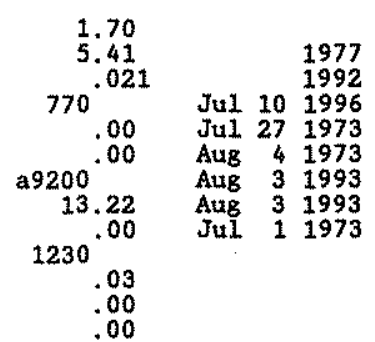

- Estimated

a-From rating curve extended.70 fts/s, on basis of slope-area measurements at 8 age helghts, 6.5 ft and $9.3 \mathrm{ft}$. 
08382810 SANTA ROSA LAKE NEAR SANTA ROSA, NM

LOCATION.--Lat 3501'47", Long 104"41'30", Guadalupe County, Hydrologic Unit 13060001, in Jose Perea Grant, near outlet gates of Senta' Rosa Dam on Pecos River, approximately 7.0 mi north of Santa Rosa, and at mile 757.2 .

DRAINAGE AREA, $--2,430 \mathrm{mi}^{2}$, approximately.

PERTOD OF RECORDS.--April 1980 to current year.

GAGE - Water-8tage recorder. Elevation of gage is National Geodetic Vertical Datum of 1929 (1ovels by U.S. Antu Corps of Engineers).

REHAFKS. -Lake is cormed by oarth and rockf111 dan on Pocos River. storage began on Apr. 22, 1980 . Capacity,

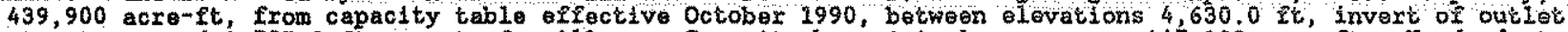
structure, and 4,797.0 rt, crest of spillway. Capacity by original survey was 447,100 acre-ft. No dead storage. Lake was created primaxily for flood, 1rrigation, and sediment control. U.s. Army Corps of Engtneers satellite telemeter at station.

COOPERATION, - -Records provided by U.S. Army Corps of Engineers.

EXTRDES FOR PERIOD OF RECORD.-Maximum contents, 120,481 acre-ft, May 8, 1987, elevat1on, 4,749.71 rt; no storage for many days, July--Sept., 1980 and June-Aug., 1981.

EXTREMS FOR CURREAT YEAR.-Maximum contents, 68,410 acreme, Dec, 31, Jan. 1 elevation, 4,736.22 ft; minimu, 3,600 ecre-1t, June 29, elevation, 4,685.86 ft.

RESERVOIR STORAGE (ACRE-FEET), WATER YEAR OCTOBER 1995 TO SEPTEMBER 1996

\begin{tabular}{|c|c|c|c|c|c|c|c|c|c|c|c|c|}
\hline AY & $\mathrm{OCT}$ & NOV & DEC & JAN & FEB & MAR & APR & MAY & JUN & JUL & AUG & SEP \\
\hline $\begin{array}{l}1 \\
2 \\
3 \\
4 \\
5\end{array}$ & $\begin{array}{r}66570 \\
66710 \\
66770 \\
66710 \\
\therefore \quad 66740\end{array}$ & $\begin{array}{l}67000 \\
66940 \\
66970 \\
67000 \\
67060\end{array}$ & $\begin{array}{l}67260 \\
67260 \\
67290 \\
67370 \\
67340\end{array}$ & $\begin{array}{l}68410 \\
68150 \\
67260 \\
66090 \\
64890\end{array}$ & $\begin{array}{l}58480 \\
58580 \\
58630 \\
58630 \\
58760\end{array}$ & $\begin{array}{r}59310 \\
59230 \\
59230 \\
59250 \\
59250\end{array}$ & $\begin{array}{l}59590 \\
59640 \\
59570 \\
59570 \\
59570\end{array}$ & $\begin{array}{l}58 \\
57 \\
55 \\
54 \\
52\end{array}$ & $\begin{array}{l}3 \\
3 \\
3 \\
3 \\
3\end{array}$ & & $\begin{array}{l}50 \\
70 \\
00 \\
00 \\
00\end{array}$ & $\begin{array}{l}4 \\
4 \\
4 \\
4\end{array}$ \\
\hline $\begin{array}{r}6 \\
7 \\
8 \\
9 \\
10\end{array}$ & $\begin{array}{l}66690 \\
66710 \\
66740 \\
66740 \\
66800\end{array}$ & $\begin{array}{l}67080 \\
67110 \\
67140 \\
67140 \\
67110\end{array}$ & $\begin{array}{l}67370 \\
67370 \\
67370 \\
67370 \\
67400\end{array}$ & $\begin{array}{l}63690 \\
62550 \\
61460 \\
60300 \\
59150\end{array}$ & $\begin{array}{l}58820 \\
58790 \\
58760 \\
58890 \\
58870\end{array}$ & $\begin{array}{l}59250 \\
59280 \\
59310 \\
59360 \\
59330\end{array}$ & $\begin{array}{l}59570 \\
59590 \\
59670 \\
59750 \\
59670\end{array}$ & $\begin{array}{l}51470 \\
50060 \\
48740 \\
47400 \\
45920\end{array}$ & $\begin{array}{l}3 \\
3 \\
3 \\
3 \\
3\end{array}$ & $\begin{array}{r}41 \\
41 \\
41 \\
44 \\
140\end{array}$ & $\begin{array}{l}0 \\
0 \\
0 \\
0 \\
0\end{array}$ & $\begin{array}{l}4 \\
4 \\
4\end{array}$ \\
\hline $\begin{array}{l}11 \\
12 \\
13 \\
14 \\
15\end{array}$ & $\begin{array}{l}66890 \\
66860 \\
66800 \\
66860 \\
66910\end{array}$ & $\begin{array}{l}66770 \\
66860 \\
66710 \\
66830 \\
66910\end{array}$ & $\begin{array}{l}67460 \\
67460 \\
67510 \\
67540 \\
67570\end{array}$ & $\begin{array}{l}58280 \\
58100 \\
58170 \\
58170 \\
58230\end{array}$ & $\begin{array}{l}58870 \\
58940 \\
58970 \\
59020 \\
59020\end{array}$ & $\begin{array}{l}593 \\
592 \\
592 \\
592 \\
595\end{array}$ & $\begin{array}{l}59 \\
59 \\
59 \\
59 \\
59\end{array}$ & $\begin{array}{l}45 \\
45 \\
41 \\
40 \\
30\end{array}$ & $\begin{array}{l}3 \\
3 \\
3 \\
2 \\
2\end{array}$ & $\begin{array}{l}2 \\
22 \\
2 \\
2 \\
2\end{array}$ & $\begin{array}{l}0 \\
0 \\
0 \\
0 \\
0\end{array}$ & $\begin{array}{l}4 \\
4 \\
4 \\
4 \\
4\end{array}$ \\
\hline $\begin{array}{l}16 \\
17 \\
18 \\
19 \\
20\end{array}$ & $\begin{array}{l}66940 \\
66970 \\
66970 \\
66940 \\
66940\end{array}$ & $\begin{array}{l}66940 \\
66970 \\
67030 \\
67060 \\
67060\end{array}$ & $\begin{array}{l}67570 \\
67800 \\
67800 \\
67800 \\
67830\end{array}$ & $\begin{array}{l}58280 \\
58280 \\
58280 \\
58280 \\
58350\end{array}$ & $\begin{array}{l}59 \\
59 \\
59 \\
59 \\
59\end{array}$ & $\begin{array}{l}59 \\
59 \\
59 \\
59 \\
59\end{array}$ & $\begin{array}{l}59 \\
59 \\
59 \\
59 \\
59\end{array}$ & & $\begin{array}{l}2 \\
2 \\
2 \\
1 \\
1\end{array}$ & $\begin{array}{l}28 \\
29 \\
29 \\
29\end{array}$ & $\begin{array}{l}0 \\
0 \\
0 \\
0 \\
0\end{array}$ & $\begin{array}{l}4 \\
4 \\
4 \\
4\end{array}$ \\
\hline $\begin{array}{l}21 \\
22 \\
23 \\
24 \\
25\end{array}$ & $\begin{array}{l}67000 \\
66890 \\
66890 \\
66860 \\
66860\end{array}$ & $\begin{array}{l}67110 \\
67140 \\
67170 \\
67230\end{array}$ & $\begin{array}{l}67890 \\
67950 \\
68000 \\
68030 \\
68120\end{array}$ & $\begin{array}{l}583 \\
584 \\
583 \\
584 \\
584\end{array}$ & $\begin{array}{l}59 \\
59 \\
59 \\
59 \\
59\end{array}$ & $\begin{array}{l}59 \\
59 \\
59 \\
59 \\
59\end{array}$ & $\begin{array}{r}5 \\
55 \\
55 \\
5 \\
55\end{array}$ & $\begin{array}{l}38 \\
38 \\
38 \\
38 \\
38\end{array}$ & $\begin{array}{r}13 \\
12 \\
10 \\
9 \\
8\end{array}$ & $\begin{array}{l}29 \\
29 \\
29 \\
29 \\
29\end{array}$ & $\begin{array}{l}0 \\
0 \\
0 \\
0 \\
0\end{array}$ & $\begin{array}{l}48 \\
4 \\
4 \\
4\end{array}$ \\
\hline $\begin{array}{l}26 \\
27 \\
28 \\
29 \\
30 \\
31\end{array}$ & $\begin{array}{l}66910 \\
66890 \\
66890 \\
66970 \\
66970 \\
67030\end{array}$ & $\begin{array}{r}67170 \\
67140 \\
67230 \\
67310 \\
67290 \\
-\end{array}$ & $\begin{array}{l}68180 \\
68150 \\
68260 \\
68290 \\
68350 \\
68410\end{array}$ & $\begin{array}{l}58460 \\
58460 \\
58460 \\
58510 \\
58480 \\
58480\end{array}$ & $\begin{array}{r}59180 \\
59150 \\
59100 \\
59230 \\
\\
-\end{array}$ & $\begin{array}{l}59540 \\
59540 \\
59540 \\
59540 \\
59540 \\
59540\end{array}$ & $\begin{array}{r}5928 \\
5923 \\
5920 \\
5918 \\
5915 \\
-\end{array}$ & $\begin{array}{l}38310 \\
38340 \\
38340 \\
38290 \\
38310 \\
38310\end{array}$ & $\begin{array}{l}68 \\
53 \\
42 \\
36 \\
39\end{array}$ & $\begin{array}{l}30 \\
30 \\
30 \\
30 \\
31 \\
31\end{array}$ & $\begin{array}{l}60 \\
80 \\
70 \\
90 \\
60 \\
90\end{array}$ & $\begin{array}{l}43 \\
39 \\
38\end{array}$ \\
\hline$A X$ & $\begin{array}{r}67030 \\
66570 \\
4735.74 \\
+630\end{array}$ & $\begin{array}{r}67310 \\
66710 \\
4735,83 \\
+260\end{array}$ & $\begin{array}{r}68410 \\
67250 \\
4736.22 \\
+1120\end{array}$ & $\begin{array}{r}68410 \\
58100 \\
4732.57 \\
-9930\end{array}$ & $\begin{array}{r}59230 \\
58480 \\
4732.86 \\
+750\end{array}$ & $\begin{array}{r}59540 \\
59230 \\
4732.98 \\
+310\end{array}$ & $\begin{array}{r}59750 \\
59150 \\
4732.83 \\
-390\end{array}$ & $\begin{array}{r}58480 \\
38290 \\
4723.40 \\
-20840\end{array}$ & $\begin{array}{r}38330 \\
3600 \\
4687.08 \\
-34380\end{array}$ & $\begin{array}{r}31720 \\
3740 \\
471971 \\
+27790\end{array}$ & $\begin{array}{r}44490 \\
32250 \\
4726.52 \\
+12770\end{array}$ & $\begin{array}{r}48340 \\
37290 \\
4722.85 \\
-7200\end{array}$ \\
\hline
\end{tabular}

CAL YR 1995 MAX 37070 MIN 19910 (ti) -26270

$\begin{array}{lllll}\text { CAL YR } 1995 & \text { MAX } 37070 & \text { MIN } 19910 & \text { (†) } & -26270 \\ \text { WTR YR } 1996 & \text { MAX } 43320 & \text { MIN } & 5190 \text { (†) } & -29110\end{array}$

(†) ELEVATION, IN FEET, AT END OF MONTH.

(†) CEANGE IN CONTENTS, IN ACRE-FEET. 
RIO GRANDE BASIN

08382830 PECOS RIVER BELOW SANTA ROSA DAM, NM

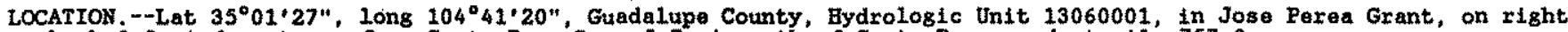
bank 0.2 mi domstream from Santa Rosa Dam, 5.7 mi north of Santa Rosa, and at mile 757.0 ,

DRATNAGE AREA. $--2,430 \mathrm{mi}^{2}$, approximately.

PERTOD OF RECORD. --Jantuary 1980 to current year.

GAGE.--Water-stage recorder. Elevation of gage $1 \mathrm{~s} 4,640 \mathrm{ft}$ above National Geodette Vertical Datum of 1929 , from topographic map. Prior to Oct. 31, 1980, at datum about 1.2 ft higher. Prior to Mar. 26 , 1982 , at site 195 ft upstream at datum 2.36 ft higher.

REMARKS.--Records good except for estimated datly discharges, which are poor. Flow completely regulated by Santa Rosa Lake (08382810) 0.2 ml upstream since Apxil 1980 . Diversions and ground-water withdrawals for irrigation of about 12000 acres, 1959 determination unstream from station. Several observations of pater temperatures were made during the year. U.S. Army Corps of Engineers satellite telemeter at station. No flow for many days.

DISCAARGE, CUBIC FEET PKR SECOND, WATER YEAR OCTOBER 1995 TO SEPTEMBER 1996

\begin{tabular}{|c|c|c|c|c|c|c|c|c|c|c|c|}
\hline OCT & NOV & DEC & JAN & FEB & MAR & AER & MAY & JUN & JUL & AUG & SEP \\
\hline $\begin{array}{r}.57 \\
.97 \\
.59 \\
.51 \\
.44\end{array}$ & $\begin{array}{l}.26 \\
.26 \\
.23 \\
.17 \\
.17\end{array}$ & $\begin{array}{l}.81 \\
.85 \\
.83 \\
.71 \\
.58\end{array}$ & $\begin{array}{l}363.0 \\
617 \\
609 \\
604\end{array}$ & $\begin{array}{r}0.43 \\
0.43 \\
0.43 \\
0.43 \\
.43\end{array}$ & $\begin{array}{l}.26 \\
.26 \\
.26 \\
.26 \\
.28\end{array}$ & $\begin{array}{l}.26 \\
.26 \\
.27 \\
.31 \\
.27\end{array}$ & $\begin{array}{l}354 \\
654 \\
687 \\
710 \\
703\end{array}$ & $\begin{array}{l}.35 \\
.37 \\
.37 \\
.37 \\
.37\end{array}$ & $\begin{array}{r}278 \\
.91 \\
.89 \\
.89 \\
.85\end{array}$ & $\begin{array}{l}.05 \\
.00 \\
.00 \\
.00 \\
.00\end{array}$ & $\begin{array}{l}.24 \\
.21 \\
.21 \\
.21 \\
.21\end{array}$ \\
\hline $\begin{array}{l}.30 \\
.17 \\
.00 \\
.00 \\
.00\end{array}$ & $\begin{array}{l}.15 \\
.22 \\
.43 \\
.61 \\
.62\end{array}$ & $\begin{array}{c}.58 \\
.55 \\
.57 \\
5.9 \\
.64\end{array}$ & $\begin{array}{l}604 \\
605 \\
603 \\
602 \\
606\end{array}$ & $\begin{array}{r}.43 \\
.43 \\
.43 \\
.43 \\
.43\end{array}$ & $\begin{array}{l}.25 \\
.25 \\
.24 \\
.24 \\
.23\end{array}$ & $\begin{array}{l}.26 \\
.29 \\
.33 \\
.29 \\
.26\end{array}$ & $\begin{array}{l}700 \\
698 \\
697 \\
695 \\
686\end{array}$ & $\begin{array}{r}.34 \\
.31 \\
.31 \\
.323^{.31}\end{array}$ & $\begin{array}{l}.68 \\
.68 \\
.68 \\
.49 \\
.45\end{array}$ & $\begin{array}{l}.00 \\
.00 \\
.11 \\
.01 \\
.00\end{array}$ & $\begin{array}{l}.21 \\
.26 \\
.25 \\
.21 \\
.21\end{array}$ \\
\hline $\begin{array}{l}.00 \\
.00 \\
.00 \\
.00 \\
.00\end{array}$ & $\begin{array}{l}.61 \\
.59 \\
.62 \\
.64 \\
.68\end{array}$ & $\begin{array}{l}.67 \\
.65 \\
.68 \\
.68 \\
.68\end{array}$ & $\begin{array}{r}473 \\
122 \\
\\
.40 \\
.37 \\
.37\end{array}$ & $\begin{array}{r}.43 \\
.43 \\
.43 \\
.43 \\
.43\end{array}$ & $\begin{array}{l}.29 \\
.31 \\
.28 \\
.29 \\
.29\end{array}$ & $\begin{array}{l}.26 \\
.26 \\
.26 \\
.26 \\
.29\end{array}$ & $\begin{array}{l}688 \\
686 \\
681 \\
682 \\
682\end{array}$ & $\begin{array}{r}968 \\
1110 \\
1100 \\
1170 \\
1200\end{array}$ & $\begin{array}{l}.02 \\
.00 \\
.20 \\
.07 \\
.00\end{array}$ & $\begin{array}{l}.00 \\
.00 \\
.00 \\
.00 \\
.02\end{array}$ & $\begin{array}{l}.21 \\
.27 \\
.26 \\
.31 \\
.23\end{array}$ \\
\hline $\begin{array}{l}.00 \\
.00 \\
.00 \\
.00 \\
.00\end{array}$ & $\begin{array}{l}.72 \\
.74 \\
.86 \\
.81 \\
.75\end{array}$ & $\begin{array}{l}.68 \\
.60 \\
.59 \\
.59 \\
.59\end{array}$ & $\begin{array}{l}.33 \\
.31 \\
.31 \\
.31 \\
.34\end{array}$ & $\begin{array}{l}.43 \\
.43 \\
.41 \\
.37 \\
.37\end{array}$ & $\begin{array}{l}.26 \\
.26 \\
.25 \\
.21 \\
.21\end{array}$ & $\begin{array}{l}.31 \\
.31 \\
.34 \\
.33 \\
.29\end{array}$ & $\begin{array}{r}263 \\
.48 \\
.51 \\
.51 \\
.48\end{array}$ & $\begin{array}{l}1180 \\
1170 \\
1150 \\
1170 \\
1090\end{array}$ & $\begin{array}{l}.00 \\
.00 \\
.00 \\
.01 \\
.00\end{array}$ & $\begin{array}{l}.04 \\
.04 \\
.04 \\
.04 \\
.04\end{array}$ & $\begin{array}{r}.24 \\
.31 \\
.31 \\
.31 \\
.28\end{array}$ \\
\hline $\begin{array}{l}.02 \\
.07 \\
.14 \\
.17 \\
.18\end{array}$ & $\begin{array}{r}.73 \\
.77 \\
.74 \\
.77 \\
.82\end{array}$ & $\begin{array}{r}.59 \\
.59 \\
.59 \\
.57 \\
.51\end{array}$ & $\begin{array}{l}.37 \\
.37 \\
.37 \\
.37 \\
.37\end{array}$ & $\begin{array}{l}.37 \\
.37 \\
.37 \\
.37 \\
.37\end{array}$ & $\begin{array}{l}.21 \\
.23 \\
.27 \\
.26 \\
.26\end{array}$ & $\begin{array}{l}.26 \\
.27 \\
.31 \\
.31 \\
.31\end{array}$ & $\begin{array}{l}.44 \\
.39 \\
.37 \\
.66 \\
.43\end{array}$ & $\begin{array}{l}819 \\
670 \\
661 \\
526 \\
587\end{array}$ & $\begin{array}{l}.00 \\
.07 \\
.09 \\
.00 \\
.00\end{array}$ & $\begin{array}{l}.04 \\
.04 \\
.04 \\
.07 \\
.12\end{array}$ & $\begin{array}{l}.31 \\
410^{.31} \\
777 \\
835\end{array}$ \\
\hline $\begin{array}{r}.23 \\
.24 \\
.21 \\
.23 \\
.26 \\
.22\end{array}$ & $\begin{array}{r}.81 \\
.86 \\
.92 \\
1.0 \\
.89 \\
-\end{array}$ & $\begin{array}{l}.51 \\
.51 \\
.58 \\
.56 \\
.56 \\
.53\end{array}$ & $\begin{array}{l}.37 \\
.37 \\
.37 \\
.37 \\
.37 \\
.44\end{array}$ & $\begin{array}{r}.30 \\
0.26 \\
0.26 \\
0.26 \\
. .- \\
. .-\end{array}$ & $\begin{array}{l}.26 \\
.24 \\
.24 \\
.24 \\
.24 \\
.26\end{array}$ & $\begin{array}{r}.33 \\
.34 \\
.35 \\
.31 \\
.37 \\
.0\end{array}$ & $\begin{array}{l}.43 \\
.38 \\
.37 \\
.26 \\
.17 \\
.26\end{array}$ & $\begin{array}{l}761 \\
766 \\
768 \\
743 \\
777 \\
\end{array}$ & $\begin{array}{l}.08 \\
.05 \\
.00 \\
.00 \\
.00 \\
.16\end{array}$ & $\begin{array}{l}.16 \\
.14 \\
.18 \\
.17 \\
.17 \\
.21\end{array}$ & $\begin{array}{l}855 \\
853 \\
851 \\
848 \\
639 \\
\end{array}$ \\
\hline $\begin{array}{l}\text { TOTAL } \\
\text { MEAN } \\
\text { MAX } \\
\text { MIN } \\
\text { AC }- \text { TT }\end{array}$ & $\begin{array}{r}18.45 \\
.61 \\
1.0 \\
.15 \\
37\end{array}$ & $\begin{array}{r}24.53 \\
.79 \\
5.9 \\
.51 \\
49\end{array}$ & $\begin{array}{r}5821.88 \\
188 \\
617 \\
.31 \\
11550\end{array}$ & $\begin{array}{r}11.39 \\
.39 \\
.43 \\
.26 \\
23\end{array}$ & $\begin{array}{r}7.89 \\
.25 \\
.31 \\
.21 \\
16\end{array}$ & $\begin{array}{r}8.87 \\
.30 \\
.37 \\
.26 \\
18\end{array}$ & $\begin{array}{r}10272.16 \\
331 \\
710 \\
20370\end{array}$ & $\begin{array}{r}18712.10 \\
624 \\
1200 \\
37121\end{array}$ & $\begin{array}{r}285.27 \\
9.20 \\
278 \\
.00 \\
566\end{array}$ & $\begin{array}{r}1.73 \\
.056 \\
.21 \\
.00 \\
3.4\end{array}$ & $\begin{array}{r}6073.57 \\
202 \\
855 \\
.21 \\
12050\end{array}$ \\
\hline
\end{tabular}

STATISTICS OF MONTBLY MEAN DATA FOR WATER YEARS 1980 - 1996, BY WATER YEAR (WY)

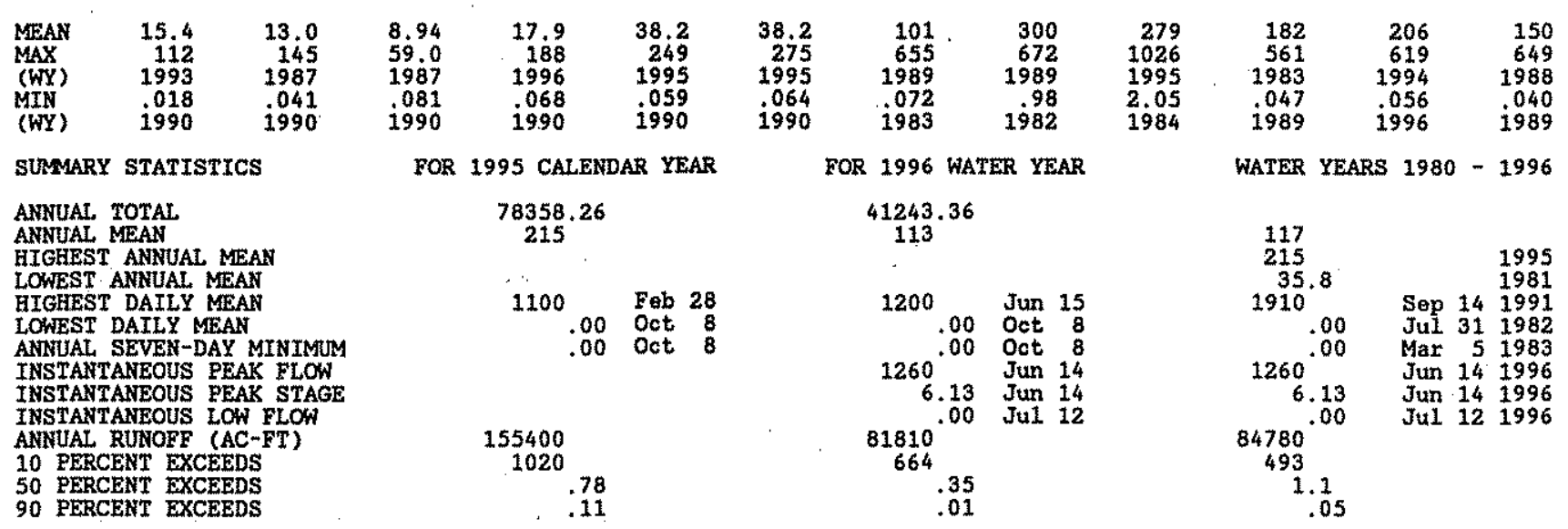

Estimated 
RIO GRANDE BASTN

08383000 PECOS RIVER AT SANTA ROSA, NM WATER-QUALITY RECORDS

LOCATION, --Samples collected $0.6 \mathrm{mi}$ downstream from discharge station.

PERIOD OF RECORD,--Water yearg 1905-07, 1959 to current yeax.

WATER QUALITX DATA, WATER YEAR OCTOBER 1995 TO SEPTEMBER 1996

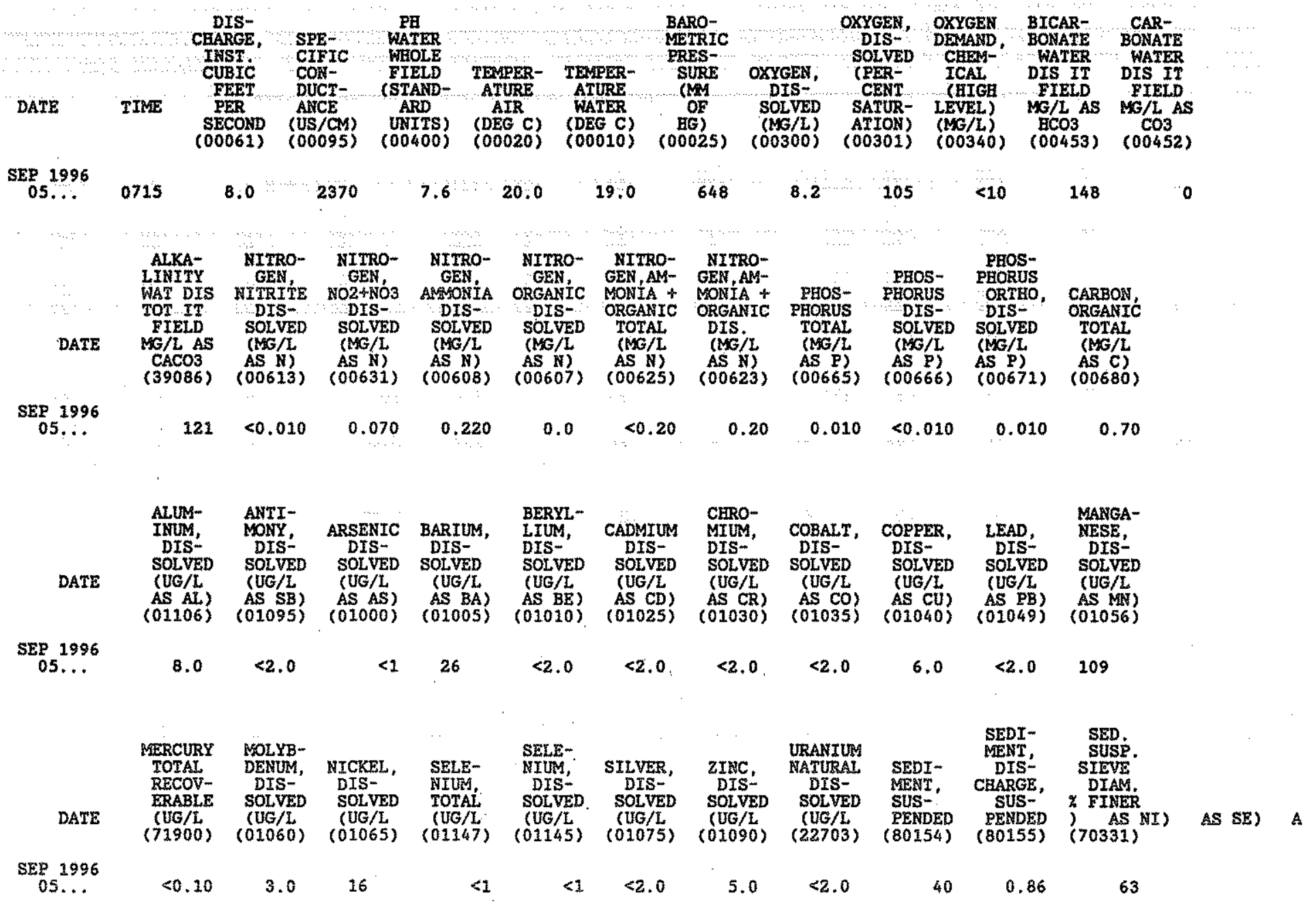


RIO GRANDE BASIN

08383500 PECOS RIVER NEAR PUERTO DE LUNA, NM

LOCATION -Lat $34^{\circ} 43^{\circ} 48^{\prime \prime}$, long $104^{\circ} 31^{\prime} 28^{\prime \prime}$, in NEKSEKNWk sec.20, T.6 N., R.23 E., Guadalupe County, Hydrologic Unit 13060001 on left bank $9.0 \mathrm{mi}$ southeast of Puerto de Luna, $17.5 \mathrm{mi}$ upstream from Sumer Dam, and at mile 719.5 . DRAINAGE AREA. $--3,970 \mathrm{mt}^{2}$, approximately (contributing area).

PERIOD OF RECORD. - -April 1938 to current year.

\section{WATER-DISCEARGE RECORDS}

REVISED RECORDS. - WSP 1512: 1939

GE. - Water-stage recorder and concrete control. Elevation of gage 1 s $4,311.34$ ft above National Geodetic Vertical Datum of 1929. Fritor to Apr. 15, 1954, datum 1.0 ft higher.

REMARKS - Water-discharge records good. Flow regulated by Santa Rosa lake (station 08382810 ) 37.7 mi upstream sinco

Apri1 1980. Diversions for irrigation of about 10,280 acres, 1970 determination, upstream from station.

Dischargo represents inflow to Lake Sumer. Several observations of water temperature were made during the year. Bureau of Reclamation satelilte telemeter at station.

AVERAGE DISCHARGE. -41 years (water years 1939-79), $209 \mathrm{ft} / \mathrm{s}, 151,400$ acre-ft/yr, prior to completion of Santa Rosa Dam.

EXTREMES OUTSIDE PERIOD OF RECORD,--Maximum flood since at least 1886 occurred June 2 , 1937 , when peak at Santa Rosa was $55,200 \mathrm{ft}^{3} / \mathrm{s}$, and peak inflow to Lake Sumer was about $75,000 \mathrm{ft}^{3} / \mathrm{s}$. Flood of July 24 , 1895 , was reported as "highest in 10 years." Other major floods occurred on June 9, 1903, Sept. 30, 1904, and May 1, 1914. DISCHARGE, CUBIC FEET PER SECOND, WATER YEAR OCTOBER 1995 TO SEPTEMBER 1996

\begin{tabular}{|c|c|c|c|c|c|c|c|c|c|c|c|c|}
\hline DAY & $\cot$ & HOV & $\mathrm{DEC}$ & JAN & FEB & MAR & APR & MAY & JUN & JUL & AUG & SEP \\
\hline $\begin{array}{l}1 \\
2 \\
3 \\
4 \\
5\end{array}$ & $\begin{array}{r}81 \\
226 \\
88 \\
82 \\
76\end{array}$ & $\begin{array}{l}80 \\
80 \\
83 \\
86 \\
86\end{array}$ & $\begin{array}{l}89 \\
90 \\
91 \\
91 \\
88\end{array}$ & $\begin{array}{r}88 \\
90 \\
246 \\
602 \\
613\end{array}$ & $\begin{array}{r}93 \\
95 \\
105 \\
94 \\
93\end{array}$ & $\begin{array}{l}82 \\
83 \\
81 \\
80 \\
80\end{array}$ & $\begin{array}{l}69 \\
62 \\
65 \\
62 \\
66\end{array}$ & $\begin{array}{r}72 \\
519 \\
646 \\
694 \\
709\end{array}$ & $\begin{array}{l}69 \\
70 \\
64 \\
61 \\
61\end{array}$ & $\begin{array}{r}830 \\
204 \\
124 \\
96 \\
83\end{array}$ & $\begin{array}{l}74 \\
60 \\
69 \\
68 \\
59\end{array}$ & $\begin{array}{l}82 \\
81 \\
76 \\
71 \\
70\end{array}$ \\
\hline $\begin{array}{r}6 \\
7 \\
8 \\
9 \\
10\end{array}$ & $\begin{array}{l}77 \\
78 \\
75 \\
75 \\
71\end{array}$ & $\begin{array}{l}85 \\
85 \\
86 \\
85 \\
83\end{array}$ & $\begin{array}{l}89 \\
90 \\
89 \\
88 \\
89\end{array}$ & $\begin{array}{l}619 \\
627 \\
633 \\
640 \\
643\end{array}$ & $\begin{array}{l}92 \\
89 \\
89 \\
89 \\
88\end{array}$ & $\begin{array}{l}79 \\
82 \\
81 \\
82 \\
83\end{array}$ & $\begin{array}{l}67 \\
69 \\
71 \\
66 \\
62\end{array}$ & $\begin{array}{l}717 \\
718 \\
723 \\
723 \\
724\end{array}$ & $\begin{array}{l}62 \\
56 \\
55 \\
56 \\
56\end{array}$ & $\begin{array}{r}76 \\
76 \\
72 \\
77 \\
489\end{array}$ & $\begin{array}{l}56 \\
58 \\
66 \\
98 \\
97\end{array}$ & $\begin{array}{r}68 \\
226 \\
177 \\
77 \\
73\end{array}$ \\
\hline $\begin{array}{l}11 \\
12 \\
13 \\
14 \\
15\end{array}$ & $\begin{array}{l}71 \\
73 \\
74 \\
74 \\
75\end{array}$ & $\begin{array}{l}84 \\
85 \\
81 \\
85 \\
82\end{array}$ & $\begin{array}{l}89 \\
87 \\
87 \\
87 \\
88\end{array}$ & $\begin{array}{l}645 \\
413 \\
189 \\
120 \\
112\end{array}$ & $\begin{array}{l}87 \\
86 \\
87 \\
87 \\
86\end{array}$ & $\begin{array}{l}82 \\
81 \\
79 \\
78 \\
80\end{array}$ & $\begin{array}{l}63 \\
67 \\
67 \\
73 \\
68\end{array}$ & $\begin{array}{l}730 \\
733 \\
734 \\
739 \\
734\end{array}$ & $\begin{array}{r}537 \\
1080 \\
1110 \\
1260 \\
1270\end{array}$ & $\begin{array}{l}429 \\
298 \\
138 \\
171 \\
128\end{array}$ & $\begin{array}{l}69 \\
64 \\
60 \\
58 \\
90\end{array}$ & $\begin{array}{r}69 \\
161 \\
163 \\
148 \\
154\end{array}$ \\
\hline $\begin{array}{l}16 \\
17 \\
18 \\
19 \\
20\end{array}$ & $\begin{array}{l}75 \\
75 \\
74 \\
74 \\
74\end{array}$ & $\begin{array}{l}81 \\
82 \\
82 \\
83 \\
83\end{array}$ & $\begin{array}{r}88 \\
95 \\
101 \\
94 \\
92\end{array}$ & $\begin{array}{r}107 \\
108 \\
103 \\
106 \\
96\end{array}$ & $\begin{array}{l}83 \\
85 \\
85 \\
83 \\
83\end{array}$ & $\begin{array}{l}82 \\
83 \\
84 \\
84 \\
85\end{array}$ & $\begin{array}{l}67 \\
69 \\
66 \\
66 \\
70\end{array}$ & $\begin{array}{r}703 \\
193 \\
103 \\
86 \\
78\end{array}$ & $\begin{array}{l}1250 \\
1190 \\
1110 \\
1110 \\
1230\end{array}$ & $\begin{array}{r}116 \\
116 \\
88 \\
80 \\
94\end{array}$ & $\begin{array}{r}101 \\
63 \\
60 \\
59 \\
59\end{array}$ & $\begin{array}{l}91 \\
83 \\
78 \\
76 \\
67\end{array}$ \\
\hline $\begin{array}{l}21 \\
22 \\
23 \\
24 \\
25\end{array}$ & $\begin{array}{l}75 \\
75 \\
75 \\
75 \\
76\end{array}$ & $\begin{array}{l}83 \\
83 \\
83 \\
83 \\
81\end{array}$ & $\begin{array}{l}92 \\
92 \\
91 \\
92 \\
91\end{array}$ & $\begin{array}{l}94 \\
94 \\
93 \\
92 \\
93\end{array}$ & $\begin{array}{l}83 \\
82 \\
83 \\
85 \\
83\end{array}$ & $\begin{array}{l}85 \\
83 \\
75 \\
69 \\
69\end{array}$ & $\begin{array}{l}71 \\
69 \\
78 \\
72 \\
72\end{array}$ & $\begin{array}{l}73 \\
70 \\
65 \\
62 \\
63\end{array}$ & $\begin{array}{l}922 \\
692 \\
674 \\
655 \\
484\end{array}$ & $\begin{array}{l}82 \\
73 \\
63 \\
60 \\
68\end{array}$ & $\begin{array}{l}55 \\
56 \\
60 \\
64 \\
95\end{array}$ & $\begin{array}{r}67 \\
68 \\
68 \\
600 \\
777\end{array}$ \\
\hline $\begin{array}{l}26 \\
27 \\
28 \\
29 \\
30 \\
31\end{array}$ & $\begin{array}{l}74 \\
73 \\
76 \\
77 \\
76 \\
76\end{array}$ & $\begin{array}{r}78 \\
78 \\
82 \\
86 \\
87 \\
---\end{array}$ & $\begin{array}{l}90 \\
90 \\
93 \\
94 \\
90 \\
89\end{array}$ & $\begin{array}{r}92 \\
101 \\
92 \\
91 \\
91 \\
90\end{array}$ & $\begin{array}{r}82 \\
80 \\
77 \\
77 \\
-\cdots \\
--\end{array}$ & $\begin{array}{l}72 \\
73 \\
73 \\
72 \\
75 \\
74\end{array}$ & $\begin{array}{r}72 \\
69 \\
69 \\
67 \\
69 \\
---\end{array}$ & $\begin{array}{l}66 \\
61 \\
62 \\
73 \\
68 \\
66\end{array}$ & $\begin{array}{l}766 \\
839 \\
91.3 \\
850 \\
851 \\
-\end{array}$ & $\begin{array}{r}164 \\
123 \\
51 \\
45 \\
46 \\
60\end{array}$ & $\begin{array}{r}138 \\
341 \\
101 \\
86 \\
78 \\
208\end{array}$ & $\begin{array}{l}867 \\
896 \\
910 \\
922 \\
899 \\
--m\end{array}$ \\
\hline $\begin{array}{l}\text { TOTAL } \\
\text { MEAN } \\
\text { MAX } \\
\text { MIN } \\
\text { AC-FT }\end{array}$ & $\begin{array}{r}2496 \\
80.5 \\
226 \\
71 \\
4950\end{array}$ & $\begin{array}{r}2491 \\
83.0 \\
87 \\
78 \\
4940\end{array}$ & $\begin{array}{r}2806 \\
90.5 \\
101 \\
87 \\
5570\end{array}$ & $\begin{array}{r}7823 \\
252 \\
645 \\
88 \\
15520\end{array}$ & $\begin{array}{r}2511 \\
86.6 \\
105 \\
77 \\
4980\end{array}$ & $\begin{array}{r}2451 \\
79.1 \\
85 \\
69 \\
4860\end{array}$ & $\begin{array}{r}2043 \\
68.1 \\
78 \\
62 \\
4050\end{array}$ & $\begin{array}{r}11807 \\
381 \\
739 \\
61 \\
23420\end{array}$ & $\begin{array}{r}19403 \\
647 \\
1270 \\
55 \\
38490\end{array}$ & $\begin{array}{r}4620 \\
149 \\
830 \\
45 \\
9160\end{array}$ & $\begin{array}{r}2670 \\
86.1 \\
341 \\
55 \\
5300\end{array}$ & $\begin{array}{r}8165 \\
272 \\
922 \\
67 \\
16200\end{array}$ \\
\hline
\end{tabular}

STATISTICS OF MONTHLY MEAN DATA FOR WATER YEARS 1980 - 1996, BY WATER YEAR (WY)

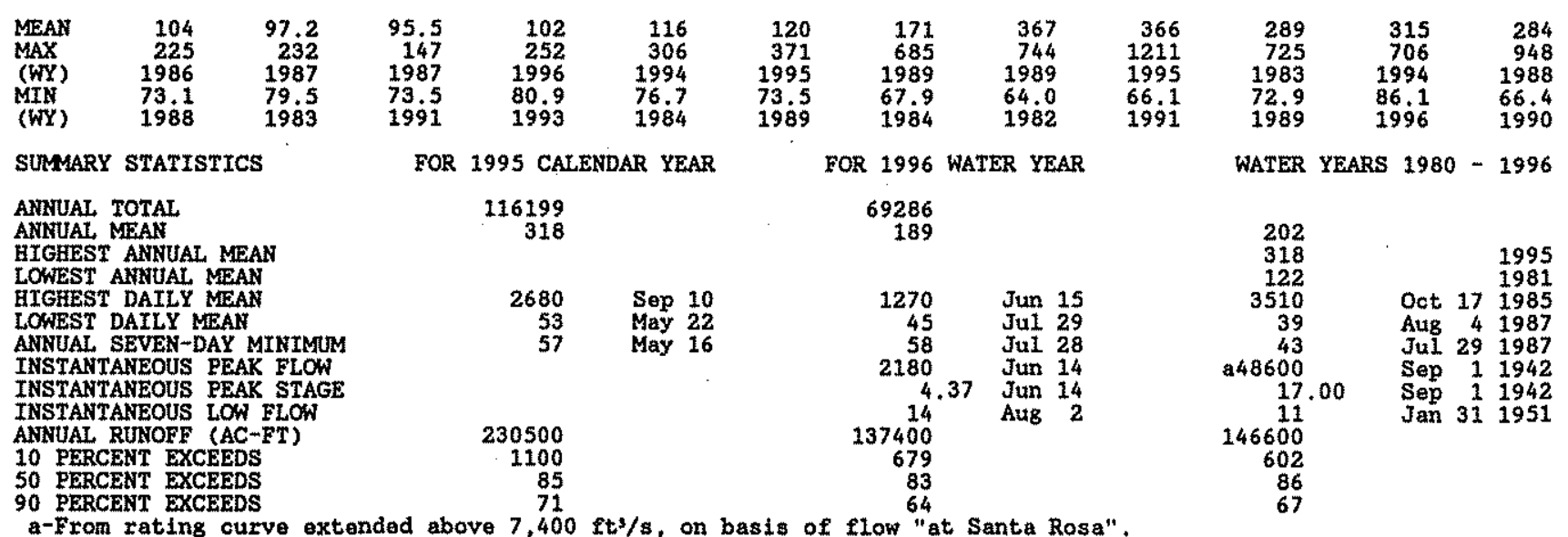

\footnotetext{
a-From rating curve extended above $7,400 \mathrm{ft} / \mathrm{s}$, on basis of flow "at Santa Rosa"
} 


\section{RIO GRANDE BASIN}

08383500 PECOS RIVER NEAR PUERTO DE LUNA, MM -- Continued

WATER-QUALITY RECORDS

PERIOD OF RECORD.--Watex years 1937-66, 1972 to current year.

WATER QUALITY DATA, WATER YEAR OCTOBER 1995 TO SEPTEMBER 1996

\begin{tabular}{|c|c|c|c|c|c|c|c|c|c|c|c|}
\hline DATE & TIME & $\begin{array}{c}\text { DYS- } \\
\text { CBARGE, } \\
\text { INST } \\
\text { CUBIC } \\
\text { FEET } \\
\text { PERR } \\
\text { SECOND } \\
\text { (00061) }\end{array}$ & $\begin{array}{l}\text { SPE- } \\
\text { CIFIC } \\
\text { CON- } \\
\text { DUCT- } \\
\text { ANCE } \\
\text { (US/CM) } \\
(00095)\end{array}$ & $\begin{array}{c}\text { PB } \\
\text { WATER } \\
\text { WHOLE } \\
\text { FIELD } \\
\text { (STARD- } \\
\text { ARD } \\
\text { UNITS) } \\
(00400)\end{array}$ & $\begin{array}{c}\text { TEMPER- } \\
\text { ATURE } \\
\text { AIR } \\
\text { (DEGC) } \\
(00020)\end{array}$ & $\begin{array}{l}\text { TEMPER- } \\
\text { ATURE } \\
\text { WATEER } \\
\text { (DEG C) } \\
(00010)\end{array}$ & $\begin{array}{c}\text { BARO- } \\
\text { METRIC } \\
\text { PRES- } \\
\text { SURE } \\
\text { (MAS } \\
\text { OF } \\
\text { BG) } \\
(00025)\end{array}$ & $\begin{array}{c}\text { OXYGEN, } \\
\text { DIS- } \\
\text { SOLVED } \\
\text { (MG/L) } \\
(00300)\end{array}$ & $\begin{array}{c}\text { OXYGEN, } \\
\text { DIS- } \\
\text { SOLVED } \\
\text { (PER- } \\
\text { CERT } \\
\text { SATUR- } \\
\text { ATION) } \\
\text { (00301) }\end{array}$ & $\begin{array}{l}\text { OXYGEN } \\
\text { DEMAND, } \\
\text { CEEM- } \\
\text { ICAL } \\
\text { (BIGH } \\
\text { LEVELL) } \\
(\text { MG/L) } \\
(00340)\end{array}$ & $\begin{array}{l}\text { COLI- } \\
\text { FORM, } \\
\text { FECAL, } \\
0.7 \\
\text { UR' } \\
\text { (COLS } \\
\text { (100 ML) } \\
(31625)\end{array}$ \\
\hline \multirow{4}{*}{$\begin{array}{c}\text { DEC } 1995 \\
05.996 \\
\text { MAR } 1996 \\
06 \ldots \\
\text { JLL... } \\
09 . . \\
\text { SEP } \\
.04 \ldots\end{array}$} & 1115 & 88 & 2920 & 8.0 & 13.5 & 9.0 & 658 & 10.3 & 104 & $<10$ & K1 \\
\hline & 1245 & 80 & 297 & 8.0 & 0.0 & 4.0 & 652 & 10.1 & 90 & $<10$ & K9 \\
\hline & 1745 & 80 & 2630 & 8.0 & 30.5 & 29.0 & 653 & 11.0 & 170 & $<10$ & $\therefore<3$ \\
\hline & 1800 & 71 & 2640 & 8.0 & 29.5 & 28.0 & 653 & 10.2 & 154 & $<10$ & $-\infty$ \\
\hline DATE & $\begin{array}{l}\text { STREP- } \\
\text { TOCOCCI } \\
\text { FECAL, } \\
\text { KF AGAR } \\
\text { (COLS. } \\
\text { PER } \\
100 \text { ML) } \\
(31673)\end{array}$ & $\begin{array}{l}\text { HARD- } \\
\text { NESS } \\
\text { TOTAL } \\
\text { (MG/L } \\
\text { AS } \\
\text { CACO3) } \\
(00900)\end{array}$ & $\begin{array}{l}\text { HARD- } \\
\text { NESS } \\
\text { NONCARB } \\
\text { DISSOLV } \\
\text { FLD, AS } \\
\text { CACO3 } \\
(M G / L) \\
(00904)\end{array}$ & $\begin{array}{l}\text { CALCIUM } \\
\text { DIS- } \\
\text { SOLVED } \\
\text { (MSG/L } \\
\text { AS CA }) \\
(00915)\end{array}$ & $\begin{array}{c}\text { MAGNE- } \\
\text { SIUM, } \\
\text { DIS- } \\
\text { SOLVED } \\
\text { (MG/L } \\
\text { AS MSG) } \\
\text { (00925) }\end{array}$ & $\begin{array}{c}\text { SODIUM, } \\
\text { DIS- } \\
\text { SOLVED } \\
\text { (MG /LA) } \\
\text { AS NA) } \\
(00930)\end{array}$ & $\begin{array}{c}\text { SODIUM } \\
\text { AD- } \\
\text { SORF- } \\
\text { TION } \\
\text { RATIO } \\
(00931)\end{array}$ & $\begin{array}{c}\text { POTAS- } \\
\text { SIUM, } \\
\text { DIS- } \\
\text { SOLVED } \\
\text { (KG/L } \\
\text { AS K) } \\
(00935)\end{array}$ & $\begin{array}{l}\text { BICAR- } \\
\text { BONATE } \\
\text { WATER } \\
\text { DIS IT } \\
\text { FIELD } \\
\text { MG/L AS } \\
\text { HCO3 } \\
(00453)\end{array}$ & $\begin{array}{c}\text { CAR- } \\
\text { BONATE } \\
\text { WATER } \\
\text { DIS IT } \\
\text { FIELD } \\
\text { MG/L AS } \\
\text { CO3 } \\
\text { (00452) }\end{array}$ & $\begin{array}{l}\text { ALKA- } \\
\text { LINITY } \\
\text { WAT DIS } \\
\text { TOT IT } \\
\text { FIELD } \\
\text { MG/L AS } \\
\text { CACO3 } \\
\text { (39086) }\end{array}$ \\
\hline $\begin{array}{l}\text { DEC } 1995 \\
051996 \\
M A R\end{array}$ & $\mathrm{~K} 4$ & 1700 & 1600 & 560 & 71 & 98 & 1 & 2.2 & 145 & 0 & 119 \\
\hline गน & $\mathrm{K} 2$ & 1700 & 1600 & 560 & 71 & 98 & 1 & 2.2 & 144 & 0 & 118 \\
\hline SEP & $\times 21$ & 1500 & 1400 & 510 & 64 & 86 & 1 & 2.6 & 112 & 0 & 92 \\
\hline $04 \ldots$ & $m$ & 1700 & 1600 & 570 & 70 & 100 & 1 & 2.4 & 122 & 0 & 100 \\
\hline DATE & $\begin{array}{c}\text { ALKA- } \\
\text { LINITY } \\
\text { LAB } \\
(\text { NGS/L } \\
\text { AS } \\
\text { CACO3) } \\
(90410)\end{array}$ & $\begin{array}{c}\text { SULFATE } \\
\text { DIS- } \\
\text { SOLVED } \\
\text { (NGG/L } \\
\text { AS SO4) } \\
(00945)\end{array}$ & $\begin{array}{l}\text { CELO- } \\
\text { RIDE, } \\
\text { DIS- } \\
\text { SOLVED } \\
\text { (MG/L } \\
\text { AS CL) } \\
(00940)\end{array}$ & $\begin{array}{l}\text { FLUO- } \\
\text { RIDE, } \\
\text { DIS- } \\
\text { SOLVED } \\
\text { (MG/L } \\
\text { AS F) } \\
(00950)\end{array}$ & $\begin{array}{c}\text { SILICA, } \\
\text { DIS- } \\
\text { SOLVED } \\
\text { (MG/L } \\
\text { AS } \\
\text { SIO2) } \\
(00955)\end{array}$ & $\begin{array}{c}\text { SOLIDS, } \\
\text { SUM OF } \\
\text { CONSTI- } \\
\text { TUENTS, } \\
\text { DIS- } \\
\text { SOLVED } \\
\text { (i.G /L) } \\
(70301)\end{array}$ & $\begin{array}{c}\text { NITRO- } \\
\text { GEN, } \\
\text { NITRATE } \\
\text { DIS-- } \\
\text { SOLVED } \\
\text { (MG/L } \\
\text { ASN } \\
(00618)\end{array}$ & $\begin{array}{c}\text { NITRO- } \\
\text { GEN, } \\
\text { NITRITE } \\
\text { DIS- } \\
\text { SOLVED } \\
\text { (MG/L } \\
\text { AS N) } \\
(00613)\end{array}$ & $\begin{array}{c}\text { NITRO- } \\
\text { GEN, } \\
\text { NO2+NO3 } \\
\text { DIS- } \\
\text { SOLVED } \\
\text { (MG/L } \\
\text { AS N) } \\
\text { (00631) }\end{array}$ & $\begin{array}{c}\text { NITRO- } \\
\text { GEN, } \\
\text { ANMONIA } \\
\text { DIS- } \\
\text { SOLVED } \\
\text { (MG/L } \\
\text { AS N } \\
(00608)\end{array}$ & $\begin{array}{c}\text { NITRO- } \\
\text { GEN, } \\
\text { ORGANIC } \\
\text { DIS- } \\
\text { SOLVED } \\
\text { (NG/L } \\
\text { AS H } \\
(00607)\end{array}$ \\
\hline $\begin{array}{l}\text { DEC } 1995 \\
05 \text { MAR } 1996\end{array}$ & 115 & 1600 & 140 & 0.50 & 13 & 2560 & - & $<0.010$ & $<0.050$ & 0.090 & 0.41 \\
\hline JuL & 121 & 1500 & 130 & 0.70 & 13 & 2450 & $-\cdots$ & $<0.010$ & $<0.050$ & 0.110 & $m$ \\
\hline $\operatorname{sep}^{09} \cdot$ & 91 & 1400 & 120 & 0.60 & 13 & 2250 & 0.130 & 0.020 & 0.150 & 0.170 & 0.03 \\
\hline $04 \ldots$ & 30 & 1600 & 140 & 0.70 & 28 & 2550 & - & $<0.020$ & 0.070 & 0.200 & 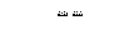 \\
\hline
\end{tabular}

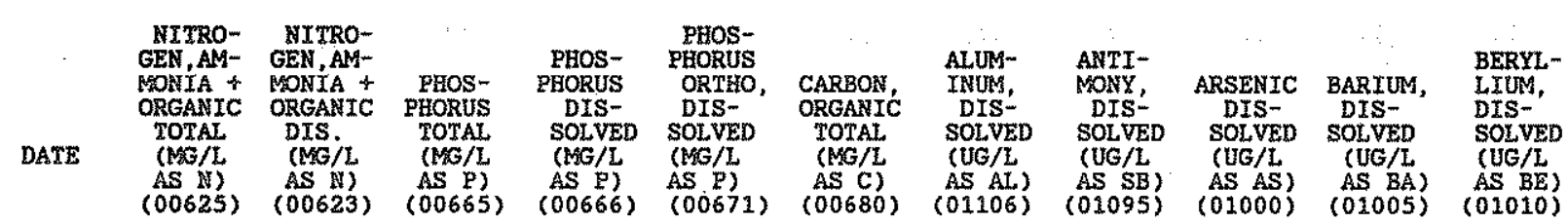

\begin{tabular}{|c|c|c|c|c|c|c|c|c|c|c|c|}
\hline $\begin{array}{l}\text { DEC } 1995 \\
055\end{array}$ & $<0.20$ & 0.50 & $<0.010$ & $<0.010^{\circ}$ & $<0.010$ & 4.3 & -- & - & -- & $\rightarrow$ & $\cdots$ \\
\hline $06 \ldots$ & $<0.20$ & $<0.20$ & $<0.010$ & $<0.010$ & $<0.010$ & 0.90 & - & -- & -- & $-m$ & - \\
\hline $09 \ldots$ & $<0.20$ & 0.20 & 0.030 & 0.020 & $<0.010$ & 1.4 & -- & -- & -- & - & -- \\
\hline & 0.30 & $<0.20$ & 0.070 & 0.010 & $<0.010$ & 2.4 & 19 & $<2.0$ & $<1$ & 58 & $<2.0$ \\
\hline
\end{tabular}


RIO GRANDE BASIN

08383500 PECOS RIVER NEAR RUERTO DE LUNA, NM -- Continued

WATER-QUALITY RECORDS

WATER QUALITY DATA, WATER YEAR OCTOBER 1995 TO SEPTEMBER 1996

\begin{tabular}{|c|c|c|c|c|c|c|c|c|c|c|c|}
\hline DATE & $\begin{array}{c}\text { BORON, } \\
\text { DIS- } \\
\text { SOLVED } \\
\text { (UG/L } \\
\text { AS B) } \\
\text { (01020) }\end{array}$ & $\begin{array}{c}\text { CADMIUM } \\
\text { DIS- } \\
\text { SOLVED } \\
\text { (UG/L } \\
\text { AS CD) } \\
\text { (01025) }\end{array}$ & $\begin{array}{l}\text { CERO- } \\
\text { MIUM, } \\
\text { DIS- } \\
\text { SOLVED } \\
\text { (UG/L } \\
\text { AS CR) } \\
\text { (01030) }\end{array}$ & $\begin{array}{c}\text { COBALT, } \\
\text { DIS- } \\
\text { SOLVED } \\
\text { (USG/L } \\
\text { AS CO) } \\
(01035)\end{array}$ & $\begin{array}{c}\text { COPPER, } \\
\text { DIS- } \\
\text { SOLVED } \\
\text { (UG/L } \\
\text { AS CU) } \\
(01040)\end{array}$ & $\begin{array}{l}\text { IRON, } \\
\text { DIS' } \\
\text { SOLVED } \\
\text { (UG/L } \\
\text { AS FE }) \\
(01046)\end{array}$ & $\begin{array}{c}\text { LEAD, } \\
\text { DIS- } \\
\text { SOLVED } \\
\text { (UG/L } \\
\text { AS PB) } \\
(01049)\end{array}$ & $\begin{array}{l}\text { MANGA- } \\
\text { NESE, } \\
\text { DIS - } \\
\text { SOLVED } \\
\text { (UG/L } \\
\text { AS MN) } \\
\text { (01056) }\end{array}$ & $\begin{array}{c}\text { MERCURY } \\
\text { TOTAL } \\
\text { RECOV- } \\
\text { ERABLE } \\
\text { (UG/L } \\
\text { AS HG) } \\
\text { (71900) }\end{array}$ & $\begin{array}{c}\text { MOLYB- } \\
\text { DENUM, } \\
\text { DIS- } \\
\text { SOLVED } \\
\text { (UG/L } \\
\text { AS MO) } \\
(01060)\end{array}$ & $\begin{array}{c}\text { NICKEL, } \\
\text { DIS- } \\
\text { SOLVED } \\
\text { (UG/L } \\
\text { AS NI) } \\
(01065)\end{array}$ \\
\hline \multirow{4}{*}{ 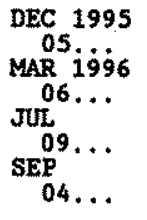 } & 110 & $-m$ & -- & -- & $-\cdots$ & $<9.0$ & - & - & $<0.10$ & -- & -- \\
\hline & 130 & -- & -- & $-\infty$ & -- & $<9.0$ & - & -- & -- & - & -- \\
\hline & 111 & - & $-\cdots$ & -- & -- & $<9.0$ & $\sim$ & -- & - & -- & -- \\
\hline & 110 & $<2.0$ & $<2.0$ & $<2.0$ & 6.0 & 21 & $<2,0$ & 7.0 & $<0.10$ & 3.0 & 15 \\
\hline \multirow{5}{*}{$\begin{array}{c}\text { DEC } 1995 \\
05 \\
\text { MAR } i 996 \\
06 \ldots \\
\text { JuL } \ldots \\
09 \ldots \\
\text { SEP } \ldots \\
04 \ldots\end{array}$} & $\begin{array}{c}\text { SELE- } \\
\text { NIUM, } \\
\text { TOTAL } \\
\text { (UG/L } \\
\text { AS SEE) } \\
\text { (01147) }\end{array}$ & $\begin{array}{l}\text { SELE- } \\
\text { NIUM, } \\
\text { DIS- } \\
\text { SOLVED } \\
\text { (UG/L } \\
\text { AS SE) } \\
(01145)\end{array}$ & $\begin{array}{c}\text { SILVER, } \\
\text { DIS- } \\
\text { SOLVED } \\
\text { (UG/L } \\
\text { AS AG) } \\
\text { (01075) }\end{array}$ & $\begin{array}{c}\text { ZINC, } \\
\text { DIS } \\
\text { SOLVED } \\
\text { (UG/L } \\
\text { AS ZN) } \\
(01090)\end{array}$ & $\begin{array}{c}\text { NITRO- } \\
\text { GEN, } \\
\text { NO2+NO3 } \\
\text { TOT. IN } \\
\text { BOT MAT } \\
\text { (MG/KG } \\
\text { AS N) } \\
(00633)\end{array}$ & $\begin{array}{l}\text { NITRO- } \\
\text { GEN, NH4 } \\
\text { TOTAL } \\
\text { IN BOT. } \\
\text { MAT. } \\
\text { (MG/KG } \\
\text { AS N } \\
\text { (00611) }\end{array}$ & $\begin{array}{l}\text { NITRO- } \\
\text { GEN, NH4 } \\
+ \text { ORG. } \\
\text { TOT IN } \\
\text { TOT MAT } \\
\text { BOT ME } \\
\text { (NS KG } \\
\text { AS N } \\
(00626)\end{array}$ & $\begin{array}{c}\text { PHOS- } \\
\text { PEORUS } \\
\text { TOTAL } \\
\text { IN BOT. } \\
\text { MAT. } \\
\text { (MG/KG } \\
\text { AS P) } \\
(00668)\end{array}$ & $\begin{array}{c}\text { ARSENTC } \\
\text { TOTAL } \\
\text { IN BOT- } \\
\text { TOM MA- } \\
\text { TERIAL } \\
\text { (UG/G } \\
\text { AS AS) } \\
(01003)\end{array}$ & $\begin{array}{c}\text { CADMIUM } \\
\text { RECOV. } \\
\text { FM BOT- } \\
\text { TOM MA- } \\
\text { TERIAL } \\
\text { (UG/G } \\
\text { AS CD) } \\
(01028)\end{array}$ & $\begin{array}{c}\text { CHRO- } \\
\text { MIUM, } \\
\text { RECOV. } \\
\text { FM BOT- } \\
\text { TOM MA- } \\
\text { TERIAL } \\
\text { (UG/G) } \\
\text { (01029) }\end{array}$ \\
\hline & $<1$ & $\because-$ & -- & $\cdots$ & $<2.0$ & 1.0 & 30 & 74 & 4 & $<1$ & 2 \\
\hline & -- & - & - & -- & -- & - & - & -- & $\cdots$ & -- & $-m$ \\
\hline & $\cdots$ & $\cdots$ & $=$ & -- & - & - & -- & $\rightarrow$ & -- & -- & $\cdots$ \\
\hline & $<1$ & $<1$ & $<2.0$ & 4.0 & $<2.0$ & 0.3 & 50 & 240 & 4 & $<1$ & 6 \\
\hline DATE & $\begin{array}{c}\text { COBALT, } \\
\text { RECOV'. } \\
\text { FM BOT- } \\
\text { TOM MA- } \\
\text { TERIAL } \\
\text { (UG /G } \\
\text { AS CO) } \\
(01038)\end{array}$ & $\begin{array}{c}\text { COPPER, } \\
\text { RECOV. } \\
\text { FM BOT- } \\
\text { TOM MA- } \\
\text { TERIAL } \\
\text { (UG/G } \\
\text { AS CU) } \\
(01043)\end{array}$ & $\begin{array}{c}\text { IRON } \\
\text { RECOV. } \\
\text { FM BOT- } \\
\text { TOM MA- } \\
\text { TERIAL } \\
\text { (UG/G } \\
\text { AS FE) } \\
(01170)\end{array}$ & $\begin{array}{c}\text { LEAD } \\
\text { RECOV. } \\
\text { FM BOT- } \\
\text { TOM MA- } \\
\text { TERIAL } \\
\text { (UG/G } \\
\text { AS PB) } \\
(01052)\end{array}$ & $\begin{array}{c}\text { MANGA- } \\
\text { NESE } \\
\text { RECOV. } \\
\text { FM BOT- } \\
\text { TOM MA- } \\
\text { TERIAL } \\
\text { (UG/G) } \\
(01053)\end{array}$ & $\begin{array}{c}\text { MERCURY } \\
\text { RECOV. } \\
\text { FH BOT- } \\
\text { TOM MA- } \\
\text { TERIAL } \\
\text { (UG/G } \\
\text { AS HG) } \\
(71921)\end{array}$ & $\begin{array}{c}\text { ZINC, } \\
\text { RECOV. } \\
\text { FM BOT- } \\
\text { TOM MA- } \\
\text { TERIAL } \\
\text { (UG/G } \\
\text { AS ZN) } \\
(01093)\end{array}$ & $\begin{array}{l}\text { URANTUM } \\
\text { NATURAL } \\
\text { DIS- } \\
\text { SOLVED } \\
\text { (UG/L } \\
\text { AS U) } \\
(22703)\end{array}$ & $\begin{array}{l}\text { SEDI- } \\
\text { MENT, } \\
\text { SUS- } \\
\text { PENDED } \\
\text { (MG/L) } \\
(80154)\end{array}$ & $\begin{array}{c}\text { SEDI- } \\
\text { MENT, } \\
\text { DIS- } \\
\text { CHARGE, } \\
\text { SUS- } \\
\text { PENDED } \\
(\text { T/DAY) } \\
(80155)\end{array}$ & $\begin{array}{r}\text { SED. } \\
\text { SUSP. } \\
\text { SIEVE } \\
\text { DIAM. } \\
\% \text { FINER } \\
\text { TTHAN } \\
\text { 062 MM } \\
(70331)\end{array}$ \\
\hline $\begin{array}{l}\text { DEC } 1995 \\
05596\end{array}$ & $<5$ & 3 & 2100 & $<10$ & 510 & $<0.01$ & 7 & - & 33 & 7.8 & 87 \\
\hline JuL $06 .$. & -- & - & - & - & -- & -- & $\cdots$ & -- & 24 & 5.2 & 48 \\
\hline $\operatorname{SEP}^{09} \ldots$ & -- & - & - & - & -- & -- & - & - & 50 & 11 & 66 \\
\hline & $<5$ & 6 & 7800 & 20 & 750 & $<0.01$ & 10 & $<2.0$ & 245 & 47 & 96 \\
\hline
\end{tabular}


RIO GRANDE BASIN

08384000 LAKE SUMNER NEAR FORT SUMNER, MM

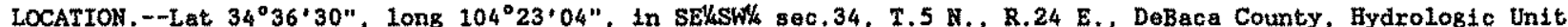
13060001 , near center of dam on Pecos River, 5.0 mi northeast of Guadalupe, $12.2 \mathrm{mi}$ northwest of Fort Sumer, and at mile 702.0 .

DRAINAGE AREA. $--4,390 \mathrm{mi}^{2}$, approximately (contributing area).

PERIOD OF RECORD.--December 1938 to September 1965 (monthend elevations and contents), October 1965 to current year. Monthend elevations September 1937 to November 1938 published in reports of Pecos River Comaission. Flevations and contents May 27 1937, to June 10, 1937, In WSP 842. Prior to October 1974, published as "Alanogordo Reservolr."

REVISED RECORDS.-WSP 1732: 1939-54 (contents). WSP 1923: 1939-53(M).

GAGE - Water-stage recorder Elevation of gage is National Geodetic Vertical Datum of 1929 (levels by Bureau of Reclamation), April 1, 1946, to Sept. 30, 1957, water-stage recorder above olevation 4, 234.25 ft, nonrecoraing gage below, Oct, 1 , 1988 to current year, water-stag recorder above elevation 4,238.00 ft, nonrecording gage. below,

REMARKS.--Lake is formed by earthfill dam; comploted and storage began in August 1937 . Capacity, 94,750 acre-ft, from capacity table dated August 1992, between olevation 4,200.0 ft, sill of outlet gate, and elevation 4, 275 .0 ft, normal operating leve1. Capacity by original survey was 132,200 acre-feet. Dead storage 2,500 acre-feet. Reservoir is used to store water for irrigation. Bureau of Reclamation satellite telemeter at station.

COOPERATION. --Records provided by Bureau of Reclamation.

EXTREMES FOR PERTOD OF RECORD.--Maximum contents, 138,300 acre-ft, May 23-30, June 1-10, July 21, Sept. 22, 23, 30, Oct. 12, Nov. 4, 5, 30, Dec. 23, 24, 1941, elevation, 4,275.00 ft; maximum elevation, 4,276.10 ft June 3, Sept. 8, 1958; no storage, July 28 to Aug. 2, 1951, elevation, 4, 200.70 ft.

EXTREMES FOR CURRENT YEAR,--Maxtmum contents, 43,320 acre-1t, Feb, 14, elovation, 4,260.84 ft; minimum, 5,190 acreft, July 9, elevation, $4,236.22 \mathrm{ft}$.

RESERVOIR STORAGE (ACRE-FEET), WATER YEAR OCTOBER 1995 TO SEPTEMBER 1996

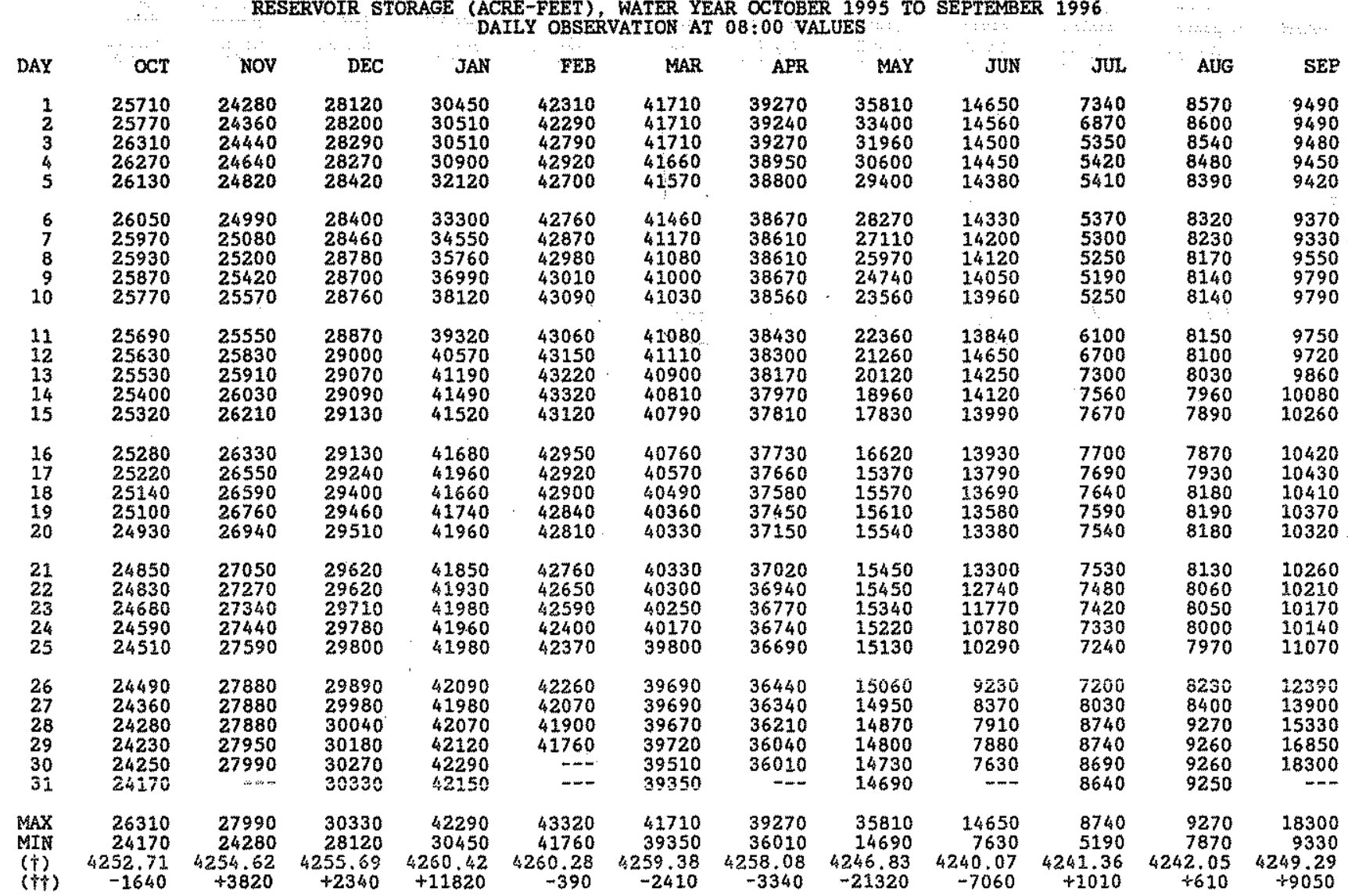

CAL. YR 1995 MAX 37070 MIN 19910 (t†) +9950

WTR YR 1996 MAX 43320 MIN $5190(+\dagger)-7510$

(i) ELEVATION, IN FEET, AT END OF MONTH

$(f)$ CHARGE IN CONTENTS, IN ACRE-FEET. 
LOCATION, - Lat $34^{\circ} 36^{\circ} 15^{\prime \prime}$, Long $104^{\circ} 23^{\prime} 14^{* \prime}$, sec.2, T.4 N., R. 24 E., DeBaca County, Hydrologic Unit 13060003, on left bank 1,200 ft downstream from Sumer Dam, 2,9 ni upstream from Salado Creok, 4.6 mi northeast of Guadalipe, 12.2 mi northwest of Fort Sumer, and at mile 701.7 .

DRAINAGE AREA. $--4,390 \mathrm{mi}^{2}$, approximately (contributing araa).

PERIOD OF RECORD --October 1912 to April 1926 . August 1926 to current year. Monthly discharge only for some perlods, published In WSP 1312. October 1944 to September 1974, published as "belon Alamogordo Dam." Prior to October 1944, published as "near Guadalupe."

REVISED RECORDS.--WSP 1512: 1932. WSP 1632: 1942. WSP 1712: 1944.

GAGE.-Water-gtage recorder and Parshall flume, with concrete control above top of flume. Elevation of gage is 4,142.99 ft above National. Geodetic Vertical Datum of 1929 (Bureau of Reclamation bench mark). Prior to Sept. 10, 1936 at stte $1.5 \mathrm{ml}$ upstream at different datum. Sept. 14, 1936, to Max. 8, 1941, and June.11 to Sept. 21. 1941, at site 0.2 mi downstream at different datums.

REMARKS. - Records good except for those below $10 \mathrm{ft} / \mathrm{s}$ whlch are poor. Flow regulated by Lake Summer (station 08384000 ) $0.3 \mathrm{mi}$ upstream, since August 1937 and Santa Rosa Lake (station 08382810) 55.5 mi upstream, 8 ince April. 1980. Diversions for irrigation of about 12,500 acres, 1959 determination, upstream from station. Severa1 observations of water temperature mere made during the year. Bureau of Reclamation satellite telemeter at station.

AVERAGE DISCFARGE.--23 years (water years 1913-25, 1927-36), $236 \mathrm{ft} 3 / \mathrm{s}, 171,000$ acre-ft/yr, prior to completion of Sumner Dam. DISCHARGE, CUBIC FEET PER SECOND, WATER YEAR OCTOBER 1995 TO SEPTEMBER 1996

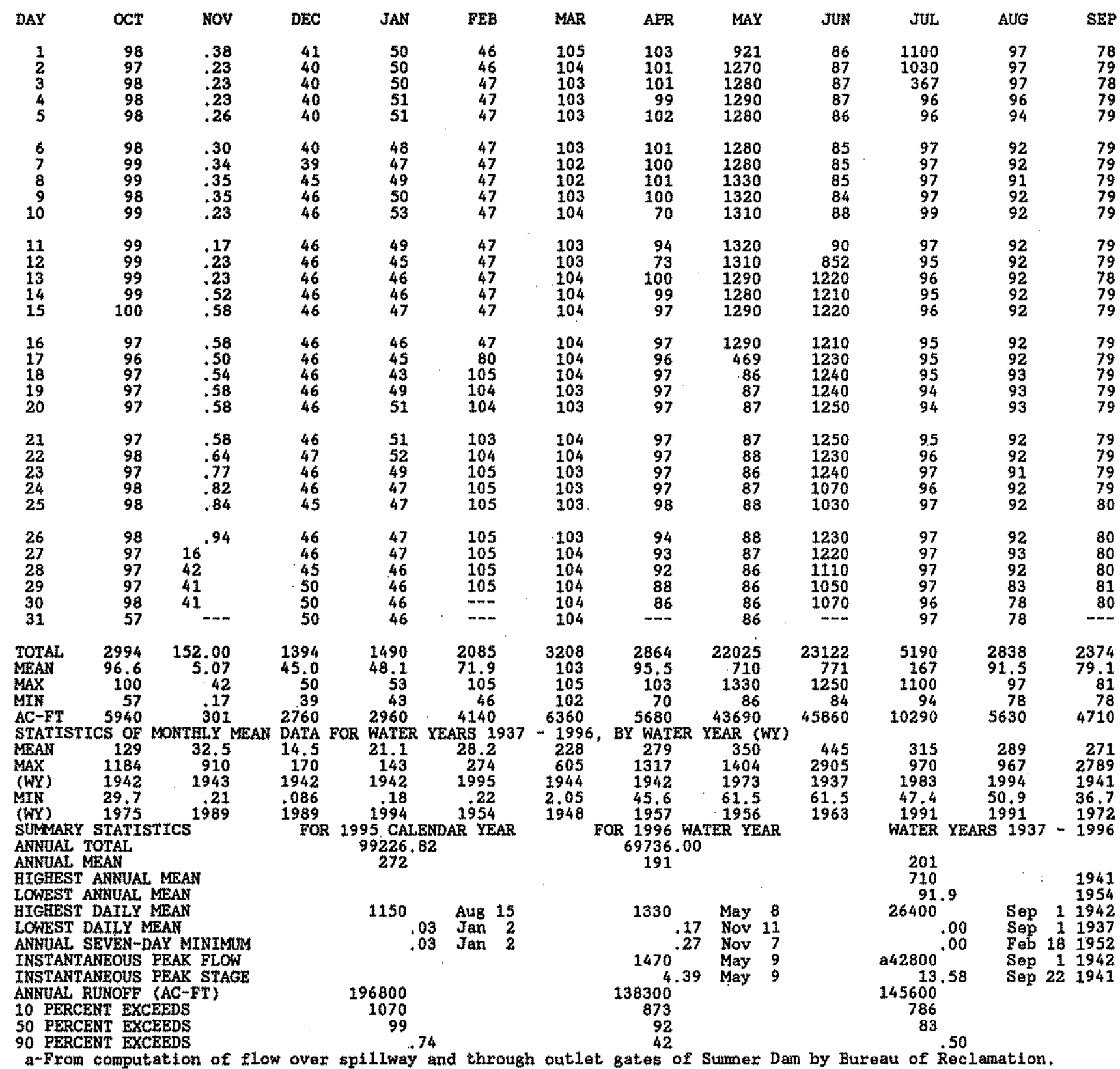


08385000 FORT SUMNER MAIN CANAL NEAR FORT SUMNER, WM

LOCATION,--Lat $34^{\circ} 30^{\prime} 30^{\prime \prime}$, Long $104^{\circ} 16^{\prime} 40^{\prime \prime}$, in SEt/4WHSWh 8ec.1, T.3 N., R.25 E., DeBaca County, Bydrologic Unit 13060003 , on right bank of concrete canal, 200 ft domstream from diversion'dam on Pecos kiver, 3.0 mi northwest of Fort Sumer, and at Pecos River mile 684.8 .

PERIOD OF RECORD, - March 1939 to February 1943 (published in WSP 1732), April 1954 to current year (monthly discharge only prior to October 1965).

GAGE,-Water-gtage recorder, Elevation of gage $18,4,034,7$ ft above Wational Geodetic Vertical Datum of 1929 (Bureau of Reclamation bench mark). Prior to March 1954, at site 2.4 mi downstream at different datum. Apri1 1954 to

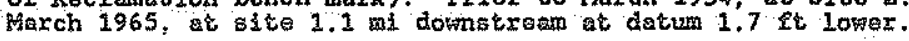

REMARKS. --Records good. Canal diverts water from Pecos River for irrigation of about 6,600 acres, 1961 determination, by the Fort Sumer Irrigation District. Several observations of water temperature were made during the yoar. No flow tor many days oach year.

DISCAARGE, CUBIC FEET PER SECOND, WATER YEAR OCTOBER 1995 TO SEPTEMBER 1996

\begin{tabular}{|c|c|c|c|c|c|c|c|c|c|c|c|c|}
\hline DAY & OCT & Nov & DEC & JAY & FEB & MAR & AFR & MAX & JuN & JuL & AUG & SEP \\
\hline $\begin{array}{l}1 \\
2 \\
3 \\
4 \\
5\end{array}$ & $\begin{array}{l}77 \\
76 \\
85 \\
84 \\
85\end{array}$ & $\begin{array}{l}5.3 \\
.00 \\
.00 \\
.00 \\
.00\end{array}$ & $\begin{array}{l}.00 \\
.00 \\
.00 \\
.00 \\
.00\end{array}$ & $\begin{array}{l}.00 \\
.00 \\
.00 \\
.00 \\
.00\end{array}$ & $\begin{array}{l}.00 \\
.00 \\
.00 \\
.00 \\
.00\end{array}$ & $\begin{array}{l}91 \\
92 \\
91 \\
91 \\
91\end{array}$ & $\begin{array}{l}88 \\
86 \\
86 \\
84 \\
86\end{array}$ & $\begin{array}{r}72 \\
82 \\
100 \\
100 \\
100\end{array}$ & $\begin{array}{l}87 \\
86 \\
86 \\
85 \\
85\end{array}$ & $\begin{array}{l}88 \\
88 \\
89 \\
88 \\
92\end{array}$ & $\begin{array}{l}94 \\
94 \\
94 \\
94 \\
94\end{array}$ & $\begin{array}{l}74 \\
73 \\
73 \\
75 \\
76\end{array}$ \\
\hline $\begin{array}{r}6 \\
7 \\
8 \\
9 \\
10\end{array}$ & $\begin{array}{l}85 \\
86 \\
64 \\
83 \\
82\end{array}$ & $\begin{array}{l}.00 \\
.00 \\
.00 \\
.00 \\
.00\end{array}$ & $\begin{array}{l}.00 \\
.00 \\
.00 \\
.00 \\
.00\end{array}$ & $\begin{array}{l}.00 \\
.00 \\
.00 \\
.00 \\
.00\end{array}$ & $\begin{array}{l}.00 \\
.00 \\
.00 \\
.00 \\
.00\end{array}$ & $\begin{array}{l}91 \\
92 \\
92 \\
92 \\
92\end{array}$ & $\begin{array}{r}86 \\
85 \\
78 \\
80 \\
79\end{array}$ & $\begin{array}{l}67 \\
81 \\
8_{95}^{.00}\end{array}$ & $\begin{array}{l}85 \\
86 \\
86 \\
85 \\
82\end{array}$ & $\begin{array}{l}92 \\
92 \\
92 \\
92 \\
91\end{array}$ & $\begin{array}{l}94 \\
90 \\
96 \\
97 \\
96\end{array}$ & $\begin{array}{l}79 \\
84 \\
92 \\
91 \\
79\end{array}$ \\
\hline $\begin{array}{l}11 \\
12 \\
13 \\
14 \\
15\end{array}$ & $\begin{array}{l}80 \\
77 \\
75 \\
73 \\
71\end{array}$ & $\begin{array}{l}.00 \\
.00 \\
.00 \\
.00 \\
.00\end{array}$ & $\begin{array}{l}.00 \\
.00 \\
.00 \\
.00 \\
.00\end{array}$ & $\begin{array}{l}.00 \\
.00 \\
.00 \\
.00 \\
.00\end{array}$ & $\begin{array}{r}.00 \\
.00 \\
73^{.00} \\
48\end{array}$ & $\begin{array}{l}91 \\
90 \\
91 \\
90 \\
90\end{array}$ & $\begin{array}{l}75 \\
86 \\
89 \\
91 \\
92\end{array}$ & $\begin{array}{l}85 \\
84 \\
84 \\
84 \\
84\end{array}$ & $\begin{array}{l}89 \\
86 \\
91 \\
93 \\
92\end{array}$ & $\begin{array}{l}87 \\
90 \\
90 \\
85 \\
84\end{array}$ & $\begin{array}{l}97 \\
98 \\
98 \\
98 \\
99\end{array}$ & $\begin{array}{r}90 \\
97 \\
99 \\
100 \\
101\end{array}$ \\
\hline $\begin{array}{l}16 \\
17 \\
18 \\
19 \\
20\end{array}$ & $\begin{array}{l}70 \\
76 \\
84 \\
84 \\
84\end{array}$ & $\begin{array}{l}.00 \\
.00 \\
.00 \\
.00 \\
.00\end{array}$ & $\begin{array}{l}.00 \\
.00 \\
.00 \\
.00 \\
.00\end{array}$ & $\begin{array}{l}.00 \\
.00 \\
.00 \\
.00 \\
.00\end{array}$ & $\begin{array}{l}54^{.00} \\
91 \\
91 \\
91\end{array}$ & $\begin{array}{l}90 \\
90 \\
91 \\
87 \\
82\end{array}$ & $\begin{array}{l}89 \\
89 \\
90 \\
89 \\
89\end{array}$ & $\begin{array}{l}85 \\
90 \\
98 \\
96 \\
95\end{array}$ & $\begin{array}{l}92 \\
91 \\
94 \\
98 \\
98\end{array}$ & $\begin{array}{l}83 \\
81 \\
77 \\
88 \\
89\end{array}$ & $\begin{array}{r}100 \\
99 \\
100 \\
102 \\
101\end{array}$ & $\begin{array}{l}98 \\
98 \\
98 \\
98 \\
47\end{array}$ \\
\hline $\begin{array}{l}21 \\
22 \\
23 \\
24 \\
25\end{array}$ & $\begin{array}{l}83 \\
83 \\
82 \\
81 \\
81\end{array}$ & $\begin{array}{l}.00 \\
.00 \\
.00 \\
.00 \\
.00\end{array}$ & $\begin{array}{l}.00 \\
.00 \\
.00 \\
.00 \\
.00\end{array}$ & $\begin{array}{l}.00 \\
.00 \\
.00 \\
.00 \\
.00\end{array}$ & $\begin{array}{l}91 \\
91 \\
91 \\
92 \\
92\end{array}$ & $\begin{array}{l}82 \\
80 \\
89 \\
89 \\
89\end{array}$ & $\begin{array}{l}90 \\
90 \\
89 \\
89 \\
89\end{array}$ & $\begin{array}{l}94 \\
94 \\
94 \\
92 \\
93\end{array}$ & $\begin{array}{l}92 \\
83 \\
88 \\
86 \\
86\end{array}$ & $\begin{array}{l}90 \\
91 \\
93 \\
93 \\
94\end{array}$ & $\begin{array}{r}100 \\
98 \\
100 \\
100 \\
99\end{array}$ & $\begin{array}{l}75 \\
80 \\
81 \\
80 \\
81\end{array}$ \\
\hline $\begin{array}{l}26 \\
27 \\
28 \\
29 \\
30 \\
31\end{array}$ & $\begin{array}{l}80 \\
80 \\
79 \\
79 \\
79 \\
79\end{array}$ & $\begin{array}{l}.00 \\
.00 \\
.00 \\
.00 \\
.00 \\
. \cdots\end{array}$ & $\begin{array}{l}.00 \\
.00 \\
.00 \\
.00 \\
.00 \\
.00\end{array}$ & $\begin{array}{l}.00 \\
.00 \\
.00 \\
.00 \\
.00 \\
.00\end{array}$ & $\begin{array}{l}89 \\
91 \\
91 \\
91 \\
\end{array}$ & $\begin{array}{l}89 \\
89 \\
89 \\
89 \\
89 \\
89\end{array}$ & $\begin{array}{r}89 \\
87 \\
86 \\
85 \\
81 \\
-\end{array}$ & $\begin{array}{l}93 \\
89 \\
87 \\
86 \\
87 \\
89\end{array}$ & $\begin{array}{r}87 \\
84 \\
86 \\
87 \\
88 \\
---\end{array}$ & $\begin{array}{l}94 \\
95 \\
94 \\
93 \\
94 \\
95\end{array}$ & $\begin{array}{l}99 \\
98 \\
95 \\
88 \\
77 \\
74\end{array}$ & $\begin{array}{r}81 \\
84 \\
83 \\
85 \\
84 \\
-\end{array}$ \\
\hline $\begin{array}{l}\text { TOTAL } \\
\text { MEAN } \\
\text { MAX } \\
\text { MIN } \\
\text { AC-FT }\end{array}$ & $\begin{array}{r}2489 \\
80.3 \\
86 \\
70 \\
4940\end{array}$ & $\begin{array}{r}5.30 \\
.18 \\
5.3 \\
.00 \\
11\end{array}$ & $\begin{array}{r}0.00 \\
.000 \\
.00 \\
.00 \\
.00\end{array}$ & $\begin{array}{l}0.00 \\
.000 \\
.00 \\
.00 \\
.00\end{array}$ & $\begin{array}{r}1267.00 \\
43.7 \\
92 \\
.00 \\
2510\end{array}$ & $\begin{array}{r}2770 \\
89.4 \\
92 \\
80 \\
5490\end{array}$ & $\begin{array}{r}2592 \\
86.4 \\
92 \\
75 \\
5140\end{array}$ & $\begin{array}{r}2580.00 \\
83.2 \\
100 \\
5100 \\
5120\end{array}$ & $\begin{array}{r}2644 \\
88.1 \\
98 \\
82 \\
5240\end{array}$ & $\begin{array}{r}2784 \\
89.8 \\
95 \\
77 \\
5520\end{array}$ & $\begin{array}{r}2963 \\
95.6 \\
102 \\
74 \\
5880\end{array}$ & $\begin{array}{r}2536 \\
84.5 \\
101 \\
47 \\
5030\end{array}$ \\
\hline
\end{tabular}

STATISTICS OF MONTHLY MEAN DATA FOR WATER YEARS 1939 - 1996, BY WATER YEAR (WY)

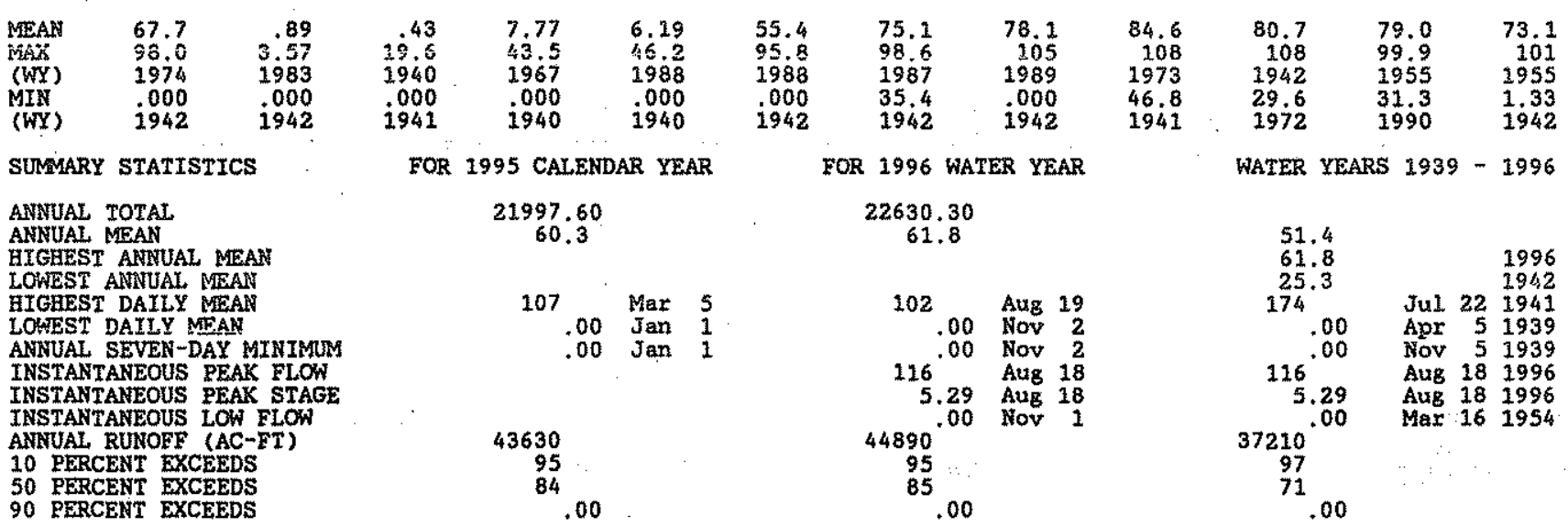


RIO GRANDE BASIX

08385500 PECOS RIVER NEAR FORT SUMNER, MM

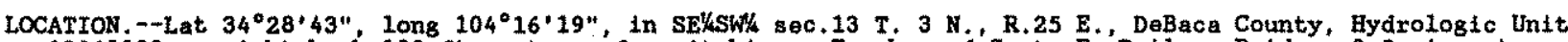
13060003 on right bank $100 \mathrm{ft}$ upstrean' from Atchison, Topeka and' Santa Fe' Railway Bridge, 0.8 mi upstream from U.S. Highway 60 and 2.5 mil downstream from Fort Sumer Diversion dam.

DRAINAGE AREA. $--5,300 \mathrm{mi}^{2}$, approximately.

PERTOD OF RECORD.--June to July 1904 , July 1904 to June 1905 (gage helghts and discharge measurements only). Daily discharges Juiy 18 to August 11,1904 are unreliable and should not be used, July 1905 to February 1910 , September 1912 to Deceaber 1913, July 1994 to current year.

GAGE.--Water-8tage recorder. Elevation of gage is 4,020 above National Geodetic Vertical Datum of 1929 , from topographic map. Prior to July 5, 1905, staff gage at site 2.5 mi upstream at different datum. July 5 , 1905 to Dec. 31, 1913, staff gage at stee $1.5 \mathrm{mi}$ upstream at different detum.

FXMARxs,--Records fair except for estimated dally discharges, which are poor. Diverston above gage for about 6,100 acres (1961 determination) pert of which are below gage. Bureau of Reclamation satelitte teiemeter at station.

EXTREMES FOR PERTOD OF RECORD,--Maximum discharge not determined, probably exceeded 53,000 fts/s, Sept. 30, 1904, gago height, $17.95 \mathrm{ft}$, from floodmarks, stte and datum then in use; minfmum dajly $0.3 \mathrm{ft} / \mathrm{s}, \mathrm{Aug}$. 17 , 1922.

EXTREMES FOR CURRENT YEAR.--Maximun daily discharge, 1,220 fts/s, May 8; mintmum datily, 0.95 fts/s, Apr. 30. DISCAARGE, CUBIC FEET PER SECOND, WATER YEAR OCTOBER 1995 TO SEPTEMBER 1996

\begin{tabular}{|c|c|c|c|c|c|c|c|c|c|c|c|c|}
\hline DAY & $\infty T$ & Nov & DEC & JAN & FEB & MAR & $A F R$ & MAY & JUt & JUL. & AUG & $\mathrm{SEF}$ \\
\hline $\begin{array}{l}1 \\
2 \\
3 \\
4 \\
5\end{array}$ & $\begin{array}{l}20 \\
68 \\
15 \\
14 \\
13\end{array}$ & $\begin{array}{r}28 \\
12 \\
8.2 \\
6.3 \\
5.2\end{array}$ & $\begin{array}{l}39 \\
39 \\
40 \\
41 \\
42\end{array}$ & $\begin{array}{l}57 \\
45 \\
55 \\
55 \\
71\end{array}$ & $\begin{array}{l}51 \\
51 \\
49 \\
46 \\
46\end{array}$ & $\begin{array}{l}14 \\
13 \\
12 \\
10 \\
9.5\end{array}$ & $\begin{array}{l}4.2 \\
3.2 \\
2.8 \\
2.8 \\
2.8\end{array}$ & $\begin{array}{r}370 \\
1010 \\
1030 \\
1050 \\
1030\end{array}$ & $\begin{array}{l}2.4 \\
2.4 \\
2.0 \\
1.9 \\
1.3\end{array}$ & $\begin{array}{r}821 \\
825 \\
523 \\
59 \\
33\end{array}$ & $\begin{array}{l}12 \\
12 \\
11 \\
11 \\
11\end{array}$ & $\begin{array}{l}2.2 \\
2.0 \\
1.6 \\
1.5 \\
1.5\end{array}$ \\
\hline $\begin{array}{r}6 \\
7 \\
8 \\
9 \\
10\end{array}$ & $\begin{array}{l}14 \\
19 \\
16 \\
15 \\
15\end{array}$ & $\begin{array}{l}4.2 \\
3.4 \\
2.9 \\
2.7 \\
2.3\end{array}$ & $\begin{array}{l}42 \\
42 \\
44 \\
47 \\
48\end{array}$ & $\begin{array}{l}60 \\
56 \\
53 \\
57 \\
57\end{array}$ & $\begin{array}{l}45 \\
45 \\
45 \\
45 \\
45\end{array}$ & $\begin{array}{l}9.8 \\
10 \\
11 \\
11 \\
11\end{array}$ & $\begin{array}{l}2.9 \\
3.2 \\
7.5 \\
3.4 \\
3.2\end{array}$ & $\begin{array}{l}1090 \\
1190 \\
1220 \\
1120 \\
1100\end{array}$ & $\begin{array}{r}e 1.0 \\
1.1 \\
1.3 \\
1.1 \\
3.5\end{array}$ & $\begin{array}{r}28 \\
23 \\
20 \\
19 \\
115\end{array}$ & $\begin{array}{l}9.6 \\
12^{9} \\
10 \\
16 \\
11\end{array}$ & $\begin{array}{l}1.5 \\
1.6 \\
7.0 \\
5.1 \\
9.1\end{array}$ \\
\hline $\begin{array}{l}11 \\
12 \\
13 \\
14 \\
15\end{array}$ & $\begin{array}{l}17 \\
19 \\
20 \\
21 \\
22\end{array}$ & $\begin{array}{l}2.0 \\
1.8 \\
1.8 \\
1.8 \\
1.6\end{array}$ & $\begin{array}{l}49 \\
49 \\
49 \\
37 \\
59\end{array}$ & $\begin{array}{l}61 \\
52 \\
53 \\
53 \\
53\end{array}$ & $\begin{array}{l}45 \\
45 \\
33 \\
37 \\
70\end{array}$ & $\begin{array}{l}9.3 \\
8.3 \\
8.4 \\
8.1 \\
8.5\end{array}$ & $\begin{array}{c}3.2 \\
4.9 \\
8.5 \\
10 \\
14\end{array}$ & $\begin{array}{l}1120 \\
1100 \\
1080 \\
1100 \\
1130\end{array}$ & $\begin{array}{c}2.1 \\
355 \\
973 \\
1040 \\
1030\end{array}$ & $\begin{array}{l}35 \\
19 \\
52 \\
20 \\
17\end{array}$ & $\begin{array}{l}13 \\
12 \\
11 \\
11 \\
12\end{array}$ & $\begin{array}{l}2.4 \\
6.3 \\
12 \\
18 \\
25\end{array}$ \\
\hline $\begin{array}{l}16 \\
17 \\
18 \\
19 \\
20\end{array}$ & $\begin{array}{l}23 \\
17 \\
13 \\
12 \\
12\end{array}$ & $\begin{array}{l}1.6 \\
1.6 \\
1.6 \\
1.5 \\
1.4\end{array}$ & $\begin{array}{l}51 \\
53 \\
51 \\
38 \\
62\end{array}$ & $\begin{array}{l}53 \\
51 \\
49 \\
48 \\
55\end{array}$ & $\begin{array}{l}86 \\
54 \\
16 \\
15 \\
14\end{array}$ & $\begin{array}{c}9.0 \\
9.3 \\
12 \\
11 \\
4.2\end{array}$ & $\begin{array}{l}7.0 \\
7.8 \\
6.6 \\
7.3 \\
7.4\end{array}$ & $\begin{array}{r}1150 \\
733 \\
80 \\
42 \\
26\end{array}$ & $\begin{array}{l}1030 \\
1040 \\
1030 \\
1020 \\
1020\end{array}$ & $\begin{array}{c}16 \\
14 \\
18 \\
8.3 \\
9.3\end{array}$ & $\begin{array}{r}15 \\
15 \\
19 \\
412 \\
42\end{array}$ & $\begin{array}{r}11 \\
9.1 \\
8.5 \\
9.4 \\
44\end{array}$ \\
\hline $\begin{array}{l}21 \\
22 \\
23 \\
24 \\
25\end{array}$ & $\begin{array}{l}12 \\
12 \\
12 \\
13 \\
13\end{array}$ & $\begin{array}{l}1.5 \\
1.5 \\
1.5 \\
1.4 \\
1.4\end{array}$ & $\begin{array}{l}52 \\
52 \\
52 \\
52 \\
52\end{array}$ & $\begin{array}{l}55 \\
56 \\
55 \\
52 \\
51\end{array}$ & $\begin{array}{l}14 \\
14 \\
15 \\
15 \\
14\end{array}$ & $\begin{array}{l}4.4 \\
9.3 \\
5.7 \\
5.5 \\
5.8\end{array}$ & $\begin{array}{l}7.4 \\
7.2 \\
6.6 \\
6.2 \\
5.9\end{array}$ & $\begin{array}{r}17 \\
13 \\
8.5 \\
6.6 \\
5.9\end{array}$ & $\begin{array}{r}1030 \\
1020 \\
1010 \\
909 \\
713\end{array}$ & $\begin{array}{r}9.7 \\
8.8 \\
8.8 \\
8.6 \\
10\end{array}$ & $\begin{array}{l}24 \\
19 \\
29 \\
22 \\
25\end{array}$ & $\begin{array}{l}3.9 \\
2.6 \\
2.0 \\
1.5 \\
1.3\end{array}$ \\
\hline $\begin{array}{l}26 \\
27 \\
28 \\
29 \\
30 \\
31\end{array}$ & $\begin{array}{l}12 \\
13 \\
12 \\
12 \\
13 \\
13\end{array}$ & $\begin{array}{l}1.6 \\
1.5 \\
7.0 \\
33 \\
38 \\
\end{array}$ & $\begin{array}{l}52 \\
52 \\
52 \\
53 \\
57 \\
57\end{array}$ & $\begin{array}{l}50 \\
51 \\
50 \\
50 \\
49 \\
50\end{array}$ & $\begin{array}{r}15 \\
15 \\
14 \\
14 \\
--- \\
---\end{array}$ & $\begin{array}{l}6.2 \\
6.1 \\
6.0 \\
5.2 \\
5.1 \\
5.6\end{array}$ & $\begin{array}{r}6.2 \\
2.9 \\
2.2 \\
1.9 \\
.95 \\
----\end{array}$ & $\begin{array}{l}4.9 \\
4.2 \\
3.9 \\
3.8 \\
3.6 \\
3.2\end{array}$ & $\begin{array}{l}987 \\
991 \\
932 \\
794 \\
813\end{array}$ & $\begin{array}{l}11 \\
16 \\
11 \\
9.8 \\
12 \\
12\end{array}$ & $\begin{array}{l}28 \\
27 \\
14 \\
12 \\
3.6 \\
2.5\end{array}$ & $\begin{array}{l}1.3 \\
1.1 \\
1.1 \\
1.1 \\
1.4\end{array}$ \\
\hline $\begin{array}{l}\text { TOTAZ } \\
\text { MEAN } \\
\text { MAX } \\
\text { MIN } \\
\text { AC-FI }\end{array}$ & $\begin{array}{r}522 \\
16.8 \\
68 \\
12 \\
1040\end{array}$ & $\begin{array}{r}180.3 \\
6.01 \\
38 \\
1.4 \\
358\end{array}$ & $\begin{array}{r}1505 \\
48.5 \\
62 \\
37 \\
2990\end{array}$ & $\begin{array}{r}1663 \\
53.6 \\
71 \\
45 \\
3300\end{array}$ & $\begin{array}{r}1013 \\
34.9 \\
86 \\
14 \\
2010\end{array}$ & $\begin{array}{r}264.3 \\
8.53 \\
14 \\
4.2 \\
524\end{array}$ & $\begin{array}{r}160.15 \\
5.34 \\
14 \\
.95 \\
318\end{array}$ & $\begin{array}{r}17845.6 \\
576 \\
1220 \\
3.2 \\
35400\end{array}$ & $\begin{array}{r}17757.1 \\
592 \\
1040 \\
1.0 \\
35220\end{array}$ & $\begin{array}{r}2792.3 \\
90.1 \\
825 \\
8.3 \\
5540\end{array}$ & $\begin{array}{r}883.7 \\
28.5 \\
412 \\
2.5 \\
1750\end{array}$ & $\begin{array}{r}196.1 \\
6.54 \\
44 \\
1.1 \\
389\end{array}$ \\
\hline
\end{tabular}


RIO GRANDE BASIN

08385522 PECOS RIVER BELON TAIBAN CREEK NEAR FORT SUMNER, RM

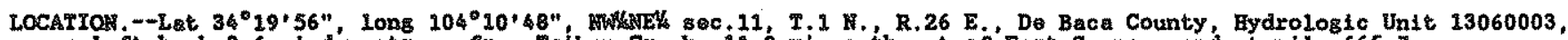

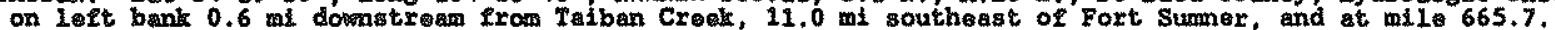

WATER-DISCEAREE RECORDS

PKRTOD OF RECORD. --August 1992 to current year.

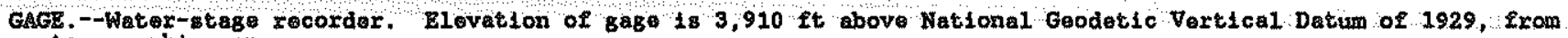
toposraphice map.

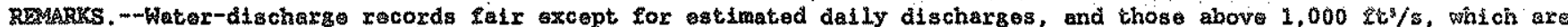

poor. Flow partis regulated by Sumer Dam (station 08384000 ) 23 mi upstream. Divarsion for irrigation of about

19, 200 cres (1959 dotermination) above station, Discharge represents in genoral, roturn flow from irrigatod

areas in Fort Sumer Irrigation Project.

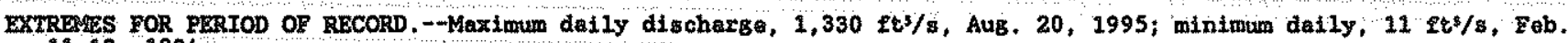

$11-13,1994$.

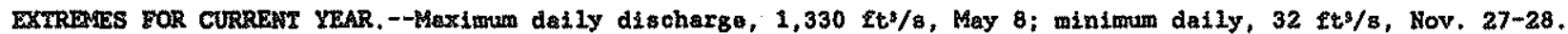
DISCBARGE, CUBIC FEET FER SECOMD, WATER YEAR OCTOBER 1995 TO SEPTEMEER 1996

\begin{tabular}{|c|c|c|c|c|c|c|c|c|c|c|c|c|}
\hline DAY & $\alpha x$ & HOV & $\mathrm{DEC}$ & JAN & FEB & MAR & APR & MAY & JUN & JUL. & AUG & SEP \\
\hline $\begin{array}{l}1 \\
2 \\
3 \\
4 \\
5\end{array}$ & $\begin{array}{r}61 \\
217 \\
68 \\
71 \\
63\end{array}$ & $\begin{array}{r}66 \\
51 \\
244 \\
\quad 40 \\
038\end{array}$ & $\begin{array}{l}53 \\
54 \\
55 \\
54 \\
52\end{array}$ & $\begin{array}{l}63 \\
65 \\
68 \\
65 \\
65\end{array}$ & $\begin{array}{r}62 \\
\quad 58 \\
62 \\
63 \\
60\end{array}$ & $\begin{array}{l}44 \\
44 \\
38 \\
40 \\
46\end{array}$ & $\begin{array}{l}47 \\
54 \\
53 \\
53 \\
62\end{array}$ & $\begin{array}{l}104 \\
1110 \\
1160 \\
1190 \\
1180\end{array}$ & $\begin{array}{l}033 \\
032 \\
030 \\
029 \\
025\end{array}$ & $\begin{array}{l}869 \\
861 \\
760 \\
152 \\
95\end{array}$ & $\begin{array}{r}123 \\
75 \\
52 \\
54 \\
47\end{array}$ & $\begin{array}{r}65 \\
58 \\
045 \\
035 \\
45\end{array}$ \\
\hline $\begin{array}{r}6 \\
7 \\
8 \\
9 \\
10\end{array}$ & $\begin{array}{l}79 \\
97 \\
74 \\
84 \\
68\end{array}$ & $\begin{array}{r}036 \\
035 \\
033 \\
031 \\
29\end{array}$ & $\begin{array}{l}53 \\
54 \\
53 \\
57 \\
58\end{array}$ & $\begin{array}{l}68 \\
63 \\
59 \\
64 \\
63\end{array}$ & $\begin{array}{l}59 \\
58 \\
57 \\
57 \\
56\end{array}$ & $\begin{array}{l}51 \\
50 \\
56 \\
62 \\
51\end{array}$ & $\begin{array}{l}57 \\
61 \\
41 \\
50 \\
41\end{array}$ & $\begin{array}{l}1220 \\
1320 \\
1330 \\
1250 \\
1220\end{array}$ & $\begin{array}{l}28 \\
28 \\
26 \\
27 \\
26\end{array}$ & $\begin{array}{r}81 \\
84 \\
103 \\
86 \\
200\end{array}$ & $\begin{array}{l}47 \\
73 \\
63 \\
50 \\
60\end{array}$ & $\begin{array}{l}42 \\
39 \\
57 \\
42 \\
38\end{array}$ \\
\hline $\begin{array}{l}11 \\
12 \\
13 \\
14 \\
15\end{array}$ & $\begin{array}{l}56 \\
58 \\
51 \\
59 \\
80\end{array}$ & $\begin{array}{l}28 \\
28 \\
27 \\
26 \\
26\end{array}$ & $\begin{array}{l}57 \\
56 \\
57 \\
59 \\
57\end{array}$ & $\begin{array}{l}64 \\
62 \\
62 \\
62 \\
62\end{array}$ & $\begin{array}{l}56 \\
56 \\
56 \\
55 \\
59\end{array}$ & $\begin{array}{l}61 \\
69 \\
70 \\
65 \\
62\end{array}$ & $\begin{array}{l}38 \\
38 \\
37 \\
39 \\
42\end{array}$ & $\begin{array}{l}1220 \\
1210 \\
1200 \\
1180 \\
1190\end{array}$ & $\begin{array}{r}30 \\
79 \\
1040 \\
1130 \\
1150\end{array}$ & $\begin{array}{r}117 \\
94 \\
137 \\
101 \\
98\end{array}$ & $\begin{array}{r}248 \\
58 \\
53 \\
42 \\
48\end{array}$ & $\begin{array}{r}40 \\
42 \\
52 \\
81 \\
254\end{array}$ \\
\hline $\begin{array}{l}16 \\
17 \\
18 \\
19 \\
20\end{array}$ & $\begin{array}{l}84 \\
51 \\
46 \\
51 \\
48\end{array}$ & $\begin{array}{l}25 \\
25 \\
25 \\
25 \\
25\end{array}$ & $\begin{array}{l}63 \\
67 \\
67 \\
62 \\
59\end{array}$ & $\begin{array}{l}62 \\
62 \\
61 \\
59 \\
62\end{array}$ & $\begin{array}{r}105 \\
90 \\
45 \\
43 \\
44\end{array}$ & $\begin{array}{l}61 \\
65 \\
57 \\
44 \\
58\end{array}$ & $\begin{array}{l}46 \\
43 \\
42 \\
48 \\
49\end{array}$ & $\begin{array}{r}1180 \\
984 \\
109 \\
49 \\
847\end{array}$ & $\begin{array}{l}1060 \\
1060 \\
1070 \\
1070 \\
1070\end{array}$ & $\begin{array}{l}79 \\
62 \\
68 \\
83 \\
68\end{array}$ & $\begin{array}{r}50 \\
45 \\
45 \\
361 \\
88\end{array}$ & $\begin{array}{l}74 \\
79 \\
80 \\
73 \\
80\end{array}$ \\
\hline $\begin{array}{l}21 \\
22 \\
23 \\
24 \\
25\end{array}$ & $\begin{array}{l}48 \\
50 \\
64 \\
58 \\
49\end{array}$ & $\begin{array}{l}25 \\
24 \\
23 \\
23 \\
23\end{array}$ & $\begin{array}{l}64 \\
62 \\
62 \\
61 \\
62\end{array}$ & $\begin{array}{l}64 \\
64 \\
66 \\
65 \\
62\end{array}$ & $\begin{array}{l}40 \\
33 \\
33 \\
38 \\
38\end{array}$ & $\begin{array}{l}44 \\
46 \\
41 \\
43 \\
43\end{array}$ & $\begin{array}{l}47 \\
47 \\
52 \\
52 \\
49\end{array}$ & $\begin{array}{l}044 \\
041 \\
038 \\
035 \\
033\end{array}$ & $\begin{array}{r}1070 \\
1080 \\
1070 \\
1030 \\
752\end{array}$ & $\begin{array}{r}65 \\
053 \\
e 54 \\
055 \\
53\end{array}$ & $\begin{array}{r}60 \\
50 \\
61 \\
63 \\
130\end{array}$ & $\begin{array}{l}64 \\
59 \\
47 \\
50 \\
51\end{array}$ \\
\hline $\begin{array}{l}26 \\
27 \\
28 \\
29 \\
30 \\
31\end{array}$ & $\begin{array}{l}45 \\
47 \\
56 \\
52 \\
54 \\
67\end{array}$ & $\begin{array}{r}23 \\
22 \\
22 \\
31 \\
49 \\
-\end{array}$ & $\begin{array}{l}63 \\
62 \\
60 \\
58 \\
62 \\
63\end{array}$ & $\begin{array}{l}64 \\
61 \\
61 \\
59 \\
57 \\
56\end{array}$ & $\begin{array}{r}34 \\
41 \\
36 \\
38 \\
--- \\
--\end{array}$ & $\begin{array}{l}48 \\
49 \\
45 \\
45 \\
52 \\
55\end{array}$ & $\begin{array}{r}43 \\
46 \\
41 \\
49 \\
49 \\
--\end{array}$ & $\begin{array}{l}033 \\
031 \\
032 \\
030 \\
032 \\
035\end{array}$ & $\begin{array}{r}995 \\
1070 \\
1050 \\
843 \\
855 \\
-\end{array}$ & $\begin{array}{r}65 \\
116 \\
112 \\
101 \\
83 \\
86\end{array}$ & $\begin{array}{r}164 \\
148 \\
108 \\
80 \\
66 \\
134\end{array}$ & $\begin{array}{r}49 \\
43 \\
53 \\
52 \\
59 \\
-\end{array}$ \\
\hline $\begin{array}{l}\text { TOEAI } \\
\text { MEAN } \\
\text { MAX } \\
\text { MIIN } \\
\text { AC }-F Y\end{array}$ & $\begin{array}{r}2056 \\
66.3 \\
217 \\
45 \\
4080\end{array}$ & $\begin{array}{r}928 \\
30.9 \\
66 \\
22 \\
1840\end{array}$ & $\begin{array}{r}1826 \\
58.9 \\
67 \\
52 \\
3620\end{array}$ & $\begin{array}{r}1940 \\
62.6 \\
68 \\
56 \\
3850\end{array}$ & $\begin{array}{r}1532 \\
52.8 \\
105 \\
33 \\
3040\end{array}$ & $\begin{array}{r}1605 \\
51.8 \\
70 \\
38 \\
3180\end{array}$ & $\begin{array}{r}1416 \\
47.2 \\
62 \\
37 \\
2610\end{array}$ & $\begin{array}{r}19837 \\
640 \\
1330 \\
30 \\
39350\end{array}$ & $\begin{array}{r}18858 \\
629 \\
1150 \\
25 \\
37400\end{array}$ & $\begin{array}{r}5041 \\
163 \\
869 \\
53 \\
10000\end{array}$ & $\begin{array}{r}2746 \\
88.6 \\
361 \\
42 \\
5450\end{array}$ & $\begin{array}{r}2848 \\
61.6 \\
254 \\
35 \\
3670\end{array}$ \\
\hline
\end{tabular}

CAL UR 1995 TOTAL 87011 MEAN 238 MAX 1330 MIN 15 AC-FT 172600 WIR XR 1996 TOTAL 59633 MEAN 163 MAX 1330 MIN 22 AC-FT 118300 
RIO GRANDE BASTN

08385522 PECOS RIVER BELON TAIBAN CREEK NBAR FORT SUMNER, NM -- Continued

WATZRR-QUALITY RECORDS

FERIOD OF RECORD. - watex years 1992 to current year.

WATER QUALITY DATA, WATER YEAR OCTOBER 1995 TO SEPTEMBER 1996

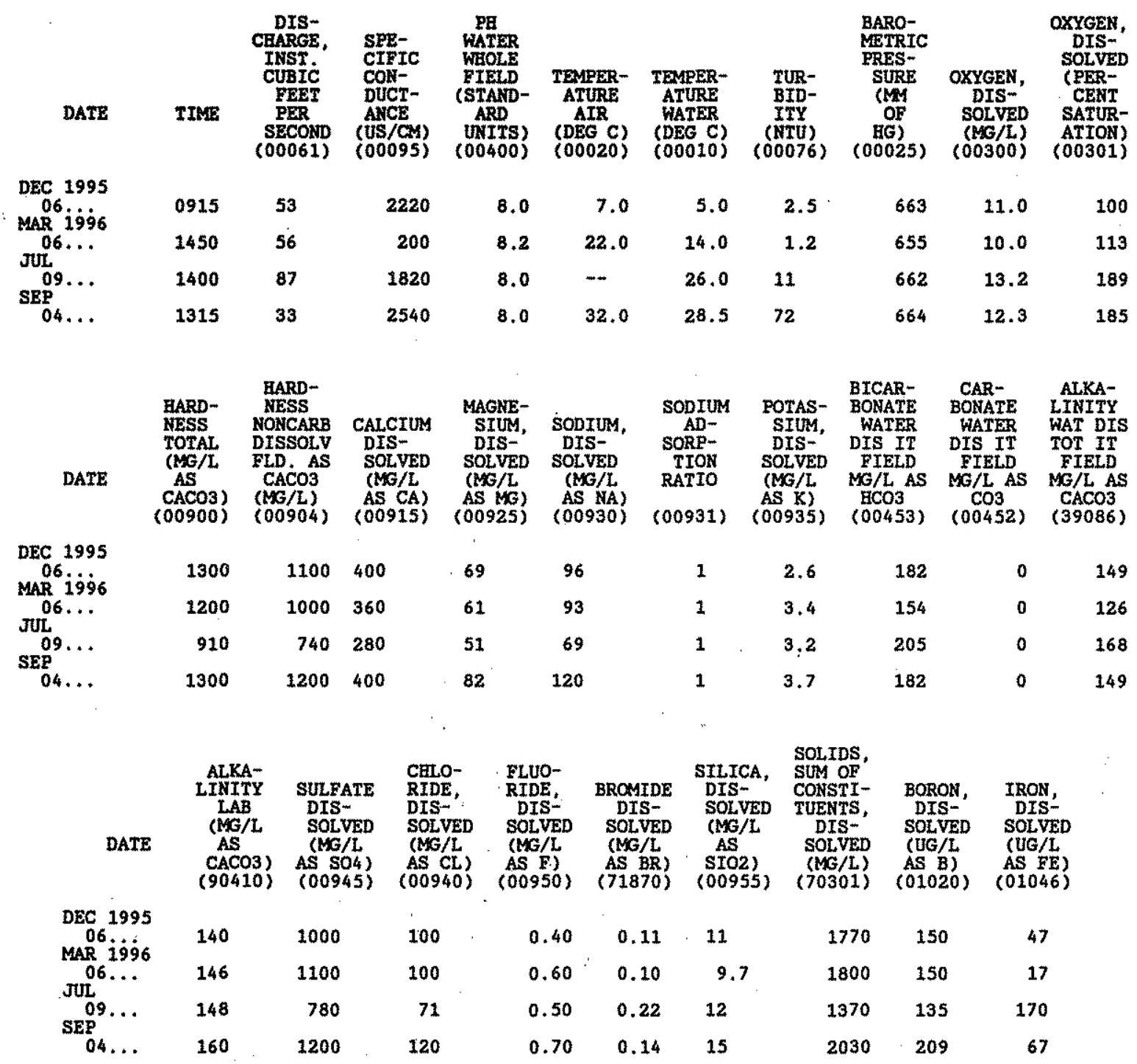


RIO GRANDE BASIN

08385630 PECOS RIVER NEAR DUNLAP, NM

LOCATION.--Lat $34^{\circ} 03^{\prime 4} 48^{\prime \prime}$, Long $104^{\circ} 18^{\circ} 22^{\prime \prime}$, In SEkWW, sec, 10, T.3 S, R.25 E., DeBaca County, Hydrologic Unit

13060003 , on left bank $1.2 \mathrm{mi}$ south of Van Eaton Ranch, 2.5 mi upstream from Arroyo de la Mora, 2.7 mi downstream from Blanco Canyon, 15 mi east of Dunlap, NM, and at mile 638.1

PERIOD OF RECORD.--August 1993 to current year,

GAGE. -Water-stage recorder. Elevation of gage is $3,760 \mathrm{ft}$ above National Geodetic Vertical Datum of 1929 , fron river profile map.

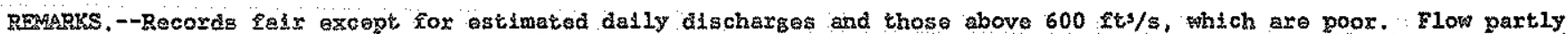
regulated by Lake Summer (station 08384000). Diverston for irrigation or about 19,100 acres (1959 dekermination) above station.

EXTREMES FOR PERIOD OF RECORD,-Maximum daily discharge, 1,320 fts/s, Sept, 9, 1995; minimum daily, 9.2 fts/s, June 12,1996 .

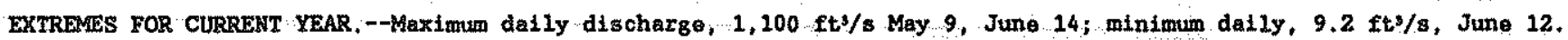
DISCHARGE, CUBIC FEET PER SECOND; WATER YEAR OCTOBER 1995 TO SEPTEMBER 1996

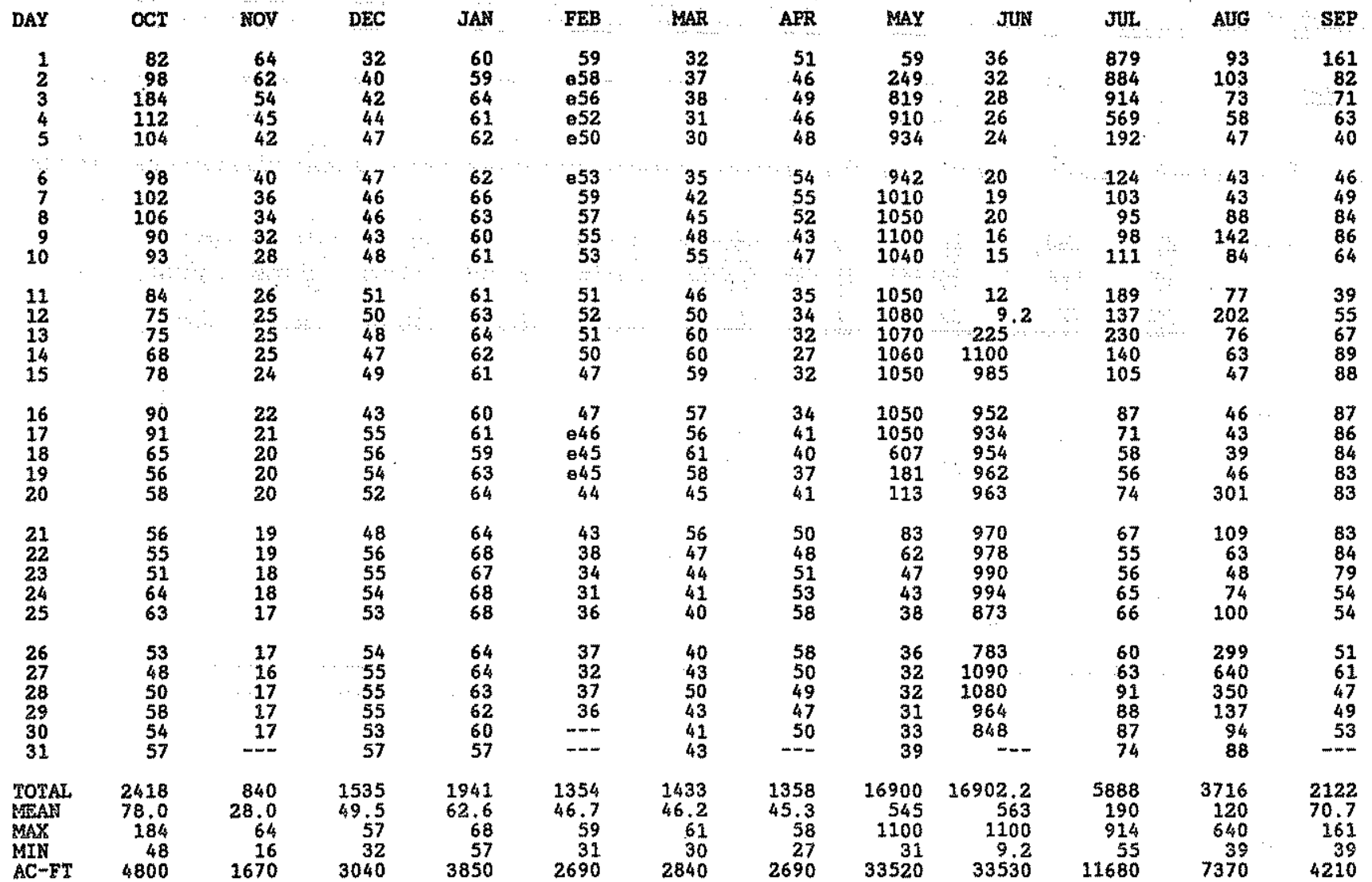

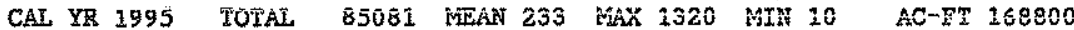
WTR YR 1996 TOTAL 56407.2 MEAN 154 MAX 1100 MIN 9.2 AC-FT 111900 
RIO GRANDE BASIN

08385648 PECOS RIVER ABOVE ACME, NM

LOCATION. --Lat $33^{\circ} 41^{\prime} 09^{\prime \prime}$, Long $104^{\circ} 18^{\prime} 59^{\prime \prime}$, in SW/ANE sec, 31, T.7 S., R. 26 E., Chaves County, Hydrologtc Unit 13060007 on left bank $0.5 \mathrm{mi}$ upstream from Eightmile Draw, $2.5 \mathrm{mi}$ upstream from boundary for Bitter Lake National Wildiffe Refuge, 4.6 miles downstream from Sand Creak and at mile 596.3.

PERIOD OF RECORD.--August 1992 to current year.

GAGE.-Water-stage recorder, Elevation of gage is 3,550 ft above National Geodetic Vertical Datum of 1929 , from topographic map.

REMARKS.--Records fair except for estimated datly discharges and those above 600 ft3/s, which are poor. Flow partly regulated by Lake Sumer (station 08384000). Diversion for 1rrigation of about 19,100 acres (1959 determination) above station.

EXTREMES FOR PERIOD OF RECORD.,-Maximum datly discharge, 1,200 ft3/s, Mar. 2, 1995; minimum daily, 1.9 ft3/s, Aug. 11,1995 .

EXTREMES FOR CURRENT YEAR.--Maximum daliy discharge, 1,170 ft3/s, May 12; minimum daily, $6.5 \mathrm{ft}^{3} / \mathrm{s}, \mathrm{June} 12$.

DISCBARGE, CUBIC FEET PER SECOND, WATER YEAR OCTOBER 1995 TO SEPTEMBER 1996

\begin{tabular}{|c|c|c|c|c|c|c|c|c|c|c|c|c|}
\hline DAY & OCT & Nov & $\mathrm{DEC}$ & JAN & FEB & MAR & AFR & MAY & JUN & JUL & AUG & SEP \\
\hline $\begin{array}{l}1 \\
2 \\
3 \\
4 \\
5\end{array}$ & $\begin{array}{r}54 \\
50 \\
62 \\
155 \\
682\end{array}$ & $\begin{array}{l}44 \\
48 \\
51 \\
54 \\
47\end{array}$ & $\begin{array}{l}20 \\
19 \\
26 \\
36 \\
40\end{array}$ & $\begin{array}{l}61 \\
65 \\
65 \\
63 \\
64\end{array}$ & $\begin{array}{l}59 \\
59 \\
60 \\
48 \\
65\end{array}$ & $\begin{array}{l}38 \\
38 \\
37 \\
40 \\
41\end{array}$ & $\begin{array}{l}35 \\
37 \\
41 \\
37 \\
43\end{array}$ & $\begin{array}{r}21 \\
19 \\
\theta 220 \\
0820 \\
0920\end{array}$ & $\begin{array}{l}39 \\
37 \\
35 \\
29 \\
25\end{array}$ & $\begin{array}{l}9910 \\
9900 \\
6850 \\
9800 \\
9550\end{array}$ & $\begin{array}{r}990 \\
58 \\
83 \\
59 \\
46\end{array}$ & $\begin{array}{r}153 \\
154 \\
86 \\
71 \\
60\end{array}$ \\
\hline $\begin{array}{r}6 \\
7 \\
8 \\
9 \\
10\end{array}$ & $\begin{array}{l}069 \\
071 \\
076 \\
086 \\
075\end{array}$ & $\begin{array}{l}43 \\
40 \\
38 \\
36 \\
32\end{array}$ & $\begin{array}{l}41 \\
44 \\
45 \\
44 \\
46\end{array}$ & $\begin{array}{l}65 \\
66 \\
68 \\
69 \\
66\end{array}$ & $\begin{array}{l}79 \\
75 \\
66 \\
61 \\
59\end{array}$ & $\begin{array}{l}38 \\
37 \\
40 \\
46 \\
48\end{array}$ & $\begin{array}{l}45 \\
45 \\
53 \\
50 \\
50\end{array}$ & $\begin{array}{r}e 950 \\
e 970 \\
e 1080 \\
\theta 1110 \\
\theta 1120\end{array}$ & $\begin{array}{l}19 \\
15 \\
13 \\
11 \\
10\end{array}$ & $\begin{array}{r}e 350 \\
255 \\
166 \\
116 \\
243\end{array}$ & $\begin{array}{r}35 \\
32 \\
48 \\
e 300 \\
144\end{array}$ & $\begin{array}{r}49 \\
41 \\
e 100 \\
140 \\
129\end{array}$ \\
\hline $\begin{array}{r}11 \\
12 \\
13 \\
14 \\
115\end{array}$ & $\begin{array}{l}061 \\
060 \\
048 \\
040 \\
046\end{array}$ & $\begin{array}{l}30 \\
28 \\
27 \\
26 \\
27\end{array}$ & $\begin{array}{l}45 \\
49 \\
51 \\
52 \\
52\end{array}$ & $\begin{array}{l}65 \\
65 \\
65 \\
67 \\
64\end{array}$ & $\begin{array}{l}56 \\
56 \\
55 \\
55 \\
54\end{array}$ & $\begin{array}{l}54 \\
57 \\
49 \\
55 \\
61\end{array}$ & $\begin{array}{l}38 \\
38 \\
30 \\
27 \\
25\end{array}$ & $\begin{array}{l}\text { e1100 } \\
\text { e } 1170 \\
\text { e1120 } \\
e 1100 \\
\text { e } 1110\end{array}$ & $\begin{array}{r}7.6 \\
6.5 \\
17 \\
0250 \\
-1100\end{array}$ & $\begin{array}{r}252 \\
e 150 \\
0200 \\
0250 \\
160\end{array}$ & $\begin{array}{r}81 \\
e 80 \\
e 110 \\
090 \\
065\end{array}$ & $\begin{array}{r}70 \\
82 \\
77 \\
242 \\
353\end{array}$ \\
\hline $\begin{array}{l}16 \\
17 \\
18 \\
19 \\
20\end{array}$ & $\begin{array}{l}e 42 \\
064 \\
063 \\
050 \\
033\end{array}$ & $\begin{array}{l}26 \\
25 \\
24 \\
23 \\
23\end{array}$ & $\begin{array}{l}53 \\
55 \\
59 \\
63 \\
63\end{array}$ & $\begin{array}{l}63 \\
61 \\
61 \\
60 \\
60\end{array}$ & $\begin{array}{l}53 \\
52 \\
67 \\
85 \\
70\end{array}$ & $\begin{array}{l}68 \\
63 \\
60 \\
63 \\
62\end{array}$ & $\begin{array}{l}23 \\
23 \\
23 \\
25 \\
25\end{array}$ & $\begin{array}{r}01080 \\
01060 \\
\times 1030 \\
0490 \\
e 320\end{array}$ & $\begin{array}{r}0950 \\
\theta 900 \\
\theta 1000 \\
\theta 1050 \\
e 1100\end{array}$ & $\begin{array}{r}114 \\
90 \\
78 \\
65 \\
54\end{array}$ & $\begin{array}{r}055 \\
0100 \\
050 \\
045 \\
045\end{array}$ & $\begin{array}{r}450 \\
-250 \\
93 \\
57 \\
49\end{array}$ \\
\hline $\begin{array}{l}21 \\
22 \\
23 \\
24 \\
25\end{array}$ & $\begin{array}{l}030 \\
029 \\
026 \\
027 \\
030\end{array}$ & $\begin{array}{l}22 \\
22 \\
21 \\
20 \\
20\end{array}$ & $\begin{array}{l}61 \\
58 \\
61 \\
62 \\
62\end{array}$ & $\begin{array}{l}61 \\
60 \\
61 \\
62 \\
61\end{array}$ & $\begin{array}{l}53 \\
47 \\
45 \\
42 \\
40\end{array}$ & $\begin{array}{l}58 \\
51 \\
55 \\
45 \\
42\end{array}$ & $\begin{array}{l}23 \\
23 \\
30 \\
28 \\
28\end{array}$ & $\begin{array}{r}207 \\
155 \\
122 \\
100 \\
81\end{array}$ & $\begin{array}{r}91130 \\
9960 \\
9920 \\
9900 \\
9910\end{array}$ & $\begin{array}{l}54 \\
57 \\
41 \\
38 \\
46\end{array}$ & $\begin{array}{r}90 \\
105 \\
67 \\
980 \\
243\end{array}$ & $\begin{array}{l}42 \\
43 \\
41 \\
33 \\
26\end{array}$ \\
\hline $\begin{array}{l}26 \\
27 \\
28 \\
29 \\
30 \\
31\end{array}$ & $\begin{array}{r}942 \\
40 \\
37 \\
37 \\
42 \\
45\end{array}$ & $\begin{array}{r}19 \\
19 \\
20 \\
20 \\
20 \\
-\cdots\end{array}$ & $\begin{array}{l}63 \\
62 \\
62 \\
62 \\
61 \\
61\end{array}$ & $\begin{array}{l}63 \\
62 \\
60 \\
61 \\
62 \\
60\end{array}$ & $\begin{array}{r}36 \\
37 \\
38 \\
36 \\
-- \\
-\end{array}$ & $\begin{array}{l}39 \\
38 \\
38 \\
40 \\
42 \\
37\end{array}$ & $\begin{array}{r}27 \\
31 \\
30 \\
25 \\
23 \\
---\end{array}$ & $\begin{array}{l}68 \\
59 \\
54 \\
51 \\
44 \\
40\end{array}$ & $\begin{array}{r}e 750 \\
e 900 \\
e 1000 \\
0950 \\
9900 \\
-\end{array}$ & $\begin{array}{r}52 \\
155 \\
54 \\
55 \\
63 \\
0100\end{array}$ & $\begin{array}{r}126 \\
e 650 \\
e 350 \\
e 200 \\
176 \\
e 200\end{array}$ & $\begin{array}{l}21 \\
21 \\
22 \\
19 \\
16 \\
-\end{array}$ \\
\hline $\begin{array}{l}\text { TOTAL } \\
\text { MEAN } \\
\text { MAX } \\
\text { MIN } \\
\text { AC }-F T\end{array}$ & $\begin{array}{r}1672 \\
53.9 \\
155 \\
26 \\
3320\end{array}$ & $\begin{array}{r}895 \\
29.8 \\
54 \\
19 \\
1780\end{array}$ & $\begin{array}{r}1578 \\
50.9 \\
63 \\
19 \\
3130\end{array}$ & $\begin{array}{r}1956 \\
63.1 \\
69 \\
60 \\
3880\end{array}$ & $\begin{array}{r}1608 \\
55.4 \\
85 \\
36 \\
3190\end{array}$ & $\begin{array}{r}1480 \\
47.7 \\
68 \\
37 \\
2940\end{array}$ & $\begin{array}{r}981 \\
32.7 \\
53 \\
23 \\
1950\end{array}$ & $\begin{array}{r}17791 \\
574 \\
1170 \\
19 \\
35290\end{array}$ & $\begin{array}{r}15934.1 \\
531 \\
1130 \\
6.5 \\
31610\end{array}$ & $\begin{array}{r}7268 \\
234 \\
910 \\
38 \\
14420\end{array}$ & $\begin{array}{r}3903 \\
126 \\
650 \\
32 \\
7740\end{array}$ & $\begin{array}{r}2990 \\
99.7 \\
450 \\
16 \\
5930\end{array}$ \\
\hline
\end{tabular}

CAL YR 1995 TOTAL 80639.9 MEAN 221 MAX 2100 MIN 1.9 AC-FT 159900

WTR YR 1996 TOTAL 58056.1 MEAN 159 MAX 1170 MIN 6.5 AC-FT 115200 
RIO GRANDE BASIN

08386000 PECOS RIVER NEAR ACME, NM

LOCATIOX, --Lat $33^{\circ} 32^{\circ} 10^{\prime \prime}$, long $104^{\circ} 22^{\prime} 34^{\prime \prime}$, in Shish gec. 14, T.9 S, R. 25 E, Chaves County, Bydrologic Unit 13060007 on right bank 3.0 mi downtream from U.s. Hi ghway 70.3 .7 mi downstream from Salt Croek. 4.7 mi southes of 14 mi northeast of Rospell and at mile $585,3$.

DRAIHACE AREA.--11,380 $\mathrm{mt}^{2}$, approximately (contributing area).

WATER-DISCEARGE RECORDS

PERIOD of RECORD.-September 1921 to June 1923, July 1937 to current year Monthly discharge only for some periods, publithed in kes 1312

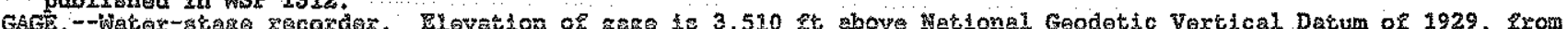
topographic mep. Prior to Nov. 1, 1938, at site on highway bridge 3 mi upstrean at various datums. Since Oct. 25,1963 , supplemental water-stage recordox at site opposito base gage at same datum.

RasARks. - Weter-discharge records good except for estimated dafly discharges, which are poor. Flow regulated by Lake Sumer (station 08384000 ) 117 mi upstroam since August 1937 and Santa Rosa lake (station 08382810 ) 172 mi upatroan Anco Apxi1 1980, Diversions for ixrigation of about 20,000 acres, 1959 determination, upstream from atation. U.S. Arwy Corps of Engineers satellite tolemeter at station. No flow at times.

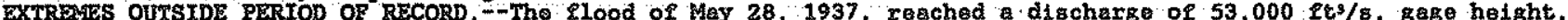
$14.82 \mathrm{rt}$, from Rloodmarks, site and datum then in use, from plope-area messurement, but may have been exceeded by the elood of oct. 1, 1904 .

DISCRAREE, CUBIC FEET PER SECOND, WATER YEAR OCTOBER 1995 TO SEPTEMBER 1996

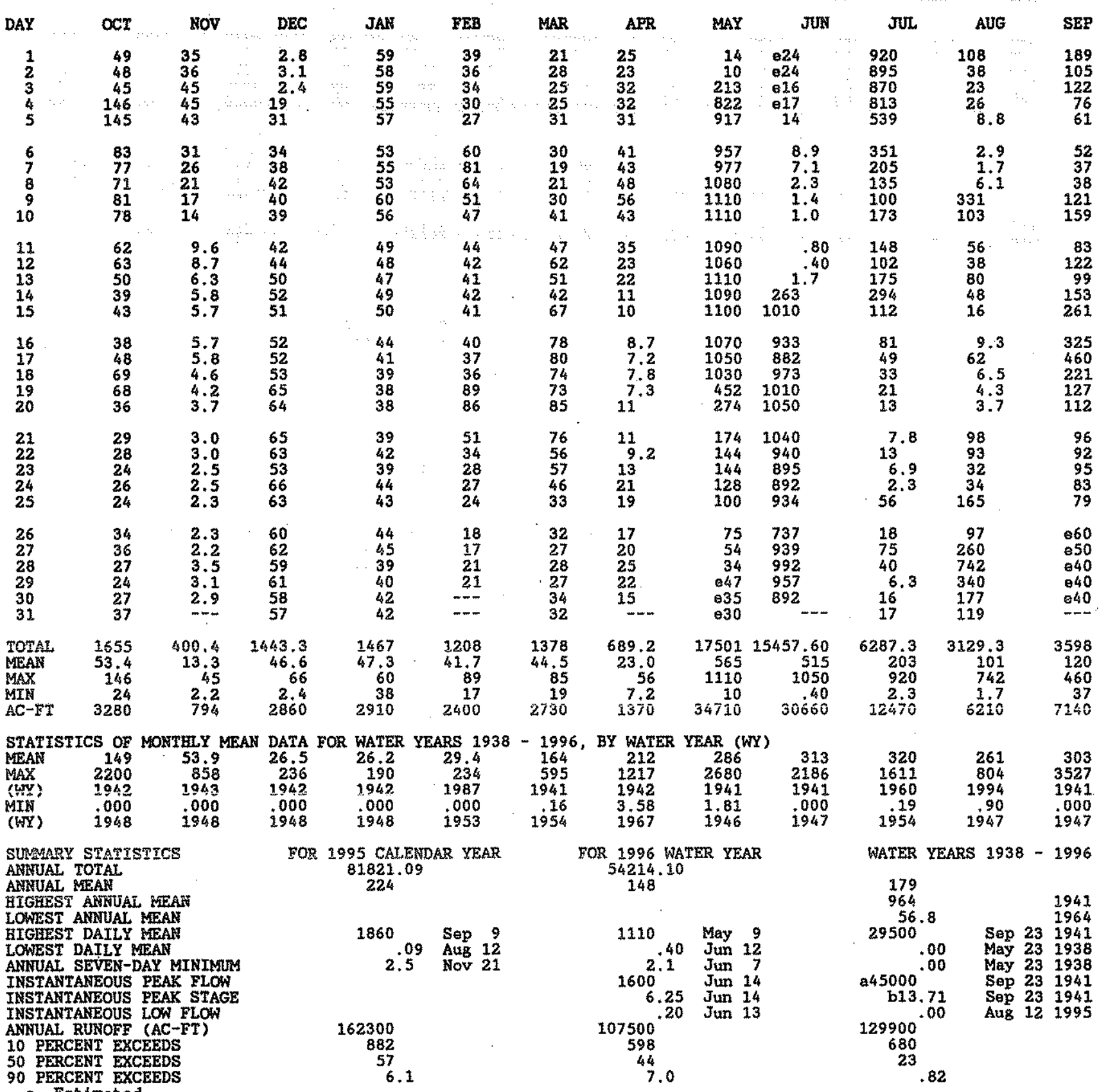

PERCENT EXCEFDS

a-From slopemarea measurement, but may have exceeded by the flood of Oct. $1,1904$.

b-From floodmarks, site and datum then in use. 
RIO GRANDE BASIN

08386000 PECOS RIVER NEAR ACME, NM -- Continued

WATER-QUALITY RECORDS

PERTOD OF RECORD. - -Water years 1937 to current year.

WATER QUALITY DATA, WATER YEAR OCTOBER 1995 TO SEPTEMBER 1996

\begin{tabular}{|c|c|c|c|c|c|c|c|c|c|c|c|}
\hline DATE & TIME & $\begin{array}{c}\text { DIS- } \\
\text { CHARGE, } \\
\text { IRST } \\
\text { CUBIC } \\
\text { FEET } \\
\text { FER } \\
\text { SECOND } \\
(00061)\end{array}$ & $\begin{array}{l}\text { SEE- } \\
\text { CIFIC } \\
\text { CON- } \\
\text { DUCT- } \\
\text { ANCE } \\
\text { (US/CM) } \\
(00095)\end{array}$ & $\begin{array}{c}\text { PE } \\
\text { WATER } \\
\text { WAOLE } \\
\text { FIELD } \\
\text { (STAND- } \\
\text { ARD } \\
\text { UNITS) } \\
\text { (00400) }\end{array}$ & $\begin{array}{l}\text { TEMPER- } \\
\text { ATURE } \\
\text { AIR } \\
(D E G C) \\
(00020)\end{array}$ & $\begin{array}{c}\text { TEMPER- } \\
\text { ATURE } \\
\text { WATER } \\
\text { (DEG C) } \\
(00010)\end{array}$ & $\begin{array}{l}\text { BARO- } \\
\text { METRIC } \\
\text { PRES- } \\
\text { SURE } \\
\text { (MM } \\
\text { OF } \\
\text { HG) } \\
(00025)\end{array}$ & $\begin{array}{c}\text { OXYGEN, } \\
\text { DIS-: } \\
\text { SOLVED } \\
(M G / L) \\
(00300)\end{array}$ & $\begin{array}{c}\text { OXYGEN, } \\
\text { DIS- } \\
\text { SOLVED } \\
\text { (PER- } \\
\text { CENT } \\
\text { SATUR- } \\
\text { ATION) } \\
\text { (00301) }\end{array}$ & $\begin{array}{l}\text { OXYGEH } \\
\text { DEMAND, } \\
\text { CEEAM- } \\
\text { ICAL } \\
\text { (BIGH } \\
\text { LEVEL) } \\
\text { (MG/L) } \\
(00340)\end{array}$ & $\begin{array}{l}\text { COLI- } \\
\text { FORM, } \\
\text { FECAL, } \\
0.7 \\
\text { UM-MH } \\
\text { (COLS. } \\
100 \mathrm{ML}) \\
(31625)\end{array}$ \\
\hline \multirow{4}{*}{$\begin{array}{c}\operatorname{DEC} 1995 \\
12 \\
\operatorname{MAR} 1996 \\
07 . . \\
\operatorname{MAY}_{02} \\
02 \ldots \\
\text { SEP } \\
05 . .\end{array}$} & 0830 & 40 & 2730 & $m$ & 3.5 & 3.0 & 676 & 11.5 & 98 & 10 & $\because-$ \\
\hline & 1500 & 20 & 3270 & 8.1 & 12.0 & 11.0 & 629 & 9.4 & 105 & 21 & $\mathrm{~K} 1$ \\
\hline & 0910 & 12 & 3980 & 8.1 & 17.5 & 12.0 & 676 & 10.4 & 110 & 22 & 67 \\
\hline & 1415 & 57 & 1880 & 8.2 & 32.5 & 29.0 & 670 & 8.4 & 126 & $<26$ & $<2$ \\
\hline DATE & $\begin{array}{c}\text { STREP- } \\
\text { TOCOCCI } \\
\text { FECAL } \\
\text { XF AGAR } \\
\text { (COLS. } \\
\text { PER } \\
100 \mathrm{ML}) \\
\text { (31673) }\end{array}$ & $\begin{array}{c}\text { HARD- } \\
\text { NESS } \\
\text { TOTAL } \\
\text { (ME/L } \\
\text { AS } \\
\text { CACO3) } \\
(00900)\end{array}$ & $\begin{array}{c}\text { HARD- } \\
\text { NESS } \\
\text { NONCARB } \\
\text { DISSOLV } \\
\text { FLD. AS } \\
\text { CACO3 } \\
(\text { MG } / L) \\
(00904)\end{array}$ & $\begin{array}{c}\text { CALCIUM } \\
\text { DIS- } \\
\text { SOLVED } \\
\text { (MEG/L } \\
\text { AS CA) } \\
\text { (00915) }\end{array}$ & $\begin{array}{c}\text { MAGNE- } \\
\text { SIUM, } \\
\text { DIS- } \\
\text { SOLVED } \\
\text { (MG/L } \\
\text { AS MG) } \\
(00925)\end{array}$ & $\begin{array}{c}\text { SODIUM, } \\
\text { DIS- } \\
\text { SOLVED } \\
\text { (MG/L } \\
\text { AS NA) } \\
(00930)\end{array}$ & $\begin{array}{c}\text { SODILM } \\
\text { AD- } \\
\text { SORP- } \\
\text { TION } \\
\text { RATIO } \\
(00931)\end{array}$ & $\begin{array}{c}\text { POTAS- } \\
\text { SIUM, } \\
\text { DIS- } \\
\text { SOLVED } \\
\text { (MG/L } \\
\text { AS K }) \\
(00935)\end{array}$ & $\begin{array}{c}\text { BICAR- } \\
\text { BONATE } \\
\text { WATER } \\
\text { DIS IT } \\
\text { FIELD } \\
\text { MG/L AS } \\
\text { BCOO } \\
(00453)\end{array}$ & $\begin{array}{c}\text { CAR- } \\
\text { BONATE } \\
\text { WATER } \\
\text { DIS IT } \\
\text { FIELL } \\
\text { MG/L AS } \\
\text { COS } \\
(00452)\end{array}$ & $\begin{array}{l}\text { ALKA- } \\
\text { LINITY } \\
\text { WAT DIS } \\
\text { TOT IT } \\
\text { FIELD } \\
\text { MG/L AS } \\
\text { CACO3 } \\
(39086)\end{array}$ \\
\hline $\begin{array}{l}\operatorname{DEC} 1995 \\
12 \\
\operatorname{MAR} \\
\operatorname{Mag6}\end{array}$ & -- & 1300 & - & 390 & 76 & 190 & 2 & 3.2 & - & -- & - \\
\hline$\underset{\mathrm{MAY}}{07 . .}$ & $<1$ & 1400 & 1300 & 410 & 88 & 250 & 3 & 3,8 & 84 & 0 & 69 \\
\hline SEP & K20 & 1800 & 1700 & 530 & 110 & 320 & 3 & 4.9 & 124 & 0 & 102 \\
\hline $05 \ldots$ & 58 & 960 & 880 & 290 & 58 & 120 & 2 & 4.2 & 96 & 0 & 79 \\
\hline DATE & $\begin{array}{c}\text { ALKA- } \\
\text { LINITY } \\
\text { IAB } \\
\text { (MG/L } \\
\text { AS } \\
\text { CACO3) } \\
(90410)\end{array}$ & $\begin{array}{c}\text { SULFATE } \\
\text { DIS- } \\
\text { SOLVED } \\
\text { (MG/L } \\
\text { AS SO4) } \\
(00945)\end{array}$ & $\begin{array}{l}\text { CELO- } \\
\text { RIDE, } \\
\text { DIS- } \\
\text { SOLVED } \\
\text { (MG/L } \\
\text { AS CL) } \\
(00940)\end{array}$ & $\begin{array}{c}\text { FLUO- } \\
\text { RrDE, } \\
\text { DIS- } \\
\text { SOLVED } \\
\text { (MG /L } \\
\text { AS F) } \\
(00950)\end{array}$ & $\begin{array}{c}\text { SILICA, } \\
\text { DIS- } \\
\text { SOLVED } \\
\text { (MG/L } \\
\text { AS } \\
\text { SIOZ) } \\
(00955)\end{array}$ & $\begin{array}{l}\text { SOLIDS, } \\
\text { SUM OF } \\
\text { CONSTI- } \\
\text { TUENTS, } \\
\text { DIS- } \\
\text { SOLVED } \\
\text { (MG/L) } \\
(70301)\end{array}$ & $\begin{array}{c}\text { NITRO- } \\
\text { GEN, } \\
\text { NITRATE } \\
\text { DIS- } \\
\text { SOLVED } \\
\text { (MG/L } \\
\text { AS N) } \\
(00618)\end{array}$ & $\begin{array}{c}\text { NITRO- } \\
\text { GEN, } \\
\text { NITRITE } \\
\text { DIS- } \\
\text { SOLVED } \\
\text { (MG/h } \\
\text { AS N) } \\
(00613)\end{array}$ & $\begin{array}{c}\text { NITRO- } \\
\text { GEN, } \\
\text { NO2+NO3 } \\
\text { DIS- } \\
\text { SOL.VED } \\
\text { (MG/L } \\
\text { AS N) } \\
(00631)\end{array}$ & $\begin{array}{c}\text { NITRO- } \\
\text { GEN } \\
\text { AMONIA } \\
\text { DIS- } \\
\text { SOLVED } \\
\text { (MGG/L } \\
\text { AS N } \\
\text { (00608) }\end{array}$ & $\begin{array}{c}\text { NITRO- } \\
\text { GEN, } \\
\text { ORGANIC } \\
\text { DIS- } \\
\text { SOLVED } \\
\text { (MG/L } \\
\text { AS N) } \\
(00607)\end{array}$ \\
\hline $\begin{array}{l}\mathrm{DEC} 1995 \\
12\end{array}$ & 102 & 1100 & 250 & 0.50 & 10 & 2080 & -- & $<0.010$ & 0.240 & 0.030 & -- \\
\hline $\begin{array}{l}\mathrm{MAR} 1996 \\
07 . \cdots \\
\mathrm{MAY}\end{array}$ & 102 & 1300 & 340 & 0.50 & 9.6 & 2440 & -- & $<0.010$ & 0.110 & 0.090 & 0.11 \\
\hline${ }_{\mathrm{SEP}}^{02} \ldots$ & 90 & 1700 & 470 & 0.60 & 9.9 & 3210 & - & $<0.010$ & $<0.050$ & $<0.015$ & -- \\
\hline 15 & 79 & 910 & 140 & 0.50 & 11 & 1580 & 0.110 & 0.010 & 0.120 & 0.060 & - \\
\hline DATE & $\begin{array}{l}\text { NITRO- } \\
\text { GEN,AM- } \\
\text { MONIA + } \\
\text { ORGANIC } \\
\text { TOTAL } \\
\text { (MG/L } \\
\text { AS N } \\
(00625)\end{array}$ & $\begin{array}{l}\text { MITRO- } \\
\text { GEN,AM- } \\
\text { MONIA + } \\
\text { ORGANIC } \\
\text { DIS. } \\
\text { (MG/L } \\
\text { AS N) } \\
(00623)\end{array}$ & $\begin{array}{c}\text { PHOS- } \\
\text { PHORUS } \\
\text { TOTAL } \\
\text { (MG/L } \\
\text { AS P) } \\
(00665)\end{array}$ & $\begin{array}{c}\text { PHOS- } \\
\text { PHORUS } \\
\text { DIS- } \\
\text { SOLVED } \\
\text { (MG/L } \\
\text { AS P) } \\
(00666)\end{array}$ & $\begin{array}{l}\text { PHOS- } \\
\text { PHORUSS } \\
\text { ORTHO, } \\
\text { DIS- } \\
\text { SOLVED } \\
\text { (MG/L } \\
\text { AS P) } \\
(00671)\end{array}$ & $\begin{array}{c}\text { CARBON, } \\
\text { ORGANIC } \\
\text { TOTAL } \\
\text { (MG/L } \\
\text { AS C) } \\
(00680)\end{array}$ & $\begin{array}{l}\text { ALUM- } \\
\text { INUM, } \\
\text { DIS- } \\
\text { SOLVED } \\
\text { (UG/L } \\
\text { AS AL) } \\
\text { (01106) }\end{array}$ & $\begin{array}{l}\text { ANTI- } \\
\text { MONY, } \\
\text { DIS- } \\
\text { SOLVED } \\
\text { (UG/L } \\
\text { AS SB) } \\
(01095)\end{array}$ & $\begin{array}{c}\text { ARSENIC } \\
\text { DIS- } \\
\text { SOLVED } \\
\text { (UG/ } \\
\text { AS AS) } \\
(01000)\end{array}$ & $\begin{array}{c}\text { BARIUM, } \\
\text { DIS- } \\
\text { SOLVED } \\
\text { (UG/L } \\
\text { AS BA) } \\
(01005)\end{array}$ & $\begin{array}{l}\text { BERYL- } \\
\text { LIUM, } \\
\text { DIS- } \\
\text { SOLVED } \\
\text { (UG/L } \\
\text { AS BE) } \\
(01010)\end{array}$ \\
\hline $\begin{array}{l}\mathrm{DEC} 1995 \\
12\end{array}$ & $<0.20$ & $<0.20$ & $<0.010$ & $<0.010$ & $<0.010$ & 1.5 & 5.0 & $<2.0$ & $<1$ & 28 & $<2.0$ \\
\hline MAY. & 0.20 & 0.20 & 0.010 & $<0.010$ & $<0.010$ & 3.4 & -- & $x_{1}$ & -- & - & -- \\
\hline SEP & 0.20 & $<0$ & 0.040 & 0.010 & $<0.010$ & & $-m$ & $-\infty$ & - & -- & - \\
\hline & 0 & $<0.20$ & 0.070 & 0.020 & 0.010 & 4.7 & 293 & $<1,0$ & 1 & 140 & \\
\hline
\end{tabular}


RIO GRANDE BASIN

08386000 PECOS RIVER NEAR ACME, MM -- Continued

WATER-QUALITY RECORDS

WATER QUALITY DATA, WATER YEAR OCTOBER 1995 TO SEPTEMBER 1996

\begin{tabular}{|c|c|c|c|c|c|c|c|c|c|c|c|}
\hline DATE & $\begin{array}{l}\text { BORON } \\
\text { DIS- } \\
\text { SOLVED } \\
\text { (UG/L } \\
\text { AS B) } \\
(01020)\end{array}$ & $\begin{array}{c}\text { CADMIUM } \\
\text { DIS - } \\
\text { SOLVED } \\
\text { (UGL/ } \\
\text { AS CD }) \\
(01025)\end{array}$ & $\begin{array}{l}\text { CHRO- } \\
\text { MIIM, } \\
\text { DIS- } \\
\text { SOLVED } \\
\text { (US/L } \\
\text { AS CR) } \\
(01030)\end{array}$ & $\begin{array}{c}\text { COBALT, } \\
\text { DIS } \\
\text { SOLVED } \\
\text { (UG/L } \\
\text { AS CO) } \\
(01035)\end{array}$ & $\begin{array}{l}\text { COPEER, } \\
\text { DIS- } \\
\text { SOLVED } \\
\text { CUE/L } \\
\text { AS CU) } \\
(01040)\end{array}$ & $\begin{array}{l}\text { IRON, } \\
\text { DIS- } \\
\text { SOLVED } \\
\text { (US/L } \\
\text { AS FE) } \\
(01046)\end{array}$ & $\begin{array}{l}\text { LEAD, } \\
\text { DIS - } \\
\text { SOLVED } \\
\text { (UE/L } \\
\text { AS PB }) \\
(01049)\end{array}$ & $\begin{array}{l}\text { MANGA- } \\
\text { NESEE, } \\
\text { DNS- } \\
\text { SOLVED } \\
\text { (UGL/ } \\
\text { AS MN) } \\
(01056)\end{array}$ & $\begin{array}{c}\text { MERCURY } \\
\text { TOTALI } \\
\text { RECOV - } \\
\text { ERABLE } \\
\text { (US/L } \\
\text { AS EG) } \\
(71900)\end{array}$ & $\begin{array}{l}\text { MOLYB- } \\
\text { DENUM, } \\
\text { DIS-' } \\
\text { SOLVED } \\
\text { (UG/L } \\
\text { AS MO) } \\
(01060)\end{array}$ & $\begin{array}{c}\text { NICKEL, } \\
\text { DIS- } \\
\text { SOLVDD } \\
\text { (US/L } \\
\text { AS NI) } \\
(01065)\end{array}$ \\
\hline \multirow{4}{*}{$\begin{array}{c}\mathrm{DEC} 1995 \\
12 \\
\mathrm{MAR} 1996 \\
07 \ldots \\
\mathrm{MAY} \\
02 \ldots \\
\mathrm{SEP} \\
05 \ldots\end{array}$} & 180 & $<2,0$ & $<2.0$ & $<2,0$ & 14 & 18 & $<2,0$ & 5.0 & $<0.10$ & $<2.0$ & 12 \\
\hline & 230 & - & -- & -- & -- & $<9.0$ & -- & -- & - & -- & -- \\
\hline & 290 & - & $-\cdots$ & - & - & $<3.0$ & -- & -- & $\cdots$ & - & - \\
\hline & 156 & $<1.0$ & $<1.0$ & 1.0 & 5.0 & 170 & $<1.0$ & 10 & $<0.10$ & 3.0 & 8.0 \\
\hline DATE & $\begin{array}{c}\text { SELE- } \\
\text { NIUMA } \\
\text { TOTAL } \\
\text { (UG/L } \\
\text { AS SE }) \\
(01147)\end{array}$ & $\begin{array}{c}\text { SELE- } \\
\text { NIUM, } \\
\text { DIS- } \\
\text { SOLVED } \\
\text { (US/L, } \\
\text { AS SE) } \\
(01145)\end{array}$ & $\begin{array}{c}\text { SILVER, } \\
\text { DIS- } \\
\text { SOLVED } \\
\text { (UG/L } \\
\text { AS AG) } \\
(01075)\end{array}$ & $\begin{array}{c}\text { ZINC, } \\
\text { DIS- } \\
\text { SOLVED } \\
\text { (UG/L/L } \\
\text { AS ZN) } \\
(01090)\end{array}$ & $\begin{array}{c}\text { NITRO- } \\
\text { GEA, } \\
\text { GO2+NO3 } \\
\text { TOT. IN } \\
\text { BOT. MAT } \\
\text { (MG/KG } \\
\text { AS N) } \\
(00633)\end{array}$ & $\begin{array}{l}\text { NITRO- } \\
\text { GEN, NHA } \\
\text { IOTAI } \\
\text { IN BOT. } \\
\text { MAT } \\
\text { MG/KG } \\
\text { AS ND) } \\
(00611)\end{array}$ & $\begin{array}{l}\text { NTrRO- } \\
\text { GEN, NH4 } \\
\text { QORG } \\
\text { TOT IN } \\
\text { BOT MAT } \\
\text { (ME/KG } \\
\text { AS N) } \\
(00626) .\end{array}$ & $\begin{array}{l}\text { PHOS- } \\
\text { PHORUS } \\
\text { TOTAL } \\
\text { IN BOT. } \\
\text { MAT. } \\
\text { MGG KG } \\
\text { AS P) } \\
\text { (00668) }\end{array}$ & $\begin{array}{l}\text { ARSENIC } \\
\text { TOOAL } \\
\text { IN BOT- } \\
\text { TOM MA- } \\
\text { TERIAL } \\
\text { UGGG } \\
\text { AS AS }) \\
(01003)\end{array}$ & $\begin{array}{l}\text { CADMIUM } \\
\text { RECOV. } \\
\text { FM BOT- } \\
\text { TOM MA- } \\
\text { TERTAL } \\
\text { (UG/G } \\
\text { AS CD } \\
(01028)\end{array}$ & $\begin{array}{c}\text { CHRO- } \\
\text { MIUUM, } \\
\text { RECOV. } \\
\text { FM BOT } \\
\text { TOM MA- } \\
\text { TERAAL } \\
\text { (UGG/G) } \\
(01029)\end{array}$ \\
\hline $\begin{array}{l}\text { DEC } 1995 \\
12\end{array}$ & $<1$ & $<<1$ & $<2.0$ & 11 & $<2,0$ & 1.0 & 50 & 78 & 2 & 2 & \\
\hline $07 \ldots$ & - & - & - & -- & -- & -- & -- & -- & - & -- & -- \\
\hline $\begin{array}{l}02 \ldots \\
\operatorname{SEP}_{05 \ldots} \ldots\end{array}$ & -- & -- & - & - & -- & - & -- & - & -- & - & -- \\
\hline DATE & 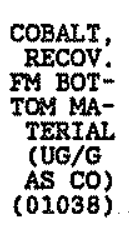 & $\begin{array}{l}\text { COPPER, } \\
\text { RECOV: } \\
\text { FM BOT- } \\
\text { TOMG MA- } \\
\text { TERIAL } \\
\text { (UG/G } \\
\text { AS CU) } \\
(01043)\end{array}$ & $\begin{array}{l}\text { IRON, } \\
\text { RECOV́ } \\
\text { ME BOT- } \\
\text { TOM MA- } \\
\text { TERIAL } \\
\text { TUG/G } \\
\text { AS FE) } \\
(01170) .\end{array}$ & $\begin{array}{c}\text { LEAD } \\
\text { RECOU. } \\
\text { FM BOT- } \\
\text { TOM MA- } \\
\text { TOERAL } \\
\text { TUG/G } \\
\text { AS PBS } \\
\text { (01052) }\end{array}$ & $\begin{array}{c}\text { MANGA- } \\
\text { MESE, } \\
\text { RECOV. } \\
\text { FE BOT- } \\
\text { TOM MA- } \\
\text { TERIAL } \\
\text { (UG/G) } \\
(01053)\end{array}$ & $\begin{array}{l}\text { MERCURY } \\
\text { RECOV. } \\
\text { FM BOT- } \\
\text { TOM MA- } \\
\text { TERIAL } \\
\text { UGG/G } \\
\text { AS HG) } \\
(71921)\end{array}$ & 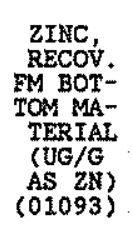 & $\begin{array}{c}\text { URANIUM } \\
\text { NATURAL } \\
\text { DIS- } \\
\text { SOLVED } \\
\text { (UG/L } \\
\text { AS U) } \\
(22703)\end{array}$ & $\begin{array}{l}\text { SEDI- } \\
\text { MENT, } \\
\text { SUS- } \\
\text { PENDED } \\
\text { (MG/LL) } \\
(80154)\end{array}$ & 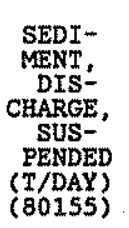 & $\begin{array}{l}\text { SED } \\
\text { SUSP. } \\
\text { SIEVE } \\
\text { DIAM. } \\
\text { \% FINER } \\
\text { THAAN } \\
\text {.062 MA } \\
(70331)\end{array}$ \\
\hline $\begin{array}{l}c 1995 \\
12 \\
12\end{array}$ & $<5$ & 2 & 1300 & $<10$ & 390 & $<0.01$ & 4 & 6.0 & 102 & 13 & 5. \\
\hline $\begin{array}{l}\text { MAR } 1996 \\
07 \ldots \\
\operatorname{MAY}\end{array}$ & -- & -- & -- & - & -- & -- & $\sim$ & -- & 54 & 2.9 & 74 \\
\hline SEP. & $-m$ & -- & - & $m$ & -- & 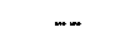 & $\ldots$ & -- & 45 & 1.4 & 58 \\
\hline 05 & $<!$ & 2 & 300 & $<3$ & 14 & $<0.01$ & 3 & 5.0 & 370 & 211 & \\
\hline
\end{tabular}


RIO GRANDE BASIN

08387000 RIO RUIDOSO AT HOLLYWOOD, NM

LOCATION - - Lat $33^{\circ} 19^{\circ} 36^{\prime \prime}$, Long $105^{\circ} 37^{\prime} 38^{\prime \prime}$, in SE\%SEKNE\% sec.25, T. 11 S., R.13 E., Lincoln County, Hydrologic Untt 13060008 , on centex pier on downstream side of bridge on Blooming Dale Road in Rutdoso Downs, $0.1 \mathrm{mi}$ north of U.S. Highway 70, 0.7 mi downstream from Gavilan Canyon, $1.7 \mathrm{mi}$ downstream from Carrizo Creok, and at mile 24.4.

DRAINAGE AREA. - - $120 \mathrm{mi}^{2}$, approximately.

PERIOD OF RECORD.--March 1953 to current YeatER-DISCHARGE RECORDS

GAGE.--Water-stage recorder. Elevation of gage is 6,420 ft above National Geodetic Vertical Datum of 1929 , from topographic map. Mar. 14, 1953 to Mar. 28, 1985, at site 0.95 mi downstream at different datum.

REMARKS. -Water-dtscharge records good except for estimated daily discharges, which are poor. Village of Ruidoso diverts from right bank $7.0 \mathrm{mi}$ upstream for municipal use and roturns a portion of this water as effluent from sewage disposal plant downstream from the gage.

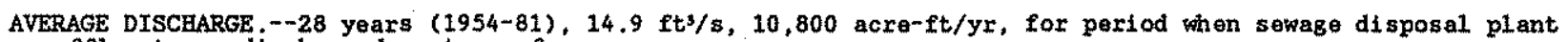
offluent was discharged upstream from gage.

EXTREMES OUTSIDE PGRIOD OF RECORD.--The flood of Sept. 29, 1941, is probably the highest stnce at least 1904 (discharge not determined).

DISCHARGE, CUBIC FEET PER SECOND, WATER YEAR OCTOBER 1995 TO SEPTEMBER 1996

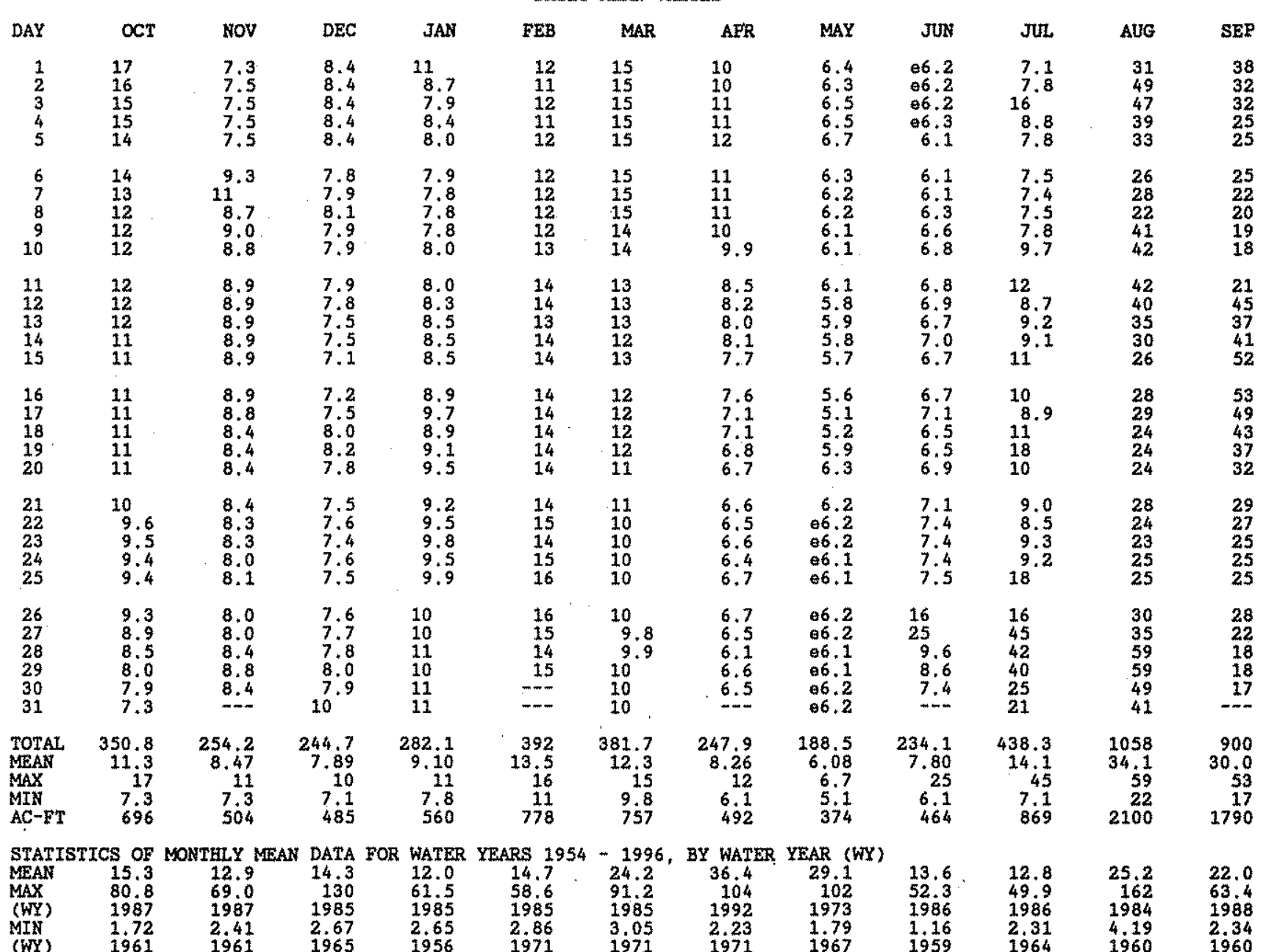

(WY) $\quad \begin{array}{lllllll}1961 & 1961 & 1965 & 1956 & 1971 & 1971 & 1971\end{array}$

FOR 1995 CALENDAR YEAR

SUMTH STATISTICS

ANNUAL TOTAL

HIGEEST ANNUAL MEAN

LOWEST ANNUAL MEAN

HIGHEST DAILY MEAN

LOWEST DAILY MEAN

ANNUAL SEVEN-DAY MINIMUM

INSTANTANEOUS PEAK FLOW

INSTANTANEOUS PEAK STAGE

INSTANTANEOUS LOW FLOW

ANNUAL RUNOFF (AC-FT)

10 PERCENT EXCEEDS

50 PERCENT EXCEEDS

90 PERCENT EXCEEDS

O Estimated

a-From rating curve extended

$b$-Site and datum then in use.
6023.3

FOR 1996 WATER YEAR 4972.3

$46 \operatorname{Max} 22$

$\begin{array}{ll}6.6 & \text { Jul } \\ 6.9 & \text { JuI } 24\end{array}$

11950

28
13
7.8

13.6

WATER YEARS 1954 - 1996

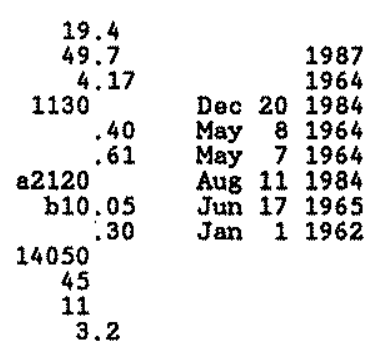


RIO GRANDE BASIN

08387000 RIO RUIDOSO AT HOLLYWOOD, MM -- Continued

WATER-QUALITY RECORDS

PERIOD OF RECORD.--Water years $1963-67,1987$ to current year.

WATER QUALITY DATA, WATER YEAR OCTOBER 1995 TO SEPTEMBER 1996

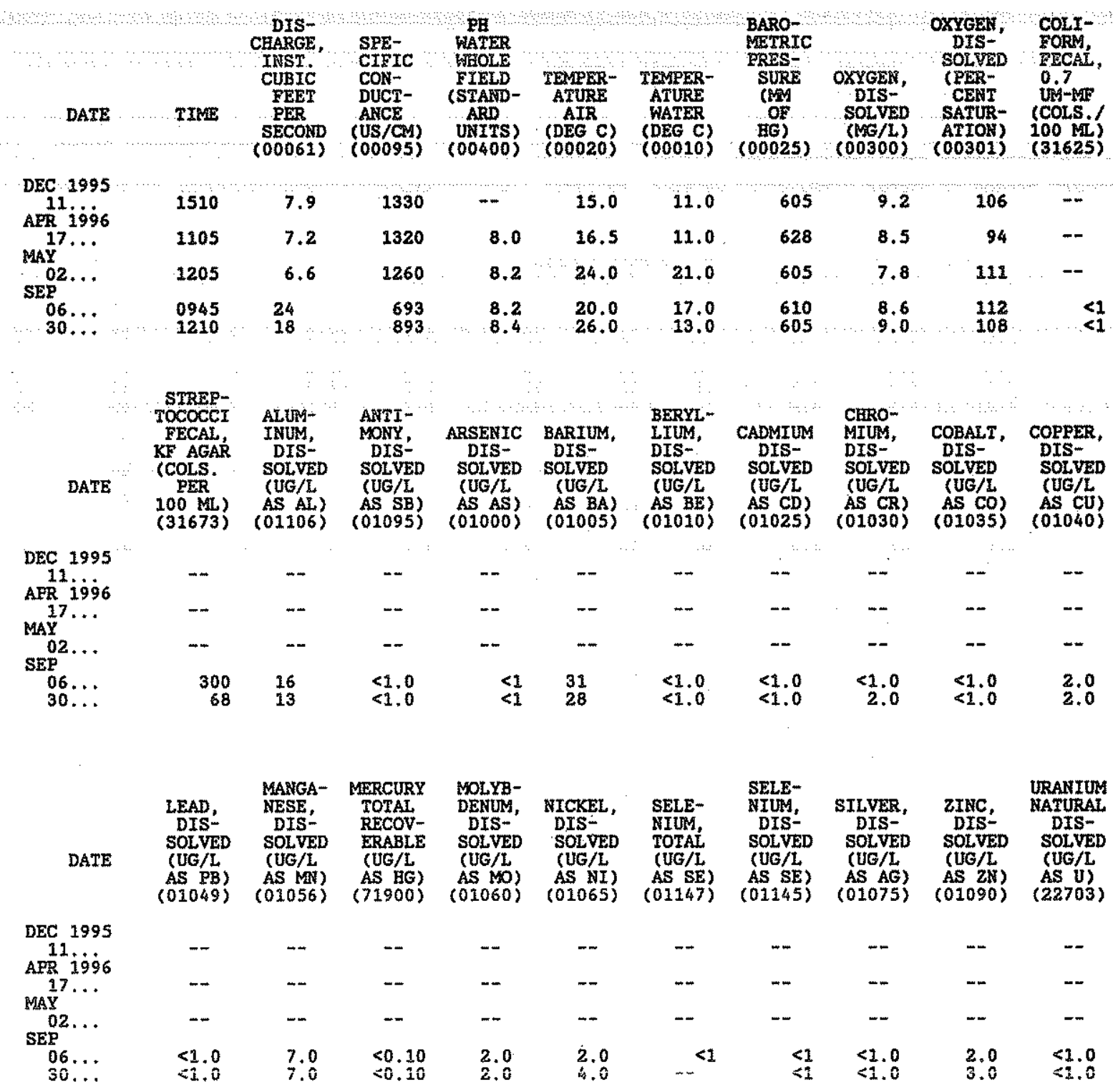


RIO GRANDE BASIN

08387600 EAGLE CREEK BELOW SOUTH FORK, NEAR ALTO, MM

LOCATION, --Lat $33^{\circ} 23^{\prime} 33^{\prime \prime}$, long $105^{\circ} 43^{\prime} 16^{\prime \prime}$, in SEKSWK sec.31, T.10 S., R.13 E., Lincoln County, Hydrologic Unit 13060008 , in Lincoln National Forest on right bank 100 ft upstream from culvert under State Road 532 , 400 ft downstream Prom South Fork, and 2.5 mi west of Alto. Mouth at Rio Ruldoso mile 11.3.

DRAINAGE AREA. $--8.14 \mathrm{mi}^{2}$.

PERIOD OF RECORD. --August 1969 to December 1980, April 1988 to current year.

GAGE.--Water-8tage recorder. Elevation of gage is 7,600 ft above National Gaodetic Vertical Datum of 1929, from toposraphic map. August 26, 1969 to Decomber 31, 1980, at site $360 \mathrm{ft}$ downstream at datum $6.0 \mathrm{ft}$ higher.

RFMARKS. --Records good. No diversions for irrigation upstream from station. Some water ts gtored in small unregulated recreational ponds on the Mescalero Apache Indian Reservation upstream. Several observations of water temperature pere made during the year. DISCHARGE, CUBIC FEET FER SECOKD, WATER YEAR OCTOBER 1995 TO SEPTEMBER 1996

\begin{tabular}{|c|c|c|c|c|c|c|c|c|c|c|c|c|}
\hline DAY & $\infty \mathrm{CI}$ & Nov & DEC & JAR & FEB & MAR & APR & MAY & $\pi$ N & JUL & AUG & SEP \\
\hline $\begin{array}{l}1 \\
2 \\
3 \\
4 \\
5\end{array}$ & $\begin{array}{l}.72 \\
.63 \\
.63 \\
.75 \\
.83\end{array}$ & $\begin{array}{l}.19 \\
.20 \\
.21 \\
.20 \\
.19\end{array}$ & $\begin{array}{l}.12 \\
.13 \\
.12 \\
.12 \\
.12\end{array}$ & $\begin{array}{l}.22 \\
.23 \\
.22 \\
.25 \\
.23\end{array}$ & $\begin{array}{l}.21 \\
.32 \\
.32 \\
.32 \\
.32\end{array}$ & $\begin{array}{l}.18 \\
.18 \\
.18 \\
.16 \\
.16\end{array}$ & $\begin{array}{l}.12 \\
.12 \\
.11 \\
.14 \\
.18\end{array}$ & $\begin{array}{l}.00 \\
.00 \\
.00 \\
.00 \\
.00\end{array}$ & $\begin{array}{l}.00 \\
.00 \\
.00 \\
.00 \\
.00\end{array}$ & $\begin{array}{l}.02 \\
.06 \\
.32 \\
.18 \\
.12\end{array}$ & $\begin{array}{l}1.5 \\
1.4 \\
2.3 \\
2.2 \\
1.8\end{array}$ & $\begin{array}{l}2.6 \\
1.8 \\
2.1 \\
1.6 \\
1.8\end{array}$ \\
\hline $\begin{array}{r}6 \\
7 \\
8 \\
9 \\
10\end{array}$ & $\begin{array}{l}.80 \\
.78 \\
.73 \\
.65 \\
.52\end{array}$ & $\begin{array}{l}.33 \\
.32 \\
.23 \\
.19 \\
.18\end{array}$ & $\begin{array}{l}.12 \\
.13 \\
.15 \\
.13 \\
.13\end{array}$ & $\begin{array}{l}.22 \\
.22 \\
.22 \\
.22 \\
.22\end{array}$ & $\begin{array}{l}.32 \\
.32 \\
.31 \\
.30 \\
.28\end{array}$ & $\begin{array}{r}.16 \\
.18 \\
.17 \\
.17 \\
.17\end{array}$ & $\begin{array}{l}.24 \\
.25 \\
.21 \\
.20 \\
.17\end{array}$ & $\begin{array}{l}.00 \\
.00 \\
.00 \\
.00 \\
.00\end{array}$ & $\begin{array}{l}.00 \\
.00 \\
.00 \\
.00 \\
.00\end{array}$ & $\begin{array}{l}.12 \\
.11 \\
.11 \\
.11 \\
.19\end{array}$ & $\begin{array}{r}1.2 \\
.98 \\
.92 \\
1.0 \\
1.0\end{array}$ & $\begin{array}{l}1.7 \\
1.2 \\
1.2 \\
1.2 \\
1.1\end{array}$ \\
\hline $\begin{array}{l}11 \\
12 \\
13 \\
14 \\
15\end{array}$ & $\begin{array}{l}.46 \\
.42 \\
.39 \\
.35 \\
.32\end{array}$ & $\begin{array}{l}.18 \\
.18 \\
.16 \\
.16 \\
.16\end{array}$ & $\begin{array}{l}.12 \\
.12 \\
.12 \\
.12 \\
.12\end{array}$ & $\begin{array}{l}.22 \\
.22 \\
.23 \\
.24 \\
.24\end{array}$ & $\begin{array}{l}.26 \\
.25 \\
.24 \\
.24 \\
.23\end{array}$ & $\begin{array}{l}.16 \\
.14 \\
.13 \\
.13 \\
.13\end{array}$ & $\begin{array}{l}.14 \\
.12 \\
.11 \\
.10 \\
.09\end{array}$ & $\begin{array}{l}.00 \\
.00 \\
.00 \\
.00 \\
.00\end{array}$ & $\begin{array}{l}.00 \\
.00 \\
.00 \\
.00 \\
.00\end{array}$ & $\begin{array}{r}.33 \\
.17 \\
.13 \\
.12 \\
.14\end{array}$ & $\begin{array}{l}.79 \\
.56 \\
.47 \\
.43 \\
.39\end{array}$ & $\begin{array}{l}2.0 \\
8.7 \\
13 \\
11 \\
11\end{array}$ \\
\hline $\begin{array}{l}16 \\
17 \\
18 \\
19 \\
20\end{array}$ & $\begin{array}{l}.31 \\
.30 \\
.27 \\
.26 \\
.25\end{array}$ & $\begin{array}{l}.16 \\
.16 \\
.15 \\
.15 \\
.15\end{array}$ & $\begin{array}{l}.12 \\
.13 \\
.13 \\
.14 \\
.14\end{array}$ & $\begin{array}{l}.24 \\
.22 \\
.21 \\
.24 \\
.23\end{array}$ & $\begin{array}{l}.21 \\
.21 \\
.21 \\
.22 \\
.25\end{array}$ & $\begin{array}{l}.13 \\
.13 \\
.13 \\
.14 \\
.13\end{array}$ & $\begin{array}{l}.09 \\
.07 \\
.05 \\
.04 \\
.03\end{array}$ & $\begin{array}{l}.00 \\
.00 \\
.00 \\
.00 \\
.00\end{array}$ & $\begin{array}{l}.00 \\
.00 \\
.00 \\
.00 \\
.00\end{array}$ & $\begin{array}{r}.16 \\
.13 \\
.12 \\
2.1 \\
1.4\end{array}$ & $\begin{array}{r}.59 \\
.71 \\
1.5 \\
1.1 \\
.58\end{array}$ & $\begin{array}{l}9.1 \\
6.7 \\
5.0 \\
4.1 \\
3.4\end{array}$ \\
\hline $\begin{array}{l}21 \\
22 \\
23 \\
24 \\
25\end{array}$ & $\begin{array}{l}.25 \\
.23 \\
.22 \\
.22 \\
.22\end{array}$ & $\begin{array}{l}.15 \\
.14 \\
.14 \\
.14 \\
.14\end{array}$ & $\begin{array}{l}.14 \\
.14 \\
.16 \\
.15 \\
.17\end{array}$ & $\begin{array}{l}.22 \\
.23 \\
.24 \\
.24 \\
.24\end{array}$ & $\begin{array}{l}.26 \\
.22 \\
.20 \\
.19 \\
.21\end{array}$ & $\begin{array}{l}.13 \\
.12 \\
.12 \\
.12 \\
.12\end{array}$ & $\begin{array}{l}.02 \\
.02 \\
.01 \\
.01 \\
.01\end{array}$ & $\begin{array}{l}.00 \\
.00 \\
.00 \\
.00 \\
.00\end{array}$ & $\begin{array}{l}.00 \\
.00 \\
.00 \\
.00 \\
.00\end{array}$ & $\begin{array}{r}.64 \\
.42 \\
.25 \\
.22 \\
1.3\end{array}$ & $\begin{array}{r}.66 \\
.64 \\
.49 \\
.49 \\
.64\end{array}$ & $\begin{array}{l}2.9 \\
2.5 \\
2.2 \\
2.3 \\
2.4\end{array}$ \\
\hline $\begin{array}{l}26 \\
27 \\
28 \\
29 \\
30 \\
31\end{array}$ & $\begin{array}{l}.22 \\
.21 \\
.20 \\
.20 \\
.20 \\
.19\end{array}$ & $\begin{array}{l}.13 \\
.13 \\
.13 \\
.13 \\
.13 \\
-\end{array}$ & $\begin{array}{l}.27 \\
.49 \\
.67 \\
.20 \\
.20 \\
.19\end{array}$ & $\begin{array}{l}.24 \\
.24 \\
.24 \\
.22 \\
.21 \\
.20\end{array}$ & $\begin{array}{l}.21 \\
.20 \\
.19 \\
.18 \\
-.- \\
--\end{array}$ & $\begin{array}{l}.12 \\
.12 \\
.12 \\
.12 \\
.12 \\
.12\end{array}$ & $\begin{array}{l}.00 \\
.00 \\
.00 \\
.00 \\
.00 \\
.0\end{array}$ & $\begin{array}{l}.00 \\
.00 \\
.00 \\
.00 \\
.00 \\
.00\end{array}$ & $\begin{array}{l}.00 \\
.20 \\
.11 \\
.06 \\
.04 \\
.0\end{array}$ & $\begin{array}{l}.65 \\
6.2 \\
3.8 \\
1.7 \\
1.9 \\
1.8\end{array}$ & $\begin{array}{l}1.4 \\
4.0 \\
5.6 \\
6.2 \\
4.6 \\
3.5\end{array}$ & $\begin{array}{l}2.5 \\
1.6 \\
1.4 \\
1.1 \\
1.0\end{array}$ \\
\hline $\begin{array}{l}\text { TOTAL } \\
\text { MEAN } \\
\text { MAX } \\
\text { MIN } \\
\text { AC-FT }\end{array}$ & $\begin{array}{r}12.73 \\
.41 \\
.83 \\
.19 \\
25\end{array}$ & $\begin{array}{r}5.21 \\
.17 \\
.33 \\
.13 \\
10\end{array}$ & $\begin{array}{r}5.31 \\
.17 \\
.67 \\
.12 \\
11\end{array}$ & $\begin{array}{r}7.06 \\
.23 \\
.25 \\
.20 \\
14\end{array}$ & $\begin{array}{r}7.20 \\
.25 \\
.32 \\
.18 \\
14\end{array}$ & $\begin{array}{r}4.39 \\
.14 \\
.18 \\
.12 \\
8.7\end{array}$ & $\begin{array}{r}2.65 \\
.088 \\
.25 \\
.00 \\
5.3\end{array}$ & $\begin{array}{l}0.00 \\
.000 \\
.00 \\
.00 \\
.00\end{array}$ & $\begin{array}{r}0.41 \\
.014 \\
.20 \\
.00 \\
.8\end{array}$ & $\begin{array}{r}25.02 \\
.81 \\
6.2 \\
.02 \\
50\end{array}$ & $\begin{array}{r}49.64 \\
1.60 \\
6.2 \\
.39 \\
98\end{array}$ & $\begin{array}{r}110.2 \\
3.67 \\
13 \\
1.0 \\
219\end{array}$ \\
\hline
\end{tabular}

STATISTICS OF MONTHLY MEAN DATA FOR WATER XEARS 1970 - 1996, BY WATER YEAR (WY)

\begin{tabular}{|c|c|c|c|c|c|c|c|c|c|c|c|c|}
\hline $\begin{array}{l}\text { MEAN } \\
\text { MAX } \\
\text { (WY) } \\
\text { MIN } \\
\text { (WX) }\end{array}$ & $\begin{array}{r}2.57 \\
14.4 \\
1975 \\
199 \\
1990\end{array}$ & $\begin{array}{l}2.07 \\
17.3 \\
1979 \\
1996\end{array}$ & $\begin{array}{r}2.17 \\
19.5 \\
1979 \\
1997 \\
1996\end{array}$ & $\begin{array}{l}1.70 \\
7.89 \\
1979 \\
192 \\
1990\end{array}$ & $\begin{array}{r}2.35 \\
8.19 \\
1979 \\
1925 \\
1996\end{array}$ & $\begin{array}{r}3.89 \\
10.6 \\
1979 \\
194 \\
1996\end{array}$ & $\begin{array}{l}5.11 \\
14.0 \\
1973 \\
1088 \\
1996\end{array}$ & $\begin{array}{l}3.99 \\
15.8 \\
1973 \\
000 \\
1996\end{array}$ & $\begin{array}{l}1.36 \\
5.94 \\
1979 \\
1014 \\
1996\end{array}$ & $\begin{array}{r}1.75 \\
5.50 \\
1990 \\
1971 \\
1971\end{array}$ & $\begin{array}{r}3.65 \\
16.3 \\
1988 \\
1931 \\
1994\end{array}$ & $\begin{array}{r}3.84 \\
9.26 \\
1974 \\
199 \\
1994\end{array}$ \\
\hline
\end{tabular}

\section{SLMMARY STATISTICS}

ANNUAL TOTAL

ALNUAL MEAN

LOWEST ANNUAL MEAN

LOWEST ANNUAL MEAN

HIGBEST DAILY MEAN

ANNUAL SEVEN-DAY MINIMIM

INSTANTAREOUS PFAK FLON

INSTANTANEOUS DEAK STAGE

INSTANTANEOUS LOW FLOW

ANNUAL RUNOFF (AC-FT)

10 PERCENT EXCEEDS

90 PKRCENT EXCEEDS
FOR 1995 CALENDAR YEAR

$\begin{array}{rlr}390.03 & & \\ 1.07 & & \\ 7.7 & \text { Fob } & 15 \\ .10 & \text { Jul } & 9 \\ .11 & \text { Jul } & 6 \\ & & \\ 774 & & \\ 3.1 & \\ .75 & \\ .14 & \end{array}$

FOR 1996 WATER YEAR

$$
229.82
$$

$$
.63
$$

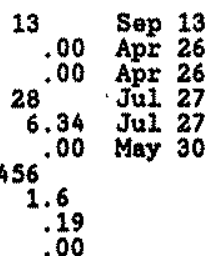

WATER YEARS 1970 - 1996

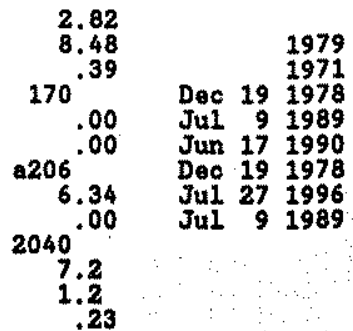

a-From rating curve extended above $40 \mathrm{ft} / \mathrm{s}$. 
RIO GRANDE BASIN

08390500 RTO BONDO AT DTANOND A RANCH, MEAR ROSWELL, MM

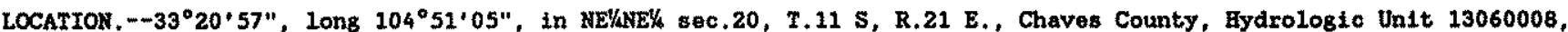
on right bank 40 ft downstream from bridge on Mossman Road at Dianond A Ranch farm, 1.3 mi $80 u t h$ of U.S. Htghway 70-380, $13 \mathrm{mi}$ upstixeam from Two Rivers Reservotr, 21 mi upstream from mouth of Rocky Arroyo, 18 mi west of Rosweli, and at mile 44.7 .

DRAINACE AREA. $--947 \mathrm{mi}^{2}$, contributing area.

EFRIOD OF RECORD.--May 1908 to August 1909, May 1939 to current year. Monthly discharge only Ior 1908-9, published In Technical Report 7. State of Nep Mexico, State Engineer Offica, "Streanflow and Reservoir Content. 1888-1954." REVISED RECORDS, - WSP 1392: Drainage area, WSP 1512: 1939-40(P), 1941, 1942-43(P), 1946(P),

GAGE - Water-stage recorder and concrete control. Elovation of gage is 4,190 ft wove National Ceodetic Vertical

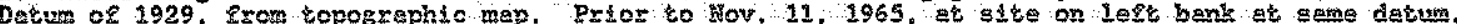

REMARKS - Records foir except for estimated daily discharges, phich are poor. Diversions and ground-water pithdrawals upstream from station for irrigation above and bolow station of about 6,500 acres, 1959

determination. Several observations of water tomperature ore made during the year... U.S. Army Corps of Engineers satelilte telemeter at station. No flow most of time.

EXTREMS OUTSIDE PERIOD OF RECORD,-A flood on June 1,1937 reached a discharge of 24,900 fts/s at Riverside, about

13 mi upstream. Other major Ploods occurred Oct. 31, 1901, Sept, 29, 30, 1904, and July 25, 1905. DISCBARGE, CUBTC FEET PER SECOND, WATER YEAR OCTOBER 1995 TO SEPTEMBER 1996

\begin{tabular}{|c|c|c|c|c|c|c|c|c|c|c|c|c|}
\hline DAY & $\mathrm{OCT}$ & nov & DEC & JAN & FEB & MAR & $A F R$ & MAY & JUN & JUL. & AUG & SEP \\
\hline $\begin{array}{l}1 \\
2 \\
3 \\
4 \\
5\end{array}$ & $\begin{array}{l}.00 \\
.00 \\
.00 \\
.00 \\
.00\end{array}$ & $\begin{array}{l}.00 \\
.00 \\
.00 \\
.00 \\
.00\end{array}$ & $\begin{array}{l}.54 \\
.00 \\
.06 \\
.00 \\
.38\end{array}$ & $\begin{array}{l}.00 \\
.00 \\
.00 \\
.00 \\
.00\end{array}$ & $\begin{array}{l}.00 \\
.00 \\
2.6 \\
5.2 \\
2.7\end{array}$ & $\begin{array}{l}3.5 \\
.00 \\
.00 \\
.00 \\
.00\end{array}$ & $\begin{array}{l}.00 \\
.00 \\
.00 \\
.00 \\
.00\end{array}$ & $\begin{array}{r}.00 \\
.00 \\
.00 \\
.00 \\
.00\end{array}$ & $\begin{array}{r}0.00 \\
.00 \\
.00 \\
.00 \\
.00\end{array}$ & $\begin{array}{r}023 \\
11 \\
7.0 \\
.86 \\
.07\end{array}$ & $\begin{array}{c}3.0 \\
5.7 \\
.00 \\
.22 \\
2.0\end{array}$ & $\begin{array}{l}12 \\
10 \\
5.0 \\
17 \\
21\end{array}$ \\
\hline $\begin{array}{r}6 \\
7 \\
8 \\
9 \\
10\end{array}$ & $\begin{array}{l}.00 \\
.00 \\
.00 \\
.00 \\
.00\end{array}$ & $\begin{array}{l}.00 \\
.00 \\
.00 \\
.00 \\
.00\end{array}$ & $\begin{array}{l}.05 \\
.00 \\
.00 \\
.00 \\
.00\end{array}$ & $\begin{array}{l}.00 \\
.00 \\
.00 \\
.00 \\
.00\end{array}$ & $\begin{array}{l}.65 \\
.01 \\
.00 \\
.21 \\
.00\end{array}$ & $\begin{array}{r}.00 \\
.00 \\
.00 \\
.00 \\
.00\end{array}$ & $\begin{array}{l}.00 \\
.00 \\
.00 \\
.00 \\
.00\end{array}$ & $\begin{array}{l}.00 \\
.00 \\
.00 \\
.00 \\
.00\end{array}$ & $\begin{array}{l}.00 \\
.00 \\
.00 \\
.00 \\
.00\end{array}$ & $\begin{array}{l}.00 \\
.00 \\
.00 \\
.00 \\
.00\end{array}$ & $\begin{array}{l}.35 \\
.18 \\
.00 \\
.03 \\
.07\end{array}$ & $\begin{array}{l}5.7 \\
5.6 \\
1.5 \\
7.5 \\
.39\end{array}$ \\
\hline $\begin{array}{l}11 \\
12 \\
13 \\
14 \\
15\end{array}$ & $\begin{array}{l}.00 \\
.00 \\
.00 \\
.00 \\
.00\end{array}$ & $\begin{array}{l}.00 \\
.00 \\
.00 \\
.00 \\
.00\end{array}$ & $\begin{array}{l}.00 \\
.00 \\
.00 \\
.00 \\
.00\end{array}$ & $\begin{array}{l}.00 \\
.00 \\
.00 \\
.00 \\
.00\end{array}$ & $\begin{array}{l}.00 \\
.00 \\
.00 \\
.00 \\
.00\end{array}$ & $\begin{array}{l}.00 \\
.00 \\
.00 \\
.00 \\
.00\end{array}$ & $\begin{array}{l}.00 \\
.00 \\
.00 \\
.00 \\
.00\end{array}$ & $\begin{array}{r}.00 \\
.00 \\
.00 \\
0.00 \\
0.00\end{array}$ & $\begin{array}{l}.00 \\
.00 \\
.00 \\
.00 \\
.00\end{array}$ & 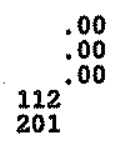 & $\begin{array}{l}.00 \\
.00 \\
.00 \\
.00 \\
.00\end{array}$ & $\begin{array}{r}91 \\
2510 \\
329 \\
174 \\
85\end{array}$ \\
\hline $\begin{array}{l}16 \\
17 \\
18 \\
19 \\
20\end{array}$ & $\begin{array}{l}.00 \\
.00 \\
.00 \\
.00 \\
.00\end{array}$ & $\begin{array}{l}.00 \\
.00 \\
.00 \\
.00 \\
.00\end{array}$ & $\begin{array}{l}.00 \\
.00 \\
.00 \\
.00 \\
.00\end{array}$ & $\begin{array}{l}.00 \\
.00 \\
.00 \\
.00 \\
.00\end{array}$ & $\begin{array}{l}.00 \\
.00 \\
.00 \\
.00 \\
.00\end{array}$ & $\begin{array}{l}.00 \\
.00 \\
.00 \\
.00 \\
.00\end{array}$ & $\begin{array}{l}.00 \\
.00 \\
.00 \\
.00 \\
.00\end{array}$ & $\begin{array}{l}0.00 \\
0.00 \\
0.00 \\
0.00 \\
0.00\end{array}$ & $\begin{array}{l}.00 \\
.00 \\
.00 \\
.00 \\
.00\end{array}$ & $\begin{array}{r}262 \\
4.0 \\
.01 \\
.00 \\
.00\end{array}$ & $\begin{array}{l}.00 \\
.00 \\
.00 \\
.00 \\
.00\end{array}$ & $\begin{array}{l}76 \\
71 \\
60 \\
49 \\
44\end{array}$ \\
\hline $\begin{array}{l}21 \\
22 \\
23 \\
24 \\
25\end{array}$ & $\begin{array}{l}.00 \\
.00 \\
.00 \\
.00 \\
.00\end{array}$ & $\begin{array}{l}.00 \\
.00 \\
.00 \\
.00 \\
.00\end{array}$ & $\begin{array}{l}.00 \\
.00 \\
.00 \\
.00 \\
.00\end{array}$ & $\begin{array}{l}.00 \\
.00 \\
.00 \\
.00 \\
.00\end{array}$ & $\begin{array}{l}.00 \\
.00 \\
.00 \\
.00 \\
.00\end{array}$ & $\begin{array}{l}.00 \\
.00 \\
.00 \\
.00 \\
.00\end{array}$ & $\begin{array}{l}.00 \\
.00 \\
.00 \\
.00 \\
.00\end{array}$ & $\begin{array}{l}0.00 \\
0.00 \\
0.00 \\
0.00 \\
0.00\end{array}$ & $\begin{array}{l}.00 \\
.00 \\
.00 \\
.00 \\
.00\end{array}$ & $\begin{array}{l}.00 \\
.00 \\
.00 \\
.00 \\
.07\end{array}$ & $\begin{array}{r}.00 \\
.00 \\
.00 \\
.00 \\
3.0\end{array}$ & $\begin{array}{l}40 \\
38 \\
33 \\
29 \\
26\end{array}$ \\
\hline $\begin{array}{l}26 \\
27 \\
28 \\
29 \\
30 \\
31\end{array}$ & $\begin{array}{l}.00 \\
.00 \\
.00 \\
.00 \\
.00 \\
.00\end{array}$ & $\begin{array}{l}.00 \\
.00 \\
.00 \\
.00 \\
.19 \\
.0\end{array}$ & $\begin{array}{l}.00 \\
.00 \\
.00 \\
.00 \\
.00 \\
.00\end{array}$ & $\begin{array}{l}.00 \\
.00 \\
.00 \\
.00 \\
.00 \\
.00\end{array}$ & $\begin{array}{c}.00 \\
.00 \\
.72 \\
3.3 \\
-.-- \\
-.-\end{array}$ & $\begin{array}{l}.00 \\
.00 \\
.00 \\
.00 \\
.00 \\
.00\end{array}$ & $\begin{array}{l}.00 \\
.00 \\
.00 \\
.00 \\
.00 \\
. .\end{array}$ & $\begin{array}{l}0.00 \\
0.00 \\
0.00 \\
0.00 \\
0.00 \\
0.00\end{array}$ & $\begin{array}{r}087^{.00} \\
e 2380 \\
e 285 \\
0177\end{array}$ & $\begin{array}{l}.00 \\
.00 \\
.00 \\
.00 \\
.00 \\
5.4\end{array}$ & $\begin{array}{l}12 \\
41 \\
23 \\
18 \\
18 \\
14\end{array}$ & $\begin{array}{l}23 \\
21 \\
19 \\
16 \\
13\end{array}$ \\
\hline $\begin{array}{l}\text { TOTAL } \\
\text { MEAN } \\
\text { MAZ } \\
\text { MIN } \\
\text { AC-FT }\end{array}$ & $\begin{array}{l}0.00 \\
.000 \\
.00 \\
.00 \\
.00\end{array}$ & $\begin{array}{r}0.19 \\
.006 \\
.19 \\
.00 \\
.4\end{array}$ & $\begin{array}{r}1.03 \\
.033 \\
.54 \\
.00 \\
2.0\end{array}$ & $\begin{array}{l}0.00 \\
.000 \\
.00 \\
.00 \\
.00\end{array}$ & $\begin{array}{r}15.39 \\
553 \\
5.2 \\
.00 \\
31\end{array}$ & $\begin{array}{r}3.50 \\
.11 \\
3.5 \\
.00 \\
6.9\end{array}$ & $\begin{array}{l}0.00 \\
.000 \\
.00 \\
.00 \\
.00\end{array}$ & $\begin{array}{l}0.00 \\
.000 \\
.00 \\
.00 \\
.00\end{array}$ & $\begin{array}{r}2929.00 \\
97.6 \\
2380 \\
5810\end{array}$ & $\begin{array}{r}626.41 \\
20.2 \\
262 \\
100 \\
1240\end{array}$ & $\begin{array}{r}140.55 \\
4.53 \\
41 \\
.00 \\
279\end{array}$ & $\begin{array}{r}3832.69 \\
128 \\
2510 \\
.39 \\
7600\end{array}$ \\
\hline
\end{tabular}

STATISTICS OF MONTALY MEAN DATA FOR WATER YEARS 1940 - 1993, BY WATER YEAR (WY)

\begin{tabular}{|c|c|c|c|c|c|c|c|c|c|c|c|c|}
\hline $\begin{array}{l}\text { MEARY } \\
\text { MAX } \\
\text { (WY) } \\
\text { MIX } \\
\text { (WY) }\end{array}$ & $\begin{array}{r}27.8 \\
458 \\
1942 \\
000 \\
1941\end{array}$ & $\begin{array}{r}17.5 \\
199 \\
1942 \\
1000\end{array}$ & $\begin{array}{r}20.0 \\
222 \\
1979 \\
000 \\
1900\end{array}$ & $\begin{array}{r}17.5 \\
160 \\
1985 \\
000 \\
1952\end{array}$ & $\begin{array}{l}13.7 \\
97.5 \\
1987 \\
.000\end{array}$ & $\begin{array}{r}14.3 \\
153 \\
1987 \\
1000 \\
1050\end{array}$ & $\begin{array}{r}28.1 \\
199 \\
1987 \\
.000 \\
1946\end{array}$ & $\begin{array}{r}30.7 \\
519 \\
1941 \\
.000 \\
1951\end{array}$ & $\begin{array}{r}24.8 \\
334 \\
1986 \\
.000 \\
1951\end{array}$ & $\begin{array}{r}28.4 \\
163 \\
1955 \\
.000 \\
1975\end{array}$ & $\begin{array}{r}41.3 \\
241 \\
1984 \\
.000 \\
1960\end{array}$ & $\begin{array}{l}53.7 \\
1090 \\
1941 \\
.000 \\
1943\end{array}$ \\
\hline
\end{tabular}

SURAARY STATISTICS

ANTEUAL TOTAL

ANNUAL, MEAR

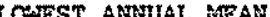

HICHEST DATLY MTAN

HIGIEST DAILY MEA

ANIEAAL SEVEN-DAY MINIMUM

ANNUAL SEVEN-DAY MINIMIM

INSTANTANEOUS PEAK FLON

INSTANTANEOUS PEAK STAG

ANNUAL RUROFF (AC-FT)

10 PERCENT EXCEEDS

50 PERCEHT EXCEEDS

50 PERCENT EXCEEDS

- Estimated

Estimatod

rating curve extended above $3,100 \mathrm{ft}^{\mathrm{s}} / \mathrm{s}$

1846.50

5.06

41. 00 Jan 16

.00
.00 Apr 27

3660

20.00
FOR 1996 WATER YEAR

7548.76
20.6

$\begin{array}{rlr}.00 & \text { Oet } & 1 \\ 6380^{\circ 00} & \text { Oct } & 1 \\ 26.02 & \text { Sep } 12 \\ & \text { Sep } 12\end{array}$

14970

12.00
WATER VEARS $1940-1993$

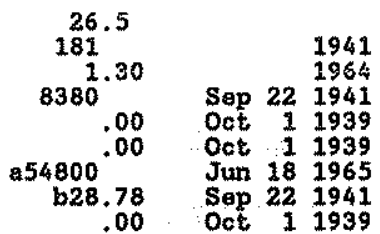

$19230^{\circ} 00$ Oct 11939 
RIO GRANDE BASIN

08390600 TWO RIVERS RESERVOTR NEAR ROSWELL, NM

LOCATION, - 08390610 Rio Hondo Reservoir: Lat $33^{\circ} 17^{\prime} 55^{\prime \prime}$, long $104^{\circ} 43^{\circ} 20^{\prime \prime}$, in SW/4SEKHEt/ sec.4, T.12 S., R.22 E. Chaves County, Hydrologtc Unit 13060008, near center' of Diamond A Dam" on Rio Hondo, 13 mi southwest of Roswell at

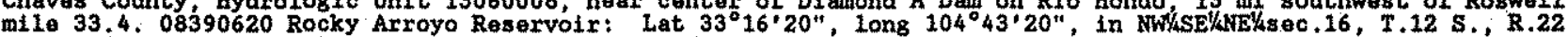
E., at left ond of Rocky Dam on Rocky Arroyo, and 14 mi southwest of Roswe11.

DRAINAGE AREA. - - 1,027 $\mathrm{mt}^{3}$; Rio Hondo, $963 \mathrm{mt}^{2}$; Rocky Arroyo, $64 \mathrm{mt}^{2}$.

PERIOD OF RECORD.--July 1963 to current year (prior to October 1965 monthend contents only). Prior to October 1966, contents at 0800 hours.

GAGE.--Water-stage recorder. Elevation of gage is National Geodetic Vertical Datum of 1929 (1evels by U.S. Army Corps of Engtneers).

REMARKS. - Two Rivers Reservoir, completed July 16,1963 , is formed by earthfill dams on $R i 0$ Eondo, which forms Rio Hondo Reservotr, and on Rocky Arroyo, which forms Rocky Arroyo Reservoir. Above elevation $3,980.0$ ft, the pools of the two reservolxs combine to form Two Rivers Reservoir with a total capacity of 163,800 acre-ft, at elevation $4,032.0 \mathrm{ft}$, crest of ungated spiliway. Capacity by original survey was 167, 900 acre-ft. Capacity of Rio Bondo Reservol $x$, 142 acre-ft, fron capacity table dated January 1990 , between elevations $3,957.0$ ft, sill of outiet gate, and 3,980.0. Capacity of Rocky Arroyo Rezervoir, 12,860 acre-ft, from capact ty table dated January 1990, between elevations $3,945,0$, sil1 of outlet gate, and $3,980,0$ ft. No dead storage in Rio Hondo Reservolr or Rocky Arroyo Reservoir. Primary objective of project is flood controi. Outlet conduits in Rocky Dam have fixed

openings. Figures given herein represent total contents at 2400 hours. U.S. Axmy Corps of Engineers satellite openings. Figures given

COOPERATION, --Records provided by U.S. Army Corps of Engineers.

EXTREMES FOR PERIOD OF RECORD.--Rto Hondo Reservolr: Maximum contents, 1, 260 acre-ft, July 29, 1965, elevation, 3,985.7 ft; no storage most of time. Rocky Arroyo Reservoir: Maximum contents, 6,090 acre-ft, June 18, 1965, elevation, $3,970.7 \mathrm{ft}$; no storage most of time.

EXTREMES FOR CURRENT YEAR.--Maximun contents, Rio Hondo Reservolr, 1,600 acre-ft, Sept. 12; elevation 3,990.70 ft; Rocky Arroyo Reservotr, 1,800 acre-ft. Sept. 13; elevation $3,962.40$ ft; no contents both reservoirs most of time.

CONTENTS, IN ACRE-FEET, AND ELEVATION, IN FEET, WATER YEAR OCTOBER 1995 TO SEPTEMBER 1996 NO CONTENTS AT 2400 HOURS DURING YEAR EXXCEPT:

RIO HONDO RESERVOIR

\begin{tabular}{|c|c|c|c|c|c|}
\hline DATE & ELEVATION & CONTENTS & DATE & ELEVATION & CONTENTS \\
\hline $\begin{array}{lr}\text { June } & 28 \\
& 29 \\
& 30 \\
\text { July } & 1 \\
& 16 \\
\text { Sep. } & 12\end{array}$ & $\begin{array}{l}3973.05 \\
3988.92 \\
3986.74 \\
3981.16 \\
3976.33 \\
3990.70\end{array}$ & $\begin{array}{r}6 \\
1240 \\
858 \\
214 \\
21 \\
1600\end{array}$ & Sep. $\begin{array}{r}13 \\
14 \\
15 \\
16 \\
17 \\
18\end{array}$ & $\begin{array}{l}3988.63 \\
3988.64 \\
3987.79 \\
3986.10 \\
3982.87 \\
3972.92\end{array}$ & $\begin{array}{r}1180 \\
1180 \\
1030 \\
760 \\
360 \\
5\end{array}$ \\
\hline
\end{tabular}

ROCKY ARROYO RESERVOIR

DATE ELEVATION CONTENTS

$\begin{array}{rlr}\text { SEP. } 12 & 3951.17 & 41 \\ 13 & 3962.40 & 1800 \\ 14 & 3958.30 & 778 \\ 15 & 39.54 .58 & 260\end{array}$


LOCATION,--Lat $33^{\circ} 18^{\prime} 05^{\prime \prime}$, Long $14^{\circ} 43^{\circ} 12^{\prime \prime}$, in NEKSEKEX gec.4, T.12 S., R.22 E., Chaves County, Hydrologic Unit 13060008 on loft bank 500 Tt downstream from outlet condult of Dianond A Dan (Two Rivers Reservoix). 13 mi southpest of Rosme11, and at mile 33,3 .

DRAINAGE AREA. $--963 \mathrm{mi}^{2}$, contributing area.

PERIOD OF RECORD. --October 1963 to current yoar.

GAGE.--Water-Btage recoxdex and concrete control. Elevation of gage is $3,949.68$ ft above Nationel Geodetio Vertical Datum of 1929 (U.S. Army Corpa of Engineors bench mark).

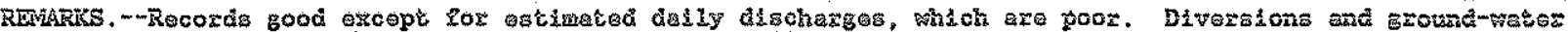
phdramals for trxigation of about 6,500 acros, 1959 dotermination, upatroam from atation. Ihis record ropresents the outelow from Two Rivers Reservoir through Di anond A Dam $0.1 \mathrm{mi}$ upstream; \&low from reasivoir cen also be discharged into Rocky Arroyo through Rocky Dam (sea RFMARKS for atation 08390600). Sovere1 obsexvations

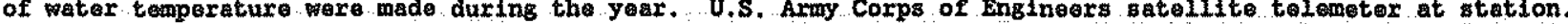
DISCEARGE, CUBIC FEET YER SECORD, WATER YEAR OCTOBER 1995 TO SEPTEMBER 1996

\begin{tabular}{|c|c|c|c|c|c|c|c|c|c|c|c|c|c|}
\hline DAY & $\mathrm{CCT}$ & MOV & DEC & JAN & $\mathrm{FHB}$ & MAR & AFR & MAY & JU: & JUL & & AUE & SEP \\
\hline $\begin{array}{l}1 \\
2 \\
3 \\
4 \\
5\end{array}$ & $\begin{array}{l}.00 \\
.00 \\
.00 \\
.00 \\
.00\end{array}$ & $\begin{array}{l}.00 \\
.00 \\
.00 \\
.00 \\
.00\end{array}$ & $\begin{array}{l}.00 \\
.00 \\
.00 \\
.00 \\
.00\end{array}$ & $\begin{array}{l}.00 \\
.00 \\
.00 \\
.00 \\
.00\end{array}$ & $\begin{array}{l}.00 \\
.00 \\
.00 \\
.00 \\
.00\end{array}$ & $\begin{array}{l}.00 \\
.00 \\
.00 \\
.00 \\
.00\end{array}$ & $\begin{array}{l}.00 \\
.00 \\
.00 \\
.00 \\
.00\end{array}$ & $\begin{array}{l}.00 \\
.00 \\
.00 \\
.00 \\
.00\end{array}$ & $\begin{array}{l}.00 \\
.00 \\
.00 \\
.00 \\
.00\end{array}$ & $\begin{array}{r}224 \\
65 \\
4.0 \\
2.5 \\
1.9\end{array}$ & $\therefore::$ & $\begin{array}{l}.00 \\
.00 \\
.00 \\
.00 \\
.00\end{array}$ & $\begin{array}{r}.32 \\
.02 \\
.00 \\
.00 \\
11.0\end{array}$ \\
\hline $\begin{array}{r}6 \\
7 \\
8 \\
9 \\
10\end{array}$ & $\begin{array}{l}.00 \\
.00 \\
.00 \\
.00 \\
.00\end{array}$ & $\begin{array}{l}.00 \\
.00 \\
00 \\
.00 \\
.00\end{array}$ & $\begin{array}{r}.00 \\
.00 \\
.00 \\
.00 \\
.00\end{array}$ & $\begin{array}{l}.00 \\
.00 \\
.00 \\
.00 \\
.00\end{array}$ & $\begin{array}{r}.00 \\
.00 \\
.00 \\
.00 \\
.00\end{array}$ & $\begin{array}{l}.00 \\
.00 \\
.00 \\
.00 \\
.00\end{array}$ & $\begin{array}{l}.00 \\
.00 \\
.00 \\
.00 \\
.00\end{array}$ & $\begin{array}{l}.00 \\
.00 \\
.00 \\
.00 \\
.00\end{array}$ & $\begin{array}{l}.00 \\
.00 \\
.00 \\
.00 \\
.00\end{array}$ & $\begin{array}{l}1.5 \\
1.3 \\
1.1 \\
1.0 \\
1.0\end{array}$ & . & $\begin{array}{l}.00 \\
.00 \\
.00 \\
.00 \\
.00\end{array}$ & $\begin{array}{l}1.3 \\
.07 \\
.00 \\
.00 \\
.00\end{array}$ \\
\hline $\begin{array}{l}11 \\
12 \\
13 \\
14 \\
15\end{array}$ & $\begin{array}{l}.00 \\
.00 \\
.00 \\
.00 \\
.00\end{array}$ & $\begin{array}{l}.00 \\
.00 \\
.00 \\
.00 \\
.00\end{array}$ & $\begin{array}{l}.00 \\
.00 \\
.00 \\
.00 \\
.00\end{array}$ & $\begin{array}{l}.00 \\
.00 \\
.00 \\
.00 \\
.00\end{array}$ & $\begin{array}{r}.00 \\
.00 \\
.00 \\
.00 \\
.00\end{array}$ & $\begin{array}{l}.00 \\
.00 \\
.00 \\
.00 \\
.00\end{array}$ & $\begin{array}{l}.00 \\
.00 \\
.00 \\
.00 \\
.00\end{array}$ & $\begin{array}{l}.00 \\
.00 \\
.00 \\
.00 \\
.00\end{array}$ & $21^{.00} .00$ & $\begin{array}{r}1.0 \\
1.0 \\
2.1 \\
57 \\
7.9\end{array}$ & & $\begin{array}{l}.00 \\
.00 \\
.00 \\
.00 \\
.00\end{array}$ & $\begin{array}{c}128^{.46} \\
49^{.03}\end{array}$ \\
\hline $\begin{array}{l}16 \\
17 \\
18 \\
19 \\
20\end{array}$ & $\begin{array}{l}.00 \\
.00 \\
.00 \\
.00 \\
.00\end{array}$ & $\begin{array}{l}.00 \\
.00 \\
.00 \\
.00 \\
.00\end{array}$ & $\begin{array}{l}.00 \\
.00 \\
.00 \\
.00 \\
.00\end{array}$ & $\begin{array}{l}.00 \\
.00 \\
.00 \\
.00 \\
.00\end{array}$ & $\begin{array}{l}.00 \\
.00 \\
.00 \\
.00 \\
.00\end{array}$ & $\begin{array}{l}.00 \\
.00 \\
.00 \\
.00 \\
.00\end{array}$ & $\begin{array}{l}.00 \\
.00 \\
.00 \\
.00 \\
.00\end{array}$ & $\begin{array}{l}.00 \\
.00 \\
.00 \\
.00 \\
.00\end{array}$ & $\begin{array}{l}.05 \\
.00 \\
.00 \\
.00 \\
.00\end{array}$ & $\begin{array}{r}189 \\
62 \\
6.5 \\
6.3 \\
6.2\end{array}$ & & $\begin{array}{l}.00 \\
.00 \\
.00 \\
.00 \\
.00\end{array}$ & $\begin{array}{r}118 \\
157 \\
150 \\
46 \\
32\end{array}$ \\
\hline $\begin{array}{l}21 \\
22 \\
23 \\
24 \\
25\end{array}$ & $\begin{array}{l}.00 \\
.00 \\
.00 \\
.00 \\
.00\end{array}$ & $\begin{array}{l}.00 \\
.00 \\
.00 \\
.00 \\
.00\end{array}$ & $\begin{array}{l}.00 \\
.00 \\
.00 \\
.00 \\
.00\end{array}$ & $\begin{array}{l}.00 \\
.00 \\
.00 \\
.00 \\
.00\end{array}$ & $\begin{array}{l}.00 \\
.00 \\
.00 \\
.00 \\
.00\end{array}$ & $\begin{array}{l}.00 \\
.00 \\
.00 \\
.00 \\
.00\end{array}$ & $\begin{array}{l}.00 \\
.00 \\
.00 \\
.00 \\
.00\end{array}$ & $\begin{array}{l}.00 \\
.00 \\
.00 \\
.00 \\
.00\end{array}$ & $\begin{array}{l}.00 \\
.00 \\
.00 \\
.00 \\
.00\end{array}$ & $\begin{array}{r}6.0 \\
2.2 \\
.00 \\
.00 \\
1.6\end{array}$ & & $\begin{array}{l}.00 \\
.00 \\
.00 \\
.00 \\
.00\end{array}$ & $\begin{array}{l}30 \\
28 \\
24 \\
21 \\
19\end{array}$ \\
\hline $\begin{array}{l}26 \\
27 \\
28 \\
29 \\
30 \\
31\end{array}$ & $\begin{array}{l}.00 \\
.00 \\
.00 \\
.00 \\
.00 \\
.00\end{array}$ & $\begin{array}{l}.00 \\
.00 \\
.00 \\
.00 \\
.00 \\
.00\end{array}$ & $\begin{array}{l}.00 \\
.00 \\
.00 \\
.00 \\
.00 \\
.00\end{array}$ & $\begin{array}{l}.00 \\
.00 \\
.00 \\
.00 \\
.00 \\
.00\end{array}$ & $\begin{array}{l}.00 \\
.00 \\
.00 \\
.00 \\
-0\end{array}$ & $\begin{array}{l}.00 \\
.00 \\
.00 \\
.00 \\
.00 \\
.00\end{array}$ & $\begin{array}{l}.00 \\
.00 \\
.00 \\
.00 \\
.00 \\
.\end{array}$ & $\begin{array}{l}.00 \\
.00 \\
.00 \\
.00 \\
.00 \\
.00\end{array}$ & $\begin{array}{l}1.00 \\
23 \\
19 \\
95 \\
\end{array}$ & $\begin{array}{l}.07 \\
.00 \\
.00 \\
.00 \\
.00 \\
.00\end{array}$ & & $\begin{array}{l}.00 \\
30 \\
8.9 \\
9.7 \\
2.8\end{array}$ & $\begin{array}{l}15 \\
11 \\
10 \\
02.2 \\
0.00 \\
-\cdots\end{array}$ \\
\hline $\begin{array}{l}\text { TORAL } \\
\text { MEAN } \\
\text { MAX } \\
\text { MIN } \\
\text { AC-FT }\end{array}$ & $\begin{array}{l}0.00 \\
.000 \\
.00 \\
.00 \\
.00\end{array}$ & $\begin{array}{l}0.00 \\
.000 \\
.00 \\
.00 \\
.00\end{array}$ & $\begin{array}{l}0.00 \\
.000 \\
.00 \\
.00 \\
.00\end{array}$ & $\begin{array}{l}0.00 \\
.000 \\
.00 \\
.00 \\
.00\end{array}$ & $\begin{array}{l}0.00 \\
.000 \\
.00 \\
.00 \\
.00\end{array}$ & $\begin{array}{l}0.00 \\
.000 \\
.00 \\
.00 \\
.00\end{array}$ & $\begin{array}{l}0.00 \\
.000 \\
.00 \\
.00 \\
00\end{array}$ & $\begin{array}{r}0.00 \\
.000 \\
.00 \\
.00 \\
00\end{array}$ & $\begin{array}{r}159.95 \\
5.33 \\
95 \\
.00 \\
317\end{array}$ & $\begin{array}{r}652.17 \\
21.0 \\
224 \\
100 \\
1290\end{array}$ & 7 & $\begin{array}{r}9.40 \\
2.56 \\
30 \\
.00 \\
957\end{array}$ & $\begin{array}{r}892.38 \\
29.7 \\
157 \\
.00 \\
1770\end{array}$ \\
\hline
\end{tabular}

STATISTICS OF MONTHLY MEAN DATA FOR WATER YEARS 1964 - 1996, BY WATER YEAR (WY)

$\begin{array}{lrrrrrrrrr}\text { MEAN } & 14.3 & 12.4 & 16.3 & 17.1 & 14.7 & 15.1 & 21.6 & 17.4 & 8.79 \\ \text { MAX } & 151 & 122 & 118 & 128 & 82.9 & 122 & 176 & 127 & 74.7 \\ \text { (WX) } & 1986 & 1987 & 1985 & 1985 & 1987 & 1987 & 1987 & 1987 & 1992 \\ \text { MIN } & 1000 & 1000 & 1000 & 1000 & 1000 & 1000 & 1000 & 1000 & 1000 \\ \text { (WY) } & 1964 & 1964 & 1964 & 1964 & 1964 & 1964 & 1964 & 1967 & 1971\end{array}$

STARAR STATTETrES

ANMUAL, TOTAL

ANRUAL MEAIV

EITERST ARYHUA MTA

LOWEST ANRUAT MSAR

HIGHST DATLY MEAP

LONEST DATLY MZAI

ANRUAL SEVEN-DAY MINTMIM

INSTANTAHEOUS DEAR FLOW

INSTANTANEOUS EEAK STAGE

INSTANTANEOUS LOW FLOW

ANNUAL RUHOFE (AC-FT)

10 PIRCENT EXCEEDS

50 PERCXNT EXCENDS

90 PERCENT EXCEEDS

FOP 1005 CAIENDAP VEAD

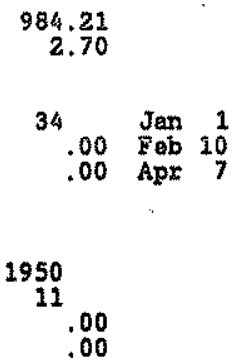

FOR 1996 WATER YEAP

1783.90
4.87

- Estimated

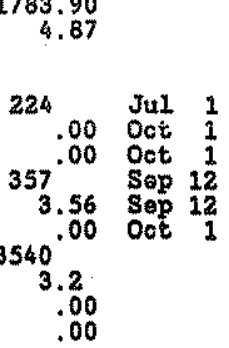

8.79
74.7
1992
000
1971

$\begin{array}{lll}7.94 & 24.5 & 26.7 \\ 52.3 & 137 & 116 \\ 1986 & 1984 & 1988 \\ 000 & 1000 & 000 \\ 1974 & 1975 & 1973\end{array}$

WATER YFARS $1964-1996$

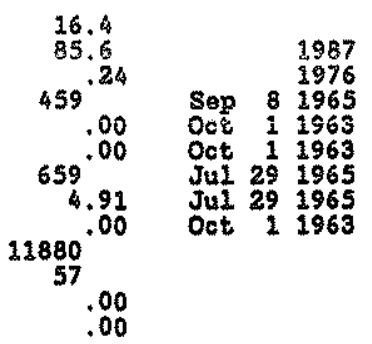




\section{RIO GRANDE BASIN}

08393500 RIO HONDO AT ROSWELL, NM

LOCATION - Lat $33^{\circ} 22^{\prime} 19^{\prime \prime}$, long $104^{\circ} 32^{\prime} 42^{\prime \prime}$, in NE/SE/ sec.7, T. 11 S., R. 24 E., Chaves County, Eydrologic Unit 13060008 , on left bank $0.3 \mathrm{mi}$ upstream' from bridge on Sunset Ave'. in Roswel1, $6.3 \mathrm{mi}$ downstream from Rocky Arroyo and $11.7 \mathrm{mi}$ upstraem from mouth. Mouth at Pecos River mile 566,0 .

DRAINAGE AREA. $-1,070 \mathrm{mi} \mathbf{1}^{2}$, approximately, (contributing area),

PERIOD OF RECORD.--Februaxy 1981 to current year. Records for June 1903 to February 1906, published in WSF 358, axe unreliable and should not be used.

GAGE.--Water-stage recordex. Elevation of gage is 3,620 ft above National Geodetic Vertical Datum of 1929, from topos raphic map.

REMARKS.--Records poor. Flow regulated by Two RIvers Reservolr (atation 08390600) 21.7 mi upstream. Diverslons and ground water athdrawals for irrigation upstream from station. Several observations of water temperature pere made during the year. U.S. Army Corps of Engineers satellite telemeter at station. No flow most of time.

DISCFARGE, CUBIC FEET PGR SECOND, WATER YEAR OCTOBER 1995 TO SEPTEMBER 1996

\begin{tabular}{|c|c|c|c|c|c|c|c|c|c|c|c|c|}
\hline DAY & $\operatorname{ocs}$ & ROV & DEC & JAN & $\mathrm{FEB}$ & MAR & AFR & MAY & JUN & JuL & AUG & SEP \\
\hline $\begin{array}{l}1 \\
2 \\
3 \\
4 \\
5\end{array}$ & $\begin{array}{l}.00 \\
.00 \\
.00 \\
.00 \\
.00\end{array}$ & $\begin{array}{l}.00 \\
.00 \\
.00 \\
.00 \\
.00\end{array}$ & $\begin{array}{l}.00 \\
.00 \\
.00 \\
.00 \\
.00\end{array}$ & $\begin{array}{l}.00 \\
.00 \\
.58 \\
.00 \\
.00\end{array}$ & $\begin{array}{l}.00 \\
.00 \\
.00 \\
.00 \\
.00\end{array}$ & $\begin{array}{l}.00 \\
.00 \\
.00 \\
.00 \\
.00\end{array}$ & $\begin{array}{l}.00 \\
.00 \\
.00 \\
.00 \\
.00\end{array}$ & $\begin{array}{l}.00 \\
.00 \\
.00 \\
.00 \\
.00\end{array}$ & $\begin{array}{l}.00 \\
.00 \\
.00 \\
.00 \\
.00\end{array}$ & $\begin{array}{r}860 \\
8100 \\
830 \\
02.0 \\
0.00\end{array}$ & $\begin{array}{l}.00 \\
.00 \\
.00 \\
.00 \\
.00\end{array}$ & $\begin{array}{r}0.00 \\
0.00 \\
0.00 \\
e .00 \\
62.0\end{array}$ \\
\hline $\begin{array}{r}6 \\
7 \\
8 \\
9 \\
10\end{array}$ & $\begin{array}{l}.00 \\
.00 \\
.00 \\
.00 \\
.00\end{array}$ & $\begin{array}{l}.00 \\
.00 \\
.00 \\
.00 \\
.00\end{array}$ & $\begin{array}{l}.00 \\
.00 \\
.00 \\
.00 \\
2.2\end{array}$ & $\begin{array}{l}.00 \\
.00 \\
.10 \\
.10 \\
.00\end{array}$ & $\begin{array}{l}.00 \\
.00 \\
.00 \\
.00 \\
.00\end{array}$ & $\begin{array}{l}.00 \\
.00 \\
.00 \\
.00 \\
.00\end{array}$ & $\begin{array}{l}.00 \\
.00 \\
.00 \\
.00 \\
.00\end{array}$ & $\begin{array}{l}.00 \\
.00 \\
.00 \\
.00 \\
.00\end{array}$ & $\begin{array}{l}.00 \\
.00 \\
.00 \\
.00 \\
.00\end{array}$ & $\begin{array}{l}0.00 \\
0.00 \\
0.00 \\
0.00 \\
0.00\end{array}$ & $\begin{array}{l}.00 \\
.00 \\
.00 \\
.00 \\
.00\end{array}$ & $\begin{array}{r}e 5.0 \\
01.0 \\
0.00 \\
0.00 \\
0.00\end{array}$ \\
\hline $\begin{array}{l}11 \\
12 \\
13 \\
14 \\
15\end{array}$ & $\begin{array}{l}.00 \\
.00 \\
.00 \\
.00 \\
.00\end{array}$ & $\begin{array}{l}.00 \\
.00 \\
.00 \\
.00 \\
.00\end{array}$ & $\begin{array}{l}.27 \\
.00 \\
.00 \\
.00 \\
.00\end{array}$ & $\begin{array}{l}.00 \\
.00 \\
.00 \\
.00 \\
.00\end{array}$ & $\begin{array}{l}.00 \\
.00 \\
.00 \\
.00 \\
.00\end{array}$ & $\begin{array}{l}.00 \\
.00 \\
.00 \\
.00 \\
.00\end{array}$ & $\begin{array}{l}.00 \\
.00 \\
.00 \\
.00 \\
.00\end{array}$ & $\begin{array}{l}.00 \\
.00 \\
.00 \\
.00 \\
.00\end{array}$ & $\begin{array}{r}.00 \\
.00 \\
0.00 \\
.00\end{array}$ & $\begin{array}{r}0.00 \\
0.00 \\
02.5 \\
e 33 \\
09.0\end{array}$ & $\begin{array}{l}.00 \\
.00 \\
.00 \\
.00 \\
.00\end{array}$ & $\begin{array}{l}0.00 \\
060 \\
010 \\
070 \\
0.00\end{array}$ \\
\hline $\begin{array}{l}16 \\
17 \\
18 \\
19 \\
20\end{array}$ & $\begin{array}{l}.00 \\
.00 \\
.00 \\
.00 \\
.00\end{array}$ & $\begin{array}{l}.00 \\
.00 \\
.00 \\
.00 \\
.00\end{array}$ & $\begin{array}{r}.00 \\
.00 \\
.00 \\
1.2 \\
.00\end{array}$ & $\begin{array}{l}.00 \\
.00 \\
.00 \\
.00 \\
.00\end{array}$ & $\begin{array}{l}.00 \\
.00 \\
.00 \\
.00 \\
.00\end{array}$ & $\begin{array}{l}.00 \\
.00 \\
.00 \\
.00 \\
.00\end{array}$ & $\begin{array}{l}.00 \\
.00 \\
.00 \\
.00 \\
.00\end{array}$ & $\begin{array}{l}.00 \\
.00 \\
.00 \\
.00 \\
.00\end{array}$ & $\begin{array}{l}.00 \\
.00 \\
.00 \\
.00 \\
.00\end{array}$ & $\begin{array}{r}070 \\
010 \\
0.00 \\
0.00 \\
9.00\end{array}$ & $\begin{array}{l}.00 \\
.00 \\
.00 \\
.00 \\
.00\end{array}$ & $\begin{array}{r}0.00 \\
0.00 \\
0.00 \\
.00 \\
.00\end{array}$ \\
\hline $\begin{array}{l}21 \\
22 \\
23 \\
24 \\
25\end{array}$ & $\begin{array}{l}.00 \\
.00 \\
.00 \\
.00 \\
.00\end{array}$ & $\begin{array}{l}.00 \\
.00 \\
.00 \\
.00 \\
.00\end{array}$ & $\begin{array}{l}.00 \\
.00 \\
.00 \\
.00 \\
.00\end{array}$ & $\begin{array}{l}.00 \\
.00 \\
.00 \\
.00 \\
.00\end{array}$ & $\begin{array}{l}.00 \\
.00 \\
.00 \\
.00 \\
.00\end{array}$ & $\begin{array}{l}.00 \\
.00 \\
.00 \\
.00 \\
.00\end{array}$ & $\begin{array}{l}.00 \\
.00 \\
.00 \\
.00 \\
.00\end{array}$ & $\begin{array}{l}.00 \\
.00 \\
.00 \\
.00 \\
.00\end{array}$ & $\begin{array}{l}.00 \\
.00 \\
.00 \\
.00 \\
.00\end{array}$ & $\begin{array}{l}0.00 \\
0.00 \\
0.00 \\
0.00 \\
0.00\end{array}$ & $\begin{array}{l}.00 \\
.00 \\
.00 \\
.00 \\
.00\end{array}$ & $\begin{array}{l}.00 \\
.00 \\
.00 \\
.00 \\
.00\end{array}$ \\
\hline $\begin{array}{l}26 \\
27 \\
28 \\
29 \\
30 \\
31\end{array}$ & $\begin{array}{l}.00 \\
.00 \\
.00 \\
.00 \\
.00 \\
.00\end{array}$ & $\begin{array}{l}.00 \\
.00 \\
.00 \\
.00 \\
.00 \\
.0\end{array}$ & $\begin{array}{l}.00 \\
.00 \\
.00 \\
.00 \\
.00 \\
.00\end{array}$ & $\begin{array}{l}.00 \\
.00 \\
.00 \\
.00 \\
.00 \\
.00\end{array}$ & $\begin{array}{l}.00 \\
.00 \\
.00 \\
.00 \\
. . . \\
.+\end{array}$ & $\begin{array}{l}.00 \\
.00 \\
.00 \\
.00 \\
.00 \\
.00\end{array}$ & $\begin{array}{l}.00 \\
.00 \\
.00 \\
.00 \\
.00 \\
0\end{array}$ & $\begin{array}{l}.00 \\
.00 \\
.00 \\
.00 \\
.00 \\
.00\end{array}$ & $\begin{array}{r}7.00 \\
e 5.0 \\
010 \\
30 \\
\ldots\end{array}$ & $\begin{array}{l}0.00 \\
0.00 \\
0.00 \\
0.00 \\
.00 \\
.00\end{array}$ & $\begin{array}{l}.00 \\
.00 \\
.00 \\
.00 \\
.00 \\
0.00\end{array}$ & $\begin{array}{l}.00 \\
.00 \\
.00 \\
.00 \\
.00 \\
-\end{array}$ \\
\hline $\begin{array}{l}\text { TOTAL } \\
\text { MEAN } \\
\text { MAX } \\
\text { MIN } \\
\text { AC-FT }\end{array}$ & $\begin{array}{l}0.00 \\
.000 \\
.00 \\
.00 \\
.00\end{array}$ & $\begin{array}{l}0.00 \\
.000 \\
.00 \\
.00 \\
.00\end{array}$ & $\begin{array}{r}3.67 \\
.12 \\
2.2 \\
.00 \\
7.3\end{array}$ & $\begin{array}{r}0.78 \\
.025 \\
.58 \\
.00 \\
1.5\end{array}$ & $\begin{array}{r}0.00 \\
.000 \\
.00 \\
.00 \\
.00\end{array}$ & $\begin{array}{l}0.00 \\
.000 \\
.00 \\
.00 \\
.00\end{array}$ & $\begin{array}{l}0.00 \\
.000 \\
.00 \\
.00 \\
.00\end{array}$ & $\begin{array}{l}0.00 \\
.000 \\
.00 \\
.00 \\
.00\end{array}$ & $\begin{array}{r}62.00 \\
2.07 \\
30 \\
.00 \\
123\end{array}$ & $\begin{array}{r}316.50 \\
10.2 \\
100 \\
.00 \\
628\end{array}$ & $\begin{array}{r}0.00 \\
.000 \\
.00 \\
.00 \\
.00\end{array}$ & $\begin{array}{r}148.00 \\
4.93 \\
70 \\
.00 \\
294\end{array}$ \\
\hline
\end{tabular}

STAIISTICS OF MONTHLY MEAN DATA FOR WATER YEARS 1981 - 1996, BY WATER YEAR (WY)

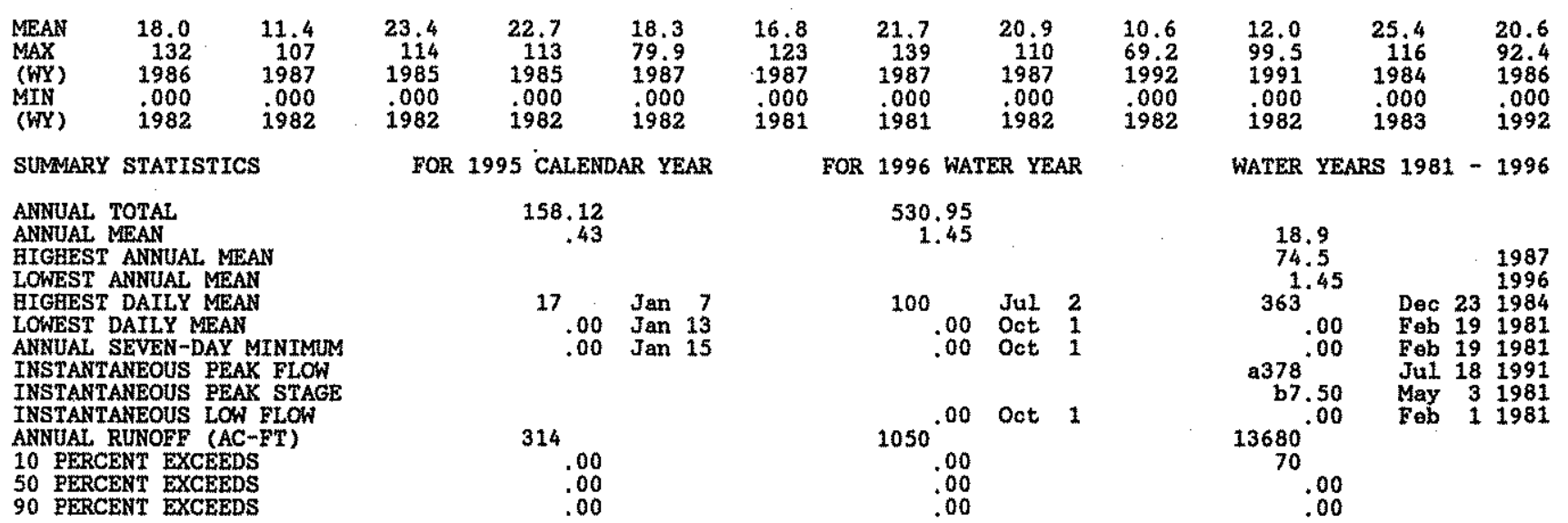

e Estimated

a-From rating curve extended above $360 \mathrm{fts} / \mathrm{s}$

b-Masimum 8 age height $7.5 \mathrm{ft}$, May 3, 1981, from floodmarks. 
RIO GRANDE BASIN

08395500 PECOS RIVAR NEAR LAKE ARTHUR, MM

LOCATION.--Lat $32^{\circ} 59^{\prime} 18^{\prime \prime}$, long $104^{\circ} 19^{\prime} 20^{*}$, in SWHEK soc.27, T.15 S., R.26 E., Chaves County, Bydrologic Unit

13060007 , on left bank $400 \mathrm{ft}$ upstream from bridge on Yuma Road, 2.5 mi east of Lake Arthur, 7 mi upstream from

Cottonwood Creek, $11 \mathrm{mi}$ northeast of Arteste, and at mile 522.0 .

DRAINAGE AREA. --14,760 $\mathrm{mi}^{2}$, approximately (contributing area).

PERIOD OF RECORD. -August 1938 to current year.

GAGE,-Water-8tage necorder and rock control. Elevation ol gage 18 3,327.07 ft above National Geodetic Vertical Datum of 1929.

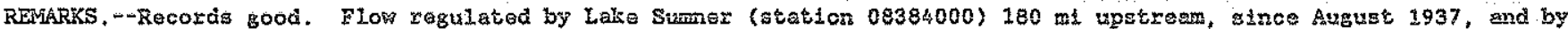
Two Rivers Reservolr (station 08390600 ) 77 mi upstream, since July 1963. Diversions and ground-water withdrawals for 1rrigation of about 124,000 acres, 1959 determination, upstream from station. Several observations of water temperature were made during the year. No flow at times.

EXTREMES OUTSIDE PERIOD OF RECORD,-F1ood of May 30,1937 , reached stage of 21.77 ft, discharge, 51,500 fts/s on basis of slope-area measurement of peak flow.

DISCHARGE, CUBTC FEET PER SECOWD, WATER YEAR OCTOBER 1995 TO SEPTEMBER 1996

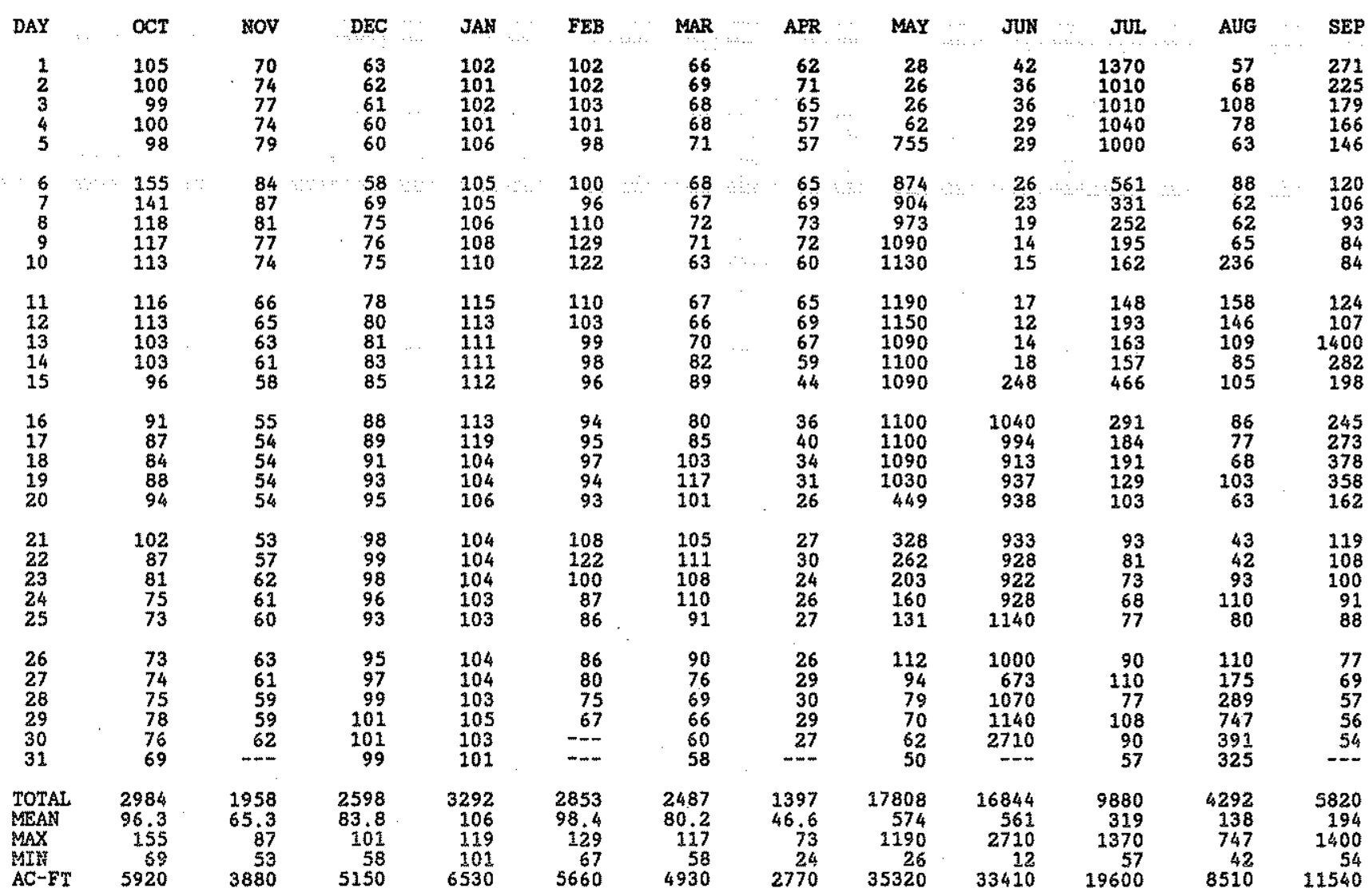

STATISTICS OF MONTHLY MEAN DATA FOR WATER YEARS 1938 - 1996, BY WATER YEAR (WY)

\begin{tabular}{|c|c|c|c|c|c|c|c|c|c|c|c|c|}
\hline $\begin{array}{l}\text { MEAN } \\
\text { MAX } \\
(W Y) \\
\text { MIN } \\
(W \mathrm{~W})\end{array}$ & $\begin{array}{r}249 \\
3701 \\
1942 \\
3.89 \\
1965\end{array}$ & $\begin{array}{r}121 \\
983 \\
1942 \\
32.0 \\
1960\end{array}$ & $\begin{array}{r}95.8 \\
546 \\
1942 \\
29.9 \\
1967\end{array}$ & $\begin{array}{r}94.8 \\
451 \\
1942 \\
34.5 \\
1055\end{array}$ & $\begin{array}{r}85.9 \\
446 \\
1942 \\
26.6 \\
1965\end{array}$ & $\begin{array}{r}178 \\
682 \\
1941 \\
16.6 \\
3967\end{array}$ & $\begin{array}{r}228 \\
1308 \\
1942 \\
7.35 \\
1957\end{array}$ & $\begin{array}{r}319 \\
3673 \\
1941 \\
11.9 \\
1975\end{array}$ & $\begin{array}{r}327 \\
2436 \\
1941 \\
4.78 \\
1977\end{array}$ & $\begin{array}{r}340 \\
1521 \\
1960 \\
1.02 \\
1954\end{array}$ & $\begin{array}{r}271 \\
913 \\
1941 \\
192 \\
1964\end{array}$ & $\begin{array}{r}375 \\
5407 \\
1941 \\
1.30 \\
1964\end{array}$ \\
\hline
\end{tabular}

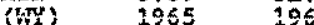

SUPAMARY STATISTICS

ANNUAL TOTAL

ANNUAL. MEAN

BIGHEST ANNUAL MEAN

LOWEST ANNUAL MEAN

HIGEEST DAILY MEAN

LOWEST DATLY MEAN

ANNUAL SEVEN-DAY MINIMUN

INSTANTANEOUS PEAK FLOW

INSTANTANEOUS PEAK STAGE

INSTANTANEOUS LOW FLOW

ANNUAL RUNOFF (AC-FT)

10 PERCENT EXCEEDS

50 PERCENT EXCEEDS

90 PERCENT EXCEEDS

$1067 \quad 1065 \quad 1065$

3967

FOR 1996 WATER YEAR

WATER YEARS 1938 - 1996

FOR 1995 CALENDAR YEAR

$\begin{array}{cc}{ }^{86302.7} & \\ 236 & \\ 1660 & \text { Sep } 11 \\ 9.7 & \text { May } 24 \\ 12 & \text { May } 21 \\ & \\ 171200 & \\ 840 & \end{array}$

72213

89

33

$$
\begin{gathered}
224 \\
1314 \\
62.2 \\
39800
\end{gathered}
$$

$\begin{array}{rrr}.00 & \text { Aug } 21 & 1947 \\ \text { Jul } 26 & 1954 \\ 496000^{\circ} & \text { Sep 24 } 1941 \\ 21.90 & \text { Sep } 241941 \\ \text { b.00 } & \text { Oct } & 1946\end{array}$

162400

666

70 
08396500 PECOS RIVER NEAR ARTESTA, NM

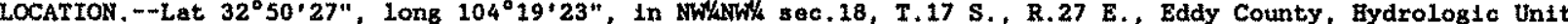
13060007 , on left bank 250 ft upstream from bridge on U.S. Highway $82,4.3$ mi east of Artesta, 7.0 mí upstream

DRAINAGE AREA. $--15,300 \mathrm{mi}^{2}$, approxtmately (contributing area).

WATER-DISCHARGE RECORDS

PERIOD OF RECORD. - September 1905 to June 1909, August 1909 to current year. Monthly discharge only for some pertods, publishod in WSP 1312 and 1712. Records for Aug. 22-31, 1934 , and October 1936 to Aprt1 1937 , pub1ished in WSP 763 and 828, respectively, are not reliable and should not be used. Prior to February 1936 , published as "near Dayton."

REVISED RECORDS,- WSP 1312 and 1512: 1913, 1915, 1917-18(M), 1920, 1923, 1931-36. WSE 1712: 1906(M), 1908-11(M), $1919,1921-23(M), 1929,1931-32(M), 1935-36(M), 1937,1939(M), 1941(M)$. Soe also PARIOD OF RECORD.

GAGE. - Water-stage recorder. Elevation of gage is $3,291.92 \mathrm{ft}$ above National Goodetic Vertical Datum of 1929 (Bureau of Reclamation bench mark). See WSP 1923 or 2123 for history of changes prior to Apr. 5 , 1941 . Apr. 5 , 1941 to Apr. 2, 1981, water-stage recorder at site $250 \mathrm{ft}$ downstream at same datum.

REMARKS. - Water-discharge records fair except for estimated dafly discharges, which are poor. Considerable flow regulation by Lake Sumer (station 08384000) since August 1937 and by Two Rivers Reservoir (station 08390600) since July 1963. Diversions and ground-water withdrawals for irrigation of about 154,000 acres, 1959 determination, upstream from station. U.S. Army Corps of Engineers satelite telemeter at station. No flow at times.

EXTREMES OUTSIDE PERIOD OF RECORD.--Greatest flood since at least 1893 occurred Oct. 2, 1904, discharge not determined; the peak inflow to Lake McMillan, which includes Rio Penasco and Fourmile Draw, was estimated at $82,000 \mathrm{ft} / \mathrm{s}$. The second highest flood occurred July 25 , 1905, discharge downstream from Rto Penasco, 50, 300 . $\mathrm{ft}^{3} / \mathrm{s}$, based on gain in storage and spill from Lake McMillan. The floods in August 1893 and $0 \mathrm{ctober} 1904$ damaged McMillan Dam and washed out Avalon Dam.

DISCHARGE, CUBIC FEET PER SECOND, WATER YSAR OCTOBER 1995 TO SEPTEMBER 1996

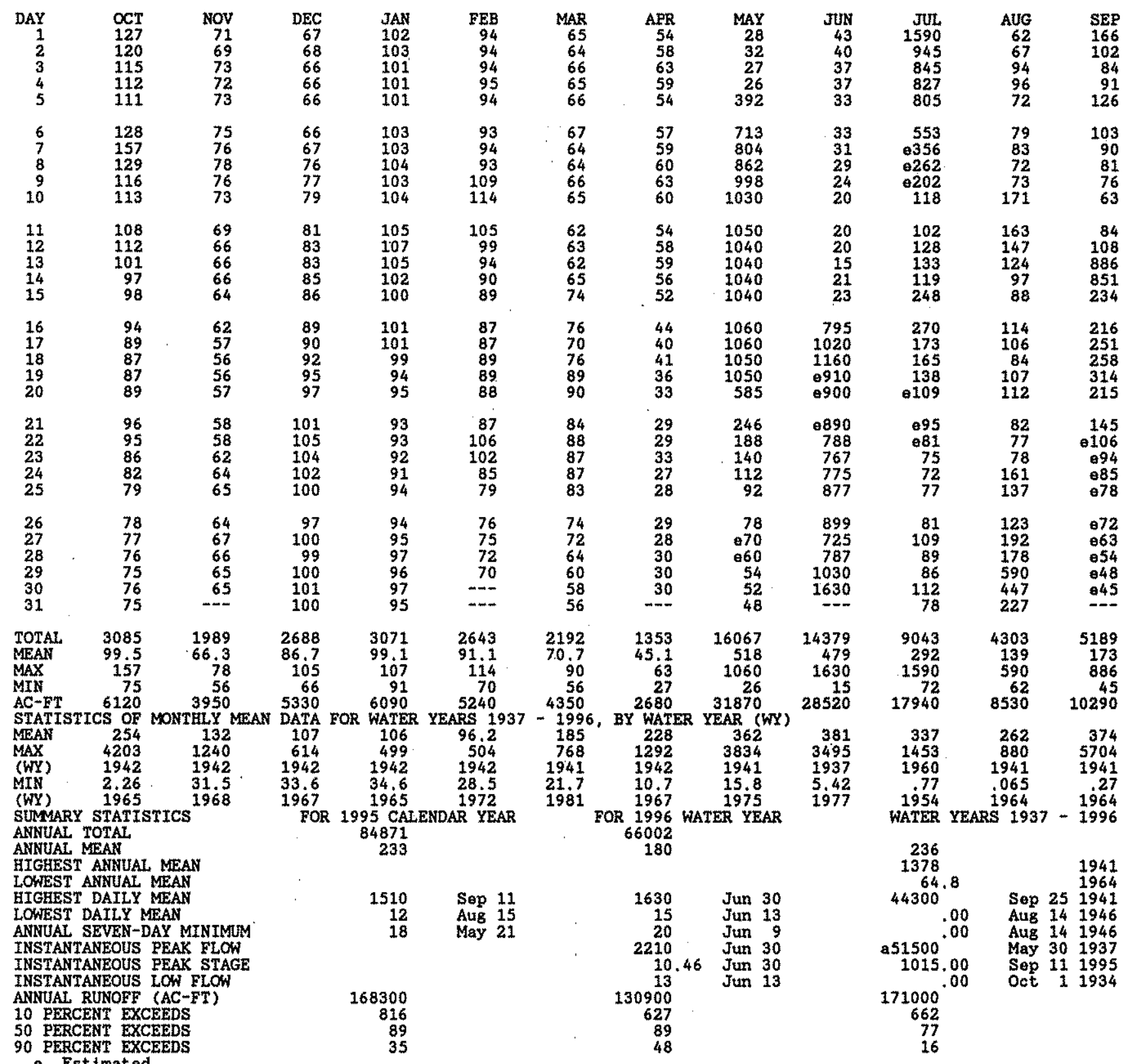

- Estimated

a-From a slope-area measurement made at a sito 15 mi upstream. 
RIO GRANDE BASIN

08396500 PECOS RIVER NEAR ARTESIA, MM -- Continued

WATER-QUALITY RECORDS

PERIOD OF RECORD, --Water years 1937 to current year.

WATER QUALITY DATA, WATER YEAR OCTOBER 1995 TO SEPTEMBER 1996

\begin{tabular}{|c|c|c|c|c|c|c|c|c|c|c|c|}
\hline DATE & TIME & $\begin{array}{c}\text { DIS- } \\
\text { CBARGE } \\
\text { INST } \\
\text { CUBIC } \\
\text { FEET } \\
\text { PERR } \\
\text { SECOND } \\
\text { (00061) }\end{array}$ & 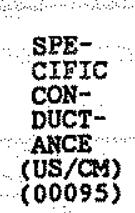 & $\begin{array}{l}\text { PH } \\
\text { WATER } \\
\text { WROEL } \\
\text { FIELD } \\
\text { (STARD- } \\
\text { AND } \\
\text { (NIIS) } \\
(00400)\end{array}$ & $\begin{array}{c}\text { TEMPER- } \\
\text { ATUEE } \\
\text { AIR } \\
(D E G C) \\
(00020)\end{array}$ & $\begin{array}{c}\text { TEMPER- } \\
\text { ATURE } \\
\text { WATER } \\
(D E G C) \\
(00010)\end{array}$ & $\begin{array}{l}\text { BARO- } \\
\text { METRIC } \\
\text { MRES } \\
\text { SURE } \\
\text { (NI } \\
\text { OF } \\
\text { HG) } \\
(00025)\end{array}$ & $\begin{array}{c}\text { OXYGEN, } \\
\text { DIS- } \\
\text { SOLWD } \\
(\text { SGG } \\
(00300)\end{array}$ & $\begin{array}{c}\text { OXYGEN, } \\
\text { DIS- } \\
\text { SOLVDD } \\
\text { (PER- } \\
\text { CEXT } \\
\text { SATUR- } \\
\text { ATION) } \\
\text { (O0301) }\end{array}$ & $\begin{array}{l}\text { OXYGEN } \\
\text { DEYAAND } \\
\text { CHES- } \\
\text { ICAL } \\
\text { (GIGH } \\
\text { LEVEL) } \\
\text { (NG/L) } \\
(00340)\end{array}$ & $\begin{array}{l}\text { COLI- } \\
\text { FORM, } \\
\text { FECAL, } \\
0.7 \\
\text { (M-MF } \\
\text { (COLS.', } \\
\text { (100 } \mathrm{ML}) \\
(31625)\end{array}$ \\
\hline DEC 1995 & 1410 & 77 & 7840 & -- & 19.5 & 12.0 & 677 & 11.4 & 123 & 59 & -- \\
\hline $12 \ldots$ & 1100 & 65 & 8410 & 8.0 & 21.0 & 13.0 & 676 & 10.4 & 114 & 75 & -- \\
\hline $4 \ldots$ & 1100 & 21 & 9490 & 8.0 & 20.0 & 24.0 & 679 & 6.8 & 94 & 130 & $<3$ \\
\hline $9 .$. & 0945 & 77 & 4510 & 8.0 & 21.0 & 24.0 & 682 & 8.0 & 108 & $<44$ & $\cdots$ \\
\hline DATE & $\begin{array}{c}\text { STREP- } \\
\text { TOCOCLI } \\
\text { FECAL } \\
\text { KF AGAR } \\
\text { (COLS. } \\
\text { gER } \\
100 \text { ME }) \\
(31673)\end{array}$ & $\begin{array}{c}\text { HARD- } \\
\text { NESS } \\
\text { TOTAL } \\
\text { (MG/L. } \\
\text { AS } \\
\text { CACO3) } \\
\text { (00900) }\end{array}$ & $\begin{array}{l}\text { HARD- } \\
\text { NAES } \\
\text { NORCARB } \\
\text { DISSOLV } \\
\text { FLD. AS } \\
\text { CACCo } \\
\text { (MSG/L) } \\
(00904)\end{array}$ & $\begin{array}{l}\text { CALCIUM } \\
\text { DIS- } \\
\text { SOLVED } \\
\text { (NSG/L } \\
\text { AS CA) } \\
(00915)\end{array}$ & $\begin{array}{c}\text { MAGNE- } \\
\text { SIUM- } \\
\text { DIS- } \\
\text { SOLVED } \\
\text { (MGL/L } \\
\text { AS MG }) \\
(00925)\end{array}$ & $\begin{array}{c}\text { SODIUM; } \\
\text { DIS } \\
\text { SOLVED } \\
\text { (MG/L } \\
\text { AS NA) } \\
(00930)\end{array}$ & $\begin{array}{c}\text { SODIUM } \\
\text { AD- } \\
\text { SORP- } \\
\text { TION } \\
\text { RATIO } \\
\text { (00931) }\end{array}$ & $\begin{array}{c}\text { POTAS- } \\
\text { SIUM, } \\
\text { DIS- } \\
\text { SOLVED } \\
\text { (MGELL } \\
\text { AS K } \\
(00935)\end{array}$ & $\begin{array}{l}\text { BICAR- } \\
\text { BONATE } \\
\text { WATER } \\
\text { DIS IT } \\
\text { FIEL } \\
\text { MG/I AS } \\
\text { BCO3 } \\
(00453)\end{array}$ & $\begin{array}{c}\text { CAR- } \\
\text { BOKATE } \\
\text { WATER } \\
\text { DIS IT } \\
\text { FIELD } \\
\text { MG/L AS } \\
\text { CO3 } \\
(00455)\end{array}$ & $\begin{array}{l}\text { AIKA- } \\
\text { LINITY } \\
\text { WAT DIS } \\
\text { TOT IT } \\
\text { FIELD } \\
\text { MG/L AS } \\
\text { CACOB } \\
(39086)\end{array}$ \\
\hline $\begin{array}{l}\text { DEC } 1995 \\
08 \text {. } \\
M A R \quad 9966\end{array}$ & -- & 2300 & -- & 580 & 210 & 1100 & 10 & 8.6 & -- & - & -- \\
\hline $\begin{array}{l}\text { MAR } 1996 \\
12 \cdots . \\
\text { JUR }\end{array}$ & $-\cdots$ & 2200 & 2100 & 580 & 1.90 & 1100 & 10 & 7.9 & 159 & 0 & 130 \\
\hline $\operatorname{sep}^{14} \cdots$ & 240 & 2400 & 2300 & 6.10 & 210 & 1300 & 12 & 14 & 127 & 0 & 104 \\
\hline $09 .$. & - & 1400 & 1300 & 350 & 120 & 610 & 7 & 6.4 & 118 & 0 & 97 \\
\hline DATE & $\begin{array}{c}\text { ALKA- } \\
\text { LINITY } \\
\text { LAB } \\
\text { (MAG/L } \\
\text { AS } \\
\text { CACO3) } \\
(90410)\end{array}$ & $\begin{array}{c}\text { SULFATE } \\
\text { DIS- } \\
\text { SOLVED } \\
\text { (MG/L } \\
\text { AS SO4) } \\
(00945)\end{array}$ & $\begin{array}{l}\text { CHLO- } \\
\text { RIDE: } \\
\text { DIS-' } \\
\text { SOLED } \\
\text { (MGG/L } \\
\text { AS CL) } \\
(00940)\end{array}$ & $\begin{array}{l}\text { FLUO- } \\
\text { RIDE, } \\
\text { DIS'- } \\
\text { SOLVDD } \\
\text { MGG/L } \\
\text { AS F) } \\
(00950)\end{array}$ & $\begin{array}{c}\text { SILICA, } \\
\text { DIS- } \\
\text { SOLVED } \\
\text { (WG/L } \\
\text { AS } \\
\text { SIO2) } \\
(00955)\end{array}$ & $\begin{array}{l}\text { SOLIDS, } \\
\text { SLMIDF } \\
\text { CONSI- } \\
\text { TUENTS, } \\
\text { DIS- } \\
\text { SOLVED } \\
\text { (MG/L) } \\
(70301)\end{array}$ & $\begin{array}{c}\text { HITRO- } \\
\text { GEN, } \\
\text { NITRAEE } \\
\text { DIS- } \\
\text { SOLVED } \\
\text { (MEG/L } \\
\text { AS N } \\
\text { (00618) }\end{array}$ & $\begin{array}{c}\text { NITRO- } \\
\text { GEN } \\
\text { NITRITE } \\
\text { DIIS- } \\
\text { SOVED } \\
\text { (ATE/L } \\
\text { AS N } \\
\text { (00613) }\end{array}$ & $\begin{array}{c}\text { HITRO- } \\
\text { GEN, } \\
\text { NO2+NO3 } \\
\text { DIS- } \\
\text { SOLVED } \\
\text { (MG/L } \\
\text { AS N } \\
(00631)\end{array}$ & $\begin{array}{c}\text { NITRO- } \\
\text { GEN } \\
\text { AMONIA } \\
\text { DIS- } \\
\text { SOLVED } \\
\text { (MG/L } \\
\text { AS N } \\
\text { (00608) }\end{array}$ & $\begin{array}{c}\text { MITRO- } \\
\text { GEN, } \\
\text { ORGANIC } \\
\text { DIS- } \\
\text { SOLVED } \\
\text { (MG/L } \\
\text { AS N } \\
(00607)\end{array}$ \\
\hline $\begin{array}{l}\mathrm{DEC} 1995 \\
08\end{array}$ & 180 & 1700 & 2000 & 0.90 & 13 & 5730 & 2.78 & 0.120 & 1.90 & 0.350 & 0.45 \\
\hline $\begin{array}{l}12 \ldots \\
\text { Juk }\end{array}$ & 143 & 1800 & 1900 & 0.80 & 9.0 & 5670 & 0.140 & 0.010 & 0.150 & 0.110 & 0.39 \\
\hline $14 \ldots$ & 108 & 2100 & 2300 & 0.70 & 13 & 6610 & -- & $<0.010$ & $<0.050$ & 0.160 & 0.04 \\
\hline $59 . .$. & 100 & 1100 & 1100 & 0.70 & 12 & 3360 & 0.570 & 0.030 & 0.600 & 0.120 & 0.18 \\
\hline DATE & $\begin{array}{c}\text { NITRO- } \\
\text { GEN, AM- } \\
\text { MONIA + } \\
\text { ORGAIIC } \\
\text { TOTAA. } \\
\text { MAG/L } \\
\text { AS N) } \\
(00625)\end{array}$ & $\begin{array}{c}\text { MITRO- } \\
\text { GEN, AM- } \\
\text { MONIA + } \\
\text { ORGANIC } \\
\text { DIS. } \\
\text { (MSG/L } \\
\text { AS N } \\
(00623)\end{array}$ & $\begin{array}{c}\text { PBOS- } \\
\text { PEOSUS } \\
\text { TORAI } \\
\text { MGG/L } \\
\text { AS P) } \\
(00665)\end{array}$ & $\begin{array}{c}\text { PHOS- } \\
\text { PHOUS } \\
\text { DIS- } \\
\text { SOLVDD } \\
\text { (AKG/L } \\
\text { AS P) } \\
(00666)\end{array}$ & $\begin{array}{l}\text { PHOS- } \\
\text { PHORUS } \\
\text { ORTHO, } \\
\text { DIS- } \\
\text { SOLVED } \\
\text { (NGG/L } \\
\text { AS P P) } \\
(00671)\end{array}$ & $\begin{array}{l}\text { CARBON, } \\
\text { ORGAAIC } \\
\text { TORAL } \\
\text { CMG/L } \\
\text { AS C) } \\
(00680)\end{array}$ & $\begin{array}{l}\text { ALUM- } \\
\text { INUM, } \\
\text { DIS- } \\
\text { SOLVED } \\
\text { (UG/L } \\
\text { AS AL) } \\
\text { (01106) }\end{array}$ & $\begin{array}{l}\text { ANTI- } \\
\text { MONY } \\
\text { DIS- } \\
\text { SOVWED } \\
\text { UGG/L } \\
\text { AS SB) } \\
(01095)\end{array}$ & $\begin{array}{c}\text { ARSENIC } \\
\text { DIS- } \\
\text { SOLWED } \\
\text { (UG/L } \\
\text { AS AS }) \\
(01000)\end{array}$ & $\begin{array}{c}\text { BARIUM, } \\
\text { DIS- } \\
\text { SOLVED } \\
\text { (UGL/ } \\
\text { AS BA) } \\
(01005)\end{array}$ & $\begin{array}{l}\text { BERYL- } \\
\text { LIUM- } \\
\text { DIS- } \\
\text { SOLYVD } \\
\text { (UGLL } \\
\text { AS BE) } \\
(01010)\end{array}$ \\
\hline $\begin{array}{l}\text { DEC } 1995 \\
08 \\
\text { MAR } 1996\end{array}$ & 0.80 & 0.80 & 0.070 & 0.030 & 0.030 & 3.8 & 7.0 & $<4.0$ & $<1$ & 37 & $<4.0$ \\
\hline${ }_{\text {sug }}^{12} \cdots$ & 0.50 & 0.50 & 0.040 & $<0.010$ & 0.010 & 3.6 & -- & - & $-\cdots$ & -- & -- \\
\hline $14 \ldots$ & 0.60 & 0.20 & 0.040 & 0.010 & $<0.010$ & 6.6 & -- & - & -- & -- & - \\
\hline $09 .$. & 0.60 & 0.30 & 0.210 & $<0.010$ & $<0.010$ & 5.5 & 7.0 & $<3.0$ & $<1$ & 103 & $<3.0$ \\
\hline
\end{tabular}


RIO GRANDE BASIN

08396500 PECOS RIVER NEAR ARTESIA, NM -- Continued

WATER-QUALITY RECORDS

WATER QUALITY DATA, WATER YEAR OCTOBER 1995 TO SEPTEMBER 1996

\begin{tabular}{|c|c|c|c|c|c|c|}
\hline $\begin{array}{l}\text { BORON, } \\
\text { DIS - } \\
\text { SOLVED } \\
\text { (UG/L } \\
\text { AS B) }\end{array}$ & $\begin{array}{c}\text { CADMIUM } \\
\text { DIS- } \\
\text { SOLVDD } \\
\text { (UG/L } \\
\text { AS CD) }\end{array}$ & $\begin{array}{l}\text { MIUM, } \\
\text { DIS- } \\
\text { SOLVED } \\
\text { (UG/L } \\
\text { AS CR) }\end{array}$ & $\begin{array}{l}\text { DI } \\
\text { SOL } \\
\text { (U } \\
\text { AS } \\
\text { COI }\end{array}$ & $\begin{array}{l}\text { DI } \\
\text { SC } \\
\text { (I } \\
\text { AS } \\
\text { Col }\end{array}$ & $\begin{array}{r}\text { IR } \\
\text { D } \\
\text { SO } \\
\text { (U) } \\
\text { AS } \\
\text { C1 }\end{array}$ & $\begin{array}{l}- \\
\text { ab } \\
\text { L } \\
\text { B) }\end{array}$ \\
\hline
\end{tabular}

MANGA- MERCURX MOLYB-

NESE, TOTAL DENUM, NICKEL,

DIS- RECOV- DIS- DIS-

SOLVED ERABLE SOLVED SOLVED

(UG/L (UG/L (UG/L (UG/L

AS (MN) AS EG) AS (O) AS NT)

$(01020)(01025) \quad(01030)(01035)$

(01040)

$(01046)$

(01049)

(01056)

$(71900)(01060)$ (01065)

DEC 1995

08

MAR 199

$\operatorname{JuN}_{14}^{12} \ldots$

410

$<4.0$

$6.0<4.0 \quad 28$

10

$<4.0 \quad 13$

$<4.0$

21

450

$m$

$+\cdots$

SEP

321

6.0

$<3.0$

$--$

$-2 \quad 40$

$+\cdots$

$-$

$<3.0 \quad 6.0<15$

3.0

$<3.0$

$<0.10$

3.0

9.0

DATE

$\begin{array}{cccc} & & & \\ \text { SELE- } & \text { NELE- } & & \\ \text { NIUM, } & \text { SILVER, } & \text { ZINC, } \\ \text { NIUM, } & \text { DIS- } & \text { DIS- } & \text { DIS- } \\ \text { TOTAL } & \text { SOLVE } & \text { SOLVED } & \text { SOLVED } \\ \text { (UG/L } & \text { (UG/L } & \text { (UG/L } & (U G / L \\ \text { AS SE) } & \text { AS SE) } & \text { AS AG) } & \text { AS ZN) } \\ (01147) & (01145) & (01075) & (01090)\end{array}$

$\begin{array}{cc}\text { NITRO- } & \text { NITRO- } \\ \text { GEN, } & \text { GEN, NH4 } \\ \text { NO2+NO3 } & \text { TOTAL } \\ \text { TOT. IN } & \text { IN BOT. } \\ \text { BOT MAT } & \text { MAT. } \\ \text { (MG/KG } & \text { (NG/KG } \\ \text { AS N) } & \text { AS N) } \\ (00633) & (00611)\end{array}$

NITRO-
GEN, NH4
+ ORG.
TOT IN
BOT MAT
(NG/KG
AS.N)
$(00626)$

PHOS-
PRORUS
TOTAL
IN BOT.
MAT.
(MG/KG
AS P)
$(00668)$

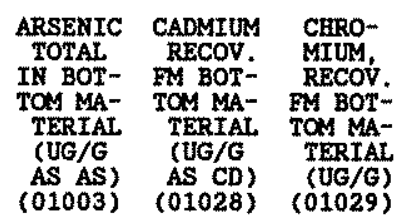

DEC 1995

MAR 1986

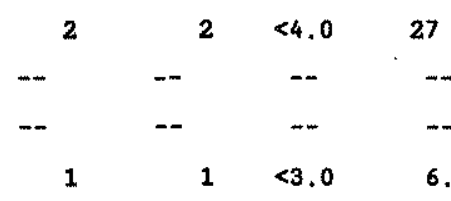

27
--
-
6.0

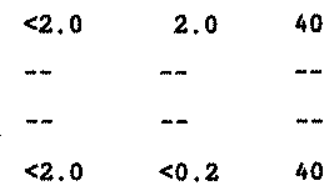

85

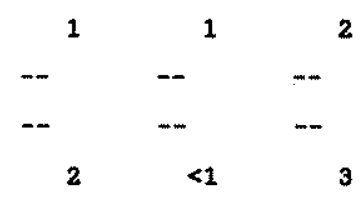

$\begin{array}{cccc}\text { COBALT, } & \text { COPEER, } & \text { IRON, } & \text { LEAD, } \\ \text { RECOV. } & \text { RECOV. } & \text { RECOV. } & \text { RECOV. } \\ \text { FM BOT- } & \text { FM BOT- } & \text { FM BOT- } & \text { FM BOT- } \\ \text { TOM MA- } & \text { TOM MA- } & \text { TOM MA- } & \text { TOM MA- } \\ \text { TERIAL } & \text { TERIAL } & \text { TERIAL } & \text { THRTAL } \\ \text { (UG/G } & \text { (UG/G } & \text { (UGG/G } & \text { (UG/G } \\ \text { AS CO) } & \text { AS CU) } & \text { AS FE) } & \text { AS PB) } \\ (01038) & (01043) & (01170) & (01052)\end{array}$

MANGA- MERCURY ZINC,

NESE: RECOV. RECOV. URANIUM

RECOV. FM BOT- EM BOT- NATURAL

FM BOT- TOM MA- TOM MA-

TOM MA- TERIAI TERIAI

(UG/G) AS EG) AS ZN)

$(01053)(71921)(01093)$

DIS-
SOLVED
SUS-

(UG/L $\mathrm{L}$

AS U) RENDED

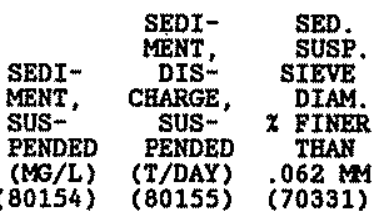

DEC 1995

MAR 1996

$12 \ldots$

गUत 14

SEP

$09 .$.

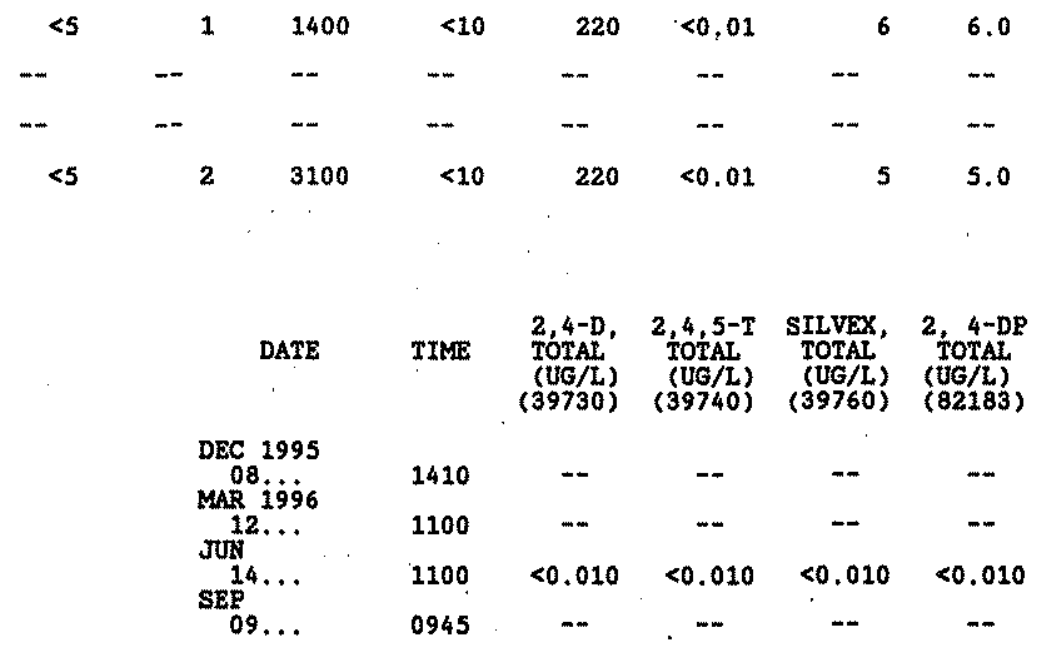


RIO GRANDE BASIN

08398500 RIO PENASCO AT DAYTON, NM

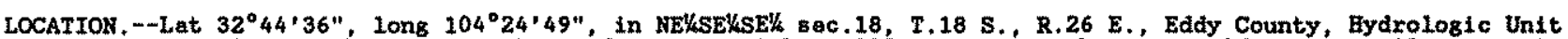
13060010 , on left bank $1.2 \mathrm{mi}$ upstream from U.S. Highway $285, i .9$ mi northwest of old Dayton railway station, 5.6 mf upstream from mouth, and $7.0 \mathrm{mi}$ south of Artesia. Mouth at Pecos River mile 496.4 .

DRAINAGE AREA, $--1,060 \mathrm{mi}^{2}$, approximately.

PERIOD OF RECORD.--Apri1 1951 to current year. Frior to Octobex 1953, published as "near Dayton."

REVISED RECORDS.-WSP 1242: 1951(M),WSP 1512: 1956,WSP 1923: 1955.

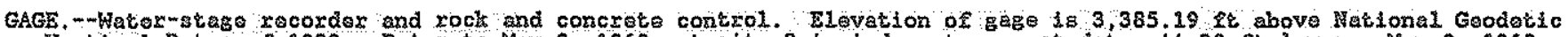
Vertical Datum of 1929. Prior to Hay 9, 1968, at site 2.4 mi downstream, at datum 44.30 ft lower. May 9 , 1968 to June 12, 1975, at present site at datum 1.98 Ft higher.

REMARKS.-Records good except for estimated dafly discharges, which are poor. Diversions ond ground-water withdrawals for irrigation of about 3,000 acres, 1959 determination, upstroam from station. Soveral observations of water temperature were made during the year. Ho flow most of $t$ ime.

EXTREMES OUTSIDE PERIOD OF RECORD,-Flood of about Sept, 22, 1941, reached a stage of about 9 ft, from floodmark, previous site and datum, discharge not determined. Peak discharge at discontinued atation "near Dunken" (station 08397600 ), about $60 \mathrm{mi}$ upstream, was $70,000 \mathrm{ft}^{3} / \mathrm{s}$, determined in 1956 , from rating curve extended above a slopearea measurement of $36,000 \mathrm{ft} / \mathrm{s}$, for peak of Oct. 6 or 7,1954 .

DISCEARGE, CUEIC FEET PER SECOND, WATER YEAR OCTOBER 1995 TO SEPTEMBER 1996

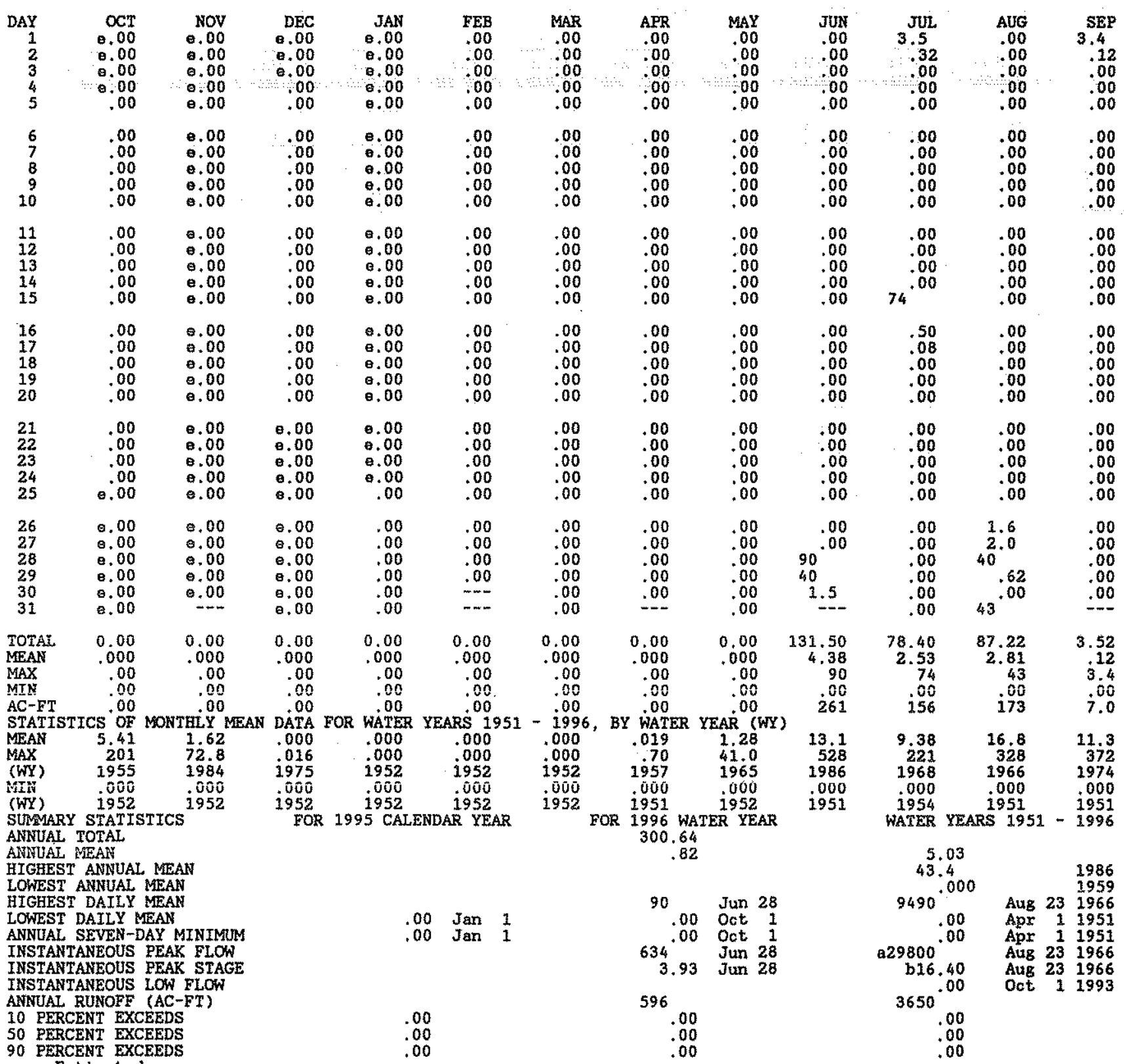

a-From rating curve extended above $7,800 \mathrm{ft} / \mathrm{s}$, on basis of slope-area measurements at $g$ age heights 6.82 ft and $7.90 \mathrm{ft}$, at previous site and datum.

b-From floodmarks, present site and datum. 
RIO GRANDE BASIN

08399500 PECOS RIVER (KAISER CAANHEL) NEAR LAKEWOOD, NM

LOCATION,--Lat $32^{\circ} 41^{\circ} 22^{\prime \prime}$, Long $104^{\circ} 17^{\prime} 53^{\prime \prime}$, in WWSEK sec.5, T.19 S., R.27 E., Eddy County, Bydrolog te Unit 13060011, on left bank 3.0 mi upstrean from high-water line of former Lake'McMi1lan, 6.0 mi northeast of Iakewood, 12 mi southeast of Artesia, and at mile 492.1 .

PERTOD OF RECORD.--May 1950 to current year. Prior to October 1954, publishod as Kaiser Lake-McMi1lan Channel near Lakewood.

GAGE.-Water-stage recorder. Elevation of gage $183,268.53$ ft above National Geodetic Vertlcal Datim of 1929 (Bureau of Reclemation bench mark). Prior to Mar. 23, 1955, at site 3.0 mi downstream at datum 7.83 ft lower. Mar. 23, 1955 to Sept. 30, 1963, at present stte at datum 2,00 ft htgher.

REMARKS.-mecords good except for estimated dally discharges, which are poor. Considerable flow regulation by Lake Sumer (station 08384000) since August 1937, and by Two Rivers Reservoir (station 08390600) since July 1963. Diversions and ground-water withdrawals for irrigation of about 170,000 acres, 1959 determination, upstream from station. Above about $1,500 \mathrm{fts} / \mathrm{s}$, flow will begin bypassing station and depending on the magnitude and duration of flow, may reach Brantley Lake (station 08401450). Several observations of water temperature were made during the year. Wo flow at times.

DISCAARGE, CUBIC FEET PER SECOND, WATER YEAR OCTOBER 1995 TO SEPTEMBER 1996

\begin{tabular}{|c|c|c|c|c|c|c|c|c|c|c|c|c|}
\hline DAY & $\alpha \mathbf{~}$ & Nov & DEC & JAN & FEB & MAR & APR & MAY & JUN & JUL & AUG & SEP \\
\hline $\begin{array}{l}1 \\
2 \\
3 \\
4 \\
5\end{array}$ & $\begin{array}{l}146 \\
133 \\
123 \\
115 \\
108\end{array}$ & $\begin{array}{l}668 \\
067 \\
071 \\
e 77 \\
079\end{array}$ & $\begin{array}{l}56 \\
57 \\
58 \\
56 \\
56\end{array}$ & $\begin{array}{l}103 \\
104 \\
104 \\
104 \\
103\end{array}$ & $\begin{array}{l}91 \\
91 \\
90 \\
90 \\
89\end{array}$ & $\begin{array}{l}58 \\
55 \\
57 \\
57 \\
55\end{array}$ & $\begin{array}{l}050 \\
053 \\
061 \\
054 \\
049\end{array}$ & $\begin{array}{r}22 \\
19 \\
20 \\
18 \\
318\end{array}$ & $\begin{array}{l}35 \\
32 \\
28 \\
24 \\
21\end{array}$ & $\begin{array}{r}1260 \\
969 \\
878 \\
857 \\
839\end{array}$ & $\begin{array}{l}44 \\
49 \\
58 \\
82 \\
62\end{array}$ & $\begin{array}{l}280 \\
176 \\
169 \\
122 \\
126\end{array}$ \\
\hline $\begin{array}{r}6 \\
7 \\
8 \\
9 \\
10\end{array}$ & $\begin{array}{l}107 \\
179 \\
138 \\
114 \\
109\end{array}$ & $\begin{array}{l}082 \\
090 \\
684 \\
078 \\
071\end{array}$ & $\begin{array}{l}55 \\
54 \\
61 \\
66 \\
68\end{array}$ & $\begin{array}{l}105 \\
104 \\
103 \\
104 \\
104\end{array}$ & $\begin{array}{r}88 \\
90 \\
87 \\
99 \\
114\end{array}$ & $\begin{array}{l}58 \\
58 \\
56 \\
60 \\
62\end{array}$ & $\begin{array}{r}053 \\
056 \\
060 \\
060 \\
059\end{array}$ & $\begin{array}{l}721 \\
789 \\
827 \\
891 \\
937\end{array}$ & $\begin{array}{r}19 \\
18 \\
015 \\
013 \\
014\end{array}$ & $\begin{array}{l}676 \\
385 \\
272 \\
210 \\
164\end{array}$ & $\begin{array}{r}53 \\
80 \\
62 \\
63 \\
105\end{array}$ & $\begin{array}{r}103 \\
83 \\
70 \\
61 \\
54\end{array}$ \\
\hline $\begin{array}{l}11 \\
12 \\
13 \\
14 \\
15\end{array}$ & $\begin{array}{r}106 \\
103 \\
103 \\
92 \\
94\end{array}$ & $\begin{array}{l}067 \\
063 \\
060 \\
058 \\
\theta 57\end{array}$ & $\begin{array}{l}72 \\
76 \\
79 \\
81 \\
83\end{array}$ & $\begin{array}{l}103 \\
108 \\
106 \\
104 \\
103\end{array}$ & $\begin{array}{r}108 \\
98 \\
93 \\
89 \\
85\end{array}$ & $\begin{array}{l}57 \\
59 \\
61 \\
63 \\
72\end{array}$ & $\begin{array}{l}75 \\
75 \\
80 \\
77 \\
62\end{array}$ & $\begin{array}{l}966 \\
948 \\
957 \\
964 \\
980\end{array}$ & $\begin{array}{l}012 \\
011 \\
010 \\
013 \\
014\end{array}$ & $\begin{array}{l}137 \\
124 \\
166 \\
131 \\
196\end{array}$ & $\begin{array}{r}220 \\
160 \\
130 \\
93 \\
76\end{array}$ & $\begin{array}{r}60 \\
104 \\
562 \\
979 \\
246\end{array}$ \\
\hline $\begin{array}{l}16 \\
17 \\
18 \\
19 \\
20\end{array}$ & $\begin{array}{l}94 \\
92 \\
85 \\
83 \\
88\end{array}$ & $\begin{array}{l}056 \\
055 \\
e 55 \\
055 \\
053\end{array}$ & $\begin{array}{l}85 \\
85 \\
87 \\
90 \\
92\end{array}$ & $\begin{array}{r}104 \\
102 \\
100 \\
96 \\
94\end{array}$ & $\begin{array}{l}83 \\
81 \\
83 \\
82 \\
82\end{array}$ & $\begin{array}{l}79 \\
75 \\
72 \\
85 \\
96\end{array}$ & $\begin{array}{l}42 \\
36 \\
39 \\
54 \\
40\end{array}$ & $\begin{array}{l}980 \\
987 \\
978 \\
982 \\
706\end{array}$ & $\begin{array}{l}e 730 \\
e 940 \\
e 970 \\
e 890 \\
e 880\end{array}$ & $\begin{array}{l}311 \\
198 \\
180 \\
174 \\
115\end{array}$ & $\begin{array}{l}93 \\
96 \\
55 \\
53 \\
81\end{array}$ & $\begin{array}{l}200 \\
241 \\
241 \\
287 \\
231\end{array}$ \\
\hline $\begin{array}{l}21 \\
22 \\
23 \\
24 \\
25\end{array}$ & $\begin{array}{r}98 \\
105 \\
93 \\
e 84 \\
677\end{array}$ & $\begin{array}{l}052 \\
054 \\
064 \\
065 \\
063\end{array}$ & $\begin{array}{r}94 \\
98 \\
100 \\
100 \\
97\end{array}$ & $\begin{array}{l}95 \\
93 \\
91 \\
91 \\
92\end{array}$ & $\begin{array}{l}81 \\
94 \\
98 \\
85 \\
70\end{array}$ & $\begin{array}{l}88 \\
90 \\
94 \\
90 \\
93\end{array}$ & $\begin{array}{l}39 \\
28 \\
45 \\
31 \\
23\end{array}$ & $\begin{array}{r}336 \\
263 \\
206 \\
165 \\
\mathrm{e} 100\end{array}$ & $\begin{array}{l}798 \\
806 \\
803 \\
827 \\
887\end{array}$ & $\begin{array}{l}97 \\
81 \\
70 \\
62 \\
71\end{array}$ & $\begin{array}{l}52 \\
34 \\
32 \\
83 \\
85\end{array}$ & $\begin{array}{r}137 \\
110 \\
96 \\
86 \\
80\end{array}$ \\
\hline $\begin{array}{l}26 \\
27 \\
28 \\
29 \\
30 \\
31\end{array}$ & $\begin{array}{l}e 72 \\
073 \\
e 73 \\
e 75 \\
e 79 \\
076\end{array}$ & $\begin{array}{r}e 61 \\
e 59 \\
57 \\
55 \\
55 \\
-\cdots\end{array}$ & $\begin{array}{r}95 \\
98 \\
99 \\
102 \\
102 \\
102\end{array}$ & $\begin{array}{l}91 \\
92 \\
94 \\
92 \\
95 \\
94\end{array}$ & $\begin{array}{r}068 \\
066 \\
062 \\
62 \\
---\end{array}$ & $\begin{array}{l}83 \\
e 72 \\
e 65 \\
e 57 \\
053 \\
050\end{array}$ & $\begin{array}{r}25 \\
23 \\
24 \\
25 \\
24 \\
---\end{array}$ & $\begin{array}{r}073 \\
065 \\
058 \\
55 \\
48 \\
43\end{array}$ & $\begin{array}{r}927 \\
808 \\
803 \\
1020 \\
11.00 \\
\end{array}$ & $\begin{array}{r}68 \\
87 \\
95 \\
69 \\
100 \\
69\end{array}$ & $\begin{array}{r}71 \\
113 \\
171 \\
413 \\
492 \\
310\end{array}$ & $\begin{array}{r}75 \\
66 \\
61 \\
52 \\
49 \\
---\end{array}$ \\
\hline $\begin{array}{l}\text { TOTAL } \\
\text { MEAN } \\
\text { MAX } \\
\text { MIN } \\
\text { AC-FT }\end{array}$ & $\begin{array}{r}3117 \\
101 \\
179 \\
72 \\
6180\end{array}$ & $\begin{array}{r}1931 \\
64.4 \\
90 \\
52 \\
3830\end{array}$ & $\begin{array}{r}2504 \\
80.8 \\
102 \\
54 \\
4970\end{array}$ & $\begin{array}{r}3078 \\
99.3 \\
108 \\
91 \\
6110\end{array}$ & $\begin{array}{r}2499 \\
86.2 \\
114 \\
62 \\
4960\end{array}$ & $\begin{array}{r}2130 \\
68.7 \\
96 \\
50 \\
4220\end{array}$ & $\begin{array}{r}1422 \\
47.4 \\
80 \\
23 \\
2820\end{array}$ & $\begin{array}{r}15422 \\
497 \\
987 \\
18 \\
30590\end{array}$ & $\begin{array}{r}13468 \\
449 \\
1100 \\
10 \\
26710\end{array}$ & $\begin{array}{r}9111 \\
294 \\
1260 \\
62 \\
18070\end{array}$ & $\begin{array}{r}3571 \\
115 \\
492 \\
32 \\
7080\end{array}$ & $\begin{array}{r}5207 \\
174 \\
979 \\
49 \\
10330\end{array}$ \\
\hline
\end{tabular}

STATISTICS OF MONTHLY MEAN DATA FOR WATER YEARS 1950 - 1996, BY WATER YEAR (WY)

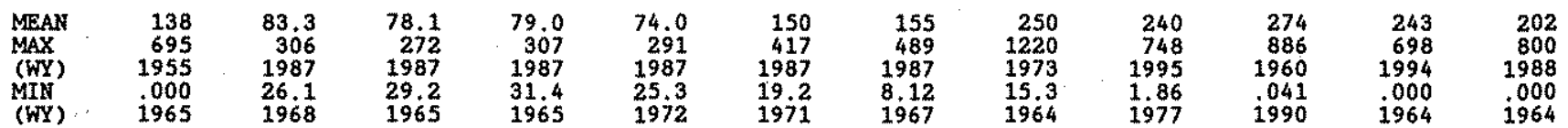

\section{SUMMARY STATISTICS}

ANNUAL TOTAL

ANNUAL MEAN

HIGHEST ANNUAL MEAN

LOWEST ANRUAL MEAN

HIGHEST DAILY MEAI

LOWEST DAILY MEAN

ANNUAZ SEVEN-DAY MINIMUM

INSTANTANEOUS PEAK FLOW

INSTANTANEOUS PEAK STAGE

INSTANTANEOUS LOW FLOW

ANNUAL RUNOFF (AC-FT)

10 PERCENT EXCEEDS

50 PERCENT EXCEEDS

90 PERCENT EXCEEDS

- Estimated
FOR 1995 CALENDAR YEAR

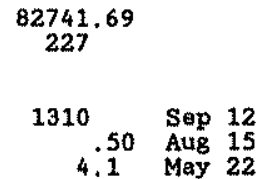

164100
800
86
27
FOR 1996 WATER YEAR

63460
173

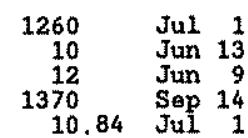

125900

685
85
WATER YEARS 1950 - 1996

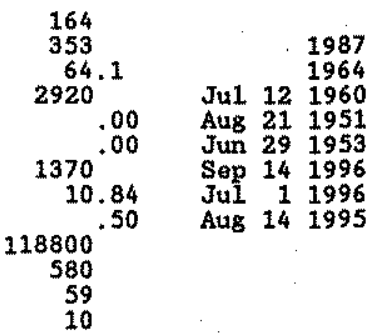




\section{RIO GRANDE BASIN}

08400000 FOURMILE DRAW REAR LAKEWOOD, NR

LOCATYON.--Lat $32^{\circ} 40^{\prime} 20^{\prime \prime}$, long $104^{\circ} 22^{\prime} 07^{\prime \prime}$, in SWhawkSEk sec.10, T.19 S., R.26 E., Eday County, Hydrologic Untc

13060011 in left $81 d e^{\prime}$ of channel 360 it downstrean from ford on Lakepood-Dayton road, 1.9 mi downstream from

U.S. Highway $285,2.8 \mathrm{mi}$ north of lasemood, $3.8 \mathrm{mi}$ upstream from mouth, and $11.5 \mathrm{mi}$ south of Artesie. Mouth at Pecos River mile 490.6 .

DRAINAGE AREA, - -265 $\mathrm{mL}^{2}$, approximately.

PERIOD OF RECORD. --October 1951 to current year.

REVISED RECORDS. --WDR WR-68-1: 1967.

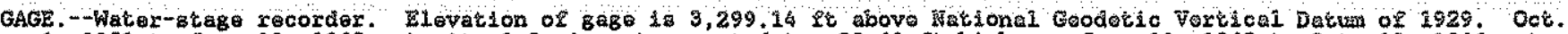

1, 1951 to June 19, 1962, at site $1.8 \mathrm{mi}$ upgtresm detura 30.61 ft higher, June 19, 1962 to 0ct. 12, 1965, at

site 410 ft upstroan at datum $6.08 \mathrm{ft}$ higher.

REMARKS. --Records good. Ho guxface diveraions upstroam erom station. Ho flow most of time.

DISCBARGE, CUBIC FEET PER SECOND, WATER YEAR OCTOBER 1995 TO SEPTERBER 1996

\begin{tabular}{|c|c|c|c|c|c|c|c|c|c|c|c|c|}
\hline DAY & $\propto T$ & NOV & DEC & JAN & FEB & MAR & AFR & $\mathrm{MAX}$ & JUस & JUL. & AUE & SEP \\
\hline $\begin{array}{ll}\mathbf{1} & \\
2 & \\
3 & \\
4 & \\
5 & \end{array}$ & $\begin{array}{l}.00 \\
.00 \\
.00 \\
.00 \\
.00\end{array}$ & $\begin{array}{l}.00 \\
.00 \\
.00 \\
.00 \\
.00\end{array}$ & $\begin{array}{l}.00 \\
.00 \\
.00 \\
.00 \\
.00\end{array}$ & $\begin{array}{l}.00 \\
.00 \\
.00 \\
.00 \\
.00\end{array}$ & $\begin{array}{l}.00 \\
.00 \\
.00 \\
.00 \\
.00\end{array}$ & $\begin{array}{l}.00 \\
.00 \\
.00 \\
.00 \\
.00\end{array}$ & $\begin{array}{l}.00 \\
.00 \\
.00 \\
.00 \\
.00\end{array}$ & $\begin{array}{l}.00 \\
.00 \\
.00 \\
.00 \\
.00\end{array}$ & $\begin{array}{l}.00 \\
.00 \\
.00 \\
.00 \\
.00\end{array}$ & $\begin{array}{l}.00 \\
.00 \\
.00 \\
.00 \\
.00\end{array}$ & $\begin{array}{l}.00 \\
.00 \\
.00 \\
.00 \\
.00\end{array}$ & $\begin{array}{l}.00 \\
.00 \\
.00 \\
.00 \\
.00\end{array}$ \\
\hline $\begin{array}{r}6 \\
7 \\
8 \\
9 \\
10\end{array}$ & $\begin{array}{l}.00 \\
.00 \\
.00 \\
.00 \\
.00\end{array}$ & $\begin{array}{l}.00 \\
.00 \\
.00 \\
.00 \\
.00\end{array}$ & $\begin{array}{l}.00 \\
.00 \\
.00 \\
.00 \\
.00\end{array}$ & $\begin{array}{l}.00 \\
.00 \\
.00 \\
.00 \\
.00\end{array}$ & $\begin{array}{l}.00 \\
.00 \\
.00 \\
.00 \\
.00\end{array}$ & $\begin{array}{l}.00 \\
.00 \\
.00 \\
.00 \\
.00\end{array}$ & $\begin{array}{l}.00 \\
.00 \\
.00 \\
.00 \\
.00\end{array}$ & $\begin{array}{l}.00 \\
.00 \\
.00 \\
.00 \\
.00\end{array}$ & $\begin{array}{l}.00 \\
.00 \\
.00 \\
.00 \\
.00\end{array}$ & $\begin{array}{l}.00 \\
.00 \\
.00 \\
.00 \\
.00\end{array}$ & $\begin{array}{l}.00 \\
.00 \\
.00 \\
.00 \\
.00\end{array}$ & $\begin{array}{l}.00 \\
.00 \\
.00 \\
.00 \\
.00\end{array}$ \\
\hline $\begin{array}{l}11 \\
12 \\
13 \\
14 \\
15\end{array}$ & $\begin{array}{l}.00 \\
.00 \\
.00 \\
.00 \\
.00\end{array}$ & $\begin{array}{l}.00 \\
.00 \\
.00 \\
.00 \\
.00\end{array}$ & $\begin{array}{l}.00 \\
.00 \\
.00 \\
.00 \\
.00\end{array}$ & $\begin{array}{l}.00 \\
.00 \\
.00 \\
.00 \\
.00\end{array}$ & $\begin{array}{l}.00 \\
.00 \\
.00 \\
.00 \\
.00\end{array}$ & $\begin{array}{l}.00 \\
.00 \\
.00 \\
.00 \\
.00\end{array}$ & $\begin{array}{l}.00 \\
.00 \\
.00 \\
.00 \\
.00\end{array}$ & $\begin{array}{l}.00 \\
.00 \\
.00 \\
.00 \\
.00\end{array}$ & $\begin{array}{l}.00 \\
.00 \\
.00 \\
.00 \\
.00\end{array}$ & $\begin{array}{r}.00 \\
.00 \\
.00 \\
32.00\end{array}$ & $\begin{array}{l}.00 \\
.00 \\
.00 \\
.00 \\
.00\end{array}$ & $\begin{array}{l}.00 \\
.00 \\
.00 \\
.00 \\
.00\end{array}$ \\
\hline $\begin{array}{l}16 \\
17 \\
18 \\
19 \\
20\end{array}$ & $\begin{array}{l}.00 \\
.00 \\
.00 \\
.00 \\
.00\end{array}$ & $\begin{array}{l}.00 \\
.00 \\
.00 \\
.00 \\
.00\end{array}$ & $\begin{array}{l}.00 \\
.00 \\
.00 \\
.00 \\
.00\end{array}$ & $\begin{array}{l}.00 \\
.00 \\
.00 \\
.00 \\
.00\end{array}$ & $\begin{array}{l}.00 \\
.00 \\
.00 \\
.00 \\
.00\end{array}$ & $\begin{array}{l}.00 \\
.00 \\
.00 \\
.00 \\
.00\end{array}$ & $\begin{array}{l}.00 \\
.00 \\
.00 \\
.00 \\
.00\end{array}$ & $\begin{array}{l}.00 \\
.00 \\
.00 \\
.00 \\
.00\end{array}$ & $\begin{array}{l}.00 \\
.00 \\
.00 \\
.00 \\
.00\end{array}$ & $\begin{array}{l}.10 \\
.00 \\
.00 \\
.00 \\
.00\end{array}$ & $\begin{array}{l}.00 \\
.00 \\
.00 \\
.00 \\
.00\end{array}$ & $\begin{array}{l}.00 \\
.00 \\
.00 \\
.00 \\
.00\end{array}$ \\
\hline $\begin{array}{l}21 \\
22 \\
23 \\
24 \\
25\end{array}$ & $\begin{array}{l}.00 \\
.00 \\
.00 \\
.00 \\
.00\end{array}$ & $\begin{array}{l}.00 \\
.00 \\
.00 \\
.00 \\
.00\end{array}$ & $\begin{array}{l}.00 \\
.00 \\
.00 \\
.00 \\
.00\end{array}$ & $\begin{array}{l}.00 \\
.00 \\
.00 \\
.00 \\
.00\end{array}$ & $\begin{array}{l}.00 \\
.00 \\
.00 \\
.00 \\
.00\end{array}$ & $\begin{array}{l}.00 \\
.00 \\
.00 \\
.00 \\
.00\end{array}$ & $\begin{array}{l}.00 \\
.00 \\
.00 \\
.00 \\
.00\end{array}$ & $\begin{array}{l}.00 \\
.00 \\
.00 \\
.00 \\
.00\end{array}$ & $\begin{array}{l}.00 \\
.00 \\
.00 \\
.00 \\
.00\end{array}$ & $\begin{array}{l}.00 \\
.00 \\
.00 \\
.00 \\
.00\end{array}$ & $\begin{array}{l}.00 \\
.00 \\
.00 \\
.00 \\
.00\end{array}$ & $\begin{array}{l}.00 \\
.00 \\
.00 \\
.00 \\
.00\end{array}$ \\
\hline $\begin{array}{l}26 \\
27 \\
28 \\
29 \\
30 \\
31\end{array}$ & $\begin{array}{l}.00 \\
.00 \\
.00 \\
.00 \\
.00 \\
.00\end{array}$ & $\begin{array}{l}.00 \\
.00 \\
.00 \\
.00 \\
.00 \\
-\end{array}$ & $\begin{array}{l}.00 \\
.00 \\
.00 \\
.00 \\
.00 \\
.00\end{array}$ & $\begin{array}{l}.00 \\
.00 \\
.00 \\
.00 \\
.00 \\
.00\end{array}$ & $\begin{array}{l}.00 \\
.00 \\
.00 \\
.00 \\
-.-\end{array}$ & $\begin{array}{l}.00 \\
.00 \\
.00 \\
.00 \\
.00 \\
.00\end{array}$ & $\begin{array}{l}.00 \\
.00 \\
.00 \\
.00 \\
.00 \\
.\end{array}$ & $\begin{array}{l}.00 \\
.00 \\
.00 \\
.00 \\
.00 \\
.00\end{array}$ & $\begin{array}{l}.00 \\
.00 \\
.00 \\
.00 \\
.00 \\
---\end{array}$ & $\begin{array}{l}.00 \\
.00 \\
.00 \\
.00 \\
.00 \\
.00\end{array}$ & $\begin{array}{l}.00 \\
.00 \\
.00 \\
.00 \\
.00 \\
.00\end{array}$ & $\begin{array}{l}.00 \\
.00 \\
.00 \\
.00 \\
.00\end{array}$ \\
\hline $\begin{array}{l}\text { TOTAL } \\
\text { MEAN } \\
\text { MAX } \\
\text { MIN } \\
\text { AC-FT }\end{array}$ & $\begin{array}{l}0.00 \\
.000 \\
.00 \\
.00 \\
.00\end{array}$ & $\begin{array}{l}0.00 \\
.000 \\
.00 \\
.00 \\
.00\end{array}$ & $\begin{array}{l}0.00 \\
.000 \\
.00 \\
.00 \\
.00\end{array}$ & $\begin{array}{l}0.00 \\
.000 \\
.00 \\
.00 \\
.00\end{array}$ & $\begin{array}{r}0.00 \\
.000 \\
.00 \\
.00 \\
.00\end{array}$ & $\begin{array}{l}0.00 \\
.000 \\
.00 \\
.00 \\
.00\end{array}$ & $\begin{array}{r}0.00 \\
.000 \\
.00 \\
.00 \\
.00\end{array}$ & $\begin{array}{r}0.00 \\
.000 \\
.00 \\
.00 \\
.00\end{array}$ & $\begin{array}{l}0.00 \\
.000 \\
.00 \\
.00 \\
.00\end{array}$ & $\begin{array}{r}32.10 \\
1.04 \\
32 \\
.00 \\
64\end{array}$ & $\begin{array}{r}0.00 \\
.000 \\
.00 \\
.00 \\
.00\end{array}$ & $\begin{array}{l}0.00 \\
.000 \\
.00 \\
.00 \\
.00\end{array}$ \\
\hline
\end{tabular}

STATISTICS OF MONTHLY MEAN DATA FOR WATER YEARS 1952 - 1996, BY WATER YEAR (WY)

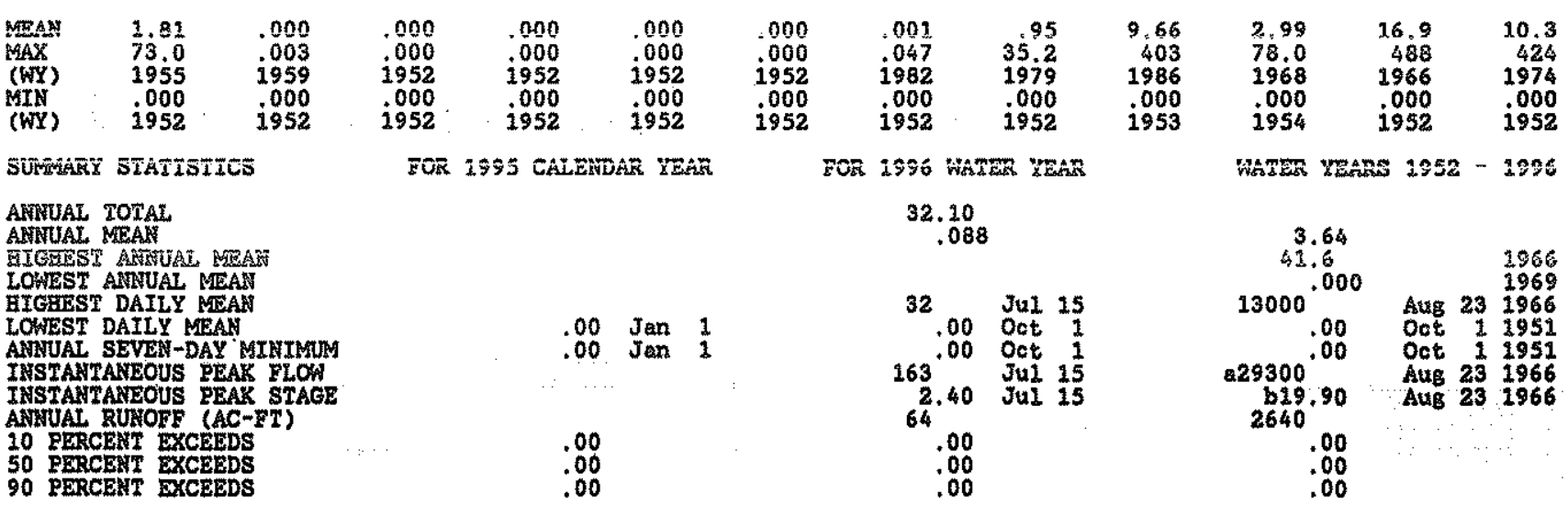

a-From rating curve axtended above $5,000 \mathrm{ft} / \mathrm{s}$, on basis of slope-area aeasurament of poek 2lop. b-From Eloodmaxks, prosent datum. 
RIO GRANDE BASIN

08401200 SOUTH SEVEN RIVERS NEAR LAKEHOOD, NM

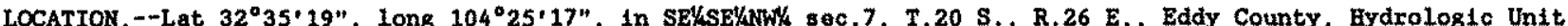
13060011 , on downstream side of center pier of bridge on U.s. Hishway $285,0.4$ mi south of Soven Rivers, 2.6 mi upstrean from mouth, and 4.0 mi southwest of lakewood. Mouth at Pecos River mile 480.9.

DRAINAGE AREA. $--220 \mathrm{mi}^{2}$, approximately.

PERIOD OF RECORD. - -October 1963 to current year.

GAGE.--Water-stage recorder. Elevation of gage is 3,280 ft above National Goodetic Vertical Datum of 1929 , from topographic map. Prior to July 8, 1965, at ste 400 ft upstream at datum 0.52 ft higher.

REMARKS, --Records good except for estimated dally discharges, which are poor. No surface diversions upstream from station, groundmatex withdrawals for 240 acres, upstream from station. Several observations of water temperature were made during the year. No flow most of time.

EXTREMES OUTSIDE PERIOD OF RECORD,-Meximum discharge since at least 1941, about 30,000 fts/s, gage ho1ght, 22.8 ft, from old debris on left bank, formex site and datum, from rating curve extended above 5,700 fts/s on basis ft, from old debris on left bank, former site and datum, from rating curve extended above
of slope-area measurement at gage height $21.8 \mathrm{ft}$. Probable date of flood, Oct. $7,1954$.

DISCEARGE, CUBIC FEET PER SECOND, WATER YEAR OCTOBER 1995 TO SEPTEMBER 1996

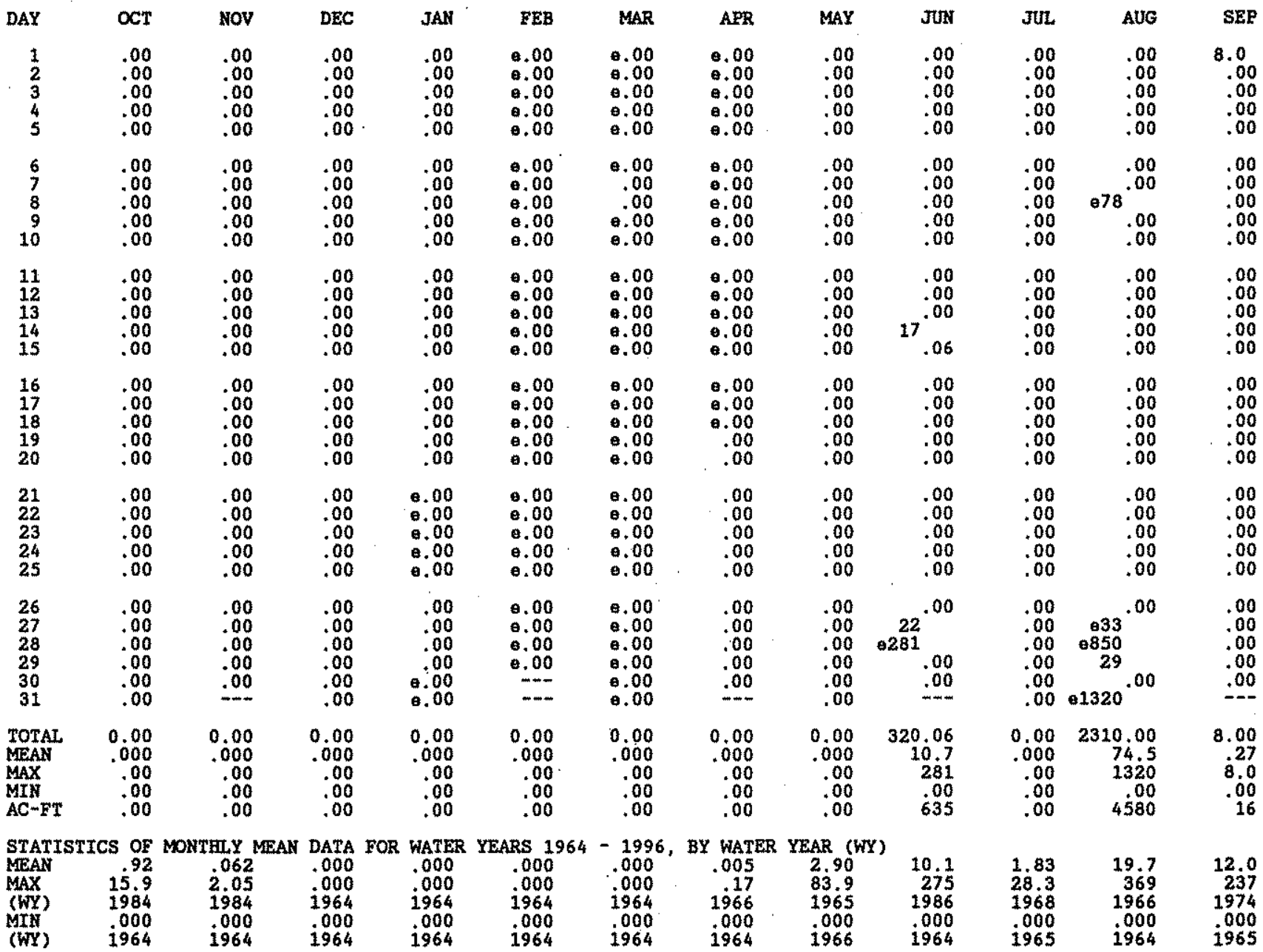

SUMMARY STATISTICS ANNUAI TOTAL

ANNUAL MEAN

ANGUEST ANNUAL MEAN

HIGREST ANNUAL MEAN

LOWEST ANNUAL MEAN

HIGHEST DAILY MFAN

AWWUAL SEVEN-DAY MINIMUM

INSTANTANEOUS PEAK FLOW

INSTANTANEOUS PEAK STAGE

INSTANTANEOUS LOW FLOW

ANNUAL RUNOFF (AC-FT)

10 PERCENT EXCEEDS

50 PERCENT EXCEED

90 PERCENT EXCEEDS

o Estimated

and 20 sating

b-From floodmaxks, present site and datum. DATL $Y$ MEAN VALUES

FOR 1995 CALENDAR YEAR EOR 1996 WATER YEAR 2638.06

186.63

$137.00 \operatorname{Jan} 30$

.00 Jan 1

370

.00
.00

00

$12800^{.00}$ Oct 1

15.76 Aus 3

230.00

.00
WATER YEARS 1964 - 1996

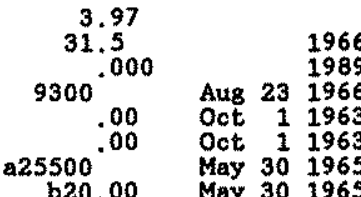

$2880^{\circ}$ .00

89 b20.00 May 30 1965

0
0
0
0
0
0
0
0
0
0
0
0
0
0
0
0
0
0
0
0
0
0
0
0
0
0
0
0
0
0
0
0
0
0
0

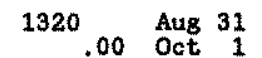

.00 
RIO GRANDE BASIN

08401450 BRANTLEY LAKE NEAR CARLSBAD, NM

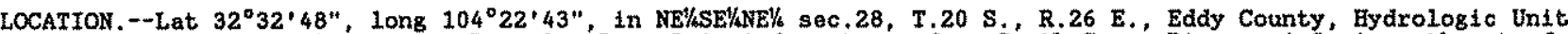
13060011 , in control tower at Brantley Dam, $2.4 \mathrm{mi}$ downstream from South Seven Rivers, 4.2 mi southeast of Seven Rivers, $6.0 \mathrm{mi}$ south of Lakewood, $11.5 \mathrm{mi}$ northwest of Carlsbad, and at mile 478.6 .

DRAINAGE AREA, $--17,650 \mathrm{mi}^{2}$, approximately (contributing area).

PERIOD OF RECORD. - -August 1988 to current year.

GAGE,-Water-stage nocorder Elevation of gage 1s $3,202.5$ ft above National Geodetic Vertical Datum of 1929 a (ievels by Bureau of Reciamation).

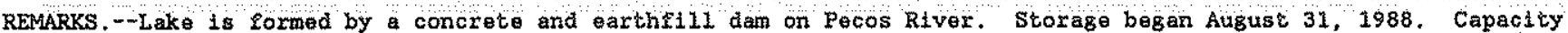
$1,008,000$ acre- ft, from capaclty table dated June 1992 , between elevations $3,202.5$ ft and $3,303.5$ ft, Dead storage 2,010 acre-ft. Lake was created primarily for irrigation storage and flood control.

COOPERATION, --Records provided by Bureau of Raclamation.

EXTREMES FOR FERIOD OF RECORD.-Maximum contents, 49, 270 acre-ft, Sept. 22-24, 1991, elevat1on, 3, 257.60 ft; minimum contents, 2,040 acre-ft, May 26, 1990, elevation, $3,224.60$ ft.

EXTREMES FOR CURRENT YEAR,--Meximum contents, 40,130 acre-ft, Oct. 1, elevation, 3,253.10 ft; minimum, 13,530 acreft, June 14, olevation, $3,240.13 \mathrm{ft}$

RESERVOIR STORAGE (ACRE-FEET), WATER YEAR OCTOBER 1995 TO SEPTEMBER 1996

DAILY OBSERVATION AT 07:00 VALUES

\begin{tabular}{|c|c|c|c|c|c|c|c|c|c|c|c|c|}
\hline$A Y$ & oct & NOV & DEC & JAN & FEB & MAR & AFR & MAY & JUK & JUL & AUG & SEP \\
\hline $\begin{array}{l}1 \\
2 \\
3 \\
4 \\
5\end{array}$ & $\begin{array}{l}40130 \\
40100 \\
40020 \\
39880 \\
39540\end{array}$ & $\begin{array}{l}24620 \\
24640 \\
24640 \\
24680 \\
24760\end{array}$ & $\begin{array}{l}26080 \\
26190 \\
26190 \\
26230 \\
26250\end{array}$ & $\begin{array}{l}28760 \\
28890 \\
28940 \\
29120 \\
29170\end{array}$ & $\begin{array}{l}32980 \\
33110 \\
33180 \\
33230 \\
33430\end{array}$ & $\begin{array}{l}32 \\
32 \\
32 \\
32 \\
32\end{array}$ & $\begin{array}{l}288 \\
285 \\
27 \\
27 \\
26\end{array}$ & $\begin{array}{l}60 \\
00 \\
90 \\
60\end{array}$ & $\begin{array}{l}30 \\
90 \\
40 \\
90 \\
20\end{array}$ & $\begin{array}{l}0 \\
0 \\
0 \\
0 \\
0\end{array}$ & & $\begin{array}{l}10 \\
30 \\
80 \\
30 \\
20\end{array}$ \\
\hline $\begin{array}{r}6 \\
7 \\
8 \\
9 \\
10\end{array}$ & $\begin{array}{l}39290 \\
38320 \\
37370 \\
36620 \\
35620\end{array}$ & $\begin{array}{l}24800 \\
24920 \\
25010 \\
25110 \\
25170\end{array}$ & $\begin{array}{l}26290 \\
26360 \\
26420 \\
26420 \\
26460\end{array}$ & $\begin{array}{l}29330 \\
29460 \\
29670 \\
29860 \\
29900\end{array}$ & $\begin{array}{l}335 \\
337 \\
338 \\
339 \\
341\end{array}$ & $\begin{array}{l}32 \\
32 \\
32 \\
32 \\
32\end{array}$ & $\begin{array}{l}26 \\
26 \\
26 \\
26 \\
25\end{array}$ & $\begin{array}{l}10 \\
80 \\
40 \\
90 \\
80\end{array}$ & $\begin{array}{l}50 \\
90 \\
70 \\
30 \\
60\end{array}$ & $\begin{array}{l}50 \\
60 \\
10 \\
80 \\
30\end{array}$ & $\begin{array}{l}70 \\
00 \\
40 \\
90 \\
60\end{array}$ & $\begin{array}{l}30 \\
10 \\
60 \\
80 \\
80\end{array}$ \\
\hline $\begin{array}{l}11 \\
12 \\
13 \\
14 \\
15\end{array}$ & $\begin{array}{l}34380 \\
33180 \\
31980 \\
30880 \\
29930\end{array}$ & $\begin{array}{l}25190 \\
25240 \\
25300 \\
25360 \\
25450\end{array}$ & $\begin{array}{l}26550 \\
26610 \\
26610 \\
26770 \\
26850\end{array}$ & $\begin{array}{l}30090 \\
30320 \\
30460 \\
30670 \\
30790\end{array}$ & $\begin{array}{l}3428 \\
3441 \\
3449 \\
3467 \\
3474\end{array}$ & $\begin{array}{l}32 \\
32 \\
32 \\
32 \\
32\end{array}$ & $\begin{array}{l}25320 \\
24900 \\
24530 \\
24070 \\
23600\end{array}$ & $\begin{array}{l}25 \\
2 . \\
23 \\
25 \\
26\end{array}$ & $\begin{array}{l}1 \\
1 \\
1 \\
1 \\
1\end{array}$ & & & $\begin{array}{l}80 \\
330 \\
510 \\
80 \\
40\end{array}$ \\
\hline $\begin{array}{l}16 \\
17 \\
18 \\
19 \\
20\end{array}$ & $\begin{array}{l}29080 \\
28020 \\
28020 \\
26080 \\
26190\end{array}$ & $\begin{array}{l}25450 \\
25550 \\
25570 \\
25590 \\
25660\end{array}$ & $\begin{array}{l}269 \\
270 \\
271 \\
272 \\
273\end{array}$ & $\begin{array}{l}309 \\
310 \\
312 \\
313 \\
315\end{array}$ & $\begin{array}{l}34 \\
34 \\
34 \\
33 \\
33\end{array}$ & $\begin{array}{l}32 \\
32 \\
31 \\
31 \\
31\end{array}$ & & & & & & $\begin{array}{l}510 \\
40 \\
30 \\
10 \\
40\end{array}$ \\
\hline $\begin{array}{l}21 \\
22 \\
23 \\
24 \\
25\end{array}$ & $\begin{array}{l}26310 \\
26210 \\
25910 \\
25660 \\
25400\end{array}$ & $\begin{array}{l}25740 \\
25780 \\
25800 \\
25820\end{array}$ & $\begin{array}{l}27490 \\
27600 \\
27730 \\
27860\end{array}$ & $\begin{array}{l}31810 \\
31930 \\
31930 \\
32100\end{array}$ & $\begin{array}{l}33580 \\
33480 \\
33380 \\
33180 \\
33030\end{array}$ & $\begin{array}{l}31 \\
31 \\
31 \\
31\end{array}$ & $\begin{array}{l}19 \\
19 \\
18 \\
18 \\
17\end{array}$ & $\begin{array}{l}3 \\
2 \\
2 \\
2\end{array}$ & $\begin{array}{l}20 \\
21 \\
22 \\
23\end{array}$ & & & $\begin{array}{l}32080 \\
32180 \\
32220 \\
32270 \\
32350\end{array}$ \\
\hline $\begin{array}{l}26 \\
27 \\
28 \\
29 \\
30 \\
31\end{array}$ & $\begin{array}{l}25150 \\
24860 \\
24700 \\
24450 \\
24410 \\
24490\end{array}$ & $\begin{array}{r}25870 \\
25950 \\
25970 \\
25990 \\
26040 \\
---\end{array}$ & $\begin{array}{l}279 \\
280 \\
282 \\
283 \\
284 \\
285\end{array}$ & $\begin{array}{l}32200 \\
32300 \\
32440 \\
32660 \\
32710 \\
32810\end{array}$ & $\begin{array}{r}32860 \\
32710 \\
32590 \\
32640 \\
\end{array}$ & $\begin{array}{l}30 \\
30 \\
29 \\
25 \\
25\end{array}$ & $\begin{array}{l}17 \\
17 \\
16 \\
16\end{array}$ & $\begin{array}{l}2 \\
2 \\
2 \\
2 \\
2\end{array}$ & $\begin{array}{c}24570 \\
25970 \\
27840 \\
29370 \\
31070 \\
---\end{array}$ & $\begin{array}{l}2 \\
2 \\
2 \\
2 \\
2 \\
2\end{array}$ & & $\begin{array}{l}40 \\
30 \\
00 \\
30\end{array}$ \\
\hline MA & $\begin{array}{r}40130 \\
24410 \\
3246.63 \\
-15670\end{array}$ & $\begin{array}{r}26040 \\
24620 \\
3247.37 \\
+1550\end{array}$ & $\begin{array}{r}28510 \\
26080 \\
3248.50 \\
+2470\end{array}$ & $\begin{array}{r}32810 \\
28760 \\
3250.33 \\
+4300\end{array}$ & $\begin{array}{r}34740 \\
32590 \\
3250.14 \\
-170\end{array}$ & $\begin{array}{r}32 \\
29 \\
3248 \\
-3\end{array}$ & $\begin{array}{r}28 \\
16 \\
3242 \\
-1\end{array}$ & $\begin{array}{r}31 \\
15 \\
3245 \\
45\end{array}$ & $\begin{array}{r}31 \\
13 \\
3249 \\
+\end{array}$ & $\begin{array}{r}39 \\
26 \\
3247\end{array}$ & $\begin{array}{r}25 \\
19 \\
3246 \\
-1\end{array}$ & $\begin{array}{r}26 \\
3249\end{array}$ \\
\hline
\end{tabular}

CAI YR 1995 MAX 43830 MTN 11230 (t+) +6280

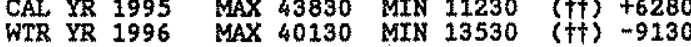

(†) ELEVATION, IN FEET, AT EHD OF MONTH.

(†) CHANGE IN CONTENTS, IN ACRE-FEET 
RIO GRANDE BASIN

08401500 PECOS RIVER BELOW BRANTLEX DAM NEAR CARLSBAD, MM

LOCATION, -Lat $32^{\circ} 32^{\prime} 38^{\prime \prime}$, long $10^{\circ} 22^{\prime} 00^{\prime \prime}$, in NE'/NWW/4E sec.27, T.20 S., R.26 E., Eddy County, Eydrologic Unit 13060011 , on left bank $0.8 \mathrm{mi}$ downstream from Brantley Dam, $3.2 \mathrm{mi}$ downstream' from South Seven Rivers, $4.7 \mathrm{mi}$ southeast of Seven Rivers, $6.4 \mathrm{mi}$ south of Lakewood, $11.0 \mathrm{mi}$ northwest of Car1sbad, and at mile 477.8 . DRAINAGE AREA. - - $17,650 \mathrm{mi}^{2}$, pproximately (contributing area).

\section{WATER-DISCHARGE RECORDS}

PERTOD OF RECORD --January 1947 to September 1950 october 1971 to current year. Prior to October 1989 published as "bolow Mator Johnson Springs " Prfor to October 1988, operated as a low-slow station only. Records prior to October 1971 not equivalent due to spring inflow between sites.

GAGE. - Water-stage recorder. Elevation of gage is $3,191.15 \mathrm{ft}$ above National Geodetic Vertical Datum of 1929 (Bureau of Reclamation reference point). Prior to October 1971, at site 1.3 mi upstream at different datum October 1971 to June 4,1985, at site 0.8 mi upstream at datum 7.29 ft higher. Prtor to October 1988, at site $0,2 \mathrm{mi}$ downstream at satne datum.

REMARKS.--Water-discharge records good except for estimated dally discharges, which are poor. Flow completely regulated by Brantley Lake (stetion 08401450) $0.8 \mathrm{mi}$ upstream since August 1988. Diversions and ground-water withdrawals for irrigation of about 173,000 acres, 1959 determination, upstream from station. Several

observations of water temperature were made during the year. Bureau of Reclamation satellite telemeter at station.

DISCHARGE, CUBIC FEET PER SECOND, WATER YEAR OCTOBER 1995 TO SEPTEMBER 1996

\begin{tabular}{|c|c|c|c|c|c|c|c|c|c|c|c|c|}
\hline DAY & $\propto \mathrm{CP}$ & Nov & $\mathrm{DEC}$ & JAN & FEB & MAR & $A P R$ & MAY & JUN & JUL & AUG & SEP \\
\hline $\begin{array}{l}1 \\
2 \\
3 \\
4 \\
5\end{array}$ & $\begin{array}{l}108 \\
130 \\
130 \\
168 \\
192\end{array}$ & $\begin{array}{l}25 \\
24 \\
24 \\
24 \\
24\end{array}$ & $\begin{array}{l}26 \\
27 \\
26 \\
26 \\
27\end{array}$ & $\begin{array}{l}28 \\
28 \\
28 \\
28 \\
19\end{array}$ & $\begin{array}{l}.54 \\
5.5 \\
1.7 \\
.69 \\
6.4\end{array}$ & $\begin{array}{r}2.5 \\
.69 \\
12.58 \\
28\end{array}$ & $\begin{array}{l}254 \\
333 \\
377 \\
337 \\
239\end{array}$ & $\begin{array}{l}142 \\
106 \\
105 \\
134 \\
151\end{array}$ & $\begin{array}{l}459 \\
438 \\
468 \\
339 \\
127\end{array}$ & $\begin{array}{r}e 19 \\
e 55 \\
e 178 \\
0263 \\
0278\end{array}$ & $\begin{array}{l}0219 \\
0290 \\
0329 \\
0261 \\
0164\end{array}$ & $\begin{array}{l}\text { e41 } \\
\text { e38 } \\
038 \\
\text { e37 } \\
\text { e37 }\end{array}$ \\
\hline $\begin{array}{r}6 \\
7 \\
8 \\
9 \\
10\end{array}$ & $\begin{array}{l}458 \\
606 \\
551 \\
583 \\
702\end{array}$ & $\begin{array}{l}24 \\
24 \\
25 \\
25 \\
23\end{array}$ & $\begin{array}{l}27 \\
28 \\
28 \\
28 \\
28\end{array}$ & $\begin{array}{l}1.5 \\
.71 \\
4.7 \\
1.7 \\
.76\end{array}$ & $\begin{array}{c}1.7 \\
.76 \\
.62 \\
6.3 \\
1.9\end{array}$ & $\begin{array}{l}32 \\
34 \\
34 \\
34 \\
34\end{array}$ & $\begin{array}{r}118 \\
95 \\
172 \\
240 \\
288\end{array}$ & $\begin{array}{l}184 \\
236 \\
258 \\
260 \\
262\end{array}$ & $\begin{array}{l}260 \\
434 \\
404 \\
330 \\
294\end{array}$ & $\begin{array}{l}e 316 \\
e 369 \\
e 502 \\
e 566 \\
0601\end{array}$ & $\begin{array}{l}e 172 \\
e 269 \\
e 372 \\
e 314 \\
0234\end{array}$ & $\begin{array}{l}040 \\
041 \\
037 \\
043 \\
045\end{array}$ \\
\hline $\begin{array}{l}11 \\
12 \\
13 \\
14 \\
15\end{array}$ & $\begin{array}{l}744 \\
739 \\
697 \\
625 \\
596\end{array}$ & $\begin{array}{l}24 \\
25 \\
26 \\
26 \\
26\end{array}$ & $\begin{array}{l}27 \\
27 \\
27 \\
27 \\
28\end{array}$ & $\begin{array}{l}.62 \\
5.1 \\
1.7 \\
5.70 \\
5.0\end{array}$ & $\begin{array}{r}6.68 \\
1.1 \\
5.6 \\
370\end{array}$ & $\begin{array}{r}34 \\
34 \\
71 \\
128 \\
149\end{array}$ & $\begin{array}{l}275 \\
260 \\
258 \\
286 \\
366\end{array}$ & $\begin{array}{l}264 \\
265 \\
266 \\
266 \\
313\end{array}$ & $\begin{array}{l}293 \\
350 \\
431 \\
335 \\
133\end{array}$ & $\begin{array}{l}0587 \\
0540 \\
6675 \\
6570 \\
0464\end{array}$ & $\begin{array}{l}e 207 \\
e 142 \\
e 150 \\
e 269 \\
0314\end{array}$ & $\begin{array}{l}049 \\
048 \\
045 \\
045 \\
044\end{array}$ \\
\hline $\begin{array}{l}16 \\
17 \\
18 \\
19 \\
20\end{array}$ & $\begin{array}{r}642 \\
666 \\
614 \\
231 \\
25\end{array}$ & $\begin{array}{l}26 \\
25 \\
25 \\
25 \\
25\end{array}$ & $\begin{array}{l}28 \\
28 \\
27 \\
27 \\
27\end{array}$ & $\begin{array}{r}1.8 \\
.54 \\
.45 \\
5.5 \\
1.9\end{array}$ & $\begin{array}{l}355 \\
187 \\
139 \\
152 \\
140\end{array}$ & $\begin{array}{l}150 \\
180 \\
145 \\
113 \\
113\end{array}$ & $\begin{array}{l}398 \\
369 \\
383 \\
396 \\
393\end{array}$ & $\begin{array}{l}341 \\
402 \\
411 \\
329 \\
293\end{array}$ & $\begin{array}{r}\theta 74 \\
060 \\
083 \\
0108 \\
0109\end{array}$ & $\begin{array}{l}0519 \\
0599 \\
0623 \\
0621 \\
0242\end{array}$ & $\begin{array}{l}285 \\
0243 \\
\theta 198 \\
0140 \\
e 152\end{array}$ & $\begin{array}{l}045 \\
045 \\
046 \\
045 \\
043\end{array}$ \\
\hline $\begin{array}{l}21 \\
22 \\
23 \\
24 \\
25\end{array}$ & $\begin{array}{l}102 \\
178 \\
199 \\
199 \\
199\end{array}$ & $\begin{array}{l}25 \\
25 \\
25 \\
25 \\
25\end{array}$ & $\begin{array}{l}27 \\
27 \\
27 \\
26 \\
26\end{array}$ & $\begin{array}{l}.77 \\
6.0 \\
1.9 \\
.79 \\
.51\end{array}$ & $\begin{array}{r}146 \\
142 \\
141 \\
147 \\
0152\end{array}$ & $\begin{array}{l}113 \\
113 \\
112 \\
110 \\
169\end{array}$ & $\begin{array}{l}392 \\
337 \\
305 \\
303 \\
276\end{array}$ & $\begin{array}{l}391 \\
713 \\
700 \\
631 \\
599\end{array}$ & $\begin{array}{l}0206 \\
0295 \\
e 356 \\
0332 \\
0206\end{array}$ & $\begin{array}{l}e 123 \\
e 189 \\
\theta 290 \\
\theta 312 \\
\theta 232\end{array}$ & $\begin{array}{r}0177 \\
0195 \\
0170 \\
083 \\
039\end{array}$ & $\begin{array}{l}044 \\
042 \\
044 \\
054 \\
066\end{array}$ \\
\hline $\begin{array}{l}26 \\
27 \\
28 \\
29 \\
30 \\
31\end{array}$ & $\begin{array}{r}199 \\
170 \\
183 \\
141 \\
61 \\
27\end{array}$ & $\begin{array}{r}25 \\
25 \\
26 \\
26 \\
26 \\
-2\end{array}$ & $\begin{array}{l}27 \\
27 \\
28 \\
28 \\
28 \\
27\end{array}$ & $\begin{array}{l}5.8 \\
1.8 \\
.68 \\
5.1 \\
1.5 \\
.66\end{array}$ & $\begin{array}{r}0153 \\
0152 \\
2.6 \\
142 \\
\end{array}$ & $\begin{array}{l}266 \\
305 \\
262 \\
188 \\
159 \\
159\end{array}$ & $\begin{array}{l}229 \\
178 \\
159 \\
187 \\
204\end{array}$ & $\begin{array}{l}558 \\
539 \\
559 \\
570 \\
568 \\
520\end{array}$ & $\begin{array}{r}0108 \\
092 \\
061 \\
020 \\
019\end{array}$ & $\begin{array}{l}0231 \\
0251 \\
e 229 \\
0175 \\
e 150 \\
0193\end{array}$ & $\begin{array}{l}836 \\
939 \\
043 \\
040 \\
940 \\
842\end{array}$ & $\begin{array}{l}968 \\
\text { e220 } \\
0318 \\
0324 \\
0375\end{array}$ \\
\hline $\begin{array}{l}\text { TOTAL. } \\
\text { MEAN } \\
\text { MAX } \\
\text { MIN } \\
\text { AC-FT }\end{array}$ & $\begin{array}{r}10865 \\
350 \\
744 \\
25 \\
21550\end{array}$ & $\begin{array}{r}748 \\
24.9 \\
26 \\
23 \\
1480\end{array}$ & $\begin{array}{r}842 \\
27.2 \\
28 \\
26 \\
1670\end{array}$ & $\begin{array}{r}189.19 \\
6.10 \\
28 \\
45 \\
375\end{array}$ & $\begin{array}{r}2560.89 \\
88.3 \\
370 \\
5080\end{array}$ & $\begin{array}{r}3284.77 \\
106 \\
305 \\
658 \\
6520\end{array}$ & $\begin{array}{r}8407 \\
280 \\
398 \\
.95 \\
16680\end{array}$ & $\begin{array}{r}11336 \\
366 \\
713 \\
105 \\
22480\end{array}$ & $\begin{array}{r}7224 \\
241 \\
468 \\
19 \\
14330\end{array}$ & $\begin{array}{r}10962 \\
354 \\
675 \\
19 \\
21740\end{array}$ & $\begin{array}{r}5628 \\
182 \\
372 \\
36 \\
11160\end{array}$ & $\begin{array}{r}2407 \\
80.2 \\
375 \\
37 \\
4770\end{array}$ \\
\hline
\end{tabular}

\begin{tabular}{|c|c|c|c|c|c|c|c|c|c|c|c|c|}
\hline $\begin{array}{l}\text { AN } \\
\text { WX } \\
\text { WI }\end{array}$ & $\begin{array}{r}\text { CS OF } \\
156 \\
412 \\
1995 \\
22.6 \\
1979\end{array}$ & $\begin{array}{l}\text { MONTHLY MEAN } \\
43.6 \\
222 \\
1992 \\
5.92 \\
1989\end{array}$ & $\begin{array}{r}\text { DATA } \\
50.7 \\
460 \\
1992 \\
1.22 \\
1995\end{array}$ & FOR & $\begin{array}{l}\text { WATER } \\
45.0 \\
297 \\
1987 \\
3.49 \\
1995\end{array}$ & $\begin{array}{c}\text { YEARS } 1972 \\
56.9 \\
300 \\
1987 \\
20.6 \\
1978\end{array}$ & $\begin{array}{r}1996 \\
76.3 \\
149 \\
1994 \\
19.1 \\
1990\end{array}$ & $\begin{array}{c}\text { BY WATER } \\
230 \\
307 \\
1986 \\
136 \\
1981\end{array}$ & $\begin{array}{c}\text { YEAR (WY) } \\
217 \\
1058 \\
1973 \\
79.9 \\
1976\end{array}$ & $\begin{array}{r}219 \\
641 \\
1992 \\
66.5 \\
1977\end{array}$ & $\begin{array}{r}225 \\
527 \\
1995 \\
11.3 \\
1976\end{array}$ & $\begin{array}{r}199 \\
305 \\
1995 \\
18.4 \\
1981\end{array}$ \\
\hline
\end{tabular}

\section{SUMMARY STATISTICS}

ANNUAL TOTAL

ANNUAT

HIGHEST ANNUAL MEAN

LOWEST ANNUAL MEAN

BIGEEST DATLY MEAN

LOWEST DAILY MEAN

ANNUAL SEVEN -DAY MINIMUM

INSTANTANEOUS PEAK FLOW

INSTANTANEOUS PEAK STAGE

INSTANTANEOUS LOW FLOW

ANNUAL RUNOFF (AC-FT)

10 PERCENT EXCEEDS

50 PERCENT EXCEEDS

90 PERCENT EXCEEDS

- Estimatec

a-From rating curve extended above $780 \mathrm{ft}^{3} / \mathrm{s}$.

b-Also occurred July 24, 1950.

c-Site and datum then in use.
FOR 1995 CALENDAR YEAR - FOR 1996 WATER YEAR
.72784 .91

199

790.30 Ju1 13

1.1 Jan 26

144400

469
150

5.0
64453.85

176

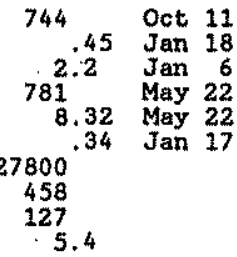

WATER YEARS 1972 - 1996

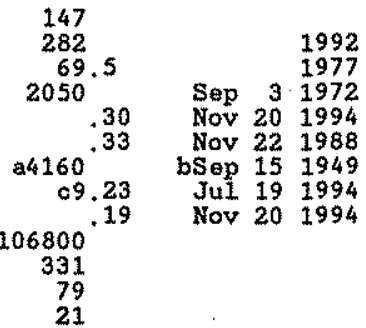




\section{RIO GRANDE BASIN}

08401500 PECOS RIVER BELOW BRANTLEY DAM NEAR CARLSBAD, NM - - Continued

WATER-QUALITY RECORDS

PERIOD OF RECORD.--Water years 1960, 1962, 1978-79, 1981 to current year.

REMARKS. -This station prior to Brantley Dam was called Fecos River below Major Johnzon Springs near Caxlsbad, KM. WATER QUALITY DATA, WATER YEAR OCTOBHR 1995 TO SEPTEMBER 1996

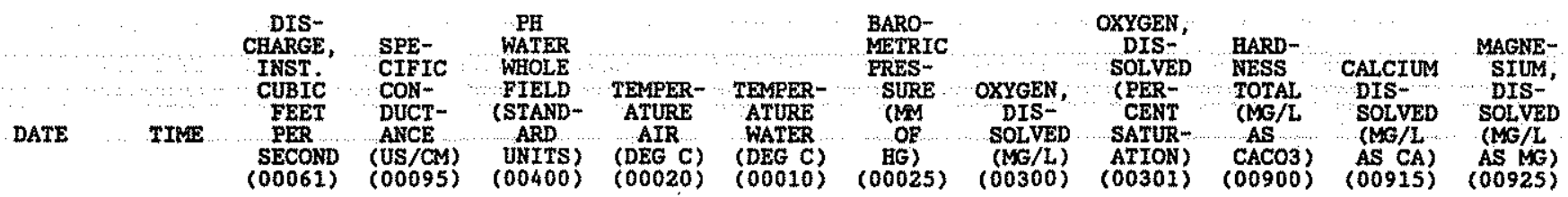

\begin{tabular}{|c|c|c|c|c|c|c|c|c|c|c|c|c|}
\hline $\begin{array}{l}\text { DEC } 1995 \\
\text { O8 } \\
\text { MAR } 1996\end{array}$ & 1025 & 28 & 5180 & -- & 14.0 & 10.5 & 680 & 10.4 & 107 & 1500 & 400 & 120 \\
\hline JuN $12 \ldots$ & 1400 & 33 & 5860 & 8.0 & 29.5 & 17.0 & 676 & 9.4 & 112 & 1700 & 450 & 140 \\
\hline $\operatorname{sEP}^{14 \ldots}$ & 1300 & 300 & 3230 & 7.9 & 31.5 & 24.0 & 682 & 11.8 & 159 & 1400 & 430 & 81 \\
\hline $09 \ldots$ & 1200 & 42 & 2860 & 7.6 & 29.0 & 27.0 & 685 & 8.3 & 118 & 1000 & 290 & 72 \\
\hline
\end{tabular}

\begin{tabular}{|c|c|c|c|c|c|c|c|c|c|c|c|}
\hline DATE & $\begin{array}{l}\text { SODIUM, } \\
\text { DIS- } \\
\text { SOLVED } \\
\text { (MG/L } \\
\text { AS NA) } \\
(00930)\end{array}$ & $\begin{array}{c}\text { SODIUM } \\
\text { AD- } \\
\text { SORP- } \\
\text { TION } \\
\text { RATIO } \\
(00931)\end{array}$ & $\begin{array}{c}\text { POTAS- } \\
\text { SIUM, } \\
\text { DIS- } \\
\text { SOLVED } \\
(\text { MG/L } \\
\text { AS K) } \\
(00935)\end{array}$ & $\begin{array}{c}\text { ALKA- } \\
\text { IINITY } \\
\text { IAB } \\
\text { (MG/L } \\
\text { AS } \\
\text { CACO3) } \\
(90410)\end{array}$ & $\begin{array}{l}\text { SULFATE } \\
\text { DIS- } \\
\text { SOLVED } \\
\text { (MG/L } \\
\text { AS } 504) \\
\text { (00945) }\end{array}$ & $\begin{array}{l}\text { CHLO- } \\
\text { RIDE, } \\
\text { DIS- } \\
\text { SOLVED } \\
\text { (MG/L } \\
\text { AS CL) } \\
(00940)\end{array}$ & $\begin{array}{l}\text { FLUO- } \\
\text { RIDE, } \\
\text { DIS - } \\
\text { SOLVED } \\
\text { (MG/L } \\
\text { AS F) } \\
(00950)\end{array}$ & $\begin{array}{l}\text { SILICA, } \\
\text { DIS- } \\
\text { SOLVED } \\
\text { (MG/L } \\
\text { AS } \\
\text { SIO2) } \\
(00955)\end{array}$ & $\begin{array}{c}\text { SOLIDS, } \\
\text { SUM OF } \\
\text { CONSTI- } \\
\text { TUENTS, } \\
\text { DIS- } \\
\text { SOLVED } \\
\text { (MG/L) } \\
(70301)\end{array}$ & $\begin{array}{l}\text { BORON, } \\
\text { DIS- } \\
\text { SOLVED } \\
\text { (UG/L } \\
\text { AS B) } \\
(01020)\end{array}$ & $\begin{array}{c}\text { IRON, } \\
\text { DIS- } \\
\text { SOLVED } \\
\text { (UG/L } \\
\text { AS FE) } \\
(01046)\end{array}$ \\
\hline
\end{tabular}

\begin{tabular}{|c|c|c|c|c|c|c|c|c|c|c|c|}
\hline $\begin{array}{l}\text { DEC } 1995 \\
08 \\
\text { MAR } 1996\end{array}$ & 650 & 7 & 3.0 & 135 & 1100 & 1100 & 0.60 & 11 & 3470 & 270 & $<12$ \\
\hline $12 \ldots$ & 690 & 7 & 6.2 & 1.27 & 1300 & 1200 & 0.70 & 9.5 & 3870 & 310 & $<12$ \\
\hline $\operatorname{SER}^{14 \ldots}$ & 260 & 3 & 4.7 & 88 & 1200 & 400 & 0.70 & 11 & 2440 & 166 & 99 \\
\hline $09 \ldots$ & 330 & 4 & 5.1 & 84 & 880 & 530. & 0.60 & 11 & 21.70 & 203 & $<9.0$ \\
\hline
\end{tabular}


RIO GRANDE BASIN

08401900 ROCKY ARROYO AT HIGEWAY BRIDGE, NEAR CARLSBAD, MM

LOCATIOK --Lat $32^{\circ} 30^{\prime} 23^{\prime \prime}$, long $104^{\circ} 22^{\prime} 26^{\prime \prime}$, in SE\%SE\% 80c.3, T.21 S. R.25 E., Eddy County, Hydrologic Untt 13060011, at dowstrean end of brtdge pier nearest left bank on U.s. Highway 285 , 2.1 mi upstream from mouth and 10 mi northwest of Carlsbad, Mouth at Pocos River mile 475.2.

DRAIHAGE AREA. $-285 \mathrm{mt}^{2}$, approxtmately.

PERIOD OF RECORD, - October 1963 to current year.

GAGE,--Water-stage recorder. Elevation of 8 age is 3,250 ft above National Geodetic Vertical Datum of 1929 , fron topographic map. Prior to Fobruary 1985, at sito $60 \mathrm{ft}$ downstream at same datum.

REMARKS. - Records good. Diverstons for irrigation of 220 acres, upstream from station. Several observations of water temperature were made during the year. No flow during water year.

EXTREMES OUTSIDE PERIOD OF RECORD.--Since about 1941 the maximum discharge probably occurred Oct. 7 , 1954. discharge, 63,600 fts/s, gage hetght, $19.2 \mathrm{ft}$, from floodmarks, on downstream end of bridge pier, by slope-area measurement at site $5 \mathrm{mi}$ upstream.

DISCEARGE, CUBIC FEET PER SECOND, WATER YEAR OCTOBER 1995 TO SEPTEMBER 1996

\begin{tabular}{|c|c|c|c|c|c|c|c|c|c|c|c|c|}
\hline DAX & CCT & Nov & DEC & JAN & FEB & MAR & APR & MAY & JUN & JUL. & AUGG & SEP \\
\hline $\begin{array}{l}1 \\
2 \\
3 \\
4 \\
5\end{array}$ & $\begin{array}{l}.00 \\
.00 \\
.00 \\
.00 \\
.00\end{array}$ & $\begin{array}{l}.00 \\
.00 \\
.00 \\
.00 \\
.00\end{array}$ & $\begin{array}{l}.00 \\
.00 \\
.00 \\
.00 \\
.00\end{array}$ & $\begin{array}{l}.00 \\
.00 \\
.00 \\
.00 \\
.00\end{array}$ & $\begin{array}{l}.00 \\
.00 \\
.00 \\
.00 \\
.00\end{array}$ & $\begin{array}{l}.00 \\
.00 \\
.00 \\
.00 \\
.00\end{array}$ & $\begin{array}{l}.00 \\
.00 \\
.00 \\
.00 \\
.00\end{array}$ & $\begin{array}{l}.00 \\
.00 \\
.00 \\
.00 \\
.00\end{array}$ & $\begin{array}{l}.00 \\
.00 \\
.00 \\
.00 \\
.00\end{array}$ & $\begin{array}{l}.00 \\
.00 \\
.00 \\
.00 \\
.00\end{array}$ & $\begin{array}{l}.00 \\
.00 \\
.00 \\
.00 \\
.00\end{array}$ & $\begin{array}{r}42 \\
4.3 \\
1.2 \\
.00 \\
.00\end{array}$ \\
\hline $\begin{array}{r}6 \\
7 \\
8 \\
9 \\
10\end{array}$ & $\begin{array}{r}.00 \\
.00 \\
.00 \\
.00 \\
.00\end{array}$ & $\begin{array}{l}.00 \\
.00 \\
.00 \\
.00 \\
.00\end{array}$ & $\begin{array}{l}.00 \\
.00 \\
.00 \\
.00 \\
.00\end{array}$ & $\begin{array}{l}.00 \\
.00 \\
.00 \\
.00 \\
.00\end{array}$ & $\begin{array}{l}.00 \\
.00 \\
.00 \\
.00 \\
.00\end{array}$ & $\begin{array}{l}.00 \\
.00 \\
.00 \\
.00 \\
.00\end{array}$ & $\begin{array}{l}.00 \\
.00 \\
.00 \\
.00 \\
.00\end{array}$ & $\begin{array}{l}.00 \\
.00 \\
.00 \\
.00 \\
.00\end{array}$ & $\begin{array}{l}.00 \\
.00 \\
.00 \\
.00 \\
.00\end{array}$ & $\begin{array}{l}.00 \\
.00 \\
.00 \\
.00 \\
.00\end{array}$ & $\begin{array}{l}.00 \\
.00 \\
.00 \\
.00 \\
.00\end{array}$ & $\begin{array}{l}.00 \\
.00 \\
.00 \\
.00 \\
.00\end{array}$ \\
\hline $\begin{array}{l}11 \\
12 \\
13 \\
14 \\
15\end{array}$ & $\begin{array}{l}.00 \\
.00 \\
.00 \\
.00 \\
.00\end{array}$ & $\begin{array}{l}.00 \\
.00 \\
.00 \\
.00 \\
.00\end{array}$ & $\begin{array}{l}.00 \\
.00 \\
.00 \\
.00 \\
.00\end{array}$ & $\begin{array}{l}.00 \\
.00 \\
.00 \\
.00 \\
.00\end{array}$ & $\begin{array}{l}.00 \\
.00 \\
.00 \\
.00 \\
.00\end{array}$ & $\begin{array}{l}.00 \\
.00 \\
.00 \\
.00 \\
.00\end{array}$ & $\begin{array}{l}.00 \\
.00 \\
.00 \\
.00 \\
.00\end{array}$ & $\begin{array}{l}.00 \\
.00 \\
.00 \\
.00 \\
.00\end{array}$ & $\begin{array}{r}.00 \\
.00 \\
.00 \\
60\end{array}$ & $\begin{array}{r}.00 \\
38.00 \\
12.00\end{array}$ & $\begin{array}{l}.00 \\
.00 \\
.00 \\
.00 \\
.00\end{array}$ & $\begin{array}{l}.00 \\
.00 \\
.00 \\
.00 \\
.00\end{array}$ \\
\hline $\begin{array}{l}16 \\
17 \\
18 \\
19 \\
20\end{array}$ & $\begin{array}{l}.00 \\
.00 \\
.00 \\
.00 \\
.00\end{array}$ & $\begin{array}{l}.00 \\
.00 \\
.00 \\
.00 \\
.00\end{array}$ & $\begin{array}{l}.00 \\
.00 \\
.00 \\
.00 \\
.00\end{array}$ & $\begin{array}{l}.00 \\
.00 \\
.00 \\
.00 \\
.00\end{array}$ & $\begin{array}{l}.00 \\
.00 \\
.00 \\
.00 \\
.00\end{array}$ & $\begin{array}{l}.00 \\
.00 \\
.00 \\
.00 \\
.00\end{array}$ & $\begin{array}{l}.00 \\
.00 \\
.00 \\
.00 \\
.00\end{array}$ & $\begin{array}{l}.00 \\
.00 \\
.00 \\
.00 \\
.00\end{array}$ & $\begin{array}{l}.70 \\
.00 \\
.00 \\
.00 \\
.00\end{array}$ & $\begin{array}{l}.00 \\
.00 \\
.00 \\
.00 \\
.00\end{array}$ & $\begin{array}{l}.00 \\
.00 \\
.00 \\
.00 \\
.00\end{array}$ & $\begin{array}{l}.00 \\
.00 \\
.00 \\
.00 \\
.00\end{array}$ \\
\hline $\begin{array}{l}21 \\
22 \\
23 \\
24 \\
25\end{array}$ & $\begin{array}{l}.00 \\
.00 \\
.00 \\
.00 \\
.00\end{array}$ & $\begin{array}{l}.00 \\
.00 \\
.00 \\
.00 \\
.00\end{array}$ & $\begin{array}{l}.00 \\
.00 \\
.00 \\
.00 \\
.00\end{array}$ & $\begin{array}{l}.00 \\
.00 \\
.00 \\
.00 \\
.00\end{array}$ & $\begin{array}{l}.00 \\
.00 \\
.00 \\
.00 \\
.00\end{array}$ & $\begin{array}{l}.00 \\
.00 \\
.00 \\
.00 \\
.00\end{array}$ & $\begin{array}{l}.00 \\
.00 \\
.00 \\
.00 \\
.00\end{array}$ & $\begin{array}{l}.00 \\
.00 \\
.00 \\
.00 \\
.00\end{array}$ & $\begin{array}{l}.00 \\
.00 \\
.00 \\
.00 \\
.00\end{array}$ & $\begin{array}{l}.00 \\
.00 \\
.00 \\
.00 \\
.00\end{array}$ & $\begin{array}{l}.00 \\
.00 \\
.00 \\
.00 \\
.00\end{array}$ & $\begin{array}{l}.00 \\
.00 \\
.00 \\
.00 \\
.00\end{array}$ \\
\hline $\begin{array}{l}26 \\
27 \\
28 \\
29 \\
30 \\
31\end{array}$ & $\begin{array}{l}.00 \\
.00 \\
.00 \\
.00 \\
.00 \\
.00\end{array}$ & $\begin{array}{l}.00 \\
.00 \\
.00 \\
.00 \\
.00 \\
.-\end{array}$ & $\begin{array}{l}.00 \\
.00 \\
.00 \\
.00 \\
.00 \\
.00\end{array}$ & $\begin{array}{l}.00 \\
.00 \\
.00 \\
.00 \\
.00 \\
.00\end{array}$ & $\begin{array}{l}.00 \\
.00 \\
.00 \\
.00 \\
.--\end{array}$ & $\begin{array}{l}.00 \\
.00 \\
.00 \\
.00 \\
.00 \\
.00\end{array}$ & $\begin{array}{l}.00 \\
.00 \\
.00 \\
.00 \\
.00 \\
.--\end{array}$ & $\begin{array}{l}.00 \\
.00 \\
.00 \\
.00 \\
.00 \\
.00\end{array}$ & $\begin{array}{r}.00 \\
308^{.00} \\
46 \\
2.7 \\
---m\end{array}$ & $\begin{array}{l}.00 \\
.00 \\
.00 \\
.00 \\
.00 \\
.00\end{array}$ & $\begin{array}{l}.00 \\
834^{.00} \\
176^{5} \\
515^{.6}\end{array}$ & $\begin{array}{l}.00 \\
.00 \\
.00 \\
.00 \\
.00 \\
.0-\end{array}$ \\
\hline $\begin{array}{l}\text { TOTAL } \\
\text { MEAN } \\
\text { MAX } \\
\text { MIN } \\
\text { AC-ET }\end{array}$ & $\begin{array}{r}0,00 \\
.000 \\
.00 \\
.00 \\
.00\end{array}$ & $\begin{array}{r}0.00 \\
.000 \\
.00 \\
.00 \\
.00\end{array}$ & $\begin{array}{l}0.00 \\
.000 \\
.00 \\
.00 \\
.00\end{array}$ & $\begin{array}{l}0.00 \\
.000 \\
.00 \\
.00 \\
.00\end{array}$ & $\begin{array}{l}0.00 \\
.000 \\
.00 \\
.00 \\
.00\end{array}$ & $\begin{array}{l}0.00 \\
.000 \\
.00 \\
.00 \\
.00\end{array}$ & $\begin{array}{l}0.00 \\
.000 \\
.00 \\
.00 \\
.00\end{array}$ & $\begin{array}{l}0.00 \\
.000 \\
.00 \\
.00 \\
.00\end{array}$ & $\begin{array}{r}417.40 \\
13.9 \\
308 \\
.00 \\
828\end{array}$ & $\begin{array}{r}50.00 \\
1.61 \\
38 \\
.00 \\
99\end{array}$ & $\begin{array}{r}1430.60 \\
46.1 \\
834 \\
.00 \\
2840\end{array}$ & $\begin{array}{r}47.50 \\
1.58 \\
42 \\
.00 \\
94\end{array}$ \\
\hline
\end{tabular}

STATISTICS OF MONTHLY MEAN DATA FOR WATER YEARS $1964-1996$, BY WATER YEAR (WY)

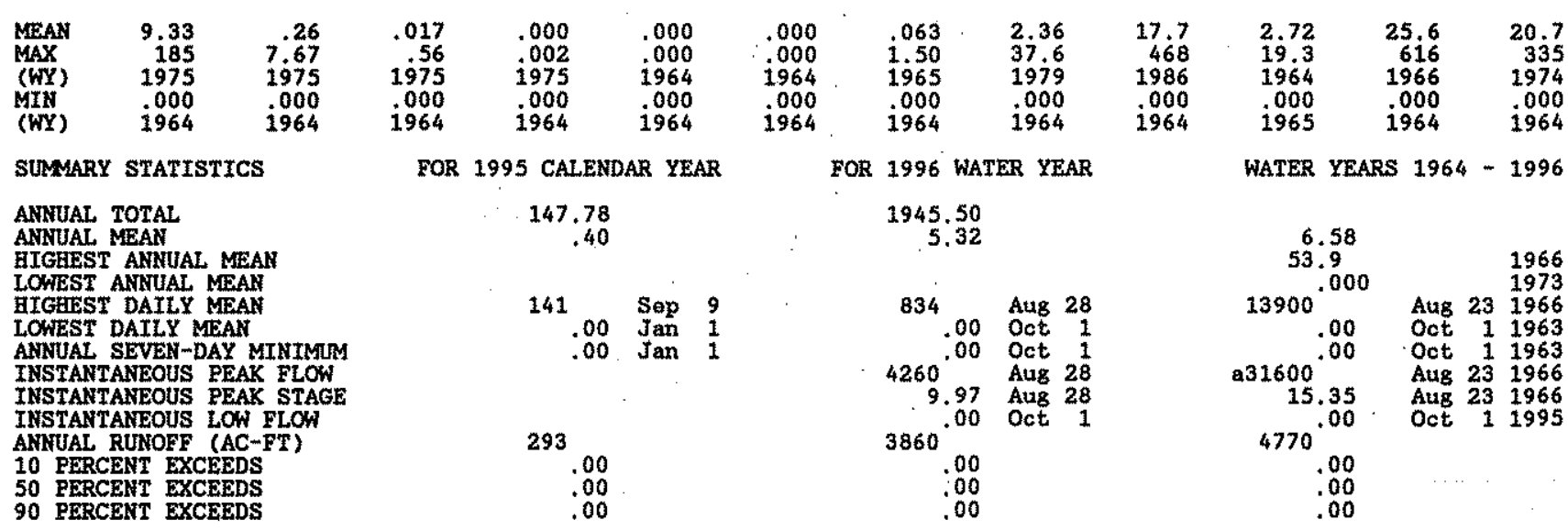

a-From rating curve extended above $8,500 \mathrm{ft} / \mathrm{s}$, on basts of slope-area measurement of peak 1 low. 
LOCATION.-Lat $32^{\circ} 30^{\prime} 40^{\prime \prime}$, long $104^{\circ} 19^{\prime} 58^{\prime \prime}$, sec.6, T.21 S., R.26 E., Eddy County, Hydrologic Unit 13060011, on right benk at damstte 3 of Carlsbad Project of Bureau of Reclamation, about 1 mi upstream from flow 1 ine of Lake

Avalon, 1.3 mi downstream from Rocky Arroyo, 8.0 mi northwest of Carlsbad, and at mile 473.8 .

DRAINAGE AREA, $-17,980 \mathrm{mi}^{2}$, approximately (contributing area)

PERIOD OF RECORD. - August 1939 to December 1940, August 1944 to current year.

REVISED RECORDS.-WSP 1512: 1946-47(M), 1948(P), 1949, 1950(P). WSP 1712: Drainage area

GAGE, - Water-stage recorder. Elevation of gage is $3,171.31 \mathrm{ft}$ above National Geodetic Vertical Datum of 1929 (levels by Bureau of Reclamation). Prior to Aug. 10, 1944 , at site 1,000 ft downstream at datum 1.00 ft higher. Aus. 10, 1944 to Dec. 31, 1966, at prosent datum 1.00 ft higher.

REAARKS.-Decords good except Eor estimated datly discharges, which are poor. Flow regulatiod by Brantley Laike

(station 08401450) $4.8 \mathrm{mi}$ upstream and other reservoirs and diversion dans, Diversions and ground-water

withdrawals for irrigation of about 17,300 acres, 1959 determination, upstream from station. Discharge

represents inflow to Lake Avalon. Several observations of water temperature were.made during the year.

EXIREMES OUTSIDE PERIOD OF RECORD.--Peaks that probably exceaded 40,000 fts/s occurred in Aug. 1893, 0ct. 2 , 1904, July 25, 1905, Apr. 17, 1915, Aus. 7, 1916, and May 30,1937 , based primarily on records for station "at

Carlsbad." Peak of May 22, 1941, was estimated at $60,000 \mathrm{ft} / \mathrm{s}$. Floods of 1893 and 1904 orlginated upstream

fxom McMillan Dam and contributed to the two failures of Avalon Dam.

DISCHARGE, CUBTC FEET PER SECOND, WATER YEAR OCTOBER 1995 TO SEPTEMBER 1996

\begin{tabular}{|c|c|c|c|c|c|c|c|c|c|c|c|c|}
\hline DAY & $\alpha C T$ & NOV & DEC & JAN & EEB & MAR & AFR & MAY & JUN & JUL & AUG & SEP \\
\hline $\begin{array}{l}1 \\
2 \\
3 \\
4 \\
5\end{array}$ & $\begin{array}{l}0107 \\
0129 \\
0130 \\
0160 \\
0185\end{array}$ & $\begin{array}{l}22 \\
23 \\
22 \\
22 \\
23\end{array}$ & $\begin{array}{l}24 \\
24 \\
24 \\
22 \\
24\end{array}$ & $\begin{array}{l}28 \\
27 \\
24 \\
24 \\
24\end{array}$ & $\begin{array}{l}1.2 \\
1.0 \\
2.9 \\
2.2 \\
1.3\end{array}$ & $\begin{array}{r}15 \\
3.4 \\
1.6 \\
1.1 \\
20\end{array}$ & $\begin{array}{l}226 \\
324 \\
367 \\
346 \\
239\end{array}$ & $\begin{array}{r}145 \\
95 \\
93 \\
117 \\
142\end{array}$ & $\begin{array}{l}477 \\
448 \\
473 \\
368 \\
152\end{array}$ & $\begin{array}{r}28 \\
52 \\
173 \\
282 \\
302\end{array}$ & $\begin{array}{l}199 \\
256 \\
305 \\
257 \\
180\end{array}$ & $\begin{array}{l}83 \\
27 \\
25 \\
25 \\
32\end{array}$ \\
\hline $\begin{array}{r}6 \\
7 \\
8 \\
9 \\
10\end{array}$ & $\begin{array}{l}0450 \\
e 590 \\
0550 \\
e 585 \\
0698\end{array}$ & $\begin{array}{l}22 \\
23 \\
23 \\
23 \\
21\end{array}$ & $\begin{array}{l}24 \\
24 \\
24 \\
24 \\
24\end{array}$ & $\begin{array}{l}7.2 \\
2.6 \\
1.4 \\
3.8 \\
2.2\end{array}$ & $\begin{array}{l}4.1 \\
2.0 \\
1.3 \\
1.1 \\
3.1\end{array}$ & $\begin{array}{l}27 \\
29 \\
29 \\
30 \\
30\end{array}$ & $\begin{array}{r}125 \\
73 \\
143 \\
219 \\
268\end{array}$ & $\begin{array}{l}169 \\
220 \\
251 \\
254 \\
259\end{array}$ & $\begin{array}{l}216 \\
453 \\
437 \\
362 \\
309\end{array}$ & $\begin{array}{l}338 \\
396 \\
533 \\
625 \\
660\end{array}$ & $\begin{array}{l}166 \\
249 \\
352 \\
318 \\
226\end{array}$ & 0 \\
\hline $\begin{array}{l}11 \\
12 \\
13 \\
14 \\
15\end{array}$ & $\begin{array}{r}9740 \\
708 \\
679 \\
580 \\
551\end{array}$ & $\begin{array}{l}22 \\
23 \\
23 \\
23 \\
23\end{array}$ & $\begin{array}{l}23 \\
23 \\
22 \\
23 \\
23\end{array}$ & $\begin{array}{l}1.2 \\
1.1 \\
2.9 \\
2.2 \\
1.3\end{array}$ & $\begin{array}{r}2.2 \\
1.2 \\
3.9 \\
2.0 \\
184\end{array}$ & $\begin{array}{r}29 \\
28 \\
52 \\
114 \\
141\end{array}$ & $\begin{array}{l}273 \\
249 \\
241 \\
266 \\
344\end{array}$ & $\begin{array}{l}259 \\
258 \\
260 \\
264 \\
305\end{array}$ & $\begin{array}{l}309 \\
360 \\
464 \\
412 \\
198\end{array}$ & $\begin{array}{l}667 \\
613 \\
768 \\
710 \\
517\end{array}$ & $\begin{array}{l}204 \\
151 \\
126 \\
238 \\
309\end{array}$ & 6 \\
\hline $\begin{array}{l}16 \\
17 \\
18 \\
19 \\
20\end{array}$ & $\begin{array}{r}603 \\
641 \\
579 \\
270 \\
24\end{array}$ & $\begin{array}{l}23 \\
24 \\
24 \\
24 \\
24\end{array}$ & $\begin{array}{l}24 \\
25 \\
26 \\
24 \\
24\end{array}$ & $\begin{array}{l}3.2 \\
1.4 \\
1.2 \\
.90 \\
2.1\end{array}$ & $\begin{array}{l}183 \\
225 \\
152 \\
181 \\
163\end{array}$ & $\begin{array}{r}144 \\
167 \\
148 \\
105 \\
99\end{array}$ & $\begin{array}{l}380 \\
365 \\
360 \\
386 \\
387\end{array}$ & $\begin{array}{l}346 \\
402 \\
425 \\
344 \\
292\end{array}$ & $\begin{array}{r}87 \\
64 \\
81 \\
124 \\
103\end{array}$ & $\begin{array}{l}561 \\
649 \\
690 \\
690 \\
344\end{array}$ & $\begin{array}{l}289 \\
247 \\
203 \\
146 \\
136\end{array}$ & $\begin{array}{l}3 \\
3 \\
3\end{array}$ \\
\hline $\begin{array}{l}21 \\
22 \\
23 \\
24 \\
25\end{array}$ & $\begin{array}{r}76 \\
161 \\
188 \\
188 \\
188\end{array}$ & $\begin{array}{l}24 \\
24 \\
24 \\
25 \\
25\end{array}$ & $\begin{array}{l}24 \\
25 \\
25 \\
25 \\
25\end{array}$ & $\begin{array}{l}1.9 \\
1.1 \\
3.4 \\
2.0 \\
1.1\end{array}$ & $\begin{array}{l}144 \\
142 \\
142 \\
1.60 \\
143\end{array}$ & $\begin{array}{r}99 \\
102 \\
98 \\
98 \\
146\end{array}$ & $\begin{array}{l}384 \\
334 \\
288 \\
289 \\
267\end{array}$ & $\begin{array}{l}361 \\
755 \\
764 \\
682 \\
639\end{array}$ & $\begin{array}{l}206 \\
296 \\
395 \\
362 \\
248\end{array}$ & $\begin{array}{l}103 \\
191 \\
269 \\
310 \\
235\end{array}$ & $\begin{array}{r}162 \\
181 \\
189 \\
126 \\
52\end{array}$ & $\begin{array}{l}42 \\
44 \\
45 \\
44\end{array}$ \\
\hline $\begin{array}{l}26 \\
27 \\
28 \\
29 \\
30 \\
31\end{array}$ & $\begin{array}{r}188 \\
165 \\
167 \\
144 \\
66 \\
24\end{array}$ & $\begin{array}{l}24 \\
25 \\
25 \\
25 \\
24 \\
-\infty\end{array}$ & $\begin{array}{l}25 \\
25 \\
24 \\
24 \\
25 \\
25\end{array}$ & $\begin{array}{l}.96 \\
2.3 \\
1.7 \\
1.2 \\
2.9 \\
2.0\end{array}$ & $\begin{array}{r}151 \\
150 \\
15 \\
138 \\
---\end{array}$ & $\begin{array}{l}237 \\
281 \\
250 \\
186 \\
147 \\
147\end{array}$ & $\begin{array}{l}219 \\
170 \\
146 \\
169 \\
193 \\
---\end{array}$ & $\begin{array}{l}598 \\
562 \\
582 \\
602 \\
601 \\
548\end{array}$ & $\begin{array}{r}125 \\
102 \\
311 \\
59 \\
28 \\
--\end{array}$ & $\begin{array}{l}211 \\
236 \\
217 \\
164 \\
129 \\
164\end{array}$ & $\begin{array}{r}55 \\
50 \\
793 \\
513 \\
32 \\
445\end{array}$ & $\begin{array}{r}45 \\
165 \\
300 \\
300 \\
344\end{array}$ \\
\hline $\begin{array}{l}\text { TOTAL } \\
\text { MEAN } \\
\text { MAX } \\
\text { MIN } \\
\text { AC-ET }\end{array}$ & $\begin{array}{r}10514 \\
339 \\
740 \\
24 \\
20850\end{array}$ & $\begin{array}{r}702 \\
23.4 \\
25 \\
21 \\
1390\end{array}$ & $\begin{array}{r}747 \\
24.1 \\
26 \\
22 \\
1480\end{array}$ & $\begin{array}{r}182.26 \\
5.88 \\
28 \\
.90 \\
362\end{array}$ & $\begin{array}{r}2302.5 \\
79.4 \\
225 \\
1.0 \\
4570\end{array}$ & $\begin{array}{r}3004.1 \\
96.9 \\
281 \\
1.1 \\
5960\end{array}$ & $\begin{array}{r}8040 \\
268 \\
387 \\
73 \\
15950\end{array}$ & $\begin{array}{r}11594 \\
374 \\
764 \\
93 \\
23000\end{array}$ & $\begin{array}{r}8029 \\
268 \\
477 \\
28 \\
15930\end{array}$ & $\begin{array}{r}11827 \\
382 \\
768 \\
28 \\
23460\end{array}$ & $\begin{array}{r}7155 \\
231 \\
793 \\
32 \\
14190\end{array}$ & $\begin{array}{r}2201 \\
73.4 \\
344 \\
25 \\
4370\end{array}$ \\
\hline
\end{tabular}

STATISTICS OF MONTHLY MEAN DATA FOR WATER YEARS 1939 - I99े, BYY WATER YEAAN (WX)

\begin{tabular}{|c|c|c|c|c|c|c|c|c|c|c|c|c|}
\hline $\begin{array}{l}\text { MEAN } \\
\text { MAX } \\
\text { (WY) } \\
\text { MIBY } \\
(W Y)\end{array}$ & $\begin{array}{r}197 \\
2609 \\
1955 \\
991 \\
1965\end{array}$ & $\begin{array}{r}73.1 \\
464 \\
1987 \\
5.71 \\
9980\end{array}$ & $\begin{array}{r}72.9 \\
421 \\
1992 \\
1.04\end{array}$ & $\begin{array}{r}63.1 \\
284 \\
1987 \\
1.98\end{array}$ & $\begin{array}{r}68.5 \\
293 \\
1987 \\
19.5\end{array}$ & $\begin{array}{r}87.0 \\
382 \\
1987 \\
17.7\end{array}$ & $\begin{array}{r}251 \\
345 \\
1945 \\
133\end{array}$ & $\begin{array}{r}195 \\
1055 \\
1973 \\
46,4\end{array}$ & $\begin{array}{r}226 \\
1892 \\
1986 \\
18.6\end{array}$ & $\begin{array}{r}247 \\
794 \\
1960 \\
10.8 \\
1976\end{array}$ & $\begin{array}{r}266 \\
2267 \\
1966 \\
21.5\end{array}$ & $\begin{array}{r}212 \\
1156 \\
1974 \\
12.3\end{array}$ \\
\hline
\end{tabular}

SUPRARY STATISTICS

ANNUAL TOTAL

GIGHEST AFHUAL REAR

LOWEST ANNUAL MEAN

HIGEEST DAILY MEAN

LOWEST DAILY MEAN

ANNUAL SEVEN-DAY MINIMUM

INSTANTANEOUS PEAX FLOW

INSTANTANEOUS PEAK STAGE

INSTANTANEOUS LOW FLOW

ANNUAL RUNOFF (AC-FT)

10 PERCENT EXCEEDS

50 PERCENT EXCEEDS

90 PERCENT EXCEEDS

90 PERCENT EXCEED

a-From rating curve extended above 25

b-From floodmarks at present datum.
FOR 1996 WATER YEAR

WATER YEARS 1939 - 1996

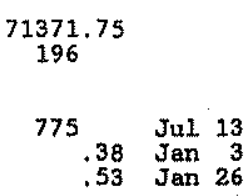

141600

458

1.2
66297.86

$793 \quad$ Aug 28

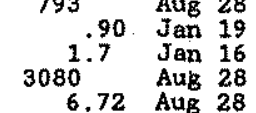

488

129

\section{4}

39000 Aug 231966

$\begin{array}{rrr}.00 & \text { Dec } 21 & 1988 \\ a 69000^{.46} & \text { Dec } 15 & 1988 \\ \text { Aug } 23 & 1966\end{array}$

b21.32 Aug 231966

$118600^{\circ}$

342

92
$66.8 \quad 1955$

5

2


RIO GRANDE BASTN

08403500 CARLSBAD MAIN CANAL AT HEAD, NEAR CARLSBAD, MM

LOCATION,--Lat $32^{\circ} 29^{\prime} 25^{\prime \prime}$, long $104^{\circ} 15^{\prime} 08^{\prime \prime}$, in NW/SW/SW// sec.12, T.21 S., R.26 E., Eddy County, Hydrologic Unit 13060011 on right bank $220 \mathrm{ft}$ downstream from headgates in Avalon Dam, and $3.3 \mathrm{mi}$ north of Carlsbad. Pecos River mile 467.2 .

PERIOD OF RECORD.--July 1939 to current year (monthly discharge only, July 1939 to September 1965). January 1941 to March 1951 published in WSP 1732.

GAGE.--Water-stage recorder and concrete control. Elevation of gage is 3,156.50 ft above National Geodetic Vertical Datum of 1929 (levels by Bureau of Reclamation). Prior to March 1951, at site 20 ft upstream at datum 0.9 ft higher.

REMARKS. - Records good except for estimated daily discharges, which are poox. Carlsbad Main Canal diverts water from Lake Avalon (station 08403800) for Irrigation of about 25,000 acres in the Carlsbad Irrigatton District. About 1,600 acres are ixrigated on the left bank, most of it upstream from gaging station 08405200 . The remaining acreage (most of which is downstream from station 08405200 ) is on the right bank. Several observations of water teroperature were made during the year. No flow for many days.

DISCBARGE, CUBIC FEET PER SECOND, WATER YEAR OCTOBER 1995 TO SEPTEMBER 1996

\begin{tabular}{|c|c|c|c|c|c|c|c|c|c|c|c|c|}
\hline DAY & $\propto C T$ & NOV & DEC & JAN & FEB & MAR & APR & MAY & JUN & JUL & AUG & SEP \\
\hline $\begin{array}{l}1 \\
2 \\
3 \\
4 \\
5\end{array}$ & $\begin{array}{r}87 \\
86 \\
95 \\
99 \\
120\end{array}$ & $\begin{array}{r}110 \\
65 \\
40 \\
29 \\
28\end{array}$ & $\begin{array}{l}21 \\
18 \\
20 \\
13 \\
24\end{array}$ & $\begin{array}{l}31 \\
19 \\
15 \\
19 \\
15\end{array}$ & $\begin{array}{l}.00 \\
.00 \\
.00 \\
.00 \\
.00\end{array}$ & $\begin{array}{r}.05 \\
.08 \\
75^{.10} \\
114\end{array}$ & $\begin{array}{l}258 \\
288 \\
310 \\
295 \\
220\end{array}$ & $\begin{array}{l}128 \\
124 \\
132 \\
143 \\
130\end{array}$ & $\begin{array}{l}117 \\
132 \\
174 \\
222 \\
261\end{array}$ & $\begin{array}{l}132 \\
157 \\
161 \\
136 \\
150\end{array}$ & $\begin{array}{l}191 \\
220 \\
219 \\
182 \\
210\end{array}$ & $\begin{array}{l}.00 \\
.00 \\
.00 \\
.00 \\
.00\end{array}$ \\
\hline $\begin{array}{r}6 \\
7 \\
8 \\
9 \\
10\end{array}$ & $\begin{array}{l}145 \\
146 \\
146 \\
146 \\
146\end{array}$ & $\begin{array}{l}24 \\
25 \\
23 \\
22 \\
21\end{array}$ & $\begin{array}{l}18 \\
15 \\
25 \\
14 \\
15\end{array}$ & $\begin{array}{c}13 \\
2.7 \\
1.3 \\
1.2 \\
.82\end{array}$ & $\begin{array}{l}.00 \\
.00 \\
.00 \\
.00 \\
.00\end{array}$ & $\begin{array}{r}113 \\
104 \\
86 \\
85 \\
86\end{array}$ & $\begin{array}{l}117 \\
118 \\
177 \\
229 \\
245\end{array}$ & $\begin{array}{l}156 \\
221 \\
190 \\
225 \\
228\end{array}$ & $\begin{array}{l}265 \\
268 \\
280 \\
237 \\
268\end{array}$ & $\begin{array}{r}206 \\
\mathrm{e} 198 \\
\mathrm{e} 247 \\
\mathrm{e} 335 \\
\mathrm{e} 311\end{array}$ & $\begin{array}{l}260 \\
298 \\
308 \\
238 \\
191\end{array}$ & $\begin{array}{l}.00 \\
.00 \\
.00 \\
.00 \\
.00\end{array}$ \\
\hline $\begin{array}{l}11 \\
12 \\
13 \\
14 \\
15\end{array}$ & $\begin{array}{l}146 \\
146 \\
146 \\
147 \\
148\end{array}$ & $\begin{array}{l}22 \\
23 \\
24 \\
23 \\
25\end{array}$ & $\begin{array}{l}21 \\
16 \\
16 \\
20 \\
20\end{array}$ & $\begin{array}{l}.39 \\
.25 \\
.14 \\
.28 \\
.16\end{array}$ & $\begin{array}{l}.00 \\
.00 \\
.00 \\
.00 \\
.00\end{array}$ & $\begin{array}{r}85 \\
106 \\
110 \\
123 \\
126\end{array}$ & $\begin{array}{l}229 \\
233 \\
235 \\
263 \\
367\end{array}$ & $\begin{array}{l}252 \\
231 \\
250 \\
267 \\
293\end{array}$ & $\begin{array}{l}294 \\
322 \\
358 \\
255 \\
177\end{array}$ & $\begin{array}{l}e 285 \\
e 331 \\
e 345 \\
e 291 \\
0268\end{array}$ & $\begin{array}{l}114 \\
127 \\
172 \\
235 \\
237\end{array}$ & $\begin{array}{l}.00 \\
.00 \\
.00 \\
.00 \\
.00\end{array}$ \\
\hline $\begin{array}{l}16 \\
17 \\
18 \\
19 \\
20\end{array}$ & $\begin{array}{l}148 \\
148 \\
148 \\
148 \\
148\end{array}$ & $\begin{array}{l}23 \\
30 \\
22 \\
25 \\
28\end{array}$ & $\begin{array}{l}15 \\
29 \\
25 \\
15 \\
15\end{array}$ & $\begin{array}{l}.16 \\
3.7^{2} \\
.00 \\
.01\end{array}$ & $\begin{array}{l}.00 \\
.00 \\
.00 \\
.00 \\
.00\end{array}$ & $\begin{array}{l}117 \\
133 \\
116 \\
133 \\
150\end{array}$ & $\begin{array}{l}351 \\
308 \\
345 \\
317 \\
336\end{array}$ & $\begin{array}{l}321 \\
364 \\
348 \\
285 \\
324\end{array}$ & $\begin{array}{l}102 \\
124 \\
172 \\
163 \\
161\end{array}$ & $\begin{array}{l}\mathrm{e} 219 \\
\mathrm{e} 238 \\
\mathrm{e} 272 \\
\mathrm{e} 238 \\
\mathrm{e} 210\end{array}$ & $\begin{array}{l}212 \\
200 \\
157 \\
143 \\
155\end{array}$ & $\begin{array}{l}.00 \\
.00 \\
.00 \\
.00 \\
.00\end{array}$ \\
\hline $\begin{array}{l}21 \\
22 \\
23 \\
24 \\
25\end{array}$ & $\begin{array}{l}148 \\
145 \\
143 \\
149 \\
150\end{array}$ & $\begin{array}{l}19 \\
25 \\
23 \\
21 \\
22\end{array}$ & $\begin{array}{l}22 \\
17 \\
15 \\
21 \\
21\end{array}$ & $\begin{array}{l}.00 \\
.00 \\
.09 \\
.00 \\
.00\end{array}$ & $\begin{array}{l}.00 \\
.00 \\
.00 \\
.00 \\
.08\end{array}$ & $\begin{array}{r}133 \\
98 \\
90 \\
120 \\
216\end{array}$ & $\begin{array}{l}288 \\
260 \\
280 \\
269 \\
229\end{array}$ & $\begin{array}{l}303 \\
319 \\
279 \\
226 \\
232\end{array}$ & $\begin{array}{l}230 \\
267 \\
275 \\
262 \\
21.3\end{array}$ & $\begin{array}{r}\mathrm{e} 238 \\
\mathrm{e} 266 \\
\mathrm{e} 238 \\
205 \\
210\end{array}$ & $\begin{array}{r}142 \\
155 \\
137 \\
134 \\
85\end{array}$ & $\begin{array}{l}123 \\
238 \\
314 \\
283\end{array}$ \\
\hline $\begin{array}{l}26 \\
27 \\
28 \\
29 \\
30 \\
31\end{array}$ & $\begin{array}{l}150 \\
150 \\
150 \\
149 \\
149 \\
133\end{array}$ & $\begin{array}{l}16 \\
28 \\
18 \\
22 \\
20 \\
\end{array}$ & $\begin{array}{l}17 \\
20 \\
12 \\
24 \\
17 \\
18\end{array}$ & $\begin{array}{l}.00 \\
.00 \\
.00 \\
.00 \\
.00 \\
.00\end{array}$ & \begin{tabular}{l}
.11 \\
.10 \\
.03 \\
.10 \\
\hdashline .2 \\
-
\end{tabular} & $\begin{array}{l}262 \\
239 \\
232 \\
195 \\
174 \\
163\end{array}$ & $\begin{array}{l}216 \\
209 \\
193 \\
179 \\
153\end{array}$ & $\begin{array}{l}209 \\
191 \\
215 \\
195 \\
165 \\
1.34\end{array}$ & $\begin{array}{r}188 \\
139 \\
84 \\
88 \\
90 \\
-\end{array}$ & $\begin{array}{l}214 \\
199 \\
172 \\
172 \\
174 \\
194\end{array}$ & $\begin{array}{r}30 \\
.00 \\
.27 \\
.00 \\
.00 \\
.02\end{array}$ & $\begin{array}{r}307 \\
306 \\
289 \\
288 \\
314\end{array}$ \\
\hline $\begin{array}{l}\text { TOTAL } \\
\text { MEAN } \\
\text { MAX } \\
\text { MIN } \\
\text { AC-FT }\end{array}$ & $\begin{array}{r}4305 \\
139 \\
150 \\
86 \\
8540\end{array}$ & $\begin{array}{r}846 \\
28.2 \\
110 \\
16 \\
1680\end{array}$ & $\begin{array}{r}579 \\
18.7 \\
29 \\
12 \\
1150\end{array}$ & $\begin{array}{r}127.90 \\
4.13 \\
31 \\
.00 \\
254\end{array}$ & $\begin{array}{r}0.42 \\
.014 \\
.11 \\
.00 \\
.8\end{array}$ & $\begin{array}{r}3784.23 \\
1.22 \\
262 \\
.05 \\
7510\end{array}$ & $\begin{array}{r}7517 \\
251 \\
367 \\
117 \\
14910\end{array}$ & $\begin{array}{r}7080 \\
228 \\
364 \\
124 \\
14040\end{array}$ & $\begin{array}{r}6188 \\
206 \\
358 \\
84 \\
12270\end{array}$ & $\begin{array}{r}7012 \\
226 \\
345 \\
132 \\
13910\end{array}$ & $\begin{array}{r}4752.29 \\
153 \\
308 \\
.00 \\
9430\end{array}$ & $\begin{array}{r}2462.00 \\
82.1 \\
314 \\
.00 \\
4880\end{array}$ \\
\hline
\end{tabular}

STATISTICS OF MONTHLY MEAN DATA FOR WATER YEARS 1939 - 1996, BY WATER YEAR (WY)

\begin{tabular}{|c|c|c|c|c|c|c|c|c|c|c|c|c|}
\hline $\begin{array}{l}\text { MEAN } \\
\text { MAX } \\
\text { (WY) } \\
\text { MIN } \\
\text { (WY) }\end{array}$ & $\begin{array}{r}81.0 \\
212 \\
1980 \\
1000 \\
1953\end{array}$ & $\begin{array}{r}4.42 \\
112 \\
1955 \\
i 000 \\
1942\end{array}$ & $\begin{array}{l}7.85 \\
172 \\
1947 \\
1900 \\
1941\end{array}$ & $\begin{array}{l}11.7 \\
120 \\
1956 \\
000 \\
1942\end{array}$ & $\begin{array}{r}23.2 \\
208 \\
1950 \\
000 \\
1941\end{array}$ & $\begin{array}{r}75.3 \\
227 \\
1940 \\
000 \\
1948\end{array}$ & $\begin{array}{r}246 \\
386 \\
1943 \\
167 \\
1967\end{array}$ & $\begin{array}{r}131 \\
228 \\
1996 \\
6.58 \\
1953\end{array}$ & $\begin{array}{r}159 \\
297 \\
1942 \\
1000 \\
1953\end{array}$ & $\begin{array}{r}201 \\
391 \\
1940 \\
000 \\
1976\end{array}$ & $\begin{array}{r}204 \\
463 \\
1943 \\
2.81 \\
1981\end{array}$ & $\begin{array}{r}140 \\
298 \\
1939 \\
1000 \\
1964\end{array}$ \\
\hline
\end{tabular}

SUMMARY STATISTICS

FOR 1995 CALENDAR YEAR

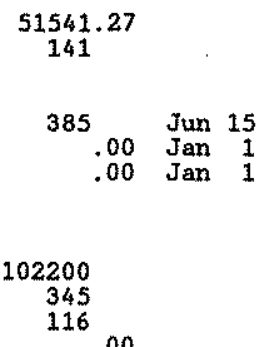

FOR 1996 WATER YEAR

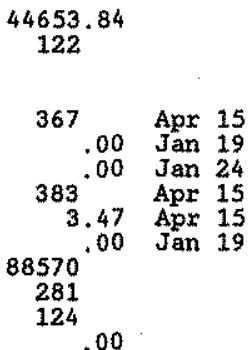

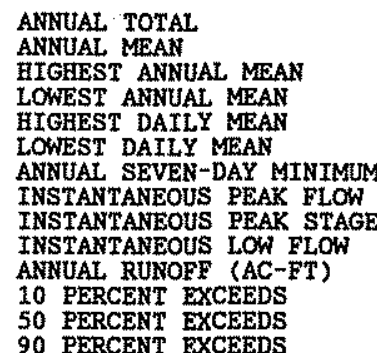

- Estimated

a-Also occurred Sept. 16, 1946.
WATER YEARS 1939 - 1996

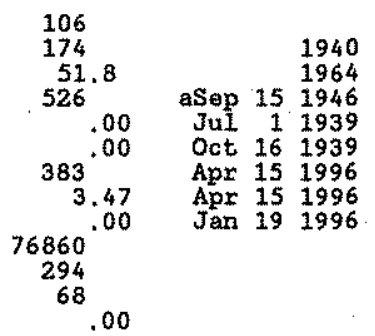


LOCATrOA, --Let $32^{\circ} 29^{\prime} 27^{\prime \prime}$, Long $104^{\circ} 15^{\prime} 05^{\prime \prime}$, in NwhSWh sec.12, T.21 S., R.26 E., Eddy County, nydrologic Unit

13060011 , on headeal1' at outlet gate of dam on Pecos River, 3.3 mi north of Carlsbad, and at mile 467.2 .

DRATMAGE AREA, $-18,070 \mathrm{mi}^{2}$, approximately (contributing area).

PERIOD OF RECORD.--January 1939 to September 1965 (monthend gage heights and contents), Octobex 1965 to current year. Monthend gage heights Januery 1919 to December 1938 in files of Pecos River Commission.

REVISED RECORDS, - WSP B98: 1939.

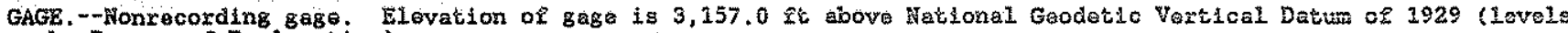
by Bureau of Reclametion).

RaAARKS, - Lake is Lormed by Avalon Dam, an earthfill structure. The original Eddy (Avalon) Dam was completed and storage began in 1891. The dam was destroyed by the Ilood of Aug. 3, 1893; repaired imediately, The dam was destroyed again Oct. 2, 1904 ; construction of present dan comenced on June 1,1906 , and was 88 percent complete June 30,1907 .... Capacity. 330 acre-, t. from capacity table put into use January 1 , 1982 , between gage heights 0.0 (sill of outlot gates) and 20.4 ft, crest of spiliway no. 2 . No dead storage. No storago allocatod to flood control. Figures given herein represent usablo contents. Water is used by Carlsbad Irrigation District.

COOPERATION.--Records provided by Carlsbad Irrigation District.

EXTREMES FOR PERIOD OR RECORD (SINCE 1938),--Maximum contents, 11,000 ecre-ft, May 22, 1941, gage height, 25.0 It; no storage at times when natural flop is passing through reservotr.

EXTREVS FOR CURRENT YaAR.-Maximum contents, 3,790 acra-ft, Aug. 31, Sept. 1-4, 13-22 elevation, 3,176.80 ft; no storage Nov. 2 to Feb. 15.

RESERVOTR STORAGE (ACRE-FEET), WATER YEAR OCTOBER 1995 TO SEPTEMBER 1996 DAILY OBSERVATION AT 08:00 VATUES

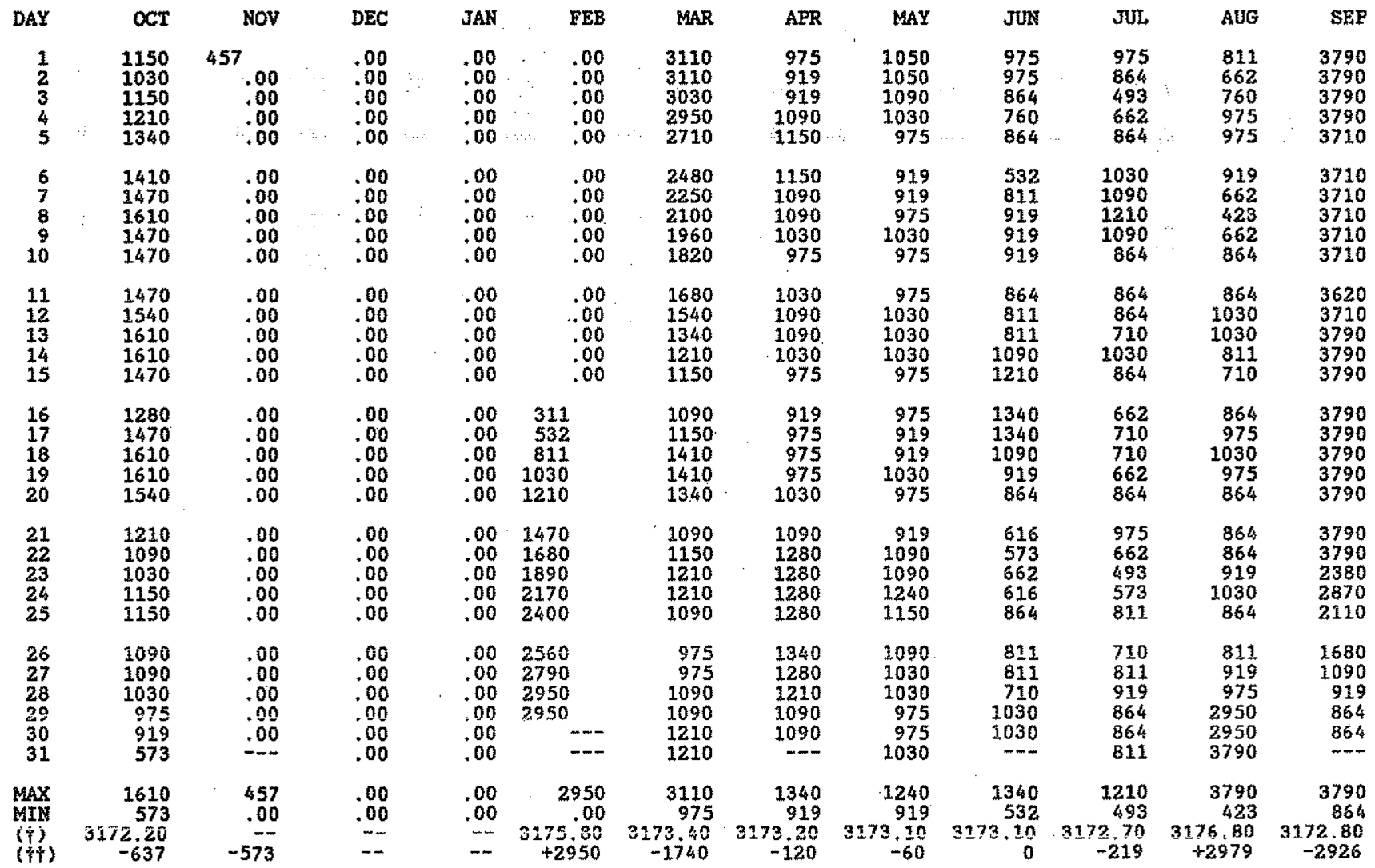

CAL YR 1995 MAX 2560. MIN .00 (†) 0

(†) ELEVATION, IR FEET, AT END OF MONTH

( $f \dagger$ CHANGE IN CONIENTS, IN ACRE-FEET. 
RIO GRANDE BASTN

08404000 PECOS RIVER BELOW AVALON DAM, NM

LOCATION, .-Lat $32^{\circ} 28^{\prime} 55^{\prime \prime}$, long $104^{\circ} 15^{\prime} 47^{\prime \prime}$, in SW/SW/4NE sec.14, T.21 S., R.26 E., Eddy County, Hydrologic Unit 13060011 , on right bank $4.800 \mathrm{ft}$ downstream from Avalon Dam, 4.5 mi northwest of Carlsbad, and at mile 466.3.

DRAINAGE AREA. $--18,080 \mathrm{mt}^{2}$, approximately (contributing area).

PERIOD OF RECORD.--January 1906 to March 1907 (published as "at Avalon"), June 1951 to current year.

GAGE. - Water-stage recorder. Elevation of gage is 3,130 ft above National Geodetic Vertical Datum of 1929 , from topographic map. January 1906 to March 1907 , nonrecording gage at site 0.5 mt upstream at different datum.

RaMaRKS.--Records good except for estimated dally discharges, which are poor. Flow completely regulated by Lake Avalon (station 08403800 ) $0.9 \mathrm{ml}$ upstream. Diversions and ground-water withdrawals upstream from station for irrigation of about 198,000 acres, 1959 determination. Station bypassed by Carlsbad Main Canal (station $08403500)$. Several observations of water temperature were made during the year. No flow most of time.

EXTREMES OUTSIDE PERIOD OF RECORD.--Flood of Oct. 2, 1904, caused in part by failure of Avalon Dam, probably exceeded $90,000 \mathrm{ft} / \mathrm{s}$, and is probably the greatest flood since 1842 . A major flood occurred Aug. 3 , 1893 , and was described as "greatest in 50 years"; it damaged McMilian Dam, then under construction, and washed out the original Avalon Dam. Another major flood occurred Aug. 7, 1916, discharge 70,000 ft3/s, at site 6.5 mi downst ream.

DISCHARGE, CUBIC FEET PER SECOND, WATER YEAR OCTOBER 1995 TO SEPTEMBER 1996

\begin{tabular}{|c|c|c|c|}
\hline $\begin{array}{r}\text { DAY } \\
1 \\
2 \\
3 \\
4 \\
5\end{array}$ & $\begin{array}{l}\text { OCT } \\
.00 \\
.00 \\
.00 \\
.00 \\
.00\end{array}$ & $\begin{array}{l}\text { Nov } \\
.00 \\
.00 \\
.00 \\
.00 \\
.00\end{array}$ & $\begin{array}{l}\text { DEC } \\
.00 \\
.00 \\
.00 \\
.00 \\
.00\end{array}$ \\
\hline $\begin{array}{r}6 \\
7 \\
8 \\
9 \\
10\end{array}$ & $\begin{array}{r}75 \\
331 \\
339 \\
329 \\
314\end{array}$ & $\begin{array}{l}.00 \\
.00 \\
.00 \\
.00 \\
.00\end{array}$ & $\begin{array}{l}.00 \\
.00 \\
.00 \\
.00 \\
.00\end{array}$ \\
\hline $\begin{array}{l}11 \\
12 \\
13 \\
14 \\
15\end{array}$ & $\begin{array}{l}325 \\
334 \\
335 \\
331 \\
319\end{array}$ & $\begin{array}{l}.00 \\
.00 \\
.00 \\
.00 \\
.00\end{array}$ & $\begin{array}{l}.00 \\
.00 \\
.00 \\
.00 \\
.00\end{array}$ \\
\hline $\begin{array}{l}16 \\
17 \\
18 \\
19 \\
20\end{array}$ & $\begin{array}{r}315 \\
328 \\
336 \\
131.55\end{array}$ & $\begin{array}{l}.00 \\
.00 \\
.00 \\
.00 \\
.00\end{array}$ & $\begin{array}{l}.00 \\
.00 \\
.00 \\
.00 \\
.00\end{array}$ \\
\hline $\begin{array}{l}21 \\
22 \\
23 \\
24 \\
25\end{array}$ & $\begin{array}{l}.00 \\
.00 \\
.00 \\
.00 \\
.00\end{array}$ & $\begin{array}{l}.00 \\
.00 \\
.00 \\
.00 \\
.00\end{array}$ & $\begin{array}{l}.00 \\
.00 \\
.00 \\
.00 \\
.00\end{array}$ \\
\hline $\begin{array}{l}26 \\
27 \\
28 \\
29 \\
30 \\
31\end{array}$ & $\begin{array}{l}.00 \\
.00 \\
.00 \\
.00 \\
.00 \\
.00\end{array}$ & $\begin{array}{l}.00 \\
.00 \\
.00 \\
.00 \\
.00 \\
.--\end{array}$ & $\begin{array}{l}.00 \\
.00 \\
.00 \\
.00 \\
.00 \\
.00\end{array}$ \\
\hline $\begin{array}{l}\text { TOTAL } \\
\text { MEAN } \\
\text { MAX } \\
\text { MIN } \\
\text { AC } \sim \text { ET }\end{array}$ & $\begin{array}{r}4142.55 \\
134 \\
339 \\
.00 \\
8220\end{array}$ & $\begin{array}{l}0.00 \\
.000 \\
.00 \\
.00 \\
.00\end{array}$ & $\begin{array}{r}0.00 \\
.000 \\
.00 \\
.00 \\
.00\end{array}$ \\
\hline
\end{tabular}

\begin{tabular}{|c|c|c|c|c|c|c|c|c|}
\hline $\begin{array}{l}\text { JAN } \\
.00 \\
.00 \\
.00 \\
.00 \\
.00\end{array}$ & $\begin{array}{l}\text { FEB } \\
.00 \\
.00 \\
.00 \\
.00 \\
.00\end{array}$ & $\begin{array}{l}\text { MAR } \\
.00 \\
.00 \\
.00 \\
.00 \\
.00\end{array}$ & $\begin{array}{l}\text { APR } \\
.00 \\
.00 \\
.00 \\
.00 \\
.00\end{array}$ & $\begin{array}{l}\text { MAY } \\
.00 \\
.00 \\
.00 \\
.00 \\
.00\end{array}$ & $\begin{array}{l}317 \\
\text { JuN } \\
306 \\
293 \\
117 \\
2.1\end{array}$ & $\begin{array}{l}\text { JUL } \\
.00 \\
.00 \\
.00 \\
.00 \\
.00\end{array}$ & $\begin{array}{l}\text { AUG } \\
.00 \\
.00 \\
.00 \\
.00 \\
.00\end{array}$ & $\begin{array}{r}\text { SEP } \\
0.00 \\
0.00 \\
0.00 \\
0.00 \\
0.00\end{array}$ \\
\hline $\begin{array}{l}.00 \\
.00 \\
.00 \\
.00 \\
.00\end{array}$ & $\begin{array}{l}.00 \\
.00 \\
.00 \\
.00 \\
.00\end{array}$ & $\begin{array}{l}.00 \\
.00 \\
.00 \\
.00 \\
.00\end{array}$ & $\begin{array}{l}.00 \\
.00 \\
.00 \\
.00 \\
.00\end{array}$ & $\begin{array}{l}.00 \\
.00 \\
.00 \\
.00 \\
.00\end{array}$ & $\begin{array}{l}.48 \\
.00 \\
.00 \\
.00 \\
.00\end{array}$ & $\begin{array}{l}172^{.00} \\
314 \\
301\end{array}$ & $\begin{array}{l}.00 \\
.00 \\
.00 \\
.00 \\
.00\end{array}$ & $\begin{array}{r}.00 \\
.00 \\
.00 \\
.00 \\
.00\end{array}$ \\
\hline $\begin{array}{l}.00 \\
.00 \\
.00 \\
.00 \\
.00\end{array}$ & $\begin{array}{l}.00 \\
.00 \\
.00 \\
.00 \\
.00\end{array}$ & $\begin{array}{l}.00 \\
.00 \\
.00 \\
.00 \\
.00\end{array}$ & $\begin{array}{l}.00 \\
.00 \\
.00 \\
.00 \\
.00\end{array}$ & $\begin{array}{l}.00 \\
.00 \\
.00 \\
.00 \\
.00\end{array}$ & $\begin{array}{l}.00 \\
.00 \\
.00 \\
.00 \\
.00\end{array}$ & $\begin{array}{l}294 \\
287 \\
274 \\
294 \\
282\end{array}$ & $\begin{array}{l}.00 \\
.00 \\
.00 \\
.00 \\
.00\end{array}$ & $\begin{array}{r}.00 \\
.00 \\
.00 \\
.00 \\
.00\end{array}$ \\
\hline $\begin{array}{l}.00 \\
.00 \\
.00 \\
.00 \\
.00\end{array}$ & $\begin{array}{l}.00 \\
.00 \\
.00 \\
.00 \\
.00\end{array}$ & $\begin{array}{l}.00 \\
.00 \\
.00 \\
.00 \\
.00\end{array}$ & $\begin{array}{l}.00 \\
.00 \\
.00 \\
.00 \\
.00\end{array}$ & $\begin{array}{l}.00 \\
.00 \\
.00 \\
.00 \\
.00\end{array}$ & $\begin{array}{l}.00 \\
.00 \\
.00 \\
.00 \\
.00\end{array}$ & $\begin{array}{l}274 \\
283 \\
294 \\
301 \\
133\end{array}$ & $\begin{array}{l}.00 \\
.00 \\
.00 \\
.00 \\
.00\end{array}$ & $\begin{array}{l}.00 \\
.00 \\
.00 \\
.00 \\
.00\end{array}$ \\
\hline $\begin{array}{l}.00 \\
.00 \\
.00 \\
.00 \\
.00\end{array}$ & $\begin{array}{l}.00 \\
.00 \\
.00 \\
.00 \\
.00\end{array}$ & $\begin{array}{l}.00 \\
.00 \\
.00 \\
.00 \\
.00\end{array}$ & $\begin{array}{l}.00 \\
.00 \\
.00 \\
.00 \\
.00\end{array}$ & $\begin{array}{l}183^{.00} \\
327 \\
335 \\
334\end{array}$ & $\begin{array}{l}.00 \\
.00 \\
.00 \\
.00 \\
.00\end{array}$ & $\begin{array}{l}1.5 \\
.13 \\
.00 \\
.00 \\
.00\end{array}$ & $\begin{array}{l}.00 \\
.00 \\
.00 \\
.00 \\
.00\end{array}$ & $\begin{array}{l}.00 \\
.00 \\
.00 \\
.00 \\
.00\end{array}$ \\
\hline $\begin{array}{l}.00 \\
.00 \\
.00 \\
.00 \\
.00 \\
.00\end{array}$ & $\begin{array}{l}.00 \\
.00 \\
.00 \\
.00 \\
.-- \\
.--\end{array}$ & $\begin{array}{l}.00 \\
.00 \\
.00 \\
.00 \\
.00 \\
.00\end{array}$ & $\begin{array}{l}.00 \\
.00 \\
.00 \\
.00 \\
.00 \\
.\end{array}$ & $\begin{array}{l}330 \\
322 \\
316 \\
315 \\
318 \\
322\end{array}$ & $\begin{array}{l}.00 \\
.00 \\
.00 \\
.00 \\
.00 \\
.\end{array}$ & $\begin{array}{l}.00 \\
.00 \\
.00 \\
.00 \\
.00 \\
.00\end{array}$ & $\begin{array}{r}.00 \\
.00 \\
2.8 \\
.12 \\
.00 \\
.00\end{array}$ & $\begin{array}{l}.00 \\
.00 \\
.00 \\
.00 \\
.00 \\
.0\end{array}$ \\
\hline $\begin{array}{l}0.00 \\
.000 \\
.00 \\
.00 \\
.00\end{array}$ & $\begin{array}{l}0.00 \\
.000 \\
.00 \\
.00 \\
.00\end{array}$ & $\begin{array}{l}0.00 \\
.000 \\
.00 \\
.00 \\
.00\end{array}$ & $\begin{array}{l}0.00 \\
.000 \\
.00 \\
.00 \\
.00\end{array}$ & $\begin{array}{r}3102.00 \\
100 \\
335 \\
.00 \\
61.50\end{array}$ & $\begin{array}{r}1035.58 \\
34.5 \\
317 \\
200 \\
2050\end{array}$ & $\begin{array}{r}3504.63 \\
113 \\
314 \\
.00 \\
6950\end{array}$ & $\begin{array}{r}2.92 \\
.094 \\
2.8 \\
.00 \\
5.8\end{array}$ & $\begin{array}{r}0.00 \\
.000 \\
.00 \\
.00 \\
.00\end{array}$ \\
\hline
\end{tabular}

STATISTICS OF MONTHLY MEAN DATA FOR WATER YEARS 1951 - 1996, BY WATER YEAR (WY)

\begin{tabular}{|c|c|c|c|c|c|c|c|c|c|c|c|}
\hline $\begin{array}{l}\text { MEAN } \\
\text { MAX } \\
\text { (WY) } \\
\text { MIN } \\
\text { (WX) }\end{array}$ & $\begin{array}{r}103 \\
2365 \\
1955 \\
0000 \\
1952\end{array}$ & $\begin{array}{r}22.4 \\
445 \\
1987 \\
0000 \\
1952\end{array}$ & $\begin{array}{r}23.7 \\
435 \\
1992 \\
000 \\
1952\end{array}$ & $\begin{array}{r}10.3 \\
237 \\
1987 \\
000 \\
1952\end{array}$ & $\begin{array}{r}11.7 \\
255 \\
1987 \\
000 \\
1952\end{array}$ & $\begin{array}{r}4.77 \\
188 \\
1987 \\
0000 \\
1952\end{array}$ & $\begin{array}{l}1.33 \\
59.6 \\
1987 \\
1000 \\
1952\end{array}$ & $\begin{array}{r}42.1 \\
739 \\
1973 \\
000 \\
1952\end{array}$ & $\begin{array}{l}58.6 \\
1832 \\
1986 \\
000 \\
1951\end{array}$ & $\begin{array}{r}32.6 \\
595 \\
1960 \\
000 \\
1951\end{array}$ & $\begin{array}{l}60.4 \\
2034 \\
1966 \\
.000 \\
1951\end{array}$ \\
\hline
\end{tabular}

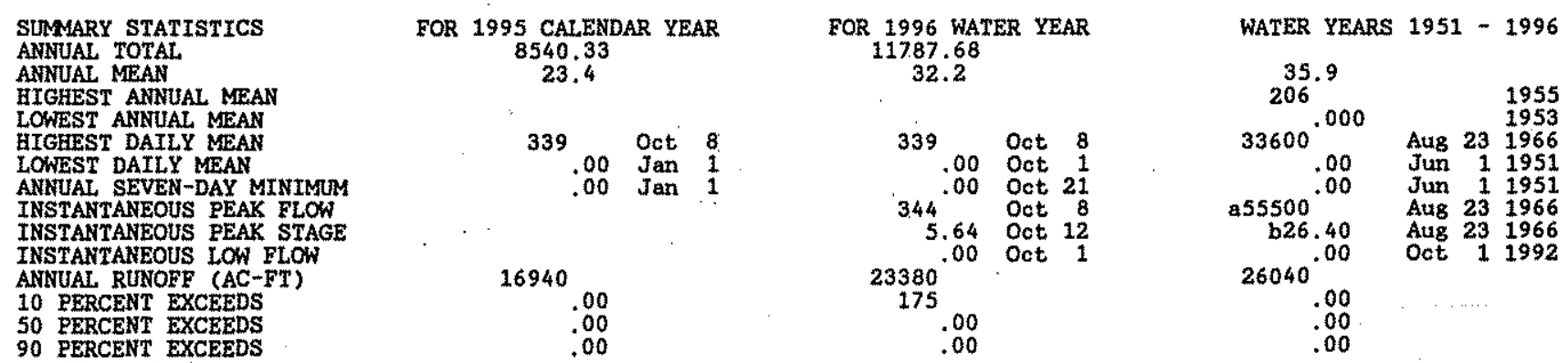

a-From rating curve extended above $33,000 \mathrm{ft} / \mathrm{s}$, on basis of computation of peak flow over Tansi11 Dam $5.8 \mathrm{mi}$ downstream.

b-From floodmarks. 
LOCATION,--Lat $32^{\circ} 24^{\circ} 24^{\prime \prime}$, long $104^{\circ} 13^{\prime} 34^{\prime \prime}$, in NE\%NW/SE\% sec.7, T.22 S., R.27 E., Eddy County, Hydrologic Unit 13060011, on downstream side of bridge on Canal Street in Carisbad, and $0.6 \mathrm{mi}$ upstream from mouth. Mouth at Pecos River mile 459,2 .

DRAINAGE AREA. $-450 \mathrm{mi}^{2}$, approximately.

PERIOD OF RECORD. - January 1973 to current year.

GAGE. - Water-stage recorder. Elevation of gage is 3,088.21 ft above National Geodetic Vertical Datum of 1929.

RWARKS, - Records good. A Soll Conservation Service Ilood-control project on lfeckberry Draw, an upstraan tributary, has some ffect on flood peaks and flow duration. Ground-water withdrawals upstream from station for irrigation of approximately 2,100 acres, 1973 determination, and for municipal supply for Carlsbad. Several observations of pater temperature were made during the year. No flow during water year.

EXTREMES OUTSIDE PERIOD OF RECORD,--The flood of Aug. 23, 1966, reached a discharge of 66,000 ets/s, as detarmined by slope-area measurement at site $1.2 \mathrm{mi}$ upstream Another flood of approximately the same magnitude occurred Sept. 20, 1941. Other major peaks occurred July 17, 1906, July 24, 1908, July 24, 1911, Apr. 16, 1915, Aug. 8, 1916, Sept. 15, 1919, Aug. 4, 1925, and May 23, 1941.

DISCHARGE, CUBIC FEET PER SECOND, WATER YEAR OCTOBER 1995 TO SERTEMBER 1996

\begin{tabular}{|c|c|c|c|c|c|c|c|c|c|c|c|c|}
\hline DAY & $\infty r$ & NOV & DEC & JAN & FEB & MAR & AER & MAY & JUN & JUL. & AUG & SEP \\
\hline $\begin{array}{l}1 \\
2 \\
3 \\
4 \\
5\end{array}$ & $\begin{array}{l}.00 \\
.00 \\
.00 \\
.00 \\
.00\end{array}$ & $\begin{array}{l}.00 \\
.00 \\
.00 \\
.00 \\
.00\end{array}$ & $\begin{array}{l}.00 \\
.00 \\
.00 \\
.00 \\
.00\end{array}$ & $\begin{array}{l}.00 \\
.00 \\
.00 \\
00 \\
00\end{array}$ & $\begin{array}{l}.00 \\
.00 \\
.00 \\
.00 \\
.00\end{array}$ & $\begin{array}{l}.00 \\
.00 \\
.00 \\
.00 \\
.00\end{array}$ & $\begin{array}{l}.00 \\
.00 \\
.00 \\
.00 \\
.00\end{array}$ & $\begin{array}{l}.00 \\
.00 \\
.00 \\
.00 \\
.00\end{array}$ & $\begin{array}{l}.00 \\
.00 \\
.00 \\
.00 \\
.00\end{array}$ & $\begin{array}{l}.00 \\
.00 \\
.00 \\
.00 \\
.00\end{array}$ & $\begin{array}{l}.00 \\
.00 \\
.00 \\
.00 \\
.00\end{array}$ & $\begin{array}{l}.00 \\
.00 \\
.00 \\
.00 \\
.00\end{array}$ \\
\hline $\begin{array}{r}6 \\
7 \\
8 \\
9 \\
10\end{array}$ & $\begin{array}{l}.00 \\
.00 \\
.00 \\
.00 \\
.00\end{array}$ & $\begin{array}{l}.00 \\
.00 \\
.00 \\
.00 \\
.00\end{array}$ & $\begin{array}{l}.00 \\
.00 \\
.00 \\
.00 \\
.00\end{array}$ & $\begin{array}{l}.00 \\
.00 \\
.00 \\
.00 \\
.00\end{array}$ & $\begin{array}{l}.00 \\
.00 \\
.00 \\
.00 \\
.00\end{array}$ & $\begin{array}{l}.00 \\
.00 \\
.00 \\
.00 \\
.00\end{array}$ & $\begin{array}{l}.00 \\
.00 \\
.00 \\
.00 \\
.00\end{array}$ & $\begin{array}{l}.00 \\
.00 \\
.00 \\
.00 \\
.00\end{array}$ & $\begin{array}{l}.00 \\
.00 \\
.00 \\
.00 \\
.00\end{array}$ & $\begin{array}{l}.00 \\
.00 \\
.00 \\
.00 \\
.00\end{array}$ & $\begin{array}{l}.00 \\
.00 \\
.00 \\
.00 \\
.00\end{array}$ & $\begin{array}{l}.00 \\
.00 \\
.00 \\
.00 \\
.00\end{array}$ \\
\hline $\begin{array}{l}11 \\
12 \\
13 \\
14 \\
15\end{array}$ & $\begin{array}{l}.00 \\
.00 \\
.00 \\
.00 \\
.00\end{array}$ & $\begin{array}{l}.00 \\
.00 \\
.00 \\
.00 \\
.00\end{array}$ & $\begin{array}{l}.00 \\
.00 \\
.00 \\
.00 \\
.00\end{array}$ & $\begin{array}{l}.00 \\
.00 \\
.00 \\
.00 \\
.00\end{array}$ & $\begin{array}{l}.00 \\
.00 \\
.00 \\
.00 \\
.00\end{array}$ & $\begin{array}{l}.00 \\
.00 \\
.00 \\
.00 \\
.00\end{array}$ & $\begin{array}{l}.00 \\
.00 \\
.00 \\
.00 \\
.00\end{array}$ & $\begin{array}{l}.00 \\
.00 \\
.00 \\
.00 \\
.00\end{array}$ & $\begin{array}{l}.00 \\
.00 \\
.00 \\
.00 \\
.00\end{array}$ & $\begin{array}{l}.00 \\
.00 \\
.00 \\
.00 \\
.00\end{array}$ & $\begin{array}{l}.00 \\
.00 \\
.00 \\
.00 \\
.00\end{array}$ & $\begin{array}{l}.00 \\
.00 \\
.00 \\
.00 \\
.00\end{array}$ \\
\hline $\begin{array}{l}16 \\
17 \\
18 \\
19 \\
20\end{array}$ & $\begin{array}{l}.00 \\
.00 \\
.00 \\
.00 \\
.00\end{array}$ & $\begin{array}{l}.00 \\
.00 \\
.00 \\
.00 \\
.00\end{array}$ & $\begin{array}{l}.00 \\
.00 \\
.00 \\
.00 \\
.00\end{array}$ & $\begin{array}{l}.00 \\
.00 \\
.00 \\
.00 \\
.00\end{array}$ & $\begin{array}{l}.00 \\
.00 \\
.00 \\
.00 \\
.00\end{array}$ & $\begin{array}{l}.00 \\
.00 \\
.00 \\
.00 \\
.00\end{array}$ & $\begin{array}{l}.00 \\
.00 \\
.00 \\
.00 \\
.00\end{array}$ & $\begin{array}{l}.00 \\
.00 \\
.00 \\
.00 \\
.00\end{array}$ & $\begin{array}{l}.00 \\
.00 \\
.00 \\
.00 \\
.00\end{array}$ & $\begin{array}{l}.00 \\
.00 \\
.00 \\
.00 \\
.00\end{array}$ & $\begin{array}{l}.00 \\
.00 \\
.00 \\
.00 \\
.00\end{array}$ & $\begin{array}{l}.00 \\
.00 \\
.00 \\
.00 \\
.00\end{array}$ \\
\hline $\begin{array}{l}21 \\
22 \\
23 \\
24 \\
25\end{array}$ & $\begin{array}{l}.00 \\
.00 \\
.00 \\
.00 \\
.00\end{array}$ & $\begin{array}{l}.00 \\
.00 \\
.00 \\
.00 \\
.00\end{array}$ & $\begin{array}{l}.00 \\
.00 \\
.00 \\
.00 \\
.00\end{array}$ & $\begin{array}{l}.00 \\
.00 \\
.00 \\
.00 \\
.00\end{array}$ & $\begin{array}{l}.00 \\
.00 \\
.00 \\
.00 \\
.00\end{array}$ & $\begin{array}{l}.00 \\
.00 \\
.00 \\
.00 \\
.00\end{array}$ & $\begin{array}{l}.00 \\
.00 \\
.00 \\
.00 \\
.00\end{array}$ & $\begin{array}{l}.00 \\
.00 \\
.00 \\
.00 \\
.00\end{array}$ & $\begin{array}{l}.00 \\
.00 \\
.00 \\
.00 \\
.00\end{array}$ & $\begin{array}{l}.00 \\
.00 \\
.00 \\
.00 \\
.00\end{array}$ & $\begin{array}{l}.00 \\
.00 \\
.00 \\
.00 \\
.00\end{array}$ & $\begin{array}{l}.00 \\
.00 \\
.00 \\
.00 \\
.00\end{array}$ \\
\hline $\begin{array}{l}26 \\
27 \\
28 \\
29 \\
30 \\
31\end{array}$ & $\begin{array}{l}.00 \\
.00 \\
.00 \\
.00 \\
.00 \\
.00\end{array}$ & $\begin{array}{l}.00 \\
.00 \\
.00 \\
.00 \\
.00 \\
.0\end{array}$ & $\begin{array}{l}.00 \\
.00 \\
.00 \\
.00 \\
.00 \\
.00\end{array}$ & $\begin{array}{l}.00 \\
.00 \\
.00 \\
.00 \\
.00 \\
.00\end{array}$ & $\begin{array}{l}.00 \\
.00 \\
.00 \\
.00 \\
- \\
.\end{array}$ & $\begin{array}{l}.00 \\
.00 \\
.00 \\
.00 \\
.00 \\
.00\end{array}$ & $\begin{array}{l}.00 \\
.00 \\
.00 \\
.00 \\
.00 \\
.\end{array}$ & $\begin{array}{l}.00 \\
.00 \\
.00 \\
.00 \\
.00 \\
.00\end{array}$ & $\begin{array}{l}.00 \\
.00 \\
.00 \\
.00 \\
.00 \\
0 .\end{array}$ & $\begin{array}{l}.00 \\
.00 \\
.00 \\
.00 \\
.00 \\
.00\end{array}$ & $\begin{array}{l}.00 \\
.00 \\
.00 \\
.00 \\
.00 \\
.00\end{array}$ & $\begin{array}{l}.00 \\
.00 \\
.00 \\
.00 \\
.00 \\
.\end{array}$ \\
\hline $\begin{array}{l}\text { TOTAL } \\
\text { MEAN } \\
\text { MAX } \\
\text { MIN } \\
\text { AC-ET }\end{array}$ & $\begin{array}{l}0.00 \\
.000 \\
.00 \\
.00 \\
.00\end{array}$ & $\begin{array}{r}0.00 \\
.000 \\
.00 \\
.00 \\
.00\end{array}$ & $\begin{array}{r}0.00 \\
.000 \\
.00 \\
.00 \\
.00\end{array}$ & $\begin{array}{r}0.00 \\
.000 \\
.00 \\
.00 \\
.00\end{array}$ & $\begin{array}{r}0.00 \\
.000 \\
.00 \\
.00 \\
.00\end{array}$ & $\begin{array}{r}0.00 \\
.000 \\
.00 \\
.00 \\
.00\end{array}$ & $\begin{array}{r}0.00 \\
.000 \\
.00 \\
.00 \\
.00\end{array}$ & $\begin{array}{l}0.00 \\
.000 \\
.00 \\
.00 \\
.00\end{array}$ & $\begin{array}{r}0.00 \\
.000 \\
.00 \\
.00 \\
.00\end{array}$ & $\begin{array}{r}0.00 \\
.000 \\
.00 \\
.00 \\
.00\end{array}$ & $\begin{array}{r}0.00 \\
.000 \\
.00 \\
.00 \\
.00\end{array}$ & $\begin{array}{r}0.00 \\
.000 \\
.00 \\
.00 \\
.00\end{array}$ \\
\hline
\end{tabular}

STATISTICS OF MONTHLY MEAN DATA FOR WATER YEARS 1973 - 1996, BY WATER YEAR (WY)

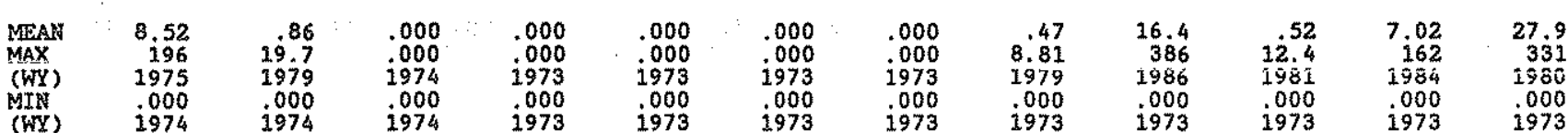

SURAARY STATISTYCS

ANNUAL TOTAL.

HIGHEST ANNUAL MEAN

LOWEST ANNUAL MEAN

HIGHEST DAILY MEAN

LOWEST DAILY MEAN

ANRUAL SEVEN-DAY MINIMUM

INSTANTANEOUS PEAK FLOW

INSTANTANEOUS PEAK STAGE

INSTANTANEOUS LOW FLOW

ANWUAL RUNOFF (AC-FT)

10 PERCENT EXCEEDS

50 PERCENT EXCEEDS

90 PERCENT EXCEEDS

a-From rating curve extended above $7,100 \mathrm{ft}^{3} / \mathrm{s}$
FOR 1995 CALENDAR YEAR FOR 1996 WATER YEAR

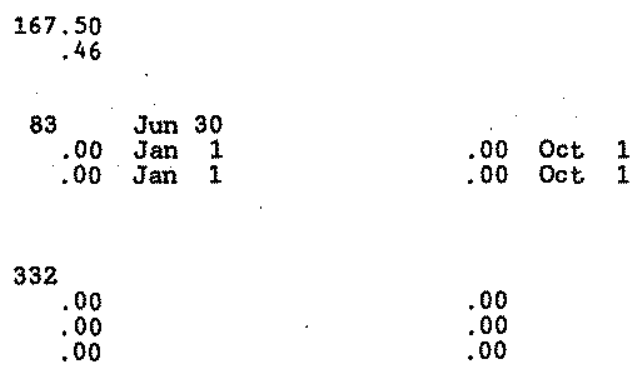

WATER YEARS 1973 - 1996

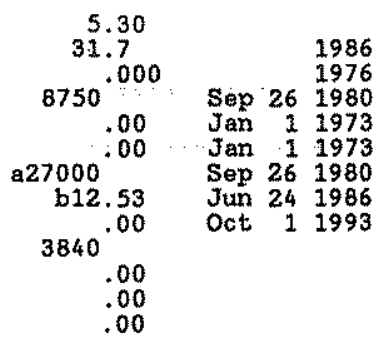


RIO GRANDE BASIN

08405200 PECOS RIVER BELOW DARK CANYON DRAW, AT CARLSBAD, MM

LOCATION.--Lat $32^{\circ} 24^{\circ} 37^{\prime \prime}$, Long $104^{\circ} 12^{\circ} 58^{\prime \prime}$, in NEKSWkNW sec.8, T.22 S., R. 27 E., Eddy County, Hydrologic Unit 13060011 , on left bank $700 \mathrm{ft}$ downstream from mouth of Dark Canyon Draw, 0.3 mi downstream from Lower Tanst11 Dam and Bataan recreational area, $0.8 \mathrm{mi}$ downstream from bridge on U.S. Highway $62-180$ in Carlsbad, and at mile 459.1 .

DRAINAGE AREA. $--18,550 \mathrm{mi}^{2}$, approximately, contributing area.

PERIOD OF RECORD.--January 1970 to current yATER-DISCHARGE RECORDS

GAGE. - Water-stage recorder and concrete controi. Elevation of gage $1 \mathrm{~s} 3,075.19 \mathrm{ft}$ above National Geodetic Vertical Datum of 1929 .

REMARKS. - Water-discharge records good. Flow regulated by Lake Avalon (station 08403800 ) 8.1 mi upstream and by several other reservoirs and up to Nov. 1982 at low stages by power plant. Power plant discontinued operatton Nov 1982 Gage ts bypassed on loft banic by Carlsbad Main Canal East which irrigates several hundred acres adjacent to and domstream from gage and on right bank by Carlsbad Main Canal South, wht ch with supplemental ground-water tharawals irrigates about 23,000 acres downstream. Diversions and ground-water witharawals upstream from station for irrigation of about 198,000 acres, 1959 determination. Bureau of Reclamation satelifte telemeter at station. No flow at times.

EXTREMES OUTSIDE PERIOD OF RECORD, - The flood of Aug. 23, 1966, reached a stage of about 22 ft, discharge not determined. (For dates of other historlcal floods see station 08404000 .) DISCAARGE, CUBIC FEET PER SECOND, WATER YEAR OCTOBER 1995 TO SEPTEMBER 1996

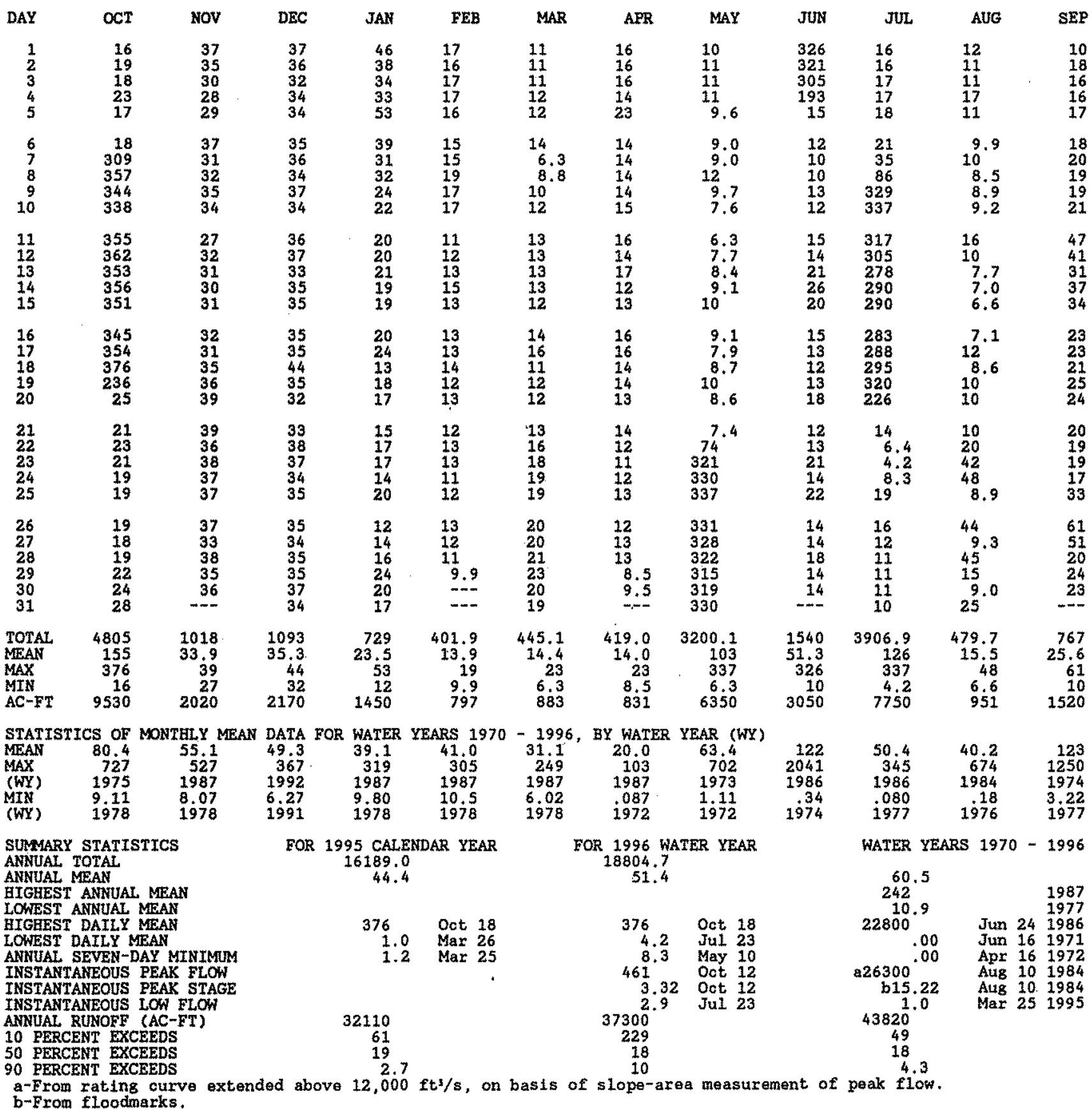


08405200 PECOS RIVER BELOW DARK CANYON DRAW, AT CARLSBAD, MM -- Continued WATER-QUALITY RECORDS

PERTOD OF RECORD.--Water years 1972 to current year.

REMARKS.--Roplaces station 08405000 Pecos River at Carlsbad, New Mexico at which sample collection was discontinued after September, 1987 . WATER QUALITY DATA, WATER YEAR OCTOBER 1995 TO SEPTEMBER 1996

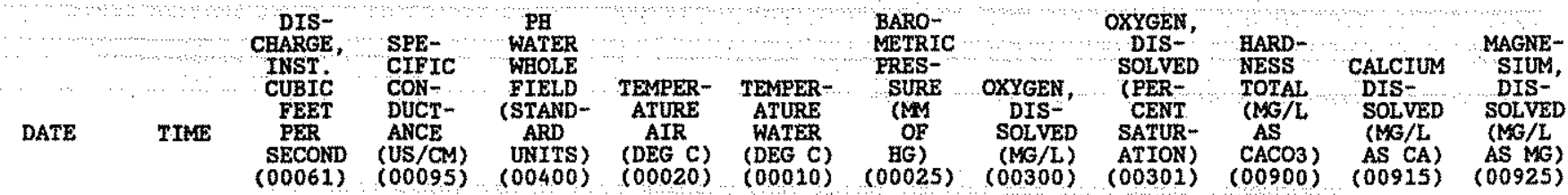

\begin{tabular}{|c|c|c|c|c|c|c|c|c|c|c|c|c|}
\hline $\begin{array}{l}\text { MAR } \\
\text { MA }\end{array}$ & 1445 & 29 & 3740 & 7.9 & 23.0 & 13.0 & 681 & 10.0 & 108 & 1300 & 320 & 110 \\
\hline $12 \ldots$ & 1515 & 19 & 3410 & 8.0 & 31.5 & 17.0 & 678 & 10.3 & 121 & 1200 & 300 & 110 \\
\hline $29 . \cdots$ & 1435 & 8.5 & 3580 & 7.7 & 20.5 & 22.0 & 690 & 9.0 & 116 & 1300 & 320 & 120 \\
\hline JuL $14 \ldots$ & 1500 & 13 & 2790 & 7.4 & 31.5 & 27.0 & 683 & 10.3 & 146 & 1200 & 340 & 78 \\
\hline $\operatorname{seP}^{25} \cdots$ & 1515 & 15 & 2120 & 7.8 & 33.0 & 30.0 & 687 & 11.9 & 177 & 850 & 250 & 54 \\
\hline 09 & 1330 & 19 & 2980 & 7.7 & 31.5 & 28.0 & 686 & 8.8 & 127 & 1200 & 300 & 110 \\
\hline
\end{tabular}

\begin{tabular}{|c|c|c|c|c|c|c|c|c|c|c|c|}
\hline & $\begin{array}{l}\text { SODIURA, } \\
\text { DIS- } \\
\text { SOLVED } \\
\text { (MG/L } \\
\text { AS NA) } \\
(00930)\end{array}$ & $\begin{array}{l}\text { SODIUM } \\
\text { AD- } \\
\text { SORP- } \\
\text { TION } \\
\text { RATIO }\end{array}$ & $\begin{array}{c}\text { POTAS- } \\
\text { SIUM, } \\
\text { DIS- } \\
\text { SOLVED } \\
\text { (MG/L } \\
\text { AS K) } \\
(00935)\end{array}$ & $\begin{array}{c}\text { ALKA- } \\
\text { LINITY } \\
\text { LAB } \\
\text { (MG/L } \\
\text { AS } \\
\text { CACO3) } \\
(90410)\end{array}$ & $\begin{array}{c}\text { SULFATE } \\
\text { DIS- } \\
\text { SOLVED } \\
\text { (MG/L } \\
\text { AS SO4) } \\
(00945)\end{array}$ & $\begin{array}{l}\text { CHLO- } \\
\text { RIDE, } \\
\text { DIS- } \\
\text { SOLVED } \\
\text { (MG/L } \\
\text { AS CL) } \\
(00940)\end{array}$ & $\begin{array}{l}\text { FLUO- } \\
\text { RIDE, } \\
\text { DIS- } \\
\text { SOLVED } \\
(M G / L \\
\text { AS F } \\
(00950)\end{array}$ & $\begin{array}{c}\text { SILICA, } \\
\text { DIS- } \\
\text { SOLVED } \\
\text { (MG/L } \\
\text { AS } \\
\text { SIOZ) } \\
(00955)\end{array}$ & $\begin{array}{l}\text { SOLIDS, } \\
\text { SUM OF } \\
\text { CONSTI- } \\
\text { TUENTS, } \\
\text { DIS- } \\
\text { SOLVED } \\
\text { (ME/L) } \\
\text { (70301) }\end{array}$ & $\begin{array}{l}\text { BORON, } \\
\text { DIS- } \\
\text { SOLVED } \\
\text { (UG/L } \\
\text { AS B) } \\
(01020)\end{array}$ & $\begin{array}{l}\text { IRON, } \\
\text { DIS- } \\
\text { SOLVED } \\
\text { (UG/L } \\
\text { AS FE) } \\
(01046)\end{array}$ \\
\hline
\end{tabular}

\begin{tabular}{|c|c|c|c|c|c|c|c|c|c|c|c|}
\hline Nov 1995 & 380 & 5 & 2.9 & 138 & 1000 & 640 & 0.60 & 8.0 & 2540 & 220 & $<9.0$ \\
\hline APR $12 \ldots$ & 310 & 4 & 4.5 & 171 & 940 & 530 & 0.70 & 13 & 2310 & 220 & $<9.0$ \\
\hline $\operatorname{Ju}^{29} \cdots$ & 370 & 4 & 4,6 & 159 & 1000 & 600 & 0.80 & 10 & 2520 & 260 & $<9.0$ \\
\hline $\mathrm{JUL}^{14 \ldots}$ & 240 & 3 & 5.1 & 94 & 950 & 360 & 0.60 & 12 & 2040 & 161 & 250 \\
\hline $\operatorname{seP}^{25} \cdots$ & 150 & 2 & 4.1 & 92 & 750 & 240 & 0.50 & 12 & 1520 & 136 & 13 \\
\hline & $33 n$ & 4 & 4.4 & 141 & 990 & 530 & 0.70 & 18 & 2370 & 237 & $<9.0$ \\
\hline
\end{tabular}


RIO GRANDE BASIN.

08405500 BLACK RIVER ABOVE MALAGA, NM

LOCATTON,--Lat $32^{\circ} 13^{\prime} 44^{\prime \prime}$, Long $104^{\circ} 09^{\circ} 02^{\prime \prime}$, in SW/4WW/4Wt/ sec.12, T.24 S., R.27 E. Eddy County, Bydrologio Unit 13060011, on right bank $0.6 \mathrm{mi}$ upstream from Black River diversion dam, $4.6 \mathrm{mi}$ west of Malaga, and $7.1 \mathrm{mi}$ upstream from mouth. Mouth at Pecos River mile 436.3.

DRAINAGE AREA. $--343 \mathrm{mi}^{2}$.

PERTOD OF RECORD.-March to December 1940, Decembex 1946 to current year.

REVISED RECORDS. -WSP 1632: 1948, 1949-50(p).

GAGE. - Water-stage recorder and concrete control. Elevation of gage is 3,070 ft above National Geodetic Vertical Datum of 1929. from topographle map. March to December 1940, water-stage recorder and Cippoletti weir at site 0.3 mit downstream at different datum.

REMARKS.--Records good except for estimated dally discharges, which are poor. Diversions and 8round-water withdrawals for irrtgation of about 1,000 acres, 1959 determination, upstream from station. Several observations of pater temperature were made during the year.

EXTREMES OUTSIDE PERIOD OF RECORD.--Flood of Sept. 20 or 21, 1941, reached a stage of 19.0 ft, present site and datum, determined in 1947 from well-defined floodmarks, discharge, 33,000 ft $3 / \mathrm{s}$, from rating curve extended above $1,400 \mathrm{ft} / \mathrm{s}$ on basis of slope-area moasurements at gage helghts $8,41 \mathrm{ft}$ and $12.60 \mathrm{ft}$.

DISCEARGE, CUBIC FEET PER SECOND, WATER YEAR OCTOBER 1995 TO SEPTEMBER 1996

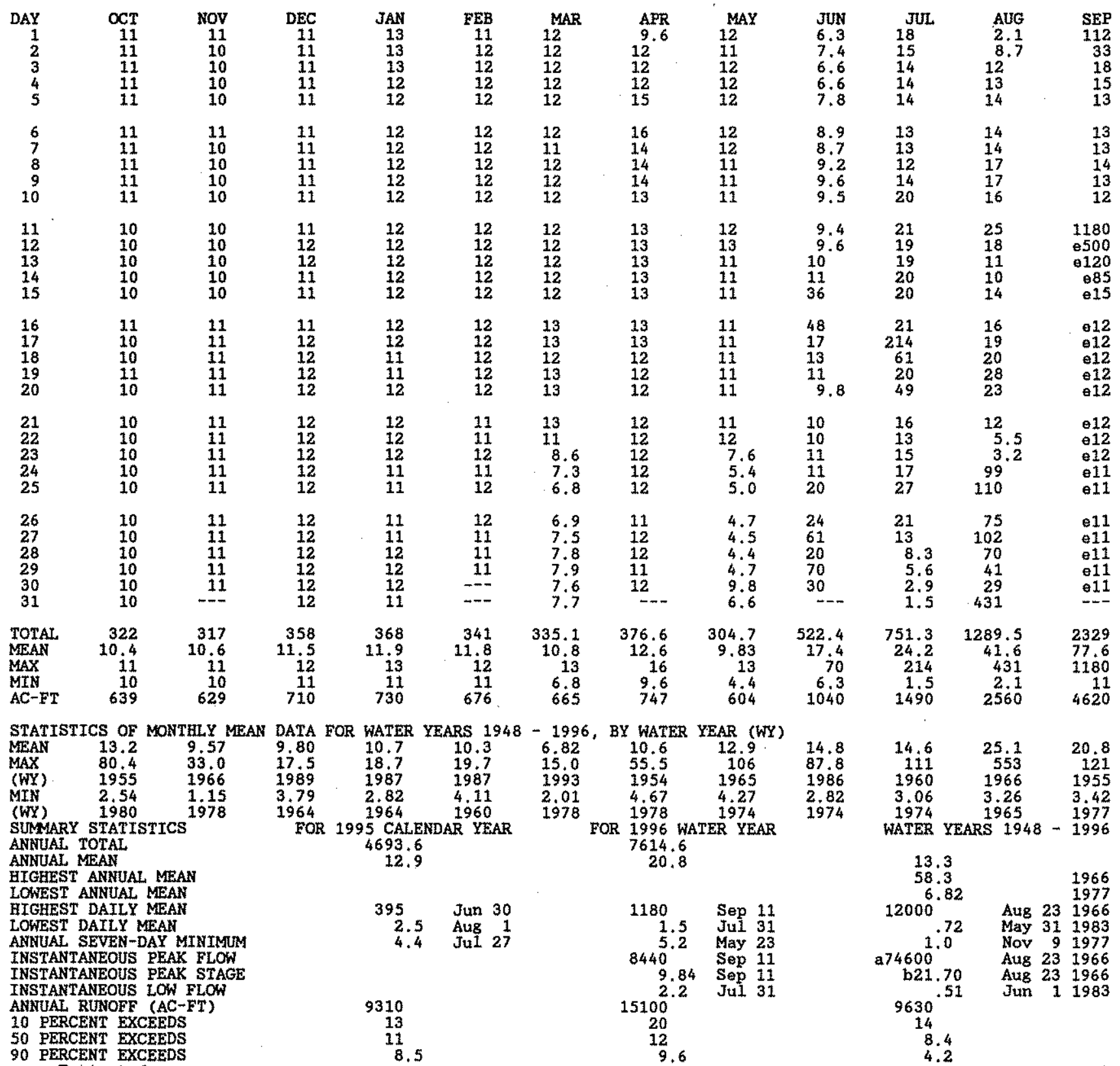

Estimatod

a-From rating curve extended above $5,900 \mathrm{ft} / \mathrm{s}$, on basis of slope-area measurements at $8 \mathrm{gge}$ helghts 12.60 ft and $21.7 \mathrm{ft}$.

b-From floodmarks. 
08406500 PECOS RIVER NEAR MALAGA, NM

LOCATrON,-Iat $32^{\circ} 12^{\prime} 26^{\prime \prime}$, long $104^{\circ} 01^{\prime} 22^{\prime \prime}$, in SW/4WWNE\% sec.19, T.24 S., R.29 E., Eddy County, Hydrologic Unit

13060011 , on right bank 3.1 mi southeast of Malaga, 4.3 mi downstream from Biack River, and at mile 432.2 .

DRATNAGE AREA. - - 19,190 $\mathrm{mi}^{2}$, approximately (contributing area).

WATER-DISCEARGE RECORDS

PERIOD OF RECORD. May 1920 to current year. Monthly discharge only for some periods, published in WSP 1312 , REVISED RECORDS, -WSP 1632: 1925, $1932-37$

CAGE-Watex-stage Iecordar. Elovation of gage is 2,895.64 ft above National Goodetic Vartical Datum of 1929. May 1, 1920 to Mar. 24,1949 , at datum 3 ft higher

REAÁks,--Weter-discharge records good. Flow regulated by meny reservolrs and diverston dams. Diversiona and ground- water withdrawals upstream from station for irrigation of about 202,000 acres, 1959 determination.

Harroún canal bypasses gage on left bank and irrigates approxitmately 1,000 acres adjacent to and downstream from sage. This bypass is not gaged.

AVRRAGE DISCHARGE,-16 years (water years 1921-36), 274 fts/s, 198,500 acre-ft/yr, prior to completion of Lake Sumer.

EXTREMES OUTSIDE PERIOD OF RECORD. - A major Elood occurred In 1904 , discharge not determined. Flood of Aug. 7 1916, reached a discharge of $70,000 \mathrm{ft} / \mathrm{s}$, at Carlsbad, 27 mi upstream. Flood in September 1919 reached a stage of $29.4 \mathrm{It}$, prosent datium, discharge, $40,400 \mathrm{ft}^{\mathrm{s}} / \mathrm{s}$.

DISCEARGE, CUBIC FEET PER SECOND, WATER YEAR OCTOBER 1995 TO SEPTEMBER 1996

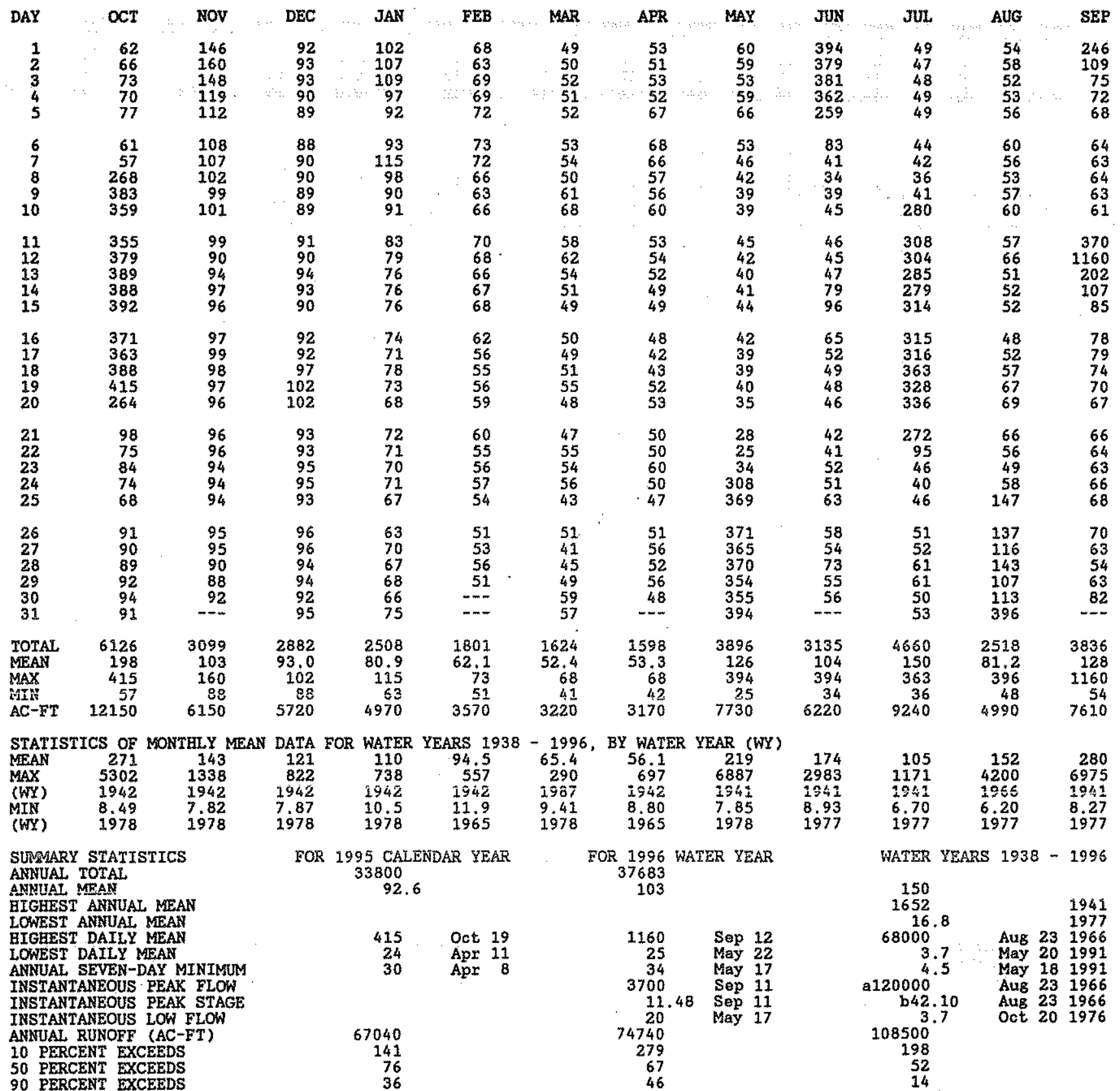

a FrCEN b-From floodinarks. 
RIO GRANDE BASIN

08406500 PECOS RIVER NEAR MALAGA, NM - Continued

WATHR-QUALITY RECORDS

PERIOD OF RECORD.--Water years 1937 to current year.

WATER QUALITY DATA, WATER YEAR OCTOEER 1995 TO SEPTEMBER 1996

\begin{tabular}{|c|c|c|c|c|c|c|c|c|c|c|c|c|}
\hline DATE & TIME & $\begin{array}{c}\text { DIS- } \\
\text { CEARGE, } \\
\text { INST. } \\
\text { CUBIC } \\
\text { FEET } \\
\text { PER } \\
\text { SECOND } \\
(00061)\end{array}$ & $\begin{array}{l}\text { SPE- } \\
\text { CIFIC } \\
\text { CON- } \\
\text { DUCT- } \\
\text { ANCE } \\
\text { (US/CM) } \\
\text { (00095) }\end{array}$ & $\begin{array}{c}\text { PH } \\
\text { WATER } \\
\text { WHOLE } \\
\text { FIELD } \\
\text { (STAND- } \\
\text { ARD } \\
\text { UNITS) } \\
\text { (00400) }\end{array}$ & $\begin{array}{c}\text { TEMPER- } \\
\text { ATURE } \\
\text { AIR } \\
\text { (DEG C) } \\
(00020)\end{array}$ & $\begin{array}{c}\text { TEMPER- } \\
\text { ATURE } \\
\text { WATER } \\
\text { (DEG C) } \\
(00010) .\end{array}$ & $\begin{array}{l}\text { BARO } \\
\text { MEYRIC } \\
\text { DRES- } \\
\text { SURE } \\
\text { (MM } \\
\text { OF } \\
\text { HG) } \\
(00025)\end{array}$ & $\begin{array}{c}\text { OXYGEN, } \\
\text { DIS- } \\
\text { SOLVED } \\
(M G / L) \\
(00300)\end{array}$ & $\begin{array}{c}\text { OXYGEN, } \\
\text { DIS- } \\
\text { SOLVED } \\
\text { (PER- } \\
\text { CENT } \\
\text { SATUR- } \\
\text { ATION) } \\
\text { (00301) }\end{array}$ & $\begin{array}{l}\text { HARD- } \\
\text { NESS } \\
\text { TOTAL } \\
\text { (MG/L. } \\
\text { AS } \\
\text { CACO3) } \\
(00900)\end{array}$ & $\begin{array}{l}\text { CALCIUM } \\
\text { DIS- } \\
\text { SOLVED } \\
(\text { MG } / L \\
\text { AS CA) } \\
(00915)\end{array}$ & $\begin{array}{c}\text { MAGNE- } \\
\text { SIUM, } \\
\text { DIS- } \\
\text { SOLVED } \\
(\text { ME/L } \\
\text { AS MG) } \\
\text { (00925) }\end{array}$ \\
\hline
\end{tabular}

\begin{tabular}{|c|c|c|c|c|c|c|c|c|c|c|c|c|}
\hline $\begin{array}{c}\mathrm{DEC} 1995 \\
01\end{array}$ & 1400 & 92 & 5710 & 8.0 & 26,0 & 13.5 & 685 & 10.3 & 112 & 1900 & 480 & 170 \\
\hline $11 \ldots$ & 1535 & 54 & 5040 & 8.1 & 28.5 & 8.5 & 687 & 9.8 & 94 & 1900 & 480 & 170 \\
\hline JuN & 1000 & 47 & 6280 & 7.8 & 26.0 & 18.5 & 687 & 8.2 & 99 & 2100 & 510 & 200 \\
\hline & 1400 & 46 & 5160 & 8.0 & 39.5 & 28.5 & 686 & 12.6 & 184 & 1800 & 510 & 130 \\
\hline & 1245 & 56 & 3640 & 7.8 & 29.0 & 28.0 & 690 & 12.9 & 185 & 1300 & 360 & 92 \\
\hline
\end{tabular}

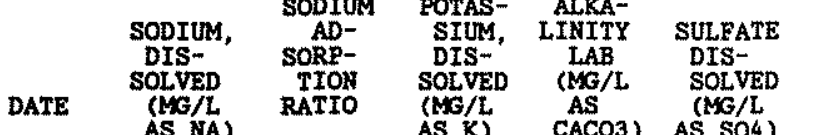

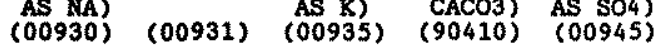

CHLO- FLUO- SILICA, SOM OF

RIDE, FLDE SILICA, SUM OF DIS - DIS- SOLVED TUENTS

SOLVED SOLVED (MG/L DIS(MG/L (MG/L AS SOLVED AS CL) ASF) SIO2) (MG/L) $\begin{array}{cccc}\text { AS CL) } & \text { AS F } & \text { STO2) } & (\mathrm{MG} / \mathrm{L}) \\ (00940) & (00950) & (00955) & (70301)\end{array}$

BORON, IRON, DIS- DISSOLVED SOLVED (UG/L (UG/L. AS B) AS FE) (01020) (01046)

\begin{tabular}{|c|c|c|c|c|c|c|}
\hline $\begin{array}{l}\mathrm{DEC} \\
01995 \\
\mathrm{MAR} \\
1996\end{array}$ & 630 & 6 & 4.2 & 190 & 1500 & 1100 \\
\hline & 590 & 6 & 8.6 & 160 & 1500 & 1000 \\
\hline $30 \ldots$ & 790 & 8 & 10 & 165 & 1700 & 1300 \\
\hline${ }_{\text {JuL }}^{10} \ldots$ & 540 & 6 & 12 & 126 & 1500 & 900 \\
\hline${ }_{\text {SEP }}^{25 \ldots}$ & 370 & 5 & 9.3 & 130 & 1100 & 620 \\
\hline & 690 & 7 & 10 & 149 & 1500 & 1200 \\
\hline
\end{tabular}

$\begin{array}{ccccc}0.70 & 13 & 4010 & 340 & <12 \\ 0.80 & 8.0 & 3850 & 360 & <12 \\ 0.90 & 9.4 & 4620 & 440 & 23 \\ 0.90 & 17 & 3690 & 296 & 150 \\ 0.70 & 16 & 2650 & 237 & <9.0 \\ 0.80 & 16 & 4140 & 390 & <15\end{array}$


RIO GRANDE BASIN

08407000 PECOS RIVER AT PIERCE CANYON CROSSING, NEAR MALAGA, MM

LOCATION,-Lat $32^{\circ} 11^{\prime} 19^{\prime \prime}$, Long $103^{\circ} 58^{\prime} 43^{\prime \prime}$, In SWhWWhW sec.27, T.24 S., R.29 E., Eddy County, Hydrologic Unit 13060011 , on right bank $550 \mathrm{ft}$ upstrean from Pierce Canyon Crossing, 6.0 mi southeast of Malaga, and at mile 425.7 .

DRAINAGE ARFA. $--19,260 \mathrm{mi}^{2}$, approximately (contributing areà).

WATER-DISCKARGE RECORDS

PERIOD OF RECORD.--July 1938 to September 1941, August 1951 to current year.

REVISED RECORDS.-WSP 898: 1938(M). WSP 1712: 1959.

GAGE,-Water-stage recorder. Elevation of gage is $2,889.18 \mathrm{ft}$ above National Geodetic vertical Datum of 1929 (levels by Bureau of Reclamation). July 1938 to Sept. 1941, at datum 1.19 ft higher.

REMARKS. -Water-discharge records good except those above 300 fts/s, which are fair. Flow regulated by many reservotrs and diversion dams Diversions and ground water withdrawals upstream from station for irrigation of about 202,000 acres, 1959 determination.

DISCHARGE, CUBIC FEET PER SECOND, WATER YEAR OCTOBER 1995 TO SEPTEMBER 1996

\begin{tabular}{|c|c|c|c|c|c|c|c|c|c|c|c|c|}
\hline DAY & $O C T$ & Hov & DEC & JAN & FEB & MAR & ARR & MAỲ & JUN & JUL & AUG & SEP \\
\hline $\begin{array}{l}1 \\
2 \\
3 \\
4 \\
5\end{array}$ & $\begin{array}{r}63 \\
64 \\
69 \\
66 \\
72\end{array}$ & $\begin{array}{l}111 \\
133 \\
133 \\
113 \\
103\end{array}$ & $\begin{array}{l}86 \\
87 \\
87 \\
86 \\
83\end{array}$ & $\begin{array}{l}92 \\
93 \\
99 \\
89 \\
83\end{array}$ & $\begin{array}{l}66 \\
59 \\
60 \\
62 \\
63\end{array}$ & $\begin{array}{l}47 \\
47 \\
47 \\
47 \\
47\end{array}$ & $\begin{array}{l}49 \\
48 \\
48 \\
48 \\
57\end{array}$ & $\begin{array}{l}50 \\
54 \\
50 \\
48 \\
57\end{array}$ & $\begin{array}{l}376 \\
362 \\
361 \\
343 \\
293\end{array}$ & $\begin{array}{l}48 \\
45 \\
46 \\
46 \\
46\end{array}$ & $\begin{array}{l}47 \\
48 \\
45 \\
46 \\
48\end{array}$ & $\begin{array}{r}327 \\
119 \\
76 \\
67 \\
66\end{array}$ \\
\hline $\begin{array}{r}6 \\
7 \\
8 \\
9 \\
10\end{array}$ & $\begin{array}{r}64 \\
60 \\
185 \\
391 \\
381\end{array}$ & $\begin{array}{r}104 \\
100 \\
99 \\
94 \\
95\end{array}$ & $\begin{array}{l}83 \\
82 \\
84 \\
83 \\
82\end{array}$ & $\begin{array}{l}81 \\
97 \\
91 \\
82 \\
80\end{array}$ & $\begin{array}{l}65 \\
65 \\
62 \\
58 \\
58\end{array}$ & $\begin{array}{l}49 \\
50 \\
47 \\
52 \\
59\end{array}$ & $\begin{array}{l}59 \\
58 \\
52 \\
48 \\
51\end{array}$ & $\begin{array}{l}51 \\
45 \\
42 \\
40 \\
37\end{array}$ & $\begin{array}{r}134 \\
72 \\
55 \\
50 \\
47\end{array}$ & $\begin{array}{r}44 \\
43 \\
41 \\
38 \\
185\end{array}$ & $\begin{array}{l}50 \\
50 \\
47 \\
48 \\
53\end{array}$ & $\begin{array}{l}62 \\
62 \\
62 \\
62 \\
61\end{array}$ \\
\hline $\begin{array}{l}11 \\
12 \\
13 \\
14 \\
15\end{array}$ & $\begin{array}{l}369 \\
386 \\
399 \\
398 \\
410\end{array}$ & $\begin{array}{l}96 \\
88 \\
86 \\
91 \\
91\end{array}$ & $\begin{array}{l}84 \\
84 \\
85 \\
86 \\
83\end{array}$ & $\begin{array}{l}78 \\
73 \\
71 \\
70 \\
70\end{array}$ & $\begin{array}{l}61 \\
61 \\
60 \\
60 \\
61\end{array}$ & $\begin{array}{l}57 \\
55 \\
51 \\
48 \\
47\end{array}$ & $\begin{array}{l}49 \\
47 \\
47 \\
45 \\
45\end{array}$ & $\begin{array}{l}42 \\
41 \\
40 \\
39 \\
41\end{array}$ & $\begin{array}{l}45 \\
44 \\
45 \\
59 \\
76\end{array}$ & $\begin{array}{l}301 \\
298 \\
287 \\
278 \\
291\end{array}$ & $\begin{array}{l}50 \\
55 \\
50 \\
47 \\
48\end{array}$ & $\begin{array}{r}65 \\
1290 \\
242 \\
110 \\
81\end{array}$ \\
\hline $\begin{array}{l}16 \\
17 \\
18 \\
19 \\
20\end{array}$ & $\begin{array}{l}389 \\
378 \\
395 \\
424 \\
339\end{array}$ & $\begin{array}{l}91 \\
93 \\
93 \\
91 \\
92\end{array}$ & $\begin{array}{l}83 \\
85 \\
89 \\
92 \\
96\end{array}$ & $\begin{array}{l}69 \\
67 \\
68 \\
70 \\
63\end{array}$ & $\begin{array}{l}60 \\
54 \\
52 \\
52 \\
52\end{array}$ & $\begin{array}{l}47 \\
47 \\
47 \\
50 \\
48\end{array}$ & $\begin{array}{l}44 \\
43 \\
40 \\
44 \\
46\end{array}$ & $\begin{array}{l}41 \\
39 \\
39 \\
39 \\
38\end{array}$ & $\begin{array}{l}69 \\
51 \\
46 \\
46 \\
45\end{array}$ & $\begin{array}{l}321 \\
292 \\
353 \\
333 \\
321\end{array}$ & $\begin{array}{l}47 \\
48 \\
49 \\
74 \\
76\end{array}$ & $\begin{array}{l}74 \\
72 \\
71 \\
68 \\
66\end{array}$ \\
\hline $\begin{array}{l}21 \\
22 \\
23 \\
24 \\
25\end{array}$ & $\begin{array}{r}145 \\
90 \\
79 \\
76 \\
67\end{array}$ & $\begin{array}{l}91 \\
91 \\
90 \\
88 \\
89\end{array}$ & $\begin{array}{l}87 \\
84 \\
85 \\
86 \\
84\end{array}$ & $\begin{array}{l}65 \\
66 \\
65 \\
65 \\
64\end{array}$ & $\begin{array}{l}56 \\
52 \\
50 \\
52 \\
50\end{array}$ & $\begin{array}{l}45 \\
48 \\
49 \\
51 \\
46\end{array}$ & $\begin{array}{l}46 \\
44 \\
50 \\
47 \\
43\end{array}$ & $\begin{array}{r}34 \\
31 \\
31 \\
201 \\
359\end{array}$ & $\begin{array}{l}43 \\
42 \\
47 \\
47 \\
52\end{array}$ & $\begin{array}{r}309 \\
149 \\
80 \\
60 \\
61\end{array}$ & $\begin{array}{l}60 \\
54 \\
48 \\
51 \\
97\end{array}$ & $\begin{array}{l}65 \\
63 \\
63 \\
64 \\
66\end{array}$ \\
\hline $\begin{array}{l}26 \\
27 \\
28 \\
29 \\
30 \\
31\end{array}$ & $\begin{array}{l}76 \\
88 \\
84 \\
83 \\
90 \\
87\end{array}$ & $\begin{array}{r}90 \\
90 \\
87 \\
83 \\
86 \\
\end{array}$ & $\begin{array}{l}85 \\
87 \\
85 \\
84 \\
84 \\
83\end{array}$ & $\begin{array}{l}59 \\
60 \\
62 \\
61 \\
61 \\
63\end{array}$ & $\begin{array}{r}48 \\
47 \\
50 \\
48 \\
-- \\
---\end{array}$ & $\begin{array}{l}46 \\
45 \\
43 \\
45 \\
50 \\
54\end{array}$ & $\begin{array}{r}43 \\
49 \\
46 \\
49 \\
46 \\
4 m-\end{array}$ & $\begin{array}{l}365 \\
356 \\
360 \\
348 \\
339 \\
367\end{array}$ & $\begin{array}{r}58 \\
50 \\
57 \\
56 \\
50 \\
\end{array}$ & $\begin{array}{l}62 \\
57 \\
58 \\
60 \\
51 \\
46\end{array}$ & $\begin{array}{r}129 \\
105 \\
124 \\
98 \\
103 \\
203\end{array}$ & $\begin{array}{r}66 \\
66 \\
58 \\
56 \\
72 \\
-\end{array}$ \\
\hline $\begin{array}{l}\text { TOTAI, } \\
\text { MEAN } \\
\text { MAX } \\
\text { MIN } \\
\text { AC-ET }\end{array}$ & $\begin{array}{r}6267 \\
202 \\
424 \\
60 \\
12430\end{array}$ & $\begin{array}{r}2882 \\
96.1 \\
133 \\
83 \\
5720\end{array}$ & $\begin{array}{r}2644 \\
85.3 \\
96 \\
82 \\
5240\end{array}$ & $\begin{array}{r}2277 \\
73.5 \\
99 \\
59 \\
4520\end{array}$ & $\begin{array}{r}1644 \\
56.7 \\
66 \\
47 \\
3260\end{array}$ & $\begin{array}{r}1511 \\
48.7 \\
59 \\
43 \\
3000\end{array}$ & $\begin{array}{r}1431 \\
47.7 \\
59 \\
40 \\
2840\end{array}$ & $\begin{array}{r}3664 \\
118 \\
367 \\
31 \\
7270\end{array}$ & $\begin{array}{r}3121 \\
104 \\
376 \\
42 \\
6190\end{array}$ & $\begin{array}{r}4650 \\
150 \\
353 \\
38 \\
9220\end{array}$ & $\begin{array}{r}2098 \\
67.7 \\
203 \\
45 \\
4160\end{array}$ & $\begin{array}{r}3742 \\
125 \\
1290 \\
56 \\
7420\end{array}$ \\
\hline
\end{tabular}

STATISTICS OF MONTHLY MEAN DATA FOR WATER YEARS 1938 - 1996, BY WATER YEAR (WY)

$\begin{array}{lrrrrrrrrrrrr}\text { MEAN } & 176 & 91.6 & 90.6 & 78.7 & 71.0 & 51.3 & 38.0 & 227 & 174 & 100 & 164 & 271 \\ \text { MAX } & 2718 & 544 & 519 & 359 & 358 & 299 & 149 & 7108 & 3040 & 1184 & 4182 & 7129 \\ \text { (WY) } & 1955 & 1987 & 1992 & 1987 & 1987 & 1987 & 1987 & 1941 & 1941 & 1941 & 1966 & 1941 \\ \text { MIN } & 8.70 & 6.77 & 9.39 & 10.6 & 12.6 & 10.1 & 7.46 & 6.35 & 7.70 & 4.43 & 5.18 & 5.73 \\ \text { (WY) } & 1978 & 1978 & 1978 & 1965 & 1965 & 1978 & 1978 & 1978 & 1971 & 1966 & 1964 & 1977\end{array}$

SUMRARY STATISTICS

ANNUAL, TOTAL

ANNUAI. MEAN

HIGHEST ANHUAD, MEAN

LOWEST ANNUAL MEAN

BIGHEST DAILY MEAN

LOWEST DAILY MEAN

ANNUAL SEVEN-DAY MINIMUM

INSTANTANEOUS PEAK FLOW

INSTANTANEOUS PEAK STAGE

INSTANTANEOUS LOW FLON

ANNUAL RUNOFF (AC-FT)

10 PERCENT EXCEEDS

50 PERCENT EXCEEDS

90 PERCENT EXCEEDS

a-From floodmarks.
FOR 1995 CALENDAR YEAR

$\begin{array}{rrr}33583 & & \\ 92.0 & & \\ & & \\ 426 & \text { Jul } & 25 \\ 29 & \text { May } & 9 \\ 34 & \text { Apr 21 } & \\ & & \\ & & \\ 66610 & & \\ 139 & & \\ 74 & & \\ 36 & & \end{array}$

FOR 1996 WATER YEAR

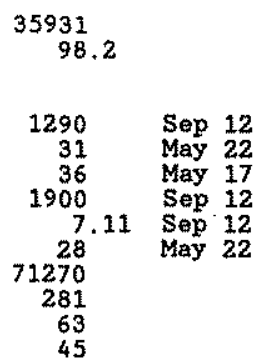

WATER YEARS 1938 - 1996

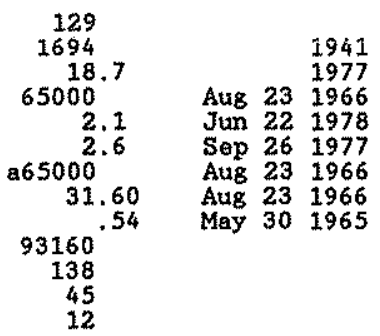


RIO GRANDE BASIN

08407000 PECOS RIVER AT PIERCE CANYON CROSSING, NEAR MALAGA, MM - continued

WATER-QUALTTY RECORDS

LOCATION. --Samples collected $0.2 \mathrm{mi}$ domstroam from streamflow gaging station.

PERIOD OF RECORD. -Water years 1938-41, 1952 to current year.

REMARKS.--No aignificant inflow botween streanflom gaging station and sampling cross-section.

WATER QUALITY DATA, WATER YEAR OCTOBER 1995 TO SEPTEMBER 1996

\begin{tabular}{|c|c|c|c|c|c|c|c|c|c|c|c|c|}
\hline DATE & TIME & $\begin{array}{c}\text { DIS- } \\
\text { CHARGE, } \\
\text { INST, } \\
\text { CUBIC } \\
\text { FEET } \\
\text { PER } \\
\text { SECOND } \\
\text { (00061) }\end{array}$ & $\begin{array}{l}\text { SPE- } \\
\text { CIFIC } \\
\text { CON- } \\
\text { DUCT- } \\
\text { ANCE } \\
\text { (US/CM) } \\
(00095)\end{array}$ & $\begin{array}{c}\text { PB } \\
\text { WATER } \\
\text { WHOLE } \\
\text { FIELD } \\
\text { (STAND- } \\
\text { ARD } \\
\text { UNITS) } \\
(00400)\end{array}$ & $\begin{array}{l}\text { TEMPER- } \\
\text { ATURE } \\
\text { AIR } \\
\text { (DEGC) } \\
(00020)\end{array}$ & $\begin{array}{c}\text { TEMPER- } \\
\text { ATURE } \\
\text { WATER } \\
\text { (DEG C) } \\
(00010)\end{array}$ & $\begin{array}{l}\text { BARO- } \\
\text { METRIC } \\
\text { ERES- } \\
\text { SURE } \\
\text { (MM } \\
\text { OF } \\
\text { HG) } \\
(00025)\end{array}$ & $\begin{array}{c}\text { OXYGEN, } \\
\text { DIS- } \\
\text { SOLVED } \\
\text { (MG/L) } \\
(00300)\end{array}$ & $\begin{array}{c}\text { OXYGEN, } \\
\text { DIS- } \\
\text { SOLVED } \\
\text { (PER- } \\
\text { CENT } \\
\text { SATUR- } \\
\text { ATION) } \\
\text { (00301) }\end{array}$ & $\begin{array}{l}\text { EARD- } \\
\text { NESS } \\
\text { TOTAL } \\
\text { (MG/L } \\
\text { AS } \\
\text { CACO3) } \\
(00900)\end{array}$ & $\begin{array}{l}\text { CALCIUM } \\
\text { DIS- } \\
\text { SOLVED } \\
\text { (MG IL } \\
\text { AS CA) } \\
(00915)\end{array}$ & $\begin{array}{c}\text { MAGN } \\
\text { SIU } \\
\text { DIS } \\
\text { SOLV } \\
(\mathrm{MG} / \\
\text { AS M } \\
(0092\end{array}$ \\
\hline 1995 & 1445 & 86 & 7330 & 8.0 & -- & 14.0 & 685 & 10.4 & 115 & 2100 & 500 & 200 \\
\hline & 1445 & 55 & 8280 & 8.2 & 31.0 & 8.0 & 687 & 10.0 & 96 & 2300 & 540 & 230 \\
\hline $30 \ldots$ & 1200 & 46 & 9160 & 7.9 & 29.0 & 21.0 & 696 & 10.4 & 132 & 2300 & 540 & 230 \\
\hline & 1500 & 46 & 7870 & 8.0 & 41.0 & 29.0 & 686 & 15.7 & 234 & 1800 & 490 & 140 \\
\hline 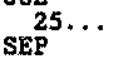 & 1345 & 58 & 5520 & 8.0 & 29.0 & 29.0 & 690 & 12.4 & 182 & 1200 & 330 & 96 \\
\hline & 1030 & 61 & 8080 & 7.9 & 32.0 & 27.0 & 694 & 8.8 & 125 & 1900 & 450 & 190 \\
\hline
\end{tabular}

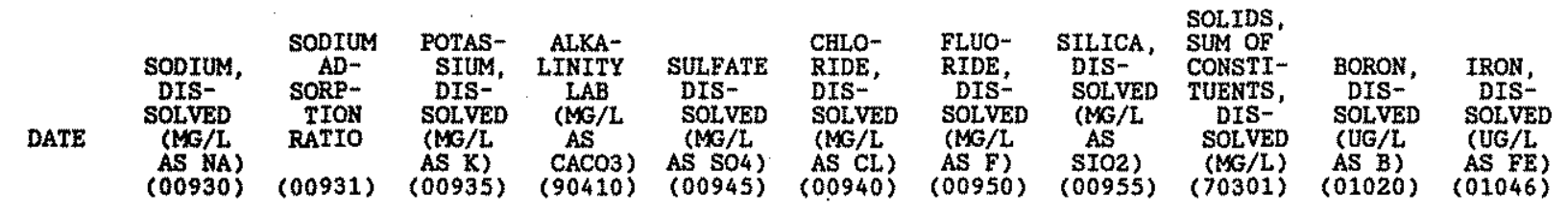

\begin{tabular}{|c|c|c|c|c|c|c|c|c|c|c|c|}
\hline $\begin{array}{l}\operatorname{DxC} 1995 \\
01.966\end{array}$ & 960 & 9 & 12 & 185 & 1500 & 1600 & 0.70 & 13 & 4900 & 410 & $<10$ \\
\hline APR & 1300 & 12 & 33 & 166 & 1900 & 2200 & 0.90 & 5.9 & 6310 & 540 & 40 \\
\hline $30 \ldots$ & 1400 & 13 & 38 & 165 & 1800 & 2300 & 0.90 & 8.5 & 6420 & 580 & $<15$ \\
\hline Ju & 1100 & 11 & 43 & 117 & 1500 & 1800 & 0.80 & 17 & 5160 & 71 & 220 \\
\hline $\operatorname{SEP}_{25} .$. & 770 & 10 & 31 & 123 & 1100 & 1200 & 0.70 & 17 & 3620 & 298 & 52 \\
\hline $10 \ldots$ & 1200 & 12 & 33 & 133 & 1500 & 1900 & 0.70 & 16 & 5370 & 485 & $<15$ \\
\hline
\end{tabular}


08407500 PECOS RIVER AT RED BLUFF, MM

LOCATrON.--Lat $32^{\circ} 04^{\circ} 30^{\prime \prime}$, long $104^{\circ} 02 \cdot 21^{\prime \prime}$, in SW/AWWHE\% sec.1, T.26 S., R. 28 E., Eddy County, Bydrologic Unit 13060011 , on $x$ ight bank at Red Bluff, $0.2 \mathrm{mi}$ downstream from Red Bluff Draw, 1.6 mi northwest of the El Paso Natural Gas (Pecos River) compressor station, $5.2 \mathrm{mi}$ north of the New Mexico-Texas State line, $5.5 \mathrm{mi}$ upstream from Delaware River, and at mile 411.2 .

DRAINAGE AREA. $--19,540 \mathrm{mi}^{2}$, approximately (contributing area).

PERIOD OF RECORD. --October 1937 to current year.

GAGE. -Water-\$tege rocorder. Elovation of gage is 2,850,05 ft above National Geodetic Vertical Datum of 1929.

REMARKS. --Discharge records good. Flow regulated by many reservoirs and diversion dams, Diversions and groundwater withdrawals upsticeam from station for irrigation of about 202,000 acres, 1959 determination. Several observations of pater temperature were made during the year.

EXTREMES OUTSIDE PERIOD OF RECORD.-Flood in October 1904 reached a stage of 28.0 ft, from information by Panhandle and Santa Fe Railway Co. (For dates of other historical floods see stations 08404000,08406500 .)

DISCEARGE, CUBIC FEET PER SECOND, WATER YEAR OCTOBER 1995 TO SEPTEMBER 1996

\begin{tabular}{|c|c|c|c|c|c|c|c|c|c|c|c|c|}
\hline DAY & oCr & Nov & DEC & JAN & FEB & MAR & APR & MAY & Jự & JuL & AUG & SEP \\
\hline $\begin{array}{l}1 \\
2 \\
3 \\
4 \\
5\end{array}$ & $\begin{array}{l}62 \\
72 \\
71 \\
71 \\
69\end{array}$ & $\begin{array}{r}93 \\
143 \\
153 \\
132 \\
108\end{array}$ & $\begin{array}{l}88 \\
89 \\
89 \\
90 \\
87\end{array}$ & $\begin{array}{r}99 \\
102 \\
107 \\
105 \\
95\end{array}$ & $\begin{array}{l}74 \\
66 \\
62 \\
67 \\
69\end{array}$ & $\begin{array}{r}51 \\
50 \\
51 \\
51 \\
51\end{array}$ & $\begin{array}{l}58 \\
54 \\
52 \\
52 \\
62\end{array}$ & $\begin{array}{l}48 \\
57 \\
56 \\
50 \\
57\end{array}$ & $\begin{array}{l}353 \\
347 \\
340 \\
336 \\
320\end{array}$ & $\begin{array}{l}55 \\
51 \\
49 \\
51 \\
50\end{array}$ & $\begin{array}{l}52 \\
49 \\
50 \\
47 \\
48\end{array}$ & $\begin{array}{r}409 \\
186 \\
90 \\
66 \\
64\end{array}$ \\
\hline $\begin{array}{r}6 \\
7 \\
8 \\
9 \\
10\end{array}$ & $\begin{array}{r}72 \\
58 \\
86 \\
361 \\
404\end{array}$ & $\begin{array}{r}105 \\
96 \\
96 \\
91 \\
90\end{array}$ & $\begin{array}{l}86 \\
85 \\
88 \\
86 \\
87\end{array}$ & $\begin{array}{r}91 \\
97 \\
109 \\
94 \\
88\end{array}$ & $\begin{array}{l}71 \\
73 \\
71 \\
64 \\
61\end{array}$ & $\begin{array}{l}52 \\
52 \\
54 \\
52 \\
63\end{array}$ & $\begin{array}{l}71 \\
70 \\
66 \\
58 \\
57\end{array}$ & $\begin{array}{l}60 \\
50 \\
44 \\
40 \\
37\end{array}$ & $\begin{array}{r}217 \\
107 \\
70 \\
58 \\
52\end{array}$ & $\begin{array}{l}50 \\
47 \\
45 \\
42 \\
94\end{array}$ & $\begin{array}{l}48 \\
51 \\
47 \\
45 \\
48\end{array}$ & $\begin{array}{l}58 \\
55 \\
69 \\
55 \\
58\end{array}$ \\
\hline $\begin{array}{l}11 \\
12 \\
13 \\
14 \\
15\end{array}$ & $\begin{array}{l}389 \\
394 \\
410 \\
418 \\
420\end{array}$ & $\begin{array}{l}91 \\
88 \\
82 \\
86 \\
89\end{array}$ & $\begin{array}{l}88 \\
89 \\
90 \\
92 \\
90\end{array}$ & $\begin{array}{l}88 \\
80 \\
76 \\
74 \\
74\end{array}$ & $\begin{array}{l}64 \\
68 \\
67 \\
65 \\
66\end{array}$ & $\begin{array}{l}69 \\
60 \\
61 \\
54 \\
52\end{array}$ & $\begin{array}{l}59 \\
53 \\
53 \\
51 \\
49\end{array}$ & $\begin{array}{l}37 \\
42 \\
40 \\
37 \\
37\end{array}$ & $\begin{array}{l}49 \\
47 \\
46 \\
55 \\
79\end{array}$ & $\begin{array}{l}326 \\
339 \\
334 \\
318 \\
316\end{array}$ & $\begin{array}{l}52 \\
48 \\
52 \\
42 \\
41\end{array}$ & $\begin{array}{r}84 \\
632 \\
625 \\
183 \\
108\end{array}$ \\
\hline $\begin{array}{l}16 \\
17 \\
18 \\
19 \\
20\end{array}$ & $\begin{array}{l}415 \\
402 \\
405 \\
427 \\
424\end{array}$ & $\begin{array}{l}89 \\
90 \\
91 \\
91 \\
91\end{array}$ & $\begin{array}{r}87 \\
90 \\
95 \\
96 \\
101\end{array}$ & $\begin{array}{l}74 \\
71 \\
69 \\
74 \\
69\end{array}$ & $\begin{array}{l}67 \\
61 \\
55 \\
54 \\
55\end{array}$ & $\begin{array}{l}52 \\
51 \\
50 \\
53 \\
55\end{array}$ & $\begin{array}{l}49 \\
48 \\
43 \\
45 \\
50\end{array}$ & $\begin{array}{l}40 \\
38 \\
36 \\
36 \\
36\end{array}$ & $\begin{array}{l}91 \\
63 \\
51 \\
49 \\
47\end{array}$ & $\begin{array}{l}346 \\
324 \\
350 \\
366 \\
341\end{array}$ & $\begin{array}{r}40 \\
41 \\
42 \\
60 \\
165\end{array}$ & $\begin{array}{l}86 \\
78 \\
77 \\
71 \\
68\end{array}$ \\
\hline $\begin{array}{l}21 \\
22 \\
23 \\
24 \\
25\end{array}$ & $\begin{array}{r}246 \\
121 \\
87 \\
84 \\
73\end{array}$ & $\begin{array}{l}90 \\
90 \\
90 \\
89 \\
89\end{array}$ & $\begin{array}{l}99 \\
92 \\
93 \\
94 \\
93\end{array}$ & $\begin{array}{l}66 \\
70 \\
69 \\
67 \\
69\end{array}$ & $\begin{array}{l}58 \\
58 \\
54 \\
56 \\
57\end{array}$ & $\begin{array}{l}50 \\
50 \\
55 \\
55 \\
55\end{array}$ & $\begin{array}{l}51 \\
49 \\
50 \\
56 \\
49\end{array}$ & $\begin{array}{r}33 \\
30 \\
29 \\
72 \\
310\end{array}$ & $\begin{array}{l}45 \\
42 \\
47 \\
50 \\
51\end{array}$ & $\begin{array}{r}355 \\
253 \\
127 \\
83 \\
78\end{array}$ & $\begin{array}{l}59 \\
53 \\
44 \\
44 \\
60\end{array}$ & $\begin{array}{l}65 \\
64 \\
62 \\
62 \\
65\end{array}$ \\
\hline $\begin{array}{l}26 \\
27 \\
28 \\
29 \\
30 \\
31\end{array}$ & $\begin{array}{l}70 \\
89 \\
86 \\
84 \\
88 \\
89\end{array}$ & $\begin{array}{r}90 \\
90 \\
89 \\
85 \\
86 \\
-\end{array}$ & $\begin{array}{l}92 \\
95 \\
94 \\
93 \\
93 \\
91\end{array}$ & $\begin{array}{l}64 \\
61 \\
68 \\
66 \\
67 \\
64\end{array}$ & $\begin{array}{r}53 \\
51 \\
52 \\
55 \\
- \\
-\end{array}$ & $\begin{array}{l}47 \\
53 \\
46 \\
48 \\
52 \\
60\end{array}$ & $\begin{array}{r}45 \\
51 \\
53 \\
50 \\
52 \\
--\end{array}$ & $\begin{array}{l}340 \\
336 \\
336 \\
335 \\
321 \\
344\end{array}$ & $\begin{array}{r}65 \\
60 \\
58 \\
71 \\
56 \\
-\end{array}$ & $\begin{array}{l}75 \\
71 \\
68 \\
72 \\
64 \\
56\end{array}$ & $\begin{array}{r}135 \\
178 \\
134 \\
114 \\
95 \\
191\end{array}$ & $\begin{array}{r}66 \\
68 \\
61 \\
53 \\
64 \\
--\end{array}$ \\
\hline $\begin{array}{l}\text { TOTAL } \\
\text { MEAAI } \\
\text { MAX } \\
\text { MIN } \\
\text { AC-ET }\end{array}$ & $\begin{array}{r}6547 \\
211 \\
427 \\
58 \\
12990\end{array}$ & $\begin{array}{r}2883 \\
96.1 \\
153 \\
82 \\
5720\end{array}$ & $\begin{array}{r}2822 \\
91.0 \\
101 \\
85 \\
5600\end{array}$ & $\begin{array}{r}2467 \\
79.6 \\
109 \\
61 \\
4890\end{array}$ & $\begin{array}{r}1794 \\
61.9 \\
74 \\
51 \\
3560\end{array}$ & $\begin{array}{r}1655 \\
53.4 \\
69 \\
46 \\
3280\end{array}$ & $\begin{array}{r}1606 \\
53.5 \\
71 \\
43 \\
31.90\end{array}$ & $\begin{array}{r}3364 \\
109 \\
344 \\
29 \\
6670\end{array}$ & $\begin{array}{r}3322 \\
111 \\
353 \\
42 \\
6590\end{array}$ & $\begin{array}{r}5196 \\
168 \\
366 \\
42 \\
10310\end{array}$ & $\begin{array}{r}2175 \\
70.2 \\
191 \\
40 \\
4310\end{array}$ & $\begin{array}{r}3752 \\
125 \\
632 \\
53 \\
7440\end{array}$ \\
\hline
\end{tabular}

STATISTICS OF WOHTHLY MEAN DATA FOR WATER YRARS 1938 - 1996, DY WATER YEAR (WN)

\begin{tabular}{|c|c|c|c|c|c|c|c|c|c|}
\hline $\begin{array}{l}\text { MEAN } \\
\text { MAX } \\
\text { (WY) } \\
\text { MIN }\end{array}$ & $\begin{array}{r}277 \\
5255 \\
1942 \\
10.0\end{array}$ & $\begin{array}{r}149 \\
1382 \\
1942 \\
6.71\end{array}$ & $\begin{array}{r}126 \\
813 \\
1942 \\
8.57\end{array}$ & $\begin{array}{r}114 \\
703 \\
1942 \\
10.7\end{array}$ & $\begin{array}{r}98.1 \\
534 \\
1942 \\
13.7\end{array}$ & $\begin{array}{r}70.5 \\
295 \\
1942 \\
7.76\end{array}$ & $\begin{array}{l}184 \\
3181 \\
1941 \\
4.30\end{array}$ & $\begin{array}{r}114 \\
1273 \\
1941\end{array}$ & $\begin{array}{r}289 \\
6521 \\
1941 \\
5.77\end{array}$ \\
\hline
\end{tabular}

SUMARY STATISTICS

ANNUAL TOTAL.

ANNUAL MEAN

HICHEST ANNUAL, MEAN

LOWEST ANNUAL MEAN

HIGHEST DAILY MEAN

LOWEST DAILY MEAN

ANNUAL SEVEN-DAY MINIMIM

INSTANTANEOUS PEAK FLOW

INSTANTANEOUS PEAK STAGE

INSTANTANEOUS LOW FLOW

ANNUAL RUNOFF (AC-FT)

10 PERCENT EXCEEDS

50 PERCENT EXCEEDS

90 PERCENT EXCEEDS
FOR 1995 CALENDAR YEAR
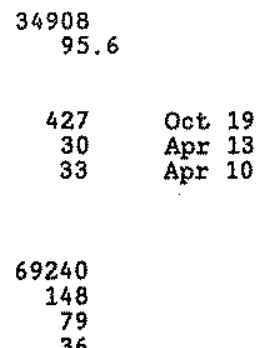

FOR 1996 WATER YEAR

37583
103

a-From rating curve extended above $32,000 \mathrm{ft} / \mathrm{s}$, on basis of slope-area measurement of peak flow.
WATER YEARS 1938 - 1996

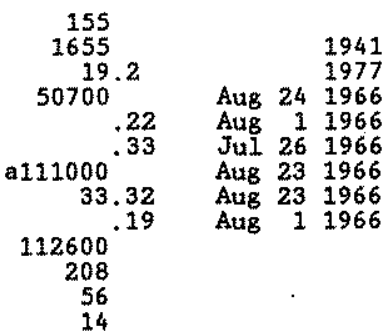

$P$.

96

58

55

84

83

86

77

68

64

53

64

752

125
632

440 
RIO GRANDE BASIN

08408500 DELAWARE RIVER NEAR RED BLUFF, NM

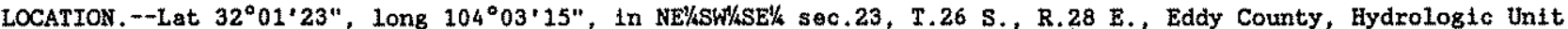
13070002 near center of channel on downstream side of pier of bridge on $\mathrm{V} . \mathrm{S}$. Highway 285 , 2.1 mi north of the New Mextco-Texas State 1ine, $3.6 \mathrm{mi}$ southwest of Red Bluff, $3.7 \mathrm{mi}$ upstream from mouth and $14 \mathrm{mi}$ south of Malaga. Mouth at Pecos River mile 405.6

DRAINAGE AREA. $--689 \mathrm{mi}^{2}$.

PERIOD OF RECORD.--Aprt1 1912 to September 1913, May 1914 to June 1915, October 1937 to current year. Pub1ished as "near Malaga" 1912-13, and as "near Angeles, Tex." 1914-15.

GAGE - Water-stage recorder Elevation of gage is 2,900.66 ft above National Geodetic Vertical Datum of 1929 (U.S. Boundary Commission post). Prior to May 1914 , at site $3,0 \mathrm{mi}$ upstrean at different datum. May 1914 to June 1915, at site $2.5 \mathrm{mi}$ downstrean at different datum.

REMARKS.--Records good except for estimated daily discharges, and those above 100 ft3/s, which are poor. One small upstream diversion. Several observations of water temperature were made during the year. No flow for many days most years.

DISCHARGE, CUBIC FEET PER SECOND, WATER YEAR OCTOBER 1995 TO SEPTEMBER 1996

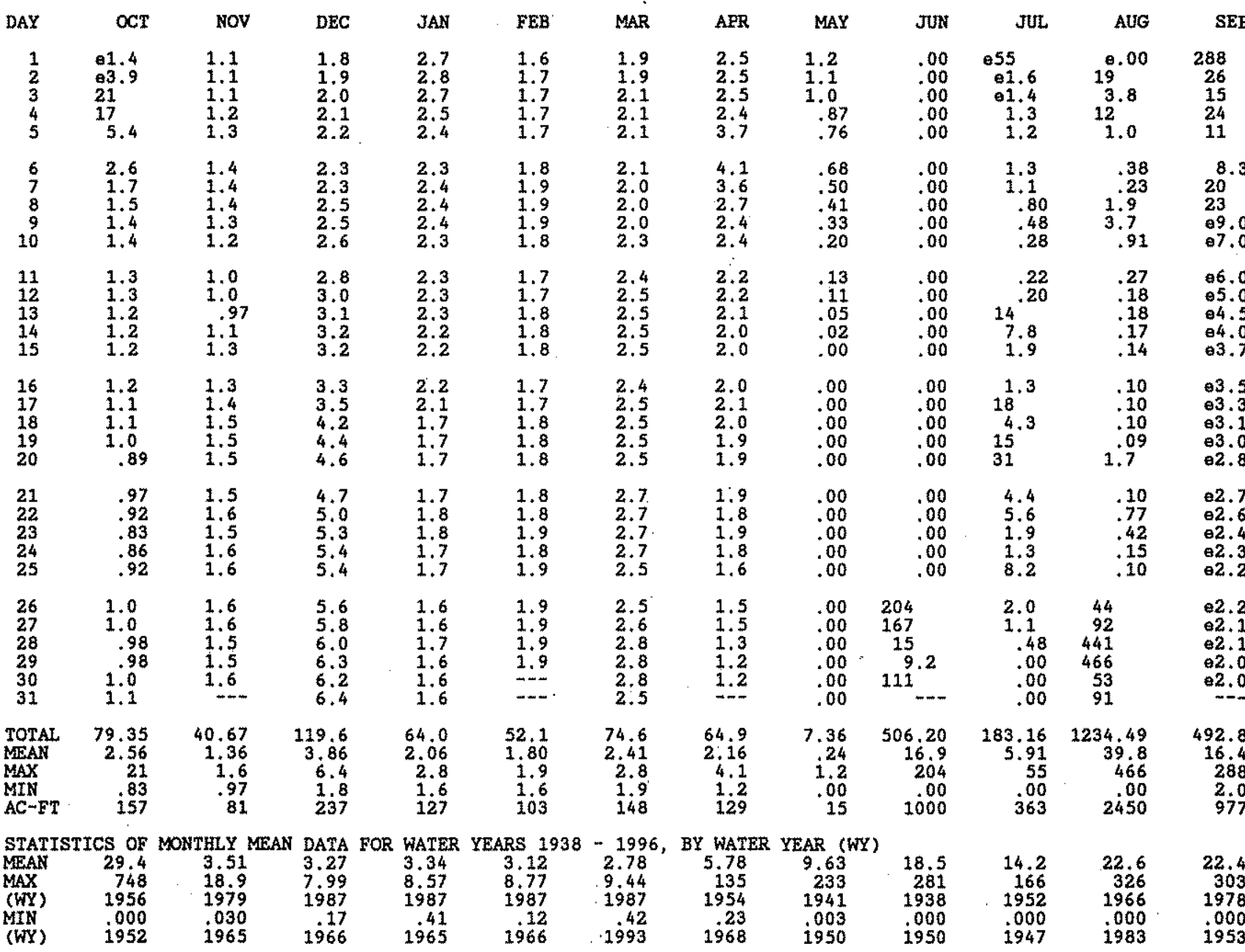

SUMMARY STATISTICS

ANNUAL TOTAL

ANNUAL MEAN

HIGKEST ANNUAL MEAN

LOWEST ANNUAL MEAN

HTGHEST DATIY MEAN

OOTEST DATIY MAN

ANNUAL SEVEN-DAY MINIMUM

INSTANTANEOUS PEAK FLOW

INSTANTANEOUS PEAK STAGE

INSTANTANEOUS LOW FLOW

ANNUAL RUNOFF (AC-FT)

10 BERCENT EXCEEDS

50 PERCENT EXCEEDS

90 PERCENT EXCEEDS

- Estimated

FOR 1995 CALENDAR YEAR

991.25

110 .00 Sep 11

1970

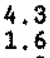

FOR 1996 WATER YEAR 2919.23

$$
\begin{array}{r}
919.23 \\
7.98
\end{array}
$$

466 Aug 29

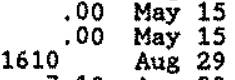

7.19 Aug 29
.00 May 14

$5790^{\circ}$

6.0

1.8
WATER YEARS 1938 - 1996

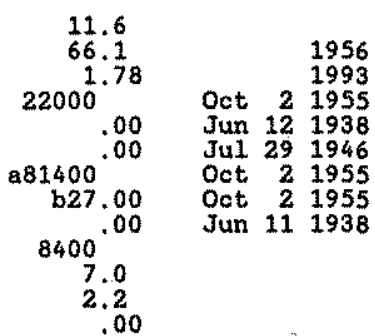

a-From rating curve extended above $6,500 \mathrm{ft} / \mathrm{s}$, on basts of slope-area measurements at 8 age heights, 12.84 ft, $17.55 \mathrm{ft}$, and $27.0 \mathrm{ft}$.

b-From floodmarks. 


\section{RIO GRANDE BASIN}

08410000 RED BLUFE RESERVOTR NEAR ORLA, TX

LOCATION,-Lat $31^{\circ} 54^{\prime} 04^{\prime \prime}$, Long $103^{\circ} 54^{\prime 3} 3$ ", Reeves County, Hydrologic Untt 13070001, at right ond of Red Blufe Dam

on the Pecos River, $2.8 \mathrm{mi}$ upstream from Salt Creek, and $5.2 \mathrm{mi}$ north of Orle.

DRAINAGE AREA. $-20,720 \mathrm{mi}^{2}$, approximately (contributing area).

PERIOD OF RECORD.-February 1937 to current year: Monthly contents only for some periods, published in WSP 1312.

GAGE.--Nonrecording gage. Datum of gage is $0.43 \mathrm{ft}$ below National Geodetic Vertical Datum of 1929 .

REMARKS.-Reservoir is formed by a rock-faced earthfill dam 9,200 ft long. The dam was comploted and storage began in September 1936. The dam and reservolr are omed and operated by the Red Bluff Watar Power Control Distx \pm ct.

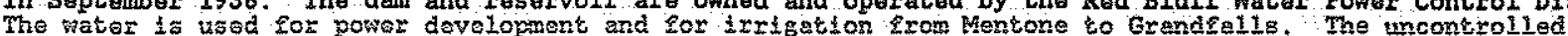
energency spiliway, $790 \mathrm{ft}$ wide, is a cut through natural ground located to the right of $x+g h t$ end of dam. The controlled service spillway is equipped with 12 tainter gates that are 25 by is ft high. Inflow is regulated by many reservoirs and diversion dams. The capacity curve is based on Geological survey topographic map and eerial photography, survey of 1986 . Figures given herein represent total contentis. Data regarding the dam and reservoir are given in the following table:

Gage haight

Capactty

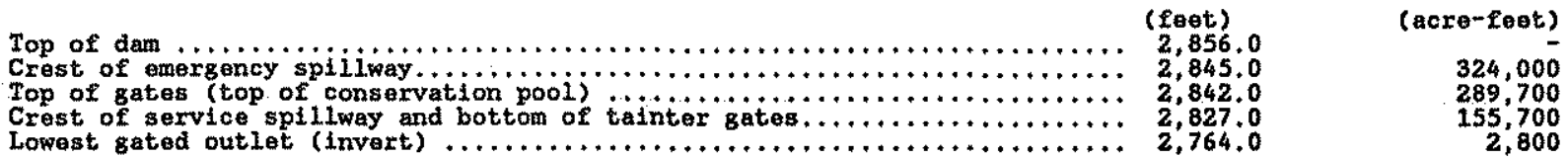

COOPERATION.--Gage-height records and capacity curve were furnished by Red Bluff Water Power and Control District.

EXTREMES FOR PERTOD OF RECORD.-Maximum contents observed, 352,000 acre-rt, Sept. 27, 28, 1941, gage hetght,

$2,846.2 \mathrm{ft}$, observed on nonrecording gage at service spillway (affected by variabie drawdown due to flow through tainter gates); minimum observed, 11,080 acreift, May 13, 1948, gage height, 2,781.4 ft.

EXTREMES (AT 0800) FOR CURRENT YEAR,--Maximum contents observed, 77,520 acre-ft, Apr. 10-12, gage he1ght, 2,812.82 ft; minimum observed, 56,690 acre-ft, Aug. 25, gage height, 2,807.31 ft.

Capacity table (gage height, in feet, and total contents, in acre-feet)

$\begin{array}{llllll}2,806.0 & 52,460 & 2,812.0 & 74,090 & 2,820.0 & 112,200 \\ 2,808.0 & 59,000 & 2,814.0 & 82,630 & 2,822.0 & 123,600 \\ 2,810.0 & 66,220 & 2,816.0 & 91,830 & 2,818,0 & 101,700\end{array}$

RESERVOIR STORAGE (ACRE-FEET), WATER YEAR OCTOBER 1995 TO SEPTEMBER 1996 DAILY OBSERVATION AT 08:00 VALUUES

\begin{tabular}{|c|c|c|c|c|c|c|c|c|c|c|c|c|}
\hline DAY & OCT & rov & DEC & JAN & FEB & MAR & APR & MAY & JUN & JUL & AUE & SEP \\
\hline $\begin{array}{l}1 \\
2 \\
3 \\
4 \\
5\end{array}$ & $\begin{array}{l}60060 \\
60410 \\
60480 \\
60550 \\
60910\end{array}$ & $\begin{array}{l}67570 \\
67600 \\
67600 \\
67570 \\
67570\end{array}$ & $\begin{array}{l}68880 \\
68990 \\
69110 \\
69260 \\
69380\end{array}$ & $\begin{array}{l}72650 \\
72800 \\
72930 \\
73050 \\
73170\end{array}$ & $\begin{array}{l}75470 \\
75510 \\
75600 \\
75680 \\
75770\end{array}$ & $\begin{array}{l}77020 \\
77020 \\
77060 \\
77060 \\
77100\end{array}$ & $\begin{array}{l}76730 \\
76730 \\
76730 \\
76770 \\
76850\end{array}$ & $\begin{array}{l}75680 \\
75350 \\
74510 \\
74010 \\
73530\end{array}$ & $\begin{array}{l}66290 \\
66370 \\
66450 \\
66370 \\
66290\end{array}$ & $\begin{array}{l}63300 \\
63340 \\
63380 \\
63380 \\
63340\end{array}$ & $\begin{array}{l}58370 \\
58030 \\
57700 \\
57530 \\
57390\end{array}$ & $\begin{array}{l}59710 \\
60770 \\
61120 \\
61220 \\
61300\end{array}$ \\
\hline $\begin{array}{r}6 \\
7 \\
8 \\
9 \\
10\end{array}$ & $\begin{array}{l}61330 \\
61370 \\
61400 \\
61440 \\
61400\end{array}$ & $\begin{array}{l}67600 \\
67570 \\
67530 \\
67450 \\
67370\end{array}$ & $\begin{array}{l}69490 \\
69610 \\
69720 \\
69840 \\
69910\end{array}$ & $\begin{array}{l}73290 \\
73410 \\
73530 \\
73690 \\
73850\end{array}$ & $\begin{array}{l}75850 \\
75890 \\
75930 \\
76010 \\
76100\end{array}$ & $\begin{array}{l}77100 \\
77100 \\
77150 \\
77150 \\
77150\end{array}$ & $\begin{array}{l}77020 \\
77190 \\
77360 \\
77440 \\
77520\end{array}$ & $\begin{array}{l}73050 \\
72560 \\
72080 \\
71600 \\
71120\end{array}$ & $\begin{array}{l}66220 \\
66070 \\
65700 \\
65180 \\
64740\end{array}$ & $\begin{array}{l}63300 \\
63270 \\
62750 \\
62180 \\
61610\end{array}$ & $\begin{array}{l}57060 \\
57030 \\
56990 \\
56960 \\
56930\end{array}$ & $\begin{array}{l}61370 \\
61440 \\
61510 \\
61580 \\
61650\end{array}$ \\
\hline $\begin{array}{l}11 \\
12 \\
13 \\
14 \\
15\end{array}$ & $\begin{array}{l}61580 \\
61790 \\
62350 \\
63190 \\
63710\end{array}$ & $\begin{array}{l}67290 \\
67220 \\
67140 \\
67060 \\
67060\end{array}$ & $\begin{array}{l}69990 \\
70070 \\
70150 \\
70270 \\
70390\end{array}$ & $\begin{array}{l}74010 \\
74130 \\
74260 \\
74380 \\
74510\end{array}$ & $\begin{array}{l}76180 \\
76270 \\
76350 \\
76430 \\
76520\end{array}$ & $\begin{array}{l}77190 \\
77190 \\
77190 \\
77230 \\
76810\end{array}$ & $\begin{array}{l}77520 \\
77520 \\
77480 \\
77480 \\
77440\end{array}$ & $\begin{array}{l}70630 \\
70150 \\
69720 \\
69300 \\
68880\end{array}$ & $\begin{array}{l}64150 \\
63640 \\
63190 \\
62970 \\
63190\end{array}$ & $\begin{array}{l}61050 \\
60770 \\
60730 \\
60700 \\
60660\end{array}$ & $\begin{array}{l}56990 \\
56990 \\
56990 \\
56990 \\
56960\end{array}$ & $\begin{array}{l}61720 \\
62240 \\
63340 \\
64370 \\
64740\end{array}$ \\
\hline $\begin{array}{l}16 \\
17 \\
18 \\
19 \\
20\end{array}$ & $\begin{array}{l}64220 \\
64890 \\
65550 \\
66220 \\
66870\end{array}$ & $\begin{array}{l}67140 \\
67260 \\
67370 \\
67490 \\
67600\end{array}$ & $\begin{array}{l}70510 \\
70630 \\
70790 \\
70950 \\
71080\end{array}$ & $\begin{array}{l}74640 \\
74760 \\
74800 \\
74840 \\
74890\end{array}$ & $\begin{array}{l}76600 \\
76640 \\
76690 \\
76730 \\
76770\end{array}$ & $\begin{array}{l}76770 \\
76730 \\
76730 \\
76690 \\
76690\end{array}$ & $\begin{array}{l}77440 \\
77400 \\
77400 \\
77360 \\
77360\end{array}$ & $\begin{array}{l}68450 \\
68060 \\
67680 \\
67290 \\
66910\end{array}$ & $\begin{array}{l}62820 \\
62460 \\
62490 \\
62530 \\
62460\end{array}$ & $\begin{array}{l}61300 \\
61300 \\
61260 \\
61220 \\
61190\end{array}$ & $\begin{array}{l}30 \\
30 \\
90 \\
90 \\
60\end{array}$ & $\begin{array}{l}64850 \\
64960 \\
65070 \\
65180 \\
65260\end{array}$ \\
\hline $\begin{array}{l}21 \\
22 \\
23 \\
24 \\
25\end{array}$ & $\begin{array}{l}67290 \\
67330 \\
67180 \\
67060 \\
66870\end{array}$ & $\begin{array}{l}67720 \\
67830 \\
67950 \\
68060 \\
68180\end{array}$ & $\begin{array}{l}71240 \\
71360 \\
71520 \\
71680 \\
71830\end{array}$ & $\begin{array}{l}74930 \\
74970 \\
75010 \\
75050 \\
75140\end{array}$ & $\begin{array}{l}76810 \\
76850 \\
76850 \\
76900 \\
76900\end{array}$ & $\begin{array}{l}76690 \\
76730 \\
76730 \\
76690 \\
76690\end{array}$ & $\begin{array}{l}77310 \\
77310 \\
77270 \\
77270 \\
77230\end{array}$ & $\begin{array}{l}66520 \\
66140 \\
65770 \\
65400 \\
65480\end{array}$ & $\begin{array}{l}62350 \\
62240 \\
62140 \\
61960 \\
62310\end{array}$ & $\begin{array}{l}61150 \\
61120 \\
60840 \\
60590 \\
60380\end{array}$ & $\begin{array}{l}56960 \\
56890 \\
56830 \\
56760 \\
56690\end{array}$ & $\begin{array}{l}65290 \\
65330 \\
65370 \\
65400 \\
65290\end{array}$ \\
\hline $\begin{array}{l}26 \\
27 \\
28 \\
29 \\
30 \\
31\end{array}$ & $\begin{array}{l}66910 \\
66990 \\
57060 \\
67180 \\
67290 \\
67490\end{array}$ & $\begin{array}{r}68300 \\
68410 \\
68530 \\
68640 \\
68760 \\
\end{array}$ & $\begin{array}{l}72000 \\
72160 \\
72320 \\
72440 \\
72560 \\
72680\end{array}$ & $\begin{array}{l}75220 \\
75260 \\
75310 \\
75350 \\
75390 \\
75430\end{array}$ & $\begin{array}{r}76940 \\
76940 \\
76900 \\
76980 \\
-\end{array}$ & $\begin{array}{l}76690 \\
76690 \\
76690 \\
76690 \\
76690 \\
76690\end{array}$ & $\begin{array}{r}77230 \\
76940 \\
76690 \\
76350 \\
76010 \\
=\end{array}$ & $\begin{array}{l}65590 \\
65740 \\
65850 \\
65990 \\
66140\end{array}$ & $\begin{array}{l}62710 \\
62900 \\
63080 \\
63150 \\
62900 \\
\end{array}$ & $\begin{array}{l}60170 \\
59920 \\
59610 \\
59290 \\
58970 \\
58670\end{array}$ & $\begin{array}{l}56790 \\
56990 \\
57450 \\
58170 \\
59010 \\
59360\end{array}$ & $\begin{array}{r}65110 \\
64920 \\
54740 \\
64560 \\
64440 \\
\end{array}$ \\
\hline $\begin{array}{c}\text { MAX } \\
\text { MIN } \\
(f) \\
(\dagger \dagger)\end{array}$ & $\begin{array}{r}67490 \\
60060 \\
2810.33 \\
+7530\end{array}$ & $\begin{array}{r}68760 \\
67060 \\
2810.66 \\
+1270\end{array}$ & $\begin{array}{r}72680 \\
68880 \\
2811.65 \\
+3920\end{array}$ & $\begin{array}{r}75430 \\
72650 \\
2812.32 \\
+2750\end{array}$ & $\begin{array}{r}76980 \\
75470 \\
2812.69 \\
+1550\end{array}$ & $\begin{array}{r}77230 \\
76690 \\
2812.62 \\
-290\end{array}$ & $\begin{array}{r}77520 \\
76010 \\
2812.46 \\
-680\end{array}$ & $\begin{array}{r}75680 \\
65400 \\
2810,00 \\
-9790\end{array}$ & $\begin{array}{r}66450 \\
61960 \\
2809.10 \\
-3320\end{array}$ & $\begin{array}{r}63380 \\
58670 \\
2807.90 \\
-4230\end{array}$ & $\begin{array}{r}59360 \\
56690 \\
2808.10 \\
+690\end{array}$ & $\begin{array}{r}65400 \\
59710 \\
2809.52 \\
+5080\end{array}$ \\
\hline
\end{tabular}

CAL YR $1995 \operatorname{MAX} 90380$ MIN 56120 (†t) -8900

WTR YR 1996 MAX 77520 MIN 56690 (†) +4480

(†) GAGE HETGHT, IN FEET, AT END OF MONTH.

(†) CHANGE IN CONTENTS, IN ACRE-FEET 
RIO GRANDE BASIN

08412500 PECOS RIVER NEAR ORLA. TX

LOCATION.--Lat $31^{\circ} 52^{\prime} 21^{\prime \prime}$. Iong $103^{\circ} 49^{\circ} 52^{\prime \prime}$, Reeves County, Eydrologic Unit 1300001, on right bank at bridge on Farm Road $652,5.5 \mathrm{mi}$ downstream form Salt Creek (Screw Bean Arroyo), $5.9 \mathrm{mi}$ northeast of Orla, and $8.5 \mathrm{mi}$ downstream from Red Bluff Reservoir.

DRAINAGE AREA. $--21,210 \mathrm{mi}$ approximately (contributing area).

WATER DISCEARGE RECORDS

PERTOD OF RECORD. - May 1937 to current year.

REVISED RECORDS, -WSP 928: 1937.

GAGE.-Water-stage recorder. Datum of gage is $2,730.86 \mathrm{ft}$ above National Geodetic Vertical Datum of 1929 . Prior to Nov. 16,1969 , at site $6.9 \mathrm{mi}$ downstream at datum $12.81 \mathrm{ft}$ lower.

REMARKS.-Water-discharge records good. Most of flow is releases from storage in Red Bluff Reservoir (station $08410000) 8.5$ mi upstream. Occastonal runoff occurs from draws between dam and station. There are many diversions above Red Bluff Reservoir for irrigation.

DISCHARGE, CUBIC FEET PER SECOND, WATER YEAR OCTOBER 1995 TO SEPTEMBER 1996 DAILY MEAN VALUES

\begin{tabular}{|c|c|c|c|c|c|c|c|c|c|c|c|c|}
\hline DAY & OCT & Nov & $\mathrm{DEC}$ & JAN & FEB & MAR & APR & MAY & JUN & JUL & AUG & SEP \\
\hline $\begin{array}{l}1 \\
2 \\
3 \\
4 \\
5\end{array}$ & $\begin{array}{l}11 \\
28 \\
13 \\
9.8 \\
9.1\end{array}$ & $\begin{array}{l}9^{9} \cdot 4 \\
109 \\
115 \\
11.4\end{array}$ & $\begin{array}{l}8.6 \\
9.8 \\
9.1 \\
9.2 \\
7.0\end{array}$ & $\begin{array}{l}11 \\
11 \\
11 \\
12 \\
10\end{array}$ & $\begin{array}{l}12 \\
15 \\
10 \\
10 \\
10\end{array}$ & $\begin{array}{l}7.4 \\
6.4 \\
4.3 \\
5.6 \\
6.1\end{array}$ & $\begin{array}{r}7.8 \\
8.0 \\
8.0 \\
7.4 \\
1.1\end{array}$ & $\begin{array}{l}223 \\
255 \\
304 \\
224 \\
227\end{array}$ & $\begin{array}{l}244 \\
245 \\
246 \\
246 \\
246\end{array}$ & $\begin{array}{l}15 \\
14 \\
13 \\
13 \\
12\end{array}$ & $\begin{array}{l}174 \\
174 \\
174 \\
176 \\
176\end{array}$ & $\begin{array}{l}9.1 \\
8.4 \\
8.6 \\
8.5 \\
8.1\end{array}$ \\
\hline $\begin{array}{r}6 \\
7 \\
8 \\
9 \\
10\end{array}$ & $\begin{array}{r}9.4 \\
9.7 \\
9.8 \\
10 \\
101\end{array}$ & $\begin{array}{r}22 \\
110 \\
118 \\
119 \\
120\end{array}$ & $\begin{array}{r}6.5 \\
5.9 \\
6.0 \\
16 \\
9.7\end{array}$ & $\begin{array}{l}11 \\
11 \\
11 \\
11 \\
11\end{array}$ & $\begin{array}{l}10 \\
11 \\
11 \\
11 \\
11\end{array}$ & $\begin{array}{l}6.1 \\
6.0 \\
5.4 \\
5.8 \\
6.9\end{array}$ & $\begin{array}{l}8.5 \\
9.2 \\
9.9 \\
10 \\
10\end{array}$ & $\begin{array}{l}286 \\
289 \\
290 \\
289 \\
264\end{array}$ & $\begin{array}{l}246 \\
246 \\
246 \\
246 \\
246\end{array}$ & $\begin{array}{r}11 \\
20 \\
267 \\
288 \\
289\end{array}$ & $\begin{array}{r}122 \\
43 \\
42 \\
267 \\
105\end{array}$ & $\begin{array}{l}8.0 \\
7.8 \\
7.6 \\
29 \\
20\end{array}$ \\
\hline $\begin{array}{l}11 \\
12 \\
13 \\
14 \\
15\end{array}$ & $\begin{array}{r}106 \\
107 \\
90 \\
12 \\
9.5\end{array}$ & $\begin{array}{r}122 \\
122 \\
103 \\
59 \\
17\end{array}$ & $\begin{array}{l}9.5 \\
9.5 \\
9.6 \\
9.6 \\
9.7\end{array}$ & $\begin{array}{l}11 \\
10 \\
11 \\
10 \\
10\end{array}$ & $\begin{array}{l}10 \\
10 \\
10 \\
11 \\
10\end{array}$ & $\begin{array}{r}7.4 \\
7.4 \\
7.4 \\
127 \\
195\end{array}$ & $\begin{array}{l}9.9 \\
9.5 \\
9.7 \\
8.7 \\
8.4\end{array}$ & $\begin{array}{l}200 \\
200 \\
200 \\
199 \\
199\end{array}$ & $\begin{array}{l}237 \\
206 \\
205 \\
206 \\
206\end{array}$ & $\begin{array}{l}288 \\
287 \\
287 \\
287 \\
298\end{array}$ & $\begin{array}{l}77 \\
54 \\
46 \\
43 \\
42\end{array}$ & $\begin{array}{l}14 \\
14 \\
11 \\
16 \\
24\end{array}$ \\
\hline $\begin{array}{l}16 \\
17 \\
18 \\
19 \\
20\end{array}$ & $\begin{array}{l}9.0 \\
8.9 \\
8.8 \\
8.4 \\
9.6\end{array}$ & $\begin{array}{l}12 \\
7.3 \\
11 \\
11 \\
10\end{array}$ & $\begin{array}{r}10 \\
9.7 \\
10 \\
9.7 \\
9.8\end{array}$ & $\begin{array}{l}11 \\
11 \\
11 \\
12 \\
12\end{array}$ & $\begin{array}{c}10 \\
10 \\
10 \\
9.9 \\
9.6\end{array}$ & $\begin{array}{r}11 \\
9.8 \\
8.7 \\
8.4 \\
8.8\end{array}$ & $\begin{array}{l}8.6 \\
8.8 \\
8.4 \\
8.4 \\
8.2\end{array}$ & $\begin{array}{l}198 \\
198 \\
198 \\
197 \\
176\end{array}$ & $\begin{array}{r}211 \\
147 \\
39 \\
31 \\
30\end{array}$ & $\begin{array}{r}1140 \\
344 \\
321 \\
298 \\
293\end{array}$ & $\begin{array}{l}42 \\
34 \\
14 \\
12 \\
73\end{array}$ & $\begin{array}{r}13 \\
11 \\
10 \\
9.7 \\
9.1\end{array}$ \\
\hline $\begin{array}{l}21 \\
22 \\
23 \\
24 \\
25\end{array}$ & $\begin{array}{l}105 \\
111 \\
112 \\
113 \\
88\end{array}$ & $\begin{array}{l}10 \\
10 \\
10 \\
10 \\
7.3\end{array}$ & $\begin{array}{l}10 \\
10 \\
10 \\
10 \\
10\end{array}$ & $\begin{array}{l}12 \\
12 \\
12 \\
11 \\
11\end{array}$ & $\begin{array}{l}9.4 \\
8.9 \\
8.5 \\
8.5 \\
8.6\end{array}$ & $\begin{array}{l}8.9 \\
8.8 \\
8.9 \\
8.5 \\
8.2\end{array}$ & $\begin{array}{l}8.4 \\
8.2 \\
8.6 \\
8.8 \\
8.3\end{array}$ & $\begin{array}{l}175 \\
175 \\
172 \\
151 \\
1.50\end{array}$ & $\begin{array}{r}30 \\
30 \\
43 \\
80 \\
526\end{array}$ & $\begin{array}{l}292 \\
291 \\
273 \\
219 \\
232\end{array}$ & $\begin{array}{l}79 \\
78 \\
55 \\
54 \\
53\end{array}$ & $\begin{array}{r}8.7 \\
9.0 \\
8.2 \\
8.9 \\
87\end{array}$ \\
\hline $\begin{array}{l}26 \\
27 \\
28 \\
29 \\
30 \\
31\end{array}$ & $\begin{array}{c}12 \\
10 \\
10 \\
10 \\
9.9 \\
10\end{array}$ & $\begin{array}{l}8.8 \\
8.9 \\
9.4 \\
9.7 \\
6.5 \\
-. .\end{array}$ & $\begin{array}{l}10 \\
10 \\
11 \\
14 \\
15 \\
11\end{array}$ & $\begin{array}{l}11 \\
11 \\
11 \\
11 \\
11 \\
10\end{array}$ & $\begin{array}{l}8.5 \\
8.1 \\
7.5 \\
7.6 \\
-\ldots \\
7 \ldots\end{array}$ & $\begin{array}{l}7.8 \\
8.9 \\
8.4 \\
8.3 \\
7.7 \\
7.7\end{array}$ & $\begin{array}{r}44 \\
138 \\
131 \\
126 \\
155 \\
-\end{array}$ & $\begin{array}{l}149 \\
149 \\
150 \\
150 \\
165 \\
254\end{array}$ & $\begin{array}{r}118 \\
136 \\
42 \\
20 \\
16 \\
-\end{array}$ & $\begin{array}{l}214 \\
158 \\
177 \\
177 \\
176 \\
174\end{array}$ & $\begin{array}{l}48 \\
11 \\
9.3 \\
20 \\
12 \\
9.7\end{array}$ & $\begin{array}{l}94 \\
94 \\
95 \\
94 \\
19 \\
---\end{array}$ \\
\hline $\begin{array}{l}\text { TOTAL. } \\
\text { MEAN } \\
\text { MAX } \\
\text { MIN } \\
\text { AC-FT }\end{array}$ & $\begin{array}{r}1170.9 \\
37.8 \\
113 \\
8.4 \\
2320\end{array}$ & $\begin{array}{r}1412.3 \\
47.1 \\
122 \\
6.5 \\
2800\end{array}$ & $\begin{array}{r}305.9 \\
9.87 \\
16 \\
5.9 \\
607\end{array}$ & $\begin{array}{r}342 \\
11.0 \\
12 \\
10 \\
678\end{array}$ & $\begin{array}{r}287.1 \\
9.90 \\
15 \\
7.5 \\
569\end{array}$ & $\begin{array}{r}543.0 \\
17.5 \\
195 \\
4.3 \\
1080\end{array}$ & $\begin{array}{r}814.7 \\
27.2 \\
155 \\
7.4 \\
1620\end{array}$ & $\begin{array}{r}6456 \\
208 \\
304 \\
149 \\
12810\end{array}$ & $\begin{array}{r}5016 \\
167 \\
526 \\
16 \\
9950\end{array}$ & $\begin{array}{r}7168 \\
231 \\
1140 \\
11 \\
14220\end{array}$ & $\begin{array}{r}2319.0 \\
74.8 \\
267 \\
9.3 \\
4600\end{array}$ & $\begin{array}{r}764.7 \\
25.5 \\
95 \\
7.6 \\
1520\end{array}$ \\
\hline
\end{tabular}

STATISTICS OF MONTHLY MEAN DATA FOR WATER YEARS 1938. - 1996, BY WATER YEAR (WY)

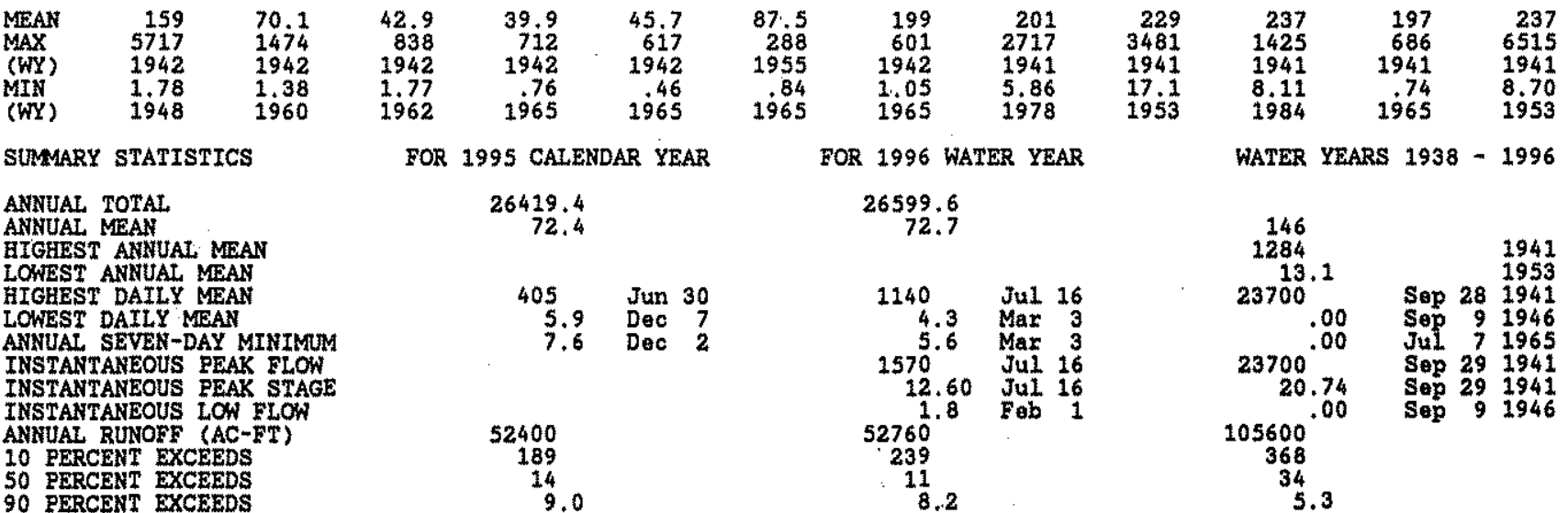




\section{RIO GRANDE BASIN}

08412500 PECOS RIVER NEAR ORLA, TX -- Continued

WATER-QUALITY RECORDS

PERIOD OF RECORD.--Chenical analyses: July 1937 to current year.

PERIOD OF DAILY RECORD.--

SPECIFIC CONDUCTANCE: July 1937 to current year.

WATER TEMPERATURE: March 1953 to current year.

RERARKS.--October 1937 to September 1969 , this station was published as 08410100 Pocos River below Red Blupe Dam

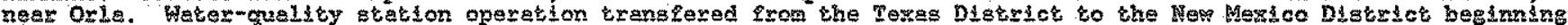
with the 1.993 watex yoar.

EXTREMES FOR PERTOD OF DAILY RECORD.--

SPECIFIC CONDUCTANCE: Maximum daily, 29,400 microsiemens May 16, 1978; minimum daily, 1,600 microsiemens Juno 19 ,

WATER TEMPERATURE: Maximum daily, $32.0^{\circ} \mathrm{C}$, Aug 4, 1991; minimun daliy, $0.0{ }^{\circ} \mathrm{C}$, many days during winter months.

EXTRERES FOR CURRENT YEAR.--

SPECIFIC CONDUCTANCE: Maximum daily, 20,500 microsiemens Sept, 10; mintmum daily, 6, 560 microstemens Aug. 10 . WATER TEMPERATURE: Maximum daily, $29.0^{\circ} \mathrm{C}$, July 2 ; minfmum daily $3.0^{\circ} \mathrm{C}$; Jan. $7, \mathrm{~F} \otimes \mathrm{b} .3,4$.

WATER-QUALITY DATA, WATER YEAR OCTOBER 1995 TO SEPTEMBER 1996

\begin{tabular}{|c|c|c|c|c|c|c|c|c|c|}
\hline DATE & TIME & $\begin{array}{c}\text { DIS- } \\
\text { CEARGE, } \\
\text { INST, } \\
\text { CUBIC } \\
\text { FEET } \\
\text { PER } \\
\text { SECOND } \\
\text { (00061) }\end{array}$ & $\begin{array}{l}\text { SPE- } \\
\text { CIFIC } \\
\text { CON- } \\
\text { DUCT- } \\
\text { ANCE } \\
\text { (US/CM) } \\
(00095)\end{array}$ & $\begin{array}{c}\text { PH } \\
\text { WATER } \\
\text { WHOLE } \\
\text { FIELD } \\
\text { (STAND- } \\
\text { ARD } \\
\text { UNITS) } \\
(00400)\end{array}$ & $\begin{array}{c}\text { TEMPER- } \\
\text { ATURE } \\
\text { AIR } \\
\text { (DEG C) } \\
(00020)\end{array}$ & $\begin{array}{l}\text { TEMPER- } \\
\text { ATURE } \\
\text { WATER } \\
\text { (DEG C) } \\
(00010)\end{array}$ & $\begin{array}{c}\text { BARO- } \\
\text { METRIC } \\
\text { PRES- } \\
\text { SURE } \\
\text { (MA } \\
\text { OF } \\
\text { BG) } \\
(00025)\end{array}$ & $\begin{array}{c}\text { OXYGEN, } \\
\text { DIS- } \\
\text { SOLVED } \\
\text { (MG/L) } \\
(00300)\end{array}$ & $\begin{array}{c}\text { OXYGEN, } \\
\text { DIS- } \\
\text { SOLVED } \\
\text { (PER- } \\
\text { CENT } \\
\text { SATUR- } \\
\text { ATION) } \\
\text { (00301) }\end{array}$ \\
\hline $\begin{array}{l}E C 1995 \\
\text { O1 } 1996\end{array}$ & 1215 & 8.6 & 10500 & 7.9 & 21.0 & 8.5 & 691 & 10.0 & 98 \\
\hline IR & 1200 & 8.0 & 10800 & 8.0 & 25.5 & 11.0 & 694 & 9.8 & 102 \\
\hline Jus & 1235 & 10 & 11800 & 7.8 & 30.0 & 22.0 & 697 & 8.6 & 112 \\
\hline $\mathrm{JUL}^{10} \ldots$ & 1145 & 247 & 9960 & 8.0 & 35.0 & 25.0 & 690 & 8.0 & 111 \\
\hline $25 \ldots$ & 1030 & 228 & 9280 & 7.6 & 24.5 & 25.5 & 696 & 11.4 & 158 \\
\hline $10 \ldots$ & 0950 & 20 & 19500 & 7.8 & 26.0 & 24.0 & 695 & 8.4 & 117 \\
\hline DATE & $\begin{array}{l}\text { EARD- } \\
\text { NESS } \\
\text { TOTAL } \\
\text { (MG/L } \\
\text { AS } \\
\text { CACO3) } \\
(00900)\end{array}$ & $\begin{array}{l}\text { EARD- } \\
\text { NESS } \\
\text { NONCARB } \\
\text { DISSOLV } \\
\text { FLD AS } \\
\text { CACO3 } \\
\text { (MG/L) } \\
(00904)\end{array}$ & $\begin{array}{c}\text { CALCIUM } \\
\text { DIS- } \\
\text { SOLVED } \\
\text { (MG /L } \\
\text { AS CA) } \\
\text { (00915) }\end{array}$ & $\begin{array}{c}\text { MAGNE- } \\
\text { SIUM, } \\
\text { DIS- } \\
\text { SOLVED } \\
\text { (MG/L } \\
\text { AS MGG) } \\
\text { (00925) }\end{array}$ & $\begin{array}{c}\text { SODTUM, } \\
\text { DIS- } \\
\text { SOLVED } \\
\text { (MG/L } \\
\text { AS NA) } \\
(00930)\end{array}$ & $\begin{array}{c}\text { SODIUM } \\
\text { AD- } \\
\text { SORP- } \\
\text { THON } \\
\text { RATHO } \\
(00931)\end{array}$ & $\begin{array}{c}\text { POTAS- } \\
\text { SIUM, } \\
\text { DIS- } \\
\text { SOLVED } \\
\text { (ME/L } \\
\text { AS K) } \\
(00935)\end{array}$ & $\begin{array}{l}\text { BICAR- } \\
\text { BONATE } \\
\text { WATER } \\
\text { DIS IT } \\
\text { FIELD } \\
\text { MG/L AS } \\
\text { HCO3 } \\
(00453)\end{array}$ & $\begin{array}{c}\text { CAR- } \\
\text { BONATE } \\
\text { WATER } \\
\text { DIS IT } \\
\text { FIELD } \\
\text { WE/L AS } \\
\text { COS } \\
(00452)\end{array}$ \\
\hline $\begin{array}{l}\mathrm{DEC} 1995 \\
011996 \\
\operatorname{MAR} 1996\end{array}$ & 2700 & 2600 & 640 & 260 & 1500 & 13 & 16 & 134 & \\
\hline APR $11 \cdots$ & 2700 & 2600 & 630 & 270 & 1600 & 13 & 32 & 155 & \\
\hline 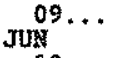 & 2700 & 2600 & 660 & 260 & 2000 & 17 & 34 & 142 & \\
\hline${ }_{\text {JUL }}^{10 . . .}$ & 2700 & 2600 & 650 & 250 & 1400 & 12 & 41 & 106 & \\
\hline $25 \ldots$ & 2400 & 2300 & 580 & 230 & 1300 & 12 & 37 & 95 & 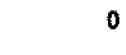 \\
\hline $10 \ldots$ & 3700 & 3600 & 940 & 340 & 4600 & 33 & 38 & 137 & \\
\hline DATE & $\begin{array}{l}\text { ALKA- } \\
\text { LINITY } \\
\text { WAT DIS } \\
\text { TOT IT } \\
\text { FIELD } \\
\text { MG/L AS } \\
\text { CACOS } \\
\text { (39086) }\end{array}$ & $\begin{array}{c}\text { AlKKA- } \\
\text { LINITYY } \\
\text { LAB } \\
\text { (MG/L } \\
\text { AS } \\
\text { CACO3) } \\
(90410)\end{array}$ & $\begin{array}{c}\text { SULFATE } \\
\text { DIS- } \\
\text { SOLVED } \\
\text { (MGG/L } \\
\text { AS SO4) } \\
(00945)\end{array}$ & $\begin{array}{l}\text { CHLO- } \\
\text { RIDE } \\
\text { DIS- } \\
\text { SOLVED } \\
\text { (MG/L } \\
\text { AS CL) } \\
(00940)\end{array}$ & $\begin{array}{l}\text { FLUO- } \\
\text { RIDE, } \\
\text { DIS } \\
\text { SOLVED } \\
(M G / L \\
\text { AS E) } \\
(00950)\end{array}$ & $\begin{array}{c}\text { SrLICA: } \\
\text { DIS- } \\
\text { SOLVED } \\
\text { (MG/L } \\
\text { AS } \\
\text { SIO2) } \\
(00955)\end{array}$ & $\begin{array}{c}\text { SOLIDS, } \\
\text { SUM OF } \\
\text { CONSTY- } \\
\text { TUENTS, } \\
\text { DIS- } \\
\text { SOLVED } \\
\text { (WGGLL) } \\
\text { (70301) }\end{array}$ & $\begin{array}{l}\text { BORON, } \\
\text { DIS- } \\
\text { SOLVED } \\
\text { (UG/L. } \\
\text { AS B) } \\
(01020)\end{array}$ & $\begin{array}{l}\text { IRON, } \\
\text { DIS } \\
\text { SOLVED } \\
\text { (UG/L } \\
\text { AS FE }) \\
\text { (01046) }\end{array}$ \\
\hline $\begin{array}{l}\text { DEC } 1395 \\
01 \text { 1996 } \\
\text { MAR } 1996\end{array}$ & 110 & - & 2100 & 2400 & 80 & 12 & 6990 & $-m$ & -- \\
\hline $\operatorname{APR}^{11} \cdots$ & 127 & -- & 2100 & 2700 & 1.0 & 9.5 & 7420 & $-m$ & $\therefore=-$ \\
\hline 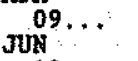 & 116 & 116 & 2300 & 3300 & 1.0 & 7. & 8630 & 1000 & $<24$ \\
\hline JuL $10 .$. & 87 & 95 & 2300 & 2400 & 1.0 & 9.4 & 7100 & 466 & $<30$ \\
\hline SEP & 78 & -- & 2200 & 2200 & 90 & 11 & 6610 & -- & -- \\
\hline $10 \ldots$ & 112 & - & 3000 & 6400 & 1.1 & 5.9 & 15400 & -- & -- \\
\hline
\end{tabular}


RIO GRANDE BASIN

08412500 PECOS RIVER NEAR ORLA, TX - Continued

WATER-QUALITY RECORDS

SPECIFIC CONDUCTANCE, US/CM O 25 DEGREES CENTIGRADE, WATER YEAR OCTOBER 1995 TO SEPTEMEER 1996

\begin{tabular}{|c|c|c|c|c|c|c|c|c|c|c|c|c|}
\hline $\begin{array}{r}\text { DAY } \\
1 \\
2 \\
3 \\
4 \\
5\end{array}$ & $\begin{array}{r}0 C T \\
13000 \\
10800 \\
16000 \\
7100 \\
9800\end{array}$ & $\begin{array}{r}\text { Nov } \\
10600 \\
10500 \\
9200 \\
9200 \\
9200\end{array}$ & 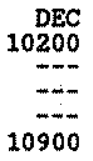 & $\begin{array}{r}\text { JAN } \\
11000 \\
11100 \\
11100 \\
12200 \\
11000\end{array}$ & $\begin{array}{r}\text { FEB } \\
11900 \\
11200 \\
12000 \\
11000 \\
11200\end{array}$ & $\begin{array}{r}\text { MAR } \\
9980 \\
9960 \\
10000 \\
10000 \\
10300\end{array}$ & $\begin{array}{r}\text { APR } \\
10900 \\
10900 \\
11000 \\
11000 \\
10600\end{array}$ & $\begin{array}{l}\text { MAY } \\
9940 \\
9890 \\
9880 \\
9920 \\
9910\end{array}$ & $\begin{array}{r}\text { JUN } \\
10300 \\
10400 \\
10300 \\
10400 \\
10400\end{array}$ & $\begin{array}{r}\text { JuL } \\
11100 \\
11500 \\
11700 \\
11600 \\
11500\end{array}$ & $\begin{array}{l}\text { AUG } \\
8560 \\
9590 \\
9580 \\
9320 \\
9500\end{array}$ & $\begin{array}{r}\text { SEP } \\
12300 \\
12100 \\
12000 \\
-- \\
11500\end{array}$ \\
\hline $\begin{array}{r}6 \\
7 \\
8 \\
9 \\
10\end{array}$ & $\begin{array}{l}10400 \\
11400 \\
11400 \\
12800 \\
10400\end{array}$ & $\begin{array}{l}9000 \\
9400 \\
9200 \\
9000 \\
9100\end{array}$ & $\begin{array}{l}11000 \\
11000 \\
11100 \\
11500 \\
10800\end{array}$ & $\begin{array}{l}11500 \\
11500 \\
11600 \\
11900 \\
12000\end{array}$ & $\begin{array}{l}11100 \\
11500 \\
11900 \\
11900 \\
12000\end{array}$ & $\begin{array}{r}10200 \\
9970 \\
10500 \\
10600 \\
10600\end{array}$ & $\begin{array}{l}10300 \\
10800 \\
11100 \\
12700 \\
12800\end{array}$ & $\begin{array}{l}9900 \\
9890 \\
9900 \\
9900 \\
9870\end{array}$ & $\begin{array}{l}10400 \\
10400 \\
10400 \\
10400 \\
11400\end{array}$ & $\begin{array}{r}11500 \\
11500 \\
9000 \\
8980 \\
9030\end{array}$ & $\begin{array}{r}9550 \\
10200 \\
10100 \\
9460 \\
6560\end{array}$ & $\begin{array}{l}11400 \\
11400 \\
11300 \\
11200 \\
20500\end{array}$ \\
\hline $\begin{array}{l}11 \\
12 \\
13 \\
14 \\
15\end{array}$ & $\begin{array}{l}10200 \\
10200 \\
10100 \\
10500 \\
10800\end{array}$ & $\begin{array}{l}9000 \\
9500 \\
9200 \\
9100 \\
9200\end{array}$ & $\begin{array}{l}10000 \\
11100 \\
11100 \\
11200 \\
11000\end{array}$ & $\begin{array}{l}12000 \\
12100 \\
12000 \\
12300 \\
12000\end{array}$ & $\begin{array}{l}12100 \\
12300 \\
12000 \\
12000 \\
12000\end{array}$ & $\begin{array}{r}11100 \\
11000 \\
11000 \\
11100 \\
9470\end{array}$ & $\begin{array}{r}12800 \\
13400 \\
13300 \\
13200\end{array}$ & $\begin{array}{r}10000 \\
9960 \\
9950 \\
10000 \\
10000\end{array}$ & $\begin{array}{l}9280 \\
9390 \\
9330 \\
9130 \\
9110\end{array}$ & $\begin{array}{l}9030 \\
9000 \\
9000 \\
9030 \\
9010\end{array}$ & $\begin{array}{l}8290 \\
8670 \\
9490 \\
9690 \\
9750\end{array}$ & $\begin{array}{r}11800 \\
20200 \\
12700 \\
10200 \\
9630\end{array}$ \\
\hline $\begin{array}{l}16 \\
17 \\
18 \\
19 \\
20\end{array}$ & $\begin{array}{l}11500 \\
11200 \\
11400 \\
11200 \\
11900\end{array}$ & $\begin{array}{r}9500 \\
10000 \\
10400 \\
10200 \\
10600\end{array}$ & $\begin{array}{l}11200 \\
12000 \\
11000 \\
10900 \\
10900\end{array}$ & $\begin{array}{l}12300 \\
12100 \\
12200 \\
12000 \\
12000\end{array}$ & $\begin{array}{l}12000 \\
10900 \\
10900 \\
10800 \\
10300\end{array}$ & $\begin{array}{r}10100 \\
-2 \\
10800 \\
10800 \\
10800\end{array}$ & $\begin{array}{l}12800 \\
12300 \\
12200 \\
12100 \\
12100\end{array}$ & $\begin{array}{l}10000 \\
10000 \\
10000 \\
10000 \\
10000\end{array}$ & $\begin{array}{r}9890 \\
9260 \\
10200 \\
9940 \\
9750\end{array}$ & $\begin{array}{r}14200 \\
8980 \\
9440 \\
9030 \\
8990\end{array}$ & $\begin{array}{r}9810 \\
9780 \\
9890 \\
10800 \\
10300\end{array}$ & $\begin{array}{r}8490 \\
10000 \\
10400 \\
10800 \\
10800\end{array}$ \\
\hline $\begin{array}{l}21 \\
22 \\
23 \\
24 \\
25\end{array}$ & $\begin{array}{r}10100 \\
9800 \\
10200 \\
9800 \\
9800\end{array}$ & $\begin{array}{r}10100 \\
10000 \\
-200 \\
10200\end{array}$ & $\begin{array}{l}11000 \\
11000 \\
10900 \\
10900 \\
11000\end{array}$ & $\begin{array}{l}11900 \\
11800 \\
11700 \\
11900 \\
11400\end{array}$ & $\begin{array}{l}10400 \\
10200 \\
10100 \\
10800 \\
10200\end{array}$ & $\begin{array}{l}10900 \\
10900 \\
10900 \\
11000 \\
11000\end{array}$ & $\begin{array}{l}12000 \\
12000 \\
11900 \\
11800 \\
11800\end{array}$ & $\begin{array}{l}10200 \\
10100 \\
10100 \\
10100 \\
10000\end{array}$ & $\begin{array}{l}9630 \\
9590 \\
9560 \\
9130 \\
7440\end{array}$ & $\begin{array}{l}8950 \\
8950 \\
8930 \\
8960 \\
8880\end{array}$ & $\begin{array}{l}9460 \\
9440 \\
9670 \\
9570 \\
9520\end{array}$ & $\begin{array}{r}10900 \\
10800 \\
10600 \\
10600 \\
8890\end{array}$ \\
\hline $\begin{array}{l}26 \\
27 \\
28 \\
29 \\
30 \\
31\end{array}$ & $\begin{array}{r}9600 \\
10600 \\
10900 \\
10900 \\
11000 \\
10600\end{array}$ & $\begin{array}{l}10400 \\
10200 \\
11000 \\
10400 \\
10100\end{array}$ & $\begin{array}{l}10500 \\
10900 \\
11000 \\
11000 \\
11500 \\
10100\end{array}$ & $\begin{array}{l}12000 \\
11200 \\
11500 \\
11800 \\
11600 \\
11100\end{array}$ & $\begin{array}{r}9990 \\
9990 \\
10000 \\
10000 \\
- \\
--\end{array}$ & $\begin{array}{l}11000 \\
10900 \\
10800 \\
10900 \\
11000 \\
11000\end{array}$ & $\begin{array}{r}11700 \\
9870 \\
9830 \\
9870 \\
9930\end{array}$ & $\begin{array}{l}10300 \\
10200 \\
10300 \\
10300 \\
10300 \\
10300\end{array}$ & $\begin{array}{r}9100 \\
9630 \\
8960 \\
9830 \\
10500\end{array}$ & $\begin{array}{l}8890 \\
8620 \\
8640 \\
8720 \\
8630 \\
8610\end{array}$ & $\begin{array}{r}9530 \\
9580 \\
10100 \\
10900 \\
14700 \\
13700\end{array}$ & $\begin{array}{l}8540 \\
8420 \\
8460 \\
8470 \\
8500\end{array}$ \\
\hline $\begin{array}{l}\text { MEAN } \\
\text { MAX } \\
\text { MIN }\end{array}$ & $\begin{array}{r}10800 \\
16000 \\
7100\end{array}$ & $\begin{array}{r}9790 \\
11000 \\
9000\end{array}$ & $\begin{array}{l}11000 \\
12000 \\
10000\end{array}$ & $\begin{array}{l}11700 \\
12300 \\
11000\end{array}$ & $\begin{array}{r}11100 \\
12300 \\
9990\end{array}$ & $\begin{array}{r}10600 \\
11100 \\
9470\end{array}$ & $\begin{array}{r}11600 \\
13400 \\
9830\end{array}$ & $\begin{array}{r}10000 \\
10300 \\
9870\end{array}$ & $\begin{array}{r}9780 \\
11400 \\
7440\end{array}$ & $\begin{array}{r}9670 \\
14200 \\
8610\end{array}$ & $\begin{array}{r}9840 \\
14700 \\
6560\end{array}$ & $\begin{array}{r}11100 \\
20500 \\
8420\end{array}$ \\
\hline
\end{tabular}

WTR YR 1996 MEAN 10600 MAX 20500 MIN 6560 WETS TEMPERATURE; DEGRES CELSIUS, WATER YEAR OCTOBER 1995 TO SEPTEMBER 1996

$$
\text { ONCE DAILY }
$$

\begin{tabular}{|c|c|c|c|c|c|c|c|c|c|c|c|c|}
\hline $\begin{array}{r}\text { DAY } \\
1 \\
2 \\
3 \\
4 \\
5\end{array}$ & $\begin{array}{r}\text { OCT } \\
21.0 \\
22.0 \\
20.0 \\
20.0 \\
20.5\end{array}$ & $\begin{array}{r}\text { Nov } \\
15.0 \\
14.0 \\
14.0 \\
14.0\end{array}$ & \begin{tabular}{r} 
DEC \\
7.0 \\
8.0 \\
\hdashline-0 \\
10.0
\end{tabular} & $\begin{array}{l}\text { JAN } \\
5.5 \\
5.0 \\
3.5 \\
6.0 \\
6.5\end{array}$ & $\begin{array}{l}\text { FEB } \\
4.5 \\
4.0 \\
3.0 \\
3.0 \\
3.5\end{array}$ & $\begin{array}{r}\text { MAR } \\
7.0 \\
7.0 \\
9.5 \\
10.0 \\
13.0\end{array}$ & $\begin{array}{r}\text { APR } \\
14.0 \\
15.0 \\
19.0 \\
17.0 \\
14.0\end{array}$ & $\begin{array}{r}\text { MAY } \\
16.0 \\
17.5 \\
18.0 \\
18.0 \\
18.0\end{array}$ & $\begin{array}{r}\text { JUN } \\
22.0 \\
24.0 \\
23.5 \\
24.0 \\
23.0\end{array}$ & $\begin{array}{l}\text { JUL } \\
27.0 \\
29.0 \\
28.5 \\
26.0 \\
27.5\end{array}$ & $\begin{array}{r}\text { AUG } \\
27.0 \\
26.0 \\
26.0 \\
26.0 \\
27.0\end{array}$ & $\begin{array}{r}\text { SEP } \\
27.0 \\
26.0 \\
25.0 \\
-25.0\end{array}$ \\
\hline $\begin{array}{r}6 \\
7 \\
8 \\
9 \\
10\end{array}$ & $\begin{array}{l}18.0 \\
16.0 \\
16.0 \\
19.0 \\
19.0\end{array}$ & $\begin{array}{l}16.0 \\
15.0 \\
14.0 \\
13.0 \\
16.0\end{array}$ & $\begin{array}{r}10.5 \\
10.0 \\
9.0 \\
7.5 \\
5.0\end{array}$ & $\begin{array}{l}5.0 \\
3.0 \\
3.5 \\
3.5 \\
4.5\end{array}$ & $\begin{array}{r}5.0 \\
7.0 \\
8.0 \\
9.5 \\
11.0\end{array}$ & $\begin{array}{r}14.5 \\
9.0 \\
7.5 \\
5.0 \\
7.0\end{array}$ & $\begin{array}{l}11.5 \\
13.0 \\
15.0 \\
17.0 \\
20.0\end{array}$ & $\begin{array}{l}18.5 \\
20.0 \\
19.0 \\
19.0 \\
20.0\end{array}$ & $\begin{array}{l}24.0 \\
24.0 \\
23.5 \\
24.0 \\
24.0\end{array}$ & $\begin{array}{l}26.5 \\
27.0 \\
26.0 \\
26.0 \\
26.0\end{array}$ & $\begin{array}{r}27.0 \\
27.0 \\
25.5 \\
24.5\end{array}$ & $\begin{array}{l}25.0 \\
25.0 \\
24.5 \\
24.5 \\
24.5\end{array}$ \\
\hline $\begin{array}{l}11 \\
12 \\
13 \\
14 \\
15\end{array}$ & $\begin{array}{l}18.0 \\
18.0 \\
18.0 \\
18.0 \\
16.5\end{array}$ & $\begin{array}{l}12.0 \\
14.0 \\
13.0 \\
13.0 \\
12.0\end{array}$ & $\begin{array}{r}5.0 \\
5.0 \\
9.5 \\
9.5 \\
9.0\end{array}$ & $\begin{array}{l}7.0 \\
7.0 \\
6.5 \\
7.5 \\
7.5\end{array}$ & $\begin{array}{r}11.0 \\
9.0 \\
8.0 \\
10.0 \\
10.0\end{array}$ & $\begin{array}{r}9.0 \\
11.5 \\
14.0 \\
15.0 \\
12.0\end{array}$ & $\begin{array}{r}19.0 \\
18.0 \\
17.0 \\
16.0\end{array}$ & $\begin{array}{l}21.5 \\
21.0 \\
21.0 \\
21.5 \\
21.0\end{array}$ & $\begin{array}{l}25.0 \\
24.0 \\
25.0 \\
24.0 \\
24.0\end{array}$ & $\begin{array}{l}27.0 \\
26.0 \\
26.0 \\
28.0 \\
27.0\end{array}$ & $\begin{array}{l}26.0 \\
26.0 \\
26.0 \\
27.0 \\
26.0\end{array}$ & $\begin{array}{l}25.0 \\
26.0 \\
22.0 \\
22.5 \\
26.0\end{array}$ \\
\hline $\begin{array}{l}16 \\
17 \\
18 \\
19 \\
20\end{array}$ & $\begin{array}{l}16.0 \\
16.0 \\
20.0 \\
17.0 \\
15.0\end{array}$ & $\begin{array}{l}13.0 \\
13.0 \\
11.0 \\
11.0 \\
15.0\end{array}$ & $\begin{array}{l}7.5 \\
7.0 \\
8.0 \\
6.5 \\
4.5\end{array}$ & $\begin{array}{r}8.0 \\
11.0 \\
6.5 \\
8.0 \\
8.0\end{array}$ & $\begin{array}{r}9.5 \\
10.0 \\
9.0 \\
10.0 \\
13.0\end{array}$ & $\begin{array}{l}19.0 \\
12.5 \\
12.0 \\
13.5\end{array}$ & $\begin{array}{l}16.0 \\
17.5 \\
18.0 \\
19.0 \\
17.0\end{array}$ & $\begin{array}{l}21.0 \\
21.0 \\
21.0 \\
22.0 \\
21.0\end{array}$ & $\begin{array}{l}25.0 \\
25.0 \\
26.5 \\
27.5 \\
27.0\end{array}$ & $\begin{array}{l}23.0 \\
25.5 \\
26.0 \\
26.0 \\
27.0\end{array}$ & $\begin{array}{l}26.0 \\
26.0 \\
26.5 \\
27.0 \\
26.0\end{array}$ & $\begin{array}{l}22.0 \\
23.0 \\
24.5 \\
23.0 \\
22.0\end{array}$ \\
\hline $\begin{array}{l}21 \\
22 \\
23 \\
24 \\
25\end{array}$ & $\begin{array}{l}16.0 \\
20.0 \\
17.0 \\
15.0 \\
16.0\end{array}$ & $\begin{array}{l}12.5 \\
14.0 \\
14.0 \\
12.0 \\
14.0\end{array}$ & $\begin{array}{l}6.0 \\
6.0 \\
5.0 \\
6.5 \\
8.0\end{array}$ & $\begin{array}{r}5.0 \\
9.5 \\
5.5 \\
10.0 \\
9.0\end{array}$ & $\begin{array}{l}16.0 \\
14.5 \\
12.5 \\
13.0 \\
15.0\end{array}$ & $\begin{array}{l}13.0 \\
14.0 \\
16.0 \\
17.0 \\
12.5\end{array}$ & $\begin{array}{l}17.0 \\
16.0 \\
17.5 \\
19.0 \\
20.0\end{array}$ & $\begin{array}{l}21.0 \\
21.5 \\
22.0 \\
22.0 \\
24.0\end{array}$ & $\begin{array}{l}27.0 \\
27.0 \\
26.5 \\
26.0 \\
22.5\end{array}$ & $\begin{array}{l}27.0 \\
27.0 \\
27.5 \\
26.0 \\
26.0\end{array}$ & $\begin{array}{l}25.5 \\
25.0 \\
25.5 \\
25.5 \\
26.0\end{array}$ & $\begin{array}{l}21.0 \\
21.5 \\
23.0 \\
24.5 \\
22.0\end{array}$ \\
\hline $\begin{array}{l}26 \\
27 \\
28 \\
29 \\
30 \\
31\end{array}$ & $\begin{array}{l}16.0 \\
18.0 \\
14.0 \\
15.0 \\
18.0 \\
20.0\end{array}$ & $\begin{array}{r}13.0 \\
11.5 \\
10.0 \\
7.5 \\
7.0 \\
\end{array}$ & $\begin{array}{l}4.5 \\
4.0 \\
4.0 \\
5.0 \\
5.0 \\
5.0\end{array}$ & $\begin{array}{l}7.0 \\
6.0 \\
7.0 \\
5.0 \\
7.5 \\
6.5\end{array}$ & $\begin{array}{r}10.0 \\
12.5 \\
10.0 \\
8.5 \\
-.- \\
-.-\end{array}$ & $\begin{array}{l}10.0 \\
11.0 \\
12.5 \\
15.0 \\
15.5 \\
15.5\end{array}$ & $\begin{array}{l}18.0 \\
17.0 \\
18.5 \\
17.0 \\
17.0\end{array}$ & $\begin{array}{l}23.0 \\
22.0 \\
21.5 \\
21.0 \\
23.0 \\
23.0\end{array}$ & $\begin{array}{l}24.5 \\
24.5 \\
24.5 \\
27.0 \\
27.5\end{array}$ & $\begin{array}{l}27.0 \\
26.0 \\
27.0 \\
27.0 \\
28.5 \\
27.0\end{array}$ & $\begin{array}{l}25.0 \\
25.0 \\
25.5 \\
23.5 \\
24.5 \\
28.5\end{array}$ & $\begin{array}{l}22.5 \\
20.0 \\
20.0 \\
19.0 \\
19.0\end{array}$ \\
\hline $\begin{array}{l}\text { MEAN } \\
\text { MAX } \\
\text { MIN }\end{array}$ & $\begin{array}{l}17.7 \\
22.0 \\
14.0\end{array}$ & $\begin{array}{r}12.9 \\
16.0 \\
7.0\end{array}$ & $\begin{array}{r}6.8 \\
10.5 \\
4.0\end{array}$ & $\begin{array}{r}6.5 \\
11.0 \\
3.0\end{array}$ & $\begin{array}{r}9.3 \\
16.0 \\
3.0\end{array}$ & $\begin{array}{r}12.0 \\
19.0 \\
5.0\end{array}$ & $\begin{array}{l}16.9 \\
20.0 \\
11.5\end{array}$ & $\begin{array}{l}20.6 \\
24.0 \\
16.0\end{array}$ & $\begin{array}{l}24.9 \\
27.5 \\
22.0\end{array}$ & $\begin{array}{l}26.7 \\
29.0 \\
23.0\end{array}$ & $\begin{array}{l}25.9 \\
28.5 \\
23.5\end{array}$ & $\begin{array}{l}23.3 \\
27.0 \\
19.0\end{array}$ \\
\hline
\end{tabular}




\section{MIMBRES RIVER BASTN}

08477110 MIMBRES RIVER AT MIMBRES, MM

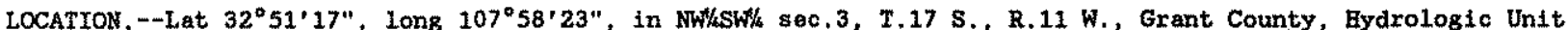

13030202 , on left bank 100 ft downstream from Willow Springs Canyon, 0.3 mi east of Mimbres, 1.1 mi downstream from Shepard Canyon, $2.5 \mathrm{mi}$ downstrearn from Bear Canyon, and at mile 73.1 .

DRAINAGE AREA, $--216 \mathrm{mi}^{2}$.

PERIOD OF RECORD.--March 1978 to current year.

GAGE -Water-stage recorder Elevation of gage is 5,920 ft above National Geodetic Vertical Datum of 1929 , from topographic map. Pxior to Jan, 17, 1979, at datum 2.29 ft higher.

REAARRS. - Records good.

DISCHARGE, CUBTC FEET PER SECOND, WATER YEAR OCTOBER 1995 TO SEPTEMBER 1996

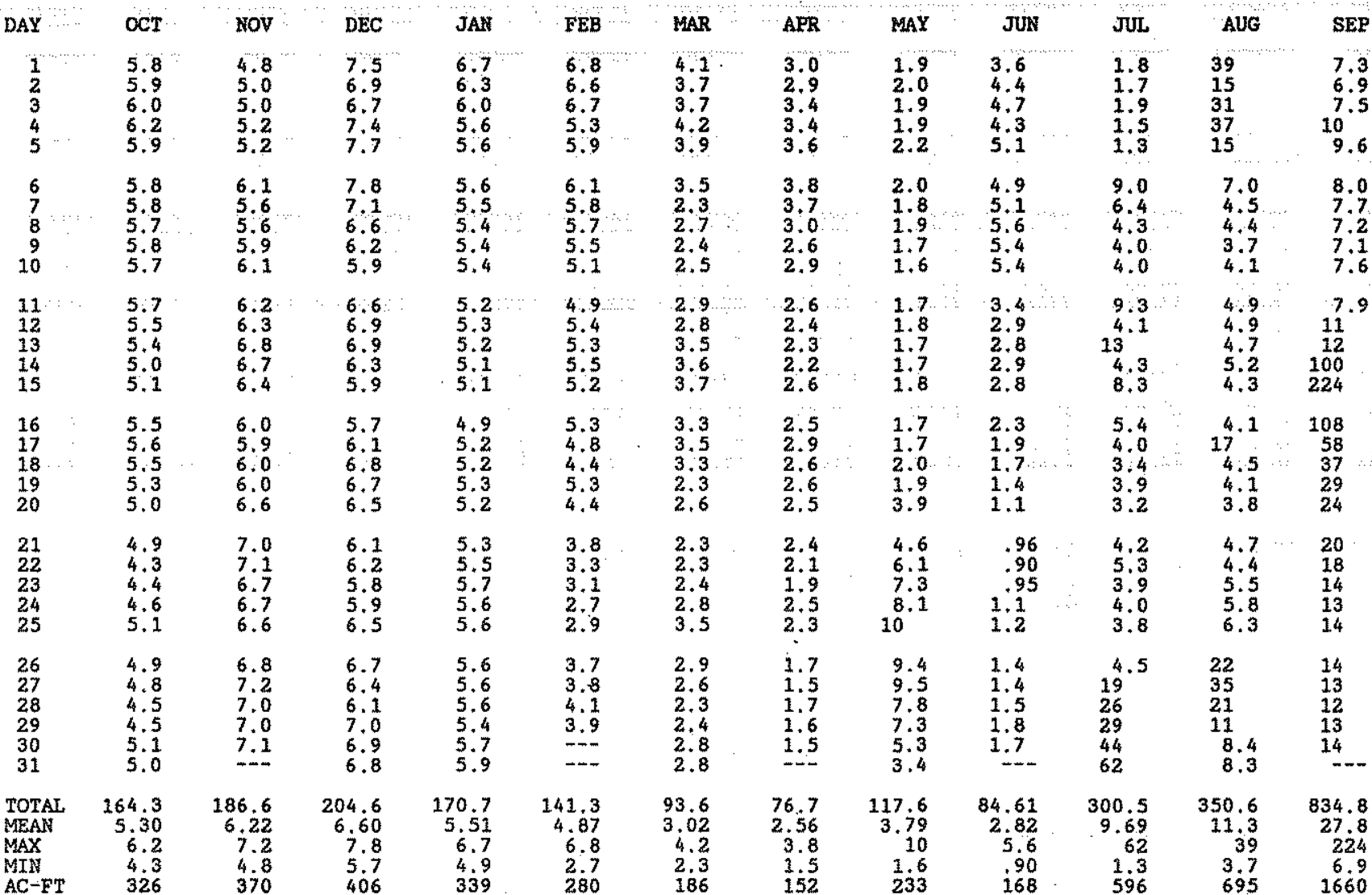

STATISTICS OF MONTHLY MEAN DATA FOR WATER YEARS 1978 - 1996, BY WATER YEAR (WY)

\begin{tabular}{|c|c|c|c|c|c|c|c|c|c|c|c|c|}
\hline $\begin{array}{l}\text { MEAY } \\
\text { MAX } \\
(W Y) \\
M I N Y \\
(W Y)\end{array}$ & $\begin{array}{l}13.6 \\
67.9 \\
1986 \\
2.56 \\
1995\end{array}$ & $\begin{array}{l}13.8 \\
43.9 \\
1979 \\
2.47 \\
1981\end{array}$ & $\begin{array}{r}37.2 \\
1.86 \\
1985 \\
3.65 \\
1981\end{array}$ & $\begin{array}{r}31.6 \\
153 \\
1993 \\
4.24 \\
1981\end{array}$ & $\begin{array}{l}31.2 \\
99.1 \\
1995 \\
3.10 \\
1981\end{array}$ & $\begin{array}{l}35.0 \\
93.2 \\
1992 \\
2.16 \\
1990\end{array}$ & $\begin{array}{l}24.5 \\
89.5 \\
1992 \\
2.34 \\
1990\end{array}$ & $\begin{array}{l}16.4 \\
64.9 \\
1992 \\
1.84 \\
1990\end{array}$ & $\begin{array}{l}8.75 \\
23.0 \\
1992 \\
2.82 \\
1996\end{array}$ & $\begin{array}{l}12.0 \\
52.1 \\
1986 \\
1.64 \\
1994\end{array}$ & $\begin{array}{r}30.6 \\
2.34 \\
1988 \\
3.30\end{array}$ & $\begin{array}{l}12.6 \\
48.6 \\
1988 \\
2.64\end{array}$ \\
\hline
\end{tabular}

SUMARY STATISTICS

ANNUAL TOTAL

ANNUAL MEAN

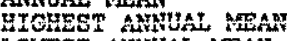

LOWEST ANNUAL MEAN

HIGHEST DAILY MEAH

LOWEST DATLY MEAN

ANDUAL SEVEN-DAY MTNIMUM

INSTANTANEOUS PEAK FLOW

INSTANTANEOUS PEAK STAGE

INSTANTANEOUS LOW ELOW

ANNUAL RUNOFY (AC-FT)

10 PERCENT EXCEEDS

50 PERCENT EXCEEDS

90 PARCENT IXCEEDS
FOR 1995 CALENDAR YEAR

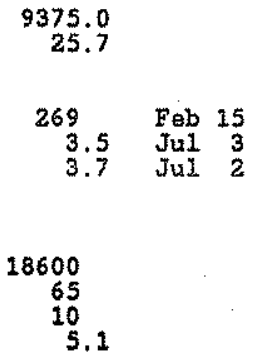

FOR 1996 WATER YEAR

$$
\begin{array}{r}
2725.91 \\
7.45
\end{array}
$$

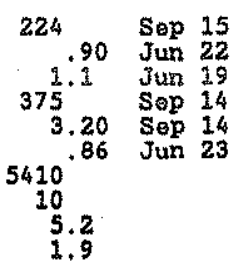

WATER YEARS 1978 - 1996

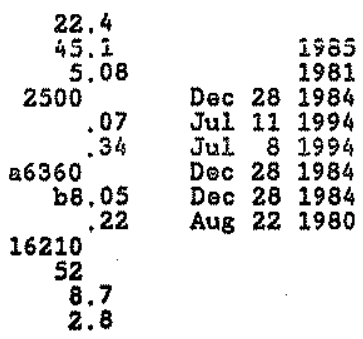

a-From sloodmarks

b-From rating curve extended above $450 \mathrm{ft} / \mathrm{s}$, on basis of slope-axea measurement at gege heights 6.70 ft and 8.05 ft. 
TULAROSA VALLEY BASIN

08480595 SALT CREEK AT RR316 ON WSMR, NM

LOCATroN, --Lat $33^{\circ} 16^{\prime} 32^{\prime \prime}$, Long $106^{\circ} 23^{\prime} 50^{\prime \prime}$, in SEKWE\% sec.16, T.12 S., R.6 E., Sierra County Eydrologic Untt 10301103 , on right bank, 360 ft upstrean from Range Road $316, .5$ mi east of Range Road 7 , and about 65 miles north of small missile range on U.S. Highway 70 .

WATER-DISCEARGE RECORDS

PERIOD OF RECORD.--August 1995 to current year.

GAGE,--Water-stage recorder. Elevation of gage is 4,020 ft above Nattonal Geodetic Vertical Datum of 1929 , from topographic map.

REMARKS, - Water-discharge records good.

EXIREMES FOR PERIOD OF RECORD. --Maximum discharge 88. fts/s, July 3, 1996, gage helght, 6.10 ft; minimum no flow at times.

EXTREMES FOR CURRENT YEAR,-Maximum discharge $88 \mathrm{ft} / \mathrm{s}, \mathrm{July} 3,1996$, gage helght, $6.10 \mathrm{ft}$; minimum discharge, $.06 \mathrm{fts} / \mathrm{s}$, Oct. 5,6

DISCHARGE, CUBIC FEET PER SECOND, WATER YEAR OCTOBER 1995 TO SEPTEMBER 1996

\begin{tabular}{|c|c|c|c|c|c|c|c|c|c|c|c|c|}
\hline DAY & OCT & Nov & DEC & JAN & FEB & MAR & APR & MAY & JUN & JUL & AUG & SEP \\
\hline $\begin{array}{l}1 \\
2 \\
3 \\
4 \\
5\end{array}$ & $\begin{array}{l}.15 \\
.12 \\
.09 \\
.08 \\
.06\end{array}$ & $\begin{array}{l}.26 \\
.26 \\
.26 \\
.26 \\
.30\end{array}$ & $\begin{array}{l}.47 \\
.49 \\
.50 \\
.49 \\
.46\end{array}$ & $\begin{array}{l}0.50 \\
0.49 \\
0.49 \\
0.51 \\
0.52\end{array}$ & $\begin{array}{r}03.0 \\
01.0 \\
0.80 \\
0.71 \\
.73\end{array}$ & $\begin{array}{l}.48 \\
.49 \\
.53 \\
.56 \\
.48\end{array}$ & $\begin{array}{l}.53 \\
.51 \\
.51 \\
.60 \\
.58\end{array}$ & $\begin{array}{l}.36 \\
.30 \\
.30 \\
.30 \\
.32\end{array}$ & $\begin{array}{r}.27 \\
.29 \\
.28 \\
.31 \\
.33\end{array}$ & $\begin{array}{r}1.1 \\
.59 \\
.49 \\
.49 \\
.49\end{array}$ & $\begin{array}{c}1.2 \\
11.53 \\
6.5 \\
1.8\end{array}$ & $\begin{array}{l}.30 \\
.29 \\
.27 \\
.28 \\
.29\end{array}$ \\
\hline $\begin{array}{r}6 \\
7 \\
8 \\
9 \\
10\end{array}$ & $\begin{array}{l}.06 \\
.08 \\
.09 \\
.09 \\
.09\end{array}$ & $\begin{array}{l}.51 \\
.44 \\
.35 \\
.31 \\
.24\end{array}$ & $\begin{array}{l}.44 \\
.47 \\
.50 \\
.42 \\
.44\end{array}$ & $\begin{array}{l}0.53 \\
0.54 \\
0.53 \\
0.51 \\
0.50\end{array}$ & $\begin{array}{l}.70 \\
.67 \\
.61 \\
.65 \\
.62\end{array}$ & $\begin{array}{l}.54 \\
.44 \\
.58 \\
.51 \\
.56\end{array}$ & $\begin{array}{l}.52 \\
.51 \\
.49 \\
.43 \\
.38\end{array}$ & $\begin{array}{r}.32 \\
.32 \\
.29 \\
.25 \\
.28\end{array}$ & $\begin{array}{l}.33 \\
.37 \\
.39 \\
.45 \\
.46\end{array}$ & $\begin{array}{r}.50 \\
.53 \\
.56 \\
.62 \\
4.0\end{array}$ & $\begin{array}{r}.51 \\
.28 \\
2.32 \\
14.1\end{array}$ & $\begin{array}{r}.27 \\
.27 \\
28.96 \\
1.0\end{array}$ \\
\hline $\begin{array}{l}11 \\
12 \\
13 \\
14 \\
15\end{array}$ & $\begin{array}{l}.09 \\
.09 \\
.10 \\
.10 \\
.11\end{array}$ & $\begin{array}{l}.22 \\
.26 \\
.28 \\
.28 \\
.29\end{array}$ & $\begin{array}{l}.46 \\
.47 \\
.45 \\
.43 \\
.45\end{array}$ & $\begin{array}{l}0.49 \\
0.50 \\
0.51 \\
0.52 \\
0.52\end{array}$ & $\begin{array}{l}.53 \\
.57 \\
.61 \\
.66 \\
.63\end{array}$ & $\begin{array}{r}.56 \\
.50 \\
.37 \\
.49 \\
.52\end{array}$ & $\begin{array}{l}.33 \\
.37 \\
.29 \\
.39 \\
.38\end{array}$ & $\begin{array}{r}.27 \\
.27 \\
.28 \\
.24 \\
.25\end{array}$ & $\begin{array}{r}.48 \\
.52 \\
.54 \\
2.4 \\
1.4\end{array}$ & $\begin{array}{l}13 \\
1.1 \\
2.67 \\
13\end{array}$ & $\begin{array}{r}5.9 \\
.36 \\
.22 \\
.17 \\
.16\end{array}$ & $\begin{array}{l}1.5 \\
9.2 \\
1.7 \\
1.5 \\
4.0\end{array}$ \\
\hline $\begin{array}{l}16 \\
17 \\
18 \\
19 \\
20\end{array}$ & $\begin{array}{l}.11 \\
.11 \\
.11 \\
.11 \\
.09\end{array}$ & $\begin{array}{l}.28 \\
.30 \\
.26 \\
.32 \\
.36\end{array}$ & $\begin{array}{l}.49 \\
.56 \\
.49 \\
.44 \\
.49\end{array}$ & $\begin{array}{l}0.50 \\
0.49 \\
0.49 \\
0.51 \\
0.52\end{array}$ & $\begin{array}{l}.59 \\
.63 \\
.56 \\
.53 \\
.59\end{array}$ & $\begin{array}{r}.55 \\
.54 \\
.52 \\
.52 \\
.54\end{array}$ & $\begin{array}{l}.40 \\
.37 \\
.32 \\
.40 \\
.30\end{array}$ & $\begin{array}{r}.21 \\
.22 \\
.23 \\
.21 \\
.20\end{array}$ & $\begin{array}{l}7.9 \\
.80 \\
.44 \\
.39 \\
.38\end{array}$ & $\begin{array}{r}1.4 \\
.94 \\
.87 \\
.83 \\
3.3\end{array}$ & $\begin{array}{rl}\frac{1.17}{8} & \\
1.1 & .28\end{array}$ & $\begin{array}{l}.80 \\
0.70 \\
0.67 \\
0.58 \\
0.55\end{array}$ \\
\hline $\begin{array}{l}21 \\
22 \\
23 \\
24 \\
25\end{array}$ & $\begin{array}{l}.11 \\
.10 \\
.13 \\
.14 \\
.17\end{array}$ & $\begin{array}{r}.35 \\
.43 \\
.41 \\
.40 \\
.43\end{array}$ & $\begin{array}{l}.51 \\
.59 \\
.58 \\
.56 \\
.52\end{array}$ & $\begin{array}{l}0.52 \\
0.52 \\
0.51 \\
0.51 \\
0.50\end{array}$ & $\begin{array}{l}.55 \\
.41 \\
.50 \\
.48 \\
.35\end{array}$ & $\begin{array}{l}.57 \\
.55 \\
.33 \\
.46 \\
.47\end{array}$ & $\begin{array}{r}.37 \\
.39 \\
.36 \\
.31 \\
.33\end{array}$ & $\begin{array}{l}.20 \\
.19 \\
.17 \\
.16 \\
.17\end{array}$ & $\begin{array}{r}.38 \\
.39 \\
.47 \\
.48 \\
.44\end{array}$ & $\begin{array}{c}5.6 \\
1.1 \\
.98 \\
17.94\end{array}$ & $\begin{array}{l}.18 \\
.16 \\
.16 \\
.15 \\
.14\end{array}$ & $\begin{array}{l}0.52 \\
0.49 \\
0.46 \\
0.43 \\
0.40\end{array}$ \\
\hline $\begin{array}{l}26 \\
27 \\
28 \\
29 \\
30 \\
31\end{array}$ & $\begin{array}{l}.19 \\
.21 \\
.22 \\
.23 \\
.26 \\
.28\end{array}$ & $\begin{array}{l}.44 \\
.45 \\
.40 \\
.43 \\
.46 \\
. .-\end{array}$ & $\begin{array}{l}.52 \\
.51 \\
.51 \\
.53 \\
.50 \\
.86\end{array}$ & $\begin{array}{r}0.50 \\
e .56 \\
01.8 \\
05.0 \\
06.3 \\
04.7\end{array}$ & $\begin{array}{r}.37 \\
.54 \\
.48 \\
.49 \\
.-. \\
.-\end{array}$ & $\begin{array}{r}.53 \\
.55 \\
.52 \\
.40 \\
.54 \\
.56\end{array}$ & $\begin{array}{r}.31 \\
.28 \\
.22 \\
.29 \\
.32 \\
-.-\end{array}$ & $\begin{array}{l}.18 \\
.22 \\
.24 \\
.24 \\
.23 \\
.25\end{array}$ & $\begin{array}{c}15^{.47} \\
2.1 \\
31 \\
4.3 \\
-\infty\end{array}$ & $\begin{array}{l}1.7 \\
0.80 \\
0.60 \\
0.50 \\
96.4 \\
32\end{array}$ & $\begin{array}{r}15 \\
5.7 \\
2.0 \\
.40 \\
.33 \\
.33\end{array}$ & $\begin{array}{r}0.40 \\
.82 \\
0.56 \\
0.44 \\
.40 \\
-.-\end{array}$ \\
\hline $\begin{array}{l}\text { TOTAL } \\
\text { MEAN } \\
\text { MAX } \\
\text { MIN } \\
\text { AC-FT }\end{array}$ & $\begin{array}{r}3.97 \\
.13 \\
.28 \\
.06 \\
7.9\end{array}$ & $\begin{array}{r}10.24 \\
.34 \\
.51 \\
.22 \\
20\end{array}$ & $\begin{array}{r}15.60 \\
.50 \\
.86 \\
.42 \\
31\end{array}$ & $\begin{array}{r}31.59 \\
1.02 \\
6.3 \\
.49 \\
63\end{array}$ & $\begin{array}{r}19.56 \\
.67 \\
3.0 \\
.35 \\
39\end{array}$ & $\begin{array}{r}15.76 \\
.51 \\
.58 \\
.33 \\
31\end{array}$ & $\begin{array}{r}11.79 \\
.39 \\
.60 \\
.22 \\
23\end{array}$ & $\begin{array}{r}7.67 \\
.25 \\
.36 \\
.16 \\
15\end{array}$ & $\begin{array}{r}73.76 \\
2.46 \\
31 \\
.27 \\
146\end{array}$ & $\begin{array}{r}133.10 \\
4.29 \\
32 \\
449 \\
264\end{array}$ & $\begin{array}{r}99.95 \\
3.22 \\
27 \\
114 \\
198\end{array}$ & $\begin{array}{r}58.05 \\
1.93 \\
28 \\
.27 \\
115\end{array}$ \\
\hline
\end{tabular}

Estimated 
PERTOD OF RECORD, - MAY 1996 to current year.

WATER QUALITY DATA, WATER YEAR OCTOBER 1995 TO SEPTEMBER 1996

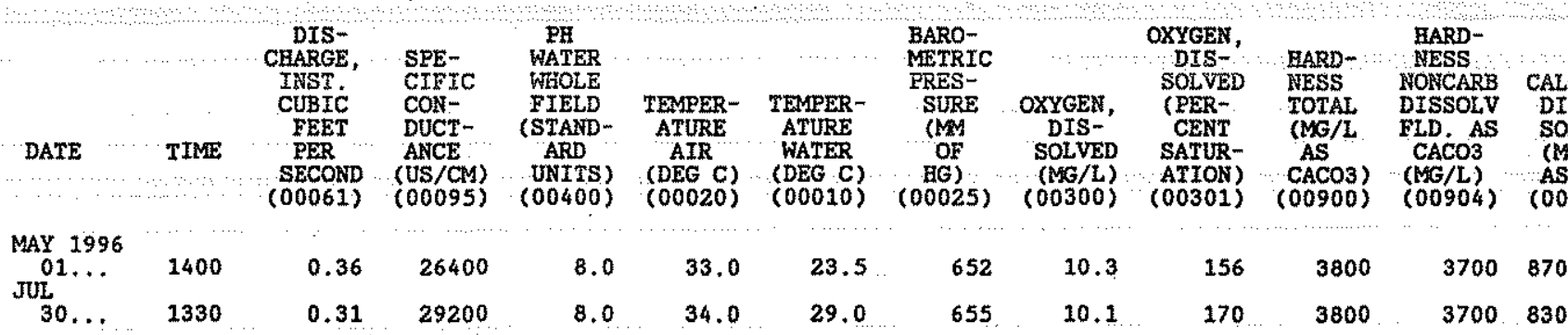

\begin{tabular}{|c|c|c|c|c|c|c|c|c|c|c|c|}
\hline DATE & $\begin{array}{c}\text { MAGNE- } \\
\text { SIUM, } \\
\text { DIS- } \\
\text { SOLVED } \\
\text { (MG/L } \\
\text { AS MG) } \\
(00925)\end{array}$ & $\begin{array}{l}\text { SODIUM, } \\
\text { DIS- } \\
\text { SOLVED } \\
\text { (NG/L } \\
\text { AS NA) } \\
(00930)\end{array}$ & $\begin{array}{l}\text { SODIUM } \\
\text { AD- } \\
\text { SORP- } \\
\text { TION } \\
\text { RATIO } \\
(00931)\end{array}$ & $\begin{array}{c}\text { POTAS- } \\
\text { SIUM, } \\
\text { DIS- } \\
\text { SOLVED } \\
(\text { ME/L } \\
\text { AS K) } \\
(00935)\end{array}$ & $\begin{array}{c}\text { BICAR- } \\
\text { BONATE } \\
\text { WATER } \\
\text { DIS IT } \\
\text { FIELD } \\
\text { DG/L AS } \\
\text { HCO3 } \\
(00453)\end{array}$ & $\begin{array}{c}\text { CAR- } \\
\text { BONATE } \\
\text { WATER } \\
\text { DIS IT } \\
\text { FIELD } \\
\text { MG/L AS } \\
\text { CO3 } \\
(00452)\end{array}$ & $\begin{array}{l}\text { ALKA- } \\
\text { IINITY } \\
\text { WAT DIS } \\
\text { TOT IT } \\
\text { FIELD } \\
\text { MG/L AS } \\
\text { CACO3 } \\
(39086)\end{array}$ & $\begin{array}{c}\text { ALKA- } \\
\text { LINITY } \\
\text { LAB } \\
\text { (AG/L } \\
\text { AS } \\
\text { CACO3) } \\
(90410)\end{array}$ & $\begin{array}{c}\text { SULFATE } \\
\text { DIS- } \\
\text { SOLVED } \\
\text { (MG/L } \\
\text { AS SO4) } \\
(00945)\end{array}$ & $\begin{array}{l}\text { CHLO- } \\
\text { RIDE, } \\
\text { DIS- } \\
\text { SOLVED } \\
\text { (HG/L } \\
\text { AS CL) } \\
(00940)\end{array}$ & $\begin{array}{c}\text { FLUO- } \\
\text { RIDE } \\
\text { DIS- } \\
\text { SOLVED } \\
\text { (MY/L } \\
\text { AS F ) } \\
(00150)\end{array}$ \\
\hline \multirow{3}{*}{$\begin{array}{c}\text { MAY } 1996 \\
01 \ldots \\
\text { JUL. } \\
30 \ldots\end{array}$} & 400 & 5000 & 35 & 130 & 149 & 0 & 122 & 125 & 3800 & 7500 & 5.3 \\
\hline & 410 & 5800 & 41 & 130 & 150 & 0 & 123 & 125 & 3600 & 9200 & 2.3 \\
\hline & $\begin{array}{c}\text { BROMIDE } \\
\text { DIS- } \\
\text { SOLVED } \\
\text { AS BR) } \\
(71870)\end{array}$ & $\begin{array}{c}\text { SILICA, } \\
\text { DIS- } \\
\text { SOLVED } \\
\text { (HG/L } \\
\text { SIO2) } \\
(00955)\end{array}$ & $\begin{array}{c}\text { SOLIDS, } \\
\text { RESIDUE } \\
\text { AT } 180 \\
\text { DEG C } \\
\text { DIS- } \\
(N G / L) \\
(70300)\end{array}$ & $\begin{array}{c}\text { SOLIDS, } \\
\text { SUM OF } \\
\text { CONSTI- } \\
\text { TUENTS, } \\
\text { DIS- } \\
\text { (NG/L) } \\
(70301)\end{array}$ & $\begin{array}{c}\text { NITRO- } \\
\text { GEN, } \\
\text { NITRITE } \\
\text { DIS- } \\
\text { SOLVED } \\
\text { AS N) } \\
(00613)\end{array}$ & $\begin{array}{c}\text { NITRO- } \\
\text { GEN, } \\
\text { NO2+NO3 } \\
\text { DIS- } \\
\text { SOLVED } \\
\text { AS N) } \\
(00631)\end{array}$ & $\begin{array}{c}\text { NITRO- } \\
\text { GEN, } \\
\text { AMNONIA } \\
\text { DTS- } \\
\text { SOLVED } \\
\text { AS N) } \\
(00608)\end{array}$ & $\begin{array}{l}\text { NITRO- } \\
\text { GEN,AM- } \\
\text { MONIA + } \\
\text { ORGANIC } \\
\text { TOTAL } \\
\text { AS N) } \\
(00625)\end{array}$ & $\begin{array}{l}\text { NITRO- } \\
\text { GEN, AN- } \\
\text { MONIA + } \\
\text { ORGANIC } \\
\text { DIS. } \\
\text { AS N) } \\
\text { (00S23) }\end{array}$ & $\begin{array}{c}\text { PHOS- } \\
\text { PEORUS } \\
\text { TOTAL } \\
\text { AS P) } \\
(00665)\end{array}$ & $\begin{array}{c}\text { PHOS- } \\
\text { PHORUS } \\
\text { DIS- } \\
\text { SOLVED } \\
\text { AS P) } \\
(00666)\end{array}$ \\
\hline \multirow{2}{*}{$\begin{array}{l}\text { MAY } 1996 \\
01 \ldots \\
\text { JUL } \\
30 \ldots\end{array}$} & 1.8 & 18 & 19200 & 17800 & $<0.010$ & 0.050 & 0.050 & 0.50 & $<0.20$ & $<0.010$ & $<0.010$ \\
\hline & 1.6 & 14 & 21200 & 20100 & $<0.010$ & 0.120 & 0.210 & $<0.20$ & $<0.20$ & $<0.010$ & $<0.010$ \\
\hline DATE & $\begin{array}{l}\text { PHOS- } \\
\text { PHORUS } \\
\text { ORTHO, } \\
\text { DIS- } \\
\text { SOLVED } \\
\text { (MG/L } \\
\text { AS P) } \\
(00671)\end{array}$ & $\begin{array}{l}\text { ALUM- } \\
\text { INUM, } \\
\text { TOTAL } \\
\text { RECOV- } \\
\text { ERABLE } \\
\text { (UG/L } \\
\text { AS AL) } \\
\text { (01105) }\end{array}$ & $\begin{array}{l}\text { ALUM- } \\
\text { INUM, } \\
\text { DIS- } \\
\text { SOLVED } \\
\text { (UG/L } \\
\text { AS AL) } \\
(01106)\end{array}$ & $\begin{array}{c}\text { ARSENIC } \\
\text { TOTAL } \\
\text { (UG/L } \\
\text { AS AS) } \\
\text { (01002) }\end{array}$ & $\begin{array}{c}\text { ARSENIC } \\
\text { DIS- } \\
\text { SOLVED } \\
\text { (UG/L } \\
\text { AS AS) } \\
(01000)\end{array}$ & $\begin{array}{l}\text { BARIUM, } \\
\text { TOTAL } \\
\text { RECOV- } \\
\text { ERABLE } \\
\text { (UG/L } \\
\text { AS BA) } \\
(01007)\end{array}$ & $\begin{array}{l}\text { BARIUM, } \\
\text { DIS - } \\
\text { SOLVED } \\
\text { (UG/L } \\
\text { AS BA) } \\
(01005)\end{array}$ & $\begin{array}{l}\text { BORON, } \\
\text { TOTAL } \\
\text { RECOV- } \\
\text { ERABLE } \\
\text { (UG/L } \\
\text { AS B) } \\
\text { (01022) }\end{array}$ & $\begin{array}{l}\text { BORON, } \\
\text { DIS- } \\
\text { SOLVED } \\
\text { (UG/C } \\
\text { AS B) } \\
(01020)\end{array}$ & $\begin{array}{l}\text { CADMIUM } \\
\text { WATER } \\
\text { UNFLTRD } \\
\text { TOTAL } \\
\text { (UG/L } \\
\text { AS CD) } \\
\text { (01027) }\end{array}$ & $\begin{array}{c}\text { CADMIUM } \\
\text { DIS- } \\
\text { SOLVED } \\
\text { (UG/L } \\
\text { AS CD) } \\
(01025)\end{array}$ \\
\hline \multirow{2}{*}{$\begin{array}{c}\text { MAY } 1996 \\
01 \ldots \\
\text { JUL } \\
30 \ldots\end{array}$} & $<0.010$ & 290 & 230 & 2 & 2 & 100 & 37 & 990 & 1000 & $<10$ & $<4.0$ \\
\hline & 0.010 & 740 & 103 & 3 & 3 & 200 & 150 & 940 & 921 & $<4$ & $<4,0$ \\
\hline DATE & $\begin{array}{l}\text { CHRO- } \\
\text { AIUAA, } \\
\text { TOTAL } \\
\text { RECOV- } \\
\text { ERABLE } \\
\text { (UG/L } \\
\text { AS CR) } \\
\text { (01034) }\end{array}$ & $\begin{array}{l}\text { CWDO- } \\
\text { MIUM, } \\
\text { DIS- } \\
\text { SOLVED } \\
\text { (UG/L } \\
\text { AS CR) } \\
(01030)\end{array}$ & $\begin{array}{l}\text { COPDLE, } \\
\text { TOTAL, } \\
\text { RECOV- } \\
\text { ERABLE } \\
\text { (UQ/L } \\
\text { AS CU) } \\
\text { (01042) }\end{array}$ & $\begin{array}{c}\text { COPPER, } \\
\text { DIS- } \\
\text { SOLVED } \\
\text { (UG/L } \\
\text { AS CU) } \\
(01040)\end{array}$ & $\begin{array}{l}\text { IROW, } \\
\text { TOTAL } \\
\text { RECOV- } \\
\text { ERABLE } \\
\text { (UG/L } \\
\text { AS FE) } \\
(01045)\end{array}$ & $\begin{array}{l}\text { IRON, } \\
\text { DIS - } \\
\text { SOLVED } \\
\text { (UG/L } \\
\text { AS FE) } \\
(01046)\end{array}$ & $\begin{array}{l}\text { LEAD, } \\
\text { TOTAL } \\
\text { RECOV- } \\
\text { ERABLE } \\
\text { (UG/L } \\
\text { AS PB) } \\
\text { (01051) }\end{array}$ & $\begin{array}{c}\text { LEAD, } \\
\text { DIS- } \\
\text { SOLVED } \\
\text { (UO/L } \\
\text { AS PB) } \\
(01049)\end{array}$ & $\begin{array}{l}\text { LIIHIUM } \\
\text { TOTAL } \\
\text { RECOV- } \\
\text { ERABLE } \\
\text { (UG/L } \\
\text { AS LI) } \\
\text { (01132) }\end{array}$ & $\begin{array}{c}\text { LITHIUM } \\
\text { DIS- } \\
\text { SOLVED } \\
\text { (UG/L } \\
\text { AS LI) } \\
(01130)\end{array}$ & $\begin{array}{l}\text { MANGA- } \\
\text { WESE. } \\
\text { TOTAL } \\
\text { RECOV- } \\
\text { ERABLE } \\
\text { (UG/L } \\
\text { AS MN) } \\
\text { (01055) }\end{array}$ \\
\hline
\end{tabular}

\begin{tabular}{|c|c|c|c|c|c|c|c|c|c|c|c|}
\hline $\begin{array}{l}71996 \\
01 \ldots\end{array}$ & 2 & 1.6 & $<4$ & $<4.0$ & 210 & 260 & $<10$ & $<10$ & 800 & 880 & 60 \\
\hline UL & $<4$ & $<4.0$ & 4 & $<4,0$ & 420 & $<60$ & $<10$ & $<4,0$ & 700 & 1100 & 80 \\
\hline
\end{tabular}


TULAROSA VALLEY BASIN

08480595 SALT CREEK AT RANGE ROAD 316 ON WSMR, IM -- Continued

WATER-QUALITY RECORDS

WATER QUALITY DATA, WATER YEAR OCTOBER 1995 TO SEPTEMBER 1996

\begin{tabular}{|c|c|c|c|c|c|c|c|c|c|c|c|}
\hline DATE & $\begin{array}{l}\text { MANGA- } \\
\text { NESE, } \\
\text { DIS- } \\
\text { SOLVED } \\
\text { (UG/L } \\
\text { AS MN) } \\
\text { (01056) }\end{array}$ & $\begin{array}{c}\text { MERCURY } \\
\text { TOTAL } \\
\text { RECOV- } \\
\text { ERABLE } \\
\text { (UG/L } \\
\text { AS EG) } \\
\text { (71900) }\end{array}$ & $\begin{array}{c}\text { MERCIIRY } \\
\text { DIS- } \\
\text { SOLVED } \\
\text { (IJG/L } \\
\text { AS HG) } \\
\text { (71890) }\end{array}$ & $\begin{array}{l}\text { SELE- } \\
\text { NIUM, } \\
\text { TOTAL } \\
\text { (UG/L } \\
\text { AS SE) } \\
\text { (01147) }\end{array}$ & $\begin{array}{c}\text { SELE- } \\
\text { NIUM, } \\
\text { DIS- } \\
\text { SOLVED } \\
\text { (UG/L } \\
\text { AS SE) } \\
(01145 \text { ) }\end{array}$ & $\begin{array}{c}\text { SILVER, } \\
\text { TOTAI } \\
\text { RECOV- } \\
\text { ERABLE } \\
\text { (UG/L } \\
\text { AS AG) } \\
(01077)\end{array}$ & $\begin{array}{c}\text { SILVER, } \\
\text { DIS- } \\
\text { SOLVED } \\
\text { (UG/L } \\
\text { AS AG) } \\
(01075 \text { ) }\end{array}$ & $\begin{array}{c}\text { STRON- } \\
\text { TIUM, } \\
\text { TOTAL } \\
\text { RECOV- } \\
\text { ERABLE } \\
\text { (UG/L } \\
\text { AS SR) } \\
\text { (01082) }\end{array}$ & $\begin{array}{c}\text { STRON- } \\
\text { TIUM, } \\
\text { DIS- } \\
\text { SOLVED } \\
\text { (UG/L, } \\
\text { AS SR) } \\
(01080)\end{array}$ & $\begin{array}{l}\text { ZINC, } \\
\text { TOTAL } \\
\text { RECOV- } \\
\text { GRABLE } \\
\text { (UG/L } \\
\text { AS ZN) } \\
\text { (01092) }\end{array}$ & $\begin{array}{c}\text { ZINC, } \\
\text { DIS } \\
\text { SOLVED } \\
\text { (UG/L } \\
\text { AS ZN) } \\
\text { (01090) }\end{array}$ \\
\hline $\begin{array}{c}\text { IAY } 1996 \\
01 \ldots\end{array}$ & 32 & 0.80 & 0.3 & 4 & 4 & $<5$ & $<4.0$ & 19000 & 19000 & $<10$ & 69 \\
\hline $30 \ldots$ & $<20$ & $<0.10$ & 0.5 & 2 & 2 & $<4$ & $<10$ & 19000 & 18000 & $<10$ & $<60$ \\
\hline
\end{tabular}

CHEMICAL ANALYSES OF ORGANIC COMPOUNDS, WATER YEAR OCTOBER 1995 TO SEPTEMBER 1996

\begin{tabular}{|c|c|c|c|c|c|}
\hline DATE & TIME & $\begin{array}{l}2,4-\mathrm{D}, \\
\text { TOTAL } \\
\text { (UG/L) } \\
(39730)\end{array}$ & $\begin{array}{l}2,4,5-T \\
\text { TOTAL } \\
\text { (UG/L) } \\
(39740)\end{array}$ & $\begin{array}{c}\text { STLVEX, } \\
\text { TOTAL } \\
\text { (UG/L) } \\
(39760)\end{array}$ & $\begin{array}{l}2, \quad 4-D P \\
\text { TOTAL } \\
\text { (UG/L) } \\
(82183)\end{array}$ \\
\hline $\begin{array}{c}\text { MAY } 1996 \\
01 \ldots \\
\text { JUL }\end{array}$ & 1400 & 0.030 & $<0.010$ & $<0.010$ & $<0.010$ \\
\hline
\end{tabular}


TULAROSA VALLEY BASIN

08481500 TULAROSA CREEK NEAR BENT, NM

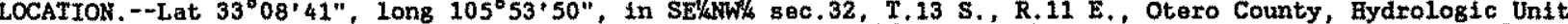
13044503 on right benk 45 ft downstream from bridge on old U.S. Highway $70,2,6$ mi west of Bent, 8,5 mi northeast of Tularosa, and at mile 19.4 .

DRAIKAGE AREA. $-120 \mathrm{mi}^{2}$, approximately.

FERIOD OF RECORD,--December 1947 to September 1996 (discontinued). Frior to October 1982 published as "Rio Tularosa near Bent".

REVISED RECORDS. - WSP $1312 ; \ldots 1949$ (M).

GAGE.--Water-stage recorder and concrete control. Elevation of gage is 5 , 450 ft above National Geodetic Vertical Datum of 1929 , from topographic map.

REMARKS,--Records good except for estimated datly discharges, which are poor. Diversions for irrigation of about 1,000 acres, 1959 determination, upstream from station.

EXIREMES OUTSIDE PERIOD OF RECORD,-A major Elood probably occurxed Sept. 3,1938, when a peak of 9,640 ft $3 / \mathrm{s}$ was computed for station approxinately 6 mi downstream near Tularosa. Anothex flood may have occurred July 2 , 1914 . DISCHARGE, CUBTC FEET PER SECOND, WATER YEAR OCTOBER 1995 TO SEFTEMBER 1996

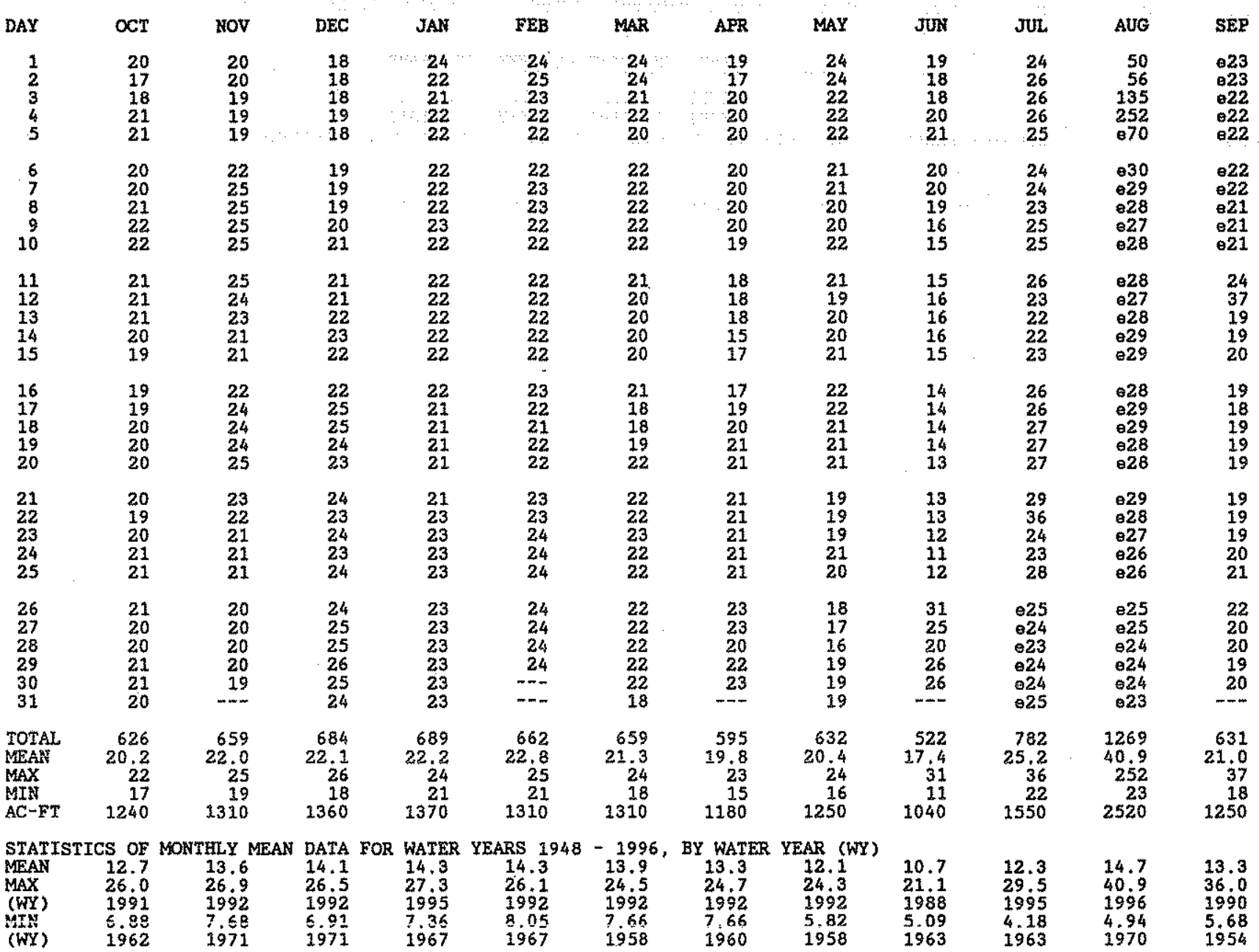
ANNUAL TOTAL.

SUMMARY STATISTICS

ANNUAL MEAN

LOWEST ANHUAL MEAN

LOWEST ANNAL, MEAN

GIGHEST DATLYY MEA

ANWUAL SEVEN-DAY MINIMUM

ANWUAL, SEVEN-DAY MINTMUM

INSTANTANEOUS PEAK STAGE

INSTANTANEOUS LOW FLOW

ANNUAL RINNOFF (AC-FT)

10 PERCENT EXCEEDS

50 PERCENT EXCEEDS

90 PERCENT EXCEEDS

Estimated
FOR 1995 CALENDAR YEAR

8241

22.6

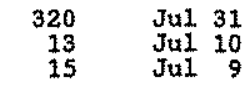

16350

27
FOR 1996 WATER YEAR

$$
\begin{array}{r}
8410 \\
23.0
\end{array}
$$

a-From rating curve extended above $160 \mathrm{ft} / \mathrm{s}$, on basis of slope-area measurement of peak flow.
b-Maximum gage height, $5.60 \mathrm{ft}$, Aug. 8, 1988 , and July 14, 1991, discharge not determined.
WATER YEARS $1948-1996$

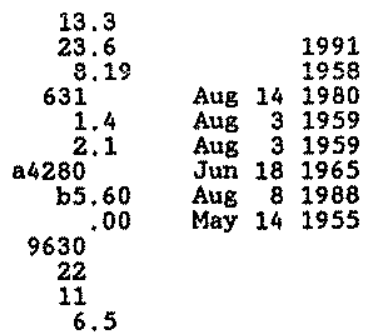


SAN JUAN RTVER BASTN

09343300 RTO BLANCO BELOW BLANCO DIVERSION DAM, NEAR PAGOSA SPRINGS, CO

LOCATION, - Lat $37^{\circ} 12^{\prime} 13^{\prime \prime}$, long $106^{\circ} 48^{\circ} 38^{\prime \prime}$, in NE/4AW/ sec.11, T.34 N., R.1 E., Archuleta County, Bydrologic Unit 14080101, on left bank 250 downstream from Blanco Diversion Dam, 1.1 mi downstream for Leche Creek, and 12 mi southeast of Fagosa Springs.

DRAINAGE AREA. $--69.1 \mathrm{mi}^{2}$.

PERTOD OF RECORD.--March 1971 to current yoar.

GAGE.--Water-stage recorder with satellite telemetry. Datum of gage is $7,858,04$ ft above sea level, (levels by Bureau of Reclanation).

REMARKS.--Records good except for Oct. 1 to Dec. 7 (unstable approach conditions at flume), and estimated daily discharges, which are poor. Flows controlled by diversion dam upstream.

COOPERATION.--Records collected and computed by Colorado Dtvtston of Water Resources and reviewed by Geological. Survey.

DISCAARGE, CUBIC FEET PER SECOND, WATER YEAR OCTOBER 1995 TO SEETEMBER 1996

\begin{tabular}{|c|c|c|c|c|c|c|c|c|c|c|c|c|}
\hline DAY & $\alpha C T$ & Nov & DEC & JAN & FEB & MAR & APR & MAY & JUN & JUL & AltG & SEP \\
\hline $\begin{array}{l}1 \\
2 \\
3 \\
4 \\
5\end{array}$ & $\begin{array}{l}43 \\
35 \\
33 \\
32 \\
30\end{array}$ & $\begin{array}{l}22 \\
20 \\
15 \\
17 \\
15\end{array}$ & $\begin{array}{r}17 \\
17 \\
016 \\
16 \\
015\end{array}$ & $\begin{array}{l}13 \\
13 \\
14 \\
15 \\
14\end{array}$ & $\begin{array}{r}14 \\
012 \\
010 \\
012 \\
14\end{array}$ & $\begin{array}{r}\theta 22 \\
e 22 \\
e 22 \\
23 \\
21\end{array}$ & $\begin{array}{l}25 \\
25 \\
24 \\
24 \\
24\end{array}$ & $\begin{array}{l}44 \\
45 \\
46 \\
47 \\
48\end{array}$ & $\begin{array}{l}29 \\
29 \\
29 \\
29 \\
30\end{array}$ & $\begin{array}{l}22 \\
22 \\
22 \\
22 \\
22\end{array}$ & $\begin{array}{l}20 \\
21 \\
20 \\
20 \\
19\end{array}$ & $\begin{array}{l}14 \\
14 \\
13 \\
13 \\
13\end{array}$ \\
\hline $\begin{array}{r}6 \\
7 \\
8 \\
9 \\
10\end{array}$ & $\begin{array}{l}30 \\
29 \\
28 \\
27 \\
25\end{array}$ & $\begin{array}{l}16 \\
16 \\
17 \\
18 \\
19\end{array}$ & $\begin{array}{r}17 \\
17 \\
16 \\
014 \\
014\end{array}$ & $\begin{array}{r}12 \\
12 \\
011 \\
12 \\
13\end{array}$ & $\begin{array}{r}15 \\
16 \\
16 \\
19 \\
020\end{array}$ & $\begin{array}{r}20 \\
820 \\
020 \\
024 \\
32\end{array}$ & $\begin{array}{l}24 \\
24 \\
25 \\
26 \\
25\end{array}$ & $\begin{array}{l}45 \\
46 \\
45 \\
44 \\
44\end{array}$ & $\begin{array}{l}29 \\
30 \\
31 \\
30 \\
30\end{array}$ & $\begin{array}{l}22 \\
22 \\
22 \\
23 \\
21\end{array}$ & $\begin{array}{l}18 \\
17 \\
17 \\
17 \\
17\end{array}$ & $\begin{array}{l}14 \\
13 \\
13 \\
12\end{array}$ \\
\hline $\begin{array}{l}11 \\
12 \\
13 \\
14 \\
15\end{array}$ & $\begin{array}{l}23 \\
23 \\
24 \\
24 \\
24\end{array}$ & $\begin{array}{l}17 \\
23 \\
30 \\
31 \\
29\end{array}$ & $\begin{array}{r}015 \\
015 \\
16 \\
17 \\
014\end{array}$ & $\begin{array}{l}13 \\
14 \\
17 \\
20 \\
19\end{array}$ & $\begin{array}{r}\text { e25 } \\
27 \\
23 \\
020 \\
\text { e25 }\end{array}$ & $\begin{array}{l}39 \\
43 \\
34 \\
31 \\
28\end{array}$ & $\begin{array}{l}25 \\
25 \\
25 \\
25 \\
23\end{array}$ & $\begin{array}{l}45 \\
45 \\
45 \\
45 \\
45\end{array}$ & $\begin{array}{l}30 \\
30 \\
30 \\
30 \\
31\end{array}$ & $\begin{array}{l}20 \\
20 \\
20 \\
20 \\
20\end{array}$ & $\begin{array}{l}16 \\
15 \\
14 \\
14 \\
14\end{array}$ & $\begin{array}{l}11 \\
15 \\
21 \\
68 \\
49\end{array}$ \\
\hline $\begin{array}{l}16 \\
17 \\
18 \\
19 \\
20\end{array}$ & $\begin{array}{l}22 \\
22 \\
21 \\
20 \\
19\end{array}$ & $\begin{array}{l}27 \\
26 \\
25 \\
24 \\
23\end{array}$ & $\begin{array}{r}e 14 \\
16 \\
015 \\
16 \\
015\end{array}$ & $\begin{array}{r}914 \\
13 \\
13 \\
15 \\
14\end{array}$ & $\begin{array}{r}930 \\
34 \\
34 \\
29 \\
34\end{array}$ & $\begin{array}{l}27 \\
27 \\
26 \\
27 \\
31\end{array}$ & $\begin{array}{l}23 \\
23 \\
23 \\
23 \\
23\end{array}$ & $\begin{array}{l}45 \\
43 \\
42 \\
42 \\
43\end{array}$ & $\begin{array}{l}30 \\
30 \\
30 \\
30 \\
29\end{array}$ & $\begin{array}{l}20 \\
20 \\
21 \\
22 \\
22\end{array}$ & $\begin{array}{c}14 \\
11 \\
9.7 \\
11 \\
11\end{array}$ & $\begin{array}{l}28 \\
38 \\
40 \\
26 \\
25\end{array}$ \\
\hline $\begin{array}{l}21 \\
22 \\
23 \\
24 \\
25\end{array}$ & $\begin{array}{l}18 \\
18 \\
15 \\
16 \\
16\end{array}$ & $\begin{array}{l}22 \\
21 \\
20 \\
19 \\
19\end{array}$ & $\begin{array}{r}15 \\
16 \\
15 \\
114 \\
13\end{array}$ & $\begin{array}{l}14 \\
13 \\
13 \\
14 \\
14\end{array}$ & $\begin{array}{r}54 \\
37 \\
31 \\
028 \\
27\end{array}$ & $\begin{array}{l}31 \\
21 \\
24 \\
24 \\
24\end{array}$ & $\begin{array}{l}23 \\
23 \\
24 \\
25 \\
26\end{array}$ & $\begin{array}{l}42 \\
37 \\
31 \\
31 \\
31\end{array}$ & $\begin{array}{l}29 \\
30 \\
29 \\
29 \\
27\end{array}$ & $\begin{array}{l}21 \\
21 \\
24 \\
23 \\
23\end{array}$ & $\begin{array}{l}12 \\
18 \\
24 \\
36 \\
29\end{array}$ & $\begin{array}{l}25 \\
25 \\
25 \\
25 \\
25\end{array}$ \\
\hline $\begin{array}{l}26 \\
27 \\
28 \\
29 \\
30 \\
31\end{array}$ & $\begin{array}{l}16 \\
16 \\
16 \\
16 \\
16 \\
16\end{array}$ & $\begin{array}{r}20 \\
17 \\
13 \\
15 \\
17 \\
--\end{array}$ & $\begin{array}{r}12 \\
11 \\
14 \\
14 \\
013 \\
14\end{array}$ & $\begin{array}{l}14 \\
15 \\
15 \\
14 \\
14 \\
14\end{array}$ & $\begin{array}{r}23 \\
20 \\
e 22 \\
23 \\
--- \\
---\end{array}$ & $\begin{array}{l}24 \\
24 \\
24 \\
24 \\
24 \\
24\end{array}$ & $\begin{array}{r}24 \\
24 \\
23 \\
22 \\
32 \\
-\end{array}$ & $\begin{array}{l}31 \\
32 \\
32 \\
32 \\
31 \\
30\end{array}$ & $\begin{array}{r}23 \\
24 \\
25 \\
23 \\
23 \\
---\end{array}$ & $\begin{array}{l}23 \\
23 \\
23 \\
22 \\
22 \\
20\end{array}$ & $\begin{array}{l}22 \\
26 \\
21 \\
20 \\
17 \\
15\end{array}$ & $\begin{array}{r}25 \\
23 \\
24 \\
22 \\
22 \\
---\end{array}$ \\
\hline $\begin{array}{l}\text { TOTAL } \\
\text { MEAN } \\
\text { MAX } \\
\text { MIN } \\
\text { AC-FI }\end{array}$ & $\begin{array}{r}713 \\
23.0 \\
43 \\
15 \\
1410\end{array}$ & $\begin{array}{r}613 \\
20+4 \\
31 \\
13 \\
1220\end{array}$ & $\begin{array}{r}463 \\
14.9 \\
17 \\
11 \\
918\end{array}$ & $\begin{array}{r}435 \\
14.0 \\
20 \\
11 \\
863\end{array}$ & $\begin{array}{r}694 \\
23.9 \\
54 \\
10 \\
1380\end{array}$ & $\begin{array}{r}807 \\
26.0 \\
43 \\
20 \\
1600\end{array}$ & $\begin{array}{r}730 \\
24.3 \\
32 \\
22 \\
1450\end{array}$ & $\begin{array}{r}12.54 \\
40.5 \\
48 \\
30 \\
2490\end{array}$ & $\begin{array}{r}858 \\
28.6 \\
31 \\
23 \\
1700\end{array}$ & $\begin{array}{r}670 \\
21.6 \\
24 \\
20 \\
1330\end{array}$ & $\begin{array}{r}555.7 \\
17.9 \\
36 \\
9.7 \\
1100\end{array}$ & $\begin{array}{r}692 \\
23.1 \\
68 \\
11 \\
1370\end{array}$ \\
\hline
\end{tabular}

STATISTICS OF MONTHLY MEAN DATA FOR WATER YEARS 1971 - 1996, BY WATER YEAR (WY)

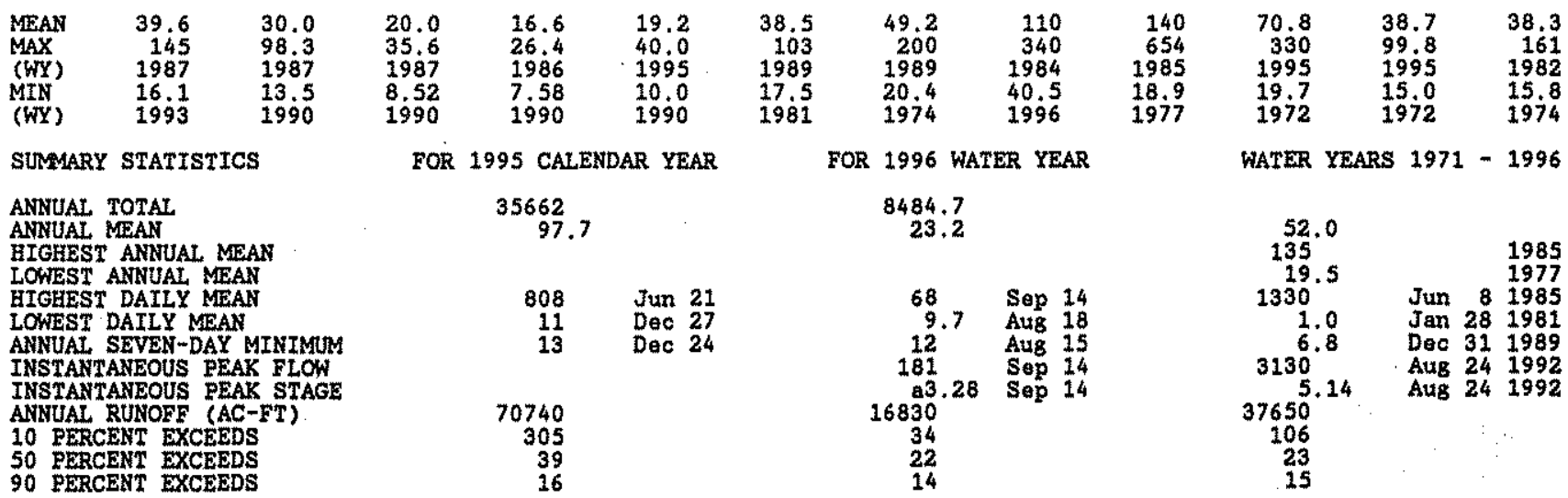

e- Estimated.

a-Maximum gage holght, $3.50 \mathrm{ft}$, Feb 10, 1996, backwater from 100. 
SAN JUAN RIVER BASIN

09344400 NAVAJO RIVER BELOW OSO DIVERSTON DAM, NEAR CHRONO, CO

LOCATION --Iat $37^{\circ} 01^{\prime} 49^{\prime \prime}$, long $106^{\circ} 44^{\prime} 14^{*}$, in NE/ sec 9, T.32 N., R.2 E., Archuleta County, Hydrologic Unit 14080101 , on left bank 600 downstream from Oso Diversion Dam, 5.8 mi east of Chromo, and 6 mi upstream from Littie Navajo River.

DRAINAGE AREA, $--100.5 \mathrm{mi}^{2}$.

PERIOD OF RECORD.--March 1971 to current year.

GAGE,-Water-gtage recorder with satellite telemetry and Parshali flume. Datum of gage is $7,665.30$ et above sea level, (levels by Buxeau of Reclamation). Prior to Sept. 5, 1979, at same site, at different datum.

REMARKS,-Racords good oxcept for estimated daliy discharges, which axe poor. Flows controlled by divarsion dem upstream.

COOPERATrON,--Records collected and compited by Colorado Division of Water Resources and reviewed by Geological Survey.

DISCHARGE, CUBIC FEET PER SECOND, WATER YEAR OCTOBER 1995 TO SEPTEMBER 1996

\begin{tabular}{|c|c|c|c|c|c|c|c|c|c|c|c|c|}
\hline DAY & $\propto T$ & Hov & $\mathrm{DEC}$ & JAN & FEB & MAR & APR & MAX & JWN & JนL & AUG & SER \\
\hline $\begin{array}{l}1 \\
2 \\
3 \\
4 \\
5\end{array}$ & $\begin{array}{l}76 \\
70 \\
66 \\
62 \\
58\end{array}$ & $\begin{array}{l}48 \\
47 \\
40 \\
41 \\
41\end{array}$ & $\begin{array}{l}29 \\
46 \\
30 \\
30 \\
36\end{array}$ & $\begin{array}{r}33 \\
\theta 32 \\
032 \\
33 \\
34\end{array}$ & $\begin{array}{r}34 \\
032 \\
030 \\
030 \\
034\end{array}$ & $\begin{array}{l}39 \\
40 \\
40 \\
42 \\
42\end{array}$ & $\begin{array}{l}43 \\
39 \\
41 \\
39 \\
38\end{array}$ & $\begin{array}{l}90 \\
88 \\
80 \\
91 \\
91\end{array}$ & $\begin{array}{l}55 \\
55 \\
57 \\
58 \\
58\end{array}$ & $\begin{array}{l}57 \\
56 \\
56 \\
58 \\
58\end{array}$ & $\begin{array}{l}34 \\
33 \\
37 \\
37 \\
32\end{array}$ & $\begin{array}{l}37 \\
36 \\
36 \\
37 \\
35\end{array}$ \\
\hline $\begin{array}{r}6 \\
7 \\
8 \\
9 \\
10\end{array}$ & $\begin{array}{l}56 \\
55 \\
53 \\
53 \\
53\end{array}$ & $\begin{array}{l}41 \\
40 \\
40 \\
40 \\
48\end{array}$ & $\begin{array}{l}35 \\
29 \\
36 \\
35 \\
35\end{array}$ & $\begin{array}{r}e 32 \\
e 30 \\
028 \\
032 \\
35\end{array}$ & $\begin{array}{r}39 \\
38 \\
e 36 \\
036 \\
39\end{array}$ & $\begin{array}{l}42 \\
38 \\
40 \\
42 \\
46\end{array}$ & $\begin{array}{l}38 \\
38 \\
38 \\
37 \\
37\end{array}$ & $\begin{array}{l}90 \\
90 \\
89 \\
89 \\
90\end{array}$ & $\begin{array}{l}59 \\
59 \\
59 \\
59 \\
59\end{array}$ & $\begin{array}{l}58 \\
58 \\
56 \\
56 \\
55\end{array}$ & $\begin{array}{l}28 \\
24 \\
25 \\
28 \\
27\end{array}$ & $\begin{array}{l}36 \\
34 \\
32 \\
32 \\
31\end{array}$ \\
\hline $\begin{array}{l}11 \\
12 \\
13 \\
14 \\
15\end{array}$ & $\begin{array}{l}52 \\
50 \\
50 \\
48 \\
47\end{array}$ & $\begin{array}{l}39 \\
43 \\
46 \\
46 \\
46\end{array}$ & $\begin{array}{l}43 \\
46 \\
40 \\
38 \\
35\end{array}$ & $\begin{array}{r}834 \\
032 \\
030 \\
030 \\
32\end{array}$ & $\begin{array}{l}39 \\
35 \\
34 \\
34 \\
40\end{array}$ & $\begin{array}{l}51 \\
48 \\
39 \\
39 \\
43\end{array}$ & $\begin{array}{l}38 \\
39 \\
38 \\
38 \\
39 \ldots\end{array}$ & $\begin{array}{l}90 \\
90 \\
90 \\
90 \\
91\end{array}$ & $\begin{array}{l}59 \\
59 \\
59 \\
59 \\
59\end{array}$ & $\begin{array}{l}55 \\
56 \\
57 \\
57 \\
57\end{array}$ & $\begin{array}{l}26 \\
25 \\
24 \\
26 \\
26\end{array}$ & $\begin{array}{l}31 \\
33 \\
34 \\
43 \\
53\end{array}$ \\
\hline $\begin{array}{l}16 \\
17 \\
18 \\
19 \\
20\end{array}$ & $\begin{array}{l}45 \\
45 \\
45 \\
44 \\
43\end{array}$ & $\begin{array}{l}40 \\
38 \\
42 \\
41 \\
42\end{array}$ & $\begin{array}{l}33 \\
35 \\
33 \\
33 \\
32\end{array}$ & $\begin{array}{r}32 \\
30 \\
e 30 \\
e 28 \\
030\end{array}$ & $\begin{array}{l}44 \\
44 \\
45 \\
43 \\
49\end{array}$ & $\begin{array}{l}46 \\
47 \\
45 \\
46 \\
51\end{array}$ & $\begin{array}{l}39 \\
39 \\
39 \\
38 \\
38\end{array}$ & $\begin{array}{l}93 \\
91 \\
90 \\
91 \\
91\end{array}$ & $\begin{array}{l}59 \\
58 \\
58 \\
56 \\
56\end{array}$ & $\begin{array}{l}55 \\
55 \\
56 \\
57 \\
57\end{array}$ & $\begin{array}{l}26 \\
30 \\
32 \\
28 \\
27\end{array}$ & $\begin{array}{l}39 \\
44 \\
61 \\
64 \\
58\end{array}$ \\
\hline $\begin{array}{l}21 \\
22 \\
23 \\
24 \\
25\end{array}$ & $\begin{array}{l}42 \\
42 \\
38 \\
43 \\
43\end{array}$ & $\begin{array}{l}41 \\
39 \\
40 \\
37 \\
39\end{array}$ & $\begin{array}{r}830 \\
32 \\
030 \\
026 \\
028\end{array}$ & $\begin{array}{l}032 \\
030 \\
028 \\
030 \\
030\end{array}$ & $\begin{array}{l}68 \\
59 \\
51 \\
47 \\
47\end{array}$ & $\begin{array}{l}46 \\
37 \\
36 \\
36 \\
38\end{array}$ & $\begin{array}{l}38 \\
38 \\
38 \\
38 \\
38\end{array}$ & $\begin{array}{l}91 \\
85 \\
82 \\
82 \\
82\end{array}$ & $\begin{array}{l}58 \\
60 \\
59 \\
58 \\
56\end{array}$ & $\begin{array}{l}50 \\
42 \\
35 \\
36 \\
36\end{array}$ & $\begin{array}{l}33 \\
36 \\
34 \\
47 \\
48\end{array}$ & $\begin{array}{l}49 \\
48 \\
46 \\
43 \\
39\end{array}$ \\
\hline $\begin{array}{l}26 \\
27 \\
28 \\
29 \\
30 \\
31\end{array}$ & $\begin{array}{l}42 \\
39 \\
38 \\
39 \\
40 \\
40\end{array}$ & $\begin{array}{r}39 \\
35 \\
43 \\
36 \\
36 \\
-\end{array}$ & $\begin{array}{r}028 \\
028 \\
030 \\
32 \\
30 \\
32\end{array}$ & $\begin{array}{r}028 \\
e 26 \\
e 32 \\
e 34 \\
35 \\
34\end{array}$ & $\begin{array}{r}43 \\
37 \\
39 \\
42 \\
--- \\
--m\end{array}$ & $\begin{array}{l}39 \\
38 \\
38 \\
38 \\
38 \\
40\end{array}$ & $\begin{array}{r}38 \\
38 \\
39 \\
39 \\
69 \\
-\end{array}$ & $\begin{array}{l}82 \\
82 \\
82 \\
82 \\
80 \\
68\end{array}$ & $\begin{array}{r}58 \\
59 \\
57 \\
56 \\
56 \\
---\end{array}$ & $\begin{array}{l}35 \\
35 \\
40 \\
42 \\
40 \\
36\end{array}$ & $\begin{array}{l}39 \\
40 \\
48 \\
58 \\
44 \\
37\end{array}$ & $\begin{array}{r}39 \\
38 \\
37 \\
37 \\
27 \\
---\end{array}$ \\
\hline $\begin{array}{l}\text { TOTAL } \\
\text { MEAN } \\
\text { MAX } \\
\text { MIN } \\
\text { AC }=\text { ET }\end{array}$ & $\begin{array}{r}1517 \\
48,9 \\
76 \\
38 \\
3010\end{array}$ & $\begin{array}{r}1234 \\
41.1 \\
48 \\
35 \\
2450\end{array}$ & $\begin{array}{r}1035 \\
33,4 \\
46 \\
26 \\
2050\end{array}$ & $\begin{array}{r}968 \\
31.2 \\
35 \\
26 \\
1920\end{array}$ & $\begin{array}{r}1188 \\
41.0 \\
68 \\
30 \\
2360\end{array}$ & $\begin{array}{r}1290 \\
41.6 \\
51 \\
36 \\
2560\end{array}$ & $\begin{array}{r}1186 \\
39.5 \\
69 \\
37 \\
2350\end{array}$ & $\begin{array}{r}2693 \\
86.9 \\
93 \\
68 \\
5340\end{array}$ & $\begin{array}{r}1737 \\
57.9 \\
60 \\
55 \\
3450\end{array}$ & $\begin{array}{r}1557 \\
50.2 \\
58 \\
35 \\
3090\end{array}$ & $\begin{array}{r}1039 \\
33.5 \\
58 \\
24 \\
2060\end{array}$ & $\begin{array}{r}1209 \\
40.3 \\
64 \\
27 \\
2400\end{array}$ \\
\hline
\end{tabular}

STATISTICS OF MONTHLY MEAN DATA FOR WATER YEARS 1971 - 2996, BY WATER YEAR (WY)

\begin{tabular}{|c|c|c|c|c|c|c|c|c|c|c|c|c|}
\hline $\begin{array}{l}\text { MEAN } \\
\text { MAX } \\
\text { (WY) } \\
\text { MIN } \\
\text { (WY) }\end{array}$ & $\begin{array}{r}54.3 \\
161 \\
1987 \\
26.3 \\
1981\end{array}$ & $\begin{array}{r}46.7 \\
132 \\
1987 \\
27.4\end{array}$ & $\begin{array}{l}38.8 \\
71.9 \\
1987 \\
21.3 \\
10.7\end{array}$ & $\begin{array}{l}35.3 \\
51.3 \\
1985 \\
19.8\end{array}$ & $\begin{array}{l}37.1 \\
52.7 \\
1986 \\
24.4\end{array}$ & $\begin{array}{r}60.9 \\
135 \\
1989 \\
32.0\end{array}$ & $\begin{array}{r}58.5 \\
183 \\
1993 \\
37.5\end{array}$ & $\begin{array}{r}132 \\
271 \\
1984 \\
86.9 \\
1996\end{array}$ & $\begin{array}{r}160 \\
720 \\
1985 \\
44.7\end{array}$ & $\begin{array}{r}95.0 \\
406 \\
1995 \\
40.2\end{array}$ & $\begin{array}{r}65.1 \\
124 \\
1982 \\
28.1 \\
1972\end{array}$ & $\begin{array}{r}59.6 \\
146 \\
1982 \\
28.4 \\
1978\end{array}$ \\
\hline
\end{tabular}

SURARY STATIETICS

ANNUAL, TOTAL

BIGEEST ANNUAL MEAR

LOWEST ANNUAL MEAN

HIGHEST DAILY MEAN

LOWEST DAILY MEAN

INSTANTANEOUS PEAK FLOW

INSTANTANEOUS PEAK STAGE

ANNUAL RINOFF (AC-FT)

10 PERCENT EXCEEDS

50 PERCERT EXCEEDS

FOR 1995 CAIEXPAP YEAP

FOR 1005 WATER YQAP

48078
132

16653
45.5

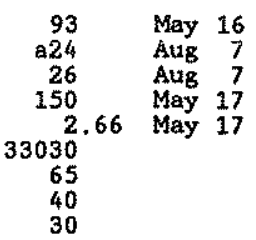

WATKR VRARS 1971 - 1996

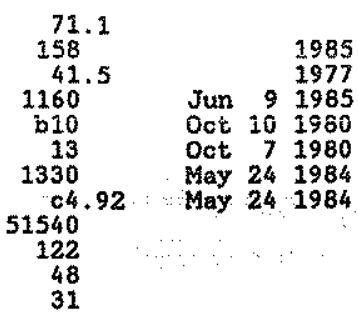

e-Estimated.

also occurred Aug 13.

b-Also occurred Oct 11, 1981.

c-Maximum gage height, $5.07 \mathrm{rt}$, Feb 13, 1994, backwater from 1ce. 
SAN JUAN RIVER BASIN

09345200 LITTLE NAVAJO RIVER BELOW LITTLE OSO DIVERSION DAM, NEAR CHRONO, CO

LOCATION,--Lat $37^{\circ} 04^{\circ} 32^{\prime \prime}$, Long $106^{\circ} 48^{\prime} 38^{\prime \prime}$, in SW sec.23, T.33 N., R. I E., Archuleta County, Hydrologic Unit 14080101 , on right bank at Little Oso Diversion Dam, $3.5 \mathrm{mi}$ northeast of Chromo, and $4.0 \mathrm{mi}$ upstream from confluence with Navajo River.

DRAINAGE AREA. $--14.2 \mathrm{ml}^{2}$.

PERTOD OF RECORD. - June 1971 to current year.

GAGE.--Water-stage recorder. Datum of gage is $7,756.10 \mathrm{ft}$ above sea level, (levels by Bureau of Reclamation).

REMARKS.-Flow controlled by diversion dam upstream. Streamflow data for water year 1994 (not proviously published), is published below. Streamflow data for water year 1996 is published on the fotioning page.

COOPERATION.--Records collected and computed by Bureau of Reclemation.

DISCAARGE, CUBIC FEET PER SECOND, WATER YEAR OCTOBER 1993 TO SEPTEMBER 1994

\begin{tabular}{|c|c|c|c|c|c|c|c|c|c|c|c|c|}
\hline DAX & $\infty \mathrm{CT}$ & nov & DEC & JAN & FEB & MAR & APR & MAY & Jux & JUL. & AuG & SEP \\
\hline $\begin{array}{l}1 \\
2 \\
3 \\
4 \\
5\end{array}$ & $\begin{array}{l}02.0 \\
02.0 \\
02.0 \\
02.0 \\
02.0\end{array}$ & $\begin{array}{l}02.0 \\
02.0 \\
02.0 \\
\theta 2.0 \\
02.0\end{array}$ & $\begin{array}{l}e 2.0 \\
e 2.0 \\
-2.0 \\
02.0 \\
02.0\end{array}$ & $\begin{array}{l}02.0 \\
02.0 \\
02.0 \\
02.0 \\
02.0\end{array}$ & $\begin{array}{l}02.0 \\
e 2.0 \\
02.0 \\
02.0 \\
02.0\end{array}$ & $\begin{array}{l}92.0 \\
02.0 \\
02.0 \\
62.0 \\
02.0\end{array}$ & $\begin{array}{l}92.0 \\
33 \\
37 \\
38 \\
15\end{array}$ & $\begin{array}{l}27 \\
27 \\
29 \\
29 \\
29\end{array}$ & $\begin{array}{l}30 \\
29 \\
30 \\
30 \\
30\end{array}$ & $\begin{array}{l}02.0 \\
02.0 \\
e 2.0 \\
\mathrm{e} 2.0 \\
\mathrm{e} 2.0\end{array}$ & $\begin{array}{l}02.0 \\
02.0 \\
02.0 \\
02.0 \\
02.0\end{array}$ & $\begin{array}{l}02.0 \\
02.0 \\
02.0 \\
02.0 \\
-2.0\end{array}$ \\
\hline $\begin{array}{r}6 \\
7 \\
8 \\
9 \\
10\end{array}$ & $\begin{array}{l}02.0 \\
02.0 \\
02.0 \\
02.0 \\
02.0\end{array}$ & $\begin{array}{l}\mathrm{e} 2.0 \\
\mathrm{e} 2.0 \\
\mathrm{e} 2.0 \\
\mathrm{e} 2.0 \\
\mathrm{e} 2.0\end{array}$ & $\begin{array}{l}02.0 \\
02.0 \\
02.0 \\
02.0 \\
02.0\end{array}$ & $\begin{array}{l}e 2.0 \\
\mathrm{e} 2.0 \\
\mathrm{e} 2.0 \\
\mathrm{e} 2.0 \\
\mathrm{e} 2.0\end{array}$ & $\begin{array}{l}2.0 \\
02.0 \\
02.0 \\
\oplus 2.0 \\
\oplus 2.0\end{array}$ & $\begin{array}{l}02.0 \\
\mathrm{e} 2.0 \\
\mathrm{e} 2.0 \\
\mathrm{e} 2.0 \\
\mathrm{e} 2.0\end{array}$ & $\begin{array}{l}9.0 \\
6.5 \\
6.7 \\
7.1 \\
7.2\end{array}$ & $\begin{array}{l}29 \\
29 \\
29 \\
28 \\
29\end{array}$ & $\begin{array}{l}30 \\
29 \\
28 \\
29 \\
29\end{array}$ & $\begin{array}{l}\ominus 2.0 \\
\theta 2.0 \\
\ominus 2.0 \\
\ominus 2.0 \\
e 2.0\end{array}$ & $\begin{array}{l}02.0 \\
02.0 \\
02.0 \\
02.0 \\
02.0\end{array}$ & $\begin{array}{l}\ominus 2.0 \\
\theta 2.0 \\
\theta 2.0 \\
\ominus 2.0 \\
\theta 2.0\end{array}$ \\
\hline $\begin{array}{l}11 \\
12 \\
13 \\
14 \\
15\end{array}$ & $\begin{array}{l}\mathrm{e} 2.0 \\
\mathrm{e} 2.0 \\
\mathrm{e} 2.0 \\
\mathrm{e} 2.0 \\
\mathrm{e} 2.0\end{array}$ & $\begin{array}{l}02.0 \\
02.0 \\
02.0 \\
02.0 \\
02.0\end{array}$ & $\begin{array}{l}\ominus 2.0 \\
e 2.0 \\
e 2.0 \\
e 2.0 \\
e 2.0\end{array}$ & $\begin{array}{l}02.0 \\
02.0 \\
02.0 \\
02.0 \\
02.0\end{array}$ & $\begin{array}{l}\mathrm{e} 2.0 \\
\mathrm{e} 2.0 \\
\mathrm{e} 2.0 \\
\mathrm{e} 2.0 \\
\mathrm{e} 2.0\end{array}$ & $\begin{array}{l}02.0 \\
02.0 \\
02.0 \\
02.0 \\
02.0\end{array}$ & $\begin{array}{l}7.2 \\
6.5 \\
6.7 \\
6.1 \\
6.5\end{array}$ & $\begin{array}{l}29 \\
28 \\
29 \\
28 \\
28\end{array}$ & $\begin{array}{l}29 \\
29 \\
28 \\
26 \\
23\end{array}$ & $\begin{array}{l}02.0 \\
02.0 \\
02.0 \\
02.0 \\
02.0\end{array}$ & $\begin{array}{l}-2.0 \\
-2.0 \\
02.0 \\
e 2.0 \\
-2.0\end{array}$ & $\begin{array}{l}02.0 \\
02.0 \\
02.0 \\
02.0 \\
\oplus 2.0\end{array}$ \\
\hline $\begin{array}{l}16 \\
17 \\
18 \\
19 \\
20\end{array}$ & $\begin{array}{l}02.0 \\
02.0 \\
02.0 \\
02.0 \\
02.0\end{array}$ & $\begin{array}{l}02.0 \\
02.0 \\
02.0 \\
02.0 \\
02.0\end{array}$ & $\begin{array}{l}\odot 2.0 \\
\theta 2.0 \\
\theta 2.0 \\
\oplus 2.0 \\
\oplus 2.0\end{array}$ & $\begin{array}{l}\mathrm{e} 2.0 \\
\theta 2.0 \\
\theta 2.0 \\
\mathrm{e} 2.0 \\
\mathrm{e} 2.0\end{array}$ & $\begin{array}{l}02.0 \\
e 2.0 \\
02.0 \\
02.0 \\
02.0\end{array}$ & $\begin{array}{l}e 2.0 \\
e 2.0 \\
e 2.0 \\
e 2.0 \\
e 2.0\end{array}$ & $\begin{array}{l}7.1 \\
6.9 \\
6.7 \\
6.7 \\
8.8\end{array}$ & $\begin{array}{l}29 \\
28 \\
28 \\
28 \\
28\end{array}$ & $\begin{array}{l}20 \\
19 \\
18 \\
20 \\
30\end{array}$ & $\begin{array}{l}22.0 \\
e 2.0 \\
02.0 \\
e 2.0 \\
02.0\end{array}$ & $\begin{array}{l}02.0 \\
02.0 \\
02.0 \\
e 2.0 \\
02.0\end{array}$ & $\begin{array}{l}\ominus 2.0 \\
\ominus 2.0 \\
\ominus 2.0 \\
\ominus 2.0 \\
\oplus 2.0\end{array}$ \\
\hline $\begin{array}{l}21 \\
22 \\
23 \\
24 \\
25\end{array}$ & $\begin{array}{l}\oplus 2.0 \\
e 2.0 \\
e 2.0 \\
02.0 \\
-2.0\end{array}$ & $\begin{array}{l}e 2.0 \\
02.0 \\
02.0 \\
02.0 \\
02.0\end{array}$ & 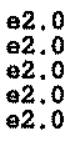 & $\begin{array}{l}02.0 \\
\theta 2.0 \\
02.0 \\
02.0 \\
02.0\end{array}$ & $\begin{array}{l}\mathrm{e} .0 \\
02.0 \\
02.0 \\
\mathrm{e} 2.0 \\
-2.0\end{array}$ & $\begin{array}{l}\mathrm{2} .0 \\
02.0 \\
\mathrm{e} 2.0 \\
\mathrm{\theta} 2.0 \\
\mathrm{e} 2.0\end{array}$ & $\begin{array}{l}9.0 \\
9.0 \\
8.6 \\
8.4 \\
8.4\end{array}$ & $\begin{array}{l}28 \\
29 \\
28 \\
29 \\
30\end{array}$ & $\begin{array}{l}02.0 \\
02.0 \\
02.0 \\
02.0 \\
02.0\end{array}$ & $\begin{array}{l}\theta 2.0 \\
e 2.0 \\
02.0 \\
92.0 \\
e 2.0\end{array}$ & $\begin{array}{l}e 2.0 \\
e 2.0 \\
e 2.0 \\
e 2.0 \\
\theta 2.0\end{array}$ & $\begin{array}{l}02.0 \\
02.0 \\
02.0 \\
\theta 2.0 \\
\theta 2.0\end{array}$ \\
\hline $\begin{array}{l}26 \\
27 \\
28 \\
29 \\
30 \\
31\end{array}$ & $\begin{array}{l}e 2.0 \\
e 2.0 \\
02.0 \\
02.0 \\
02.0 \\
02.0\end{array}$ & $\begin{array}{l}e 2.0 \\
e 2.0 \\
e 2.0 \\
e 2.0 \\
e 2.0 \\
-\end{array}$ & $\begin{array}{l}\mathrm{e} 2.0 \\
\mathrm{e} 2.0 \\
e 2.0 \\
\mathrm{e} 2.0 \\
\mathrm{e} 2.0 \\
\mathrm{e} 2.0\end{array}$ & $\begin{array}{l}\mathrm{e} .0 \\
02.0 \\
\mathrm{e2.0} \\
\mathrm{e} 2.0 \\
\mathrm{e} 2.0 \\
\mathrm{e2.0}\end{array}$ & $\begin{array}{l}e 2.0 \\
e 2.0 \\
e 2.0 \\
-\ldots \\
-\ldots\end{array}$ & $\begin{array}{l}e 2.0 \\
-2.0 \\
e 2.0 \\
e 2.0 \\
e 2.0 \\
e 2.0\end{array}$ & $\begin{array}{r}8.8 \\
9.0 \\
9.4 \\
27 \\
27 \\
----\end{array}$ & $\begin{array}{l}29 \\
30 \\
30 \\
30 \\
30 \\
30\end{array}$ & $\begin{array}{l}e 2.0 \\
02.0 \\
02.0 \\
02.0 \\
02.0 \\
-.--\end{array}$ & $\begin{array}{l}e 2.0 \\
02.0 \\
02.0 \\
\theta 2.0 \\
\theta 2.0 \\
02.0\end{array}$ & $\begin{array}{l}02.0 \\
02.0 \\
02.0 \\
02.0 \\
02.0 \\
02.0\end{array}$ & $\begin{array}{l}02.0 \\
02.0 \\
02.0 \\
02.0 \\
02.0 \\
-\end{array}$ \\
\hline $\begin{array}{l}\text { TOTAL. } \\
\text { MEAN } \\
\text { MAX } \\
\text { MIN } \\
\text { AC-FT }\end{array}$ & $\begin{array}{r}62.0 \\
2.00 \\
2.0 \\
2.0 \\
123\end{array}$ & $\begin{array}{r}60.0 \\
2.00 \\
2.0 \\
2.0 \\
119\end{array}$ & $\begin{array}{r}62.0 \\
2.00 \\
2.0 \\
2.0 \\
123\end{array}$ & $\begin{array}{r}62.0 \\
2.00 \\
2.0 \\
2.0 \\
123\end{array}$ & $\begin{array}{r}56.0 \\
2.00 \\
2.0 \\
2.0 \\
111\end{array}$ & $\begin{array}{r}62.0 \\
2.00 \\
2.0 \\
2.0 \\
123\end{array}$ & $\begin{array}{r}355.3 \\
11.8 \\
38 \\
2.0 \\
705\end{array}$ & $\begin{array}{r}891 \\
28.7 \\
30 \\
27 \\
1770\end{array}$ & $\begin{array}{r}556.0 \\
18.5 \\
30 \\
2.0 \\
1100\end{array}$ & $\begin{array}{r}62.0 \\
2.00 \\
2.0 \\
2.0 \\
12.3\end{array}$ & $\begin{array}{r}62.0 \\
2.00 \\
2.0 \\
2.0 \\
123\end{array}$ & $\begin{array}{r}60.0 \\
2.00 \\
2.0 \\
2.0 \\
119\end{array}$ \\
\hline
\end{tabular}

STATISTICS OF MONTHLY MEAN DATA FOR WATER YEARS 1971 - 1994, BY WATER YEAR (WY)

\begin{tabular}{|c|c|c|c|c|c|c|c|c|c|c|c|c|}
\hline $\begin{array}{l}\text { MEAN } \\
\text { MAX } \\
\text { (WY) } \\
\text { MIN } \\
\text { (WY) }\end{array}$ & $\begin{array}{l}6.64 \\
49.9 \\
1987 \\
1.47 \\
1976\end{array}$ & $\begin{array}{l}5.23 \\
46.4 \\
1987 \\
1.00 \\
1990\end{array}$ & $\begin{array}{r}2.80 \\
8.82 \\
1987 \\
.47 \\
1990\end{array}$ & $\begin{array}{l}2.47 \\
5.82 \\
1987 \\
1.02\end{array}$ & $\begin{array}{l}2.55 \\
6.53 \\
1986 \\
1.03 \\
1990\end{array}$ & $\begin{array}{l}8.22 \\
32.0 \\
1985 \\
1.95 \\
1977\end{array}$ & $\begin{array}{l}14.1 \\
45.7 \\
1989 \\
4.19\end{array}$ & $\begin{array}{l}25.0 \\
66.3 \\
1973 \\
4.86 \\
1977\end{array}$ & $\begin{array}{l}18.9 \\
29.3 \\
1983 \\
1.87 \\
1977\end{array}$ & $\begin{array}{r}7.23 \\
17.1 \\
1983 \\
198\end{array}$ & $\begin{array}{r}4.01 \\
9.25 \\
1986 \\
.47\end{array}$ & $\begin{array}{l}3.95 \\
17.3 \\
1982 \\
1.02 \\
1972\end{array}$ \\
\hline
\end{tabular}

SUMMARY STATISTICS

ANNUAL TOTAL

ANNUAL MEAN

GIGHEST ANNUAL MEAN

LOWEST ANNUAL MEAN

HIGHEST DAILY MEAN

LOWEST DAILY MEAN

ANNUAL SEVEN-DAY MINIMUM

INSTANTANEOUS PEAK FLOW

ANNUAL RUNOFE (AC-FT)

10 PERCENT EXCEEDS

50 PERCENT EXCEEDS

90 PERCENT EXCEEDS

e-Estimated.

a-Occurs many times some years.

b-Also occurred Oct $21,1988$.

c-Gage height not determined.
FOR 1993 CALENDAR YEAR FOR 1994 WATER YEAR

2350.3

$\begin{array}{rrr}4208.4 & & \\ 11.5 & & \\ 70 & \text { Apr } & 22 \\ \text { a2.0 } & \text { JuI } & 29 \\ 2.0 & \text { Oct } & 1 \\ & & \\ 8350 & & \\ 28 & & \\ 3.3 & & \\ 2.0 & & \end{array}$

2350.3
6.44

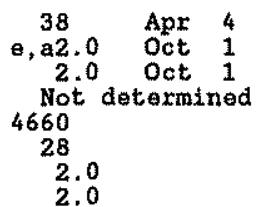

WATER YEARS 1971 - 1994

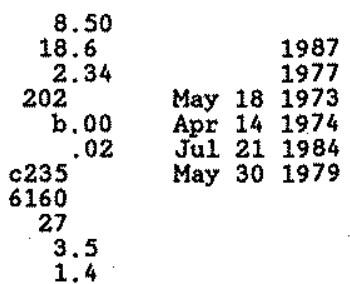



DISCEARGE, CUBIC FEET PER SECOND, WATER YEAR OCTOBER 1995 TO SEPTEMBER 1996 DAILY MEAN VALUES

\begin{tabular}{|c|c|c|c|c|c|c|c|c|c|c|c|c|}
\hline DAY & OCT & wov & $\mathrm{DEC}$ & JAN & $\mathrm{FEB}$ & MAR & APR & MAY & JUN & JUL & AUG & SEP \\
\hline $\begin{array}{l}1 \\
2 \\
3 \\
4 \\
5\end{array}$ & $\begin{array}{l}3.6 \\
3.0 \\
3.0 \\
2.9 \\
2.8\end{array}$ & $\begin{array}{l}3.0 \\
2.6 \\
1.7 \\
2.5 \\
2.0\end{array}$ & $\begin{array}{l}2.4 \\
2.1 \\
2.0 \\
2.1 \\
2.1\end{array}$ & $\begin{array}{r}2.0 \\
1.9 \\
01.9 \\
01.9 \\
01.9\end{array}$ & $\begin{array}{l}01.9 \\
01.9 \\
81.9 \\
01.9 \\
01.9\end{array}$ & $\begin{array}{l}3.5 \\
3.5 \\
3.6 \\
3.6 \\
3.6\end{array}$ & $\begin{array}{r}10 \\
6.9 \\
8.4 \\
7.0 \\
7.0\end{array}$ & $\begin{array}{l}31 \\
30 \\
29 \\
29 \\
29\end{array}$ & $\begin{array}{l}6.9 \\
6.3 \\
5.4 \\
4.8 \\
4.4\end{array}$ & $\begin{array}{l}8.6 \\
4.8 \\
2.8 \\
2.2 \\
2.1\end{array}$ & $\begin{array}{l}1.3 \\
1.2 \\
1.6 \\
1.5 \\
1.2\end{array}$ & $\begin{array}{l}.98 \\
.98 \\
.88 \\
.88 \\
1.1\end{array}$ \\
\hline $\begin{array}{r}6 \\
7 \\
8 \\
9 \\
10\end{array}$ & $\begin{array}{l}2.9 \\
2.8 \\
2.8 \\
2.6 \\
2.5\end{array}$ & $\begin{array}{l}2.4 \\
2.2 \\
2.2 \\
2.5 \\
2.5\end{array}$ & $\begin{array}{l}2.1 \\
2.0 \\
2.2 \\
1.7 \\
1.9\end{array}$ & $\begin{array}{r}\text { e1.9 } \\
01.9 \\
81.9 \\
81.9 \\
01.9\end{array}$ & $\begin{array}{r}01.9 \\
1.9 \\
2.0 \\
2.1 \\
2.4\end{array}$ & $\begin{array}{l}3.8 \\
3.6 \\
3.8 \\
4.1 \\
4.6\end{array}$ & $\begin{array}{l}4.6 \\
6.7 \\
6.5 \\
7.4 \\
7.2\end{array}$ & $\begin{array}{l}32 \\
31 \\
28 \\
26 \\
25\end{array}$ & $\begin{array}{l}4.1 \\
3.9 \\
3.6 \\
3.3 \\
3.0\end{array}$ & $\begin{array}{l}2.0 \\
2.1 \\
5.8 \\
6.9 \\
3.2\end{array}$ & $\begin{array}{c}1.1 \\
.88 \\
.98 \\
1.2 \\
1.2\end{array}$ & $\begin{array}{r}1.4 \\
98 \\
.88 \\
.70 \\
.70\end{array}$ \\
\hline $\begin{array}{l}11 \\
12 \\
13 \\
14 \\
15\end{array}$ & $\begin{array}{l}2.5 \\
2.5 \\
2.5 \\
2.5 \\
2.4\end{array}$ & $\begin{array}{l}1.7 \\
2.9 \\
3.2 \\
3.3 \\
3.2\end{array}$ & $\begin{array}{l}1.9 \\
2.0 \\
2.1 \\
2.2 \\
2.0\end{array}$ & $\begin{array}{l}01.9 \\
01.9 \\
01.9 \\
01.9 \\
01.9\end{array}$ & $\begin{array}{l}2.4 \\
2.2 \\
2.2 \\
2.5 \\
2.0\end{array}$ & $\begin{array}{l}4.9 \\
6.1 \\
5.2 \\
4.8 \\
4.4\end{array}$ & $\begin{array}{l}7.2 \\
7.4 \\
7.2 \\
8.2 \\
7.2\end{array}$ & $\begin{array}{l}24 \\
24 \\
25 \\
25 \\
24\end{array}$ & $\begin{array}{l}3.0 \\
2.9 \\
2.9 \\
3.2 \\
3.9\end{array}$ & $\begin{array}{l}3.3 \\
4.4 \\
3.8 \\
2.9 \\
9.9\end{array}$ & $\begin{array}{l}.98 \\
.98 \\
.88 \\
.79 \\
.79\end{array}$ & $\begin{array}{l}1.2 \\
2.1 \\
1.5 \\
3.0 \\
4.8\end{array}$ \\
\hline $\begin{array}{l}16 \\
17 \\
18 \\
19 \\
20\end{array}$ & $\begin{array}{l}2.5 \\
2.5 \\
2.5 \\
2.5 \\
2.5\end{array}$ & $\begin{array}{l}3.0 \\
2.8 \\
2.5 \\
2.5 \\
2.5\end{array}$ & $\begin{array}{l}2.1 \\
2.1 \\
2.1 \\
2.1 \\
2.0\end{array}$ & $\begin{array}{r}01.9 \\
e 1.9 \\
01.9 \\
01.9 \\
01.9\end{array}$ & $\begin{array}{l}5.1 \\
4.4 \\
4.6 \\
4.3 \\
4.3\end{array}$ & $\begin{array}{l}4.4 \\
4.6 \\
4.4 \\
4.6 \\
5.1\end{array}$ & $\begin{array}{l}7.2 \\
7.4 \\
7.6 \\
7.6 \\
6.3\end{array}$ & $\begin{array}{l}23 \\
23 \\
21 \\
19 \\
18\end{array}$ & $\begin{array}{l}3.2 \\
2.8 \\
2.6 \\
2.4 \\
2.0\end{array}$ & $\begin{array}{l}4.6 \\
6.5 \\
3.8 \\
3.0 \\
2.5\end{array}$ & $\begin{array}{l}.88 \\
.88 \\
.88 \\
.79 \\
.79\end{array}$ & $\begin{array}{l}2.5 \\
2.5 \\
3.8 \\
4.1 \\
4.8\end{array}$ \\
\hline $\begin{array}{l}21 \\
22 \\
23 \\
24 \\
25\end{array}$ & $\begin{array}{l}2.4 \\
2.5 \\
.98 \\
2.6 \\
2.4\end{array}$ & $\begin{array}{l}2.4 \\
2.2 \\
2.2 \\
1.9 \\
2.0\end{array}$ & $\begin{array}{l}2.0 \\
2.0 \\
2.1 \\
2.0 \\
1.9\end{array}$ & $\begin{array}{l}\text { e1.9 } \\
\text { e1.9 } \\
01.9 \\
\text { e1.9 } \\
\text { e1.9 }\end{array}$ & $\begin{array}{l}5.8 \\
4.6 \\
4.3 \\
4.1 \\
4.1\end{array}$ & $\begin{array}{l}6.7 \\
9.0 \\
9.2 \\
7.6 \\
6.3\end{array}$ & $\begin{array}{l}2.9 \\
2.2 \\
5.4 \\
5.4 \\
5.2\end{array}$ & $\begin{array}{l}16 \\
15 \\
13 \\
12 \\
12\end{array}$ & $\begin{array}{l}2.0 \\
2.0 \\
2.0 \\
2.0 \\
2.0\end{array}$ & $\begin{array}{l}2.2 \\
2.0 \\
1.9 \\
1.7 \\
1.7\end{array}$ & $\begin{array}{l}1.3 \\
1.4 \\
1.3 \\
2.8 \\
2.8\end{array}$ & $\begin{array}{l}3.9 \\
2.9 \\
2.2 \\
1.9 \\
1.6\end{array}$ \\
\hline $\begin{array}{l}26 \\
27 \\
28 \\
29 \\
30 \\
31\end{array}$ & $\begin{array}{l}2.2 \\
2.2 \\
2.4 \\
2.4 \\
2.2 \\
2.2\end{array}$ & $\begin{array}{l}2.4 \\
1.2 \\
1.1 \\
1.5 \\
2.2\end{array}$ & $\begin{array}{l}1.9 \\
1.9 \\
1.7 \\
1.7 \\
1.7 \\
1.9\end{array}$ & $\begin{array}{l}e 1.9 \\
e 1.9 \\
01.9 \\
01.9 \\
01.9 \\
01.9\end{array}$ & $\begin{array}{l}3.9 \\
3.9 \\
3.8 \\
3.8 \\
---\end{array}$ & $\begin{array}{l}6.1 \\
6.9 \\
8.8 \\
9.2 \\
8.0 \\
9.6\end{array}$ & $\begin{array}{r}5.2 \\
5.2 \\
5.1 \\
5.2 \\
30 \\
-\end{array}$ & $\begin{array}{r}11 \\
9.9 \\
9.2 \\
8.4 \\
7.8 \\
7.2\end{array}$ & $\begin{array}{l}2.0 \\
2.0 \\
2.0 \\
2.0 \\
2.8\end{array}$ & $\begin{array}{l}1.7 \\
1.7 \\
2.0 \\
1.6 \\
1.5 \\
1.5\end{array}$ & $\begin{array}{l}1.9 \\
2.0 \\
1.5 \\
1.7 \\
1.3 \\
1.1\end{array}$ & $\begin{array}{l}1.5 \\
1.4 \\
1.4 \\
1.3 \\
1.2\end{array}$ \\
\hline $\begin{array}{l}\text { TOTAL } \\
\text { MEAN } \\
\text { MAX } \\
\text { MIN } \\
\text { AC-FT }\end{array}$ & $\begin{array}{r}78.28 \\
2.53 \\
3.6 \\
i 98 \\
155\end{array}$ & $\begin{array}{r}70.3 \\
2.34 \\
3.3 \\
1.1 \\
139\end{array}$ & $\begin{array}{r}62.0 \\
2.00 \\
2.4 \\
1.7 \\
123\end{array}$ & $\begin{array}{r}59.0 \\
1.90 \\
2.0 \\
1.9 \\
117\end{array}$ & $\begin{array}{r}92.1 \\
3.18 \\
5.8 \\
1.9 \\
183\end{array}$ & $\begin{array}{r}173.6 \\
5.60 \\
9.6 \\
3.5 \\
344\end{array}$ & $\begin{array}{r}216.8 \\
7.23 \\
30 \\
2.2 \\
430\end{array}$ & $\begin{array}{r}637.5 \\
20.6 \\
32 \\
7.2 \\
1260\end{array}$ & $\begin{array}{r}95.4 \\
3.18 \\
6.9 \\
2.0 \\
189\end{array}$ & $\begin{array}{r}104.7 \\
3.38 \\
9.9 \\
1.5 \\
208\end{array}$ & $\begin{array}{r}39.90 \\
1.29 \\
2.8 \\
.79 \\
79\end{array}$ & $\begin{array}{r}59.08 \\
1.97 \\
4.8 \\
.70 \\
117\end{array}$ \\
\hline
\end{tabular}

STATISTICS OF MONTHLY MEAN DATA FOR WATER YEARS 1971 - 1996, BY WATER YEAR (WY)

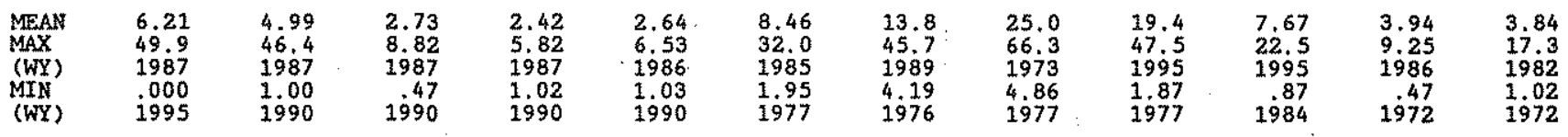

SUMMARY STATISTICS

ANNUAL TOTAL

ANIGUAL MEAN ANNUL MEAT

LOWEST ANNUAL MEAN

LOWEST ANNUAL MEAN

LOWEST DAILY MEAN

ANNUAL SEVEN-DAY MINIMUM

INSTANTANEOUS PEAK FLON

ANNUAL RUYOFF (AC-FT)

10 PERCENT EXCEEDS

50 PERCENT EXCEEDS

-Escimated.

a-Also occurred Sep 10

FOR 1995 CALENDAR YEAR

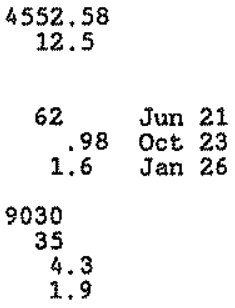

FOR 1996 WATER YEAR

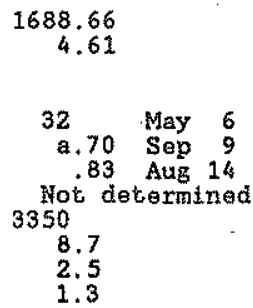

WATER YEARS 1971 - 1996

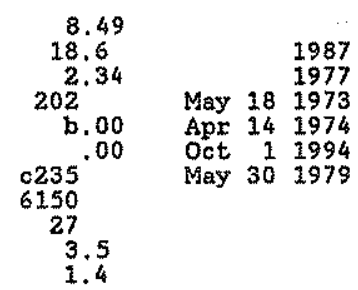

b-Also occurred Oet 21, 1988 and Oct 1 to Nov 3, 1994

c-Gage helght not determined. 
SAN JUAN RIVER BASTN

09346400 SAN JUAN RIVER NEAR CARRACAS, CO

LOCATroN.--Lat $37^{\circ} 00^{\prime} 49^{\prime \prime}$, long $107^{\circ} 18^{\prime} 42^{\prime \prime}$, in SE/4SW sec.17, T.32 N., R.4 W. Archuleta County, Hydrologic Unit 14080101 , on right bank just upstream from flow line of Navajo Reservoir, ' 3 mi northwest of Carracas, 7.2 mi upstream from Piedra River, and at mile 332.8 .

DRAINAGE AREA. $-1,230 \mathrm{mi}^{2}$, approximately.

PERIOD OF RECORD. - October 1961 to current year. Water-qual1ty data avallable, July 1969 to August 1973 . Sediment data available, August 1.973 .

GAGE.-Water-stage recorder and crest-stage gage. Elevation of gage is 6,090 ft above National Geodetic Vertical Datum of 1929 , from river-profile map.

REMARKS.--Records falr except for estimated dally discharges, which are poor. Diversions for irrigation of about 11,000 acres upstroam from station. High-water diversions upstroam from station into Rio Grande basin through Azotea tunnel (08284160) began in March 1971. Several observations of spectfic conductance and water temperature were obtained and are published in "Water resources data for Colorado."

EXTREMES OUTSIDE PERIOD OF RECORD.--Major floods occurred Sept. 5 or 6, 1909; Oct. 5, 1911; June 29, 1927. DISCHARGE, CUBIC FEET PER SECOND, WATER YEAR OCTOBER 1995 TO SEPTEMBER 1996

\begin{tabular}{|c|c|c|c|c|c|c|c|c|c|c|c|c|c|}
\hline $\begin{array}{r}\text { DAY } \\
1 \\
2 \\
3 \\
4 \\
5\end{array}$ & $\begin{array}{l}\alpha C T \\
402 \\
328 \\
292 \\
261 \\
242\end{array}$ & $\begin{array}{l}\text { Nov } \\
145 \\
165 \\
169 \\
141 \\
139\end{array}$ & $\begin{array}{l}\text { DEC } \\
\theta 150 \\
\theta 150 \\
\theta 140 \\
\theta 140 \\
\theta 140\end{array}$ & $\begin{array}{l}\text { JAN } \\
0120 \\
0115 \\
0115 \\
0125 \\
0130\end{array}$ & $\begin{array}{l}\text { FEB } \\
0135 \\
0135 \\
0115 \\
0120 \\
0130\end{array}$ & $\begin{array}{l}\text { MAR } \\
182 \\
163 \\
162 \\
182 \\
189\end{array}$ & $\begin{array}{l}\text { APR } \\
227 \\
296 \\
302 \\
279 \\
279\end{array}$ & $\begin{array}{r}\text { MAY } \\
0700 \\
9800 \\
0960 \\
01150 \\
1310\end{array}$ & $\begin{array}{l}\text { JUN } \\
453 \\
446 \\
483 \\
453 \\
434\end{array}$ & $\begin{array}{l}\text { JUL } \\
374 \\
282 \\
242 \\
192 \\
174\end{array}$ & & $\begin{array}{l}\text { AUfG } \\
124 \\
119 \\
118 \\
121 \\
124\end{array}$ & $\begin{array}{r}\text { SEP } \\
94 \\
90 \\
84 \\
92 \\
92\end{array}$ \\
\hline $\begin{array}{r}6 \\
7 \\
8 \\
9 \\
10\end{array}$ & $\begin{array}{l}227 \\
224 \\
216 \\
208 \\
208\end{array}$ & $\begin{array}{l}141 \\
144 \\
144 \\
144 \\
156\end{array}$ & $\begin{array}{l}e 135 \\
0130 \\
127 \\
128 \\
119\end{array}$ & $\begin{array}{l}e 125 \\
e 120 \\
e 120 \\
e 130 \\
e 135\end{array}$ & $\begin{array}{l}0145 \\
0145 \\
0145 \\
0150 \\
0155\end{array}$ & $\begin{array}{l}0190 \\
e 180 \\
\theta 175 \\
\theta 180 \\
\theta 190\end{array}$ & $\begin{array}{l}275 \\
270 \\
307 \\
369 \\
456\end{array}$ & $\begin{array}{l}1460 \\
1480 \\
1310 \\
1350 \\
1300\end{array}$ & $\begin{array}{l}410 \\
439 \\
394 \\
360 \\
364\end{array}$ & $\begin{array}{l}161 \\
131 \\
148 \\
208 \\
251\end{array}$ & & $\begin{array}{r}111 \\
94 \\
90 \\
88 \\
92\end{array}$ & 90 \\
\hline $\begin{array}{l}11 \\
12 \\
13 \\
14 \\
15\end{array}$ & $\begin{array}{l}202 \\
197 \\
190 \\
186 \\
182\end{array}$ & $\begin{array}{l}177 \\
141 \\
156 \\
172 \\
175\end{array}$ & $\begin{array}{l}126 \\
142 \\
156 \\
165 \\
162\end{array}$ & $\begin{array}{l}0125 \\
0125 \\
0130 \\
0135 \\
0135\end{array}$ & $\begin{array}{l}0165 \\
0160 \\
0160 \\
0175 \\
186\end{array}$ & $\begin{array}{l}2195 \\
0205 \\
0215 \\
0210 \\
0205\end{array}$ & $\begin{array}{l}440 \\
405 \\
382 \\
381 \\
344\end{array}$ & $\begin{array}{l}1470 \\
1410 \\
1520 \\
1460 \\
1580\end{array}$ & $\begin{array}{l}324 \\
289 \\
270 \\
270 \\
326\end{array}$ & $\begin{array}{l}28 \\
24 \\
28 \\
19 \\
18\end{array}$ & & $\begin{array}{l}94 \\
82 \\
72 \\
66 \\
64\end{array}$ & $\begin{array}{r}74 \\
100 \\
110 \\
168 \\
350\end{array}$ \\
\hline $\begin{array}{l}16 \\
17 \\
18 \\
19 \\
20\end{array}$ & $\begin{array}{l}179 \\
176 \\
172 \\
172 \\
169\end{array}$ & $\begin{array}{l}172 \\
166 \\
156 \\
159 \\
150\end{array}$ & $\begin{array}{l}130 \\
121 \\
1.46 \\
132 \\
135\end{array}$ & $\begin{array}{l}0135 \\
0130 \\
0115 \\
0130 \\
0140\end{array}$ & $\begin{array}{l}204 \\
231 \\
239 \\
240 \\
232\end{array}$ & $\begin{array}{l}0190 \\
0195 \\
e 200 \\
e 200 \\
0200\end{array}$ & $\begin{array}{l}333 \\
333 \\
323 \\
303 \\
279\end{array}$ & $\begin{array}{l}1640 \\
1720 \\
1670 \\
1530 \\
1480\end{array}$ & $\begin{array}{l}369 \\
314 \\
271 \\
234 \\
212\end{array}$ & $\begin{array}{l}15 \\
18 \\
25 \\
24 \\
16\end{array}$ & & $\begin{array}{l}66 \\
64 \\
67 \\
69 \\
63\end{array}$ & $\begin{array}{l}281 \\
232 \\
235 \\
311 \\
249\end{array}$ \\
\hline $\begin{array}{l}21 \\
22 \\
23 \\
24 \\
25\end{array}$ & $\begin{array}{l}164 \\
159 \\
153 \\
138 \\
141\end{array}$ & $\begin{array}{l}148 \\
147 \\
141 \\
141 \\
127\end{array}$ & $\begin{array}{r}135 \\
0130 \\
0115 \\
080 \\
095\end{array}$ & $\begin{array}{l}0130 \\
0135 \\
0135 \\
0130 \\
0125\end{array}$ & $\begin{array}{l}306 \\
408 \\
300 \\
241 \\
228\end{array}$ & $\begin{array}{l}e 200 \\
\theta 210 \\
-225 \\
e 240 \\
e 235\end{array}$ & $\begin{array}{l}279 \\
257 \\
261 \\
359 \\
548\end{array}$ & $\begin{array}{r}1320 \\
1180 \\
1110 \\
886 \\
873\end{array}$ & $\begin{array}{l}208 \\
243 \\
316 \\
243 \\
212\end{array}$ & $\begin{array}{l}15 \\
12 \\
14 \\
13 \\
12\end{array}$ & & $\begin{array}{r}67 \\
81 \\
145 \\
261 \\
341\end{array}$ & $\begin{array}{r}224 \\
e 225 \\
e 220 \\
e 210 \\
e 210\end{array}$ \\
\hline $\begin{array}{l}26 \\
27 \\
28 \\
29 \\
30 \\
31\end{array}$ & $\begin{array}{l}146 \\
147 \\
144 \\
138 \\
138 \\
138\end{array}$ & $\begin{array}{r}132 \\
132 \\
e 100 \\
0110 \\
0130 \\
--2\end{array}$ & $\begin{array}{l}0110 \\
0110 \\
0110 \\
0115 \\
0125 \\
0130\end{array}$ & $\begin{array}{l}0120 \\
0115 \\
0130 \\
0140 \\
0140 \\
0140\end{array}$ & $\begin{array}{l}220 \\
187 \\
172 \\
186 \\
--- \\
--m\end{array}$ & $\begin{array}{l}e 225 \\
e 210 \\
0200 \\
0195 \\
200 \\
208\end{array}$ & $\begin{array}{r}788 \\
947 \\
1090 \\
788 \\
-730 \\
\end{array}$ & $\begin{array}{l}775 \\
666 \\
533 \\
485 \\
477 \\
477\end{array}$ & $\begin{array}{l}201 \\
277 \\
385 \\
349 \\
324 \\
\end{array}$ & $\begin{array}{l}12 \\
13 \\
12 \\
12 \\
15 \\
14\end{array}$ & & $\begin{array}{l}226 \\
174 \\
223 \\
171 \\
141 \\
115\end{array}$ & $\begin{array}{l}210 \\
8210 \\
0205 \\
0195 \\
0185 \\
-\end{array}$ \\
\hline $\begin{array}{l}\text { TOEAL } \\
\text { MEAN } \\
\text { MAX } \\
\text { MTN } \\
\text { AC-ET }\end{array}$ & $\begin{array}{r}6039 \\
195 \\
402 \\
138 \\
11980\end{array}$ & $\begin{array}{r}4420 \\
147 \\
177 \\
100 \\
8770\end{array}$ & $\begin{array}{r}4029 \\
130 \\
165 \\
80 \\
7990\end{array}$ & $\begin{array}{r}3975 \\
128 \\
140 \\
115 \\
7880\end{array}$ & $\begin{array}{r}5615 \\
194 \\
408 \\
115 \\
11140\end{array}$ & $\begin{array}{r}6156 \\
199 \\
240 \\
162 \\
12210\end{array}$ & $\begin{array}{r}12630 \\
421 \\
1090 \\
227 \\
25050\end{array}$ & $\begin{array}{r}36082 \\
1164 \\
1720 \\
477 \\
71570\end{array}$ & $\begin{array}{r}9873 \\
329 \\
483 \\
201 \\
19580\end{array}$ & $\begin{array}{r}583 \\
18 \\
37 \\
12 \\
1157\end{array}$ & & $\begin{array}{r}3733 \\
120 \\
341 \\
63 \\
7400\end{array}$ & $\begin{array}{r}5070 \\
169 \\
350 \\
72 \\
10060\end{array}$ \\
\hline $\begin{array}{l}\text { STATIST } \\
\text { MEAN } \\
\text { MAX } \\
\text { (WX) } \\
\text { MIN } \\
\text { (WY) } \\
\text { SLUMARY } \\
\text { ANMUAL } \\
\text { ANNUAL } \\
\text { HIGHEST } \\
\text { LOWEST } \\
\text { HTGHEST } \\
\text { LOWEST } \\
\text { ANNUAL } \\
\text { INSTANT } \\
\text { INSTANT } \\
\text { ANNUAL } \\
\text { I0 PERC } \\
\text { SO EERC } \\
90 \text { PERC }\end{array}$ & $\begin{array}{l}\text { ICS OF } \\
314 \\
932 \\
1987 \\
106 \\
1979 \\
\text { STATIS } \\
\text { TOTAL } \\
\text { MEAN } \\
\text { ANNUAL } \\
\text { ANNUAL } \\
\text { DATLY } \\
\text { DATLY ME } \\
\text { SEVEN-DA } \\
\text { ANEOUS } \\
\text { ANEOUS } \\
\text { RUNOFF } \\
\text { ENT EXCE } \\
\text { CENT EXCF } \\
\text { ENT EXCE }\end{array}$ & $\begin{array}{l}\text { MONTHLY MEAN } \\
248 \\
983 \\
1987 \\
104 \\
1990 \\
\text { TICS } \\
\\
\text { MEAN } \\
\text { MEAN } \\
\text { MEAN } \\
\text { EAN } \\
\text { AY MINIMUM } \\
\text { PEAK FLOW } \\
\text { PEAK STAGE } \\
\text { (AC-FT) } \\
\text { EEDS } \\
\text { EEDS } \\
\text { EEDS }\end{array}$ & $\begin{array}{c}\text { DATA } \\
176 \\
406 \\
1987 \\
72.9 \\
1990 \\
\text { FOR }\end{array}$ & $\begin{array}{c}\text { FOR WATER } \\
159 \\
296 \\
1987 \\
74.7 \\
1990 \\
1995 \mathrm{CA} E \mathrm{E} \\
354184 \\
970\end{array}$ & $\begin{array}{c}\text { YEARS } 1971 \\
202 \\
481 \\
1986 \\
85.0 \\
1990 \\
\text { INDAR YEAR }\end{array}$ & $\begin{array}{c}-1996, \\
615 \\
1369 \\
1995 \\
134 \\
1977\end{array}$ & $\begin{array}{c}\text { BY WATER } \\
1122 \\
2524 \\
1979 \\
233 \\
1977 \\
\text { FOR } 1996 \text { We } \\
103453 \\
283\end{array}$ & $\begin{array}{c}\text { YEAR (WY) } \\
1767 \\
3195 \\
1973 \\
395 \\
1977 \\
\text { ATER YEAR }\end{array}$ & $\begin{array}{r}1874 \\
4080 \\
1985 \\
251 \\
1977\end{array}$ & $\begin{array}{r}690 \\
2427 \\
1995 \\
132 \\
1972 \\
\text { WATER } \\
\text { a649 } \\
1191 \\
200 \\
\text { b6700 } \\
\text { c28 } \\
39 \\
\text { d8590 } \\
\text { f8 } \\
470300 \\
1750 \\
287 \\
110\end{array}$ & $\begin{array}{r}1 \\
6 \\
1 \\
\text { YEARS }\end{array}$ & $\begin{array}{c}330 \\
733 \\
1993 \\
69.0 \\
1972 \\
\text { is } 1971\end{array}$ & $\begin{array}{rr} & 1985 \\
& 1977 \\
12 & 1985 \\
14 & 1974 \\
14 & 1978 \\
6 & 1995 \\
6 & 1995\end{array}$ \\
\hline
\end{tabular}

e-Estimated. Azotea Tunnel.

b-Also maximum datly discharge for period of record.

c-Minimum daily discharge for period of record, about $5 \mathrm{ft} / \mathrm{s}$, Dec 10, 1961, result of fxeezeup.

d-Maximum discharge and stage for pertod of record, $9,730 \mathrm{ft} / \mathrm{s}$, Sep 6,1970 , 8 age height, 8.34 ft, from rating curve extended above $6,000 \mathrm{ft}^{3} / \mathrm{s}$, on basis of slope-area measurement of peak flow.

f-Maximum gage height for statistical period, and period of record, 9.63 ft, Jan. 4, 1994, backwater from 1ce. 
LOCATION.--Lat $37^{\circ} 05^{\prime} 18^{\prime \prime}$, Long $107^{\circ} 23^{\prime} 50^{\prime \prime}$, in NEKSWk sec,21, T.33 N., R.5 W., Archuleta County, Hydrologic Unit 14080102 , on left bank 3 mi downstream from Ignacto Creek, 4.6 mi northeast of Arboles post office, and 2.5 mi upstream from Navajo Reservoir.

DRAINAGE AREA, $--629 \mathrm{mi}^{2}$.

PERTOD OF RECORD.--August 1962 to current year. Gage operated 1895-99, 1910-27 at a site 7.5 mi downstream at altitude 6,000 ft. Low-flow records probabiy not oquivalent. Watar-quality data avallable, November to August 1973 .

GAGE - Wator-stage recordex. Elevation of gago 12 6, 147.52 ft above Natjonal Goodetic Vertical Datum of 1929, from Colorado state Highway Department bench mark.

REMARKS,--Records fair except for estimated daily discharges, which are poor. Diversions for irrigation of about 2,800 acres upstream from station. Several observations of specific conductance and watier tomperature were obtained and are published in "Supplemental WatermQuality Data for Gaging Stations" section of this report.

EXTREMES OUTSIDE PERTOD OF RECORD.--Major floods occurred Sept. 5 or 6, 1909, and Oct. 5, 1911. DISCHARGE, CUBIC FEET PER SECOND, WATER YEAR OCTOBER 1995 TO SEPTEMBER 1996 DAILY MEAN VALUES

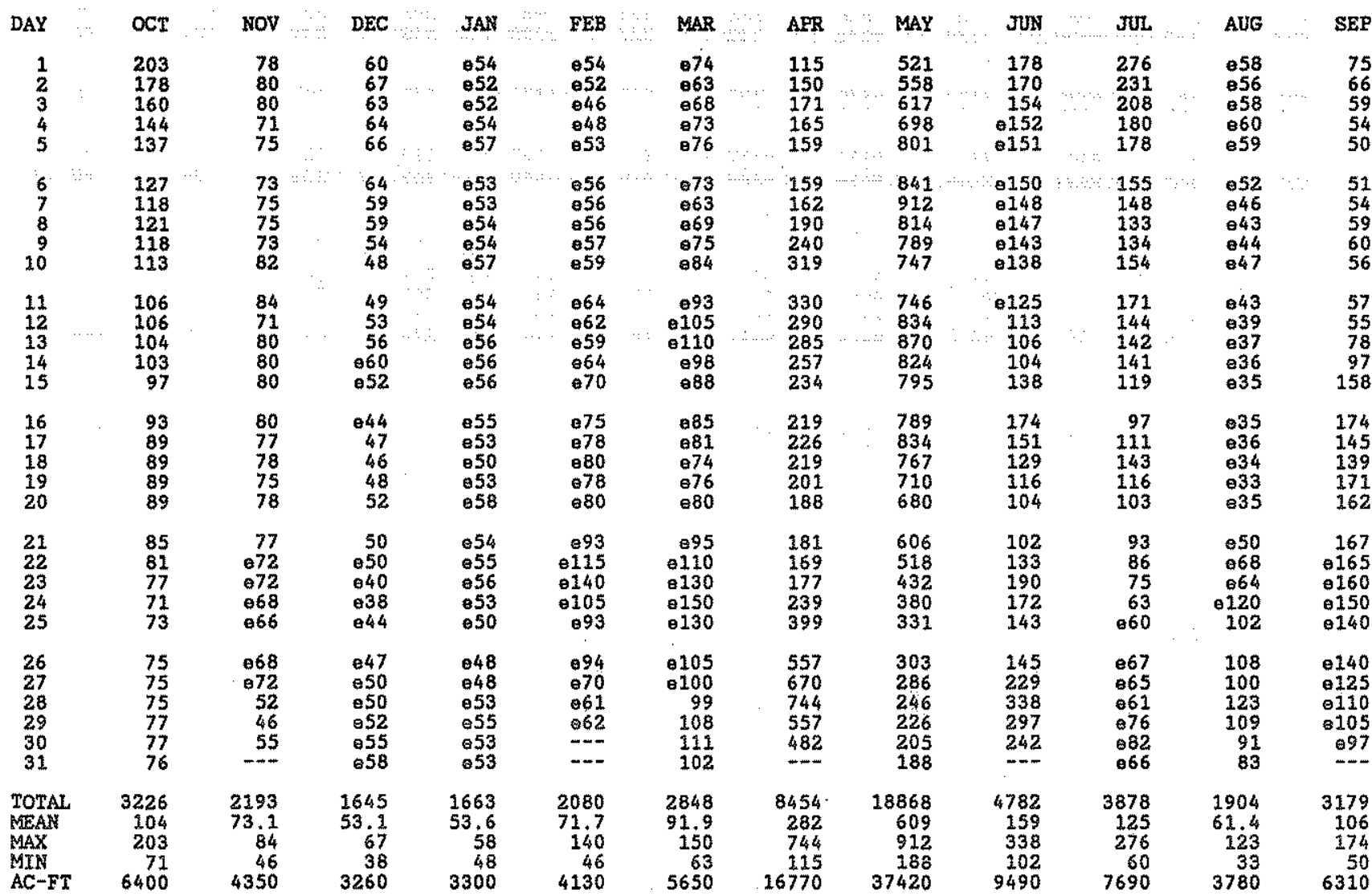

STATISTICS OF MONYZLY MEAN DATA FOR WATER YEARS 1953 - 1996, BY WATER YEAR (WY)

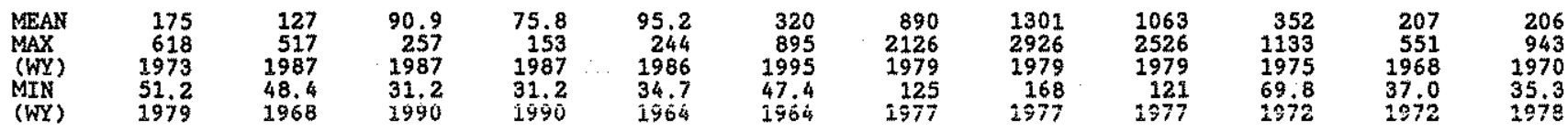

\section{SURRARY STATISTICS}

ANNUAL TOTAL

ANNUAL MEAN

GIGEEST ANHWAI MEAN

LOWEST ANNUAL MEAN

HIGHEST DAILY MEAN

LOWEST DAILY MEAN

ANNUAL SEVER-DAY MINIMUM

INSTANTANEOUS PEAK FLOW

INSTANTANEOUS PEAK STAGE

ANNUAL, RUNOFF (AC-FT)

10 PERCENT EXCEEDS

50 PERCENT EXCEEDS

90 EERCENT EXCEEDS
FOR 1995 CALENDAR YEAR

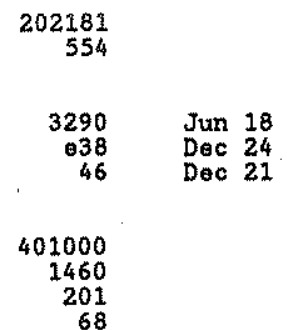

FOR 1996 WATER YEAR

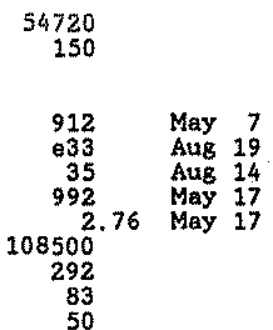

WATER YEARS 1963 - 1996

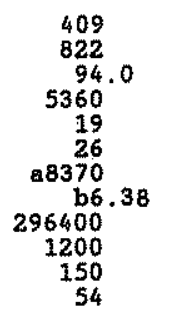

EP

e-Estimated.

a-From rating curve extended above $4,400 \mathrm{ft} / \mathrm{s}$, on basis of slope-area measurement of peak flow.

$b-G a g e$ height, $6.38 \mathrm{ft}$, recorded, $7.55 \mathrm{ft}$ from floodnarks. 
SAN JUAN RIVER BASIN

09354500 LOS RTNOS RIVER AT LA BOCA, CO

LOCATION - Lat $37^{\circ} 00^{\prime} 34^{* *}$, Long $107^{\circ} 35^{\prime} 56^{*}$, in NEKNWK soc.22, T.32 N., R.7 W., La Plata County, Hydrologic Unit 14080101 on downstream end of right abutment of the Denver \& Rio Grande Western Railroad Co. bridge, at southeast edge of La Boca, $0.1 \mathrm{mi}$ upstream from Spring Creek, and 2 mi upstream from maximum elovation of Navajo Reservoir.

DRATHAGE AREA. $-510 \mathrm{~m}^{2}$, approximately.

PERIOD OF RECORD. -October 1950 to current year. Monthly discharge only for some pertods, published in WSP 1733 . Water-quality dat availablo, July 1969 to August 1973, January 1988 to September 1991.

GAGE.- Water-stage recorder. Datum of gage is 6,143.59 ft above National Geodetic Vertical Datum of 1929.

REMARKS.--Records fair except for estimated daily discharges, which ere poor. Flow regulated by Vallecito Reservolr (station 09353000 ) 24 mi upstream since April 1941. Diversions for irrigation of about 33,000 acres upstream

from station. Several measurements of specific conductance and water temperature were obtained and are published in the "Supplemental Water-Quality Data For Gaging Stations" section of this report.

EXTREMES OUTSIDE PERIOD OF RECORD.--A flood on Oct. 5, 1911 has not yet been exceeded. DISCAARGE, CUBIC FEET PER SECOND, WATER YEAR OCTOBER 1995 TO SEPTEMBER 1996

\begin{tabular}{|c|c|c|c|c|c|c|c|c|c|c|c|c|}
\hline DAY & OCT & NOV & DEC & JAN & FEB & MAR & APR & MAY & JUN & JUL & AUG & SEP \\
\hline $\begin{array}{l}1 \\
2 \\
3 \\
4 \\
5\end{array}$ & $\begin{array}{l}e 330 \\
e 330 \\
e 320 \\
0200 \\
e 200\end{array}$ & $\begin{array}{l}77 \\
64 \\
56 \\
55 \\
53\end{array}$ & $\begin{array}{r}85 \\
45 \\
45 \\
044 \\
43\end{array}$ & $\begin{array}{l}053 \\
049 \\
049 \\
653 \\
055\end{array}$ & $\begin{array}{l}052 \\
050 \\
047 \\
044 \\
048\end{array}$ & $\begin{array}{l}105 \\
107 \\
105 \\
107 \\
116\end{array}$ & $\begin{array}{r}\mathrm{a} 53 \\
50 \\
50 \\
53 \\
56\end{array}$ & $\begin{array}{l}28 \\
25 \\
30 \\
51 \\
67\end{array}$ & $\begin{array}{l}107 \\
107 \\
112 \\
110 \\
105\end{array}$ & $\begin{array}{l}254 \\
179 \\
161 \\
139 \\
128\end{array}$ & $\begin{array}{l}101 \\
107 \\
12.5 \\
127 \\
119\end{array}$ & $\begin{array}{l}092 \\
080 \\
e 74 \\
065 \\
060\end{array}$ \\
\hline $\begin{array}{r}6 \\
7 \\
8 \\
9 \\
10\end{array}$ & $\begin{array}{l}\theta 180 \\
0180 \\
\theta 180 \\
\theta 160 \\
0135\end{array}$ & $\begin{array}{l}53 \\
49 \\
49 \\
49 \\
56\end{array}$ & $\begin{array}{r}43 \\
044 \\
044 \\
043 \\
043\end{array}$ & $\begin{array}{l}652 \\
050 \\
050 \\
048 \\
047\end{array}$ & $\begin{array}{l}e 53 \\
e 52 \\
052 \\
052 \\
054\end{array}$ & $\begin{array}{l}125 \\
132 \\
130 \\
115 \\
105\end{array}$ & $\begin{array}{l}52 \\
52 \\
51 \\
49 \\
54\end{array}$ & $\begin{array}{l}59 \\
60 \\
67 \\
73 \\
75\end{array}$ & $\begin{array}{l}110 \\
110 \\
108 \\
120 \\
122\end{array}$ & $\begin{array}{l}123 \\
110 \\
127 \\
380 \\
192\end{array}$ & $\begin{array}{r}118 \\
108 \\
97 \\
91 \\
107\end{array}$ & $\begin{array}{l}059 \\
062 \\
066 \\
070 \\
067\end{array}$ \\
\hline $\begin{array}{l}11 \\
12 \\
13 \\
14 \\
15\end{array}$ & $\begin{array}{l}115 \\
113 \\
115 \\
120 \\
113\end{array}$ & $\begin{array}{l}55 \\
52 \\
49 \\
48 \\
46\end{array}$ & $\begin{array}{r}044 \\
43 \\
46 \\
048 \\
049\end{array}$ & $\begin{array}{l}047 \\
047 \\
047 \\
047 \\
048\end{array}$ & $\begin{array}{l}56 \\
60 \\
62 \\
63 \\
69\end{array}$ & $\begin{array}{r}99 \\
93 \\
93 \\
99 \\
154\end{array}$ & $\begin{array}{l}56 \\
55 \\
60 \\
65 \\
43\end{array}$ & $\begin{array}{l}73 \\
76 \\
89 \\
93 \\
97\end{array}$ & $\begin{array}{l}118 \\
117 \\
115 \\
142 \\
158\end{array}$ & $\begin{array}{l}128 \\
134 \\
179 \\
167 \\
161\end{array}$ & $\begin{array}{l}97 \\
93 \\
89 \\
85 \\
89\end{array}$ & $\begin{array}{r}966 \\
066 \\
\theta 78 \\
e 105 \\
\theta 150\end{array}$ \\
\hline $\begin{array}{l}16 \\
17 \\
18 \\
19 \\
20\end{array}$ & $\begin{array}{r}112 \\
95 \\
89 \\
87 \\
79\end{array}$ & $\begin{array}{l}50 \\
51 \\
51 \\
49 \\
48\end{array}$ & $\begin{array}{r}49 \\
050 \\
048 \\
047 \\
046\end{array}$ & $\begin{array}{l}048 \\
051 \\
048 \\
048 \\
047\end{array}$ & $\begin{array}{l}69 \\
66 \\
67 \\
61 \\
60\end{array}$ & $\begin{array}{r}164 \\
158 \\
104 \\
95 \\
97\end{array}$ & $\begin{array}{l}36 \\
37 \\
37 \\
29 \\
22\end{array}$ & $\begin{array}{r}97 \\
91 \\
91 \\
89 \\
101\end{array}$ & $\begin{array}{l}138 \\
135 \\
123 \\
110 \\
108\end{array}$ & $\begin{array}{l}148 \\
161 \\
145 \\
175 \\
146\end{array}$ & $\begin{array}{l}107 \\
107 \\
1.07 \\
115 \\
108\end{array}$ & $\begin{array}{l}\otimes 200 \\
0180 \\
0165 \\
0190 \\
0200\end{array}$ \\
\hline $\begin{array}{l}21 \\
22 \\
23 \\
24 \\
25\end{array}$ & $\begin{array}{r}129 \\
136 \\
101 \\
93 \\
87\end{array}$ & $\begin{array}{l}43 \\
47 \\
48 \\
45 \\
43\end{array}$ & $\begin{array}{l}44 \\
043 \\
041 \\
045 \\
050\end{array}$ & $\begin{array}{l}046 \\
047 \\
048 \\
047 \\
047\end{array}$ & $\begin{array}{l}75 \\
71 \\
64 \\
56 \\
55\end{array}$ & $\begin{array}{l}93 \\
91 \\
94 \\
97 \\
97\end{array}$ & $\begin{array}{l}23 \\
15 \\
12 \\
63 \\
72\end{array}$ & $\begin{array}{r}101 \\
99 \\
93 \\
105 \\
120\end{array}$ & $\begin{array}{l}125 \\
172 \\
141 \\
133 \\
132\end{array}$ & $\begin{array}{l}130 \\
128 \\
113 \\
105 \\
99\end{array}$ & $\begin{array}{l}110 \\
112 \\
120 \\
182 \\
193\end{array}$ & $\begin{array}{l}0190 \\
0190 \\
0185 \\
0180 \\
0170\end{array}$ \\
\hline $\begin{array}{l}26 \\
27 \\
28 \\
29 \\
30 \\
31\end{array}$ & $\begin{array}{l}81 \\
81 \\
81 \\
81 \\
81 \\
81\end{array}$ & $\begin{array}{r}45 \\
43 \\
043 \\
045 \\
045 \\
---\end{array}$ & $\begin{array}{l}050 \\
046 \\
048 \\
050 \\
054 \\
054\end{array}$ & $\begin{array}{l}044 \\
043 \\
046 \\
e 50 \\
051 \\
e 52\end{array}$ & $\begin{array}{r}60 \\
56 \\
71 \\
91 \\
-\cdots \\
--\end{array}$ & $\begin{array}{r}93 \\
93 \\
68 \\
060 \\
057 \\
055\end{array}$ & $\begin{array}{r}30 \\
26 \\
28 \\
32 \\
27 \\
---\end{array}$ & $\begin{array}{l}120 \\
125 \\
120 \\
120 \\
110 \\
108\end{array}$ & $\begin{array}{l}137 \\
226 \\
323 \\
218 \\
171 \\
-\end{array}$ & $\begin{array}{l}117 \\
1.22 \\
115 \\
115 \\
110 \\
101\end{array}$ & $\begin{array}{l}144 \\
135 \\
128 \\
120 \\
e 115 \\
0105\end{array}$ & $\begin{array}{l}0165 \\
0150 \\
0135 \\
0125 \\
0110 \\
--1\end{array}$ \\
\hline $\begin{array}{l}\text { TOTAL } \\
\text { MEAN } \\
\text { MAX } \\
\text { MIN } \\
\text { AC-FT }\end{array}$ & $\begin{array}{r}4285 \\
138 \\
330 \\
79 \\
8500\end{array}$ & $\begin{array}{r}1507 \\
50.2 \\
77 \\
43 \\
2990\end{array}$ & $\begin{array}{r}1434 \\
46.3 \\
54 \\
41 \\
2840\end{array}$ & $\begin{array}{r}1505 \\
48,5 \\
55 \\
43 \\
2990\end{array}$ & $\begin{array}{r}1736 \\
59.9 \\
91 \\
44 \\
3440\end{array}$ & $\begin{array}{r}3201 \\
103 \\
164 \\
55 \\
6350\end{array}$ & $\begin{array}{r}1288 \\
42.9 \\
72 \\
12 \\
2550\end{array}$ & $\begin{array}{r}2653 \\
85.6 \\
125 \\
25 \\
5260\end{array}$ & $\begin{array}{r}4153 \\
138 \\
323 \\
105 \\
8240\end{array}$ & $\begin{array}{r}4592 \\
148 \\
380 \\
99 \\
9110\end{array}$ & $\begin{array}{r}3561 \\
115 \\
193 \\
85 \\
7060\end{array}$ & $\begin{array}{r}3695 \\
123 \\
200 \\
59 \\
7330\end{array}$ \\
\hline
\end{tabular}

STATISTICS OF MONTRLY MEAN DATA FOR WATER YEARS 1951 - 1996, BY WATER YEAR (WY)

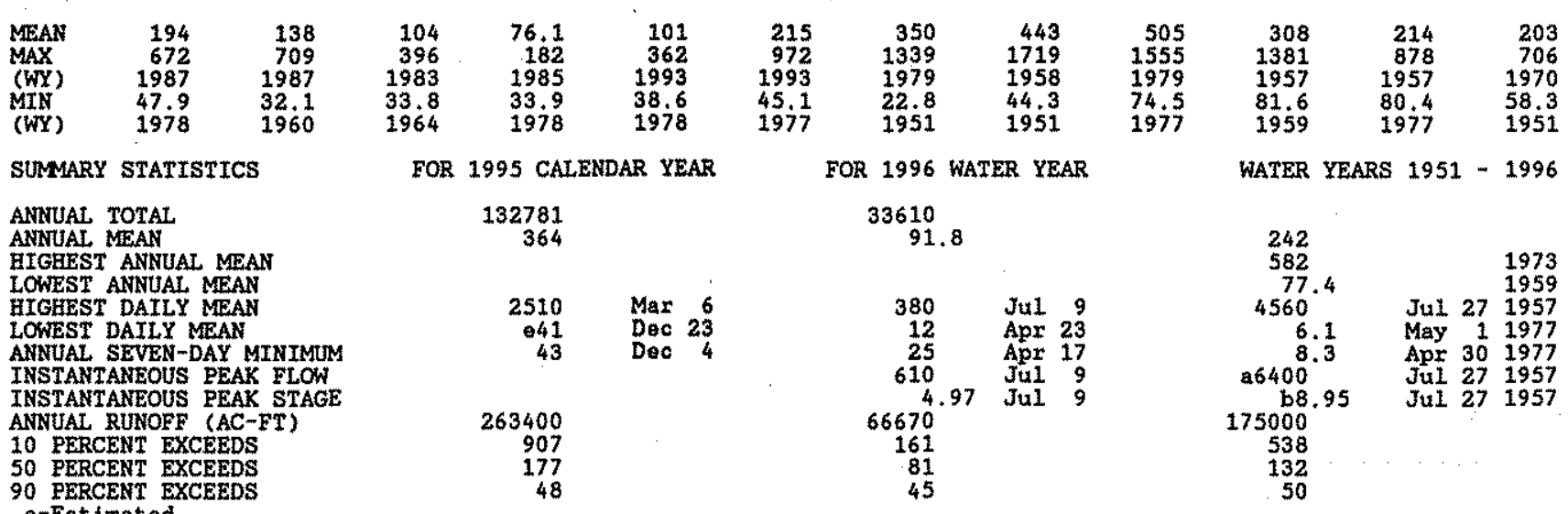
90 PERCENT EXCEFDS e-Estimated.

b-Maximum gage height, $9.00 \mathrm{ft}$, backwater from 1ce, sometime during pertod, Doc 23,1990 to Jan 17,1991 . 
SAN JUAN RIVER BASIN

09355000 SERING CREEK AT LA BOCA, CO

LOCATION.-Lat $37^{\circ} 00^{\prime} 40^{\prime \prime}$, Long $107^{\circ} 35^{\prime} 47^{\prime \prime}$, in SEKSW sec.15, T.32 N., R.7 W., La Plata County, Eydrologic Unit 14080101 , on $r$ ight bank in an excavated channel, 0.2 mi upstream from mouth, and 0.2 mi east of La Boca.

DRAINAGE AREA. $-58 \mathrm{mi}^{2}$, approximately.

PERIOD OF RECORD.--October 1950 to current year. Monthly discharge only for some pertods, published in WSP 1733, Water-quality data available, May 1974, January 1986 to September 1991.

GAGE. - Water-gtage recorder. Elevation of gage is 6,160 ft above National Geodet.1c Vertical Datum of 1929 , from topographic mas.

REMARKS.--Records fair except for estinated dally discharges, which are poor. Part of flow is return weste from Irrigation. Nearly all irrigation in this basin is water diverted from the Los Pinos River near Bayfield, Co. which causes a considerable chang in the annual pattern and natural flow Several measurements of specific conductance and water temperature wore obtained and are published fn the "Supplemental Water-Quality Data Fox Gaging stations" section of this report.

DISCHARGE, CUBIC FEET EER SECOND, WATER YEAR OCTOBER 1995 TO SEFTEMBER 1996

\begin{tabular}{|c|c|c|c|c|c|c|c|c|c|c|c|c|}
\hline DAY & $\infty r$ & Nov & DEC & JAN & FEB & MAR & APR & MAY & JUN & JUL & ALG & SEP \\
\hline $\begin{array}{l}1 \\
2 \\
3 \\
4 \\
5\end{array}$ & $\begin{array}{l}68 \\
65 \\
60 \\
48 \\
46\end{array}$ & $\begin{array}{l}5.5 \\
6.7 \\
5.1 \\
4.7 \\
4.7\end{array}$ & $\begin{array}{r}03.3 \\
03.3 \\
03.2 \\
2.7 \\
03.0\end{array}$ & $\begin{array}{l}02.6 \\
02.5 \\
02.4 \\
e 2.5 \\
02.6\end{array}$ & $\begin{array}{l}\mathrm{e} 2.2 \\
\mathrm{e} 2.1 \\
\mathrm{e} 2.0 \\
\mathrm{e} 1.8 \\
\mathrm{e} 2.0\end{array}$ & $\begin{array}{l}02.3 \\
02.4 \\
02.4 \\
02.4 \\
02.5\end{array}$ & $\begin{array}{l}2.0 \\
2.0 \\
2.0 \\
2.0 \\
2.3\end{array}$ & $\begin{array}{l}017 \\
019 \\
e 21 \\
024 \\
e 26\end{array}$ & $\begin{array}{l}53 \\
52 \\
51 \\
48 \\
43\end{array}$ & $\begin{array}{r}156 \\
91 \\
87 \\
85 \\
85\end{array}$ & $\begin{array}{l}108 \\
120 \\
138 \\
122 \\
101\end{array}$ & $\begin{array}{r}135 \\
138 \\
142 \\
0140 \\
e 122\end{array}$ \\
\hline $\begin{array}{r}6 \\
7 \\
8 \\
9 \\
10\end{array}$ & $\begin{array}{l}48 \\
53 \\
53 \\
47 \\
46\end{array}$ & $\begin{array}{l}4.7 \\
4.7 \\
4.7 \\
4.7 \\
5.8\end{array}$ & $\begin{array}{r}03.1 \\
3.0 \\
63.0 \\
03.0 \\
\mathrm{e} .0\end{array}$ & $\begin{array}{l}\mathrm{e} 2.4 \\
\mathrm{e} 2.3 \\
\mathrm{e} 2.4 \\
\mathrm{e} 2.2 \\
\mathrm{e} 2.2\end{array}$ & $\begin{array}{l}\ominus 2.2 \\
\mathrm{e} 2.1 \\
\mathrm{e} 2.1 \\
\mathrm{e} 2.1 \\
\mathrm{e} 2.2\end{array}$ & $\begin{array}{l}02.5 \\
e 2.4 \\
02.3 \\
e 2.4 \\
02.5\end{array}$ & $\begin{array}{l}2.3 \\
2.0 \\
2.3 \\
2.0 \\
2.0\end{array}$ & $\begin{array}{l}\mathrm{e} 28 \\
\mathrm{e} 28 \\
\mathrm{e} 28 \\
\mathrm{e} 29 \\
\mathrm{e} 28\end{array}$ & $\begin{array}{l}40 \\
39 \\
40 \\
46 \\
46\end{array}$ & $\begin{array}{r}88 \\
85 \\
91 \\
132 \\
118\end{array}$ & $\begin{array}{l}109 \\
116 \\
115 \\
118 \\
131\end{array}$ & $\begin{array}{r}156 \\
150 \\
\mathrm{e} 138 \\
\mathrm{e} 118 \\
\mathrm{e} 117\end{array}$ \\
\hline $\begin{array}{l}11 \\
12 \\
13 \\
14 \\
15\end{array}$ & $\begin{array}{l}48 \\
48 \\
45 \\
42 \\
43\end{array}$ & $\begin{array}{l}5.9 \\
4.4 \\
4.3 \\
4.7 \\
4.4\end{array}$ & $\begin{array}{l}e 3.0 \\
02.9 \\
03.0 \\
03.0 \\
02.9\end{array}$ & $\begin{array}{l}\text { e2.2 } \\
e 2.1 \\
\mathrm{e} 2.1 \\
\mathrm{e} 2.1 \\
\mathrm{e} 2.1\end{array}$ & $\begin{array}{l}\mathrm{e} .2 .2 \\
\mathrm{e} 2.2 \\
-2.2 \\
\mathrm{e} 2.2 \\
\mathrm{\theta} 2.3\end{array}$ & $\begin{array}{l}e 2.6 \\
e 2.7 \\
02.8 \\
02.7 \\
e 2.6\end{array}$ & $\begin{array}{l}2.3 \\
2.0 \\
3.0 \\
3.0 \\
2.3\end{array}$ & $\begin{array}{l}\text { e29 } \\
\text { e31 } \\
832 \\
833 \\
\text { e34 }\end{array}$ & $\begin{array}{l}45 \\
45 \\
49 \\
54 \\
63\end{array}$ & $\begin{array}{l}103 \\
108 \\
130 \\
129 \\
125\end{array}$ & $\begin{array}{l}124 \\
122 \\
116 \\
113 \\
116\end{array}$ & $\begin{array}{r}0120 \\
011.8 \\
166 \\
222 \\
154\end{array}$ \\
\hline $\begin{array}{l}16 \\
17 \\
18 \\
19 \\
20\end{array}$ & $\begin{array}{l}43 \\
38 \\
39 \\
38 \\
39\end{array}$ & $\begin{array}{l}4.0 \\
4.0 \\
4.0 \\
4.3 \\
4.0\end{array}$ & $\begin{array}{r}92.8 \\
82.9 \\
02.9 \\
02.8 \\
2.7\end{array}$ & $\begin{array}{l}\mathrm{e} 2.2 \\
\mathrm{e} 2.3 \\
\theta 2.2 \\
\mathrm{e} 2.1 \\
\mathrm{e} 2.1\end{array}$ & $\begin{array}{l}82.3 \\
02.3 \\
02.3 \\
02.3 \\
02.4\end{array}$ & $\begin{array}{l}e 2.5 \\
e 2.5 \\
e 2.5 \\
e 2.5 \\
e 2.5\end{array}$ & $\begin{array}{r}21 \\
3.7 \\
3.3 \\
3.3 \\
3.0\end{array}$ & $\begin{array}{r}035 \\
635 \\
34 \\
35 \\
38\end{array}$ & $\begin{array}{l}58 \\
57 \\
55 \\
56 \\
58\end{array}$ & $\begin{array}{l}125 \\
134 \\
126 \\
134 \\
126\end{array}$ & $\begin{array}{l}125 \\
127 \\
131 \\
122 \\
125\end{array}$ & $\begin{array}{r}32 \\
28 \\
27 \\
e 27 \\
e 25\end{array}$ \\
\hline $\begin{array}{l}21 \\
22 \\
23 \\
24 \\
25\end{array}$ & $\begin{array}{l}43 \\
22 \\
7.4 \\
5.9 \\
5.5\end{array}$ & $\begin{array}{r}3.7 \\
4.0 \\
3.7 \\
3.3 \\
03.2\end{array}$ & $\begin{array}{l}\text { e2.6 } \\
\text { e2.6 } \\
\text { e2.4 } \\
\text { e2.1 } \\
\text { e2.3 }\end{array}$ & $\begin{array}{l}\mathrm{e} 2.1 \\
\mathrm{e} 2.1 \\
\mathrm{e} 2.1 \\
\mathrm{e} 2.0 \\
\mathrm{e} 2.1\end{array}$ & $\begin{array}{l}e 2.5 \\
e 2.6 \\
e 2.5 \\
22.5 \\
e 2.4\end{array}$ & $\begin{array}{l}\theta 2.5 \\
e 2.7 \\
e 2.8 \\
\theta 2.9 \\
\theta 2.8\end{array}$ & $\begin{array}{r}4.0 \\
3.3 \\
3.0 \\
5.0 \\
12\end{array}$ & $\begin{array}{l}40 \\
46 \\
43 \\
43 \\
48\end{array}$ & $\begin{array}{l}61 \\
85 \\
71 \\
68 \\
64\end{array}$ & $\begin{array}{l}e 112 \\
e 105 \\
e 102 \\
e 102 \\
e 106\end{array}$ & $\begin{array}{l}127 \\
134 \\
135 \\
221 \\
201\end{array}$ & $\begin{array}{l}\text { e21 } \\
018 \\
016 \\
\text { e14 } \\
\text { e12 }\end{array}$ \\
\hline $\begin{array}{l}26 \\
27 \\
28 \\
29 \\
30 \\
31\end{array}$ & $\begin{array}{l}5.5 \\
5.5 \\
4.7 \\
5.1 \\
5.1 \\
4.7\end{array}$ & $\begin{array}{l}03.3 \\
e 3.2 \\
03.2 \\
03.3 \\
03.3 \\
-\ldots\end{array}$ & $\begin{array}{l}e 2.5 \\
e 2.4 \\
e 2.3 \\
e 2.4 \\
e 2.5 \\
e 2.7\end{array}$ & $\begin{array}{l}e 1.9 \\
01.9 \\
e 2.0 \\
02.1 \\
02.2 \\
02.2\end{array}$ & $\begin{array}{l}02.4 \\
e 2.3 \\
02.2 \\
\mathrm{e} 2.2 \\
---\end{array}$ & $\begin{array}{r}02.7 \\
02.6 \\
e 2.5 \\
02.4 \\
2.3 \\
2.0\end{array}$ & $\begin{array}{l}\text { e19 } \\
022 \\
023 \\
018 \\
015 \\
-\end{array}$ & $\begin{array}{l}52 \\
51 \\
48 \\
52 \\
52 \\
52\end{array}$ & $\begin{array}{r}69 \\
141 \\
157 \\
101 \\
102 \\
-\end{array}$ & $\begin{array}{l}107 \\
104 \\
111 \\
109 \\
109 \\
104\end{array}$ & $\begin{array}{l}157 \\
152 \\
144 \\
146 \\
142 \\
140\end{array}$ & $\begin{array}{r}010 \\
09.8 \\
99.1 \\
99.0 \\
99.3 \\
\end{array}$ \\
\hline $\begin{array}{l}\text { TOTAL } \\
\text { MEAN } \\
\text { MAX } \\
\text { MIN } \\
\text { AC-FT }\end{array}$ & $\begin{array}{r}1071.4 \\
34.6 \\
68 \\
4.7 \\
2130\end{array}$ & $\begin{array}{r}129.5 \\
4.32 \\
6.7 \\
3.2 \\
257\end{array}$ & $\begin{array}{r}86.3 \\
2.78 \\
3.3 \\
2.1 \\
173\end{array}$ & $\begin{array}{r}68.3 \\
2.20 \\
2.6 \\
1.9 \\
135\end{array}$ & $\begin{array}{r}65.1 \\
2.24 \\
2.6 \\
1.8 \\
129\end{array}$ & $\begin{array}{r}78.2 \\
2.52 \\
2.9 \\
2.0 \\
155\end{array}$ & $\begin{array}{r}192.1 \\
6.40 \\
23 \\
2.0 \\
381\end{array}$ & $\begin{array}{r}1111 \\
35.8 \\
52 \\
17 \\
2200\end{array}$ & $\begin{array}{r}1917 \\
63.9 \\
157 \\
39 \\
3800\end{array}$ & $\begin{array}{r}3429 \\
111 \\
156 \\
85 \\
6800\end{array}$ & $\begin{array}{r}4098 \\
132 \\
221 \\
101 \\
8130\end{array}$ & $\begin{array}{r}2403.2 \\
80.1 \\
222 \\
9.0 \\
4770\end{array}$ \\
\hline
\end{tabular}

STATISTICS OF MONTHLY MEAN DATA FOR WATER YEARS 1951 - 1996, BY WATER YEAR (WY)

\begin{tabular}{|c|c|c|c|c|c|c|c|c|c|c|c|c|}
\hline $\begin{array}{l}\text { MEAN } \\
\text { MAX } \\
(W Y) \\
\text { MIN } \\
(W X)\end{array}$ & $\begin{array}{l}35.3 \\
87.9 \\
1973 \\
5.25 \\
1978\end{array}$ & $\begin{array}{l}10.8 \\
29.6 \\
1956 \\
3.68 \\
1978\end{array}$ & $\begin{array}{l}5.50 \\
20.4 \\
1985 \\
1.74 \\
1960\end{array}$ & $\begin{array}{l}4.70 \\
19.3 \\
1980 \\
2.04 \\
1973\end{array}$ & $\begin{array}{l}10.3 \\
54,8 \\
1980 \\
2.24 \\
1996\end{array}$ & $\begin{array}{l}19.0 \\
89.7 \\
1979 \\
2.52 \\
1996\end{array}$ & $\begin{array}{l}13.7 \\
41.1 \\
1979 \\
3.77 \\
1978\end{array}$ & $\begin{array}{l}39.6 \\
64.5 \\
1992 \\
15.7 \\
1978\end{array}$ & $\begin{array}{l}57.7 \\
79.3 \\
1986 \\
24.4 \\
1977\end{array}$ & $\begin{array}{r}67.2 \\
111 \\
1996 \\
21.2 \\
1977\end{array}$ & $\begin{array}{r}67.5 \\
132 \\
1996 \\
32.1 \\
1977\end{array}$ & $\begin{array}{l}59.4 \\
92.0 \\
1983 \\
26.5 \\
1951\end{array}$ \\
\hline
\end{tabular}

\section{SUMMARY STATISTICS}

ANNUAR TOTAL.

ARHUAL MEAN

HIGHEST ANNUAL MEAN

LOWEST ANNUAZ MEAN

GIGESST DAILY MEAN

LOWEST DAILY MEAN

ANNUAL SEVEN-DAY MINTMIM

INSTANTANEOUS PEAK FLOW

INSTANTANEOUS PEAK STAGE

ANNUAL RUNOFF (AC-FT)

10 PERCENT EXCEEDS

90 PERCENT EXCEEDS
FOR 1995 CALENDAR YEAR

$\begin{aligned} 14120.4 & \\ 38.7 & \\ & \\ 918 & \text { Mar } 6 \\ 02.1 & \text { Dec 24 } \\ 2.3 & \text { Dec 23 } \\ & \\ 28010 & \\ 75 & \\ 25 & \\ 3.3 & \end{aligned}$

FOR 1996 WATER YEAR

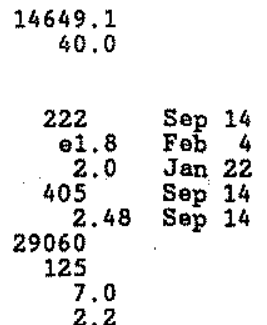

WATER YEARS 1951 - 1996

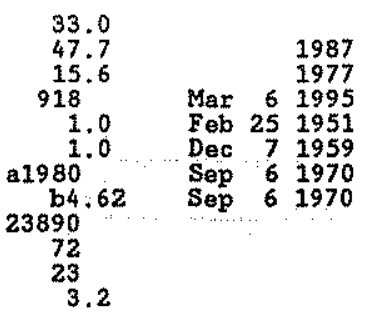

o-Estimated.

a-From rating curve extended above $160 \mathrm{ft}^{3} / \mathrm{s}$, on basis of fleld estimate of peak flow.

b-Maximum gage height, $5.98 \mathrm{ft}$, Mar 9, 1960, backwater from ice. 
SAN JUAN RIVER BASTN

09355100 NAVAJO RESERVOTR NEAR ARCHULETA, MM

LOCATION.--Lat $36^{\circ} 48^{\circ} 28^{\prime \prime}$, long $107^{\circ} 36^{\circ} 31^{\prime \prime}$, in SW/SE\% sec.18, T. 30 N., R.7 W. San Juan County, Eydrologic Unit 14080101 in gate shaft of outlet works structure near right abutment of Navajo Dam on San Juan River, 5.5 m1 east of Archuleta, $33 \mathrm{mi}$ east of Farmington, and at mile 298,6 .

DRAINAGE AREA. $--3,230 \mathrm{mi}^{3}$, approximataly.

GAGE.--Water-stage recorder. Datum of gage is National Geodetic Vertical Datum of 1929.

PERIOD OF RECORD.--June 1962 to current year. Prior to October 1968 dead storage included.

REMARKS.-- Reservoir is formed by earth rock-fill dam, completed in June 1963; storage began June $27,1962$. Capacity, 1,708,600 acre-ft botween elevation 5,720 ft upstream toe of dam and 6,085 ft crest of spiliway. Usable capacity $1,696,000$ acre-ft above elevation $5,774.9$ ft minimum operating level. Dead storage below elevation $5,774.9 \mathrm{ft}$ is 12,600 acre-ft. Figures given herein are usable contents. Reservoir is used for irrigation storage, river regulation, desiling, flood control, and recreation.

COOPERATION, --Records provided by Bureau of Reclamation.

EXXREMES FOR PERIOD OF RECORD. --Maximum contents, 1,731,000 acre-ft, July 2-4, 1973, elevation, 6,087.25 ft; minimum contents after June 1964 (initial filling period), 234, 300 acre-ft, Mar. 10, 11, 1965, elevation, 5,906.36 ft.

EXTREMES FOR CURRENT YEAR.--Maximum contents, 1,555,600 acre-ft, Oct. 1, elevation, 6,075.64 ft; minimum contents, $1,202,800$ acre-ft, Sept. 30, elevation, $6,048,40 \mathrm{ft}$.

Capacity table (elevation, in feet, and contents, in thousands of acre-feet)

$\begin{array}{lrrrrrrr}6.015 & 864.5 & 6,035 & 1,056.7 & 6,055 & 1,281.3 & 6,075 & 1,546.2 \\ 6,020 & 910.1 & 6,040 & 1,109.4 & 6,060 & 1,343.5 & 6,080 & 1,619.5 \\ 6,025 & 957.2 & 6,045 & 1,164.3 & 6,065 & 1,408.3 & 6,085 & 1,696.0 \\ 6,030 & 1,006.0 & 6,050 & 1,221.6 & 6,070 & 1,475.8 & 6,090 & 1,775.7\end{array}$

RESERVOIR STORAGE (ACRE-FEET), WATER YEAR OCTOBER 1995 TO SEPTEMBER 1996

DAIL.Y OBSERVATION AT 24:00 VALUES

\begin{tabular}{|c|c|c|c|c|c|c|c|c|c|c|c|c|}
\hline$A Y$ & OCT & nov & $\mathrm{DEC}$ & JAN & FEB & MAR & AER & MAY & JUN & JUL & AUG & SEP \\
\hline $\begin{array}{l}1 \\
2 \\
3 \\
4 \\
5\end{array}$ & $\begin{array}{l}1555600 \\
1555000 \\
1554400 \\
1553500 \\
1552600\end{array}$ & $\begin{array}{l}152 \\
152 \\
152 \\
152 \\
152\end{array}$ & $\begin{array}{l}149 \\
149 \\
149 \\
149 \\
148\end{array}$ & $\begin{array}{l}14 \\
14 \\
14 \\
14 \\
14\end{array}$ & $\begin{array}{l}145 \\
144 \\
144 \\
144\end{array}$ & $\begin{array}{l}1 \\
1 \\
1 \\
1 \\
1\end{array}$ & & $\begin{array}{l}1 \\
1 \\
1 \\
1 \\
1\end{array}$ & & & & $\begin{array}{l}900 \\
100 \\
300 \\
200 \\
300\end{array}$ \\
\hline $\begin{array}{r}6 \\
7 \\
8 \\
9 \\
10\end{array}$ & $\begin{array}{l}155 \\
155 \\
155 \\
154 \\
154\end{array}$ & $\begin{array}{l}15 \\
15 \\
15 \\
15\end{array}$ & & $\begin{array}{l}1 \\
1 \\
1 \\
1\end{array}$ & $\begin{array}{l}14 \\
14\end{array}$ & & & & & & & $\begin{array}{l}00 \\
00 \\
00 \\
00 \\
00\end{array}$ \\
\hline $\begin{array}{l}11 \\
12 \\
13 \\
14 \\
15\end{array}$ & $\begin{array}{l}154 \\
154 \\
154 \\
154\end{array}$ & $\begin{array}{l}15 \\
15 \\
15\end{array}$ & $\begin{array}{l}34 \\
14 \\
14 \\
14\end{array}$ & $\begin{array}{l}14 \\
14 \\
14 \\
14 \\
14\end{array}$ & $\begin{array}{l}14 \\
14\end{array}$ & & & & & & & $\begin{array}{l}00 \\
00 \\
00 \\
00 \\
00\end{array}$ \\
\hline $\begin{array}{l}16 \\
17 \\
18 \\
19 \\
20\end{array}$ & $\begin{array}{l}154 \\
154 \\
153 \\
153\end{array}$ & $\begin{array}{l}15 \\
15 \\
15\end{array}$ & $\begin{array}{l}14 \\
14 \\
14 \\
14\end{array}$ & $\begin{array}{l}14 \\
14 \\
14 \\
14 \\
14\end{array}$ & $\begin{array}{l}14 \\
14\end{array}$ & $\begin{array}{l}14 \\
14 \\
14 \\
14\end{array}$ & & & & & & $\begin{array}{l}1213600 \\
1213500 \\
1212900 \\
1212500 \\
1211800\end{array}$ \\
\hline $\begin{array}{l}21 \\
22 \\
23 \\
24\end{array}$ & $\begin{array}{l}1536 \\
1535 \\
1534\end{array}$ & $\begin{array}{l}15 \\
15 \\
15\end{array}$ & $\begin{array}{l}14 \\
14 \\
14\end{array}$ & & & & & & & & & \\
\hline $\begin{array}{l}26 \\
27 \\
28 \\
29 \\
30\end{array}$ & $\begin{array}{l}1532000 \\
1531300 \\
1530300 \\
1529300 \\
1528400\end{array}$ & $\begin{array}{r}1700100 \\
1499000 \\
1497800 \\
1496700 \\
1495600 \\
-\end{array}$ & $\begin{array}{l}14 \\
14 \\
14 \\
140 \\
14 \\
14\end{array}$ & $\begin{array}{l}14 \\
14 \\
1.4 \\
14 \\
14 \\
14\end{array}$ & $\begin{array}{r}1441000 \\
1440600 \\
1440200 \\
\end{array}$ & $\begin{array}{l}14 \\
14 \\
14 \\
14 \\
14 \\
14\end{array}$ & $\begin{array}{r}1427600 \\
1428800 \\
1430300 \\
1431800 \\
1432100\end{array}$ & & $\begin{array}{r}1336700 \\
1337000 \\
1336600 \\
1337600 \\
\end{array}$ & & & $\begin{array}{l}1205200 \\
1204600 \\
1203900 \\
1203200 \\
1202800\end{array}$ \\
\hline $\begin{array}{l}\text { MAX } \\
\text { MIN } \\
(\neq)\end{array}$ & $\begin{array}{r}1555600 \\
1528400 \\
6073.74 \\
-27200\end{array}$ & $\begin{array}{r}1527200 \\
1495600 \\
6071.40 \\
-32800\end{array}$ & $\begin{array}{r}1494200 \\
1461500 \\
6068.96 \\
-34100\end{array}$ & $\begin{array}{r}1460800 \\
1450700 \\
6068.13 \\
-10800\end{array}$ & $\begin{array}{c}1450200 \\
1440200 \\
6067.37 \\
-10500\end{array}$ & $\begin{array}{r}1439500 \\
1430300 \\
6066.64 \\
-9900\end{array}$ & $\begin{array}{r}14321 \\
14264 \\
6066 \\
+18\end{array}$ & $\begin{array}{l}3300 \\
9.21 \\
2800\end{array}$ & $\begin{array}{l}00 \\
00 \\
51 \\
10\end{array}$ & $\begin{array}{c}1337200 \\
1291500 \\
6055.81 \\
-46100\end{array}$ & $\begin{array}{r}1234 \\
6051 \\
-568\end{array}$ & $\begin{array}{l}1232900 \\
1202800 \\
6048.40\end{array}$ \\
\hline
\end{tabular}

CAL YR 1995 MAX 1610400 MIN 1378600 (†t) +70400

WTR YR 1996 MAX 1555600 MIN 1202800 ( + † $)-352800$

(t) ELEVATION, IN FEET, AT END OF MONTH.

$(\dagger)$ CHANGE IN CONTENTS, IN ACRE-FEET. 
SAN JUAN RIVER BASIN

09355500 SAN JUAN RIVER NEAR ARCEULETA, NM

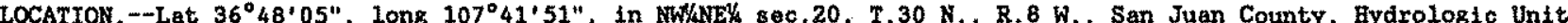
14080101, on lest bank $0.5 \mathrm{mi}$ upstream from Gobernador Canyon, 0.8 mi northeast of Archuleta, 7.2 mi downstream from Navajo Dam, and at mile 291.4

DRAIKAGE AREA. $--3,260 \mathrm{mi}^{2}$, approximately.

PIRIOD OF RECORD. --December 1954 to current year.

REVISED RECORDS.--The annual runoff for the 1958 water year as published in table 2 , WSP 1733 , is 455,000 acre-ft, GAGE correct value is $1,455,000$ acrom

-Water-stage recorder. Elevation of gage is $5,653 \mathrm{ft}$ above National Geodetic Vertical Datum of 1929 , from

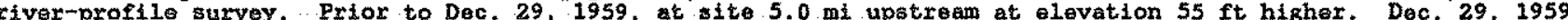

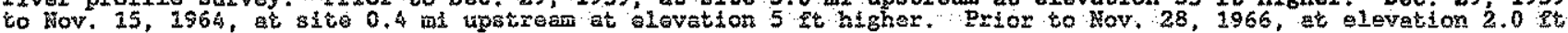
higher.

REMARKS, - - Hater-discharge records good. Flow completely regulated by Navajo Reservoir (station 09355100) 7 mi upstream except for minor inflow from 30 mi2 Intervening drainage area, High-water diversions through Azotea tunnel (station 08284160) into Rio Grande basin began in March 1971. Diversions for irrigation of about 47,000 acres upstream from station. Releases from Navajo Reservoir, beginning in January 1976, for use on Navajo Indian Irrigation Project bypass gage in tumel on left bank. See tabulation below for monthly and annuel releases as Surnished by Bureau of Reclametion. Nationel Weather Service satellite telemetex at station. DISCHARGE, CUBIC FEET PER SECOND, WATER YEAR OCTOBER 1995 TO SEPTEMBER 1996

\begin{tabular}{|c|c|c|c|c|c|c|c|c|c|c|c|c|}
\hline $\begin{array}{r}\text { DAY } \\
1 \\
2 \\
3 \\
4 \\
5\end{array}$ & $\begin{array}{l}\text { OCT } \\
815 \\
803 \\
810 \\
804 \\
803\end{array}$ & $\begin{array}{l}\text { Nov } \\
778 \\
773 \\
769 \\
771 \\
771\end{array}$ & $\begin{array}{l}\text { DEC } \\
780 \\
778 \\
778 \\
778 \\
795\end{array}$ & $\begin{array}{r}\text { JAN } \\
511 \\
510 \\
504 \\
506 \\
507\end{array}$ & $\begin{array}{l}\text { FEB } \\
502 \\
498 \\
493 \\
493 \\
494\end{array}$ & $\begin{array}{l}\text { MAR } \\
510 \\
510 \\
508 \\
510 \\
509\end{array}$ & $\begin{array}{l}\text { APR } \\
487 \\
487 \\
487 \\
485 \\
488\end{array}$ & $\begin{array}{l}\text { MAX } \\
498 \\
488 \\
495 \\
494 \\
493\end{array}$ & $\begin{array}{l}\text { JUN } \\
2410 \\
2440 \\
2450 \\
2440 \\
2390\end{array}$ & $\begin{array}{l}\text { JUL } \\
647 \\
630 \\
607 \\
607 \\
608\end{array}$ & $\begin{array}{l}\text { AUG } \\
624 \\
632 \\
630 \\
631 \\
633\end{array}$ & $\begin{array}{l}\text { SEP } \\
609 \\
608 \\
608 \\
608 \\
609\end{array}$ \\
\hline $\begin{array}{r}6 \\
7 \\
8 \\
9 \\
10\end{array}$ & $\begin{array}{l}807 \\
808 \\
808 \\
804 \\
804\end{array}$ & $\begin{array}{l}771 \\
771 \\
774 \\
775 \\
777\end{array}$ & $\begin{array}{l}834 \\
801 \\
796 \\
795 \\
790\end{array}$ & $\begin{array}{l}512 \\
510 \\
509 \\
508 \\
387\end{array}$ & $\begin{array}{l}493 \\
490 \\
493 \\
493 \\
493\end{array}$ & $\begin{array}{l}513 \\
494 \\
511 \\
512 \\
509\end{array}$ & $\begin{array}{l}487 \\
490 \\
493 \\
493 \\
489\end{array}$ & $\begin{array}{l}493 \\
490 \\
487 \\
487 \\
485\end{array}$ & $\begin{array}{l}2440 \\
2440 \\
2430 \\
2430 \\
2450\end{array}$ & $\begin{array}{l}613 \\
608 \\
608 \\
608 \\
608\end{array}$ & $\begin{array}{l}629 \\
633 \\
635 \\
641 \\
636\end{array}$ & $\begin{array}{l}602 \\
603 \\
607 \\
607 \\
610\end{array}$ \\
\hline $\begin{array}{l}11 \\
12 \\
13 \\
14 \\
15\end{array}$ & $\begin{array}{l}803 \\
801 \\
800 \\
802 \\
799\end{array}$ & $\begin{array}{l}772 \\
773 \\
774 \\
778 \\
778\end{array}$ & $\begin{array}{l}778 \\
749 \\
749 \\
754 \\
749\end{array}$ & $\begin{array}{l}232 \\
244 \\
241 \\
241 \\
241\end{array}$ & $\begin{array}{l}493 \\
493 \\
488 \\
487 \\
487\end{array}$ & $\begin{array}{r}507 \\
499 \\
499 \\
499 \\
495\end{array}$ & $\begin{array}{r}487 \\
493 \\
502 \\
501 \\
500\end{array}$ & $\begin{array}{l}489 \\
487 \\
485 \\
482 \\
482\end{array}$ & $\begin{array}{l}2520 \\
2580 \\
2470 \\
2440 \\
2450\end{array}$ & $\begin{array}{l}607 \\
606 \\
607 \\
610 \\
613\end{array}$ & $\begin{array}{l}629 \\
626 \\
626 \\
626 \\
626\end{array}$ & $\begin{array}{l}608 \\
608 \\
606 \\
644 \\
620\end{array}$ \\
\hline $\begin{array}{l}16 \\
17 \\
18 \\
19 \\
20\end{array}$ & $\begin{array}{l}785 \\
776 \\
777 \\
776 \\
772\end{array}$ & $\begin{array}{l}780 \\
775 \\
776 \\
781 \\
778\end{array}$ & $\begin{array}{l}744 \\
742 \\
763 \\
797 \\
825\end{array}$ & $\begin{array}{l}242 \\
251 \\
249 \\
249 \\
252\end{array}$ & $\begin{array}{l}487 \\
477 \\
476 \\
474 \\
480\end{array}$ & $\begin{array}{l}500 \\
501 \\
496 \\
490 \\
490\end{array}$ & $\begin{array}{l}499 \\
501 \\
495 \\
490 \\
488\end{array}$ & $\begin{array}{l}482 \\
484 \\
493 \\
495 \\
499\end{array}$ & $\begin{array}{l}2450 \\
2440 \\
2440 \\
2440 \\
2430\end{array}$ & $\begin{array}{l}615 \\
613 \\
613 \\
610 \\
608\end{array}$ & $\begin{array}{l}626 \\
630 \\
627 \\
628 \\
623\end{array}$ & $\begin{array}{l}622 \\
621 \\
624 \\
628 \\
631\end{array}$ \\
\hline $\begin{array}{l}21 \\
22 \\
23 \\
24 \\
25\end{array}$ & $\begin{array}{l}773 \\
771 \\
771 \\
768 \\
771\end{array}$ & $\begin{array}{l}778 \\
780 \\
781 \\
782 \\
779\end{array}$ & $\begin{array}{l}800 \\
795 \\
785 \\
788 \\
788\end{array}$ & $\begin{array}{l}250 \\
252 \\
252 \\
252 \\
363\end{array}$ & $\begin{array}{l}499 \\
501 \\
503 \\
499 \\
500\end{array}$ & $\begin{array}{l}493 \\
490 \\
492 \\
491 \\
490\end{array}$ & $\begin{array}{l}483 \\
484 \\
487 \\
486 \\
486\end{array}$ & $\begin{array}{l}494 \\
494 \\
496 \\
493 \\
490\end{array}$ & $\begin{array}{l}2430 \\
2420 \\
2410 \\
2240 \\
1900\end{array}$ & $\begin{array}{l}609 \\
609 \\
608 \\
608 \\
609\end{array}$ & $\begin{array}{l}622 \\
619 \\
620 \\
624 \\
610\end{array}$ & $\begin{array}{l}634 \\
634 \\
631 \\
634 \\
636\end{array}$ \\
\hline $\begin{array}{l}26 \\
27 \\
28 \\
29 \\
30 \\
31\end{array}$ & $\begin{array}{l}769 \\
771 \\
774 \\
769 \\
772 \\
771\end{array}$ & $\begin{array}{l}778 \\
779 \\
778 \\
778 \\
780 \\
---\end{array}$ & $\begin{array}{l}659 \\
526 \\
500 \\
501 \\
507 \\
512\end{array}$ & $\begin{array}{l}493 \\
489 \\
490 \\
498 \\
499 \\
501\end{array}$ & $\begin{array}{r}504 \\
507 \\
507 \\
510 \\
-\cdots- \\
-\cdots\end{array}$ & $\begin{array}{l}487 \\
487 \\
487 \\
484 \\
487 \\
487\end{array}$ & $\begin{array}{l}484 \\
476 \\
471 \\
478 \\
490 \\
\end{array}$ & $\begin{array}{r}490 \\
493 \\
768 \\
1280 \\
1730 \\
2220\end{array}$ & $\begin{array}{r}1540 \\
1150 \\
801 \\
659 \\
670 \\
-\end{array}$ & $\begin{array}{l}608 \\
610 \\
614 \\
628 \\
617 \\
622\end{array}$ & $\begin{array}{l}609 \\
631 \\
607 \\
603 \\
606 \\
611\end{array}$ & $\begin{array}{l}635 \\
640 \\
639 \\
642 \\
648 \\
-\end{array}$ \\
\hline $\begin{array}{l}\text { TOTAL } \\
\text { MEAN } \\
\text { MAX } \\
\text { MIN } \\
\text { AC-FT } \\
(1)\end{array}$ & $\begin{array}{r}24437 \\
788 \\
815 \\
788 \\
48470 \\
6200\end{array}$ & $\begin{array}{r}23288 \\
776 \\
782 \\
759 \\
46190 \\
0\end{array}$ & $\begin{array}{r}22736 \\
733 \\
834 \\
500 \\
45100 \\
0\end{array}$ & $\begin{array}{r}11745 \\
379 \\
512 \\
232 \\
23300 \\
0\end{array}$ & $\begin{array}{r}14314 \\
494 \\
510 \\
474 \\
28390 \\
0\end{array}$ & $\begin{array}{r}15437 \\
498 \\
513 \\
454 \\
30620 \\
2300\end{array}$ & $\begin{array}{r}14667 \\
489 \\
502 \\
471 \\
29090 \\
13000\end{array}$ & $\begin{array}{r}19236 \\
621 \\
2220 \\
482 \\
38150 \\
31300\end{array}$ & $\begin{array}{r}65200 \\
2173 \\
2580 \\
659 \\
129300 \\
39500\end{array}$ & $\begin{array}{r}18988 \\
613 \\
647 \\
606 \\
37660 \\
40500\end{array}$ & $\begin{array}{r}9353 \\
624 \\
641 \\
603 \\
8390 \\
8600\end{array}$ & $\begin{array}{r}18656 \\
622 \\
648 \\
602 \\
37000 \\
22300\end{array}$ \\
\hline $\begin{array}{l}\text { SUMEARY } \\
\text { ANNUAL } \\
\text { ANNUAL } \\
\text { HIGEEST } \\
\text { LOWEST } \\
\text { HIGHEST } \\
\text { LOWEST } \\
\text { ANNUAL } \\
\text { INSTANT } \\
\text { INSTANTA } \\
\text { INSTANTA } \\
\text { ANNUAL } \\
10 \text { PERCE } \\
50 \text { PERCE } \\
90 \text { PERCE }\end{array}$ & $\begin{array}{l}\text { STATIS } \\
\text { TOTAL } \\
\text { MEANY } \\
\text { ANHUAL } \\
\text { ANNUAL } \\
\text { DAILY } \\
\text { DAILY } \\
\text { SEVEN-D } \\
\text { ANEOUS } \\
\text { ANEOUS } \\
\text { ANEOUS } \\
\text { RUNOFF } \\
\text { ENT EXC } \\
\text { ENT EXC } \\
\text { ENT EXC }\end{array}$ & $\begin{array}{l}\text { TICS } \\
\text { MEAN } \\
\text { MEAN } \\
\text { MEAN } \\
\text { IEAN } \\
\text { AY MINTMUM } \\
\text { PEAK FLOW } \\
\text { PEAK STAGE } \\
\text { LOW FLOW } \\
\text { (AC-FT) } \\
\text { EEDS } \\
\text { EEDS } \\
\text { EEDS }\end{array}$ & FOR & $\begin{array}{c}1995 \text { CAL } \\
586958 \\
1608\end{array}$ & $\begin{array}{lr}\operatorname{Jun} 6 \\
\operatorname{Jan} 20 \\
\operatorname{Jan} 18\end{array}$ & & $\begin{array}{c}2580 \\
232 \\
242 \\
2630 \\
5.24 \\
219 \\
531700 \\
807 \\
609 \\
487\end{array}$ & $\begin{array}{l}\operatorname{Jun} 12 \\
\operatorname{Jan} 11 \\
\operatorname{Jan} 11 \\
\operatorname{Jun} 11 \\
\operatorname{Jun} 11 \\
\operatorname{Jan} 11\end{array}$ & $\therefore$ & $\begin{array}{c}\text { WATER YEARS } \\
\text { 21200 } \\
2686 \\
280 \\
6420 \\
30 \\
108 \\
\text { b18900 } \\
\text { c11.00 } \\
8.0 \\
869100 \\
2620 \\
689 \\
408\end{array}$ & $\begin{array}{ll}J_{u n} & 2 \\
\text { Mar } & 1 \\
\text { Jan } & 1 \\
\text { Jul } & 2 \\
\text { Ju1 } & 2 \\
\text { Feb } & 2\end{array}$ & $\begin{array}{ll} & 1987 \\
& 1963 \\
21 & 1955 \\
12 & 1964 \\
10 & 1963 \\
27 & 1957 \\
27 & 1957 \\
28 & 1963\end{array}$ \\
\hline
\end{tabular}

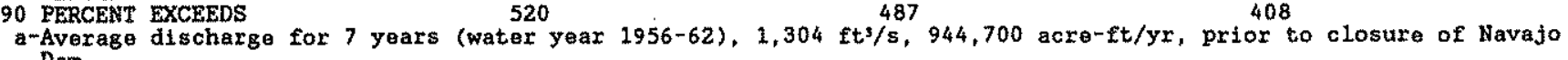
Dam.

b-Site and datum then in use.

c-Maximum discharge since construction of Navajo Dam in 1962, 6,500 fts/s, June 20, 1965, gage helght 4.75 ft. $(\dagger)$ DISCHARGE, IN ACRE-ET, THROUGE NAVAJO INDIAN IRRIGATION TUNNEL. 
SAN JUAN RIVER BASTN

09355500 SAN JUAN RIVER NEAR ARCHULETA, MM -- Cont 1 nued

WATER-QUALITY RECORDS

PERIOD OF RECORD.-Water years 1955 to current year.

WATER QUALITY DATA, WATER YEAR OCTOBER 1995 TO SEPTEMBER 1996

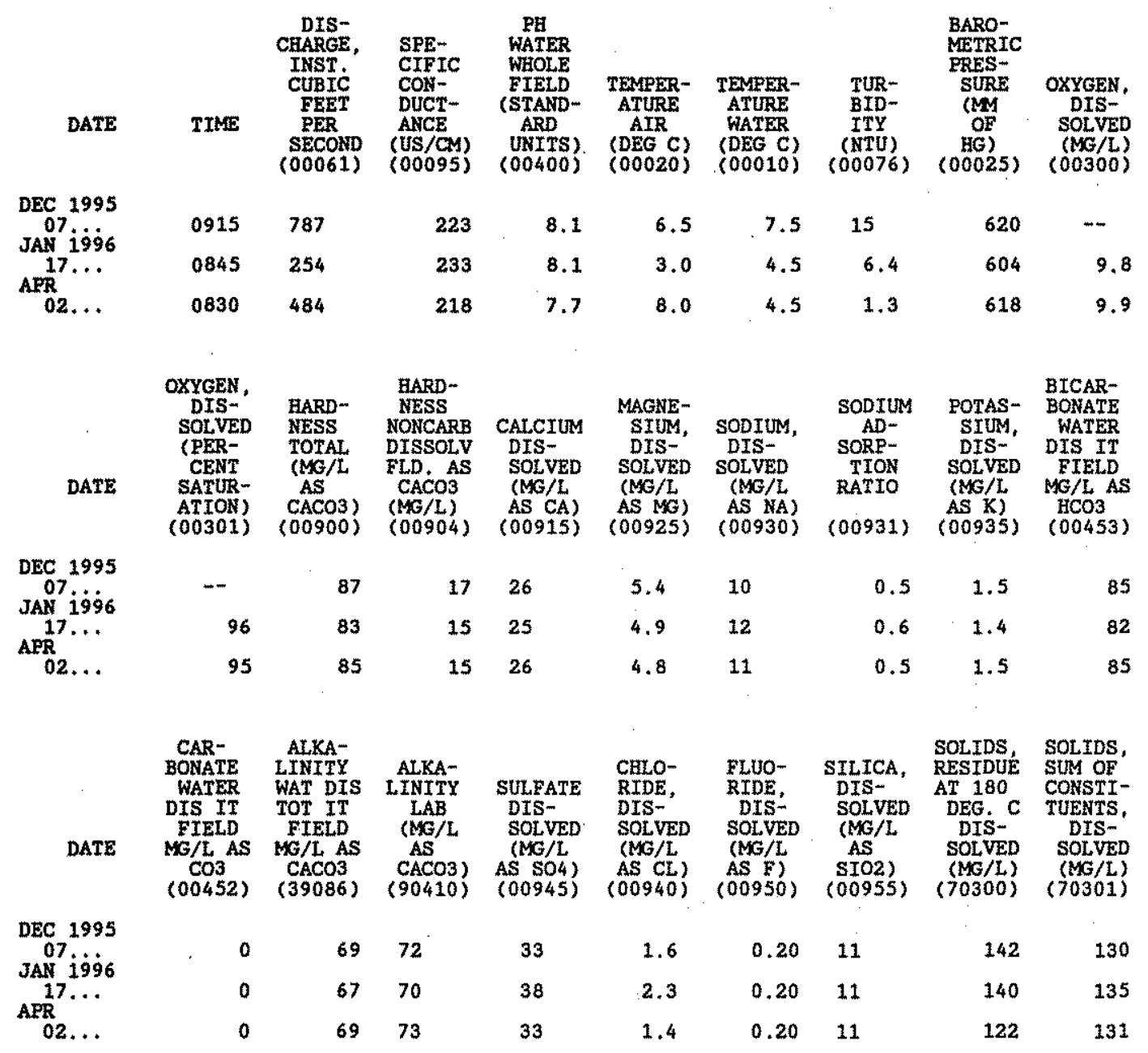


SAN JUAN RIVER BASIN

09363500 ANIMAS RIVER NEAR CEDAR HILL, NM

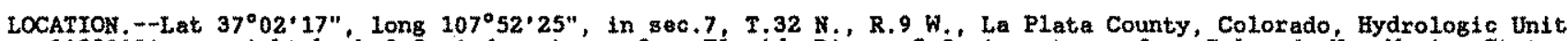
14080104 , on right bank $0.8 \mathrm{mi}$ downstream from Fiorida River, 2.5 mi upstream from Colorado-New Mexico State line, $8.5 \mathrm{ml}$ north of Cedar Hill, and at mile 32.9 .

DRAINAGE AREA. $--1,090 \mathrm{mi}^{2}$, approximately.

PERIOD OF RECORD - - October 1933 to current year. Monthly discharge only for October and November 1933 , published in WSP 1313

REVISED RECOEDS, - WSP 2563,1940 and 1946 (monthly Pigures only).

GAGE. -Water-stage recorder. Elevation of gage is 5,960 ft above National Geodetic Vertical Datum of 1929 , from topographic map. Prior to Sept. 14, 1937, at datum between $1.52 \mathrm{ft}$ and 1.36 ft higher. Sept. 15, 1937 , to Sept. 30 , 1946, at datum 1.36 ft higher.

RERARKS. -Water-discharge records good except for estimated daily discharges, which are poor, Diversions for irrigetion of about 20,000 acres upstream from stacton. During tater years 1944-49, Twin Rocks Canal diverted upstream from station for irrigation downstream. Silght regulation by Lemon Dam about 30 mi upstream on Floride River since November 1963 (capacity, 40,100 acre-ft). Satelilte telemeter at station.

EXIRERES OUTSEDE PERIOD OF RECORD. - A major flood occurred in Octobar 1911 at this location. DISCAARGE, CUBIC FEET PER SECOND, WATER YEAR OCTOBER 1995 TO SEPTEMBER 1996

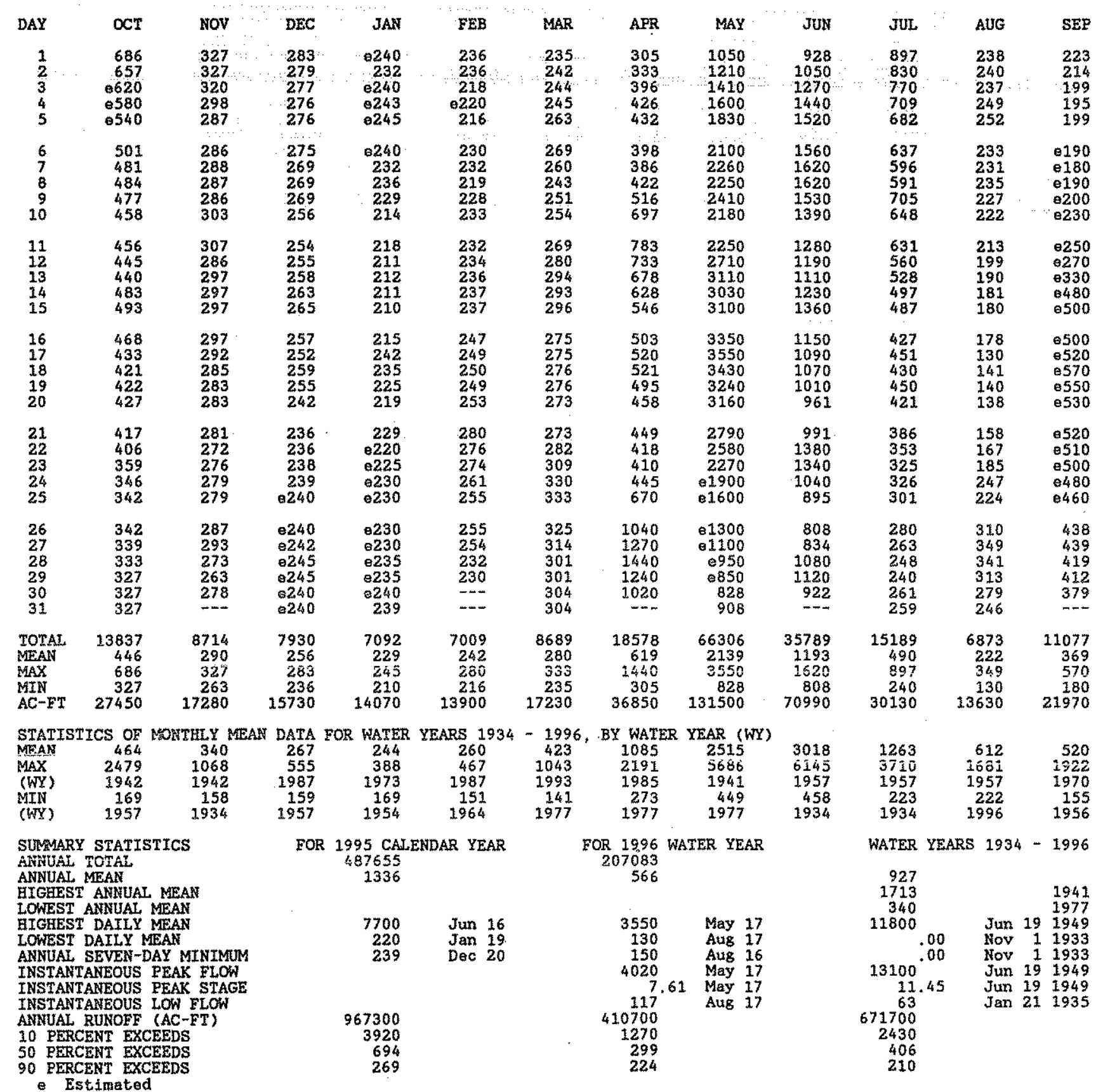


SAN JUAN RIVER BASIN

09363500 ANTMAS RIVER NEAR CEDAR EILL, NM -- Continued

PERIOD OF RECORD.--Water years 1943, 1945, 1958-59, 1969-73, 1975, 1987 to current year.

WATER QUALITY DATA, WATER YEAR OCTOBER 1995 TO SEPTEMBER 1996

\begin{tabular}{|c|c|c|c|c|c|c|c|c|c|c|c|c|}
\hline ATE & TIME & $\begin{array}{c}\text { DIS- } \\
\text { CHARGE, } \\
\text { INST. } \\
\text { CUEIC } \\
\text { FEET } \\
\text { EER } \\
\text { SECOND } \\
(00061)\end{array}$ & $\begin{array}{l}\text { SPE- } \\
\text { CIFIC } \\
\text { CON- } \\
\text { DUCT- } \\
\text { ANCE } \\
\text { (US/CM) } \\
(00095 \text { ) }\end{array}$ & $\begin{array}{c}\text { PA } \\
\text { WATER } \\
\text { WHOLE } \\
\text { FIELD } \\
\text { (STAND- } \\
\text { ARD } \\
\text { UNITS) } \\
\text { (00400) }\end{array}$ & $\begin{array}{c}\text { TEMPER- } \\
\text { ATURE } \\
\text { AIR } \\
(\text { DEG C) } \\
(00020)\end{array}$ & $\begin{array}{c}\text { TEMPER- } \\
\text { ATURE } \\
\text { WATER } \\
\text { (DEG C) } \\
(00010)\end{array}$ & $\begin{array}{l}\text { BARO- } \\
\text { METRIC } \\
\text { FRES- } \\
\text { SURE } \\
\text { (MM } \\
\text { OF } \\
\text { HG) } \\
(00025)\end{array}$ & $\begin{array}{c}\text { OXYGEN, } \\
\text { DIS - } \\
\text { SOLVED } \\
(M G / L) \\
(00300)\end{array}$ & $\begin{array}{c}\text { OXYGEN, } \\
\text { DIS- } \\
\text { SOLVED } \\
\text { (PER- } \\
\text { CENT } \\
\text { SATUR- } \\
\text { ATION) } \\
\text { (00301) }\end{array}$ & $\begin{array}{l}\text { OXYGEN } \\
\text { DEMAND, } \\
\text { CHEM- } \\
\text { ICAL } \\
\text { (HIGE } \\
\text { LEVEL) } \\
\text { (MG/L) } \\
\text { (00340) }\end{array}$ & $\begin{array}{l}\text { COLI - } \\
\text { FORM, } \\
\text { FECAL, } \\
0.7 \\
\text { UM-ME } \\
\text { (COLS. } \\
100 \mathrm{ML}) \\
(31625)\end{array}$ & $\begin{array}{c}\text { STRER } \\
\text { TOCOCCI } \\
\text { FECAL } \\
\text { KFAGAR } \\
\text { (COLS. } \\
\text { PER } \\
100 \mathrm{ML}) \\
(31673)\end{array}$ \\
\hline
\end{tabular}

\begin{tabular}{|c|c|c|c|c|c|c|c|c|c|c|c|c|}
\hline $\begin{array}{l}\mathrm{DEC} 1995 \\
07 \mathrm{~F}\end{array}$ & 1145 & 269 & 640 & 8.3 & 9.0 & 3.5 & 620 & -- & $-m$ & $<10$ & $\mathrm{k7}$ & 41 \\
\hline AER $18 . \cdots$ & 0930 & 252 & 654 & 8.2 & $-11,5$ & 0.0 & 613 & 11.9 & 102 & 13 & $\mathrm{~K} 1$ & 36 \\
\hline${ }_{\operatorname{SEP}}^{02} \ldots$ & 1115 & 346 & 549 & 7.9 & 17.0 & 10.0 & 612 & 10.4 & 115 & $<10$ & 18 & 50 \\
\hline $04 \ldots$ & 0900 & 200 & 708 & 8.3 & 12.0 & 15.0 & 617 & 7.6 & 94 & $<10$ & -- & 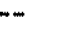 \\
\hline
\end{tabular}

\begin{tabular}{|c|c|c|c|c|c|c|c|c|c|c|c|c|}
\hline DATE & $\begin{array}{l}\text { HARD- } \\
\text { NESS } \\
\text { TOTAL } \\
\text { (MG/L } \\
\text { AS } \\
\text { CACO3) } \\
(00900)\end{array}$ & $\begin{array}{l}\text { HARD- } \\
\text { NESS } \\
\text { NONCARB } \\
\text { DISSOLV } \\
\text { FLD. AS } \\
\text { CACO3 } \\
(\text { MG/L }) \\
(00904)\end{array}$ & $\begin{array}{l}\text { CALCIUM } \\
\text { DIS- } \\
\text { SOLVED } \\
(M G / 1 \\
\text { AS CA) } \\
(00915)\end{array}$ & $\begin{array}{c}\text { MAGNE- } \\
\text { SIUM, } \\
\text { DIS- } \\
\text { SOLVED } \\
\text { (MG/L } \\
\text { AS ME) } \\
(00925)\end{array}$ & $\begin{array}{l}\text { SODIUM, } \\
\text { DIS- } \\
\text { SOLVED } \\
\text { (MG/L } \\
\text { AS NA) } \\
(00930)\end{array}$ & $\begin{array}{c}\text { SODIUM } \\
\text { AD- } \\
\text { SORP- } \\
\text { TION } \\
\text { RATIO } \\
(00931)\end{array}$ & $\begin{array}{c}\text { POTAS- } \\
\text { SIUM, } \\
\text { DIS - } \\
\text { SOLVED } \\
\text { (MG/L } \\
\text { AS K) } \\
(00935)\end{array}$ & $\begin{array}{c}\text { BICAR- } \\
\text { BONATE } \\
\text { WATER } \\
\text { DIS IT } \\
\text { FIELD } \\
\text { MG/L AS } \\
\text { HCO3 } \\
(00453)\end{array}$ & $\begin{array}{c}\text { CAR- } \\
\text { BONATE } \\
\text { WATER } \\
\text { DIS IT } \\
\text { FIELD } \\
\text { MG/L AS } \\
\text { CO3 } \\
(00452)\end{array}$ & $\begin{array}{c}\text { ALKA- } \\
\text { LINITY } \\
\text { WAT DIS } \\
\text { TOT IT } \\
\text { FIELD } \\
\text { MG/L AS } \\
\text { CACO3 } \\
(39086)\end{array}$ & $\begin{array}{c}\text { ALKA- } \\
\text { LINITY } \\
\text { LAB } \\
\text { (MG/L } \\
\text { AS } \\
\text { CACO3) } \\
(90410)\end{array}$ & $\begin{array}{c}\text { SULFAT } \\
\text { DIS- } \\
\text { SOLVE } \\
\text { (MG/L } \\
\text { AS SO4 } \\
\text { CO0945 }\end{array}$ \\
\hline $\begin{array}{l}\text { वC } 1995 \\
07 \text { i }\end{array}$ & 270 & 120 & 85 & 14 & 26 & 0.7 & 3.0 & 170 & 9 & 155 & 159 & 130 \\
\hline $\begin{array}{l}18 \ldots \\
18 \ldots \\
\mathrm{FR}\end{array}$ & 300 & 150 & 95 & 15 & 28 & 0.7 & 3.9 & 1.77 & 0 & 145 & 150 & 160 \\
\hline${ }_{1 E P}^{02} \ldots$ & 240 & 110 & 77 & 12 & 20 & 0.6 & 3.0 & 155 & 0 & 127 & 131 & 130 \\
\hline & 270 & 100 & 83 & 16 & 38 & 1 & 5.0 & 209 & 0 & 172 & 177 & 140 \\
\hline
\end{tabular}

\begin{tabular}{|c|c|c|c|c|c|c|c|c|c|c|c|c|}
\hline & $\begin{array}{l}\text { CHLO- } \\
\text { RIDE, } \\
\text { DIS- } \\
\text { SOLVED } \\
\text { (MG/L } \\
\text { AS CL) } \\
(00940)\end{array}$ & $\begin{array}{l}\text { FLUO- } \\
\text { RIDE, } \\
\text { DIS- } \\
\text { SOLVED } \\
(M G / L \\
\text { AS F) } \\
(00950)\end{array}$ & $\begin{array}{l}\text { SILICA, } \\
\text { DIS } \\
\text { SOLVED } \\
\text { (MG/L } \\
\text { AS } \\
\text { SIO2) } \\
(00955)\end{array}$ & $\begin{array}{c}\text { SOLIDS, } \\
\text { SUM OF } \\
\text { CONSTI- } \\
\text { TUENTS, } \\
\text { DIS- } \\
\text { SOLVED } \\
(M G / L) \\
(70301)\end{array}$ & $\begin{array}{c}\text { NTTRO- } \\
\text { GEN, } \\
\text { NITRATE } \\
\text { DIS- } \\
\text { SOLVED } \\
\text { (MG/L } \\
\text { ASN) } \\
\text { (00618) }\end{array}$ & $\begin{array}{c}\text { NITRO- } \\
\text { GEN, } \\
\text { NITRITE } \\
\text { DIS- } \\
\text { SOLVED } \\
\text { (MG/L } \\
\text { AS N) } \\
(00613)\end{array}$ & $\begin{array}{c}\text { NITRO- } \\
\text { GEN, } \\
\text { NO2+NO3 } \\
\text { DIS- } \\
\text { SOLVED } \\
\text { (MG/L } \\
\text { AS N) } \\
\text { (00631) }\end{array}$ & $\begin{array}{c}\text { NITRO- } \\
\text { GEN, } \\
\text { AMMONIA } \\
\text { DIS- } \\
\text { SOLVED } \\
\text { (MG/L } \\
\text { AS N) } \\
(00608)\end{array}$ & $\begin{array}{c}\text { NITRO- } \\
\text { GEN, } \\
\text { ORGANIC } \\
\text { DIS- } \\
\text { SOLVED } \\
(M G / L \\
\text { AS N) } \\
(00607)\end{array}$ & $\begin{array}{l}\text { NITRO- } \\
\text { GEN,AM- } \\
\text { MONIA + } \\
\text { ORGANIC } \\
\text { TOTAL } \\
\text { (MG/L } \\
\text { AS N) } \\
(00625)\end{array}$ & $\begin{array}{c}\text { NI3RO- } \\
\text { GEN,AM- } \\
\text { MONIA + } \\
\text { ORGANIC } \\
\text { DIS. } \\
\text { (MG/L } \\
\text { AS N) } \\
(00623)\end{array}$ & $\begin{array}{r}P B \\
\text { PHO } \\
\text { TO } \\
(\mathrm{M} \\
\mathrm{AS} \\
100\end{array}$ \\
\hline
\end{tabular}

\begin{tabular}{|c|c|c|c|c|c|c|c|c|c|c|c|c|}
\hline $\begin{array}{l}\text { DEC } 1995 \\
07.01 \\
\text { JAN } 1996\end{array}$ & 21 & 0.50 & 8.5 & 383 & 0.410 & 0.020 & 0.430 & 0.050 & -- & $<0.20$ & $<0.20$ & 0.040 \\
\hline$\frac{18}{A P R}$ & 23 & 0.40 & 9.7 & 424 & 0.480 & 0.020 & 0.500 & 0.060 & -- & $<0.20$ & $<0.20$ & 0.030 \\
\hline $\operatorname{SEP}^{02} \ldots$ & 16 & 0.40 & 7.2 & 343 & 0.120 & 0.020 & 0.140 & 0.040 & 0.16 & $<0.20$ & 0.20 & 0.020 \\
\hline $04 \ldots$ & 36 & 0.50 & 7.5 & 429 & 0.090 & 0.010 & 0.100 & $<0.015$ & $-\infty$ & 0.20 & $<0.20$ & 0.020 \\
\hline
\end{tabular}

\begin{tabular}{|c|c|c|c|c|c|c|c|c|c|c|}
\hline $\begin{array}{c}\text { PHOS- } \\
\text { PHORLS } \\
\text { DIS- } \\
\text { SOLVED } \\
\text { (MG/L } \\
\text { AS P) } \\
(00666)\end{array}$ & $\begin{array}{l}\text { PHORUS } \\
\text { ORTHO, } \\
\text { DTS- } \\
\text { SOLVED } \\
\text { (MG/L } \\
\text { AS P) } \\
(00671)\end{array}$ & $\begin{array}{c}\text { CARBON, } \\
\text { ORGANIC } \\
\text { TOTAL } \\
\text { (MG/L } \\
\text { AS C) } \\
(00680)\end{array}$ & $\begin{array}{l}\text { ALUM- } \\
\text { INUM, } \\
\text { DIS- } \\
\text { SOLVED } \\
\text { (UG/L } \\
\text { AS AL) } \\
\text { (01106) }\end{array}$ & $\begin{array}{l}\text { ANII- } \\
\text { MONY, } \\
\text { DIS- } \\
\text { SOLVED } \\
\text { (UG/L } \\
\text { AS SB) } \\
\text { (01095) }\end{array}$ & $\begin{array}{c}\text { ARSENIC } \\
\text { DIS- } \\
\text { SOLVED } \\
\text { (UG/L } \\
\text { AS AS) } \\
(01000)\end{array}$ & $\begin{array}{l}\text { BARIUM, } \\
\text { DIS- } \\
\text { SOLVED } \\
\text { (UG/L } \\
\text { AS BA) } \\
\text { (0100S) }\end{array}$ & $\begin{array}{l}\text { BERYL- } \\
\text { LIUM, } \\
\text { DIS- } \\
\text { SOLVED } \\
\text { (UG/L } \\
\text { AS BE) } \\
(01010 \text { ) }\end{array}$ & $\begin{array}{l}\text { BORON, } \\
\text { DIS - } \\
\text { SOLVED } \\
(\text { UG/L } \\
\text { AS B) } \\
(01020)\end{array}$ & $\begin{array}{c}\text { CADMIUM } \\
\text { DIS- } \\
\text { SOLVED } \\
\text { (UG/L } \\
\text { AS CD) } \\
(01025)\end{array}$ & $\begin{array}{l}\text { CH } \\
\text { MI } \\
\text { DI } \\
\text { SO } \\
\text { (UI } \\
\text { AS }\end{array}$ \\
\hline
\end{tabular}

DEC 1995

JAN 1996
$\quad 18 \ldots$
APR
$\quad 02 \ldots$
$\quad 04 \ldots$

$\begin{array}{lr}0.020 & 0.020 \\ 0.020 & 0.020 \\ 0.030 & 0.010 \\ 0.010 & <0.010\end{array}$

2.

2.1

$2.9 \quad 18$

$-$

$<1.0$

$\begin{array}{rr}<1 & 82 \\ -- & \\ <1 & 68 \\ <1 & 104\end{array}$

$\begin{array}{rr}<1.0 & 80 \\ -- & 60 \\ <1.0 & 60 \\ <1.0 & 119\end{array}$

$<1.0<1.0$

$2.2 \quad 5.0<1.0$

$<1.0<1.0$ 
SAN JUAN RIVER BASIN

09363500 ANIMAS RIVER NEAR CEDAR BILL, NM -- Continued

WATER-QUALITY RECORDS

WATER QUALITY DATA, WATER YEAR OCTOBER 1995 TO SEPTEMBER 1996

\begin{tabular}{|c|c|c|c|c|c|c|c|c|c|c|c|}
\hline DATE & $\begin{array}{l}\text { COBALT, } \\
\text { DIS- } \\
\text { SOLVED } \\
\text { (UE / } \\
\text { AS CO) } \\
(01035)\end{array}$ & $\begin{array}{l}\text { COPPER, } \\
\text { DIS- } \\
\text { SOLVED } \\
\text { (UG/L } \\
\text { AS CU) } \\
(01040)\end{array}$ & $\begin{array}{l}\text { IRON, } \\
\text { DIS- } \\
\text { SOLVED } \\
\text { (UG/L } \\
\text { AS FE) } \\
(01046)\end{array}$ & $\begin{array}{l}\text { LEAD, } \\
\text { DIS- } \\
\text { SOLVED } \\
\text { (US/L) } \\
\text { AS PS) } \\
(01049)\end{array}$ & $\begin{array}{l}\text { MANGA- } \\
\text { NESE, } \\
\text { DIS-, } \\
\text { SOLVED } \\
\text { (USG/L } \\
\text { AS Haw ) } \\
(01056)\end{array}$ & $\begin{array}{c}\text { MERCURY } \\
\text { TOTAL } \\
\text { RECOV- } \\
\text { ERABLE } \\
\text { UCG/L } \\
\text { AS GG ) } \\
(71900)\end{array}$ & $\begin{array}{c}\text { MOLYB- } \\
\text { DENGM, } \\
\text { DIS- } \\
\text { SOLVED } \\
\text { (UGG/L, } \\
\text { AS NWO } \\
(01060)\end{array}$ & $\begin{array}{l}\text { NICKEL, } \\
\text { DIS- } \\
\text { SOLVED } \\
\text { (UG/L } \\
\text { AS HI ) } \\
(01065)\end{array}$ & $\begin{array}{c}\text { SELE- } \\
\text { IIUM, } \\
\text { TOTAL } \\
\text { (UG/L) } \\
\text { AS SE) } \\
(01147)\end{array}$ & $\begin{array}{l}\text { SELE- } \\
\text { NIUMS- } \\
\text { DIS- } \\
\text { SOLVED } \\
\text { (UG/L } \\
\text { AS SE) } \\
\text { (01145) }\end{array}$ & $\begin{array}{l}\text { SILVER, } \\
\text { DIS- } \\
\text { SOLVED } \\
\text { (UG AL } \\
\text { AS AG) } \\
(01075)\end{array}$ \\
\hline $\begin{array}{l}\text { Ec } 1995 \\
\text { of }\end{array}$ & $<1.0$ & 4.0 & 8.0 & $<1.0$ & 47 & $<0.10$ & 2.0 & 2.0 & $<1$ & $<1$ & $<1.0$ \\
\hline & - & -- & 7.0 & $\cdots$ & $\cdots$ & - & - & -- & $\because-$ & -- & $\cdots$ \\
\hline$\ldots$ & $<1.0$ & 2.0 & 5.0 & $<1.0$ & 55 & $<0.10$ & 1.0 & 2.0 & $<1$ & $<1$ & $<1.0$ \\
\hline$\ldots$ & $<1.0$ & 2.0 & 5.0 & $<1.0$ & 40 & $<0.10$ & 2.0 & 3.0 & $<1$ & $<1$ & $<1.0$ \\
\hline DATE & 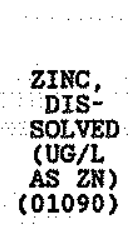 & $\begin{array}{l}\text { HITRO- } \\
\text { GEN, } \\
\text { OO2+NO3 } \\
\text { TOT. IN } \\
\text { BOT.MAT } \\
\text { (MG/KG } \\
\text { AS N) } \\
(00633)\end{array}$ & $\begin{array}{l}\text { NITRO- } \\
\text { GEN, NH } \\
\text { TOTAL } \\
\text { IN BOT, } \\
\text { BAT, } \\
\text { MA/KG } \\
\text { AS N } \\
(00611)\end{array}$ & $\begin{array}{l}\text { NTTRO- } \\
\text { GEN, NB } \\
+ \text { ORG } \\
\text { OOT IN } \\
\text { BOT MAT } \\
\text { (MG/KG } \\
\text { AS N } \\
(00626)\end{array}$ & $\begin{array}{l}\text { PHOS- } \\
\text { FHORUS } \\
\text { TOTAL } \\
\text { IN BOT. } \\
\text { MAT. } \\
\text { (MMA/KG } \\
\text { AS P) } \\
(00668)\end{array}$ & $\begin{array}{c}\text { ARSENIC } \\
\text { TOTAL } \\
\text { IN BOT- } \\
\text { TOM MA- } \\
\text { TERAL } \\
\text { (UG } / \mathrm{G} \\
\text { AS AS) } \\
(01003)\end{array}$ & $\begin{array}{l}\text { CADMIUM } \\
\text { RECOV } \\
\text { FM BOT- } \\
\text { TOM MA- } \\
\text { TERIAL } \\
\text { (UG/G } \\
\text { AS CD) } \\
(01028)\end{array}$ & $\begin{array}{l}\text { CERO- } \\
\text { MIUM, } \\
\text { RECOV. } \\
\text { FM BOT- } \\
\text { TOM MA- } \\
\text { TERIAL } \\
\text { (UG/G) } \\
(01029)\end{array}$ & $\begin{array}{l}\text { COBALT, } \\
\text { RECOV: } \\
\text { FM BOT- } \\
\text { FOM MA- } \\
\text { TORIAL } \\
\text { TEG/G } \\
\text { AS CO) } \\
\text { (01038) }\end{array}$ & $\begin{array}{l}\text { COPPER, } \\
\text { RECOV. } \\
\text { FM BOT- } \\
\text { TOM MA- } \\
\text { TERIAL } \\
\text { (UG /G } \\
\text { AS CU) } \\
(01043)\end{array}$ & $\begin{array}{c}\text { IRON, } \\
\text { RECOV. } \\
\text { FM BOT- } \\
\text { FOM MA- } \\
\text { TOM MAL } \\
\text { TERIAL } \\
\text { CUG/G } \\
\text { AS FE) } \\
(01170)\end{array}$ \\
\hline $\begin{array}{l}E C 1995 \\
07\end{array}$ & 25 & 4.0 & 230 & 1600 & 580 & 7 & 2 & $<1$ & $<5$ & 13 & 720 \\
\hline $\begin{array}{l}18 \cdots \\
A P R\end{array}$ & $-\cdots$ & - & -- & - & -- & - & $\cdots$ & -- & $\cdots$ & $\cdots$ & -- \\
\hline $02 \ldots$ & 15 & -- & -- & -- & - & -- & -- & -- & -- & -- & -- \\
\hline $04 \ldots$ & 3.0 & $\cdots$ & -- & - & - & -- & -- & -- & -- & -- & $\cdots$ \\
\hline DATE & 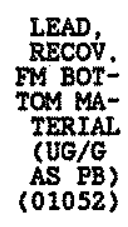 & $\begin{array}{l}\text { MANGA- } \\
\text { MESE, } \\
\text { RECOV. } \\
\text { MEBOT- } \\
\text { TOM MA- } \\
\text { TERIAL } \\
\text { TEG/G) } \\
\text { (OI053) }\end{array}$ & $\begin{array}{l}\text { MERCURY } \\
\text { RECOV. } \\
\text { ME BOT- } \\
\text { TOM MA- } \\
\text { TERIAI } \\
\text { (UG/G } \\
\text { AS BG) } \\
(71921)\end{array}$ & 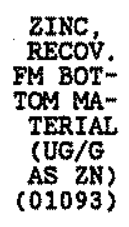 & $\begin{array}{l}\text { RADIUM } \\
226, \\
\text { DIS- } \\
\text { SOLVED, } \\
\text { RADON' } \\
\text { METHOD } \\
\text { (PCI/L) } \\
\text { (09511) }\end{array}$ & $\begin{array}{l}\text { RA-226 } \\
2 \text { SITMA } \\
\text { WATER, } \\
\text { DISSS } \\
\text { (PCI L }) \\
(76001)\end{array}$ & $\begin{array}{l}\text { URANIUM } \\
\text { NATURAL } \\
\text { DIS- } \\
\text { SOLVED } \\
\text { CUG/L } \\
\text { AS U) } \\
(22703)\end{array}$ & $\begin{array}{l}\text { URANIUM } \\
\text { NATURAL } \\
\text { 2 SIGMA } \\
\text { WATER, } \\
\text { DISS; } \\
\text { (UG/L) } \\
\text { (75990) }\end{array}$ & $\begin{array}{l}\text { SEDI- } \\
\text { MENT, } \\
\text { SUS- } \\
\text { PENDED } \\
\text { (MG/L) } \\
(80154)\end{array}$ & $\begin{array}{c}\text { SEDI- } \\
\text { MENT, } \\
\text { DIS- } \\
\text { CHARGE, } \\
\text { SUS- } \\
\text { PENDED } \\
\text { PEN/DAY } \\
(80155)\end{array}$ & $\begin{array}{l}\text { SED. } \\
\text { SUSP. } \\
\text { SIEVE } \\
\text { DIAM. } \\
\text { DINER } \\
\text { FINAN } \\
\text { THE2 MMM } \\
(70331)\end{array}$ \\
\hline DEC 1995 & 30 & 1200 & 0.03 & 460 & 0.07 & 0.020 & 1.1 & 0.0 & 13 & 9.4 & 5 \\
\hline ER & -- & -- & -- & -- & $\cdots$ & $\cdots$ & - & - & 8 & 5.4 & 67 \\
\hline 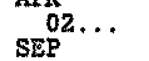 & -- & - & - & $\cdots$ & $m$ & -- & 1.0 & -- & 16 & 15 & 67 \\
\hline & $\cdots$ & -- & - & -- & -- & -- & & $\cdots$ & 0 & & \\
\hline
\end{tabular}


SAN JUAN RIVER BASIN

09364500 ANIMAS RIVER AT FARMINGTON, NM

LOCATTON --Lat $36^{\circ} 43^{\prime} 17^{\prime \prime}$, long $108^{\circ} 12^{\prime} 05^{\prime \prime}$, in SW/4Wt/ sec.15, T.29 N., R.13 W. San Juan County, Hydrologic Unit 14080104, In Boyd City Park, on right bank $900 \mathrm{ft}$ upstream from bridge on Miller Ave, 0.4 mi downstream from bridge on U.S. Highway 64 in Farmington, and $1.5 \mathrm{mi}$ upstraam from mouth.

DRAINAGE AREA. $--i, 360 \mathrm{mi}^{2}$, 'approximately.

WATER-DISCHARGE RECORDS

PERIOD OF RECORD. --June 1904 to October 1905 (published as "near Farmington"), September 1912 to current year. Monthly discharge onty for some periods published in WSP 1313.

REVISED RECORDS. - -WSP 1243: 1931. WSP 1313: 1913

GAGE. - Water-stage recorder. Elevation of gage is $5,280 \mathrm{ft}$ above National Geodetic Vertical Datum of 1929 , from topographic map. Prior to Nov. 1 , 1905, non recording gage at old bridge $0.1 \mathrm{mi}$ upstream at different datum. Sept. 17, 1912, to Oct. 4, 1938, water-stage recorder at site 0.8 mf downstrean at lower datums (datum lowered 2.0 ft Aug. 15, 1927, and raised $0.2 \mathrm{ft}$ Dec. 16, 1929). Oct. 5, 1938, to Nov. 1, 1973, at site 900 ft downstream at datum $1.74 \mathrm{ft}$ lower.

RDMARKS. - Water-discharge records good except for estimated dally discharges, which are poor. Dtversions for irrigation of about 30,000 acres upstream from station. Satellite telerneter at station.

EXTREMES OUTSIDE PERIOD OF RECORD - -Maximum flood accurred Oct. 6,1911 , when a stage of about 16.5 ft was reached (datum in use Oct. 1938 to Nov. 1973). Flood of Sept. 6, 1909, reached a stage of 11.1 ft, 1904-5 site and datum (discharge, about 19,000 $f \mathrm{t}^{\mathrm{s} / \mathrm{s}) \text {. }}$

DISCHARGE, CUBIC FEET PER SECOND, WATER YEAR OCTOBER 1995 TO SEETEMBER 1996 DAILY MEAN VALUES

\begin{tabular}{|c|c|c|c|c|c|c|c|c|c|c|c|c|}
\hline DAY & OCT & SOV & DEC & JAN & FEB & MAR & APR & MAY & JUN & JUL & AUG & SEP \\
\hline $\begin{array}{l}1 \\
2 \\
3 \\
4 \\
5\end{array}$ & $\begin{array}{l}662 \\
623 \\
563 \\
478 \\
491\end{array}$ & $\begin{array}{l}259 \\
246 \\
240 \\
223 \\
212\end{array}$ & $\begin{array}{l}\mathbf{e} 213 \\
\mathbf{e 2 0 9} \\
\mathbf{e 2 0 7} \\
\mathbf{e 2 0 6} \\
\mathbf{e 2 0 6}\end{array}$ & $\begin{array}{l}0170 \\
0162 \\
e 170 \\
0173 \\
0175\end{array}$ & $\begin{array}{l}\text { e166 } \\
\text { e166 } \\
0140 \\
0148 \\
0147\end{array}$ & $\begin{array}{l}275 \\
275 \\
279 \\
283 \\
289\end{array}$ & $\begin{array}{l}220 \\
199 \\
202 \\
267 \\
284\end{array}$ & $\begin{array}{r}640 \\
791 \\
986 \\
1200 \\
1440\end{array}$ & $\begin{array}{r}607 \\
662 \\
883 \\
1120 \\
1220\end{array}$ & $\begin{array}{l}705 \\
683 \\
655 \\
592 \\
538\end{array}$ & $\begin{array}{l}40 \\
15 \\
1.5 \\
2.0 \\
6.0\end{array}$ & $\begin{array}{l}69 \\
58 \\
58 \\
59 \\
39\end{array}$ \\
\hline $\begin{array}{r}6 \\
7 \\
8 \\
9 \\
10\end{array}$ & $\begin{array}{l}485 \\
491 \\
429 \\
397 \\
371\end{array}$ & $\begin{array}{l}222 \\
222 \\
227 \\
222 \\
231\end{array}$ & $\begin{array}{l}\text { e205 } \\
\text { e199 } \\
0199 \\
\text { e199 } \\
\text { e186 }\end{array}$ & $\begin{array}{l}\text { e170 } \\
0162 \\
0166 \\
0159 \\
\text { e144 }\end{array}$ & $\begin{array}{l}0167 \\
e 173 \\
0161 \\
0169 \\
0174\end{array}$ & $\begin{array}{l}303 \\
297 \\
278 \\
259 \\
258\end{array}$ & $\begin{array}{l}228 \\
228 \\
240 \\
264 \\
377\end{array}$ & $\begin{array}{l}1730 \\
1950 \\
1980 \\
2060 \\
1970\end{array}$ & $\begin{array}{l}1320 \\
1450 \\
1490 \\
1440 \\
1300\end{array}$ & $\begin{array}{l}494 \\
476 \\
443 \\
514 \\
511\end{array}$ & $\begin{array}{r}18 \\
.14 \\
.00 \\
.00 \\
.28\end{array}$ & $\begin{array}{l}37 \\
18 \\
11 \\
21 \\
9.3\end{array}$ \\
\hline $\begin{array}{l}11 \\
12 \\
13 \\
14 \\
15\end{array}$ & $\begin{array}{l}349 \\
327 \\
330 \\
329 \\
355\end{array}$ & $\begin{array}{l}261 \\
236 \\
234 \\
244 \\
240\end{array}$ & $\begin{array}{l}0199 \\
e 183 \\
0188 \\
0193 \\
0195\end{array}$ & $\begin{array}{l}0147 \\
0145 \\
0143 \\
0142 \\
0141\end{array}$ & $\begin{array}{r}\text { e250 } \\
e 261 \\
e 265 \\
266 \\
268\end{array}$ & $\begin{array}{l}256 \\
266 \\
277 \\
287 \\
288\end{array}$ & $\begin{array}{l}532 \\
523 \\
481 \\
472 \\
458\end{array}$ & $\begin{array}{l}1900 \\
2350 \\
2880 \\
2880 \\
2890\end{array}$ & $\begin{array}{r}1140 \\
1030 \\
986 \\
952 \\
1300\end{array}$ & $\begin{array}{l}530 \\
417 \\
387 \\
387 \\
349\end{array}$ & $\begin{array}{r}24 \\
4.6 \\
.00 \\
.00 \\
.00\end{array}$ & $\begin{array}{r}17 \\
28 \\
69 \\
201 \\
388\end{array}$ \\
\hline $\begin{array}{l}16 \\
17 \\
18 \\
19 \\
20\end{array}$ & $\begin{array}{l}354 \\
319 \\
323 \\
314 \\
316\end{array}$ & $\begin{array}{l}234 \\
226 \\
215 \\
221 \\
224\end{array}$ & $\begin{array}{l}\text { e187 } \\
\text { e182 } \\
\text { e189 } \\
\text { e185 } \\
\text { e172 }\end{array}$ & $\begin{array}{l}0143 \\
0162 \\
0155 \\
0156 \\
0147\end{array}$ & $\begin{array}{l}276 \\
283 \\
286 \\
286 \\
288\end{array}$ & $\begin{array}{l}284 \\
259 \\
261 \\
264 \\
261\end{array}$ & $\begin{array}{l}315 \\
293 \\
287 \\
277 \\
260\end{array}$ & $\begin{array}{l}3130 \\
3400 \\
3340 \\
3130 \\
3030\end{array}$ & $\begin{array}{r}1110 \\
921 \\
891 \\
858 \\
793\end{array}$ & $\begin{array}{l}305 \\
288 \\
275 \\
267 \\
273\end{array}$ & $\begin{array}{l}.00 \\
.00 \\
.00 \\
.00 \\
.00\end{array}$ & $\begin{array}{l}407 \\
401 \\
415 \\
459 \\
431\end{array}$ \\
\hline $\begin{array}{l}21 \\
22 \\
23 \\
24 \\
25\end{array}$ & $\begin{array}{l}307 \\
311 \\
332 \\
318 \\
300\end{array}$ & $\begin{array}{l}222 \\
214 \\
209 \\
210 \\
208\end{array}$ & $\begin{array}{l}0166 \\
0166 \\
0168 \\
e 169 \\
\theta 170\end{array}$ & $\begin{array}{l}0154 \\
0165 \\
0175 \\
e 180 \\
0180\end{array}$ & $\begin{array}{l}310 \\
318 \\
315 \\
298 \\
289\end{array}$ & $\begin{array}{l}249 \\
249 \\
262 \\
279 \\
292\end{array}$ & $\begin{array}{l}239 \\
217 \\
188 \\
183 \\
239\end{array}$ & $\begin{array}{l}2730 \\
2410 \\
2170 \\
1760 \\
1310\end{array}$ & $\begin{array}{r}808 \\
1100 \\
1310 \\
988 \\
771\end{array}$ & $\begin{array}{l}230 \\
205 \\
166 \\
125 \\
109\end{array}$ & $\begin{array}{r}.00 \\
.00 \\
173\end{array}$ & $\begin{array}{l}386 \\
375 \\
346 \\
327 \\
303\end{array}$ \\
\hline $\begin{array}{l}26 \\
27 \\
28 \\
29 \\
30 \\
31\end{array}$ & $\begin{array}{l}307 \\
305 \\
295 \\
288 \\
287 \\
286\end{array}$ & $\begin{array}{r}208 \\
223 \\
e 223 \\
\theta 203 \\
\theta 193\end{array}$ & $\begin{array}{l}e 170 \\
e 172 \\
e 175 \\
e 175 \\
e 170 \\
e 170\end{array}$ & $\begin{array}{l}e 180 \\
e 180 \\
e 180 \\
e 175 \\
e 172 \\
e 170\end{array}$ & $\begin{array}{l}289 \\
292 \\
281 \\
268 \\
--- \\
-\cdots\end{array}$ & $\begin{array}{l}286 \\
271 \\
252 \\
249 \\
254 \\
242\end{array}$ & $\begin{array}{r}535 \\
871 \\
1050 \\
1060 \\
739\end{array}$ & $\begin{array}{r}1030 \\
861 \\
707 \\
584 \\
518 \\
595\end{array}$ & $\begin{array}{l}641 \\
615 \\
806 \\
952 \\
784\end{array}$ & $\begin{array}{l}85 \\
65 \\
55 \\
42 \\
34 \\
53\end{array}$ & $\begin{array}{r}123 \\
179 \\
184 \\
167 \\
124 \\
99\end{array}$ & $\begin{array}{l}295 \\
299 \\
289 \\
274 \\
255\end{array}$ \\
\hline $\begin{array}{l}\text { TOTAL } \\
\text { MEAN } \\
\text { MAX } \\
\text { MIN } \\
\text { AC-FT }\end{array}$ & $\begin{array}{r}11642 \\
376 \\
662 \\
286 \\
23090\end{array}$ & $\begin{array}{r}6752 \\
225 \\
261 \\
193 \\
13390\end{array}$ & $\begin{array}{r}5773 \\
186 \\
213 \\
166 \\
11450\end{array}$ & $\begin{array}{r}5043 \\
163 \\
180 \\
141 \\
10000\end{array}$ & $\begin{array}{r}7000 \\
241 \\
318 \\
140 \\
13880\end{array}$ & $\begin{array}{r}8384 \\
270 \\
303 \\
242 \\
16630\end{array}$ & $\begin{array}{r}11728 \\
391 \\
1060 \\
183 \\
23260\end{array}$ & $\begin{array}{r}58352 \\
1882 \\
3400 \\
518 \\
115700\end{array}$ & $\begin{array}{r}30248 \\
1008 \\
1490 \\
607 \\
60000\end{array}$ & $\begin{array}{r}10258 \\
331 \\
705 \\
34 \\
20350\end{array}$ & $\begin{array}{r}1246.52 \\
40.2 \\
184 \\
.00 \\
2470\end{array}$ & $\begin{array}{r}6344.3 \\
211 \\
459 \\
9.3 \\
12580\end{array}$ \\
\hline
\end{tabular}

STATISTICS OF MONTHLY MEAN DATA FOR WATER YEARS $1914-1996$, BX WATER YEAR (WY)

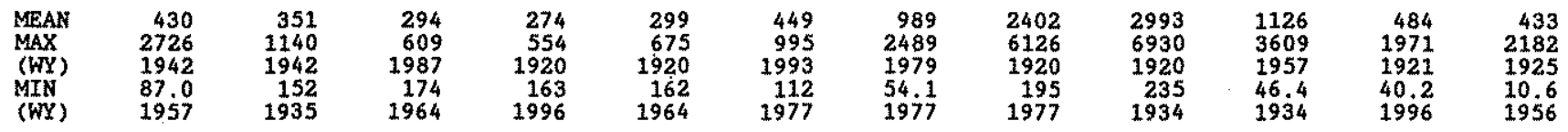

\section{SUMMARY STATISTICS}

ANEUAL TOTAL

ANNUAL MEAN

BIGHEST ANNUAL MEAN

LOWEST ANNUAL MEAN

HIGHEST DAILY MEAN

LOWEST DAILY MEAN

ANHUAL SEVEN-DAY MINIMIM

INSTANTANEOUS PEAK FLOW

INSTANTANEOUS PEAK STAGE

INSTANTANEOUS LOW FLOW

ANNUAL RUNOFF (AC-FT)

10 PERCENT EXCEEDS

50 PERCENT EXCEEDS

90 PERCENT EXCEEDS

- Estimated

a-From rating curve extended above $10,000 \mathrm{fts} / \mathrm{s}$.
FOR 1995 CALENDAR YEAR

\begin{tabular}{rrrrr}
457501 & \multicolumn{5}{c}{162770.82} \\
1253 & & 445 & \\
& & & \\
7460 & Jun 16 & 3400 & May 17 \\
166 & Dec 21 & .00 & Aus 8 \\
169 & Dec 20 & .00 & Aug 13 \\
& & 7.49 & May 17 \\
& & $322900^{\circ}$ & Aug & \\
907500 & & 1040 & & \\
3770 & & 261 & \\
644 & & 58 &
\end{tabular}

WATER YEARS 1914 - 1996

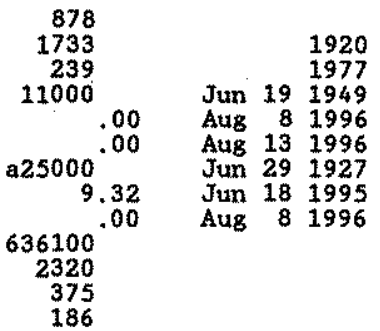


PERIOD OF RECORD.--Water years 1940 to current year.

WATER QUALITY DATA, WATER YEAR OCTOBER 1995 TO SEPTEMBER 1996

\begin{tabular}{|c|c|c|c|c|c|c|c|c|c|c|c|c|}
\hline DATE & TIRE & $\begin{array}{c}\text { DIS- } \\
\text { CEARGE } \\
\text { INSS } \\
\text { CUBIC } \\
\text { EEET } \\
\text { PER } \\
\text { SECORD } \\
(00061)\end{array}$ & $\begin{array}{l}\text { SPE- } \\
\text { CIFYC } \\
\text { CON- } \\
\text { DUCT- } \\
\text { ANCE } \\
\text { (US/CR) } \\
(00095)\end{array}$ & $\begin{array}{c}\text { PH } \\
\text { WATER } \\
\text { WROLE } \\
\text { FIELD } \\
\text { (STARD- } \\
\text { ARD } \\
\text { UNITS) } \\
(00400)\end{array}$ & $\begin{array}{c}\text { TEMPER- } \\
\text { ATURE } \\
\text { AIR } \\
(\text { DEG C) } \\
(00020)\end{array}$ & $\begin{array}{c}\text { TEMPER- } \\
\text { ATURE } \\
\text { WATER } \\
\text { (DEG C) } \\
\text { (00010) }\end{array}$ & $\begin{array}{c}\text { BARO- } \\
\text { NETRIC } \\
\text { ERES- } \\
\text { SURE } \\
\text { (NDI } \\
\text { OF } \\
\text { HG) } \\
(00025)\end{array}$ & $\begin{array}{c}\text { OXYGER, } \\
\text { DIS- } \\
\text { SOLVED } \\
\text { (BGG/L) } \\
(00300)\end{array}$ & $\begin{array}{l}\text { OXYGEN, } \\
\text { DYS- } \\
\text { SOLVED } \\
\text { (DER- } \\
\text { CENT } \\
\text { SATUR- } \\
\text { ATION) } \\
\text { (O0301) }\end{array}$ & $\begin{array}{l}\text { OXYGEN } \\
\text { DEMAND. } \\
\text { CHERA } \\
\text { ICAL } \\
\text { (GIGEE } \\
\text { LEVEL) } \\
\text { (MES/L) } \\
(00340)\end{array}$ & $\begin{array}{l}\text { COLI- } \\
\text { FORM, } \\
\text { FECAL, } \\
0.7 \\
\text { UN-MF } \\
\text { (COLS } \\
100 \mathrm{ML}) \\
\text { (31625) }\end{array}$ & $\begin{array}{l}\text { STREP- } \\
\text { TOCOCCI } \\
\text { FECAIL, } \\
\text { KF AGAR } \\
\text { (COLS. } \\
\text { PER } \\
100 \text { MiL) } \\
(31673)\end{array}$ \\
\hline $\begin{array}{c}\text { OCT } 1995 \\
03 \ldots \\
\text { DEC }\end{array}$ & 1600 & 508 & 480 & -- & 19.0 & 16.0 & -- & - & -- & -- & $=$ & - \\
\hline JAN 1996 & 0830 & 269 & 774 & 8.2 & 0.0 & 3.5 & 630 & 11.9 & 109 & $<10$ & $<14$ & 47 \\
\hline $16 \ldots$ & 1430 & 261 & 792 & 8,2 & 12.0 & 2.0 & 625 & 12.1 & 107 & 10 & $<1$ & $<1.5$ \\
\hline $\begin{array}{c}01 \ldots \\
\operatorname{SEP}\end{array}$ & 1415 & 22.0 & 636 & 8.6 & 22.5 & 14.5 & 629 & 12.5 & 149 & $<10$ & $\mathrm{~K} 2$ & 35 \\
\hline $04 \ldots$ & 1315 & 72 & 905 & 8.3 & 27.5 & 22.0 & 631 & 9.0 & 125 & $<10$ & -- & -- \\
\hline & & & & & & & & & & & & \\
\hline DATE & $\begin{array}{l}\text { HARD- } \\
\text { NEESS } \\
\text { TOTAL } \\
\text { (MG/L } \\
\text { AS } \\
\text { CACO3) } \\
(00900)\end{array}$ & $\begin{array}{c}\text { HARD- } \\
\text { NESS } \\
\text { NOACARB } \\
\text { DISSOLV } \\
\text { FLD.AS AS } \\
\text { CACO3 } \\
(\text { NGG/L) } \\
(00904)\end{array}$ & $\begin{array}{c}\text { CALCIUM } \\
\text { DIS } \\
\text { SOLVED } \\
\text { (MG L } \\
\text { AS CA } \\
(00915)\end{array}$ & $\begin{array}{c}\text { MAGNE- } \\
\text { SIUM, } \\
\text { DIS- } \\
\text { SOLVED } \\
\text { (MG/L } \\
\text { AS MGS) } \\
(00925)\end{array}$ & $\begin{array}{c}\text { SODIUM, } \\
\text { DIS- } \\
\text { SOLVEDD } \\
\text { (MG/L } \\
\text { AS NA) } \\
(00930)\end{array}$ & $\begin{array}{c}\text { SODIUM } \\
\text { AD- } \\
\text { SORP- } \\
\text { TION } \\
\text { RATIO } \\
(00931)\end{array}$ & $\begin{array}{c}\text { POTAS- } \\
\text { SIUM, } \\
\text { DIS- } \\
\text { SOLVED } \\
\text { (MG/L } \\
\text { AS K) } \\
(00935)\end{array}$ & $\begin{array}{c}\text { BICAR- } \\
\text { BONATE } \\
\text { WATER } \\
\text { DIS IT } \\
\text { FIELD } \\
\text { WG/L AS } \\
\text { HCO3 } \\
(00453)\end{array}$ & $\begin{array}{c}\text { CAR- } \\
\text { BONATE } \\
\text { WATER } \\
\text { DIS IT } \\
\text { FIELD } \\
\text { MG/L AS } \\
\text { CO3 } \\
(00452)\end{array}$ & $\begin{array}{l}\text { ALKA- } \\
\text { LINITY } \\
\text { WAT DIS } \\
\text { TOT IT } \\
\text { YIELD } \\
\text { MS/L AS } \\
\text { CACOS } \\
(39086)\end{array}$ & $\begin{array}{c}\text { ALXA- } \\
\text { LINITY } \\
\text { LAB } \\
\text { (MGS/L } \\
\text { AS } \\
\text { CACO3) } \\
(90410)\end{array}$ & $\begin{array}{c}\text { SULFATE } \\
\text { DIS- } \\
\text { SOLVED } \\
\text { (NGG/L } \\
\text { AS SO4) } \\
(00945)\end{array}$ \\
\hline $\begin{array}{c}\mathrm{OCT} 1995 \\
03 \ldots \\
\mathrm{DEC}\end{array}$ & -- & - & $-\infty$ & - & -- & - & $-\cdots$ & - & $\ldots$ & -- & $\ldots$ & -- \\
\hline${ }_{\text {JAN }}^{06} i 996$ & 310 & 1.50 & 100 & 15 & 39 & 1 & 3.0 & 202 & 0 & 166 & 173 & 180 \\
\hline${ }_{\mathrm{APR}}^{16} \cdots$ & 300 & 130 & 96 & 15 & 34 & 0.9 & 3.0 & 207 & 0 & 170 & 273 & 190 \\
\hline SEP & 270 & 140 & 85 & 13 & 31 & 0.8 & 3.0 & 129 & 11 & 123 & 114 & 170 \\
\hline $04 \ldots$ & 350 & 170 & 110 & 18 & 55 & 1 & 4.0 & 205 & 5 & 176 & 158 & 250 \\
\hline DATE & $\begin{array}{l}\text { CHLO- } \\
\text { RIDE, } \\
\text { DIS- } \\
\text { SOLVED } \\
\text { (MS/L } \\
\text { AS CL) } \\
(00940)\end{array}$ & $\begin{array}{c}\text { FLUO- } \\
\text { RIDE, } \\
\text { DIS- } \\
\text { SOLVED } \\
(\text { MG/L } \\
\text { AS E) } \\
(00950)\end{array}$ & $\begin{array}{c}\text { SILICA, } \\
\text { DIS - } \\
\text { SOLVED } \\
\text { (MG /L } \\
\text { AS } \\
\text { SIO2) } \\
(00955)\end{array}$ & $\begin{array}{c}\text { SOLIDS, } \\
\text { SLM OF } \\
\text { CONSTI- } \\
\text { TUENTS , } \\
\text { DIS- } \\
\text { SOLVED } \\
\text { (MG/L) } \\
\text { (70301) }\end{array}$ & $\begin{array}{c}\text { NITRO- } \\
\text { GEN } \\
\text { NITRATE } \\
\text { DIS- } \\
\text { SOLVED } \\
\text { (MEL/L } \\
\text { AS N) } \\
\text { (00618) }\end{array}$ & $\begin{array}{c}\text { NITRO- } \\
\text { GEN, } \\
\text { NITRITE } \\
\text { DIS- } \\
\text { SOLVED } \\
\text { (MG/L } \\
\text { AS N) } \\
(00613)\end{array}$ & $\begin{array}{c}\text { NITRO- } \\
\text { GEN, } \\
\text { NO2+NO3 } \\
\text { DIS- } \\
\text { SOLVED } \\
\text { (MG/L } \\
\text { AS N } \\
(00631)\end{array}$ & $\begin{array}{c}\text { MITRO- } \\
\text { GEN } \\
\text { ANMONIA } \\
\text { DIS- } \\
\text { SOLVED } \\
\text { (MG/L } \\
\text { AS N) } \\
(00608)\end{array}$ & $\begin{array}{c}\text { NITRO- } \\
\text { GER, } \\
\text { ORGANIC } \\
\text { DIS- } \\
\text { SOLVED } \\
\text { (NG/L } \\
\text { AS NS } \\
(00607)\end{array}$ & $\begin{array}{l}\text { MITRO- } \\
\text { GEN,AM- } \\
\text { MONIA + } \\
\text { ORGANIC } \\
\text { TOTAL } \\
\text { (NG/L } \\
\text { AS N) } \\
(00625)\end{array}$ & $\begin{array}{l}\text { NITRO- } \\
\text { GEN,AM- } \\
\text { MONIA + } \\
\text { ORGANIC } \\
\text { DIS. } \\
\text { (NG/L. } \\
\text { AS N) } \\
(00623)\end{array}$ & $\begin{array}{c}\text { PHOS- } \\
\text { PHORUS } \\
\text { TOTAL } \\
\text { (MG/L } \\
\text { AS P) } \\
(00665)\end{array}$ \\
\hline $\begin{array}{c}0{ }^{1} 1995 \\
03 \ldots\end{array}$ & -- & $\sim$ & - & -- & $-m$ & -- & $-m$ & - & $\cdots$ & -- & $-\cdots$ & - \\
\hline JAN 1966 & 22 & 0.50 & 5.0 & 465 & 0.200 & 0.010 & 0.210 & $<0.015$ & -- & $<0.20$ & $<0.20$ & $<0.010$ \\
\hline${ }_{\mathrm{APR}}^{16} \cdots$ & 24 & 0.40 & 8.0 & 474 & 0.370 & 0.010 & 0.380 & 0.020 & $\cdots$ & $<0.20$ & $<0.20$ & 0.030 \\
\hline $\operatorname{sip}_{0 .}^{01 . .}$ & 19 & 0.40 & 5.3 & 401 & -- & 0.020 & $<0.050$ & 0.020 & 0.18 & $<0.20$ & 0.20 & 0.020 \\
\hline 04 & 31 & 0.50 & 4.9 & 580 & - & $<0.010$ & 0.090 & $<0.015$ & $-m$ & 0.20 & $<0.20$ & 0.040 \\
\hline
\end{tabular}

\begin{tabular}{|c|c|c|c|c|c|c|c|c|c|c|c|}
\hline DATE & $\begin{array}{c}\text { FHOS- } \\
\text { PHORUS } \\
\text { DIS- } \\
\text { SOLVED } \\
\text { (MU/L } \\
\text { AS P) } \\
(00666)\end{array}$ & $\begin{array}{l}\text { PEOS- } \\
\text { EHORUS } \\
\text { ORTHO, } \\
\text { DYS-- } \\
\text { SOLVED } \\
\text { (MSG/L } \\
\text { AS P) } \\
(00671)\end{array}$ & $\begin{array}{l}\text { CARBON, } \\
\text { ORGAMIC } \\
\text { TOTAL } \\
\text { (MSG/L } \\
\text { AS C) } \\
(00680)\end{array}$ & $\begin{array}{l}\text { ALUA- } \\
\text { INUM, } \\
\text { DIS- } \\
\text { SOLVED } \\
\text { (UG/L } \\
\text { AS AL) } \\
\text { (01106) }\end{array}$ & $\begin{array}{l}\text { ANYT- } \\
\text { MONY, } \\
\text { DIS- } \\
\text { SOLVED } \\
\text { (UQ/L } \\
\text { AS SB) } \\
\text { (01095) }\end{array}$ & $\begin{array}{c}\text { ARSENIC } \\
\text { DIS } \\
\text { SOLVED } \\
\text { (UE/L } \\
\text { AS AS) } \\
\text { (01000) }\end{array}$ & $\begin{array}{c}\text { BARYUM, } \\
\text { DIS- } \\
\text { SOLVED } \\
\text { (USG/L } \\
\text { AS BA) } \\
(01005)\end{array}$ & $\begin{array}{l}\text { EEREL- } \\
\text { LIUM, } \\
\text { DIS- } \\
\text { SOLVED } \\
\text { (UE/L } \\
\text { AS BE) } \\
(01010)\end{array}$ & $\begin{array}{l}\text { BORON, } \\
\text { DIS- } \\
\text { SOLVED } \\
\text { (UE/L } \\
\text { AS B) } \\
(01020)\end{array}$ & $\begin{array}{c}\text { CADMIUM } \\
\text { DIS-- } \\
\text { SOLVED } \\
\text { (UE/L } \\
\text { AS CD) } \\
\text { (01025) }\end{array}$ & $\begin{array}{l}\text { CEPO- } \\
\text { MIUM, } \\
\text { DIS- } \\
\text { SOLVED } \\
\text { (UG/L } \\
\text { AS CR) } \\
(01030)\end{array}$ \\
\hline $\begin{array}{l}1995 \\
03 \ldots \\
C\end{array}$ & -- & $-\infty$ & - & $m-$ & - & -- & - & - & $=-$ & - & -- \\
\hline N6 1996 & $<0.010$ & $<0.010$ & 2.4 & 7.0 & $<1.0$ & $<1$ & 81 & $<1,0$ & 90 & $<1.0$ & 1.0 \\
\hline gR 16 & 0.020 & $<0,010$ & 3.3 & - & -- & $m$ & -- & $\rightarrow$ & 90 & - & -- \\
\hline $01 \ldots$ & $<0.010$ & 0.020 & 3.6 & 9.0 & $<1.0$ & $<1$ & 59 & $<1.0$ & 70 & $<1.0$ & $<1.0$ \\
\hline A & 0.020 & $<0.010$ & 2.2 & 6.0 & $<1.0$ & $<1$ & 107 & $<1.0$ & 111 & $<1.0$ & 1.0 \\
\hline
\end{tabular}


SAN JUAN RIVER BASIN

09364500 ANIMAS RIVER AT FARMINGTON, NM -- Continued

WATER-QUALITY RECORDS

WATER QUALITX DATA, WATER YEAR OCTOBER 1995 TO SEPTEMBER 1996

\begin{tabular}{|c|c|c|c|c|c|c|c|c|c|c|c|}
\hline DATE & $\begin{array}{c}\text { COBALT, } \\
\text { DIS- } \\
\text { SOLVED } \\
\text { (UG/L } \\
\text { AS CO) } \\
\text { (01035) }\end{array}$ & $\begin{array}{c}\text { COPPER, } \\
\text { DIS- } \\
\text { SOLVED } \\
\text { (UG/L } \\
\text { AS CU) } \\
\text { (01040) }\end{array}$ & $\begin{array}{c}\text { IRON, } \\
\text { DIS- } \\
\text { SOLVED } \\
\text { (UG/L } \\
\text { AS FE) } \\
\text { (01046) }\end{array}$ & $\begin{array}{c}\text { LEAD, } \\
\text { DIS- } \\
\text { SOLVED } \\
\text { (UG/L } \\
\text { AS FB) } \\
(01049)\end{array}$ & $\begin{array}{l}\text { MANGA- } \\
\text { NESE, } \\
\text { DIS- } \\
\text { SOLVED } \\
\text { (UG/L } \\
\text { AS MN) } \\
\text { (01056) }\end{array}$ & $\begin{array}{c}\text { MERCIJRY } \\
\text { TOTAL } \\
\text { RECOV- } \\
\text { ERABLE } \\
\text { (UG/L } \\
\text { AS RG) } \\
(71900)\end{array}$ & $\begin{array}{c}\text { MOLYB- } \\
\text { DENUM, } \\
\text { DIS- } \\
\text { SOLVED } \\
\text { (UG/L } \\
\text { AS MO) } \\
(01060)\end{array}$ & $\begin{array}{c}\text { NICKEL, } \\
\text { DIS- } \\
\text { SOLVED } \\
\text { (UG/L } \\
\text { AS NI) } \\
\text { (01065) }\end{array}$ & $\begin{array}{l}\text { SELE- } \\
\text { NIUM, } \\
\text { TOTAL } \\
\text { (UG/L } \\
\text { AS SE) } \\
\text { (01147) }\end{array}$ & $\begin{array}{l}\text { SELE- } \\
\text { NIUM, } \\
\text { DIS- } \\
\text { SOLVED } \\
\text { (UG/L } \\
\text { AS SE) } \\
\text { (01145) }\end{array}$ & $\begin{array}{c}\text { SILVER, } \\
\text { DIS- } \\
\text { SOLVED } \\
\text { (UG/L } \\
\text { AS AG) } \\
\text { (01075) }\end{array}$ \\
\hline \multirow{5}{*}{$\begin{array}{c}\text { OCT } 1995 \\
03 . \ldots \\
\text { DEC } \\
06 \ldots \\
\text { JAH } 1996 \\
16 \ldots \\
\text { AER } \ldots \\
01 \ldots \\
\text { SEP } \\
04 \ldots \\
04 \ldots\end{array}$} & -- & - m & $=-$ & $m$ & -- & -- & $=-$ & -- & $-m$ & -m & -- \\
\hline & $<1,0$ & 4.0 & 11 & $<1.0$ & 51 & $<0,10$ & 2.0 & 3.0 & $<1$ & $<1$ & $<1.0$ \\
\hline & $-\infty$ & $-\cdots$ & 6.0 & $-\infty$ & -- & - & -- & -- & $-\cdots$ & -- & -- \\
\hline & $<1.0$ & 2.0 & 7.0 & $<1.0$ & 46 & $<0.10$ & 2.0 & 2.0 & $<1$ & $<1$ & $<1.0$ \\
\hline & $<1.0$ & 2.0 & $<3.0$ & $<1.0$ & 55 & $<0.10$ & 3.0 & 3.0 & $<1$ & $<1$ & $<1.0$ \\
\hline DATE & $\begin{array}{l}\text { ZINC, } \\
\text { DIS- } \\
\text { SOLVED } \\
\text { (UG/L } \\
\text { AS ZN) } \\
\text { (01090) }\end{array}$ & $\begin{array}{c}\text { NITRO- } \\
\text { GEN, } \\
\text { NO2+NO3 } \\
\text { TOT. IN } \\
\text { BOT MAT } \\
\text { (MG/KG } \\
\text { AS N) } \\
\text { (00633) }\end{array}$ & $\begin{array}{c}\text { NITRO- } \\
\text { GEN,NB4 } \\
\text { TOTAL } \\
\text { IN BOT. } \\
\text { MAT. } \\
\text { (MG/KG } \\
\text { AS N) } \\
\text { (00611) }\end{array}$ & $\begin{array}{l}\text { NITRO- } \\
\text { GEN, NHA } \\
+ \text { ORG. } \\
\text { TOT IN } \\
\text { BOT MAT } \\
\text { (MG/KG } \\
\text { AS N) } \\
(00626)\end{array}$ & $\begin{array}{c}\text { PHOS- } \\
\text { PHORUS } \\
\text { TOTAL } \\
\text { IN BOT. } \\
\text { MAT } \\
\text { (MG/KG } \\
\text { AS P) } \\
(00668)\end{array}$ & $\begin{array}{c}\text { ARSENIC } \\
\text { TOTAL } \\
\text { IN BOT- } \\
\text { TOM MA- } \\
\text { TERIAL } \\
\text { (UG/G } \\
\text { AS AS) } \\
\text { (01003) }\end{array}$ & $\begin{array}{c}\text { CADMIUM } \\
\text { RECOV. } \\
\text { FM BOT- } \\
\text { TOM MA- } \\
\text { TERIAL. } \\
\text { (UG/G } \\
\text { AS CD) } \\
(01028)\end{array}$ & $\begin{array}{c}\text { CHRO- } \\
\text { MIUM, } \\
\text { RECOV. } \\
\text { FM BO'- } \\
\text { TOM MA- } \\
\text { TERIAL } \\
\text { (UG/G) } \\
(01029)\end{array}$ & $\begin{array}{c}\text { COBALT, } \\
\text { RECOV'. } \\
\text { FM BOT- } \\
\text { TOM MA- } \\
\text { TERIAL } \\
\text { (UG/G } \\
\text { AS CO) } \\
\text { (01038) }\end{array}$ & $\begin{array}{c}\text { COPPER, } \\
\text { RECOV' } \\
\text { FM BOT- } \\
\text { TOM MA- } \\
\text { TERIAL } \\
\text { (UG/G } \\
\text { AS CU) } \\
(01043)\end{array}$ & $\begin{array}{l}\text { IRON } \\
\text { RECOV. } \\
\text { FM BOT- } \\
\text { TOM MA- } \\
\text { TERIAL } \\
\text { (UG/G } \\
\text { AS FE) } \\
(01170)\end{array}$ \\
\hline $\mathrm{CT}$ & & & & & & & & & & & \\
\hline $\mathrm{DEC}^{03} \cdots$ & -- & -- & $-\cdots$ & -- & - & -- & -- & - & $m$ & - & -- \\
\hline JAN & 20 & 2.0 & 63 & 1000 & 390 & 6 & $<1$ & $<1$ & $<5$ & 12 & 700 \\
\hline APR & - & -- & - & $-m$ & -- & -- & -- & -- & $m$ & -- & -- \\
\hline $01 \ldots$ & 4.0 & -- & $\cdots$ & $\cdots$ & -- & -- & - & $-m$ & - & - & -- \\
\hline $04 \ldots$ & 4.0 & -- & - & -- & -- & - & $-\infty$ & -- & - & -- & - \\
\hline DATE & $\begin{array}{c}\text { LEAD } \\
\text { RECOV. } \\
\text { FM BOT- } \\
\text { TOM MA* } \\
\text { TERIAL } \\
\text { (UG/G } \\
\text { AS PB) } \\
(01052)\end{array}$ & $\begin{array}{c}\text { MANGA- } \\
\text { MESE, } \\
\text { RECOV. } \\
\text { FM BOT- } \\
\text { FMA MA- } \\
\text { TOM TERAL } \\
\text { (UG/G) } \\
\text { (01053) }\end{array}$ & $\begin{array}{c}\text { MGRCURY } \\
\text { RECOV. } \\
\text { FM BOT- } \\
\text { TOM MA- } \\
\text { TERIAL } \\
\text { (UGG/G } \\
\text { AS GG) } \\
\text { (71921) }\end{array}$ & $\begin{array}{c}\text { ZINC, } \\
\text { RECOU. } \\
\text { FM BOT- } \\
\text { TOM MA- } \\
\text { TERIAL } \\
\text { (UG G } \\
\text { AS ZN) } \\
(01093)\end{array}$ & $\begin{array}{c}\text { RADrUM } \\
226, \\
\text { DIS- } \\
\text { SOLVED, } \\
\text { RADON } \\
\text { METHOD } \\
(\mathrm{PCI} / \mathrm{L}) \\
(09511)\end{array}$ & $\begin{array}{c}\text { RA-226 } \\
\text { 2 SIGMA } \\
\text { WATER, } \\
\text { DISS, } \\
\text { (PCI/L) } \\
(76001)\end{array}$ & $\begin{array}{c}\text { URANIUM } \\
\text { NATURAL } \\
\text { DIS- } \\
\text { SOLVED } \\
\text { (UG/L } \\
\text { AS U) } \\
\text { (22703) }\end{array}$ & $\begin{array}{l}\text { URANIUM } \\
\text { NATURAL } \\
2 \text { SIGMA } \\
\text { WATER, } \\
\text { DISS, } \\
\text { (UG/L) } \\
\text { (75990) }\end{array}$ & $\begin{array}{l}\text { SEDI- } \\
\text { MENT, } \\
\text { SUS- } \\
\text { PENDED } \\
\text { (MG/L) } \\
(80154)\end{array}$ & $\begin{array}{c}\text { SEDI- } \\
\text { MENT', } \\
\text { DIS- } \\
\text { CEARGE, } \\
\text { SUS- } \\
\text { FENDED } \\
\text { (T/DAY) } \\
(80155)\end{array}$ & $\begin{array}{l}\text { SED. } \\
\text { SUSP. } \\
\text { SIEVE } \\
\text { DIAM. } \\
\% \text { FINER } \\
\text { THAA } \\
\text { O062 MMA } \\
(70331)\end{array}$ \\
\hline $\begin{array}{c}\text { OCT } 1995 \\
03 \ldots\end{array}$ & $m$ & $\cdots$ & -- & $\rightarrow-$ & -- & $\cdots$ & - & -- & -- & -- & -- \\
\hline JAN 1966 & 30 & 960 & 0.03 & 120 & 0.08 & 0.020 & 1.7 & 0.0 & 30 & 22 & 34 \\
\hline $\begin{array}{l}16 \ldots \\
\text { APR }\end{array}$ & -+ & -- & -- & - & $-\infty$ & -- & - & -- & 31 & 22 & 62 \\
\hline $01 \ldots$ & -- & -- & -- & - & - & -- & 2.0 & -- & 22 & 3 & 64 \\
\hline $04 \ldots$ & $-\cdots$ & $-\cdots$ & -- & - & -- & -- & 3.0 & - & 36 & 7.0 & 53 \\
\hline
\end{tabular}


LOCATYON. - Lat $36^{\circ} 43^{\prime} 22^{\prime \prime}$, long $108^{\circ} 13^{\prime} 30^{\prime \prime}$, in WW/4EK sec.17, T.29 N., R. 13 W., San Juan County, Hydrologic Unit 14080105, on left bank $360 \mathrm{ft}$ downstream from highway bridge on State Highway 371 in Farmington, 4,000 ft downstream from Animas River, $2.3 \mathrm{mi}$ upstream from La plata River, and at mile 251.4.

DRAINAGE ARFA, $--7,240 \mathrm{mi}^{2}$, approximately.

PERIOD OF RECORD.--June to December 1904, January 1905 to September 1906 (gage heights and discharge measurements only), September 1912 to curxent year. Monthly discharge only for sone periods, published in WSP 1313.

Discharge records for January to December 1905 , published in wsP 175 , are unreliable and should not bo used. REVISED RECORDS - WSP 1119: Drainage area. WSP 1243: 1938. WSP 1313: 1905, 1914. See also PERIOD OF RECORD. GAGE-Water-stage recorder, Datum of gage is $5,230,37$ ft above National Geodetic Vertical Datum of 1929 . See WSP 1313 or 1733 for history of changes prior to Hov. $19,1933$.

REMARLS,-Records good except fox ostimated dally discharges, which are poor. Since June 1962 Ilow 18 partly controlled by operation of Navajo Reservoir (station 09355100) 50 mi upstream. Diversions upstream srom station for irrigation of about 86,000 acres, 4,000 of which is irrigated by Farmers Mutual ditch, which diverts from Animes River and bypasses this station; ditch flow not included in record. At times this ditch may be supplied partly or entiroly by diversion from $\mathrm{San} J u a n$ River downstream from this station. Satelilto teleater at station.

EXIREMES OUTSIDE PERIOD OF RECORD,-Maximum flood occurred Oct. 6, 1911. Flood of Sept. 6, 1909, reached a sage of about 12.3 Rt, zite and datum in use May to September 1906.

DISCHARGE, CUBIC FEET PIR SECOND, WATER YEAR OCTOBER 1995 TO SEPTEMBER 1996

\begin{tabular}{|c|c|c|c|c|c|c|c|c|c|c|c|c|}
\hline DAY & $\mathrm{OCT}$ & Nov & $\therefore$ DEC & $\therefore$ JAN & FEB & $\therefore \quad M A R$ & AFR & MAY & JUN & JUL & AUUS & SEP \\
\hline $\begin{array}{l}1 \\
2 \\
3 \\
4 \\
5\end{array}$ & $\begin{array}{l}1430 \\
1370 \\
1370 \\
1320 \\
1270\end{array}$ & $\begin{array}{l}1110 \\
1110 \\
1100 \\
1130 \\
2190\end{array}$ & $\begin{array}{l}1270 \\
1260 \\
1260 \\
1260 \\
1250\end{array}$ & $\begin{array}{r}923 \\
886 \\
\mathrm{e} 890 \\
886 \\
913\end{array}$ & $\begin{array}{r}8880 \\
e 880 \\
8880 \\
866 \\
816\end{array}$ & $\begin{array}{l}839 \\
870 \\
876 \\
905 \\
896\end{array}$ & $\begin{array}{r}696 \\
582 \\
562 \\
e 548 \\
e 550\end{array}$ & $\begin{array}{l}1110 \\
1330 \\
1650 \\
1930 \\
2160\end{array}$ & $\begin{array}{l}3040 \\
3070 \\
3220 \\
3390 \\
3500\end{array}$ & $\begin{array}{l}1720 \\
1340 \\
1220 \\
1100 \\
1020\end{array}$ & $\begin{array}{l}533 \\
527 \\
526 \\
538 \\
535\end{array}$ & $\begin{array}{l}651 \\
659 \\
654 \\
629 \\
608\end{array}$ \\
\hline $\begin{array}{r}6 \\
7 \\
8 \\
9 \\
10\end{array}$ & $\begin{array}{l}1250 \\
1190 \\
1160 \\
1140 \\
1140\end{array}$ & $\begin{array}{l}1190 \\
1220 \\
1260 \\
1250 \\
1270\end{array}$ & $\begin{array}{l}1310 \\
1280 \\
1280 \\
1270 \\
1260\end{array}$ & $\begin{array}{r}923 \\
917 \\
891 \\
.872 \\
884\end{array}$ & $\begin{array}{l}8840 \\
8840 \\
8830 \\
\theta 820 \\
6810\end{array}$ & $\begin{array}{l}925 \\
916 \\
864 \\
858 \\
874\end{array}$ & $\begin{array}{l}557 \\
580 \\
664 \\
678 \\
820\end{array}$ & $\begin{array}{l}2470 \\
2700 \\
2760 \\
2820 \\
2760\end{array}$ & $\begin{array}{l}3550 \\
3660 \\
3720 \\
3690 \\
3570\end{array}$ & $\begin{array}{r}962 \\
889 \\
855 \\
944 \\
924\end{array}$ & $\begin{array}{l}535 \\
533 \\
526 \\
612 \\
588\end{array}$ & $\begin{array}{l}624 \\
576 \\
625 \\
590 \\
578\end{array}$ \\
\hline $\begin{array}{l}11 \\
12 \\
13 \\
14 \\
15\end{array}$ & $\begin{array}{l}1120 \\
1080 \\
1040 \\
1050 \\
1130\end{array}$ & $\begin{array}{l}1310 \\
1260 \\
1240 \\
1250 \\
1270\end{array}$ & $\begin{array}{l}1250 \\
1240 \\
1220 \\
1270 \\
1290\end{array}$ & $\begin{array}{r}0700 \\
0570 \\
598 \\
615 \\
580\end{array}$ & $\begin{array}{l}0800 \\
9800 \\
9790 \\
790 \\
800\end{array}$ & $\begin{array}{l}872 \\
827 \\
832 \\
870 \\
865\end{array}$ & $\begin{array}{r}1070 \\
1060 \\
1020 \\
947 \\
839\end{array}$ & $\begin{array}{l}2670 \\
3010 \\
3390 \\
3420 \\
3450\end{array}$ & $\begin{array}{l}3490 \\
3470 \\
3340 \\
3340 \\
3620\end{array}$ & $\begin{array}{r}1010 \\
843 \\
817 \\
851 \\
816\end{array}$ & $\begin{array}{r}655 \\
0510 \\
6450 \\
424 \\
422\end{array}$ & $\begin{array}{r}608 \\
594 \\
641 \\
938 \\
1240\end{array}$ \\
\hline $\begin{array}{l}16 \\
17 \\
18 \\
19 \\
20\end{array}$ & $\begin{array}{l}1140 \\
1060 \\
1070 \\
1060 \\
1080\end{array}$ & $\begin{array}{l}1280 \\
1260 \\
1250 \\
1260 \\
1260\end{array}$ & $\begin{array}{l}1230 \\
1240 \\
1280 \\
1310 \\
1360\end{array}$ & $\begin{array}{r}577 \\
e 640 \\
e 610 \\
589 \\
628\end{array}$ & $\begin{array}{l}808 \\
820 \\
823 \\
858 \\
840\end{array}$ & $\begin{array}{l}860 \\
919 \\
898 \\
851 \\
799\end{array}$ & $\begin{array}{l}696 \\
661 \\
612 \\
589 \\
567\end{array}$ & $\begin{array}{l}3600 \\
3780 \\
3780 \\
3650 \\
3590\end{array}$ & $\begin{array}{l}3540 \\
3390 \\
3350 \\
3340 \\
3310\end{array}$ & $\begin{array}{r}750 \\
740 \\
1160 \\
765 \\
712\end{array}$ & $\begin{array}{l}412 \\
427 \\
444 \\
455 \\
456\end{array}$ & $\begin{array}{l}1150 \\
1150 \\
1210 \\
1280 \\
1240\end{array}$ \\
\hline $\begin{array}{l}21 \\
22 \\
23 \\
24 \\
25\end{array}$ & $\begin{array}{l}1070 \\
1090 \\
1190 \\
1170 \\
1150\end{array}$ & $\begin{array}{l}1260 \\
1230 \\
1230 \\
1230 \\
1230\end{array}$ & $\begin{array}{l}1310 \\
1290 \\
1260 \\
1240 \\
1220\end{array}$ & $\begin{array}{r}598 \\
608 \\
575 \\
565 \\
583\end{array}$ & $\begin{array}{l}888 \\
899 \\
905 \\
888 \\
831\end{array}$ & $\begin{array}{l}778 \\
779 \\
735 \\
748 \\
771\end{array}$ & $\begin{array}{l}519 \\
493 \\
480 \\
470 \\
539\end{array}$ & $\begin{array}{r}3400 \\
\mathrm{e} 3180 \\
\mathrm{e} 2960 \\
2590 \\
2180\end{array}$ & $\begin{array}{l}3330 \\
3510 \\
3700 \\
3410 \\
2960\end{array}$ & $\begin{array}{l}636 \\
581 \\
533 \\
525 \\
519\end{array}$ & $\begin{array}{r}450 \\
505 \\
924 \\
879 \\
1220\end{array}$ & $\begin{array}{r}1140 \\
1120 \\
1070 \\
1020 \\
978\end{array}$ \\
\hline $\begin{array}{l}26 \\
27 \\
28 \\
29 \\
30 \\
31\end{array}$ & $\begin{array}{l}1170 \\
1140 \\
1120 \\
1110 \\
1120 \\
1110\end{array}$ & $\begin{array}{l}1240 \\
1260 \\
1260 \\
1250 \\
1250 \\
-\cdots\end{array}$ & $\begin{array}{l}1230 \\
940 \\
0950 \\
0970 \\
0990 \\
0980\end{array}$ & $\begin{array}{l}0760 \\
9810 \\
6820 \\
9830 \\
6850 \\
e 870\end{array}$ & $\begin{array}{l}860 \\
850 \\
849 \\
834 \\
-1-\end{array}$ & $\begin{array}{l}743 \\
715 \\
669 \\
669 \\
670 \\
644\end{array}$ & $\begin{array}{r}908 \\
1440 \\
1630 \\
1720 \\
1270 \\
-\end{array}$ & $\begin{array}{l}1860 \\
1640 \\
1410 \\
2000 \\
2450 \\
2860\end{array}$ & $\begin{array}{l}2410 \\
2170 \\
2010 \\
1770 \\
1530 \\
-\end{array}$ & $\begin{array}{l}522 \\
501 \\
504 \\
511 \\
517 \\
526\end{array}$ & $\begin{array}{r}1240 \\
1060 \\
1200 \\
1030 \\
851 \\
670\end{array}$ & $\begin{array}{l}1060 \\
964 \\
961 \\
911 \\
879 \\
-\end{array}$ \\
\hline $\begin{array}{l}\text { TOTAL } \\
\text { MEAN } \\
\text { MAX } \\
\text { MIN } \\
\text { AC-FT }\end{array}$ & $\begin{array}{r}35910 \\
1158 \\
1430 \\
1040 \\
71230\end{array}$ & $\begin{array}{r}36910 \\
1230 \\
1310 \\
1100 \\
73210\end{array}$ & $\begin{array}{r}37770 \\
1218 \\
1360 \\
940 \\
74920\end{array}$ & $\begin{array}{r}22961 \\
741 \\
923 \\
565 \\
45540\end{array}$ & $\begin{array}{r}24395 \\
841 \\
905 \\
790 \\
48390\end{array}$ & $\begin{array}{r}25337 \\
817 \\
925 \\
644 \\
50260\end{array}$ & $\begin{array}{r}23767 \\
792 \\
1720 \\
470 \\
47140\end{array}$ & $\begin{array}{r}82560 \\
2663 \\
3780 \\
1110 \\
163800\end{array}$ & $\begin{array}{r}95400 \\
3180 \\
3720 \\
1530 \\
189200\end{array}$ & $\begin{array}{r}25313 \\
817 \\
1720 \\
501 \\
50210\end{array}$ & $\begin{array}{r}20137 \\
650 \\
1240 \\
412 \\
39940\end{array}$ & $\begin{array}{r}26348 \\
878 \\
1280 \\
576 \\
52260\end{array}$ \\
\hline
\end{tabular}

SIEATISTICS OF MONTHLX MEAN DATA FOR WATER TEARS 1931" 1996 , DY WATER YEAR (WY)

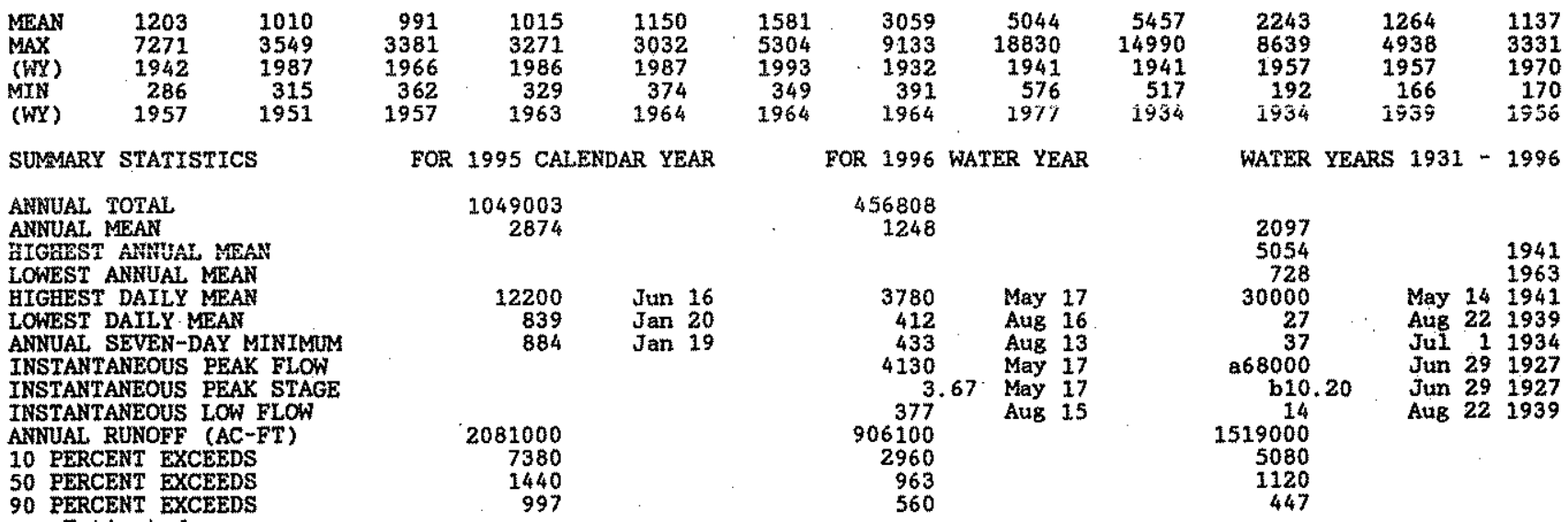

90 PERCENT EXCEEDS

- Estimated

a-Site and datum then in use.

b-From rating curve extended above $37,000 \mathrm{ft}^{3} / \mathrm{s}$. 
SAN JUAN RIVER BASIN

09366500 LA PLATA RIVER AT COLORADO-NEW MEXICO STATE LINE

LOCATrON --Lat $36^{\circ} 59^{\prime} 51^{\prime \prime}$, long $108^{\circ} 11^{\prime} 17^{\prime \prime}$, in NW/sEk sec.10, T.32 N., R.13 W. La Plata County, Colorado, flydrolog 10 Unit 14080105 , on right bank at Colorado-New Mexico State line, 0.2 mi downstream from Ponds Arroyo, and 4.8 mi north of La Piata, NM.

DRATKAGE AREA. $--331 \mathrm{mt}^{2}$.

PERIOD OF RECORD:--January 1920 to current year. Monthly discharge only for some periods, published in WSP 1313.

REVISED RECORDS. - -WSP 1313: $1934(\mathrm{M}), 1936(\mathrm{M})$.

GAGE. - Water-stage recorder with satellite telemetry. Datum of gage is $5,975.15$ ft above National Geodetic Vertical Datum of 1929. See WSP 1713 or 1733 for history of changes prior to Mar. 17, 1934. Mar. 17, 1934 to July 1 , 1996, water-stage recorder at same site, and at datum 3.12 ft higher.

REMARKS.--Records good except for estimated datly discharges, which are poor. Diversions upstream from station for irrigation of about 15,000 acres, most of which are upstream from station.

COOPERATION.--Records collected and computed by Colorado Division of Water Resources and reviewed by Geological Survey.

DISCAARGE, CUBIC FEET PER SECOND, WATER YEAR OCTOBER 1995 TO SEETEMBER 1996

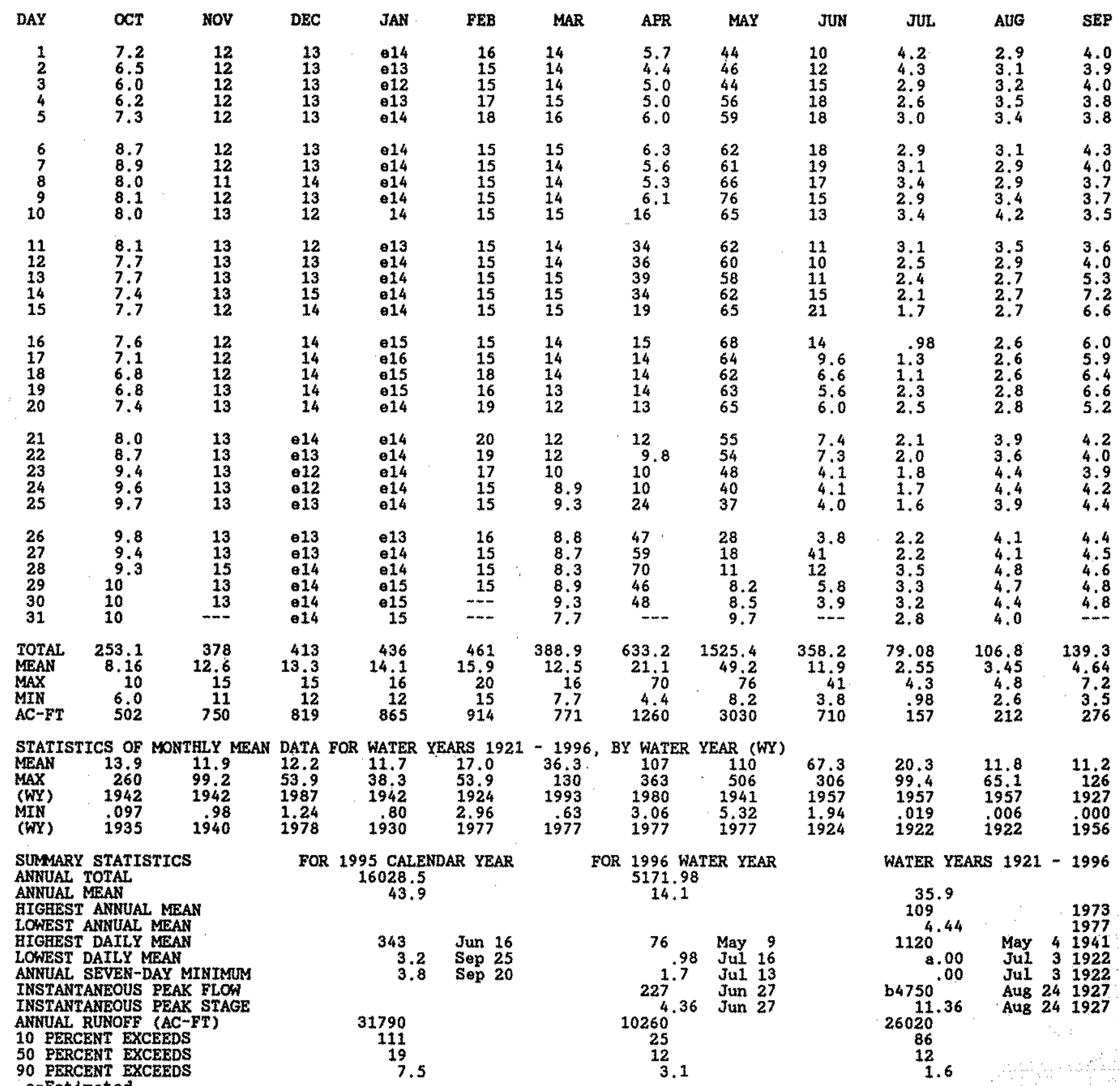

\footnotetext{
a-Estimated.
}

b-From rating curve extended above $750 \mathrm{ft} / \mathrm{s}$, on basis of slope area measurement of peak flow, at datum then in use. 


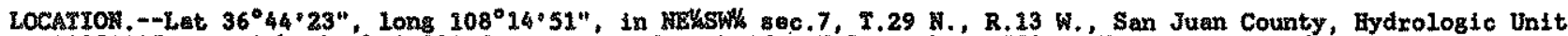
14080105 , on right bank 1,300 te upstrean \&rom bxidge U'.s. 'gighway 550 in'Farmington, and i, 800 ft upstream from mouth.

DRAIMAGE AREA. --583 m $^{2}$.

EERTOD OP RECORD. - m 1938 to curreat yoar.

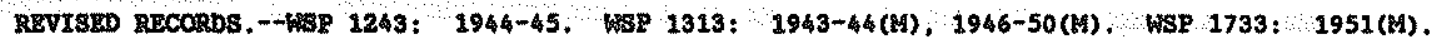

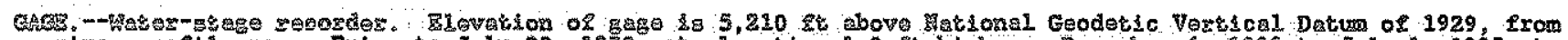

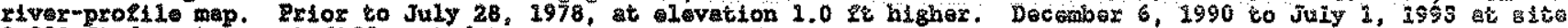
1,000 it downtreen at dieforont dectum.

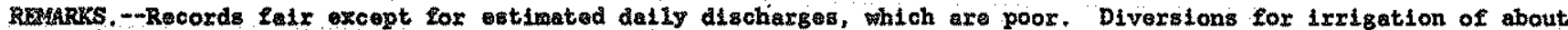
24,000 scres upstrean srom station. Sevoral obsezvations of pater temporatuxe pore made during the year.

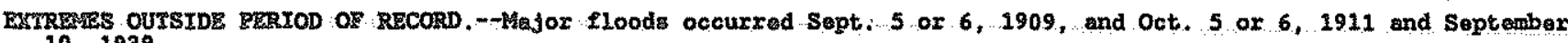
$10,1939$.

DTSCHARGE, CUBIC FEET PER SECOND, MATER YEAR OCTOBER 1995 TO SEETEMEER 1996

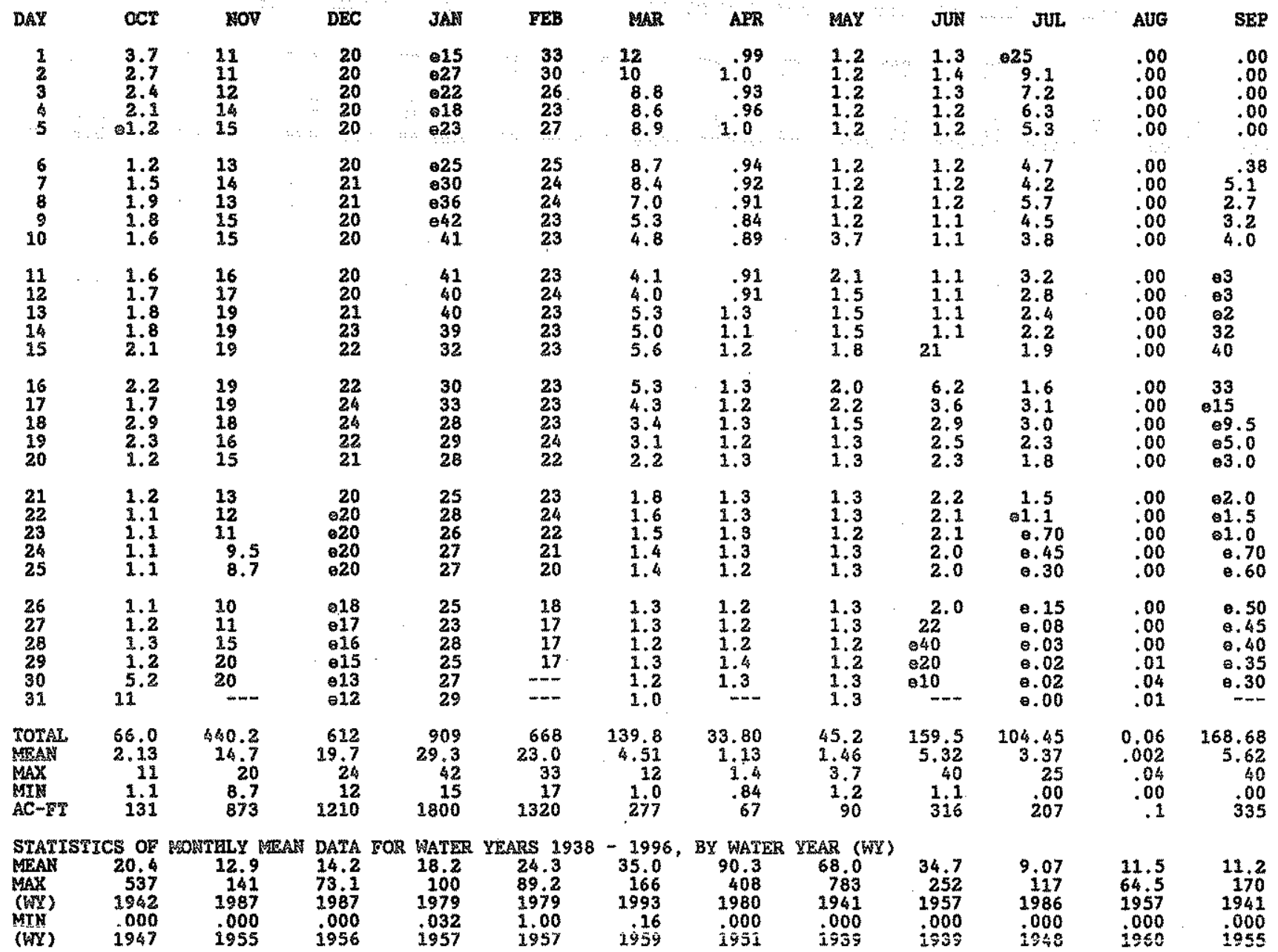

SURAARY STATISTTCS ARTUAL, TOKAZ

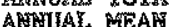

BTGEIEST AHAUAL BTAN

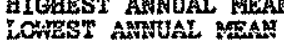

HIGHEST DAILY MEAN

HIGEEST DAILY MEA
LOWEST DAILX MEAN

ANTUAL SEVEA-DAY MINIMUM

INSTAKTANEOUS PEAK FLOS

IMSTANTAFEOUS PEAK STAGE

INSTANTAYEOUS LOW FLOW

ANQUAL RUROFF (AC-FT)

10 PFRCENT EXCEEDS

50 PERCENT EXCEEDS

90 PERCENT EXCEEDS

- Estimatad

a-From floodmarks.

b-From rating curve extended on basis of slope-area measurement of peak flow.
FOR 1995 CALENDAR YEAR FOR 1996 WATER YEAR

$$
\begin{aligned}
& 11741.31 \\
& 32.2
\end{aligned} 346.69
$$

300 Mar 6 .26 Aug 14

$42 \quad J \operatorname{Jan} 9$

$$
\begin{array}{r}
.00 \\
71.00 \quad \text { JuI } 31 \\
\text { Jul } 31
\end{array}
$$$$
71,10 \mathrm{Jul}
$$

23290

108
19

19.92
WATER YEARS 1938 - 1996

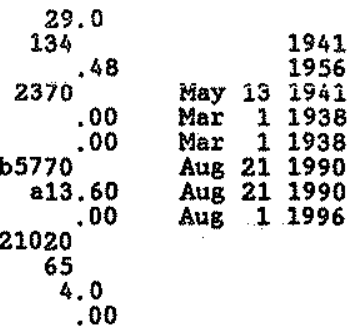


SAN JUAN RIVER BASIN

09367540 SAN JUAN RIVER MEAR FRUITLAND, NM

WATER-QUALITY RECORDS

LOCATroN. - Lat $36^{\circ} 44^{\circ} 25^{\prime \prime}$, long $108^{\circ} 24^{\circ} 09^{\prime \prime}$, In NW S SE S sec. 10, T. 29 N., R, 15 W. San Juan County, Bydrologic

Unte 14080105 , on right bank 300 ft downstream from Four Corners Powerplant highway bridge, 0 , mi west of

Fruitiand, $10 \mathrm{ml}$ downstream from La Plata River, $14.0 \mathrm{mi}$ upstream from Chaco River, and at milo 239.

DRAINAGE AREA. $--8,010 \mathrm{mi}^{2}$, approximately.

PERIOD OF RECORD.--Watex years 1978 to September 1996 (discontinued).

REMARKS. --Discharges estimated from discharge records at 09365000 San Juan River at Farmington, which is

approximately 11 miles upstrean and station 09367500 La Plate River near Farmington which is approximately 8.7

miles upstroam.

WATER QUALITY DATA, HATER YEAR OCTOBER 1995 TO SEPTEMBER 1996

\begin{tabular}{|c|c|c|c|c|c|c|c|c|c|c|}
\hline DATE & TIME & $\begin{array}{c}\text { DIS- } \\
\text { CEARGE, } \\
\text { INST. } \\
\text { CUBIC } \\
\text { FEET } \\
\text { PER } \\
\text { SECOND } \\
(00061)\end{array}$ & $\begin{array}{l}\text { SPE- } \\
\text { CIFIC } \\
\text { CON- } \\
\text { DUCT- } \\
\text { ANCE } \\
\text { (US/CM) } \\
(00095)\end{array}$ & $\begin{array}{c}\text { PH } \\
\text { WATER } \\
\text { WHOLE } \\
\text { FIELD } \\
\text { (STAND- } \\
\text { ARD } \\
\text { UNITS) } \\
(00400)\end{array}$ & $\begin{array}{l}\text { TEMPER- } \\
\text { ATURE } \\
\text { AIR } \\
\text { (DEG C) } \\
(00020)\end{array}$ & $\begin{array}{c}\text { TEMPER- } \\
\text { ATURE } \\
\text { WATER } \\
\text { (DEG C) } \\
(00010)\end{array}$ & $\begin{array}{c}\text { TUR- } \\
\text { BID- } \\
\text { ITX } \\
\text { (NTU) } \\
(00076)\end{array}$ & $\begin{array}{c}\text { BARO- } \\
\text { METRIC } \\
\text { PRES- } \\
\text { SURE } \\
\text { (MRI } \\
\text { OF } \\
\text { HG) } \\
(00025)\end{array}$ & $\begin{array}{c}\text { OXYGEN, } \\
\text { DIS- } \\
\text { SOLVED } \\
(M G / L) \\
(00300)\end{array}$ & $\begin{array}{c}\text { OXYGEN, } \\
\text { DIS- } \\
\text { SOLVED } \\
\text { (RER- } \\
\text { CENT } \\
\text { SATUR- } \\
\text { ATIOH) } \\
\text { (00301) }\end{array}$ \\
\hline $\begin{array}{c}\text { OCT } 1995 \\
\text { OS } \ldots \\
\text { DEC }\end{array}$ & 1100 & E1200 & 453 & $-m$ & 18.0 & 9.0 & - & - & - & - \\
\hline JAN 1996 & 1330 & E1270 & 524 & 8.6 & 12.5 & 6.0 & 21 & 635 & 11.2 & 108 \\
\hline${ }_{A P R}^{18}, \ldots$ & 1500 & E659 & 764 & 8.6 & 0.0 & 0.5 & 38 & 634 & 13.4 & 112 \\
\hline $04 \ldots$ & 0830 & E394 & 528 & 7.8 & 12.0 & 10.0 & 11 & 634 & 9.6 & 103 \\
\hline DATE & $\begin{array}{l}\text { HARD- } \\
\text { NESS } \\
\text { TOTAL, } \\
\text { (MG/L } \\
\text { AS } \\
\text { CACO3) } \\
(00900)\end{array}$ & $\begin{array}{l}\text { HARD- } \\
\text { NESS } \\
\text { NONCARB } \\
\text { DISSOLV } \\
\text { FLD. AS } \\
\text { CACO3 } \\
\text { (MG/L) } \\
(00904)\end{array}$ & $\begin{array}{l}\text { CALCIUM } \\
\text { DIS- } \\
\text { SOLVED } \\
\text { (MG/L } \\
\text { AS CA) } \\
\text { (O0915) }\end{array}$ & $\begin{array}{c}\text { MAGNE- } \\
\text { SIUM, } \\
\text { DIS- } \\
\text { SOLVED } \\
\text { (MG/L } \\
\text { AS MG) } \\
(00925)\end{array}$ & $\begin{array}{l}\text { SODIUM, } \\
\text { DIS- } \\
\text { SOLVED } \\
\text { (MG/L } \\
\text { AS NA) } \\
(00930)\end{array}$ & $\begin{array}{c}\text { SODIUM } \\
\text { AD- } \\
\text { SORP- } \\
\text { TION } \\
\text { RATIO } \\
(00931)\end{array}$ & $\begin{array}{c}\text { POIAS- } \\
\text { SIUM, } \\
\text { DIS- } \\
\text { SOLVED } \\
\text { (MG/L } \\
\text { AS K) } \\
(00935)\end{array}$ & $\begin{array}{c}\text { BICAR- } \\
\text { BONATE } \\
\text { WATER } \\
\text { DIS IT } \\
\text { FIELD } \\
\text { MG/L AS } \\
\text { BCO3 } \\
(00453)\end{array}$ & $\begin{array}{c}\text { CAR- } \\
\text { BONATE } \\
\text { WATER } \\
\text { DIS IT } \\
\text { FIELD } \\
\text { MG/L AS } \\
\text { CO3 } \\
(00452)\end{array}$ & $\begin{array}{c}\text { ALKA- } \\
\text { LINITY } \\
\text { WAT DIS } \\
\text { TOT IT } \\
\text { FTELD } \\
\text { MG/L AS } \\
\text { CACO3 } \\
(39086)\end{array}$ \\
\hline
\end{tabular}

\begin{tabular}{|c|c|c|c|c|c|}
\hline $\begin{array}{c}\text { OCT } 1995 \\
05 \ldots \\
\operatorname{DFC}^{2} \ldots\end{array}$ & $-m$ & -- & - & $m$ & - \\
\hline${ }_{\text {JAN }}^{06} 19 \dot{9} 6$ & 190 & 77 & 59 & 11 & 32 \\
\hline${ }_{\mathrm{APR}}^{18 \ldots}$ & 290 & 140 & 88 & 16 & 54 \\
\hline & 170 & 64 & 54 & 8.7 & 34 \\
\hline
\end{tabular}

$\begin{array}{ccccc}-- & -- & -- & & -\cdots \\ 1 & 2.2 & 129 & 6 & 115 \\ 1 & 2.6 & 162 & 6 & 143 \\ 1 & 1.8 & 130 & 0 & 106\end{array}$

$\begin{array}{ccc}\text { ALKA- } & \\ \text { LINITY } & \text { SULFATE } \\ \text { LAB } & \text { DIS- } & \text { DID } \\ \text { (NG/L } & \text { SOLVED } & \text { SOL } \\ \text { AS } & (\text { MG } / L & ( \\ \text { CACO3) } & \text { AS SOL) } & \text { A } \\ (90410) & (00945) & (009\end{array}$

CELO-
RIDE,
DIS-
SOLVED
(MG/L.
AS CL)
$(00940)$

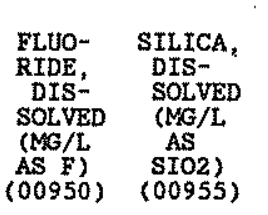

SOLIDS, AT 180 DEG. C DIS$(M G / L)$ $(70300)$

SOLIDS,
SUM OF
CONSTI-
TUENTS,
DIS-
SOLVED
(MG/L)
$(70301)$

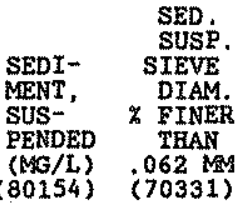

OCres 1995<smiles>[R16]1CCC[AsH2]1</smiles>

06 ig்

18 ..

APR

$04 \ldots$

117

1.09

$\begin{array}{ll}\ldots & \ldots \\ 130 & 10 \\ 220 & 17 \\ 140 & 12\end{array}$

$\begin{array}{ll}0.30 & 8.4 \\ 0.30 & 8.9 \\ 0.30 & 6.4\end{array}$

-. $\quad$ -

334

512

328

$\begin{array}{lll}- & 15 & 40 \\ 322 & -- & - \\ 492 & -- & -- \\ 321 & -- & -\end{array}$


LOCATION - - Lat $36^{\circ} 46^{\circ} 52^{\prime \prime}$, long $108^{\circ} 41^{\circ} 23^{\prime \prime}$, in SE/ sec.25, T.30 N, R. 18 W., San Juan County, Eydrologic Unit 14080105 , on right bark $500 \mathrm{ft}$ upstream from bridge on U.S. Bighway $666^{\prime}$ in Shiprock, 3 mi dowstream from Chaco at mile 215 .

DRAINAGE AREA, $-12,900 \mathrm{mi}^{i}$, epproximately.

\section{WATER-DISCHARGE RECORDS}

PERIOD OF RECORD.--January to Octobex 1911, February 1927 to current year Monthly or yearly discharge only for some periods, published in WSP 1313

REVISED DECORDS',-WSP 1243: 1931, 1934-38, 1951, WSP 1313: 1911, 1933,.WDR NM-78-1: 1977.

GAGE.-Water-stage recorder, Datum of gage is 4,850 rt above Hational Godat 10 Vertical Datum of 1929 (rtverprosile survey). Prior to Apr. 6, 1922 , nonrecording gage and Apr. 7, 1922, to Oct. 25, 1933, water-stage recorder, at site 3 mi upstream at different datum. oct. 26, 1933, to Sept. 30, 1936 , water-stage recorder at present site at detum $3,31 \mathrm{ft}$ higher and oct. 1, 1936, to Sept. 30, 1952 , at datum 1.77 It higher. Supplementary water-stage recorders at nearby sites, same datum, used at times. Water-stage recorder, at site 4 mi upstream Sept. 1994 .

RaMARXS. -Water-discharge records good except for estimated daily discharges, which are Fair, since 1962 flow partiy regulated by Wavajo Reservoir (station 09355100). Diversions for irrigation of about 118,000 ecres upstream from station. Ungaged canals bypass station on both right and left banks, though some of bypass flow is returned to river downstream from gage. Satellite telemeter at station.

EXTREAES OUTSIDE PERYOD OF RECORD,-Maximum flood occurred Oct. 6 , 1911 , and reached a stage of 22 ft, site and datum then in use.

DISCRARGE, CUBIC FEET PER SECOND, WATER YEAR OCTOBER 1995 TO SEPTEMBER 1996

\begin{tabular}{|c|c|c|c|c|c|c|c|c|c|c|c|c|}
\hline $\begin{array}{r}\text { DAY } \\
1 \\
2 \\
3 \\
4 \\
5\end{array}$ & $\begin{array}{r}\text { OCT } \\
1240 \\
1230 \\
1130 \\
1120 \\
1100\end{array}$ & $\begin{array}{r}\text { NOV } \\
1040 \\
983 \\
977 \\
975 \\
978\end{array}$ & $\begin{array}{l}\text { DEC } \\
1100 \\
1070 \\
1060 \\
1050 \\
1040\end{array}$ & $\begin{array}{l}\text { JAN } \\
824 \\
820 \\
788 \\
770 \\
799\end{array}$ & $\begin{array}{l}\text { FEB } \\
862 \\
897 \\
823 \\
774 \\
759\end{array}$ & $\begin{array}{l}\text { MAR: } \\
709 \\
761 \\
760 \\
793 \\
788\end{array}$ & $\begin{array}{l}\text { APR } \\
469 \\
470 \\
394 \\
393 \\
467\end{array}$ & $\begin{array}{r}\text { MAY } \\
691 \\
777 \\
843 \\
1010 \\
1210\end{array}$ & $\begin{array}{r}\text { JUN } \\
\text { e1600 } \\
\text { e2000 } \\
82500 \\
\text { e2900 } \\
\text { e } 3240\end{array}$ & $\begin{array}{r}\text { JUL } \\
\text { 1500 } \\
\text { e1250 } \\
\mathrm{e} 1060 \\
981 \\
847\end{array}$ & $\begin{array}{l}\text { AUG } \\
155 \\
144 \\
142 \\
145 \\
158\end{array}$ & $\begin{array}{l}\text { SEP } \\
402 \\
375 \\
378 \\
311 \\
261\end{array}$ \\
\hline $\begin{array}{r}6 \\
7 \\
8 \\
9 \\
10\end{array}$ & $\begin{array}{r}1150 \\
1110 \\
1060 \\
1010 \\
876\end{array}$ & $\begin{array}{r}990 \\
1050 \\
1050 \\
1060 \\
1060\end{array}$ & $\begin{array}{l}1040 \\
1110 \\
1080 \\
1070 \\
1060\end{array}$ & $\begin{array}{l}885 \\
884 \\
864 \\
855 \\
855\end{array}$ & $\begin{array}{l}771 \\
763 \\
771 \\
806 \\
803\end{array}$ & $\begin{array}{l}746 \\
761 \\
729 \\
718 \\
707\end{array}$ & $\begin{array}{l}510 \\
498 \\
511 \\
539 \\
429\end{array}$ & $\begin{array}{l}1530 \\
1930 \\
2170 \\
2070 \\
2270\end{array}$ & $\begin{array}{l}\text { e } 3220 \\
\text { e } 3210 \\
\text { e } 3260 \\
\text { e3250 } \\
\text { e } 3100\end{array}$ & $\begin{array}{l}749 \\
687 \\
663 \\
581 \\
592\end{array}$ & $\begin{array}{l}140 \\
161 \\
218 \\
227 \\
369\end{array}$ & $\begin{array}{l}398 \\
450 \\
393 \\
329 \\
330\end{array}$ \\
\hline $\begin{array}{l}11 \\
12 \\
13 \\
14 \\
15\end{array}$ & $\begin{array}{l}871 \\
853 \\
817 \\
812 \\
827\end{array}$ & $\begin{array}{l}1090 \\
1090 \\
1060 \\
1050 \\
1050\end{array}$ & $\begin{array}{l}1050 \\
1050 \\
1020 \\
1040 \\
1090\end{array}$ & $\begin{array}{r}783 \\
609 \\
5 \quad 587 \\
5 \quad 591 \\
591\end{array}$ & $\begin{array}{r}814 \\
810 \\
801 \\
794 \\
788\end{array}$ & $\begin{array}{l}713 \\
716 \\
696 \\
719 \\
773\end{array}$ & $\begin{array}{l}587 \\
823 \\
741 \\
738 \\
684\end{array}$ & $\begin{array}{l}1950 \\
2060 \\
2710 \\
3090 \\
2970\end{array}$ & $\begin{array}{l}02950 \\
02990 \\
03100 \\
02800 \\
02950\end{array}$ & $\begin{array}{l}630 \\
622 \\
531 \\
550 \\
590\end{array}$ & $\begin{array}{l}356 \\
296 \\
239 \\
167 \\
158\end{array}$ & $\begin{array}{r}295 \\
362 \\
376 \\
932 \\
1640\end{array}$ \\
\hline $\begin{array}{l}16 \\
17 \\
18 \\
19 \\
20\end{array}$ & $\begin{array}{l}932 \\
903 \\
847 \\
847 \\
842\end{array}$ & $\begin{array}{l}1060 \\
1040 \\
1040 \\
1090 \\
1120\end{array}$ & $\begin{array}{l}1080 \\
1020 \\
1050 \\
1050 \\
1080\end{array}$ & $\begin{array}{l}582 \\
531 \\
635 \\
600 \\
537\end{array}$ & $\begin{array}{l}785 \\
790 \\
790 \\
795 \\
811\end{array}$ & $\begin{array}{l}771 \\
764 \\
794 \\
766 \\
710\end{array}$ & $\begin{array}{l}576 \\
408 \\
387 \\
384 \\
382\end{array}$ & $\begin{array}{l}3090 \\
3380 \\
3550 \\
3390 \\
3220\end{array}$ & $\begin{array}{l}e 3100 \\
e 2870 \\
e 2750 \\
\oplus 2690 \\
e 2540\end{array}$ & $\begin{array}{l}454 \\
538 \\
835 \\
845 \\
722\end{array}$ & $\begin{array}{l}156 \\
143 \\
147 \\
154 \\
157\end{array}$ & $\begin{array}{l}1260 \\
1090 \\
1100 \\
1390 \\
1280\end{array}$ \\
\hline $\begin{array}{l}21 \\
22 \\
23 \\
24 \\
25\end{array}$ & $\begin{array}{l}812 \\
804 \\
940 \\
975 \\
997\end{array}$ & $\begin{array}{l}1120 \\
1100 \\
1080 \\
1090 \\
1090\end{array}$ & $\begin{array}{l}1120 \\
1070 \\
1060 \\
1040 \\
1030\end{array}$ & $\begin{array}{l}513 \\
498 \\
512 \\
550 \\
572\end{array}$ & $\begin{array}{l}739 \\
784 \\
781 \\
759 \\
730\end{array}$ & $\begin{array}{l}703 \\
706 \\
677 \\
663 \\
669\end{array}$ & $\begin{array}{l}364 \\
296 \\
282 \\
286 \\
265\end{array}$ & $\begin{array}{r}3140 \\
2660 \\
\mathrm{e} 2250 \\
\mathrm{\theta} 1900 \\
\mathrm{~B} 1550\end{array}$ & $\begin{array}{l}02700 \\
02800 \\
03000 \\
e 2600 \\
02400\end{array}$ & $\begin{array}{l}535 \\
465 \\
419 \\
371 \\
239\end{array}$ & $\begin{array}{r}159 \\
170 \\
535 \\
1210 \\
1210\end{array}$ & $\begin{array}{r}1100 \\
932 \\
887 \\
862 \\
823\end{array}$ \\
\hline $\begin{array}{l}26 \\
27 \\
28 \\
29 \\
30 \\
31\end{array}$ & $\begin{array}{r}994 \\
1080 \\
1050 \\
1030 \\
1030 \\
1030\end{array}$ & $\begin{array}{l}1090 \\
1090 \\
1120 \\
1100 \\
1090 \\
-\end{array}$ & $\begin{array}{r}1020 \\
995 \\
865 \\
765 \\
771 \\
809\end{array}$ & $\begin{array}{l}586 \\
795 \\
833 \\
856 \\
859 \\
868\end{array}$ & $\begin{array}{l}694 \\
705 \\
709 \\
707 \\
-- \\
-\end{array}$ & $\begin{array}{l}652 \\
633 \\
579 \\
546 \\
490 \\
477\end{array}$ & $\begin{array}{r}324 \\
731 \\
983 \\
1120 \\
907 \\
-m .\end{array}$ & $\begin{array}{r}01350 \\
01080 \\
01040 \\
1000 \\
0980 \\
01050\end{array}$ & $\begin{array}{l}82100 \\
82200 \\
01900 \\
e 1600 \\
\theta 1520\end{array}$ & $\begin{array}{l}211 \\
203 \\
192 \\
172 \\
169 \\
154\end{array}$ & $\begin{array}{r}1210 \\
1220 \\
1260 \\
673 \\
544 \\
429\end{array}$ & $\begin{array}{l}864 \\
810 \\
770 \\
747 \\
-\end{array}$ \\
\hline $\begin{array}{l}\text { TOTAL } \\
\text { MEAN } \\
\text { MAX } \\
\text { AIN } \\
\text { AC-FT }\end{array}$ & $\begin{array}{r}30319 \\
978 \\
1740 \\
804 \\
60140\end{array}$ & $\begin{array}{r}31823 \\
1061 \\
1120 \\
975 \\
63120\end{array}$ & $\begin{array}{r}31755 \\
1024 \\
1120 \\
765 \\
62990\end{array}$ & $\begin{array}{r}21832 \\
704 \\
885 \\
498 \\
43300\end{array}$ & $\begin{array}{r}22615 \\
780 \\
897 \\
694 \\
44860\end{array}$ & $\begin{array}{r}21689 \\
700 \\
794 \\
477 \\
43020\end{array}$ & $\begin{array}{r}15948 \\
532 \\
1120 \\
265 \\
31630\end{array}$ & $\begin{array}{r}61911 \\
1997 \\
3550 \\
691 \\
122800\end{array}$ & $\begin{array}{r}79840 \\
2661 \\
3260 \\
1520 \\
158400\end{array}$ & $\begin{array}{r}18357 \\
592 \\
1500 \\
154 \\
36410\end{array}$ & $\begin{array}{r}12452 \\
402 \\
1260 \\
140 \\
24700\end{array}$ & $\begin{array}{r}21943 \\
731 \\
1640 \\
261 \\
43520\end{array}$ \\
\hline $\begin{array}{l}\text { STATIS } \\
\text { MEAN } \\
\text { MAAN } \\
\text { (WY) } \\
\text { MIN } \\
\text { (WY) }\end{array}$ & $\begin{array}{r}\text { CS OF } \\
1267 \\
8370 \\
1942 \\
247 \\
1957\end{array}$ & $\begin{array}{c}\text { MONTHLY MEAN } \\
1108 \\
3997 \\
1987 \\
365 \\
1935\end{array}$ & $\begin{array}{r}\text { DATA } \\
1073 \\
3420 \\
1966 \\
386 \\
1957\end{array}$ & $\begin{array}{c}\text { FOR WATER } \\
1082 \\
3169 \\
1966 \\
390 \\
1963\end{array}$ & $\begin{array}{c}\text { YEARS } 1935 \\
1233 \\
3314 \\
1987 \\
395 \\
1964\end{array}$ & $\begin{array}{c}-1996 \\
1649 \\
5099 \\
1993 \\
359 \\
1964\end{array}$ & $\begin{array}{l}Y \text { WATER } \\
3080 \\
9275 \\
1937 \\
274 \\
1977\end{array}$ & $\begin{array}{c}\text { YEAR (WY) } \\
4918 \\
19790 \\
1941 \\
268 \\
1977\end{array}$ & $\begin{array}{r}5365 \\
15540 \\
1941 \\
630 \\
1977\end{array}$ & $\begin{array}{r}2236 \\
8869 \\
1957 \\
185 \\
1963\end{array}$ & $\begin{array}{r}1300 \\
5171 \\
1957 \\
126 \\
1939\end{array}$ & $\begin{array}{l}118 \\
332 \\
193 \\
44 . \\
195\end{array}$ \\
\hline
\end{tabular}

SUMARX STATISTICS ALEUAL TOTAL

ANNUAL MEAN

ATGHEST ANNUAL MEAN

LOWEST ANTUAL MEAN

MIGHEST DAILY MEAN

LOWEST DAILY MEAN

ANNUAL SEVEN-DAY MINIMUM

INSTANTANEOUS PEAK ELOW

INSTANTANEOUS PEAK STAGE

INSTANTANEOUS LOW FLON

ANNUAJ RUNOFF (AC-ET)

10 PERCEN? EXCEEDS

50 EERCENT EXCEEDS

90 PERCENT EXCEEDS

- Estimated

a-Also occurred AuB. 26, 1939.
FOR 1995 CALENDAR YEAR 1010233
2768

$11700 \quad$ Jun 17
765
Dec 29

$\begin{array}{ll}814 & \text { Dec } 29 \\ & \text { Sep } 20\end{array}$

2004000

6910

1280
872
FOR 1996 WATER YEAR 370484

$$
\begin{array}{r}
3012 \\
1012
\end{array}
$$

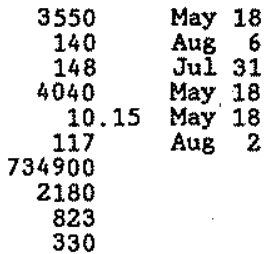

WATER YEARS 1935 - 1996

$$
\begin{array}{r}
2125 \\
5324 \\
702
\end{array}
$$$$
33300
$$$$
8.0
$$$$
\begin{array}{r}
13 \\
80000
\end{array}
$$$$
14.02 \text { Jun } 191995
$$$$
1540000
$$$$
5070
$$$$
\begin{aligned}
& 5070 \\
& 1150
\end{aligned}
$$ 
SAN JUAN RIVER BASIN

09368000 SAN JUAN RIVER AT ȘEIPROCK, NM - Continued

WATER-QUALITY RECORDS

PERIOD OF RECORD. -Water years $1941-45,1951$ to current year.

WATER QUALITY DATA, WATER YEAR OCTOBER 1995 TO SEPTEMBER 1996

\begin{tabular}{|c|c|c|c|c|c|c|c|c|c|c|c|}
\hline DATE & TIME & $\begin{array}{c}\text { DIS- } \\
\text { CEARGE, } \\
\text { INST, } \\
\text { CURIC } \\
\text { FEET } \\
\text { PER } \\
\text { SECOND } \\
(00061)\end{array}$ & $\begin{array}{l}\text { SPE- } \\
\text { CIFIC } \\
\text { CON- } \\
\text { DUCT- } \\
\text { ANCE } \\
\text { (US/CA) } \\
(00095)\end{array}$ & $\begin{array}{c}\text { PH } \\
\text { WATER } \\
\text { WHOLE } \\
\text { FIELD } \\
\text { (STAND- } \\
\text { ARD } \\
\text { UNITS) } \\
(00400)\end{array}$ & $\begin{array}{c}\text { TEMPER- } \\
\text { ATURE } \\
\text { AIR } \\
(D E G C) \\
(00020)\end{array}$ & $\begin{array}{c}\text { TEMPER- } \\
\text { ATURE } \\
\text { WATER } \\
\text { (DEG C) } \\
(00010)\end{array}$ & $\begin{array}{l}\text { BARO- } \\
\text { METRIC } \\
\text { PRES- } \\
\text { SURE } \\
\text { (MM } \\
\text { OF } \\
\text { HG) } \\
(00025)\end{array}$ & $\begin{array}{c}\text { OXYGEN, } \\
\text { DIS - } \\
\text { SOLVED } \\
\text { (MG/L) } \\
(00300)\end{array}$ & $\begin{array}{c}\text { OXYGEN, } \\
\text { DIS- } \\
\text { SOLVED } \\
\text { (PER- } \\
\text { CENT } \\
\text { SATUR- } \\
\text { ATION) } \\
\text { (00301) }\end{array}$ & $\begin{array}{l}\text { OXYGEN } \\
\text { DEMAND, } \\
\text { CHEM- } \\
\text { ICAL } \\
\text { (EIGH } \\
\text { LEVEL) } \\
(\text { MG/L) } \\
(00340)\end{array}$ & $\begin{array}{l}\text { COLI- } \\
\text { FORM, } \\
\text { FECAL, } \\
0.7 \\
\text { UMM-MF } \\
\text { (COLS.' } \\
100 \mathrm{ML} \text { ) } \\
(31625)\end{array}$ \\
\hline $\begin{array}{l}{ }_{04} 1995 \\
\text { DEC } \ldots\end{array}$ & 1450 & 1080 & 465 & -- & 18.0 & 14.0 & -- & -- & -- & $-\ldots$ & - \\
\hline JAN 1996 & 1200 & 1040 & 586 & 8.1 & 5.0 & 5.5 & 641 & 12.6 & 119 & 11 & $<14$ \\
\hline $\mathrm{ARR}^{17} \ldots$ & 1245 & 619 & 805 & 8.1 & 7.0 & 3.5 & 623 & 12.5 & 116 & $<10$ & $<7$ \\
\hline $\operatorname{SEP}^{03 \ldots}$ & 1130 & 394 & 604 & 8.3 & 15.0 & 13.0 & 635 & 9.8 & 112 & 20 & 20 \\
\hline $03 \ldots$ & 1230 & 368 & 553 & 8.3 & 26.0 & 20.5 & 640 & 7.9 & 105 & 14 & - \\
\hline
\end{tabular}

$\begin{array}{cccccc}\text { PER } & \text { AS } & \text { CACO3 } & \text { (MG/L } & \text { (MG/L } & \text { (MG/L } \\ 100 \mathrm{ML}) & \mathrm{CACO3}) & (\mathrm{MG} / \mathrm{L}) & \mathrm{AS} C A) & \mathrm{AS} M G) & \text { AS NA) }\end{array}$

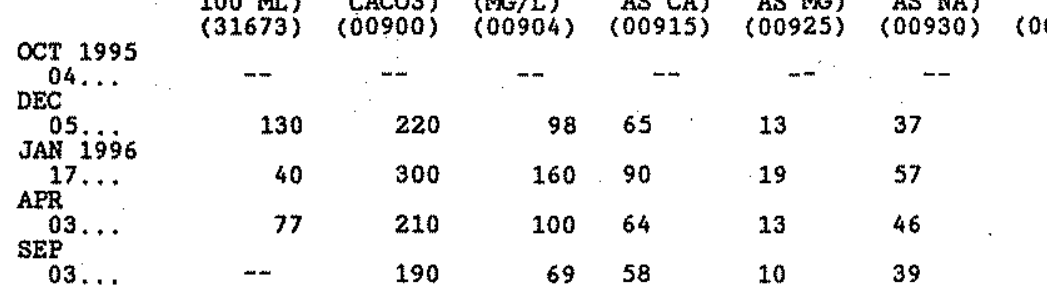

\begin{tabular}{|c|c|c|c|c|c|c|c|c|c|c|}
\hline $\begin{array}{c}\text { STREP- } \\
\text { TOCOCCI } \\
\text { FECAL } \\
\text { KF AGAR } \\
\text { (COLS. } \\
\text { PER } \\
100 \mathrm{ML}) \\
(31673)\end{array}$ & $\begin{array}{l}\text { HARD- } \\
\text { NESS } \\
\text { TOTAL } \\
\text { (MG/L } \\
\text { AS } \\
\text { CACO3) } \\
(00900)\end{array}$ & $\begin{array}{l}\text { BARD- } \\
\text { NESS } \\
\text { NONCARB } \\
\text { DISSOLV } \\
\text { FLD AS } \\
\text { CACO3 } \\
(M G / L) \\
(00904)\end{array}$ & $\begin{array}{l}\text { CALCIUM } \\
\text { DIS- } \\
\text { SOLVED } \\
(M G / L \\
\text { AS CA }) \\
\text { (00915) }\end{array}$ & $\begin{array}{c}\text { MAGNE- } \\
\text { SIUM, } \\
\text { DIS- } \\
\text { SOLVED } \\
\text { (MG/L } \\
\text { AS MG) } \\
(00925)\end{array}$ & $\begin{array}{l}\text { SODIUM, } \\
\text { DIS- } \\
\text { SOLVED } \\
\text { (MG/L } \\
\text { AS NA) } \\
(00930)\end{array}$ & $\begin{array}{c}\text { SODIUM } \\
\text { AD- } \\
\text { SORP- } \\
\text { TION } \\
\text { RATIO } \\
(00931)\end{array}$ & $\begin{array}{c}\text { POTAS- } \\
\text { SILM, } \\
\text { DIS- } \\
\text { SOLVED } \\
\text { (MG/L } \\
\text { AS X) } \\
(00935)\end{array}$ & $\begin{array}{c}\text { BICAR- } \\
\text { BONATE } \\
\text { WATER } \\
\text { DIS IT } \\
\text { FIELD } \\
\text { MG/L AS } \\
\text { HCO3 } \\
(00453)\end{array}$ & $\begin{array}{c}\text { CAR- } \\
\text { BONATE } \\
\text { WATER } \\
\text { DIS IT } \\
\text { FIELD } \\
\text { MG/L AS } \\
\text { CO3 } \\
(00452)\end{array}$ & $\begin{array}{c}\text { ALKA- } \\
\text { LINITY } \\
\text { WAT DIS } \\
\text { TOT IT } \\
\text { FIELD } \\
\text { MG/L AS } \\
\text { CACO3 } \\
(39086)\end{array}$ \\
\hline
\end{tabular}

\begin{tabular}{|c|c|c|c|c|}
\hline $\begin{array}{c}\text { ALKA- } \\
\text { LINITY } \\
\text { IAB } \\
\text { (MG/1 } \\
\text { AS } \\
\text { CACO3) } \\
(90410)\end{array}$ & $\begin{array}{c}\text { SULFATE } \\
\text { DIS- } \\
\text { SOLVED } \\
\text { (MG/L } \\
\text { AS SO4) } \\
\text { (00945) }\end{array}$ & $\begin{array}{l}\text { CHLO- } \\
\text { RIDE, } \\
\text { DIS- } \\
\text { SOLVED } \\
\text { (MG/L } \\
\text { AS CL) } \\
(00940)\end{array}$ & $\begin{array}{l}\text { FLUO- } \\
\text { RIDE, } \\
\text { DIS- } \\
\text { SOLVED } \\
\text { (MG/L } \\
\text { AS F) } \\
(00950)\end{array}$ & $\begin{array}{c}\text { SILICA, } \\
\text { DIS- } \\
\text { SOL.VED } \\
\text { (MG/L } \\
\text { AS } \\
\text { SIO2) } \\
(00955)\end{array}$ \\
\hline
\end{tabular}

\begin{tabular}{|c|c|c|c|c|c|}
\hline , & SO & $\begin{array}{l}\mathrm{SO} \\
\mathrm{SO} \\
\mathrm{AS} \\
00\end{array}$ & 3 & LA & $\begin{array}{l}T \\
\text { A } \\
0\end{array}$ \\
\hline
\end{tabular}

OCT 1995
04
DEC

$(90410)(00945) \quad(00940)$

1
1
1
1
1

NITRO-
GEN,
DTRATE
DIS-
SOLVED
(MG/L
AS N)
OO618)

$\begin{array}{llll}-- & - & -- & - \\ 2.3 & 144 & 0 & 118 \\ 2.6 & 175 & 2 & 148 \\ 2.4 & 137 & 0 & 112 \\ 2.4 & 143 & 0 & 117\end{array}$

\begin{tabular}{|c|c|c|c|c|c|c|c|c|c|c|c|}
\hline $04 \ldots$ & -- & $m-$ & $m$ & -- & -- & -- & -- & -- & -+ & $-\infty$ & -- \\
\hline 05. & 12.5 & 150 & 12 & 0.30 & 7.5 & 359 & 0.180 & 0.010 & 0.190 & $<0.015$ & 0.30 \\
\hline$\frac{17 \ldots}{A P R}$ & 151 & 240 & 20 & 0.30 & 7.3 & 526 & 0.400 & 0.010 & 0.410 & $<0.015$ & 0.20 \\
\hline SEP & 115 & 170 & 14 & 0.30 & 3.5 & 381 & -- & $<0,010$ & 0.080 & $<0.015$ & 0.30 \\
\hline $03 \ldots$ & 121 & 140 & 11 & 0.30 & 10 & 342 & - & $<0.010$ & 0.180 & $<0.015$ & 0.40 \\
\hline
\end{tabular}

\begin{tabular}{|c|c|c|c|c|c|c|c|c|c|c|c|}
\hline DATE & $\begin{array}{l}\text { NITRO- } \\
\text { GEN, AM- } \\
\text { MONIA+ } \\
\text { ORGANIC } \\
\text { DIS. } \\
\text { (MG/L } \\
\text { AS N) } \\
(00623)\end{array}$ & $\begin{array}{c}\text { PHOS- } \\
\text { PHORUS } \\
\text { TOTAL } \\
\text { (MG/L } \\
\text { AS P) } \\
(00665)\end{array}$ & $\begin{array}{c}\text { PHOS- } \\
\text { PHORUS } \\
\text { DIS- } \\
\text { SOLVED } \\
\text { (MG/L } \\
\text { AS P) } \\
(00666)\end{array}$ & $\begin{array}{l}\text { PHOS- } \\
\text { PHORUS } \\
\text { ORTHO, } \\
\text { DIS- } \\
\text { SOLVED } \\
\text { (MG/L } \\
\text { AS P) } \\
(00671)\end{array}$ & $\begin{array}{l}\text { CARBON, } \\
\text { ORGANIC } \\
\text { TOTAL } \\
\text { (NG/L } \\
\text { AS C) } \\
(00680)\end{array}$ & $\begin{array}{l}\text { ALLU- } \\
\text { INLM, } \\
\text { DIS- } \\
\text { SOLVED } \\
\text { (UG/L } \\
\text { AS AL) } \\
\text { (01106) }\end{array}$ & $\begin{array}{l}\text { ANTI- } \\
\text { MONY, } \\
\text { DIS- } \\
\text { SOLVED } \\
\text { (UG/L } \\
\text { AS SB) } \\
(01095)\end{array}$ & $\begin{array}{c}\text { ARSENIC } \\
\text { DIS - } \\
\text { SOLVED } \\
\text { (UG/L } \\
\text { AS AS) } \\
(01000)\end{array}$ & $\begin{array}{l}\text { BARIUM, } \\
\text { DIS- } \\
\text { SOLVED } \\
\text { (UG/L } \\
\text { AS BA) } \\
(01005)\end{array}$ & $\begin{array}{l}\text { BERYL- } \\
\text { LIUM, } \\
\text { DIS- } \\
\text { SOLVED } \\
\text { (UG/L } \\
\text { AS BE) } \\
(01010)\end{array}$ & $\begin{array}{c}\text { BORON, } \\
\text { DIS- } \\
\text { SOLVED } \\
\text { (UG/L. } \\
\text { AS B) } \\
\text { (01020) }\end{array}$ \\
\hline $\begin{array}{c}\text { OCT } 1995 \\
04 \ldots \\
D E C\end{array}$ & - & $-\cdots$ & - & $m$ & -- & $-\cdots$ & - & - & -- & $\cdots$ & $m$ \\
\hline JAN $1 \dot{9} 96$ & 0.20 & 0.030 & $<0.010$ & $<0.010$ & 3.8 & 16 & $<1.0$ & $<1$ & 69 & $<1.0$ & 50 \\
\hline${ }_{\text {APR }}^{17} \cdots$ & $<0.20$ & 0.020 & $<0.010$ & $<0.010$ & 3.9 & -- & -- & -- & -- & -- & 80 \\
\hline${ }_{\operatorname{SEF}}^{03} \ldots$ & 0.30 & 0.020 & $<0.010$ & $<0.010$ & 4.1. & - & -- & -- & $m$ & $-m$ & 50 \\
\hline $03 \ldots$ & 0.20 & 0.100 & 0.020 & 0.020 & 5.6 & 6.0 & $<1.0$ & 1 & 76 & $<1.0$ & 55 \\
\hline
\end{tabular}


09368000 SAN JUAN RIVER AT SHIPROCK, MM -- Continued

WATER-QUALITY RECORDS

WATER QUALITY DATA, WATER YEAR OCTOBER 1995 TO SEPTEMBER 1996

\begin{tabular}{|c|c|c|c|c|c|c|c|c|c|c|c|}
\hline DATE & $\begin{array}{c}\text { CADMIUUA } \\
\text { DIS - } \\
\text { SOLVED } \\
\text { (UG/L) } \\
\text { AS CD } \\
(01025)\end{array}$ & $\begin{array}{l}\text { CGRO- } \\
\text { MIUM, } \\
\text { DIS- } \\
\text { SOLVED } \\
\text { (UG/L } \\
\text { AS CR) } \\
\text { (01030) }\end{array}$ & $\begin{array}{c}\text { COBALT, } \\
\text { DIS - } \\
\text { SOLVED } \\
\text { (UG/L) } \\
\text { AS CO) } \\
\text { (01035) }\end{array}$ & $\begin{array}{l}\text { COPPER, } \\
\text { DIS- } \\
\text { SOLVED } \\
\text { (UG/L } \\
\text { AS CU) } \\
(01040)\end{array}$ & $\begin{array}{l}\text { IRON, } \\
\text { DIS- } \\
\text { SOLVED } \\
\text { (UG/E } \\
\text { AS FE) } \\
(01046)\end{array}$ & $\begin{array}{l}\text { LEAD, } \\
\text { DIS } \\
\text { SOLVED } \\
\text { (UG/L } \\
\text { AS PB }) \\
(01049)\end{array}$ & $\begin{array}{l}\text { MANGA- } \\
\text { NESE, } \\
\text { DIS - } \\
\text { SOLVED } \\
\text { (UE/L } \\
\text { AS MAH) } \\
(01056)\end{array}$ & $\begin{array}{l}\text { MERCURY } \\
\text { TOTAL } \\
\text { RECOV- } \\
\text { MRABLE } \\
\text { (UE/L } \\
\text { AS ERG) } \\
\text { (71900) }\end{array}$ & $\begin{array}{l}\text { MOLYB- } \\
\text { DENUA, } \\
\text { DIS } \\
\text { SOLYED } \\
\text { (UC/A } \\
\text { AS POO) } \\
\text { (01060) }\end{array}$ & $\begin{array}{l}\text { NICXELL, } \\
\text { DIS- } \\
\text { SOLVED } \\
\text { (UE/L } \\
\text { AS HI) } \\
\text { (01065) }\end{array}$ & $\begin{array}{c}\text { SELE- } \\
\text { NIUM, } \\
\text { TOTAL } \\
\text { TUG/ } \\
\text { AS SE) } \\
\text { A1147) }\end{array}$ \\
\hline
\end{tabular}

\begin{tabular}{|c|c|c|c|c|c|c|c|c|c|c|c|}
\hline${ }_{04}^{O C T} 1995$ & - & - & $\cdots$ & - & $-\cdots$ & -- & - & - & - & - & $\rightarrow$ \\
\hline ES & $<1.0$ & $<1.0$ & $<1.0$ & 3.0 & 17 & $<1.0$ & 26 & $<0.10$ & 1.0 & 3.0 & $<1$ \\
\hline$\frac{17 .}{17}$ & - & -- & -- & -- & 8.0 & - & $-\infty$ & -- & -- & $-\cdots$ & -- \\
\hline${ }_{\operatorname{sep}}^{03} \ldots$ & -- & -- & -- & $\because-$ & 16 & $m$ & - & -- & $m$ & -- & -- \\
\hline $03 \ldots$ & $<1.0$ & $<1.0$ & $<1.0$ & 2.0 & $<3.0$ & $<1.0$ & 4.0 & $<0.10$ & 2.0 & 2.0 & $<1$ \\
\hline
\end{tabular}

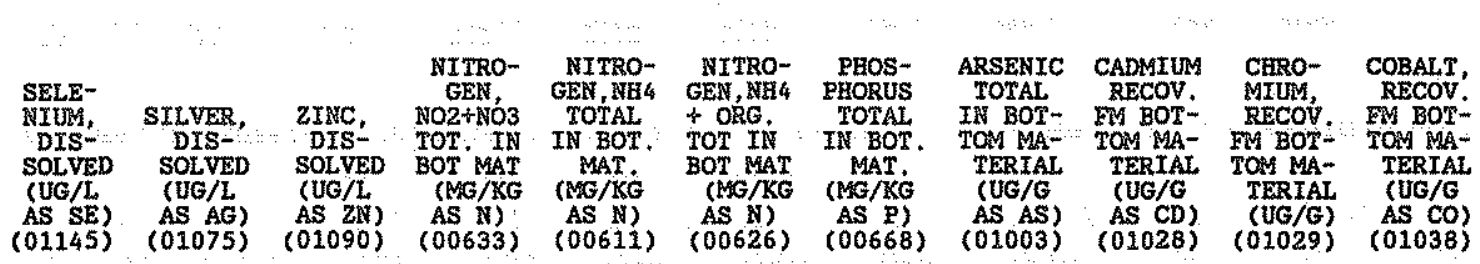

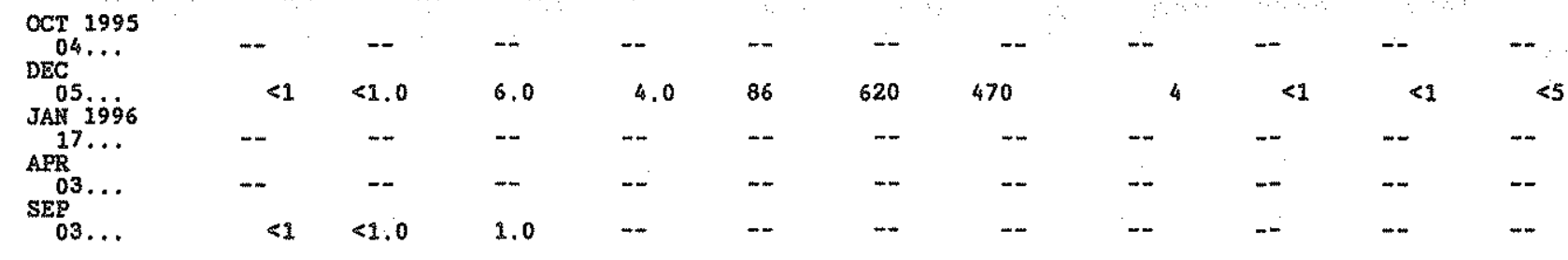

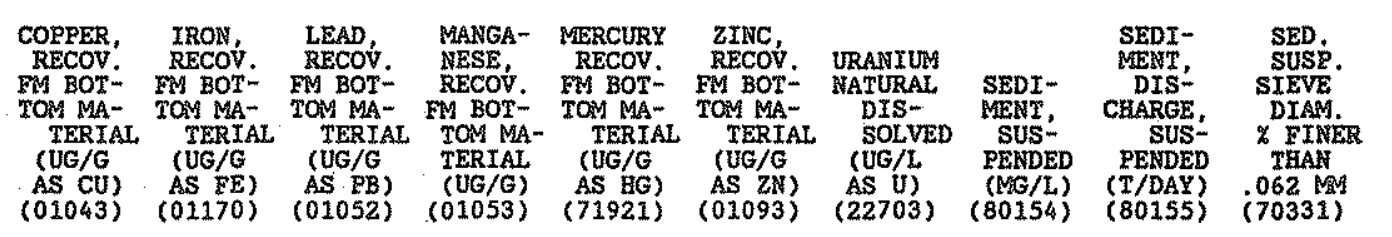

\begin{tabular}{|c|c|c|c|c|c|c|c|c|c|c|}
\hline $\begin{array}{c}\text { OCT } 1995 \\
04 \ldots\end{array}$ & -- & - & -- & - & -- & $\cdots$ & $-\cdots$ & - & -- & -- \\
\hline${ }_{\text {JAN }}^{05} \mathrm{ig} 96$ & 4 & 630 & $<10$ & 170 & 0.01 & 10 & 2.0 & 33 & 92 & 80 \\
\hline $17 \ldots$ & -- & -- & $m$ & - & -- & -- & - & 54 & 90 & 82 \\
\hline${ }_{\text {SEP }}^{03} \ldots$ & $-\cdots$ & -- & -- & -- & -- & $-\infty$ & -- & 26 & 28 & 76 \\
\hline 03. & -- & -- & -- & -- & $-\infty$ & -- & 2.0 & 340 & 346 & 98 \\
\hline
\end{tabular}


SAN JUAN RIVER BASIN

09368000 SAN JUAN RIVER AT SAIPROCK, FM - - Continued

WATER-QUALITY RECORDS

CHEMICAL ANALYSES OF ORGANIC COMPOUNDS, WATER YEAR OCTOBER 1995 TO SEPTEMBER 1996

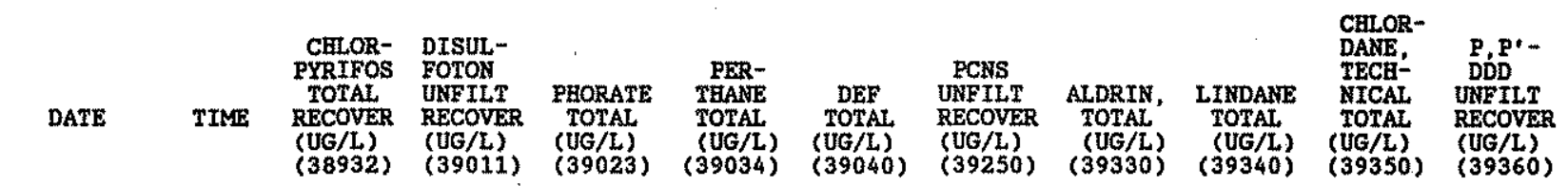

OCT 1995

$04 \ldots$

DEC

JAN 1996

1450

(39250)

(39350)

39360)

AFR ...

03.

$\mathrm{SEP}_{03} \ldots$

1200

$\begin{array}{ll}- & -- \\ -- & --\end{array}$

$-$

$-\infty$

1245

- -

1130

1230

$<0.010$

$<0.010$

$<0.010$

$<0.100$

$<0.010$

$<0.100$

$<0.010$

$<0.010$

$<0.100$

$<0.010$

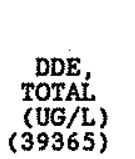

$\begin{array}{ll}\text { P, P'- } & \\ \text { DDT } \\ \text { UNFILT } \\ \text { RECOVER } \\ \text { RLDRIN } \\ \text { TOTAL }\end{array}$

ENDO-
SULEAN,
I
TOTAL
(UG/L)
$(39388)$

ENDRIN
WATER
UNFLTRD
REC
(UG/L)
(39390)

$\begin{array}{cc}\text { TOX- } \\ \text { ETHION, } & \text { APHENE, } \\ \text { TOTAL: } & \text { TOTAL, } \\ \text { (UG/L) } & \text { (UG/L) } \\ (39398) & (39400)\end{array}$

HEPTA-
CHLOR,
TOTAL,
(UG/L)
(39410)

HEPTA-
CHLOR
EPOXIDE
TOTAL
(UG/L)
$(39420)$

METH-

OXY-

TOTAL, TOTÁ

$\begin{array}{ll}(\mathrm{UG} / \mathrm{L}) & (\mathrm{UG} / \mathrm{L}) \\ (39370) & (39380)\end{array}$

..

OCt 1995

DEC

JAN 1996

JAR 1996

APR

SEP.

$03 . .$.

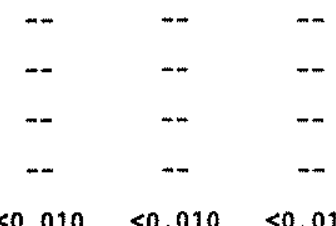

$-$
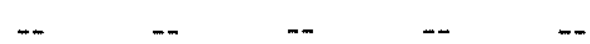

$-$

$--$

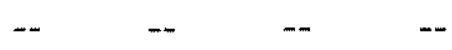

$-$

$-\infty$

$-$

--

$<0.010$

$<0.010<0.010$

$<0.010$

$<0.010<0.010<1.00$

$<0.010$

$-\infty$

$<0.010$

$<0.100$

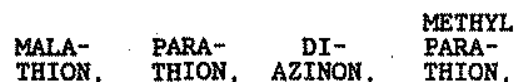

TOTAL, TOTAL, TOTAL, TOTAL

TUL, TOTAL,

(39530)

$\begin{array}{rr}(\mathrm{UG} / \mathrm{L}) & (\mathrm{UG} / \mathrm{L}) \\ (39540) & (39570)\end{array}$

(UG/L)

$(39600)$

2,4-D, 2,4,5-T : MrREX, SILVEX,
TOTAL
(UG /L)

$\begin{array}{cccc}(\mathrm{UG} / \mathrm{L}) & (\mathrm{UG} / \mathrm{L}) & (\mathrm{UG} / \mathrm{L}) & (\mathrm{UG} / \mathrm{L}) \\ (39730) & (39740) & (39755) & (39760)\end{array}$

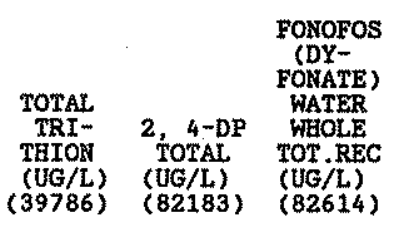

OCT 1995

$\mathrm{DEC}^{04}$

JAN 1996

JAN 1996

APR

SER

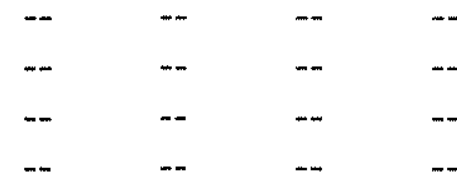

$\begin{array}{ccc}-- & -- & - \\ -- & -- & - \\ -- & -- & - \\ <0.010 & <0.010 & -\end{array}$

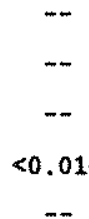

$-$

$03 \ldots$

$<0.010$

$<0,010<0.010<0.010$

--

--

$<0.010$

$-$

-.

$<0.010$ 
SAN JUAN RIVER BASIN

09371010 SAN JUAN RIVER AT FOUR CORNERS, CO

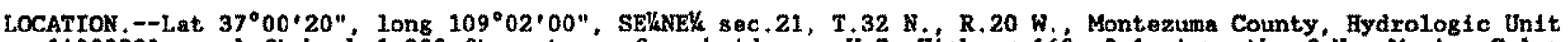
14080201 , on left bank 1,300 ft upstream from bridge on U.S. Bighway 160, 0.1 mi north of New Moxico-Colorado State line, $1.0 \mathrm{mi}$ east of Four Corners Monument, $3.0 \mathrm{mi}$ downstrean from Mancos River, and at mile 187.2 .

DRAINAGE AREA. $-14,600 \mathrm{mi} 2$, approximately.

WATER-DISCEARGE RECORDS

PERIOD OF RECORD, -October 1977 to current year.

GAGE.-Wator-gtage recordor. Elovation of gage is 4,900 ft above Narionel Gooderic Vortical Darum of 1929 , Erom topographic map.

RFMARKS.-Water-discharge records good except for estimated daily discharges, which are poor. Flow partly regulated by Navajo Reservoir (09355100). DISCEARGE, CUBIC FEET PER SECOND, WATER YEAR OCTOBER 1995 TO SEPTEMBER 1996

\begin{tabular}{|c|c|c|c|c|c|c|c|c|c|c|c|c|}
\hline DAY & OCT & WoV & DEC & JAN & FEB & MAR & APR & MAY & JUW & JUL. & AUG & SEP \\
\hline $\begin{array}{l}1 \\
2 \\
3 \\
4 \\
5\end{array}$ & $\begin{array}{l}1310 \\
1340 \\
1250 \\
1250 \\
1180\end{array}$ & $\begin{array}{l}1110 \\
1100 \\
1070 \\
1070 \\
1070\end{array}$ & $\begin{array}{l}1170 \\
1140 \\
1120 \\
1120 \\
1110\end{array}$ & $\begin{array}{l}879 \\
877 \\
856 \\
832 \\
846\end{array}$ & $\begin{array}{l}964 \\
991 \\
923 \\
846 \\
828\end{array}$ & $\begin{array}{r}780 \\
841 \\
853 \\
872 \\
886\end{array}$ & $\begin{array}{l}579 \\
608 \\
556 \\
523 \\
535\end{array}$ & $\begin{array}{r}858 \\
e 840 \\
0950 \\
\mathrm{e} 1150 \\
\mathrm{e} 1500\end{array}$ & $\begin{array}{l}2550 \\
2780 \\
3000 \\
3100 \\
3400\end{array}$ & $\begin{array}{r}1530 \\
1540 \\
1190 \\
1070 \\
971\end{array}$ & $\begin{array}{r}\$ 220 \\
+220 \\
+210 \\
+210 \\
-220\end{array}$ & $\begin{array}{l}6510 \\
6470 \\
6430 \\
6410 \\
e 360\end{array}$ \\
\hline $\begin{array}{r}6 \\
7 \\
8 \\
9 \\
10\end{array}$ & $\begin{array}{r}1230 \\
1170 \\
1120 \\
1100 \\
972\end{array}$ & $\begin{array}{l}1070 \\
1140 \\
1120 \\
1130 \\
1140\end{array}$ & $\begin{array}{l}1100 \\
1170 \\
1130 \\
1120 \\
1110\end{array}$ & $\begin{array}{l}927 \\
926 \\
911 \\
896 \\
898\end{array}$ & $\begin{array}{l}837 \\
847 \\
842 \\
895 \\
912\end{array}$ & $\begin{array}{l}844 \\
862 \\
843 \\
804 \\
796\end{array}$ & $\begin{array}{l}564 \\
551 \\
525 \\
569 \\
532\end{array}$ & $\begin{array}{r}01780 \\
01900 \\
02200 \\
02160 \\
02300\end{array}$ & $\begin{array}{r}3440 \\
3380 \\
3440 \\
3420 \\
3290\end{array}$ & $\begin{array}{l}894 \\
824 \\
756 \\
691 \\
607\end{array}$ & $\begin{array}{l}0200 \\
0220 \\
0260 \\
0340 \\
0400\end{array}$ & $\begin{array}{l}0430 \\
6530 \\
0510 \\
6450 \\
6400\end{array}$ \\
\hline $\begin{array}{l}11 \\
12 \\
13 \\
14 \\
15\end{array}$ & $\begin{array}{r}1020 \\
1000 \\
957 \\
898 \\
938\end{array}$ & $\begin{array}{l}1150 \\
1180 \\
1140 \\
1140 \\
1130\end{array}$ & $\begin{array}{l}1100 \\
1120 \\
1090 \\
1110 \\
1140\end{array}$ & $\begin{array}{l}869 \\
690 \\
630 \\
632 \\
636\end{array}$ & $\begin{array}{l}924 \\
915 \\
905 \\
898 \\
893\end{array}$ & $\begin{array}{l}796 \\
814 \\
761 \\
753 \\
805\end{array}$ & $\begin{array}{l}579 \\
908 \\
819 \\
798 \\
752\end{array}$ & $\begin{array}{l}\odot 2200 \\
\bullet 2150 \\
e 2700 \\
-3100 \\
-2910\end{array}$ & $\begin{array}{l}3160 \\
3110 \\
3040 \\
2910 \\
3150\end{array}$ & $\begin{array}{l}580 \\
689 \\
641 \\
579 \\
549\end{array}$ & $\begin{array}{l}e 390 \\
e 370 \\
e 320 \\
0260 \\
0190\end{array}$ & $\begin{array}{r}9380 \\
0450 \\
0700 \\
1040 \\
1580\end{array}$ \\
\hline $\begin{array}{l}16 \\
17 \\
18 \\
19 \\
20\end{array}$ & $\begin{array}{r}1030 \\
1010 \\
953 \\
951 \\
951\end{array}$ & $\begin{array}{l}1140 \\
1140 \\
1110 \\
1170 \\
1180\end{array}$ & $\begin{array}{l}1140 \\
1100 \\
1100 \\
1100 \\
1130\end{array}$ & $\begin{array}{l}637 \\
631 \\
689 \\
688 \\
656\end{array}$ & $\begin{array}{l}898 \\
905 \\
898 \\
885 \\
925\end{array}$ & $\begin{array}{l}801 \\
794 \\
813 \\
789 \\
753\end{array}$ & $\begin{array}{l}648 \\
556 \\
501 \\
456 \\
406\end{array}$ & $\begin{array}{l}3080 \\
3290 \\
3540 \\
3480 \\
3280\end{array}$ & $\begin{array}{l}3330 \\
3070 \\
2930 \\
2830 \\
2770\end{array}$ & $\begin{array}{r}507 \\
1040 \\
997 \\
1070 \\
965\end{array}$ & $\begin{array}{l}0190 \\
0180 \\
0190 \\
0190 \\
0190\end{array}$ & $\begin{array}{l}1790 \\
1390 \\
1310 \\
1420 \\
1550\end{array}$ \\
\hline $\begin{array}{l}21 \\
22 \\
23 \\
24 \\
25\end{array}$ & $\begin{array}{r}946 \\
974 \\
1050 \\
1140 \\
1160\end{array}$ & $\begin{array}{l}1180 \\
1170 \\
1130 \\
1150 \\
1150\end{array}$ & $\begin{array}{l}1170 \\
1120 \\
1100 \\
1090 \\
1100\end{array}$ & $\begin{array}{l}615 \\
607 \\
576 \\
611 \\
667\end{array}$ & $\begin{array}{l}839 \\
863 \\
880 \\
869 \\
837\end{array}$ & $\begin{array}{l}725 \\
743 \\
723 \\
690 \\
700\end{array}$ & $\begin{array}{l}e 390 \\
6350 \\
0330 \\
\theta 330 \\
0310\end{array}$ & $\begin{array}{l}3220 \\
2770 \\
2520 \\
2260 \\
1880\end{array}$ & $\begin{array}{l}2770 \\
2920 \\
3220 \\
3250 \\
2790\end{array}$ & $\begin{array}{r}856 \\
719 \\
557 \\
431 \\
e 350\end{array}$ & $\begin{array}{r}0200 \\
0220 \\
0650 \\
01070 \\
1100\end{array}$ & $\begin{array}{l}1360 \\
1240 \\
1170 \\
1120 \\
1020\end{array}$ \\
\hline $\begin{array}{l}26 \\
27 \\
28 \\
29 \\
30 \\
31\end{array}$ & $\begin{array}{l}1120 \\
1200 \\
1160 \\
1140 \\
1120 \\
1110\end{array}$ & $\begin{array}{l}1160 \\
1160 \\
1190 \\
1170 \\
1160 \\
=-\end{array}$ & $\begin{array}{r}1100 \\
1110 \\
955 \\
856 \\
823 \\
839\end{array}$ & $\begin{array}{r}675 \\
956 \\
1070 \\
1020 \\
929 \\
952\end{array}$ & $\begin{array}{l}771 \\
789 \\
788 \\
785 \\
\ldots \ldots \\
-\ldots . .\end{array}$ & $\begin{array}{l}700 \\
685 \\
646 \\
604 \\
609 \\
627\end{array}$ & $\begin{array}{l}0360 \\
0680 \\
1080 \\
1250 \\
1140 \\
-\end{array}$ & $\begin{array}{l}1500 \\
1220 \\
1150 \\
1080 \\
1530 \\
2030\end{array}$ & $\begin{array}{l}2250 \\
2390 \\
2410 \\
1880 \\
1610 \\
-\end{array}$ & $\begin{array}{l}0300 \\
0270 \\
e 250 \\
e 240 \\
0230 \\
0230\end{array}$ & $\begin{array}{l}1360 \\
1230 \\
1400 \\
1260 \\
1020 \\
\text { e750 }\end{array}$ & $\begin{array}{r}0950 \\
01030 \\
0980 \\
0910 \\
0850 \\
-\end{array}$ \\
\hline $\begin{array}{l}\text { MOTAL } \\
\text { MEAN } \\
\text { MAX } \\
\text { MIN } \\
\text { AC-FT }\end{array}$ & $\begin{array}{r}33750 \\
1089 \\
1340 \\
898 \\
66940\end{array}$ & $\begin{array}{r}34120 \\
1137 \\
1190 \\
1070 \\
67680\end{array}$ & $\begin{array}{r}33683 \\
1087 \\
1170 \\
823 \\
66810\end{array}$ & $\begin{array}{r}24284 \\
783 \\
1070 \\
576 \\
48170\end{array}$ & $\begin{array}{r}25352 \\
874 \\
991 \\
771 \\
50290\end{array}$ & $\begin{array}{r}2.3712 \\
765 \\
886 \\
604 \\
47030\end{array}$ & $\begin{array}{r}18185 \\
606 \\
1250 \\
310 \\
36070\end{array}$ & $\begin{array}{r}66528 \\
2146 \\
3540 \\
840 \\
132000\end{array}$ & $\begin{array}{r}87590 \\
2920 \\
3440 \\
1610 \\
173700\end{array}$ & $\begin{array}{r}22123 \\
714 \\
1540 \\
230 \\
43880\end{array}$ & $\begin{array}{r}15230 \\
491 \\
1400 \\
180 \\
30210\end{array}$ & $\begin{array}{r}26740 \\
891 \\
1790 \\
360 \\
53040\end{array}$ \\
\hline
\end{tabular}

STATISTICS OF MONTELY MEAN DATA FOR WATER YEARS 1978 - 1996, BY WATER YEAR (WY)

\begin{tabular}{|c|c|c|c|c|c|c|c|c|c|c|c|c|}
\hline $\begin{array}{l}\text { MEAN } \\
\text { MAY } \\
\text { (WY) } \\
\text { MIN } \\
(W Y)\end{array}$ & $\begin{array}{r}1273 \\
2959 \\
1987 \\
634 \\
1978\end{array}$ & $\begin{array}{r}1418 \\
3732 \\
1987 \\
838 \\
1980\end{array}$ & $\begin{array}{r}1462 \\
3466 \\
1987 \\
799 \\
1990\end{array}$ & $\begin{array}{r}1547 \\
3300 \\
1987 \\
760 \\
1990\end{array}$ & $\begin{array}{r}1666 \\
3365 \\
1987 \\
739 \\
1990\end{array}$ & $\begin{array}{r}2187 \\
5454 \\
1993 \\
707 \\
1990\end{array}$ & $\begin{array}{r}3146 \\
7893 \\
1979 \\
606 \\
1996\end{array}$ & $\begin{array}{r}4730 \\
10220 \\
1979 \\
1030 \\
1981\end{array}$ & $\begin{array}{r}5314 \\
10370 \\
1979 \\
1236 \\
1989\end{array}$ & $\begin{array}{r}2520 \\
5846 \\
1979 \\
714 \\
1996\end{array}$ & $\begin{array}{r}1361 \\
3016 \\
1986 \\
259 \\
1978\end{array}$ & $\begin{array}{r}1328 \\
3243 \\
1986 \\
467 \\
1989\end{array}$ \\
\hline
\end{tabular}

SUMAARY STATISTTCS

AMNUAL, TOTAL.

ANNUAL MEAN

HIGHEST ANNUAL MEAR

IOWEST ANNUAL MEAN

HIGEEST DAILY MEAN

LOWEST DAILY MEAN

ANHUAL SEVEN-DAY MINIMUM

INSTANTANEOUS PEAK FLOW

INSTANTANEOUS PEAK STAGE

INSTANTAREOUS LOW FLOW

ANNUAL RUNOFF (AC-FT)

10 PERCENT EXCEEDS

50 PERCEN' EXCEEDS

90 PERCENT EXCEEDS
FOR 1995 CALENDAR YEAR

$\begin{array}{rr}1065148 & \\ 2918 & \\ & \\ 12100 & \text { Jun } 19 \\ 816 & \text { Jan } 25 \\ 859 & \text { Jan } 19 \\ & \\ & \\ 2113000 & \\ 7200 & \\ 1340 & \\ 937 & \end{array}$

FOR 1996 WATER YEAR

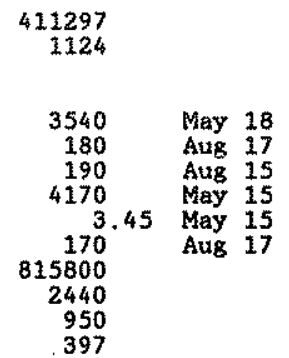

WATER YEARS 1978 - 1996

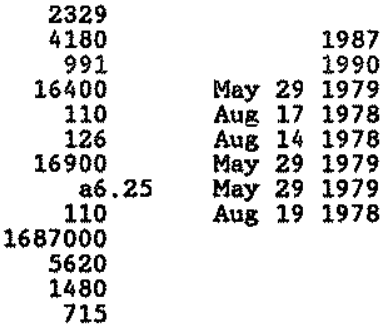

- Estimated

a-Maximum 8 age height, $14.43 \mathrm{ft}$, Dec, 12, 1978 (backwater from 1ce). 
SAN JUAN RIVER BASIN

09371010 SAN JUAN RIVER AT FOUR CORNERS, CO -- Continued

WATER-QUALITY RECORDS

PERIOD OF RECORD.-Water years 1978-81, 1985 to current year.

WATER QUALITY DATA, WATER YEAR OCTOBER 1995 TO SEPTEMBER 1996

\begin{tabular}{|c|c|c|c|c|c|c|c|c|c|}
\hline DATE & TIME & $\begin{array}{c}\text { DIS- } \\
\text { CBARGE, } \\
\text { INST: } \\
\text { CUBIC } \\
\text { FEET } \\
\text { PER } \\
\text { SECOND } \\
(00061)\end{array}$ & $\begin{array}{l}\text { SPE- } \\
\text { CIFIC } \\
\text { CON- } \\
\text { DUCT- } \\
\text { ANCE } \\
\text { (US/CM) } \\
(00095)\end{array}$ & $\begin{array}{c}\text { PH } \\
\text { WATER } \\
\text { WHOLE } \\
\text { FIELD } \\
\text { (STAND- } \\
\text { ARD } \\
\text { UNITS) } \\
(00400)\end{array}$ & $\begin{array}{l}\text { TEMPER- } \\
\text { ATURE } \\
\text { AIR } \\
\text { (DEG C) } \\
(00020)\end{array}$ & $\begin{array}{l}\text { TEMPER- } \\
\text { ATURE } \\
\text { WATER } \\
\text { (DEG C) } \\
(00010)\end{array}$ & $\begin{array}{l}\text { TUR- } \\
\text { BID- } \\
\text { ITY } \\
\text { (NTU) } \\
(00076)\end{array}$ & $\begin{array}{l}\text { BARO- } \\
\text { METRIC } \\
\text { FRES- } \\
\text { SURE } \\
\text { (MM } \\
\text { OF } \\
\text { HG) } \\
(00025)\end{array}$ & $\begin{array}{c}\text { OXYGEN, } \\
\text { DIS- } \\
\text { SOLVED } \\
\text { (MG/L) } \\
(00300)\end{array}$ \\
\hline \multirow{4}{*}{$\begin{array}{c}\text { OCT } 1995 \\
04 \ldots \\
\text { DEC } \\
08 \\
\text { JAN } 1996 \\
19 . \ldots \\
\text { APR } \\
03 \ldots\end{array}$} & 0915 & 1250 & 453 & $-m$ & 18.0 & 12.5 & $-\infty$ & $m$ & -- \\
\hline & 0930 & 1140 & 630 & 8.4 & 2.0 & 4.0 & 9.0 & 646 & -- \\
\hline & $: 1000$ & 660 & 878 & 8.3 & 0.5 & 0.5 & 26 & 640 & 11.9 \\
\hline & 0900 & 560 & 675 & 8.0 & 13.5 & 12.0 & 8.0 & 642 & 8.9 \\
\hline DATE & $\begin{array}{c}\text { OXYGEN, } \\
\text { DIS- } \\
\text { SOLVED } \\
\text { (PER- } \\
\text { CENT } \\
\text { SATUR- } \\
\text { ATION) } \\
\text { (00301) }\end{array}$ & $\begin{array}{l}\text { HARD- } \\
\text { NESS } \\
\text { TOTAL } \\
\text { (MG/L } \\
\text { AS } \\
\text { CACO3) } \\
(00900)\end{array}$ & $\begin{array}{l}\text { HARD- } \\
\text { NESS } \\
\text { NONCARB } \\
\text { DISSOLV } \\
\text { FLD.AS } \\
\text { CACO3 } \\
(\text { MG/L) } \\
(00904)\end{array}$ & $\begin{array}{l}\text { CALCIUM } \\
\text { DIS - } \\
\text { SOLVED } \\
\text { (MG/L } \\
\text { AS CA) } \\
(00915)\end{array}$ & $\begin{array}{c}\text { MAGNE- } \\
\text { SIUM, } \\
\text { DIS- } \\
\text { SOLVED } \\
\text { (MG/L } \\
\text { AS MG) } \\
(00925)\end{array}$ & $\begin{array}{c}\text { SODIUM, } \\
\text { DIS- } \\
\text { SOLVED } \\
\text { (MG/L } \\
\text { AS NA) } \\
(00930)\end{array}$ & $\begin{array}{c}\text { SODIUM } \\
\text { AD- } \\
\text { SORP- } \\
\text { TION } \\
\text { RATIO } \\
(00931)\end{array}$ & $\begin{array}{c}\text { POTAS- } \\
\text { SIUM, } \\
\text { DIS- } \\
\text { SOLVED } \\
\text { (MG/L } \\
\text { AS K) } \\
(00935)\end{array}$ & $\begin{array}{l}\text { BICAR- } \\
\text { BONATE } \\
\text { WATER } \\
\text { DIS IT } \\
\text { FTELD } \\
\text { MG/L AS } \\
\text { HCO3 } \\
(00453)\end{array}$ \\
\hline \multirow{4}{*}{$\begin{array}{c}\text { OCT } 1995 \\
04 \ldots \\
\text { DEC } \\
08 \ldots \\
\text { JAN } 1996 \\
19 \ldots \\
\text { APR } \\
03 \ldots\end{array}$} & -- & -- & - & -- & - & -- & -- & -- & $-\infty$ \\
\hline & $-m$ & 230 & 110 & 66 & 15 & 39 & 1 & 2.4 & 136 \\
\hline & 99 & 330 & 180 & 96 & 21 & 66 & 2 & 2.6 & 180 \\
\hline & 98 & 230 & 120 & 67 & 16 & 50 & 1 & 2.6 & 137 \\
\hline DATE & $\begin{array}{c}\text { CAR- } \\
\text { BONATE } \\
\text { WATER } \\
\text { DIS IT } \\
\text { FIELD } \\
\text { MG/L AS } \\
\text { CO3 } \\
(00452)\end{array}$ & $\begin{array}{l}\text { ALKA- } \\
\text { LINITY } \\
\text { WAT DIS } \\
\text { TOT IT } \\
\text { FIELD } \\
\text { MG/L AS } \\
\text { CACO3 } \\
(39086)\end{array}$ & $\begin{array}{c}\text { ALKA- } \\
\text { LINITY } \\
\text { LAB } \\
(\mathrm{MG} / \mathrm{L} \\
\text { AS } \\
\text { CACO3) } \\
(90410)\end{array}$ & $\begin{array}{l}\text { SULFATE } \\
\text { DIS } \\
\text { SOLVED } \\
\text { (MG/L } \\
\text { AS SO4) } \\
(00945)\end{array}$ & $\begin{array}{l}\text { CHLO- } \\
\text { RIDE, } \\
\text { DIS- } \\
\text { SOLVED } \\
\text { (MG/L } \\
\text { AS CL) } \\
(00940)\end{array}$ & $\begin{array}{l}\text { FLUO- } \\
\text { RIDE, } \\
\text { DIS- } \\
\text { SOLVED } \\
\text { (MG/L } \\
\text { AS F) } \\
(00950)\end{array}$ & $\begin{array}{l}\text { SILICA, } \\
\text { DIS- } \\
\text { SOLVED } \\
\text { (MG/L } \\
\text { AS } \\
\text { SIO2) } \\
(00955)\end{array}$ & $\begin{array}{l}\text { SOL.TDS, } \\
\text { RESTDUE } \\
\text { AT } 180 \\
\text { DEG.C } \\
\text { DIS- } \\
\text { SOLVED } \\
\text { (MG/L) } \\
(70300)\end{array}$ & $\begin{array}{c}\text { SOLIDS, } \\
\text { SUM OF } \\
\text { CONSTI- } \\
\text { TUENTS, } \\
\text { DIS- } \\
\text { SOLVED } \\
\text { (MG/L) } \\
(70301)\end{array}$ \\
\hline $\begin{array}{l}\operatorname{OCT} 1995 \\
04 \ldots \\
\mathrm{DEC}^{\circ}\end{array}$ & -- & -- & - & $-m$ & -- & $-m$ & - & - & -- \\
\hline$\stackrel{08}{\text { JAN }} 19 \dot{9} 6$ & 5 & 119 & 125 & 170 & 12 & 0.30 & 7.1 & 400 & 384 \\
\hline $\operatorname{APR}^{19}, \ldots$ & 0 & 148 & 156 & 280 & 23 & 0.30 & 6.8 & 604 & 584 \\
\hline $03 \ldots$ & 0 & 112 & 116 & 210 & 13 & 0.30 & 3.7 & 432 & 430 \\
\hline
\end{tabular}




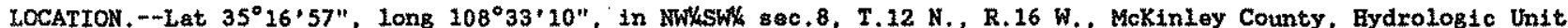
15020004 , on Zuni Indian Reservation, on left bank at mouth of Nutria Canyon, 0.9 mi upseream erom Nutria diversion dem, $1.3 \mathrm{mi}$ northeast of Upper Nutria, and $10.4 \mathrm{mi}$ northwest of Rameh.

DRATNAGE AREA. $--71.4 \mathrm{mi}^{2}$.

PERIOD OF RECORD. - - October 1969 to current year.

REVISED RECORDS. -WDR MM-78-1: 1977 .

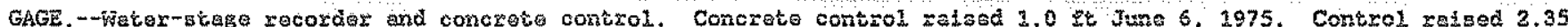
fit June 28, 2984 . Elavation of gage is 6,860 2t above Nationel Goodetfo Vertical Datum of ig29, from topographic map.

REMARKS, --Records fair except for ostimated deily discharges, which are poor. Soveral observationg of water temperature were mede during the year. No flow Oct. 1-20, 1969.

DISCHARGE, CUBIC FEET PER SECOND, WATER YEAR OCTOBER 1995 TO SEPTEMBER 1996

\begin{tabular}{|c|c|c|c|c|c|c|c|c|c|c|c|c|}
\hline DAX & $\cos$ & Nov & $\mathrm{DEC}$ & JAN & FEB & MAR & APR & MAY & JUN & JUL & AUU: & SEP \\
\hline $\begin{array}{l}1 \\
2 \\
3 \\
4 \\
5\end{array}$ & $\begin{array}{l}.20 \\
.18 \\
.15 \\
.14 \\
.13\end{array}$ & $\begin{array}{l}.28 \\
.36 \\
.33 \\
.39 \\
.41\end{array}$ & $\begin{array}{l}.22 \\
.22 \\
.22 \\
.22 \\
.22\end{array}$ & $\begin{array}{l}.18 \\
.14 \\
.12 \\
.14 \\
.14\end{array}$ & $\begin{array}{l}.17 \\
.19 \\
.14 \\
.12 \\
.14\end{array}$ & $\begin{array}{l}.21 \\
.20 \\
.19 \\
.23 \\
.29\end{array}$ & $\begin{array}{r}.36 \\
.36 \\
.31 \\
.36 \\
.36\end{array}$ & $\begin{array}{l}.59 \\
.60 \\
.73 \\
.69 \\
.68\end{array}$ & $\begin{array}{l}.45 \\
.41 \\
.36 \\
.34 \\
.31\end{array}$ & $\begin{array}{l}.29 \\
.14 \\
.12 \\
.11 \\
.10\end{array}$ & $\begin{array}{l}.09 \\
.07 \\
.07 \\
.06 \\
.06\end{array}$ & $\begin{array}{l}.10 \\
.10 \\
.09 \\
.68 \\
.42\end{array}$ \\
\hline $\begin{array}{r}6 \\
7 \\
8 \\
9 \\
10\end{array}$ & $\begin{array}{l}.15 \\
.15 \\
.15 \\
.15 \\
.15\end{array}$ & $\begin{array}{l}.36 \\
.36 \\
.31 \\
.31 \\
.31\end{array}$ & $\begin{array}{l}.21 \\
.22 \\
.22 \\
.22 \\
.22\end{array}$ & $\begin{array}{r}.13 \\
.12 \\
.12 \\
.13 \\
.14\end{array}$ & $\begin{array}{l}.15 \\
.16 \\
.13 \\
.14 \\
.13\end{array}$ & $\begin{array}{l}.31 \\
.26 \\
.22 \\
.18 \\
.18\end{array}$ & $\begin{array}{l}.31 \\
.36 \\
.36 \\
.36 \\
.43\end{array}$ & $\begin{array}{r}.72 \\
.73 \\
.74 \\
.75 \\
.80\end{array}$ & $\begin{array}{l}.27 \\
.25 \\
.24 \\
.20 \\
.18\end{array}$ & $\begin{array}{l}.08 \\
.09 \\
.10 \\
.23 \\
.18\end{array}$ & $\begin{array}{l}.05 \\
.05 \\
.06 \\
.06 \\
.06\end{array}$ & $\begin{array}{l}.13 \\
.12 \\
.10 \\
.10 \\
.10\end{array}$ \\
\hline $\begin{array}{l}11 \\
12 \\
13 \\
14 \\
15\end{array}$ & $\begin{array}{l}.15 \\
.15 \\
.15 \\
.15 \\
.15\end{array}$ & $\begin{array}{l}.31 \\
.30 \\
.28 \\
.31 \\
.29\end{array}$ & $\begin{array}{l}.22 \\
.18 \\
.18 \\
.26 \\
.27\end{array}$ & $\begin{array}{l}.14 \\
.14 \\
.12 \\
.12 \\
.12\end{array}$ & $\begin{array}{l}.13 \\
.14 \\
.16 \\
.16 \\
.15\end{array}$ & $\begin{array}{l}.19 \\
.20 \\
.19 \\
.20 \\
.26\end{array}$ & $\begin{array}{l}.51 \\
.43 \\
.51 \\
.51 \\
.31\end{array}$ & $\begin{array}{l}.80 \\
.85 \\
.91 \\
.90 \\
.95\end{array}$ & $\begin{array}{l}.16 \\
.14 \\
.13 \\
.15 \\
.23\end{array}$ & $\begin{array}{l}.17 \\
.14 \\
.14 \\
.12 \\
.13\end{array}$ & $\begin{array}{l}.05 \\
.05 \\
.05 \\
.04 \\
.04\end{array}$ & $\begin{array}{l}.11 \\
23^{.16} \\
42 \\
15\end{array}$ \\
\hline $\begin{array}{l}16 \\
17 \\
18 \\
19 \\
20\end{array}$ & $\begin{array}{r}.15 \\
.18 \\
.17 \\
.15 \\
.15\end{array}$ & $\begin{array}{l}.26 \\
.26 \\
.26 \\
.26 \\
.26\end{array}$ & $\begin{array}{l}.24 \\
.34 \\
.27 \\
.21 \\
.22\end{array}$ & $\begin{array}{r}.13 \\
0.14 \\
0.14 \\
0.16 \\
0.14\end{array}$ & $\begin{array}{l}.20 \\
.22 \\
.22 \\
.22 \\
.23\end{array}$ & $\begin{array}{l}.20 \\
.18 \\
.17 \\
.15 \\
.15\end{array}$ & $\begin{array}{l}.31 \\
.36 \\
.37 \\
.37 \\
.39\end{array}$ & $\begin{array}{l}1.1 \\
1.1 \\
1.1 \\
1.3 \\
1.3\end{array}$ & $\begin{array}{l}.18 \\
.12 \\
.10 \\
.09 \\
.08\end{array}$ & $\begin{array}{l}2.0 \\
2.0 \\
.20 \\
.14 \\
.12\end{array}$ & $\begin{array}{l}.04 \\
.04 \\
.03 \\
.03 \\
.04\end{array}$ & $\begin{array}{r}.85 \\
3.37 \\
10^{3.4} \\
1.0\end{array}$ \\
\hline $\begin{array}{l}21 \\
22 \\
23 \\
24 \\
25\end{array}$ & $\begin{array}{l}.16 \\
.18 \\
.18 \\
.18 \\
.19\end{array}$ & $\begin{array}{l}.26 \\
.26 \\
.23 \\
.22 \\
.22\end{array}$ & $\begin{array}{l}.21 \\
.18 \\
.13 \\
.17 \\
.15\end{array}$ & $\begin{array}{r}0.13 \\
0.13 \\
0.14 \\
.13 \\
.14\end{array}$ & $\begin{array}{l}.25 \\
.24 \\
.24 \\
.18 \\
.16\end{array}$ & $\begin{array}{l}.15 \\
.15 \\
.18 \\
.22 \\
.22\end{array}$ & $\begin{array}{l}.43 \\
.39 \\
.41 \\
.45 \\
.51\end{array}$ & $\begin{array}{l}1.2 \\
1.1 \\
.99 \\
.97 \\
.88\end{array}$ & $\begin{array}{l}.09 \\
.08 \\
.07 \\
.06 \\
.06\end{array}$ & $\begin{array}{l}.11 \\
.09 \\
.09 \\
.10 \\
.08\end{array}$ & $\begin{array}{l}14 \\
12 \\
45 \\
1.3 \\
1.4\end{array}$ & $\begin{array}{l}.37 \\
.24 \\
.20 \\
.18 \\
.16\end{array}$ \\
\hline $\begin{array}{l}26 \\
27 \\
28 \\
29 \\
30 \\
31\end{array}$ & $\begin{array}{l}.22 \\
.22 \\
.22 \\
.25 \\
.26 \\
.26\end{array}$ & $\begin{array}{l}.24 \\
.23 \\
.22 \\
.22 \\
.22 \\
-\end{array}$ & $\begin{array}{r}.14 \\
.13 \\
.13 \\
.15 \\
.17 \\
.17\end{array}$ & $\begin{array}{l}.16 \\
.13 \\
.16 \\
.16 \\
.16 \\
.17\end{array}$ & $\begin{array}{l}.17 \\
.21 \\
.21 \\
.22 \\
-\ldots \\
-.\end{array}$ & $\begin{array}{l}.26 \\
.22 \\
.22 \\
.31 \\
.36 \\
.36\end{array}$ & $\begin{array}{r}.53 \\
.59 \\
.55 \\
.52 \\
.59 \\
.\end{array}$ & $\begin{array}{l}.80 \\
.77 \\
.74 \\
.65 \\
.59 \\
.53\end{array}$ & $\begin{array}{l}.07 \\
.07 \\
.08 \\
.07 \\
.37 \\
-\end{array}$ & $\begin{array}{l}.08 \\
.09 \\
.09 \\
.09 \\
.09 \\
.09\end{array}$ & $\begin{array}{l}.61 \\
.32 \\
.32 \\
.21 \\
.14 \\
.11\end{array}$ & $\begin{array}{l}.24 \\
.20 \\
.17 \\
.15 \\
.14\end{array}$ \\
\hline $\begin{array}{l}\text { TOTAL } \\
\text { MEAN } \\
\text { MAX } \\
\text { MIN } \\
\text { AC-FT }\end{array}$ & $\begin{array}{r}5.42 \\
.17 \\
.26 \\
.13 \\
11\end{array}$ & $\begin{array}{r}8.53 \\
.28 \\
.41 \\
.22 \\
17\end{array}$ & $\begin{array}{r}6.31 \\
.20 \\
.34 \\
.13 \\
13\end{array}$ & $\begin{array}{r}4.32 \\
.14 \\
.18 \\
.12 \\
8.6\end{array}$ & $\begin{array}{r}5.18 \\
.18 \\
.25 \\
.12 \\
10\end{array}$ & $\begin{array}{r}6.81 \\
.22 \\
.36 \\
.15 \\
14\end{array}$ & $\begin{array}{r}12.61 \\
.42 \\
.59 \\
.31 \\
25\end{array}$ & $\begin{array}{r}26.56 \\
.86 \\
1.3 \\
.53 \\
53\end{array}$ & $\begin{array}{r}5.41 \\
.18 \\
.45 \\
.06 \\
11\end{array}$ & $\begin{array}{r}7.60 \\
.25 \\
2.0 \\
.08 \\
15\end{array}$ & $\begin{array}{r}75.45 \\
2.43 \\
45 \\
.03 \\
i .50\end{array}$ & $\begin{array}{r}99.88 \\
3.33 \\
42 \\
.09 \\
198\end{array}$ \\
\hline
\end{tabular}

STATISTICS OF MORTRLY MEAN DATA FOR WATER YEARS 1970 - 1996, BY WATER YEAR (WY)

\begin{tabular}{|c|c|c|c|c|c|c|c|c|c|c|c|c|}
\hline $\begin{array}{l}\text { MEAN } \\
\text { MAX } \\
\text { (WY) } \\
\text { MIN } \\
\text { (WY) }\end{array}$ & $\begin{array}{r}.39 \\
2.43 \\
1973 \\
.028 \\
1994\end{array}$ & $\begin{array}{r}.67 \\
5.43 \\
1995 \\
.023 \\
2978\end{array}$ & $\begin{array}{r}.89 \\
3.76 \\
1984 \\
.019 \\
1978\end{array}$ & $\begin{array}{l}1.26 \\
18.9 \\
1993 \\
1058 \\
1976\end{array}$ & $\begin{array}{l}6.34 \\
57.1 \\
1995 \\
084 \\
1971\end{array}$ & $\begin{array}{r}32.5 \\
135 \\
1993 \\
19 \\
1972\end{array}$ & $\begin{array}{r}34.5 \\
187 \\
1973 \\
112 \\
1976\end{array}$ & $\begin{array}{l}3.71 \\
33.8 \\
1973 \\
1087 \\
1976\end{array}$ & $\begin{array}{r}.34 \\
1.33 \\
1973 \\
031 \\
1984\end{array}$ & $\begin{array}{r}.58 \\
3.52 \\
1982 \\
.015 \\
1993\end{array}$ & $\begin{array}{l}1.30 \\
7.15 \\
1992 \\
1038 \\
1971\end{array}$ & $\begin{array}{r}.49 \\
3.33 \\
1996 \\
1033 \\
1983\end{array}$ \\
\hline
\end{tabular}

(WY) $1994-1978$

WATER YEARS 3970 - 2996

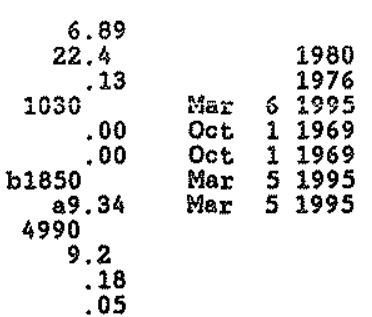

FOR 1995 CALEMDAR YEAR

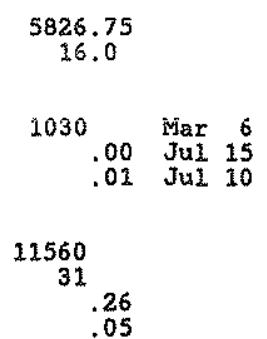

FOR 1996 WATER YEAR

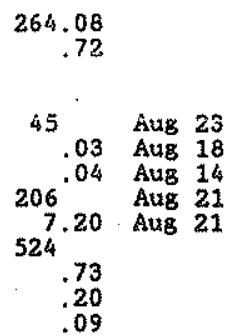

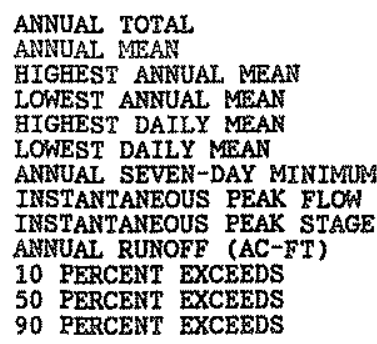

- Estimated

Q-Detum then in uxo.

b- From rating curve extended above $470 \mathrm{ft} / \mathrm{s}$; maximum gage helght, 7.90 ft, Max. $12,1985$. 
LITTLLE COLORADO RIVER BASIN

09386950 ZUNI RIVER ABOVE BLACK ROCK RESERVOTR, NM

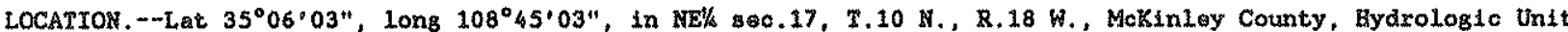
15020004 , on Zunt Indian Reservation, on left bank dopmstream from highway bridge on $S t a t e$ Highway 36 , 0.8 mi upstream from flow line of Black Rock Reservoir, 2.3 mi northeast of Black Rock, and 5.9 mi northeast of Zun1 Rueblo.

DRAIHAGE AREA. $--848 \mathrm{mt}^{2}$, of which $13 \mathrm{mit}^{2}$ is non-contributing.

PERIOD OF RECORD.--October 1969 to current year. Prior to October 1974 published as "above Zuni Resarvolr."

GAGE, - Water-stage recordex, crest-stage gage, and concrete control. Elevatton of gage is 6,480 ft above Nattonal Goodatic Vertical Datum of 1929 , from topographic map.

REMARXs. - Records faix oxcept for estlmated dally dischaxges, which axe poor. Several observations of water temperature were made during the year. No slow fox many days.

DISCRARGE, CUBIC FEET PER SECOND, WATER YEAR OCTOBER 1995 TO SEPTEMBER 1996

\begin{tabular}{|c|c|c|c|c|c|c|c|c|c|c|c|c|}
\hline DAY & oct & MOV & $\mathrm{DEC}$ & JANH & FEB & MAR & AlPR & MAY & Jun & JUL. & AUC & SER \\
\hline $\begin{array}{l}1 \\
2 \\
3 \\
4 \\
5\end{array}$ & $\begin{array}{l}.00 \\
.00 \\
.00 \\
.00 \\
.00\end{array}$ & $\begin{array}{r}.17 \\
.66 \\
1.1 \\
1.0 \\
.94\end{array}$ & $\begin{array}{l}.17 \\
.15 \\
.13 \\
.11 \\
.08\end{array}$ & $\begin{array}{r}.59 \\
.57 \\
0.56 \\
0.56 \\
0.53\end{array}$ & $\begin{array}{l}.14 \\
.12 \\
.28 \\
.23 \\
.17\end{array}$ & $\begin{array}{l}7.3 \\
7.2 \\
6.8 \\
5.3 \\
3.9\end{array}$ & $\begin{array}{l}8.9 \\
8.3 \\
6.9 \\
5.2 \\
6.0\end{array}$ & $\begin{array}{l}.06 \\
.00 \\
.00 \\
.00 \\
.00\end{array}$ & $\begin{array}{l}.00 \\
.00 \\
.00 \\
.00 \\
.00\end{array}$ & $\begin{array}{l}.00 \\
.00 \\
.00 \\
.00 \\
.00\end{array}$ & $\begin{array}{l}.00 \\
.00 \\
.00 \\
.00 \\
.00\end{array}$ & $\begin{array}{l}.00 \\
.00 \\
.00 \\
.00 \\
.00\end{array}$ \\
\hline $\begin{array}{r}6 \\
7 \\
8 \\
9 \\
10\end{array}$ & $\begin{array}{l}.00 \\
.00 \\
.00 \\
.00 \\
.00\end{array}$ & $\begin{array}{l}1.4 \\
1.1 \\
1.0 \\
.72 \\
.71\end{array}$ & $\begin{array}{l}.34 \\
.54 \\
.23 \\
.30 \\
.31\end{array}$ & $\begin{array}{l}.52 \\
.45 \\
.45 \\
.44 \\
.45\end{array}$ & $\begin{array}{l}.22 \\
.24 \\
.23 \\
.23 \\
.23\end{array}$ & $\begin{array}{l}3.7 \\
5.1 \\
6.8 \\
6.5 \\
4.0\end{array}$ & $\begin{array}{l}7.1 \\
7.5 \\
7.2 \\
5.3 \\
1.4\end{array}$ & $\begin{array}{l}.00 \\
.00 \\
.00 \\
.00 \\
.00\end{array}$ & $\begin{array}{l}.00 \\
.00 \\
.00 \\
.00 \\
.00\end{array}$ & $\begin{array}{l}.00 \\
.00 \\
.00 \\
.00 \\
.00\end{array}$ & $\begin{array}{l}.00 \\
.00 \\
.00 \\
.00 \\
.00\end{array}$ & $\begin{array}{l}.00 \\
.00 \\
.00 \\
.00 \\
.00\end{array}$ \\
\hline $\begin{array}{l}11 \\
12 \\
13 \\
14 \\
15\end{array}$ & $\begin{array}{l}.00 \\
.00 \\
.00 \\
.00 \\
.00\end{array}$ & $\begin{array}{l}.69 \\
.68 \\
.57 \\
.45 \\
.43\end{array}$ & $\begin{array}{l}.29 \\
.28 \\
.76 \\
.39 \\
.62\end{array}$ & $\begin{array}{l}.44 \\
.42 \\
.42 \\
.43 \\
.45\end{array}$ & $\begin{array}{r}.23 \\
.23 \\
.23 \\
.44 \\
1.1\end{array}$ & $\begin{array}{l}4.7 \\
5.0 \\
7.6 \\
8.2 \\
7.2\end{array}$ & $\begin{array}{r}.92 \\
.79 \\
.73 \\
10^{.73} \\
6.9\end{array}$ & $\begin{array}{l}.00 \\
.00 \\
.00 \\
.00 \\
.00\end{array}$ & $\begin{array}{l}.00 \\
.00 \\
.00 \\
.00 \\
.00\end{array}$ & $\begin{array}{l}.00 \\
.00 \\
.00 \\
.00 \\
.00\end{array}$ & $\begin{array}{l}.00 \\
.00 \\
.00 \\
.00 \\
.00\end{array}$ & $\begin{array}{l}.00 \\
.00 \\
.00 \\
.00 \\
.00\end{array}$ \\
\hline $\begin{array}{l}16 \\
17 \\
18 \\
19 \\
20\end{array}$ & $\begin{array}{l}.00 \\
.00 \\
.00 \\
.00 \\
.00\end{array}$ & $\begin{array}{l}.15 \\
.22 \\
.40 \\
.41 \\
.31\end{array}$ & $\begin{array}{r}.92 \\
.88 \\
1.0 \\
.99 \\
.83\end{array}$ & $\begin{array}{l}.59 \\
.65 \\
.63 \\
.65 \\
.65\end{array}$ & $\begin{array}{l}1.5 \\
2.1 \\
1.3 \\
1.4 \\
1.5\end{array}$ & $\begin{array}{l}8.5 \\
8.2 \\
6.2 \\
7.3 \\
6.8\end{array}$ & $\begin{array}{l}.55 \\
.55 \\
.47 \\
.27 \\
.00\end{array}$ & $\begin{array}{l}.00 \\
.00 \\
.00 \\
.00 \\
.00\end{array}$ & $\begin{array}{l}.00 \\
.00 \\
.00 \\
.00 \\
.00\end{array}$ & $\begin{array}{l}.09 \\
.00 \\
.00 \\
.00 \\
.00\end{array}$ & $\begin{array}{l}.00 \\
.00 \\
.00 \\
.00 \\
.00\end{array}$ & $\begin{array}{l}.00 \\
.00 \\
.00 \\
.00 \\
.00\end{array}$ \\
\hline $\begin{array}{l}21 \\
22 \\
23 \\
24 \\
25\end{array}$ & $\begin{array}{l}.00 \\
.00 \\
.00 \\
.00 \\
.00\end{array}$ & $\begin{array}{l}.35 \\
.36 \\
.36 \\
.35 \\
.32\end{array}$ & $\begin{array}{r}.37 \\
0.45 \\
0.52 \\
0.55 \\
.57\end{array}$ & $\begin{array}{l}.84 \\
.57 \\
.70 \\
.51 \\
.08\end{array}$ & $\begin{array}{l}1.1 \\
2.6 \\
5.5 \\
4.9 \\
4.2\end{array}$ & $\begin{array}{l}10 \\
12 \\
11 \\
8.5 \\
9.2\end{array}$ & $\begin{array}{l}.00 \\
.00 \\
.00 \\
.00 \\
.00\end{array}$ & $\begin{array}{l}.00 \\
.00 \\
.00 \\
.00 \\
.00\end{array}$ & $\begin{array}{l}.00 \\
.00 \\
.00 \\
.00 \\
.00\end{array}$ & $\begin{array}{l}.00 \\
.00 \\
.00 \\
.00 \\
.00\end{array}$ & $\begin{array}{l}.00 \\
.00 \\
.00 \\
.00 \\
.00\end{array}$ & $\begin{array}{l}.00 \\
.00 \\
.00 \\
.00 \\
.00\end{array}$ \\
\hline $\begin{array}{l}26 \\
27 \\
28 \\
29 \\
30 \\
31\end{array}$ & $\begin{array}{l}.20 \\
.48 \\
.52 \\
.45 \\
.39 \\
.39\end{array}$ & $\begin{array}{r}.29 \\
.24 \\
.22 \\
.19 \\
.19 \\
\end{array}$ & $\begin{array}{r}0.58 \\
0.59 \\
.60 \\
.60 \\
.59 \\
.58\end{array}$ & $\begin{array}{r}.05 \\
.06 \\
.03 \\
0.03 \\
.04 \\
.10\end{array}$ & $\begin{array}{l}4.4 \\
5.4 \\
6.2 \\
6.3 \\
-m \\
m-\infty\end{array}$ & $\begin{array}{r}10 \\
8.2 \\
8.5 \\
7.8 \\
9.9 \\
9.9\end{array}$ & $\begin{array}{l}.00 \\
.00 \\
.00 \\
.00 \\
.03 \\
.03\end{array}$ & $\begin{array}{l}.00 \\
.00 \\
.00 \\
.00 \\
.00 \\
.00\end{array}$ & $\begin{array}{l}.00 \\
.00 \\
.00 \\
.00 \\
.00 \\
.0\end{array}$ & $\begin{array}{l}.00 \\
.00 \\
.00 \\
.00 \\
.00 \\
.00\end{array}$ & $\begin{array}{l}.00 \\
.00 \\
.00 \\
.00 \\
.00 \\
.00\end{array}$ & $\begin{array}{l}.00 \\
.00 \\
.00 \\
.00 \\
.00 \\
\end{array}$ \\
\hline $\begin{array}{l}\text { TOTAL } \\
\text { MEAN } \\
\text { MAX } \\
\text { MIN } \\
\text { AC-FT }\end{array}$ & $\begin{array}{r}2.43 \\
.078 \\
.52 \\
.00 \\
4.8\end{array}$ & $\begin{array}{r}15.98 \\
: 53 \\
1.4 \\
.15 \\
32\end{array}$ & $\begin{array}{r}15.32 \\
4.49 \\
1.0 \\
.08 \\
30\end{array}$ & $\begin{array}{r}13.44 \\
.43 \\
.84 \\
.03 \\
27\end{array}$ & $\begin{array}{r}52.72 \\
1.82 \\
6.3 \\
12 \\
105\end{array}$ & $\begin{array}{r}231.3 \\
7.46 \\
12 \\
3.7 \\
459\end{array}$ & $\begin{array}{r}85.01 \\
2.83 \\
10 \\
.00 \\
169\end{array}$ & $\begin{array}{r}0.06 \\
.002 \\
.06 \\
.00 \\
.1\end{array}$ & $\begin{array}{r}0.00 \\
.000 \\
.00 \\
.00 \\
.00\end{array}$ & $\begin{array}{r}0.09 \\
.003 \\
.09 \\
.00 \\
.2\end{array}$ & $\begin{array}{r}0.00 \\
.000 \\
.00 \\
.00 \\
.00\end{array}$ & $\begin{array}{r}0.00 \\
.000 \\
.00 \\
.00 \\
.00\end{array}$ \\
\hline
\end{tabular}

STATISTICS OF MONTHLY MEAN DATA FOR WATER YEARS 1970 - 1996, BY WATER YEAR (WY)

\begin{tabular}{|c|c|c|c|c|c|c|c|c|c|c|c|c|}
\hline $\begin{array}{l}\text { MEAR } \\
\text { MAX } \\
\text { (WY) } \\
\text { MIN } \\
\text { (WY) }\end{array}$ & $\begin{array}{l}1.67 \\
12.6 \\
1984 \\
1000 \\
1974\end{array}$ & $\begin{array}{l}1.48 \\
13.7 \\
1984 \\
000 \\
1071\end{array}$ & $\begin{array}{l}1.35 \\
5.87 \\
1984 \\
1013\end{array}$ & $\begin{array}{r}3.06 \\
41.9 \\
1993 \\
1971\end{array}$ & $\begin{array}{r}11.1 \\
73.4 \\
1980 \\
33 \\
1970\end{array}$ & $\begin{array}{r}45.9 \\
263 \\
1985 \\
166\end{array}$ & $\begin{array}{r}55.3 \\
308 \\
1973 \\
.009 \\
1970\end{array}$ & $\begin{array}{l}5.70 \\
65.3 \\
1973 \\
.002 \\
1906\end{array}$ & $\begin{array}{r}.20 \\
1.97 \\
1979 \\
1000 \\
j 970\end{array}$ & $\begin{array}{l}3.04 \\
25.6 \\
1977 \\
000 \\
1971\end{array}$ & $\begin{array}{l}6.23 \\
23.6 \\
1977 \\
1000 \\
1986\end{array}$ & $\begin{array}{r}2.67 \\
17.5 \\
1984 \\
1000 \\
1979\end{array}$ \\
\hline
\end{tabular}

SUMAARY STATISTICS

ARNUAL TOTAL

ANRUAL MEAN

BIGHEST ANWUAL MEAN

LOWEST ANNUAL MEAN

HIGREST DAILY MEAN

LOWEST DAILY MEAN

ANNUAL SEVEN-DAY MINTMUM

INSTANTAREOUS PEAK FLOW

INSTARTANEOUS PEAK STAGE

INSTANTANEOUS LOW FLOW

ANYUAL RUNOFF (AC-FT)

10 PERCENT EXCEEDS

50 PERCENT BXCEEDS

90 PERCENT EXCEEDS
FOR 1995 CALENDAR YEAR

FOR 1996 WATES YEAR

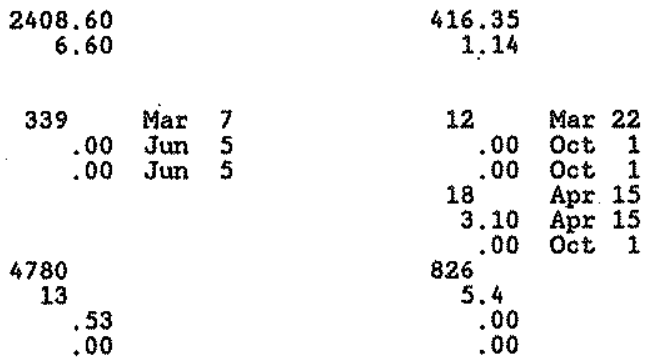

WATER YEARS 1970 - 1996

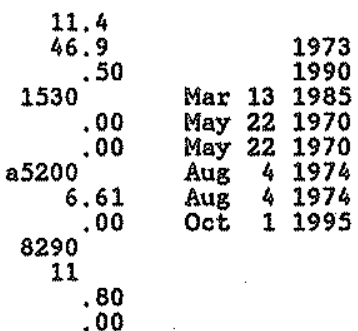

- Estimated

a-From rating curve extended above $670 \mathrm{ft} / \mathrm{s}$ on basis of slope-area measurements at gage heights 4.05 it, $3.95 \mathrm{ft}$ and $6.61 \mathrm{ft}$. 
LOCATION.-Lat $33^{\circ} 03^{\prime 4} 40^{\prime \prime}$, long $108^{\circ} 32^{\prime} 12^{\prime \prime}$, in NEKWW sec.30, T.14 S., R.16 W., Grant County, Hydrologic Unit 15040001, on left bank at Hooker damsite, 1.6 mi upstresm from Mogollon Creek, 7 mi. northeast of Gila, and at mile 572.5 .

DRAINAGE AREA. $--1,864 \mathrm{mit}^{2}$.

PERIOD OF RECORD.--April to December 1914, December 2927 to current year. Monthly discharge only December 1927 to Septembex 1930, published in WSE 1313.

REVISED RECORDS.--WSP 1283: Dratnage aroa. WSP 1313: 1944 (M), 1949 (M), WDR WM-78-1: 1977.

GAGE. -Water-stage recordex, Datum of gage is 4,654.8 ft above National Geodetic Vertical Datum of 1929, (riverprofile survey). Prior to Dec. 31 , 1928 , at site 5 mi upstrean at different datum. Dec. 31 , 1928 , to Jan. 7 , 1942 , at site $200 \mathrm{ft}$ upstream at datum 1.00 ft higher. Prior to Feb. 28, 1994 at datum 1.00 ft higher.

REMARKS.--Records good. "Diversions for irrigation of about 500 acres upstream from station. Several observations of water temperature were made during the year. National Weather Service satellite telemeter at station.

EXTREMES OUTSIDE PERIOD OF RECORD.--Other major Lloods occurred in November 1905, December 1906 , and January 1916. DISCRARGE, CUBIC FEET PER SECOND, WATER YEAR OCTOBER 1995 TO SEPTEMBER 1996

\begin{tabular}{|c|c|c|c|c|c|c|c|c|c|c|c|c|}
\hline DAY & $\cos$ & Mov & DEC & JAN & EEB & $M A R$ & APR & MAY & JUN & JuL & AUG & SEP \\
\hline $\begin{array}{l}1 \\
2 \\
3 \\
4 \\
5\end{array}$ & $\begin{array}{l}65 \\
63 \\
64 \\
64 \\
64\end{array}$ & $\begin{array}{l}67 \\
68 \\
68 \\
67 \\
65\end{array}$ & $\begin{array}{l}70 \\
70 \\
69 \\
71 \\
70\end{array}$ & $\begin{array}{l}68 \\
69 \\
68 \\
68 \\
67\end{array}$ & $\begin{array}{l}77 \\
95 \\
88 \\
79 \\
74\end{array}$ & $\begin{array}{l}64 \\
64 \\
64 \\
65 \\
66\end{array}$ & $\begin{array}{l}56 \\
56 \\
57 \\
56 \\
56\end{array}$ & $\begin{array}{l}44 \\
42 \\
43 \\
43 \\
42\end{array}$ & $\begin{array}{l}27 \\
25 \\
25 \\
24 \\
23\end{array}$ & $\begin{array}{r}106 \\
59 \\
53 \\
58 \\
57\end{array}$ & $\begin{array}{l}71 \\
82 \\
76 \\
83 \\
93\end{array}$ & $\begin{array}{l}210 \\
169 \\
160 \\
191 \\
154\end{array}$ \\
\hline $\begin{array}{r}6 \\
7 \\
8 \\
9 \\
10\end{array}$ & $\begin{array}{l}65 \\
65 \\
65 \\
66 \\
66\end{array}$ & $\begin{array}{l}68 \\
68 \\
68 \\
67 \\
68\end{array}$ & $\begin{array}{l}68 \\
68 \\
71 \\
71 \\
69\end{array}$ & $\begin{array}{l}67 \\
68 \\
67 \\
67 \\
66\end{array}$ & $\begin{array}{l}71 \\
69 \\
68 \\
68 \\
68\end{array}$ & $\begin{array}{l}64 \\
64 \\
63 \\
62 \\
62\end{array}$ & $\begin{array}{l}56 \\
57 \\
56 \\
56 \\
55\end{array}$ & $\begin{array}{l}40 \\
40 \\
38 \\
38 \\
37\end{array}$ & $\begin{array}{l}22 \\
21 \\
24 \\
26 \\
26\end{array}$ & $\begin{array}{l}47 \\
41 \\
40 \\
47 \\
47\end{array}$ & $\begin{array}{l}77 \\
64 \\
59 \\
59 \\
93\end{array}$ & $\begin{array}{r}128 \\
108 \\
96 \\
90 \\
116\end{array}$ \\
\hline $\begin{array}{l}11 \\
12 \\
13 \\
14 \\
15\end{array}$ & $\begin{array}{l}66 \\
66 \\
66 \\
66 \\
67\end{array}$ & $\begin{array}{l}68 \\
68 \\
69 \\
69 \\
68\end{array}$ & $\begin{array}{l}69 \\
70 \\
70 \\
70 \\
69\end{array}$ & $\begin{array}{l}64 \\
63 \\
63 \\
63 \\
63\end{array}$ & $\begin{array}{l}66 \\
65 \\
68 \\
73 \\
75\end{array}$ & $\begin{array}{l}61 \\
60 \\
62 \\
62 \\
62\end{array}$ & $\begin{array}{l}54 \\
54 \\
54 \\
52 \\
52\end{array}$ & $\begin{array}{l}37 \\
35 \\
34 \\
33 \\
33\end{array}$ & $\begin{array}{l}24 \\
23 \\
24 \\
26 \\
29\end{array}$ & $\begin{array}{r}50 \\
67 \\
71 \\
96 \\
172\end{array}$ & $\begin{array}{l}92 \\
90 \\
79 \\
69 \\
63\end{array}$ & $\begin{array}{r}135 \\
161 \\
210 \\
652 \\
2160\end{array}$ \\
\hline $\begin{array}{l}16 \\
17 \\
18 \\
19 \\
20\end{array}$ & $\begin{array}{l}66 \\
66 \\
66 \\
66 \\
67\end{array}$ & $\begin{array}{l}69 \\
70 \\
70 \\
70 \\
71\end{array}$ & $\begin{array}{l}70 \\
71 \\
70 \\
70 \\
69\end{array}$ & $\begin{array}{l}63 \\
64 \\
64 \\
63 \\
63\end{array}$ & $\begin{array}{l}73 \\
71 \\
69 \\
68 \\
66\end{array}$ & $\begin{array}{l}62 \\
62 \\
62 \\
62 \\
60\end{array}$ & $\begin{array}{l}52 \\
52 \\
53 \\
52 \\
52\end{array}$ & $\begin{array}{l}32 \\
32 \\
31 \\
29 \\
29\end{array}$ & $\begin{array}{l}30 \\
28 \\
27 \\
24 \\
22\end{array}$ & $\begin{array}{r}178 \\
169 \\
124 \\
93 \\
80\end{array}$ & $\begin{array}{l}59 \\
56 \\
55 \\
69 \\
85\end{array}$ & $\begin{array}{r}1350 \\
841 \\
524 \\
352 \\
259\end{array}$ \\
\hline $\begin{array}{l}21 \\
22 \\
23 \\
24 \\
25\end{array}$ & $\begin{array}{l}66 \\
65 \\
65 \\
65 \\
65\end{array}$ & $\begin{array}{l}71 \\
71 \\
70 \\
70 \\
70\end{array}$ & $\begin{array}{l}69 \\
69 \\
68 \\
68 \\
68\end{array}$ & $\begin{array}{l}63 \\
62 \\
63 \\
63 \\
63\end{array}$ & $\begin{array}{l}64 \\
64 \\
64 \\
64 \\
67\end{array}$ & $\begin{array}{l}60 \\
60 \\
62 \\
59 \\
57\end{array}$ & $\begin{array}{l}51 \\
50 \\
50 \\
48 \\
47\end{array}$ & $\begin{array}{l}28 \\
28 \\
27 \\
27 \\
27\end{array}$ & $\begin{array}{l}22 \\
22 \\
22 \\
21 \\
21\end{array}$ & $\begin{array}{l}64 \\
57 \\
53 \\
69 \\
71\end{array}$ & $\begin{array}{l}121 \\
168 \\
358 \\
402 \\
295\end{array}$ & $\begin{array}{l}201 \\
165 \\
141 \\
125 \\
122\end{array}$ \\
\hline $\begin{array}{l}26 \\
27 \\
28 \\
29 \\
30 \\
31\end{array}$ & $\begin{array}{l}65 \\
66 \\
66 \\
66 \\
66 \\
66\end{array}$ & $\begin{array}{r}71 \\
70 \\
70 \\
70 \\
70 \\
\end{array}$ & $\begin{array}{l}69 \\
69 \\
68 \\
68 \\
68 \\
68\end{array}$ & $\begin{array}{l}62 \\
62 \\
62 \\
62 \\
62 \\
62\end{array}$ & $\begin{array}{r}66 \\
66 \\
66 \\
65 \\
--\infty \\
-\infty\end{array}$ & $\begin{array}{l}57 \\
57 \\
56 \\
57 \\
56 \\
57\end{array}$ & $\begin{array}{r}47 \\
47 \\
46 \\
44 \\
43 \\
---\end{array}$ & $\begin{array}{l}26 \\
27 \\
27 \\
26 \\
26 \\
26\end{array}$ & $\begin{array}{r}22 \\
25 \\
57 \\
56 \\
83 \\
---\end{array}$ & $\begin{array}{l}56 \\
56 \\
60 \\
69 \\
72 \\
68\end{array}$ & $\begin{array}{l}257 \\
237 \\
416 \\
353 \\
247 \\
243\end{array}$ & $\begin{array}{l}121 \\
118 \\
110 \\
104 \\
101 \\
--\infty\end{array}$ \\
\hline $\begin{array}{l}\text { TOTAL } \\
\text { MEAN } \\
\text { MAX } \\
\text { MIN } \\
\text { AC-ET }\end{array}$ & $\begin{array}{r}2030 \\
65.5 \\
67 \\
63 \\
4030\end{array}$ & $\begin{array}{r}2069 \\
69.0 \\
71 \\
65 \\
4100\end{array}$ & $\begin{array}{r}2147 \\
69.3 \\
71 \\
68 \\
4260\end{array}$ & $\begin{array}{r}1994 \\
64.3 \\
69 \\
62 \\
3960\end{array}$ & $\begin{array}{r}2037 \\
70.2 \\
95 \\
64 \\
4040\end{array}$ & $\begin{array}{r}1891 \\
61.0 \\
66 \\
56 \\
3750\end{array}$ & $\begin{array}{r}1561 \\
52.0 \\
57 \\
43 \\
3100\end{array}$ & $\begin{array}{r}1027 \\
33,2 \\
44 \\
26 \\
2040\end{array}$ & $\begin{array}{r}851 \\
28.4 \\
83 \\
21 \\
1690\end{array}$ & $\begin{array}{r}2350 \\
75.8 \\
178 \\
40 \\
4660\end{array}$ & $\begin{array}{r}571 \\
147 \\
416 \\
55 \\
9070\end{array}$ & $\begin{array}{r}9374 \\
312 \\
2160 \\
90 \\
18590\end{array}$ \\
\hline $\begin{array}{l}\text { STATIS } \\
\text { MEAN } \\
\text { MAX } \\
\text { (WY) } \\
\text { MIN } \\
\text { (WY) }\end{array}$ & $\begin{array}{r}\text { ICS OF } \\
119 \\
994 \\
1973 \\
29.1 \\
1957\end{array}$ & $\begin{array}{l}\text { MONTHLY MEAN } \\
99.0 \\
726 \\
1995 \\
47.8 \\
1951\end{array}$ & $\begin{array}{l}\text { DATA } \\
171 \\
1632 \\
1979 \\
50.1 \\
1954\end{array}$ & $\begin{array}{c}\text { FOR WATER } \\
178 \\
1810 \\
1993 \\
50.0 \\
1954\end{array}$ & $\begin{array}{c}\text { YEARS } 1928 \\
242 \\
1204 \\
1993 \\
50.9 \\
1954\end{array}$ & $\begin{array}{c}-1996, \\
318 \\
1049 \\
1985 \\
53.9 \\
1971\end{array}$ & $\begin{array}{c}\text { BY WATER } \\
221 \\
903 \\
1973 \\
49.2 \\
1971\end{array}$ & $\begin{array}{c}\text { YEAR (WY) } \\
143 \\
716 \\
1973 \\
33.1 \\
1996\end{array}$ & $\begin{array}{r}60.6 \\
249 \\
1992 \\
23.5 \\
1974\end{array}$ & $\begin{array}{r}65.1 \\
225 \\
1986 \\
22.3 \\
1971\end{array}$ & $\begin{array}{r}141 \\
901 \\
1988 \\
37.5 \\
1956\end{array}$ & $\begin{array}{r}145 \\
960 \\
1988 \\
24.0 \\
1956\end{array}$ \\
\hline \multicolumn{3}{|c|}{$\begin{array}{l}\text { SUJMARY STATISTICS } \\
\text { ANNUAL TOTAL } \\
\text { ANNUAL MEAN } \\
\text { GIGHEST ANNUAL MEAN } \\
\text { LOWEST ANHUAI MEAN } \\
\text { HIGHEST DAILY MEAN } \\
\text { LOWEST DAILY MEAN } \\
\text { ANNUAL SEVEN-DAY MINIMUM } \\
\text { INSTANTANEOUS FEAK FLOW } \\
\text { INSTANTANEOUS PEAK STAGE } \\
\text { IKSTANTANEOUS IOW FLOW } \\
\text { ANNUAL RUNOFF (AC-FT) } \\
10 \text { PERCENT EXCEEDS } \\
\text { 50 PERCENT EXCEEDS } \\
90 \text { PERCENT EXCEEDS }\end{array}$} & FOR & $\begin{array}{r}149100 \\
459 \\
79 \\
51\end{array}$ & $\begin{array}{lr}\text { Fob } & 15 \\
\text { Jul } & 13 \\
\text { Jul } & 9\end{array}$ & & $\begin{array}{c}2160 \\
21 \\
22 \\
2530 \\
4.62 \\
18 \\
63280 \\
121 \\
65 \\
29\end{array}$ & $\begin{array}{lr}\text { Sep } & 15 \\
\text { Jun } & 7 \\
\text { Jun } & 20 \\
\text { Sep } & 15 \\
\text { Sep } & 15 \\
\text { Jun } & 7\end{array}$ & & $\begin{array}{c}\text { WATER YEARS } \\
159 \\
477 \\
47.8 \\
23400 \\
15 \\
16 \\
\mathrm{a} 35200 \\
\mathrm{~b} 13.00 \\
14 \\
115000 \\
317 \\
74 \\
40\end{array}$ & $\begin{array}{ll}\text { Dec } & 28 \\
\text { Jul } & 16 \\
\text { Jul } & 14 \\
\text { Dec } & 28 \\
\text { Dec } & 28 \\
\text { Jul } & 15\end{array}$ & $\begin{array}{ll} & 1979 \\
& 1956 \\
8 & 1984 \\
6 & 1971 \\
4 & 1971 \\
8 & 1984 \\
8 & 1984 \\
5 & 1971\end{array}$ \\
\hline
\end{tabular}

a-From rating curve extended above $7,000 \mathrm{ft} 3 / \mathrm{s}$ on basis of slope-area measurement at gage height 12.5 ft; meximum gage height, 17.2 ft from floodmark, Sept. $29,1941$. b-From floodmarks. 
GILA RIVER BASIN

09430600 NOGOLLON CREEK NEAR CLIFF, MM

(Hydrologic bench-mark station)

LOCATION.--Lat $33^{\circ} 10^{\circ} 00^{\prime \prime}$, long $108^{\circ} 38^{\circ} 57^{\prime \prime}$, in SE/sse\% sec.13, T.13 S., R.18 W., Grant County, Hydrologic Unit

15040001, on right bank 0.3 mi downstream from Rain Creok, 0.8 mi downstream from Gila Wilderness Boundary, 12 mi upstream from mouth, and $14 \mathrm{mi}$ north of Cliff.

DRAINAGE AREA. $--69 \mathrm{mi}^{2}$.

WATER-DISCEARGE RECORDS

PERIOD OF RECORD. -March 1967 to curront yoar.

GAGE.-Water-stage recorder. Elevation of gage is 5,440 ft above National Geodetic Vertical Datum of 1929 , from topographic map.

REMARKS. -Water-discharge records good except for estinated daily discharges, which are poor. DISCRARGE, CUBIC FEET PER SECOND, WATER YEAR OCTOBER 1995 TO SEPTEMBER 1996 DAILY MEAN VALUES

\begin{tabular}{|c|c|c|c|c|c|c|c|c|c|c|c|c|}
\hline DAY & OCT & Nov & DEC & JAN & FEB & MAR & APR & MAY & JUN & JUL & AUt & SEP \\
\hline $\begin{array}{l}1 \\
2 \\
3 \\
4 \\
5\end{array}$ & $\begin{array}{l}1.0 \\
.82 \\
.58 \\
.37 \\
.21\end{array}$ & $\begin{array}{l}.57 \\
1.1 \\
1.4 \\
1.2 \\
1.2\end{array}$ & $\begin{array}{l}2.2 \\
2.2 \\
2.3 \\
2.3 \\
2.3\end{array}$ & $\begin{array}{l}3.3 \\
2.9 \\
3.6 \\
3.8 \\
2.8\end{array}$ & $\begin{array}{l}12 \\
12 \\
9.3 \\
6.7 \\
5.8\end{array}$ & $\begin{array}{l}2.8 \\
2.7 \\
2.7 \\
2.6 \\
2.6\end{array}$ & $\begin{array}{l}1.8 \\
1.7 \\
1.6 \\
1.5 \\
2.2\end{array}$ & $\begin{array}{l}.54 \\
.47 \\
.32 \\
.22 \\
.12\end{array}$ & $\begin{array}{l}.00 \\
.00 \\
.00 \\
.00 \\
.00\end{array}$ & $\begin{array}{l}7.0 \\
10 \\
15 \\
9.1 \\
5.4\end{array}$ & $\begin{array}{l}24 \\
21 \\
15 \\
40 \\
17\end{array}$ & $\begin{array}{r}18 \\
5.3 \\
7.1 \\
5.8 \\
2.6\end{array}$ \\
\hline $\begin{array}{r}6 \\
7 \\
8 \\
9 \\
10\end{array}$ & $\begin{array}{l}.11 \\
.20 \\
.22 \\
.13 \\
.09\end{array}$ & $\begin{array}{l}2.6 \\
4.1 \\
2.2 \\
1.6 \\
1.2\end{array}$ & $\begin{array}{l}2.3 \\
2.4 \\
3.1 \\
2.8 \\
2.7\end{array}$ & $\begin{array}{l}2.9 \\
2.7 \\
2.5 \\
2.4 \\
2.4\end{array}$ & $\begin{array}{l}6.4 \\
6.8 \\
5.8 \\
4.7 \\
4.1\end{array}$ & $\begin{array}{l}2.5 \\
2.5 \\
2.5 \\
2.5 \\
2.5\end{array}$ & $\begin{array}{l}2.3 \\
2.0 \\
1.7 \\
1.6 \\
1.5\end{array}$ & $\begin{array}{l}.08 \\
.02 \\
.00 \\
.00 \\
.00\end{array}$ & $\begin{array}{l}.00 \\
.00 \\
.00 \\
.00 \\
.00\end{array}$ & $\begin{array}{l}11 \\
11 \\
21 \\
13 \\
56\end{array}$ & $\begin{array}{r}9.8 \\
7.2 \\
5.8 \\
9.9 \\
108\end{array}$ & $\begin{array}{l}1.8 \\
1.8 \\
1.7 \\
1.6 \\
1.6\end{array}$ \\
\hline $\begin{array}{l}11 \\
12 \\
13 \\
14 \\
15\end{array}$ & $\begin{array}{l}.05 \\
.02 \\
.00 \\
.00 \\
.00\end{array}$ & $\begin{array}{l}1.2 \\
1.7 \\
1.7 \\
1.7 \\
1.5\end{array}$ & $\begin{array}{l}2.6 \\
2.5 \\
2.5 \\
2.4 \\
2.2\end{array}$ & $\begin{array}{l}2.0 \\
2.0 \\
1.9 \\
2.0 \\
1.8\end{array}$ & $\begin{array}{l}3.8 \\
3.2 \\
4.6 \\
6.9 \\
5.3\end{array}$ & $\begin{array}{l}2.3 \\
2.2 \\
2.1 \\
2.2 \\
2.3\end{array}$ & $\begin{array}{l}1.5 \\
1.7 \\
1.7 \\
1.7 \\
1.6\end{array}$ & $\begin{array}{l}.00 \\
.00 \\
.00 \\
.00 \\
.00\end{array}$ & $\begin{array}{l}.00 \\
.00 \\
.00 \\
.00 \\
.00\end{array}$ & $\begin{array}{r}25 \\
58 \\
76 \\
77 \\
136\end{array}$ & $\begin{array}{r}142 \\
48 \\
2.7 \\
2.6 \\
3.9\end{array}$ & $\begin{array}{r}5.3 \\
4.4 \\
10 \\
213 \\
198\end{array}$ \\
\hline $\begin{array}{l}16 \\
17 \\
18 \\
19 \\
20\end{array}$ & $\begin{array}{l}.00 \\
.00 \\
.00 \\
.00 \\
.01\end{array}$ & $\begin{array}{l}1.4 \\
1.4 \\
1.4 \\
1.4 \\
1.3\end{array}$ & $\begin{array}{l}2.3 \\
3.2 \\
3.0 \\
2.7 \\
3.0\end{array}$ & $\begin{array}{l}2.1 \\
2.3 \\
2.5 \\
2.2 \\
2.3\end{array}$ & $\begin{array}{l}4.1 \\
3.5 \\
3.4 \\
3.2 \\
2.9\end{array}$ & $\begin{array}{l}2.3 \\
2.3 \\
2.2 \\
2.1 \\
2.0\end{array}$ & $\begin{array}{r}1.5 \\
1.3 \\
1.1 \\
1.0 \\
.94\end{array}$ & $\begin{array}{l}.00 \\
.00 \\
.00 \\
.00 \\
.00\end{array}$ & $\begin{array}{l}.00 \\
.00 \\
.00 \\
.00 \\
.00\end{array}$ & $\begin{array}{r}151 \\
145 \\
0110 \\
e 100 \\
095\end{array}$ & $\begin{array}{r}7.5 \\
6.4 \\
6.2 \\
8.6 \\
33\end{array}$ & $\begin{array}{l}40 \\
10 \\
1.9 \\
1.3 \\
1.2\end{array}$ \\
\hline $\begin{array}{l}21 \\
22 \\
23 \\
24 \\
25\end{array}$ & $\begin{array}{l}.09 \\
.16 \\
.10 \\
.34 \\
.56\end{array}$ & $\begin{array}{l}1.3 \\
1.3 \\
1.3 \\
1.3 \\
1.3\end{array}$ & $\begin{array}{l}2.8 \\
2.7 \\
3.1 \\
3.2 \\
3.0\end{array}$ & $\begin{array}{l}2.2 \\
2.3 \\
2.0 \\
3.3 \\
5.4\end{array}$ & $\begin{array}{l}2.6 \\
2.5 \\
2.5 \\
2.5 \\
2.4\end{array}$ & $\begin{array}{l}1.9 \\
1.9 \\
1.9 \\
1.8 \\
2.0\end{array}$ & $\begin{array}{r}1.0 \\
1.1 \\
1.1 \\
.99 \\
.90\end{array}$ & $\begin{array}{l}.00 \\
.00 \\
.00 \\
.00 \\
.00\end{array}$ & $\begin{array}{r}.00 \\
.00 \\
.00 \\
.00 \\
.00\end{array}$ & $\begin{array}{l}990 \\
075 \\
070 \\
065 \\
060\end{array}$ & $\begin{array}{l}428 \\
153 \\
162 \\
222 \\
265\end{array}$ & $\begin{array}{r}1.7 \\
2.2 \\
1.3 \\
7.1 \\
12\end{array}$ \\
\hline $\begin{array}{l}26 \\
27 \\
28 \\
29 \\
30 \\
31\end{array}$ & $\begin{array}{l}.55 \\
.59 \\
.63 \\
.64 \\
.59 \\
.61\end{array}$ & $\begin{array}{l}1.5 \\
1.9 \\
1.9 \\
2.0 \\
2.2 \\
-=-\end{array}$ & $\begin{array}{l}2.9 \\
3.0 \\
2.9 \\
3.0 \\
2.9 \\
2.9\end{array}$ & $\begin{array}{l}2.5 \\
1.8 \\
1.9 \\
1.7 \\
1.9 \\
2.1\end{array}$ & $\begin{array}{l}2.6 \\
3.0 \\
3.0 \\
2.9 \\
\ldots .2 \\
\ldots-\end{array}$ & $\begin{array}{l}2.0 \\
2.0 \\
1.9 \\
1.8 \\
1.8 \\
1.8\end{array}$ & $\begin{array}{l}.79 \\
.66 \\
.59 \\
.42 \\
.50 \\
.0-\end{array}$ & $\begin{array}{l}.00 \\
.00 \\
.00 \\
.00 \\
.00 \\
.00\end{array}$ & $\begin{array}{l}.00 \\
.00 \\
.00 \\
.00 \\
.00 \\
.0\end{array}$ & $\begin{array}{r}055 \\
e 53 \\
050 \\
045 \\
43 \\
29\end{array}$ & $\begin{array}{r}237 \\
217 \\
158 \\
111 \\
83 \\
39\end{array}$ & $\begin{array}{r}13 \\
11 \\
9.8 \\
9.1 \\
8.7 \\
-\end{array}$ \\
\hline $\begin{array}{l}\text { TOTAL } \\
\text { MEAN } \\
\text { MAX } \\
\text { MIN } \\
\text { AC-FT }\end{array}$ & $\begin{array}{r}8.67 \\
.28 \\
1.0 \\
.00 \\
17\end{array}$ & $\begin{array}{r}47.87 \\
1.60 \\
4.11 \\
.57 \\
95\end{array}$ & $\begin{array}{r}83.4 \\
2.69 \\
3.2 \\
2.2 \\
1.65\end{array}$ & $\begin{array}{r}77.5 \\
2.50 \\
5.4 \\
1.7 \\
1.54\end{array}$ & $\begin{array}{r}138.5 \\
4.78 \\
12 \\
2.4 \\
275\end{array}$ & $\begin{array}{r}68.5 \\
2.21 \\
2.8 \\
1.8 \\
136\end{array}$ & $\begin{array}{r}39.99 \\
1.33 \\
2.3 \\
.42 \\
79\end{array}$ & $\begin{array}{r}1.77 \\
.057 \\
.54 \\
.00 \\
3.5\end{array}$ & $\begin{array}{l}0.00 \\
.000 \\
.00 \\
.00 \\
.00\end{array}$ & $\begin{array}{r}1766.5 \\
57.0 \\
151 \\
5.4 \\
3500\end{array}$ & $\begin{array}{r}2593.6 \\
83.7 \\
428 \\
2.6 \\
5140\end{array}$ & $\begin{array}{r}608.3 \\
20.3 \\
213 \\
1.2 \\
1210\end{array}$ \\
\hline
\end{tabular}

STATISTICS OF MONTELY MEAN DATA FOR WATER YEARS 1967 - 1996, BY WATER YEAR (WY)

\begin{tabular}{|c|c|c|c|c|c|c|c|c|c|c|c|c|}
\hline $\begin{array}{l}\text { MEAN } \\
\text { MAX } \\
\text { (WY) } \\
\text { MIN } \\
\text { (WY) }\end{array}$ & $\begin{array}{r}22.4 \\
237 \\
1973 \\
14 \\
1980\end{array}$ & $\begin{array}{r}19.0 \\
166 \\
1979 \\
1.07 \\
1971\end{array}$ & $\begin{array}{r}48.6 \\
410 \\
1979 \\
1.03 \\
1974\end{array}$ & $\begin{array}{r}38,1 \\
298 \\
1993 \\
1.14 \\
1971\end{array}$ & $\begin{array}{r}61.5 \\
211 \\
1.968 \\
1.44 \\
1.971\end{array}$ & $\begin{array}{r}71.1 \\
272 \\
1978 \\
1.33 \\
1971\end{array}$ & $\begin{array}{r}56.6 \\
182 \\
1973 \\
.90 \\
1971\end{array}$ & $\begin{array}{r}29.1 \\
160 \\
1992 \\
057 \\
1996\end{array}$ & $\begin{array}{l}3.57 \\
24.1 \\
1992 \\
000 \\
1971\end{array}$ & $\begin{array}{l}7.83 \\
57.0 \\
1996 \\
1900 \\
1980\end{array}$ & $\begin{array}{l}17.8 \\
83.7 \\
1996 \\
1.02 \\
1975\end{array}$ & $\begin{array}{r}16.2 \\
120 \\
1975 \\
193 \\
1987\end{array}$ \\
\hline
\end{tabular}

SUMMARY STATISTICS

ANNUAL TOTAL

ANNUAL MEAN

HIGHEST ANNUAL MEAN

LOWEST ANNUAL MEAN

HIGHEST DAILY MEAN

LOWEST DAILY MEAN

ANNUAL SEVEN-DAY MINIMLM

INSTANTANEOUS PEAK FLOW

INSTANTANEOUS REAK STAGE

INSTANTANEOUS LOW FLOW

ANNUAL RUNOFE (AC-FT)

10 PERCENT EXCEEDS

50 PERCENT DXCEEDS

90 PERCENT EXCEEDS

- Estimated
FOR 1995 CALENDAR YEAR

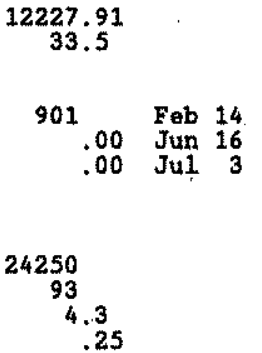

FOR 1996 WATER YEAR

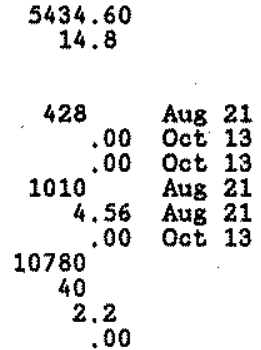

WATER YEARS 1967 - 1996

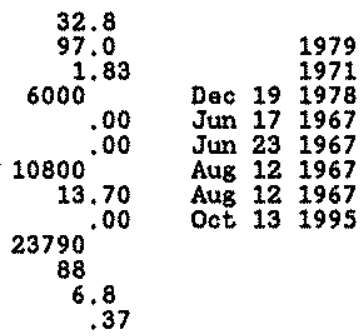


GILA. RIVER BASIN

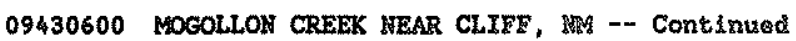
WATER-QUALITY RECORDS

PERIOD OF RECORD. --Water years 1967 to current year.

WATER QUALITY DATA, WATER YEAR OCTOBER 1995 TO SERIESBER 1996

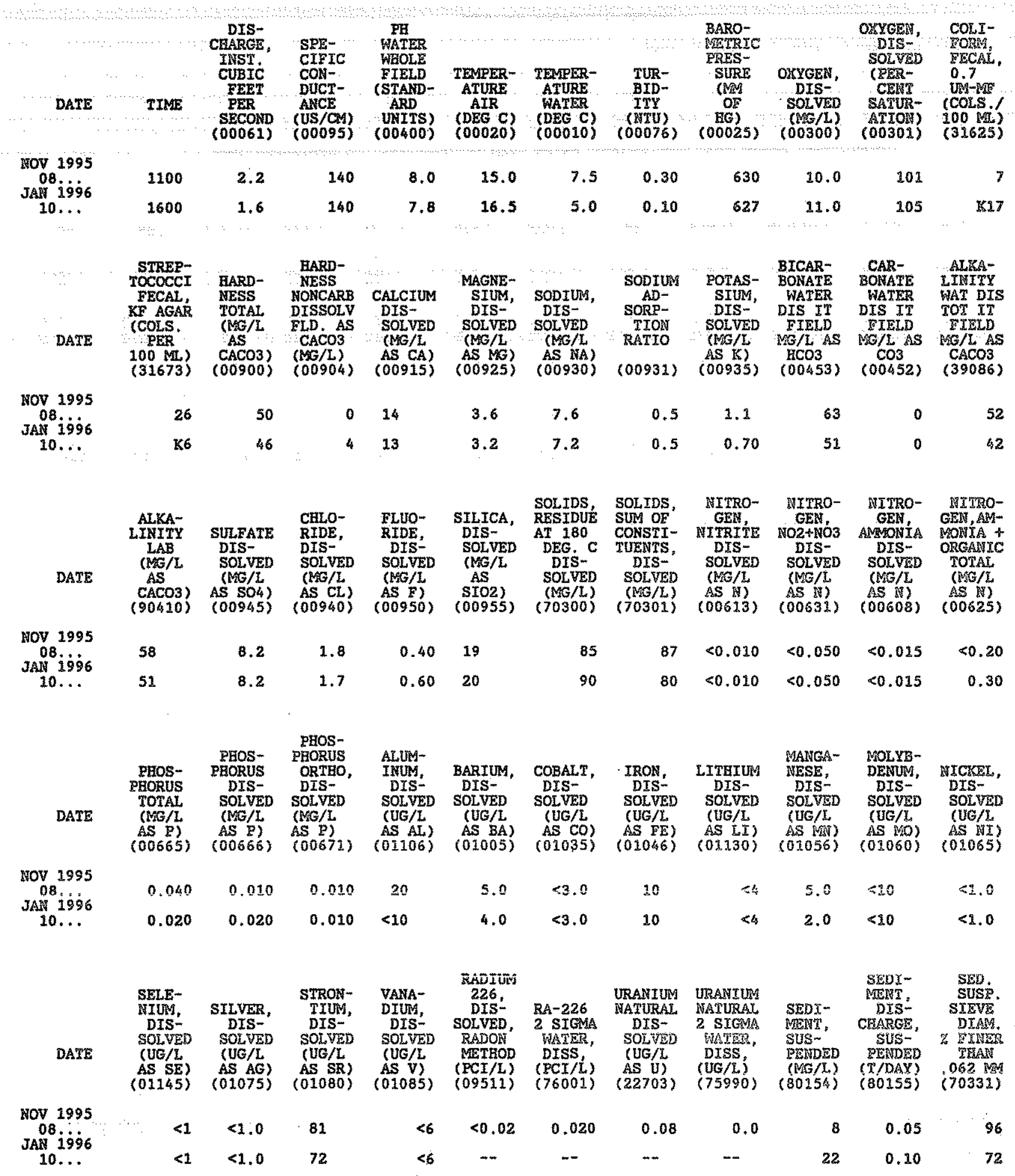


GILA RIVER BASIN

09431500 GILA RIVER NEAR REDROCK, NM

LOCATION.--Lat $32^{\circ} 43^{\prime} 37^{\prime \prime}$, Long $108^{\circ} 40^{\prime} 30^{\prime \prime}$, in $w / 4$ sec.23, T.18 5., R.18 W., Grant County, Hydrologic Unit 15040002, on left bank $0.2 \mathrm{mi}$ downstream from Copper Canyon, $0.2 \mathrm{mi}$ upstream from lower end of box canyon, $4.7 \mathrm{mi}$ northeast of Redrock, $14 \mathrm{mi}$ downstream from Mangas Creek, and at mile 539.2 .

DRAINAGE AREA. $--2,829 \mathrm{~mL}^{2}$.

WATER-DISCHARGE RECORDS

PERIOD OF RECORD. - September 1904 to February 1905 (gage heights only). May 1905 to Decenber 1906 , January to December 1907 and July to October 1908 (gage heights only). November 1908 to December 1910 , January ig11 to January 1912 and May to June 1912 (gage heights only). August 1912 to September 1955 , October 1962 to current year. Monthly or annual discharge only some periods, published in WSP 1313 . Published as "near C1iff" $1904-7$. REVISED RECORDS.-WSP 1213: 1906, 1911-15, 1931, 1936-37, 1939, 1941, 1944, 1945(P), 1946(M), 1947. WSP 1283: Drainage area. WSP 1926: 1955. WDR NM-78-1: 1977

GAGE - - Water-stage recorder. Elevation of gage is 4,090 ft above National Geodetic Vertical Datum of 1929 , from plane table survey. Prior to Dec. 31, 1907 , nonrecording gage at site 13.5 mi upstream at different datum. May plane table survey 16,1909 nonrecording gage nocording 8 age at site 13.5 mi upstream at different datum. May 14,1908 , to July 16,1909 , nonrecording 8 age at site 0.2

REMARKS. - Watex-discharge records good except for estimated daily discharges, which are poor. Diversions for irrigation of about 5,000 acres upstream from atation. Gage height and rain gage satelifte telemeter at station. DISCAARGE, CUBIC FEET PER SECOND, WATER YEAR OCTOBER 1995 TO SEPTEMBER 1996

\begin{tabular}{|c|c|c|c|c|c|c|c|c|c|c|c|c|}
\hline DAX & OCT & Mov & DEC & JAN & FEB & MAR & APR & MAY & JuN & JUL & AUG & SEP \\
\hline $\begin{array}{l}1 \\
2 \\
3 \\
4 \\
5\end{array}$ & $\begin{array}{l}74 \\
66 \\
63 \\
61 \\
57\end{array}$ & $\begin{array}{l}70 \\
76 \\
80 \\
83 \\
86\end{array}$ & $\begin{array}{l}87 \\
86 \\
85 \\
85 \\
83\end{array}$ & $\begin{array}{r}70 \\
89 \\
88 \\
89 \\
103\end{array}$ & $\begin{array}{r}92 \\
108 \\
117 \\
116 \\
111\end{array}$ & $\begin{array}{l}84 \\
83 \\
75 \\
64 \\
81\end{array}$ & $\begin{array}{l}66 \\
64 \\
62 \\
45 \\
43\end{array}$ & $\begin{array}{l}42 \\
43 \\
40 \\
25 \\
21\end{array}$ & $\begin{array}{c}20 \\
19 \\
18 \\
12 \\
8.9\end{array}$ & $\begin{array}{l}23 \\
34 \\
47 \\
73 \\
45\end{array}$ & $\begin{array}{r}100 \\
106 \\
105 \\
94 \\
105\end{array}$ & $\begin{array}{l}286 \\
286 \\
238 \\
261 \\
226\end{array}$ \\
\hline $\begin{array}{r}6 \\
7 \\
8 \\
9 \\
10\end{array}$ & $\begin{array}{l}56 \\
54 \\
52 \\
53 \\
55\end{array}$ & $\begin{array}{l}94 \\
94 \\
96 \\
94 \\
82\end{array}$ & $\begin{array}{l}83 \\
63 \\
72 \\
68 \\
71\end{array}$ & $\begin{array}{l}68 \\
68 \\
88 \\
84 \\
85\end{array}$ & $\begin{array}{r}104 \\
102 \\
98 \\
95 \\
92\end{array}$ & $\begin{array}{l}80 \\
81 \\
76 \\
75 \\
72\end{array}$ & $\begin{array}{l}46 \\
67 \\
70 \\
67 \\
64\end{array}$ & $\begin{array}{l}30 \\
36 \\
35 \\
34 \\
33\end{array}$ & $\begin{array}{l}8.2 \\
14 \\
18 \\
19 \\
19\end{array}$ & $\begin{array}{l}61 \\
68 \\
57 \\
37 \\
45\end{array}$ & $\begin{array}{r}96 \\
89 \\
82 \\
79 \\
516\end{array}$ & $\begin{array}{l}192 \\
169 \\
142 \\
132 \\
139\end{array}$ \\
\hline $\begin{array}{l}11 \\
12 \\
13 \\
14 \\
15\end{array}$ & $\begin{array}{l}56 \\
55 \\
40 \\
45 \\
55\end{array}$ & $\begin{array}{l}74 \\
75 \\
75 \\
79 \\
88\end{array}$ & $\begin{array}{l}66 \\
64 \\
64 \\
81 \\
82\end{array}$ & $\begin{array}{l}70 \\
88 \\
86 \\
87 \\
89\end{array}$ & $\begin{array}{l}91 \\
90 \\
91 \\
93 \\
96\end{array}$ & $\begin{array}{l}61 \\
73 \\
70 \\
72 \\
76\end{array}$ & $\begin{array}{l}63 \\
59 \\
59 \\
47 \\
48\end{array}$ & $\begin{array}{l}32 \\
19 \\
15 \\
23 \\
30\end{array}$ & $\begin{array}{r}19 \\
20 \\
22 \\
021 \\
020\end{array}$ & $\begin{array}{r}78 \\
128 \\
108 \\
234 \\
246\end{array}$ & $\begin{array}{l}232 \\
168 \\
145 \\
129 \\
114\end{array}$ & $\begin{array}{r}145 \\
297 \\
241 \\
1020 \\
2260\end{array}$ \\
\hline $\begin{array}{l}16 \\
17 \\
18 \\
19 \\
20\end{array}$ & $\begin{array}{l}57 \\
57 \\
54 \\
54 \\
55\end{array}$ & $\begin{array}{r}88 \\
93 \\
99 \\
101 \\
100\end{array}$ & $\begin{array}{l}83 \\
70 \\
74 \\
89 \\
90\end{array}$ & $\begin{array}{l}87 \\
86 \\
87 \\
89 \\
89\end{array}$ & $\begin{array}{l}98 \\
94 \\
94 \\
90 \\
88\end{array}$ & $\begin{array}{l}73 \\
70 \\
69 \\
67 \\
69\end{array}$ & $\begin{array}{l}47 \\
49 \\
57 \\
56 \\
55\end{array}$ & $\begin{array}{l}28 \\
28 \\
26 \\
18 \\
15\end{array}$ & $\begin{array}{r}020 \\
019 \\
20 \\
019 \\
018\end{array}$ & $\begin{array}{l}305 \\
224 \\
192 \\
179 \\
156\end{array}$ & $\begin{array}{r}101 \\
90 \\
164 \\
123 \\
116\end{array}$ & $\begin{array}{r}1080 \\
618 \\
423 \\
332 \\
281\end{array}$ \\
\hline $\begin{array}{l}21 \\
22 \\
23 \\
24 \\
25\end{array}$ & $\begin{array}{l}56 \\
42 \\
45 \\
64 \\
63\end{array}$ & $\begin{array}{l}93 \\
91 \\
92 \\
74 \\
65\end{array}$ & $\begin{array}{l}86 \\
87 \\
68 \\
69 \\
69\end{array}$ & $\begin{array}{l}88 \\
90 \\
87 \\
86 \\
83\end{array}$ & $\begin{array}{l}85 \\
83 \\
79 \\
72 \\
73\end{array}$ & $\begin{array}{l}65 \\
66 \\
51 \\
54 \\
68\end{array}$ & $\begin{array}{l}39 \\
56 \\
58 \\
56 \\
54\end{array}$ & $\begin{array}{l}14 \\
19 \\
22 \\
22 \\
22\end{array}$ & $\begin{array}{l}18 \\
19 \\
17 \\
13 \\
13\end{array}$ & $\begin{array}{r}130 \\
0110 \\
\theta 100 \\
0110 \\
120\end{array}$ & $\begin{array}{l}589 \\
372 \\
296 \\
377 \\
340\end{array}$ & $\begin{array}{l}248 \\
223 \\
204 \\
196 \\
189\end{array}$ \\
\hline $\begin{array}{l}26 \\
27 \\
28 \\
29 \\
30 \\
31\end{array}$ & $\begin{array}{l}63 \\
62 \\
63 \\
52 \\
54 \\
59\end{array}$ & $\begin{array}{r}67 \\
79 \\
80 \\
82 \\
88 \\
---\end{array}$ & $\begin{array}{l}87 \\
90 \\
90 \\
82 \\
65 \\
68\end{array}$ & $\begin{array}{l}67 \\
66 \\
86 \\
84 \\
85 \\
82\end{array}$ & $\begin{array}{r}82 \\
83 \\
84 \\
84 \\
\ldots-\infty\end{array}$ & $\begin{array}{l}67 \\
64 \\
62 \\
60 \\
66 \\
68\end{array}$ & $\begin{array}{r}52 \\
48 \\
33 \\
39 \\
43 \\
---\end{array}$ & $\begin{array}{l}23 \\
16 \\
13 \\
16 \\
19 \\
20\end{array}$ & $\begin{array}{l}13 \\
13 \\
25 \\
25 \\
26 \\
-\end{array}$ & $\begin{array}{r}115 \\
87 \\
80 \\
81 \\
85 \\
83\end{array}$ & $\begin{array}{l}403 \\
337 \\
384 \\
429 \\
341 \\
353\end{array}$ & $\begin{array}{l}179 \\
172 \\
163 \\
154 \\
173 \\
-\end{array}$ \\
\hline $\begin{array}{l}\text { TOTAL } \\
\text { MEAN } \\
\text { MAX } \\
\text { MIN } \\
\text { AC-ET }\end{array}$ & $\begin{array}{r}1742 \\
56.2 \\
74 \\
40 \\
3460\end{array}$ & $\begin{array}{r}2538 \\
84.6 \\
101 \\
65 \\
5030\end{array}$ & $\begin{array}{r}2407 \\
77.6 \\
90 \\
63 \\
4770\end{array}$ & $\begin{array}{r}2594 \\
83.7 \\
103 \\
66 \\
5150\end{array}$ & $\begin{array}{r}2685 \\
92.6 \\
117 \\
72 \\
5330\end{array}$ & $\begin{array}{r}2162 \\
69.7 \\
84 \\
51 \\
4290\end{array}$ & $\begin{array}{r}3612 \\
53.7 \\
70 \\
33 \\
3200\end{array}$ & $\begin{array}{r}779 \\
25.1 \\
43 \\
13 \\
1550\end{array}$ & $\begin{array}{r}536.1 \\
17.9 \\
26 \\
8.2 \\
1060\end{array}$ & $\begin{array}{r}3441 \\
111 \\
305 \\
23 \\
6830\end{array}$ & $\begin{array}{r}6975 \\
225 \\
589 \\
79 \\
13830\end{array}$ & $\begin{array}{r}10669 \\
356 \\
2260 \\
132 \\
21160\end{array}$ \\
\hline
\end{tabular}

STATISTICS OF MONTHLY MEAN DATA FOR WATER YEARS 1963 - 1996, BY WATER YEAR (WY)

\begin{tabular}{|c|c|c|c|c|c|c|c|c|c|c|c|c|}
\hline $\begin{array}{l}\text { MEAN } \\
\text { MAX } \\
\text { (WY) } \\
\text { MrN } \\
\text { (WY) }\end{array}$ & $\begin{array}{r}207 \\
1768 \\
1973 \\
27.6 \\
1974\end{array}$ & $\begin{array}{r}154 \\
911 \\
1995 \\
55.1 \\
1974\end{array}$ & $\begin{array}{r}355 \\
2200 \\
1979 \\
60.0 \\
1981\end{array}$ & $\begin{array}{r}338 \\
2987 \\
1993 \\
64.9 \\
1971\end{array}$ & $\begin{array}{r}438 \\
1692 \\
1993 \\
53.8 \\
1971\end{array}$ & $\begin{array}{r}500 \\
1438 \\
1978 \\
40.0 \\
1971\end{array}$ & $\begin{array}{r}311 \\
1155 \\
1973 \\
41.2 \\
1971\end{array}$ & $\begin{array}{r}198 \\
1068 \\
1992 \\
25.1 \\
1996\end{array}$ & $\begin{array}{r}62.4 \\
278 \\
1992 \\
12.0 \\
1974\end{array}$ & $\begin{array}{r}75.6 \\
287 \\
1986 \\
15.6 \\
1978\end{array}$ & $\begin{array}{r}212 \\
1182 \\
1988 \\
40.9 \\
1994\end{array}$ & $\begin{array}{r}227 \\
1315 \\
3975 \\
22.2 \\
1978\end{array}$ \\
\hline
\end{tabular}

SUMMARY STATISTICS

ANNUAL TOTAL

ANNUAL. MEAN

HIGHEST ANNUAL MEAN

LOWEST ANNUAL MEAN

BIGHEST DAILY MEAN

COWEST DATLY MEAN

ANNUAL, SEVEN-DAY MINTMUM

INSTANTANEOUS PEAK ELOW

INSTANTANEOUS PEAK STAGE

INSTANTANEOUS LOW FLOW

ANNUAL RUNOFF (AC-FT)

10 PERCENT EXCEEDS

SO PERCENT EXCEEDS

90 PFRCENT HXCERDS
FOR 1995 CALENDAR YEAR

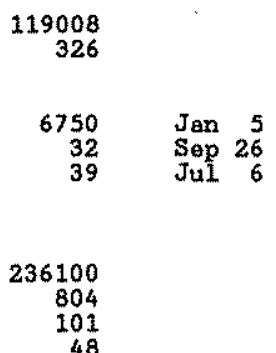

FOR 1996 WATER YEAR

38140.1

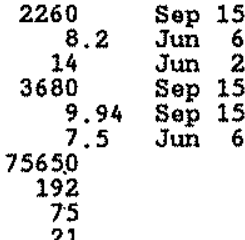

WATER YEARS 1963 - 1996

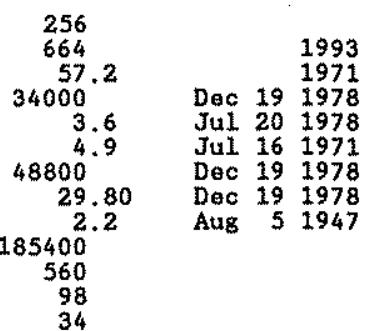

- Estimatod 
GILA RIVER BASIN

09431500 GILA RIVER NEAR REDROCX, MM -- Continued

WATER-QUALITY RECORDS

PERIOD OF RECORD.--Water years 1967 to current year.

WATER QUALITY DATA, WATER YEAR OCTOBER 1995 TO SEFTEMBER 1996

\begin{tabular}{|c|c|c|c|c|c|c|c|c|c|c|c|}
\hline DATE & TIME & $\begin{array}{l}\text { DIS- } \\
\text { CHARGE, } \\
\text { INST } \\
\text { CUBIC } \\
\text { FEET } \\
\text { PER } \\
\text { SECOND } \\
(00061)\end{array}$ & $\begin{array}{l}\text { SEE } \\
\text { CIFIC } \\
\text { CON- } \\
\text { DUCT- } \\
\text { ANCE } \\
\text { (US/CM) } \\
(00095)\end{array}$ & $\begin{array}{c}\text { PH } \\
\text { WATER } \\
\text { WROLLE } \\
\text { FIELD } \\
\text { (STAND- } \\
\text { ARD } \\
\text { URITS) } \\
(00400)\end{array}$ & $\begin{array}{c}\text { TEMPER- } \\
\text { ATURE } \\
\text { AIR } \\
\text { (DEG C) } \\
(00020)\end{array}$ & $\begin{array}{c}\text { TEMPER- } \\
\text { ATURE } \\
\text { WATER } \\
\text { (DEG C) } \\
(00010)\end{array}$ & $\begin{array}{l}\text { TUR- } \\
\text { BID } \\
\text { ITY } \\
(\text { NTU) } \\
(00076)\end{array}$ & $\begin{array}{c}\text { BARO- } \\
\text { METEE } \\
\text { PRES- } \\
\text { SURE } \\
\text { (MAI } \\
\text { OF } \\
\text { HG) } \\
(00025)\end{array}$ & $\begin{array}{r}\text { OXYGEN, } \\
\text { DIS- } \\
\text { SOLVED } \\
\text { (MG/L) } \\
(00300)\end{array}$ & $\begin{array}{c}\text { OXYGER, } \\
\text { DIS- } \\
\text { SOLVED } \\
\text { (RER- } \\
\text { CENT } \\
\text { SATUR- } \\
\text { ATION) } \\
(00301)\end{array}$ & $\begin{array}{l}\text { OXYGER } \\
\text { DERAAND, } \\
\text { CHEM- } \\
\text { ICAL } \\
\text { (HIGR } \\
\text { LEVEL) } \\
\text { (RKG/L) } \\
(00340)\end{array}$ \\
\hline \multirow{6}{*}{ 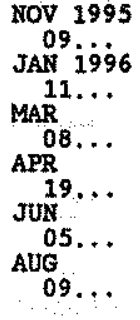 } & 1300 & 98 & 521 & 8.2 & 22.0 & 13.0 & 3.6 & 656 & 9.7 & 107 & $<10$ \\
\hline & 1630 & 71 & 425 & 8.8 & 20.0 & 20.0 & 0.50 & 664 & 9.7 & 123 & $<10$ \\
\hline & 1330 & 83 & 402 & 8.2 & 15.0 & 16.5 & 3.3 & 666 & 10.2 & 120 & $<10$ \\
\hline & 1230 & 58 & 430 & 8.1 & 17.0 & 23.5 & 1.0 & 659 & 8.0 & 109 & 11 \\
\hline & 1530 & 9.4 & 421 & 8.5 & 38.5 & 28.5 & 1.1 & 656 & 9.6 & 145 & $<10$ \\
\hline & 1030 & 84 & 405 & 8.3 & 28.0 & 24.5 & 69 & 664 & 7.0 & 97 & 31 \\
\hline DATE & $\begin{array}{c}\text { COLI- } \\
\text { FORM, } \\
\text { FECAL, } \\
0.7 \\
\text { WM-MF } \\
\text { (COLS. } \\
100 \mathrm{ML}) \\
(31625)\end{array}$ & $\begin{array}{l}\text { STREP- } \\
\text { TOCOCCI } \\
\text { FECAL, } \\
\text { KF AGAR } \\
\text { (COLS, } \\
\text { PER } \\
100 \text { ML) } \\
(31673)\end{array}$ & $\begin{array}{c}\text { HARD- } \\
\text { NESS } \\
\text { TOTAL. } \\
\text { (MG/L } \\
\text { AS } \\
\text { CACO3) } \\
(00900)\end{array}$ & $\begin{array}{l}\text { HARD- } \\
\text { NESS } \\
\text { NONCARB } \\
\text { DISSOLV } \\
\text { FLD. AS } \\
\text { CACO3 } \\
(\text { MG/L) } \\
(00904)\end{array}$ & $\begin{array}{c}\text { CALCIUM } \\
\text { DIS- } \\
\text { SOLVED } \\
\text { (MG/L } \\
\text { AS CA) } \\
(00915)\end{array}$ & $\begin{array}{c}\text { MAGNE- } \\
\text { SIUM, } \\
\text { DIS- } \\
\text { SOLVED } \\
\text { (MG/L } \\
\text { AS MG) } \\
(00925)\end{array}$ & $\begin{array}{c}\text { SODIUM, } \\
\text { DIS- } \\
\text { SOLVED } \\
\text { (MGGL } \\
\text { AS NA) } \\
(00930)\end{array}$ & $\begin{array}{c}\text { SODIUM } \\
\text { AD- } \\
\text { SORP- } \\
\text { TION } \\
\text { RATIO } \\
(00931)\end{array}$ & $\begin{array}{c}\text { POTAS- } \\
\text { SIUM, } \\
\text { DIS- } \\
\text { SOLVED } \\
(\mathrm{NG} / \mathrm{L} \\
\text { AS K) } \\
(00935)\end{array}$ & $\begin{array}{c}\text { BICAR- } \\
\text { BONATE } \\
\text { WATER } \\
\text { DIS IT } \\
\text { FIELD } \\
\text { WG/L AS } \\
\text { HCO3 } \\
(00453)\end{array}$ & $\begin{array}{c}\text { CAR- } \\
\text { BONATE } \\
\text { WATER } \\
\text { DIS IT } \\
\text { FIELD } \\
\text { NG/L AS } \\
\text { CO3 } \\
(00452)\end{array}$ \\
\hline Nov 1995 & 30 & 14 & 140 & 0 & 42 & 8.5 & 29 & 1 & 2.1 & 145 & 19 \\
\hline $\operatorname{MAR}^{11} \cdots$ & 30 & K17 & 150 & 37 & 44 & 8.7 & 30 & 1 & 2.1 & 132 & 0 \\
\hline $\begin{array}{l}08 \ldots \\
A P R\end{array}$ & 64 & 21 & 130 & 0 & 40 & 8.3 & 31 & 1 & 2.0 & 166 & 0 \\
\hline JUN $19 .$. & $\mathrm{K} 18$ & K8 & 150 & 3 & 45 & 8.7 & 34 & 1 & 2.2 & 177 & 0 \\
\hline $\begin{array}{c}05 . . \\
\text { AUG }\end{array}$ & 39 & -- & 140 & 15 & 40 & 9.3 & 35 & 1 & 2.6 & 118 & 16 \\
\hline $09 .$. & 210 & 290 & 130 & 0 & 38 & 7.9 & 30 & 1 & 2.9 & 174 & 0 \\
\hline & & & & & & & & & & & \\
\hline DATE & $\begin{array}{l}\text { ALKA- } \\
\text { LINITY } \\
\text { WAT DIS } \\
\text { TOT ITT } \\
\text { FIELD } \\
\text { NGSL AS } \\
\text { CACOS } \\
(39086)\end{array}$ & $\begin{array}{c}\text { ALKA- } \\
\text { LINITY } \\
\text { LAB } \\
\text { (MG/L } \\
\text { AS } \\
\text { CACO3) } \\
(90410)\end{array}$ & $\begin{array}{c}\text { SULFATE } \\
\text { DIS- } \\
\text { SOLVED } \\
\text { (NG L } \\
\text { AS SO4) } \\
(00945)\end{array}$ & $\begin{array}{l}\text { CHLO- } \\
\text { RIDE, } \\
\text { DIS- } \\
\text { SOLVED } \\
\text { (MSG/L } \\
\text { AS CL) } \\
(00940)\end{array}$ & $\begin{array}{l}\text { FLUO- } \\
\text { RIDE, } \\
\text { DIS- } \\
\text { SOLVED } \\
\text { (MG/L } \\
\text { AS F) } \\
(00950)\end{array}$ & $\begin{array}{c}\text { SOLIDS, } \\
\text { RESIDUE } \\
\text { AT. } 180 \\
\text { DEG. C } \\
\text { DIS - } \\
\text { SOLVED } \\
\text { (NGG/L) } \\
(70300)\end{array}$ & $\begin{array}{c}\text { SOLIDS, } \\
\text { SUR OF } \\
\text { CONSTI- } \\
\text { TUENTS, } \\
\text { DIS- } \\
\text { SOLVED } \\
(\text { MG } / L) \\
(70301)\end{array}$ & $\begin{array}{l}\text { RESIDUE } \\
\text { TOTAL } \\
\text { AT 10S } \\
\text { DEG.CC, } \\
\text { SUS- } \\
\text { PENDED } \\
\text { (ME/L) } \\
(00530)\end{array}$ & $\begin{array}{l}\text { NITRO- } \\
\text { GEN, ARY- } \\
\text { MONIA + } \\
\text { ORGANIC } \\
\text { TOTAL } \\
\text { (NGELL } \\
\text { AS N) } \\
(00625)\end{array}$ & $\begin{array}{c}\text { PBOS- } \\
\text { PHORUS } \\
\text { TOTAL } \\
\text { (NGS/L } \\
\text { AS P) } \\
(00665)\end{array}$ & $\begin{array}{c}\text { ANTI- } \\
\text { BONY } \\
\text { TORAL } \\
\text { (UG/L } \\
\text { AS SB) } \\
(01097)\end{array}$ \\
\hline $\begin{array}{l}\text { NoV } 1995 \\
09 \\
\text { JAN } 19966\end{array}$ & 151 & 14.9 & 39 & 12 & 1.9 & 256 & 225 & 37 & $<0.20$ & 0.060 & $<1$ \\
\hline${ }_{M A R}^{11} \cdots$ & 108 & 149 & 41 & 14 & 2.1 & 270 & 207 & 4 & 0.21 & 0.300 & $<1$ \\
\hline APR & 136 & 145 & 37 & 14 & 2 & 255 & 216 & 10 & 0.21 & 0.030 & $<1$ \\
\hline JuN & 145 & 154 & 43 & 14 & 2.0 & 268 & 236 & 6 & 0.23 & 0.020 & $<1$ \\
\hline AUG $05 .$. & 123 & 126 & $\begin{array}{l}61 \\
35\end{array}$ & .5 & 2 & 280 & 23 & 5 & 0.31 & $<0.020$ & $<1$ \\
\hline & 143 & 146 & 35 & 14 & 1.9 & 266 & 215 & 206 & 0.53 & 0.360 & \\
\hline
\end{tabular}


GILA RIVER BASIN

09431500 GILA RIVER NEAR REDROCK, NM - - Continued

WATER-QUALITY RECORDS

WATER QUALITY DATA, WATER YEAR OCTOBER 1995 TO SEPTEMBER 1996

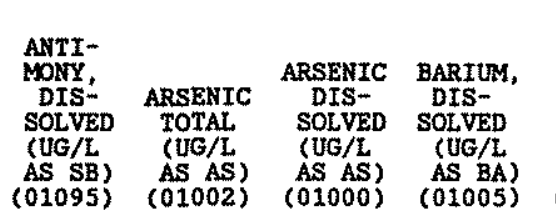

$\begin{array}{ll}\text { BERYL- } & \\ \text { LILM, } & \text { BERYL- } \\ \text { TOTAL } & \text { LIUM, } \\ \text { RECOV- } & \text { DIS- } \\ \text { ERABLE } & \text { SOLVED } \\ \text { (UG/L } & \text { (UG/L } \\ \text { AS BE) } & \text { AS BE) } \\ \text { (01012) } & (01010)\end{array}$
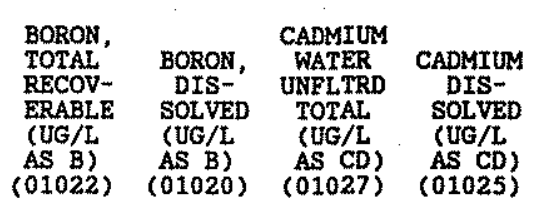

CHRO-

MIUM,

TOTAL.

RECOV-

ERABLE

(UG/L)

AS CR)

$(01095)(01002)(01000)(01005)$

$\begin{array}{lccc}<10 & <0.50 & 40 & 40 \\ <10 & <0.50 & 20 & 50 \\ <10 & <0.50 & 50 & 30 \\ -- & <0.50 & 50 & 50 \\ <10 & <0.50 & 50 & 54 \\ <10 & 1.3 & 50 & 43\end{array}$

40
50
30
50
54
43

$\begin{array}{rrr}<1 & <1.0 & <1 \\ <1 & <1.0 & <1 \\ <1 & <1.0 & <1 \\ <1 & <1.0 & <1 \\ <1 & <1.0 & 2 \\ <1 & <1.0 & 3\end{array}$

\begin{tabular}{|c|c|c|c|}
\hline Nov 1995 & $<1.0$ & 2 & 1 \\
\hline $\begin{array}{l}\text { JAN } 1996 \\
11 \ldots \\
\text { MAR }\end{array}$ & $<1.0$ & 1 & 2 \\
\hline & $<1,0$ & 1 & 1 \\
\hline & $<1.0$ & $<1$ & 1 \\
\hline & $<1.0$ & 1 & 1 \\
\hline $09 \ldots$ & $<1.0$ & 2 & 2 \\
\hline DATE & $\begin{array}{l}\text { CERO- } \\
\text { MIUM, } \\
\text { DIS- } \\
\text { SOLVED } \\
\text { (UG/L } \\
\text { AS CR) } \\
(01030)\end{array}$ & $\begin{array}{c}\text { COPPER, } \\
\text { TOTAL. } \\
\text { RECOV- } \\
\text { ERABLE } \\
\text { (UG/1. } \\
\text { AS CU) } \\
\text { (01042) }\end{array}$ & $\begin{array}{l}\text { COPPER, } \\
\text { DIS } \\
\text { SOLVED } \\
\text { (UG/L } \\
\text { AS CU) } \\
(01040)\end{array}$ \\
\hline
\end{tabular}

20
18
17
18
18
28
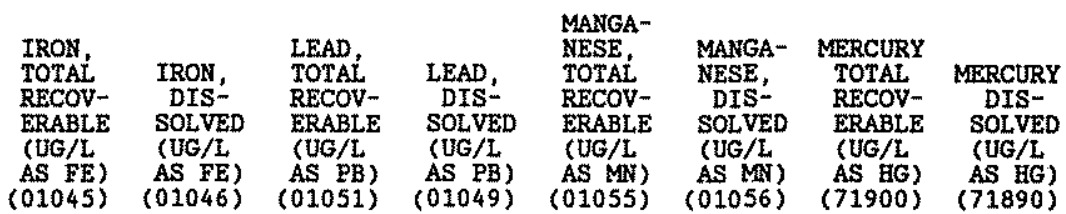

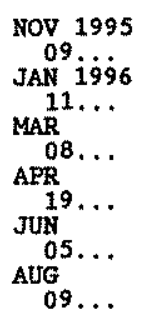

\begin{tabular}{|c|c|c|c|c|c|c|c|c|c|c|}
\hline 1.2 & 8 & $<1.0$ & 340 & 6.0 & 1 & $<1,0$ & 20 & 2.0 & $<0.10$ & $<0.1$ \\
\hline$<1.0$ & 2 & $<1.0$ & 90 & 7.0 & $<1$ & $<1.0$ & 10 & 5.0 & $<0.10$ & $<0.1$ \\
\hline$<1,0$ & 2 & 2.0 & 170 & 5.0 & $<1$ & $<1.0$ & 10 & 5.0 & $<0.10$ & $<0.1$ \\
\hline$<1.0$ & 3 & 4.0 & 120 & 11 & $<1$ & $<1.0$ & 10 & 3.0 & $<0.10$ & $<0.1$ \\
\hline 1.2 & 2 & 2.0 & so & 6.0 & $<1$ & $<1.0$ & $<10$ & 3.0 & $<0.10$ & $<0.1$ \\
\hline 1.2 & 19 & 1.0 & 50000 & $<3.0$ & 6 & $<1.0$ & 210 & 3.0 & $<0.10$ & $<0.1$ \\
\hline
\end{tabular}

NICKEL,

TOTAL"

RECOV- DIS-

ERABLE SOLVED

(UG/L (UG/L

$\begin{array}{cc}\text { AS NI) } & \text { AS NI) } \\ (01067) & (01065)\end{array}$

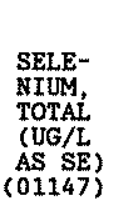

SELE-
NIUM,
DIS-
SOLVED
(UG/L
AS SE)
$(01145)$

SILVER,
DIS-
SOLVED
(UG/L
AS AG)
$(01075)$

ZINC,
TOTAL
RECOV-
ERABLE
(UG/L
AS ZN)
$(01092)$

ZINC,
DIS-
SOLVED
(UG/L
AS ZN)
(01090)

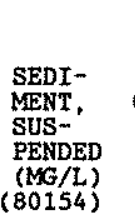

$\begin{array}{cc}\text { SEDI- } & \text { SED. } \\ \text { MENT, } & \text { SUSP. } \\ \text { DIS- } & \text { SIEVE } \\ \text { CHARGE, } & \text { DIAM. } \\ \text { SUS- } & \times \text { FINER } \\ \text { PENDED } & \text { THAN } \\ \text { (T/DAY) } & .062 \mathrm{MM} \\ \text { (B0155) } & (70331)\end{array}$

NOV 1995
09
JAN 1996
$11 \ldots$
MAR $\ldots$
$08 \ldots$
APR
$19 \ldots$
JUN $\ldots$
$05 \ldots$
AUG $\ldots$

$<100<10$
$<100<10$
$<100<10$
$<100<10$
$<100<10$
$<100<10$

$<1$

$<1$

$<1.0$

9.0

$$
29
$$

7.7

1.9

$<1.0$

20

$<3.0$

10

$<10<3.0$

16

3.6

4.0

42

6.6

0.46

$<10$

5.0

18

$<3.0 \quad 219 \quad 50$

49
72
77
28
61
94


GILA RIVIR BASIN

09432000 GILA RIVER BELOW BLUE CREEK, NEAR VIRDEN, NM

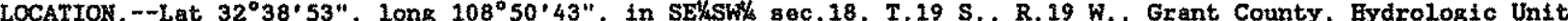
15040002 , on laft bank at head of canyon, 1.4 mi downstream from Blue Creek, 10 mi east of Virden, and 16 mi upstrean Erom How Hexico-Arizone State line.

DRAINAGE AREA. --3,203 mi2, excluding Animas River basin,

PERIOD OF RECORD. - May to November 1914, March to September 19is, July 1927 to current year. July 1927 to

May 1931 monthly dischargo only, publishod in WSP 1313 computed as sum of flow lit Virden Bridge, 9 mi

downstream, and in Sunset Canal. Published as "Gila River near Duncan, Ariz," $1914-15$ and as "Gila River at

Fuller's Ranch, neax Duncan, Ariz, ". 1931-38.

REVISED RECORDS.-WSP 1283: Dralnage area, WSP 1313: 1929, 1931-32(M).

GAGE,-Water-gtage recorder. Elevation of gage $1 \mathrm{~s} 3,875 \mathrm{ft}$ above sea level, from river-profile map. May 11 , 1914, to Sept. 30, 1915, at s1te 6 mi domstream, 1,000 st upstream from intake of Sunset Canal. June 1 to July 7, 1931, nonrecording gage at present site and datum, Since April 18, 1980, gupplementary gage on Ioft bank 800 ft downtream at same datum. Since June 1980, crest-stage gages supplementary gage sito. Since Nov. 1990, water-8toge recorder at supplomentary gago.

REMARKS,--Records fair. Station is above all Duncan Valley diversions. Diversions for irrigation of about 6,200 acres above station.

AVRAGE DISCHARGE.--69 (water years 1928-96), $215 \mathrm{ft} / \mathrm{s}, 155,800$ acre-ft/yr; median of yearly mean discharges, $150 \mathrm{ft}^{3} / \mathrm{s}, 109,000$ acre-ft/yr.

DISCHARGE, CUBIC FEET PER SECOND, WATER YEAR OCTOBER 1995 TO SEPTEMBER 1996 DATLY MEAN VALUES

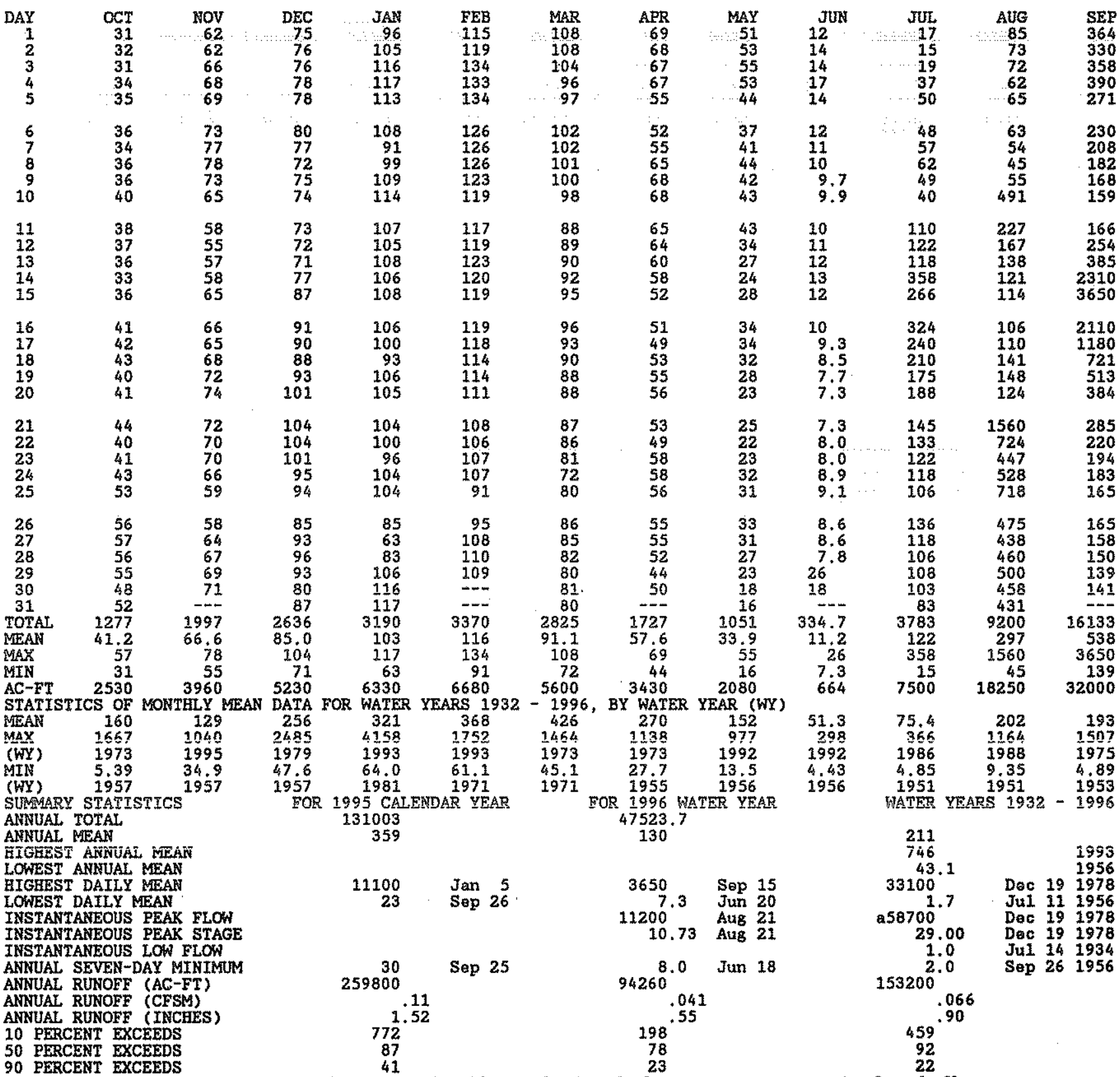

a-From rating curve extended above $38,000 \mathrm{ft} / \mathrm{s}$, on basis of slope-area measurement of peak flon. 
GILA RTVER BASIN

09442680 SAN FRANCISCO RIVER NEAT RESERVE, NM

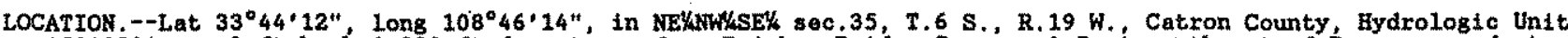
15040004 , on left bank $1,300 \mathrm{ft}$ downstream from Rainbow Bridge Canyon, 1.7 mi northwest of Reserve, and at mile 563.1 .

DRAINAGE AREA. $--350 \mathrm{~mL}^{2}$, approxtmately.

PERIOD OF RECORD.--March 1959 to current year.

REVISED RECORDS. --WDR NM-78-1; 1977. WDR NM-84-1: 1973, 1979-80.

GAGE. -Water-stage recorder. Elovation of gage is 5,820 ft above National Geodetic Vextical Datum of 1929 , from topographic map. Prior to Dec. 15, 1972 at site 1,800 ft upstream at different datum.

REMARKS.--Records fair except for estimated dally discharges, which are poor. Posstble minor regulation by Lune Lake, 27 mi upstream. Diversions for irrigation of about 280 acres upstream from station. sevaral observations of water temperature were made during the year. U.S. Geological Survey satelifte telemeter at station.

EXTREMES OUTSIDE PERIOD OF RECORD.--Maximum stage, about $15 \mathrm{ft}$, as determined in 1962 from old floodmarks. Major floods of Nov. 26, 1905 and Dec. 3, 1906, exceeded 20,000 fts/s at Alma (downstream). See WSP 1313.

DISCHARGE, CUAIC FEET PER SECOND, WATER YEAR OCTOBER 1995 TO SEPTEMBER 1996

\begin{tabular}{|c|c|c|c|c|c|c|c|c|c|c|c|c|}
\hline $\mathrm{DAY}$ & oct & NOV & DEC & JAN & FEB & MAR & AFR & MAY & JUN & JUL & AUtG & SEP \\
\hline $\begin{array}{l}1 \\
2 \\
3 \\
4 \\
5\end{array}$ & $\begin{array}{l}8.5 \\
5.5 \\
5.9 \\
3.5 \\
5.5\end{array}$ & $\begin{array}{l}3.3 \\
6.8 \\
5.1 \\
5.1 \\
7.4\end{array}$ & $\begin{array}{l}8.8 \\
6.7 \\
6.3 \\
6.2 \\
6.2\end{array}$ & $\begin{array}{r}9.7 \\
9.4 \\
9.0 \\
11.5 \\
8.5\end{array}$ & $\begin{array}{l}12 \\
8.1 \\
12 \\
11 \\
9.7\end{array}$ & $\begin{array}{l}8.5 \\
8.2 \\
8.2 \\
8.2 \\
4.7\end{array}$ & $\begin{array}{l}6.2 \\
7.0 \\
8.7 \\
6.9 \\
6.2\end{array}$ & $\begin{array}{r}3.6 \\
3.0 \\
3.7 \\
4.0 \\
03.9\end{array}$ & $\begin{array}{l}2.1 \\
1.7 \\
1.9 \\
1.6 \\
1.6\end{array}$ & $\begin{array}{r}6.9 \\
7.1 \\
6.3 \\
07.7 \\
07.3\end{array}$ & $\begin{array}{l}2.5 \\
4.2 \\
7.3 \\
6.5 \\
4.6\end{array}$ & $\begin{array}{r}4.7 \\
4.0 \\
3.7 \\
3.0 \\
16\end{array}$ \\
\hline $\begin{array}{r}6 \\
7 \\
8 \\
9 \\
10\end{array}$ & $\begin{array}{l}4.5 \\
5.2 \\
2.8 \\
2.9 \\
5.1\end{array}$ & $\begin{array}{l}6.8 \\
6.2 \\
5.3 \\
5.8 \\
5.7\end{array}$ & $\begin{array}{l}6.3 \\
8.8 \\
9.2 \\
6.7 \\
6.0\end{array}$ & $\begin{array}{l}8.5 \\
9.8 \\
8.7 \\
8.8 \\
9.4\end{array}$ & $\begin{array}{l}6.6 \\
8.6 \\
9.4 \\
8.0 \\
8.9\end{array}$ & $\begin{array}{l}6.7 \\
8.0 \\
6.8 \\
7.1 \\
8.9\end{array}$ & $\begin{array}{l}8.0 \\
8.2 \\
8.0 \\
7.2 \\
3.2\end{array}$ & $\begin{array}{l}03.8 \\
03.7 \\
03.9 \\
03.6 \\
03.9\end{array}$ & $\begin{array}{l}1.6 \\
1.3 \\
1.6 \\
1.8 \\
2.1\end{array}$ & $\begin{array}{l}98.0 \\
15 \\
19 \\
18 \\
015\end{array}$ & $\begin{array}{r}3.5 \\
3.5 \\
5.1 \\
12 \\
6.6\end{array}$ & $\begin{array}{r}11 \\
5.0 \\
2.5 \\
5.1 \\
6.5\end{array}$ \\
\hline $\begin{array}{l}11 \\
12 \\
13 \\
14 \\
15\end{array}$ & $\begin{array}{l}4.0 \\
4.7 \\
2.0 \\
1.4 \\
3.5\end{array}$ & $\begin{array}{l}5.6 \\
6.2 \\
6.4 \\
6.1 \\
6.6\end{array}$ & $\begin{array}{l}5.9 \\
5.4 \\
6.6 \\
6.9 \\
6.7\end{array}$ & $\begin{array}{l}8.0 \\
7.8 \\
9.0 \\
9.3 \\
8.9\end{array}$ & $\begin{array}{l}8.8 \\
8.3 \\
7.3 \\
8.4 \\
8.6\end{array}$ & $\begin{array}{l}9.7 \\
8.7 \\
5.5 \\
4.2 \\
5.3\end{array}$ & $\begin{array}{l}3.1 \\
4.1 \\
5.1 \\
5.6 \\
6.5\end{array}$ & $\begin{array}{r}3.0 \\
3.9 \\
4.2 \\
03.9 \\
04.0\end{array}$ & $\begin{array}{l}1.9 \\
1.9 \\
2.2 \\
3.4 \\
5.1\end{array}$ & $\begin{array}{c}09.0 \\
.87 \\
01.1 \\
01.3 \\
5.7\end{array}$ & $\begin{array}{l}6.1 \\
3.1 \\
3.0 \\
2.8 \\
2.3\end{array}$ & $\begin{array}{l}6.0 \\
7.1 \\
15 \\
45 \\
14\end{array}$ \\
\hline $\begin{array}{l}16 \\
17 \\
18 \\
19 \\
20\end{array}$ & $\begin{array}{l}2.1 \\
3.0 \\
2.1 \\
3.5 \\
2.7\end{array}$ & $\begin{array}{l}6.8 \\
7.4 \\
6.7 \\
6.6 \\
6.5\end{array}$ & $\begin{array}{l}5.1 \\
8.5 \\
8.5 \\
7.9 \\
7.9\end{array}$ & $\begin{array}{l}8.2 \\
8.9 \\
8.1 \\
8.3 \\
8.9\end{array}$ & $\begin{array}{l}7.7 \\
7.5 \\
9.5 \\
9.0 \\
7.0\end{array}$ & $\begin{array}{l}5.7 \\
6.3 \\
7.2 \\
6.9 \\
6.6\end{array}$ & $\begin{array}{l}3.8 \\
4.5 \\
4.0 \\
5.0 \\
4.4\end{array}$ & $\begin{array}{r}03.9 \\
03.6 \\
03.1 \\
2.9 \\
3.7\end{array}$ & $\begin{array}{l}4.6 \\
3.9 \\
3.0 \\
2.5 \\
2.9\end{array}$ & $\begin{array}{l}2.3 \\
.87 \\
1.3 \\
.82 \\
.88\end{array}$ & $\begin{array}{r}2.0 \\
1.9 \\
18 \\
4.2 \\
4.0\end{array}$ & $\begin{array}{l}11 \\
6.6 \\
5.6 \\
5.4 \\
5.6\end{array}$ \\
\hline $\begin{array}{l}21 \\
22 \\
23 \\
24 \\
25\end{array}$ & $\begin{array}{l}4.2 \\
3.6 \\
3.5 \\
2.8 \\
2.7\end{array}$ & $\begin{array}{l}6.1 \\
6.5 \\
6.5 \\
7.6 \\
6.5\end{array}$ & $\begin{array}{l}7.0 \\
6.8 \\
8.4 \\
7.0 \\
8.7\end{array}$ & $\begin{array}{l}8.0 \\
9.6 \\
8.4 \\
7.8 \\
8.8\end{array}$ & $\begin{array}{l}6.5 \\
6.2 \\
5.9 \\
4.5 \\
7.6\end{array}$ & $\begin{array}{l}6.8 \\
6.6 \\
5.5 \\
6.0 \\
6.2\end{array}$ & $\begin{array}{l}5.9 \\
7.4 \\
6.8 \\
5.6 \\
4.0\end{array}$ & $\begin{array}{l}3.5 \\
2.4 \\
1.9 \\
2.8 \\
2.0\end{array}$ & $\begin{array}{l}2.8 \\
2.8 \\
2.6 \\
3.2 \\
3.0\end{array}$ & $\begin{array}{l}.88 \\
.90 \\
.94 \\
.96 \\
.91\end{array}$ & $\begin{array}{r}2.6 \\
6.3 \\
10 \\
9.1 \\
7.9\end{array}$ & $\begin{array}{l}5.3 \\
5.6 \\
5.6 \\
3.1 \\
7.4\end{array}$ \\
\hline $\begin{array}{l}26 \\
27 \\
28 \\
29 \\
30 \\
31\end{array}$ & $\begin{array}{l}5.5 \\
6.0 \\
4.2 \\
3.1 \\
3.7 \\
5.9\end{array}$ & $\begin{array}{l}6.2 \\
6.0 \\
5.7 \\
5.9 \\
7.8 \\
\end{array}$ & $\begin{array}{l}7.1 \\
6.7 \\
7.3 \\
7.9 \\
7.8 \\
8.8\end{array}$ & $\begin{array}{l}7.3 \\
7.3 \\
8.1 \\
9.9 \\
9.9 \\
8.9\end{array}$ & $\begin{array}{r}9.8 \\
10 \\
9.8 \\
8.8 \\
--\end{array}$ & $\begin{array}{l}5.8 \\
5.2 \\
5.8 \\
4.6 \\
5.1 \\
5.7\end{array}$ & $\begin{array}{l}2.8 \\
2.3 \\
2.3 \\
4.0 \\
4.3 \\
-.-7\end{array}$ & $\begin{array}{l}3.2 \\
3.6 \\
3.2 \\
3.1 \\
3.0 \\
2.9\end{array}$ & $\begin{array}{l}2.5 \\
3.9 \\
5.0 \\
5.6 \\
6.4 \\
-.-\end{array}$ & $\begin{array}{l}.82 \\
.86 \\
1.3 \\
1.5 \\
2.3 \\
2.4\end{array}$ & $\begin{array}{r}11 \\
8.6 \\
7.2 \\
6.1 \\
6.3 \\
5.6\end{array}$ & $\begin{array}{l}4.4 \\
6.4 \\
4.9 \\
4.2 \\
3.8 \\
\end{array}$ \\
\hline $\begin{array}{l}\text { TOTAL } \\
\text { MEAN } \\
\text { MAX } \\
\text { MIN } \\
\text { AC-ET }\end{array}$ & $\begin{array}{r}123.6 \\
3.99 \\
8.5 \\
1.4 \\
245\end{array}$ & $\begin{array}{r}187.2 \\
6.24 \\
7.8 \\
3.3 \\
371\end{array}$ & $\begin{array}{r}224.1 \\
7.23 \\
9.2 \\
5.1 \\
445\end{array}$ & $\begin{array}{r}272.2 \\
8.78 \\
11 \\
7.3 \\
540\end{array}$ & $\begin{array}{r}245.5 \\
8.47 \\
12 \\
4.5 \\
487\end{array}$ & $\begin{array}{r}204.7 \\
6.60 \\
9.7 \\
4.2 \\
406\end{array}$ & $\begin{array}{r}161.1 \\
5.37 \\
8.7 \\
2.3 \\
320\end{array}$ & $\begin{array}{r}104.9 \\
3.38 \\
4.2 \\
1.9 \\
208\end{array}$ & $\begin{array}{r}86.5 \\
2.88 \\
6.4 \\
1.3 \\
172\end{array}$ & $\begin{array}{r}148.21 \\
4.78 \\
19 \\
.82 \\
294\end{array}$ & $\begin{array}{r}183.9 \\
5.93 \\
18 \\
1.9 \\
365\end{array}$ & $\begin{array}{r}233.5 \\
7.78 \\
45 \\
2.5 \\
463\end{array}$ \\
\hline
\end{tabular}

STATISTICS OF MONTHLY MEAN DATA FOR WATER YEARS $1959 \div 1996$, BY WATER YEAR (WY)

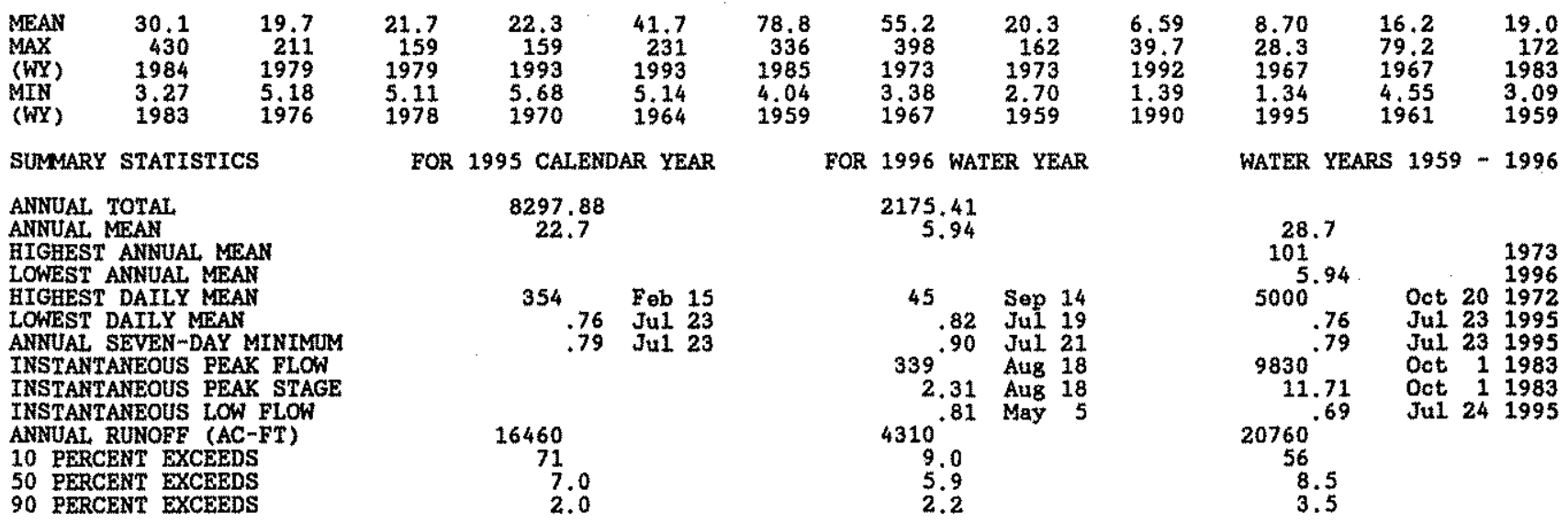

e Estimatod 


\section{GILA RIVER BASIN}

09442692 TULAROSA RIVER ABOVE ARACON, MI

LOCATION. --Lat $33^{\circ} 53^{\prime} 29^{\prime \prime}$, long $108^{\circ} 30^{\prime} 54^{*}$, in NE/4WW sec.9, T.5 S., R. 16 W., Catron County, Bydrologic Untt 15040004 , on right bank $0.4 \mathrm{mi}$ upstream from first diversion, $i .4$ mi northeast of Aragon, and 8 mi upstream from Apache Creak.

DRAINAGE AREA, $--94 \mathrm{mi}^{2}$.

PERIOD OF RECORD.--July 1966 to September 1996 (discontinued). 1955 to 1965 at site 0.6 mi upstrean (drainage area, $\left.89 \mathrm{mi}^{2}\right)$, annual maximum only.

REVISED RECORD, - -WDR NM-78-1: 1977.

GAGE. - Water-stage recorder and concrete control. Elevation of gage is 6,750 ft above National Geodetic Vertical Datum of 1929 , from topographic map.

REMARKS - -Records good.

DISCEARGE, CUBIC FEET PER SECOND, WATER YEAR OCTOBER 1995 TO SEPTEYBER 1996

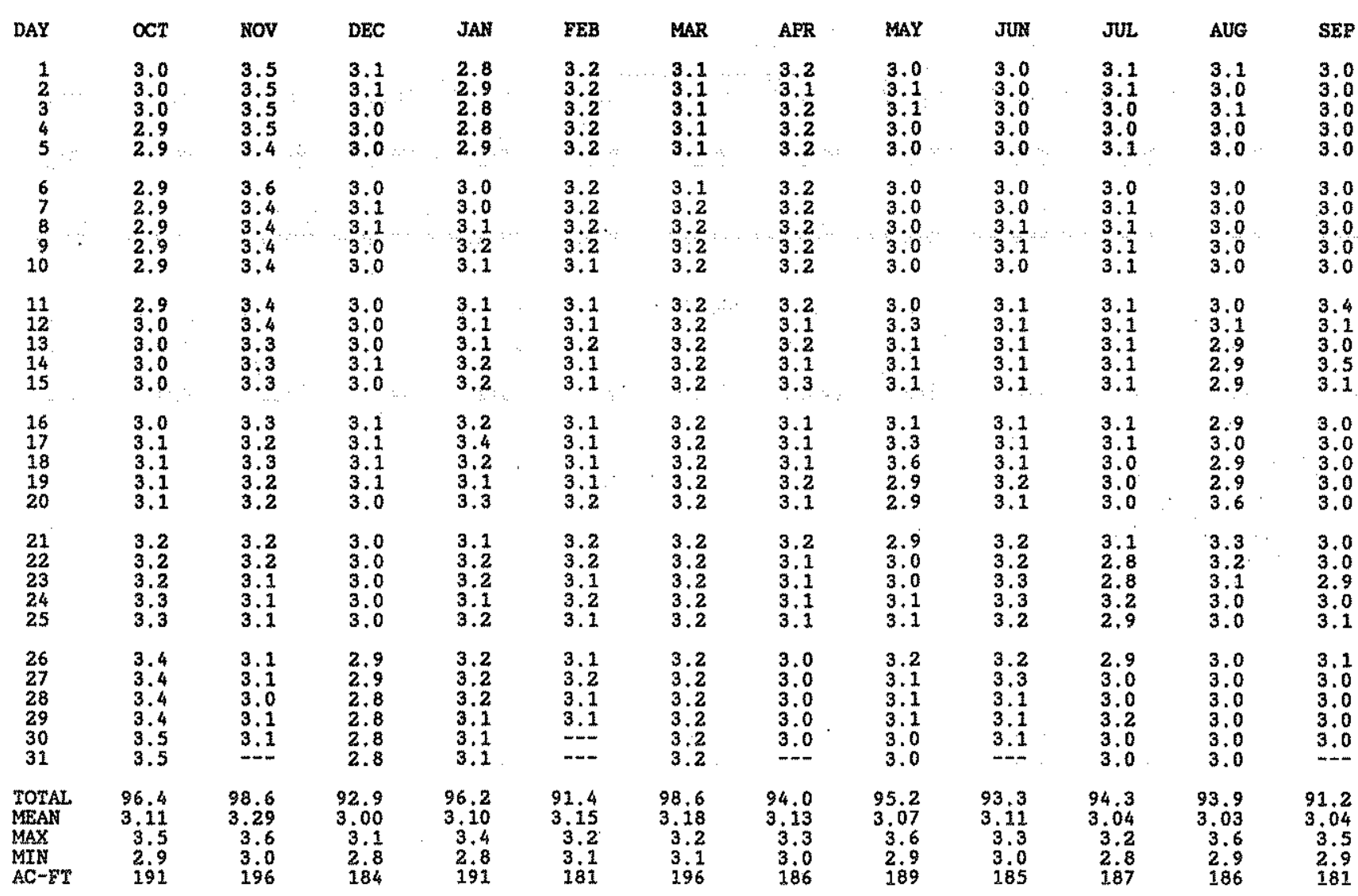

STATISTYCS OF MONTHLY MEAN DATA FOR WATER YEARS 1966 - 1996, BY WATER YEAR (WY)

\begin{tabular}{|c|c|c|c|c|c|c|c|c|c|c|c|c|}
\hline $\begin{array}{l}\text { MAAN } \\
M A Y \\
\text { (WY) } \\
\text { MIN } \\
\text { (WY) }\end{array}$ & $\begin{array}{l}3,34 \\
20.6 \\
1984 \\
2.59 \\
1983\end{array}$ & $\begin{array}{l}3.02 \\
3.87 \\
1973 \\
2.62 \\
1984\end{array}$ & $\begin{array}{l}3.42 \\
7.72 \\
1985 \\
2.63 \\
1984\end{array}$ & $\begin{array}{l}3.29 \\
10.4 \\
1993 \\
2.32 \\
1984\end{array}$ & $\begin{array}{l}4.15 \\
10.8 \\
1968 \\
2.49 \\
1984\end{array}$ & $\begin{array}{l}4.99 \\
17.2 \\
1.985 \\
2.41 \\
1984\end{array}$ & $\begin{array}{l}4.88 \\
24.2 \\
1973 \\
2.33 \\
1984\end{array}$ & $\begin{array}{l}3.02 \\
3.64 \\
1992 \\
2.66 \\
1984\end{array}$ & $\begin{array}{l}2.89 \\
3.51 \\
1995 \\
2.25 \\
1994\end{array}$ & $\begin{array}{l}3.02 \\
5.12 \\
1992 \\
2.45 \\
1986\end{array}$ & $\begin{array}{l}2.98 \\
3.41 \\
1987 \\
2.47 \\
1986\end{array}$ & $\begin{array}{l}3.00 \\
3.65 \\
1976 \\
2.49 \\
1968\end{array}$ \\
\hline
\end{tabular}

SUMMARY STATISTICS

ANNUAI, TOTAL

ANNUAI MEAN

HIGHEST ANNUAL MEAN

LOWEST ANDUAI MEAN

FITGEST DAILY MEAN

LOWEST DAILY MEAN

ANNUAL SEVEN-DAY MINIMIN

INSTANTANEOUS PEAK FLOW

INSTANTANEOUS PEAK STAGE

INSTANTANEOUS LOW FLOW

ANNUAL RUNOFF (AC-FT)

10 PERCENT EXCEEDS

50 PERCENT EXCEEDS

90 PERCENT EXCEEDS
FOR 1995 CALENDAR YEAR

$\begin{array}{cc}1275.0 & \\ 3.49 & \\ & \\ 53 & \text { Feb } 15 \\ 2.8 & \text { Dec } 28 \\ 2.9 & \text { Dec } 25 \\ & \\ 2530 & \\ 3.5 & \\ 3.1 & \\ 3.0 & \end{array}$

FOR 1996 WATER YEAR

1136.0

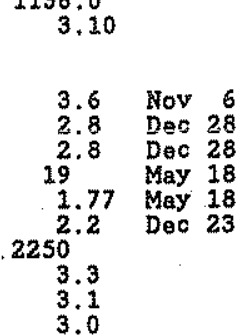

WATER YEARS 1966 - 1996

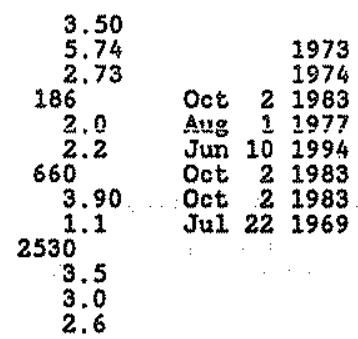


GILA RIVER BASIN

09444000 SAN FRANCISCO RIVER NEAR GLENWOOD, NM

LOCATION, --Lat $33^{\circ} 14^{\circ} 48^{\prime \prime}$, long $108^{\circ} 52^{\prime} 47^{\prime \prime}$, In NEKWWK sec, 23, T, 12 S., R.20 W., Catron County, Hydrologic Unit 15040004 , on left bank $0,2 \mathrm{mi}$ upstream from hot springs, $5 \mathrm{ml}$ south of Glenwood, 6 mi downstream from Whitewater Crook, and at mile 511.5 .

DRAINAGE' AREA $=-1,653 \mathrm{mi}^{2}$

PERTOD OF RFCORD - October 1927 to current year. Monthly discharge only for some periods, published in WSP 1313 . REVISED RECORDS:--WSP 1213: 1931, 1934, 1936-37, 1940-42, 1943-44(M), 1945-47, WSP 1283: Drainage area, WDR AM78-1: 1977. WDR NM-79-1: 1973, 1975-77 (P)'.

GAGE. - Water-stage recorder and crest-stage gage. Elevation of gage is 4,560 ft above National Geodetic Vertical Datum of 1929, from topographic map. Prior to Feb. 15, 1934, at 5 ite 4.5 mi upstream at datum 98.82 ft higher. REMARKS.--Records fair except for estinated daily discharges, which are poor. Diversions for irrigation of about 2,000 acres upstream from station. Gage height and rain gage satellite telemeter at station.

EXTREMES OUTSIDE PERIOD OF RECORD,--Major floods probably occurred Jan. 19 and Oct. 14.1916 when discharges of $60,000 \mathrm{ft}^{3} / \mathrm{s}$ or greater were computed for station at Clifton, AZ. On Nov, 26, 1905, a peak of 25, 000 ft's/s was measured (by float-area method) at station at Alma (about 12 mi upstream, drainage area, 1,560 mi2); a imilar measurement of $21,000 \mathrm{ft} / \mathrm{s}$ was made at the Alma station for peak of Dec. 3.1906.

DISCBARGE, CUBIC FEET RER SECOND, WATER YEAR OCTOBER 1995 TO SEPTEMBER 1996

\begin{tabular}{|c|c|c|c|c|c|c|c|c|c|c|c|c|}
\hline DAY & $\alpha C r$ & NOV & $D E C$ & JAN & FEB & MAR & APR & MAY & JUN & JUL & ALG & SEP \\
\hline $\begin{array}{l}1 \\
2 \\
3 \\
4 \\
5\end{array}$ & $\begin{array}{l}46 \\
39 \\
34 \\
29 \\
24\end{array}$ & $\begin{array}{l}21 \\
24 \\
24 \\
24 \\
24\end{array}$ & $\begin{array}{l}24 \\
23 \\
23 \\
25 \\
25\end{array}$ & $\begin{array}{l}35 \\
33 \\
33 \\
33 \\
34\end{array}$ & $\begin{array}{l}45 \\
47 \\
46 \\
45 \\
43\end{array}$ & $\begin{array}{l}35 \\
34 \\
35 \\
34 \\
36\end{array}$ & $\begin{array}{l}22 \\
22 \\
20 \\
21 \\
23\end{array}$ & $\begin{array}{l}16 \\
15 \\
16 \\
19 \\
21\end{array}$ & $\begin{array}{l}13 \\
11 \\
11 \\
13 \\
14\end{array}$ & $\begin{array}{l}27 \\
28 \\
33 \\
31 \\
30\end{array}$ & $\begin{array}{l}28 \\
28 \\
23 \\
25 \\
26\end{array}$ & $\begin{array}{l}35 \\
36 \\
36 \\
36 \\
34\end{array}$ \\
\hline $\begin{array}{r}6 \\
7 \\
8 \\
9 \\
10\end{array}$ & $\begin{array}{l}23 \\
19 \\
18 \\
18 \\
18\end{array}$ & $\begin{array}{l}25 \\
26 \\
27 \\
27 \\
27\end{array}$ & $\begin{array}{l}26 \\
28 \\
30 \\
30 \\
30\end{array}$ & $\begin{array}{l}35 \\
35 \\
35 \\
35 \\
35\end{array}$ & $\begin{array}{l}43 \\
41 \\
40 \\
41 \\
41\end{array}$ & $\begin{array}{l}34 \\
33 \\
31 \\
32 \\
31\end{array}$ & $\begin{array}{l}24 \\
24 \\
23 \\
21 \\
19\end{array}$ & $\begin{array}{l}21 \\
20 \\
18 \\
17 \\
17\end{array}$ & $\begin{array}{l}13 \\
12 \\
14 \\
14 \\
15\end{array}$ & $\begin{array}{l}26 \\
50 \\
50 \\
62 \\
54\end{array}$ & $\begin{array}{r}51 \\
21 \\
20 \\
24 \\
369\end{array}$ & $\begin{array}{l}35 \\
33 \\
34 \\
33 \\
36\end{array}$ \\
\hline $\begin{array}{l}11 \\
12 \\
13 \\
14 \\
15\end{array}$ & $\begin{array}{l}19 \\
19 \\
19 \\
20 \\
20\end{array}$ & $\begin{array}{l}27 \\
27 \\
26 \\
26 \\
25\end{array}$ & $\begin{array}{l}30 \\
31 \\
31 \\
30 \\
29\end{array}$ & $\begin{array}{l}36 \\
36 \\
36 \\
36 \\
35\end{array}$ & $\begin{array}{l}41 \\
40 \\
40 \\
41 \\
39\end{array}$ & $\begin{array}{l}32 \\
29 \\
27 \\
25 \\
26\end{array}$ & $\begin{array}{l}21 \\
23 \\
24 \\
24 \\
23\end{array}$ & $\begin{array}{l}18 \\
16 \\
15 \\
14 \\
15\end{array}$ & $\begin{array}{l}14 \\
13 \\
14 \\
18 \\
17\end{array}$ & $\begin{array}{r}70 \\
76 \\
105 \\
70 \\
80\end{array}$ & $\begin{array}{l}e 74 \\
e 53 \\
049 \\
045 \\
044\end{array}$ & $\begin{array}{l}34 \\
16 \\
17 \\
18 \\
19\end{array}$ \\
\hline $\begin{array}{l}16 \\
17 \\
18 \\
19 \\
20\end{array}$ & $\begin{array}{l}20 \\
20 \\
19 \\
19 \\
17\end{array}$ & $\begin{array}{l}24 \\
22 \\
23 \\
23 \\
23\end{array}$ & $\begin{array}{l}30 \\
33 \\
33 \\
33 \\
34\end{array}$ & $\begin{array}{l}34 \\
36 \\
36 \\
36 \\
36\end{array}$ & $\begin{array}{l}40 \\
40 \\
40 \\
36 \\
35\end{array}$ & $\begin{array}{l}23 \\
24 \\
23 \\
23 \\
21\end{array}$ & $\begin{array}{l}19 \\
20 \\
19 \\
19 \\
21\end{array}$ & $\begin{array}{l}16 \\
16 \\
13 \\
15 \\
15\end{array}$ & $\begin{array}{l}19 \\
21 \\
21 \\
19 \\
18\end{array}$ & $\begin{array}{l}80 \\
072 \\
064 \\
052 \\
046\end{array}$ & $\begin{array}{l}040 \\
\oplus 36 \\
\oplus 51 \\
\oplus 43 \\
040\end{array}$ & $\begin{array}{l}19 \\
19 \\
19 \\
19 \\
20\end{array}$ \\
\hline $\begin{array}{l}21 \\
22 \\
23 \\
24 \\
25\end{array}$ & $\begin{array}{l}16 \\
20 \\
19 \\
19 \\
19\end{array}$ & $\begin{array}{l}23 \\
23 \\
21 \\
21 \\
21\end{array}$ & $\begin{array}{l}34 \\
34 \\
34 \\
35 \\
35\end{array}$ & $\begin{array}{l}36 \\
35 \\
35 \\
36 \\
37\end{array}$ & $\begin{array}{l}35 \\
34 \\
34 \\
36 \\
35\end{array}$ & $\begin{array}{l}18 \\
21 \\
22 \\
23 \\
24\end{array}$ & $\begin{array}{l}21 \\
17 \\
18 \\
16 \\
18\end{array}$ & $\begin{array}{l}14 \\
13 \\
12 \\
13 \\
12\end{array}$ & $\begin{array}{l}14 \\
14 \\
15 \\
15 \\
16\end{array}$ & $\begin{array}{l}054 \\
e 47 \\
040 \\
033 \\
025\end{array}$ & $\begin{array}{r}e 280 \\
e 135 \\
096 \\
e 124 \\
090\end{array}$ & $\begin{array}{l}19 \\
18 \\
18 \\
22 \\
34\end{array}$ \\
\hline $\begin{array}{l}26 \\
27 \\
28 \\
29 \\
30 \\
31\end{array}$ & $\begin{array}{l}19 \\
20 \\
22 \\
22 \\
18 \\
21\end{array}$ & $\begin{array}{l}22 \\
24 \\
24 \\
24 \\
24 \\
--\end{array}$ & $\begin{array}{l}35 \\
35 \\
35 \\
35 \\
34 \\
35\end{array}$ & $\begin{array}{l}38 \\
37 \\
38 \\
38 \\
38 \\
37\end{array}$ & $\begin{array}{r}37 \\
38 \\
36 \\
35 \\
- \\
--\infty\end{array}$ & $\begin{array}{l}23 \\
22 \\
24 \\
24 \\
22 \\
22\end{array}$ & $\begin{array}{r}16 \\
15 \\
21 \\
22 \\
20 \\
---\end{array}$ & $\begin{array}{l}15 \\
17 \\
15 \\
15 \\
17 \\
13\end{array}$ & $\begin{array}{r}15 \\
18 \\
48 \\
32 \\
32 \\
\end{array}$ & $\begin{array}{l}27 \\
26 \\
27 \\
24 \\
27 \\
25\end{array}$ & $\begin{array}{l}53 \\
37 \\
37 \\
36 \\
35 \\
35\end{array}$ & $\begin{array}{l}e 27 \\
e 25 \\
e 26 \\
e 24 \\
e 22 \\
---\end{array}$ \\
\hline $\begin{array}{l}\text { TOTAL } \\
\text { MEAN } \\
\text { MAX } \\
\text { MIN } \\
\text { AC-FT }\end{array}$ & $\begin{array}{r}675 \\
21.8 \\
46 \\
16 \\
1340\end{array}$ & $\begin{array}{r}722 \\
24.1 \\
27 \\
21 \\
1430\end{array}$ & $\begin{array}{r}959 \\
30.9 \\
35 \\
23 \\
1900\end{array}$ & $\begin{array}{r}1105 \\
35.6 \\
38 \\
33 \\
2190\end{array}$ & $\begin{array}{r}1144 \\
39.4 \\
47 \\
34 \\
2270\end{array}$ & $\begin{array}{r}833 \\
26.9 \\
36 \\
18 \\
1650\end{array}$ & $\begin{array}{r}616 \\
20.5 \\
24 \\
15 \\
1.220\end{array}$ & $\begin{array}{r}489 \\
15.8 \\
21 \\
12 \\
970\end{array}$ & $\begin{array}{r}523 \\
17.4 \\
48 \\
11 \\
1040\end{array}$ & $\begin{array}{r}1461 \\
47.1 \\
105 \\
24 \\
2900\end{array}$ & $\begin{array}{r}2048 \\
66.1 \\
369 \\
20 \\
4060\end{array}$ & $\begin{array}{r}783 \\
26.1 \\
36 \\
16 \\
1550\end{array}$ \\
\hline
\end{tabular}

STATISTICS OF MONTHLY MEAN DATA FOR WATER YEARS 1928 - 1996, BY WATER YEAR (WY)

\begin{tabular}{|c|c|c|c|c|c|c|c|c|c|c|c|c|}
\hline $\begin{array}{l}\text { MEAN } \\
\text { MAX } \\
\text { (WY) } \\
\text { MIN } \\
\text { (WX) }\end{array}$ & $\begin{array}{l}85.9 \\
2026 \\
1984 \\
9.77 \\
1966\end{array}$ & $\begin{array}{r}49.7 \\
520 \\
1979 \\
10.8 \\
1957\end{array}$ & $\begin{array}{l}88.7 \\
1068 \\
1979 \\
12.9 \\
1954\end{array}$ & $\begin{array}{r}103 \\
1568 \\
1993 \\
13.5 \\
1956\end{array}$ & $\begin{array}{r}132 \\
1034 \\
1993 \\
14.9 \\
1956\end{array}$ & $\begin{array}{r}198 \\
1036 \\
1985 \\
11.3 \\
1959\end{array}$ & $\begin{array}{r}145 \\
1049 \\
1973 \\
10.2 \\
1957\end{array}$ & $\begin{array}{r}77.5 \\
593 \\
1973 \\
8.65 \\
1956\end{array}$ & $\begin{array}{r}28.9 \\
146 \\
1992 \\
5.70 \\
1956\end{array}$ & $\begin{array}{r}37.9 \\
108 \\
1930 \\
13.2 \\
1963\end{array}$ & $\begin{array}{r}77.0 \\
392 \\
1957 \\
13.7 \\
1960\end{array}$ & $\begin{array}{r}59.1 \\
368 \\
1988 \\
7.66 \\
1956\end{array}$ \\
\hline
\end{tabular}

SUMMARY STATISTICS

ANNUAL TOTAL

ANNIAL MEAN

BIGREST ANNUAL MEAN

LOWEST ANNUAL MEAN

HIGHEST DAILY MEAN

LOWEST DAILY MEAN

ANNUAL SEVEN-DAY MINIMUM

TNSTANTANEOUS PEAK FLOW

INSTANTANEOUS PEAK STAGE

TNSTANTANEOUS LOW FLOW

ANNUAL RUNOFF (AC-FT)

10 PERCENT EXCEEDS

50 PERCENT EXCEEDS

90 PERCENT EXCEEDS

- Estimated
FOR 1995 CALENDAR YEAR

$\begin{aligned} 43168 & \\ 118 & \\ & \\ 2120 & \text { Feb } 16 \\ 16 & \text { Oct } 21 \\ 18 & \text { Jul } 30 \\ & \\ & \\ 85620 & \\ 361 & \\ 34 & \\ 20 & \end{aligned}$

FOR 1996 WATER YEAR

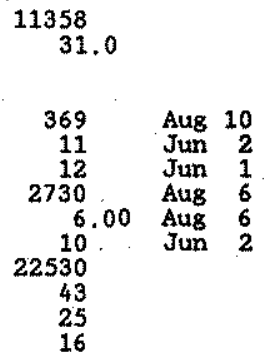

WATER YEARS 1928 - 1996

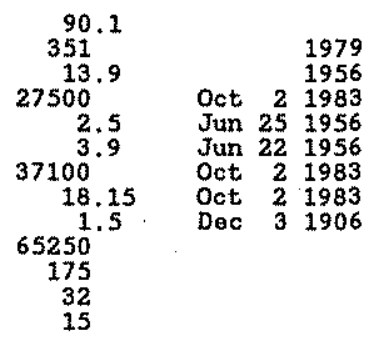




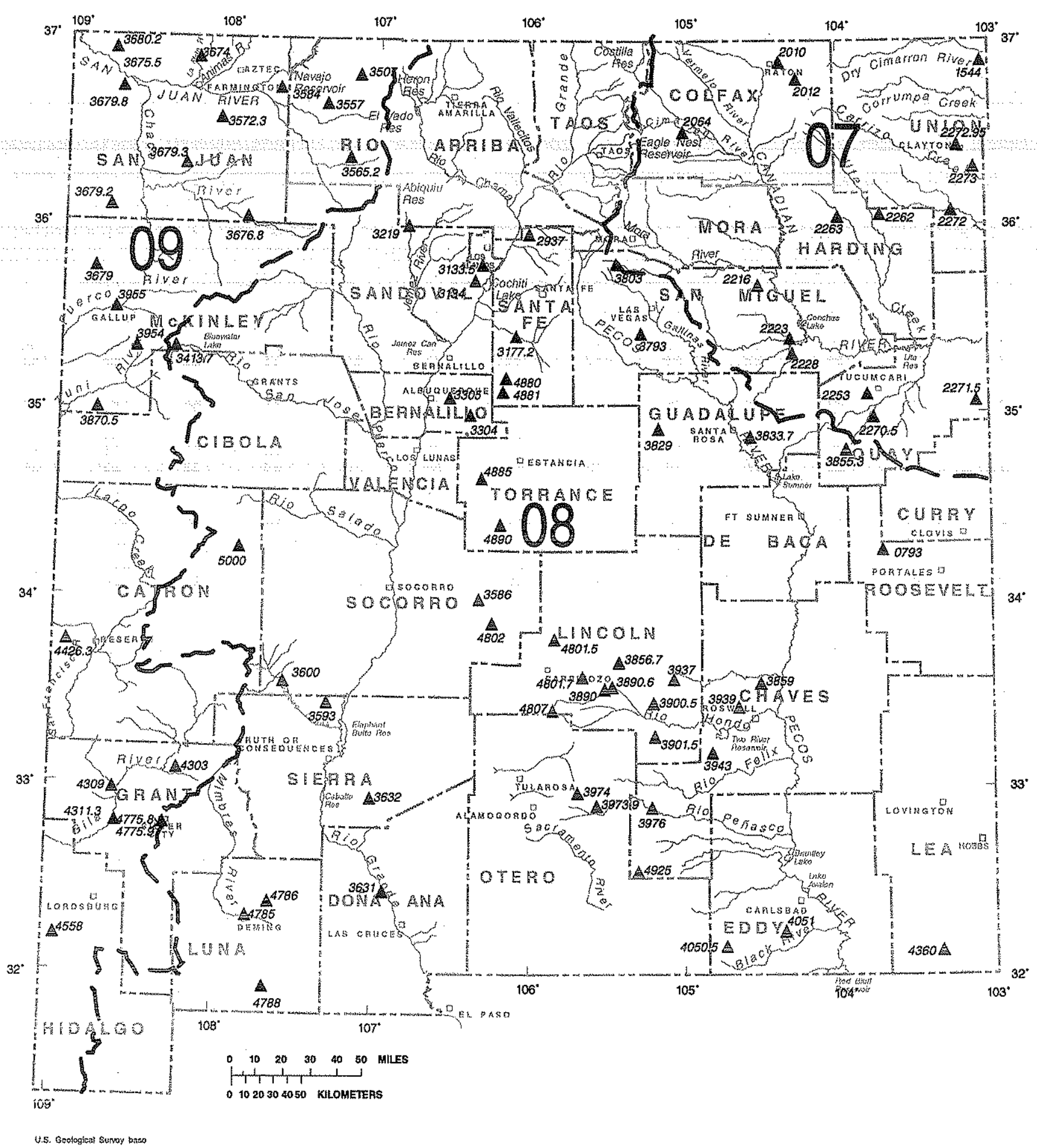

EXPLANATION

07 LOWER MISSISSIPPI RIVER BASIN NUMBER 4057 $\triangle$ CRESTSSTAGE STATION AND ABBREVATED AUMBER

08 WESTERN GULF OF MEXICO BASIN NUMBER Complete national station number is: 08

09 COLORADO RIVER BASIN NUMBER

Basin number + station number

- RIVER BASIN BOUNDARY

Figure 7.--Location of partial-record stations. 
DISCHARGE AT PARTIAL-RECORD STATIONS AND MISCELLANEOUS SITES

As the number of streams on which streanflow information is likely to be destred far exceeds the number of tream-gaging stations feasible to operate at one time, the Geological Survey collocts limited streamelow data at sites other than stream-gaging stations. When limited streanflow data are collected on a systematic basis over a period of years for use In hydrologic analyses, the site at which the data are collected 1s called a partial-record station. Data collected at these partial-record stations are usable in floodflow analyses. In addition, discharge messurements are made at other sites not included in the partial-record program. These measurements are generally made in times of drought or flood to give better areal coverage to those events. Those measurements and others collected for some special reason are called measurements at miscellaneous sites.

Records collected at partial-record stations aro presented in two tables. The first is a table of annual maximum stage and discharge at crest-stage stations. Discharge measurements mede at miscellaneous sites for both low flow and high flow are given in the second table.

\section{Crest-gtage partial-record gtations}

The following table contains annual maximum discharge for crest-stage stations. A crest-stage gage is a device that wil register the peak gtage occurring between inspections of the gage. A stage-discharge relation for each gage is developed from discharge measurements made by indirect measurenents of peak flow or by current meter. The date of the maximum discharge is not always certain but is usually determined by comparison with noarby continuousrecord stations, weathor records, or local inquixy. Only the maximum discharge for each year is given. Information on scme loper floods may have been obtained, and discharge measurements made for purposes of establishing the atage-discharge rolation, but these are not published herein. The year given in the pertod of record colum represents the first year of period oxtending through the current year unless otherwise noted. For some stations, publication of discharge is delayed panding definition of stage-discharge relationship. Published maximums are for water years.

Annual maximum discharge at crest-stage partial-record stations

\begin{tabular}{|c|c|c|c|c|c|c|c|c|}
\hline \multirow[t]{2}{*}{$\begin{array}{l}\text { Station name } \\
\text { and } \\
\text { number }\end{array}$} & \multirow[t]{2}{*}{$\begin{array}{c}\text { Location } \\
\text { and } \\
\text { drainage area }\end{array}$} & \multirow[t]{2}{*}{$\begin{array}{l}\text { Period } \\
\text { of } \\
\text { record }\end{array}$} & $\begin{array}{l}\text { Water yes } \\
\text { Date. }\end{array}$ & $\frac{\operatorname{ar} 1996 \pi}{\text { Gage }}$ & $\begin{array}{l}\text { aximum } \\
\text { chis } \\
\left(f t^{3} / s\right)\end{array}$ & $\frac{\text { Period of }}{\text { Date }}$ & 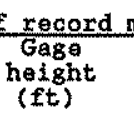 & 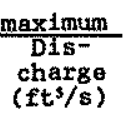 \\
\hline & & & AS RIVER & BASIN & & & & \\
\hline $\begin{array}{l}\text { Carrizozo Creek } \\
\text { near Kenton, oK. } \\
(07154400)\end{array}$ & $\begin{array}{l}\text { Lat } 36^{\circ} 52 \cdot 55^{\prime \prime}, \text { long } 103^{\circ} 01^{\prime} 05^{\prime \prime}, \\
\text { Union County, Hydrologic Unit } \\
11040001 \text {, wder bridge on } \\
\text { Nam Moxico State Highway } 406 \text {; } \\
4 \mathrm{mi} \text { southwest of Kenton, ox. } \\
\text { Drainage area is } 111 \text { mi2. }\end{array}$ & $1953-$ & $07-25-96$ & 5.85 & 2,500 & $07-06-58$ & 12.22 & 15,600 \\
\hline $\begin{array}{l}\text { Raton Creak at } \\
\text { Raton. } \\
(07201000)\end{array}$ & $\begin{array}{l}\text { Lat } 36^{\circ} 55^{\prime} 38^{\prime \prime}, \text { long } 104^{\circ} 26^{\prime} 22^{\prime \prime}, \\
\text { Colfax County, Bydrologic } \\
\text { Unit } 11080001,60 \text { ft upstraam } \\
\text { from bridge on State Bighway } \\
72 \text { at Raton, Drainago aroa } \\
\text { is } 14.4 \mathrm{mi} \text {. }\end{array}$ & $1953-96 \mathrm{~g}$ & $07-27-96$ & 1.16 & 201 & $06-17-65$ & 14.80 & 3,990 \\
\hline $\begin{array}{l}\text { Chicorica Creak } \\
\text { tributary near } \\
\text { Raton. } \\
(07201200)\end{array}$ & $\begin{array}{l}\text { Lat } 36^{\circ} 49^{\prime} 41^{\prime \prime} \text {, long } 104^{\circ} 19^{\circ} 58^{\prime \prime} \\
\text { Colfax Count, Hydrologic Unit } \\
11080001 \text {, upstream from culvert } \\
\text { on U.S. Highway } 64-87,7.7 \mathrm{mt} \\
\text { southeast of Raton. Drainage } \\
\text { area is } 5.18 \mathrm{mt2} \text {. }\end{array}$ & $1971-968$ & $07-27-96$ & 9.14 & 475 & $08-05-82$ & 18,30 & 1,340 \\
\hline $\begin{array}{l}\text { Clear Creek near } \\
\text { Ute Park. } \\
\text { (07206400) }\end{array}$ & $\begin{array}{l}\text { Lat } 36^{\circ} 31 \cdot 35^{\prime \prime}, \text { long } 105^{\circ} 10^{\prime} 30^{\prime \prime} \\
\text { Colfax County, Hydrologic Init } \\
11080002 \text {, } 0.25 \text { mi upstream } \\
\text { from mouth, and } 4 \text { mi southwest } \\
\text { of Ute Park. Drainage area is } \\
7.44 \text { mi2. }\end{array}$ & $\begin{array}{l}1962-67 \text { * } \\
1968-968\end{array}$ & $06-02-96$ & 2.49 & 49 & $06-18-65$ & 3.05 & 151 \\
\hline $\begin{array}{l}\text { Lagartija Crook } \\
\text { tributary near } \\
\text { Sanchez } \\
\text { (07221600) }\end{array}$ & $\begin{array}{l}\text { Lat } 35^{\circ} 39^{\prime} 21^{\prime \prime} \text {, long } 104^{\circ} 24^{\circ} 57^{\prime \prime}, \\
\text { San Miguel County, Hydrolog tc } \\
\text { Unit } 11080003 \text { at bridge on } \\
\text { State Highway } 419 ; 0.9^{\circ} \text { mi } \\
\text { northeast of Sanchez, } \\
\text { Drainage area is } 1.19 \mathrm{mil} \text {. }\end{array}$ & $1961-96 g$ & $07-25-96$ & 4.41 & 730 & $05-11-94$ & 5.83 & 1,500 \\
\hline $\begin{array}{l}\text { Trementina Creak } \\
\text { at Tromentina. } \\
(07222300)\end{array}$ & $\begin{array}{l}\text { Lat } 35^{\circ} 29^{\prime} 28^{\prime \prime}, \text { Long } 104^{\circ} 24^{\circ} 59^{\prime \prime} \\
\text { San Miguel County, Bydrologic } \\
\text { Unit } 1 \text { 1080005, at bridge on } \\
\text { State Elghway } 419 \text {; at } \\
\text { Trementina. Dratnage area } \\
\text { ig } 63.9 \text { mi2. }\end{array}$ & $1959-$ & $07-25-96$ & (e) & $(+)$ & $09-11-65$ & 12.00 & 14,100 \\
\hline
\end{tabular}


DISCHARGE AT PARTIAL-KECORD STATIONS AND MISCELLANEOUS SITES

Annual maximum discharge at crest-stage partial-record stations

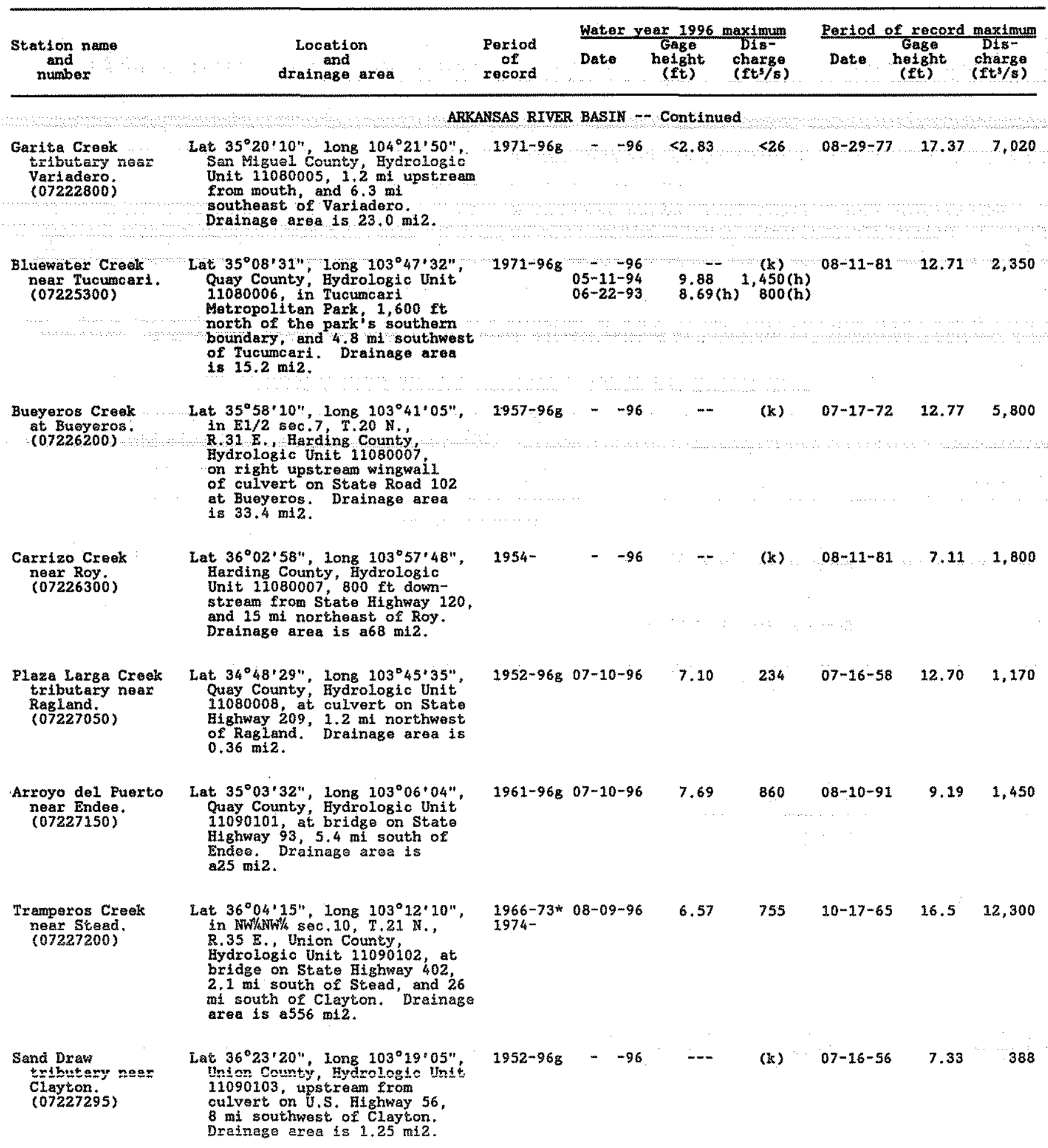


DISCHARGE AT RARTIAL-RECORD STATIONS AND MISCELLANEOUS STTES

Annual maximum discharge at crest-stage partial-record stations

ARKANSAS RIVRR BASIN -- Continued

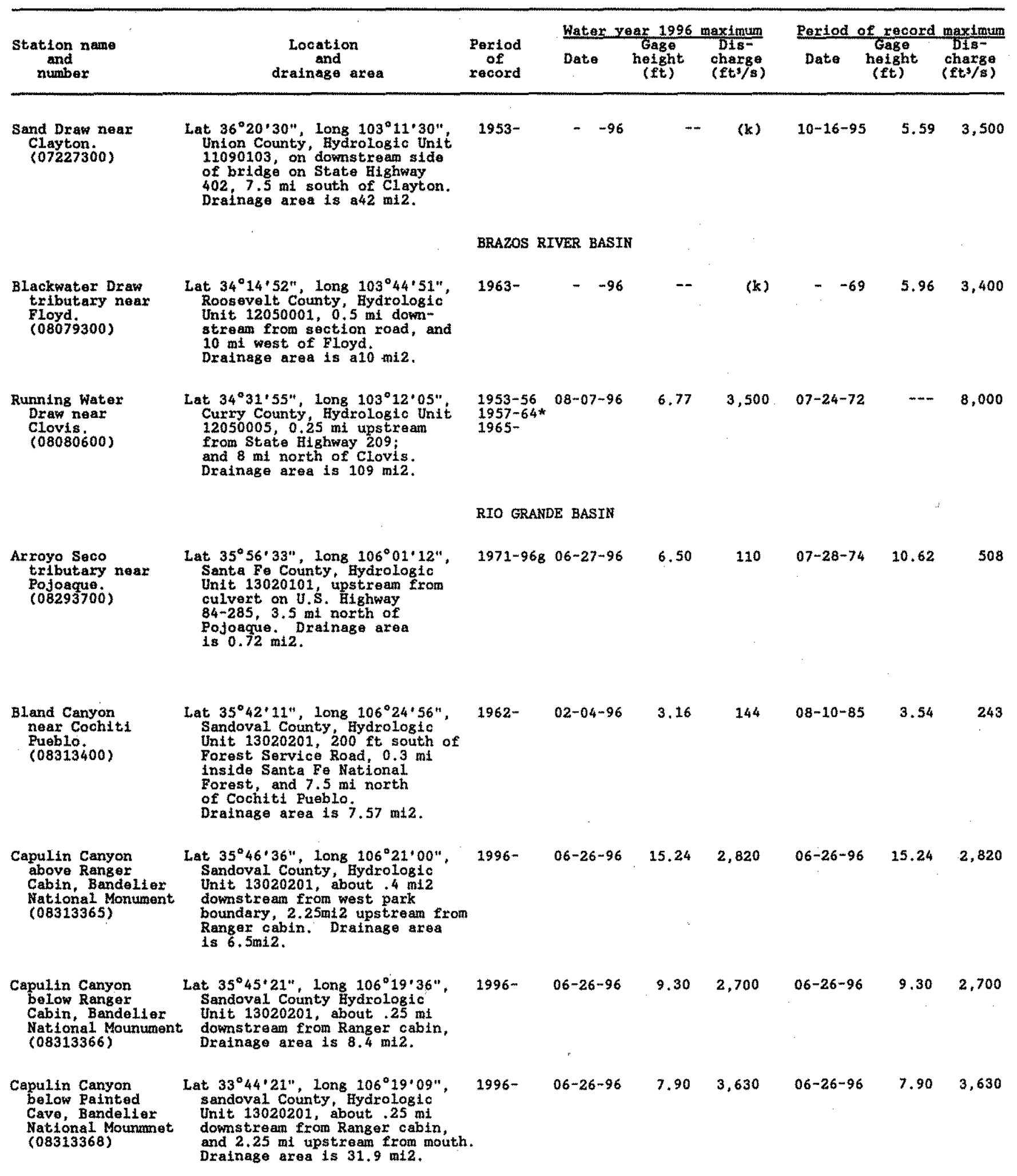




\begin{tabular}{|c|c|c|c|c|c|c|c|c|}
\hline & & & Water & year 1996 & $\operatorname{maximum}$ & Pertod & of record & maximum \\
\hline $\begin{array}{l}\text { Station name } \\
\text { and } \\
\text { numbor }\end{array}$ & $\begin{array}{l}\text { Location } \\
\text { and } \\
\text { drainage araa }\end{array}$ & $\begin{array}{l}\text { Period } \\
\text { of } \\
\text { record }\end{array}$ & Date & $\begin{array}{l}\text { Gage } \\
\text { hetght } \\
\text { (ft) }\end{array}$ & $\begin{array}{l}\text { Dig- } \\
\text { charge } \\
(\mathrm{ft} / \mathrm{s})\end{array}$ & Date & $\begin{array}{l}\text { Gage } \\
\text { height } \\
\text { (ft) }\end{array}$ & $\begin{array}{c}\text { Dts- } \\
\text { charge } \\
\left(\mathrm{ft}^{3} / \mathrm{s}\right)\end{array}$ \\
\hline
\end{tabular}

RIO GRARDE BASIN -- Continued

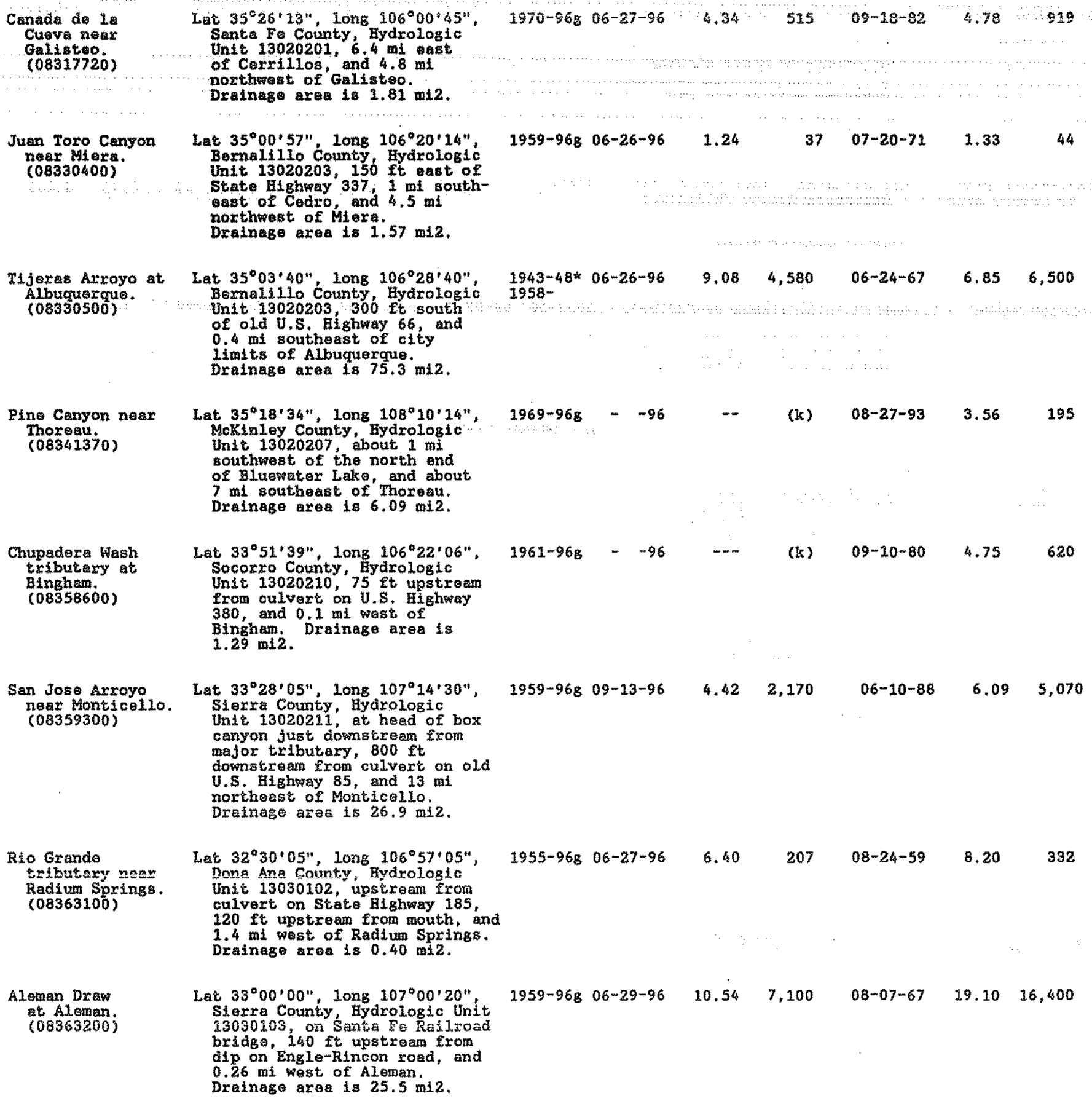


DISCHARGE AT PARTIAL-RECORD STATIONS AND MISCELLANEOUS STTES

Annual maximum dischaxge at crest-stage partial record stations

\begin{tabular}{|c|c|c|c|c|c|c|c|c|}
\hline $\begin{array}{l}\text { Station name } \\
\text { and } \\
\text { number }\end{array}$ & $\begin{array}{l}\text { Location } \\
\text { and } \\
\text { drainage area }\end{array}$ & $\begin{array}{l}\text { Perlod } \\
\text { of } \\
\text { record }\end{array}$ & $\begin{array}{l}\text { Water y } \\
\text { Date }\end{array}$ & 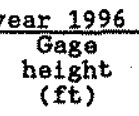 & $\begin{array}{l}\text { maximum } \\
\text { Dis } \\
\text { charge } \\
\left(\mathrm{ft}^{3} / \mathrm{s}\right)\end{array}$ & $\frac{\text { Pertod of }}{\text { Date }}$ & $\begin{array}{l}\frac{\text { record }}{\text { Gege }} \\
\text { height } \\
\text { (ft) }\end{array}$ & $\frac{\max i m u m}{\text { Dis- }}$ \\
\hline & \multicolumn{8}{|c|}{ RIO GRANDE BASIR - - Continuod } \\
\hline $\begin{array}{l}\text { Tecolote Croek } \\
\text { at Tecolote. } \\
(08379300)\end{array}$ & 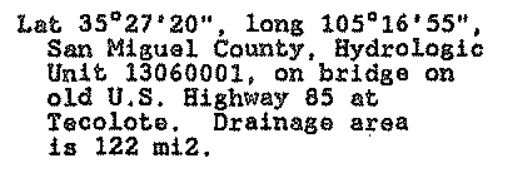 & $1954-$ & $07-10-96$ & 7.66 & 1,910 & $08-17-61$ & 12.92 & 12,300 \\
\hline $\begin{array}{c}\text { Sandoval Canyon } \\
\text { at Gallinas. } \\
(08380300)\end{array}$ & $\begin{array}{l}\text { Lar } 35^{\circ} 41^{\prime} 19^{\prime \prime} \text {, long } 105^{\circ} 21^{\prime} 17^{\prime \prime}, \\
\text { Som Miguel County, Hydrologic } \\
\text { Unt } 13060001 \text {, about } 500 \text { ft } \\
\text { upstream from culvert on State } \\
\text { gighway } 65 \text {, at north odge } \\
\text { of Galinnas. Drainage area } \\
\text { is } 7.6 \text { mi2. }\end{array}$ & $\begin{array}{l}1957 \\
1961-96 \mathrm{~g}\end{array}$ & $-\quad-96$ & -- & (m) & $08-01-66$ & 5.26 & 2,530 \\
\hline $\begin{array}{l}\text { Pecos Rivor } \\
\text { tributary near } \\
\text { Pintada. } \\
(08382900)\end{array}$ & $\begin{array}{l}\text { Lat } 34^{\circ} 58^{\circ} 06^{\prime \prime} \text {, long } 105^{\circ} 05^{\prime} 38^{\prime \prime}, \\
\text { Guadalupe County, Hydrologic } \\
\text { Unit } 13060001 \text { in Anton Chico } \\
\text { Grant 1, 500 ft south of } \\
\text { Interstate Highway } 40 \text {, } \\
\text { and } 6.8 \mathrm{mi} \text { north of Pintada. } \\
\text { Dralnage area is } 16.0 \mathrm{mi} \text {. }\end{array}$ & $1961-968$ & $06-26-96$ & $(\theta)$ & $(+)$ & $07-39-71$ & 4.80 & 6,600 \\
\hline $\begin{array}{l}\text { Pecos Rtver } \\
\text { tributary near } \\
\text { Fuerto de Luna. } \\
(08383370)\end{array}$ & 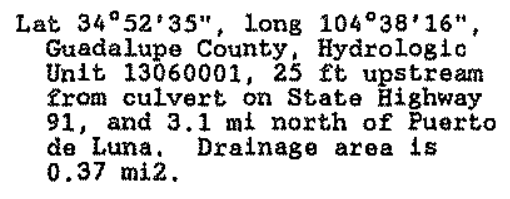 & $1961-968$ & $09-14-96$ & 7.39 & 118 & $08-23-87$ & 15.89 & 2,000 \\
\hline $\begin{array}{l}\text { Alamosa Creok } \\
\text { tributary noax } \\
\text { Jordan. } \\
\text { (08385530) }\end{array}$ & $\begin{array}{l}\text { Lat } 34^{\circ} 47^{\prime} 44^{\prime \prime} \text {, long } 103^{\circ} 58^{\circ} 07^{\prime \prime}, \\
\text { Quay Cotunty, Hydrologic Untt } \\
13060004 \text {, 500 ft upstrean from } \\
\text { dip on State Highay is6, } \\
\text { and } 6.9 \mathrm{mt} \text { west of Jordan. } \\
\text { Drainage area is } 9.71 \mathrm{mi}^{2} \text {. }\end{array}$ & $1962-968$ & $07-10-96$ & 1.44 & 5.8 & $07-11-72$ & 6.86 & 2,850 \\
\hline $\begin{array}{l}\text { Aragon Creok } \\
\text { trtbutary near } \\
\text { Encinoso } \\
\left(08385670^{\circ}\right)\end{array}$ & $\begin{array}{l}\text { Lat } 33^{\circ} 43^{\prime} 35^{\prime \prime}, \text { long } 105^{\circ} 31^{\prime} 43^{\prime \prime}, \\
\text { Lincoln County, Hydrologic } \\
\text { Unit } 13060005,0.3 \text { mi upstream } \\
\text { from wooden bridge on dirt } \\
\text { road, } 1.2 \text { mi. north of State } \\
\text { Highway } 246 \text {, and } 4.3 \text { mi. west } \\
\text { of Encinoso. Drainage area } \\
\text { is } 6.07 \text { m12. }\end{array}$ & $1961-968$ & $09-11-96$ & 4.00 & 590 & $09-06-61$ & 5.10 & 1,610 \\
\hline $\begin{array}{l}\text { Salt Crook } \\
\text { tributary neax } \\
\text { Roswell. } \\
(08385900)\end{array}$ & 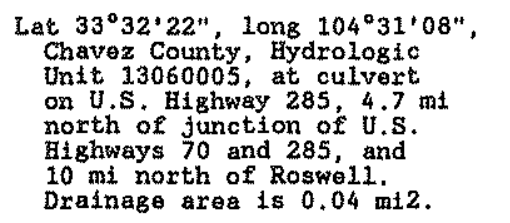 & $1952-968$ & $06-28-96$ & 1.10 & 13 & $08-11-77$ & 3.75 & 73 \\
\hline $\begin{array}{l}\text { Rto Bontso } \\
\text { tributary noar } \\
\text { Fort Stanton. } \\
(08389060)\end{array}$ & $\begin{array}{l}\text { Lat } 33^{\circ} 31^{\prime} 15^{\prime \prime} \text {, long } 105^{\circ} 28^{\prime} 05^{\prime \prime}, \\
\text { Lincoln County, Hydrologic } \\
\text { Unit } 13060008, \text { at culvert on } \\
\text { U.S. Htghway } 380,150 \text { ft } \\
\text { upstream from mouth, and } 3.5 \\
\text { mi northeast of Fort Stanton. } \\
\text { Drainage area is } 0.72 \text { mi2. }\end{array}$ & $1955-968$ & $-\quad-96$ & $-\infty$ & (k) & $09-30-82$ & 6.40 & 512 \\
\hline $\begin{array}{l}\text { Rio bondo } \\
\text { tributary at } \\
\text { TInnie. } \\
(08390050)\end{array}$ & $\begin{array}{l}\text { Lat } 33^{\circ} 22^{\prime} 36^{\prime \prime}, \text { Long } 105^{\circ} 13^{\circ} 01^{\prime \prime}, \\
\text { Incoln County, Hydrolog } 1 \mathrm{c} \\
\text { Unit } 13060008 \text {, upstream from } \\
\text { culvert on U.S. Highway } 70-380, \\
0.5 \text { mi east of junction of } \\
\text { U.S. Highway } 70-380 \text { and State } \\
\text { Bighway } 368 \text {, and at Tinnie. } \\
\text { Drainage area is } 0.23 \mathrm{mi2} \text {. }\end{array}$ & $1971-96 z$ & $06-27-96$ & 3.48 & 18 & $09-07-72$ & 10.80 & 420 \\
\hline
\end{tabular}




\begin{tabular}{|c|c|c|c|c|c|c|c|c|}
\hline & & & Water & an 1996 & maximum & Pertod & of recoro & maximum \\
\hline $\begin{array}{l}\text { Station name } \\
\text { and } \\
\text { number. }\end{array}$ & $\begin{array}{l}\text { Location } \\
\text { arainage aren }\end{array}$ & $\begin{array}{l}\text { Period } \\
\text { of } \\
\text { record }\end{array}$ & Date & $\begin{array}{l}\text { Gage } \\
\text { height } \\
\text { (ft). }\end{array}$ & $\begin{array}{c}\text { Dis } \\
\text { charge } \\
\text { (ets/s) }\end{array}$ & Date & $\begin{array}{l}\text { Gage } \\
\text { height } \\
\text { (ft) }\end{array}$ & $\begin{array}{c}\text { Dis } \\
\text { charge } \\
(\mathrm{ft} / \mathrm{s})\end{array}$ \\
\hline
\end{tabular}

RIO GRANDE BASIN - Continued

Gallo Caryoa near Picacho (08390150) Pancho Cabyon
near Arabol. (08393700)

\section{near Rospell. (08393900)}

Elght 440 Draw

\section{Twin Butto Canyon tributary near Roswell. \\ (08394300)}

Ryatt Canyon near Cloudcroft. (08397400)

\section{Lagt Chence Canyon tributaxy near Carlsbad Caverna (08405050)}

\section{Mogley Canyon near Whitos \\ City.}

(08405100)

\section{Antelope Drew neaz Jal.} $(08436000)$

$$
\begin{aligned}
& \text { Croek at } \\
& \text { Stlver City. } \\
& \text { (08477590) }
\end{aligned}
$$$$
\text { inos Altos }
$$

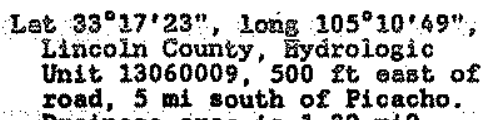

Lat $33^{\circ} 17 \cdot 23^{\prime \prime}, 1003105^{\circ} 10^{\prime} 49^{\prime \prime}$ Incoln County, Eydrologic Unit 13060009,500 It aas of road, 5 wi south of Picacho. Drainage siea $1 \mathrm{~s} 1.32 \mathrm{mi2}$.
Let $33^{\circ} 30^{\prime} 36^{\prime \prime}$, Long $105^{\circ} 11$ '38",
Lincoln County, Hydrolog ic Inincolin County, Eydrologic Untt 13060008,200 ft dow stream from dip on Stat mouth of Arabola.

Drainage arsa is $16.7 \mathrm{mil}$.

Let $33^{\circ} 24^{\circ} 05^{\prime \prime}$, long $104^{\circ} 37^{\prime} 54^{\prime \prime}$, Chavez County, Hydrolog ic Unit $13060008,6.5 \mathrm{mi}$. of Rozmell. Drainago are is $397 \mathrm{mid} 2$

Lat $33^{\circ} 10^{\circ} 34^{\prime \prime}$, Long $104^{\circ} 51 \cdot 30^{\prime \prime}$, Chaver Cotmity, Fydrologic Unit 13060009, about 0.1 mi upstrasm from mouth, and about 22 mi southwest of Rosmel.1. Drainage ara is $5.01 \mathrm{miz}$.

Lat $32^{\circ} 56^{\circ} 06^{\prime \prime}$, Long $105^{\circ} 37^{\prime} 37^{\prime \prime}$ Otero County, Bydrologic Unit $13060010,0.5$ mi south of U.S. Highway 82 , and 7 mi ast of cloudcrost. Drainago ares is. $3.08 \mathrm{miz}$

Let $32^{\circ} 17^{\prime} 30^{\prime \prime}$, Long $104^{\circ} 36^{\prime} 20^{\prime \prime}$, Eddy County, Bydrologic Unit 13060011 , upstream from culvert on State Highway 137 , $0.1 \mathrm{mi}$ north of road to Sitting Bull Falls, and $12.5 \mathrm{mi}$ northwest of Carlsbad Cavarns. Dratnage araa $\$ 80.2$ का2

Lat $32^{\circ} 15^{\prime} 27^{\prime \prime}$, Long $104^{\circ} 22^{\prime} 43^{\prime \prime}$ Eddy County, Bydrologic

Unte 13060011, 600 it

dosistrean Exon dip on Dark Canyon zoad, and 5.5 wis north of Whitiog City. Drainago axe $1 \mathrm{~B} 14.6$ ind2.

Lat $32^{\circ} 09^{\prime} 18^{\prime \prime}$, Long $103^{\circ} 21 ' 51^{\prime \prime}$, Laa County, Eydrologis Untt 13070007 . $0.4 \mathrm{mi}$ gouth of State Highwey 128 , and 10.7

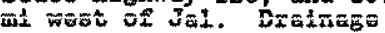
area is 20 mi2.

MIMBRES BASIN

Lat $32^{\circ} 46^{\circ} 41^{\prime \prime}$, long $108^{\circ} 16^{\circ} 41^{\prime \prime}$, Grant County, Bydrologic Unit 13030202,190 ft upstream from Twolfth streot bridge in Silver city. Drainage area is $10,0 \mathrm{mi2}$.

Lat $32^{\circ} 46^{\circ} 52^{\prime \prime}$, long $108^{\circ} 16^{\prime} 04^{\prime \prime}$ Grant County, Bydrologic Unit 13030202 , domitrean from U.S Highway 180 in Silver City. preinage area is $4.63 \mathrm{mi}$.
$1941 \cdot 09-13-96 \quad 13.90 \quad \cdots 40 \quad 07-13-91 \quad 17.80 \quad 10.300$

$1968-968 \quad 06-14-96$

3.60

290

$09-08-95$

$9.60 \quad 5,900$

$1953-96 \mathrm{~g} \quad 06-27-96$

2.21

109

$06-27-96$

2.21

109

$1959-968 \quad 08-25-96$

3.29

160

$08-23-6$

7.77

683

$09-12-96$

$5.33 \quad 1.750$

$05-30-65$

$13.70 \quad 16,400$

$1963-96 \mathrm{~g} \quad 0 \mathrm{~B}-19-96 \quad 1.28$

$$
4.0
$$

$07-30-94$

4.85

530
$3.64 \quad 1.180 \quad 08-11-60$

$6.01 \quad 2,670$

$08-11-60$

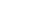

$4.09 \quad 3,700$ 
DISCHARGE AT PARTTAL-RECORD STATIONS AND MISCELLANEOUS STTES

Annual maximum discharge at crest-stage partial-record stations

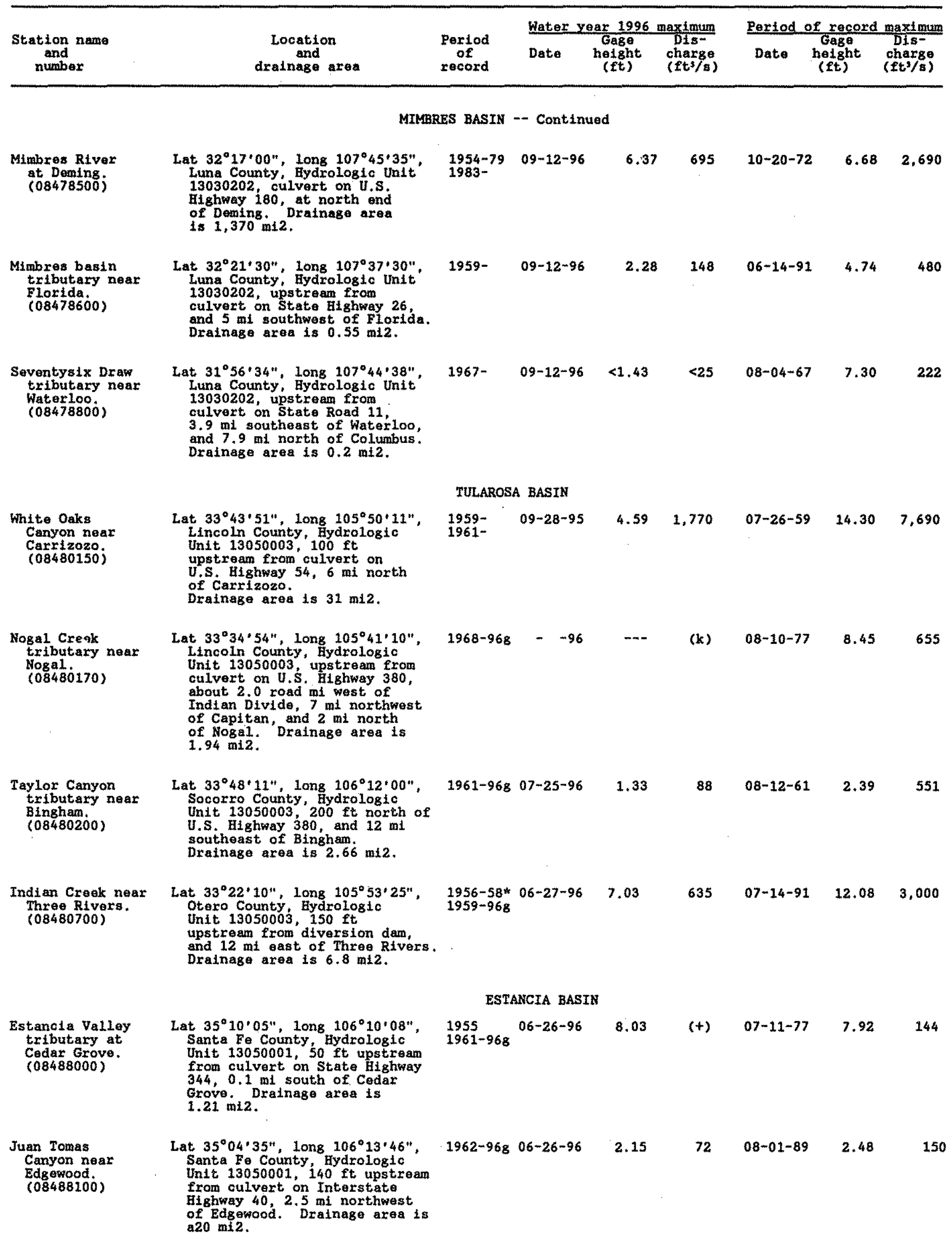




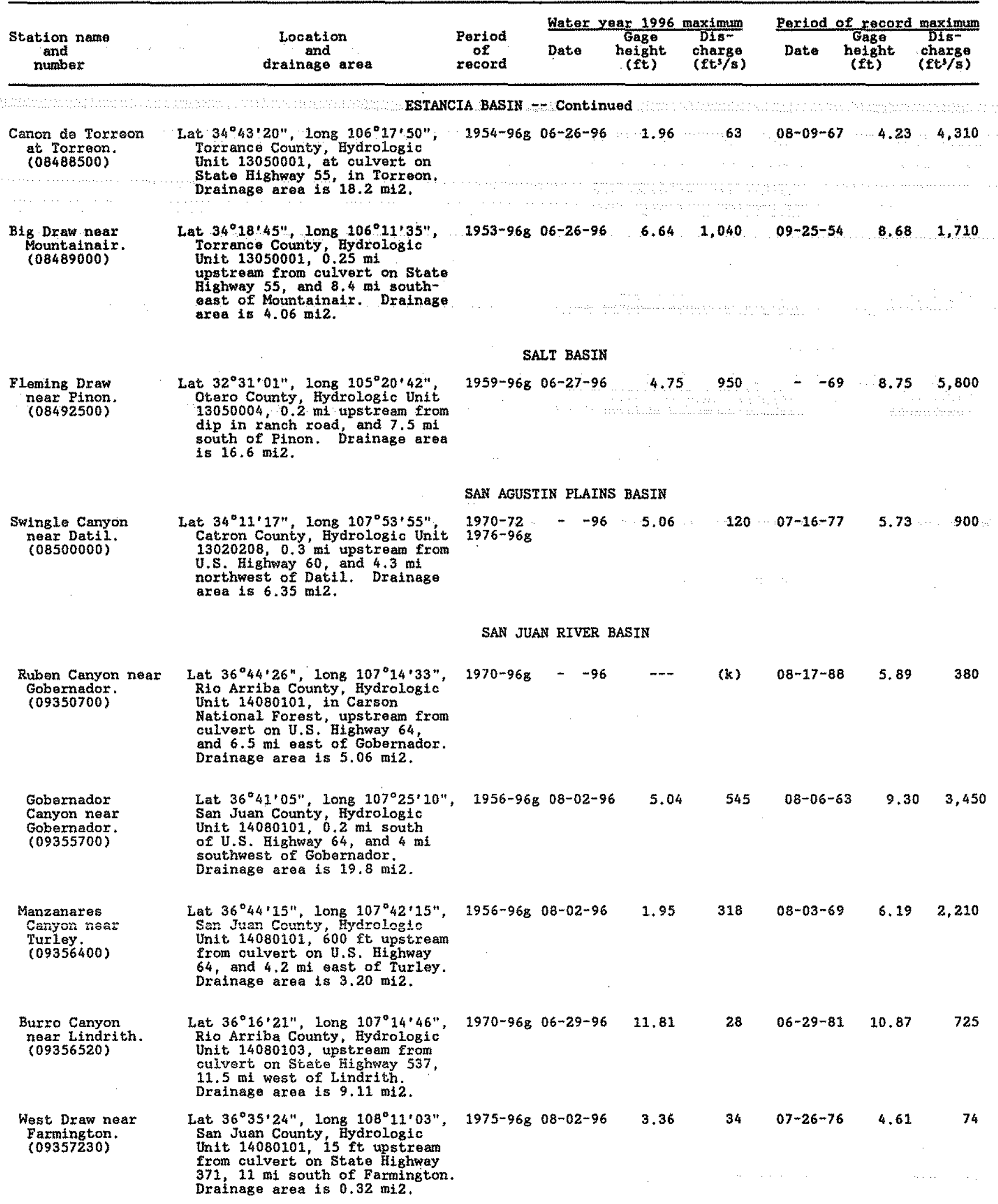


DISCEARGE AT RARTIAL-RECORD STATTONS AND MISCELLANEOUS SITES

Annual maximum discharge at crest-stage partial-record stations

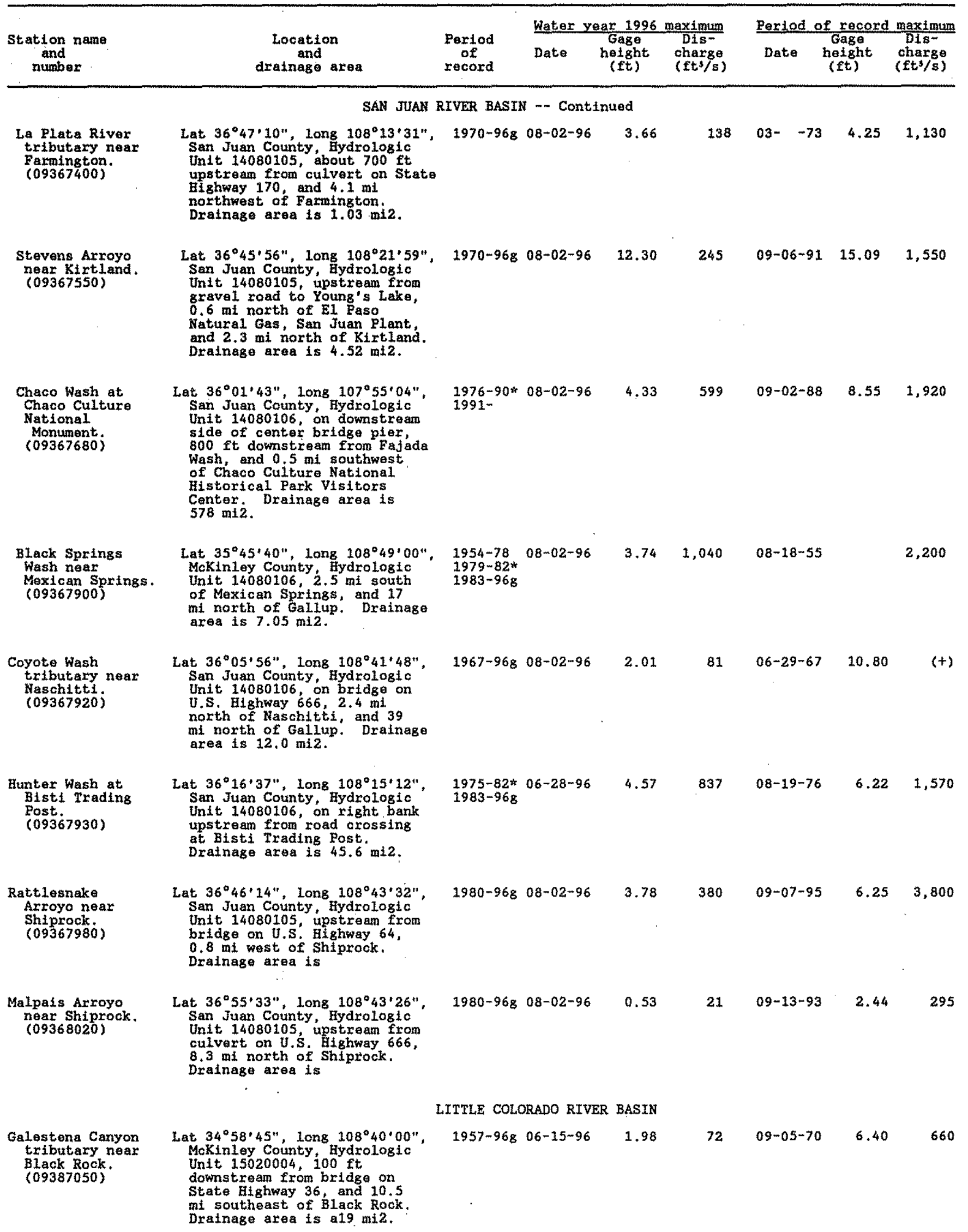


Measurements at Miscellaneous Sites

Measurements of streamflow at polnts other than gaging stations are given in the following table.

Discharge Measurenents Made at Miscellaneous Sites during Water Year 1996

\begin{tabular}{|c|c|c|c|c|c|c|}
\hline \multirow[b]{2}{*}{ Stream } & \multirow[b]{2}{*}{ Trtbutary to } & \multirow[b]{2}{*}{ Location } & \multirow[b]{2}{*}{$\begin{array}{c}\text { Drainago } \\
\text { area } \\
\text { (m士2) }\end{array}$} & \multirow{2}{*}{$\begin{array}{l}\text { Measured } \\
\text { previously } \\
\text { (water } \\
\text { years) }\end{array}$} & \multicolumn{2}{|c|}{ Measuraments } \\
\hline & & & & & Date & $\begin{array}{c}\text { Discharge } \\
(\mathrm{fts} / \mathrm{s})\end{array}$ \\
\hline \multicolumn{7}{|c|}{ RIO GRANDE BASIN } \\
\hline $\begin{array}{l}\text { La Cienega } \\
\text { Stream }\end{array}$ & $\underset{\text { Raver }}{\text { Re }}$ & 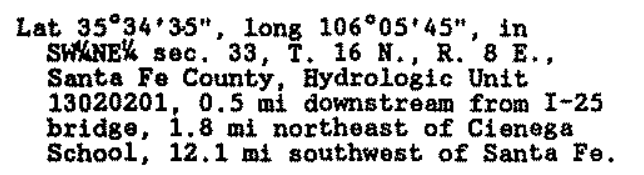 & $-\cdots$ & $\begin{array}{l}1986 \\
1989 \\
1991\end{array}$ & $\begin{array}{l}02-20-96 \\
05-10-96 \\
07-22-96\end{array}$ & $\begin{array}{l}0.71 \\
0.59 \\
0.59\end{array}$ \\
\hline $\begin{array}{l}\text { Lea Lake Drain } \\
08394018\end{array}$ & Pecos River & 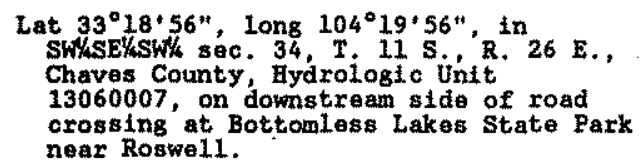 & -- & $1976-$ & $\begin{array}{l}10-04-95 \\
01-11-96 \\
04-16-96 \\
07-22-96\end{array}$ & $\begin{array}{l}5.33 \\
7.91 \\
6.38 \\
8.08\end{array}$ \\
\hline $\begin{array}{c}\text { Blue Springs } \\
08405450\end{array}$ & Black River & 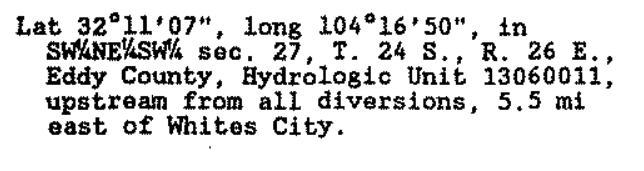 & $-\infty$ & $\begin{array}{l}1907 \\
1919-20 \\
1923 \\
1935 \\
1952-70 \\
1974-\end{array}$ & $\begin{array}{l}10-12-95 \\
01-16-96 \\
07-22-96\end{array}$ & $\begin{array}{l}12.9 \\
13.9 \\
13.2\end{array}$ \\
\hline $\begin{array}{c}\text { Castle Springs } \\
08405490\end{array}$ & Black River & 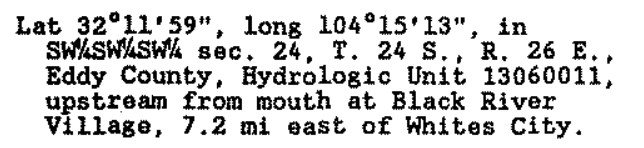 & $-\infty$ & 1975 - & $\begin{array}{l}10-12-95 \\
01-16-96 \\
07-22-96\end{array}$ & $\begin{array}{l}0.40 \\
0.32 \\
0.23\end{array}$ \\
\hline \multicolumn{7}{|c|}{ GILA RIVER BASIN } \\
\hline $\begin{array}{c}\text { Mangas Creak } \\
09431100\end{array}$ & Gila River & $\begin{array}{l}\text { Lat } 32^{\circ} 50^{\prime} 48^{\prime \prime}, \text { long } 108^{\circ} 30^{\prime} 57^{\prime \prime} \text { in } \\
\text { NWhE\% sec. 8, T. } 17 \mathrm{~s}, \text { R. in W. } \\
\text { Grant County, Hydrologic Untt } \\
15040002 \text {, } 0.4 \mathrm{mi} \text { northwest of Mangas } \\
\text { Springs. }\end{array}$ & 177 & $1970-$ & $\begin{array}{l}10-05-95 \\
12-07-95 \\
03-07-96 \\
06-03-96\end{array}$ & $\begin{array}{l}6.44 \\
6.38 \\
5.72 \\
4.82\end{array}$ \\
\hline
\end{tabular}


Water-quality partial-record stations and water-quality miscellaneous sttes are surface-water locations where chemical-quality, biological, and/or sediment data are collected on a limited frequency over a short period of years or once only for use in hydrologic investigations. Continuous streanflow recording gages are not located at these stations or sites.

WATER QUALITY DATA, WATER YEAR OCTOBER 1995 TO SEPTEMBER 1996

RIO GRANDE BASIN

08329000 JEMEZ RTVER BELOW JEMEZ CANYON DAM, NM

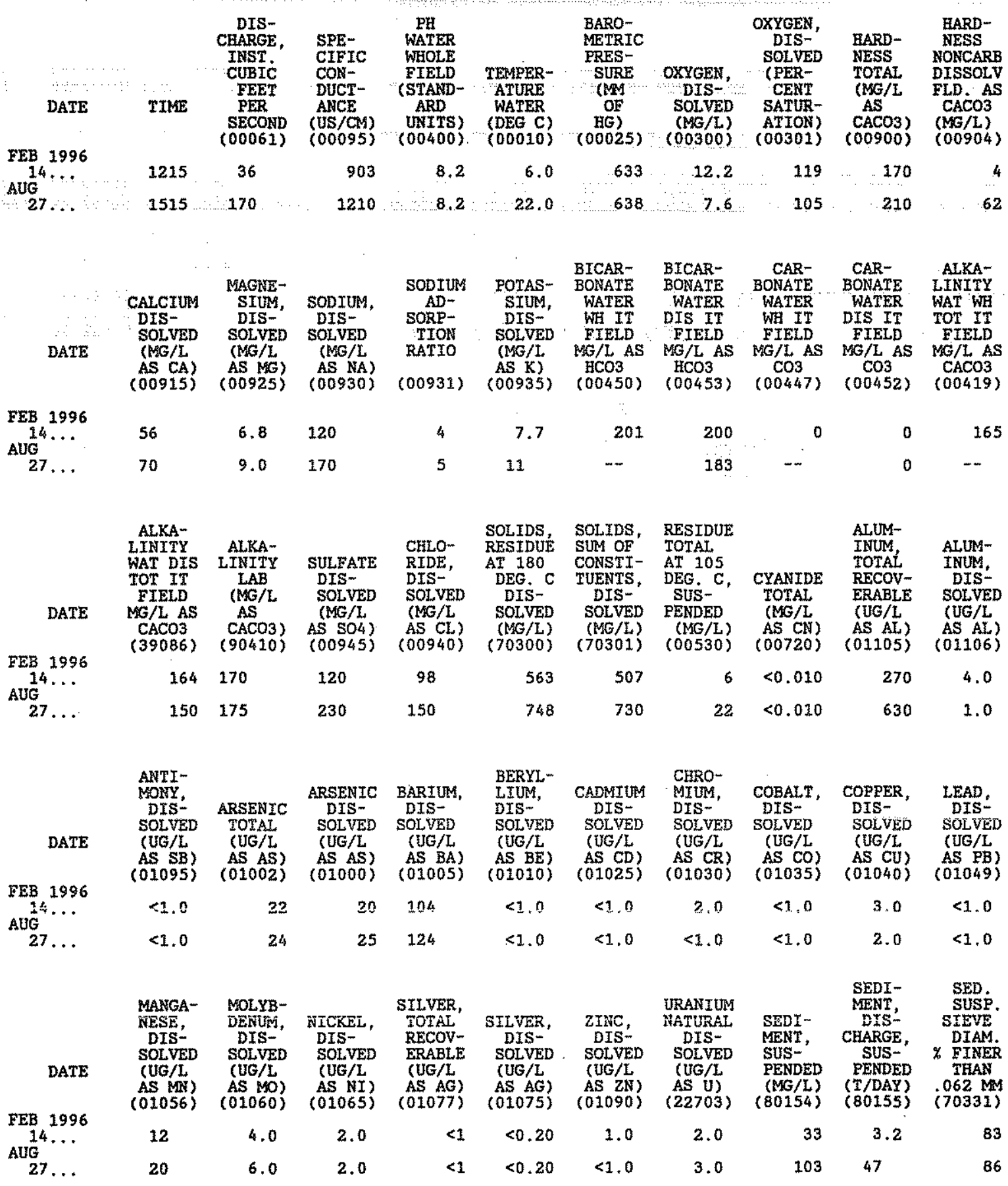


ANALYSES OF SAMPLES COLLECTED AT WATER-QUALITY PARTIAL-RECORD STATIONS AND MISCELLANEOUS SITES

WATER-QUALITY DATA, WATER YEAR OCTOBER 1995 TO SEPTEMRER 1996

RIO GRANDE BASIN -- Continued

08329928 RIO GRANDE NEAR ALAMEDA, NM

\begin{tabular}{|c|c|c|c|c|c|c|c|c|c|c|}
\hline DATE & TIME & $\begin{array}{c}\text { DIS- } \\
\text { CHARGE, } \\
\text { INST, } \\
\text { CUBIC } \\
\text { FEET } \\
\text { PER } \\
\text { SECOND } \\
(00061)\end{array}$ & $\begin{array}{l}\text { SPE- } \\
\text { CIFIC } \\
\text { CON- } \\
\text { DUCT- } \\
\text { ANCE } \\
\text { (US/CM) } \\
(00095)\end{array}$ & $\begin{array}{c}\text { PH } \\
\text { WATER } \\
\text { WROLE } \\
\text { FIELD } \\
\text { (STAND- } \\
\text { ARD } \\
\text { UNITS) } \\
(00400)\end{array}$ & $\begin{array}{c}\text { TEMPER- } \\
\text { ATURE } \\
\text { HATER } \\
\text { (DEGC) } \\
(00010)\end{array}$ & $\begin{array}{c}\text { BARO- } \\
\text { METRIC } \\
\text { PRES- } \\
\text { SURE } \\
\text { (MM } \\
\text { OF } \\
\text { HG) } \\
(00025)\end{array}$ & $\begin{array}{c}\text { OXYGEN, } \\
\text { DIS- } \\
\text { SOLVED } \\
\text { (MG/L) } \\
(00300)\end{array}$ & $\begin{array}{c}\text { OXYGEN, } \\
\text { DIS- } \\
\text { SOLVED } \\
\text { (PER- } \\
\text { CENT } \\
\text { SATUR- } \\
\text { ATION) } \\
\text { (00301) }\end{array}$ & $\begin{array}{l}\text { HARD- } \\
\text { NESS } \\
\text { TOTAL } \\
\text { (MG/L } \\
\text { AS } \\
\text { CACO3) } \\
(00900)\end{array}$ & $\begin{array}{l}\text { HARD- } \\
\text { NESS } \\
\text { NONCARB } \\
\text { DISSOLV } \\
\text { FLD. AS } \\
\text { CACOO3 } \\
(\mathrm{MG} / \mathrm{L}) \\
(00904)\end{array}$ \\
\hline CT 1995 & 1430 & 740 & 352 & 7.8 & 21.0 & 635 & 7.8 & 106 & 120 & - \\
\hline $\begin{array}{l}13 . . \\
1996 \\
\operatorname{MAX}\end{array}$ & 0900 & 1260 & 362 & 8.1 & 4.5 & 642 & 10.6 & 97 & 130 & - \\
\hline $19 .$. & 1500 & 490 & 371 & 7.9 & -- & 631 & - & - & 130 & - \\
\hline $26 \ldots$ & 1445 & 760 & 388 & 8.0 & 26.0 & 640 & 6.5 & 96 & 130 & 47 \\
\hline DATE & $\begin{array}{l}\text { CALCIUM } \\
\text { DIS - } \\
\text { SOLVED } \\
\text { (MG/L } \\
\text { AS CA) } \\
(00915)\end{array}$ & $\begin{array}{c}\text { MAGNE- } \\
\text { SIUM, } \\
\text { DIS-- } \\
\text { SOLVED } \\
\text { (MG/L } \\
\text { AS MG) } \\
\text { (00925) }\end{array}$ & $\begin{array}{c}\text { SODIUM, } \\
\text { DIS- } \\
\text { SOLVED } \\
\text { (MG/L } \\
\text { AS NA) } \\
(00930)\end{array}$ & $\begin{array}{c}\text { SODIUM } \\
\text { AD- } \\
\text { SORP- } \\
\text { TION } \\
\text { RATIO } \\
(00931)\end{array}$ & $\begin{array}{c}\text { POTAS- } \\
\text { SIUM, } \\
\text { DIS- } \\
\text { SOLVED } \\
\text { (MG/L } \\
\text { AS K) } \\
(00935)\end{array}$ & $\begin{array}{c}\text { BICAR- } \\
\text { BONATE } \\
\text { WATER } \\
\text { WH IT } \\
\text { FIELD } \\
\text { MG/L AS } \\
\text { HCO3 } \\
(00450)\end{array}$ & $\begin{array}{c}\text { BICAR- } \\
\text { BONATE } \\
\text { WATER } \\
\text { DIS IT } \\
\text { FIELD } \\
\text { MG/L AS } \\
\text { HCO3 } \\
(00453)\end{array}$ & $\begin{array}{c}\text { CAR- } \\
\text { BONATE } \\
\text { WATER } \\
\text { WHI IT } \\
\text { FIELD } \\
\text { MG/L AS } \\
\text { CO3 } \\
(00447)\end{array}$ & $\begin{array}{c}\text { CAR- } \\
\text { BONATE } \\
\text { WATER } \\
\text { DIS IT } \\
\text { FIELD } \\
\text { MG/L AS } \\
\text { CO3 } \\
(00452)\end{array}$ & $\begin{array}{c}\text { ALKA- } \\
\text { LINITY } \\
\text { WAT WH } \\
\text { TOT IT } \\
\text { FIELD } \\
\text { MG/L AS } \\
\text { CACO3 } \\
(00419)\end{array}$ \\
\hline${ }_{0 \mathrm{~T}} 1995$ & 38 & 7.2 & 21 & 0.8 & 3.0 & 137 & -- & 0 & -- & 112 \\
\hline${ }_{\operatorname{MAY}}^{13} \ldots$ & 41 & 7.5 & 24 & 0.9 & 3.1 & 139 & - & 0 & - & 114 \\
\hline $19 . .$. & 40 & 7.5 & 24 & 0.9 & 3.2 & 143 & -- & 0 & - & 117 \\
\hline 26. & 43 & 6.4 & 27 & 1 & 3.5 & -- & 106 & -- & 0 & - \\
\hline DATE & $\begin{array}{l}\text { ALKA- } \\
\text { LINITY } \\
\text { WAT DIS } \\
\text { TOT IT } \\
\text { FIELD } \\
\text { MG/L AS } \\
\text { CACO3 } \\
(39086)\end{array}$ & $\begin{array}{c}\text { ALKA- } \\
\text { LINITY } \\
\text { LAB } \\
(M G / L \\
\text { AS } \\
\text { CACO3) } \\
(90410)\end{array}$ & $\begin{array}{c}\text { SULFATE } \\
\text { DIS- } \\
\text { SOLVED } \\
\text { (MG/L } \\
\text { AS SO4) } \\
(00945)\end{array}$ & $\begin{array}{l}\text { CHLO- } \\
\text { RIDE, } \\
\text { DIS- } \\
\text { SOLVED } \\
\text { (MG/L } \\
\text { AS CL) } \\
(00940)\end{array}$ & $\begin{array}{c}\text { SOLIDS, } \\
\text { RESIDUE } \\
\text { AT } 180 \\
\text { DEG. C } \\
\text { DIS- } \\
\text { SOLVED } \\
\text { (MG/L) } \\
(70300)\end{array}$ & $\begin{array}{c}\text { SOLIDS, } \\
\text { SUM OF } \\
\text { CONSTI- } \\
\text { TUENTS, } \\
\text { DIS } \\
\text { SOLVED } \\
\text { (MG/L) } \\
(70301)\end{array}$ & $\begin{array}{l}\text { RESIDUE } \\
\text { TOTAL } \\
\text { AT 105 } \\
\text { DEG. C, } \\
\text { SUS- } \\
\text { PENDED } \\
\text { (MG/L) } \\
(00530)\end{array}$ & $\begin{array}{l}\text { CYANIDE } \\
\text { TOTAL } \\
\text { (MG/L } \\
\text { AS CN }) \\
(00720)\end{array}$ & $\begin{array}{l}\text { ALUM- } \\
\text { INUM, } \\
\text { TOTAL } \\
\text { RECOV- } \\
\text { ERABLE } \\
\text { (UG/L. } \\
\text { AS AL) } \\
(0110.5)\end{array}$ & $\begin{array}{l}\text { ALUM- } \\
\text { INUM, } \\
\text { DIS- } \\
\text { SOLVED } \\
\text { (UG/L } \\
\text { AS AL) } \\
(01106)\end{array}$ \\
\hline $\begin{array}{l}\text { OCT } 1995 \\
02 \\
\text { FEB } 1996\end{array}$ & - & 113 & 52 & 6.4 & 218 & 195 & 73 & $<0.010$ & 1300 & 4.0 \\
\hline${ }_{M A Y}^{13} \ldots$ & $m$ & 119 & 47 & 9.4 & 233 & 200 & 22 & $<0.010$ & 250 & 28 \\
\hline $19 \ldots$ & $\cdots$ & 120 & 58 & 7. & 206 & 210 & 39 & $<0.010$ & 55 & 2.0 \\
\hline & 87 & 121 & 79 & 11 & 238 & 222 & 4100 & $<0.010$ & 50000 & 5.0 \\
\hline
\end{tabular}

\begin{tabular}{|c|c|c|c|c|c|c|c|c|c|c|}
\hline & $\begin{array}{l}\text { ANTI- } \\
\text { MONY, } \\
\text { DIS- } \\
\text { SOLVED } \\
\text { (UG/L } \\
\text { AS SB) } \\
\text { (01095) }\end{array}$ & $\begin{array}{c}\text { ARSENIC } \\
\text { TOTAL } \\
\text { (UG/L } \\
\text { AS AS) } \\
(01002)\end{array}$ & $\begin{array}{c}\text { ARSENIC } \\
\text { DIS } \\
\text { SOLVED } \\
\text { (UG/L } \\
\text { AS AS) } \\
(01000)\end{array}$ & $\begin{array}{c}\text { BARIUM, } \\
\text { DIS- } \\
\text { SOLVED } \\
\text { (UG/L. } \\
\text { AS BA) } \\
(01005)\end{array}$ & $\begin{array}{l}\text { BERYL- } \\
\text { LIUM, } \\
\text { DIS- } \\
\text { SOLVED } \\
\text { (UG/L } \\
\text { AS BE) } \\
(01010)\end{array}$ & $\begin{array}{c}\text { CADMIUM } \\
\text { DIS- } \\
\text { SOLVED } \\
\text { (UG/L } \\
\text { AS CD) } \\
(01025)\end{array}$ & $\begin{array}{l}\text { CHRO- } \\
\text { MIUM, } \\
\text { DIS - } \\
\text { SOLVED } \\
\text { (UG/L } \\
\text { AS CR) } \\
\text { (01030) }\end{array}$ & $\begin{array}{l}\text { COBALT, } \\
\text { DIS- } \\
\text { SOLVED } \\
\text { (UG/L } \\
\text { AS CO) } \\
\text { (01035) }\end{array}$ & $\begin{array}{l}\text { COPPER, } \\
\text { DIS- } \\
\text { SOLVED } \\
\text { (UG/L } \\
\text { AS CU) } \\
(01040)\end{array}$ & $\begin{array}{l}\text { LEAD, } \\
\text { DIS- } \\
\text { SOLVED } \\
\text { (UG/L } \\
\text { AS PB) } \\
(01049)\end{array}$ \\
\hline
\end{tabular}

\begin{tabular}{|c|c|c|c|c|c|c|c|c|c|c|}
\hline $\begin{array}{l}\operatorname{CT}_{02} 1995 \\
\text { FEB } j 996\end{array}$ & $<1.0$ & 2 & 2 & 66 & $<1.0$ & $<1.0$ & 1.0 & $<1.0$ & 2.0 & $<1.0$ \\
\hline $133 \ldots$ & $<1.0$ & 3 & 2 & 63 & $<1.0$ & $<1.0$ & 1.0 & $<1.0$ & 1.0 & $<1.0$ \\
\hline AUG 19. & $<1.0$ & 2 & 2 & 65 & $<1.0$ & $<1.0$ & $<1.0$ & $<1.0$ & 1.0 & $<1.0$ \\
\hline & $<1.0$ & 3 & 3 & 90 & $<1.0$ & $<1.0$ & $<1.0$ & $<1.0$ & 1.0 & $<1.0$ \\
\hline
\end{tabular}


WATOR-QUALITY DATA, WATER YEAR OCTOBER 1995 TO SEPTEMBER 1996

RIO GRANDE BASIN -- Continued

08329928 RIO GRANDE MEAR ALAMEDA, KM

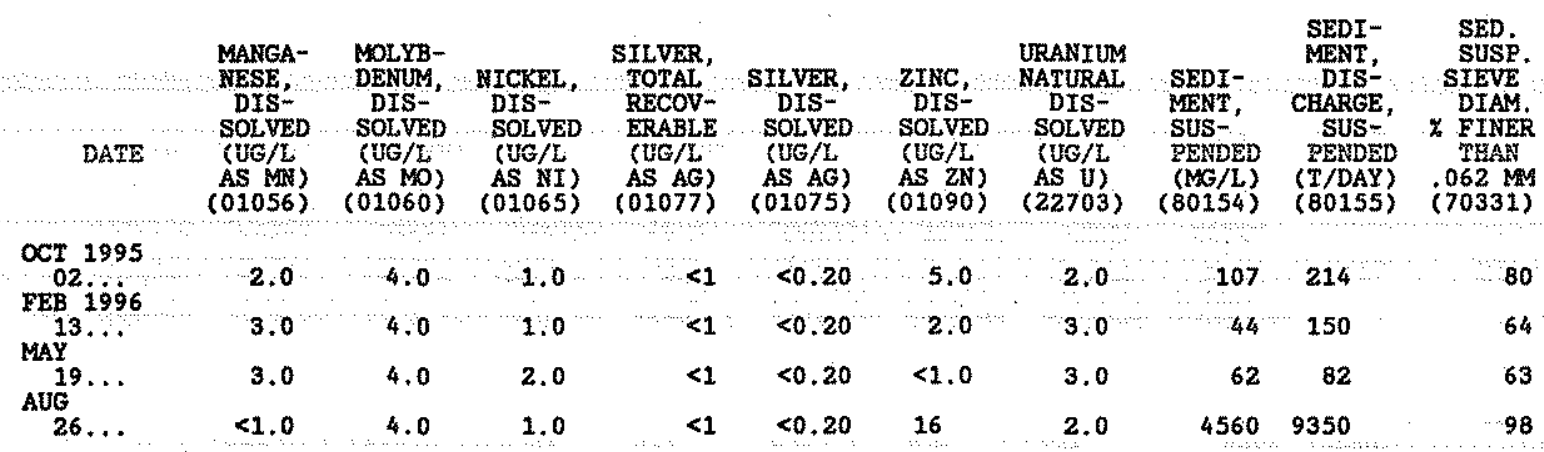

08330075 CITY OF ALBQ LIFT STATION 32 AT ALBQ, NM

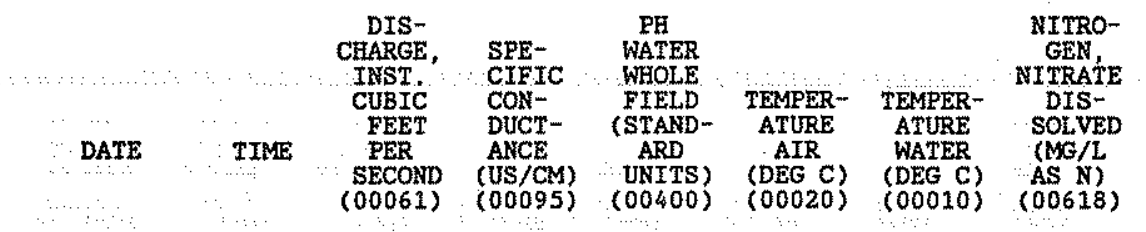

$\begin{array}{ccccccccc}\text { JAN } 1996 & 0912 & \text { E0.50 } & 528 & 52 & 7.5 & 10.0 & 12.5 & 0.250\end{array}$

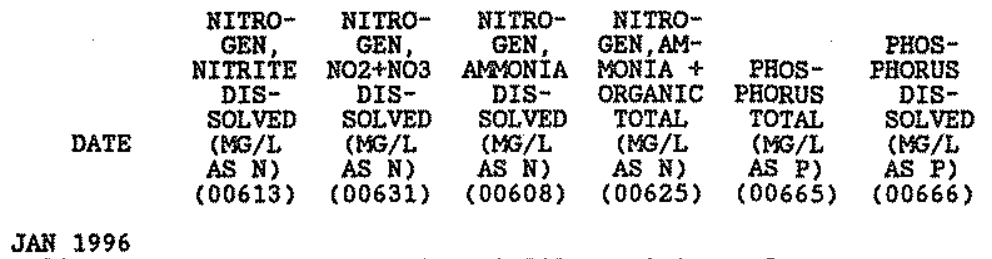

$\begin{array}{ccccccc}\text { JAN } 1996 & 0.040 & 0.290 & 0.310 & 1.1 & 2.00 & 2.10\end{array}$

08330150 RIO GRANDE AT RIO BRAVO BRTDGE BRTDGE NEAR ALBUQUERQUE, NM

\begin{tabular}{|c|c|c|c|c|c|c|c|c|c|c|}
\hline DATE & TIME & $\begin{array}{c}\text { DIS- } \\
\text { CHARGE, } \\
\text { INST. } \\
\text { CUBIC } \\
\text { FEET } \\
\text { BEE } \\
\text { SECOND } \\
(00061)\end{array}$ & $\begin{array}{l}\text { SPE- } \\
\text { CIFIC } \\
\text { CON- } \\
\text { DUCT- } \\
\text { ANCE } \\
\text { (US/CM) } \\
(00095)\end{array}$ & $\begin{array}{c}\text { PH } \\
\text { WATER } \\
\text { WHOLE } \\
\text { FIELD } \\
\text { (STAND } \\
\text { ARD } \\
\text { UNITS) } \\
\text { (00400) }\end{array}$ & $\begin{array}{c} \\
\text { TEMPER- } \\
\text { ATURE } \\
\text { WATER } \\
\text { (DEG C) } \\
(00010)\end{array}$ & $\begin{array}{c}\text { BARO- } \\
\text { METRIC } \\
\text { PRES- } \\
\text { SURE } \\
\text { (RE } \\
\text { OF } \\
\text { HG) } \\
(00025)\end{array}$ & $\begin{array}{c}\text { OXYGEN, } \\
\text { DYS- } \\
\text { SOLVED } \\
(\mathrm{MG} / 1) \\
(00300)\end{array}$ & $\begin{array}{c}\text { OXYGEN, } \\
\text { DIS- } \\
\text { SOLVED } \\
\text { (PER- } \\
\text { CENT } \\
\text { SATUR- } \\
\text { ATION) } \\
\text { (O0301) }\end{array}$ & $\begin{array}{l}\text { HARD- } \\
\text { NESS } \\
\text { TOTAL } \\
\text { (MG/L } \\
\text { AS } \\
\text { CACO3) } \\
(00900)\end{array}$ & $\begin{array}{c}\text { HARD- } \\
\text { NESS } \\
\text { NONCARB } \\
\text { DISSOLV } \\
\text { ELD. AS } \\
\text { CACO3 } \\
\text { (NG/L) } \\
\text { (O0904) }\end{array}$ \\
\hline
\end{tabular}

\begin{tabular}{|c|c|c|c|c|c|c|c|c|c|c|}
\hline $\begin{array}{l}\text { OCT } 1995 \\
03 \\
\text { FEB }\end{array}$ & 0845 & 790 & 388 & 7.7 & 14.0 & 639 & 8.3 & 96 & 130 & -- \\
\hline $\begin{array}{l}13 \ldots \\
M A Y\end{array}$ & 1300 & 1150 & 368 & 8.1 & 6.0 & 644 & 10.7 & 102 & 130 & $-\infty$ \\
\hline$\stackrel{20}{A}^{n} \ldots$ & 0845 & 460 & 381 & 8.0 & 16.5 & 637 & 8.0 & 98 & 130 & $=$ \\
\hline $27 \ldots$ & 0830 & 650 & 366 & 7.8 & 21.0 & 642 & 7.0 & 94 & 130 & 40 \\
\hline
\end{tabular}


ANALYSES OF SAMPLES COLLECTED AT WATER-QUALITY RARTIAL-RFCORD STATIONS AND MISCELLANEOUS SITES

WATER-QUALITY DATA, WATER YEAR OCTOBER 1995 TO SEPTEMBER 1996

RIO GRANDE BASIN - - Continued

08330150 RIO GRANDE AT RIO BRAVO BRIDGE BRIDGE NEAR ALBUQUERQUE, MM

\begin{tabular}{|c|c|c|c|c|c|c|c|c|c|c|}
\hline DATE & $\begin{array}{c}\text { CALCIUM } \\
\text { DIS- } \\
\text { SOLVED } \\
(\text { MG/L } \\
\text { AS CA) } \\
(00915)\end{array}$ & $\begin{array}{c}\text { MAGNE- } \\
\text { SIUM, } \\
\text { DIS- } \\
\text { SOLVED } \\
\text { (MG/L } \\
\text { AS MG) } \\
(00925)\end{array}$ & $\begin{array}{c}\text { SODIUM, } \\
\text { DIS- } \\
\text { SOLVED } \\
\text { (MG/L } \\
\text { AS NA) } \\
(00930)\end{array}$ & $\begin{array}{c}\text { SODIUM } \\
\text { AD- } \\
\text { SORP- } \\
\text { TION } \\
\text { RATIO } \\
(00931)\end{array}$ & $\begin{array}{c}\text { POTAS- } \\
\text { SIUM, } \\
\text { DIS- } \\
\text { SOLVED } \\
(\mathrm{MG} / \mathrm{L} \\
\text { AS K) } \\
(00935)\end{array}$ & $\begin{array}{c}\text { BICAR- } \\
\text { BONATE } \\
\text { WATER } \\
\text { WH IT } \\
\text { FIELD } \\
\text { MG/L AS } \\
\text { HCO3 } \\
(00450)\end{array}$ & $\begin{array}{c}\text { BICAR- } \\
\text { BONATE } \\
\text { WATER } \\
\text { DIS IT } \\
\text { FIELD } \\
\text { MG/L AS } \\
\text { HCO3 } \\
(00453)\end{array}$ & $\begin{array}{c}\text { CAR- } \\
\text { BONATE } \\
\text { WATER } \\
\text { WI IT } \\
\text { FIELD } \\
\text { MG/L AS } \\
\text { CO3 } \\
(00447)\end{array}$ & $\begin{array}{c}\text { CAR- } \\
\text { BONATE } \\
\text { WATER } \\
\text { DIS IT } \\
\text { FIELD } \\
\text { MG/L AS } \\
\text { CO3 } \\
(00452)\end{array}$ & $\begin{array}{c}\text { ALKA- } \\
\text { LINITY } \\
\text { HAT WH } \\
\text { TOT IT } \\
\text { FIELD } \\
\text { NG/L AS } \\
\text { CACO3 } \\
(00419)\end{array}$ \\
\hline $\begin{array}{l}\text { OCT } 1995 \\
\text { O3 } \\
\text { FEB } 1996\end{array}$ & 40 & 7.3 & 23 & 0.9 & 3.0 & 143 & -- & 0 & $-m$ & 117 \\
\hline MAY & 41 & 7.6 & 25 & 0.9 & 3.0 & 140 & -- & 0 & -- & 115 \\
\hline$u_{\operatorname{tug}}^{20} \ldots$ & 41 & 7.7 & 24 & 0.9 & 3.1 & 146 & $-\infty$ & 0 & $m$ & 120 \\
\hline $27 \ldots$ & 41 & 6.6 & 23 & 0.9 & 3.2 & -- & 109 & $\cdots$ & 0 & -- \\
\hline DATE & $\begin{array}{c}\text { ALKA- } \\
\text { LINITY } \\
\text { WAT DIS } \\
\text { TOT IT } \\
\text { FIELD } \\
\text { MG/L AS } \\
\text { CACO3 } \\
(39086)\end{array}$ & $\begin{array}{c}\text { ALKA- } \\
\text { LINITY } \\
\text { LAB } \\
(\mathrm{MG} / \mathrm{L} \\
\mathrm{AS} \\
\text { CACO3) } \\
(90410)\end{array}$ & $\begin{array}{c}\text { SULFATE } \\
\text { DIS } \\
\text { SOLVED } \\
\text { (MG/L } \\
\text { AS SO4) } \\
(00945)\end{array}$ & $\begin{array}{l}\text { CHLO- } \\
\text { RIDE, } \\
\text { DIS- } \\
\text { SOLVED } \\
\text { (MG/L } \\
\text { AS CL) } \\
(00940)\end{array}$ & $\begin{array}{c}\text { SOLIDS, } \\
\text { RESIDUE } \\
\text { AT } 180 \\
\text { DEG. C } \\
\text { DIS- } \\
\text { SOLVED } \\
(M G / L) \\
(70300)\end{array}$ & $\begin{array}{c}\text { SOLIDS, } \\
\text { SUM OF } \\
\text { CONSTI- } \\
\text { TUENTS, } \\
\text { DIS- } \\
\text { SOLVED } \\
\text { (MG/L) } \\
(70301)\end{array}$ & $\begin{array}{l}\text { RESIDUE } \\
\text { TOTAL } \\
\text { AT } 105 \\
\text { DEG. C, } \\
\text { SUS- } \\
\text { PENDED } \\
\text { (MG/L) } \\
(00530)\end{array}$ & $\begin{array}{c}\text { CYANIDE } \\
\text { TOTAL } \\
\text { (MG/L } \\
\text { AS CN) } \\
(00720)\end{array}$ & $\begin{array}{l}\text { ALUM- } \\
\text { INUM, } \\
\text { TOTAL } \\
\text { RECOV- } \\
\text { ERABLE } \\
\text { (UG/L } \\
\text { AS AL) } \\
\text { (01105) }\end{array}$ & $\begin{array}{l}\text { ALUM- } \\
\text { INUM, } \\
\text { DIS- } \\
\text { SOLVED } \\
\text { (UG/L } \\
\text { AS AL) } \\
\text { (01106) }\end{array}$ \\
\hline $\begin{array}{c}\text { OCT } 1995 \\
\text { O3 } \\
\text { FEB } 1996\end{array}$ & $-\infty$ & 117 & 53 & 6.5 & 223 & 203 & 78 & $<0.010$ & 1800 & 2.0 \\
\hline $13 \ldots$ & -- & 121 & 48 & 9.7 & 2.38 & 203 & 37 & $<0.010$ & 550 & 22 \\
\hline${ }_{\text {AUG }}^{20} \ldots$ & -- & 124 & 59 & 7.6 & 266 & 214 & 1 & $<0.010$ & 990 & 15 \\
\hline $27 \ldots$ & 89 & 110 & 69 & 9.0 & 196 & 205 & 2580 & $<0.010$ & 32000 & 3.0 \\
\hline
\end{tabular}

\begin{tabular}{|c|c|c|c|c|c|c|c|c|c|c|}
\hline DATE & $\begin{array}{l}\text { ANTI- } \\
\text { MONY, } \\
\text { DIS- } \\
\text { SOLVED } \\
\text { (UG/L } \\
\text { AS SB) } \\
\text { (01095) }\end{array}$ & $\begin{array}{c}\text { ARSENIC } \\
\text { TOTAL. } \\
\text { (UG/L. } \\
\text { AS AS) } \\
(01002)\end{array}$ & $\begin{array}{c}\text { ARSENIC } \\
\text { DIS- } \\
\text { SOLVED } \\
\text { (UG/L } \\
\text { AS AS) } \\
(01000)\end{array}$ & $\begin{array}{l}\text { BARIUM, } \\
\text { DIS- } \\
\text { SOLVED } \\
\text { (UG/L } \\
\text { AS BA) } \\
(01005)\end{array}$ & $\begin{array}{l}\text { BERYL- } \\
\text { LIUM, } \\
\text { DIS- } \\
\text { SOLVED } \\
\text { (UG/L } \\
\text { AS BE) } \\
\text { (01010) }\end{array}$ & $\begin{array}{c}\text { CADMIUM } \\
\text { DIS- } \\
\text { SOLVED } \\
\text { (UG /L } \\
\text { AS CD) } \\
\text { (01025) }\end{array}$ & $\begin{array}{l}\text { CHRO- } \\
\text { MIJM, } \\
\text { DIS- } \\
\text { SOLVED } \\
\text { (UG/L } \\
\text { AS CR) } \\
(01030)\end{array}$ & $\begin{array}{c}\text { COBALT, } \\
\text { DIS- } \\
\text { SOLVED } \\
\text { (UG/L } \\
\text { AS CO) } \\
(01035)\end{array}$ & $\begin{array}{c}\text { COPPER, } \\
\text { DIS- } \\
\text { SOLVED } \\
\text { (UG/L } \\
\text { AS CU) } \\
(01040)\end{array}$ & $\begin{array}{l}\text { LEAD, } \\
\text { DIS- } \\
\text { SOLVED } \\
\text { (UG/L } \\
\text { AS PB) } \\
(01049 \text { ) }\end{array}$ \\
\hline $\begin{array}{c}\text { OCT } 1995 \\
03.1996 \\
\text { FEB } 1996\end{array}$ & $<1,0$ & (ector & 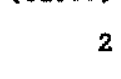 & 69 & $<1.0$ & $<1.0$ & 1.0 & $<1.0$ & 2.0 & $<1.0$ \\
\hline$\frac{13}{M A Y}$ & $<1.0$ & 2 & 2 & 66 & $<1.0$ & $<1: 0$ & 2.0 & $<1,0$ & 2.0 & $<1.0$ \\
\hline $\mathrm{UG}^{20} \ldots$ & $<1.0$ & 2 & 2 & 66 & $<1.0$ & $<1.0$ & $<1.0$ & $<1.0$ & 1.0 & $<1.0$ \\
\hline-7 & $<1,0$ & 3 & 3 & 89 & $<1.0$ & $<1,0$ & $<1.0$ & $<1.0$ & 1.0 & $<1.0$ \\
\hline
\end{tabular}

\begin{tabular}{|c|c|c|c|c|c|c|c|c|c|c|}
\hline DATE & $\begin{array}{c}\text { MANGA- } \\
\text { NESE, } \\
\text { DIS- } \\
\text { SOLVED } \\
\text { (UG/L } \\
\text { AS MN) } \\
(01056)\end{array}$ & $\begin{array}{l}\text { MOLYB- } \\
\text { DENUM, } \\
\text { DIS- } \\
\text { SOLVED } \\
\text { (UG/L } \\
\text { AS MO) } \\
(01060)\end{array}$ & $\begin{array}{l}\text { NICKEL, } \\
\text { DIS- } \\
\text { SOLVED } \\
\text { (UG/L. } \\
\text { AS NI) } \\
(01065)\end{array}$ & $\begin{array}{c}\text { SILVER, } \\
\text { TOTAL } \\
\text { RECOV- } \\
\text { BRABLE } \\
\text { (UG/L } \\
\text { AS AG) } \\
(01077)\end{array}$ & $\begin{array}{c}\text { SILVER, } \\
\text { DIS- } \\
\text { SOLVED } \\
\text { (UG/L } \\
\text { AS AG) } \\
(01075)\end{array}$ & $\begin{array}{c}\text { ZINC, } \\
\text { DIS- } \\
\text { SOLVED } \\
\text { (UG/L } \\
\text { AS ZN) } \\
(01090)\end{array}$ & $\begin{array}{l}\text { URANIUM } \\
\text { NATURAL } \\
\text { DIS- } \\
\text { SOLVED } \\
\text { (UG/L } \\
\text { AS U) } \\
(22703)\end{array}$ & $\begin{array}{l}\text { SEDT- } \\
\text { MENT, } \\
\text { SUS- } \\
\text { PENDED } \\
(M G / L) \\
(80154)\end{array}$ & $\begin{array}{c}\text { SEDI- } \\
\text { MEHT } \\
\text { DIS- } \\
\text { CEARGE, } \\
\text { SUS- } \\
\text { PENDED } \\
\text { (T/DAY) } \\
(80155)\end{array}$ & $\begin{array}{c}\text { SED. } \\
\text { SUSE. } \\
\text { SIEVE } \\
\text { DIAM. } \\
\text { × FINER } \\
\text { THAN } \\
062 \mathrm{MM} \\
(70331)\end{array}$ \\
\hline $\begin{array}{l}\text { CT } 1995 \\
\text { EB } 1996\end{array}$ & $<1.0$ & 4.0 & $<1.0$ & $<1$ & $<0.20$ & 1.0 & 2.0 & 110 & 235 & 90 \\
\hline $13 \ldots$ & 2.0 & 4.0 & 2.0 & $<1$ & $<0.20$ & 8.0 & 3.0 & 66 & 205 & 64 \\
\hline$U_{G}^{20} \ldots$ & 2.0 & 5.0 & 2.0 & $<1$ & $<0.20$ & $<1.0$ & 3.0 & 76 & 94 & 73 \\
\hline & $<1,0$ & 4.0 & 1.0 & $<1$ & $<0.20$ & $<1.0$ & 2.0 & 2770 & 4870 & 9 \\
\hline
\end{tabular}


ANALYSES OF SAMPLES COLLECTED AT WATER-QUALITY PARTIAL,-RECORD STATIONS AND MISCELLANEOUS SITES

WATER-QUALITY DATA, WATER YEAR OCTOBER 1995 TO SERTEMBER 1996

RIO GRANDE BASIN - Continued

08333450 ARROYO CHAVEZ NR SAN LUIS, MM

\begin{tabular}{|c|c|c|c|c|c|}
\hline DATE & TIME & $\begin{array}{l}\text { ENDING } \\
\text { TIME }\end{array}$ & $\begin{array}{c}\text { DIS- } \\
\text { CHARGE, } \\
\text { IN } \\
\text { CUBIC } \\
\text { FEET } \\
\text { PER } \\
\text { SECOND } \\
(00060)\end{array}$ & $\begin{array}{l}\text { SEDI- } \\
\text { MENT, } \\
\text { SUS- } \\
\text { PENDED } \\
\text { (MNG/L) } \\
(80154)\end{array}$ & $\begin{array}{c}\text { SEDI } \\
\text { LOAD } \\
\text { (TONS) }\end{array}$ \\
\hline $\begin{array}{l}1995 \\
6-16\end{array}$ & 1557 & 1657 & 8.5 & 59000 & 56 \\
\hline $2-22$ & 1912 & 2037 & 1.4 & 63000 & 14 \\
\hline $4-24$ & 1753 & 1813 & 2.6 & 78000 & 8.1 \\
\hline $5-25$ & 1852 & 1917 & 1.1 & 62000 & 3.3 \\
\hline $\begin{array}{c}8-28 \\
1996\end{array}$ & 1051 & 1120 & 4.2 & 59000 & 14 \\
\hline $6-26$ & 1601 & 1701 & 9.4 & 108000 & 114 \\
\hline $8-28$ & 1346 & 1400 & $\therefore 6.7$ & 67500 & 13 \\
\hline-28 & $\cdots \quad 1446$ & 1456 & $\therefore \quad 4.1$ & 29500 & 2.7 \\
\hline $0-30$ & 1547 & 1607 & 5.4 & 52000 & 10 \\
\hline-07 & 1957 & 2012 & 7.4 & 122000 & 25 \\
\hline-09 & 2146 & 0039 & 14 & 38000 & 173 \\
\hline-09 & 2015 & $\therefore \quad 2100$ & 18 & 29000 & 45 \\
\hline-07 & 1605 & 1645 & 2.3 & 60500 & 10 \\
\hline-14 & 1513 & 1649 & 4.3 & 52200 & 40 \\
\hline-17 & 1513 & 1826 & 3.3 & 34000 & 10 \\
\hline-26 & 0249 & 0337 & 3.0 & 40000 & 11 \\
\hline
\end{tabular}

08351225 VOLCANO HILL WASH NR CORREO, NM

\begin{tabular}{|c|c|c|c|c|c|}
\hline DATE & TIME & $\begin{array}{l}\text { ENDING } \\
\text { TIME }\end{array}$ & $\begin{array}{c}\text { DIS- } \\
\text { CHARGE, } \\
\text { IN } \\
\text { CUBIC } \\
\text { FEET } \\
\text { PER } \\
\text { SECOND } \\
(00060)\end{array}$ & $\begin{array}{l}\text { SEDI- } \\
\text { MENT, } \\
\text { SUS- } \\
\text { PENDED } \\
\text { (MG/L) } \\
(80154)\end{array}$ & $\begin{array}{l}\text { SEDI } \\
\text { MENT } \\
\text { LOAD } \\
\text { (TONS) }\end{array}$ \\
\hline $\begin{array}{l}\text { AUG } 1995 \\
16-16 \\
\text { AUG }\end{array}$ & 1910 & 2045 & 42 & 34700 & 259 \\
\hline $\operatorname{SEP}^{25-26}$ & 2200 & 0003 & 43 & 31800 & 315 \\
\hline $\begin{array}{c}08-08 \\
\text { JUN } 1996\end{array}$ & 1852 & 2015 & 12 & 24000 & 45 \\
\hline JUL & 1341 & 1441 & 55 & 44000 & 272 \\
\hline JUL & 2337 & 2347 & 5.6 & 30500 & 3.1 \\
\hline$\underset{A v G}{15-15}$ & 1250 & 1450 & 3.5 & 38500 & 30 \\
\hline $\begin{array}{l}09-09 \\
\text { AUG }\end{array}$ & 1504 & 1657 & 43 & 38000 & 345 \\
\hline${ }_{A U G}^{22-22}$ & 0125 & 0149 & 1.9 & 36000 & 3.1 \\
\hline $\operatorname{SEP}^{22-23}$ & 2.233 & 0100 & 1.9 & 22500 & 12 \\
\hline $\operatorname{SEP}^{12-12}$ & 1423 & 1523 & 10 & 36500 & 41 \\
\hline $\operatorname{SEP}^{13-13}$ & 1353 & 1553 & 62 & 32500 & 453 \\
\hline $14-14$ & 0229 & 0341 & 21 & 31000 & 88 \\
\hline
\end{tabular}


ANALYSES OF SAMPLES COLLECTED AT WATER-QUAL.ITY PARTIAL-RECORD STATIONS AND MISCELLANEOUS SITES

WATER-QUALITY DATA, WATER YEAR OCTOBER 1995 TO SEPTENBER 1996

RIO GRANDE BASIN - - Continued

08362500 RTO GRANDE BLW CABALLO DAM, NM

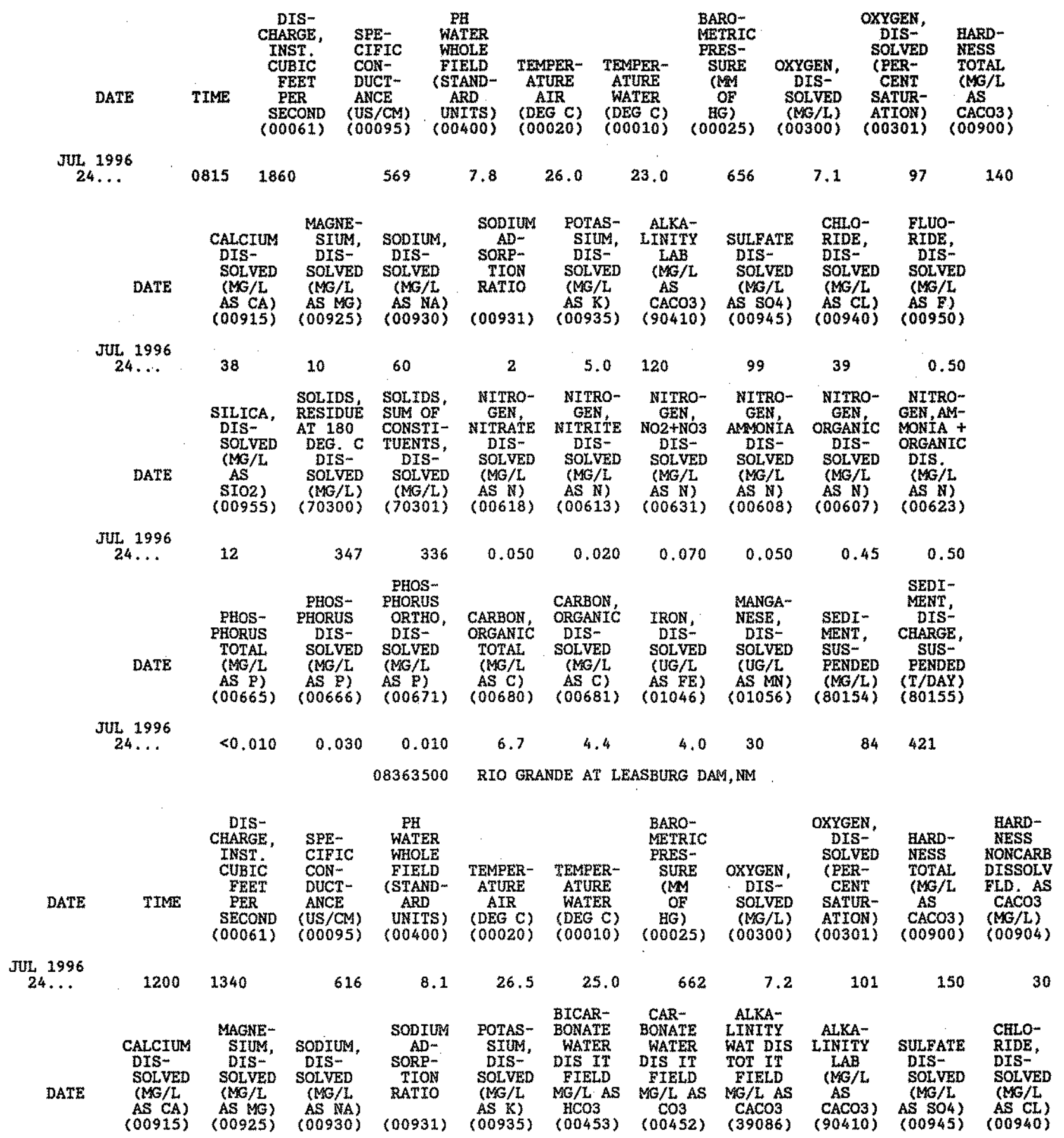

JUL 1996

$24 \ldots$

43

11

64

2

$5.3 \quad 150$

0

$123 \quad 141$

110 
WATER-QUALITY DATA, WATER YEAR OCTOBER 1995 TO SEPTEMBER 1996

RIO GRANDE BASIN - Continued

08363500 . RIO GRANDE AT LEASBURG DAM, RM

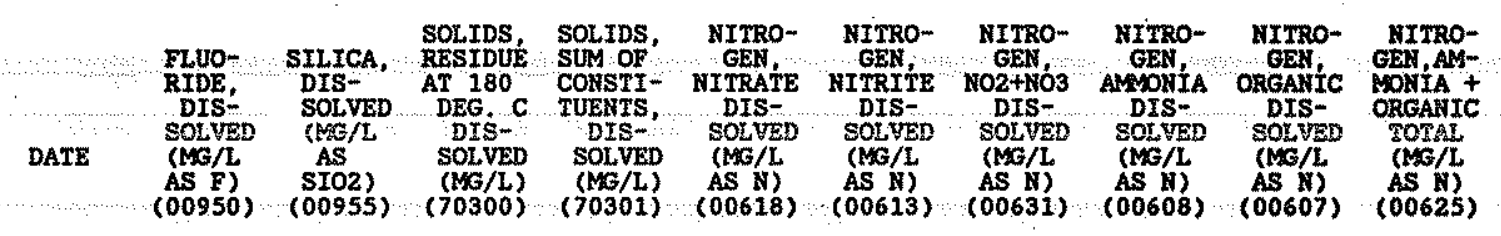

JUL 1996

374

365

0.080

0.010

0.090

0.040

0.16

0.60

\begin{tabular}{|c|c|c|c|}
\hline & \multirow{5}{*}{$\begin{array}{l}\text { NITRO- } \\
\text { GEN,AM- } \\
\text { MONIA + } \\
\text { ORGANIC } \\
\text { DIS } \\
\text { (MG/L } \\
\text { AS N) } \\
(00623)\end{array}$} & \multirow{5}{*}{$\begin{array}{c}\text { PHOS- } \\
\text { PEORUS } \\
\text { TOTAL } \\
\text { (MG/L } \\
\text { AS P) } \\
(00665\end{array}$} & \\
\hline & & & PHO \\
\hline & & & DIS- \\
\hline & & & 促 \\
\hline & & & \\
\hline
\end{tabular}

FHOS-

PHORUS

ORTHO, CARBOY CARBON

DIS-

ORGANIC

IRON NESE

AS P)

(MG/L $\quad$ (MG/L.

AS C)

AS C)

(UG/L

AS FE) AS DA

$(01056)$

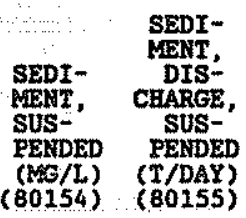

JUL 1996

$0.20 \quad 0.140<0.010$

0.010

6.5

3.7

$<3.0$

1.0

262948 
The following water - cuality tables for miscellaneous sites in the Rto Grande bastn are 1 dentified by 15-digit latitude-longitude site numbers are in order by ascending site numbers as shown in parenthesis after the site names. The inorganic analyses tables are followed by the organic-compotmd analyses table for these sites. This departure from the normal downstream order for surface water sites was taken to for these sites. This departure from the normal downstream order for surface water sites was taken to

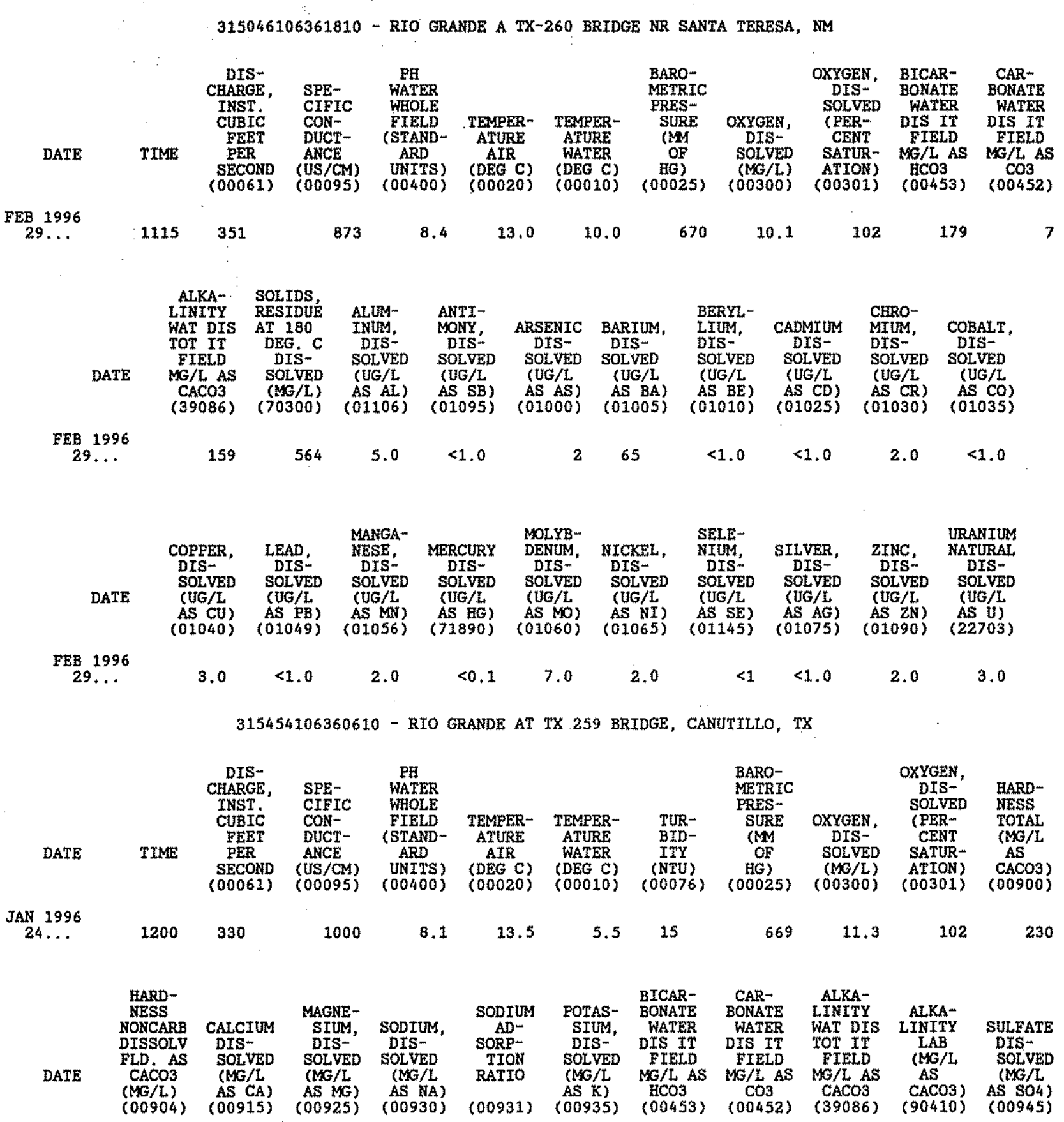


ANALYSES OF SAMPLES COLLECTED AT WATER-QUALITY PARTIAL-RECORD STATIONS AND MISCELIANEOUS SITES

WATER-QUALITY DATA, WATER YSAR OCTOBER 1995 TO SEPTEMBER 1996

RIO GRANDE BASIN -- Continued

315454106360610 - RIO GRANDE AT TX 259 BRIDGE, CANUTILLO, TX

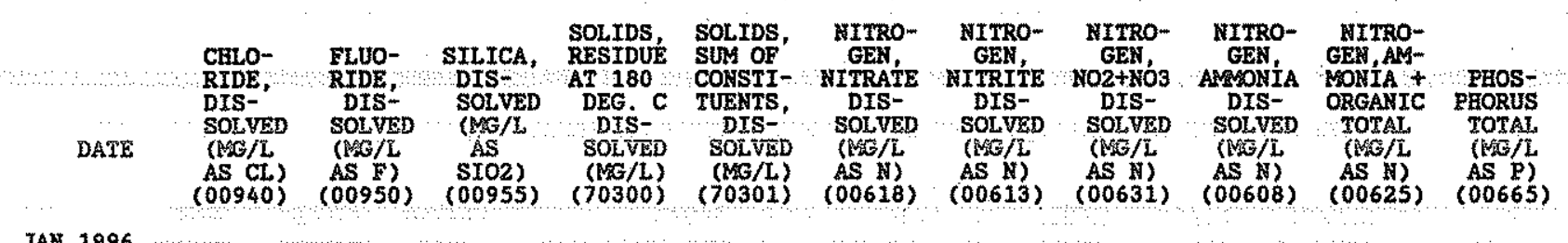

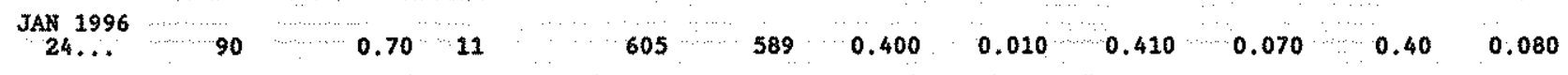

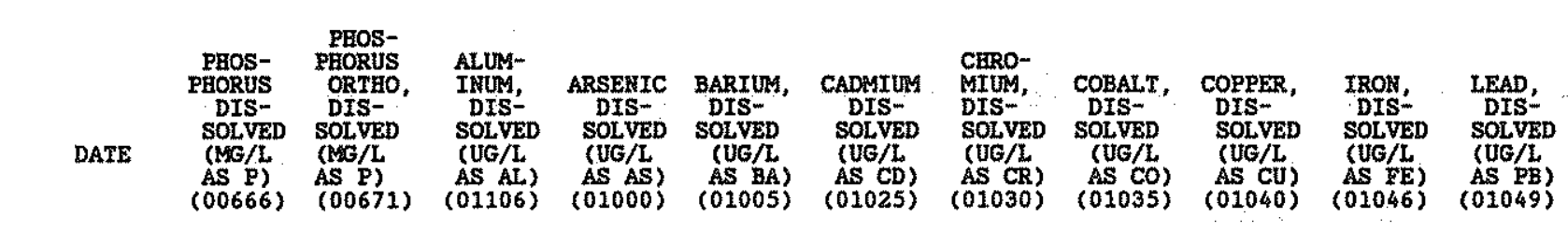

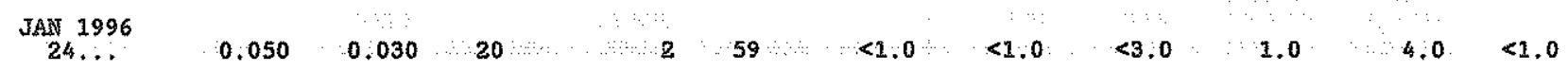

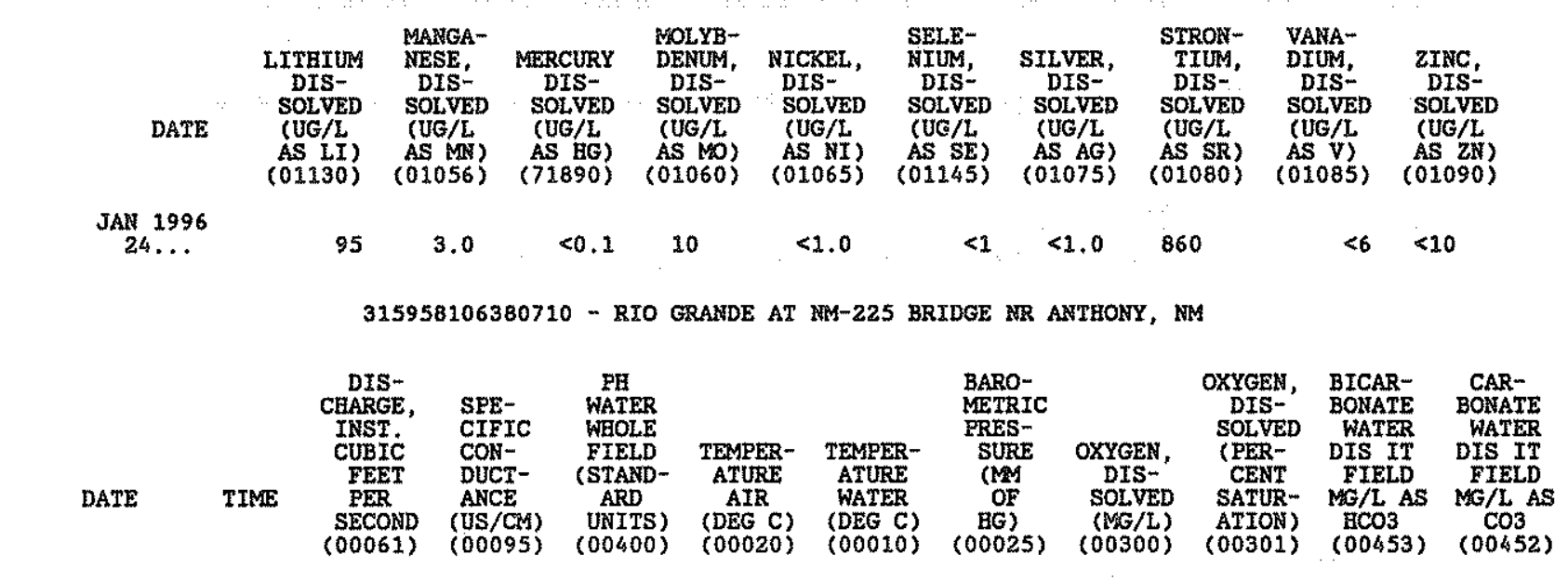

EEB 1996

$\begin{array}{cccccccccccccc}\mathrm{EB} & 1996 & 1415 & 245 & 880 & 8.2 & 14.5 & 13.0 & 665 & 9.8 & 107 & 197 & 0\end{array}$

\begin{tabular}{|c|c|c|c|c|c|c|c|c|c|c|}
\hline DATE & $\begin{array}{l}\text { ALKA- } \\
\text { LINITY } \\
\text { WAT DIS } \\
\text { TOT IT } \\
\text { FIELD } \\
\text { MG/L AS } \\
\text { CACO3 } \\
\text { (39086) }\end{array}$ & $\begin{array}{l}\text { SOLIDS, } \\
\text { RESIDUE } \\
\text { AT } 180 \\
\text { DEE. C } \\
\text { DIS- } \\
\text { SOLVED } \\
(M G / L) \\
(70300)\end{array}$ & $\begin{array}{l}\text { ALUN- } \\
\text { INUM, } \\
\text { DYS- } \\
\text { SOLNED } \\
\text { (UG/L } \\
\text { AS AL) } \\
\text { (01106) }\end{array}$ & $\begin{array}{l}\text { ANTI- } \\
\text { MONY, } \\
\text { DIS- } \\
\text { SOLVED } \\
\text { (UG/L } \\
\text { AS SB) } \\
\text { (01095) }\end{array}$ & $\begin{array}{c}\text { ARSENIC } \\
\text { DIS- } \\
\text { SOLVED } \\
\text { (UG/L } \\
\text { AS AS) } \\
(01000)\end{array}$ & $\begin{array}{l}\text { BARIUM, } \\
\text { DIS- } \\
\text { SOLVED } \\
\text { (UG/L } \\
\text { AS BA }) \\
(01005)\end{array}$ & $\begin{array}{l}\text { BERYL- } \\
\text { LIUM, } \\
\text { DIS- } \\
\text { SOLVED } \\
\text { (UG/L } \\
\text { AS BE) } \\
\text { (01010) }\end{array}$ & $\begin{array}{c}\text { CABMIUM } \\
\text { DIS- } \\
\text { SOLVED } \\
\text { (UG/L } \\
\text { AS CD) } \\
(01025)\end{array}$ & $\begin{array}{l}\text { CERO- } \\
\text { MIUM, } \\
\text { DIS- } \\
\text { SOLVED } \\
\text { (UG/L } \\
\text { AS CR) } \\
(01030)\end{array}$ & $\begin{array}{c}\text { COBALT, } \\
\text { DIS- } \\
\text { SOLVED } \\
\text { (UG/L } \\
\text { AS CO) } \\
(01035)\end{array}$ \\
\hline
\end{tabular}

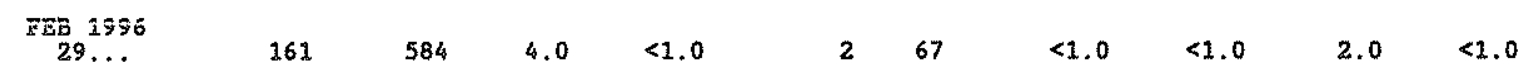

\begin{tabular}{|c|c|c|c|c|c|c|c|c|c|c|}
\hline DATE & $\begin{array}{l}\text { COPEER, } \\
\text { DIS- } \\
\text { SOLVED } \\
\text { (UG/L } \\
\text { AS CU) } \\
(01040)\end{array}$ & $\begin{array}{c}\text { LEAD, } \\
\text { DIS } \\
\text { SOLVED } \\
\text { (UG/L } \\
\text { AS PB) } \\
(01049)\end{array}$ & $\begin{array}{c}\text { MANGA- } \\
\text { NESE, } \\
\text { DIS- } \\
\text { SOLVED } \\
\text { (UG/L } \\
\text { AS MN) } \\
\text { (01056) }\end{array}$ & $\begin{array}{c}\text { MERCURY } \\
\text { DIS- } \\
\text { SOLVED } \\
\text { (UG/L } \\
\text { AS HG) } \\
(71890)\end{array}$ & $\begin{array}{l}\text { MOLYB- } \\
\text { DENUM, } \\
\text { DIS- } \\
\text { SOLVED } \\
\text { (UO/L } \\
\text { AS WO) } \\
(01060)\end{array}$ & $\begin{array}{l}\text { NICKEL, } \\
\text { DIS- } \\
\text { SOLVED } \\
\text { (UG/L } \\
\text { AS NI) } \\
(01065)\end{array}$ & $\begin{array}{l}\text { SELE- } \\
\text { NIUM, } \\
\text { DIS- } \\
\text { SOLVED } \\
\text { (UG/L } \\
\text { AS SE) } \\
(01145)\end{array}$ & $\begin{array}{c}\text { SILVER, } \\
\text { DIS- } \\
\text { SOLVED } \\
\text { (UG/L } \\
\text { AS AG) } \\
\text { (01075) }\end{array}$ & $\begin{array}{l}\text { ZINC, } \\
\text { DIS } \\
\text { SOLVED } \\
\text { (UG/L } \\
\text { AS ZN) } \\
\text { (01090) }\end{array}$ & $\begin{array}{c}\text { URANIUM } \\
\text { XATURAL } \\
\text { DIS- } \\
\text { SOLVED } \\
\text { (UG/L } \\
\text { AS U) } \\
\text { (22703) }\end{array}$ \\
\hline
\end{tabular}

TEB 1996

$29 \ldots$

$2.0<1.0 \quad 11$

$<0.1 \quad 7.0$

2.0

$<1<1,0$

4.0

3.0 
ANALYSES OF SAMPLES COLLECTED AT WATER-QUALITY FARTIAL-RECORD STATIONS AND MISCELLANEOUS SITES

WATER-QUALITY DATA, WATER YEAR OCTOBER 1995 TO SEPTEMBER 1996

RIO GRANDE BASIN - Continued

320610106393110 - DEL, RIO DRAIN AT LEVEE ROAD NEAR VADO, MM

\begin{tabular}{|c|c|c|c|c|c|c|c|c|c|c|c|}
\hline DATE & TIME & $\begin{array}{c}\text { DIS- } \\
\text { CHARGE, } \\
\text { INST } \\
\text { CUBIC } \\
\text { FEET } \\
\text { PER } \\
\text { SECOND } \\
(00061)\end{array}$ & $\begin{array}{l}\text { SPE- } \\
\text { CIFIC } \\
\text { CON- } \\
\text { DUCT- } \\
\text { ANCE } \\
\text { (US/CM) } \\
(00095)\end{array}$ & $\begin{array}{c}\text { PH } \\
\text { WATER } \\
\text { WHOLE } \\
\text { FIELD } \\
\text { (STAND- } \\
\text { ARD } \\
\text { UNITS) } \\
(00400)\end{array}$ & $\begin{array}{c}\text { TEMPER- } \\
\text { ATURE } \\
\text { AIR } \\
(\text { DEG C) } \\
(00020)\end{array}$ & $\begin{array}{c}\text { TEMPER- } \\
\text { ATURE } \\
\text { WATER } \\
\text { (DEG C) } \\
(00010)\end{array}$ & $\begin{array}{c}\text { BARO- } \\
\text { METRIC } \\
\text { ERES- } \\
\text { SURE } \\
\text { (MM } \\
\text { OF } \\
\text { HG) } \\
(00025)\end{array}$ & $\begin{array}{c}\text { OXYGEN, } \\
\text { DIS- } \\
\text { SOLVED } \\
\text { (MG/L) } \\
(00300)\end{array}$ & $\begin{array}{c}\text { OXYGES, } \\
\text { DIS- } \\
\text { SOLVED } \\
\text { (PER- } \\
\text { CENT } \\
\text { SATUR- } \\
\text { ATION) } \\
\text { (00301) }\end{array}$ & $\begin{array}{c}\text { BICAR- } \\
\text { BONATE } \\
\text { WATER } \\
\text { DIS IT } \\
\text { FIELD } \\
\text { MG/L AS } \\
\text { HCO3 } \\
(00453)\end{array}$ & $\begin{array}{c}\text { CAR- } \\
\text { BONATE } \\
\text { WATERR } \\
\text { DIS IT } \\
\text { FIELLD } \\
\text { MG/L AS } \\
\text { CO3 } \\
(00452)\end{array}$ \\
\hline $\begin{array}{l}\text { B } 1996 \\
28 \ldots\end{array}$ & 1215 & 38 & 1160 & 8.1 & 17.0 & 13.0 & 665 & 9.3 & 102 & 244 & 0 \\
\hline
\end{tabular}

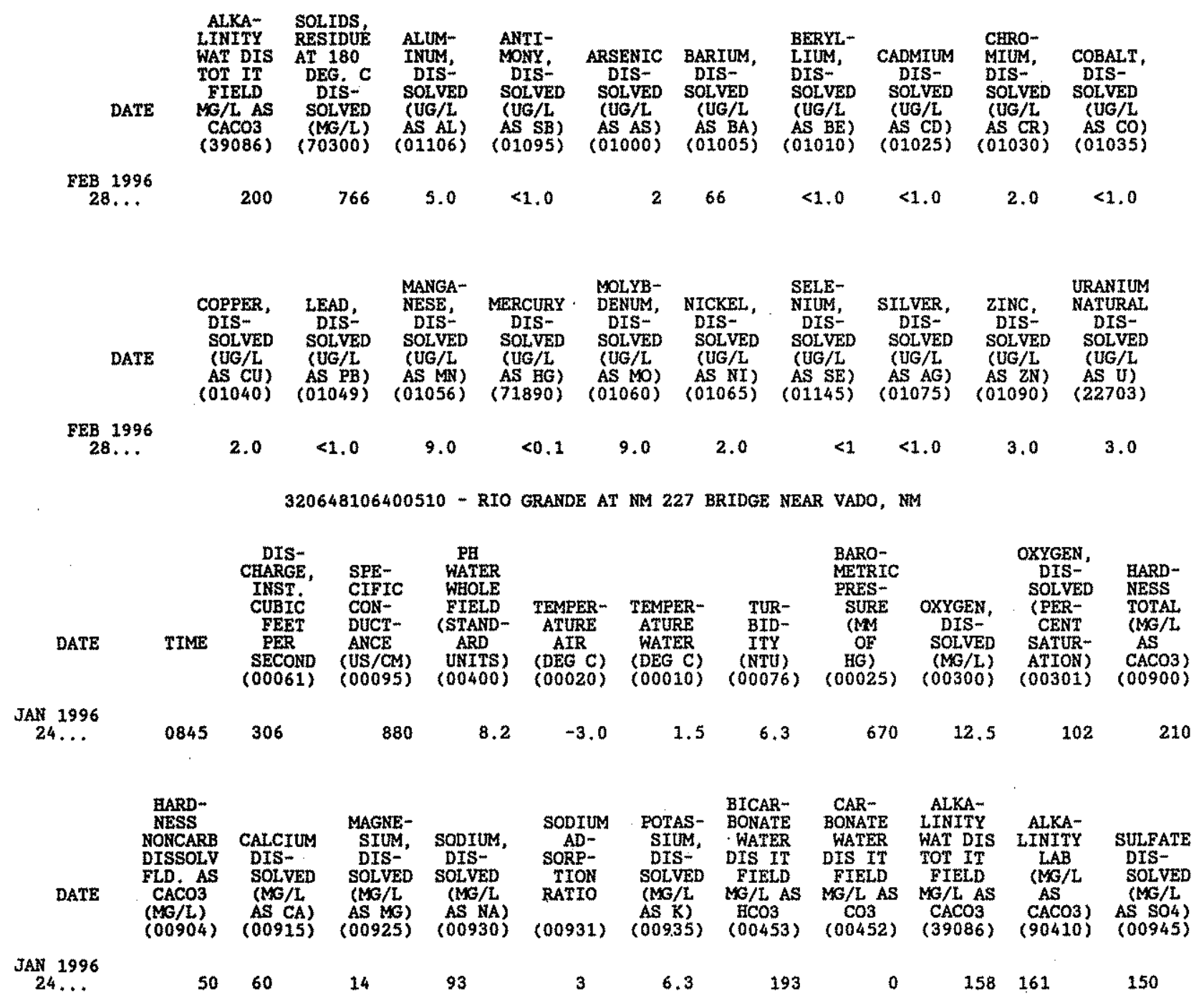


ANALYSES OF SAMPLES COLLECTED AT WATER-QUALITY PARTIAL-RECORD STATIONS AND MISCELLANEOUS SITES

WATER-QUALITY DATA, WATER YEAR OCTOBAR 1995 TO SEPTEMBER 1996

RIO GRANDE BASIN -- Continued

320648106400510 - RIO GRANDE AT NM 227 BRIDGE NEAR VADO, NM

\begin{tabular}{|c|c|c|c|c|c|c|c|c|c|c|c|}
\hline DATE & $\begin{array}{l}\text { CAIL- } \\
\text { RIDE, } \\
\text { DIS- } \\
\text { SOLVED } \\
\text { (HS/L } \\
\text { AS CL) } \\
(00940)\end{array}$ & $\begin{array}{l}\text { FLUO- } \\
\text { RIDE, } \\
\text { DIS- } \\
\text { SOLVED } \\
\text { (AES/L } \\
\text { AS F) } \\
(00950)\end{array}$ & $\begin{array}{c}\text { SILICA, } \\
\text { DIS } \\
\text { SOLVED } \\
\text { (MG/L } \\
\text { AS } \\
\text { SIO2) } \\
(00955)\end{array}$ & $\begin{array}{l}\text { SOLIDS, } \\
\text { RESIDUE } \\
\text { AT } 180 \\
\text { DEG C } \\
\text { DIS- } \\
\text { SOLVED } \\
\text { (MG/L) } \\
(70300)\end{array}$ & $\begin{array}{l}\text { SOLIDS, } \\
\text { SUN OF } \\
\text { CONSTI- } \\
\text { TUENTS, } \\
\text { DIS - } \\
\text { SOL YED } \\
(\text { WG/L }) \\
(70301)\end{array}$ & $\begin{array}{l}\text { NITRO- } \\
\text { GEN, } \\
\text { NITRATE } \\
\text { DIS- } \\
\text { SOLVED } \\
\text { (ME/I } \\
\text { AS N) } \\
\text { (00618) }\end{array}$ & $\begin{array}{c}\text { NITRO- } \\
\text { GEN, } \\
\text { NITRITE } \\
\text { DIS- } \\
\text { SOLVED } \\
\text { (MG/L } \\
\text { AS N) } \\
\text { (00613) }\end{array}$ & $\begin{array}{l}\text { NITRO- } \\
\text { GEN, } \\
\text { NO2+NO3 } \\
\text { DIS- } \\
\text { SOLVED } \\
\text { (NGS/L, } \\
\text { AS N) } \\
(00631)\end{array}$ & $\begin{array}{c}\text { NITRO- } \\
\text { GEN, } \\
\text { AMONIA } \\
\text { DIS- } \\
\text { SOLVED } \\
\text { (MEL/L } \\
\text { AS N } \\
(00608)\end{array}$ & $\begin{array}{l}\text { NITRO- } \\
\text { GEN, AN- } \\
\text { MONIA + } \\
\text { ORGANIC } \\
\text { TOTAL } \\
\text { (HG/L } \\
\text { AS N) } \\
(00625)\end{array}$ & $\begin{array}{l}\text { PHOS- } \\
\text { PHORUS } \\
\text { TORAL. } \\
\text { (NG/L. } \\
\text { AS P) } \\
(00665)\end{array}$ \\
\hline$\cdots$ & 74 & 0.60 & 8.7 & 524 & 505 & 0.490 & 0.020 & 0.510 & 0.020 & & \\
\hline
\end{tabular}

DATE

$\begin{array}{ccc}\text { PHOS- } & \text { PHOS- } \\ \text { PEORUS } & \text { ALUM- } \\ \text { PHORUS } & \text { ORTHO, INUM, } \\ \text { DIS- } & \text { DIS- } & \text { DIS- } \\ \text { SOLVED } & \text { SOLVED } & \text { SOLVED } \\ (\text { ME } / L & (M G / L & (U G / L \\ \text { AS P) } & \text { AS P) } & \text { AS RL) } \\ (00666) & (00671) & (01106)\end{array}$

$\begin{array}{ccc}\text { ARSFWIC } & \text { BARTUM, } & \text { CADMIUM } \\ \text { DIS- } & \text { DIS- } & \text { DIS- } \\ \text { SOLVED } & \text { SOLVED } & \text { SOLVED } \\ \text { (UG/L } & \text { (UG/L } & \text { (UG/L } \\ \text { ASAS) } & \text { AS BA) } & \text { AS CD) } \\ (01000) & (01005) & (01025)\end{array}$

CHRO-

MIUM, COBALT, COPPER, IRON, LEAD,

DIS- DIS- DIS - DIS - DIS

SOLVED SOLVED SOLVED SOLVED SOLVED

(UG/L (UG/L $\quad$ (UG/L $\quad$ (UG/L

AS P) AS AS AS AS AS CO AS CU) AS FE) AS PB)

\begin{tabular}{|c|c|c|c|c|c|c|c|c|c|c|c|}
\hline $\begin{array}{c}\text { JAN } 1.996 \\
24 \ldots\end{array}$ & 0.040 & $\therefore 0.030$ & $\therefore 10$ & 2 & 67 & $<1.0$ & $<1.0$ & $<3.0$ & $<1,0$ & 4.0 & $<1.0$ \\
\hline & DATE & $\begin{array}{l}\text { 1.ITEIUM } \\
\text { DIS- } \\
\text { SOLVED } \\
\text { (UG/L } \\
\text { AS LI) } \\
\text { (01130) }\end{array}$ & $\begin{array}{l}\text { MANGA- } \\
\text { NESE, } \\
\text { DIS- } \\
\text { SOLVED } \\
\text { (UG/L } \\
\text { AS MN) } \\
(01056)\end{array}$ & $\begin{array}{c}\text { MERCURX } \\
\text { DIS- } \\
\text { SOLVED } \\
\text { (UG/L } \\
\text { AS HG) } \\
(71890)\end{array}$ & $\begin{array}{c}\text { MOLYB- } \\
\text { DENUM, } \\
\text { DIS- } \\
\text { SOLVED } \\
\text { (UG/1 } \\
\text { AS MO) } \\
\text { (01060) }\end{array}$ & $\begin{array}{l}\text { NICKEL, } \\
\text { DIS- } \\
\text { SOLVED } \\
\text { (UG/L } \\
\text { AS NI) } \\
(01065)\end{array}$ & $\begin{array}{l}\text { SELE- } \\
\text { NIUM, } \\
\text { DIS- } \\
\text { SOLVED } \\
\text { (UG/L } \\
\text { AS SE) } \\
(01145)\end{array}$ & $\begin{array}{c}\text { SILVER, } \\
\text { DIS- } \\
\text { SOLVED } \\
\text { (UG/L } \\
\text { AS AG) } \\
(01075)\end{array}$ & $\begin{array}{c}\text { STRON- } \\
\text { TIUM, } \\
\text { DIS- } \\
\text { SOLVED } \\
\text { (UG/L } \\
\text { AS SR) } \\
(01080)\end{array}$ & $\begin{array}{l}\text { VANA- } \\
\text { DIUM, } \\
\text { DIS- } \\
\text { SOLVED } \\
\text { (UG/L } \\
\text { AS V) } \\
(01085)\end{array}$ & $\begin{array}{l}\text { ZIHC, } \\
\text { DIS- } \\
\text { SOLVED } \\
\text { (UG/L } \\
\text { AS ZN) } \\
(01090)\end{array}$ \\
\hline
\end{tabular}

JAN 1996

$24 \ldots$

84

$2.0<0.1 \quad 20$

$<1.0$

$<1<1.0 \quad 770$

$<6<10$

321317106471510 - RIO GRANDE BELOW MESILLA DAM NR SANTO TOMAS, NM

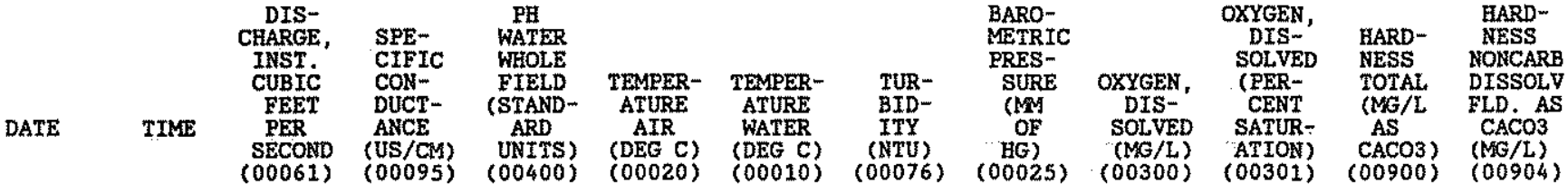

\begin{tabular}{|c|c|c|c|c|c|c|c|c|c|c|c|c|}
\hline $\begin{array}{c}\text { JAN } 1996 \\
23 . \cdots \\
\end{array}$ & 1445 & 315 & 850 & 8.2 & 9.5 & 8.5 & 21 & 666 & 10.7 & 105 & 210 & 48 \\
\hline $28 \ldots$ & 0810 & 139 & 746 & 8.2 & 3.0 & 8.0 & $m-$ & 660 & 8.9 & 87 & $m$ & -- \\
\hline
\end{tabular}

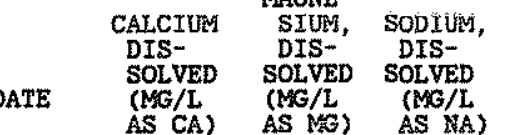

(00915) (00925) (00930)

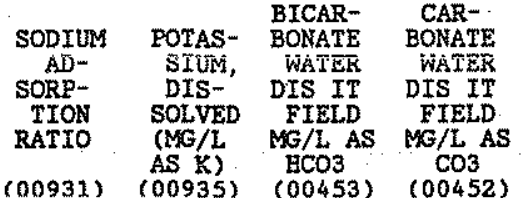

ALXA-

LINITY ALKA- CELO-

WAT DIS LINTIY SULFATE RIDE,

TOT IT LAB DIS- DIS-

FIELD (MG/L SOLVED SOLVED

MG/L AS AS $\quad$ ASG/L (MS/L

$\begin{array}{llllllllll}(00931) & (00935) & (00453) & (00452) & (39086) & (90410) & (00945) & (00940)\end{array}$

\begin{tabular}{|c|c|c|c|c|c|c|c|c|c|c|c|}
\hline $\begin{array}{c}\text { JAN } 1996 \\
23 . \cdots \\
\text { FEB }\end{array}$ & 59 & 14 & 93 & 3 & 6.6 & 192 & 0 & 157 & 160 & 3.50 & 77 \\
\hline $28 \ldots$ & - & -- & -- & -- & -- & 270 & 0 & 139 & -- & -- & $-\infty$ \\
\hline
\end{tabular}


ANALYSES OF SAMPLES COLLECTED AT WATER-QUALITY PARTIAL-RECORD STATIONS AND MISCELLANEOUS SITES

WATER-QUALITY DATA, WATER YEAR OCTOBER 1995 TO SEPTEMBER 1996

RIO GRANDE BASIN -- Continued

321317106471510 - RIO GRANDE BELOW MESILLA DAM NR SANTO TOMAS, NM

DATE

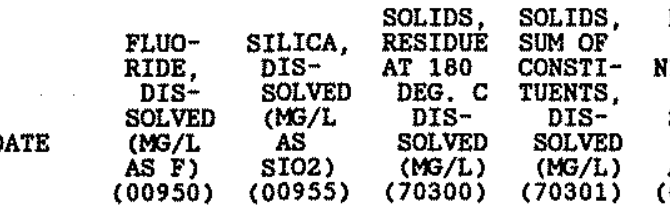

NITRO- NITRO- NITRO- NITRO-

GEN GEN GEN GEN,AM

ITRTTE NO2+Nó3 AMMONIA MONIA +

(MG/L (MG/L (MG/L (MG/L $\quad(M G / L$

AS N) AS N) AS N) AS N) AS P)

PHOS-

PHORUS ORTHO

DIS - DIS-

SOLVED SOLVED

(MG/L $\quad$ (MG/L

AS P) AS P)

$\begin{array}{cccccccccccc}\begin{array}{l}\text { JAN } 1996 \\ 23 \ldots\end{array} & 0.60 & 8.9 & 524 & 507 & <0.010 & 0.620 & 0.020 & 0.40 & 0.090 & 0.050 & 0.030 \\ \text { FEB } & & -\ldots & 466 & - & \ldots & \ldots- & \ldots & \ldots & \ldots & \ldots & \ldots\end{array}$

DATE

$\begin{array}{cccc}\text { ALUM- } & \text { ANTI- } & & \\ \text { INIM, } & \text { MONY, } & \text { ARSENIC } & \text { BARIUM, } \\ \text { DIS- } & \text { DIS- } & \text { DIS- } & \text { DIS- } \\ \text { SOLVED } & \text { SOLVED } & \text { SOLVED } & \text { SOLVED } \\ \text { (UG/L } & \text { (UG/L } & \text { (UG } / L & (U G / L \\ \text { AS AL) } & \text { AS SB) } & \text { AS AS) } & \text { AS BA }) \\ (01106) & (01095) & (01000) & (01005)\end{array}$

BERYL-

MIUM, COBALT, COPPER, IRON, LEAD,

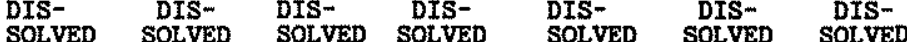

(UG/L (UG/L (UG/L (UG/L) (UG/L (UG/L (UG/L

AS BE) AS CD) AS CR) AS CO) AS CU) AS FE) AS PB

$(01106)(01095)(01000)(01005)(01010)(01025)(01030)(01035) \quad(01040) \quad(01046) \quad(01049)$

\begin{tabular}{|c|c|c|c|c|c|c|c|c|c|c|c|}
\hline $\begin{array}{c}23 \ldots \\
28 \ldots\end{array}$ & $\begin{array}{l}20 \\
6.0\end{array}$ & $\begin{array}{l}-- \\
<1,0\end{array}$ & 2 & $\begin{array}{l}65 \\
64\end{array}$ & $<1.0$ & $\begin{array}{l}<1,0 \\
<1,0\end{array}$ & $\begin{array}{r}<1.0 \\
2.0\end{array}$ & $\begin{array}{l}<3.0 \\
<1.0\end{array}$ & $\begin{array}{r}<1.0 \\
2.0\end{array}$ & 4.0 & $\begin{array}{l}<1,0 \\
<1,0\end{array}$ \\
\hline
\end{tabular}

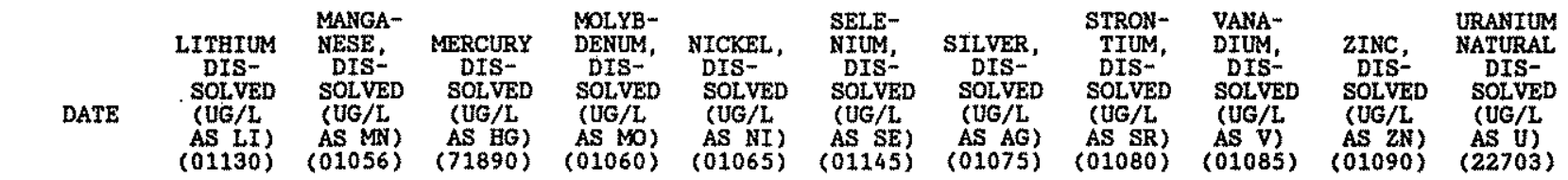

\begin{tabular}{|c|c|c|c|c|c|c|c|c|c|c|c|}
\hline${ }_{F E B}^{23} \cdots$ & 84 & 4.0 & $<0.1$ & $<10$ & $<1.0$ & $<1$ & $<1.0$ & 760 & $<6$ & $<10$ & - \\
\hline $28 \ldots$ & -- & 2.0 & $<0.1$ & 6.0 & 2.0 & $<1$ & $<1,0$ & -- & -- & 3.0 & 3.0 \\
\hline
\end{tabular}

321551106492910 - RIO GRANDE AT CALLE DEL NORTE BRDG NR MESILLA, NM

\begin{tabular}{|c|c|c|c|c|c|c|c|c|c|c|c|c|}
\hline DATE & TIME & $\begin{array}{c}\text { DIS- } \\
\text { CHARGE, } \\
\text { INST. } \\
\text { CUBIC } \\
\text { FEET } \\
\text { PER } \\
\text { SECOND } \\
(00061)\end{array}$ & $\begin{array}{l}\text { SPE- } \\
\text { CIEIC } \\
\text { CON- } \\
\text { DUCT- } \\
\text { ANCE } \\
\text { (US/CM) } \\
(00095)\end{array}$ & $\begin{array}{c}\text { PH } \\
\text { WATER } \\
\text { WHOLE } \\
\text { FIELD } \\
\text { (STAND- } \\
\text { ARD } \\
\text { UNITS) } \\
\text { (00400) }\end{array}$ & $\begin{array}{c}\text { TEMPER- } \\
\text { ATURE } \\
\text { AIR } \\
(\text { DEG C) } \\
(00020)\end{array}$ & $\begin{array}{c}\text { TEMPER- } \\
\text { ATURE } \\
\text { WATER } \\
\text { (DEG C) } \\
(00010)\end{array}$ & $\begin{array}{l}\text { BARO- } \\
\text { METRIC } \\
\text { PRES- } \\
\text { SURE } \\
\text { (MM } \\
\text { OF } \\
\text { HG) } \\
(00025)\end{array}$ & $\begin{array}{c}\text { OXYGEN, } \\
\text { DIS - } \\
\text { SOLVED } \\
(M G / L) \\
(00300)\end{array}$ & $\begin{array}{c}\text { OXYGEN, } \\
\text { DIS- } \\
\text { SOLVED } \\
\text { (PER- } \\
\text { CENT } \\
\text { SATUR- } \\
\text { ATION) } \\
\text { (O0301) }\end{array}$ & $\begin{array}{l}\text { KARD- } \\
\text { NESS } \\
\text { TOTAL } \\
\text { (MG/L } \\
\text { AS } \\
\text { CACO3) } \\
(00900)\end{array}$ & $\begin{array}{c}\text { CALCIUM } \\
\text { DIS- } \\
\text { SOLVED } \\
\text { (MG/1 } \\
\text { AS CA) } \\
(00915)\end{array}$ & $\begin{array}{c}\text { MAGNE- } \\
\text { SIUM, } \\
\text { DIS- } \\
\text { SOLVED } \\
\text { (MG/L } \\
\text { AS MG) } \\
\text { (00925) }\end{array}$ \\
\hline
\end{tabular}

$\begin{array}{ccc}\text { AltG } 1996 & & \\ 06 \ldots & 0830 & 1120 \\ 07 \ldots & 0800 & 1290 \\ 20 \ldots & 1330 & 1680\end{array}$

$\begin{array}{lllll}623 & 8.3 & -- & 23.0 & - \\ 611 & 8.2 & - & 24.0 & - \\ 595 & 8.1 & 35.0 & 27.0 & 664\end{array}$

664
664

$$
\begin{array}{ll}
7.0 & - \\
7.3 & -1 \\
7.1 & 103 \\
6.3 & 86
\end{array}
$$

$\begin{array}{ll}150 & 42 \\ 140 & 40 \\ 130 & 36 \\ 140 & 38\end{array}$

$$
\begin{array}{ll}
42 & 11 \\
40 & 10 \\
36 & 9.5 \\
38 & 10
\end{array}
$$

\begin{tabular}{|c|c|c|c|c|c|c|c|c|c|c|c|c|}
\hline $\mathrm{ATE}$ & $\begin{array}{l}\text { CARBON, } \\
\text { ORGANIC } \\
\text { TOTAL } \\
(M G / L \\
\text { AS C) } \\
(00680)\end{array}$ & $\begin{array}{l}\text { CARBON, } \\
\text { ORGANIC } \\
\text { DIS- } \\
\text { SOLVED } \\
\text { (MG/L } \\
\text { AS C) } \\
(00681)\end{array}$ & $\begin{array}{l}\text { ALUM- } \\
\text { INUM, } \\
\text { TOTAL } \\
\text { RECOV- } \\
\text { ERABLE } \\
\text { (UG/L } \\
\text { AS AL) } \\
\text { (01105) }\end{array}$ & $\begin{array}{l}\text { ALUM- } \\
\text { INUM, } \\
\text { DIS- } \\
\text { SOLVED } \\
\text { (UG/L } \\
\text { AS AL) } \\
\text { (01106) }\end{array}$ & $\begin{array}{l}\text { ANTI- } \\
\text { MONY, } \\
\text { TOTAL } \\
\text { (UG/L } \\
\text { AS SB) } \\
\text { (01097) }\end{array}$ & $\begin{array}{l}\text { ANTI- } \\
\text { MONY, } \\
\text { DIS- } \\
\text { SOLVED } \\
\text { (UG/L } \\
\text { AS SB) } \\
(01095)\end{array}$ & $\begin{array}{l}\text { BARIUM, } \\
\text { DIS- } \\
\text { SOLVAD } \\
\text { (UG/L } \\
\text { AS BA) } \\
(01005)\end{array}$ & $\begin{array}{l}\text { BERYL- } \\
\text { LIUM, } \\
\text { TOTAL } \\
\text { RECOV- } \\
\text { ERABLE } \\
\text { (UG/L } \\
\text { AS BE) } \\
\text { (01012) }\end{array}$ & $\begin{array}{l}\text { BERYL- } \\
\text { LIUM, } \\
\text { DIS-- } \\
\text { SOLVED } \\
\text { (UG/L } \\
\text { AS BE) } \\
\text { (01010) }\end{array}$ & $\begin{array}{l}\text { CADMIUM } \\
\text { WATER } \\
\text { UNFLTRD } \\
\text { TOTAL } \\
\text { (UG/L } \\
\text { AS CD) } \\
(01027 \text { ) }\end{array}$ & $\begin{array}{c}\text { CADMIUM } \\
\text { DIS- } \\
\text { SOLVED } \\
\text { (UG/L } \\
\text { AS CD) } \\
\text { (01025) }\end{array}$ & $\begin{array}{l}\text { CHRO- } \\
\text { MIUM, } \\
\text { TOTAL. } \\
\text { RECOV- } \\
\text { ERABLE } \\
\text { (UG/L } \\
\text { AS CR) } \\
\text { (01034) }\end{array}$ \\
\hline
\end{tabular}

AUG 1996

$\begin{array}{llll}06 \ldots & 6.5 & 3.3 & 1900 \\ 07 \ldots & 6.3 & 3.3 & 1900 \\ 20 \ldots & 6.0 & 3.5 & 2700 \\ 21 \ldots & 6.2 & 3.3 & 2500\end{array}$

$\begin{array}{rrr}25 & 1 & <1.0 \\ 21 & 1 & 2.0 \\ 6.0 & <1 & 1.0 \\ 5.0 & <1 & <1.0\end{array}$

$\begin{array}{lll}73 & <10 & <1.0 \\ 68 & <10 & <1.0 \\ 63 & <10 & <1.0 \\ 66 & <10 & <1.0\end{array}$

$<1.0$
$<1.0$
$<1.0$
$<1.0$

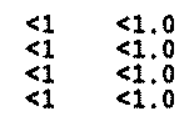


WATER-QUALITY DATA, WATER YEAR OCTOBER 1995 TO SEPTEMBER 1996

RIO GRANDE BASIN -- Continued

321551106492910 - RIO GRANDE AT CALLE DEL NORTE BRDG NR MESILLA, MM

\begin{tabular}{|c|c|c|c|c|c|c|c|c|c|c|c|}
\hline DATE & $\begin{array}{l}\text { CHRO- } \\
\text { MIUM, } \\
\text { DIS- } \\
\text { SOLVED } \\
\text { (UG/L } \\
\text { AS CR) } \\
(01030)\end{array}$ & $\begin{array}{l}\text { COBALT, } \\
\text { TOTAL } \\
\text { RECOV- } \\
\text { ERABLE } \\
\text { (UG/L } \\
\text { AS CO) } \\
(01037)\end{array}$ & $\begin{array}{l}\text { COBALT, } \\
\text { DIS- } \\
\text { SOLVED } \\
\text { (UUG/L } \\
\text { AS CO) } \\
(01035)\end{array}$ & $\begin{array}{c}\text { COPPER, } \\
\text { TOTAL } \\
\text { RECOV- } \\
\text { ERABLE } \\
\text { (UE/L } \\
\text { AS CU) } \\
\text { (01042) }\end{array}$ & $\begin{array}{l}\text { COPPER, } \\
\text { DIS- } \\
\text { SOLVED } \\
(\text { UG } / L \\
\text { AS CIS) } \\
(01040)\end{array}$ & $\begin{array}{l}\text { LEAD, } \\
\text { TOTAL } \\
\text { RECOV- } \\
\text { GRABLE } \\
\text { (UG/L } \\
\text { AS PB) } \\
\text { (01051) }\end{array}$ & $\begin{array}{l}\text { LEAD, } \\
\text { DIS- } \\
\text { SOLVED } \\
\text { (UG/1. } \\
\text { AS PB) } \\
(01049)\end{array}$ & $\begin{array}{l}\text { MANGA- } \\
\text { NESE, } \\
\text { TOTAL } \\
\text { RECOV-- } \\
\text { GRABLE } \\
\text { (UG/L } \\
\text { AS MiH) } \\
\text { (01055) }\end{array}$ & $\begin{array}{l}\text { MANGA- } \\
\text { NESE, } \\
\text { DIS- } \\
\text { SOLVED } \\
\text { (UG/L } \\
\text { AS MA) } \\
\text { (01056) }\end{array}$ & $\begin{array}{l}\text { MGRCURY } \\
\text { TOFAL } \\
\text { RECOV- } \\
\text { TRABLE } \\
\text { (UE/L } \\
\text { AS HG) } \\
\text { (71900) }\end{array}$ & $\begin{array}{l}\text { MRRCURY } \\
\text { DIS } \\
\text { SOLVED } \\
\text { (UC/L } \\
\text { AS HG) } \\
\text { (71890) }\end{array}$ \\
\hline $\begin{array}{l}\text { AUG } 1996 \\
06 \ldots \\
07 \ldots \\
20 \ldots \\
21 \ldots\end{array}$ & $\begin{array}{r}2.0 \\
2.0 \\
<1.0 \\
1.0\end{array}$ & $\begin{array}{r}<1 \\
\frac{1}{6} \\
1\end{array}$ & $\begin{array}{l}<1.0 \\
<1.0 \\
<1.0 \\
<1.0\end{array}$ & $\begin{array}{r}3 \\
3 \\
2 \\
2\end{array}$ & $\begin{array}{l}6.0 \\
3.0 \\
2.0 \\
2.0\end{array}$ & $\begin{array}{l}2 \\
2 \\
4 \\
2\end{array}$ & $\begin{array}{l}<1.0 \\
<1.0 \\
<1.0 \\
<1.0\end{array}$ & $\begin{array}{r}220 \\
180 \\
230 \\
170\end{array}$ & $\begin{array}{r}<1.0 \\
<1.0 \\
1.0 \\
<1.0\end{array}$ & $\begin{array}{l}<0 \\
<0 \\
<0 \\
<0\end{array}$ & $\begin{array}{l}<0.1 \\
<0.1 \\
<0.1 \\
<0.1\end{array}$ \\
\hline DATE & $\begin{array}{l}\text { MOLYB- } \\
\text { DENUM, } \\
\text { TOTAL } \\
\text { RECOV- } \\
\text { FRABLE } \\
\text { (UG/L } \\
\text { AS MO) } \\
(01062)\end{array}$ & $\begin{array}{c}\text { MOLYB- } \\
\text { DENUM, } \\
\text { DIS- } \\
\text { SOLVED } \\
\text { (UG/L } \\
\text { AS MO) } \\
(01060)\end{array}$ & $\begin{array}{l}\text { NICKEL, } \\
\text { TOTAL } \\
\text { RECOV- } \\
\text { FRABLE } \\
\text { (UG/L } \\
\text { AS NT) } \\
\text { (01067) }\end{array}$ & $\begin{array}{c}\text { NICKEL, } \\
\text { DIS- } \\
\text { SOLVED } \\
\text { (UG/L } \\
\text { AS NI) } \\
(01065)\end{array}$ & $\begin{array}{l}\text { SELE- } \\
\text { NIUM, } \\
\text { TOTAL } \\
\text { (UG/L } \\
\text { AS SE) } \\
\text { (01147) }\end{array}$ & $\begin{array}{l}\text { SELE- } \\
\text { NIUM, } \\
\text { DIS- } \\
\text { SOLVED } \\
\text { (UG/L } \\
\text { AS SE) } \\
\text { (01145) }\end{array}$ & $\begin{array}{c}\text { SILVER, } \\
\text { TOTAL } \\
\text { RECOV- } \\
\text { ERABLE } \\
\text { (UG/L } \\
\text { AS AG) } \\
\text { (01077) }\end{array}$ & $\begin{array}{c}\text { SILVER, } \\
\text { DIS- } \\
\text { SOLVED } \\
\text { (UG/L } \\
\text { AS AG) } \\
(01075)\end{array}$ & $\begin{array}{l}\text { ZINC, } \\
\text { TOTAL } \\
\text { RECOV- } \\
\text { ERABLE } \\
\text { (UG/L } \\
\text { AS ZN) } \\
(01092)\end{array}$ & $\begin{array}{c}\text { ZINC, } \\
\text { DIS } \\
\text { SOLVED } \\
\text { (UG/L } \\
\text { AS ZN) } \\
(01090)\end{array}$ & $\begin{array}{l}\text { URANIUM } \\
\text { NATURAL } \\
\text { DIS- } \\
\text { SOLVED } \\
\text { (UG/L } \\
\text { AS U) } \\
(22703)\end{array}$ \\
\hline $\begin{array}{l}\text { UG } 1996 \\
06 \ldots \ldots \\
07 \ldots \\
20 \ldots \\
21 \ldots\end{array}$ & $\begin{array}{l}4 \\
4 \\
4 \\
4\end{array}$ & $\begin{array}{l}6.0 \\
6.0 \\
6.0 \\
6.0\end{array}$ & $\begin{array}{l}2 \\
3\end{array}$ & $\begin{array}{l}2.0 \\
2.0 \\
2.0 \\
2.0\end{array}$ & $\begin{array}{l}<1 \\
<1 \\
<1 \\
<1\end{array}$ & $\begin{array}{l}<1 \\
<1 \\
<1 \\
<1\end{array}$ & $\begin{array}{l}<1 \\
<1 \\
<1 \\
<1 .\end{array}$ & $\begin{array}{l}<1,0 \\
<1,0 \\
<1,0 \\
<1,0\end{array}$ & $\begin{array}{r}20 \\
20 \\
20 \\
<10\end{array}$ & $\begin{array}{l}6.0 \\
8.0 \\
6.0\end{array}$ & $\begin{array}{l}2.0 \\
2.0 \\
2.0 \\
2.0\end{array}$ \\
\hline
\end{tabular}

321739106485910 - CITY OF LAS CRUCES WASTE WATER TREATMENT PLANT, NM

\begin{tabular}{|c|c|c|c|c|c|c|c|c|c|c|c|c|}
\hline DATE & TIME & $\begin{array}{l}\text { SEE- } \\
\text { CIFIC } \\
\text { CON- } \\
\text { DUCT- } \\
\text { ANCE } \\
\text { (US/CM) } \\
(00095)\end{array}$ & $\begin{array}{c}\text { FE } \\
\text { WATER } \\
\text { WHOLE } \\
\text { FIELD } \\
\text { (STAND- } \\
\text { ARD } \\
\text { UNITS) } \\
\text { (00400) }\end{array}$ & $\begin{array}{l}\text { TEMPER- } \\
\text { ATURE } \\
\text { AIR } \\
\text { (DEG C) } \\
(00020)\end{array}$ & $\begin{array}{c}\text { TEMPER- } \\
\text { ATURE } \\
\text { WATER } \\
\text { (DEG C) } \\
(00010)\end{array}$ & $\begin{array}{l}\text { BARO- } \\
\text { METRIC } \\
\text { PRES- } \\
\text { SURE } \\
\text { (MM } \\
\text { OF } \\
\text { HG) } \\
(00025)\end{array}$ & $\begin{array}{c}\text { OXYGEN, } \\
\text { DIS- } \\
\text { SOLVED } \\
(\text { MG/L) } \\
(00300)\end{array}$ & $\begin{array}{c}\text { OXYGEN, } \\
\text { DIS - } \\
\text { SOLVED } \\
\text { (PER- } \\
\text { CENT } \\
\text { SATUR- } \\
\text { ATION) } \\
\text { (00301) }\end{array}$ & $\begin{array}{l}\text { HARD- } \\
\text { NESS } \\
\text { TOTAL } \\
\text { (MG/I. } \\
\text { AS } \\
\text { CACO3) } \\
(00900)\end{array}$ & $\begin{array}{l}\text { CALCIUM } \\
\text { DIS- } \\
\text { SOLVED } \\
\text { (NG/L } \\
\text { AS CA) } \\
(00915)\end{array}$ & $\begin{array}{c}\text { MAGNE- } \\
\text { SIUM, } \\
\text { DIS- } \\
\text { SOLVED } \\
\text { (MG/L } \\
\text { AS NG) } \\
\text { (00925) }\end{array}$ & $\begin{array}{l}\text { CARBON, } \\
\text { ORGANIC } \\
\text { TOTAL. } \\
\text { (MG/L } \\
\text { AS C) } \\
(00680)\end{array}$ \\
\hline $\begin{array}{c}U G 1996 \\
06 \ldots \\
07 \ldots \\
20 \ldots \\
21 \ldots\end{array}$ & $\begin{array}{l}0950 \\
0950 \\
1100 \\
1130\end{array}$ & $\begin{array}{l}1260 \\
1260 \\
1220 \\
1210\end{array}$ & $\begin{array}{l}7.5 \\
7.4 \\
7.3 \\
7.3\end{array}$ & $\begin{array}{l}m- \\
-- \\
33.0 \\
27.5\end{array}$ & $\begin{array}{l}29.0 \\
28.0 \\
32.0 \\
28.0\end{array}$ & $\begin{array}{l}m \\
-\overline{6} \\
663 \\
663\end{array}$ & $\begin{array}{l}6.7 \\
6.4 \\
6.4 \\
6.3\end{array}$ & $\begin{array}{r}- \\
102 \\
93\end{array}$ & $\begin{array}{l}250 \\
250 \\
250 \\
250\end{array}$ & $\begin{array}{l}72 \\
72 \\
70 \\
71\end{array}$ & $\begin{array}{l}18 \\
18 \\
18 \\
18\end{array}$ & $\begin{array}{l}7.6 \\
5.8 \\
8.9 \\
6.6\end{array}$ \\
\hline
\end{tabular}

\begin{tabular}{|c|c|c|c|c|c|c|c|c|c|c|c|}
\hline DATE & $\begin{array}{l}\text { CARBON, } \\
\text { ORGANIC } \\
\text { DIS- } \\
\text { SOLVED } \\
\text { (MG/L } \\
\text { AS C) } \\
(00681)\end{array}$ & $\begin{array}{l}\text { ALUM- } \\
\text { INUM, } \\
\text { TOTAL } \\
\text { RECOV- } \\
\text { ERABLE } \\
\text { (UG/L } \\
\text { AS AL) } \\
\text { (01105) }\end{array}$ & $\begin{array}{c}\text { ALUM- } \\
\text { INUM, } \\
\text { DIS- } \\
\text { SOLVED } \\
\text { (UG/L } \\
\text { AS AL) } \\
(01106)\end{array}$ & $\begin{array}{l}\text { ANTI- } \\
\text { MONY, } \\
\text { TOTAL } \\
\text { (UG/L } \\
\text { AS SB) } \\
\text { (01097) }\end{array}$ & $\begin{array}{l}\text { ANTL- } \\
\text { MONY, } \\
\text { DIS- } \\
\text { SOLVED } \\
\text { (UG/L } \\
\text { AS SB) } \\
\text { (01095) }\end{array}$ & $\begin{array}{l}\text { BARIUM, } \\
\text { DIS- } \\
\text { SOLVED } \\
\text { (UG/L } \\
\text { AS BA) } \\
(01005)\end{array}$ & $\begin{array}{l}\text { BERYL- } \\
\text { LIUM, } \\
\text { TOTAL } \\
\text { RECOV- } \\
\text { ERABLE } \\
\text { (UG/L } \\
\text { AS BE) } \\
\text { (01012) }\end{array}$ & $\begin{array}{l}\text { BERYL- } \\
\text { LIUM, } \\
\text { DIS- } \\
\text { SOLVED } \\
\text { (UG/L } \\
\text { AS BE) } \\
\text { (01010) }\end{array}$ & $\begin{array}{l}\text { CADMIUM } \\
\text { WATER } \\
\text { UNFLTRD } \\
\text { TOTAL } \\
\text { (UG/L } \\
\text { AS CD) } \\
(01027)\end{array}$ & $\begin{array}{c}\text { CADMIUM } \\
\text { DIS-- } \\
\text { SOLVED } \\
\text { (UG/L } \\
\text { AS CD) } \\
\text { (01025) }\end{array}$ & $\begin{array}{l}\text { CHRO- } \\
\text { MIUM, } \\
\text { TOTAL } \\
\text { RECOV- } \\
\text { ERABLE } \\
\text { (UG/L } \\
\text { AS CR) } \\
(01034)\end{array}$ \\
\hline $\begin{array}{c}\text { AIUC } 1996 \\
06 \ldots \\
07 \ldots \\
20 \ldots \\
21 \ldots\end{array}$ & $\begin{array}{l}5.1 \\
5.0 \\
5.5 \\
5.0\end{array}$ & $\begin{array}{l}30 \\
30 \\
40 \\
40\end{array}$ & $\begin{array}{l}17 \\
15 \\
13 \\
13\end{array}$ & $\begin{array}{l}<1 \\
<1 \\
<1 \\
<1\end{array}$ & $\begin{array}{l}<1.0 \\
<1.0 \\
<1.0 \\
<1.0\end{array}$ & $\begin{array}{l}8.0 \\
8.0 \\
9.0 \\
8.0\end{array}$ & $\begin{array}{l}<10 \\
<10 \\
<10 \\
<10\end{array}$ & $\begin{array}{l}<1.0 \\
<1.0 \\
<1.0 \\
<1.0\end{array}$ & $\begin{array}{l}<1 \\
<1 \\
<1 \\
<1\end{array}$ & $\begin{array}{l}<1,0 \\
<1.0 \\
<1.0 \\
<1,0\end{array}$ & $\begin{array}{l}<1 \\
<1 \\
<1 \\
<1\end{array}$ \\
\hline DATE & $\begin{array}{l}\text { CHRO- } \\
\text { MIUM, } \\
\text { DIS- } \\
\text { SOLVED } \\
\text { (UG/L } \\
\text { AS CR) } \\
\text { (01030) }\end{array}$ & $\begin{array}{c}\text { COBAET, } \\
\text { TOTAL } \\
\text { RECOV- } \\
\text { ERABLE } \\
\text { (UG/L } \\
\text { AS CO) } \\
(01037)\end{array}$ & $\begin{array}{l}\text { COBALT, } \\
\text { DIS- } \\
\text { SOLVED } \\
\text { (UE/I } \\
\text { AS CO) } \\
(01035)\end{array}$ & $\begin{array}{c}\text { COFEER, } \\
\text { TOTAL } \\
\text { RECOV- } \\
\text { ERABLE } \\
\text { (UG/I } \\
\text { AS CU) } \\
(01042)\end{array}$ & $\begin{array}{l}\text { COPPER, } \\
\text { DIS- } \\
\text { SOLVED } \\
\text { (UGG/L } \\
\text { AS CU) } \\
(01040)\end{array}$ & $\begin{array}{l}\text { LEAD, } \\
\text { TOTAL } \\
\text { RECOV- } \\
\text { ERABLE } \\
\text { (UG/L } \\
\text { AS PB) } \\
\text { (01051) }\end{array}$ & $\begin{array}{l}\text { LEAD, } \\
\text { DIS } \\
\text { SOLVED } \\
\text { (UG/L } \\
\text { AS PB) } \\
(01049 \text { ) }\end{array}$ & $\begin{array}{l}\text { MANGA- } \\
\text { NESE, } \\
\text { TOTALL } \\
\text { RECOV- } \\
\text { ERABLE } \\
\text { (UG/L } \\
\text { AS MN) } \\
\text { (01055) }\end{array}$ & $\begin{array}{l}\text { MAFGA- } \\
\text { NESE, } \\
\text { DIS- } \\
\text { SOLVED } \\
\text { (UG/L } \\
\text { AS MN) } \\
\text { (01056) }\end{array}$ & $\begin{array}{l}\text { MEKCURY } \\
\text { TOTAL } \\
\text { RECOV- } \\
\text { ERABLE } \\
\text { (UG/L } \\
\text { AS HG) } \\
(71900)\end{array}$ & $\begin{array}{l}\text { MERCURY } \\
\text { DIS- } \\
\text { SOLVED } \\
\text { (UG/L } \\
\text { AS HG) } \\
\text { (71890) }\end{array}$ \\
\hline $\begin{array}{c}\text { AUG } 1996 \\
06 \ldots \\
07 \ldots \\
20 \ldots \\
21 \ldots\end{array}$ & $\begin{array}{l}1.0 \\
2.0 \\
1.0 \\
2.0\end{array}$ & $\begin{array}{l}<1 \\
<1 \\
<1 \\
<1\end{array}$ & $\begin{array}{l}<1,0 \\
<1.0 \\
<1.0 \\
<1.0\end{array}$ & $\begin{array}{l}1 \\
1 \\
2 \\
2\end{array}$ & $\begin{array}{l}3.0 \\
2.0 \\
2.0 \\
2.0\end{array}$ & $\begin{array}{l}<1 \\
<1 \\
<1 \\
<1\end{array}$ & $\begin{array}{l}<1.0 \\
<1.0 \\
<1.0 \\
<1.0\end{array}$ & $\begin{array}{l}50 \\
40 \\
50 \\
40\end{array}$ & $\begin{array}{l}32 \\
30 \\
39 \\
35\end{array}$ & $\begin{array}{l}<0.10 \\
<0.10 \\
<0.10 \\
<0.10\end{array}$ & $\begin{array}{l}<0.1 \\
<0.1 \\
<0.1 \\
<0.1\end{array}$ \\
\hline
\end{tabular}


ANALYSES OF SAMPLES COLLECTED AT WATER-QUALITY RARTIAL-RECORD STATIONS AND MISCELLANEOUS SITES

WATER-QUALITY DATA, WATER YEAR OCTOBER 1995 TO SEPTEMBER 1996

RIO GRANDE BASIN - Continued

321739106485910 - CITY OF LAS CRUCES WASTE WATER TREATMENT PLANT, NM

\begin{tabular}{|c|c|c|c|c|c|c|c|c|c|c|c|}
\hline DATE & $\begin{array}{l}\text { MOLYB- } \\
\text { DENUM, } \\
\text { TOTAI. } \\
\text { RECOV- } \\
\text { ERABLE } \\
\text { (UG/L } \\
\text { AS MO) } \\
\text { (01062) }\end{array}$ & $\begin{array}{c}\text { YOLYB- } \\
\text { DENTU, } \\
\text { DIS - } \\
\text { SOLVED } \\
\text { (UG/L } \\
\text { AS MO) } \\
(01060)\end{array}$ & $\begin{array}{c}\text { NICKEL, } \\
\text { TOTAL } \\
\text { RECOV- } \\
\text { ERABLE } \\
\text { (UG/L } \\
\text { AS NI) } \\
\text { (01067) }\end{array}$ & $\begin{array}{c}\text { NICKEL, } \\
\text { DIS- } \\
\text { SOLVED } \\
\text { (UG/L } \\
\text { AS NI) } \\
\text { (01065) }\end{array}$ & $\begin{array}{c}\text { SELE- } \\
\text { NIUM, } \\
\text { TOTAL } \\
\text { (UG/L } \\
\text { AS SE) } \\
\text { (01147) }\end{array}$ & $\begin{array}{l}\text { SELE- } \\
\text { NIUM, } \\
\text { DIS- } \\
\text { SOLVED } \\
\text { (UG/L } \\
\text { AS SE) } \\
\text { (01145) }\end{array}$ & $\begin{array}{c}\text { SILVER, } \\
\text { TOTAL, } \\
\text { RECOV- } \\
\text { ERABLE } \\
\text { (UG/L } \\
\text { AS AG) } \\
(01077 \text { ) }\end{array}$ & $\begin{array}{c}\text { SILVER, } \\
\text { DIS- } \\
\text { SOLVED } \\
\text { (UG/L } \\
\text { AS AG) } \\
(01075)\end{array}$ & $\begin{array}{c}\text { ZINC, } \\
\text { TOTAL } \\
\text { RECOV- } \\
\text { ERABLE } \\
\text { (UG/L } \\
\text { AS ZN) } \\
(01092)\end{array}$ & $\begin{array}{c}\text { ZINC, } \\
\text { DIS - } \\
\text { SOLVED } \\
\text { (UG/L } \\
\text { AS ZN) } \\
(01090)\end{array}$ & $\begin{array}{c}\text { URANTUM } \\
\text { NATURAL } \\
\text { DHS- } \\
\text { SOLVED } \\
\text { (UG/L } \\
\text { AS U) } \\
\text { (22703) }\end{array}$ \\
\hline $\begin{array}{l}\text { UG } 1996 \\
06 \ldots \\
07 \ldots \\
20 \ldots \\
21 \ldots\end{array}$ & $\begin{array}{l}23 \\
23 \\
27 \\
27\end{array}$ & $\begin{array}{l}27 \\
28 \\
31 \\
31\end{array}$ & $\begin{array}{l}2 \\
1 \\
2 \\
1\end{array}$ & $\begin{array}{l}3.0 \\
3.0 \\
4.0 \\
4.0\end{array}$ & $\begin{array}{l}<1 \\
<1 \\
<1\end{array}$ & $\begin{array}{l}<1 \\
<1 \\
<1\end{array}$ & $\begin{array}{l}<1 \\
<1 \\
<1\end{array}$ & $\begin{array}{l}<1.0 \\
<1.0 \\
<1.0\end{array}$ & $\begin{array}{r}30 \\
20 \\
<10 \\
10\end{array}$ & $\begin{array}{l}16 \\
16 \\
14\end{array}$ & $\begin{array}{l}10 \\
10 \\
11 \\
10\end{array}$ \\
\hline
\end{tabular}

321745106492510 - RIO GRANDE BELOW PICACEO BRIDGE NR LAS CRUCES, IM

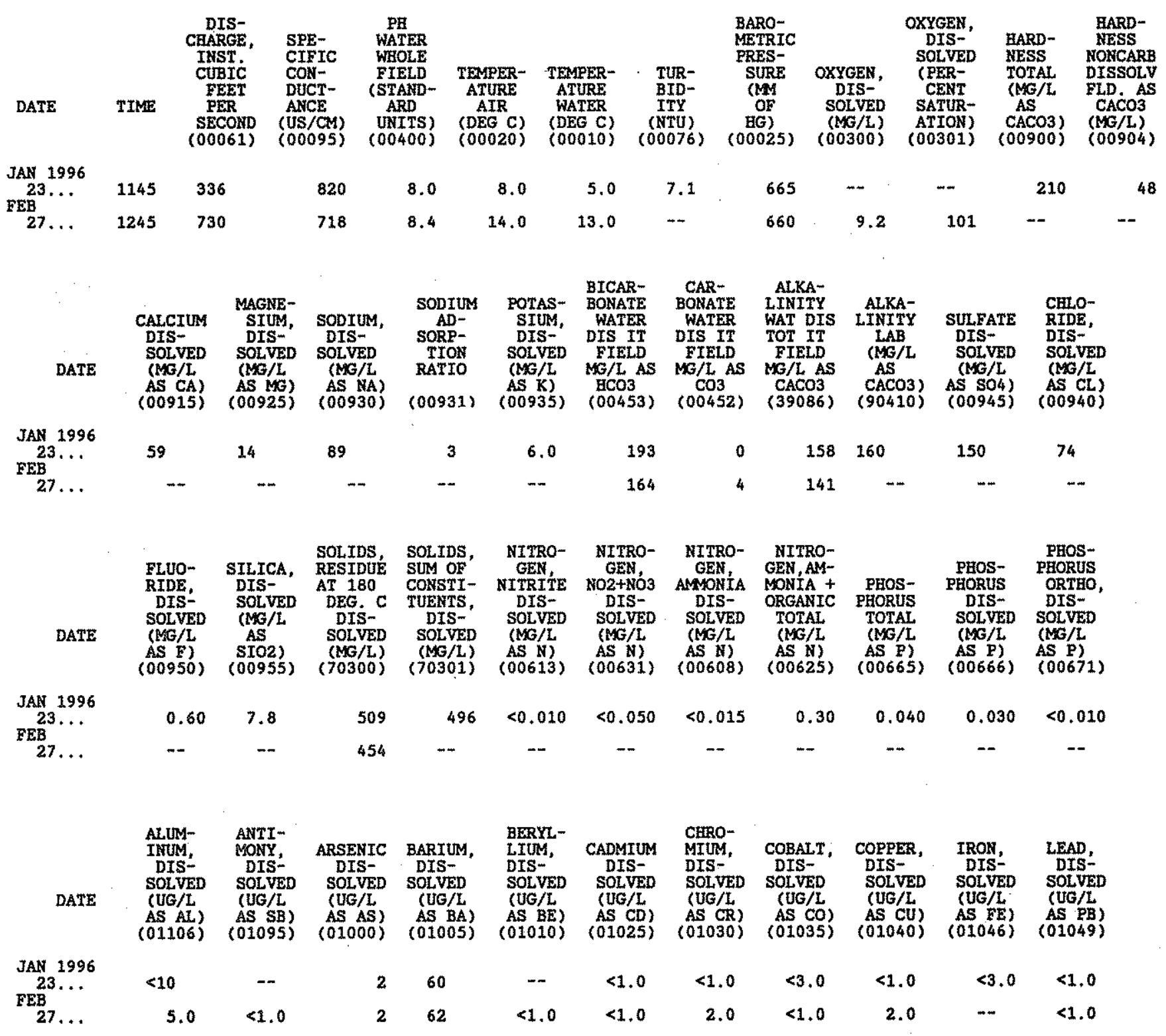


WATER-QUALITY DATA, WATER YEAR OCTOBER 1995 TO SEPTEMBER 1996

RIO GRANDE BASIN - Continued

321745106492510 - RIO GRANDE BELOW PICACHO BRIDGE NR LAS CRUCES, NM

\begin{tabular}{|c|c|c|c|c|c|c|c|c|c|c|c|}
\hline DATE & $\begin{array}{c}\text { LITEIUM } \\
\text { DIS- } \\
\text { SOLVED } \\
\text { (UG } / L \\
\text { AS LI) } \\
\text { (01130) }\end{array}$ & $\begin{array}{c}\text { MANGA- } \\
\text { NESE, } \\
\text { DIS- } \\
\text { SOLVED } \\
\text { (UE/L } \\
\text { AS MA) } \\
(01056)\end{array}$ & $\begin{array}{l}\text { MERCURY } \\
\text { DIS- } \\
\text { SOLVED } \\
\text { (UG/L } \\
\text { AS HG) } \\
(71890)\end{array}$ & $\begin{array}{c}\text { MOLYB- } \\
\text { DENUM, } \\
\text { DIS- } \\
\text { SOLVED } \\
\text { (UST/L } \\
\text { AS MO) } \\
\text { (01060) }\end{array}$ & $\begin{array}{l}\text { NICKEL, } \\
\text { DIS- } \\
\text { SOLVED } \\
\text { (UG/L } \\
\text { AS NI) } \\
(01065)\end{array}$ & $\begin{array}{c}\text { SELE- } \\
\text { NIUU, } \\
\text { DIS- } \\
\text { SOLVED } \\
\text { (UG/L } \\
\text { AS SE) } \\
\text { (O1145) }\end{array}$ & $\begin{array}{c}\text { SILVGR, } \\
\text { DIS- } \\
\text { SOLVED } \\
\text { (UG } / 2 \\
\text { AS AG) } \\
\text { (01075) }\end{array}$ & $\begin{array}{c}\text { STRON- } \\
\text { TIUM, } \\
\text { DIS- } \\
\text { SOLVED } \\
\text { (UG/L } \\
\text { AS SR) } \\
\text { (01080) }\end{array}$ & $\begin{array}{l}\text { VANA- } \\
\text { DIUM, } \\
\text { DIS- } \\
\text { SOLVED } \\
\text { (UG / } \\
\text { AS V) } \\
\text { (01085) }\end{array}$ & $\begin{array}{c}\text { ZINC } \\
\text { DIS- } \\
\text { SOLVED } \\
\text { (UUG } / L \\
\text { AS ZN) } \\
(01090)\end{array}$ & $\begin{array}{l}\text { URANIUN } \\
\text { YATURAL } \\
\text { DIS- } \\
\text { SOLVED } \\
\text { (UG/L } \\
\text { AS U) } \\
(22703)\end{array}$ \\
\hline & & & & & & & & & & & \\
\hline $23 \ldots$ & 77 & 4.0 & 80. & $<10$ & $<1.0$ & $<1$ & $<1,0$ & 730 & $<6$ & $<10$ & -- \\
\hline & -- & 3.0 & $<0.1$ & 6.0 & 2.0 & $<1$ & $<1.0$ & 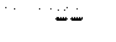 & -- & 2.0 & 3. \\
\hline
\end{tabular}

321837106493810 - RIO GRANDE AT PYCACHO BRDG NR LAS CRUCES, NM

\begin{tabular}{|c|c|c|c|c|c|c|c|c|c|c|c|c|}
\hline DATE & TIME & $\begin{array}{c}\text { DIS- } \\
\text { CHARGE, } \\
\text { INST } \\
\text { CUBIC } \\
\text { FEET } \\
\text { PER } \\
\text { SECOND } \\
(00061)\end{array}$ & $\begin{array}{l}\text { SPE- } \\
\text { CIFIC } \\
\text { CON- } \\
\text { DUCT- } \\
\text { ANCE } \\
\text { (US/CM) } \\
(00095)\end{array}$ & $\begin{array}{c}\text { PH } \\
\text { WATER } \\
\text { WEOLE } \\
\text { FIELD } \\
\text { (STAND- } \\
\text { ARD } \\
\text { UNITS) } \\
\text { (00400) }\end{array}$ & $\begin{array}{l}\text { TEMPER- } \\
\text { ATURE } \\
\text { AIR } \\
\text { (DEG C) } \\
(00020)\end{array}$ & $\begin{array}{l}\text { TEMEER- } \\
\text { ATURE } \\
\text { WATER } \\
\text { (DEG C) } \\
\text { (00010) }\end{array}$ & $\begin{array}{c}\text { BARO- } \\
\text { METRIC } \\
\text { FRES- } \\
\text { SURE } \\
\text { (MM } \\
\text { OF } \\
\text { HG) } \\
(00025)\end{array}$ & $\begin{array}{c}\text { OXYGEN, } \\
\text { DIS- } \\
\text { SOLVED } \\
(\text { NG/L) } \\
(00300)\end{array}$ & $\begin{array}{c}\text { OXYGEN, } \\
\text { DIS- } \\
\text { SOLVED } \\
\text { (PER- } \\
\text { CENT } \\
\text { SATUR- } \\
\text { ATION) } \\
\text { (OOBOI) }\end{array}$ & $\begin{array}{l}\text { HARD- } \\
\text { NESS } \\
\text { TOTAL } \\
\text { (MG/L } \\
\text { AS } \\
\text { CACO3) } \\
(00900)\end{array}$ & $\begin{array}{c}\text { CALCIUN } \\
\text { DIS- } \\
\text { SOLVED } \\
\text { (MG/L } \\
\text { AS CA) } \\
(00915)\end{array}$ & $\begin{array}{c}\text { MAGNE } \\
\text { SIUM } \\
\text { DIS- } \\
\text { SOLVE } \\
\text { (MG/L } \\
\text { AS MG } \\
100925\end{array}$ \\
\hline $\begin{array}{c}\text { UG } 1996 \\
06 \ldots \\
07 \ldots \\
20 \ldots \\
21 \ldots\end{array}$ & $\begin{array}{l}1250 \\
1130 \\
0845 \\
1300\end{array}$ & $\begin{array}{l}1180 \\
1060 \\
1140 \\
1140\end{array}$ & $\begin{array}{l}623 \\
620 \\
595 \\
603\end{array}$ & $\begin{array}{l}8.2 \\
8.2 \\
8.0 \\
8.3\end{array}$ & $\begin{array}{l}- \\
21.0 \\
28.5\end{array}$ & $\begin{array}{l}31.5 \\
24.0 \\
24.0 \\
25.5\end{array}$ & $\begin{array}{l}-7 \\
-\overline{663} \\
663\end{array}$ & $\begin{array}{l}7.3 \\
7.4 \\
6.6 \\
6.9\end{array}$ & $\begin{array}{r}- \\
91 \\
98\end{array}$ & $\begin{array}{l}150 \\
150 \\
130 \\
140\end{array}$ & $\begin{array}{l}42 \\
41 \\
35 \\
39\end{array}$ & $\begin{array}{c}11 \\
11 \\
9.5 \\
10^{.5}\end{array}$ \\
\hline
\end{tabular}

\begin{tabular}{|c|c|c|c|c|c|c|c|c|c|c|c|c|}
\hline DATE & $\begin{array}{l}\text { CARBON, } \\
\text { ORGANIC } \\
\text { TOTAL } \\
\text { (NG/L } \\
\text { AS C) } \\
(00680)\end{array}$ & $\begin{array}{l}\text { CARBON, } \\
\text { ORGANIC } \\
\text { DIS- } \\
\text { SOLVED } \\
\text { (MG/L } \\
\text { AS C) } \\
\text { (00681) }\end{array}$ & $\begin{array}{l}\text { ALUM- } \\
\text { INUM, } \\
\text { TOTAI, } \\
\text { RECOV- } \\
\text { ERABLE } \\
\text { (UG/L, } \\
\text { AS AL) } \\
\text { (01105) }\end{array}$ & $\begin{array}{l}\text { ALIUM- } \\
\text { INUM, } \\
\text { DIS- } \\
\text { SOLVED } \\
\text { (UG/I. } \\
\text { AS Ad) } \\
\text { (01106) }\end{array}$ & $\begin{array}{l}\text { ANIT- } \\
\text { MONY, } \\
\text { TOFAL } \\
\text { (UG/L } \\
\text { AS SB) } \\
\text { (01097) }\end{array}$ & $\begin{array}{l}\text { ANTI- } \\
\text { MONY, } \\
\text { DIS- } \\
\text { SOLVED } \\
\text { (UG/E } \\
\text { AS SB) } \\
\text { (01095) }\end{array}$ & $\begin{array}{l}\text { BARIUM, } \\
\text { DIS- } \\
\text { SOLVED } \\
\text { (UG/L } \\
\text { AS BA) } \\
(01005)\end{array}$ & $\begin{array}{l}\text { BERYL- } \\
\text { LIUM, } \\
\text { TOTAL } \\
\text { RECOV- } \\
\text { ERABLE } \\
\text { (UG/L } \\
\text { AS BE) } \\
\text { (01012) }\end{array}$ & $\begin{array}{l}\text { BERYL } \\
\text { LIUM, } \\
\text { DIS- } \\
\text { SOLVED } \\
\text { (UG/L } \\
\text { AS BE) } \\
\text { (01010) }\end{array}$ & $\begin{array}{l}\text { CADMIUM } \\
\text { WATER } \\
\text { UNFLTRD } \\
\text { TOTAL } \\
\text { (UG/L } \\
\text { AS CD) } \\
\text { (01027) }\end{array}$ & $\begin{array}{c}\text { CADMIUM } \\
\text { DIS- } \\
\text { SOLVED } \\
\text { (UG/L } \\
\text { AS CD) } \\
(01025)\end{array}$ & $\begin{array}{l}\text { CERO- } \\
\text { MIUM, } \\
\text { TOTAL } \\
\text { RECOV- } \\
\text { ERABLE } \\
\text { (UG/L } \\
\text { AS CR) } \\
\text { (01034) }\end{array}$ \\
\hline $\begin{array}{l}\text { UG } 1996 \\
06 \ldots \\
07 \ldots \\
20 \ldots \\
21 \ldots\end{array}$ & $\begin{array}{l}5.1 \\
6.9 \\
6.4 \\
6.1\end{array}$ & $\begin{array}{l}3.4 \\
3.3 \\
3.3 \\
3.3\end{array}$ & $\begin{array}{l}1500 \\
1800 \\
2600 \\
1700\end{array}$ & $\begin{array}{l}19 \\
20 \\
10 \\
6.0\end{array}$ & $\begin{array}{r}1 \\
1 \\
<1 \\
1\end{array}$ & $\begin{array}{r}1.0 \\
<1.0 \\
<1.0 \\
<1.0\end{array}$ & $\begin{array}{l}72 \\
70 \\
62 \\
68\end{array}$ & $\begin{array}{l}<10 \\
<10 \\
<10 \\
<10\end{array}$ & $\begin{array}{l}<1,0 \\
<1.0 \\
<1.0 \\
<1.0\end{array}$ & $\begin{array}{l}<1 \\
<1 \\
<1 \\
<1\end{array}$ & $\begin{array}{l}<1.0 \\
<1.0 \\
<1.0 \\
<1.0\end{array}$ & $\begin{array}{r}<1 \\
1 \\
2 \\
1\end{array}$ \\
\hline
\end{tabular}

\begin{tabular}{|c|c|c|c|c|c|c|c|c|c|c|c|}
\hline DATE & $\begin{array}{l}\text { CHRO- } \\
\text { MIUM, } \\
\text { DIS- } \\
\text { SOLVED } \\
\text { (UG/L } \\
\text { AS CR) } \\
(01030)\end{array}$ & $\begin{array}{c}\text { COBALT, } \\
\text { TOTAL } \\
\text { RECOV- } \\
\text { ERABLE } \\
\text { (UG/L } \\
\text { AS CO) } \\
(01037)\end{array}$ & $\begin{array}{c}\text { COBALT, } \\
\text { DHS- } \\
\text { SOLVED } \\
\text { (UG/L } \\
\text { AS CO) } \\
(01035)\end{array}$ & $\begin{array}{c}\text { COPPER, } \\
\text { TOTAL } \\
\text { RECOV- } \\
\text { ERABLE } \\
\text { (UG/L } \\
\text { AS CU) } \\
(01042)\end{array}$ & $\begin{array}{l}\text { COPYER, } \\
\text { DIS- } \\
\text { SOLVED } \\
\text { (UG/L } \\
\text { AS CU) } \\
(01040)\end{array}$ & $\begin{array}{l}\text { LEAD, } \\
\text { TORAL } \\
\text { RECOV- } \\
\text { ERABL. } \\
\text { (UG/L } \\
\text { AS DB) } \\
(01051)\end{array}$ & $\begin{array}{l}\text { LEAD, } \\
\text { DIS- } \\
\text { SOLVED } \\
\text { (UG/L } \\
\text { AS DB) } \\
(01049)\end{array}$ & $\begin{array}{l}\text { MANGA - } \\
\text { NESE, } \\
\text { TOTAL } \\
\text { RECOV- } \\
\text { ERABLE } \\
\text { (UG/L } \\
\text { AS MN) } \\
\text { (01055) }\end{array}$ & $\begin{array}{l}\text { MANGA- } \\
\text { NESE, } \\
\text { DIS- } \\
\text { SOLVED } \\
\text { (UG/L } \\
\text { AS MN) } \\
(01056)\end{array}$ & $\begin{array}{c}\text { MERCURX } \\
\text { TOTAL } \\
\text { RECOV- } \\
\text { ERABLE } \\
\text { (UG/L } \\
\text { AS HC) } \\
\text { (71900) }\end{array}$ & $\begin{array}{c}\text { MORCURY } \\
\text { DIS- } \\
\text { SOLVED } \\
\text { (UE/L } \\
\text { AS HC) } \\
(71890)\end{array}$ \\
\hline $\begin{array}{l}\text { UE } 1996 \\
06 \ldots \\
07 \ldots \\
20 \ldots \\
21 \ldots\end{array}$ & $\begin{array}{r}1.0 \\
2.0 \\
<1.0 \\
2.0\end{array}$ & $\begin{array}{r}<1 \\
<1 \\
2 \\
<1\end{array}$ & $\begin{array}{l}<1.0 \\
<1.0 \\
<1.0 \\
<1.0\end{array}$ & $\begin{array}{l}2 \\
3 \\
3 \\
2\end{array}$ & $\begin{array}{l}4.0 \\
3.0 \\
1.0 \\
1.0\end{array}$ & $\begin{array}{l}1 \\
1 \\
3 \\
1\end{array}$ & $\begin{array}{l}<1,0 \\
<1.0 \\
<1.0 \\
<1.0\end{array}$ & $\begin{array}{l}200 \\
200 \\
180 \\
160\end{array}$ & $\begin{array}{r}1.0 \\
<1.0 \\
<1.0 \\
1.0\end{array}$ & $\begin{array}{l}<0.10 \\
<0.10 \\
<0.10 \\
<0.10\end{array}$ & $\begin{array}{l}<0.1 \\
<0.1 \\
<0.1 \\
<0.1\end{array}$ \\
\hline
\end{tabular}

\begin{tabular}{|c|c|c|c|c|c|c|c|c|c|c|c|}
\hline DATE & $\begin{array}{l}\text { MOLYB- } \\
\text { DEMUMA, } \\
\text { TOTAL } \\
\text { RECOV- } \\
\text { ERABLE } \\
\text { (UG /L } \\
\text { AS NO) } \\
\text { (01062) }\end{array}$ & $\begin{array}{c}\text { MOLYB- } \\
\text { DENUM, } \\
\text { DIS- } \\
\text { SOLVED } \\
\text { (UG/L } \\
\text { AS MO) } \\
(01060)\end{array}$ & $\begin{array}{c}\text { NICKELL, } \\
\text { TOTAL, } \\
\text { RECOV- } \\
\text { ERABLE } \\
\text { (UG/L } \\
\text { AS NI) } \\
\text { (01067) }\end{array}$ & $\begin{array}{l}\text { NICKEL, } \\
\text { DIS- } \\
\text { SOLVED } \\
\text { (UG/L } \\
\text { AS NY) } \\
\text { (01065) }\end{array}$ & $\begin{array}{l}\text { SELE- } \\
\text { NIUM, } \\
\text { TOTAL } \\
\text { (UE/I } \\
\text { AS SE) } \\
(01147)\end{array}$ & $\begin{array}{c}\text { SEY.- } \\
\text { NYUM, } \\
\text { DIS- } \\
\text { SOLVED } \\
\text { (UE/L } \\
\text { AS SE) } \\
\text { (O1145) }\end{array}$ & $\begin{array}{c}\text { SIYYED, } \\
\text { TOTALE } \\
\text { RECOV- } \\
\text { ERABLE } \\
\text { (UG/L } \\
\text { AS AG) } \\
(01077)\end{array}$ & $\begin{array}{c}\text { SILVER, } \\
\text { DIS- } \\
\text { SOLVED } \\
\text { (UE/I } \\
\text { AS AG) } \\
(01075)\end{array}$ & $\begin{array}{l}\text { ZINC, } \\
\text { TOTAL } \\
\text { RECOV- } \\
\text { BRABLE } \\
\text { (USG/L } \\
\text { AS ZN) } \\
(01092)\end{array}$ & $\begin{array}{l}\text { ZINC, } \\
\text { DIS- } \\
\text { SOLVED } \\
\text { (UG/L } \\
\text { AS ZN) } \\
\text { (01090) }\end{array}$ & $\begin{array}{l}\text { UAAPYILA } \\
\text { NATURAL } \\
\text { DIS } \\
\text { SOLVED } \\
\text { (UE/L } \\
\text { AS U) } \\
\text { (22703) }\end{array}$ \\
\hline $\begin{array}{c}\text { ALE } 1996 \\
06 \ldots \\
07 \ldots \\
20 \ldots \\
21 \ldots\end{array}$ & $\begin{array}{l}4 \\
4 \\
5 \\
5\end{array}$ & $\begin{array}{l}6.0 \\
6.0 \\
5.0 \\
6.0\end{array}$ & $\begin{array}{l}3 \\
2 \\
3 \\
2\end{array}$ & $\begin{array}{l}3.0 \\
1.0 \\
2.0 \\
2.0\end{array}$ & $\begin{array}{l}<1 \\
<1 \\
<1 \\
<1\end{array}$ & $\begin{array}{l}<1 \\
<1 \\
<1 \\
<1\end{array}$ & $\begin{array}{l}<1 \\
<1 \\
<1 \\
<1\end{array}$ & $\begin{array}{l}<1.0 \\
<1.0 \\
<1.0 \\
<1.0\end{array}$ & $\begin{array}{r}10 \\
<10 \\
<10 \\
<10\end{array}$ & $\begin{array}{l}4.0 \\
2.0 \\
2.0 \\
2.0\end{array}$ & $\begin{array}{l}2.0 \\
2.0 \\
2.0 \\
2.0\end{array}$ \\
\hline
\end{tabular}


ANALYSES OF SAMPLES COLLECTED AT WATER-QUALITY PARTIAL-RECORD STATIONS AND MISCELLANEOUS SITES

WATER-QUALITX DATA, WATER YEAR OCTOBER 1995 TO SEPTEMBER 1996

RIO GRANDE BASIN -- Continued

322841106551010 - RIO GRANDE BELOW LEASBURG DAM, NM

\begin{tabular}{|c|c|c|c|c|c|c|c|c|c|c|c|c|}
\hline DATE & TIME & $\begin{array}{c}\text { DIS- } \\
\text { CEARGE; } \\
\text { INST } \\
\text { CUBIC } \\
\text { FEET } \\
\text { PER } \\
\text { SECOND } \\
(00061)\end{array}$ & $\begin{array}{l}\text { SPE- } \\
\text { CIFIC } \\
\text { CON- } \\
\text { DUCT- } \\
\text { ANCE } \\
\text { (US/CM) } \\
(00095)\end{array}$ & $\begin{array}{c}\text { PH } \\
\text { WATER } \\
\text { WHOLE } \\
\text { FIELD } \\
\text { (STAKD- } \\
\text { ARD } \\
\text { UNITS) } \\
(00400)\end{array}$ & $\begin{array}{c}\text { TEMPER- } \\
\text { ATURE } \\
\text { AIR } \\
\text { (DEG C) } \\
(00020)\end{array}$ & $\begin{array}{c}\text { TEMPER- } \\
\text { ATURE } \\
\text { WATER } \\
\text { (DEG C) } \\
(00010)\end{array}$ & $\begin{array}{l}\text { TUR- } \\
\text { BID- } \\
\text { ITY } \\
\text { (NTU) } \\
\text { (00076) }\end{array}$ & $\begin{array}{c}\text { BARO- } \\
\text { METRIC } \\
\text { FRES- } \\
\text { SURE } \\
\text { (MM } \\
\text { OF } \\
\text { HG) } \\
(00025)\end{array}$ & $\begin{array}{c}\text { OXYGEN, } \\
\text { DIS- } \\
\text { SOLVED } \\
(M G / L) \\
(00300)\end{array}$ & $\begin{array}{c}\text { OXYGEN, } \\
\text { DIS- } \\
\text { SOLVED } \\
\text { (PER } \\
\text { CENT } \\
\text { SATUR- } \\
\text { ATION) } \\
\text { (00301) }\end{array}$ & $\begin{array}{l}\text { BARD- } \\
\text { NESS } \\
\text { TOTAL } \\
\text { (MG/L } \\
\text { AS } \\
\text { CACO3) } \\
(00900)\end{array}$ & $\begin{array}{l}\text { HARD- } \\
\text { NESS } \\
\text { NONCARB } \\
\text { DISSOL. } \\
\text { FLD AS } \\
\text { CACOS } \\
(M G / L) \\
(00904)\end{array}$ \\
\hline $\begin{array}{l}\text { JAN } 1996 \\
23 . \ldots \\
\text { FEB }\end{array}$ & 0915 & 313 & 810 & 8.5 & 5.5 & 4.5 & 3.3 & 662 & 10.5 & 94 & 200 & 49 \\
\hline $27 \ldots$ & 0800 & E789 & 714 & 8.1 & 5.0 & 7.5 & -- & 660 & 9.8 & 95 & $\cdots$ & 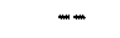 \\
\hline
\end{tabular}

$\begin{array}{ccc} & \text { MAGNE- } \\ \text { CALCIUM } & \text { SIUM, } & \text { SODIUM, } \\ \text { DIS- } & \text { DIS- } & \text { DIS- } \\ \text { SOLVED } & \text { SOLVED } & \text { SOLVED } \\ (M G / L & \text { (MG/L } & \text { (NG/L } \\ \text { ASCA) } & \text { AS MG) } & \text { AS NA) } \\ (00915) & (00925) & (00930)\end{array}$

\begin{tabular}{|c|c|c|c|c|c|c|c|}
\hline $\begin{array}{l}\text { SODIUM } \\
\text { AD- } \\
\text { SORP- } \\
\text { TION } \\
\text { RATIO }\end{array}$ & $\begin{array}{c}\text { POTAS- } \\
\text { SIUM, } \\
\text { DIS- } \\
\text { SOLVED } \\
(\mathrm{MG} / \mathrm{L} \\
\text { AS K } \\
(00935)\end{array}$ & $\begin{array}{l}\text { BICAR- } \\
\text { BONATE } \\
\text { WATER } \\
\text { DIS IT } \\
\text { FIELD } \\
\text { MG/L AS } \\
\text { HCO3 } \\
(00453)\end{array}$ & $\begin{array}{c}\text { CAR- } \\
\text { BONATE } \\
\text { WATER } \\
\text { DIS IT } \\
\text { FIELD } \\
\text { MG/L AS } \\
\text { CO3 } \\
(00452)\end{array}$ & $\begin{array}{c}\text { ALKA- } \\
\text { LINITY } \\
\text { WAT DIS } \\
\text { TOT IT } \\
\text { FIELD } \\
\text { MG/L AS } \\
\text { CACO3 } \\
(39086)\end{array}$ & $\begin{array}{c}\text { ALKA } \\
\text { LINITY } \\
\text { IAB } \\
\text { (MG/L } \\
\text { AS } \\
\text { CACO3) } \\
(90410)\end{array}$ & $\begin{array}{r}\text { SU] } \\
\text { D } \\
\text { S } \\
\text { (1 } \\
\text { AS } \\
\text { (0. }\end{array}$ & VED \\
\hline
\end{tabular}

\begin{tabular}{|c|c|c|c|c|c|c|c|c|c|c|c|}
\hline $\begin{array}{c}\text { JAN } 1996 \\
23 \\
23\end{array}$ & 57 & 14 & 85 & 3 & 5.9 & 185 & 0 & 152 & 158 & 140 & 67 \\
\hline $27 \ldots$ & -- & -- & -- & -- & -- & 153 & 11 & 143 & -- & -- & -- \\
\hline DATE & $\begin{array}{l}\text { ELUO- } \\
\text { RIDE, } \\
\text { DIS- } \\
\text { SOLVED } \\
\text { (MG/L } \\
\text { AS F) } \\
(00950)\end{array}$ & $\begin{array}{c}\text { SILICA, } \\
\text { DIS- } \\
\text { SOLVED } \\
\text { (MG/L } \\
\text { AS } \\
\text { SIO2) } \\
\text { (00955) }\end{array}$ & $\begin{array}{l}\text { SOLIDS, } \\
\text { RESIDUE } \\
\text { AT } 180 \\
\text { DEG C } \\
\text { DIS- } \\
\text { SOLVED } \\
(M G / L) \\
(70300)\end{array}$ & $\begin{array}{c}\text { SOLIDS, } \\
\text { SUM OF } \\
\text { CONSTI- } \\
\text { TUENTS, } \\
\text { DIS- } \\
\text { SOLVED } \\
(\mathrm{MG} / \mathrm{L}) \\
(70301)\end{array}$ & $\begin{array}{c}\text { NITRO- } \\
\text { GEN, } \\
\text { NITRITE } \\
\text { DIS- } \\
\text { SOLVED } \\
\text { (MG/L } \\
\text { AS N) } \\
(00613)\end{array}$ & $\begin{array}{c}\text { NITRO- } \\
\text { GEN, } \\
\text { NO2+NO3 } \\
\text { DIS- } \\
\text { SOLVED } \\
\text { (MG/L } \\
\text { ASN) } \\
(00631)\end{array}$ & $\begin{array}{c}\text { NITRO- } \\
\text { GEN, } \\
\text { AMONYA } \\
\text { DTS- } \\
\text { SOLVED } \\
\text { (MG/L } \\
\text { AS N) } \\
(00608)\end{array}$ & $\begin{array}{l}\text { NTTRO- } \\
\text { GEN,AM- } \\
\text { MONIA + } \\
\text { ORGANIC } \\
\text { TOTAL } \\
\text { (MG/L } \\
\text { AS N) } \\
(00625)\end{array}$ & $\begin{array}{c}\text { PHOS- } \\
\text { PHORUS } \\
\text { TOTAL. } \\
\text { (MG/L } \\
\text { AS P) } \\
(00665)\end{array}$ & $\begin{array}{c}\text { PHOS- } \\
\text { PHORUS } \\
\text { DIS- } \\
\text { SOLVED } \\
\text { (MG/L } \\
\text { AS D) } \\
(00666)\end{array}$ & $\begin{array}{c}\text { PHOS- } \\
\text { PEORUS } \\
\text { ORTHO, } \\
\text { DIS- } \\
\text { SOLVED } \\
(\text { MG/L } \\
\text { AS P) } \\
(00671)\end{array}$ \\
\hline JAN 1996 & 0.60 & 7.5 & 487 & 469 & $<0.010$ & $<0.050$ & $<0.015$ & 0.40 & 0.050 & 0.030 & $<0.010$ \\
\hline $27 \ldots$ & -- & -- & 458 & $-\infty$ & - & - & -- & -- & - & -- & -- \\
\hline
\end{tabular}

$\begin{array}{cccc}\text { ALUM- } & \text { ANTT- } & & \\ \text { INUM, } & \text { MONY, } & \text { ARSENIC } & \text { BARIUM, } \\ \text { DIS- } & \text { DIS } & \text { DIS- } & \text { DIS- } \\ \text { SOLVED } & \text { SOLVED } & \text { SOLVED } & \text { SOLVED } \\ \text { (UG/L } & \text { (UG/L } & \text { (UG/L } & \text { (UG/L } \\ \text { AS AL) } & \text { AS SB) } & \text { AS AS) } & \text { AS BA) } \\ (01106) & (01095) & (01000) & (01005)\end{array}$

BERYL- $\quad$ CHRO

LIUM, CADMIUM MIUM, COBALT, COPPER, IRON, LEAD,

DIS- DIS- DIS- DIS- DIS- DIS- DIS-

SOLVED SOLVED SOLVED SOLVED SOLVED SOLVED SOLVED

(UG/L (UG/L (UG/L W (UG/L (UG/L (UG/L (UG/L

AS BE) AS CD) AS CR) AS CO) AS CU) AS FE) AS PB)

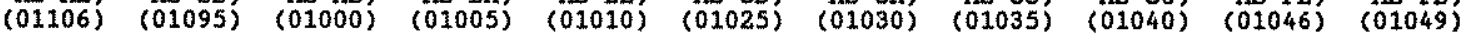

\begin{tabular}{|c|c|c|c|c|c|c|c|c|c|c|c|}
\hline $\mathrm{FEB}^{23} \cdots$ & $<10$ & - & 2 & 65 & $-m$ & $<1.0$ & $<1,0$ & $<3,0$ & $<1.0$ & $<3.0$ & $<1,0$ \\
\hline $27 \ldots$ & 5.0 & $<1,0$ & 2 & 73 & $<1.0$ & $<1.0$ & 2.0 & $<1,0$ & 1.0 & - & $<1.0$ \\
\hline
\end{tabular}




\begin{tabular}{|c|c|c|c|c|c|c|c|c|c|c|c|c|}
\hline DATE & TIME & $\begin{array}{c}\text { DIS- } \\
\text { CBARGE, } \\
\text { INSTS } \\
\text { CUEIC } \\
\text { FEET } \\
\text { PER } \\
\text { SECOND } \\
\text { (00061) }\end{array}$ & $\begin{array}{l}\text { SPE- } \\
\text { CIFIC } \\
\text { CON } \\
\text { DUCT- } \\
\text { ANCE } \\
(\text { US } / \mathrm{CM}) \\
(00095)\end{array}$ & $\begin{array}{c}\text { PH } \\
\text { WATER } \\
\text { WEOLE } \\
\text { FIELD } \\
\text { (STAND- } \\
\text { ARD } \\
\text { UNITS) } \\
(00400)\end{array}$ & $\begin{array}{c}\text { TEMPER - } \\
\text { ATURER } \\
\text { WATER } \\
\text { (DEG C) } \\
(00010)\end{array}$ & $\begin{array}{c}\text { BARO- } \\
\text { METIC } \\
\text { PRES } \\
\text { SRRE } \\
\text { SRE } \\
\text { (NOF } \\
\text { OOF } \\
(00025)\end{array}$ & $\begin{array}{c}\text { OKYGEN, } \\
\text { DIS- } \\
\text { SOLVED } \\
(\mathrm{MG} / \mathrm{L}) \\
(00300)\end{array}$ & $\begin{array}{c}\text { OXYGEN, } \\
\text { DIS- } \\
\text { SOLVED } \\
\text { (PER- } \\
\text { CENT } \\
\text { SATUR- } \\
\text { ATION) } \\
\text { (OO301) }\end{array}$ & $\begin{array}{l}\text { HARD- } \\
\text { NESS } \\
\text { TOTALL } \\
\text { (MAS/L } \\
\text { AS } \\
\text { CACOS) } \\
(00900)\end{array}$ & $\begin{array}{l}\text { CALCIUM } \\
\text { DIS } \\
\text { SOLED } \\
\text { (KSGLL } \\
\text { ASCAA) } \\
(00915)\end{array}$ & $\begin{array}{c}\text { MAGNE- } \\
\text { SIMM- } \\
\text { DIS- } \\
\text { SOVVED } \\
\text { MOGELL } \\
\text { AS MG) } \\
(00025)\end{array}$ & $\begin{array}{c}\text { SODIUM, } \\
\text { DIS- } \\
\text { SOLVED } \\
\text { (NGG/L } \\
\text { AS NA }) \\
(00930)\end{array}$ \\
\hline $\begin{array}{c}C^{C T} 1995 \\
04 \ldots\end{array}$ & 0930 & 550 & $\cdots 450$ & 7.5 & 14.5 & 635 & 7.7 & 91 & 140 & 43 & 7.3 & 32 \\
\hline DATE & $\begin{array}{l}\text { SODIUM } \\
\text { AD- } \\
\text { SORP- } \\
\text { TION } \\
\text { RATIO } \\
\text { (00931) }\end{array}$ & $\begin{array}{c}\text { POTAS- } \\
\text { SIUM, } \\
\text { DIS- } \\
\text { SOLVED } \\
\text { MGGL/ } \\
\text { AS LS) } \\
(00935)\end{array}$ & $\begin{array}{c}\text { BICAR- } \\
\text { BONATE } \\
\text { WATER } \\
\text { WH IT } \\
\text { FIELD } \\
\text { MG/L AS } \\
\text { HCO3 } \\
(00450)\end{array}$ & $\begin{array}{c}\text { CAR- } \\
\text { BONATE } \\
\text { WATER } \\
\text { WA IT } \\
\text { FIELD } \\
\text { MG/L AS } \\
\text { CO3 } \\
(00447)\end{array}$ & $\begin{array}{c}\text { ALKA- } \\
\text { LINITY } \\
\text { WAT WH WT } \\
\text { TOT IT } \\
\text { FIELD } \\
\text { MG/L AS } \\
\text { CACO3 } \\
(00419)\end{array}$ & $\begin{array}{c}\text { ALKA- } \\
\text { LINITY } \\
\text { LAB } \\
\text { (MAG/L } \\
\text { AS } \\
\text { CACO3) } \\
(90410) .\end{array}$ & $\begin{array}{c}\text { SULFATE } \\
\text { DIS- } \\
\text { SOLVDD } \\
\text { (MG/L } \\
\text { AS SO4) } \\
(00945)\end{array}$ & $\begin{array}{l}\text { CHLO- } \\
\text { RIDE, } \\
\text { DIS- } \\
\text { SOLVED } \\
\text { (MG/L } \\
\text { AS CL) } \\
(00940)\end{array}$ & $\begin{array}{c}\text { SOLIDS } \\
\text { RESIDUE } \\
\text { AT 180 } \\
\text { DEG. } \\
\text { DES- } \\
\text { SOLVED } \\
\text { (MG/L) } \\
(70300)\end{array}$ & $\begin{array}{l}\text { SOLIDS, } \\
\text { SUM OF' } \\
\text { CONSTI- } \\
\text { TUENST, } \\
\text { DIS - } \\
\text { SOVED } \\
(M G / L) \\
(70301)\end{array}$ & $\begin{array}{l}\text { RESIDUE } \\
\text { TOOAL } \\
\text { AT I0S } \\
\text { DEG. C. } \\
\text { SUS- } \\
\text { DEDED } \\
\text { (MG/L) } \\
(00530)\end{array}$ & $\begin{array}{c}\text { CYANIDE } \\
\text { TOOALA } \\
\text { (NG/L } \\
\text { AS CN) } \\
(00720)\end{array}$ \\
\hline
\end{tabular}

$\begin{array}{ccccccccccccc}04 \ldots & 1 & 4.0 & 149 & 0 & 122 & 120 & 60 & 15 & 261 & 235 & 75 & <0.010\end{array}$

DATE

$04 \ldots$

OCT 1995

$04, \ldots$

\section{ALUM-}

INUM, ALUM- ANTI-

ALUM- ANTI

ERABLE

(UG/L.

(AS AL)

INUM,
DIS
SOLVED

(UG/L.

(UG/L (UG/L

(01106)

1500

2.0

$<1.0$

(01002)

ARSENIC
TOTAL
(UG/L
AS AS)
$(01002)$

ARSENIC BARIUM,
DIS-
DIS-

SOLVED SOLVED

(UG/L) (UG/L

AS AS) AS BA)
$(01000)(01005)$

BERYL-

- CHRO-

LIUM, CADMIUM MIUM, COBALT, COPEER,

SOIVED DIS- DIS- DIS- DIS-

(UG/L SOLVED SOLVED SOLVED SOLVED

(UG/L) (UG/L (UG/L (UG/L (UG/L

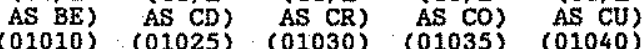

$$
1500
$$

\section{.}

MANGA- MOLYB-

LEAD, NESE, DENUM, NICKEL, DIS- DIS- DIS- DISSOLVED SOLVED SOLVED SOLVED $\begin{array}{llll}\text { (UG/L } & \text { (UG/L } & \text { (UG/L } & \text { (UG/L } \\ \text { AS PB) AS MN) AS NO) AS NI) }\end{array}$ $\begin{array}{llll}(01049) & (01056) & (01060) & (01065)\end{array}$

$$
\begin{array}{llll}
<.0 & 2.0 & 7.0 & 1.0
\end{array}
$$

1. 0

SILVER,

TITAR, URANIUM

SILVER,

DIS-

GRABLE

(UG/L

AS AG) (UG/L

$A S$ AG)
$(01077) \quad(01075)$

\section{DIS-}

SOLVED

(US $Z$ LN)

$(01090)$
$<1.0<1.0$

1.0

$<1.0$

2.0
DIS-

DIS-

SOLVED SUS

(UG/L PENDED

AS U)

(22703)

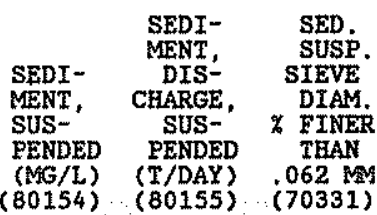

2.0

$115 \quad 171$

87 
ANALYSES OF SAMPLES COLLECTED AT WATER-QUALITY PARTIAL-RECORD STATIONS AND MISCELLANEOUS STTES

WATER-QUALITY DATA, WATER YEAR OCTOBER 1995 TO SEPTEMBER 1996

RTO GRANDE BASIN -- Continued

345547106405510 - ATRISCO RTVRRSTDE DRAIN AT MOUTE NEAR ISLETA, NM

\begin{tabular}{|c|c|c|c|c|c|c|c|c|c|c|}
\hline DATE & TIME & $\begin{array}{c}\text { DIS- } \\
\text { CEARGE, } \\
\text { INST } \\
\text { CUBIC } \\
\text { FEET } \\
\text { PER } \\
\text { SECOND } \\
(00061)\end{array}$ & $\begin{array}{l}\text { SPE- } \\
\text { CIFIC } \\
\text { CON- } \\
\text { DUCT- } \\
\text { ANCE } \\
\text { (US/CM) } \\
(00095)\end{array}$ & $\begin{array}{c}\text { PH } \\
\text { WATER } \\
\text { WHOLE } \\
\text { FTELD } \\
\text { (STAND- } \\
\text { ARD } \\
\text { UNITS) } \\
\text { (00400) }\end{array}$ & $\begin{array}{l}\text { TEMPER- } \\
\text { ATURE } \\
\text { WATER } \\
\text { (DEG C) } \\
(00010)\end{array}$ & $\begin{array}{l}\text { BARO- } \\
\text { METRIC } \\
\text { PRES } \\
\text { SURE } \\
\text { (MM } \\
\text { OF } \\
\text { EG) } \\
(00025)\end{array}$ & $\begin{array}{c}\text { OXYGEN, } \\
\text { DIS- } \\
\text { SOLVED } \\
(M G / L) \\
(00300)\end{array}$ & $\begin{array}{c}\text { OXYGEN, } \\
\text { DIS- } \\
\text { SOLVED } \\
\text { (PER- } \\
\text { CENT } \\
\text { SATUR- } \\
\text { ATION) } \\
\text { (00301) }\end{array}$ & $\begin{array}{l}\text { HARD- } \\
\text { NESS } \\
\text { TOTAL } \\
\text { (MG/L } \\
\text { AS } \\
\text { CACO3) } \\
(00900)\end{array}$ & $\begin{array}{l}\text { HARD- } \\
\text { NESS } \\
\text { NONCARB } \\
\text { DISSOLV } \\
\text { FLD AS } \\
\text { CACO3 } \\
(M G / L) \\
(00904)\end{array}$ \\
\hline $\begin{array}{l}\text { OCT } 1995 \\
\text { OS } \\
\text { FEB } 1996\end{array}$ & 1315 & 46 & 430 & 7.4 & 18.5 & 640 & 7.1 & 91 & 150 & $\cdots$ \\
\hline $\mathrm{MAY}^{15} \ldots$ & 1330 & 55 & 421 & 8.0 & 7.0 & 639 & 9.8 & 97 & 150 & 23 \\
\hline UG & 1500 & 34 & 446 & 8.4 & 21.5 & 638 & -- & -- & 160 & - \\
\hline $28, \ldots$ & 1330 & 56 & 441 & 8.0 & 23.5 & 645 & 7.2 & 101 & 160 & 32 \\
\hline DATE & $\begin{array}{l}\text { CALCIUM } \\
\text { DIS- } \\
\text { SOLVED } \\
\text { (NG/L } \\
\text { AS CA) } \\
(00915)\end{array}$ & $\begin{array}{c}\text { MAGNE- } \\
\text { SIUM, } \\
\text { DIS- } \\
\text { SOLVED } \\
\text { (MG/L } \\
\text { AS MG) } \\
\text { (00925) }\end{array}$ & $\begin{array}{l}\text { SODIUM, } \\
\text { DIS } \\
\text { SOLVID } \\
\text { (MG/L } \\
\text { AS NA) } \\
(00930)\end{array}$ & $\begin{array}{l}\text { SODIUM } \\
\text { AD- } \\
\text { SORP- } \\
\text { TION } \\
\text { RATIO } \\
(00931)\end{array}$ & $\begin{array}{c}\text { POTAS- } \\
\text { SIUM, } \\
\text { DIS- } \\
\text { SOLVED } \\
\text { (MG/L } \\
\text { AS K) } \\
(00935)\end{array}$ & $\begin{array}{c}\text { BICAR- } \\
\text { BONATE } \\
\text { WATER } \\
\text { WH IT } \\
\text { FIELD } \\
\text { MG/L AS } \\
\text { HCO3 } \\
(00450)\end{array}$ & $\begin{array}{c}\text { BICAR- } \\
\text { BONATE } \\
\text { WATER } \\
\text { DIS IT } \\
\text { FIELD } \\
\text { MG/L AS } \\
\text { BCO3 } \\
(00453)\end{array}$ & $\begin{array}{c}\text { CAR } \\
\text { BONATE } \\
\text { WATER } \\
\text { WHIT } \\
\text { FIELD } \\
\text { MG/L AS } \\
\text { CO3 } \\
(00447)\end{array}$ & $\begin{array}{c}\text { CAR- } \\
\text { BONATE } \\
\text { WATER } \\
\text { DIS IT } \\
\text { FIELD } \\
\text { MG/L AS } \\
\text { CO3 } \\
(00452)\end{array}$ & $\begin{array}{c}\text { ALKA- } \\
\text { LINITY } \\
\text { WAT WH } \\
\text { TOT IT } \\
\text { FIELD } \\
\text { MG/L AS } \\
\text { CACO3 } \\
(00419)\end{array}$ \\
\hline
\end{tabular}

\begin{tabular}{|c|c|c|c|}
\hline $\begin{array}{c}\text { OCT } 1995 \\
05\end{array}$ & 47 & 7.2 & 30 \\
\hline $\begin{array}{c}\text { FEB } 1996 \\
15 . \\
\text { MAY }\end{array}$ & 46 & 7.4 & 28 \\
\hline${ }_{\text {AUG }}^{21} \ldots$ & 50 & 7.6 & 32 \\
\hline & 50 & 7.8 & 31 \\
\hline
\end{tabular}

(

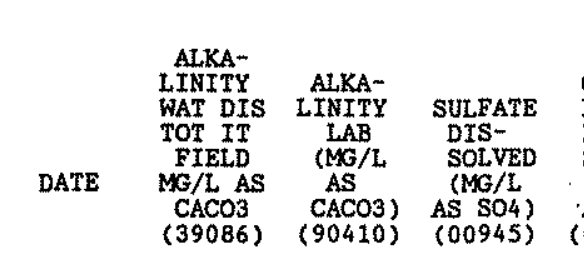

$\begin{array}{rrrrrrr}1 & 3.9 & 160 & -- & 0 & -- & 131 \\ 1 & 3.6 & 159 & 149 & 0 & 0 & 130 \\ 1 & 3.6 & 148 & \ldots & 7 & - & 133 \\ 1 & 4.3 & \ldots- & 152 & \cdots & 0 & --\end{array}$

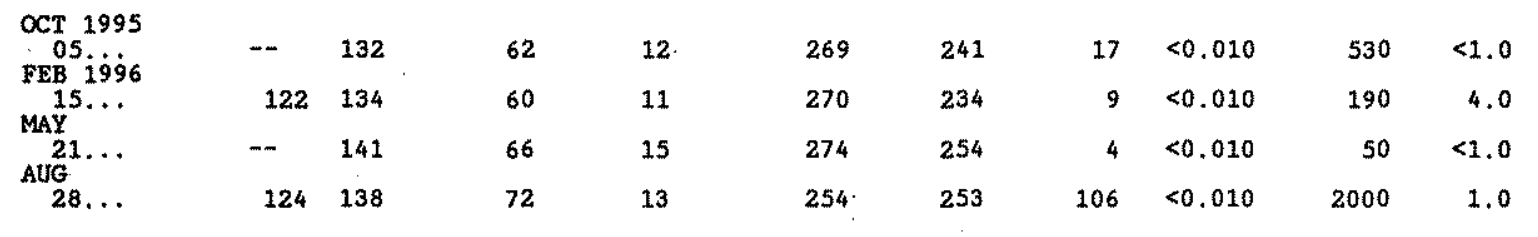

\begin{tabular}{|c|c|c|c|c|c|c|c|c|c|c|}
\hline DATE & $\begin{array}{c}\text { ANTI- } \\
\text { MONY, } \\
\text { DIS- } \\
\text { SOLVED } \\
\text { (UG/L } \\
\text { AS SB) } \\
(01095)\end{array}$ & $\begin{array}{l}\text { ARSENIC } \\
\text { TOTAL } \\
\text { (UG/L } \\
\text { AS AS) } \\
(01002)\end{array}$ & $\begin{array}{c}\text { ARSENIC } \\
\text { DIS- } \\
\text { SOLVED } \\
\text { (UG/L } \\
\text { AS AS) } \\
(01000)\end{array}$ & $\begin{array}{l}\text { BARIUM, } \\
\text { DIS- } \\
\text { SOLVED } \\
\text { (UG/L } \\
\text { AS BA) } \\
(01005)\end{array}$ & $\begin{array}{l}\text { BERYL- } \\
\text { LIUM, } \\
\text { DIS- } \\
\text { SOLVED } \\
\text { (UG/L } \\
\text { AS BE) } \\
(01010)\end{array}$ & $\begin{array}{c}\text { CADMIUM } \\
\text { DIS- } \\
\text { SOLVED } \\
\text { (UG/L } \\
\text { AS CD) } \\
(01025)\end{array}$ & $\begin{array}{l}\text { CHRO- } \\
\text { MIUM, } \\
\text { DIS- } \\
\text { SOLVED } \\
\text { (UE/L } \\
\text { AS CR) } \\
(01030)\end{array}$ & $\begin{array}{c}\text { COBALT, } \\
\text { DIS- } \\
\text { SOLVED } \\
\text { (UG/L } \\
\text { AS CO) } \\
\text { (01035) }\end{array}$ & $\begin{array}{l}\text { COPPLR, } \\
\text { DIS- } \\
\text { SOLVED } \\
\text { (UG/L } \\
\text { AS CU) } \\
(01040)\end{array}$ & $\begin{array}{l}\text { LEAD, } \\
\text { DIS- } \\
\text { SOLVED } \\
\text { (UG/L } \\
\text { AS PB) } \\
(01049)\end{array}$ \\
\hline $\begin{array}{l}\text { OCT } 1995 \\
\text { OS } \\
\text { FEB } 1996\end{array}$ & $<1,0$ & 5 & 5 & 87 & $<1.0$ & $<1,0$ & 2.0 & $<1.0$ & 2.0 & $<1.0$ \\
\hline MAY & $<1.0$ & 4 & 4 & 87 & $<1.0$ & $<1,0$ & 1.0 & $<1.0$ & 2.0 & $<1.0$ \\
\hline$\underset{A U G}{21} \ldots$ & $<1.0$ & 4 & 4 & 82 & $<1.0$ & $<1,0$ & 1.0 & $<1.0$ & 2.0 & $<1.0$ \\
\hline $28 \ldots$ & $<1.0$ & 5 & 5 & 98 & $<1.0$ & $<1.0$ & $<1.0$ & $<1.0$ & 1.0 & $<1.0$ \\
\hline
\end{tabular}


ANALYSES OF SAMPLES COLLECTED AT WATER-QUALITY PARTIAL-RECORD STATIONS AND MISCELLANEOUS STTES

WATER-QUALITY DATA, WATER YEAR OCTOBER 1995 TO SEPTEMBER 1996

RTO GRANDE BASIN - - Continued

345547106405510 - ATRISCO RIVERSIDE DRAIN AT NOUTH NEAR ISLETA, MM

\begin{tabular}{|c|c|c|c|c|c|c|c|c|c|c|}
\hline DATE & $\begin{array}{l}\text { MANGA- } \\
\text { MESE } \\
\text { DIS- } \\
\text { SOLVED } \\
\text { (UG/L } \\
\text { AS MN) } \\
(01056)\end{array}$ & $\begin{array}{l}\text { MOLYB- } \\
\text { DENUM, } \\
\text { DYS-- } \\
\text { SOLVED } \\
\text { (UG/L } \\
\text { AS MO) } \\
(01060)\end{array}$ & $\begin{array}{l}\text { NICKEL, } \\
\text { DIS- } \\
\text { SOLVED } \\
\text { (UG/L } \\
\text { AS NI) } \\
\text { (01065) }\end{array}$ & $\begin{array}{c}\text { SILVER, } \\
\text { TOTAL' } \\
\text { RECOV- } \\
\text { ERABLE } \\
\text { (UG/L } \\
\text { AS AG) } \\
(01077)\end{array}$ & $\begin{array}{c}\text { SrLVER, } \\
\text { DIS- } \\
\text { SOLVED } \\
\text { (UG/L } \\
\text { AS AG) } \\
(01075)\end{array}$ & $\begin{array}{c}\text { ZINC, } \\
\text { DIS } \\
\text { SOLVED } \\
\text { (UG/L } \\
\text { AS ZN) } \\
(01090)\end{array}$ & $\begin{array}{c}\text { URANIUM } \\
\text { NATURAL } \\
\text { DIS - } \\
\text { SOIVED } \\
\text { (UG/L } \\
\text { AS U) } \\
\text { (22703) }\end{array}$ & $\begin{array}{l}\text { SEDI- } \\
\text { MENT, } \\
\text { SUS- } \\
\text { PENDED } \\
\text { (MG/L) } \\
(80154)\end{array}$ & $\begin{array}{c}\text { SEDI- } \\
\text { MENT, } \\
\text { DIS- } \\
\text { CHARGE, } \\
\text { SUS- } \\
\text { PENDED } \\
\text { (T/DAY) } \\
\text { (80155) }\end{array}$ & $\begin{array}{l}\text { SED } \\
\text { SUSP. } \\
\text { SIEVE } \\
\text { DIAM. } \\
\% \text { FINER } \\
\text { THAN } \\
\text { O062 MM } \\
\text { (70331) }\end{array}$ \\
\hline CT 1995 & 45 & 5.0 & 1.0 & $<1$ & $<0.20$ & 1.0 & 2.0 & 73 & 9.1 & 68 \\
\hline $\begin{array}{l}15.996 \\
15 Y\end{array}$ & 89 & 4.0 & 1.0 & $<1$ & $<0.20$ & 2.0 & 3.0 & 39 & 5.7 & 9 \\
\hline $21 \ldots$ & 45 & 5.0 & 2.0 & $<1$ & $<0.20$ & 1.0 & 3.0 & 31 & 2.8 & 66 \\
\hline & 64 & 6.0 & 2.0 & $<1$ & $<0.20$ & 2.0 & 2.0 & 193 & 29 & 61 \\
\hline
\end{tabular}

345550106404810 - ALBUQUERQUE RIVERSIDE DRATN AT NOUTH RR ISLETA, NM

\begin{tabular}{|c|c|c|c|c|c|c|c|c|c|c|}
\hline DATE & TIME & $\begin{array}{c}\text { DIS- } \\
\text { CHARGE, } \\
\text { INST: } \\
\text { CUBIC } \\
\text { FEET } \\
\text { PER } \\
\text { SECOND } \\
(00061)\end{array}$ & $\begin{array}{l}\text { SPE- } \\
\text { CIFIC } \\
\text { CON- } \\
\text { DUCT- } \\
\text { ANCE } \\
\text { (US /CM) } \\
(00095)\end{array}$ & $\begin{array}{c}\text { PH } \\
\text { WATER } \\
\text { WAOLE } \\
\text { FIELD } \\
\text { (STAND- } \\
\text { ARD } \\
\text { UNITS) } \\
(00400)\end{array}$ & $\begin{array}{l}\text { TEMPER- } \\
\text { ATURE } \\
\text { WATER } \\
\text { (DEG C) } \\
(00010)\end{array}$ & $\begin{array}{c}\text { BARO- } \\
\text { METRIC } \\
\text { PRES- } \\
\text { SURE } \\
\text { (MM } \\
\text { OF } \\
\text { HG) } \\
(00025)\end{array}$ & $\begin{array}{c}\text { OXYGEN, } \\
\text { DIS- } \\
\text { SOLVED } \\
\text { (MG/L) } \\
(00300)\end{array}$ & $\begin{array}{c}\text { OXYGEN, } \\
\text { DIS- } \\
\text { SOLVED } \\
\text { (PER- } \\
\text { CEN } \\
\text { SATUR- } \\
\text { ATION) } \\
\text { (00301) }\end{array}$ & $\begin{array}{l}\text { HARD- } \\
\text { NESS } \\
\text { TOTAL } \\
\text { (MG/L } \\
\text { AS } \\
\text { CACO3) } \\
(00900)\end{array}$ & $\begin{array}{l}\text { HARD- } \\
\text { NESS } \\
\text { NONCARB } \\
\text { DISSOLV } \\
\text { FLD.AS } \\
\text { CACO3 } \\
\text { (MG/L) } \\
(00904)\end{array}$ \\
\hline $\begin{array}{l}\text { CT } 1995 \\
05\end{array}$ & 0930 & 164 & 435 & 7.7 & 14.0 & 640 & 7.5 & 87 & 150 & $-\cdots$ \\
\hline$\frac{15}{4 Y}$ & 1115 & 51 & 460 & 7.9 & 10.5 & 640 & 7.0 & 75 & 150 & 10 \\
\hline${ }_{J G}^{21} \ldots$ & 1300 & 94 & 433 & 8.0 & 18.5 & 641 & 7.4 & 94 & 150 & - \\
\hline $28 \ldots$ & 1145 & 178 & 412 & 7.9 & 21.5 & 646 & 6.4 & 86 & 160 & 39 \\
\hline DATE & $\begin{array}{l}\text { CALCIUM } \\
\text { DIS } \\
\text { SOLVED } \\
\text { (RSGL } \\
\text { AS CA) } \\
(00915)\end{array}$ & $\begin{array}{c}\text { MAGNE- } \\
\text { SIUM, } \\
\text { DIS- } \\
\text { SOLVED } \\
\text { (MG/L } \\
\text { AS MG) } \\
(00925)\end{array}$ & $\begin{array}{c}\text { SODIUM, } \\
\text { DIS - } \\
\text { SOLVED } \\
\text { (MGE/L } \\
\text { AS HA ) } \\
(00930)\end{array}$ & $\begin{array}{c}\text { SODIUM } \\
\text { AD- } \\
\text { SORP- } \\
\text { TION } \\
\text { RATIO } \\
(00931)\end{array}$ & $\begin{array}{c}\text { POTAS- } \\
\text { SIUM, } \\
\text { DIS- } \\
\text { SOLVED } \\
\text { (MG /L } \\
\text { AS K }) \\
(00935)\end{array}$ & $\begin{array}{l}\text { BICAR- } \\
\text { BONATE } \\
\text { WATERR } \\
\text { WHIIT } \\
\text { FIELD } \\
\text { MG/L AS } \\
\text { HCOO3 } \\
(00450)\end{array}$ & $\begin{array}{c}\text { BICAR- } \\
\text { BONARE } \\
\text { WATER } \\
\text { DIS IT } \\
\text { FIELD } \\
\text { MG/LAS } \\
\text { HCO3 } \\
(00453)\end{array}$ & $\begin{array}{c}\text { CAR- } \\
\text { BONATE } \\
\text { WATER } \\
\text { WH IT } \\
\text { FIELD } \\
\text { MG/L AS } \\
\text { CO3 } \\
(00447)\end{array}$ & $\begin{array}{c}\text { CAR- } \\
\text { BONATE } \\
\text { WATER } \\
\text { DIS IT } \\
\text { FIELD } \\
\text { MG/L AS } \\
\text { CO3 } \\
(00452)\end{array}$ & $\begin{array}{c}\text { ALKA- } \\
\text { LINITY } \\
\text { WAT WH } \\
\text { TOT IT } \\
\text { FIELD } \\
\text { MGLL AS } \\
\text { CACO3 } \\
(00419)\end{array}$ \\
\hline 051996 & 47 & 7.7 & 27 & 1 & 3.7 & 159 & - & 0 & $-\infty$ & 130 \\
\hline $15 . \cdots$ & 48 & 7.6 & 33 & 1 & 4.3 & 172 & 172 & 0 & 0 & 141 \\
\hline${ }_{J G}^{21} \cdots$ & 48 & 8.1 & 29 & 1 & 3.6 & 364 & -- & 0 & -- & 134 \\
\hline $28 \ldots$ & 50 & 8.2 & 28 & 1 & 4.0 & - & 146 & $\cdots$ & 0 & -- \\
\hline DATE & $\begin{array}{l}\text { ALKA- } \\
\text { LINITY } \\
\text { WAT DIS } \\
\text { TOT IT } \\
\text { FIELD } \\
\text { WG/L AS } \\
\text { CACO3 } \\
\text { (39086) }\end{array}$ & $\begin{array}{c}\text { ALKA- } \\
\text { ETNITY } \\
\mathrm{LAB} \\
(\mathrm{MGG} / \mathrm{L} \\
\mathrm{AS} \\
\mathrm{CACO} \\
(90410)\end{array}$ & $\begin{array}{c}\text { SULFATE } \\
\text { DIS- } \\
\text { SOLVED } \\
\text { (MA/L } \\
\text { AS SO4) } \\
(00945)\end{array}$ & $\begin{array}{l}\text { CHLO- } \\
\text { DIDE, } \\
\text { DIS- } \\
\text { SOLVED } \\
\text { (MG/L } \\
\text { AS CL) } \\
(00940)\end{array}$ & $\begin{array}{l}\text { SOLIDS, } \\
\text { RESIDUE } \\
\text { AT } \$ B 0 \\
\text { DEG. C } \\
\text { DIS - } \\
\text { SOLVED } \\
(M G / L) \\
(70300)\end{array}$ & $\begin{array}{c}\text { SOLIDS, } \\
\text { SUM OF } \\
\text { CONSTI- } \\
\text { TUENTS, } \\
\text { DIS- } \\
\text { SOLVED } \\
\text { (MES/L) } \\
\text { (70301) }\end{array}$ & $\begin{array}{l}\text { RESIDUE } \\
\text { TOTAL } \\
\text { AT IOJ } \\
\text { DEG, C, } \\
\text { SUS - } \\
\text { PENDED } \\
\text { (MG/L) } \\
(00530)\end{array}$ & $\begin{array}{l}\text { CYANIDE } \\
\text { TOTAL } \\
\text { (MG/L } \\
\text { AS CA }) \\
(00720)\end{array}$ & $\begin{array}{l}\text { ALUM- } \\
\text { INUM } \\
\text { TOTAL } \\
\text { RECOV- } \\
\text { ERABLE } \\
\text { (UGG/L } \\
\text { AS AL) } \\
\text { (01105) }\end{array}$ & $\begin{array}{l}\text { ALUM- } \\
\text { INUNA, } \\
\text { DIS- } \\
\text { SOLVED } \\
\text { (UG/L } \\
\text { AS AL) } \\
\text { (01106) }\end{array}$ \\
\hline $\begin{array}{l}\text { CT } 1995 \\
051996 \\
B B\end{array}$ & $-\cdots$ & 130 & 60 & 9.5 & 255 & 233 & 45 & $<0.010$ & 1300 & 1.0 \\
\hline$\frac{15}{A Y} \ldots$ & 141 & 142 & 61 & 15 & 293 & 254 & 12 & $<0.010$ & 80 & 4.0 \\
\hline${\underset{u G}{21} \cdots}^{21}$ & $-\cdots$ & 138 & 65 & 11 & 222 & 245 & 69 & $<0.010$ & 980 & $<1.0$ \\
\hline & 119 & 134 & 73 & 10 & 242 & 245 & 612 & $<0.010$ & 8600 & 2.0 \\
\hline
\end{tabular}


ANALYSES OF SAMPLES COLLECTED AT WATER-QUALITX PARTIAL-RECORD STATIONS AND MISCELLANEOUS SITES

WATER-QUALITY DATA, WATER YEAR OCTOBER 1995 TO SEPTEMBER 1996

RIO GRANDE BASIN -- Continued

345550106404810 - ALBUQUERQUE RIVERSIDE DRAIN AT MOUTH NR ISLETA, NM

\begin{tabular}{|c|c|c|c|c|c|c|c|c|c|c|}
\hline DATE & $\begin{array}{c}\text { ANTI- } \\
\text { MONY, } \\
\text { DIS- } \\
\text { SOLVED } \\
\text { (UG/L } \\
\text { AS SB) } \\
(01095)\end{array}$ & $\begin{array}{l}\text { ARSENIC } \\
\text { TOTAL } \\
\text { (UG/L } \\
\text { AS AS) } \\
(01002)\end{array}$ & $\begin{array}{c}\text { ARSENIC } \\
\text { DIS- } \\
\text { SOLVED } \\
\text { (UG/L } \\
\text { AS AS) } \\
(01000)\end{array}$ & $\begin{array}{c}\text { BARIUM, } \\
\text { DIS- } \\
\text { SOLVED } \\
\text { (UG/L } \\
\text { AS BA }) \\
(01005)\end{array}$ & $\begin{array}{l}\text { BERYL- } \\
\text { LIUM, } \\
\text { DIS- } \\
\text { SOLVED } \\
\text { (UG/L } \\
\text { AS BE) } \\
\text { (01010) }\end{array}$ & $\begin{array}{c}\text { CADMIUM } \\
\text { DIS- } \\
\text { SOLVED } \\
\text { (UG/L } \\
\text { AS CD) } \\
\text { (01025) }\end{array}$ & $\begin{array}{l}\text { CRRO- } \\
\text { MIUM, } \\
\text { DIS- } \\
\text { SOLVED } \\
\text { (UG/L } \\
\text { AS CR) } \\
\text { (01030) }\end{array}$ & $\begin{array}{l}\text { COBALT, } \\
\text { DIS- } \\
\text { SOLVED } \\
\text { (UG/L } \\
\text { AS CO) } \\
\text { (01035) }\end{array}$ & $\begin{array}{l}\text { COPPER, } \\
\text { DrS-- } \\
\text { SOLVED } \\
\text { (UG/L } \\
\text { AS CU) } \\
\text { (01040) }\end{array}$ & $\begin{array}{l}\text { LEAD, } \\
\text { DIS- } \\
\text { SOLVED } \\
\text { (UG/L } \\
\text { AS PB) } \\
(01049)\end{array}$ \\
\hline $\begin{array}{l}1995 \\
05\end{array}$ & $<1.0$ & 3 & 4 & 79 & $<1.0$ & $<1.0$ & 2.0 & $<1.0$ & 1.0 & $<1.0$ \\
\hline & $<1.0$ & 5 & 5 & 107 & $<1.0$ & $<1.0$ & 1.0 & $<1.0$ & 1.0 & $<1.0$ \\
\hline $21 \ldots$ & $<1.0$ & 4 & 3 & 81 & $<1.0$ & $<1.0$ & 1.0 & $<1.0$ & 1.0 & $<1.0$ \\
\hline & $<1,0$ & 4 & 4 & 103 & $<1.0$ & $<1.0$ & $<1.0$ & $<1.0$ & 1.0 & $<1.0$ \\
\hline
\end{tabular}

$\begin{array}{ccccc}\text { MANGA- } & \text { MOLYB- } & & \text { SILVER, } \\ \text { NESE, } & \text { DENUM, } & \text { NICKEL, } & \text { TOTAL, } & \text { SILVER, } \\ \text { DIS- } & \text { DIS- } & \text { DIS- } & \text { RECOV- } & \text { DIS- } \\ \text { SOLVED } & \text { SOLVED } & \text { SOLVED } & \text { ERABLE } & \text { SOLVED } \\ \text { (UG/L } & \text { (UG/L } & \text { (UG/L } & \text { (UG/L } & \text { (UG/L } \\ \text { AS MN) } & \text { AS MO) } & \text { AS NI) } & \text { AS AG) } & \text { AS AG) } \\ (01056) & (01060) & (01065) & (01077) & (01075)\end{array}$

$\begin{array}{cc} & \text { URANIUM } \\ \text { ZINC, } & \text { NATURAL } \\ \text { DIS- } & \text { DIS- } \\ \text { SOLVED } & \text { SOLVED } \\ \text { (UG/L } & \text { (UG/L } \\ \text { AS ZN) } & \text { AS U) } \\ (01090) & (22703)\end{array}$

$\begin{array}{lcc} & \text { SEDI- } & \text { SED. } \\ \text { MENT, } & \text { SUSP. } \\ \text { SEDI- } & \text { DIS- } & \text { SIEVE } \\ \text { MENT, } & \text { CHARGE, } & \text { DIAM. } \\ \text { SUS- } & \text { SUS- } & \% \text { FINER } \\ \text { PENDED } & \text { PENDED } & \text { THAN } \\ \text { (MNG/L) } & (\text { TIDAY) } & \text { (062 MM } \\ (80154) & (80155) & (70331)\end{array}$

OCT 1995
05.59
FEB 1996
$15 \ldots$
MAY $21 \ldots$
AUG
$\quad 28$

$\begin{array}{ccc}18 & 5.0 & 2.0 \\ 351 & 6.0 & 2.0 \\ 32 & 5.0 & 2.0 \\ 4.0 & 5.0 & 2.0\end{array}$

$\begin{array}{ll}<1 & <0.20 \\ <1 & <0.20 \\ <1 & <0.20 \\ <1 & <0.20\end{array}$

1.0

2.0

$\left(\frac{10}{20}\right.$

44

76

5.0

4.0

17

2.4

89

$104 \quad 26$

79

345612106403310 - BASS LAKE AT ISLETA LAKES, NM

$630 \quad 303$

92

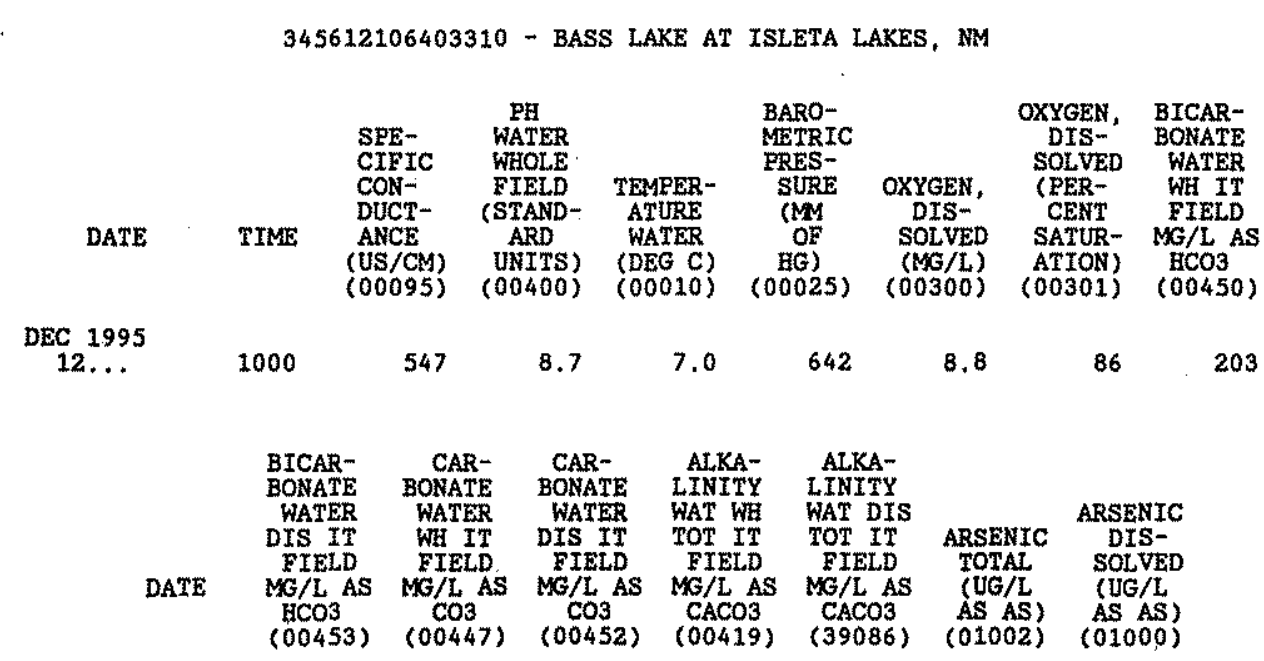

DEC 1995

$12 .$.

200

10

8

182

178

3

2 
ANALYSES OF SAMPLES COLLECTED AT WATER-QUALITY RARTIAL-RECORD STATIONS AND MISCELLANEOUS SITES

WATER-QUALITY DATA, WATER YEAR OCTOBER 1995 TO SEPTEMBER 1996

RIO GRANDE BASIN -- Continued

345705106405210 - RIO GRANDE AT I25 BRIDGE NR ISLETA PUEBLO, NM

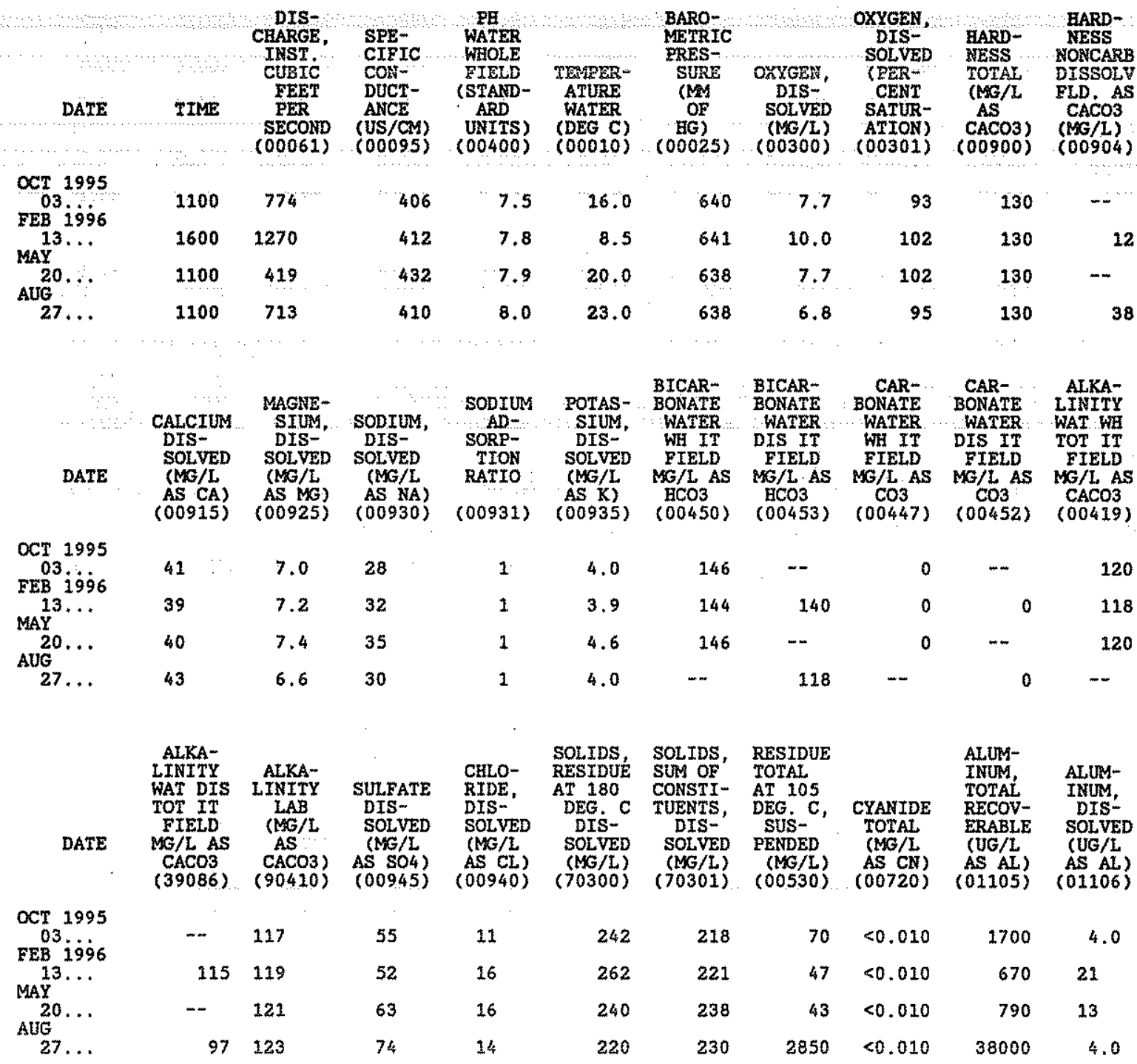

\begin{tabular}{|c|c|c|c|c|c|c|c|c|c|c|}
\hline DAT & $\begin{array}{c}\text { ANTY- } \\
\text { MONY, } \\
\text { DIS- } \\
\text { SOLVED } \\
\text { (UE, } \\
\text { AS SB) } \\
\text { (01095) }\end{array}$ & $\begin{array}{c}\text { ARSENIC } \\
\text { TOTAL } \\
\text { (UE/L } \\
\text { AS AS) } \\
(01002)\end{array}$ & $\begin{array}{c}\text { ARSENIC } \\
\text { DIS- } \\
\text { SOLVED } \\
\text { (JUE, } \\
\text { AS AS) } \\
(01000)\end{array}$ & $\begin{array}{c}\text { BARIUM, } \\
\text { DIS- } \\
\text { SOLVED } \\
\text { (IIS/I } \\
\text { AS BA) } \\
(01005)\end{array}$ & $\begin{array}{l}\text { BERYL- } \\
\text { LIUM, } \\
\text { DIS- } \\
\text { SOLVED } \\
\text { (JE' } \\
\text { AS BE) } \\
\text { (01010) }\end{array}$ & $\begin{array}{c}\text { CADMIUM } \\
\text { DIS- } \\
\text { SOLVED } \\
\text { (USG/I } \\
\text { AS CD) } \\
(01025)\end{array}$ & $\begin{array}{l}\text { CHRO- } \\
\text { MIUM, } \\
\text { DIS- } \\
\text { SOLVED } \\
\text { (IUG/T } \\
\text { AS CR) } \\
(01030)\end{array}$ & $\begin{array}{l}\text { COBALT, } \\
\text { DIS- } \\
\text { SOLVED } \\
\text { (YYE, } \\
\text { AS CO) } \\
(01035)\end{array}$ & $\begin{array}{c}\text { COPPER, } \\
\text { DIS- } \\
\text { SOLVED } \\
\text { (YSE/L } \\
\text { AS CU) } \\
(01040)\end{array}$ & $\begin{array}{c}\text { LEAD, } \\
\text { DIS- } \\
\text { SOLVED } \\
\text { (UE/L } \\
\text { AS PB) } \\
(01049)\end{array}$ \\
\hline
\end{tabular}

\begin{tabular}{|c|c|c|c|c|c|c|c|c|c|c|}
\hline $\begin{array}{c}\text { OCT } 1995 \\
\text { O3 } 1996 \\
\text { FEB }\end{array}$ & $<1.0$ & 3 & 3 & 63 & $<1.0$ & $<1,0$ & 2.0 & $<1.0$ & 2.0 & $<1.0$ \\
\hline $\mathrm{MAY}$ & $<1.0$ & 3 & 3 & 64 & $<1,0$ & $<1,0$ & 1.0 & $<1,0$ & 2.0 & $<1.0$ \\
\hline${ }_{\text {AUG }}^{20} \ldots$ & $<1,0$ & 3 & 3 & 59 & $<1,0$ & $<1,0$ & 1.0 & $<1.0$ & 1.0 & $<1.0$ \\
\hline 27 & $<1,0$ & 4 & 3 & 87 & $<1,0$ & $<1.0$ & $<1.0$ & $<1,0$ & 1.0 & $<1,0$ \\
\hline
\end{tabular}


ANALYSES OF SAMPLES COLLECTED AT WATER-QUALITY PARTIAL-RECORD STATIONS AND MISCELLANEOUS SITES

WATER-QUALITY DATA, WATER YEAR OCTOBER 1995 TO SEPTEMBER 1996

RIO GRANDE BASIN -- Continued

345705106405210 - RIO GRANDE AT I2S BRIDGE NR ISLETA PUEBLO, NM

\begin{tabular}{|c|c|c|c|c|c|c|c|c|c|}
\hline $\begin{array}{l}\text { MANGA- } \\
\text { NESE, } \\
\text { DIS- } \\
\text { SOLVED } \\
\text { (UG/L } \\
\text { AS MN) }\end{array}$ & $\begin{array}{l}\text { MOLYB- } \\
\text { DENUM, } \\
\text { DIS- } \\
\text { SOLVED } \\
\text { (UG/L } \\
\text { AS MO) } \\
\text { (01060) }\end{array}$ & $\begin{array}{c}\text { NICKEL, } \\
\text { DIS- } \\
\text { SOLVED } \\
\text { (UE/L } \\
\text { AS NI) } \\
\text { (01065) }\end{array}$ & $\begin{array}{c}\text { SILVER, } \\
\text { TOTAL } \\
\text { RECOV- } \\
\text { ERABLE } \\
\text { (UG/L } \\
\text { AS AG) } \\
(01077)\end{array}$ & $\begin{array}{c}\text { SILVER, } \\
\text { DIS- } \\
\text { SOLVED } \\
\text { (UG/L } \\
\text { AS AG) } \\
(01075)\end{array}$ & $\begin{array}{l}\text { ZINC, } \\
\text { DIS- } \\
\text { SOLVED } \\
\text { (UG/L } \\
\text { AS ZN) } \\
(01090)\end{array}$ & $\begin{array}{c}\text { NATU } \\
\text { DI } \\
\text { SOL } \\
\text { (US } \\
\text { AS } \\
(227\end{array}$ & $\begin{array}{l}\text { SEDI- } \\
\text { MENT, } \\
\text { SUS- } \\
\text { PENDED } \\
(M G / L) \\
(80154)\end{array}$ & $\begin{array}{c}\text { SEDI- } \\
\text { MENT, } \\
\text { DIS- } \\
\text { CHARGE, } \\
\text { SUS- } \\
\text { PENDED } \\
\text { (T/DAY) } \\
(80155)\end{array}$ & $\begin{array}{r}\text { SED. } \\
\text { SUSP. } \\
\text { SIEVE } \\
\text { DIAM. } \\
\times \text { FINER } \\
\text { THAN } \\
\text { THA MM } \\
(70331)\end{array}$ \\
\hline
\end{tabular}

OCT 1995

FEB 19396

FEB 199

MAY

$20 \ldots$

$$
3.0
$$

$6.0<1.0$

$\begin{array}{ll}<1 & <0.20 \\ <1 & <0.20 \\ <1 & <0.20 \\ <1 & <0.20\end{array}$

2.0
5.0
3.0
2.0

2.0

$127 \quad 265$

3.0

$59 \quad 203$

$\begin{array}{lll}3.0 & 66 & 75\end{array}$

$2.0 \quad 3300 \quad 6340$

(0631)

27 ...

$<1.0$

5.0

2.0

$<0.20$

2,0

79

54

54

350104106401110 - AL.BUQUERQUE WASTEWATER TREATMENT PLANT OUTFALL, MM

\begin{tabular}{|c|c|c|c|c|c|c|c|c|c|c|}
\hline DATE & TIME & $\begin{array}{c}\text { DIS- } \\
\text { CHARGE, } \\
\text { IN } \\
\text { CUBIC } \\
\text { FEET } \\
\text { FER } \\
\text { SECOND } \\
(00060)\end{array}$ & $\begin{array}{l}\text { SEE- } \\
\text { CIFIC } \\
\text { CON- } \\
\text { DUCT- } \\
\text { ANCE } \\
\text { (US/CM) } \\
(00095)\end{array}$ & $\begin{array}{c}\text { PE } \\
\text { WATER } \\
\text { WHOLE } \\
\text { FIELD } \\
\text { (STAND- } \\
\text { ARD } \\
\text { UNITS) } \\
\text { (00400) }\end{array}$ & $\begin{array}{c}\text { TEMPER- } \\
\text { ATURE } \\
\text { WATER } \\
\text { (DEG C) } \\
(00010)\end{array}$ & $\begin{array}{c}\text { BARO- } \\
\text { METRIC } \\
\text { PRES- } \\
\text { SURE } \\
\text { (MM } \\
\text { OF } \\
\text { HG) } \\
(00025)\end{array}$ & $\begin{array}{c}\text { OXYGEN, } \\
\text { DIS- } \\
\text { SOLVED } \\
\text { (MG/L) } \\
(00300)\end{array}$ & $\begin{array}{c}\text { OXYGEN, } \\
\text { DIS- } \\
\text { SOLVED } \\
\text { (PER- } \\
\text { CENT } \\
\text { SATUR- } \\
\text { ATION) } \\
\text { (00301) }\end{array}$ & $\begin{array}{l}\text { RARD- } \\
\text { NESS } \\
\text { TOTAL } \\
\text { (MG/L } \\
\text { AS } \\
\text { CACO3) } \\
(00900)\end{array}$ & $\begin{array}{l}\text { HARD- } \\
\text { NESS } \\
\text { NONCARB } \\
\text { DISSOLV } \\
\text { FLD. AS } \\
\text { CACO3 } \\
(M G / L) \\
(00904)\end{array}$ \\
\hline $\begin{array}{r}1995 \\
6 \\
1996\end{array}$ & 1130 & 89 & 808 & 6.6 & 24.5 & 640 & 4.3 & 62 & 130 & 29 \\
\hline & 1500 & 87 & 833 & 6.9 & 11.0 & 637 & 6.8 & 74 & 130 & 18 \\
\hline & 1120 & 78 & 794 & 7.0 & 26.0 & 638 & 6.4 & 95 & 130 & -- \\
\hline$\ldots$ & 1200 & 81 & 764 & 7.1 & 28.5 & 645 & 5.6 & 86 & 130 & 0 \\
\hline DATE & $\begin{array}{l}\text { CALCIUM } \\
\text { DIS- } \\
\text { SOLVED } \\
\text { (MG/L } \\
\text { AS CA) } \\
\text { (00915) }\end{array}$ & $\begin{array}{c}\text { MAGNE- } \\
\text { SIUM, } \\
\text { DIS- } \\
\text { SOLVED } \\
\text { (MG/L } \\
\text { AS MG) } \\
(00925)\end{array}$ & $\begin{array}{l}\text { SODIUM, } \\
\text { DIS- } \\
\text { SOLVED } \\
\text { (MS/L } \\
\text { AS NA) } \\
(00930)\end{array}$ & $\begin{array}{c}\text { SODIUM } \\
\text { AD- } \\
\text { SORP- } \\
\text { TION } \\
\text { RATIO } \\
(00931)\end{array}$ & $\begin{array}{c}\text { POTAS- } \\
\text { SIUM, } \\
\text { DIS- } \\
\text { SOLVED } \\
\text { (MG/L } \\
\text { AS K) } \\
(00935)\end{array}$ & $\begin{array}{c}\text { BICAR- } \\
\text { BONATE } \\
\text { WATER } \\
\text { WHIT } \\
\text { FIELD } \\
\text { MG/L AS } \\
\text { HCO3 } \\
(00450)\end{array}$ & $\begin{array}{c}\text { BICAR- } \\
\text { BONATE } \\
\text { WATER } \\
\text { DIS IT } \\
\text { FIELD } \\
\text { MG/L AS } \\
\text { ECO3 } \\
(00453)\end{array}$ & $\begin{array}{c}\text { CAR- } \\
\text { BONATE } \\
\text { WATER } \\
\text { WH IT } \\
\text { FIELD } \\
\text { MG/L AS } \\
\text { CO3 } \\
(00447)\end{array}$ & $\begin{array}{c}\text { CAR- } \\
\text { BONATE } \\
\text { WATER } \\
\text { DIS IT } \\
\text { FIELD } \\
\text { MG/L AS } \\
\text { CO3 } \\
(00452)\end{array}$ & $\begin{array}{c}\text { ALKA- } \\
\text { LINITY } \\
\text { WAT WH } \\
\text { TOT IT } \\
\text { FIELD } \\
\text { MG/L AS } \\
\text { CACO3 } \\
(00419)\end{array}$ \\
\hline
\end{tabular}

\begin{tabular}{|c|c|c|c|c|c|c|c|c|c|c|}
\hline $\begin{array}{l}\text { OCT } 1995 \\
\text { O6 } \\
\text { FEB } 1996\end{array}$ & 42 & 6.2 & 110 & 4 & 14 & 128 & 124 & & & 105 \\
\hline${ }_{\mathrm{MAY}}^{15} \cdots$ & 40 & 6.3 & 100 & 4 & 15 & 139 & 132 & & & 114 \\
\hline$\underset{\text { AUG }}{22 . .}$ & 41 & 6.1 & 110 & 4 & 14 & 149 & $\rightarrow$ & & -- & 122 \\
\hline $29 \ldots$ & 42 & 6.1 & 100 & 4 & 14 & $-m$ & 166 & - & & - \\
\hline
\end{tabular}

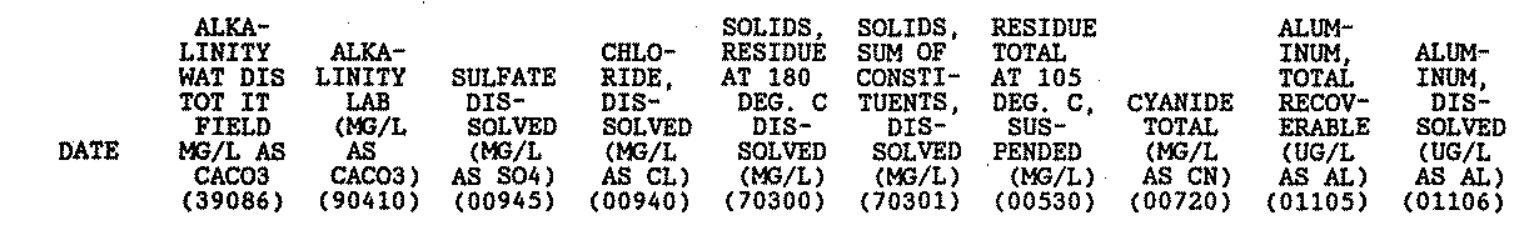

\footnotetext{
OCT 1995

FEB 1996

$15 . \cdots$

$\underset{\text { AUG }}{22} \ldots$

$29 .$.
}

$\begin{array}{cr}102 & 80 \\ 108 & 99 \\ -- & 123 \\ 136 & 118\end{array}$

$\begin{array}{ll}93 & 92 \\ 94 & 89 \\ 90 & 85 \\ 90 & 88\end{array}$

$\begin{array}{ll}541 & 420 \\ 532 & 413 \\ 486 & 420 \\ 452 & 422\end{array}$
$7<0.010$
$17<0.010$
$3 \quad 50.008$
$3<0.010$

$150 \quad 22$

$180 \quad 20$

88

$\begin{array}{ll}90 & 20 \\ 60 & 16\end{array}$ 
WATER-QUALITY DATA, WATER YEAR OCTOBER 1995 TO SEPTEMBER 1996

RIO GRANDE BASIN -- Continued

350104106401110 - ALBUQUERQUE WASTEWATER TREATMENT PLANT OUTFALL, NM

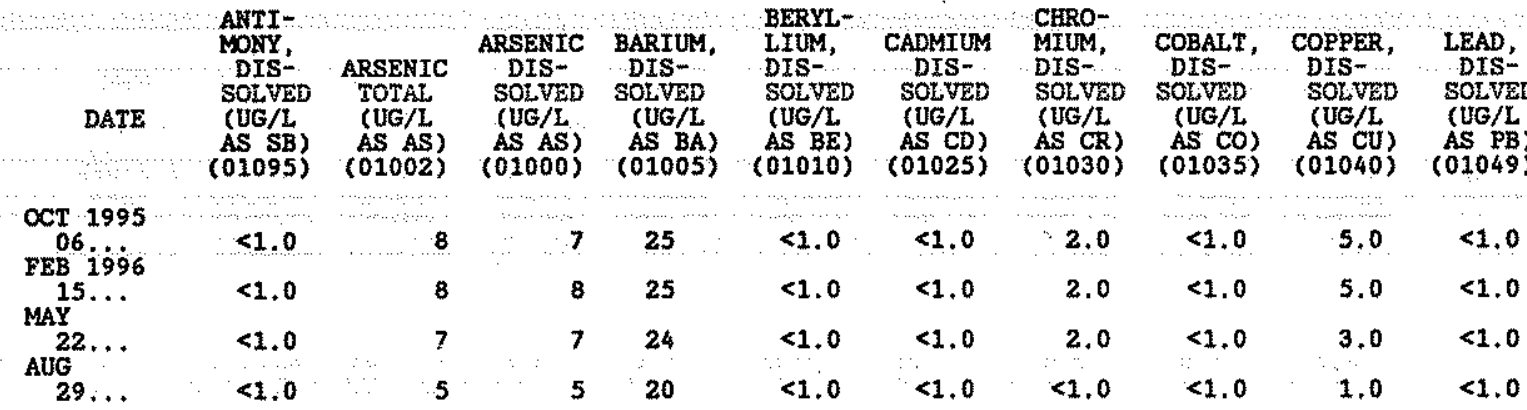

MANGA- MOLYB-

DERYM

DIS- DENASOLVED SOLVED (UG/L) (US $\mathrm{MO}$

$(01056)$ $(01060)$
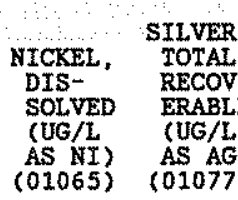

$<1.0$

1.0

$<1.0$

OCT 1995 FEB 1966 FEB 19 MAY 15. $\underset{\text { AUG }}{22}$... $29 .$.

$\begin{array}{ll}18 & 28 \\ 20 & 26 \\ 24 & 29 \\ 42 & 34\end{array}$

$\begin{array}{llll}4.0 & 1 & <0.20 & 23 \\ 5.0 & 1 & <0.20 & 30 \\ 3.0 & <1 & <0.20 & 29 \\ 4.0 & <1 & <0.20 & 19\end{array}$

$<1.0$

$<1.0$

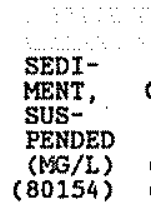

SEDI- SED.

MENT, SUSP.

CHARGE, DIAM.

SUS- \% FINER

PES-O F FINER

(T/DAY) .062 (80155) (70331)

350547106411610 - ALBUQUERQUE RSD BLW GATE N OF CENTRAL BLVD, NM

\begin{tabular}{|c|c|c|c|c|c|c|c|c|c|c|}
\hline & 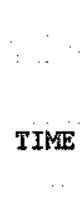 & $\begin{array}{c}\text { DIS- } \\
\text { CHARGE, } \\
\text { INST } \\
\text { CUBIC } \\
\text { EEET } \\
\text { PER } \\
\text { SECOND } \\
(00061)\end{array}$ & $\begin{array}{l}\text { SPE- } \\
\text { CIFIC } \\
\text { CON- } \\
\text { DUCT- } \\
\text { ANCE } \\
\text { (US/CM) } \\
(0005)\end{array}$ & $\begin{array}{c}\text { FH } \\
\text { WATER } \\
\text { WHOLE } \\
\text { FIELD } \\
\text { (STAND- } \\
\text { ARD } \\
\text { UNITS) } \\
(00400)\end{array}$ & $\begin{array}{c}\text { TEMPER- } \\
\text { ATURE } \\
\text { WATER } \\
\text { (DEG C) } \\
\text { (00010) }\end{array}$ & $\begin{array}{l}\text { BARO- } \\
\text { METRIC } \\
\text { PRES-: } \\
\text { SURE } \\
\text { (MN } \\
\text { OF } \\
\text { HG) } \\
(00025)\end{array}$ & $\begin{array}{c}\text { OXYGEN, } \\
\text { DIS- } \\
\text { SOLVED } \\
(M G / L) \\
(00300)\end{array}$ & $\begin{array}{c}\text { OXYGEN, } \\
\text { DIS- } \\
\text { SOLVED } \\
\text { (PER- } \\
\text { CENT } \\
\text { SATUR- } \\
\text { ATION) } \\
\text { (00301) }\end{array}$ & $\begin{array}{l}\text { FARD- } \\
\text { NESS } \\
\text { TOTAL } \\
\text { (MG/L } \\
\text { AS } \\
\text { CACO3) } \\
(00900)\end{array}$ & $\begin{array}{l}\text { HARD- } \\
\text { NESS } \\
\text { NONCARB } \\
\text { DISSOLV } \\
\text { FLD. AS } \\
\text { CACO3 } \\
(\text { MG/L) } \\
(00904)\end{array}$ \\
\hline
\end{tabular}

\begin{tabular}{|c|c|c|c|c|}
\hline \multicolumn{4}{|l|}{$\begin{array}{c}\text { OCT } 1995 \\
05.996 \\
F E B ~ 1996 \\
15 \ldots \\
\text { MAY } \\
21 \ldots \\
\text { AUG }\end{array}$} & 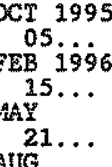 \\
\hline
\end{tabular}

$\begin{array}{rr}1130 & 131 \\ 0850 & 78 \\ 1000 & 577 \\ 0930 & 87\end{array}$

405
401
401
400

7.6
7.8
7.9
8.0

$\begin{array}{rr}15.0 & 640 \\ 6.5 & 639 \\ 15.5 & 640 \\ 21.0 & 644\end{array}$

8.1
9.4
7.7
6.8

$\begin{array}{ccc}96 & 140 & - \\ 91 & 140 & 17 \\ 92 & 140 & -- \\ 91 & 150 & 31\end{array}$

\begin{tabular}{|c|c|c|}
\hline $\begin{array}{c}\text { CAICTUMA } \\
\text { DrS- } \\
\text { SOLVED } \\
\text { (MG/L } \\
\text { AS CA }) \\
(00915)\end{array}$ & $\begin{array}{c}\text { MAGHE- } \\
\text { STUA, } \\
\text { DIS- } \\
\text { SOLVED } \\
\text { (MG/L } \\
\text { AS ME) } \\
(00925)\end{array}$ & $\begin{array}{c}\text { SODrüs, } \\
\text { DIS- } \\
\text { SOLVED } \\
\text { (NG/L } \\
\text { AS HA) } \\
(00930)\end{array}$ \\
\hline
\end{tabular}

$\begin{array}{ccc} & & \\ \text { SODIUN } & \text { POTAS- } & \text { BICAR- } \\ \text { ADN } & \text { SISAT, } & \text { WATER } \\ \text { SORP- } & \text { DIS } & \text { WHA IT } \\ \text { TION } & \text { SOLVED } & \text { FIELD } \\ \text { RATIO } & (\text { MG } / L & \text { MG/L AS } \\ & \text { AS K) } & \text { HCO3 } \\ (00931) & (00935) & (00450)\end{array}$
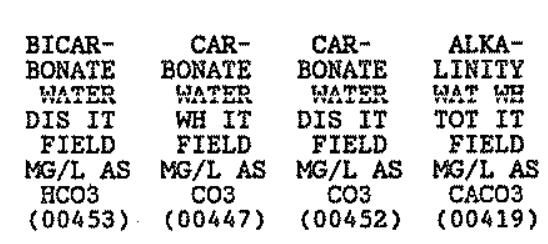

$\begin{array}{cccc}\text { OCT } 1995 & & & \\ 05 \ldots 9 & 44 & 7.6 & 24 \\ \text { FEB } 1996 & 45 & 7.6 & 25 \\ 15 \ldots & 45 & 7.8 & 26 \\ \text { MAY } \ldots & 44 & 8.1 & 26 \\ \text { AUG } \ldots & 44 \ldots & 48 & 8.1\end{array}$

$\begin{array}{ll}0.9 & 3.3 \\ 0.9 & 2.9 \\ 0.9 & 3.1 \\ 0.9 & 3.6\end{array}$

154

$-$

(00452)

$\begin{array}{ll}9.6 & 80 \\ 4.5 & 76 \\ 9.1 & 79 \\ 5.5 & 74\end{array}$


ANALYSES OF SAMRLES COLLECTED AT WATER-QUALITY PARTIAL-RICORD STATIONS AND MISCELLANEOUS SITES

WATER-QUALITY DATA, WATER YEAR OCTOBER 1995 TO SERTEMBER 1996

RIO GRANDE BASIN - - Continued

350547106411610 - ALBUQUERQUE RSD BLW GATE N, OF CERTRAL BLVD, NM

\begin{tabular}{|c|c|c|c|c|c|c|c|c|c|c|}
\hline DATE & $\begin{array}{c}\text { ALKA- } \\
\text { LINITY } \\
\text { WAT DIS } \\
\text { TOT IT } \\
\text { FIELD } \\
\text { WG/L AS } \\
\text { CACOS } \\
(39086)\end{array}$ & $\begin{array}{c}\text { ALKA- } \\
\text { LINITY } \\
\text { LAB } \\
\text { (MG/L } \\
\text { AS } \\
\text { CACO3) } \\
(90410)\end{array}$ & $\begin{array}{c}\text { SULFATE } \\
\text { DIS- } \\
\text { SOLVED } \\
(M G / L \\
\text { AS SO4) } \\
(00945)\end{array}$ & $\begin{array}{l}\text { CHLO- } \\
\text { RIDE, } \\
\text { DIS- } \\
\text { SOLVED } \\
\text { (MG/L } \\
\text { AS CL) } \\
(00940)\end{array}$ & $\begin{array}{c}\text { SOLIDS } \\
\text { RESIDUE } \\
\text { AT } 180 \\
\text { DEG. C } \\
\text { DIS- } \\
\text { SOLVED } \\
\text { (MG/L) } \\
(70300)\end{array}$ & $\begin{array}{c}\text { SOLIDS, } \\
\text { SUM OF } \\
\text { CONSTI- } \\
\text { TUENTS, } \\
\text { DIS- } \\
\text { SOLVED } \\
\text { (MG/L) } \\
\text { (70301) }\end{array}$ & $\begin{array}{l}\text { RESIDUE } \\
\text { TOTAL } \\
\text { AT 105 } \\
\text { DEG. C, } \\
\text { SUS- } \\
\text { PENDED } \\
(\text { MG/L) } \\
(00530)\end{array}$ & $\begin{array}{c}\text { CYANIDE } \\
\text { TOTAL. } \\
\text { (MG/L } \\
\text { AS CN) } \\
(00720)\end{array}$ & $\begin{array}{l}\text { ALUM- } \\
\text { INUM, } \\
\text { TOTAL } \\
\text { RECOV- } \\
\text { ERABLE } \\
\text { (UG/L } \\
\text { AS AL) } \\
\text { (01105) }\end{array}$ & $\begin{array}{c}\text { ALUM- } \\
\text { INUM, } \\
\text { DIS- } \\
\text { SOLVED } \\
\text { (UG/L } \\
\text { AS AL) } \\
\text { (01106) }\end{array}$ \\
\hline $\begin{array}{l}1995 \\
51996\end{array}$ & -- & 1.25 & 57 & 7.4 & 239 & 219 & 21 & $<0.010$ & 690 & 2.0 \\
\hline & 126 & 131 & 56 & 9.5 & 252 & 221 & 8 & $<0.010$ & 60 & 1.0 \\
\hline & -- & 130 & 62 & 8.7 & 242 & 229 & 1 & $<0.010$ & 1400 & $<1.0$ \\
\hline & 122 & 130 & 73 & 7.3 & 254 & 239 & 920 & $<0.010$ & 14000 & 2.0 \\
\hline
\end{tabular}

\begin{tabular}{|c|c|c|c|c|c|c|c|c|c|c|}
\hline DATE & $\begin{array}{c}\text { ANTI- } \\
\text { MONY, } \\
\text { DIS:- } \\
\text { SOLVED } \\
\text { (UG/L } \\
\text { AS SE) } \\
\text { (01095) }\end{array}$ & $\begin{array}{c}\text { ARSENIC } \\
\text { TOTAL } \\
\text { (UG/L } \\
\text { AS AS) } \\
(01002)\end{array}$ & $\begin{array}{c}\text { ARSENIC } \\
\text { DIS- } \\
\text { SOLVED } \\
\text { (UG/L } \\
\text { AS AS) } \\
(01000)\end{array}$ & $\begin{array}{l}\text { BARIUM, } \\
\text { DIS- } \\
\text { SOLVED } \\
\text { (UG/L } \\
\text { AS BA) } \\
(01005)\end{array}$ & $\begin{array}{l}\text { BERYL- } \\
\text { LIUM, } \\
\text { DIS - } \\
\text { SOLVED } \\
\text { (UG/L } \\
\text { AS BE) } \\
(01010)\end{array}$ & $\begin{array}{c}\text { CADMIUM } \\
\text { DIS- } \\
\text { SOLVED } \\
\text { (UG/L } \\
\text { AS CD) } \\
(01025)\end{array}$ & $\begin{array}{l}\text { CHRO- } \\
\text { MIUM, } \\
\text { DIS- } \\
\text { SOLVED } \\
\text { (UG/L } \\
\text { AS CR) } \\
(01030)\end{array}$ & $\begin{array}{c}\text { COBALT, } \\
\text { DIS- } \\
\text { SOLVED } \\
\text { (UG/L } \\
\text { AS CO) } \\
(01035)\end{array}$ & $\begin{array}{l}\text { COPPER, } \\
\text { DIS- } \\
\text { SOLVED } \\
\text { (UG/L } \\
\text { AS CU) } \\
(01040)\end{array}$ & $\begin{array}{c}\text { LEAD, } \\
\text { DIS - } \\
\text { SOLVED } \\
\text { (UG/L } \\
\text { AS PB) } \\
(01049)\end{array}$ \\
\hline $\begin{array}{l}\text { OCT } 1995 \\
\text { OS } \\
\text { FEB } 1996\end{array}$ & $<1.0$ & 3 & 3 & 75 & $<1.0$ & $<1,0$ & 2.0 & $<1,0$ & 1,0 & $<1.0$ \\
\hline MAY 15. & $<1.0$ & 3 & 3 & 92 & $<1.0$ & $<1,0$ & 1.0 & $<1.0$ & 1.0 & $<1,0$ \\
\hline${ }_{\text {Aug }}^{21} \ldots$ & $<1.0$ & 3 & 3 & 75 & $<1.0$ & $<1.0$ & 1.0 & $<1.0$ & 1.0 & $<1.0$ \\
\hline 28 & $<1,0$ & 3 & 3 & 95 & $<1,0$ & $<1,0$ & $<1,0$ & $<1.0$ & $<1.0$ & $<1.0$ \\
\hline
\end{tabular}

$\operatorname{OcT} 1995$

FEB 1996

$15 . .$.

MAY $21 .$.

AUG 28

$\begin{array}{cccc} & \text { MANGA- } & \text { NOLYB- } \\ & \text { NESE, } & \text { DENUM, } & \text { NICKEL, } \\ \text { DIS- } & \text { DIS- } & \text { DIS- } \\ \text { SOLVED } & \text { SOLVED } & \text { SOLVED } \\ & (\text { UG } / L & \text { (UG/L } & \text { (UG/L } \\ \text { AS MH) } & \text { AS MO) } & \text { AS NI) } \\ (01056) & (01060) & (01065)\end{array}$

$\begin{array}{cccc}\text { SILVER, } & & & \\ \text { TOTAL, } & \text { SILVER, } & \text { ZINC, } & \text { URANTUM } \\ \text { NATURAL } \\ \text { RECOV- } & \text { DIS } & \text { DIS- } & \text { DIS- } \\ \text { ERABL. } & \text { SOLVED } & \text { SOL.VED } & \text { SOLVED } \\ \text { (UG/L } & (U G / L & \text { (UG/L } & \text { (UG/L } \\ \text { AS AG) } & \text { AS AG) } & \text { AS ZN) } & \text { AS US) } \\ (01077) & (01075) & (01090) & (22703)\end{array}$

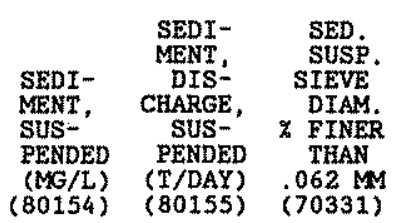

$\begin{array}{rrr}3.0 & 4.0 & <1.0 \\ 6.0 & 4.0 & 1.0 \\ 6.0 & 4.0 & 2.0 \\ <1.0 & 4.0 & 1.0\end{array}$

$\begin{array}{rrr}<1 & <0.20 & 4.0 \\ <1 & <0.20 & <1.0 \\ <1 & <0.20 & 1.0 \\ <1 & <0.20 & <1.0\end{array}$

3.0

$\begin{array}{rc}65 & 23 \\ 14 & 2.9 \\ 145 & -- \\ 1080 & 254\end{array}$

75

65

64

93 
ANALYSES OF SAMPLES COLLECTED AT WATER-QUALITY PARTIAL-RECORD STATIONS AND MISCELLANEOUS SITES

WATER-QUALITY DATA, WATER YEAR OCTOBER 1995 TO SEPTEMBER 1996

RIO GRANDE BASIN - - Continued

351153106383510 - UPPER CORRALES RSD AT MOUTH NEAR CORRALES, NM

$\begin{array}{cl}\text { DIS- } & \\ \text { CHARGE, } & \text { SPE- } \\ \text { INST } & \text { CIFIC } \\ \text { CUBIC } & \text { CON- } \\ \text { FEET } & \text { DUCT- } \\ \text { PER } & \text { ANCE } \\ \text { SECOND } & \text { (US/CM) } \\ (00061) & (00095)\end{array}$
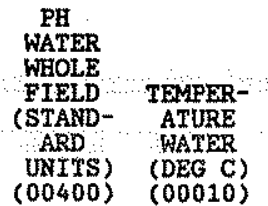

BARO-

METRIC

PRES-

SURE

OF

HG)

$(00025)$

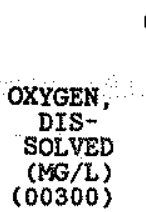

OXYGEN,
DIS-
SOLVED
(PER-
CENT
SATUR-
AIION)
(00301)

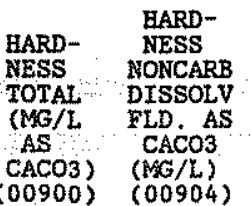

OCT 1995

FEB 1996

MAY

$21 \ldots$

$1330 \quad 36$

375

77.9

$17.5 \therefore 630$

$8.5 \quad 108$

(00904)

AUG $28 \ldots$

$1400 \quad 21$

374

$13.5 \quad 633$

11.4

132

$13.5 \quad 638$

7.1

379

7.9

20.5

642

6.0

82

80

$\begin{array}{rr}130 & -19 \\ 140 & - \\ 140 & - \\ 150 & 48\end{array}$

$\begin{array}{cccc}\text { OCT } 1995 & & & \\ 04.96 & 41 & 6.9 & 23 \\ \text { FEB } 1996 & 44 & 7.1 & 23 \\ \underset{14}{M A} & 44 & 7.6 & 24 \\ \underset{21}{21 .} & 43 & 7.9 & 23 \\ 28 \ldots & 47 & & \end{array}$

47

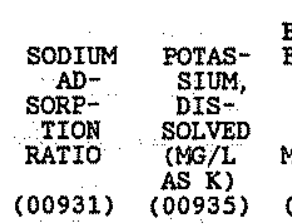

BICAR-
BONATE
WATER
WH IT
FIELD
MG/L AS
HCO 3
$(00450)$

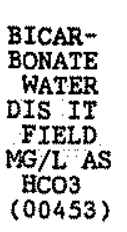

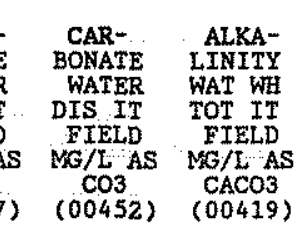

$\begin{array}{lllllll}0.9 & 3.2 & 142 & - & 0 & -- & 116 \\ 0.8 & 3.0 & 134 & 130 & 7 & 8 & 122 \\ 0.9 & 3.1 & 122 & -m & 0 & \cdots & 100 \\ 0.8 & 3.3 & -\cdots & 124 & - & 0 & --\end{array}$
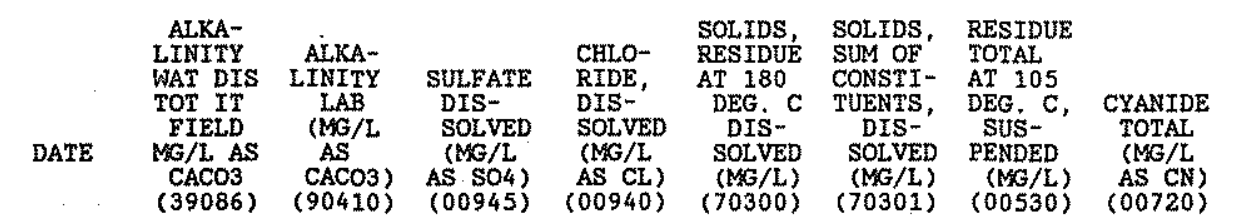

ALUM-

INUM, ALUM-

TOTAL INUM,

RECOV - DIS:

ERABLE SOLVED

(UG/L) (UG/L

AS AL) AS AL)

OCT 1995

FEB $19 \dot{9} 6$

14 .

MAY 21.

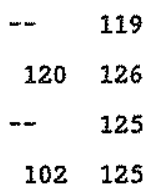

55

$7.0 \quad 235$

206

$20<0.010$

570

2.0

$8.6 \quad 239$

210

$6<0.010$

40

4.0

AUG 28 .

$102 \quad 125$

58

8. 3

210

55

$<0.010$

750

$<1.0$

$6.4 \quad 242$

220

456

$<0.010$

5600

2.0

ANTI-

DIS

DIS-

DATE

(UG/L

$\mathrm{AS} S \mathrm{SB})$
$(01095)$

ARSENIC
TOTAL
(UG/L
AS AS)
$(01002)$

ARSENTC DARIUA,
DIS- DIS-

SOLVED SOLVED

(UG/L (UG/L
AS CO) AS CII)

As CO) (
$(01000) \quad(01005)$
BERYL-

LIUA,

DIS-

SOLVED

(UG/L

AS $P B$ )

$(01010)$

\begin{abstract}
$<1.0$
\end{abstract}
$<1.0$

$<1.0$

CADMTILA
DIS--
SOLVED
(UG/L
(01025)

CERO-

CALRO-
MIIS
DIS-

DIS- DIS-

(UG/L

CORPER, LEAD,

SOLVED SOLVED

(01025) (01030) (01035) (01040) (01049)

$1.0<1.0$

$1.0<1.0$

$1.0<1.0$

OCT 1995

FEB 19496

$\begin{array}{llll}<1.0 & 3 & 3 & 78 \\ <1.0 & 3 & 3 & 86 \\ <1.0 & 2 & 2 & 74 \\ <1.0 & 3 & 3 & 99\end{array}$

$<1.0$
$<1.0$
$<1.0$
$<1.0$

$\begin{aligned} 1.0 & <1.0 \\ 1.0 & <1.0 \\ <1.0 & <1.0 \\ <1.0 & <1.0\end{aligned}$

MAY

$21 \ldots$
AUG
$28 \ldots$

$<1.0$

$<1.0$

$1.0<1.0$ 
ANALYSES OF SAMPLES COLLECTED AT WATER-QUALITY PARTIAL-RECORD STATIONS AND MISCELLANEOUS STTES

WATER-QUALITY DATA, WATER YEAR OCTOBER 1995 TO SERTEMBER 1996

RIO GRANDE BASIN -- Continued

351153106383510 - UPPER CORRALES RSD AT MOUTH NEAR CORRALES, NM

DATE

SILVER.

IJRANIUM

NESE, DENUM, NICKEL, TOTAL SILVER,

DTS- DTS-

RECOV-

DIS-

DIS

MATURAI

(UG/L

(UG $/ \mathrm{L}$

SOLVED

DIS-

(UTG/L (UG/L

(UG/L) (UG/L

AS AG) AS AG)

AS ZN)

As U)

(01090) (

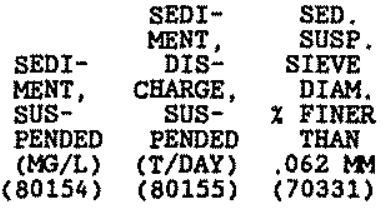

OCT 1995

FEB 1996

$(01056)$

$(01060) \quad(01065)$

$\begin{array}{lrrrrrr}4.0 & 4.0 & 1.0 & <1 & <0.20 & 1.0 & 2.0 \\ 44 & 4.0 & <1.0 & <1 & <0.20 & <1.0 & 3.0 \\ 12 & 4.0 & 2.0 & <1 & <0.20 & 3.0 & 3.0 \\ 3.0 & 4.0 & 2.0 & <1 & <0.20 & <1.0 & 2.0\end{array}$

53

5.275

$14 \ldots$

$21 .$.

28 ...

3.0

4.0

2.0

$<1.0$

2. 0

12

$0.66 \quad 71$

351533106354610 - RIO RANCHO WASTEWATER TREATMENT PLANT NO.2 OUTFALL, MM

$\begin{array}{cl}\text { DIS- } & \\ \text { CHARGE, } & \text { SPE- } \\ \text { IN } & \text { CTFIC } \\ \text { CUBIC } & \text { CON- } \\ \text { FEET } & \text { DUCT- } \\ \text { PER } & \text { ANCE } \\ \text { SECOND } & \text { (US/CM) } \\ (00060) & (00095)\end{array}$
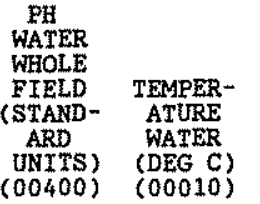

BARO-

METRIC

METRIC

OXYGEN,
DIS-
SOLVED
(MG/L)
$(00300)$

OXYGEN,

HARD- KARD-

DOLSS

$\begin{array}{lll}\text { SPER- } & \text { NOSS NOLCARB } \\ & \text { DISSOLV }\end{array}$

SURE
(MM OXYGEN
DIS-

OF

HG)

SATUR-

$(00301)$

$(M G / L$

$\mathrm{AS}$
$\mathrm{CACO})$
$(\mathrm{MG} / \mathrm{L})$

FLD. AS

$\begin{array}{ll}\text { CACO3) } & (\mathrm{MG} / \mathrm{L}) \\ (00900) & (00904)\end{array}$

OCT 1995

FEB 19696

$16 \ldots$

1000

$2.8 \quad 707$

$7.0 \quad 23.0 \quad 640$

$6.2 \cdot 87$

$22, \ldots$

0900

3.0

712

17.0

6.7

0900

1.1766

7.3

23.5

641

2.5

775

7.5

28.0

636
643

$\begin{array}{cr}6.7 & 83 \\ -- & -\end{array}$

$\begin{array}{rrr}47 & -- \\ 38 & 0 \\ 46 & -- \\ 53 & & 0\end{array}$
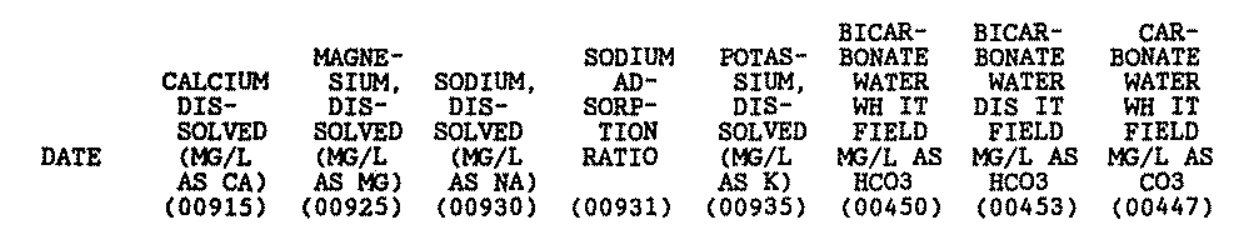

CAR- ALKA-

LNATE LINITY

DIS IT TOT IT

FIELD

$M G / L$ AS $M G / L$ AS

CO3 CACO3

15

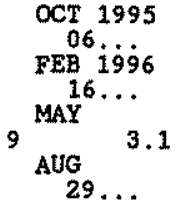

15
12

$2.3 \quad 120$

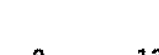

\section{3}

154

$2.0 \quad 130$

17

$2.6 \quad 140$

913

154

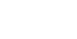

(2047)

(00419)

\begin{tabular}{|c|c|c|c|c|c|c|c|c|c|c|}
\hline DATE & $\begin{array}{l}\text { ALKA- } \\
\text { LINITY } \\
\text { WAT DIS } \\
\text { TOT IT } \\
\text { FIELD } \\
\text { MG/L AS } \\
\text { CACO3 } \\
(39086)\end{array}$ & $\begin{array}{c}\text { ALKA- } \\
\text { LINITY } \\
\text { LAB } \\
\text { (MG/L } \\
\text { AS } \\
\text { CACO3) } \\
(90410)\end{array}$ & $\begin{array}{c}\text { SULFATE } \\
\text { DIS- } \\
\text { SOLVED } \\
\text { (MG/L } \\
\text { AS SO4) } \\
(00945)\end{array}$ & $\begin{array}{l}\text { CHLO- } \\
\text { RIDE, } \\
\text { DIS- } \\
\text { SOLVED } \\
\text { (NG/L } \\
\text { AS CL) } \\
(00940)\end{array}$ & $\begin{array}{c}\text { SOL.IDS, } \\
\text { RESTDUE } \\
\text { AT } 180 \\
\text { DEG. C } \\
\text { DIS.- } \\
\text { SOLVED } \\
(M G / L) \\
(70300)\end{array}$ & $\begin{array}{c}\text { SOLIDS, } \\
\text { SUM OF } \\
\text { CONSTI- } \\
\text { TUENTS, } \\
\text { DIS } \\
\text { SOLVED } \\
\text { (MG/L) } \\
\text { (70301) }\end{array}$ & $\begin{array}{l}\text { RESIDUE } \\
\text { TOTAL. } \\
\text { AT IOS } \\
\text { DEG. C, } \\
\text { SUS- } \\
\text { PENDED } \\
\text { (MG/L) } \\
(00530)\end{array}$ & $\begin{array}{c}\text { CYANIDE } \\
\text { TOTAL } \\
\text { (MG/L } \\
\text { AS CN }) \\
(00720)\end{array}$ & $\begin{array}{l}\text { ALUM- } \\
\text { INUM, } \\
\text { TOTAL } \\
\text { RECOV- } \\
\text { ERABLE } \\
\text { (UG/L } \\
\text { AS AL) } \\
\text { (0110S) }\end{array}$ & $\begin{array}{l}\text { ALUM- } \\
\text { INUM, } \\
\text { DIS- } \\
\text { SOLVED } \\
\text { (UG/L. } \\
\text { AS AL) } \\
\text { (01106) }\end{array}$ \\
\hline $\begin{array}{l}\text { OCT } 1995 \\
\text { O6 } \\
\text { FEB } 1996\end{array}$ & - & 126 & 80 & 53 & 463 & 359 & 14 & $<0.010$ & 310 & 30 \\
\hline${ }_{M A Y}^{16} \cdots$ & 108 & 114 & 80 & 50 & 474 & 353 & 12 & $<0.010$ & 290 & 29 \\
\hline${ }_{U G}^{22} \ldots$ & $m-$ & 131 & 94 & 60 & 480 & 390 & 3 & $<0.010$ & 150 & 30 \\
\hline 29. & 167 & 180 & 96 & 56 & 486 & 427 & $<1$ & $<0.010$ & 90 & 42 \\
\hline
\end{tabular}


WATER-QUALITY DATA, WATER YEAR OCTOBER 1995 TO SEPTEMBER 1996

RIO GRANDE BASIN - - Continued

351533106354610 - RIO RANCEO WASTEWATER TREAMMENT PLANT NO.2 OUTFALL, MM

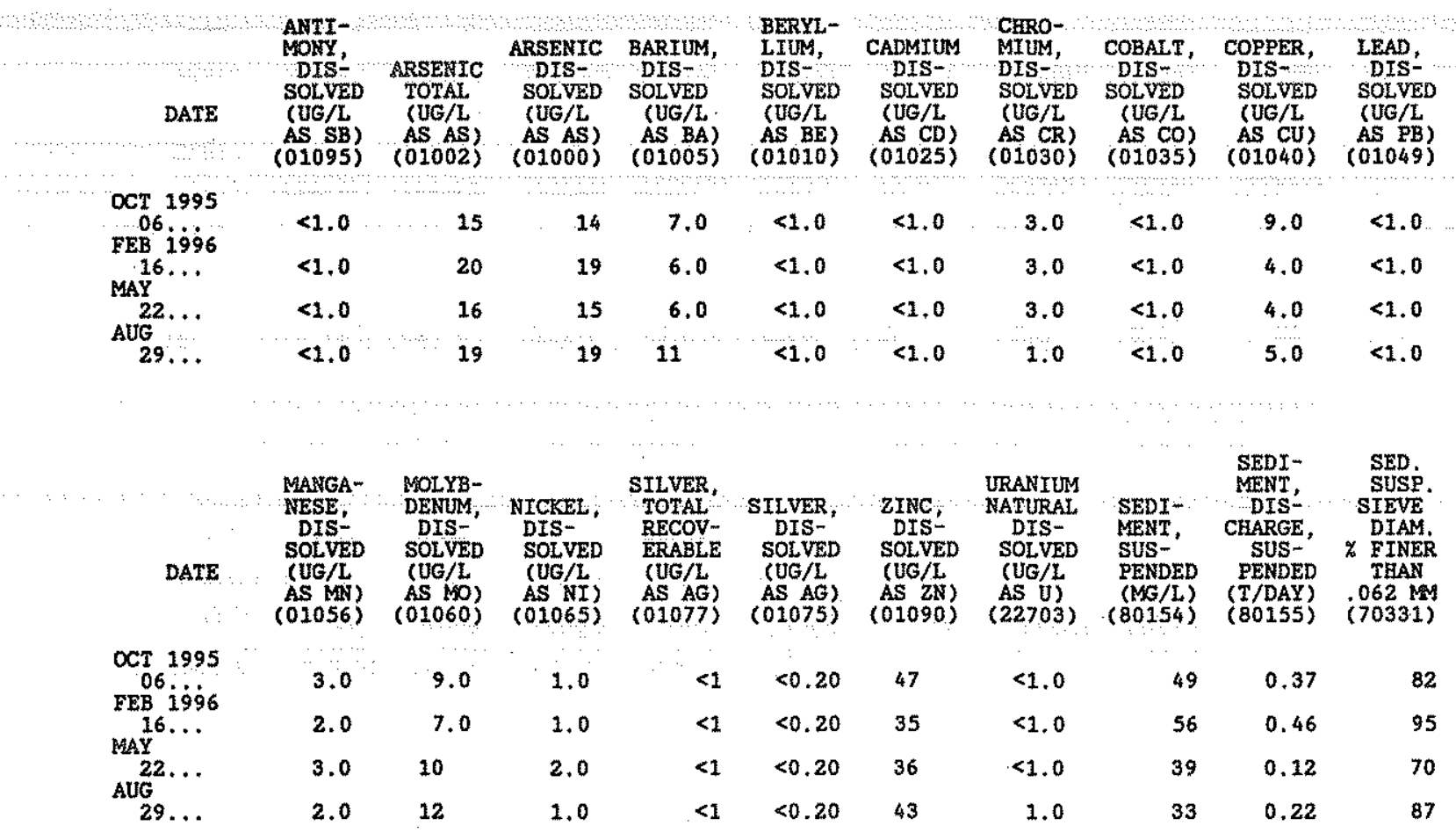

351827106333710 - BERNALILLO WASTEWATER TREATMENT PLANT OUTFALL, NM

\begin{tabular}{|c|c|c|c|c|c|c|c|c|c|c|}
\hline$E$ & TIME & $\begin{array}{c}\text { DIS- } \\
\text { CHARGE, } \\
\text { IN } \\
\text { CUBIC } \\
\text { FEET } \\
\text { PER } \\
\text { SECOND } \\
(00060)\end{array}$ & $\begin{array}{l}\text { SPE- } \\
\text { CIFIC } \\
\text { CON- } \\
\text { DUCT- } \\
\text { ANCE } \\
\text { (US/CM) } \\
(00095)\end{array}$ & $\begin{array}{c}\text { PH } \\
\text { WATER } \\
\text { WHOLE } \\
\text { FIELD } \\
\text { (STAND- } \\
\text { ARD } \\
\text { UNITS) } \\
(00400)\end{array}$ & $\begin{array}{c}\text { TEMPGR- } \\
\text { ATURE } \\
\text { WATER } \\
\text { (DEG C) } \\
(00010)\end{array}$ & $\begin{array}{l}\text { BARO- } \\
\text { METRIC } \\
\text { PRES- } \\
\text { SURE } \\
\text { (MM } \\
\text { OF } \\
\text { HG) } \\
(00025)\end{array}$ & $\begin{array}{c}\text { OXYGEN, } \\
\text { DIS- } \\
\text { SOLVED } \\
(\mathrm{NG} / \mathrm{L}) \\
(00300)\end{array}$ & $\begin{array}{c}\text { OXYGEN, } \\
\text { DIS- } \\
\text { SOLVED } \\
\text { (PER- } \\
\text { CENT } \\
\text { SATUR- } \\
\text { ATION) } \\
\text { (OO301) }\end{array}$ & $\begin{array}{l}\text { HARD- } \\
\text { NESS } \\
\text { TOTAL } \\
\text { (WG/L } \\
\text { AS } \\
\text { CACO3) } \\
(00900)\end{array}$ & $\begin{array}{l}\text { HARD- } \\
\text { NESS } \\
\text { NONCARB } \\
\text { DISSOLV } \\
\text { FLD AS } \\
\text { CACO3 } \\
\text { (NG/L) } \\
(00904)\end{array}$ \\
\hline
\end{tabular}

\begin{tabular}{|c|c|c|c|c|c|c|c|c|c|c|}
\hline OCt 1995 & 0830 & 0.71 & 1420 & 7.2 & 19.0 & 640 & 4.7 & 61 & 250 & - \\
\hline $16 \ldots$ & 0800 & 0.88 & 1390 & 7.3 & 14.0 & 640 & 5.0 & 58 & 240 & 120 \\
\hline $\begin{array}{l}22 \ldots \\
\text { AUG }\end{array}$ & 0800 & 0.64 & $\$ 360$ & 7.5 & 21.5 & 635 & 5.0 & 69 & 240 & $-\infty$ \\
\hline $29 \ldots$ & 0830 & 0.75 & 1270 & 7.6 & 25.0 & 643 & 6.4 & 93 & 230 & 100 \\
\hline DATE & $\begin{array}{c}\text { CALCIUM } \\
\text { DIS- } \\
\text { SOLVED } \\
\text { (NG/L } \\
\text { AS CA) } \\
(00915)\end{array}$ & $\begin{array}{c}\text { MAGNE- } \\
\text { SIUM, } \\
\text { DIS- } \\
\text { SOLVED } \\
\text { (MG/L } \\
\text { AS MG) } \\
(00925)\end{array}$ & $\begin{array}{c}\text { SODIUM, } \\
\text { DIS- } \\
\text { SOLVED } \\
\text { (RGS/L } \\
\text { AS NA) } \\
(00930)\end{array}$ & $\begin{array}{c}\text { SODIUM } \\
\text { AD- } \\
\text { SURF- } \\
\text { TION } \\
\text { RATIO } \\
(00931)\end{array}$ & $\begin{array}{c}\text { POTAS- } \\
\text { SIUM, } \\
\text { DIS- } \\
\text { SOLVED } \\
\text { (MG/L } \\
\text { AS X) } \\
(00935)\end{array}$ & $\begin{array}{c}\text { BICAR- } \\
\text { BONATE } \\
\text { WATER } \\
\text { WE II } \\
\text { FIELD } \\
\text { MG/L AS } \\
\text { HCO3 } \\
\text { (00450) }\end{array}$ & $\begin{array}{c}\text { BICAR- } \\
\text { BONATE } \\
\text { WATER } \\
\text { DIS II } \\
\text { FIELD } \\
\text { BG/L AS } \\
\text { HCO3 } \\
(00453)\end{array}$ & $\begin{array}{c}\text { CAR- } \\
\text { BONATE } \\
\text { WATER } \\
\text { WII II } \\
\text { EIELD } \\
\text { ME/L AS } \\
\text { CO3 } \\
(00447)\end{array}$ & $\begin{array}{c}\text { CAR- } \\
\text { BONATE } \\
\text { WATER } \\
\text { DIS II } \\
\text { FIELD } \\
\text { MGSLAS AS } \\
\text { CO3 } \\
(00452)\end{array}$ & $\begin{array}{l}\text { ALXA- } \\
\text { LINITY } \\
\text { WAT WH } \\
\text { IOT II } \\
\text { FIELD } \\
\text { WG/L AS } \\
\text { CACO3 } \\
(00419)\end{array}$ \\
\hline
\end{tabular}

\begin{tabular}{|c|c|c|c|c|c|c|c|c|c|c|}
\hline $\operatorname{CEt}_{06} 1.995$ & 80 & 12 & 170 & $\stackrel{5}{.}$ & 16 & 177 & - & c & -- & 145 \\
\hline $\begin{array}{l}16 B \\
16 \ldots 96 \\
M A Y\end{array}$ & 77 & 12 & 170 & 5 & 17 & 149 & 150 & 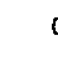 & 0 & 122 \\
\hline $\begin{array}{c}22 . \cdots \\
\text { AUG }\end{array}$ & 77 & 11 & 180 & 5 & 16 & 188 & -- & 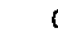 & -- & 154 \\
\hline $29 \ldots$ & 74 & 11 & 170 & 5 & 17 & -- & 153 & $-m$ & c & - \\
\hline
\end{tabular}


ANALYSES OF SAMPLES COLLECTED AT WATER-QUALITY PARTIAL-RECORD STATIONS AND MISCELLANEOUS SITES

WATER-QUALITY DATA, WATER YEAR OCTOBER 1995 TO SEPTEMBER 1996

RIO GRANDE BASTH -- Continued

351827106333710 - BERNALILLO WASTENATER TREATMENT PLANT OUTFALL, NM

\begin{tabular}{|c|c|c|c|c|c|c|c|c|c|c|}
\hline DATE & $\begin{array}{c}\text { ALKA- } \\
\text { LINITY } \\
\text { WAI DIS } \\
\text { TOT IT } \\
\text { FIRLD } \\
\text { WG/L AS } \\
\text { CACO3 } \\
\text { (39086) }\end{array}$ & $\begin{array}{c}\text { ALKA- } \\
\text { LIKITY } \\
\text { LAB } \\
\text { (ME/L } \\
\text { AS } \\
\text { CACO3) } \\
(90410)\end{array}$ & $\begin{array}{c}\text { SULFATE } \\
\text { DIS } \\
\text { SOLVED } \\
(\mathrm{NGG/L} \\
\text { AS SO4) } \\
(00945)\end{array}$ & $\begin{array}{l}\text { CHLO- } \\
\text { RIDE, } \\
\text { DIS- } \\
\text { SOLVED } \\
(\text { HG/L } \\
\text { AS CL, } \\
(00940)\end{array}$ & $\begin{array}{l}\text { SOLIDS, } \\
\text { RESIDUE } \\
\text { AT } 180 \\
\text { DEG.C } \\
\text { DIS-- } \\
\text { SOLVED } \\
(M G / L) \\
(70300)\end{array}$ & $\begin{array}{c}\text { SOLIDS, } \\
\text { SUM OF } \\
\text { CONSTI- } \\
\text { TUENTS, } \\
\text { DIS- } \\
\text { SOLVED } \\
(\mathrm{MG} / \mathrm{L}) \\
(70301)\end{array}$ & $\begin{array}{l}\text { RESIDUE } \\
\text { TOTAL } \\
\text { AT } 105 \\
\text { DEG. C, } \\
\text { SUS- } \\
\text { PENDED } \\
(\mathrm{MGE} / \mathrm{L}) \\
(00530)\end{array}$ & $\begin{array}{c}\text { CYANIDE } \\
\text { TOTAL } \\
\text { (ME/L } \\
\text { AS CN }) \\
(00720)\end{array}$ & $\begin{array}{l}\text { ALUD- } \\
\text { INUM, } \\
\text { TOTAL } \\
\text { RECOV- } \\
\text { BRABLE } \\
\text { (UG/L } \\
\text { AS AL, } \\
\text { (01105) }\end{array}$ & $\begin{array}{l}\text { ALUM- } \\
\text { INUM, } \\
\text { DIS- } \\
\text { SOLVED } \\
\text { (UG/L } \\
\text { AS AL,) } \\
\text { (01106) }\end{array}$ \\
\hline $\begin{array}{l}\text { C7 } 1995 \\
06\end{array}$ & $\rightarrow$ & 147 & 140 & 220 & 860 & 725 & $<1$ & 0.019 & 70 & 48 \\
\hline $16 \ldots$ & 123 & 132 & 140 & 230 & 882 & 719 & 6 & 50.005 & 90 & 19 \\
\hline$U_{U G}^{22} \ldots$ & -- & 160 & 140 & 230 & 834 & 747 & 2 & E0.007 & 80 & 48 \\
\hline & 125 & 137 & 130 & 220 & 796 & 697 & 3 & 0.016 & 60 & 30 \\
\hline
\end{tabular}

\begin{tabular}{|c|c|c|c|c|c|c|c|c|c|c|}
\hline DATE & $\begin{array}{l}\text { ANTI- } \\
\text { MONY, } \\
\text { DIS- } \\
\text { SOLVED } \\
\text { (UG/L } \\
\text { AS SB) } \\
(01095)\end{array}$ & $\begin{array}{c}\text { ARSERIC } \\
\text { TOTAL } \\
\text { (UG/L } \\
\text { AS AS) } \\
(01002)\end{array}$ & $\begin{array}{c}\text { ARSENIC } \\
\text { DIS- } \\
\text { SOLVED } \\
\text { (UG/L } \\
\text { AS AS) } \\
(01000)\end{array}$ & $\begin{array}{c}\text { BARIUM, } \\
\text { DIS- } \\
\text { SOLVED } \\
\text { (UG/L } \\
\text { AS BA) } \\
(01005)\end{array}$ & $\begin{array}{l}\text { BERYL- } \\
\text { LIUM, } \\
\text { DIS- } \\
\text { SOLVED } \\
\text { (UG/L } \\
\text { AS BE) } \\
\text { (01010) }\end{array}$ & $\begin{array}{c}\text { CADMIUM } \\
\text { DIS } \\
\text { SOLVED } \\
\text { (UG/L } \\
\text { AS CD) } \\
\text { (01025) }\end{array}$ & $\begin{array}{l}\text { CHRO- } \\
\text { MIUM, } \\
\text { DIS- } \\
\text { SOLVED } \\
\text { (UG/L } \\
\text { AS CR) } \\
\text { (01030) }\end{array}$ & $\begin{array}{l}\text { COBALT, } \\
\text { DIS- } \\
\text { SOLVED } \\
\text { (UG/L } \\
\text { AS CO) } \\
\text { (01035) }\end{array}$ & $\begin{array}{l}\text { COPPER, } \\
\text { DIS- } \\
\text { SOLVED } \\
\text { (UG/L } \\
\text { AS CU) } \\
\text { (01040) }\end{array}$ & $\begin{array}{c}\text { LEAD, } \\
\text { DIS - } \\
\text { SOLVED } \\
\text { (UG/L } \\
\text { AS PB) } \\
\text { (01049) }\end{array}$ \\
\hline $\begin{array}{l}0 T 1995 \\
066\end{array}$ & $<1.0$ & 16 & 16 & 23 & $<1.0$ & $<1.0$ & 3.0 & $<1.0$ & 3.0 & $<1.0$ \\
\hline $16 \ldots$ & $<1.0$ & 16 & 15 & 22 & $<1.0$ & $<1.0$ & 2.0 & $<1.0$ & 3.0 & $<1.0$ \\
\hline${ }_{1 G}^{A 2} \ldots$ & $<1,0$ & 13 & 12 & 25 & $<1.0$ & $<1.0$ & 3.0 & $<1.0$ & 2.0 & $<1.0$ \\
\hline & $<1.0$ & 15 & 12 & 33 & $<1.0$ & $<1.0$ & $<1.0$ & $<1.0$ & 2.0 & $<1.0$ \\
\hline
\end{tabular}

\begin{tabular}{|c|c|c|c|c|c|c|c|c|c|c|}
\hline DATE & $\begin{array}{l}\text { MANGA- } \\
\text { NESE, } \\
\text { DIS- } \\
\text { SOLVED } \\
\text { (UG/L } \\
\text { AS MN) } \\
\text { (01056) }\end{array}$ & $\begin{array}{l}\text { MOLYB- } \\
\text { DENUM, } \\
\text { DIS- } \\
\text { SOLVED } \\
\text { (UG/L } \\
\text { AS MO) } \\
(01060)\end{array}$ & $\begin{array}{c}\text { MICKEL, } \\
\text { DIS- } \\
\text { SOLVED } \\
\text { (UG/L } \\
\text { AS NI }) \\
(01065)\end{array}$ & $\begin{array}{c}\text { SILVER, } \\
\text { TOTAL } \\
\text { RECOV- } \\
\text { RRABLE } \\
\text { (UG/L } \\
\text { AS AG) } \\
(01077)\end{array}$ & $\begin{array}{c}\text { SILVER, } \\
\text { DIS- } \\
\text { SOLVED } \\
\text { (UG/L } \\
\text { AS AG) } \\
(01075)\end{array}$ & $\begin{array}{l}\text { ZINC, } \\
\text { DIS'- } \\
\text { SOLVED } \\
\text { (UG } / \mathrm{L} \\
\text { AS ZN) } \\
(01090)\end{array}$ & $\begin{array}{l}\text { URANIUM } \\
\text { NATURAL } \\
\text { DIS- } \\
\text { SOLVED } \\
\text { (UG/L } \\
\text { AS U) } \\
\text { (22703) }\end{array}$ & $\begin{array}{l}\text { SEDI- } \\
\text { MENT, } \\
\text { SUS- } \\
\text { PENDED } \\
(M G / L) \\
(80154)\end{array}$ & $\begin{array}{c}\text { SEDI- } \\
\text { MEDNT, } \\
\text { DIS- } \\
\text { CHARGE, } \\
\text { SUS- } \\
\text { PENDED } \\
\text { (T/DAY) } \\
\text { (80155) }\end{array}$ & $\begin{array}{r}\text { SED. } \\
\text { SUSE. } \\
\text { SIEVE } \\
\text { DIAM. } \\
\times \text { FINER } \\
\text { FIHAN } \\
\text { THA MM } \\
(70331)\end{array}$ \\
\hline $\begin{array}{l}2595 \\
06\end{array}$ & 26 & 3.0 & 3.0 & $<1$ & $<0.20$ & 14 & 3.0 & 49 & 0.09 & 81 \\
\hline & 10 & 2.0 & 2.0 & 2 & $<0.20$ & 28 & 2.0 & 67 & 0.16 & 88 \\
\hline $22 \ldots$ & 2.0 & 1.0 & 3.0 & $<1$ & $<0.20$ & 21 & 3.0 & 32 & 0.05 & 79 \\
\hline & 1.0 & 3.0 & 3.0 & $<1$ & $<0.20$ & 34 & 2.0 & 55 & 0.11 & 61 \\
\hline
\end{tabular}

351921106332710 - RIO GRANDE AT HWY 44 AT BERHALILLO, NM

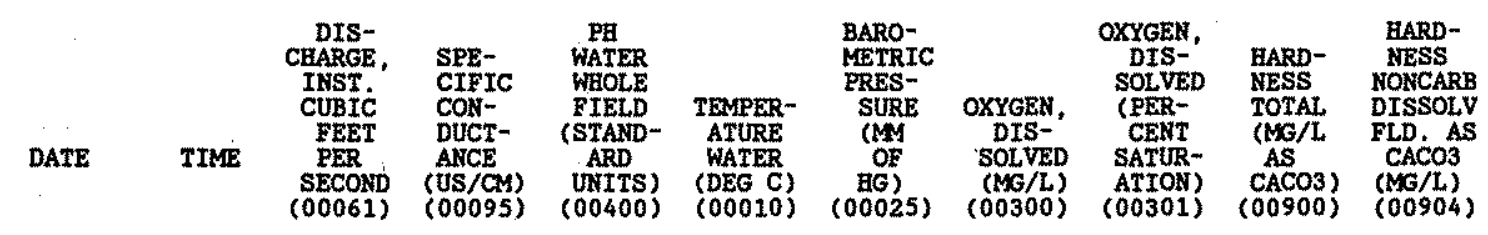

\begin{tabular}{|c|c|c|c|c|c|c|c|c|c|c|}
\hline $\begin{array}{l}\text { OCT } 1995 \\
\text { O2 } \\
\text { FEB } 1996\end{array}$ & 1245 & 806 & 354 & 7.5 & 17.0 & 636 & 8.8 & 110 & 130 & -- \\
\hline $12 \ldots$ & 1300 & 1160 & 355 & 8.3 & 5.0 & 643 & 13.4 & 125 & 130 & 15 \\
\hline${ }_{\mathrm{AUG}}^{19} \ldots$ & 1145 & 599 & 365 & 7.8 & 16.5 & 631 & $m$ & $-\infty$ & 130 & -- \\
\hline $26 \ldots$ & 1130 & 808 & 388 & 7.5 & 22.0 & 640 & 6.7 & 92 & 140 & 44 \\
\hline
\end{tabular}


WATER-QUALITY DATA, WATER YEAR OCTOBER 1995 TO SEPTEMBER 1996

RIO GRANDE BASIN -- Continued

351921106332710 - RIO GRANDE AT HWY 44 AT BERNALILLO, MM

\begin{tabular}{|c|c|c|c|c|c|c|c|c|c|c|}
\hline DATE & $\begin{array}{c}\text { CALCIUM } \\
\text { DIS- } \\
\text { SOLVED } \\
\text { (NAG/L } \\
\text { AS CA) } \\
(00915)\end{array}$ & $\begin{array}{c}\text { MAGHE- } \\
\text { SIUM, } \\
\text { DIS- } \\
\text { SOLVED } \\
\text { (MG/L } \\
\text { AS MS }) \\
\text { (00925) }\end{array}$ & $\begin{array}{c}\text { SODIUM, } \\
\text { DIS- } \\
\text { SOLVED } \\
\text { (MGGL } \\
\text { AS NA) } \\
(00930)\end{array}$ & $\begin{array}{c}\text { SODIUM } \\
\text { AD- } \\
\text { SORP- } \\
\text { TIOND } \\
\text { RATIO } \\
\text { (00931) }\end{array}$ & $\begin{array}{c}\text { POTAS- } \\
\text { SIUM, } \\
\text { DIS- } \\
\text { SOLVED } \\
\text { (MG/L } \\
\text { AS K) } \\
(00935)\end{array}$ & $\begin{array}{c}\text { BICAR- } \\
\text { BONATE } \\
\text { WATER } \\
\text { WH IT } \\
\text { ETELD } \\
\text { MG/L AS } \\
\text { BCO3 } \\
(00450)\end{array}$ & $\begin{array}{l}\text { BICAR- } \\
\text { BONATE } \\
\text { WATER } \\
\text { DIS IT } \\
\text { WIELD } \\
\text { WGLL AS } \\
\text { BCOO3 } \\
(00453)\end{array}$ & $\begin{array}{c}\text { CAR- } \\
\text { BONATE } \\
\text { WATER } \\
\text { WE IT } \\
\text { TIELD } \\
\text { WELA AS } \\
\text { COS } \\
(00447)\end{array}$ & $\begin{array}{c}\text { CAR- } \\
\text { BONATE } \\
\text { WATER } \\
\text { DIS IT } \\
\text { FIELD } \\
\text { ME/L AS } \\
\text { COS } \\
(00452)\end{array}$ & $\begin{array}{c}\text { ALXA- } \\
\text { LINITY } \\
\text { WAT WR } \\
\text { TOT IT } \\
\text { YrELD } \\
\text { MG/L AS } \\
\text { CACO3 } \\
(00419)\end{array}$ \\
\hline $\begin{array}{c}\mathrm{OCT} 1995 \\
02 \ldots\end{array}$ & 39 & 7.1 & 21 & 0.8 & 2.9 & 126 & - & 0 & - & 103 \\
\hline $\begin{array}{l}\mathrm{FEB} 1996 \\
12 \ldots\end{array}$ & 39 & 7.2 & 24 & 0.9 & 3.0 & 138 & 131 & 0 & 3 & 113 \\
\hline $19,$. & 39 & 7.6 & 23 & 0.9 & 3.1 & 143 & $\cdots$ & 0 & $-m$ & 117 \\
\hline $26 \ldots$ & 44 & 7.2 & 25 & 0.9 & 3.3 & -- & 117 & -- & 0 & $-\cdots$ \\
\hline DATE & $\begin{array}{l}\text { ALKA- } \\
\text { LINITY } \\
\text { WAT DIS } \\
\text { TOT IT } \\
\text { FIELD } \\
\text { MG/L AS } \\
\text { CACO3 } \\
(39086)\end{array}$ & $\begin{array}{c}\text { ALKA- } \\
\text { LINITY } \\
\text { IAAB } \\
\text { (MG/L } \\
\text { AS } \\
\text { CACO3) } \\
(90410)\end{array}$ & $\begin{array}{c}\text { SULFATE } \\
\text { DIS- } \\
\text { SOLVED } \\
\text { (NGGL } \\
\text { AS SO4) } \\
(00945)\end{array}$ & $\begin{array}{l}\text { CHLO- } \\
\text { RIDE, } \\
\text { DIS- } \\
\text { SOLVED } \\
\text { (MG/L } \\
\text { AS CL) } \\
(00940)\end{array}$ & $\begin{array}{c}\text { SOLIDS, } \\
\text { RESIDUE } \\
\text { AT } 180 \\
\text { DEG, C } \\
\text { DIS- } \\
\text { SOLVED } \\
(M G / L) \\
(70300)\end{array}$ & $\begin{array}{c}\text { SOLYSS, } \\
\text { SUM OF } \\
\text { CONSTI- } \\
\text { TUENTS, } \\
\text { DIS- } \\
\text { SOLVED } \\
\text { (MG/L) } \\
(70301)\end{array}$ & $\begin{array}{l}\text { RESIDUE } \\
\text { TOTAL } \\
\text { AT } 105 \\
\text { DEG C, } \\
\text { SUS- } \\
\text { PENDED } \\
\text { (MG/L) } \\
(00530)\end{array}$ & $\begin{array}{l}\text { CXANIDE } \\
\text { TOTAL } \\
\text { (MG } / L \\
\text { AS CN) } \\
(00720)\end{array}$ & $\begin{array}{l}\text { ALUM- } \\
\text { INUM, } \\
\text { TOTAL } \\
\text { RECOV- } \\
\text { ERABLE } \\
\text { (UG/L } \\
\text { AS AL) } \\
\text { (01105) }\end{array}$ & $\begin{array}{l}\text { ALUM- } \\
\text { INUM, } \\
\text { DIS- } \\
\text { SOLVED } \\
\text { (UG/L } \\
\text { AS AL) } \\
\text { (O1106) }\end{array}$ \\
\hline OCT 1995 & -- & 113 & 51 & 5.7 & 208 & 189 & 35 & $<0.010$ & 980 & 2.0 \\
\hline${ }_{\mathrm{MAY}} \cdots$ & 112 & 118 & 46 & 9.2 & 232 & 199 & 11 & $<0.010$ & 190 & 18 \\
\hline AUG & - & 120 & 56 & 6.7 & 186 & 206 & 21 & $<0.010$ & 350 & 22 \\
\hline $26, \ldots$ & 96 & 115 & 76 & 9.6 & 182 & 223 & 1930 & $<0,010$ & 23000 & 3.0 \\
\hline DATE & $\begin{array}{c}\text { ANTI- } \\
\text { MONY, } \\
\text { DIS - } \\
\text { SOLVED } \\
\text { (UG/L } \\
\text { AS SB) } \\
(01095)\end{array}$ & $\begin{array}{c}\text { ARSENIC } \\
\text { TOTAL } \\
\text { (UG/L } \\
\text { AS AS) } \\
(01002)\end{array}$ & $\begin{array}{c}\text { ARSENIC } \\
\text { DIS- } \\
\text { SOLVED } \\
\text { (UG/L } \\
\text { AS AS) } \\
\text { (01000) }\end{array}$ & $\begin{array}{l}\text { BARIUR, } \\
\text { DIS- } \\
\text { SOLVED } \\
\text { (UGG/L } \\
\text { AS BA) } \\
(01005)\end{array}$ & $\begin{array}{l}\text { BERYL- } \\
\text { LIUR, } \\
\text { DIS- } \\
\text { SOLVED } \\
\text { (UG/L } \\
\text { AS BE) } \\
(01010)\end{array}$ & $\begin{array}{c}\text { CADMIUM } \\
\text { DIS- } \\
\text { SOLVED } \\
\text { (UG/L } \\
\text { AS CD) } \\
\text { (01025) }\end{array}$ & $\begin{array}{l}\text { CHRO- } \\
\text { MIUM, } \\
\text { DIS- } \\
\text { SOLVED } \\
\text { (UG/L } \\
\text { AS CR) } \\
(01030)\end{array}$ & $\begin{array}{c}\text { COBALT, } \\
\text { DIS- } \\
\text { SOLVED } \\
\text { (UG /L } \\
\text { AS CO) } \\
(01035)\end{array}$ & $\begin{array}{c}\text { COPPER, } \\
\text { DIS- } \\
\text { SOLVED } \\
\text { (UE/L } \\
\text { AS CU) } \\
\text { (01040) }\end{array}$ & $\begin{array}{l}\text { LEAD, } \\
\text { DIS- } \\
\text { SOLVED } \\
\text { (UG/L } \\
\text { AS PB) } \\
\text { (01049) }\end{array}$ \\
\hline $\begin{array}{l}\text { OCT } 1995 \\
02 \\
\end{array}$ & $<1.0$ & 2 & 2 & 77 & $<1.0$ & $<1.0$ & 1.0 & $<1,0$ & $<1.0$ & $<1.0$ \\
\hline${ }_{M A Y}^{12} \cdots$ & $<1.0$ & 2 & 2 & 62 & $<1.0$ & $<1.0$ & 1.0 & $<1.0$ & 1.0 & $<1.0$ \\
\hline AUG $^{19} \cdots$ & $<1.0$ & 2 & 2 & 63 & $<1,0$ & $<1.0$ & 1.0 & $<1.0$ & 1.0 & $<1,0$ \\
\hline $26 \ldots$ & $<1.0$ & 3 & 2 & 86 & $<1.0$ & $<1.0$ & $<1.0$ & $<1.0$ & 1.0 & $<1.0$ \\
\hline
\end{tabular}

\begin{tabular}{|c|c|c|c|c|c|c|c|c|c|c|}
\hline & $\begin{array}{l}\text { MANGA- } \\
\text { RESE, }\end{array}$ & MOLYB & & $\begin{array}{l}\text { SILVER, } \\
\text { TOTAL }\end{array}$ & & zIne, & URA & & $\begin{array}{l}\text { SEDI- } \\
\text { MENT, } \\
\text { DIS- }\end{array}$ & $\begin{array}{l}\text { SED. } \\
\text { SUSE. } \\
\text { SIEVE. }\end{array}$ \\
\hline & $\begin{array}{l}\text { DIS- } \\
\text { SOLVED } \\
\text { (UG/L } \\
\text { AS MN) } \\
\text { (01056) }\end{array}$ & $\begin{array}{c}\text { DIS- } \\
\text { SOLVED } \\
\text { (UC/L } \\
\text { AS MO) } \\
(01060)\end{array}$ & $\begin{array}{l}\text { SOLVED } \\
\text { (UG/Lt } \\
\text { AS NT) } \\
(01065)\end{array}$ & $\begin{array}{l}\text { DECOV- } \\
\text { BRABLE } \\
\text { (UG/L } \\
\text { AS AG) } \\
(01077)\end{array}$ & $\begin{array}{c}\text { DISS- } \\
\text { SOLVED } \\
\text { (UG/L } \\
\text { AS AG) } \\
\text { (01075) }\end{array}$ & $\begin{array}{l}\text { DIS- } \\
\text { SOLVED } \\
\text { (UG/L } \\
\text { AS ZR ) } \\
\text { (01090) }\end{array}$ & $\begin{array}{l}\text { DIS- } \\
\text { SOLVED } \\
\text { (UE/L } \\
\text { AS U) } \\
(22703)\end{array}$ & $\begin{array}{l}\text { SUS - } \\
\text { EENDED } \\
\text { (NS/L) } \\
\text { (80154) }\end{array}$ & $\begin{array}{c}\text { CAAREE } \\
\text { SUS- } \\
\text { PEADED } \\
(\mathrm{T} / \mathrm{DAY}) \\
(80155)\end{array}$ & $\begin{array}{c}x \text { FINNER } \\
\text { IHAN } \\
.062 \text { MA4 } \\
(70331)\end{array}$ \\
\hline
\end{tabular}

OCT 1995

OD 1996
FEB $12 \ldots$
MAY
$19 . \ldots$
AUG $\ldots$
$26 \ldots$

$\begin{array}{rrr}4.0 & 5.0 & <1.0 \\ 9.0 & 4.0 & 1.0 \\ 8.0 & 4.0 & 2.0 \\ <1.0 & 4.0 & 2.0\end{array}$

$\begin{array}{rrr}<1 & <0.20 & 1.0 \\ <1 & <0.20 & 1.0 \\ <1 & <0.20 & 1.0 \\ <1 & <0.20 & <1.0\end{array}$

2.0

$\begin{array}{rrr}68 & 148 & 86 \\ 19 & 60 & 78 \\ 54 & 87 & 73 \\ 2330 & 5090 & 92\end{array}$


ANALYSES OF SAMPLES COLLECTED AT WATER-QUALITY PARTIAL-RECORD STATIONS AND MISCELLANEOUS SITES

CHEMICAL ANALYSES OF ORGANIC COMPOUNDS, WATER YEAR OCTOBER 1995 TO SEPTEMBER 1996

RIO GRANDE BASIN - - Continued

315046106361810 - RIO GRANDE A TX-260 BRIDGE NR SANTA TERESA, NM

\begin{tabular}{|c|c|c|c|c|c|c|c|c|c|c|}
\hline DATE & TIME & $\begin{array}{l}\text { FROP- } \\
\text { CELOR, } \\
\text { WATER, } \\
\text { DISS, } \\
\text { REC } \\
\text { (UG/L) } \\
(04024)\end{array}$ & $\begin{array}{l}\text { BUTYL- } \\
\text { ATE, } \\
\text { WATER, } \\
\text { DISS, } \\
\text { REC } \\
\text { (UG/L) } \\
(04028)\end{array}$ & $\begin{array}{l}\text { SI- } \\
\text { MAZINE, } \\
\text { WATER, } \\
\text { DISS, } \\
\text { REC } \\
\text { (UG/L) } \\
(04035)\end{array}$ & $\begin{array}{l}\text { ERO- } \\
\text { METON, } \\
\text { WATER, } \\
\text { DISS, } \\
\text { REC } \\
\text { (UG/L) } \\
(04037)\end{array}$ & $\begin{array}{l}\text { DEETHYL } \\
\text { ATRA- } \\
\text { ZINE, } \\
\text { WATER, } \\
\text { DISS, } \\
\text { REC } \\
\text { (UG/L) } \\
(04040)\end{array}$ & $\begin{array}{l}\text { CYANA- } \\
\text { ZINE, } \\
\text { WATER, } \\
\text { DISS, } \\
\text { REC } \\
\text { (UG/L) } \\
(04041)\end{array}$ & $\begin{array}{c}\text { FONOFOS } \\
\text { WATER } \\
\text { DISS } \\
\text { REC } \\
\text { (UG/L) } \\
\text { (04095) }\end{array}$ & $\begin{array}{c}\text { ALPHA } \\
\text { BHC } \\
\text { DIS- } \\
\text { SOLVED } \\
\text { (UG/L) } \\
(34253)\end{array}$ & $\begin{array}{c}\text { P,P' } \\
\text { DDE } \\
\text { DISSOLV } \\
\text { (UG/L) } \\
(34653)\end{array}$ \\
\hline $\begin{array}{c}\text { EB } 1996 \\
29 \ldots\end{array}$ & 111.5 & $<0.007$ & $<0.002$ & 0.007 & E0.006 & $<0.002$ & $<0.004$ & $<0.003$ & $<0.002$ & $<0.006$ \\
\hline DATE & $\begin{array}{c}\text { CHLOR- } \\
\text { PYRIFOS } \\
\text { DIS- } \\
\text { SOLVED } \\
\text { (UG/L) } \\
(38933)\end{array}$ & $\begin{array}{l}\text { LINDANE } \\
\text { DIS- } \\
\text { SOLVED } \\
\text { (UG/L) } \\
(39341)\end{array}$ & $\begin{array}{c}\text { DI- } \\
\text { ELDRIN } \\
\text { DIS } \\
\text { SOLVED } \\
\text { (UG/L) } \\
\text { (39.381) }\end{array}$ & $\begin{array}{l}\text { METO- } \\
\text { LACHLOR } \\
\text { WATER } \\
\text { DISSOLV } \\
\text { (UG/L) } \\
\text { (39415) }\end{array}$ & $\begin{array}{l}\text { MALA- } \\
\text { THION, } \\
\text { DIS- } \\
\text { SOLVED } \\
\text { (UG/L) } \\
(39532)\end{array}$ & $\begin{array}{l}\text { PARA- } \\
\text { THION, } \\
\text { DIS- } \\
\text { SOLVED } \\
\text { (UG/L) } \\
\text { (39542) }\end{array}$ & $\begin{array}{c}\text { DI- } \\
\text { AZINON, } \\
\text { DIS- } \\
\text { SOLVED } \\
\text { (UG/L) } \\
(39572)\end{array}$ & $\begin{array}{l}\text { ATRA- } \\
\text { ZINE, } \\
\text { WATER, } \\
\text { DISS, } \\
\text { REC } \\
\text { (UG/L) } \\
(39632)\end{array}$ & $\begin{array}{c}\text { SILVEX, } \\
\text { DIS- } \\
\text { SOLVED } \\
\text { (UG/L) } \\
(39762)\end{array}$ & $\begin{array}{l}\text { ALA- } \\
\text { CEILOR, } \\
\text { WATER, } \\
\text { DISS, } \\
\text { REC, } \\
\text { (UG/L) } \\
(46342)\end{array}$ \\
\hline $\begin{array}{c}\text { EB } 1996 \\
29 \ldots\end{array}$ & $<0,004$ & $<0.004$ & $<0.001$ & E0.004 & $<0,005$ & $<0.004$ & $<0.002$ & $<0.001$ & $<0.021$ & $<0.002$ \\
\hline DATE & $\begin{array}{c}\text { ACETO- } \\
\text { CHLOR, } \\
\text { WATER } \\
\text { FLTRD } \\
\text { REC } \\
(\text { UGG/L) } \\
(49260)\end{array}$ & $\begin{array}{c}\text { METRI- } \\
\text { BUZIN } \\
\text { SENCOR } \\
\text { WATER } \\
\text { DISSOLV } \\
(\text { UG/L) } \\
(82630)\end{array}$ & $\begin{array}{c}\text { 2,6-DI- } \\
\text { ETHYLL } \\
\text { ANILINE } \\
\text { WAT FLT } \\
0,7 \mathrm{U} \\
\text { GF, REC } \\
(\mathrm{UG} / \mathrm{L}) \\
(82660)\end{array}$ & $\begin{array}{c}\text { TRI- } \\
\text { FLUR- } \\
\text { ALIN } \\
\text { WAT FLT } \\
0.7 \mathrm{U} \\
\text { GF, REC } \\
\text { (UG/L) } \\
(82661)\end{array}$ & $\begin{array}{c}\text { ETHAL- } \\
\text { FL.UR- } \\
\text { ALIN } \\
\text { WAT FLT } \\
0.7 \mathrm{U} \\
\text { GE, REC } \\
\text { (UG/L) } \\
\text { (82663) }\end{array}$ & $\begin{array}{l}\text { PHORATE } \\
\text { WATER } \\
\text { FLTRD } \\
0.7 \mathrm{U} \\
\text { GF, REC } \\
\text { (UG/L) } \\
(82664)\end{array}$ & $\begin{array}{c}\text { TER- } \\
\text { BACIL } \\
\text { WATER } \\
\text { FLTRD } \\
0.7 \mathrm{U} \\
\text { GF, REC } \\
(\mathrm{UGG} / \mathrm{L}) \\
(82665)\end{array}$ & $\begin{array}{c}\text { LIN- } \\
\text { URON } \\
\text { WATER } \\
\text { FLTRD } \\
0.7 \mathrm{U} \\
\text { GE, REC } \\
\text { (UG/L) } \\
(82666)\end{array}$ & $\begin{array}{c}\text { METHYL } \\
\text { PARA- } \\
\text { THION } \\
\text { WAT FLT } \\
0.7 \mathrm{U} \\
\text { GF, REC } \\
\text { (UG/L) } \\
(82667)\end{array}$ & $\begin{array}{c}\text { EPTC } \\
\text { WATER } \\
\text { FLTRD } \\
0.7 \mathrm{U} \\
\text { GF, REC } \\
\text { (UG/L) } \\
(82668)\end{array}$ \\
\hline $\begin{array}{l}\text { BB } 1996 \\
29 \ldots\end{array}$ & $<0.002$ & $<0.004$ & $<0.003$ & $<0.002$ & $<0.004$ & $<0.002$ & $<0.007$ & $<0.002$ & $<0.006$ & $<0.002$ \\
\hline DATE & $\begin{array}{c}\text { PEB- } \\
\text { ULATE } \\
\text { WATER } \\
\text { FILTRD } \\
0.7 \mathrm{U} \\
\text { GF, REC } \\
\text { (UG/L) } \\
(82669)\end{array}$ & $\begin{array}{c}\text { TEBU- } \\
\text { THIURON } \\
\text { WATER } \\
\text { FLTRR } \\
0.7 \mathrm{U} \\
\text { GF, REC } \\
\text { (UG/L) } \\
(82670)\end{array}$ & $\begin{array}{c}\text { MOL- } \\
\text { INATE } \\
\text { WATER } \\
\text { FLTRD } \\
0.7 \mathrm{~J} \\
\text { GF, REC } \\
\text { (UG/L) } \\
(82671)\end{array}$ & $\begin{array}{l}\text { ETHO- } \\
\text { PROP } \\
\text { WATER } \\
\text { FLTRD } \\
0.7 \mathrm{U} \\
\text { GF, REC } \\
\text { (UG/L) } \\
(82672)\end{array}$ & $\begin{array}{c}\text { BEN- } \\
\text { FLUR- } \\
\text { ALIN } \\
\text { WAT FLD } \\
0.7 \mathrm{U} \\
\text { GE, REC } \\
\text { (UG/L) } \\
(82673)\end{array}$ & $\begin{array}{c}\text { CARBO- } \\
\text { FURAN } \\
\text { WATER } \\
\text { FLTRD } \\
0.7 \mathrm{U} \\
\text { GF, REC } \\
\text { (UG/L) } \\
(82674)\end{array}$ & $\begin{array}{c}\text { TER- } \\
\text { BUFOS } \\
\text { WATER } \\
\text { FLTRD } \\
0.7 \mathrm{U} \\
\text { GE, REC } \\
\text { (UG/L) } \\
\text { (82675) }\end{array}$ & $\begin{array}{c}\text { FRON- } \\
\text { AMIDE } \\
\text { WATER } \\
\text { FLTRD } \\
0.7 \mathrm{U} \\
\text { GF, REC } \\
\text { (UG/L) } \\
(82676)\end{array}$ & $\begin{array}{c}\text { DISUL- } \\
\text { FOTON } \\
\text { WATER } \\
\text { FLTRD } \\
0.7 \mathrm{U} \\
\text { GF, REC } \\
\text { (UG/L) } \\
(82677)\end{array}$ & $\begin{array}{c}\text { TRIAL- } \\
\text { LATE } \\
\text { WATER } \\
\text { FLTRD } \\
0.7 \mathrm{U} \\
\text { GF, REC } \\
\text { (UG/L) } \\
(82678)\end{array}$ \\
\hline EB 1996 & $<0.004$ & $<0.010$ & $<0.004$ & $<0.003$ & $<0.002$ & $<0.003$ & $<0.013$ & $<0.003$ & $<0.017$ & $<0.001$ \\
\hline
\end{tabular}

\begin{tabular}{|c|c|c|c|c|c|c|c|c|c|}
\hline ATE & $\begin{array}{c}\text { PRO- } \\
\text { PANIL } \\
\text { WATER } \\
\text { FLTRRD } \\
0.7 \text { U } \\
\text { GE, REC } \\
\text { (UGG/L) } \\
\text { (82679) }\end{array}$ & $\begin{array}{c}\text { CAR- } \\
\text { BARYL } \\
\text { WATER } \\
\text { FLTRD } \\
0.7 \mathrm{U} \\
\text { GF, REC } \\
\text { (UGG/L) } \\
(82680)\end{array}$ & $\begin{array}{c}\text { THIO- } \\
\text { BENCARB } \\
\text { WATER } \\
\text { FLTRRD } \\
0.7 \text { U } \\
\text { GF, REC } \\
\text { (UGG/L) } \\
\text { (82681) }\end{array}$ & $\begin{array}{c}\text { DCPA } \\
\text { WATER } \\
\text { FLTRD } \\
0.7 \mathrm{v} \\
\text { GE, REC } \\
\text { (UG/L) } \\
(82682)\end{array}$ & $\begin{array}{l}\text { PENDI- } \\
\text { METH- } \\
\text { ALIN } \\
\text { WAT FLT } \\
0.7 \text { U } \\
\text { GE, REC } \\
\text { (UG /L) } \\
\text { (82683) }\end{array}$ & $\begin{array}{c}\text { NAPROP- } \\
\text { AMIDE } \\
\text { WATER } \\
\text { FLTRD } \\
0.7 \text { U } \\
\text { GF, REC } \\
\text { (UG/L) } \\
(82684)\end{array}$ & $\begin{array}{c}\text { PRO- } \\
\text { PARGITE } \\
\text { WATER } \\
\text { FLTRDD } \\
0.7 \mathrm{U} \\
\text { GE, REC } \\
\text { (UG/L) } \\
\text { (82685) }\end{array}$ & $\begin{array}{c}\text { METHYL } \\
\text { AZIN- } \\
\text { PHOS } \\
\text { WAT FLT } \\
0.7 \mathrm{U} \\
\text { GF, REC } \\
\text { (UG/L) } \\
\text { (82686) }\end{array}$ & $\begin{array}{c}\text { PER- } \\
\text { METHRIN } \\
\text { CIS } \\
\text { WAT FIT } \\
0.7 \mathrm{U} \\
\text { GF, REC } \\
\text { (UG/L) } \\
(82687)\end{array}$ \\
\hline & & & & & & & & & \\
\hline & $<0,004$ & $<0.003$ & $<0.002$ & 0.008 & $<0,004$ & $<0.003$ & $<0.013$ & $<0.001$ & $<0.005$ \\
\hline
\end{tabular}




\begin{tabular}{|c|c|c|c|c|c|c|c|c|c|c|}
\hline DATE & TIME & $\begin{array}{l}\text { PROP-, } \\
\text { CRLOR, } \\
\text { WATER, } \\
\text { DISS, } \\
\text { REC } \\
\text { (UG/L) } \\
\text { (04024) }\end{array}$ & $\begin{array}{l}\text { BUTYL- } \\
\text { ATE, } \\
\text { ATTRE, } \\
\text { DISS, } \\
\text { REC } \\
(\text { UGG } / \mathrm{L}) \\
(04028)\end{array}$ & $\begin{array}{l}\text { SI- } \\
\text { MAZNE, } \\
\text { HATER; } \\
\text { DISS, } \\
\text { REC } \\
\text { (UG/L) } \\
(04035)\end{array}$ & $\begin{array}{l}\text { PRO- } \\
\text { METON, } \\
\text { WATER, } \\
\text { DISS, } \\
\text { REC } \\
\text { (UG/L) } \\
(04037)\end{array}$ & $\begin{array}{c}\text { DEETRYL } \\
\text { ARRA- } \\
\text { ZNAE, } \\
\text { WATER, } \\
\text { DISS, } \\
\text { REC , } \\
\text { (UG/L) } \\
(04040)\end{array}$ & $\begin{array}{c}\text { CYANA- } \\
\text { ZINE, } \\
\text { WATEX, } \\
\text { DISS, } \\
\text { REC } \\
(U G / L) \\
(04041)\end{array}$ & $\begin{array}{c}\text { FONOFOS } \\
\text { WATRR } \\
\text { DISS } \\
\text { REC } \\
\text { (UG/L) } \\
(04095)\end{array}$ & $\begin{array}{c}\text { ALPBA } \\
\text { BEC } \\
\text { DIS- } \\
\text { SoLVED } \\
(\text { UEG/L) } \\
(34253)\end{array}$ & $\begin{array}{c}\mathrm{P}, \mathrm{R}^{\prime} \\
\text { DDE } \\
\text { DISSOV } \\
(\mathrm{UGG/L}) \\
(34653)\end{array}$ \\
\hline $\begin{array}{l}8 \mathrm{~B} 1996 \\
29 \ldots\end{array}$ & 1415 & $<0.007$ & $<0.002$ & 0.007 & E0.005 & $<0,002$ & $<0.004$ & $<0,003$ & $<0.002$ & $<0.006$ \\
\hline DATE & $\begin{array}{c}\text { CHLOR- } \\
\text { PYRIFOS } \\
\text { DIS- } \\
\text { SOLVED } \\
\text { (UG/L) } \\
(38933)\end{array}$ & $\begin{array}{l}\text { LINDANE } \\
\text { DIS- } \\
\text { SOLVED } \\
\text { (UG/L) } \\
\text { (39341) }\end{array}$ & $\begin{array}{c}\text { DI- } \\
\text { ELDRIN } \\
\text { DIS- } \\
\text { SOLVED } \\
\text { (UG/L) } \\
(39381)\end{array}$ & $\begin{array}{l}\text { METO- } \\
\text { LACELOR } \\
\text { WATER } \\
\text { DASSOLV } \\
\text { (UG/L) } \\
(39415)\end{array}$ & $\begin{array}{l}\text { MALA- } \\
\text { THION, } \\
\text { DIS- } \\
\text { SOLVED D } \\
\text { (UG/L) } \\
(39532)\end{array}$ & $\begin{array}{c}\text { PARA- } \\
\text { THION, } \\
\text { DIS- } \\
\text { SOLVED } \\
\text { (UG/L) } \\
(39542)\end{array}$ & $\begin{array}{c}\text { DI- } \\
\text { AZINON, } \\
\text { DIS- } \\
\text { SOLVD } \\
\text { (UG/L) } \\
(39572)\end{array}$ & $\begin{array}{l}\text { ATRA- } \\
\text { ZINEE, } \\
\text { WATRK, } \\
\text { DISS, } \\
\text { REC } \\
\text { (UGG/L) } \\
(39632)\end{array}$ & $\begin{array}{c}\text { SILVEX, } \\
\text { DISE } \\
\text { SOLVED } \\
\text { (UG/L) } \\
(39762)\end{array}$ & $\begin{array}{l}\text { ALA- } \\
\text { CELLR, } \\
\text { WATER, } \\
\text { DISS, } \\
\text { REC, } \\
\text { (UGG/L) } \\
\text { (46342) }\end{array}$ \\
\hline $\begin{array}{c}\text { FEB } 1996 \\
29 \ldots\end{array}$ & $<0.004$ & $<0.004$ & $<0.001$ & E0.004 & $<0.005$ & $<0.004$ & $<0.002$ & $<0.001$ & $<0.021$ & $<0.002$ \\
\hline DATE & $\begin{array}{c}\text { ACETO- } \\
\text { CCLOR, } \\
\text { WATER } \\
\text { FLTRD } \\
\text { REC } \\
(\mathrm{GG} / \mathrm{L}) \\
(49260)\end{array}$ & $\begin{array}{l}\text { METRI- } \\
\text { BUZIN } \\
\text { SENCOR } \\
\text { WATER } \\
\text { DISSOLV } \\
\text { (UG/L) } \\
(82630)\end{array}$ & $\begin{array}{c}2,6-D I- \\
\text { ETHYL } \\
\text { ANILINE } \\
\text { WAT FLT } \\
0.7 \mathrm{U} \\
\text { GF, REC } \\
\text { (UGGLL) } \\
(82660)\end{array}$ & $\begin{array}{l}\text { TRI- } \\
\text { FLUR- } \\
\text { ALIN } \\
\text { WAT FLT } \\
0.7 \text { U } \\
\text { GF, REC } \\
\text { (UGG/L) } \\
(82661)\end{array}$ & $\begin{array}{l}\text { ETHAL- } \\
\text { FLUR- } \\
\text { ALIN } \\
\text { WAT FLT } \\
0.7 \text { U } \\
\text { GE REC } \\
\text { (UG/L) } \\
(82663)\end{array}$ & $\begin{array}{c}\text { PHORATE } \\
\text { WATER } \\
\text { FLTRD } \\
0.7 \mathrm{U} \\
\text { GF REC } \\
\text { (UG/L) } \\
(82664)\end{array}$ & $\begin{array}{l}\text { TER- } \\
\text { BACTL } \\
\text { WATER } \\
\text { FLTRD } \\
0.7 \mathrm{U} \\
\text { GF REC } \\
\text { (UGGL) } \\
(82665)\end{array}$ & $\begin{array}{l}\text { LIN- } \\
\text { URON } \\
\text { WATER } \\
\text { ELTRD } \\
0.7 \text { V } \\
\text { GE, REC } \\
\text { (UGL/) } \\
(82666)\end{array}$ & $\begin{array}{c}\text { METHYL } \\
\text { PARA- } \\
\text { THON } \\
\text { WAT FLT } \\
0.7 \mathrm{~V} \\
\text { GE; REC } \\
\text { (GGGL) } \\
(82667)\end{array}$ & $\begin{array}{c}\text { EPTC } \\
\text { WATRR } \\
\text { FLTRD } \\
0.7 \mathrm{U} \\
\text { GE, REC } \\
\text { (UG/L) } \\
(82668)\end{array}$ \\
\hline $\begin{array}{c}1996 \\
29 \ldots\end{array}$ & $<0.002$ & $<0.004$ & $<0.003$ & $<0.002$ & $<0.004$ & $<0.002$ & $<0.007$ & $<0.002$ & $<0.006$ & $<0.002$ \\
\hline DATE & $\begin{array}{c}\text { PEB- } \\
\text { ULATE } \\
\text { WATER } \\
\text { FILTRD } \\
0.7 \mathrm{U} \\
\text { GE REC } \\
\text { (UE, RE) } \\
(82669)\end{array}$ & $\begin{array}{c}\text { TEBU- } \\
\text { THIURON } \\
\text { WATER } \\
\text { FLTRD } \\
0.7 \mathrm{U} \\
\text { GF, REC } \\
\text { (UG/L) } \\
(82670)\end{array}$ & $\begin{array}{c}\text { MOL- } \\
\text { INATE } \\
\text { WATER } \\
\text { FLTRD } \\
0.7 \mathrm{U} \\
\text { GF, REC } \\
\text { (UG/L) } \\
(82671)\end{array}$ & $\begin{array}{l}\text { ETHO- } \\
\text { PROP } \\
\text { WATER } \\
\text { FLTRD } \\
0.7 \mathrm{U} \\
\text { GF, REC } \\
\text { (UG/L) } \\
(82672)\end{array}$ & $\begin{array}{c}\text { BEN- } \\
\text { FLUR- } \\
\text { ALIN } \\
\text { WAT FLD } \\
0.7 \mathrm{U} \\
\text { GF REC } \\
(\mathrm{UGG} / \mathrm{L}) \\
(82673)\end{array}$ & $\begin{array}{l}\text { CARBO- } \\
\text { FURAN } \\
\text { WATER } \\
\text { FLTRD } \\
0.7 \mathrm{U} \\
\text { GF REC } \\
\text { (UGGLL) } \\
(82674)\end{array}$ & $\begin{array}{l}\text { TER-- } \\
\text { BUFOS } \\
\text { WATER } \\
\text { FLTRD } \\
0.7 \mathrm{U} \\
\text { GF REC } \\
\text { (UG'R) } \\
(82675)\end{array}$ & $\begin{array}{l}\text { PRON- } \\
\text { AMIDE } \\
\text { WMAER } \\
\text { FLTRD } \\
0.7 \mathrm{U} \\
\text { GF, REC } \\
\text { (UGG/) } \\
(82676)\end{array}$ & $\begin{array}{l}\text { DISUL- } \\
\text { FOTON } \\
\text { WATER } \\
\text { FLTRD } \\
0.7 \mathrm{U} \\
\text { GE }, \text { REC } \\
\text { (UGGL) } \\
(82677)\end{array}$ & $\begin{array}{l}\text { TRIAL- } \\
\text { LATE } \\
\text { WATER } \\
\text { FLTRD } \\
0.7 \mathrm{U} \\
\text { GE, REC } \\
(\mathrm{UG} / \mathrm{L}) \\
(82678)\end{array}$ \\
\hline $29 \ldots$ & 0.004 & .010 & $<0.004$ & .003 & 0.002 & .003 & .013 & 303 & 017 & 01 \\
\hline
\end{tabular}

\begin{tabular}{|c|c|c|c|c|c|c|c|c|c|}
\hline DATE & $\begin{array}{c}\text { PRO- } \\
\text { PANIL } \\
\text { WATER } \\
\text { FLTRD } \\
0.7 \mathrm{U} \\
\text { GE, REC } \\
\text { (UG/L) } \\
(82679)\end{array}$ & $\begin{array}{c}\text { CAR- } \\
\text { BARYL } \\
\text { WATER } \\
\text { FLTRD } \\
0.7 \mathrm{y} \\
\text { GE, REC } \\
\text { (UG/L) } \\
(82680)\end{array}$ & $\begin{array}{l}\text { THIO- } \\
\text { BENCARB } \\
\text { WATER } \\
\text { FLTRD } \\
0.7 \mathrm{~V} \\
\text { GE, REC } \\
\text { (UG/L) } \\
(82681)\end{array}$ & $\begin{array}{c}\text { DCPA } \\
\text { WATER } \\
\text { FLTRDD } \\
0.7 \mathbb{Z} \\
\text { GF, REC } \\
\text { (UG/L) } \\
(82682)\end{array}$ & $\begin{array}{l}\text { PENDI- } \\
\text { METHE- } \\
\text { ALIN } \\
\text { WAT FET } \\
0.7 \mathrm{UI} \\
\text { GE, REC } \\
\text { (UG/L) } \\
(82683)\end{array}$ & $\begin{array}{l}\text { NAPROP- } \\
\text { AMIDE } \\
\text { WATER } \\
\text { FLTRD } \\
0,7 \mathrm{U} \\
\text { GF, REC } \\
\text { (UG/L) } \\
(82684)\end{array}$ & $\begin{array}{c}\text { PRO- } \\
\text { PARGTYE } \\
\text { WATER } \\
\text { FLTRD } \\
0.7 \mathrm{U} \\
\text { GF, REC } \\
\text { (UG/L) } \\
(82685)\end{array}$ & $\begin{array}{c}\text { METHYL } \\
\text { AZIII- } \\
\text { PHOS } \\
\text { WAT FLT } \\
0.7 \mathrm{U} \\
\text { GE, REC } \\
\text { (UG/L) } \\
(82686)\end{array}$ & $\begin{array}{c}\text { PER- } \\
\text { RETTRN } \\
\text { CIS } \\
\text { WAT FIT } \\
0.7 \mathrm{U} \\
\text { GE \& REC } \\
\text { (UG/L) } \\
(82687)\end{array}$ \\
\hline & $<0$. & $<0.003$ & $<\tilde{u} . \tilde{0} \bar{Z} \bar{Z}$ & 0.009 & $<0.004$ & $=0.003$ & $<0.01 .3$ & .00 & .005 \\
\hline
\end{tabular}


ANALYSES OF SAMPLES COLLECTED AT WATER-QUALITY PARTTAL-RECORD STATIONS AND MISCELLANEOUS SITES

CHEMICAL ANALYSES OF ORGANIC COMPOUNDS, WATER YEAR OCTOBER 1994 TO SEPTEMBER 1995

RIO GRANDE BASIN - Continued

320610106393110 - DEL RTO DRAIN AT LEVEE ROAD NEAR VADO, NM

\begin{tabular}{|c|c|c|c|c|c|c|c|c|c|c|}
\hline DATE & TrME & $\begin{array}{l}\text { PROP- } \\
\text { CHLOR, } \\
\text { WATER, } \\
\text { DISS, } \\
\text { REC } \\
\text { (UG/L) } \\
(04024)\end{array}$ & $\begin{array}{l}\text { BUTYL- } \\
\text { ATE, } \\
\text { WATER, } \\
\text { DISS, } \\
\text { REC } \\
\text { (UG/L) } \\
(04028)\end{array}$ & $\begin{array}{l}\text { SI- } \\
\text { MAZINE, } \\
\text { WATER, } \\
\text { DISS, } \\
\text { REC } \\
\text { (UG/L) } \\
(04035)\end{array}$ & $\begin{array}{l}\text { FRO- } \\
\text { METON, } \\
\text { WATER, } \\
\text { DISS, } \\
\text { REC } \\
\text { (UG/L) } \\
(04037)\end{array}$ & $\begin{array}{c}\text { DEETHYL } \\
\text { ATRA- } \\
\text { ZINE, } \\
\text { WATER, } \\
\text { DISS, } \\
\text { REC } \\
(\text { UG } / L) \\
(04040)\end{array}$ & $\begin{array}{l}\text { CYANA- } \\
\text { ZINE, } \\
\text { WATER, } \\
\text { DISS, } \\
\text { REC } \\
\text { (UG/L) } \\
(04041)\end{array}$ & $\begin{array}{c}\text { FONOFOS } \\
\text { WATER } \\
\text { DISS } \\
\text { REC } \\
\text { (UG/L) } \\
(04095)\end{array}$ & $\begin{array}{c}\text { ALFHA } \\
\text { BEC } \\
\text { DIS- } \\
\text { SOLVED } \\
\text { (UG/L) } \\
\text { (34253) }\end{array}$ & $\begin{array}{c}\text { P,P' } \\
\text { DDE } \\
\text { DISSOLV } \\
\text { (UG/L) } \\
\text { ( } 34653 \text { ) }\end{array}$ \\
\hline $\begin{array}{c}\text { EB } 1996 \\
28 \ldots\end{array}$ & 1215 & $<0.007$ & $<0.002$ & $\mathrm{E} 0.004$ & 50.006 & $<0,002$ & $<0.004$ & $<0.003$ & $<0.002$ & 50.002 \\
\hline DATE & $\begin{array}{c}\text { CHLOR- } \\
\text { PYRIFOS } \\
\text { DIS- } \\
\text { SOLVED } \\
\text { (UG/L) } \\
(38933)\end{array}$ & $\begin{array}{l}\text { LINDANE } \\
\text { DIS- } \\
\text { SOLVED } \\
\text { (UG/L) } \\
(39341)\end{array}$ & $\begin{array}{c}\text { DI- } \\
\text { BLDRIN } \\
\text { DIS- } \\
\text { SOLVED } \\
\text { (UG/L) } \\
(39381)\end{array}$ & $\begin{array}{l}\text { METO- } \\
\text { LACELOR } \\
\text { WATER } \\
\text { DISSOLV } \\
\text { (UG/L) } \\
(39415)\end{array}$ & $\begin{array}{l}\text { MALA- } \\
\text { THION, } \\
\text { DIS- } \\
\text { SOLVED } \\
(\text { UG/L) } \\
(39532)\end{array}$ & $\begin{array}{l}\text { PARA- } \\
\text { THION, } \\
\text { DIS- } \\
\text { SOLVED } \\
\text { (UG/L) } \\
(39542)\end{array}$ & $\begin{array}{c}\text { DI- } \\
\text { DZINON, } \\
\text { DIS- } \\
\text { SOLVED } \\
\text { (UG/L) } \\
(39572)\end{array}$ & $\begin{array}{l}\text { ATRA- } \\
\text { ZINE, } \\
\text { WATER, } \\
\text { DISS, } \\
\text { REC } \\
\text { (UG/L) } \\
(39632)\end{array}$ & $\begin{array}{c}\text { SILVEX, } \\
\text { DIS- } \\
\text { SOLVAD } \\
\text { (1JG/L) } \\
(39762)\end{array}$ & $\begin{array}{l}\text { AlAA- } \\
\text { CEILOR, } \\
\text { WATER, } \\
\text { DISS, } \\
\text { REC, } \\
\text { (UG/L) } \\
\text { (46342) }\end{array}$ \\
\hline $\begin{array}{c}\text { FEB } 1996 \\
28 \ldots\end{array}$ & $<0.004$ & $<0.004$ & $<0.001$ & 0.005 & $<0.005$ & $<0.004$ & $<0.002$ & $<0.001$ & $<0.021$ & $<0.002$ \\
\hline DATE & $\begin{array}{l}\text { ACETO- } \\
\text { CHLOR, } \\
\text { WATER } \\
\text { FLTRD } \\
\text { REC } \\
\text { (UG/L) } \\
\text { (49260) }\end{array}$ & $\begin{array}{c}\text { METRI- } \\
\text { BUZIN } \\
\text { SENCOR } \\
\text { WATER } \\
\text { DISSOLV } \\
\text { (UG/L) } \\
(82630)\end{array}$ & $\begin{array}{c}2,6-D I- \\
\text { ETHYL } \\
\text { ANILINE } \\
\text { WAT FLT } \\
0.7 \text { U } \\
\text { GF, REC } \\
\text { (UG/L) } \\
(82660)\end{array}$ & $\begin{array}{c}\text { TRI- } \\
\text { FLUR- } \\
\text { ALIN } \\
\text { WAT FLT } \\
0.7 \text { U } \\
\text { GE, REC } \\
\text { (UG/L) } \\
(82661)\end{array}$ & $\begin{array}{c}\text { ETHAL- } \\
\text { FL,UR- } \\
\text { Al,IN } \\
\text { WAT FLT } \\
0.7 \text { U } \\
\text { GF, REC } \\
\text { (UG/L) } \\
(82663)\end{array}$ & $\begin{array}{c}\text { PHORATE } \\
\text { WATER } \\
\text { FLTRD } \\
0.7 \text { U } \\
\text { GF, REC } \\
(\mathrm{UG} / \mathrm{L}) \\
(82664)\end{array}$ & $\begin{array}{c}\text { TER- } \\
\text { BACIL } \\
\text { WATER } \\
\text { FLTRD } \\
0.7 \text { U } \\
\text { GF, REC } \\
\text { (UG/L) } \\
(82665)\end{array}$ & $\begin{array}{l}\text { LIN- } \\
\text { URON } \\
\text { WATER } \\
\text { FLTRD } \\
0.7 \text { U } \\
\text { GE, REC } \\
\text { (UG/L) } \\
(82666)\end{array}$ & $\begin{array}{c}\text { METHYL } \\
\text { PARA- } \\
\text { THION } \\
\text { WAT FLT } \\
0.7 \text { U } \\
\text { GF, REC } \\
\text { (UG/L) } \\
(82667)\end{array}$ & $\begin{array}{c}\text { EPTC } \\
\text { WATER } \\
\text { FLTRD } \\
0.7 \text { U } \\
\text { GF, REC } \\
\text { (1G/L) } \\
(82668)\end{array}$ \\
\hline $\begin{array}{c}\text { FEB } 1996 \\
28 \ldots\end{array}$ & $<0.002$ & $<0.004$ & $<0.003$ & $<0.002$ & $<0.004$ & $<0.002$ & $<0.007$ & $<0.002$ & $<0.006$ & $<0.002$ \\
\hline DATE & $\begin{array}{c}\text { PEB- } \\
\text { ULATE } \\
\text { WATER } \\
\text { FILTRD } \\
0.7 \text { U } \\
\text { GF, REC } \\
\text { (UG/L) } \\
\text { (82669) }\end{array}$ & $\begin{array}{c}\text { TEBU- } \\
\text { THIURON } \\
\text { WATER } \\
\text { FLTRO } \\
0.7 \text { U } \\
\text { GF, REC } \\
\text { (UG/L) } \\
\text { (82670) }\end{array}$ & $\begin{array}{c}\text { MOL- } \\
\text { INATE } \\
\text { WATER } \\
\text { FLTRD } \\
0.7 \text { U } \\
\text { GF, REC } \\
\text { (UG/L) } \\
(82671)\end{array}$ & $\begin{array}{l}\text { ETHO- } \\
\text { PROP } \\
\text { WATER } \\
\text { FLTRD } \\
0.7 \text { U } \\
\text { GF, REC } \\
(\mathrm{UG} / \mathrm{L}) \\
(\mathrm{B2672})\end{array}$ & $\begin{array}{c}\text { BEN- } \\
\text { FLUR- } \\
\text { ALIN } \\
\text { WAT FLD } \\
0,7 \text { U } \\
\text { GF, REC } \\
(\text { UG/L) } \\
(82673)\end{array}$ & $\begin{array}{l}\text { CARBO- } \\
\text { FURAN } \\
\text { WATER } \\
\text { FLTRD } \\
0.7 \text { U } \\
\text { GF, REC } \\
(\mathrm{UG} / \mathrm{L}) \\
(82674)\end{array}$ & $\begin{array}{c}\text { TER- } \\
\text { BUFOS } \\
\text { WATER } \\
\text { FLTRD } \\
0.7 \mathrm{U} \\
\text { GE, REC } \\
\text { (UG/L) } \\
(82675)\end{array}$ & $\begin{array}{l}\text { PRON- } \\
\text { AMIDE } \\
\text { WATER } \\
\text { FLTRD } \\
0.7 \text { U } \\
\text { GF, REC } \\
(\text { UG/L) } \\
(82676)\end{array}$ & $\begin{array}{l}\text { DISUL- } \\
\text { FOTON } \\
\text { WATER } \\
\text { FLTRD } \\
0.7 \text { U } \\
\text { GE. REC } \\
(U G / L) \\
(82677)\end{array}$ & $\begin{array}{l}\text { TRIAL- } \\
\text { LATE } \\
\text { WATER } \\
\text { FLTRD } \\
0.7 \mathrm{U} \\
\text { GF, REC } \\
(\mathrm{UG} / \mathrm{L}) \\
(82678)\end{array}$ \\
\hline $\begin{array}{c}2 \mathrm{~B} 1996 \\
28 \ldots\end{array}$ & 0.004 & $<0.010$ & $<0.004$ & $<0.003$ & $<0.002$ & $<0.003$ & $<0.013$ & $<0.003$ & $<0.017$ & $<0.001$ \\
\hline
\end{tabular}

\begin{tabular}{|c|c|c|c|c|c|c|c|c|c|}
\hline DATE & $\begin{array}{c}\text { PRO- } \\
\text { PANIL } \\
\text { WATER } \\
\text { FLTRD } \\
0.7 \text { U } \\
\text { GE; REC } \\
(U G / L) \\
(82679)\end{array}$ & $\begin{array}{c}\text { CAR- } \\
\text { BARYL } \\
\text { WATER } \\
\text { FLTRD } \\
0.7 \text { U } \\
\text { GF, REC } \\
\text { (UG/L) } \\
(82680)\end{array}$ & $\begin{array}{l}\text { THIO- } \\
\text { BEHCARB } \\
\text { WATER } \\
\text { FLTRD } \\
0.7 \text { U } \\
\text { GF, REC } \\
\text { (UG/L) } \\
\text { (82681) }\end{array}$ & $\begin{array}{c}\text { DCPA } \\
\text { WATER } \\
\text { FLTRD } \\
0.7 \mathrm{U} \\
\text { GE, REC } \\
(\mathrm{UG} / \mathrm{L}) \\
(82682)\end{array}$ & $\begin{array}{l}\text { PENDI- } \\
\text { METH- } \\
\text { ALIN } \\
\text { WAT FLT } \\
0.7 \text { U } \\
\text { GF, REC } \\
(U G / L) \\
(82683)\end{array}$ & $\begin{array}{c}\text { NAPROP- } \\
\text { AMIDE } \\
\text { WATER } \\
\text { FLTRD } \\
0.7 \text { U } \\
\text { GF, REC } \\
\text { (UG/L) } \\
\text { (B2684) }\end{array}$ & $\begin{array}{c}\text { PRO- } \\
\text { PARGITE } \\
\text { WATER. } \\
\text { FLTRD } \\
0.7 \text { U } \\
\text { GF, REC } \\
\text { (UG/L) } \\
\text { (82685) }\end{array}$ & $\begin{array}{l}\text { METHYL } \\
\text { AZIN- } \\
\text { PHOS } \\
\text { HAT FLT } \\
0.7 \text { U } \\
\text { GE, REC } \\
(U G / L) \\
(82686)\end{array}$ & $\begin{array}{c}\text { PER- } \\
\text { METHRIN } \\
\text { CIS } \\
\text { WAT FLT } \\
0.7 \text { U } \\
\text { GF, REC } \\
(\mathrm{UG} / L) \\
(82687)\end{array}$ \\
\hline
\end{tabular}

EAB 1996

$<0.004<0.003<0.002 \quad \mathrm{E} 0.003<0.004<0.003<0.013<0.001<0.005$ 
CHEMTCAL ANALYSES OF ORGANIC COMPOUNDS, WATER YEAR OCTOBER 1994 TO SEPTEMBER 1995

RIO GRAWDE BASIX - - Continued

321317106471510 - RIO GRANDE BELOW MESILIA DAM NR SANTO TOMAS, MM

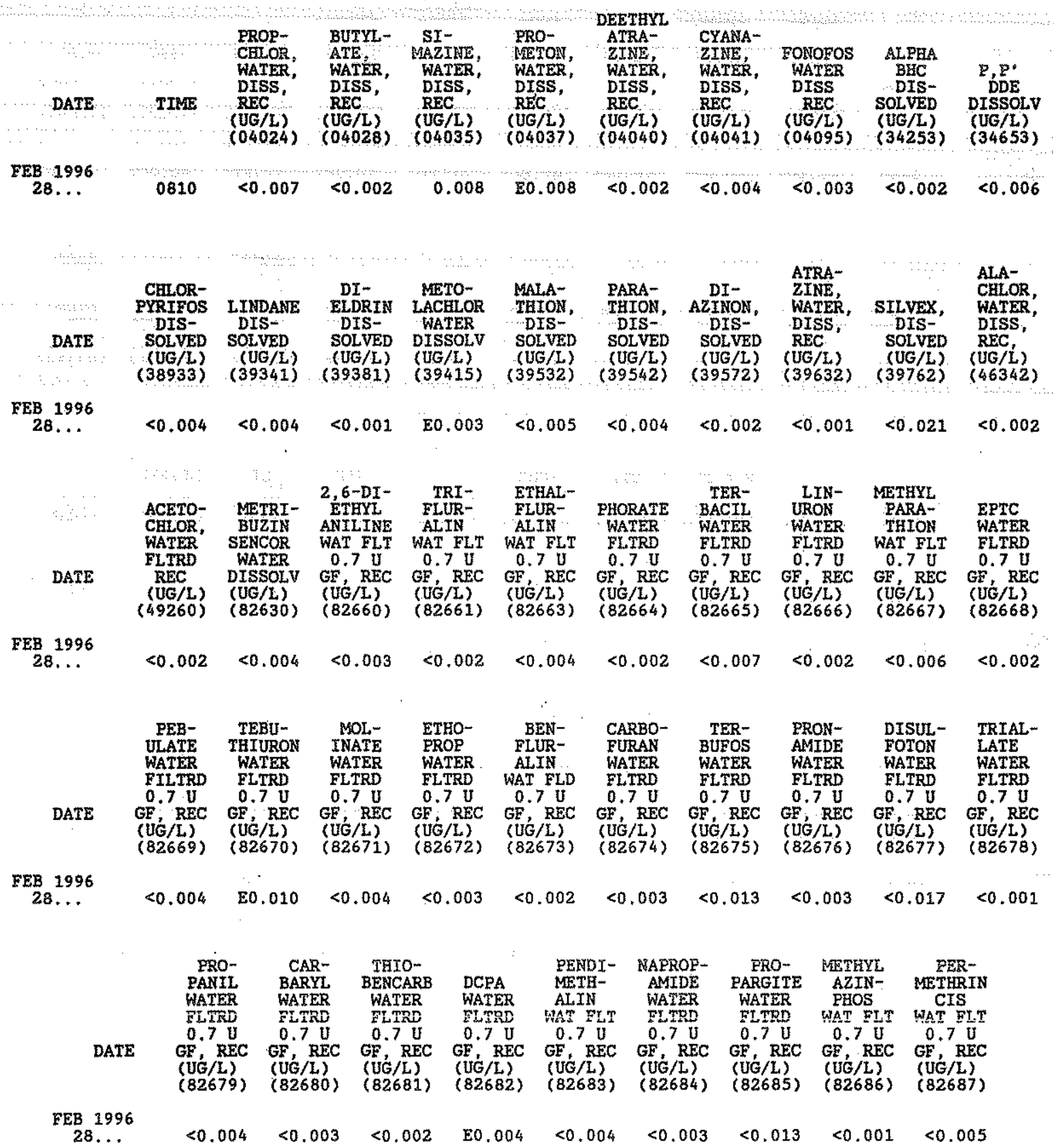


ANALYSES OF SAMPLES COLLECTED AT WATER-QUALITY RARTIAL-RECORD STATIONS AND MISCELLANEOUS SITES

CHDMICAL ANALYSES OF ORGANIC COMPOUIDS, WATER YEAR OCTOBER 1994 TO SEPTEMBER 1995

RIO GRANDE BASIN -- Continued

321745106492510 - RIO GRANDE BELOW PICACBO BRIDGE NR LAS CRUCES,

\begin{tabular}{|c|c|c|c|c|c|c|c|c|c|c|}
\hline DATE & TIME & $\begin{array}{l}\text { FROP- } \\
\text { CEL.OR, } \\
\text { WATER, } \\
\text { DISS, } \\
\text { REC } \\
\text { (UG/L) } \\
(04024)\end{array}$ & $\begin{array}{l}\text { BUTYL- } \\
\text { ATE, } \\
\text { WATKR, } \\
\text { DISS, } \\
\text { REC } \\
(U G / L) \\
(04028)\end{array}$ & $\begin{array}{l}\text { SI- } \\
\text { MAZINE, } \\
\text { WATER, } \\
\text { DISS, } \\
\text { REC } \\
\text { (UG/L) } \\
(04035)\end{array}$ & $\begin{array}{l}\text { PRO- } \\
\text { METON, } \\
\text { WATER, } \\
\text { DISS, } \\
\text { REC } \\
(U G / L) \\
(04037)\end{array}$ & $\begin{array}{c}\text { DEETHYL } \\
\text { ATRA- } \\
\text { ZINE, } \\
\text { WATER, } \\
\text { DISS, } \\
\text { REC } \\
(\text { UG/L) } \\
(04040)\end{array}$ & $\begin{array}{l}\text { CYANA- } \\
\text { ZINE, } \\
\text { WATER, } \\
\text { DISS, } \\
\text { REC } \\
\text { (UG/L) } \\
(04041)\end{array}$ & $\begin{array}{l}\text { FONOFOS } \\
\text { WATER } \\
\text { DISS } \\
\text { REC } \\
\text { (UG/L) } \\
\text { (04095) }\end{array}$ & $\begin{array}{c}\text { ALPEA } \\
\text { BHC } \\
\text { DIS- } \\
\text { SOLVED } \\
\text { (UG/L) } \\
\text { (34253) }\end{array}$ & $\begin{array}{c}\text { P.P' } \\
\text { DDE } \\
\text { DISSOLV } \\
\text { (UG/L) } \\
(34653)\end{array}$ \\
\hline $\begin{array}{l}1996 \\
27 \ldots\end{array}$ & 1245 & $<0.007$ & $<0.002$ & 0.007 & E0.008 & $<0.002$ & $<0.004$ & $<0.003$ & $<0,002$ & $<0.006$ \\
\hline
\end{tabular}

\begin{tabular}{|c|c|c|c|c|c|c|c|c|c|c|}
\hline DATE & $\begin{array}{c}\text { CELOR- } \\
\text { PYRIFOS } \\
\text { DIS- } \\
\text { SOLVED } \\
\text { (UG/L) } \\
(38933)\end{array}$ & $\begin{array}{c}\text { LINDANE } \\
\text { DIS- } \\
\text { SOLVED } \\
\text { (UG/L) } \\
(39341)\end{array}$ & $\begin{array}{c}\text { DI- } \\
\text { ELDRIN } \\
\text { DIS- } \\
\text { SOLVED } \\
\text { (UG/L) } \\
(39381)\end{array}$ & $\begin{array}{l}\text { METO- } \\
\text { LACHLOR } \\
\text { WATER } \\
\text { DISSOLV } \\
\text { (UG/L) } \\
\text { (39415) }\end{array}$ & $\begin{array}{l}\text { MALA- } \\
\text { THION, } \\
\text { DIS- } \\
\text { SOLVED } \\
\text { (UG/L) } \\
(39532)\end{array}$ & $\begin{array}{l}\text { PARA- } \\
\text { THION, } \\
\text { DIS-" } \\
\text { SOLVED } \\
\text { (UG/L) } \\
(39542)\end{array}$ & $\begin{array}{c}\text { DI- } \\
\text { AZINON, } \\
\text { DIS- } \\
\text { SOLVED } \\
\text { (UG/L) } \\
\text { (39572) }\end{array}$ & $\begin{array}{l}\text { ATRA- } \\
\text { ZINE, } \\
\text { WATER, } \\
\text { DISS, } \\
\text { REC } \\
(\mathrm{UG} / \mathrm{L}) \\
(39632)\end{array}$ & $\begin{array}{c}\text { SILVEX, } \\
\text { DIS- } \\
\text { SOLVED } \\
\text { (UG/L) } \\
(39762)\end{array}$ & $\begin{array}{l}\text { ALA- } \\
\text { CHLOR, } \\
\text { WATER, } \\
\text { DISS, } \\
\text { REC, } \\
\text { (UG/Ĺ) } \\
\text { (46342) }\end{array}$ \\
\hline $\begin{array}{l}2 \mathrm{~B} 1996 \\
27 \ldots\end{array}$ & $<0.004$ & $<0.004$ & $<0.001$ & E0.003 & $<0.005$ & $<0.004$ & $<0.002$ & EO:003 & $<0.021$ & $<0.002$ \\
\hline DATE & $\begin{array}{c}\text { ACETO- } \\
\text { CHLOR, } \\
\text { WATER } \\
\text { FLTRD } \\
\text { REC } \\
\text { (UG/L) } \\
(49260)\end{array}$ & $\begin{array}{l}\text { METRI- } \\
\text { BUZIN } \\
\text { SENCOR } \\
\text { WATER } \\
\text { DISSOLV } \\
(U G / L) \\
(82630)\end{array}$ & $\begin{array}{c}2,6-D I- \\
\text { ETHYY } \\
\text { ANILINE } \\
\text { WAT FLT } \\
0.7 \mathrm{U} \\
\text { GF, REC } \\
\text { (UG/L) } \\
(82660)\end{array}$ & $\begin{array}{c}\text { TRI- } \\
\text { FLUR- } \\
\text { ALIN } \\
\text { WAT FLT } \\
0.7 \mathrm{U} \\
\text { GE REC } \\
\text { (UG/L) } \\
\text { (82661) }\end{array}$ & $\begin{array}{l}\text { ETHAL- } \\
\text { FLUR- } \\
\text { ALIN } \\
\text { WAT FLT } \\
0.7 \mathrm{U} \\
\text { GF, REC } \\
\text { (UG/L) } \\
(82663)\end{array}$ & $\begin{array}{l}\text { PHORATE } \\
\text { WATER } \\
\text { FLTRD } \\
0.7 \text { U } \\
\text { GF, REC } \\
\text { (UG/L) } \\
(82664)\end{array}$ & $\begin{array}{c}\text { TER- } \\
\text { BACIL } \\
\text { WATER } \\
\text { FLTRD } \\
0.7 \text { U } \\
\text { GE, REC } \\
\text { (U⿺/L) } \\
(82665)\end{array}$ & $\begin{array}{c}\text { LIN- } \\
\text { URON } \\
\text { WATER } \\
\text { FLTRD } \\
0.7 \text { U } \\
\text { GF, REC } \\
\text { (UG/L) } \\
(82666)\end{array}$ & $\begin{array}{c}\text { METHYL } \\
\text { PARA- } \\
\text { THION } \\
\text { WAT FLT } \\
0.7 \mathrm{U} \\
\text { GF, REC } \\
\text { (UG/L) } \\
(82667)\end{array}$ & $\begin{array}{l}\text { FPTC } \\
\text { WATER } \\
\text { FLTRD } \\
0.7 \mathrm{U} \\
\text { GF, REC } \\
\text { (UGG/L) } \\
(82668)\end{array}$ \\
\hline $\begin{array}{l}\text { EB } 1996 \\
27 \ldots\end{array}$ & $<0.002$ & $<0.004$ & $<0.003$ & $<0.002$ & $<0.004$ & $<0.002$ & $<0.007$ & $<0.002$ & $<0.006$ & $<0.002$ \\
\hline DATE & $\begin{array}{c}\text { PEB- } \\
\text { ULATE } \\
\text { WATER } \\
\text { FILTRD } \\
0.7 \mathrm{U} \\
\text { GF, REC } \\
\text { (UGG/L) } \\
(82669)\end{array}$ & $\begin{array}{c}\text { TEBU- } \\
\text { TEIURON } \\
\text { WATER } \\
\text { FLTRD } \\
0.7 \mathrm{U} \\
\text { GF, REC } \\
\text { (UG／L) } \\
\text { (82670) }\end{array}$ & $\begin{array}{c}\text { MOL- } \\
\text { INATE } \\
\text { WATER } \\
\text { FLTRD } \\
0.7 \mathrm{U} \\
\text { GF, REC } \\
\text { (UG/L) } \\
(82671)\end{array}$ & $\begin{array}{l}\text { ETHO- } \\
\text { PROP } \\
\text { WATER } \\
\text { FLTRD } \\
0.7 \mathrm{U} \\
\text { GF, REC } \\
\text { (UGG/L) } \\
(82672)\end{array}$ & $\begin{array}{c}\text { BEN- } \\
\text { FLUR- } \\
\text { ALIN } \\
\text { WAT FLD } \\
0.7 \mathrm{U} \\
\text { GE REC } \\
\text { (UG/L) } \\
(82673)\end{array}$ & $\begin{array}{c}\text { CARBO- } \\
\text { FURAN } \\
\text { WATER } \\
\text { FLTRD } \\
0.7 \mathrm{U} \\
\text { GF, REC } \\
(\mathrm{UG} / \mathrm{L}) \\
(82674)\end{array}$ & $\begin{array}{c}\text { TER- } \\
\text { BUFOS } \\
\text { WATER } \\
\text { FLTRD } \\
0.7 \mathrm{U} \\
\text { GE, REC } \\
\text { (UGG/L) } \\
\text { (82675) }\end{array}$ & $\begin{array}{c}\text { PRON- } \\
\text { AMIDE } \\
\text { WATER } \\
\text { FLTRD } \\
0.7 \mathrm{U} \\
\text { GE, REC } \\
\text { (UG/L) } \\
(82676)\end{array}$ & $\begin{array}{l}\text { DISUL- } \\
\text { FOTON } \\
\text { WATER } \\
\text { FLTRD } \\
0.7 \mathrm{~V} \\
\text { GE, REC } \\
\text { (UG/L) } \\
(82677)\end{array}$ & $\begin{array}{l}\text { TRIAL- } \\
\text { LATE } \\
\text { WATER } \\
\text { FLTRD } \\
0.7 \mathrm{U} \\
\text { GE: REC } \\
\text { (UG/L) } \\
(82678)\end{array}$ \\
\hline . & & & 004 & co. & 0.002 & 0.003 & 60. & 60. & 0.017 & \\
\hline
\end{tabular}

\begin{tabular}{|c|c|c|c|c|c|c|c|c|c|}
\hline DATE & $\begin{array}{c}\text { PRO- } \\
\text { PANIL } \\
\text { WATER } \\
\text { FLTRD } \\
0.7 \mathrm{U} \\
\text { GF, REC } \\
\text { (UG/L) } \\
(82679)\end{array}$ & $\begin{array}{c}\text { CAR- } \\
\text { BARYL } \\
\text { WATER } \\
\text { FLTRD } \\
0.7 \mathrm{U} \\
\text { GE;REC } \\
\text { (UG/L) } \\
(82680)\end{array}$ & $\begin{array}{c}\text { THIO- } \\
\text { BENCARB } \\
\text { WATERR } \\
\text { FLTRD } \\
0.7 \mathrm{U} \\
\text { GF. REC } \\
\text { (UGG/L) } \\
(82681)\end{array}$ & $\begin{array}{l}\text { DCPA } \\
\text { WATER } \\
\text { FLTRD } \\
0.7 \mathrm{U} \\
\text { GE, REC } \\
\text { (UG/L) } \\
(82682)\end{array}$ & $\begin{array}{l}\text { DENDI- } \\
\text { METH- } \\
\text { ALIN } \\
\text { WAT FLT } \\
0.7 \mathrm{U} \\
\text { GF REC } \\
\text { (UG/L) } \\
(82683)\end{array}$ & $\begin{array}{c}\text { NAEROP- } \\
\text { AMIDE } \\
\text { WATER } \\
\text { FLTRD } \\
0.7 \text { U } \\
\text { GF, REC } \\
(\text { UG/L) } \\
(82684)\end{array}$ & $\begin{array}{c}\text { PRO- } \\
\text { PARGITE } \\
\text { WATER } \\
\text { FLTRD } \\
0.7 \text { U } \\
\text { GE, REC } \\
\text { (UG/L) } \\
\text { (B2685) }\end{array}$ & $\begin{array}{c}\text { METHYL } \\
\text { AZTN- } \\
\text { PHOS } \\
\text { WAT FLT } \\
0.7 \mathrm{~V} \\
\text { GF, REC } \\
(\mathrm{UG} / \mathrm{L}) \\
(82686)\end{array}$ & $\begin{array}{c}\text { PER- } \\
\text { METHRIN } \\
\text { CIS } \\
\text { WAT FLT } \\
0.7 \mathrm{U} \\
\text { GE, REC } \\
\text { (UG/L) } \\
(82687)\end{array}$ \\
\hline $\begin{array}{l}199 \\
27 \ldots\end{array}$ & 0.004 & 0.003 & 0.002 & 0.003 & $<0.004$ & $<0.003$ & 0.013 & $<0.001$ & $<0.005$ \\
\hline
\end{tabular}


CHDICAL, ANALYSES OF ORGANIC COMPOUNDS, WATER YEAR OCTOBER 1995 TO SEPTEMBER 1996

RIO GRANDE BASIN -- Continued

322841106551010 - RIO GRANDE BELON LEASBURG DAM, MM

\begin{tabular}{|c|c|c|c|c|c|c|c|c|c|c|}
\hline DATE & TIHE & 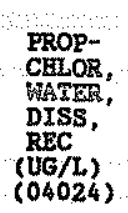 & $\begin{array}{l}\text { BUTYL- } \\
\text { ATE, } \\
\text { WETTE, } \\
\text { DISS, } \\
\text { REC } \\
\text { (UG/L) } \\
(\text { (OA02B) }\end{array}$ & 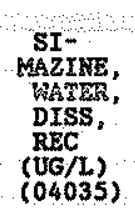 & 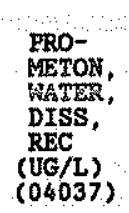 & $\begin{array}{c}\text { DEETHYL } \\
\text { ATRA } \\
\text { ZINE, } \\
\text { WATR, } \\
\text { DISS, } \\
\text { REC, } \\
\text { (UGG/L) } \\
(04040)\end{array}$ & 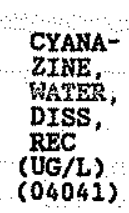 & $\begin{array}{c}\text { FONOFOS } \\
\text { WATES } \\
\text { DISS } \\
\text { REC } \\
(\text { UG/L) } \\
(04095)\end{array}$ & $\begin{array}{c}\text { ALPEA } \\
\text { BEEC } \\
\text { DIS- } \\
\text { SOLVED } \\
(U G / L) \\
(34253)\end{array}$ & $\begin{array}{c}9, p^{\prime} \\
\text { DDE } \\
\text { pISSOV } \\
\text { (UG/L) } \\
\text { (34653) }\end{array}$ \\
\hline $\begin{array}{l}2896 \\
27 \ldots\end{array}$ & 0800 & $<0.007$ & $<0.002$ & 0.008 & E0.008 & E0.003 & $<0.004$ & $<0,003$ & $<0.002$ & $<0.006$ \\
\hline DATE & $\begin{array}{c}\text { CELOR- } \\
\text { PYRIFO } \\
\text { DIS- } \\
\text { SOLVED } \\
\text { (UE/L) } \\
(38933)\end{array}$ & $\begin{array}{l}\text { LINDARE } \\
\text { DDS - } \\
\text { SOLED } \\
\text { (UG/L) } \\
(39341)\end{array}$ & $\begin{array}{c}\text { DI- } \\
\text { ELDIN } \\
\text { DIS - } \\
\text { SOLVED } \\
\text { (GGL) } \\
(39381)\end{array}$ & $\begin{array}{c}\text { METO- } \\
\text { LACROR } \\
\text { GATER } \\
\text { DISSOLV } \\
\text { (UG/) } \\
(39415)\end{array}$ & $\begin{array}{c}\text { MALA- } \\
\text { TMEON, } \\
\text { DIS- } \\
\text { SOLVED } \\
\text { (UG/L) } \\
(39532)\end{array}$ & $\begin{array}{c}\text { PARA- } \\
\text { TEION } \\
\text { DIS- } \\
\text { SOLVDD } \\
(U G / L) \\
(39542)\end{array}$ & $\begin{array}{c}\text { DI- } \\
\text { AZINNON, } \\
\text { DIS } \\
\text { SOLED } \\
(U G / L) \\
(39572)\end{array}$ & $\begin{array}{l}\text { ATRA- } \\
\text { ZINEE, } \\
\text { WATRR, } \\
\text { DISS, } \\
\text { REC } \\
\text { (UG/L) } \\
(39632)\end{array}$ & $\begin{array}{c}\text { SILVEX, } \\
\text { DIS- } \\
\text { SOLVDD } \\
\text { (UG /LI) } \\
(39762)\end{array}$ & $\begin{array}{l}\text { ALA- } \\
\text { CELOR, } \\
\text { WATRR, } \\
\text { DISS, } \\
\text { REC, } \\
\text { (UGG/L) } \\
(46342)\end{array}$ \\
\hline $\begin{array}{l}\text { SB } 1996 \\
27 \ldots\end{array}$ & .004 & $<0.004$ & $<0.001$ & E0,004 & $<0.005$ & $<0.004$ & $<0.002$ & E0.003 & $<0.021$ & $<0.002$ \\
\hline DATE & $\begin{array}{c}\text { ACETO- } \\
\text { CHLOR, } \\
\text { WATERR } \\
\text { FLTRD } \\
\text { REC } \\
(\mathrm{UGG} / \mathrm{L}) \\
(49260)\end{array}$ & $\begin{array}{l}\text { RETRI- } \\
\text { BEUIN } \\
\text { SEEMCOR } \\
\text { WIATRR } \\
\text { DISSOLV } \\
\text { (UG/L) } \\
(82630)\end{array}$ & 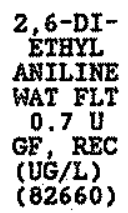 & 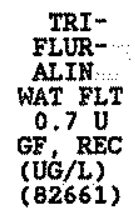 & $\begin{array}{l}\text { ETHAL- } \\
\text { FLUR- } \\
\text { ALIN } \\
\text { WAT FLT } \\
0.7 \mathrm{U} \\
\text { GF REC } \\
(U G / L) \\
(82663)\end{array}$ & $\begin{array}{c}\text { PHORATE } \\
\text { WATER } \\
\text { FLTRD } \\
0.7 \mathrm{U} \\
\text { GF, REC } \\
\text { (UG/L) } \\
(82664)\end{array}$ & 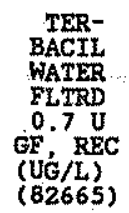 & $\begin{array}{l}\text { LIN- } \\
\text { URON } \\
\text { WATER } \\
\text { ELTRD } \\
0.7 \mathrm{U} \\
\text { GE, REC } \\
(\mathrm{BG} / \mathrm{L}) \\
(82666)\end{array}$ & $\begin{array}{l}\text { METHYL } \\
\text { PARA- } \\
\text { THION } \\
\text { WAT } F L T \\
0.7 \\
\text { GE } \\
\text { GE, REC } \\
\text { (UG/L) } \\
(82667)\end{array}$ & $\begin{array}{c}\text { EPTC } \\
\text { WATER } \\
\text { FLTTD } \\
0.7 \mathrm{U} \\
\text { GE, REC } \\
\text { (UG/L) } \\
(8266)\end{array}$ \\
\hline $\begin{array}{c}F E B \quad 1996 \\
27 \ldots\end{array}$ & $<0.002$ & $<0.004$ & $<0.003$ & $<0.002$ & $<0.004$ & $<0.002$ & $<0.007$ & $<0.002$ & $<0.006$ & $<0.002$ \\
\hline DATE & $\begin{array}{c}\text { PEB- } \\
\text { ULATE } \\
\text { WATER } \\
\text { FILTRD } \\
0.7 \mathrm{U} \\
\text { GF, REC } \\
\text { (UG/L) } \\
(82669)\end{array}$ & $\begin{array}{c}\text { TEBU- } \\
\text { THIURON } \\
\text { WHARR } \\
\text { ELTRD } \\
0.7 \mathrm{U} \\
\text { GF, REC } \\
\text { (U⿺𠃊)L } \\
(82670)\end{array}$ & 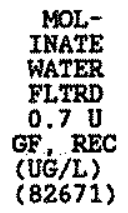 & $\begin{array}{c}\text { ETHO- } \\
\text { RROP } \\
\text { WATR } \\
\text { WLTRD } \\
\text { FLT } \\
0.7 \mathrm{U} \\
\text { GF REC } \\
\text { (UGLL) } \\
(82672)\end{array}$ & $\begin{array}{c}\text { BEN- } \\
\text { FLUR- } \\
\text { AAIN } \\
\text { WAT FLD } \\
0.7 \mathrm{U} \\
\text { GF, REC } \\
\text { (UG }(8 / L) \\
(82673)\end{array}$ & $\begin{array}{c}\text { CARBO- } \\
\text { FURAN } \\
\text { WATRR } \\
\text { FLTRD } \\
0.7 \mathrm{U} \\
\text { GF, REC } \\
\text { (UG/) } \\
(82674)\end{array}$ & $\begin{array}{l}\text { TER- } \\
\text { BUROS } \\
\text { WATRR } \\
\text { FLTRD } \\
0.7 \mathrm{U} \\
\text { GF REC } \\
\text { GÉ RE/L) } \\
(82675)\end{array}$ & $\begin{array}{c}\text { PRON- } \\
\text { AMIDE } \\
\text { AMATER } \\
\text { FLTRD } \\
0.7 \mathrm{U} \\
\text { GF, REC } \\
\text { (UG/L) } \\
(82676)\end{array}$ & 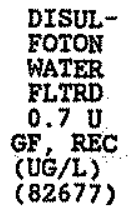 & $\begin{array}{l}\text { TRIAL- } \\
\text { TATE - } \\
\text { WATERR } \\
\text { FLTRD } \\
0.7 \mathrm{U} \\
\text { GE, REC } \\
\text { (UG/L) } \\
(82678)\end{array}$ \\
\hline • & 0.0 & E0,0 & & 0.0 & 0.002 & 0.003 & 013 & $<0.003$ & 0.017 & 0.001 \\
\hline
\end{tabular}

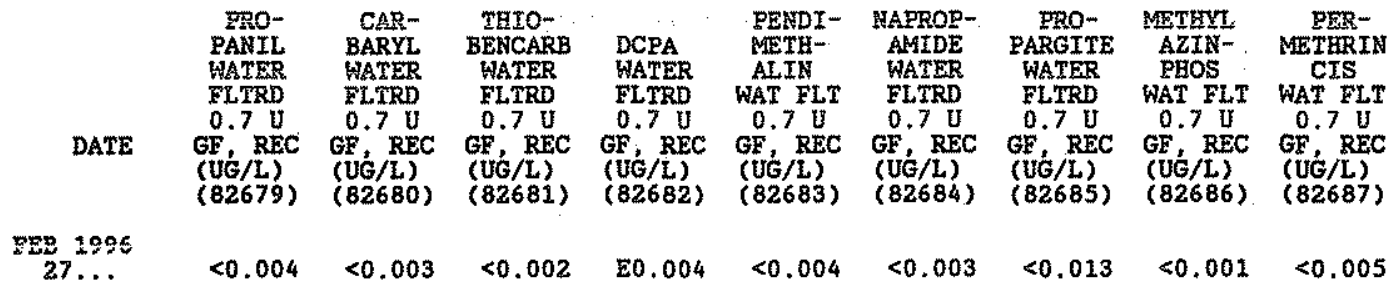


The following water -quality tables for miscellaneous sites in the Tularosa valley Basin are ldentified by 15-digit latitude-longitude sito numbers are in order by ascending site numbers as shown in parenthesis after the site names. The inorganic analyses tables are followed by the organic-compound analyses table for these sites. This departure from the normal domstream order for surface water sites was taken to facilitate locating these sites in this report and for comparing results for the same group of analyses.

330716106234510 - SALT CREBK 3 AT RANGE ROAD 6 ON WSMR, MM

\begin{tabular}{|c|c|c|c|c|c|c|c|c|c|c|c|c|}
\hline DATE & TIME & $\begin{array}{c}\text { DIS- } \\
\text { CHARGE, } \\
\text { INST. } \\
\text { CUBIC } \\
\text { FEET } \\
\text { PER } \\
\text { SECOND } \\
(00061)\end{array}$ & $\begin{array}{l}\text { SPE- } \\
\text { CIFIC } \\
\text { CON- } \\
\text { DUCY- } \\
\text { ANCE } \\
\text { (US/CM) } \\
(00095)\end{array}$ & $\begin{array}{c}\text { PR } \\
\text { WATER } \\
\text { WHOLE } \\
\text { FIELD } \\
\text { (STAND- } \\
\text { ARD } \\
\text { UNTTS) } \\
(00400)\end{array}$ & $\begin{array}{c}\text { TEMPER- } \\
\text { ATURE } \\
\text { ATR } \\
(\mathrm{DEGC}) \\
(00020)\end{array}$ & $\begin{array}{c}\text { TEMPER- } \\
\text { ATURE } \\
\text { MATER } \\
\text { (DEG C) } \\
(00010)\end{array}$ & $\begin{array}{l}\text { BARO- } \\
\text { METRIC } \\
\text { PRES- } \\
\text { SURE } \\
\text { (MM } \\
\text { OF } \\
\text { HG) } \\
(00025)\end{array}$ & $\begin{array}{c}\text { OXYGEN, } \\
\text { DIS- } \\
\text { SOLVED } \\
(M G / L) \\
(00300)\end{array}$ & $\begin{array}{c}\text { OXYGEN, } \\
\text { DIS- } \\
\text { SOLVED } \\
\text { (PER- } \\
\text { CENT } \\
\text { SATUR- } \\
\text { ATION) } \\
\text { (OO301) }\end{array}$ & $\begin{array}{l}\text { EARD- } \\
\text { MESS } \\
\text { TOTAL } \\
\text { (MG/L } \\
\text { AS } \\
\text { CACO3) } \\
(00900)\end{array}$ & $\begin{array}{l}\text { HARD- } \\
\text { NESS } \\
\text { NONCARB } \\
\text { DISSOLV } \\
\text { FLD. AS } \\
\text { CACO3 } \\
(\text { WG/L) } \\
(00904)\end{array}$ & $\begin{array}{l}\text { CALCIUM } \\
\text { DIS- } \\
\text { SOLVED } \\
\text { (MG/L } \\
\text { AS CA) } \\
(00915)\end{array}$ \\
\hline $\begin{array}{c}\text { UL. } 1996 \\
29 \ldots\end{array}$ & 1130 & 0.19 & 26800 & 9.1 & 31.5 & 26.0 & 660 & 12.0 & 188 & 3800 & 3700 & 840 \\
\hline DAPE & $\begin{array}{c}\text { MAGNE- } \\
\text { SIUM, } \\
\text { DIS- } \\
\text { SOLVED } \\
\text { (MG/L } \\
\text { AS MG) } \\
\text { (00925) }\end{array}$ & $\begin{array}{c}\text { SODIUM, } \\
\text { DIS- } \\
\text { SOLVED } \\
\text { (MG/L } \\
\text { AS NA) } \\
(00930)\end{array}$ & $\begin{array}{c}\text { SODIUM } \\
\text { AD- } \\
\text { SORP- } \\
\text { TION } \\
\text { RATIO } \\
(00931)\end{array}$ & $\begin{array}{c}\text { POTAS- } \\
\text { STUM, } \\
\text { DIS- } \\
\text { SOLVED } \\
\text { (MG/L } \\
\text { AS K) } \\
(00935)\end{array}$ & $\begin{array}{c}\text { BICAR- } \\
\text { BONATE } \\
\text { WATER } \\
\text { DIS IT } \\
\text { FIELD } \\
\text { MG/L AS } \\
\text { BCO3 } \\
(00453)\end{array}$ & $\begin{array}{c}\text { CAR- } \\
\text { BONATE } \\
\text { WATER } \\
\text { DIS IT } \\
\text { FIELD } \\
\text { MG/L AS } \\
\text { CO3 } \\
(00452)\end{array}$ & $\begin{array}{c}\text { ALKA- } \\
\text { LINITY } \\
\text { WAT DIS } \\
\text { TOT IT } \\
\text { FIELD } \\
\text { MG/L AS } \\
\text { CACOB } \\
(39086)\end{array}$ & $\begin{array}{c}\text { ALKA- } \\
\text { LINITY } \\
\text { LAB } \\
\text { (MG/L } \\
\text { AS } \\
\text { CACO3) } \\
(90410)\end{array}$ & $\begin{array}{c}\text { SULFATE } \\
\text { DIS- } \\
\text { SOLVED } \\
(M G / L \\
\text { AS SO4) } \\
(00945)\end{array}$ & $\begin{array}{l}\text { CHLO- } \\
\text { RIDE, } \\
\text { DIS- } \\
\text { SOLVED } \\
\text { (NG/L } \\
\text { AS CL) } \\
(00940)\end{array}$ & $\begin{array}{l}\text { FLUO- } \\
\text { RIDE, } \\
\text { DIS- } \\
\text { SOLVGD } \\
\text { (MG/L } \\
\text { AS F) } \\
(00950)\end{array}$ & $\begin{array}{c}\text { BROMTDE } \\
\text { DIS- } \\
\text { SOLVED } \\
\text { (MG/L } \\
\text { AS BR) } \\
(71870)\end{array}$ \\
\hline $\begin{array}{c}\text { UL } 1996 \\
29 \ldots\end{array}$ & 400 & 5300 & 38 & 120 & 21 & 24 & 57 & 47 & 3800 & 8100 & 1.6 & 1.2 \\
\hline
\end{tabular}

\begin{tabular}{|c|c|c|c|c|c|c|c|c|c|c|c|}
\hline DATE & $\begin{array}{l}\text { SILICA, } \\
\text { DIS- } \\
\text { SOLVED } \\
\text { (MG/L } \\
\text { AS } \\
\text { sIO2) } \\
(00955)\end{array}$ & $\begin{array}{l}\text { SOLIDS, } \\
\text { RESTDUE } \\
\text { AT } 180 \\
\text { DEG. C } \\
\text { DIS- } \\
\text { SOLVED } \\
(M G / L) \\
(70300)\end{array}$ & $\begin{array}{c}\text { SOLIDS, } \\
\text { SUM OF } \\
\text { CONSTI- } \\
\text { TUENTS, } \\
\text { DIS- } \\
\text { SOLVED } \\
(\mathrm{WG} / \mathrm{L}) \\
(70301)\end{array}$ & $\begin{array}{c}\text { NITRO- } \\
\text { GEN, } \\
\text { NITRITE } \\
\text { DIS- } \\
\text { SOLVED } \\
(\text { ME /L } \\
\text { AS N) } \\
(00613)\end{array}$ & $\begin{array}{c}\text { NITRO- } \\
\text { GEN, } \\
\text { NO2+NO3 } \\
\text { DIS- } \\
\text { SOLVED } \\
\text { (MO/L } \\
\text { AS N) } \\
(00631)\end{array}$ & $\begin{array}{c}\text { NITRO- } \\
\text { GEN, } \\
\text { AMONIA } \\
\text { DIS- } \\
\text { SOLVED } \\
\text { (MG/L } \\
\text { ASN) } \\
(00608)\end{array}$ & $\begin{array}{c}\text { NITRO- } \\
\text { GEN, } \\
\text { ORGANIC } \\
\text { DIS- } \\
\text { SOLVED } \\
\text { (ME/L } \\
\text { AS H) } \\
(00607)\end{array}$ & $\begin{array}{l}\text { MTTRO- } \\
\text { GEN,AM- } \\
\text { MONIA + } \\
\text { ORGANIC } \\
\text { TOTAL, } \\
\text { (MG/L } \\
\text { AS N) } \\
(00625)\end{array}$ & $\begin{array}{l}\text { NITRO- } \\
\text { GEN,AM- } \\
\text { MONIA + } \\
\text { ORGANIC } \\
\text { DIS. } \\
\text { (MG/L } \\
\text { AS N) } \\
\text { (00623) }\end{array}$ & $\begin{array}{c}\text { PHOS- } \\
\text { PHORtSS } \\
\text { TOTAL } \\
\text { (ME/L } \\
\text { AS E) } \\
(00665)\end{array}$ & $\begin{array}{c}\text { PHOS- } \\
\text { PHORUS } \\
\text { DIS - } \\
\text { SOLVED } \\
\text { (NG/L } \\
\text { AS P) } \\
(00666)\end{array}$ \\
\hline 601 & 1.5 & 19500 & 18600 & $<0.010$ & 0.110 & 0.210 & 0.79 & 1.0 & 1.0 & $<0,010$ & \\
\hline
\end{tabular}

\begin{tabular}{|c|c|c|c|c|c|c|c|c|c|c|c|}
\hline DATE & $\begin{array}{l}\text { PHOS- } \\
\text { PHORUS } \\
\text { ORTHO, } \\
\text { DIS - } \\
\text { SOLVED } \\
(M G / L \\
\text { AS P) } \\
(00671)\end{array}$ & $\begin{array}{l}\text { AL,UM- } \\
\text { INUM, } \\
\text { TOTAL } \\
\text { RECOV- } \\
\text { ERABLE } \\
\text { (UG/L } \\
\text { AS AL) } \\
\text { (01105) }\end{array}$ & $\begin{array}{l}\text { ALUM- } \\
\text { INUM, } \\
\text { DIS- } \\
\text { SOLVED } \\
\text { (UG/L } \\
\text { AS AL) } \\
\text { (01106) }\end{array}$ & $\begin{array}{c}\text { ARSENIC } \\
\text { TOTAL } \\
\text { (UG/L } \\
\text { AS AS) } \\
(01002)\end{array}$ & $\begin{array}{c}\text { ARSENIC } \\
\text { DIS - } \\
\text { SOLVED } \\
\text { (UG/L } \\
\text { AS AS) } \\
(01000)\end{array}$ & $\begin{array}{c}\text { BARIUM, } \\
\text { TOTAL } \\
\text { RECOV- } \\
\text { ERABLE } \\
\text { (UG/L } \\
\text { AS BA) } \\
(01007)\end{array}$ & $\begin{array}{l}\text { BARIUM, } \\
\text { DIS- } \\
\text { SOLVED } \\
\text { (UG/L } \\
\text { AS BA) } \\
(01005)\end{array}$ & $\begin{array}{l}\text { BORON, } \\
\text { TOTAL. } \\
\text { RECOV-. } \\
\text { ERABLE } \\
\text { (UG/L } \\
\text { AS B) } \\
\text { (01022) }\end{array}$ & $\begin{array}{c}\text { BORON, } \\
\text { DIS - } \\
\text { SOLVED } \\
\text { (UG/L } \\
\text { AS B) } \\
(01020)\end{array}$ & $\begin{array}{c}\text { CADMIUM } \\
\text { WATER } \\
\text { UNFLTRD } \\
\text { TOTAL } \\
\text { (UG/L } \\
\text { AS CD) } \\
(01027)\end{array}$ & $\begin{array}{c}\text { CADMIUM } \\
\text { DIS- } \\
\text { SOLVED } \\
\text { (UG/L } \\
\text { AS CD) } \\
(01025)\end{array}$ \\
\hline $\begin{array}{c}\text { JUL } 1996 \\
29 \ldots\end{array}$ & $<0.010$ & 40 & 29 & 4 & 3 & 200 & 150 & 1000 & 964 & $<4$ & $<4.0$ \\
\hline \multirow{2}{*}{$\begin{array}{c}\text { JUL } 1996 \\
29 \ldots\end{array}$} & $\begin{array}{l}\text { CHRO- } \\
\text { MIUM, } \\
\text { TOTAL } \\
\text { RECOV- } \\
\text { HRABLE } \\
\text { (UUG/L } \\
\text { AS CR) } \\
\text { (01034) }\end{array}$ & $\begin{array}{l}\text { CERO- } \\
\text { MIUM, } \\
\text { DIS- } \\
\text { SOLVED } \\
\text { (UG/L } \\
\text { AS CR) } \\
(01030)\end{array}$ & $\begin{array}{c}\text { COPPER, } \\
\text { TOTAL } \\
\text { RECOV- } \\
\text { ERABLE } \\
\text { (UUG/L } \\
\text { AS CU) } \\
\text { (01042) }\end{array}$ & $\begin{array}{l}\text { COPPER, } \\
\text { DIS- } \\
\text { SOLVED } \\
\text { (UG/L } \\
\text { AS CU) } \\
(01040)\end{array}$ & $\begin{array}{l}\text { IRON, } \\
\text { TOTAL } \\
\text { RECOV- } \\
\text { ERABLE } \\
\text { (UG/L } \\
\text { AS FE) } \\
\text { (01045) }\end{array}$ & $\begin{array}{c}\text { IRON, } \\
\text { DIS- } \\
\text { SOLVED } \\
\text { (UG/L } \\
\text { AS FE) } \\
(01046)\end{array}$ & $\begin{array}{l}\text { LEAD, } \\
\text { TOTAL } \\
\text { RECOV- } \\
\text { ERABLE } \\
\text { (UG/L } \\
\text { AS PB) } \\
\text { (01051) }\end{array}$ & $\begin{array}{l}\text { LEAD, } \\
\text { DIS- } \\
\text { SOLVED } \\
\text { (UG/L } \\
\text { AS RB) } \\
\text { (01049) }\end{array}$ & $\begin{array}{l}\text { LITHIUM } \\
\text { TOTAL } \\
\text { RECOV- } \\
\text { ERABLE } \\
\text { (UG/L } \\
\text { AS LI) } \\
\text { (01132) }\end{array}$ & $\begin{array}{c}\text { LITHIUM } \\
\text { DIS- } \\
\text { SOLVED } \\
\text { (UG/L } \\
\text { AS LI) } \\
\text { (01130) }\end{array}$ & $\begin{array}{l}\text { MANGA- } \\
\text { NESE, } \\
\text { TOTAL } \\
\text { RECOV- } \\
\text { BRABLE } \\
\text { (UG/L. } \\
\text { AS MNI) } \\
\text { (01055) }\end{array}$ \\
\hline & $<4$ & $<4.0$ & $<4$ & $<4.0$ & $<10$ & $<60$ & $<10$ & $<4.0$ & 660 & 940 & 130 \\
\hline DATE & $\begin{array}{l}\text { MANGA- } \\
\text { WESE, } \\
\text { DIS' } \\
\text { SOLVBD } \\
\text { (UG/L } \\
\text { AS MN) } \\
(01056)\end{array}$ & $\begin{array}{l}\text { MERCURY } \\
\text { TOTAL } \\
\text { RECOV- } \\
\text { ERABLE } \\
\text { (UG/L } \\
\text { AS EG) } \\
(71900)\end{array}$ & $\begin{array}{c}\text { MERCURY } \\
\text { DIS- } \\
\text { SOLVED } \\
\text { (UG/L } \\
\text { AS HC) } \\
(71890)\end{array}$ & $\begin{array}{l}\text { SELE- } \\
\text { NIUM, } \\
\text { TOTAL } \\
\text { (UG/L } \\
\text { AS SE) } \\
(01147)\end{array}$ & $\begin{array}{l}\text { SELE- } \\
\text { NIUM, } \\
\text { DIS- } \\
\text { SOLVED } \\
\text { (UG/L } \\
\text { AS SE) } \\
\text { (01145) }\end{array}$ & $\begin{array}{c}\text { SILVER, } \\
\text { TOTAL } \\
\text { RECOV- } \\
\text { ERABLE } \\
\text { (UG/L } \\
\text { AS AG) } \\
\text { (01077) }\end{array}$ & $\begin{array}{c}\text { SILVER, } \\
\text { DIS - } \\
\text { SOLVED } \\
\text { (UG/L } \\
\text { AS AG) } \\
(01075)\end{array}$ & $\begin{array}{c}\text { STRON- } \\
\text { TIUM, } \\
\text { TOTAL } \\
\text { RECOV- } \\
\text { ERABLE } \\
\text { (UG/L } \\
\text { AS SR) } \\
\text { (01082) }\end{array}$ & $\begin{array}{c}\text { STRON- } \\
\text { TIUM, } \\
\text { DIS-- } \\
\text { SOLVED } \\
\text { (UG/L } \\
\text { AS SR) } \\
(01080)\end{array}$ & $\begin{array}{l}\text { ZINC, } \\
\text { TOTAL } \\
\text { RECOV- } \\
\text { ERABLE } \\
\text { (UG/L } \\
\text { AS ZNA) } \\
\text { (01092) }\end{array}$ & $\begin{array}{c}\text { ZINC, } \\
\text { DIS } \\
\text { SOLVED } \\
\text { (UG/L } \\
\text { AS 2N) } \\
\text { (01090) }\end{array}$ \\
\hline $\begin{array}{c}\text { JUL } 1996 \\
29 \ldots\end{array}$ & 110 & 0.40 & $<0.1$ & 2 & 2 & $<4$ & $<10$ & 16000 & 17000 & $<10$ & 76 \\
\hline
\end{tabular}




\begin{tabular}{|c|c|c|c|c|c|c|c|c|c|c|c|c|}
\hline DAEE & TIME & $\begin{array}{c}\text { DIS- } \\
\text { CEARGE, } \\
\text { INST: } \\
\text { CUBIC } \\
\text { FEE2 } \\
\text { EER } \\
\text { SECOND } \\
(00061)\end{array}$ & $\begin{array}{l}\text { SPE- } \\
\text { CIFIC } \\
\text { CON- } \\
\text { DUC?- } \\
\text { ANCE } \\
\text { (US/CM) } \\
\text { (00095) }\end{array}$ & $\begin{array}{c}\text { PH } \\
\text { WATER } \\
\text { WHOLE } \\
\text { FIELD } \\
\text { (STARD- } \\
\text { ARD } \\
\text { UNITS) } \\
(00400)\end{array}$ & $\begin{array}{c}\text { TEMPER- } \\
\text { ATURE } \\
\text { AIR } \\
\text { (DEG C) } \\
(00020)\end{array}$ & $\begin{array}{l}\text { TEMPER- } \\
\text { ATURE } \\
\text { WATER } \\
\text { (DEG C) } \\
(00010)\end{array}$ & $\begin{array}{l}\text { BARO- } \\
\text { NIETRIC } \\
\text { PRES- } \\
\text { SURE } \\
\text { (DOI } \\
\text { OF } \\
\text { BG) } \\
(00025)\end{array}$ & $\begin{array}{c}\text { OXYGEN, } \\
\text { DIS- } \\
\text { SOLVED } \\
(\text { WG } / 1) \\
(00300)\end{array}$ & $\begin{array}{c}\text { OXYGEN, } \\
\text { DIS- } \\
\text { SOLVED } \\
\text { (PER- } \\
\text { CERI } \\
\text { SATUR- } \\
\text { ATION) } \\
\text { (O0301) }\end{array}$ & $\begin{array}{l}\text { EARD- } \\
\text { NESS } \\
\text { TOTAL } \\
\text { (MUS } / 2 \\
\text { AS } \\
\text { CACO3) } \\
(00900)\end{array}$ & $\begin{array}{l}\text { HARD-- } \\
\text { NESS } \\
\text { NONCARB } \\
\text { DISSOLV } \\
\text { FLD AS } \\
\text { CACO3 } \\
(\text { ME/L) } \\
(00904)\end{array}$ & $\begin{array}{c}\text { CALCIUM } \\
\text { DIS- } \\
\text { SOLVED } \\
\text { (MS } 11 \\
\text { AS CA }) \\
\text { (00915) }\end{array}$ \\
\hline JUL 1996 & 1330 & 0.08 & 26000 & 9.2 & 35.0 & 33,5 & 651 & 9.2 & 166 & 3400 & 3300 & 710 \\
\hline
\end{tabular}

\begin{tabular}{|c|c|c|c|c|c|c|c|c|c|c|c|c|}
\hline DATE & $\begin{array}{c}\text { MAGNE- } \\
\text { SIUM, } \\
\text { DIS- } \\
\text { SOLVED } \\
\text { (MG/L } \\
\text { AS MG) } \\
(00925)\end{array}$ & $\begin{array}{c}\text { SODTUM, } \\
\text { DIS- } \\
\text { SOLVED } \\
\text { (MG/L } \\
\text { AS NA) } \\
(00930)\end{array}$ & $\begin{array}{c}\text { SODIUM } \\
\text { AD- } \\
\text { SORP- } \\
\text { TION } \\
\text { RATIO } \\
(00931)\end{array}$ & $\begin{array}{c}\text { POTAS- } \\
\text { SIUM, } \\
\text { DIS- } \\
\text { SOLVED } \\
\text { (MG/L } \\
\text { AS K) } \\
(00935)\end{array}$ & $\begin{array}{c}\text { BICAR- } \\
\text { BONATE } \\
\text { WATER } \\
\text { DIS IT } \\
\text { FIELD } \\
\text { MG/L AS } \\
\text { HCO3 } \\
(00453) .\end{array}$ & $\begin{array}{c}\text { CAR- } \\
\text { BONATE } \\
\text { WATER } \\
\text { DIS IT } \\
\text { FIELD } \\
\text { ME/L AS } \\
\text { CO3 } \\
(00452)\end{array}$ & $\begin{array}{c}\text { ALKA- } \\
\text { LINITY } \\
\text { WAT DIS } \\
\text { TOT IT } \\
\text { FIELD } \\
\text { ME/L AS } \\
\text { CACO3 } \\
(39086)\end{array}$ & $\begin{array}{c}\text { ALKA- } \\
\text { LINITY } \\
\text { LAB } \\
\text { (MG/L } \\
\text { AS } \\
\text { CACO3) } \\
(90410)\end{array}$ & $\begin{array}{c}\text { SULFATE } \\
\text { DIS- } \\
\text { SOLVED } \\
\text { (MG/L } \\
\text { AS SO4) } \\
(00945)\end{array}$ & $\begin{array}{l}\text { CHLO- } \\
\text { RIDE, } \\
\text { DIS } \\
\text { SOLVED } \\
\text { (MG/L } \\
\text { AS CL) } \\
(00940)\end{array}$ & $\begin{array}{l}\text { FLUO- } \\
\text { RIDE, } \\
\text { DIS- } \\
\text { SOLVED } \\
\text { (MG/L } \\
\text { AS F) } \\
(00950)\end{array}$ & $\begin{array}{c}\text { BROMIDE } \\
\text { DIS- } \\
\text { SOLVED } \\
\text { (MG/L } \\
\text { AS BR) } \\
(71870)\end{array}$ \\
\hline $\begin{array}{c}\text { JL } 1996 \\
29 \ldots\end{array}$ & 380 & 5300 & 40 & 130 & 20 & 19 & 48 & 40 & 3400 & 8200 & 1.6 & 1.2 \\
\hline
\end{tabular}

\begin{tabular}{|c|c|c|c|c|c|c|c|c|c|c|c|}
\hline DATE & $\begin{array}{l}\text { SILICA, } \\
\text { DIS- } \\
\text { SOLVED } \\
\text { (NG/L } \\
\text { AS } \\
\text { SIO2) } \\
(0095)\end{array}$ & $\begin{array}{l}\text { SOLIDS, } \\
\text { RESIDUE } \\
\text { AT I80 } \\
\text { DEG. C } \\
\text { DIS- } \\
\text { SOLVED } \\
\text { (MG/L) } \\
(70300)\end{array}$ & $\begin{array}{c}\text { SOLIDS, } \\
\text { SUM OF } \\
\text { CONSTY- } \\
\text { TUENTS, } \\
\text { DIS- } \\
\text { SOEVED } \\
\text { (MG/L) } \\
(70301)\end{array}$ & $\begin{array}{c}\text { NITRO- } \\
\text { GEN } \\
\text { NITRTTE } \\
\text { DIS- } \\
\text { SOLVED } \\
\text { (MG/L } \\
\text { AS N) } \\
\text { (00613) }\end{array}$ & $\begin{array}{c}\text { NITRO- } \\
\text { GEN } \\
\text { NO2+Nó3 } \\
\text { DIS- } \\
\text { SOLVED } \\
\text { (MG/L } \\
\text { AS N) } \\
\text { (00631) }\end{array}$ & $\begin{array}{c}\text { NITRO- } \\
\text { GEN, } \\
\text { AMONIA } \\
\text { DIS- } \\
\text { SOLVED } \\
\text { (MG/I } \\
\text { AS N) } \\
(00608)\end{array}$ & $\begin{array}{c}\text { NITRO- } \\
\text { GEN, } \\
\text { ORGANIC } \\
\text { DIS- } \\
\text { SOLVED } \\
\text { (MG/L } \\
\text { AS N) } \\
(00607)\end{array}$ & $\begin{array}{l}\text { NITRO- } \\
\text { GEN,AM- } \\
\text { MONIA + } \\
\text { ORGANIC } \\
\text { TOTAL } \\
\text { (MG/L } \\
\text { AS N }) \\
(00625)\end{array}$ & $\begin{array}{l}\text { NITRO- } \\
\text { GEN, AM- } \\
\text { MONIA + } \\
\text { ORGANYC } \\
\text { DIS. } \\
\text { (MG/L } \\
\text { AS N) } \\
\text { (00623) }\end{array}$ & $\begin{array}{l}\text { PHOS- } \\
\text { PHORUS } \\
\text { TOTAL. } \\
\text { (MG/L } \\
\text { AS P) } \\
(00665)\end{array}$ & $\begin{array}{c}\text { PHOS- } \\
\text { FHORUS } \\
\text { DIS } \\
\text { SOLVED } \\
\text { (ME/L } \\
\text { AS P) } \\
\text { (00666) }\end{array}$ \\
\hline $\begin{array}{c}\text { UL } 1996 \\
29 \ldots\end{array}$ & 0.90 & 18600 & 18200 & $<0.010$ & $=0.120$ & 0.190 & 0.61 & 0.80 & 0.80 & $<0.010$ & $<0.010$ \\
\hline DATE & $\begin{array}{c}\text { PHOS- } \\
\text { FHORUS } \\
\text { ORTHO, } \\
\text { DIS- } \\
\text { SOLVED } \\
(\text { MG /L } \\
\text { AS P) } \\
(00671)\end{array}$ & $\begin{array}{l}\text { ALUM- } \\
\text { INUM, } \\
\text { TOTAL } \\
\text { RECOV- } \\
\text { ERABLE } \\
\text { (UG/L } \\
\text { AS AL) } \\
\text { (01105) }\end{array}$ & $\begin{array}{l}\text { ALUM- } \\
\text { INUM, } \\
\text { DIS- } \\
\text { SOLVED } \\
\text { (UG/L } \\
\text { AS Al) } \\
\text { (01106) }\end{array}$ & $\begin{array}{c}\text { ARSENIC } \\
\text { TORAL } \\
\text { (UG/L } \\
\text { AS AS) } \\
(01002)\end{array}$ & $\begin{array}{c}\text { ARSENIC } \\
\text { DIS- } \\
\text { SOLVED } \\
\text { (UG/L } \\
\text { AS AS) } \\
(01000)\end{array}$ & $\begin{array}{c}\text { BARIUM, } \\
\text { TOTAL } \\
\text { RECOV } \\
\text { ERABLE } \\
\text { (UTG/L } \\
\text { AS BA) } \\
(01007)\end{array}$ & $\begin{array}{l}\text { BARIUM, } \\
\text { DIS- } \\
\text { SOLVED } \\
\text { (UG/L } \\
\text { AS BA) } \\
(01005)\end{array}$ & $\begin{array}{l}\text { BORON, } \\
\text { TOTAL } \\
\text { RECOV- } \\
\text { ERABLE } \\
\text { (UG/L } \\
\text { AS B) } \\
\text { (01022) }\end{array}$ & $\begin{array}{c}\text { BORON, } \\
\text { DIS- } \\
\text { SOLVED } \\
\text { (UG/L } \\
\text { AS B) } \\
\text { (01020) }\end{array}$ & $\begin{array}{c}\text { CADMIUM } \\
\text { WATER } \\
\text { UNFLTRD } \\
\text { TOTAL } \\
\text { (UG/L } \\
\text { AS CD) } \\
(01027)\end{array}$ & $\begin{array}{c}\text { CADMIUM } \\
\text { DIS- } \\
\text { SOLVED } \\
\text { (UG/L } \\
\text { AS CD) } \\
(01025)\end{array}$ \\
\hline $\begin{array}{c}121996 \\
29 \ldots\end{array}$ & 0.010 & 2200 & 15 & 2 & 2 & 200 & 150 & 930 & 878 & $<4$. & $<4.0$ \\
\hline DATE & $\begin{array}{l}\text { CHRO- } \\
\text { MIUM, } \\
\text { TOTAL } \\
\text { RECOV- } \\
\text { ERABLE } \\
\text { (UG/L } \\
\text { AS CR) } \\
\text { (01034) }\end{array}$ & $\begin{array}{l}\text { CHRO- } \\
\text { BIUM, } \\
\text { DIS - } \\
\text { SOEVED } \\
\text { (UG/L } \\
\text { AS CR) } \\
\text { (01030) }\end{array}$ & $\begin{array}{c}\text { COPEER, } \\
\text { TOTAL } \\
\text { RECOV- } \\
\text { ERABLE } \\
\text { (UG/L } \\
\text { AS CU) } \\
(01042)\end{array}$ & $\begin{array}{c}\text { COPFER, } \\
\text { DIS- } \\
\text { SOLYED } \\
\text { (UG/L } \\
\text { AS CU) } \\
(01040)\end{array}$ & $\begin{array}{l}\text { IRON, } \\
\text { TOTAL } \\
\text { RECOV- } \\
\text { ERABLE } \\
\text { (UG/L } \\
\text { AS EE) } \\
\text { (01045) }\end{array}$ & $\begin{array}{l}\text { IRON, } \\
\text { DIS - } \\
\text { SOLVED } \\
\text { (UG/L } \\
\text { AS FE) } \\
(01046)\end{array}$ & $\begin{array}{c}\text { LEAD, } \\
\text { TOTAL } \\
\text { RECOV- } \\
\text { BRABLE } \\
\text { (UG/L } \\
\text { AS EB) } \\
(01051)\end{array}$ & $\begin{array}{c}\text { LEAD, } \\
\text { DIS- } \\
\text { SOLYED } \\
\text { (UG/L } \\
\text { AS PB) } \\
(01049)\end{array}$ & $\begin{array}{l}\text { LITEIUM } \\
\text { TOTAL } \\
\text { RECOV- } \\
\text { ERABLE } \\
\text { (UG/L } \\
\text { AS LI) } \\
(01132)\end{array}$ & $\begin{array}{c}\text { LITGIUM } \\
\text { DIS- } \\
\text { SOLVED } \\
\text { (UG/L } \\
\text { AS LI) } \\
\text { (01130) }\end{array}$ & $\begin{array}{l}\text { MANGA- } \\
\text { NESE, } \\
\text { TOTAL } \\
\text { RECOV- } \\
\text { BRABLE } \\
\text { (UG/L } \\
\text { AS MN) } \\
\text { (01055) }\end{array}$ \\
\hline $\begin{array}{c}\text { JUL } 1996 \\
29 \ldots\end{array}$ & $<4$ & $<4.0$ & 7 & $<4.0$ & 1900 & $<60$ & $<10$ & $<4.0$ & 620 & 910 & 130 \\
\hline DATE & $\begin{array}{l}\text { MANGA- } \\
\text { WESE, } \\
\text { DIS- } \\
\text { SOLVED } \\
\text { (UG/L } \\
\text { AS MN) } \\
\text { (01056) }\end{array}$ & $\begin{array}{c}\text { MERCURY } \\
\text { TOTAL } \\
\text { RECOV- } \\
\text { ERABLE } \\
\text { (UG/L } \\
\text { AS HG) } \\
(71900)\end{array}$ & $\begin{array}{c}\text { MERCIIRY } \\
\text { DIS- } \\
\text { SOLVED } \\
\text { (UG/L } \\
\text { AS HG) } \\
(71890)\end{array}$ & $\begin{array}{l}\text { SELE- } \\
\text { NIUM, } \\
\text { TOTAL } \\
\text { (UG/L } \\
\text { AS SE) } \\
\text { (O1147) }\end{array}$ & $\begin{array}{l}\text { SELE- } \\
\text { NIUM, } \\
\text { DIS- } \\
\text { SOLVED } \\
\text { (UG/L } \\
\text { AS SE) } \\
\text { (OL145) }\end{array}$ & $\begin{array}{c}\text { SILVGR, } \\
\text { TOTAL } \\
\text { RECOV- } \\
\text { ERABLE } \\
\text { (UG/L } \\
\text { AS AG) } \\
(01077 \text { ) }\end{array}$ & $\begin{array}{c}\text { SILVER, } \\
\text { DIS- } \\
\text { SOLVED } \\
\text { (UG/L } \\
\text { AS AG) } \\
\text { (01075) }\end{array}$ & $\begin{array}{c}\text { STROL- } \\
\text { TIUM, } \\
\text { TOTAL } \\
\text { RECOV- } \\
\text { GRABLE } \\
\text { (UG/L } \\
\text { AS SR) } \\
\text { (O1082) }\end{array}$ & $\begin{array}{c}\text { STRON- } \\
\text { TIUM, } \\
\text { DIS- } \\
\text { SORVED } \\
\text { (UG/L } \\
\text { AS SR) } \\
\text { (01080) }\end{array}$ & $\begin{array}{l}\text { ZINC, } \\
\text { TOTAI } \\
\text { RECOV- } \\
\text { ERABIL } \\
\text { (UG/L } \\
\text { AS ZN) } \\
(01092)\end{array}$ & $\begin{array}{c}\text { ZINC, } \\
\text { DIS- } \\
\text { SOLVED } \\
\text { (UG/L } \\
\text { AS ZN) } \\
(01090)\end{array}$ \\
\hline $\begin{array}{c}\text { JUL, } 1996 \\
29 \ldots\end{array}$ & 36 & $<0.10$ & $<0.1$ & 2 & 1 & $<4$ & $<10$ & 14000 & 16000 & 20 & $<60$ \\
\hline $30 \ldots$ & $<20$ & 0.10 & . & 2 & 2 & $<4$ & $<10$ & 19000 & 18000 & $<10$ & $<60$ \\
\hline
\end{tabular}


ANALYSES OF SAMPLES COLLECTED AT WATER-QUALITY RARTIAL-RECORD STATIONS AND MISCELLANEOUS SITES

WATER-QUALITY DATA, WATER YEAR CCTOBER 1995 TO SEPTEMBER 1996

TULAROSA VALLEY BASTN -- Continued

331657106185010 - MALPAIS MARSH NR OSCURA, MM

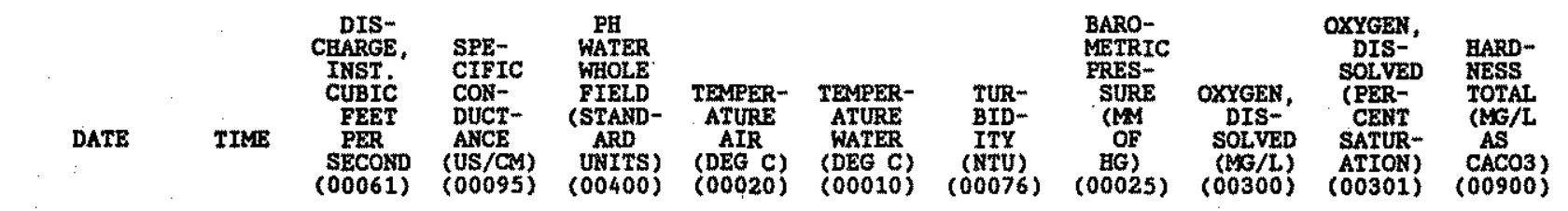

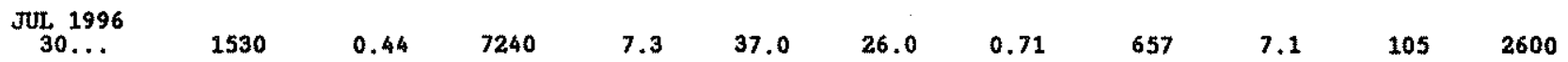

\begin{tabular}{|c|c|c|c|c|c|c|c|c|c|c|c|}
\hline DAEE & $\begin{array}{l}\text { CALCIUM } \\
\text { DIS- } \\
\text { SOLVED } \\
\text { (MG/L } \\
\text { AS CA) } \\
(00915)\end{array}$ & $\begin{array}{c}\text { MAGNE- } \\
\text { SIUM, } \\
\text { DIS- } \\
\text { SOLVED } \\
\text { (MG/L } \\
\text { AS MG) } \\
(00925)\end{array}$ & $\begin{array}{c}\text { SODIUM, } \\
\text { DIS- } \\
\text { SOLVED } \\
\text { (MA/L } \\
\text { AS NA) } \\
(00930)\end{array}$ & $\begin{array}{c}\text { SODIUN } \\
\text { AD- } \\
\text { SORP- } \\
\text { TION } \\
\text { RATIO } \\
(00931)\end{array}$ & $\begin{array}{c}\text { POTAS- } \\
\text { SIUM, } \\
\text { DIS- } \\
\text { SOLVED } \\
\text { (MG/L } \\
\text { AS K) } \\
(00935)\end{array}$ & $\begin{array}{c}\text { ALKA- } \\
\text { LINITY } \\
\text { LAB } \\
\text { (NG/L } \\
\text { AS } \\
\text { CACO3) } \\
(90410)\end{array}$ & $\begin{array}{c}\text { SULFATE } \\
\text { DIS- } \\
\text { SOLVED } \\
\text { (MG/L } \\
\text { AS SO4) } \\
(00945)\end{array}$ & $\begin{array}{l}\text { CHLO- } \\
\text { RIDE, } \\
\text { DTS- } \\
\text { SOLVED } \\
\text { (MG/L } \\
\text { AS CL) } \\
(00940)\end{array}$ & $\begin{array}{l}\text { FLUO- } \\
\text { RIDE, } \\
\text { DIS- } \\
\text { SOLVED } \\
\text { (MG/L } \\
\text { AS F) } \\
(00950)\end{array}$ & $\begin{array}{c}\text { BROMIDE } \\
\text { DIS- } \\
\text { SOLVED } \\
\text { (MG/L } \\
\text { AS BR) } \\
\text { (71870) }\end{array}$ & $\begin{array}{l}\text { SILICA, } \\
\text { DIS- } \\
\text { SOLVED } \\
\text { (NG/L } \\
\text { AS } \\
\text { SIO2) } \\
(00955)\end{array}$ \\
\hline $\begin{array}{l}1996 \\
30 \ldots\end{array}$ & 740 & 180 & 750 & 6 & 8.6 & 71 & 2200 & 1500 & 1.5 & 0.35 & 28 \\
\hline
\end{tabular}

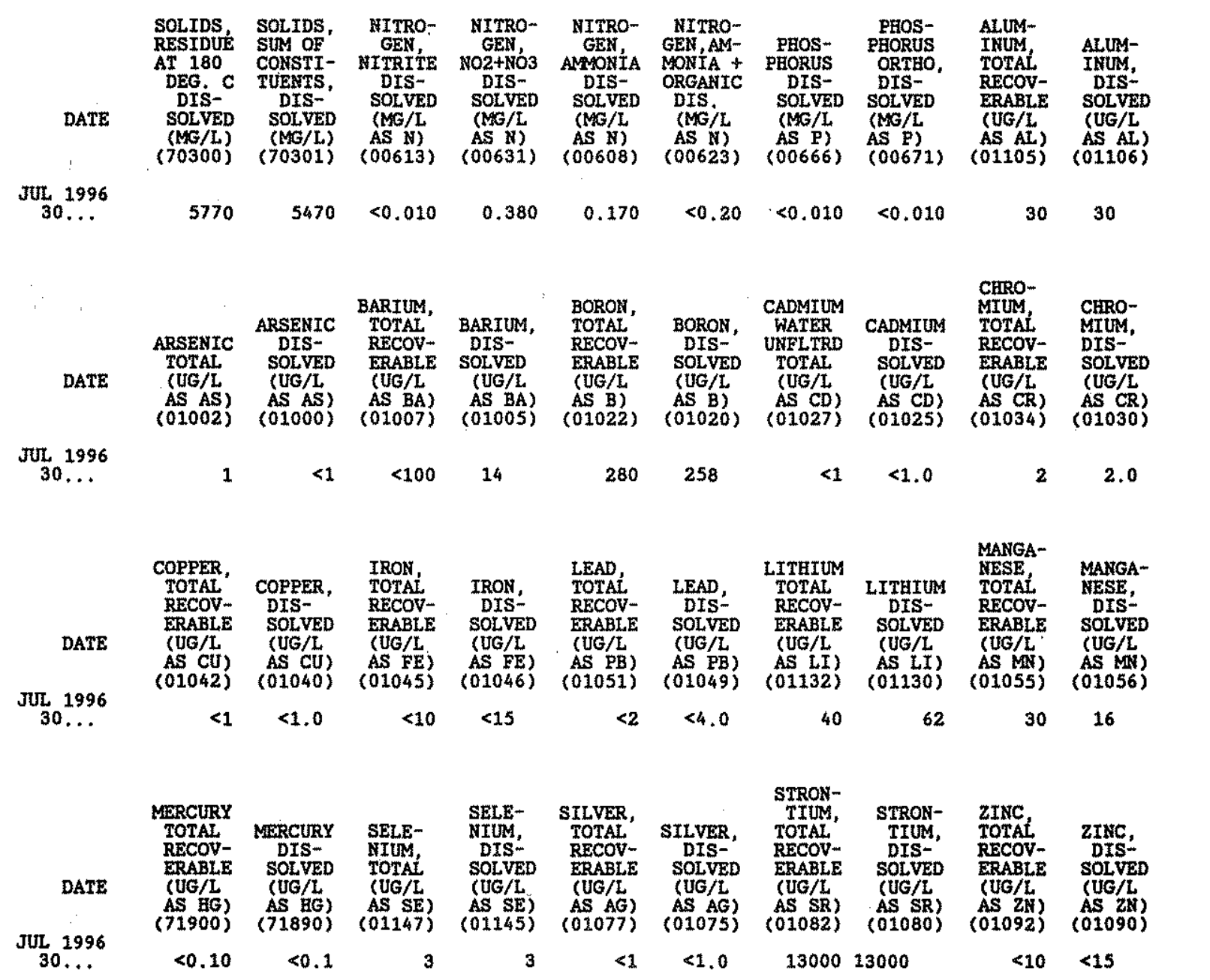




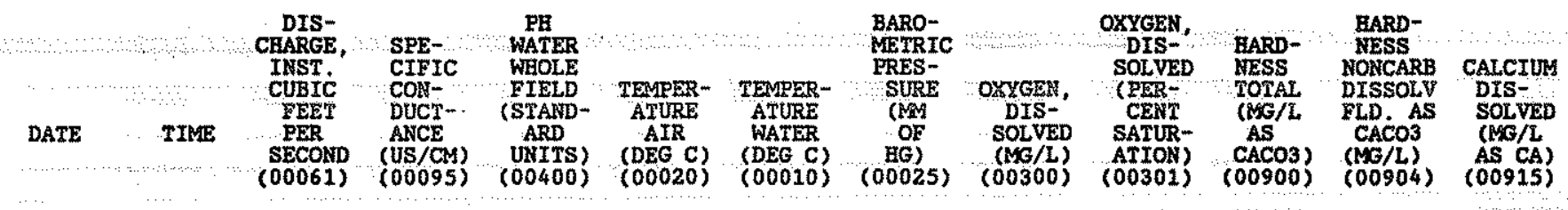

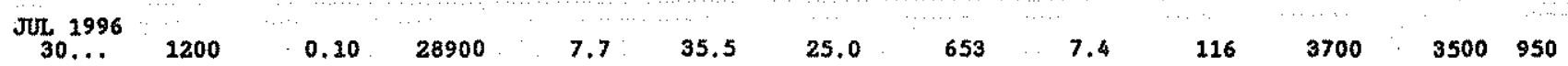

\begin{tabular}{|c|c|c|c|c|c|c|c|c|c|c|c|}
\hline DATE & $\begin{array}{c}\text { MAGNE- } \\
\text { SIUM, } \\
\text { DIS- } \\
\text { SOLVED } \\
\text { (MG/L } \\
\text { AS MG) } \\
(00925)\end{array}$ & $\begin{array}{l}\text { SODIUM, } \\
\text { DIS- } \\
\text { SOLVED } \\
\text { (MSSL } \\
\text { AS NA) } \\
(00930)\end{array}$ & $\begin{array}{l}\text { SODIUM } \\
\text { AD- } \\
\text { SORP- } \\
\text { TION } \\
\text { RATIO } \\
(00931)\end{array}$ & $\begin{array}{l}\text { POTAS- } \\
\text { SIUM, } \\
\text { DIS-: } \\
\text { SOLVED } \\
\text { (MG/L } \\
\text { AS K) } \\
(00935)\end{array}$ & $\begin{array}{l}\text { BICAR- } \\
\text { BOHATE } \\
\text { WATER } \\
\text { DIS IT } \\
\text { FIELD } \\
\text { WGG AS } \\
\text { BCOS } \\
(00453)\end{array}$ & $\begin{array}{c}\text { CAR- } \\
\text { BONATE } \\
\text { WATER } \\
\text { DIS IT } \\
\text { FIELD } \\
\text { MG/L AS } \\
\text { CO3 } \\
(00452)\end{array}$ & $\begin{array}{c}\text { ALKA- } \\
\text { LINITYY } \\
\text { WAT DIS } \\
\text { TOT IT } \\
\text { FIELD } \\
\text { MGL AS } \\
\text { CACOS } \\
(39086)\end{array}$ & $\begin{array}{c}\text { ALKA- } \\
\text { LINITY } \\
\text { LAB } \\
\text { (MG/L } \\
\text { AS } \\
\text { CACO3) } \\
(90410)\end{array}$ & $\begin{array}{c}\text { SULFATE } \\
\text { DIS- } \\
\text { SOLVED } \\
(\mathrm{MG} / \mathrm{L} \\
\text { AS SO4) } \\
(00945)\end{array}$ & $\begin{array}{l}\text { CHLO- } \\
\text { RIDE, } \\
\text { DIS- } \\
\text { SOLVED } \\
\text { (HG/L } \\
\text { AS CL) } \\
(00940)\end{array}$ & $\begin{array}{c}\text { FLUO- } \\
\text { RIDE, } \\
\text { DIS- } \\
\text { SOLVED } \\
\text { (ME/L } \\
\text { AS F) } \\
(00950)\end{array}$ \\
\hline $\begin{array}{c}\text { JUL } 1996 \\
30 \ldots\end{array}$ & 310 & 5700 & 41 & 140 & 225 & 0 & 184 & 187 & 3200 & 9200 & 4.5 \\
\hline DATE & $\begin{array}{c}\text { BROMTDE } \\
\text { DIS - } \\
\text { SOLVED } \\
\text { (MG/L } \\
\text { AS BR) } \\
\text { (71870) }\end{array}$ & $\begin{array}{l}\text { SILICA, } \\
\text { DIS } \\
\text { SOLVED } \\
\text { (MG/L } \\
\text { AS } \\
\text { SIO2) } \\
(00955)\end{array}$ & $\begin{array}{l}\text { SOLIDS, } \\
\text { RESIDUE } \\
\text { AT } 180 \\
\text { DEG.CC } \\
\text { DIS- } \\
\text { SOLVED } \\
\text { (MG/L) } \\
(70300)\end{array}$ & $\begin{array}{c}\text { SOLIDS, } \\
\text { SUM OF } \\
\text { CONSTI- } \\
\text { TUENTS, } \\
\text { DIS-- } \\
\text { SOLVED } \\
\text { (MG/L) } \\
(70301)\end{array}$ & $\begin{array}{c}\text { NITRO- } \\
\text { GEN } \\
\text { NITRITE } \\
\text { DIS- } \\
\text { SOLVED } \\
\text { (MS/L } \\
\text { AS N) } \\
(00613)\end{array}$ & $\begin{array}{c}\text { NITRO- } \\
\text { GEN } \\
\text { NO2+NO3 } \\
\text { DIS- } \\
\text { SOLVED } \\
\text { (MG/L } \\
\text { AS N) } \\
(00631)\end{array}$ & $\begin{array}{c}\text { NITRO- } \\
\text { GEN } \\
\text { AMONIA } \\
\text { DIS- } \\
\text { SOLVED } \\
\text { (MGS/L } \\
\text { AS N) } \\
(00608)\end{array}$ & $\begin{array}{l}\text { NITRO- } \\
\text { GEN,AM- } \\
\text { MONIA + } \\
\text { ORGANIC } \\
\text { TOTAL } \\
\text { (MSG/L } \\
\text { AS H) } \\
(00625)\end{array}$ & $\begin{array}{l}\text { NITRO- } \\
\text { GEN,AM- } \\
\text { MONIA + } \\
\text { ORGANIC } \\
\text { DIS. } \\
\text { (MGA } \\
\text { AS A) } \\
(00623)\end{array}$ & $\begin{array}{l}\text { PHOS- } \\
\text { PHORUS } \\
\text { TOTAL } \\
\text { (MGL } \\
\text { AS P) } \\
(00665)\end{array}$ & $\begin{array}{c}\text { PHOS- } \\
\text { PRORUS } \\
\text { DIS- } \\
\text { SOLVED } \\
\text { (MEG/L } \\
\text { AS P) } \\
(00666)\end{array}$ \\
\hline $\begin{array}{c}\text { JuL } 1996 \\
30 \ldots\end{array}$ & 1.9 & 29 & 20600 & 19700 & $<0.010$ & 0.120 & 0.220 & $<0.20$ & $<0.20$ & $<0.010$ & $<0.010$ \\
\hline DATE & $\begin{array}{l}\text { PHOS- } \\
\text { PHORUS } \\
\text { ORTHO, } \\
\text { DIS- } \\
\text { SOLVED } \\
\text { (MESLL } \\
\text { AS } \$ \text { ) } \\
\text { (00671) }\end{array}$ & $\begin{array}{l}\text { ALUM- } \\
\text { INUM, } \\
\text { TOTAL } \\
\text { RECOV- } \\
\text { ERABLE } \\
\text { (UG/L } \\
\text { AS AL) } \\
\text { (01105) }\end{array}$ & $\begin{array}{l}\text { ALUM- } \\
\text { INUM, } \\
\text { DIS- } \\
\text { SOLVED } \\
\text { (UG/L } \\
\text { AS AL) } \\
\text { (01106) }\end{array}$ & $\begin{array}{l}\text { ARSENIC } \\
\text { TOTAL } \\
\text { (UG/L } \\
\text { AS AS) } \\
(01002)\end{array}$ & $\begin{array}{c}\text { ARSENIC } \\
\text { DIS- } \\
\text { SOLVED } \\
\text { (UG/L } \\
\text { AS AS) } \\
(01000)\end{array}$ & $\begin{array}{c}\text { BARIUM, } \\
\text { TOTAL } \\
\text { RECOV- } \\
\text { ERABLE } \\
\text { (UG/L } \\
\text { AS BA) } \\
(01007)\end{array}$ & $\begin{array}{c}\text { BARIUM, } \\
\text { DIS- } \\
\text { SOLVED } \\
\text { (UG/L } \\
\text { AS BA) } \\
\text { (01005) }\end{array}$ & $\begin{array}{l}\text { BORON, } \\
\text { TOTAL } \\
\text { RECOV- } \\
\text { ERABLE } \\
\text { (UG/L } \\
\text { AS B) } \\
\text { (01022) }\end{array}$ & $\begin{array}{l}\text { BORON, } \\
\text { DIS-' } \\
\text { SOLVED } \\
\text { (UG/L } \\
\text { AS B) } \\
\text { (01020) }\end{array}$ & $\begin{array}{c}\text { CADMIUM } \\
\text { WATER } \\
\text { UNFLTRD } \\
\text { TOTAL } \\
\text { (UG/L } \\
\text { AS CD) } \\
\text { (01027) }\end{array}$ & $\begin{array}{c}\text { CADMIUM } \\
\text { DIS- } \\
\text { SOLVED } \\
\text { (UG } / \mathrm{L} \\
\text { AS CD) } \\
\text { (01025) }\end{array}$ \\
\hline $\begin{array}{c}J U L, 1996 \\
30 \ldots\end{array}$ & 0.010 & 90 & 64 & 3 & 2 & $<100$ & 78 & 680 & 632 & $<4$ & $<4.0$ \\
\hline DATE & $\begin{array}{l}\text { CERO- } \\
\text { GIIUA, } \\
\text { TOTAL } \\
\text { RECOV- } \\
\text { ERABLE } \\
\text { (UG/L } \\
\text { AS CR) } \\
\text { (01034) }\end{array}$ & $\begin{array}{l}\text { CHEO- } \\
\text { MIUM, } \\
\text { DIS- } \\
\text { SOIVED } \\
\text { (UG/L } \\
\text { AS CR) } \\
(01030)\end{array}$ & $\begin{array}{c}\text { COPPER, } \\
\text { TOTAL, } \\
\text { RECOV- } \\
\text { ERABIE } \\
\text { (UG/L } \\
\text { AS CU) } \\
\text { (01042) }\end{array}$ & $\begin{array}{c}\text { COPPER, } \\
\text { DIS- } \\
\text { SOLVED } \\
\text { (UG } / \bar{L} \\
\text { AS CU) } \\
\text { (01040) }\end{array}$ & $\begin{array}{c}\text { IRON, } \\
\text {,TOTAL } \\
\text { RECOV- } \\
\text { ERABLE } \\
\text { (UG/L } \\
\text { AS FE) } \\
(01045)\end{array}$ & $\begin{array}{l}\text { IRON, } \\
\text { DIS- } \\
\text { SOLVED } \\
\text { (UG/L } \\
\text { AS FE) } \\
(01046)\end{array}$ & $\begin{array}{l}\text { LEAD } \\
\text { TOTAL } \\
\text { RECOV- } \\
\text { ERABLE } \\
\text { (UG/L } \\
\text { AS PB) } \\
(01051)\end{array}$ & $\begin{array}{c}\text { LEAD, } \\
\text { DIS- } \\
\text { SOLVED } \\
\text { (UG/L } \\
\text { AS PB }) \\
(01049)\end{array}$ & $\begin{array}{l}\text { LTTHIUM } \\
\text { TOTAL } \\
\text { RECOV- } \\
\text { REABLE } \\
\text { (UG/L } \\
\text { AS LI) } \\
\text { (01132) }\end{array}$ & $\begin{array}{c}\text { LITHYUM } \\
\text { DIS- } \\
\text { SOLVUED } \\
\text { (UE/L } \\
\text { AS LI) } \\
(01130)\end{array}$ & $\begin{array}{l}\text { MANGA- } \\
\text { NESE } \\
\text { TOTAL } \\
\text { RECOV- } \\
\text { RRABLE } \\
\text { (UG/L } \\
\text { AS MN) } \\
\text { (01055) }\end{array}$ \\
\hline $\begin{array}{c}\text { JUL, } 1996 \\
30 \ldots\end{array}$ & $<4$ & $<4.0$ & $<4$ & $<4.0$ & 20 & $<60$ & $<10$ & $<4.0$ & 770 & 1200 & 150 \\
\hline DATE & $\begin{array}{l}\text { MAAEGA- } \\
\text { NESE, } \\
\text { DIS- } \\
\text { SOLVED } \\
\text { (UG/L } \\
\text { AS NNN) } \\
\text { (01056) }\end{array}$ & $\begin{array}{l}\text { MERCURY } \\
\text { TOTAL } \\
\text { RECOV- } \\
\text { ERABLE } \\
\text { (UG/L } \\
\text { AS HG) } \\
\text { (71900) }\end{array}$ & $\begin{array}{c}\text { MERCURY } \\
\text { DIS- } \\
\text { SOLVED } \\
\text { (UG/L } \\
\text { AS HG) } \\
(71890)\end{array}$ & $\begin{array}{l}\text { SELE- } \\
\text { NIUM, } \\
\text { TOTAL: } \\
\text { (UG/L } \\
\text { AS SE) } \\
\text { (01147) }\end{array}$ & $\begin{array}{c}\text { SELE- } \\
\text { NIUM, } \\
\text { DIS- } \\
\text { SOLVED } \\
\text { (UG/L } \\
\text { AS SE) } \\
(01145)\end{array}$ & $\begin{array}{c}\text { SILVER, } \\
\text { TOTAL, } \\
\text { RECOV- } \\
\text { ERABLE } \\
\text { (UG/L } \\
\text { AS AG) } \\
\text { (01077) }\end{array}$ & $\begin{array}{c}\text { SILVER, } \\
\text { DIS- } \\
\text { SOLVED } \\
\text { (UG/L } \\
\text { AS AG) } \\
(01075)\end{array}$ & $\begin{array}{l}\text { STRON- } \\
\text { TIUM, } \\
\text { TOTAL, } \\
\text { RECOVV- } \\
\text { ERABLE } \\
\text { (UG/L } \\
\text { AS SR) } \\
\text { (01082) }\end{array}$ & $\begin{array}{c}\text { STRON- } \\
\text { TIUM, } \\
\text { DIS- } \\
\text { SOLVED } \\
\text { (UG/L } \\
\text { AS SR) } \\
(01080)\end{array}$ & $\begin{array}{l}\text { ZINC, } \\
\text { TOTAL } \\
\text { RECOV- } \\
\text { ERABLE } \\
\text { (UG/L } \\
\text { AS ZN) } \\
\text { (01092) }\end{array}$ & $\begin{array}{l}\text { ZINC, } \\
\text { DIS- } \\
\text { SOLVED } \\
\text { (UG/L } \\
\text { AS ZN) } \\
(01090)\end{array}$ \\
\hline $\begin{array}{l}3 J_{L} 1996 \\
30 \ldots\end{array}$ & 150 & $<0.10$ & 0.2 & $<1$ & $<1$ & $<4$ & $<10$ & 19000 & 20000 & $<10$ & $<60$ \\
\hline
\end{tabular}


ANALYSES OF SAMPLES COLLECTED AT WATER-QUALITY RARTIAL-RECORD STATIONS AND MISCELLANEOUS SITES

WATER-QUALITY DATA, WATER YEAR OCTOBER 1995 TO SEPTEMBER 1996

TULAROSA VALLEY BASIN - Continued

332528106170710 - LOWER MOUND SPRING POND NR OSCURA, NM

\begin{tabular}{|c|c|c|c|c|c|c|c|c|c|c|c|c|c|c|c|c|c|c|c|}
\hline \multirow[b]{2}{*}{$\begin{array}{c}\text { AUG } 1996 \\
01 \ldots\end{array}$} & \multicolumn{2}{|c|}{$\begin{array}{l}\text { SPE- } \\
\text { CIFIC } \\
\text { CON- } \\
\text { DUCT- } \\
\text { AHCE } \\
\text { (US/CM) } \\
\text { (00095) }\end{array}$} & \multicolumn{2}{|c|}{$\begin{array}{c}\text { TEMPER- } \\
\text { ATURE } \\
\text { AIR } \\
\text { (DEG C) } \\
(00020)\end{array}$} & \multicolumn{2}{|c|}{$\begin{array}{l}\text { TEMPER- } \\
\text { ATURE } \\
\text { WATEER } \\
\text { (DEG C) } \\
(00010)\end{array}$} & \multicolumn{2}{|c|}{$\begin{array}{c}\text { TUR- } \\
\text { BID- } \\
\text { ITY } \\
\text { (NTU) } \\
(00076)\end{array}$} & \multicolumn{2}{|c|}{$\begin{array}{c}\text { FRES- } \\
\text { SURE } \\
\text { (MM } \\
\text { OF } \\
\text { HG) } \\
(00025)\end{array}$} & \multicolumn{2}{|c|}{$\begin{array}{c}\text { OXYGEN, } \\
\text { DIS- } \\
\text { SOLVED } \\
\text { (MG/L) } \\
(00300)\end{array}$} & \multicolumn{2}{|c|}{$\begin{array}{l}\text { SOLVED } \\
\text { (PER- } \\
\text { CENT } \\
\text { SATUR- } \\
\text { ATION) } \\
(00301)\end{array}$} & \multicolumn{2}{|c|}{$\begin{array}{l}\text { HARD-- } \\
\text { NESS } \\
\text { TOTAL } \\
\text { (MG/L } \\
\text { AS } \\
\text { CACO3) } \\
(00900)\end{array}$} & \multicolumn{2}{|c|}{$\begin{array}{l}\text { CALCIUM } \\
\text { DIS- } \\
\text { SOLVED } \\
\text { (HG/L } \\
\text { AS CA) } \\
\text { (00915) }\end{array}$} & $\begin{array}{r}\text { MAC } \\
\text { SI } \\
D I \\
\text { SOI } \\
(\mathrm{MX} \\
\mathrm{AS} \\
\mathrm{COOS}\end{array}$ \\
\hline & 1520 & 6140 & & 2.5 & & 8.0 & & .2 & & 652 & & 2.0 & & 184 & & 800 & 830 & & 180 \\
\hline DATE & $\begin{array}{c}\text { SODIUM, } \\
\text { DIS- } \\
\text { SOLVDD } \\
\text { (NG/L } \\
\text { AS NA) } \\
(00930)\end{array}$ & $\begin{array}{r}\text { SO } \\
\text { SO } \\
\text { I } \\
\text { RA } \\
100\end{array}$ & $\begin{array}{l}\text { TIUM } \\
\text { AD- } \\
\text { RP- } \\
\text { ION } \\
\text { FIO } \\
931)\end{array}$ & $\begin{array}{r}P C \\
S \\
S D \\
S \\
\text { AS } \\
100\end{array}$ & $\begin{array}{l}\text { CAS- } \\
\text { UMM, } \\
\text { [S- } \\
\text { VED } \\
\text { O/L } \\
\text { K) } \\
\text { 35) }\end{array}$ & $\begin{array}{r}\text { AL } \\
\text { LII } \\
(S \\
\text { CA } \\
60\end{array}$ & 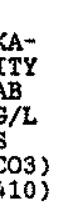 & $\begin{array}{l}\text { SUL } \\
\text { DI } \\
\text { SO } \\
\text { (M } \\
\text { AS } \\
100\end{array}$ & $\begin{array}{l}\text { EATE } \\
\text { S- } \\
\text { LVED } \\
\text { G/L } \\
\text { SO4) } \\
945)\end{array}$ & $\begin{array}{l}\text { CE } \\
\text { RI } \\
\text { DI } \\
\text { SO } \\
(M \\
\text { AS } \\
C O O\end{array}$ & $\begin{array}{l}\text { LO- } \\
\text { DE } \\
\text { S- } \\
\text { LVED } \\
\text { G } / \mathrm{L} \\
\mathrm{CL}) \\
940)\end{array}$ & $\begin{array}{c}\text { FL } \\
\text { RI } \\
\text { D } \\
\text { SOI } \\
\text { (M } \\
\text { AS } \\
C 00\end{array}$ & $\begin{array}{l}\text { JO- } \\
\text { OE } \\
\text { IS- } \\
\text { CVED } \\
\text { G/L } \\
\text { F) } \\
950)\end{array}$ & $\begin{array}{r}\text { BRO } \\
\text { D } \\
\text { SO } \\
\text { (M } \\
\text { AS } \\
\angle 71\end{array}$ & $\begin{array}{l}\text { UIDE } \\
\text { S- } \\
\text { VED } \\
\text { BL } \\
\text { BR) } \\
70 \text { ) }\end{array}$ & $\begin{array}{l}\text { SIL } \\
\text { DI } \\
\text { SO } \\
\text { (N) } \\
\text { A } \\
\text { SI } \\
\text { COO }\end{array}$ & $\begin{array}{l}\text { ICA, } \\
S- \\
\text { LVED } \\
G / L \\
S \\
02) \\
955)\end{array}$ & $\begin{array}{l}\text { SOI } \\
\text { RES } \\
\text { AT } \\
\text { D } \\
\text { I } \\
\text { SC } \\
(I)\end{array}$ & $\begin{array}{l}\text { IDS, } \\
\text { IDUE } \\
180 \\
\text { G. C } \\
\text { IS- } \\
\text { LVED } \\
\text { G/L) } \\
300 \text { ) }\end{array}$ \\
\hline $\begin{array}{c}\mathrm{AUG} 1996 \\
01 \ldots\end{array}$ & 460 & & 4 & & .8 & 33 & & 250 & & 90 & & & 1.5 & & 41 & 24 & & & 5150 \\
\hline DATE & $\begin{array}{c}\text { SOLIDS, } \\
\text { SUM OF } \\
\text { CONSTI- } \\
\text { TUENTS, } \\
\text { DIS- } \\
\text { SOLVED } \\
\text { (MG/L) } \\
(70301)\end{array}$ & $\begin{array}{r}\text { NI } \\
G \\
N I T \\
D \\
S O \\
(M \\
A S \\
100\end{array}$ & $\begin{array}{l}\text { RRO- } \\
\text { NN, } \\
\text { RTE } \\
\text { SS- } \\
\text { VED } \\
\text { /L } \\
\text { N) } \\
\text { S13) }\end{array}$ & $\begin{array}{r}N I \\
G \\
\text { NO2 } \\
D \\
\text { SO } \\
\text { AM } \\
\text { AS } \\
100\end{array}$ & $\begin{array}{l}\text { RO- } \\
\text { No } \\
\text { NO3 } \\
\text { S- } \\
\text { VED } \\
\text { iL } \\
\text { N) } \\
\text { 31) }\end{array}$ & $\begin{array}{r}N I \\
G \\
A M \\
D \\
S O \\
(M \\
A S \\
100\end{array}$ & $\begin{array}{l}\text { CRO- } \\
\text { EN, } \\
\text { ONIA } \\
\text { CS- } \\
\text { VED } \\
\text { (/L } \\
\text { N) } \\
508)\end{array}$ & $\begin{array}{r}\text { NI } \\
G \\
\text { ORG } \\
D \\
\text { SO } \\
(M \\
\text { AS } \\
100\end{array}$ & $\begin{array}{l}\text { TRO- } \\
\text { EN, } \\
\text { ANIC } \\
\text { IS } \\
\text { LVED } \\
\text { G/L } \\
\text { N) } \\
607 \text { ) }\end{array}$ & $\begin{array}{l}\text { NI } \\
\text { GEN } \\
\text { MON } \\
\text { ORG } \\
\text { DI } \\
\text { M } \\
\text { AS } \\
100\end{array}$ & $\begin{array}{l}\text { TRO- } \\
\text { AM- } \\
\text { IA + } \\
\text { ANIC } \\
\text { S. } \\
\text { G/L } \\
\text { N) } \\
623)\end{array}$ & $\begin{array}{r}\text { PHC } \\
\text { PHO } \\
\text { D } \\
\text { SOI } \\
\text { (M } \\
\text { AS } \\
\text { COO }\end{array}$ & $\begin{array}{l}\text { DS- } \\
\text { RUS } \\
\text { (S- } \\
\text { VED } \\
\text { F/L } \\
\text { P) } \\
(66)\end{array}$ & $\begin{array}{r}\mathrm{PH} \\
\mathrm{PHO} \\
\mathrm{OR} \\
\mathrm{DI} \\
\mathrm{SOL} \\
(\mathrm{MG} \\
\mathrm{AS} \\
\mathrm{CO}\end{array}$ & $\begin{array}{l}\text { S- } \\
\text { US } \\
\text { Ho, } \\
\text { ED } \\
\text { in } \\
\text { i1) }\end{array}$ & $\begin{array}{l}\text { AL } \\
\text { IN } \\
\text { TO } \\
\text { REC } \\
\text { ER } \\
\text { (US } \\
\text { AS } \\
\text { CO1 }\end{array}$ & $\begin{array}{l}\text { LM- } \\
\text { IM, } \\
\text { CAL. } \\
\text { COV- } \\
\text { ABLE } \\
\text { G/L. } \\
\text { AL.) } \\
(05)\end{array}$ & & $\begin{array}{l}\text { UM- } \\
\text { IUM, } \\
\text { IS- } \\
\text { LVED } \\
\text { IG/L } \\
\text { AL, } \\
106)\end{array}$ \\
\hline $\begin{array}{c}\text { AUG } 1996 \\
01 \ldots\end{array}$ & 4930 & & .010 & & 050 & & 170 & & 0.13 & & 0.30 & $<0$ & .010 & $<0$ & 010 & & $<10$ & & 4 \\
\hline DATE & $\begin{array}{c}\text { ARSENIC } \\
\text { TOTAL } \\
\text { (UG/L } \\
\text { AS AS) } \\
(01002)\end{array}$ & $\begin{array}{r}\text { ARS } \\
\text { D } \\
\text { SO } \\
\text { (U } \\
\text { AS } \\
\text { CO1 }\end{array}$ & $\begin{array}{l}\text { ENIC } \\
\text { IS- } \\
\text { VED } \\
\text { G/L } \\
\text { AS ) } \\
000 \text { ) }\end{array}$ & $\begin{array}{c}\text { BAR } \\
\text { TO } \\
\text { RE } \\
\mathrm{ER} \\
\text { (D) } \\
\text { AS } \\
\text { CO1 }\end{array}$ & $\begin{array}{l}\text { UM, } \\
\text { AL, } \\
\text { OV- } \\
\text { BLEE } \\
\text { BL } \\
\text { BA. } \\
075\end{array}$ & $\begin{array}{r}\text { BAR } \\
\text { DI } \\
\text { SOL } \\
\quad \text { I } \\
\text { AS } \\
\angle 01\end{array}$ & $\begin{array}{l}\text { UM, } \\
\text { ED } \\
\text { ED } \\
\text { BA) } \\
\text { O5) }\end{array}$ & $\begin{array}{l}\text { BO } \\
\text { TO } \\
R E \\
\text { ER } \\
\text { (U } \\
\text { AS } \\
\text { CO1 }\end{array}$ & $\begin{array}{l}\text { RON, } \\
\text { TAL } \\
\text { COV. } \\
\text { ABLE } \\
\text { G/L } \\
\text { B) } \\
\text { O22) }\end{array}$ & $\begin{array}{r}\mathrm{BO} \\
\mathrm{D} \\
\mathrm{SO} \\
(\mathrm{L} \\
\mathrm{AS} \\
\mathrm{CO1}\end{array}$ & $\begin{array}{l}\text { RON } \\
\text { IS } \\
\text { L.VED } \\
\text { IG/L } \\
\text { B) } \\
020)\end{array}$ & $\begin{array}{c}\text { CADL } \\
\text { WA } \\
\text { UNFI } \\
\text { TO' } \\
\text { (LY } \\
\text { AS } \\
\text { (O10 }\end{array}$ & $\begin{array}{l}\text { AIUM } \\
\text { TER } \\
\text { TRD } \\
\text { CAL } \\
\text { ज/L } \\
\text { CD } \\
275\end{array}$ & $\begin{array}{r}\text { CAD } \\
\text { D } \\
\text { SO } \\
Y U \\
\text { AS } \\
C 01\end{array}$ & $\begin{array}{l}\text { IIUM } \\
\text { S- } \\
\text { VED } \\
\text { (L } \\
\text { CD) } \\
25)\end{array}$ & $\begin{array}{c}\mathrm{CH} \\
\mathrm{MI} \\
\mathrm{TO} \\
\mathrm{RE} \\
\mathrm{ER} \\
\mathrm{CU} \\
\mathrm{AS} \\
\mathrm{CO}\end{array}$ & $\begin{array}{l}\text { RO- } \\
\text { UM, } \\
\text { TAL } \\
\text { COV- } \\
\text { ABL.E } \\
\text { G/L } \\
\text { CR) } \\
034)\end{array}$ & $\begin{array}{l}\mathrm{CH} \\
\mathrm{MI} \\
\mathrm{DI} \\
\mathrm{SC} \\
(\mathrm{L} \\
\mathrm{AS} \\
\mathrm{CO}\end{array}$ & $\begin{array}{l}\text { RO- } \\
\text { UM, } \\
\text { S- } \\
\text { LVED } \\
\text { G/L } \\
\text { CR) } \\
030 \text { ) }\end{array}$ \\
\hline $\begin{array}{c}\text { AUG } 1996 \\
01 \ldots\end{array}$ & $<1$ & & $<1$ & & 100 & 1 & & & 280 & & 74 & & $<1$ & & .0 & & 3 & & 1.0 \\
\hline DATE & $\begin{array}{c}\text { COPPER, } \\
\text { TOTAL } \\
\text { RECOV- } \\
\text { ERABLE } \\
\text { (UG/L } \\
\text { AS CU) } \\
(01042)\end{array}$ & $\begin{array}{r}\text { COP } \\
\text { DI } \\
\text { SO } \\
\text { (U } \\
\text { AS } \\
\text { CO1 }\end{array}$ & $\begin{array}{l}\text { ER, } \\
\text { SVED } \\
\text { G/1 } \\
\text { CU) } \\
040)\end{array}$ & $\begin{array}{l}\text { IR } \\
\text { TO } \\
\text { RE } \\
\text { ER } \\
\text { CU } \\
\text { AS } \\
101\end{array}$ & $\begin{array}{l}\text { DN, } \\
\text { CAL } \\
\text { COV- } \\
\text { ABLE } \\
\text { J/L } \\
\text { FE) } \\
45)\end{array}$ & $\begin{array}{l}\text { IR } \\
\text { D } \\
\text { SO } \\
\text { (U } \\
\text { AS } \\
\text { CO1 }\end{array}$ & $\begin{array}{l}\text { SN, } \\
\text { (S- } \\
\text { (VED } \\
\text { iL } \\
\text { FE) } \\
\text { (46) }\end{array}$ & $\begin{array}{l}\text { LE } \\
\text { TO } \\
\text { RE } \\
\text { ER } \\
\text { (U } \\
\text { AS } \\
\text { C01 }\end{array}$ & $\begin{array}{l}\mathrm{AD}, \\
\mathrm{TAL} \\
\mathrm{COV}- \\
\mathrm{ABLE} \\
\mathrm{G} / \mathrm{L} \\
\mathrm{PB}) \\
051)\end{array}$ & $\begin{array}{r}\text { LE } \\
\text { D } \\
\text { SO } \\
\text { CU } \\
\text { AS } \\
C 01\end{array}$ & $\begin{array}{l}A D \\
\text { IS } \\
\text { VED } \\
\text { IG/L } \\
P B) \\
049)\end{array}$ & $\begin{array}{c}\text { LITE } \\
\text { TO' } \\
\text { REC } \\
\text { ER } \\
\text { (LUC } \\
\text { AS } \\
\text { C } 01\end{array}$ & $\begin{array}{l}\text { IUM } \\
\text { AL } \\
O \text { OV- } \\
\text { ALEE } \\
\text { (L. } \\
\text { L.I) } \\
(32)\end{array}$ & $\begin{array}{r}\text { LIT } \\
\mathrm{D} \\
\text { SO } \\
6 \mathrm{U} \\
\mathrm{AS} \\
\mathrm{C}\end{array}$ & $\begin{array}{l}\text { IIUM } \\
\text { S- } \\
\text { VED } \\
\text { (L } \\
\text { LI) } \\
30)\end{array}$ & $\begin{array}{l}\text { MA } \\
\text { NE } \\
\text { TO } \\
\text { RE } \\
\text { ER } \\
\text { (U } \\
\text { AS } \\
\text { CO1 }\end{array}$ & $\begin{array}{l}\text { RGA- } \\
\text { SE, } \\
\text { TAL } \\
\text { COV- } \\
\text { ABLE } \\
\text { G/L } \\
\text { MN } \\
055)\end{array}$ & $\begin{array}{r}M A \\
\mathrm{NE} \\
\mathrm{D} \\
\mathrm{SC} \\
\mathrm{CL} \\
\mathrm{AS} \\
\mathrm{C} 01\end{array}$ & $\begin{array}{l}\text { NGA- } \\
\text { SE, } \\
\text { IS- } \\
\text { LVED } \\
\text { G/L } \\
\text { MN) } \\
056)\end{array}$ \\
\hline $\begin{array}{c}\text { AUG } 1996 \\
01 \ldots\end{array}$ & $<1$ & & 1.0 & & $<10$ & & & & $<4$ & & 4.0 & & 50 & & 69 & & 20 & & 5.0 \\
\hline DATE & $\begin{array}{l}\text { MERCURY } \\
\text { TOTAL } \\
\text { RECOV- } \\
\text { BRABLE } \\
\text { (UG/L } \\
\text { AS EG ) } \\
\text { (71900) }\end{array}$ & $\begin{array}{r}\text { MER } \\
D \\
\text { SO } \\
(\mathrm{U} \\
\text { AS } \\
(71\end{array}$ & $\begin{array}{l}\text { JURY } \\
\text { IS- } \\
\text { VED } \\
y / L \\
\text { HG) } \\
390 \text { ) }\end{array}$ & $\begin{array}{l}\text { SE } \\
\text { NI } \\
\text { TO } \\
\text { (U } \\
\text { AS } \\
\text { Co1 }\end{array}$ & $\begin{array}{l}\mathrm{E}- \\
\mathrm{M} \\
\mathrm{AL} \\
\mathrm{AL} \\
\mathrm{SE} \\
\mathrm{LE} \\
(47)\end{array}$ & $\begin{array}{r}\text { SE } \\
\text { NI } \\
\text { D } \\
\text { SO } \\
(\mathrm{U} \\
\mathrm{AS} \\
\mathrm{C} 1\end{array}$ & 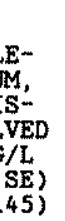 & $\begin{array}{r}\text { SIL } \\
\text { TO } \\
\text { RE } \\
\text { ER } \\
\text { LL } \\
\text { AS } \\
\text { Co1 }\end{array}$ & $\begin{array}{l}\text { VER, } \\
\text { TAL } \\
\text { COV- } \\
A B L E \\
\text { S/L } \\
A G) \\
077 \text { ) }\end{array}$ & $\begin{array}{r}\text { SIL } \\
\text { D } \\
\text { SO } \\
(\mathrm{U} \\
\mathrm{AS} \\
\mathrm{C} 01\end{array}$ & $\begin{array}{l}\text { VER, } \\
\text { IS } \\
\text { LED } \\
G / L \\
A G) \\
075)\end{array}$ & $\begin{array}{l}\text { STF } \\
\text { T } \\
\text { TO } \\
\text { REC } \\
\text { ER } \\
\text { (UTC } \\
\text { AS } \\
\text { C010 }\end{array}$ & $\begin{array}{l}\text { ON- } \\
\text { UM, } \\
\text { AL } \\
\text { OOV- } \\
\text { BLE } \\
\text { (L } \\
\text { SR) } \\
82 \text { ) }\end{array}$ & $\begin{array}{r}\text { ST } \\
T \\
\mathrm{D} \\
\text { SO } \\
\text { (U } \\
\text { AS } \\
\text { C01 }\end{array}$ & $\begin{array}{l}\text { ON- } \\
\text { UM, } \\
\text { S- } \\
\text { VED } \\
\text { /L } \\
\text { SR) } \\
80 \text { ) }\end{array}$ & $\begin{array}{l}\text { ZI } \\
\text { TO } \\
\text { RE } \\
\text { ER } \\
\text { (U } \\
\text { AS } \\
\text { COI }\end{array}$ & $\begin{array}{l}\text { VC, } \\
\text { IAL } \\
\text { COV- } \\
\text { BLE } \\
\text { J/L } \\
\text { ZN) } \\
92)\end{array}$ & & $\begin{array}{l}\text { NC, } \\
\text { IS- } \\
\text { LVED } \\
\text { G/L } \\
\text { ZN) } \\
090 \text { ) }\end{array}$ \\
\hline
\end{tabular}

AUG 1996
$<0.1$
$<0.1$
1
$1<1$
$<1.0 \quad 1100012000$
$<10<15$ 
CHEMICAL, ANALYSES OF ORGANIC COAEOUNDS, WATER YEAR OCTOBER 1995 TO SEPTEMBER 1996

TULAROSA VALLEY BASIN -- Continued

330716106234510 - SALT CREEK 3 AT RANGE ROAD 6 ON WSWR, KM

\begin{tabular}{|c|c|c|c|c|}
\hline DATE & TIME & $\begin{array}{c}2,4-D, \\
\text { TÓTAL } \\
\text { (UG/L) } \\
(39730)\end{array}$ & $\begin{array}{c}2,4,5-T \\
\text { TOTALA } \\
\text { (UG/L) } \\
(39740)\end{array}$ & $\begin{array}{l}2, \quad 4-D F \\
\text { ToTAL } \\
\text { (UG/L) } \\
\text { (E2IB3) }\end{array}$ \\
\hline 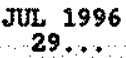 & 1130 & $<0.010$ & $<0,010$ & $<0.010$ \\
\hline
\end{tabular}

331158106265710 - SALT CREEK NR NW-50 ON WSMR, WM

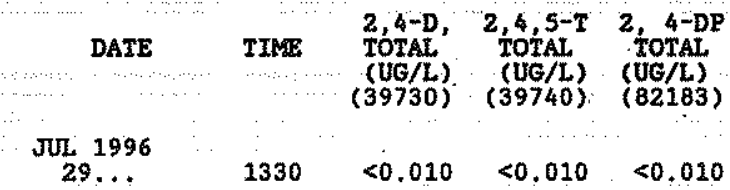

331657106185010 - MALPAIS MARSH NR OSCURA, MM

\begin{tabular}{|c|c|c|c|c|}
\hline DATE & TIME & $\begin{array}{c}2,4-D, \\
\text { TOTAL } \\
\text { (UG/L) } \\
(39730)\end{array}$ & $\begin{array}{c}2,4,5-\mathrm{T} \\
\text { TOTAL } \\
\text { (UG/L) } \\
(39740)\end{array}$ & $\begin{array}{l}2, \quad 4-\mathrm{DP} \\
\text { TOTAL } \\
\text { (UG/L) } \\
(82183)\end{array}$ \\
\hline $\begin{array}{c}\text { UL } 2996 \\
30 \ldots\end{array}$ & 1530 & $<0,010$ & $<0.010$ & 010 \\
\hline
\end{tabular}

332057106211310 - SALT CREEK 4 AT RANGE ROAD 7 ON WSMR, MM

$\begin{array}{ccccc}\text { DATE } & \text { TIME } & \begin{array}{c}2,4-D \\ \text { TOTAL, } \\ \text { (UG/L) } \\ (39730)\end{array} & \begin{array}{c}2,4,5-T \\ \text { TOTAL } \\ \text { (UG/L) } \\ (39740)\end{array} & \begin{array}{c}2,4-D P \\ \text { TOTAL } \\ (\text { UG } / L) \\ (82183)\end{array} \\ \text { JUL } 1996 & 2200 & <0.010 & <0.010 & <0.010 \\ 30 \ldots & 200 & & \end{array}$

332528106170710 - LOWER NOUND SERING POND NR OSCURA, MM

\begin{tabular}{|c|c|c|c|c|}
\hline DATE & TIME & $\begin{array}{c}2,4-D, \\
\text { TOTAL } \\
\text { (UG/L) } \\
(39730)\end{array}$ & $\begin{array}{c}2,4,5-T \\
\text { TOTAL } \\
\text { (UG/L) } \\
(39740)\end{array}$ & $\begin{array}{c}2, \quad 4-D P \\
\text { TOTAL } \\
\text { (UG/L) } \\
\text { (82183) }\end{array}$ \\
\hline & 1520 & $<0.010$ & $<0.010$ & $<0.010$ \\
\hline
\end{tabular}




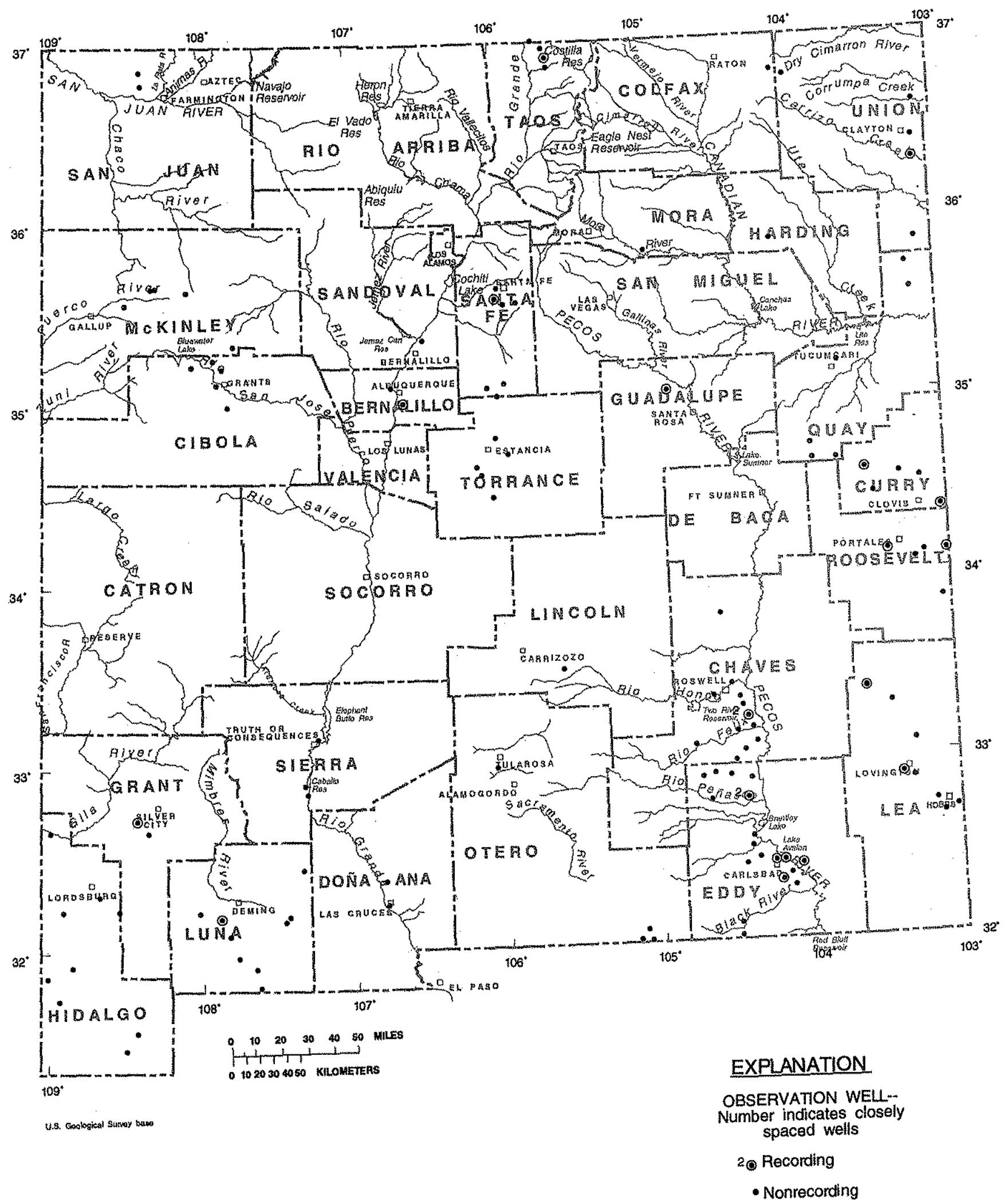

Figure 8.--Location of observation wells. 
350256106390801 , Local number, 10N.03E.32.314.

LOCATION,-Lat $35^{\circ} 02^{\prime} 56^{\prime \prime}$, Long $106^{\circ} 39^{\circ} 08^{\prime \prime}$, Hydrologic Unit 13020203. Owner: Ctty of Albuquerque.

AQUTFER. - Santa Fo Group.'

WELL CHARACTERISTICS. --Drilled water-table observation well, diameter $16 \mathrm{in}$, depth $764 \mathrm{ft}$, perforated $188-764 \mathrm{ft}$.

INSTRUMENTATION.--Digital recorder, 1-hour punch.

DATUM.--Elevation of land-surface datum is 4,941 ft above National Geodetic Vertical Datum of 1929.

Measuring point: Top of casing, $3.00 \mathrm{ft}$ above land-surface datum.

REMARKS.--Lost several days of record, due to recorder malfunction.

PERIOD OF RECORD - 1982 to current year. water level measured, 25.07 ft below land-surface datum, Jan. 5, 1987;

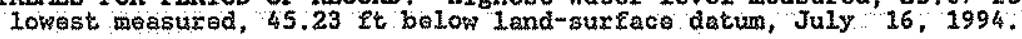

WATER LEVEL, IN FEET BELON LAND-SURFACE DATUM, WATER YEAR OCTOBER 1995 TO SEFTEMBER 1996

\begin{tabular}{|c|c|c|c|c|c|c|c|c|c|c|c|c|}
\hline DAY & $\alpha r$ & Nov & $\mathrm{DEC}$ & JAl & FEB & MAR & APR & MAY & JWN & JUL & AUG & SEP \\
\hline $\begin{array}{r}5 \\
10 \\
15 \\
20 \\
25 \\
\text { EOM }\end{array}$ & $\begin{array}{r}38.90 \\
38.92 \\
38.95 \\
38.61 \\
38.03 \\
37.25\end{array}$ & $\begin{array}{l}37.73 \\
37.70 \\
37.83 \\
36.84 \\
37.24 \\
37.33\end{array}$ & $\begin{array}{l}36.86 \\
36.05 \\
36.48 \\
36.08 \\
35.83 \\
35.39\end{array}$ & $\begin{array}{l}35.32 \\
35.54 \\
35.33 \\
35.46 \\
35.06 \\
35.04\end{array}$ & $\begin{array}{l}34.61 \\
35.06 \\
35.44 \\
35.81 \\
36.75 \\
36.41\end{array}$ & $\begin{array}{l}36.44 \\
36.88 \\
37.88 \\
37.44 \\
37.02 \\
36.96\end{array}$ & $\begin{array}{l}37.47 \\
37.15 \\
38.09 \\
38.16 \\
37.62 \\
37.53\end{array}$ & $\begin{array}{l}38.44 \\
40.21 \\
39.48 \\
39.90 \\
41.78 \\
40.86\end{array}$ & $\begin{array}{l}40.77 \\
40.47 \\
41.65 \\
41.07 \\
40.51 \\
40.17\end{array}$ & $\begin{array}{l}40.23 \\
39.69 \\
38.82 \\
39.65 \\
40.53 \\
41.22\end{array}$ & $\begin{array}{l}40.81 \\
41.24 \\
41.20 \\
40.38 \\
39.81 \\
38.70\end{array}$ & $\begin{array}{r}39.22 \\
38.44 \\
38.28 \\
37.81 \\
- \\
-.-\end{array}$ \\
\hline
\end{tabular}

WTR YEAR 1996 HIGBEST 34.61 FEB 5, 1996 LOWEST 42.83 AUG 3, 1996

351051106395304 . Local number, 11N.03E.18.411.

LOCATION. - Lat $35^{\circ} 10^{\circ} 51^{\prime \prime}$, Long $106^{\circ} 39^{\prime} 53^{\prime \prime}$, Hydrologic Untt 13020203. Owner: C1ty of Albuquerque.

AQUIFER CHARACTERISTICS, -Drilled water-table observation well, casing dianeter 6 in., with 2 tn., F.V.C. piezometer set at 980 ft. casing 18 screened from 870 to $1,050 \mathrm{ft}$.

INSTRUMENTATION.--Monthly steel-tape measurements.

DATUM. - Elevation of land-surface datum is 4,995 ft above National Geodetic Vertical Datum of 1929.

Measuring point: Top of 2 in. P.V.C., $1.80 \mathrm{ft}$, above land-surface datum.

PERIOD OF RECORD, -1982 to current year.

EXTREMES FOR PERIOD OF RECORD.-Fighest water level measured, 28.29 ft below land-surface datum, Feb. 22, 1984 ;

lowest measured; 43.71 ft below land-surface datun, June 27,1996 .

WATER LEVEL, IN FEET BELOW LAND-SURFACE DATUM, WATER YEAR OCTOBER 1995 TO SEPTEMBER 1996

\begin{tabular}{|c|c|c|c|c|c|c|c|c|c|c|c|}
\hline$A T E$ & $\begin{array}{l}\text { WATER } \\
\text { LEVEL }\end{array}$ & DATE & $\begin{array}{l}\text { WATER } \\
\text { LEVEL }\end{array}$ & DATE & $\begin{array}{l}\text { WATER } \\
\text { LEVEL }\end{array}$ & DATE & $\begin{array}{l}\text { WATER } \\
\text { LEVEL }\end{array}$ & DATE & $\begin{array}{l}\text { WATER } \\
\text { LEVEL }\end{array}$ & DATE & $\begin{array}{l}\text { WATER } \\
\text { LEVEL }\end{array}$ \\
\hline $\begin{array}{l}30 \\
29\end{array}$ & $\begin{array}{l}41.73 \\
42.44\end{array}$ & $\begin{array}{l}\text { NOV } 28 \\
\text { MAX } 30\end{array}$ & $\begin{array}{l}41.17 \\
43.25\end{array}$ & $\begin{array}{l}\text { DEC } \\
\text { JUNE } 27\end{array}$ & 43.71 & $\begin{array}{l}\text { JAN } 26 \\
\text { JULY } 31\end{array}$ & $\begin{array}{l}40.77 \\
43.60\end{array}$ & $\begin{array}{ll}\text { FEB } & 26 \\
\text { AUG } & 30\end{array}$ & $\begin{array}{l}40.99 \\
43.59\end{array}$ & $\begin{array}{l}\text { MAR } 26 \\
\text { SEP } 26\end{array}$ & $\begin{array}{l}41.23 \\
43.25\end{array}$ \\
\hline
\end{tabular}

\section{CHAVES COUNTY}

334138104343801. (formerly 334645104344501 ) Local number, 07S.23E.23.24431.

LOCATION --Lat $33^{\circ} 46^{\circ} 45^{\prime \prime}$, Long $104^{\circ} 34^{\circ} 45^{\prime \prime}$, Hydrologic Unit' $13060005^{\circ}$ Owner: Ted Nelson.

AOUIFER. - - San Andres liméstone.

WELL CHARACTERISTICS, --Drilied artesian irrtgation well, diameter 14 in., depth 436 ft.

INSTRLMENTATION. --Pertodic steel-tape measurements.

DATUM. - Elevation of land-surface datum is 3,810 fi above National Geodettc Vertical Datun of 1929.

Measuring point: Lower outer odge of mouth of discharge pipe, 3.71 ft above land-surface datum.

PERIOD OF RECORD. - May 1951 to Mar. 1960, Jan, 1962 to Jan. 1966, Jan. 1968 to current year.

EXTREMES FOR PERIOD OF RECORD,--Highest water leve1 measured, 239.83 ft below land-surface datun, May 26, 1951,

Lowest measured, 290.80 ft below land-surface datum. Aug. 21,1978 .

WATER LEVEL, IN FEET BELOW LAHD-SURFACE DATITA, WATER YEAR OCTOBER 1995 TO SEETEMBER 1996

$\begin{array}{ll}\text { DATE } & \text { WATER } \\ \text { LEVEL } \\ \text { Jan. } 24 & 266.55 \\ \text { AUg. } 15 & 261.00\end{array}$


GROUND-WATER LEVELS

CHAVES COUNTY

Roswoll Basin

332615104303601 . Local number, 10S.24E.21.212.

LOCATION.-Lat $33^{\circ} 26^{\circ} 15^{\prime \prime}$, Long $10^{\circ} 30^{\prime} 36^{\prime \prime}$, Hydrologic Untt 1306000 . Owner: Pecos Valley Artestan Conservancy District.

AQUIFER, --San Andres Limestone. WELL CHARACTERISTICS. --Drilled artesian observation well completed in San Andres Limestone, diameter 10 in,, depth
$324 \mathrm{ft}$.

INSTRUMENTATION --Monthly steel-tape measurements

DATUM.--Elevation of land-surface datum is 3,580,65 ft above National Geodetic Vertical Datum of 1929.

Measuring point: Top of recorder shelf, $3.60 \mathrm{ft}$ above land-surface datum.

REMARKS.--Recorder removed Nov. 26,1990 . Monthly steel-tape measurements.

PERIOD OF RECORD. -June 1940 to current year.

EXTRFMES FOR PERIOD OF RECORD.--Highest water level measured, 6.06 ft below land-surface datum, Jan. 19 , 1946 ; lowest measured, $74.40 \mathrm{ft}$ below land-surface datum, July 30,1977 .

WATER LEVEL, IN FEET BELOW LAND-SURFACE DATUM, WATER YEAR OCTOBER 1995 TO SEPTEMBER 1996

$\begin{array}{llllllllll}\text { DATE } & \text { WATER } & & \text { WATEL } & \text { WATE } & \text { WATER } & \text { WATER } & \text { WATER } & \text { WATER } & \text { WATER } \\ \text { LEVEL } & \text { DATE } & \text { LEVEL } & \text { DATE } & \text { LEVEL } & \text { DATE } & \text { WEVEL }\end{array}$

$\begin{array}{llllllllllll}\text { OCT } 25 & 36.70 & \text { NOV } 27 & 34.30 & \text { DEC } 26 & 33.00 & \text { JAN } 25 & 31.80 & \text { FEB } 26 & 32.30 & \text { MAR } 25 & 34.20\end{array}$

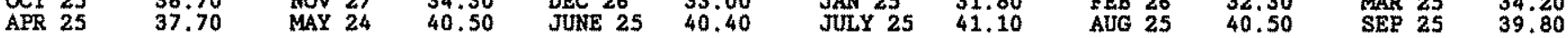

332255104360401 . Local number, 11S.23E.03.342.

LOCATION. - Lat $33^{\circ} 22^{\prime} 55^{\prime \prime}$, Long $104^{\circ} 36^{\circ} 04^{\prime \prime}$, Hydrologic Unit 13060008 . Owner: J. L. Mask.

AQUIFER. - San Andres Limestone.

WELL CHARACTERISTICS. - Drilled artestan observation

INSTRUMENTATION.--Periodic steel-tape measurements.

DATMM.--Elevation of land-surface datum is $3,725 \mathrm{ft}$ above National Grodetic Vertical Datum of 1929 ,

Measuring point: Top of casing 0.50 ft above land-surface datum.

PERIOD OF RECORD.--Mar. 1952 to current year.

EXTREMES FOR PERIOD OF RECORD. - - Highest water level measured, 156.97 ft below land-surface datum, Mar. 11, 1952;

lowest measured, $198.96 \mathrm{ft}$ below land-surface datum, Oct. $18,1985$.

WATER LEVEL, IN FEET BELOW LAND-SURFACE DATUM, WATER YEAR OCTOBER 1995 TO SEPTEMBER 1996

$$
\text { DATE WATER }
$$

Jan. $30 \quad 171.13$

$\begin{array}{ll}\text { Jan. } 30 & 171.13 \\ \text { Aug. } 15 & 176.91\end{array}$

331914104253701. (formerly 331930104261001) Local number, 11S.25E.29.34333.

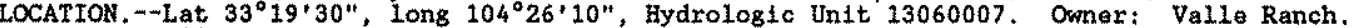

AQUIFER, - -VaIley Fill

WELL CRARACTERISTICS.-Drilled water-table irrigation we11, diameter $16 \mathrm{in.}$, depth $160 \mathrm{ft}$, cased to $160 \mathrm{ft}$

INSTRUMENTATION. -- Feriodic ste日1-tape measurements.

DATUM. --Elevation of land-surface datum is 3,535 ft above National Geodetic Vertical Datum of 1929.

Measuring point: Edge of pung base, southeast corner, at land-surface datum.

PERIOD OF RECORD.--Aug. 1974 to current year.

EXTREMES FOR PERIOD OF RECORD.--Highest water level measured, 12.30 ft below land-surface datum, Aug. 19, 1991;

lowest measured, 21.72 ft below land-surface datum, Aug. 26, 1980.

WATER LEVEL, IN FEET BELOW LAND-SURFACE DATUM, WATER YEAR OCTOBER 1995 TO SEPTEMBER 1996

$\begin{array}{cc}\text { DATE } & \text { WATER } \\ \text { LEVEL } \\ \text { Jan. } 30 & 14.41 \\ \text { Aug. } 16 & \text { pumping }\end{array}$

331705104262801 . (formerly 332200104270001 ) Local number, 12S.25E.09.422.

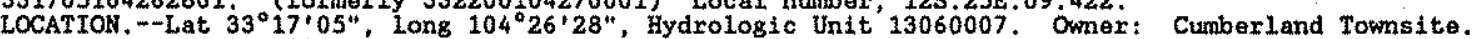

AQUIFER. --Valley Fi11.

WELL CHARACTERISTICS,-Drilled watex-table unused well, diameter $10 \mathrm{in}$, reported depth $90 \mathrm{ft}$, cased to $90 \mathrm{ft}$. INSTRUMENTATION. --Periodic ste日l-tape measurements.

DATUM. --Elevation of land-surface datum is $3,564 \mathrm{ft}$ above National Geodetic Vertical Datum of 1929.

Measuring point: Top of $3 / 4 \mathrm{in}$. collar, $0.62 \mathrm{ft}$ above land-surface datum.

PERIOD OF RECORD.-May 1937 to current year.

lowest measured, $83.06 \mathrm{ft}$ below land-surface datum, Aug. 21, 1973 .

WATER LEVEL, IN FEET BELOW LAND-SURFACE DATUM, WATER YEAR OCTOBER 1995 TO SEPTEMBER 1996

$$
\text { DATE WATER }
$$

Jan. $30 \quad 66.51$

Aug. $16 \quad 69.43$ 


\section{GROUND-WATER IEVELS}

CHAVES COUNTY

Roswell Basin

331525104245201. (formerly 331205104245101) Local number, 12S.25E.23.344.

LOCATION - Lat $33^{\circ} 15^{\prime} 25^{\prime \prime}$, Long $104^{\circ} 24^{\prime} 52^{\prime \prime}$, Hydrologic Unit 13060007 . Omer: Pecos Valley Artesian Conservancy District.

AOUTFER, - -San Andres Limestone.

WELL CHARACTERISTICS,-Drilled artesian observation wel1, diamoter 9 to 7 in., depth 930 ft, 9 in, casing 0-304 ft, 7 in, casing $304-714 \mathrm{ft}$.

TNSTRIMENTATION - Digital recorder 1-hour punch.

DATUM.--Elevation of land-surface datiun is 3,539 ft above National Geodetic Vertical Datum of 1929.

Measuring point: Top of recorder shelf, $2.90 \mathrm{ft}$ above land-surface datum.

REMARKS - Lost racord duo to rocorder malrunction

PERIOD OF RECORD. -Jan. 1966 to current year.

EXTREMES FOR PERIOD OF RECORD.--Highest water level measured, 4.97 ft below land-surface datum, Fob. 9, 1993;

lowest measured, 199.68 ft below land-surface datum, June $20,1978$.

WATER LEVEL, IN FEET BELOW LAND-SURFACE DATUM, WATER YEAR OCTOBER 1995 TO SEPTEMBER 1996 DAILY HIGHEST VALUES

\begin{tabular}{|c|c|c|c|c|c|c|c|c|c|c|c|c|}
\hline DAY & $\infty \mathrm{CT}$ & Nov & DEC & JAN & FEB & MAR & APR & MAY & JUN & JUL & AUG & SEP \\
\hline $\begin{array}{r}5 \\
10 \\
15 \\
20 \\
25 \\
\mathrm{EOA}\end{array}$ & $\begin{array}{l}38.07 \\
36.83 \\
36.87 \\
41.90 \\
37.39 \\
34.37\end{array}$ & $\begin{array}{l}27.91 \\
24.47 \\
23.27 \\
21.07 \\
18.67 \\
16.11\end{array}$ & $\begin{array}{r}16.65 \\
14.33 \\
12.54 \\
10.50 \\
9.61 \\
10.90\end{array}$ & $\begin{array}{l}8.74 \\
7.54 \\
7.33 \\
7.23 \\
8.55 \\
8.41\end{array}$ & $\begin{array}{r}7.86 \\
9.84 \\
14.84 \\
17.57 \\
14.33 \\
20.85\end{array}$ & $\begin{array}{l}26.71 \\
25.89 \\
32.47 \\
48.61 \\
68.38 \\
92.98\end{array}$ & $\begin{array}{r}95.75 \\
95.25 \\
93.51 \\
-. \\
-\end{array}$ & $\begin{array}{r}88.77 \\
114.92 \\
130.30\end{array}$ & $\begin{array}{r}126.14 \\
119.70 \\
112.85 \\
114,47 \\
112.98 \\
89.79\end{array}$ & $\begin{array}{r}--- \\
107.40 \\
108.49 \\
104.69 \\
111.36 \\
97.03\end{array}$ & $\begin{array}{l}95.07 \\
84.95 \\
90.47 \\
94.26 \\
90.25 \\
58.48\end{array}$ & $\begin{array}{l}58.39 \\
67.47 \\
50.28 \\
60.39 \\
60.11 \\
48.75\end{array}$ \\
\hline
\end{tabular}

WIR YEAR 1996 HIGHEST 6.80 JAN 19, 1996 LOWEST 135.02 JUN 7,1996

331524104245101 Local number, $12 \mathrm{~S}, 25 \mathrm{E}, 23,344 \mathrm{~A}$

LOCATrON - Lat $33^{\circ} 15^{\circ} 24^{\circ}$, long $204^{\circ} 24^{\prime} 5 i^{\prime \prime}$, Hydrologic Unit 13060007. Omer: Pecos Valley Artesian Conservancy District.

AQUIFER. - Valley Fill.

WELl CHARACTERISTICS, -Drilled water-table observation well, dianeter 7 in., total depth 231 Pt, cased to total depth, perforated $105-231 \mathrm{ft}$

INSTRUMENTATION, --Digital recorder, 1-hour punch.

DATUM.--Elevation of land-surface datum is 3,540 ft above National Geodetic Vertical Datum of 1929.

Measuring point: Top of recorder shelf 2.90 st above land-surface datum.

REMARKS. - Lost several days of record, due to recorder malfunction.

PERIOD OF RECORD. -1942 to current year.

EXTREMES FOR PERIOD OF RECORD,-Highest water level measured, 101.62 ft below land-surface datum, Apr. 9 , 1995 ; lowest measured, 111,17 below lend"surface datum, Sep. 22, 1980 .

WATER LEVEL, IN FEET BELOW LAND SURFACE DATUM, WATER YEAR OCTOBER 1995 TO SEPTEMBER 1996 DAILY HTGHEST VALUES

\begin{tabular}{|c|c|c|c|c|c|c|c|c|c|c|c|c|}
\hline AY & OCT & NOV & DEC & JAN & FEB & MAR & $\mathrm{APR}$ & MAY & JUN & JUL & AlvG & SEP \\
\hline $\begin{array}{r}5 \\
10 \\
15 \\
20 \\
25\end{array}$ & $\begin{array}{l}103.10 \\
103.10 \\
103.06 \\
103.02 \\
102.96 \\
102.91\end{array}$ & $\begin{array}{l}102.89 \\
102.84 \\
102.88 \\
102.87 \\
102.77 \\
102.65\end{array}$ & $\begin{array}{l}102.68 \\
102.60 \\
102.62 \\
102.47 \\
102.51 \\
102.25\end{array}$ & $\begin{array}{l}102.40 \\
102.25 \\
102.22 \\
102.25 \\
102.02 \\
102.00\end{array}$ & $\begin{array}{l}102.06 \\
102.01 \\
101.91 \\
101.89 \\
101.83 \\
101.75\end{array}$ & $\begin{array}{l}101.69 \\
101.69 \\
101.63 \\
101.64 \\
101.62 \\
101.55\end{array}$ & $\begin{array}{l}101.61 \\
101.37 \\
101.46 \\
101.32 \\
101.35 \\
101.35\end{array}$ & $\begin{array}{l}101.41 \\
101.53 \\
101.48 \\
101.45 \\
101.47 \\
101.52\end{array}$ & $\begin{array}{l}101.56 \\
101.58 \\
101.62 \\
101.70 \\
101.76 \\
101.84\end{array}$ & $\begin{array}{r}101.88 \\
101.96 \\
102.00 \\
102.01 \\
\end{array}$ & 102.34 & $\begin{array}{l}102.44 \\
102.51 \\
102.47 \\
102.51 \\
102.46 \\
102.54\end{array}$ \\
\hline
\end{tabular}

WTR YEAR 1996 HIGHEST 102.32 APR 20, $1996 \quad$ LOWEST $103.23 \quad$ OCT $19,20,1996$

331213104241601. (Eormerly 331216104241701) Local number, 13S.25E.12.311.

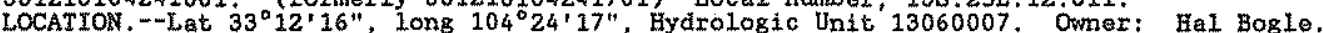

AQUIFER - -ALluvium WEL CHARACTERISTICS.--Drilled water-table irrigation well, diameter 13 in., depth 190 ft.

INSTRUMENTATION. --Periodic ste日l-tape measurements.

DATUM. - Elevation of land-surface datum is 3,506 ft above National Geodetic Vertical Datum of 1929.

Measuring point: Top of casing, 0.80 ft wove iand-surfeco datum.

REMARKS. - " $\mathrm{s}$ " indicates neaxby well pumping.

PERIOD OF RFCORD - -Jan. 1939 to current year.

EXTREMES FOR PERTOD OF RECORD,--Highest water level measured, 16.23 ft below land-surface datum, Feb, 3 , 1942 ;

lowest measured, 90.13 ft below land-surface datum, Aug. 27, 1984 .

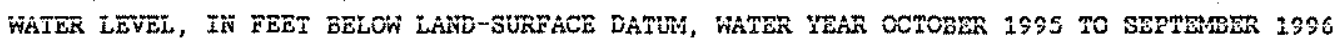

$$
\text { DATE } \quad \text { WATER }
$$

Jan. $30 \quad 82.32$

Aug. $16 \quad 89.15$ 


\section{GROUND-WATER LEVELS}

Roswel1 Basin

331002104254701. (formerly 331002104272001) Local number, 13S.2SE.27.211.

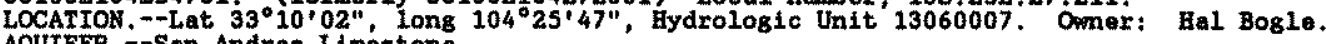

AQUIFER. - San Andres Limestone.

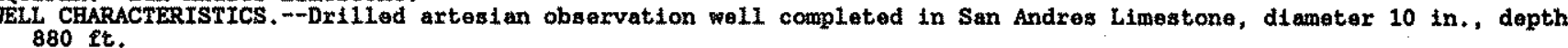

IMSTRUMENTATION --Monthly steel-tape measurements

DATUM.--Elevation of land-surface datum 1: $3,523.76 \mathrm{ft}$ above National Geodetic Vextical Datum of 1929.

Measuring point: Top of recorder shelf $3.59 \mathrm{ft}$ above land-surface datum.

REMARKS - -Recorder removed Kov, 25, 1990. Monthly stee1-tape measurements.

PERTOD OF RECORD. -1940 to current year.

EXTREMES FOR EERIOD OF RECORD.--Highest water lovel moasured, 1.06 ft above land-surface datum, Dec. 27, 1993;

lowest measured, $198,30 \mathrm{ft}$ below land-surface datum, July 18,1980 .

WATER LEVEL, IN FEET BELON LAND-SURFACE DATUM, WATER YEAR OCTOBER 1995 TO SEETEMBER 1996

\begin{tabular}{|c|c|c|c|c|c|c|c|c|c|c|c|}
\hline DATE & $\begin{array}{l}\text { WATER } \\
\text { LEVII. }\end{array}$ & DATE & $\begin{array}{l}\text { WATER } \\
\text { LEVEL }\end{array}$ & DATE & $\begin{array}{l}\text { WATER } \\
\text { LEVEL }\end{array}$ & DATE & $\begin{array}{l}\text { WATER } \\
\text { LEVEL }\end{array}$ & DATE & $\begin{array}{l}\text { WATERR } \\
\text { LEVEL }\end{array}$ & DATE & $\begin{array}{l}\text { WATER } \\
\text { LEVEL }\end{array}$ \\
\hline $\begin{array}{ll}\mathrm{CT} & 25 \\
\mathrm{PR} & 25\end{array}$ & $\begin{array}{r}36.09 \\
132.68\end{array}$ & $\begin{array}{ll}\text { MOV } & 27 \\
\text { MAY } & 24\end{array}$ & $\begin{array}{r}10.28 \\
124.41\end{array}$ & $\begin{array}{l}\text { DEC } 26 \\
\text { JUNE } 25\end{array}$ & $\begin{array}{r}1.72 \\
136.43\end{array}$ & $\begin{array}{l}\text { JAN } 25 \\
\text { JUL } 25\end{array}$ & $\begin{array}{r}2.91 \\
136.61\end{array}$ & $\begin{array}{ll}\text { EEB } & 26 \\
\text { AUG } & 26\end{array}$ & $\begin{array}{l}10.54 \\
95.01\end{array}$ & $\begin{array}{ll}\text { MAR } 25 \\
\text { SAP } 26\end{array}$ & $\begin{array}{l}96.81 \\
63.65\end{array}$ \\
\hline
\end{tabular}

330702104402401. (formerly 330700104402501) Local number, 14S.23E.08.144.

LOCATION.--Lat $33^{\circ} 07^{\prime} 00^{\prime \prime}$, Long $104^{\circ} 40^{\prime} 25^{\prime \prime}$, Hydrologic Unit $13060009^{\circ}$ Omer: M. D. Kincatd.

AQUTFER. - San Andres Limestone

WELL CHARACTERISTICS.--Drilled artesian stock well, diameter 8 in, depth $460 \mathrm{ft}$, casing inforration not avallable. INSTRIMENTATION. --Periodic steel-tape measurements.

DATUM. - Elevation of land-surface datum is $3,844 \mathrm{ft}$ above National Geodetic Vertical Datum of 1929.

Measuring point: Top of casing 1.00 ft above land-surface datum.

PERIOD OF RECORD --Apr. 1940 to current year.

EXTREMES FOR PERIOD OF RECORD,--Highest water level measured, 257.55 ft below land-surface datum, Feb. 9, 1943;

lowest measured, 327.34 ft below land-surface datum, Aug. 27,1967 .

WATER LEVEL, IN FEET BELOW LAND-SURFACE DATUM, WATER YEAR OCTOBER 1995 TO SEPTEMBER 1996

$\begin{array}{ll}\text { DATE } & \text { WATER } \\ \text { LEVEL } \\ \text { Jan. } 29 & 282.56 \\ \text { Aug. } 16 & 285.80\end{array}$

330646104173301. (formerly 330640104174501) Local number, 14S.26E.12.431331.

LOCATION. - Lat $33^{\circ} 06^{\prime} 40^{\prime \prime}$, long $10^{\circ} 17^{\prime} 45^{\prime \prime}$, Hydrologtc Untt 13060007 . Owner: C. B. Donaghay.

AQUIFER. --Va11ey F $\$ 11$.

WELL CHARACTERISTCS.--Drilled water-table 1rrigation we11, diameter 13 in., depth 125 ft, cased $0-125$ ft, perforated $50-115 \mathrm{ft}$

INSTRUMENTATION.--Feriodic steel-tape measurements.

DATUM. - Elevation of land-surface datum is $3,396.4$ ft above National Geodetic Vertical Datum of 1929.

Measuring point: Top of casing at land-surface datum.

PERIOD OF RECORD. - Jan. 1940 to current year.

EXTREMES FOR PERIOD OF RECORD.--Highest water level measured, 12,50 ft below land-surface datum, Jan. 22, 1942;

lowest measured, 23,77 ft below land-surface datum, Aus, 25, 1967 .

WATER LEVEL, IN FEET BELON LAND-SURFACE DATUM, WATER YEAR OCTOBER 1995 TO SEPTEMBER 1996

DATE WATER

Jan. $29 \quad 16.99$

Aug. $16 \quad 16.44$

330404104221201 . Local number, 14S.26E.30.44444.

LOCATION. - Lat $33^{\circ} 04^{\circ} 04^{\prime \prime}$, long $104^{\circ} 22^{\prime} 12^{\prime \prime}$, Hydrologic Untt 13060007. Omer; Bartlett.

AOUITER -- San Andres Limestone.

WELL CHARACTERISTICS. -DDrilled artesian observation well, diameter $85 / 8$ in., depth $1,150 \mathrm{ft}$, cased to $740 \mathrm{ft}$, open hole $740-1,150$ ft.

INSTRUMENTATIÓN.--Perłodic steel-tape measurements.

DATUM. - Elevation of land-surface datum is $3,484 \mathrm{ft}$ above National Geodetic Vertical Datum of 1929.

Measuring polnt: Top of casing $1.00 \mathrm{ft}$ above land-surface datum.

PERIOD OF RECORD. - Fob. 1970 to current year.

EXTREMES FOR PERIOD OF RECORD. - -Haghest water level measured, 58,10 ft below land-surface datum, Feb. 11 , 1993 ;

loweat measured, $276.99 \mathrm{ft}$ below land-surface datum, Aug. 17, 1995.

WATER LEVEL, IN FEET BELOW LAND-SURFACE DATUM, WATER YEAR OCTOBER 1995 TO SEPTEMBER 1996

DATE WATER

Jan, $29 \quad 71.26$

Aug. $16 \quad 218.88$ 
350346107521201. (formerly 350400107510501) Local number, 10N.10W.26.331.

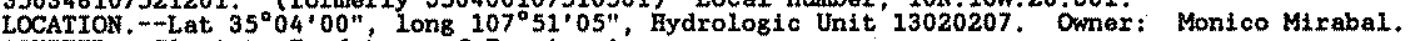
AQUIFER. -Glorieta Sandstone of Permian Age,

WELL CHARACTERISTICS.--Drilled artesian Irxigation we11, diameter 16 in., depth $216 \mathrm{ft}$

INSTRUMENTATION. - Periodic steel-tape measurements.

DATUM.-Elevation of land-surface datum $1 \$ 6,455$ ft above National Geodetic Vertical Datum of 1929.

Heasturing point: Top of $1 / 2$ in hole in pump base, 1,00 ft above land-surface datum.

PERIOD OF RECORD - $-\mathrm{Feb}, 1952$ to current year.

EXTREMES FOR EERIOD OF RECORD.-HIghest pater level measured, 22. 18 ft below land-surface datum, Feb. 21, 1952 ;

lowere messured, 34.69 st bolow land-surface datim, Jan. 17,1977 .

WATER LEVEL, IN EEET BELOW LAND-SURFACE DATUM, WATER YEAR OCTOBER 1995 TO SEPTEMBQR 1996

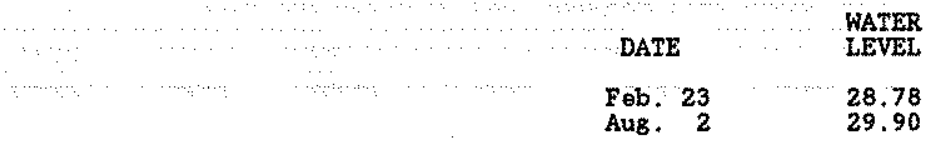

350923107522701. (formerly 350925107523001) Local number, 11N.10W.27.242.

LOCATION, - - Lat $35^{\circ} 09^{\circ} 25^{\prime \prime}$, long $107^{\circ} 52^{\prime} 30^{\prime \prime}$, Rydrologic Untt 13020207. Owner: City of Grants.

AQUIFER. - San Andres Limestone.

WELL CHARACTERISTICS,--Drilled water-table unused well, diameter 16 to 12 in, depth 158 ft, perforated 50 to

150 ft.

DATUM. --Elevation of land-surface datum is 6,480 fit above National Goodetic vertical Datum of 1929 .

Measuring point: Top of casing at land-surface datum

PERTOD OF RECORD. - -Feb. 1953 to current year.

EXTREMES FOR PERIOD OF RECORD, - Hilghest water level measured, 19.23 ft below land-surface datum, Sep. 29, 1988;

lowest measured, $39.08 \mathrm{st}$ below land-surface datum, Aug: 1, 1972 .

WATIX LEVEL, IN FEET BELOW LAND-SURFACE DATUM, WATER YEAR OCTOBER 1995 TO SEPTEMBER 1996

$\begin{array}{cr}\text { DATE } & \text { WATER } \\ \text { FEb. } 23 & \text { LEVEL } \\ \text { AUB. } & 21.53\end{array}$

$\begin{array}{ll}\text { Feb. } & 23 \\ \text { Aug. } & 21\end{array}$

351304107543701." (rormerly 351400107524201) Local number, 12N.10W.29.434.

LOCATION. -Lat $35^{\circ} 1^{\prime} 00^{\circ}$, Long $107^{\circ} 52^{\prime} 42^{\prime \prime}$, Hydrologio Unit' $13020207^{2}$. Owmer: Plains Electric.

AOUIFER, - -San Andres limestone.

WELL CHARACTERISTICS,-Dr111ed artesian unused we11, diameter 18 in, reported depth 205 ft, cased 0-150 ft, perforated $93-130$ it

TNSTRUMENTATYON - Feriodic steel-tape measurements.

DATUM.--Elevation of land-surface datum is 6,552 fit above National Geodetic Vertical Datum of 1929.

Measuring point: Lower edge of hole in north side of casing, 2.20 ft above land-surface datum.

PERIOD OF RECORD.--OCt. $1944, \mathrm{Feb} .1946$ to current year.

EXTREMES FOR EERIOD OF RECORD. - -Highest water level measured, 65.46 ft below land-surface datum, Oct. 14, 1944;

lowest measured, $107.61 \mathrm{ft}$ below land-surface datum, Aug. 6, 1975.

WATER LEVEL, IN FEET BELOW LAND-SURFACE DATUM, WATER YEAR OCTOBER 1995 TO SEPTEMBER 1996

$\begin{array}{ll}\text { DATE } & \begin{array}{l}\text { WATER } \\ \text { LEVEL }\end{array} \\ \text { Fob. 23 } & 77.96 \\ \text { Aug. 2 } & 78.26\end{array}$

351651107594501. (Formerly 351650107535001) Local number, 12N.11W.09.424.

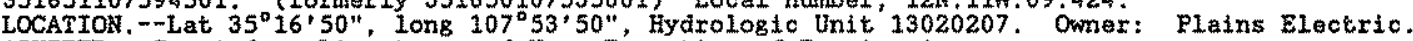

AQUIFER.-Sen Andres Limestone and Yeso Formation or Permian Age.

WELL CHARACTERISTICS. -Drilled artesian unused well, diameter 16 in., reported depth 505 ft, 16 in. casing to $175 \mathrm{ft}, 12 \mathrm{in}$. casing to $325 \mathrm{ft}$.

INSTRUMENTATION.--Monthly steel-tape measurements.

DATHA. - Elevation of land-surface datum is 6,642 ft above National Goodetic Vertical Datum of 1929 .

Megeuring point: Top of cestng 3.05 ft above 1and-sureace detum.

PERTOD OF RECORD. - May. 1946 to current year.

EXTREMES FOR PERIOD OF RECORD.-Highest water Level measured, 86.69 ft below landwsurface datum, Sep. 29, 1988; lowest measured, $274.81 \mathrm{ft}$ below land-surface datum, Jan. 23,1984 .

WATER LEVEL, IN FEET BELOW LAND-SURFACE DATUM, WATER YEAR OCTOBER 1995 TO SERTEMBER 1996

\begin{tabular}{|c|c|c|c|c|c|c|c|c|c|c|c|}
\hline DATE & $\begin{array}{l}\text { WATEK } \\
\text { LEVEL }\end{array}$ & DATE & $\begin{array}{l}\text { WATEK } \\
\text { LEVEL }\end{array}$ & DATE & $\begin{array}{l}\text { WATEK } \\
\text { LEVEL }\end{array}$ & DATE & $\begin{array}{l}\text { WAEER } \\
\text { LEVEL }\end{array}$ & DATE & $\begin{array}{l}\text { WATHEK } \\
\text { LEVEL }\end{array}$ & DATE & $\begin{array}{l}\text { WATER } \\
\text { LEVEL }\end{array}$ \\
\hline $\begin{array}{ll}\text { CT } & 30 \\
\mathrm{PR} & 16\end{array}$ & $\begin{array}{l}89.75 \\
93.81\end{array}$ & $\begin{array}{l}\text { NOV } 21 \\
\text { MAY } 21\end{array}$ & $\begin{array}{l}90.82 \\
93.35\end{array}$ & $\begin{array}{l}\text { DEC } \\
\text { JUNE } 18\end{array}$ & 92.44 & $\begin{array}{l}\text { JAN } 18 \\
\text { JULY } 23\end{array}$ & $\begin{array}{l}92.51 \\
93.06\end{array}$ & $\begin{array}{ll}\text { FEB } & 21 \\
\text { AUG } & 20\end{array}$ & $\begin{array}{l}93.10 \\
94.64\end{array}$ & $\begin{array}{ll}\text { MAR } 19 \\
\text { SEP } 25\end{array}$ & $\begin{array}{l}93.43 \\
95.37\end{array}$ \\
\hline
\end{tabular}


GROUND-WATER LEVELS

CIBOLA COUNTY

Grants-Bluewater Area

351630107572801 (formerly 351637107584501 ) Local number, $12 N .11 \mathrm{~W} .14 .213$.

LOCATION. - Lat $35^{\circ} 16^{\circ} 37^{\prime \prime}$, long $107^{\circ} 58^{\prime} 45^{\prime \prime}$, Hydrologic Unit' 13020207. Owner: Duane Berryh111.

AQUIFER.--San Andres Liméstone and Yeso Formation of Permian Age.

WELL CHARACTERISTICS. --Drilled test well, diameter $4 \mathrm{in}$, depth $130.4 \mathrm{ft}$.

INSTRUMENTATION. --Periodic steel-tape measurements.

DATUM. - Elevation of land-surface datum is $6,605 \mathrm{ft}$ above National Geodetic Vertical Datum of 1929.

Measuring point: Top of casing, $3.70 \mathrm{ft}$ above land-surface datum.

PERIOD OF RECORD.--June 1949 to current year.

EXTREMES FOR PERIOD OR RECORD.--Highest water Level measured, 81.74 ft below land-surface datum, Sep. 25, 1986;

lowest measured, $101.39 \mathrm{ft}$ below land-surface datum, June 10, 1954.

WATER LEVEL, IN FEET BELOW LAND-SURFACE DATUM, WATER YEAR OCTOBER 1995 TO SEPTEMBER 1996

DATE WATER

Feb. $23 \quad 83.29$

83.29
Aus. 2

\section{COLFAX COUNTY}

364522104034501 . (formerly 364500104031501) Local number, 29N.27E.16.222.

LOCATION. -Lat $36^{\circ} 45^{\circ} 00^{\prime \prime}$, long $10^{\circ} 03^{\prime} 15^{\prime \prime}$, Hydrologic Unit 11040001. Owner: John King.

AQUTFER, --Alluvi um.

WELL CBARACTERISTICS. --Drilled water-table unused well, diameter $8 \mathrm{in}$, depth $120 \mathrm{ft}$, cased to $20 \mathrm{ft}$.

INSTRUMENTATION. --Perlodic steel-tape measurements.

DATUM. - Filevation of land-surface datum is $6,821.5$ ft above National Geodetic Vertical Datum of 1929.

Measuring point: Top of casing, $1.50 \mathrm{ft}$ above 1and-surface datum.

PERIOD OF RECORD. - Feb. 1957 to Feb. 1969, Feb. 1971 to current year.

EXTREMES FOR PERIOD OF RECORD.--Highest water level measured, $4.65 \mathrm{ft}$ below land-surface datum, Feb. 3, 1960;

lowest measured, $9.37 \mathrm{ft}$ below land-surface datum, Aug. 13, 1975 .

WATER LEVEL, IN FEET BELOW LAND-SURFACE DATUM, WATZR YEAR OCTOBER 1995 TO SEETEḾEER 1996

$\begin{array}{cr}\text { DATE } & \begin{array}{r}\text { WATER } \\ \text { ILVEL }\end{array} \\ \text { Jan. 23 } & 8.39 \\ \text { July 24 } & 8.84\end{array}$

COSTILLA COUNTY (in Colorado)

Sunshine Valley

370004105402201. (formerly 370009105410001) Local number, 01N.74W.33.322.

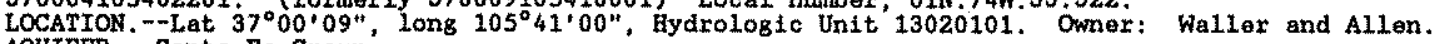

AQUTFER. - - Santa Fe Group.

WELI CHARACTERISTICS. --Drilled water-table unused well, dianeter $15 \mathrm{ln}$, depth $232 \mathrm{ft}$, casing information not avallabie.

INSTRUMENTATION. --Periodic steel-tape measurements.

DATUM.--Elevation of land-surface datum is 7,495 ft above National Geodetic Vertical Datum of 1929.

Measuring point: Edge of hole inside pump base, $2.00 \mathrm{ft}$ above land surface-datun (since 1971).

PERIOD OF RECORD - Feb. 1966 to current year.

EXTREMES FOR PERIOD OF RECORD.-- Eighest water level measured, $101.82 \mathrm{ft}$ below land-surface datum, Aug. 26, 1968; lowest measured, $139.24 \mathrm{ft}$ below land-surface datum, Sep. $2,1982$.

WATER LEVEL, IN FEET BELOW LAND-SURFACE DATUM, WATER YEAR OCTOBER 1995 TO SEPTEMBER 1996

DATE WATER

Feb. $15 \quad 111.17$

Aug. $5 \quad 138.40$ 
341836103052001 . Local number, 01N.37E.17.113133

LOCATION, --Lat $34^{\circ} 18^{\prime} 53^{\prime \prime}$, long $103^{\circ} 05^{\prime} 26^{\prime \prime}$, Hydrologic Unit 12050002, Omner: Don Oppliger.

AQULF CHARACTERISTICS. --DFilled water-toble unused mo11, dimeter 16 in., depth 373 st, screened 293-373 ft. INSTRUNENTATION, --Digital recorder, 1-hour punch.

DATUM.--Elevation of land-aurface datum is 4,113 ft above National Gaodetic Vertical Datum of 1929.

Measuring point: Top edge of recorder ghelter apron, 3.93 \&t ebove land-surface datum.

REMARKS. - Lost record due to recorder malfunction.

EERTOD OF RECORD.--Jan. 1982 to current year.

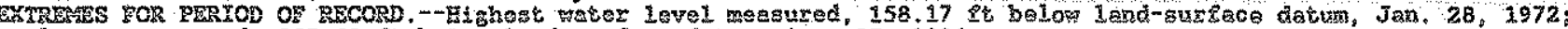

lowest measured, $257.63 \mathrm{ft}$ belog land-8uxface datum, Aug. 27, 1996.

WATER LEVEL, IN FEET BELON LAND-SURFACE DATUM, WATER YEAR OCTOBER 1995 TO SEPTOMBER 1996

\begin{tabular}{|c|c|c|c|c|c|c|c|c|c|c|c|c|}
\hline $\mathrm{AY}$ & $\infty$ & ROV & DEC & JAN & FEB & MAAR & APR & MAY & JUN & JuL. & AUG & SEP \\
\hline $\begin{array}{r}5 \\
10 \\
15 \\
20 \\
25 \\
\text { ECO4 }\end{array}$ & $\begin{array}{l}246.74 \\
246.65 \\
246.61 \\
246.48 \\
246.45 \\
246.43\end{array}$ & $\begin{array}{l}246.33 \\
246.15 \\
246.38 \\
246.41 \\
246.16 \\
246.14\end{array}$ & $\begin{array}{l}246.10 \\
246.13 \\
246.32 \\
246.02 \\
246.15 \\
245.67\end{array}$ & $\begin{array}{l}246.12 \\
245.70 \\
245.72 \\
245.73 \\
245.42 \\
245.55\end{array}$ & $\begin{array}{r}245.56 \\
=- \\
-\cdots \\
-\cdots\end{array}$ & $\begin{array}{r}248.17 \\
248.55 \\
248.79 \\
247.39\end{array}$ & $\begin{array}{l}247.24 \\
247.15 \\
247.21 \\
247.61 \\
248.53 \\
248.73\end{array}$ & $\begin{array}{r}248.25 \\
248.91 \\
248.52 \\
-\end{array}$ & $\begin{array}{r}--- \\
--0 \\
252.68 \\
253.37 \\
253.70\end{array}$ & $\begin{array}{l}254.16 \\
254.96 \\
254.96 \\
254.87 \\
255.50 \\
255.86\end{array}$ & $\begin{array}{l}256.37 \\
256.77 \\
256.92 \\
257.30 \\
257.37 \\
256.55\end{array}$ & $\begin{array}{l}256.31 \\
256.26 \\
256.03 \\
256.12 \\
255.87 \\
256.08\end{array}$ \\
\hline
\end{tabular}

WTR YEAR 1996 HIGEST 245.30 JAN 17, 1996 LOWEST 257.63 AUG 27, 1996

342358103093601 . LOCEI number, O2N.36E.15.11111.

LOCATION, - Lat $34^{\circ} 23^{\prime} 58^{\prime \prime}$, Long $103^{\circ} 09^{\prime} 36^{\prime \prime}$, Eydrologle Unit 12050002 . Owner: Anne Humphreys.

AQUIFER. - Ogallala formation.

WELL CEARACTERISTICS.--Drilled water-table irrigation well; diameter, depth and casing information not available.

INSTRUMENTATION. --Periodic steel-tape measurements.

DATUM. - Elovation of land-surface datum 18 4,227 ft abovo National Goodetic Vortical Datum of 1929.

Moasuring point: Top of concrete base 1.20 ft above land-surface datum.

PERIOD OF RECORD. --Jan. 1974 to current yoar.

EXTREMES FOR PERIOD OF RECORD. --Highest water level measured, 266.89 ft below land-surfece datum, Jan, 4, 1974;

lovest measured, 291.29 Lt below land-surfece datum, Aug. 6, 1985.

WATER LEVEL, IN FEET BELOW LAND-SURFACE DATUM, WATER YEAR OCTOBER 1995 TO SEPTEMBER 1996

$\begin{array}{lr}\text { DATE } & \text { WATER } \\ \text { LEVEL } \\ \text { Jan. } 10 & 288.42 \\ \text { Aug. } 9 & 290.95\end{array}$

342736103203701. (Formerly 342815103270001) Local number, 03N.34E.23.433133.

LOCATION, --Lat $34^{\circ} 27^{\prime} 36^{\prime \prime}$, long $103^{\circ} 20^{\prime} 37^{\prime \prime}$, Bydrologic Unit 12050001. Owner: Archie Baker.

AQUIFER. - Ogallala formation.

WELL CHARACTERISTICS.-Drilled mater-table unused we11, diameter $16 \mathrm{ln}$, depth $418 \mathrm{ft}$, cased to 418 ft, perforated 365-418 ft.

INSTRUMENTATION. --Periodic stool-tape measurements.

DATUM.--Elevation of land-surface datum is 4,432 ft above National Geodetic Vertical Datum of 1929.

Mensuring point: Top of casing, 0.40 ft abovo lend-surface datum.

PERIOD OF RECORD.-Apr. 1954 to current year.

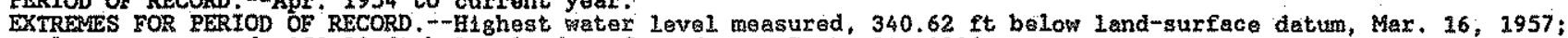

lonest mezsured, 359.39 ft below land-surface datum, Sept. 24, 1996.

WATER LEVEL, IN FEET BELON LAND-SURFACE DATUM, WATER YEAR OCTOBER 1995 TO SEPTEMBER 1996

$$
\text { DATE WATER }
$$

Jan. il $35 \overline{9} .20$

Sep. $24 \quad 359.39$


GROUND-WATER LEVELS

CURRY COUNTY

Clovis area

343347103345001 . Local number, 04N.32E. 22.111.

LOCATYON. - Lat $34^{\circ} 33^{\prime} 47^{\prime \prime}$, long $103^{\circ} 34^{\circ} 50^{\prime \prime}$, Eydrologic Untt 12050001. Owner: Noel Dougherty. AQUIFER - - Ogallala formation.

WELL CHARACTERISTICS. -Drilled water-table observation well, diameter $16 \mathrm{ln}$, depth $401 \mathrm{ft}$

INSTRUMENTATION.--Continuous strip-chart recorder.

DATUM.--Elevation of land-surface datum is 4,587 ft above National Geodetic Vertical Datum of 1929

Measuring point: Edge of recorder shelter, $3.50 \mathrm{ft}$ above land surface datum.

REMARKS. - Recorder installed Aug. 1988. Lost record due to recorder malfunction.

PERIOD OF RECORD. - -Jan. 1980 to current year.

EXTREMES FOR PERIOD OF RECORD.--Highest water level measured, 297.64 ft above land-surface datum, Feb. 26, 1995;

lowest measured, 309.92 ft below land-surface datun, Jan.' 9, 1981.

WATER LIEVEL, IN FEET BELOW LAND-SURFACE DATUM, WATER YEAR OCTOBER 1995 TO SEPTEMBER 1996 DAILY HIGHEST VALUES

\begin{tabular}{|c|c|c|c|c|c|c|c|c|c|c|c|c|}
\hline DAY & $\infty r$ & Nov & DEC & JAN & FEB & MAR & APR & MAX & JUN & JUL & AUtG & SEP \\
\hline $\begin{array}{r}5 \\
10 \\
15 \\
20 \\
25 \\
\text { EOM }\end{array}$ & $\begin{array}{l}297.63 \\
297.76 \\
297.68 \\
297.60 \\
297.58 \\
297.48\end{array}$ & $\begin{array}{l}297.48 \\
297.29 \\
297.64 \\
297.68 \\
297.42 \\
297.47\end{array}$ & $\begin{array}{l}297.47 \\
297.53 \\
297.72 \\
297.45 \\
297.67 \\
297.06\end{array}$ & $\begin{array}{l}297.72 \\
297.30 \\
297.44 \\
297.49 \\
297.21 \\
297.31\end{array}$ & $\begin{array}{l}297.45 \\
297.38 \\
297.43 \\
297.41 \\
297.33 \\
297.42\end{array}$ & 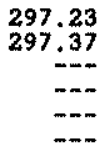 & & & $\begin{array}{r}--- \\
297.42 \\
297.49 \\
---\end{array}$ & $\begin{array}{l}297.45 \\
297.55 \\
297.52 \\
297.46 \\
297.50 \\
297.41\end{array}$ & \begin{tabular}{r}
297.38 \\
297.40 \\
-9 \\
\hdashline- \\
-
\end{tabular} & $\begin{array}{r}--- \\
-\cdots \\
297.20 \\
297.42\end{array}$ \\
\hline
\end{tabular}

WTR YEAR 1996 HIGHEST 296.84 JAN 17, 1996 LOWEST 298.08 OCT 19,20, 1995

343615103123801 . Local number, 0.5N.35E.35.313.

LOCATION. - Lat $34^{\circ} 36^{\circ} 15^{*}$, long $103^{\circ} 12^{\prime} 38^{\prime \prime}$, Hydrologic Unit 12050005 . Owner: S. W. Pipkin.

AQUIFER. - Ogallala formation.

WELL CRARACTERISTICS,--Drillod water-table irrigation woll, diameter $16 \mathrm{ln}$, depth $527 \mathrm{ft}$

INSTRUMENTATION. --Periodic steel-tape measurements.

DATUM.--Elevation of land-surface datum is 4,504 ft above National Geodetic Vertical Daturn of 1929.

Measuring point: Top of casing 0.50 ft above Land-surface datum.

PERIOD OF RECORD - - Jan. 1954 to current year.

EXTREMES FOR PERIOD OF RECORD.--Highest water level measured, $376.40 \mathrm{ft}$ below land-surface datum, Mar. 26, 1954; lowest maasured, 450.61 ft below land-surface datum, Aug.' 9, 1996.

WATER LEVEL, IN FEET BELOW LAND-SURFACE DATUM, WATER YEAR OCTOBER 1995 TO SEPTEMBER 1996

$\begin{array}{cr}\text { DATE } & \begin{array}{r}\text { WATER } \\ \text { LEVEL }\end{array} \\ \text { Jan. 9 } & 449.60 \\ \text { Aug. 9 } & 450.61\end{array}$

DONA ANA COUNTY

Rincon and Mestilla Valleys

322203106484101 . (formerly 3222.10106483001) Local number, 22S.01E.26.411.

LOCATION. - Lat $32^{\circ} 22^{\prime} 10^{\prime \prime}$, Long $106^{\circ} 48^{\prime} 30^{\prime \prime}$, Eydrologic Unit 13030102. Owner: H. Wortheim.

AQUTFER.--Valley Fill.

WELL CHARACTERISTICS.--Drilled water-table irrigation well, diameter $18 \mathrm{fn}$., depth $107 \mathrm{ft}$, cased to $107 \mathrm{ft}$

DATUM.--Elevation of land-surface datum is 3,920 ft above National Geodetic Vertical Datum of 1929.

Measuring point: Top of east side of casing, $1.50 \mathrm{ft}$ above land-surface datum.

PERIOD OF RECORD.--Apr. 1957 to current year.

EXTREMES FOR PERIOD OF RECORD.---Highest water level measured, 9.67 ft below landusurface datum, July 23, 1993;

lowest measured, $25.57 \mathrm{ft}$ below land-surface datum, Apr. 25, 1957 .

WATER LEVEL, IN FEET BELOW LAND-SURFACE DATUM, WATER YEAR OCTOBER 1995 TO SEPTEMBER 1996

$\begin{array}{ll}\text { DATE } & \text { WATER } \\ \text { LEVEL } \\ \text { Feb. 9 } & 11.92 \\ \text { Aug. 29 } & 10.37\end{array}$


321606106462901. (formerly 321620106461501) Local number, 23S.02E.31.213.

LOCATIOR. --Lat $32^{\circ} 16^{\circ} 20^{\prime \prime}$, long $106^{\circ} 46^{\prime} 1^{\prime \prime}$, Hydrologic Unit 13030102. Owner: New Mexico State University.

AQUIFER --Valley Fill. -Drilled water-tablo irrigation well, diameter $14 \mathrm{in}$, reported depth $70 \mathrm{ft}$, cased to $70 \mathrm{ft}$. INSTRUMERTATION, --Poriodic steel-tape measurements.

DATUM. - -Elevation of Iand-surface datum $1 \$ 3,880$ \&t above National Geodetic Vertical Datum of 1929.

Measuring point: Top of $5 / 8$ in. hole in pump base, 1.08 et above land-surface datum.

PERIOD OF RECORD. - Fob. 1948 , Apr. 1957 to current year.

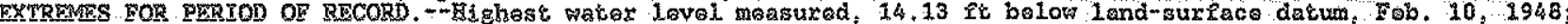

lowest measured, $29.12 \mathrm{ft}$ below land-surface datum, Jan. $7,1958$.

WATER LEVEL, IN FEET BELOW LARD-SURFACE DATUM, WATER YEAR OCTOBER 1995 TO SEPTERERR 1996

$\begin{array}{cc}\text { DATE } & \text { MATER } \\ \text { LEVEL } \\ \text { Eob. } 15 & 19.08\end{array}$

EDDY COUNTY

325516104404601. (qormerly 325510104410001) Locel number, 16S.23E.15.322333

LOCATION.--Lat $32^{\circ} 5^{\prime} 1^{\prime \prime}$, Long $104^{\circ} 41^{\prime} 00^{\prime \prime}$, Hydrologtc Unde 13060007 . Owner: D. W. Runyan.

AOUIFER. -Yoso Eormation

WELE CHARACTERISTICS, -Drilled ol1 test well, used for stock water, diameter 10 in, depth 1,458 It, cased.

INSTRUMENTATION. --Periodic steel-tape measurements.

DATUM. - Elevation of land-surface datum is 3,807 ft above National Geodetic vertical Datum of 1929.

Measuring point: Top of casing $0.70 \mathrm{ft}$ above land-surface datum

PERTOD OF RECORD.--Jan, 1951 to Jan. 1965, Fob. 1970 to Aug, 1971, Jan. 1974 to current year.

EXTREMES FOR PERIOD OF RECORD.--Highest water level measured, 225, 16 ft below land-surface datum, Jan. 12, 1951;

lowest measured, 277,60 It below land-surface datum, Aug, s, 1971.

WATER LEVEL, IN FEET BELOW LAND-SUREACE DATUM, WATER YEAR OCTOBER 1995 TO SEPTEMBER 1996

$\begin{array}{cr}\text { DATE } & \text { WATER } \\ \text { LEVEL } \\ \text { Jan. 26 } & 231.74 \\ \text { Aug. 16 } & 235.77\end{array}$

325702104352801 . (formexly 325735104360701 ) Local number, 16S.24E.04. 411341 .

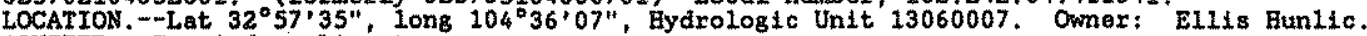

AQUIFER.--San Andres Limestone.

WELL CHARACTERISTICS.-Drilled artesian irrigation well, diameter not avallable, depth $610 \mathrm{ft}$

INSTRUMENTATION.---Periodic steel-tape measurements

DATUM. - Elevation of land-surface datum 1s 3,624 ft above National Geodetic Vertical Datum of 1929.

Measuring point: Southriest side of pump, $1.50 \mathrm{ft}$ above land-surface datum.

PERTOD OF RRCORD:--Jan. 1969 to current yoar.

EXTREMES FOR PERIOD OF RECORD.--Highest water level measured, 52.48 ft below land-surface datum, Jan. 29, 1996;

Lopest measured, 100.54 ft below land-surface datum, Aug. 27, 1974.

WATER LEVEL, IN FEET BELOW LAND-SURFACE DATUM, WATER YEAR OCTOBER 1995 TO SERTEMBER 1996

$\begin{array}{cc}\text { DATE } & \text { WATER } \\ \text { LEVEL } \\ \text { Jan. 29 } & 52.48 \\ \text { Aug. 16 } & \text { punping }\end{array}$

325638104274801 . Local number, 16S, 25E.11.111131A.

LOCATION,-Lat $32^{\circ} 56^{\circ} 38^{*}$, Long $10^{\circ} 27^{\circ} 48^{\prime \prime}$, Bydrologic Unit 13060007. Owner: Pecos Valley Artesian Conservancy District.

AQUTFER. - Valley F111.

WELL CHARACTERISTICS.--Drilled water-table observation woll, dtemeter 7 in., depth 171 st, casing 0-171 $5 t$, perforated $94-170 \mathrm{rc}$

INSTRIUENTATION.-Recorder removed Nov, 27, 1990. Monthly steel-tape messurements.

DATUM. - Elovation of land-surface datum is 3,450 ft above National Geodetic Vertical Datum of 1929.

Maasuring point: Top of recordex shelf 3.00 ft above land-surface datur.

PERIOD OF RECORD -1964 to current year.

EXTPEMES FOR PERIOD OF pECORD. --Híghest weter level measured, 39.90 ft below land-surface datum, Feb. 18, 1966;

lowest measured, $64,72 \mathrm{ft}$ below land-surface datum, July $24,1986$.

WATER LEVEL, IN FEET BELOW LAND-SURFACE DATUM, WATER YEAR OCTOBER 1995 TO SEPTEMBER 1996

\begin{tabular}{|c|c|c|c|c|c|c|c|c|c|c|c|}
\hline DAI & $\begin{array}{l}\text { WATER } \\
\text { LEVEL }\end{array}$ & DATE & $\begin{array}{l}\text { WATER } \\
\text { LEVEL }\end{array}$ & DATE & $\begin{array}{l}\text { WATER } \\
\text { LEVEL }\end{array}$ & DATE & $\begin{array}{l}\text { WATER } \\
\text { LEVEL }\end{array}$ & DATE & $\begin{array}{l}\text { WATER } \\
\text { LEVEI }\end{array}$ & DATE & $\begin{array}{l}\text { WATER } \\
\text { LEVELE }\end{array}$ \\
\hline $\begin{array}{l}23 \\
R \\
19\end{array}$ & $\begin{array}{l}61.07 \\
61.26\end{array}$ & $\begin{array}{ll}\text { NOV } 20 \\
\text { MAY } 16\end{array}$ & $\begin{array}{l}60.53 \\
61.68\end{array}$ & $\begin{array}{l}\text { DEC } 15 \\
\text { JURE } 21\end{array}$ & $\begin{array}{l}60.16 \\
62.19\end{array}$ & $\begin{array}{l}\text { JAN } 19 \\
\text { JULY } 19\end{array}$ & $\begin{array}{l}59.77 \\
62.31\end{array}$ & $\begin{array}{ll}\text { FEB } & 20 \\
\text { AUG } 20\end{array}$ & $\begin{array}{l}59.62 \\
61.89\end{array}$ & $\begin{array}{l}\text { MAR } 21 \\
\text { SEP } 20\end{array}$ & $\begin{array}{l}60.33 \\
61.51\end{array}$ \\
\hline
\end{tabular}


GROUND-WATER LEVELS

EDDY COUNTY

325450104251101 (formex1y 325445104253501) Local number, 16S.26E.19.21113.

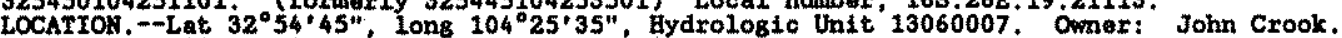

AQUIFER -VHALACIERISTICS.-Drillod water-table irrigation well, diametex 16 in., depth 160 ft.

INSTRUMINTATIOA. --Perlodic ateel-tape measurements.

DATUM.-Elevation of land-surface datum 183,399 ft above National Geodetic vertical Datum of 1929.

Measuring point: $1 / 2 \mathrm{in}$. by $3 \mathrm{in}$. vertical lot under pump base, at land-surface datum.

PERTOD OF RECORD. -Jan. 1969 to current year.

EXTRDMES FOR PERIOD OF RECORD,--Highest water level measuxed, 82.60 ft below land-8urface datum, Jan. 16, 1969;

lowest measured, 140.89 ft below land-surface datum, Aug. 6,1992 .

WATER LEVEL, IN FEET BELOW LAND-SURFACE DATUM, WATER YEAR OCTOBER 1995 TO SERTEMBER 1996

DATE WATER

Jan. $29 \quad 105.32$

$\begin{array}{ll}\text { Jan. } & 29 \\ \text { Aug. } 16 & 117.09\end{array}$

324838104435301. (former1y 324831104435701) Local number, 17S.23E.30.12344

LOCATION. -Lat $32^{\circ} 48^{\prime} 31^{\prime \prime}$, long $104^{\circ} 43^{\prime} 57^{* t}$, Fydrologte Unit 13060007. Owner: village of Hope.

AOUIPBR.--San Andres Limestone.

WLLE CHARACTERISTICS. --Drilled artesian public-supply pell, diameter 16 in., depth $600 \mathrm{ft}$, cased to 558 ft, perforated $498-558 \mathrm{ft}$

IMSTRUMENTATIOH. --Perlodic steel-tape measurements.

DATUM. --Elevation of land-surface datum is 4,085 fit above National Geodetic vertical Datum of 1929

Measuring point: Top of 2 in. pipe on north side of concrete base, 2.00 ft above land-surface datum.

PERIOD OF RECORD - -DeC. 1968 , Jan. 1970 to current year.

EXTREMS FOR PERIOD OF RECORD.--Highest water level measured, 514.85 ft below land-surface datum, Jan. 27, 1988,

lowast measured, $553.18 \mathrm{ft}$ belos land-surface datum, Aug. 7, 1974 .

MATHR LEVEL, IN FEET BELOW LAND-SURFACE DATUM, WATER YEAR OCTOBER 1995 TO SEPTEMBER 1996

$\begin{array}{cr}\text { DATE } & \begin{array}{r}\text { WATER } \\ \text { LEVEI }\end{array} \\ \text { Jan. } 26 & 540.80 \\ \text { Sept. } 4 & 534.53\end{array}$

324620104255001. (former1y 324624104244501) Local number, 18S.26E.06.442A.

LOCATION, --Lat $32^{\circ} 46^{\circ} 20^{\prime \prime}$, Long $104^{\circ} 24^{\circ} 45^{\prime \prime}$. Hydrologic Unit 13060007 , Owner: pecos Valley Artesian Conservancy District.

AQUIFER. - -San Andres Limestone.

WELL CAARACTERISTICS, -Dr111ed artestan observation wo11, dameter 9 tn., depth 1,008 ft, cased to 726 ft. INSTRUMENTATION, - -Digital recorder, 1-hour punch

DATUM.--Elevation of land-surface datum is $3,402.1$ ft above National Geodetic vertical Datum of 1929.

Measuring polnt: Top of recorder shelf, $3.40 \mathrm{ft}$ above land-surface datum.

REMARKS. - Lost record due to recorder malfunction.

PERIOD OF RECORD. -June 1961 to current year.

EXTREMES FOR PERIOD of RECORD. - - Highest pater level measured, 62.57 ft below land-surface datum, Feb. 20, 1989 ; lowest measured, $209.15 \mathrm{ft}$ below land-surface datum, July 31-Aug. $2,1966$.

WATER LEVEL, IN FEET BELOW LAND-SURFACE DATUM, WATER YEAR OCTOBER 1995 TO SEPTEMBER 1996 DAILY HIGHEST VALUES

\begin{tabular}{|c|c|c|c|c|c|c|c|c|c|c|c|c|}
\hline DAY & OCT & Nov & DEC & JAH & FEB & MAR & APR & MAY & JIN & JUL & AUG & $\mathbf{S E}$ \\
\hline $\begin{array}{r}5 \\
10 \\
15 \\
20 \\
25 \\
\mathrm{DOM}\end{array}$ & $\begin{array}{l}119.72 \\
116.04 \\
111.25 \\
107.62 \\
105.04 \\
101.98\end{array}$ & $\begin{array}{l}99.46 \\
97.71 \\
96.56 \\
94.53 \\
92.96 \\
91.38\end{array}$ & $\begin{array}{l}90.40 \\
88.93 \\
87.45 \\
85.71 \\
84.43 \\
83.08\end{array}$ & $\begin{array}{l}81.95 \\
80.72 \\
80.02 \\
78.89 \\
78.13 \\
77.48\end{array}$ & $\begin{array}{l}76.18 \\
76.08 \\
76.06 \\
77.80 \\
77.23 \\
78.71\end{array}$ & $\begin{array}{r}81.87 \\
84.20 \\
98.39 \\
104.34 \\
108.47 \\
113.85\end{array}$ & $\begin{array}{l}118.64 \\
119.19 \\
123.33 \\
128.66 \\
129.11 \\
126.66\end{array}$ & $\begin{array}{l}125.89 \\
129.16 \\
132.04 \\
138.12 \\
144.87 \\
147.45\end{array}$ & $\begin{array}{l}146.10 \\
144.78 \\
146.24 \\
145.60 \\
146.69 \\
141.20\end{array}$ & $\begin{array}{r}140.52 \\
142.25 \\
147.17 \\
149.35 \\
. . . \\
\ldots-\end{array}$ & $\begin{array}{r}\ldots \\
144.78 \\
132.26\end{array}$ & $\begin{array}{l}124.5 \\
125.5 \\
118.1 \\
121 . \\
127.2 \\
126.8\end{array}$ \\
\hline
\end{tabular}

WTR YEAR 1996 HIGHEST 75.71 FEB 13, 1996 LOWEST 155.80 JUL 25,1996 
324620104255101 Local number, $18 \mathrm{~S}, 26 \mathrm{E}, 06.442 \mathrm{~B}$

LOCATION.--Lat $32^{\circ} 46^{\prime} 20^{\prime \prime}$, long $10^{\circ} 24^{\prime} 45^{\prime \prime}$, Hydrologic Unit 13060007. Omer: Pecos Valley Axtesian Conservancy District.

AQUIFER - -Villey Fill.

WELL CAARACTERISTICS.--Drilled water-table observation well, diameter 7 in, depth $246 \mathrm{ft}$, casing 0-246 ft. INSTRUMENTATION. --Digital recorder, 1 -hour punch.

DATUM.--Elevation of land-surface datum is $3,402 \mathrm{ft}$ above National Geodetic Vertical Datum of 1929. Measuring point: Top of recorder shelf, 2.70 It above land-surface datum.

REMARKS.--Lost record due to recorder malfunction.

PERYOD OF BSCORD. - -1963 to curront yeer.

EXTREMES FOR PERIOD OF RECORD.--Highest water level measured, 106.83 ft below land-surface datum, Jan. 7, 1974;

lowest measured, 142.37 ft below land-surface datum, Aus. $16 \& 17,1996$. WATER LEVEL, IN FEET BELOW LAND-SURFACE DATUM, WATER YEAR OCTOBER 1995 TO SEPTEMBER 1996

\begin{tabular}{|c|c|c|c|c|c|c|c|c|c|c|c|c|}
\hline$A Y$ & OCT & NOV & DEC & JAN & FEB & MAR & APR & MAY & JUN & JUL & AUG & \\
\hline $\begin{array}{r}5 \\
10 \\
15 \\
20 \\
25 \\
80 M\end{array}$ & $\begin{array}{r}139.36 \\
- \\
135.52 \\
134.48\end{array}$ & $\begin{array}{l}133.75 \\
132.90 \\
132.38 \\
131.63 \\
130.57 \\
129.83\end{array}$ & $\begin{array}{l}129.28 \\
128.55 \\
127.98 \\
127.22 \\
126.80 \\
125.62\end{array}$ & $\begin{array}{l}125.58 \\
124.73 \\
124.25 \\
123.86 \\
123.07 \\
122.60\end{array}$ & $\begin{array}{l}122.31 \\
121.91 \\
121.64 \\
121.45\end{array}$ & $\begin{array}{l}121.00 \\
121.18 \\
121.77 \\
122.84 \\
123.87 \\
125.48\end{array}$ & $\begin{array}{l}126.93 \\
127.68 \\
129.10 \\
129.83 \\
130.73 \\
131.74\end{array}$ & $\begin{array}{l}132.51 \\
132.99 \\
133.47 \\
134.21 \\
135.14 \\
136.10\end{array}$ & $\begin{array}{l}136 . \\
137 . \\
137 \\
137 \\
139 \\
140 .\end{array}$ & $\begin{array}{l}39 \\
67 \\
14 \\
36 \\
86 \\
09\end{array}$ & $\begin{array}{l}141.55 \\
142.07 \\
142.13 \\
142.11 \\
142.08 \\
142.08\end{array}$ & \\
\hline
\end{tabular}

WTR YEAR 1996 EIGHEST 121.00 MAR 5, 1996 LOWEST 142.37 AUG 16,17, 1996

324325104233001 . Local number, 18S.26E.28,122111.

LOCATION. - Lat $32^{\circ} 43^{\prime} 25^{\prime \prime}$, long $104^{\circ} 2^{\prime} 30^{\prime \prime}$, Hydrologio Unit 13060011. Owner: Town of Dayton.

AQUIFER . - Valley Fill.

WELI CHARACTERISTICS.--Drilled water-table observation well, diameter $8 \mathrm{in}$, depth 250 ft, cased to 182 ft, casing slottod $92-182$ st.

INSTRUMENTATION, --Monthly steel-tape measurements.

DATUM. - Elevation of land-surfece datum is 3,403 ft above National Geodetic Vextical Datum of 1929.

Measuring point: Top of casing, $0.06 \mathrm{ft}$ above land-surface datum.

REMARKS, --Recorder removed Nov. $27,1990$.

PERIOD OF RECORD, - -Aug. 1951 to current year.

EXTREMES FOR PERIOD OF RECORD.- -Highest water level measured, 59.79 ft below land-surface datum, Feb. 5, 1952;

lowest measured, 124.87 rt below land-surface datum, Feb. 25, 1982 and Sept. 20, 1996.

WATER LEVEL, IN FEET BELOW LAND-SURFACE DATUM, WATER YEAR OCTOBER 1995 TO SEPTEMBER 1996

\begin{tabular}{|c|c|c|c|c|c|c|c|c|c|c|c|}
\hline DATE & $\begin{array}{l}\text { WATER } \\
\text { LEVEL }\end{array}$ & DATE & $\begin{array}{l}\text { WATER } \\
\text { LEVEL }\end{array}$ & DATE & $\begin{array}{l}\text { WATER } \\
\text { LEVEL }\end{array}$ & DATE & $\begin{array}{l}\text { WATER } \\
\text { LEVEL }\end{array}$ & DATE & $\begin{array}{l}\text { WATER } \\
\text { LEVEL }\end{array}$ & DATE & $\begin{array}{l}\text { WATER } \\
\text { LEVEL }\end{array}$ \\
\hline $\begin{array}{ll}\text { OCT } & 23 \\
\text { APR } & 19\end{array}$ & $\begin{array}{l}124.39 \\
124.52\end{array}$ & $\begin{array}{l}\text { NOV } 20 \\
\text { MAY } \\
\text { 16 }\end{array}$ & $\begin{array}{l}124.45 \\
124.56\end{array}$ & $\begin{array}{l}\text { DEC } 15 \\
\text { JUNE } 21\end{array}$ & $\begin{array}{l}124.52 \\
124.70\end{array}$ & $\begin{array}{l}\text { JAN } 11 \\
\text { JULY } 19\end{array}$ & $\begin{array}{l}124.57 \\
124.69\end{array}$ & $\begin{array}{l}\text { FEB } 20 \\
\text { AUG } 20\end{array}$ & $\begin{array}{l}124.44 \\
124.85\end{array}$ & $\begin{array}{l}\text { MAR } 21 \\
\text { SEP } 20\end{array}$ & $\begin{array}{l}124.52 \\
124.87\end{array}$ \\
\hline
\end{tabular}

323705104225501 . Local number, 19S.26E.33.41224.

LOCATION,--Lat $32^{\circ} 37^{\prime} 05^{\prime \prime}$, Long $104^{\circ} 22^{\prime} 55^{\prime \prime}$, Hydrologic Unit 13060011 . Owner: L. T. Lewis.

AQUIFER. -Alluvium.

WELL CHARACTERISTICS. --Drilled water-table unused irrigation well, diameter 14 in., depth 225 it.

INSTRUMENTATION. --Poriodic steol-tape measurements.

DATUM. - Elevation of land-surface datum is 3,282 ft above National Geodetic Vertical Datum of 1929.

Measuring point: Top of 1 in. hole, in north side of pump base, $0.95 \mathrm{ft}$, above land-surface datum.

PERIOD OF RECORD.--Jan. 1938 to current year.

EXTREAES FOR PERIOD OF RECORD.--Highest water level measured, 35.48 te bolow land-surface datum, Aug, 19, 1991;

lowest measured, 124.00 ft below land-surface datum, Jan. 9 , ig84.

WATER LEVEL, IN FEET BELOW LAND-SURFACE DATUM, WATER YEAR OCTOBER 1995 TO SEPTEMBER 1996

$\begin{array}{lc}\text { DATE } & \text { WATER } \\ \text { JaEVL } \\ \text { Jan. } 29 & 36.41 \\ \text { Aug. } 16 & \text { pumping }\end{array}$

323542104242701. (5ormer1y 323540104232001) Local number, 20S.26E.08.121111.

LOCATION. - Lat $32^{\circ} 35^{\prime} 40^{\prime \prime}$, Iong $10^{\circ} 23^{\prime} 20^{\prime \prime}$, Hydrologic Unit 13060011 . Owmer: Moutry.

AQUIFER. - Valley FiII

WELL CHARACTERISTICS.--Drilled water-table trrigation well, diameter 13 in., depth 346 rt, casing information not avatlable.

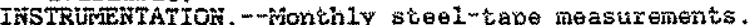

DATUM.--Elevation of land-surface datum is $3,286 \mathrm{ft}$ above National Geodetic Verticel Datum of 1929.

Measuring point: Top of basal flange of pump head, $0.20 \mathrm{ft}$ above land-surface datum.

PERTOD OF RECORD.--Jan. 1938 to current year.

EXTREMES FOR PERIOD OF RECORD, - - Highest water level measured, 25.47 ft below land-surface datum, May 26, 1992;

lowest measured, $90.25 \mathrm{ft}$ below land-surface datum, Aug. 8, 1977.

WATER LEVEL, IN FEET BELOW LAND-SURFACE DATUM, WATER YEAR OCTOBER 1995 TO SEPTEMBER 1996

$\begin{array}{llllllllll}\text { WATER } & \text { WATE } & \text { WEVEL } & \text { WATE } & \text { WATER } & \text { WATER } & \text { WATER } & \text { WATER } & \text { WATER } \\ \text { LEVEL } & \text { DATE } & \text { LEVEL } & \text { DATE } & \text { LEVEL } & \text { DATE } & \text { LEVELL }\end{array}$

$\begin{array}{llllllllllll}\text { OCT } 23 & 32.86 & \text { NOV 20 } & 33.38 & \text { DEC 15 } & 33.27 & \text { JAN 19 } & 32.70 & \text { FEB } & -- & \text { MAR } \\ \text { APR } 19 & 33.31 & \text { MAY 16 } & 34.25 & \text { JUNE 21 } & 38.47 & \text { JULY 19 } & 33.67 & \text { AUG } 20 & 35.94 & \text { SEP } 20 & 33.30\end{array}$


GROUND-WATER LEVELS

EDDY COUNTY

Carlsbad Area

322637104142301. (formerly 322652104141901) Local number, 21S.26E.36.221.

LOCATION. - Lat $32^{\circ} 26^{\circ} 52^{\prime \prime}$, long $104^{\circ} 14^{\prime} 19^{*}$, Hydrologic Unit 13060011 . Owner: City of Carlsbad.

AQUIFER - - Capitan I.tmestone.

WELL CHARACTERISTICS.--Drilied qater-table muntcipal well, diameter 20 in., depth 327 ft, casing $0-290$ ft.

INSTRUMENTATION.--Digital recorder, 1-hour punch.

DATUM.--Elevation of land-gurface datum $\pm \mathrm{s} 3,121.84$ ft ebove National Geodetic Vertical Datum of 1929.

Measurting point: Top of recorder shelf, 4.14 ft above land-surface datum.

REMARKS --Records good.

PERIOD OF RECORD - -April 1962 to current year.

EXTREAES FOR TERIOD OF RECORD. - -Highest water lovel measured, 16.98 ft below land-surface datum, June 14, 1987;

lowest measured, 26.07 ft below land-surface datum, Aus. 2, 1974.

WATER LEVEL, IN FEET BELOW LAND-SURFACE DATUM, WATGR YEAR OCTOBER 1995 TO SEPTEMBER 1996 DAILY HIGHEST VALUES

\begin{tabular}{|c|c|c|c|c|c|c|c|c|c|c|c|c|}
\hline DAY & $\propto \mathbf{C T}$ & Nov & $\mathrm{DEC}$ & JAN & EEB & MAR & APR & MAY & JUN & JUL & AUG & SEP \\
\hline $\begin{array}{r}5 \\
10 \\
15 \\
20 \\
25 \\
\text { EOM }\end{array}$ & $\begin{array}{l}22.98 \\
22.84 \\
22.61 \\
22.63 \\
22.57 \\
22.47\end{array}$ & $\begin{array}{l}22.55 \\
22.38 \\
22.48 \\
22.51 \\
22.43 \\
22.38\end{array}$ & $\begin{array}{l}22.39 \\
22.45 \\
22.40 \\
22.32 \\
22.39 \\
22.12\end{array}$ & $\begin{array}{l}22.27 \\
22.34 \\
22.45 \\
22.42 \\
22.40 \\
22.54\end{array}$ & $\begin{array}{l}22.72 \\
22.71 \\
22.79 \\
22.86 \\
22.99 \\
22.99\end{array}$ & $\begin{array}{l}22.97 \\
22.97 \\
22.87 \\
22.99 \\
22.95 \\
23.15\end{array}$ & $\begin{array}{l}23.12 \\
22.95 \\
23.12 \\
23.08 \\
23.32 \\
23.38\end{array}$ & $\begin{array}{l}23.56 \\
23.60 \\
23.62 \\
23.87 \\
23.64 \\
23.55\end{array}$ & $\begin{array}{l}23.48 \\
23.58 \\
23.50 \\
23.52 \\
23.60 \\
23.46\end{array}$ & $\begin{array}{l}23.48 \\
23.61 \\
23.43 \\
23.34 \\
23.44 \\
23.54\end{array}$ & $\begin{array}{l}23.49 \\
23.53 \\
23.73 \\
23.48 \\
23.31 \\
22.92\end{array}$ & $\begin{array}{l}22.78 \\
22.81 \\
22.23 \\
22.22 \\
22.09 \\
22.27\end{array}$ \\
\hline
\end{tabular}

WTR YEAR 1996 HIGHEST 22.09 SEP 25, 1996 LOWEST 24.04 MAY 21, 1996

322636104125801. (former1y 322640104165801) Local number, 21S.27E.32.112411.

LOCATION. -Lat $32^{\circ} 26^{*} 40^{\prime \prime}$, long $104^{\circ} 12^{\prime} 58^{*+}$, Hydrologic Unit 13060011. Ownex: L. E. Loman.

AQUIFR. - Capitan Ltmestóne.

WELL CHARACTERISTICS,--Drilied water-table domestic well, diameter 12 in, reported depth 305 ft.

INSTRUMENTATION. --Periodic steel-tape measurements.

DATUM.--Elevation of land-surface datum is 3,112 ft above National Geodettc Vertical Datum of 1929.

Measuring point: Top of casing, $0.40 \mathrm{ft}$ above land-surface datum.

PERTOD OF RECORD. - Oct. 1947 to current year.

EXTREMES FOR PERIOD OF RECORD.--Highest water level measured, 4.64 ft below land-surface datum, Jan. 17, 1950;

lowest measured, $17.35 \mathrm{ft}$ below land-surface datum, Alug. 9, 1974.

WATER LEVEL, IN FEET BELOW LAND-SURFACE DATLM, WATER YEAR OCTOBER 1995 TO SEPTEMBER 1996

$\begin{array}{cc} & \text { WATER } \\ \text { DATE } & \text { LEVEL } \\ \text { Jan. } & \text { not measured } \\ \text { Aug. } & \text { not measured }\end{array}$

322712104074501. (formerly 322710104073901) Local number, 21S.28E.30.14123.

LOCATrON. - Lat $32^{\circ} 27^{\prime} 1^{\prime \prime}$, long $104^{\circ} 07^{\prime} 39^{\prime \prime}$, Hydrologic Unit 13060011 . Owmer: Forrest M 11 ler.

AOUIFER --Capitan Limestóne.

WELI CHARACTERISTICS,-Drilied exploration well, diameter $85 / 8-51 / 2$ in., reported depth 1,060 ft, plugged back, total depth 906 ft.

INSTRUMENTATION, -Digital rocorder, 1-hour punch

DATUM. - Elevation of land-surface datum is 3,181.71 ft above National Geodetic Vertical Datum of 1929.

Measuring point: Top of casing $1.64 \mathrm{ft}$ above land-surface datum.

REMARKS. - -Records good.

PERIOD OF RECORD - -1963 to current year

EXTREMES FOR PERIOD OF RECORD,--Highest water level measured, 88.13 ft below land-surface datum, June 29, 1987 ;

lowest measured, 98.68 ft below land-surface datum, Aug. 3, 1974 .

WATER LEVEL, IN FEET BELOW LAND SURFACE DATUM, WATER YEAR OCTOBER 1995 TO SEPTEMBER 1996 DAILY HIGHEST VALUES

\begin{tabular}{|c|c|c|c|c|c|c|c|c|c|c|c|c|}
\hline DAX & OCT & Nov & DEC & JAN & FEB & MAR & APR & MAY & JUN & JUL & AUG & SEP \\
\hline $\begin{array}{r}5 \\
10 \\
15 \\
20 \\
25 \\
\text { EOM }\end{array}$ & $\begin{array}{l}93.84 \\
93.72 \\
93.46 \\
93.40 \\
93.25 \\
93.18\end{array}$ & $\begin{array}{l}93.26 \\
93.08 \\
93.20 \\
93.21 \\
93.13 \\
93.10\end{array}$ & $\begin{array}{l}93.10 \\
93.20 \\
93.06 \\
93.04 \\
93.12 \\
92.86\end{array}$ & $\begin{array}{l}92.98 \\
93.08 \\
93.15 \\
93.16 \\
93.17 \\
93.27\end{array}$ & $\begin{array}{l}93.42 \\
93.36 \\
93.41 \\
93.48 \\
93.68 \\
93.73\end{array}$ & $\begin{array}{l}93.66 \\
93.70 \\
93.62 \\
93.76 \\
93.59 \\
93.80\end{array}$ & $\begin{array}{l}93.88 \\
93.69 \\
93.82 \\
93.79 \\
94.06 \\
94.14\end{array}$ & $\begin{array}{l}94.16 \\
94.22 \\
94.21 \\
94.44 \\
94.37 \\
94.25\end{array}$ & $\begin{array}{l}94.15 \\
94.26 \\
94.29 \\
94.16 \\
94.21 \\
94.17\end{array}$ & $\begin{array}{l}94.17 \\
94.30 \\
94.28 \\
94.06 \\
94.28 \\
94.26\end{array}$ & $\begin{array}{l}94.27 \\
94.31 \\
94.36 \\
94.32 \\
94.20 \\
93.79\end{array}$ & $\begin{array}{l}93.61 \\
93.60 \\
93.06 \\
93.01 \\
93.03 \\
93.22\end{array}$ \\
\hline
\end{tabular}

WTR YEAR 1996 HIGHEST 92.86 DEC 31, $1996 \quad$ LOWEST 94.57 MAY 21, 1996 


\section{GROUND-WATER LEVELS}

EDDX COUNTY

Carlsbad Area

322120104151501. Local number, 22S, 26E, 25.333333. (formerly 22S. 26E. 36.111A)

LOCATION. - Lat $32^{\circ} 21^{\circ} 20^{\prime \prime}$, Long $104^{\circ} 15^{\prime} 15^{\prime \prime}$, Hydrologic Unit 13060011. Owner: Carlsbad Airfield.

AQUIEER. - ALLuVium.

WELL CHARACTERISTICS.-Drilled water-table unused pe11, diemeter 12 in, depth 260 ft, cased to 260 ft.

INSTRLAENTATION, $--D \pm g i$ tal recorder, 1 -hour punch.

DATUM.--Elevation of land-surface datum is 3,225 ft above National Geodetic Vertical Datum of 1929.

Measuring point: Top of casing, 0.40 ft above land-surface datum.

REMARKS:-Records good.

PERTOD OF RECORD.--July 1942 to current yoar.

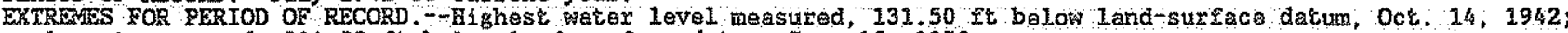

lowest measured, $214.82 \mathrm{ft}$ below land-surface datum, Sep. 15, 1978 ,

WATER LEVEL, IN EEET BELOW LAND-SURFACE DATUM, WATER YEAR OCTOBER 1995 TO SEPTEMBER 1996 DATIY HIGHEST VALUES

\begin{tabular}{|c|c|c|c|c|c|c|c|c|c|c|c|c|}
\hline DAY & OCT & Nov & DEC & JAN & FEB & MAR & APR & MAX & JUN & JUL & AUE & SER \\
\hline $\begin{array}{r}5 \\
10 \\
15 \\
20 \\
25 \\
\text { EOM }\end{array}$ & $\begin{array}{l}159.28 \\
158.37 \\
157.23 \\
156.30 \\
155.27 \\
153.96\end{array}$ & $\begin{array}{l}152.99 \\
151.88 \\
151.24 \\
150.79 \\
150.18 \\
149.43\end{array}$ & $\begin{array}{l}148.90 \\
148.33 \\
147.78 \\
147.24 \\
147.04 \\
146.26\end{array}$ & $\begin{array}{l}146.63 \\
146.79 \\
146.83 \\
146.56 \\
146.31 \\
146.13\end{array}$ & $\begin{array}{l}146.21 \\
145.93 \\
145.86 \\
145.69 \\
145.64 \\
145.75\end{array}$ & $\begin{array}{l}146.18 \\
146.45 \\
146.45 \\
146.97 \\
147.15 \\
147.78\end{array}$ & $\begin{array}{l}149.11 \\
150.84 \\
153.21 \\
155.00 \\
155.66 \\
156.91\end{array}$ & $\begin{array}{l}158.12 \\
158.42 \\
158.63 \\
157.89 \\
158.48 \\
159.63\end{array}$ & $\begin{array}{l}160.49 \\
161.28 \\
160.94 \\
160.41 \\
160.85 \\
161.99\end{array}$ & $\begin{array}{l}162.71 \\
162.65 \\
163.01 \\
163.50 \\
163.46 \\
164.47\end{array}$ & $\begin{array}{l}164.43 \\
163.38 \\
161.99 \\
161.39 \\
161.13 \\
159.96\end{array}$ & $\begin{array}{l}158.70 \\
157.59 \\
156.26 \\
155.23 \\
154.25 \\
154.86\end{array}$ \\
\hline
\end{tabular}

WTR YEAR 1996 HIGREST 145.58 FEB 18, $1996 \therefore$ LOWEST 164.67 AUG 4, 1996

322238104101801. (formerly 322231104131001). Local number, 22S.27E.22.421333.

LOCATION -Lat $32^{\circ} 22^{\circ} 31^{\prime \prime}$, Long $104^{\circ} 10^{\prime} 10^{\prime \prime}$, Hydrologic Unt 13060011 . Owner: Enea Grandi.

AQUIFER - -ALluvium.

WELL CHARACTERISTICS.--Drilled water-table irrigation well, diameter 16 in., reported depth 150 ft.

INSTRUMENTATION. - - Periodic steel-tape measurements.

DATUM.--Elevation of land-surface datum is 3,100 ft above National Geodetic vertical Datum of 1929

Measuring point: Top of casing, $1.20 \mathrm{ft}$ above land-surface datum.

PERIOD OF RECORD.--Sep. 1947 to Aug. 1968, Jan. 1970 to current year.

EXTREMES FOR PERIOD OF RECORD,-Highest water ievel measured, 21.43 ft below landmsurface datum, Sep. 15 , 1950 ;

lowest measured, $81.10 \mathrm{Et}$ below land-surface datum, Aug. 8, 1977

WATER LEVEL, IN FEET BELOW LAND-SURFACE DATUM, WATER YEAR OCTOBER 1995 TO SEPTEMBER 1996

$\begin{array}{cc}\text { DATE } & \begin{array}{l}\text { WATER } \\ \text { LEVEL }\end{array} \\ \text { Jan. } 24 & \\ \text { Aug. 1.5 } & 28.95 \\ & 28.44\end{array}$

321741104204901. (formerly 321721104204801) Local number, 23S.25E.24.213.

LOCATION. - Lat $32^{\circ} 17^{\prime} 21^{\prime \prime}$, Long $104^{\circ} 20^{\prime} 48^{\prime \prime}$, Hydrologic Unit 13060011. Owner: City of Carlsbad.

AQULL CHARACTRRISTICS.--Drilied water-table observation well, diameter 16 in, 0-20 ft, open hole 20-900 ft,

INSTRUMENTATION. --Digital recorder, 1-hour punch.

DATUM. --Elavation of land-surface datum is 3,501.7 ft above National Geodettc Vertical Datum of 1929.

Measuring point: Top of casing, 1.17 ft above land-surface datum.

REMARKS. - Records good.

PERIOD OF RECORD. -1963 to current year.

EXTREMIES FOR PERIOD OF RECORD.--Highest water level measured, 369.53 ft below land-surface datum, June 27 , 1986 ;

lowest measured, 404.06 st below land-surface datum, July 10, 1974.

WATER LEVEL, IN FEET BELOW LAND-SURFACE DATUM, WATER YEAR OCTOBER 1995 TO SEPTEMBER 1996 DATLY HIGHEST VALUES

\begin{tabular}{|c|c|c|c|c|c|c|c|c|c|c|c|c|}
\hline DAY & OCT & NOV & $\mathrm{DEC}$ & JAN & FEB & MAR & AIR & MAY & JUN & JUL & AUG & SEP \\
\hline $\begin{array}{r}5 \\
10 \\
15 \\
20 \\
25 \\
\text { EOM }\end{array}$ & $\begin{array}{l}400.76 \\
400.83 \\
400.75 \\
400.69 \\
400.63 \\
400.55\end{array}$ & $\begin{array}{l}400.56 \\
400.43 \\
400.58 \\
400.52 \\
400.53 \\
400.34\end{array}$ & $\begin{array}{l}400.37 \\
400.42 \\
400.56 \\
400.17 \\
400.40 \\
400.16\end{array}$ & $\begin{array}{l}400.22 \\
400.33 \\
400.39 \\
400.58 \\
400.46 \\
400.48\end{array}$ & $\begin{array}{l}400.64 \\
400.60 \\
400.67 \\
400.71 \\
400.86 \\
400.95\end{array}$ & $\begin{array}{l}400.95 \\
401.14 \\
400.89 \\
401.05 \\
400.96 \\
401.17\end{array}$ & $\begin{array}{l}401.35 \\
401.17 \\
401.42 \\
401.38 \\
401.67 \\
401.92\end{array}$ & $\begin{array}{l}402.06 \\
402.02 \\
402.13 \\
402.93 \\
403.00 \\
402.74\end{array}$ & $\begin{array}{l}402.74 \\
402.81 \\
402.69 \\
402.61 \\
401.82 \\
401.62\end{array}$ & $\begin{array}{l}401.60 \\
401.83 \\
401.63 \\
401.46 \\
401.65 \\
401.78\end{array}$ & $\begin{array}{l}401.58 \\
401.57 \\
401.64 \\
401.57 \\
401.43 \\
401.03\end{array}$ & $\begin{array}{l}400 \\
400\end{array}$ \\
\hline
\end{tabular}

WTR YEAR 1996 HIGEEST 391.29 SEP 12, 1996 LOWEST 403,26 MAY 21,22, 1996 
GROUND-WATER LEVELS

EDDY COUNTY

Carlsbad Area

321939104113301. (fommerly 321930104113301) Local number, 23S.27E.09.211124.

LOCATION - - Lat $32^{\circ} 19^{\circ} 30^{\prime \prime}$, Long $104^{\circ} 11^{\prime} 33^{\prime \prime}$, Bydrolog te Unit 13060011. Omer: H. C. Bindel.

AQUTFBR .-Alluvium.

WELL CBARACTERISTICS.--Dr111ed water-table irrigation wel1, diameter 16 in., depth $200 \mathrm{ft}$.

INSTRINENTATION. --Periodic stee1-tape measurements

DATUM.--Elevation of Land-surface datum is $3,143 \mathrm{ft}$ above National Geodetic Vertical Datum of 1929.

Measuring point: Top of casing, under pump base, $1.25 \mathrm{ft}$ above land-surface datum.

PERTOD OF RECORD - July 1949 to Nov. 1955, Jan. 1971 to current year.

EXTREMES EOR PERIOD OF RECORD. - - H1 ghest water level measured, 41.70 ft below land-surface datum, Sep. 15, 1950;

lowest measured, $60.92 \mathrm{ft}$ below land-surface datum, Jan. 13, 1976.

WATER LEVEL, IN FEET BELOW LAND-SURFACE DATUM, WATER YEAR OCTOBER 1995 TO SEPTEMBER 1996

$\begin{array}{cc}\text { DATE } & \begin{array}{l}\text { WATER } \\ \text { LEVEL }\end{array} \\ & \\ \text { Jan. 26 } & 56.04 \\ \text { Aug. 15 } & 55.48\end{array}$

320604104284101 . (formerly 320602104285201) Local number, 25S.24E.27.421121.

LOCATION, - Lat 32 $06^{\circ} 02^{\prime \prime}$, Long 104\%28'52", Hydrologic Unit' 13060011. Owner: Walker Hood.

AQUIFER. --Alluvium.

WELL CHARACTERISTICS.--Drilled water-table irrigation well, diameter 16 in., depth $101 \mathrm{ft}$, uncased.

INSTRUMENTATION. --Periodic steel-tape measurements.

DATUM. - Elevation of land-surface datum is 3,701 ft above National Geodetic Vertical Datum of 1929.

Measuring point: Northwest corner of pump base, $1.00 \mathrm{ft}$ above land-surface datum.

PERIOD OF RECORD.-Apr. 1952 to Aug. 1967, Jan. 1969 to current year.

EXTREMES FOR PERIOD OF RECORD.--Highest water leve1 measured, 50,12 ft below land-sunface datum, Aus. 22, 1988; lowest measured, $85.10 \mathrm{ft}$ below land-surface datum, Aug. 25, 1967 .

WATER LEVEL, IN FEET BELOW LAND-SURFACE DATUM, WATER YEAR OCTOBER 1995 TO SEPTEMBER 1996

$\begin{array}{cc}\text { DATE } & \begin{array}{r}\text { WATER } \\ \text { LEVEL }\end{array} \\ \text { Jan. 26 } & 57.18 \\ \text { Aug. 15 } & \text { pumping }\end{array}$

320316104294301 . (formerly 320257104295201) Local number, 26S.24E.09.443111.

LOCATION. --Lat $32^{\circ} 03^{\prime} 16^{\prime \prime}$, Long $14^{\circ} 29^{\prime} 43^{\prime \prime}$, Hydrologic Unit 13060011. Owner: John Mayes.

WQUIFER. -AIIUvium.

INSTRUMENTATION. - Perjodic steo1-tape measurements.

DATUM.--Elevation of land-surface datum is $3,749.4^{*}$ ft above National Geodetic Vertical Datum of 1929.

Measuring point: Top of air-line flange support, $1.40 \mathrm{ft}$ above land surface datum.

PERIOD OF RECORD - Apr. 1952 to current year.

EXTREMES FOR PERIOD OF RECORD,--Highest water level measured, 35.31 ft below land-surface datum, Aug. 22, 1988;

lowest measured, $54.98 \mathrm{ft}$ below land-surface datum, Sep. 8, 1965.

WATER LEVEL, IN FEET BELOW LAND-SURFACE DATUM, WATER YEAR OCTOBER 1995 TO SEPTEMBER 1996

DATE WATER

Jan. $26 \quad 43.55$

Aug. $15 \quad 44.78$

GRANT COUNTY

Mimbres Basin

324245108175603. Local number, 185.14W.28.143B.

LOCATION. - Lat $32^{\circ} 42^{*} 45^{\prime \prime}$, Long $108^{\circ} 17^{\prime} 56^{*}$, Eydrologic Untt 13030202. Owner: Exxon Corp.

AQUTFER. - Alluvium.

WELL CHARACTERISTICS.-Drilled water-table unused irrigation well, dianeter 6 in., depth unknown.

INSTRUMENTATION. --Periodic steel-tape measurements.

DATUM. - Elevation of land-surface datum is 5,800 ft above National Geodetic Vertical Datum of 1929.

Measuring point: $3 / 4 \mathrm{tn}$. hole in cover plate, at land-surface datum.

RGMARKS. - - " s" indtcates nearby well pumping.

PERIOD OF RECORD. - Mar. 1984 to current year.

EXTREMES FOR PERIOD OF RECORD.--Highest water level measured, 268.84 ft below land-surface datum, Jan. 14, 1986; lowest measured, $404.60 \mathrm{~s}$ ft below land-surface datum, Jan. 6,1994 .

WATER LEVEL, IN FEET BELOW LAND-SURFACE DATUM, WATER YEAR OCTOBER 1995 TO SEPTEMBER 1996

$\begin{array}{lr}\text { DATE } & \begin{array}{r}\text { WATER } \\ \text { LEVEL }\end{array} \\ \text { Jan. } 18 & 386.78 \\ \text { July } 1 & 388.06\end{array}$




\section{GROUND-WATER LEVELS}

\section{GRANT COUNTY \\ Silver City Aree}

324600108222501 . Local number, 185.15W.11.323.

LOCATION, - Lat 32 $46^{\circ} 00^{\prime \prime}$, long $108^{\circ} 22^{\prime} 25^{\prime \prime}$, Bydrologic Unit 15040002, Omer: Tow of Silver City. AQUIFER - - Gila Conglomerate.

WELI CHARACTERISTICS.--Drilied water-table unused we11, diameter 12 in, depth 580 ft.

IASTRUMTNTATIOA --Digitel rocorder, 1-hour punch.

DATUM. -Elevation of land-surface datim is $5,845 \mathrm{ft}$ above National Geodetic vertical Datum of 1929 .

Measuring potnt: Top of 12 in. casing, 1.50 it above land-surface datum.

REMARKS - Lost zeveral days of record, due to recorder malfunction.

PERIOD OF RECORD,-Mar. 1957 to current year.

EXTREAES FOR PERTOD OF RECORD. --Highest watex love1 measured, 262.34 ft below land-surface datum, Max. 3 , 1962;

lowest maasured, 294.52 te below lend-strrece detum, Apr. 20, 1986.

WATER LEVEL, IN FEET BELON LAND-SURFACE DATUM, WATER YEAR OCTOBER 1995 TO SEPTEMBER 1996 DAILY HICHEST VALUES

\begin{tabular}{|c|c|c|c|c|c|c|c|c|c|c|c|c|}
\hline DAY & $\therefore \quad \alpha \operatorname{cct}$ & NOV & DEC & JAN & FEB & $\therefore \quad M A R$ & APR & MAY & JUN & JUL & AUG & SEP \\
\hline $\begin{array}{r}5 \\
10 \\
15 \\
20 \\
25 \\
\text { EOM }\end{array}$ & $\begin{array}{l}291.57 \\
292.06 \\
292.42 \\
292.62 \\
292.81 \\
293.00\end{array}$ & $\begin{array}{l}293.10 \\
292.89 \\
292.95 \\
293.00 \\
292.90 \\
292.87\end{array}$ & $\begin{array}{l}292.88 \\
293.00 \\
292.80 \\
292.71 \\
293.00 \\
292.46\end{array}$ & $\begin{array}{l}292.80 \\
292.72 \\
292.72 \\
292.64 \\
292.61 \\
292.53\end{array}$ & $\begin{array}{l}292.91 \\
292.69 \\
292.61 \\
292.62 \\
292.47 \\
292.72\end{array}$ & $\begin{array}{l}292.53 \\
292.73 \\
292.51 \\
292.62 \\
292.47 \\
292.59\end{array}$ & $\begin{array}{l}292.61 \\
292.47 \\
292.73 \\
292.60 \\
292.73 \\
292.65\end{array}$ & $\begin{array}{l}293.13 \\
293.14 \\
293.08 \\
293.32 \\
293.46 \\
293.77\end{array}$ & $\begin{array}{l}293.89 \\
294.00 \\
294.02 \\
293.83 \\
293.80 \\
293.84\end{array}$ & $\begin{array}{l}293.63 \\
293.68 \\
293.62 \\
293.53 \\
293.53 \\
293.44\end{array}$ & $\begin{array}{l}293.39 \\
293.41 \\
293.38 \\
293.34 \\
293.28 \\
293.24\end{array}$ & $\begin{array}{l}293.26 \\
293.37 \\
293.20 \\
293.30 \\
293.14 \\
293.22\end{array}$ \\
\hline
\end{tabular}

WTR YEAR 1996 EIGEEST 291.34 OCT 4, 1995 LOWEST 294.22 JUN 13, 1996

GUADALUPE COUNTY

Santa Rosa Area

350414104485101 . Local number, 10N.20E.28.2214.

LOCATION. - Lat $35^{\circ} 04^{\prime} 14^{\prime \prime}$, long $104^{\circ} 48^{\prime} 51^{\prime \prime}$, Hydrologic Unit 13060001. Owner: Town of Santa Rosa,

AQUIFER. - -San Andres Limestone.

WELL CHARACTERISTICS, --Drilled wate-table observation well, diameter $123 / 4$ in., castng $0-514$ ft, 10 3/4 in. 505-575 ft, casing perforated 515-575 ft

INSTRUMENTATIÓN. - -Digital recorder, 1 -hour punch.

DATUM.--Elevation of land-surface datum is 5,162,7 ft above National Geodetic Vertical Datum of 1929

Measuring point: Top of casing $1.10 \mathrm{ft}$ above land-surface datum.

REMARKS.--Records good.

PERIOD OF RECORD. - May 1977 to current yoar.

EXTREMES FOR PERIOD OF RECORD.--Highest water leve1 measured, 343.67 ft below land-5urface datum, July 27 , 1992 ;

lowest measured, $362.36 \mathrm{ft}$ below land-surface datum, Apr, 12, 1978 .

WATER LEVEL, IN FEET BELOW LAND-SURFACE DATUM, WATER YEAR OCTOBER 1995 TO SEPTEMBER 1996 DAILY BIGHEST VALUES

\begin{tabular}{|c|c|c|c|c|c|c|c|c|c|c|c|c|}
\hline DAY & ocr & Hov & DEC & JAN & FEB & MAR & AER & MAY & JUN & JUL & AUG & SEP \\
\hline $\begin{array}{l}5 \\
10 \\
15 \\
20 \\
25\end{array}$ & $\begin{array}{l}345.62 \\
345.81 \\
345.99 \\
346.21 \\
346.29 \\
346.48\end{array}$ & $\begin{array}{l}346.67 \\
346.82 \\
347.15 \\
347.32 \\
347.43 \\
347.55\end{array}$ & $\begin{array}{l}347.74 \\
347.92 \\
348.14 \\
348.20 \\
348.44 \\
348.45\end{array}$ & $\begin{array}{l}348.74 \\
348.77 \\
348.92 \\
349.01 \\
349.10 \\
349.32\end{array}$ & $\begin{array}{l}349.45 \\
349.60 \\
349.75 \\
349.80 \\
349.89 \\
350.08\end{array}$ & $\begin{array}{l}350.14 \\
350.33 \\
350.47 \\
350.64 \\
350.78\end{array}$ & $\begin{array}{l}351.05 \\
351.14 \\
351.34 \\
351.41 \\
351.45 \\
351.58\end{array}$ & $\begin{array}{l}351.84 \\
352.00 \\
352.11 \\
352.51 \\
352.73 \\
352.77\end{array}$ & $\begin{array}{l}353.07 \\
353.24 \\
353.00 \\
353.13 \\
353.61 \\
353.22\end{array}$ & $\begin{array}{l}353.14 \\
352.78 \\
352.50 \\
352.38 \\
352.57 \\
352.37\end{array}$ & $\begin{array}{l}352.39 \\
352.46 \\
352.57 \\
352.59 \\
352.47 \\
351.90\end{array}$ & $\begin{array}{l}351.90 \\
351.95 \\
351.68 \\
351.64 \\
351.59 \\
351.81\end{array}$ \\
\hline
\end{tabular}

WTR YEAR 1996 MIGHEST 345.48 OCT 4,1995 LOWEST 354.09 JUN 27,1996

BARDTNG COUNTY

Roy Area

355352104054201 . Local number, $19 \mathrm{~N} .27 \mathrm{E} .05 .334$

LOCATION. - Lat $35^{\circ} 53^{\circ} 52^{\prime \prime}$, long $104^{\circ} 05^{\circ} 42^{\prime \prime}$, Hydrologic Unit 11080007. Owner: Town of Roy.

AQUIFER - - Ogallala formation.

WELL CHARACTERISTICS --Drilled water-table muntctpal well, diameter 10 in, depth 75 ft, cased to 75 gt.

THSTRLEENTATYON - PorLodic steel-tape messurements.

DATUM, - Elevation of land-surface datum is 5,658 It above National Geodetic Verttcal Datum of 1929.

Measuring point: $3 / 4 "$ plugged hole, east" stde, 1.50 ft above land-surface datum.

REMARKS. - - Submersible pump installed in 1984.

QERTOD OE RECORD. - Jan. 1967 to present.

EXTREMES FOR PERTOD OF RECORD, --Hil thest water level measured, 48.34 ft below land-surface datum, Jan. 18 , 1983,

lowest measured, 55.76 ft below land-surface datum, Aug. 19, 1987.

WATER LEVEL, IN FEET BELOW LAND-SURFACE DATUM, WATER YEAR OCTOBER 1995 TO SEPTEMBER 1996

$\begin{array}{cc}\text { DATE } & \begin{array}{l}\text { WATER } \\ \text { LEVEL }\end{array} \\ \text { Jan. } 17 & 50.12 \\ \text { JU. } 25 & 50.35\end{array}$


GROUND-WATER LEVELS

\section{HIDALGO COUNTY
Virden Valley}

324051108594101. (formerly 324053108594101) Local number, 19S.21W.03.414.

LOCATION. - Lat $32^{\circ} 40^{\prime} 51^{\prime \prime}$, Long $108^{\circ} 59^{\prime} 41^{\prime \prime}$, Hydrologic Unit' 15040002. Owner: Jones, Clouse, and Jensen.

AQUIFER - -Valley F111.

WELI, CHARACTERISTICS.--Drilled water-table irrigation we11, diameter 20 in., depth 72 ft.

INSTRUMENTATION.--Periodtc steel-tape toasurements.

DATUM. - Elevation of land-surface datum is $3,750 \mathrm{ft}$ above National Geodetic Vertical Datum of 1929.

Measuring polnt: Hole inside pump shell, $0,90 \mathrm{ft}$ above land-surface datum.

PERTOD OF RECORD.--Jan. 1959 to current year.

EXTREMES FOR PERIOD OF RECORD.-Highest water level meastured, 7.50 ft below land-surface datum, Jan. 11, 1993 ;

lowest measured, $15.79 \mathrm{ft}$ below land-surface datum, Aug. $4,1978$.

WATER LEVEL, IN FEET BELOW LAND-SURFACE DATUM, WATER YEAR OCTOBER 1995 TO SEPTEMBER 1996

$\begin{array}{lr}\text { DATE } & \begin{array}{r}\text { WATER } \\ \text { LEVEL }\end{array} \\ \text { Jan. } 24 & 12.94 \\ \text { July } 8 & 13.77\end{array}$

\section{GIDALGO COUNTY \\ Lordsburs Ares}

321849108392001 . (formerly 321848108391401 ) Local number, 23S.18W.12.333.

LOCATION. - Lat $32^{\circ} 18^{\prime} 49^{\prime \prime}$, Long $108^{\circ} 39^{\prime} 20^{\prime \prime}$, Hydrologic Untt 15040003. Owner: R. T. McDonald.

AQUIEER . - Alluvium.

WELL CHARACTERISFICS.--Drilled water-table trrtgation wel.1, diameter 12 in., depth 220 ft. perforations $100-220 \mathrm{ft}$

INSTRLMENTATION. --Periodic steel-tape measurements.

DATUM. - Elevation of land-surface datum is 4,240 ft above National Geodetic Vertical Datum of 1929 ,

Measuring point: End of entry port pipe, 1.50 ft above land-surface detum.

PERIOD OF RECORD.-Apr. 1957 to current year.

EXIREMES FOR PERIOD OF RECORD. --H1ghest water lovel measured, 100.02 ft below land-surface datum, Jan. 11, 1958;

lowest measured, $190.45 \mathrm{ft}$ below land-surface datum, Aug. 7, 1981 .

WATER LEVEL, IN FEET BELOW LAND-SURFACE DATUM, WATER YEAR OCTOBER 1995 TO SEPTEMBER 1996

$\begin{array}{lr}\text { DATE } & \begin{array}{r}\text { WATER } \\ \text { LEVEL }\end{array} \\ \text { Jan, 23 } & 156.96 \\ \text { July } 9 & 166.24\end{array}$

321248108331401. (formerly 321257108331201) Local number, 24S.17W.14.442.

LOCATION, - Lat $32^{\circ} 12^{\prime} 48^{\prime \prime}$, long $108^{\circ} 33^{\prime} 14^{\prime \prime}$. Hydrologic Untt 15040003 . Owmer: E. W. Richens.

AQUIFER - - Alluvium.

WELL CHARACTERISTICS, --Drilled water-table frrigation well, diameter 18 in., depth $420 \mathrm{ft}$

INSTRINENTATION. --Perlodic steel-tape measurements.

DATUM. - -Elevation of Land-surface datum is 4,265 ft above National Geodetic Vertical Datum of 1929.

Measuring point: Top of casing $1.00 \mathrm{ft}$ above land-surface datum.

PERTOD OF RECORD - May 1955 to current year.

EXTREMES FOR PERIOD OF RECORD. - Highest water Level measured, 78.97 ft below land-surface datum, Jan. 7 , 1981 ;

lowest measured, $114.90 \mathrm{ft}$ below land-surface datun, Jan. 15,1970 .

WATER LEVEL, IN FEET BELOW LAND-SURFACE DATUM, WATER YEAR OCTOBER 1995 TO SEPTEMBER 1996

$\begin{array}{ll} & \text { WATER } \\ \text { DATE } & \text { LEVEL } \\ \text { Jan, 22 } & 86.98 \\ \text { July } 8 & 90.97\end{array}$

\section{HIDALGO COUNTY}

Animas Valiey

321624108504001. (formerly 321540108514101) Local number, 23S.20W.25.422.

LOCATrON. - Lat $32^{\circ} 16^{\prime} 24^{\prime \prime}$, Long $108^{\circ} 50^{\prime 4} 40^{\prime \prime}$, Hydrologtc Unit 15040003 . Owner: Kerr Cattle Co.

AQUIF ER. - -Alluvium.

WELL CHARACTERISTICS. -Drilled water-table irrigation we11, dianeter 16 in., depth $150 \mathrm{ft}$.

INSTRUMENTATION. --Periodic steel-tape measurements.

DATIM.-Elevation of land-surface datum is 4,150 ft above National Geodetic Vertical Datum of 1929 .

Measuring point: Top of casing 0.40 ft above land-surface datum.

PERTOD OF RECORD - May 1948 to current year.

EXTREMES FOR EERIOD OF RECORD. - Highest water level measured, 31.36 ft below land-surface datun, May 21 , 1948 ;

lowest measured, $54.82 \mathrm{ft}$ below land-surface datum, July 9,1996 .

WATER LEVEL, IN FEET BELOW LAND-SURFACE DATUM, WATER YEAR OCTOBER 1995 TO SEPTEMBER 1996

$\begin{array}{ll}\text { DATE } & \text { WATER } \\ \text { LEVEL } \\ \text { Jan. 23 } & 53.71 \\ \text { July } 9 & 54.82\end{array}$




\section{BIDALGO COUNTY}

315610108483901. (formexly 315645108493501) Local number, 27s.19W.20.343.

LOCATION, - Lat $31^{\circ} 56^{\prime} 10^{\prime \prime}$, Long $108^{\circ} 49^{\prime} 35^{\prime \prime}$, Eydrologic Unit 15040003. Owmer: Felix Gauthier.

AQUIFER. - -Alluvium.

WELE CHARACTERTSTCS,--Drilled watex-table irrigation well, diameter 16 in., depth 358 ft, cesed to 358 ft.

INSTRUAEATATIOA. --Pordodic steel-tape measuremontis,

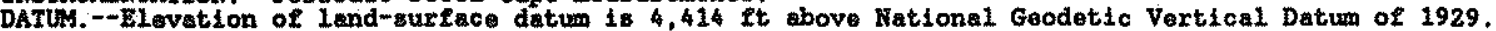

Hesaring point: Top edge of 1 1/4 in. pipe in concrete pump base, 1.25 ft above land-surface datum.

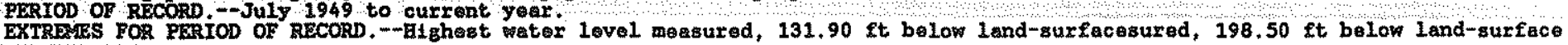

WAFE UEVER, IN FEET BELON LAHD-SURPACE DATUA, WATER YEAR COTOBER 1995 TO SEETAWBR 1996

\begin{tabular}{|c|c|}
\hline DATE & $\begin{array}{l}\text { WATEER } \\
\text { LEVEL }\end{array}$ \\
\hline$J_{\text {July }} 23$ & $\begin{array}{r}179.79 \\
189.20\end{array}$ \\
\hline
\end{tabular}

315738109004001, Local number, 27S.21W.17.124.

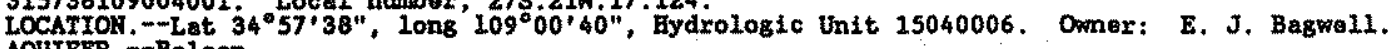

AQUIFER . - Bolson.

WELL CRARACTERISTYCS.-Drilled weter-tablo frrigation well, dianeter 16 in., depth 220 rt

INSTRURENATION. --Periodic steol-tape measurement:

DAFUA,-Elevation of land-surface datum is 4,020 et above National Geodetic Vertical Datum of 1929.

Hogsuring potnt: Holo in weat stde of pump base, 1.00 ft above land-surface datum.

PRRIOD OF RECORD,-Jan. 1978, Jan, 1980, July 1984 to current year.

EXTREXES FOR PERIOD OF RECORb. -- Highest pater level measured, 120.98 ft above land-surface datun, Jan. 10, 1980 ;

lowest measured, $126.69 \mathrm{ft}$ below land-surface datuin, July 10, 1996.

WATER LEVEL, IN FEET BELOW LAND-SURFACE DATUM, WATER YEAR OCTOBER 1995 TO SEPTERER 1996

$\begin{array}{lr}\text { DATE } & \text { WATER } \\ \text { IEVEL } \\ \operatorname{Jan} .24 & 125.93\end{array}$

Jan. $24 \quad 125.93$

315048109010201. (Formex1y 315010108570001 ) Local number, 28S.21W.30.222.

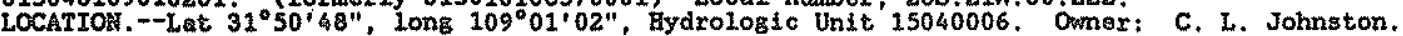

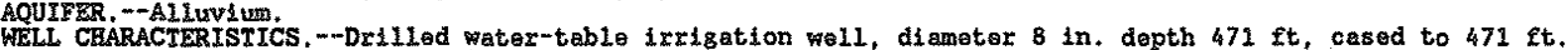

INSTRUATHTATON, --POXiodic atool-tape mosurement:

DATHA. --Elovation of land-surface datum is 4,128 fit above National Geodetic Vertical Datum of 1929.

Measuring point: Fole in wast side of casing, 0.70 ft abovo land-surface datum.

PERIOD OF RECORD. - Jan. 1968 to current year,

EXTREYAS FOR PERIOD OF RECORD,--Highest water level measured, 110.88 ft bolow land-surface datum, Jan. 15, 1969;

lowest mensured, 124.93 ft below land-aurface datum, July 16,1984 .

WATER LEVBL, IN FEET BELOW LAND-SURFACE DATUM, WATER YEAR OCTOBER 1995 TO SEPTEMBER 1996

$\begin{array}{lr}\text { DATE } & \text { WATER } \\ \text { LEVEL } \\ \text { Jan. } 24 & 121.76 \\ \text { July } 10 & 122.46\end{array}$

\section{RIDALGO COUNTY}

313502108275001 . Local number, 31S. 16W.33.233.

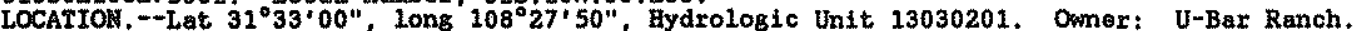

AOUTYER, - Alluvium.

WELL CBARACTERISTICS.--Dx1110d mater-table observation we11, diameter 16. in., depth 654 ft.

INSTRUNENTATYOA, --PexLodic steol-tape measurenents.

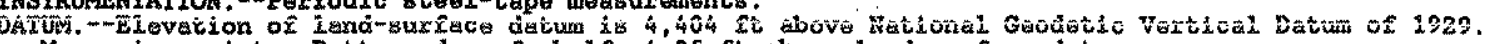

Masuring point: Botton odge of shelf, \&,05 ft abovo land-surface detum.

PERIOD OF RECORD.--Jan, 1965 to current year.

EXTREAES EOR PERIOD OF RECORD, --Highast watex level measured, 44.66 It below land-surface datum, Apr. 18-20, 1973;

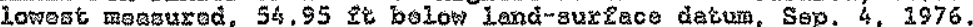

WATER LEVEL, IN FEET BELOA LAND-SURFACE DATUM, WATER YEAR OCTOBER 1995 TO SEPTEMBER 1996

$\begin{array}{cr}\text { DATE } & \text { WATER } \\ \text { Jan. } 22 & \text { LEVEL } \\ \text { Ju1y } 8 & 47.16 \\ & 47.14\end{array}$


312938108302301 . Local number, 325. 16W. 30.134

LOCATION. - Lat $31^{\circ} 29^{\prime} 38^{\prime \prime}$, Long 108 $30^{\circ} 23^{\prime \prime}$, Hydrologte Untt 13030201. Owner: C. C. Edwards.

AQUIFER. - -ALluvium.

GELI CHARACTERISTICS.--DrtLled water-table irrigation vell, diameter 16 in., depth $150 \mathrm{ft}$.

INSTRUMENTATIOH.--Periodic steel-tape measurements.

DATUM.--Elevation of land-surface datum is 4,490 ft shove National Geodetic Vertical Datum of 1929

Measuring point: Top of $3 / 4 \mathrm{in}$. pipe nipple inside pump thell, 1.45 ft above land-surface datum.

PARTOD OF RECORD - -Mar. 1952 to current year.

EXTRDAES FOR FERIOD OF RECORD.--HAghest pator lovel measured, 85.11 ft belor land-8urface datun, Mar. 27, 1952:

lowest measured, 129.10p ft below land-surface datum, Aug, 20, 1962.

WATER LEVEL, IN FEET BELON LAMD-SURFACE DATUM, WATER YEAR OCTOBER 1995 TO SEPTEMBER 1996

$\begin{array}{cc}\text { DATE } & \begin{array}{l}\text { WATER } \\ \text { LEVEL }\end{array} \\ \operatorname{Jan}, 22 & 86.31 \\ \text { July } 8 & 86,41\end{array}$

LEA COUNTY

Tatum-Lovington-Fobbs Area

332115103403301. Local number, 11S.32E.24.113.

LOCATION,-Lat $33^{\circ} 21^{\circ} 15^{\prime \prime}$, long $103^{\circ} 40^{\circ} 3^{*}$, Fydrologic Unit 12080001. Owner: Paul Hamilton.

AQUIFER. - Ogallala formation.

WELL. CHARACTERISTICS.--Drilled pater-table observation well, diametex $41 / 2 \mathrm{in}$., depth $110 \mathrm{ft}$.

IHSTRURENTATION, --Digital recorder, 1-hour punch.

DATUM. - Elevation of land-surface datum is 4,336 ft above National Geodetic Vertical Datum of 1929.

Measuring point: Top of casing, $0.70 \mathrm{ft}$. above land-surface datum.

REMARKS. - - Records good.

PERIOD OF RECORD.--Oct. 1977 to current year.

EXTREMES FOR PERIOD OF RECORD.--Highest water level measured, 59.74 ft above land-murface datun, Oct. 3 , 1993 ;

lonest measured, 62.67 ft below land-surface datum, Apr. 19, 1993 .

WATER LEVEL, IN FEET BELOW LAND-SURFACE DATUM, WATER YEAR OCTOBER 1995 TO SEPTEMBER 1996

$\begin{array}{rr}\text { DAY } & \text { CC } \\ 5 & 61.5 \\ 10 & 61.5 \\ 15 & 61.5 \\ 20 & 61.5 \\ 25 & 61.5 \\ \text { EOM } & 61.5\end{array}$

WTR YEAR 1996

CT
61.55
61.54
61.53
61.52
61.52
61.52

HOV
61.51
61.51
61.50
61.50
61.49
61.49

$\begin{array}{rr}\text { DEC } & \text { JAN } \\ 61.49 & 61.56 \\ 61.48 & 61.51 \\ 61.48 & 61.52 \\ 61.52 & 61.53 \\ 61.55 & 61.49 \\ 61.54 & 61.50\end{array}$

FEB
61.52
61.53
61.51
61.48
61.48
61.48

AIGHEST
61.50

61.48
61.48 DAILY BIGEEST VALUES

331713103283301. (formerly 331740103285001) local number, 12S.34E.11.421

LOCATIOH - Lat $33^{\circ} 7^{\prime} 22^{\prime \prime}$, long $103^{\circ} 28^{\prime} 50^{\prime \prime}$. Hydrologic Untt $12080006^{\circ}$ Owner. A. D. Jones.

AQUTFER. - Ogallala formation.

WELL CEARACTERISTICS.--Drilied water-table unused well, diameter 15 in., depth 87 ft

INSTRUMENTATION. --Perlodic steel-tape measurements.

DATUM,--Elevation of land-surface datum is 4,144 ft above National Geodetic Vertical Datum of 1929.

Measuring point: Top of concrete pump base, 0.80 ft above land-surface datum.

PERTOD OF RECORD.-May 1949 to current year.

EXTREMES FOR PERIOD OF RECORD.--Highest water level measured, 29.57 ft below 1and-surface datum, May 24, 1949;

lowest measured, $34.14 \mathrm{ft}$ below land-surface datum, Aug. 17,1983 .

WATER LEVEL, IN FEET BELOW LAND-SURFACE DATUM, WATER YEAR OCTOBER 1995 TO SEPTEMBER 1996

$\begin{array}{cc}\text { DATE } & \text { WATER } \\ \text { LEVEL } \\ \text { Jan. } 2 & 31.42 \\ \text { Aug. } 7 & 31.65\end{array}$

330458103251001. (formerly 330455103251301) Local number, 14S.35E.28.1111.

LOCATrON, - Lat $35^{\circ} 04^{\prime} 55^{\prime \prime}$, long $103^{\circ} 25^{\circ} 13^{\prime \prime}$, Hydrologic init 12080003. Owner: Paul Fisher.

AOUIFER - -Ogallala formation.

WELL CHARACTERISTICS, --Drilled water-table well, diameter 5 in., depth 137 ft.

INSTRLAENTATION. --periodic stee1-tape measurements.

DATUM.--Elovation of land-surface datum is 4,031 ft above National Geodetic Vertical Datum of 1929.

Measuring point: Top of casing, $2.00 \mathrm{ft}$ above land-surface datum.

PERIOD OF RECORD. - J Jan. 1983 to current year.

EXTREMES FOR PARIOD OF RECORD.--Highest water love1 measured, 43.05 ft below land-surface datum, Jan, 5, 1994;

lowest measured, 44.73 ft below land-surface datum, Aug. 7, 1996.

WATER LEVEL, IN FEET BELOW LAND-SURFACE DATUM, WATER YEAR OCTOBER 1995 TO SEPTEMBER 1996

$$
\text { DATE WATER }
$$

$\begin{array}{ll}\text { Jan. } 3 & 43.50 \\ \text { Aug. } 7 & 44.73\end{array}$




\section{GROUND-WATER LEVELS}

LEA COUNTY

Tatum-Lovington-Hobbs Area

330405103194501. (former1y 330400103193401) Local number, 14S.36E.32.12121.

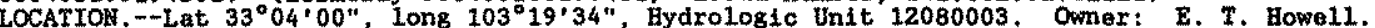

AOUTFER - - Ogallala formation.

WELL CEARACTERISTICS.--Drilled water-table irrigation well, diameter 16 in., depth and casing information not avallable

INSTRUMENTATION, --Periodic steel-tape measurements.

DATUM. - - Llevation of land-surface datum is 3,990 it above National Geodetic Vertical Datum of 1929.

Messuring point: Top of concrete pump base, 0,50 st above land-surface datum.

PERIOD OF RECORD,-Jan. 1949 to Jan. 1950, Jan. 1971 to current year.

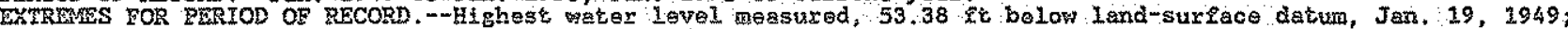

lowest measured, 73,62 ft below land-surface datum, Jan. 3, 1996.

WATER LEVEL, IN FEET BELON LAND-SURFACE DATUM, WATER YEAR OCTOBER 1995 TO SEPTEMBER 1996

\begin{tabular}{|c|c|}
\hline DATE & $\begin{array}{l}\text { WATER } \\
\text { LEVEL }\end{array}$ \\
\hline $\begin{array}{l}\text { an. } 3 \\
\text { ug. } 7\end{array}$ & $\begin{array}{l}73.62 \\
71.10\end{array}$ \\
\hline
\end{tabular}

325730103213901. (formerly 325703103213201) Local number, 16S.36E.04.322.

LOCATION, - Lat $32^{\circ} 57^{\prime} 03^{\prime *}$, Long $103^{\circ} 21^{\prime} 32^{\prime \prime}$, Hydrologic Unit 12080003. Owner: City of Lovington,

AOUIFER. - Ogallala cormation.

WELL CHARACTERISTICS,--Dxilled water-table unused well, diameter 13 in, depth 212 ft, perforated $80-208$ ft INSTRUMENTATION, - Digital recorder, 1-hour punch.

DATUM.--Elovation of land-surface datun is $3,926 \mathrm{ft}$ above National Geodetic Vertical Datun of 1929

Measuring point: Top of shelf, 4,00 ft ebove land-surface datum.

REMARKS - -Records good.

PERIOD OF RECORD. - Aug. 1971 to current year.

EXTREMES FOR PERIOD OF RECORD.-Highest water level measured, 57.67 ft below land-surface datum, Feb. 5, 1995;

lowest measured, 67.11 ft below land-surface datum, Aug. $24,1971$.

WATER LEVEL, IN FEET BELOW LAND-SURFACE DATUM, WATER YEAR OCTOBER 1995 TO SEPTEMBER 1996

\begin{tabular}{|c|c|c|c|c|c|c|c|c|c|c|c|c|}
\hline DAY & OCT & Mov & DEC & JAN & FEB & MAR & APR & MAY & JUN & JUL & AUSG & SEP \\
\hline $\begin{array}{r}5 \\
10 \\
15 \\
20 \\
25 \\
\mathrm{EOH}\end{array}$ & $\begin{array}{l}58.40 \\
58.38 \\
58.33 \\
58.29 \\
58.24 \\
58.21\end{array}$ & $\begin{array}{l}58.19 \\
58.14 \\
58.16 \\
58.15 \\
58.12 \\
58.10\end{array}$ & $\begin{array}{l}58.10 \\
58.11 \\
58.10 \\
58.09 \\
58.11 \\
58.03\end{array}$ & $\begin{array}{l}58.07 \\
58.04 \\
58.03 \\
58.02 \\
57.99 \\
57.99\end{array}$ & $\begin{array}{l}58.00 \\
57.96 \\
57.94 \\
57.91 \\
57.89 \\
57.90\end{array}$ & $\begin{array}{l}57.86 \\
57.90 \\
57.88 \\
57.90 \\
57.91 \\
57.95\end{array}$ & $\begin{array}{l}58.00 \\
58.00 \\
58.04 \\
58.03 \\
58.05 \\
58.08\end{array}$ & $\begin{array}{l}58.13 \\
58.17 \\
58.22 \\
58.25 \\
58.25 \\
58.30\end{array}$ & $\begin{array}{l}58.30 \\
58.33 \\
58.38 \\
58.38 \\
58.37 \\
58.40\end{array}$ & $\begin{array}{l}58.37 \\
58.39 \\
58.40 \\
58.38 \\
58.38 \\
58.35\end{array}$ & $\begin{array}{l}58.32 \\
58.31 \\
58.30 \\
58.30 \\
58.26 \\
58.22\end{array}$ & $\begin{array}{l}58.19 \\
58,17 \\
58.12 \\
58.10 \\
58.04 \\
58.03\end{array}$ \\
\hline
\end{tabular}

WTR YZAR 1996 BYGHEST 57.83 MAR 17, 1996 LOWEST 58.45 OCT 1, 1995

325658103200001 Local number, 16S.37E.11.11111.

LOCATION, -lat $32^{\circ} 56^{\circ} 58^{\prime \prime}$, long $103^{\circ} 20^{\circ} 00^{\prime \prime}$, Hydrologic Unit 12080003. Owner: H. J, Taylor.

AQUIFER. - Ogallala formation.

WELI CHARACTERISTICS.-Drilled water-table irrigation well, diameter 16 in., reported depth 118 ft,

INSTRIMENTATION.--Periodic steel-tape measurements.

DATUM. --Elevation of land-surface datum is 3,900 ft above National Geodetic Vertical Datum of 1929.

Measuring point: Top of 1 in. hole in southwest side of pump, 1.34 ft above land-surface datum,

PERTOD OF RECORD - Jan 1949 to current year.

EXTRESS FOR PERIOD OF RECORD.--Highest water level measured, 31.93 ft below land-surface datum, Jan. 23, 1949;

lowast measured, 78.64 ft below land-surface datum, Jan. 3, 1979.

WATER LEVEL, IN FEET BELON LAND-SURFACE DATUM, WATER YEAR OCTOBER 1995 TO SEPTEMBER 1996

$\begin{array}{cc}\text { DATE } & \text { WATER } \\ \text { JEVEL } 9 & \\ \text { Aug. } 7 & 56.59 \\ & 67.42\end{array}$

325132103112501 tonal number, $17 \mathrm{~S} .38 \mathrm{E} .07 .111311$

LOCATION. - Lat 32 $51^{\prime} 32^{\prime \prime}$, Long $103^{\circ} 11^{\prime} 25^{\prime \prime}$, Fydrologic Unit 12080003, Omex; L. R. Sebings.

AQUTFER - - Og日1101 Pormation.

WELL CBARACTERISTICS.--Drilled water-table frrigation well, diameter 16 in., reported depth 125 ft.

INSTRUHENTATION, - - Pertodic steel-tepe measurements.

DATUM.--Elevation of land-surface datum is 3,740 it above National Geodetic Vartical Dartu or 1929

Measuring point: Edge of pipe on west side of pump, 0.95 ft above land-surface datum.

PERIOD OF RECORD. - July 1951 so current year.

EXTREMES FOR PERIOD OF RECORD,--Highest water level measured, 35.59 ft below land-surface datum, Mar. 21, 1952,

lowest measured, 74.15 ft below land-surface datum, July $22,1980$.

WATER LEVEL, IN FEET BELOW LAND-SURFACE DATUM, WATER YEAR OCTOBER 1995 TO SEPTEMBER 1996

DATE WATER

Jan. $10 \quad 68.28$


GROUND-WATER LEVELS

LEA COUNTY

Tatum-Lovington Hobbs Area

324745103082001 . Local number, 17S.38E. 34.113143.

LOCATION. - Lat $32^{\circ} 47^{\prime} 45^{\prime \prime}$, Long $103^{\circ} 08^{\prime} 20^{\prime \prime}$, Bydrologic Untt 12080003. Omer: W. E. Busby.

AQUTFER. - Ogallala formation.

WELL CHARACTERISTICS,-Drilled water-table irrigation well, diameter 12 in, depth 125 ft, cased to 90 ft. INSTRUMENTATION, --Poriodic steel-tape measurements.

DATUM. --Elevation of land-surface datum is $3,660 \mathrm{ft}$ above National Geodetic vertical Datum of 1929.

Measuring point: Top of casing, $0.40 \mathrm{ft}$ above land-surface datum.

PERIOD OF RECORD. -Nov. 1943 to current year.

EXTREMES FOR PERIOD OF RECORD, - -Highest water level measured, 24.78 ft below land-surface datum, Jan. 15, 1944;

lowest measured, $68.25 \mathrm{ft}$ below land-surface datum, Aug. 7, 1996.

WATER LEVEL, IN FEET BELOW LAND-SURFACE DATUM, WATER YEAR OCTOBER 1995 TO SEPTEMBER 1996

$\begin{array}{ll}\text { DATE } & \text { WATER } \\ \text { LEVEL } \\ \text { Jan. } 10 & 67.17 \\ \text { Aug. } 7 & 68.25\end{array}$

\section{LINCOLN COUNTY}

333241105341101. (formerly 333242105340701) Local number, 09S.14E.10.13221. LOCATION. - Lat $33^{\circ} 32^{\prime 4} 42^{\prime \prime}$, Long $105^{\circ} 34^{\circ} 07^{\prime \prime}$, Hydrologic Unit 13060008. Owner: Village of Capttan. AOUIFER --Mancos Shale of Late Cretaceous age.

WELI CHARACTERISTICS.--Drilled water-table municipal well, diameter 8 in., depth 324 ft, cased to $271 \mathrm{ft}$. INSTRUMENTATION. --Periodic steel-tape measurements.

DATIM.--Elevation of land-surface datum is $6,340 \mathrm{ft}$ above National Geodetic Vertical Datum of 1929.

Measurting point: Top of breather hole on west side of pump base, 1.00 ft above landmsurface datum.

PERIOD OF RECORD - - June 1955 to current year.

EXTREMES FOR PERIOD OF RECORD.--Highest water level measured, 36.56 ft below land"surface datum, Jan. 28, 1993;

lowest measured, 69.77 ft below land-surface datum, Nov. 28, 1956.

WATER LEVEL, IN FEET BELOW LAND-SURFACE DATUM, WATER YEAR OCTOBER 1995 TO SEPTEMBER 1996

$\begin{array}{cc}\text { DATE } & \begin{array}{l}\text { WATER } \\ \text { LEVEL }\end{array} \\ \text { Jan. 29 } & 39.02 \\ \text { Aug. 15 } & 37.99\end{array}$

332110105092501. (formerly 332157105094101) Local number, 115.18E.15.33313.

LOCATION. - Lat 33 $21^{\circ} 02^{\prime \prime}$, long $105^{\circ} 09^{\prime} 41^{\prime \prime}$, Hydrologic Unit 13060008 . Owner: Lincoln County Livestock Co.

AQUTFE. - Yeso formation of Permian age.

WELL CHARACTERISTICS, -Drilled water-table domestic and stock well, diameter 12 in., depth 125 ft, cased to 110 ft.

INSTRUMENTATION. - Periodic stee1-tape measurements

DATUM. - Elovation of land-sturface datum is $4,989 \mathrm{ft}$ above National Geodetic Vertical Datum of 1929

Measuring point: Top of castng, $0.50 \mathrm{ft}$ above land-sturface datum.

PERIOD OF RECORD.--Oct. 1955 to current year.

EXTREMES FOR PERIOD OF RECORD. --Bighest water level measured, 44.43 ft below Land-sureface datum, Aug. 18, 1988 ;

lowest measured, $60.18 \mathrm{ft}$ below land-surface datum, Jan. 15, 1959 .

WATER LEVEL, IN FEET BELOW LAND-SURFACE DATUM, WATER YEAR OCTOBER 1995 TO SEPTEMBER 1996

$\begin{array}{cc}\text { DATE } & \begin{array}{l}\text { WATER } \\ \text { LEVEL }\end{array} \\ \text { Jan. } 29 & 47.96 \\ \text { Aug. } 15 & 47.03\end{array}$

LUNA COUNTY

Nutt-Hockett

322927107220101, (formerly 322930107221001) Local number, 21S.05W.08.444.

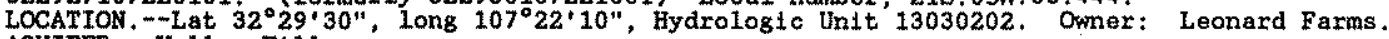

AQUIFER - -Valley F 111 .

WELI, CHARACTERISTICS,-Dr11led water-tablo irrigation wel1, diameter 16 in., depth 435 ft, cased to 435 ft.

INSTRUMENTATION. --Periodic steel-tape measurements.

DATUM. - Elevation of land-surface datum is 4,530 ft above National Geodetic Vertical Datum of 1929.

Measuring point: Hole in NE side of pump shell, 1.60 ft above land-surface datum.

PERTOD OF RECORD - Nov. 1961 to current year.

EXTREMES FOR PERIOD OF RECORD. - Highest water level measured, 102.06 ft below land-surface datum, Jan. 17, 1962;

lowest measured, 205.42 ft below land-surface datum, Jan.' 17, 1996 .

WATER LEVEL, IN EEET BELOW LAND-SURFACE DATUM, WATER YEAR OCTOBER 1995 TO SEPTEMBER 1996

$\begin{array}{lc}\text { DATE } & \text { WATER } \\ \text { LEVEL } \\ \text { Jan. } 17 & 205.42 \\ \text { July } 3 & \text { pumping }\end{array}$


GROUND-WATER LEVELS

LUNA COUNTY

Mimbres Valley

321352107493901 . Local number, 24S.10W.12.431.

LOCATION, --Lat $32^{\circ} 13^{\circ} 52^{\prime \prime}$. Long $107^{\circ} 4^{\prime} 39^{\prime \prime}$. Hydrologic Unit 13030202. Owner: Steve Hrna.

AQUIFER.--Bolson depositis.

WELL CHARACTERISTICS.--Dug and drilled water-table unused well, diameter $36 \mathrm{ln}$, reported depth 132 ft. INSTRURENTATION:--Continuous strip-chart recorder.

DArug, -Elevation of land-surface datum is 4,330 ft above National Geodetic Vertical Datum of 1929

Measuring point: Top of rocorder shelter shelf, 1.36 ft above land-surface datum.

REMARKS --Recorder re-installed Jan.26, 1994 . Lost several days of record due to recorder malfunction PERIOD OF RECORD - Apr. 1939 to current year.

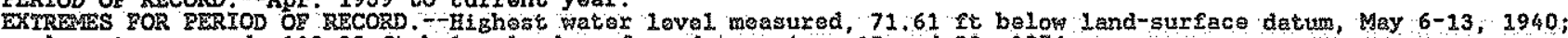

lopest measured, 113,30 ft below land-surface datum, Aug, 12 and $20,1976$.

WATER LEVEL, IN FEET BELOW LAND-SURFACE DATUM, WATER YEAR OCTOBER 1995 TO SEPTEMBBER 1996 DAILY BIGHEST VALUES

\begin{tabular}{|c|c|c|c|c|c|c|c|c|c|c|c|c|}
\hline DAY & OCT & Nov & DEC & JAN & FEB & MAR & AFR & MAY & JUN & JUL & AUG & SEP \\
\hline $\begin{array}{r}5 \\
10 \\
15 \\
20 \\
25 \\
\text { EOM }\end{array}$ & $\begin{array}{l}101.63 \\
101.78 \\
101.73 \\
101.87 \\
101.87 \\
101.85\end{array}$ & $\begin{array}{l}101.80 \\
101.54 \\
101.54 \\
101.51 \\
101.39 \\
101.34\end{array}$ & $\begin{array}{r}101.27 \\
101.33 \\
101.22 \\
-. \\
-\end{array}$ & 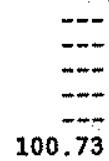 & $\begin{array}{l}100.95 \\
100.92 \\
100.98 \\
101.24 \\
101.28 \\
101.52\end{array}$ & $\begin{array}{r}101.58 \\
101.78 \\
101.88 \\
102.13\end{array}$ & $\begin{array}{r}-\overline{-} \\
102.69 \\
102.72\end{array}$ & $\begin{array}{r}--- \\
=- \\
=- \\
=- \\
103.42\end{array}$ & $\begin{array}{l}103.43 \\
103.44 \\
103.42 \\
103.37 \\
103.38 \\
103.42\end{array}$ & $\begin{array}{l}103.28 \\
103.30 \\
103.26 \\
103.20 \\
103.19 \\
103.16\end{array}$ & $\begin{array}{l}103.23 \\
103.24 \\
103.29 \\
103.32 \\
103.33 \\
103.35\end{array}$ & $\begin{array}{r}103.36 \\
103.36 \\
103.05 \\
103.06\end{array}$ \\
\hline
\end{tabular}

WTR YEAR 1996 घIGHEST 100.70 FEB 1, 1996 ... LOWEST 103.59 MAY 29, 1996

321328107565301. (formerly 321415107565501) Local number, 24S.11W.14.122.

LOCATION,--Lat $32^{\circ} 13^{\prime} 28^{\prime \prime}$, long $107^{\circ} 56^{\prime} 55^{\prime \prime}$, Hydrologic Unit 13030202 . Owner: Charles Waldrop.

AOUIFER. --Bolson deposits.

WELL CHARACTERISTICS,--Drilled water-table Lrrigation woll, diameter 12 in., reported depth 350 ft, cased to 198 ft.

INSTRUMENTATION.-Periodic steol-tape measurements.

DATUM.--Elevation of iand-surface datum is $4,405 \mathrm{ft}$ above National Geodetic Vertical Datum of 1929

Measuring point: Top of $1 \mathrm{in}$. hole in punp base, $0.80 \mathrm{ft}$ above land-surface datum.

PERTOD OF RECORD. - -July 1951 to current year.

EXTREMES FOR PERIOD OF RECORD.--H1ghest water level measured, $107.66 \mathrm{ft}$ below land-surface datum, Jan. 23, 1952;

lowest measured, 228.00 ft below land-surface datum, May 11, 1956.

WATER LEVEL, IN FEET BELOW LAND-SURFACE DATUM, WATER YEAR OCTOBER 1995 TO SEPTEMBER 1996

$\begin{array}{cr}\text { DATE } & \begin{array}{r}\text { WATER } \\ \text { LEVEL }\end{array} \\ \text { Jan. } 16 & 172.00 \\ \text { July } 2 & 176.74\end{array}$

321010107260201. (formerly 321015107260501) Local number, 25S.06W.02.111.

LOCATION, - -Lat $32^{\circ} 10^{\prime} 15^{\prime \prime}$, long 107 $26^{\circ} 05^{\prime \prime}$, Hydrologic Unit, 13030202 . Owner: C, W. Johnson, Jr.

AQUIFER. - -Bolson deposits.

WELL CHARACTERISTICS,--Drilled artesian irrigation well, diameter 16 in., depth 235 ft, perforated 180-235 ft,

gravel packed.
INSTRUMENTATION.--Periodic steel-tape measurenents.

DATUM.--Elevation of land-surface datum is $4,090 \mathrm{ft}$ above National. Geodetic Vertical. Datum of 1929.

Measuring point: Top of castng, $1.30 \mathrm{ft}$ above land-surface datum.

PERIOD OF RECOrD. - May 1952 to current year.

EXTRERES FOR PERIOD OF RECORP.--Highest water level measured, 0.45 ft below land-surface datum, Mar. 14 , 1953 ;

lowest measured, 117.66 it below land-surface datum, Aug. 6,1980 .

WATER LEVEL, IN FEET BELON LAND-SURFACE DATUM, WATER YEAR OCTOBER 1995 TO SEPTEMBER 1996

$\begin{array}{ll} & \text { WATER } \\ \text { DATE } & \text { LEVEL } \\ \text { Jan. } 17 & 21.49 \\ \text { JuIY } 3 & 31.24\end{array}$

320918107293301, (former1y 320915104294501) Locel number, 25S.06W.07.211.

LOCATION. - Lat $32^{\circ} 09^{\prime} 15^{\prime \prime}$, Iong $107^{\circ} 29^{\circ} 45^{\prime \prime}$, Hydrologic Unit 13030202. Owner: H. C. Telles.

AQUIFER - -Bolson deposit's.

WELL CHARACTERISTICS, -Drilled water-table irrigation well, diameter 16 in., depth 230 ft, cased to 230 ft.

INSTRUMENTATION. --Periodic steol-tape measurements.

DATUM--Elovation of land-sureace datun is 4,084.22 ft above National Geodettc Vertical Datum of 1929.

Measuring potnc: Hole in pump base, 1.20 St above land"surface datum (since Jan. 15, 1966).

PFTROD OF RECORD - -Jan. 1953 to current year.

EXTREAGE FOR DERIOD OF RECORD.--Highest watex level measured, 65.34 ft below land-surface datum, Mar. 14, 1953 ;

lowest measured, $1.22 .16 \mathrm{ft}$ below land-surface datum, Aug. $13,1970$.

WATER LEVEL, IN FEET BELOW LAND-SURFACE DATUM, WATER YEAR OCTOBER 1995 TO SEPTEMBER 1996

$\begin{array}{ll}\text { DATE } & \text { WATER } \\ \text { LEVEL } \\ \text { Jan. } 17 & 82.39 \\ \text { July } 3 & 82.33\end{array}$


GROUND-WATER LEVELS

LUNA COUNTY

Mimbres Valley

320647107490701. Local number, 25S.09W.19.31331.

LOCATION, -Lat $32^{\circ} 26^{\circ} 47^{*}$, Long $107^{\circ} 49^{\circ} 07^{\prime \prime}$, Hydrologic Unit 13030202. Omer: Tryon.

AQUIFER. --Alluvium.

WELL CBARACTERISTICS.--Drilled water-table unused irrigation weil, diameter 14 in,, depth 240 ft, cased to $240 \mathrm{ft}$, perforated $80-240 \mathrm{ft}$.

INSTRUMENTATION. --Pertodic steei-tape measurements.

DATM. - Elevation of land-surface datum is 4,070 ft above National Geodetic Vertical Datum of 1929.

Measuring point: Top of casing, $1.00 \mathrm{ft}$ above land-surface datum.

PQRTOD OF RECORD. - July 1959 to current year.

EXTREMES FOR PERIOD OF RECORD.--Highest water level measured, 98.68 ft below land-surface datum, Feb. 10, 1959; lowest measured, $221.86 \mathrm{ft}$ below land-surface datum, Aug. 20, 1996.

WATER LEVEL, IN FEET BELON LAND-SURFACE DATUM, WATER YEAR OCTOBER 1995 TO SEPTEMBER 1996

\begin{tabular}{|c|c|c|c|c|c|c|c|c|c|c|c|}
\hline DATE & $\begin{array}{l}\text { WATER } \\
\text { LEVEL }\end{array}$ & DATE & $\begin{array}{l}\text { WATER } \\
\text { LEVEL }\end{array}$ & DATE & $\begin{array}{l}\text { WATER } \\
\text { LEVEL }\end{array}$ & DATE & $\begin{array}{l}\text { WATER } \\
\text { LEVEL }\end{array}$ & DATE & $\begin{array}{l}\text { WATER } \\
\text { LEVEEL }\end{array}$ & DATE & $\begin{array}{l}\text { WATERR } \\
\text { LEVEL }\end{array}$ \\
\hline 8 & $\begin{array}{l}211.50 \\
201.68\end{array}$ & NOV $_{\text {MAY }} 23$ & $\overline{215.26}$ & $\begin{array}{l}\text { DEC } 20 \\
\text { JUNE } 20\end{array}$ & $\begin{array}{l}195.00 \\
218.66\end{array}$ & $\begin{array}{l}\text { JAN } 25 \\
\text { JULY } 19\end{array}$ & $\begin{array}{l}193.50 \\
220.02\end{array}$ & $\begin{array}{l}\text { FEB } \\
\text { AUG } 2\end{array}$ & $\overline{221.86}$ & $\begin{array}{l}\text { MAR } 5 \\
\text { SEP } 23\end{array}$ & $\begin{array}{r}198.40 \\
215.19\end{array}$ \\
\hline
\end{tabular}

315517107375001 (formerly 315525107374501) Local number, 27S.08W.35.122.

LOCATION. - Lat $31^{\circ} 55^{\prime} 25^{\prime \prime}$, long 107 $37^{\prime} 45^{\prime \prime}$, Hydrologic Unit 13030202 . Omer: M. M. Gibson.

AQUIFER.--Bolson deposit's

WELI CBARACTERISTICS.--Drilled water-table unused irrigation well, diameter 12 to 8 in., depth 550 ft, cased to $550 \mathrm{ft}$, perforated $155-550 \mathrm{ft}$.

INSTRUMENTATION. --Pexiodic steel-tape measurements.

DATUM.--Elevation of land-surface datum is 4,070 ft above National Geodetic Vertical Datum of 1929.

Measuring point: Top of casing, $0.20 \mathrm{ft}$ above land-surface datum.

PERIOD OF RECORD. --July 1952 to current year.

EXTREMES FOR PERIOD OF RECORD.--Highest water level measured, 20.84 ft below land-surface datum, Mar. 16, 1953; lowest measured, $119.34 \mathrm{ft}$ below land-surface datum, Aug. $3,1981$.

WATER LEVEL, IN FEET BELOW LAND-SURFACE DATUM, WATER YEAR OCTOBER 1995 TO SEPTEMBER 1996

$\begin{array}{ll}\text { DATE } & \text { WATER } \\ \text { Jan. } 16 & \text { LEVEL } \\ \text { July } 2 & 75.56 \\ & 81.84\end{array}$

315903107424501. (former1y 315905107425001 ) Local number, 27s. 09W. 01.431

LOCATTON. - Lat $31^{\circ} 59: 05^{\prime \prime}$, long $107^{\circ} 42^{\prime} 50^{\prime \prime}$, Bydrologic Unit 13030202 , Owner: I. G. Burns

AQUIFER. - Valley Fill.

WELL CHARACTERISTICS.--Drilled water-table irrigation well, diameter $16 \mathrm{in}$, depth $62 \mathrm{ft}$, cased to $62 \mathrm{ft}$. INSTRIMENTATION.--Periodic steel-tape measurements.

DATUM.--Elevation of Iand-surface datum is $4,135 \mathrm{ft}$ above National Geodetic Vertical Datum of 1929.

Measuring point: Top odge of rectangular hole in pump base, 0.65 ft above land-surface datum.

PERIOD OF RECORD. -Jan. 1954 to current year.

EXTREMES FOR PERIOD OF RECORD, - - Highest water level measured, 30.61 ft below land-surface datum, Jan. 19, 1954;

lowest measured, 47.26 ft below land-surface datum, Aug. 11, 1977.

WATER LEVEL, IN FEET BELOW LAND-SURFACE DATUM, WATER YEAR OCTOBER 1995 TO SEPTEMBER 1996

$\begin{array}{ll}\text { DATE } & \text { WATER } \\ \text { LEVEL } \\ \text { Jan. } 16 & 38.71 \\ \text { July } 2 & 38.87\end{array}$

314942107361001 . (formerly 314938107371401) Local number, 28S.08W.36.411.

LOCATION, --Lat $31^{\circ} 49^{\prime} 38^{\prime \prime}$, long $107^{\circ} 37^{\prime} 4^{\prime \prime}$, Hydrologic Untt 13030202 . Owner: M. R. Hemley.

AQUTFER.--Bolson deposits.

WELL CHARACTERISTICS.--Drilled pater-table irrigation well, diameter $16 \mathrm{ln}$, depth $250 \mathrm{ft}$, cased to $250 \mathrm{ft}$.

INSTRUMBNTATION. --Periodic \& teel-tape measurements.

DATUM. - Elevation of land-surface datum is $4,008 \mathrm{ft}$ above National Geodetic vertical Datum of 1929.

Measuring point: Top of casing, $1.85 \mathrm{ft}$ above land-surface datum.

PERTOD OF RECORD - Jan. 1961 to current year.

EXTREMES FOR PERIOD OF RECORD,--Highest water level measured, $8.18 \mathrm{ft}$ below land-surface datum, Aug. 2, 1983 ;

lowest measured, 27.85 ft below land-surface datum, Jan. 14, 1966.

WATER LEVEL, IN FEET BELOW LAND-SURFACE DATUM, WATER YEAR OCTOBER 1995 TO SEPTEMBER 1996

$\begin{array}{ll}\text { DATE } & \text { WATER } \\ \text { LEVEL } \\ \text { Jan. } 16 & 15.67 \\ \text { July } 2 & 16.15\end{array}$




\section{GROUND-WATER LEVELS}

McKINLEY COUNTY

San Juan Basin

352023107473201. Local number, 13N.09W.21.4123.

LOCATrON. - Lat $35^{\circ} 20^{\prime} 23^{\prime \prime}$, long $107^{\circ} 47^{\prime} 32^{\prime \prime}$, Hydrologic Unit 13020207. Omer: Nabor Marquez.

WQUIFER --Morrison Formation. INSTRUNENTATION, - Monthly steel-tape measurements

DATUR.--Elevation of land-surface datum is 6,785 it above National Geodetic vertical Datum of 1929.

Measuring point: Top of casing, 0.80 ft above land-surface datum.

PERIOD OF RECORD,-July 1955 to current year.

EXTRERES FOR RERIOD OF RECORD. - Highest water level measured, 58.30 ft bolow land-surface datum, Feb. 22, 1978 ;

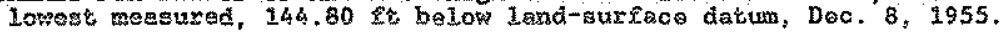

WATER LEVEL, IN FEET BELOW LAND-SURFACE DATUR, WATER YEAR OCTOBER 1995 TO SEPTIMBER 1996

\begin{tabular}{|c|c|c|c|c|c|c|c|c|c|c|c|}
\hline ATE & $\begin{array}{l}\text { WATER } \\
\text { LEVEL }\end{array}$ & DATE & $\begin{array}{l}\text { WATER } \\
\text { LEVEL }\end{array}$ & DATE & $\begin{array}{l}\text { WATER } \\
\text { LEVEL }\end{array}$ & DATE & $\begin{array}{l}\text { WATER } \\
\text { LEVEL }\end{array}$ & DATE & $\begin{array}{l}\text { WATER } \\
\text { LEVEL }\end{array}$ & DA & $\begin{array}{l}\text { WATER } \\
\text { LEVEL }\end{array}$ \\
\hline R 16 & $\begin{array}{l}86.37 \\
87.34\end{array}$ & $\begin{array}{l}\text { MOV } 21 \\
\text { MAY } 21\end{array}$ & $\begin{array}{l}86.62 \\
87.58\end{array}$ & $\begin{array}{l}\text { DEC } \\
\text { JUNE } 18\end{array}$ & 87.75 & $\begin{array}{l}\text { JAN } 18 \\
\text { JULY } 23\end{array}$ & 87.90 & AUG 20 & 89.63 & $\begin{array}{l}\text { MAR } 19 \\
\text { SEP } 25\end{array}$ & 90.13 \\
\hline
\end{tabular}

353645108011501 . Local number, 16N.11W.17.4322

LOCATION. - Lat $35^{\circ} 36^{\circ} 45^{\prime \prime}$, Long $108^{\circ} 01^{\prime} 15^{\prime \prime}$, Hydrologic Unit 14080106. Owner: Navajo Nation.

AQUIFER. - -Gallup Sandstone.

WELL CHARACTERISTICS.--Drillod water-table well, diameter $65 / 8$ in., depth 570 ft, cased to

$570 \mathrm{ft}$ perforated $470-570 \mathrm{ft}$.

INSTRUMENTATION:--Periodic steo1-tape measurements.

DATUM,-Elevation of land-surface datun is 7,070 fi above National Geodetic Vertical Datum of 1929

Measuring point: Top of castng, 0.53 rt above land-surface datum.

PERIOD OF RECORD. - -July 1959 to current year.

EXTREMES FOR PERTOD OF RECORD.--Highest water leve1 measured, 254,76 ft below land-surface datum, Feb. 23, 1996;

lowest measured, $318.28 \mathrm{ft}$ below land-surface datum, July 21,1982 .

WATER LEVEL, IN FEET BELOW LAND-SURFACE DATIM, WATER YEAR OCTOBER 1995 TO SEPTEMBER 1996

$\begin{array}{ll}\text { DATE } & \begin{array}{l}\text { WATER } \\ \text { LEVEL }\end{array} \\ \text { Feb. } 23 & 254.76 \\ \text { July } 31 & 255.73\end{array}$

353521108284901 Local number, $16 \mathrm{~N}, 16 \mathrm{~W}, 25,142$.

LOCATION, - Lat $35^{\circ} 35^{\prime} 21^{\prime \prime}$, Long $108^{\circ} 28^{\prime} 49^{\prime \prime}$, Hydrologic Unit 15020006. Owner: Navajo Nation.

AOUYFER -..-Entrada Sandstone.

WELL CHARACTERISTICS,-Drilied water-table well, dameter $83 / 4$ in., depth 1,052 ft, cased to 1,052 It, pertorated $628-896,974-1033 \mathrm{It}$.

INSTRUMENTÁTION. --Pexlodic steei-tape measurements.

DATUM.--Elevation of land-surface datum is 7,115 ft above National Geodetic Vertical Datum of 1929.

Moasuring point: Hole in cover plate, 0.80 ft above land-surface dacum.

PERIOD OF RECORD.--Oct. 1965 to current year.

EXTREMES FOR PERIOD OF RECORD, - Highest water level measured, 125.55 ft below land-surface datum, Feb. 2, 1995;

lowest measured, $160.64 \mathrm{ft}$ below land-surface datum, Feb. 20,1985 .

WATER LEVEL, IN EEET BELOW LAND-SURFACE DATUM, WATER YEAR OCTOBER 1995 TO SEFTEMBER 1996

DATE WATER

Feb. $23 \quad 147.76$

July 31

354235108170702, Local number, 17N.14W.13.1144B.

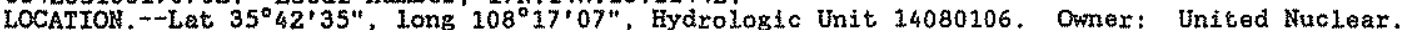

AOUTFER - Morrison Samâstone.

WELL CHARACTERISTICS.-Drilled water-table well, diametex $85 / 8$ in. 0-2, 225 te, total depth 2,225 ft. Percorated $1,820-2,225 \mathrm{ft}$.

INSTRUMENTATION. --Periodic stee1-tape measurements.

DATUM,-Elevation of land-surface datun is $6,757.70$ t above Nattonal Geodetic Vertical Datun of 1929.

Measuring point: $3 / 8$ in. plug, 0.80 ft above land-surface datum.

PERIOD OF RECCORD.--Aug. 1982 to current year.

EXTREMES FOR RERIOD OF RECORD. - -Highest water level measured, 245.39 ft below land-surface datum, July 31 , 1996 ;

lowest measured, 350.38 te below land-surface datum, Oct. $8,1986$.

WATER LEVEL, IN FEET BELOW LANDMSURFACE DATUM, WATER YEAR OCTOBER 1995 TO SEPTEMBER 1996

DATE WATER

Feb. $23 \quad 246.86$

July $31 \quad 245.39$ 
GROUND-WATER LEVELS

MCKINLEY COUNTY

San Juan Basin

354235108170703 . Local number, 17N $14 \mathrm{~W} .13 .1144 \mathrm{C}$

LOCATION. - Lat $35^{\circ} 42^{\prime} 35^{\prime \prime}$, long $108^{\circ} 17^{\circ} 07^{\prime \prime}$, Hydrologic Unit 14080106. Owner: Untted Nuclear.

AQUIFER. - -Dakota Sandstone.

WELL CHARACTERISTICS.-Drilled water-table weil, diameter $85 / 8 \mathrm{in}, 0-54 \mathrm{ft}, 6 \mathrm{f} / 8 \mathrm{in} .54-1,728 \mathrm{ft}$. Perforated $1,587-i, 728 \mathrm{ft}$.

INSTRUMENTATION, --Periodic steel-tape measurements.

DATUM.--Elevation of land-surface datum is $6,757.70 \mathrm{ft}$ above National Geodetic Vertical Datum of 1929.

Measuring point: $3 / 8$ in. plug, 0.80 ft above land-surface datum.

PERTOD OF RECORD.--Aug. 1982 to current year.

EXTREMES FOR PERIOD OF RECORD.--Highest water Level measured, 76.21 ft below land-surface datum, Aug. 4, 1982, lowest measured, 126.35 ft below land-surface datum, July 11, 1994 .

WATER LEVEL, IN FEET BELOW LAND-SURFACE DATUM, WATER YEAR OCTOBER 1995 TO SEPTEMBER 1996

$\begin{array}{cr}\text { DATE } & \text { WATER } \\ \text { LEVEL } \\ \text { Fob. 23 } & 125.97 \\ \text { July 31 } & 125.94\end{array}$

OTERO COUNTY

Tularosa-Alamogordo Area

330321106011101 . (formerly 330324106011201) Local number, 14S.10E.31.144.

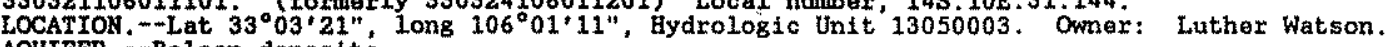

AQUIFER.--Bolson deposits.

WELL CHARACTERISTICS. - -Drilled water-table frrigation well, depth $230 \mathrm{ft}$, dtameter 17 in, casing 0-130 ft. INSTRUMENTATION.--Periodic steel-tape measurements.

DATUM.--Elevation of land-surface datum is 4,450 ft above National Geodetic Vertical Datum of 1929.

Measuring point: Top edge of $1 \mathrm{in}$. hole in pump base, $0.70 \mathrm{ft}$ above land-surface datum.

PERIOD OF RECORD. - Apr. 1952 to current year.

EXTREMES FOR PERIOD OF RECORD.---Highest water level measured, $73.75 \mathrm{ft}$ below land-surface datum, Apr. 8, 1952 ; lowest measured, 134.21 ft below land-surface datum, Aug. 3,1978 .

WATER LEVEL, IN FEET BELOW LAND-SURFACE DATUM, WATER YEAR OCTOBER 1995 TO SEFTEMBER 1996

$\begin{array}{lc}\text { DATE } & \text { WATER } \\ \text { LEVEL } \\ \text { Feb. } & \text { not moasured } \\ \text { Aug. 23 } & 93.18\end{array}$

\section{Crow Flats Bastn}

(Salt Basin)

320657105061501 . Local number, 25s.18E.21.233.

LOCATION. --Lat $32^{\circ} 06^{\circ} 57^{\prime \prime}$, Long $105^{\circ} 06^{\prime} 15^{\prime \prime}$, Hydrologtc Unit 13050004. Omer: Gene Lew1s.

AQUIFER. - Bolson deposits.

WELL CHARACTERISTICS.-Drilled water-table irrigation well, diameter $16 \mathrm{in.}$, depth unknown.

INSTRUMENTATION, --Periodic steel-tape measurements.

DATUM.--Elevation of land-surface datum is 3,690 ft above National Geodetic Vertical Datum of 1929.

Measuring point: Top of casing $0.50 \mathrm{ft}$ above land-surface datum.

PERIOD OF RECORD. - Apr. 1956 to current year.

EXTREMES FOR PERIOD OF RECORD.--Highest water level measured, 68.80 ft below land-surface datum, Apr. 20, 1956; lowest measured, $101.55 \mathrm{ft}$ below land-surface datum, Sep. 15, 1983 .

WATER LEVEL, IN FEET BELOW LAND-SURFACE DATUM, WATER YEAR OCTOBER 1995 TO SEPTEMBER 1996

$\begin{array}{cc}\text { DATE } & \text { WATER } \\ \text { LEVEL } \\ \text { Jan. } 26 & 89.07 \\ \text { Aug. 15 } & 91.67\end{array}$

320138105063101 (formerly 320650105034801) Local number, 26S.18E.21.331.

LOCATION. -1, lat $32^{\circ} 01^{\prime} 38^{\prime \prime}$, Long $105^{\circ} 06^{\prime} 31^{\prime \prime}$, Hydrologic Unit 13050004 . Owner: Frank Gentry.

AQUIFER. --Bolson deposits.

WELL CHARACTERISTICS. -Drilled water-table irrfgation well, diameter $18 \mathrm{in.}$, depth $544 \mathrm{ft}$.

INSTRUMENTATION. --Perlodic steel-tape measurements.

DATUM. - Elevation of land-surface datum is 3,655 ft above National Geodettc Vertical Datum of 1929.

Measuring point: Top of casing, $2.50 \mathrm{ft}$ above land-surface datum.

PERIOD OF RECORD --Jan 1971 to current year.

EXTREMES FOR PERIOD OF RECORD.--Highest water level measured, $51.08 \mathrm{ft}$ below land-surface datum, Jan. 8, 1973; lowest measured, 82.94 ft below land-surface datum, Aug. 17, 1978.

WATER LEVEL, IN FEET BELOW LAND-SURFACE DATUM, WATER YEAR OCTOBER 1995 TO SEPTEMBER 1996

DATE WATER

$\begin{array}{lr}\text { Jan. } 26 & 55.33 \\ \text { Aug. } 15 & 68.68\end{array}$


Crow Flats Basin

(Salt Basin)

$32000810506 \$ 501$, Locel number, 26S.18E.33.133. LOCATYON.-Int $32^{\circ} 00^{\circ} 08^{\prime \prime}$, Long $105^{\circ} 06^{\prime} 45^{\prime \prime}$, Hydrologic Unit 13050004 , Omer: J. W. Et11. AQUIFER. - Bone Spring Limestone. WELt CBARACTRRIStICS.-Drilled watex-table used irxigation we11, diameter 14 in., depth 435 et.

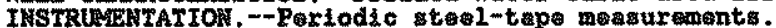

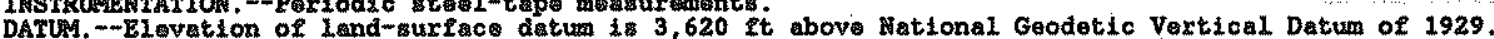

Heasuring point: Fop of casing, 0.80 ft. above land-surface datum.

PXRIOD OF RECORD - $\mathrm{Kab} 1956$ to current yoar

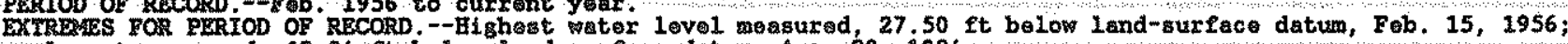

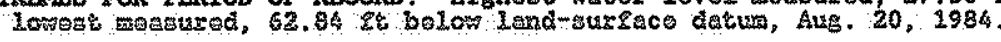

WATER LEVEL, IN FEET BELOW LAND-SURFACE DATUM, WATER YEAR OCTOBER 1995 TO SEPTEMBER 1996

$\begin{array}{lr}\text { DATE } & \text { WATER } \\ \text { Jan. } 26 & \text { LEVEL } \\ \text { AuB. } 15 & 50.12 \\ & 61.76 \\ & \\ & \text { QUAY COUNTY } \\ & \text { BOUSE AYE }\end{array}$

343848103555801 . Loce1 number, 05A $28 \mathrm{E} .23 .222232$.

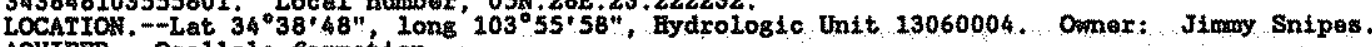

AQUIFER. --Ogallala Lormation.

WELL CBARACTERISTICS.--Drilied water-teble stock well, diameter $6 \mathrm{ln}$, depth $93.5 \mathrm{ft}$.

IUSTRINENTATION, ---Poriodic steol-tapo measurements.

DATUM --Elavation of land-surface datum is 4,788 it abovo Hational Geoderic Vortical Datum of 1929

PSesuring point: Top of casing, west side, 2,00 ft above land-surface datum.

FERIOD OF RECORD.-Jan. 1968 to current year.

EXTREMES FOR PERIOD OF RECORD.--Highest water level measured, 74.50 ft below land-surface datum, Sep. 15, 1994;

lopest messured, $84.22 x$ ft below land-surface datum, Feb. 18, 1972 .

WATER LEVEL, IN FEET BELON LAND-SURFACE DATUM, WATER YEAR OCTOBER 1995 TO SEPTEMBER 1996

\begin{tabular}{|c|c|}
\hline DATE & $\begin{array}{l}\text { WATER } \\
\text { LEVEL }\end{array}$ \\
\hline $\begin{array}{ll}\text { Mar. } & 14 \\
\text { Sap. } & 24\end{array}$ & $\begin{array}{l}74.52 \\
74.58\end{array}$ \\
\hline
\end{tabular}

343855103482901 (Formerly 343810103463001 ) LOcal number, 05N.30E.18.331311.

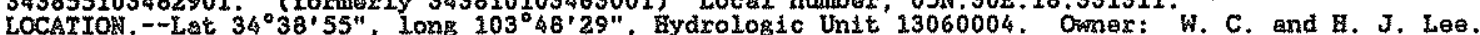
AQUTEER. -Ogallala formation.

WELL CHARACTERISTICS,-Drilled water-tablo irrigation well, diameter 16 in., depth 75 ft, casod to 60 it. INSTRUREATATOR, --Pariodic steol-tape moasuraments.

DATUA.--Elevation of land-surface datum is 4,630 ft above National Geodetic vertical Datum of 1929.

Measuring point: Top of concrete pump base, $0.50 \mathrm{ft}$ above land-surfaco datum.

PERTOD OF RECORD. --Ray. 1944 to current yoar.

EXTREASS FOR PERIOD OF RECORD, - Highest mater leve1 measured, 34.76 ft below land-8urface datum, Mar. 28, 1946;

lowest measured, $51.49 \mathrm{ft}$ below land-surface datum, Aug. 11, 1969.

WATER LEVEL, IN FEET BELON LAND-SURFACE DATUM, WATER YEAR OCTOBER 1995 TO SEPTEMBER 1996

DATE WATER

Max. $14 \quad 48.67$

Sep. $24 \quad 48.44$

344406103555501 . Local nutber, 06N.281.13.33333.

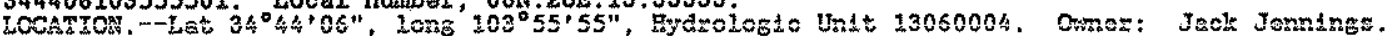

AOUIFER. - Alluvium.

WELL CEARACTERISTICS, --Drilled domestic well, diameter $16 \mathrm{in}$, depth $131 \mathrm{ft}$

INSTRUAENTATION.--Periodic steel-tape measurements.

DATtP. - Elevation of land-surface datum is 4,816 fi above Hational Geodetic Vertical Datum of 1929.

Measuring point: $3 / 4 \mathrm{in}$. hole in cover plate, $0.40 \mathrm{ft}$ above land-surface datum.

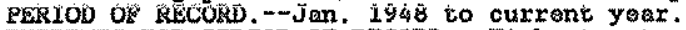

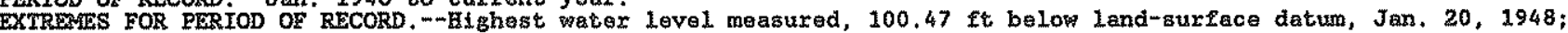

lowset messured, 120.20 ft below land-surfece datum, Seg. 24, 1996.

WATER LEVEL, TN FEEI BELOW LAND-SURFACE DATUM, WATER YEAR OCTOBER 1995 TO SEPTEABER 1996

$\begin{array}{cr}\text { DATE } & \text { WATER } \\ \text { LEVEL } \\ \text { Mar. } 14 & 119.88 \\ \text { Sep. } 24 & 120.20\end{array}$


GROUND-WATER LEVELS

QUAY COUNTY
Lower Canadian

351040103433602 , Local number, 11K.30E.14.144D.

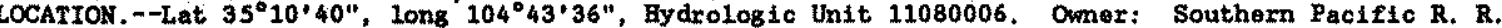

AQUIFER.-ALIUvium.

INSTRUMENTATION, --Pertodic steel-tape measurements.

DATUM.--Elovation of land-surface datum is 4,080 ft above Rational Geodetic Vertical Datum of 1929

Measuring polnt: Top of $1.5 \mathrm{in}$. pipe axtenston, 4.20 ft above land-surface datum.

PERIOD OF RECORD.--July 1952 to current year.

EXTREMES FOR FERIOD OF RECORD.--Highest water level measured, 21.20 ft below land-surface datum, Sep. 9, 1963;

lopest measured, $137.66 \mathrm{ft}$ below land-surface datum, Dec. 16, 1952.

WATER LEVIL, IN FEET BELOM LAND-SURFACE DATUM, WATER YEAR OCTOBER 1995 TO SEPTEMBER 1996

$\begin{array}{cr}\text { WATER } \\ \text { DATE } & \text { LEVEL } \\ \text { Jan. } 18 & 47.50 \\ \text { July } 25 & 57.55\end{array}$

QUAY COUNYY

Northern Eigh P.lains

353239103111301 . Local number, 15N.35E.11.21222.

LOCATION. -Lat $35^{\circ} 32^{\prime} 39^{\prime \prime}$, Long $103^{\circ} 1^{\prime} 1^{\prime \prime}$, Eydrologic Unit 11080006. Owner: J, L. Santh.

AQUIFER - Alluvium.

WELL CAARACTERISTICS.--Drilled water-table frrigation well, dianeter 12 in., depth $175 \mathrm{ft}$.

INSTRUMENTATION, --Poriodic ateal-tape measurements.

DATUM. - Elevation of land-surface datum is $4,126 \mathrm{ft}$ above National Geodetic Vertical Datum of 1929.

Measuring point: $21 / 2 \mathrm{tn}$. hole, in east side of casing, 1.20 ft above land-surface daturs.

PERTOD OF RECORD.--July 1971 to current year.

EXTRDNES FOR PERIOD OF RECORD,--Highest watex level measured, 88,83 ft below land-surface datum, July 26, 1995;

lopest measured, $114.67 \mathrm{ft}$ below land-surface datum, Feb, 5, 1974 .

WATER LEVEL, IN FEET BELON LAND-SURFACE DAFUM, WATER YEAR OCTOBER 1995 TO SEPTEMBER 1996

$\begin{array}{lc}\text { DATE } & \begin{array}{c}\text { WATER } \\ \text { LEVEL }\end{array} \\ \text { Jan. } & \text { not measured } \\ \text { July } 24 & 89.20\end{array}$

354238103132301 Local number, 17N.35E. 16.221.

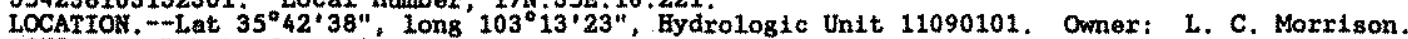

AQUTFER - -Dakoto formetion

WELI CHARACTERISTICS.-Drilied mater-table. irrigation well, dianeter unknown, depth 250 ft.

INSTRUNENTATION. --Poriodic stee1-tape measurements.

DATUM. - Elevation of Land-surface datum $184,465 \mathrm{ft}$ above National Geodetic Vertical Datum of 1929

Messuring point: Hole in south side of pump base, $2.00 \mathrm{ft}$. above land-surface datum.

PERIOD OF RECORD - -OCt. 1967 to current year.

EXTREMES FOR PERIOD OF RECORD.--Hitghest water level measured, 159.30 ft below land-surface datum, Apr. 10, 1991.

lowest measured, $171.59 \mathrm{ft}$ below land-surface datum, Sep. 19, 1988.

WATFR LEVEL, IN FEET BELOW LAND-SURFACE DATUM, WATER YEAR OCTOBER 1995 TO SEPTEMBER 1996

$$
\begin{array}{lc}
\text { DATE } & \text { WATER } \\
\text { LEVEL } \\
\text { Jan. } & \text { not measured } \\
\text { July } 24 & 169.01
\end{array}
$$

\section{ROOSEVELT COUNTY}

341014103264401 Local number, 01S 33E. 35.434344.

LOCATION, - - Lat $34^{\circ} 10^{\circ} 14^{\prime \prime}$, Long $103^{\circ} 26^{\circ} 44^{*}$, Hydrologic Unit 12050002, Owner: Jones

AQUTPER. - Alluvium.

WELI CHARACTERISTICS, --Dri11ed water-table unused irrigation woll, dianeter 16 in., depth 84 ft. INSTRLABHTATION, --Dig1tal recorder, 1-hour punch.

DATUM. - Elovation of land-aurface datum is 4,066 ft above National Geodetic Vertical Datum of 1929

Measuring point: Top of 1.80 in. shaft hole, in center of pump, 2.80 ft above land-surface datum. REMARKS - -Recorder installed Apr. 25, 1996. Lost record several days due to recorder malfunction. PERIOD of RECORD --Apr. to current year.

EXTREMES FOR PERIOD OF RECORD, mighest pater level measured, 66.37 ft below

land-surface datum, Apr. 25, 1996; lowest measured, 66.72 ft below land-surface datim, Sep. 27, 1996 WATER LEVEL, IN FEET BELOW LAND-SURFACE DATUM, WATER YEAR OCTOBER 1995 TO SEPTEMBER 1996

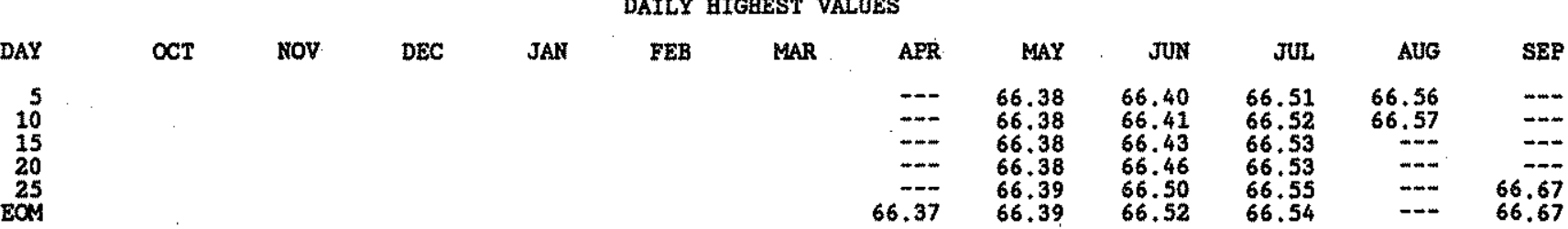

WTR YBAR 1996 HTCBEST 66.37 AFR 25, $1996 \quad$ LONEST 66.72 SEP 27, 1996 
341037103254501 . Local number, 01S.33E. 36.23111.

LOCATION. - Lat $34^{\circ} 10^{\prime} 37^{\prime \prime}$, Long $103^{\circ} 25^{\prime} 4^{\prime \prime}$, Hydrologic Unit 12050002. Owner: State of New Mexico.

AQUIFER. - Alluvium.

WELL CHARACTERISTICS. -Drilled water-table unused we11, diameter 18 in., depth 105 ft.

INSTRUMENTATION, --Digital recorder, 1-hour punch.

DATUM.--Elevation of land-surface datum is 4,048 ft above National Geodetic Vertical Datum of 1929.

Measuring point: Top of casing 1.95 ft above land-surface datum.

REMARKS.--Lost record, several deys, due to recorder malfunction, Recorder removed Apr. $25,1996$.

PERIOD OF RECORD - - Jan 1952 to current year.

EXTREMES FOR PERIOD OF RECORD.--Highest water level measured, 38.19 ft below land-surface datum, Jan. 25 , 1952 ;

lowest measured, 86.42 xi below land-surface datum, Jan, 17,1984 .

WATER LEVEL, IN FEET BELOW LAND-SURFACE DATUM, WATER YEAR OCTOBER 1995 TO SEPTEMBER 1996

\begin{tabular}{|c|c|c|c|c|c|c|c|c|c|c|c|c|}
\hline DAY & OCT & Nov & DEC & JAN & FEB & MAR & APR & $\therefore$ MAY & JUN & JUL. & AUG & SEP \\
\hline $\begin{array}{r}5 \\
10 \\
15 \\
20 \\
25 \\
\text { EOM }\end{array}$ & $\begin{array}{l}84.62 \\
84.64 \\
84.64 \\
84.62 \\
84.66 \\
84.68\end{array}$ & $\begin{array}{l}84.69 \\
84.69 \\
84.73 \\
84.75 \\
84.71 \\
84.71\end{array}$ & $\begin{array}{l}84.75 \\
84.72 \\
84.80 \\
84.79 \\
84.82 \\
84.73\end{array}$ & $\begin{array}{r}84.80 \\
84.77 \\
84.73 \\
-\ldots \\
-\ldots \\
-. .\end{array}$ & & $\begin{array}{r} \\
84.79 \\
84.81 \\
84.82\end{array}$ & $\begin{array}{l}-m \\
-\cdots \\
-\cdots \\
-\cdots\end{array}$ & & & & & \\
\hline
\end{tabular}

WIR YEAR 1996 HIGEEST 84.57 OCT 1,3,4, 1995 LOWEST 84.96 JAN 15, 1996

340732103145001 . Local number, 02S.35E.23.11113.

LOCATION. -Lat $34^{\circ} 07^{\prime} 32^{\prime \prime}$, Long $13^{\circ} 4^{\prime} 5^{\prime \prime}$, Hydrologic Unit 12050001. Owmer: Herman Gres,

AQUIFER, - -Alluvium.

WELL CHARACTERISTICS. - Drilled water-table unused Irrigation woll, diameter 10 in., depth 80 ft.

INSTRUMENTATION. --Periodic stoel-tape measurements.

DATUM,--Elevation of lend-surface datum is $3,961 \mathrm{ft}$ above National Geodetic Vertical Datum of 1929.

Measuring point: Top of $1.5 \mathrm{in}$.: shaft holo, in center of punp, $2.80 \mathrm{ft}$ above land-surface datum.

PERTOD OF RECORD. - July 1949 to current year.

EXTREMES FOR RERIOD OF RECORD.--Highest water leve1 measured, 21.32 ft below land-surface datum, Mar. 27, 1951;

lowest measured, $56.33 \mathrm{ft}$ below land-surface datum, Aug. 8, 1996.

WATER LEVEL, IN FEET BELOW LAND-SURFACE DATUM, WATER YEAR OCTOBER 1995 TO SEPTEMBER 1996

$\begin{array}{cr}\text { DATE } & \text { WATER } \\ \text { LEVEL } \\ \text { Jan. } 10 & \\ \text { Aug. } 8 & 57.58 \\ & 57.59\end{array}$

340753103083101. Local number, 02S.36E.14.311111.

LOCATION, - Lat $34^{\circ} 07^{\prime} 53^{\prime \prime}$, Long $103^{\circ} 08^{\prime} 31^{\prime \prime}$. Hydrologtc Unit 12050001. Owner: Rogers,

AQUIFER. - Alluvium.

WELL CHARACTERISTICS. --Drilled water-tablo observation well, diameter $16 \mathrm{in}$, depth $151 \mathrm{ft}$.

INSTRUMENTATION. - - Periodic steel-tape measurements.

DATUM. - -Elevation of land-surface datum is 3,938 ft above National Geodetic Vertical Datum of 1929.

Measuring point: Top of casing, 2.00 ft above land-surface datum.

PERTOD OF RECORD. - Jan. 1975 to current year.

lowest measured, $79.44 \mathrm{ft}$ below land-surface datum, July 25,1990 .

WATER LEVEL, IN FEET BELOW LAND-SURFACE DATUM, WATER YEAR OCTOBER 1995 TO SEFTEMBER 1996

$\begin{array}{cc}\text { DATE } & \text { WATER } \\ \text { LEVEL } \\ \text { Jan. } 9 & 79.06 \\ \text { Aug. } 8 & \text { pumping }\end{array}$

340844103055001 . Locai number, 025. 37E.007.432222.

LOCATION, -l.at $34^{\circ} 08^{\prime} 44^{\prime \prime}$, long $103^{\circ} 05^{\prime} 50^{\prime \prime}$, Hydrologic unit 12050001. Owner: Rogers.

AQUIFER. - - Ogallala Formation.

WELL CHARACTERISTICS.--Drilled water-table unused Arrigation well, diameter 13.5 in., depth 204 ft, cased to 204 ft, percorated $151-204$ st.

INSTRUMENTATION, - -Digital recorder, 1-hour punch.

DATUR.--Elevation of land-surface datum is 3,982 ft abovo National Geodetic Vertical Datum of 1929.

Measuring point: Edge of recorder shelter, $3,00 \mathrm{ft}$. above land-surface datum.

REMARKS.--Recorder installed June 2, 1992 . Lost record, due to recorder malfuncation.

PERIOD OF RECORD. - June 1992 to current year.

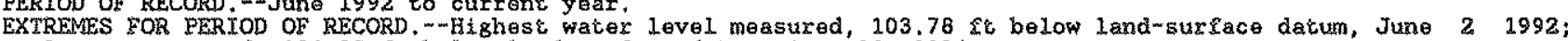
lowest measured, 126.82 ft below land-surface datum, Aug. 16, 1996.

WATER LEVEL, IN FEET BELOW LAND-SURFACE DATUM, WATER YEAR OCTOBER 1995 TO SEFTEMBER 1996

\begin{tabular}{|c|c|c|c|c|c|c|c|c|c|c|c|c|}
\hline DAY & $\infty \mathrm{N}$ & Nov & DEC & JAN & FEB & MAR & APR & MAY & JUN & JUL & AUG & SEP \\
\hline $\begin{array}{r}5 \\
10 \\
15 \\
20 \\
25 \\
\text { EOM }\end{array}$ & $\begin{array}{l}119.19 \\
119.22 \\
119.14 \\
119.05 \\
119.22 \\
119.02\end{array}$ & $\begin{array}{l}118.99 \\
118.97 \\
119.70 \\
119.09 \\
118.98 \\
118.74\end{array}$ & $\begin{array}{l}118.78 \\
118.56 \\
118.49 \\
118.42 \\
118.36 \\
118.19\end{array}$ & $\begin{array}{l}118.02 \\
118.15 \\
118.00 \\
117.85 \\
117.74 \\
117.71\end{array}$ & $\begin{array}{l}117.57 \\
118.31 \\
117.59 \\
117.43 \\
117.35 \\
120.77\end{array}$ & & & & $\begin{array}{r}--- \\
\overline{--} \\
\overline{--} \\
123.84 \\
122.28\end{array}$ & $\begin{array}{l}123.42 \\
125.35 \\
125.36 \\
126.00 \\
126.06 \\
126.08\end{array}$ & $\begin{array}{r}126.09 \\
126.10 \\
124.25 \\
123.53 \\
123.20\end{array}$ & $\begin{array}{l}123.06 \\
123.99 \\
123.19 \\
123.34 \\
123.04 \\
123.06\end{array}$ \\
\hline
\end{tabular}

WTR YEAR 1996 HIGHEST 117.35 FEB 2, 1996 LOWEST 126.82 AUG 16, 1996 
GROUND-WATER LEVELS

ROOSEVELT COUNTY

Causey-Lingo Area

334700103030601. (formerly 335655103032001) Local number, 06S.38E.21.233131.

LOCATION. - Lat $33^{\circ} 47^{\circ} 00^{*}$, long $103^{\circ} 03^{\circ} 11^{\prime \prime}$, Hydrologic Unit 12050001. Owner: c. c. Harvey.

AQUIFER. --Undifferentiated Cretaceous rocks.

WELL CHARACTERISTICS,--Drilled water-table irrigation well, dianeter $16 \mathrm{in}$, depth $140 \mathrm{ft}$, cased to $140 \mathrm{ft}$, casing slotted 100-140 ft.

INSTRUMENTATION. --Periodic steel-tape measurements.

DATUM.--Elevation of land-surface datum is $3,939 \mathrm{ft}$ above National Geodetic Vertical Datum of 1929.

Measuring point: Top of $1 \mathrm{in}$. hole in north side of pump, $2.10 \mathrm{ft}$ above land-surface datum.

REMARKS. - " " means well pumping during messurement.

PERIOD OF RECORD.--JJan. 1956 to current year.

EXTREMES FOR PERIOD OF RECORD.--Highest water Level measured, 87.18 ft below land-surface datum, Jan. 13, 1956;

lowest measured, 115.21p ft below land-surface datum, Aug. 11, 1976.

WATER LEVEL, IN FEET BELOW LAND-SURFACE DATUM, WATER YEAR OCTOBER 1995 TO SEPTEMBER 1996

$\begin{array}{cc}\text { DATE } & \text { WATER } \\ \text { LEVEL } \\ \text { Jan. 10 } & 92.40 \\ \text { Aug. 8 } 8 & 95.52\end{array}$

SANDOVAL COUNTY

Bernalillo Area

352121106285501. (formerly 352235106282401 ) Local number, 13N.04E.12.112.

LOCATION. - Lat $35^{\circ} 22^{\prime} 35^{\prime \prime}$, Long $106^{\circ} 28^{\prime} 24^{\prime \prime}$, Hydrologic Unit 13020201. Ower: John Bowers.

AQUTFER.--Valley Fill

WELL CHARACTERISTICS,--Drilled water-table 1rrigation well, diameter $12 \mathrm{ln}$, depth $50 \mathrm{ft}$, cased.

INSTRUMENTATION. -- Periodic stee1-tape measurements.

DATUM.-Elevation of land-surface datum is 5,117 ft above National Geodetic Vertical Datum of 1929.

Measuring point: Lower inside adge of hole in south side of casing $0.45 \mathrm{ft}$ above land-surface datum.

PERIOD OF RECORD. - -Jan. 1976 to current year.

EXTREMES FOR PERTOD OF RECORD.--Highest water level measured, 20.57 ft below land-surface datum, July 18, 1991;

lowest measured, 25.27 ft below land-surface datum, Jan. 31, 1978 .

WATER LEVEL, IN FEET BELOW LAND-SURFACE DATUM, WATER YEAR OCTOBER 1995 TO SEPTEMBER 1996

$$
\begin{array}{cc}
\text { DATE } & \text { WATER } \\
\text { FebEL } & \text { not measured } \\
\text { JuIy } 30 & 26.67
\end{array}
$$

SAN JUAN COUNTY

San Juan Basin

364534108292701 . Local number, 29N.15W.02,232.

LOCATION. - Lat $36^{\circ} 5734^{\prime \prime}$, long $108^{\circ} 92270^{\prime \prime}$, Hydrologic Unit 14080105. Owner: Myrl Harper.

AQUIFER. - -Alluvium.

WELL CHARACTERISTICS.--Drilled water-table well, diameter $10 \mathrm{in}$, depth $37 \mathrm{ft}$, cased to $37 \mathrm{ft}$.

INSTRLMENTATION. - Periodic steel-tape measurements.

DATUM. --Elevation of land-surface datum is 5,045 ft above National Geodetic Vertical Datum of 1929.

Measuring point: Top of casing, $1.05 \mathrm{ft}$ above land-surface datum.

PERIOD OF RECORD.--Apr. 1992 to current year.

EXTREMES FOR PERIOD OF RECORD.--Highest water level measured, 7.53 ft below land-surface datum, July 30,1996 ;

lowest measured, 10.04 ft below land-surface datum, Feb. 22, 1996.

WATER LEVEL, IN FEET BELOW LAND-SURFACE DATUM, WATER YEAR OCTOBER 1995 TO SEPTEMBER 1996

$\begin{array}{cr}\text { DATE } & \text { WATER } \\ \text { LEVEEL } \\ \text { Feb. } 22 & 10.04 \\ \text { July } 30 & 7.53\end{array}$

364744108225001 . Local number, 30N.15W.23.4411.

LOCATION. - Lat $36^{\circ} 47^{\prime 44} "$ ", long $108^{\circ} 22^{\prime} 50^{\prime \prime}$, Hydrologtc Untt 14080105. Owner: B.L.M.
AQUIFER. - Pictured Cliff's Sandstone.

WELI CHARACTERISTICS.--Drilled water-table weil, dtameter $5 \mathrm{in}$, depth $729.5 \mathrm{ft}$, cased to $729.5 \mathrm{ft}$, perforated $613-729.5 \mathrm{ft}$.

INSTRUMENTATION. --Periodic steei-tape measurements.

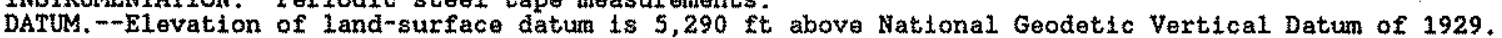

Measuring point: Top of casing, 2.00 ft above land-surface datum.

FERTOD OF RECORD.-Feb. 1978 to current year. lowest measured, $161.45 \mathrm{ft}$ below Land-surface datur, July 30 , 1996 .

WATER LEVEL, IN FEET BELOW LAND-SURFACE DATUM, WATER YEAR OCTOBER 1995 TO SEPTEMBER 1996

$\begin{array}{cr}\text { DATE } & \begin{array}{r}\text { WATER: } \\ \text { LEVEL }\end{array} \\ \text { Feb. 22 } & 159.39 \\ \text { July } 30 & 161.45\end{array}$


GROUND-WATER LEVELS

\section{SANTA FE COUNTY \\ Estancta Valley}

350534106024801. (former1y 350525106025001) Local number, 10N.08E.13.1332.

LOCATION, - - Lat $35^{\circ} 05^{\prime 3} 4^{\prime \prime}$, Long 106 02,48", Hydxologic Untt 13050001. Owner: W. R. Irby.

AQUIFER. - Valley Fill.

WELL CHARACTERISTICS.--Drł1led water-table irrigation well, diameter 16 in., reported depth 513 ft.

IKSTRUMENTATION. --Periodic steel-tape measurements.

DATUM. --Elevation of lend-surface datum is 6,274 it above National Geodetic Vertical Datum of 1929.

Heasuring point: Lower inside edge of hole in south side of casing. 0.45 ft above land-8urface datum.

PERIOD OF RECORD - Feb. 1950 to current year.

EXIRPYES FOR RERIOD OF RECORD,-Highest pater level measured, 86,75 ft below land-surface datua, Feb, 22 , 1950 ;

lowest measurad, 181.55 p fi bolow land-suxface datum, Aug. 4, 1969.

WATER LEVEL, IN FEET BELOW LAND-SURFACE DATUM, WATER YEAR OCTOBER 1995 TO SEPTEMBMR 1996

$\begin{array}{ll}\text { DATE } & \text { WATER } \\ \text { LEVEL } \\ \text { Fob, } 21 & 145.89 \\ \text { Aug. } 1 & \text { pumping }\end{array}$

350344106004601. (formerly 350340106005001) Local number, 10N.09E.29.1334.

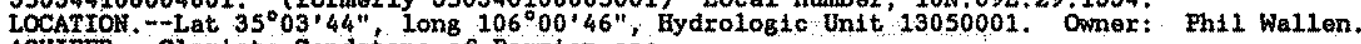

AQUTFER. -Glorieta Sandstone of Permian age.

WELL CRARACTERISTICS.--Drilled water-table irrigation well, diameter 14 in., reported depth 200 et, cased to 140 ft.

INSTRUMENTATION, --Pexiodic steel-tape measurements.

DATUM. - Elevation of land surface datum is 6,248 ft above National Geodetic Vertical Datum of 1929 .

Masuring point: Top edge of 3 in. pipe on north side of pump, 1.30 ft above land-surface datum.

PERIOD OF RECORD.--Feb. 1949 to cuxxent yeax.

EXTREAES FOR PERIOD OF RECORD,-Highest water level measured, 55.00 ft below land-surface datum, May 4 , 1949 ;

lowest measured, $133.50 \mathrm{ft}$ below land-surface datum, Aug. $1,1996$.

WATER LEVEL, IN FEET BELOW LAND-SURFACE DATUM, WATER YEAR OCTOBER 1995 TO SEPTEMBER 1996

DATE WATER

$\begin{array}{ll}\text { Feb. } 21 & \text { pumping } \\ \text { Aug. } 1 & 133.50\end{array}$

350859106002901 , Local number, 11N.09E.29.143.

LOCATION. - Lat $35^{\circ} 08^{\prime} 59^{\prime \prime}$, Long $106^{\circ} 00^{\prime} 29^{\prime \prime}$. Hydrologic Untt 13050001 . Owner: King Bros.

AQUIFER. - Alluvium.

WELL CRARACTERISTICS.--Drilled water-table unused 1rrigation well, dieneter 15 in.. depth unknown.

INSTRUMENTATION. -- Periodic steel-tape measurements.

DATUM. - Elevation of land-surface datum is 6,274 ft above Netional Geodetic vertical Datum of 1929.

Measuring point: Top of casing, 0.80 ft above land-surface datum.

PERTOD OF RECORD, - July 1986 to current year.

EXTREMIES FOR PERIOD OF RECORD.--Highest water level measured, 125.93 ft below land-surface datum, Apr. 1, 1987;

lowest measured, 137.55 ft below land-surface datum, Aug. 1, 1996.

WATER LEVEL, IN FEET BELOW LAND-SURFACE DATUM, WATER YEAR OCTOBER 1995 TO SEPTERBER 1996

DATE WATER

$\begin{array}{lr}\text { Fob. } 21 & 136.83 \\ \text { Aug. } & 1\end{array}$

Santa Fe Area

353636106021001 . Local number, 16N.08E. 13.444.

LOCATION, - Let $35^{\circ} 36^{\prime} 36^{\prime \prime}$, Long $106^{\circ} 02^{\prime} 10^{\prime \prime}$, Hydrologic Unit 13020201. Omer: Herold Nelson.

AUUUTER. - Tresucue Formation of Santa Fe Group.

WELL CHARACTERISTICS.-Drilled domestic well, diameter $61 / 2$ in., depth 337 It.

INSTRURENTATION. --Periodic steel-tape measurements.

DATUR.--Elevation of land-surface datum 156,400 fit above National Geodetic Vextical Datum of 1929.

Heasuring point: Top of casing, 0.70 st ebove land-surface datiun.

PERIOD OF RECORD. - Feb, 1972 to current year.

EXTREXES FOR PERIOD OF RECORD.--Highest water leve1 measured, 256.04 ft belon land-surface datum, Jan, 20, 1982;

lowest measured, $263.54 \mathrm{Ft}$ below land-surface dacum, Fob. 15,1994 .

WATER LEVEL, IN FEET BELOW LAND-SURFACE DATUM, WATER YEAR OCTOBER 1995 TO SEPTENBER 1996

$\begin{array}{lc}\text { DATE } & \text { WATER } \\ \text { IEVEL } \\ \text { Feb. 14 } & 262.54 \\ \text { Aug. } & \text { not measured }\end{array}$


GROUND-WATER LEVELS

SANTA FE COUNTY

Santa Fe Area

353516106035801 . Local number, 16N.08E.26.32112.

LOCATION,-Lat $35^{\circ} 35^{\prime} 16^{\prime \prime}$, long $106^{\circ} 03^{\prime} 58^{\prime \prime}$, Bydrologic Unit 13020201. Owner: State Highway Dept.

AQUIFER. - Tesuque Formation of Santa $\mathrm{Fe}$ Group.

WELL CHARACTERISTICS.--Drilled water-table unused irrigation woll, dfameter $10 \mathrm{in.,} \mathrm{depth} 160 \mathrm{ft}$, cased to $160 \mathrm{ft}$ parforated $125-160 \mathrm{ft}$.

INSTRUMENTATION.--Digitial recorder, 1-hour punch.

DATUM,--Elevation of land-gurface datum is 6,285 ft above National Geodetic Vertical Datum of 1929.

Measuring point: Top of casing, $1.25 \mathrm{ft}$ above land-surface datum.

REMARKS, - -Records good.

PERIOD OF RECORD.--July 1973 to current year.

EXTREMES FOR PERIOD OF RECORD.--Highest water level measured, 125.62 ft below land-surface datum, June 11, 1973 ;

lowest measured, $130.80 \mathrm{ft}$ below land-surface datum, Jul. $10,1996$.

WATER LEVEL, IN FEET BELOW LAND-SURFACE DATUM, WATER YEAR OCTOBER 1995 TO SEPTEMBER 1996 DAILY HIGHEST VALUES

\begin{tabular}{|c|c|c|c|c|c|c|c|c|c|c|c|c|}
\hline DAX & $\alpha \mathrm{CT}$ & NOV & DEC & JAN & FEB & MAR & APR & MAY & JUN & JUL & AUG & SEP \\
\hline $\begin{array}{r}5 \\
10 \\
15 \\
20 \\
25 \\
\text { EOM }\end{array}$ & $\begin{array}{l}130.36 \\
130.35 \\
130.33 \\
130.31 \\
130.34 \\
130.34\end{array}$ & $\begin{array}{l}130.37 \\
130.40 \\
130.42 \\
130.41 \\
130.36 \\
130.38\end{array}$ & $\begin{array}{l}130.42 \\
130.40 \\
130.40 \\
130.41 \\
130.46 \\
130.35\end{array}$ & $\begin{array}{l}130.44 \\
130.42 \\
130.44 \\
130.50 \\
130.36 \\
130.44\end{array}$ & $\begin{array}{l}130.52 \\
130.55 \\
130.51 \\
130.53 \\
130.48 \\
130.49\end{array}$ & $\begin{array}{l}130.48 \\
130.50 \\
130.50 \\
130.51 \\
130.51 \\
130.52\end{array}$ & $\begin{array}{l}130.56 \\
130.45 \\
130.49 \\
130.42 \\
130.50 \\
130.47\end{array}$ & $\begin{array}{l}130.50 \\
130.54 \\
130.51 \\
130.53 \\
130.51 \\
130.54\end{array}$ & $\begin{array}{l}130.53 \\
130.54 \\
130.55 \\
130.54 \\
130.55 \\
130.59\end{array}$ & $\begin{array}{l}130.59 \\
130.60 \\
130.61 \\
130.60 \\
130.52 \\
130.54\end{array}$ & $\begin{array}{l}130.57 \\
130.57 \\
130.55 \\
130.56 \\
130.57 \\
130.58\end{array}$ & $\begin{array}{l}130.59 \\
130.61 \\
130.65 \\
130.63 \\
130.58 \\
130.63\end{array}$ \\
\hline
\end{tabular}

WTR YEAR 1996 HIGHEST 130.27 OCT 3, 1995 LOWEST 130.80 JUL 10, 1996

353735105581201. (formerly 353753105580501) Local number, 16N.09E.10.42114.

LOCATION. - Lat $35^{\circ} 37^{\prime} 53^{\prime \prime}$, long $105^{\circ} 58^{\circ} 05^{\prime \prime}$, Eydrologic Unit 33020201 . Owner: Paul Ragel.

AQUIFER. - Ancha Formation of Santa Fe Group.

WELL CHARACTERISTICS,--Drilled domest \pm c weli, diameter 6 in, depth 243 ft.

INSTRUMENTATION. --Periodic steel-tape measurements.

DATUM. --Elevation of land-surface datum $1 \mathrm{~s} 6,820 \mathrm{ft}$ above National Geodettc Vertical Datum of 1929 .

Measuring point: $1 / 2 \mathrm{in}$. plug in cover plate, 6.00 ft below land-surface datum,

PERTOD OF RECORD.-Aug. 1957 to current year.

EXTREMES FOR PERIOD OF RECORD.--Highest water level measured, 149.52 ft below land-surface datum, Dec. 11 , 1957 ; lowest measured, $230.44 \mathrm{ft}$ below land-surface datum, Aug. 22, 1994 .

WATER LEVEL, IN FEET BELOW LAND-SURFACE DATUM, WATER YEAR OCTOBER 1995 TO SEPTEMBER 1996

$\begin{array}{lc}\text { DATE } & \begin{array}{c}\text { WATER } \\ \text { LEVEL }\end{array} \\ \text { Feb. } 14 & 229.30 \\ \text { Aug. } & \text { not measured }\end{array}$

354013105580601. (formerly 354005105574501) Local number, 17N.09E.27.441.

LOCATION. -Lat $35^{\circ} 40^{\prime} 05^{\circ}$, long $105^{\circ} 57^{\prime} 45^{\prime \prime}$, Hydrologic Unit 13020201. Owner: U.S. Indian School.

AQUIFER. - Testuque Formation of Santa Fe Group.

WELL CHARACTERISTICS,-Drilled water-table frrigation well, diameter 8 in., depth 989 ft.

INSTRUMENTATION. --Periodic steel-tape measurements.

DATUM.--Elevation of land-surface datum is $6,848 \mathrm{ft}$ above National Geodetic Verttcal Datum of 1929.

Measuring point: Top of casing $2.70 \mathrm{ft}$ below land-surface datum.

PERIOD OF RECORD. --Dec. 1951 to current year.

EXTREMES FOR PERIOD OF RECORD, - -H1ghest water level measured, 102.33 ft below land-surface datum, Dec. 27, 1951;

lowest measured, 231.17 ft below land-surface datum, Feb. 14, 1996.

WATER LEVEL, IN FEET BELOW LAND-SURFACE DATUM, WATER YEAR OCTOBER 1995 TO SEPTEMBER 1996

$\begin{array}{lc}\text { DATE } & \text { WATER } \\ \text { LEVEL } \\ \text { Feb. } 14 & 231.17 \\ \text { Aug. } & \text { not measured } \\ & \\ & \text { SIERRA CoUn'y } \\ \text { Hot Springs Area }\end{array}$

331002107150001 , Local number, $13 \mathrm{~S}, 04 \mathrm{~W}, 21.213$

LOCATION. - Lat $33^{\circ} 10^{\circ} 02^{\prime \prime}$, Long $107^{\circ} 15^{\circ} 00^{\prime \prime}$, Hydrolog to Untt 13030101. Owner: Unknown.

AOUIFER - -ALIUviun.

WELL CHARACTERISTICS.--Drilled water-table unused well, dlameter 13 in., depth unknown.

INSTRUMENTATION. - Pertodic steel-tape measurements.

DATUM.--Elevation of land-surface datum is $4,355 \mathrm{ft}$ above National Geodetic Vertical Datum of 1929.

Measurting point: 1 in. hole in west side of pump base, and $1.50 \mathrm{ft}$ above land-surface datum.

PERIOD OF RECORD. - Feb. 1972 to current year.

EXTREMES FOR PERIOD OF RECORD, -Htghest water level measured, 48.84 ft below land-surface datum, July 27 , 1992 ,

lowest measured, $65.56 \mathrm{ft}$ below land-surface datum, Feb. 25, 1972

' WATER LEVEL, IN FEET BELOW LAND-SURFACE DATLM, WATER YEAR OCTOBER 1995 TO SEPTEMBER 1996

$\begin{array}{ll}\text { DATE } & \text { WATER } \\ \text { LEVEL } \\ \text { Feb. } 9 & 49.16 \\ \text { Aug. } 30 & 45.06\end{array}$


GROUND-WATER LEVELS

STERRA COUNTY

Hot Springs Ares

325921107185101 (formerly 325550107184001 ) Local number, 15S.05W.24.312.

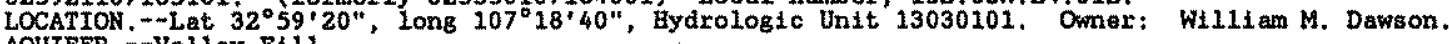

AQUIFER.--Valley Fi11.

WELL CHARACTERISTICS.--Drilled water-teble irrigation well, diameter $16 \mathrm{in}$, depth and casing information not avadlable.

INSTRUMENTATION, --Periodic ste日1-tape measurements.

DATUM.--Elovation of land-surface datum is 4,279 ft above National Geodetic Vextlcal Datum of 1929.

Measuring point: Top of casing, $1.20 \mathrm{ft}$ above land-surface datum.

PERIOD OF RECORD. - Mey 1974 to current year.

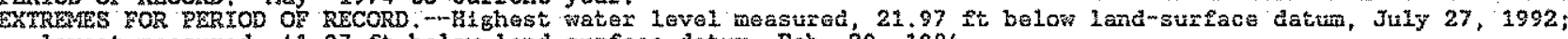

lowest measured, 41.97 ft below land-surface datum, Fob. 29, 1984 .

WATER LEVEL, IN FEET BELOW LAND-SURFACE DATUM, WATER YEAR OCTOBER 1995 TO SEPTEMBER 1996

$\begin{array}{ll}\text { DATE } & \text { WATER } \\ \text { LEVEL } \\ \text { Fob. } 9 & 38.47 \\ \text { Aug. } 30 & 40.65\end{array}$

\section{Rincon Valley}

325340107183001 . (formerly 325350107175501 ) Local number, 16S.05W.25.211.

LOCATION, - Lat $32^{\circ} 53^{\prime} 35^{\prime \prime}$, Long $107^{\circ} 17^{\prime} 55^{\prime \prime}$, Hydrolog tc Unit 13030102. Owmer: U.S. Government.

AQUIFER. - -Valley F111.

WELL CHARACTERISTICS.--Drilled water-table unused we11, diameter $10 \mathrm{in}$, depth $32 \mathrm{ft}$, cased to $32 \mathrm{ft}$ INSTRUMENTATION.--Pertodic steel-tape measurements.

DATUM.--Elevation of land-surface datum is 4,198 ft above National Geodetic Vextical Datum of 1929.

Measuring point: Top of casing, $3.00 \mathrm{ft}$ above land-surface datun.

PERIOD OF RECORD.--Jan. 1961 to current year.

EXTREMES FOR PERIOD OF RECORD, - -Highest water level measured, 11,29 ft below land-surface datum, Feb. 12, 1987,

lowest measured, $25.95 \mathrm{ft}$ below land-surface datum, Jan. 6, 1966.

WATER LEVEL, IN FEET BELOW LAND-SURFACE DATUM, WATER YEAR OCTOBER 1995 TO SEFTEMBER 1996

$\begin{array}{lr}\text { DATE } & \text { WATER } \\ \text { Feb. } 9 & 16.26 \\ \text { Aug. } 30 & 18.76 \\ & \\ & \text { TAOS COUNTY } \\ & \text { Sunshine Valley }\end{array}$

365035105360501, (formerly 365036105355301) Local number, 30N.13E.18.1121.

LOCATrON - - Let $36^{\circ} 50^{\prime} 35^{\prime \prime}$. Long $105^{\circ} 36^{\circ} 05^{\prime \prime}$, Hydralogic Unit 13020101. Owner: U, S, Government.

AQUIFER,--Valley Fi11.

INSTRUMENTATTON. - -Periodic steel-tape measurements.

DATUM. - - Elevation of land-surface datum is 7,597 ft above National Geodetic vertical Datum of 1929 .

Measuring point: Top of casing, $2.00 \mathrm{ft}$ above land-surface datum.

PERIOD OF RECORD, --Sep. 1973 to current year.

EXTREMES FOR PERIOD OF RECORD, -mighest water level measured, 63.50 Et below landmurface datum, Jan. 16 , 1994 ;

lowest measured, 77.33 ft below land-surface datum, Aug. 9, 1978.

WATER LEVEL, IN FEET BELOW LAND-SURFACE DATUM, WATER YEAR OCTOBER 1995 TO SEPTEMBER 1996

DATE WATER

Eob. $16 \quad 68.68$

365644105363501, (formerly 365650105370001) Local number, 01S.74W.24.244.

LOCATION, - - Lat $36^{\circ} 56^{\prime 44 "}$, Long $105^{\circ} 36^{\prime} 35^{\prime \prime}$, Hydrologic Unit 13020101. Owner: Dimmitt.

AQUTFER, - -Valley F111.

WELI CHARACTERISTICS,-Drilled water-table irrigation well, diameter 16 in, depth $270 \mathrm{ft}$.

IASTRUMENTATION, - - Periodic steel-tape measurements.

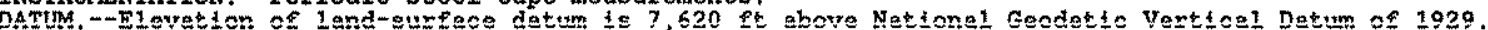

Measuring potni: Top of casing 3.00 it above land-surface datum.

REMARKS.--iost record due to recorder malfunction.

PERTOD OF RECORD.--June 1955 to current year.

ENTREMES FOR PERIOD OF RECORD.--Highest water level measured, 182.78 ft below land-surface datum, Jan. 17, 1996

lowest measured, 213.53 ft below land-surface datum, Aug. 10, 1965.

WATEK LEVEL, IN FEET BELOW LANDD-SURFACE DATUN, WATEK YEAR OCTOBER 1995 TO SEFTEMEEK 1996 DAILY HIGHEST VALUES

\begin{tabular}{|c|c|c|c|c|c|c|c|c|c|c|c|c|}
\hline DAY & OCT & NOV & DEC & JAN & FEB & MAR & APR & MAY & JUN & JUL & AUtG & SEF \\
\hline $\begin{array}{l}5 \\
10 \\
15 \\
20 \\
25\end{array}$ & $\begin{array}{l}183.94 \\
184.17 \\
184.19 \\
184.16 \\
183.96 \\
183.68\end{array}$ & $\begin{array}{l}183.87 \\
183.39 \\
183.97 \\
183.98 \\
183.84 \\
183.76\end{array}$ & $\begin{array}{l}183.70 \\
183.89 \\
183.77 \\
183.45 \\
183.82 \\
183.13\end{array}$ & $\begin{array}{l}183.47 \\
183.60 \\
183.68 \\
183.34 \\
183.10 \\
183.21\end{array}$ & $\begin{array}{l}183.87 \\
183.73 \\
183.66 \\
183.48 \\
183.28 \\
183.47\end{array}$ & $\begin{array}{l}183.31 \\
183.87 \\
183.48 \\
183.69 \\
183.43 \\
183.66\end{array}$ & $\begin{array}{r}183.72 \\
183.42 \\
183.71 \\
183.40 \\
183.50 \\
-.-\end{array}$ & $\begin{array}{l}183.67 \\
183.74 \\
183.67 \\
183.52 \\
183.50 \\
183.68\end{array}$ & $\begin{array}{l}183.80 \\
183.87 \\
183.82 \\
183.83 \\
183.87 \\
184.07\end{array}$ & $\begin{array}{l}183.86 \\
183.95 \\
183.91 \\
183.94 \\
184.02 \\
184.00\end{array}$ & $\begin{array}{l}183.83 \\
183.99 \\
184.01 \\
184.01 \\
183.94 \\
183.83\end{array}$ & $\begin{array}{l}183.78 \\
184.07 \\
183.79 \\
183.98 \\
183.83 \\
184.02\end{array}$ \\
\hline
\end{tabular}

WTR YEAR 1996 BIGEEST 182.78 JAN 17, 1996 LOWEST 184.39 OCT 20, 1995 


\section{GROUND-WATER LEVELS}

TAOS COUNTY

Sunshine Valley

365410105345601. (formerly 365410105354501) Local number, 02s.73W.05.244.

LOCATYON, -Lat $36^{\circ} 54^{\prime} 10^{\prime \prime}$, Long $105^{\circ} 34^{\circ} 56^{\prime \prime}$, Eydrologic Untt 13020101. Onmer: Bert Quintana.

AQUIFER - - Alluvium.

WELL CRARACTERISTICS, --Drilled water-table domestic and stock well, dianeter 6 in., depth unknown.

INSTRUMENTATION. --Periodic stee1-tape measurements.

DATUM. --Elevation of land-surface datum is 7,590 ft above National Geodetic Vertical Datum of 1929.

Measuring point: I in. hole in plate over casing, $1.00 \mathrm{ft}$ above land-surface datim.

PERTOD OF RECORD.-F $-\mathrm{Fb}$. 1974 to current year.

EXTREMES FOR PERIOD OF RECORD.--Highest water leve1 measured, 63.86 ft bolow land-surface datum, Aug. 11, 1995 . lowest measured, $84.78 \mathrm{ft}$ below land-surface datum, Jan. 27,1977 .

WATER LEVEL, IN FEET BELOW LAND-SURFACE DATUM, WATER YEAR OCTOBER 1995 TO SEPTENBER 1996

$\begin{array}{cc}\text { DATE } & \text { WATER } \\ \text { LEVEL }\end{array}$

\section{TORRANCE COUNTY}

Estancia Valley

343443106024401. Local number, 04N.09E.07.334

LOCATION. - Lat $34^{\circ} 34^{\circ} 43^{\prime \prime}$, Long $106^{\circ} 02 \cdot 44^{\prime \prime}$. Hydrologic Untt 13050001. Owner: Frank1in Development.

AQUIFER. - -Valley Fill.

WELL CEARACTERISTICS, - Drilled water-table unused well, dianeter 16 in, reported depth 163 ft.

INSTRUMENTATION. --Periodic steel-tape measurements

DATUM, --Elevation of land-surface datun is 6,118 ft above National Geodetic Vertical Datum of 1929

Measuring point: Hole in northwest side of pump base, $1.50 \mathrm{ft}$ above land-surface datum.

PERIOD OF RECORD - - Feb. 1956 to current year.

EXTREMES FOR PERIOD OF RECORD.--Highest water level measured, 54.70 ft below land-surface datum, Feb. 10, 1958 ,

lowest measured, 100.39 ft below land-surface datum, Aug. 24,1995 .

WATER LEVEL, IN FEET BELOW LAND-SURFACE DATUM, WATER YEAR OCTOBER 1995 TO SEPTEMBER 1996

$\begin{array}{lc}\text { DATE } & \begin{array}{c}\text { WATER } \\ \text { LEVEL }\end{array} \\ \text { Feb. 21 } & 94.10 \\ \text { Aug. } 1 & \text { pumptng }\end{array}$

344016106070901. (Formerly 344016106064701) Local number, 05N.08E.08.424.

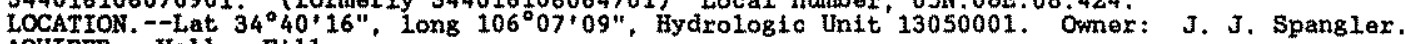

AQUIFER. - Valloy Fill.

WELL CHARACTERISTICS.-Drilled water-table trrigation well, diameter 16 tn., reported depth 204 ft, cased to 98 ft. INSTRUMENTATION. --Pertodic steel-tape measurements.

DATUM. --Elevation of land-surface datum is $6,218 \mathrm{ft}$ above National Geodetic Vertical Datum of 1929.

Measuring point: $3 / 4$ in. Inch plug in south side of discharge pipe, 1.80 ft above land-surface datum.

PERIOD OF RECORD. - Mar. 1948 to current year.

EXTREMES FOR PERIOD OF RECORD.-Highest water lovel measured, 62.03 ft below land-surface datum, Mar. 23, 1948;

lowest measured, 129.74 ft below land-surface datum, Sep. $17,1986$.

WATER LEVEL, IN FEET BELOW LAND-SURFACE DATUM, WATER YEAR OCTOBER 1995 TO SEPTEMBER 1996

\begin{tabular}{ll} 
DATE & \multicolumn{1}{c}{ WATER } \\
LEVEL
\end{tabular}

344234106070601 (formerly 344234106074901) Local number, 06N.08E.32.212.

LOCATION, -lat $34^{\circ} 42^{\prime} 4^{\prime \prime}$, Long $106^{\circ} 07^{\prime} 06^{\prime \prime}$, Hydrologic Unit 13050001 . Owner: Robert McMath.

AQUTFER. --Valley Fill.

WELL CHARACTERISTICS.-Drilled water-table irrigation wel1, dianeter 18 in., reported depth 209 ft, cased to 84 ft.

INSTRUMENTATION, --periodic steel-tape measurements.

DATUM. - Elevation of land-surface datum is $6,174 \mathrm{ft}$ above National Geodetic Vertical Datum of 1929.

Measuring point: Top of $11 / 2 \mathrm{in}$. hole in pump base, $0.04 \mathrm{ft}$ above land-surface datum.

PERIOD OF RECORD - - Feb. 1947 to current year.

EXTREMES FOR PERIOD OF RECORD.--Highest water level measured, 23.22 ft below land-surface datun, Feb. 18 , 1947 ,

lowest measured, $84.64 \mathrm{ft}$ below land-surface datum, July 27,1992 .

WATER LEVEL, IN FEET BELOW LAND-SURFACE DATUM, WATER YEAR OCTOBER 1995 TO SEPTEMBER 1996

$\begin{array}{ll}\text { DATE } & \text { WATER } \\ \text { LEVEL }\end{array}$

Fob. $21 \quad 80.90$

Aug. 1 pumping 


\section{GROUND-WATER LEVELS}

TORRANCE COUNTY

344604105574601 (Pormarly 344622105575501 ) Local number, 06N.09E.11.211.

LOCATYON. - Lat $34^{\circ} 46^{\circ} 04^{\prime \prime}$, Long $105^{\circ} 57^{\circ} 46^{*}$, Hydrologic Unte 13050001 . Owner: Paragon Corp.

AQUIFER. - Valley Fill.

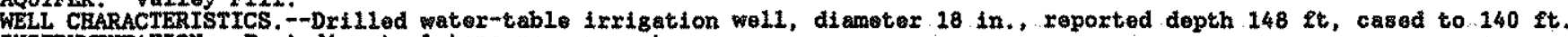

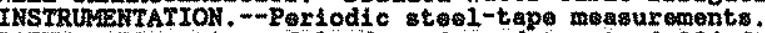

DATU. --Elevetion of land-zurfac datum is 6,086 ft above Fational Geodetic vertical Datum os 1929 .

Measuring point: Top of casing, 0.75 ft above land-surface datum.

PERIOD OF RHCORD May 1949 to current year.

EXTREMES FOR PFRIOD OF RECORD,-Bighest Watex level measured, 5.07 ft belon land-sureace datum, May 4 , 1949 ;

lowest meanured, 28.65 ft below Land-sur\&ace datum, July i3, 1994 .

WATER LEVEL, IN FEET BELON LAAD-SURFACE DATUM, WATER YEAR OCTOBER 1995 TO SEPTEMBER 1996

\begin{tabular}{|c|c|}
\hline DATE & $\begin{array}{l}\text { WATER } \\
\text { LEVEL }\end{array}$ \\
\hline$\because 21$ & $\begin{array}{r}18.25 \\
19.97\end{array}$ \\
\hline
\end{tabular}

344842106032701, Local numbir, 07N. 08E.25.121.

LOCATION.-Lat $34^{\circ} 48^{\prime} 43^{\prime \prime}$, Long $106^{\circ} 03^{\circ} 22^{\prime \prime}$, Eydrologke Unte 13050001. Owner: M. D. Brooks.

AQUIFER. - Alluvium.

WELL CHARACTERISTICS.--Drilled water-table unused irrigation mell, diameter 16 in., depth 200 It.

INSTRUNENTATION. - Periodic steel-tape measurements:

DATUM,--Elovation of lend-surface dation is 6,131 et above National Geodetic Vertical Datum of 1929

Measuring point: Top of casing, 0.00 ft above land-surface datum.

RTMARKS - "s" Andicatea noaxby voli pumping.

PERIOD OF RECORD - FOb. 1962 to current year,

EXTREMES FOR PERIOD OF RECORD, - Highest water level measured, 25.30 ft below land-gurface datum, Feb. 7 , 1962, loweat measured, 65.71 ft below land-surface datum, May 21, 1986.

WATER LEVEL, IN FEET BELOW LAND-SURFACE DATUR, WATER YEAR OCTOBER 1995 TO SEPTEMBER 1996

$\begin{array}{lc}\text { DATE } & \text { WATER } \\ \text { LEVEL }\end{array}$

\section{UWION COUNTY}

Clayton Area

355144103041201. (Formerly 360940103083501) Local number, 19:.36E.23.244.

LOCATIOA, - -Lat $35^{\circ} 51^{\prime} 44^{\prime \prime}$, Iong $103^{\circ} 04^{\circ} 12^{\prime \prime}$, Hydrologic Untt' 11090102 . Owner: Stevens.

AQUIFER - -Dakota and Purgatoire formation.

WELL CIARACTERISTICS, --Drilled water-table unused pell, diameter 14 in. depth $206 \mathrm{ft}$

INSTRUMTETATION, --Periodic steol-tape measurements.

DATUM. --Elevation of land-surface datum is 4,326 ft abova National Geodetic Vortical Datum of 1929.

Measuring point: Top of casting, $1.00 \mathrm{ft}$ above land-surface datum.

EXTREMES FOR PERIOD OF RECORD.--1ighest water level measured, 145.22 ft below land-surface datum, Mar. 17, 1971,

lopest measured, $158.58 \mathrm{~s}$ ft below land-surface datum, Aug, 19, 1987.

WATER LEVEL; IN FEET BELOW LAND-SURFACE DATUM, WATER YEAR OCTOBER 1995 TO SEPTEMBER 1996

$$
\begin{array}{lc}
\text { DATE } & \text { WATER } \\
\text { LEVEL } \\
\text { Jan. } & \text { not massurad } \\
\text { July 24 } & 150.36
\end{array}
$$




\section{GROUND-WATER LEVELS}

UNION COUNTY

Clayton Area

361847103064701. (formarly 361910103170501) Local number, 24N.36E.17.244.

LOCATION. - Lat $36^{\circ} 18^{\prime} 47^{\prime \prime}$. Long $103^{\circ} 06^{\circ} 47^{\prime \prime}$, Hydrologic Unit 11090103. Omner: Glen Burroms.

AQUIFER. - - Ogallala formation.

WELL CHARACERRISTICS.--Dr1lled water-table unused well, diameter 20 in., depth 231 ft.

INSTRURENTATION, --Continuous strip-chart rocorder.

DATUM.--Elevation of land-surface datim is 4,707 it above National Geodetio Vertical Datum of 1929.

Measuring point: Top of casing, $1.95 \mathrm{ft}$ above land-surface datum.

REMARKS. - - Records good.

PERIOD OF RECORD.--May 1968 to cuxrent year.

EXTREYES FOR PERIOD OF RECORD, - - Highest water level measured, $81.38 \mathrm{ft}$ below Land-surface datum, May 8 , 1968 ;

lowest measured, 99.40 ft below land-surface datum, Sep. 27, 1996 .

WATER LEVEL, IN FEET BELOW LAND-SURFACE DATUM, WATER YEAR OCTOBER 1995 TO SEPTEXEER 1996

\begin{tabular}{|c|c|c|c|c|c|c|c|c|c|c|c|c|}
\hline DAY & $\propto C T$ & Nov & $\mathrm{DEC}$ & JAE & FEB & MAR & AFR & MAY & Jus & JuL & AUG & SEP \\
\hline $\begin{array}{r}5 \\
10 \\
15 \\
20 \\
25 \\
\text { EOM }\end{array}$ & $\begin{array}{l}98.38 \\
98.40 \\
98.38 \\
98.35 \\
98.35 \\
98.34\end{array}$ & $\begin{array}{l}98.36 \\
98.31 \\
98.41 \\
98.45 \\
98.40 \\
98.39\end{array}$ & $\begin{array}{l}98.43 \\
98.54 \\
98.47 \\
98.44 \\
98.50 \\
98.40\end{array}$ & $\begin{array}{l}98.50 \\
98.45 \\
98.46 \\
98.50 \\
98.45 \\
98.47\end{array}$ & $\begin{array}{l}98.54 \\
98.56 \\
98.55 \\
98.55 \\
98.60 \\
98.59\end{array}$ & $\begin{array}{l}98.58 \\
98.73 \\
98.64 \\
98.65 \\
98.68 \\
98.69\end{array}$ & $\begin{array}{l}98.77 \\
98.70 \\
98.79 \\
98.74 \\
98.87 \\
98.81\end{array}$ & $\begin{array}{l}98.86 \\
98.93 \\
98.93 \\
98.96 \\
98.88 \\
98.91\end{array}$ & $\begin{array}{l}98.93 \\
98.96 \\
98.97 \\
99.04 \\
99.07 \\
99.08\end{array}$ & $\begin{array}{l}99.11 \\
99.17 \\
99.11 \\
99.15 \\
99.15 \\
99.18\end{array}$ & $\begin{array}{l}99.20 \\
99.20 \\
99.19 \\
99.23 \\
99.22 \\
99.24\end{array}$ & $\begin{array}{l}99.29 \\
99.30 \\
99.23 \\
99.30 \\
99.25 \\
99.34\end{array}$ \\
\hline
\end{tabular}

WTR YRAR 1996 BIGESST 98.27 OCT 4. 1995 LOWEST $99.40 \quad$ SEP 27, 1996

362540103095001 . Local number, 25N.35E.02.441.

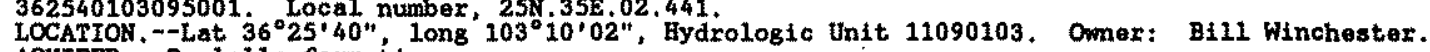

AQUIFER. - - Ogalaila formation.

WELL CHARACTERISTICS.--Drf1led water-table irrigation woll, diameter uniknown, depth $185 \mathrm{ft}$.

INSTRIRENTATION. --Pexiodic steel-tape measurements.

DATUM.--Elevation of land-surface datum is 4,984 ft above National Geodetic Vertical Datum of 1929.

Measurting point: Plugged hole in pump base, $1.70 \mathrm{ft}$ above land-surface datum.

PERIOD OF RECORD. -Dec. 1965 to current year.

EXTREMES FOR PERIOD OF RECORD.--Highest water level measured, 91.14 ft below land-surface datum, Jan. 9, 1989;

lowest measured, $106.85 \mathrm{ft}$ below land-surface datum, Fob. 2, 1971 .

WATER LEVEL, IN FEET BELOW LAND-SURFACE DATUM, WATER YEAR OCTOBER 1995 TO SEPTEABER 1996

DATE WATER

Jan. $24 \quad 95.82$

July $24 \quad 98: 45$

363410103064801 , Local number, 27N.36E.17.434.

LOCATION, - - Lat $36^{\circ} 34^{\circ} 10^{\prime \prime}$, Long $103^{\circ} 06^{\circ} 4^{\prime \prime}$, Hydrologic Unit 11100101, Omer: Paul Carter. AQUIFER. - Ogalalla formation.

WELL CHARACTERISTICS.--Drilled pater-table trrigation we11, diameter 16 in., depth $200 \mathrm{ft}$.

INSTRUMENTATION.---Pertodic steel-tape measurements.

DATUM. - -Elevation of land-surface datum is 4,837 ft above Nattonal Geodetic Vertical Datum of 1929.

Measuring point: Top of casing, north side, 1.20 ft above land-surface datum.

PERIOD OF RECORD. - Fob. 1967 to current year.

EXTREMES FOR PERIOD OF RECORD, --titghest water lovel measured, $81.16 \mathrm{ft}$ below land-surface datum, Jan, 21, 1975;

lowest measured, $97.44 \mathrm{ft}$ below land-șurface datum, Jan, 26, 1993.

WATER LEVEL, IN FEET BELOW LAND-SURFACE DATUM, WATER YEAR OCTOBER 1995 TO SEPTEMBER 1996

$\begin{array}{cc}\text { DATE } & \text { WATER } \\ \text { LEVEL } \\ \text { Jan. } 23 & 96.26 \\ \text { July } 24 & 94.89\end{array}$

Capulin Area

364444104000201 (formerly 364430103595501 ) Local number, 29N. 28E.18.341.

LOCATIO: - - Lat $36^{\circ} 44^{\prime} 44^{\prime \prime}$, long $104^{\circ} 00^{\circ} 02^{\prime \prime}$, Hydrologic Unit 11040001 . Ommex: C1ty of Raton.

AQUIFER, --Cinders.

WELL CHARACTERISTICS.--Drflled watex-table irrigation well, diameter $16 \mathrm{in.}$, depth $78 \mathrm{ft}$.

INSTRUMENTATION. --Poriodic steel-tape measurements.

DATUM.--Elevation of land-surface datum is $6,820.8$ ft above National Geodetic Vertical Datum of 1929.

Measuring point: Edge of 2 in. hole in west side of steel plate, at land-surface datum.

REYARKS.--" "p" indicates poll pumping during measurement.

PERIOD OF RECORD.--JuLy 1951 , Aug. 1958 to current year.

EXTRFA4S FOR FERIOD OF RECORD. --Eighest water leve1 measured, 28.01 ft belon land-murface datum, Feb. 8, 1974;

lowest measured, 53.38p ft below land-surface datum, Aug. 7, 1985 .

WATER LEVEL, IH FEET BELOW LAND-SURFACE DATUM, WATER YEAR OCTOBER 1995 TO SEPTEMBRR 1996

$\begin{array}{cc}\text { DATE } & \begin{array}{c}\text { WATER } \\ \text { LEVEL }\end{array} \\ \text { Jan. } 23 & 34.88 \\ \text { July } 24 & 34.07\end{array}$


434

QUALITY OF GROUND WATER

EXPLANATION OF GEOLOGIC UNIT (AQUIFER) CODES (LISTED FROM YOUNGEST TO OLDEST AGE) U-UPPER, M-MIDDLE, L-LOWER: 000 EXRV-UNKNOWN, Extrusive Rocks; 110 AVMB-Cenozolc, Quaternary, Alluvium, Bolson Deposits and other Surface Deposits; 110 BLSN-Cenozoic, Quaternary, Bolson Fil1; 400 PCAB-Paleozodc, Precambrian, Precambrian Erathem.

REMARKS.--Ground Water sites in this table are segregated by county which appear alphabetically. The sites are then listed in ascending well numbers that are explained at the beginning of this report.

BERNALILLO COUNTY

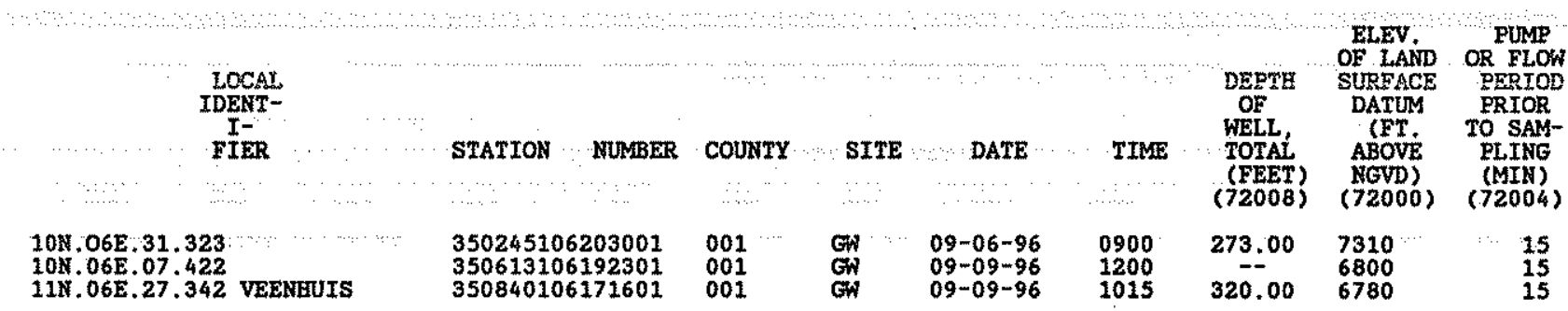

$10 \mathrm{~N}, 06 \mathrm{E}, 07,422 \quad 09-09-96$

$13 \mathrm{H} .06 \mathrm{E} .27 .342 \mathrm{VEE} 09-09-96$

$10 \quad 810$

\section{PB}

WATER

(STAND

ARD

UNITS)

TEMPER - TEMPERATURE AIR (DEG C) (00020)

ATURE WATER (DEG C)

\section{3 \\ 14.0}

$$
17.5
$$

15.5

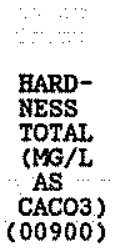

HARD -

NESS

YONCARB CALCIUM

DISSOLV DIS-

FLD AS SOLVED

CACO3 $\quad$ (MG/L

$\mathrm{CACO}$
(MG/L)
$(\mathrm{MS} / \mathrm{CA}$

$(00904) \quad(00915)$

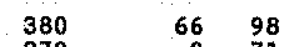

$\begin{array}{rrrr}270 & 2 & 0 & 71\end{array}$

240130

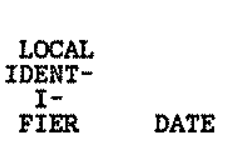

MAGNE-

STUM, SODIUN,

DIS- DIS-

SOLVED SOLVED

(MG/L (MG/L

AS MG) AS NA)
$(00925) \quad(00930)$

SODIUM

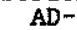

SOMP-

TION

RATIO

(00931)

POTAS-
SIUM,
DIS-
SOLVED
(MG/L
AS K)
$(00935)$

BICAR-

BONATE

WATER

DIS IT

FIELD

$\mathrm{MG} / \mathrm{L}$ AS

$\mathrm{HCO} 3$
$(00453)$

CAR-
BONATE
WATER
DIS IT
FIELD
MG/L AS
CO3
$(00452)$

AIKA-

L.INITY ALKA-

WAT DIS LINITY

TOT T

TOT I

$M G / L$ AS

/L AS AS

CACO3 CACO3)

$\begin{array}{lll}0.6 & 2.3 & 379\end{array}$

$10 \mathrm{~N} .06 \mathrm{E} .31 .323 \quad 09-06-96 \quad 32$

$10 \mathrm{~N} .06 \mathrm{E} .07 .422 \quad 09-09-96$

$\begin{array}{ll}32 & 25 \\ 22 & 75 \\ 21 & 21\end{array}$

2.5

2.0

344

311321

$282 \quad 284$

167168
10N.06E.31.323 09-06-96

10N. 06E.07.422 09-09-96

11N.06E,27.342 VEE 09-09m96

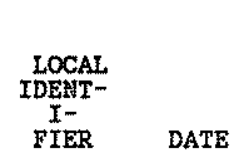

10N.06E.31.323 $\quad 09-06-96$

$\begin{array}{ll}10 \mathrm{~N} .06 \mathrm{E} .07 .422 & 09-09-96 \\ 11 \mathrm{~N} .06 \mathrm{E} .27 .342 \mathrm{VEF} & 09-09-96\end{array}$

SUEFATE
DIS-
SOLVED
(MG/L
AS SO4)
$(00945)$

CHLO- FLUO- SILICA,

DIS-

SOLVED

(MG/L

A.S CL)
$(00940)$

59

89
100

38

59
140

NITRO-

GEN, AM- PHOS

MONIA + PHORUS

( $A S$ S $/ L$

(00623)

SOLVED

(MG/L)

AS P)
$(00666)$

$<0.20$

0.30
$<0.010$

0.010
DIS - SOLVED

SOLVED $\quad(M G / L$

(NG/L

(00950) (00955)

$\begin{array}{ll}0.40 & 18 \\ 0.60 & 12 \\ 0.20 & 19\end{array}$

PHOS-

PHORUS

AS $\mathrm{P})$

( $M G / L$

0.010

1.5
2.3
2.0

$0.020 \quad 2.0$
AS F) STO2)

SOLIDS,

SUM OF

CONSTI-

TUENTS,

DIS-

SOLVED

$(\mathrm{MG} / \mathrm{L})$

(70301)

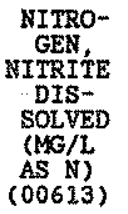

NITRO-

GEN,

AMNONTA

DIS- DIS-

SOLVD SOLVED

(MG/L

(00631) (00608)

$\begin{array}{rrrr}473 & <0.010 & 0.860 & 0.020 \\ 504 & <0.010 & 1.60 & 0.030 \\ 566 & <0.010 & 7.20 & <0.015\end{array}$

ORTHO, CARBOY ARSEKIC BORON, IRON MANGA-

DIS- ' ORGANIC AR DIS-

DIS DOA DIS- DIS- DIS-

sutir

(UG/L

(UTe/L

(UT)

(UG/I

AS FE) $(\mathrm{NG} / \mathrm{L}$

(00680) (01000) (01020) (01046) (0. MN)

$<\frac{1}{<1}$

61
125
61

$<3.0<<1.0$

$<3.0<1.0$ 
QUALITY OF GROUND WATER

WATER-QUALITY DATA, WATER YEAR OCTOBER 1995 TO SEPTEMBER 1996

DONA ANA COUNTY

$$
\begin{gathered}
\text { LOCAL } \\
\text { IDENT- } \\
\text { I- } \\
\text { FIER }
\end{gathered}
$$

$25 \mathrm{~S} .06 \mathrm{E} .19 .443$

22S. 05E.28.234 T-34

$22 \mathrm{~S} .05 \mathrm{E} .28 .142 \mathrm{~B} \mathrm{~T}-37$

$22 \mathrm{~S} .05 \mathrm{E} .28 .142 \mathrm{~A} T-35$

$22 \mathrm{~S} .05 \mathrm{E} \cdot 28.122 \mathrm{~T}-29$

21S.04E.14.114 (HTA-3)

21S.04E. 10.411B (HTA-11)

21S. 04E. 10.411C (BTA-10A)

2.15. 04E $10.322($ ETA-12)

320645106215101
322201106260201
322205106261801
322208106261401
322213106261301
322910106303601

322910106303601

322941106311301

322941106311502

013
013
013
013

013
013
013

013

$\begin{array}{ll}013 & \text { G } \\ 013 & \text { G } \\ 013 & \text { G } \\ 013 & \text { G } \\ 013 & \\ 013 & \text { G } \\ 013 & \text { G } \\ 013 & \text { G } \\ 013\end{array}$

013 GN 01-18-96

013 G $04-03-96$

GW $\begin{aligned} & 06-12-96 \\ & 09-11-96\end{aligned}$

GW $01-19-96$

$01-19-96$
$04-04-96$

$04-12-96$

$06-12-96$
$09-11-96$

01-18-96

$04-03-96$

$06-12-96$
$09-11-96$
DEFTH ELEV TO TOP OF LAND

SAMPLE DATUM

INTER- DAT.

VAL ABOVE

(FT) NGVD)

(72015) (72000)

$\begin{array}{ll}290 & = \\ 253 & = \\ 250 & = \\ 248 & =\end{array}$

1300 110BLSN

400.00

$-$

1000

1420 110BLS

$110 B L S N$

157.00

$\begin{array}{lll}157.00 & -\cdots & 5150\end{array}$

$157.00 \quad=-5150$

$1040 \quad 400 \mathrm{PCAB}$

1515 400 PCMB

157.00

$400 \mathrm{PCMB}$

$\begin{array}{ll}1530 & 400 \mathrm{PCMB} \\ 1550 & 400 \mathrm{PCMB}\end{array}$

$\begin{array}{ll}1550 & 400 \mathrm{PCMB} \\ 1045 & 400 \mathrm{PCMB}\end{array}$

86.10

86.10

86.10

86.10

$-$

5150

1230 400РCMB

82.00

$0907 \quad 400 \mathrm{PCM}$

$0930 \quad 400 \mathrm{PCM}$

$1215 \quad 400 \mathrm{PCMB}$

82.00

82.00

82.00

$\begin{array}{ll}84 & 5690 \\ 84 & 5690\end{array}$

$\begin{array}{ll}84 & 5690 \\ 84 & 5690\end{array}$

$1207 \quad 400 \mathrm{PCMB}$

157.10

$1400 \quad 400 \mathrm{PCMB}$ 157.10

$1330 \quad 400 \mathrm{PCMB}$ 157.10

157.10

$$
-
$$

$1400 \quad 400 \mathrm{PCMB}$

155
155
155
155
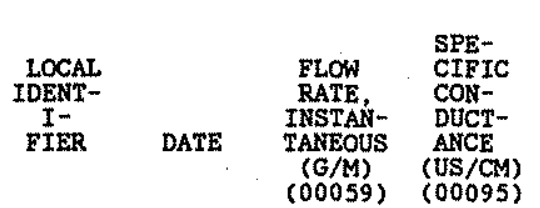

PH

WHOLE

FIELD

(STAND

ARD

$(00400)$

TEMPER-
ATURE
AIR
(DEG C)
$(00020)$

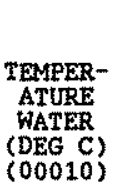

TUR-
BID-
ITY
(NTU)
(00076)

HARD-
NESS
TOTAL
(MG/L
AS
CACO3)
$(00900)$

CALCIUM
DIS-
SOLVED
(MG/L
AS CA)
(00915)

MAGNE-

STUM,

DIS-

\begin{tabular}{|c|c|c|}
\hline $\begin{array}{l}25 \mathrm{~S} .06 \mathrm{E} \cdot 19.443 \\
22 \mathrm{~S} .05 \mathrm{E} \cdot 28.234 \mathrm{~T}-3 \\
22 \mathrm{~S} .05 \mathrm{E} \cdot 28.142 \mathrm{~B} \mathrm{~T}- \\
22 \mathrm{~S} .05 \mathrm{E} \cdot 28.142 \mathrm{~A} \mathrm{~T}- \\
22 \mathrm{~S} .05 \mathrm{E} .28 .122 \mathrm{~T}-2\end{array}$ & $\begin{array}{l}09-12-96 \\
04-01-96 \\
04-01-96 \\
04-01-96 \\
04-01-96\end{array}$ & $\begin{array}{r}1000 \\
=- \\
=- \\
=-\end{array}$ \\
\hline $21 \mathrm{~S} .04 \mathrm{E} .14 .114 \mathrm{KHT}$ & $\begin{array}{l}01-19-96 \\
04-04-96 \\
06-12-96 \\
09-11-96\end{array}$ & $\begin{array}{l}=- \\
=-\end{array}$ \\
\hline $21 \mathrm{~S} .04 \mathrm{E} .10 .411 \mathrm{~B}$ (E & $\begin{array}{l}01-18-96 \\
04-03-96 \\
06-12-96 \\
09-11-96\end{array}$ & $\begin{array}{l}=- \\
-- \\
-\end{array}$ \\
\hline $21 \mathrm{~S} .04 \mathrm{E}, 10.411 \mathrm{C}(\mathrm{H}$ & $\begin{array}{l}01-19-96 \\
04-04-96 \\
06-12-96 \\
09-11-96\end{array}$ & $\begin{array}{l} \pm \\
\overline{-}\end{array}$ \\
\hline $21 \mathrm{~S} .04 \mathrm{E} .10 .322 \mathrm{HT}$ & $\begin{array}{l}01-18-96 \\
04-03-96 \\
06-12-96 \\
09-11-96\end{array}$ & $\begin{array}{l}=- \\
=- \\
=\end{array}$ \\
\hline
\end{tabular}

SOLVED

(MG/L

AS MG)
$(00925)$

$\begin{array}{ll}7.9 & - \\ 7.8 & 27.0 \\ 7.9 & 25.0 \\ 7.9 & 26.5 \\ 7.7 & 26.0 \\ 7.4 & -- \\ 7.3 & -- \\ 7.3 & -- \\ 7.2 & - \\ 7.3 & -- \\ 7.2 & -- \\ 7.3 & -- \\ 7.2 & - \\ 7.3 & -- \\ 7.4 & -- \\ 7.3 & -- \\ 7.3 & - \\ 7.2 & -- \\ 7.0 & -- \\ 7.2 & -- \\ 7.2 & -\end{array}$

$\begin{array}{ll}24.0 & - \\ 23.0 & = \\ 23.5 & = \\ 24.0 & = \\ 24.0 & - \\ 19.0 & -\end{array}$

$=$

19.0

$21.0 \quad 0.91$

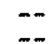

$=$

$--$

$-\cdots$

$\ddot{-}$

$-$

$-$

$-$

$\begin{array}{lll}290 & 82 & 20 \\ 290 & 83 & 19\end{array}$

$\begin{array}{lll}290 & 85 & 20\end{array}$

21.0 - 280

$\begin{array}{llll}19.0 & 2.5 & 390 & 110\end{array}$

$19.5 \quad 1.6 \quad 370 \quad 100$

$\begin{array}{llll}20.0 & -. & 390 & 110 \\ 20.0 & -- & 360 & 100\end{array}$

20
19
20
19

1.5
3.6

19.0

$19.0 \quad 3.6$

$350 \quad 95$

37010

350

100
96

28
28
29

29
27

28
27

27
28
27

$\begin{array}{rrr}09-11-96 & = & 990\end{array}$

$\begin{array}{ll}18.5 & 0.39 \\ 19.5 & 0.75\end{array}$

$400 \quad 110$

$390 \quad 110$

420120

30
29
30
29 
QUALITY OF GROUND WATER

WATER-QUALITY DATA, WATER YEAR OCTOBER 1995 TO SEPTEMBER 1996

DONA ANA COUNTY -- Continued

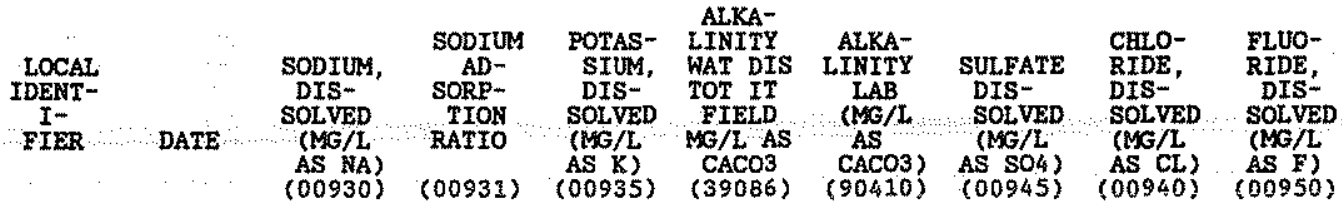

\begin{tabular}{|c|c|c|c|c|c|c|c|c|c|}
\hline $\begin{array}{l}25 \mathrm{~S} .06 \mathrm{E} .19 .443 \\
22 \mathrm{~S} .05 \mathrm{E} \cdot 28.234 \mathrm{~T}-3 \\
225.05 \mathrm{E} .28 .142 \mathrm{~B} T- \\
22 \mathrm{~T} .05 \mathrm{E} .28 .142 \mathrm{~T}- \\
22 \mathrm{~T} .05 \mathrm{E} .28,122 \mathrm{~T}-2\end{array}$ & $\begin{array}{l}09-12-96 \\
04-01-96 \\
04-01-96 \\
04-01-96 \\
04-01-96\end{array}$ & - & $\begin{array}{l}-\cdots \\
=- \\
= \\
=-\end{array}$ & $=$ & $\begin{array}{l}-76 \\
-- \\
-\end{array}$ & $\frac{-}{--}$ & $=$ & $\overline{--}$ & $\begin{array}{l}-- \\
-- \\
--\end{array}$ \\
\hline $21 \mathrm{~S} .04 \mathrm{E} .14 .114$ (HT & $\begin{array}{l}01-19-96 \\
04-04-96 \\
06-12-96 \\
09-11-96\end{array}$ & $\begin{array}{l}58 \\
57 \\
57 \\
58\end{array}$ & $\begin{array}{l}1 \\
1 \\
1 \\
2\end{array}$ & $\begin{array}{l}0.80 \\
0.80 \\
0.80 \\
1.0\end{array}$ & $=$ & $\begin{array}{l}229 \\
229 \\
229 \\
228\end{array}$ & $\begin{array}{l}130 \\
130 \\
130 \\
130\end{array}$ & $\begin{array}{l}27 \\
26 \\
26 \\
28\end{array}$ & 5.6 \\
\hline $21 \mathrm{~S} .04 \mathrm{E} .10 .411 \mathrm{~B}$ (H & $\begin{array}{l}01-18-96 \\
04-03-96 \\
06-12-96 \\
09-11-96\end{array}$ & $\begin{array}{l}58 \\
58 \\
58 \\
57\end{array}$ & $\begin{array}{l}1 \\
1 \\
1 \\
1\end{array}$ & $\begin{array}{l}1.1 \\
1.0 \\
1.0 \\
1.2\end{array}$ & $=$ & $\begin{array}{r}254 \\
254 \\
253 \\
254\end{array}$ & $\begin{array}{l}160 \\
170 \\
170 \\
170\end{array}$ & $\begin{array}{l}34 \\
34 \\
33 \\
32\end{array}$ & $\begin{array}{l}5.0 \\
4.7\end{array}$ \\
\hline $21 \mathrm{~S} .04 \mathrm{E} \cdot 10.411 \mathrm{C}$ (E) & $\begin{array}{l}01-19-96 \\
04-04-96 \\
06-12-96 \\
09-11-96\end{array}$ & $\begin{array}{l}59 \\
57 \\
59 \\
59\end{array}$ & $\begin{array}{l}1 \\
1 \\
1 \\
1\end{array}$ & $\begin{array}{l}1.4 \\
1.2 \\
1.3 \\
1.5\end{array}$ & $=$ & $\begin{array}{l}248 \\
248 \\
247 \\
248\end{array}$ & $\begin{array}{l}160 \\
160 \\
160 \\
160\end{array}$ & $\begin{array}{l}32 \\
33 \\
31 \\
32\end{array}$ & \\
\hline $21 \mathrm{~s} .04 \mathrm{E} \cdot 10.322 \mathrm{( \textrm {HT }}$ & $\begin{array}{l}01-18-96 \\
04-03-96 \\
06-12-96 \\
09-11-96\end{array}$ & $\begin{array}{l}65 \\
64 \\
66 \\
65\end{array}$ & $\begin{array}{l}1 \\
1 \\
1 \\
1\end{array}$ & $\begin{array}{l}1.3 \\
1.1 \\
1.2 \\
1.4\end{array}$ & $\begin{array}{l}- \\
-- \\
--\end{array}$ & $\begin{array}{l}284 \\
280 \\
280 \\
281\end{array}$ & $\begin{array}{l}200 \\
200 \\
200 \\
200\end{array}$ & $\begin{array}{l}33 \\
31 \\
31 \\
31\end{array}$ & 4.3 \\
\hline
\end{tabular}

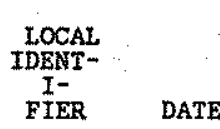

25S.06E.19.443 09-12-96 22S.05E.28.234 T-3 $04-01-96$ 22S.05E.28.142B T- 04 01-96 $22 \mathrm{~S} .05 \mathrm{E} .28 .142 \mathrm{~A} \mathrm{~T}-04-01-96$ 22S.05E.28.122 T-2 04-01-96

21S.04E. 14.114 (HT 01-19-96 $04-04-96$
$06-12-96$ $06-12-96$
$09-11-96$

21S.04E. $10.411 \mathrm{~B}$ (H) 01-18-96 $04-03-96$ $06-12-96$ 09-11-96

21S. 04E, $10.411 \mathrm{C}$ (B $01-19-96$ $04-04-96$ $06-12-96$ 09-11-96

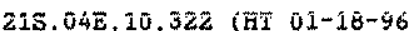
$04-03-96$ $06-12-96$ $06-12-96$

$04-112-96$
$06-12-96$
$09-11-96$

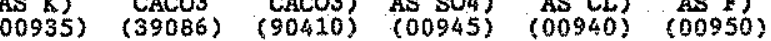
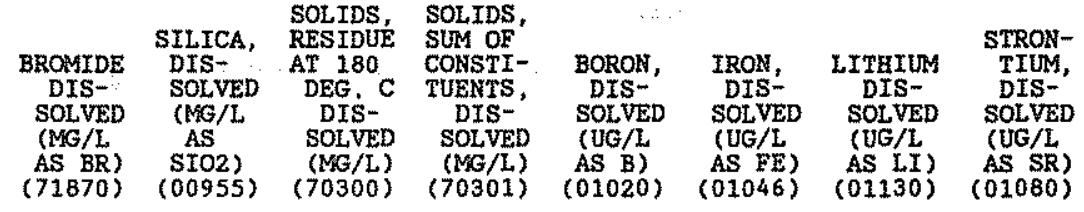

$$
\begin{array}{llll}
-z & -z & =- & = \\
-z & =- & = & = \\
-z & =- & = & = \\
-- & -- & - &
\end{array}
$$

0.3224

$0.31 \quad 25$

$\begin{array}{ll}0.35 & 25 \\ 0.32 & 25\end{array}$

0.34

0.36

$0.28 \quad 25$

$0.41 \quad 25$

$\begin{array}{ll}0.37 & 24 \\ 0.39 & 25\end{array}$

$\begin{array}{ll}0.39 & 25 \\ 0.34 & 25\end{array}$

$0.37 \quad 25$

$0.34 \quad 24$

$0.48 \quad 25$

$0.49 \quad 26$

$=-$
$=-$
$=-$
--

$\overline{--}$

484

484

487
485

572
574

574
584

570

553
568
558

558

638

633

648 $\begin{array}{lll}- & = & = \\ = & = & = \\ -- & = & = \\ - & - & =\end{array}$

$<3.0 \quad 23 \quad 260$

$\begin{array}{lll}3.0 & 21 & 260\end{array}$

$<3.0 \quad 23 \quad 260$

$<3.0 \quad 16 \quad 270$

$\begin{array}{rll}4.0 & 16 & 270 \\ 4.0 & 18 & 270\end{array}$

$<3.0 \quad 15 \quad 270$

$<3.0 \quad 15 \quad 250$

\begin{tabular}{rrr}
3.0 & 15 & 250 \\
12 & 11 & 240 \\
\hline & 13 & 260
\end{tabular}

$<3.0$ is 250

$<3.0 \quad 19 \quad 290$

$<3.0 \quad 16 \quad 280$

$\begin{array}{lll}11 & 18 & 290 \\ <3.0 & 19 & 280\end{array}$ 
QUALITY OF GROUND WATER

WATER-QUALITY DATA, WATER YEAR OCTOBER 1995 TO SEPTEMBER 1996

LINCOLN COUNTY

$$
\begin{gathered}
\text { LOCAL } \\
\text { IDENT- } \\
\text { I- } \\
\text { FIER }
\end{gathered}
$$

$\begin{array}{lll}\text { STATION NUMBER } & \text { COUNTY } \\ & & \\ 332420106173901 & 027 & \\ 332501106171201 & 027 & 5 \\ 332508106173401 & 027 & 5 \\ 332535106170501 & 027 & 5 \\ 332606106172001 & 027 & \end{array}$
DEAD ORYX MOUND SPRTHG WEST MOUND SPRING NR OSCUR MOUND SPRING (UPPER POND) NORTH NOUND SERING NR OSCU

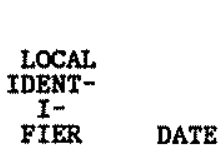

SOUTH MOUND SPRING 07-31-96 DEAD ORYX MOUND SP $07-31-96$ WEST MOIND SPRING 08-01-96 MOUND SPRING (UPPE 08-01-9 NORTH YOUND SPRING 08-01-96

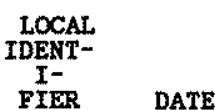

SOUTH MOUND SPRING 07-31-96 DEAD ORYX MOUND SP 07-31-96 WEST MOUND SERING 08-01-96 MOUND SPRING (UPPE 08-01-96 NORTE MOUND SPRING 08-01-96

LOCAL.
IDENT-
I-
FIER $\quad$ DATE

SOUTH MOUND SERING 07-31-96 DEAD ORYX MOUND SP 07-31-96

\begin{tabular}{|c|c|c|c|c|c|c|c|}
\hline $\begin{array}{c}\text { TEMPER- } \\
\text { ATURE } \\
\text { WATER } \\
\text { (DEG C) } \\
(00010)\end{array}$ & $\begin{array}{c}\text { TUR- } \\
\text { BID- } \\
\text { ITY } \\
\text { (NTU) } \\
(00076)\end{array}$ & $\begin{array}{l}\text { BARO- } \\
\text { METRIC } \\
\text { PRES- } \\
\text { SURE } \\
\text { (MM } \\
\text { OF } \\
\text { HG) } \\
(00025)\end{array}$ & $\begin{array}{c}\text { OXYGEN, } \\
\text { DIS- } \\
\text { SOLVED } \\
(M G / L) \\
(00300)\end{array}$ & $\begin{array}{l}\text { HARD- } \\
\text { NESS } \\
\text { TOTAL } \\
\text { (MG/L } \\
\text { AS } \\
\text { CACO3) } \\
(00900)\end{array}$ & $\begin{array}{l}\text { CALCIUM } \\
\text { DIS- } \\
\text { SOLVED } \\
\text { (MG/L } \\
\text { AS CA }) \\
(00915)\end{array}$ & $\begin{array}{c}\text { MAGNE- } \\
\text { SIUM, } \\
\text { DIS- } \\
\text { SOLVED } \\
\text { (MG/L } \\
\text { AS MG) } \\
(00925)\end{array}$ & $\begin{array}{c}\text { SODIUM, } \\
\text { DIS- } \\
\text { SOLVED } \\
\text { (MG /L } \\
\text { AS NAA) } \\
(00930)\end{array}$ \\
\hline $\begin{array}{l}22.0 \\
25.0 \\
20.5 \\
19.5 \\
27.0\end{array}$ & $\begin{array}{c}22 \\
6.6 \\
160 \\
19 \\
2.0\end{array}$ & $\begin{array}{l}655 \\
655 \\
654 \\
652\end{array}$ & $\begin{array}{l}1.7 \\
7.7 \\
1.1 \\
2.3 \\
7.7\end{array}$ & $\begin{array}{l}2400 \\
2300 \\
2200 \\
2300 \\
2700\end{array}$ & $\begin{array}{l}710 \\
670 \\
660 \\
680 \\
780\end{array}$ & $\begin{array}{l}140 \\
150 \\
140 \\
140 \\
170\end{array}$ & $\begin{array}{l}330 \\
340 \\
300 \\
350 \\
350\end{array}$ \\
\hline
\end{tabular}
WEST MOUND SPRING 08-01-96 MOUND SPRING (UPPE 08-01-96 NORTE MOUND SPRING 08-01-96

$\begin{array}{cccc}\text { SODIUM } & \text { POTAS- } & \text { ALKA- } & \\ \text { AD- } & \text { SIUM, } & \text { LINITY } & \text { SULFATE } \\ \text { SORP- } & \text { DIS- } & \text { LAB } & \text { DIS- } \\ \text { TION } & \text { SOLVED } & \text { (MG/L } & \text { SOLVED } \\ \text { RATIO } & \text { (MG/L } & \text { AS } & \text { (MG/L } \\ & \text { AS K) } & \text { CACO3) } & \text { AS SO4 }) \\ (00931) & (00935) & (90410) & (00945)\end{array}$

CRLO-
RIDE,
DIS-
SOLVED
(MG/L
AS CL)

FLUO-
RIDE,
DIS-
SOLVED
(MG/L
AS F)

BROMID
DIS-
SOLVED
(MG/L
AS BR
$(71870)$
0.32
0.25
0.32
0.36
0.31

SILICA, DIS-SOLVED (MG/L AS (00955)

$\begin{array}{rrrr}3 & 4.9 & 120 & 1900 \\ 3 & 4.8 & 58 & 2000 \\ 3 & 4.7 & 121 & 1900 \\ 3 & 4.6 & 115 & 1900 \\ 3 & 6.4 & 47 & 2300\end{array}$

690
720
600
700
790

1.1
1.1
1.2
1.3

27

\begin{tabular}{|c|c|c|c|c|c|c|c|}
\hline $\begin{array}{l}\text { SOLIDS, } \\
\text { RESIDUEE } \\
\text { AT } 180 \\
\text { DEG.CC } \\
\text { DIS-- } \\
\text { SOLVED } \\
\text { (MG/L) } \\
(70300)\end{array}$ & $\begin{array}{c}\text { SOL.IDS, } \\
\text { SUM OE } \\
\text { CONSTI- } \\
\text { TUENTS, } \\
\text { DIS-- } \\
\text { SOLVED } \\
\text { (MG/L) } \\
(70301)\end{array}$ & $\begin{array}{c}\text { NITRO- } \\
\text { GEN, } \\
\text { NITRATE } \\
\text { DIS- } \\
\text { SOLVED } \\
\text { (MG/L } \\
\text { AS N) } \\
(00618)\end{array}$ & $\begin{array}{c}\text { NITRO- } \\
\text { GEN, } \\
\text { NITRITE } \\
\text { DIS- } \\
\text { SOLVED } \\
\text { (ME/L } \\
\text { AS N } \\
(00613)\end{array}$ & $\begin{array}{c}\text { NITRO- } \\
\text { GEN, } \\
\text { NO2+NÓ3 } \\
\text { DIS- } \\
\text { SOLVED } \\
\text { (MG/L } \\
\text { AS N) } \\
(00631)\end{array}$ & $\begin{array}{c}\text { NITRO- } \\
\text { GEN } \\
\text { ANAONIA } \\
\text { DIS- } \\
\text { SOLVED } \\
\text { (MG/L } \\
\text { AS N) } \\
(00608)\end{array}$ & $\begin{array}{l}\text { GEN, } \\
\text { MONI } \\
\text { ORGA } \\
\text { DIS } \\
\text { (ME } \\
\text { AS } \\
0006\end{array}$ & $\begin{array}{c}\text { PHOS- } \\
\text { PHORUS } \\
\text { DIS- } \\
\text { SOLVED } \\
\text { (MG/L } \\
\text { AS P) } \\
(00666)\end{array}$ \\
\hline $\begin{array}{l}426 \\
440 \\
408 \\
430\end{array}$ & $\begin{array}{l}3950 \\
3720 \\
3890 \\
4460\end{array}$ & 0.08 & $\begin{array}{r}0.1 \\
<0.0 \\
0.0 \\
0.1 \\
<0.0\end{array}$ & $\begin{array}{r}0.3 \\
0.0 \\
0.1 \\
0.7 \\
<0.0\end{array}$ & & 0.30 & \\
\hline
\end{tabular}

21
28

28

$\begin{array}{lll}1.4 & 0.31 & 21\end{array}$


QUALITY OF GROUND WATER

WATER-QUALITY DATA, WATER YEAR OCTOBER 1995 TO SEPTEMBER 1996

LINCOLN COUNTY -- Continued

\begin{tabular}{|c|c|c|c|c|c|c|c|c|c|}
\hline $\begin{array}{l}\text { LOCAL } \\
\text { IDENT- } \\
\text { I- } \\
\text { FIER }\end{array}$ & DATE & $\begin{array}{l}\text { PHOS- } \\
\text { PHORUS } \\
\text { ORTHO, } \\
\text { DIS- } \\
\text { SOLVED } \\
\text { (ME/L } \\
\text { AS P) } \\
(00671)\end{array}$ & $\begin{array}{c}\text { ARSENIC } \\
\text { DIS- } \\
\text { SOLVED } \\
\text { (UG/L } \\
\text { AS AS) } \\
(01000)\end{array}$ & $\begin{array}{c}\text { BARIUM, } \\
\text { DIS- } \\
\text { SOLVED } \\
\text { (UG/L } \\
\text { AS BA }) \\
(01005)\end{array}$ & $\begin{array}{l}\text { BORON, } \\
\text { DIS- } \\
\text { SOLVED } \\
\text { (UG/L } \\
\text { AS B) } \\
(01020)\end{array}$ & $\begin{array}{c}\text { CADMIUM } \\
\text { DIS- } \\
\text { SOLVED } \\
\text { (UE/L } \\
\text { AS CD) } \\
(01025)\end{array}$ & $\begin{array}{l}\text { CHRO- } \\
\text { MIUM, } \\
\text { DIS- } \\
\text { SOLVED } \\
\text { (UG/I } \\
\text { AS CR) } \\
\text { (01030) }\end{array}$ & $\begin{array}{c}\text { COPPER, } \\
\text { DIS - } \\
\text { SOLVED } \\
\text { (UG/L } \\
\text { AS CU) } \\
(01040)\end{array}$ & $\begin{array}{l}\text { IRON } \\
\text { TOTAL } \\
\text { RECOV- } \\
\text { ERABLE } \\
\text { (UG/L } \\
\text { AS FE) } \\
(01045)\end{array}$ \\
\hline SLR & $\begin{array}{l}07 \\
07 \\
08 \\
08 \\
08\end{array}$ & $\begin{array}{r}<0 . \\
<0 . \\
0 . \\
<0 . \\
<0 .\end{array}$ & $\begin{array}{l}<1 \\
<1 \\
<1 \\
<1 \\
<1\end{array}$ & $\begin{array}{l}15 \\
17 \\
17 \\
10 \\
19\end{array}$ & $\begin{array}{l}207 \\
193\end{array}$ & $\begin{array}{l}<1.0 \\
<1.0 \\
<1.0 \\
<1.0\end{array}$ & $\begin{array}{l}2 . \\
<1 . \\
<1 . \\
<1 .\end{array}$ & $\begin{array}{l}<1.0 \\
<1.0 \\
<1.0 \\
<1.0\end{array}$ & $\begin{array}{r}240 \\
90 \\
6000 \\
30 \\
10\end{array}$ \\
\hline
\end{tabular}

\begin{tabular}{|c|c|c|c|c|c|c|c|c|c|}
\hline $\begin{array}{c}\text { LOCAL } \\
\text { IDENT- } \\
\text { I- } \\
\text { IIER }\end{array}$ & DATE & $\begin{array}{l}\text { IROA, } \\
\text { DIS- } \\
\text { SOLVED } \\
\text { (UG/I } \\
\text { AS FE) } \\
(01046)\end{array}$ & $\begin{array}{l}\text { LEAD, } \\
\text { DIS- } \\
\text { SOLVED } \\
\text { (UG/L } \\
\text { AS PB) } \\
(01049)\end{array}$ & $\begin{array}{c}\text { LITHIUM } \\
\text { DIS- } \\
\text { SOLVED } \\
\text { (UG/L } \\
\text { AS LI) } \\
\text { (01130) }\end{array}$ & $\begin{array}{l}\text { MANGA- } \\
\text { NESE, } \\
\text { DIS- } \\
\text { SOLVED } \\
\text { (UG/1. } \\
\text { AS ME) } \\
\text { (01056) }\end{array}$ & $\begin{array}{c}\text { MARCURY } \\
\text { DIS- } \\
\text { SOLVED } \\
\text { (UC/L } \\
\text { AS HC) } \\
\text { (71890) }\end{array}$ & $\begin{array}{l}\text { SELE- } \\
\text { NIUM, } \\
\text { DIS- } \\
\text { SOLVED } \\
\text { (UG/L } \\
\text { AS SE) } \\
\text { (01145) }\end{array}$ & $\begin{array}{c}\text { SILVER, } \\
\text { DIS- } \\
\text { SOLVED } \\
\text { (UG/L } \\
\text { AS AG) } \\
(01075)\end{array}$ & $\begin{array}{c}\text { STRON- } \\
\text { TIUM, } \\
\text { DIS- } \\
\text { SOLVED } \\
\text { (UG/L } \\
\text { AS SR) } \\
\text { (01080) }\end{array}$ \\
\hline
\end{tabular}

SOUTH MOUND SPRING $07-31-96$

120

$<4.0$
$<4.0$
$<4.0$
$<4.0$
$<4.0$

$\begin{array}{rr}43 & 6 \\ 53 & 12 \\ 49 & 13 \\ 52 & <4 \\ 66 & 8\end{array}$

$6.0<0.1$

$<0.1$
$<0.1$
$<0.1$
$<0.1$

3
3
$<1$
3
1

$<1.0 \quad 9000$

$<1.0 \quad 8400$

$<1.0 \quad 9200$ MOUND SPRING (UPPE 08-01-96

$<15 \quad<4.0$

$\begin{aligned} 4.0 & <0.1 \\ 8.0 & <0.1\end{aligned}$

$<1.0 \quad 10000$

LOCAL
IDENT-
I-
FIER

SOUTH NOUND SFRING NR OSCU

DEAD ORYX MOUND SPRTNG NR

WEST MOUND SPRING NR OSCUR

MOUND SPRING (UPPER POND)

NORTH MOUND SRRING NR OSCU
DATE
$07-31-96$
$07-31-96$
$08-01-96$
$08-01-96$
$08-01-96$
TIME

TÓTA (UG/L

$(39730)$

$2,4,5-\mathrm{T} \quad 2,4-\mathrm{DP}$

TOTAL

(UG/L)

TOTAL

(UG/L)

$<0.010$

$<0.010$

$<0.010$

$1630<0.010<0.010<0.010$

$<0.010$

$<0.010$

$<0.010$

$<0.010$

$<0.010$

$<0.010<0.010<0.010$ 
QUALITY OF GROUND WATER

WATER-QUALITY DATA, WATER YEAR OCTOBER 1995 TO SEPTEMBER 1996

OTERO COUNTY

\begin{tabular}{|c|c|c|c|c|c|c|c|c|c|}
\hline $\begin{array}{c}\text { LOCAL } \\
\text { IDENT- } \\
\text { I- } \\
\text { FIER }\end{array}$ & STATION & NUMBER & COUNTY & SITE & DATE & TIME & $\begin{array}{l}\text { GEO- } \\
\text { LOGIC } \\
\text { UNIT }\end{array}$ & $\begin{array}{l}\text { SPE- } \\
\text { CIFIC } \\
\text { CON- } \\
\text { DUCT- } \\
\text { ANCE } \\
\text { (US/CM) } \\
(00095)\end{array}$ & $\begin{array}{c}\text { PH } \\
\text { WATER } \\
\text { WEOLE } \\
\text { FIELD } \\
\text { (STAND- } \\
\text { ARD } \\
\text { UNITS) } \\
(00400)\end{array}$ \\
\hline CNG N & 3317151 & 06 & 035 & SP & -96 & 1345 & 110AVMB & $\$ 700$ & 7.5 \\
\hline
\end{tabular}

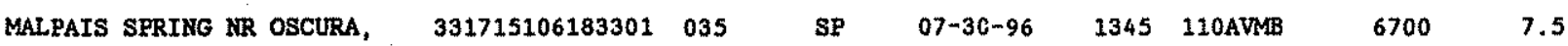

MALPAIS SFRING NR 07-30-96

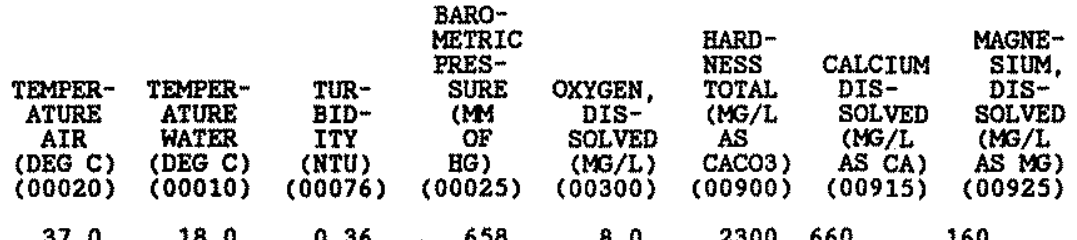

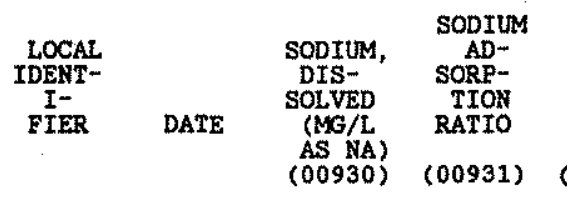

$\begin{array}{cccccc}\text { POTAS- } & \text { ALKA- } & & \text { CHLO- } & \text { FLUO- } \\ \text { SIUM, } & \text { LINITY } & \text { SULFATE } & \text { RIDE, } & \text { RIDE, } & \text { BROMIDE } \\ \text { DIS- } & \text { LAB } & \text { DIS- } & \text { DIS- } & \text { DIS- } & \text { DIS- } \\ \text { SOLVED } & \text { (MG/L } & \text { SOLVED } & \text { SOLVED } & \text { SOLVED } & \text { SOLVED } \\ \text { (MG/L } & \text { AS } & \text { (MG/L } & \text { (MG/L } & \text { (MG/L } & \text { (MG/L } \\ \text { AS K) } & \text { CACO3) } & \text { AS SO4) } & \text { AS CL) } & \text { AS F) } & \text { AS BR) } \\ (00935) & (90410) & (00945) & (00940) & (00950) & (71870)\end{array}$

MALPAIS SPRING NR $\quad 07-30-96 \quad 680$

6

8.254

1900

1300

$1.2 \quad 0.39$

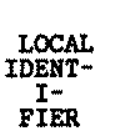

DATE $\begin{gathered}\text { SILICA, } \\ \text { DIS- } \\ \text { SOLVED } \\ \text { (MG/L } \\ \text { AS } \\ \text { SIO2) } \\ (00955)\end{gathered}$

SOLIDS, SOLIDS, NITRO- NITRO- NITRO- NITRO-

RESTDUE SUM OF GEN, GEN, GEN, GEN,AM- PHOSAT 180 CONSTI- NITRITE NO2+NÓ3 AMMONIA MONIA + PHORUS DEG. C TUENTS, DIS- DIS- DIS- ORGANIC DISDIS- DIS- SOLVED SOLVED SOLVED DIS. SOLVED SOLVED SOLVED (MG/L (MG/L (MG/L (MG/L (MG/L (MG/L) (MG/L) AS N AS N AS N) AS N) AS P) $\begin{array}{lllllllll} & (00955) & (70300) & (70301) & (00613) & (00631) & (00608) & \text { (00623) (00666) }\end{array}$

MALPAIS SPRING NR 07-30-96 28

5200

4800

$<0.010$

3.20

$0.160<0.20<0.010$

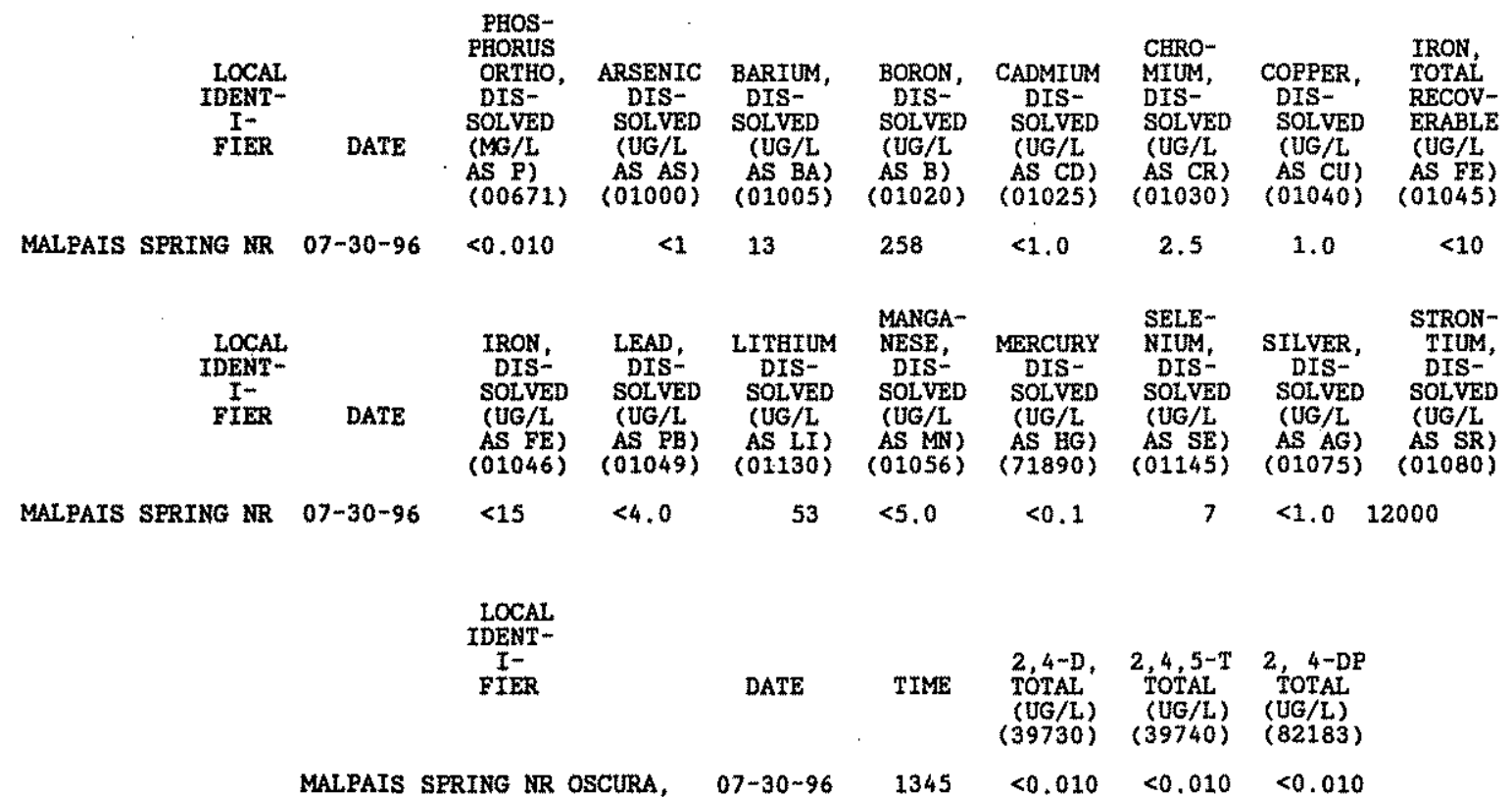


QUALITY OF GROUND WATER

WATER-QUALITY DATA, WATER YEAR OCTOBER 1995 TO SEPTEMBER 1996

RIO ARRIBA COUNTY

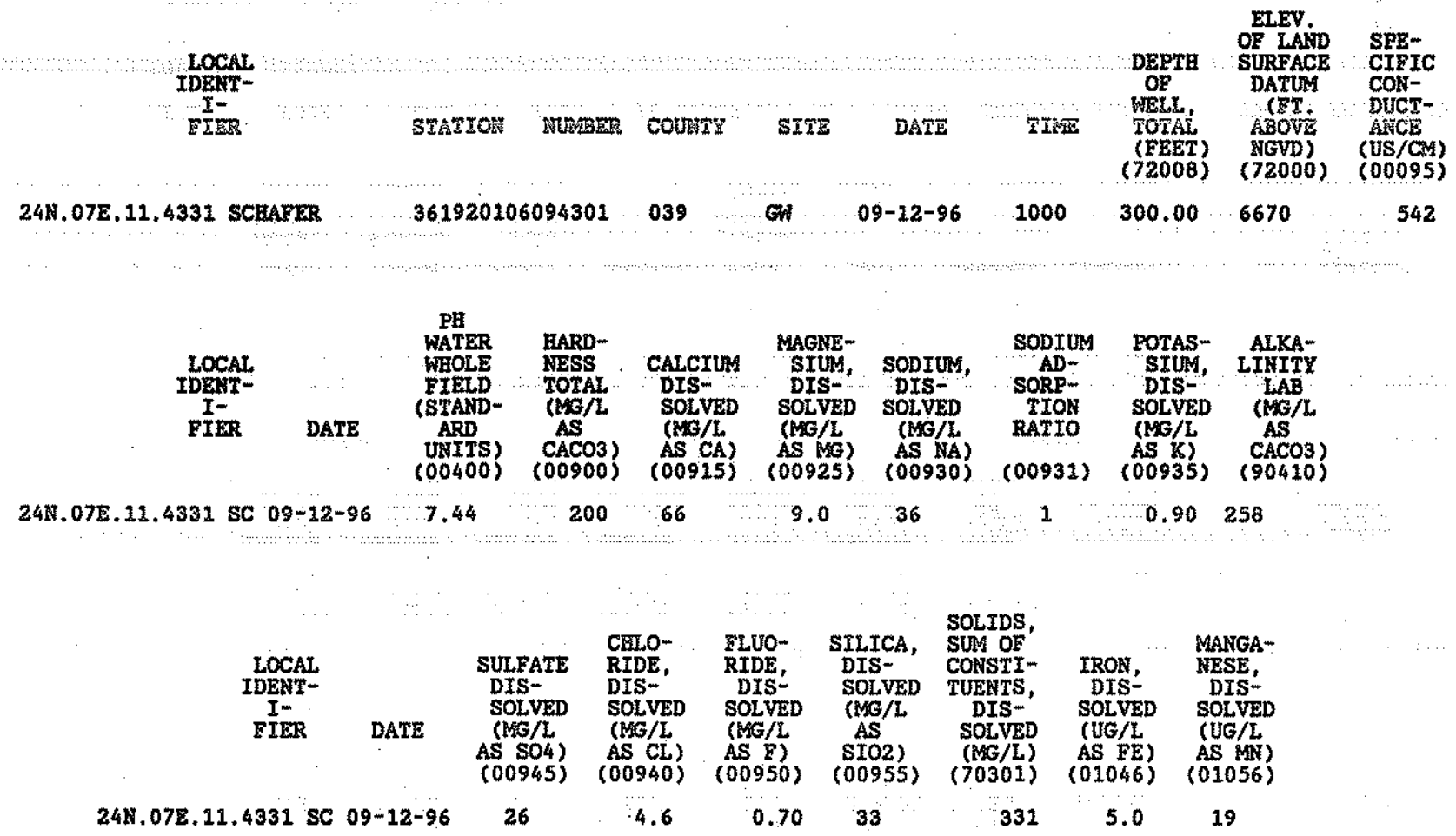


RIO GRANDE BASIN

Rio Grande Seepage Investigation

REACE. -The seepage investigation was conducted along a 62.4 mile reach frow the Rto Grande below Leasburg Dam near Radium Spxings, New Mexico to the Rio Grande at El Paso, Texas (08364000). Rtver miles are referenced upstream from the Rio Grande at El Raso, Texas; whtch is designated as river mile $1,249.9$.

FREVIOUS INVESTIGATIONS.--A aeepage Investigation of the reach between the gaging gtation "below Caballo Dam" $(08362500)$ and aite $0.3 \mathrm{ml}$ upatream from the gaging station "at El Paso". (08364000) was conducted by the U.S. Geological Survey on February 12-13, 1974. A seepage investigation of the reach from m110 $1,312.3$ to $1,249.9$ wes conducted on January $5-6,1986$, January 10-11, 1989, Jantary 9-10, 1990, January 8-9, 1991. A seepage investigation of the reach from mile $1,312.3$ to $1,277.8$ was conducted on December 17, 1991, and January 26-27, 1993.

DATE.--January 23-24, 1996.

WEATHER. -Weather was favorable for the seepage investigation; no prectpitation occurred. Temperature extrenes at Las Cruces, Nen Mexico ranged from low of -7 degrees Colsius on January 24 to high of 18 degreas Celsius on January 23 ,

STREAMFLW. - The seepage inveatigation was conducted during a scheduled winter release from Caballo Reservoir of approximately $300 \mathrm{ft} / \mathrm{s}$. Discharge measurements indicate a net seepage $10 \mathrm{ss}$ of $67.3 \mathrm{ft} / \mathrm{s}$, from $\mathrm{xtver}$ mile $1,312.3$ to river mile 1,249.9. Indicated gains and losses throughout the reach are shown in the following table. Tributary flow recorded as inflow is considered a contribution and not a gain; no outflow (diversions) occurred during the investigation. Evaporation from the water surface of the river in January ts considered negligible.

REMARKS-The seepage investigation is rated good based upon steady streamelow conditions. Recorded river stage in the Rio Grande at MM-227 Bridge near Vado, Now Mexico (river mile 1.277.8), indicateg steady stage with a slight change in gage-height from 0.09 feet on January 23 at 1630 to 0.07 feet on January 24 at 0910 . Individual. discharge measurements were rated good (within 5 percent) to fair (within 8 percent) throughout most of the stream reach. Recent channel excavation occurred from river mile 1,296,5 to rtver mile $1,293.2$ and at river mile $1,259.3$. The channel excavation resulted in poor channel conditions; altered streambed profiles may effect sepage results. Accuracy of dischargo measurements needs to be considered when evaluating indicated galns and $1088 \theta$.

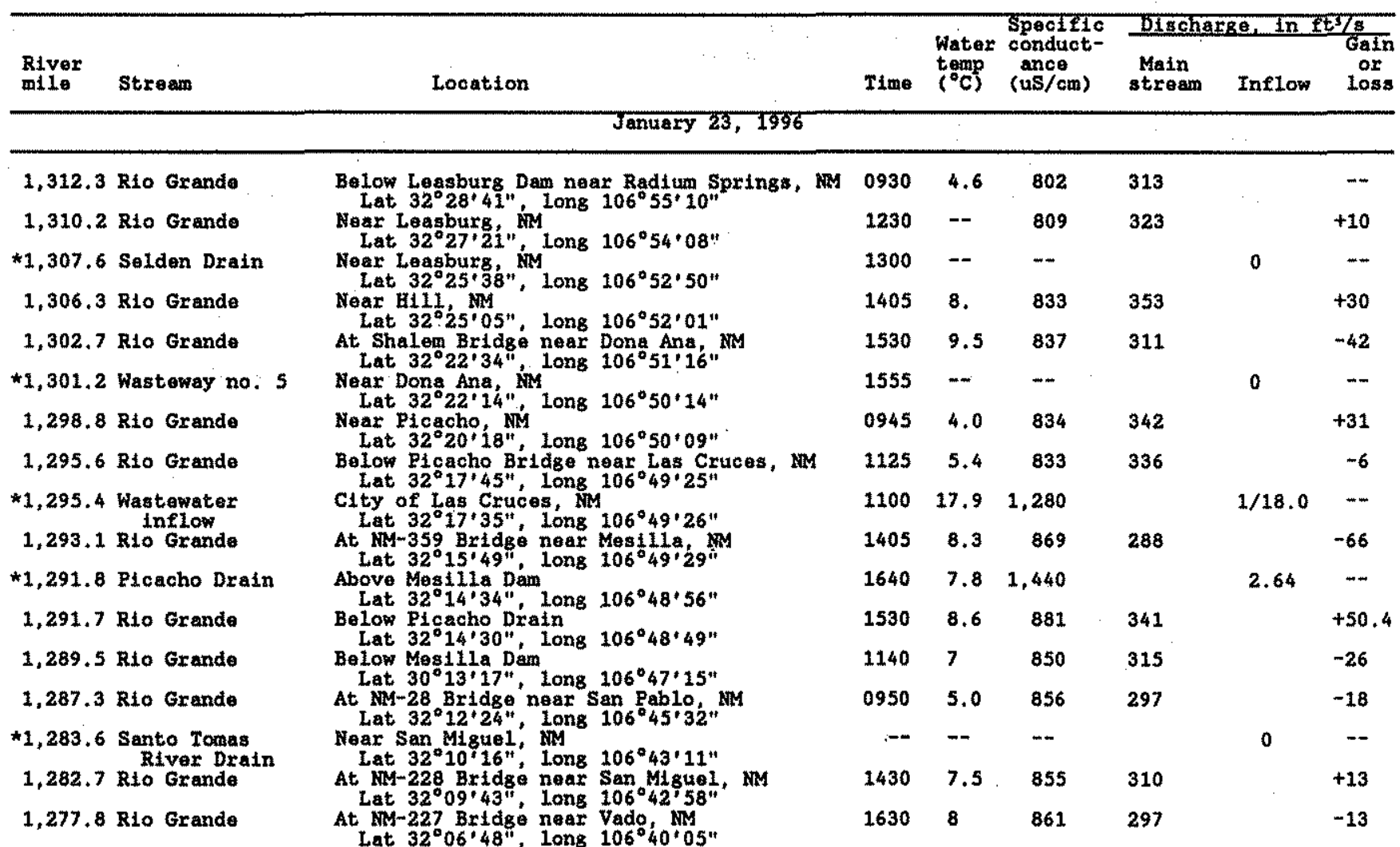




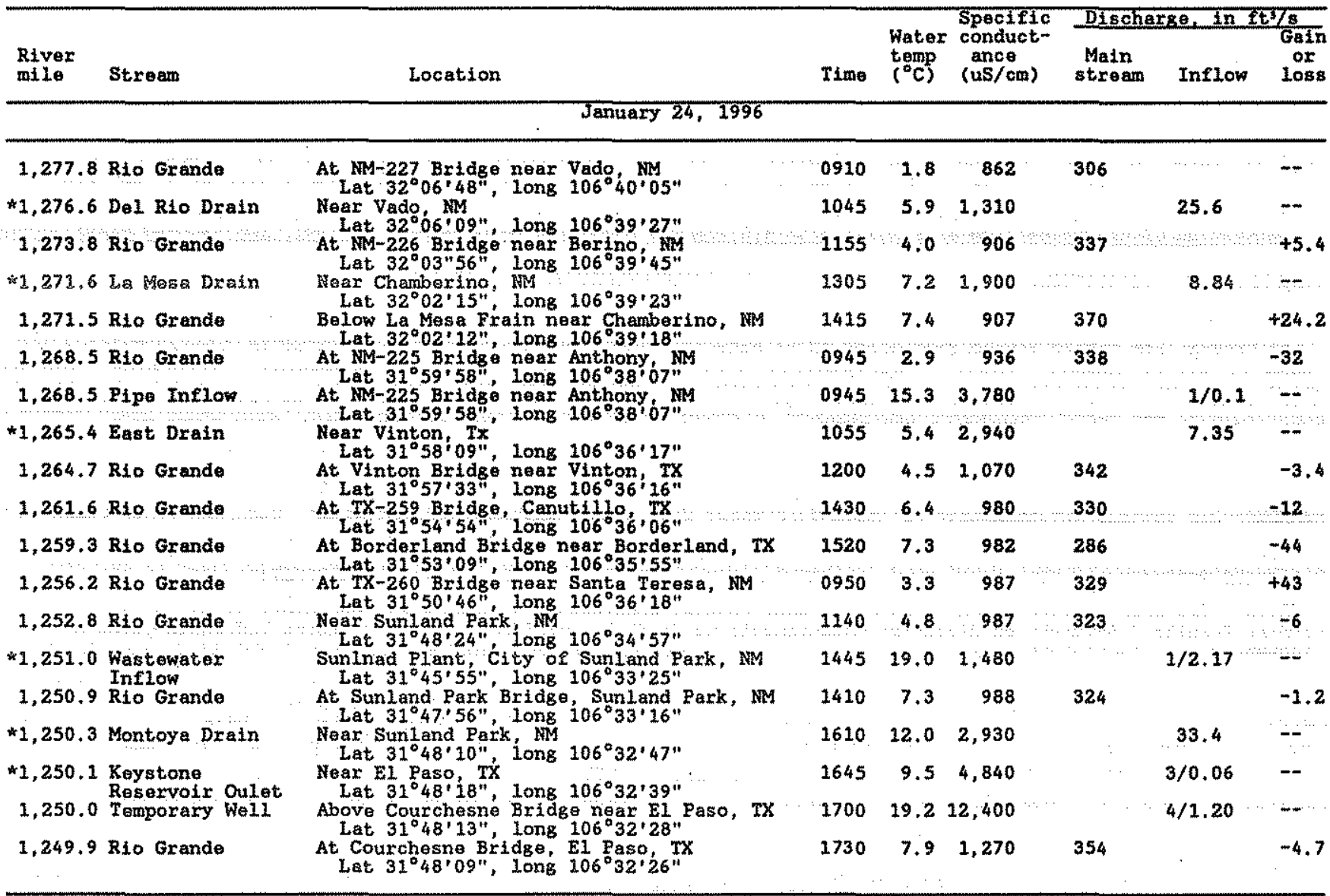

* River mile at mouth of draln or point of discharge.

1/ Reported discharge

2) Estimated discherge

3/ Storm drain inflow

4) Temporary well inflow to the Rto Grande at lept bank approximately 400 feet upstream from the Courchesne Bridge. Shallow wells were pumped for the purpose of dewatering at road construction sites along Doniphan Drive. 
Abiquiu Reservoir near Abiquiu. . . . . . . . . Acadeny Acres drain at Albuquerque.......... Access to WATSTORE data $\ldots \ldots \ldots \ldots \ldots \ldots \ldots \ldots$ Accuracy of the records.

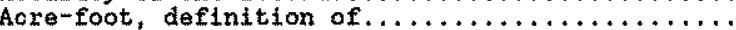

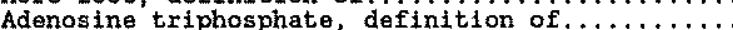
Alamosa Creek tributary near Jordan (crest) ...... Alemen Draw at Aleman (crest) ................

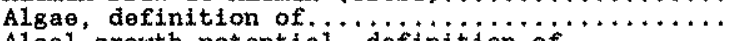
Alsal growth potential, definition of .......... miscellaneous sites

water quality partial-record stations....... 361-398

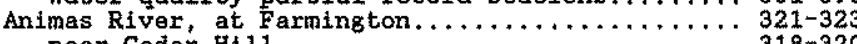
near Cedar Hil1..................... 318 320

Antelope Draw near Jal (crest)............. Aquifer, definition of . . . . . . . . . . . . . . Aragon Creek tributary near Encinoso (crest).... Arroyo $19 \mathrm{~A}$ at Albuquerque $\ldots \ldots \ldots \ldots \ldots \ldots$

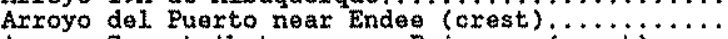
Arroyo Seco tributary near Pojoacue (crest)......

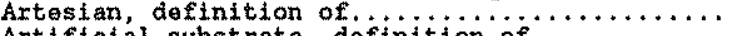

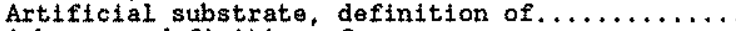

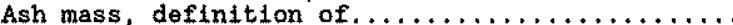

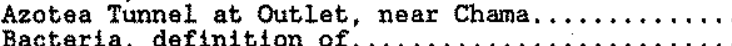
Bacterta, definition of $\ldots \ldots \ldots \ldots \ldots \ldots \ldots \ldots$

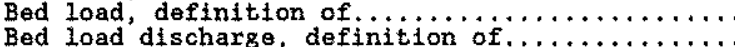

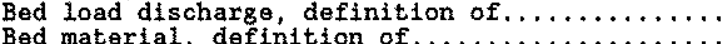
Bernalillo County, ground-water levels in..........

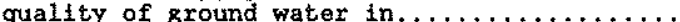

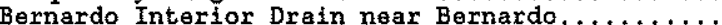

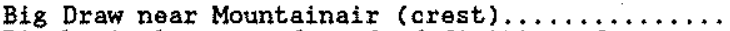

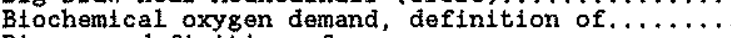
Biomass, definition of.

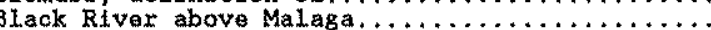
Black Springs Wash near Mexican Springs (crest). Blackwater Draw tributary near Floyd (crest)..... Bland Canyon near Cochiti Pueblo (crest)........

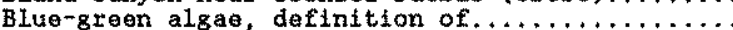

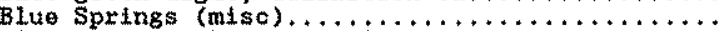
Bluewater Creek, above Biuewater Dam,

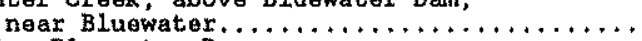
below Bluewater Dam.

near Tucumcari (crest).

mear Blusater.

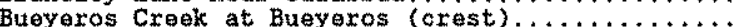

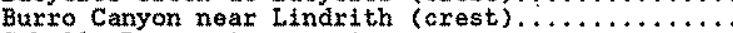

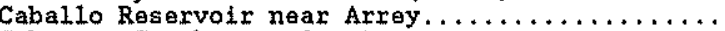

Cabresto Creek near Questa.

Campus Wash at Albuquercue $\ldots \ldots \ldots \ldots \ldots \ldots \ldots$

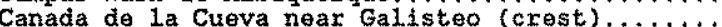

Cenadian River at Logan.

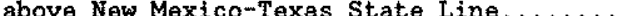

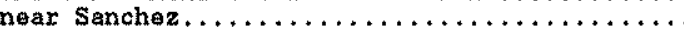

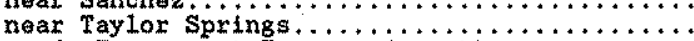

Canon de Torreon at Torreon (crest) . . . . . . . . .

Capultn Canyon above Ranger Cabin, Bandeifer

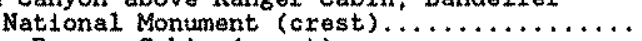

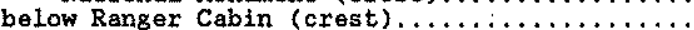

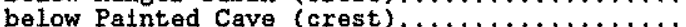
Carlsbad Main Canal at Head, near Carisbad.......

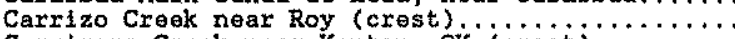

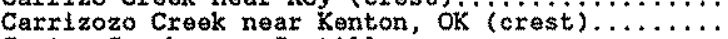

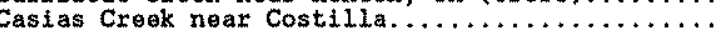
Castle Springs (misc).

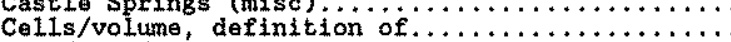

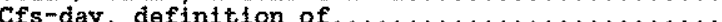
Chaco Wash at Chaco Culture Nationa Monument

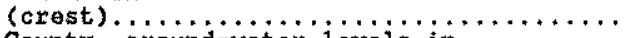

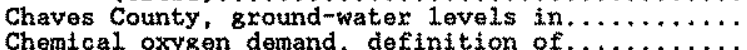

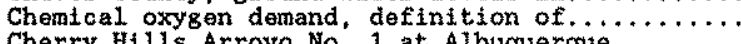

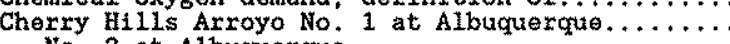
No. 2 at Albuquerque............ . . . . . . . Chicorica Creek tributary near Raton (crest) .... Chlorophy 11, definttion of.

Chupadera Wash tributary at Bingham (crest).....

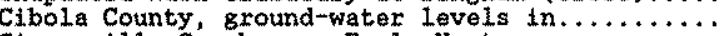

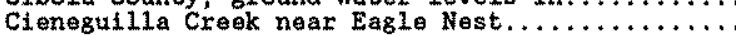
35
Cimarron River, at Springer..............

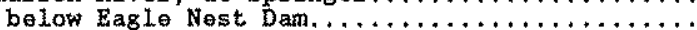
near Cimarron.

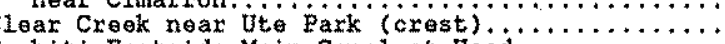
Cochiti Eastside Matn Canal at Head........... Cochiti lake near Cochiti Fueblo $\ldots \ldots \ldots \ldots \ldots \ldots$ Colfax County, groundwater levels in......... Color untt, definition of . . . . . . . . . . . .

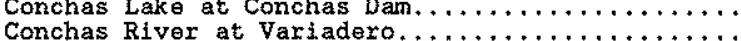

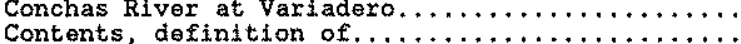

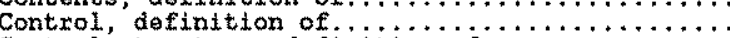
Control' structure, defintion of . . . . . . . . .

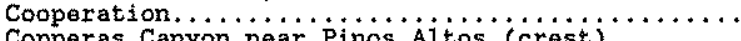
Copperas Canyon neax Pinos Altos (crest) ........ Costilla County, Co, ground-water levels in.....

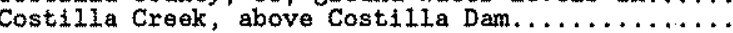

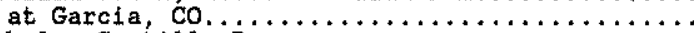
below Costilla Dan. . . . . . . . . . . . .

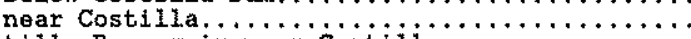

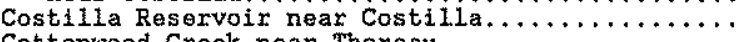

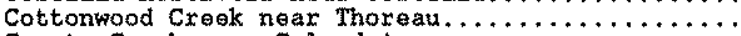
Coyote Creek near Golondrinas . . $\ldots \ldots \ldots \ldots \ldots \ldots$ Coyote Wash tributary near Naschitt 1 (crest).... Crest-stage parttal-record stations...
Cubtc feet per second, per square mili,

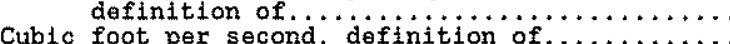
Cublc foot per second, definition of $\ldots \ldots \ldots \ldots \ldots$ Dark Canyon Draw at Carlsbad. . . . . . . . . . .

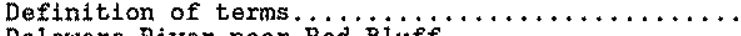

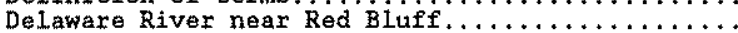

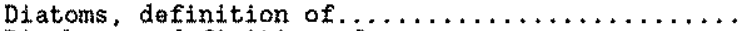

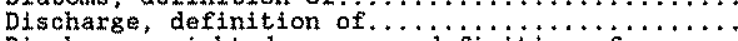
Discharge-weighted average, definition of .......

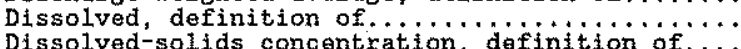
Dissolved-solids concentiation, definition of...

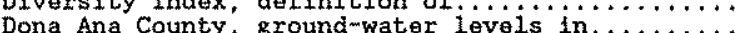
quality of ground water in . . . . . . . . . . Downstream-oxder system. . . . . . . . . . . . Drainage area, definition of $\ldots \ldots \ldots \ldots \ldots \ldots \ldots$

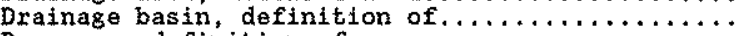
Dry mass, definition of.

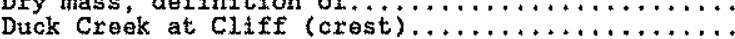

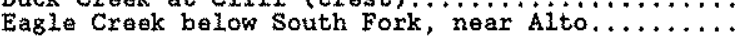
Eagle Nest Lake near Eagle Nest............. Eagle Tail Ditch near Maxwell.

Eddy County, ground-water levels in ............

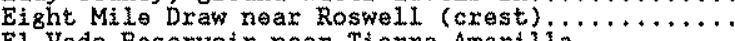
E1 Vado Reservolir near Tzerra Amardila. $\ldots \ldots \ldots$ Embudo Creek at Dixon. . . . . . . . . . . . . . . . Estancta Valley tributary at Cedar Grove (crest). Explanation of the records $\ldots \ldots \ldots \ldots \ldots \ldots \ldots$ Fecal streptococcal bacteria, definttion of $\ldots \ldots$ Fleming Draw near pinon (crest) ............. Fort Sumner Main Canal near Fort Sumer..........

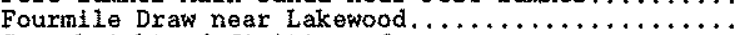
Gage height, definition of.

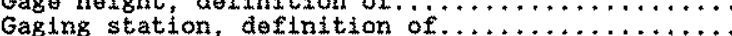
Galostena Canyon trtbutary near Black Rock (crest)

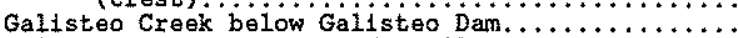
Galisteo Reservoix near Cerrillos........... Gallinas Creek near Montezuma.

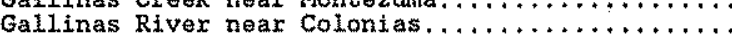

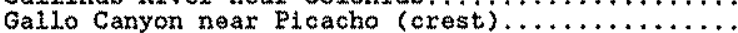
Garita Creek tributary near Variadero (crest).... Gila River, below Blue Creek near Virden........

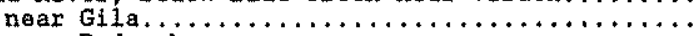

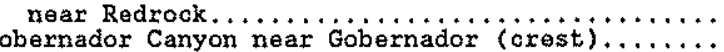
Grant County, groundwwater levels in.......... Grant Line Arroyo.

at Villa del Oso at Albuquerque.......... Green algae, definition of

Ground water levels.

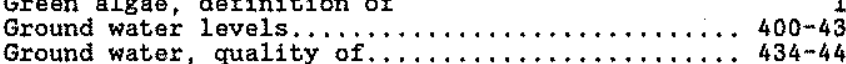

$39-40$

347

Guadalupe County, ground-watex leveis in $\ldots \ldots \ldots$ 


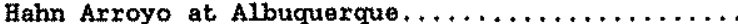

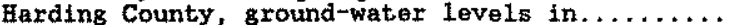

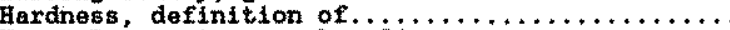
Heron Reservoir near Los ojos ................... Hidalgo County, ground-water levels in ........... of fmantown Church outlet, No. 1 at Albuquerque. No. 2 at Albuquerque..................... Los Ojos.

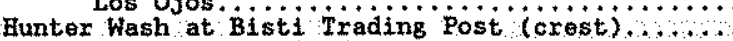
Hyatt Canyon near Cloudcroft (crest) Eydrologic bonch-mark station, definition of............

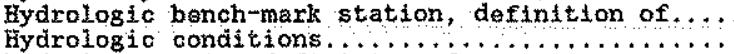

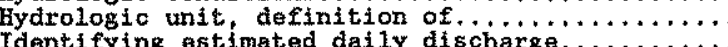
Identifying estimated daily discharge $\ldots \ldots \ldots \ldots \ldots$

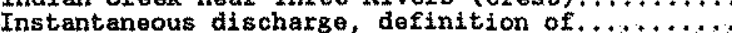

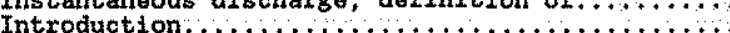

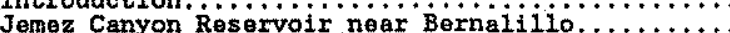

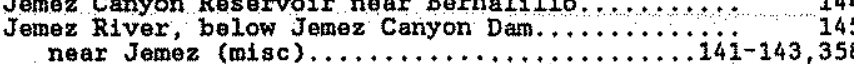
Juan Tomes Canyon near Edgewood (crest)........... 35

Juan Toro Canyon near Miera (crest) . . ............... La Cueva Canal below la Cueva . . . . . . . . . . . .

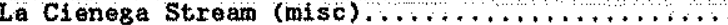
Ladera Arroyo at Albuquerque $\ldots \ldots \ldots \ldots \ldots \ldots \ldots$. Lagartija Croek tributary near Sanchez (crest)...

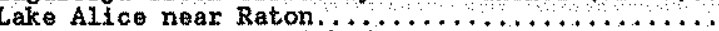

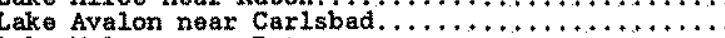

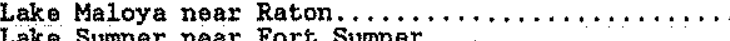
Lake Sumex neax Fort

lakes and reservotrs:

Abiquiu Reservolir near Ablquiu. . . . . . . . .

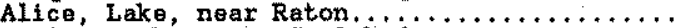

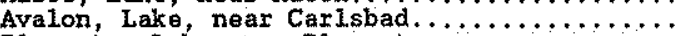

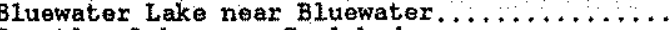

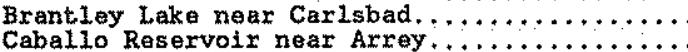

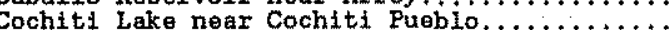
Conchas Lake at Conchas Dam

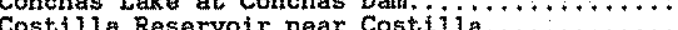
Eaglo Nest haire near Eaglo Nest... Elophant Butte Reservoir at Elephant Butte...

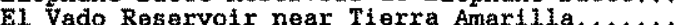

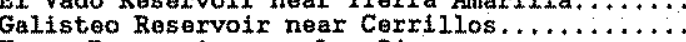

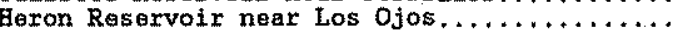
Maloya, Lake, near Raton.

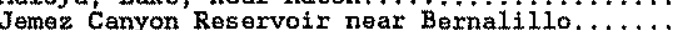

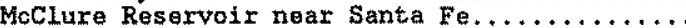

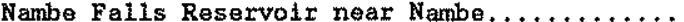

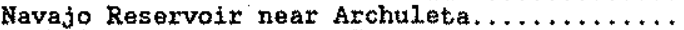
Nichols Reservoir noar Santa Fe.

Red Bluff Reservoir near Orla, $\mathrm{TX} . \ldots \ldots \ldots \ldots$ Rio Hondo Reservoir.

Rocky Arroyo Reservot

Santa Rosa Lake near Santa Rosa...............

Sumer, Lake, near Fort Sumner. . . . . . . . . . .

Two Rivers Reservolr near Rosweil . . . . . . . . .

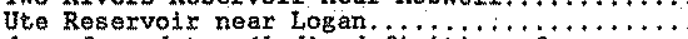

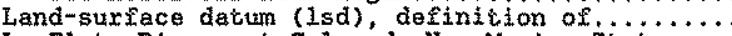

La Plata River, at Colorado-New Mexico State

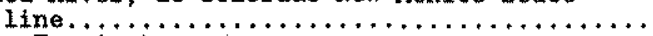

a plata River tributary neax farmington (crest).

Lest Chance Canyon tributary near Carlsbad

Caverns (crest)

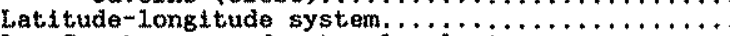

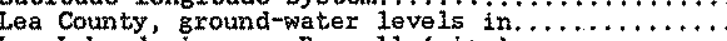
Lea lake drain near Roswell (misc).

Litile Navajo River below Lietle oso diversion

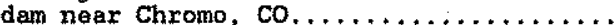

Lincoln County, ground-water leveis in.............

Llano ditch near Questa $\ldots \ldots \ldots \ldots \ldots \ldots \ldots \ldots \ldots$

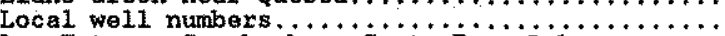

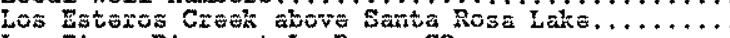
Los Pinos River at La Boca, Co.

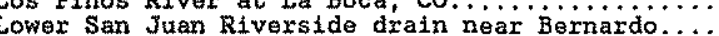
Luna Councy, ground-water levels in............ Mai1 Hollow near Luna (crest)

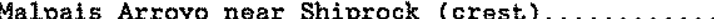

Mangas Creek near Cliff (crest) (misc) ............

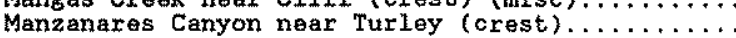

Mep of New Mexico showing location of: Hydrologic units....................

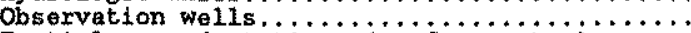
Partial-record stations (surface water)........ Surface-water gaging stations........... Water-quality gaging stations............. McClure Reservoir near Santa Fe. $\ldots \ldots \ldots \ldots \ldots$ Mean concentration, definition of ............

Mean discharge, definition of $\ldots \ldots \ldots \ldots \ldots \ldots$ Measuring point (MP), definition of $\ldots \ldots \ldots \ldots \ldots$

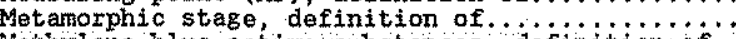
Methylene blue active substances, derinition os. Micrograms per gram, definition of $\ldots \ldots \ldots \ldots \ldots$ Milk Ranch Canyon near Fort Wingate (crest) . . . . Miligrams per liter, definttion of .......... Mimbres Besin tributary near Floride (crest). .... Mimbres River, at Deming (crest) $\ldots \ldots \ldots \ldots \ldots \ldots$ at Mimbres $\ldots \ldots \ldots \ldots \ldots \ldots \ldots \ldots \ldots \ldots$

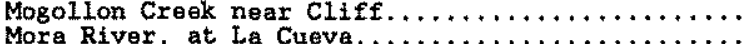
near Golondrinas $\ldots \ldots \ldots \ldots \ldots \ldots \ldots \ldots \ldots \ldots \ldots \ldots \ldots$

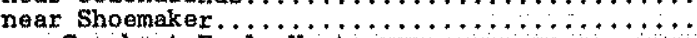

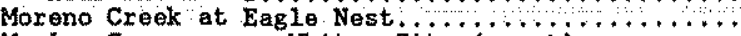
Mos ley Canyon near whites city (crest)..........

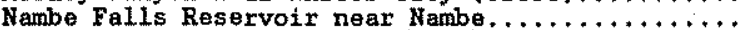
National Geodetic Vertical Datum of $1929 \ldots \ldots \ldots$ National Geodetic Vertical Datum of $1929 \ldots \ldots \ldots \ldots$ Natural substrate, definition of $\ldots \ldots \ldots \ldots \ldots$

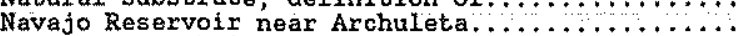
Navajo River below Oso diversion dam near Chromo, Co.

Nichlos Reservoir near santa pe $\ldots \ldots \ldots \ldots \ldots$ Nogal Creek tributaxy near Nogai (crest)......... North Camino Arroyo tributary at Albuquerque.......... orth Floodway Channel, at Albuquerque.......... near Alameda. $\ldots \ldots \ldots \ldots \ldots \ldots$ On-site measurements and sample collection. $\ldots$ Organic mass, definition of . . . . . . . . . .

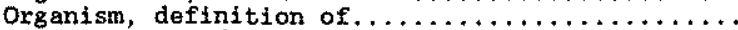

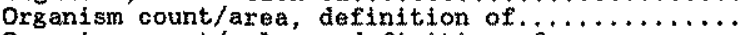
Organism count/volume, definition of ..........

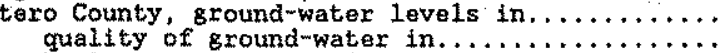
ancho Canyon neax Arabela (crest) $\ldots \ldots \ldots \ldots \ldots \ldots$

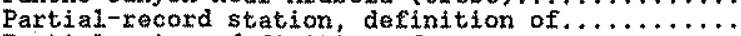

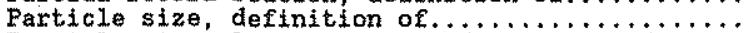
Particle size classtfication, definition of.....

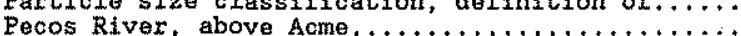
above Canon del Uta near Colonias...........

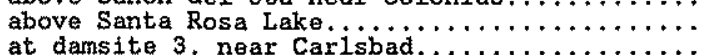
at damsite 3 , near Carisbad...

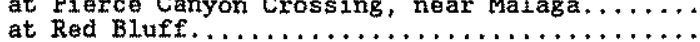

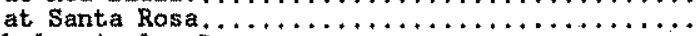

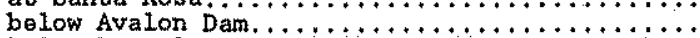

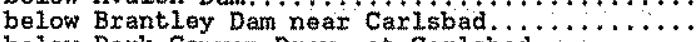

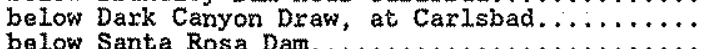

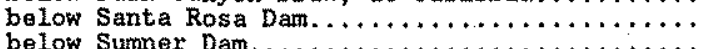
below Taiban Creeic near Fore Sumer...........

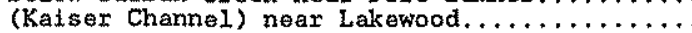

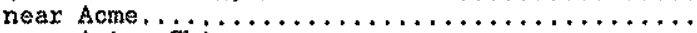

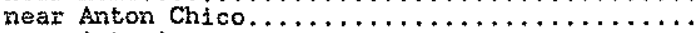
near Artesia $\ldots \ldots \ldots \ldots \ldots \ldots \ldots \ldots \ldots \ldots \ldots$

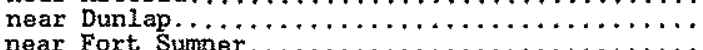

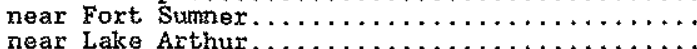
near Malaga.......................

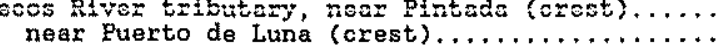

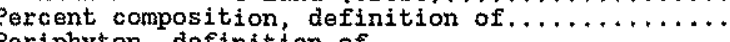
Periphyton, definition of $\ldots \ldots \ldots \ldots \ldots \ldots \ldots \ldots$

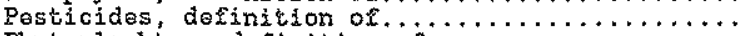

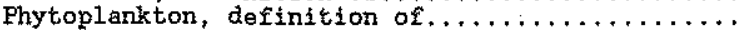

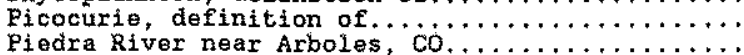

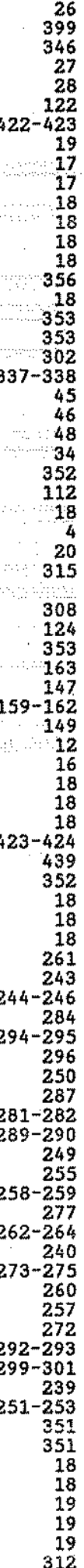


Pine Canyon near Thoreau (crest) $\ldots \ldots \ldots \ldots \ldots \ldots$ Pino Arroyo at Ventura at Albuquerque.............

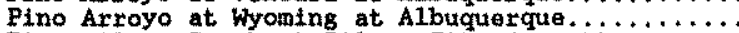
Pinos Altos Creek at Silver City (crest).............

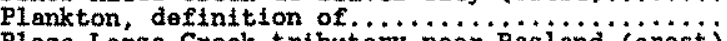
Plaza Larga Creak tributary near Ragland (crest).

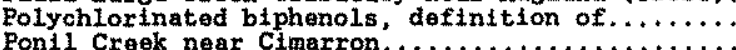

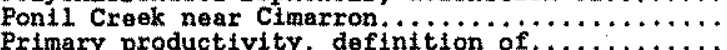
Publications on techniques of water-resources investigations.

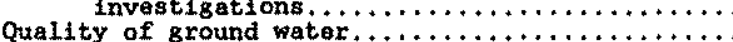

Quality of ground water. $\ldots \ldots \ldots \ldots \ldots \ldots$

Quay County, ground-water levels in $\ldots \ldots \ldots \ldots \ldots$

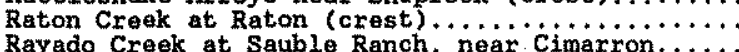

Rayado Creek at Sauble Ranch, near Cimarron......

Records of ground-water levels, explanation of...

Records of staund and water discharge explanation of.................................

Records of surface-water quality, explanation of.

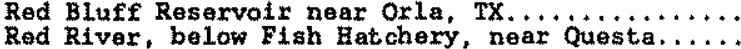

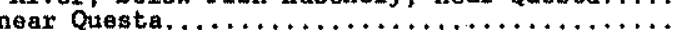

Remark codes.

Reservotrs (seo Lakes and reservotrs)

Revral to Creek near Logan.

Rio Arriba County, ground-water levels.......... lanco below Blanco dtversion dan

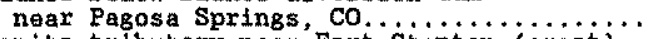

Rto Bonito tributary near Fort stanton (crest)...

Rio Chama, above Abiquiu Reservoir.............. below Abiquiu Dam

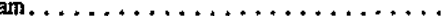

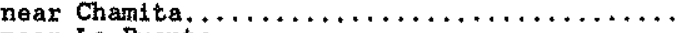

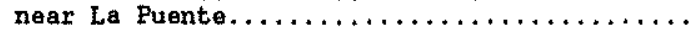

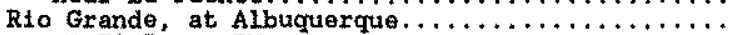

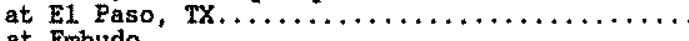

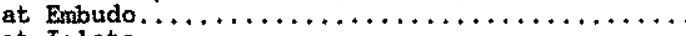
at Isleta $\ldots \ldots \ldots \ldots \ldots \ldots \ldots \ldots$ at Otowi Bridge,

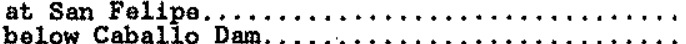

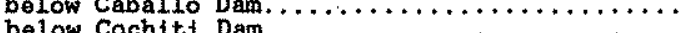

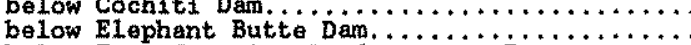

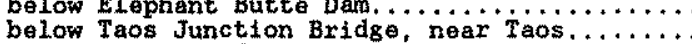

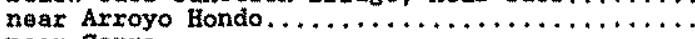

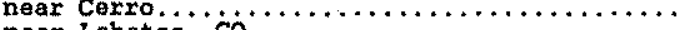
near Lobatos, $\mathrm{CO}$

Rio Grande Conveyance........................... $63-65$ 208-209

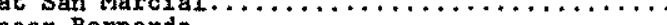

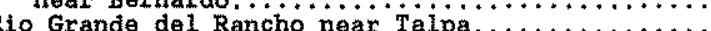

Rio Grande del Rancho near Talpa. $\ldots \ldots \ldots \ldots \ldots \ldots \ldots$

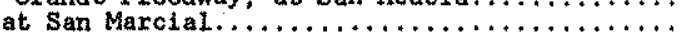
near Bernardo.

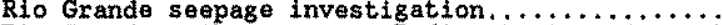

Rio Grande tributary near Radiun springs (crest).

Rio Guadalupe at Box Canyon, near Jemez...........

Rto Bondo, at $\mathrm{Di}$ amond $A$ Ranch, near Rosweii.......

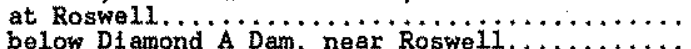
near Valdez (tributary to Rlo Grande).............

Rio Hondo Reservoir . . . . . . . . . . . . . . . .

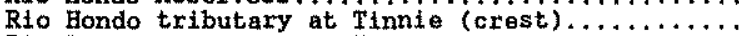

Rio Lucero near Arroyo seco. . . . . . . . . . . .

Rio Mora near Terrero .

Rio Nambe below Nambe Fai is $D$ am, no nar Nambe........

Rio Nutria near Ramah. . . . . . . . . . . . . . . .

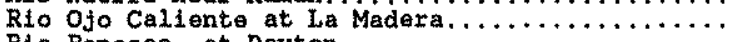

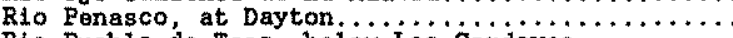

Rio Pueblo de Taos, below los Cordovas...

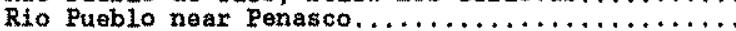

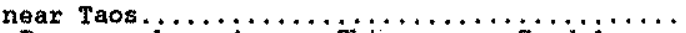

Rto Puerco, above Arroyo Chico, near Guadaiupe..

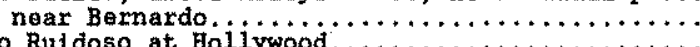

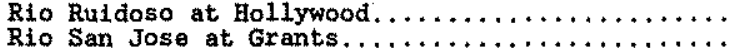

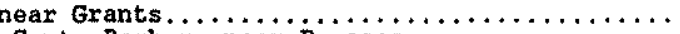

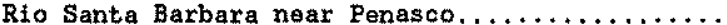

Rito de los Frijoles in Bandelior National

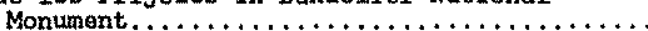

350 Rocky Arroyo at Highway Bridge, near Carlsbad....

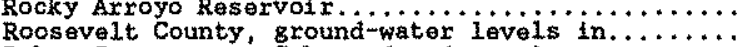
Ruben Canyon near Gobernador (crest) ............. Rumning water Draw near Clovis (crest)........... Salt Creok at $R R$ 316 on WSMR.... $\ldots \ldots \ldots \ldots \ldots \ldots$ San Francisco Rtver, near Glenwood...............

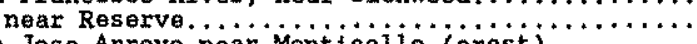
San Jose Arroyo near Monticello (crest) .......... San Juan River, at Farmington. ................ at Four Corners, Co $\ldots \ldots \ldots \ldots \ldots \ldots \ldots \ldots \ldots$

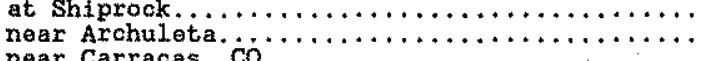

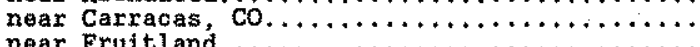
near Fruitland....... Sand Draw tributary neax Clayton (crest)........... Sandoval Canyon at Gallinas (crest) . . . . . . . . . Sandoval County, ground-water levels in............ San Juan County, groundwwater levels in...........

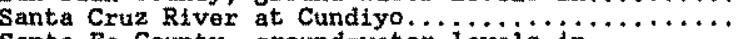
Santa Fe County, ground-water leveis in.............. Santa Fe River, above Cochtt Lake.............. Santa Rosa Lake near santa Rosa................

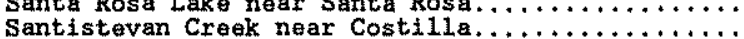

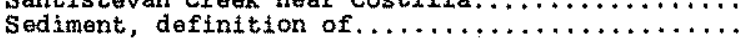
explanation of program. Seventys $1 x$ Draw tributary near Waterioo (crest). Sierra County, ground-water levels in........... Sili Main Canal at Head . $\ldots \ldots \ldots \ldots \ldots \ldots \ldots$ Sixmile Crook near Eagle Nest...

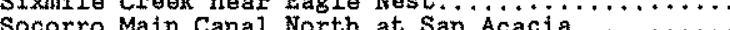
Sodium adsorption ratio, definition of...............

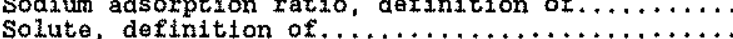
Solute, definition of...

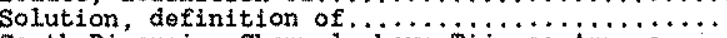
South Diversion Channel above Tijeras Arroyo near Albuquerque.

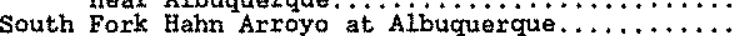
South Seven Rivers near Lakewood................. Spectal notworks and programs $\ldots \ldots \ldots \ldots \ldots \ldots \ldots$

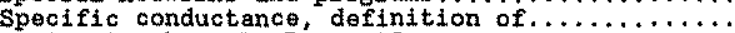
Spring Creek at La Boca, Co . . . . . . . . . . . . . . . . Stage-discharge relation, definition of......
Station-identification numbers, downstream-

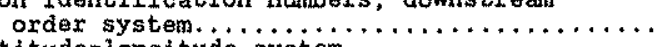

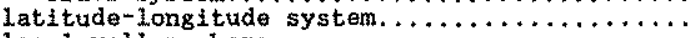
local well numbers.

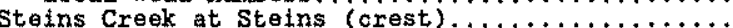

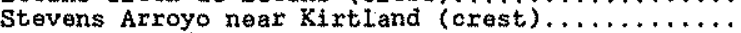

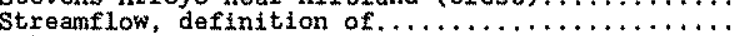
Substrate, definition of.

Surmary of hydrologic conditions: streamfiow..... surface water quald ty $\ldots \ldots \ldots \ldots \ldots \ldots \ldots \ldots$ ground-water levels.

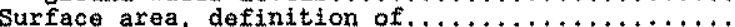

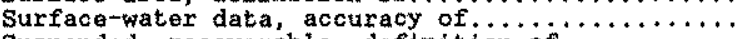
Suspended, recoverable, deftnition of............ Suspended, total, defintition of..
Suspended sediment, definition of

Suspended-sediment concentration, definition of.. Suspended-sediment discharge, definition of...... Suspended-sediment load, definition of...........

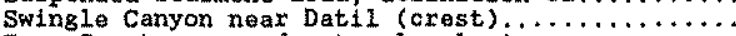
Taylor Canyon tributary near Bingham (crest) $\ldots \ldots$

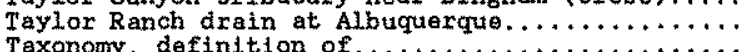
Texonomy, defindtion of....................................

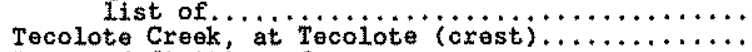

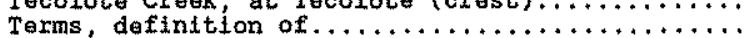
Thermograph, definttion of $\ldots \ldots \ldots \ldots \ldots \ldots \ldots \ldots \ldots$ Tijeras Arroyo, at Albuquerque (crest) ............. near Albuquerque.

Time-weighted average, derinition of $\ldots \ldots \ldots \ldots \ldots$ Tons per acre-foot, definition of . . . . . . . . . .

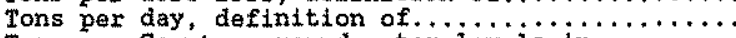

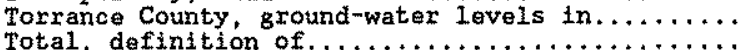


INDEX collform bacteria, definition of $\ldots \ldots \ldots \ldots \ldots \ldots$
discharge, defintition of $\ldots \ldots \ldots \ldots \ldots \ldots \ldots \ldots \ldots$

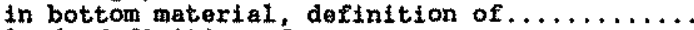

load, definition of.

load, dollnition or . $\ldots \ldots \ldots \ldots \ldots \ldots \ldots \ldots$

organisan count, definition

recoverable, definition of ...........................

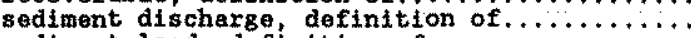

sediment load, defintition of.

Tramperos creok near stead (crest) $\ldots \ldots \ldots \ldots \ldots$ Tramway floodway Channel at Albuquerque

Trementina Creek at Trementina (crest).

Tritium notwork, explanation of

Tularosa Croek noar Bent.

Tularosa River above Aragon.

Twin Butte Canyon tributary near Rosweii (crest).

Two Rivers Reservoir near Roswell

Union County, ground-water levels in $\ldots \ldots \ldots \ldots$
16 Ute Creek near Logan. . . . . . . . . . . . . .

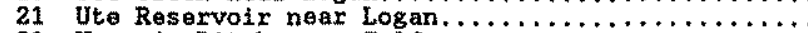

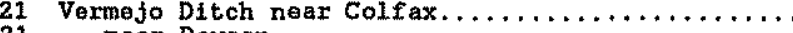

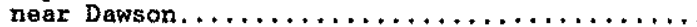

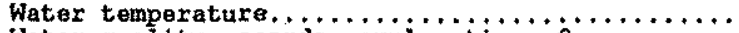

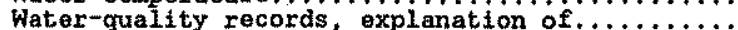

Water year, definition of.

Water year, definition of $\ldots \ldots \ldots \ldots \ldots \ldots \ldots \ldots$

Weighted average, definition of $\ldots \ldots \ldots \ldots \ldots$

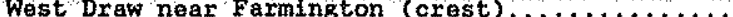

Wet mass, definition of $\ldots \ldots \ldots \ldots \ldots \ldots \ldots \ldots$

White Oaks Canyon near Carrizozo (crost) .........

W1Llow Creek, above Heron Reservoir near los Ojos

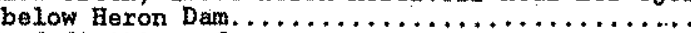

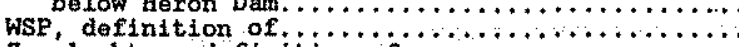

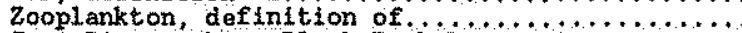

Zuni River, above Black Rock Reservolr.........
54
$55-57$ 